\title{
MINUTES OF THE 43rd GENERAL ASSEMBLY OF THE EUROPEAN ASSOCIATION FOR THE STUDY OF DIABETES
}

\author{
held in Amsterdam, The Netherlands on 20 September 2007 at 18:15 \\ Present: Drs. E. $\quad$ Ferrannini $\quad$ (President) \\ G. Spinas (Honorary Treasurer) \\ D. L. Eizirik (Honorary Secretary) \\ E. Gale (Editor-in-Chief, Diabetologia) \\ V. Jörgens (Executive Director)
}

The President welcomed everyone to the 43rd General Assembly.

\section{MINUTES, 42nd GENERAL ASSEMBLY 2006}

Since there were no comments, the minutes were approved unanimously and officially signed as a correct record.

\section{REPORTS}

\section{a) President}

Dr. Ferrannini reported that Dr. DeFronzo would give the Claude Bernard Lecture in 2008.

The President reported that the European Foundation for the Study of Diabetes (EFSD) was continuing to be very successful, with many partnerships being maintained.

The President announced that Dr. R. Heine would not be standing for President from 2008-2011 and that he had resigned from the Board of the European Foundation for the Study of Diabetes with immediate effect. The election of the EASD President for the term of office 2008-2011 will take place in the $87^{\text {th }}$ Council meeting before the Annual Meeting in Rome.

The President reported that Dr. P. Cryer had requested cooperation with EASD regarding the preparation of a Clinical Practice Guideline entitled 'Evaluation and Management of Adult Hypoglycemic Disorders' which is organised by the European Society of Endocrinology. He explained that the Executive Committee had unanimously decided to expand the remit of EASD to include issuing of consensus statements, formulating guidelines etc. To achieve this, ad hoc committees composed of expert colleagues from Europe and USA will be set up to evaluate the issues critically. Dr. Ferrannini said a communication strategy was needed and that the EASD membership would be informed so that anyone who was especially interested could contact the relevant committee. The information will also be put on the web pages of EASD and in Diabetologia.

Dr. Ferrannini reported on the ad hoc committee meeting for clinical trials which had taken place in Düsseldorf on 5-6 September and had been chaired by Dr. D. Matthews. He said the Executive Committee had unanimously decided to change the ad hoc committee into a sub-committee, which should consist of Drs. B. Charbonnel, J. Ludvigsson, D. Matthews [Chair], M. Nauck, A. Scheen, A. Siebenhofer, C. Tack and S. Heller.

The President reported on relations with the European Agency for the Evaluation of Medicinal Products (EMEA). EMEA is presently inviting a number of professional organisations to participate in discussions and to provide advice on clinical trials and pharmacovigilance. Dr. Ferrannini said that Dr. Smith had attended 3 meetings with EMEA. Dr. Smith reported that it had been decided that more European clinical trials were needed and he suggested that EASD had an important role to play in this matter. Dr. Ferrannini reported that the Executive Committee had unanimously decided to set up a sub-committee to be chaired by Dr. Smith and to include Drs C. Bailey, A. Barnett and J. Nolan. It was 
further decided that all information should be sent to Dr. Gale.

\section{b) Honorary Treasurer}

Dr. Spinas reported that income had decreased slightly due to a decrease in registration fees and donations. On the expenditure side, salary costs decreased slightly as there were no costs for copy-editing in Düsseldorf. At the end of 2006 EASD transferred 2.5 million Euro to EFSD to support diabetes research.

Dr. Spinas brought his report to an end by thanking the EASD staff, in particular Mrs. A. Klee, for the professional administration of the accounts.

The President thanked the Honorary Treasurer for his diligence and asked if there were any questions. There were none.

\section{c) Honorary Auditors}

The President asked the Honorary Auditors to formally discharge the accounts. Drs. Tiedge and Katsilambros confirmed that the accounts had been checked carefully and were in perfect order. Dr. Ferrannini asked for the vote to accept the accounts.

The accounts were accepted unanimously (one abstention).

\section{d) Honorary Secretary}

Dr. Eizirik reported that in 20072179 abstracts had been received, an increase of 249 compared to the previous year. The main countries submitting abstracts are the UK, USA, Germany and Italy. In 2006, a trial questionnaire had been introduced which produced positive feedback and which would be continued in a simpler form, the goal being to have a more representative sampling. In 2007, an Evening Poster Reception had been introduced, when there would be the opportunity for one-to-one discussions. For the Annual Meeting in 2007, 183 applications for Travel grants had been received and 138 accepted in the value of $€ 76,094$. A total of 23 Stayment Grants were awarded.

Dr. Eizirik said future challenges included maintaining and improving the academic traditions of the founding members and preserving the unique transparency and scientific quality of EASD Annual Meetings.

Dr. Eizirik closed his report by thanking all members of the EASD staff for their outstanding help and support with the organisation of the EASD Annual Meetings during the past three years. He wished his successor, Dr. M. Stumvoll, good luck.

Dr. Ferrannini thanked Dr. Eizirik for the meticulous and enthusiastic work he had done.

\section{e) Editor-in-Chief, Diabetologia}

Dr. Gale reported that a total of 1742 articles had been submitted to Diabetologia, an increase of $18 \%$. The largest number of submissions in 2006 originated from the USA, followed by the UK, Japan and Germany. The acceptance rate for all articles was $20.8 \%$ and that for original articles had decreased to $18.6 \%$. The Impact Factor in 2006 was 5.247. The Editor-in-Chief said the journal was coming out on time. He expressed his thanks to his team in Bristol.

Dr. Ferrannini thanked Dr. Gale for the excellent work he had done. He said a sub-committee had been appointed by the Executive Committee to evaluate potential candidates for the position of Editor-in-Chief.

\section{f) Chair, Postgraduate Education Sub-committee}

Dr. Nolan reported that two postgraduate courses (in Jena and Loipersdorf) had been held since the Annual Meeting in Copenhagen and three were planned for 2008 (in Jena, Wroclaw and Belgrade). Possible future courses included Bulgaria, Turkey, Latvia, Romania and Bosnia-Herzogovina.

Dr. Nolan reported that one course had been organised in St Petersburg and he particularly thanked Dr. Boulton for the excellent organisation. Dr. Nolan reported that Dr. Boulton, as Chair of the Extra-European Postgraduate Courses, was scheduling the Third Joint EASD/ADA/IDF Advanced Clinical Postgraduate Course in the Management of Diabetes and its Complications in the South American Region, Brasilia from 1-3 November 2007.

Dr. Nolan said the committee was working on developing a new web-based CME series of initiatives which would start in 2007. The educational sessions will be prepared for viewing on the website. As this initiative develops, it is hoped to arrange debates between experts at short notice on controversial topics as they arise.

Dr. Nolan thanked Dr. Czupryniak for his close co-operation.

Dr. Ferrannini thanked Dr. Nolan for his valuable work.

\section{ELECTIONS}

\section{a) Honorary Secretary 2007-2010}

The Council's election of Dr. M. Stumvoll was unanimously approved with one abstention.

\section{b) Editor-in-Chief extension until 2009}

The extension of the Editor-in-Chief's term of office until 2009 was unanimously approved with one abstention. 


\section{c) Council Members 2008-2011}

The following Council Members were unanimously elected by the General Assembly:

Drs. T. Battelino (Slovenia) - two abstentions,

D. Matthews (UK) - one abstention,

J. Philippe $(\mathrm{CH})$ - one abstention and

J. Zierath $(\mathrm{S})$ - one abstention.

\section{STUDY GROUPS}

Dr. Eizirik reported that there were 15 Study Groups, 13 of which had attended the Study Group Forum in 2006. All Study Groups are allowed to make proposals for symposia, with the Programme Committee making the final choice. The Honorary Secretary reiterated the rule that under no circumstances should a meeting of a Study Group be held during the period of the EASD Annual Meeting. A Study Group on cardiovascular disease which was introduced to the membership last year is now officially endorsed as a Study Group of the Association. Another group of researchers asked for a Study Group on genetics and diabetes. The group was asked to describe their activities in an article in the News Section of Diabetologia. The matter will be on the agenda of the $87^{\text {th }}$ Council meeting and the $44^{\text {th }}$ General Assembly.

\section{HONORARY MEMBERSHIP}

Drs. L. Orci and G. Reaven were unanimously elected by the General Assembly.

\section{ANY OTHER BUSINESS}

There was no other business.

Dr. Ferrannini thanked the outgoing member of the Executive, Dr. D. L. Eizirik, for his dedication and presented him with the Albert Renold Medal.

Dr. Ferrannini also thanked the outgoing members of the Council: Drs. K. Dahl Jørgensen, R. J. Heine, B. Mankovsky and S. Sasson.

Dr Ferrannini thanked the industry for their support. He also expressed his sincere gratitude to the Local Organising Committee, in particular to Drs. Heine and Tack and Mrs. L. van "t Hull for their outstanding contribution to the organisation of the 43rd EASD Annual Meeting. He again thanked Dr. D. L. Eizirik and the members of the Programme Committee for their hard work with regard to the scientific programme. The President warmly thanked the EASD team in Düsseldorf for their kind and efficient help.

Dr Ferrannini brought the General Assembly to a close at 19:00. 


\section{Agenda for the 44th General Assembly of the European Association for the Study of Diabetes}

to be held in the Da Vinci Hall at the Nuova Fiera di Roma, Rome, Italy on 10 September 2008 at 18:15 hours

\section{Minutes of the 43rd General Assembly, Amsterdam, the Netherlands 2007}

\section{Reports}
a) President
b) Honorary Treasurer
Dr. E. Ferrannini
c) Honorary Auditors
Dr. G. Spinas
Dr. N. Katsilambros
d) Honorary Secretary
Dr. M. Tiedge
e) Editor in Chief, Diabetologia
Dr. M. Stumvoll
f) Chair, Postgraduate Education
Dr. E. Gale
Dr. J. Nolan

Sub-committee

\section{Elections}
a) President
in place of
2008-2011

Dr. E. Ferrannini

b) Vice President

in place of

2008-2011

Dr. U. Smith

c) Editor-in-Chief

Extension:

2009-2010

Dr. E. Gale
d) Honorary Auditors 2008-2011

in place of

Drs N. Katsilambros and

M. Tiedge
e) Council Members 2009-2012

in place of

Dr. V. Pirags (Latvia)

Dr. F. Pociot (DK)

Dr. M. Porta (I)

\section{Study Groups}

\section{Honorary Membership}

6. Membership Fee. Members joining on 1 July to pay for 18 months, thus, giving access to reduced Annual Meeting registration fees for 2 years.

\section{Any other business}




\title{
$44^{\text {th }}$ EASD Annual Meeting of the European Association for the Study of Diabetes
}

\author{
Rome, Italy, 8 - 11 September 2008
}

\author{
Abstracts \\ Index of Oral Sessions \\ OP 1 New treatments in type 2 diabetes \\ OP 2 Genome-wide scans in type 2 diabetes \\ OP 3 Continuous glucose monitoring system \\ OP 4 Mechanisms and new markers of macrovascular \\ disease \\ OP 5 Beta cell development and regeneration \\ OP 6 Fat traffic and parking \\ OP 7 Novel oral agents for type 2 diabetes \\ OP 8 Nephropathy: development and treatment \\ OP 9 Prediction of diabetes \\ OP 10 High glucose, oxidative stress and cellular \\ signalling \\ OP 11 Genetics: type 2 diabetes \\ OP 12 Inflammation, metabolism, cross-talk \\ OP 13 DPP-IV inhibitors \\ OP 14 Obesity: new insights and new therapies \\ OP 15 Molecular mechanisms in insulin signalling \\ OP 16 Nephropathy: experimental treatments \\ OP 17 Dynamics of beta cell exocytosis \\ OP 18 Type 1 diabetes mellitus epidemiology \\ OP 19 Cardiomyopathy and dysfunction of the heart \\ OP 20 Diabetes in childhood \\ OP 21 Incretins: action and secretion \\ OP 22 Brain and peripheral metabolism \\ OP 23 Lipotoxicity in beta cells \\ OP 24 Genetics of type 1 diabetes and complications \\ OP 25 GLP-1 based therapies \\ OP 26 Health care models across Europe \\ OP 27 Microangiopathy: glucose toxicity and \\ inflammation \\ OP 28 The triumvirate gets fatty \\ OP 29 Glucagon and effects of other hormones \\ OP 30 Metabolic signals in beta cells \\ OP 31 Insulin and analogue treatment \\ OP 32 Type 2 diabetes mellitus epidemiology \\ OP 33 Retinopathy: experimental treatments \\ OP 34 Adipokines: from bone to heart \\ OP 35 Endothelium cross-talk \\ OP 36 Apoptotic mechanisms in beta cells \\ OP 37 Thiamine and microangiopathy \\ OP 38 Prevention of type 2 diabetes \\ OP 39 Hypoglycaemia and the brain \\ OP 40 Classical and sophisticated education \\ OP 41 Experimental and clinical immunology \\ OP 42 Monogenic diabetes \\ OP 43 Neural pathways and neuropathy
}

OP 44 Nutrition and diet

OP 45 Insulin signalling and glucose transport

OP 46 Low grade inflammation and endothelial function

OP 47 Novel mechanisms in insulin secretion

OP 48 Programming of metabolism

\section{Index of Poster Sessions}

PS 1 Genetics of autoimmunity

PS 2 Genetics of type 1 diabetes

PS 3 Monogenic diabetes

PS 4 Single gene influence on type 2 diabetes risk

PS 5 Multiple gene influence on type 2 diabetes risk

PS 6 Genetic regulation of insulin action

PS 7 Genetic influence on beta cell function

PS 8 Genetic influence on insulin sensitivity

PS 9 Prediction of type 1 diabetes

PS 10 Environmental factors and diabetes

PS 11 Screening and prediction of type 2 diabetes

PS 12 Animal models for type 2 diabetes

PS 13 Classification of type 1 diabetes

PS 14 Incidence of type 1 diabetes

PS 15 Cardiovascular risk

PS 16 Glycated haemoglobin and diabetes management

PS 17 Epidemiology and genetics of body weight

PS 18 Epidemiology of type 2 diabetes complications

PS 19 CVD and mortality in diabetes

PS 20 Diabetes and ethnicity

PS 21 Quality of life and diabetic complications

PS 22 Beta cells from stem cells

PS 23 Beta cell regeneration

PS 24 Beta cell proliferation

PS 25 regulation in beta cells

PS 26 Beta cell exocytosis

PS 27 Beta cells in type 2 diabetes

PS 28 Metabolic signals in the beta cell

PS 29 Modulators of insulin secretion

PS 30 Lipid effects in the beta cell

PS 31 Cytokine toxicity in beta cells

PS 32 Glucotoxicity in beta cells

PS 33 Apoptotic mechanisms in beta cells

PS 34 Inflammation and ROS in beta cells

PS 35 ER stress in the beta cell

PS 36 Experimental immunology

PS 37 Autoimmunity against GAD

PS 38 Clinical immunology

PS 39 Autoantibodies and regulatory T-cells in type 1 diabetes 


\begin{tabular}{|c|c|c|}
\hline PS & 40 & Clinical islet and pancreas transplantation \\
\hline PS & 41 & Experimental islet transplantation \\
\hline PS & 42 & Intestinal glucose absorption and metabolism \\
\hline PS & 43 & Insulin action: liver and skeletal muscle \\
\hline PS & 44 & $\begin{array}{l}\text { ROS and mitochondria: effects on insulin secretion } \\
\text { and action }\end{array}$ \\
\hline PS & 45 & Vascular effects of insulin \\
\hline PS & 46 & Regulation of incretin secretion and action \\
\hline PS & 47 & $\begin{array}{l}\text { Animal models: altered glycaemia and insulin } \\
\text { resistance }\end{array}$ \\
\hline PS & 48 & Regulation of insulin secretion \\
\hline PS & 49 & Other hormones \\
\hline PS & 50 & Exercise physiology \\
\hline PS & 51 & Hypoglycaemia \\
\hline PS & 52 & $\begin{array}{l}\text { Drug effects on insulin sensitivity and insulin } \\
\text { action }\end{array}$ \\
\hline PS & 53 & Mechanisms of insulin resistance: cellular studies \\
\hline PS & 54 & Insulin sensitivity and resistance: human subjects \\
\hline PS & 55 & $\begin{array}{l}\text { Insulin sensitivity and glucose metabolism in } \\
\text { humans: various mechanisms }\end{array}$ \\
\hline PS & 56 & Adipokines \\
\hline PS & 57 & Adiponectin \\
\hline PS & 58 & Animal models of obesity \\
\hline PS & 59 & Ectopic fat: liver and pancreas \\
\hline PS & 60 & Lipids and lipoproteins \\
\hline PS & 61 & Ectopic fat muscle \\
\hline PS & 62 & Mitochondrial function and metabolism \\
\hline PS & 63 & Animal models of type 2 diabetes \\
\hline PS & 64 & Cytokines: IL- 1 and others \\
\hline PS & 65 & AMPK and ERS \\
\hline PS & 66 & Obesity \\
\hline PS & 67 & Oxidative stress \\
\hline PS & 68 & Cytokines: TNF alpha and related cytokines \\
\hline PS & 69 & Weight regulation \\
\hline PS & 70 & Inflammation and fat \\
\hline PS & 71 & Adipocyte and related biology \\
\hline PS & 72 & Fat distribution \\
\hline PS & 73 & Lipid oriented therapies \\
\hline PS & 74 & GLP-1 based treatments: clinical and experimental \\
\hline PS & 75 & Novel GLP- 1 based treatments \\
\hline PS & 76 & Exenatide: clinical trials \\
\hline PS & 77 & Exenatide: real life clinical practice \\
\hline PS & 78 & Liraglutide \\
\hline PS & 79 & Liraglutide clinical studies \\
\hline PS & 80 & $\begin{array}{l}\text { Mechanisms involved in treatment with DPP-IV } \\
\text { inhibitors }\end{array}$ \\
\hline PS & 81 & Sitagliptin and vildagliptin \\
\hline PS & 82 & Treatment with PPARy agonists \\
\hline PS & 83 & Novel oral agents \\
\hline PS & 84 & Traditional oral therapies \\
\hline PS & 85 & Diabetes in childhood \\
\hline PS & 86 & Nutrition and diet \\
\hline PS & 87 & Insulin therapy: acronym studies \\
\hline PS & 88 & Insulin therapy in type 1 diabetes \\
\hline PS & 89 & $\begin{array}{l}\text { Insulin therapy: body weight and cardiovascular } \\
\text { disease }\end{array}$ \\
\hline PS & 90 & Insulin pumps \\
\hline PS & 9 & Insulin therapy: others \\
\hline
\end{tabular}

PS 92 Insulin and costs

PS 93 New treatments

PS 94 Diabetes in daily practice

PS 95 Cognitive and psychological aspects

PS 96 Diabetes treatment and exercise

PS 97 Health care: national surveys

PS 98 Health care: others

PS 99 Monitoring type 2 diabetes

PS 100 Monitoring type 1 diabetes

PS 101 Diabetes at the hospital

PS 102 Costs of diabetes

PS 103 Quality of life

PS 104 Education

PS 105 Pregnancy in type 1 diabetes mellitus

PS 106 Pregnancy: inflammation and pathogenetic mechanisms

PS 107 Diabetic pregnancy: complications

PS 108 Gestational diabetes: metabolism

PS 109 Gestational diabetes: outcomes

PS 110 Diabetic microangiopathy: pathogenetic mechanisms

PS 111 Genetics of complications

PS 112 Diabetic retinopathy screening: clinical aspects

PS 113 Experimental nephropathy: inflammation, oxidative stress and growth factors

PS 114 Screening and investigation of kidney disease

PS 115 Human diabetic nephropathy: risk factors, cardiovascular disease and anaemia

PS 116 Human diabetic nephropathy: markers and associated factors

PS 117 Treating diabetic nephropathy

PS 118 Hypertension: treatment

PS 119 Hypertension and cardiovascular risk

PS 120 Autonomic neuropathy: clinical features

PS 121 Neuropathy: experimental and clinical treatments

PS 122 Neuropathy: screening and associated features

PS 123 Diabetic foot: pathogenetic mechanisms

PS 124 Diabetic foot: assessment

PS 125 Diabetic foot: treatment

PS 126 Oxidative stress and endothelial function

PS 127 Diabetes related changes in endothelial gene expression

PS 128 Glycotoxic metabolites

PS 129 AGE-receptors

PS 130 Impairment and repair of endothelial function

PS 131 Predictors of macrovascular disease

PS 132 Macrovascular disease: risk factors, treatment and costs

PS 133 Cardiac complications

PS 134 Metabolic risk and cardiovascular complications

PS 135 Predictors of vascular complications

PS 136 Complications: animal models and pathogenic mechanisms

PS 137 Screening methods for diabetic complications

PS 138 Dyslipidaemia mediated complications

PS 139 Non-classical risk factors and markers of inflammation 


\section{OP 01 New treatments in type 2 diabetes}

\section{1}

XOMA 052, an anti-IL-1 $\beta$ antibody, in a double-blind, placebocontrolled, dose escalation study of the safety and pharmacokinetics in patients with type 2 diabetes mellitus - a new approach to therapy M.Y. Donath ${ }^{1}$, C. Weder ${ }^{1}$, J. Whitmore ${ }^{2}$, R.J. Bauer ${ }^{2}$, K. Der $^{2}$, P.J. Scannon ${ }^{2}$, C.A. Dinarello ${ }^{3}$, A.M. Solinger ${ }^{2}$

${ }^{1}$ Endocrinoloy \& Diabetes, University Hospital Zurich, Switzerland, ${ }^{2}$ Clinical Development, XOMA (US) LLC, Berkeley, United States,

${ }^{3}$ University of Colorado Health Sciences Center, Denver, United States

Background and aims: The cytokine interleukin-1 $\beta$ (IL-1 $\beta$ ) is involved in the pathology of many inflammatory diseases, and blocking of the IL-1 pathway shows efficacy in both animal and human disease. Recent evidence points to the role of elevated IL- $1 \beta$ in glucose-induced beta-cell apoptosis and dysfunction. Clinical data has also shown IL-1 receptor inhibition improving glycemic control and beta-cell function, thus validating this pathway as a target for therapy. The Human Engineered ${ }^{\mathrm{mix}}$ anti-IL-1 $\beta$ antibody, XOMA 052, was developed for the treatment of inflammatory diseases and has a very high affinity $\left(\mathrm{K}_{\mathrm{D}}=300 \mathrm{fM}\right)$ and activity for human IL-1 $\beta$ (hIL-1 $\left.\beta\right)$.

Materials and methods: Two clinical studies in Type 2 Diabetes Mellitus patients with inadequate control of their hyperglycemia (FBS $>126 \mathrm{mg} / \mathrm{dL}$ and HgAlc $\geq 7.5 \%$ but $\leq 12 \%$ ) evaluated a total of 72 subjects after single IV slow infusions of XOMA 052 over a dose range beginning at $0.01 \mathrm{mg} / \mathrm{kg}$ and escalating at half-logs in six equal cohorts. Each 6-subject treatment cohort in the two parallel studies contained 5 active drug-treated subjects and 1 placebotreated subject. Each subject was evaluated for safety, pharmacokinetics of the study drug, and various diabetes- and inflammation-related parameters for 2-3 months after their single infusion. These parameters will be used to assess clinical activity and include inflammatory markers such as CRP and IL-6 levels, and key measures of glucose control such as FBS and HgAlc.

Results: Infusion of study drug was well tolerated without any evidence of any serious or drug-related adverse events during the study. Clearance of study drug was consistent with that for a human IgG with a $15-21$ day $\mathrm{T}^{1} 12$.

Conclusion: In this first-in-disease study of XOMA 052, an anti-IL-1 $\beta$ antibody, no evidence of safety or pharmacokinetic issues for XOMA 052 was present. This therapeutic agent with the projected profile may be able to be used on a once every month or longer schedule. Further investigation of dosing schedule and initial clinical signals will need to be performed. Supported by: XOMA (US) LLC

\section{2}

Improvement in glycaemic control by Gastric Electrical Stimulation (TANTALUS $^{\mathrm{TM}}$ ) in overweight subjects with type 2 diabetes (T2DM) B. Guerci ${ }^{1}$, E. Renard ${ }^{2}$, R. Prager ${ }^{3}$, B. Ludvik ${ }^{4}$, A. Hamann ${ }^{5}$

${ }^{1}$ Hopital Jeanne-d'Arc, Nancy, France, ${ }^{2}$, Hopital Lapeyronie, Montpellier, France, ${ }^{3}$ Hietzing Hospital, Vienna, Austria, ${ }^{4}$ Medical University of Vienna, Austria, ${ }^{5}$ Diabetes Klinik, Bad Nauheim, Germany

Background: The TANTALUS System (MetaCure Ltd.) is a minimally invasive implantable gastric stimulation modality that does not exhibit malabsorptive or restrictive characteristics. The device automatically detects food intake and applies GCM (Gastric Contractility Modulation) signals to the stomach antrum to induce early satiety. Retrospective analysis of previous studies indicated that T2DM subjects poorly controlled with oral anti-diabetic medication are likely to benefit from the treatment.

Aims: To prospectively investigate the potential effect of the TANTALUS System therapy on glycemic control and weight change in obese T2DM subjects.

Clinical characteristics and methods: In this European multi-center, openlabel pilot study, 19 T2DM obese subjects ( $12 \mathrm{M}, 7$ F, Age: $53 \pm 2$ years, BMI: $38.1 \pm 0.8 \mathrm{~kg} / \mathrm{m}^{2}, \mathrm{HbAlc} 8.0 \pm 0.1 \%$ (Mean $\pm \mathrm{SEM}$ ) treated with oral anti-diabetic medications, but with poor glycemic control, were implanted laparoscopically with the TANTALUS System.

Results: To date, 16 out of 19 subjects have completed 3 months of treatment and show a mean significant reduction in HbAlc from $8.0 \pm 0.1 \%$ to $6.8 \pm 0.2 \%(\mathrm{P}<0.01)$. This was accompanied by a mean significant decrease in body weight from $109.6 \pm 4.5 \mathrm{Kg}$ to $105.5 \pm 4.7 \mathrm{Kg}(\mathrm{P}<0.01)$, and in waist cir- cumference from $123.3 \pm 2.8 \mathrm{~cm}$ to $118.4 \pm 2.9 \mathrm{~cm}(\mathrm{P}<0.01)$. The $\mathrm{HbAlc}$ reduction was sustained also after 6 months of treatment $(\mathrm{N}=7) . \mathrm{R}^{2}$ of the correlation between changes in body weight and $\mathrm{HbAlc}$ was $0.31(\mathrm{P}=0.02)$. Weight reduction contributed only $31 \%$ of the decrease in HbAlc.

Conclusion: Interim results with the TANTALUS System suggest that this stimulation regime can potentially improve glucose homeostasis and induce weight loss in obese T2DM subjects on oral anti-diabetic therapy with poor glycemic control. As weight reduction was only minimaly associated with major $\mathrm{HbAlc}$ decrease the observed beneficial TANTALUS effects might be associated with weight-independent mechanism(s).

Supported by: Metacure Limited

\section{3}

Rimonabant improves glycaemic control and associated cardiometabolic risk factors in insulin-treated type 2 patients

P.A. Hollander ${ }^{1}$, A. Amod ${ }^{2}$, L.E. Litwak ${ }^{3}$

${ }^{1}$ Baylor Endocrine Center, Baylor University Medical Center, Dallas, United States, ${ }^{2}$ Centre for Diabetes, Endocrinology and Metabolic Diseases, Chatsmed Garden Hospital, Durban, South Africa, ${ }^{3}$ Department of Endocrinology, Hospital Italiano de Buenos Aires, Argentina

Background and aims: Rimonabant, the first selective cannabinoid type-1 $\left(\mathrm{CB}_{1}\right)$ receptor antagonist, has demonstrated a significant beneficial effect on a comprehensive set of cardiometabolical risk parameters in patients with type 2 diabetes (T2DM) naïve to antidiabetic therapy or treated with metformin or sulphonylurea. To further evaluate rimonabant $20 \mathrm{mg} /$ day in an advanced stage of T2DM, rimonabant was studied in patients receiving insulin monotherapy.

Materials and methods: Entry criteria for this 48 -week, double-blind, placebo-controlled trial included $\mathrm{HbAlc} \geq 7 \%$, insulin monotherapy for $\geq 3$ months; there was no requirement for BMI. Primary outcome was change from baseline in $\mathrm{HbAlc}$. Secondary outcomes included change in high-density lipoprotein (HDL)-cholesterol, triglycerides, body weight and waist circumference.

Results: In total, 368 patients were randomised between rimonabant (181) and placebo (187). At baseline: mean body mass index (BMI), $34.6 \mathrm{~kg} / \mathrm{m}^{2}$; mean total daily insulin dose (MTDID), $83.5 \pm 50.5$ IU; T2DM duration, $14.4 \pm 7.7$ years; insulin treatment, $6.2 \pm 4.9$ years. In the intention-to-treat population, mean baseline $\mathrm{HbAlc}(9.1 \pm 1.5 \%)$ was reduced significantly more with rimonabant vs placebo $(-0.89 \pm 1.34 \%$ vs $-0.24 \pm 1.13 \%$; $p<0.0001)$. There was significantly greater improvement in high-density lipoprotein cholesterol $(p<0.0001)$ and triglyceride $(p=0.02)$ levels, weight $(p<0.0001)$ and waist circumference $(\mathrm{WC})(\mathrm{p}<0.0001)$ with rimonabant vs placebo (Table). The observed effect on $\mathrm{HbAlc}$ appeared consistent in additional analysis across baseline BMI strata (Figure). The safety profile of rimonabant was consistent with previous studies in T2DM with more gastrointestinal (nausea $11.0 \%$ vs $1.6 \%$, diarrhoea $7.3 \%$ vs $6.4 \%$ ), nervous system (dizziness $10.1 \%$ vs $8.1 \%$ ) and psychiatric (anxiety $14.0 \%$ vs $4.3 \%$, depressed $\operatorname{mood} 10.1 \%$ vs $4.3 \%$ ) events reported with rimonabant vs placebo. Similar rates of serious adverse events were reported in both treatment groups.

Conclusion: This study in patients with advanced T2DM, receiving insulin, confirmed the clinically significant effect of rimonabant on glycaemic control and associated cardiometabolical risk factors.

Mean (SD) changes from baseline

\begin{tabular}{llll}
\hline Outcome & & $\begin{array}{l}\text { Placebo } \\
\mathbf{n}=\mathbf{1 8 6}\end{array}$ & $\begin{array}{l}\text { Rimonabant 20 } \mathbf{~ m g} \\
\mathbf{n}=\mathbf{1 7 9}\end{array}$ \\
\hline HDL-C, mM & Baseline & $1.32(0.4)$ & $1.32(0.3)$ \\
& \% change & $-7.1(13.4)$ & $3.1(13.5)$ \\
\hline Triglycerides, $\mathrm{mM}$ & Baseline & $2.7(4.4)$ & $2.7(3.9)$ \\
& \% change & $7.6(40.6)$ & $-4.0(53.0)$ \\
\hline Weight, kg & Baseline & $95.3(20.5)$ & $97.4(20.7)$ \\
& Change & $0.1(3.5)$ & $-2.5(4.0)$ \\
\hline WC, cm & Baseline & $110(15)$ & $112(14)$ \\
& Change & $0.2(6.9)$ & $-2.8(8.6)$ \\
\hline
\end{tabular}




\section{Descriptive analysis (mean [SD]) of HbA1c at Day 336 by $B M I$ subgroup}

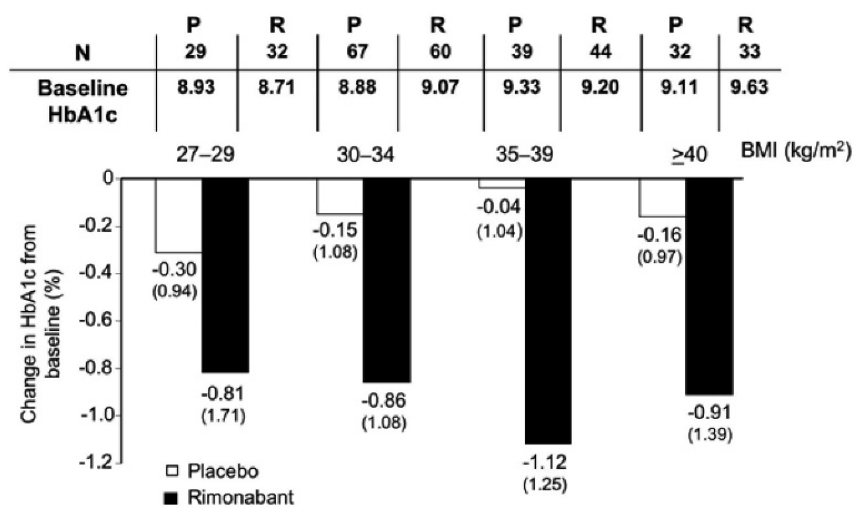

Supported by: sanofi-aventis

\section{4}

Repeat-dosing of oral insulin (Capsulin) in persons with type 2 diabetes T.P. Broke-Smith ${ }^{1}$, S.D. Luzio ${ }^{2}$, A. Lockett ${ }^{1}$, R.R.C. New $^{1}$, D.R. Owens ${ }^{2}$ ${ }^{1}$ Diabetology Limited, Cobham, ${ }^{2}$ Diabetes Research Unit, Cardiff University, Penarth, United Kingdom

Background and aims: A 10 day repeat dose study to investigate twice-daily dosing with the oral insulin (Capsulin ${ }^{\mathrm{TM}}$ ) in the treatment of type 2 diabetes. Materials and methods: 16 subjects (12 male) participated; mean (SD) age 60.2 (5.5) years, BMI 28.3 (3.4) kg/m2, HbAlc 7.5 (1.3) \%. All subjects received 150U Capsulin BID given approximately one hour prior to breakfast and the evening meal for 10 consecutive days, during which self-monitored blood glucose (SMBG) readings were conducted 5-times a day (fasting, 2-hr after breakfast, before dinner, 2-hr after dinner, at bedtime). Prior to study entry, subjects were on stable doses of oral hypoglycaemic agents (OHA) for at least 3 months and had suboptimal glycaemic control. During the study, all OHAs apart from metformin were withdrawn ( 9 out of 16 subjects dropped at least one OHA; 14 remained on metformin; 2 were treated with Capsulin alone). Pre-study and post study screening occurred around 5 days prior and post the dosing period respectively.

Results: During the repeat dosing period SMBG readings remained within the recommended range $(6-11 \mathrm{mmol} / \mathrm{L})$. Daily fluctuations in SMBG as assessed by the mean amplitude of glycaemic excursion (MAGE) analysis were reduced over the study period. Analysis of postprandial glucose (PPG) excursions indicated that by day 10 the proportion of post-meal values which fell within the ADA recommended range $(<8.9 \mathrm{mmol} / \mathrm{L})$ had increased from 9.7\% (day 2) to $35.5 \%$ (day 10) ( $\mathrm{p}=0.027$ ). This effect was more pronounced during the evening meals where the proportion of data points that fell within the recommended range increased from $6.3 \%$ (day 2) to $43.8 \%$ (day 10 ) $(\mathrm{p}=0.009)$. These observations were associated with significant improvements in HbAlc $(7.38 \pm 1.14 \%$ to $7.16 \pm 0.86 \%, \mathrm{p}<0.05)$, weight $(84.9 \pm 11.9$ to $84.2 \pm 11.6 \mathrm{~kg}, \mathrm{p}<0.05)$ and triglycerides $(1.83 \pm 0.88$ to $1.53 \pm 0.60 \mathrm{mmol} / \mathrm{l}$, $\mathrm{p}<0.05)$ between pre-study screen and study close. No serious adverse events and no hypoglycaemia were reported.

Conclusion: The ability for Capsulin to potentially improve glycaemic control throughout the day (including mealtimes) without inducing hypoglycaemia may be due to its route of delivery via the hepatic portal system, thereby mimicking endogenous insulin secretion.

\section{5}

An open label, two period, placebo controlled, multiple dose study to evaluate the effect of food on pharmacokinetic and pharmacodynamic of IN-105 $10 \mathrm{mg}$ in type 2 diabetes mellitus patients

H. Iyer ${ }^{1}$, A.P. Khedkar ${ }^{1}$, S. Suryanarayan ${ }^{1,2}$, A. Anand ${ }^{1}$, A. Vedala ${ }^{1}$,

S. Krishnamurthy ${ }^{1}$, A.S. Arvind ${ }^{3}$, A. Eshwaraiah ${ }^{3}$, S. Savale ${ }^{3}$, K.M. Prasanna Kumar $^{4}$, S.R. Arvind ${ }^{5}$, S.S. Srikanta ${ }^{6}$

${ }^{1}$ Research and Development, Biocon Limited, Bangalore, India, ${ }^{2}$ Visiting faculty, Department of Molecular Medicine and Surgery, Karolinska Institute, Stockholm, Sweden, ${ }^{3} \mathrm{Clinical}$ research, Clinigene International limited, Bangalore, India, ${ }^{4}$ M.S.Ramaiah Medical College and Hospital, Bangalore, India, ${ }^{5}$ Diacon Hospital and Research Centre, Bangalore, India, ${ }^{6}$ Diabetology, Samatvam Science and Research for Human Welfare Trust, Bangalore, India

Background and aims: IN-105 is a modified insulin analog which, on oral administration, showed glucose lowering activity in normal healthy volunteers. The aim of this study was to evaluate safety and effect of meal on pharmacokinetics and pharmacodynamics of IN-105 in type II diabetics.

Materials and methods: 14 type II diabetic patients, with HbAlc between 7.5 to $9.5 \%$, were enrolled in an open label, two treatment, two period placebo-controlled study, conducted to assess pharmacokinetics and pharmacodynamics of multiple doses of "IN-105" tablets [10 mg dose]. In period 1 , subjects were given placebo tablets $20 \mathrm{~min}$ prior to meal and a metformin tablet was given 30 min post meal. 3 tablets were administered during the single day period: one each prior to breakfast, lunch and dinner. A washout period of 7 days was maintained between period 1 and period 2. In period 2, the same patients were dosed with $10 \mathrm{mg} I N-105$ prior to breakfast, lunch and dinner at the same times as in period 1 . The patients were given $600 \mathrm{kcal}$ meal for breakfast, lunch and dinner and $200 \mathrm{kcal}$ after the final sample of the day was collected. Immunoreactive insulin levels in plasma were measured along with the c-peptide and glucose levels.

Results: A significant drop in plasma glucose levels was observed after each dose of IN-105 as compared to that of placebo. Average maximum \% drop in glucose after administration of morning, afternoon and evening doses of IN105 tablets ( $10 \mathrm{mg}$ dose) were 16,23 and $18 \%$ respectively. The corresponding values for drop in glucose after administration of placebo were 2, 5 and $7 \%$. Further average $\mathrm{T}_{\min }$ values for corresponding doses of IN-105 were between 30-40 min. The average $C_{\max }$ for total insulin obtained after administration of morning, afternoon, evening doses of IN-105 (10mg) were $137 \pm 33,112 \pm 21$, $69 \pm 13 \mathrm{mIU} / \mathrm{L}$. The corresponding values of $\mathrm{C}_{\text {max }}$ for total insulin after administration of placebo were 46.54, 53.42, $42.65 \mathrm{mIU} / \mathrm{L}$. The $\mathrm{C}_{\max }$ values for total insulin, in period 2, reduced in the order of morning $>$ afternoon $>$ evening dose. The data on $\mathrm{C}_{\max }$ and $\mathrm{C}_{\min }$ for C-peptide after administration of morning, afternoon and evening doses of IN-105 and placebo treatment indicates that the higher levels of total insulin after administration of IN-105 tablet are due to the absorption of IN-105 administered orally. The IN-105 tablet formulation $(10 \mathrm{mg}$ dose $)$ administered three times a day was well tolerated in presence of multiple meals administered 20 min after each dose in type II diabetes mellitus patients.

Conclusion: IN-105, $10 \mathrm{mg}$ dose was well tolerated by type II diabetic patients. Glucose lowering effect was found to be proportional to insulin exposure. The plasma exposure of IN-105 was reduced from morning to evening. The glucodynamic effect was however, was similar for morning and evening dose.

\section{6}

Effects of grapeseed extract in type 2 diabetic subjects: a double blind randomised placebo controlled trial looking at the effects upon inflammatory and metabolic markers P. Kar ${ }^{1}$, D. Laight ${ }^{2}$, K.M. Shaw ${ }^{1}$, M.H. Cummings ${ }^{1}$

${ }^{1}$ Academic Department of Diabetes \& Endocrinology, Queen Alexandra Hospital,Portsmouth, ${ }^{2}$ School of Pharmacy, University of Portsmouth, United Kingdom

Background: Vascular inflammation is thought to be a possible contributor to the pathology of type 2 diabetes and cardiovascular disease. Recent attention has focused upon the beneficial effect of plant-based products containing flavonoids, which have been shown to have anti-inflammatory properties. Studies have also highlighted the potentially beneficial platelet-dependent antithrombotic and anti-inflammatory properties of flavonoid rich products such as grape seed extracts (GSE). Moreover experimental work in animal models have suggested GSE improves metabolic markers such as cholesterol. 
Aim: This study aimed to test the hypothesis that GSE may improve inflammatory and metabolic markers of vascular risk in high-risk cardiovascular subjects with T2DM.

Methods: 32 T2DM patients, diet or tablet controlled, were given 600mg GSE and placebo, for 4 weeks each, in a double-blinded randomized crossover trial. Markers of inflammation (hsCRP;Urinary ACR) and metabolism (fructosamine, lipid profile) were measured at baseline and after intervention with GSE or placebo.

Results: Baseline characteristics of patients(16 M, 16F): Age $61.81 \pm 6.36$ years (mean $+/$ - $1 \mathrm{SD}$ ); BMI $30.24 \pm 5.92 \mathrm{~kg} / \mathrm{m} 2$; diabetes duration $5.92 \pm 2.14$ years; HbAlc $6.81 \pm 1.04 \%$; total cholesterol of $4.51 \pm 0.95 \mathrm{mmol} / \mathrm{l}$, HDLcholesterol $1.88 \pm 1.01 \mathrm{mmol} / \mathrm{l}$ and triglycerides $1.19 \pm 0.3 \mathrm{mmol} / \mathrm{l}$.

Significant reductions were noted in fructosamine $(282.2 \pm 40.87 \mu \mathrm{mol} / \mathrm{l}$ vs. $272.87 \pm 50.19 \mu \mathrm{mol} / \mathrm{l} ; \mathrm{p}=0.0004)$ and hsCRP $(3.22 \pm 3.65 \mathrm{mg} / \mathrm{l}$ vs. $1.95 \pm 2.2$ $\mathrm{mg} / \mathrm{l} ; \mathrm{p}=0.0006)$, post GSE intervention. A reduction in total cholesterol (4.51 $\pm 0.96 \mathrm{mmol} / 1$ vs. $4.33 \pm 0.99 \mathrm{mmol} / \mathrm{l})$ was also observed $(\mathrm{p}=0.05)$. Triglyceride $(1.88 \pm 1 \mathrm{mmol} / \mathrm{l}$ vs. $1.69 \pm 0.99 \mathrm{mmol} / \mathrm{l} ; \mathrm{p}=0.2957)$, HDL-cholesterol $(1.19$ $\pm 0.3 \mathrm{mmol} / \mathrm{l}$ vs. $1.22 \pm 0.32 \mathrm{mmol} / \mathrm{l} ; \mathrm{p}=0.1441)$ and urinary ACR levels ( 1.1 \pm 0.5 vs. $1.2 \pm 0.5 ; \mathrm{p}=0.4734$ ) did not show any significant changes. Multiple correlation analysis did not show any correlation between the GSE mediated changes shown in vascular and metabolic markers.

Conclusion: GSE significantly improved inflammatory and metabolic markers in high-risk cardiovascular subjects (T2DM) over a 4-week period.

Large prospective studies are required to examine whether these changes in short term surrogate markers of vascular risk with GSE translate into beneficial clinical outcome implications in T2DM patients.

Supported by: Novonordisk

\section{OP 02 Genome-wide scans in type 2 diabetes}

\section{7}

Analysis of genome-wide scans identifies potential novel common susceptibility loci for central obesity

C.M. Lindgren ${ }^{1}$, I.M. Heid ${ }^{2}$, J.C. Randall ${ }^{1}$, C. Lamina ${ }^{2}$, E.K. Speliotes ${ }^{3}$, The GIANT Consortium

${ }^{1}$ Wellcome Trust Centre for Human Genetics, University of Oxford, United Kingdom, ${ }^{2}$ German Research Center for Environmental Health, Helmholtz Zentrum Muenchen, Institute of Epidemiology, Neuherberg, Germany, ${ }^{3}$ Harvard University and MIT, Broad Institute, Boston, United States

Background: Recently, FTO and one other locus located 188 kilobases downstream of MC4R were the first loci identified harboring common variants with an unequivocal impact on common obesity predisposition. In contrast, no specific associations have been reported to central obesity (in particular the amount of visceral/abdominal fat), which is especially relevant to metabolic disease (Type 2 Diabetes, Cardiovascular Disorder etc.). Anthropometric measures of central obesity can be used as physiological proxies of fat distribution and are more directly correlated to adverse metabolic outcomes including diabetes risk than BMI. Due to imperfect correlation with overall obesity (r2 0.8), measures of central obesity can be used to identify genetic variants specific to regulation of fat distribution. Heritability estimates are still $\sim 60 \%$ for waist circumference (WC) and $\sim 45 \%$ for waist-hip ratio (WHR) after correcting for BMI. Thus we set out to undertake global identification of common genetic variants and pathways influencing individual patterns of fat distribution and central obesity through large-scale genome-wide association meta-analysis of WC and WHR.

Methods: We have carried out a meta-analysis of 13 GWA scans across $\sim 29,000$ individuals of European ancestry and include individuals from Germany (KORA), Sweden and Finland (DGI, FUSION), the US (PLCO, NHS), the UK (WTCCC T2D, HT, CHD, BC58, NBS and GEM-EPIC), Switzerland (CoLaus), and Italy (SardiNIA). First we excluded SNPs with HWE1\% and high imputation quality scores $(>.3 \mathrm{MACH} 2 \mathrm{QTL}-\mathrm{R} 2 \mathrm{HAT},>.9$ posterior probability, >.4 SNPTEST proper_info). The meta-analysis of the association results was performed using METAL.

Results: The analysis of the waist-related phenotypes has identified several novel loci associated with fat distribution phenotypes; we observed 15 independent loci for WC and 3 for WHR with p-values $<1 \mathrm{x} 10-6$. We strongly confirmed the association of the FTO and MC4R, which accounted for the best $\mathrm{p}$-values for WC ( $\mathrm{p}<5 \times 10-13$ and $\mathrm{p}<2 \times 10-8$, respectively) but was less associated with WHR ( $p<5 \times 10-6$ and $p<2 \times 10-5$, respectively). Several of the nominally associated loci have strong biological credentials and appear be distinct from BMI signals. For example, at least two variants that show an association with WHR $(\mathrm{p}<2 \times 10-5$ and $\mathrm{p}<3 \times 10-5)$ are involved in the central neuronal signalling pathways.

Conclusion: Our results suggest there are genetic variants with a primary effect on central obesity (WC/WHR) that differ from those with an effect on overall obesity (weight/BMI). We are currently expanding the GWA metaanalysis to other cohorts attempting to replicate our findings $(\mathrm{n} 22,000)$. These results indicate that multiple common variants influence central obesity in humans and that genome-wide association analysis with large numbers of individuals will aid in the identification of such variants.

Supported by: UK Medical Research Council, Wellcome Trust, Diabetes UK, Cancer Research, BDA, UK Research and Development, European Commission, Academy of Finland, British Heart Foundation, National Institutes of Health, Novartis, GlaxoSmithKline, German National Genome Research Net

\section{8}

Meta-analysis of genome-wide association data for weight from over 32000 individuals

J.C. Randall ${ }^{1}$, C.M. Lindgren ${ }^{1}$, E.K. Speliotes ${ }^{2}$, S. Li $^{3}$, The GIANT

Consortium

${ }^{1}$ Wellcome Trust Centre for Human Genetics, University of Oxford, United Kingdom, ${ }^{2}$ Broad Institute, Harvard and MIT, Boston, MA, United States,

${ }^{3}$ Epidemiology Unit, MRC, Cambridge, United Kingdom

Background and aims: Obesity is increasingly prevalent worldwide, and is reaching epidemic levels in some western countries. Health risks associated with obesity have long been established and include an increased risk of de- 
veloping a number of metabolic diseases as well as a shortened life expectancy. Adult weight is highly heritable (heritability $70 \%$ ), but the genetic variants that predispose to this trait are not yet well-characterized, and those loci that have been identified have had modest effects. To increase power to detect common variants with modest effect sizes, we performed a meta-analysis of 13 genome-wide association studies that each contributed association data on anthropometric measures as part of the GIANT consortium.

Materials and methods: All the populations studied are of European ancestry and include individuals from Germany (KORA), Sweden and Finland (DGI \& FUSION), the USA (PLCO \& NHS), the UK (WTCCC BC58, CHD, HT, NBS, T2D; \& GEM EPIC), Switzerland (CoLaus), and Italy (SardiNIA); and together constitute more than 32,000 individuals. Each study excluded SNPs with HWE $<10-6$ and then imputed to obtain probable genotypes for approximately 2.5 million SNPs in the CEU HapMap reference panel. Each study then inverse-normal transformed the weight data separately for each gender before performing gender-stratified association testing, in which each SNP was tested for association with the transformed weight phenotype using an additive model with age as a covariate. We then performed QC on those results and excluded SNPs with a minor allele frequency $<1 \%$ or with low imputation quality scores. Finally, we performed a meta-analysis of the association results using METAL to generate a single summary p-value for each SNP.

Results: This meta-analysis represents the largest whole-genome association study of weight performed to date. Our preliminary analysis has identified 18 independent loci associated with adult weight at levels of genome-wide significance $(\mathrm{p}<1 \times 10-6)$. Top hits include FTO ( $\mathrm{p}<6 \times 10-15), H M G A 2(\mathrm{p}<6 \times 10-$ 10), MC4R ( $<<8 \times 10-9), Z B T B 38(\mathrm{p}<2 \times 10-7)$, and GDF5 ( $\mathrm{p}<4 \times 10-7)$, confirming previously known associations at these loci for BMI (FTO, MC4R) and showing novel associations with weight for several loci previously shown to be associated with height (HMGA2, ZBTB38, GDF5). A number of the remaining 13 loci identified by our meta-analysis as being associated with weight also have modest levels of association with either BMI or height, while a handful appear to be unique to weight. Of those, several of the strongest hits are implicated in biologically interesting pathways such as lipid metabolism, glycogen storage, and neural signaling. Additionally, gender-stratified metaanalyses indicate strong sexual dimorphism for some loci.

Conclusion: These results are consistent with previously known associations for obesity and height, with the most associated locus for weight being a known BMI locus and the second most associated being a height locus. Loci that share a high level of association between weight and height may in fact be associated with overall body size. The numerous additional associated loci found continue to support the hypothesis that multiple common variants influence body weight and that genome-wide association analysis with large numbers of individuals can identify such variants.

Supported by: UK Medical Research Council, Wellcome Trust, Diabetes UK, Cancer Research, BDA, UK Research and Development, European Commission, Academy of Finland, British Heart Foundation, Novartis Institutes for BioMedical Research, GlaxoSmithKline, German National Genome Research Net

\section{9}

First-generation scan of genome-wide association data allowing for epistasis prioritises multiple interacting candidate loci in T2D J.T. Bell ${ }^{1}$, N. Timpson ${ }^{1}$, N.W. Rayner ${ }^{1}$, E. Zeggini ${ }^{1}$, A.P. Morris ${ }^{1}$, M.I. McCarthy'2, Wellcome Trust Case Control Consortium, UK, UK Type Two Diabetes Consortium

${ }^{1}$ Wellcome Trust Centre for Human Genetics, ${ }^{2}$ Oxford Centre for Diabetes, Endocrinology and Metabolism, Oxford University, United Kingdom

Background and aims: Genome-wide association (GWA) provides an opportunity to discover genetic variation involved in complex human traits and has yielded evidence of novel genes associated with T2D. GWA data allow for a systematic search of joint genetic effects including epistasis, though such analyses are computationally and methodologically challenging. We undertook a series of interaction analyses within data from the Wellcome Trust Case Control Consortium (WTCCC) for T2D (1924 cases and 2938 controls) with the aim of developing interaction analysis as a strategy for prioritising pairs of markers for follow-up.

Materials and methods: We first took novel T2D loci implicated by GWA analyses and assessed pairwise interaction within these and between these and previously known T2D loci. We then performed a two-dimensional, genomewide, gene/gene effect scan using a joint two-locus test of association (including main and epistatic effects) in a subset of 70,236 markers tagging common SNP variation at $\mathrm{r}^{2}$ of $>0.2$ and $\mathrm{MAF} \geq 0.05$. These were derived from the tagging of 393,242 SNPs from WTCCC Affy chip data which passed initial stringent quality control. Top results from this were assessed for additive-by-additive epistasis alone (1df test PLINK).

Results: There was no evidence for interaction between novel loci for T2D from GWA analysis or between these and previously known T2D loci ( $p$ value $\leq 0.05$ ). In stage two, we examined $2.34 \times 10^{9}$ comparisons involving pairs of inter-chromosomal SNPs and found that 79 pairs show evidence for two-locus association at a Bonferroni-corrected $P$-value $\leq 0.05$ (uncorrected $P$-value $\leq 2.14 \times 10^{-11}$ ). All of the 79 pairs involved loci that showed evidence for joint effects on T2D in cooperation with TCF7L2 (rs11196205), which has strong single-locus effects. Within these, 4 loci showed association results suggestive of epistasis, where the two-locus association results independent of the main effect at TCF7L2 were significant $\left(p\right.$-value $\left.<10^{-5}\right)$, but contrasted with the single point main-effects at the 4 loci $\left(p\right.$-value $\left.>10^{-2}\right)$. Three of these were genic; contained within coding regions for BMP7 (rs1998190), GNAQ (rs10512091) and FYCO1 (rs1552489). Other SNPs of biological interest were found within GLP1R (rs910171), FTO (rs1421090/rs9926289), KCNJ6 (rs6517434) and CDK5R1 (rs9348440). Relaxing significance thresholds to a Bonferroni-corrected $P$-value $\leq 0.15$, exposed further evidence for joint effects involving rs9926289 in FTO, in cooperation with SNPs on chromosomes 2 and 5. In further analysis of the top 79 pairs, 5 exhibited $p$-values < $10^{-5}$ for additive-by-additive epistasis alone. The strongest evidence for epistatic effect with TCF7L2 in this stage ( $p$-value $2.9 \times 10^{-5}$ ) was found with $R F$ PL4B (rs1935683), a locus not previously implicated in T2D aetiology.

Conclusion: Our findings allow us to prioritize pairs of markers for a second stage scan, involving more detailed epistatic models in an extended data set of additional SNPs and independent samples. Supported by: WTCCC

\section{0}

Stratified analysis of the Wellcome Trust Case Control Consortium scan for type 2 diabetes reveals susceptibility loci that may affect age of diagnosis

I. Fernandez-Cadenas ${ }^{1,2}$, I. Prokopenko ${ }^{2}$, N.J. Timpson ${ }^{2,3}$, V. Boraska ${ }^{2,4}$ N.W. Rayner ${ }^{2}$, A.T. Hattersley ${ }^{5}$, T.M. Frayling ${ }^{5}$, E. Zeggini ${ }^{2}$, C.M. Lindgren ${ }^{2,6}$, M.I. McCarthy ${ }^{2,6}$

${ }^{1}$ Neurovascular Research Laboratory, Institut de Recerca, Vall d'Hebron Hospital, Barcelona, Spain, ${ }^{2}$ Wellcome Trust Centre for Human Genetics, University of Oxford, United Kingdom, ${ }^{3}$ Translational Epidemiology, MRC Centre for Causal Analyses in Translational Epidemiology, Bristol University, United Kingdom, ${ }^{4}$ Department of Biology, University of Split, Department of Biology, Croatia, ${ }^{5}$ Peninsula Medical School, Exeter, United Kingdom, ${ }^{6}$ Oxford Centre for Diabetes, Endocrinology and Metabolism (OCDEM) University of Oxford, United Kingdom

Background and aims: We hypothesized that age of diagnosis (AOD) in type 2 diabetes (T2D) may be genetically influenced.

Materials and methods: We used the Wellcome Trust Case Control Consortium genome-wide association (GWA) scan data for T2D (1,924 T2D cases and 2,938 controls, typed on the 500k Affymetrix chip). We analyzed 529 subjects with AOD $<45$ years vs. controls, 1395 subjects with AOD $\geq 45$ years vs. controls. In addition, we analyzed the difference between effect estimates in cases with $\mathrm{AOD}<45$ vs. cases with $\mathrm{AOD} \geq 45$ years. The cut-off of $\mathrm{AOD}$ of 45 years was used in keeping with previously published studies. We used PLINK and STATA software for the analysis of these data sets.

Results: The T2D-associated genes FTO and TCF7L2 were associated with T2D regardless of AOD stratum. In cases with $\mathrm{AOD}<45$ years, the strongest association with T2D was identified $\sim 10 \mathrm{~kb}$ downstream from THBS2 ( $\mathrm{rs} 10806665, \mathrm{p}=4.6 \times 10^{-10}$ ), followed by a locus $\sim 10 \mathrm{~kb}$ downstream from TSHZ2 ( $r 6068566, \mathrm{p}=4.8 \times 10^{-7}$ ). In addition we found a new intergenic region $\sim 150 \mathrm{~kb}$ upstream from MYR 8 and TNFSF $13 B$ in $A O D<45$ cases associated with T2D ( $\left.r s 1924340, \mathrm{p}=2.2 \times 10^{-6}\right)$. In cases with $\mathrm{AOD} \geq 45$ years, the strongest signals of association with T2D were identified in TCF7L2 (rs7901695, $\mathrm{p}=9.24 \times 10^{-12}$ ), followed by an intergenic region $\sim 25 \mathrm{~kb}$ upstream

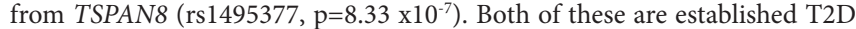
gene regions. When we compared differences in T2D association signals between cases with AOD $<45$ vs cases with AOD $\geq 45$ years, the locus close to TSHZ2 showed statistical evidence for difference in T2D association between AOD strata $\left(\mathrm{rs} 6068566, \mathrm{p}_{\text {diff }}=1.38 \times 10^{-5}\right)$.

Conclusion: Stratified analysis of T2D association by AOD appears to yield differential profiles of genetic association indicating a genetic predisposition associated with age. This may have importance for the design and interpretation of future association studies for T2D. 


\section{1}

Genome-wide association (GWA) analysis in $\mathbf{4 0 0 0}$ members of a Finnish birth cohort identifies common variants associated with fasting insulin levels and related metabolic traits

M. Kaakinen ${ }^{1}$, I. Prokopenko², W.N. Rayner ${ }^{2}$, C. Lindgren ${ }^{2}$, N. Timpson ${ }^{2}$, A. Pouta ${ }^{1}$, A. Ruokonen ${ }^{1}$, A.-L. Hartikainen ${ }^{1}$, N. Freimer ${ }^{3}$, P. Elliott ${ }^{4}$, M. Daly ${ }^{5}$, C. Sabatti ${ }^{6}$, L. Peltonen ${ }^{7,8}$, M.-R. Järvelin ${ }^{4}$, M.I. McCarthy ${ }^{2}$ ${ }^{1}$ Institute of Health Sciences, University of Oulu, Finland, ${ }^{2} \mathrm{WTCHG}$, University of Oxford, United Kingdom, ${ }^{3}$ Center for Neurobehavioral Genetics, University of California, Los Angeles, United States, ${ }^{4}$ Dept of Epidemiology and Public Health, Imperial College London, United Kingdom, ${ }^{5}$ Massachusetts Institute Of Technology, The Broad Institute, Boston, United States, ${ }^{6}$ Depts. of Human Genetics and Statistics, University of California, Los Angeles, United States, ${ }^{7}$ Institute of Molecular Medicine, Biomedicum Helsinki, Finland, ${ }^{8}$ Sanger Institute, Wellcome Trust Genome Campus, Hinxton, United Kingdom

Background and aims: Identification of genetic variants influencing the concentrations of fasting insulin levels and related metabolic traits may deepen our understanding of the genetic pathways regulating metabolic traits relevant to the development of type 2 diabetes (T2D).

Materials and methods: 6007 individuals from the Northern Finland Birth Cohort of 1966 (NFBC1966) representing a homogeneous population, underwent exhaustive phenotypic and clinical characterisation at age 31 . Of these, genomewide association data (Illumina 370K) were available for 4628. After excluding subjects with established diabetes, pregnant woman, non-fasting subjects and outliers $( \pm 3 S D)$ for fasting glucose or insulin, a final set of 316870 SNPs and 4042 subjects were available for analysis. Insulin, HOMA-B and HOMA-IR values were log transformed: association analyses evaluated the additive model and were adjusted by gender.

Results: GWA analysis of fasting insulin levels showed the strongest association at rs2969344, $(\mathrm{p}=4.4 \times 10-7)$ on chromosome 2 , approximately $15 \mathrm{~kb}$ from the nearest predicted transcript [GenBank accession BG259880]. Additional association signals of interest were found within the SYN3 (synapsin III isoform IIIa, chr.22, $\mathrm{p}=2.7 \mathrm{x} 10-6$ ) and PANK1 (pantothenate kinase 1 isoform beta, chr7, $\mathrm{p}=1.1 \times 10-5)$ genes. Unsurprisingly, given the strong correlation between fasting insulin and HOMA-IR, these same SNPs showed associations of similar strength with HOMA-IR measures ( $\mathrm{p}=1.2 \times 10-6$ and $2.2 \times 10-6$ respectively). For HOMA-B (beta-cell function), the most promising novel association was observed at $\mathrm{rs} 132689(\mathrm{p}=2.0 \times 10-7)$, close to the APOL3 (apolipoprotein L3 isoform 3) gene. An additional signal for beta-cell function was detected close to the G6PC2 gene (rs563694: $\mathrm{p}=5.8 \mathrm{x} 10-6$ ), this being driven by the strong (and previously-reported) association with fasting glucose levels seen in this sample ( $\mathrm{rs560887,} \mathrm{p}=4 \mathrm{x} 10-9)$. The PANK1 SNPs showing modest association with insulin levels were also significantly ( $\mathrm{p} \sim 4 \mathrm{x} 10$ 5) associated with HOMA-B. None of the known T2D-susceptibility genes showed strong signals for HOMA-B (eg TCF7L2, rs7903146 p=0.04).

Conclusion: GWA analysis of these three related metabolic traits, in over 4000 young non-diabetic adults has revealed several promising association signals distinct from those previously shown to be involved in predisposition to T2D. Further replication and meta-analysis efforts are underway to substantiate these findings, but the indications are that analyses of quantitative traits such as these will allow identification of additional loci impacting on metabolic regulation in human populations.

Supported by: Academy of Finland, NIH/NHLBI USA

\section{2}

Meta-analysis of genome-wide association data involving 6,100 adults of European origin identifies common variants associated with fasting glucose levels

I. Prokopenko ${ }^{1,2}$, Y. Aulchenko ${ }^{3}$, M. Kaakinen ${ }^{4}$, A. Dehghan ${ }^{3}$, S. Service ${ }^{5}$, N. Freimer ${ }^{5}$, P. Elliott ${ }^{6}$, M.-R. Jarvelin ${ }^{6}$, L. Peltonen ${ }^{7,8}$, C.M. van Duijn ${ }^{3}$, M.I. McCarthy ${ }^{1,2}$

${ }^{1}$ WTCHG, University of Oxford, United Kingdom, ${ }^{2}$ Ocdem, University of Oxford, United Kingdom, ${ }^{3}$ Dept. Epidemiology \& Biostatistics, Erasmus MC, Rotterdam, Netherlands, ${ }^{4}$ Institute of Health Sciences, University of Oulu, Finland, ${ }^{5}$ Center for Neurobehavioral Genetics, University of California, Los Angeles, United States, ${ }^{6}$ Dept. of Epidemiology and Public Health, Imperial College of London, United Kingdom, ${ }^{7}$ Biomedicum, Institute of Molecular Medicine, Helsinki, Finland, ${ }^{8}$ Sanger Insitute, Wellcome Trust Genome Campus, Hinxton, United Kingdom

Background and aims: Identification of common variants influencing fasting glucose concentrations will provide novel clues to the mechanisms involved in the maintenance of glucose homeostasis, and complement casecontrol association approaches for the detection of the genes influencing risk of type 2 diabetes.

Materials and methods: We analysed genome-wide association (GWA) data from $\sim 6,100$ European individuals of Caucasian origin. GWA data were available from 4042 subjects from the Northern Finland Birth Cohort (NFBC1966) who had been tested for fasting glucose levels at age 31 (Illumina 370k), and 2058 subjects from the ERGO sample, a population-based cohort study in the Netherlands (mean (SD) age: 64.06( \pm 5.89): Illumina $317 \mathrm{~K})$. Those with established diabetes, pregnant woman, non-fasting subjects and outliers $( \pm 3 \mathrm{SD})$ had been excluded from the analysis. Association analyses were adjusted by gender and age (ERGO sample) and glucose values log-transformed. Meta-analysis was performed using the inverse-variance weighted method with a fixed effects model.

Results: In these samples, we were able to replicate two previous associations with fasting glucose. At rs2971671, close to the glucokinase gene $(\sim 230 \mathrm{~kb}$ from the variants previously described), we detected a per-allele effect of $\sim 0.06 \mathrm{mmol} / 1 \quad(\mathrm{p}=4.8 \times 10-7)$. An effect of similar size was also detected at SNP rs560887, near to G6PC2 (glucose-6 phosphatase catalytic subunit 2) $(\mathrm{p}=6.8 \times 10-10)$. The most striking association pointing towards a novel quantitative trait locus was observed near to the MTNR1B gene encoding the melatonin receptor $1 \mathrm{~b}$ (per-allele effect $\sim 0.06 \mathrm{mmol} / \mathrm{l}$ in NFBC1966 and $\sim 0.11 \mathrm{mmol} / 1$ in ERGO, $\mathrm{p}=3.7 \mathrm{x} 10-11$ ). Other promising signals (both $\mathrm{p}$ $\sim 3 \times 10-6$ ) were observed for SNPs in the vicinity of the CNTNAP2 (contactin associated protein-like 2) and $P B X 1$ (pre-B-cell leukemia transcription factor 1) genes, both of which have substantial functional credibility as candidates. Evidence of association for known T2D-susceptibility genes was muted, the strongest signal being for rs7903146 within TCF7L2 ( $\mathrm{p}=1.8 \mathrm{x} 10-5)$ : this effect was stronger in the (more elderly) Rotterdam cohort than in the Finnish sample (per-allele effect $\sim 0.09$ and $\sim 0.03 \mathrm{mmol} / 1$ respectively).

Conclusion: As well as confirming previous associations, these GWA data have identified several novel signals related to fasting glucose. Efforts at largescale replication and meta-analysis of these findings currently underway should reveal which of these have a robust, genuine effect on glucose levels in adults. Both physiological (glucose-sensing) and pathological (diabetesrelated) mechanisms seem to be implicated in the determination of glucose levels.

Supported by: European Union 


\section{OP 03 Continuous glucose monitoring system}

\section{3}

Effectiveness of continuous glucose monitoring during pregnancy: results of a randomised clinical trial

H.R. Murphy ${ }^{1}$, G. Rayman ${ }^{1}$, K. Lewis ${ }^{1}$, S. Kelly ${ }^{2}$, B. Johal ${ }^{2}$, D. Fowler ${ }^{1}$, K. Duffield ${ }^{3}$, R.C. Temple ${ }^{4}$

${ }^{1}$ Diabetes centre, Ipswich Hospital, ${ }^{2}$ Obstetrics and Gynaecology, Ipswich Hospital, ${ }^{3}$ Elsie Bertram Diabetes centre, Norfolk and Norwich University Hospital NHS Trust, Norwich, United Kingdom, ${ }^{4}$ Elsie Bertram Diabetes centre, Norfolk and Norwich University Hospital NHS, United Kingdom

Background and aims: To evaluate the effectiveness of Continuous Glucose Monitoring (CGM) during pregnancy on maternal glycaemic control, infant birth weight and risk of macrosomia in women with type 1 and type 2 diabetes.

Materials and methods: A prospective, open label randomised controlled trial comparing standard antenatal care with and without CGM in 2 secondary care multidisciplinary obstetric diabetes clinics in the United Kingdom. CGM was used as an educational tool to inform decision making and future therapeutic changes at 4-6 weekly intervals during pregnancy. All other aspects of antenatal care were equal between the 2 groups. 71 women with type $1(n=46)$ and type $2(n=25)$ diabetes were randomised to antenatal care with CGM $(n=38)$ or without supplementary CGM $(n=33)$. All statistical analyses were performed on an intention-to-treat basis.

Results: Women randomised to CGM had lower mean HbA1c between 3236 weeks gestation compared to women in standard care $(5.8 \% \pm 0.6$ versus $6.4 \% \pm 0.7 ; \mathrm{p}=0.007)$. Compared to infants of standard care mothers, infants of the CGM mothers had a lower median birth weight centile (69\% versus $93 \% ; \mathrm{p}=0.009$ ) and reduced risk of macrosomia (Odds ratio $0.36,95 \%$ Confidence Interval 0.13 to $0.98 ; \mathrm{p}=0.05$ ).

Conclusion: Use of CGM during pregnancy is associated with improved glycaemic control, lower infant birth weight and reduced risk of macrosomia. This is the first trial to demonstrate the effectiveness of continuous glucose monitoring on glycaemic control and pregnancy outcome.

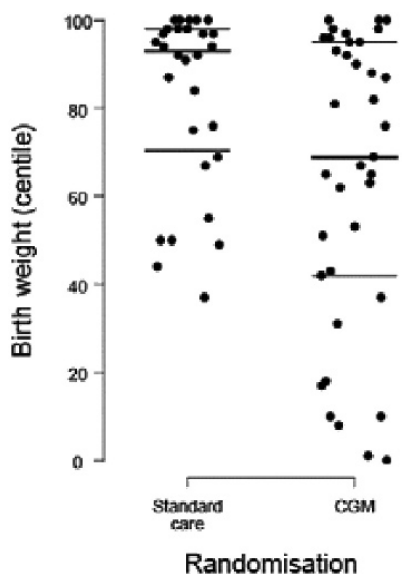

Figure. Distribution of the individual customised birth weight centiles for $67 / 69$ infants of mothers randomised to supplementary continuous glucose monitoring (CGMS) or standard antenatal care. Lines mark the medians (thick lines) and interquartile range (thin lines)

Supported by: Ipswich Hospital Diabetes Centre Charity Research Fund, Diabetes UK, Medtronic UK

\section{4}

Glycaemic rises after waking up in response to an alarm clock during the night in type 1 diabetic patients can be avoided by experienced nurses drawing blood in a hospital setting as shown by continuous glucose monitoring $\left(\right.$ GlucoDay $^{\circledR}$ )

C. Berndt ${ }^{1}$, L. Köthe ${ }^{1}$, B. Nawrodt ${ }^{1}$, B. Mraz ${ }^{2}$, A. Patzelt-Bath ${ }^{3}$, M.A. Nauck ${ }^{1}$; ${ }^{1}$ Diabeteszentrum, Bad Lauterberg im Harz, Germany, ${ }^{2}$ A. Menarini Diagnostics, Berlin, Germany, ${ }^{3}$ Diagnostica, Berlin-Chemie AG, Berlin, Germany

Background and aims: It is not known, to which degree waking up in response to an alarm clock evokes a stress reaction that may lead to acute elevations in glucose concentrations and to false estimates of overnight glucose profiles. Therefore, it was the aim of the present study, to compare, by use of continuous glucose monitoring, overnight glucose profiles obtained by patients with type 1 diabetes themselves (i.e. waking up in response to an alarm clock, and performing glucose self-measurements), or by experienced nurses, in a prospective, randomized trial.

Materials and methods: 30 type 1 diabetic patients participated. They were 49 \pm 13 years old (21 male and 9 female patients), their BMI was $27.7 \pm 4.2 \mathrm{~kg} / \mathrm{m}^{2}$, and their diabetes had been diagnosed $24 \pm 11$ years earlier. Treatment was with continuous subcutaneous insulin infusion $(n=9)$ or intensified conventional regimens $(n=21)$, daily insulin doses were $43.7 \pm 17.6 \mathrm{IU} / \mathrm{d}$. In randomized order, each participant participated (a) in one night, during which they were instructed to set their alarm clock for glucose measurements to be performed by the patient themselves at midnight, 2 a.m., and 4 a.m, and (b) in another night, when an experienced nurse was scheduled to perform capillary blood glucose determinations as gently as possible (dimmed light, little noise etc.). (c) The night between these two experiments, the patients were left undisturbed. In addition, venous blood was taken for the analysis of stress hormones. Throughout the study, patients were equipped with a GlucoDay device for continuous glucose monitoring (CGM; 2 periods of $48 \mathrm{~h}$ ). CGM data obtained between 15 min before and $30 \mathrm{~min}$ after the times specified for glucose measurement were analysed by repeated-measures analysis of variance.

Results: After waking up in response to an alarm clock, CGM-determined glucose concentrations rose by $15.7 \pm 6.1 \mathrm{mg} / \mathrm{dl}$ after $9 \mathrm{~min}$ at $2 \mathrm{a} . \mathrm{m}$. and by $17.7 \pm 6.3 \mathrm{mg} / \mathrm{dl}$ at $4 \mathrm{p} . \mathrm{m}$. ( $\mathrm{p}=0.0003)$, whereas only minor increments were elicited with gently taking blood by nurses $(4.8 \pm 4.4 \mathrm{mg} / \mathrm{dl}$ at $2 \mathrm{a} . \mathrm{m}$. and 0.0 $\pm 3.8 \mathrm{mg} / \mathrm{dl}$ at 2 and 4 a.m.). Pulse rates and adrenaline concentrations were higher $(\mathrm{p}<0.05)$ in response to alarm clocks.

Conclusion: Waking up in response to an alarm clock leads to a stressful arousal reaction that will lead to erroneously high glucose concentrations. Exact night-time glucose profiles may require hospitalization and gentle procedures for taking blood avoiding such conditions. Continuous glucose monitoring with GlucoDay is able to demonstrate short-lived increments in glucose concentrations in response to stressful arousal. Supported by: A. Menarini Diagnostics, Germany

\section{5}

A cross over study on patient reported outcomes of continuous glucose monitoring (CGM) comparing real time access to glucose values with retrospective analysis

N. Hermanns ${ }^{1}$, G. Gulde ${ }^{1}$, B. Kulzer ${ }^{1}$, H. Eberle ${ }^{1}$, E. Pradler ${ }^{2}$, A. Patzelt-Barth ${ }^{3}$, T. Haak

${ }^{1}$ Research Institute of Diabetes Academy Mergentheim, FIDAM, Bad Mergentheim, Germany, ${ }^{2}$ Menarini, diagnostics, Berlin, Germany, ${ }^{3}$ BerlinChemie, AG, Berlin, Germany

Background and aims: The results of CGM can be reported to patients either in real time or retrospectively. This randomized cross over trial examines the effect of CGM with real time access to glucose data (RTA) vs. a CGM with a retrospective analysis (RA) of glucose data on satisfaction with CGM and other patient reported outcomes

Materials and methods: Participants used the CGM device (GlucoDay", Menarini Diagnostics, Florence, Italy) twice. In one condition patients got real time access to current glucose values and in the other condition glucose values were only reported retrospectively. The order of these two conditions was randomized. At baseline, after the first and the second trial, subjects completed questionnaires about perceived satisfaction with CGM (CGM-SAT, with a total score of satisfaction with CGM, with a perceived benefit scale and a perceived disadvantage scale).They also completed the Problem Areas in Diabetes (PAID), and the state anxiety scale (State Version STAI). 
Results: In this study 50 type 1 diabetic patients (age $41.7 \pm 12.3$ yrs., diabetes duration $14.75 \pm 11.9$ yrs., $48 \%$ female, Alc $8.1 \% \pm 1.5 \%$, years of education $10.3 \pm 2,1 \mathrm{yrs})$ participated. At baseline patients perceived CGM as rather advantageous, after RA and RTA the perceived benefits were reduced (baseline: $47.8 \pm 10.5$; RA: $38.4 \pm 17.2$; RTA: $37.0 \pm 16.8, \mathrm{p}<.01$ ), but there was no significant difference between RA and RTA. Interestingly the same was true for perceived disadvantages of CGM (Baseline: $22.9 \pm 10.6$; RA: $18.7 \pm 10.6$; RTA: $19.3 \pm 12.6 \mathrm{p}<.05)$. The total scale of the GCM SAT showed a similar course (Baseline: $101.0 \pm 16.0$; RA: $95.7 \pm 20.2$; RTA: $93.6 \pm 22.8$ p $>.01$ ). There was also no significant effect on diabetes related distress or state anxiety.

Conclusion: After the experience of CGM the possible benefits were perceived as a little bit less positive and potential disadvantages were perceived less negative. Thus experience of CGM corrected expected benefits and disadvantages. But there was no specific significant negative or positive effect of RA vs. RTA on satisfaction with CGM. Furthermore CGM with RA or RTA had no significant effect on the course of diabetes related distress or state anxiety. Exposing type 1 diabetic patients to their current glucose values doesn't seem to have a specific negative impact on the appraisal of CGM or more generic patient reported outcomes.

Supported by: Berlin Chemie

\section{6}

\section{WITHDRAWN}

\section{7}

\section{Assessing glycaemic excursions by continuous glucose monitoring system (CGMS) in patients with type 2 diabetes treated with insulin secretagogues}

Y. $\mathrm{Li}^{1}$, L. Xu${ }^{1}$, J. Shen ${ }^{2}$, J. Ran ${ }^{2}$, Y. Zhang ${ }^{3}$, M. Wang' ${ }^{2}$ L. Yan ${ }^{1}$, Z. Fu

${ }^{1}$ Department of Endocrinology, Sun Yat-Sen Memorial Hospital,

${ }^{2}$ Department of Endocrinology, Southern Hospital, ${ }^{3}$ Department of

Endocrinology, Guangzhou Red Cross Hospital, Guangzhou, China

Background and aims: Patients with similar $\mathrm{HbA}_{1 c}$ may differ in glycemic excursion profiles. The aim of this study was to explore glycemic excursions in patients who had similar glycemic control when treated with different insulin secretagogues.

Materials and methods: A total of 60 newly diagnosed patients were enrolled into this multi-center, open-labelled, parallel study and randomly (1:1:1) assigned to receive repaglinide $(\mathrm{Rg})$, glimepiride $(\mathrm{Gm})$ and gliclazide (Gli). The initial dosage for $\mathrm{Rg}$, Gm and Gli were $1 \mathrm{mg}$ tid, $1 \mathrm{mg} \mathrm{qd}$, and 30mg qd, respectively, and were titrated to reach the fasting blood glucose (FBG) target of $\leq 7 \mathrm{mmol} / \mathrm{L}$. Glucose concentration was monitored by CGMS for 72 hours at the end of a 4-week treatment period.

Results: Patients' age, $\mathrm{BMI}, \mathrm{FBG}, \mathrm{HbA}_{1 \mathrm{c}}$ and glycosylated serum protein were comparable among the three treatment groups at baseline. After treatment, mean glycemic values during 72 hours were similar in the three groups. Mean amplitude of glycemic excursions (MAGE) was significantly lower in Rg as compared with Gm and Gli ( $p=0.006$ and 0.007 , respectively), and no difference existed between Gm and Gli $(p=0.581)$. The same tendency was found in mean maximal glycemic values ( $\operatorname{Rg} v s$. Gm, $p=0.016$; $\operatorname{Rg} v s$. Gli, $p=0.016$; $\mathrm{Gm} v s$. Gli, $p=0.948$ ). Mean minimal glycemic values were similar in the three groups. The percentages of time when patients experiencing hypoglycemia $(<$ $3.9 \mathrm{mmol} / \mathrm{L}$ ) and hyperglycemia $(>7.8$ and $>11.1 \mathrm{mmol} / \mathrm{L}$ ) were comparable. The exposure to hyperglycemia tended to be the shortest with Rg.

Conclusion: Despite similar glucose control, glycemic excursion profiles can be different with different insulin secretagogues. Repaglinide causes less glyc- emic excursions than glimepiride and gliclazide. CGMS can be used as a tool to monitor glycemic excursions and help patient to achieve accurate glycemic control.

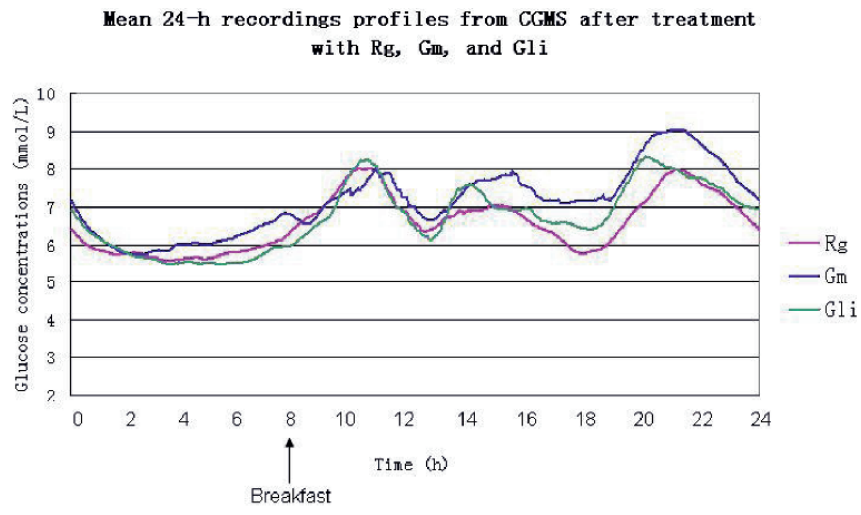

\section{8}

Diabetes and sleep apnoea - lessons learnt from continuous glucose monitoring in type 2 diabetic patients with sleep-disordered breathing M. Pallayova, V. Donic, Z. Tomori

Department of Physiology and Sleep Laboratory, PJ Safarik University School of Medicine, Kosice, Slovakia

Background and aims: There is compelling evidence that severe sleep-disordered breathing (SDB) and coexisting diabetes significantly increase cardiovascular morbidity and mortality. Given the enormous cardiovascular consequences of type 2 diabetes it is important to understand the development of the hyperglycemic state and to develop identification and intervention strategies. Our aim was to discern the glycemic consequences of sleep apnea in type 2 diabetic state.

Materials and methods: Thirty type 2 diabetic patients on diet or stable oral hypoglycemic therapy (19 men; mean age $55.5 \pm 7.8$ years, BMI $34.7 \pm 7.4$, duration of diabetes $3.0 \pm 1.7$ years, $\mathrm{HbA}_{1 \mathrm{c}} / \mathrm{HPLC}$ method $7.1 \pm 0.79 \%$ ) with objectively assessed SDB of various grades of severity underwent continuous glucose monitoring (CGM) for several days. Subjects were divided into four subgroups according to their apnea-hypopnea index (AHI): $[\mathrm{A}]$ normal with an AHI $<5 / h$, [B] with mild SDB (AHI 5-15/h), [C] with moderate SDB (AHI $15-30 / h$ ), and $[D]$ with severe sleep apnea $(A H I \geq 30 / h)$. The primary study outcome measures included indices of nocturnal glucose variability (overnight glucose standard deviation $\mathrm{SD}_{\text {overnight }}$, coefficient of variance $\mathrm{CV}$, mean of nocturnal glucose differences MOND) and mean nocturnal glucose values. The differences between groups were tested using ANOVA. Correlations between two continuous variables were analyzed using Spearman's rank correlation.

Results: 111 nocturnal glucose profiles showed the most stable overnight glucose control in $[\mathrm{A}]$ ( $\mathrm{n}=11 ; 38$ nocturnal CGM profiles; AHI 1.6 $\pm 1.0 / \mathrm{h}, \mathrm{BMI}$ $31.7 \pm 8.7, \mathrm{SD}_{\text {overnight }} 0.53 \pm 0.22$, CV $9.35 \pm 3.92 \%$, MOND $2.16 \pm 0.87 \mathrm{mmol} / \mathrm{l}$, mean nocturnal glycemia $5.8 \pm 1.08 \mathrm{mmol} / \mathrm{l})$. The most labile nocturnal glucose control was observed in [D] $(\mathrm{n}=10 ; 32$ nocturnal CGM profiles; AHI $57.8 \pm 16.0 / \mathrm{h}$, BMI $36.9 \pm 6.5, \mathrm{SD}_{\text {overnight }} 1.02 \pm 0.4$, CV $12.81 \pm 4.91 \%$, MOND $3.96 \pm 1.55 \mathrm{mmol} / \mathrm{l}$, mean nocturnal glycemia $8.15 \pm 1.76 \mathrm{mmol} / \mathrm{l})$. ANOVA showed significantly increased nocturnal glucose variability in $[\mathrm{C}]$ as compared to $[A](\mathrm{p}=0.008)$, in $[\mathrm{D}]$ as compared to $[\mathrm{B}](\mathrm{p}=0.01)$, and in $[\mathrm{D}]$ as compared to $[\mathrm{A}](\mathrm{p}<0.001)$. Despite no significant difference in HbAlc be-

\begin{tabular}{lllll}
\hline & Repaglinide $(\mathrm{Rg})$ & Glimepiride $(\mathrm{Gm})$ & Gliclazide $(\mathrm{Gli})$ & $\mathrm{p}$-value \\
\hline Mean glycemic values $(\mathrm{mmol} / \mathrm{L})$ & $6.55 \pm 1.17$ & $7.13 \pm 1.79$ & $6.71 \pm 1.28$ & 0.463 \\
\hline MAGE $(\mathrm{mmol} / \mathrm{L})$ & $4.28 \pm 1.02$ & $5.54 \pm 1.55$ & $5.94 \pm 2.37$ & 0.011 \\
\hline Mean maximal glycemic values $(\mathrm{mmol} / \mathrm{L})$ & $11.73 \pm 1.56$ & $13.84 \pm 2.92$ & $13.53 \pm 3.02$ & 0.028 \\
\hline Mean minimal glycemic values $(\mathrm{mmol} / \mathrm{L})$ & $3.28 \pm 1.03$ & $3.57 \pm 1.01$ & $3.44 \pm 0.72$ & 0.658 \\
\hline Percentage of time $(\%)$ & & & & \\
\hline BG $<3.9 \mathrm{mmol} / \mathrm{L}$ & $2.0(0-44)$ & $0.0(0-58)$ & $1.0(0-35)$ & 0.699 \\
BG $>7.8 \mathrm{mmol} / \mathrm{L}$ & $18.0(1-52)$ & $27.0(2-91)$ & $18.5(2-95)$ & 0.749 \\
BG $>11.1 \mathrm{mmol} / \mathrm{L}$ & $1.0(1-11)$ & $3.0(0-40)$ & $2.5(0-35)$ & 0.168 \\
\hline
\end{tabular}

Values are presented as Mean \pm SD or Median (min-max).

If $\mathrm{p}<0.05$, there is a difference among three groups; If $\mathrm{p} \geq 0.05$, there is no difference among three groups. 
tween subgroups, ANOVA showed significant difference in mean nocturnal glycemia between $[A]$ and $[D],[A]$ and $[C],[B]$ and $[D]$, and $[B]$ and $[C]$. Evaluation of CGM- and parallel sleep and respiratory data showed strong positive correlations between AHI and parameters of nocturnal glucose variability $(\mathrm{p}<0.005)$ and a strong positive correlation of AHI with mean nocturnal glucose values $(r=0.6 ; p<0.001)$. We observed strong negative correlations of mean nocturnal glucose values with both mean oxygen saturation $(r=-$ $0.59 ; \mathrm{p}<0.001)$ and minimum oxygen saturation levels $(r=-0.54 ; p<0.001)$.

Conclusion: The results suggest that moderate to severe sleep apnea accompanied by severe oxygen desaturation is associated with significantly increased nocturnal glucose variability and worse overnight glucose control as compared to type 2 diabetic state without SDB. Our findings emphasise need for multidisciplinary approach to diabetes care with early diagnosis and treatment of comorbidities, which might aggravate glucose control. With the rapid increase in incidence of coexisting diabetes and sleep apnea, it is important to ensure that patients are treated as effectively as possible in order to achieve sustained glycemic control and, thereby, minimise the impact of the condition.

\section{OP 04 Mechanisms and new markers of macrovascular disease}

\section{9}

Reduced circulating endothelial progenitor cell number in young adult insulin-resistant healthy subjects

A. Dei Cas ${ }^{1}$, V. Spigoni ${ }^{1}$, D. Ardigò ${ }^{1}$, G. Pedrazzi ${ }^{2}$, L. Franzini ${ }^{1}$, E. Derlindati ${ }^{1}$, L. Monti ${ }^{3}$, L. Gnudi ${ }^{4}$, I. Zavaroni ${ }^{1}$

${ }^{1}$ Department of Internal Medicine and Biomedical Sciences, University of Parma, Italy, ${ }^{2}$ Department of Public Health, University of Parma, Italy, ${ }^{3}$ Department of Internal Medicine, Istituto Scientifico San Raffaele, Milan, Italy, ${ }^{4}$ Department of Diabetes and Endocrinology, Cardiovascular Division, King's College, London, United Kingdom

Background and aims: Insulin-resistance (IR) associates with a cluster of metabolic and hemodynamic abnormalities, endothelial dysfunction and increased cardiovascular (CV) risk. Circulating Endothelial Progenitor Cells (EPCs) are considered to play a key role in vessel repair following endothelial damage. The number of EPCs inversely correlates with the presence of glucose intolerance, diabetes, cardiovascular risk factors, $\mathrm{CV}$ morbidity and mortality. However, it remains unclear whether the presence of insulin-resistance is associated to a variation in the number of EPCs in healthy subjects with normal glucose tolerance.

Materials and methods: To address this issue, circulating EPC number was determined in 122 healthy subjects $(73 \mathrm{M} / 49 \mathrm{~F}$; age $37 \pm 8$ yrs; BMI $24.6 \pm 4.2$ $\left.\mathrm{Kg} / \mathrm{m}^{2}\right)$. All subjects were normotensive and not taking any medication. Glucose tolerance test was performed in all subjects with the determination of plasma glucose and insulin. Subjects were classified as insulin resistant on the basis of fasting plasma insulin as a surrogate marker of insulin resistance. Fasting blood samples were drawn in all subjects for the determination of EPC number, lipid profile, white blood count (WBC), high sensitive C-reactive protein (hsCRP), uric acid and PAI-1. EPCs were identified as cell positive for CD34, CD133 and Kinase insert Domain Receptor (KDR) cell-surface antigens by flow cytometry analysis. Isotype Immunoglobulins (IgG1) were used as controls. EPC number is express respectively as percentage of cells positive for KDR within the CD133+ cells (early EPCs) and KDR+ within the CD133+ and CD34+ cells (triple EPCs) within the lymphomonocyte population. Data were analysed with Spearman correlation and differences among groups were evaluated with non parametric tests for ordered alternatives (Jonkheere-Terpstra) and $\chi^{2}$ test.

Results: Early EPC number was greater in female $(\mathrm{p}<0.05)$ and significantly inversely correlated with BMI $(\mathrm{r}=-0,18 ; \mathrm{p}<0.05)$, waist circumference $(\mathrm{r}=-$ $0,2 ; \mathrm{p}<0.05)$, diastolic and systolic blood pressure $(\mathrm{r}=-0,23 ; \mathrm{p}<0.01 ; \mathrm{r}=-0,21$; $\mathrm{p}<0.05)$, creatinine $(\mathrm{r}=-0,18 ; \mathrm{p}<0.05)$, uric acid $(\mathrm{r}=-0,24 ; \mathrm{p}<0.005)$, gGT $(\mathrm{r}=-$ $0,24 ; \mathrm{p}<0.005)$, PAI-1 $(\mathrm{r}=-0.197 ; \mathrm{p}<0.05)$ and fasting insulinaemia $(\mathrm{r}=-0,2$; $\mathrm{p}<0.05)$. The number of triple EPCs inversely correlated with body weight $(\mathrm{r}=-$ $0,18 ; \mathrm{p}<0.05)$, monocytes $(\mathrm{r}=-0,2 ; \mathrm{p}<0.05)$, uric acid $(\mathrm{r}=-0,28 ; \mathrm{p}<0.005)$, gGT $(r=-0,3 ; p<0.005)$, GPT $(r=-0,2 ; p<0.05)$ ed insulinaemia $(r=-0,2 ; p<0.05)$. By dividing in tertiles the subjects on the basis of fasting plasma insulin, subjects belonging to the third tertile (insulin resistant) showed reduced EPC number with respect to the first tertile (insulin-sensitive) $(\mathrm{p}<0.05)$. Insulin tertiles were inversely associated with EPC tertiles $(\mathrm{p}<0.05)$.

Conclusion: Insulin resistant subjects showed reduced EPC number which inversely correlated also with the cluster of abnormalities linked to the insulin resistance syndrome. These data suggest a possible role for EPCs as early markers of CV risk in insulin-resistant subjects.

Supported by: EU Project Multiknowledge

\section{0}

Diminished endothelial progenitor cells are related to glycaemic control in type 1 diabetic children

G.H. Schernthaner ${ }^{1}$, B. Rami ${ }^{2}$, M. Satler ${ }^{1}$, F. Hoellerl ${ }^{1}$, C. Höbaus ${ }^{1}$, E. Schober ${ }^{3}$, G. Schernthaner ${ }^{4}$

${ }^{1}$ Medicine II, Medical University of Vienna, ${ }^{2}$ Pediatrics and Adolescent Medicine, Medical University of Vienna, ${ }^{3}$ Pediatrics and Adolescenf Medicine, Medical University of Vienna, ${ }^{4}$ Medicine I, Rudolfstiftung Hospital Vienna, Austria

Background and aims: The risk of dying of cardiovascular disease (CVD) before the age of 40 is increased nearly 20 -fold in patients with type 1 diabetes 
mellitus (T1DM) compared with nondiabetic subjects. One main contributor to the increased CVD risk might be unsatisfactory glycemic control, which emerges from the very beginning of T1DM, thus in childhood. However, the mechanisms mediating this increased risk are not completely understood. Endothelial progenitor cells (EPC) are bone marrow derived cells circulating in the peripheral blood. They are involved in animal adult neovascularisation and (animal and human) endothelial homeostasis and may predict CVD morbidity and mortality in humans. Thus, we performed a longitudinal study in T1DM children, enumerated EPC and studied the associations of the latter.

Materials and methods: In total, 120 children, 90 with T1DM and 30 control children (CO, were included. The patients and controls were carefully matched and did not differ for age ( $13.5 \pm 2.8$ years), gender ( $48.5 \%$ female) and BMI $(21.2 \pm 3.6 \mathrm{~kg} / \mathrm{m} 2)$. Circulating pluripotent progenitor cells (CPC; $\mathrm{CD} 34+/ \mathrm{CD} 133+)$ and EPC (CD34+/CD133+/CD309+) were enumerated by flow cytometry out of peripheral blood specimen. All laboratory tests were performed at the beginning of the study and one year thereafter, when the patients were scheduled for their routine follow up examination. Statistical analysis included unpaired and paired student's t test, correlation analysis as well as multivariate linear regression modelling, as appropriate. In order to analyse changes of variables during the one year observation time, delta values were calculated.

Results: EPC were significantly reduced in children with T1DM compared to $\mathrm{CO}(607 \pm 368$ vs $1037 \pm 504, \mathrm{p}<0.001)$. Other significant differences were found in systolic blood pressure $(112 \pm 14$ vs $101 \pm 8 \mathrm{mmHg}, \mathrm{p}<0.001)$ and glucose levels ( $150 \pm 77$ vs $90 \pm 8 \mathrm{mg} / \mathrm{dl}, \mathrm{p}<0.001)$, each T1DM vs CO. Multivariate regression analysis revealed that $\mathrm{HbAlc}$ was the strongest independent predictor of EPC levels (Beta $=-0.319, \mathrm{p}=0.003$ ). The overall glycemic control at the beginning as well as at the end of the study was satisfying, and not different ( $7.8 \pm 1.2$ vs $7.8 \pm 1.2$ rel.\%, $\mathrm{p}=\mathrm{ns})$ and in our 90 patients with T1DM none presented with neuropathy, nephropathy, retinopathy or CVD. However, looking up the patients individually, we had changes of HbAlc levels during the one year follow up of -4.30 and +3.10 rel. \%, respectively. When calculating the mean change of EPC per 0.10 rel. \% HbAl, we obtained an increase of mean EPC in 93 cells per each reduction of HbAlc of 0.1 rel \% and vice versa.

Conclusion: In conclusion, this is the first study providing evidence for diminished numbers of endothelial progenitor cells in children with type 1 diabetes mellitus. Since low endothelial progenitor cells are important predictors of future cardiovascular morbidity and mortality in nondiabetic high risk patients, these new findings could be relevant for the understanding of the high cardiovascular risk of type 1 diabetic children. The observed significant increase of EPC in diabetic children with improved diabetic control within 1 year suggest that optimization of glycemic control could be relevant in reducing the high CVD burden in these patients.

Supported by: Austrian Diabetes Association Research Prize 2006

\section{1}

Circulating progenitor cells in the natural history of type 2 diabetes mellitus

G. Fadini, S. de Kreutzenberg, C. Agostini, A. Tiengo, A. Avogaro Dept Clinical and Experimental Medicine, University of Padova, Italy

Background and aims: Cardiovascular disease (CVD) in type 2 diabetes is attributed to the biochemical effects of hyperglycemia and to the coexistence of additional risk factors. Depletion of bone marrow-derived circulating progenitor cells has been recently proposed as another mechanism of CVD induction in diabetes. The present study was undertaken to describe quantitatively progenitor cell de-modulation during the natural history of type 2 diabetes.

Materials and methods: CD34+ cells were chosen as the simplest phenotype of circulating immature cells likely derived from the bone marrow. We searched our database for subjects having circulating CD34+ cell count determined and carbohydrate metabolism status known. We identified 425 individuals, which were divided into 7 categories according to carbohydrate metabolism status (normal glucose tolerance, NGT; impaired fasting glucose, IFG; impaired glucose tolerance, IGT; newly diagnosed type 2 diabetes, „DM new") and diabetes duration ( $0-9 ; 10-19 ; \geq 20$ years). These categories were examined as ideally describing the natural history of type 2 diabetes development and progression. Rough data of CD34+ cell count were plotted as variation with respect to NGT patients. Adjusted CD34+ cell variation was derived from non-standardized regression coefficients of a multivariable analysis taking into account all possible confounders.
Results: The analysis of rough data (continue line) revealed that, in comparison with NGT, CD34+ cells were significantly $\left(^{*}\right)$ reduced as early as in IGT subjects, and had a first nadir at time of diagnosis. In patients with $0-19$ years of diabetes duration a slight increase or stabilization of CD34+ cell count was recorded. A second deeper nadir was evident after 20 years of diabetes. Full adjustment to clear out possible confounders from this trend (including age, concomitant risk factors, prevalent cardiovascular disease, and medications), confirmed that CD34+ cell count are deeply reduced at time of diagnosis $(\dagger)$, partially recover during the subsequent $0-19$ years, and dip again after at least 20 year of diabetes duration (dashed line).

Conclusion: This study describes the time course level of CD34+ progenitor cells, likely to be derived from bone marrow, across categories of patients reflecting the natural history of type 2 diabetes. A sort of bone marrow "stunning" may mark the clinical onset of type 2 diabetes. While a partial recovery occurs during subsequent years, bone marrow reserve is exhausted in the long term. Recognition and treatment of this early abnormality may help preserving longer marrow function, thus preventing CVD.

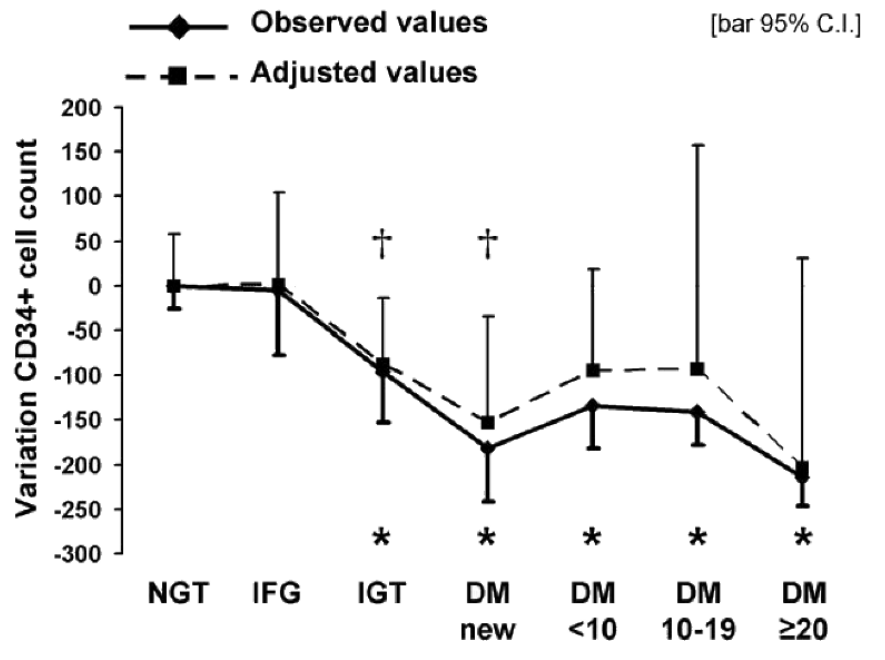

22

Increased levels of endothelial microparticles CD144 positives in type 2 diabetic patients with coronary noncalcified plaques evaluated by multidetector computed tomography

S. Bernard ${ }^{1}$, R. Loffroy ${ }^{2}$, A. Sérusclat ${ }^{2}$, L. Boussel ${ }^{2}$, E. Bonnefoy ${ }^{3}$,

C. Thévenon ${ }^{4}$, D. Revel ${ }^{2}$, P. Moulin ${ }^{1}$, P. Douek ${ }^{2}$;

${ }^{1}$ Endocrinology Diabetology, Cardiovascular Hospital-HCL, Bron,

${ }^{2}$ Radiology, Cardiovascular Hospital-HCL, Bron, ${ }^{3}$ Intensive Care Unit, Cardiovascular Hospital-HCL, Bron, ${ }^{4}$ Cecil, Université Claude Bernard Lyon I, France

Background and aims: The combination of both morphological and cellular markers of subclinical atherosclerosis, in addition to conventional risk factors, may help to improve cardiovascular prevention in type 2 diabetic patients. The aim of our cross-sectional study was to evidence a putative increase in endothelial (EMP) or platelet (PMP) microparticles, in type 2 diabetic patients with coronary noncalcified plaques detected by multidetector CT (MDCT).

Materials and methods: Microparticles and coronary MDCT were assessed in 56 type 2 diabetic patients with different cardiovascular risk levels. EMP and PMP were detected by cell-specific monoclonal antibodies using flux cytometry.

Results: Both EMP ( $\rho=0.35, p=0.022)$ and PMP $(\rho=0.34, p=0.022)$ were correlated with CRP. EMP were elevated in patients with acute coronary syndromes (ANOVA, $\mathrm{p}=0.034$ ). EMP count was significantly higher in presence of noncalcified plaques (ANOVA, $\mathrm{p}=0.01$ ). This increase in EMP in noncalcified plaque carriers remained significant $(\mathrm{p}=0.05)$ after adjustment for coronary heart disease and hsCRP. By contrast, there was no association of PMP count with noncalcified plaques and no difference in PMP count between patients with and without acute coronary syndrome. No significant association between either EMP and PMP counts and mixed or calcified plaques was observed.

Conclusion: We report for the first time an association between plasma EMPCD144+ and coronary noncalcified plaques assessed by MDCT in a population of type 2 diabetic patients. EMP might be used as a surrogate marker 
of unstable plaques, and might help to improve cardiovascular prediction in diabetic patients with intermediate risk.

Supported by: Hospices Civils de Lyon, CNRS, Astra-Zeneca

\section{3}

Retinal microvascular changes and risk of coronary heart disease in hypertensive diabetic and non-diabetic individuals: an ASCOT substudy C. Stettler ${ }^{1,2}$, N. Witt ${ }^{2}$, R. Tapp ${ }^{2}$, A. Stanton ${ }^{2}$, S. Thom ${ }^{2}$, S. Allemann ${ }^{1,3}$, T. Tillin'², A.D. Hughes ${ }^{2}$, N. Chaturvedi ${ }^{2}$

${ }^{1}$ University Hospital Bern, Division of Endocrinology, Diabetes and Clinical Nutrition, Bern, Switzerland, ${ }^{2}$ Imperial College London, International Centre for Circulatory Health, NHLI, United Kingdom, ${ }^{3}$ University of Bern, Institute of Social and Preventive Medicine, Switzerland

Background and aims: The role of arteriolar retinal changes as a marker of cardiovascular disease is widely accepted, but the importance of venular changes has only recently been emphasised. We aimed to assess differences in the retinal microvasculature between diabetic and non-diabetic hypertensive individuals with multiple cardiovascular risk factors, and to assess whether their prognostic value differs between diabetic and non-diabetic individuals. Materials and methods: This was a substudy of the Anglo-Scandinavian Cardiac Outcomes Trial (ASCOT), a randomised controlled multicenter trial assessing the effect of two antihypertensive regimens on coronary heart disease (CHD) endpoints. In addition to hypertension, individuals had to have at least 3 additional risk factors out of: diabetes mellitus (DM); left-ventricular hypertrophy; ECG abnormalities; peripheral arterial disease; previous stroke or transient ischaemic attack; male sex; age > 55 years; micro- or proteinuria; smoking; dyslipidemia; family history of premature CHD. Retinal analyses were performed on digital $30^{\circ}$ images of superior and inferior temporal fields. Multiple linear regression was used to compare DM and non-DM groups, logistic regression was used to assess the association of retinal parameters with CHD events, all models were adjusted for age, sex, body mass index, smoking, antihypertensive and lipid-lowering treatment.

Results: The study included 720 individuals (160 DM, 560 non-DM) with 44 CHD events. Mean age \pm SD was $61.4 \pm 8.4$ and $61.5 \pm 7.7$ years $(\mathrm{p}=0.88)$, body mass index was $30.5 \pm 5.4 \mathrm{~kg} / \mathrm{m}^{2}$ and $28.8 \pm 4.3 \mathrm{~kg} / \mathrm{m}^{2}$, respectively $(\mathrm{p}<0.001)$. Antihypertensive treatment was equally distributed across the two groups but more patients used a lipid-lowering agent in the non-DM group (56.6 vs. $32.5 \%, \mathrm{p}<0.001)$. Venular length to diameter ratio (LDR) was smaller in DM compared with non-DM $(10.6 \pm 5.0$ vs $11.4 \pm 5.0$ pixels, $p=0.04)$, venular vessels being shorter in DM compared with non-DM $(461.5 \pm 184.1$ vs $495.3 \pm 196.9$ pixels, $\mathrm{p}=0.04)$, while diameters were comparable $(42.5 \pm 5.7$ and $41.9 \pm 4.9$ pixels, $\mathrm{p}=0.18)$. Arteriolar LDR also differed between DM and non-DM ( $13.3 \pm 4.1$ vs $14.7 \pm 5.0, p=0.001)$, the arterioles being shorter (447.2 \pm 103.4 vs $466.8 \pm 127.5$ pixels, $\mathrm{p}=0.05)$ and wider $(29.4 \pm 3.1$ vs $28.3 \pm 3.1$ pixels, $\mathrm{p}<0.001)$. Venular LDR was negatively associated with the risk to develop a first coronary event in DM ( $\mathrm{p}=0.007)$ but not in non-DM $(\mathrm{p}=0.90)$. A similar association was also found between venular length and coronary events in DM $(\mathrm{p}=0.008)$ but not in non-DM $(\mathrm{p}=0.46)$. Venular diameter was not associated with the risk of CHD in either group and no associations were found between arteriolar LDR, diameter, or length and CHD.

Conclusion: In this treated hypertensive population diabetic people had shorter retinal vessels compared with non-diabetic individuals. Shorter venular length predicted CHD events in diabetic but not in non-diabetic individuals. These findings suggest that changes in retinal venular architecture may be useful in CHD prediction in diabetic individuals.

Supported by: Swiss National Science Foundation

\section{4}

Changes in bone metabolism biomarkers in the ADOPT study S.E. Kahn ${ }^{1}$, W.H. Herman ${ }^{2}$, R.R. Holman ${ }^{3}$, S.M. Haffner ${ }^{4}$, B. Zinman ${ }^{5}$, R.P. Aftring ${ }^{6}$, B.G. Kravitz ${ }^{6}$, G. Paul7, N.P. Jones ${ }^{8}$, J.M. Lachin', G.F. Viberti ${ }^{10}$, for the ADOPT Study Group

${ }^{1}$ VA Puget Sound Health Care System, Seattle, United States, ${ }^{2}$ University of Michigan, Ann Arbor, United States, ${ }^{3}$ Diabetes Trials Unit, OCDEM, Churchill Hospital, Oxford, United Kingdom, ${ }^{4}$ Medicine / Clinical Endocrinology, University of Texas Health Science Center, San Antonio, United States, ${ }^{5}$ Mount Sinai Hospital, Toronto, Canada, ${ }^{6}$ Metabolic Medicine Development Center, GlaxoSmithKline, King of Prussia, United States, ${ }^{7}$ Biomedical Data Services, GlaxoSmithKline, King of Prussia, United States, ${ }^{8}$ GlaxoSmithKline, Harlow, United Kingdom, ${ }^{9}$ The George Washington
University, Rockville, United States, ${ }^{10}$ King's College London School of Medicine, United Kingdom

Background and aims: ADOPT was a long-term randomized clinical trial comparing the effects of rosiglitazone (RSG), glibenclamide (GLIB) and metformin (MET) monotherapy on glycaemic control in patients with type 2 diabetes. Post-hoc safety data analysis revealed an increased risk for fracture in women treated with RSG compared with those treated with GLIB or MET. This analysis measured bone biomarkers in samples from the ADOPT study. Materials and methods: Paired stored baseline and 12-month serum samples available from 1605 participants were assayed for selected bone metabolism biomarkers. Paired samples ( $46.6 \%$ of ADOPT participants at one year) were available from 299, 291 and 326 males and 250, 214 and 225 females in the RSG, GLIB and MET groups, respectively.

Results: More than $70 \%$ of the females were postmenopausal. Baseline characteristics (gender, menopausal status, glycaemia, blood pressure and lipids) in the subset of patients with available paired samples were similar to the overall study population.

Baseline values were similar between treatment groups for each analyte. Data were analyzed by gender and menopausal status. The pattern of change by treatment was similar for the male and female subgroups. Geometric means of the baseline 25 -hydroxyvitamin D levels were low across all groups (60 $\mathrm{nmol} / \mathrm{l}[24 \mathrm{ng} / \mathrm{ml}])$.

\begin{tabular}{llll}
\hline & \multicolumn{3}{l}{ \% Change from Baseline (n) } \\
\hline Analyte & RSG & GLIB & MET \\
\hline $\begin{array}{l}\text { Aminoterminal propeptide of } \\
\text { type I collagen (P1NP) }\end{array}$ & $-10.0(542)$ & $-2.1(502)^{\star}$ & $-17.3(547)^{\star}$ \\
\hline $\begin{array}{l}\text { C-telopeptide of collagen cross- } \\
\text { links (CTX) }\end{array}$ & $2.1(491)$ & $-3.9(454)^{\star}$ & $-8.3(496)^{\star}$ \\
\hline Parathyroid hormone (PTH) & $-18.3(492)$ & $2.7(462)^{\star}$ & $-14.5(510)^{*}$ \\
\hline Estradiol & $-11.5(427)$ & $-2.6(426)^{\star}$ & $-3.2(450)^{\star}$ \\
\hline 25-Hydroxyvitamin D & $-4.0(532)$ & $-1.2(493)$ & $7.2(543)^{\star}$ \\
\hline
\end{tabular}

${ }^{*}$ Statistically significant at level 0.05 compared with RSG, adjusted for multiple comparisons.

Conclusion: While the clinical relevance of these data is uncertain, the significant changes in C-telopeptide of collagen cross-links (CTX) and estradiol with RSG compared with GLIB and MET are directionally consistent with adverse effects on bone metabolism. Targeted interventions may be useful in mitigating the increased fracture risk with type 2 diabetes and thiazolidinedione (TZD) therapy; however, information on the effects of antidiabetic agents, and TZDs in particular, on bone metabolism from prospectively designed studies is required before making specific therapeutic recommendations.

Supported by: GlaxoSmithKline 


\section{OP 05 Beta cell development and regeneration}

\section{5}

The transcription factor RFX3 is critical for beta cell specification and differentiation

A. Ait-Lounis ${ }^{1}$, C. Bonal ${ }^{2}$, E. Barras ${ }^{1}$, P. Herrera ${ }^{2}$, W. Reith ${ }^{1}$

${ }^{1}$ Pathology and Immunology, ${ }^{2}$ Genetic Medicine and Development,

University of Geneva Medical School, Switzerland

Background and aims: We have recently reported that mice lacking the transcription factor RFX3 exhibit a strong reduction in the number of cells expressing glucagon, insulin and ghrelin, whereas the number of cells expressing pancreatic polypeptide (PP) are markedly increased. This phenotype was documented mainly at stage E19.5, resulting into an absolute reduction of endocrine hormone-expressing cells.

Materials and methods: To further understand the temporal and cell-type specific role of RFX3 we have performed a detailed analysis of the pancreatic endocrine defects that arise in Rfx3-/- mice during development, from the appearance of endocrine progenitor cells to their subsequent differentiation into specific cell lineages.

Results: The earliest marker of endocrine progenitor cells - the transcription factor Neurogenin3 (Ngn3) - was found to be expressed in normal numbers of cells and with a normal pattern during development. In contrast, significant reductions in the numbers of insulin and glucagon positive cells became evident by stage E15.5, during the secondary transition. The increase in PPpositive cells was observed as early as stage E13.5. We next examined the expression pattern of factors involved in early commitment to the alpha and/ or beta cell lineages (mafB, arx, pax4, nkx2.2, nkx6.1) and of factors implicated in the subsequent differentiation and maturation of alpha (brn4, mafB) and beta cells (nkx6.1, pdx1, Iapp). The expression of mafB, which is implicated in the initial commitment of both alpha and beta cells, was strongly reduced throughout development in $\mathrm{Rf} \times 3$-/- mice. The same was observed for all mature alpha cell markers examined, suggesting that the deficiency in RFX3 leads to an early block in alpha cell specification. In contrast, the numbers of cells expressing the beta cell marker Nkx6.1 were completely unaffected throughout development. Interestingly, the majority (70\%) of these beta-lineage committed cells presented a maturation defect characterized by a marked reduction in insulin, IAPP and slc2a2 expression. Moreover, a fraction of these "immature" beta cells exhibited abnormal or "ectopic" PP expression. These results suggest that, in contrast to the early specification defect observed for alpha cells, the absence of RFX3 affects a later step in the specification or maturation of beta cells. The latter finding prompted us to explore a potential role of RFX3 in maintaining beta-cell maturity by performing siRNA knockdown experiments with the mouse insulinoma cell line MIN6. The inhibition of RFX3 expression in MIN6 cells led to a reduction in the expression of insulin and several mature beta cells marker, including pdx1, IAPP and slc2a .

Conclusion: Taken together, the combined in vivo and in vitro results suggest that RFX3 is essential for both the terminal differentiation of beta cells and the maintenance of their mature phenotype.

Supported by: ALFEDIAM, Sir Jules Thorn Foundation, Roche Research Foundation and an EFSD/Lilly research programme

\section{6}

TCF7L2 regulates cell proliferation and differentiation in the developing pancreatic epithelium

G. da Silva Xavier, R. An, A. McDonald, M.K. Loder, G.A. Rutter

Cell Biology, Imperial College, London, United Kingdom

Background and aims: Polymorphisms in the human TCF7L2 gene have been shown in recent whole genome studies to be associated with an increased risk of type 2 diabetes. TCF7L2, a transcription factor regulated by the Wnt $/ \beta$-catenin signaling pathway, has previously been implicated in the regulation of cell proliferation and differentiation. In this study we investigate the potential role of TCF7L2 in the regulation of embryonic pancreas development using E13.5 pancreatic epithelial explants or INSr $\alpha / \beta$ cells.

Materials and methods: siRNA against rat TCF7L2 were designed according to criteria set-out by Tuschl et al and synthesised using Ambion's Silencer siRNA kit (Ambion, TX). INSr $\alpha / \beta$ cells were kindly provided by Dr.Thomas
Mandrup-Poulsen and were cultured as previously described. Cells were transfected with siRNA using TransIT ${ }^{\mathrm{TM}}$-TKO (Mirus Bio Corporation, Madison), according to the manufacturer's instructions. Pancreatic epithelia were isolated from E13.5 rat embryos and were treated with $500 \mathrm{nM}$ control or anti-TCF7L2 siRNA, or adenovirus encoding eGFP or hTCF7L2 (100 MOI), for ten days prior to RNA extraction. Primers for real-time PCR analysis were designed using Primer Express 3.0 (Applied Biosystems, CA) using rat mRNA sequences on the Ensembl database (http://www.ensembl.org/). Sequence specificity for all primers was verified using BLAST (http://www. ncbi.nlm.nih.gov/blast/Blast.cgi). Real time-PCR was performed on an ABIPrism Fast 7500 device (Applied Biosystems, CA).

Results: TCF7L2 silencing in E13.5 rat epithelia led to a reduction of TCF7L2 mRNA of $44.3 \pm 2.04 \%$, and complete blockade ( $n=3$ separate preparations with three pancreatic epithelia in each preparation) of development of endocrine buds and insulin-expressing cells after ten days of culture. The expression of a number of genes that are associated with differentiated endocrine cells was significantly decreased in comparison with control, namely preproinsulin $(98.8 \pm 3.58 \%)$, prohormone convertase $1(62.6 \pm 20.4 \%)$, and $2(73.6 \pm 17.2 \%)$, HNF- $4 \alpha(74.9 \pm 12.5 \%)$, and HNF-1 $\beta(85.6 \pm 9.46 \%)$, and the cell proliferation marker, ki67 (75.2 $\pm 1.57 \%)$, whilst preproglucagon gene expression was enhanced $(179 \pm 7.46 \%)$. These phenomen were reversed by over-expression of TCF7L2 in E13.5 pancreatic epithelia that had been treated with siRNA. E13.5 epithelial cultures over-expressing TCF7L2 develop endocrine buds two days earlier than cultures treated with a control virus, and the size of the epithelial cores were $1.89 \pm 0.28$-times larger than control epithelial cultures $(n=9)$. By contrast in INSr $\alpha / \beta$ cells the effect of silencing TCF7L2 on preproinsulin gene expression was minimal even though preproglucagon gene expression was increased compared to control, as observed in the E13.5 epithelial cultures.

Conclusion: TCF7L2 is expressed during pancreatic development and is required for normal $\beta$-cell development and $\beta$-cell specific gene expression from E13.5 in pancreatic epithelial explants. Thus, decreases in TCF7L2 may contribute to an elevated risk of developing diabetes by affecting the development of $\beta$-cells from pancreatic islet progenitor cells. Further studies will be required to determine whether effect of TCF7L2 silencing on endocrine cell development is due to effects on the proliferation of progenitor cells, stalling of the differentiation programme, or a combination of both.

Supported by: The Wellcome Trust, JDRF and an EFSD Albert Renold Fellowship

\section{7}

Role of cadherin-mediated cell-cell adhesion in transdifferentiation of pancreatic acinar cells

K. Minami ${ }^{1}$, H. Okano ${ }^{1}$, S. Seino ${ }^{1,2}$

${ }^{1}$ Division of Cellular and Molecular Medicine, ${ }^{2}$ Division of Diabetes, Metabolism, and Endocrinology, Kobe University Graduate School of Medicine, Kobe, Japan

Background and aims: Recent studies have revealed unexpected plasticity of adult cells in their differentiation capacity. We have demonstrated that insulin-secreting cells can be generated from pancreatic acinar cells of adult mouse in vitro, using Cre/loxP-based direct cell lineage tracing. However, little is known of the mechanism of the transdifferentiation. Our aim is to clarify the mechanism of transdifferentiation of pancreatic acinar cells into insulin-secreting cells.

Materials and methods: Pancreatic acinar cells were isolated from streptozotocin-injected ( $200 \mathrm{mg} / \mathrm{kg}$, i.p.) mice, and cultured in suspension with 20 $\mathrm{ng} / \mathrm{ml} \mathrm{EGF}$ in the presence or absence of signaling inhibitors (PD98059 for MEK, SB203580 for p38 MAPK, SP600125 for JNK, and LY294002 for PI3kinase). Changes in mRNA expression of genes were quantified by real-time RT-PCR analysis. Activation of intracellular signaling was evaluated by immunoblotting of phospho-specific antibodies.

Results: Enzymatic dissociation of pancreas caused destruction of cadherin-mediated cell-cell adhesion and activation of EGF receptor in pancreatic acinar cells. The isolated acinar cells formed smooth spheroids within a few days in suspension culture. Insulin expression gradually increased during the culture. Genes characteristic of pancreatic $\beta$-cells also were induced. Insulin secretion in the newly-made cells was stimulated by $\mathrm{KCl}(30 \mathrm{mM})$, glibenclamide $(100 \mathrm{nM})$, and glucose $(20 \mathrm{mM})$. However, in the presence of LY294002 $(10 \mu \mathrm{M})$, induction of the genes characteristic of pancreatic $\beta$-cells was significantly suppressed and no insulin secretion was detected, demonstrating that PI3-kinase is essential for the transdifferentiation of pancreatic acinar cells into insulin-secreting cells. Interestingly, we found that spheroid formation 
was blocked in the presence of the PI3-kinase inhibitor, suggesting that cellcell interaction is involved in the transdifferentiation process. Indeed, when spheroid formation was inhibited by the addition of neutralizing antibody against E-cadherin, the induction of genes required for glucose-induced insulin secretion was significantly suppressed. Moreover, we found that Ecadherin and $\beta$-catenin, major components of cadherin-mediated cell-cell adhesion in isolated acinar cells, were degraded in the presence of LY294002, while removal of the inhibitor upregulates both E-cadherin and $\beta$-catenin, thereby leading to transdifferentiation into insulin-secreting cells.

Conclusion: Destruction and remodeling of cadherin-mediated cell-cell adhesion is critical in transdifferentiation of pancreatic acinar cells into insulinsecreting cells, in which the PI3-kinase pathway plays an essential role. Supported by: MEXT, JDRF

\section{8}

p2 $7^{\mathrm{kip} 1}$ is a critical checkpoint for adult mouse islet regeneration during hyperglycaemia adaptation

B.R.S. Hsu, S.-H. Fu, S.-T. Chen

Internal Medicine, Chang-Gung Memorial Hospital, Taoyuan Hsien, Taiwan

Background and aims: Enhancing adult islet regeneration is a potential means of treating diabetes mellitus. However, it is not yet clear which mechanism controls adult islet cell regeneration. We intend to study the role of p2 $7^{\text {kip } 1}$ on adult mouse islet regeneration.

Materials and methods: We first cultured 12 weeks old male C57BL/6 mouse islets and used polyacrylamide gel electrophoresis (PAGE), western blotting and immunostaining to investigate the effect of glucose on changes of cell cycle proteins. We then used p27kip1 gene silencing to study the effect of p27 kip1 on growth-related proteins and function of adult pancreatic islet cells.

Results: We used PAGE, western blotting and immunostaining to determine profiles of cell cycle proteins of adult mouse islets and found that $\mathrm{p} 27^{\mathrm{kip} 1}$ (G1) G0 checkpoint) reduced $52 \%$ and $39 \%$ and cyclin B1 (G2/M) elevated 7 -fold and 12 -fold after one day (day 1 ) of culture in $5 \mathrm{mM}$ and $20 \mathrm{mM}$ glucose, respectively, when compared to that of day 0 . The levels of $\mathrm{p} 27^{\mathrm{kip} 1}$ protein of islets cultured in both 5 and $20 \mathrm{mM}$ glucose elevated progressively during cultivation. The elevation slope of $\mathrm{p} 27^{\mathrm{kip} 1}$ of islets cultured in $20 \mathrm{mM}$ glucose was only $64 \%$ of that of islets cultured in $5 \mathrm{mM}$ glucose. Proteins of D1 (G1/ S), B1 (G2/M), and FoxM1 of islets cultured in $5 \mathrm{mM}$ decreased gradually during cultivation. On the contrary, D1, B1, and FoxM1 of islets cultured in $20 \mathrm{mM}$ increased progressively. We then used p $27^{\text {kipl }}$ gene silencing to study the effect of $\mathrm{p} 27^{\mathrm{kip} 1}$ on growth-related proteins and function of adult pancreatic islet cells. Lentivirus carrying small interfering RNA duplex (siRNA) reduced $78 \%(\mathrm{n}=6)$ and $81 \%(\mathrm{n}=6)$ of $\mathrm{p} 27^{\text {kip } 1}$ protein in adult male mice islets after 3 and 7 days of cultivation, respectively. Proteins of D1, B1, and FoxM1 in $\mathrm{p} 27^{\mathrm{kip} 1}$ gene silencing islets were 3.0, 1.5, and 1.9 times and 1.0, 1.3, and 2.8 times of that of the control islets after 3 and 7 days culture, respectively. Glucose-stimulation insulin secretion tests revealed that stimulation index (SI) and insulin secretion (IS, ng/islet $\times 60 \mathrm{~min}$ ) of $\mathrm{p} 27^{\mathrm{kip} 1}$ gene silencing islets did not differ significantly from that of control islets at 3 and 7 days of culture (Day 3: gene-silencing islet: $\mathrm{SI}=2.6 \pm 0.3(\mathrm{n}=6)$, $\mathrm{IS}=0.19 \pm 0.02$; control islet: $\mathrm{SI}=2.8 \pm 0.4(\mathrm{n}=10), \mathrm{IS}=0.20 \pm 0.02)$ (Day 7: gene-silencing islet: $\mathrm{SI}=2.4 \pm 0.3$ $(\mathrm{n}=6), \mathrm{IS}=0.24 \pm 0.09$; control islet: $\mathrm{SI}=2.6 \pm 0.3(\mathrm{n}=12), \mathrm{IS}=0.17 \pm 0.02)$.

Conclusion: Suppression of $\mathrm{p} 27^{\text {kip1 }}$ rapidly up-regulates growth-related cell cycle proteins including D1, B1, and FoxM1 in adult mouse islets. Our data suggest that $\mathrm{p} 27^{\mathrm{kip} 1}$ is a critical checkpoint for adult mouse islet regeneration during hyperglycemia adaptation.

Supported by: NSC96-2314-B-182A-029-MY2

\section{9}

Role of EGF-receptor signalling in physiological beta cell mass expansion E. Hakonen ${ }^{1}$, J. Ustinov ${ }^{1}$, P. Ormio ${ }^{1}$, N. De Medts ${ }^{2}$, L. Bouwens ${ }^{2}$,

P.J. Miettinen ${ }^{1}$, T. Otonkoski

${ }^{1}$ Biomedicum Stem Cell Center, Hospital for Children and Adolescents, University of Helsinki, Finland, ${ }^{2}$ Cell Differentiation Unit, Free University of Brussels, Belgium

Background and aims: Better understanding of beta-cell regeneration mechanisms is important for the generation of new therapeutic approaches for diabetes. Beta-cell mass is known to increase in response to insulin demand and during pancreatic regeneration induced by injury. Epidermal growth factor receptor (EGF-R) signaling is required for normal pancreatic beta-cell de- velopment and proliferation. In this study we investigated the role of EGF-R signaling in physiological situations of beta-cell mass expansion.

Materials and methods: E1-DN mice, which have a kinase negative EGFreceptor under the Pdx-1 promoter, and wild type (WT) mice were subjected to high fat diet (HF-diet) or partial pancreatic duct ligation (PDL). The mice were kept on HF-diet or standard chow for 8 weeks. BrdU was added to the drinking water $(1 \mathrm{mg} / \mathrm{ml})$ for long-term labeling of proliferating beta-cells. Intraperitoneal glucose tolerance test (IPGTT) was done on weeks 4 and 8 . Morphometrical analysis of the pancreata was performed at 8 weeks. In the PDL model the pancreatic duct of 8 weeks old E1 and WT mice was partially ligated. After one week the tail and head parts of the pancreata were collected. Morphometrical analysis of the pancreata was performed. Beta-cell mass, islet size and distribution were analyzed.

Results: After 8 weeks on HF-diet E1-DN mice $(n=6)$ were clearly diabetic in IPGTT ( $2 \mathrm{~h}$ b-gluc $=26 \mathrm{mmol} / \mathrm{L})$ compared to E1-DN mice $(\mathrm{n}=5)$ on standard chow $(2 \mathrm{~h}$ b-gluc $=15 \mathrm{mmol} / \mathrm{L}, \mathrm{p}<0.01)$. WT mice on HF-diet $(\mathrm{n}=6)$ had mildly impaired glucose tolerance in IPGTT ( $2 \mathrm{~h} \mathrm{~b}$-gluc $=14 \mathrm{mmol} / \mathrm{L}$ ) compared to WT mice $(n=6)$ on standard chow $(2 \mathrm{~h} b$-gluc $=10 \mathrm{mmol} / \mathrm{L}, \mathrm{p}<0.01)$. HF-fed WT mice could increase their beta-cell surface area by $67 \%(n=3, p<0.05)$ compared with those on standard chow, while the response in HF-fed E1-DN mice was only $19 \%(n=3)$. WT mice had $30 \%$ more proliferating beta-cells compared to E1-DN mice $(\mathrm{p}<0.05)$. Surprisingly, we did not detect any significant difference in beta-cell proliferation between WT mice on standard chow vs. WT mice on HF-diet or between the two E1-DN groups. There was no difference in beta-cell apoptosis (immunohistochemistry for activated caspase 3) between the four groups. After partial duct ligation beta-cell surface area in the tail part of pancreas was increased by $300 \%$ compared to the head part of pancreas in WT mice $(n=3)$ and by $389 \%$ in E1-DN mice $(n=3)$. However the total beta-cell surface area, islet number and size in the tail part remained smaller in E1-DN mice (islet size 2.6 vs. 3.7 um$^{2}$, islet number 3.7 vs. 5.9 islets $/ \mathrm{mm}^{2}, \mathrm{n}=3$ in each group).

Conclusion: When fed on HF-diet, WT mice showed marked beta-cell hyperplasia, whereas the hyperplastic response of E1-DN mice was insufficient to maintain normoglycaemia. Partial duct ligation induced islet neogenesis in both WT and E1-DN mice. However, the number and size of the regenerating islets remained smaller in the E1-DN mice. These results suggests that EGF-R signaling is required for beta-cell mass expansion both in HF-diet induced insulin resistance and in pancreatic regeneration after partial duct ligation. Supported by: Academy of Finland/JDRF, SAVEBETA project of the EU FP6, Diabetes research Foundation, Foundation of Pediatric Research

\section{0}

Attempted beta cell regeneration from replication independent sources in a rat model of type 2 diabetes

E. Manesso' ${ }^{1}$, G.M. Toffolo ${ }^{1}$, Y. Saisho ${ }^{2}$, A.E. Butler ${ }^{2}$, R. Galasso 2 , P.C. Butler ${ }^{2}$, C. Cobelli ${ }^{1}$

${ }^{1}$ Department of Information Engineering, University of Padova, Italy, ${ }^{2}$ Department of Medicine, Larry Hillblom Islet Research Center, UCLA David Geffen School of Medicine, Los Angeles, United States

Background and aims: Type 2 diabetes (T2DM) is characterized by decreased beta cell mass, increased beta cell apoptosis and islet amyloid derived from islet amyloid polypeptide (IAPP). The human IAPP transgenic (HIP) rat reproduces this islet phenotype reaching a steady state beta cell deficit comparable to humans with T2DM at $\sim 12$ months of age. There is controversy as to whether a replication independent (RI) pathway for beta cell formation exists, and if so, if there is attempted beta cell regeneration through this mechanism to offset beta cell loss in T2DM. We addressed this by developing a model for beta cell turnover that resolves input from replication (R) and RI sources and applying it to non-diabetic wild type (WT) versus T2DM HIP rats.

Materials and methods: Beta cell mass (fractional insulin positive area $\mathrm{x}$ pancreas weight), $M[\mathrm{mg}]$, the frequency of beta cell replication (Ki67 and insulin positive cells), R [\%], and beta cell apoptosis (TUNEL and insulin positive cells), A [\%], were measured in WT $(n=20)$ and HIP $(n=22)$ rats age 2, 5, 10 and 18 months. To establish conversion factors for both beta cell replication and apoptosis from frequency to rate, rat islet cells were cultured for 16-48 hrs and imaged by time-lapse video-associated microscopy and then immunostained for insulin and Ki67 or TUNEL. We modeled beta cell turnover by a dynamic equation supposing either the absence (M1) or the presence (M2) of a replication independent source (RI $[\mathrm{mg} / \mathrm{month}])$ of beta cell formation i.e. $\mathrm{M} 1 \mathrm{~d} \mathrm{dM}($ age $) /$ dage $=\left[\alpha_{\mathrm{R}} \cdot \mathrm{R}(\right.$ age $)-\alpha_{\mathrm{A}} \cdot \mathrm{A}($ age $\left.)\right] \cdot \mathrm{M}($ age $) ; \mathrm{M} 2$ : 
$\mathrm{dM}($ age $) /$ dage $=\left[\alpha_{\mathrm{R}} \cdot \mathrm{R}(\right.$ age $)-\alpha_{\mathrm{A}} \cdot \mathrm{A}($ age $\left.)\right] \cdot \mathrm{M}($ age $)+\mathrm{RI}($ Age $)$, where $\alpha_{\mathrm{R}}$ and $\alpha_{\mathrm{A}}$ are conversion factors from frequency to rate.

Results: Changes in beta cell mass in both WT and HIP rats required beta cell formation from RI. From 2-18 months of age beta cell mass increased by $52 \mathrm{mg}$ in WT, and decreased by $15 \mathrm{mg}$ in HIP rats. This loss was due to a $~ 30$ fold greater loss of beta cells through apoptosis in HIP versus WT rats $(2,472$ Vs $92 \mathrm{mg}$ ), but the extent of the loss was offset by a 30 fold increase in new cell formation from RI in HIP versus WT rats $(2,267 \mathrm{vs} 77 \mathrm{mg})$ and a more modest contribution from R (190 vs $67 \mathrm{mg}$ ).

Conclusion: By use of novel model for beta cell turnover we conclude, (1) in the WT rat beta cell turnover includes comparable input from replication and replication independent sources, (2). In the HIP rat model of T2DM, increased beta cell apoptosis is partially countered by a marked increase in beta cell formation, but the contribution from replication independent sources is ten fold greater than that from replication. These data imply that in this model of T2DM in rats there is a substantial adaptive capacity for beta cell regeneration from replication independent sources that is overcome by apoptosis. Supported by: Juvenile Diabetes Research Foundation, US National Institute of Health, Larry Hillblom Foundation

\section{OP 06 Fat traffic and parking}

\section{1}

Metabolic adaptations in the early stage of insulin resistance measured in vivo by ${ }^{31} \mathrm{P}$ and ${ }^{1} \mathrm{H}$ MRS in skeletal muscle

N.M.A. van den Broek ${ }^{1}$, H.M.M. De Feyter ${ }^{1,2}$, K. Nicolay ${ }^{1}$, J.J. Prompers ${ }^{1}$

${ }^{1}$ Department of Biomedical Engineering, Eindhoven University of Technology, Netherlands, ${ }^{2}$ Departement of Diagnostic Radiology, Yale University, New Haven, United States

Background and aims: Current knowledge indicates that the pathology of insulin resistance involves metabolic alterations in several tissues, including skeletal muscle. Recent experiments have shown that mitochondrial oxidative phosphorylation and expression of genes involved in oxidative phosphorylation are reduced in muscle of insulin-resistant patients. Decreased muscle fat oxidation can favor the accumulation of intramyocellular lipids (IMCL), which has been associated with reduced insulin sensitivity. Therefore, impairments of mitochondrial oxidative capacity might be a major risk factor predisposing individuals to develop insulin resistance. We investigated skeletal muscle oxidative capacity and IMCL content in a rat model of early, lifestyle-induced insulin resistance using in vivo ${ }^{1} \mathrm{H}$ and ${ }^{31} \mathrm{P}$ magnetic resonance spectroscopy (MRS).

Materials and methods: Ten, 14 week old Wistar rats were put on a high fat diet (HFD, 45.7 En\% fat) for 2 weeks (HF) and a control group of 10 Wistar rats received normal chow (9 En\% fat) (NC). After an oral glucose tolerance test (OGTT) $(1 \mathrm{~g} / \mathrm{kg}$ body weight), IMCL was measured separately in both glycolytic (TA1) and oxidative (TA2) tibialis anterior muscle (TA) with in vivo ${ }^{1} \mathrm{H}$ MRS and oxidative capacity was measured in mixed TA with in vivo ${ }^{31} \mathrm{P}$ MRS. Data were analyzed statistically by applying two-tailed t-tests. Data are presented as means $\pm \mathrm{SD}$.

Results: At 16 weeks of age (weight HF: 426 g, NC:385 g), the HF animals showed a significantly lower ratio for the area under the curve (AUC) for glucose and the AUC for insulin (figure 1a, b) during the OGTT compared to $\mathrm{NC}$ animals $\left(\mathrm{AUC}_{\text {glucose }} / \mathrm{AUC}_{\text {insulin }}: 016 \mathrm{mM} \cdot \mathrm{h} / \mathrm{pM} \cdot \mathrm{h}\right.$ vs $0.033 \mathrm{mM} \cdot \mathrm{h} / \mathrm{pM} \cdot \mathrm{h}$ respectively). This indicates that 2 weeks of HFD induces insulin resistance. IMCL levels were about 3 times higher in HF compared to NC rats for both voxels (TA1: $0.23 \pm 0.05 \%$ vs. $0.07 \pm 0.03 \%, p<0.0001$ and TA2: $0.46 \pm 0.08$ $\%$ vs. $0.14 \pm 0.04 \%, \mathrm{p}<0.0001$ ) (figure $1 \mathrm{c}$ ).

The phosphocreatine recovery time constant, $\tau_{\mathrm{PCr}}$ which is a measure for oxidative capacity, was shorter in HF compared to NC animals ( $70.7 \pm 8.3 \mathrm{~s}$ and $85.7 \pm 12.1 \mathrm{~s}$, respectively, $\mathrm{p}=0.005$ ) (figure $1 \mathrm{~d}$ ).

Conclusion: We found that, in a rat model of early, HFD-induced insulin resistance, IMCL levels were elevated in TA. Interestingly, in vivo skeletal muscle oxidative capacity was higher in the rats with glucose intolerance compared to control rats. This can be explained as an adaptation to overfeeding of the mitochondria. Persistent overfeeding might have more detrimental effects in the long term. As part of a longitudinal study, we will study the same animals also during a more advanced stage of insulin resistance. In conclusion, mitochondrial dysfunction does not play a role in the early stage of insulin resistance in a validated animal model of lifestyle-induced insulin resistance. a

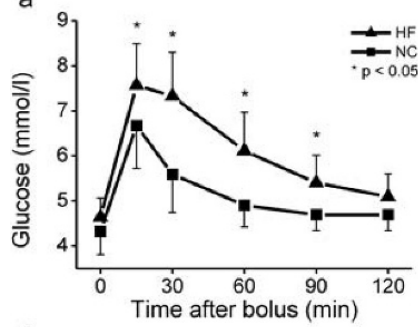

c
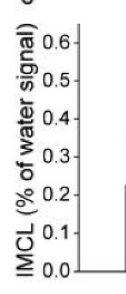

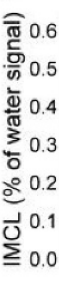
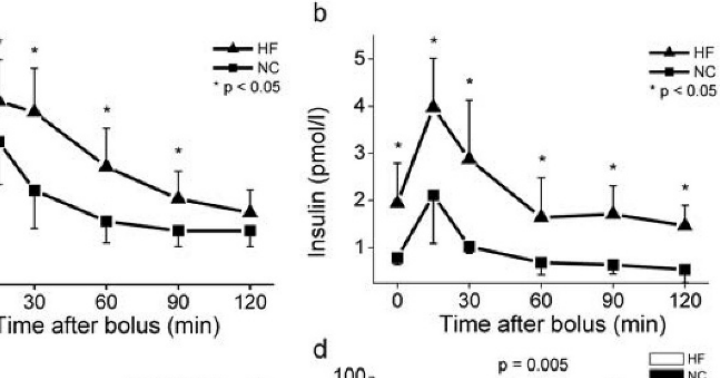

Figure 1. Glucose (a) and insulin (b) concentrations during OGTT. Mean IMCL (in glycolytic (TA1) and oxidative (TA2) TA) (c) and $\tau_{\mathrm{PCr}}$ for $\mathrm{HF}$ and $\mathrm{NC}$ rats ion TA. Data are presented as means $\pm \mathrm{SD}$ 


\section{2}

Regulation of fat storage in human subcutaneous adipose tissue over a 24-hour period

F. Karpe, T. Ruge, L. Hodson, B. Fielding, S.M. Humphreys, L. Dennis, J. Cheeseman, K.N. Frayn

Oxford Centre for Diabetes Endocrinology and Metabolism, University of Oxford, United Kingdom

Background and aims: Human adipose tissue stores fat from chylomicron triglycerides (TG), very low density lipoprotein (VLDL) TG and directly from non-esterified fatty acids. The contribution of these different sources for fatty acid storage in adipose tissue and how that changes in response nutritional state is not well known.

Materials and methods: Abdominal subcutaneous adipose tissue FA handling was studied over a $24 \mathrm{~h}$ period in eight healthy men by the combination of arterio-venous blood sampling, quantification of blood flow and the use of stable isotope-labelled FA tracers in response to three nutritionally similar meals with $5 \mathrm{~h}$ intervals.

Results: The efficiency of adipose tissue FA uptake increased with each meal, and the chylomicron-TG was the dominating source of FA. The initial fractional extraction of chylomicron-TG increased from $21 \pm 4$ to $47 \pm 8 \%(\mathrm{p}=0.03)$ between the first and the last meal. Although lipoprotein lipase rate of action increased with time (approximately 2 -fold), there was an even greater increase in the efficiency of FA re-esterification (approximately 3-fold), which led to a reduced spillover fraction of chylomicron-derived FA, from $77 \pm 15$ to $34 \pm 7 \%(\mathrm{p}=0.04)$ comparing the end of the first and the third meal period, respectively. Glucose extraction by the tissue was not enhanced by meal sequence. Uptake of VLDL-derived FA was particularly noticeable in the last meal period but spillover of VLDL-derived FA was only seen in the fasting state.

Conclusion: There is a considerable induction of fat storage capacity in the healthy human adipose tissue that is not appreciated in short-term studies. Supported by: British Heart Foundation

\section{3}

Lipidomic characterisation of the human liver: diacylglycerols but not ceramides associate with liver fat content

A. Kotronen ${ }^{1}$, T. Seppänen-Laakso ${ }^{2}$, J. Westerbacka ${ }^{1}$, J. Arola ${ }^{3}$,

A.-L. Ruskeepää ${ }^{2}$, M. Orešič ${ }^{2}$, H. Yki-Järvinen ${ }^{1}$

${ }^{1}$ Department of Medicine, University of Helsinki, Finland, ${ }^{2}$ Technical Research Centre of Finland, Espoo, Finland, ${ }^{3}$ Department of Pathology, University of Helsinki, Finland

Background and aims: Studies in animals have shown that fat may induce insulin resistance via ceramides or diacylglycerols. Dietary interventions and tracer studies in humans suggest dietary saturated fatty acids and saturated fatty acids formed by de novo lipogenesis associate with liver fat. There are no data regarding levels of ceramides or diacylglycerols or individual triacylglyerols and their fatty acid composition in the human liver.

Materials and methods: In the present study, we approached these questions by analyzing human liver biopsies from 16 subjects, whose liver fat, as determined by histology, ranged between 1 and $90 \%$. Increased liver fat ( $>10 \%$ macrovesicular steatosis, $\mathrm{n}=8$ ) was due to non-alcoholic causes. In each liver biopsy, concentrations of 253 lipids were determined using ultra performance liquid chromatography coupled to mass spectrometry (UPLC/MS), and fatty acid composition using gas chromatography.

Results: The number of double bonds in triacylglycerols were negatively related to the correlation coefficients between individual triacylglycerols/total triglyceride and liver fat $\%(r=-0.57, \mathrm{p}<0.0001)$, implying that the more saturated the triacylglycerols, the more fat the liver contains. Analyses of fatty acids revealed that liver fat content was negatively related to the concentrations of long polyunsaturated fatty acids, e.g. arachidonic (20:4n-6, $r=-0.70$, $\mathrm{p}=0.002)$ and docosahexanoic $(22: 6 \mathrm{n}-3, \mathrm{r}=-0.63, \mathrm{p}=0.009)$ acids, and positively to saturated and monounsaturated fatty acids, such as palmitic (16:0, $\mathrm{r}=0.69, \mathrm{p}=0.003)$ and oleic $(18: 1 \mathrm{n}-9, \mathrm{r}=0.69, \mathrm{p}=0.004)$ acids, and to SCD1 activity $(18: 1 / 18: 0, r=0.70, p=0.003)$ in the liver. Liver fat content was not related to total ceramide $(r=0.21$, NS) or sphingomyelin $(r=0.07, \mathrm{NS})$ content normalized to phospholipids, but was positively related to hepatic phospholipid-normalized diacylglycerol content $(\mathrm{r}=0.71, \mathrm{p}=0.002)$. The absolute amount of palmitate (16:0) was not related to ceramides ( $r=0.02$, NS).

Conclusion: The results suggest that hepatic fat accumulation due to nonalcoholic causes is associated with the enrichment of saturated and monoun- saturated fatty acids, and with depletion of polyunsaturated fatty acids. Levels of diacylglycerols but not ceramides increase with increasing liver fat content.

\section{4}

Changes of mitochondrial function and hepatocellular lipid content in relation to insulin sensitivity in women with a history of gestational diabetes

A. Kautzky-Willer ${ }^{1}$, T. Prikoszovich ${ }^{1}$, C. Winzer ${ }^{1}$, A.I. Schmid ${ }^{2,3}$, J. Szendroedi2,, , M. Chmelik ${ }^{2,3}$, G. Pacini ${ }^{5}$, M. Krssak ${ }^{3}$, E. Moser ${ }^{3}$, S. Trattnig ${ }^{3}$, P. Nowotny ${ }^{1}$, W. Waldhäusl ${ }^{1}, \mathrm{M}$. Roden ${ }^{2,4}$

${ }^{1}$ Department of Internal Medicine III, Division of Endocrinology and Metabolism, Medical University of Vienna, Austria, ${ }^{2}$ Karl-Landsteiner Institute of Endocrinology and Metabolism, Vienna, Austria, ${ }^{3} \mathrm{High}-$ Field Magnetic Resonance Centre of Excellence, Medical University of Vienna, Austria, ${ }^{4} 1$ st Medical Department, Hanusch Hospital, Vienna, Austria, ${ }^{5}$ Institute of Biochemical Engineering, National Research Council, Padova, Italy

Background and aims: Excessive lipid storage and reduced mitochondrial ATP-synthesis are present in type 2 diabetics with insulin resistance (T2DM) and their relatives. Women with a history of gestational diabetes (pGDM) show subtle alterations in glucose metabolism frequently with features of the metabolic syndrome and represent an important group at increased risk for development of T2DM. Here, we tested the hypothesis that changes of mitochondrial function and hepatocellular lipid content relate to glucose metabolism also in women with pGDM.

Materials and methods: We investigated 23 pGDM and 8 women with normal glucose tolerance during their pregnancies $(\mathrm{CON})$ at $4-5$ years after delivery. All women were glucose tolerant and comparable regarding age, BMI and physical activity. The glucose tolerance status and the insulin sensitivity were obtained by an oral (75g-oGTT; OGIS) and intravenous glucose tolerance test (IVGTT; $\mathrm{S}_{\mathrm{I}}$ ), respectively. The intramyocellular (IMCL) as well as the hepatocellular (HCL) lipid contents were determined by ${ }^{1} \mathrm{H}$ MR spectroscopy, myocellular fluxes through ATP synthase (fATP) and creatine kinase (fCK) by ${ }^{31} \mathrm{P}$ MR spectroscopy. Insulin resistance was defined by an OGIS lower than $463 \mathrm{ml} \cdot \mathrm{min}^{-1} \cdot \mathrm{m}^{-2}$.

Results: pGDM showed a higher body fat mass $(24.5 \pm 1.3$ vs. $18.0 \pm 1.4 \mathrm{~kg}$; $\mathrm{p}=0.02)$, but lower HDL-cholesterol $(\mathrm{p}=0.02)$ and insulin sensitivity $(447 \pm 14$ vs. $\left.508 \pm 31 \mathrm{ml} \cdot \mathrm{min}^{-1} \cdot \mathrm{m}^{-2} ; \mathrm{p}=0.05\right)$ versus CON. Their fATP was $13 \%$ lower compared with CON (10.5 \pm 0.9 vs. $12.1 \pm 0.5 \mu$ mol.g muscle $\left.{ }^{-1} \cdot \mathrm{min}^{-1} ; \mathrm{p}<0.05\right)$, while no difference was observed in fCK. IMCL was elevated only in insulinresistant women and $69 \%$ higher than in insulin-sensitive women $(0.91 \pm 0.08$ vs. $0.54 \pm 0.08 \%$; $\mathrm{p}<0.003)$. HCL was increased more than twofold in $\mathrm{pGDM}$ versus $\mathrm{CON}$ ( $3.6 \pm 0.8$ vs. $1.5 \pm 0.3 \%$; $\mathrm{p}<0.02)$, as well as in insulin-resistant versus insulin-sensitive subjects ( $4.0 \pm 1.1$ vs. $2.0 \pm 0.5 \%$; $<<0.03$ ). fATP correlated with plasma adiponectin ( $\mathrm{p}<0.04 ; \mathrm{r}=0.44)$ in all women and with usCRP in the insulin-resistant subgroup $(\mathrm{p}<0.004 ; \mathrm{r}=0.74)$. HCL related to body fat mass $(\mathrm{p}<0.01 ; \mathrm{r}=0.50)$ and insulin sensitivity $\left(\mathrm{S}_{\mathrm{I}}, \mathrm{p}=0.050 ; \mathrm{r}=-0.46\right)$.

Conclusion: Glucose tolerant women with a history of gestational diabetes and blunted insulin sensitivity exhibit lower muscular mitochondrial function and augmented hepatic lipid accumulation suggesting the operation of similar pathogenic mechanisms as in modifications in relatives of T2DM.

\section{5}

High intramuscular triglyceride concentration and low turnover distinguish pre-diabetes from obesity in humans L. Perreault, B.C. Bergman, M.C. Playdon, R.H. Eckel University of Colorado Health Sciences Center, Aurora, United States

Background and aims: High intramuscular triglyceride (IMTG) concentration has been repeatedly observed in people with, or at risk for, type 2 diabetes. Whether IMTG is causative, or simply a marker, of processes underlying diabetes is controversial. Therefore, the current study examined intramuscular triglyceride (IMTG) concentration, as well as its saturation and turnover, in people with pre-diabetes before and after exercise.

Materials and methods: A pre-diabetes group $(\mathrm{PD} ; \mathrm{n}=20)$ and normal glucose tolerant control group (NGT; $\mathrm{n}=19$ ) were matched for age and anthropometry. Insulin action and secretion were assessed using an intravenous glucose tolerance test (IVGTT). [U-13C]palmitate was infused for 4 hours prior and throughout 1.5 hours of treadmill walking at 50\% VO2max. IMTG 
concentration was measured by GC/MS, and turnover by GC/C/IRMS, from muscle biopsies taken immediately before and after exercise. Muscle biopsies were dissected free of visible fat and lyophilized, after which lipid fractions were isolated using solid phase extraction and derivatized to fatty acid methyl esters prior to analysis.

Results: Fasting and 2-hour glucose concentrations were higher in PD vs. NGT (fasting [glucose] $106 \pm 1.8$ vs. $92 \pm 1.2 \mathrm{mg} / \mathrm{dl}$, $2 \mathrm{~h}$ [glucose] $137 \pm 10.3 \mathrm{vs.}$ $101 \pm 4.5 \mathrm{mg} / \mathrm{dl}, \mathrm{p}<0.05$ for both), by study design, otherwise age ( $57 \pm 1.4 \mathrm{vs}$. $58 \pm 1.6$ years), body mass index ( $32 \pm 1.0$ vs. $31 \pm 1.0 \mathrm{~kg} / \mathrm{m} 2)$, body fat $(35 \pm 1.8$ vs. $37 \pm 1.8 \%$ ), and $\mathrm{VO} 2 \mathrm{max}(25 \pm 1.3$ vs. $22 \pm 1.3 \mathrm{ml} \mathrm{O} / \mathrm{kg} / \mathrm{min}$ ) were not different. Furthermore, whole-body substrate use and circulating concentrations of insulin, glucagon, free fatty acids, glycerol, lactate and catecholamines were not different between groups at rest or during exercise. Insulin action (Si) at baseline was similar between the groups, but insulin secretion (AIR \& DI) was significantly lower in PD (AIR $146 \pm 24$ vs. $428 \pm 67 \mu \mathrm{U} / \mathrm{ml}$, DI $375 \pm 60$ vs. $1231 \pm 145 \times 10-4 / \mathrm{min}, \mathrm{PD}$ vs. NGT, $\mathrm{p}<0.01$ for both). Basal IMTG concentration was higher $(43.1 \pm 5.2$ vs. $23.5 \pm 6.3 \mu \mathrm{g} / \mathrm{mg}$ dry weight, $\mathrm{p}=0.04)$ and turnover lower $(0.24 \pm 0.04$ vs. $0.37 \pm 0.5 \% / \mathrm{hr}, \mathrm{p}=0.05)$ in PD vs. NGT, whereas no such differences were seen for diacylglycerol (DAG) at baseline. No differences were observed in IMTG or DAG concentration or turnover during exercise, or in IMTG and DAG saturation at any time point. IMTG turnover during exercise correlated with $\mathrm{DI}$, but not $\mathrm{Si}$, in both groups $(\mathrm{R} 2=-0.438$, $\mathrm{p}=0.03$ ). Analyses were performed using repeated measures ANOVA.

Conclusion: The current study demonstrates that in groups matched for obesity, higher IMTG concentration and lower IMTG turnover, distinguish pre-diabetes from NGT. Diacylglycerol and fatty acid saturation of intramuscular lipids do not appear a major point of differentiation. Insulin secretion, rather than insulin action, was lower in pre-diabetes and correlated with IMTG turnover. How, or if, IMTG turnover influences insulin secretion, or vice versa, remains to be determined.

Supported by: NIH DK064811

\section{6}

Intravenous AICAR administration reduces hepatic glucose output and inhibits whole-body lipolysis in type 2 diabetes patients H. Boon ${ }^{1}$, M. Bosselaar ${ }^{2}$, S.F.E. Praet ${ }^{3}$, E.E. Blaak ${ }^{1}$, W.H.M. Saris ${ }^{1}$, A.J.M. Wagenmakers ${ }^{4}$, S.L. McGee ${ }^{5}$, C.J. Tack ${ }^{2}$, P. Smits ${ }^{2,6}$, L.J.C. van Loon ${ }^{1,3}$ ${ }^{1}$ Department of Human Biology, Maastricht University, Netherlands, ${ }^{2}$ Department of General Internal Medicine, Radboud University Nijmegen Medical Centre, Netherlands, ${ }^{3}$ Department of Movement Science,

Maastricht University, Netherlands, ${ }^{4}$ School of Sport and Exercise Sciences, University of Birmingham, United Kingdom, ${ }^{5}$ Department of Physiology, University of Melbourne, Australia, ${ }^{6}$ Department of Pharmacology and Toxicology, Radboud University Nijmegen Medical Centre, Netherlands

Background and aims: The AMP-activated protein kinase (AMPK) pathway is intact in type 2 diabetes patients and is seen as a target for the treatment of this disease. Here, for the first time, we assessed the effect of the AMPK activator 5-aminoimidazole-4-carboxamide riboside (AICAR) on both glucose and fatty acid metabolism in vivo in type 2 diabetes patients.

Material and methods: Stable isotope methodology with blood and muscle biopsy sampling were combined to assess glucose and fatty acid kinetics following continuous intravenous infusion of AICAR $\left(0.75 \mathrm{mg} \cdot \mathrm{kg}^{-1} \cdot \mathrm{min}^{-1}\right)$ and/ or saline in 10 male, type 2 diabetes patients (age: $64 \pm 2 y$; BMI: $28 \pm 1 \mathrm{~kg} / \mathrm{m}^{2}$ ). Results: Plasma glucose rate of appearance (Ra) was reduced following AICAR administration, while plasma glucose rates of disappearance (Rd) were equal in the AICAR and control test. Consequently, blood glucose disposal (Rd expressed as \% of Ra) was increased following AICAR infusion $(\mathrm{P}<0.001)$. Accordingly, a greater decline in plasma glucose concentration was observed following AICAR infusion $(\mathrm{P}<0.001)$. Plasma FFA Ra and $\mathrm{Rd}$ were both significantly reduced in response to AICAR infusion, which was accompanied by a significant net decline in plasma FFA concentration. Although the phosphorylation state of AMPK in skeletal muscle was not increased, we observed a significant rise in the phosphorylation of acetyl-CoA carboxylase (ACC; $\mathrm{P}<0.001$ ).

Conclusion: We conclude that intravenous AICAR administration reduces hepatic glucose output and augments glucose disposal, thereby lowering blood glucose concentrations in vivo in type 2 diabetes patients. Furthermore, AICAR administration stimulates hepatic fatty acid oxidation and/or inhibits whole-body lipolysis, thereby reducing plasma FFA concentrations. Supported by: Dutch Diabetes Research Foundation

\section{OP 07 Novel oral agents for type 2 diabetes}

\section{7}

Use of a GLUT4 translocation assay strategy to identify new insulin sensitisers with efficacy in vitro and in vivo

J.C. Molero ${ }^{1}$, N. Konstantopoulos ${ }^{1}$, C. Hohnen-Behrens ${ }^{2}$, J. Ye², D. Segal ${ }^{1}$, G. Collier ${ }^{1}$, K. Walder ${ }^{1}$, D.E. James ${ }^{2}$

${ }^{1}$ School of Exercise and Nutrition Sciences, Deakin University, Waurn Ponds, ${ }^{2}$ Diabetes and Obesity Program, Garvan Institute of Medical Research, Darlinghurst, Australia

Background and aims: The aim of the current study was to identify new insulin insulin sensitising agents from a small molecule library using a GLUT4 translocation assay screening strategy.

Materials and methods: We employed a fluorescence-based HA-GLUT4 translocation assay to determine the amount of GLUT4 present at the cell surface of differentiated rat L6 myotubes. The cells were rendered insulinresistant using $0.3 \mathrm{mM}$ palmitate for $16 \mathrm{~h}$ (68\% decrease in GLUT4 content at the cell surface compared with myotubes incubated with $100 \mathrm{nM}$ insulin for $20 \mathrm{~min}$ in the absence of palmitate). Insulin resistant L6 myotubes were then treated with small molecules from a library of $\sim 1500$ compounds at 10 $\mu \mathrm{M}$ for $60 \mathrm{~min}$. Positive hits were selected for their ability to increase GLUT4 content at the cell surface. Hits were further tested for their ability to reverse other models of insulin resistance in cultured 3T3-L1 adipocytes: chronic insulin stimulation ( $10 \mathrm{nM}$ for $48 \mathrm{~h}$ ), dexamethasone (500 $\mathrm{nM}$ for 8 days) or glucose oxidase ( $100 \mathrm{mU}$ for $2 \mathrm{~h}$ ). To test the in vivo efficacy of the lead compound, we examined its effect on glucose tolerance in insulin resistant high fat fed (HFF) mice (60\% calories from saturated fat for 6 weeks). The lead compound was administered with the food for the last 4 weeks.

Results: Using this cell-based GLUT4 translocation assay, we identified 8 compounds that reversed the inhibitory effect of palmitate on insulin stimulated GLUT4 translocation in L6 myotubes. The magnitude of the protective effect was variable between the compounds: $30-40 \%$ rescue, $\mathrm{p}<0.05$ (compounds designated as VVP086, VVP412 and VVP708); 60-70\% rescue, $\mathrm{p}<0.01$ (VVP326, VVP593, VVP600 and VVP912); $>80 \%$ rescue, $\mathrm{p}<0.005$ (VVP443). These compounds did not have a significant effect on GLUT4 translocation in the absence of insulin. We then tested the efficacy of these compounds on several models of insulin resistance in 3T3-L1 adipocytes. While several compounds were able to reverse insulin resistance induced by either chronic insulin stimulation, dexamethasone or glucose oxidase, VVP326, the berberine analog palmatine, achieved a significant effect in all models tested. We examined the effects of palmatine on glucose homeostasis in HFF mice. Palmatine $(50 \mathrm{mg} / \mathrm{Kg}$ ) administered with the food for 4 weeks ameliorated glucose intolerance in HFF mice $(23 \%$ decrease in the area under the curve compared with control HFF mice $\mathrm{p}<0.05, \mathrm{n}=8$ /group). This effect was accompanied by a $55 \%$ reduction in body weight gain $(\mathrm{p}<0.01)$.

Conclusion: The GLUT4 translocation assay successfully identified a number of new compounds with insulin sensitising properties. Palmatine showed efficacy in all cellular insulin resistant models tested and ameliorated the glucose intolerance associated with high fat feeding in mice. The GLUT4 translocation assay is a useful screen for novel insulin sensitising agents and the hit compounds identified in this study represent potential targets for the development of new diabetes therapeutics.

Supported by: Australian Research Council Linkage Program and Verva Pharmaceuticals

\section{8}

PPAR-sparing insulin sensitizers; path for development and clinical evaluation

J.R. Colca ${ }^{1}$, W.G. McDonald ${ }^{1}$, R. Malapaka ${ }^{2}$, H.E. Xu${ }^{2}$, R.F. Kletzien ${ }^{1}$

${ }^{1}$ Metabolic Solutions Dev Co, Kalamazoo, ${ }^{2}$ Van Andel Research Institute, Grand Rapids, United States

Background and aims: We have proposed that development of insulin sensitizer therapies has been delayed because of focus on nuclear receptor activation. Further, superior pharmacological profiles, [e.g., less plasma volume expansion (and edema) as well as greater reductions in blood pressure and corrections of dyslipidemia] might be obtained by minimizing direct activation of nuclear receptors. These studies seek to establish whether a series of 
insulin sensitizers could be established that limit or avoid interaction with nuclear receptors. We also aim to directly benchmark a prototype "PPARsparing" compound against pioglitazone in the clinic.

Materials and methods: Sixty novel analogs were generated and evaluated for PPAR $\gamma$ binding activity with a florescence polarization assay (Invitrogen) using rosiglitazone as a positive control. Selected compounds were tested for anti-diabetic activity in KKAy mice. Compounds $(10-100 \mathrm{mg} / \mathrm{kg} /$ day) were given by oral gavage for 4 days and fasting plasma glucose, insulin, and triglycerides were measured. To determine whether these compounds might interact with other PPAR transcription factors, AlphaScreen assays were set up with twelve nuclear receptor cofactor peptides for PPAR $\gamma$, PPAR $\alpha$, and PPAR $\delta$ using positive controls rosiglitazone, GW7647, and GW0742, respectively. A preclinical package was developed for testing a prototype PPARsparing analog in human clinical trials. Phase IA (single dose) and Phase IB (seven doses) were completed in comparison to $45 \mathrm{mg}$ pioglitazone in normal human volunteers.

Results: The novel analogs demonstrated a range in binding to PPAR $\gamma$ as compared to rosiglitazone. Five analogs demonstrated little to no binding to PPAR $\gamma$ even at $100 \mathrm{uM}$ compound. Forty percent of the novel analogs have demonstrated anti-diabetic pharmacology in vivo. The ability of the analogs in this series to lower circulating levels of glucose, insulin, and triglycerides in KKAy mice demonstrates no correlation with PPAR $\gamma$ binding. The PPARsparing analogs with anti-diabetic activity were also tested in the AlphaScreen assays for all three PPAR subtypes. The PPAR $\gamma$ sparing nature of the compounds established from initial direct binding studies was confirmed. None of the compounds interacted with PPAR $\delta$. The interaction with PPAR $\alpha$ was either similar to or less than pioglitazone. A preclinical support package was created for the prototype PPAR-sparing analog that supports clinical testing at up to $50 \%$ higher exposure relative to pioglitazone (and its active metabolites) for 28 days. Phase IA and Phase IB clinical studies were completed demonstrating no safety concerns, a twelve hour half-life, steady state circulating levels of active drug after two days of dosing, and the expected increase in circulating biomarker, adiponectin.

Conclusion: We conclude that it is possible to create PPAR sparing compounds with anti-diabetic pharmacology. We are now exploring the clinical pharmacology of the prototype PPAR-sparing compound vs pioglitazone in non-diabetic and diabetic patients. We are also evaluating whether selective mitochondrial interactions might predict more optimal insulin sensitizing pharmacology.

Supported by: Metabolic Solutions Development Company

\section{9}

Cycloset (Quick-Release Bromocriptine Mesylate), a novel centrally acting treatment for type 2 diabetes

A.H. Cincotta ${ }^{1}$, J.M. Gaziano ${ }^{2}$, M. Ezrokhi ${ }^{1}$, R. Scranton ${ }^{1}$

${ }^{1}$ Veroscience, Tiverton, United States, ${ }^{2}$ Harvard Medical School, Boston, United States

Background and aims: Oral administration of Cycloset (quick release - bromocriptine mesylate), a dopamine D2 receptor agonist, represents a new therapeutic approach in the management of type 2 diabetes (DM). Reduced dopaminergic tone in the hypothalamus is associated with and drives the obese insulin resistant state which is restored to the lean insulin sensitive state with appropriate timed delivery of bromocriptine. In the diabetic state, morning administration of Cycloset re-estabishes the daily peak of dompamingeric activity that changes neuroendocrine output to improve hepatic and muscle insulin resistance, primarily following a meal. Consequently, the Cycloset effect on gly- cemic control may be additive to a variety of peripherally acting oral hypoglycemic agents (OHA). The aim of this study was to determine the long term effectiveness of Cycloset versus placebo in improving glycemic control among subjects with DM currently treated with various peripherally acting OHA. Materials and methods: The Cycloset Safety Trial was a one year, 3070 subject, 2:1 randomized trial to assess the overall safety of Cycloset versus placebo among subjects with DM. Within this study design, several pre-specified efficacy analyses were conducted to assess changes in glycemic control after 24 weeks of treatment among subgroups of subjects receiving one or two oral OHAs and having an $\mathrm{HbAlc}$ (A1C) of $\geq 7.5 \%$ and $\leq 10 \%$ at baseline. Subgroups were defined as a) all subjects on one or two OHAs of any type (N: Cycloset: 261, placebo 151), b) subjects on metformin (MET) \pm another OHA (N: Cycloset: 181, placebo 101), and c) subjects on sulfonylurea (SU) \pm another OHA (N: Cycloset: 176, placebo 106). Additional post-hoc analyses included subjects on thiazolidinediones \pm OHA and subjects on metformin plus glyburide therapy. Study investigators were instructed to alter the dose or regimen of concomitant DM therapy if needed to adhere to ADA target glucose goals. Change from baseline in A1C was determined by ANCOVA. The proportion reaching an $\mathrm{AlC}$ of $\leq 7.0 \%$ at Week 24 was compared between the two treatment groups using the Cochran-Mantel-Haenzsel test.

Results: The mean A1C at baseline was 8.3, mean age was 58,63\% were male, and the mean body mass index was $33 \mathrm{~kg} / \mathrm{m}^{2}$. Among subjects on any $\mathrm{OHA}$, the between-group difference in change from baseline AlC was $-0.6(\mathrm{P}<0.0001)$ in favor of Cycloset. More Cycloset subjects reached a target $\mathrm{HbAlc}$ of $\leq 7.0(32 \%$ vs. $10 \%$, respectively; $\mathrm{p}=0.0001)$. Among subjects on MET $\pm \mathrm{OHA}$ or $\mathrm{SU} \pm$ OHA, A1C was significantly reduced in favor of Cycloset $(-0.70, p<0.0001 ;-0.6$, $\mathrm{p}<0.0001$, respectively). Also more Cycloset subjects reached a goal $\mathrm{AlC}$ of $\leq 7.0$ compared to placebo (MET subgroup: Cycloset 36\%, placebo 10\%; $\mathrm{p}=0.0001$ and SU subgroup: Cycloset 34\%, placebo $10 \%$; $\mathrm{p}=0.0001$ ). The mean between group change in $\mathrm{A} 1 \mathrm{C}$ over this treatment period also favored Cycloset versus placebo treated subjects receiving thiazolidinediones \pm OHA $(-0.91 ; \mathrm{p}=0.003)$ or metformin plus glyburide $(-0.85 ; \mathrm{p}=0.0001)$. Cycloset treated subjects were less likely to intensify their concomitant diabetes therapy across all subgroups.

Conclusion: Cycloset, a centrally acting drug, represents a new oral diabetes treatment effective across a broad patient population as adjunctive therapy to a wide variety of OHAs to improve glycemic control.

Supported by: Veroscience and S2 Therapeutics

\section{0}

Efficacy and safety of dapagliflozin in a dose-ranging monotherapy study of treatment-naïve patients with type 2 diabetes

J.F. List ${ }^{1}$, V. Woo ${ }^{2}$, E. Morales ${ }^{3}$, W. Tang ${ }^{1}$, F.T. Fiedorek ${ }^{1}$

${ }^{1}$ Bristol-Myers Squibb, Princeton, United States, ${ }^{2}$ Diabetes Research \& Treatment Centre, Health Sciences Centre, Winnipeg, Canada, ${ }^{3}$ Centro de Investigación Cardiometabólica, Aguascalientes, Mexico

Background and aims: Dapagliflozin, a novel oral antidiabetic agent that selectively inhibits the renal sodium-glucose co-transporter 2, is being developed for the treatment of patients with type 2 diabetes mellitus (T2DM). Treatment-naïve T2DM patients were enrolled in a 12 -wk, randomized, double-blind, parallel-group, placebo ( $\mathrm{PBO}$ )-controlled, dose-ranging trial to evaluate the efficacy and safety of dapagliflozin.

Material and methods: After a 2-wk lead-in phase, 389 patients were randomized in equal ratios to once-daily dapagliflozin $2.5,5,10,20$, or $50 \mathrm{mg}$, metformin (Met) XR $750 \mathrm{mg}$ titrated to $1500 \mathrm{mg}$, or PBO.

Results: Mean reductions from baseline in A1C at wk 12 were statistically significant for all dapagliflozin treatment groups compared with PBO (Table).

Table. Adjusted Mean Decreases from Baseline at Wk 12.

\begin{tabular}{|c|c|c|c|c|c|c|c|}
\hline & \multicolumn{7}{|c|}{ Dapagliflozin dose (mg) } \\
\hline & $\begin{array}{l}2.5 \\
\mathrm{n}=59 \\
\end{array}$ & $\begin{array}{l}5 \\
\mathrm{n}=58 \\
\end{array}$ & $\begin{array}{l}10 \\
\mathrm{n}=47\end{array}$ & $\begin{array}{l}20 \\
\mathrm{n}=59\end{array}$ & $\begin{array}{l}50 \\
\mathrm{n}=56\end{array}$ & $\begin{array}{l}\mathrm{PBO} \\
\mathrm{n}=54\end{array}$ & $\begin{array}{l}\text { Met } \\
\mathrm{n}=56\end{array}$ \\
\hline Baseline A1C (\%) (SD) & $7.7(0.8)$ & $8(0.9)$ & $8(0.8)$ & $7.7(0.9)$ & $7.8(1.0)$ & $7.9(0.9)$ & $7.7(0.8)$ \\
\hline Change in $\mathrm{A} 1 \mathrm{C}(\%)$ at wk 12 & $-0.71 \mathrm{a}$ & $-0.72 \mathrm{a}$ & $-0.85 a$ & $-0.55 \mathrm{a}$ & $-0.90 \mathrm{a}$ & -0.18 & -0.73 \\
\hline Baseline FPG (mg/dL) (SD) & $145(34)$ & $153(48)$ & $148(38)$ & $149(41)$ & $153(42)$ & $150(46)$ & $143(33)$ \\
\hline Change in FPG $(\mathrm{mg} / \mathrm{dL})$ at wk 12 & $-16.2 \mathrm{~b}$ & $-19.3 c$ & $-21.1 \mathrm{c}$ & $-24.4 \mathrm{~d}$ & $-30.5 \mathrm{e}$ & -5.8 & -18.0 \\
\hline $\begin{array}{l}\text { Change in PPG } \mathrm{mg} \cdot \mathrm{min} / \mathrm{dL} \\
{[95 \% \mathrm{CI}] \text { at wk } 12}\end{array}$ & $\begin{array}{l}-9382 \\
{[-11420} \\
-7344]\end{array}$ & $\begin{array}{l}-8478 \\
{[-10200} \\
-6756]\end{array}$ & $\begin{array}{l}-10,149 \\
{[-12215} \\
-8082]\end{array}$ & $\begin{array}{l}-7053 \\
{[-8913} \\
-5194]\end{array}$ & $\begin{array}{l}-10,093 \\
{[-12024} \\
-8162]\end{array}$ & $\begin{array}{l}-3182 \\
{[-5086} \\
-1277]\end{array}$ & $\begin{array}{l}-5891 \\
{[-7775,} \\
-4008]\end{array}$ \\
\hline
\end{tabular}

a $P<0.01$ for dapagliflozin vs $P B O$.

${ }^{\mathrm{b}} \mathrm{NS}$ vs $\mathrm{PBO},{ }^{\mathrm{c}} P<0.01,{ }^{\mathrm{d}} P<0.001,{ }^{\mathrm{e}} P<0.0001$ vs $P B O$. 
Dapagliflozin also lowered fasting plasma glucose (FPG) and postprandial glucose (PPG), as measured by oral glucose tolerance testing (75 g, $3 \mathrm{hr}$ ), without increasing insulin concentrations. No statistical comparisons were applied to the Met arm. Laboratory monitoring revealed no clinically meaningful changes in serum sodium, potassium, or creatinine, or in serum or urinary calcium. By wk 12 dapagliflozin was associated with 0.1 to $0.2-\mathrm{mEq} /$ $\mathrm{L}$ increases above baseline in mean serum magnesium across all doses and a $0.2-\mathrm{mg} / \mathrm{dL}$ increase above baseline in mean serum phosphate at the highest doses. Serum uric acid declined by approximately $1.0 \mathrm{mg} / \mathrm{dL}$ for all dapagliflozin doses tested. The most common adverse events in dapagliflozin -treated patients were urinary tract infection, nausea, dizziness, headache, fatigue, back pain and nasopharyngitis. The rate of reported hypoglycemic events was higher than PBO but similar to Met, but no hypoglycemic events with a documented glucose level $\leq 50 \mathrm{mg} / \mathrm{dL}$ were reported.

Conclusion: Once-daily administration of dapagliflozin improved glycemic control at all doses tested compared with $\mathrm{PBO}$, without adverse short-term safety and tolerability signals.

Supported by: Bristol-Myers Squibb

\section{1}

Effect of a selective and reversible hepatic CPT1 inhibitor on HOMA index and fasting blood glucose in diabetic type 2 patients

G. Valentini, M. Bianchetti, S. Pace, P. Carminati

Medical Department, Sigma-tau, Pomezia, Italy

Background and aims: Type II diabetes is characterized by insulin resistance that can impair the adipose tissue response to insulin, with consequent great availability of free fatty acids and increase in mitochondrial $\beta$-oxidation. This results in an increased hepatic gluconeogenesis rate by activation of pyruvate carboxylase and an alteration of glucose homeostasis. In type II diabetic patients with this impaired metabolic status, increased hepatic gluconeogenesis is one of the major factors responsible for fasting hyperglycemia. The selective hepatic inhibition of CPT1, the outer mitochondrial membrane enzyme that catalyzes the transport of fatty acids into the mitochondrial matrix, can be effective in reducing hepatic gluconeogenesis and, therefore, a possible strategy in the therapy of diabetes. Teglicar is a new agent, characterized by a reversible and selective inhibitory activity on hepatic CPT1. After defining its safety and pharmacokinetic (PK) profiles in several studies in healthy volunteers, a study in diabetic type II patients was conducted, having as primary objective safety and tolerability and as secondary, PK and pharmacodynamics (PD).

Materials and methods: Double-blind, randomized, placebo-controlled, multiple-dose study for 15 days in 40 male and female type II diabetic subjects. ANOVA was applied to AUC ${ }_{(0-t z)}$ on day $1, \mathrm{AUC}_{\mathrm{ss}}$ on days 14 and 15 and peak concentration $\left(\mathrm{C}_{\max }\right)$ after dose correction and logarithmic pretransformation; PD and safety parameters were evaluated descriptively.

Results: Despite the study being dimensioned for safety and tolerability and not for efficacy, evidence of efficacy was obtained at dose of $450 \mathrm{mg} /$ day. In particular, HOMA index was calculated after an overnight fast at baseline (day 1) and after the last day of treatment (day 16), demonstrating significant improvement $(\mathrm{p}<00.4)$ in tissue insulin resistance in subjects treated with 450 $\mathrm{mg} /$ day of Teglicar (decrease from $4.1 \pm 3.1$ on day 1 to $3.0 \pm 1.9$ on day 16) compared to placebo (increase from $4.9 \pm 2.6$ on day 1 to $5.7 \pm 2.9$ on day 16 ). Subjects treated with $450 \mathrm{mg} /$ day of ST1326 showed a mean decrease in fasting blood glucose from day 1 to day 16 of $16 \mathrm{mg} / \mathrm{dl}$, compared to $4 \mathrm{mg} / \mathrm{dl}$ of subjects in placebo group. The pharmacokinetics of Teglicar, defined here and in previous studies carried out in healthy volunteers, were characterized by slow absorption and a slow elimination process $\left(t_{1 / 2}\right.$ ranging between 18 and $35 \mathrm{~h}$ ), which led to accumulation in plasma as consequence of daily dosing. Based on an average $t_{1 / 2}$ of $25 \mathrm{~h}$, according to standard rules, steady-state conditions may be assumed by day 5 . AUC after $24 \mathrm{~h}$ increased about two to three times from first administration to steady-state condition. Teglicar was well tolerated, with no clinically relevant changes in vital signs, clinical laboratory results, or ECG findings in any of the studies so far conducted.

Conclusion: Glucose homeostasis is regulated by complex mechanisms, thus administration of Teglicar is expected to induce only very slight modifications after such short-term administration. Nevertheless, the statistically significant results obtained on reduction of tissue insulin resistance and a clear tendency to reduce fasting blood glucose make Teglicar a promising tool in the control of fasting blood glucose in those patients where other glucoselowering drugs fail to control nocturnal hepatic glucose production.

\section{2}

A novel glucokinase activator RO4389620 improved fasting and postprandial plasma glucose in type 2 diabetic patients

J. Zhi' ${ }^{1}$, S. Zhai ${ }^{1}$, M.-E. Mulligann', J. Grimsby ${ }^{1}$, C. Arbet-Engels ${ }^{1}$, M. Boldrin ${ }^{1}$, R. Balena ${ }^{1}$

${ }^{1}$ Hoffmann-La Roche Inc., Nutley, United States, ${ }^{2}$ Pharma Development, F. Hoffmann-La Roche Ltd., Basel, Switzerland

Background and aims: RO4389620 is a nonessential, mixed-type activator of glucokinase (GK). It binds to an allosteric site on GK, which leads to an increase in the catalytic effectiveness of GK to metabolize glucose, and increases the glucose phosphorylation capacity of pancreatic beta-cells and hepatocytes. Thus, RO4389620 is proposed as a novel treatment of type 2 diabetes. In a completed single-ascending dose study in healthy volunteers, RO4389620 effectively lowered fasting plasma glucose in a dose-related fashion. The aim of the present study was to assess the safety, tolerability, pharmacokinetics (PK), and pharmacodynamics (PD) following multiple-ascending doses (MAD) of RO4389620. Food effect on RO4389620 PK/PD was also examined.

Material and methods: This was a double-blind, randomized, placebo-controlled, MAD study. Fifty-nine T2D patients, either treatment naïve (on diet and exercise) or previously treated with oral antidiabetic drugs, were recruited. They were given RO4389620 or placebo as a single dose on day 1 and multiple doses from day 3 to day 8 , at doses from 10, 25, 50, 100, and, $200 \mathrm{mg}$ BID for $5 \frac{1}{2}$ days and $200 \mathrm{mg}$ QD for 6 days, with a parallel dose-escalation design. Blood and urine samples were collected for PK analysis. PD assessments (fasting and postprandial 24-h AUC) included plasma glucose, insulin, C-peptide, glucagon, and GLP-1 on pretreatment day (baseline) and postdose up to day 8 . Non-compartmental methods were applied to compute the $\mathrm{PK}$ and PD parameters.

Results: The plasma exposure of RO4389620 was dose-proportional over the range tested with no appreciable drug accumulation or food-effect. The 24-h postprandial glucose AUCs at steady-state yielded dose-dependent reductions from baseline, up to $35.5 \%$ at the highest dose. There was also a dose-dependent decrease of fasting plasma glucose with a reduction (from baseline) up to $32.5 \%$ after $5 \frac{1}{2}$ days of treatment. A relative increase in insulin secretion was observed at high doses. RO4389620 was well tolerated. The most common adverse event was headache, with no clear dose-related pattern. No clinically significant laboratory, vital sign, or ECG abnormalities were reported. Mild or moderate hypoglycemia with rapid recovery after sugar-containing drinks or scheduled meals was the only dose-limiting adverse event.

Conclusion: Multiple doses of RO4389620 in T2D patients consistently showed a rapid, dose-dependent glucose reduction over 24 hours resulting from fasting and postprandial plasma glucose decreases, demonstrating its potential utility in the treatment of type 2 diabetes. Other than mild or moderate hypoglycemia, RO4389620 was safe and well tolerated. 


\section{OP 08 Nephropathy: development and treatment}

\section{3}

Progression of diabetic nephropathy is predicted by increased oxidative stress and decreased deglycation

P.J. Beisswenger ${ }^{1}$, S.K. Howell ${ }^{1}$, B.S. Szwergold ${ }^{1}$, S.S. Rich ${ }^{2}$, G.B. Russell ${ }^{3}$, Y.-K. Kim ${ }^{4}$, M. Mauer ${ }^{4}$

${ }^{1}$ Medicine, Dartmouth Medical School, Hanover, ${ }^{2}$ Public Health Sciences, University of Virginia, Charlottesville, ${ }^{3}$ Public Health Sciences, Wake Forest University, Winston-Salem, ${ }^{4}$ Pediatric Nephrology, University of Minnesota, Minneapolis, United States

Background and aims: Oxidative stress (OS) has been proposed as a key initiator of diabetic complications, primarily by inactivating Glyceraldehyde-3Phosphate Dehydrogenase (GAPDH) and increasing vascular damage, while the enzyme Fructosamine-3-Kinase (FN3K) can reverse damaging non-enzymatic glycation. Since OS has not been shown to predict progression of biopsy proven diabetic nephropathy (DN), and FN3K has not been explored in this clinical setting, we studied OS and FN3K in cultured skin fibroblasts (SF) from subjects with Type 1 Diabetes (T1D), discordant for progression or non-progression of DN.

Materials and methods: From 125 subjects, 25 fast progressors (P), 25 nonprogressors (NP) (from the highest and lowest quintiles of mesangial expansion scores and degree of albuminuria) and 25 controls (CON) were studied. Cells were cultured in physiologic $(5 \mathrm{mM})$ and high $(25 \mathrm{mM})$ glucose. OS was measured by fluorescence (FU) of 2',7'-dichlorodihydrofluorescein diacetate, and GAPDH activity was measured by an arsenolysis reaction. GAPDH and FN3K mRNA was measured by RT-PCR, while Poly (ADP-ribose) polymerase (PARP) activity was measured by a P32 NAD assay. A repeated measures ANOVA was used with covariates to determine independent effects of group (P, NP, CON) and glucose condition on the above parameters.

Results: OS was significantly $(\mathrm{P}=0.025)$ greater in $\mathrm{P}$ relative to $\mathrm{CON}$ in 5 and in $25 \mathrm{mM}$ glucose $(750 \pm 166 \mathrm{FU}$ vs $623 \pm 148 \mathrm{FU}$, and $679 \pm 154 \mathrm{FU}$ vs $580 \pm 140 \mathrm{FU})$, while CON and NP were not significantly different $(714 \pm 191$ and $654 \pm 152 \mathrm{FU}$ ) under these conditions. Increasing glucose to $25 \mathrm{mM}$ resulted in a significant $(\mathrm{P}<0.001)$ decrease in OS $(698 \pm 176$ vs $640 \pm 153 \mathrm{FU})$. GAPDH activity was significantly $(\mathrm{P}=0.01)$ lower in $\mathrm{P}(986.8 \pm 173.5)$ than in CON $(1145.3 \pm 273.1)$ in 5 and $25 \mathrm{mM}$ glucose, but did not differ $(\mathrm{P}=0.15)$ between NP (1056.2 \pm 188.8$)$ and CON, while $25 \mathrm{mM}$ glucose significantly decreased overall GAPDH activity $(\mathrm{p}=0.006)$. There were no significant differences between DN groups and CON in GAPDH mRNA ( $\mathrm{P}=0.30)$, which was increased by $25 \mathrm{mM}$ glucose. FN3K mRNA levels were significantly $(\mathrm{P}=0.04)$ lower in $\mathrm{P}(0.42 \pm 0.21)$ than in CON $(0.52 \pm 0.25)$ in $5 \mathrm{mM}$ glucose, while $\mathrm{NP}(0.50 \pm 0.16)$ did not differ from CON or P. Increasing glucose to $25 \mathrm{mM}$ decreased overall FN3K mRNA $(\mathrm{P}=0.0001)$ in the combined $\mathrm{DN}$ groups and CON, while it increased PARP. PARP increase was greater in P (18.9 and 19.1) compared with NP or CON (14-16); but did not achieve statistical significance.

Conclusion: Cells from nephropathy progressors show increased oxidative stress, decreased GAPDH activity, and increased PARP relative to controls, in contrast with non-progressors. GAPDH mRNA increased, suggesting that post-translational change led to decreased enzyme activity. Levels of FN3K mRNA were lower in P than in NP or CON, suggesting impaired deglycation in P. High glucose suppressed GAPDH activity, increased PARP, and decreased FN3K, but led to a previously unobserved decrease in OS in SF. These results directly link OS, decreased GAPDH activity, and impaired deglycation, to DN in human diabetic patients. Since these differences did not require high glucose in vitro, they could be secondary to inherent genetic traits or metabolic memory.

Supported by: NIDDK R01 DK62995

\section{4}

Can diabetic kidney disease develop in the absence of a history of elevated urinary albumin excretion?

J. Karalliedde, R. Ghosal, G. Viberti

Diabetes, King's College London, United Kingdom

Background and aims: Elevated urinary albumin excretion rate (AER) accompanies the development of nephropathy in diabetes. However the validity of AER as a predictor and marker of kidney disease in diabetes has been questioned by reports of renal impairment, as measured by estimated glomerular filtration rate or histology, in the absence of a history of elevated AER. Aims: To establish whether diabetic kidney disease (DKD) with significant renal functional impairment can develop in the absence of a history of elevated AER.

Materials and methods: We performed a comprehensive retrospective analysis and audit of electronic patient records and patient notes of all type 1 and type 2 diabetic patients attending a specialist diabetes renal unit at Guy's and St Thomas' Hospital foundation trust between January 2005 and January 2007. DKD impaired renal function was defined at two different serum creatinine levels of $>150 \mu \mathrm{mol} / \mathrm{l}$ or $>300 \mu \mathrm{mol} / \mathrm{l}$ and as current history of renal replacement therapy [RRT] such as regular dialysis or transplantation. Elevated AER was defined as: albumin:creatinine ratio $\geq 2.5 \mathrm{mg} / \mathrm{mmol}$ in men and $\geq 3.5 \mathrm{mg} / \mathrm{mmol}$ in women and / or AER $\geq 20 \mathrm{mcg} / \mathrm{min}$ on timed overnight urine collections and /or positive urine protein dipstick. All electronic and medical case notes were verified and double checked independently by two investigators.

Results: Six hundred and twelve diabetic patients had a serum creatinine $>150 \mathrm{umol} / \mathrm{l}$ or were receiving RRT $(\mathrm{n}=62)$. Ninety six $(52 \mathrm{M} 47 \mathrm{~F})$ patients had type 1 diabetes (T1DM) and $516(272 \mathrm{M} 108 \mathrm{~F})$ patients had type 2 diabetes $(\mathrm{T} 2 \mathrm{DM})$. Of these patients, $55(\mathrm{RRT}=22)$ with $\mathrm{T} 1 \mathrm{DM}$ and $154(\mathrm{RRT}=40)$ patients with T2DM had serum creatinine value $>300 \mu \mathrm{mol} / 1$ or were receiving RRT. At time of diagnosis of confirmed elevated AER in T1DM, mean (SD), age was 47.4 (12.4) years and duration of diabetes was 24.6(11.8) years. In the T1DM cohort 5 patients had clinical and histological evidence of nondiabetic renal disease. All patients who had developed DKD had a history of albuminuria prior to developing renal impairment whether a cut-off of serum creatinine $>150 \mu \mathrm{mol} / \mathrm{l}$ or $>300 \mu \mathrm{mol} / \mathrm{l}$ was used. In T2DM at diagnosis of elevated AER, mean (SD), age was 65.4(10) years, with duration of diabetes of 9.9 (8.3) years. In the T2DM cohort of 516 patients, 88 had clinical/histological evidence of non-diabetic renal disease. Of the remaining 428 patients, 14 had incomplete clinical records and had to be excluded from the analysis. Only 2 patients of the remaining 414 had no recorded evidence of raised AER preceding the development of renal impairment. When a serum creatinine threshold of $>300 \mu \mathrm{mol} / \mathrm{l}$ was used to define renal failure all patients with evidence of DKD had a documented history of preceding raised AER.

Conclusion: In a large clinic based cohort of diabetic patients with overt diabetic kidney disease a history of elevated AER almost invariably precedes the development of significant renal impairment. AER therefore remains a critical diagnostic tool to identify diabetic patients at risk of renal failure.

\section{5}

The combination of aliskiren, a direct renin inhibitor, and losartan in patients with type 2 diabetes and nephropathy

H.-H. Parving ${ }^{1,2}$, F. Persson ${ }^{3}$, J.B. Lewis ${ }^{4}$, E.J. Lewis ${ }^{5}$, N.K. Hollenberg ${ }^{6}$ ${ }^{1}$ Department of Medical Endocrinology, Rigshospitalet, Copenhagen, Denmark, ${ }^{2}$ Faculty of Health Science, Aarhus University, Denmark, ${ }^{3}$ Steno Diabetes Center, Gentofte, Denmark, ${ }^{4}$ School of Medicine, Vanderbilt University, Nashville, United States, ${ }^{5}$ Medical Center, Rush University, Chicago, United States, ${ }^{6}$ Brigham and Women's Hospital, Harvard Medical School, Boston, United States

Background and aims: We evaluated the renoprotective effects of dual blockade of the renin-angiotensin system by adding aliskiren, a novel oral direct renin inhibitor to treatment with maximal recommended dose of losartan (100 mg daily) and optimal antihypertensive therapy in hypertensive type 2 diabetic patients with nephropathy.

Materials and methods: 599 patients were enrolled in this multi-national, randomized, double blind study. After a 3-month open-label run-in period on losartan $100 \mathrm{mg}$ daily, patients were randomized to receive 6 months treatment with placebo or aliskiren ( $150 \mathrm{mg}$ daily for 3 months followed by forced titration to $300 \mathrm{mg}$ daily for another 3 months). The primary outcome was reduction in early morning urinary albumin creatinine ratio at 6 months. Results: The baseline characteristics in the two groups were similar. Aliskiren $300 \mathrm{mg}$ daily reduced the mean urinary albumin creatinine ratio by $20 \%$ (95\% confidence interval 9-30) $(\mathrm{P}<0.001)$ compared to placebo. The number of patients with $\geq 50 \%$ reduction in urinary albumin creatinine ratio at the end of the study was $24.7 \%$ versus $12.5 \%$ for aliskiren and placebo respectively $(\mathrm{P}<0.001)$. A small difference in blood pressure of $2 / 1 \mathrm{mmHg}$ occurred during aliskiren treatment $(\mathrm{P}=0.08)$. The decline in kidney function tended to be smaller with aliskiren $(\mathrm{P}=0.07)$. The total number of adverse and serious adverse events was similar between groups. 
Conclusion: Aliskiren is renoprotective independent of its blood-pressurelowering effect in hypertensive type 2 diabetic patients with nephropathy receiving recommended renoprotective treatment, and has a safety profile comparable to placebo. Supported by: Novartis

\section{6}

Urinary liver-type fatty acid- binding protein increases with levels of albuminuria in type 1 diabetic patients, and is reduced with ACE inhibition

S. Nielsen ${ }^{1}$, T. Sugaya ${ }^{2}$, L. Tarnow ${ }^{1}$, K.J. Schjoedt ${ }^{1}$, A.S. Astrup ${ }^{1}$, T. Baba ${ }^{3}$, H.-H. Parving ${ }^{4}$, P. Rossing

${ }^{1}$ Steno Diabetes Center, Gentofte, Denmark, ${ }^{2}$ St. Marianna University School of Medicine, Kanagawa, Japan, ${ }^{3}$ Internal Medicine 3, Fukushima Medical University, Japan, ${ }^{4}$ Department of Medical Endocrinology, Rigshospitalet, University Hospital of Copenhagen, Denmark

Background and aims: Urinary liver-type fatty acid-binding protein (uLFABP) is a marker of tubulointerstitial inflammation, and has been suggested to be a marker for chronic renal disease. We studied the level of u-LFABP in type 1 diabetic patients with different albuminuria level. Subsequently we evaluated the effect of renoprotective treatment with ACE inhibition on uLFABP in patients with diabetic nephropathy.

Materials and methods: Three groups of Caucasians with type 1 diabetes (T1D) from Steno Diabetes Center: 58 with normoalbuminuria $(\mathrm{N})(\mathrm{u}-$ albumin $<30 \mathrm{mg} / 24 \mathrm{~h}), 45$ with persistent microalbuminuria (MA)(30-300 $\mathrm{mg} / 24 \mathrm{~h}$ ) and 45 with persistent macroalbuminuria $(\mathrm{DMN})(\geq 300 \mathrm{mg} / 24 \mathrm{~h})$. Control group consisted of 55 healthy individuals (C). Groups were matched by gender and duration of diabetes (> 30 years). In 56 T1D patients with DMN a randomized crossover trial consisting of three treatment periods was performed, 7 patients did not complete all visits. After 2 months wash-out period (baseline), patients were treated in random order with lisinopril 20, 40, and $60 \mathrm{mg}$ once daily, each dose for 2 months and furosemide in fixed doses. Results: In the cross sectional study median levels (IQR) of u-LFABP ( $\mu \mathrm{g} / \mathrm{g}$ creatinine) in spot urine samples were significantly higher in $\mathrm{N}$ vs. C (2.6 $(1.3-4.1)$ vs. $1.9(0.8-3.0), \mathrm{p}=0.02)$ and were increasing with increasing levels of albuminuria: MA, 4.2 (1.8-8.3); DMN: 71.2 (8.1-123.4), $\mathrm{p}<0.05$ for all comparisons. U-LFABP is correlated with urinary albumin/creatinine ratio (UACR) $(\mathrm{r}=0.58, \mathrm{p}<0.001)$, eGFR $(\mathrm{r}=-0.58 \mathrm{p}<0.001)$ in patients with DMN and with UACR in MA $(r=0.44, p=0.002)$. No correlations between $u-L F A B P$ and age or $\mathrm{HbAlc}$ and no difference between sexes.

In the intervention study baseline urinary albumin excretion rate (UAER) were [geometric mean ( $95 \%$ CI)] 362 (240 to 545) mg/24 hours. All doses of lisinopril significantly reduced UAER, u-LFABP from baseline. Reductions ( $95 \% \mathrm{CI}$ ) in UAER from baseline were 63\% (55 to 69), 71 (66 to 76 ), and 70 (64 to 75 ) with increasing doses of lisinopril ( $\mathrm{p}<0.001$ vs baseline, $\mathrm{p}<0.05$ for 40 and $60 \mathrm{vs} 20 \mathrm{mg}$ ). At baseline u-LFABP was $12.69(3.88-49.82) \mu \mathrm{g} / 24 \mathrm{~h}$. Reductions in u-LFABP was 43\% (15-62), 46 (19-64) and 40 (11-60) with increasing doses of lisinopril (ns between doses). This reduction in u-LFABP was associated with the changes in BP and UAER.

Conclusion: Early and progressive rise in tubulointerstitial damage as reflected by u-LFABP occurs in type 1 diabetic patients associated with albuminuria. ACE inhibition has a beneficial effect both on glomerular and tubulointerstitial markers of damage.

\section{7}

New onset diabetes mellitus in white coat, masked and sustained hypertension

P. Gamba, F. Paleari, M. Bombelli, F. Ganz, A. De Mattheis,

F. Quarti-Trevano, D. Fodri, L. Primitz, M. Volpe, L. Magni, R. Facchetti, R. Sega, G. Grassi, G. Mancia

Università Milano-Bicocca, Ospedale San Gerardo, Monza, Milan, Italy

Background and aims: In the general population, the increase of "office" and "out of office" blood pressure (BP) is accompanied by an increase of metabolic variables, included blood glucose. Aim of the present study is to investigate the long term risk of white coat (WCH), masked $(\mathrm{MH})$ and sustained hypertension (SH) of developing impaired fasting glucose (IFG) and diabetes mellitus (DM).

Materials and methods: In a sample of 1400 subjects of the PAMELA Study, randomly selected from the general population of Monza (Milan, Italy), strat- ified for gender and decades of age (25 to 74 years), we measured 1) office BP (mercury sphygmomanometric technique), 2) 24 hour BP mean by ambulatory blood pressure (Spacelabs 90207, automatic oscillometric measurements every 20 min during the 24-hours), 3) plasma glucose (radioenzymatic method). The condition of WCH was identified when "office" BP was $\geq 140$ $\mathrm{mmHg}$ systolic or $90 \mathrm{mmHg}$ diastolic and 24 hour BP mean was $<125 \mathrm{mmHg}$ systolic or $79 \mathrm{mmHg}$ diastolic. MH was identified when "office" BP was $<140$ $\mathrm{mmHg}$ systolic or $90 \mathrm{mmHg}$ diastolic and 24 hour BP mean was $\geq 125 \mathrm{mmHg}$ systolic or $79 \mathrm{mmHg}$ diastolic. SH was identified when both "office" and 24 hour BP mean were over the threshold values, derived from previous analysis of the BP distribution curves in the general PAMELA population. The subjects were re-analyzed 10 years later, to identify the new cases of IFG and DM, defined when fasting blood glucose was $\geq 110 \mathrm{mg} / \mathrm{dL}$ and $\geq 126 \mathrm{mg} / \mathrm{dl}$ (or use of antidiabetic drugs) respectively, being $<110 \mathrm{mg} / \mathrm{dL}$ and $<126 \mathrm{mg} / \mathrm{dL}$ respectively at the first examination.

Results: WCH, MH and SH were identified respectively in 225 (16.1\%), 124 $(8.9 \%)$ and $293(20.9 \%)$ subjects of the original sample. At the second examination we found 101 and 53 new cases of respectively IFG and DM. As compared to subjects with both "office" and 24 hour mean BP normal (normotensives), $\mathrm{WCH}, \mathrm{MH}$ and $\mathrm{SH}$ have higher age and gender-adjusted risk of developing a new IFG (HR 3.715, 2.852 and 2.96 respectively, $\mathrm{p}<0.005$ ). Statistical significance remains also after a further adjustment for antihypertensive treatment and baseline glycemia. The age and gender adjusted risk of developing a new DM was also significantly higher in $\mathrm{WCH}, \mathrm{MH}$ and $\mathrm{SH}$ as compared to normotensives (HR 2.876, 2.71, 2.228 respectively, $\mathrm{p}<0.05$ ). Statistical significance disappeared after further adjustment for antihypertensive treatment and baseline glycemia. Independent contributors in the development of DM were: antihypertensive treatment, baseline blood glucose, cholesterol, tryglicerides and body mass index.

Conclusion: The abnormality of either "office" or 24 hour mean BP is associated with an enhanced risk of developing IFG and DM. This may contribute to the increased cardiovascular hazard characterizing not only SH but also $\mathrm{WCH}$ and $\mathrm{MH}$.

\section{8}

Cardiovascular risk is associated with night-time rather than daytime blood pressure in normoalbuminuric type 2 diabetic patients H. Haller ${ }^{1}$, A. Januszewicz' ${ }^{2}$, S. Ito ${ }^{3}$, J.L. Izzo Jr ${ }^{4}$, S. Katayama ${ }^{5}$, A. Mimran ${ }^{6}$, A.J. Rabelink ${ }^{7}$, E. Ritz ${ }^{8}$, L.M. Ruilope ${ }^{9}$, L.C. Rump ${ }^{10}$, G.C. Viberti ${ }^{11}$ ${ }^{1}$ Abteilung Nephrologie, Medizinische Hochschule Hannover, Germany, ${ }^{2}$ Department of Hypertension, Institute of Cardiology, Warsaw, Poland, ${ }^{3}$ Department of Clinical Medicine, Division of Nephrology, Endocrinology, and Vascular Medicine, Tohoku University, Sendai, Japan, ${ }^{4}$ Department of Medicine, Erie County Medical Center, Buffalo, United States, ${ }^{5}$ The Fourth Department of Medicine, Saitama Medical School, Japan, ${ }^{6}$ Medicine Interne, Hospital Lapeyronie, Montpellier, France, ${ }^{7}$ Department of Nephrology and Hypertension, Leiden University, Netherlands, ${ }^{8}$ Department of Nephrology, University of Heidelberg, Germany, ${ }^{9}$ Division of Hypertension, Hospital 12 de Octubre, Madrid, Spain, ${ }^{10}$ Medical Clinic, Marienhospital Herne, University Clinic Ruhr-University-Bochum, Germany, ${ }^{11}$ Division of Medicine, KCL Guy's Hospital, London, United Kingdom

Background and aims: Whether night-time blood pressure (BP) values outperform daytime BP values in cardiovascular risk prediction is controversial.

Materials and methods: We monitored 24hr ambulatory BP (ABPM) in 1137 (46\%M) Type 2 diabetic patients (T2DM), a random subgroup of the 4449 T2DM with normoalbuminuria and at least one additional cardiovascular risk factor enrolled in the Randomised Olmesartan And Diabetes MicroAlbuminuria Prevention (ROADMAP) study. Mean 24 hour, daytime and nighttime systolic BP (SBP) and diastolic BP (DBP) and the night-to-day BP ratios were evaluated in relation to cardiovascular risk factors at baseline. Results: Mean (SD) age in this population was 57.9 (8.9) yrs, duration of diabetes 6.0 (6.0) yrs and 93\% of patients had office $\mathrm{BP} \geq 130 / 80 \mathrm{mmHg}$ or were receiving anti-hypertensive treatment. Both mean daytime and nighttime SBP and DBP levels correlated significantly ( $<<0.01$ for all) with history of cardiovascular disease; presence of metabolic syndrome; albumin to creatinine ratio (ACR); fasting plasma glucose (PG) concentration. Moreover daytime and night-time SBP also correlated significantly ( $\mathrm{p}<0.01$ for all) with $10 \mathrm{yr}$ risk of $\mathrm{CHD}$, duration of diabetes, and $\mathrm{HbAlc}$ levels. After adjusting daytime for night-time BP values, daytime SBP correlated significantly only with presence of metabolic syndrome $\left(\mathrm{r}_{\mathrm{s} \text {, artial }}=0.07, \mathrm{p}<0.01\right.$ by mean of partial Spearman rank correlation) and $\mathrm{PG}$ concentrations $\left(\mathrm{r}_{\mathrm{s} \text {,partial }}=0.06\right.$, 
$\mathrm{p}<0.01)$. By contrast after adjusting night-time for daytime BP values, nighttime SBP was significantly associated with $10 \mathrm{yr}$ risk of $\mathrm{CHD}\left(\mathrm{r}_{\mathrm{spartial}}=0.10\right.$, $\mathrm{p}<0.001)$, history of cardiovascular disease $\left(\mathrm{r}_{\text {s.partial }}=0.11, \mathrm{p}<0.0001\right), \mathrm{ACR}$ $\left(\mathrm{r}_{\mathrm{s}, \text { partial }}=0.13, \mathrm{p}<0.0001\right)$, presence of metabolic syndrome $\left(\mathrm{r}_{\mathrm{s} \text {,partial }}=0.09\right.$, $\mathrm{p}<0.01)$ and duration of diabetes $\left(\mathrm{r}_{\text {s.partial }}=0.07, \mathrm{p}<0.01\right)$.

Conclusion: In this group of T2DM with normoalbuminuria night-time BP adjusted for daytime values correlated significantly with a greater number of cardiovascular risk factors than daytime BP adjusted for night-time values. These results suggest that night-time BP may be a better predictor of cardiovascular risk than daytime $\mathrm{BP}$ and require confirmation from prospective studies.

Supported by: Daiichi-Sankyo

\section{OP 09 Prediction of diabetes}

\section{9}

Perinatal factors and immunological markers at birth are associated to type 1 diabetes risk - a population-based study

S. Eising ${ }^{1,2}$, B. Carstensen ${ }^{1}$, K. Skogstrand ${ }^{2,3}$, D.M. Hougaard ${ }^{2}$,

B. Norgaard Pedersen ${ }^{2}$ J. Nerup ${ }^{1,4}$, F. Pociot ${ }^{1,4}$

${ }^{1}$ Steno Diabetes Center, Gentofte, Denmark, ${ }^{2}$ Department of Clinical

Biochemistry, Statens Serum Institut, Copenhagen, Denmark, ${ }^{3}$ Department of Epidemiology and Social Medicine, NANEA, Aarhus, Denmark,

${ }^{4}$ Department of Clinical Sciences, Lund University/ CRC, Malmø, Sweden

Background: Reliable prediction is a prerequisite for developing and testing prevention strategies for type 1 diabetes (T1D). Epidemiological studies found perinatal factors (birth weight BW, maternal age) being associated to increased T1D risk. These findings could not be confirmed in previous Danish data.

Aims: To test the impact of perinatal and other demographic data on T1D risk in a large population-based case-control study, and to test for interaction with immunological markers.

Materials and methods: 2265 T1D cases from the birth cohort 1981 - 2002 diagnosed before 2004/05/01 were identified via National Patient Registry. Cases were validated against independent registry and hospital records. Cases and two controls per case (together $\mathrm{n}=6795$ ) were matched by place and date of birth. Birth data, gender, parental age at birth, and information on parental diabetes, for cases and controls were collected from Danish registers (Patient Registry, Birth Register). Measurements of cytokines and MBL, CrP, TREM-1, adiponectin, leptin were available from 6143 samples, measured by use of flowmetric Luminex xMAP technology. We used conditional logistic regression for matched sets to analyse the case-control outcome (SAS 9.1 proc phreg).

Results: Maternal diabetes (HR 5.00, $\mathrm{p}<0.0001,95$ per cent CI 3.02-8.28), paternal diabetes (HR 6.96, p<0.0001, CI 4.76-10.19), BW (HR 1.11 per kg, $\mathrm{p}<0.0174$, CI 1.02-1.20), birth length (HR 1.02 per $\mathrm{cm}, \mathrm{p}<0.05$, CI 1.00-1.04), and maternal age at delivery (HR 1.13 per 10 years, $\mathrm{p}<0.036$, CI 1.01-1.26), were significantly associated to an increased T1D risk. In the randomly selected subsets from cytokine measurements, only parental diabetes (HR mother 5.13, CI 3.231-8.128; father 6.40, CI 4.49-9.11, $\mathrm{p}<0.0001$ ) and maternal age (HR 1.15 per 10 years; $\mathrm{p}<0.0278$; CI 1.02-1.31) remained significant. Maternal age under 25 years was associated to an increased T1D risk (HR $1.34, \mathrm{p}<0.0001$, CI 1.18-1.51), while paternal age under 25 years was associated to a reduced T1D risk (HR 0.76, $\mathrm{p}<0.0024$, CI 0.63-0.91). By classification of the dataset into two groups by age at T1D onset (below and above 10 years of age), a significant association of BW (HR 1.31 per kg, $\mathrm{p}<0.0162$, $1.05-1.62)$ to T1D risk was only detected in the young onset group. In small newborns, every 10 fold increase in $\mathrm{CrP}(\mathrm{ug} / \mathrm{ml})$ and in adiponectin $(\mathrm{ug} / \mathrm{ml})$ were associated to a decreased T1D risk (HR 0.72, $\mathrm{p}<0.0052$, CI $0.57-0.91$ and HR 0.54, $\mathrm{p}<0.0160$, CI 0.33-0.89, respectively). A similar effect was seen for $\mathrm{CrP}$ (HR 0.62, p $<0.0005$, CI 0.48-0.81) in premature babies. Every 10 fold increase in IL-10 (pg/ml) was in small babies associated to an increased T1D risk (HR 1.37, $\mathrm{p}<0.0031, \mathrm{CI} 1.11-1.69$ ), corresponding to IL-10 effects in the whole dataset as previously described by our group.

Conclusion: We confirm for the Danish population that perinatal factors like BW and maternal age are associated to T1D risk. Effects are small and can only be detected in large datasets, or in subsets such as children with T1D onset below the age of 10 . Significant interactions were detected for CrP, adiponectin, and IL-10 with low birth weight and for $\mathrm{CrP}$ with gestational age. Supported by: Danish Medical Research Council, Danish Diabetes Association, P.Carl Petersen Foundation, EFSD/Pfizer grant, E. and A.Danielsens Foundation, Sehested Hansen Foundation, Danish Medical Research Foundation, Danish Study Group for Childhood Diabetes

\section{0}

Immunological changes in subjects positive for diabetes-associated autoantibodies at preclinical and clinical stages of type 1 diabetes mellitus

V. Popova, K. Zak, B. Mankovsky

Diabetes, Institute of Endocrinology, Kiev, Ukraine

Background and aims: The presence of diabetes-associated autoantibodies (DAAb) provides the opportunity to predict type 1 diabetes mellitus in 
healthy persons. However, immunological changes occurring during the progression of disease from preclinical to clinical stages are not fully understood. Therefore, the aim of this study was to monitor the immunological changes in subjects positive for DAAb at preclinical and clinical stages of type 1 diabetes mellitus compared to subjects negative for DAAb.

Materials and methods: We prospectively studied 294 children aged $12.34 \pm 0.82$ years for the period over 10 years. All of those subjects were normoglycemic at the baseline. There was the family history of type 1 diabetes mellitus in the first degree relatives in 226 children and 70 children without such a history were examined as the control group. The measurements of $\mathrm{DAAb}$ titers (IAA, GADA and IA-2A) were carried out by radioimmunoassay at least twice at baseline and then in the course of progression from preclinical to clinical stages of type 1 diabetes mellitus. We performed the repeated measurements of leucocytic composition, immunophenotype of lymphocytes (CD3+, CD4+, CD8+, CD20+ and CD56+ cells) by FACS-analysis. The concentration of circulating cytokines (IL-1 $\alpha, \beta$, IL-4, IL-6, IL-8, IL-16, FNO $\alpha$ and IFN $\gamma$ ) was measured by immunoenzymic method (ELISA).

Results: An elevated titer of autoantibodies to GADA and IA-2A was found in 94 children (42\%). The overt type 1 diabetes mellitus was diagnosed in 47 children positive for DAAb at the different terms of follow-up after primary examination (from 6 months until 9 years - the mean time was 30,9 $\pm 3,2$ months since the first examination). In the group of children negative for $\mathrm{DAAb}$ only one child developed diabetes. We found the significant decrease of absolute numbers of CD3+, CD4+ and CD56+ cells and IL-4 concentration along with elevated levels of IL-6, IL-8, FNO $\alpha$ and IFN- $\gamma$ in the group of children positive for DAAb at preclinical stage of diabetes compared to DAAb negative ones. The number of CD $3+$ cells was $0,79 \pm 0,05 \cdot 10^{9} / 1,1,02 \pm 0,06 \cdot 10^{9} / 1$, $1,39 \pm 0,09 \cdot 10^{9} / 1$; CD $4+$ cells - $0,52 \pm 0,03 \cdot 10^{9} / 1,0,67 \pm 0,04 \cdot 10^{9} / 1,0,98 \pm 0,04 \cdot 10^{9} / 1$, and CD56+ cells - 0,18 $\pm 0,02 \cdot 10^{9} / 1,0,24 \pm 0,03 \cdot 10 / 1,0,29 \pm 0,02 \cdot 10^{9} / 1$, in those children positive and negative for DAAb and control group, respectively, $\mathrm{p}<0.05$ for all comparisons between children positive for DAAb and those negative for DAAb and controls. These changes of immunological indices between those groups became less pronounced after the onset of the clinically overt diabetes. It was an association between immunological changes and the progression to clinical stage of diabetes - the more decreased the number of $\mathrm{CD} 3+, \mathrm{CD} 4+\mathrm{CD} 56+$ cells and plasma IL-4 levels the faster the progression of type 1 diabetes was registered.

Conclusion: The measurement of the numbers of CD3+, CD4+ and CD56+ cells and IL-4 plasma concentration could be used as the test to predict the progression of type 1 diabetes from subclinical to clinical stages in children positive for DAAb.

\section{1}

Dysregulation of lipid and amino acid metabolism precedes islet autoimmunity in children who later progress to type 1 diabetes M. Oresic ${ }^{1}$, S. Simell ${ }^{2}$, M. Sysi-Aho ${ }^{1}$, K. Nantö-Salonen ${ }^{2}$, T. SeppänenLaakso $^{1}$, V. Parikka ${ }^{2}$, A. Hekkala ${ }^{3}$, I. Mattila ${ }^{1}$, M. Katajamaa ${ }^{1}$, P. Keskinen ${ }^{4}$, T. Simell ${ }^{2}$, R. Veijola ${ }^{3}$, J. Ilonen ${ }^{5}$, M. Knip ${ }^{6}$, O. Simell ${ }^{2}$

${ }^{1}$ VTT Technical Research Centre of Finland, Espoo, ${ }^{2}$ Department of Pediatrics, University of Turku, ${ }^{3}$ Department of Pediatrics, University of Oulu, ${ }^{4}$ Department of Pediatrics, University of Tampere, ${ }^{5}$ Department of Clinical Microbiology, University of Kuopio, ${ }^{6} \mathrm{Hospital}$ for Children and Adolescents, University of Helsinki, Finland

Background and aims: To explore etiopathogenesis and prevention possibilities of type 1 diabetes (T1D), over 8000 children carrying HLA-conferred genetic risk for T1D have been followed since birth at 3- to 12-month intervals for development of T1D-related autoantibodies (ICA, IAA, GADA and IA-2A) and overt T1D in Type 1 Diabetes Prediction and Prevention Study (DIPP), launched in Finland in 1994. As changes in the concentrations of metabolites reflect responses in biological systems to the interaction between genes and the environment, we hypothesized that extended serum metabolite patterns might differ between children remaining autoantibody negative (nonprogressors) and children who later develop autoantibodies and progress to T1D (progressors), and that the changes might reflect etiopathogenetic events that precede development of autoimmunity.

Materials and methods: Serum metabolite profiles were compared between 56 children who progressed to T1D and 73 nonprogressors, who remained permanently healthy and autoantibody negative and were matched with the progressors for time and site of birth, gender, and HLA risk group. The 129 children contributed 1196 samples. Additionally, the cord blood samples of 39 children, 15 of whom progressed to diabetes before the age of 12 years were investigated. Lipidome was analyzed using ultra performance liquid chromatography coupled to mass spectrometry (UPLC/MS). 53 lipids were measured in each of the 1196 samples, and 249 lipids were identified in each of the 39 cord blood samples. Serum samples were also analyzed by two dimensional gas chromatography coupled to time of flight mass spectrometry (GCxGC-TOF/MS). The metabolomic analysis was performed on a subset of DIPP study samples from 13 progressors and 26 matched nonprogressors. The final metabolomics dataset consisted of 75 identified metabolites measured in each of the 419 samples.

Results: The individuals who developed disease have reduced serum levels of succinate $(\mathrm{P}=0.042)$, citrate $(\mathrm{P}=0.018)$ and phosphatidylcholine $(\mathrm{P}=0.0041)$ at birth, significantly reduced levels of multiple antioxidant ether phospholipids throughout follow-up and increased levels of proinflammatory lysophosphatidylcholines several months prior to seroconversion to autoantibody positivity $(\mathrm{P}=0.034)$. Ketoleucine was decreased 20 -fold within the period of 9-18 months prior to seroconversion to IAA $(\mathrm{P}=0.0093)$ and 2.5 -fold within the succeeding 9-month period $(\mathrm{P}=0.015)$. Elevation of glutamate was associated with the subsequent appearance of autoantibodies to glutamic acid decarboxylase (GADA) and to IAA. The metabolic profile was partially normalized following the seroconversion.

Conclusion: Dysregulation of metabolism precedes $\beta$ cell autoimmunity and overt T1D. Our findings imply that metabolic interventions during the preautoimmune period may be used as a potential strategy for T1D prevention. Supported by: JDRF, Tekes FinnWell Program, Academy of Finland, Sigrid Juselius Foundation, Päivikki and Sakari Sohlberg Foundation, Signe and Ane Gyllenberg Foundation, Diabetes Research Foundation, Finland, and a Specified Government Transfer to Turku University Hospital

\section{2}

Low levels of insulin-like growth factor binding protein-1 is associated with development of type 2 diabetes: a prospective observational study U. Petersson ${ }^{1}$, C. Östgren ${ }^{1}$, L. Brudin ${ }^{1}$, K. Brismar ${ }^{2}$, P.M. Nilsson ${ }^{3}$

${ }^{1}$ Medical and Health Sciences, Department of Medical and Health Sciences, Linköping University, Sweden, ${ }^{2}$ Department of Molecular Medicine and Surgery, Department of Medical and Health Sciences, Linköping University, Solna, Sweden, ${ }^{3}$ Department of Clinical Sciences Medicine, Lund University Hospital, Malmö, Sweden

Background and aims: To explore the association between IGFBP-1 at baseline and the development of impaired glucose tolerance (IGT), and type 2 diabetes in a defined middle-aged population.

Materials and methods: A cross-sectional population-based screening study was conducted in 1989-1990 including baseline data on 664 non-diabetic subjects aged 40-59. Anthropometric data were collected and blood samples were analyzed for blood glucose, serum lipids and serum insulin. Blood specimens were frozen at baseline and analyzed for IGF-I, IGFBP-1 and C-reactive protein (CRP). At the follow-up in 2006, through primary care medical records, the incidence of type 2 diabetes and IGT, was registered.

Results: During the 17-year observation period 42 subjects (6.3\%) developed type 2 diabetes/IGT. Subjects in the lowest quintile of IGFBP-1 $(<24 \mu \mathrm{g} / \mathrm{l})$ at baseline had an incidence of diabetes of $12.1 \%$, while in the highest quintile of IGFBP-1 $(\geq 59 \mu \mathrm{g} / \mathrm{l})$ the incidence was $1.4 \%$. Cox proportional hazard model regression analyses were used for incidence of type 2 diabetes, corrected for age and gender with regard to IGFBP-1, CRP and waist circumference. Subjects in the lowest IGFBP-1 quintile showed an independent increased risk of developing type 2 diabetes or IGT, hazard ratio (HR) 3.09; CI 95\% (1.099.10, $\mathrm{p}<0.041)$. For CRP and waist circumference the corresponding figures were HR 6.39; CI 95\% (2.41-17.0, $\mathrm{p}<0.001)$ and HR 3.37; CI 95\% (1.48-7.7, $\mathrm{p}<0.001)$, respectively.

Conclusion: Low levels of IGFBP-1 predicted the development of type 2 diabetes or IGT in a defined middle-aged population. The association was independent of CRP and abdominal obesity. 


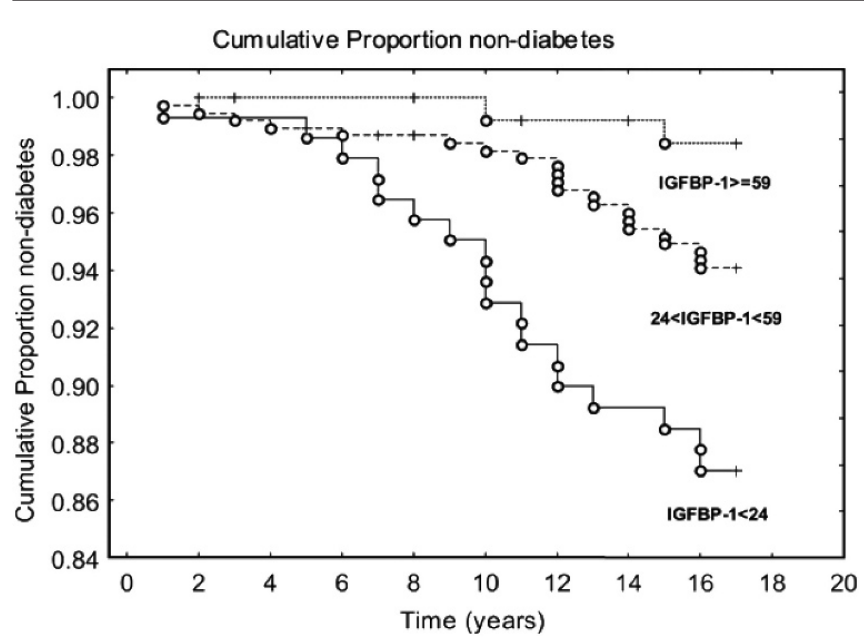

Supported by: Kalmar County Council and the Medical Research Council of South eastern Sweden

\section{3}

Interactions between genes and birth size for development of type 2 diabetes

N. Pulizzi ${ }^{1}$, V. Lyssenko ${ }^{2}$, A. Jonsson ${ }^{2}$, C. Osmond ${ }^{2}$, E. Kajantie², D. Barker², L.C. Groop ${ }^{2}$ J.G. Eriksson ${ }^{2}$

${ }^{1}$ Clinical sciences-Diabetes and endocrinology, Lund Universitet, Malmö, Sweden, ${ }^{2}$ Clinical sciences-Diabetes and endocrinology, Lund Universitet, Malmö, Sweden

Background and aims: A small body size at birth is a well-recognized risk factor for type 2 diabetes (T2D). Previous studies have shown that there is an interaction between early environmental factors and genes in the development of T2D. Recently several whole genome-wide association studies for T2D have identified a number of novel loci (TCF7L2, IGF2BP2, HHEX, CDKN2A/2B, CDKAL1 and JAZF1) increasing susceptibility to the disease. Our study aimed to investigate the potential influence of these genetic variants on birth weight and the possible interaction between genes and birth size on development of T2D.

Materials and methods: 2003 subjects, (M/F: 928/1075), 56-70 yrs old, from the Helsinki Birth Cohort Study (HBCS), were genotyped by Taq Man allelic discrimination assay, and common variants detected by ABI PRISM 7900. Logistic regression analysis was used to test the effect of genetic variants on risk of T2D adjusted for age, gender and BMI.

Results: 311 (15.6\%) subjects had developed T2D. Subjects with low birth weight had an increased risk of developing T2D (OR 1.43 [1.11-2.0], p=0.008). Only the HHEX gene influenced birth weight (AA: $3495 \pm 452$ g; AG: 3426 \pm 460 g; GG: $3422 \pm 466 \mathrm{~g}, \mathrm{p}<0.05)$. Common variants in the TCF7L2, HHEX and JAZF1 genes predicted T2D in subjects born in the lowest tertile of birth weight (OR 1.71 [1.2-2.45], $\mathrm{p}=0.003 ; 2.22$ [1.17-4.18], $\mathrm{p}=0.014) ; 1.64$ [1.0072.67], $\mathrm{p}=0.04$, respectively), whereas variants in the IGF2BP2 gene predicted independently of birth weight future T2D (OR 1.71 [1.15-2.56], $\mathrm{p}=0.008$ ). High-risk genotype ( $>7$ alleles) carriers, whose insulin secretion was reduced compared to the low-risk ( $<4$ alleles) (corrected insulin response, $3.54 \pm 1.2$ vs. $3.76 \pm 1.07, \mathrm{p}=0.019$; disposition index, $1.36 \pm 1.22$ vs. $1.57 \pm 1.1, \mathrm{p}=0.02$ ), had also a 2 -fold increase of T2D risk (1.98 [1.33-2.92], $\mathrm{p}=0.0006)$, and this risk was highest in individuals from the lowest tertile of birth weight (OR 3.87 [1.96-7.65], $\mathrm{p}=0.00009)$ demonstrating a strong interaction between genetic variants and birth weight for prediction of future T2D $\left(\mathrm{p}_{\text {inter }}=0.0002\right)$. Neither insulin secretion nor insulin resistance were clearly shown to influence this interaction in our cohort

Conclusion: Common variants in T2D susceptibility genes interact with birth weight to increase future risk of T2D; this interaction cannot be fully explained by their effects on insulin secretion and action.

Supported by: EFSD Clinical Research Grant

\section{4}

Development of multimarker algorithms for the prediction of type 2 diabetes from a $5 \mathrm{yr}$ prospective study of 6,784 Danish people (Inter99) J.A. Kolberg' ${ }^{1}$, R. Gerwien ${ }^{1}$, E.J. Moler ${ }^{1}$, M.W. Rowe ${ }^{1}$, M. Urdea ${ }^{1}$, M.P. McKenna $^{1}$, T. Hansen ${ }^{2}$, O. Pedersen ${ }^{2}$, K. Borch-Johnsen ${ }^{2}$, T. Jorgensen ${ }^{3}$ ${ }^{1}$ Tethys Bioscience, Emeryville, United States, ${ }^{2}$ Steno Diabetes Center, Copenhagen, Denmark, ${ }^{3}$ Research Centre for Prevention and Health, Glostrup University Hospital, Copenhagen, Denmark

Background and aims: Improved identification of subjects at high risk for development of Type 2 diabetes (T2D) would allow more aggressive preventive interventions to be targeted towards those individuals who would benefit most. The aim of the study was to develop a multivariate serum biomarker algorithm to predict 5 year risk of T2D. Subjects were selected from the Inter99 cohort, a population-based primary prevention study of cardiovascular disease and T2D in middle-aged Danes.

Materials and methods: Among subjects with BMI $\geq 25 \mathrm{~kg} / \mathrm{m}^{2}$ and age $\geq$ $39 \mathrm{yrs}$ at baseline, all 160 individuals who progressed to T2D (converters: age $51 \pm 6 \mathrm{yrs}$; BMI $31 \pm 4 \mathrm{~kg} / \mathrm{m}^{2}$ ) during 5 yrs were compared with $472 \mathrm{ran}$ domly selected non-converters (age $48 . \pm 6$ yrs; BMI $29 \pm 4 \mathrm{~kg} / \mathrm{m}^{2}$ ). 92 protein biomarkers were measured in each subject's baseline fasting serum sample using an ultrasensitive Molecular Counting Technology (MCT). MCT protein assays had dynamic ranges of $10^{2}$ to $10^{3}$, intra-plate CVs of $\leq 5 \%$, and sensitivities ranging from sub-pg/mL to $\mathrm{ng} / \mathrm{mL}$ using $\sim 1 \mu \mathrm{l}$ of serum per assay. Additional laboratory measures included fasting plasma glucose, serum insulin, cholesterol (total, HDL, LDL) and triglycerides.

Results: Data from one half of the 632 subjects were used for marker selection and development of the algorithms (training set). Univariate and multivariate statistical analyses were conducted employing protein biomarker concentrations and clinical annotations. Markers were prioritized on the basis of performance in univariate and small $(\leq 6)$ multivariate logistic models, and on frequency of selection under bootstrap resampling using heuristic model building methods (e.g., forward selection). The bootstrap estimates of Area Under the Receiver Operator Characteristic Curve (AUC) for nested 9-, 7and 5 -marker models using the best predictors were $\sim 0.76$ for 5 year risk of developing diabetes. When the same algorithms were applied to the second half of the data (testing set) to validate the models, similar performance $($ AUC $=0.75)$ was achieved. An algorithm developed using all 632 subjects and utilizing $<15$ serum biomarkers had an AUC of 0.78 . The performance of this algorithm was significantly better than fasting plasma glucose, $\mathrm{HbAlc}$, fasting insulin, BMI and gender-adjusted waist and was similar in performance to $2 \mathrm{hr}$ glucose.

Conclusion: These results suggest that algorithms using panels of serum biomarkers can predict the risk of developing type 2 diabetes in a general population and provide additional information beyond current clinical methods. 


\section{OP 10 High glucose, oxidative stress and cellular signalling}

\section{5}

The relation of diabetes-associated membrane fusion processes to impaired brain function: effect of gabapentin treatment

T. Kuchmerovska ${ }^{1}$, V. Gumenyuk ${ }^{2}$, A. Klimenko', I. Trikash ${ }^{2}$

${ }^{1}$ Coenzymes, A.V. Palladin Institute of Biochemistry, ${ }^{2}$ Neurochemistry, A.V. Palladin Institute of Biochemistry, Kiev, Ukraine

Background and aims: Alterations in neurotransmission may directly or indirectly contribute to diabetes-associated pathophysiology. Regulated exocytosis forms the basis for many intracellular signaling processes e.g. neurotransmitter release. $\mathrm{Ca}^{2+}$-triggered fusion of synaptic vesicles (SVs) with the presynaptic plasma membranes (PM) is regarded as a final step of exocytosis. Calcium-dependent SVs fusion's disturbances may be a pathophysiological mechanism in diabetes. It is known that effect of gabapentin (GP) can be realized via regulation of GABA and others neurotransmission. The study was aimed to investigate the diabetes-induced alterations and GP effect in the $\mathrm{Ca}^{2+}$-triggered fusion of SVs with target membranes.

Materials and methods: STZ-induced $(60 \mathrm{mg} / \mathrm{kg}$ of body weight, i.p.) diabetic rats were treated with GP $(50 \mathrm{mg} / \mathrm{kg}$, i.p.) for 1 mo following 4 wks of untreated diabetes. SVs and PM were isolated from rat brain synaptosomes. SVs size was monitored by dynamic light scattering using the Zetasizer Nano system (Malvern 4700, UK). Fusion studies were performed in cell-free model system using fluorescent dye octadecylrhodamine B (R18) incorporated into SVs membranes at self-quenching concentration. The fusion of SVs, containing marker R18, with target membranes was detected by dequenching of the probe fluorescence. The $\mathrm{Ca}^{2+}$-dependent SVs fusion was carried out on heterotypic and homotypic membrane systems in synaptosomal cytosolic proteins media. PM were overloaded with cholesterol by methyl- $\beta$-cyclodextrin (MCD).

Results: We identified that suspension of SVs from control and diabetic brains were abounded in the fractions of the particles with the average size about 50 and $80 \mathrm{~nm}$ respectively. It was found that in diabetic rats the rate of SVs fusion with PM (heterotypic) in the presence of $\mathrm{Ca}^{2+}$ and synaptosomal cytosolic proteins was decreased as it is evident from lowering of fluorescence from $23 \%$ in control to $14.5 \%$ in diabetes, $\mathrm{p}<0.05$. Diabetes-induced reduction in fusion is likely to be associated with alteration of PM cholesterol that can play a critical role in this process. To verify this idea we overloaded the PM by cholesterol using MCD:cholesterol mixture. PM cholesterol level in control was $0.67 \pm 0.06 \mu \mathrm{M} / \mathrm{mg}$ of protein as compared with $0.75 \pm 0.07$ in diabetes $(\mathrm{p}<0.05)$. After overloading PM by cholesterol its level in control was $0.88 \pm 0.07 \mu \mathrm{M} / \mathrm{mg}$ of protein, $\mathrm{p}<0.05$. The fusion ability of cholesterolsaturated PM as well as PM of diabetic rats was reduced to the same extent. The opposite effect has been shown in the rate of SVs fusion with each other (homotypic): in diabetes the fluorescence signal was achieved up to $30 \%$ vs $25 \%$ in contol, $\mathrm{p}<0.05$. Following GP treatment the rate of SVs fusion with target membranes was partially normalized.

Conclusion: The findings suggest that diabetes may cause failures in realization of the last step of exocytosis - membrane fusion. These alterations may be associated with increased cholesterol content of synaptosomal PM that modifies the structure and function of membrane-bounded proteins and affects membrane fluidity and fusibility. Our data imply that GP is involved in the exocytosis more likely acting on proteins that provide SVs fusion. Thus, presynaptic fusion machinery may be considered as a viable therapeutic target for development of new drugs for the treatment of diabetes-related CNS abnormalities.

\section{6}

Association of impaired brain protein ADP-ribosylation with mitochondrial dysfunction in diabetes: evidence for the beneficial role of acetyl-L-carnitine supplementation

I. Shymanskyy, O. Zolochevska, G. Donchenko, A. Klimenko,

T. Kuchmerovska

Coenzymes, O.V. Palladin Institute of Biochemistry, Kiev, Ukraine

Background and aims: An excessive and sustained increase in reactive oxygen species (ROS) formation and oxidative stress have been implicated in the pathogenesis of diabetic complications. ROS-mediated enhancement of
ADP-ribosylation of nuclear and extra-nuclear proteins has been also shown to underlie several neuropathological aspects of diabetes mellitus. Since mitochondrial dysfunction might be associated with enhanced generation of free radicals, the present study was designed to investigate whether mitochondrial ROS overproduction is involved in the activation of protein mono- and polyADP-ribosylation in diabetic brain. To this end, the effects of acetyl-L-carnitine (ALC), mitochondria-specific neuroprotective nutrient, were assessed. Materials and methods: All studies were carried out after 4 weeks of STZinduced diabetes $(60 \mathrm{mg} / \mathrm{kg}$ of body weight, i.p.) in rats treated for 14 days with or without ALC $(150 \mathrm{mg} / \mathrm{kg}$, i.p.). The mitochondrial membrane potential $\left({ }_{\Delta} \psi_{\mathrm{m}}\right)$ was assayed spectrofluorimetrically with rhodamine123 (Rh123). ADP-ribosylation was measured by incorporation of labelled ADP-ribose from $\left[\mathrm{U}-{ }^{14} \mathrm{C}\right] \mathrm{NAD}^{+}$to proteins. Mitochondrial ROS production was analysed using 2', ',-dichlorodihydrofluorescein diacetate which is converted to the fluorescent 2',7'-dichlorofluorescein (DCF) upon oxidation.

Results: The major diabetes-related changes that occur to brain mitochondria comprise decreased ${ }_{\Delta} \psi_{\mathrm{m}}(21.7 \pm 0.19 \%$ elevation of Rh123 fluorescence, $\mathrm{p}<0.01$ ) with concomitant $25.4 \pm 0.21 \%$ decline in brain ATP content without pronounced alterations in the total sum of ATP and ADP as compared to control $(\mathrm{p}<0.05)$. Brain mitochondria isolated from diabetic rats generated more reactive oxygen species as DCF fluorescence level was approximately $30 \%$ higher than in control. Administration of ALC to diabetic rats diminished the development of cellular respiration defects strongly attenuating the loss of $\psi_{\mathrm{m}}$ with corresponding restoration of ATP content. It also reversed the diabetes-associated DCF-sensitive mitochondrial ROS production (by 23\%), thus indicating that the efficiency of mitochondrial electron transport had improved after the treatment. The ability of ALC to counteract energy imbalances and ROS generation in mitochondria may account for the decrease in elevated protein ADP-ribosylation found in diabetes $(56.4 \pm 3.9,98.3 \pm 7.2$, $240.3 \pm 20.4$ vs. $67.4 \pm 5.1,115.8 \pm 9.1,293.5 \pm 22.4$ and $48.3 \pm 3.2,89.0 \pm 7.0$, $234.8 \pm 19.8 \mathrm{pmol}\left[\mathrm{U}-{ }^{14} \mathrm{C}\right]$ ADP-ribose/mg of protein respectively in brain synaptosomes, mitochondria and nuclei of ALC-treated vs. diabetic and control rats, $\mathrm{p}<0.05)$. Probably due to increased consumption of $\mathrm{NAD}^{+}$in ADP-ribosylation of proteins, diabetes exhibited accelerated $\mathrm{NAD}^{+}$catabolism in brain (29.5\% reduction, $\mathrm{p}<0.05)$. ALC treatment of diabetic rats did not affect hyperglycaemia or brain polyol pathway activity as reflected by sorbitol levels but did increase $\mathrm{NAD}^{+}$levels.

Conclusion: Thus, mitochondria may be an important therapeutic target for ameliorating diabetes-related brain failures when energy depletion is accompanied by oxidative stress and ROS-induced activation of general ADPribosylation. Because ALC is unlikely to be a potent radical scavenger or a direct inhibitor of ADP-ribosylating enzymes, these data suggest that ALC protects against excessive ADP-ribosylation through mitochondria-mediated mechanisms.

\section{7}

The impact of oscillating glucose and the metabolic memory effect on oxidative stress in human endothelial cells

C.R. Radford, B. Schisano, P.G. McTernan, A. Ceriello

Diabetes and Metabolism, CSRI University of Warwick, Coventry, United Kingdom

Background and aims: The 'cellular memory' phenomenon, which occurs after hyperglycaemic stress, has been suggested as a mechanism for the long term complications of diabetic stress. Our previous studies have shown that human endothelial cells which have been exposed to high glucose, followed by a phase of normal glucose, maintain a cellular stress response referred to as the 'cellular memory' effect. Cellular stress can be mediated through activating transcriptional factors such as NRF2 which modulates anti-oxidant defenses in cells and has been shown to be activated by high glucose. NRF2 can also activate NQO1 which also has an anti-oxidant role. Therefore the aim of this study was to (1) determine in the effect of oscillating glucose on oxidative glucose stress compared with chronic high glucose in human endothelial cells (HUVECs) and (2) to identify whether the cells retain the cellular memory effect following oscillating glucose which is subsequently normalized. Materials and methods: HUVECs were cultured in either normal glucose (5 $\mathrm{mmol} / \mathrm{L})$, high glucose $(30 \mathrm{mmol} / \mathrm{L})$, oscillating glucose $(12 \mathrm{~h}$ high glucose, $12 \mathrm{~h}$ normal glucose) for $21 \mathrm{~d}$, or $14 \mathrm{~d}$ oscillating glucose ( $12 \mathrm{~h}$ high glucose, $12 \mathrm{~h}$ normal glucose) followed by $7 \mathrm{~d}$ normal glucose. Protein was extracted using a standard protocol and samples were analysed by Western blotting. Results: Exposure of endothelial cells to three weeks oscillating glucose levels resulted in considerable upregulation of both NRF2 and NQO1, as compared to exposure to either continuous normal or high glucose conditions. Further- 
more, there was a three fold elevation of these anti-oxidative proteins even after glucose levels had been normalised for one week following oscillating conditions $(\mathrm{p}>0.05)$. This suggests oscillating glucose conditions imparts a cellular memory, resulting in sustained elevation of these proteins. General markers of high glucose stress, such as Bax, were also found to be elevated to a higher degree in oscillating glucose compared to constant high glucose. TIGAR, a protein which protects against oxidative stress was elevated two fold in oscillating glucose compared to constant high glucose. This effect was maintained after normalisation, supporting the cellular memory phenomenon seen with anti-oxidative proteins.

Conclusion: In summary, exposure to oscillating glucose induces extensive levels of oxidative stress mediated through NRF2, NQO1, BAX and TIGAR compared with chronic, high glucose treated HUVEC cells; and this stress results in a cellular memory effect. Furthermore, in addition to continuous hyperglycaemia, type 2 diabetic individuals are often exposed to postprandial oscillations in glucose levels. Our current findings support the theory that daily oscillations in blood glucose levels may be a contributing factor for the long-term complications associated with type 2 diabetes.

\section{8}

High glucose acutely impairs the nitric oxide/ cyclic GMP/ PKG/ VASP pathway in arterial vascular smooth muscle cells: a putative mechanism of vascular damage induced by glycaemic spikes

I. Russo, M. Viretto, P. Del Mese, G. Doronzo, C. Giordanino, L. Mattiello, G. Anfossi, M. Trovati

Diabetes Unit, Department of Clinical and Biological Sciences, University of Turin, San Luigi Gonzaga Hospital, Orbassano - Torino, Italy

Background and aims: In type 2 diabetes, blood glucose excursions are considered a risk factor for cardiovascular events and are correlated with intima-media thickness. Oxidative stress has been claimed to play a role in the relationships between blood glucose excursions and vascular damage. Previous studies evaluated the effects of glucose spikes on vascular endothelium: less attention has been given to their influence on vascular smooth muscle cells (VSMC), which play a pivotal role in vascular tone and remodelling. VSMC are targets of nitric oxide (NO), which induces relaxation and inhibits proliferation. NO downstream molecules are cyclic GMP (cGMP), cGMPdependent protein kinase (PKG) and Vasodilator Stimulated Phosphoprotein (VASP), which is phosphorylated by PKG at Ser-239. Aim of this study is to evaluate in VSMC whether high glucose acutely affects the NO/cGMP/ PKG/VASP pathway, and whether oxidative stress is involved in this phenomenon.

Materials and methods: In rat aortic VSMC obtained as primary culture in our laboratory and pre-incubated for $120 \mathrm{~min}$ with 5, 15 and $25 \mathrm{mM}$ glucose, we measured by western blot the expression of total VASP and the extent of VASP phosphorylation at Ser-239 in response to a 60-min incubation with $100 \mu \mathrm{M}$ of the NO donor Sodium Nitroprusside (SNP) or with $500 \mu \mathrm{M}$ of the permeable cGMP analogue 8-Br-cGMP. Experiments $(n=6)$ have been repeated in the presence of a 30-min pre-incubation with the antioxidant mixture Superoxide Dismutase (SOD) $(300 \mathrm{U} / \mathrm{ml})$ and Catalase $(250 \mathrm{U} / \mathrm{ml})$ $(\mathrm{n}=6)$. Data are expressed as mean \pm SEM.

Results: High glucose did not modify total VASP expression, but significantly impaired VASP phosphorylation at Ser-239 in response to SNP and 8-BrcGMP. In particular, in the presence of glucose 5, 15 and $25 \mathrm{mM}$, VASP phosphorylation -expressed as fold increase vs baseline- was i)in response to SNP: $682.3 \pm 40.2,308.8 \pm 31.1$ and $184.1 \pm 24.3$, respectively (ANOVA, $\mathrm{p}=0.0001$ ); ii)in response to 8 -Br-cGMP: $978.5 \pm 38.8,325.5 \pm 33.3$ and $201.0 \pm 51.6$, respectively (ANOVA, $\mathrm{p}=0.0001$ ). Incubation with SOD/Catalase prevented the inhibiting effect exerted by high glucose on VASP phosphorylation in response to SNP and 8-Br-cGMP, supporting the role of oxidative stress: in particular, in VSMC incubated with SOD/Catalase, VASP phosphorylation in the presence of $15 \mathrm{mM}$ glucose (fold increase vs baseline) was $665.7 \pm 26.31$ in response to SNP and $824.0 \pm 31.01$ in response to 8 - $\mathrm{Br}-\mathrm{cGMP}$ ( $\mathrm{p}=0.0001$ for both vs values in the absence of SOD/Catalase).

Conclusion: A short-term VSMC exposure to high glucose impairs via oxidative stress the NO/cGMP/PKG/VASP pathway, as indicated by the reduced phosphorylation at Ser-239 of VASP, a protein deeply involved in vascular relaxation and in inhibition of VSMC proliferation. Since exposure to antioxidants prevents the impairment of VASP phosphorylation in response to both SNP and 8-Br-cGMP induced by high glucose, we can state that PKG activity is negatively affected by oxidative stress. In conclusion, our study provides a further link between rapid increases of glucose concentrations, oxidative stress and mechanisms involved in vascular damage, inducing to suppose that glucose spikes reduce not only $\mathrm{NO}$ availability by influencing endothelial function, but also NO action on arterial VSMC.

Supported by: Piedmont Region

\section{9}

Postmeal hyperglycaemia induced oxidative stress is associated with cardio-vascular dysfunction in patients with type 2 diabetes - assessed by tissue Doppler and Wave-Intensity

H. von Bibra ${ }^{1}$, A. Ceriello ${ }^{2}$, T. Siegmund ${ }^{1}$, P.-M. Schumm-Draeger ${ }^{1}$

${ }^{1}$ Endocrinology, Academic Hospital Bogenhausen, München, Germany, ${ }^{2}$ Diabetology, Warwick Medical School, Coventry, United Kingdom

Background and aims: Given the high prevalence and prognostic relevance of diastolic myocardial dysfunction in people with type 2 diabetes, we tested the hypothesis that postmeal hyperglycemia induced generation of oxidative stress is associated with cardio-vascular dysfunction by using tissue Doppler and the new Wave-Intensity technique.

Materials and methods: In 46 healthy controls (C) (mean age $60 \pm 10$ years) and 31 individuals with type 2 diabetes (D) (mean age $58 \pm 5$ years) without hypertension and overt cardiovascular disease, the effects of a carbohydrate breakfast (48 g) on oxidative stress and cardiac and vascular function were assessed in the fasting state and 2 hours after the meal. At both time-points, serum glucose, insulin and lipids were determined in addition to nitrotyrosine as marker of oxidative stress. Global left ventricular function was assessed by tissue Doppler as systolic (Vs) and diastolic (Ve) myocardial velocities averaged from 6 basal segments. Traditional parameters of carotid arterial stiffness and the early systolic peak (W1) of Wave-Intensity, a measure of blood wave energy transfer into the systemic circulation, were recorded by a combined echo-tracking Doppler-system (ALOKA SSD-5500, Tokyo).

Results: In D (HbAlc 7.0 $\pm 1.2 \%$, HOMA-IR 9.6 \pm 9.0 ), the postmeal increase of glucose $(p<0.016)$, insulin $(p<0.03)$ and nitrotyrosine $(0.33 \pm 0.37$ vs $0.26 \pm 0.42 \mathrm{nM}, \mathrm{p}<0.001)$ was significantly higher than in $\mathrm{C}$. Postmeal lipid levels were unchanged so that triglycerides remained higher and HDL lower in $D$ than in $C(p<0.04)$. Ve was lower in $D$ than in $C(7.9 \pm 1.5 \mathrm{vs} 9.9 \pm 1.4 \mathrm{~cm} /$ $\mathrm{s}, \mathrm{p}<0.001)$ and $\mathrm{Vs}$ was similar in both groups. Coincidently, the rate pressure product $(9009 \pm 2647 \mathrm{vs} 7853 \pm 1438 \mathrm{mmHg} / \mathrm{min}, \mathrm{p}<0.007)$ and stiffness parameters $(\mathrm{p}<0.04)$ were higher in D. W1 was higher as well $(11842 \pm 8466$ vs $\left.7949 \pm 3911 \mathrm{mmHgm} / \mathrm{s}^{3}, \mathrm{p}<0.02\right)$ and had a significant correlation with the rate pressure product $(\mathrm{r}=0.669, \mathrm{p}<0.001)$, taking the latter as indicator of myocardial oxygen requirement and W1 as a measure of energy transfer into the stiffened arterial system. In multivariate regression models, independent predictors of fasting Ve were age, HOMA-IR and the postmeal changes of nitrotyrosine $\left(\mathrm{R}^{2} 0.474\right)$, for postmeal Ve the postmeal changes of nitrotyrosine alone $\left(R^{2} 0.354\right)$ and for W1 the rate pressure product and HOMA-IR $\left(\mathrm{R}^{2} 0.534\right)$.

Conclusion: In people with type 2 diabetes, postmeal hyperglycemia induced oxidative stress is associated with diastolic dysfunction and increased myocardial oxygen requirements and energy transfer into the stiffened vascular bed already at rest. Given the known impairments of perfusion and intramitochondrial energy production in the diabetic heart, this myocardial mismatch of energy demand and supply bears the progredient risk of myocardial energy exhaustion, as indicated by diastolic dysfunction. In order to avoid this risk, respective therapeutic strategies should be developed.

\section{0}

The relationship between plasma osteoprotegerin levels, coronary artery calcification score and arterial stiffness in asymptomatic type $\mathbf{2}$ diabetes C. Jung ${ }^{1}$, W. Lee ${ }^{2}$, Y. Bae ${ }^{2}$, S. Kim², S. Jo ${ }^{3}$, E. $\mathrm{Lee}^{3}$, J. Mok ${ }^{1}$, K. Oh ${ }^{3}$, C. Kim², S. Park ${ }^{3}$, S. Kim ${ }^{3}$

${ }^{1}$ Endocrinology and Metabolism, Soonchunhyang University College of Medicine, Bucheon, ${ }^{2}$ Endocrinology and Metabolism, Kangbuk Samsung Hospital, Seoul, ${ }^{3}$ Endocrinology and Metabolism, Kangbuk Samsung, Seoul, Republic of Korea

Background and aims: Contemporary studies have shown similarities between vascular and skeletal calcification suggesting a regulatory role for osteogenic and calcitropic factors in the development of cardiovascular disease. Elevated OPG levels are associated with the progression of vascular calcification and the presence and severity of CAD. Coronary calcium is a good marker for atherosclerosis and correlates with the total extent of prevalent atherosclerosis. Coronary calcium score (CACS) has been shown to predict 
CHD events in non-diabetic group. In addition, pulse wave velocity (PWV) is a well-known indicator of arteriosclerosis, and it may predict cardiovascular risk. Because type 2 diabetes increases the risk of coronary atherosclerosis and $\mathrm{CAD}$, the aim of this study was to evaluate the relationship between plasma OPG levels, the extent of coronary artery calcification and PWV.

Materials and methods: One hundred three patients (male; 60, female; 43) who were treated with type 2 diabetes in Kangbuk Samsung Hospital, Sungkyunkwan University, Seoul, South Korea were enrolled in this study. The subjects underwent both CACS by MDCT (Philips Brilliance 40) and brachial-ankle PWV from November 2006 to December 2007. Plasma OPG levels were measured by enzyme-linked immunosorbent assay. The concentrations of $\mathrm{HbAlc}$, lipid profile, hsCRP and other basic laboratory parametes were determined in serum.

Results: The mean age was $57.8 \pm 11.4$ years, BMI was $25.1 \pm 3.6 \mathrm{~kg} / \mathrm{m}^{2}$ and DM duration $6.6 \pm 5.8$ years. The mean OPG levels were $685.3 \pm 278.8 \mathrm{pg} / \mathrm{ml}$. The mean CACS was $96.4 \pm 261.9$ and PWV was $1,516 \pm 371 \mathrm{~cm} / \mathrm{sec}$. The CACS showed significant correlation with OPG levels, PWV ( $\mathrm{r}=0.353, \mathrm{p}=0.004$, $\mathrm{r}=0.305, \mathrm{p}=0.006)$ in all subjects. Also, OPG levels showed significant correlation with PWV ( $r=0.304, \mathrm{p}=0.023)$. The CACS, OPG and other CHD risk factors such as lipid profiles, hsCRP, DM duration, $\mathrm{HbA}_{1 \mathrm{C}}$, smoking, blood pressure didn't show significant correlation in all subjects. Also, when the subjects were analyzed according to the severity of CACS (CAC score $>400$ or $\leq 400$ ), only OPG levels and PWV showed different levels significantly between groups $(p=0.046, p=0.005)$. Plasma OPG levels were significantly higher in severe CACS group. Of the 6 patients who showed severe CACS, 4 showed abnormal myocardial perfusion and positive treadmill test.

Conclusion: This study showed CACS and plasma OPG had significant correlation. Therefore, CACS measured by MDCT might be helpful assessing of coronary atherosclerosis and measurement of OPG merits further investigation as a simple test for identifying asymptomatic type 2 diabetic patients. Supported by: Korean Diabetes Association

\section{OP 11 Genetics: type 2 diabetes}

\section{1}

Novel type 2 diabetes susceptibility loci and measures of glycaemic control in a population based study of healthy children

C. Langenberg ${ }^{1}$, R.J.F. Loos ${ }^{1}$, S. Brage ${ }^{1}$, L.B. Sardinha ${ }^{2}$, L.B. Andersen ${ }^{3}$, U. Ekelund ${ }^{1}$, N.J. Wareham ${ }^{1}$

${ }^{1}$ Institute of Metabolic Sciences, MRC Epidemiology Unit, Cambridge, United Kingdom, ${ }^{2}$ Faculty of Human Movement, Technical University, Lisbon, Portugal, ${ }^{3}$ Department of Sports Medicine, Norwegian School of Sport Sciences, Oslo, Norway

Background and aims: Genome-wide association studies have recently associated previously unsuspected common genetic variants with type 2 diabetes (T2D), and several of these have since been shown to affect $B$-cell function in studies of adults. Associations with measures of glucose homeostasis in childhood or adolescence have not been investigated, but may show whether genetic susceptibility to T2D is already apparent early in life. Our objective was to examine associations of novel T2D loci with fasting glucose and insulin, and the homeostasis model of assessment for $\mathrm{B}$-cell function (HOMA-B) and insulin resistance (HOMA-IR) in European children.

Materials and methods: Variants linked to CDKN2A/B, CDKAL1, IGF2BP2, SLC30A8, chromosome 11p12, and HHEX were genotyped in Danish and Estonian Caucasian children of the European Youth Heart Study, a population-based study of 2,027 girls and boys aged between 9-10 and 15-16 years. Multivariate linear regression was used to investigate associations of genetic variants with fasting plasma glucose and insulin, and HOMA-B and HOMAIR, adjusting for age, sex, country and laboratory.

Results: Variants in CDKAL1 (G-minor allele of rs7756992, C-minor allele of rs10946398) were each significantly associated with lower levels of HOMA$\mathrm{B}$, with differences between minor and common homozygotes of $10.0 \%$ $(\mathrm{p}=0.011)$ and $8.3 \%(\mathrm{p}=0.019)$, respectively. The $\mathrm{G}$-minor allele of rs7756992 was also weakly associated with higher levels of insulin $(\mathrm{p}=0.055)$. These findings support previous studies of adults linking these variants in CDKAL1 to altered $\beta$-cell function and insulin secretion. In addition, we found that the C-major allele of rs13266634 in SLC30A8 was associated with higher fasting glucose $(\mathrm{p}=0.016)$, in line with recent case-control studies showing increased odds of T2D in adult carriers of the C-major allele. None of these associations differed significantly by sex, country or age group. Other candidates from recent GWA studies did not show any such relationships.

Conclusion: This is the first study to show that CDKAL1 and SLC30A8 may influence susceptibility to T2D from an early age. Future studies with detailed measures of insulin processing and secretion in children and adults will help to understand how and when in life these genes operate to influence the risk of T2D.

\section{2}

Metabolic effects of novel type 2 diabetes genes in a population aged 18-75 years

A. Jonsson ${ }^{1}$, B. Isomaa 2 , T. Tuomi ${ }^{3}$, V. Lyssenko ${ }^{1}$, L. Groop ${ }^{1,3}$

${ }^{1}$ Clinical Sciences, Diabetes \& Endocrinology, Lund University, Malmö, Sweden, ${ }^{2}$ Folkhälsan Research Center, Biomedicum, Folkhälsan Genetic Institute, Helsinki, Finland, ${ }^{3}$ Department of Medicine, University Hospital, University of Helsinki, Finland

Background and aims: Recently, several whole genome association scans (WGASs) for type 2 diabetes (T2D) have identified a number of novel T2D loci. Although many of them have been suggested to influence $\beta$-cell function, the quantitative effects of these variants on 1) BMI, 2) insulin secretion, 3) insulin action, and 4) FFA in a representative population is not known and formed the basis for the study carried out as part of population-based PPPBotnia Study (Prevalence, Prediction and Prevention of Diabetes), including 8-10\% of the population aged $18-75$ years.

Materials and methods: 3,500 individuals from the PPP-Botnia Study (age 49.3 \pm 16.0 , BMI 26.4 $\pm 4.4 ; 2,903$ with normal glucose tolerance (NGT), 395 with impaired fasting glucose and/or impaired glucose tolerance (IFG/IGT) and 202 with T2D) were genotyped for common variations in IGF2BP2 (rs4402960 and rs1470579), CDKAL1 (rs9460545), CDKN2A/2B (rs10811661), HHEX (rs1111875), FTO (rs9939609), SLC30A8 (rs11558471), TCF2 (rs4430796), TCF7L2 (rs7903146), KCNJ11 (rs5219) and PPARG (rs1801282). Insulin sensitivity was assessed with Matsuda Insulin sensitivity 
index (ISI). Insulin secretion was measured with corrected insulin response (CIR) and beta-cell function as disposition index (DI), i.e. insulin secretion adjusted for the degree of insulin sensitivity. Linear regression analysis was used to test genotype-phenotype correlations.

Results: In all non-diabetic individuals, variants in CDKAL1 (C-allele, CIR $\mathrm{p}=5.97 \times 10^{-5}$ and DI; $\left.\mathrm{p}=3.49 \times 10^{-5}\right)$, HHEX (G-allele, CIR $\mathrm{p}=1.33 \times 10^{-2}$ and DI $\mathrm{p}=7.60 \times 10^{-3}$ ), KCNJ11 (T-allele, CIR $\mathrm{p}=3.73 \times 10^{-2}$ ), and TCF2 (A-allele, CIR $\mathrm{p}=2.60 \times 10^{-2}$ ) were associated with reduced insulin secretion. In NGT, risk genotype carriers in CDKAL1 (CIR, $\mathrm{p}=1.86 \times 10^{-4}$ and $\left.\mathrm{DI} \mathrm{p}=5.24 \times 10^{-4}\right)$, HHEX (CIR, $\mathrm{p}=2.56 \times 10^{-2}$ and DI, $\mathrm{p}=7.92 \times 10^{-3}$ ) and CDKN2A/2B (A-allele, CIR $\mathrm{p}=2.95 \times 10^{-2}$ and DI, $\left.\mathrm{p}=3.06 \times 10^{-2}\right)$ had reduced $\beta$-cell function. $P P A R G$ CC-carriers had lower insulin sensitivity $\left(\mathrm{p}=1.89 \times 10^{-2}\right)$ compared to $\mathrm{G}$-allele carriers. In the whole cohort, the FTO AA-genotype carriers had $1.07 \mathrm{~kg} / \mathrm{m}^{2}$ higher BMI than TT-carriers $\left(\mathrm{p}=6.52 \times 10^{-5}\right)$ which resulted in an extra 1.9 $\mathrm{kg}$ weight increase per A-allele. Furthermore, CDKAL1 and IGF2BP2 risk carriers showed impaired postprandial suppression of FFA levels $(\mathrm{p}<0.05)$. Carriers of more than 7 risk alleles from genes previously shown to influence insulin secretion (CDKAL1, HHEX, CDKN2A/2B, KCNJ11, TCF2, TCF7L2 and IGF2BP2) had lower insulin secretion (CIR $-22.7 \% \mathrm{p}=2.38 \times 10^{-4}$ and DI $\left.-22.0 \% \mathrm{p}=3.63 \times 10^{-4}\right)$ than carriers of less than 5 risk-alleles.

Conclusion: Most novel common variants increase susceptibility to T2D by influencing $\beta$-cell function, possibly mediated through alterations in FFA stimulated mechanisms. The exceptions are FTO and PPARG, which confer risk of T2D by influencing BMI and insulin sensitivity.

Supported by: Swedish Research Council, including a Linné grant and EFSD Clinical Research Grant

\section{3}

Variants in the JAZF1, CDC123/CAMK1D, and TSPAN8 loci associate with impaired insulin response in a population-based sample of 4,516 glucose-tolerant middle-aged people

N. Grarup ${ }^{1}$, G. Andersen ${ }^{1}$, N.T. Krarup ${ }^{1}$, A. Albrechtsen ${ }^{2}$, O. Schmitz ${ }^{3,4}$, T. Jørgensen ${ }^{5}$, K. Borch-Johnsen ${ }^{5,6}$, T. Hansen ${ }^{1}$, O. Pedersen ${ }^{1,7}$

${ }^{1}$ Genetics, Steno Diabetes Center, Copenhagen, Denmark, ${ }^{2}$ Department of Biostatistics, University of Copenhagen, Denmark, ${ }^{3}$ Department of Endocrinology and Diabetes, Aarhus University Hospital, Denmark, ${ }^{4}$ Department of Clinical Pharmacology, University of Aarhus, Denmark, ${ }^{5}$ Research Centre for Prevention and Health, Glostrup University Hospital, Denmark, ${ }^{6}$ Epidemiology, Steno Diabetes Center, Copenhagen, Denmark, ${ }^{7}$ Faculty of Health Sciences, University of Aarhus, Denmark

Background and aims: We evaluated the impact on diabetes-related intermediary traits of common novel type 2 diabetes-associated gene variants in the JAZF1, CDC123/CAMK1D, TSPAN8, THADA, ADAMTS9, and NOTCH2 loci which were recently identified by meta-analysis of genome-wide association data.

Materials and methods: We genotyped the JAZF1 (rs864745), CDC123/ CAMK1D (rs12779790), TSPAN8 (rs7961581), THADA (rs7578597), ADAMTS9 (rs4607103), and NOTCH2 (rs10923931) variants in 6,083 middleaged subjects with available DNA from the population-based Inter99 cohort who had all been subjected to an oral glucose tolerance test (OGTT).

Results: In 4,516 glucose-tolerant individuals, homozygous carriers of the minor diabetes-associated G-allele of the CDC123/CAMK1D rs12779790 variant showed an $18 \%$ decreased insulinogenic index $\left(10-27 \% ; P=4 \times 10^{-5}\right)$, an $18 \%$ decreased corrected insulin response $\left(8.1-29 \% ; P=5 \times 10^{-4}\right)$, and a $13 \%$ decreased ratio of area under the serum-insulin and plasma-glucose curves during an OGTT (AUC-insulin/AUC-glucose) $\left(5.8-20 \% ; P=4 \times 10^{-4}\right)$. Carriers of the diabetes-protective $\mathrm{C}$-allele of JAZF1 rs864745 had an allele-dependent $3 \%$ increase in BIGTT-AIR $(0.9-4.3 \%$; $P=0.003)$. Furthermore, the diabetesassociated C-allele of TSPAN8 associated with decreased levels of corrected insulin response (4.5\% [0.5-8.4]; $P=0.03)$, of AUC-insulin/AUC-glucose ratio (3.9\% [1.2-6.7]; $P=0.005)$, and of insulinogenic index $(5.2 \%$ [1.9-8.6\%]; $P=0.002)$. Associations were confirmed when taking the effect of insulin sensitivity into account by constructing an OGTT-based disposition index or when performing multivariate analysis of insulin release and insulin sensitivity. Similar associations were found when including all 5,964 treatment-naïve individuals from the Inter99 cohort.

Conclusion: If replicated our data suggest that type 2 diabetes at-risk alleles in the JAZF1, CDC123/CAMK1D, and TSPAN8 loci associate with various OGTT-based surrogate measures of insulin release emphasising the contribution of abnormal pancreatic $\beta$-cell function in the pathogenesis of type 2 diabetes.

\section{4}

Large-scale type 2 diabetes association analysis of low-frequency nonsynonymous coding variants in the HNF4A gene including up to 17,600 individuals B. Jafar Mohammadi ${ }^{1}$, C.J. Groves ${ }^{1}$, B. Herrera ${ }^{1,2}$, T.M. Frayling ${ }^{3}$, A.T. Hattersley ${ }^{3}$, A.L. Gloyn ${ }^{1}$, M.I. McCarthy ${ }^{1,2}$

${ }^{1}$ Oxford Centre for Diabetes, Endocrinology and Metabolism, University of Oxford, ${ }^{2}$ Wellcome Trust Centre for Human Genetics, University of Oxford, ${ }^{3}$ Institute of Biomedical and Clinical Science, Peninsula Medical School, Exeter, United Kingdom

Background and aims: Despite the tremendous increase in our understanding of genetics of Type 2 Diabetes (T2DM) over the past 18 months, the familial aggregation seen in T2DM remains poorly explained. Low frequency variants, which crucially are likely to have been missed by both genome-wide association and linkage scans, may explain this "heritability gap". Genes already implicated in diabetes risk are logical candidates within which to start the search for low frequency, incompletely-penetrant variants that may make substantial contributions to diabetes predisposition. This study focused on HNF4A and two rare non-synonymous variants, T130I (rs1800961) and V255M (minor allele frequencies [MAF], 0.05 and 0.001 respectively), for which previous small-scale studies had provided suggestive, but not genome-wide significant, evidence for association. This study aims to establish, through large-scale, well-powered association analysis, the true contribution of these variants to diabetes risk.

Materials and methods: The HNF4A T130I and V255M variants were genotyped on the ABI 7900HT platform. Genotyping was carried out in between 15,546 (T130I: 6,079 cases, 9,467 controls) and 17,628 individuals (V255M: 5,011 cases, 12,617 controls) of UK British/Irish ancestry. We conducted association testing using an exact implementation of the Cochran-Armitage (CA) trend test (StatXact 6).

Results: Genotyping success rates were $98.7 \%$ for the T130I variant and $97.9 \%$ for the V255M variant with no genotyping error revealed by discordancy testing. Neither variant showed any evidence of association with diabetes in these analyses. For T130I, using the common homozygote as reference, an $\mathrm{OR}_{\mathrm{Het}}$ of 0.93 (95\%CI 0.81-1.06) and an $\mathrm{OR}_{\mathrm{Hom}}$ of 0.86 (95\% CI 0.65-1.13) was observed (case AF: $3.32 \%$; control AF: $3.14 \%, p=0.30$ ). For V255M an $\mathrm{OR}_{\mathrm{Het}}$ of 1.68 (95\% CI 0.62-5.35) was obtained and no rare homozygotes were detected (case AF: $0.06 \%$; control AF: $0.09 \%, \mathrm{p}=0.33$ ).

Conclusion: Although both these non-synonymous coding variants in a gene implicated in diabetes pathogenesis, have been previously reported to be associated with T2DM susceptibility, the large-scale, well-powered association analyses performed here indicate that these findings are not robust. This study once again emphasises the importance of using adequately powered studies when investigating associations, especially where the frequency of the susceptibility allele is low.

\section{5}

Is there a unifying genetic factor predisposing to the metabolic syndrome?

M. Sjögren ${ }^{1}$, M. Orho-Melander ${ }^{1}$, A. Jonsson ${ }^{1}$, M. Svensson' ${ }^{1}$, G. Berglund ${ }^{2}$, P. Nilsson ${ }^{2}$, L. Groop ${ }^{1,3}$, V. Lyssenko

${ }^{1}$ Clinical Sciences, Diabetes \& Endocrinology, Lund University, Malmö, Sweden, ${ }^{2}$ Clinical Sciences, Medicine, Lund University, Malmö, Sweden, ${ }^{3}$ Program of Molecular Medicine, University of Helsinki, Finland

Background and aims: The metabolic syndrome (MetS) is defined as cluster of factors contributing to increased risk of cardiovascular disease and type 2 diabetes. Heritability of MetS is relatively high, indicating a genetic component but environmental factors, such as physical inactivity and westernized high fat diet are also important. No unifying mechanism for MetS has to date been identified. One approach could be to search for common genetic susceptibility loci. Our aim was therefore to study whether common variations in 20 genes previously associated with either T2D or insulin resistance/obesity also would increase risk for MetS.

Materials and methods: Association between 21 SNPs (TCF7L2 rs7903146, PPARg rs1801282, KCNJ11 s5219, HHEX rs111875, IGF2BP2 rs4402960, CDKAL1 rs7754840, FTO rs9939609, CDKN2A rs10811661, SLC30A8 rs13266634, WSF1 rs10010131, CAPN10 rs3792267 and rs2975760, UCP2 rs659366, IRS1 rs1801278, PPARGC1A rs8192678, B1AR rs1801253, B2AR rs1042714, B3AR rs4994, PTPN1 rs3787348, ENPP1 rs1044498 and GCKR rs1260326) and risk of developing MetS was investigated in 16064 non-dia- 
betic individuals from the Malmoe preventive project (MPP) followed prospectively for a mean time of $23 \pm 4$ years. The definition of MetS in our study includes at least three of the following features: obesity (BMI $\geq 30 \mathrm{~kg} / \mathrm{m}^{2}$ ), dyslipidemia (triglycerides $\geq 1.7 \mathrm{mmol} / \mathrm{l}$ or lipid lowering treatment), hypertension (blood pressure $\geq 140 / 90 \mathrm{mmHg}$ or anti-hypertensive medication) and hyperglycemia (fasting plasma glucose $\geq 5.6 \mathrm{mmol} / \mathrm{l}$ or overt diabetes). The risk of developing MetS was calculated by logistic regression adjusted for age and gender, when appropriate.

Results: Polymorphisms in TCF7L2 rs7903146 (OR = 1.11 [1.04-1.17], $\mathrm{p}=$ 0.001), FTO rs9939609 (OR = 1.08 [1.02-1.14], $\mathrm{p}=0.006)$, IGF2BP2 $\mathrm{rs} 4402960$ $(\mathrm{OR}=1.07$ [1.01-1.13], $\mathrm{p}=0.023)$ and WSF1 rs10010131 $(\mathrm{OR}=1.07$ [1.02$1.13], p=0.010)$ increased the risk of developing MetS in individuals unaffected at baseline. The FTO rs9939609 (OR = 1.09 [1.03-1.16], p = 0.004) was associated with development of obesity, GCKR rs1260326 (OR $=1.15$ [1.09-1.22], $\left.\mathrm{p}=1.43 \times 10^{-6}\right)$ with development of dyslipidemia and TCF7L2 rs7903146 (OR $=1.17$ [1.09-1.25], $\left.\mathrm{p}=9.15 \times 10^{-6}\right)$, IGF2BP2 $\mathrm{rs} 4402960(\mathrm{OR}$ $=1.10[1.03-1.18], \mathrm{p}=0.004), C D K A L 1$ rs7754840 $(\mathrm{OR}=1.12[1.04-1.20], \mathrm{p}$ $=0.002)$ and SLC30A8 rs13266634 $(\mathrm{OR}=1.12$ [1.05-1.19], $\mathrm{p}=0.001)$ with increased risk of developing hyperglycemia. None of the studied polymorphisms was associated with all sub-components of MetS.

Conclusion: A number of common studied variants predispose to MetS by influencing specific sub-components but do not identify a unifying pathophysiological etiology.

\section{6}

The fasting plasma glucose-associated G6PC2/ABCB11 rs560887 variant influences pancreatic beta cell function in studies of 10,317 Danes

T. Nielsen ${ }^{1}$, C.S. Rose ${ }^{1}$, N. Grarup ${ }^{1}$, N.T. Krarup ${ }^{1}$, A. Albrechtsen ${ }^{2}$,

K. Borch-Johnsen, ${ }^{3,4}$, T. Jørgensen ${ }^{4}$, T. Lauritzen ${ }^{5}$, A. Sandbæk $k^{5}$, T. Hansen ${ }^{1}$, O. Pedersen ${ }^{1,6}$

${ }^{1}$ Genetics, Steno Diabetes Center, Gentofte, Denmark, ${ }^{2}$ Department of Biostatistics, University of Copenhagen, Denmark, ${ }^{3}$ Epidemiology, Steno Diabetes Center, Gentofte, Denmark, ${ }^{4}$ Research Centre for Prevention and Health, Glostrup University Hospital, Denmark, ${ }^{5}$ Department of General Practice, University of Aarhus, Denmark, ${ }^{6}$ Department of Health Science, University of Aarhus, Denmark

Background and aims: An association between fasting plasma glucose (FPG) and variants in a locus containing the G6PC2 (glucose-6-phosphatase, catalytic subunit 2) and $A B C B 11$ (ATP-binding cassette, subfamily $\mathrm{B}$, member $11)$ genes has been reported. Since G6PC2 is expressed predominantly in the pancreatic $\beta$-cells, variants in G6PC2 may also contribute to susceptibility to type 2 diabetes and insulin release. Thus, the aim of the present study was to extensively characterise diabetes-related metabolic traits in carriers of the G6PC2 rs560887 variant and to assess association with type 2 diabetes.

Materials and methods: The rs560887 polymorphism was genotyped in 5,924 middle-aged Danes from the population-based Inter99 study, 369 young, healthy subjects, and additionally in 3,514 type 2 diabetic patients and 510 glucose-tolerant subjects from the Danish ADDITION study and Steno Diabetes Center.

Results: Besides decreased FPG the A-allele of rs560887 variant showed association with lower levels of serum insulin $(p=0.0003)$ and serum C-peptide $\left(p=1 \times 10^{-5}\right)$ after 30 minutes during an oral glucose tolerance test in 5,808 treatment-naïve subjects from the Inter99 study. Furthermore, the A-allele of the rs560887 variant was associated with a decreased glucose-stimulated insulin release as assessed by the insulinogenic index $\left(p=4 \times 10^{-5}\right)$. In a study of 369 young, healthy subjects we replicated the association with FPG $(p=0.01)$, and found a borderline association with lowered acute insulin response during an intravenous glucose tolerance test $(p=0.06)$. Yet, in a statistically wellpowered case-control study of 3,856 type 2 diabetic cases and 4,918 glucosetolerant control subjects, we observed no association with type 2 diabetes.

Conclusion: The G6PC2 rs560887 variant influences FPG and decreases glucose-stimulated insulin release implying an impaired pancreatic $\beta$-cell function in carriers of the minor A-allele.

\section{OP 12 Inflammation, metabolism, cross-talk}

\section{7}

Modulation of TACE activity by high fat diet and metabolic stimuli L. Fiorentino, M. Cavalera, F. Tornei, R. Amoruso, R. Menghini, R. Lauro, M. Federici

Internal Medicine, University of Rome, Italy

Background and aims: Obesity progressively leads to an increase of white adipose mass and to the recruitment of macrophages to the visceral adipose tissue, thus establishing a low grade, systemic inflammatory status that arises from deregulated production and release of inflammatory cytokines and free fatty acids. The mechanisms underlying obesity-driven inflammation are not fully understood, but it has been shown that nutritional fatty acids activate Toll-like receptor 4 and trigger low grade inflammation and mild endotoxemia in experimental models that recapitulate some effects of high fat diet on insulin sensitivity. TNF- $\alpha$ is the most potent pro-inflammatory cytokine produced by adipose tissue, and initiate the low-grade inflammatory response characteristic of obesity. It is regulated at a post-translational level by TACE (TNF- $\underline{\alpha}$ Converting Enzyme), a transmembrane sheddase that releases soluble TNF- $\alpha$ from the membrane-bound form. Recent studies have suggested that the activity of TACE, and of its inhibitor Timp3, may be altered in patients affected by chronic metabolic and inflammatory diseases as levels of soluble forms of many TACE substrates like TNF- $\alpha$, TNFR-1, and IL6R resulted increased in these patients. Thus, we sought evidence in vivo and in vitro for an increased activation of TACE in the inflammatory status characteristic of high fat diet-induced obesity.

Materials and methods: To gain insight into TACE activation in an animal model of obesity, we compared wild-type mice fed a standard or a high fat diet for different periods of time, and measured TACE activity using a fluorogenic TACE substrate encompassing TNF- $a$ cleaving site. For in vitro studies, we treated murine hepatocytes and pre-hepatocytes with different metabolic stimuli, and then proceed to TACE activity measurement.

Results: To test the hypothesis that excessive fat intake results in increased TACE activity we fed wild type mice with standard chow or high fat diet for 20 weeks. We analyzed TACE and Timp3 expression in liver, muscle and white adipose tissue (WAT), by Real-Time PCR and Western blot, and showed that TACE levels were significantly increased in HFD-fed mice, while Timp3 levels resulted significantly reduced ( $\mathrm{p}<0.05$ for all). This prompted us to analyze the shedding activity of TACE in the same tissues, using a fluorimetric assay. TACE activity was significantly higher in liver, muscle and fat of HFD-fed animals compared to the control ones ( $\mathrm{p}<0.01$ for all). Next, we analyze more in details the metabolic stimuli that are able to activate TACE; to this aim, we used in vitro cultured pre-hepatocytes and hepatocytes, and treated them with palmitic acid (PA), endotoxin (LPS) and, as a positive control, TPA, a known activator of TACE. The shedding activity of TACE was significantly increased in both cell lines, mostly by LPS and, to a lesser extent, by PA treatment.

Conclusion: Our studies demonstrated that modulation of TACE activity occurs in an in vivo model of obesity, partially explaining the increased production of TNF- $\alpha$ and other pro-inflammatory cytokines, and consequently the low grade inflammatory status characteristic of obesity. Moreover, other metabolic stimuli, such as free fatty acids and endotoxin, which are released from the expanding WAT, are also able to activate TACE shedding activity. Our results suggested that modulation of TACE activity is a new pathway to be investigated for development of agents acting against obesity.

\section{8}

TIMP3 controls diet-induced inflammation and metabolic disorders in mice

M. Federici ${ }^{1}$, R. Menghini ${ }^{1}$, R. Amoruso ${ }^{1}$, V. Marzano ${ }^{1}$, S. Menini ${ }^{2}$,

L. Fiorentino ${ }^{1}$, F. Tornei ${ }^{1}$, A. Urbani ${ }^{1}$, G. Pugliese ${ }^{1}$, R. Lauro ${ }^{1}$

${ }^{1}$ Dept. of Internal Medicine, University of Tor Vergata, Rome, ${ }^{2}$ Dept. of Clinical Sciences, University of Rome La Sapienza, Italy

Background and aims: Obesity is associated with low-grade inflammatory state which prompts to diabetes and atherosclerosis. We found that activity and expression of Tissue inhibitor of Metalloproteinase 3 (TIMP3) are 
decreased in humans and mice affected by obesity. TIMP3 is an inhibitor of metalloproteinases such as MMP9 and TNF-alpha converting enzyme (TACE), of VEGFR and EGFR activation. Alteration of TIMP3 activity may impact on processes such as inflammation, angiogenesis, cell proliferation and fibrosis, all potentially relevant in obesity and its metabolic and vascular complications. Because obesity is associated with increased macrophage accumulation in different tissues, we used a transgenic approach under control of CD68 promoter to reconstitute TIMP3 levels in vivo and we analyzed its effects on metabolic homeostasis.

Materials and methods: Transgenic ( $\mathrm{Tg}$ ) mice were identified by RT-PCR and confirmed by Southern blot. In Tg mice, TIMP3 overexpression was confirmed by RT-PCR, Western blot and Reverse Zymography in spleen, liver, peripheral blood monocytes and bone marrow derived macrophages, treated or not with LPS. WT and Tg mice fed standard chow or a high fat diet (HFD, $60 \% \mathrm{cal}$ from fat) were subjected to metabolic and molecular phenotypization.

Results: After 20 weeks of High Fat Diet (HFD), we observed that Tg mice compared with WT littermates showed a significant improved glucose tolerance and insulin sensitivity, measured by IPGTT and IPITT ( $\mathrm{p}<0.01$ for all, $\mathrm{n}=8$ per group). Insulin, leptin, AST and ALT were lower and adiponectin increased in Tg mice compared with WT littermates ( $<0.01$ for all, $n=8$ per group). Histology of white adipose tissue (WAT), liver, kidney and aorta showed accumulation of lipids, intense fibrosis and increased inflammatory markers in WT compared with Tg mice. Expression profiling for metabolic and inflammatory genes revealed that Tg compared with WT have significant lower levels for MCP1, CXCL16, IL6, SOCS3 and SCD1 in WAT, and for SCD1, FAS, Srebp and G6Pase in liver ( $\mathrm{p}<0.01$ for all, $\mathrm{n}=5)$. Tg mice showed also lower levels of pJNK, and pIKK and pEGFR and higher levels of pAMPK in liver. To further elucidate the mechanisms involved in Timp3 rescue of hepatic steatosis we analyzed liver homogenates by shotgun proteomics (ESILC-qTOF-MS/MS) identifying 62 differentially expressed proteins in WT compared with Tg mice, including acyl-CoA synthetase long-chain family member 1 or ACSL1 (5-fold higher in WT vs Tg, p $<0.001$ ), Fatty Acid Binding Protein-1 or FABP1 (2.2 higher in WT vs Tg, $\mathrm{p}<0.01)$ and Retinaldehyde Dehydrogenase 1 or RALDH1 (1.9 higher in WT vs Tg, $\mathrm{p}<0.01$ ), all confirmed by western blot. Analysis of results by IPA software revealed that WT significantly differed from $\mathrm{Tg}$ mice for pathways including 1.Fatty acid Metabolism, 2.Glycolysis/Gluconeogenesis and 3.Fatty Acids elongation in mitochondria.

Conclusion: Our data indicate that macrophage specific overexpression of TIMP3 protects from HFD induced inflammation and metabolic disorders. Since TIMP3 related pathways play a role in linking inflammation to disruption of metabolic homeostasis, we suggest that TIMP3 should be investigated by a therapeutic point of view in obesity and its metabolic and vascular complications.

Supported by: Italian Society of Diabetes-SID

\section{9}

P-selectin glycoprotein ligand-1 (PSGL-1) deficient mice are protective for insulin resistance induced by high-fat diet

D. Hirota, K. Shikata, C. Sato, M. Sasaki, S. Nishishita, S. Miyamoto, R. Kodera, N. Kajitani, H. Makino

Department of Medicine and Clinical Science, Okayama University Graduate School of Medicine Dentistry and Pharmaceutical Sciences, Okayama, Japan

Background and aims: There have been accumulating evidences that inflammation in adipose tissue are involved in the mechanism of insulin resistance in obesity. Macrophages and proinflammatory cytokines are increased in visceral adipose tissues of obese people and animal models. However, the recruitment pathway of monocyte/macrophage into visceral adipose tissues has not been clarified. We have recently screened the gene expression profiles of adhesion molecules in adipose tissues of obese mice using DNA microarray and found that P-selectin glycoprotein ligand-1 (PSGL-1) is up-regulated in both $\mathrm{db} / \mathrm{db}$ mice and C57/BL6 mice fed a high-fat diet (HFD). We also found that CD68, MCP-1 and P-selectin are concomitantly increased in adipose tissues. PSGL-1 is known to express on both leukocytes and endothelial cells and has binding capacity to P-, L- and E-selectin. We found PSGL-1 is expressed on both endothelial cells and macrophages in adipose tissues. These data suggest that PSGL-1-selectin pathway may mediate the recruitment of macrophages into adipose tissues. The aim of this study is to clarify the role of PSGL-1 in macrophage infiltration and insulin resistance in obesity using PSGL-1 deficient $(\mathrm{KO})$ mice.
Materials and methods: Seven week-old male PSGL-1 KO mice $(\mathrm{n}=8)$ and C57/BL6 mice (wild type mice, $n=7$ ) were fed a HFD. Metabolic data were measured, and an intraperitoneal insulin tolerance test (ITT) and an intraperitoneal glucose tolerance test (IPGTT) were performed at 10 weeks after start of HFD. We measured HbAlc, serum lipids, insulin, leptin, adiponectin. We examined the expression of proinflammatory cytokines, chemokines and adhesion molecules and infiltration of macrophages in epididymal white adipose tissues (WAT). We also measured the size of adipocytes. PSGL-1 KO mice and wild type mice with normal fat diet were used for controls.

Results: There was no significant difference in body weight (BW) (Mean values of PSGL-1-KO and wild type mice fed a HFD; 39.2 vs $36.9 \mathrm{~g}$ ), weight of epididymal WAT per BW (4.5 vs $4.8 \%$ ), fasting blood glucose ( 247.0 vs 260.4 $\mathrm{mg} / \mathrm{dl}$ ) and HbAlc (3.0 vs $3.1 \%$ ) between PSGL-1 KO and wild type mice fed a HFD. Significant reductions were observed in total cholesterol (134.8 vs $189.1 \mathrm{mg} / \mathrm{dl}$ ), LDL cholesterol ( $15.4 \mathrm{vs} 34.1 \mathrm{mg} / \mathrm{dl}$ ), triglyceride ( 40.5 vs 62.5 $\mathrm{mg} / \mathrm{dl}$ ), free fatty acid (672.8 vs $1070.5 \mu \mathrm{Eq} / \mathrm{l})$, fasting insulin (1.05 vs 1.45 $\mathrm{ng} / \mathrm{ml}$ ) and leptin (6.4 vs $11.9 \mathrm{ng} / \mathrm{ml}$ ) in PSGL-1 KO mice as compared with wild type mice fed a HFD. The size of adipocytes in epididymal WAT was also significantly decreased in PSGL-1 KO mice as compared with wild type mice fed a HFD. Glucose tolerance was significantly improved in PSGL-1 KO mice in ITT and in IPGTT. The expression of MCP-1, F4/80, leptin and NOS2 (iNOS) were decreased in PSGL-1 KO mice as compared with wild type mice fed a HFD. Serum adiponectin level was increased in PSGL-1 KO mice but there was no statistical significance.

Conclusion: PSGL-1 deficient mice revealed decreased macrophage infiltration into adipose tissues, improved adipocyte hypertrophy and improved insulin resistance induced by high fat diet. These results suggest that the PSGL1 -selectin pathway promotes the recruitment of macrophages into adipose tissue. PSGL-1 might be a novel target for the prevention of diabetes in obese people.

Supported by: Ministry of Education, Science, Culture, Sports and Technology of Japan

\section{0}

Changes in gut microbiota control intestinal permeability-induced inflammation in obese and diabetic mice through unexpected dependent mechanisms

P.D. Cani ${ }^{1}$, O. Rottier ${ }^{1}$, Y. Guiot ${ }^{2}$, A. Neyrinck ${ }^{1}$, L. Geurts ${ }^{1}$, N. Delzenne ${ }^{1}$

${ }^{1}$ Unit PMNT, ${ }^{2}$ Unit of Pathology, Université catholique de Louvain, Brussels, Belgium

Background and aims: Recently, we and others have proposed that gut microbiota would favor the occurrence of the metabolic diseases associated with obesity. In the light of the role of gut microbiota for the development of metabolic diseases, we reported that high fat feeding was associated with metabolic endotoxaemia, and a lower Bifidobacterium spp caecal content in mice. Moreover, we found that selective increases of Bifidobacterium spp in gut microbiota improve high fat diet induced diabetes in mice through a mechanism associated with endotoxaemia. We and other have demonstrated that obese and diabetic mice ( $o b / o b$ and HF-fed) display enhanced intestinal permeability, and are characterised by a metabolic endotoxaemia and a low grade inflammation. Hence, we tested the hypothesis that specific and selective changes in gut microbiota control the intestinal permeability and the occurrence of inflammation and obesity-associated metabolic diseases.

Materials and methods: Since bifidobacteria have been reported to reduce plasma endotoxin and improve gut barrier function by an unknown mechanism, we specifically changes gut microbiota of $o b / o b$ mice by the mean of prebiotics (oligofructose, Ob-OFS) for 5 weeks.

Results: Ob-OFS fed mice exhibit a significantly lower intestinal permeability as measured by an oral Dx-FITC challenge. We found a strong improved intestinal mucosal integrity as shown by the epithelial intestinal tight-junction (TJ) proteins ZO1 and Occludin (mRNA and immunohistochemistry analysis) compared with normal chow-fed $o b / o b$ mice (Ob-CT). This phenomenon was associated with a dramatic decreased inflammatory tone (lower plasma endotoxin levels, plasma and liver cytokines concentrations, liver macrophages infiltration and oxidative stress) as compared to Ob-CT mice. Interestingly, OFS feeding significantly increased jejunum and colon proglucagon mRNA concentrations, and was positively correlated with a higher portal plasma GLP-1 and GLP-2 levels. To identify whether the gut microbiota induced proglucagon mRNA expression was involved in the improved intestinal permeability of Ob-OFS mice, we performed multiple correlations analysis between TJ proteins and intestinal proglucagon mRNA. We found that proglucagon mRNA were significantly and positively correlated with TJ 
mRNA, and were negatively correlated with in vivo intestinal permeability. All these results were independ of the OFS-induced lower food intake, since all the parameters measured in the energy pair fed group were equivalent to the $\mathrm{Ob}-\mathrm{CT}$ fed mice.

Conclusion: Together, these findings suggest that gut microbiota participate to the inflammatory phenotype of $o b / o b$ mice. In addition, these data demonstrate that a selective modulation in gut microbiota improve intestinal permeability, inflammatory markers, obesity and diabetes, via several unexpected mechanisms. We propose that the gut microbiota controls and increases endogenous production of the intestinotrophic proglucagon derived peptides GLP-2, and consequently improves gut barrier functions. These new findings demonstrate that it would be useful to develop specific therapeutic strategies using GLP-2 or by modifying gut microbiota to control intestinal permeability and obesity related metabolic disorders.

\section{1}

Transgenic expression of human C-reactive protein suppresses adiponectin and induces insulin resistance in spontaneously hypertensive rats

O. Oliyarnyk ${ }^{1}$, H. Malinska ${ }^{1}$, M. Maxova ${ }^{1}$, L. Kazdova ${ }^{1}$, M. Pravenec ${ }^{2}$ ${ }^{1}$ Department of Metabolism and Diabetes, Institute for Clinical and Experimental Medicine, ${ }^{2}$ Institute of Physiology, Academy of Sciences, Prague, Czech Republic

Background and aims: C-reactive protein (CRP), a marker and mediator of inflammation, is now recognized as central to the development of cardiovascular disease. Growing evidence indicate that CRP may also participate in the development of metabolic syndrome-related disease, but the mechanism of CRP implication in insulin resistance is not well understood. We tested hypothesis that increased expression of human CRP in adipose tissue affects age-related change in glucose tolerance, adiponectin levels, and insulin resistance of peripheral tissues in one year old transgenic spontaneously hypertensive rats (SHR), a well established animal model of genetic hypertension, dyslipidemia, and insulin resistance. Keeping in mind that chronic inflammation can contribute to increased oxidative stress, we measured liver antioxidant enzymes activity, reduced glutathione (GSH), and lipid peroxidation products (LPO) levels.

Materials and methods: One year old male SHR expressing the human CRP gene (SHR Tg) under control of the fat-specific aP2 promoter were used. Control group comprised age-matched genetically identical rats with absence of the transgene (SHR). Tissue sensitivity to insulin action was measured in vitro without or with insulin $(250 \mu \mathrm{U} / \mathrm{ml})$ according to basal and insulinstimulated ${ }^{14} \mathrm{C}$-U-glucose incorporation into muscle glycogen or adipose tissue lipids. Adiponectin was determined by EIA kit (B-Bridge Inc, USA).. Triacylglycerols (TAG) and glucose concentration were determined by kits of Pliva Lachema, Czech Republic. NEFA level was measured using kit of Roche Diagnostics, Germany. Antioxidant enzymes activities, GSH and LPO products concentrations were determinated by kits of Cayman Chemical, USA and Randox Laboratories, UK.

Results: There were no significant differences in body weight, weight of epididymal fat pads, serum NEFA, fasting blood glucose levels or adipose tissue insulin sensitivity between rats with and without the CRP transgene. The transgenic expression of CRP was associated with a significant increase in serum TAG $(0.84 \pm 0.05$ vs $0.64 \pm 0.037 \mathrm{mmol} / \mathrm{l}, \mathrm{p}<0.05)$, serum insulin levels after glucose load (insulinemia in $30 \mathrm{~min} .: 9.1 \pm 0.4$ vs $6.0 \pm 0.1 \mathrm{mmol} / \mathrm{l}, \mathrm{p}<0.05$; in $60 \mathrm{~min} .: 10.3 \pm 1.6$ vs $6.2 \pm 0.18 \mathrm{mmol} / \mathrm{l}, \mathrm{p}<0.02$ ), liver TAG accumulation $(4.30 \pm 0.56$ vs $6.89 \pm 0.56 \mathrm{mmol} / \mathrm{g}, \mathrm{p}<0.05)$ and markedly decrease in insulin stimulated glycogenesis in muscle ( $174 \pm 8$ vs $278 \pm 35 \mathrm{nmol} / \mathrm{g} / 2 \mathrm{~h}, \mathrm{p}<0.05)$. The SHR Tg had markedly decreased levels of serum adiponectin $(2.37 \pm 0.31$ vs $4.32 \pm 0.59 \mu \mathrm{g} / \mathrm{ml}, \mathrm{p}<0.05)$, reduced liver glutathione peroxidase activity ( 388 \pm 29 vs $546 \pm 27 \mu \mathrm{mol} \mathrm{GSH} / \mathrm{min} / \mathrm{mg}$ protein, $\mathrm{p}<0.01)$ and $\mathrm{GSH}$ concentration $(15.9 \pm 1.0$ vs $21.8 \pm 0.9 \mu \mathrm{mol} / \mathrm{mg}$ protein, $\mathrm{p}<0.01)$. Liver LPO products levels were elevated in SHR Tg rats in comparison to control SHR: conjugated dienes $26.5 \pm 1.3$ vs $22.4 \pm 1.1 \mathrm{nmol} / \mathrm{mg}$ protein, $\mathrm{p}<0.05$; thiobarbithuric acid reactive substances $1.17 \pm 0.09 \mathrm{vs} 0.81 \pm 0.07 \mathrm{nmol} / \mathrm{mg}$ protein, $\mathrm{p}<0.01$.

Conclusion: It can be concluded that fat-specific overexpression of human CRP promotes skeletal muscle insulin resistance, liver triglyceride accumulation and lipoperoxidation in the one year old SHR rats. Our data support hypothesis that this effect of transgenic CRP expression could be related to lower adiponectin secretion under these conditions.

Suppported by: Ministry of Health of the Czech Republic

\section{2}

Pioglitazone treatment in subjects with impaired glucose tolerance: inhibition of cytokine gene and protein expression from mononuclear cells and cross-talk with adipocytes

P.D. Reaven, W. Zhang, E. Schwartz, P. Permana

Endocrinology, Phoenix VA Health System, Phoenix, United States

Background and aims: Infiltration by monocytes and lymphocytes in the artery wall and their production of a variety of inflammatory factors contributes to the development of atherosclerosis. Previous studies have demonstrated that therapy with thiazolidinediones (TZDs) might play a protective role not only by improving metabolic disorders but also by inhibiting inflammation. However, the specific in vivo effects of TZDs on individual mononuclear cell types is unclear, as is whether these circulating cells from TZD treated individuals are less able to induce inflammation in other tissues.

Materials and methods: To address these questions, we first confirmed that inflammation, as assessed by IL- 6 gene and protein expression, was increased in monocytes and lymphocytes isolated (using sequential percoll and histopaque gradient centrifugations) from subjects with IGT $(n=13)$ compared to age and BMI matched individuals with normal glucose tolerance $(n=13)$. We then randomized 67 IGT subjects to 5 months of placebo or pioglitazone (PIO) therapy $(45 \mathrm{mg} / \mathrm{d})$ and assessed effects on mononuclear cell gene expression. Inflammatory effects of conditioned media from both monocytes and lymphocytes were also tested on human adipocytes.

Results: Plasma IL-6 levels were increased and gene expression and protein secretion in the condition media of IL-6 was increased nearly 3 -fold ( $\mathrm{p}<$ 0.05 ) in both monocytes and lymphocytes from IGT subjects, confirming enhanced inflammatory activity in this group. After 5-months of therapy, subjects receiving PIO had lower $(\mathrm{p}<0.05)$ triglycerides and higher HDL cholesterol than subjects receiving placebo $(\mathrm{p}<0.05)$. While IL-1b, IL-6 and IL-8 gene expression from freshly isolated monocytes was similar in both groups at baseline, gene and protein expression levels of these factors after PIO therapy were significantly lower ( $\mathrm{p}<0.05$ for all). Conditioned media from monocytes from PIO (but not placebo) treated subjects still contained significantly less secreted protein even when stimulated for $16 \mathrm{hrs}$ with LPS. Similarly, gene expression, and protein secretion into condition media, of IL-2, IL-6 and IL-8 were lower in lymphocytes isolated from PIO treated subjects. Interestingly, for all subjects, cytokine secretion was strongly and inversely related to plasma HDL levels at follow-up $(\mathrm{r}=0.3-0.8, \mathrm{P}<0.05)$. As we have previously demonstrated that factors secreted from macrophages can greatly enhance adipocyte inflammation, we compared the effects of conditioned media from monocytes and lymphocytes on human adipocyte inflammation. Gene expression of IL-6, IL-8 and MCP-1 were all increased (8-38-fold) in human adipocytes conditioned with media from monocytes or lymphocytes from placebo treated subjects, while these were reduced by nearly $50 \%$ when exposed to conditioned media from monocytes or lymphocytes from PIO subjects.

Conclusion: These results demonstrate 1) that both peripheral monocytes and lymphocytes demonstrate greater inflammation in IGT subjects (even when matched with subjects of equal weight); 2) that PIO therapy can reduce gene and protein expression from both these inflammatory cell types; and 3) that treatment with PIO can potentially reduce the inflammatory cross-talk between inflammatory cells and adipose tissue which may in turn contribute to metabolic improvements resulting from PIO therapy.

Supported by: Office of Research and Development, Medical Research Service, Department of Veterans Affairs and Takeda Pharmaceutical Inc. 


\section{OP 13 DPP-IV inhibitors}

\section{3}

Two-year treatment with sitagliptin and initial combination therapy of sitagliptin and metformin provides substantial and durable glycaemic control in patients with type 2 diabetes

D.S. Qi ${ }^{1}$, R. Teng ${ }^{2}$, M. Jiang ${ }^{1}$, M.J. Davies ${ }^{3}$, K.D. Kaufman ${ }^{1}$, J.M. Amatruda ${ }^{1}$, D. Williams-Herman ${ }^{1}$

${ }^{1}$ Clinical Research, Merck Research Laboratories, Rahway, ${ }^{2}$ BARDS, Merck Research Laboratories, Rahway, ${ }^{3}$ Medical Communications, Merck Research Laboratories, Rahway, United States

Background and aims: Long-term efficacy and safety of sitagliptin and initial combination therapy with sitagliptin and metformin were assessed in patients (pts) with type 2 diabetes (T2DM) and inadequate glycemic control (A1C $7.5 \%$ to $11 \%$ ) on diet and exercise.

Materials and methods: After completing the initial, double-blind, 54-wk phase of the study, consenting pts continued in a double-blind, 50-wk extension. Pts initially randomized to active treatments remained on these treatments during the extension: sitagliptin $100 \mathrm{mg} / \mathrm{metformin} 2000 \mathrm{mg}$ (S100/ M2000), sitagliptin $100 \mathrm{mg} /$ metformin $1000 \mathrm{mg}$ (S100/M1000), metformin $2000 \mathrm{mg}$ (M2000), metformin $1000 \mathrm{mg}$ (M1000) [all as divided doses], and sitagliptin $100 \mathrm{mg}$ q.d. (S100); pts initially randomized to placebo were switched to M2000 at Wk 24. This report presents 104-wk results for pts who were randomized to active treatment and who entered the extension. The primary efficacy analyses of this population required at least one measurement in the extension (all-patients-treated [APT] cohort), imputed missing data using LOCF, and excluded data from pts after they initiated glycemic rescue therapy.

Results: Of the original 1091 randomized pts, 915 received active treatment, 587 of these entered the extension and 402 were included in the APT analysis. In the APT cohort, mean baseline A1C was 8.6\% (range 8.5\% to 8.7\%). Mean A1C changes from baseline (\%) at Wk 104 were -1.7 (S100/M2000, $\mathrm{n}=105)$, $-1.4(\mathrm{~S} 100 / \mathrm{M} 1000, \mathrm{n}=96),-1.3(\mathrm{M} 2000, \mathrm{n}=87),-1.1(\mathrm{M} 1000, \mathrm{n}=64)$, and -1.2 $(\mathrm{S} 100, \mathrm{n}=50)$. The proportions of patients with an $\mathrm{AlC}<7 \%$ at Wk 104 were 60\% (S100/M2000), 45\% (S100/M1000), 45\% (M2000), 28\% (M1000), and 32\% (S100). For FPG and 2-hr postmeal glucose, respectively, mean changes from baseline $(\mathrm{mmol} / \mathrm{L})$ at Wk 104 were -3.2 and -6.1 in the S100/M2000 group, -2.8 and -5.3 in the S100/M1000 group, -2.4 and -4.8 in the M2000 group, -2.3 and -4.0 in the M1000 group, and -1.5 and -4.1 in the $\mathrm{S} 100$ group. For pts who completed treatment through Wk 104, mean $\mathrm{HbA}_{1 c}$ changes from baseline (\%) were -1.7 (S100/M2000, n=77), -1.5 (S100/M1000, $\mathrm{n}=64),-1.7$ (M2000, n=53), -1.4 (M1000, n=26), and -1.4 (S100, n=22). Glycemic response was generally durable over time across treatments. All treatments were generally well tolerated. Conclusion: Over 2 yrs, sitagliptin and the initial combination of sitagliptin and metformin provided substantial and durable glycemic improvements and were well tolerated in pts with T2DM.

Supported by: Merck \& Co., Inc.

\section{4}

Vildagliptin is safe and well tolerated in patients with mild or moderate renal impairment

T. Thuren ${ }^{1}$, S. Byiers ${ }^{2}$, P. Mohideen ${ }^{3}$, M. Goodman ${ }^{3}$

${ }^{1}$ CVM-TA, Global CD\&MA, Novartis Pharmaceuticals Corporation, East Hanover, United States, ${ }^{2}$ Biostatistics, Global CD\&MA, Novartis Pharma AG, Basel, Switzerland, ${ }^{3}$ CVM-TA, Global CD \& MA, Novartis Pharmaceuticals Corporation, East Hanover, United States

Background and aims: Vildagliptin is a potent and selective DPP-4 inhibitor that has been shown to enhance the physiological effects of incretin hormones thereby increasing islet alpha- and beta-cell responsiveness to glucose.
At marketed doses of 50mg once or twice-daily vildagliptin effectively reduces $\mathrm{HbA}_{1 c}$ and is well tolerated. Although vildagliptin is not predominantly renally excreted, it is not approved in Europe for moderate and severe renal impairment due to insufficient clinical experience at this time.

The purpose of this abstract is to report on the prevalence of renal impairment in the vildagliptin global development program and to compare the safety and efficacy profiles of the marketed doses of vildagliptin in patients with renal impairment compared to those with normal renal function.

Materials and methods: Safety data from pooled vildagliptin monotherapy studies were used and glomerular filtration rate (GFR) was estimated using the MDRD method. Renal impairment was defined by categories of 60 to $<90 \mathrm{~mL} / \mathrm{min} / 1.73 \mathrm{~m}^{2}$ (mild) and 30 to $<60 \mathrm{ml} / \mathrm{min} / 1.73 \mathrm{~m}^{2}$ (moderate). The number of patients with severe renal impairment $\left(<30 \mathrm{ml} / \mathrm{min} / 1.73 \mathrm{~m}^{2}\right)$ were too small to allow an assessment of safety in this population.

Results: 927 patients receiving the marketed doses of vildagliptin as monotherapy had mild $(\mathrm{n}=825)$ or moderate $(\mathrm{n}=102)$ renal impairment, compared to 853 vildagliptin-treated patients with normal renal function. In patients with normal renal function or mild renal impairment, incidence of any adverse event (AE) was similar among patients receiving vildagliptin 50mg either once or twice-daily, rosiglitazone, pioglitazone or placebo, but lower than those who received metformin. In patients with moderate renal impairment, the incidence of any $\mathrm{AE}$ was similar among all active treatment groups, but higher than in the placebo group.

As expected, patients with normal renal function tended to have somewhat lower incidences of serious AEs and discontinuations due to adverse events, compared to patients with impaired renal function. Importantly, the incidences of these events in patients with renal impairment, were similar across the treatment groups (vildaglpitin vs comparators). Additionally, there were no adverse changes in renal function over time following long term vildaglitpin treatment. The $\mathrm{HbAlc}$ lowering of vildagliptin $50 \mathrm{mg}$ twice-daily was similar in patients with mild renal impairment $(\Delta=-1.06 \pm 0.10)$ compared with those with normal renal function $(\Delta=-0.89 \pm 0.09)$.

Conclusion: Half of all patients in the global development program exposed to the marketed doses of vildagliptin as monotherapy had either mild or moderate renal impairment at study baseline. Vildagliptin was effective and well tolerated in this population.

\section{5}

Vildagliptin improves alpha cell glucose sensing in patients with type 2 diabetes

B. Ahrén ${ }^{1}$, A. Schweizer ${ }^{2}$, S. Dejager ${ }^{3}$, B.E. Dunning ${ }^{4}$, M. Persson ${ }^{5}$, J.E. Foley ${ }^{6}$

${ }^{1}$ Clinical Sciences, Lund University, Sweden, ${ }^{2}$ Methodology \& Innovation, Novartis Pharma AG, Basel, Switzerland, ${ }^{3}$ Clinical Research, Novartis Pharmaceuticals, Rueil Malmaison, France, ${ }^{4}$ Diabetes \& Metabolism, PharmaWrite, Princeton, United States, ${ }^{5}$ Clinical Sciences, Malmö, Lund University, Malmö, Sweden, ${ }^{6}$ Clinical Research, Novartis Pharmaceuticals, E Hanover, United States

Background and aims: Vildagliptin is a potent and selective DPP-4 inhibitor that improves glycemic control in patients with type 2 diabetes (T2DM) by increasing islet responsiveness to glucose. A previous study demonstrated that vildagliptin improved glycemic control when added to high dose insulin monotherapy in patients with T2DM and reduced the frequency and severity of hypoglycemia. Hence we hypothesized that vildagliptin would increase the glucagon response to hypoglycemia while suppressing the glucagon response to hyperglycemia.

Materials and methods: This single-center, double-blind, randomized, placebo (PBO)-controlled crossover study assessed the glucagon response to hyper- and hypoglycemic stimuli. Thirty patients $(24 \mathrm{M}, 6 \mathrm{~F})$ with T2DM (baseline BMI, AIC and FPG $=28.0 \mathrm{~kg} / \mathrm{m}^{2}, 6.3 \%$ and $7.4 \mathrm{mM}$, respectively)

\begin{tabular}{|c|c|c|c|c|c|c|}
\hline & Vilda50 mg QD & Vilda50 mg BID & Met $\leq 2 \mathrm{~g} / \mathrm{d}$ & Rosi8 mg/d & Pio30 mg/d & PBO \\
\hline \multicolumn{7}{|c|}{ Normal renal function $\left(\mathrm{GFR} \geq 90 \mathrm{~mL} / \mathrm{min} / 1.73 \mathrm{~m}^{2}\right)$} \\
\hline n $(\%)$ & $223(54.5)$ & $630(45.9)$ & $67(26.6)$ & $151(56.6)$ & $137(63.4)$ & $160(46.1)$ \\
\hline Any AE \% & 54.3 & 60.3 & 74.6 & 64.9 & 50.4 & 63.8 \\
\hline \multicolumn{7}{|c|}{ Mild renal insufficiency (GFR $60-<90 \mathrm{~mL} / \mathrm{min} / 1.73 \mathrm{~m}^{2}$ ) } \\
\hline n (\%) & $163(39.9)$ & $662(48.2)$ & $165(65.5)$ & $104(39.0)$ & $75(34.7)$ & $162(46.7)$ \\
\hline Any AE \% & 55.2 & 65.3 & 76.4 & 61.5 & 48.0 & 57.4 \\
\hline \multicolumn{7}{|c|}{ Moderate renal insufficiency (GFR $30-<60 \mathrm{~mL} / \mathrm{min} / 1.73 \mathrm{~m}^{2}$ ) } \\
\hline n (\%) & $23(5.6)$ & $79(5.8)$ & $20(7.9)$ & $12(4.5)$ & $4(1.9)$ & $25(7.2)$ \\
\hline Any AE & 82.6 & 68.4 & 70.0 & 75.0 & 75.0 & 44.0 \\
\hline
\end{tabular}


A

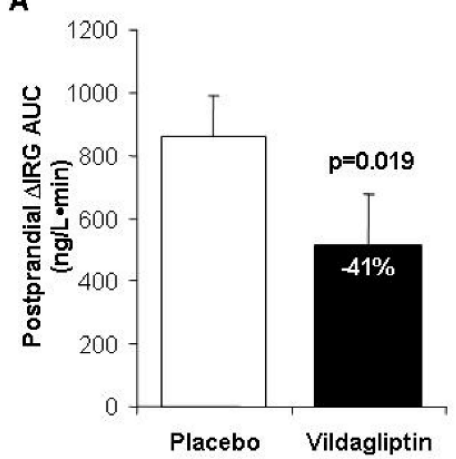

B

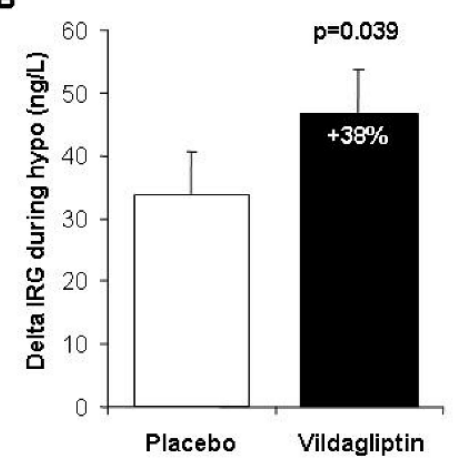

C

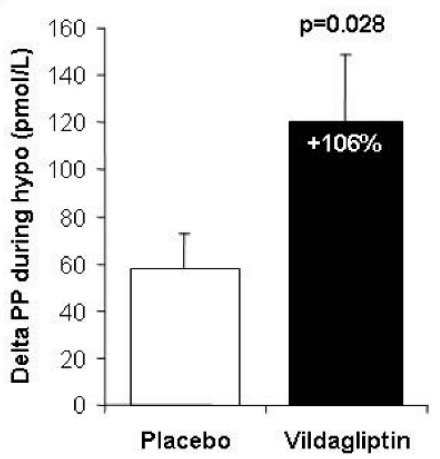

received vildagliptin $100 \mathrm{mg}$ qd or $\mathrm{PBO}$ for 4 wks in random order with a 4wk washout between treatment periods. On Day 28 of treatment, patients underwent standard meal tests followed by hyperinsulinemic, stepped glucose clamps (7.5, 5.0 and $2.5 \mathrm{mM}$ glucose). During the hypoglycemic step, plasma levels of epinephrine, norepinephrine and cortisol were measured to assess sympathoadrenal activation, and pancreatic polypeptide (PP) was assessed as an index of parasympathetic activation.

Results: Plasma glucose levels were lower with vildagliptin than with PBO throughout the meal test and during the hyperglycemic clamp, but were superimposable during the euglycemic and hypoglycemic clamps. As shown below, vildagliptin significantly reduced inappropriate glucagon secretion (by $41 \%$ ) during standard meal tests (Panel A), confirming previous results, and plasma glucagon levels were significantly lower during the hyperglycemic and euglycemic clamps with vildagliptin vs. $\mathrm{PBO}$ (data not shown). In contrast, during hypoglycemia, the increase in plasma glucagon levels (Panel B) and PP levels (Panel C) were significantly greater with vildagliptin than with $\mathrm{PBO}(+38 \%$ for glucagon, $+106 \%$ for PP). The increases in plasma epinephrine, norepinephrine and cortisol during hypoglycemia were essentially identical during the two treatment periods, demonstrating that the degree of stress induced by (the identical degrees of) hypoglycemia did not differ between treatments.

Conclusion: Vildagliptin enhances $\alpha$-cell sensitivity to both the suppressive effects of hyperglycemia and the stimulatory effects of hypoglycemia. The increase in PP seen during hypoglycemia in the vildagliptin treatment period suggests that vagal mechanisms contribute to the glucagon response to hypoglycemia.

Supported by: Novartis

\section{6}

Efficacy and safety of alogliptin and glyburide combination therapy in patients with type 2 diabetes

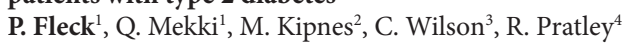

${ }^{1}$ Clinical Research, Takeda Global Research \& Development Center Inc., Deerfield, ${ }^{2}$ Medicine, Christus Santa Rosa Medical Center, San Antonio, ${ }^{3}$ Biometrics Statistics, Takeda Global Research and Development Center Inc., Deerfield, ${ }^{4}$ Medicine, University of Vermont, Burlington, United States

Background and aims: Alogliptin (ALO) is a potent and highly selective DPP-4 inhibitor being developed for the treatment of type 2 diabetes mellitus (T2DM). Sulfonylureas, a class of antidiabetic agents, act by increasing insulin release from $\beta$-cells in a glucose-independent fashion but are associated with decreased efficacy over time. Therefore, the addition of alogliptin to an ongoing sulfonylurea regimen may result in improved glycemic control. This randomized, double-blind, placebo (PBO)-controlled study was designed to assess the efficacy and safety of ALO in combination with glyburide (GLY) in patients with T2DM inadequately controlled on sulfonylurea monotherapy. Material and methods: Patients $(\mathrm{N}=500$; mean age $=57$ years; $71 \%$ White; $48 \%$ men; mean baseline $\mathrm{HbA1c}=8.1 \%$; mean duration of $\mathrm{T} 2 \mathrm{DM}=8$ years) were randomized to receive ALO $12.5 \mathrm{mg}(\mathrm{n}=203)$, ALO $25 \mathrm{mg}(\mathrm{n}=198)$, or PBO $(n=99)$ once daily in combination with their ongoing GLY regimen (mean dose $=12 \mathrm{mg} /$ day) for 26 weeks after participating in a 4-week singleblind $\mathrm{PBO}$ run-in/stabilization period. The primary endpoint was change from baseline in $\mathrm{HbAlc}$ at week 26 (or last observation) in the intent-to-treat population.

Results: A total of 363 patients completed the study (ALO $12.5 \mathrm{mg}, 75.4 \%$; ALO $25 \mathrm{mg}$ 74.7\%; PBO, 62.6\%). At week 26, least-squares (LS) mean changes from baseline in $\mathrm{HbAlc}$ were significantly $(P<.001)$ greater for ALO $12.5 \mathrm{mg}(-0.38 \%)$ and ALO $25 \mathrm{mg}(-0.52 \%)$ vs PBO (+0.01\%) (Figure). The changes from baseline in HbA1c were similar regardless of age $(<65$ or $\geq 65$ years), BMI ( $<30$ or $\geq 30 \mathrm{~kg} / \mathrm{m}^{2}$ ), or ethnicity (Hispanic or non-Hispanic) and were greater in patients with higher baseline HbA1c. A significantly $(P<.001)$ greater proportion of patients achieved a $\geq 1 \%$ reduction in $\mathrm{HbAlc}$ for ALO $12.5 \mathrm{mg}(28.6 \%)$ and ALO $25 \mathrm{mg}$ (30.0\%) vs PBO (8.7\%). LS mean changes from baseline in fasting plasma glucose were greater for both ALO $12.5 \mathrm{mg}$ $(-0.26 \mathrm{mmol} / \mathrm{L} ; P=.241)$ and ALO $25 \mathrm{mg}(-0.46 \mathrm{mmol} / \mathrm{L} ; P=.072)$ vs $\mathrm{PBO}$ $(+0.12 \mathrm{mmol} / \mathrm{L})$ at week 26 . LS mean changes from baseline in weight and lipids at week 26 were not clinically meaningful. The proportions of patients who experienced $\geq 1$ adverse event (ALO $12.5 \mathrm{mg}, 63.5 \%$; ALO $25 \mathrm{mg}, 63.1 \%$; $\mathrm{PBO}, 53.5 \%$ ) or who discontinued the study due to an adverse event (ALO $12.5 \mathrm{mg}, 2.5 \%$; ALO $25 \mathrm{mg}, 2.0 \%$; PBO, 2.0\%) were similar across groups. The proportion of subjects who experienced $\geq 1$ serious adverse event was low across groups (ALO $12.5 \mathrm{mg}, 5.4 \%$; ALO $25 \mathrm{mg}$, 5.6\%; PBO, 2.0\%). Hypoglycemia was reported in $11.1 \%$ of patients for PBO, $15.8 \%$ for ALO 12.5 $\mathrm{mg}$, and $9.6 \%$ for ALO $25 \mathrm{mg}$.

Conclusion: ALO added to ongoing GLY monotherapy significantly improved glycemic control in patients with T2DM without a clinically meaningful increase in weight gain or the incidence of hypoglycemia.

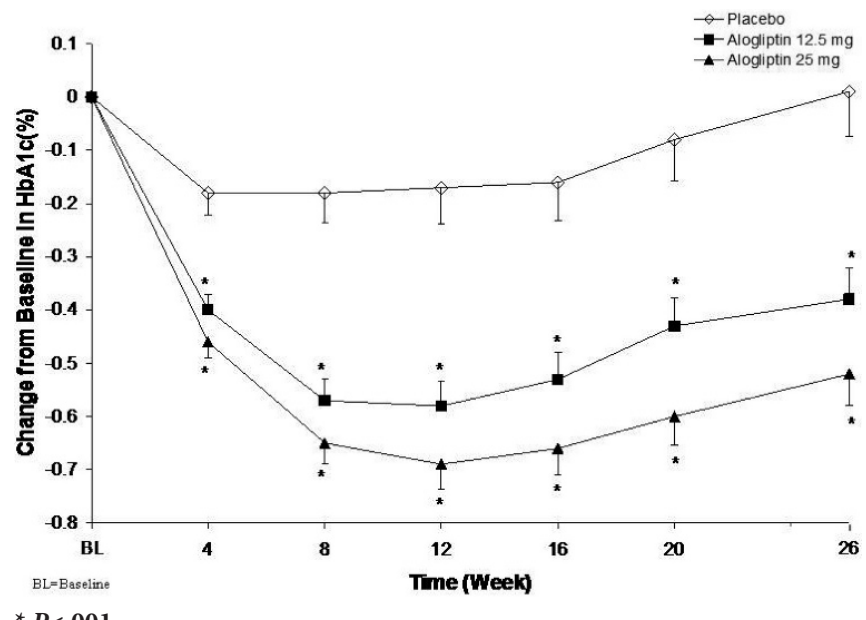

${ }^{\star} \boldsymbol{P}<.001$

Values are least-squares mean (SE)

Supported by: Takeda Global Research \& Development Center

\section{7}

Addition of alogliptin to insulin therapy reduces $\mathrm{HbA}_{1 \mathrm{c}}$ without increasing weight gain or hypoglycaemia in patients with type 2 diabetes M. Rendell ${ }^{1}$, J. Rosenstock ${ }^{2}$, J. Gross ${ }^{3}$, P. Fleck ${ }^{4}$, C. Wilson ${ }^{4}$, Q. Mekki ${ }^{4}$ ${ }^{1}$ Creighton Diabetes Center, Creighton University, Omaha, United States, ${ }^{2}$ University of Texas Southwestern Medical Center at Dallas, United States, ${ }^{3}$ Hospital de Clinicas de Porto Alegre, Brazil, ${ }^{4}$ Takeda Global Research \& Development Center Inc., Deerfield, United States

Background and aims: Alogliptin (ALO) is a potent and highly selective DPP-4 inhibitor being developed for the treatment of type 2 diabetes (T2DM). Insulin therapy is most effective at achieving and maintaining glycemic control when used in combination with oral antidiabetic agents in patients with T2DM. This randomized, double-blind, placebo (PBO)-controlled study as- 
sessed the efficacy and safety of ALO added to conventional insulin therapy (INS) in patients with T2DM inadequately controlled on INS alone or on INS with metformin (MET).

Materials and methods: Patients $(\mathrm{N}=390$; mean age $=55$ years; $65 \%$ White; $41 \%$ men; mean baseline $\mathrm{HbA} 1 \mathrm{c}=9.3 \%$; mean duration of $\mathrm{T} 2 \mathrm{DM}=13$ years; mean insulin dose $=57 \mathrm{IU} /$ day) were randomized to receive ALO $12.5 \mathrm{mg}$ $(\mathrm{n}=131)$, ALO $25 \mathrm{mg}(\mathrm{n}=129)$, or PBO $(\mathrm{n}=130)$ once daily as an add-on to INS for 26 weeks after a 4-week single-blind PBO run-in/stabilization period. Patients already taking MET ( $59 \%$; mean dose $=1733 \mathrm{mg} /$ day) continued this therapy unchanged. The primary endpoint was change from baseline in $\mathrm{HbAlc}$ at week 26 (or last observation) in the intent-to-treat population.

Results: A total of 215 patients completed the study (ALO $12.5 \mathrm{mg}, 63 \%$; ALO $25 \mathrm{mg} \mathrm{60 \% ;} \mathrm{PBO,} \mathrm{42 \% ).} \mathrm{At} \mathrm{week} \mathrm{26,} \mathrm{least-squares} \mathrm{(LS)} \mathrm{mean} \mathrm{changes}$ from baseline in $\mathrm{HbAlc}$ were significantly greater $(P<.001)$ for ALO $12.5 \mathrm{mg}$ $(-0.63 \%)$ and ALO $25 \mathrm{mg}(-0.71 \%)$ vs PBO $(-0.13 \%)$ (Figure). The changes from baseline in $\mathrm{HbAlc}$ were similar regardless of age ( $<65$ or $\geq 65$ years), BMI $\left(<30\right.$ or $\geq 30 \mathrm{~kg} / \mathrm{m}^{2}$; mean $\left.=32.4 \mathrm{~kg} / \mathrm{m}^{2}\right)$, ethnicity (Hispanic or non-Hispanic), or background therapy (MET or none). LS mean changes from baseline in fasting plasma glucose were $+0.13 \mathrm{mmol} / \mathrm{L}$ for ALO $12.5 \mathrm{mg}(P=.662)$ and $-0.65 \mathrm{mmol} / \mathrm{L}$ for ALO $25 \mathrm{mg}(P=.030)$ vs $+0.32 \mathrm{mmol} / \mathrm{L}$ for PBO. At week 26, no significant differences from PBO in LS mean changes from baseline in weight or lipids were observed for either ALO dose. The proportions of patients who experienced $\geq 1$ adverse event (ALO $12.5 \mathrm{mg}, 67.9 \%$; ALO 25 $\mathrm{mg}$, 66.7\%; PBO, 73.6\%) or $\geq 1$ serious adverse event (ALO $12.5 \mathrm{mg}, 6.1 \%$; ALO $25 \mathrm{mg}, 5.4 \%$; PBO, 4.7\%) were similar across groups. The proportion of patients who discontinued the study due to an adverse event was small (ALO $12.5 \mathrm{mg}, 0.8 \%$; ALO $25 \mathrm{mg}, 4.7 \%$; PBO, 3.1\%). Incidence of severe hypoglycemia was very low [ALO $12.5 \mathrm{mg}(0 \%)$, ALO $25 \mathrm{mg}(0.8 \%), \mathrm{PBO}(1.6 \%)$ ], and mean daily insulin dose was unchanged from baseline across groups.

Conclusion: ALO added to ongoing INS therapy (with or without MET) significantly improved glycemic control in patients with T2DM inadequately controlled on INS without increasing weight gain or hypoglycemia.

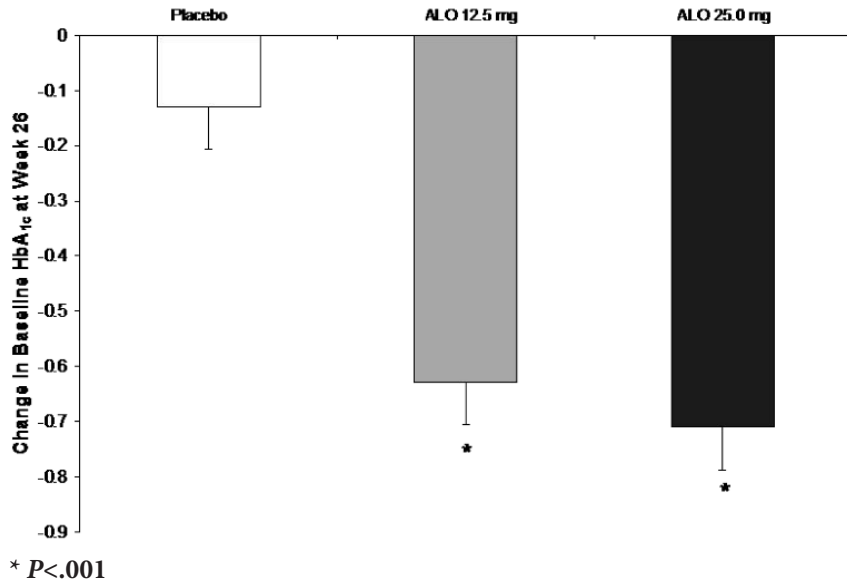

Values are least-squares mean (SE)

Supported by: Takeda Global Research \& Development Center

\section{8}

Initial combination therapy with saxagliptin and metformin improves glycaemic control compared with either monotherapy alone in drugnaïve patients with type 2 diabetes

R. Chen ${ }^{1}$, A. Pfützner ${ }^{2}$, M. Jadzinsky ${ }^{3}$, E. Paz-Pacheco ${ }^{4}$, Z. Xu1 ${ }^{1}$, E. Allen ${ }^{1}$ ${ }^{1}$ Bristol-Myers Squibb, Princeton, United States, ${ }^{2}$ Institute for Clinical Research and Development, Mainz, Germany, ${ }^{3}$ Department of Nutrition, Durand Hospital, Buenos Aires, Argentina, ${ }^{4}$ Department of Medicine, The Medical City, Pasig City, Philippines

Background and aims: Saxagliptin is a potent modulator of key hormones that regulate glucose homeostasis, specifically designed to provide selective, durable inhibition of the dipeptidyl peptidase-4 (DPP-4) enzyme. In this study, the efficacy and safety of the initial combination of saxagliptin plus immediate-release metformin (MET) were compared with saxagliptin or MET monotherapy in drug-naïve patients with type 2 diabetes (T2D) and inadequate glycemic control.
Materials and methods: This 24-wk, double-blind, active-controlled trial (CV181-039) randomized 1306 patients with T2D, $\mathrm{HbA}_{1 c} 8 \%-12 \%$ and age 18-77 y. Patients were randomized to 1 of 4 treatment arms: saxagliptin $5 \mathrm{mg}+$ MET $500 \mathrm{mg}$ (S5/MET), saxagliptin $10 \mathrm{mg}+$ MET $500 \mathrm{mg}$ (S10/ MET), saxagliptin $10 \mathrm{mg}$ + placebo (S10), or MET $500 \mathrm{mg}+$ placebo (MET). Saxagliptin was dosed once daily. From wk 1-5, in the S5/MET, S10/MET, and MET treatment arms, metformin was up-titrated based on fasting plasma glucose (FPG) levels in $500-\mathrm{mg} / \mathrm{d}$ increments as tolerated to a maximum of $2000 \mathrm{mg} / \mathrm{d}$. The primary end point was the $\mathrm{HbA}_{1 c}$ change from baseline at wk 24. Changes in FPG, PPG, and HOMA-2 $\beta$ were also evaluated. Efficacy analyses for continuous variables were performed using an ANCOVA model with last observation carried forward (LOCF) methodology.

Results: Treatment groups were well balanced for baseline demographics and diabetes characteristics: 49\% male, mean age 52 y, mean duration of T2D 1.7 y, mean $\mathrm{HbA}_{1 \mathrm{c}}$ 9.5\%, mean FPG $200.6 \mathrm{mg} / \mathrm{dL}$. Final mean metformin total daily doses in the S5/MET, S10/MET, and MET arms were $1790 \mathrm{mg}, 1776 \mathrm{mg}$, and $1817 \mathrm{mg}$, respectively. At 24 wks, patients randomized to S5/MET and S10/MET demonstrated significant reductions in $\mathrm{HbA}, \mathrm{FPG}$, and PPG, and increases in HOMA-2 $\beta$ (a measure of $\beta$-cell function) from baseline compared with either monotherapy (Table).

\begin{tabular}{lllll}
\hline & S5/MET & S10/MET & S10 & MET \\
\hline HbA1c $^{*}(\%)$ & $-2.53 \mathrm{a}, \mathrm{b}$ & $-2.49 \mathrm{a}, \mathrm{b}$ & -1.69 & -1.99 \\
\hline FPG $^{*}(\mathrm{mg} / \mathrm{dL})$ & $-59.8 \mathrm{a}, \mathrm{c}$ & $-62.2 \mathrm{a}, \mathrm{b}$ & -30.9 & -47.3 \\
\hline $\begin{array}{l}\mathrm{PPG}^{*} 120 \text { min post-OGTT } \\
(\mathrm{mg} / \mathrm{dL})\end{array}$ & $-137.9 \mathrm{f}, \mathrm{b}$ & $-137.3 \mathrm{~d}, \mathrm{~b}$ & -106.3 & -96.8 \\
\hline HOMA-2 $\beta^{*}(\%)$ & $33.0 \mathrm{a}, \mathrm{e}$ & $38.0 \mathrm{a}, \mathrm{b}$ & 18.2 & 22.6 \\
\hline $\begin{array}{l}\text { Patients who achieved HbA1c } \\
<7.0 \%(\%)\end{array}$ & $60.3 \mathrm{a}, \mathrm{b}$ & $59.7 \mathrm{a}, \mathrm{b}$ & 32.2 & 41.1 \\
\hline
\end{tabular}

${ }^{*}$ Adjusted mean change from baseline.

$\mathrm{aP}<.0001$ vs $\mathrm{S} 10$.

$\mathrm{bP}<.0001$ vs $\mathrm{MET}$.

$\mathrm{cP}=.0002$ vs $\mathrm{MET}$.

$\mathrm{dP}=.0002$ vs $\mathrm{S} 10$.

$\mathrm{eP}=.0004$ vs MET.

$\mathrm{fP}=.0001$ vs $\mathrm{S} 10$.

A numerically greater decrease in $\mathrm{HbA}_{1 c}$ was seen in patients with a baseline $\mathrm{HbA}_{1 \mathrm{c}} \geq 10 \%$ (adjusted mean change from baseline $-3.33 \%,-3.25 \%,-2.52 \%$, and $-2.73 \%$ for S5/MET, S10/MET, S10, and MET, respectively). Overall, the occurrence of adverse events was similar across all treatment groups: S5/ MET 55.3\%, S10/MET 57.3\%, S10 53.4\%, and MET 58.5\%. Occurrences of hypoglycemic events were few (S5/MET 3.4\%, S10/MET 5.0\%, S10 1.5\%, and MET $4.0 \%$ ).

Conclusion: In drug-naïve patients with T2D, initial combination therapy of saxagliptin and metformin produced statistically significant and clinically meaningful improvements in glycemic parameters compared with either saxagliptin or metformin alone, and was well tolerated.

Supported by: Bristol-Myers Squibb Company and AstraZeneca 


\section{OP 14 Obesity: new insights and new therapies}

\section{9}

Leptin is released from human skeletal muscle in vivo E. Wolsk-Jackson ${ }^{1}$, H. Mygind ${ }^{1}$, T. Hansen ${ }^{2}$, B.K. Pedersen ${ }^{1}$, G. van Hall ${ }^{2}$ ${ }^{1}$ Centre of Inflammation and Metabolism, ${ }^{2}$ Department of Biomedical Sciences, Rigshospitalet, Copenhagen, Denmark

Background and aims: Leptin has been characterized as an adipokine released from adipose tissue. Furthermore human in vivo leptin concentrations seem to be correlated with total body fat mass. Recent reports indicate that leptin may also be produced by skeletal muscle, however a characterization of the leptin release from adipose tissue versus skeletal muscle has never been investigated. Our aim was to;

- Characterise and quantify the release of leptin from leg skeletal muscle and from anterior abdominal wall adipose tissue.

-Quantify the possible contribution of leptin from skeletal muscle to systemic circulation compared to the concomitant contribution from subcutaneous adipose tissue.

Materials and methods: Eight healthy male volunteers were recruited in this observational study. After an overnight fast, subjects reported to the lab where they remained fasting, supine and sedentary during the entire study. The femoral artery and vein, as well as the superficial epigastric vein were cannulated allowing arterio-venous blood sampling over the lower extremity and abdominal subcutaneous tissue bed. The femoral venous catheter was placed retrograde, bypassing the saphenous vein, thus minimizing subcutaneous blood contamination. Blood flow was measured using 133-Xenon washout, and Doppler ultrasound in the abdomen and groin, respectively. This allowed quantification of leptin flux across the aforementioned tissue beds according to Fick's principle. Blood was collected every $30 \mathrm{~min}$. for three hours, followed by hourly sampling for the remaining three hours. All data listed are pooled geometric means \pm SD.

Results: Leptin concentrations were $1067 \pm 105 \mathrm{pg} \mathrm{ml}^{-1}, 1391 \pm 136 \mathrm{pg} \mathrm{ml}^{-1}$, and $1783 \pm 244 \mathrm{pg} \mathrm{ml}^{-1}$, for artery, leg vein, and abdominal vein, respectively. There was a significant difference between arterio-venous samples from both leg $(\mathrm{p}=0.02)$ and adipose tissue $(\mathrm{p}<0.0001)$, indicating that both organs released leptin to the systemic circulation throughout the trial. There was not any significant change over time within groups. Leptin flux across the adipose tissue and leg averaged $1250 \pm 308 \mathrm{pg} \mathrm{min}^{-1} 100 \mathrm{~g}^{-1}$ and $89796 \pm 25020 \mathrm{pg} \mathrm{min}^{-1}$, respectively.

Conclusion: In agreement with our aims, we showed that leptin not only was released into the systemic arterial circulation from adipose tissue, but also from the lower extremity. Although our femoral catheter placement minimizes subcutaneous adipose tissue contribution to the leptin flux observed, it cannot be ruled out as a contributor. Calculations were performed using a "worst-case" scenario that the leg flux observed was due to leg adipose tissue. Assuming that leptin release pr. mass unit from adipose tissue in leg and abdomen is equal, approximations using tissue composition obtained through DXA scans reveal that adipose contribution to the flux observed maximally

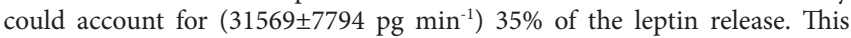
would leave the remaining $65 \%$ to be accounted for by skeletal muscle, equalling a release of $834 \mathrm{pg} \mathrm{min}^{-1} 100 \mathrm{~g}^{-1}$ tissue. Conclusively, it seems plausible that skeletal muscle is a major contributor to human leptin production. Supported by: Danish National Research Foundation, Danish Medical Research Council, Commission of the European Communities, A.P.Moller foundation

\section{0}

A common MC4R variant is associated with higher intakes of total energy and dietary fat, long-term weight change, and obesity risk in women

L. Qi, P. Kraft, D. Hunter, F. Hu

Nutrition and Epidemiology, Harvard School of Public Health, Boston, United States

Background and aims: Melanocortin-4 receptor (MC4R) regulates energy balance. The common variants near to MC4R gene were recently associated with obesity risk (Loos et al. Nature Genet. 2008). We examined the associations of the MC4R gene variants with energy intakes, adiposity and the longitudinal changes of anthropometrical measures.
Materials and methods: The associations of MC4R SNPs rs17782313 (T>C) and rs $17700633(\mathrm{G}>\mathrm{A}$ ) with dietary intakes (total energy, fat, and carbohydrates) and longitudinal measures of adiposity (1976 to 2004) were analyzed in 5,724 women (1,533 with type 2 diabetes) from the Nurses' Health Study. Results: Women with CC genotype of $\mathrm{rs} 17782313 \mathrm{had} 84 \mathrm{kcal} / \mathrm{d}, 4.6 \mathrm{~g} / \mathrm{d}$, and $1.8 \mathrm{~g} / \mathrm{d}$ higher intakes in total energy $(\mathrm{P}=0.028)$, total fat $(\mathrm{P}=0.008)$, and saturated fat $(\mathrm{P}=0.007)$ compared with those carrying TT genotype, adjusting for age, BMI, and diabetes status. Per allele-C of SNP rs17782313 was significantly associated with $0.34 \mathrm{~kg} / \mathrm{m} 2$ higher $\mathrm{BMI}(\mathrm{P}=0.002), 0.73 \mathrm{~cm}$ higher waist circumference $(\mathrm{P}=0.01)$, and $12 \%(1 \%-26 \%)$ greater obesity risk (measured in 1986). Adjustment for dietary factors (total energy and fat) did not appreciably change the SNP-adiposity associations. Carrying allele- $\mathrm{C}$ was also associated with moderate but statistically significant difference $(0.2 \mathrm{~kg} / \mathrm{m} 2)$ in tenyear (1976-1986) changes of BMI ( $\mathrm{P}=0.028)$. Age-specific analyses indicated that the genetic difference in BMI was significant across all the age groups and was most evident in women aged 50-54.9 years (Figure; ${ }^{*}$ : $\mathrm{P}<0.05$ ). In addition, body size (height) significantly modified the associations between rs17782313 and BMI (P for interaction=0.03). The allelic difference in BMI was $0.9,0.6$, and $0.2 \mathrm{~kg} / \mathrm{m} 2$ respectively in women with height of greater than $165 \mathrm{~cm}, 160-164.9 \mathrm{~cm}$, and less than $160 \mathrm{~cm}$. Analyses restricting to non-diabetic women generated similar results.

Conclusion: The common SNP rs17782313 in MC4R gene was significantly associated with higher intakes of total energy and dietary fat (total and saturated), long-term weight change, and obesity risk. Body size may modify the genetic effects.

\section{$\diamond \mathbf{T T} \quad \square \mathbf{T C}+\mathbf{C C}$}

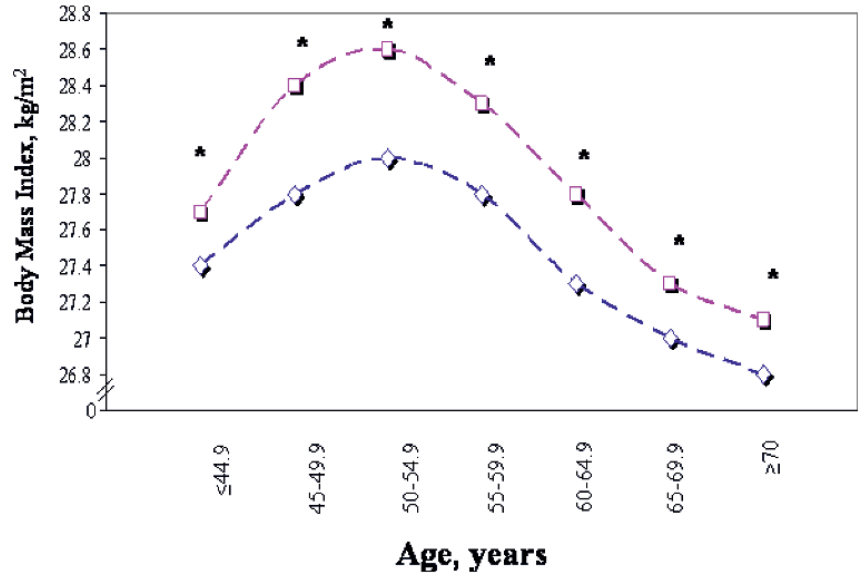

Supported by: American Heart Association Scientist Development Award

\section{1}

Development of leptin resistance during long-term substitutive therapy on children with congenital lipodystrophy

J. Beltrand ${ }^{1,2}$, N. Lahlou ${ }^{3}$, N. Tubiana Rufi, M. Polak ${ }^{5}$, M. De Kerdanet ${ }^{6}$, F. Huet ${ }^{7}$, D. Lacombe ${ }^{8}$, D. Chevenne ${ }^{1}$, C. Lévy Marchal ${ }^{1}$

${ }^{1}$ U690, Inserm, Paris, France, ${ }^{2}$ Hopital Robert Debré, Paris, France, ${ }^{3}$ UF Hormonologie pédiatrique, Hopital Saint Vincent de Paul, Paris, France, ${ }^{4}$ Endocrinologie pédiatrique, Hopital Robert Debré, Paris, France, ${ }^{5}$ Endocrinologie pédiatrique, Hopital Necker, Paris, France, ${ }^{6}$ Endocrinologie pédiatrique, Hopital Sud, Rennes, France, ${ }^{7}$ Pédiatrie, Hopital d’enfants, Dijon, France, ${ }^{8}$ Génétique médicale, Hopital Pellegrin, Bordeaux, France

Background and aims: It has been proposed that leptin could be used as a treatment against metabolic complications in patients with congenital lipodistrophy (CLD). The benefit of short-term replacement therapy on dyslipidemia, steatosis and insulin resistance has been previously reported in children with CLD but the persistence of such an effect on a long-term basis is unknown.

Materials and methods: The efficacy of long-term leptin replacement therapy (24 months) has been evaluated in children with CLD. R-metHU leptin was initially given (subcutaneous injections) at a daily "physiological" dose $(0.06 \mathrm{mg} / \mathrm{kg} / \mathrm{d})$ and increased up to $0.12 \mathrm{mg} / \mathrm{kg} / \mathrm{d}$ if necessary. Patients were evaluated every 4 months. Criteria for a positive response to treatment were defined as a reduction in liver volume by $30 \%$ or more (CT scan) and/or an improvement in insulin sensitivity $(\mathrm{M})$ measured during a euglycemic hyper- 
insulinemic clamp by at least $30 \%$ and/or a reduction in fasting triglyceride (TG) levels by $40 \%$ or more in comparison to baselines values. Antibodies against leptin were measured with a specific home-made radio-binding assay.

Results: Eight non-diabetic children (6 boys, 2 girls, 6-16 years old) were included in the study. In 3 patients, improvement of TG levels was sustained for up to 24 months (TG: M0 2,23+/- 0,9 to M24: $1,43+/-0,8$ ) associated with an increase in insulin sensitivity (M: M0 4,6 mg/kg FFM/mn to 7,3 mg/kg $\mathrm{FFM} / \mathrm{mn}$ ) and a marked decrease in hepatic volume (M0 $5087+/-618 \mathrm{~mm} 3$ to M24: $3153+/-392 \mathrm{~mm} 3$ ). In 2 patients, leptin dosage needed to be increased in order to maintain these effects. In 2 other patients, the increase in the leptin dosage failed to maintain the benefit on insulin sensitivity whereas the effect on liver volume was still observed after 24 months of therapy (6752 $\mathrm{mm} 34713 \mathrm{~mm} 3$ and 5714 to $2759 \mathrm{~mm} 3$ ). In 3 patients, no benefit at all was observed and one patient developed overt diabetes despite leptin concentration above the physiological range $(133+/-90 \mathrm{ng} / \mathrm{ml})$. In all children antibodies against leptin were detectable after 4 month with levels parallel to the increase in leptin dosage.

Conclusion: The benefit of leptin replacement therapy on metabolic complications of CLD was fully maintained in 3 patients whereas a partial or a null response was observed in 5 patients despite a 150 to $200 \%$ increase in the leptin dosage. This resistance to leptin therapy in patients with CLD has never been previously reported and the present observation argues for the contribution, at least in part, of an immunological reaction. Further investigations are crucial since leptin replacement remains the only therapeutical hope in this disease with a life-threatening prognosis.

Supported by: Institut national de la santé et de la recherche médicale, France

\section{2}

Responsiveness to leptin restored by amylin in diet-induced obese (DIO) rats: magnitude and mechanisms of synergy

J. Roth, J. Koda, B. Roland, R. Cole, T. Coffey, C. Cronister, C. Weyer,

A. Baron, D. Parkes

Research, Amylin Pharmaceuticals, Inc., San Diego, United States

Background and aims: Previously, we reported that single dose combination therapy with rat amylin $(100 \mu \mathrm{g} / \mathrm{kg} / \mathrm{d})$ and murine leptin $(500 \mu \mathrm{g} / \mathrm{kg} / \mathrm{d})$ elicited greater body weight (BW) loss in leptin resistant DIO rats than predicted by the sum of monotherapy-induced BW loss, a finding consistent with amylin restoration of responsiveness to leptin.

Materials and methods: Here, we used a $3{ }^{\star} 4$ factorial design to define the interaction of a lower range of doses of amylin $(0,10,50 \mu \mathrm{g} / \mathrm{kg} / \mathrm{d})$ and leptin $(0$, $5,25,125 \mu \mathrm{g} / \mathrm{kg} / \mathrm{d}$ ) for BW loss following $4 \mathrm{w}$ of subcutaneous (SC) osmotic infusion in DIO rats. This design enabled a Response Surface Methodology (RSM) analysis and statistical testing for synergy.

Results: At $4 \mathrm{w}$, the RSM revealed a synergistic effect of the amylin+leptin combination $(\mathrm{P}<0.03)$, at doses lower than previously reported. The highest dose combination (amylin 50+leptin 125) induced a maximal mean BW loss of $-14.8 \pm 3.2 \%$ (vehicle corrected). Leptin resistance in DIO rodents has been attributed to impaired hypothalamic leptin signaling, chiefly within the arcuate nucleus (Arc). We hypothesized that amylin modulates hypothalamic sensitivity to leptin. Hypothalamic pSTAT-3 immunoreactive (IR) cells stimulated by leptin $(15 \mathrm{mg} / \mathrm{kg}$, IP) were quantified in DIO rats pre-treated with either vehicle, amylin $(100 \mu \mathrm{g} / \mathrm{kg} / \mathrm{d}, \mathrm{SC})$ or food restriction by pair-feeding (PF) to amylin rats for $7 \mathrm{~d}$, and in lean rats maintained on low fat diet. Compared to lean controls, vehicle-treated DIO rats had reduced pSTAT-3 IR in both the $\operatorname{Arc}(-46 \%, \mathrm{p}<0.0001)$ and the ventromedial hypothalamus (VMH; $-45 \%, \mathrm{p}<0.0001)$. Amylin pre-treatment for $7 \mathrm{~d}$ increased leptin-induced pSTAT-3 IR in the VMH relative to vehicle (by $40 \% ; \mathrm{p}=0.015$ ) and PF controls (by $50 \%, \mathrm{p}=0.004$ ), but not in the Arc.

Conclusion: These findings formally confirm BW loss synergy between amylin and leptin in DIO rodents and reveal a novel neural correlate for synergy, whereby amylin, via hindbrain signaling, upregulates leptin VMH signaling. Unexpectedly, restoration of PSTAT-3 signaling in Arc does not appear to be necessary for expression of this effect.

\section{3}

Enhanced weight loss following pramlintide/metreleptin combination treatment in obese subjects: clinical evidence for restoration of leptin responsiveness by amylin agonism

J. Koda ${ }^{1}$, C. Weyer ${ }^{1}$, S. Smith ${ }^{2}$, E. Ravussin ${ }^{2}$, J. Mitchell ${ }^{1}$, N. Kesty ${ }^{1}$, K. Shan ${ }^{1}$, A. Baron ${ }^{1}$

${ }^{1}$ Amylin Pharmaceuticals, Inc., San Diego, United States, ${ }^{2}$ Biomedical Research, Louisiana State University, Baton Rouge, United States

Background and aims: Numerous clinical trials have failed to demonstrate significant weight loss with human recombinant leptin (metreleptin) monotherapy. In diet-induced obese rats, prior or concomitant amylin agonism restores responsiveness to leptin, yielding marked synergistic weight loss.

Materials and methods: We conducted a 24 -wk, randomised, active-drugcontrolled, double-blind clinical study in 177 obese or overweight subjects (Enrolled; 63\% female; $39 \pm 8 \mathrm{y} ; 32.0 \pm 2.1 \mathrm{~kg} / \mathrm{m}^{2} ; 93.3 \pm 13.2 \mathrm{~kg}$; mean $\pm \mathrm{SD}$ ). During a 4-wk lead-in period, subjects were treated with the amylin agonist pramlintide (180 $\mu \mathrm{g}$ BID for $2 \mathrm{wks}$; then $360 \mu \mathrm{g}$ BID) and diet (40\% calorie deficit). Subjects (N=139) completing the lead-in and achieving 2-8\% weight loss by Wk 4 were randomised 2:2:1 to pramlintide (360 $\mu \mathrm{g}$ BID) + metreleptin (5 mg BID), pramlintide+placebo or metreleptin+placebo for $20 \mathrm{wks}$.

Results: Following the 4-wk lead-in, 9\% of the subjects did not achieve $2 \%$ weight loss. By Wk 20 (Evaluable $\mathrm{N}=93$ ), pramlintide+metreleptin elicited significant reductions in weight from enrollment $(12.7 \pm 0.9 \%$; LS mean \pm SE) compared to pramlintide $(8.4 \pm 0.9 \% ; \mathrm{P}<0.001)$ or metreleptin following the pramlintide lead-in $(8.2 \pm 1.3 \%$; $<0.01)$. From Wk $12-20$, weight loss had stabilised with pramlintide or metreleptin (rate of weight change $-0.05 \mathrm{~kg} / \mathrm{wk}$ or $-0.02 \mathrm{~kg} / \mathrm{wk}$; both $\mathrm{P}>0.05$ ), but not with pramlintide+metreleptin (rate of weight change $-0.20 \mathrm{~kg} / \mathrm{wk} ; \mathrm{P}<0.001$ ). The proportion of subjects achieving $\geq 10 \%$ weight loss was greater with pramlintide+metreleptin (56\%) than with pramlintide $(35 \%)$ or metreleptin $(21 \%)$. The most common side effects with pramlintide+metreleptin were injection site adverse events and nausea, which were mostly mild to moderate.

Conclusion: This is the first demonstration of a significant and robust weight loss effect of leptin in subjects with general obesity and is consistent with preclinical studies showing amylin+leptin synergy. Further clinical development of pramlintide+metreleptin combination treatment is warranted as part of a novel, integrated neurohormonal approach to obesity pharmacotherapy.

\section{4}

Anti-obesity effect of highly purified eicosapentaenoic acid in mice with high-fat/high-sucrose diet-induced obesity: possible role of hepatic lipogenesis

A. Sato ${ }^{1}$, M. Itoh ${ }^{2}$, M. Nakakuki ${ }^{1}$, M. Ohta ${ }^{1}$, H. Kawano ${ }^{1}$, K. Mizuguchi ${ }^{1}$, T. Suganami ${ }^{2}$, Y. Ogawa ${ }^{2}$

${ }^{1}$ Pharmaceutical Research Center, Mochida Pharmaceutical Co., Ltd., Shizuoka, Japan, ${ }^{2}$ Department of Molecular Medicine and Metabolism, Medical Research Institute, Tokyo Medical and Dental University, Tokyo, Japan

Background and aims: Obesity is a major risk factor of coronary heart disease (CHD). A large-scale, prospective, randomized clinical trial has recently revealed that the addition of highly purified eicosapentaenoic acid (EPA), one of the major $n-3$ polyunsaturated fatty acids in fish oil, to statin therapy significantly reduces the incidence of $\mathrm{CHD}$. Here we investigated the anti-obesity effect of highly purified EPA in mice with diet-induced obesity.

Material and methods: 10 -week-old male C57BL/6 mice were fed standard $\operatorname{diet}(\mathrm{N})$, high-fat/high-sucrose diet (HF/HS), high-fat diet (HF), or HF/HS or HF containing $5 \% \mathrm{EPA}$ (HF/HS+EPA or HF+EPA). Body weight, tissue weight, expression of mRNAs for enzymes related to $\beta$-oxidation, glycolysis, and lipogenesis in white adipose tissue (WAT), liver, and skeletal muscle, hepatic triglyceride (TG) secretion, and $\mathrm{O}_{2}$ consumption were measured.

Results: After 20-week feeding, body weight was significantly higher in both $\mathrm{HF} / \mathrm{HS}$ and $\mathrm{HF}$ groups than $\mathrm{N}$ group. Both $\mathrm{HF} / \mathrm{HS}$ and HF groups also showed increased hepatic weight and TG content $(P<0.01 \mathrm{vs.} \mathrm{N} \mathrm{group).} \mathrm{The}$ HF/HS+EPA group showed significant reduction in body weight relative to HF/HS group $(P<0.01)$, which was accompanied by the reduction in visceral (epididymal, mesenteric, and retroperitoneal) and subcutaneous (inguinal) fat weights $(P<0.01)$. The HF/HS+EPA group was protected from HF/HSinduced increase in liver weight $(P<0.01)$. By contrast, there was no such reduction of body and liver weight in $\mathrm{HF}+\mathrm{EPA}$ group relative to HF group. Increased $\mathrm{O}_{2}$ consumption and respiratory exchange ratio were observed in 
HF/HS+EPA group relative to HF/HS group after 6 weeks of feeding $(P<$ 0.01 ). In this study, HF/HS+EPA group did not show appreciable increases in mRNA expression of enzymes related to $\beta$-oxidation in WAT, liver, and skeletal muscle, those related to glycolysis in liver and skeletal muscle, and lipogenic enzymes in WAT, relative to HF/HS group. On the other hand, expression of mRNAs for hepatic lipogenic enzymes such as ACC-1, FAS, and SCD-1 was markedly increased in HF/HS group relative to $\mathrm{N}$ group, which was significantly suppressed by treatment with EPA $(P<0.01)$. In this study, there was no significant increase in mRNA expression of hepatic lipogenic enzymes in HF group relative to $\mathrm{N}$ group. The hepatic TG secretion rate in HF/ HS+EPA group was significantly lower than that in HF/HS group $(P<0.05)$. Analysis of fatty acid composition in very low density lipoprotein (VLDL) obtained from HF/HS+EPA group revealed significant decrease in palmitic (16:0), palmitoleic (16:1) and oleic (18:1) acids, but increase in stearic (18:0), linoleic $(18: 2)$ acids, and EPA relative to HF/HS group $(P<0.05)$.

Conclusion: This study demonstrates that treatment with EPA attenuates $\mathrm{HF} / \mathrm{HS}$-induced obesity and hepatic steatosis in parallel with the reduction of hepatic lipogenesis. Given that EPA does not affect HF-induced obesity with no marked increase in hepatic lipogenesis, the data of this study suggest that anti-obesity effect of EPA is related to reduced amount and altered composition of fatty acids delivered from the liver to the adipose tissue via VLDL.

\section{OP 15 Molecular mechanisms in insulin signalling}

\section{5}

Subcellular localisation determines protein kinase zeta function in 3T3L1 adipocytes

C. Blouin ${ }^{1}$, K.K. Takane ${ }^{2}$, A. Garcia-Ocana ${ }^{2}$, I. Dugail ${ }^{1}$, E. Hajduch ${ }^{1}$

${ }^{1}$ Centre de Recherches des Cordeliers, INSERM U872, Paris, France,

${ }^{2}$ Division of Endocrinology, University of Pittsburgh, United States

Background and aims: Atypical Protein kinase Cs (aPKCs) are involved in a wide variety of biological processes and data suggest an important role in insulin signalling and stimulation of glucose uptake in adipocytes and skeletal muscle. However, under certain circumstances, particularly in the presence of the sphingomyelin lipid derivative ceramide, aPKCs are also able to negatively regulate important proteins for insulin signalling, like protein kinase $\mathrm{B}$ (PKB), a key component of the signalling cascade that induces an increase in glucose uptake. The exact mechanism of this inhibition begins to be understood and is the result of a lipid-induced association between the aPKC zeta (PKC $)$ and $\mathrm{PKB}$, leading to the sequestration of the latter kinase in a repressed state within specific plasma membrane domains called caveolae. The aim of this study is to figure out how $\mathrm{PKC}$ can act positively or negatively on the insulin signalling pathway, depending of the stimulus by using different $\mathrm{PKC} \zeta$ mutants in 3T3-L1 adipocytes.

Materials and methods: We used adenovirus vectors containing either wild-

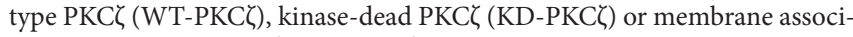

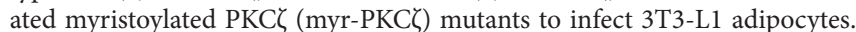
Subcellular localization of these mutants were carried out and we have evaluated the impact of insulin and ceramide on PKB activity.

Results: With the help of these mutants, we wanted to evaluate the importance of PKCל subcellular localization in the differential effects of insulin and ceramide on the insulin signalling pathway. Although both WT- and $\mathrm{KD}-\mathrm{PKC \zeta}$ mutants were preferentially present in the cytosol, all membranes fractions, including detergent resistant membranes (DRM) containing caveolae were enriched with the myr-PKCל mutant. Insulin, which induced the recruitment and phosphorylation of $\mathrm{PKC} \zeta$ to the plasma membrane, did not alter the partitioning between caveolar enriched membranes and other membrane fractions. Ceramide prevented insulin-induced PKB activation in WT- and myr-PKCל expressing cells and induced the phosphorylation of

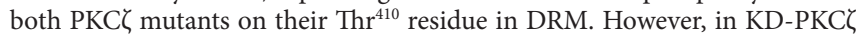
mutant expressing cells, PKC $\zeta$ did not distribute in DRM, and PKB activation by insulin was near to normal in the presence of ceramide. Importantly, in myr-PKC $\zeta$ expressing cells, the constitutive presence of $\mathrm{PKC} \zeta$ in caveolar enriched fraction did not mimic the negative effect of ceramide on insulininduced PKB activation, indicating the importance of the lipid to activate PKC $\zeta$ for the transduction of its signal.

Conclusion: All together, these findings indicate that activation of PKC $\mathrm{in}$ response of insulin or ceramide occurs in different subcellular membrane domains, leading the kinase to different actions on the insulin signalling pathway.

Supported by: INSERM

\section{6}

Nelfinavir-induced adipocyte insulin resistance is caused by impairment in $\mathrm{PKB} / \mathrm{Akt}$ sensing of $\mathrm{PI}_{3,45} \mathrm{P}_{3}$

A. Maissel ${ }^{1}$, I. Kachko ${ }^{1}$, L. Mazor ${ }^{1}$, R. Ben-Romano ${ }^{1}$, R. Watson ${ }^{2}$, J. Pessin ${ }^{2}$, N. Bashan ${ }^{1}$, A. Rudich ${ }^{1}$

${ }^{1}$ Department of Clinical Biochemistry, Ben-Gurion University, Beer Sheva, Israel, ${ }^{2}$ Department of Pharmacological Sciences, Stony Brook University, Stony Brook, United States

Background and aims: HIV protease inhibitors frequently cause insulin resistance and loss of adipose tissue, but the mechanisms remain largely unknown. In response to nelfinavir, adipocytes manifest insulin resistance characterized by normal signaling to PI 3-kinase (as detected in-vitro), but impaired activation of $\mathrm{PKB} / \mathrm{Akt}$ and stimulation of glucose uptake. In this study we aimed to dissect out the signaling defect at the level of PKB/Akt phosphorylation, caused by nelfinavir.

Materials and methods: A single-cell assay was used, based on 3T3-L1 adipocytes electroporated with a 3Xmyc-GLUT4-GFP reporter along with 
constitutively active PI 3-kinase or PKB/Akt, assessed by laser confocal microscopy. This was complemented by cell population analyses using Western blotting.

Results: Utilizing a single cell assay, we observed that nelfinavir treatment impaired the insulin-stimulated translocation and membrane-fusion of the myc-GLUT4-GFP tagged reporter. Signaling downstream of PKB/Akt (to GSK3, and to other PKB/Akt substrates, including AS160) was decreased, confirming that defective $\mathrm{PKB} /$ Akt activation is rate-limiting for signal propagation, and that $\mathrm{PKB} / \mathrm{Akt}$ function is required for insulin-stimulated GLUT4 translocation. However, insulin-induced generation of phosphatidylinositol (PI)-3,4,5-trisphohphate (PIP3) was comparable between nelfinavir-treated and control cells. Consistent with a nelfinavir induced inhibition of PKB/Akt activation, expression of a membrane-targeted catalytic subunit of PI 3-kinase induced phosphorylation of PKB/Akt and of GSK3, and mycGLUT4-GFP translocation in the absence of insulin in control, but not in nelfinavir-treated cells. Conversely, a membrane targeted and constitutively active $\mathrm{PKB} /$ Akt mutant was normally phosphorylated on S473 and T308 (confirming intact PKB/Akt kinases activity), and induced myc-GLUT4-GFP translocation even in nelfinavir-treated cells. Finally, nelfinavir treatment results in elevated expression of endogenous TRB3 (nearly 2.5 fold, $p=0.001$ ), a putative $\mathrm{PKB} / \mathrm{Akt}$ inhibitor protein.

Conclusion: A constitutively active PKB/Akt, but not PI 3-kinase normally induces GLUT4 translocation in adipocytes, suggesting that nelfinavir induces insulin resistance by directly interfering with PIP3-dependent activation of $\mathrm{PKB} /$ Akt.

Supported by: Israel Science Foundation

\section{7}

Downregulation of Sam68 inhibits insulin action by decreasing insulin receptor signalling

S. Najib, C. Gonzalez-Yanes, M. Martinez de Ubago, V. Sánchez-Margalet Medical Biochemistry and Moleculr Biology, Virgen Macarena University Hospital, Seville, Spain

Background and aims: Sam68, is an RNA binding protein with a proposed role as an adaptor protein in signal transduction based on the modular structure containing different domains for protein-protein interaction. We have previously found that Sam68 is tyrosine phosphorylated by the insulin receptor and recruited to signaling complexes in the PI3K and Ras pathways via interaction with $\mathrm{SH} 2$ and $\mathrm{SH} 3$ domain containing proteins. In the present study we have investigated the possible contribution of Sam68 in insulin receptor signaling by decreasing Sam68 expression.

Materials and methods: We have studied the role of Sam68 on insulin action and insulin receptor signaling pathways by decreasing Sam68 expression using an antisense oligonucleotide in $\mathrm{CHO}$ cells overexpressing insulin receptor (CHO-IR). A sense oligonucleotide was used as a control. We have investigated DNA synthesis by thymidine incorporation assay, and we have investigated insulin receptor signalling studying the phosphorylation of MEK, Erk-1-2, Akt and GSK-3.

Results: Downregulation of Sam68 expression decreased insulin stimulated DNA synthesis and insulin receptor signaling pathways in CHO-IR. Insulin stimulated thymidine incorporation was inhibited by $50 \%$, and this effect was correlated with a decrease in insulin receptor signalling. More precisely, both the insulin-stimulated PI3K and MAPK activation were decreased in CHOIR. Thus, the insulin-stimulated phosphoryation of MEK, MAPK, PKB, and GSK-3 were decreased by inhibiting the expression of Sam68 in CHO-IR cells.

Conclusion: These results provide evidence for a role of Sam68 in insulin action and signalling, and confirm the participation of Sam68 in the signal transduction pathways of the insulin receptor.

Supported by: Ministerio de Sanidad y Consumo, Spain

\section{8}

Synergy of SIRT1 with insulin signalling

L. Pirola, S. Fröjdö, C. Durand, H. Vidal

Medical Faculty BP12, INSERM U870, Oullins Cedex, France

Background and aims: Sir2 and its mammalian homolog SIRT1, that are proteins deacetylases belonging to the sirtuin family, underlie the control of genetic programs determining longevity in eukaryotes by acting as a stress resistance factor. Under caloric-restriction (CR) conditions, in which energy supply is limited - yet not to the stage of inducing starvation, Sir2/SIRT1 is considered to be the major genetic target. CR is, at present, the only experimental intervention known to prolong lifespan in eukaryotes. As CR is associated to enhanced peripheral insulin sensitivity, lower insulinaemia and improved glycaemic control, we sought to determine whether SIRT1 plays a direct role in the modulation of the insulin signalling cascade in muscle cells, that constitute the principal glucose-disposing tissue of the body.

Materials and methods: Adenoviruses expressing myc-SIRT1, IRS1 and myc-IRS2 have been constructed using the pVmAdcDNA3 system. Delivery of small interfering RNA in 293 cells was achieved by calcium phosphate transfection. The insulin-induced activation of PKB was monitored by measuring the PKB phosphorylation at Serine 473, with anti-phospho-specific antibodies.

Results: 293 cells transiently co-overexpressing myc-SIRT1 and myc-PKB displayed increased endogenous PKB and overexpressed myc-PKB Serine 473 phosphorylation when stimulated with $1000 \mathrm{nM}$ insulin. To reproduce this observation in physiologically relevant insulin-responsive cells, we constructed a SIRT1-expressing adenovirus allowing SIRT-1 overexpression in L6 muscle cells and human primary myotubes. Upon adenoviral-mediated overexpression of SIRT1, both in human primary myotubes and L6 cells, insulin-induced PKB phosphorylation was increased. Reciprocally, RNA interference of SIRT1 in 293 cells substantially decreased insulin-induced PKB Serine 473 phosphorylation after $10 \mathrm{nM}$ insulin stimulation. To test at which level of the insulin signalling cascade SIRT1 may act upon, we performed co-immunoprecipitation experiments and showed that SIRT1 interacts with IRS- 1 and IRS- 2 in an insulin dependent manner. Whether IRS proteins functionality and tyrosine phosphorylation status is enhanced through this interaction is being currently investigated.

Conclusion: SIRT1 have been proposed to improve glucose disposal and exert its metabolic-promoting effects through several pathways, including i) positive modulation of PGC 1-alpha via deacetylation and ii) transcriptional suppression of the protein tyrosine phosphatase PTP1B. Here, we show that SIRT1 also directly participate in the positive modulation of insulin signalling, in 293 and muscle cell lines, possibly by modulating the function of IRS1 and IRS-2 via a physical association induced by insulin. Supported by: ARD/PNRD

\section{9}

A novel role for Synaptotagmins in insulin-regulated glucose uptake in the 3T3-L1 adipocyte

S.C.M. Miller ${ }^{1,2}$, J.M.C. Connell', G.W. Gould ${ }^{2}$

${ }^{1}$ Faculty of Medicine, University of Glasgow, ${ }^{2}$ Faculty of Biomedical and Life Sciences, University of Glasgow, Scotland, United Kingdom

Background and aims: When insulin binds to its receptor at the surface of an adipocyte, myocyte or cardiomyocyte, the ensuing cascade of protein-protein and protein-lipid interactions leads to the translocation of a GLUT4-containing vesicle (GSV) to the plasmalemma (PM) where it docks, binds and fuses. In this way GLUT4 is delivered to its intended site of action, and glucose can enter the cell. The exact molecular machinery which governs GSV-PM fusion is incompletely characterised, but it is known that cognate interaction between the v-SNARE VAMP2, and t-SNAREs Syntaxin4(Stx4) and SNAP23 is required for effective GLUT4 delivery. GSV-PM fusion is known to be disrupted in adipocytes from individuals with Type-2 diabetes, and this disruption is proposed to contribute to insulin resistance.

SNARE-mediated fusion events are best understood in neurons and neuroendocrine cells, where an isoform of the Synaptotagmin (SYT) family, is known to bind to the assembled SNARE complex, and both target and vesicle membranes. Thereafter the opposing thermodynamic forces between adjacent lipid bilayers are overcome and membrane fusion can occur. We hypothesised that in a similar way a SYT isoform may bind the VAMP2/Stx4/ SNAP23 SNARE complex, and thus influence GSV-PM fusion. We therefore investigated the role of SYT isoforms in insulin-dependent glucose uptake in the adipocyte.

Materials and methods, and results: Using RTPCR, the expression of SYTs was confirmed in cultured 3T3-L1 adipocytes. SYT7 and SYT11 were shown to be the predominant isoforms, and SYT11 expression is upregulated as cells differentiate and acquire insulin-sensitivity. Both SYT7 and SYT11 proteins were observed in intracellular membrane fractions alongside GLUT4, and SYT7 was observed at the PM. siRNA-mediated knockdown of SYT7 was seen to potentiate glucose uptake, whereas SYT11 knockdown led to abrogated insulin-stimulated glucose uptake and reduced intracellular GLUT4. 
Finally SYT7 can be seen to bind VAMP2 and Stx4/SNAP23 in vitro whereas SYT11 does not.

Conclusion: These findings are consistent with a novel role for SYTs in the adipocyte, and we propose that SYT7 may influence the GSV-PM fusion machinery, and SYT11 may regulate intracellular GLUT4 trafficking and availability.

Supported by: MRC, DRWF, Diabetes UK, Chief Scientists Office (Scottish Government)

\section{0}

Role of AMPK in IL-6 release from isolated mouse skeletal muscle

S. Glund ${ }^{1}$, J.T. Treebak ${ }^{2}$, Y.C. Long ${ }^{1}$, R. Barres ${ }^{1}$, B. Viollet ${ }^{3}$,

J.F. Wojtaszewski ${ }^{2}$, J.R. Zierath ${ }^{1}$

${ }^{1}$ Molecular Medicine and Surgery, Karolinska Institutet, Stockholm, Sweden, ${ }^{2}$ Department of Exercise and Sports Sciences, University of Copenhagen,

Denmark, ${ }^{3}$ Institute Cochin, Université Paris Descartes, Paris, France

Background and aims: Interleukin-6 (IL-6) is released from skeletal muscle during exercise, and has consequently been implicated to mediate beneficial effects on whole body metabolism. Using 5-aminoimidazole-4-carboxamide-1- $\beta$-4-ribofuranoside (AICAR), a pharmacological activator of AMPK, we tested the hypothesis that AMPK modulates IL- 6 release from isolated skeletal muscle. We also tested whether AICAR suppresses IL-6 release via an AMPK-dependent pathway in animal models with impaired AMPK signaling

Materials and methods: Skeletal muscle from AMPKa2 kinase dead (KD) transgenic, AMPKa1 knockout (KO) and AMPK $\gamma 3 \mathrm{KO}$ mice and respective wild-type littermates was incubated in vitro, in the absence or presence of 2 $\mathrm{mM}$ AICAR. Skeletal muscle from wild-type mice was also incubated with the AMPK activator A-769662.

Results: Incubation of mouse extensor digitorum longus (EDL) and soleus muscles for $2 \mathrm{~h}$ was associated with a profound, ionomycin-independent IL6 mRNA production and protein release, which was suppressed by AICAR $(\mathrm{P}<0.001)$. Basal IL-6 release from soleus was increased between AMPKa2 $\mathrm{KD}$ and $\mathrm{AMPKa} 1 \mathrm{KO}$ and their respective wild-type littermates $(\mathrm{P}<0.05)$, suggesting AMPK participates in the regulation of basal IL-6 release from oxidative muscle. The effect of AICAR on muscle IL-6 release was similar between AMPK $\alpha 2 \mathrm{KD}$, AMPKa1 KO and AMPK $\gamma 3 \mathrm{KO}$ mice and their respective wild-type littermates $(\mathrm{P}<0.001)$, indicating AICAR-mediated suppression of IL- 6 release is independent of AMPK function. IL- 6 release from soleus, but not EDL muscle, was reduced $40 \%$ by A-769662 stimulation, further supporting the finding that the AICAR effects on muscle IL- 6 release are for the most part unspecific.

Conclusion: Using transgenic and knockout mouse models to perturb AMPK, we provide evidence that AMPK-dependent pathways regulate IL6 release from isolated oxidative skeletal muscle. We show in vitro AICARstimulation blunts IL-6 mRNA expression and protein release independent of functional AMPK-signaling in mouse skeletal muscle. Our results provide evidence for a role of AMPK in the regulation of IL-6 release from oxidative skeletal muscle.

\section{OP 16 Nephropathy: experimental treatments}

\section{1}

Protective roles of cholecystokinin against progression of diabetic nephropathy: cholecytokinin receptor deficiency enhances renal injuries after induction of diabetes

S. Miyamoto, K. Shikata, M. Sasaki, S. Nishishita, C. Sato, H. Kataoka, R. Kodera, D. Hirota, N. Kajitani, H. Makino

Department of Medicine and Clinical Science, Okayama University

Graduate School of Medicine, Dentistry and Pharmaceutical Sciences, Japan

Background and aims: We have recently reported that blockade of inflammatory axis of ICAM-1 activation to macrophage infiltration ameliorates diabetic nephropathy in ICAM-1 deficient (KO) mice, indicating that inflammatory mechanism is involved in the pathogenesis of diabetic nephropathy. DNA microarray analyses demonstrated that cholecystokinin (CCK) is upregulated in the kidney of diabetic wild type mice but not increased in diabetic ICAM-1 KO mice. These data suggest the involvement of CCK in development of nephropathy. CCK is a peptide with various functions including gastrointestinal homeostasis and neurotransmission, however, little is known about renal effects of CCK. Two types of CCK receptors, types A and B, have been identified in kidney tissues and cultured macrophages. We have also reported that deficiency of both CCK-A and B receptors accelerates renal injuries in 5/6 nephrectomized model, and that CCK-peptide suppressed the expression of proinflammatory cytokines in cultured macrophages. The aim of this study is to clarify the role of CCK in the pathogenesis of diabetic nephropathy using CCK receptor deficient mice.

Materials and methods: We induced diabetes by streptozotocin (STZ) in CCK-A receptor deficient, CCK-B receptor deficient (B-DM) and CCK-A and $B$ receptor double deficient mice $(A B-D M)$ and wild type mice (WT$\mathrm{DM})$. Non diabetic control mice were injected with citrate buffer. Renal functions and renal pathology were evaluated over a period of 3 months. We examined the expression of ICAM-1, CD-68 and type-IV collagen in kidney tissues by quantitative real-time RT-PCR and immunostaining. Gene expression profile were evaluated using DNA microarray.

Results: In non-diabetic mice, there was no difference of $\mathrm{HbAlc}$, blood pressure (BP) and albuminuria in all 4 strains. In diabetic mice, there was no significant difference of $\mathrm{HbAlc}$ in 4 strains. AB-DM showed markedly increased albuminuria as compared with WT-DM (Mean value; 474 vs. 154 $\mathrm{mg} / \mathrm{gCr}, \mathrm{P}<0.001)$. There was no significant difference of $\mathrm{BP}$ between $\mathrm{AB}-\mathrm{DM}$ and WT-DM (108 vs. $106 \mathrm{mmHg}$ ). Mesangial matrix index was significantly greater in AB-DM than in WT-DM ( 17.5 vs. $10.3 \%, \mathrm{p}<0.01)$. The intensity of type-IV collagen in glomeruli was higher in AB-DM as compared with WTDM. Gene expression of ICAM-1 and CD-68 were significantly increased in AB-DM as compared with WT-DM ( $<<0.001$ and $\mathrm{p}<0.05$, respectively). Gene expression of CCK was increased in diabetic group, whereas there was no difference between AB-DM and WT-DM. DNA microarray analysis indicated that various kinds of proinflammatory genes including cytokines, chemokines and adhesion molecules were up-regulated in $\mathrm{AB}-\mathrm{DM}$ as compared with WT-DM.

Conclusion: Our results have shown that deficiency of both CCK-A and B receptors enhances inflammatory reactions and tissue injuries in the kidney after induction of diabetes. These findings suggest that anti-inflammatory effects of CCK in the development of diabetic nephropathy were largely abrogated in CCK-A and B receptor double deficient mice. In conclusion, CCK is increased in diabetic kidney tissues and may play protective roles against progression of diabetic nephropathy through anti-inflammatory effects. CCK might be beneficial for the treatment of diabetic nephropathy.

\section{2}

Exendin-4 ameliorates diabetic nephropathy via anti-inflammatory and anti-ischaemic effects without change of blood glucose level in type 1 diabetic rats

R. Kodera, H. Kataoka, K. Shikata, C. Sato, M. Sasaki, S. Nishishita, S. Miyamoto, D. Hirota, N. Kajitani, H. Makino

Department of Medicine and Clinical Science, Okayama University Graduate School of Medicine Dentistry and Pharmaceutical Sciences, Japan 
Background and aims: Glucagon like peptide-1 (GLP-1) is known to have various extrapancreatic effects in addition to enhancement of insulin secretion. GLP-1 receptor has been shown to express in the kidney suggesting that GLP-1 may directly act on kidney via GLP-1 receptor. Exendin-4 is a DPPIV-resistant long-acting GLP-1 analog and synthetic exendin-4 is used for the therapy of type 2 diabetes. The aim of this study is to investigate the renoprotective effects of exendin- 4 independent of blood glucose lowering actions using streptozotocin induced diabetic rats.

Materials and methods: Five-week old male Sprague-Dawley rats were divided into four groups: non-diabetic: ND, non-diabetic rats treated with exendin- $4: \mathrm{ND}+\mathrm{EX}$, diabetic rats without treatment: DM, diabetic rats treated with extendin-4: DM+EX. Rats were administered intraperitoneally with exendin $-4(10 \mu \mathrm{g} / \mathrm{kg} / \mathrm{day}, \mathrm{ND}+\mathrm{EX}$ and $\mathrm{DM}+\mathrm{EX})$ or vehicle (ND and DM) every day for 8 weeks. To evaluate the effects of exendin-4, we measured urinary albumin excretion and creatinine clearance and examined the expression of GLP-1 receptor, TGF- $\beta 1$, ICAM-1, MCP-1, CD68, hypoxia-inducible factor (HIF) in kidney tissues by quantitative real-time RT-PCR and immunostaining.

Results: GLP-1 receptor was expressed in glomeruli, tubular epithelium and arterioles. Exendin-4 significantly reduced urinary albumin excretion

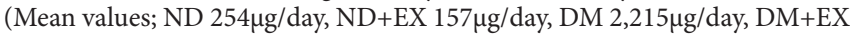
$713 \mu \mathrm{g} /$ day, $\mathrm{DM}$ vs $\mathrm{DM}+\mathrm{EX}: \mathrm{P}<0.05)$ without change of blood glucose levels (HbAlc; ND 3.7\%, ND+EX 3.8\%, DM 10.4\%, DM+EX 9.9\%, DM vs DM+EX: n.s.) and blood pressure (systolic blood pressure; ND 115mmHg, ND+EX $114 \mathrm{mmHg}$, DM 120 mmHg, DM+EX 123mmHg: n.s.). Hyperfiltration in diabetic rats were improved by treatment with exendin- 4 . Exendin- 4 significantly attenuated mesangial matrix expansion (mesangial matrix index; DM 9.2\% vs DM+EX 5.4\%: $\mathrm{P}<0.05$ ) and also significantly reduced mRNA expression of TGF- $\beta 1$ (Ratio to ND; ND 100\%, ND+EX 103\%, DM 262\%, DM+EX $143 \%$, DM vs DM+EX: P<0.05), ICAM-1 (ND 100\%, ND+EX 120\%, DM $283 \%$, DM+EX 142\%, DM vs DM+EX: P<0.05) and HIF (ND 100\%, ND+EX $104 \%$, DM 160\%, DM+EX 106\%, DM vs DM+EX: $\mathrm{P}<0.05)$ in kidney tissues. Expression of CD68 and MCP-1 were reduced by exendin-4, however, there was no statistical significance. Immunohistochemical analysis revealed that macrophage infiltration into kidney tissues in diabetic rats was suppressed by treatment with exendin- 4 .

Conclusion: Exendin- 4 ameliorated albuminuria and renal tissue injuries without change of blood glucose level and blood pressure in type 1 diabetic rats. Excendin-4 decreased the expressions of ICAM-1 and HIF suggesting that excendin- 4 ameliorated diabetic nephropathy through anti-inflammatory and anti-ischemic effects. Extendin-4 may exert novel extrapancreatic effects of reno-protection beyond blood glucose lowering effect.

Supported by: Ministry of Education, Science, Culture, Sports and Technology of Japan

\section{3}

Deletion of the receptor for advanced glycation end products, RAGE, protects from diabetic nephropathy and atherosclerosis

K.A.M. Jandeleit-Dahm ${ }^{1}$, J. Yong ${ }^{1}$, L. Jiaze ${ }^{1}$, D. Barit ${ }^{1}$, A. Soro-Paavonen ${ }^{1}$, S. Giunti ${ }^{1}$, A. Watson ${ }^{1}$, A. Bierhaus ${ }^{2}$, T. Allen ${ }^{1}$, M. Cooper $^{1}$

${ }^{1}$ Albert Einstein JDRF Centre for Diabetes Complications, Diabetes \& Metabolism Division, The Baker Heart Research Institute, Melbourne, Australia, ${ }^{2}$ Medicine and Clinical Chemistry, University of Heidelberg, Germany

Diabetic nephropathy remains the leading cause of end-stage renal disease in the Western World and diabetes associated macrovascular disease accounts for the increased morbidity and mortality in diabetes. There is increasing evidence that the multiligand receptor RAGE, plays a pivotal role in development and progression of diabetic nephropathy and macrovascular disease. We have previously shown that inhibition of AGE accumulation in the kidney and aorta can attenuate renal injury and atherosclerosis in diabetic apoE KO mouse. In this study we investigated if RAGE deletion protects from diabetic nephropathy and atherosclerosis.

ApoE KO and RAGE/apoE double KO mice were rendered diabetic by streptozotocin $(55 \mathrm{mg} / \mathrm{kg}$ in 5 daily injections ip)and followed for 20 weeks. At the end of the study, blood pressure was measured by tail cuff method and blood and urine were collected for analysis of glycated hemoglobin ( $\mathrm{GHb}$, HPLC)and plasma lipids as well as albuminuria. The aorta was assessed for plaque area after Sudan IV staining. The kidneys were analysed for structural renal injury including assessment of glomerulosclerosis index, mesangial area and tubulointerstitial injury. Extracellular matrix expression (collagen IV and fibronectin) as well as expression of pro-inflammatory and profibrotic growth factors, cytokines and chemokines were investigated by RT-PCR.
The results are presented in the Table below. Data are presented as mean \pm $\mathrm{SEM}^{*} \mathrm{p}<0.05$ vs control apoE KO, \# $\mathrm{p}<0.05$ vs diabetic apoE KO, $\ddagger \mathrm{p}<0.05$ vs control RAGE/apoE KO.

\begin{tabular}{lllll}
\hline & $\begin{array}{l}\text { Control } \\
\text { apoEKO }\end{array}$ & $\begin{array}{l}\text { Control } \\
\text { RAGE/ } \\
\text { apoE KO }\end{array}$ & $\begin{array}{l}\text { Diabetic } \\
\text { apoE KO }\end{array}$ & $\begin{array}{l}\text { Diabetic } \\
\text { RAGE/ } \\
\text { apoE KO }\end{array}$ \\
\hline Plaque area \% & $4.3 \pm 0.4$ & $2.3 \pm 0.4$ & $14.9 \pm 1.7^{*}$ & $4.9 \pm 0.4$ \\
\hline Mesangial area \% & $0.34 \pm 0.02$ & $0.41 \pm 0.01^{*}$ & $0.50 \pm 0.01^{*} 0.45 \pm 0.01 \#$ \\
\hline $\begin{array}{l}\text { Collagen IV } \\
\text { (fold expression) }\end{array}$ & $1.0 \pm 0.1$ & $0.5 \pm 0.1$ & $1.6 \pm 0.3^{*}$ & $0.9 \pm 0.1 \#$ \\
\hline $\begin{array}{l}\text { Fibronectin } \\
\text { (fold expression) }\end{array}$ & $1.4 \pm 0.1$ & $0.8 \pm 0.1$ & $2.9 \pm 0.3^{*}$ & $1.1 \pm 0.2 \#$ \\
\hline $\begin{array}{l}\text { MCP-1 (fold } \\
\text { expression) }\end{array}$ & $1.0 \pm 0.1$ & $0.4 \pm 0.1^{*}$ & $3.5 \pm 0.4^{*}$ & $1.5 \pm 0.2 \#$ \\
\hline $\begin{array}{l}\text { NFkappaB subunit } \\
\text { p65 (fold expression) }\end{array}$ & $1.0 \pm 0.1$ & $0.7 \pm 0.1$ & $2.9 \pm 0.7^{*}$ & $0.9 \pm 0.2$ \\
\hline $\begin{array}{l}\text { Albuminuria } \\
\text { microgram/24 hours }\end{array}$ & $49 \pm 6$ & $73 \pm 11$ & $188 \pm 20^{*}$ & $212 \pm 20$ \\
\hline
\end{tabular}

RAGE deletion attenuates renal structural injury and atherosclerosis in the diabetic apoE KO mouse, a model of diabetes accelerated atherosclerosis and renal disease. These changes were associated with reduced expression of proinflammatory and profibrotic growth factors in the kidney and aorta, including MCP-1 and the p65 subunit of NF kappaB. By contrast, albuminuria was not significantly attenuated by RAGE deletion.

Supported by: JDRF Centre grant and the National Health \& Medical Research Council of Australia

\section{4}

Functional expression of TRPV4 in human collecting duct (HCD) cells: a role in diabetic nephropathy

P.E. Squires ${ }^{1}$, C.E. Hills ${ }^{2}$, P.M. Ronco ${ }^{3}$, R. Bland ${ }^{1}$

${ }^{1}$ Biological Science, University of Warwick, Coventry, United Kingdom, ${ }^{2}$ Infection, Immunity and Inflammation, University of Leicester, United Kingdom, ${ }^{3}$ Unite INSERM 489, Universite Paris, France

Background and aims: According to the World Health Organisation (WHO Fact sheet 312, Sept 2006) diabetes is among the leading causes of kidney failure with a $10-20 \%$ mortality. When hyperglycaemia spills over into the urine (glucosuria), loss of glucose causes osmotic drag and diuresis and exposes the kidney to various physical and nutrient stresses. In collecting duct epithelium, sodium absorption is linked to cell volume regulation and depends upon detection of these mechanical stimuli. Prolonged exposure of renal epithelial to osmotic changes and increased fluid flow has led to a number of suggestions concerning the mechanisms involved in stimulus-response coupling. One mechanism involves the calcium permeable transient receptor potential-vanilloid-4 (TRPV4) channel. Gated by changes in osmolarity or membrane stretch, these mechano-sensitive channels reputedly regulate cell volume via a $\mathrm{Ca}^{2+}$ dependent mechanism. In the present study, mechanical stimulation was employed as a surrogate form of osmotic stress to elucidate a role for TRPV4 in cell volume regulation in a novel model system of the human collecting duct.

Materials and methods: RT-PCR and western blot analysis were used to determine mRNA and protein expression for TRPV4 in a human collecting duct (HCD) cell line. Fura-2-microfluorimetry was used to assess generation of touch evoked calcium transients. Knockdown of TRPV4 expression was achieved using a TRPV4 specific siRNA and the siLentGene U6 Casette RNA Interferance System different siRNA protocols.

Results: Bands corresponding to TRPV4 were identified from HCD mRNA and protein. Mechanical stimulation of a single HCD cell evoked a transient increase in cytosolic calcium $\left(\left[\mathrm{Ca}^{2+}\right]\right.$. $)$ that propagated between neighbouring cells within a cluster (5 separate experiments). Touch evoked changes in $\left[\mathrm{Ca}^{2+}\right]$, were still observed under $\mathrm{Ca}^{2+}$-free conditions, however the basal to peak amplitude of the response was only $35 \%$ of that obtained in the presence of extracellular calcium $(P<0.05 \mathrm{n}=6)$. Pre-incubation with the $\mathrm{Ca}^{2+}$-ATPase inhibitor thapsigargin $(1 \mu \mathrm{M})$ was used to assess contribution of intracellular $\mathrm{Ca}^{2+}$-stores on touch-evoked changes in $\left[\mathrm{Ca}^{2+}\right]_{i}$. Whilst removal of extracellular calcium alone, failed to negate the generation of touch evoked $\mathrm{Ca}^{2+}$ transients, the chelation of extracellular calcium in conjunction with the depletion of $\mathrm{Ca}^{2+}$-stores completely prevented touch-evoked changes in $\left[\mathrm{Ca}^{2+}\right]_{\mathrm{i}}$ 
$(P<0.05 ; \mathrm{n}=6)$. Confirmation of a role for TRPV4 in the generation of touch evoked $\mathrm{Ca}^{2+}$-transients was assessed by siRNA knockdown. Transient transfection of HCD cells with TRPV4 siRNA reduced TRPV4 protein expression by approximately $40 \%(n=4 ; P<0.01$ compared to control), whilst mechanical stimulation of individual RFP-tagged anti-TRPV4 cells failed to significantly elevate $\left[\mathrm{Ca}^{2+}\right]_{\mathrm{i}}$, compared to control cells observed in the same field of view under identical culture conditions.

Conclusion: These data confirm a functional role for TRPV4 in generation of touch evoked $\mathrm{Ca}^{2+}$-signals between coupled cells of the human collecting duct and suggest that knockdown of TRPV4 expression inhibit the cells ability to both detect and respond to osmotic signals via a $\mathrm{Ca}^{2+}$-dependent mechanism. These data have implications for altered fluid and electrolyte handling in the diabetic kidney.

Supported by: Diabetes, Endocrine and Immersion Trust

\section{5}

PARP inhibition counteracts multiple manifestations of nephropathy associated with type 1 diabetes

I.G. Obrosova ${ }^{1}$, W. Xu ${ }^{2}$, B. Slusher ${ }^{2}$, J. Zhang ${ }^{2}$, U. Julius ${ }^{3}$, V.R. Drel ${ }^{1}$

${ }^{1}$ Pennington Biomedical Research Center, Louisiana State University, Baton Rouge, United States, ${ }^{2}$ Pharmacology, Eisai Inc., Baltimore, United States,

${ }^{3}$ Medical Clinic III, University Hospital, Dresden, Germany

Background and aims: Evidence for the important role of poly(ADP-ribose) polymerase (PARP) activation in the pathogenesis of diabetes mellitus and diabetic complications is emerging. PARP activation manifest by increased poly(ADP-ribose) immunoreactivity and poly(ADP-ribosyl)ated protein expression is clearly manifest in the renal cortex of Type 1 diabetic rodents. The purpose of this work was to evaluate the role for PARP in early diabetic nephropathy using rat STZ-diabetic model.

Materials and methods: The experiments have been performed in rats with 12-wk duration of STZ-diabetes treated with or without two structurally diverse PARP inhibitors, 1,5-isoquinolinediol (ISO) and 10-(4-methyl-piperazin-1-ylmethyl)-2H-7-oxa-1,2-diaza-benzo[de] anthracen-3-one (GPI15427, Eisai Inc.), at the doses of $3 \mathrm{mg} \mathrm{kg}^{-1} \mathrm{~d}^{-1}$ i.p. and $30 \mathrm{mg} \mathrm{kg}^{-1} \mathrm{~d}^{-1}$ p.o., respectively, for 10 weeks after first 2 weeks without treatment. PARP activity in the renal cortex was assessed by immunohistochemistry and Western blot analysis of poly(ADP-ribosyl) ated proteins. Urinary albumin excretion, and renal concentrations of vascular endothelilal growth factor, endothelin1 , transforming growth factor- $\beta 1$, tumor necrosis factor- $\alpha$, and monocyte chemotactic protein-1 were evaluated by ELISA. Renal podocyte loss, PASpositive substances, and collagen- $\alpha 1$ (IY) were assessed by immunohistochemistry, and endothelin (B) receptor and advanced glycation end product expression by Western blot analyses.

Results: Final body weights were similarly reduced and blood glucose concentrations similarly increased in untreated and ISO- or GPI-15427-treated STZ-diabetic rats compared with controls. Urinary albumin excretion was increased $\sim 4$-fold in diabetic rats vs controls and this increase was essentially prevented by both ISO and GPI-15427. Both ISO and GPI-15427 alleviated diabetes-associated PARP activation in glomerular and tubular compartments of rat renal cortex. Kidney concentrations of vascular endothelial growth factor and endothelin-1, two important players in diabetic nephropathy, were increased in diabetic rats vs controls and this increase was completely (endothelin-1) or essentially (VEGF) prevented by PARP inhibition. ISO and GPI-15427 prevented diabetes-induced upregulation of renal endothelin (B) receptor. Both PARP inhibitors reduced diabetes-associated podocyte loss, and accumulation of transforming growth factor- $\beta$ 1 , collagen- $\alpha 1$ (IY), PAS-positive substances, and methylglyoxal-, but not glycol-derived advanced glycation end-products in the renal cortex. Elevation of renal concentrations of two inflammatory markers, tumor necrosis factor- $\alpha$ and monocyte chemotactic protein-1, was reduced although not completely prevented.

Conclusion: PARP activation is an important factor in early kidney disease associated with Type 1 diabetes. Assessment of the effects of PARP inhibition on chronic diabetic nephropathy is in progress.

Supported by: R21DK070720, JDRF

\section{6}

Deficiency in the angiotensin II type 1a receptor subtype decreases renal injury in diabetic mice

A. Koitka, A. Somasegeram, P. Sivakumaran, A. Mibus, A. Watson, P. Koh, M.C. Thomas, M.E. Cooper, T.J. Allen

Diabetes \& Metabolism, Baker Heart Research Institute, Melbourne, Australia

Background and aims: Angiotensin II (AII) plays a key pathophysiological role in the progression of diabetic renal disease. Agents that block the binding of AII to the AT1 receptor are an important therapeutic strategy to prevent renal damage in patients with diabetes. However, the specific role of AT1 receptors in diabetic renal disease remains to be defined. Two subtypes of the AT1 receptor, AT1a and AT1b, have been identified in the kidney. Because pharmacological AT1 receptor antagonists block both AT1a and AT1b receptors, it has been difficult to separate their distinct functions.

Materials and methods: We used genetically modified mice deficient in AT1a receptor (AT1a KO) or AT1b receptor (AT1b KO) to address the mechanism of RAS blockade-induced renoprotection in a diabetic model. Diabetes was induced by injection of streptozotocin $\left(60 \mathrm{mg} . \mathrm{kg}^{-1}\right.$. day ${ }^{-1}, 5$ daily intraperitoneal injections) in 6-week old male AT1a KO, AT1b KO and appropriate wild-type (WT) mice. In addition, control and streptozotocin-induced diabetic WT mice were randomised to the AT1 receptor antagonist, candesartan cilexetil (10 mg. $\mathrm{kg}^{-1}$.day ${ }^{-1}$ in drinking water) or no treatment for 20 weeks. Renal fibrosis was assessed by standard histological methods. Kidney function included measurement of urinary albumin excretion.

Results: Twenty weeks of diabetes in WT mice was associated with renal hypertrophy and an increase in glomerulosclerosis, tubulointerstitial fibrosis and albuminuria. The progression of diabetic kidney disease was attenuated in diabetic AT1a KO mice but not in diabetic AT1b KO mice. Deficiency in the AT1 a receptor subtype conferred similar renoprotection to that seen with candesartan in the diabetic WT mice (Table 1) as assessed by a similar reduction in albumineria and glomerular and tubulointerstitial injury.

Conclusion: Our results provide direct evidence that the renoprotective effects of candesartan in diabetic mice are predominantly mediated by blockade of the AT1a receptor. Given the clear benefits observed in AT1a KO mice and the negative effect of AT1b gene deletion on renal function, the potential utility of selective AT1a blockade deserves to be further explored.

\begin{tabular}{|c|c|c|c|c|c|}
\hline & Control WT & Diabetic WT & $\begin{array}{c}\text { Diabetic WT + } \\
\text { Candesartan }\end{array}$ & Diabetic AT1a KO & Diabetic AT1b KO \\
\hline \multicolumn{6}{|l|}{ Metabolic parameters } \\
\hline Body weight (g) & $30 \pm 1$ & $26 \pm 1 *$ & $25 \pm 1 *$ & $27 \pm 1 *$ & $23 \pm 1 * f$ \\
\hline Glucose $(\mathrm{mM})$ & $9.0 \pm 0.7$ & $26.5 \pm 1.4^{*}$ & $26.7 \pm 1.8^{*}$ & $24.0 \pm 1.4^{*}$ & $25.2 \pm 1.5^{*}$ \\
\hline Blood pressure $(\mathrm{mmHg})$ & $111 \pm 4$ & $119 \pm 6$ & $88 \pm 12 * f$ & $99 \pm 4 f$ & $115 \pm 2$ \\
\hline \multicolumn{6}{|l|}{ Renal function } \\
\hline Kidney mass $\left(g / \mathrm{m}^{2}\right)$ & $36.0 \pm 0.6$ & $53.0 \pm 0.9 *$ & $50.8 \pm 1.9 *$ & $44.4 \pm 1.4^{*} f$ & $57.4 \pm 1.4 * f$ \\
\hline Glomerulosclerosis & $1.3 \pm 0.1$ & $3.1 \pm 0.3^{*}$ & $1.9 \pm 0.3 f$ & $1.9 \pm 0.2 f$ & $2.7 \pm 0.3 *$ \\
\hline Tubulointerstitial area & $7.5 \pm 0.4$ & $13.9 \pm 0.4^{*}$ & $6.2 \pm 0.5 t$ & $8.3 \pm 0.7 f$ & $12.1 \pm 0.3^{*}$ \\
\hline Urine Albumin $(\mu \mathrm{g} / 24 \mathrm{hrs})$ & $131 \pm 14$ & $399 \pm 42^{*}$ & $268 \pm 37 * f$ & $293 \pm 19 * f$ & $561 \pm 53^{*} f$ \\
\hline
\end{tabular}

Data are expressed as means \pm SEM.

$* P<0.05$ vs. control WT group; $f P<0.05$ vs. diabetic WT group ( $n=6-12 /$ group)

Supported by: JDRF and ALFEDIAM 


\section{OP 17 Dynamics of beta cell exocytosis}

\section{7}

Both the first and second phases of glucose-induced insulin secretion involve insulin granules newly recruited to the plasma membrane H. Takahashi ${ }^{1}$, T. Shibasaki ${ }^{1}$, S. Seino ${ }^{1,2}$

${ }^{1}$ Division of Cellular and Molecular Medicine, ${ }^{2}$ Division of Diabetes, Metabolism and Endocrinology, Kobe University Graduate School of Medicine, Kobe, Japan

Background and aims: Insulin granule exocytosis includes several steps such as recruitment, docking, and fusion to the plasma membrane. Using a total internal reflection fluorescence microscopy (TIRFM) system, we have shown that fusion of insulin granules to the plasma membrane occurs in three modes, based on the dynamics of the granules. These modes are 1) old face: predocked granules are fused to the plasma membrane by stimulation. 2) restless newcomer: granules are newly recruited by stimulation and immediately fused to the plasma membrane without docking. 3) resting newcomer: granules are newly recruited by stimulation, docked, and then fused to the plasma membrane. In this study, we investigated granule dynamics in the first and second phases of glucose-induced insulin secretion.

Materials and methods: To monitor insulin granule dynamics, insulin fused to fluorescent protein Venus (insulin-Venus) was constructed. Insulin-Venus was introduced to isolated mouse pancreatic $\beta$-cells by adenovirus infection. Insulin granule dynamics in the infected cells was examined by sequential treatment of $16.7 \mathrm{mM}$ glucose and $60 \mathrm{mM} \mathrm{K}^{+}$stimulation. In condition A, cells were stimulated with $16.7 \mathrm{mM}$ glucose for $20 \mathrm{~min}$.; after a $10 \mathrm{~min}$ interval of incubation in $2.8 \mathrm{mM}$ glucose, the cells were stimulated with 60 $\mathrm{mM} \mathrm{K}^{+}$for $5 \mathrm{~min}$. In condition B, cells were stimulated with $60 \mathrm{mM} \mathrm{K}^{+}$for 5 min.; after a $10 \mathrm{~min}$ interval of incubation in $2.8 \mathrm{mM}$ glucose, the cells were stimulated with $16.7 \mathrm{mM}$ glucose for $20 \mathrm{~min}$. Insulin granule dynamics was monitored by TIRFM and analyzed.

Results: In cells stimulated with high glucose, fusion events occurred in a biphasic manner, most of them restless newcomer in both the first and the second phases. In contrast, in cells stimulated with $\mathrm{K}^{+}$, fusion events occurred immediately and transiently, more than $60 \%$ of them old face. In $\beta$-cells stimulated with $16.7 \mathrm{mM}$ glucose after $\mathrm{K}^{+}$stimulation, restless newcomer fusion events still occurred in a biphasic manner, similar to $\beta$-cells stimulated with glucose without initial $\mathrm{K}^{+}$stimulation. On the other hand, in $\beta$-cells stimulated with $60 \mathrm{mM} \mathrm{K}^{+}$after glucose stimulation, fusion events also occurred transiently and were mainly old face. In addition, the fusion sites in the $\beta$-cell induced by $\mathrm{K}^{+}$stimulation differed from those induced by glucose stimulation.

Conclusion: Our data indicate that the mode of insulin granule dynamics involved in the first phase of glucose-induced exocytosis differs from that involved in $\mathrm{K}^{+}$-triggered exocytosis, suggesting that $\mathrm{K}^{+}$stimulation and glucose stimulation induce fusion of granules from distinct pools. The data also demonstrate that the readily releasable pool (RRP) involved in the first phase of glucose-induced insulin secretion does not necessarily comprise predocked granules, indicating that some granules in pancreatic $\beta$-cells a distance away from the plasma membrane are readily releasable.

\section{8}

The role of depolarisation in the initiation of insulin secretion M. Willenborg, K. Hatlapatka, I. Rustenbeck

Institute of Pharmacology and Toxicology, Technical University of Braunschweig, Germany

Background and aims: The relevance of plasma membrane depolarization and the resulting $\mathrm{Ca}^{2+}$ influx for the initiation of insulin secretion was explored

Materials and methods: NMRI mouse islets and beta cells were used to measure insulin secretion by perifusion and ELISA, membrane potential by patch clamping (perforated patch) and cytosolic free calcium concentration $\left(\left[\mathrm{Ca}^{2+}\right]\right.$ ) by microfluorimetry of Fura 2PE-loaded islets.

Results: The depolarization by $\mathrm{KCl}$ followed closely the theoretical values as calculated by the Goldman equation. In contrast to the $\mathrm{K}_{\mathrm{ATP}}$ channel-blocking sulfonylurea, tolbutamide, $\mathrm{KCl}$ depolarization did not induce a visible action potential spiking. The depolarization by $15 \mathrm{mmol} / \mathrm{l} \mathrm{KCl}(21 \mathrm{mV})$ correspond- ed to the plateau depolarization by $500 \mu \mathrm{M}$ tolbutamide, that by $40 \mathrm{mmol} / \mathrm{l}$ $\mathrm{KCl}(41 \mathrm{mV})$ corresponded to the peak values of tolbutamide-induced action potentials. Nifedipine abolished tolbutamide-induced action potentials but had only a minimal effect on the depolarization by $40 \mathrm{mmol} / \mathrm{l} \mathrm{KCl}$. Action potential spiking was also abolished by diazoxide, even before the onset of repolarization. Apparently, the influx of $\mathrm{Ca}^{2+}$ during $\mathrm{KCl}$ depolarization is not reflected by action potential spiking because of a compensatory charge efflux through the open $\mathrm{K}^{+}$channels. Thus, depolarization by $15 \mathrm{mmol} / \mathrm{l}$, but not by $40 \mathrm{mmol} / \mathrm{l} \mathrm{KCl}$ is equivalent to the depolarizing effect of $\mathrm{K}_{\text {ATP }}$ channel closure. In contrast to $40 \mathrm{mmol} / \mathrm{l} \mathrm{KCl}$, which induced a massive secretory response in the presence of $5 \mathrm{mmol} / \mathrm{l}$ glucose, $15 \mathrm{mmol} / \mathrm{l} \mathrm{KCl}$ was only minimally effective under this condition, even though an increase in $\left[\mathrm{Ca}^{2+}\right]_{c}$ could be clearly shown. The $\left[\mathrm{Ca}^{2+}\right]_{c}$ measurements suggest the participation of intracellular $\mathrm{Ca}^{2+}$ stores as a reason for the different insulinotropic characteristics of the two $\mathrm{KCl}$ concentrations.

Conclusion: The influx of $\mathrm{Ca}^{2+}$ caused by a moderate depolarization, as is typically produced by a physiological or pharmacological $\mathrm{K}_{\mathrm{ATP}}$ channel closure, is only weakly effective to initiate insulin secretion. $\mathrm{K}_{\text {ATP }}$ channel-independent signalling may play a major role for the full expression of the first phase.

\section{9}

Glucose can increase insulin secretion by controlling $\mathrm{Ca}^{2+}$ influx and oscillations of cytosolic $\mathrm{Ca}^{2+}$ in beta cells independently of $\mathrm{K}_{\mathrm{ATP}}$ channels M.A. Ravier ${ }^{1}$, M. Nenquin ${ }^{1}$, T. Miki², S. Seino ${ }^{2}$, J.-C. Henquin ${ }^{1}$ ${ }^{1}$ Endocrinology and Metabolism, University of Louvain, Brussels, Belgium, ${ }^{2}$ Cellular and Molecular Medicine, University of Kobe, Japan

Background and aims: Glucose-induced insulin secretion is classically attributed to the cooperation of two major mechanisms in $\beta$-cells. The indispensable triggering signal, a rise in cytosolic $\left[\mathrm{Ca}^{2+}\right]_{\mathrm{c}^{c}}$, results from a $\mathrm{K}_{\text {ATP }}$ channel-dependent depolarization that leads to acceleration of $\mathrm{Ca}^{2+}$ influx through voltage-dependent $\mathrm{Ca}^{2+}$ channels. Amplifying signals, which do not directly involve $\mathrm{K}_{\mathrm{AтP}}$ channels, then augment the secretory response without further increasing $\mathrm{Ca}^{2+}$. In $\beta$-cells, $\mathrm{K}_{\text {ATP }}$ channels are composed of a pore (Kir6.2) and a regulatory subunit (SUR1). In this study we have evaluated the ability of glucose to control insulin secretion in islets lacking $\mathrm{K}_{\text {ATP }}$ channels because of a deletion of Kir6.2.

Materials and methods: Islets were isolated from 1-year and 2-week old control and Kir6.2 knockout (KO) mice, and studied $2 \mathrm{~h}$ after isolation (fresh) or after $18 \mathrm{~h}$ of culture in 5 or $10 \mathrm{mmol} / 1$ glucose. Insulin secretion and $\left[\mathrm{Ca}^{2+}\right]_{c}$ were measured in parallel, in perifused islets usually obtained from the same animals.

Results: Kir6.2KO islets were insensitive to diazoxide and tolbutamide, which indicates that they really lack functional $\mathrm{K}_{\mathrm{ATP}}$ channels. During perifusion with $1 \mathrm{mmol} / \mathrm{l}$ glucose, fresh adult Kir6.2KO islets showed only marginally elevated basal $\left[\mathrm{Ca}^{2+}\right]_{\mathrm{c}}$ and insulin secretion compared with controls. Upon stimulation with $15 \mathrm{mmol} / \mathrm{l}$ glucose, $\left[\mathrm{Ca}^{2+}\right]_{\mathrm{c}}$ only transiently increased, so that insulin secretion was only doubled (as compared to $>20$-fold increase in controls), in spite of a well functioning amplifying pathway (evidenced in the presence of $30 \mathrm{mmol} / \mathrm{l} \mathrm{KCl}$ ). Culture in $10 \mathrm{mmol} / \mathrm{l}$ glucose increased basal insulin secretion and markedly improved acute glucose-induced insulin secretion in Kir6.2KO islets (200\% of controls whose response was unchanged by culture). These changes in Kir6.2KO islets were unexpectedly due to an increase in average $\left[\mathrm{Ca}^{2+}\right]_{c}$ which entirely depended on extracellular $\mathrm{Ca}^{2+}$. Culture in $5 \mathrm{mmol} / \mathrm{l}$ glucose did not influence basal $\left[\mathrm{Ca}^{2+}\right]_{\mathrm{c}}$, but unmasked biphasic $\left[\mathrm{Ca}^{2+}\right]_{c}$ and insulin acute responses to high glucose. Oscillations of $\left[\mathrm{Ca}^{2+}\right]_{\mathrm{c}}$ were present in Kir6.2KO islets perifused with $1 \mathrm{mmol} / \mathrm{l}$ glucose (never in control islets) and were accelerated but shortened by $15 \mathrm{mmol} / \mathrm{l} \mathrm{glu}$ cose. Similar results were obtained with 2-week old Kir6.2KO islets tested in the presence of forskolin. Particularly striking were the biphasic increases in $\left[\mathrm{Ca}^{2+}\right]_{\mathrm{c}}$ and insulin secretion produced by high glucose in islets studied freshly and after culture in $5 \mathrm{mmol} / \mathrm{l}$ glucose. They were unambiguously caused by a $\mathrm{K}_{\mathrm{ATP}}$ channel-independent stimulation of $\mathrm{Ca}^{2+}$ influx through voltagedependent $\mathrm{Ca}^{2+}$ channels.

Conclusion: Kir6.2KO $\beta$-cells down-regulate insulin secretion by maintaining low $\left[\mathrm{Ca}^{2+}\right]_{c}$ but overnight culture unmasks their intrinsic glucose-responsive phenotype mainly by increasing $\left[\mathrm{Ca}^{2+}\right]_{c}$. The results support models implicating a $\mathrm{K}_{\text {ATP }}$ channel-independent amplifying pathway in glucose-induced insulin secretion, and show that $\mathrm{K}_{\mathrm{ATP}}$ channels are not the only possible transducers of metabolic effects on the triggering $\mathrm{Ca}^{2+}$ signal. 


\section{0}

Mitochondrial reactive oxygen species are obligatory signals for glucoseinduced insulin secretion

C. Leloup

Metabolism, Plasticity and Mitochondria, UMR CNRS 5241, Toulouse,

France

Background and aims: insulin secretion involves a complex cascade of events in which the mitochondria plays a pivotal role in the generation of signals coupling glucose detection to insulin secretion. Studies on the mitochondrial generation of reactive oxygen species (ROS) generally focus on chronic nutrient exposure and consequent deleterious effects of ROS. Here, we investigate whether an early moderate and transient mitochondrial ROS production linked to glucose-induced increased respiration might act as a signal to monitor insulin secretion.

Materials and methods: all investigations were carried out on freshly isolated static or perifused islets. Basal glucose was $5.5 \mathrm{mM}$ and stimulated concentration $16.7 \mathrm{mM}$. All pharmacological products had been tested for their toxic effects. ROS production was measured using H2DCFDA probe; ATP, $\mathrm{NADH}$, lipid peroxydation with classical biochemical kit, insulin secretion using ELISA. Ca2+ imaging was performed using the Fura-2probe.

Results: Transient glucose increase from 5.5 to $16.7 \mathrm{mM}$ stimulated ROS generation that was reversed by antioxidants. Strikingly, insulin secretion was dose-dependently blunted by antioxidants and highly correlated with ROS level. The $\beta$-cells incapacity to secrete insulin in response to glucose with antioxidants induced inhibition was associated with the maintenance of high levels of ATP and NADH. Then, we investigated the mitochondrial origin of ROS (mROS) as the triggering signal. Insulin release was mimicked by the mitochondrial complex blockers antimycin and rotenone that generate mROS. Both adding antioxidants to mitochondrial blockers or to glucose in order to lower mROS reversed insulin secretion. Imaging $\mathrm{Ca} 2+$ in perifused islets with glucose or with the complex blocker I rotenone showed in both cases a $\mathrm{Ca} 2+$ mobilization which exhibited different profiles. It occurred in the first $3 \mathrm{~min}$ with the glucose $16.7 \mathrm{mM}$ and after $10 \mathrm{~min}$ with the mitochondrial blocker, after a first slight decrease. Although this might be due to a different kinetic of mROS production, insulin secretion exhibited a similar profile whatever the stimulus, glucose or rotenone. These data suggest that part of the mROS signaling might operate downstream Ca2+ mobilization, at least in the 10 first min. In both cases, the mROS scavenger trolox was showed to inhibit $\mathrm{Ca} 2+$ mobilization. Whatever, any toxic effects were present as showed by lipid peroxydation evaluations and re-secretion tests using these pharmacological approaches.

Conclusion: Altogether these complementary and cohesive results demonstrate that mROS production is an obligatory stimulus for glucose induced insulin secretion.

Supported by: funding PNRD 2006 HYPOGLUROS

\section{1}

Single nucleotide polymorphism rs13266634 modifies the zinc transport activity of SLC30A8/ZnT-8 in clonal pancreatic beta cells

F. Chimienti ${ }^{1}$, M.B. Wheeler ${ }^{2}$, T.J. Nicholson ${ }^{3}$, E.A. Bellomo ${ }^{3}$, N.

Wijesekara ${ }^{2}$, A.I. Tarasov ${ }^{3}$, T. Taneja ${ }^{3}$, R. Sladek ${ }^{4}$, S.A. Baldwin ${ }^{5}$, J. Baldwin ${ }^{5}$, G.A. Rutter ${ }^{3}$

${ }^{1}$ Mellitech, CEA Grenoble, France, ${ }^{2}$ Dept of Physiology, University of Toronto, Canada, ${ }^{3}$ Dept of Cell Biology, Imperial College, London, United Kingdom, ${ }^{4}$ Dept of Human Genetics, Mc Gill University, Montreal, Canada, ${ }^{5}$ Institute of Membrane and Systems Biology, University of Leeds, United Kingdom

Background and aims: A recent genome-wide association study identified rs13266634 single nucleotide polymorphism (SNP) in the SLC30A8 gene as associated with type 2 diabetes. This SNP induces a non synonymous transition from arginine to tryptophan at position 325 of the secretory granule zinc transporter ZnT-8, expressed largely in pancreatic B- and alpha cells. Correspondingly, the $\mathrm{C}$ "at risk" genotype is linked to impaired $\beta$-cell function in vivo. Here, we investigated whether the SNP affects $\mathrm{Zn}^{2+}$ and $\mathrm{Ca}^{2+}$ ion homeostasis in pancreatic $ß$-cells ex vivo.

Materials and methods: We used plasmid or adenovirus-mediated delivery systems to overexpress either form of the human protein in clonal MIN6 Bcells. Subcellular localization was studied by confocal immunofluorescence, and steady-state levels of free $\mathrm{Zn}^{2+}$ ions were measured with $\mathrm{Zn}^{2+}$-specific fluorescent probes. Free cytosolic $\mathrm{Ca}^{2+}$ concentrations were measured using
Fura-red. Plasma membrane potential (Vm) and whole-cell KATP channel conductance (GKATP) were recorded in patch-clamps

Results: Homology modeling indicated that R325 lies in the loop connecting the second $ß$-strand and the second alpha-helix of the predicted cytosolic domain. It thus sits at the dimer interface, close to the binuclear $\mathrm{Zn}^{2+}$ clusters identified in the cytosolic domain of the bacterial homologue, YiiP. Overexpressed in clonal $ß$-cells, the R325 variant displayed unaltered subcellular localization, being confined largely to secretory granules with a minor component on the plasma membrane. However, R325 ZnT8-expressing cells displayed elevated cytosolic $\mathrm{Zn}^{2+}$ uptake rates, assessed using FluoZin3 or RhodZin, but decreased $\mathrm{Zn}^{2+}$ accumulation into secretory granules (Zinquin). R-variant expressing cells displayed elevated cytosolic free $\mathrm{Ca}^{2+}$ concentrations in response to glucose or KCl. Finally, ZnT8 silencing decreased $\mathrm{Zn}^{2+}$ influx into the cytosol. No differences were noticeable in glucose-mediated closure of KATP channels or membrane depolarization between cells over-expressing R- or W-325 ZnT8

Conclusion: These data suggest that the R325W SNP affects the zinc transport efficiency of $\mathrm{ZnT}$, and may result in altered $\mathrm{B}$ cell intracellular $\mathrm{Zn}^{2+}$, and possibly $\mathrm{Ca}^{2+}$, homeostasis. Moreover, by using differentially-localised $\mathrm{Zn}^{2+}$ probes, we show that the $\mathrm{R}$ form catalyses $\mathrm{Zn}^{2+}$ uptake into granules less efficiently than the $\mathrm{W}$-form. This may result either from lower intrinsic transporter activity of $\mathrm{R} 325-\mathrm{ZnT} 8$, or from a relatively weakened interaction with an associated protein. Importantly, we now provide direct evidence for a role for endogenous $\mathrm{ZnT}$ in $\mathrm{Zn} 2+$ uptake across the plasma membrane. We speculate that $\mathrm{Zn}^{2+}$ influx are both enhanced by the $\mathrm{R} v s \mathrm{~W}$ form but that inhibitory effects of $\mathrm{Zn}^{2+}$ on exocytosis may lead to defective insulin secretion. At the same time, decreased uptake of $\mathrm{Zn}^{2+}$ into granules likely causes the less efficient processing of proinsulin described in carriers of the at-risk C-allele. Finally, alterations of $\mathrm{Zn}^{2+}$ transport into insulin granules may also have implications for the paracrine regulation of glucagon secretion. Supported by: Wellcome Trust, MRC and EU ("Savebeta")

\section{2}

Beta cell heterogeneity in vivo: functional characterisation and involvement in pancreatic islet plasticity M. Karaca' ${ }^{1}$ J. Castel ${ }^{1}$, C. Tourrel-Cuzin ${ }^{1}$, A. Geant ${ }^{2}$, M. Brun 2 , M. Rodriguez $^{2}$, B. Lockhart ${ }^{2}$, A. Ktorza ${ }^{2}$, C. Magnan ${ }^{1}$, C. Kargar ${ }^{2}$ ${ }^{1}$ Universite Paris7 Denis Diderot, CNRS UMR 7059, ${ }^{2}$ IdRS, Maladies Métaboliques, Paris, France

Background and aims: Using PSA-NCAM as a marker for sorting B-cell sub-populations in non diabetic Wistar rats, we previously identified 2 B-cell sub-populations: a population highly expressing PSA-NCAM ( $\mathrm{B}^{+}$-cells) and highly responsive to glucose (60\% of total B-cells) and a population poorly expressing PSA-NCAM (B-cells) and poorly responsive to glucose (40\% of total B-cells). The aim of the present work was to explore the functional and the gene expression profiles of the 2 sub-populations. In order to evaluate the physiological relevance of these populations we also measured the $\mathrm{B}^{+} / \mathrm{B}^{-}$-cell ratio in a model of type 2 diabetes with reduced $B$-cell mass and function, the ZDF rat and in a model of increased insulin demand, the non diabetic Wistar rat infused with glucose during $48 \mathrm{~h}$ (HG/HI).

Materials and methods: Islet cell suspensions from adult rats were sorted by flow cytometry according to their PSA-NCAM surface labelling. Insulin release was measured by perifusion in a system allowing concomitant recording of intracytoplasmic $\mathrm{Ca}^{2+}$ influx $\left(\left[\mathrm{Ca}^{2+}\right] \mathrm{i}\right)$ by microfluorimetry. cAMP content and/or insulin release in response to glucose $(5.5$ or $16.7 \mathrm{mM})$ and/or 10nM GLP-1, 10mM leucine, were studied using batch-incubated cells. The gene expression profile was analyzed by quantitative real-time RT-PCR.

Results: In $\mathrm{B}^{+}$-cells, insulin release in response to $16.7 \mathrm{mM}$ glucose was 8 -fold higher than in $\mathrm{B}$-cells and was correlated with a high $\left[\mathrm{Ca}^{2+}\right] \mathrm{i}$, contrarily to $\mathrm{B}$-cells. In batch-incubated $\mathrm{B}^{+}$-cells, insulin secretion in response to $16.7 \mathrm{mM}$ glucose was strongly potentiated by GLP-1, in parallel with a huge increase in cAMP content, whereas in $\mathrm{B}$-cells insulin release and cAMP content remained very low under all conditions. Moreover, leucine elicited a sustained insulin response in $\mathrm{B}^{+}$-cells but not in $\mathrm{B}^{-}$-cells. The analysis of gene expression profile of the $2 \mathrm{~B}$-cell populations showed that many genes related to $\mathrm{B}$-cell maturation (PDX1, Nkx6.1, NeuroD, Insulin), metabolic activity (Glut2, GK), pumps/ion channels (Sur-1, Kir6.2, SERCA2b and3, Cav1.2, Znt8), signaling (PC1/3 and 2, PKA) and adhesion/exocytosis machinery (Connexin36, Integrin $\beta 1$, SNAP25, VAMP2, Rab3A) were upregulated in $\mathrm{B}^{+}$-cells compared to B-cells, while genes such as NGN3, TCF7L2, involved in early B-cell differentiation were expressed at higher levels in B-cells. Glucose infusion in Wistar rats resulted in increased B-cell mass and gain of function which was 
correlated with an excess of $\mathrm{B}^{+}$-cells compared to $\mathrm{B}^{-}$-cells (80\% versus $20 \%$ ). In 12 week-old $\mathrm{ZDF}$ fa/fa rats the $\mathrm{B}$-cells were largely predominant $(70 \%$ of total B-cells) in high contrast with ZDF lean control non diabetic rats of the same age in which they represented only $40 \%$ of the total B-cells.

Conclusion: We have identified and characterized 2 B-cell sub-populations with quite different functional and gene expression profiles. The data suggest that $\mathrm{B}^{-}$-cells did not achieve functional maturity and that the proportion of $\mathrm{B}^{+} / \mathrm{B}^{-}$-cells varies according to the prevailing insulin demand but in opposite ways in non diabetic and diabetic rats. This suggests that pancreatic islet plasticity may involve changes not only in the B-cell mass but also in the distribution between the $2 \mathrm{~B}$-cell sub-populations and that the inability to increase the $\mathrm{B}^{+}$-cell sub-population may be part of the inadequate insulin response to insulin resistance in diabetics.

\section{OP 18 Type 1 diabetes mellitus epidemiology}

\section{3}

How does the parental age-at-onset of type 1 diabetes (T1D) affect the risk of T1D in the offspring? A comparison of two large cohorts (age-atonset 0-17 years and 15-39 years) in Finland

V. Harjutsalo ${ }^{1}$, N. Lammi ${ }^{1}$, M. Karvonen ${ }^{1}$, J. Tuomilehto ${ }^{1,2}$ ${ }^{1}$ Department of Health Promotion and Chronic Disease Prevention, Diabetes Unit, National Public Health Institute, ${ }^{2}$ Department of Public Health, University of Helsinki, Finland

Background and aims: Number of studies have detected sex-specific transmission of type 1 diabetes (T1D) from one generation to the next. Age at onset of T1D in the proband has been known to strongly influence the recurrence risk of T1D in the first-degree relatives. There are few data available on the recurrence risk of T1D among the offspring of probands with young adult-onset T1D and no reports comparing the risk of T1D between the offspring of parents with early-onset T1D and those of parents with young adult-onset T1D. The aim of this study was to examine the wide range effect of the parental age at onset of T1D on the recurrence risk of T1D in offspring stratifying the offspring data by the sex of the T1D parents.

Materials and methods: The offspring $(n=9,158)$ of a population-based cohort of probands with early-onset T1D ( $0-17$ years) $(n=5,144)$ and a population-based cohort of T1D among 15-39 years old (young adult-onset T1D, $\mathrm{n}=3,389$ ) were studied for recurrence of T1D. Offspring with both parents affected were excluded. Life table method was used to estimate the cumulative incidence of T1D. The analyses were carried out stratifying the data by the age at diagnosis of T1D in the parent in 5-year age groups (0-4, 5-9, 10-14, 15-19 and 20-39). Based on the preliminary analysis ages 20-39 were pooled together. Analyses were carried out separately in the offspring of fathers and mothers with T1D.

Results: During 127,174 follow-up years, 92 offspring of parents with young adult-onset T1D and 299 offspring of early-onset T1D were affected with T1D by the end of the year 2006. In all offspring with one diabetic parent, the overall cumulative incidence to develop T1D by the age of 15 years was $4.9 \%$ (95\% CI 4.4-5.4) and by the age of 20 years 5.8\% (95\% CI 5.2-6.4). A young age at onset of T1D in diabetic mothers did not increase the risk of T1D in the offspring. The overall cumulative risk by age 15 years was $4.1 \%(95 \% \mathrm{CI}$ 3.4-4.8) in all offspring of diabetic mothers irrespective of maternal age at onset of diabetes. On the contrary, each 5-year increase in the age at onset of T1D in the fathers until 25 years decreased the risk of T1D in their offspring. The cumulative risk by age 15 years in the offspring of the fathers with T1D was $12.9 \%$ (95\% CI 8.0-17-4), 8.7\% (95\% CI 6.5-10.8) , 6.0\% (95\% CI 4.7-7.3) , $4.7 \%$ (95\% CI 3.1-6.2) and 3.1 \% (95\% CI 1.9-4.4) for age at onset groups 0-4, 5-9, 10-14, 15-19 and 20-39 years, respectively.

Conclusion: Maternal age at onset of diabetes did not have an impact on the recurrence risk of T1D in their offspring. On the contrary, the risk of T1D in

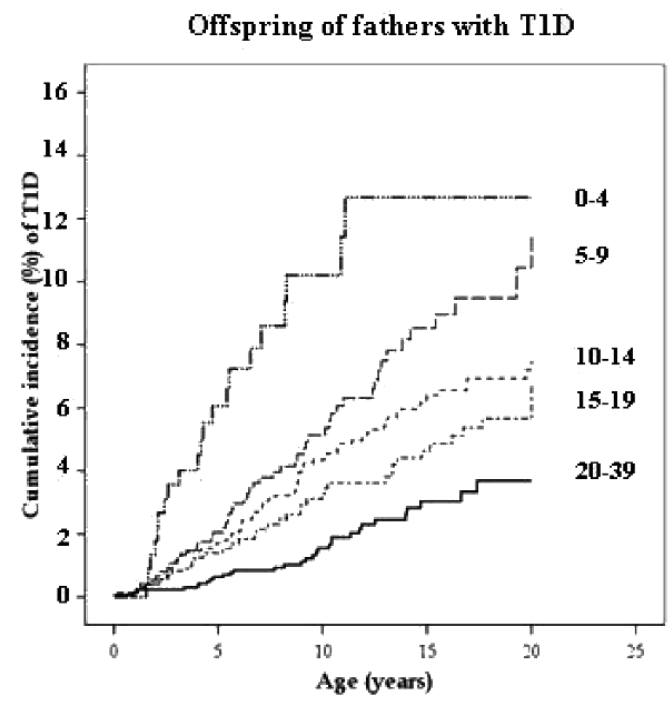

Offspring of mothers with T1D

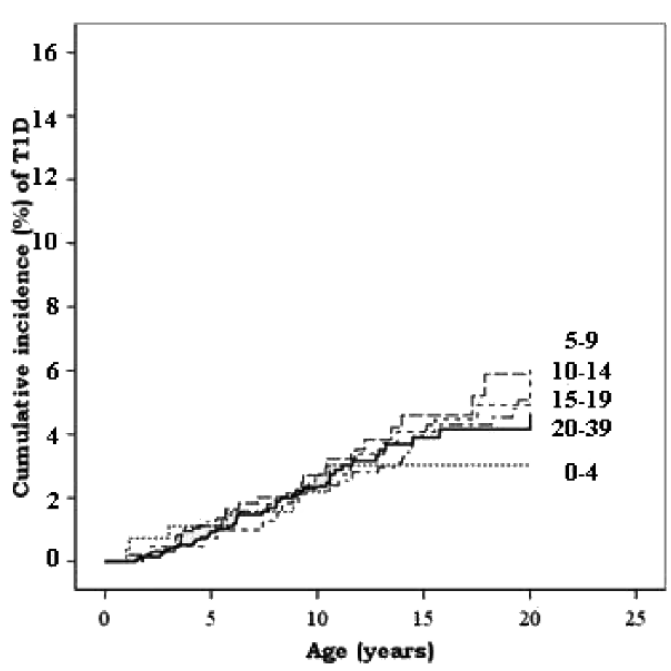

Cumulative incidence of T1D in the offspring of patients with T1D according to age at onset of T1D 
the offspring of the fathers with T1D decreased progressively with increasing age at onset of fathers. The decrease, however, levelled off around 25 years of age at onset.

\section{4}

Incidence trends for childhood type 1 diabetes in Europe during 19892003 and projection of numbers to 2025

C.C. Patterson ${ }^{1}$, G. Dahlquist ${ }^{2}$, E. Gyürüs ${ }^{3}$, G. Soltész ${ }^{3}$ on behalf of the EURODIAB Study Group

${ }^{1}$ Department of Epidemiology \& Public Health, Queen's University Belfast, United Kingdom, ${ }^{2}$ Department of Clinical Sciences Paediatrics, Umeå University, Sweden, ${ }^{3}$ Department of Paediatrics, Pécs University, Hungary

Background and aims: To describe fifteen-year trends in incidence of chidhood type 1 diabetes in European centres and to use these data to predict future burden of childhood diabetes in European countries.

Materials and methods: Twenty population-based EURODIAB registers in 17 countries registered 29,311 new cases of type 1 diabetes diagnosed before the age of 15 years during a fifteen year period 1989-2003. Each centre validated completeness of acsertainment. Age-specific log linear rates of increase were estimated in five geographic regions of relatively homogeneous incidence, and these trends were used in conjunction with published incidence rates and population projections to make predictions of the numbers of new cases throughout Europe in the years 2010, 2015, 2020 and 2025.

Results: Ascertainment rates were better than $90 \%$ in most registers throughout the period. All but two registers showed significant increases in incidence, and in the six registers where there were significant differences in the rate of increase between age groups it was lowest in relative terms in 10-14 year olds. When centres were amalgamated into five regions the rates of increase were largest among 0-4 year olds in central and eastern Europe. The number of new cases in Europe in 2005 is estimated as 14,700, and this total is divided between the 0-4 yr, 5-9 yr and 10-14 yr age groups in the ratio $23 \%, 35 \%$ and $42 \%$, respectively. By the year 2025 the predictions show almost a doubling in the yearly numbers of new cases to 29,000 but this change is not shared evenly between the age-groups and the percentage distribution across the three age groups is predicted to become rather more uniform at $31 \%, 37 \%$ and $32 \%$, respectively.

Conclusion: A near doubling in absolute numbers of new cases of type 1 diabetes in European children is to be anticipated in the next 20 years if current trends continue. A disproportionate change over time with more new cases in the youngest age groups is predicted and the differences in different part of Europe are indicated to be less pronounced. Resources to meet this changing pattern must be made available.

\section{5}

Childhood BMI trajectories and the risk for type 1 and type 2 diabetes diagnosed in young adulthood

N.M. Lammi ${ }^{1}$, E.V. Moltchanova ${ }^{1}$, P.A. Blomstedt ${ }^{1}$, J.G. Eriksson ${ }^{1,2}$, J. Tuomilehto ${ }^{1,3}$, M. Karvonen ${ }^{1}$

${ }^{1}$ Department of Health Promotion and Chronic Disease Prevention, National Public Health Institute, Helsinki, ${ }^{2}$ Department of General Practise and Primary Health Care, University of Helsinki, ${ }^{3}$ Department of Public Health, University of Helsinki, Finland

Background and aims: The rate of growth during infancy and childhood can influence the risk for type 1 diabetes (T1DM) and type 2 diabetes (T2DM). Subjects with childhood-onset T1DM are heavier and taller during infancy, and they also seem to grow faster during infancy than their healthy peers. Conversely, patients with T2DM are smaller up to two years of age and they have accelerated growth after infancy compared with children who remain non-diabetic. The aim of this case-control study was to examine the childhood growth trajectories in individuals diagnosed with T1DM and T2DM between ages 15-39 years.

Materials and methods: Patients with diabetes, aged 15-39 years at diagnosis, were identified from the Finnish national healthcare registers, and classified as T1DM or T2DM utilizing the register information as well as their medical statements when necessary. Two non-diabetic, sex- and age-matched control subjects were obtained for each case from the National Population Registry. The original childhood welfare records including anthropometric measurements were collected for the cases and controls from the healthcare archives nationwide. Only the case-control pairs with sufficient growth data recorded were included in the analysis. A quadratic curve was estimated (in accordance with the widely accepted ICP-growth model).

Results: The results of the analysis including 224 case-control pairs of T1DM (161 with 1 control and 63 with 2 controls) indicated that the maximum BMI between ages 0-2 and the minimum BMI between ages 3-11 were similar in cases with T1DM and control subjects. The age of the minimum BMI between ages 3-11 was lower in individuals with T1DM but the result was not statistically significant (Figure 1a). The analysis of young adult-onset T2DM was based on data from 69 case-control pairs (54 with 1 control and 15 with 2 controls). (Figure 1b) The maximum BMI between ages $0-2$ years was similar between cases and controls. The risk for T2DM was strongly affected by the minimum BMI between ages 3-11 years: $1 \mathrm{~kg} / \mathrm{m} 2$ rise in the BMI increased the risk of T2DM by $59 \%(\mathrm{p}<0.05)$. The excess weight gain in individuals who later developed young adult-onset T2DM began already at childhood after the age of the minimum BMI (Figure 1b).

Conclusion: Childhood growth does not affect the risk for young adult-onset T1DM contrary to the previous findings in childhood-onset T1DM. The etiology of young adult-onset T1DM may therefore be different from childhood-onset T1DM.The profound effect of the childhood growth on the risk for T2DM was confirmed, and it seems that the pattern of childhood growth may be even more important than birth size in predicting the future risk for T2DM.

\section{a. Avg BMI curves of T1DM cases \& controls}

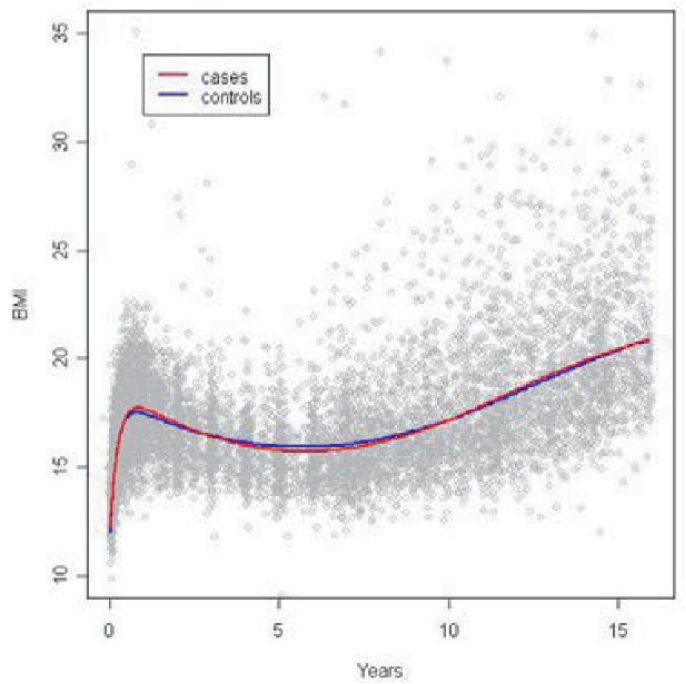

b. Avg BMI curves of T2DM cases \& controls

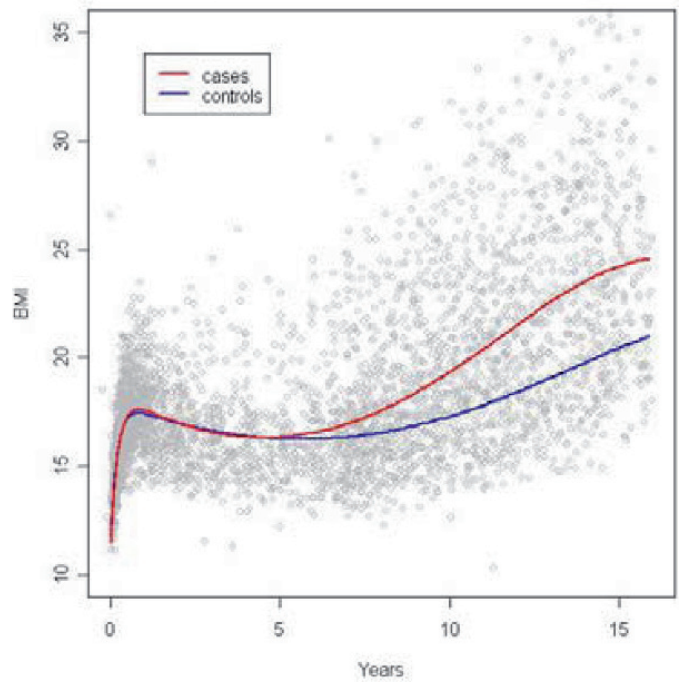

Supported by: National Institutes of Health and the Academy of Finland 


\section{6}

Modern-day clinical course of type 1 diabetes mellitus. The DCCT/EDIC experience 1983-2008

D.M. Nathan ${ }^{1}$, B. Zinman², P.A. Cleary ${ }^{3}$, J.-Y.C. Backlund ${ }^{3}$, R. Miller ${ }^{4}$, T. Orchard ${ }^{4}$

${ }^{1}$ Diabetes Unit, Massachusetts General Hospital, Boston, United States, ${ }^{2}$ Centre for Diabetes, Mount Sinai Hospital, Toronto, Canada, ${ }^{3}$ The Biostatistics Center, The George Washington University, Rockville, United States, ${ }^{4}$ Epidemiology, University of Pittsburgh, United States

Background and aims: The DCCT/EDIC has collected clinical data, including treatments and outcomes, for twenty-five years and provides the opportunity to describe the clinical course of type 1 diabetes mellitus (T1DM) in the latter part of the 20th and early 21 st century. We describe the clinical care, metabolic results and the long-term complications and outcomes that can be expected with current diabetes therapy, comparing and contrasting the DCCT/EDIC conventional (CONV) and intensive (INT) therapy groups. In addition, data collected in the Epidemiology of Diabetes Complications (EDC) study, a population-based observational study, provide the results of conventional therapy in an unselected population with T1DM.

Materials and methods: Although not population-based, the DCCT/EDIC cohort has the advantage of being current, distributed in the US and Canada, and compete in its followup, with $92 \%$ of the baseline cohort ( $96 \%$ of survivors) followed for a mean of 19.5 years. DCCT CONV treatment $(n=730)$ was designed to represent the standard of clinical care at the time. At the end of the DCCT in 1993, all CONV subjects were offered intensive therapy. While the DCCT CONV treatment group and the EDC cohort $(n=161$, selected to have similar baseline age, diabetes duration, and complications to the DCCT), reflect contemporaneous clinical care and outcomes, the clinical care and outcomes in the INT group $(\mathrm{N}=711)$ represent what we can expect of diabetes care going forward. The long-term complications presented here include proliferative retinopathy (PDR), albumin excretion rate $(\mathrm{AER})>300$ $\mathrm{mg} / 24 \mathrm{~h}$, and cardiovascular disease (CVD: fatal and non-fatal myocardial infarction or stroke, death secondary to CVD, subclinical myocardial infarction , confirmed angina, or cardiac revascularization).

Results: The frequencies of serious complications in the DCCT CONV treatment group by diabetes duration are shown in the figure. The cumulative incidences of PDR, AER > $300 \mathrm{mg} / 24 \mathrm{~h}$, and CVD are $49.7 \%, 25.1 \%$, and $14 \%$, respectively, after 30 years diabetes duration. In the population based EDC, the corresponding values are very similar at $47 \%, 17 \%$, and $14 \%$. The cumulative incidences with intensive therapy are substantially lower with $21.1 \%$ with PDR, $8.6 \%$ with AER $>300 \mathrm{mg} / 24 \mathrm{~h}$, and $8.5 \%$ with CVD after 30 years duration.

Conclusion: This more current data provides a more accurate assessment of the long-term outcomes of T1DM with current care and should be used in models evaluating heath outcomes of and quality of life for T1DM. The long-term outlook for patients with T1DM is projected to improve further as intensive therapy is applied throughout their diabetes duration.

Estimated Cumulative Incidence CVD, Renal, PDR among DCCT/EDIC Patients

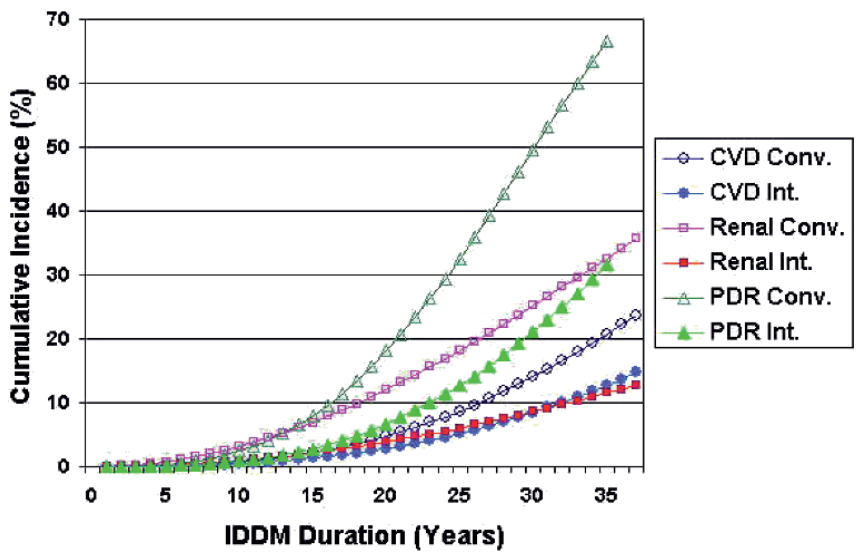

Supported by: Division of Diabetes, Endocrinology and Metabolic Diseases of the National Institute of Diabetes and Digestive and Kidney Diseases and the General Clinical Research Centers Program, National Center for Research Resources.

\section{7}

Validating a coronary heart disease prediction model for type 1 diabetes J.C. Zgibor ${ }^{1}$, K. Ruppert ${ }^{1}$, S. Soedamah-Muthu' ${ }^{2}$, T.J. Orchard ${ }^{1}$,

M.S. Roberts ${ }^{3}$

${ }^{1}$ Epidemiology, University of Pittsburgh, United States, ${ }^{2}$ Epidemiology, Julius Center for Health Sciences and Primary Care, Utrecht, Netherlands, ${ }^{3}$ Medicine, University of Pittsburgh, United States

Background and aims: Previous analyses demonstrated that existing coronary heart disease (CHD) risk prediction models do not predict events well in type 1 diabetes (T1D). Therefore, our objective was to create CHD risk prediction models specific to T1D and to validate the models in a separate cohort of people with type 1 diabetes.

Materials and methods: Development of the CHD prediction model used data from the Pittsburgh Epidemiology of Diabetes Complications Study (EDC). EDC subjects had T1D diagnosed between 1950-1980 and received their first study exam between 1986-1988, and have been followed biennially since. The final cohort for model development consisted of 603 subjects and 46 incident events. Hard CHD was defined as CHD death, fatal or non-fatal MI or Q-waves. Baseline CHD risk factors were tested bivariately and introduced into a Weibull model. The model was externally validated using the EURODIAB Prospective Complications Study (PCS) population. The EURODIAB PCS is a hospital based multi-center study designed to examine risk factors for micro and macrovascular complications in type 1 diabetes subjects in 31 centers from 16 countries across Europe. Baseline exams took place in 1989-91 on 3250 subjects diagnosed with type 1 diabetes before age 36 who were on continuous insulin treatment within one year of diagnosis. Follow-up exams were conducted on 2328 participants 6-8 years later. Of these participants, 53 had incident hard CHD events at follow-up.

Results: The following risk equations were developed.

Males: $X=1.8738-0.9452$ (log white blood cell count) - 1.0625 (presence of microalbuminuria) $+1.4808(\log$ HDLc) -0.2286 (sqrt diabetes duration at baseline) Females: $\mathrm{X}=21.62-4.4331$ (waist/hip ratio) - 1.594(log nonHDLc) - 0.023 (systolic blood pressure) - 0.498 (use of blood pressure medication) - 0.705 (sqrt diabetes duration at baseline). Following this calculation, the probability of not having an event was calculated using

$\mathrm{S}_{i}(t)=\exp \left\{-\left[t_{i} \mathrm{e}^{-\beta \mathrm{xx} i}\right]^{1 / \sigma}\right\}$ where $\sigma=0.6417$ for males and $\sigma=0.6215$ for females. $1-\mathrm{S}_{\mathrm{i}}(10)=$ probability of an event in 8 years. Results of external validation included comparison between expected and actual events applying the newly developed type 1 diabetes CHD prediction model to data from the EURODIAB Study. The outcome of interest was the same although fatal cases were based solely on death certificate information. In males, the model performed well with a c statistic of 0.77 . Events were slightly overpredicted in the tenth decile. The female model also performed well with a c statistic was 0.78 , however the model overpredicted events in the tenth decile.

Conclusion: This is the first clinically practical model developed to predict $\mathrm{CHD}$ events for type 1 diabetes that was externally validated in an independent type 1 diabetes population. This model can be used as a tool to enhance both patient and provider knowledge about CHD risk.

Supported by: National Institutes of Health and the American Diabetes Association Junior Faculty Award. EURODIAB PCS was supported by grants from the Wellcome Trust, the European Community and Diabetes UK

\section{8}

Future burden of diabetes in Denmark; the National Diabetes Register B. Carstensen ${ }^{1}$, P. Ottosen ${ }^{2}$

${ }^{1}$ Epidemiology, Steno Diabetes Center, Gentofte, ${ }^{2}$ Medical statistics, National Board of Health, Copenhagen, Denmark

Background and aims: For planning purposes it is of paramount interest to predict the likely number of future diabetes cases in the Danish population. Therefore we want to estimate incidence and mortality rates in order to predict future diabetes prevalence and life time risk in the Danish population. Materials and methods: The National Diabetes Register covers the period 1995-2006 inclusive, and holds all cases of diabetes in Denmark alive in this period. Based on this we derived age-specific incidence rates and mortality rates for diabetes patients, and based on population data from statistics Denmark we derived mortality rates for non-diabetic persons. These were used to estimate future prevalences and lifetime risk of diabetes in the Danish population.

Results: There were 359,000 cases in the register, 233,000 included after 1995. We saw an an annual increase in incidence rates of $5 \%$ until 2003 and after 
that an annual decrease of $5 \%$. Mortality rates decreased $3.6 \%$ per year. The lifetime risk of diabetes was found to be $28 \%$ for women and $31 \%$ for men, assuming the current rates prevail at the 2003 in the future. The peak prevalence in the population will be $22 \%$ for both sexes, attained at age 75 for men and age 85 for women.

Conclusion: We have observed a significant decrease in incidence rates. Even though the anticipated lifetime risk for currently born is around $30 \%$, this is likely to decrease if the incidence rates continue to decrease. This is however difficult to asses with the rather short time span observed.

\section{OP 19 Cardiomyopathy and dysfunction of the heart}

\section{9}

Microarray expression analysis in delayed cardioprotection: the effect of exercise, AICAR or metformin and the possible role of AMP-activated protein kinase (AMPK)

L. Solskov ${ }^{1}$, N.E. Magnusson ${ }^{1}$, S.B. Kristiansen ${ }^{2}$, H.E. Bøtker², T.T. Nielsen², O. Schmitz ${ }^{3,1}$, S. Lund ${ }^{3}$

${ }^{1}$ Department of Pharmacology, Aarhus University, ${ }^{2}$ Department of Cardiology, Aarhus University Hospital, Skejby Sygehus, ${ }^{3}$ Medical Research Laboratory and Medical Department M (Endocrinology and Diabetes), Aarhus University Hospital, Denmark

Background and aims: AMP-activated protein kinase (AMPK) is an enzyme which acts as an energy sensor in various cell types and has been indicated to play an important protective role in the ischaemic heart. It has previously been demonstrated that exercise, 5-aminoimidazole-4-carboxamide-1- $\beta$ D-ribofuranoside (AICAR) and metformin, all known activators of AMPK, induce delayed preconditioning which protects the heart against ischaemiareperfusion injury. The aim was to determine the effect of exercise, AICAR and metformin on rat left ventricular gene expression profile and to demonstrate possible interactions in different pathways.

Materials and methods: Rats were divided into the following groups: 1) an exercise group trained on a treadmill for one hour, 2) an AICAR group given a single injection of AICAR, 3) a metformin group given a single oral dose of metformin. Sedentary rats were used as controls. 24 hours later hearts were removed and gene expression profiles were analysed in tissue from the left ventricle using affymetrix gene chip probe arrays. Ingenuity Pathway Analysis software was used to analyse regulated genes and regulatory pathways. To reduce low intensity genes in the analysis a cut-off limit was chosen at a fold change $\geq 2.0$ and signal $\geq 100$.

Results: Each gene chip identified 30.000 different probesets of which Ingenuity identified approximately 12.000 genes. The total numbers of up- and downregulated genes in the three groups are shown in table 1. Ingenuity indentified 17 pathways all regulated by exercise, AICAR and metformin. 5 of these are summarized in table 1 including top genes involved. Among these genes HIF1A (hypoxia inducible factor 1 alpha), NR2C2 (nuclear receptor subfamily 2) and EIF2S3 (eukaryotic translation initiation factor 2) have all previously been indicated to play a role in the protection against myocardial ischaemiareperfusion injury.

Conclusion: This study demonstrates that exercise, AICAR and metformin, all known activators of AMPK, regulate several identical pathways in the rat myocardium with the most overlapping genes observed in the exercise and AICAR group. These results may provide insight into the molecular mechanisms of delayed cardioprotection.

Table1: Regulated genes (presented by gene names) representing relevant pathways

\begin{tabular}{llll}
\hline- & Exercise & AICAR & Metformin \\
\hline Total up-regulated genes & 84 & 131 & 248 \\
\hline Total down-regulated genes & 19 & 39 & 141 \\
\hline Pathways & - & - & - \\
\hline PPARa/RXRa Activation & GHR $\uparrow$ & GHR $\uparrow$ & NR2C2 $\uparrow$ \\
\hline VEGF Signaling & HIF1A $\uparrow$ & HIF1A $\uparrow$ & EIF2S3 $\downarrow$ \\
\hline Axonal Guidance Signaling & CFL2 $\uparrow$ & CFL2 $\uparrow$ & PRKD1 \\
\hline Leukocyte Extravasation Signaling & ROCK1 $\uparrow$ & ROCK1 $\uparrow$ & ACTN1 $\downarrow$ \\
\hline Cardiac $\beta$-adrenergic Signaling & PPP1CB $\uparrow$ & PPP1CB $\uparrow$ & PKIB $\uparrow$ \\
\hline
\end{tabular}

110

HMGB1- a new prognostic marker in the development of diabetic cardiomyopathy?

M. Andrassy ${ }^{1}$, C. Volz ${ }^{1}$, Z. Kaya ${ }^{1}$, A. Bierhaus ${ }^{2}$, H. Katus ${ }^{1}$

${ }^{1}$ Department of Cardiology, Department of Medicine III, ${ }^{2}$ Endocrinology and Clinical Chemistry, Department of Medicine I, Heidelberg, Germany

Background and aims: Diabetes is one of the most important comorbid factors in patients with acute myocardial infarction (MI). The association of dia- 
betes with higher incidence, severity and complexity of cardiac diseases compared to euglycemic patients is poorly understood. We studied the impact of High-mobility group box 1 (HMGB1), a newly recognized innate danger signal for the initiation of host defense and tissue repair, and the receptor for advanced glycation endproducts (RAGE) on the pathogenesis of early and late phase complications of diabetic cardiomyopathy.

Materials and methods: Immunohistochemical expression analyses of HMGB1 was analyzed in reperfused left ventricles in an animal model of type 1 diabetes mellitus followed by studies on the influence of the HMGB1RAGE axis on the development of cardiac inflammation, fibrosis and apoptosis next to its influence on compensatory hypertrophy of the viable myocardium at the early and late phase of diabetic cardiomyopathy. The influence of HMGB1-RAGE axis on cardiac function was assessed in 2-dimensional echocardiography.

Results: Diabetes (DM) induced an enhanced HMGB1 expression in the myocardium of mice in comparison to healthy control mice. Furthermore, diabetic mice subjected to I/R injury (IR) displayed significantly pronounced cardiac remodelling compared to healthy mice with $\mathrm{I} / \mathrm{R}$ or diabetes in the absence of I/R. Treatment with the functional HMGB1 antagonist HMGB1 box A significantly reduced cardiac remodeling in diabetic mice with $I / R$ injury. Survival rates during the study period of 10 weeks were significantly lower in the group with diabetes alone versus control mice, while the DM+ I/R group displayed the highest mortality rate. Treatment of the DM + I/R group with the functional HMGB1 antagonist box A significantly enhanced the survival rate compared to the non-treated $\mathrm{DM}+\mathrm{I} / \mathrm{R}$ group pointing to an essential role of HMGB1 in the development of diabetic cardiomyopathy. Echocardiography revealed significant reduction of the fractional shortening (FS) of the left ventricles of diabetic mice despite unchanged left ventricular diameter compared to sham operated control mice. The DM+ I/R group demonstrated significantly enhanced remodelling with reduced FS and LV dilation compared to untreated I/R group or the DM group without I/R. Treatment with the HMGB1 antagonist box A showed a drastic improved recovery of cardiac function in the $\mathrm{DM}+\mathrm{I} / \mathrm{R}$ group compared with the untreated $\mathrm{DM}+\mathrm{I} / \mathrm{R}$ group.

Conclusion: We propose that HMGB1/RAGE-interaction is a key component initiating and sustaining an inflammatory response following ischemic stress eventually leading to the development of diabetic cardiomyopathy.

\section{1}

Cardiomyopathy, glycogen accumulation and low AMP -activated protein kinase $\gamma 2$ expression in the heart of transgenic mice with activated polyamine catabolism

E. Pirinen ${ }^{1,2}$, M. Merentie ${ }^{1}$, M.-M. Ryhänen ${ }^{1}$, M. Hedman ${ }^{1,2}$, S. Heinonen ${ }^{1}$, P. Korpisalo ${ }^{1}$, A. Virkamäki ${ }^{3}$, J. Kuusisto ${ }^{4}$, J. Jänne ${ }^{1}$, S. Ylä-Herttuala ${ }^{1}$, M. Laakso ${ }^{2}$

${ }^{1}$ Department of Biotechnology and Molecular Medicine, University of Kuopio, ${ }^{2}$ Department of Medicine, University of Kuopio, ${ }^{3}$ Minerva Foundation Institute for Medical Research, University of Helsinki, ${ }^{4}$ Cardiology Unit, Kuopio University Hospital, Kuopio, Finland

Background and aims: Mutations in the $\gamma 2$ subunit of AMP -activated protein kinase (AMPK) produce glycogen storage hypertrophic cardiomyopathy with Wolff-Parkinson-White syndrome in man, often evolving systolic dysfunction resembling dilated cardiomyopathy (DCM). We have previously shown that transgenic mice overexpressing spermidine/spermine $\mathrm{N}^{1}$-acetyltransferase (SSAT), the key enzyme in the catabolism of polyamines, have severely reduced fat mass, increased insulin sensitivity and improved glucose tolerance. In this study, we investigated the mechanisms of cardiac dysfunction in these mice.

Materials and methods: Echocardiographic measurements were accomplished in female SSAT (tg) and wild-type (wt) mice using a high-frequency ultrasound imaging system (Vevo 770). Blood pressure was measured in female SSAT and wt mice with a tail-cuff system (CODA $2^{\mathrm{TM}}$ system). Renal perfusion of female SSAT and wt mice was analyzed with Acuson sequoia 512 ultrasound device using Doppler mode with administration of ultrasound contrast agent. Gene expression levels in the hearts were analyzed using quantitative RT-PCR. Glycogen content was analyzed microfluorometrically after $\mathrm{KOH}$ hydrolysis.

Results: Echocardiography revealed that SSAT mice have dyskinetic wall motions of the left ventricles and impaired pump function since ejection fraction (tg: $57.9 \pm 4.0$ vs. wt: $71.7 \pm 4.3 \%, \mathrm{p}<0.001$ ) and fractional shortening (tg: $30.2 \pm 2.8$ vs. wt: $40.3 \pm 3.7 \%, \mathrm{p}<0.01$ ) were significantly reduced. In addition, left ventricle mass (tg: $5.9 \pm 0.7$ vs. wt: $5.1 \pm 0.1 \mathrm{mg} / \mathrm{g}, \mathrm{p}<0.05$ ), left ventricle di- astolic volume (tg: $74.4 \pm 10.7$ vs. wt: $55.5 \pm 2.5 \mu \mathrm{l}, \mathrm{p}<0.01$ ), left ventricle systolic volume (tg: $31.1 \pm 3.2$ vs. wt: $15.7 \pm 2.0 \mu \mathrm{l}, \mathrm{p}<0.001$ ), left ventricle diastolic internal diameter (tg: $4.1 \pm 0.3$ vs. wt: $3.6 \pm 0.1 \mathrm{~mm}, \mathrm{p}<0.01$ ) and left ventricle systolic internal diameter (tg: $2.9 \pm 0.1$ vs. wt: $2.2 \pm 0.1 \mathrm{~mm}, \mathrm{p}<0.001$ ) were significantly increased. In contrast, systolic (tg: $94 \pm 16$ vs. wt: $128 \pm 6 \mathrm{mmHg}$, $\mathrm{p}<0.01$ ) and diastolic (tg: $58 \pm 12$ vs. wt: $89 \pm 6 \mathrm{mmHg}, \mathrm{p}<0.01$ ) arterial blood pressure and renal cortex perfusion (tg: $0.82 \pm 0.05$ vs. wt: $0.95 \pm 0.03$ cortex/artery ratio, $\mathrm{p}<0.01$ ) were significantly reduced. Glycogen content in the hearts of SSAT mice was significantly increased in the fed state (tg: $66 \pm 21$ vs. wt: $35 \pm 14 \mathrm{mmol} / \mathrm{g}$ prot, $\mathrm{p}<0.001$ ). Message RNA level of AMPK $\gamma 2$ subunit (tg: $0.72 \pm 0.02$ fold to wt, $\mathrm{p}<0.001$ ) was significantly decreased in the hearts of SSAT mice compared to wt mice.

Conclusion: Activated polyamine catabolism leads to DCM. The underlying mechanisms seem to be related to the accumulation of glycogen in the heart and altered cardiac energy metabolism, especially downregulation of AMPK $\gamma 2$ subunit in heart muscle, mimicking human glycogen-storage cardiomyopathy.

Supported by: Academy of Finland

\section{2}

Impaired glucose tolerance exacerbates the abnormal myocardial metabolic response to pacing in subjects with dilated cardiomyopathy A. Natali ${ }^{1}$, S. Baldi ${ }^{1}$, A. Gastaldelli ${ }^{2}$, D. Ciociaro ${ }^{2}$, S. Frascerra ${ }^{1}$, A. De Caterina ${ }^{2}$, P. Marraccini ${ }^{2}$, E. Ferrannini ${ }^{1}$, F. Recchia ${ }^{2}$, D. Neglia ${ }^{2}$ ${ }^{1}$ Department of Internal Medicine, University of Pisa, ${ }^{2}$ Institute of Clinical Physiology, C.N.R., Pisa, Italy

Background and aims: Studies in animal models and in man have shown that dilated cardiomyopathy (DCM) under resting conditions is characterized by a shift of myocardial substrate metabolism towards carbohydrates at the expense of fatty substrates. During a metabolic stress (pacing), it has been documented that DCM patients fail to increase myocardial glucose uptake. Since an abnormal glucose homeostasis is rather common in patients with DCM, we hypothesised that it could be responsible for some of the observed myocardial metabolic defects.

Materials and methods: In 10 patients with DCM (ejection fraction $<50 \%$ ), 5 with normal (NGT) and 5 with impaired glucose tolerance (IGT), we measured coronary blood flow (by intravascular ultrasonometry) and transmyocardial artero-venous differences of oxygen and metabolites at rest, during pacing (up to $130 \mathrm{~b} / \mathrm{min}$ ) and during $30 \mathrm{~min}$ of recovery. Lactate exchange (by ${ }^{13} \mathrm{C}$-lactate) and oleate oxidation (from ${ }^{3} \mathrm{H}$-oleate/ ${ }^{3} \mathrm{H}_{2} \mathrm{O}$ conversion) were also measured. Metabolic fluxes are normalized per gram of myocardial tissue (LV mass was measured by echography).Glucose, lactate and FFA were converted in ATP equivalents assuming 36, 18 and $131 \mathrm{~mol}$ per mol, respectively.

Results: Atrial pacing induced a similar rate-pressure product plateau in IGT $\left(11,410 \mathrm{mmHg}^{\star} \mathrm{b} / \mathrm{min}\right)$ and NGT $(12,089)$ and a similar increment in coronary blood flow $(+0.26$ and $+0.25 \mathrm{ml} / \mathrm{min} / \mathrm{g}$ ). In response to pacing, myocardial glucose and lactate uptake did not change in either NGT or IGT patients. During recovery, the uptake of these substrates increased in NGT (from $5.6 \pm 1.2$ to $11.6 \pm 2.8 \mu \mathrm{mol}$ of $\mathrm{ATP} / \mathrm{g} / \mathrm{min}, \mathrm{p}<0.05$ ) but decreased in IGT (from $10.5 \pm 1.7$ to $6.5 \pm 0.8 \mu \mathrm{mol}$ of $\mathrm{ATP} / \mathrm{g} / \mathrm{min}, \mathrm{p}<0.05)$. Furthermore, in the latter group a significant increase in lactate release occurred during the pacing (from 62 to $142 \mathrm{pmol} / \mathrm{g} / \mathrm{min}, \mathrm{p}<0.05$ ). While the myocardium of the IGT patients sustained the pacing work by increasing free fatty acids oxidation (from $61 \pm 16$ to $124 \pm 2 \mathrm{nmol} / \mathrm{g} / \mathrm{min}, \mathrm{p}<0.05)$, in the NGT patients there was a rapid and prolonged increase in $\mathrm{B}$-OH-butyrate uptake (from $131 \pm 55$ to $211 \pm 95$ $\mathrm{nmol} / \mathrm{g} / \mathrm{min}$ ). The enhanced reliance on lipid oxidation during pacing and recovery of the IGT patients was confirmed by the gas exchange measurements, which gave estimates of ATP derived from lipids (from 11.2 \pm 5.6 to $18.3 \pm 4.5$ $\mu \mathrm{mol}$ of ATP $/ \mathrm{g} / \mathrm{min}$ ) similar to the estimates obtained from the conversion of ${ }^{3} \mathrm{H}$-oleate to ${ }^{3} \mathrm{H}_{2} \mathrm{O}$ (from $8.0 \pm 2.1$ to $16.3 \pm 0.3 \mu \mathrm{mol}$ of ATP $/ \mathrm{g} / \mathrm{min}$ ).

Conclusion: Patients with DCM and IGT show a more severe impairment in the ability to use carbohydrate for an acute increase in myocardial work and are heavily dependent on fatty substrate utilisation. The presence of IGT in DCM exasperates a (presumably) primary myocardial metabolic defect in substrate handling. 


\section{3}

Myocardial steatosis is an independent predictor of diastolic dysfunction in type 2 diabetes mellitus

R.W. van der Meer ${ }^{1}$, L.J. Rijzewijk², M. Diamant ${ }^{2}$, J.J. Bax ${ }^{3}$, J.A. Romijn ${ }^{4}$, J.W.A. Smit ${ }^{4}$, A. de Roos ${ }^{1}$, H.J. Lamb ${ }^{1}$

${ }^{1}$ Radiology, Leiden University Medical Center, ${ }^{2}$ Endocrinology, VU university Medical Center, Amsterdam, ${ }^{3}$ Cardiology, Leiden University Medical Center, ${ }^{4}$ Endocrinology, Leiden University Medical Center, Netherlands

Background and aims: Type 2 diabetes mellitus (T2DM) is a major risk factor for cardiovascular disease. Increasing evidence is emerging indicating that lipid oversupply to cardiomyocytes plays a role in the development of diabetic cardiomyopathy, by causing lipotoxic injury and myocardial steatosis. Therefore, the aim of this study was to compare myocardial triglyceride content and myocardial function in patients with uncomplicated T2DM and age- and body mass index (BMI) matched healthy subjects and to study the associations between myocardial triglyceride content and heart function.

Materials and methods: Myocardial triglyceride content and myocardial function were measured in $41 \mathrm{~T} 2 \mathrm{DM}$ patients (mean \pm SE age: $57 \pm 1$ years, BMI: $28.1 \pm 0.6$, and HbAlc $7.2 \pm 0.2$, free of cardiac ischaemia (verified by dobutamine stress echocardiography) and 30 healthy age- and BMI matched volunteers by proton magnetic resonance (MR) spectroscopy (fig 1) and MR imaging, respectively.

Results: Myocardial triglyceride content was significantly higher in T2DM patients compared to healthy volunteers $(0.96 \pm 0.07$ vs. $0.65 \pm 0.05 \%$, p $<$ $0.05)$. Systolic function did not significantly differ between both groups. Indices of diastolic function, including left ventricular E/A ratio and E peak deceleration, were significantly impaired in T2DM compared to healthy subjects ( $1.24 \pm 0.06$ vs. $1.08 \pm 0.04$ and $4.4 \pm 0.3 v s .3 .6 \pm 0.2 \mathrm{ml} / \mathrm{s}^{2} \times 10^{-3}$ respectively, $\mathrm{p}<0.05)$. Multivariable analysis indicated that myocardial triglyceride content was associated with $\mathrm{E} / \mathrm{A}$ and $\mathrm{E}$ peak deceleration, independently of diabetic state, age, BMI, heart rate, visceral fat and diastolic blood pressure. Conclusion: Myocardial triglyceride content is increased in uncomplicated T2DM and associated with impaired left ventricular diastolic function, independently of age, BMI, heart rate, visceral fat, and diastolic blood pressure.
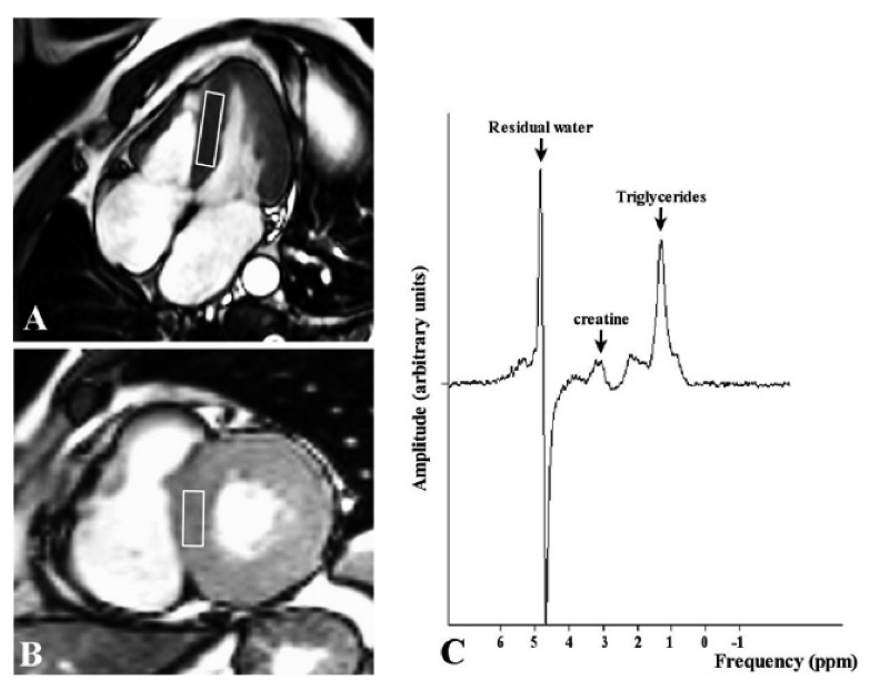

Fig. 1. Proton Magnetic Resonance Spectroscopy. Spectroscopic volume localization in the interventricular septum on 4-chamber (A) and shortaxis (B) views. Panel C shows a typical water suppressed $1 \mathrm{H}$ spectrum of myocardial tissue located in the interventricular septum. Peak heights are in arbitrary units

\section{4}

Effect of pioglitazone versus metformin on myocardial function, metabolism and triglyceride content in men with well-controlled type 2 diabetes and verified absence of cardiac ischaemia L.J. Rijzewijk ${ }^{1}$, R.W. van der Meer², H.J. lamb², H.W.A. Jong ${ }^{3}$, M. Lubberink ${ }^{3}$, J.A. Romijn ${ }^{4}$, J.J. Bax ${ }^{5}$, A. de Roos' ${ }^{2}$, O. Kamp ${ }^{6}$, W.J. Paulus ${ }^{7}$, A.A. Lammertsma ${ }^{3}$, J.W.A. Smit ${ }^{4}$, M. Diamant ${ }^{1}$

${ }^{1}$ Diabetescenter, VU medical center, Amsterdam, ${ }^{2}$ Radiology, Leiden University Medical Center, ${ }^{3}$ Nuclear Medicine \& PET Research, VU medical center, Amsterdam, ${ }^{4}$ Endocrinology, Leiden University Medical Center, ${ }^{5}$ Cardiology, Leiden University Medical Center, ${ }^{6}$ Cardiology, VU medical center, Amsterdam, Netherlands, ${ }^{7}$ Physiology, VU medical center, Amsterdam, Netherlands

Background and aims: Cardiac disease is the leading cause of mortality in type 2 diabetes mellitus (T2DM). Pioglitazone (PIO) therapy is associated with potential benefits but also with an elevated risk of heart failure. In the PIRAMID study (Pioglitazone Influence on tRiglyceride Accumulation in the Myocardium In Diabetes) we determined the effects of PIO, versus metformin (MET), on myocardial function, perfusion, glucose and fatty acid metabolism in T2DM patients. In addition, myocardial and liver triglyceride content were determined.

Materials and methods: Seventy-eight $\mathrm{T} 2 \mathrm{DM}$ patients (mean $\pm \mathrm{SE}$ : age $56.6 \pm 0.6 \mathrm{y} ; \mathrm{HbA}_{1 \mathrm{c}} 7.1 \pm 0.1 \%$; BMI $28.7 \pm 0.4 \mathrm{~kg} / \mathrm{m}^{2}$ ), without cardiac ischaemia, as verified by dobutamine-stress echocardiography, were randomly assigned to PIO (30 mg daily) or MET (2000 mg daily) and matching placebo during 24 weeks. The primary endpoint was change from baseline in cardiac function, relative to myocardial metabolic and perfusion changes, measured by magnetic resonance imaging, proton MR spectroscopy and positronemission tomography using $\left[{ }^{15} \mathrm{O}\right]-\mathrm{H}_{2} \mathrm{O},\left[{ }^{11} \mathrm{C}\right]$-palmitate in the fasting state and $\left[{ }^{18} \mathrm{~F}\right]$-fluoro-2-deoxy-glucose under euglycaemic hyperinsulinaemic conditions.

Results: Seventy-one patients completed the study. No patient developed congestive heart failure. Both therapies similarly improved glycaemic control and systolic blood pressure. PIO increased body weight $(\mathrm{p}=0.001)$ and enddiastolic volume $(\mathrm{p}<0.001)$ without changing cardiac index or left ventricular mass. Only PIO improved diastolic function $(\mathrm{p}=0.034)$, in association with improved whole-body insulin sensitivity $(\mathrm{p}<0.02)$, changes in myocardial fatty-acid uptake $(p<0.02)$ and oxidation $(p<0.03)$. However, these functional changes were unrelated to increased myocardial glucose uptake. MET also improved insulin sensitivity $(\mathrm{p}=0.033)$ but reduced myocardial glucose uptake $(\mathrm{p}=0.005)$ and fatty acid oxidation $(\mathrm{p}=0.024)$, without affecting myocardial function. MET increased, whereas PIO decreased, plasma lactate $(\mathrm{p}=0.001$, between groups). Neither treatment affected myocardial triglyceride content or perfusion. However, PIO but not MET decreased liver triglyceride content ( $\mathrm{p}=0.001$, between groups).

Conclusion: In the absence of cardiac ischaemia, PIO therapy may have beneficial cardiac effects in T2DM patients, both by directly affecting cardiac metabolism and by inducing systemic metabolic changes related to improvement in insulin sensitivity.

Supported by: Lilly, The Netherlands 


\section{OP 20 Diabetes in childhood}

\section{5}

A mouse model of neonatal diabetes caused by the $\mathrm{K}_{\mathrm{ATP}}$ channel mutation Kir6.2-V59M

C.A. Girard ${ }^{1}$, T.F. Wunderlich ${ }^{2}$, K. Shimomura ${ }^{1}$, S. Collins ${ }^{3}$, S. Kaizik ${ }^{1}$, P. Proks ${ }^{1}$, F. Abdulkader ${ }^{3,4}$, A. Clark ${ }^{3}$, L. Bentley ${ }^{5}$, J. Galvanovskis ${ }^{3}$, R. Cox ${ }^{5}$, P. Rorsman ${ }^{3}$, J.C. Bruning ${ }^{2}$, F.M. Ashcroft ${ }^{1}$

${ }^{1}$ Department of Physiology,Anatomy and Genetics, University of Oxford, United Kingdom, ${ }^{2}$ Department of Mouse Genetics and Metabolism, University of Cologne, Germany, ${ }^{3}$ The Oxford Center for Diabetes, Endocrinology and Metabolism, Churchill Hospital, Oxford, United Kingdom, ${ }^{4}$ Department of Physiology and Biophysics, Institute of Biomedical Sciences, Sao Paulo, Brazil, ${ }^{5} \mathrm{MRC}$ Harwell, Medical Research Council, Harwell, United Kingdom

Background and aims: Gain-of-function mutations in Kir6.2, the pore-forming subunit of ATP-sensitive potassium channels ( $\mathrm{K}_{\text {ATP }}$ channels) are a common cause of human neonatal diabetes. Around $20 \%$ of patients also exhibit muscle weakness and developmental delay, a condition known as intermediate DEND syndrome, usually due to mutation of valine 59 to methionine (V59M). Using Cre-loxP technology, we generated a mouse expressing the Kir6.2-V59M mutation specifically in pancreatic beta-cells (V59M mice) in order to determine its contribution to the development of neonatal diabetes observed in humans.

Materials and methods: Five week old mice (Wild-Type, V59M, RIP-Cre, ROSA) were used in this study. Hormone concentrations were determined after overnight fasting. Insulin secretion and intracellular calcium measurements were assessed on isolated islets stimulated with either high glucose concentration or sulphonylurea drugs. Morphometric analyses were performed on wax-embedded pancreases. Glucose and ATP sensitivity of mutant $\mathrm{K}_{\mathrm{ATP}}$ channels were determined by electrophysiology on isolated beta-cells.

Results: V59M mice developed severe diabetes soon after birth: plasma glucose was $>30 \mathrm{mM}$ and insulin was undetectable. Isolated islets failed to secrete insulin in response to glucose, due to a reduced sensitivity of $\mathrm{K}_{\text {ATP }}$ currents in V59M beta-cells to inhibition by glucose and ATP ( IC $_{50} 270 \mu \mathrm{M}$, vs. $25 \mu \mathrm{M}$ for wild-type beta-cells). However, the sulphonylurea tolbutamide closed V59M $\mathrm{K}_{\mathrm{ATP}}$ channels and stimulated insulin release. Unexpectedly, morphometric analysis revealed that V59M islets also displayed an abnormal morphology, reduced beta-cell number ( $20 \%$ less), and lower insulin content.

Conclusion: Our findings demonstrate that expression of the V59M mutation in beta-cells alone is sufficient to recapitulate the neonatal diabetes observed in humans, and explain why human patients with this mutation can be successfully transferred from insulin injections to sulphonylurea therapy. Supported by: Wellcome Trust, the MRC, the EU and BioSim, the Royal Society, and the Deutsche Forschungsgemeinschaft, EMBO for a short-term fellowship

\section{6}

The use of prophylactic dose of alfacalcidol maintains remission regardless of children's age in new onset type 1 diabetes - 2 years observation

R. Piekarski, Y. Azab, A. Bury, L. Szewczyk

Pediatric Endocrinology and Neurology, Medical University in Lublin, Lublin, Poland

Background and aims: In recent years studies focused on prevention of type 1 diabetes mellitus (DM) and possibility of beta-cell protection at the clinical diagnosis of DM. Some recent studies have demonstrated beneficial role of vit. D3 analogue in human beta-cell activity maintenance at the early stages of DM. In this study have been continued the observations concerning the role of vit. D3 analogue with the aim of beta cells functional state evaluation.

Materials and methods: The study was performed in 54 children and adolescents aged $4-18$ years ( 21 children $<7$ year old and $33 \geq 7$ year old ) who received insulin and vit D3 analogue (alfacalcidol - 1alpha-OH-vitamin D3) in daily dose $0.25 \mu \mathrm{g}$. 37 peers with type $1 \mathrm{DM}$ who received only insulin therapy without vit. D3 administration were the control group. After two years therapy we compared the level of $\mathrm{HbAlc}$, body mass, daily insulin dose in both groups.

Results: There are no differences in mean body mass and in metabolic control of diabetes reported by mean HbAlc level at the onset of the DM and after two years observation of these patients among studied groups. How- ever, daily insulin dose in $60 \%$ of patients who received vit. D3 therapy after two years observation were below $0.5 \mathrm{u} / \mathrm{kg}$. There are no differences in insulin requirement regardless the children's age. On the contrary, insulin requirement in the group of patients without vit. D3 treatment significantly increased $(\mathrm{p}<0.01)$ after two years observation (from $0.37 \mathrm{u} / \mathrm{kg}$ to $0.78 \mathrm{u} / \mathrm{kg}$ ). In this group remission rate $(<0.5 \mathrm{u} / \mathrm{kg})$ in only $6 \%$ was found. There are disturbances in calcium homeostasis in the group of children treated with alfacalcidol not observed.

Conclusion: 1 . In $60 \%$ of children treated alfacalcidol insulin requirement after two years therapy with vit. D3 analogue was below $0.5 \mathrm{u} / \mathrm{kg}$. 2 . There are no differences in insulin requirement in the group of children treated with alfacalcidol related to the age. 3. Children without vit. D3 administration had twofold increase of insulin requirement after two years observation from clinical diagnosis. 4. Our observations suggest, that alfacalcidol could play a role in prevention of further beta-cells destruction. Supported by: Grant MNiSzW no N N407 310033

\section{7}

Metabolic control in diabetic children and adolescents - a trend analysis using multi-centre data

J. Rosenbauer ${ }^{1}$, E. Gerst $1^{2}$, C. Kastendieck ${ }^{3}$, M. Grabert ${ }^{4}$, S. Hofer ${ }^{5}$, W. Rabl ${ }^{6}$, H. Gröbe ${ }^{7}$, R. Holl ${ }^{4}$ on behalf of the DPV Wiss Initiative

${ }^{1}$ Institute of Biometrics and Epidemiology, German Diabetes Centre, Düsseldorf, Germany, ${ }^{2}$ Paediatric Clinic, University Hospital München, Germany, ${ }^{3}$ Children's Hospital, Hospital Bremen North, Germany, ${ }^{4}$ Institute of Epidemiology, Ulm University, Germany, ${ }^{5}$ Children's Hospital, University Hospital Innsbruck, Austria, ${ }^{6}$ Children's Hospital, University Hospital München, Germany, ${ }^{7}$ Children's Hospital, Hospital Nürnberg South, Germany

Background and aims: During the past decade considerable efforts have been undertaken in diabetes care to intensify insulin therapy and to improve patient education. The issue, whether treatment results have contemporaneously improved, has great public health importance. $\mathrm{HbAlc}$ is the most important parameter to assess long-term metabolic control and quality of diabetes care. Evaluation of metabolic control is complicated by methodical problems (laboratory specific normal ranges for $\mathrm{HbAlc}$ ) and the great number of correlating predictors.

Materials and methods: Within the framework of the quality assurance project DPV German and Austrian diabetes centres document relevant parameters of diabetes care using the DPV software. Twice a year anonymous data are transmitted for central plausibility checks and analysis to provide centres with benchmarking reports. Further, this anonymous data base is available for epidemiologic-scientific research. This analysis is based on data of 27360 patients from 268 diabetes centres documented during 1995-2007. $\mathrm{HbAlc}$ values were converted to the international DCCT standard using the multiple-of-the-mean method. For each patient, the median of all HbAlc measurements during the most recent treatment year was evaluated. Data were analysed by descriptive statistics and generalized linear mixed regression models including various relevant factors (year and month of treatment, age, sex, diabetes duration, foreign ethnicity, treatment modality, size and kind of centre) as fixed and treatment centre as random effect to account for variation between diabetes centres.

Results: Crude median $\mathrm{HbA1c}$ decreased from $8.0 \%$ (interquartile range: $2.0 \%$ ) in 1995 to $7.6 \%$ (interquartile range: $1.7 \%$ ) in 2007. Multivariate regression analysis showed treatment year, age, sex, diabetes duration, foreign ethnicity, month of Hbalc measurement, and mode of insulin treatment to be significant predictors of metabolic control $(\mathrm{p}<0.001)$, adjusting for variation between diabetes centres. Size and kind of diabetes centre ( $<100$ vs. $\geq 100$ children treated or regular vs. rehab hospital, respectively) had no significant effect $(\mathrm{p}>0.5)$. Trend analysis indicated that mean HbAlc decreased significantly by $0.7 \%$ per year on average in relative terms, corresponding to an absolute average decrease by $0.06 \%$.

Conclusion: Applying a complex statistical model this study showed a slight improvement in metabolic control in diabetic children and adolescents during the past decade. This improvement was not fully explained by changes in the mode of insulin treatment. Possibly, improved patient education during the last decade may account for this trend, at least in part. Using observational data the study can not prove observed associations to reflect causal effects on the metabolic control.

Supported by: German Ministry of Health, German Diabetes Foundation, German Diabetes Association, NAFDM, Dr. Bürger-Büsing Foundation, German Medical Association, Novo Nordisk, Germany 


\section{8}

Intensive care admissions for acute diabetic complications of children and adolescents in England and Wales

J. Bodansky ${ }^{1}$, R.C. Parslow ${ }^{2}$, R.G. Feltbower ${ }^{3}$, P.A. Mckinney ${ }^{3}$

${ }^{1}$ General medicine, The Diabetes Centre, ${ }^{2}$ Peadiatric Epidemiology Unit,

Centre for Epidemiology and Biostatistics, ${ }^{3}$ Paediatric Epidemiology Group,

Centre for Epidemiology and Biostatistics, Leeds, United Kingdom

Background and aims: The incidence of type 1 diabetes in young people is increasing and places a growing burden on healthcare providers. The treatment of severely ill children and adolescents in intensive care is very expensive. There is evidence of self-harm or neglect of their condition in teenage patients, especially males, some of whom may require intensive care. Nationally available data on children admitted to paediatric intensive care (PICU) with acute diabetic complications are now available for the UK. The aim of this study was to examine patient demographics and outcomes of these children and adolescents in England and Wales, using a high quality national dataset.

Materials and methods: The Paediatric Intensive Care Audit Network (PICANet) collects data on all admissions to paediatric intensive care in England and Wales. Admissions between April 2003 and March 2007 whose primary diagnosis was related to diabetes were identified by expert clinical review of coded data. Demographic information included age at admission, sex, ethnic group (south Asian or not, determined by two name classification algorithms) and socioeconomic status. Crude and risk-adjusted mortality (using the Paediatric Index of Mortality (PIM)) and length of stay (LOS) were used as outcome measures. Descriptive statistics and logistic regression were used to analyse the data.

Results: 330 diabetic children and adolescents were admitted to a PICU in the 4 year period, with 341 admissions overall representing $0.6 \%$ of all PICU admissions. There was an excess of female admissions (56\% vs. 44\%) with a M: F ratio of $0.78: 1$ in this cohort of patients compared with $1.33: 1$ in the PICU population overall (chi square 24.39 , 1df, $\mathrm{p}<0.001$ ). $89 \%$ of admissions were diagnosed with diabetic ketoacidosis (DKA) (57\% female, $43 \%$ male). $40 \%$ of admissions were aged 11-15 years. The proportion of admissions increased with lower socioeconomic status following a similar pattern to all PICU admissions. There were significantly fewer admissions of South Asians ( $7 \%$ vs. $11 \%$, chi-square $4.44,1 \mathrm{df}, \mathrm{p}=0.035)$. Five deaths ( $1.47 \%$ of admissions) occurred in this period, all female, four with DKA, and one with hypoglycaemia. PIM predicted 27 deaths based on presenting physiology. The risk-adjusted odds of mortality for admission with a diabetes-related condition was $0.22, p=0.002,95 \%$ CI, 0.09-0.56. Median length of stay was 2 days (IQR 2-3) compared with 3 days (IQR 2-6) in the rest of the PICU population.

Conclusion: Admission to PICU for diabetes related conditions is proportionately higher in females despite higher incidence of type 1 diabetes in males nationally (M: F ratio 1.2:1) and in PICU admissions generally. Despite high PICU survival overall, there is an unexpected sex bias in mortality. The apparently low risk-adjusted mortality may relate to using base excess as a predictive component of the model, which is usually corrected rapidly in this group of patients. Avoiding acute diabetic complications would reduce the admission rate to costly intensive care beds, which are usually in short supply. Supported by: Department of Health; Health Commission Wales Specialised Services

\section{9}

\section{High prevalence of cardiovascular risk factors in youths with type 2 diabetes: the search for diabetes in a youth case-control study}

D. Dabelea ${ }^{1}$, N.A. West ${ }^{1}$, E.J. Mayer-Davis ${ }^{2}$, R.B. D;Agostino Jr. ${ }^{3}$, S. Marcovina ${ }^{4}$, A.D. Liese $^{5}$, P.S. Zeitler ${ }^{6}$, S.R. Daniels ${ }^{6}$, R.F. Hamman ${ }^{1}$ ${ }^{1}$ Preventive Medicine and Biometrics, University of Colorado Denver, ${ }^{2}$ Nutrition, University of North Carolina at Chapel Hil, ${ }^{3}$ Public Health Sciences, Wake Forest University, Winston-Salem, ${ }^{4}$ Northwest Lipid Reserach Laboratories, Univeristy of Washington, Seattle, ${ }^{5}$ Epidemiology, Arnold School of Public Health, Columbia, ${ }^{6}$ Pediatrics, University of Colorado, Denver, CO, United States

While the increased risk of cardiovascular disease (CVD) in adults with Type 2 Diabetes (T2D) compared to the general population has been well documented, data on CVD risk factor profile in youth with T2D remain rela- tively sparse. We evaluated case/control differences in CVD risk factor profile among 331 multi-ethnic participants (38\% African American, 16\% Hispanic, $46 \%$ non-Hispanic white), age 10-22 years, in the SEARCH for Diabetes in Youth Case-Control Study. T2D $(n=116)$ and control $(n=215)$ youth were recruited in Colorado and South Carolina. Compared with controls, youth with T2D had higher age- and race/ethnicity-adjusted prevalence of hypertension (blood pressure $\geq 95^{\text {th }}$ percentile or medication: $27 \%$ vs $5 \%, \mathrm{p}<.0001$ ), obesity (body mass index-BMI $\geq 95^{\text {th }}$ percentile: $86 \%$ vs $26 \%$, $p<.0001$ ), large waist circumference $\left(\geq 90^{\text {th }}\right.$ percentile : $82 \%$ vs $\left.22 \%, \mathrm{p}<.0001\right)$, low HDLc $(\leq 35$ $\mathrm{mg} / \mathrm{dl}: 25 \%$ vs $5 \%, \mathrm{p}<.0001$ ), high triglycerides (TG $\geq 150 \mathrm{mg} / \mathrm{dl}: 27 \%$ vs $6 \%, \mathrm{p}<.0001$ ), and high urinary albumin:creatinine ratio (ACR $\geq 30 \mu \mathrm{g} / \mathrm{mg}$ : $17 \%$ vs $7 \%, p=.02$ ). There was little difference among youth with and without T2D in the prevalence of high LDLc ( $14 \%$ vs $10 \%, \mathrm{p}=.29)$. The distribution of the total number of CVD risk factors $(0,1,2,3$, or $4+)$ in youth was also significantly different $(\mathrm{p}<.0001)$, with a less favorable CVD risk profile for T2D youth (figure). T2D youth also had a less favorable profile of non-traditional CVD risk factors. This included higher apolipoprotein B (79 vs. 61 $\mathrm{mg} / \mathrm{dl}$ ) and lower LDL particle density (0.27 vs $0.28 \mathrm{Rf})$, even after adjusting for differences in BMI ( $p<0.0001$ for each), as well as higher fibrinogen (404 vs $319 \mathrm{mg} / \mathrm{dl}, \mathrm{p}<0.0001)$ and IL6 levels ( $13 \mathrm{vs} 6 \mathrm{pg} / \mathrm{ml}, \mathrm{p}=0.02)$, even after adjustment for both BMI and $\mathrm{HbAlc}$. In conclusion, when compared with healthy adolescents, youth with T2D have a higher prevalence of many CVD risk factors. Obesity is an important contributor to a less favorable CVD risk profile; however, additional mechanisms, such as hyperglycemia and inflammation, may also play a role. These data highlight the need for early prevention and treatment strategies aimed at reducing the prevalence of CVD risk factors in youth with T2D.

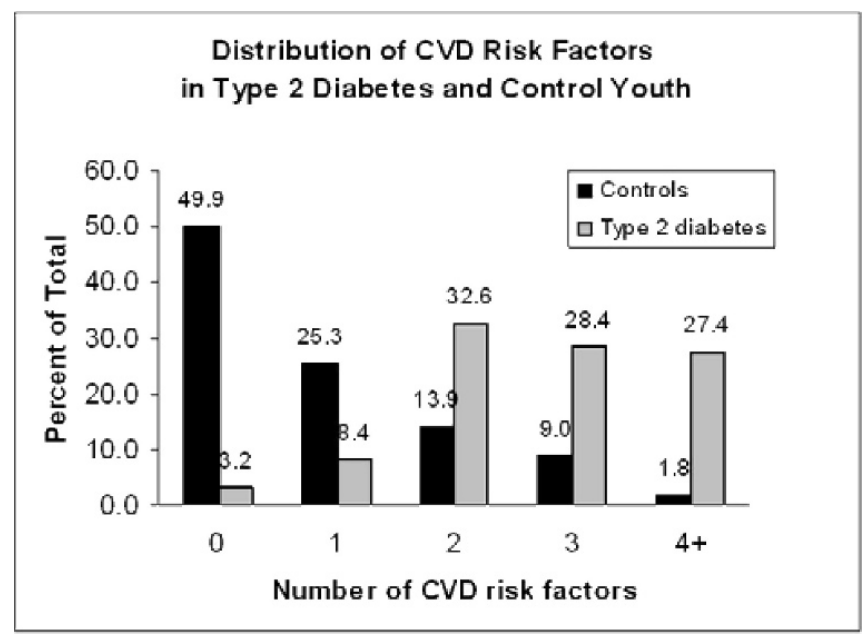

Supported by: National Institute Of Diabetes And Digestive And Kidney Diseases

\section{0}

Ethnic differences in cardiovascular risk profile in an overweight/obese multiethnic paediatric cohort

I. von Rosenstiel ${ }^{1}$, M. van Vliet ${ }^{1}$, R.K. Schindhelm², D.P.M. Brandjes ${ }^{3}$, J.H. Beijnen ${ }^{4}$, M. Diamant ${ }^{5}$

${ }^{1}$ Paediatrics, Slotervaart Hospital, Amsterdam, ${ }^{2}$ Clinical Chemistry, Isala Clinics, Zwolle, ${ }^{3}$ Internal Medicine, Slotervaart Hospital, Amsterdam, ${ }^{4}$ Pharmacy \& Pharmacology, Slotervaart Hospital, Amsterdam, ${ }^{5}$ Diabetes Centre, VU University Medical Centre, Amsterdam, Netherlands

Background and aims: Among children, the global prevalence of obesity and all components of the metabolic syndrome (MetS), including glucose intolerance, insulin resistance, dyslipidaemia and hypertension is increasing at an alarming rate. However, differences in prevalence of these cardiovascular risk factors between different ethnic groups are unknown. In the present study, we determined the variation in cardiovascular risk profile and the association of insulin resistance with cardiometabolic risk factors according to ethnicity in a cohort of overweight/obese children.

Materials and methods: An oral glucose tolerance test was performed in 516 consecutive overweight/obese Dutch children of multiethnic origin, from an obesity out-patient clinic of a community hospital (mean age $11 \pm 3$ yrs; $n=285$ boys). Anthropometric parameters and fasting blood samples were collected. 
The prevalence of (components of) MetS (according to a previously defined paediatric definition) and insulin resistance (defined by the Homeostasis Model Assessment for Insulin Resistance [HOMA-IR] $\geq 3.5$ ) were determined. Data were analysed after stratification for ethnic group.

Results: Major ethnic groups were Dutch native (18.4\%), Turkish (28.1\%), Moroccan (25.8\%), Hindu (4.3\%) and a group with other or mixed origins (23.4\%, i.e. with parents of different origins). MetS was established in $18.6 \%$, impaired glucose tolerance in 3.2\%, low HDL-cholesterol in 31.2\%, high triglycerides in $17.4 \%$ and hypertension in $22.9 \%$. Of all children, $4.3 \%$ did not meet the obesity criterion of MetS. Turkish children had the highest mean waist circumference $(P<0.05)$ and prevalences of MetS $(26.9 \%)$, low HDLcholesterol (37.9\%), hypertension (29.7\%) and insulin resistance $(54.9 \%)$, whereas in Moroccan children, insulin resistance was least frequently present (37.4\%), as compared to other ethnic groups (all $P<0.05$ ). Although the prevalences of the various MetS-components in Moroccan children did not significantly differ from other ethnic groups, mean plasma HDL-cholesterol was higher, while triglycerides and systolic blood pressure were lower in these children $(P<0.01, P<0.001$ and $P<0.05$, respectively) as compared to other ethnic groups. In addition, apart from obesity, $60.6 \%$ of Moroccan children $(P<0.01)$, relative to $40.0 \%$ of Turkish children $(P<0.01)$ were free of MetScomponents, compared to $48.7 \%$ of their peers of other origins. HOMA-IR was associated with MetS and its separate components, after adjustment for ethnicity and BMI. When observing these relations in Dutch native, Turkish and Moroccan children separately (after adjustment for BMI), HOMA-IR was associated with at least two MetS-components (apart from obesity).

Conclusion: In a Dutch cohort of overweight/obese children, Turkish children showed a higher prevalence of cardiovascular risk factors relative to their peers of other ethnicities. In contrast, children of Moroccan background had a relatively favourable cardiovascular risk profile. Long-term studies are needed to establish whether our findings are compatible with actual risk of future cardiometablic disease in the different ethnic populations.

\section{OP 21 Incretins: action and secretion}

\section{1}

The effects of jugular vs. portal GLP-1 during hyperglycaemic clamps in rats

M. Perabo ${ }^{1,2}$, B.A. Aulinger ${ }^{1}$, E.E. Elfers ${ }^{1}$, J. Heyl ${ }^{1}$, K. Ellis ${ }^{1}$, R.J. Seeley ${ }^{3}$, D.A. D'Alessio ${ }^{1}$

${ }^{1}$ Medicine - Division of Endocrinology, University of Cincinnati, Cincinnati, United States, ${ }^{2}$ Medizinische Klinik und Poliklinik 2, Ludwig-MaximiliansUniversitaet, Muenchen, Germany, ${ }^{3}$ Psychiatry, University of Cincinnati, United States

Background and aims: Glucagon-like-peptide 1 (GLP-1) is secreted by intestinal L-cells after nutrient ingestion and augments insulin secretion from pancreatic beta-cells in a glucose-dependent manner, thus contributing to the incretin effect. Its very low circulating levels, the moderate relative increment over fasting levels and the rapid degradation by ubiquitous dipeptidylpeptidase-IV have opened discussion as to whether GLP-1 acts through an endocrine mechanism or rather mediates its effect by activation of a neuroendocrine pathway. Previous studies have shown the presence of the GLP-1 receptor (GLP-1r) in the portal vein (PV) and suggested that those receptors are necessary for mediation of glucose tolerance by GLP-1. We sought to determine whether insulin secretion differs after GLP-1 administration into the PV vs. a systemic infusion into the jugular vein (JV) during fixed and constant glycemia.

Materials and methods: Catheters were implanted into the JV, PV and carotid artery of Long-Evans rats weighing 300-320g. After 7-10 days of recovery with animals returning to their pre-surgical BW, the vascular catheters were connected to infusion pumps allowing administration of test substances, and blood draws under conscious and freely moving conditions. All rats had a glucose target of $210 \mathrm{mg} / \mathrm{dl}$ that was maintained by a variable infusion of $20 \%$ dextrose for $120 \mathrm{~min}$. Blood samples for determination of glucose and insulin were taken at fixed intervals. After reaching steady-state glycemia a GLP-1 infusion of $7.5 \mathrm{pmol} / \mathrm{kg} \mathrm{BW} / \mathrm{min}$ was started at $60 \mathrm{~min}$ and was raised to 12.5 and $25 \mathrm{pmol} / \mathrm{kg} / \mathrm{min}$ at 80 and $100 \mathrm{~min}$, respectively.

Results: Before GLP-1 was started (50-60 min) glucose and insulin were not different between the JV and PV group ( $214 \pm 4$ vs. $216 \pm 4 \mathrm{mg} / \mathrm{dl} ; 318 \pm 29$ vs. $283 \pm 33 \mathrm{pM}$ ). There was no increase of insulin secretion above the hyperglycemic baseline during infusion of the lowest GLP-1 dose in either group. However, the two higher doses of GLP-1 resulted in a significant increase of arterial insulin concentrations with both JV and PV infusion. The insulin response was significantly greater in animals receiving JV vs. PV GLP-1 ( $679 \pm 112$ and $1178 \pm 235 \mathrm{pM}$ vs. $396 \pm 47$ and $577 \pm 71 \mathrm{pM} ; \mathrm{p}<0.05)$. Consistent with this, the glucose infusion needed to maintain the glucose clamp was significantly higher in JV vs. PV during the last $20 \mathrm{~min}$ of the clamp (5.1 \pm 0.4 vs. $3.3 \pm 0.4, \mathrm{p}<0.005)$.

Conclusion: These data suggest that the GLP-1r previously described in the wall of the PV does not mediate the effects of GLP-1 on insulin secretion. However the significant difference in the GLP-1:insulin dose-response between JV and PV administration indicates a differential fate of GLP-1 in the systemic and portal circulation. Specifically these data support a central role of the liver in inactivation of endogenous GLP-1 such that a lesser amount is available for interaction with target organs through the arterial circulation. Since supraphysiologic amounts of GLP-1 were infused in this study our results raise questions as to the amount of endogenously secreted GLP-1 is available to the general circulation after meals. We conclude that an alternative mechanism, beyond PV or endocrine signalling, is required to explain how GLP-1 physiologically mediates its incretin effect.

Supported by: NIH - NIDDK

\section{2}

The effect of delayed incretin clearance on fasting and postprandial glucose turnover in subjects with impaired fasting glucose A. Vella ${ }^{1}$, C. Dalla Man², G. Bock ${ }^{1}$, R. Basu ${ }^{1}$, P.D. Giesler' ${ }^{1}$ J.M. Laugen ${ }^{1}$, G. Toffolo ${ }^{2}$, C. Cobelli², R.A. Rizza ${ }^{1}$

${ }^{1}$ Endocrinology, Mayo Clinic, Rochester, United States, ${ }^{2}$ Informatics, University of Padua, Italy

Background and aims: Abnormal concentrations of the incretin hormone Glucagon-like Peptide-1 (GLP-1) have been associated with type 2 diabetes and impaired fasting glucose (IFG) in some, but not all, studies. It is uncer- 
Data are shown as mean + SEM

\begin{tabular}{|c|c|c|c|c|c|c|}
\hline \multirow[t]{2}{*}{ Area above basal } & \multicolumn{2}{|c|}{$\begin{array}{l}\text { Endogenous Glucose Production } \\
\text { (mol/kg per } 6 \mathrm{~h})\end{array}$} & \multicolumn{2}{|c|}{$\begin{array}{l}\text { Glucose Disappearance } \\
\text { (mol/kg per } 6 \mathrm{~h})\end{array}$} & \multicolumn{2}{|c|}{ Meal Ra (mol/kg per $6 \mathrm{~h})$} \\
\hline & Placebo & Sitagliptin & Placebo & Sitagliptin & Placebo & Sitagliptin \\
\hline Baseline & $-2.66 \pm 0.12$ & $-3.24 \pm 0.20$ & $2.66 \pm 0.36$ & $2.61 \pm 0.44$ & $5.86 \pm 0.42$ & $6.64 \pm 0.38$ \\
\hline At 6 weeks & $-2.45 \pm 0.30$ & $-3.29 \pm 0.17$ & $2.41 \pm 0.67$ & $2.68 \pm 0.28$ & $5.74 \pm 0.63$ & $6.58 \pm 0.32$ \\
\hline$P$ & 0.41 & 0.86 & 0.5 & 0.81 & 0.14 & 0.85 \\
\hline
\end{tabular}

tain if these abnormalities contribute directly to the pathogenesis of IFG and impaired glucose tolerance (IGT). GLP-1 stimulates insulin secretion in the presence of hyperglycemia; however, it is unknown if this effect is extant at the concentrations observed in IFG. Dipeptidyl peptidase-4 (DPP-4) inhibitors provide an opportunity to modulate fasting and postprandial incretin hormone concentrations and examine their effects on glucose turnover in non-diabetic humans.

Materials and methods: We studied 20 subjects with IFG using a double blind, placebo-controlled parallel group design. At the time of study enrollment, subjects ate a standardized, labeled mixed meal consisting of $50 \mathrm{~g}$ of bacon, 2 scrambled eggs and $75 \mathrm{~g}$ of Jell-O labeled with $\left[1-{ }^{13} \mathrm{C}\right]$-glucose. [6$\left.{ }^{3} \mathrm{H}\right]$ glucose was infused intravenously to measure the systemic rate of meal appearance (Meal $\mathrm{Ra})$. Infused $\left[6,6{ }^{2} \mathrm{H}_{2}\right]$ glucose enabled measurement of endogenous glucose production (EGP) and glucose disappearance (Rd). Subsequently, subjects were randomized to daily treatment with $100 \mathrm{mg}$ of sitagliptin or placebo. After a 6-week treatment period, the mixed meal test was repeated.

Results: Subjects with IFG who received placebo did not experience any change in fasting $(5.92 \pm 0.08$ vs. $5.87 \pm 0.12 \mathrm{Mmol} / \mathrm{L}, P=0.69)$ and postprandial glucose concentrations $(0.375 \pm 0.0062$ vs. $0.337 \pm 0.0074$ mol per $6 \mathrm{~h}, P=0.25)$. Glucose fluxes did not change over the study period. Treatment with sitagliptin did not alter fasting $(5.79 \pm 0.15$ vs. $5.81 \pm 0.11 \mathrm{Mmol} / \mathrm{L}, P$ $=0.91)$ or significantly decrease postprandial glucose concentrations $(0.54 \pm$ 0.0132 vs. $0.42 \pm 0.0091 \mathrm{~mol}$ per $6 \mathrm{~h}, P=0.09)$. Sitagliptin was associated with a decrease in fasting glucagon concentrations $(60.5 \pm 5.9$ vs. $58.2 \pm 5.9 \mathrm{ng} / \mathrm{L}, P$ $<0.01)$. EGP, Rd and MRa were unchanged from baseline values.

Conclusion: The use of a DPP-4 inhibitor to raise endogenous incretin hormone concentrations was not associated with significant effect on glucose metabolism in subjects with IFG. Therefore, in contrast to their effects in type 2 diabetes, DPP-4 inhibitors do not significantly lower glucose concentrations. Whether this effect is proportional to the degree of hyperglycemia during treatment remains to be determined.

Supported by: Dr. Vella is supported by DK078646 and Dr. Rizza by DK29953

\section{3}

Obestatin, a peptide derived from preproghrelin, stimulates insulin secretion without affecting glucagon or somatostatin output

E.M. Egido, R.A. Silvestre, R. Hernández, J. Marco

Hospital Puerta de Hierro and Department of Physiology, Universidad

Autónoma de Madrid, Spain

Background and aims: Obestatin is a 23 -amino acid peptide derived from preproghrelin, purified from stomach extracts and detected in peripheral plasma. In contrast to ghrelin, obestatin has been reported to inhibit appetite and gastric motility, but these effects remain controversial. Obestatin is mainly produced in the A cells of the oxyntic mucosa and is also present in the pancreas, where it colocalizes with ghrelin in the peripheral region of pancreatic islets, a distribution distinct for alpha and beta cells. We have previously observed that obestatin dose-dependently potentiated the insulin response to glucose. In the present study, we have investigated the effect of obestatin on insulin, glucagon and somatostatin secretion, both under basal and stimulated conditions, in the isolated perfused rat pancreas.

Materials and methods: Rat obestatin (Bachem, Switzerland) was infused at $1 \mathrm{nM}$, a concentration that, in our perfused pancreas system, has been shown to potentiate glucose-induced insulin release. Insulin secretion was stimulated by increasing the perfusate glucose concentration (from 5.5 to $9 \mathrm{mM}$ or from $9 \mathrm{mM}$ to $13 \mathrm{mM}$ ) and by infusing arginine $(5 \mathrm{mM})$, Na-tolbutamide $(100 \mathrm{mg} / \mathrm{l})$ or carbachol $(5 \mu \mathrm{M})$. The effect of obestatin on glucose-induced insulin release was also tested in the presence of diazoxide $(300 \mu \mathrm{M})$. Insulin, glucagon and somatostatin were measured by RIA.

Results: $1 \mathrm{nM}$ obestatin potentiated by $50 \%(\mathrm{p}<0.05)$ the insulin response to glucose (an increase from 5.5 to $9 \mathrm{mM}$ ) and to tolbutamide, both of which are known to stimulate beta cell output by inducing the blockade of ATP- dependent K+ channels. Furthermore, the insulinotropic effect of obestatin on glucose-induced insulin secretion was not observed in the presence of diazoxide, an agent known to open these $\mathrm{K}+$ channels. These results suggest that beta cell ATP-dependent $\mathrm{K}+$ channels might be sensitive to obestatin. Obestatin did not affect the insulin response induced by increasing the glucose concentration from 9 to $13 \mathrm{mM}(260 \pm 23$ vs. $280 \pm 41 \mathrm{ng} / 15 \mathrm{~min}$; $\mathrm{p}=0.66)$, thus indicating that, at high glucose concentrations, the beta cells are less responsive to obestatin than at normal glucose levels. Basal insulin secretion was unaffected either at $5.5 \mathrm{mM}$ or at $9 \mathrm{mM}$ glucose. Obestatin did not modify the insulin response to carbachol, thus indicating that this peptide is not a general inhibitor of insulin output, acting at a crucial step in the insulin secretion-coupling mechanism. Finally, obestatin potentiated the insulin response to arginine, while it did not modify glucagon or somatostatin responses to this amino acid, thus suggesting that the insulinotropic effect of obestatin represents a direct effect on the beta cell, not mediated by an alpha- or deltacell paracrine effect.

Conclusion: Our results allow us to speculate about a role of obestatin in the control of beta cell secretion. As an insulinotropic agent, its potential antidiabetic effect may be worthy of investigation.

Supported by: Ministerio de Educación y Ciencia, Spain

\section{4}

Peripheral $\mathrm{N}$-acetylated cholecystokinin-8 administration has more potent effect in reducing short term food intake than peptide $\mathrm{YY}$ in mice F.P.M. O'Harte, P.A. Frizelle, P.R. Flatt

School of Biomedical Sciences, University of Ulster, Coleraine, Northern Ireland, United Kingdom

Background and aims: The neuropeptide hormone cholecystokinin octapeptide (CCK-8), along with the gut hormone peptide YY (PYY) are peripheral hormones which help regulate short-term feeding and satiety. Both peptides have short circulating biological half-lives due to the actions of peptidase enzymes. Here we examined the effects of CCK- 8 and a stable N-terminally modified analogue N-acetylated-CCK-8 (N-Ac-CCK(26-33)amide) on food intake in mice and compared their actions with those of the C-terminally truncated peptide N-Ac-CCK(26-31), as well as PYY(1-36) and PYY(3-36). Materials and methods: Food intake was examined using 12-14 week-old male Swiss TO mice, which were gradually habituated over 3-weeks to $3 \mathrm{~h}$ (10.00-13.00 h) of voluntary food intake per day. Mice had access to water $a d$ libitum. The dose-dependent effects on food intake of native CCK-8, N-AcCCK-8, the C-terminally truncated peptide N-Ac-CCK $(26-31)$, PYY (1-36) and PYY(3-36) were examined in this model. Groups of eight $21 \mathrm{~h}$ fasted mice (body weight $29.5 \pm 0.1 \mathrm{~g}$ mean \pm sem) were injected intraperitoneally $(10.00 \mathrm{~h})$ with peptide $(1,5,10,25,50$ and $100 \mathrm{nmol} / \mathrm{kg})$ or saline control (10 $\mathrm{ml} / \mathrm{kg}$ ) and immediately allowed access to food. Cumulative food intake was measured at times 30, 60, 90, 120, 150 and $180 \mathrm{~min}$.

Results: Food intake studies showed that saline-treated control Swiss TO mice $(\mathrm{n}=8)$ consumed (mean \pm sem) between $1.5 \pm 0.1 \mathrm{~g}$ and $3.3 \pm 0.2 \mathrm{~g}$ per mouse at 30-180 min. CCK-8 dose-dependently reduced food intake by $23-$ $97 \%$ over the range of concentrations $(5-100 \mathrm{nmol} / \mathrm{kg}$ ) compared to saline controls $(\mathrm{P}<0.05$ to $\mathrm{P}<0.001$, Student's t-test $)$. N-Ac-CCK- $8(5-100 \mathrm{nmol} / \mathrm{kg})$ was more effective that native CCK- 8 at reducing food intake by $52-99 \%$ up to 180 post injection and had a more protracted action at the later time points. At all concentrations tested the C-terminally truncated CCK peptide analogue N-Ac-CCK(26-31) failed to reduce food intake at any time point (30-180 $\mathrm{min}$ ) compared to the saline treated controls, indicating the importance of an intact C-terminal region for retention of biological activity. Both PYY(1-36) and PYY(3-36) dose-dependently reduced food intake from 10$100 \mathrm{nmol} / \mathrm{kg}$ (but not at 1 and $5 \mathrm{nmol} / \mathrm{kg}$ ) by $34-48 \%(\mathrm{P}<0.05$ to $\mathrm{P}<0.01)$ and $48-54 \%(\mathrm{P}<0.05$ to $\mathrm{P}<0.001)$, respectively. The $\mathrm{N}$-terminally truncated PYY(3-36) was only slightly more effective than the intact PYY(1-36) but both peptides were significantly less potent than equivalent concentrations of either native sulphated CCK-8 or N-Ac-CCK-8. These data indicate that 
N-terminal acetylation of CCK-8 leads to a significantly enhanced and prolonged satiating activity compared to native CCK-8. This improved activity is most likely due to enhanced resistance to aminopeptidases, which helps to prolong the circulating half-life. In contrast, results with N-Ac-CCK(26-31) demonstrate that removal of two C-terminal amino acids from CCK- 8 results in a total loss of activity.

Conclusion: The CCK-8 analogue N-Ac-CCK(26-33)amide is significantly more effective than equivalent doses of PYY at suppressing food intake, indicating the potential of stable $\mathrm{N}$-terminally modified CCK analogues as future agents for treatment of obesity and related metabolic disorders. Supported by: Department of Education \& Learning, Northern Ireland

\section{5}

Oleoylethanolamide stimulates glucagon-like peptide-1 secretion from intestinal L cells in a GPR119 receptor-dependant manner

L.M. Lauffer ${ }^{1}$, R. Iakoubov ${ }^{1}$, P.L. Brubaker ${ }^{1,2}$

${ }^{1}$ Physiology, University of Toronto, Canada, ${ }^{2}$ Medicine, University of Toronto, Canada

Background and aims: Intestinal L cells secrete glucagon-like peptide-1 (GLP-1) in response to nutrients, especially long-chain fatty acids, e. g. oleic acid (OA); GLP-1 then increases both insulin secretion and satiety. We have previously shown that the endogenously-produced long-chain fatty acid derivate, oleoylethanolamide (OEA), increases GLP-1 secretion from the mouse intestinal L cell line (GLUTag). Additionally, OEA regulates food intake and body weight by an unknown mechanism. Excitingly, OEA has been identified as a ligand of the recently de-orphanized $G$ protein-coupled receptor GPR119, acting through the cAMP/PKA pathway. We detected GPR119 mRNA in mouse (GLUTag) and human (NCI-H716) L cell lines, as well as rat intestinal cells, mouse ileum and human colon. We therefore hypothesized that OEA-stimulated GLP-1 release is GPR119-dependent.

Materials and methods: GLUTag and NCI-H716 L cell lines were used for cAMP and GLP-1 RIA-based secretion bioassays. For in vivo studies, fasted male Wistar rats (180-250 g) were anesthetized and injected with the dipeptidylpeptidase-4 inhibitor sitagliptin (5mg/kg i.v.), to prevent GLP-1 degradation. The abdominal cavity was then opened and OEA $(10 \mu \mathrm{M})$ or vehicle was infused into a $10 \mathrm{~cm}$ distal ileal pouch. Blood samples were collected over 1 hour. Bioactive GLP-1, insulin and glucose plasma levels were detected by ECL-based microassay, ELISA and glucose analyzer, respectively.

Results: Treatment of GLUTag cells with OEA (5-15 $\mu \mathrm{M})$ increased cAMP levels, by up to $13 \pm 5 \%$ ( $\mathrm{p}<0.05$ vs. control; $\mathrm{n}=13-19)$, and induced a dosedependent increase in GLP-1 secretion, by up to $106 \pm 19 \%$ ( $p<0.001$ vs. control; $\mathrm{n}=6-9)$. OEA treatment $(10-15 \mu \mathrm{M})$ also increased GLP-1 secretion from NCI-H716 cells, by up to $302 \pm 62 \%$ ( $p<0.01$ vs. control; $n=4)$. OEA is known to be rapidly degraded to OA by a naturally occurring fatty acid amide hydrolase (FAAH), and we have previously shown that OA stimulates GLP-1 secretion through PKCzeta. Therefore, GLUTag cells were pre-treated with the FAAH inhibitor URB597, causing an increase in the OEA-induced cAMP response, by $28 \pm 2 \%$ ( $<<0.01$ vs. control; $\mathrm{n}=4$ ) and the GLP- 1 response, by $119 \pm 38 \%(\mathrm{p}<0.01$ vs. OEA treatment alone; $n=6)$, suggesting that OEA degradation to OA decreases its effects on GLP-1 secretion. To isolate the role of GPR119 in OEA signaling, GLUTag cells were pretreated with siRNAs to induce specific GPR119 knockdown. OEA failed to increase cAMP levels in siRNA-treated GLUTag cells $(n=5)$, in parallel with a $32 \%$ reduction in GLP-1 secretion $(n=3)$, suggesting a major contribution of GPR119 to OEA-induced GLP-1 secretion. Finally, to determine the effects of OEA on the L cell in vivo, OEA or vehicle were administered directly into the rat ileum. Basal plasma bioactive GLP- 1 levels were $5 \pm 1 \mathrm{pg} / \mathrm{ml}(\mathrm{n}=5-6)$ and did not differ between groups. OEA $(10 \mu \mathrm{M})$ induced a robust increase in GLP-1 secretion, by up to $160 \pm 22 \%$ at $60 \mathrm{~min}$ ( $\mathrm{p}<0.05$ vs. basal; $\mathrm{p}<0.01$ vs. vehicle). Basal plasma insulin and glucose levels $(62 \pm 11 \mathrm{pg} / \mathrm{ml}$ and $126 \pm 12 \mathrm{mg} / \mathrm{dl}$, respectively) were not different between the groups and did not change during the course of the treatment.

Conclusion: In summary, these findings demonstrate that OEA mediates GLP-1 secretion through the novel G protein-coupled receptor, GPR119, in mouse and human $\mathrm{L}$ cell lines and in vivo. These studies provide support for the use of OEA as a nutriceutical approach to enhance GLP-1 secretion in type 2 diabetic patients.

Supported by: L. M. L. is supported by an EFSD Albert Renold Fellowship, R. I. is supported by postdoctoral fellowship from the Banting and Best Diabetes Centre, University of Toronto, and P. L. B. by the Canada Research Chairs program and operating grant from the Canadian Diabetes Association

\section{6}

The Rho GTPase, Cdc42, regulates glucagon-like peptide-1 secretion from the enteroendocrine $L$ cell

G.E. Lim ${ }^{1}$, P.L. Brubaker ${ }^{1,2}$

${ }^{1}$ Physiology, University of Toronto, ${ }^{2}$ Medicine, University of Toronto, Canada

Background and aims: Glucagon-like peptide-1 (GLP-1) is secreted from intestinal endocrine L cells, whereupon its main role is to enhance glucosedependent insulin release. We have recently demonstrated that, like the beta cell, depolymerization of the actin cytoskeleton enhances secretagoguestimulated GLP-1 release from the L cell. Moreover, secretagogue-induced secretion was also accompanied by a transient depolymerization of the actin cytoskeleton. Cdc42 has been found to regulate actin dynamics and hormone release from the beta cell; therefore, we hypothesized that Cdc42 similarly controls GLP-1 secretion, in addition to regulating actin dynamics, in the L cell.

Materials and methods: GLUTag cells, which are immortalized murine L cells, were used in all experiments. RT-PCR was performed to determine mRNA expression of various Rho GTPases. Following treatment with $10^{-8} \mathrm{M}$ insulin (INS) for 5 - $60 \mathrm{~min}$, GLUTag cells were immediately lysed, and lysates were incubated with GST-tagged PAK-PBD proteins, which bind to activated Rho GTPases. Immunoblot analysis was used to quantify the amount of activated protein. Cells were also transfected with two targeting siRNAs against Cdc42 (100nM final concentration), and western blotting was used to confirm decreased protein expression as well as to examine ERK1/2 phosphorylation. Finally, normal or transfected cells were treated with INS for up to $2 \mathrm{hr}$, and GLP-1 secretion was measured by radioimmunoassay. Statistical analysis was performed by one- or two-way ANOVA, following by appropriate post-hoc tests, and significance was achieved when $\mathrm{p}<0.05$.

Results: RT-PCR confirmed the expression of mRNA transcripts for the Rho GTPases, Cdc42 and Rac1 in GLUTag cells. Following stimulation with INS, a 1.8 -fold increase $(p<0.05)$ in Cdc42 activation was detected at $t=15 \mathrm{~min}$. In contrast, Racl activation was not detected at any time point. We have previously demonstrated that treatment of GLUTag cells with INS activates the actin-severing enzyme cofilin as early as 5 min following treatment, in addition to inducing actin depolymerization (as detected by an increase in the ratio of G:F actin) by $\mathrm{t}=15 \mathrm{~min}$. In light of this temporal pattern of events, we examined the secretion kinetics of GLP-1. After treatment with INS for 15, 30,60 , and $120 \mathrm{~min}$, a 1.4 -fold increase $(\mathrm{p}<0.05)$ in insulin-induced GLP-1 release was observed after $60 \mathrm{~min}$. To determine the role of $\mathrm{Cdc} 42$ in insulininduced GLP-1 release, GLUTag cells were transfected with siRNAs against Cdc42, which resulted in a $31 \%$ decrease $(\mathrm{p}<0.01)$ in Cdc42 protein expression, and a complete abrogation of insulin-induced GLP-1 secretion. Finally, we examined the activation of ERK1/2 in Cdc42-knockdown cells, as we have previously demonstrated a requirement for the MEK-ERK1/2 pathway in INS-induced GLP-1 secretion. Interestingly, a 2.5 -fold $(\mathrm{p}<0.05)$ increase in basal ERK1/2 phosphorylation was detected, and this was accompanied by a 1.8 -fold $(\mathrm{p}<0.05)$ increase in basal GLP-1 release.

Conclusion: In summary, we demonstrate for the first time, a role for the Rho GTPase, Cdc42, in regulating basal and insulin-induced GLP-1 secretion from the intestinal $\mathrm{L}$ cell. These studies also indicate that $\mathrm{Cdc} 42$ acts in concert with changes in the actin cytosekelton, and may also interact with the MEK-ERK1/2 pathway.

Supported by: G. E. L. is supported by a Frederick Banting and Charles Best Canada Graduate Scholarship - Doctoral Award from the Canadian Institutes of Health Research, and P.L. B. by the Canada Research Chairs Program and operating grant from the Canadian Diabetes Association 


\section{OP 22 Brain and peripheral metabolism}

\section{7}

Glucose homeostasis is altered in transgenic mice with brain specific knockout of the mitochondrial enzyme glutamate dehydrogenase F. Frigerio ${ }^{1}$, S. Carobbio ${ }^{1}$, V. Mlynarik ${ }^{2}$, R. Gruetter ${ }^{2}$, P. Maechler ${ }^{1}$ ${ }^{1}$ Department of Cell Physiology and Metabolism, University of Geneva, ${ }^{2}$ Biomedical Imaging Center, EPFL, Lausanne, Switzerland

Background and aims: Glutamate dehydrogenase (GDH) is a mitochondrial enzyme that catalyzes the reversible transformation of glutamate into alphaketoglutarate. GDH represents a key link between catabolic and biosynthetic pathways in several tissues and in particular in the central nervous system (CNS). Astrocytes take up the neurotransmitter glutamate from the intersynaptic space and GDH converts glutamate into alpha-ketoglutarate, thereby providing energy to the cells. The aim of the present study was to investigate the importance of GDH in the brain and potential connections with systemic metabolic homeostasis.

Materials and methods: Taking advantage of our Glud1 lox/lox mice, we generated CNS specific GDH knockout mice, crossing GDH-exon 7 floxed mice with Nestin-Cre expressing mice. Then, we characterized the knockout mice in comparison to wild types, measuring metabolic parameters and hormone levels.

Results: Brain specific knockout mice are viable and fertile, but show a significant reduction in body weights compared to wild types $(-20 \%$ at 6 months, $\mathrm{p}<0.001$ ). We could exclude intrauterine growth retardation, pointing to post-natal growth impairment. Respiration from brain mitochondria was impaired upon GDH dependent glutamate stimulation, while preserved when induced by succinate. Calorimetric recording did not reveal differences in energy intake and expenditure between the two groups. Epididymal fat was about 30\% larger in knockout mice, while lean mass was lower. DEXA scan measurements confirmed the elevated body fat mass $(+23 \%)$ in knockout mice. Interestingly, glucose tolerance test (ipGTT) showed elevated glucose excursions in brain GDH null mice; although they exhibited higher insulin sensitivity. We also compared glucagon and leptin plasma levels in fed and starved animals. Knockout mice had higher leptin levels in both starved $(+212 \%)$ and fed state $(+69 \%)$ but normal glucagon levels. This complex phenotype indicated energy imbalance possibly driven by modification in brain metabolism. In particular, the absence of GDH could impair the energy balance between neurons and glial cells. To test this, we performed preliminary NMR studies measuring metabolite levels directly in the brain of living animals. Glutamate and glutamine concentrations were higher in knockout mice, indicating impaired catabolism of these metabolites. As an alternative energy source, the brain could increase glucose consumption. Accordingly, brain glucose levels were lower in knockouts.

Conclusion: The absence of GDH in the brain altered whole body energy balance and storage resulting in glucose intolerance. We hypothesize that impaired CNS glutamate metabolism affected energetic fluxes in the brain, with outcomes on whole body energy metabolism modifying glucose homeostasis.

Supported by: Swiss National Science Foundation

\section{8}

Intrahypothalamic circuitry regulating hypothalamic fuel sensing to induce insulin sensitivity or insulin resistance

S. Luo, M. Ezrokhi, Y. Trubitsyna, A.H. Cincotta

Research and Development, VeroScience, Tiverton, United States

Background and aims: The hypothalamic arcuate nuclei are capable of sensing and responding to free fatty acid (FFA) levels during feeding times of day in rodents. Depending if the FFA are re-esterified and stored or oxidized in the arcuate, these nuclei initiate signaling to the liver that stimulates insulin suppression of glucose output or not, respectively. However, little is known of what endogenous hypothalamic factors govern the arcuate response to the FFA molecule to elicit either its subsequent action or inaction. We have previously demonstrated that increased noradrenergic tone at the ventromedial hypothalamus $(\mathrm{VMH})$ can elicit a marked peripheral insulin resistance and obesity and is characteristic of these states. Here we investigate the possible effects of increased VMH noradrenergic tone on the peripheral glucose response to intracerebroventricular FFA administration during the feeding time of day in rats.

Materials and methods: Female Sprague-Dawley rats were implanted with dual cannulas directed to the third ventricle and the VMH. Animals were divided into three groups and assigned treatment to either chronic VMH vehicle and subsequent acute icv oleic acid (OA) infusion, chronic VMH NE and acute icv OA infusion, or chronic VMH vehicle and acute icv vehicle infusion. Animals were infused continuously for 6 weeks with either norepinephrine (NE) $(25 \mathrm{nmol} /$ hour $)(\mathrm{N}=6)$, or vehicle $(\mathrm{N}=12)$ to the $\mathrm{VMH}$. At 2 weeks after the initiation of VMH infusion, animals were infused icv with oleic acid ( $30 \mathrm{nM})$ or vehicle during the feeding time of day and blood samples were taken at 2-4 hours afterwards. After 6 weeks of VMH infusion, such groups of animals were subjected to an insulin suppression test (insulin, somatostatin and glucose infusion rates $1 \mathrm{mU} / \mathrm{kg}^{\star} \mathrm{min}, 3 \mu \mathrm{g} / \mathrm{kg}^{\star} \mathrm{min}$, and 10 $\mathrm{mg} / \mathrm{kg}^{\star} \mathrm{min}$ respectively) and steady state plasma insulin (SSPI) and plasma glucose levels (SSPG) were determined. In a similarly designed separate study, animals from these three groups were subjected to an euglycemic-hyperinsulinemic clamp.

Results: After 2 weeks of VMH infusion, The VMH vehicle/ icv vehicle infused rats exhibited plasma glucose and insulin levels of $120 \pm 5 \mathrm{mg} / \mathrm{dL}$ and $1.0 \mathrm{ng} / \mathrm{ml}$, respectively at 3 hours after the icv vehicle infusion. The VMH vehicle/icv OA infused rats by contrast exhibited plasma glucose and insulin levels of $100 \pm 3 \mathrm{mg} / \mathrm{dL}$ and $1.1 \mathrm{ng} / \mathrm{ml}(\mathrm{P}=0.013)$. The $\mathrm{VMH}$ NE infusion completely blocked the effect of icv OA on plasma glucose without altering the plasma insulin level $(123 \pm 8 \mathrm{mg} / \mathrm{dL}$ and $1.0 \mathrm{ng} / \mathrm{ml}$, respectively, $\mathrm{P}=0.007)$. Such VMH NE infusion for 6 weeks again completely blocked the effect of acute icv OA infusion on SSPG (VMH vehicle/icv vehicle SSPG: $175 \pm 15$ $\mathrm{mg} / \mathrm{dL}$; VMH vehicle/icv OA SSPG: $124 \pm 14 \mathrm{mg} / \mathrm{dL} ; \mathrm{VMH}$ NE/icv OA SSPG $234 \pm 38 \mathrm{mg} / \mathrm{dL} ; \mathrm{P}=0.007)$ without altering SSPI $(0.95,0.91$, and 1.28 , respectively) during an insulin suppression test. Finally, in such animals subjected to an euglycemic-hyperinsulinemic clamp, VMH NE / icv OA infusion, relative to the VMH Vehicle / icv OA infusion reduced clamp glucose infusion rate needed to maintain euglycemia by $29 \%$; $\mathrm{p}=0.018$.

Conclusion: Central lipid sensing mechanisms that regulate peripheral insulin sensitivity and hepatic glucose output are strictly controlled by VMH NE tone. Increases in VMH NE tone can completely abrogate the normal central lipid sensing response to OA during feeding and have important implications for understanding the neuroendocrine regulation of peripheral fuel metabolism and insulin resistance syndrome.

\section{9}

Restored hypothalamic leptin signalling to Koletsky rats increases hepatic insulin sensitivity G.J. Morton ${ }^{1}$, J. German 1 , F. Kim ${ }^{1}$, C.J. Rhodes ${ }^{2}$, M.W. Schwartz ${ }^{1}$ ${ }^{1}$ Medicine, University of Washington, Seattle, ${ }^{2}$ Medicine, University of Chicago, United States

Background and aims: We recently reported that using adenoviral gene therapy to selectively restore leptin receptors to the hypothalamic arcuate nucleus (ARC) of leptin receptor-deficient Koletsky $\left(f a^{k} / f a^{k}\right)$ rats improves whole-body insulin sensitivity. To better understand the mechanism mediating this effect, we used the glucose clamp technique to determine whether hypothalamic leptin action regulates hepatic glucose production (HGP), glucose utilization (GU), or both.

Materials and methods: To accomplish this, obese Koletsky rats received a bilateral microinjection of adenovirus (Ad) expressing either the signaling form of the leptin receptor, lepr ${ }^{b}$ (Ad-LEPR-B) or the reporter gene, GFP (AdGFP), directed to the hypothalamic arcuate nucleus (ARC). Six days later, at a time when there were no differences in mean daily food intake or body weight between groups, animals were subjected to an euglycemic-hyperinsulinemic clamp.

Results: Confirming our previous findings that hypothalamic leptin signaling increases peripheral insulin sensitivity, the rate of glucose infusion required to maintain euglycemia was significantly increased in animals that received Ad-LEPR-B compared to Ad-GFP ( $5.30 \pm 0.31$ vs. $4.25 \pm 0.42 \mathrm{mg} / \mathrm{kg} /$ min; $\mathrm{p}<0.05)$. Using tracer dilution techniques to measure rates of glucose appearance $(\mathrm{Ra})$ and disappearance $(\mathrm{Rd})$, we found that while there was no effect on $\mathrm{Rd}(8.95 \pm 0.45$ vs. $9.43 \pm 0.43 \mathrm{mg} / \mathrm{kg} / \mathrm{min}$; $\mathrm{p}=\mathrm{ns})$, insulin-induced suppression of HGP during the clamp was increased in Ad-LEPR-B-treated animals compared to Ad-GFP-treated controls $(44.7 \pm 0.5 \%$ vs. $34.4 \pm 4.4 \%$; $\mathrm{p}<0.05)$. Enhanced insulin-mediated suppression of HGP was accompanied by reduced hepatic expression of two key gluconeogenic genes, G6Pase and Pepck, by $80 \%$ and $51 \%$, respectively ( $<<0.05$ for each). Similarly, in separate 
groups of animals, peripheral administration of glucose suppressed G6Pase and Pepck mRNA levels to a greater extent (by 15\% and 25\%, respectively; $\mathrm{p}<0.05$ for each) in animals treated with Ad-LEPR-B than Ad-GFP. In a parallel study, we also found that restoring leptin receptors to the ARC of Koletsky rats increased insulin-induced tyrosine phosphorylation of IRS-1 and serine phosphorylation of AKT (markers of IRS-PI3K-AKT activation) in the liver by $51 \%$ and $49 \%$ respectively, compared to Ad-GFP animals ( $\mathrm{p}<0.05$ for each), whereas insulin receptor signal transduction was not altered in skeletal muscle.

Conclusion: Taken together, these findings demonstrate that hypothalamic leptin signaling increases peripheral insulin sensitivity via enhanced insulinmediated suppression of HGP. Our data suggest that the mechanism mediating this effect involves increased hepatic insulin signal transduction via the IRS-PI3K pathway, which in turn inhibits gluconeogenic gene expression. Supported by: the Diabetes Endocrinology Research Center, University of Washington and the Naomi Berrie Investigator in Diabetes Research Award

\section{0}

\section{Increased insulin sensitivity in mice lacking inhibitory leptin receptor} signals

M. Björnholm ${ }^{1}$, R.Z. Tom ${ }^{1}$, S. Glund ${ }^{1}$, E. Vieira ${ }^{1}$, M.G. Myers Jr², M. Gilbert ${ }^{1}$

${ }^{1}$ Dept of Molecular Medicine and Surgery, Karolinska Institutet, Stockholm, Sweden, ${ }^{2}$ Dept of Medicine, University of Michigan, Ann Arbor, United States

Background and aims: Leptin regulates food intake and neuroendocrine function by activating the long form of the leptin receptor $(\mathrm{LRb})$ in the brain. Furthermore, leptin regulates lipid and glucose metabolism to improve whole body insulin sensitivity. In addition to stimulating positive signals via Jak2 and STAT3, activation of LRb promotes the phosphorylation of LRb Tyr985, which mediates both positive and inhibitory signals. In order to understand the contribution of LRb Tyr985-mediated signals to leptin action in vivo, we have mutated LRb Tyr985 in mice ( $l / l$ mice). These $l / l$ mice display decreased body weight and adiposity and consequently low leptin levels. The aim of this study is to investigate the mechanisms of enhanced insulin sensitivity in these leptin receptor mutated $l / l$ mice.

Materials and methods: We have performed glucose tolerance test and euglycemic-hyperinsulinemic clamp in female $+/+$ and $l / l$ mice. Insulin-stimulated glucose transport and insulin signaling have been assessed in isolated soleus and extensor digitorum longus muscle. Liver, adipose tissue and skeletal muscle have been collected for further analysis of expression profiles and insulin signal transduction.

Results: Female $l / l$ mice have normal blood glucose levels under fed and fasted conditions. The glucose profile under a glucose tolerance test is also normal in the $l / l$ mice. Conversely, insulin concentration in the blood is decreased $30-35 \%$ at baseline and 15 minutes post a glucose injection indicating increased insulin sensitivity in the $/ / l$ mice. Euglycemic-hyperinsulinemic clamps on conscious mice show increased hepatic insulin sensitivity and increased peripheral glucose utilization in $l / l$ mice. We further examined whether the insulin sensitivity in the $l / l$ mice is due to improved insulin signaling and glucose transport in skeletal muscle. The $l / l$ mice have normal insulin-stimulated glucose transport in isolated soleus and extensor digitorum longus muscle. Moreover, insulin-stimulated phosphorylation of Akt Ser473, a key component in the pathway to insulin-stimulated glucose uptake, is also unchanged.

Conclusion: In conclusion, the $l / l$ mice display increased whole body insulin sensitivity with an increased suppression of hepatic glucose production and peripheral glucose utilization under a euglycemic-hyperinsulinemic clamp. Further studies to identify the mechanisms of improved insulin sensitivity in LRb Tyr985 mutated mice are required.

Supported by: Swedish Research Council and Novo Nordisk Research Foundation

\section{1}

A two-week central leptin infusion is not sufficient to induce hypothalamic leptin- or insulin-resistance but is able to induce hypothalamic PTP-1B expression

F. Berthou ${ }^{1}$, C. Rouch ${ }^{2}$, A. Gertler ${ }^{3}$, J. Djiane ${ }^{1}$, K. Gerozissis ${ }^{2}$, M. Taouis ${ }^{1}$ ${ }^{1}$ Laboratoire de Neuroendocrinologie Moléculaire de la Prise Alimentaire, INRA / Université Paris Sud XI, Orsay, France, ${ }^{2}$ Laboratoire de Physiopathologie de la Nutrition, Université Paris VII, Paris, France, ${ }^{3}$ The Institute of Biochemistry, Food Science and Nutrition, The Hebrew University of Jerusalem, Rehovot, Israel

Background and aims: To regulate energy and glucose homeostasis, leptin and insulin act through their respective receptors on hypothalamic nuclei. Obesity is generally associated to a leptin-resistance state characterized by hyperleptinemia with a loss of leptin-dependent food intake (FI) control. We have recently demonstrated in human neuroblastoma cell line that overexposure to leptin induced both leptin- and insulin-resistance. Here, we hypothesized that a central leptin resistance induced by provoked hyperleptinemia may lead to reduced insulin sensitivity at the hypothalamic level. This may suggest a plausible link between leptin resistance and insulin resistance, and latest may contribute to the onset of type 2 diabetes. To assess the role of leptin on insulin hypothalamic sensitivity, leptin-dependent signaling pathways were either activated by leptin infusion or blocked by leptin antagonist infusion.

Materials and methods: Adult male Wistar rats received chronic central leptin $(0.25 \mu \mathrm{g} / \mathrm{ml})$ or leptin antagonist $(1 \mu \mathrm{g} / \mu \mathrm{l})$ infusion in the third ventricle for 14 days, via osmotic pumps. Body weight (BW) and FI were daily measured. After sacrifice, blood sample, hypothalamus and liver were collected and glycemia, insulinemia and leptinemia determined. Protein lysates from hypothalamus and liver were subjected to a western-blotting analysis.

Results: BW gain and FI were significantly increased in animals treated with leptin antagonist as compared to controls. As expected, central leptin infusion led to a significant reduction of FI and BW. Leptin also caused an important decrease of glycemia, insulinemia and leptinemia whereas leptin antagonist increased leptinemia. Furthermore, central leptin infusion led to a significant increase of hypothalamic STAT-3 (signal transducer and activator of transcription 3) phosphorylation. This is in good agreement with the reduction in BW and FI, but indicates that 2 weeks of central leptin infusion are not sufficient to induce hypothalamic leptin resistance. In contrast, leptin antagonist did not affect STAT-3 phosphorylation. We have also clearly shown that this treatment does not affect neither hypothalamic nor hepatic IR protein expression. Interestingly, we report a significant increase in PTP-1B (phosphotyrosine phosphatase) expression subsequent to leptin treatment. This may indicate a progressive installation of leptin resistance and most likely insulin resistance since it has been reported that this phosphatase negatively affects both leptin and insulin signaling pathways.

Conclusion: We show that a two-week leptin central infusion is not sufficient to induce leptin resistance and to affect hypothalamic or hepatic IR expression. However, this treatment was able to induce PTP-1B expression at the protein level which may constitute an early sign of the progressive onset of leptin and/or insulin resistance.

\section{2}

Mitochondrial reactive oxygen species and brain glucose sensing: dysregulation in the insulin resistant obese Zucker rat A.-L. Colombani ${ }^{1}$, L. Carneiro ${ }^{1}$, C. Magnan ${ }^{2}$, A. Galinier ${ }^{1}$, A. Benani ${ }^{1}$, L. Casteilla ${ }^{1}$, L. Pénicaud ${ }^{1}$, C. Leloup ${ }^{1}$

${ }^{1}$ Métabolisme Plasticité Mitochondrie, UMR 5241 CNRS-UPS, Toulouse, ${ }^{2}$ Physiopathologie de la nutrition, UMR 7059 CNRS-UDD, Paris, France

Background and aims: It is now well etablished that the brain has a critical role for both monitoring and regulating the energy needs of the body. In conditions of obesity and non-insulin-dependent diabetes mellitus (NIDDM), the brain's ability to respond to alterations in glucose metabolism becomes aberrant. We aimed at understanding the abnormalities in cerebral glucose sensing in insulin resistant, hyperinsulinic state. The link between substrates, mitochondrial reactive oxygen species (mROS) production and their deleterious effects has been already highlighted. Emerging data now demonstrate also a physiological role for mROS. Therefore, we have developed the hypothesis that hypothalamic glucose sensing involves mROS signalling pathway and we recently demonstrated their implication in vivo. In the Zücker rat model, hypothalamic glucose sensing in vivo is altered, showing a hypersen- 
sitivity to a lower dose of glucose than in lean rats $(3 \mathrm{mg} / \mathrm{kg}$ vs $9 \mathrm{mg} / \mathrm{kg})$. We questioned in this study whether the mROS signalling as well as the redox status might be involved in this abnormal response.

Materials and methods: In vivo model of brain glucose sensing consists of a glucose load ( $9 \mathrm{mg} / \mathrm{kg}$ or $3 \mathrm{mk} / \mathrm{kg}$ for testing hypersensitivity) towards the brain via the carotid. This induces an increased hypothalamic electrical activity and a peak of insulin 1 min later. Multiunit recordings were made in arcuate nucleus. mROS were quantified with the H2DCFDA probe and redox status by evaluating the oxidized glutathione (HPLC). Complex IV and aconitase activities were determined on whole tissue. Insulin was measured by ELISA. Mitochondrial respiration was evaluated by oxygraphy on permeabilized tissue. Each group consists of 6-9 rats.

Results: Hypothalamic mitochondrial oxidative potential (complex IV activity) is significantly higher in this obese model and the aconitase activity, a mitochondrial negatively ROS-sensitive enzyme, is significantly (40\%) downregulated compared to lean rats. Glutathione is although more oxidized in obese, insulino-resistant rat compared to the lean rat. Finally, the hypersensitivity to $3 \mathrm{mg} / \mathrm{kg}$ which is only observed in obese group is clearly linked to an exacerbated production of hypothalamic mROS: restoration of the redox status (monoester ethyl glutathione in the third ventricle) in obese Zücker rats completely reverses the abnormalities observed, i.e., hypersensitivity to glucose in terms of insulin secretion is loss and mROS signalling is normalized.

Conclusion: In the obese insulino-resistant Zücker rat, abnormal hypothalamic glucose sensing may be due to mROS signalling, leading to an increase in the overall electrical activity of the hypothalamus and then the insulin secretion. Normalization of the redox state with glutathione supplementation in the hypothalamus of insulino-resistant rats restores the physiological responses identical to that of lean rats. Oxygraphic measures on hypothalamic tissues point out a major role of insulin in these abnormal responses, suggesting that hyperinsulinism in vivo accelerates the mitochondrial activity. Supported by: PNRD 2006, France

\section{OP 23 Lipotoxicity in beta cells}

\section{3} Free fatty acid-induced endoplasmic reticulum stress signalling and
apoptosis in human pancreatic islets

L.C.L. Ladrière ${ }^{1}$, M. Igoillo-Esteve ${ }^{1}$, D.A. Cunha ${ }^{1}$, J.-P. Brion ${ }^{2}$, R. Lupi ${ }^{3}$, P. Marchetti ${ }^{3}$, D.L. Eizirik ${ }^{1}$, M. Cnop ${ }^{1,4}$

${ }^{1}$ Laboratory of Experimental Medicine, Université Libre de Bruxelles, Belgium, ${ }^{2}$ Laboratory of Histology and Neuropathology, Université Libre de Bruxelles, Belgium, ${ }^{3}$ Department of Endocrinology and Metabolism, Metabolic Unit, University of Pisa, Italy, ${ }^{4}$ Division of Endocrinology, Erasmus Hospital, Brussels, Belgium

Background and aims: Free fatty acids (FFA) cause pancreatic beta cell apoptosis and may contribute to beta cell loss in type 2 diabetes via the induction of endoplasmic reticulum (ER) stress. We have previously shown that oleate and palmitate trigger an ER stress response in rat beta cells. Both FFA activate the ATF6 branch, but palmitate induces more apoptosis and activates the IRE1 and PERK pathways more potently. Salubrinal (sal), a selective inhibitor of eIF2 $\alpha$ dephosphorylation, has been used to protect other cell types against ER stress-mediated apoptosis. In INS-1E cells and primary rat beta cells, however, sal markedly potentiates the deleterious effects of oleate and palmitate. The aim of this study was to examine whether FFA induce ER stress in human islets and whether enhanced PERK-eIF2 $\alpha$ signaling by sal protects or exacerbates FFA effects in human $\beta$ cells.

Materials and methods: Human pancreatic islets were cultured in the presence of oleate or palmitate $(0.5 \mathrm{mM}, 1 \% \mathrm{BSA})$, with or without sal $(75 \mu \mathrm{M})$. Islet viability and $\beta$ cell morphology were assessed by Hoechst 33342/propidium iodide staining and electron microscopy. Gene expression was analyzed by real time RT-PCR and corrected for $\beta$-actin expression levels.

Results: The saturated FFA palmitate caused human islet cell death $(19 \pm 4 \%$ vs control $9 \pm 2 \%, 72 \mathrm{~h}$ treatment, $\mathrm{n}=8, \mathrm{p}<0.05$ vs control), while the unsaturated oleate did not. Sal alone was no toxic but it potentiated FFA toxicity (oleate + sal $17 \pm 2 \%$ cell death, palmitate + sal $26 \pm 6 \%, p<0.05$ ). FFA induced cytoplasmic accumulation of triglycerides and dilation of the ER in INS-1E and human islet cells (electron microscopy after $48 \mathrm{~h}$ exposure). The combination of FFA and sal lead to mitochondrial morphological changes and signs of apoptotic cell death. The PERK-dependent ER stress markers ATF3 and CHOP were induced by palmitate (ATF3: 2.7- and 2.6-fold increase compared to control after 24 and 48h; CHOP: 1.8 - and 2.9-fold; $\mathrm{n}=4-5, \mathrm{p}<0.05$ ). Sal alone did not induce these markers but markedly potentiated oleate and palmitate effects (ATF3: oleate+sal 1.9- and 1.8-fold increase after 24-48h, $\mathrm{p}<0.05$, palmitate + sal 7.9 -fold increase, $48 \mathrm{~h}, \mathrm{p}<0.01$; CHOP: oleate + sal 4 -fold increase, $48 \mathrm{~h}, \mathrm{p}<0.05$, palmitate + sal 3.7 - and 9.5 -fold increase after $24-48 \mathrm{~h}$, $\mathrm{p}<0.05)$. Palmitate also induced the ER chaperone BiP $(1.7$ - and 2.2 -fold after 24 and $48 \mathrm{~h}, \mathrm{p}<0.05)$, and this was potentiated by sal ( 4.8 -fold, $48 \mathrm{~h}, \mathrm{p}<0.01)$. Activation of XBP1 by its alternative splicing by IRE1 was not observed with FFA, and the combination of FFA+sal decreased splicing $(\mathrm{p}<0.05)$.

Conclusion: Palmitate, but not oleate, triggers ER stress (characterized by PERK and ATF6 signaling) and apoptosis in human islets. The activation of the PERK-eIF2 $\alpha$ pathway by the chemical sal sensitizes islets to oleate, suggesting that this pathway plays an important role in FFA-induced ER stress and human islet demise. Intervention strategies to prevent lipotoxic $\beta$-cell loss should avoid prolonged eIF2 $a$ phosphorylation.

\section{4}

Endoplasmic reticulum stress signalling contributes to palmitate- but not to cytokine-induced beta cell death

M. Åkerfeldt, J. Chan, T. Biden, R. Laybutt

Diabetes and Obesity, Garvan Institute of Medical Research, Sydney, Australia

Background and aims: Increased pancreatic $\beta$-cell death is implicated in the reduced $\beta$-cell mass of both type 1 and type 2 diabetes. Cytokines [interleukin-1 $\beta$ (IL-1 $\beta$ ) and $\gamma$-interferon (IFN- $\gamma$ )] and fatty acids are likely mediators of $\beta$-cell death in type 1 and type 2 diabetes, respectively. However, the mechanisms by which $\beta$-cells are destroyed remain to be clarified. In this study, we tested whether endoplasmic reticulum (ER) stress and the unfolded protein response (UPR) were necessary for the increased cell death in INS-1 cells following exposure to cytokines (IL- $1 \beta$ and IFN- $\gamma$ ) or the saturated fatty acid, palmitate. Furthermore, we investigated whether the UPR was activated in $\beta$-cells of the non-obese diabetic (NOD) mouse model of type 1 diabetes. 
Materials and methods: INS- 1 cells were treated with the chemical chaperones phenyl butyric acid (PBA, $2.5 \mathrm{mM}$ ) or trimethylamine $\mathrm{N}$-oxide (TMAO, $100 \mathrm{mM})$ and thereafter exposed to IL- $1 \beta(50 \mathrm{U} / \mathrm{ml})+\mathrm{IFN}-\gamma(100 \mathrm{U} / \mathrm{ml})$ or palmitate ( $0.4 \mathrm{mM}$ coupled to $0.92 \% \mathrm{BSA}$ ) for 6 or $24 \mathrm{~h}$. Rat pancreatic islets were treated with PBA followed by exposure to cytokines. The role of the proapoptotic transcription factor $\mathrm{CHOP}$ in cytokine- and palmitate-induced apoptosis was investigated in INS-1 cells in which CHOP protein levels were reduced using siRNA. Apoptosis was measured using an apoptosis ELISA. Expression of UPR genes was evaluated using Western blotting and real-time PCR. Islets were isolated from C57BL/6J (control) and NOD mice (before and after the onset of diabetes), and mRNA levels assessed by real-time PCR. XBP-1 splicing was also assessed.

Results: Cytokines and palmitate triggered ER stress in INS-1 $\beta$-cells as evidenced by increased phosphorylation of PERK and EIF2 $\alpha$ as well as increased expression of ATF4 and CHOP. Both cytokines and palmitate increased JNK phosphorylation, but only palmitate increased XBP-1 splicing in INS-1 cells. PBA and TMAO act as chemical chaperones to reduce the load of unfolded proteins in the ER by improving folding capacity and trafficking of mutant proteins. PBA attenuated cytokine- and palmitate-induced ER stress as indicated by reduced PERK and EIF2 $\alpha$ phosphorylation as well as reduced mRNA and protein levels of ATF4 and CHOP. Additionally, PBA reduced JNK phosphorylation in response to palmitate, but not in response to cytokines. Strikingly, this was associated with a complete block of palmitateinduced cell death $(\mathrm{p}<0.05)$ but did not affect cytokine-induced cell death. Similar results were found with TMAO. Moreover, siRNA-mediated reduction of CHOP protected against palmitate-, but not against cytokine-induced cell death. In cultured rat islets, PBA-mediated reduction in UPR gene activation had no effect on cytokine-induced cell death. Importantly, in islets from NOD mice, both before and after the onset of diabetes, ER stress was absent as indicated by reduced (BiP, GRP94, ATF4, Edem1, Erp72, p58, XBP-1 splicing; all $\mathrm{p}<0.05)$ and unchanged $(\mathrm{CHOP}) \mathrm{mRNA}$ levels of UPR genes.

Conclusion: We conclude that although both cytokines and palmitate induce ER stress in cultured $\beta$-cells, UPR activation is selectively necessary for lipotoxicity and not for cytokine-induced cell death. These data suggest that ER stress is responsible for $\beta$-cell death in type 2 diabetes and that inhibition of $\mathrm{CHOP}$ protects against lipotoxicity. Different mechanisms operate in type 1 diabetes, with $\beta$-cell death possibly mediated via ER stress-independent activation of JNK.

Supported by: National Health and Medical Research Council Australia

\section{5}

Molecular mechanism of diet effect on pancreatic beta cell preservation in diabetic $d b / d b$ mice: evidence for anti-oxidative stress mechanism Y. Kanda, M. Shimoda, K. Tawaramoto, F. Kawasaki, M. Hashiramoto, K. Nakashima, M. Matsuki, K. Kaku

Diabetes and Endocrine Division, Kawasaki Medical School, Kurashiki-shi, Japan

Background and aims: It is well known that diet therapy is important at all levels of diabetes prevention. The aim of this study is to assess the molecular mechanism for preventive effect of diet on beta cell damage in obese diabetic animal model $\mathrm{db} / \mathrm{db}$ mice.

Materials and methods: Male BKS.Cg-+ Lepr $r^{d b} /+\operatorname{Lepr}^{\mathrm{db}} / \mathrm{Jcl}(d b / d b)$ mice were divided into 2 groups with or without diet therapy (DT) $(n=5)$. Daily food intake of DT(+) mice was restricted to that of the control BKS.Cg-m $+/+\operatorname{Lepr}^{d b} / \mathrm{Jcl}(\mathrm{db} / \mathrm{m})$ mice previously estimated. DT was implemented for 6 weeks from 6 weeks of age. Body weight (BW), fasted blood glucose (FBG), insulin (FIRI), TG and FFA were measured at 6,8 and 12 weeks of age, and intraperitoneal insulin tolerance test (ipITT:1IU/kg BW) was performed at 12 weeks. The beta-cell mass and cell proliferation/apoptosis were assessed by histological analysis including proliferating cell number antigen (PCNA) and 4-hydroxynonenal modified protein (4-HNE) immunostaining of the islet tissue. Gene expressions specific for the core area of pancreatic islet were analyzed by Laser Capture Microdissection method and real time RTPCR. Primer pairs encoding genes associated with pancreatic hormones, cell proliferation, apoptosis, cell cycle, and oxidative stress were prepared, and real-time RT-PCR with Sybr Green was applied. Each gene expression was relatively quantified by the comparative $\mathrm{Ct}$ method with each result in the 18 SrRNA as a control.

Results: Food intake in $d b / m$ mice was $50 \%$ in $d b / d b$ mice. BW, FBG and FIRI in DT(+) group were significantly lower than those in DT(-) group of mice, but were significantly greater than in $\mathrm{db} / \mathrm{m}$ mice at 12 weeks of age (BW: $34.0 \pm 0.07,49.4 \pm 0.45,27.3 \pm 0.38 \mathrm{~g}, \mathrm{p}<0.0001$, FBG :106.5 $\pm 10.1,200.7 \pm 29.9$,
$66.6 \pm 4.8 \mathrm{mg} / \mathrm{dl}, \mathrm{p}<0.05$, FIRI : $12.9 \pm 1.0,17.5 \pm 2.3,0.46 \pm 0.01 \mathrm{ng} / \mathrm{ml}, \mathrm{p}<0.05$, respectively). TG and FFA level in $\mathrm{DT}(+) d b / d b$ mice were significantly lower than those in DT(-) mice, but no difference was observed between DT(+) and control $\mathrm{db} / \mathrm{m}$ mice. The ipITT at $30 \mathrm{~min}$ was $77.6 \pm 3.4 \%$ in DT(+) mice, $107 \pm 7.3 \%$ in DT(-) mice, $60.4 \pm 1.6 \%$ in $d b / m$ mice ( $<<0.05$, respectively). The pancreatic beta cell mass was greater in $\mathrm{DT}(+)$ mice than that in $\mathrm{DT}(-)$ mice (7.8 \pm 1.7 vs. $4.4 \pm 1.0 \mathrm{mg}, \mathrm{p}<0.05)$. Beta cell gene expression for insulin was decreased in DT( +$) d b / d b$ mice. Cycline E and ERK1 genes related with cell proliferation and CAD gene related with promotion of apoptosis were significantly up-regulated at 8 week, but Cycline E and ERK1 genes were downregulated at 12 weeks of age in $\mathrm{DT}(-) d b / d b$ mice. On the other hand, DT significantly increased Cyclin E gene expression, and down-regulated CAD gene expression at 12 weeks. Anti-apoptotic bcl-2 gene expression was significantly increased by DT. NADPH oxidase gene and TNF gene related with oxidative stress and inflammatory process were markedly down-regulated in DT(+) mice. Morphometric results for PCNA and 4-HNE observed corresponded to the data of gene expression analysis.

Conclusion: DT increases insulin sensitivity and preserves pancreatic beta cell function in diabeteic $d b / d b$ mice. Our results strongly suggest that DT prevents beta cell loss by suppressing cell apoptosis probably through antioxidative stress mechanism.

\section{6}

Mononuclear cell infiltration of intra-pancreatic fat and decreased islet beta cell area in human type 2 diabetes

M. Galli, S. Torri, M. Bugliani, M. Masini, N. Funel, R. Lupi, S. Del Guerra, V. D’Aleo, U. Boggi, F. Filipponi, D. Campani, L. Marselli, F. Dotta, P. Marchetti

Endocrinology and Metabolism, Metabolic Unit, Pisa, Italy

Background and aims: Increased accumulation of visceral fat (AVF) is associated with type 2 diabetes (T2D), but the factors linking AVF and the pathophysiological mechanisms leading to T2D are still unclear. We assessed the relationships between intra-pancreatic fat (IPF, quantified by light microscopy) and several features of beta-cells in human T2D.

Materials and methods: Pancreata were obtained from 26 non-diabetic (ND) and 17 T2D organ donors, matched for age and body mass index. Immunohistochemistry studies were performed on pancreatic samples. Islets were isolated by enzymatic digestion and gradient purification. Glucosestimulated (3.3 and $16.7 \mathrm{mmol} / \mathrm{l}$ ) insulin secretion was assessed ex-vivo by the batch incubation technique. Beta-cell apoptosis was quantified by electron microscopy. MessengerRNA expression of selected genes was performed by quantitative RT-PCR.

Results: IPF accumulation was higher in T2D $(11.7 \pm 11.3 \%)$ than ND $(5.8 \pm 7.4 \%, \mathrm{p}<0.05)$ samples, and beta-cell area was lower in T2D $(59.7 \pm 20.4 \%)$ than in ND $(81.9 \pm 9.8 \%, \mathrm{p}<0.05)$. However, no significant correlation was found between IPF amount and beta-cell area. The number of macrophages and mast cells (immunostaining by anti-CD68 and anti-CD117 antibodies, respectively) was significantly ( $\mathrm{p}=0.01)$ higher in IPF of T2D than ND ( $\mathrm{n}=7$ each group, $23.7 \pm 9.6$ vs $14.0 \pm 6.5 / 100$ adipocytes nuclei). The number of infiltrating mononuclear cells $(\mathrm{MC})$ was correlated with beta-cell depletion in T2D $(\mathrm{p}<0.001)$, but it did not affect ex-vivo insulin secretion. When islets from ND were exposed for 1 week to cytokines of MC origin (IL-6, $5 \mathrm{pg} / \mathrm{ml}$; TNFalpha, $5 \mathrm{pg} / \mathrm{ml}$; or a combination of both), decreased glucose-stimulated insulin secretion $(65 \pm 22 \%$ of control ND cells, $\mathrm{p}<0.05)$, increased apoptosis ( $7.0 \pm 1.0$ vs $1.1 \pm 0.6 \%, \mathrm{p}<0.05)$ and reduced PDX-1 expression $(79 \pm 20 \%$ of control ND, $\mathrm{p}<0.05$ ) were observed with IL-6 plus TNFalpha.

Conclusion: 1) IPF amount was higher in T2D than ND, but it did not correlate with beta-cell area or ex-vivo islets function; 2) mononuclear cell infiltration of IPF was higher in T2D pancreata and was associated with reduced beta-cell area; 3) cytokines of MC origin impaired isolated islets function and survival. Therefore, the presence of an "inflamed" intrapancreatic fat tissue might affect the beta-cell in human T2D, representing a possible link between AVF and beta-cell defects. 


\section{7}

Gene expression regulated by pioglitazone and exenatide in normal and diabetic human islets exposed to lipotoxicity

H. Ghanaat-pour, Å. Sjöholm

Karolinska Institutet, Stockholm South Hospital, Department of Internal

Medicine, Stockholm, Sweden

Background and aims: Chronically elevated free fatty acids (FFAs) are believed to have a detrimental effect on glucose-stimulated insulin secretion (GSIS), insulin resistance, $\beta$-cell failure, and $\beta$-cell apoptosis in type 2 diabetes. Whether FFAs alter beta-cell function and apoptosis in synergy with glucose or separately, remains controversial. It is still unclear weather $\beta$-cell failure is induced by increased FFAs alone or together with genetic factors, since most of the previous studies have been performed in animal models with a genetic predisposition to diabetes/obesity. The objective of the present study was to investigate the global alterations in gene expression induced by long term exposure to palmitate, pioglitazone (a TZD), and/or exenatide (an incretin mimetic) in human diabetic and non-diabetic pancreatic islets (at physiological glucose concentration). Also, to investigate the possible protective effect of these drugs against the $\beta$-cell lipotoxicity, which is still debated. Materials and methods: Gene expression analysis using Affymetrix micoarray measurements of mRNA from human diabetic and non-diabetic pancreatic islets were performed after $48 \mathrm{hrs}$ of culture in $6 \mathrm{mM}$ glucose, $0.125 \mathrm{mM}$ palmitate, $10 \mathrm{mM}$ pioglitazone and/or $1 \mathrm{nM}$ exenatide.

The differentially regulated transcripts were annotated and then clustered according to functional annotation. Cluster analyses were performed as described in GeneSpring 7.3, NetAffx and other databases.

Results: Gene expression profiling revealed alterations in several functional categories, such as epigenetic regulation of gene expression, cell proliferation and differentiation, metabolism, response to stimulus, transport, and signal transduction. Palmitate, pioglitazone and/or exenatide appear to have a substantial influence on epigenetic processes, e.g. stable alterations in gene expression potential that arise during development and cell proliferation. Pancreatic polypeptide $(P P Y)$, Epidermal growth factor receptor (EGFR), Thioredoxin interacting protein (TXNIP), Carboxyl ester lipase (CEL), Growth arrest and DNA-damage-inducible, beta $(G A D D 45 B)$, Glyceraldehyde-3phosphate dehydrogenase (GAPDH), Defender against cell death 1(DAD1), Regenerating islet-derived 3 alpha (REG3A), Transcription factor 7-like 2 (TCF7L2)(TCF4), Aryl hydrocarbon receptor nuclear translocator (ARNT), LIM homeobox 4 (LHX4), ISL1 transcription factor, LIM/homeodomain, (ISL1) are some of the interesting genes that were differently regulated in our study. The apoptotic pathway was not majorly influenced by either pioglitazone or exenatide in the presence of palmitate. The function and survival of $\beta$-cell seems to be controlled directly through the epigenetic control of gene expression rather than a direct effect on the apoptotic pathway. FFAs seem to contribute and play an important role in the development of $\beta$-cell failure in synergy with hyperglycemia, in obese individuals, or in subjects who are genetically more predisposed to type 2 diabetes.

Conclusion: Considering that the nutritional state directly induces the epigenetic modifications, pioglitazone and exenatide appear to normalize these epigenetic misregulations and may protect the $\beta$-cell from lipotoxic insult. The epigenetic modifications of the genome provide new promising targets for clinical diagnostics and also therapeutic purposes in the treatment of type 2 diabetes.

\section{8}

Mice expressing kinase negative PKCdelta in insulin secreting cells are protected from fatty acid - induced beta cell failure

F. Ranta ${ }^{1}$, A.M. Hennige ${ }^{1}$, I. Heinzelmann ${ }^{1}$, D. Avram ${ }^{1}$, S.Z. Lutz H. Braumüller ${ }^{2}$, F. Bosch ${ }^{3}$, R. Lammers ${ }^{1}$, S. Ullrich ${ }^{1}$, H.-U. Häring ${ }^{1}$ Internal Medicine IV, University Hospital of Tübingen, Germany, ${ }^{2}$ Dermatology, University Hospital of Tübingen, Germany, ${ }^{3}$ Center of Animal Biotechnology and Gene Therapy, Universitat Autonoma de Barcelona, Spain

Background and aims: The ubiquitously expressed PKCdelta is known as a pro-apoptotic serine/threonine kinase that can be activated by diverse stimuli including oxidative stress or death receptor ligands. In insulin secreting cells, controversial findings on the role of PKCdelta have been published over the years. Although diverse observations suggest that PKC isoforms others than PKCdelta are essential for proper glucose-induced insulin secretion, the second phase of secretion was impaired in PKCdelta null mice. More consistent- ly, free fatty acid and IL1beta-activated apoptotic cell death has been found to depend on PKCdelta activation. Evidence accumulated showing that translocation into the nucleus of a cleaved, constitutively active form of PKCdelta is essential for apoptosis. Here, transgenic mice over-expressing a kinase negative (KN) PKCdelta in beta-cells were generated to study the role of PKCdelta in glucose homeostasis and beta-cell survival and function.

Materials and methods: Mice were fed a high fat diet (HFD) for 8 weeks. Blood glucose and insulin was determined after a glucose load $(2 \mathrm{~g} / \mathrm{kg}$ body weight, GTT) and insulin secretion was measured in isolated islets after static incubations using insulin ELISA and RIA kits. Apoptotic cell death was assessed using attached islet cell cultures with nuclear TUNEL/TOPRO3 DNAstaining. Beta-cell mass was estimated after immunohistochemical staining for insulin in pancreatic cryo-sections.

Results: Blood glucose and insulin levels were not different in animals fed a standard diet. After HFD, GTT was impaired in wild- type (wt) mice but significantly improved in KN-PKCdelta littermates. This improvement was accompanied by increased insulin levels in vivo while insulin tolerance was not significantly different between wt and transgenic mice. In isolated islets, glucose-induced insulin secretion as well as secretion augmented by palmitate, oleate, phorbol ester and forskolin in the presence of $12 \mathrm{mM}$ glucose was not significantly different. As expected, palmitate but not oleate stimulated apoptotic cell death from $5.3 \%$ to $10.9 \%$ in wt islet cell cultures. In islet cells overexpressing KN-PKCdelta, the apoptotic rate was not affected by palmitate. In accordance, islets of KN-PKCdelta transgenic mice were significantly bigger in size than wt islets.

Conclusion: Thus, depletion of PKCdelta signalling improves glucose homeostasis after HFD due to reduced beta-cell death that finally results in increased beta-cell mass.

Supported by: DFG 


\section{OP 24 Genetics of type 1 diabetes and complications}

\section{9}

Identification of type 1 diabetes genes in the MHC region by a novel approach combining genetic data with information on functional pathways

C.A. Brorsson ${ }^{1}$, N. Tue Hansen ${ }^{2}$, K. Lage ${ }^{2}$, R. Bergholdt ${ }^{1}$, S. Brunak ${ }^{2}$, F. Pociot $^{1}$

${ }^{1}$ Steno Diabetes Center, Gentofte, ${ }^{2}$ Center for Biological Sequence Analysis, Technical University of Denmark, Lyngby, Denmark

Background and aims: We have developed a novel method that combines SNP genotyping data with protein-protein interaction (ppi) networks to identify new genes that contribute to the risk of developing type 1 diabetes (T1D). We focused on genes located within the MHC region on chromosome 6. Using this new approach their contribution to the development of T1D could be analysed independently of the known LD with the HLA-DRB1, $-D Q A 1,-D Q B 1$ genes, which is usually a hurdle in detecting genes in this region with smaller contributions.

Materials and methods: We have tested and validated this approach on a dataset generated by the Type 1 Diabetes Genetics Consortium. This comprised of $3000 \mathrm{SNPs}$ located in the $4 \mathrm{Mb} \mathrm{MHC}$-region. Initially a subset of genotypes from $1300 \mathrm{~T} 1 \mathrm{D}$ families were analysed. Association to T1D was tested using the Transmission Disequilibrium Test (TDT) and the SNP with the lowest $\mathrm{p}$-value in each gene of this region was chosen and corresponding proteins were mapped to ppi networks for identification of interaction partners. The association testing and resulting interacting protein modules were statistically evaluated using permutation. We then expanded the dataset to include 2363 T1D families genotyped for the same 3000 SNPs. Association to T1D was again tested using TDT. The dataset was stratified into HLA-DRB1 highrisk and low-risk genotypes to detect if these genotype risk-groups confer different risk of T1D due to different ppi networks including candidates both within and outside the MHC-region.

Results: 151 genes were mapped within the protein interaction networks and their interaction partners were identified. Five protein interaction modules reached statistical significance using this approach. They contained proteins encoded both within and outside of the MHC region. Many of the identified proteins are well known in T1D pathogenesis, but the modules also contain additional candidates that have been implicated in beta-cell development and diabetic complications. Results from the stratified analysis show different significant protein interaction modules between the high, moderate and low HLA risk-groups.

Conclusion: In conclusion the extensive LD within the MHC-region makes it important to develop new methods for analysing genotyping data for identification of additional risk genes for T1D. The results from the stratified analysis are highly interesting and indicate that individuals with different HLA-DRB1 risk genotypes differ at the ppi network level. Different candidates for conferring risk in the different groups are thereby directly suggested. We believe that this new approach combining genetic data with knowledge about functional pathways could provide new insight into the mechanisms underlying T1D. Supported by: EFSD/JDRF/NovoNordisk grant

\section{0}

Genome-wide linkage scan for type 1 diabetes: The Type 1 Diabetes Genetics Consortium

S.S. Rich ${ }^{1}$, W.-M. Chen ${ }^{1}$, P. Concannon ${ }^{1}$, H.A. Erlich ${ }^{2}$, C. Julier ${ }^{3}$, G. Morahan ${ }^{4}$, J. Nerup ${ }^{5}$, F. Pociot ${ }^{5}$, J.A. Todd ${ }^{6}$

${ }^{1}$ Center for Public Health Genomics, University of Virginia, Charlottesville, United States, ${ }^{2}$ Human Genetics, Roche Molecular Systems, Pleasanton, United States, ${ }^{3}$ Institute Pasteur, Paris, France, ${ }^{4}$ Diabetes Research Centre, Western Australia Institute for Medical Research, Perth, Australia, ${ }^{5}$ Steno Diabetes Center, Steno Diabetes Center, Gentofte, Denmark, ${ }^{6}$ Diabetes Inflammation Laboratory, Cambridge University, United Kingdom

Background and aims: Type 1 diabetes (T1D) is an autoimmune disease characterized by an autoimmune destruction of the pancreatic beta cells. T1D aggregates in families and risk is thought to be due to multiple genes and undetermined environmental triggers. While the greatest contributors to T1D genetic risk reside in the human MHC (HLA regions), other regions of the genome are recognized to be important. The T1DGC has launched a major effort to identify genes of major effect (or clusters of genes) using a linkage strategy.

Materials and methods: A genome-wide linkage scan of 6,000 SNPs were analyzed from 2508 affected sib pairs (ASP) from 2396 families in the T1DGC. These ASP families were obtained from 9 different cohorts in Asia-Pacific (AP), North America (NA), Europe (EU), United Kingdom (UK), the Joslin Diabetes Center (JOS), Sardinia (SAR), Denmark (DAN), Human Biological Data Interchange (HBDI), and the British Diabetes Association (BDA). Data were cleaned using PedCheck, GRR and Merlin software. at the T1DGC Coordinating Center using PedCheck and GRR software. The average information content across the genome (0.86) was good.

Results: Nonparametric linkage analyses revealed regions on chromosomes $2 q, 6 p, 6 q$, and $11 p$ that significantly (LOD > 3.0) supported linkage. Several regions on chromosome 19 exhibit suggestive evidence (LOD between 2.5 - 2.9) for linkage. The region on $6 \mathrm{p}$ (HLA) is consistently seen in all linkage scans in T1D. The regions on $6 \mathrm{q}$ and $11 \mathrm{p}$ had been previously reported in some, but not all, independent linkage scans. The large sample size afforded by the CIDR $6 \mathrm{~K}$ dataset allowed us to further explore possible heterogeneity within the data. Three models were considered - allele sharing at HLA, sex strata, and age at onset strata (with a cutoff at age 5). The regions on 2q, $6 \mathrm{q}$, and $11 \mathrm{p}$ show similar trends in all strata suggesting they are not strongly dependent on linkage at HLA. In contrast, the region on chromosome 19 appears to be dependent on linkage at HLA. At $68 \mathrm{cM}$ on chromosome 19, LOD $=2.84$ among the 1233 ASPs that share 2 alleles IBD at HLA; however, $\mathrm{LOD}=0.01$ among the 1011 ASPs that share 0 or 1 alleles at HLA. The most significant findings under either "homogeneity" or any of the three heterogeneity (stratified) models were determined. Age at onset heterogeneity appears to play only a minor role in these data, but this result may reflect the limited number of ASPs $(\mathrm{N}=290)$ in the case that both sibs have age at onset less than 5 years.

Conclusion: These results suggest that there are important regions of the genome that may be important in defining genetic susceptibility to type 1 diabetes, either in isolation (chromosome 19) or in presence of stratification based upon HLA or gender. These effects are intriguing but require additional sample size (more ASPs) to confirm/refute these initial findings and determine the identify of specific genes residing in these regions.

Supported by: NIDDK and JDRF

\section{1}

Lower risk HLA genotypes account for the rising incidence of type 1 diabetes

S. Fourlanos ${ }^{1}$, M.D. Varney ${ }^{2}$, B.D. Tait ${ }^{2}$, G. Morahan ${ }^{3}$, M.C. Honeyman ${ }^{1}$, P.G. Colman ${ }^{4}$, L.C. Harrison ${ }^{1}$

${ }^{1}$ Autoimmunity \& Transplantation Division, The Walter \& Eliza Hall Institute of Medical Research, Melbourne, ${ }^{2}$ Victorian Transplantation and Immunogenetics Service, Melbourne, ${ }^{3}$ Genetics Department, Western Australian Institute for Medical Research, Perth, ${ }^{4}$ Dept of Diabetes \& Endocrinology, The Royal Melbourne Hospital, Melbourne, Australia

Background and aims: The incidence of childhood-onset type 1 diabetes (T1D) increased progressively over the last half century and has been attributed to environment, implying a lesser role for genetic susceptibility. If the environment increasingly impacts on the expression of T1D then the contribution of genetic susceptibility in newly diagnosed cases may change over time and, for the same degree of genetic susceptibility, age at diagnosis may decrease over time. To examine these possibilities, HLA-DRB1 genes known to confer risk for T1D were analysed in relation to year of birth and age at diagnosis over the last 5 decades.

Materials and methods: Caucasian subjects $(n=462)$ from the Australian T1D DNA Repository diagnosed with T1D before age 18 between 1950 2005 were DRB1 genotyped. Subjects were grouped by decade of birth or diagnosis (1950-1969, 1970-79 1980-89, 1990-99 and 2000-05) and from the highest $(\mathrm{DR} 3,4)$ to intermediate $(\mathrm{DR} 3,3$ or DR4,4; DR4,X or DR3,X) to the lowest (DRX,X, where $\mathrm{X}$ denotes a non-3 or non-4 allele) HLA genotypes for T1D risk.

Results: Mean age at diagnosis, 8.5 years (SD 4.5y), did not differ across decades. The proportion of the highest risk genotype, DR3,4, decreased significantly over time from $79 \%$ in $1950-1969$ to $28 \%$ in $2000-05$ ( $p<0.0001$ ) (Figure 1). On the other hand, the proportion of the heterozygous intermediate risk genotypes DR4, $\mathrm{X}$ and $\mathrm{DR} 3, \mathrm{X}$ increased significantly over this period; taken together, they increased from $20 \%$ to $48 \%$ ( $\mathrm{p}=0.0002$ ). Matched for DRB1 genotype and decade of birth, subjects were analysed for age at diagnosis. 
There was no change across time in age at diagnosis for the high risk DR3,4 genotype, but for the intermediate risk genotypes, DR4,4 and DR3,3, DR4,X and DR3,X, age at diagnosis significantly decreased over time. The incidence of childhood-onset T1D in Australia has doubled in the last 20 years, from 11.3 cases per 100000 person years in 1985 to 23.2 in 2002. The contribution of DRB1 genotypes to the increasing incidence of T1D was examined. We related the number of incident cases to specific DRB1 genotypes in the 1980s versus the current decade. From this analysis it is apparent the population incidence (cases per 100000 person years) of T1D in subjects with the highest risk genotype DR3,4 has remained unchanged (5.3 vs. 6.5). In contrast, the number of cases with intermediate risk genotypes DR3,3 or DR4,4 (1.3 vs. 4.9) and DR3,X or DR4,X (4.4 vs. 11.1) has increased, accounting for the increase in disease incidence.

Conclusion: The rising incidence and decreasing age at diagnosis of T1D is accounted for by the impact of environment on children with lower risk HLA class 2 genes, who previously would not have developed T1D in childhood.

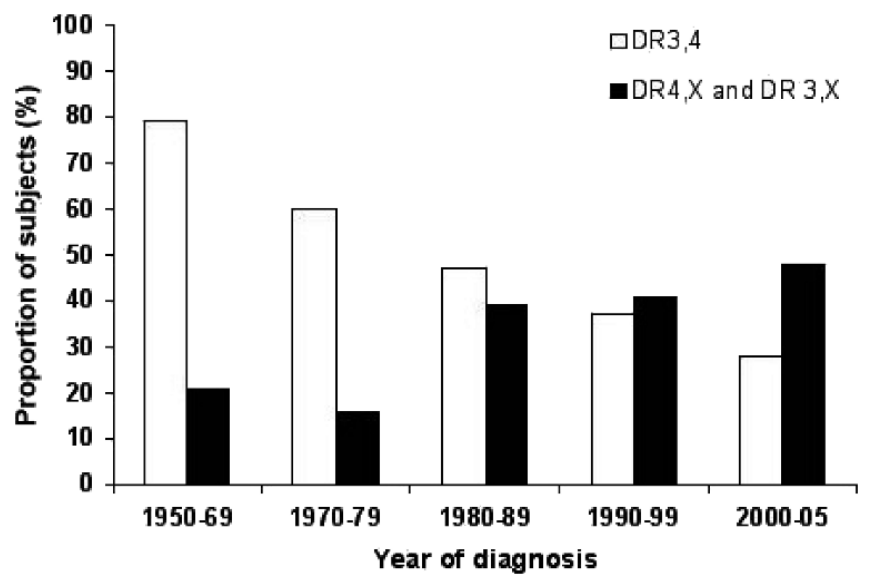

Supported by: National Health and Medical Research Council of Australia

\section{2}

Genome-wide association study using pooled DNA identifies region associated with kidney disease attributed to type 1 diabetes

J.K. DiStefano, M.P. Millis, K. Anchors, D.W. Craig

Diabetes, Cardiovascular and Metabolic Diseases Division, Translational

Genomics Research Institute, Phoenix, AZ, United States

Background and aims: Genetic factors play a major role in the progression of kidney disease in diabetes. To validate genes with significant effects on susceptibility to end-stage renal disease (ESRD), we performed a whole genome association study using pooled DNA from Caucasian individuals ascertained for type 1 diabetes.

Materials and methods: We utilized the Illumina Infinium II HumanHap 550 beadchip platform to genotype 555,352 SNPs in DNA pools comprised of 547 cases with ESRD and 549 controls with diabetes duration $>20$ years and no evidence of nephropathy. Pooled probe intensity was used to predict mean allele frequency (MAF) for each locus. Individual genotyping was performed using the iPLEX assay in conjunction with the MassARRAY platform (Sequenom)

Results: We identified 2,870 markers showing substantial differences in MAF (5.0-10.7\%) between pools. Among these, we selected 103 markers for validation based on overall ranking, proximity to annotated genes, or proximity to previously identified candidate genes, and genotyped them in the individuals comprising each pool. We observed the strongest evidence for association between ESRD and markers in the carnosinase I gene (CNDP1). In specific, rs11151964, which produces a glycine to arginine substitution at position 6 in CNDP1, was strongly associated with ESRD (OR $=1.72$ [1.29-2.29] per copy of A allele; $\left.\mathrm{P}=2.00 \times 10^{-4}\right)$. In addition, markers rs 17817077 and rs12964208, both located in intron 1 , were also associated with ESRD (OR=1.38 [1.151.65] per copy of A allele; $\mathrm{P}=6.00 \times 10^{-4}$ and $\mathrm{OR}=1.24$ [1.05-1.48] per copy of A allele; $\mathrm{P}=0.01$, respectively).

Conclusion: Marker rs11151964 represents a novel locus for modulating risk for diabetic ESRD, providing evidence supporting a role for CNDP1 and the carnosine pathway in the development of renal complications associated with diabetes.

\section{Sib pair linkage analysis identifies susceptibility loci for proliferative retinopathy in type 1 diabetes (The FinnDiane study)}

K.A.A. Hietala ${ }^{1,2}$, C. Forsblom ${ }^{1,3}$, M. Lehto ${ }^{1}$, M. Parkkonen ${ }^{1}$, P. Summanen ${ }^{2}$, P.-H. Groop ${ }^{1,3}$

${ }^{1}$ Department of Diabetes Genetics, Folkhalsan Research Center, Helsinki,

${ }^{2}$ Department of Ophthalmology, Helsinki University Central Hospital, ${ }^{3}$ Division of Nephrology, Department of Medicine, Helsinki University Central Hospital, Finland

Background and aims: Diabetic nephropathy and diabetic proliferative retinopathy (PDR) cluster in families, an observation that cannot be accounted for by environmental factors alone. It is obvious that genetic factors play a role and therefore, the aim of this study was to to find susceptibility loci for PDR by sib pair linkage analysis in siblings with type 1 diabetes (T1D).

Materials and methods: The FinnDiane Study has so far examined around 4800 patients with T1D. Among them, we identified 180 families with at least two siblings with T1D. Insulin treatment had been initiated within one year of diagnosis in all the patients and $98 \%$ were younger than 40 at the onset of diabetes. The patients with an onset $>40$ years of age (9/361) had all a sibling with an age at onset $\leq 40$ years. Ophthalmic records were obtained for $94 \%$ and fundus photographs for $68 \%$ of these patients. The PDR-status was graded based on fundus photographs and/or repeated clinical ophthalmic examinations using the ETDRS-grading scale, in which values $\geq 61$ represent PDR. Patients $(n=361)$ as well as their non-diabetic relatives $(n=316)$ were genotyped. Genomic DNA was extracted from whole blood using PureGene ${ }^{\circ}, \mathrm{Ge}-$ nomic DNA Purification Kit (Gentra Systems, Minnesota, USA). The DNA samples were genotyped using ABI 3730 DNA analyzer (Applied Biosystems, CA, USA) with ABI Linkage Mapping Set MD-10 V2.5 (Genethon map). A total of 367 autosomal markers with a mean interval of 9.6 (SD 4.1) cM and 77.7\% heterozygosity were used. A Modified Haseman-Elston regression of full sib pairs (SIBPAL-program, Case Western Reserve University, Cleveland, $\mathrm{OH}, \mathrm{USA}$ ) treated the binary variable PDR as continuous by designating the values 2 for affected and 1 for unaffected diabetic siblings. Duration of diabetes was included as a covariate. Empirical p-values were obtained by up to $10^{6}$ permutations, and converted to pointwise LOD scores

Results: The mean age at onset of diabetes was 15.5 (SD 11.5) years and duration 25.4 (SD 12.4) years. PDR was found in 118 (33\%) patients. Mean number of generations was 2.0 (SD 0.5), pedigree size 5.0 (SD 1.74) and number of siblings 2.6 (SD 1.0) in a sibship. The highest singlepoint LODscore (2.7) was observed on chromosome 10p12 and the highest multipoint LOD-score (3.01) was observed on 19q13.43.

Conclusion: The present study offers evidence of linkage for diabetic proliferative retinopathy on chromosomes 10p12 and 19q13.43. Interestingly the linkage at 19q13.43 replicates an earlier finding regarding severe retinopathy in patients with type 2 diabetes (Hallman, et al. 2007).

Supported by: Wilhelm and Else Stockmann Foundation, Finnish Eye Foundation, Eye and Tissue Bank Foundation, Folkhälsan Research Foundation

\section{4}

Independent predictive roles of genetic variants of aldose reductase on cardio-renal complications in Chinese with type 2 diabetes Y. Wang ${ }^{1}$, W.Y. So ${ }^{1}$, M.C.Y. Ng$^{1}$, X.L. Yang ${ }^{1}$, R.C.W. $\mathrm{Ma}^{1}$, A.P.S. Kong ${ }^{1}$, P.C.Y. Tong ${ }^{1,2}$, J.C.N. Chan ${ }^{1,2}$

${ }^{1}$ Medicine and Therapeutics, The Chinese University of Hong Kong, ${ }^{2}$ Hong Kong Institute of Diabetes and Obesity, The Chinese University of Hong Kong, China

Background and aims: Aldose reductase gene (ALR2) has been implicated in the development of diabetic complications. We previously reported the independent risk association of type 2 diabetic nephropathy with z-2 allele of the 5 '- $(\mathrm{CA})_{\mathrm{n}}$ microsatellite and C-106T promoter polymorphisms of the aldose reductase (ALR2) gene using case-control design. In this expanded cohort, we examined their predictive roles on new onset of cardio-renal complications using a prospective design.

Materials and methods: In this 8-year prospective cohort of 1074 type 2 diabetic patients (59\% male, median age: 61 years; disease duration: 7 years) with a total observation period of 8592 person-years, none had clinical evidence of coronary heart disease (CHD) or chronic kidney disease (CKD) at 
recruitment. Renal endpoint was defined as new onset of estimated glomerular filtration rate $(\mathrm{eGFR})<60 \mathrm{ml} / \mathrm{min} / 1.73 \mathrm{~m}^{2}$, hospitalizations with dialysis or death due to renal disease while CHD was defined as hospitalizations with myocardial infarction, ischemic heart disease or related deaths. The 5'-(CA)n and C-106T polymorphisms were examined by PCR followed by capillary electrophoresis and digestion with $\mathrm{B} f a \mathrm{I}$, respectively.

Results: After controlling for baseline risk factors and use of medications, ALR2 z-2 allele of (CA) microsatellite carriers had increased risk of renal [hazard ratio, 1.53 ; 95 percent confidence interval, $(1.14-2.05) \mathrm{p}=0.005$ ] or combined cardio-renal endpoints [1.31 (1.01-1.72) $\mathrm{p}=0.047]$. Carriers of ALR2 C-106T polymorphism also had increased risk of renal [1.54 (1.152.07) $\mathrm{p}=0.004]$ and cardio-renal endpoint [1.49 (1.14-1.95) $\mathrm{p}=0.004]$. Compared to non-carriers, patients with 2 risk conferring genotypes had 2-fold increased risk of renal [2.41 (1.57-3.70) $\mathrm{p}<0.001]$ and combined cardio-renal endpoints [1.94 (1.29-2.91) $\mathrm{p}=0.002]$.

Conclusion: In Chinese type 2 diabetic patients, genetic polymorphisms of the ALR2 independently predict new onset of renal and combined cardiorenal endpoints, the latter largely mediated through renal disease.

Supported by: CUHK Strategic Grant, Hong Kong Research Grant Committee Earmarked Grant, and the Hong Kong Innovation and Technology Fund

\section{OP 25 GLP-1 based therapies}

\section{5}

Dose range effects of the new once daily GLP-1 receptor agonist AVE0010 added to metformin in type 2 diabetes

J. Rosenstock ${ }^{1}$, R.E. Ratner ${ }^{2}$, G. Boka ${ }^{3}$

${ }^{1}$ Clinical Research, Dallas Diabetes and Endocrine Center, Dallas, United States, ${ }^{2}$ MedStar Research Institute, Hyattsville, United States, ${ }^{3} \mathrm{Clinical}$ Investigations, Metabolism \& Diabetes, sanofi-aventis R\&D, Antony, France

Background and aims: The effects of glucagon-like peptide-1 (GLP-1) receptor agonists are dependent on the dose concentrations. This randomized, 13week, double-blind, placebo-controlled, parallel-group study evaluated the dose-response effect of AVE0010, a new GLP-1 receptor agonist, in patients with type 2 diabetes (T2DM) treated with metformin.

Materials and methods: T2DM patients ( $\mathrm{N}=542)$ with $\mathrm{HbA}_{1 \mathrm{c}} \geq 7 \%$ and $<9 \%$ on metformin (1.6-1.9 g/day) were randomized to add-on therapy with subcutaneous $\operatorname{AVE} 0010(5,10,20$, and $30 \mu \mathrm{g}$ QD or BID) or matching placebo (total daily doses in the BID groups were $10,20,40$, and $60 \mu \mathrm{g}$, respectively). Primary endpoint was change in $\mathrm{HbA}_{1 \mathrm{c}}$ from baseline. Secondary endpoints included body weight, fasting plasma glucose (FPG), mean change in averaged self-monitored 7-point plasma glucose (MSMPG), post-prandial plasma glucose $\mathrm{AUC}_{[0-4 \mathrm{~h}]}, 2$-h post-prandial glucose changes, and post-prandial glucagon $\left.\mathrm{AUC}_{[0-4 \mathrm{~h}]}\right]^{\text {. }}$

Results: Baseline characteristics were similar among groups: mean age $56 \pm$ 9 years, diabetes duration $6.6 \pm 5$ years, $\mathrm{HbA}_{1 \mathrm{c}} 7.5 \pm 0.6 \%$, and BMI $31.9 \pm$ $4 \mathrm{~kg} / \mathrm{m}^{2} .489$ patients $(90.2 \%)$ completed the study. Despite mildly elevated baseline $\mathrm{HbA}_{1 \mathrm{c}}$ there were significant placebo-subtracted decreases in mean $\mathrm{HbA}_{1 \mathrm{c}}$ from baseline (Table). Percentages of patients with $\mathrm{HbA}_{1 \mathrm{c}}<7 \%$ at study-end were: $47 \%, 52 \%, 68 \%$, and $69 \%$ for $5,10,20$, and $30 \mu \mathrm{g} \mathrm{QD}$; and $51 \%, 65 \%, 62 \%$, and $77 \%$ for $5,10,20$, and $30 \mu \mathrm{g}$ BID vs $32 \%$ on placebo. Least squares mean weight reductions ranged from -2.0 to $-3.5 \mathrm{~kg}$ with 5 $\mu \mathrm{g}$ and $30 \mu \mathrm{g}$ QD, respectively, and -2.1 to $-3.9 \mathrm{~kg}$ with $5 \mu \mathrm{g}$ and $30 \mu \mathrm{g}$ BID, respectively, vs $-1.9 \mathrm{~kg}$ with placebo. Least squares mean placebo-subtracted body weight and specific metabolic parameter changes are presented in the Table. Adverse events included transient, dose-dependent nausea (from $7.3 \%$ to $35.2 \%$ with 5 and $30 \mu \mathrm{g}$ QD, respectively, and from $7.5 \%$ to $33.3 \%$ with 5 and $30 \mu \mathrm{g}$ BID, respectively, vs $4.6 \%$ with placebo). Vomiting occurred in $3.6 \%$ to $18.5 \%$ with $5 \mu \mathrm{g}$ and $30 \mu \mathrm{g}$ QD, respectively, and $5.7 \%$ to $9.3 \%$ with $5 \mu \mathrm{g}$ and $20 \mu \mathrm{g}$ BID, respectively, vs $0.9 \%$ with placebo. No cases of severe hypoglycaemia were reported. Few patients discontinued treatment due to adverse events $(1.8 \%$ to $11.1 \%$ with QD and 0 to $14.8 \%$ with BID vs $1.8 \%$ with placebo).

Conclusion: The GLP-1 agonist, AVE0010, significantly improved glycaemic control with meaningful $\mathrm{HbA}_{1 \mathrm{c}}$ reductions in patients with T2DM inadequately controlled with metformin. A clear dose-response relationship was seen within both QD and BID regimens, with similar efficacy between QD and BID. These results highlight the promising efficacy, safety and tolerability profile of a new once-daily GLP-1 agonist for the management of T2DM.

Placebo-Subtracted Weight and Metabolic Changes with AVE0010

\begin{tabular}{|c|c|c|c|c|c|c|c|c|}
\hline & $\begin{array}{l}5 \mu g \text { QD } \\
(n=55)\end{array}$ & $\begin{array}{l}10 \mu \mathrm{g} Q D \\
(\mathrm{n}=51)\end{array}$ & $\begin{array}{l}20 \mu g \text { QD } \\
(\mathrm{n}=53)\end{array}$ & $\begin{array}{l}30 \mu g \text { QD } \\
(n=52)\end{array}$ & $\begin{array}{l}5 \mu \mathrm{g} \text { BID } \\
(\mathrm{n}=51)\end{array}$ & $\begin{array}{l}10 \mu \mathrm{g} \text { BID } \\
(\mathrm{n}=54)\end{array}$ & $\begin{array}{l}20 \mu \mathrm{g} \text { BID } \\
(\mathrm{n}=52)\end{array}$ & $\begin{array}{l}30 \mu \mathrm{g} B I D \\
(\mathrm{n}=53)\end{array}$ \\
\hline $\mathrm{HbA}_{1 \mathrm{c}}(\%)$ & $-0.28^{\star}$ & $-0.31^{\star}$ & $-0.50^{\star * \star}$ & $-0.57^{\star * \star}$ & $-0.47^{\star * *}$ & $-0.59^{* * *}$ & $-0.57^{\star * *}$ & $-0.69^{* * *}$ \\
\hline Weight (kg) & $-0.05^{\mathrm{ns}}$ & $-0.45^{\mathrm{ns}}$ & $-1.07^{\star}$ & $-1.53^{\star * \star}$ & $-0.16^{\mathrm{ns}}$ & $-0.27^{\mathrm{ns}}$ & $-0.67^{\mathrm{ns}}$ & $-1.95^{\star * *}$ \\
\hline FPG (mmol/L) & $-0.41^{\mathrm{ns}}$ & $-0.33^{\mathrm{ns}}$ & $-0.59^{\mathrm{ns}}$ & $-0.81^{\star}$ & $0.02^{\mathrm{ns}}$ & $-0.77^{\star}$ & $-0.92^{\star * *}$ & $-1.21^{\star * *}$ \\
\hline MSMPG (mmol/L) & $-0.70^{*}$ & $-0.74^{*}$ & $-1.21^{\star * *}$ & $-1.24^{* * *}$ & $-0.35^{\mathrm{ns}}$ & $-1.07^{\star * *}$ & $-1.30^{* * *}$ & $-1.55^{\star * \star}$ \\
\hline $\begin{array}{l}\text { Post-prandial plasma } \\
\text { glucose AUC } \\
\left(^{[0-4 \mathrm{~h}]}\right. \\
\left.\mathrm{h}^{*} \mathrm{mmol} / \mathrm{L}\right)^{+} \\
\end{array}$ & $-5.20^{*}$ & $-8.59^{* *}$ & $-9.09^{* *}$ & $-11.63^{\star * *}$ & $-4.19^{n s}$ & $-9.26^{* *}$ & $-11.64^{\star * *}$ & $-13.41^{\star * *}$ \\
\hline $\begin{array}{l}\text { 2-h post-prandial plasma } \\
\text { glucose }(\mathrm{mmol} / \mathrm{L})^{+}\end{array}$ & $-1.71^{\star}$ & $-3.16^{\star * *}$ & $-3.24^{\star * *}$ & $-3.92^{\star * *}$ & $-1.60^{*}$ & $-3.10^{* * *}$ & $-3.71^{\star * *}$ & $-4.20^{\star * *}$ \\
\hline $\begin{array}{l}\text { Post-prandial glucagon } \\
\text { AUC }_{[0-4 \mathrm{~h}]}\left(\mathrm{h}^{*} \mathrm{ng} / \mathrm{L}\right)^{+}\end{array}$ & $-57.58^{*}$ & $-75.26^{\star *}$ & $-82.97^{\star *}$ & $-75.97^{*}$ & $-57.91^{\star}$ & $-59.99^{*}$ & $-63.42^{\star}$ & $-73.61^{\star}$ \\
\hline$n s=$ non - significant & ${ }^{*} p<0.05$ & ${ }^{* *} p<0.001$ & ${ }^{* * *} p<0.0001$ & 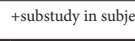 & & e test & & \\
\hline
\end{tabular}

Supported by: sanofi-aventis 


\section{6}

Exenatide once weekly elicits sustained glycaemic control and weight loss over 52 weeks

J. Buse ${ }^{1}$, D. Drucker 2 , K. Taylor 3 , T. Kim³, K. Wilhelm³, D. Kendall', M. Trautmann ${ }^{4}$, D. Zhuang ${ }^{3}$, L. Porter ${ }^{3}$

${ }^{1}$ University of North Carolina Chapel Hill, Chapel Hill, United States,

${ }^{2}$ University of Toronto, Canada, ${ }^{3}$ Amylin Pharmaceuticals, Inc., San

Diego, United States, ${ }^{4}$ Lilly Research Laboratories, Eli Lilly and Company,

Indianapolis, United States

Background and aims: The incretin mimetic exenatide improves glycaemic control and reduces body weight when administered twice daily (BID) in patients with type 2 diabetes. In a 30-wk randomised, open-label trial (DURATION-1: Diabetes Therapy $\underline{\mathbf{U}}$ tilisation: $\underline{R}$ esearching Changes in $\mathrm{Hb} \underline{\mathbf{A}}_{1 \mathrm{C}}$, Weight, and Other Factors Through Intervention with Exenatide ONce Weekly) treatment with a once weekly formulation (QW) of exenatide resulted in greater improvements in $\mathrm{HbA}_{1 \mathrm{C}}[-1.9 \%$ vs. $-1.5 \%]$ and fasting plasma glucose (FPG) $[-2.3 \mathrm{mmol} / \mathrm{L}$ vs. $-1.4 \mathrm{mmol} / \mathrm{L}]$ than twice daily exenatide (BID) in patients ( $\mathrm{N}=295$, ITT) with type 2 diabetes. Both groups lost $\sim 4 \mathrm{~kg}$ by wk 30 . Following the 30 -wk study, 258 (87.5\%) subjects entered an openended treatment with exenatide QW.

Materials and methods: Here we describe results following $22 \mathrm{wks}$ of follow-up treatment in 241 (evaluable) patients (prior to 52-wk study: baseline $\mathrm{HbA}_{1 \mathrm{C}} 8.3 \pm 1.0 \%$, FPG $9.4 \pm 2.4 \mathrm{mmol} / \mathrm{L}$, weight $102 \pm 20 \mathrm{~kg}$, BMI $35.1 \pm 5.0 \mathrm{~kg} /$ $\mathrm{m}^{2}$, diabetes duration $6.8 \pm 5.2 \mathrm{y}$; mean $\pm \mathrm{SD}$ ).

Results: Improvements in both $\mathrm{HbA}_{1 \mathrm{C}}$ and FPG were sustained in patients receiving exenatide QW $(n=120)$ for 52 wks [A1C: $-2.0 \pm 0.1 \%$; FPG $-2.6 \pm 0.2$ $\mathrm{mmol} / \mathrm{L}$ (LS mean $\pm \mathrm{SE}$ )], while patients who switched from exenatide BID to exenatide QW ( $\mathrm{n}=121)$ achieved similar glycaemic control by wk 52 $\left[\mathrm{HbA}_{1 \mathrm{C}}\right.$ : $-2.0 \pm 0.1 \%$; FPG $\left.-2.4 \pm 0.2 \mathrm{mmol} / \mathrm{L}\right]$. In both groups $\sim 3$ in 4 patients achieved $\mathrm{HbA}_{1 \mathrm{C}} \leq 7.0 \%$, while over half achieved $\mathrm{HbA}_{1 \mathrm{C}} \leq 6.5 \%$. Patients in both groups lost $\sim 4 \mathrm{~kg}$ by wk 52 . Both exenatide QW patients and those that switched from exenatide BID to exenatide QW had clinically significant reductions in systolic $(-5.7 \pm 1.2$ and $-4.0 \pm 1.4 \mathrm{mmHg}$, respectively; ITT analysis) and diastolic blood pressure $(-2.2 \pm 0.8$ and $-2.1 \pm 0.8 \mathrm{mmHg})$ at wk 52 . Serum lipid profiles improved in both cohorts after $30 \mathrm{wks}$, but to a greater extent in subjects who received exenatide QW. However, patients who switched from exenatide BID to exenatide QW ( $\mathrm{n}=121)$ exhibited comparable changes in serum lipids by wk 52 (total cholesterol: $-0.25 \pm 0.07$ vs $-0.23 \pm 0.07 \mathrm{mmol} /$ L; LDL: $-0.09 \pm 0.06$ vs $-0.07 \pm 0.06 \mathrm{mmol} / \mathrm{L}$; HDL: $-0.02 \pm 0.02$ vs $-0.04 \pm 0.02$ $\mathrm{mmol} / \mathrm{L}$; triglycerides: $-15 \%$ vs $-13 \%$; exenatide QW vs exenatide BID). Exenatide QW was well tolerated. Nausea was predominantly mild and transient during the 30-wk study; cumulative incidence of patients reporting an episode of nausea was $35 \%$ in the exenatide BID cohort and $26 \%$ in the exenatide QW cohort. During the 22-wk follow-up, the incidence of nausea was $\sim 7 \%$ in the ITT population. No hypoglycaemia occurred in patients not using a concomitant sulphonylurea, and no major hypoglycaemia was observed, regardless of background therapy.

Conclusion: This study demonstrated sustained glucose control and weight loss over 52 wks with exenatide QW. Patients switching from exenatide BID to exenatide QW experienced improvements in glycaemic control.

\section{7}

Comparison of single and combined exenatide and metformin treatment on metabolic parameters and body composition in insulin-resistant obese women with polycystic ovary syndrome

R. Bhushan ${ }^{1}$, O.S. Marrioneaux ${ }^{1}$, M. Bhushan ${ }^{1}$, D.C. Vernor ${ }^{1}$,

K.E. Elkind-Hirsch ${ }^{2}$

${ }^{1}$ Metabolic Center of Louisiana Research Foundation, Baton Rouge,

${ }^{2}$ Woman's Health Research Institute, Baton Rouge, United States

Background and aims: Given that a disturbed balance between insulin sensitivity and pancreatic $\beta$-cell activity is found in women with polycystic ovary syndrome (PCOS), early recognition and intervention such as weight loss and/or pharmacological intervention may prevent or delay the development of diabetes. In order to do so, we evaluated exenatide and metformin, alone and in combination, on metabolic profiles, body weight and fat distribution, and hormonal parameters in obese insulin-resistant women with PCOS.

Materials and methods: Sixty obese oligo-ovulatory women with PCOS were randomized to 1 of 3 treatment groups: metformin (MET; $1000 \mathrm{mg} \mathrm{BID);} \mathrm{ex-}$ enatide (EX; 10 mcg BID) or combined (COM; metformin 1000 mg BID; exenatide $10 \mathrm{mcg}$ BID) for 24 weeks. An oral glucose tolerance test (SI ${ }_{\text {OGTT }}$ ) was performed with measurement of glucose $(\mathrm{G})$ and insulin (I) before and after the course of treatment. Insulin sensitivity and $\beta$-cell function measures were computed from G-I values obtained during the $\mathrm{SI}_{\text {OGTT }}$ Sex steroids (testosterone, sex hormone binding globulin [SHBG], free androgen index [FAI]) and adiponectin levels were determined in the fasting sample.

Results: Insulin resistance was confirmed in all study participants. Forty-two (70\%) patients completed the study per protocol. COM therapy was superior to either single agent therapy in improving insulin sensitivity and reducing abdominal girth as shown in the table below. Both exenatide treatment groups were more effective in promoting weight loss and reducing androgen excess than metformin alone ( $\mathrm{p}=0.003)$.

Conclusion: Treatment with exenatide and metformin is more effective than either medication alone in improving metabolic profiles, increasing adiponectin levels and reducing body weight, abdominal girth, and hyperandrogenism. A marked decrease in central adiposity in the COM population is noted, which could partly explain the improvements in G-I parameters and adiponectin levels. The amelioration of these factors alone could lead to the delay in progression of DM2; it is enough, at least, to warrant a prospective study.

Baseline and Post-Treatment Data

\begin{tabular}{|c|c|c|c|c|c|c|}
\hline \multirow[t]{2}{*}{ Parameter } & \multicolumn{2}{|l|}{ Exenatide } & \multicolumn{2}{|l|}{ Metformin } & \multicolumn{2}{|c|}{ Combination } \\
\hline & Baseline & Post-Treatment & Baseline & Post-Treatment & Baseline & Post-Treatment \\
\hline HOMA-IR & $7.04+/-0.96$ & $5.2+/-0.7$ & $6.03+/-0.96$ & $5.7+/-0.7$ & $4.3+/-1.0$ & $3.5+/-0.7$ \\
\hline $\mathrm{SI}_{\mathrm{OGTT}}$ & $1.7+/-0.22$ & $2.2+/-0.3$ & $2.1+/-0.22$ & $2.5+/-0.3$ & $2.5+/-0.23$ & $3.2+/-0.3$ \\
\hline CIRgp & $2.3+/-0.2$ & $1.7+/-0.3$ & $1.4+/-0.2$ & $2.0+/-0.3$ & $1.6+/-0.2$ & $1.3+/-0.3$ \\
\hline ISSI & $335+/-63$ & $395+/-112$ & $284+/-65$ & $232+/-116$ & $233+/-65$ & $516+/-117$ \\
\hline Adiponectin (ng/dL) & $5.4+/-0.8$ & $5.5+/-0.8$ & $4.9+/-0.8$ & $6.4+/-0.8$ & $5.5+/-0.8$ & $6.7+/-0.7$ \\
\hline Absolute Weight $(\mathrm{kg})$ & $110.5+/-6.0$ & $107.3+/-6.0$ & $113.4+/-7.0$ & $111.8+/-6.0$ & $112+/-8.0$ & $106.4+/-6.0$ \\
\hline BMI $\left(\mathrm{kg} / \mathrm{m}^{2}\right)$ & $40.3+/-2.0$ & $39.3+/-2.0$ & $43.3+/-2.0$ & $42.3+/-2.0$ & $40.9+/-2.0$ & $39.2+/-2.0$ \\
\hline Abdominal Girth $(\mathrm{cm})$ & $120.4+/-4.5$ & $119.6+/-4.3$ & $123.4+/-4.3$ & $123.9+/-4.4$ & $122+/-4.4$ & $116+/-4.3$ \\
\hline FAI (U) & $16.3+/-3.5$ & $11.9+/-1.4$ & $12.6+/-2.4$ & $11.4+/-1.3$ & $10.4+/-2.4$ & $5.7+/-1.3$ \\
\hline
\end{tabular}

Supported by: Amylin Pharmaceuticals/Eli Lilly \& Company 


\section{8}

Significantly better glycaemic control/weight reduction with human GLP-1 analogue liraglutide, than with insulin glargine: all as add-on to metformin + sulphonylurea in type 2 diabetes

D. Russell-Jones ${ }^{1}$, A. Vaag ${ }^{2}$, O. Schmitz ${ }^{3}$, B. Sethi ${ }^{4}$, N.M. Lalic ${ }^{5}$, S. Antic ${ }^{6}$, M. Zdravkovic ${ }^{7}$, G.M. Ravn ${ }^{8}$, R. Simo ${ }^{9}$

${ }^{1}$ Endocrinology \& Metabolism, Royal Surrey County Hospital,

Guildford, United Kingdom, ${ }^{2}$ Steno Diabetes Center, Gentofte, Denmark,

${ }^{3}$ Endocrinology \& Metabolism, University of Aarhus, Denmark,

${ }^{4}$ Endocrinology \& Metabolism, Care Hospitals, Hyderabad, India,

${ }^{5}$ Endocrinology \& Metabolism, School of Medicine, Belgrade, Serbia,

${ }^{6}$ Endocrinology \& Metabolism, Diabetes and Metabolic Diseases

Clinic, Nis, Serbia, ${ }^{7}$ International Medical Affairs, Novo Nordisk A/S,

Bagsvaerd, Denmark, ${ }^{8}$ Statistics, Novo Nordisk A/S, Bagsvaerd, Denmark,

${ }^{9}$ Endocrinology \& Metabolism, Vall d'Hebron University Hospital,

Barcelona, Spain

Background and aims: This 26-week, randomised trial compared the efficacy and safety of liraglutide (1.8 mg, once daily; OD), liraglutide placebo and open label insulin glargine, all as add-on to metformin (1 mg, twice daily; BID) and glimepiride (2-4 mg OD).

Materials and methods: In total, 581 subjects were randomised (mean age $57.5 \pm 9.9$ ); mean body mass index $30.5 \pm 5.3 \mathrm{~kg} / \mathrm{m}^{2}$, mean $\mathrm{HbA}_{1 c} 8.2 \pm 0.9 \%$ ).

Results: Liraglutide + metformin + glimepiride reduced $\mathrm{HbA}$ more than glargine and placebo in combination with metformin and glimepiride (ANCOVA, $p=0.0015$ and $p<0.0001)$ and more of the subjects in the liraglutide group reached $\mathrm{HbA}_{1 c} \leq 6.5 \%(p<0.0001)$. Estimated weight difference between the liraglutide and glargine groups was $-3.4 \mathrm{~kg}(p<0.0001)$ and $-1.4 \mathrm{~kg}$ between the liraglutide and placebo groups $(p=0.0001)$. Glargine was individually titrated according to a patient-driven algorithm, and the average dose of glargine was $24 \mathrm{IU} /$ day at the end of trial. Most common adverse events in the liraglutide group were gastrointestinal disorders (mainly nausea). Nausea occurred in approximately $15 \%$ of subjects in the liraglutide group, but was transient. Frequency of minor hypoglycaemic episodes $(<3.1 \mathrm{mmol} / \mathrm{L})$ were similar for the liraglutide and glargine groups and more frequent than in the placebo group, but did not reach statistical significance. Five subjects reported major hypoglycaemic events in the liraglutide group (only one required medical assistance, and none resulted in coma or seizures). Presence of liraglutide antibodies was $9.8 \%$.

Conclusion: Liraglutide added to metformin and a sulphonylurea (SU) was generally well tolerated and statistically significantly improved glycaemic control, and reduced body weight in comparison to metformin and an SU alone and to glargine added to metformin and an SU.

\section{9}

Liraglutide, a human GLP-1 analogue, substantially reduces $\mathrm{HbA}_{1 \mathrm{c}}$ in subjects with type 2 diabetes, irrespective of $\mathrm{HbA}_{1 \mathrm{c}}$ at baseline A. Vaag', M.A. Nauck 2 , M. Brandle 3 , S. Colagiuri ${ }^{4}$, O. Schmitz, M. Zdravkovic ${ }^{6}$, K. Hermansen ${ }^{7}$, A. Garber ${ }^{8}$, B. Zinman ${ }^{9}$

${ }^{1}$ Steno Diabetes Centre, Gentofte, Denmark, ${ }^{2}$ Diabeteszentrum, Bad Lauterberg im Harz, Germany, ${ }^{3}$ Kantonsspital, St Gallen, Switzerland, ${ }^{4}$ Metabolic Health, University of Sydney, Australia, ${ }^{5}$ Endocrinology and Diabetes, University of Aarhus, Denmark, ${ }^{6}$ International Medical Affairs, Novo Nordisk A/S, Bagsvaerd, Denmark, ${ }^{7}$ Endocrinology, Aarhus University Hospital, Denmark, ${ }^{8}$ Baylor College of Medicine, Methodist Hospital, Houston, United States, ${ }^{9}$ Leadership Sinai Centre for Diabetes, Mount Sinai Hospital, Toronto, Canada

Background and aims: Baseline $\mathrm{HbA}_{1 c}$ is an important factor when deciding on the pharmacological agent to use and can also determine the response to treatment. The aim of this analysis was to examine the impact of baseline $\mathrm{HbA}_{1 c}$ on the responses to liraglutide, a once-daily human GLP-1 analogue. Materials and methods: Data on glycaemic control from four 26-week trials and one 52-week LEAD (Liraglutide Effect and Action in Diabetes) study were stratified by baseline $\mathrm{HbA}_{1 c}$ quartiles (Table).

\begin{tabular}{|c|c|c|c|c|c|}
\hline \multirow{2}{*}{$\begin{array}{l}\text { Baseline } \\
\text { characteristic }\end{array}$} & \multicolumn{5}{|l|}{ Trial } \\
\hline & LEAD 1 & LEAD 2 & LEAD 3 & LEAD 4 & LEAD 5 \\
\hline Subjects & $\mathrm{n}=695$ & $\mathrm{n}=725$ & $\mathrm{n}=746$ & $\mathrm{n}=543$ & $\mathrm{n}=232$ \\
\hline $\begin{array}{l}\text { Concomitant } \\
\text { OADs }\end{array}$ & $\begin{array}{l}\text { glimepiride } \\
2-4 \mathrm{mg}\end{array}$ & $\begin{array}{l}\text { metformin } \\
2 \mathrm{~g}\end{array}$ & - & $\begin{array}{l}\text { metformin } \\
2 \mathrm{~g}+\text { rosigli- } \\
\text { tazone } 8 \mathrm{mg}\end{array}$ & $\begin{array}{l}\text { metformin } \\
2 \mathrm{~g}+ \\
\text { glimepiride } \\
2-4 \mathrm{mg}\end{array}$ \\
\hline $\begin{array}{l}\mathrm{HbA}_{1 \mathrm{c}} \\
\text { (mean, \%) }\end{array}$ & 8.4 & 8.3 & 8.3 & 8.3 & 8.6 \\
\hline $\begin{array}{l}\mathrm{HbA}_{1 \mathrm{c}}(\text { mean, } \\
\text { quartile } 1)\end{array}$ & 7.3 & 7.3 & 7.0 & 7.2 & 7.2 \\
\hline $\begin{array}{l}\mathrm{HbA}_{1 \mathrm{c}} \text { (mean, } \\
\text { quartile 2) }\end{array}$ & 8.1 & 8.0 & 7.7 & 7.9 & 7.9 \\
\hline $\begin{array}{l}\mathrm{HbA}_{1 \mathrm{c}} \text { (mean, } \\
\text { quartile 3) } \\
\end{array}$ & 8.8 & 8.7 & 8.4 & 8.6 & 8.5 \\
\hline $\begin{array}{l}\mathrm{HbA}_{1 \mathrm{c}} \text { (mean, } \\
\text { quartile 4) }\end{array}$ & 9.8 & 9.8 & 9.7 & 10.3 & 9.5 \\
\hline $\begin{array}{l}\text { Bodyweight } \\
\text { (mean, kg) }\end{array}$ & 81.9 & 88.1 & 92.6 & 96.3 & 85.5 \\
\hline
\end{tabular}

Results: Changes in $\mathrm{HbA}_{1 \mathrm{c}}$ (mean (SE)) from baseline to end of trial are shown (Figure). Liraglutide added to oral antidiabetic agents (OADs) resulted in clinically meaningful reductions in $\mathrm{HbA}_{1 \mathrm{c}}$ across all baseline $\mathrm{HbA}_{1 \mathrm{c}}$ quartiles. The effect of baseline $\mathrm{HbA}_{1 c}$ on actual $\mathrm{HbA}_{1 c}$ reduction was confirmed by an analysis of the change in $\mathrm{HbA}_{1 \mathrm{c}}$ with treatment, previous $\mathrm{OAD}$ treatment and baseline $\mathrm{HbA}_{1 c}$ as explanatory variables $(p<0.0001)$. Treatment with liraglutide + metformin + rosiglitazone (LEAD 4) resulted in the largest decrease in $\mathrm{HbA}_{1 \mathrm{c}}$ compared with other regimens, irrespective of baseline

\begin{tabular}{llll}
\hline & $\begin{array}{l}\text { Liraglutide 1.8+ } \\
\text { metformin + glimepiride }\end{array}$ & $\begin{array}{l}\text { Placebo + metformin + } \\
\text { glimepiride }\end{array}$ & $\begin{array}{l}\text { Glargine + metformin + } \\
\text { glimepiride }\end{array}$ \\
\hline Final $\mathrm{HbA}_{1 \mathrm{c}} \%(\mathrm{SD})$ & $7.0(1.0)$ & $8.1(1.3)$ & $7.2(0.9)$ \\
$\mathrm{Change} \mathrm{HbA}_{1 \mathrm{c}} \%(\mathrm{SE})$ & $-1.33(0.09)^{*} \dagger$ & $-0.24(0.11)$ & $-1.09(0.09)$ \\
\hline$\% \mathrm{HbA}_{1 \mathrm{c}} \leq 6.5 \%$ & $37.1^{*} \dagger$ & 10.9 & 23.6 \\
\hline Weight change, $\mathrm{kg}(\mathrm{SE})$ & $-1.81(0.33)^{*} \dagger$ & $-0.42(0.39)$ & $1.62(0.33)$ \\
\hline Final FPG, mmol/L (SD) & $7.7(2.2)$ & $10.0(2.9)$ & $7.4(2.1)$ \\
Change FPG, mmol/L & $-1.6^{*}$ & 0.5 & -1.8 \\
\hline \% reporting minor hypo events & 27.4 & 16.7 & 28.9 \\
\hline \% reporting nausea & 13.9 & 3.5 & 1.3 \\
\hline \% of subjects reaching week 26 FPG & & & $2.6(1.1)$ \\
titration targets & & & $5.2(2.2)$ \\
$5.6 \mathrm{mmol} / \mathrm{L}$ (\% subjects) & & &
\end{tabular}

$6.7 \mathrm{mmol} / \mathrm{L}(\% \mathrm{subjects})$

${ }^{*}=$ statistically significant in comparison to metformin + glimepiride, $p<0.05$

$\dagger=$ statistically significant in comparison to glargine + metformin + glimepiride, $p<0.05$

FPG, fasting plasma glucose

Supported by: Novo Nordisk A/S 
$\mathrm{HbA}_{1 c} \cdot\left(-1.0 \%,-1.1 \%,-1.4 \%\right.$, and $-2.3 \%$ in baseline $\mathrm{HbA}_{1 \mathrm{c}}$ quartiles $1,2,3$ and 4 , respectively).

Conclusion: Liraglutide reduces $\mathrm{HbAlc}$ irrespective of $\mathrm{HbA}_{1 \mathrm{c}}$ at baseline, and the reduction is greater with higher baseline $\mathrm{HbAlc}$, as seen with other drugs.

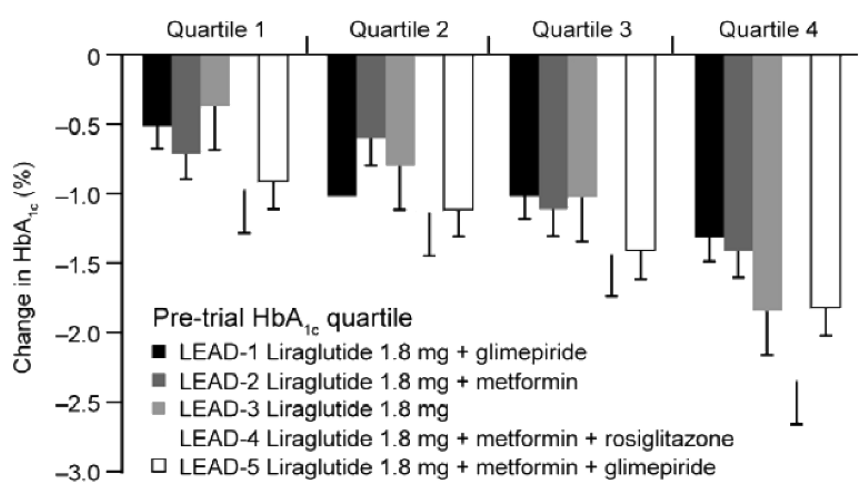

Supported by: Novo Nordisk A/S

\section{0}

Surgical treatment for non-obese type 2 diabetic subjects: results from 1-year prospective study

B. Geloneze ${ }^{1}$, S.R. Geloneze ${ }^{1}$, C. Fiori ${ }^{1}$, M. Tambascia ${ }^{1}$, F.F. Hirsch ${ }^{1}$, C. Stabe ${ }^{1}$, A.C. Felici ${ }^{1}$, B.D. Astiarraga ${ }^{1}$, E. Chaim ${ }^{2}$, J.C. Pareja ${ }^{2}$ ${ }^{1}$ Laboratory of Investigation on Metabolism and Diabetes - LIMED, University of Campinas, ${ }^{2}$ Obesity and Metabolic Surgery Unit, University of Campinas, Brazil

Background and aims: Most patients who undergo Roux-en-Y gastric bypass (RYGB) experience rapid resolution of type 2 diabetes (T2DM). These results come from more than gastric restriction and weight loss, indicating the gut as a incretin mediator. Goto-Kakizaki T2DM rats underwent duodenal exclusion, a stomach-preserving RYGB that excludes the proximal intestine, ameliorates glycaemic control suggesting that a proximal intestine bypass could be considered as an incretin-based diabetic treatment.

Materials and methods: To test this hypothesis we performed a duodenal exclusion in 15 non-obese T2DM subjects from less than 10 years, age:38-58y, no evidence of autoimmunity (negative anti-GAD), significant endogenous insulin production (C-peptide $>1 \mathrm{ng} / \mathrm{mL}$ ), and documented use of insulin, but for less than 8 years. Clinical 1-year follow-up included self-monitoring blood glucose assessment to further adjustment on medical therapy based on current international guidelines.

Results: The major finding of this study was a reduction on insulin requirement (2/15 remain taking insulin at 1-year) along with improvement on fasting, postprandial glucose, and A1C levels. At baseline, all the patients had A1C above 7\%, but 5 patients at 2 months, 6 at 6 months, and 5 at 1 -year had A1C less than $7 \%$. No changes in BMI, \% of fat mass or fat distribution were observed. Insulin sensitivity (KITT) remained unchanged during 1-year follow-up.

Conclusion: Surgical treatment for T2DM bypassing a segment of proximal intestine in the duodenal exclusion procedure is an effective treatment for non-obese subjects independently of weight loss and changes on insulin sensitivity. We hypothesized that this clinical improvement is mediated by an enhanced incretin effect. These findings suggest a potential role of the proximal intestine in the pathogenesis of T2DM and the possibility of a new incretinbased surgical approach for the management of non-obese diabetic subjects.

\begin{tabular}{llllll}
\hline & Baseline & 2 months & 6 months & 12 months & Anova \\
\hline BMI $(\mathrm{kg} / \mathrm{m} 2)$ & $27.2(2.1)$ & $25.2(2.7)$ & $25.8(3.1)$ & $26.5(2.7)$ & NS \\
\hline \% Fat mass & $28.5(7.1)$ & $27.1(8.9)$ & $26.6(7.1)$ & $25.4(4.5)$ & NS \\
\hline $\begin{array}{l}\text { Fasting Glucose } \\
\text { (mg/dL) }\end{array}$ & $182(61)$ & $151(45)$ & $156(42)$ & $155(45)$ & $\mathrm{p}<0.05$ \\
\hline A1C $(\%)$ & $8.9(1.5)$ & $7.3(0.9)$ & $8.1(1.2)$ & $7.9(0.8)$ & $\mathrm{p}<0.05$ \\
\hline KITT $(\% / m i n)$ & $0.8(0.5)$ & ------- & $0.8(0.5)$ & $1.1(0.9)$ & $\mathrm{NS}$ \\
\hline $\begin{array}{l}\text { Patients taking } \\
\text { insulin }\end{array}$ & $15 / 15$ & $2 / 15$ & $1 / 15$ & $2 / 15$ & $\mathrm{p}<0.01$ \\
\hline
\end{tabular}

Supported by: Ethicon Endo-Surgery
OP 26 Health care models across Europe

\section{1}

ROMEO (Rethink Organization to iMprove Education and Outcomes). A 4-year multicentre randomised controlled trial of group care for the management of type 2 diabetes

M. Trento on behalf of the ROMEO investigators

Internal Medicine, Laboratory of Clinical Pedagogy, University of Turin, Italy

Background and aims: We showed previously (Diabetes Care 27;670:2004) that Type 2 diabetes (T2DM) is managed effectively by continuous systemic group education (Group Care), which substitutes traditional one-to-one visits with sessions centred on hands-on and problem-solving activities, role playing and discussions on motivation, diabetes acceptance, psychosocial and coping strategies. Sessions last 40-50 minutes, are held every 3-4 months and are followed by brief individual consultations with a physician. A multicentre clinical trial was organised to ascertain if Group Care would perform as well in diabetes clinics throughout Italy.

Patients and Methods: 815 patients with non insulin-treated T2DM from 13 Italian clinics were centrally randomised to either Group or one-to-one Care. Operators were specifically trained and provided with a detailed operating manual and educational materials. At baseline, the two treatment groups were similar by age, gender, known duration of diabetes, BMI, HbAlc, lipids, quality of life, knowledge of diabetes and health behaviours, as assessed by questionnaires. Analysis was by intention-to-treat. 592 patients from 11 clinics completed the 4-year follow-up. Results are expressed as differences $(95 \%$ CI) between treatment groups, after adjusting for participating centre, gender, family history for diabetes, schooling, occupation, years of attendance in clinic and baseline values of the relevant variables.

Results: After 4 years, the patients followed by Group Care had lower HbAlc $[-1.49 \%(-1.63 /-1.34)]$, fasting blood glucose $[-19.1 \mathrm{mg} / \mathrm{dl}(-27.1 /-11.1)]$, weight $[-3.15 \mathrm{Kg}(-4.21 /-2.08)]$, BMI $[-1.09(-1.56 /-0.62)]$, systolic [-4.4 $\mathrm{mmHg}(-7.3 /-1.5)]$ and diastolic $[-3.3 \mathrm{mmHg}(-5.2 /-1.3)]$ blood pressure, triglyceride $[-44.8 \mathrm{mg} / \mathrm{dl}(-56.0 /-33.5)]$ and total cholesterol $[-25.7 \mathrm{mg} / \mathrm{dl}$ $(-31.8 /-19.5)$, and higher HDL cholesterol $[+5.2 \mathrm{mg} / \mathrm{dl}(+3.5 /+7.0)]$ than those followed by traditional care. They also had better quality of life [-16.8 $(-18.3 /-15.2)]$, knowledge $[+10.6(+8.9 /+12.3)]$ and health behaviours $[+4.2$ $(+3.8 /+4.5)]$. All differences were significant at $\mathrm{p}<0.001$.

Conclusion: Group Care produces better clinical, cognitive and psychological outcomes than traditional one-to-one care when applied within a structured systemic programme. However, it requires re-organization of working practices, which may account for failure of two participating clinics in implementing it.

Supported by: EFSD-Novo Nordisk grant

\section{2}

Clinical effectiveness of oral antidiabetic drugs and insulin in the management of type 2 diabetes in UK primary care - a UK population based study

P. McEwan ${ }^{1}$, P. Sharplin ${ }^{1}$, M. Baldwin ${ }^{1}$, M. Evans ${ }^{2}$

${ }^{1} \mathrm{CRC}$, Cardiff Research Consortium, ${ }^{2}$ School of Medicine, University Hospital of Wales, Cardiff, United Kingdom

Background and aims: The maintenance of long term glycaemic control is important in limiting the complications associated with type 2 diabetes (T2D), driving clinical practice toward the attainment of near normoglycaemia $\left(\mathrm{HbA}_{1 c}<7 \%\right)$. Injected insulin is the most effective glucose lowering agent; however, current clinical practice results in a stepwise escalation of oral antidiabetic drugs (OADs) prior to insulin initiation. This pattern of therapy may result in patients being exposed to unacceptable prolonged hyperglycaemia essentially through delaying the initiation of insulin. The aim of this retrospective cohort based study was to establish evidence that the introduction of insulin earlier in the course of the disease has better outcomes than sequential OAD therapy.

Materials and methods: We analysed the UK THIN (The Health Improvement Network) general practice database between 2001 and 2006. Patients were stratified by maximum concurrent number of oral therapies. All patients were analysed by $\mathrm{HbA}_{1 \mathrm{c}}$ history, relative to the time point of first, sec- 
ond and third line therapy initiation. Patients progressing to insulin were also analysed by $\mathrm{HbA}_{1 c}$ history, relative to the time of insulin initiation.

Results: The table details the number of patients and mean HbAlc categorized by insulin status and maximum number of OAD usage.

\begin{tabular}{lllllll}
\hline OAD usage & None & One & Two & Three & Four & Total \\
\hline Non-insulinised & & & & & & \\
\hline Number & 40,924 & 49,534 & 31,433 & 7,473 & 864 & 130,228 \\
Mean Age & 72 & 70 & 71 & 66 & 64 & 71 \\
Proportion male & 0.52 & 0.50 & 0.54 & 0.57 & 0.64 & 0.52 \\
HbA1c* & 6.4 & 7.1 & 7.6 & 7.9 & 8.2 & 7.1 \\
\hline Insulinised & & & & & & \\
Number & & 4,641 & 9,404 & 2,465 & 68 & 16,578 \\
Mean Age & & 70 & 69 & 67 & 63 & 68 \\
Proportion male & & 0.52 & 0.52 & 0.56 & 0.69 & 0.52 \\
HbAlc** & & 9.2 & 9.6 & 9.7 & 9.9 & 9.6 \\
\hline
\end{tabular}

$\mathrm{HbA}_{1 \mathrm{c}}{ }^{*}$ as recorded in 2nd quarter of 2006; $\mathrm{HbA}_{1 \mathrm{c}}{ }^{* *}$ as recorded in the quarter prior to insulin initiation. Prior to insulin initiation patients receiving OADs typically had an increase in $\mathrm{HbA}_{1 \mathrm{c}}$ of $0.92 \%$ in the previous year; mean $\mathrm{HbA}_{1 c}$ was 8.58, 8.73, 9.01 and 9.51 in the 9-12 month 6-9, 3-6 and 0-3 month period prior to insulinisation respectively. Patients switching to insulin after one OAD with baseline $\mathrm{HbA}_{1 \mathrm{c}}$ levels $>7.5 \%$; or after 2 OADs with baseline $\mathrm{HbA}_{1 \mathrm{c}}$ levels $>8.5 \%$ showed mean reductions of $1.5 \%$ and $2 \%$, respectively; similar patients switching to further OADs had mean reductions of $1 \%$ and $1.5 \%$ respectively.

Conclusion: On average, patients start insulin with $\mathrm{HbA}_{1 \mathrm{c}}$ levels $>9 \%$. These patients had been poorly controlled for a long period of time prior to insulin initiation. Increased escalation of OADs is associated with higher mean levels of $\mathrm{HbA}_{1 \mathrm{c}}$ at insulin initiation. Therefore, starting insulin above a threshold $\mathrm{HbA}_{1 \mathrm{c}}$ of $8.5 \%$ is unlikely to achieve a target $\mathrm{HbA}_{1 \mathrm{c}}<7 \%$. Finally, these results confirm that insulin is the most effective $\mathrm{HbA}_{1 c}$ lowering therapy in T2D. Supported by: sanofi-aventis U.K. Group

\section{3}

Improvement in the management of people with diabetes living in France between 2001 and 2007

M. Varroud-Vial ${ }^{1}$, N. Thammavong ${ }^{2}$, I. Romon ${ }^{2}$, A. Weill ${ }^{3}$, M. Chantry ${ }^{4}$, G.R. Auleley ${ }^{5}$, C. Fournier ${ }^{6}$, N. Poutignat ${ }^{7}$, B. Detournay ${ }^{8}$, E. Eschwege ${ }^{9}$, D. Simon ${ }^{10}$, S. Halimi ${ }^{11}$, P. Lecomte ${ }^{12}$, A. Penfornis ${ }^{13}$, A. Fagot-Campagna ${ }^{2}$ ${ }^{1}$ Diabetes Department, CH Sud Francilien, Corbeil-Essonnes, ${ }^{2}$ Epidemiology, INVS, Saint-Maurice, ${ }^{3}$ Medical Department, CNAMTS, Paris, ${ }^{4}$ Medical Department, CNAM, Creteil, ${ }^{5}$ Health Care Department, CANAM, Paris, ${ }^{6}$ Medical Department, INPES, Paris, ${ }^{7}$ Medical Department, HAS, Saint-Denis, ${ }^{8}$ Epidemiology, CEMKA EVAL, Bourg La Reine, ${ }^{9}$ Epidemiology, INSERM, Villejuif, ${ }^{10}$ Diabetes Department, CHU La Pitié, Paris, ${ }^{11}$ Diabetes Department, CHU, Grenoble, ${ }^{12}$ Diabetes Department, CHU, Tours, ${ }^{13}$ Diabetes Department, CHU, Besancon, France

Background and aims: To compare reimbursed diabetes care for adults in mainland France in 2001 and 2007.

Materials and methods: 7676 subjects older than 18 years have been randomly selected in august 2007 from the claims of the French national Health Insurance System among people reimbursed of an oral hypoglycemic agent (OHA) or insulin on at least one occasion during the last 3 months. Data on reimbursed examinations and treatments have been collected and compared with data obtained with the same methodology in 2001 for the 9787 subjects included in the first ENTRED study.

Results: Gender (male $=52 \%$ ) and mean age (65 years) were similar in 2001 and 2007, with a greater proportion of persons older than 75 years in 2007 (women $35 \%$ vs $28 \%$, men $25 \%$ vs $17 \%$ ). The assessment of glycemic control improved : 3 measures of $\mathrm{HbAlc}$ during the previous year was performed for $30 \%$ of patients in 2001 vs $39 \%$ in 2007 , and at least one measure for $77 \%$ in 2001 vs $87 \%$ in 2007 . The biological follow-up also improved : at least one measurement of creatinin $71 \%$ vs $80 \%$, of microalbuminuria $18 \%$ vs $26 \%$, of blood lipids $63 \%$ vs $72 \%$. An EKG had been performed for $30 \%$ of patients in 2001 vs $34 \%$ in 2007 and fundus examination moderately increased from $43 \%$ to $45 \%$. The proportion of patients attending a private diabetes specialist was $11 \%$ in 2001 and 2007, but refferals to a cardiologist increased from $28 \%$ to $33 \%$. Treatments of hyperglycemia were more aggressive in 2007: one OHA was prescribed to $47 \%$ of patients in 2001 vs $42 \%$ in 2007 , two or more OHA to $33 \%$ vs $35 \%$, insulin \pm OHA to $20 \%$ vs $23 \%$. Prescription of metformine in- creased from $48 \%$ in 2001 to $59 \%$ in 2007, while prescription of sulfonylureas decreased from $60 \%$ to $48 \%$. The management of CV risk factors became also more aggressive : prescription of antiplatelet therapy increased from $24 \%$ to $32 \%$, of lipid-lowering agents from $39 \%$ to $55 \%$ (statin from $23 \%$ to $44 \%$ ), of ACE inhibitors/sartans from $44 \%$ to $57 \%$ and of diuretics from $37 \%$ to $41 \%$. Conclusion: Although hospital care data are not yet available in this study, resulting in a partial estimation of the quality of follow-up, this study describes the management of french people with diabetes living in France based on two successive national representative samples and using similar methods. The quality of surveillance and therapeutic practices improved between 2001 and 2007, with a better implementation of the French and European guidelines. However, there are still important gaps between practices and guidelines concerning the measurements of $\mathrm{HbAlc}$, microalbuminuria and fundus examinations. This study will be completed with data on outcomes obtained from self administred questionnaire surveys sent to patients and physicians.

\section{4}

Clinical and patient reported outcomes in 5 European countries at 6 months following insulin initiation

A. Simpson ${ }^{1}$, C. Nicolay ${ }^{2}$, A. Goday ${ }^{3}$, H. Smih ${ }^{4}$, M.A. Charles ${ }^{5}$, A. Liebl ${ }^{6}$, M. Benroubi ${ }^{7}$, S. Jones ${ }^{8}$, A. Tynan ${ }^{4}$

${ }^{1}$ EU Medical, Eli Lilly, Surrey, United Kingdom, ${ }^{2}$ EU Medical, Eli Lilly, Bad Homburg, Germany, ${ }^{3}$ Endocrinology, Hospital Del Mar, Barcelona, Spain, ${ }^{4}$ Health Outcomes, Eli Lilly, Surrey, United Kingdom, ${ }^{5}$ Dept de Recherche, INSERM, Paris, France, ${ }^{6}$ Centre for Diabetes and Metabolism, Fachklinik Bad Heilbrunn, Germany, ${ }^{7}$ Diabetes Centre, General Hospital, Athens, Greece, ${ }^{8}$ The Academic Centre, James Cook University Hospital, Middlesborough, United Kingdom

Background and aims: INSTIGATE is a prospective observational study in 5 European countries (France, Germany, Greece, Spain, UK) investigating patients with type 2 diabetes who initiate insulin during usual care.

Materials and methods: Data on medication, clinical outcomes, health related quality of life (HRQoL) and resource use are collected when patients initiate insulin at baseline (BL) and at regular intervals for 24 months (m). This abstract describes observed clinical and patient reported outcomes in the $6 \mathrm{~m}$ following insulin initiation.

Results: Data were collected from 1153 patients at BL and 1051 patients at $6 \mathrm{~m}$. Patients initiated the following regimens; basal only 537 (46.6\%), mixtures $301(26.1 \%)$, short acting $168(14.6 \%)$, basal bolus $123(10.7 \%)$ and other regimens $22(1.9 \%)$. In Germany, more patients initiated intensive regimens; $49 \%$ started short acting and $23 \%$ basal bolus regimens. In other countries the majority of patients started either a basal only regimen (56\%) or a mixture (31\%). At $6 \mathrm{~m} 107$ patients (10.2\%) had changed regimen.

In all countries mean $\mathrm{HbAlc}$ was lower at $6 \mathrm{~m}$ however, with the exception of Germany, the majority of patients still had a HbAlc level higher than the EASD/ADA target.

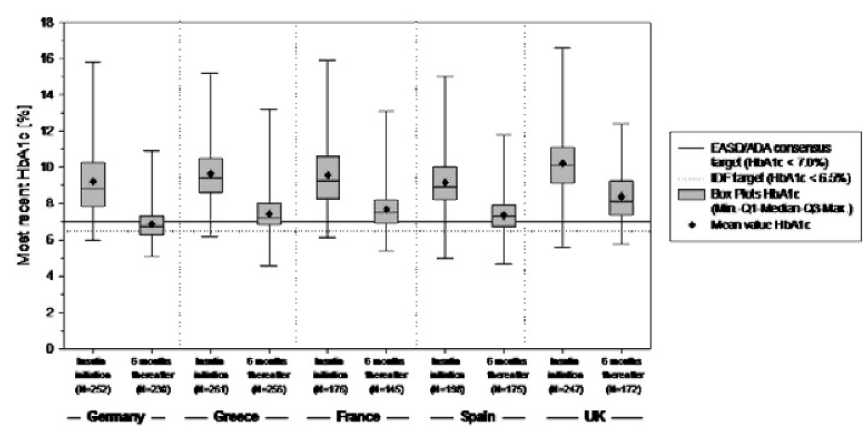

Figure 1. Most recent HbAlc (\%) - distribution by country

Changes were observed in Fasting Blood Glucose (FBG); from an overall mean of $12.2 \mathrm{mmol} / \mathrm{l}$ at $\mathrm{BL}$ to $7.8 \mathrm{mmol} / \mathrm{l}$ at $6 \mathrm{~m}$, the largest difference in mean FBG being in Greece (12.9 to $7.8 \mathrm{mmol} / \mathrm{l})$ and the smallest in the UK (13.2 to $10.2 \mathrm{mmol} / \mathrm{l}$ ). There were also changes in other clinical outcomes (table 1 ). The number of reported episodes of hypoglycaemia increased; a total of 215 were reported in the $3 \mathrm{~m}$ prior to $\mathrm{BL}$ and 737 were reported in the $3 \mathrm{~m}$ prior to the $6 \mathrm{~m}$ visit. 
Table 1: Other clinical outcomes - all countries

\begin{tabular}{llllll}
\hline & \multicolumn{2}{l}{ Baseline } & & \multicolumn{3}{l}{6 months } \\
\cline { 2 - 3 } & mean & SD & & mean & SD \\
\hline Fasting blood glucose $(\mathrm{mmol} / \mathrm{l})$ & 12.2 & 4.32 & & 7.8 & 2.47 \\
\hline Systolic blood pressure $(\mathrm{mm} \mathrm{Hg})$ & 137.1 & 16.90 & & 133.3 & 15.20 \\
\hline Diastolic blood pressure $(\mathrm{mm} \mathrm{Hg})$ & 79.3 & 10.44 & & 78.4 & 15.20 \\
\hline HDL $(\mathrm{mmol} / \mathrm{l})$ & 1.3 & 0.40 & 1.3 & 0.35 \\
\hline LDL $(\mathrm{mmol} / \mathrm{l})$ & 2.9 & 1.04 & 2.7 & 0.86 \\
\hline Total cholesterol $(\mathrm{mmol} / \mathrm{l})$ & 5.1 & 1.23 & 4.7 & 0.98 \\
\hline Triglycerides $(\mathrm{mmol} / \mathrm{l})^{*}$ & 1.9 & $1.3-2.7$ & 1.5 & $1.1-2.0$ \\
\hline
\end{tabular}

${ }^{*}$ figures reported are median and inter-quartile range as per standard reporting practice

Using the Euroquol VAS score, individual patient reported HRQoL improved by a median of 5 points (population median scores were; 62 at BL and 75 at $6 \mathrm{~m})$. Median intra patient changes were higher in Germany and Greece (10 points) and lowest in France (0 points).

Conclusion: The observations indicate improvements in metabolic control following insulin initiation. HRQoL was also seen to improve, in spite of the additional demands associated with injectable therapy.

Supported by: Eli Lilly and Amylin Pharmaceuticals

\section{5}

Glycaemic and blood pressure control seems harder to improve than lipid control in type 2 diabetic patients: comparison of two surveys over 5 years in Belgium

N. Paquot ${ }^{1}$, H. Vandenberghe ${ }^{2}$, A.J. Scheen ${ }^{3}$, C.E. De Block ${ }^{4}$, L.F. Van Gaal ${ }^{4}$ ${ }^{1}$ Diabetes, University of Liege, ${ }^{2} \mathrm{WPO}$, Pfizer WPO Belgium, Brussels,

${ }^{3}$ Diabetes, University of Liège, ${ }^{4}$ Diabetology, Antwerp University Hospital, Belgium

Background and aims: In order to tackle cardiovascular risk factors and prevent complications in type 2 diabetes (T2DM), European guidelines have set clear goals and recommendations for treatment in terms of glycaemic, blood pressure (BP) and lipid control. The purpose of this repeated survey over five years was to investigate the evolution in the extent to which T2DM patients received treatment and achieved the goals for glycated haemoglobin (HbAlc), total cholesterol, LDL (low density lipoprotein) cholesterol and BP as recommended by the European Task Force (ETF) for cardiovascular disease prevention.

Material and methods: A non-interventional, cross-sectional survey (OCAPI [Optimizing Cardiovascular Prevention in Diabetes]) was conducted in 2001 and repeated in 2006, which enrolled T2DM patients above 40 years $(952$ and 1108 , respectively) at endocrinology departments spread throughout Belgium. Data was collected based on information present in the medical record of T2DM patients visiting the physician consecutively over a 2-month period. The first survey applied the goals of the second ETF (goals were respectively $6.5 \%, 190 \mathrm{mg} / \mathrm{dL}, 115 \mathrm{mg} / \mathrm{dL}$ and $130 / 85 \mathrm{mmHg}$ for $\mathrm{HbAlc}$, total and LDL-cholesterol and BP); the second survey these of the third ETF (6.1\%, $175 \mathrm{mg} / \mathrm{dL}, 100 \mathrm{mg} / \mathrm{dL}$ and 130/80 mmHg). Still, for HbAlc we assessed the achievement of a more realistic target of $<7 \%$ (as proposed by the American Diabetes Association) in both surveys.

Results: Insulin was used more widely in 2006 (74\% of T2DM patients) compared to 2001 ( $46 \%$; $<<0.001)$, as were biguanides ( $58 \%$ vs $47 \%$; $\mathrm{p}<0.001)$. Sulfonylureas were less used $(17 \%$ vs $43 \%$; $<<0.001)$ and the proportion of glitazone users remained low in 2006 (4\%; not measured in 2001). Over five years, more patients received antihypertensive treatment (79\% vs $65 \%$; $\mathrm{p}<0.001$ ) and the use of statins had doubled ( $63 \%$ vs $29 \%$; $<<0.001$ ). Although a higher proportion of T2DM patients reached the ETF goals for total cholesterol ( $52 \%$ in 2006 vs $29 \%$ in 2001; p $<0.001)$ and LDL cholesterol $(56 \%$ vs $43 \%$; $\mathrm{p}<0.001$ ) - even with more stringent goals in 2006 -, there was no similar improvement in BP ( $21 \%$ versus $19 \% ; \mathrm{p}=0.472)$, more particularly because persistence of high systolic BP levels in numerous T2DM patients. Glycaemic control even deteriorated (37\% vs $59 \%$ below the HbA1c level of $7 \% ; \mathrm{p}<0.001$ ) when the two surveys are compared, despite apparently more aggressive glucose-lowering therapy. The latter observation might be related to longer duration of diabetes ( 13 years vs 9 years; $p<0.001$ ) and higher body mass index $\left(32 \mathrm{~kg} / \mathrm{m}^{2}\right.$ vs $\left.29 \mathrm{~kg} / \mathrm{m}^{2} ; \mathrm{p}<0.001\right)$ of the population in the second survey.
Conclusion: In a repeated cross-sectional survey over a 5 year period (20012006) in Belgium, HbA1c and systolic BP levels remained difficult to control in T2DM patients and the percentage of patients at goal did not increase despite more intensive pharmacological therapy - whereas lipid goal achievement markedly improved thanks to a greater use of statins. Although partially explained by the demographic characteristics (more advanced diabetes and more overweight in the second survey), the apparent decline in glycaemic control needs further investigation and stresses the fact that even more stringent treatment is necessary to meet the goals set by the guidelines.

\section{6}

Diabetes care practice variation among outpatient diabetes clinics: an urgent need for quality improvement initiatives

A. Nicolucci ${ }^{1}$, M.C.E. Rossi ${ }^{1}$, A. Arcangeli ${ }^{2}$, A. Cimino ${ }^{3}$, G. De Bigontina ${ }^{4}$, C. Giorda ${ }^{5}$, I. Meloncelli ${ }^{6}$, I. Otranto ${ }^{1}$, F. Pellegrini ${ }^{1}$, B. Pomili ${ }^{1}$, U. Valentini ${ }^{3}$, G. Vespasiani ${ }^{6}$

${ }^{1}$ Department of Clinical Pharmacology and Epidemiology, Consorzio Mario Negri Sud, S. Maria Imbaro (CH), ${ }^{2}$ A.S. Diabetes and Metabolic Diseases, AUSL 4, Prato, ${ }^{3}$ Diabetes Unit, A.O. Spedali Civili, Brescia, ${ }^{4}$ Diabetes Unit, Cadore Hospital, Dolomiti-Belluno, ${ }^{5}$ Diabetes and Metabolism Unit, ASL 8, Chieri (TO), ${ }^{6}$ Diabetes Unit, Madonna del Soccorso Hospital, S. Benedetto del Tronto (AP), Italy

Background and aims: Practice variation is generally considered as an important source of inappropriate care as well as of resource consumption. The Associazione Medici Diabetologi promoted a study to evaluate the extent to which process and intermediate outcome indicators varied among diabetes clinics throughout Italy.

Materials and methods: Eighty-six clinics were involved, all using electronic medical record systems. Process and intermediate outcomes indicators were identified and a software was developed, enabling the extraction of the information needed for the profiling of quality of care. Data were centrally analyzed anonymously. Between centers variability was investigated using multilevel models adjusted for patient age, gender, and clustering effect.

Results: Overall, 114,249 patients with T2DM seen during 2004 were evaluated. Between centers variability in the median proportion of patients with at least one value registered during the year (process measures) and variation in the percentage of patients reaching specific therapeutic targets (outcome measures) is shown in the table.

Rates of use of specific drug classes also markedly varied among centers: statins were used on average in $22 \%$ of the patients, but such a proportion ranged between $7 \%$ and $50 \%$ in the different centers. On the same line, ACE-inhibitors were prescribed on average to $21 \%$ of the patients, but the prescription rate ranged between $7 \%$ and $40 \%$.

Conclusion: The high level of variability in process and outcomes of diabetes care calls for intensive efforts devoted to enhance the delivery of care. Ensuring more homogeneous levels of care through a wider adoption of existing evidence should represent a priority to reduce the clinical and economical burden of diabetes. Ongoing activities in Italy are specifically devoted to reduce between centers variability by providing reference standards identified on the basis of the "best performers" approach.

Between centers variability

\begin{tabular}{llll}
\hline $\begin{array}{l}\text { Process indicators: } \\
\text { \% of patients with at } \\
\text { least one measure of }\end{array}$ & $\begin{array}{l}\text { Median } \\
{\left[\mathbf{1 0}^{\text {th }}-\mathbf{9 0}^{\text {th }}\right.} \\
\text { percentile }]\end{array}$ & $\begin{array}{l}\text { Outcome } \\
\text { indicators: \% of } \\
\text { patients with }\end{array}$ & $\begin{array}{l}\text { Median } \\
{\left[\mathbf{1 0}^{\text {th }}-\mathbf{9 0}^{\text {th }}\right.} \\
\text { percentile }]\end{array}$ \\
\hline HbA1c & 93.2 & HbA1c $\leq 7 \%$ & 40.7 \\
& {$[64.8-96.9]$} & & {$[21.0-59.3]$} \\
\hline Blood pressure & 91.1 & Blood & 35.2 \\
& {$[18.4-98.1]$} & $\begin{array}{l}\text { pressure } \leq 130 / 85 \\
\text { mmHg }\end{array}$ & {$[20.5-50.7]$} \\
\hline Lipid profile & 66.6 & LDL-C $<100 \mathrm{mg} / \mathrm{dl}$ & 30.1 \\
& {$[14.4-89.7]$} & & {$[22.9-37.3]$} \\
\hline Microalbuminuria & 37.4 & & \\
\hline
\end{tabular}




\section{OP 27 Microangiopathy: glucose toxicity and inflammation}

\section{7}

High glucose-conditioned medium from endothelial cells enhances apoptosis in human retinal pericytes

E. Berrone, E. Beltramo, S. Tarallo, M. Porta

Internal Medicine, University of Turin, Italy

Background and aims: In diabetic retinopathy, pericyte survival is dependent, among other factors, on interactions with endothelial cells (EC), but little is known about the activation of pericyte apoptosis by EC in the presence of elevated glucose concentrations. We previously reported that human retinal pericytes (HRP) seem to be not affected by exposure to constant high glucose concentrations, while their apoptosis is strongly enhanced by culture on matrix produced by EC in high glucose. The purpose of this study was to evaluate HRP response to soluble factors released by EC in the medium in the presence of physiological or high glucose concentrations.

Materials and methods: Human umbilical vein EC were cultured in physiological (5.6 mM, G5.6) or high (28 mM, G28) glucose for 7 days; media from last 2 days of culture were collected and filtered (EC-CM). HRP were then cultured for 7 days in normal or high glucose only, or in normal glucose with the addition of 25 or $50 \%$ EC-CM. DNA fragmentation was measured by ELISA to assess cell apoptosis. In parallel, the expression of two genes involved in apoptosis, bax and $b c l-2$, was determined by RT-PCR. HRP morphology in the various conditions was evaluated by phase-contrast microscopy.

Results: Apoptosis increased significantly in the presence of 50\% EC-CM produced in high glucose $(1.584 \pm 0.293$ vs $0.202 \pm 0.104$ abs of controls in physiological glucose only, $\mathrm{p}=0.005)$, but not in high glucose only $(0.164 \pm$ $0.065, \mathrm{p}=\mathrm{NS}$ vs control), nor with EC-CM produced in normal glucose $(25 \%$ G5.6 EC-CM: $0.292 \pm 0.429$, p=NS vs control; 50\% G5.6 EC-CM: $0.112 \pm$ $0.063, \mathrm{p}=\mathrm{NS}$ vs control). As regards mRNA expression, there was a clear overexpression of $\mathrm{Bax}$ in the presence of $50 \% \mathrm{EC}-\mathrm{CM}$ produced in high glucose only ( $241.5 \pm 47.7 \%$ of G5.6, $\mathrm{p}=0.01)$. Bcl- 2 mRNA was again unchanged in all conditions, except in HRP cultured in 25-50\% EC-CM produced in high glucose, where there was an evident reduction (25\% G28 EC-CM: $72.8 \pm 1.7 \%$ of G5.6 p $=0.000 ; 50 \%$ G28 EC-CM: $60.8 \pm 7.1 \%$ of G5.6 $p=0.002)$. Finally, cell morphology showed modifications in the presence of $50 \%$ EC-CM produced in high glucose only, in particular the typical apoptotic aspect with cell shrinkage, cytosolic vacuoles and nucleus condensation.

Conclusion: Conditioned medium from EC cultured in high glucose increases significantly HRP apoptosis and Bax expression, while reducing bcl2. Instead, conditioned medium from EC cultured in normal glucose does not influence HRP apoptosis. This suggests that soluble factors derived from EC are likely to play a critical role in the apoptotic response of HRP. Further elucidations about the effects of combined hyperglycaemia and EC-HRP interactions could be useful to investigate the pathogenesis of diabetic retinopathy.

\section{8}

High glucose concentration leads to differential expression of tight junction proteins in human retinal pigment epithelial cells M. Villarroel, J. Matesanz, M. García, C. Hernández, R. Simó Diabetes and Metabolism Research Unit, Institut de Recerca Hospital Universitari Vall d'Hebron, Barcelona, Spain

Background and aims: The breakdown of the blood-retinal barrier (BRB) due to the disruption of the tight junctions is one of the early features of diabetic retinopathy. In fact, there are two BRBs: the internal BRB, which is constituted by the endothelial cell tight junctions in the capillary bed, and the external BRB which is constituted by the tight junctions located in retinal pigment epithelial (RPE) cells. Whereas the impairment of the proteins involved in the disruption of the tight junctions of the internal BRB have been extensively studied, the information regarding this issue in RPE is scanty and there are no studies in which the expression of main tight junction proteins has been assessed simultaneously. The aim of the study is to determine the change of expression (mRNA and protein) of occludin, zonula ocludens-1 (ZO-1) and claudin-1 in RPE cells using two different glucose concentrations: $5.5 \mathrm{mM}$ (mimicking euglycemia) and $25 \mathrm{mM}$ (mimicking hyperglycemia).

Materials and methods: ARPE-19, a spontaneously immortalized human $\mathrm{RPE}$ cell line, was cultured at $37^{\circ} \mathrm{C}$ under $5 \% \mathrm{CO}_{2}$ in medium (DMEM/F12) supplemented with $10 \%$ fetal calf serum containing $5.5 \mathrm{mM}$ D-glucose or 25 $\mathrm{mM} D$-glucose. The medium was changed every 3 days. Cells were cultured for 7, 14 and 21 days. Occludin (OCLN), zonula occludens (ZO-1, TJP1) and claudin-1 (CLDN1) production was studied in Western blot (primary antibodies from Zymed Lab, 1:1000). Bands were transformed to densitometric values in a calibrated densitometer (GS-800 BIO-RAD) and normalized by beta-actin values. Real-Time PCR RQ values were obtained in ABI Prism 7000 SDS software (Applied Biosystems) using TaqMan assays for OCLN Hs00170162_m1, TJP1 Hs00268480_m1 and CLN1 Hs00221623_m1, and beta-actin was used as the endogenous reference gene (Hs9999903_m1, Applied Byosystems). All the measurements were performed at 7, 14 days and 21 days. In the same experimental conditions, cytoxicity (LDH, Roche Applied Science) was assessed. All the results are expressed in arbitrary units after correction for beta-actin.

Results: All the tight junction proteins evaluated reached the maximum concentration at day 14. Occludin mRNA expression and protein content was similar in 5.5 and $25 \mathrm{mM}$ of D-glucose at 7, 14 and 21 days. ZO-1 mRNA levels were similar in cultures maintained in $25 \mathrm{mM}$ of D-glucose to those with $5.5 \mathrm{mM}$, but a decrease in protein content was observed in $25 \mathrm{mM}$ at 14 ( 0.53 vs. 0.27$)$ and 21 days ( 0.28 vs. 0.09). By contrast high glucose concentration $(25 \mathrm{mM})$ produced a clear upregulation in claudin-1 mRNA expression and protein content at day 14 (mRNA levels: 1.16 vs.1.49. Protein content: 0.14 vs. 0.28 ) that persisted at 21 (mRNA levels: 1.03 vs. 2.29. Protein content: 0.92 vs. 1.14).

Conclusion: High glucose concentration leads to differential expression of tight junction proteins in human RPE cells. The functional consequences as well as the clinical applicability of these findings require further investigation.

\section{9}

Possible involvement of cortisol-induced MR activation in diabetic nephropathy - effect of high glucose on cortisol production in human mesangial cells

Y. Matsuzawa, J. Saito, H. Ito, M. Omura, T. Nishikawa

Medicine, Yokoham Rosai Hospital, Yokohama, Japan

Background and aims: We had already reported that human mesangial cells (Msg) had the ability to produce aldosterone (Aldo), similarly to the adrenal gland and that the addition of high glucose and/or LDL to the incubation mixture enhanced Aldo production, suggesting the involvement of local Aldo production in the progression of nephropathy via autocrine/paracrine action on Msg. On the other hand, there were recent studies reporting that the nuclear receptor LXR plays a central role in sugar- or lipid-induced metabolic changes, directly regulating the cortisol (F)-metabolizing enzymes 11ßHSD1 and $11 \beta \mathrm{HSD}-2$. Therefore, we examined the regulation of F production in Msg and the involvement of LXR.

Materials and methods: We cultured Msg (BioWhittaker Inc.) in MCDB131 medium, determined the levels of LXR $\alpha$, LXR $\beta, 11 \beta \mathrm{HSD}-1$, and $11 \beta \mathrm{HSD}$ 2 mRNA expression by quantitative RT-PCR, and examined the effects of culture conditions, such as the LDL concentration, glucose concentration, and presence or absence of stimulation with angiotensin II (AII), on the levels of each mRNA expression. We also examined the effect of the transfection of an siRNA against LXR $\beta$ on $11 \beta \mathrm{HSD}-1$ and $11 \beta \mathrm{HSD}-2$ mRNA expression.

Results: 1) Stimulation with AII reduced LXR $\alpha$ and LXR $\beta$ mRNA expression. 2) In low-glucose $(5.5 \mathrm{mM})$ medium, the level of $11 \beta \mathrm{HSD}-2 \mathrm{mRNA}$ expression was 5 to 6 times higher than that of 11ßHSD-1 mRNA expression. In the presence of LDL, stimulation with AII slightly enhanced 11ßHSD-1 and markedly reduced 11ßHSD-2 mRNA expressions. 3) The level of 11ßHSD-2 mRNA expression was lower in high- $(25 \mathrm{mM})$ than in low-glucose medium. The level of $11 \beta$ HSD-1 mRNA expression in high- was similar to that in lowglucose medium, but markedly increased at high glucose and LDL levels under AII stimulation. 4) Transfection with a siRNA against LXR $\beta$ to inhibit its function enhanced $11 \beta \mathrm{HSD}-1$ and reduced $11 \beta \mathrm{HSD}-2$ mRNA expressions. Conclusion: We first confirmed the presence of LXR, 11ßHSD-1, and $11 \beta \mathrm{HSD}-2$ in human Msg, and showed that F production and its metabolic regulation occurred locally in Msg. In Msg, AII stimulation enhanced $11 \beta \mathrm{HSD}-1$ and reduced $11 \beta \mathrm{HSD}-2 \mathrm{mRNA}$ expressions. The results of the experiment with siRNA suggest that the expression of these enzymes is regulated through changes in LXR induced by AII, and that locally increased F seems to bind directly to the mineralocorticoid receptor (MR) like as binding of aldosterone to MR, particularly in the presence of high concentrations of glucose. 


\section{0}

Interaction between the renin-angiotensin system (RAS) and advanced glycation end products (AGES) in the development of diabetic nephropathy

K.C. Sourris, A.L. Mibus, A. Koitka, B.E. Harcourt, A.L.Y. Tan,

M.E. Cooper, J.M. Forbes

JDRF Albert Einstein Centre for Diabetes Complications, Baker Heart

Research Institute, Melbourne, Australia

Background and aims: The RAS influences local hemodynamics in the diabetic kidney, acting through the binding of the hormone angiotensin II (Ang II) to two receptor subtypes, AT1 and AT2. In addition, hyperglycaemia facilitates the irreversible modification of proteins by sugars, resulting in advanced glycation end-products (AGEs), which are known metabolic contributors to diabetic nephropathy (DN). Therefore, the aim of this study was to elucidate the renal interactions between the RAS and AGE pathways once thought to be separate mediators of progressive DN.

Materials and methods: We investigated specific opposing interactions between the RAS and AGEs both in-vivo and in-vitro. In-vitro, primary mesangial cells were chronically treated for 7 days in high glucose $(25 \mathrm{mM}$ with $10^{-7} \mathrm{M}$ Angiotensin II (Ang II) or 100ug/mL AGE-modified BSA (AGE-BSA). Some groups of cells also were concomitantly administered AT1 (Valsartan, $10^{-7} \mathrm{M}$ ) or AT2 receptor (PD123312, $10^{-6} \mathrm{M}$ ) antagonists. Gene expression by real-time PCR, nuclear factor- $\mathrm{\kappa B}$ activation (NF- $\mathrm{\kappa B}$ ) by DNA binding assay and reactive oxygen species (ROS) generation by flow cytometric analysis were determined. In vivo, mice deficient in either angiotensin receptor-1a (AT-1a) or the receptor for AGEs (RAGE) and their background strains; AT1-WT and C57BL/ 6 respectively ( $\mathrm{n}=10$ per group) were studied with and without streptozotocin induced diabetes $(60 \mathrm{mg} / \mathrm{kg})$. At 24 weeks, peripheral blood and renal tissues were collected for analysis. In vivo, gene expression by real-time PCR, nuclear factor- $\mathrm{\kappa B}$ activation (NF- $\mathrm{\kappa B}$ ) by DNA binding assay and ROS generation by chemiluminescence assay were determined .

Results: Ang II exposure increased the AT1, AT2 and RAGE receptors when compared to control treated cells with a decline in expression for AT1 and AT2 exhibited in the presence of their respective inhibitors. AGE-BSA exposure also increased expression of the AT1 receptor and RAGE, however, a decrease in AT2 receptor was observed when compared to BSA alone. Furthermore, gene expression of AT1 was not altered by either AT1 or AT2 inhibition, although AGE-induced up-regulation of RAGE was ameliorated by Valsartan. NF- $\mathrm{kB}$ activity was significantly increased by treatment with AGEs but not Ang II. Importantly, this was significantly reduced in the presence of AT1 and AT2 inhibitors $(\mathrm{p}<0.05)$. Furthermore, AGE treatment of mesangial cells induced a significant increase in ROS $(p<0.05)$ relative to the control and this was significantly reduced in the presence of AT2 inhibitor $(\mathrm{p}<0.05)$. In-vivo, gene expression of both AT1 and AT2 receptors and RAGE were elevated in the kidneys from diabetic control mice, which was not seen in diabetic RAGE-/- mice. In addition an increase in RAGE gene expression was detected in the kidneys of diabetic AT-1a -/- and AT-WT groups. . Renal nuclear NF- $\mathrm{KB}$ activity was reduced with diabetes in C57BL/6 and RAGE/- mice whilst it remained elevated in diabetic AT-WT and AT1-a-/- mice. Diabetes induced an increase in renal ROS production which was not seen in RAGE-/- mice.

Conclusion: We conclude that there are interactions between the RAS and advanced glycation in the development of diabetic nephropathy. Importantly, we postulate that RAGE appears to be a common and key modulator of RAS mediators which each contribute to the development of diabetic nephropathy.

Supported by: JDRF

\section{1}

Effects of different LDL particles on inflammatory molecules in human mesangial cells

A. Solini ${ }^{1}$, E. Santini ${ }^{1}$, S. Baldi ${ }^{1}$, R. Lupi ${ }^{2}$, S. Madec ${ }^{1}$, D. Chimenti ${ }^{1}$,

E. Ferrannini ${ }^{1}$

${ }^{1}$ Department of Internal Medicine, ${ }^{2}$ Department of Endocrinology and Nephrology, University of Pisa, Italy

Background and aims: Inflammation is regarded as a key mechanism underlying glomerular damage even in chronic glomerulopathies. LDL may increase the expression of several cytokines in renal tissues, contributing in the development of glomerular disease. However, the relative role of native and glycated LDL in promoting an inflammatory response has been only partially elucidated. This study evaluates the inflammatory and proapoptotic effect of different LDL particles in human mesangial cells (HMC), defining the intracellular signalling steps potentially responsible for these effects.

Materials and methods: HMC were stimulated with 25 and $100 \mu \mathrm{g} / \mathrm{ml} \mathrm{na-}$ tive (nLDL), oxidized (oxLDL) and glycated LDL (glyLDL) for 30, 60, 180 min, and 6 and 24 hrs for apoptosis. IL-6, CD40 and macrophage migration inhibitory factor (MIF) expression was measured by RealTime PCR and immunostaining. Supernatants were analysed for IL-6, soluble CD40 (sCD40L), fibronectin and laminin content by ELISA. Early and late apoptosis were determined by FACS. ERK1/2 and JNK2/3 activation was assessed by WB analysis.

Results: IL- 6 mRNA was rapidly upregulated by all the three species (after 30 min, with 25 and $100 \mu \mathrm{g} / \mathrm{ml}$ nLDL: $7 \pm 0.2$ and $13.4 \pm 0.5$; with 25 and $100 \mu \mathrm{g} /$ ml oxLDL: $8.7 \pm 0.2$ and $16.3 \pm 1.3$; with 25 and $100 \mu \mathrm{g} / \mathrm{ml}$ glyLDL: $7.0 \pm 0.2$ and $17.1 \pm 0.8$ fold above unstimulated cells (unst); all $\mathrm{p}<0.001$ ), and decreased within $180 \mathrm{~min}$. CD40 was upregulated by oxLDL and glyLDL with a good dose-response relationship (after $30 \mathrm{~min}$, with 25 and $100 \mu \mathrm{g} / \mathrm{ml}$ oxLDL: $2.8 \pm 0.4$ and $5.7 \pm 0.6$; with 25 and $100 \mu \mathrm{g} / \mathrm{ml}$ glyLDL: $2.3 \pm 0.3$ and $6.7 \pm 1.1$ fold above unst, both $\mathrm{p}<0.01$ ), whereas nLDL had a slower onset of effect (after $60 \mathrm{~min}$, with 25 and $100 \mu \mathrm{g} / \mathrm{ml} \mathrm{nLDL}: 2.1 \pm 0.6$ and $3.6 \pm 0.6$ fold above unst, $\mathrm{p}<0.01)$. mRNA upregulation was paralleled by an increased release of both IL-6 (after $30 \mathrm{~min}$, from $50 \pm 4$ to $138 \pm 11$ with $\mathrm{nLDL}, 131 \pm 17$ with oxLDL, $160 \pm 12$ with $100 \mu \mathrm{g} / \mathrm{ml}$ glyLDL all $\mathrm{p}<0.001$ ) and sCD40L (after $30 \mathrm{~min}$, from $4.7 \pm 0.6$ to $8.7 \pm 0.6$ with $\mathrm{nLDL}, 15.3 \pm 2.1$ with oxLDL, $19.3 \pm 2.3$ with $100 \mu \mathrm{g} / \mathrm{ml}$ glyLDL, all $\mathrm{p}<0.001)$. MIF mRNA was potently stimulated by glyLDL and, expecially, by oxLDL (after $60 \mathrm{~min}: 15.6 \pm 1.7$ with $100 \mu \mathrm{g} / \mathrm{ml}$ oxLDL and $6.8 \pm 1.1$ fold above unst with $100 \mu \mathrm{g} / \mathrm{ml}$ glyLDL, both $\mathrm{p}<0.001$ ) while $100 \mu \mathrm{g} / \mathrm{ml} \mathrm{nLDL}$ minimally influenced it (1.9 \pm 0.9 fold above unst). Immunostaining for MIF after treatment with oxLDL and glyLDL confirmed the observation. All LDL species activated both pathways; however, while JNK2/3 phosphorylation did not differ among the three, ERK1/2 phosphorylation was more strongly upregulated by oxLDL than either $\mathrm{nLDL}$ or glyLDL. nLDL did not influence fibronectin release (after $60 \mathrm{~min}$ : from $60 \pm 11$ to $66 \pm 8 \mu \mathrm{g} / \mathrm{ml} / 1.5 \times 10^{5} \mathrm{cells}$ ), while oxLDL and glyLDL increased it (to $103 \pm 6$ with oxLDL and $96 \pm 9 \mu \mathrm{g} / \mathrm{ml} /$ $1.5 \times 10^{5}$ cells with $100 \mu \mathrm{g} / \mathrm{ml}$ glyLDL, $\left.\mathrm{p}<0.001\right)$. The same effect was observed on laminin. LDL induced a small increase in apoptosis (unst: $15.32 \pm 0.88 ; 100$ $\mu \mathrm{g} / \mathrm{ml}$ nLDL: $19.60 \pm 1.65$; oxLDL: $24.25 \pm 1.91$; glyLDL: $23.67 \pm 1.47 \%$, p $<0.001$ by ANOVA) only after $24 \mathrm{hrs}$. No effect on late apoptosis was observed for any of the LDL species.

Conclusion: In HMC, expression and release of IL-6 and CD40 is similarly stimulated by both native and modified LDL, while MIF is more strongly stimulated by oxLDL than glyLDL. With regard to the pattern of mesangial expansion, fibronectin and laminin are significantly upregulated by oxLDL and glyLDL. Apoptosis, if modest, is induced by all species.

\section{2}

Involvement of sialoadhesin-positive macrophages in microinflammation in the pathogenesis of diabetic nephropathy N. Kajitani, K. Shikata, R. Nagase, M. Sasaki, S. Miyamoto, R. Kodera, D. Hirota, C. Sato, J. Wada, S. Nishishita, H. Makino

Medicine and Clinical Science, Okayama University Graduate School of Medicine, Dentistry and Pharmaceutical Science, Japan

Background and aims: Low grade inflammation, i.e. microinflammation, is involved in the pathogenesis of diabetic nephropathy. Phenotypic change of mesangial cell is known to play a pivotal role in accumulation of extracellular matrix in diabetic nephropathy, although the state of activation and phenotype of macrophages infiltrated into diabetic kidney are still remained unclear. Sialoadhesin $(\mathrm{Sn})$ is a macrophage-restricted cellular interaction molecule and a member of sialic acid binding immunoglobulin-like lectins. $\mathrm{Sn}$ is expressed on activated macrophage but not on monocyte. There have been several reports that $\mathrm{Sn}$-positive macrophages play a role in development of chronic inflammatory diseases including rheumatoid arthritis and atherosclerosis. The aim of this study is to evaluate the phenotypic change of macrophages in diabetic kidney and to clarify the role of Sn-positive macrophage in the pathogenesis of diabetic nephropathy.

Materials and methods: Diabetes was induced in 4-week old male SD rats $(n=25)$ by injection with streptozotocin (STZ). Non-diabetic control rats were injected with citrate buffer. Insulin-treated group was injected with NPH insulin every day. Kidneys were harvested from diabetic and control rats at 1, 4, 12, 24 weeks after injection with STZ or buffer. Renal tissues were examined by immunostaining with specific antibodies to pan macrophage (ED1), resident macrophage (ED2), Sn-positive macrophage (ED3), and MHC class II 
(OX-6). Expression of $\alpha$-smooth muscle actin (SMA) was investigated as a marker for the activation of mesangial cell. We also examined the expression of TGF- $\beta 1$ and Type IV collagen and histological changes. Moreover, we examined the effects of high-glucose condition or TNF- $\alpha$, IL- 4 and IL- 6 on gene expression of $\mathrm{Sn}$ in cultured human monocytes (THP-1 cells).

Results: ED1 positive macrophages in diabetic glomeruli were significantly increased at 1 week after the induction of diabetes and the cell number was maintained at same level during 24weeks. ED2 positive macrophages in the diabetic glomeruli revealed no significant difference with non-diabetic rats. The expression of Sn-positive macrophages (ED3) increased gradually from 4 weeks after induction of diabetes and revealed highest at 24 weeks. ED1 or ED3 positive cells are also positive for OX-6. Insulin treatment decreased total and Sn-positive macrophages in the glomeruli of diabetic rats to the same level of control rats. Sn-positive macrophages were concomitantly increased with expression of $\alpha$-SMA, TGF- $\beta 1$ and type IV collagen and with expansion of mesangial matrix area. Gene expression of $\mathrm{Sn}$ was significantly increased in THP- 1 cells under the existence of TNF- $\alpha$. Expression of Sn was not changed by IL- 6 and IL-4. High-glucose condition per se did not induce Sn expression in THP-1 cells.

Conclusion: Phenotypic changes were observed in both macrophage and mesangial cell in glomeruli of diabetic rats. Sn-positive macrophages were increased in diabetic glomeruli concomitantly with expression of $\alpha$-SMA and progression of renal injuries. TNF- $\alpha$ might play a role in Sn expression on macrophages. Sn-positive macrophage is known to contribute to development of chronic inflammation. Our data suggest that $\mathrm{Sn}$-positive macrophage may contribute to the development of microinflammation in the pathogenesis of diabetic nephropathy.

Supported by: Ministry of Education, Science, Culture, Sports and Technology of Japan

\section{OP 28 The triumvirate gets fatty}

\section{3}

MicroRNA expression in human adipose tissue

N. Kloting ${ }^{1}$, K. Ruschke ${ }^{1}$, P. Kovacs ${ }^{2}$, M.R. Schön ${ }^{3}$, M. Stumvoll' ${ }^{1}$, M. Bluher ${ }^{1}$ ${ }^{1}$ Medical Clinic III, University of Leipzig, ${ }^{2}$ Interdisciplinary Centre for Clinical Research (IZKF), University of Leipzig, ${ }^{3}$ Department of Surgery, Karlsruhe, Germany

Background and aims: MicroRNAs (miRNAs) are small, noncoding RNAs, that play important regulatory roles in a variety of diverse biological processes, including development, differentiation, apoptosis as well as fat metabolism. In mammals, miRNAs have been shown to modulate adipocyte differentiation and insulin secretion. Therefore, we performed a global miRNA gene expression assay in adipose tissue from different depots of lean and obese individuals.

Materials and methods: Paired samples of visceral and subcutaneous adipose tissue were obtained from 15 Caucasian men $(\mathrm{n}=8)$ and women $(\mathrm{n}$ = 7) who underwent open abdominal surgery. The gene expression of 155 miRNAs was carried out using the TaqMan ${ }^{\circ}$ MicroRNA Assays Human Panel Early Access Kit (Applied Biosystems, Darmstadt, Germany).

Results: In total, we identified $106(68 \%)$ miRNAs out of 155 miRNAs that are expressed in both visceral and subcutaneous fat. We found that $7 \mathrm{miR}-$ NAs significantly differing between men and women and seventeen miRNAs (11\%) were significantly up-regulated in visceral compared to subcutaneous fat. Surprisingly, we found no miRNA which is exclusively expressed in either fat depot. Univariate correlation analyses revealed that several metabolic parameters, including visceral fat area, $\mathrm{HbAlc}$, fasting plasma glucose, Leptin, Adiponectin as well as serum cholesterol are associated with expression levels of microRNA 17-5p, -132, -99a, -134, 181a, -145, and miRNA -197.

Conclusion: In summary, expression of selected miRNAs in human adipose tissue show sexual dimorphism and a fat depot specific expression pattern. In addition miRNA expression in human fat correlates with parameters of glucose homeostasis, obesity and fat distribution. Supported by: Klinische Forschergruppe(KFO-152 MB)

\section{4}

Regional differences in gene expression between subcutaneous and visceral adipose tissue

D. Guoxian

Department of Geriatrics, Nanjing Medical University First affiliated Hospital, Nanjing, China

Background and aims: Obese individuals with most of their fat stored in visceral tissue generally suffer greater adverse metabolic consequences than similarly obese subjects with fat stored predominantly in subcutaneous tissue. Further study was needed to explore differentially expressed genes that might be relevant to the determination of fat distribution and the associated metabolic sequelae in subcutaneous adipose tissue (SAT) and visceral adipose tissue (VAT).

Materials and methods: SAT and VAT were obtained from patients (all Chinese) attending the Department of Surgery for abdominal surgeries. A total of 73 samples were analyzed from patients $(n=39)$ aged from 17 to 78 . All subjects were not treated with any hormones. The study protocol was approved by the local research ethics committee. total RNA was isolated from paired samples of SAT and VAT from 30 adults ( 15 normal (BMI $=22.78 \pm 1.63$ $\left.\mathrm{kg} / \mathrm{m}^{2}\right)$ and 15 obese patients $\left(B M I=28.25 \pm 1.24 \mathrm{~kg} / \mathrm{m}^{2}\right)$ Expression differences between SAT and VAT were assessed using the paired Student's $t$ test and independent-samples $t$ test was used in other two samples comparison. Linear relationships were assessed by least square regression analysis. SPSS11.0 was used as statistical software.

Results: Depot-related gene expression: Adiponectin and monocyte chemoattractant protein-1 (MCP-1) mRNA expression had no difference between SAT and VAT either in normal or obese patients, while leptin mRNA expression, in both groups of subjects, was higher in SAT than in VAT (the values of VAT/SAT were 0.7 and 0.7 in normal and obese subjects respectively). In normal subjects, tumor necrosis factor- $\alpha$ (TNF- $\alpha$ ) was expressed equally both in SAT and VAT. However, in obese subjects, TNF- $a$ was expressed 1.6-fold in VAT compared to SAT. Compared with SAT, toll like receptor 4 (TLR4) expression in VAT was lower in normal patients(VAT/SAT $=0.8$ ); however, TLR4 expression in VAT was higher in obese patients (VAT/SAT=1.2). Glu- 
cocorticoid receptor (GR) mRNA expressed higher in SAT than in VAT in normal patients, while expressed no difference in obese patients. No differences were found between SAT and VAT for peroxisome proliferator-activated receptor- $\gamma$ (PPAR- $\gamma)$ and $11 \beta$-hydroxysteroid dehydrogenate type 1 (11 $\beta$ HSD1) mRNA expression neither in normal nor obese patients. Associations between metabolic and anthropometric parameters and TLR4 mRNA level: There was a positive correlation between plasma glucose concentrations and the TLR4 mRNA expression in VAT $\left(\mathrm{r}^{2}=0.3536, \mathrm{p}=0.0005\right)$. The ratio of VAT and SAT TLR4 mRNA expression correlated positively with BMI $\left(\mathrm{r}^{2}=0.1974\right.$, $\mathrm{p}=0.0139)$. Relationship between TLR4 and GR mRNA level: GR mRNA expression was positively correlating with TLR4 in both SAT $\left(\mathrm{r}^{2}=0.6285\right.$, $\mathrm{p}<0.0005)$ and VAT $\left(\mathrm{r}^{2}=0.2532, \mathrm{p}=0.0054\right)$. Expression of TLR4 and TNF- $\alpha$ mRNA in primary culture of human adipocytes: VAT was obtained from 5 normal subjects for primary culture. TLR4 mRNA was remarkably increased at the end stage of differentiation (1.5-fold, $\mathrm{p}<0.01)$. Treatment of differentiated adipocytes with $500 \mu \mathrm{g} / \mathrm{ml} \mathrm{FFAs}$ for $24 \mathrm{~h}$ significantly induced TNF- $\alpha$ mRNA expression 2.5 -fold $(\mathrm{p}<0.01)$

Conclusion: Depot-specific differences in mRNA levels of TLR4 and TNF- $\alpha$, GR, Leptin were present in people. The increased GR expression and activated TLR4 pathway in human adipose tissue may reflect a causal role in visceral fat accumulation during adult.

Supported by: National Natural Science Foundation of China

\section{5}

The cytokine Interleukin-18 is involved in the regulation of lipogenic gene expression in liver

R. Stienstra, M.G. Netea, A. Hijmans, C.J. Tack

General Internal Medicine, UMC St Radboud University, Nijmegen, Netherlands

Background and aims: The development of obesity leads to increased levels of pro-inflammatory cytokines resulting in a chronic low grade inflammation. Obesity-induced inflammation is associated with metabolic abnormalities including insulin resistance. However, not all pro-inflammatory cytokines unequivocally participate in the development of obesity-induced inflammation. Whereas TNFa induces insulin resistance, interleukin-18 (IL-18) appears to have opposite metabolic effects. While the pro-inflammatory cytokine IL-18 has initially been identified as a potent inducer of interferon gamma production, recent studies have revealed an important role of IL-18 in controlling energy metabolism and improving insulin sensitivity. Animals lacking IL-18 spontaneously developed obesity and insulin resistance and treatment with recombinant IL-18 partly reversed these metabolic abnormalities. Although not much is known about the tissue-specific effects of IL-18 in controlling the development of obesity and insulin resistance, it has been demonstrated that liver expresses has high steady state levels of IL-18. Therefore, the aim of this study was to learn more about the specific metabolic role of IL-18 in liver.

Materials and methods: We performed immunohistochemistry and applied microarray analysis to compare gene expression patterns in the liver from Wild type and IL-18-/- animals. In addition, the metabolic effects of recombinant IL-18 treatment in Wild type mice were studied.

Results: Immunohistochemical analysis revealed that hepatic IL-18 is localized in macrophages and hepatocytes. Microarray analysis to compare hepatic gene expression profiles of Wild type and IL-18 -/- animals uncovered that numerous genes involved in lipogenesis including SCD-1, Fatty Acid Synthase (FAS), ATP citrate lyase (ACLY) and SPOT-14 were significantly elevated in IL-18-/- mice. In line with increased lipogenic gene expression levels in liver, hepatic triglyceride content was elevated in IL-18-/- animals compared to Wild type mice. In addition, plasma analysis showed a significantly increase in plasma triglycerides concentrations in IL-18 -/- animals which were mainly carried in VLDL particles. Interestingly, treatment of Wild type mice with recombinant IL-18 (rIL-18) led to suppression of many lipogenic genes in liver suggesting direct regulation by IL-18. The suppression of lipogenesis by rIL-18 was accompanied by an improvement of insulin sensitivity in Wild type mice.

Conclusion: IL-18 is localized in hepatocytes and Kupffer cells and plays an important role in controlling the hepatic expression of key genes involved in lipogenesis. As such, IL-18 may be an important link between the development of fatty liver and the induction of insulin resistance.

Supported by: Dutch Diabetes Research Foundation

\section{6}

Increased liver fat in type 2 diabetes patients is associated with decreased hepatic glucose uptake and phosphorylation rate: studies using PET and MR spectroscopy

J.W. Smit ${ }^{1}$, L.J. Rijzewijk ${ }^{2}$, R.W. van der Meer ${ }^{3}$, M. Lubberink ${ }^{4}$, J.A. Romijn ${ }^{1}$, J.J. Bax ${ }^{5}$, A. de Roos 3 , A.A. Lammertsma ${ }^{4}$, H.J. Lamb ${ }^{3}$, M. Diamant ${ }^{2}$ ${ }^{1}$ Endocrinology, Leiden University Medical Center, ${ }^{2}$ Diabetescenter, VU University Medical Center, Amsterdam, ${ }^{3}$ Radiology, Leiden University Medical Center, ${ }^{4}$ Nuclear medicine and PET research, VU University Medical Center, Amsterdam, ${ }^{5}$ Cardiology, Leiden University Medical Center, Netherlands

Background and aims: Type 2 diabetes mellitus (T2DM) is characterised by insulin resistance and lipid accumulation in non-adipose tissues, in particular hepatic steatosis. The aim of the present study was to investigate whether the amount of liver fat content, as measured by proton $\left[{ }^{1} \mathrm{H}\right]$-magnetic resonance spectroscopy $\left(\left[{ }^{1} \mathrm{H}\right]\right.$-MRS) influences hepatic glucose uptake and the rate of glucose phosphorylation, assessed by positron-emission tomography (PET) using $\left[{ }^{18} \mathrm{~F}\right]$-fluoro-2-deoxy-glucose $\left[{ }^{18} \mathrm{FDG}\right]$ in males with uncomplicated T2DM and healthy males with normal glucose metabolism.

Materials and methods: Twenty-two men with uncomplicated T2DM treated with sulfonylureum monotherapy (mean \pm SD: age $58 \pm 4 \mathrm{yrs}$; BMI $28 \pm 4 \mathrm{~kg} \cdot \mathrm{m}^{2}$; $\mathrm{HbAlc} 7.1 \pm 0.9 \%)$ and 14 age- and BMI-matched healthy men underwent $\left[{ }^{1} \mathrm{H}\right]-\mathrm{MRS}$ and $\left[{ }^{18} \mathrm{FDG}\right]-\mathrm{PET}$ of the liver, the latter under euglycaemic hyperinsulinaemic clamp conditions to determine whole-body insulin sensitivity, and MR-measurements of visceral fat. T2DM patients were divided in two groups according to median (10.3\%) liver fat content, to create a T2DM-low $(\mathrm{n}=10)$ versus T2DM-high $(\mathrm{n}=12)$ liver fat group. A two tissue compartment model was used to quantify the net ${ }^{18} \mathrm{FDG}$ uptake (hepatic glucose uptake $=$ net ${ }^{18} \mathrm{FDG}$ uptake - plasma glucose) and the rate of hepatic glucose phosphorylation.

Results: Controls had similar hepatic fat content as T2DM-low subjects. Whole-body insulin resistance was highest in T2DM-high, relative to T2DM-low and controls $(0.48 \pm 0.58,0.76 \pm 0.34$ and $1.29 \pm 0.57 \mathrm{mmol} / \mathrm{ml} /$ min.pmol/l respectively; one-way ANOVA $\mathrm{p}=0.001)$. T2DM-high, relative to controls had the lowest hepatic glucose uptake $(17 \pm 70$ versus $33 \pm 15$ $\mathrm{nmol} / \mathrm{ml} / \mathrm{min} ; \mathrm{p}=0.003$ and the lowest rate of hepatic glucose phosphorylation $\left(0.00464 \pm 0.00222\right.$ versus $\left.0.00736 \pm 0.00317 \mathrm{~min}^{-1} ; \mathrm{p}=0.028\right)$. Hepatic glucose uptake was negatively correlated with $\operatorname{HbA}_{1 c}(\mathrm{r}=-0,524, \mathrm{p}=0.001)$, visceral fat content $(\mathrm{r}=-0.548, \mathrm{p}=0.001)$, alanine aminotransferase $(\mathrm{r}=-0.428$, $\mathrm{p}=0.009)$ and non-esterified fatty acid (NEFA) levels during clamp $(\mathrm{r}=-0.518$, $\mathrm{p}=0.001$ ). Similarly, hepatic glucose phosphorylation was inversely associated with $\mathrm{HbA}_{1 \mathrm{c}}(\mathrm{r}=-0,451, \mathrm{p}=0.006)$, visceral fat content $(\mathrm{r}=-0.506, \mathrm{p}=0.002)$ and NEFA levels during clamp $(\mathrm{r}=-0.442, \mathrm{p}=0.008)$. Stepwise multivariate analysis in patients revealed liver fat content and $\mathrm{HbAlc}$ to be independent determinants of hepatic glucose uptake $\left(\mathrm{R}^{2}=0.52, \mathrm{p}<0.001\right)$.

Conclusion: By combining liver $\left[{ }^{1} \mathrm{H}\right]$-MRS and $\left[{ }^{18} \mathrm{FDG}\right]$-PET we were able to show that in patients with uncomplicated and well-controlled T2DM, increased liver fat is associated with impaired hepatic glucose uptake and a decreased glucose phosphorylation rate. Liver fat content appeared the strongest predictor of decreased hepatic glucose uptake.

\section{7}

Differential effects of diets rich in medium- versus long-chain triacylglycerols on muscular lipid and acylcarnitine content do not affect glucose tolerance in rats

J. De Vogel ${ }^{1,2}$, J. Hoeks ${ }^{2}$, S.M. Houten ${ }^{3}$, S. Timmers ${ }^{1,2}$, M.K. Hesselink ${ }^{4}$, P. Schrauwen ${ }^{1,2}$

${ }^{1}$ Nutrition and Health, TI Food \& Nutrition, Wageningen, ${ }^{2}$ Department of Human Biology, NUTRIM, Maastricht University, ${ }^{3}$ Laboratory Genetic Metabolic Diseases, Academic Medical Centre, Amsterdam, ${ }^{4}$ Department of Movement Sciences, NUTRIM, Maastricht University, Netherlands

Background and aims: High-fat long-chain triacylglycerol (HFLCT) diets are associated with obesity, diabetes and insulin resistance, amongst others via accumulation of triacylglycerol and acylcarnitine levels in skeletal muscle. Medium-chain triacylglycerols (MCT) are more readily degraded and absorbed in the small intestine than LCT's and are preferentially $\beta$-oxidized. Therefore, we studied the effects of a HFMCT diet on muscle lipid storage and metabolism, and whole body glucose tolerance.

Materials and methods: During eight weeks we subjected rats to a low fat (10 energy\% from fat) LCT control, a HFLCT or an isocaloric HFMCT diet (47 
energy $\%$ from fat). Whole body glucose tolerance was determined after a $6 \mathrm{hr}$ fast with an intra peritoneal glucose load of $1.5 \mathrm{~g} / \mathrm{kg}$. Muscle triacylglycerol content was analyzed in freeze-dried and dissected muscle fibers using gas chromatography. Furthermore, acylcarnitine profiles were studied as a marker of metabolic overload to muscle mitochondria using mass spectrometry. Results: No differences in body weight gain $(\mathrm{p}=0.946)$ or net energy uptake $(\mathrm{p}=0.15)$ were observed between the diets. Only the HFLCT increased the level of triacylglycerol compared to controls $(2.06 \pm 0.17$ vs $1.36 \pm 0.12$ and $1.49 \pm 0.16 \mu \mathrm{g} / \mathrm{mg}$ freeze dried muscle in HFLCT, control and HFMCT respectively, $\mathrm{p}=0.014 ; \mathrm{n}=7-9$ ). The HFLCT but not the HFMCT diet increased the total amount of skeletal muscle long-chain acylcarnitines (C14:0, C16:0, C18:0, C18:1, C20:1) compared with the control diet $(\mathrm{p}<0.05)$. Whole body glucose tolerance was not significantly changed after either high fat diet compared with control $(802 \pm 40,772 \pm 18$ and $886 \pm 18$ AUC, control, HFLCT and HFMCT, respectively, $\mathrm{n}=5-6$ ).

Conclusion: A diet rich in LCT, in contrast to MCT, increases intramyocellular lipid and acylcarnitine content compared to a low fat control diet. Despite these marked differences in muscular fat content, however, whole body glucose tolerance was similar between diets. This might suggest that accumulation of triacylglycerol and acylcarnitine in muscle not necessarily leads to whole body glucose intolerance.

Supported by: TI Food and Nutrition, an alliance of major Dutch food industries, TNO Quality of Life, Zeist, Maastricht University, Wageningen University and Research Centre, Dutch government.

\section{8}

Evaluation of intramyocellular and subsarcolemmal mitochondrial structure and function in type 2 diabetes

E. Phielix ${ }^{1}$, P. Schrauwen ${ }^{1}$, E.M. Kooi ${ }^{2}$, V.B. Schrauwen-Hinderling ${ }^{1,2}$, M.K.C. Hesselink ${ }^{3}$

${ }^{1}$ Human Biology, ${ }^{2}$ Radiology, ${ }^{3}$ Human Movement Sciences, Maastricht University, Netherlands

Background and aims: Mitochondrial dysfunction has been implicated in the development of type 2 diabetes mellitus (T2DM). Here we examined mitochondrial content, size and density in relation to in vivo mitochondrial function in T2DM patients, first-degree relatives (FDR) of diabetic patients, and control subjects.

Materials and methods: Nine T2DM patients, 11 FDR and 8 control subjects were included, all matched for age and BMI (age (y): $62.4 \pm 1.4,60.4 \pm 0.9$ and $60.0 \pm 0.7$; BMI (kg/m2): $28.8 \pm 0.8,29.9 \pm 1.2$ and $27.2 \pm 0.5$; respectively). Insulin sensitivity was determined with a hyperinsulinemic-euglycemic clamp combined with indirect calorimetry. In vivo mitochondrial function was determined as rate constant $(\mathrm{k})$ of phosphocreatine recovery post-exercise using 31phosphorus magnetic resonance spectroscopy (31P-MRS). We analyzed intramyofibrillar (IMF)- and subsarcolemmal (SS) mitochondrial size (mean mitochondrial area $(\mu \mathrm{m} 2)$ ), content (number of mitochondria per cell area $(\# / \mu \mathrm{m} 2)$ ) and density (mitochondrial size relative to myofibrillar area for IMF mitochondria and subsarcolemmal area for SS mitochondria (\%)) by electron microscopy in muscle tissue from the $\mathrm{m}$. vastus lateralis.

Results: Insulin sensitivity was lower in T2DM compared to control subjects (insulin-stimulated Rd: $9.2 \pm 2.2$ v.s. $25.0 \pm 3.0$; $\mathrm{p}<0.05$ ), with intermediate values for FDR $(17.1 \pm 2.7, \mathrm{~ns})$. In vivo mitochondrial function was reduced in T2DM patients and FDR compared to control subjects $(0.031 \pm 0.003$, $0.032 \pm 0.002$ vs. $0.045 \pm 0.005 \mathrm{~s}-1 ; \mathrm{p}<0.05$; respectively). Between groups, no differences were found for IMF and SS mitochondrial size, content and density. However, SS mitochondrial content seemed to be lower in T2DM compared to control subjects $(1.3 \pm 0.2$ vs. $2.3 \pm 0.3 \# / \mu \mathrm{m} 2$; $\mathrm{p}=0.13)$, with intermediate values for FDR $(1.8 \pm 0.3 \# / \mu \mathrm{m} 2)$, but these differences did not reach statistical significance. Joint examination of all three groups revealed that in vivo mitochondrial function correlated positively with SS mitochondrial content (Spearman's rho: $0.5 ; \mathrm{p}<0.05)$. IMF mitochondrial density correlated positively with basal fat oxidation $(\mu \mathrm{mol} / \mathrm{kg} / \mathrm{min})$ (Spearman's rho: $0.5 ; \mathrm{p}<0.05)$.

Conclusion: From these preliminary results no significant differences were found for IMF and SS mitochondrial size, content and density between groups. Additional subjects are currently enrolled to investigate if SS mitochondrial content may underlie reduced in vivo mitochondrial function observed in type 2 diabetes.

Supported by: Dutch Diabetes Research Foundation

\section{OP 29 Glucagon and effects of other hormones}

\section{9}

A study of glucagon secreting cells by a novel whole pancreas tissue slice approach: NADH desensitization of $\alpha$ cell KATP channel contributes to glucose-inhibited glucagon release

Y.-C. Huang ${ }^{1,2}$, M. Rupnik ${ }^{2}$, H.Y. Gaisano ${ }^{1}$

${ }^{1}$ Medicine, University of Toronto, Canada, ${ }^{2}$ Medical faculty, Institute of Physiology, University of Maribor, Slovenia

Background and aims: Glucagon is released from islet $\alpha$ cells at low blood glucose while insulin is released from $\beta$ cells when glucose levels rise. In $\beta$ cells, ATP-sensitive potassium (KATP) channels act as the major link between glucose metabolism and insulin secretion. Given that $a$ cells also express KATP channels, it remains elusive why glucagon secretion in a cells ceases when glucose metabolism is at the highest. As anaerobic glycolysis is more essential in a cells compared to $\beta$ cells, we tested the hypothesis that intermediates derived from glucose metabolism such as NADH could modulate KATP channel sensitivity to ATP.

Materials and methods: We have surmounted the a cell culture limitations by employing the whole pancreas slice preparation, a technique that preserves the islet architecture and cell-to-cell communication. We optimized whole cell patch clamp approach to readily distinguish a cells, thereby boosting the a cell yield. Statistics is shown as mean \pm s.d., $n$ indicates the number of $\alpha$ cells tested.

Results: $\alpha$ cells within tissue slices displayed apparent A-type K+ current and $\mathrm{Na}+$ current which were previously found to be diminished or even completely absent in the conventional dispersed a cells system. Voltage-activated $\mathrm{Na}+$ channel half inactivation potential was measured to be $-41.4 \pm 7.8 \mathrm{mV}$ $(\mathrm{n}=31)$, consistent with previous studies, and the $\mathrm{Na}+$ current amplitude in a cells yielded $287 \pm 117 \mathrm{pA}(\mathrm{n}=31)$. a cell sizes were distributed at $3.9 \pm 1.0$ $\mathrm{pF}(\mathrm{n}=65)$. Two voltage dependent Ca2+ current (VDCC), likely to be attributed by the reported low- and high-VDCCs, were observed. The mean $\mathrm{Ca} 2+$ current amplitude averaged $-26 \pm 9 \mathrm{pA}$ and $-41 \pm 12 \mathrm{pA}$, and each peaked at $-26 \pm 5 \mathrm{mV}$ and $-1 \pm 5 \mathrm{mV}$ respectively $(\mathrm{n}=34)$. a cell membrane capacitance increased when $[\mathrm{Ca} 2+]$ i was elevated, indicating exocytotic activity. $72 \%$ of cells examined increased in membrane capacitance after trains of depolarization and all cells responded to photo-release of caged Ca2+. KATP channels opened readily when dialyzed with ATP free pipette solution, although the maximal KATP conductance between the different a cells varied significantly. $43 \%$ of cells reached a mean peak conductance of $7 \pm 2 \mathrm{nS}$ at $384 \pm 183 \mathrm{~s}$ and $38 \%$ cells reached an average conductance of $15 \pm 1 \mathrm{nS}$ at $295 \pm 176 \mathrm{~s}$. $17 \%$ of cells revealed almost no KATP conductance throughout the dialysis. The peak conductance indicates the fraction of the KATP channels on the plasma membrane, while time to reach the peak KATP conductance indicates overall KATP channel sensitivity to ATP. NADH modulated a cell KATP channel sensitivity to ATP. When dialyzing $1 \mathrm{mM}$ NADH but ATP free pipette solution into a cells, 79\% KATP conductances reached an average peak conductance of $4 \pm \ln$ S after $218 \pm 129$ s

Conclusion: Our data validated the feasibility of studying a cells in whole pancreas tissue slice by examining the a cell electrophysiological properties. We further confirmed that glucagon exocytosis is calcium dependent. a cells can potentially be divided into discrete subpopulations according to their maximum KATP conductance. NADH has not only reduced the available fraction of KATP conductance but the sensitivity of the remaining fraction of KATP channels was also reduced. We concluded that glucose-induced inhibition of glucagon release is in part due to ATP desensitization of KATP channels by NADH.

Supported by: JDRF, AD-FUTURA ARRS

\section{0}

Interleukin- 6 regulates pancreatic glucagon production and the expansion of $\alpha$ cell mass in response to high fat feeding H. Ellingsgaard ${ }^{1}$, J.A. Ehses ${ }^{1}$, E. Hammar ${ }^{2}$, L. van Lommel ${ }^{3}$, R. Quintens ${ }^{3}$, G. Martens ${ }^{4}$, J. Kerr-Conte ${ }^{5}$, F. Pattou ${ }^{5}$, T. Berney ${ }^{2}$, D. Pipeleers ${ }^{4}$, P. Halban², F.C. Schuit ${ }^{3}$, M.Y. Donath ${ }^{1}$

${ }^{1}$ Division of Endocrinology and Diabetes, University Hospital Zürich, Switzerland, ${ }^{2}$ Department of Genetic Medicine and Development, University Medical Center, Geneva, Switzerland, ${ }^{3}$ Department of Molecular 
Cell Biology, Katholieke University Leuven, Belgium, ${ }^{4}$ Diabetes Research Center, Brussels Free University, Belgium, ${ }^{5}$ Cell Therapy of Diabetes, INSERM, Lille, France

Background and aims: Interleukin-6 (IL-6) is systemically elevated in obesity and is a predictive factor to develop type 2 diabetes. The relevance and actions of IL- 6 on pancreatic islet function and mass are unclear. Pancreatic islet pathology in type 2 diabetes is characterized not only by reduced betacell function and mass but also by increased proportion of alpha-cells relative to beta-cells, in addition to alpha-cell dysfunction.

Materials and methods: Detection of the IL- 6 receptor was done by gene array and quantitative RT-PCR. Glucagon and insulin mRNA and secretion were assessed in human islets by RT-PCR and RIA for glucagon and insulin. Furthermore, Ki67, BrdU and TUNEL staining determined the effects of IL-6 on islet cell turnover and function. Glucose tolerence, insulin sensitivity, and morphometric quantification of endocrine cells were assessed in WT and IL$6 \mathrm{KO}$ mice after 8 weeks and 18 weeks of high fat diet feeding.

Results: Here we show that the pancreatic islet, specifically the alpha-cell, is a primary target of IL- 6 actions. Beginning with investigating the tissuespecific expression pattern of the IL- 6 receptor in both mice and rats, we find the highest expression of the IL- 6 receptor in the endocrine pancreas, with highest expression on the alpha-cell. The islet IL- 6 receptor is functional, and IL-6 acutely increases glucagon mRNA and glucagon secretion, with no acute effect on insulin secretion. Furthermore, IL-6 stimulates alpha-cell proliferation, inhibits apoptosis, and regulates alpha-cell mass. Using IL-6 KO mice fed a high fat diet for 8 weeks, we find that IL- 6 is required for high fat diet induced increased alpha-cell mass, an effect that occurs early in response to diet change. Further, after 18 weeks of high fat diet feeding, IL-6 KO mice without expansion of alpha-cell mass display increased fed glycemia due to impaired insulin secretion, despite decreased systemic glucagon, unchanged insulin sensitivity, and similar body weights.

Conclusion: Thus, we conclude that IL-6 regulates the expansion of pancreatic alpha-cell mass which may be needed for functional beta-cell compensation in response to high fat diet feeding.

Supported by: University of Leuven and Zurich, SNF, EFSD/MSD programme, and JDRF

\section{1}

Elevated plasma level of preptin in newly diagnosed type 2 diabetic subjects

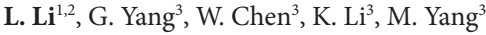

${ }^{1}$ The Key Laboratory of Laboratory Medical Diagnostics in Ministry of Education, Chongqing Medical University, ${ }^{2}$ Department of Clinical Biochemistry, Chongqing Medical University, ${ }^{3}$ Department of Endocrinology, the Second Affiliated Hospital, Chongqing Medical University, China

Background and aims: Preptin is a new regulatory peptide that consists of 34 -amino acid derived from proinsulin-like growth factor II (proIGF-II). It is synthesized by pancreatic $\beta$-cells and secreted along with insulin and amylin. Previous study has demonstrated that preptin enhanced pancreas secretion of insulin. Infusion of isolated pancreas with anti-preptin antibodies significantly reduced glucose-mediated insulin secretion. However, the physiological effects of preptin on the regulation of body metabolism are unknown, especially its role in the pathogenesis of diabetes and insulin resistance (IR ). In this study we investigate whether plasma preptin level is different in patients with impaired glucose tolerance (IGT) and type 2 diabetes mellitus (T2DM). Materials and methods: Fifty patients with T2DM, 56 subjects with IGT, and 54 sex- and age-matched normal controls participated in the study. Plasma preptin levels were measured with a radioimmunoassay. The relationship between plasma preptin levels and anthropometric and metabolic parameters was also assessed.

Results: Plasma preptin levels were found to be increased in patients with T2DM compared with IGT and controls $(456 \pm 14$ vs. $416 \pm 13$ and $398 \pm 13$ ng/ L, $P<0.05$ and $P<0.01)$. There were significantly difference between male and female in plasma preptin levels ( $403 \pm 10$ vs. $432 \pm 10 \mathrm{ng} / \mathrm{L}, P<0.05$ ). Fasting plasma preptin was found to correlate positively with DBP $(r=0.20$, $P<0.01)$, TG $(r=0.24, P<0.01)$, TC $(r=0.24, P<0.01)$, HDL-C $(r=0.18, P<0.05)$, FFA $(r=0.21, P<0.01)$, 2hOGTT $(r=0.18, P<0.05), \mathrm{HbA}_{1} \mathrm{c}(r=0.19, P<0.01)$, and HOMA- $_{\text {IR }}(r=0.13, P<0.05)$ in simple regression analysis in the pooled data, while in multiple stepwise regression analysis, only DBP, TG, HDL-C and FFA were independent related factors with plasma preptin levels. The multiple regression equation was: $Y_{\text {preptin }}=147.959+1.876 \mathrm{X}_{\mathrm{DBP}}+17.827 \mathrm{X}_{\mathrm{TG}}+$
$47.869 \mathrm{X}_{\mathrm{HDL}-\mathrm{L}}+59.964 \mathrm{X}_{\mathrm{FFA}}$. The plasma preptin levels was also significantly associated with T2DM even after controlling for anthropometric variables, blood pressure and lipid profile. Increasing levels of preptin showed a significant linear trend and were independently associated with T2DM, especially when concentrations were analysised both by Mow Mean Scores and Cochran-Armitage Trend Test.

Conclusion: Our results of elevated preptin in newly diagnosed T2DM indicate that preptin may play a role in the pathogenesis of T2DM. However, further experiments need to be done to clarify the role of preptin. Supported by: National Natural Science Foundation of China and Chongqing Municipal Education Commission

\section{2}

$\mathrm{T} 2$ and $\mathrm{T} 3$ stimulate glucose-induced insulin secretion in INS-1e cells and human islets

A. Antonelli ${ }^{1}$, E. Santini ${ }^{1}$, M. Bugliani ${ }^{2}$, E. Barsotti ${ }^{1}$, S. Ferrari ${ }^{1}$, P. Fallahi ${ }^{1}$, P. Marchetti ${ }^{2}$, F. Goglia ${ }^{3}$, E. Ferrannini ${ }^{1}$

${ }^{1}$ Dept.Int.Med.,Metabolic Unit, University of Pisa, Italy, ${ }^{2}$ Dept.Int.

Med.,Diabetic Unit, University of Pisa, Italy, ${ }^{3}$ Dip.Scienze Biologiche ed Ambientali, University of Sannio, Benevento, Italy

Background and aims: Recent evidence suggests that 3,5-diiodo-L-thyronine (T2), a naturally occurring iodothyronine, is biologically active. Whether T2 exerts any effect on $B$-cells is unknown.

Materials and methods: INS-1e cells were incubated (for 48 hours) in fresh RPMI 1640 medium containing $11 \mathrm{mM}$ glucose in the presence or absence of different concentrations $[0.1 \mathrm{nM}, 1 \mathrm{nM}, 0.1$ micromolar $(\mathrm{mcM}), 1 \mathrm{mcM}$ and $10 \mathrm{mcM}]$ of T2 or triiodothyronine (T3). After washing, cells were incubated for 60 min with KRB-HEPES buffer containing 3.3, 7.5, 11.0 or $20 \mathrm{mM}$ glucose. The supernatants were collected for insulin determination and cellular insulin content was determined from acid-ethanol extracts. Pancreatic islets from human donors were isolated and cultured by standard procedures. The islets were then washed and incubated at $37^{\circ} \mathrm{C}$ for $45 \mathrm{~min}$ in $\mathrm{KRB}$ solution containing $3.3 \mathrm{mM}$ glucose, either with or without the addition of T3 or T2 (0.1 nM, $0.1 \mathrm{mcM}, 1 \mathrm{mcM})$.

Results: In INS-1e cells, at medium glucose concentrations of 11.0 and 20.0 $\mathrm{mM}$, insulin secretion and content were significantly $(\mathrm{p}<0.01$ by ANOVA) stimulated by T2 in the range $1 \mathrm{nM}-0.1 \mathrm{mcM}$, while an inhibition $(\mathrm{p}<0.01)$ was observed with higher concentrations $(1-10 \mathrm{mcM})$. T3 showed a similar pattern of stimulation, with a significant $(\mathrm{p}<0.01)$ stimulation of insulin release and content at $0.1-1 \mathrm{mcM}$ and a significant inhibition at higher T3 concentrations $(10 \mathrm{mcM})$. In human pancreatic islets, insulin secretion was significantly $(\mathrm{p}<0.01)$ stimulated by $0.1 \mathrm{nM}$ and $0.1 \mathrm{mcM} \mathrm{T} 2$, while an inhibition was observed with the highest concentration $(1 \mathrm{mcM})$. A slight increase of insulin secretion was observed with $1 \mathrm{mcM}$ T3.

Conclusion: In INS-1e cells and human islets, both T2 and T3 dose-dependently modulate glucose-induced insulin secretion, lower hormone concentrations being stimulatory and higher concentrations being inhibitory. These effects are detectable with lower T2 than T3 concentrations.

\section{3}

Subcutaneous fat shows higher thyroid hormone receptor- $a$ gene expression and higher TR $1: \mathrm{TR} \alpha 2$ ratio than omental fat F.J. Ortega ${ }^{1}$, J.M. Moreno-Navarrete ${ }^{1}$, V. Ribas ${ }^{2}$, E. Esteve ${ }^{1}$, J. Vendrell ${ }^{3}$, W. Ricart ${ }^{1}$, A. Zorzano ${ }^{2}$, J.M. Fernandez-Real ${ }^{1}$

${ }^{1}$ Unidad de Diabetes, Endocrinología y Nutrición, Hospital of Girona, Spain, ${ }^{2}$ Departament de Bioquímica i Biología Molecular, Institute for Research in Biomedicine (IRB), CIBERDEM, Barcelona, Spain, ${ }^{3}$ Unidad de Diabetes, Endocrinología y Nutrición, Hospital of Tarragona, Spain

Background and aims: Differential thyroid hormone receptor alpha (TRa) gene expression and, specifically, TRa1:TR $\alpha 2$ ratio, may explain some of regional differences in human adipose tissues. As a central regulatory factor of lipogenic and lipolytic pathways, the gene encoding TRal and the TRal: $\mathrm{TR} \propto 2$ ratio was identified as a candidate gene for explaining regional differences between intra-abdominal and subcutaneous human adipose tissue. Materials and methods: In 84 samples of visceral and subcutaneous adipose tissue from 42 participants (men or women; lean or obese), we investigated TR $a$ gene expression, TR 1 and TR $\alpha 2$ mRNA levels and the TR 1 :TR 22 ratio (assessed by RT-PCR). Arginine-serine-rich proteins (SF2), heterogeneous nuclear ribonucleoprotein $\mathrm{H} 1$ (hnRNP H1) and heterogeneous nuclear ribo- 
nucleoprotein A1 (hnRNP A1), three splicing proteins involved in alternative and constitutive TRa pre-mRNA splicing, and Spot 14 (S14), one of the most relevant target genes for TR $\alpha 1$ activated by $\mathrm{T} 3$, gene expression were also studied.

Results: TRa gene expression was increased $\sim 1.45$-fold in subcutaneous vs. visceral fat. TRa1 mRNA was also increased $~ 1.81$-fold while the TRa1:TRa2 ratio was $\sim 2.18$-fold higher in subcutaneous than in visceral fat. S14 gene expression was increased by $\sim 2.31$-fold in subcutaneous vs. visceral adipose tissue and correlated significantly with TRa gene expression, TRal mRNA presence and, more significantly, with the TRa1:TRa2 ratio in both tissues. Moreover, hnRNP A1:SF2 ratio was decreased by $\sim 1.31$-fold.

Conclusion: Subcutaneous fat shows higher thyroid hormone receptor- $\alpha$ gene expression and higher TR $\alpha 1: T R \alpha 2$ ratio than omental fat. Supported by: Ministerio de Educación y Ciencia

\section{4}

Human islet amyloid peptide induced apoptosis of human liver cell line HepG2 via endoplasmic reticulum stress

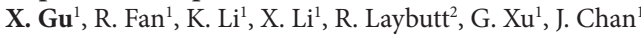

${ }^{1}$ Medicine and Therapeutics, The Chinese University of Hong Kong, SAR,

China, ${ }^{2}$ Diabetes and Obesity Research Program, Garvan Institute of

Medical Research, St Vincent's Hospital, Sydney, Australia

Background and aims: In states of insulin resistance, increased insulin requirement will lead to increased production of islet amyloid peptide (IAPP). Many studies have provided evidence that human IAPP (hIAPP) is cytotoxic in islets and the brain both in vitro and in vivo. Although liver is one of the important insulin resistant targeted organs in type 2 diabetes, the effects of hIAPP on human liver cells remain unclear. In the present study we aim to examine the hypothesis that hIAPP disrupts the homeostasis of the endoplamic reticulum (ER) in liver cells and this promotes ER stress and apoptosis.

Materials and methods: HepG2 human liver cells were exposed to varying concentrations of hIAPP for 24 hours and MTT assay was conducted to measure the cell viability. Cell Immunofluorescent staining with anti-caspase 3 antibody and DAPI were used to monitor apoptosis. Protein expression level of cleaved caspase 3 was also tested by Western Blot. ER homeostasis of HepG2 cells was monitored over a time course of hIAPP treatment using markers of the ER stress response pathway, including phosphorylation of PERK and eIF2 $\alpha$, and spliced XBP-1 by Western Blot. Other markers of the ER stress response pathway, including CHOP and BIP were measured by real time RT-PCR.

Results: hIAPP lead to cytotoxity in HepG2 cells in dose-dependent pattern with the $\mathrm{IC}_{50}$ of $7.74 \mu \mathrm{mol} / \mathrm{l}$. hIAPP $(4 \mu \mathrm{mol} / \mathrm{l}$ and $8 \mu \mathrm{mol} / \mathrm{l})$ induced typical apoptosis of HepG2 cells as evidenced by increased anti-caspase 3 immunofluorescent staining and characteristic condensed apoptotic nuclei stained with DAPI. The expression of cleaved caspase- 3 was also highly up regulated after hIAPP treatment by Western blot. Incubation with hIAPP $(4 \mu \mathrm{mol} / \mathrm{l}$ and $8 \mu \mathrm{mol} / \mathrm{l})$ resulted in phosphorylation of PERK with the peak at 2 hours after treatment and phosphorylation of eIF2 $\alpha$ with the peak at 4 hours after treatment. The expression of XBP-1 was also up regulated with the peak at 12 hours after treatment. However, incubation HepG2 cells with hIAPP (4 $\mu \mathrm{mol} / \mathrm{l}$ and $8 \mu \mathrm{mol} / \mathrm{l})$ did not significantly change the expression levels of $\mathrm{CHOP}$ and BIP.

Conclusion: These data demonstrated that hIAPP induced apoptosis and disrupted ER homeostasis in HepG2 liver cells.

\section{OP 30 Metabolic signals in beta cells}

\section{5}

Live imaging of glucose homeostasis in MIN6 cells and primary beta cells by fluorescence microscopy

M.T. Kaminski, S. Lenzen, S. Baltrusch

Institute of Clinical Biochemistry, Hannover Medical School, Germany

Background and aims: An increase in the intracellular glucose concentration is the stimulating signal for insulin release in pancreatic beta cells. In addition, changes in the intracellular glucose concentration mediate protein expression and modifications. The recently developed FLII ${ }^{12}$ Pglu $600 \mu-\delta 6$ (FLIPglu) sensor based on FRET (fluorescence resonance energy transfer) is a fusion construct specifically detecting glucose. We could successfully established the FLIPglu sensor to monitor in real-time the intracellular glucose concentration in insulin-secreting cells. The aim of this study was to analyze glucose uptake and metabolism with the FLIPglu sensor in insulin-secreting MIN6 cells as well as in primary beta cells.

Materials and methods: The FLIPglu sensor was stably expressed in insulin-secreting MIN6 cells. Primary rat and mouse beta cells were transduced with a recombinant adenovirus expressing the FLIPglu sensor. The dynamic changes in the intracellular glucose concentration were analyzed in a perifusion chamber in the absence and presence of glucose and selective inhibitors of glucose uptake and metabolism, respectively, by fluorescence microscopy. FRET was calculated offline as enhanced yellow fluorescence protein (EYFP)/ enhanced cyan fluorescence protein (ECFP) emission intensity ratio upon ECFP excitation.

Results: In MIN6 cells as well as in primary beta cells expressing the FLIPglu sensor changes in the intracellular glucose concentration were positively correlated with the detected changes in the EYFP/ECFP ratio upon ECFP excitation. Thus, glucose homeostasis in a living single cell could be described by calculating the rates of sugar uptake and metabolism, respectively. Glucose uptake of MIN6 cells was significantly lower upon addition of Krebs Ringer medium containing $10 \mathrm{mM}$ of the glucose analogue 3-O-methylglucose in addition to $10 \mathrm{mM}$ glucose in comparison to $10 \mathrm{mM}$ glucose alone. In contrast, no significant changes in the glucose uptake rate were observed, when the cells were perifused with a mixture of $10 \mathrm{mM}$ glucose and $10 \mathrm{mM} 2$-deoxyglucose. Glucose consumption in insulin-secreting cells was investigated by calculating the decrease of the EYFP/ECFP ratio after glucose removal. Incubation of MIN6 cells in Krebs Ringer medium supplemented with 50 $\mathrm{mM}$ mannose, which reduces the phosphorylation of glucose caused in the presence of $10 \mathrm{mM}$ glucose a significant decrease in the glucose metabolism compared to incubation at $10 \mathrm{mM}$ glucose alone.

Conclusion: Alterations in glucose homeostasis in insulin-secreting cells could be monitored using the FLIPglu sensor. Furthermore, generation of the recombinant adenoviral FLIPglu sensor construct will open the way to further elucidating glucose homeostasis in living primary beta cells of healthy individuals and those with type 2 diabetes mellitus.

\section{6}

Protein kinase B (PKB/Akt) $\alpha$ and $\beta$ are required for normal metabolic regulation in the periphery but dispensable for maintenance of islet mass F. Buzzi ${ }^{1}$, L. Xu' ${ }^{1}$, R.A. Zuelligi', S.B. Boller ${ }^{1}$, G.A. Spinas ${ }^{1}$, O. Tschopp ${ }^{1,2}$, D. Hynx ${ }^{2}$, B.A. Hemmings' ${ }^{2}$, M. Niessen ${ }^{1}$

${ }^{1}$ Endocrinology and Diabetes, University Hospital Zurich, ${ }^{2}$ Department for Growth Control, Friedrich Miescher Institute for Biomedical Research, Basel, Switzerland

Background and aims: Adaptation of islet mass and function relative to metabolic demand is required to avoid the development of type 2 diabetes. PKB is considered to be a key target downstream of IRS2 in the regulation of $\beta$-cell mass. Particularly, constitutive activation of the $\alpha$ isoform is sufficient to increase islet mass. Strikingly, only loss of the $\mathrm{PKB} \beta$ isoform leads to a mild metabolic phenotype, whereas PKB $\alpha$ and $\gamma$ were found not to be required for metabolic regulation. Aim of this project is to better characterise the role of $\mathrm{PKB}$ isoforms in the regulation of islet mass.

Materials and methods: Phenotypic analysis of mice (between one and six months old) deficient for individual PKB isoforms and comparison with wild type littermates: assessment of body weight, random blood glucose, insulin and glucagon; analysis of glucose-stimulated insulin secretion in isolated is- 
lets; immunostaining of pancreatic sections to determine islet mass, $\alpha$ - and $\beta$-cell mass, proliferation and apoptosis.

Results: $P k b \alpha^{-1-}$ mice were smaller $(30.4 \pm 0.7 \mathrm{~g}$ vs. $36.7 \pm 1.7 \mathrm{~g}, \mathrm{n}=6-7, P<0.05)$, had lower blood glucose $(5.4 \pm 0.3 \mathrm{mM}$ vs. $6.3 \pm 0.7 \mathrm{mM}, \mathrm{n}=6, P<0.05)$ and increased serum glucagon $(0.22 \pm 0.1 \mathrm{pg} / \mathrm{ml}$ vs. $0.03 \pm 0.02 \mathrm{pg} / \mathrm{ml}, \mathrm{n}=3-4$, n.s.). Analysis of pancreatic sections revealed a trend toward increased $\alpha$-cell mass (1.79 \pm 0.81 fold, $n=3-4, n . s$.$) and proliferation (2.91 \pm 0.92$ fold, $n=3-4$, n.s.). $\beta$-Cell mass and proliferation were unchanged. $P k b \beta \%$ mice were smaller ( $31.6 \pm 2.1$ vs. $41.9 \pm 1.6 \mathrm{~g}, \mathrm{n}=14, P<0.05)$, had higher blood glucose levels $(11.8 \pm 1.0 \mathrm{mM}$ vs. $6.7 \pm 0.3 \mathrm{mM}, \mathrm{n}=11-14, P<0.05)$ and increased serum insulin $(15.8 \pm 2.2 \mathrm{ng} / \mathrm{ml}$ vs. $3.9 \pm 0.9 \mathrm{ng} / \mathrm{ml}, \mathrm{n}=13, P<0.05)$. Furthermore, islet mass was significantly increased $(2.82 \pm 0.64$ fold, $n=4-6, P<0.05)$ with higher $\beta$-cell proliferation ( $2.92 \pm 1.19$ fold, $n=4-5$, n.s.). $\alpha$-Cell mass and proliferation were unchanged. Data were obtained from 5-6 months-old male mice, and analysis of younger mice and females gave similar results. $P k b \gamma^{-1}$ mice showed no metabolic or islet phenotype.

Conclusion: Previous studies had suggested that the metabolic PKB function is not redundant in the periphery and that only $\mathrm{PKB} \beta$ is required for normal insulin sensitivity. Although our results confirm this phenotype for $P k b \beta$

- mice, we have found lower blood glucose levels combined with increased glucagon levels and $\alpha$-cell proliferation in $P k b \alpha^{-1-}$ mice. Lower blood glucose levels might be due to higher insulin sensitivity in the absence of PKBa. However, lower than normal blood glucose levels and compensatory increase in $\alpha$-cell mass have also been observed in mice deficient for the glucagon receptor. PKB $\alpha$ might therefore be required for normal glucagon signalling. Importantly, PKB-deficient mice do not show loss of islet mass, in contrast to the phenotype of IRS2-deficient mice which display severe loss of $\beta$-cells at similar age. Unlike in the periphery, PKB function might therefore be redundant in $\beta$-cells. However, our results are also consistent with a recently proposed model which suggests that PKBa is required for compensatory expansion but not maintenance of $\beta$-cell mass.

Supported by: Takeda Research Foundation

\section{7}

In situ characterisation of plasma membrane PtdIns(4)P and PtdIns (4,5) $P_{2}$ turnover in individual insulin secreting cells

A. Wuttke, A. Tengholm

Medical Cell Biology, Uppsala University, Sweden

Background and aims: The phospholipid phosphatidylinositol 4-phosphate [PtdIns(4)P] is a key component of cellular membranes. It is generated from phosphatidylinositol via PI4-kinases and serves as precursor for the generation of phosphatidylinositol 4,5-bisphosphate $\left[\operatorname{PtdIns}(4,5) \mathrm{P}_{2}\right]$, which in turn is important in a variety of cellular processes, including insulin secretion. Little is known about the regulation of PtdIns(4)P. The aim of this study was to investigate the kinetics of the plasma membrane pools of PtdIns(4)P and PtdIns $(4,5) \mathrm{P}_{2}$ by in situ imaging the molecules in individual $\beta$-cells.

Materials and methods: PtdIns(4)P was measured with the PH domains from the oxysterol binding protein or four-phosphate-adaptor protein 1 fused to cyan fluorescent protein (OSBP-CFP, FAPP1-CFP), whereas PtdIns $(4,5) \mathrm{P}_{2}$ was recorded with the yellow fluorescent protein-tagged $\mathrm{PH}$ domain from phospholipase C- $\delta$ (PLC $\delta$-YFP). Localization and translocation of the biosensors were analyzed with confocal and evanescent wave microscopy in individual MIN6 $\beta$-cells. The kinetics of phosphoinositide synthesis and breakdown were determined after plasma membrane permeabilization with $\alpha$-toxin.

Results: Confocal microscopy demonstrated a strong localization of the PtdIns(4)P-binding probes to the Golgi complex, consistent with the important role of the lipid in this organelle, but there was no distinct binding to the plasma membrane. In contrast, evanescent wave microscopy revealed a clear plasma membrane localization of both PtdIns(4)P-binding probes. This membrane localization was lost when the cells were permeabilized in ATPfree solution. Addition of $0.25-3 \mathrm{mM}$ ATP caused dose-dependent reassociation of the PtdIns(4)P biosensors to the plasma membrane. Simultaneous imaging of PtdIns $(4,5) \mathrm{P}_{2}$ and PtdIns(4)P revealed that PtdIns(4)P was often formed at lower ATP concentrations than $\operatorname{PtdIns}(4,5) \mathrm{P}_{2}$ and that the ATPinduced translocation of the PtdIns(4)P-sensing constructs usually preceded that of the PtdIns $(4,5) \mathrm{P}_{2}$ biosensor. Inhibition of type III PI4-kinases with 200 $\mu \mathrm{M}$ LY294002 abolished the synthesis of both PtdIns(4)P and PtdIns(4,5) $\mathrm{P}_{2}$. However, while the fluorescence of the PtdIns(4)P biosensor OSBP-CFP started to decrease instantly with biphasic kinetics ( $\tau=34 \pm 4 \mathrm{~s}$ and $395 \pm 139$ $\mathrm{s}, \mathrm{n}=11)$, the PtdIns $(4,5) \mathrm{P}_{2}$ biosensor dissociated from the membrane in a monophasic manner ( $\tau=291 \pm 47 \mathrm{~s}, \mathrm{n}=9)$ after a delay of $39 \pm 3 \mathrm{~s}$.
Conclusion: Simultaneous imaging of the plasma membrane pools of PtdIns(4)P and PtdIns(4,5) $\mathrm{P}_{2}$ with evanescent wave microscopy demonstrates that the lipids undergo rapid ATP-dependent turnover with distinct kinetics and that type III PI4-kinases are essential for their synthesis. The rapid turnover of the lipids should allow fast and local generation of second messengers with maintenance of appropriate phosphoinositide levels for insulin secretion.

\section{8}

Crucial role for mitochondrial calcium signals in metabolism-secretion coupling in the pancreatic b-cell

A.C. Wiederkehr ${ }^{1}$, K.-S. Park ${ }^{1}$, O. Dupont ${ }^{1}$, T. Pozzan ${ }^{2}$, C.B. Wollheim ${ }^{1}$ ${ }^{1}$ Department of Cell Physiology and Metabolism, University of Geneva, Switzerland, ${ }^{2}$ Department of Biomedical Sciences, University of Padua, Italy

Background and aims: Cytosolic calcium signals are relayed into the mitochondria where calcium activates matrix dehydrogenases reflected by increases in $\mathrm{NAD}(\mathrm{P}) \mathrm{H}$. However calcium function in the organelle remains obscure. In the pancreatic b-cell this connection between calcium and metabolism is further complicated by the fact that increased nutrient availability raises $\mathrm{NAD}(\mathrm{P}) \mathrm{H}$ levels prior to calcium activation. Here we have evaluated the role of mitochondrial calcium by expressing a calcium buffering protein in the organelle.

Materials and methods: A number of adenovirus-based probes were employed in this study: Ad-cytoAequorin (cytosolic calcium), Ad-mitoAequorin (mitochondrial calcium), Ad-Luciferase (cytosolic ATP), Ad-mitoAlpHi (mitochondrial $\mathrm{pH}$ ). Kinetic changes of these parameters during metabolism-secretion coupling were analysed 2-3 days after infection of either INS-1E cells or intact rat islets. We have further generated an adenovirus expressing the calcium binding protein S100G fused to a mitochondrial targeting sequence (Ad-mitoS100G) to buffer the mitochondrial calcium rises.

Results: The activation of islet $\beta$-cells by glucose results in a rapid onset of the net ATP response followed by a slow increase reaching new steady-state levels 10-15 minutes following glucose activation. In the absence of extra cellular calcium, which prevents both the cytosolic and mitochondrial calcium signal, the ATP response was similar over the first 5 minutes but strongly inhibited thereafter. The results underline the importance of calcium signals in the generation of the high energy compound and coupling factor ATP. Neither alkalinisation of the mitochondrial $\mathrm{pH}$ (associated with an increase in delta $\mathrm{pH}$ ) nor hyperpolarisation of the electrical gradient across the inner mitochondrial membrane evoked by glucose, were altered by the calcium signal. Therefore calcium specifically affects ATP generation in the $\beta$-cell without influencing the electrochemical potential, the driving force for the ATP-synthase. To investigate the role of mitochondrial calcium specifically, we massively over-expressed the calcium binding protein S100G in the mitochondrial matrix using Ad-mitoS100G. Over-expression of the transgene indeed buffered mitochondrial calcium responses to glucose by $60 \%$, while the rise due to plasma membrane depolarization $(30 \mathrm{mM} \mathrm{KCl})$ was lowered by $50 \%$ in INS-1E cells. Mitochondrial calcium buffering also reduced the glucose dependent increase of insulin secretion by $56 \%$, whereas the $\mathrm{KCl}$ response was only slightly reduced by $16 \%$. General cell function was unaffected by mitochondrial calcium buffering, in as much as basal insulin secretion and insulin content were not changed.

Conclusion: Our results demonstrate that mitochondrial calcium plays a central role in metabolism-secretion coupling possibly affecting the generation of a number of mitochondria-derived coupling factors including ATP. Supported by: EuroDia and the Swiss National Science Foundation

\section{9}

Beta-arrestin 1: a new molecular partner essential for long term GLP-1 actions in pancreatic beta cells

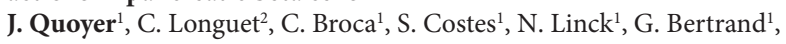
J. Bockaert ${ }^{1}$, S. Dalle ${ }^{1}$

${ }^{1}$ Team Avenir, Physiopathology of beta-cells, INSERM U661, CNRS 5203, Université de Montpellier (IFR3), Institut de Génomique Fonctionnelle, Montpellier, France, ${ }^{2}$ Department of Medicine, University of Toronto, Canada

Background and aims: Therapeutic strategies for the treatment of type 2 diabetes based on activating the G-protein coupled GLP-1 receptor on pancreatic beta-cells and enhancing GLP-1 actions are intensively developed. Classically, upon GLP-1 receptor activation adenylyl cyclase is activated by 
Gas coupling and cAMP is generated. This leads in turn to cAMP-dependent activation of second messenger pathways such as PKA and ERK1/2 (p44/42 MAP kinase) pathways which are known to play key roles in beta-cell function and survival. Here, we investigated the role of beta-arrestin 1, a scaffold protein known to mediate desensitization, internalization and signaling of G-protein coupled receptor, on the spatiotemporal regulation of the ERK1/2 pathway linked to GLP-1 receptor activation.

Materials and methods: MIN6 cell line, isolated rat islets and human islets were used. Beta-arrestin 1 was invalidated by siRNA. Protein expression and phosphorylation of ERK1/2 and Bad were analysed by western blot. Cellular distribution of beta-arrestin 1 and ERK1/2 was determined by subcellular fractionation and confocal microscopy.

Results: Beta-arrestin 1 was found to be expressed in mouse, rat and human pancreatic beta-cells. We found that GLP-1 $(10 \mathrm{nM})$-activated receptor stimulates the ERK1/2 signaling cascade by two temporally distinct pathways dependent, respectively, on Gas/PKA and beta-arrestin 1. Gas/PKA-dependent ERK activation was rapid, transient (maximum stimulation observed at 5 min), and blocked by H89 (a PKA inhibitor, $10 \mu \mathrm{M}$ ) but insensitive to siRNAmediated depletion of beta-arrestin 1. By contrast, beta-arrestin 1-dependent ERK1/2 activation was slower but more sustained in duration. It was insensitive to $\mathrm{H} 89$ but was completely blocked by the depletion of beta-arrestin 1 by siRNA. Remarkably, following GLP-1 stimulation, ERK1/2 associate with beta-arrestin 1 and remain located in the beta-cell cytoplasm. This allows activated ERK1/2 to favour the serine 112 phosphorylation state of the proapoptotic Bcl-2 family member Bad resulting in its proapoptotic inactivation. Conclusion: These findings show for the first time the existence of a betaarrestin 1-dependent, G-protein-independent, pathway linked to the GLP-1 receptor leading to sustained ERK1/2 activation. We propose that such signaling pathway may contribute to the special long term efficacy of GLP-1 in beta-cells and may serve as a prototype for a generation of new therapeutic GLP-1 receptor ligands.

\section{0}

Effect of GLP-1 on AMPK, mTOR and p38 MAPK pathways in pancreatic beta cells

K. Bellmann, M. Barbuta, A. Marette

CRML, Centre Hospitalier de l'Université Laval, Québec, Canada

Background and aims: In type 2 diabetes, a decline in functional beta-cell mass causes insulin deficiency leading to hyperglycemia. Insulin secretion from beta-cells is driven by glucose and also in great part by incretins, such as GLP-1, released by the L-cells of the intestine. Indeed, it is thought that GLP1 is a major contributor to insulin release after an oral glucose challenge. In addition to its positive effect on insulin secretion, GLP-1 also improves betacell growth and survival. However, the signaling pathways involved in GLP-1 action on beta-cell function and proliferation remain poorly understood. It has been proposed that AMPK, a key regulator of cellular energy homeostasis, is negatively associated with insulin release. Furthermore, activation of AMPK down-regulates the mTOR pathway, a nutrient sensor and positive effector of beta-cell growth. On the other hand, it has been shown that activation of the p38 MAP kinase pathway is implicated in insulin gene transcription. Until now, only few studies have explored the effect of GLP-1 on mTOR and p38 MAPK signaling in pancreatic beta-cells and whether these signaling pathways are linked to AMPK. In this study, we have therefore evaluated the effects of the incretin GLP-1 on the phosphorylation of AMPK, p38 MAPK and the mTOR pathway in beta-cell lines.

Materials and methods: Several beta-cell lines (RINm5F, $\beta$-TC3 and MIN-6) were used to evaluate the effect of GLP-1 on AMPK, p38 MAPK, mTOR and its downstream effector S6K1.

Results: GLP-1 was found to transiently reduce AMPK, as revealed by its phosphorylation on Thr172 in all cell lines tested. Maximal inhibition of AMPK activation was observed after 30 minutes of GLP-1 treatment and returned to baseline levels $2 \mathrm{~h}$ post-incretin treatment. Whereas GLP- 1 failed to modulate mTOR phosphorylation, a $2 \mathrm{~h}$ treatment with the incretin was shown to increase the phosphorylation of its downstream effector S6K1 and this activation was maintained until $4 \mathrm{~h}$ of GLP- 1 treatment. Interestingly, we also observed a transient increase in p38 MAPK phosphorylation reaching a peak at $1 \mathrm{~h}$ post GLP-1 treatment followed by a slow decline until $4 \mathrm{~h}$ after incretin exposure.

Conclusion: Since activation of AMPK has been reported to inhibit insulin secretion our data suggest that the transient reduction of AMPK phosphorylation following GLP-1 treatment may contribute to the incretin action on insulin release. Moreover, the observed activation of p38 MAPK may increase insulin gene transcription. On the other hand, the increase in S6K1 activation may contribute to the glucose-dependent effect of GLP-1 on beta-cell proliferation.

Supported by: Merck Frosst Postdoctoral Fellowship Programme 


\section{OP 31 Insulin and analogue treatment}

\section{1}

Good overnight closed-loop glucose control in children and adolescents with type 1 diabetes following ingestion of large, rapidly and slowly absorbed evening meal

R. Hovorka ${ }^{1}$, C.L. Acerini' ${ }^{2}$, J. Allen ${ }^{1}$, L.J. Chassin ${ }^{1}$, A.F. Larsen ${ }^{1}$, D. Mundt ${ }^{2}$, A. De Palma ${ }^{2}$, M.E. Wilinska ${ }^{1}$, D.B. Dunger ${ }^{2}$

${ }^{1}$ Institute of Medical Sciences, University of Cambridge, ${ }^{2}$ Department of Paediatrics, University of Cambridge, United Kingdom

Background and aims: In type 1 diabetes (T1D), large evening meal is accompanied by large prandial insulin dose increasing the risk of nocturnal hyper- and hypoglycaemia due $\mathrm{CHO} /$ prandial insulin mismatch. We investigated overnight closed-loop (CL) insulin delivery in children and adolescents with T1D to evaluate the performance of CL following ingestion of rapidly and slowly absorbed large dinner.

Materials and methods: Six children and adolescents with T1D treated by CSII (M 1; age $15.1 \pm 2.2$ years; BMI $22.8 \pm 4.5 \mathrm{~kg} / \mathrm{m}^{2}$; duration diabetes $6.6 \pm 4.4$ years; A1C 9.1 $\pm 2.4 \%$; basal dose $27 \pm 13 \mathrm{U} /$ day; bolus dose $27 \pm 18 \mathrm{U} /$ day; mean \pm SD) received overnight $C L$ at a clinical facility on two separate nights 1 - 4 wks apart. In random order, the subjects ate either rapidly or slowly absorbed dinner matched for carbohydrates $(\mathrm{CHO})$ (rapid vs slow: $\mathrm{CHO} 129 \pm 34$ vs $129 \pm 34$ g; glycaemic load $113 \pm 29$ vs $40 \pm 8$; energy distribution $68: 12: 20$ vs $54: 29: 17 \%$ CHO:fat:protein; recommended daily energy intake $32 \pm 6$ vs $41 \pm 11 \%)$. Following dinner and prandial insulin ( $17 \pm 6$ vs $17 \pm 7$ U; rapid vs slow) at 18:00h, CL was performed between 18:30 - 08:00 the next day. Realtime subcutaneous (sc) continuous glucose monitoring (CGM) data was fed into a model predictive controller (MPC) every 15 minutes, which calculated sc insulin infusion for manually adjusted insulin pump. Blood glucose (BG) was sampled every $15 \mathrm{~min}$ and measured with $3 \mathrm{~h}$ delay.

Results: CGM data. Following rapidly absorbed dinner, $4 \mathrm{~h}$ incremental glucose area-under-curve (IAUC) was significantly higher (Table). This was accompanied by a non-significant increase in CL insulin delivery. From midnight, glucose excursions were comparable, see Figure showing median (IQ range). Overall, overnight glucose control was good and comparable between two study nights as assessed by mean glucose, time in glucose target 3.9 8.0mM, Kovatchev's Low BG Index (LBGI), Chassin's Grade A+B efficacy rating, and Grade $\mathrm{E}+\mathrm{F}$ hypo/hyperglycaemia risk index (Table). $B G$ data. Compared to CGM data, similar glucose control was observed except higher LBGI [1.4 (0.3 - 3.5) vs $1.3(0.9-1.5)]$ due to CGM overestimation of low glucose values.

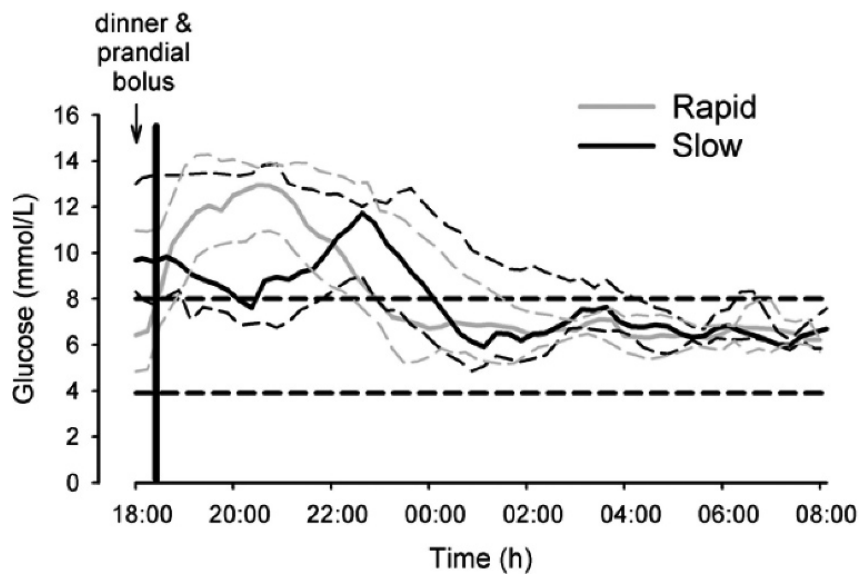

Conclusion: In children and adolescents with T1D, 12h overnight CL with MPC results in safe and efficacious glucose control following the ingestion of large evening meal irrespective of its composition.
Comparison of CL following rapidly and slowly absorbed dinner [mean \pm SD or median (IQR)]

\begin{tabular}{llll}
\hline & Rapid & Slow & $\mathrm{P}$ \\
\hline Glucose at 18:30 $(\mathrm{mM})$ & $9.0 \pm 4.4$ & $11.1 \pm 5.2$ & $\mathrm{NS}$ \\
\hline $\begin{array}{l}\text { Glucose IAUC 18:00 to 22:00 } \\
\text { (mM/240min) }\end{array}$ & $798 \pm 767$ & $-396 \pm 517$ & 0.03 \\
\hline Overnight glucose (mM) & $8.4 \pm 1.9$ & $8.6 \pm 2.2$ & $\mathrm{NS}$ \\
\hline Time in target 3.9 - 8.0mM (\%) & $59(49-63)$ & $61(43-68)$ & $\mathrm{NS}$ \\
\hline LBGI & $0.1(0.0-1.1)$ & $0.2(0.0-0.3)$ & $\mathrm{NS}$ \\
\hline Grade A+B (\%) & $45(40-52)$ & $51(38-65)$ & $\mathrm{NS}$ \\
\hline Grade E+F (\%) & $0(0-11)$ & $1(0-1)$ & $\mathrm{NS}$ \\
\hline Overnight insulin infusion (U/h) & $1.6 \pm 0.5$ & $1.4 \pm 0.6$ & $\mathrm{NS}$ \\
\hline $\begin{array}{l}\text { Insulin infusion 18:00 to 22:00 } \\
\text { (U/240min) }\end{array}$ & $9.2 \pm 4.0$ & $6.7 \pm 4.1$ & $\mathrm{NS}$ \\
\hline
\end{tabular}

Supported by: JDRF (Artificial Pancreas Project), EFSD/LifeScan grant, and NIHR Cambridge Biomedical Research Centre

\section{2}

Marked improvement of glycaemic control, and more time in euglycaemia without increase of hypoglycaemia with CIPII compared to CSII in type 1 diabetes

S.J.J. Logtenberg ${ }^{1}$, N. Kleefstra ${ }^{1,2}$, S.T. Houweling ${ }^{2}$, K.H. Groenier ${ }^{3}$, H.J.G. Bilo ${ }^{1,4}$

${ }^{1}$ Diabetes Centre, Isala Clinics, Zwolle, ${ }^{2}$ Medical Research Group, Langerhans, Zwolle, ${ }^{3}$ General Practice, UMCG, Groningen, ${ }^{4}$ Internal Medicine, UMCG, Groningen, Netherlands

Background and aims: To investigate whether continuous intraperitoneal insulin infusion (CIPII) delivered with an implantable pump is safe and provides equal or superior glycemic control with less episodes of hypoglycemia as compared to intensive subcutaneous insulin therapy (SC; multiple dose injections (MDI) or continuous subcutaneous insulin infusion (CSII)) in poorly controlled type 1 diabetes patients.

Materials and methods: Open-label, randomized controlled cross-over trial. After a 3-month qualification phase, during which optimization of insulin therapy was targeted, patients who had a $\mathrm{HbA}_{1 c}>7.5 \%$ and/or $\geq 5$ hypoglycemic events/week were randomized to receive either 6 months CIPII followed by 6 months SC or vice versa with a washout phase (max. 4 weeks) in between. Treatment adjustments were done according to protocol. IP insulin was delivered through an implantable pump (MIP 2007C, Medtronic). Glycemic control was assessed after 3 and 6 months in both phases with $\mathrm{HbA}_{1}$; frequency of hypoglycemic events $(<4.0 \mathrm{mmol} / \mathrm{L})$ as measured with self monitoring of blood glucose; time spent in euglycemia $(4.0-10.0 \mathrm{mmol} / \mathrm{L})$ as measured with continuous glucose monitoring. General linear models were used to test differences, taking treatment order into account.

Results: Baseline characteristics: 11 males, 13 females; mean (SD) age 43.6 (11.8) years; mean diabetes duration 22.6 (10.6) years; mean HbA 8.6 (1.1) $\%$; $\mathrm{HbA}_{1 \mathrm{c}}>7.5 \%$ in 20 subjects; hypoglycemic events $\geq 5 /$ week in 14 subjects. After randomization groups were comparable regarding clinical characteristics. One subject was lost to follow-up (start IP insulin).

Outcomes: $\mathrm{HbA}_{1 c}$ at different points in the study are shown in Figure 1 for both groups. $\mathrm{HbA}_{1 \mathrm{c}}$ improvement with CIPII was better compared to SC after both 3 and 6 months ( $\mathrm{p}=0.006$ and $\mathrm{p}=0.025$, respectively).

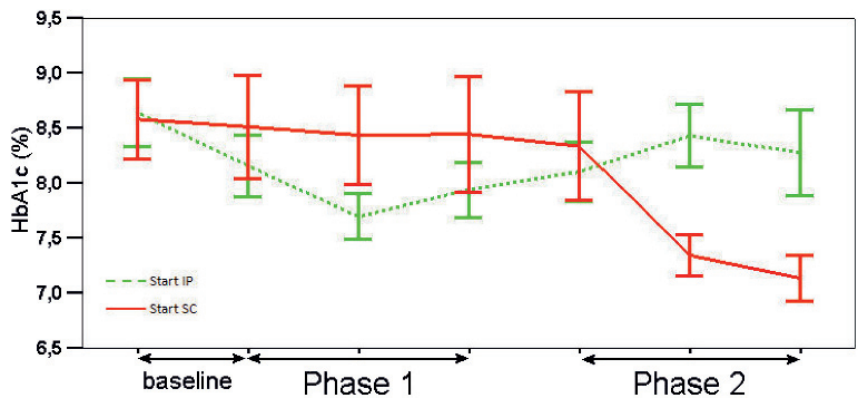

Figure 1: HbAlc (\%) at different time points in study for both groups. 
Mean (SD) number of hypoglycemic events during the baseline phase was 4.1 (2.7) per week. During the CSII phase this was 4.0 (2.6), as compared to 3.5 (2.3) per week in the CIPII phase $(\mathrm{p}=0.073)$.

Mean percentage of time spent in euglycemia at baseline was 45.1 (12.9) \%. After 6 months CIPII and SC, this percentage was 52.9 (19.6) and 41.8 (18.5), respectively $(\mathrm{p}=0.003)$.

Conclusion: CIPII improved glycemic control without increase (trend toward decrease) of hypoglycemic events and increasing time spent in euglycemia. Therefore, in selected subjects with type 1 diabetes, CIPII will be of use for those that do not reach acceptable glycemic control on MDI or CSII. Supported by: Medtronic Minimed

\section{3}

\section{Effect of the long-acting insulin analogue detemir on IGF-I axis in} humans

F. Porcellati ${ }^{1}$, P. Candeloro ${ }^{1}$, P. Rossetti ${ }^{1}$, C.G. Fanelli ${ }^{1}$, N. Ricci Busciantella ${ }^{1}$, E. Ghigo ${ }^{2}$, G.B. Bolli ${ }^{1}$

${ }^{1}$ Internal Medicine, University of Perugia, ${ }^{2}$ Internal Medicine, University of Turin, Italy

Background and aims: The IGF-I system plays a critical role in cellular survival and proliferation. Recent evidence from both physiologic and clinical studies, suggests that it may also be important in the processes underlying carcinogenesis. Elevated circulating IGF-I concentrations have been associated with an increased risk of colon, breast, lung and prostate cancer. Insulin may be an important mediator of IGF-I effects on carcinogenesis through inhibition of hepatic synthesis of IGF binding proteins-1 (IGFBP-1), which, in turn, regulate IGF-I bioavailability. Insulin detemir (ID), with respect to regular human insulin (RHI), has a lower affinity for both insulin and IGF1 receptors. Nevertheless ID is formulated at a four time greater concentration on molar basis vs RHI, with resulting much higher plasma insulin concentrations. Therefore it is possible that ID and RHI exert differential actions in the modulation of IGF-1 axis. The aim of our study was to assess the effects of an i.v. infusion of equipotent doses of ID and RHI on plasma concentrations of IGF-I, IGFBP-1, and IGFBP-3. Ten normal, non-diabetic subjects (M/F 6/4, age $36 \pm 7$ years, BMI $22.9 \pm 2.6 \mathrm{~kg} / \mathrm{m} 2$ ) were studied on 2 occasions at random, during i.v. infusion of either ID $(2 \mathrm{mU} / \mathrm{kg} / \mathrm{min}$ and $4 \mathrm{mU} / \mathrm{kg} / \mathrm{min} 240-300$ $\mathrm{min}$ ) or RHI ( $1 \mathrm{mU} / \mathrm{kg} / \mathrm{min} 0-240 \mathrm{~min}$ and $2 \mathrm{mU} / \mathrm{kg} / \mathrm{min} 240-300 \mathrm{~min})$ with variable glucose infusion (GIR) to clamp plasma glucose (PG) at $90 \mathrm{mg} / \mathrm{dl}$. Results: PG was not different between studies $(\mathrm{p}>0.2)$. Plasma insulin concentrations were on average 11 times greater with ID than HI (AUCs: $845 \pm 90$ $\mu \mathrm{U} / \mathrm{ml} / \mathrm{min}$ vs $83 \pm 20 \mu \mathrm{U} / \mathrm{ml} / \mathrm{min}$ ). GIR with ID was lower than RHI during the first $105 \mathrm{~min}(\mathrm{p}<0.05)$ but later was no different until the end of studies $(9 \pm 0.5$ vs $9.5 \pm 0.3 \mathrm{mg} / \mathrm{Kg} / \mathrm{min}, \mathrm{p}>0.2)$. There were no significant changes in plasma GH concentrations during both ID and RHI infusion, without any differences between the two insulins $(\mathrm{p}>0.2)$. Similarly plasma IGF-I concentrations (ID $147 \pm 16$, HI $155 \pm 15 \mathrm{ng} / \mathrm{ml}$ ) and BP-3 (ID 2,6 $\pm 0,2$ RHI 2,5 $\pm 0,2$ $\mu \mathrm{g} / \mathrm{ml})(\mathrm{p}>0.2)$. In contrast insulin infusion resulted in a suppression of plasma BP-1 concentrations (only data of 6 out of 10 subjects are reported)

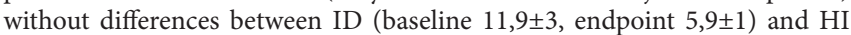
(baseline $11,1 \pm 2,4$, endpoint $4,4 \pm 1$ ) (ng/ml, $\mathrm{p}>0.2)$.

Conclusion: the greater plasma insulin concentrations obtained with ID exert similar effects on IGF-I axis as compared to RHI. Additional studies are needed to confirm these results in patients with insulin-treated diabetes mellitus.

\section{4}

Evaluation of long-term clinical benefits associated with early versus late initiation of insulin in type $\mathbf{2}$ diabetes patients in the UK

E. Sarpong ${ }^{1}$, C. Pratoomsoot ${ }^{2}$, G. Goodall ${ }^{2}$, R. Hayes ${ }^{1}$, W.J. Valentine ${ }^{2}$

${ }^{1}$ Eli Lilly and Company, Indianapolis, United States, ${ }^{2}$ Health Economics and Outcomes Research, IMS Health, Basel, Switzerland

Background and aims: Recent data have shown that, despite poor glycaemic control, initiation of insulin is frequently delayed (by up to 8 years) and this may have a substantial effect on clinical outcomes in patients. The aim of this study was to evaluate the long-term clinical impact of delaying insulin initiation.

Materials and methods: A published and non-product specific diabetes model was used to project long-term clinical outcomes associated with type 2 diabetes in terms of life expectancy, cumulative incidence and time free of complications. The model utilises findings from landmark clinical and epidemiological studies to simulate the long-term incidence and progression of diabetes and its associated complications. Cohort details were derived from published data on UK patients failing glycaemic control with OADs (mean age 64.2 years, duration of diabetes 3.8 years, HbAlc $9.07 \%$ and BMI 30.1 kg.m 2). The base case analysis compared the clinical outcomes of an immediate initiation of insulin versus a delay of eight years. In both scenarios (early and late), initiation of insulin was assumed to decrease $\mathrm{HbAlc}$ by $1.37 \%$ and was associated with an overall hypoglycaemic event rate of 570 per 100 patient years.

Results: Early initiation of insulin was associated with an improvement in average life expectancy of 0.46 years versus delayed insulin initiation $(9.94 \pm$ 0.32 versus $9.48 \pm 0.30$ years). Patients experienced both an increase in the mean time free of complications and a reduced incidence of complications overall. A summary of the clinical benefits associated with early versus delayed initiation of insulin over patient lifetimes, expressed in terms of time to onset of complications (years), is given in the table below. A reduction in the lifetime cumulative incidence of complications was also associated with early insulin initiation versus delay in insulin initiation (data not shown). Additional analyses demonstrated that reducing the length of the delay for the initiation of insulin still resulted in substantial clinical benefits. Even when the delay was reduced to 2, 4 or 6 years there were still notable clinical benefits. Conclusion: Published data indicates that there is a substantial number of type 2 diabetes patients on oral combination therapy in poor glycaemic control in the UK. In our modelling analysis, immediate initiation of insulin therapy was associated with improvements in survival and diabetes-related complication rates compared to delayed insulin therapy in this group of patients.

Time to onset of selected complications (years)

\begin{tabular}{llll}
\hline Complication & No delay & $\begin{array}{l}\text { Insulin delayed } \\
\text { by } 8 \text { years }\end{array}$ & Difference \\
\hline Acute myocardial infarction & 8.7 years & 8.0 years & +0.7 years \\
\hline Stroke & 9.0 years & 8.6 years & +0.4 years \\
\hline Severe vision loss & 9.6 years & 9.1 years & +0.5 years \\
\hline End-stage renal disease & 9.9 years & 9.4 years & +0.5 years \\
\hline Lower-limb amputation & 9.8 years & 9.3 years & +0.5 years \\
\hline
\end{tabular}

Supported by: Eli Lilly and Company

\section{5}

Adding a single dose of insulin glulisine at breakfast or main meal to basal insulin and oral antidiabetic therapy; which patients of the OPAL study benefit most?

M. Lankisch ${ }^{1}$, K. Ferlinz ${ }^{2}$, W.A. Scherbaum ${ }^{3}$, for the OPAL Study Group ${ }^{1}$ Medizinische Klinik III, Kardiologie, Herzzentrum Wuppertal, Wuppertal, Germany, ${ }^{2}$ sanofi-aventis Deutschland GmbH, Berlin, Germany, ${ }^{3}$ HeinrichHeine-University, Düsseldorf, Germany

Background and aims: Most patients with Type 2 diabetes (T2DM) treated with oral antidiabetic drugs (OADs), will ultimately require insulin therapy to maintain tight glycaemic control. While a single daily injection of longacting basal insulin glargine (GLAR) and OAD therapy is highly effective, a more intensive insulin regimen may be required if $\mathrm{HbA}_{1 \mathrm{c}}$ targets are not met. In patients on GLAR+OADs, we show the results of adding a single daily prandial dose of insulin glulisine (GLU) at breakfast or main meal.

Materials and methods: A 26-wk, randomised, multicentre study was conducted in T2DM patients ( $\mathrm{n}=316$ per protocol set), poorly controlled ( $\mathrm{HbA}_{1}$ $>6.5-9.0 \%$ ) on previous GLAR+OADs. Patients (FBG $\leq 6.7 \mathrm{mmol} / \mathrm{L}[\leq 120$ $\mathrm{mg} / \mathrm{dL}]$ ) were stratified by the main meal (breakfast, lunch or dinner), determined by the highest postprandial blood glucose (BG) level, and randomised to GLU+GLAR+OADs (GLAR as basal insulin) with GLU given at breakfast $(n=162)$ or main meal $(n=154)$. Here, we describe the patient profiles at baseline between the two arms. The primary aim was to demonstrate equivalence in baseline to endpoint $\mathrm{HbA}_{1}$ change between both arms.

Results: Baseline demographics for the overall, breakfast and main meal groups were similar (Table). Patients had an average age $>60 \mathrm{yrs}$, obese $\left(>30 \mathrm{~kg} / \mathrm{m}^{2}\right)$ and had suboptimally controlled T2DM (FBG $\sim 6 \mathrm{mmol} / \mathrm{L}$ and $\left.\mathrm{HbA}_{1 \mathrm{c}} \sim 7.3 \%\right)$. Overall, $\mathrm{HbA}_{1 \mathrm{c}}$ significantly improved from baseline to endpoint $(\mathrm{p}<0.0001)$, while both arms were equivalent in terms of $\mathrm{HbA}_{1 c}$ change (equivalence margin $=0.4 \%$; Table). $B G$ values improved significantly within 
each arm for most pre- and post-meal timepoints. Overall, 30.7\% of patients achieved $\mathrm{HbA}_{1 \mathrm{c}} \leq 6.5 \%$ (27.8\% breakfast and $33.8 \%$ main meal arms; $\mathrm{p}=0.21$ ). For those patients with baseline $\mathrm{HbA}_{1 c}>7.0 \%, 44.1 \%$ achieved $\mathrm{HbA}_{1 c} \leq 7.0 \%$ at endpoint ( $36.5 \%$ breakfast and $52.2 \%$ main meal arms; $p=0.028$; Table). Mean GLAR dose was unchanged (baseline vs endpoint: 31 vs $32 \mathrm{U} /$ day breakfast and 27 vs $27 \mathrm{U} /$ day main meal arms) whereas GLU dose increased (starting dose of $5 \mathrm{U} /$ day vs 11 and $12 \mathrm{U} /$ day, respectively). The rate of on-treatment hypoglycaemia $(B G \leq 3.3 \mathrm{mmol} / \mathrm{L}[\leq 60 \mathrm{mg} / \mathrm{dL}])$ was 3.21 events/patient year ( $n=393$ safety set) for the overall group (2.72 and 3.69 events/patient year for the breakfast and main meal arms, respectively).

Conclusion: Here, we identify and describe the profile of patients with suboptimally controlled T2DM on basal insulin who are potential candidates for intensification of their GLAR+OADs regimen by adding 1 injection of prandial insulin. In these patients, adding GLU at breakfast is equivalent to GLU given at main meal in lowering $\mathrm{HbA}_{1 \mathrm{c}}$ with similar rates of hypoglycaemia between the two treatment arms. For patients with $\mathrm{HbA}_{1 c}>7.0 \%$ at baseline, a trend towards a higher number of patients achieving $\mathrm{HbA}_{1 c} \leq 7.0 \%$ at endpoint was seen in the main meal versus breakfast arm.

\begin{tabular}{|c|c|c|c|}
\hline $\begin{array}{l}\text { Patients with } \mathrm{HbA} 1 \mathrm{c}>6.5-9.0 \% \\
\text { at baseline }\end{array}$ & $\begin{array}{l}\text { Overall } \\
\mathrm{N}=316\end{array}$ & $\begin{array}{l}\text { Breakfast } \\
\mathrm{N}=162\end{array}$ & $\begin{array}{l}\text { Main meal } \\
\mathrm{N}=154\end{array}$ \\
\hline Age (years) & $63.3 \pm 9.2$ & $62.7 \pm 92$ & $64.0 \pm 9.1$ \\
\hline BMI (kg/m2) & $31.3 \pm 5.1$ & $31.6 \pm 5.2$ & $30.9 \pm 5.0$ \\
\hline Diabetes duration (years) & $10.5 \pm 7.1$ & $10.6 \pm 7.1$ & $10.4 \pm 7.0$ \\
\hline HbAlc (\%) & $7.32 / 6.99^{*}$ & $7.35 / 7.03^{*}$ & $7.29 / 6.94^{*}$ \\
\hline $\begin{array}{l}\text { Adjusted mean difference in } \\
\text { HbA1c }(\%) \text { change between } \\
\text { groups }(95 \% \mathrm{CI})\end{array}$ & - & \multicolumn{2}{|c|}{$0.048(-0.115 ; 0.211)$} \\
\hline \multicolumn{2}{|c|}{$\begin{array}{l}\text { Patients achieving HbAlc } \leq 6.5 \% \text { at } 97 \text { [30.7] } \\
\text { endpoint }(\mathrm{n})[\%]\end{array}$} & $45[27.8]$ & $52[33.8]$ \\
\hline$\overline{\mathrm{FBG}}(\mathrm{mmol} / \mathrm{L})[\mathrm{mg} / \mathrm{dL}]$ & $\begin{array}{l}6.0 / 6.4 \\
{[109 / 116]^{*}} \\
\end{array}$ & $\begin{array}{l}6.1 / 6.6 \\
{[110 / 118]^{*}}\end{array}$ & $\begin{array}{l}6.0 / 6.3 \\
{[108 / 113] \dagger}\end{array}$ \\
\hline $\begin{array}{l}\text { Patients with } \mathrm{HbA1c}>7.0 \% \text { at } \\
\text { baseline (n) }\end{array}$ & 188 & 96 & 92 \\
\hline $\begin{array}{l}\text { Patients with } \mathrm{HbA} 1 \mathrm{c}>7.0 \% \text { at } \\
\text { baseline achieving HbA1c } \leq 7.0 \% \\
\text { at endpoint (n) [\%] }\end{array}$ & $83[44.1]$ & $35[36.5] \ddagger$ & $48[52.2] \ddagger$ \\
\hline \multicolumn{4}{|c|}{$\begin{array}{l}\text { Within-group change: }{ }^{\star} \mathrm{p}<0.0001 ; \dagger \mathrm{p}<0.05 \text {. Between-group change: } \\
\ddagger \mathrm{p}=0.028 \text {. Data are mean (baseline/endpoint) unless otherwise stated }\end{array}$} \\
\hline \multicolumn{4}{|l|}{ Supported by: sanofi-aventis } \\
\hline
\end{tabular}




\section{OP 32 Type 2 diabetes mellitus epidemiology}

\section{7}

EUropean Core Indicators in Diabetes

F. Storms ${ }^{1}$, A. Fagot Campagna ${ }^{2}$

${ }^{1} \mathrm{EBM}, \mathrm{CBO}$ the Dutch Institute for Quality in Health Care, Utrecht, Netherlands, ${ }^{2}$ Département des Maladies Chroniques et Traumatismes, Institut de Veille Sanitaire, Saint Maurice, France

The aim of Eucid is to collect and compare data about risk factors for diabetes, complications and quality of care indicators in member or future member countries of the European Union.

Methods: 19 countries provided data for a list of 36 indicators by age-band, which were representative at a regional or a national level, for 2004, 2005 or 2006. Methods (surveys, registries, administrative databases) were provided. Data were age-standardized for comparisons performed in the general population.

Results: - Among the least available indicators, incidence of blindness in people with diabetes was provided by only 4 countries, and impaired fasting glucose in general population by 2 .

- The standardized prevalence of diabetes varied from $2.6 \%$ in Finland to $7.6 \%$ in Cyprus; crude incidence of diabetes (0-14 yrs) from 11 in Spain to 60 per 100,000 in Finland; standardized prevalence of overweight (25-74 yrs) from $37 \%$ in Germany to $60 \%$ in Cyprus; standardized mortality rates linked with diabetes from 7 in Luxembourg to 56 per 100,000 in Finland. - Among people with diabetes ( $>25 \mathrm{yrs}$ ), process indicators ranged: for $\mathrm{HbAlc}$ testing once a year, from $51 \%$ in Ireland to $99 \%$ in the Netherlands, France and Belgium; for lipid testing, from $45 \%$ in Ireland to $99 \%$ in the Netherlands; for microalbuminuria testing, from $25 \%$ in Finland to $97 \%$ in the Netherlands; for fundus examination, from $12 \%$ in Ireland to $84 \%$ in the Netherlands.

Risk factors in people with diabetes varied: HbAlc $>7 \%, 32 \%$ in Sweden and Ireland to $83 \%$ in Cyprus; total cholesterol $>5 \mathrm{mmol} / 1,14 \%$ in Ireland to $68 \%$ in Cyprus; microalbuminuria, 9\% in Finland to $41 \%$ in England; blood pressure $>140 / 90 \mathrm{mmHg}, 17 \%$ in France to $46 \%$ in Sweden; smoking, $10 \%$ in Ireland to $37 \%$ in Denmark.

- Complication incidence rates were: dialysis and transplantation, 4 in Cyprus to 149 per 100,000 in Scotland; stroke, 37 in Cyprus to 2675 in Germany; myocardial infarction, 21 in Cyprus to 2135 in Austria; major amputation, 78 in Scotland to 574 in Spain.

Conclusion: While European epidemiologic systems can provide diabetes indicators, major indicators as blindness are still missing. Most of the European countries achieve remarkable good testing of people with diabetes. Risk factors and outcomes vary across countries, reflecting a mixture of genetic background, societal and cultural factors, organisation of diabetes care as well as public health policies. The results of Eucid will be used within countries to try to influence these policies.

The complete report can be downloaded at www.eucid.eu.

Supported by: DG-SANCO, Health and Consumer Protection Directorate General, Luxembourg

\section{8}

Implications of diabetes mellitus for temporal changes in left ventricular geometry and function. The MONICA/KORA Augsburg Cohort Study M.R.P. Markus ${ }^{1}$, J. Stritzke ${ }^{1}$, J. Wellman ${ }^{2}$, S. Duderstadt ${ }^{1}$, W. Lieb ${ }^{1}$, A. Luchner ${ }^{3}$, A. Döring ${ }^{4}$, U. Keil ${ }^{2}$, H. Schunkert ${ }^{1}$, H.W. Hense ${ }^{2}$ ${ }^{1}$ Medical Clinic II, University of Lübeck Medical School, ${ }^{2}$ Institute of Epidemiology and Social Medicine, University Hospital of Münster, ${ }^{3} \mathrm{Clinic}$ and Policlinic for Internal Medicine II, University Hospital of Regensburg, ${ }^{4}$ Institute of Epidemiology, GSF - National Research Center for Environment and Health, Neuherberg, Germany

Background and aims: Several studies suggest that diabetes mellitus is one of the most important risk factors for the development of systolic and diastolic heart failure. However, details of the mechanisms of this process are still unclear. We investigated the long-term consequences of diabetes on the heart in a prospective population-based study.

Materials and methods: The population-based MONICA/KORA-Augsburg cohort study provided anthropometric, biochemical and echocardiographic data for 1005 individuals examined during a baseline survey in 1994/95 and a follow-up investigation ten years later. We defined three groups of individuals: who were persistently, at both examinations, non diabetics (nonDM, i. e.; defined as no history of diabetes and not using medications for glycemic control at both examinations; $\mathrm{n}=811$ ), who were persistently diabetic (PDM, i. e., defined as disease known to the patient as reported by standard questionnaire or the use of antiglycemic medications or insulin or non-fasting glucose blood levels $>=200 \mathrm{mg} / \mathrm{dl} ; \mathrm{n}=34$ ) and who presented incident diabetes at follow-up (incDM; $n=19$ ). We evaluated prospectively the impact of diabetes on the 10-year changes in left ventricular (LV) mass indexed to height ${ }^{2.7}$ (in $\mathrm{g} / \mathrm{m}^{2.7}$ ), left ventricular end-diastolic diameter (LVEDD in $\mathrm{mm}$ ), wall thickness $(\mathrm{WT}=$ septal + posterior wall thickness in $\mathrm{mm})$, relative wall thickness (RWT, WT in mm divided by in $\mathrm{mm}$ ), left atrial diameter (LA in $\mathrm{mm}$ ) and the ratio of early diastolic peak transmitral flow and early diastolic peak myocardial relaxation velocities (E/EM) using linear regression models adjusting for age and sex and other relevant confounders including systolic blood pressure.

Results: For the relative changes over the ten years period, there was a positive trend across the three groups for LV mass ${ }^{2.7}(\mathrm{p}=0.011), \operatorname{LVEDD}(\mathrm{p}=0.002)$ and LA ( $\mathrm{p}=0.020)$. Specifically, compared the mean difference with the nonDM group, the PDM group, had a bigger relative increase of $12.6 \%$ (95\% confidence interval: $4.20 \%$ to $20.9 \%, \mathrm{p}=0.003)$ in the $\mathrm{LV}$ mass ${ }^{2.7}, 6.0 \%(2.70 \%$ to $9.30 \%, \mathrm{p}<0.001)$ in LVEDD and $6.50 \%(1.90 \%$ to $11.1 \%, \mathrm{p}=0.006)$ in LA. Finally, in 2004/5, the PDM group showed a higher ratio of E/EM than the nonDM group (11.6 [10.5 to 12.8 ] versus 9.83 [9.66 to 10.0], $\mathrm{p}=0.002$ ).

Conclusion: Over a period of ten years, incident and prevalent diabetes mellitus are associated with significant changes in left ventricular geometry and mass, mostly attributable to a pattern of eccentric remodelling. Left atrial enlargement and a worse diastolic function were also observed. All associations were independent of cofactors and confounders.

Supported by: German Research Foundation, the Federal Ministry of Education and Research and Competence Network of Heart Failure, and the University of Lübeck Medical School. The KORA research platform (KORA: Cooperative Research in the Region of Augsburg) and the MONICA Augsburg studies were initiated and financed by the GSF - National Research Center for Environment and Health, which is founded by the Federal Ministry of Education and Research and by the Bavarian Ministry of Finance

\section{9}

Complement $\mathrm{C} 3$, but not $\mathrm{C} 4$, is independently associated with the metabolic syndrome and prevalent cardiovascular disease: the CODAM study M.M.J. van Greevenbroek ${ }^{1}$, C.J.H. van der Kallen ${ }^{1}$, I. Ferreira ${ }^{2}$, C.G. Schalkwijk ${ }^{1}$, E.E. Blaak ${ }^{3}$, E.J.M. Feskens ${ }^{4}$, C.D.A. Stehouwer ${ }^{1}$ ${ }^{1}$ Dept of Internal Medicine / Cardiovascular Research Institute Maastricht (CARIM), Maastricht University, ${ }^{2}$ Dept of Internal Medicine / Dept of Clinical Epidemiology and Medical Technology Assessment, Maastricht University Hospital, ${ }^{3}$ Dept of Human Biology/Nutrition and Toxicology Research Institute Maastricht (NUTRIM), Maastricht University, ${ }^{4}$ Dept of Human Nutrition, Wageningen University, Netherlands

Background and aims: Increased plasma concentrations of complement C3 and C4 have been implicated in cardiovascular disease (CVD). This association may, at least partly, be related to their increase in the metabolic syndrome (MetS). C3 plays a pivotal role in complement activation via the classical, the alternative, as well as the mannan-binding lectin pathway. C4, on the other hand, is a component of the classical and the lectin pathway.

Research question: Are plasma concentrations of $\mathrm{C} 3$ and $\mathrm{C} 4$ independently associated with (components of) the MetS and, if yes, are they also independently associated with CVD?

Materials and methods: Relations between C3, C4, the MetS (NCEP-ATP 2005) and CVD were studied in the CODAM (cohortstudy on diabetes and atherosclerosis Maastricht) population. CODAM subjects $(n=574)$ were selected from a large population-based cohort on the basis of an increased risk of type 2 diabetes and CVD. Associations of C3 and C4 (z-scores) with each component of the MetS were analysed using linear regression (Model(s) A: C3 or $\mathrm{C} 4$ as independent variables, adjusted for sex and age, with each component of the MetS as dependent variables; Model(s) B (full model): as model $\mathrm{A}$ but with $\mathrm{C} 3$ and $\mathrm{C} 4$ as independent variables). Associations with presence of the MetS and CVD were determined using logistic regression analyses. Results: C3 and C4 were higher $(\mathrm{p}<0.001)$ in plasma of subjects with the MetS ( $\mathrm{n}=312 ; \mathrm{C} 3,1.94 \pm 0.31 \mathrm{~g} / \mathrm{L}$ and C4, $0.30 \pm 0.07 \mathrm{~g} / \mathrm{L})$ than in those without ( $\mathrm{n}=259 ; \mathrm{C} 3,1.63 \pm 0.28 \mathrm{~g} / \mathrm{L}$, and C4, $0.28 \pm 0.07 \mathrm{~g} / \mathrm{L})$. C3 and C4 were significantly associated with most components of the MetS (Model(s) A); waist 
(C3, $\mathrm{p}<0.001 ; \mathrm{C} 4, \mathrm{p}<0.001),[\ln ]$ triglycerides $(\mathrm{C} 3 \mathrm{p}<0.001 ; \mathrm{C} 4, \mathrm{p}=0.001)$, HDL-cholesterol (inversely; $\mathrm{C} 3, \mathrm{p}<0.001 ; \mathrm{C} 4, \mathrm{p}<0.001$ ), blood pressure (C3, $\mathrm{p}<0.001 ; \mathrm{C} 4, \mathrm{P}=0.002)$, and fasting glucose $(\mathrm{C} 3, \mathrm{p}<0.001 ; \mathrm{C} 4, \mathrm{p}=0.3)$. $\mathrm{C} 3$ and $\mathrm{C} 4$ were also associated with a marker of inflammation, a common feature of the metabolic syndrome ( $[\ln ]$ CRP, $C 3, \mathrm{p}<0.001 ; \mathrm{C} 4, \mathrm{p}<0.001)$. Next, when C3 and $\mathrm{C} 4$ were included simultaneously in the full regression models only $\mathrm{C} 3$, but not $\mathrm{C}$, was independently associated with the individual components of the MetS. In contrast, both C3 and C4 contributed significantly to prediction of [ln]CRP. In a full logistic regression model, C3 was significantly associated with the MetS, while C4 was not (Odds Ratio (OR) [95\% CI] C3 $=3.81$ [2.92-4.98], OR C4 $=0.87$ [0.70-1.08]. Lastly, C3 was associated with prevalent CVD in a full model $(\mathrm{n}=144$; OR C3=1.63 [1.31-2.04], while $\mathrm{C} 4$ was not (OR C4= $0.88[0.70-1.10])$.

Conclusion: In the CODAM population, $\mathrm{C} 3$ and $\mathrm{C} 4$ both show strong associations with various components of the MetS, and with the syndrome itself, but after mutual adjustments only $\mathrm{C} 3$ was independently associated with (components of) the MetS. Moreover C3, but not C4, is independently associated with prevalent CVD in the CODAM population, despite our finding of independent associations of both C3 and C4 with inflammation.

Supported by: Netherlands Organisation for Scientific Research and the Dutch Diabetes Research Foundation

\section{0}

Cytokines, advanced glycation end-products, leptin, adiponectin and diabetes: a nested case-control study

P.Z. Zimmet, D.J. Magliano, B. De Courten, J. Forbes, J.E. Shaw

The Baker IDI Heart and Diabetes Institute, Melbourne, Australia

Background and aims: Low grade, chronic inflammation, cytokines and advanced glycation end-products (AGEs) have all been suggested to be involved in the pathogenesis of diabetes. However, the extent to which any one of these factors is independent of the others in relation to the development of diabetes is unclear. We examined the prospective relationship of markers of inflammation (high sensitivity $\mathrm{C}$ reactive protein (CRP), migratory inhibitory factor (MIF), interleukin 6 (IL6), intracellular adhesion molecule (ICAM), leptin, adiponectin and serum levels of advanced glycation end-products (AGEs), and the soluble receptor for AGE - sRAGE with incident diabetes in a nested case-control study from the Australian, Diabetes, Obesity and Lifestyle Study (AusDiab).

Materials and methods: AusDiab was a population-based national study established in 1999/2000 with a five year follow-up in 2004/2005. An oral glucose tolerance test was performed at both time points. A nested case-control study was conducted on 224 cases of incident diabetes and 233 controls, who remained free of diabetes at the five year follow-up. CRP was measured in stored fasted sera from baseline (1999/2000) using a chemiluminescent immunoturbidimetric assay. IL6, MIF and sRAGE were measured using high sensitivity ELISA, and ICAM, leptin and adiponectin were measured using a bioplex assay. AGEs concentrations were determined using an $\mathrm{Ne}$ carboxymethyllysine sensitive monoclonal in an 'in house' indirect ELISA. Multivariate logistic regression was used to explore the relationship between inflammatory markers, cytokines and diabetes, adjusted for age and sex. CRP, MIF, IL6, adiponectin, leptin, sRAGE, and AGEs were tested in models using sex-specific derived quartiles or tertiles, and in a continuous form.

Results: Mean levels of cytokines were significantly different between cases and controls (age and sex adjusted) for leptin $(\mathrm{p}<0.01)$, CRP $(\mathrm{p}<0.01)$, sRAGE ( $\mathrm{p}=0.03)$ and ICAM $(\mathrm{p}<0.01)$, but not for AGEs $(\mathrm{p}=0.61)$, adiponectin ( $\mathrm{p}=0.09)$, IL6 ( $\mathrm{p}=0.12)$, and MIF ( $\mathrm{p}=0.52)$. In age and sex adjusted logistic regression, those with CRP levels in the top tertile (vs. bottom) were more likely to have diabetes [OR 95\% CI: 2.3 (1.4-3.6)]. This relationship remained significant after adjustment for sRAGE or AGEs, but lost significance with adjustment for BMI or waist circumference. Similarly, in age-sex adjusted models, those in the top quartile (vs. bottom) of ICAM [OR: 1.9 (1.1-3.3)], were more likely to develop diabetes but with further adjustment for BMI, this lost significance Those in the top quartile (vs. bottom) of sRAGE were less likely to develop diabetes [OR: $0.6(0.4-1.0)$ ] in age-sex adjusted models but this lost significance after adjustment for waist circumference or BMI. In age-sex adjusted models, those in the top quartile of leptin [2.3 (1.3 - 4.3)] or the top quartile of leptin-waist ratio [OR: $1.9(1.1-3.6)]$ were more likely to develop diabetes.

Conclusion: Elevated levels of circulating sRAGE appear to be associated with a lowering of diabetes risk, while CRP increased the risk of diabetes. Such associations were independent of age and sex and each other, but not from obesity. Furthermore, leptin-waist ratio, but not any of the other in- flammatory markers or cytokines, was independently associated with incident diabetes.

Supported by: Future Forum Research Grant

\section{1}

Glycaemic control and cardiovascular complications and death in type 2 diabetes: an observational study in 17,691 patients

K. Eeg-Olofsson ${ }^{1}$, J. Cederholm², P.M. Nilsson ${ }^{3}$, B. Zethelius ${ }^{2}$,

S. Gudbjörnsdottir ${ }^{1}$, B. Eliasson ${ }^{1}$

${ }^{1}$ Dept of Medicine, University of Gothenburg, ${ }^{2}$ Dept of Public Health and Caring Sciences, Uppsala University, ${ }^{3}$ Dept of Clinical Sciences Medicine, Lund University, Sweden

Background and aims: The role of improved glycaemic control in reducing cardiovascular disease (CVD) complications in diabetes is currently under debate. The aim of this study was to describe the association between glycaemic control and cardiovascular complications in type 2 diabetic patients using data from the Swedish National Diabetes Register.

Materials and methods: 17,691 type 2 diabetic patients, age 18 to 85 years and no previous coronary heart disease (CHD) or stroke, were identified 1997-1998 and followed for six years. A Cox regression analysis was performed with $\mathrm{HbAlc}$ as predictor and first incident fatal or non-fatal CHD $(n=2280)$, stroke $(n=1376), C V D(n=3339)$ and all cause mortality $(n=1707)$ as outcome measures. Endpoint events were retrieved by data linkage to the Swedish Cause of Death and Hospital Discharge Registers. Case fatality rate was calculated by logistic regression.

Results: Mean baseline characteristics: age $64.4 \pm 11.5$ years, diabetes duration $8.4 \pm 7.3$ years, HbAlc (DCCT standardized) $7.6 \pm 1.3 \%$; $49.1 \%$ used antihypertensive treatment and $10.8 \%$ were treated with lipid lowering drugs, $13.9 \%$ were smokers and $21.4 \%$ had microalbuminuria (MA). Increasing HbAlc by one unit increased the risk of CHD by $13 \%$, hazard ratio (HR) 1.13 (95\% CI 1.10-1.17), after adjustment for age, sex, diabetes duration, smoking, MA, antihypertensive and lipid-lowering treatment. The risk of stroke, CVD and all cause mortality also increased, HR 1.09 (1.05-1.14), HR 1.12 (1.09-1.15) and HR 1.12 (1.08-1.16), respectively. Comparing patients at different HbA1c intervals, with $\mathrm{HbAlc} 6.0-6.9 \%$ as reference, showed that patients with $\mathrm{HbAlc}$ 7.0-7.9\% had 33\% higher risk (HR CI 1.18-1.49) of fatal or non-fatal CHD, adjusted for age and sex. Patients with HbAlc 8.0-8.9\% had 56\% higher risk (HR CI 1.37-1.77) whereas patients with HbAlc 5.0-5.9\% had 8\% lower risk, although not statistically significant (HR CI 0.76-1.11). The same patterns were seen regarding stroke and CVD. An HbAlc increase by one unit also increased the risk of a CVD event to be fatal, odds ratio 1.07 (CI 1.003-1.14). Conclusion: This large observational study of an unselected sample of type 2 diabetic patients in clinical practice, demonstrates a gradually increasing risk of CHD, stroke, CVD and all cause mortality with increasing HbAlc levels independently of well known risk factors.

\section{2}

Progression from normal glucose tolerance to impaired fasting glycaemia and impaired glucose tolerance: differential roles of insulin resistance and beta cell dysfunction

K. Færch ${ }^{1}$, A. Vaag 2 , J.J. Holst ${ }^{3}$, O. Pedersen ${ }^{4,5}$, C. Glümer ${ }^{6}$,

K. Borch-Johnsen ${ }^{1,5}$

${ }^{1}$ Department of Epidemiology, Steno Diabetes Center, ${ }^{2}$ Department of Pathophysiology, Steno Diabetes Center, ${ }^{3}$ Department of Biomedical Sciences, The Panum Institute, Copenhagen, ${ }^{4}$ Department of Molecular Genetics, Steno Diabetes Center, Gentofte, ${ }^{5}$ Faculty of Health Sciences, University of Aarhus, ${ }^{6}$ Research Centre for Prevention and Health, Capital Region of Denmark, Glostrup, Denmark

Background and aims: We aimed at examining whether defects in insulin sensitivity and/or insulin secretion differed between different groups of prediabetic individuals and most importantly whether these defects were apparent in the normoglycaemic state five years prior to the development of isolated impaired fasting glycaemia (i-IFG), isolated impaired glucose tolerance (i-IGT) and combined IFG/IGT.

Material and methods: Prospective data from the Danish population-based non-pharmacological intervention study Inter99 were used. Individuals with normal glucose tolerance (NGT) at baseline and i-IFG, i-IGT, IFG/IGT or normal glucose tolerance (NGT) at the 5-year follow up were included $(n=3,145)$. Estimates of insulin sensitivity (ISI), acute insulin response (AIR) 
and beta cell function (Disposition Index, DI = ISI - AIR) were calculated at baseline and after 5 years of follow up based on glucose and insulin levels during an OGTT.

Results: Five years prior to the pre-diabetic diagnosis individuals who later developed i-IFG exhibited insulin resistance, defective insulin secretion and consequently reduced DI ( $\mathrm{p}<0.05$ vs. NGT, Table 1$)$. Those who subsequently progressed to i-IGT were more insulin resistant $(\mathrm{p}<0.05)$, but had normal AIR ( $\mathrm{p}=0.877$ vs. NGT), and thereby the same reduction in DI $(\mathrm{p}=0.768)$ as those developing i-IFG. At the 5-year follow-up, those with i-IGT had lower insulin sensitivity $(\mathrm{p}<0.001)$, equally reduced $\operatorname{AIR}(\mathrm{p}=0.186)$, and consequently more pronounced beta cell dysfunction $(\mathrm{p}<0.001)$ than those with i-IFG. IFG/IGT individuals exhibited worse defects than the other pre-diabetic groups.

Conclusion: Defective absolute insulin secretion followed by insulin resistance characterise the transition to i-IFG, while insulin resistance with secondary beta cell failure is likely to cause the development of i-IGT. The pre-diabetic states i-IFG and i-IGT may thereby have different aetiological origins with implications for future prevention and treatment of overt type 2 diabetes.

Table 1 Characteristics of individuals with incident i-IFG, i-IGT, IFG/IGT or NGT $(n=3,145)$.

\begin{tabular}{llllll}
\hline \multicolumn{1}{c}{$\begin{array}{l}\text { NGT } \rightarrow \\
\text { NGT } \\
(\mathrm{n}=2,842)\end{array}$} & $\begin{array}{l}\text { NGT } \rightarrow \\
\text { i-IFG } \\
(\mathrm{n}=83)\end{array}$ & $\begin{array}{l}\text { NGT } \rightarrow \\
\text { i-IGT } \\
(\mathrm{n}=192)\end{array}$ & $\begin{array}{l}\text { NGT } \rightarrow \\
\text { IFG/IGT } \\
(\mathrm{n}=28)\end{array}$ & $p<0.05$ \\
\hline \multicolumn{7}{l}{ Baseline (NGT state) } \\
\hline ISI $^{1}$ & $2.78(0.02)$ & $2.45(0.10)$ & $2.21(0.06)$ & $2.22(0.16)$ & a, b, c, d \\
AIR & $795(7.4)$ & $682(43.1)$ & $816(28.4)$ & $611(75.1)$ & a, c, d, f \\
DI $^{1}$ & $1983(21.0)$ & $1455(89.9)$ & $1501(61.5)$ & $1163(26.8)$ & a, b, c, f \\
\hline 5-year follow-up (pre-diabetic state) & & \\
\hline ISI $^{1}$ & $2.81(0.02)$ & $2.12(0.09)$ & $1.48(0.04)$ & $1.38(0.10)$ & a, b, c, d, e \\
AIR & $810(8.3)$ & $694(47.5)$ & $789(31.2)$ & $623(82.8)$ & a, b, c, f \\
DI & $2049(22.2)$ & $1213(76.8)$ & $916(37.7)$ & $743(82.0)$ & a, b, c, d, e, f \\
\hline
\end{tabular}

Means (SE) or ${ }^{1}$ geometric means (SE). P values are adjusted for age, gender, intervention, BMI, height, waist circumference and physical activity. a) i-IFG vs. NGT, b) i-IGT vs. NGT, c) IFG/IGT vs. NGT, d) i-IGT vs i-IFG, e) IFG/ IGT vs. i-IFG, f) IFG/IGT vs. i-IGT.

Supported by: Ministry of Science, Technology and Innovation, the Novo Nordisk Foundation, the Foundation of Gerda and Aage Haensch and the EXGENESIS grant from the European Union

\section{OP 33 Retinopathy: experimental treatments}

\section{3}

Candesartan attenuates vascular pathology in Ren-2 rats with diabetic retinopathy

J.L. Wilkinson-Berka ${ }^{1}$, G. Tan ${ }^{1}$, M.E. Cooper ${ }^{2}$, A. Miller ${ }^{1}$

${ }^{1}$ Immunology, Monash University, Melbourne, ${ }^{2}$ JDRF Danielle Alberti Centre for Diabetic Complications, Diabetes and Metabolism Division, Baker Heart Research Institute, Melbourne, Australia

Background and aims: Angiotensin II blockade is currently being evaluated as a treatment for diabetic retinopathy. We aimed to determine if the angiotensin type 1 receptor (AT1-R) blocker, candesartan, improves vascular pathology and modulates the expression of angiogenic and inflammatory mediators and renin-angiotensin system components in retina of rats with hypertension and diabetes.

Materials and methods: Streptozotocin diabetes was induced in transgenic Ren-2 rats, which are hypertensive and overexpress renin and angiotensin II in extra-renal tissues including the retina. At 8 weeks of age, female Ren-2 rats were randomised to the following groups; (1) non-diabetic control, (2) diabetic control and (3) diabetic + candesartan $(5 \mathrm{mg} / \mathrm{kg} /$ day, gavage). Animals were studied for 1 and 20 weeks of diabetes. $\mathrm{N}=6$ to 11 rats per group. Systolic blood pressure (SBP) was measured by a tail cuff method. Leukostasis was evaluated by counting adherent leukocytes in whole retina following in vivo perfusion with Concavanalin A. Acellular capillaries (devoid of pericytes and endothelial cells) were counted per high powered field of retina following typsin digestion of whole retina. Real-time PCR was performed for vascular endothelial growth factor (VEGF), intracellular adhesion molecule1 (ICAM-1), osteopontin, (pro)renin receptor, AT1a-R and aldosterone synthase.

Results: SBP was similar in non-diabetic and diabetic Ren-2 rats after 1 (241 $\pm 5.1 \mathrm{mmHg}$, non-diabetic; $239 \pm 6.3 \mathrm{mmHg}$, diabetic) and 20 weeks (230.3 $\pm 2.4 \mathrm{mmHg}$, non-diabetic; $235.4 \pm 3.6 \mathrm{mmHg}$ diabetic). Candesartan slightly reduced SBP in 1 week diabetic Ren-2 rats $(208.5 \pm 5.6 \mathrm{mmHg})$ and normalised SBP in 20 week diabetic Ren-2 rats $(123.1 \pm 3.6 \mathrm{mmHg})$ to Sprague Dawley control $(122.4 \pm 2.9 \mathrm{mmHg})$. In the 1 week study, retinal leukostasis was increased in diabetic Ren-2 rats (249.8 $\pm 59.1 /$ retina) compared to agematched non-diabetic control $(30.1 \pm 7.8 /$ retina, $\mathrm{P}<0.01)$. Candesartan reduced leukostasis in diabetic Ren-2 rats to the level of non-diabetic control (29.0 5 5.6/retina, $\mathrm{P}<0.01$ to diabetic control). Candesartan was similarly effective in the 20 week study. Acellular capillaries were increased in diabetic Ren-2 rats (4.66 $\pm 0.56 /$ field) compared to non-diabetic Ren- 2 rats $(1.37 \pm 0.16 /$ field), and in diabetic rats were reduced with candesartan to the level of nondiabetic control $(1.22 \pm 0.19, \mathrm{P}<0.005$ to diabetic control). Candesartan attenuated changes in diabetes-related gene expression of retinal VEGF, ICAM-1 and renin-angiotensin system components.

Conclusion: These findings indicate that the AT1-RB, candesartan, attenuates retinal vascular pathology in hypertensive diabetic Ren- 2 rats consistent with a potentially retinoprotective effect of this agent in diabetic retinopathy. Supported by: Astra Zeneca

\section{4}

Diabetic retinal neurodegeneration is associated with mitochondrial oxidative stress and it is ameliorated by angiotensin receptor blocker K.C. Silva, M.A.B. Rosales, S.K. Biswas, J.B. Lopes de Faria, J.M. Lopes de Faria

Faculty of Ciencies Medicine, State University of Campinas, Brazil

Background and aims: In addition to be a very frequent disease in patients with diabetes mellitus (DM), hypertension is the major risk factor associated with the development of diabetic retinopathy (DR). DR displays features of neurodegeneration disease with loss of neural cells and glial reaction. Oxidative stress mechanisms were described to be involved into the pathogenesis of DR. This investigation sought to determine whether hypertension exacerbates the oxidative stress induced-apoptosis in neural retinal cells and if high blood pressure control abrogates neurodegeneration in the retina from diabetic hypertensive rats.

Materials and methods: Streptozotocin induced diabetes was carried out in spontaneously hypertensive rats (SHR) and compared with normotensive 
control Wystar Kyoto rats (WKY). Diabetic SHR rats were randomized to receive no antihypertensive treatment (Sd) or an angiotensin receptor blocker (ARB) antihypertensive drug (losartan, $200 \mathrm{mg} / \mathrm{L}$ ). After 12 weeks, rats were sacrificed and the retinas were collected. At least five animals were used in each group for each experiment. The results were compared by analysis of variance (ANOVA) followed by Fisher's protected least-significant difference test.

Results: Apoptosis of retinal cells, evaluated by terminal deoxynucleotidyl transferase (TdT)-mediated dUTP-biotin nick-end labeling (TUNEL) assay, were higher in diabetic WKY compared with non diabetic control $(2.3 \pm 0.9 \mathrm{vs}$ $0.55 \pm 0.1, \mathrm{p}=0.03)$ and the concomitance of hypertension and diabetes further increased retinal apoptotic cells $(4.4 \pm 2.0, p=0.0003)$. Apoptotic cells showed neural and glial markers as indicated by specific antigens. The biochemistry marker of apoptosis, caspase- 8 expression, was elevated only in diabetic SHR rats $(\mathrm{p}=0.01)$. The superoxide production in retinal tissue was increased in presence of DM ( $10 \pm 3$ vs $22 \pm 6$ for control and diabetic WKY, $\mathrm{p}=0.03)$ and accentuated in diabetic hypertensive rats $(40 \pm 10, \mathrm{p}=0.0002)$. The reduced glutathione, an important antioxidative defense, was found reduced in diabetic SHR ( $140 \pm 54$ vs $56 \pm 10$ for control SHR and Sd, $\mathrm{p}=0.0005)$. As a result, an extensive retinal oxidative damage, estimated by nitrotyrosine (NT) and 8-hydroxy 2'deoxiguanosine (8-OHdG) expressions, were higher in diabetic WKY $(2.0 \pm 0.1$ vs $3.2 \pm 0.2,6.0 \pm 0.2$ vs $11 \pm 0.5$ for NT and 8 -OHdG, $\mathrm{p}<0.0001$ and $p=0.003$, respectively) and worsened in diabetic SHR rats $(3.7 \pm 0.26$ and $30 \pm 2.6$ for NT and 8-OHdG, $\mathrm{p}<0.0001$ ). To evaluate a possible role of mitochondria in superoxide production, the expression of $\mathrm{Bcl}-2$, an antiapoptotic mitochondrial protein and uncoupling protein-2 (UCP-2) were evaluated by western blot assays. The retinal expressions of Bcl-2 and UCP-2 were diminished in both diabetic groups $(67 \pm 7 v s 42 \pm 17$ and $60 \pm 9 v s 45 \pm 7, \mathrm{p}<0.05$ for Bcl-2; $42 \pm 27$ vs $36 \pm 7$ and $53 \pm 14$ vs $36 \pm 2, \mathrm{p}=0.03$ for UCP-2, for control vs diabetic WKY and control vs diabetic SHR, respectively). The antihypertensive treatment with losartan reestablished all the evaluated parameters.

Conclusion: These observations suggest that hypertension exacerbates the mitochondrial oxidative stress-induced apoptosis of retinal cells and the ARB ameliorated the retinal neurodegeneration in a model that combine diabetes and hypertension. This beneficial effect may be due to reestablishment of oxidative retinal status by reducing superoxide production and improving the antioxidative defense.

Supported by: the State of São Paulo Research Foundation (FAPESP), Kamila Cristina Silva was in receipt of a scholarship from the National Council for Scientific and Technological Development (CNPq)

\section{5}

Activation of angiotensin II signalling pathways in high glucose-treated rat retinas

F. Pricci ${ }^{1}$, L. Gaddini ${ }^{1}$, M. Villa ${ }^{1,2}$, A. Matteucci ${ }^{1}$, C. Mallozzi ${ }^{1}$,

A.M. Di Stasi ${ }^{1}$, F. Malchiodi-Albedi ${ }^{1}$, T.C. Petrucci ${ }^{1}$

${ }^{1}$ Cell Biology and Neuroscience, Istituto Superiore di Sanità, ${ }^{2}$ Medical Retina Unit, G.B. Bietti Foundation for the Study and Research in Ophthalmology, Rome, Italy

Background and aims: The vascular impairment in Diabetic Retinopathy (DR) have been deeply studied in the past. On the contrary, little is known about the neuroglial alterations, which may be present also before overt vasculopathy, as recently observed. Neuroglial damage could be linked to the excess glucose disposal which can activate several signalling pathways. Among these, the retinal renin-angiotensin system (RAS) could play a pivotal role in view of its well-known alterations in diabetic retina and its action on vasculature and tissue remodelling.

Aim of this study was to verify the possible involvement of RAS signalling in neuroglial dysfunction in an in vitro experimental model of diabetic retinopathy.

Materials and methods: We used retinal tissue cultures explanted from Sprague-Dawley rats and exposed to normal glucose (NG: $5.5 \mathrm{mM}$ ), high glucose (HG: $30 \mathrm{mM}$ ), iso-osmolar mannitol (M: $5.5 \mathrm{mM}$ glucose $+24.5 \mathrm{mM}$ mannitol) for $48 \mathrm{~h} \pm$ the Angiotensin-Converting Enzyme (ACE)-inhibitor enalaprilat $(200 \mu \mathrm{M})$.

Results: In retinal cultures exposed to HG, but not to NG or M, Western blot (WB) analysis revealed an increase in protein tyrosine phosphorylation, especially in specific bands identified as proline-rich tyrosine kinase 2

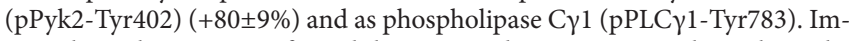
munohistochemistry confirmed the increased pPyK2 in HG, throughout the retinal layers, in both glial and neuronal cells. In NG, in contrast, positivity was only detected in endothelial cells. An increased Glial Fibrillar Acidic Pro- tein (GFAP) reactivity was also observed by immunohistochemistry and WB, suggesting a process of reactive gliosis. Further analysis in HG conditions showed an increase in c-src and lyn tyrosine kinase activities $(40 \% \pm 17$ and $63 \% \pm 5$, respectively), and an activation of both p44/42 MAPK (Erk 1/2) and transcription factor pCREB-Ser133. Enalaprilat reverted both the increase in protein tyrosine phosphorylation and activation of CREB, in agreement with the hypothesis that RAS signalling pathway was activated in HG. RAGE (a receptor for Advanced Glycosylation End-products-AGEs) expression was evaluated to verify if the observed modifications were related to AGEs in HG-treated retina. No induction of the receptor was observed. In contrast, treatment with glycated BSA $(200 \mu \mathrm{g} / \mathrm{ml})$ effectively upregulated RAGE.

Conclusion: These results strongly suggest that RAS signalling pathway is activated in HG-treated retinas and may have a role in diabetic neuroglial impairment

Supported by: grant from Istituto Superiore di Sanità, Rome, Italy

\section{6}

KIOM-79 long-term treatment inhibits the development of diabetic retinopathy and AGEs accumulation in retinal tissue

J. Kim, C.-S. Kim, E.J. Sohn, Y.M. Lee, D.S. Jang, J.S. Kim

Department of Herbal Pharmaceutical Development, Korea Institue of Oriental Medicine, Daejeon, Republic of Korea

Background and aims: KIOM-79 is the $80 \%$ ethanol extracts of parched puerariae radix, gingered Magnoliae cortex, Glycyrrhizae radix, and Euphorbiae radix. Our previous studies reported that KIOM-79 inhibits advanced glycation end products (AGEs) formation and prevents diabetic nephropathy in STZ induced diabetic rats. In this study, we evaluate the potential preventive effect of KIOM-79 on diabetic retinopathy in Zucker diabetic fatty (ZDF) rat, an animal model of type II diabetes.

Material and methods: Six week-old male ZDF rats were treated with KIOM$79(50 \mathrm{mg} / \mathrm{kg}$ body weight) once a day orally for 13 weeks. The retinas were collected at 13 weeks and examined the retinal vascular damage using wholemount preparation of the retinas.

Results: Serum levels of AGEs by ELISA were significantly reduced in ZDF rats treated with KIOM-79 ( $<<0.05$ vs vehicle). And the immunohistochemical analysis was shown that the numbers of AGEs positive cells were significantly reduced in ZDF rats treated with KIOM-79 ( $<<0.05$ vs vehicle). In periodic acid-Schiff staining of trypsin-digested retinal preparations, severe changes included pericytes ghost, acellular formation of capillaries were observed in ZDF rats, but in ZDF rats treated with KIOM-79, these changes were observed rarely. Furthermore, Fluorescein angiography of retina injected with fluorescein-dextran revealed that the changes of retinal vasculature (non-perfusion of fluorescein, fluorescein leakage and vessel narrowing) were significantly reduced in ZDF rats treated with KIOM-79 compared with ZDF rats treated with vehicle $(\mathrm{p}<0.05)$.

Conclusion: These results indicate that the treatment with KIOM-79 is useful in inhibiting the AGEs accumulation in retinal tissue and has a preventive effect on the development of diabetic retinopathy. 

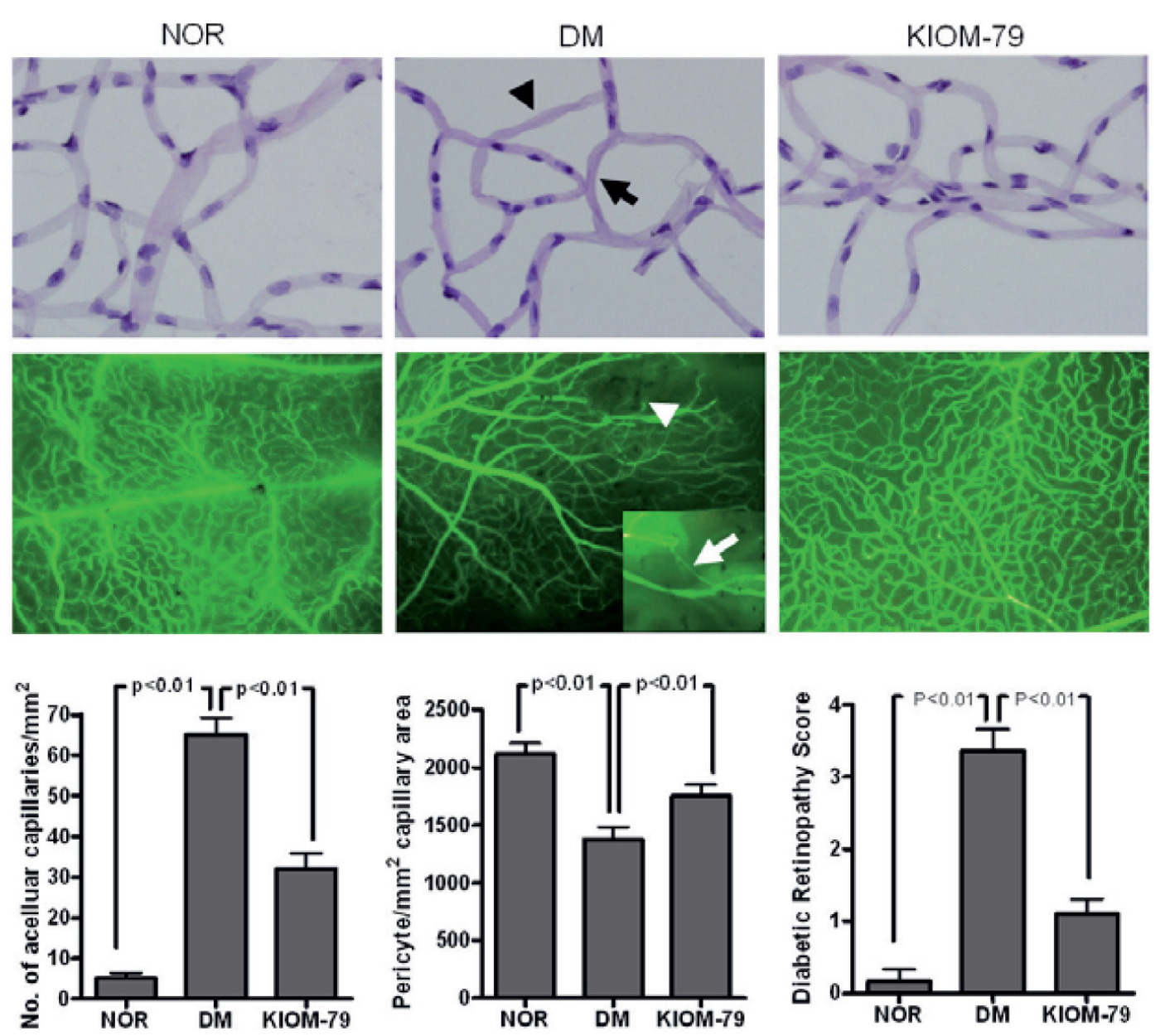

Figure 1. Effects of KIOM-79 on retinal vascular damage. In retinal vessel pattern analysis (upper panels), pericyte ghost (arrow) and acelluar capillary (arrowhead) were observed in ZDF rats. In fluorescein angiography (middle panels), arrowhead and arrow indicate non-perfusion areas and vessel narrowing (magnified inset), respectively. Quantitative analysis of acelluar formatmion, pericyte ghost and retinopathy score of retinal vessels revealed that KIOM-79 inhibits the acelluar formation and reduces the pericyte loss. Nor, normal lean rat; DM, ZDF rat; KIOM-79, ZDF rat treated with KIOM-79. All data were expressed as mean \pm SD

Supported by: the Korea Institute of Oriental Medicine

\section{7}

Long-term siRNA strategy regulates fibronectin overexpression and improves vascular lesions in retinas of diabetic rats

S. Roy ${ }^{1}$, S. Nasser ${ }^{1}$, D. Graves ${ }^{2}$, S. Roy ${ }^{1}$

${ }^{1}$ Medicine and Ophthalmology, Boston University School of Medicine, ${ }^{2} \mathrm{Oral}$ Biology, Boston University School of Dental Medicine, United States

Background and aims: To regulate abnormal gene expression associated with diabetic retinopathy, a long-term microvascular complication of diabetes, a sustained gene regulatory strategy is much desired. Having recently shown siRNA approach to be effective in long-term cell culture studies in reducing high glucose-induced fibronectin (FN) overexpression, in this study, we have investigated the efficacy of siRNA strategy in vivo as a long-term modulatory strategy for regulating altered gene expression in retinal vascular cells of diabetic rats. Time course studies conducted in rat retinas indicated siRNA efficacy up to 6 weeks.

Materials and methods: To determine long-term efficacy of siRNA strategy, three intravitreal injections at $1.5,3$, and 4.5 months of diabetes were performed with $3 \mu \mathrm{M}$ siRNA targeted to FN transcript in streptozotocin-induced diabetic rats. Control rats were either uninjected or injected with scrambled siRNA. After 1.5 months following the third injection, that is, at the end of 6 months of diabetes, retinal FN protein expression, basement membrane (BM) thickness, pericyte loss and acellular capillaries were assessed. At the time of sacrifice, blood samples were collected and evaluated for HbAlc level.

Results: Western blot analyses showed a significant increase in FN expression in retinas of diabetic rats compared to that of non-diabetic control rats (188 \pm
$4.2 \%$ of control vs. $100 \pm 7.4 \%$ of control, $\mathrm{p}<0.002)$. FN expression was significantly downregulated in retinas of diabetic rats injected with siRNA compared to those of uninjected diabetic control eyes and diabetic eyes injected with scrambled siRNA ( $145 \pm 9.9 \%$ of control vs. $188 \pm 4.2 \%$ of control, $\mathrm{p}=$ $0.03 ; 145 \pm 9.9 \%$ of control vs. $183 \pm 5.7 \%$ of control, $\mathrm{p}=0.032$, respectively). Electron microscopy analysis showed a significant increase in BM thickening in the diabetic rats compared to those of non-diabetic control rats $(62.5 \pm 5$ $\mathrm{nm}$ vs. $51.5 \pm 4.8 \mathrm{~nm}, \mathrm{p}<0.001)$. A significant reduction of retinal capillary BM thickness was observed in eyes injected with siRNA compared to those of uninjected diabetic control eyes and diabetic eyes injected with scrambled siRNA $(56.4 \pm 2.8 \mathrm{~nm}$ vs. $62.5 \pm 5.0 \mathrm{~nm}, \mathrm{p}=0.03 ; 56.4 \pm 2.8 \mathrm{~nm}$ vs. $63.7 \pm$ $3.9 \mathrm{~nm}, \mathrm{p}<0.01$, respectively). Both acellular capillaries and pericyte loss were significantly increased in the diabetic rats $(194 \pm 21 \%$ of control; $154 \pm 16 \%$ of control; $\mathrm{p}<0.01$, respectively), and long-term FN-siRNA treatment partially but significantly reduced the number of acellular capillaries and pericyte loss compared to those of diabetic $(122 \pm 14 \%, 118 \pm 12 \%$ of control, $\mathrm{p}<0.02$, respectively) or diabetic rats treated with scrambled siRNA $(116 \pm 21 \%, 109 \pm 15 \%$ of control, $\mathrm{p}<0.04$, respectively). Increased $\mathrm{HbAlc}$ levels confirmed hyperglycemic condition in the diabetic rats compared to non-diabetic control rats ( $11.5 \pm 1.9 \%$ vs. $5.4 \pm 0.8 \%$, respectively).

Conclusion: Results from this study suggest that siRNA approach may have potential for long-term beneficial effect on vascular lesions associated with diabetic retinopathy.

Supported by: National Eye Institute, NIH 


\section{8}

Increased retinal HSP27 expression in early experimental diabetes D. Burt, S. Pinach, E. Berrone, I. Miceli, M. Porta, P. Cavallo Perin, G. Gruden

Department of Internal Medicine, University of Turin, Italy

Background and aims: Diabetic retinopathy is characterised by microvascular abnormalities, apoptosis, and loss of retinal ganglion cells (RGC). Diabetes is known to induce oxidative stress in the retina and this has been pathogenetically associated with retinal neurodegeneration. Heat shock protein 27 (HSP27), a member of the small family of heat shock proteins, exists in a phosphorylated/non phosphorylated state (HSP27/P-HSP27). The expression of HSP27 is upregulated under various conditions of stress including oxidative stress and HSP27 exhibits cytoprotective activity. The aim of this work was to determine whether retinal HSP27 expression is increased in experimental diabetes in parallel with markers of oxidative stress.

Materials and methods: Eight-week old C57BL/6J mice $(\mathrm{n}=7)$ were rendered diabetic by intraperitoneal injection of streptozotocin (55 $\mathrm{mg} \mathrm{kg}-1$ ). Animals were considered diabetic if blood glucose levels were over $250 \mathrm{mg} / \mathrm{dl}$ three weeks after the injection. Mice $(n=5)$ sham-injected with sodium citrate buffer were used as non-diabetic control animals. Ten weeks after the onset of diabetes the animals were sacrificed, eyes enucleated, and retinas extracted. Blood glucose and glycated haemoglobin levels were measured by glucose-oxidase and latex method, respectively. HSP27 mRNA levels were determined by RT-real-time PCR on RNA extracts from whole retinas. HSP27 and P-HSP27 expression were evaluated by immunohistochemistry using specific anti-HSP27 and anti-phospho-HSP27 antibodies. The signal was revealed using the LSAB+ system-HRP, quantified blinded by two independent observers, and expressed as percentage area of positive staining in 7 randomly selected fields per section. Expression of nitrotyrosine and $\mathrm{Cu} / \mathrm{Zn}$ superoxide-dismutase (SOD), markers of oxidative stress, was assessed by immunohistochemistry and immunofluorescence, respectively.

Results: Blood glucose and glycated haemoglobin were significantly higher in diabetic animals as compared to non-diabetic control animals. In the whole retina from diabetic animals there was a significant $(\mathrm{p}<0.05)$ fourfold increase in HSP27 mRNA levels as compared to controls. Furthermore, immunohistochemical analysis showed that both HSP27 and P-HSP27 expression were significantly greater in the retina from diabetic mice than in the retina from control animals (HSP27: $21.6 \pm 3.4$ vs. $3.9 \pm 1.8, \mathrm{p}<0.005$; P-HSP27: $26.4 \pm 17.6$ vs. $5.14 \pm 2.9$; percentage area, $\mathrm{p}<0.05)$ and localised to the RGC layer. Double immunostaining for Thy-1, a RGC specific marker, confirmed that HSP27 was overexpressed predominantly by RGC. In the retinas from diabetic mice there was also a significant increase in the expression of markers of oxidative stress (nitrotyrosine: $18.3 \pm 6.6$ vs. $3.5 \pm 1.5 ; \mathrm{p}<0.001 ; \mathrm{Cu} / \mathrm{Zn}$ SOD: $25.5 \pm 4.3$ vs. $2.3 \pm 2.87$; percentage area, $\mathrm{p}<0.0001$ ) localized to the RGC layer.

Conclusion: In early experimental diabetes there is an overexpression of both total and phosphorylated HSP27 in RGC that parallels enhanced expression of markers of oxidative stress. Induction of HSP27 may represent a defence mechanism in response to increased oxidative stress in the diabetic retina.

\section{OP 34 Adipokines: from bone to heart}

\section{9}

Reversible induction of insulin resistance by adipokines in human megakaryocytes

A.J. Gerrits, E. Gitz, A.C. Koekman, J.-W.N. Akkerman

Department of Clinical Chemistry and Haematology, University Medical Center Utrecht, Netherlands

Background and aims: Diabetes mellitus (DM) patients have a 2-8 fold increased risk for cardiovascular disease (CVD) and suffer from microvascular (nephropathy, retinopathy) and macrovascular (peripheral arterial disease) complications. We demonstrated previously in normal platelets that insulin signals through Insulin Receptor Substrate-1 (IRS-1) and inhibits $\mathrm{Ca}^{2+}$ mobilization by interfering with the suppression of cAMP, a platelet inhibitor. Platelets from type $2 \mathrm{DM}$ patients have become insulin resistant and their platelets aggregate better than controls. This hyperaggregability might be a cause for the increased CVD risk. In the present project we search for the cause of this insulin resistance. Obese subjects have abnormal levels of plasma adipokines and are prone to develop insulin resistance. Platelets show only minor protein synthesis and their properties are determined during their synthesis by the megakaryocyte. We investigated whether adipokines change the synthesis of platelet properties in the megakaryocyte.

Materials and methods: In suspensions of megakarocytic CHRF-288-11 cells incubated with adipokines and plasma from an obese individual, we measured (i) insulin inhibition of thrombin-induced $\mathrm{Ca}^{2+}$ mobilization, (ii) aggregation induced by arachidonic acid, (iii) IRS-1 signaling to protein kinase $\mathrm{B} \alpha, \beta(\mathrm{AKT} 1,2)$ induced by insulin.

Results: Insulin (0.1-100nM) inhibited $\mathrm{Ca}^{2+}$ mobilization by $20 \pm 7 \%$ at $1 \mathrm{nM}$ $(\mathrm{p}<0.05)$ and by $47 \pm 3 \%$ at $100 \mathrm{nM}(\mathrm{p}<0.0005)$. Inhibition was mimicked by prostacyclin and blocked by an adenylyl cyclase inhibitor confirming that insulin controls cAMP. Incubation with adipokines in concentrations 10 fold the physiological range showed that leptin, resistin, PAI- 1 and RBP- 4 but not IL-6, TNF $\alpha$ and visfatin made cells resistant to insulin for up to 7 days. After a $2 \mathrm{hrs}$ incubation insulin sensitivity was rescued by cantharidin, but longer incubation made cells unresponsive to this serine phosphatase inhibitor. The protein synthesis inhibitors puromycin and cycloheximide failed to change the rapid induction of insulin resistance, revealing a transcription-independent mechanism. The adipokine-induced insulin resistance revealed at the level of $\mathrm{Ca}^{2+}$ also showed at the level of protein kinase $\mathrm{B}$ activation and made aggregation of megakaryocytes insensitive to inhibition by insulin. A preliminary experiment with blood from an obese subject showed that her plasma could replace the insulin resistance inducing adipokines whereas plasma from lean subjects could not.

Conclusion: Leptin, resistin, PAI- 1 and RBP-4 induce insulin resistance in megakaryocytes by interfering with IRS-1. The resistance is reversible during short, and irreversible during prolonged incubation indicative for a transcription-independent and -dependent mechanism. This is a first report of insulin resistance induced by adipokines in the precursor of platelets, the megakaryocyte, and a possible explanation for the hyperactivity of platelets and increase in CVD risk when obese subjects develop type 2 diabetes mellitus.

Supported by: Dutch Diabetes Research Foundation

\section{0}

Adiponectin and skeletal muscle: pathophysiological implications during stress

J. Jortay ${ }^{1}$, M. Senou ${ }^{2}$, L. Noel ${ }^{1}$, M.-C. Many ${ }^{2}$, S.M. Brichard ${ }^{1}$

${ }^{1}$ Unit of Endocrinology and Metabolism, Université catholique de Louvain, ${ }^{2}$ Unit of Experimental Morphology, Université catholique de Louvain, Brussels, Belgium

Background and aims: Adiponectin ( $\mathrm{ApN})$ is a hormone exclusively secreted by adipocytes under normal conditions. This adipokine exhibits insulin-sensitizing and anti-inflammatory properties as well as modulatory effects on oxidative stress thereby thwarting several disorders belonging to the metabolic syndrome. We have recently shown that ApN was induced in tibialis anterior muscle of mice injected with lipopolysaccharide (LPS) and in $\mathrm{C} 2 \mathrm{C} 12$ myotubes cultured in the presence of pro-inflammatory cytokines. 
We have hypothesized that this expression of ApN in muscle could be viewed as a local protective mechanism to counteract excessive inflammatory reactions and oxidative damage.

To test this hypothesis, we examined whether muscles of ApN-knockout (ApN-KO) mice exhibit higher degree of oxidative stress and apoptosis than those of wild-type (WT) mice when challenged by LPS and whether these abnormalities may be corrected by local administration of ApN.

Materials and methods: ApN-KO mice (with virtually undetectable circulating $\mathrm{ApN}$ ) were used and compared to WT mice. Mice were injected with ip lipopolysaccharide (LPS) or saline. $24 \mathrm{~h}$ later, mice were killed and tibialis anterior muscles were collected for immunohistochemistry. In additional experiments, muscle transfer of $\mathrm{ApN}$ gene was performed in anesthetized ApN$\mathrm{KO}$ mice by injection of ApN cDNA containing-plasmid (pcDNA3.1-ApN) followed by electroporation in one tibialis anterior, the contro-lateral muscle being electroporated by a control plasmid. Ten days after electroporation, ApN was overexpressed only in the muscle injected with ApN cDNA without any rise in circulating levels of the adipokine. Mice were then challenged by LPS as described above.

Results: We first confirmed that WT mice displayed a positive labelling for ApN in myocytes after LPS, but not after saline, injection. We next found that muscles of ApN-KO exhibited myotube degenerescence when compared to WT mice, especially after challenge by LPS. We searched for the underlying mechanisms. Even in the basal state (after saline), myotubes of ApNKO displayed a positive immunolabelling for 3 markers of oxidative stress (peroxiredoxin 3/5 and heme oxygenase-1) as well as for a lipid peroxidation product (hydroxynonenal). The pro-inflammatory cytokine (TNF-alpha) and a marker of apoptosis (caspase-6) were also already present in the basal state in muscle of these ApN-KO mice, but not in those of WT mice. After LPS injection, immunoreactivity for these markers was much more stronger in muscle of ApN-KO mice, while being only slightly detected in WT mice. Finally, we demonstrated that transfer of ApN gene prevents muscle damage in ApN-KO mice challenged by LPS. Thus, muscular electroporation with pcDNA3.1-ApN markedly reduced the expression of oxidative markers, hydroxynonenal, TNF-alpha and caspase- 6 when compared to the contro-lateral untreated muscle.

Conclusion: The presence of ApN appears to be crucial to counteract locally excessive and deleterious inflammatory reactions, oxidative stress and subsequent apoptosis. These results may have repercussions in the pathogenesis of the metabolic syndrome where chronic inflammation and oxidative stress play determinant roles.

Supported by: a fellowship from the FRIA (BELGIUM)

\section{1}

Distribution and characteristics of adiponectin $\mathrm{R} 1$ and $\mathrm{R} 2$ receptor mRNA gene expression in human visceral and subcutaneous adipose tissue

J.H. Pinkney ${ }^{1}$, S.P.Y. Wong ${ }^{1}$, D. Kerrigan ${ }^{2}$, K. Kos ${ }^{1}$, J.P.H. Wilding ${ }^{1}$

${ }^{1}$ Obesity Research Group, University of Liverpool, ${ }^{2}$ Bariatric Surgery,

University Hospital Aintree, Liverpool, United Kingdom

Background and aims: Adiponectin (ADN) is secreted by adipose tissue, and exerts metabolic actions in liver, skeletal muscle and vascular tissues. Reduced expression of ADN may contribute to development of type 2 diabetes. ADN acts through two receptors, R1 and R2 expressed in target tissues, and R2 is the main receptor in liver and muscle. These receptors acivate AMPK and have been shown to play a role in the regulation of fatty-acid and glucose metabolism. Whilst there is paucity of data from human studies on AT-derived ADN receptor expression, a disruption of the R2 gene in mice was shown to mimic features of human Type 2 diabetes. The aim of this study was to characterise expression of ADN receptors in human subcutaneous and visceral adipose tissue regions.

Materials and methods: Samples of subcutaneous (SCAT) and visceral adipose tissue (VAT) were obtained from 18 lean volunteers and 39 obese well characterised subjects undergoing bariatric surgery. R1 and R2 mRNA were quantified by RT-PCR using QuantiTect ${ }^{\circ}$ Multiplex PCR (Qiagen, Crawley, UK), with prevalidated primers and Quantiprobes ${ }^{\circ}$, on a ROTORGENE2000 analyser (Corbett Research, Cambridge, UK). B-actin was the housekeeping gene.

Results: $\mathrm{R} 2$ receptor/ $\beta$-actin expression ratios indicated far more abundant expression of R2 than R1 in both VAT (median (interquartiles) 7.6(5.410.5) $\mathrm{X} 10^{-3}$ vs $\left.2.07(0.53-7.9) \times 10^{-5} \mathrm{p}<0.001\right)$ and SCAT $\left(8.1(6.1-10.7) \times 10^{-3}\right.$ vs $\left.2.1(0.3-8.4) \times 10^{-5} ; \mathrm{p}<0.001\right)$. However, there were no differences in $\mathrm{R} 1$ or R2 mRNA expression between lean and obese subjects $(0.38<\mathrm{p}<0.77)$, unlike adiponectin mRNA; and expression was unaffected by age or gender. R1 expression in VAT correlated inversely with serum $\mathrm{ADN}(\mathrm{r}=-0.37 ; \mathrm{p}<0.01)$, but neither R1 in SCAT, nor R2 in VAT or SCAT correlated with serum levels. In marked contrast to findings with serum ADN and expression of ADN mRNA, receptor mRNA expression in VAT and SCAT was unrelated to plasma insulin, insulin sensitivity, BMI or waist circumference (all $p>0.05$ ). When examined against a panel of adipokines, R1 and R2 expression in VAT and R1 in SCAT correlated with expression of MMIF-1 mRNA (all $r \geq 0.35 ; \mathrm{p}<0.02$ ).

Conclusion: R2 is the predominant receptor isoform expressed in human VAT and SCAT, and is expressed at similar levels at both sites. In marked contrast to ADN itself, receptor expression shows no apparent relationships with metabolic syndrome parameters. Our study does therefore not confirm the proposed pivotal role of $\mathrm{R} 2$ in the pathogenesis of insulin resistance found in mice. ADN receptors in adipose tissue may therefore play a separate role in rodent and mice adipose tissue physiology. Further insight is necessary in the autocrine and paracrine effects of ADN in human adipose tissue. Supported by: Diabetes UK and University of Liverpool Research and Development Fund

\section{2}

RNA oligonucleotides improve metabolic syndrome by targeting retinol binding protein 4 gene in high fat fed mice

Y. $\operatorname{Tan}^{1}$, R. Dale ${ }^{2}$, L.-Q. Sun' ${ }^{2}$ M.A. Kamal ${ }^{1}$, X. Qu

${ }^{1}$ Medical and Molecular Biosciences, University of Technology Sydney, Australia, ${ }^{2}$ Research \& Development, Oligos Etc.,Inc, Wilsonville, United States

Background and aims: Retinol binding protein 4 (RBP4) is a newly discovered adipokine, which plays a role in insulin resistance and obesity. The aim of this study was to examine whether down-regulation of the RBP4 expression could impact on abnormalities of glucose and lipid metabolism seen in metabolic syndrome.

Materials and methods: RNA oligonucleotides (RNA oligos) were designed to be complementary to the 5'-untranslated region, start codon or termination regions of the RBP4 gene and chemically modified with 2'-O-methyl and a 3'-butanol tag. In vitro activity of the RNA oligonucleotides was determined in 3T3-L1 adipocytes with the aid of Lipofectamine ${ }^{\mathrm{rw}} 2000$ and assayed by Real-time PCR. High fat diet (HFD) fed mice were used. 3 groups of HFD mice $(\mathrm{n}=8)$ received treatment with saline $(5 \mathrm{ml} / \mathrm{kg}$, HFD control), rosiglitizoe (RSG, $10 \mathrm{mg} / \mathrm{kg}$ ) or RNA oligos $(25 \mathrm{mg} / \mathrm{kg}$ ) every second day for 4 weeks. By the end of the treatment, animals were subjected to insulin tolerance test (i.p. ITT $1 \mathrm{U} / \mathrm{kg}$ ) and glucose tolerance test (i.p. GTT $2 \mathrm{~g} / \mathrm{kg}$ ). The levels of serum glucose, insulin, triglycerides (TG) and non-esterified fatty acids (NEFAs) were measured. The RBP4 levels in the serum and adipose tissue and the level of glucose transport 4 (Glut 4) in skeletal muscles were examined by Western blotting.

Results: RBP4 mRNA levels in RNA oligos transfected 3T3-L1 cells were significantly lower compared with control cells, indicating that the expression of RBP4 mRNA was inhibited by the RNA oligos. In vivo study showed that RSG and RNA oligo treatments did not affect whole body weight but significantly reduced the weight of abdominal adipose tissues $(1.01 \pm 0.15$ vs $0.71 \pm 0.10$ and $0.64 \pm 0.05 \mathrm{~g}, \mathrm{P}<0.05)$. Compared with the control, both RSG and RNA oligos treatment significantly reduced blood glucose levels $(8.72$ \pm 0.24 vs $6.47 \pm 0.20$ and $6.58 \pm 0.48 \mathrm{mmol}, \mathrm{P}<0.001$ and 0.01 , respectively) and the corresponding values for serum insulin were $15.62 \pm 2.87,6.70 \pm 1.60$ and $9.88 \pm 1.37 \mu \mathrm{U} / \mathrm{ml}$, respectively $(\mathrm{p}<0.05)$. Following an i.p. injection of glucose, there were significant reductions in area under curve (AUC) of the blood glucose concentration-time profiles in RSG and RNA oligos treated mice. The AUC[glu] was $2617 \pm 287$ for HFD control vs $1907 \pm 105$ and 2043 $\pm 112, \mathrm{p}<0.05)$. RSG and RNA oligos treatments also enhanced insulin-mediated glucose disposal during i.p. ITT: AUC [glu] was $925 \pm 61$ vs $745 \pm 74$ and $795 \pm 27$, respectively. Both RSG and RNA oligos attenuated the rise in NEFA associated with HFD feeding $(0.62 \pm 0.04$ and $0.64 \pm 0.08 \mathrm{mmol}$ compared with $0.79 \pm 0.07 \mathrm{mmol}$ in HFD mice). RNA oligos but not RSG significantly reduced serum TG level $(0.42 \pm 0.03 \mathrm{mmol}$ in RNA oligo group vs $0.52 \pm 0.07$ in RSG group and $0.55 \pm 0.06 \mathrm{mmol}$ in HFD group, $\mathrm{P}<0.05$ ). Western blotting revealed that RNA oligos significantly inhibited the expression of RBP4 in adipose tissue and enhanced GLUT4 expression in skeletal muscle. Although RSG up-regulated GLUT 4 expression in muscle it had no significant effect on RBP4 expression in adipose tissues. Both RNA oligos and RSG significantly reduced the circulating RBP4 levels.

Conclusion: RNA oligonucleotides targeting RBP4 could improve hyperlipidaemia and glucose-intolerant conditions associated with HFD feeding, sug- 
gesting that RBP4 may be a new molecular target for potential treatment of metabolic syndrome and potentially for prevention of type 2 diabetes. Supported by: University of Technology Sydney and Oligos Etc., Inc

\section{3}

Relationship between serum retinol binding protein 4 and liver fat content in subjects without known diabetes

H. Yan ${ }^{1}$, X. Gao ${ }^{1}$, M. Liu 1 , Q. Gu' ${ }^{2}$ B. Zhang ${ }^{3}$, X. Li $^{4}$

${ }^{1}$ Endocrinology and Metabolism, Zhongshan Hospital,Fudan University, Shanghai, ${ }^{2}$ Geriatrics, Zhongshan Hospital,Fudan University, Shanghai,

${ }^{3}$ Geriatrics, 10th People's Hospital of Shanghai, Tongji University, Shanghai, ${ }^{4}$ Endocrinology and Metabolism, Dalian University Affiliated Zhongshan Hospital, Dalian, China

Background and aims: Previous studies have shown that adipose-derived serum retinol-binding protein 4 (RBP4) levels are increased in insulin-resistant mouse models and in subjects with insulin resistance or type 2 diabetes. However, fewer studies researched the association of serum RBP4 and liver fat content. The purpose of this study was to investigate the relationship between serum RBP4 and liver fat content in subjects with nonalcoholic fatty liver disease (NAFLD)and without known diabetes .

Materials and methods: 106 patients diagnosed as fatty liver by ultrasonography (male/female:61/45; aged $47.44 \pm 14.16$ years) were enrolled. Subjects with known diabetes, chronic virus hepatitis, and those with alcohol consumption $(\geq 30 \mathrm{~g} / \mathrm{d}$ in male and $\geq 20 \mathrm{~g} / \mathrm{d}$ in female) were excluded. Anthropometrics and laboratory tests including lipid profile, OGTT glucose and insulin level after $75 \mathrm{~g}$ glucose load were measured. Serum RBP4 was detected by ELISA and validated by quantitative Western blotting. Insulin resistance was represented by HOMA-IR in all patients. Insulin Sensitivity index (ISI) was determined by hyperglycemic clamp technique in 17 subjects. Liver CT scan was used to determine fat content in the whole liver.

Results: Liver fat content in all subjects distributed from $0 \%$ to $34.72 \%$. When liver fat increased by quartile order $[<3.11 \%(\mathrm{n}=26), \geq 3.11 \% \sim<6.34 \%$ $(\mathrm{n}=27), \geq 6.34 \% \sim<10.82 \%(\mathrm{n}=27), \geq 10.82 \%(\mathrm{n}=26)]$, serum RBP4 level was $47.09 \pm 1.19,53.11 \pm 8.74,52.81 \pm 1.52,53.54 \pm 9.86 \mathrm{mg} / \mathrm{L}$ respectively $(p>0.05)$. Pearson correlation analysis demonstrated that RBP4 levels associated with liver fat content $(r=0.202, p=0.049)$, waist circumference $(r=0.209, p=0.041)$, $\operatorname{WHR}(r=0.347, p=0.001)$, HDL-c $(r=-0.356, p=0.001)$, triglyceride $(r=0.322$, $p=0.002)$, systolic blood pressure $(r=0.226, p=0.027)$, diastolic blood pressure $(r=0.228, p=0.025)$ and $\operatorname{sex}(r=-0.409, p=0.000)$. However, there were no significant associations of RBP4 and age, BMI, hip circumference, total cholesterol, LDL-c, fasting and 2 hour serum glucose and insulin, HOMA-IR, ISI(all $p>0.05$ ). Multiple linear regression analysis revealed that RBP4 was correlated with $\operatorname{WHR}(p=0.018)$, but not with liver fat content $(p=0.437)$ ( table 1).

Table 1 Multiple Linear Regression Analysis of RBP4

\begin{tabular}{|c|c|c|c|c|}
\hline Model & $\begin{array}{l}\text { Unstandardized } \\
\text { Coefficients B }\end{array}$ & $\begin{array}{l}\text { Unstandardized } \\
\text { Coefficients Std. } \\
\text { Error }\end{array}$ & $\begin{array}{l}\text { Standardized } \\
\text { Coefficients } \\
\text { Beta }\end{array}$ & $\begin{array}{l}\mathrm{p} \\
\text { value }\end{array}$ \\
\hline WHR & 67.837 & 28.019 & 0.424 & 0.018 \\
\hline Waist circumference & -0.389 & 0.213 & -0.337 & 0.071 \\
\hline Sex & -4.819 & 2.590 & -0.203 & 0.066 \\
\hline HDL-c & -6.937 & 4.975 & -0.154 & 0.167 \\
\hline Triglyceride & 1.308 & 1.038 & 0.134 & 0.212 \\
\hline $\begin{array}{l}\text { Systolic blood } \\
\text { pressure }\end{array}$ & 0.060 & 0.074 & 0.106 & 0.414 \\
\hline Liver fat content & 0.173 & 0.222 & 0.092 & 0.437 \\
\hline $\begin{array}{l}\text { Diastolic blood } \\
\text { pressure }\end{array}$ & 0.053 & 0.141 & 0.051 & 0.707 \\
\hline
\end{tabular}

Conclusion: Our results showed that RBP4 was associated with WHR,but not with liver fat content. It suggested that the level of RBP4 was mainly effected by visceral fat accumulation.

\section{4}

Epicardial adipose tissue gene expression in metabolic syndrome M. D’Adamo ${ }^{1}$, V. Guglielmi ${ }^{1}$, A. Bellia ${ }^{1}$, B. Micchelini ${ }^{1}$, S. Costa ${ }^{1}$, B. Carteni ${ }^{1}$, C. Del Giudice ${ }^{2}$, G. Donadel ${ }^{1}$, D. Lauro ${ }^{1}$, M. Federici ${ }^{1}$, L. Chiarielloº R. Lauro ${ }^{1}$, P. Sbraccia ${ }^{1}$

${ }^{1}$ Internal Medicine, University of Rome Tor Vergata, ${ }^{2}$ Surgery, University of Rome Tor Vergata, Italy

Background and aims: Epicardial Adipose Tissue (EAT) is situated predominantly on the right-ventricular free wall and the left-ventricular apex, has been shown to have a high capacity for non-esterified fatty acid (NEFA) release and is proposed as a source of this preferred metabolite for the myocardium. Furthermore EAT accumulation has been related to clinical and anthropometrical parameters of Metabolic Syndrome.

Aim of our study was to evaluate adipokines expression pattern and macrophage infiltration rate of EAT in metabolic syndrome.

Materials and methods: To this aim we studied 10 subjects who underwent cardiac surgery for either coronary artery by-pass graft (CABPG) or valvular disease: 5 patients with metabolic syndrome $(3 \mathrm{M} / 2 \mathrm{~F}$; BMI $27.6 \pm 2.6$; waist 98.5 $\pm 7.0 ; 5 / 5$ Hypertensive on treatment; $3 / 5$ dyslipidemic; $4 / 5$ CABP) (MS) e 5 patients without metabolic syndrome (3M/2F; BMI $26.8 \pm 2.8$; waist 91.2 $\pm 12.37 ; 3 / 5$ Hypertensive on treatment; No dyslipidemia, 2/5 CABP) (No-MS).

EAT biopsy ( $\sim 0.5 \mathrm{~g})$ was performed during surgery and total RNA was extracted, reverse transcribed, amplified and, by means of Real-time PCR, we evaluated the expression level of major adipokines (adiponectin, leptin,TNFa, IL-6), and macrophage markers CD68 and CD14.

Results: Expression levels of insulin-sensitizing-adipokine adiponectin were significantly lower in the MS group compared to control group (27.3 \pm 3.5 vs $43.7 \pm 4.6$ relative amounts, $p<0.02$ ), whereas leptin expression levels were higher in the MS group ( $77.6 \pm 15.5$ vs $32.7 \pm 4.6$ relative amounts, $\mathrm{p}<0.05)$. Inflammatory cytokines TNF- $\alpha$ and IL- 6 show a similar expression pattern in the two groups, with MS group showing very high expression levels compared to No-MS group (TNF- $\alpha 147.7 \pm 21.5$ vs $44.7 \pm 9.6$ relative amounts, $\mathrm{p}<0.005$; IL-6 $55.8 \pm 7.5$ vs $12.7 \pm 2.6$, relative amounts $\mathrm{p}<0.01$ ).

These data, along with an increased expression of macrophage markers (CD68 108.7 \pm 12.5 vs $12.4 \pm 2.6$ relative amounts, $\mathrm{p}<0.005$; CD14 $53.8 \pm 8.5$ vs $9.7 \pm 2.6$ relative amounts, $\mathrm{p}<0.01$ ), reveals a large macrophage infiltration in EAT from subjects with Metabolic Syndrome.

Conclusion: These data suggest that: 1) EAT has an adipocytokine expression pattern similar to abdominal visceral adipose tissue; 2) the increased expression of inflammatory cytokine in EAT of subjects with Metabolic Syndrome, might contribute to the cardiovascular component of the Syndrome. 


\section{OP 35 Endothelium cross-talk}

\section{5}

Visceral adipose stromal secretome triggers early events in atherothrombotic endothelial injury

F. Hanzu ${ }^{1}$, M. Palomo ${ }^{2}$, M. Diaz-Ricart ${ }^{2}$, P. Gomez ${ }^{3}$, M. Garaulet ${ }^{3}$, S. Casas ${ }^{1}$, R. Gomis ${ }^{1}$

${ }^{1}$ Diabetes and Obesity Laboratory.Endocrinology and Nutrition Unit, Hospital Clinic /IDIBAPS, Barcelona, ${ }^{2}$ Hemotherapy and Haemostasis, Hospital Clinic, Barcelona, Spain, ${ }^{3}$ Nutrition, University of Murcia, Spain

Background and aims: Central abdominal obesity is a major risk factor for atherothrombotic morbidity/mortality. Dysfunction of endothelium is a key event in the initial step of cardiovascular disease. This study aims to determinate the direct role of adipose tissue secretome fractions from obese subjects in the early atherothrombotic endothelial injury and to identify biomolecules implicated.

Materials and methods: Abdominal subcutaneous and visceral (omental) adipose tissue was obtained from morbid (BMI: 40-45 kg/m2), obese males $(n=6)$, without other cardiometabolic associated risk factors (no alteration of glucose metabolism, no dyslipidemia, no major cardiovascular disease) undergoing bariatric surgery. Fat pads were separated in stromal and adipocyte cell fraction. Resulted cell types were incubated with nonfetal medium (24h) to obtain the secretome. Stromal/adipocytes, respectively visceral/subcutaneous conditioned medium (CM) containing the secretome of adipose tissue fractions were further utilised. Human endothelial cells (EC) were grown, respecting the Jaffe method, using human sera. Studies were carried out using EC monolayer and extracellular matrix (ECM) generated by EC. Semiconfluent EC were incubated for $24 \mathrm{~h}$ with $100 \%$ CM or have been cocultured for 7 days with a medium containing 20\% CM - of adipose tissue fractions from morbid obese. Expression of adhesion molecules in EC and prothrombotic ECM proteins were determinate by immunogold labelling and qRT-PCR. Images were analysed by computerized morphometry. Cellular adhesion on ECM under flow conditions was studied. Cellular proliferation was assessed through analysis on light microscopy and cell cycle evaluation using PI staining flow cytometry on a FACScan (FACS Calibur).

Results: Exposure of EC to adipose tissue CM fractions induces an increased presence of adhesion receptors on EC monolayer ( $s V C A M-1, s I C A M-1, s E$ $C A M-1)$ and expression in prothrombotic ( $v$. Willebrand factor) ECM proteins and platelet adhesion under reologic flow conditions. qRT-PCR shows increased mRNA levels for all corresponding genes. Highest expression was induced by obese visceral stromal secretome fraction. Microscopic analysis of EC exposed to CM fractions shows alteration in cell morphology. Cell cycle analysis by flowcytomery using DNA histograms with coefficients of variations $<7 \%$ are indicating a charactestic pattern of cell cycle for control EC with $20.2+/-2.6 \%$ of cells in S+G2M phase and an heterogeneous pattern for EC exposed to CM fractions. A tendency to increase cell death after exposure to visceral stromal conditioned medium was observed.

Conclusion: Results of this study indicate the causative role of stromal, nonadipocyte cell fraction secretome of visceral morbid obese adipose tissue in the initiating endothelial injury events of atherothrombotic disease. Those, advancing in understanding of early endothelial injury mechanism in obesity, will permit future target suppression of various proinflammatory cascades in adipocytes which specifically represent an exciting new therapeutic opportunity for cardiovascular diseases.

Supported by: Ministerio de Educacion y Ciencia, Ministerio de Sanidad y Consumo, CIBER de Diabetes y Enfermedades Metabólicas Asociadas, Instituto de Salud Carlos III

\section{6}

Differences in vascular activity between Exenatide and GLP-1 in rat conduit arteries ex vivo

T. Nyström ${ }^{1}$, D. Nathanson ${ }^{1}$, Ö. Tütüncü ${ }^{1}$, A. Bulhak ${ }^{2}$, J. Pernow ${ }^{2}$, A. Sjöholm ${ }^{1}$

${ }^{1}$ South Hospital, Karolinska Institutet, ${ }^{2}$ Karolinska University Hospital, Karolinska Institutet, Stockholm, Sweden

Background and aims: Glucagon-like peptide-1 (GLP-1) is a promising drug decreasing blood glucose in type 2 diabetes. In addition, its salutary effects in endothelial dysfunction, heart failure and myocardial infarction are of high interest. Recently, we demonstrated that Exenatide rapidly activate endothe- lial nitric oxide synthase in endothelial cells. Exenatide is a GLP-1 mimetic which exerts its effects via the GLP-1 receptor with almost the same potency as for GLP-1 in terms of lowering blood glucose. However, it remains elusive whether Exenatide exerts any vasoactive effects.

The aim of this study was to investigate whether Exenatide affects basal vascular tone, compared to GLP-1, and protects against endothelial dysfunction in rat femoral arterial rings.

Materials and methods: Thirty-eight male Sprague-Dawley rats were anesthetized and killed by excision of the heart. The femoral arteries were removed and put in organ baths. The contractile function of the vascular segments was tested by administration of phenylephrine $\left(10^{-5} \mathrm{~mol} / \mathrm{l}\right)$. Endothelium-dependent and endothelium-independent relaxations were determined by administration of acetylcholine (ACh) and sodium nitroprusside (SNP) added to the organ baths at cumulatively increasing concentrations $\left(10^{-9}-10^{-5} \mathrm{~mol} / \mathrm{l}\right.$ and $\left.10^{-9}-10^{-6} \mathrm{~mol} / \mathrm{l}\right)$, respectively. Exenatide and GLP-1 were added to the organ baths at cumulative increasing concentrations $\left(10^{-13}-10^{-9}\right.$ and $10^{-13}-10^{-8}$ $\mathrm{mol} / \mathrm{l}$, respectively) during baseline tension to evaluate contractile effects per se. In separate parallel experiments, endothelial dysfunction was induced by incubating with a triglyceride rich fat emulsion (Intralipid") at two different concentrations 0.5 and $2 \%$ for 20 minutes. To investigate whether Exenatide protects against endothelial dysfunction, Exenatide $\left(10^{-9} \mathrm{~mol} / \mathrm{l}\right)$ was added to the organ baths prior to Intralipid ${ }^{\circ}$

Results: ACh and SNP induced concentration-dependent relaxations, demonstrating a valid working model. Exenatide applied during basal tension was without any discernable contractile or relaxant effect on the femoral artery rings. The maximum relaxation induced by Exenatide on the pre-contracted rings was only $3 \%$, compared to $18 \%, 87 \%$ and $100 \%$ relaxations induced by GLP-1, ACh and SNP, respectively $(P<0.001)$. Preincubation with 0.5 and $2 \%$ Intralipid" elicited a significant reduction in ACh-induced relaxation by $29 \%$ and $35 \%$, respectively $(P<0.01)$. No such reduction was seen in SNPinduced vasorelaxation, revealing an endothelium-dependent dysfunction by Intralipid". Exenatide did not protect against Intralipid"induced endothelial dysfunction. Finally, to ascertain that the Exenatide preparation we used was biologically active, both Exenatide $\left(10^{-11} \mathrm{~mol} / \mathrm{l}\right)$ and GLP-1 $\left(10^{-9} \mathrm{~mol} / \mathrm{l}\right) \mathrm{sig}$ nificantly increased insulin secretion from rat islets in vitro.

Conclusion: We show in a rat model that there is a significant difference in vasorelaxant properties between Exenatide and GLP-1. Furthermore, Exenatide could not confer any acute protective effects against lipid-induced endothelial dysfunction. In conclusion, Exenatide may not have the same influence on vascular tissue as GLP-1.

Supported by: EFSD/Servier grant

\section{7}

Exendin-4 upregulates eNOS phosphorylation and promotes proliferation of human coronary artery endothelial cells through the PI3K/Akt pathway

Ö. Tütüncï̈ ${ }^{1}$, D. Nathanson ${ }^{2}$, Å. Sjöholm², T. Nyström², Q. Zhang ${ }^{1}$

${ }^{1}$ Karolinska Institutet, Research Center, Stockholm South Hospital,

${ }^{2}$ Karolinska Institutet, Internal Medicine, Stockholm South Hospital, Sweden

Background and aims: Endothelial cells have a robust capacity to grow and participate in angiogenesis, which underlies the maintenance of intimal layer integrity. We previously showed that endothelial cells express the GLP-1 receptor and that GLP-1 ameliorates endothelial dysfunction in type 2 diabetic patients with coronary heart disease. Exendin-4 is a stable GLP-1 receptor agonist and has been approved for clinical use against type 2 diabetes. In contrast to its effect on metabolism and endothelial function, the role of GLP-1 and its analogue on endothelial cell growth is not known. We therefore examined the effect of exendin- 4 on endothelial cell growth as well as eNOS phosphorylation regulation in human coronary artery endothelial cells (HCAECs).

Materials and methods: HCAECs were incubated with or without (10 nM) exendin-4 in serum-deficient medium in the presence of $5 \mathrm{mM}$ glucose for $48 \mathrm{~h}$. Phosphorylation and expression of endothelial nitric oxide synthase (eNOS) and Akt were examined after $48 \mathrm{~h}$ incubation by Western blotting using anti-phospho-eNOS (Ser-1177), anti-eNOS, anti-phospho-Akt (Ser 473), and anti-Akt antibodies, respectively. Beta-actin was used as loading control. ${ }^{3} \mathrm{H}$-thymidine incorporation was assayed in 96-well plates as a measure of DNA synthesis after $48 \mathrm{~h}$ incubation with the reagents. Cell viability was assessed using Trypan-blue exclusion.

Results: Exendin-4 treatment (10 nM, $48 \mathrm{~h}$ ) promoted DNA synthesis in HCAECs after $48 \mathrm{~h}$ incubation in $5 \mathrm{mM}$ glucose, evaluated by thymidine in- 
corporation, and neogenesis was confirmed by an increased cell number. To address the involvement of eNOS in proliferation, Western blotting with the use of antibodies against phospho-eNOS and eNOS was performed. Longterm treatment with exendin- 4 enhanced the phosphorylation state of eNOS even when normalized for eNOS expression. We next examined the effects of the eNOS inhibitor L-NAME and the PI3K inhibitor LY294002 to determine whether exendin-4-induced cell proliferation was eNOS- and PI3K-dependent. Exendin-4-induced proliferation was completely inhibited by treatment with $1 \mathrm{mM} \mathrm{L-NAME}$ and partially suppressed by $2 \mu \mathrm{M}$ LY294002, suggesting involvement of eNOS and PI3K/Akt in exendin-4-induced proliferation. Exendin-4-induced eNOS phosphorylation was also inhibited by treatment with L-NAME and LY294002, respectively. These data suggest that Akt is involved in exendin-4-induced eNOS phosphorylation. Akt, which lies downstream of $\mathrm{PI} 3 \mathrm{~K}$, is known to activate eNOS and thereby trigger NO production. Effects of exendin-4 on Akt phosphorylation were next examined. Western blotting with the use of antibodies against phospho-Akt and Akt revealed that exendin-4 significantly increased Akt phosphorylation in HCAECs, even when normalized for total Akt, and the exendin-4-induced Akt phosphorylation was inhibited by treatment with LY294002.

Conclusion: We have shown that PI3K/Akt pathway contributes to exendin-4-induced HCAEC proliferation and eNOS activation. The PI3K/Akt pathway is known to play important roles in endothelial cells, including cell survival and migration. These novel findings add yet other beneficial properties of exendin-4, increasing its clinical utility in type 2 diabetic patients in whom endothelial dysfunction is a salient feature that adversely affect their survival.

\section{8}

Interaction between beta cells and their capillaries: impact of hyperglycaemia. An in vivo model using chimeras

C.N. Chougnet, S. Calderari, H. Kempf, J.-M. Gasc, P. Corvol, E. Larger Chaire de médecine expérimentale INSERM U833, Collège de France, Paris, France

Background and aims: The islets of Langerhans have a dense vascular network, with highly specialized endothelial cells. Animal data suggest that failure to maintain an intact islet vasculature may be a key feature in the course of type 2 diabetes. To study the relationships between beta and endothelial cells, we created pancreatic chimeras. We grafted embryonic chick pancreases under the kidney capsule of severe combined immuno-deficient (SCID) mice. These inter species grafts enabled us to separately study endocrine (of avian origin) and endothelial (of murine origin) cells. Using this model, we have studied the impact of hyperglycemia on the interaction between endocrine and endothelial cells.

Materials and methods: Seven week-old female mice were made diabetic by a single intraperitoneal injection of streptozotocin $(160 \mathrm{mg} / \mathrm{kg}$, in citrate buffer) and only the mice with random blood glucose level above $200 \mathrm{mg} / \mathrm{dl}$ were further studied. Embryonic chick pancreases, obtained at day 14 post-incubation, were microdissected and grafted under the kidney capsule of SCID mice, one embryonic pancreas per mouse. Two weeks later, the transplanted pancreas was analyzed. The macroscopic vascularization was observed after a systemic injection of fluorescein. The microscopic graft vascularization, endocrine and exocrine differentiation were analyzed by immunohistochemistry. To better characterize the interaction between mouse and chick cells, we used an avian-specific retrovirus, the RCAS (Replication-Competent Avian sarcoma-leukosis virus long terminal repeat with a Splice acceptor) system, which can be used for stable transfection of dividing avian cells. This study was carried out along the principles of laboratory animal care.

Results: 1) In control mice, two weeks after transplantation, the pancreatic mass had increased, and the graft was well vascularized. The pancreas was well differentiated with insulin, glucagon and amylase positive cells. The microvascularization was dense and of murine origin as detected by nestin immunohistochemistry, an endothelial marker that is specific for mouse, as shown in control experiments. 2) In hyperglycemic mice, the pancreatic development was decreased, whereas beta cell density was increased. In all experiments $(n=5)$, we observed large blood-filled spaces suggesting abnormal blood vessels that were however not lined by murine endothelial, nestin-positive, cells. 3) In the first control RCAS experiments, we used an RCAS carrying the gene encoding the green fluorescent protein (RCAS-GFP). Chick pancreases were infected in vitro with the RCAS-GFP for 1 hour before transplantation. Two weeks later, we detected green fluorescence in the entire graft, whereas no fluorescence was detected in mouse tissues.
Conclusion: To study the interaction between beta cells and endothelial cells, we have developed a new and original model of inter-species transplantation that allowed us to create chimeras. We obtained chimeric pancreases consisted of chicken endocrine cells and mouse endothelial cells. Hyperglycemia induced large vascularization defects. Furthermore, the RCAS system should provide tools to alter specifically the development of beta cells, but not that of their capillaries.

\section{9}

Impaired vascular insulin signalling in subcutaneous microvascular endothelial cells in type 2 diabetes

S. Gogg, U. Smith, P.-A. Jansson

Department of Molecular and Clinical Medicine, Sahlgrenska Academy, University of Göteborg, The Lundberg Laboratory for Diabetes Research, Göteborg, Sweden

Background and aims: The culture of microvascular endothelial cells (MVEC) from different organs is a widely used experimental model. However, the methodological problems with extraction and culture of subcutaneous MVEC from small tissue samples have not been resolved. In this study, we established a method for extraction and culture of human MVEC from small tissue biopsies obtained from the subcutaneous adipose tissue (SAT).

We have previously shown that insulin-resistance is associated with an impaired insulin signaling, due to a reduced expression of the key docking protein IRS-1, in human subcutaneous adipose tissue. Therefore, we also studied the effect of insulin in subcutaneous MVEC from both healthy and type 2 diabetic (T2D) subjects.

Materials and methods: Subcutaneous needle biopsies were obtained from T2D patients and healthy control subjects. The tissue $(4 \mathrm{~g})$ was incubated in sterile medium containing collagenase. After $50 \mathrm{~min}$ the digest was filtered through a sterile mesh and centrifuged. Two fractions were obtained, the floating isolated adipose cells and the lower fraction containing the stromalvascular cells. The latter were seeded in endothelial growth media and the MVEC were selected by using the Dynabeads magnetic cell sorting system (CD 31 Microbeads). Immunofluorescense with von Willebrand factor, uptake of acetylated LDL, the cobblestone morphology and tube formation were used to characterize the cells. The Western blot and RT-PCR techniques were applied for protein- and gene expression analysis.

Results: We found that insulin receptor expression was low but there was a clear insulin-stimulated tyrosine phosphorylation of the receptor in both T2D and control MVEC. IRS-1 protein expression was markedly decreased in MVEC from T2D subjects. This reduction was also reflected by the reduced activation and serine phosphorylation of the downstream protein PKB/Akt. We also found an increased basal and insulin-stimulated phosphorylation of the MAP kinases, ERK1/2, in cells from diabetic subjects compared to controls. Interestingly, endothelin-1 (ET-1) mRNA levels were significantly increased in the MVEC from diabetic subjects. Addition of ET-1 to the culture medium increased basal MAPK phosphorylation in both control and T2D MVEC. PD98059, a specific MAPK inhibitor, prevented the effect of ET-1 and also decreased the activation of MAPK. Furthermore, the addition of the $\mathrm{ET}_{\mathrm{A}}$ and $\mathrm{ET}_{\mathrm{B}}$ receptor anatagonists, $\mathrm{BQ} 123$ and $\mathrm{BQ788}$, decreased the effect of ET-1 on MAPK activation.

Conclusion: We describe a method for primary MVEC culture obtained from small SAT samples. We found that MVEC from T2D patients have a reduced IRS-1 protein expression and an impaired insulin-stimulated $\mathrm{PKB} /$ Akt activation, whereas activation of ERK1/2 was increased in the basal state. Consistent with this we also found increased ET-1 mRNA levels in cells from diabetic subjects. Increased expression of ET-1 could be responsible for the MAPK activation seen in MVEC of T2D subjects, since this effect was prevented by PD98059 indicating the involvement of ERK1/2 activation in the ET-1 pathway. ET-1 has been shown to regulate insulin signaling by decreasing the tyrosine phosphorylation and expression of IRS-1 in adipose tissue. It is possible that similar mechanisms occur in the MVEC of SAT.

Supported by: Swedish Medical Research Council, Novo Nordisk Foundation, Swedish Diabetes Association, Torsten and Ragnar Söderbergs Foundations, Inga-Britt and Arne Lundberg Foundation and the European Community 


\section{0}

Effects of hyperglycaemia on Nrf2 and p53 antioxidant transcriptional activity in HUVECS

B. Schisano, C.R. Radford, A.L. Harte, K.C. Mc Gee, P.G. McTernan, A. Ceriello

Diabetes and Metabolism, CSRI University of Warwick, Coventry, United Kingdom

Background and aims: Cellular stress initiates a pro-oxidant response within cells which in turn activates transcriptional factors such as NRF2 and p53. Nrf2 has been shown to induce NQO1 and recent studies have identified a p53 induced anti-oxidant gene, TIGAR. However p53 may have a second role, suppressing the Nrf2-dependent transcription of antioxidant genes. This negative control on the Nrf2 transactivation appears to be aimed to prevent the generation of a too strong antioxidant intracellular environment that could hinder the induction of apoptosis. These evidences suggest p53 as a key factor for the cell's survival after exposure to cellular stress, a condition also present in hyperglycaemic. The aim of this study is to investigate whether hyperglycaemia can trigger an Nrf2/p53 mediated oxidative stress response and, more specifically, what the effects of chronic versus oscillating hyperglycaemia are on this pathway in HUVECs.

Materials and methods: HUVECs were grown for 3 weeks either in $5 \mathrm{mmol} /$ 1 glucose, with $25 \mathrm{mmol} / \mathrm{l}$ mannitol for osmolarity normalisation, or in 30 $\mathrm{mmol} / \mathrm{l}$ glucose or alternating 12 hours in normal glucose $(5 \mathrm{mmol} / \mathrm{l})$ with 12 hours in high glucose $(30 \mathrm{mmol} / \mathrm{l})$. Equal amounts of protein lysates $(20 \mu \mathrm{g})$ were used for protein expression analysis by western blotting.

Results: Cells exposed to oscillating glucose (O-GLC) showed a significantly elevated protein expression of TIGAR compared with cells treated with normal $(5 \mathrm{mM})$ glucose (N-GLC) and high glucose (H-GLC) (TIGAR: O-GLC $3.5 \pm 0.3$ OD units vs N-GLC $1.0 \pm 0.3$ OD units*, O-GLC $3.5 \pm 0.3$ OD units vs $\mathrm{H}$-GLC $1.9 \pm 0.3 \mathrm{OD}^{*} ;{ }^{*} p<0.05$ data). A significant difference in TIGAR protein expression was also found between cell cultured in normal glucose compared with high glucose (N-GLC $1.0 \pm 0.3$ OD units vs H-GLC $1.9 \pm 0.3$ OD units $p<0.05)$. Bax was significantly increased in oscillating glucose compared with either normal glucose or high glucose (BAX: N-GLC 1.5 \pm 0.3 OD units vs O-GLC: $2.7 \pm 0.3$ OD units ${ }^{* *}$; H-GLC $1 . .8 \pm 0.6^{\star}$ OD units vs O-GLC: $2.7 \pm 0.5$ OD units ${ }^{*}{ }^{*} p<0.05 ;{ }^{*} p<0.01$ ); Nrf2 was significantly increased in both oscillating and high glucose, compared to normal glucose HUVECs (Nrf2: N-GLC $0.5 \pm 0.2$ OD units vs O-GLC: $1.1 \pm 0.3$ OD units*; H-GLC $1.1 \pm 0.3^{\star}$ OD units vs N-GLC $0.5 \pm 0.2$ OD units ${ }^{*} ;{ }^{*} p<0.05 ;{ }^{* *} p<0.01$ ), but no significant difference was observed between high and oscillating glucose $(\mathrm{H}-$ GLC $1.1 \pm 0.3$ OD vs O-GLC: $1.1 \pm 0.3$ OD units $p=$ N.S). Furthermore NQO1 showed only a modest increase in oscillating glucose compared with either normal or high glucose treated HUVECs (NQO1: N-GLC $0.7 \pm 0.3$ OD units vs O-GLC: $1.0 \pm 0.3 \mathrm{OD}$ units*; H-GLC $0.5 \pm 0.2$ OD units Vs O-GLC: $1.0 \pm 0.3$ OD units ${ }^{*}{ }^{*} \mathrm{p}=$ N.S).

Conclusion: Our findings indicate that oscillating and chronic high glucose induces a p53 mediated intracellular antioxidant response that limits the induction of Nrf2 antioxidant genes such as NQO1. Additionally, this is the first study to indicate that hyperglycaemia triggers TIGAR mediated induction of a p53 anti-oxidant response which further appears dependent on the length and pattern of glucose exposure. This may result in a more stressful condition for the cell which has clear implications for hyperglycaemic conditions induced in type 2 diabetes and cardiovascular disease.

\section{OP 36 Apoptotic mechanisms in beta cells}

\section{1}

Alterations in microRNA expression contribute to cytokine-induced pancreatic $\beta$-cell dysfunction

E. Roggli, S. Gattesco, R. Regazzi

DBCM, University of Lausanne, Lausanne, Switzerland

Background and aims: In Type I diabetes mellitus pancreatic $\beta$-cells are the target of an autoimmune reaction and are exposed to proinflammatory cytokines, such as IL- $1 \beta$, TNF $\alpha$ and IFN $\gamma$. Chronic exposure to cytokines has a detrimental impact on pancreatic $\beta$-cell functions leading to reduction in insulin content, defects in insulin secretion and sensitization towards apoptosis. In this study, we investigated the possible involvement of microRNAs in this phenomenon. MicroRNAs form a recently discovered class of noncoding RNAs that regulate gene expression by sequence-specific inhibition of target mRNA translation.

Materials and methods: The mouse insulin-secreting cell line MIN6 was exposed to IL-1 $\beta$ or to a mixture of cytokines including IL-1 $\beta$, TNF $\alpha$ and IFN $\gamma$, after which microRNAs expression was profiled by microarray analysis. The results were confirmed by quantitative Real time-PCR measurements both in the insulin-producing cell lines MIN6 and INS-1E, and in isolated human pancreatic islets. The microRNAs induced by cytokines were over-expressed individually in MIN6 cells to test for their impact on the synthesis and secretion of insulin and for their effect on $\beta$-cells apoptosis. Moreover, to evaluate the contribution of microRNAs to IL- $1 \beta$-mediated $\beta$-cell dysfunction, the rise in the expression of each microRNA elicited by the cytokine was prevented using antisense oligonucleotides (antagomiRs).

Results: We observed that IL- $1 \beta$ and TNFa increase the expression of miR$21, \mathrm{miR}-34 \mathrm{a}$ and miR-146. We found that the induction of miR-34a triggered by the cytokines was linked to the activation of the $\mathrm{p} 53$ pathway while the rise in the expression of miR-146 was due to the activation of the NFkB signaling cascade. Overexpression of these three microRNAs led to defects in insulin synthesis, insulin secretion and apoptosis that reproduce the effects of IL-1ß. Moreover, transfection of the cells with antagomirs, that prevent the rise of miR-34a or miR-146 elicited by IL-1 $\beta$, partially protected the cells from $c y-$ tokine-induced apoptosis.

Conclusion: Taken together, our findings suggest that at least part of the deleterious effects of cytokines on $\beta$-cell functions may be attributed to alterations in the level of specific microRNAs.

Supported by: University of Lausanne

\section{2}

High glucose activates hypoxia inducible factors 1 and 2 in cultured rat islets and INS1-E cell monolayers

M. Bensellam, J.-C. Jonas

Unit of Endocrinology and Metabolism, Université catholique de Louvain, Brussels, Belgium

Background: We have previously tested the effects of a $18 \mathrm{~h}$ culture in 2, 5, 10 and $30 \mathrm{mmol} / \mathrm{l}$ glucose (G2, G5, G10 and G30) on the transcriptome of cultured rat islets and have identified 18 clusters with distinct glucose-dependent mRNA profiles. Of these, genes that were up-regulated between G10 and G30 are particularly interesting as they may contribute to beta-cell glucotoxicity or protection against it. Besides genes involved in the unfolded protein response, this cluster contains most glycolytic enzymes and other genes typically induced by hypoxia, such as Adrenomedullin $(A d m)$.

Hypothesis and aims: High glucose, which increases $\mathrm{O}_{2}$ consumption in beta-cells, may induce hypoxia and thereby cause glucotoxicity. In this study, we tested whether overnight culture in high glucose activates the hypoxiainducible transcription factor HIF in cultured rat beta-cells.

Materials and methods: Male Wistar rat islets were precultured for one week in serum-free RPMI medium containing $10 \mathrm{mmol} / \mathrm{l}$ glucose (G10) and $5 \mathrm{~g} / \mathrm{l}$ BSA, during which islets with signs of central necrosis were systematically discarded. INS1-E cells were cultured as monolayers (passage 72 to 80 ) in the presence of $10 \%$ FBS. Rat islets and INS1-E cells ( $70 \%$ confluence) were then cultured $18 \mathrm{~h}$ in the presence of increasing glucose concentrations (G2, G5, G10 and G30) with various test substances and at different $\mathrm{O}_{2}$ concentrations ( $1 \%, 20 \%$ or $60 \%$ corresponding to a $\mathrm{pO}_{2}$ of $7.6,152$ and $456 \mathrm{mmHg}$ ). The 
protein levels of HIF1 $\alpha$ and HIF2 $\alpha$ regulated subunits and their dimerization partner HIF1 $\beta$ (also called ARNT) were measured by Western blot. Gene to Tbp mRNA ratios were determined by real-time RT-PCR. The results are means \pm S.E. for 3-6 experiments.

Results: Compared with G2, culture in G30 induced a significant $\sim 4$-fold increase in the protein levels of HIF1 $\alpha$ and HIF2 $\alpha$ in INS1-E cell nuclear extracts. These changes were accompanied by nuclear translocation without changes in total protein levels of HIF1 $\beta$. High glucose also significantly upregulated the Gene to Tbp mRNA ratio for several HIF target genes (Adm, Glyceraldehyde phosphate dehydrogenase, Aldolase A, Lactate dehydrogenase $A)$. These glucose effects, which were confirmed in whole rat islets, were mimicked by a $6 \mathrm{~h}$ exposure to a low $\mathrm{pO}_{2}\left(1 \% \mathrm{O}_{2}\right)$ or by a $18 \mathrm{~h}$ treatment with 10-30 $\mu \mathrm{mol} / \mathrm{l} \mathrm{CoCl} \mathrm{Cl}_{2}$ a known activator of HIF. In contrast, they were almost completely inhibited by $60 \% \mathrm{O}_{2}$ or by various agents that inhibit $\mathrm{Ca}^{2+}$ influx and insulin secretion $(250 \mu \mathrm{mol} / 1$ diazoxide, $1 \mu \mathrm{mol} / \mathrm{l}$ nimodipine and $1 \mu \mathrm{mol} / 1$ clonidine) and thereby likely reduce without suppressing the glucose stimulation of beta-cell $\mathrm{O}_{2}$ consumption. In comparison with HIF target genes, Thioredoxin interacting protein (Txnip), one of the most glucose-responsive genes in cultured rat islets, was regulated in a completely different manner. Thus, the Txnip to Tbp mRNA ratio was not increased by $\mathrm{CoCl}_{2}$ and $1 \% \mathrm{O}_{2}$, and its increase by $\mathrm{G} 30$ was not reduced by $60 \% \mathrm{O}_{2}$ and was markedly enhanced by diazoxide, nimodipine and clonidine.

Conclusion: High glucose induces HIF $1 \alpha$ and HIF2 $\alpha$ protein stabilization, HIF $1 / 2 \alpha-H I F 1 \beta$ dimer translocation to the nucleus, and increased expression of glycolytic enzymes and other HIF target genes in cultured rat beta-cells. These effects likely result from the glucose stimulation of ATP utilization and $\mathrm{O}_{2}$ consumption which may induce beta-cell hypoxia. These effects could contribute to in vitro beta-cell glucotoxicity not only in whole islets but also in insulin-secreting cells cultured as monolayers.

\section{3}

Role of mitochondrial ROS for increased incidence of autoimmune diabetes induced by natural variant of the $F_{0} F_{1}$-ATP synthesis (ATP8) gene

L. Wester Rosenlöf ${ }^{1}, \mathrm{X} . \mathrm{Yu}^{1}$, C. Koch², H. Weiss ${ }^{2}$, M. Tiedge' ${ }^{2}$, S. Ibrahim ${ }^{1}$ ${ }^{1}$ Immunegenetics, University of Rostock, ${ }^{2}$ Medical Biochemistry and Molecular Biology, University of Rostock, Germany

Background and aims: Conplastic mouse strains with FVB mitochondria on $\mathrm{NOD} / \mathrm{Lt}$ J background (NOD-mt ${ }^{\mathrm{FV} / \mathrm{N}}$ ) showed significantly higher incidence of diabetes compared to NOD/LtJ. Three genes are polymorphic between the NOD and FVB mitochondrial genomes from which the ATP8 subunit of the $\mathrm{F}_{\mathrm{o}} \mathrm{F}_{1}$-ATP synthesis complex gene most likely affects the diabetes incidence. It was the aim of our study to elucidate the mechanism by which the ATP8 variant affects beta cell and immune cell function in C57BL/6J-mt ${ }^{\mathrm{FVB} / \mathrm{N}} \mathrm{NOD}-$ $\mathrm{mt}^{\mathrm{FVB} / \mathrm{N}}, \mathrm{NOD} / \mathrm{Lt} \mathrm{J}$ and C57BL/6J-mt ${ }^{\mathrm{AKR} / \mathrm{J}}$ conplastic strains.

Materials and methods: The activity of the $\mathrm{F}_{0} \mathrm{~F}_{1}$-ATP synthesis complex, mitochondrial ROS production and the ratio between state 3: state 4 mitochondrial respirations were measured in normoglycaemic mice. Serum concentrations of insulin-autoantibodies (IAA) in NOD-mt ${ }^{\mathrm{FVB} / \mathrm{N}}$ and $\mathrm{NOD} / \mathrm{Lt} J$ mice were monitored over a 40 week period. Immune cell repertoire, activation marker expression, apoptosis and number of cell cycles followed $\mathrm{T}$ cell stimulation in vitro were analysed by flow cytometry. For lipotoxicity studies C57BL/6J-mt$\mathrm{FVB} / \mathrm{N}$ and C57BL/6J-mt ${ }^{\mathrm{AKR} / \mathrm{J}}$ were fed a high fat diet for 12 weeks. The mice were analysed for blood glucose, serum insulin, glucose tolerance, insulin sensitivity and glucose-stimulated insulin secretion from isolated islets.

Results: The ATP8 polymorphism does not affect the in vitro activity of the $\mathrm{F}_{0} \mathrm{~F}_{1}$-ATP synthesis complex on the NOD/LtJ and C57BL/6J background. It also does not affect the mitochondrial state 3: state 4 respiration, but the mitochondrial ROS production was significantly higher $(\mathrm{p}<0.05)$ in FVB derived mitochondria both on the NOD/LtJ and C57BL/6J background. A significantly higher IAA production $(\mathrm{p}<0.05)$ was found in the NOD-mtFVB/N mice compared to the NOD/Lt] mice, but no significant differences in leukocyte repertoire or consequences of in vitro $\mathrm{T}$ cell activation (activation marker expression, number of cell division or apoptosis) could be detected on NOD/LtJ or C57BL/6J background. Feeding C57BL/6J-mt $\mathrm{m}^{\mathrm{FVB} / \mathrm{N}}$ mice with a high fat diet resulted in a significant impairment of glucose tolerance, lower serum insulin in fasted state and lower glucose stimulated insulin secretion in isolated islets. Blood glucose, body weight and insulin sensitivity was not affected in comparison to the C57BL/6J-mt ${ }^{\mathrm{AKR} / \mathrm{J}}$ control strain.

Conclusion: Our data indicate that the ATP8 variant leads to beta cell dysfunction and ROS production in particular under conditions of lipotoxicity. The beta cell dysfunction accelerates autoimmunity on the NOD backround without compromising the cellular immune system by the ATP8 mutation. The study emphasizes the role of mitochondrial ROS for the susceptibility of beta cells to autoimmune destruction.

Supported by: MRTN-CT-2002-005693 and FORUN 2008-02/11

\section{4}

Sustained expression of spliced XBP-1 is deleterious to primary beta cells F. Allagnat ${ }^{1}$, F. Christulia ${ }^{1}$, P. Pirot ${ }^{1}$, S. Lortz ${ }^{2}$, D.L. Eizirik ${ }^{1}$, A.K. Cardozo ${ }^{1}$ ${ }^{1}$ Laboratory of Experimental Medicine, ULB, Brussels, Belgium, ${ }^{2}$ Institute of Clinical Biochemistry, Hannover Medical School, Hannover, Germany

Background and aims: A tight control of endoplasmic reticulum (ER) homeostasis is important for beta cell function and survival. IL- $1 \beta+$ IFN- $\gamma$ deplete beta cell ER Ca2+ stores, leading to ER stress and apoptosis. Cytokine-induced ER stress is characterized by induction of the IRE-1 $\alpha$ and PERK pathways and by a defective ATF6 and XBP-1 activation. ATF6 and XBP-1 are required for the up-regulation of ER-chaperones, and their impaired activation may deprive beta cells from a crucial defense mechanism against ER stress. Against this background, we overexpressed the spliced (active) form of XBP-1 in beta cells and examined whether this increases their resistance to cytokine-induced ER-stress and apoptosis.

Materials and methods: Recombinant adenoviruses expressing the spliced (active) form of mouse XBP-1s (AdXBP-1s) and, as controls, enhanced green fluorescence protein (AdeGFP), luciferase (AdLUC), truncated spliced mouse XBP-1 (AdtXBP-1s) and truncated unspliced (inactive) mouse XBP-1 (AdtXBP-1u) were used to infect dispersed rat islets and FACS purified beta cells. Expression of XBP-1s and key downstream genes and proteins were measured by real time RT-PCR and/or Western blot. Luciferase assays were performed with a construct containing the unfolded protein response (UPR) element, a binding site for XBP-1. Cell viability was evaluated by HO 342 and propidium iodide. Cells were exposed to IL- $1 \beta(50 \mathrm{U} / \mathrm{ml})+\mathrm{IFN}-\gamma(500$ $\mathrm{U} / \mathrm{ml})$, cyclopiazonic acid $(\mathrm{CPA}, 25 \mu \mathrm{M})$ or tunicamycin $(5 \mu \mathrm{g} / \mathrm{ml})$.

Results: Following infection of dispersed rat islet cells there was an efficient expression of mouse XBP-1s at the mRNA (XBP-1s/GAPDH: non-infected $3 \pm 2$; AdeGFP 1 \pm 1 ; AdXBP-1 146 \pm 55 ) and protein levels (10-fold higher in AdXBP-1s-infected cells than in non-infected or AdeGFP-infected cells). The exogenous XBP-1s protein was active as shown by a 17-fold induction of the UPR reporter in cells infected with AdXBP-1s, as compared to non-infected cells $(n=8 ; p<0.001)$. On the other hand, no induction was found in cells infected with AdeGFP or AdtXBP-1u. AdXBP-1s also induced a significant increase in the expression of the XBP-1s target genes EDEM-1 and PDI-P5 (EDEM-1/GAPDH: non-infected 0.14 \pm 0.04 ; AdXBP-1 0.28 \pm 0.08 ; PDI-P5/ GAPDH: non-infected 1.14 \pm 0.32 ; AdXBP-1 2.01 $\pm 0.58, \mathrm{n}=4-5, \mathrm{p}<0.05)$. Unexpectedly, overexpression of XBP-1s induced apoptosis in dispersed islets cells. Apoptosis was related to XBP-1s activity, since it was not observed in cells infected with AdLUC or AdtXBP-1u (\% apoptosis: non-infected 18 \pm 2 ; AdLUC 19 \pm 2 ; AdtXBP-1u 18 \pm 1 ; AdXBP-1s 34 \pm 1 ; p $<0.02$ for AdXBP-1s vs. non-infected). Moreover, when comparing different viruses there was a significant correlation between XBP- 1 s activity and apoptosis $(\mathrm{R} 2=0.66 ; \mathrm{p}<0.04)$. These pro-apoptotic effects of sustained XBP-1s expression were confirmed in FACS purified beta cells. Overexpression of XBP-1s did not augment IL- $1 \beta$ + IFN- $\gamma$ or CPA-induced islet cell death, while it increased tunicamycin-induced death. The increased apoptosis levels observed in cells overexpressing XBP-1s was not due to ER stress, since there was no induction of ER stress markers as evaluated by real time PCR.

Conclusion: Sustained overexpression of XBP-1s is pro-apoptotic in beta cells, suggesting that a tight control of the ER stress pathways is crucial to preserve beta cell viability.

Supported by: JDRF and FNRS

\section{5}

Recombinant human prolactin (rhPRL) promotes human beta cell survival by inhibiting apoptosis

L. Labriola ${ }^{1}$, L.F. Terra ${ }^{1}$, M.H. Garay-Malpartida ${ }^{1,2}$, T.R. Mares-Guia ${ }^{1}$, M.C. Sogayar ${ }^{1,3}$

${ }^{1}$ Nucleo de Terapia Celular e Molecular, University of São Paulo, ${ }^{2}$ Each, University of São Paulo, ${ }^{3}$ Biochemistry, Chemistry Institute- University of São Paulo, Brazil

Background and aims: Transplantation of pancreatic islets isolated from organ donors constitutes a promising alternative treatment for typel diabetes, 
however, it is severely limited by the shortage of organ donors. Ex-vivo expansion of islet cell cultures appears as an attractive strategy, however, maintenance of human islets has been a difficult task since islet fate in culture is, in part, determined by the balance between pro- and anti-apoptotic mediators. Previous results from our laboratory have demonstrated a significant beneficial effect of rhPRL treatment on cell proliferation and secretory function of human islet primary cultures. Therefore, in the present study, we set out to explore the role of rhPRL in human beta-cell survival.

Materials and methods: Human pancreatic islets were isolated after ductal distension of the pancreas and tissue digestion with Liberase HI, following the automated method of Riccordi et al, with some modifications. After isolation, islet cultures were pre-treated in the presence or in the absence of rhPRL $(300 \mathrm{ng} / \mathrm{mL})$ and then subjected to serum starvation or cytokines treatment for 24 or $48 \mathrm{~h}$. Insulin beta cell content was analyzed by Newport Green staining and apoptosis was evaluated by DNA fragmentation (propidium iodide staining) and phosphatidyl serine externalization (Annexin-V staining). Both were quantified by flow cytometry (FACS) analysis. We also analyzed the expression of anti- and pro-apoptotic Bcl-2 gene family members by qRT-PCR. Additionaly, using a specific synthetic substrate, we also evaluated caspase activity by fluorimetric assays.

Results: In order to establish whether rhPRL treatment would increase beta-cells survival, primary cultures of human pancreatic islets were treated with rhPRL and subjected to serum starvation or cytokines treatment. The proportion of simultaneous Newport green- and propidium iodide-positive cells was significantly lower in the presence of $\operatorname{rhPRL}(\mathrm{p}<0.05)$ under both serum-deprived and cytokine-treated conditions, when compared to control cells. Accordingly, a decreased rate of phosphatidyl serine externalization was observed upon rhPRL treatment under both cell death-inducing conditions, when compared to control cells $(\mathrm{p}<0.05)$. In order to identify the mechanisms through which lactogens induce beta-cell survival, we studied the mRNA ratio between the anti-apoptotic Bcl-2 family and the pro-apoptotic Bax genes. Stimulation of human islet cells with rhPRL increased the Bcl-2 family/Bax mRNA ratio compared to control cells under both cell death-inducing situations. Moreover, caspase activity was lower in islet cultures which were pretreated with the lactogen and then subjected to cytokines treatment than in cytokines-treated cells.

Conclusion: Our study provides, for the first time, relevant evidence for a protective effect of lactogens on both serum deprivation- and cytokine-induced human beta-cell apoptosis. Furthermore, the gene expression results suggest that rhPRL improves beta-cell survival, at least in part, by inhibition of cell death pathways controlled by the Bcl-2 gene family members.

Supported by: FAPESP, CNPq, FINEP, PRP-USP

\section{6}

Up-regulation of GLP-1 from alpha cells - a protective mechanism against diabetes?

A.K. Hansen ${ }^{1}$, T.B. Bödvarsdottir ${ }^{1}$, D.N.E. Lykkegaard ${ }^{1}$, R. Heller ${ }^{2}$, J.J. Holst ${ }^{3}$, A.E. Karlsen ${ }^{1}$

${ }^{1}$ Diabetes Biology, Novo Nordisk A/S, Maaloev, ${ }^{2}$ Developmental Biology,

HRI, Gentofte, ${ }^{3}$ Biomedical Sciences, University of Copenhagen, Denmark

Background and aims: It is well established that GLP-1 has proliferative and anti-apoptotic effects on pancreatic beta-cells. The neighbouring alpha-cells produce proglucagon peptides and thus may liberate intact GLP-1 if prohormone convertase enzyme 1/3 (PC 1/3) is present. This alpha-cell GLP-1 can have paracrine effects on beta-cells. We hypothesise that alpha-cell GLP-1 may be up-regulated in situations when an increase in the functional beta-cell mass is needed, such as diabetes. The gerbil Psammomys obesus (P. obesus) is an animal model of nutritionally induced diabetes and was in this study used to investigate the hypothesis that alpha-cell GLP-1 is up-regulated during the development of diabetes.

Materials and methods: Diabetes was introduced in P. obesus by transferring animals from a low energy (LE) to a high-energy (HE) diet. Control animals were maintained on LE diet. Animals were categorised as diabetic when blood glucose levels exceeded $10 \mathrm{mM}$. Animals on $\mathrm{HE}$ diet that did not develop diabetes were categorised as non-responders. At the time of sacrifice blood samples were drawn from the portal vein and the aorta and the plasma concentrations of bioactive GLP-1 were measured. From a subset of animals the whole pancreas was removed, snap-frozen and subsequently analysed for intact GLP-1 content. From another subset of animals the islets were isolated and plated in a 96-well plate with 10 islets/well. After overnight incubation the medium was removed and analysed for levels of glucagon and intact GLP1. Islets from control animals were incubated in $3 \mathrm{mM}$ or $30 \mathrm{mM}$ glucose for
6 days and the medium was analysed for intact GLP-1. Sections of paraffinized pancreas from control P. obesus were stained for glucaogn, insulin and PC $1 / 3$.

Results: P. obesus that had been fed a HE diet and were diabetic had increased levels of intact GLP-1 in the portal vein compared to control animals (8.5 $\pm 1.1 \mathrm{pM}(\mathrm{n}=32)$ and $3.6 \pm 0.3 \mathrm{pM}(\mathrm{n}=12)$, respectively). For non-responders the level was $4.3 \pm 0.5 \mathrm{pM}(\mathrm{n}=10)$. For all animals the level of intact GLP-1 in the aorta was below the detection limit of the assay. We found a significantly increased content of intact GLP-1 in pancreas tissue from diabetic animals compared to control animals $(11.4 \pm 2.2 \mathrm{pmol} / \mathrm{g}$ tissue $(\mathrm{n}=9)$ and $3.9 \pm 1.0$ $\mathrm{pmol} / \mathrm{g}$ tissue $(\mathrm{n}=3)$, respectively). Interestingly, the non-responders seemed to have further increased pancreas content of intact GLP-1 (38.2 $\pm 1.4 \mathrm{pmol} / \mathrm{g}$ tissue $(n=2))$. We found that islets isolated from diabetic animals released more intact GLP-1 relative to glucagon than islets from control animals (GLP-1/glucagon ratio 0.02 and 0.0034 respectively). The bioactivity of the produced GLP-1 was confirmed by activation of the human GLP-1 receptor expressed in BHK cells. In vitro mimic of the diabetic condition (high glucose) led to a $\sim 6$ fold higher release of intact GLP-1 from control islets compared to control islets maintained in low glucose. Triple fluorescent staining of P. obesus pancreas sections verified the presence of PC1/3 in some glucagon cells.

Conclusion: In this study we find up-regulation of the active form of GLP-1 from alpha-cells in P. obesus when the animals are challenged with a diabetogenic diet. Our results are in agreement with the hypothesis that GLP-1 released from alpha-cells is involved in promoting beta-cell mass and function under challenging circumstances such as diabetes. 


\section{OP 37 Thiamine and microangiopathy}

\section{7}

Thiamine and benfotiamine normalize apoptosis of human retinal pericytes on high glucose-conditioned extracellular matrix

E. Beltramo, E. Berrone, S. Tarallo, M. Porta

Dept Internal Medicine, University of Turin, Italy

Background and aims: Thickening of basement membrane and pericyte loss are well-known early events in the pathogenesis of diabetic retinopathy. In previous experiments, we reported reduced adhesion, but no changes in apoptosis, in bovine retinal pericytes cultured on extracellular matrices (ECMs) produced by human endothelial cells in high hexose concentrations, probably due to glycation of matrix proteins. Subsequently, we showed that human retinal pericyte (HRP) apoptosis is strongly affected by matrix produced in high glucose probably due to impairment of the Bcl-2/Bax ratio. The behaviour of human cells was therefore different from that observed with bovine pericytes, underlining the importance of establishing species-specific cell models. For this reason, we created an immortalized human retinal pericyte line (BmiHRP), with the same morphology and metabolic characteristics of the wildtype cells (WT-HRP) from which it was derived.The aim of this study was to verify if thiamine and its lipophilic derivative benfotiamine, which have been shown to counteract glucose-induced apoptosis in vascular cells, are able to correct enhanced HRP apoptosis in the presence of high glucose-conditioned ECMs.

Materials and methods: Conditioned ECMs were obtained by growing human endothelial cells (HUVEC) for 7 days in culture media containing physiological $(5.6 \mathrm{mmol} / \mathrm{l})$ or high $(28 \mathrm{mmol} / \mathrm{l}) \mathrm{D}$-glucose with or without 50 and $100 \mu \mathrm{mol} / \mathrm{l}$ thiamine and benfotiamine. Cells were then lysed and ECM fixed by $\mathrm{NH} \mathrm{OH}$. Wild-type (WT-HRP) and immortalized HRP (Bmi-HRP) were cultured for 7 days in physiological glucose only on these conditioned ECMs. Pericyte apoptosis was evaluated measuring DNA fragmentation by ELISA; the expression of two molecules known to be involved in glucose-mediated apoptosis, Bcl-2 with a pro-proliferating and Bax with a pro-apoptotic function, was measured by RT-PCR and their concentration by ELISA. A decrease in the ratio $\mathrm{Bcl}-2 / \mathrm{Bax}$ is a marker of increased apoptosis.

Results: Both types of pericytes showed the same behaviour. Apoptosis of HRP was greatly enhanced by high glucose-conditioned matrix in comparison with physiological glucose ECMs (WT-HRP: $1.23 \pm 0.48$ abs units vs $0.06 \pm 0.02, \mathrm{p}=0.002$; Bmi-HRP: $2.12 \pm 0.24$ vs $0.05 \pm 0.01, \mathrm{p}=0.000)$ and this was confirmed by $\mathrm{Bcl}-2 / \mathrm{Bax}$ ratio, both for mRNA expression (WT-HRP $0.43 \pm 0.21$ vs $1.62 \pm 0.69, \mathrm{p}=0.006$; Bmi-HRP: $0.47 \pm 0.09$ vs $1.01 \pm 0.22, \mathrm{p}=0.000$ ) and protein concentration (WT-HRP $0.02 \pm 0.01$ vs $0.11 \pm 0.07, \mathrm{p}=0.02$; BmiHRP: $0.09 \pm 0.03$ vs $0.20 \pm 0.12, p=0.03$ ). Both thiamine and benfotiamine were able to reverse this effect: DNA fragmentation in WT-HRP and Bmi-HRP cultured on ECMs produced by HUVEC in high glucose plus thiamine and benfotiamine at both concentrations was fully normalized, as well as Bcl-2/ Bax expression and concentration.

Conclusion: Thiamine and benfotiamine are able to counteract the apoptotic effect of high glucose-conditioned ECM on human retinal pericytes, probably reducing the glycation of matrix proteins, which, in turn, can lead to damaging interactions between pericytes and their surrounding microenvironment and, finally, to the onset of diabetic retinopathy.

\section{8}

Effects of high glucose and thiamine on the balance between matrix metalloproteinases and their tissue inhibitors in vascular cells S. Tarallo, E. Beltramo, E. Berrone, P. Dentelli, M. Porta Internal Medicine, University of Turin, Italy

Background and aims: Capillary pericytes disappear early and selectively in the course of diabetic retinopathy. Perycite survival depends on interactions with extracellular matrix (ECM) proteins, which are degraded by matrix metalloproteinases (MMP). Elevated glucose concentrations can influence ECM synthesis and degradation, acting on the expression of MMPs and their tissue inhibitors, TIMPs. We reported previously on reduced adhesion of pericytes cultured on ECM produced by endothelium in high glucose and its correction by thiamine. In this paper, we now aimed at verifying the effects of thiamine and benfotiamine on the expression and activity of MMP-2, MMP-9 and TIMPs in human vascular cells in high glucose.
Materials and methods: Human retinal pericytes (HRP) and umbilical vein endothelial cells (HUVEC) were cultured in $5.6 \mathrm{mM}$ or $28 \mathrm{mM}$ glucose, with or without thiamine (T) or benfotiamine (BT). MMP-2, MMP-9, TIMP-1, TIMP-2 and TIMP-3 mRNA expression was determined by RT-PCR and activity by gelatin zymography and activity by gelatin zymography; TIMP-1 concentrations were measured by ELISA.

Results: In HRP, MMP-2 activity, though not expression, increased in high glucose $(123.7 \pm 11.8 \%$ of G5.6, $\mathrm{p}<0.05)$ and was reduced by thiamine $(94.5$ $\pm 11.7 \%$ of G5.6, $\mathrm{p}<0.05)$ and benfotiamine $(78.4 \pm 26.9 \%$ of $\mathrm{G} 5.6, \mathrm{p}<0.05)$; TIMP-1 expression increased in high glucose plus thiamine $(118.7 \pm 30.0 \%$ of G5.6, p < 0.05) and benfotiamine (118.2 $\pm 30.2 \%$ of G5.6); MMP-9 was not expressed. In HUVEC, MMP-9 and MMP-2 expression did not change in high glucose, while their activity increased (MMP-2: $130.2 \pm 24.9 \%$ of G5.6, $\mathrm{p}<0.05$; MMP-9: $120.0 \pm 19.1 \%$ of G5.6, $<<0.05$ ); thiamine and benfotiamine had no effects. TIMP-1 expression was unchanged in HUVEC. Neither glucose nor thiamine modified TIMP-2 and TIMP-3 expression. TIMP-1 concentrations did not change in either HRP or HUVEC.

Conclusion: High glucose may imbalance the MMP/TIMP regulation, leading to increased turnover of ECM. Thiamine and benfotiamine may correct the increase in MMP-2 activity due to high glucose in human pericytes, while increasing TIMP-1.

\section{9}

High glucose causes a decrease in expression of thiamine transporters in human proximal tubule epithelial cells in vitro

J.R. Larkin, P.J. Thornalley

Clinical Sciences Research Institute, University of Warwick, Coventry, United Kingdom

Background and aims: It has been shown recently that both type 1 and type 2 diabetic patients have markedly decreased plasma concentrations of thiamine whilst maintaining nutritional sufficiency of the vitamin. Low plasma thiamine concentration was linked inversely to renal clearance of thiamine - attributed to decreased re-uptake of thiamine in the renal tubule epithelium. Similar decreases in plasma thiamine concentration and increases in thiamine clearance were found in streptozotocin-induced diabetic rats, and were also associated with decreased expression and activity of the thiamine pyrophosphate-dependent enzyme transketolase. Transketolase is a critical rate-controlling enzyme of the pentose phosphate pathway which helps resist adverse effects of high glucose concentration and the development of nephropathy and other microvascular complications in diabetes. In diabetic patients, low plasma thiamine concentration was also linked inversely with abnormally high plasma levels of soluble vascular cell adhesion molecule-1 - a marker of microvascular and macrovascular disease. The cause of increased thiamine clearance in diabetes remains unknown. Thiamine clearance is normally minimised by efficient re-uptake of thiamine from the glomerular filtrate by the thiamine transporters of renal proximal tubule epithelial cells. The aim of this study is to investigate if hyperglycaemia changes the expression of the thiamine transporter THTR-1 in human renal tubule epithelial cells in vitro.

Materials and methods: The HK-2 cell line was used as a model source of human proximal tubule epithelium cells. HK-2 cells were cultured in DMEM/F-12 medium supplemented with $10 \%$ foetal bovine serum and $23 \mathrm{nM}$ thiamine - a typical physiological level - for five days before harvesting. Cultures contained either a low glucose concentration $(5 \mathrm{mM})$ or high glucose concentration $(26 \mathrm{mM})$. After culture, cells were lysed and the membrane fraction separated by centrifugation $\left(20,000 \mathrm{xg}, 30 \mathrm{~min}, 4^{\circ} \mathrm{C}\right)$. Proteins of the membrane fraction were separated by $12 \%$ SDS-PAGE, transferred to nitrocellulose membrane and blotted with anti-THTR-1 antibodies (Alpha Diagnostics), stripped of antibody and re-blotted for the housekeeping protein $\beta$-actin with a second anti- $\beta$-actin antibody (AbCam, UK). Western blotting results are expressed as a THTR- $1 / \beta$-actin band intensity ratio.

Results: Thiamine transporter antigen was present in HK-2 cell membrane extracts as a protein of ca. $50 \mathrm{kDa}$ in SDS-PAGE analysis. The THTR-1/ $\beta$ actin band intensity ratio was decreased $58 \%$ in cultures of HK-2 cells with high glucose concentration, relative to low glucose concentration controls. Intensity ratio: low glucose 0.255 ; high glucose $0.107(\mathrm{p}<0.01, \mathrm{n}=4)$.

Conclusion: High glucose concentration decreased the expression of the thiamine transporter in human proximal tubule HK-2 epithelial cells in vitro. This is expected to decrease uptake of thiamine across the tubular epithelium. A similar effect of hyperglycaemia in vivo may contribute to the increased clearance and plasma deficiency of thiamine in experimental and clinical diabetes.

Supported by: University of Warwick 


\section{0}

Thiamine status and risk of early renal function decline in type 1 diabetic patients

A. Adaikalakoteswari ${ }^{1}$, B. Perkins ${ }^{2}$, A.S. Krolewski ${ }^{3}$, N. Rabbani ${ }^{1}$,

P.J. Thornalley ${ }^{1}$

${ }^{1}$ Clinical Sciences Research Institute, University of Warwick, Coventry,

United Kingdom, ${ }^{2}$ Toronto General Hospital, University of Toronto, Canada,

${ }^{3}$ Section on Genetics and Epidemiology, Joslin Diabetes Center, Boston,

United States

Background and aims: Discovery of novel metabolic factors linked to diabetes may inform interventions for treating the early phenotypes of diabetic nephropathy: microalbuminuria (MA) and early renal function decline (ERFD). In view of the observed plasma thiamine deficiency in diabetes, we aimed to identify association of thiamine status with the onset of MA and with subsequent ERFD.

Materials and methods: Of 943 subjects recruited in 1991 with normoalbuminuria (NA) as part of the $1^{\text {st }}$ Joslin Kidney Study, 86 of 109 who developed new onset MA had sufficient (>10y) follow-up after onset and had stored urine available from within 3 years of onset. We ranked them according to glomerular filtration rate (GFR) slope estimated by cystatin-C measured at least 3 times over a mean of 12 years. The outer tertiles of GFR slope were selected to produce a study group of 52 subjects with MA: 25 had ERFD defined as GFR loss exceeding 3.3\% per year, while 27 had stable renal function. Urine from 30 subjects with longstanding NA was used for comparison. Plasma and urine were analysed for thiamine content by pre-column derivatization HPLC with fluorimetric detection. $\log _{10}$ transformed plasma and urinary thiamine concentration $(\mathrm{nM})$ and thiamine fractional excretion TFE (\%) data were analysed parametrically.

Results: Although markedly lower as compared to the non-diabetes literature, the mean $\log$ [plasma thiamine concentration] was not significantly different between MA and NA groups (1.0131 vs 0.9815). There was, however, a significant increase of log[plasma thiamine concentration] in MA subjects with ERFD compared to those with stable renal function (0.964 vs 1.148; $\mathrm{P}<0.05)$. However, the urinary thiamine measures showed higher concentration and excretion in MA than in NA groups: the mean $\log$ [urinary thiamine/creatinine] was 1.409 vs $1.076(\mathrm{P}<0.001)$ and the $\log [\mathrm{TFE}]$ was 2.961 vs $2.688(\mathrm{P}<0.001)$. In addition, those with MA and ERFD had higher urinary thiamine concentration and excretion as compared to those with stable renal function: the mean $\log$ [urinary thiamine/creatinine] was 1.534 vs 1.204 , respectively $(\mathrm{P}<0.02)$ and the $\log [\mathrm{TFE}]$ was $-2.685 \mathrm{vs}-2.833$, respectively $(\mathrm{P}<0.05)$. These differences were not confounded by age or sex. In a logistic regression model, $\log$ [urinary thiamine concentration] and glycated haemoglobin $\mathrm{HbA}_{1 c}$ were independently associated with ERFD.

Conclusion: We conclude that increased urinary thiamine is associated both with processes that lead to the onset of microalbuminuria and furthermore to processes that lead to initiation of early renal function decline. In light of evidence in animal models of diabetes in which thiamine supplementation prevents the development of early stage nephropathy, these clinical results imply that thiamine supplementation in humans may represent one approach to preventing the onset of microalbuminuria and the initiation of renal function loss in patients with type 1 diabetes.

Supported by: an NIH project grant
OP 38 Prevention of type 2 diabetes

\section{1}

Role of a diabetes risk score in screening obese patients for metabolic syndrome, dysglycaemic states and cardiovascular risk

E. Cosson, E. Hamo Tchatchouang, M. Nguyen, H. Ba, I. Banu, P. Valensi

Department of Endocrinology, Diabetology, Nutrition, Paris Nord

University - CRNH IdF, Paris, France

Background and aims: The Findrisk score has been developed in a Finnish population to evaluate the 10 -yr risk of incident diabetes and validated subsequently in another Finnish cohort. An association has also been reported between this score and glycemic disorders and metabolic syndrome. The aim of this study was to evaluate whether this score correlates at the time of evaluation with metabolic syndrome, dysglycemic states and cardiovascular risk in patients with $B M I \geq 25 \mathrm{~kg} / \mathrm{m}^{2}$.

Materials and methods: An oral glucose tolerance test was performed in 1274 patients referred consecutively to our department (Jan 1998-Oct 2005) for overweight or obesity. Prediabetes and diabetes were defined according to WHO 1999 criteria, and metabolic syndrome according to IDF criteria. After exclusion of 328 patients without available score or with undetermined metabolic syndrome, and of 26 patients with fasting blood glucose (BG) $\geq 7 \mathrm{mM}$, 920 patients ( 769 women), $39 \pm 15$ years, BMI $37 \pm 7 \mathrm{~kg} / \mathrm{m}^{2}$, were included.

Results: Prediabetes (impaired glucose tolerance, impaired fasting glucose or both) and diabetes were found in $20.5 \%$ and $4.4 \%$ of the population, respectively. The patients were categorized according to their diabetes risk score value $(<10,10-14,15-19$, and $>19)$. Increasing score categories correlated with age, the prevalence of metabolic syndrome, BMI, waist circumference, systolic, diastolic and pulse blood pressure, HbA1c, fasting and 120-min after glucose load BG and insulinemia, HOMA insulin resistance index, triglycerides, LDL-cholesterol, alanine aminotransferase and gamma-glutamyl transpeptidase levels ( $p<0.01$ to 0.001$)$. These correlations were still significant after age adjustment $(\mathrm{p}<0.001)$. The prevalence of prediabetes and diabetes increased with increasing score categories $(2.7,11.3,62.5$, and $60 \%$; $\mathrm{p}<0.001$, and 0.0 , $1.5,7.2$, and $30.2 \% ; \mathrm{p}<0.001$, respectively). The increase of HOMA index with score categories was independent of the presence or not of metabolic syndrome, with a higher value when metabolic syndrome was present.

Conclusion: In overweight or obese patients, 1- the Findrisk score correlates with current metabolic syndrome, insulin resistance, glycemic status, and cardiovascular risk; 2- this score may be useful to select the patients to be screened for these conditions.

\section{2}

Perceived risk of type 2 diabetes in Australian women with a recent history of gestational diabetes mellitus

M.K. Morrison ${ }^{1}$, C.C. Collins ${ }^{2}$, J.M. Lowe ${ }^{2}$

${ }^{1}$ Health \& Education Division, Diabetes Australia-NSW, Sydney, ${ }^{2}$ Faculty of Health, University of Newcastle, Australia

Background and aims: Despite the well documented risks of type 2 diabetes in women with a history of Gestational Diabetes Mellitus (GDM), there is some evidence to suggest that women with prior GDM do not perceive themselves to be at increased risk. The aim of this study was to describe the risk perceptions of a national sample of Australian women with a recent history of GDM.

Materials and methods: Participants were women aged 18 years and over, diagnosed with GDM between 2003-2005, sampled from the National Diabetes Services Scheme (NDSS) database. Cross sectional data was collected via written postal survey and included a self-reported risk perception scale.

Results: Of the 4098 women invited, 1372 eligible women consented to participate (response rate $36 \%$ ). Respondents who were currently pregnant or subsequently diagnosed with type 2 diabetes were excluded from analysis $(n=196)$. Mean age $( \pm S D)$ was $35.6 \pm 4.9 y r, 72 \%$ were Australian born, $19 \%$ spoke a language other than English and 1.1\% were indigenous Australians. Up to 3 years post GDM, mean $( \pm$ SD) self-reported BMI was $27.1 \pm 6.36$ and more than half $(57 \%)$ of the participants were overweight or obese. Thirty two percent $(32 \%)$ of women perceived that they were low or very low risk for developing type 2 diabetes, $42 \%$ moderate risk, $20 \%$ high and $6 \%$ very high risk. Using logistic regression analysis, factors associated with a high or very high level of perceived risk were BMI $>25 \mathrm{~kg} / \mathrm{m}^{2}$ (OR $\left.4.395 \% \mathrm{CI}[2.9,6.1]\right)$, a family history of diabetes (OR $3.695 \% \mathrm{CI}[2.6,5.2]$ ), use of insulin during 
pregnancy (OR 1.9 95\% CI[1.3, 2.6]) and being born in Australia (OR 1.5 $95 \% \mathrm{CI}[1.0,2.2])$. No two-way interactions between these factors were significant and the model was a good fit using Hosmer and Lemeshow test. Conclusion: Although women with known risk factors for type 2 diabetes such as obesity and family history were more likely to perceive their risk as high or very high, the majority of this sample of women with previous GDM did not perceive that they were high risk for type 2 diabetes. Interestingly women who used insulin during pregnancy or were Australian born were more likely to perceive their risk as high. Overall these results suggest a need for increased awareness of GDM as an independent risk factor for type 2 diabetes.

Supported by: DAA Unilever Post-Graduate Research Scholarship

\section{3}

Metabolic syndrome as guide to treatment of people with IGT with acarbose: a post hoc analysis of the STOP-NIDDM trial results M. Hanefeld ${ }^{1}$, A. Karasik ${ }^{2}$, C. Koehler ${ }^{1}$, T. Westermeier ${ }^{3}$, G. Saunders ${ }^{4}$, J.-L. Chiasson ${ }^{5}$

${ }^{1}$ Center for Clinical Studies, GWT-TUD GmbH, Dresden, Germany, ${ }^{2}$ Department of Nuclear Medicine, Chaim Sheba Medical Center, Tel Hashomer, Israel, ${ }^{3}$ Bayer Vital GmbH, Leverkusen, Germany, ${ }^{4} \mathrm{G}$. Saunders Enterprises Inc., Drayton Valley, Canada, ${ }^{5}$ Department of Medicine, Université de Montréal, Canada

Background and aims: The metabolic syndrome (MetS) is associated with an odds ratio of $\sim 4$ for development of diabetes. The aim of the study was to analyse the independent and joint effects of the components of MetS on the incidence of diabetes in people with impaired glucose tolerance (IGT) and to assess the number needed to treat (NNT) with acarbose by MetS status.

Materials and methods: Double-blind placebo controlled trial, 1,368 patients, follow-up time 3.3 years. MetS by ATP III definition with fasting plasma glucose of $>=6.1 \mathrm{mmol} / \mathrm{l}$ as limit for impaired fasting glucose (IFG), post hoc analysis of the STOP-NIDDM trial.

Results: IFG, hypertriglyceridemia, low HDL-cholesterol and hypertension as single traits significantly increased the risk for diabetes with hazard ratio (HR) of 1.5, 1.4, 1.3 and 1.2 resp. Overall MetS resulted in a HR of 1.6 $(\mathrm{p}<0.0001)$.

The annual incidence for diabetes in the placebo group with MetS was $18.7 \%$ vs. $11.2 \%$ without MetS.

Acarbose reduced the incidence to $13.5 \%$ and $9.4 \%$ resp. resulting in a number needed to treat (NNT) for patients without MetS of 16.5 and with MetS of 5.8. In multivariate analysis with established risk factors as continuous variables and MetS and treatment group as categorical variable acarbose, FPG, post challenge PG, Hba1C, triglycerides and leucocytes were independent predictors of diabetes but not MetS.

Conclusion: MetS components except large waist independently predict diabetes in people with IGT but in combination confer no higher risk as expected from single traits. Acarbose reduces incidence of diabetes in people with and without MetS. However those with MetS have a distinctly higher benefit. Thus MetS labels a very high risk group in people with IGT which should have a priority for medical intervention.

Supported by: Bayer

\section{4}

ACTos NOW for the Prevention of Diabetes (ACT NOW) Study D. Tripathy ${ }^{1}$, M.A. Banerji ${ }^{2}$, G.A. Bray ${ }^{2}$, T.A. Buchanan ${ }^{2}$, S. Clement ${ }^{2}$, R.R. Henry' ${ }^{2}$ A. Kitabchi' ${ }^{2}$, S. Mudaliar ${ }^{2}$, N. Musi ${ }^{1}$, R. Ratner ${ }^{2}$, P. Reaven ${ }^{2}$, D. Schwenke ${ }^{2}$, F. Stentz ${ }^{2}$, R.A. DeFronzo ${ }^{1}$

${ }^{1}$ Diabetes, Medicine, University of Texas Health Science Center at San Antonio, United States, ${ }^{2}$ Diabetes, Medicine, ACT NOW Investigators, San Antonio, United States

Background: Impaired glucose tolerance (IGT) is a prediabetic state characterized by insulin resistance and impaired insulin secretion. $~ 10 \%$ of IGT individuals have evidence of diabetic retinopathy (DPP) and peripheral neuropathy has been reported in $5-10 \%$ of IGT subjects in other studies. Therefore, interventions targeting IGT have assumed added clinical importance. Methods: ACT NOW is a randomized double-blind, placebo-controlled study to examine whether treatment of IGT with pioglitazone (PIO) can prevent/delay development of type 2 diabetes mellitus (T2DM). 602 IGT subjects (age $=52.3 \mathrm{y} ; \mathrm{BMI}=34.3 \mathrm{~kg} / \mathrm{m}^{2}, 42 \%$ male; $\mathrm{FPG}=105 \pm 0.3 \mathrm{mg} \%$; $2-\mathrm{h}$ PG
[OGTT] $\left.=168 \pm 1 \mathrm{mg} \% ; \mathrm{HbA}_{1 \mathrm{c}}=5.5 \pm 0.01 \%\right)$ were recruited in 8 US centers over 2 years and followed for an additional 2 years or until development of diabetes. IGT subjects were required to have FPG $\geq 95 \mathrm{mg} \%$ and at least one other high risk characteristic. All subjects had OGTT with insulin/C-peptide, lipid profile, inflammatory markers, and ankle-arm blood pressure. At 5 centers carotid IMT, DEXA, and FSIVGTT were performed. At entry, insulin sensitivity (Matsuda Index [MI] and $\mathrm{S}_{\mathrm{I}}$ ) was reduced by $48 \%(\mathrm{p}<0.0001)$ and insulin secretion/insulin resistance (disposition) index was decreased by $78 \%$ $(\mathrm{p}<0.0001)$ compared to 102 age/BMI/gender-matched normal-glucose-tolerant controls identified during screening. IGT subjects were randomized to PIO (45 mg/day) or placebo (PLAC) and returned every 3 months for FPG/ $\mathrm{HbA}_{1 \mathrm{c}}$ and annually for OGTT. Diagnosis of diabetes (FPG $\geq 126$ or 2-h PG $\geq 200$ ) was confirmed by repeat OGTT. At time of diagnosis of diabetes or at study end (4 years), all studies were repeated.

Results: 45 PLAC-treated subjects developed diabetes vs 10 PIO-treated subjects (6.8\% vs $1.5 \%$ per year), representing a $78 \%$ decrease in IGT conversion to T2DM ( $\mathrm{p}<0.000001) .42 \%$ of individuals in PIO vs $28 \%$ in PLAC returned to NGT at study end $(\mathrm{P}<0005)$, while the 2 -h $\mathrm{PG}$ decreased by $28 \pm 0.4$ vs $13 \pm 0.3$ in PIO vs PLAC $(\mathrm{P}<0.005)$. Insulin sensitivity (Matsuda Index, $4.3 \pm$ 0.4 to $7.1 \pm 0.6, \mathrm{P}<0.0005)$ and insulin secretion/insulin resistance index $(3.1$ \pm 0.2 to $5.1 \pm 0.4, \mathrm{P}<0.0005)$ improved in PIO and were unchanged in PLAC. Weight gain (3.9 vs $0.8 \mathrm{~kg})$ and edema (22 vs $15 \%)$ were more frequent in PIO. There were 2 cases of CHF (PIO- 1, PLAC-1). There were 13 bone fractures (8 in PIO and 5 in PLAC) and all were related to trauma.

Conclusion: Pioglitazone markedly reduced, by $78 \%$, the conversion of IGT to T2DM in association with improved beta cell function and reduced insulin resistance. Pioglitazone was safe and well tolerated during this 4 year study. Supported by: Takeda Pharmaceuticals 


\section{OP 39 Hypoglycaemia and the brain}

\section{5}

Automated detection of hypoglycaemia induced EEG-changes recorded by subcutaneous electrodes in type 1 diabetic subjects - the brain as a biosensor

C.B. Juhl ${ }^{1}$, K. Højlund ${ }^{2}$, R. Elsborg ${ }^{3}$, M.K. Poulsen², P. Selmar',

C. Christiansen ${ }^{5}$, H. Beck-Nielsen ${ }^{2}$

${ }^{1}$ Department of Medicine, Esbjerg Sygehus, Esbjerg, ${ }^{2}$ Department of Endocrinology, Odense University Hospital, ${ }^{3}$ Hyposafe A/S, Lyngby, ${ }^{4}$ Department of Neurophysiology, Roskilde Sygehus, ${ }^{5}$ Nordic Bioscience A/S, Copenhagen, Denmark

Background and aims: Recurrent and severe hypoglycemia is a complication to tight glycemic control in patients with type 1 diabetes. Hypoglycemia unawareness is a common condition associated with increased risk of severe hypoglycemia. Cerebral hypoglycemia increases theta-activity and reduces alpha activity in the electroencephalogram (EEG) as recorded by standard scalp electrodes. The aim of the present study was, in order to develop a hypoglycemia alarm, to test the hypothesis that specific changes in the EEG during hypoglycemia can be recorded by subcutaneous electrodes and processed by a general mathematical algorithm and that a significant hypoglycemia signal can be obtained before the development of severe cognitive failure.

Materials and methods: Fifteen patients with type 1 diabetes were studied. Subcutaneous electrodes were placed at the area of $\mathrm{Cz}$ and $\mathrm{C} 3$ and EEG signals were recorded during insulin-induced hypoglycemia. The cognitive function was evaluated by repeated cognitive testing and the counterregulatory hormones glucagon, adrenalin and noradrenalin were measured. EEG signals were subjected to quantitative analysis by an automated mathematical algorithm with a predefined threshold of hypoglycemia. The study was terminated when the subjects showed obvious signs of cognitive dysfunction as judged by the investigator.

Results: During the experiment all subjects experienced symptoms of hypoglycemia. Counterregulatory hormones increased significantly from plasma glucose $=3.0 \mathrm{mmol} / 1$ to the termination of the study. Significant EEG-changes were detected by the automated mathematical algorithm in all subjects. Plasma glucose at the time of EEG changes was $2.5 \pm 0.3 \mathrm{mmol} / \mathrm{l}$ (range $2.0-3.4 \mathrm{mmol} / \mathrm{l})$. At the time of study termination plasma glucose was $2.0 \pm 0.3 \mathrm{mmol} / \mathrm{l}$ (range $1.6-2.7 \mathrm{mmol} / \mathrm{l}$ ) and all subjects had reduced cognitive function. Significant EEG changes occurred 29 \pm 28 minutes (range 3 113 minutes) before termination of the study. In 13 out of 15 subjects EEG changes occurred before significant loss of cognition.

Conclusion: Previously defined changes in EEG following hypoglycemia were confirmed by recordings from subcutaneous electrodes. The changes could be detected by the automated algorithm applied. The time from significant EEG changes to loss of cognition was, in most cases, sufficient for the patient to correct hypoglycemia.

Supported by: Hyposafe A/S

\section{6}

The medial amygdala; part of the central hypoglycaemia-sensing network?

L. Zhou, Z. Sang, S.N. Brown, R.J. McCrimmon

Internal Medicine (Endo), Yale University, New Haven, United States

Background and aims: Specialized glucose-sensing neurons within the ventromedial hypothalamus ( $\mathrm{VMH}$ ) are recognized to play a major role in the detection of hypoglycaemia. In rodents pharmacological or genetic manipulation of VMH glucose-sensing neurons has a marked effect on counterregulatory responses to acute hypoglycaemia. We recently demonstrated that the activity of glucose-sensing neurons in the VMH was significantly modulated by Urocortin III (UCNIII) via its action on Corticotrophin Releasing Hormone Receptor type-2 (CRHR2). The nerve terminals that release UCN III into the VMH arise from cell bodies in the medial amygdala (MeA). This region is also known to contain glucokinase mRNA, which suggested that the MeA may form an additional part of a glucose-sensing network linking the amygdala to the hypothalamus.

Materials and methods: To further investigate this hypothesis acute hyperinsulinemic $(20 \mathrm{mU} / \mathrm{kg} / \mathrm{min})$ hypoglycaemia $(\sim 3.9 \mathrm{mmol} / \mathrm{l})$ studies were performed in two groups of chronically catheterized male Sprague-Dawley rats. Thirty minutes prior to the start of the mild hypoglycemia clamp one group of rats $(\mathrm{n}=6)$ were microinjected with 2-deoxyglucose, (2-DG, a non-metabolizable form of glucose, $10 \mathrm{mM}$ solution at $0.04 \mu \mathrm{min}^{-1}$ for $30 \mathrm{~min}$ ) into the MeA. The control group $(n=6)$ were injected with aECF at same infusion rate. Blood for hormone analysis was taken under basal and experimental conditions. On completion of the clamp the rats were euthanasized, after which their brains were perfused for later immunostaining.

Results: Additional glucopenic stress amplify the glucagon $(181 \pm 34 \mathrm{pg} / \mathrm{ml}$ vs $111 \pm 18 \mathrm{pg} / \mathrm{ml} ; \mathrm{P}<0.05)$ and epinephrine $(2337 \pm 637 \mathrm{pg} / \mathrm{ml}$ vs $1101 \pm 252$ $\mathrm{pg} / \mathrm{ml} ; \mathrm{p}<0.05)$ responses to hypoglycaemia compared to control group.

Conclusion: These studies suggest that the MeA is an additional glucosesensing center that contributes to the detection of hypoglycemia and initiation of a glucose counterregulatory response. Moreover, the UCNIII/CRHR2 neural pathway may provide a link between hypothalamic and amygdala glucose-sensing neurons that allows for a more integrated response to acute hypoglyaemia.

Supported by: NIDDK, JDRF Center for the Study of Hypoglycemia at Yale and the NIDDK - supported Diabetes Endocrinology Research Center

\section{7}

Habituation of regional brain activation during hypoglycaemia in Type 1 Diabetes: a $\left[{ }^{15} \mathrm{O}\right]-\mathrm{H}_{2} \mathrm{O}$ PET study

M.M. Teh ${ }^{1}$, J.T. Dunnn ${ }^{2}$, Y. Samarasinghe ${ }^{1}$, P. Choudhary ${ }^{1}$, P.K. Marsden ${ }^{2}$,

L.J. Reed ${ }^{3}$, S.A. Amiel ${ }^{1}$

${ }^{1}$ Department of Diabetes and Endocrinology, King's College London School of Medicine, ${ }^{2}$ PET Imaging Centre, St Thomas' Hospital, London, ${ }^{3}$ King's College London, Institute of Psychiatry, United Kingdom

Background and aims: Central mechanisms of hypoglycaemia awareness (HA) are not well understood, although neuroimaging studies of brain glucose metabolism have shown differences in activation in multiple brain networks during hypoglycaemia. We investigated the central correlates of HA in healthy subjects and type 1 diabetes (T1DM) using $\left[{ }^{15} \mathrm{O}\right]-\mathrm{H}_{2} \mathrm{O}$ positron emission tomography, to measure regional changes in brain perfusion. To identify differences in central mechanisms of hypoglycaemia awareness between men with T1DM and HA and hypoglycaemia naive male volunteers, using high resolution $\left[{ }^{15} \mathrm{O}\right]-\mathrm{H}_{2} \mathrm{O}$ PET, together with monitoring of symptoms and counterregulatory hormones.

Materials and methods: Twelve sequential $\left[{ }^{15} \mathrm{O}\right]-\mathrm{H}_{2} \mathrm{O}$ PET brain scans were obtained in 10 healthy non-diabetic male and 7 hypoglycaemia aware male type 1 diabetic subjects. These were acquired at $10 \mathrm{~min}$ intervals before, during and after a one-stepped hypoglycaemic clamp. Hypoglycaemia symptoms, counterregulatory hormonal responses and mood were measured after each scan. PET data were analysed with statistical parametric mapping (SPM).

Results: Despite a greater degree of hypoglycaemia in the T1DM group (2.4 $\pm 0.3 \mathrm{mmol} / \mathrm{l}$ vs $2.7 \pm 0.3 \mathrm{mmol} / \mathrm{l}, \mathrm{p}<0.01)$, adrenaline responses were less $(9.9$ $\mathrm{nmol} / \mathrm{l} / 50 \mathrm{~min}$ vs $23.4 \mathrm{nmol} / \mathrm{l} / 50 \mathrm{~min}, \mathrm{p}<0.05)$; cortisol responses were flattened $(-55.6 \mathrm{nmol} / \mathrm{l} / 50 \mathrm{~min}$ vs $938 \mathrm{nmol} / \mathrm{l} / 50 \mathrm{~min}, \mathrm{p}<0.02)$ and changes in symptom scores were not different ( $38 \mathrm{vs} 43, \mathrm{p}=0.64$ for adrenergic scores, 44 vs $26, \mathrm{p}=0.06$ for neuroglycopenic). The diabetic group felt more drowsy ( 7.1 vs 3.6, $\mathrm{p}=0.02)$; troubled $(-1.2$ vs $-4.4, \mathrm{p}<0.05)$ and sick ( 6.6 vs $2.8, \mathrm{p}<0.05)$. In the cerebral responses to hypoglycaemia, healthy subjects showed a stronger response in the amygdala and the sub-genual anterior cingulate cortex, than T1DM. In contrast, a stronger dorsolateral prefrontal cortical response was seen in the T1DM subjects ( $<<0.05$, cluster corrected).

Conclusion: The non-diabetic group achieved a greater counterregulatory endocrine response associated despite being exposed to a lesser hypoglycaemia challenge. Increased activation of the anterior cingulate and the amygdala in this group may underpin these differences in autonomic activation and between the two groups. The attenuation of responses in the T1DM subjects suggests habituation within brain networks which subserve stress responses in T1DM subjects. In contrast, the increased prefrontal responses to hypoglycaemia in this group suggest engagement of conscious and effortful problem solving responses to this stressor.

Supported by: JDRF 


\section{8}

BOLD brain activation in response to a cognitive challenge during hypoglycaemia among T1DM subjects

A.M. Jacobson ${ }^{1}$, N. Bolo ${ }^{2}$, G. Musen ${ }^{1}$, D. Simonson ${ }^{3}$, K. Weinger ${ }^{1}$, A. Raji ${ }^{3}$, P. Renshaw ${ }^{2}$

${ }^{1}$ Psychiatry, Joslin Diabetes Center, Boston, ${ }^{2}$ Psychiatry, McLean Hospital, Belmont, ${ }^{3}$ Medicine, Brigham and Womens Hospital, Boston, United States

Background and aims: Acute hypoglycemia is known to impair cognitive ability. Working memory, which is involved in maintaining and manipulating information for immediate retrieval, is greatly affected by hypoglycemia. Working memory plays an important role in complex cognitive skills such as driving and maintaining a list of items or actions. Despite the impact of hypoglycemia on working memory little is known about the underlying brain activation mechanisms that support this function. The aim of this study was to evaluate brain activation and cognitive performance during laboratory-induced hypoglycemia in both T1DM patients (who commonly experience at least mild hypoglycemic episodes as a consequence of the disease) and nondiabetic control patients.

Materials and methods: Using blood oxygenation level dependent (BOLD) functional magnetic resonance imaging at $3 \mathrm{~T}$ during a hyperinsulinemic clamp, we compared brain activation responses to a working memory task (WMT) in patients with T1DM $(\mathrm{n}=7 ; 5 \mathrm{M}$; age $37 \pm 12)$ and non-diabetic controls $(\mathrm{n}=7,7 \mathrm{M}$; age $30.4 \pm 8)$ during euglycemic $(5.0 \mathrm{mmol} / \mathrm{l})$ and hypoglycemic $(2.8 \mathrm{mmol} / \mathrm{l})$ conditions. Cognitive performance on the WMT was assessed by percent correct and reaction time.

Results: During euglycemia, we observed brain BOLD activation responses to the WMT $(\mathrm{P}<0.05)$ that were similar for both groups. Activation was decreased during hypoglycemia in comparison to euglycemia for both groups $(\mathrm{P}<0.001)$. However, T1DM patients showed less of a drop in activation during hypoglycemia when compared to controls. Specifically, this effect was found for parts of the dorsolateral prefrontal cortex (superior and inferior frontal gyri), and limbic cingulate regions $(\mathrm{P}<0.001)$, when comparing regional \%BOLD signal changes by ANOVA. No significant differences in task performance were found between either subject groups or glycemic conditions.

Conclusion: Decreases in task-related BOLD activation during hypoglycemia were less pronounced for patients with T1DM compared to non-diabetic controls while behavioral performance was conserved. This suggests that cerebral metabolism or physiology was altered in patients, possibly as the result of an adaptation of brain metabolism to antecedent hypoglycemic episodes or to hyperglycemia. To achieve the same level of cognitive performance T1DM patients maintained higher levels of brain activation than controls during hypoglycemia. This suggests that the patients' brain energetic metabolism underlying activation was increased in order to sustain the same level of cognitive performance.

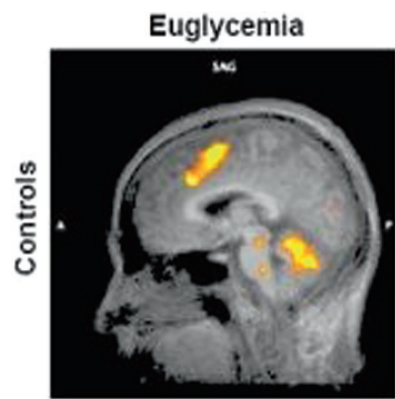

Hypoglycemia
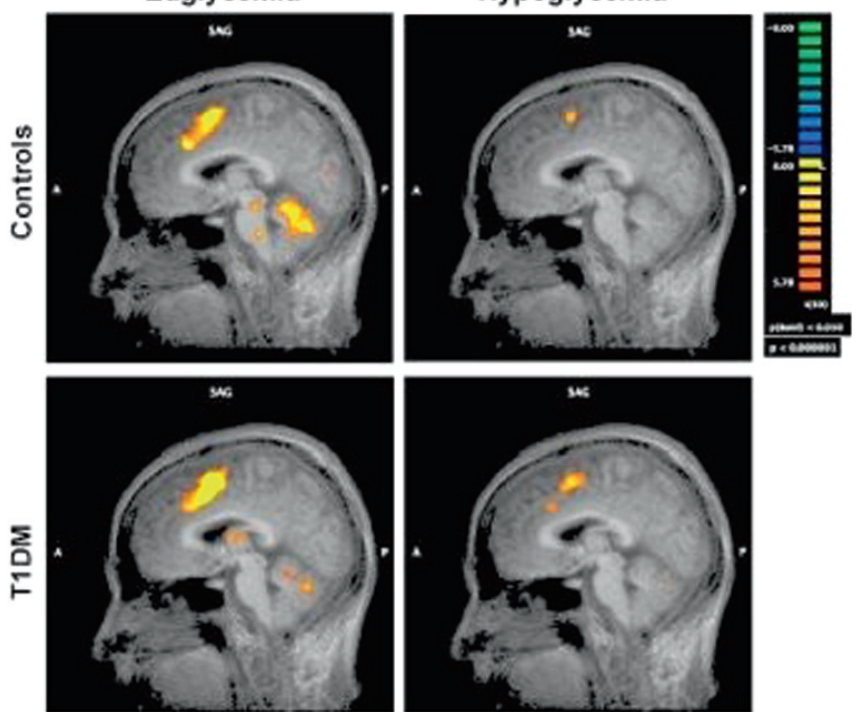

BOLD brain activation for T1DM and Control Subjects in Response to the WMT during euglycemia and hypoglyemia

Supported by: NIH, Herbert Graetz Fund

\section{OP 40 Classical and sophisticated education}

\section{9}

Long term effects of a new treatment and education programme for insulin treated diabetic patients with hypoglycaemia problems (HyPOS) B. Kulzer ${ }^{1}$, N. Hermanns ${ }^{1}$, T. Kubiak², M. Krichbaum ${ }^{1}$, T. Haak ${ }^{1}$ ${ }^{1}$ Research Institute of Diabetes Academy Mergentheim, FIDAM, Bad Mergentheim, ${ }^{2}$ Psychological Department, University of Greifswald, Greifswald, Germany

Background and aims: HyPOS is a treatment and education program for insulin treated diabetic patients. It is conducted in group sessions (4 - 6 participants) and consists of 5 lessons. It's focussed on causes and improvement of hypoglycaemia unawareness, modification of dysfunctional glycaemic targets and health beliefs related to development and chronification of hypoglycaemia problems. Short term evaluation of HyPOS showed a significant improvement of hypoglycaemia awareness and a significant reduction of mild hypoglycaemic episodes after 6 months compared to a control group. This study examines the long-term effect of HyPOS on the incidence of severe hypoglycaemic episodes (defined as requiring glucose or glucagon injections). Materials and methods: 135 type 1 diabetic patients (HbAlc $7.3 \pm 0.9 \%$; diabetes duration $21.6 \pm 11.0$ yrs., age $46.0 \pm 12.8$ yrs) participated in this study. In this follow-up assessment 2 years after the end of treatment $82.3 \%$ of the original sample (66 patients in the control group, CG; participation rate $82.5 \%$ vs. 69 patients in HyPOS, participation rate $82.1 \%$ p=.95) participated: The mean follow up time was highly comparable in HyPOS and the CG (CG $24.5 \pm 2.7$ vs. HyPOS $24.3 \pm 3.2$ months; $\mathrm{p}=.62$ ). Hypoglycaemia awareness and incidence of severe hypoglycaemia were assessed by questionnaire and validated by telephone interview.

Results: Severe hypoglycaemic episodes were reduced from 0.87 events per patient and year at baseline to 0.14 events per patient and year in HyPOS. In the control group event rate per patient and year were reduced from 0.87 to 0.27 . The reduction was significantly greater in HyPOS than in the CG $(\mathrm{p}=.04)$. Proportion of patients with at least one severe hypoglycaemic episode was also significantly reduced in HyPOS compared to CG (HyPOS from $37 \%$ to $13 \%$; KG from $40.9 \%$ to $27.3 \%$ ). The hazard ration for a severe hypoglycaemic episode was 0.4 (0.16- $0.96 \mathrm{p}=.04)$ in HyPOS compared to CG. Thus relative risk for having a severe hypoglycaemic episode was reduced by $60 \%$. The course of glycaemic control was highly comparable (HyPOS baseline $7.2 \pm 0.9 \%$ to $7.1 \pm 0.9 \%$; CG baseline $7.4 \% \pm 1.0$ to $7.3 \pm 1.1 ; \mathrm{p}=.84$ ).

Conclusion: Severe hypoglycaemic episodes are a cause of diabetes related distress and also a potentially dangerous complication in diabetic patients. Furthermore they are also causing high costs, because of the necessary emergency treatment. Participation in HyPOS could reduce the incidence of severe hypoglycaemic episodes in a long term follow-up, without compromising glycaemic control. Therefore HyPOS seems to be an effective tool for treating insulin treated diabetic patients with hypoglycaemic problems. Supported by: Berlin Chemie

\section{0}

Comparison between the "Diabetes Interactive Diary" system and standard carbohydrate counting education: an open label, multicentre, randomised study

G. Vespasiani ${ }^{1}$, M.C. Rossi ${ }^{2}$, A. Nicolucci², P. Di Bartolo ${ }^{3}$, C. Sardu ${ }^{3}$,

D. Bruttomesso ${ }^{4}$, M. Dal Pos ${ }^{4}$, E. Zarra ${ }^{5}$, A. Girelli ${ }^{5}$, F.J. Ampudia ${ }^{6}$, D. Kerr A. Ceriello ${ }^{8}$, C.M. De la Questa ${ }^{9}$, B. Pomili ${ }^{2}$, D. Horwitz ${ }^{10}$

${ }^{1}$ Diabetes Unit, Madonna del Soccorso Hosp., S. Benedetto del Tronto, Italy, ${ }^{2}$ Clinical Pharmacology and Epidemiology, Consorzio Mario Negri Sud, S. Maria Imbaro, Italy, ${ }^{3}$ Diabetes Unit, Presidio Osp., Ravenna, Italy, ${ }^{4}$ Clinical and Experimental Medicine, Policlinico Universitario, Padova, Italy, ${ }^{5}$ Diabetes Unit, Spedali Civili, Brescia, Italy, ${ }^{6}$ Endocrinology, Hosp. Clínico Universitario, Valencia, Spain, ${ }^{7}$ Diabetes and Endocrine Centre, Royal Bournemouth Hospital, Bournemouth Dorset, United Kingdom, ${ }^{8}$ Warwick Medical School, University of Warwick, Coventry, United Kingdom, ${ }^{9}$ Endocrinology, Hosp. Universitario Virgen Macarena, Sevilla, Spain, ${ }^{10}$ LifeScan Inc., Milpitas, United States

Background and aims: Carbohydrates $(\mathrm{CHO})$ counting is an effective strategy to promote dietary freedom, quality of life, and glycaemic control, but its 
widespread use is limited by the complex educational approach. The Interactive Diary for Diabetes (DID) is an electronic diary, a bolus calculator and a telemedicine system based on the communication between patient and specialist by SMS through mobile phones. It enables patients to match the insulin to desired $\mathrm{CHO}$ intake on a meal by meal basis without the complexities of the standard education needed to teach $\mathrm{CHO}$ counting. The aim of this study was to evaluate whether the DID could represent a valid alternative to standard $\mathrm{CHO}$ counting education in terms of metabolic and weight control.

Materials and methods: Adults with type 1 diabetes were randomised to the DID educational program (Group A, N=67) or to standard education for CHO counting (Group B, N=63). Patients randomised to Group A attended a 2 weeks course to learn the use of DID. Patients in Group B received a standard education lasting up to 3-months, to learn how to adjust insulin doses on the basis of $\mathrm{CHO}$ content of the meals. Patients were seen after 3 and 6 months from randomization. During the 3 study visits data on $\mathrm{HbAlc}$, fasting blood glucose (FBG), body weight, blood pressure and lipid profile were collected. A hierarchical linear model for repeated measurements was used to assess differences over time between groups.

Results: Out of 130 patients enrolled, 80 individuals completed the study and were included in this analysis. The mean age of participants was of $36.3 \pm 9.8$, while the mean diabetes duration was of $16.6 \pm 10.7$ years; $49.2 \%$ of the patients were males, and $21.7 \%$ were treated with CSII. After six months of follow, HbAlc levels decreased in both groups (Group A: from $8.1 \pm 0.8$ to $7.7 \pm 0.8$; Group B: from $8.35 \pm 0.7$ to $8.0 \pm 1.3$; between groups comparison $\mathrm{p}=0.8$ ). Body weight was almost unchanged in Group A (from $68.1 \pm 11.8$ to $68.9 \pm 13.4 \mathrm{~kg}$ ), while it increased from $69.0 \pm 13.4$ to $70.8 \pm 13.6 \mathrm{~kg}$ in Group B $(\mathrm{p}=0.34)$. FBG decreased in Group A (from $175 \pm 82$ to $165 \pm 71 \mathrm{mg} / \mathrm{dl}$ ), while it increased in Group B (from $181 \pm 59$ to $195 \pm 81 \mathrm{mg} / \mathrm{dl}$ ) $(\mathrm{p}=0.23)$. No changes in blood pressure and lipid profile were detected.

Conclusion: These preliminary results suggest that the use of DID is at least as effective as the traditional educational approach to $\mathrm{CHO}$ counting, but allows to avoid the complexities of $\mathrm{CHO}$ counting, thus allowing dietary freedom to a larger proportion of individuals with T1DM. The final analysis including all randomized patients will provide more accurate estimates of the comparative benefits of the two educational approaches.

Supported by: Me.Te.Da. srl

\section{1}

Randomised controlled evaluation of "modular" versus "full course" patient education programmes for insulin-treated patients with type 2 diabetes and previous experience with patient education

M.A. Nauck, H. Schulze, C. Nagel-Reuper, S. Golla

Diabeteszentrum Bad Lauterberg, Bad Lauterberg im Harz, Germany

Background and aims: Patients with type 2 diabetes need education programs at different stages of their disease. Repeated participation in similar education programs with unchanged composition and content may reduce motivation. Therefore, we have developed a "modular" patient education program that allows the patients and their treatment teams to select topics (covered over 1-2 h) of specific interest to their actual situation. The aim of the present evaluation is to demonstrate the effectiveness of our ",modular" patient education program in comparison to repeated participation in a standard course.

Materials and methods: Hospitalized patients with type 2 diabetes treated with insulin (mostly intensified regimens) were randomized to another standard patient education course or a ,modular" patient education program ( $\mathrm{n}=75$ for both). 71 patients participated in the standard course, 72 in the "modular" education (men/women: 28/43; 31/42; age $61 \pm 10 / 62 \pm 8$ years; BMI $34.5 \pm 5.9 / 34.5 \pm 6.2 \mathrm{~kg} / \mathrm{m}^{2}$; diabetes duration $15 \pm 9 / 16 \pm 8$ years.; insulin treatment for $7 \pm 6 / 8 \pm 5$ years; insulin dose $96 \pm 57 / 93 \pm 67 \mathrm{IU} / \mathrm{d} ; \mathrm{HbA}_{1 \mathrm{c}}$ $8.7 \pm 1.5 / 8.1 \pm 1.4 \%$; means \pm SD). Immediately after the educational measures, diabetes-related knowledge and patient satisfaction were assessed. One year later, $\mathrm{HbA}_{1 c}$, body weight, and achievement of individually chosen treatment goals were determined. Statistics: ANOVA, if appropriate for repeated measures; Fisher's exact test. The hypothesis was that "modular" patient education was at least non-inferior (drop in $\mathrm{HbA}_{1 \mathrm{c}}$ between baseline and one year later not different by more than $0.4 \%$ ).

Results: 62 patients could be evaluated after a repeated standard course, and 63 patients after modular patient education. $\mathrm{HbA}_{1 c}$ significantly dropped in both groups $(-0.8[-1.1 /-0.5] \% /-0.6[-0.9 /-0.2] \% ; \mathrm{p}<0.0001$; mean $\pm 95 \%$ confidence interval), with no significant difference between groups $(\mathrm{p}=0.48)$. Non-inferiority criteria were met. Body weight remained constant in both groups (no significant changes or differences). The duration of patient educa- tion measures was shorter for ,,modular" training than for standard courses $(10.4 \pm 2.2 \mathrm{~h}$ vs. $15.9 \pm 2.5 \mathrm{~h}, \mathrm{p}<0.0001)$. The amount of time required for patient education measures significantly correlated with the duration of the hospital stay $\left(\mathrm{r}^{2}=0.342, \mathrm{p}<0.0001\right)$. Duration of hospital treatment tended to be shorter with "modular" training $(11.9 \pm 3.5$ vs. $13.0 \pm 4.1$ days; $p=0.064)$. Patient satisfaction was good and not different comparing both approaches. Conclusion: Regarding metabolic and body weight control results were similar for „modular" and standard patient education, while the time required for educational measures was less for "modular" education. Since the time required for educational measures is a significant determinant of total treatment duration, ,,modular" patient education may be of interest to patients in specialized hospital departments and practices. Supported by: Lilly Deutschland, Bad Homburg, Germany

\section{2}

Integrated diabetes health care network (IDN) in telemedicinesupported health care delivery

E. Salzsieder ${ }^{1}$, P. Augstein ${ }^{1}$, L. Vogt ${ }^{1}$, K.D. Kohnert ${ }^{1}$, P. Boddenberg ${ }^{2}$, E. Lewin ${ }^{2}$, V. Heuzeroth ${ }^{3}$

${ }^{1}$ Research, Institute of Diabetes, Karlsburg, ${ }^{2}$ Management, PHTS

Telemedizin, Düsseldorf, ${ }^{3}$ Management, BKK Taunus, Frankfurt, Germany

Background and aims: Progress in basic and clinical research has introduced the development of new strategies for treatment of diabetes. The innovative Karlsburg Diabetes Management System (KADIS ${ }^{\circ}$ ) was developed and experimentally verified over almost two decades. When combined with telemedicine-based communication (TeleDIAB ${ }^{\circ}$ ) and continuous glucose monitoring (CGM), KADIS" has the potential to provide effective, evidence-based support to doctors in their daily efforts to optimize glycemic control. It was the aim of this project to demonstrate the feasibility of improving diabetes control in conventional diabetes care by implementing KADIS ${ }^{\circ}$ and TeleDIAB in an integrated diabetes health care network.

Materials and methods: Based on a contract for integrated health care provision (IV contract) between the health care insurance company BKK Taunus, the telemedicine provider PHTS Düsseldorf, the Diabetes Service Center and the Institute of Diabetes Karlsburg KADIS-based decision support and telemedicine-based communication technology (TeleDIAB ${ }^{*}$ ) was implemented into an integrated health care network to facilitate information exchange between patients, physicians, and health care professionals under daily life conditions. The interactive diabetes management system KADIS" enables individualized evaluation of glucose profiles in association with metabolic interventions (Metabolic Fingerprint) and to display onlinetrended patient-specific metabolic data and simulates the outcome of treatment recommendations. The Metabolic Fingerprint characterizes glycemic status by visualization of relationships between the continuously monitored glucose profiles and causal endogenous and exogenous factors and enabled evidence-based identification of "weak points" in glycemic control. Using KADIS"-based simulation, physician recommendation are generated in the form of patient-focused decision support that enables elimination of weak points. The outcome of KADIS"-based support in routine diabetes care was evaluated by comparing $\mathrm{HbAlc}$ levels and 24-hours glucose profiles before and after the intervention.

Results: At present there are 384 diabetic patients permanently included into the integrated diabetes health care network, which are treated by 132 General Practitioners and 30 Diabetes Specialists. Application of KADIS based decision support reduced $\mathrm{HbAlc}$ during the follow-up by $0.2 \%$ ( $7.1 \%$ to $6.9 \%)$ after 3 months and by $0.4 \%$ ( $7.1 \%$ to $6.7 \%)$ after 6 months in routine diabetes care. The reduction in $\mathrm{HbAlc}$ was strongly related to significantly improved 24-hours glucose profiles.

Conclusion: Application of KADIS in combination with CGM and telemedicine-based communication in integrated health care networks has high potential for improving outpatient diabetes care and management considering daily life conditions.

Supported by: German Federal Ministry of Education and Research, regional Ministry of Education, Science, and Culture Mecklenburg-Vorpommern 


\section{OP 41 Experimental and clinical immunology}

\section{3}

Cd4 $\mathrm{T}$ cell reactivity to the major diabetes autoantigen Slc30A8 in newly diabetic subjects

H.W. Davidson, J. Rockell, R. Wagner, J.M. Wenzlau, D. Zipris, J.C. Hutton, P.A. Gottlieb

Barbara Davis Center for Childhood Diabetes, University of Colorado Denver, Aurora, United States

Background and aims: ZnT8 (Slc30A8), an islet-specific zinc transporter is a major target of humoral autoimmunity in type $1 \mathrm{~A}$ diabetes, with more than $60 \%$ of patients having $\mathrm{ZnT} 8$-autoantibodies at diagnosis. As the generation of high affinity class-switched antibodies is a CD4 T cell dependent process it is likely that disease associated ZnT8-specific T cells are also present in some or all newly diabetic individuals. These may potentially be targets of immunotherapies designed to prevent or ameliorate the course of disease. We therefore investigated whether ZnT8-specific, gamma interferon producing $\mathrm{CD} 4 \mathrm{~T}$ cells are amplified in the peripheral blood of diabetic subjects.

Materials and methods: Peripheral blood mononuclear cells were obtained from 21 newly diabetic subjects and 12 age and HLA matched controls in accordance with institutionally approved human subjects protocols. PBMCs $(1,000,000)$ were cultured for $48 \mathrm{~h}$ with 23 pools of overlapping 20 -mer peptides ( 2 peptides / incubation at $10 \mu \mathrm{g} / \mathrm{ml}$ spanning the entire 369 amino acid primary sequence of ZnT8). After replating into 96 well plates coated with anti gamma interferon antibody (300,000 cells/ well) the reactive precursor frequency was identified by ELISPOT analysis. Data was normalized to the stimulation index relative to a DMSO control. Responses to the T1D antigens proinsulin, GAD and IA2 and the mitogen ConA served as positive controls. Non-parametric statistical analyses (Mann Whitney) were performed using GraphPad Prism 4 software. The ability of individual 15 mer peptides to bind recombinant HLA DR3 and DR4 molecules were assessed using Proimmue REVEAL technology. Individual HLA DRB1 and DQA and DQB haplotypes were determined by a PCR based sequence specific oligonucleotide probe system (Roche).

Results: DR3 binding sites were focused in the hydrophobic core (aa100-200) and C-terminal (aa268-369) domain respectively. 16 DR4 binding sites were mapped 8 of which were also found in the C-terminal region. When considered as a group, significant ELISPOT responses were detected in diabetic subjects relative to controls to 3 peptides in the $\mathrm{C}$-terminal cytosolic region and a fourth in the second cytosolic loop ( $\mathrm{p}<0.03-0.05, \mathrm{n}=21$ ). Individually, 14 of the 21 T1D subjects carried high risk DR3 and or DR4 alleles and ELISPOT responses to the defined DR3 or 4 binding regions in these subjects varied from one to all (mean 5.6 peptides). Only 2 of the 21 diabetic subjects showed no response to any ZnT8 peptide $(\mathrm{SI}<3)$.

Conclusion: ZnT8 is an important autoantigen recognized by T-cells as well as autoantibodies in type 1 diabetes. The T-cell responses appeared to be focused in the same region as the autoantibody response, a feature that is also observed in other diabetes autoantigens such as GAD and IA2. Positive responses were seen with peptides in regions where binding to HLA molecules associated with diabetes susceptibility (DR3 and DR4) were evident, but also to other peptides suggesting participation of other class II binding specificities.

Supported by: JDRF

\section{4}

T-lymphocyte autoimmune activity measured at the single cell level is associated with residual $\beta$-cell function in type 1 diabetes

C. Pfleger, G. Meierhoff, H. Kolb, N.C. Schloot on behalf of the p520/521 Study Group

Institute for Clinical Diabetes Research, German Diabetes Center, Leibniz Institute at Heinrich-Heine-University, Düsseldorf, Germany

Background and aims: In the context of a large placebo controlled intervention trial in recent type 1 diabetes we have tested the hypothesis that the activation status of autoimmune $\mathrm{T}$ cells in peripheral blood is related to the quantity or activity of insulin-producing islet cells as reflected by residual B-cell function.

Materials and methods: 50 adult (mean age $27.26 \pm 8.1$ years) and 49 pediatric patients (mean age $10.9 \pm 2.8$ years) with recent onset type 1 diabetes were included in the DiaPep277 Trial and received 0.2/1.0/2.5 mg or 0.2/1.0mg DiaPep277, respectively or placebo at $0,4,26$ and 52 weeks. We investigated the secretion of Th 1 associated IFN- $\gamma$ and Th2 associated interleukin (IL)-5, IL-10 and IL-13 by peripheral mononuclear cells (PBMC) upon stimulation with diabetes related antigens peptide fragment of Islet Antigen 2 (pIA2), heat shock protein 60 (hsp60) or glutamic acid decarboxylase (GAD65), at the single cell level by ELISPOT according to the Peakman protocol. The area under the curve (AUC) of serum C-peptide concentrations after stimulation with glucagon was used as measure of residual $B$-cell function. Since sex, age, BMI, DiaPep277 treatment, HLA and HbAlc represented potential confounder; we adjusted for these factors in a regression model analyzing the relation of cytokine response and $B$-cell function.

Results: At baseline, mean two months after diagnosis, PBMC of adults showed a positive association of residual $\beta$-cell function with IL-13 cytokine secretion upon stimulation with $\mathrm{pIA} 2(\mathrm{p}=0.04)$, whereas there was a negative association with IFN- $\gamma$ secretion $(\mathrm{p}=0.038)$. Likewise, PBMC of children at baseline (two months after diagnosis) also exhibited a positive association of residual $B$-cell function with IL-13 secretion in response to stimulation with $\mathrm{pIA} 2(\mathrm{p}=0.034)$. The same was found after stimulation with hsp60 $(\mathrm{p}=0.024)$, but IFN- $\gamma$ secretion was not related to AUC C-peptide levels.

At 8 weeks, both age groups had preserved an association of AUC C-peptide concentrations with cytokine secretion. In adults, there was a positive association with the IL-13 response to hsp60 ( $\mathrm{p}=0.038)$. In children, there was a positive association with the IL-5 response to hsp60 ( $\mathrm{p}=0.044)$ and a negative association for the secretion of IFN $-\gamma$ in response to pIA2 $(\mathrm{p}=0.047)$ and GAD65 ( $\mathrm{p}=0.045)$.

After 30 weeks, only children exhibited some association between residual ßcell function, for the IL-13 response upon stimulation of PBMC with GAD65 $(p=0.019)$. At 56 or 78 weeks neither adults nor children showed an association of autoimmune responsiveness and AUC C-peptide concentrations.

Conclusion: Cytokine secretion of PBMC upon stimulation with ß-cell antigens was associated with $B$-cell function during the first months after diagnosis of type 1 diabetes and lasted longer in children compared to adults, which can be interpreted as a more aggressive immune response and/or insulitis in pediatric patients. Increased IFN- $\gamma$ response was associated with lower $\beta$-cell function whereas increased IL-5 or IL-13 response was associated with better $\beta$-cell function except for the latest time point. The loss of $\beta$-cell function related PBMC responses over time may be due to regression of insulitis activity or caused by exogenous insulin treatment that may dampen reactivity of immune cells to $B$-cell antigens.

Supported by: We thank Andromeda for providing DiaPep277.

\section{5}

Estrogens prevent the development of auto-immune diabetes in NOD mice

P. Gourdy ${ }^{1,2}$, L. Pham Van Linh ${ }^{3}$, M.-L. Ahui ${ }^{3}$, D. Damotte ${ }^{4}$, F. Bayard ${ }^{2}$, J.-F. Arnal' ${ }^{2}$, A. Herbelin ${ }^{3}$

${ }^{1}$ Service de Diabétologie, Maladies Métaboliques et Nutrition, CHU Rangueil, Toulouse, ${ }^{2}$ Inserm u858, Institut de Médecine moléculaire de Rangueil, Toulouse, ${ }^{3}$ UMR8147, CNRS et Université Paris Descartes, Hôpital Necker, Paris, ${ }^{4}$ Service de d'Anatomie et de Cytologie Pathologique, Hotel-Dieu, Paris, France

Background and aims: Although the incidence of diabetes is higher in female than in male non obese diabetic (NOD) mice, the influence of estrogens in this prototypic model of auto-immune diabetes has not been clearly identified. However, in the last years, estrogens were demonstrated to modulate immune responses, natural killer $\mathrm{T}$ (NKT) and regulatory $\mathrm{T}$ (Treg) lymphocyte biology, and also to prevent pancreatic beta cells from apoptosis. Thus, we aimed to study whether in vivo exposure to estrogens influences diabetes development in NOD mice.

Materials and methods: Wild type, $\mathrm{RAG}^{-/}$, Ja18 ${ }^{--}$(NKT-deficient) and $\mathrm{CD} 28^{-/-}$(Treg-deficient) NOD female mice were used. The effect of chronic $17 \beta$-estradiol (E2) administration was assessed in female mice implanted with subcutaneous pellets delivering a physiological and constant dose of the hormone $(80 \mu \mathrm{g} / \mathrm{kg} /$ day). The influence of endogenous estrogens was assessed by the comparison of ovariectomized mice with intact sham-operated mice. At least 15 mice per group were included in each experiment.

Results: Chronic E2 administration from 4 to 30 weeks of age exerted a strong preventive effect against spontaneous diabetes incidence in NOD female mice. Indeed, whereas $80 \%$ of the untreated control mice developed diabetes during the follow-up period, none of the E2-treated mice were affected $(\mathrm{p}<0.001)$. A similar protective effect was observed in the model of ac- 
celerated diabetes induced by cyclophosphamide in NOD mice (diabetes appeared in $60 \%$ of control mice, but in none of the E2-treated mice, $\mathrm{p}<0.001$ ). Histological analysis of pancreatic islets demonstrated that $\mathrm{E} 2$ treatment was associated with a significant reduction in spontaneous insulitis in 10-weekold mice. Furthermore, the titers of anti-insulin antibodies were significantly lower in both 17- and 30-week-old E2-treated mice. Then, splenocytes from 30-week-old NOD mice chronically treated or not with E2 were transferred to $\mathrm{RAG}^{-/}$NOD mice and induced diabetes in a similar extent, suggesting that E2 treatment does not influence the development of diabetogenic cells. Thus, to study the respective involvement of NKT and Treg cells in the preventive effect of estrogens, $\mathrm{Ja}_{18^{-/-}}$and $\mathrm{CD} 28^{-/-} \mathrm{NOD}$ mice were treated with E2. Interestingly, the hormone exerted a full protective effect in both models, ruling out a crucial role of these lymphocyte subpopulations. Finally, we demonstrated a significant protective effect of endogenous estrogens. Indeed, ovariectomy at 4 weeks of age led to an acceleration of the apparition of diabetes in both the spontaneous and the cyclophosphamide-induced models of diabetes in NOD mice.

Conclusion: Our results demonstrate that chronic exposure to estrogens prevents insulitis and the development of diabetes in NOD mice. Although the mechanisms mediating this beneficial effect remain to be definitely elucidated, we ruled out the involvement of NKT and Treg lymphocytes. This protective effect could explain the age-related gender dimorphism in type 1 diabetes incidence recently reported by Scandinavian epidemiological studies. Finally, activation of the estrogen pathway could represent a new research area for diabetes prevention.

\section{6}

Mesenchymal stem cells protect NOD mice from diabetes by recovery of regulatory $\mathrm{T}$ cells

A.-M. Madec ${ }^{1}$, E. AbouMrad ${ }^{1}$, R. Mallone ${ }^{2}$, G. Afonso ${ }^{3}$, A. ElJaafari ${ }^{4}$,

C. Thivolet ${ }^{1}$;

${ }^{1}$ Faculte de Medecine Lyon-Sud, INSERM U.870/INRA 1235, Oullins, ${ }^{2}$ Hopital St Vincent de Paul, INSERM U.561, Paris, ${ }^{3}$ Hopital St Vincent de Paul, INSERM U.561, Paris, ${ }^{4}$ Hopital Edouard Herriot, EA 4061, Lyon, France

Background and aims: Due to their modulatory $\mathrm{T}$ cells properties and/or the immunosuppressive local milieu that they create, Mesenchymal Stem Cells (MSCs) are considered as beneficial agents for autoimmune disease therapy. Here, we examined the capacity of MSCs isolated from BALB-b mouse bone marrow to prevent autoimmune diabetes in the NOD mouse model.

Materials and methods: Prevention of insulitis or clinical diabetes was evaluated after a single I.V. injection of 103 to 106 MSCs in a 4wk-old female NOD or following their co-injection with 5.106 diabetogenic T cells in 8-12wk old male irradiated NOD mice. Five days after cell cotranfer, we analyzed the frequency of $\mathrm{CD} 4+\mathrm{FOXP} 3+$ expression within spleen $\mathrm{T}$ cell population from NOD mice treated by 106 MSCs. FoxP3 mRNA levels were also quantified. In vivo cell homing was assessed by monitoring CFE labeled T cells or MSCs. In vitro, cell proliferation and cytokines production were assessed by adding graded (102 to 105 per well) doses of irradiated MSCs to insulin B9-23 peptide (ins B9-23) specific T cell lines (7.5.105 per well) in the presence of 1.5.106 irradiated splenocytes pulsed with 10 microM ins B9-23.

Results: During cotransfer experiments, 105 or 106 MSCs reduced the capacity of diabetogenic cells to infiltrate pancreatic islets and to transfer diabetes $(\mathrm{p}<0.001)$. Although they did not modify $\mathrm{T}$ cell homing to the thymus, spleen or pancreatic lymph nodes, MSCs preferentially migrated to spleen and pancreatic lymh nodes to reach a statistically significant peak five days after cell transfer. Injection of diabetogenic $\mathrm{T}$ cells resulted in a decrease of FoxP3+ cells, which was recovered by cotransfer of MSCs and diabetogenic cells as assessed by $\mathrm{CD} 4+\mathrm{FoxP} 3+$ staining $(2.9 \pm 0.3 \%$ versus $4.9 \pm 1.2 \%$; $\mathrm{p}<0.01)$ and measurement of FoxP3 mRNA levels (mean \pm SD relative expression rate, $2.32 \pm 0.3$ versus $5.23 \pm 0.5 ; \mathrm{p}<0.001$ ). Finally, MSCs inhibited ins B9-23 antigen-specific proliferative responses in vitro in a dose-dependent fashion from $75 \%$ with 102 MSCs to $100 \%$ with 105 MSCs. This effect correlated with an increase in IL-10 production from $94 \pm 20 \mathrm{pg} / \mathrm{mL}$ to $1041 \pm 96 \mathrm{pg} / \mathrm{mL}$ respectively.

Conclusion: Taken together, our results strongly suggest that MSCs inhibit autoimmune beta cell destruction in NOD mouse by recovery of regulatory T cells. MSCs should thus offer a novel immunotherapeutic approach for autoimmune diabetes and islet transplantation.

Supported by: INSERM/INRA/UCBL

\section{OP 42 Monogenic diabetes}

\section{7}

Recessive mutations in the INS gene; a novel common cause of permanent neonatal diabetes in consanguineous pedigrees

E.L. Edghill, J. Locke, S.E. Flanagan, A.-M. Patch, L.W. Harries, S. Ellard, A.T. Hattersley

Institute of biomedical and clinical science, Peninsula Medical School, Exeter, United Kingdom

Background and aims: Permanent Neonatal Diabetes (PNDM) diagnosed in the first 6 months of life is commonly caused by heterozygous mutations in KCNJ11, ABCC8 or INS genes. Recessive mutations in GCK and ABCC8 can also cause non-syndromic PNDM but recessive INS mutations have not been described. We hypothesised that homozygous mutations in the INS gene could cause PNDM and these would be most likely in subjects born to consanguineous parents.

Materials and methods: We investigated 25 patients of consanguineous descent with non-syndromic PNDM diagnosed before 6 months of life. We screened INS, GCK, KCNJ11 and ABCC8 by direct sequencing. In addition we used Multiplex Ligation-dependent Probe Amplification (MLPA) to test for INS deletions.

Results: We identified 9 mutations in the known genes; 5 homozygous GCK mutations, 2 homozygous ABCC8 mutations and 2 heterozygous KCNJ11 mutations. We also identified five novel homozygous mutations in INS; M1I $(\mathrm{n}=2), \mathrm{Q} 62 \mathrm{X}, \mathrm{IVS} 2-15 \mathrm{G}>\mathrm{A}$ and c.-331C $>\mathrm{G}(\mathrm{n}=3)$ in 7 patients. MLPA revealed a homozygous INS gene deletion (M1_Q62del), after PCR of exon 1 and exon 2 consistently failed. The c.-331C $>$ G variant and the IVS2-15G $>$ A variant was not found in control population subjects. Patients with a homozygous mutation are diagnosed earlier $(\mathrm{p}=0.005)$ and have a much lower birth weight $(\mathrm{p}>0.0001)$ [median age of diagnosis 0.85 weeks $(0.14-7.1)$, centile birth weight $\left.<1^{\text {st }}(0.0002-0.3)\right]$ compared to heterozygous INS mutation carriers [ $(9$ weeks, $0-26)$, centile $<5(0-87)]$.

Conclusion: We have shown that homozygous mutations in INS are a novel cause of PNDM and are the most common cause (32\%) of recessively inherited non-syndromic PNDM. The genetic aetiology can now be defined in the majority of patients with PNDM from consanguineous families. Supported by: European Union and the Wellcome Trust

\section{8}

Atypical endoplasmic reticulum (ER) stress in cell and animal models of MODY3

C. Kirkpatrick, M. Baquié, H. Wang, B. Gauthier, C.B. Wollheim

Dept. of Cell Physiology and Metabolism, University of Geneva, Switzerland

Background and aims: ER stress occurs when the protein folding and processing capacity of the ER is perturbed, either by increased protein load or by interference with the folding machinery. The typical response of a cell to ER stress is to upregulate transcription of ER chaperones (such as BiP) and the transcription factors Atf 4 and Xbpl. Pancreatic $\beta$-cells are particularly susceptible to ER stress and evidence for its involvement in diabetes has been observed in multiple animal models and in human disease (Wolfram and Wolcott-Rallison syndromes). We examined whether ER stress also occurs in MODY3, caused by mutations in the transcription factor Hnfla, using both cultured cells and diabetic mouse islets carrying a dominant-negative (DN) variant of Hnfla. The transgenic mice express the dominant-negative gene only in the $\beta$-cells, which have been observed to have dilated ER cisternae and fewer mature secretory granules, providing ultrastructural evidence that ER function may be perturbed in this model.

Materials and methods: INS $\mathrm{r} 9$ cells expressing DN-Hnfla under the control of the Tet-On system were cultured in RPMI with doxycycline to induce the transgene, or cyclopiazonic acid (CPA) to induce ER stress. Mouse islets were isolated by collagenase digestion and either cultured for 24 hours in RPMI to allow recovery from the stress of isolation or immediately used for RNA extraction. Q-RT-PCR was performed on cell and islet extracts. Cell viability was analysed by MTT assay.

Results: INS DN-Hnfla cells showed an atypical ER stress response on induction of the transgene, with increased mRNA levels of SERCA2b, the ER-associated transcription factors Atf4 and CHOP and the pro-apoptotic gene PUMA, but decreased levels of other ER stress markers including BiP, calreticulin, protein disulphide isomerase (PDI), Xbpl and the $\beta$-cell specific 
SERCA3. In cells with ER stress induced by CPA, upregulation of some of the ER stress genes (BiP, calreticulin, PDI and SERCA2b) was attenuated when DN Hnfla was induced. A consensus Hnfla binding site was found in the $\mathrm{Xbp} 1$ promoter region, suggesting that this gene may be directly regulated by Hnfla. INS DN-Hnfla cells were more sensitive to CPA-induced cell death when the transgene was induced. Similarly, when ER stress gene expression was compared between DN-Hnfla and control mouse islets, expression of $\mathrm{Xbp} 1, \mathrm{BiP}$, calreticulin, PDI and SERCA3 was lower in the DN-Hnfla islets. Conclusion: These data show that in cell and animal models of MODY 3 levels of ER chaperone and folding enzyme mRNA are attenuated at the basal level and in response to chemically induced ER stress. However, mRNA levels of other ER stress-associated genes (Atf4, CHOP and SERCA2b) were more abundant in the presence than the absence of DN-Hnfla in the cell model, unlike the expected ER stress response where chaperones and transcription factors are upregulated simultaneously. Insufficient chaperone production as a result of DN-Hnfla expression may sensitise the ER of these cells to stress, making them more prone to apoptosis. The results suggest that DN-Hnfla directly downregulates Xbp1 expression, and as a consequence of Xbp1 loss the $\beta$-cells have lower levels of ER chaperones and are sensitised to ER stress.

\section{9}

Permanent neonatal diabetes caused by an in-frame deletion in the $\mathrm{N}$-terminus of Kir6.2

T.J. Craig ${ }^{1}$, K. Shimomura ${ }^{1}$, S. Flanagan ${ }^{2}$, S. Ellard ${ }^{2}$, A. Hattersley², F.M. Ashcroft ${ }^{1}$

${ }^{1}$ Physiology, University of Oxford, ${ }^{2}$ Institute of Biomedical and Clinical Science, Peninsula Medical School, Exeter, United Kingdom

Background and aims: We identified a novel mutation in KCNJ11 (Kir6.2), the pore-forming subunit of the $\mathrm{K}_{\mathrm{ATP}}$ channel, that causes permanent neonatal diabetes mellitus (PNDM), and characterised its functional consequences. This mutation results in an in-frame deletion of 5 amino acids (A28 to R32) from the N-terminus of Kir6.2.

Materials and methods: Electrophysiology of $\mathrm{K}_{\mathrm{ATP}}$ channel function, surface expression assays, and protein-protein interaction assays were performed in Xenopus oocytes. Oocytes were coinjected with cRNA coding for SUR1 and mutant or wild-type Kir6.2. In some experiments we used Kir6.2 $\Delta$ C, a Cterminal 36-amino acid truncated Kir6.2, which traffics to the cell surface in the absence of SUR1.

Results: Deletion of residues 28-32 produced a 6-fold decrease in the $\mathrm{IC}_{50}$ for ATP inhibition of Kir6.2/SUR1 currents. This can be attributed to an increase in the channel open probability (Po) from 0.23 (wild-type) to 0.8 (mutant; $\mathrm{P}<0.05$ ). The mutation had no effect on the Po or ATP sensitivity of Kir6.2 $\Delta \mathrm{C}$ currents. Thus these data suggest deletion of residues 28-32 disrupts the interaction between Kir6.2 and SUR1. The first five transmembrane domains (TMD0) and the linker between transmembrane domains 5 and 6 (CL3 linker), are required for the ability of SUR1 to influence the Po (and thereby ATP sensitivity) of Kir6.2. When mutant Kir6.2 $\Delta \mathrm{C}$ was coexpressed with TMD0, there was no effect on the channel properties, and co-immunoprecipitation studies showed no difference in binding of TMD0 and Kir6.2 $\triangle \mathrm{C}$. However, when Kir6.2 $\Delta$ C was coexpressed with TMD0+CL3 of SUR1, the 5aa deletion caused a significant $(\mathrm{P}<0.05)$ increase in Po: from 0.58 for wild-type Kir6.2 to 0.74 for mutant. Thus it is likely the 5 aa deletion mutation disrupts an interaction between the N-terminus of Kir6.2 and the CL3 linker of SUR1.

Conclusion: We show here that an in-frame deletion (A28 to R32) in KCNJ11 can cause neonatal diabetes. This deletion selectively disrupts an interaction between the CL3 linker of SUR1 and Kir6.2, thereby increasing the Po of the $\mathrm{K}_{\text {ATP }}$ channel and reducing its sensitivity to inhibition by ATP. Supported by: Wellcome Trust

\section{0}

Mitochondrial A3243G DNA mutation leads to decreased insulin sensitivity in skeletal muscle accompanied by failing beta cell dysfunction

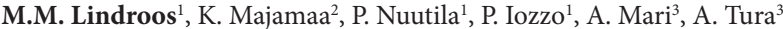

${ }^{1}$ Turku PET Centre, University of Turku, Finland, ${ }^{2}$ Department of

Neurology, University of Turku, Finland, ${ }^{3}$ Institute of biomedical

engineering, National Research Council, Pisa, Italy

Background and aims: Diabetes in adulthood is characterized by low insulin sensitivity but most of the gene variants associated with diabetes affect the $\beta$ - cell insulin secretion. Also mitochondrial DNA point mutation A3243G leads finally to failing insulin secretion while most of its carriers develop diabetes. The aim of this study was to assess the role of insulin sensitivity in this most common form of mitochondrial diabetes.

Materials and methods: Eight non-diabetic A3243G carriers with a proper glycaemic control $(\mathrm{Hb}-1 \mathrm{AC}<6.1 \%)$, seven patients with overt A3243G diabetes and 13 age and physical activity matched healthy subjects were studied. Skeletal muscle and whole body insulin sensitivity was measured using positron emission tomography and $2-\left[{ }^{18} \mathrm{~F}\right]$ fluoro-2-deoxyglucose tracer during eugycemic hyperinsulinemia.Parameters of $\beta$ - cell function were derived from an oral glucose tolerance test by mathematical modelling based on Cpeptide deconvolution. In particular, $\beta$ - cell glucose sensitivity was calculated as the slope of the dose-response function relating glucose concentration to insulin secretion. A parameter quantifying early insulin release was also obtained from the model.

Results: Among the A3243G mutation carries whole body insulin sensitivity was $30 \%$ lower than those in healthy subjects, and was similar to the values in the patients with overt A3243G diabetes $(P=0,003)$. As compared to the healthy subjects the skeletal muscle insulin sensitivity was decreased by $62 \%$ both in the A3243G mutation carries and in the patients with overt diabetes $\left(55 \pm 9,21 \pm 5\right.$, and $\left.21 \pm 4 \mu \mathrm{mol} \cdot \mathrm{min}^{-1} \cdot \mathrm{kg}^{-1}, P=0,0031\right) \cdot \beta$ - cell glucose sensitivity was progressively impaired across the groups, being highest in healthy subjects, decreased in A3243G carries and lowest in the A3243G patients with overt diabetes $\left(137 \pm 15,82 \pm 15\right.$ and $\left.22 \pm 14 \mathrm{pmol} \cdot \mathrm{min}^{-1} \cdot \mathrm{m}^{-2} \cdot \mathrm{mM}^{-1}, P=0,002\right)$ Additionally, as compared to controls and to non-diabetic A3243G carries, early insulin release was decreased in the $\mathrm{A} 3243 \mathrm{G}$ patients with overt diabetes $\left(686 \pm 82,902 \pm 228\right.$ and $\left.103 \pm 50 \mathrm{pmol} \mathrm{m}^{-2} \mathrm{mM}^{-1} P=0,0014\right)$.

Conclusion: Subjects harbouring the A3243G mutation have decreased muscle and whole body insulin sensitivity before the glucose control is compromised, wheras beta-cell function deteriorates gradually and is markedly impaired in overt mitochondrial diabetes.

Supported by: Academy of Finland 


\section{OP 43 Neural pathways and neuropathy}

\section{1}

Measures of autonomic nervous system function in the diabetes control and complications trial/epidemiology of diabetes interventions and complications study (DCCT/EDIC)

R. Pop-Busui ${ }^{1}$, P.A. Low ${ }^{2}$, B.H. Waberski ${ }^{3}$, C.L. Martin ${ }^{4}$, E.L. Feldmam ${ }^{5}$, J.W. Albers ${ }^{6}$, C.L. Sommer ${ }^{7}$, P.A. Cleary ${ }^{3}$, W.H. Herman ${ }^{6}$

${ }^{1}$ Division of Metabolism Endocrinology and Diabetes, University of Michigan, Ann Arbor, ${ }^{2}$ Neurology, Mayo Clinic, Rochester, ${ }^{3}$ The Biostatistics Center, The George Washington University, Rockville, ${ }^{4}$ Michigan Diabetes Research and Training Center, University of Michigan, Ann Arbor, ${ }^{5}$ Neurology, University of Michigan, Ann Arbor, ${ }^{6}$ University of Michigan Health Systems, University of Michigan, Ann Arbor, ${ }^{7}$ Autonomic Reading Center, Mayo Clinic, Rochester, United States

Background and aims: The DCCT demonstrated that intensive diabetes treatment (INT) significantly slowed the decline in R-R variation compared to conventional therapy (CONV) in type 1 diabetes. Longitudinal EDIC followup has shown that the former INT group continues to have a lower cumulative incidence of retinopathy and nephropathy years after DCCT completion, despite similar levels of glycemic control. Using the long-term follow-up data from the DCCT/EDIC cohort, we evaluated whether the former INT group continues to experience a lower risk of developing cardiac autonomic neuropathy (CAN) compared to the former CONV group.

Materials and methods: All tests of CAN performed during the DCCT (R-R response to paced breathing, Valsalva ratio and postural testing) were conducted in 1,001 DCCT subjects ( $\mathrm{N}=504 \mathrm{INT}$; $\mathrm{N}=497 \mathrm{CONV}$ ) during the 13th and 14th year of EDIC follow-up. The Wilcoxon rank-sum test estimated the treatment group differences in the change in each measure from DCCT closeout to EDIC. Changes in measured variables within groups were compared using analysis of covariance, with adjustments made for closeout values, age, sex, and use of beta-blockers.

Results: In EDIC year 13-14, R-R variation was significantly higher in the INT (29.8 \pm 19.1$)$ compared with the CONV (26.1. \pm 17.2$)$ group $(\mathrm{p}=0.0012)$. The change in R-R variation from DCCT closeout was $-11.3 \pm 18.5$ in the INT and $-13.1 \pm 18.3$ in the CONV and the difference in Valsalva ratio was $-0.20 \pm 0.40$ in the INT and $-0.27 \pm 0.42$ in the CONV $(p<0.05)$. Based on the DCCT definition of CAN as R-R $<15,24.5 \%$ of the INT group were diagnosed with CAN compared with $30.2 \%$ in the CONV group in EDIC year 13$14,(\mathrm{p}<0.05)$. Additional ongoing analyses of $\mathrm{R}-\mathrm{R}$ variation as well as Valsalva and postural testing will further evaluate the rate of CAN development and odds of emergent CAN during EDIC.

Conclusion: During EDIC, CAN as measured by R-R response to paced breathing, progressed in both groups, but the prevalence of CAN in year 1314 remained significantly higher in the former CONV group than in the INT group. The benefits of former intensive therapy extend to measures of CAN 14 years after trial end.

Supported by: National Institute of Diabetes and Digestive and Kidney Diseases, General Clinical Research Centers Program, National Center for Research Resources

\section{2}

Raised thalamic blood volume in painful diabetic neuropathy: clues to the pathogenesis of neuropathic pain in diabetes?

D. Selvarajah ${ }^{1}$, I.D. Wilkinson ${ }^{2}$, P.D. Griffiths ${ }^{2}$, C.J. Emery ${ }^{1}$, S. Tesfaye ${ }^{1}$

${ }^{1}$ Diabetes Research Department, Royal Hallamshire Hospital, Sheffield,

${ }^{2}$ Academic Unit of Radiology, University of Sheffield, United Kingdom

Background and aims: The pathogenesis of pain in diabetic neuropathy (DN) remains unknown and as a result there are no fully effective treatments. The thalamus plays a crucial role in modulating and processing all sensory information to the brain. Recently there have been reports of thalamic neuronal dysfunction in patients with painless-DN, and we sought to investigate if these were accompanied with abnormalities in blood flow.

Materials and methods: Eighteen right-handed, male subjects with type 1 diabetes and 5 healthy volunteers (HV) were recruited. Detailed neurophysiological assessments and pain symptom scores were undertaken. Based on these assessments subjects were divided into no-DN $(n=6)$, painful- $(n=5)$ and painless-DN $(n=7)$. Cerebral parenchymal perfusion was assessed by tracking the passage of an iv bolus of contrast agent (gadolinium-chelate) using a multi time point, $\mathrm{T} 2{ }^{\star}$ weighted EPI sequence on a $1.5 \mathrm{~T}$ magnetic resonance imaging system. Haemodynamic anatomical regions supplied by the anterior cerebral artery [ACA], middle cerebral artery [MCA] and posterior cerebral artery $[\mathrm{PCA}]$ ) were studied. We also looked at perfusion through the thalamus using the caudate nucleus as a control.

Results: In all three vascular territories, in the resting state and assuming equivalent intracranial resonance properties, diabetic subjects had larger relative cerebral blood volume ( $\mathrm{rCBV}$ ) compared to $\mathrm{HV}$ (ACA mean $\pm \mathrm{SD}$, $264.4 \pm 55.2$ vs $205.3 \pm 57.2$, p=0.05; MCA $244.6 \pm 43.3$ vs $189.0 \pm 55.2$, $\mathrm{p}=0.03$; PCA $248.4 \pm 43.8$ vs $190.8 \pm 36.5, \mathrm{p}=0.01$ ). However, there was a non significant trend towards longer bolus transit time and greater relative cerebral blood flow $(\mathrm{rCBF})$ in diabetic subjects. Subjects with painful-DN had significantly higher thalamic rCBV compared to the other study groups (painful DN, 234.3 \pm 45.1 , No-DN, 196.5 \pm 78.7 , painless DN, 203.1 $\pm 171.7 ; \mathrm{p}=0.045$ ). Subjects with painful-DN also had the longest contrast bolus transit time compared with other groups. In contrast, subjects with painless $\mathrm{DN}$ had lowest thalamic rCBF compared to other groups. There were no significant differences in the perfusion characteristics of the caudate nucleus between subgroups.

Conclusion: In this preliminary study, we found that diabetic subjects had global increased rCBV in all major cerebral artery territories. This suggests impaired vasoreactivity with a persistent resting state cerebral vasodilation, similar to observation in the diabetic kidney. Painful-DN is accompanied by increased thalamic blood volume; in keeping with previous studies demonstrating increased peripheral nerve blood flow. Conversely, subjects with painless-DN lowest thalamic blood flow suggesting a greater degree of microvascular impairment. As similar observations were not found in the caudate nucleus, this suggests that unique pathophysiological microvascular changes are occurring within the thalamus that may provide important clues to the pathogenesis of neuropathic pain in diabetes.

Supported by: Diabetes UK

\section{3}

Association of reduced glyoxalase- 1 activity and painful peripheral neuropathy in diabetes mellitus patients

I. Konrade ${ }^{1}$, M. Dambrova ${ }^{2}$, E. Skapare ${ }^{3}$, J. Klovins ${ }^{4}$, A. Lejnieks ${ }^{5}$,

S.B. Stoyanov ${ }^{6}$, P.M. Humpert ${ }^{6}$, P.P. Nawroth ${ }^{6}$, A. Bierhaus ${ }^{6}$

${ }^{1}$ Dep. of Endocrinology, Riga Stradins University, Latvia, ${ }^{2}$ Laboratory of Pharmacology, Latvian Institute of Organic Synthesis, Riga, Latvia, ${ }^{3}$ Faculty of Pharmacy, Riga Stradins University, Latvia, ${ }^{4}$ Genome Center, Latvian Biomedical Research and Study Centre, Riga, Latvia, ${ }^{5}$ Clinic "Linezers", Riga Stradins University, Latvia, ${ }^{6}$ Department of Medicine I and Clinical Chemistry, University of Heidelberg, Germany

Background and aims: Glyoxalase-1 (GO-1) is a central part of the cellular defense to detoxify reactive dicarbonyls such as methylglyoxal, glyoxal and other alpha-oxoaldehydes, which are potent precursors of Advanced Glycation Endproduct (AGEs). Experimental animal models have provided evidence that reduced detoxification of AGEs by GO-1, engagement of the receptor RAGE and RAGE-dependent sustained activation of the proinflammatory transcription factor NF- $\mathrm{KB}$ significantly contribute to functional deficits in diabetic neuropathy (DN). Since we have recently demonstrated that GO-1 controlled reactions modulate pain in rodent models of DN, we studied the relationship between GO-1 activity and painful peripheral DN in patients with type- 1 and type- 2 diabetes.

Materials and methods: GO-1 enzymatic activity was determined in blood samples of 70 type- 1 diabetic patients ( $56 \%$ (39) female, age $38.6 \pm 14.9$ years; $\left.\mathrm{HbA}_{1 \mathrm{c}} 9.06 \pm 2.1 \%\right)$ and 67 age- and sex-matched non-diabetic controls as well as in 66 type- 2 diabetic patients (56\% (37) female, age $55.6 \pm 9.8$ years; $\left.\mathrm{HbA}_{1 c} 8.31 \pm 1.91 \%\right)$ and 48 matched age- and sex-non-diabetic controls. All patients were diagnosed for peripheral DN by neuropathy disability score and by measuring current perception threshold. Patients were deemed to have naturopathic pain, if pain for which no other cause could be found lasted for at least 6 months and at least $40 \mathrm{~mm}$ on the $100-\mathrm{mm}$ visual analogue scale was scored. GO-1 activity was measured in blood samples by a standardized spectrophotometrical assay. Blood samples were also tested for lipids, C-peptide and inflammatory markers. In addition, all samples were screened for the presence of three SNPs (rs2736654, rs1130534 and rs1049346) in the GO-1 gene using MALDI TOF mass spectrometry.

Results: In non-diabetic control subjects, the average GO-1 activity was $7.46 \mu \mathrm{mol} / \mathrm{min} / \mathrm{ml}$. In type 1 diabetic patients with $(\mathrm{n}=35)$ and without $(\mathrm{n}=35)$ painful peripheral DN the activity of GO-1 was $6.73 \pm 1.03 \mu \mathrm{mol} / \mathrm{min} / \mathrm{ml}$ and $8.16 \pm 1.13 \mu \mathrm{mol} / \mathrm{min} / \mathrm{ml}(\mathrm{p}<0.001)$, respectively. In type- 2 diabetic patients 
with $(\mathrm{n}=30)$ and without $(\mathrm{n}=37)$ painful peripheral $\mathrm{DN}, \mathrm{GO}-1$ activity was $6.87 \pm 1.21 \mu \mathrm{mol} / \mathrm{min} / \mathrm{ml}$ and $8.0 \pm 1.23 \mu \mathrm{mol} / \mathrm{min} / \mathrm{ml}$ ( $\mathrm{p}<0.001$ ), respectively. Even though GO-1 activity in both, type-1 and type-2 diabetes correlated positively with two SNPs studied (rs1130534 and rs1049346), none of them was associated with painful DN. In multivariate models, only reduced activity of GO- 1 was associated with painful $\mathrm{DN}(\beta=-0.45, \mathrm{p}<0.001 \beta$ in type $1 \mathrm{DM}$ patients and $\beta=-0.33, p<0.01$ in type $2 \mathrm{DM}$ patients).

Conclusion: Our results demonstrate for the first time that decreased GO-1 enzyme activity in blood samples of both, type 1 and type 2 DM patients is associated with painful DN.

Supported by: EFSD Albert Renold Fellowship (IK) and JDRF

\section{4}

Neurochemical abnormalities within sensory pathways in the brain in diabetic neuropathy

R.A. Gandhi ${ }^{1}$, D. Selvarajah ${ }^{1}$, C. Emery ${ }^{1}$, I. Wilkinson ${ }^{2}$, S. Tesfaye ${ }^{1}$

${ }^{1}$ Diabetes Research Unit, Royal Hallamshire Hospital, Sheffield, ${ }^{2}$ Academic

Unit of Radiology, University of Sheffield, United Kingdom

Background and aims: Pain is the most distressing symptom of diabetic peripheral neuropathy $(\mathrm{DN})$. Although clear pathological abnormalities have been identified at the level of the peripheral nerve and spinal cord in $\mathrm{DN}$, there is only limited understanding of why some patients suffer severe chronic pain whilst others have painless symptoms. A better understanding of the pathogenesis of painful and painless DN is crucial for the development of novel, mechanism-based treatments. There is growing evidence that central mechanisms play an important role.

Materials and methods: 110 subjects with type 1 diabetes $(20$ no DN, 30 subclinical DN, 30 painful DN and 30 painless DN) and 20 healthy volunteers (HV) underwent detailed clinical and neurophysiological assessments (Dyck's NIS(LL)+7 staging criteria). Proton magnetic resonance spectroscopy of the left thalamic nucleus and somatosensory cortex was performed to measure established markers ( $\mathrm{N}$-acetylaspartate [NAA]) of neuronal function using long echo time (LET) and neuronal integrity using short echo time (SET) sequences.

Results: In the thalamus, at LET (Fig. 1), subjects with painless DN had significantly lower NAA:Creatine ratio $(1.55 \pm 0.22[$ mean \pm SD] $)$ compared to other

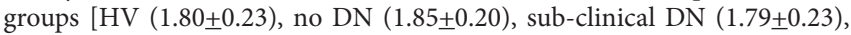
painful DN $(1.75 \pm 0.19)$, ANOVA $p<0.001]$. There were no significant intergroup differences at SET. In the somatosensory cortex, no inter-group differences were seen at LET. At SET (Fig. 2), the painless DN group had lower NAA $(0.62 \pm 0.08)$, compared to HV $(0.73 \pm 0.15)$ and no DN $(0.68 \pm 0.13)$, $\mathrm{p}=0.03$. Subjects with painful DN had intermediate levels $(0.66 \pm 0.10)$.

Conclusion: These results imply change in neuronal physiology or function, rather than neuronal loss, within the thalamus (the gateway to the somatosensory cortex) in painless but not painful DN. It is possible that relative preservation of thalamic neuronal function is necessary for the transmission of abnormal peripheral signals to higher centres and the perception of chronic pain in DN. The results within the somatosensory cortex, in contrast, are suggestive of neuronal loss in subjects with DN and may be reflecting local cerebral parenchymal atrophy.

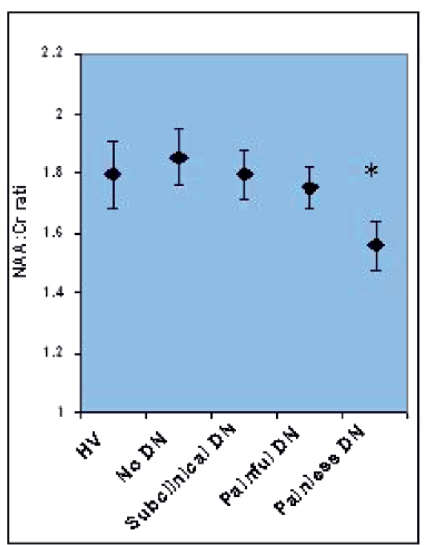

Fig. 1. Thalamus NAA:CR ratio (LET)

Error bars represent $95 \% \mathrm{CI}$

Supported by: Diabetes UK

\section{OP 44 Nutrition and diet}

\section{5}

Independent role of fatty acid profile and insulin resistance in steatosis: a population-based study in elderly men

U. Risérus, H. Petersson, T. Cederholm, B. Vessby

Public health and caring sciences, Uppsala university, Sweden

Background and aims: Fatty liver (steatosis) has been proposed to promote insulin resistance and metabolic disorders. Steatosis may partly be driven by dietary and endogenously produced fatty acids. The link between steatosis and fatty acid profile is however unexplored in large populations, and it is unclear if such potential associations may be independent of insulin resistance and obesity. We aimed to investigate the associations between liver fat biomarkers and serum fatty acid profile, and examined if such associations may be explained by insulin resistance, obesity or other related metabolic and hormonal factors.

Materials and methods: In a population-based cross-sectional sample $(n=583)$ of 70-year old men (Uppsala Longitudinal Study of Adult Men; ULSAM) with complete data on serum fatty acid profile, alanine aminotransferase (ALT) concentrations (as marker of steatosis), directly measured insulin sensitivity (determined by hyperinsulinemic euglycemic clamp), adiponectin concentrations and lifestyle factors (abdominal obesity, i.e. waist circumference, physical activity, smoking and alcohol consumption) was investigated. In addition, to estimate endogenous fatty acid metabolism, fatty acid desaturase activities (Stearoyl-CoA desaturase (SCD), $\Delta 6$-desaturase, and $\Delta 5$-desaturase) were estimated by product-to-precursor fatty acid ratios in cholesteryl esters. Associations were assessed by univariate and multivariate linear regression, and P-values below are standardized (1 SD) regression coefficients.

Results: A fatty acid profile in serum cholesteryl esters characterized by increased proportions of palmitoleic, oleic, $\gamma$-linolenic and dihomo- $\gamma$-linolenic acid, and decreased proportions of linoleic acid, as well as increased activities of $\Delta 6$-desaturase and SCD-1 were significantly related to ALT concentrations, even after adjusting for lifestyle factors and insulin resistance (multivariate model 1: all $\mathrm{P}$-values $\mathrm{P}<0.05$ ). These relationships remained if subjects with diabetes and cardiovascular disease were excluded $(n=391)$. Serum linoleic acid proportions mainly reflect dietary intake of this fatty acid, whereas monounsatuared fatty acids and dihomo- $\gamma$-linolenic acid mainly reflect dietary intake of saturated fat and/or SCD-1 activity and $\Delta 5$-desaturase activity, respectively. In a second multivariate model where additional metabolic factors were added, only SCD-1 activity index (multivariate model 2: $\mathrm{P}=0.0009$ ) and insulin sensitivity (model 2: $\mathrm{P}<0.0001$ ) were independent determinats of steatosis, whereas waist circumference, triglycerides, high density lipoprotein cholesterol, non-esterified fatty acids, and adiponectin were not significant predictors.

Conclusion: Although dietary biomarkers such as serum linoleic acid were inversely related to steatosis, especially strong independent associations between steatosis and endogenous fatty acids were evident in this population of elderly men. Interestingly, the latter associations were independent of insulin resistance, obesity and lifestyle factors. In summary, a high SCD-1 actvity index and insulin resistance were the two major independent determinants of steatosis. Controlled studies will be required to investigate causality and to further examine the role of dietary fatty acids and insulin resistance in steatosis.

Supported by: NordForsk: Nordic Centre of Excellence on Food, Nutrition and Health (SYSDIET)

\section{6}

Efficacy and safety of low-carbohydrate, mediterranean, and low-fat diet strategies for weight loss - a two year dietary intervention randomized controlled trial (DIRECT)

I. Shai ${ }^{1}$, D. Schwarzfuchs ${ }^{2}$, Y. Henkin ${ }^{3}$, D. Shahar ${ }^{1}$, I. Greenberg ${ }^{1}$, R. Golan ${ }^{1}$, D. Fraser ${ }^{1}$, O. Tangi-Rozental ${ }^{1}$, Z. Schwartz ${ }^{2}$, E. Sheiner ${ }^{2}$, J. Thiery ${ }^{4}$, G.M. Fiedler ${ }^{4}$, M. Blüher ${ }^{4}$, M. Stumvoll ${ }^{4}$, M.J. Stampfer ${ }^{5}$

${ }^{1}$ Epidemiology, Ben Gurion University, Beer-Sheva, Israel, ${ }^{2}$ Medicine, Nuclear Research Center Negev, Dimona, Israel, ${ }^{3}$ Cardiology, Soroka University Medical Center, Beer-Sheva, Israel, ${ }^{4}$ Medicine, University of Leipzig, Germany, ${ }^{5}$ Medicine, Harvard University, Boston, United States

Background and aims: Trials comparing effectiveness and safety of alternative diets are frequently limited by short follow-up and high dropout rates. 
Materials and methods: In a two-year Dietary Intervention Randomized Controlled Trial (DIRECT) we randomized 322 moderately obese (age $=52 \mathrm{yrs}$, body-mass-index $=31 \mathrm{~kg} / \mathrm{m}^{2}, 86 \%$ men) to one of three diets: low-fat restricted-calorie, Mediterranean restricted-calorie or low-carbohydrate non-restricted-calorie. The trial was conducted in an isolated workplace, with an integrated clinic. We displayed daily diet-group-specific colored food labels in the exclusive workplace cafeteria.

Results: Retention was 95\% after one year and $85 \%$ after two, with no adverse events. In repeated dietary analyses, the Mediterranean diet-group consumed the highest dietary fiber and monounsaturated/saturated fat ratio $(\mathrm{P}<.05)$. The low- carbohydrate diet-group consumed the least carbohydrates, highest fat, protein and cholesterol, and had higher urinary ketones $(\mathrm{P}<.05)$. Caloric deficit was similar among groups. Weight loss was $2.9 \mathrm{~kg}$ for low-fat, $4.4 \mathrm{~kg}$ for Mediterranean, and $4.7 \mathrm{~kg}$ for low-carbohydrate, $\mathrm{P}$ diet-group ${ }^{*}$ time $<.001$ $(-3.3 \mathrm{~kg},-4.6 \mathrm{~kg}$, and $-5.5 \mathrm{~kg}$ respectively among 272 who completed the 2 -year intervention). Favorable changes in liver function biomarkers, adiponectin, leptin, and c-reactive-protein were similar. Triglycerides and high-densitylipoprotein-cholesterol were more favorable in the low-carbohydrate, with $20 \%$ reduction in total-cholesterol:high-density-lipoprotein-cholesterol ratio compared to $12 \%$ for low-fat $(\mathrm{P}=.01)$. Changes in fasting glucose and insulin were more favorable on Mediterranean diet among 36 diabetics (P-interaction diabetes ${ }^{\star}$ Mediterranean-diet ${ }^{*}$ time $<.001$ for glucose).

Conclusion: This long-term trial suggests that Mediterranean and low-carbohydrate diets are effective safe alternatives to low-fat. The more favorable effects on lipids (low-carbohydrate) and on glycemic control (Mediterranean) suggest that personal preferences and metabolic considerations can govern individualized tailoring of dietary intervention.

Supported by: Nuclear Research Center Negev (NRCN); Robert C. and Veronica Atkins Research Foundation; S. Daniel Abraham International Center for Health and Nutrition, Ben-Gurion University, Israel

\section{7}

The effects of dietary fat modification on metabolic markers of the metabolic syndrome - insights from the LIPGENE Dietary Intervention Study

A.C. Tierney ${ }^{1}$, H.L. Gulseth ${ }^{2}$, C. Defoort ${ }^{3}$, J.A. Lovegrove ${ }^{4}$, E. Blaak ${ }^{5}$,

J. Lopez-Miranda ${ }^{6}$, A. Dembinska-Kiec ${ }^{7}$, B. Vessby ${ }^{8}$, C.A. Drevon',

H.M. Roche ${ }^{10}$

${ }^{1}$ School of Public Health and Population Sciences, University College Dublin, Ireland, ${ }^{2}$ Dept Nutrition, University of Oslo, Norway, ${ }^{3}$ Nutrition Humaine et lipids, INSERM, Marseille, France, ${ }^{4}$ Hugh Sinclair Unit of Human Nutrition, University of Reading, United Kingdom, ${ }^{5}$ Dept Human Biology, Maastricht University, Netherlands, ${ }^{6}$ Lipid and Athersclerosis Unit, University of Cordoba, Spain, ${ }^{7}$ Dept Clinical Biochemistry, JUMC, Krakow, Poland, ${ }^{8}$ Dept of Public Health and Caring Sciences/Clinical Nutrition and Metabolism, Uppsala University, Sweden, ${ }^{9}$ Department of Nutrition, Univesity of Oslo, Norway, ${ }^{10} \mathrm{~S}$ chool of Public Health and Population Sciences, Conway Institute of Biomolecular and Biomedical Research, Dublin, Ireland

Background and aims: Evidence suggests that dietary fatty acid composition affects insulin sensitivity. The LIPGENE Human Dietary Intervention Study is a multi-centre, pan-European, randomised, controlled trial with the aim of determining the relative efficacy of reducing dietary SFA consumption, by altering quality and reducing the quantity of dietary fat, on metabolic risk factors of the metabolic syndrome (MetS).

Materials and methods: 417 free-living subjects with the MetS received one of four dietary treatments for 12 weeks: (1) High-fat (38\% energy) SFA-rich diet (HFSFA); (2) High-fat (38\% energy), MUFA-rich diet (HFMUFA); (3) Low-fat (28\% energy), high-complex carbohydrate diet (LFHCC) and (4) Low-fat (28\% energy), high-complex carbohydrate diet, with $1.24 \mathrm{~g} / \mathrm{d}$ LC n-3 PUFA (LFn-3PUFA). An IVGTT, lipoprotein analysis, cytokines, adhesion molecules, coagulation factors and isoprostane levels were determined preand post-intervention.

Results: Dietary fat modification had no significant effect on insulin sensitivity $\left(\mathrm{S}_{\mathrm{I}}\right)$, or any of the other IVGTT parameters, HOMA-IR or QUICKI. The effect of the four dietary treatments was determined in volunteers with a habitual high- or low-fat intake pre-intervention, defined as being above or below the median (36\% of Total Energy). $\mathrm{S}_{\mathrm{I}}$ was significantly lower following the high-fat, SFA-rich diet $(\mathrm{P}=0.021)$ in subjects with a habitual low-fat intake pre-intervention. The sensitivity to SFA was more applicable to females wherein $\mathrm{S}_{\mathrm{I}}$ was reduced following the high-fat, SFA rich diet. HOMA-IR was also reduced in females following the high-fat MUFA diet $(\mathrm{P}<0.05)$. Plasma
TAG concentrations showed a significant diet ${ }^{\star}$ time interaction $(\mathrm{P}=0.006)$, whereby plasma TAG concentrations were significantly reduced following the HFSFA diet and the LFn-3PUFA diet $(\mathrm{P}=0.018$ and $\mathrm{P}=0.005$, respectively). These effects were more apparent in males. Plasma total HDL-cholesterol concentrations showed a significant diet ${ }^{\star}$ time interaction $(\mathrm{P}=0.005)$, whereby HDL-cholesterol levels were significantly increased by the two highfat diets, HFSFA and HFMUFA post-intervention $(\mathrm{P}<0.0005$ and $\mathrm{P}=0.006$, respectively). Reducing dietary SFA did not alter markers of inflammation or coagulation associated with insulin resistance.

Conclusion: The effectiveness of dietary fat modification was dependent upon pre-intervention dietary fat intake. Several markers of lipid metabolism were improved particularly by the low-fat diet with LC n-3 PUFA supplementation. Also interestingly the lipid lowering efficacy of the LC n-3 PUFA intervention was most evident in males.

Supported by: LIPGENE is an EU Integrated Project funded under the Framework 6 Food Safety and Quality Programme in 2003

\section{8}

Effect of high and low glycaemic index diets on skeletal muscle and liver substrate storage and utilisation during exercise

M.I. Trenell ${ }^{1,2}$, P. Thelwall ${ }^{2}$, K. Thomas ${ }^{3}$, E. Stevenson ${ }^{3}$

${ }^{1}$ Clinical Medical Sciences, Newcastle University, ${ }^{2}$ Newcastle Magnetic Resonance Centre, Newcastle University, ${ }^{3}$ Sports Science, Northumbria University, Newcastle upon Tyne, United Kingdom

Background and aims: Carbohydrates which are rapidly absorbed into the circulation, termed high glycemic index, produce higher peak circulating glucose levels and require greater peak insulin production than carbohydrates which are absorbed slower, termed low glycemic index. Postprandial hyperglycemia and hyperinsulinaemia hold significant implications for substrate storage and oxidation in people with or without adequate glycemic control. Insulin promotes glycogen storage but high circulating levels may hold sustained effects on adipose tissue lipolysis, decreasing the supply of lipids to skeletal muscle for oxidation. The reduced capacity to oxidise lipids places a greater reliance upon anaerobic energy sources, thus reducing metabolic flexibility. The aim of this study was to evaluate the effect of high and low glycemic index foods upon substrate storage and oxidation in both skeletal muscle and liver during exercise. Materials and methods: Nine male endurance trained cyclists (age $33 \pm 6.9$ yrs, mass $76.3 \pm 7.0 \mathrm{~kg}, \mathrm{VO} 2 \max 61.5 \pm 5.0 \mathrm{ml} \cdot \mathrm{kg} \cdot \mathrm{min}-1$ ) took part in a randomised cross-over design study. Participants completed a 90 min cycle at $70 \% \mathrm{VO} 2 \mathrm{max}$ and were then provided with a high or low glycemic index (73 vs. 35) mixed meal diet to be consumed over the following $12 \mathrm{~h}$. Carbohydrate was provided at $8 \mathrm{~g} \cdot \mathrm{kg}$ body mass with $11 \%$ protein and $17 \%$ fat content. The following day after a 10 hour fast, the 90 min cycle was repeated and metabolic parameters measured. $1 \mathrm{H}$ and 13C Magnetic resonance spectroscopy were used to evaluate intra-myocellular triglyceride and glycogen content of the vastus lateralis and glycogen content of the liver immediately before and after exercise. Blood samples were collected at $15 \mathrm{~min}$ intervals throughout exercise. Substrate oxidation was calculated from expired air samples.

Results: Mean power output held for the 90 minute cycle was $247 \pm 13$ watts. Circulating NEFA concentrations were significantly reduced throughout exercise following the high compared to the low glycemic index diet. Intra-myocellular lipid oxidation during exercise was significantly increased following a high in comparison with the low glycemic index diet ( $1.6 \pm 0.2$ vs. $1.0 \pm 0.3$ $\mu \mathrm{mol} / \mathrm{g})$. There were no differences in the oxidation of glycogen in skeletal muscle ( $68 \pm 5$ vs. $76 \pm 8$ mmol.l) or liver ( $71 \pm 4$ vs. $65 \pm 8$ mmol.l) between the high and low glycemic index trials. There were no differences in whole body substrate oxidation, heart rate, glucose, lactate or insulin concentrations during exercise following high or low glycemic index recovery diets.

Conclusion: (1) The consumption of high glycemic index carbohydrates in recovery from exercise reduce NEFA availability during exercise and increases reliance on intra-myocellular lipid as a substrate source during moderate intensity exercise. (2) The glycemic index of carbohydrates consumed during a $24 \mathrm{~h}$ recovery period does not influence utilisation of glycogen by skeletal muscle or liver. In combination, these results suggest that mixed meals differing only in glycemic index influence the supply and oxidation of lipid during exercise independent of carbohydrate oxidation in people with adequate glucose control. Further studies investigating this effect in people with impaired glucose control are warranted.

Supported by: SUGiRs, Dr Trenell is funded by Diabetes UK RD Lawrence Fellowship 


\section{OP 45 Insulin signalling and glucose transport}

\section{9}

\author{
AHNAK - a novel regulator of GLUT4 gene expression: role in insulin \\ resistance \\ D. Ben Yosef, M. Armoni, C. Harel, E. Karnieli \\ Endocrinology, Diabetes and Metabolism, Rambam Medical Center, \\ Technion, Haifa, Israel,
}

Background and aims: Obesity and type 2 diabetes (DM2) are associated with reduced GLUT4 expression and function in adipocytes, hyperlipidemia and insulin resistance. We have previously shown that high levels of arachidonic acid (AA) repress GLUT4 promoter activity via specific prompter region (GLUT4-P) but the mediator remained elusive. Our aim was to identify the protein/s mediating GLUT4 gene repression upon chronic exposure to $\mathrm{AA}$ and examine their role in diabetes 1 .

Methods and results: Nuclear extracts from AA-treated H9C2 cardiomyocytes were subjected to GLUT4-P-biotin-avidin affinity column, followed by mass spectrometry analysis. This analysis detected that AHNAK, a giant phosphoprotein known to be involved in calcium and lipid metabolism, is attached to GLUT4-P. Transient co-expression in primary adipocytes of expression vectors for either C-terminal, middle or $\mathrm{N}$ terminal parts of AHNAK, resulted in $60 \pm 5 \%, 70 \pm 3 \%$ and $80 \pm 3 \%,(p<0.05)$ repression of GLUT4-P activity, respectively. Repression could be partially relieved by insulin (100 nM, $24 \mathrm{hrs}$ ). ChIP assay showed specific binding of AHNAK middle part to GLUT4-P. Silencing of AHNAK gene in adipocytes, resulted in significant 2-fold increase in cellular Glut4 protein levels, and protection of cellular Glut4 protein against AA-induced depletion. In cardiac and skeletal muscle biopsies obtained from STZ diabetic rats, AHNAK mRNA levels were increased $290 \pm 30 \%$ and $270 \pm 15 \%(\mathrm{p}<0.05)$, respectively, compared to control. Interestingly, upon 8 days insulin therapy AHNAK mRNAs returned to control level.

Conclusion: Increased cellular level of AHNAK is associated with reduced GLUT4 expression, and contributes to lipotoxicity-induced insulin resistance. Our data introduce AHNAK as a novel regulator of GLUT4 gene expression and a potential molecular target for diabetes and obesity treatment.

\section{0}

Direct effect of inhibition of TCF7L2 expression on insulin action in cultured human skeletal muscle cells

A.E. Brown, S.J. Yeaman, M. Walker

Institute of Cellular Medicine, Newcastle University, United Kingdom

Background and aims: The recent genome-wide association studies have highlighted a number of genes, variants within which are associated with the development of type 2 diabetes. There is increasing evidence that variants within these genes, in particular, TCF7L2, may be associated with reduced insulin sensitivity. TCF7L2 is a member of the Wnt signalling pathway and is important in regulating gene expression. A recent study reported that $T C$ F7L2 is one of several genes in the Wnt signalling pathway that is downregulated in the gastrocnemius muscle of transgenic mice who over accumulate glycogen in muscle but the mechanisms behind this are unclear. Therefore, the aim of this study is to examine whether TCF7L2 plays a role in insulin action in skeletal muscle.

Materials and methods: Expression of TCF7L2 in cultured myoblasts and myotubes was determined by quantitative PCR and Western blotting. Cultured myoblasts were transfected with short interfering RNAs (siRNAs) specific for TCF7L2 in order to inhibit expression of this gene. All studies described were performed $48 \mathrm{~h}$ after transfection. Quantitative PCR and Western blotting were employed to assess the extent of inhibition of TCF7L2 expression compared with cells transfected with a scrambled control. Insulinstimulated glucose uptake and glycogen synthesis was measured to assess the effects of TCF7L2 inhibition on insulin action. Insulin signalling was examined using antibodies specific for phospho-PKB and GSK3.

Results: Expression of TCF7L2 in cultured human skeletal muscle cells was confirmed by quantitative PCR and Western blotting. This showed that expression of TCF7L2 is reduced by 2.5 -fold in myotubes compared with myoblasts. Transfection of myoblasts with siRNAs resulted in a $72 \%$ reduction in TCF7L2 expression compared with the scrambled control. Inhibition of TCF7L2 was confirmed at the protein level by Western blot- ting. Both basal and insulin-stimulated glycogen synthesis were significantly increased by TCF7L2 inhibition. Basal levels were increased in the TCF7L2 inhibited cells compared to the scrambled controls $(169.7 \pm 28.2$ vs $123.3 \pm 23.2 \mathrm{pmol} / \mathrm{min} / \mathrm{mg} ; \mathrm{p}=0.01)$. Insulin-stimulated glycogen synthesis also increased from $341.5 \pm 45.6 \mathrm{pmol} / \mathrm{min} / \mathrm{mg}$ to $515.1 \pm 51.5$ (vs scrambled control, $\mathrm{p}=0.0004)$ respectively. An increase in insulin-stimulated glucose uptake was also observed in TCF7L2-inhibited cells compared with the scrambled control $(90.1 \pm 15.3 \mathrm{pmoln} / \mathrm{min} / \mathrm{mg}$ vs $54.8 \pm 4.7, \mathrm{p}=0.03$, respectively). Insulin signalling, as assessed by the phosphorylation state of PKB ${ }^{\text {Ser473 }}$ and GSK- $3^{\text {Ser21/9 }}$, was normal in cells transfected with TCF7L2 siRNAs. mRNA expression of the glucose transporter, GLUT1, was significantly increased in TCF7L2-inhibited cells compared to scrambled control, $(\mathrm{p}=0.008)$.

Conclusion: We have confirmed that TCF7L2 is expressed in cultured human skeletal muscle cells. By inhibiting expression of this gene, both basal and insulin-stimulated glycogen synthesis and insulin-stimulated glucose uptake are significantly increased without any measurable differences in the activation of intermediates in the insulin signalling pathway. These effects on glucose metabolism may be mediated via increases in the expression of the basal glucose transporter, GLUT1.

Supported by: Diabetes Research and Wellness Foundation

\section{1}

Inhibition of phosphoinositide 5-phosphatase Sac3 links insulin signalling with GLUT4 translocation in 3T3L1 adipocytes

A. Shisheva, D. Sbrissa, O. Ikonomov

Physiology, Wayne State University, Detroit, United States

Background and aims: Our previous studies have shown that silencing of the sole enzyme for phosphatidylinositol (PtdIns) $(3,5) \mathrm{P}_{2}$ synthesis, PIKfyve, and/or its associated activator ArPIKfyve reduces insulin-regulated glucose transport and GLUT4 surface arrival in 3T3L1 adipocytes, concomitant with a decrease in a $\operatorname{Ptd} \operatorname{Ins}(3,5) \mathrm{P}_{2}$ intracellular pool. These data implicate $\operatorname{PtdIns}(3,5) \mathrm{P}_{2}$ as a positive regulator in insulin responsiveness. Concordantly, insulin action increases $\operatorname{PtdIns}(3,5) \mathrm{P}_{2}$ production associated with intracellular membranes derived from 3T3L1 adipocytes in in vitro labeling assays. However, insulin-dependent regulation at the level of the PIKfyve activity and/or ArPIKfyve-PIKfyve association has not been observed. Recently we have identified a novel 5-phosphatase, Sac3, to hydrolyze $\operatorname{PtdIns}(3,5) \mathrm{P}_{2}$, and to physically associate with the PIKfyve-ArPIKfyve protein complex in different cell types, including 3T3L1 adipocytes. In this study we have examined whether perturbations in Sac3 protein and/or activity result in gain of insulin responsiveness and whether Sac3 hydrolyzing activity is targeted by insulin action in 3T3L1 adipocytes.

Materials and methods: Sac3 protein levels were manipulated by electroporating 3T3L1 adipocytes with Sac3 siRNAs or with cDNAs of GFP-Sac3 ${ }^{\text {WT }}$ and the GFP-Sac3 $3^{\mathrm{D} 488 \mathrm{~A}}$ phosphatase-deficient mutant. Electroporations with control siRNAs and cDNAs were run in parallel. To study GLUT4 dynamics, cDNAs of HA-GLUT4-GFP or (Myc) $)_{7}$-GLUT4 were co-electroporated. The phosphatase activity associated with immunopurified Sac3 was determined by the malachite-green assay in the presence of different $\mathrm{C}_{8}$-phosphoinositide substrates. Glucose transport and the surface accumulation of ectopicallyexpressed GLUT4 were determined by the 2-deoxyglucose assay and quantitative immunofluorescence microscopy in non-permeabilized cells, respectively.

Results: Silencing of Sac3 (by $\sim 70 \%$ ) resulted in significant gain of insulininduced 2-deoxyglucose transport at both submaximal ( $1 \mathrm{nM} ; 132 \%)$ and maximal (100 nM; 128\%) insulin doses, without altering the basal levels. Sac3 depletion was also associated with a significant increase (140\%) in surface HA-GLUT4-GFP (measured by the cell-surface-to-total ratio) under submaximally activating insulin doses. The percentage of the (Myc) $)_{7}$-GLUT4expressing cells that displayed a complete plasma membrane rim in response to insulin increased by $32 \%$ upon co-expression of the phosphatase inactive GFP-Sac3 ${ }^{\text {D488A }}$ mutant. In contrast, expression of enzymatically active GFPSac ${ }^{\mathrm{WT}}$ inhibited the (Myc)-GLUT4 arrival to the cell surface. Importantly, in response to acute insulin there was a marked decrease ( 2-fold) of in vitro Sac3 phosphatase activity towards $\operatorname{PtdIns}(3,5) \mathrm{P}_{2}$, but not $\operatorname{PtdIns}(4,5) \mathrm{P}_{2}$ or PtdIns $(3,4,5) \mathrm{P}_{3}$ substrates.

Conclusion: Our observations in 3T3L1 adipocytes suggest that acute insulin increases the PIKfyve/ArPIKfyve-produced PtdIns $(3,5) \mathrm{P}_{2}$ signal by inhibiting the PtdIns $(3,5) \mathrm{P}_{2}$ hydrolyzing activity of the Sac3 phosphatase. Silencing of Sac3 or expression of an inactive Sac3 mutant potentiates insulin responsiveness of GLUT4 translocation and glucose transport presumably by 
further increases in PtdIns $(3,5) \mathrm{P}_{2}$ levels. Thus, pharmacological inhibition of Sac3 activity may improve insulin responsiveness. Supported by: NIH/NIDDKD

\section{2}

Adrenaline induces insulin resistance by altering glycogen synthase phosphorylation in human skeletal muscle

J.W. Eriksson ${ }^{1,2}$, T. Ruge ${ }^{3}$, Y.-C. Lai ${ }^{4}$, M.K. Svensson ${ }^{5}$, J. Jensen ${ }^{4}$

${ }^{1}$ Institute of Medicine, Lundberg Laboratory for Diabetes Research, Gothenburg University, Sweden, ${ }^{2}$ Clinical Development, AstraZeneca R\&D, Mölndal, Sweden, ${ }^{3}$ Surgery, Umeå University, Sweden, ${ }^{4}$ Physiology, National Institute of Occupational Health, Oslo, Norway, ${ }^{5}$ Institute of Medicine, Nephrology, Gothenburg University, Sweden

Background and aims: Sympathoadrenergic activation with accompanying elevation of circulating catecholamines is known to antagonize insulin action, and it contributes to insulin resistance during psychological or physical stress. In the present study, we investigated the effect of adrenaline in vivo on insulin-mediated glucose and fat metabolism and on regulation of protein kinase B (PKB) and glycogen synthase (GS) phosphorylation in human skeletal muscle.

Materials and methods: Ten healthy subjects $(5 \mathrm{M} / 5 \mathrm{~F}$, age $31 \pm 2$, mean $\pm \mathrm{SE})$ received a $240 \mathrm{~min}$ i.v infusion of adrenaline $(0.05 \mu \mathrm{g} / \mathrm{kg} / \mathrm{min}$, to mimic a high physiological level) and saline, respectively, on separate days according to a cross-over protocol. After $120 \mathrm{~min}$, a hyperinsulinemic euglycemic clamp $\left(56 \mathrm{mU} / \mathrm{m}^{2} / \mathrm{min}\right)$ was started and it continued until $240 \mathrm{~min}$. Skeletal muscle biopsies ( $\mathrm{m}$ tibialis anterior) were performed at 120 and $240 \mathrm{~min}$, but also on the following day. Muscle GS and PKB activities were investigated, including insulin-mediated changes in phosphorylation status.

Results: Adrenaline infusion increased blood glucose concentrations by $\approx$ $50 \%$ compared to saline $(\mathrm{p}<0.01)$. FFA, glycerol, TG and lactate levels were also elevated by adrenaline $(\mathrm{p}<0.05)$. The hyperinsulinemic clamp normalized blood glucose and suppressed FFA (to the same levels with or without adrenaline) within $30 \mathrm{~min}$. However, glucose infusion rate during the last hour was $\approx 60 \%$ lower during adrenaline infusion compared to saline (M-value $\left.4.2 \pm 0.5 \mathrm{vs} 11.2 \mathrm{mg} \cdot \mathrm{kg} \mathrm{FFM}^{-1} \cdot \mathrm{min}^{-1}, \mathrm{p}<0.001\right)$. Insulin infusion increased skeletal muscle PKB Ser ${ }^{473}$ phosphorylation and co-infusion of adrenaline did not alter this. Adrenaline alone did not influence PKB Ser ${ }^{473}$ phosphorylation. Insulin increased GS fractional activity and decreased GS $\mathrm{Ser}^{641}$ and Ser ${ }^{645,649,653,657}$ phosphorylation. In the presence of adrenaline, insulin did neither activate glycogen synthase or dephosphorylate GS Ser ${ }^{641}$. GS $\mathrm{Ser}^{7}$ phosphorylation was not influenced by adrenaline. The glycogen content was reduced in skeletal muscle the day after adrenaline infusion.

Conclusion: Adrenaline did not influence insulin-stimulated PKB phosphorylation but completely blocked insulin-mediated GS activation and Ser ${ }^{641}$ dephosphorylation. Adrenaline-mediated hyperglycemia was normalized by insulin within $30 \mathrm{~min}$, but glucose utilisation during the hyperinsulinemic euglycemic clamp was reduced by $60 \%$. The data suggest that altered glycogen synthase phosphorylation and activity in skeletal muscle contribute to whole-body insulin resistance in situations with sympathoadrenergic activation.

Supported by: Swedish Diabetes Association, Swedish Research Council, Aktieselskabet Freia Chokolade Fabriks Medisinske Fond, Novo Nordisk Foundation

\section{OP 46 Low grade inflammation and endothelial function}

\section{3}

Inflammation and endothelial dysfunction in children with type 1 diabetes mellitus

B. Rami ${ }^{1}$, T. Hoertenhuber ${ }^{2}$, M. Elhenicky ${ }^{2}$, C. Hoebaus ${ }^{2}$, E. Schober ${ }^{1}$, G. Schernthaner ${ }^{3}$, G.-H. Schernthaner ${ }^{2}$

${ }^{1}$ Pediatrics and Adolescenf Medicine, Medical University of Vienna, ${ }^{2}$ Medicine II, Medical University of Vienna, ${ }^{3}$ Medicine I, Rudolfstiftung Hospital Vienna, Austria

Background and aims: Advances in diabetes treatment have resulted in a decline of the microvascular complications, namely neuropathy and retinopathy, in patients with childhood onset type 1 diabetes mellitus (T1DM) but also in T2DM. However, recent studies have proven, that despite the dramatic improvements in treatment, the macrovascular disease (MVD) morbidy and mortality in patients with T1DM compared to the healthy background population stayed at an advanced level, resulting in a total loss of lifetime between 5 and 20 years. Since the mechanisms are not completely understood, we decided to investigate inflammation and endothelial dysfunction in children with T1DM and to compare the results to healthy control subjects.

Materials and methods: We included 175 children, 140 with T1DM, 35 served as control group (CO). As a general marker of subclinical systemic vascular inflammation, we chose C-reactive protein (CRP), as early marker of endothelial dysfunction, we investigated endothelial progenitor cells (EPC). EPC are cells that circulate in the peripheral blood and are involved in neovascularisation, endothelial dysfunction and predict MVD morbidity and mortality in humans. Circulating pluripotent progenitor cells (CPC; CD34+/CD133+) and EPC (CD34+/CD133+/CD309+) were enumerated by direct immunostaining technique and recorded on a flow cytometer. Statistical analysis included unpaired student's t test, correlation analysis as well as multivariate linear regression modelling, as appropriate.

Results: The patients (T1DM) and controls (CO) were matched for age ( $14 \pm 3$ years) and gender $(49.2 \%$ female). EPC but not CPC were significantly impaired in children with T1DM compared to CO $(609 \pm 359$ vs $1137 \pm 504$, $\mathrm{p}<0.001)$. Other significant differences were found in systolic blood pressure ( $115 \pm 15$ vs $101 \pm 8 \mathrm{mmHg}, \mathrm{p}<0.001)$, pulse ( $88 \pm 16$ vs $80 \pm 11, \mathrm{p}=0.001)$, LDL-cholesterol ( $90 \pm 28$ vs $83 \pm 15 \mathrm{mg} / \mathrm{dl}, \mathrm{p}=0.03)$, and glucose levels (154 \pm 83 vs $90 \pm 8 \mathrm{mg} / \mathrm{dl}, \mathrm{p}<0.001)$, and CRP $(112 \pm 14$ vs $101 \pm 8 \mathrm{mg} / \mathrm{dl}, \mathrm{p}<0.001)$, each T1DM vs CO. Patients (T1DM vs CO) did not differ for diastolic blood pressure $(63 \pm 9 \mathrm{mmHg})$, size $(161 \pm 15 \mathrm{~cm})$, weight $(57 \pm 18 \mathrm{~kg})$, BMI $(22 \pm 4$ $\mathrm{kg} / \mathrm{m} 2)$, total cholesterol $(176 \pm 33 \mathrm{mg} / \mathrm{dl})$, triglyceride $(116 \pm 92 \mathrm{mg} / \mathrm{dl})$, HDL-cholesterol $(64 \pm 15 \mathrm{mg} / \mathrm{dl})$, VLDL-cholesterol $(20 \pm 11 \mathrm{mg} / \mathrm{dl})$, serum creatinine $(0.8 \pm 0.4 \mathrm{mg} / \mathrm{dl})$, GGT $(17 \pm 7 \mathrm{U} / \mathrm{l})$, ASAT $(25 \pm 10 \mathrm{U} / \mathrm{l})$ and ALAT $(31 \pm 8 \mathrm{U} / \mathrm{l})$ (given are mean of all patients). Multivariate regression analysis revealed that $\mathrm{HbAlc}$ was the single strongest predictor of EPC levels in our study cohort (beta $=-0.355, \mathrm{p}<0.001)$ and that BMI and triglyceride levels were the strongest predictors of CRP levels (beta $=0.264, \mathrm{p}<0.001$; beta $=0.189$, $\mathrm{p}=0.009$, respectively). Remarkably, EPC and CPR were not associated with one another.

Conclusion: In conclusion, this is the first study investigating both inflammation and endothelial dysfunction (by EPC measurements) in children with type 1 diabetes mellitus. EPC are significantly reduced and CRP levels are elevated in children with T1DM. The missing association of CRP and EPC in our study cohort, suggests a from inflammation independent demetrial effect on EPC in children with T1DM.

Supported by: Austrian Diabetes Association Research Prize 2006 awarded by the Austrian Diabetes Association 


\section{4}

Endothelial dysfunction and inflammation partially explain the association between soluble RAGE and cardiovascular disease and albuminuria in type 1 diabetes: The EURODIAB Study

J.W.M. Nin' ${ }^{1}$ I. Ferreira ${ }^{2}$, C.G. Schalkwijk ${ }^{1}$, M.H. Prins ${ }^{3}$, N. Chaturvedi ${ }^{4}$, J.H. Fuller ${ }^{5}$, C.D.A. Stehouwer ${ }^{2}$

${ }^{1}$ Department of Internal Medicine, University of Maastricht, Netherlands, ${ }^{2}$ Department of Internal Medicine, University Hospital Maastricht, Netherlands, ${ }^{3}$ Department of Clinical Epidemiology \& MTA, University Hospital Maastricht, Netherlands, ${ }^{4}$ National Heart and Lung Institute, Imperial College London, United Kingdom, ${ }^{5}$ Department of Epidemiology and Public Health, Royal Free and University College London Medical School, United Kingdom

Background and aims: The interaction between AGEs and the receptor for AGE (RAGE) have been proposed as a potential mechanism linking hyperglycaemia to vascular complications in diabetes, possibly through the increased inflammatory activity and endothelial dysfunction they provoke. We have therefore investigated 1) whether soluble RAGE (sRAGE) is associated with increased cardiovascular disease (CVD) and microvascular complications (i.e. albuminuria, retinopathy) in type 1 diabetic patients; and 2) the extent to which any such associations are explained by markers of inflammation (CRP, TNFa, IL6), endothelial dysfunction (sVCAM-1, sE-selectin) and AGEs (CML, CEL and pentosidine).

Materials and methods: The study population consisted of 477 subjects (234 women; mean age $42 \pm 10$ yrs) from the EURODIAB Prospective Complications Study. We used linear regression analyses to investigate the differences in sRAGE levels (expressed by the regression coefficients $\beta$ (in $\mathrm{ng} / \mathrm{ml}$ ) and respective $95 \% \mathrm{CI}$ ) between subjects with vs. without complications; this was first analyzed with adjustments for age, sex, duration of diabetes, HbAlc and traditional risk factors (smoking, BMI, dyslipidemia, MAP), and then with successive adjustments for the potential pathophysiological mechanisms listed above.

Results: Subjects with CVD ( $\mathrm{n}=116)$ had greater levels of sRAGE than those without CVD (who also did not have any microvascular complications; $\mathrm{n}=178$ ). However, this difference was mainly observed in men, but not in women: $\beta=0.19$ (95\%CI:0.01;0.37) and $\beta=0.08$ (95\%CI:-0.06;0.22, $\mathrm{p}_{\text {sex-in- }}$

$=0.014)$, respectively. In men, these differences were attenuated after teraction
further adjustment for markers of endothelial function $[\beta=0.15$ (95\%CI:$0.02 ; 0.32)]$ and of inflammation $[\beta=0.14$ (95\%CI:-0.02;0.31)]. sRAGE was also increased in the presence and across the levels of severity of albuminuria: $\beta=0.02$ (95\%CI:-0.08;0.12) and $\beta=0.09$ (95\%CI:-0.01;0.19) in subjects with micro- $(\mathrm{n}=72)$ and macro- $(\mathrm{n}=112)$ vs. normo-albuminuria $(\mathrm{n}=293)$, respectively ( $\mathrm{p}$-trend $=0.077$ ). These differences were greatly attenuated after adjustments for endothelial dysfunction $[\beta=-0.01$ (95\%CI:-0.11;0.09) and $\beta=0.05$ (95\%CI:-0.05;0.15), p-trend $=0.336]$ and inflammation $[\beta=-0.02$ (95\%CI:-0.11;0.08) and $\beta=0.05$ (95\%CI:-0.05;0.14), p-trend $=0.375]$. Finally, sRAGE was also increasingly greater (but not significantly so) in the presence and across the severity of retinopathy: $\beta=0.03$ ( $95 \% \mathrm{CI}:-0.06 ; 0.13)$ and $\beta=0.08$ (95\%CI:-0.03;0.18) in subjects with non-proliferative $(\mathrm{n}=123)$ and proliferative $(\mathrm{n}=141)$ vs. without retinopathy $(\mathrm{n}=213)$, respectively ( $\mathrm{p}$-trend $=0.145)$. Conclusion: sRAGE is associated with greater CVD (particularly among men) and albuminuria in type 1 diabetic subjects, and these associations may be explained, at least in part, by greater inflammatory activity and endothelial dysfunction.

\section{5}

Endothelial dysfunction and low-grade inflammation precede the increase in urinary albumin excretion in type 1 diabetes: a 20 -yr longitudinal study

I. Ferreira ${ }^{1}$, P. Hovind ${ }^{2}$, C.G. Schalkwijk ${ }^{1,3}$, H.-H. Parving ${ }^{4,5}$, P. Rossing ${ }^{2}$, C.D.A. Stehouwer ${ }^{1,3}$

${ }^{1}$ Department of Internal Medicine, University Hospital Maastricht, Netherlands, ${ }^{2}$ Steno Diabetes Center, Gentofte, Denmark, ${ }^{3}$ Cardiovascular Research Institute Maastricht, University of Maastricht, Netherlands, ${ }^{4}$ Department of Medical Endocrinology, University Hospital of

Copenhagen, Denmark, ${ }^{5}$ Faculty of Health Science, University of Aarhus, Denmark

Background and aims: Microalbuminuria is a predictor of widespread severe microangiopathy and macroangiopathy in type 1 diabetic patients. Patients with microalbuminuria show generalized dysfunction of the vascular endothelium and chronic inflammation, but it is not known to what extent these pathophysiological mechanisms may, in fact, precede the development of microalbuminuria. We have therefore investigated, in a 20 -year prospective study, 1) whether the longitudinal developments of markers of inflammation (CRP and sICAM-1) and endothelial dysfunction (sICAM-1 and sVCAM-1) (main determinants) were associated with urinary albumin excretion (UAE) (main outcome); and 2) the extent to which the increase in those markers preceded the increase in UAE.

Materials and methods: Study population consisted on 277 type 1 diabetic patients (114 women) from an outpatient diabetic clinic (Gentofte, Denmark), who were consecutively admitted, upon diagnosis, between Sep'79Aug'84 (mean age at diagnosis $27.5 \pm 13.8$ yrs; 216 adults). These patients attended the outpatient clinic every 3 or 4 months, throughout a total follow-up period of $>20$ yrs [median follow-up duration $=18$ yrs (range 1.021.5)], as part of their routine evaluation. This included the assessments of UAE (mg/24h), HbAlc, BMI, blood pressure, creatinine, cholesterol, and smoking status. CRP, sICAM1 and sVCAM1 were recently measured in blood samples collected throughout the whole longitudinal period. We used generalized estimating equations (GEEs) to investigate, longitudinally, with concomitant and time-lag models, the associations hypothesized and results hereby obtained are expressed by longitudinal regression coefficients (respective $95 \% \mathrm{CI}$ ). All analyses were adjusted for sex, age and the other risk factors assessed (listed above).

Results: Per year, UAE increased by $6.1 \%$, CRP by $1.2 \%$, sICAM- 1 by $1.0 \%$ (all $\mathrm{p}<0.01$ ), whereas no increase was observed in sVCAM-1 levels. In a concomitant model (i.e. the association between the determinants and outcome assessed at the same time point), the longitudinal development of $\log$ CRP and sICAM-1 (but not of sVCAM-1) was positively and independently associated with $\log \mathrm{UAE}[\beta=0.067(0.001 ; 0.134), \mathrm{p}=0.042$ and $\beta=0.369$ $(0.186 ; 0.552), p<0.001$, respectively]. When a 2 -yr time-lag model was fitted (i.e. the association between the determinants at a given time point with UAE 2 -yrs later), only sICAM- 1 was positively associated with $\log \mathrm{UAE}[\beta=0.277$ $(0.017 ; 0.538), \mathrm{p}=0.037]$. In a 4 -yr time-lag model, however, the association between sICAM and logUAE was attenuated and was no longer significant: $\beta=0.204(-0.077 ; 0.486, \mathrm{p}=0.155)$.

Conclusion: In type 1 diabetes, UAE, endothelial dysfunction and lowgrade inflammation (as reflected by CRP and sICAM-1) are inter-related and progress in parallel. In, addition, endothelial dysfunction/inflammation precedes (by 2 to 4 years) the increased of UAE, and therefore may predict the development of (micro)albuminuria in type 1 diabetes.

\section{6}

Role of reactive oxygen species in mediation of sphingomyelinase induced vascular dysfunction

Z. Benyo ${ }^{1}$, A. Magyar ${ }^{1}$, P. Örsy ${ }^{1}$ Á. Donkó ${ }^{2}$, B. Horváth ${ }^{1}$

${ }^{1}$ Institute of Human Physiology and Clinical Experimental Research, ${ }^{2}$ Department of Physiology, Semmelweis University, Budapest, Hungary

Background and aims: Sphingolipids, derived from sphingomyelin metabolism, have been implicated as mediators of inflammatory processes in diabetes. Sphingomyelinase (SMase) catalyzes the conversion of sphingomyelin to ceramide, a biologically active compound and precursor of other sphingolipid mediators. In the present study we aimed to analyze the influence of SMase activity on vascular functions and to assess the role of reactive oxygen species (ROS) in mediating its effects.

Materials and methods: Carotid artery segments were prepared from wild type (WT) and transgenic mice overexpressing CuZn superoxide dismutase (SOD-tg) or deficient in the NAD(P)H oxidase isoform NOX2 (NOX2-ko). Vasoconstrictor responses to $124 \mathrm{mM} \mathrm{K}^{+}$or $10 \mu \mathrm{M}$ phenylephrine (Phe) as well as endothelium dependent relaxations induced by acetylcholine (ACh) after precontraction with $10 \mu \mathrm{M}$ Phe have been analyzed in vitro by isometric tension recording. Phe- and ACh-induced tension changes have been normalized for the $\mathrm{K}^{+}$-induced contraction and the precontraction, respectively. Superoxide production has been visualized with the fluorescent dye dihydroethidium (DHE) and determined in the vascular endothelial and smooth muscle layers by confocal microscopy.

Results: Incubation of the vessels with $10 \mu \mathrm{M}$ ceramide for 30 minutes resulted in a strong increase of DHE staining both in the endothelial and smooth muscle layers indicating the stimulation of superoxide production by both cell types. The changes of the vascular reactivity induced by incubation with $0.5 \mathrm{U} / \mathrm{ml}$ neutral SMase (nSMase) are summarized in Table I. The contractile effect of $124 \mathrm{mM} \mathrm{K}^{+}$was similar in WT, SOD-tg and NOX2-ko vessels and remained unaltered after incubation with nSMase. In contrast, nSMase reduced 
Table I. Vascular reactivity in the different experimental groups before and after nSMase incubation

\begin{tabular}{lllllll}
\hline Vasoactive agent & $\begin{array}{l}\text { WT, before } \\
\text { nSMase }\end{array}$ & $\begin{array}{l}\text { WT, after } \\
\text { nSMase }\end{array}$ & $\begin{array}{l}\text { NOX2-ko, } \\
\text { before nSMase }\end{array}$ & $\begin{array}{l}\text { NOX2-ko, } \\
\text { after nSMase }\end{array}$ & $\begin{array}{l}\text { SOD-tg, } \\
\text { before nSMase }\end{array}$ & $\begin{array}{l}\text { SOD-tg, } \\
\text { after nSMase }\end{array}$ \\
\hline $\mathbf{1 2 4} \boldsymbol{m} \boldsymbol{M} \boldsymbol{K}^{+}(\boldsymbol{m} \boldsymbol{N})$ & $5.3 \pm 0.5$ & $5.9 \pm 0.5$ & $5.4 \pm 0.3$ & $5.7 \pm 0.4$ & $4.9 \pm 0.4$ & $4.6 \pm 0.5$ \\
\hline $\mathbf{1 0} \boldsymbol{\mu} \boldsymbol{M} \boldsymbol{P h} \boldsymbol{( \% )}$ & $75 \pm 2$ & $60 \pm 2^{* *}$ & $69 \pm 7$ & $49 \pm 8^{* * *}$ & $69 \pm 5$ & $75 \pm 5$ \\
\hline $\mathbf{1 0} \boldsymbol{n} \boldsymbol{M}$ Ach $(\%)$ & $-43 \pm 8$ & $-33 \pm 7^{\star *}$ & $-58 \pm 7$ & $-43 \pm 3^{*}$ & $-65 \pm 2^{*}$ & $-42 \pm 2^{* * *}$ \\
\hline
\end{tabular}

Phe-induced contraction in WT and NOX2-ko but not in SOD-tg vessels. Interestingly, the relaxant effect of ACh under resting conditions was significantly $\left({ }^{\#} \mathrm{p}<0.05\right)$ stronger in SOD-tg vessels as compared to WT controls and NOX2-ko vessels also showed a tendency of enhanced relaxations indicating that superoxide production may constitutively suppress NO-mediated vasodilatations. Incubation of the vessels with nSMase resulted in a reduction of ACh-induced relaxations in all experimental groups.

Conclusion: Enhanced activity of the SMase pathway stimulates endothelial superoxide release and suppresses endothelium-dependent relaxations of the carotid artery but there is apparently no causal relationship between these effects. On the other hand, sphingolipid mediators also stimulate superoxide generation of the vascular smooth muscle and diminish adrenoreceptor-mediated contractions without influencing the contractile effect of cell membrane depolarization. Since SMase decreased Phe-induced contractions in NOX2-ko but not in SOD-tg carotid arteries its effect appears to be mediated by NOX2-independent superoxide production.

Supported by: an EFSD/Servier grant and the Hungarian OTKA (K62375)

\section{OP 47 Novel mechanisms in insulin secretion}

\section{7}

Syndapin I/PACSIN 1 participates in inositol hexakisphosphate-induced endocytosis via its $\mathrm{SH} 3$ domain in beta cells

S.-N. Yang ${ }^{1}$, G. Yang ${ }^{1}$, J. Yu ${ }^{1}$, B. Qualmann ${ }^{2}$, M.M. Kessels ${ }^{2}$, L. Yu ${ }^{1}$, P.-O. Berggren ${ }^{1}$;

${ }^{1}$ The Rolf Luft Research Center for Diabetes and Endocrinology, Karolinska Institutet, Stockholm, Sweden, ${ }^{2}$ Institute for Biochemistry I, Friedrich Schiller University Jena, Germany

Background and aims: Endocytosis is a fundamental cellular process. It works in concert with exocytosis to ensure adequate exchange between the intracellular milieu and extracellular environment, and thereby cellular homeostasis. Endocytic-exocytic cycles guarantee structural and functional components in the plasma membrane, cytoplasm and nucleus by exquisitely recycling membrane proteins and lipids. Syndapin I/PACSIN 1 has been identified as a new member of the family of endocytic proteins. It harbors an $\alpha$-helical region forming an F-BAR domain at its $\mathrm{N}$-terminus, two arginine-proline-phenylalanine (NPF) motifs in its middle and a Src homology 3 (SH3) domain at its C-terminus. The $\mathrm{SH} 3$ domain interacts with dynamin 1, synaptojanin, synapsin I and the neural Wiskott-Aldrich syndrome protein. Such SH3 domain-specific interactions play a crucial role in dynamin-mediated endocytosis. The molecular details of the endocytic machinery in the $\beta$ cell have not been well clarified. In the present study, we expressed the syndapin I SH3 domain in $\beta$ cells to disrupt the interaction between endogenous syndapin I/PACSIN 1 and other endocytic proteins in a dominant-negative fashion and subsequently evaluated inositol hexakisphosphate (InsP $\left.{ }_{6}\right)$-induced endocytosis in these syndapin I SH3 domain-expressing cells.

Materials and methods: Experiments were performed in mouse $\beta$-cells and a series of insulin-secreting cell lines using patch-clamp recording, immunoblot analysis, immunostaining and confocal microscopy.

Results: Immunoblot analysis and immunofluorescence labeling/confocal microcopy show that mouse islet $\beta$ cells as well as insulin-secreting HIT-T15, INS-1, MIN6-m9 and RINm5F cells exhibit specific syndapin I/PACSIN 1 immunoreactivity. Capacitance measurements reveal that dominant-negative interference with endogenous syndapin I/PACSIN 1 protein complexes by expression of the syndapin I SH3 domain significantly slows down endocytosis elicited by intracellular application of InsP ${ }_{6}$. However, patch clamp analysis illustrates that incorporation of GST-syndapin I SH3 domain into the mouse $\beta$ cell does not influence whole cell $\mathrm{Ca}^{2+}$ currents.

Conclusion: Our data demonstrate that the key endocytic protein syndapin I/PACSIN 1 is present in $\beta$ cells. Its SH3 domain specifically mediates InsP $\mathrm{P}_{6}$ induced $\beta$ cell endocytosis. However it is unable to convey appreciable impact on voltage-gated $\mathrm{Ca}^{2+}$ channel activity in $\beta$ cells, although $\mathrm{Ca}_{\mathrm{v}} 1.2$ and $\mathrm{Ca}_{\mathrm{v}} 1.3$ pore-forming subunits are equipped within proline-rich SH3 binding sites. In the physiological context, glucose rapidly elevates a series of signaling molecules, such as ATP, $\mathrm{Ca}^{2+}$ and $\operatorname{InsP}_{6}$, in pancreatic $\beta$ cells which ultimately results in insulin exocytosis followed by endocytosis. Interestingly, Ins $\mathrm{P}_{6}$ not only promotes insulin exocytosis, but also facilitates $\beta$ cell endocytosis through syndapin I/PACSIN 1 , which obviously plays an important role in the maintenance of pancreatic $\beta$ cell function. Hence, this novel molecular mechanism mediating the stimulatory effect of InsP $_{6}$ on endocytosis provides insights into how $\beta$ cells preserve structures and components in their different subcellular compartments to guarantee cellular homeostasis.

Supported by: Swedish Foundation for Strategic Research, the Swedish Society of Medicine, Fredrik and Ingrid Thuring's Foundation, Åke Wiberg's Foundation, Swedish Alzheimer Association., Magn. Bergvall's Foundation, Funds of Karolinska Institutet, the Deutsche Forschungsgemeinschaft (DFG), the EFSD/ Novartis grant, Novo Nordisk Foundation, the Swedish Research Council, the Swedish Diabetes Association, Berth von Kantzow's Foundation, the Family Erling-Persson Foundation, and EuroDia 


\section{8}

Tmem27 role in islets physiology

J. Altirribaa ${ }^{1,2}$, S. Casas ${ }^{1}$, A. Barberà ${ }^{1}$, R. Gasa ${ }^{1,2}$, M.J. Ramírez ${ }^{3}$, S. Ros ${ }^{4}$, A. Gutiérrez ${ }^{3}$, M.C. Ruiz de Villa ${ }^{5}$, M. Julià ${ }^{1,2}$, R. Gomis ${ }^{1,2}$

${ }^{1}$ Diabetes and Obesity Laboratory, IDIBAPS, ${ }^{2}$ CIBER de Diabetes y

Enfermedades Metabólicas Asociadas, CIBERDEM, ${ }^{3}$ Experimental

Laboratory of Nephrology and Transplantation, Hospital Clinic, ${ }^{4}$ Institute

for Research in Biomedicine, Scientific Park, ${ }^{5}$ Department of Statistics, UB,

Barcelona, Spain

Background and aims: Tmem27 is an HNF1a target expressed in $\beta$ cells, whose role is controversial. While one group concluded that an increased expression induces pancreatic $\beta$ cell proliferation, another one stated that its rise enhances glucose stimulated insulin exocytosis. We aim to study Tmem27 to characterize its function and biochemistry in the islets.

Materials and methods: Wistar rats were treated with tungstate for 40 days. At the end of the treatment, islet proliferation was assessed by immunohistochemistry and in isolated islets glucose induced insulin secretion, Tmem 27 and insulin expression were determined. Control islets were infected with Ad-Tmem $27-m y c$ and Ad- $\beta$-Gal to determine glucose induced insulin secretion and proliferation. TMEM27, INSULIN, SNAPIN, CYCLIN E1, CDK 2, CYCLIN D1 and CDK 6 levels were assessed in human islets by qPCR, establishing its correlation. Protein extracts of Ad-Tmem27-myc INS-1E cells were treated with heparinase, calf intestinal phosphatase, N-glycosidase F or O-glycosidase. Colocalisation analysis of Ad-Tmem27-myc with membrane, Golgi, endoplasmic reticulum and insulin were performed. Islets, hepatocytes and mouse proximal tubular renal cells were isolated and infected with Ad-Tmem27-myc.

Results: Tmem 27 function: Islets from treated (T) rats compared to untreated (UT) show decreased mRNA levels of insulin (fold change -2.26 p<0.01) and Tmem 27 (fold change $-3.08 \mathrm{p}<0.01$ ) with a significant correlation between both $(\rho=0.72 p<0.001)$. Moreover, $T$ animals have decreased glucose induced insulin secretion (UT 140.42 vs T $62.56 \mathrm{IU} /$ islet/90 min, $\mathrm{p}<0.01$ ) and morphometric analysis reveals increased islet proliferation (UT 4.03 vs T 32.90 nuclei PCNA $+/ \mathrm{mm}^{2}$ islet $\mathrm{p}<0.001$ ). Therefore, treatment leads to a decrease expression of Tmem 27 similarly to what happens with insulin expression, secretion and insulinemia; whereas the islet proliferation is increased. Control rat islets infected with Tmem 27 (compared to $\beta$-Gal infection) show an increased glucose induced insulin secretion ( 20.87 vs $40.88 \mathrm{IU} /$ islet $/ 90 \mathrm{~min}$ $[\beta-G a l$ vs Tmem27] $p<0.05)$, whereas there is no differences in islet proliferation. In human islets, TMEM27 expression strongly correlates with INSULIN $(\rho=0.87 \mathrm{p}<0.01)$ and SNAPIN $(\rho=0.73 \mathrm{p}<0.05)$ but no significant correlation is found with CYCLIN E1, CDK 2, CYCLIN D1 and CDK 6. Tmem27 biochemistry: Confocal analysis of INS-1E infected with Tmem 27 shows that Tmem27 is present in Golgi, plasma membrane and colocalizes with a $30 \%$ of insulin granules. Characterization of Tmem 27 fragments by Western blot demonstrates that in INS-1E Tmem27 is N-glycosilated but not O-Glycosilated, and neither heparinazed nor phosphorilated. Finally, infection of islets, hepatocytes and proximal tubular cells (tissues where Tmem 27 expression have been described) with Tmem 27 proves that islets and proximal tubular cells have the capacity for cleaving this protein, whereas hepatocytes don't. Conclusion: Our results support the hypothesis that Tmem 27 role in $\beta$ cell function is involved in insulin secretion but not in $\beta$ cell proliferation. Moreover, they suggest that islets and proximal tubular cells can cleave Tmem27. Supported by: MEC, CIBER de Diabetes y Enfermedades Metabólicas Asociadas is an ISCIII project

\section{9}

Low, but physiological, concentration of GLP-1 stimulates insulin secretion independent of the cAMP-protein kinase A pathway M. Shigeto ${ }^{1}$, M. Katsura ${ }^{2}$, M. Matsuda ${ }^{1}$, S. Ohkuma ${ }^{2}$, K. Kaku ${ }^{1}$ ${ }^{1}$ Diabetes and Endocrine Division,Department of Medicine, ${ }^{2}$ Pharmacology, Kawasaki Medical School, Kurashiki, Japan

Background and aims: Glucagon-like peptide-1 (GLP-1) concentration used in previous in vitro experiments was far from the level of in vivo. Alteration of plasma GLP-1 concentration at pM order lowers blood glucose concentration. The postprandial GLP-1 concentration is only $20 \mathrm{pM}$, even in the presence of DPP-IV inhibitors. This slight change in GLP-1 concentration, however, has a large impact on blood glucose concentration. The aim of this study is to examine GLP-1 action mechanism at a physiological concentration on pancreatic insulin secretion in vitro.
Materials and methods: MIN6 cells (passages 20 - 30) were cultured in modified DMEM in tissue culture flasks. For determination of insulin secretion, the cells were preincubated for $30 \mathrm{~min}$ in $7.0 \mathrm{mM}$ glucose containing KRB-HEPES buffer. The cells were pretreated with verapamil, dantrolene and BAPTA for $60 \mathrm{~s}$ before GLP-1 stimulation. KT5720 was added $20 \mathrm{~min}$ before stimulation.

Results: A high concentration of GLP-1 (10 nM) stimulated intracellular cAMP accumulation and insulin secretion was significantly inhibited by KT5720, a selective inhibitor of PKA. A relatively lower concentration of GLP-1 (1 pM) also increased insulin secretion significantly. The GLP-1 dosedependency on insulin secretion was significantly observed at GLP-1 concentrations greater than $100 \mathrm{pM}$, but not significant over the range of $1 \mathrm{pM}$ to $100 \mathrm{pM}$ of GLP-1. The intracellular accumulation of cAMP was increased by treatment with $100 \mathrm{nM}$ forskolin, and also was increased by addition of 100 pM or more GLP-1 with a dose-dependent manner. On the other hand, low concentrations of GLP-1 ( 10 pM) failed to increase the intracellular cAMP accumulation. Insulin secretion stimulated by $10 \mathrm{nM}$ GLP-1 was significantly, but not completely, inhibited by $100 \mathrm{nM}$ KT5720. In contrast, insulin secretion induced by 1 pM GLP-1 was not affected by KT5720, suggesting an existence of the cAMP-PKA-independent pathway. The time course of insulin secretion showed a marked increase at 420-660 s after an addition of $1 \mathrm{pM}$ GLP-1 (Phase 1), following with oscillations in insulin release (Phase 2). This increase in insulin secretion was completely inhibited by addition of BAPTA, an intracellular $\mathrm{Ca}^{2+}$ chelating agent, but not by KT5720. The first half of Phase 1 insulin secretion was inhibited by addition of $3 \mu \mathrm{M}$ verapamil, a Ltype voltage-dependent calcium channel blocker. A similar waveform of insulin secretion was noted even in the absence of extracellular $\mathrm{Ca}^{2+}$. The latter half of Phase 1 was inhibited by $1 \mu \mathrm{M}$ dantrolene, a blocker of calcium efflux from endoplasmic reticulum, and Phase 2 insulin secretion also was partially inhibited. Simultaneous addition of dantrolene and verapamil completely inhibited Phase 1 insulin secretion.

Conclusion: The present data clearly demonstrate that low GLP-1 concentrations comparable to in vivo blood concentrations promoted insulin secretion independent of the cAMP-PKA pathway. This effect is dependent on the intracellular $\mathrm{Ca}^{2+}$ concentration. Thus our results strongly suggest the possibility that the signal-transducing pathway of GLP-1 action on pancreatic insulin secretion involves both cAMP-PKA-dependent and -independent mechanisms, which are dependent on GLP-1 concentration. Studies of GLP1 at physiologic concentrations may lead to new developments in studies of pancreatic $\beta$-cell function.

\section{0}

Role of mTOR in inhibition of glucose-induced insulin secretion by glucocorticoids in rat INS-1E cells

M.M.L. Linssen ${ }^{1}$, B. Guigas ${ }^{1}$, J. Kriek ${ }^{1}$, G.C.M. van der Zon ${ }^{1}$, M. Diamant ${ }^{2}$, D.M. Ouwens ${ }^{1}$

${ }^{1}$ Molecular Cell Biology, Leiden University Medical Center, Netherlands, ${ }^{2}$ Endocrinology, Vrije Universteit Medical Center, Diabetes Center, Amsterdam, Netherlands

Background and aims: Synthetic Glucocorticoids (GCs) are generally prescribed anti-inflammatory and immunosuppressive agents to treat for example Rheumatoid Arthritis, Asthma and many other diseases, because of their very potent characteristics. A great disadvantage of these therapeutics is the fact that an excess of GCs can lead to unfavourable changes in glucose-, lipidand protein metabolism associated with the development of insulin resistance and overt diabetes. One of the signalling pathways affected by GCs is the mammalian target of rapamycin (mTOR) pathway, in which GCs inhibit mTOR via induction of REDD1 and activation of tuberous sclerosis complex 2 (TSC2). mTOR has also been implicated in the regulation of beta-cell survival. Here, we determined whether dysregulation of the mTOR pathway contributes to inhibition of glucose-stimulated insulin secretion (GSIS) by glucocorticoids in INS-1E cells.

Materials and methods: INS-1E rat insulinoma cells were incubated with either Prednisolone (700 nmol/l) or Dexamethasone (300 nmol/l) (20hrs). After 2 hrs glucose- and serum starvation, cells were exposed to glucose (2.5 or $20 \mathrm{mmol} / \mathrm{l}, 30 \mathrm{~min}$ ) and insulin secretion and protein expression were determined. For gene silencing, cells were transduced with lentiviral vectors encoding rat specific shRNA.

Results: Stimulation of INS-1E cells with glucose, led to a 2.8 -fold induction of GSIS. This response was abrogated $30 \%$ by the mTOR inhibitor rapamycin. Exposure with dexamethasone and prednisolone reduced GSIS up to $40 \%$. Furthermore, the glucose-induced stimulation of mTOR-Ser2481 phospho- 
rylation, and its downstream target ribosomal protein S6-Ser236 and 4EBP1-Thr70 were markedly reduced in cells treated with GCs. No differences between prednisolone and dexamethasone were observed. The inhibitory effects of GCs were absent in cells in which TSC2 was silenced by shRNA. Similar results were obtained in cells lacking FK506 binding protein 38 (FKBP38), another upstream regulator of mTOR-activity.

Conclusion: Both prednisolone and dexamethasone reduced glucose induced insulin secretion and phosphorylation of mTor and its downstream targets $4 \mathrm{E}-\mathrm{BP} 1$ and ribosomal protein S6 in insulin secreting cells. Silencing the mTOR regulators FKBP38 or TSC2 abolished the inhibitory effects of GCs. These findings implicate an important role of mTor as a signalling intermediate in the unfavourable effects of prednisolone and dexamethasone on insulin secretion.

Supported by: Dutch Top Institute Pharma

\section{OP 48 Programming of metabolism}

\section{1}

Early programming of islets antioxidant activities and mitochondrial function by maternal low protein diet

N.H. Theys, B. Reusens, C. Remacle

Institute of Life Sciences, Université catholique de Louvain, Louvain-laNeuve, Belgium

Background and aims: Foetal environment can determine long-term susceptibility to Type 2 diabetes. We have shown that the vulnerability of the foetal and adult $\beta$-cells against reactive oxygen species (ROS) can be influenced by the nutritional status of the mother during gestation and lactation. Moreover, new findings indicated that programmed mitochondrial dysfunction may be a key adaptation of the foetus to survive in utero in an altered nutritional environment. In this context, our aim was to investigate whether maternal low protein diet may programme the antioxidant activity and the mitochondrial function in the islets of the progeny.

Materials and methods: Wistar rats were fed a control diet containing $20 \%$ protein (C group) or an isocaloric low protein diet (LP) containing $8 \%$ protein during gestation only (LPR group) or until weaning (LPT group), after which offspring received the control diet. The antioxidant capacity was studied in 21.5 day-old foetuses and at 5 days, 28 days, 6 weeks, and 3 months in isolated islets from male rats. The mitochondrial function was studied at 3 months in C and LPR progeny.

Results: In foetal islets, SOD (superoxide dismutase) activity was not influenced by the maternal LP diet whereas GPX (glutathione peroxidase) activity was significantly lower in LP foetuses $(\mathrm{p}<0.05)$. CAT (catalase) activity was very low at this stage and was unaffected by the LP diet. The gene expression of antioxidant enzymes was unchanged in LP foetal islets compared to C. In the newborn, SOD activity was lower in LPR offspring compared to C. At 3 months, this activity was higher in LPR $(\mathrm{p}=0.05)$ and in LPT islets $(\mathrm{p}<0.001)$. This could be linked to an increased SOD1 mRNA level in both progeny $(\mathrm{p}<0.05)$ and a SOD2 expression slightly higher in LPT islets. CAT activity was so low for many samples from LP animals that it could not be detected, particularly in LPT islets. Moreover, the level of CAT transcripts was well below $50 \%$ in islets from LP animals compared to controls $(\mathrm{p}<0.05$ vs LPT; $\mathrm{p}<0.01$ vs LPR). Lower GPX activity was measured in LPR newborns $(\mathrm{p}<0.01)$ as well as at 28 days in both LPR and LPT islets $(\mathrm{p}<0.01)$. The lower GPX activity was no more observed thereafter. A significant increase in $\mathrm{c}$ Myc expression ( $\mathrm{p}<0.05$ vs LPT; $\mathrm{p}<0.01$ vs LPR) and a reduced insulin mRNA level $(\mathrm{p}<0.05)$ were observed in LPR and LPT islets. PDX1 (pancreatic and duodenal homeobox-1) expression was unchanged. In response to $16.7 \mathrm{mM}$ glucose, ATP production was blunted in LPR islets $(\mathrm{p}=0.07)$ while the insulin secretion was unaffected. The expression of mtDNA encoded gene ATP synthase (subunit 6) declined in LP rats $(\mathrm{p}<0.05)$ while the expression of subunit of complex I (ND4L) was higher $(\mathrm{p}<0.01)$. The expression of genes coding for enzymes involved in the TCA cycle was also affected with a reduced in citrate synthase and malate deshydrogenase transcripts $(\mathrm{p}<0.05)$.

Conclusion: Consequences of the maternal LP diet on the antioxidant status of the offspring provide evidence that the environment during early life exerts considerable long-term effects. An increased SOD activity may reflect an efficient metabolisation of superoxide anion supply from mitochondrial electron transport chain. this will finally result in an increased $\mathrm{H} 2 \mathrm{O} 2$ production which can be involved in overexpression of $\mathrm{c}$-Myc in $\beta$-cells, that may cause downregulation of insulin gene expression. Our study highlighted that mitochondria are important targets linking molecular and physiological impacts of the foetal programming.

\section{2}

Maternal obesity influences the phenotype of the offspring due to altered intrauterine milieu and modifies the wiring of the POMC cells in the arcuate nucleus in mice

B. Sarman ${ }^{1}$, M. Shanabrough ${ }^{1}$, E. Borok ${ }^{1}$, Z.-W. Liu ${ }^{1}$, J. Bruening ${ }^{2}$,

X.-B. Gao ${ }^{1}$, T. Horvath ${ }^{1}$

${ }^{1}$ Department of Obstetrics, Gynecology and Reproductive Sciences, Yale University, School of Medicine, New Haven, United States, ${ }^{2}$ Department of Mouse Genetics and Metabolism, Institute for Genetics, University of Cologne and Center of Molecular Medicine Cologne, Germany

Background and aims: We tested the hypothesis whether the altered perinatal metabolic milieu due to maternal hyperglycemia and/or obesity in 
$\mathrm{C} 57 \mathrm{Bl} / 6$ mice leads to a metabolic phenotype in the offspring and if it modifies the wiring of the POMC (pro-opio-melanocortin) cells the main anorexigenic component in the arcuate nucleus.

Materials and methods: All POMC cells expressed GFP driven by the POMC promoter. Data is expressed as the average \pm standard deviation (SD). We placed 8 weeks old female mice on either high fat (HF) or low fat normal diet (ND). After six weeks the body weights (bw:g) and plasma glucose levels (gl:mg/dl) of the groups were significantly different, (bw: HF: 26,74 \pm 1,3 vs. ND: $22,74 \pm 1,2 ; \mathrm{p}=0,005$; gl: HF: $200,86 \pm 20,18$ vs ND: $118,17 \pm 2,99$ $\mathrm{p}<0.001)$. Females from both groups were set up as breeders and the bw of these litters were monitored. At weaning, all progeny were placed on ND until postnatal day 60 . At day 60 the 2 groups were divided into subgroups maintained on either ND (ND/nd, HF/nd) or HF diet (ND/hf, HF/hf). We monitored the bw for 12 weeks and at day 144 we measured glucose, insulin and body fat. At days 60 and 144 mice from each litter were processed for synaptic analysis of POMC.

Results: Offspring from mothers on HF diet weighed more than those from ND dams during lactation. After weaning, when all offspring were on ND the bw difference diminished within two weeks, and remained indistinguishable until day 60 . At the end of the study, HF/hf offspring gained more weight than any other group in the study; their plasma glucose was significantly higher $(\mathrm{p}<0,01)$. The synaptic input organization of POMC neurons from 21 day old mice from mothers kept on HF diet was significantly different from that of ND reared mice: the ratio of inhibitory to excitatory synapses was shifted towards inhibitory contacts in the offspring of HF fed compared to ND mothers. At day 60 similar differences were seen in the input organization of POMC cells even though there were no weight differences between groups. The analysis of the wiring of POMC cells at day 144 is ongoing.

Conclusion: Our results confirm that the mouse (like the rat) is an excellent model to study the molecular and neurobiological mechanisms through which maternal obesity results in metabolic phenotype in the offspring. We showed that overfeeding of mothers does not result in a obesity of the offspring while they are kept on ND, however, they are vulnerable to diet-induced obesity. Further, synaptic input organization of the melanocortin cells is altered in offspring from obese mothers which favors a suppressed melanocortin tone. This change likely contributes to diet induced obesity. Supported by: NIH

\section{3}

Progression of body composition during a eight-years follow-up study of adults born SGA

T. Meas ${ }^{1,2}$, S. Deghmoun ${ }^{2}$, E. Carreira ${ }^{2}$, C. Levy-Marchal ${ }^{3,4}$

${ }^{1}$ Internal medicine, Université, Paris $7,{ }^{2}$ U690, INSERM U690, ${ }^{3}$ INSERM,

U690, ${ }^{4}$ Université Paris 7, Denis Diderot, Paris, France

Background and aims: Increased fat mass has been reported both in children and adults born small for gestational age (SGA). The theory of the "developmental mismatch" suggests that the predictive adaptative response during the phase of developmental plasticity under poor in-utero conditions assists the survival of the fetus and optimizes the phenotype for the probable environment of the mature organism. When the post-natal environment is different, this mismatch increases the risk of metabolic disease. This theory predicts that fat mass, which is considerably reduced in SGA newborns, would increase after birth and that the excess of body fat in young adults born SGA would be more pronounced with increasing age.

Materials and methods: Subjects were selected from a community-based cohort where 389 subjects born full-term SGA (Birth Weight $<10^{\text {th }}$ percentile) were considered as exposed to fetal growth restriction and 462 adapted for gestational age (AGA) subjects, $\left(25^{\text {th }}<\mathrm{BW}<75^{\text {th }}\right.$ percentile) as not exposed. Anthropometric measurements were measured at 22 and $30 \mathrm{yr}$ of age and body fat was evaluated by multifrequency bioelectrical-impedancemetry at $30 \mathrm{yr}$.

Results: Ponderal index at birth was significantly lower in the SGA group $\left(24.2 \pm 2.6\right.$ vs $\left.26.5 \pm 1.6 \mathrm{~kg} / \mathrm{m}^{3} ; \mathrm{p}=0.001\right)$. At 22 and 30 years of age, mean body mass index (BMI) was not significantly different between the two groups, however, both groups gained weight, BMI and waist circumference. The proportion of obese subjects at $30 \mathrm{yr}$ was significantly increased in the SGA group $(12.1 \%$ versus $6.5 \%, \mathrm{p}=0.02)$. During the $8 \mathrm{yr}$-follow-up the progression of BMI was significantly greater in SGA $(1.8 \pm 2.6$ versus $1.4 \pm 2.6 \mathrm{~kg} / \mathrm{m} 2$; $\mathrm{p}=0.03)$. At $30 \mathrm{yr}$, percent body fat was significantly higher in SGA $(23.5 \pm$ 8.7 versus $21.9 \pm 8.0 \%, \mathrm{p}=0.01$ ), which difference persisted after adjustment on age, gender and gain in BMI and for the same gain in BMI body fat was significantly further increased in the SGA group in comparison to the AGA $(\mathrm{p}=0.02$ for the statistical interaction).
Conclusion: SGA subjects when adults have an accelerated gain in BMI resulting in a higher body fat content. Our data suggest that the excess of fat mass in SGA subjects could result not only from catch-up in early infancy but also from a long-term and persistent effect of "developmental mismatch". Supported by: Agence National de la Recherche and INSERM

\section{4}

Prevalence and predictors of overweight in offspring with a first-degree relative with type 1 diabetes

S. Hummel, S. Kreichauf, M. Pflüger, C. Winkler, M. Hummel, A.G. Ziegler Immunology, Diabetes Research Institute, Munich, Germany

Background and aims: Offspring of mothers with type 1 diabetes (T1D) have been reported to be at increased risk for overweight. This analysis was performed to study the prevalence of overweight in a prospective cohort of offspring with a first-degree relative (FDR) with (T1D) and to identify predictors of overweight in this population.

Materials and methods: Data on weight and height were collected at age 2, 5 and 8 years from 1483 children born in Germany between 1989 and 2005 and participating in the prospective BABYDIAB $(\mathrm{n}=1214)$ or BABYDIET study $(n=269)$. All children had a FDR with T1D, including 910 with a mother with T1D. Overweight was defined as BMI percentile $\geq 90$, adjusted for sex and age. Data on birth weight/height and smoking during pregnancy were collected at birth, breastfeeding data at age 3 months, 9 months and 2 years. To identify predictors of overweight, a multiple logistic regression analysis was performed.

Results: At 2 and 5 years of age, prevalence and risk for overweight was significantly higher in offspring of mothers with T1D compared to offspring of non-diabetic mothers ( $14.4 \%$ vs. $10.5 \%$, OR $1.4 ; 95 \% \mathrm{CI}: 1.04-2.0, \mathrm{p}=0.03$ and $13.1 \%$ vs, $7.2 \%$, OR $1.9 ; 95 \%$ CI: $1.2-3.1, p=0.004)$ while at 8 years prevalence for overweight was comparable in offspring of mothers with and without T1D (12.4\% vs. 11.4\%). Risk for overweight throughout childhood was increased in offspring of non-diabetic mothers who were large for gestational age (LGA) and decreased if they were breastfed $\geq 26$ weeks. In contrast, in offspring of mothers with T1D, LGA status and breast feeding affected risk for overweight at early ages but not at 8 years. Smoking during pregnancy had no effect on weight development in both offspring of mothers with and without T1D.

Conclusion: These data suggest that in offspring of mothers with T1D risk for overweight is increased only during the first years of life compared to offspring of mothers without T1D and that reducing birth weight and increasing breastfeeding duration would help to decrease the prevalence of overweight during childhood in both offspring of mothers with and without T1D. Supported by: German Research Foundation, the Juvenile Diabetes Research Foundation and the Foundation "Children with type 1 diabetes" 


\section{PS 001 Genetics of autoimmunity}

\section{5}

SUR mutation in type 1 diabetes: autoimmunity prevents sulfonylurea rescue of diabetes caused by SUR1 mutation. Results from the Hvidøre Study Group

S. Pörksen ${ }^{1}$, L. Laborie ${ }^{2}$, L. Nielsen ${ }^{1}$, H. de Wet $^{3}$, E. Schvarcz ${ }^{4}$, T. Sandal ${ }^{2}$, J. Åman $^{5}$, P. Swift ${ }^{6}$, P. Hougaard 7 , F. Ashcroft ${ }^{3}$, A. Molven ${ }^{8}$, M. Knip', L. Hansen ${ }^{1}$, P.R. Njølstad ${ }^{2}$, H.B. Mortensen ${ }^{1}$

${ }^{1}$ Department of paediatrics, Glostrup University Hospital, Glostrup, Denmark, ${ }^{2}$ Department of Clinical Medicine, University of Bergen, Norway, ${ }^{3}$ Laboratory of Physiology, The University, Oxford, United Kingdom, ${ }^{4}$ Department of Medicine, University Hospital, Ørebro, Sweden, ${ }^{5}$ Department of paediatrics, University Hospital, Ørebro, Sweden, ${ }^{6}$ Children's Hospital, Leicester Royal Infirmery, Leicester, United Kingdom, ${ }^{7}$ Department of Statistics, University of Southern Denmark, Odense, Denmark, ${ }^{8}$ The Gade Institute, University of Bergen, Bergen, Norway, ${ }^{9}$ Hospital for Children and Adolescents, University of Helsinki, Helsinki, Finland

Background and aims: Idiopathic diabetes presents without type 1 diabetesassociated autoantibodies. Absence of autoantibodies is also a characteristic of neonatal diabetes due to mutations in KCNJ11 or ABCC8. We compared disease progression of autoantibody-negative and autoantibody-positive children during the first 12 months after disease onset, and investigated whether mutations in the KCNJ11, ABCC8, HNF1A, HNF4A or INS genes can explain some cases of idiopathic diabetes.

Materials and methods: We followed 261 newly diagnosed diabetic children and adolescents from 18 centers in 15 countries. Residual $\beta$-cell function, proinsulin release, blood glucose levels, $\mathrm{HbA}_{1 \mathrm{c}}$, insulin requirement and autoantibodies were measured at 1,6 and 12 months after diagnosis. The genes KCNJ11, ABCC8, HNF1A, HNF4A and INS were sequenced in subjects autoantibody-negative at diagnosis.

Results: Twenty-four patients (9.2\%) tested negative for GADA, IA-2A, and ICA autoantibodies after one month. Autoantibody-negative subjects did not differ in sex, ethnicity, age, HLA or INS-VNTR genotypes. They had higher residual $\beta$-cell function $(P=0.002)$, lower blood glucose excursions $(P=$ $0.004)$, received less insulin $(P=0.05)$ and lower $\operatorname{HbA}_{1 c}(P=0.02) 12$ months after diagnosis. None had mutations in KCNJ11 but one patient had a heterozygous mutation leading to the substitution of arginine (CGC) by cysteine (TGC) at residue 1530 of the $A B C C 8$ gene encoding SUR1. He was 13 years old at diagnosis and had no relatives with diabetes. He became positive for IA- $2 \mathrm{~A}$ antibodies 6 months after diagnosis. Functional analyses showed that the channel was highly sensitive to sulphonylureas. However, there was no effect of sulfonylurea treatment after 4 weeks on $1.0-1.2 \mathrm{mg} / \mathrm{kg} / 24 \mathrm{~h}$. None of the 6 autoantibody negative patients with a family history of diabetes had mutations in HNF1A or HNF4A. There were no INS gene mutations in the autoantibody negative group.

Conclusion: GADA, IA-2A, and ICA autoantibody-negative children have slower disease progression including preservation of residual $\beta$-cell function and improved glycaemic control 12 months after diagnosis. Mutations within $A B C C 8$ may be a, so far, unidentified reason for autoantibody negative childhood onset diabetes, also after the neonatal period. Superimposed type 1 diabetes in the present case may leave SU treatment ineffective.

\section{6}

Correlation between the soluble levels of cytotoxic T-lymphocyte associated antigen 4 (CTLA-4) and polymorphisms of CTLA-4 and programmed death receptor 1 (PD1) in type 1 diabetes

F. Perez-Bravo ${ }^{1}$, S. Momim ${ }^{1}$, B. Angel ${ }^{1}$, E. Codner ${ }^{2}$, E. Carrasco ${ }^{3}$ ${ }^{1}$ Genetic Epidemiology, INTA-University of Chile, Santiago, Chile, ${ }^{2}$ Endocrinology, IDIMI- Hospital San Borja Arriarán. Medicine Faculty, Santiago, ${ }^{3}$ Diabetes Unit, Hospital San Juan de Dios. Faculty of Medicine, Santiago, Chile

Background and aims: Multiple genes are likely to be involved in type-1 diabetes and other immune diseases. In particular, type 1 diabetes may be influenced by Programmed Death-1 (PD-1) receptor, an inhibitory molecule which has been demonstrated to play a role in the regulation of immune responses. To explore the contribution of the PD-1 gene polymorphisms involved in type-1 diabetes (T1D) as well as the relationship between the PD-1 gene and sCTLA4 concentrations.
Materials and methods: 189 incident cases of type 1 diabetes children and 235 healthy children less 15 years old were included in this study. Haplotypes for the polymorphic restriction sites of the PD- 1 gene and CTLA- 4 were obtained by DNA amplification using PCR, followed by the restriction fragment length polymorphism (RFLP) method. Screening for soluble CTLA4 was done using an ELISA assay. The statistical analysis was performed using the online SHESIS package.

Results: We found that PD-1.9 T/T may play a protective role in the development of T1D. Our results show that the sCTLA- 4 was different among patients and controls $(2.57 \pm 0.18 \mathrm{ng} / \mathrm{ml}$ versus $1.18 \pm 0.57, \mathrm{p}<0.001)$. In T1D the sCTLA- 4 levels were different when we considered the allele dosage of CTLA-4 polymorphism, showing a modified effect of $\mathrm{G}$ allele on the sCTLA4 concentrations $\left({ }^{\star} \mathrm{P}<0.04\right)$

Table 1: PD1 haplotypes and CTLA- 4 in T1D patients.

Conclusion: Consistent with the higher serum sCTLA-4 levels observed in other autoimmune diseases, our results suggest that sCTLA-4 may be a risk factor for T1D. Our data suggest a possible regulation effect of the "G" CTLA4 allele over sCTLA- 4 across the PD-1 protector or susceptibility genotype.

\begin{tabular}{lll}
\hline $\begin{array}{l}\text { PD 1.5 - PD 1.9 } \\
\text { Haplotype }\end{array}$ & $\begin{array}{l}+49 \text { A/G } \\
\text { CTLA-4 Dosage }\end{array}$ & $\begin{array}{l}\text { Concentrations of } \\
\text { sCTLA-4 }(\mathrm{ng} / \mathrm{ml})\end{array}$ \\
\hline $\mathrm{T}-\mathrm{T}$ & $\mathrm{A}$ & $0.25 \pm 0.12$ \\
\hline $\mathrm{T}-\mathrm{T}$ & $\mathrm{G}$ & $0.57 \pm 0.31$ \\
\hline $\mathrm{T}-\mathrm{C}$ & $\mathrm{A}$ & $2.50 \pm 1.70$ \\
\hline $\mathrm{T}-\mathrm{C}$ & $\mathrm{G}$ & $5.89 \pm 3.21^{*}$ \\
\hline $\mathrm{C}-\mathrm{C}$ & $\mathrm{A}$ & $1.68 \pm 1.09$ \\
\hline $\mathrm{C}-\mathrm{C}$ & $\mathrm{G}$ & $2.38 \pm 0.53^{*}$ \\
\hline
\end{tabular}

Supported by: FONDECYT grant 1060790

\section{7}

Can HLA-DQ, PTPN22- and CTLA4 polymorphisms explain the putative association between childhood atopic dermatitis and lower subsequent risk of type 1 diabetes?

L.C. Stene ${ }^{1}$, K.S. Rønningen ${ }^{1}$, M. Bjørnvold ${ }^{2}$, D.E. Undlien ${ }^{3,4}$, G. Joner ${ }^{5,6}$ ${ }^{1}$ Division of Epidemiology, Norwegian Institute of Public Health, Oslo, ${ }^{2}$ Institute of Medical Genetics, Faculty division, Ullevål university hospital, University of Oslo, ${ }^{3}$ Department of Medical Genetics, Ullevål university hospital, Oslo, ${ }^{4}$ Institute of Medical Genetics, Faculty Division, Ullevål university hospital, University of Oslo, ${ }^{5}$ Department of Pediatrics, Ullevål university hospital, Oslo, ${ }^{6} \mathrm{Faculty}$ of Medicine, University of Oslo, Norway

Background and aims: Established susceptibility loci for type 1 diabetes are important in immune regulation and may play a role also in atopic disorders, potentially explaining the inverse association between atopic dermatitis and subsequent risk for type 1 diabetes reported in several studies. Some previous studies have investigated whether susceptibility genes for atopy are associated with type 1 diabetes or vice versa, but no previous study has studied the role of genetic factors where information on both diabetes and atopy has been available in the same individuals. We aimed to directly assess whether HLA-DQ, CTLA4 and PTPN22 genes could explain the putative association between childhood atopic dermatitis and lower subsequent risk of type 1 diabetes.

Materials and methods: We designed a case-control study with 339 incident cases of type 1 diabetes identified in The Norwegian childhood diabetes registry, and 985 population-based control children. DNA was collected, and physician-diagnosed childhood atopic dermatitis was ascertained by a questionnaire administered to the parents of children with and without type 1 diabetes.

Results: The previously reported association between atopic dermatitis and lower risk of type 1 diabetes was confirmed (odds ratio: $0.61,95 \%$ confidence interval: $0.40-0.95)$ and this was consistent in sub-groups defined by HLADQ, CTLA4 and PTPN22 genotypes. The odds ratio was essentially not influenced by adjustment for genetic variation at these loci (adjusted odds ratio: $0.55,95 \%$ confidence interval: $0.32-0.92$ ), showing that these genetic factors could not explain the observed inverse relation between atopic dermatitis and type 1 diabetes.

Conclusion: In this first study of its kind, we demonstrated directly that the observed inverse association between atopic dermatitis and type 1 diabetes could not be explained by established diabetes susceptibility genes HLA-DQ, CTLA4 or PTPN22. 
Supportet by: Research Council of Norway, Norwegian Diabetes Association, Aker Diabetes Research Foundation, Novo Nordisk Fonden, TINE Norwegian Diaries, Novo Nordisk Scandinavia, Eastern Regional Health Authorities

\section{8}

Genetic predisposition for celiac disease in children with type 1 diabetes mellitus

J. Pedras ${ }^{1}$, P. Jarosz-Chobot ${ }^{2}$, J. Polanska ${ }^{3}$, M. Minkina-Pedras ${ }^{4}$, U. Siekiera ${ }^{5}$ ${ }^{1}$ Department of Cardiology, District Hospital, Tychy, ${ }^{2}$ Depart. of Pediatric, Endocrinology and Diabetes, The Medical University of Silesia, Katowice, ${ }^{3}$ The Faculty of Automatic Control, Electronics and Computer Science, The Silesian University of Technology, Gliwice, ${ }^{4}$ Depart. of Pediatric, Endocrinology and Diabetes, Upper Silesia Center Health of Child, Katowice, ${ }^{5} \mathrm{HLA}$ and Immunology Lab, Regional Blood Center, Katowice, Poland

Background and aims: Type 1 diabetes mellitus (T1DM) and celiac disease (CD) frequently occur together. Some genetic polymorphisms may significantly modify the risk of CD in subjects with T1DM. The aim of the study was to evaluate genetic predisposition in class HLA (locus DRB1, DQB1) and by gene polymorphisms of tumor necrosis factor- $\alpha$ (TNF- $\alpha$ ), interferon- $\gamma$ (IFN$\gamma)$, interleukin-6 (Il-6), interleukin-10 (Il-10).

Materials and methods: 33 children (18 girls and 15 boys) with positive tissue transglutaminase tTG were selected during a routine screening carried out in 513 children with T1DM. In all cases, small intestine biopsy was performed. HLA was identified in the locus DR and DQ. HLA alleles and polymorphisms were determined using PCR with sequence-specific primers. This group was compared to the group of children with T1DM only. The following factors were similar in both groups: number of patient, sex, onset of T1DM. Results: According to biopsy results silent $\mathrm{CD}$ was diagnosed in 24 children (72.72\%) cases and latent form CD in $9(27.27 \%)$ cases. Mean age at onset of T1DM was $6.41 \pm 3.27$ years. CD was diagnosed after $15.34 \pm 16.08$ months of T1DM duration. Haplotype DRB1 ${ }^{\star} 03 \mathrm{DQB1} 1^{\star} 02$ was present in 25 children $(75.76 \%)$. Alleles DRB1 and DQB1 were significantly more frequent $(\mathrm{p}=$ 0.014; $\mathrm{p}=0,006$ respectively) in T1DM with CD than in patients with T1DM only. Another common haplotype for CD - DRB1 ${ }^{\star} 04 \mathrm{DQB1} 1^{\star} 03$ was found in 17 children (51.51\%). Only in 4 cases no haplotypes mentioned were present. We found positive statistical significance in TNF- $\alpha-308 \mathrm{~A}$ genotype between the examined group and the group with T1DM $(\mathrm{p}=0.024)$. We did not find association between IFN- $\gamma$, Il- 6 and Il-10 polimorphisms and celiac susceptibility.

Conclusion: $\mathrm{CD}$ in children with T1DM seems to be modified by presence of haplotype DRB $1^{\star} 03 \mathrm{DQB} 1^{*} 02$ and by a variant of allele TNF- $\alpha-308 \mathrm{~A}$. Supported by: Grant KBN 3T11FO1029

\section{9}

Common variants in or around the JAZF1, CDKN2A/2B, CDKAL1 and WFS1 genes discriminate between autoimmune and non-autoimmune diabetes

E. Bakhtadze ${ }^{1}$, E. Lindholm ${ }^{1}$, V. Lyssenko ${ }^{1}$, H.J. Arnkvist ${ }^{2}$, J. Bolinder ${ }^{3}$, J.W. Eriksson ${ }^{4,5}$, S. Gudbjörnsdottir ${ }^{4}$, L. Nyström ${ }^{5}$, M. Landin-Olsson ${ }^{6}$, L.C. Groop ${ }^{1}$

${ }^{1}$ Clinical sciencies-Diabetes and Endocrinology, Lund University, Malmö, ${ }^{2}$ Department of Clinical and Experimental Medicine, Linköping University, Linköping, ${ }^{3}$ Department of Medicine, Karolinska University, Stockholm, ${ }^{4}$ Department of Medicine, Sahlgrenska university hospital, Göteborg, ${ }^{5}$ Department of Medicine, Umeå University, ${ }^{6}$ Endocrinology, Lund University, Sweden

Background and aims: To study whether common variants in novel T2D genes can discriminate between autoimmune and non-autoimmune diabetes in young (15-34 years) and middle-aged (40-59 years) subjects.

Materialsandmethods:Wegenotyped thefollowingSNPs:PPARG(rs1801282), IGF2BP2 (rs4402960), WFS1 (rs10010131), CDKAL1 (rs7754840), JAZF1 (rs864745), CDKN2A/2B (rs10811661), HHEX (rs1111875) and KCNJ11 (rs5912) and measured GADA, IA-2A antibodies and fasting C-peptide concentrations in 1,642 young (15-34 years) and 1,619 middle-aged (40-59 years Swedish diabetic patients. Patients were classified as $\mathrm{Ab}+$ if one antibody was positive and negative (Ab-) if they had no antibodies.

Results: Altogether, $67 \%$ of the young and $13 \%$ of the elderly patients were $\mathrm{Ab}+$. Young $\mathrm{Ab}+$ patients had lower prevalence of JAZF1 (recessive model)
AA ( $23 \%$ vs. $32 \%$; $=0.0003$ ) and CDKN2A/2B (recessive model) TT ( $67 \%$ vs. $74 \%$; $=0.0078$ ) than young $\mathrm{Ab}$ - patients. Middle-aged $\mathrm{Ab}+$ patients had a decreased prevalence of WFS1 (recessive model) GG (29\% vs. 36\%; $\mathrm{p}=0.045)$ and CDKAL1 (recessive model) CC ( $8 \%$ vs. $15 \%$; $\mathrm{p}=0.02$ ) genotypes.

Conclusion: The lower frequency of variants in (or close to) JAZF1, CDKN2/ A2, WSF1 and CDKAL1 in antibody-positive individuals suggest that the mechanisms by these variants increase susceptibility to T2D do not involve autoimmune phenomenons.

Supported by: Juvenile Diabetes Foundation-Wallenberg Diabetes Research Program, Swedish diabetes Association, Swedish Medical Research Council, Albet Påhlsson Foundation

\section{0}

Assosiation of $(\mathrm{C} 1858 \mathrm{~T})$ polymorphism in the PTPN22 gene and latent autoimmune diabetes in adults (LADA) at Ukrainian population T. Tikhonova ${ }^{1}$, S. Shtandel ${ }^{2}$, M. Fedetz ${ }^{3}$, O. Khijnyak ${ }^{1}$, N. Kravchun ${ }^{4}$, T. Levchenko ${ }^{1}$, O. Fedetz ${ }^{5}$ N. Velikikh ${ }^{1}$, H. Svetlova-Kovalenko ${ }^{2}$ ${ }^{1}$ Department of Age Endocrinology, Institute of Endocrine Pathology Problems, Kharkov, Ukraine, ${ }^{2}$ Laboratory of Pathophisiology and Medical Genetics, Institute of Endocrine Pathology Problems, Kharkov, Ukraine, ${ }^{3}$ Department of Cellular Biology and Immunology, Institute of Parasitology and Biomedicine, Granada, Spain, ${ }^{4}$ Department Pharmakotherapy of Endocrine Diseases, Institute of Endocrine Pathology Problems, Kharkov, Ukraine, ${ }^{5}$ Department of Cell Biology, Clinical Laboratory, Kharkov, Ukraine

Background and aims: The Protein Tyrosine Phosphatase Non-receptor type 22 (PTPN22) gene encodes for lymphoid-specific phosphatase, the powerful T-cell activation inhibitor. Today many studies have showed an association between the C1858T polymorphism (Arg620Trp) of the PTNP22 gene and type 1 diabetes mellitus (T1DM) in various populations. The aim of the study was to examine the association of the PTNP22 gene polymorphism C1858T with the predisposition to latent autoimmune diabetes in adults (LADA) in Ukrainian population.

Materials and methods: The study was performed in the group, that consist of 321 patients with T1DM classical clinical form, 38 individuals with LADA (age of manifestation $45.52 \pm 1.83 \mathrm{yrs}$, with anti islet, GADA antibodies and low C-peptide levels), 57 patients with type 2 diabetes mellitus (T2DM) and 253 healthy Kharkov inhabitants. The GADA, IA2A and ICA antibodies were measured with RIA. The presence of different variants of the PTPN22 gene polymorphism was estimated using the DNA sequencing method. Genotype and allelic distributions of the $\mathrm{C} 1858 \mathrm{~T}$ polymorphism were comparing using the $\chi^{2}$ test.

Results: LADA patients had the higher C1858T polymorphism of PTPN22 gene frequency, than T1DM, T2DM and control data in Kharkov population: Trp/Trp genotype was $21.05 \%$ vs $6.54 \%\left(\chi_{\text {LADA-TIDM }}^{2}=7.78 ; \mathrm{p}=0.005\right), 5.26 \%$ $\left(\chi_{\text {LADA-T2DM }}^{2}=4.12 ; \mathrm{p}=0.042\right), 0.79 \%\left(\chi_{\text {LADA-control }}^{2}=35.00 ; \mathrm{p}=0.000\right)$, respectively; $1858 \mathrm{~T}$ allele frequency was $43.42 \%$ vs $21.96 \%\left(\chi_{\text {LADA-TIDM }}^{2}=15.89 ; \mathrm{p}=0.000\right)$, $28.07 \%\left(\chi_{\text {LADA-T2DM }}^{2}=4.12 ; \mathrm{p}=0.042\right), 13.86 \%\left(\chi_{\text {LADA-control }}^{2}=37.71 ; \mathrm{p}=0.000\right)$, respectively. Data of our study data suggested more powerful association C1858T polymorphism of PTPN22 gene in LADA patients in comparison to those with T1DM: for Trp/Trp genotype $-\mathrm{OR}_{\mathrm{LADA}}=33.47(6.75-165.67)$ vs $\mathrm{OR}_{\mathrm{T} 1 \mathrm{DM}}=8.79(2.03-37.71)$; for $1858 \mathrm{~T}$ allele $-\mathrm{OR}_{\mathrm{LADA}}=4.78(2.82-8.02) \mathrm{vs}$ $\mathrm{OR}_{\mathrm{TIDM}}=1.75(1.28-2.40)$.

Conclusion: It has been shown, that C1858T polymorphism of PTPN22 gene like in T1DM plays the important role in the LADA genetic predisposition in Ukrainian population. More powerful the polymorphism association with LADA in comparison to T1DM was shown also. 


\section{1}

Genetic risk factors for patients with Latent Autoimmune Diabetes (LADA) including insulin resistance genes. Results from the HUNT Study E. Pettersen ${ }^{1}$, V. Grill ${ }^{2}$, K. Kvaløy ${ }^{3}$, F. Skorpen ${ }^{2}$

${ }^{1}$ Norwegian University of Science and Technology, Institute of cancer research and molecular medicine, Levanger, Norway, ${ }^{2}$ Norwegian University of Science and Technology, Institute of cancer research and molecular medicine, Trondheim, Norway, ${ }^{3}$ Norwegian University of Science and Technology, Institute of public health and general practice, Levanger, Norway

Background and aim: Previous studies have indicated that Latent Autoimmune Diabetes of the Adult (LADA) shares some genetic risk with type 1 diabetes (T1D). However, data from the North Trøndelag Health (HUNT) study, containing cross-sectional data from 64,500 men and women (aged $>20$ ), indicate that phenotypes of LADA and type 2 diabetes (T2D) are similar and that insulin resistance factors such as obesity are strong risk factors for both. These findings suggest that LADA shares genetic features with T2D including propensity for insulin resistance. This notion was tested by comparing genetic associations in T1D, T2D and LADA patients.

Materials and methods: DNA samples collected from the HUNT study (1995-96) were used for analysis. We analysed 10 single nucleotide polymorphisms (SNPs) within six candidate genes for T1D and 22 SNPs within 21 candidate genes for insulin resistance and T2D in 120 T1D patients, 126 LADA patients, 1090 T2D patients and 1503 age and gender matched healthy non diabetic controls. Patients starting insulin treatment within six months of diagnosis were classified as T1D if they also were antiGAD positive or had fasting C-peptid levels $<150 \mathrm{pmol} / \mathrm{l}$. Patients were classified as LADA if they were antiGAD positive and had not been treated with insulin within 12 months of diagnosis. T2D cases were antiGAD negative and without insulin treatment within one year of diagnosis. The results were adjusted for gender, age and BMI by logistic regression.

Results: INS (rs689 and rs3842753) and PTPN22 (rs2476601) were strongly associated with T1D $\left(\mathrm{p}=6.9^{\star} 10^{-5}, \mathrm{p}=0.0002\right.$ and $\mathrm{p}=1.5^{\star} 10^{-5}$ respectively) but did not show association with LADA ( $\mathrm{p}=0.60, \mathrm{p}=0.37$ and $\mathrm{p}=0.16)$. For the T2D associated genes, the CC/CT genotypes of rs7961581 (upstream of TSPAN8) was associated with both T2D $(\mathrm{p}=0.033, \mathrm{OR}=1.21$ [1.02-1.44]) and LADA ( $\mathrm{p}=0.0097, \mathrm{OR}=1.68$ [1.13-2.48]). Likewise, the obesity-linked AA/AC genotypes of rs8050126 (FTO) was associated with T2D $(\mathrm{p}=0.0051, \mathrm{OR}=1.31$ $[1.08-1.58])$ as well as with LADA $(\mathrm{p}=0.0052, \mathrm{OR}=1.97$ [1.23-3.17]). The TT/ TC genotype within the strongly T2D associated TCF7L2 gene (rs7903146) were not associated with LADA $(\mathrm{p}=0.14, \mathrm{OR}=1.35$ [0.91-2.00]) but strongly with T2D $\left(\mathrm{p}=4.8^{\star} 10^{-14}, \mathrm{OR}=1.97[1.65-2.35]\right)$ in our study.

Conclusion: The genetic susceptibility to develop LADA is partly distinct from both T1D and T2D, and includes an insulin resistance associated gene. Supported by: Nord-Trøndelag Health Study (The HUNT study), collaboration between The HUNT Research Centre, Faculty of Medicine, The Norwegian University of Science and Technology (NTNU), The National Institute of Public Health and The Nord-Trøndelag County Council

\section{PS 002 Genetics of type 1 diabetes}

\section{2}

Killer cell immunoglobulin-like receptor genes confer susceptibility to type 1 diabetes in patients belonging to a 1-14 year age group and not in a 15-34 year age group

S.K. Sedimbi, C.B. Sanjeevi

Molecular Medicine and Surgery, Karolinska Institutet, Stockholm, Sweden

Background: Natural Killer (NK) cells are potent modulators of innate (and adaptive) immune responses. Killer Ig-like receptors (KIR) are a group of activatory and inhibitory receptors expressed on NK cells, which bind to specific motifs on Human Leukocyte Antigen (HLA) Class I molecules. The activation or inhibition of NK cell activity depends on the overall signal generated as a result of the dynamic balance between activatory and inhibitory KIRs. Aims: i) To test the KIR gene frequencies in Swedish children with type 1 diabetes (T1DM) and healthy controls and ii) To test whether presence (or absence) of certain KIR genes in combination with their putative HLA-C ligands ( $\mathrm{C} 1$ and $\mathrm{C} 2$ ) confers susceptibility (or protection) to the disease.

Subjects and methods: A total of 350 consecutively diagnosed type 1 diabetes patients (of which 173 patients were in 1-14 year age group and 177 patients were in 15-34 year age group) and 293 healthy age and sex matched controls $(0-14$ years $=152$ and $15-34$ years $=141)$ were used for genotyping 16 KIR genes and HLA-C1 and C2 ligands using PCR-sequence specific primer (SSP) methodology. Statistical analyses were performed using Chi-square test and Fischer's exact test as applicable.

Results: i) $1-14$ year age group: KIRs 2DL2 ( $\mathrm{OR}=1.59$; $95 \% \mathrm{CI}=1.00$ 2.51; $\mathrm{P}=0.04), 2 \mathrm{DL} 3(\mathrm{OR}=1.85 ; 95 \% \mathrm{CI}=1.01-3.38 ; \mathrm{P}=0.04)$, and $2 \mathrm{DS} 2$ $(\mathrm{OR}=1.68 ; 95 \% \mathrm{CI}=1.06-2.66) ; \mathrm{P}=0.02)$ are positively associated and 3DS1 $(\mathrm{OR}=0.47 ; 95 \% \mathrm{CI}=0.30-0.74 ; \mathrm{P}=0.001)$ is negatively associated with the disease in the 0-14 year age group. But we failed to see any of this association in patients in the 15-35 year age group. In 0-14 year age group, stratification analyses of KIR genes with their putative HLA-C ligands showed the combinations 2DL2+/HLA-C1- $(\mathrm{OR}=2.25 ; \mathrm{P}=0.02)$; and 2Dl3-/HLA-C1 $(\mathrm{OR}=2.17 ; \mathrm{P}=0.03)$ were positively and $2 \mathrm{DL} 3-/ \mathrm{HLA}-\mathrm{C} 1+(\mathrm{OR}=0.49 ; \mathrm{P}=0.02)$; $2 \mathrm{DS} 1-/ \mathrm{HLA}-\mathrm{C} 2+(\mathrm{OR}=0.60 ; \mathrm{P}=0.02) ; 2 \mathrm{DS} 2+/ \mathrm{HLAC} 1-(\mathrm{OR}=0.56 ; \mathrm{P}=0.04)$; $2 \mathrm{DS} 2-/ \mathrm{HLAC} 1+(\mathrm{OR}=0.62 ; \mathrm{P}=0.03)$ were negatively associated.

Conclusion: KIR genes are important in conferring susceptibility (and/or protection) to T1DM. Presence of HLA-C ligands (C1 and C2) confers protection to the disease when seen in association with the respective KIRs they bind to. Their absence, in the presence of the corresponding KIR genes confers susceptibility. From the functional perspective, presence of a ligand for an inhibitory KIR on the target cell delivers an inhibitory signal to the NK cell, leading to tolerance. Similar to the classical HLA association, age appears to be a very important factor in determining the role of KIR genes susceptibility to T1DM. The age group 1-14 years, being much more susceptible compared to that of 15-34 years.

Supported by: Swedish Medical Research Council, Swedish Diabetes Association, Bran Diabetes Fund, Svenska Diabetes Stiftelsen and Karolinska Institute

\section{3}

Ctla-4 polymorphism and lymphocyte caspase- 3 expression in Chilean children with type 1 diabetes (T1D)

B. Angel ${ }^{1}, \mathrm{M}$. Arredondo ${ }^{2}$, E. Codner ${ }^{3}$, E. Carrasco ${ }^{4}$, F. Dotta ${ }^{5}$,

F. Pérez-Bravo ${ }^{1}$

${ }^{1}$ Genetic Epidemiology Laboratory, INTA-University of Chile, Santiago, ${ }^{2}$ Micronutrients, INTA-University of Chile, Santiago, Chile, ${ }^{3}$ Endocrinology, IDIMI-University of Chile, Santiago, ${ }^{4}$ Diabetes Unit, San Juan de Dios Hospital-University of Chile, Santiago, ${ }^{5}$ Diabetes Unit. Department of Internal Medicine, University of Siena, Italy

Background and aims: Cytotoxic T lymphocyte-associated antigen (CTLA4) is a critical down-regulatory molecule expressed on T cells. Several polymorphism of the CTLA-4 gene has been associated with autoimmune diseases. Apoptosis events of $\beta$ cells is the most important and final step in the development of autoimmune diabetes. The activation of induced cell death is the major event and caspase 3 represent the main protein for the apoptotic machinery. To characterize a genotype of CTLA4 in children with T1D and to correlate it with caspase 3 expression in peripheral mononuclear cells (PMCs) simulating "in vitro" the glucose effect on the Caspase 3 expression. Materials and methods: CTLA- 4 polymorphisms were determined by PCR in 260 T1D children and 255 controls. Caspase 3 expression was determined 
by semiquantitative RT-PCR and Western Blot. The "in vitro" study included 11 T1D of recent diagnosis (first 15 days) and 11 controls. Caspase 3 mRNA expression by RT-PCR and their activity by colorimetric assay were analyzed.

Results: No differences in the genotypes frequencies were observed when comparing T1D (+49A/G: A/A:45; A/G:42; G/G:13\%;) v/s controls (+49A/G: A/A:43; A/G:42; G/G:15\%); $p=0,768$. A decreasing of mRNACasp3/ $\beta$-Actin expression was observed in T1D in the presence of glucose: 0.55 in basal and 0.24 at $14 \mathrm{mM}$ and a slight diminution in the Casp3 activity: 1.0 in Basal and 0.9 at $14 \mathrm{mM}$ in carriers of $\mathrm{G}$ allele and an increase of the mRNA/ $\beta$-Actin expression ( 0.4 in Basal and 0.83 to at $14 \mathrm{mM}$ ) in non carriers. Non significant changes in relation to the glucose stimulus were observed in control subject. Conclusion: T1D's PMCs of recent diagnosis, carrying G allele in $+49 \mathrm{~A} / \mathrm{G}$ polymorphisms of CTLA4, present an important decrease in the expression and activity of the major executioner of lymphocyte apoptosis in comparison to the non carriers.

Supported by: FONDECYT 1060790

\section{4}

\section{Abnormal monocyte-related signatures in type 1 diabetes; a study of identical twins \\ R.D.G. Leslie ${ }^{1}$, H. Beyan ${ }^{1}$, H.A. Drexhage ${ }^{2}$, H.J. De Witt ${ }^{2}$ \\ ${ }^{1}$ Centre for Diabetes and Metabolic Medicine, Institute of Cell and \\ Molecular Science, London, United Kingdom, ${ }^{2}$ Immunology, Erasmus MC, Rotterdam, Netherlands}

Background and aims: Type 1 diabetes (T1DM) is probably due to an aggressive adaptive immune response but the innate immune system could be relevant since monocytes infiltrate the islets. We initially detected changes in T1DM monocytes including a cluster of altered genes expression profiles (unpublished).

Aim: Our present aim was to identify monocyte-related biomarkers in identical twin pairs discordant for T1DM (only one twin with diabetes) to determine and validate monocyte gene clusters which are diabetes-associated or associated with disease-susceptibly.

Materials and methods: We studied 14 identical twin pairs (all discordant for T1DM) 6 male; mean age of 37 years (range17-53), mean disease duration 20.5 years (7-44) and 13 normal identical twin pairs, 7 male; mean age of 37 (23-55 years). Peripheral blood mononuclear cells (PBMC) were isolated using Ficoll. Monocytes were isolated from PBMC by MACS-CD14 separation. Real-time quantitative PCR was preformed on extracted RNA for 14 candidate genes identified using Affymetrix plus appropriate controls including Able gene (housekeeping gene).

Results: Abnormal expression levels of 11 monocyte genes were validated, encompassing changes in monocyte pro-inflammatory signatures, MAP kinase pathway, cell adhesion/motility and immune regulation. Abnormal levels of gene expression were detected either in the diabetic twin alone (e.g. FABP5, MAPK6, CD9, CDC42, STX1A), or in both diabetic and non-diabetic twins (e.g. CCL2, CCL7, DHRS, HSP70, SDR, DHRS, PTPN7, EMP1)(all differences $\mathrm{p}<0.02$ ); the latter having altered expression of a different gene set but gene expression levels all being strongly correlated between twins of a pair $(\mathrm{r}$ $>0.78$ ) and not altered in type 2 diabetes excluding a hyperglycaemic effect. Conclusion: In summary, these results both identify and validate monocyte signatures of altered gene expression levels associated with T1DM which are either familial or disease specific, and in neither case associated with type 2 diabetes. Thus we identified both disease biomarkers as well as potential targets for therapeutic intervention.

Supported by: British Diabetic Twin Trust and JDRF

\section{5}

European Type 1 Diabetes Genetics Network (ET1DGN): preliminary results from Spain

A.M. Wägnerer,2, A. Santana ${ }^{3}$, F.J. Ampudia ${ }^{4}$, J. Argente ${ }^{5}$, L. Castaño ${ }^{6}$, R. Corripio ${ }^{7}$, B. García ${ }^{8}$, M. Hernández ${ }^{9,10}$, A. de Leiva ${ }^{11}$, D. Mauricio ${ }^{9,11}$, F.J. Nóvoa ${ }^{1}$, T. Pedro ${ }^{4}$, M.J. Rodríguez ${ }^{1}$, M. Rodríguez ${ }^{12}$, F. Vázquez ${ }^{6}$ on behalf of the Spanish Type 1 Diabetes Genetics Network, ET1DGN and T1DGC

${ }^{1}$ Endocrinology, Hospital Universitario Insular de Gran Canaria, Las Palmas de Gran Canaria, Spain, ${ }^{2}$ Steno Diabetes Center, Gentofte, Denmark, ${ }^{3}$ Mathematics, Universidad de Las Palmas de Gran Canaria, Spain, ${ }^{4}$ Endocrinology, Hospital Clínico, Valencia, Spain, ${ }^{5}$ Endocrinology, Hospital Niño Jesús, Madrid, Spain, ${ }^{6}$ Endocrinology, Hospital de Cruces, Barakaldo, Spain, ${ }^{7}$ Paediatrics, Consorci Hospitalari Parc Taulí, Sabadell, Spain, ${ }^{8}$ Paediatrics, Hospital Severo Ochoa, Madrid, Spain, ${ }^{9}$ Endocrinology, Hospital Arnau de Vilanova, Lleida, Spain, ${ }^{10}$ Endocrinology, Hospital Universitario de Canarias, Sta Cruz de Tenerife, Spain, ${ }^{11}$ Endocrinology, Hospital de la Santa Creu i Sant Pau, Barcelona, Spain, ${ }^{12}$ Paediatrics, Hospital Miguel Servet, Zaragoza, Spain

Background and aims: ET1DGN is the European branch of the Type 1 Diabetes Genetics Consortium (T1DGC), created to study the genetics and pathogenesis of type 1 diabetes. A total of 1232 affected sibpair (ASP) families have been included in Europe between 2004 and 2007. The aim of this study is to present the first results of the Spanish families.

Materials and methods: A total of 151 ASP families (610 members) have been included in Spain. Phenotypic and antibody information is available from most of the families and genetic information, from more than half of them. This includes HLA genotyping (118 families), a 6000 SNP genome scan $(\mathrm{N}=85)$ and SNP genotyping in genes previously described to be associated with type 1 diabetes $(\mathrm{N}=70)$. Association was analysed using multiple TDTsib-TDT and linkage analysis was performed using non-parametric linkage (Merlin 1.1.1).

Results: Mean age of onset was $15.1+/-8.7$ (1-42) years, age at examination $30.7+/-10.8$ years, $18.8 \%$ of the affected siblings had associated autoimmune diseases, $57.3 \%$ had positive anti-GAD, $35.5 \%$ positive anti-IA2 and $24.8 \%$ were positive for both antibodies. High risk HLA alleles were present in $68.6 \%$ (DR3) and $48.6 \%$ (DR4) of the affected siblings, whereas $9.4 \%$ showed the low-risk allele DRB $1^{*} 0701$. Linkage analysis showed a predominant LOD of $6,4\left(\mathrm{p}=2.84^{*} 10^{-8}\right)$ on the short arm of chromosome 6 , as well as LOD above 1 on chromosomes 2, 10, 17 and 21. The TDT-sib-TDT analysis of the genome scan data showed most significant association with markers on chromosomes $1\left(\mathrm{rs} 1252133\right.$, unadjusted $\mathrm{p}=7.25{ }^{*} 10^{-4)}, 3\left(\mathrm{rs} 1996818, \mathrm{p}=8.5{ }^{*} 10^{-4}\right.$, $15\left(\mathrm{rs} 11433, \mathrm{p}=5.35^{\star} 10^{-4)}\right.$ and $18\left(\mathrm{rs} 635269, \mathrm{p}=1.61{ }^{\star} 10^{-4}\right)$.

Of the genes previously described to be associated with type 1 diabetes, the following showed a non-corrected, significant $(p<0,05)$ association with the disease in the Spanish families: CEACAM21, CTLA4, CXCL12, IL2RA, IL4R, INS and VDR.

Conclusion: Although HLA accounts for most of the genetic risk of type 1 diabetes also in Spanish ASP families, other genes seem to be play a role, too. Further stratification by HLA may clarify this point. Supported by: NIDDK and JDRF

\section{6}

Follow-up analysis of genome-wide association data and replication identifies additional susceptibility loci for type 1 diabetes

S.F.A. Grant ${ }^{1,2}$, H.-Q. Qu ${ }^{3}$, J.P. Bradfield ${ }^{1}$, L. Marchand ${ }^{3}$, C.E. Kim ${ }^{1}$, R. Grabs ${ }^{3}$, S.P. Taback ${ }^{4}$, M.L. Lawson ${ }^{5}$, R.M. Chiavacci ${ }^{1}$, C.A. Stanley ${ }^{6}$, S.E. Kirsch ${ }^{7}$, A.D. Paterson ${ }^{8}$, D.S. Monos ${ }^{9}$, C. Polychronakos ${ }^{3}$, H. Hakonarson ${ }^{1,2}$

${ }^{1}$ Center for Applied Genomics, Children's Hospital of Philadelphia, United States, ${ }^{2}$ Pediatrics, University of Pennsylvania, Philadelphia, United States, ${ }^{3}$ Pediatrics and Human Genetics, McGill University, Montreal, Canada, ${ }^{4}$ Pediatrics and Child Health, University of Manitoba, Winnipeg, Canada, ${ }^{5}$ Division of Endocrinology, Children's Hospital of Eastern Ontario, Ottawa, Canada, ${ }^{6}$ Division of Endocrinology, Children's Hospital of Philadelphia, United States, ${ }^{7}$ Pediatrics, Markham-Stouffville Hospital, Markham, Canada, ${ }^{8}$ Public Health Sciences, Hospital for Sick Kids, Toronto, Canada, ${ }^{9}$ Pathology and Laboratory Medicine, Children's Hospital of Philadelphia, United States

Background and aims: Type 1 diabetes (T1D) is a common, strongly heritable disease that most often manifests in childhood. Recent genome wide 
association studies have revealed a number of new genes associated with the disease. We carried out a follow-up strategy to uncover additional novel T1D risk loci.

Materials and methods: We selected 1,192 independent single nucleotide polymorphisms (SNPs) with at least a nominally significant $P$-value (but excluding SNPs in the MHC region) from a combination of our data generated on 563 T1D probands and 1,146 controls plus 483 complete T1D family trios of the same ancestry, using the Illumina HumanHap550 SNP chip. We then genotyped these SNPs using the Illumina GoldenGate platform in an independent cohort of 939 nuclear T1D families from Montreal and the T1DGC. Subsequently we looked across all three cohorts plus the publicly available Wellcome Trust Case Control Consortium dataset for T1D to identify SNPs in loci that were both not previously described and nominally significant across all cohorts.

Results: We selected five loci for further investigation, which we queried in a T1D dataset from Toronto using an independent matched control dataset from Philadelphia both of which were genotyped on the HumanHap550K SNP chip. Two of the five loci were also significantly associated with T1D in this cohort and, interestingly, resided in genes that were biologically relevant to autoimmunity.

Conclusion: We have identified two novel loci that are associated with T1D across five different Caucasian cohorts. The identity of this loci will be presented.

Supported by: JDRF and Genome Canada through the Ontario Genomics Institute. H.Q.Q. is supported by a fellowship from the Canadian Institutes of Health Research. We acknowledge the use of DNA samples from the Type 1 Diabetes Genetics Consortium, funded by NIH grant

\section{7}

The 3q21-region is showing moderate evidence for association with type 1 diabetes in three Finnish family datasets

A.-P. Laine ${ }^{1}$, R. Veijola ${ }^{2}$, O. Simell ${ }^{3}$, M. Knip ${ }^{4,5}$, J. Ilonen

${ }^{1}$ Immunogenetics laboratory, University of Turku, ${ }^{2}$ Department of

Paediatrics, University of Oulu, ${ }^{3}$ Department of Paediatrics, University

of Turku, ${ }^{4} \mathrm{Hospital}$ for Children and Adolescents, University of Helsinki,

${ }^{5}$ Department of Paediatrics, University of Tampere, Finland

Background and aims: IDDM9-region on chromosome $3 \mathrm{q}$ has shown suggestive evidence for linkage to type 1 diabetes (T1D) in some but not all genome scans. In a previous study we have reported linkage to and association with the 3q21-region in Finnish datasets. LOD scores of 3.4 and 2.5 (markers D3S1589 and D3S3606 respectively) were found in a dataset of 121 Finnish T1D multiplex families. An association (transmission/disequilibrium test (TDT) p-value 0.0002) was found with a dataset of 384 Finnish T1D simplex families using marker AFM203wd10, located $~ 500 \mathrm{~kb}$ downstream of the linked marker D3S1589. In an attempt to confirm the observed association, we have analyzed six SNP markers at a region spanning $518 \mathrm{~kb}$ around the AFM203wd10.

Materials and methods: The analyzed SNP markers rs777498, rs12485336, rs11718344, rs17693752 are located upstream of the AFM203wd10 and markers rs10755080 and rs7649075 downstream of the AFM203wd10. The respective distances between above-mentioned markers and AFM203wd10 in kilobases are $-286,-118,-8.2,-1.4,+7$ and +232 . None of the marker alleles in any of the SNPs showed notable correlation with the AFM203wd10 alleles, $\mathrm{r}^{2}$ was less than 0.5 for every marker interval. The SNP markers were genotyped in both the original simplex family dataset and in an additional, independent set of 330 Finnish simplex families and TDT analysis was performed using TDTPHASE program. The additional dataset was genotyped for the AFM203wd10 microsatellite and analyzed for TDT.

Results: In the original dataset the SNP markers showed increased transmission in TDT, $(53 \%, 55 \%, 57 \%, 54 \%, 56 \%, 54 \%$ respectively towards downstream) but did not reach significance when tested individually and showed weaker association than the AFM203wd10 (64\%). Haplotypes of the SNP markers did show association of the same magnitude as the AFM203wd10 which rules out genotyping error as the source of the original finding. In contrast to the original dataset, the additional Finnish dataset of 330 simplex families did not show significantly increased transmission in AFM203wd10 (53\%) or in the SNPs $(52 \%, 54 \%, 54 \%, 53 \%, 51 \%, 50 \%$ respectively towards downstream). SNP haplotypes upstream of the AFM203wd10 did show significantly increased transmission (strongest p-value 0.0005) and certain three-marker haplotypes in consecutive markers rs12485336, rs11718344 and rs17693752 showed significant association in both datasets (not corrected for multiple testing). The Finnish multiplex dataset of 121 families showed close to nominal association in TDT when one sib in each family was used in the analysis $(58 \%, \mathrm{p}=0.07)$. The same allele as in the original simplex family dataset showed increased transmission, the combined association showed stronger significance than the original simplex families alone $(61 \%, \mathrm{p}=0.00007)$. Conclusion: Although this initial follow-up study on the 3q21-region did not undoubtedly confirm or exclude the existence of a T1D locus at this region in the Finnish population, it provided enough proof to warrant a more definitive association study to be carried out in an expanded genomic region with an extensive SNP marker coverage.

\section{8}

Containment of the susceptibility region Iddm8 and sequencing of functional relevant candidate genes in the LEW.1AR1-iddm rat, an animal model of human type 1 diabetes

T. Arndt ${ }^{1}$, H. Weiss ${ }^{2}$, A. Jörns ${ }^{1}$, S. Lenzen ${ }^{1}$, E. Cuppen ${ }^{3}$, H.J. Hedrich ${ }^{4}$, D. Wedekind ${ }^{4}$

${ }^{1}$ Hannover Medical School, Institute of Clinical Biochemistry, Germany, ${ }^{2}$ University of Rostock, Institute of Medical Biochemistry and Molecular Biology, Rostock, Germany, ${ }^{3}$ Hubrecht Institute, Functional genomics and bioinformatics, Utrecht, Netherlands, ${ }^{4}$ Hannover Medical School, Institute for Laboratory Animal Science, Germany

Background and aims: The LEW.1AR1-iddm rat is an animal model of human type 1 diabetes mellitus (T1DM) which arose through a spontaneous mutation within the MHC congenic inbred strain LEW.1AR1. Earlier genetic analyses using a (BN x LEW.1AR1-iddm) x LEW.1AR1-iddm backcross population (Diabetes incidence 6.5\%) revealed three diabetes susceptibility loci: Iddm8 and Iddm9 (RNO1q45-54, RNO1p11-q11) as well as Iddm1 $($ RNO20p12 = MHCII region). Linkage analysis of a (PAR x LEW.1AR1iddm) x LEW.1AR1-iddm backcross population (Diabetes incidence 13.5\%) confirmed two susceptibility regions (Iddm 1 und Iddm8). It was the aim of the study, (a) to narrow down the region Iddm8 using KASPar analysis, and (b) to sequence candidate genes in the region Iddm8 and Iddm9, to identify genes which are involved in T1DM pathogenesis.

Materials and methods: Fine mapping of the Iddm8 region was performed by SNP dependent KASPar (KBiosciences Allele Specific PCR) analysis to make use of both backcross populations. In parallel potential candidate genes were sequenced, which are also relevant for human T1DM and T2DM. Sequencing was performed with a 3730XL DNA (Applied Biosystems) sequencer and analysed using the PolyPhred program.

Results: With the aid of the SNP dependent KASPar method the identified susceptibility region Iddm 8 could be reduced from $40 \mathrm{Mb}$ to $3 \mathrm{Mb}$. Thereby a further containment of possible candidate genes could be achieved to reduce the length of the region for sequencing. Up to now 54 potential candidate genes were sequenced in total.

Additional sequencing showed that in the LEW.1AR1-iddm rat, genes which are mutated in other T1DM animal models, namely Ian5 (BB-DP rat) and cblb (KDP rat), are not affected. The AIRE gene (RNO 20), which is important for human T1DM pathogenesis, showed two SNPs in the coding region in comparison to the congenic strain LEW.1WR1.

Conclusion: Initially it was not possible to narrow down the susceptibility region Iddm8 on RNO1 of the LEW.1AR1-iddm rat using micro satellite markers. The KASPar method, however, offered a new possibility for fine mapping using single nucleotide polymorphisms (SNP). The smaller Iddm 8 region contains further genes, which have been considered as functionally relevant candidate genes for human T1DM, such as Anxal (Annexin 1) and Pdcd11 (programmed cell death 11). It is also interesting that in the Iddm 8 region some more genes could be identified, which are probably involved at the pathogenesis of human T2DM, such as HHEX (hematopoietically expressed homeobox) and TCF7L2 (transcription factor 7-like 2, T-cell specific). Thus, this approach provides the chance to identify the mutation responsible for T1DM development in the animal model. 


\section{PS 003 Monogenic diabetes}

\section{9}

Identification of a novel beta cell glucokinase (GCK) promoter mutation $(-71 G>C)$ which reduces promoter activity

D. Gasperikova ${ }^{1}$, N.D. Tribble ${ }^{2}$, J. Stanik ${ }^{1,3}$, M. Huckova ${ }^{1}$, N. Misovicova ${ }^{4}$, M. van de Bunt ${ }^{2}$, L. Kuklisova ${ }^{1}$, B. Barrow ${ }^{2}$, L. Barak ${ }^{5}$, R. Dobransky ${ }^{6}$, E. Bereczkova ${ }^{7}$, K. Colclough ${ }^{8}$, S. Ellard ${ }^{8}$, I. Klimes ${ }^{1}$, A.L. Gloyn ${ }^{2}$ ${ }^{1}$ DIABGENE \& Diabetes Laboratory, Institute of Experimental Endocrinology, Bratislava, Slovakia, ${ }^{2}$ Oxford Centre for Diabetes, Endocrinology \& Metabolism, University of Oxford, United Kingdom, ${ }^{3}$ Children Diabetes Centre at 1st Pediatric Department, Comenius University School of Medicine, Bratislava, Slovakia, ${ }^{4}$ Clinical Genetics, Jesenius School of Medicine, Martin, Slovakia, ${ }^{5}$ Children Diabetes Center at 1st Pediatric Department, Comenius University School of Medicine, Bratislava, Slovakia, ${ }^{6}$ Diabetelogy Outpatient Department, Reimanus Hospital, Presov, Slovakia, ${ }^{7}$ District Hospital, Children Endocrinology Outpatient Department, Dunajska Streda, Slovakia, ${ }^{8}$ Institute of Biomedical \& Clinical Sciences, Peninsula Medical School, Exeter, United Kingdom

Background and aims: Mutations in the gene encoding the $\beta$-cell enzyme glucokinase (GCK) cause maturity-onset diabetes of the young (MODY). Over 200 mutations have been identified in the coding region of the gene but no mutations to date have been found in the GCK promoter region. The aim of our study was to screen the GCK $\beta$-cell promoter region in 74 (68 SK + 6 UK) families with a phenotype suggesting a defect in glucokinase but with no abnormality of the GCK coding sequence.

Materials and methods: The $\beta$-cell GCK promoter was sequenced on an ABI 3130 and ABI 3700 respectively. The GCK promoter sequence ( 1000bp upstream of the ATG translation initiation site) was examined for $\beta$-cell specific transcription factor binding sites using TRANSFAC. The effect of the novel mutation on GCK promoter activity was assessed using site directed mutagenesis and a luciferase expression system (normalized to control renilla expression) in INS- 1 cells.

Results: A novel -71G $>C$ mutation in the $\beta$-cell GCK promoter was identified in 6 unrelated families from Slovakia $(n=5)$ and the UK $(n=1)$. Family testing established that the mutation co-segregated with elevated fasting plasma glucose levels $(>5.5 \mathrm{mmol} / \mathrm{l})$ in a total of 39 affected individuals. To determine the mechanism for this mutation, the human $\beta$-cell GCK promoter was examined for $\beta$-cell specific transcription factor binding sites. The novel - $71 \mathrm{G}>\mathrm{C}$ mutation maps directly to a potential transcriptional activator binding site for Sp1 and is only $4 \mathrm{bp}$ downstream of a USF2 site. The Pal- 1 consensus binding site, which has been previously shown in rodents to be essential for $\beta$-cell GCK expression, is only 10bp away. The full length promoter $(1031 \mathrm{bp})$ was required for maximal activity. Site directed mutagenesis $(-71 \mathrm{G}>\mathrm{C})$ in short $(-430 \mathrm{bp})$ and long $(-1031 \mathrm{bp})$ promoter sequences reduced promoter activity by 2 fold and 4 fold ( $\mathrm{p}=3.6 \times 10^{-6}$ and $1.9 \times 10^{-6}$ respectively). Interestingly, the decrease in activity seen between these fragments was highly significant $\mathrm{p}=3.4 \times 10^{-8}$ suggesting that this mutation potentially impacts on multiple regions.

Conclusion: We have identified a novel $(-71 \mathrm{G}>\mathrm{C})$ mutation in the $\beta$-cell GCK promoter which co-segregates with elevated fasting glucose levels in 6 unrelated families. This mutation significantly reduces the gene expression in vitro. To ensure correct diagnosis of potential GCK-MODY cases analysis of the $\beta$-cell GCK promoter should be performed.

Supported by: grants in Slovakia MZ.2005/15-NEDU-01, APVV-51-014205, CENDO, and BITCET and in Oxford by the Medical Research Council and Diabetes UK (ALG)

\section{0}

Variable clinical picture of mutations in the ABCC8 gene: can it be modified by the sensitivity to insulin or the islet-specific antibodies? T. Klupa ${ }^{1}$, I. Kowalska ${ }^{2}$, K. Wyka ${ }^{3}$, A.-M. Patch ${ }^{4}$, J. Skupien ${ }^{1}$, A. Noczynska ${ }^{5}$, S. Ellard ${ }^{4}$, A. Hattersley ${ }^{4}$, J. Sieradzki ${ }^{1}$, W. Mlynarski ${ }^{3}$, M.T. Malecki ${ }^{1}$ ${ }^{1}$ Department of Metabolic Diseases, Jagiellonian University, Krakow, Poland, ${ }^{2}$ Department of Endocrinology, Medical University of Bialystok, Poland, ${ }^{3}$ Department of Children's Diseases, Medical University of Lodz, Poland, ${ }^{4}$ Institute of Biomedical and Clinical Science, Peninsula Medical School, Exeter, United Kingdom, ${ }^{5}$ Department of Endocrinology, Medical University of Wroclaw, Poland

Background and aims: Mutations in the ABCC8 gene encoding the SUR1 subunits of the beta-cell KATP channel cause permanent neonatal diabetes mellitus (PNDM). In addition, they may also result in other diabetic phenotypes. The aim of the study was to determine the contribution of ABCC8 gene to neonatal diabetes in Poland and to examine factors that may influence its clinical phenotype

Materials and methods: The Nationwide Registry of Neonatal Diabetes in Poland includes patients diagnosed before 6 months of age. ABCC8 gene mutations were detected by direct sequencing. We also performed the hyperinsulinemic euglycemic clamp before and after sulphonylurea therapy and tested for islet-specific antibodies in diabetic mutation carriers.

Results: We sequenced ABCC 8 gene mutations in 16 Kir6.2 negative patients with neonatal diabetes, 14 permanent and 2 relapsed transient neonatal diabetes. We identified 4 diabetic probands (25\% of all examined cases) with heterozygous missense mutations: F132V, R826W, V86A; and one compound heterozygote with two, $\mathrm{N} 23 \mathrm{H}$ and R826W, mutations. Two mutations, F132V and V86A, occurred de novo in their carriers. In addition, a father of the R826W mutation carrier had adult onset diabetes. Three carriers of R826W and 2 carriers of $\mathrm{N} 23 \mathrm{H}$ mutations were non-diabetic (range 24-63 yrs). There were striking differences in the clinical picture of SUR1 related diabetes: the carrier of two mutations, $\mathrm{N} 23 \mathrm{H}$ and $\mathrm{R} 286 \mathrm{~W}$, was controlled on diet alone (HbAlc 7.2\%), while the F132V mutation carrier was on 47 units of insulin/ day (HbAlc 11.7\%). We performed the hyperinsulinemic euglycemic clamp in 3 diabetic subjects. Interestingly, we observed a variable degree of resistance to insulin: from moderate $(\mathrm{M}=5.5$ and $5.6 \mathrm{mg} / \mathrm{kg} / \mathrm{min}$, respectively, in the two R826 mutation carriers) to severe $(\mathrm{M}=2.6 \mathrm{mg} / \mathrm{kg} / \mathrm{min}$ in the $\mathrm{F} 132 \mathrm{~V}$ mutation carrier). In one R826 mutation carrier, a significant improvement in sensitivity to insulin was seen after the transfer to sulphonylurea (SU) with the M parameter increasing by $>3.0 \mathrm{mg} / \mathrm{kg} / \mathrm{min}$. In his father, with the same R826W mutation, there was a moderate improvement in insulin sensitivity ( $\mathrm{M}$ rose $1.1 \mathrm{mg} / \mathrm{kg} / \mathrm{min}$ ) and the daily insulin dose substantially reduced on SU, but could not be completely stopped. There was no response to SU treatment in the most insulin resistant F132V carrier of the mutation despite 6 months of high dose SUs ( $>1 \mathrm{mg} / \mathrm{kg}$ of glibenclamide). Auto-antibodies were only present in one diabetic mutation carrier, V86A, although they did not seem to influence the clinical picture as she transferred off insulin to SU.

Conclusion: Mutations in SUR1 are the second commonest cause of PNDM in Poland. The clinical phenotype and treatment response of SUR1 diabetic mutation carriers is very heterogenous. This appears to be modified by variable sensitivity to insulin and the presence of other variants in the gene. Although islet-specific antibodies are present in some mutation carriers, their role is uncertain.

Supported by: Polish Ministry of Science

\section{1}

Detection of the whole gene deletion mutation of the HNF-1b gene in Japanese patients with MODY

N. Iwasaki ${ }^{1}$, T. Yamamoto ${ }^{2}$, A. Watanabe ${ }^{3}$, M. Tsurumi ${ }^{4}$, K. Asai $^{4}$, K. Mizuno ${ }^{4}$, T. Shimada ${ }^{3}$, M. Ogata ${ }^{1}$, M. Takizawa ${ }^{1}$, Y. Iwamoto ${ }^{1}$ ${ }^{1}$ Diabetes Center, Tokyo Women's Medical University, ${ }^{2}$ International Research and Educational Institute for Integrated Medical Science, Tokyo Women's Medical University, ${ }^{3}$ Division of Clinical Genetics, Nippon Medical School, ${ }^{4}$ First department of Internal Medicine, Nippon Medical School, Tokyo, Japan

Background and aims: Mutations in the HNF- $1 \beta(H N F 1 B)$ gene are the cause of one form of maturity-onset diabetes of the young (MODY5). Genomic deletions of $H N F 1 B$ have recently been shown to account for one third of mutations causing renal cysts and diabetes syndrome. We have screened our panel of MODY patients with diabetes and kidney disease for deletion of HNF1B. Materials and methods: We studied 7 Japanese subjects with clinical diagnosis of MODY, and renal cysts or renal dysfunction without retinopathy. Direct sequencing showed no mutations in the coding region of $H N F 1 B$. We tested for complete and partial deletions of $H N F 1 B$ by using quantitative multiplex PCR of short fluorescent fragments (QMPSF) (Diabetes 2005, 3126). Then, we confirmed the deletion and determined its extent by comparative genome hybridization (CGH) using the Human Genome CGH micro-array 244A (Agilent, USA), which contains 243504 probes at a $6.4 \mathrm{~kb}$ interval, and with 8 probes in $H N F 1 B$.

Results: We found two subjects who were hemizygous for HNF1B by QMPSF. The following CGH Micro-array revealed that the deleted region was spanning $1.3 \mathrm{Mb}$ at $17 \mathrm{q} 12$ including $H N F 1 B$ as well as TRIP3, AATF, $A C A C A$, and LHX1. An exactly the same $1.3 \mathrm{Mb}$ region, that encompassed by the segmental duplications those known to be hot spots for genomic rearrangements was deleted in both subjects. The clinical features of the two 
patients were: patient 1 - non-obese male diagnosed with diabetes at 19 years of age, successfully treated with sulphonylureas, renal cysts, hyperuricemia, hepatic steatosis, hypercholesterolemia were pointed out. The ultrasonography revealed a pancreas with normal size. Father had diabetes; and patient 2 - a non-obese female presented with diabetic ketoacidosis at 24 years of age. The ultrasonography revealed renal cysts and a defect in the body and tail of the pancreas. The angiography disclosed the absence of a part of pancreatic artery indicating that this defect was caused by genetic malformation. The grand mother had diabetes.

Conclusion: We have identified two Japanese subjects with diabetes and renal cysts due to $1.3 \mathrm{Mb}$ deletion including $H N F 1 B$ out of 7 subjects studied. The cases show various phenotype other than renal cysts and diabetes. This study indicates the importance of screening for the deletion of HNF1B in subjects with diabetes and kidney disease showing no mutations by routine screening of $H N F 1 B$

Supported by: Grant-in-Aids for Scientific Research

\section{2}

Insulin gene mutations in children with diabetes who tested negative for T1D autoantibodies

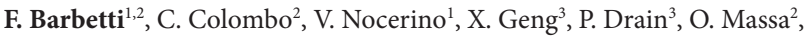
M. Viscardi ${ }^{4}$, D. Iafusco ${ }^{5}$, G. Chiumello ${ }^{4}$, F. Meschi ${ }^{4}$, R. Bonfanti ${ }^{4}$

${ }^{1}$ Internal Medicine, University of Tor Vergata, Rome, Italy, ${ }^{2}$ Laboratory of Molecular Endocrinology, Bambino Gesù Pediatric Hospital, Rome, Italy, ${ }^{3}$ Cell Biology and Physiology, University of Pittsburgh School of Medicine, Pittsburgh, United States, ${ }^{4}$ Pediatric Diabetes, H San Raffaele, Milan, Italy, ${ }^{5}$ Pediatric Diabetes, Second University of Naples, Italy

Background and aims: Heterozygous mutations of insulin (INS) gene are found in patients with neonatal/infancy-onset diabetes. We previously demonstrated that INS gene mutations cause permanent diabetes by endoplasmic reticulum stress-induced apoptosis of the beta cells. Probands of the Italian series who carry insulin mutations show diabetes in isolation with onset between 1 to 6 months from birth. However, the affected parent of a familial case bearing the R65C (or R89C) mutation presented with diabetes at 4 years of age. Aim of this work was to screen the INS gene in patients referred to HSR Paediatric Diabetes Clinic in the period 2003-2006, who tested negative for T1D autoantibodies.

Materials and methods: We identified 9 patients with diabetes onset above 1 year of age, who were previously classified as patients with T1D on clinical ground, but tested negative for ICA, GAD, IA-A2, IAA autoantibodies at diagnosis and -later on - for zinc transporter 8 (ZnT8) autoantibodies.

Results: We screened the INS gene in 7 patients whose genomic DNA was available. We detected two INS gene mutations: the already described GB8S (or G32S) and the novel Signal-A23S. The GB8S mutant had diabetes onset at $2 \mathrm{y}, 10 \mathrm{~m}$ of age with detectable $C$ peptide at outset $(0.49 \mathrm{ng} / \mathrm{ml})$, and after $2 \mathrm{y}(0.34 \mathrm{ng} / \mathrm{ml})$; presently, his insulin dose is $0.5 \mathrm{U} \mathrm{Kg}-1 \mathrm{~d}-1$. The individual with the Signal-A23S mutation presented with typical symptoms of diabetes (polyuria, polydipsia) when he was 6 y $8 \mathrm{~m}$ old $(\mathrm{HBA} 1 \mathrm{c}=11 \%)$. Insulin was started and continued for 6 months; during the following 2 years the patient went off/on insulin several times. Currently, he is on low insulin dose $(0.2 \mathrm{U}$ Kg-1 d-1). C-peptide measured 11 and 24 months after onset of hyperglycemia was 1.32 and $0.7 \mathrm{ng} / \mathrm{ml}$, respectively. We have engineered the insulin mutations causing diabetes into a human insulin fluorescent reporter, human Ins-C-GFP, and characterized alterations in its maturation, trafficking, and secretion in live beta cells caused by the mutations.

Conclusion: We conclude that INS gene mutations can be found in children previuosly classified as T1D who tested negative for five T1D autoantibodies and with detectable levels of C-peptide long after diabetes diagnosis.

\section{3}

\section{Searching for MODY-X}

R.M. Roccasecca ${ }^{1}$, A. Thompson ${ }^{1}$, K. Stals ${ }^{2}$, W. Bottomley ${ }^{1}$, C. Cordina ${ }^{1}$, S. Ellard ${ }^{2}$, A. Hattersley ${ }^{2}$, I. Barroso ${ }^{1}$

${ }^{1}$ Metabolic Disease Group, Wellcome Trust Sanger Institute, Cambridge, ${ }^{2}$ Institute of Biomedical and Clinical Sciences, Peninsula Medical School, Exeter, United Kingdom

Background and aims: Maturity Onset Diabetes of the Young is an autosomal dominant disease characterized by early onset of type 2 diabetes, usually before 25 years, caused principally by insulin secretion and action defects. So far, mutations or chromosomal rearrangements in six genes, GCK, IPF1, HNF1A, HNF4A, HNF1B and NEUROD1, have been identified as responsible for the disease. Most studies have identified mutations in HNF1A, GCK and $H N$ F4A as the most common causes of MODY although the prevalence of mutations in each gene varies across populations. In our study we investigated a cohort of MODY patients known not to have mutations in HNF1A, GCK and $H N F 4 A$ to investigate the prevalence of coding mutations in the rarer genes associated with MODY: IPF1, HNF1B and NEUROD1. We also sequenced four additional candidate genes: TCF7L2, NNT, PCIF1 and TMEM27 to further investigate the possibility of these genes being involved in this disease. Materials and methods: Genomic DNA from a cohort of 81 British patients diagnosed with MODY, and pre-screened for mutations in HNF1A, GCK and HNF4A, was PCR amplified using gene specific primers covering exons and splice junctions. PCR fragments were sequenced using Big Dye Terminator chemistry and mutations were confirmed on fresh genomic DNA aliquots. Sequence trace analysis was performed using Mutation Surveyor ${ }^{\mathrm{TM}}$ software. $^{\mathrm{T}}$ Results: In this cohort we detected mutations in HNF1B in 5\% of the patients, while the prevalence of mutations in NEUROD1 and IPF1 was $3 \%$ and $1 \%$, respectively. We detected two previously described amino acid substitution polymorphisms in TCF7L2 that were present in $14 \%$ of patients and three non-synonymous variants in NNT in a total of $11 \%$ of the patients. No coding variants were found in PCIF1 and TMEM27.

Conclusion: In a cohort of 81 MODY patients without mutations in the most common MODY genes (HNF1A, GCK, HNF4A) approximately 9\% of patients presented with mutations in HNF1B (5\%), NEUROD1 (3\%) or IPF1 (1\%). This highlights the importance of screening for mutations in these genes, once mutations in the more commonly mutated genes has been excluded as the likely cause of MODY. Our study also demonstrated that in the remaining $91 \%$ of our patients (MODY X) mutations in TCF7L2, NNT, PCIF1 and TMEM27 do not account for the observed phenotype.

\section{4}

Prevalence of maturity onset diabetes of the young (MODY) in Sardinian overweight/obese children not selected according to family history for diabetes

M.G. Baroni ${ }^{1}$, V.M. Cambuli ${ }^{1,2}$, S. Romeo $^{1}$, T. Congiu ${ }^{1}$, M. Incani ${ }^{1}$, F. Sentinelli ${ }^{1}$, S. Filetti ${ }^{2}$, M.C. Musiu ${ }^{3}$, M.G. Cavallo ${ }^{4}$, E. Cossu ${ }^{1}$, S. Loche ${ }^{3}$ ${ }^{1}$ Endocrinology and Metabolism, Department of Medical Sciences, University of Cagliari, Monserrato, ${ }^{2}$ Internal Medicine, Department of Clinical Sciences, University of Rome "La Sapienza", Rome, ${ }^{3}$ Paediatric Endocrine Unit, Regional Hospital for Microcitaemia, Cagliari, ${ }^{4}$ Division of Endocrinology, Department of Clinical and Medical Therapy, University of Rome "La Sapienza", Rome, Italy

Background and aims: Childhood obesity is increasing worldwide. In children and adolescents, overweight can accelerate the clinical onset of any type of diabetes. In case of excess of body weight, it is advisable to screen children for impaired glucose regulation (IGR). Of the various causes of IGR in children and adolescents, maturity onset diabetes of the young (MODY) needs to be considered. MODY represents a proportion (2-5\%) of the total cases of diabetes and it is characterized by an autosomal dominant mode of inheritance. The relative distribution of the different forms of MODY depends on the population investigated, although in all studies mutations in GCK and HNFlalfa are the two most prevalent, accounting for almost $80 \%$ of all cases in Caucasian populations. Nothing is known about the presence of MODY in Sardinian population, a region, together with Finland, with the highest prevalence of type 1 diabetes in the world. Aim of this study was to evaluate the prevalence of MODY in a cohort of overweight/obese children and adolescents affected by IGR (any subclasses: impaired fasting glycaemia, IFG; impaired glucose tolerance, IGT; diabetes mellitus, DM).

Populations and methods: We select subjects affected by IGR from a population of overweight/obese children and adolescents (age 4-18) consecutively screened by OGTT (1.75 g of glucose per kg, max $75 \mathrm{~g}$; samples at baseline and after 120 minutes). We recorded metabolic measures (glycaemia, insulinaemia, lipids etc.) for phenotyping of the subjects and further stratification. Once excluded type 1 diabetes by the presence of auto-antibodies, the subjects were screened for the two most prevalent forms of MODY, searching for GCK (MODY2) and HNF-1alfa (MODY3) mutations. This analysis was perform regardless of the presence of known diabetes in the families. Results: 49 children with IGR were identified. Of these, 33 presented IFG (67.3\%), 10 IGT (20.4\%), 3 combined glucose intolerance (CGI, 6.1\%), 3 diabetes $(6.1 \%)$. In 5 hyperglycaemic children (2 IFG, 1 IGT, 2 DM) we found autoimmunity against beta-cells. In particular, in the 2 children with diabetes 
both GADA and IA-2 were present. Once excluded this two cases with overt type 1 diabetes, we performed the genetic analysis in the other 47 children and adolescents: in 2 cases of IFG (4.25\%) we found HNF-1alfa mutations. One children (male, 12 years old, obese, pubertal) carried the Gly31Asp mutation on exon 1 . The other children (male, 7 years old, obese, pre-pubertal) carried the Thr354Met mutation on exon 5. These mutations were confirmed in the mothers of both children. No hyperglycaemic children were positive for GCK mutations.

Conclusion: Our data shown that MODY could be present in children also in absence of a known family history for diabetes and in presence of a typical type 2 diabetes phenotype. In our population MODY3 was present in $4.25 \%$ of cases. Further research in obese and/or normal weight children will be useful to clarify the prevalence of MODY within IGR/DM subjects in the Sardinian population.

\section{5}

The second "de novo" activating glucokinase mutation (V452L) in a patient with developmental delay

A.L. Cuesta-Muñoz ${ }^{1}$, T. Meissner ${ }^{2}$, N. Cobo-Vuilleumier ${ }^{1}$, M. Maringa ${ }^{3}$, M.A. García-Gimeno ${ }^{4}$, M.J. Castro-Santiago ${ }^{1}$, J.C. Aledo ${ }^{1}$, J. Weber ${ }^{5}$, P. Sanz ${ }^{6}$ ${ }^{1}$ Center for the Study of Pancreatic Beta-cell Diseases, IMABIS Foundation, Málaga, Spain, ${ }^{2}$ Department of General Pediatrics, University Children's Hospital Düsseldorf, Germany, ${ }^{3}$ Human Genetics Practice, Bonn, Germany, ${ }^{4}$ Institute of Biomedicine of Valencia, Consejo Superior de Investigaciones Científicas, Valencia, Spain, ${ }^{5}$ Integragen, Bonn, Germany, ${ }^{6}$ Institute of Biomedicine of Valencia, Consejo Superior de Investigaciones Científica, Valencia, Spain

Background and aims: The glycolytic enzyme Glucokinase (GK) is considered the "glucose sensor" in the pancreatic $\beta$-cells since it regulates glucose stimulated insulin secretion. Activating mutations in the glucokinase gene (GCK) result in Monogenic Hyperinsulinism (HI-GCK).

Materials and methods: The child was the first offspring of healthy German parents and no consanguinity. Family history was negative for hyperinsulinism or diabetes. There was marked macrosomia of the newborn with a birth weight of 5860g and a length of $60 \mathrm{~cm}$. At the third day of life the patient was admitted to the ICU of the local children hospital due to hypoglycemia and was treated with i.v. glucose. Hyperinsulinism suspected. At two weeks of age the i.v. glucose was stopped and changed to oral carbohydrates in addition to frequent feeding. The patient was finally discharged with diagnosis of "transient hyperinsulinism" without special diet or medication and with glucose concentrations of $2.6-3.3 \mathrm{mmol} / \mathrm{l}$. At the age of 3 years he was sent to the neurology outpatient clinic because of speech delay and mental retardation. Glucose monitoring revealed persistence of low glucose concentrations (2.5$3.6 \mathrm{mmol} / \mathrm{l}$ ) with low glucose levels particulary after the night fast.

Results: Genetic studies of the proband showed a novel activating mutation in GCK (V452L). Both, mother and father were negative for this mutation. Functional studies of V452L showed over 3 fold more affinity for glucose than that for Wild-type ( $2.5 \mathrm{vs.} 7.6 \mathrm{mmol} / \mathrm{l})$, and over 2.5 times more efficient (16.33 vs. $7.36 \mathrm{sec}^{-1} \mathrm{x} \mathrm{mmol}^{-1} \mathrm{x} \mathrm{l}^{-1}$ ). A fasting test revealed different insulin concentrations (0.2-6.7 mU/l) at 14,16 and 18 hours of fasting without severe hypoglycaemia, After 12 hours glucose was $46 \mathrm{mg} / \mathrm{dl}$ and at $18 \mathrm{~h}$ was $2.2 \mathrm{mmol} / \mathrm{l}$ (lowest glucose concentration). The OGTT revealed reactive hypoglycemia at $120 \mathrm{~min}(2.1 \mathrm{mmol} / \mathrm{l})$. With diazoxide treatment the mean and the morning glucose concentration increased.

Conclusion: This is the third case of a "de novo" GCK activating mutation out of ten described so far (30\%), and the second case of a patient with "de novo" HI-GCK activating with mental retardation. Therefore the high frequency of "de novo" GCK activating mutation along with the high risk of developing mental retardation means that any newborn with hypoglicemia not resolved in the first two weeks of life, should be tested for GCK mutations.

Supported by: MEC (P.I3); Dirección General de Investigación Cientifica y Técnica. Junta de Andalucía. Spanish Novo Nordisk Pharma

\section{6}

Structural changes in the repeated region of the carboxyl-ester lipase (CEL) gene and the development of diabetes

K. Fjeld ${ }^{1,2}$, J. Torsvik ${ }^{1,2}$, S. Johansson ${ }^{1}$, J. Ek ${ }^{3}$, S. Flanagan ${ }^{4}$, A. Johansen ${ }^{3}$, H. Ræder', O. Pedersen ${ }^{3}$, A.T. Hattersley ${ }^{4}$, T. Hansen ${ }^{3}$, A. Molven ${ }^{6,7}$, P.R. Njølstad ${ }^{1,5}$

${ }^{1}$ Department of Clinical Medicine, University of Bergen, Norway, ${ }^{2}$ Centre for Medical Genetics and Molecular Medicine, Haukeland University Hospital, Bergen, Norway, ${ }^{3}$ Steno Diabetes Center, Gentofte, Denmark, ${ }^{4}$ Institute of Biomedical and Clinical Sciences, Peninsula Medical School, Exeter, United Kingdom, ${ }^{5}$ Department of Pediatrics, Haukeland University Hospital, Bergen, Norway, ${ }^{6}$ Gades Institute, University of Bergen,

Norway, ${ }^{7}$ Department of Pathology, Haukeland University Hospital, Bergen, Norway

Background and aims: We have recently described a novel syndrome of exocrine and endocrine pancreatic dysfunction caused by mutations in a variable number of tandem repeats (VNTR) located in exon 11 of the carboxyl-ester lipase (CEL) gene. Sequencing of CEL has proven very challenging due to the repetitive nature of the GC-rich VNTR. The aims of this study were to develop a simple and robust method to search for mutations in the CEL VNTR and to use this method in the screening of diabetic subjects for $C E L$ exon 11 sequence variations.

Materials and methods: We developed an assay which consisted of a multiplex PCR followed by DNA fragment analysis. The method could reveal insertions/deletions in the proximal repeats of the CEL VNTR and enabled simultaneous determination of the total number of repeats for each allele. A material of 241 diabetic MODY probands (95 from Denmark and 146 from UK), who had tested negative for mutations in the common MODY genes, were screened with the method. Mutation analyses were also performed by DNA sequencing in all Danish samples to assure consistency of results. Southern blotting was used to analyze suspected CEL gene rearrangements detected by the assay.

Results: We did not find any new cases with single-base deletions in the proximal repeats of the CEL VNTR, suggesting that such mutations will remain a rare cause of MODY. In the Danish MODY material, we identified one family with a VNTR consisting of only three repeats, whereas the normal number is 12-20. Further investigation of the family showed that five of seven individuals with the short VNTR had diabetes and one had impaired glucose tolerance. Family members with normal VNTR length were normoglycemic. There was no co-segregation between genotype and exocrine dysfunction in the family. In the total material, we observed six individuals who had three different VNTR lengths revealed by fragment analysis. This suggests that some individuals harbor three copies of the CEL VNTR. By sequencing and Southern blot analysis we confirmed the existence of an additional copy of the CEL VNTR in both affected subjects and in healthy controls. In addition, we found evidence that the duplication may occur in at least two different forms and that it may involve the neighboring CEL pseudogene.

Conclusion: Frame-shift mutations in the CEL VNTR seem to be a rare cause of MODY. Our data indicate that alleles with very few VNTR repeats may predispose to diabetes in some families. Interestingly, we also found evidence of novel rearranged CEL alleles, both in normal subjects and in diabetes patients, and we are currently performing further studies to pinpoint the exact structure, size and possible functional implication of the rearrangements. Supported by: Translational Research Fund of the University of Bergen, Haukeland University Hospital, Innovest, Norwegian Research Council

\section{7}

Pancreatic exocrine dysfunction in diabetes: functional characterisation of the carboxyl-ester lipase gene

J. Torsvik ${ }^{1,2}$, B.B. Johansson ${ }^{1,2}$, S. Johansson ${ }^{1,2}$, J. Saraste ${ }^{3}$, P.R. Njølstad ${ }^{1,4}$, A. Molven ${ }^{5,6}$

${ }^{1}$ Department of Clinical Medicine, University of Bergen, ${ }^{2}$ Center for Medical Genetics and Molecular Medicine, Haukeland University Hospital, Bergen, ${ }^{3}$ Department of Biomedicine, University of Bergen, ${ }^{4}$ Department of Pediatrics, Haukeland University Hospital, ${ }^{5}$ Gade Institute, University of Bergen, ${ }^{6}$ Department of Pathology, Haukeland University Hospital, Bergen, Norway

Background and aims: We have described a novel syndrome of exocrine and endocrine pancreatic dysfunction caused by mutations in the carboxyl-ester lipase (CEL) gene. CEL is expressed mainly in the acinar tissue of the pan- 
creas. The protein is secreted from the pancreas into the digestive tract where it plays a role in cholesterol and lipid-soluble vitamin ester hydrolysis and absorption. We identified mutations in the last exon of $C E L$, localized in a coding region consisting of a variable number of tandem repeats (VNTR). Both mutations were single base deletions that lead to a frameshift, predicting a new protein $\mathrm{C}$-terminus. Patients with these mutations suffer from exocrine pancreatic dysfunction and diabetes with beta-cell failure. The pathogenic effect of the mutant CEL protein is unknown.

Materials and methods: The mutant and wild-type CEL genes were cloned in a mammalian expression vector and stably transfected into HEK293 cells. The recombinant proteins were studied using SDS-PAGE and Western blotting, and their subcellular distribution was analyzed by immunofluorescense. Results: Both the normal and the mutant CEL proteins were secreted from the HEK293 cells into the growth medium at high levels. Real-time PCR experiments showed that the mRNA levels of the two transfected genes were nearly identical. In cells expressing wild-type CEL, the protein tended to colocalize with the endoplasmatic reticulum (ER) and the Golgi compartment. However, in the cells expressing mutant CEL, the protein appeared to cluster in unidentified organelle-like structures in the cytoplasm and it also partly co-localized with the ER. One hypothesis is that the mutant protein follows a secretion route different from the classical ER to Golgi pathway used by wild type CEL. Experiments in which the protein synthesis is blocked by cycloheximide are ongoing to study whether the protein clusters are proteins that are marked for degradation or if they persist in the cytoplasm as a part of the secretion pathway of the protein.

Conclusion: Patients with a mutation in the VNTR of the CEL-gene suffer from diabetes and exocrine deficiency. The disease mechanism could involve impaired secretion or increased degradation of the protein. An alternative explanation is ER stress due to improper glycosylation as the wild type CEL protein is heavily $\mathrm{O}$-glycosylated in the $\mathrm{C}$-terminal end which is affected by the mutations.

Supported by: Translational Research Fund of the University of Bergen, Haukeland University Hospital, Innovest, Norwegian Research Council

\section{8}

Weight and glycaemic responses to 6 months Exenatide treatment in 9 Alström syndrome subjects with type 2 diabetes

R.B. Paisey, D. Hodge, L. Bower

Diabetes, Torbay Hospital, Torquay, United Kingdom

Background and aims: Alström syndrome is a rare autosomal recessive syndrome associated with insulin resistant young onset type 2 diabetes. Regular NHS sponsored clinics with Alström UK have allowed audit of antidiabeteic therapy.

Materials and methods: Local Research and Ethics permission was obtained to study insulin and glucose responses to meals in 9 Alström subjects with intractable obesity and poor glycaemic control, despite varying combinations of Metformin and Glitazones. Three had also received insulin. Exenatide was added, oral agents continued and insulin reduced to twice daily long acting preparations, then withdrawn after 3 months if possible.

Results: Severe hyperinsulinaemia was confirmed in all cases(C peptide levels 2006-7614 pmol/l, one hour after a high carbohydrate breakfast). All had intractable obesity and poor glycaemic control (weight $89.8+/-16.7 \mathrm{Kg}$, BMI $34.2+/-3.5$, and HbAlc $9.3+/-1.6 \%)$. All agreed with the addition of Exenatide to oral hypoglycaemic agents and reducing insulin dosage in 3 cases. After 6 months of 10 micrograms BD subcutaneous Exenatide there were significant improvements in weight and glycaemic control (weight $84.0+/-13.9 \mathrm{Kg}$, $\mathrm{P}<0.01$ : HbAlc $7.9+/-2.0 \%, \mathrm{P}=0.013$ ). The only significant adverse effect was nausea in 4 cases. Insulin was successfully withdrawn in two cases.

Conclusion: Significant weight reductions and improvement in glycaemic control were sustained for 6 months by the addition of Exenatide treatment in 9 type 2 diabetic Alström subjects with proven hyperinsulinism.

Supported by: National Clinical Services Group, Department of Health, UK, and Alström Syndrome UK

\section{PS 004 Single gene influence on type 2 diabetes risk}

\section{9}

The role of genetic variation in the cannabinoid type 1 receptor gene (CB1R) in the pathophysiology of human obesity

D. Schleinitz ${ }^{1}$, Y. Böttcher ${ }^{2}$, S. Carmienke ${ }^{2}$, A. Tönjes ${ }^{2}$, J. Berndt ${ }^{1}$,

N. Klöting ${ }^{2}$, K. Ruschke 2 , G.H. Scholz ${ }^{3}$, M. Blüher ${ }^{2}$, M. Stumvoll², P. Kovacs ${ }^{1}$

${ }^{1}$ Interdisciplinary Center for Clinical Research, University of Leipzig,

${ }^{2}$ Medical Department III, University of Leipzig, ${ }^{3}$ Medical Department I,

Hospital St. Elisabeth, Leipzig, Germany

Background and aims: The endocannabinoid system has been suspected to contribute to the association of visceral fat accumulation with metabolic diseases. Our recent findings suggest that abdominal fat accumulation is a critical correlate of the dysregulation of the peripheral endocannabinoid system in human obesity. Therefore, the cannabinoid type 1 receptor gene $(C B 1 R)$ was investigated as a plausible obesity candidate gene by testing whether genetic variation within the $C B 1 R$ might affect obesity and pathophysiologically relevant traits.

Materials and methods: The $C B 1 R$ was sequenced (exons, exon-intron boundaries, 5' and 3' UTRs) in DNA samples from 48 non-related Caucasian subjects to identify genetic variants. Eight variants were identified, 4 of which were novel. Seven variants including HapMap (www.hapmap.org) tagging single nucleotide polymorphisms (SNPs) and representing their linkage disequilibrium groups were genotyped for subsequent association studies in 2 independent cohorts with detailed metabolic testing: German Caucasians $\left(\mathrm{N}=1100\right.$; mean age $50 \pm 14$ years, mean BMI $\left.29.3 \pm 5.7 \mathrm{~kg} / \mathrm{m}^{2}\right)$ and an isolated population of Sorbs from Germany ( $\mathrm{N}=933$; mean age $48 \pm 16$ years, mean BMI $27.0 \pm 4.9 \mathrm{~kg} / \mathrm{m}^{2}$ ), totalling 2033 subjects.

Results: In a case control study including a subgroup of 304 lean $(\mathrm{BMI}<25 \mathrm{~kg} /$ $\left.\mathrm{m}^{2}\right)$ vs. 307 obese $\left(\mathrm{BMI}>30 \mathrm{~kg} / \mathrm{m}^{2}\right)$ subjects from the German Caucasian cohort, none of the SNPs was associated with obesity. However, in the entire study cohort of German Caucasians, several SNPs (rs806368, rs806369, rs806370, rs12720071) were significantly associated with obesity-related quantitative traits (\% body fat, serum leptin, fasting plasma insulin) (all $P<0.05$ after adjusting for age, sex and body mass index-BMI). Furthermore, in the isolated population of Sorbs, rs806368 was significantly associated with obesity in a case-control study including a subgroup of 344 obese and 211 lean Sorbs (adjusted $P<0.05$ ). Consistent with this, the rs 806368 also showed association with BMI and fasting plasma glucose (all adjusted $P<0.05$ ).

Conclusion: Although modest, the association of $C B 1 R$ genetic variants with obesity and pathophysiologically relevant traits in 2 independent populations indicates its potential role in the pathophysiology of human obesity. Since CB1 system is a known target for obesity treatment, our data provoke further studies to reveal pharmacogenetic aspects of the $C B 1 R$ genetic variation.

\section{0}

Variation in the transcription factor 7-like 2(TCF7L2) does not play a significant role in conferring type 2 diabetes risk in Japanese Americans D. Gable ${ }^{1}$, B. Pierce ${ }^{2}$, P. Talmud ${ }^{3}$, K. Lee ${ }^{3}$, S. Humphries', M. Austin ${ }^{2}$ ${ }^{1}$ Department of Diabetes and Endocrinology, St Mary's Hospital, London, United Kingdom, ${ }^{2}$ Department of Epidemiology and Institute for Public Health Genetics, School of Public Health and Community Medicine, Seattle, United States, ${ }^{3}$ Centre for Cardiovascular Genetics, University College London, United Kingdom

Background and aims: Specific alleles of a tetranucleotide repeat (DG10S478) in the TCF7L2 gene were originally found to be associated with type 2 diabetes in samples from Iceland, Denmark and the USA, explaining a high proportion of the cases (Genotype population attributable risk 21\%). This finding has since been replicated in Indian Asians and Black Afro-Caribbeans. This study aimed to estimate the impact of this variant in a sample of Japanese ancestry

Materials and methods: We determined DG10S478 genotype in a sample of 609 Japanese American Family Study [JAFS] participants. JAFS was designed to investigate risk factors for coronary heart disease, diabetes, and the insulin resistance syndrome in Japanese American families. The Megabace (Amersham Biosciences.UK) genotyping program was used to determine purified (Ampure magnetic bead system [Agentcourt MA. USA]) PCR product length. 
Results: The expected four alleles were seen but frequencies were significantly different to those reported in caucasian studies, the common allele being +8 rather than +0 . The frequency of the previously identified risk allele [not +0 ] was not different between those with and without type 2 diabetes. In the parental generation, compared to $[+0]$, the $[$ not +0$]$ allele was associated with an odds ratio of 2.39 [0.30-18.68: $\mathrm{p}=0.73$ ] for type 2 diabetes. By contrast, compared to the most common allele in this Japanese American sample, $[+8]$ the hazard ratio associated with the combined rare alleles [not +8$]$ was 0.82 [0.35-1.93: $\mathrm{p}=0.21]$. The frequency of the combination, "any allele other than the common allele", is much lower in Japanese Americans (0.06[0.03-0.05]) than that reported in caucasian studies (Range 0.24-0.34) [2;5]. A genotype population attributable fraction based on this odds ratio for the combined rare allele $[\mathrm{Not}+8]$ in this Japanese American sample is only $1.0 \%$.

Conclusion: Although, the previously identified risk allele [not +0$]$ and the rare alleles of this Japanese American sample, [not+8] may be associated with differences in T2DM risk, they have a much lower frequency in this ethnic group. The sequence variants [rs12255372, C>T; rs7903146G $>$ T] that mark the risk haplotype in Caucasians and Indian Asians are also much rarer in samples of Japanese ancestry. This means that variation in TCF7L2 is unlikely to contribute, to the same magnitude, to differences in genetic risk of T2DM in a population of Japanese Ancestry.

Supported by: British Heart Foundation/NIH

\section{1}

Fine-mapping type 2 diabetes causal variants on chromosome 9p21 in 2000 UK cases and 3000 unselected controls

A. Morris ${ }^{1}$, I. Prokopenko ${ }^{2}$, P. Akan ${ }^{3}$, H. Lango ${ }^{4}$, E. Zeggini' ${ }^{2}$, T.M. Frayling ${ }^{4}$, P. Deloukas ${ }^{3}$, A.T. Hattersley ${ }^{4}$, M.I. McCarthy ${ }^{2}$

${ }^{1}$ Genetic Epidemiology, Wellcome Trust Centre for Human Genetics, Oxford, United Kingdom, ${ }^{2}$ Type 2 Diabetes Group, Wellcome Trust Centre for Human Genetics, Oxford, United Kingdom, ${ }^{3}$ Genotyping Group, Wellcome Trust Sanger Institute, Hinxton, United Kingdom, ${ }^{4}$ University of Exeter, United Kingdom

Background and aims: One of the most interesting regions to emerge from the recent genome wide association scans for type 2 diabetes (T2D) lies on chromosome 9p21. The region is devoid of annotated genes, but is $\sim 100 \mathrm{~kb}$ from CDKN2A and $2 B$, and contains at least two independent signals for $\mathrm{T} 2 \mathrm{D}$, and one for coronary artery disease. The stronger signal of association with T2D ( $\mathrm{p}<10^{-7}$ in meta-analysis) mapped to a $\sim 10 \mathrm{~kb}$ interval flanked by two recombination hotspots. Multi-locus analysis of this region revealed a two-SNP haplotype (rs10811661 and rs10757283) that is more strongly associated with T2D $\left(\mathrm{p}=5.8 \times 10^{-8}\right)$ than either SNP alone. This suggests that these polymorphisms may tag a rarer risk haplotype which harbours a causative allele, or that there may be multiple causal variants in the region. Our aim is to dissect the haplotype association and fine-map causal variants within the region.

Materials and methods: To address this hypothesis, we have sequenced the $\sim 10 \mathrm{~kb}$ region flanking the associated two-SNP haplotype in a reference panel of 96 samples: 16 CEPH individuals and 80 T2D cases, 40 of them carrying the two high-risk alleles at rs10811661 and rs10757283. A total of 30 SNPs were identified, 5 of which are not present in Phase II HapMap. Haplotypes were reconstructed in the reference panel and subsequently used to impute genotypes at all 30 SNPs in the entire case-control sample. The GENEBPM algorithm was then used to jointly test for T2D association with all SNPs across the region by clustering haplotypes according to their similarity in terms of allelic-makeup and risk of carrying causal variants.

Results: There is overwhelming evidence of T2D association $\left(\log _{10}\right.$ Bayes' factor of 8.52), with haplotypes grouped into three distinct risk classes: highrisk haplotypes ( $26 \%$ frequency, posterior mean odds ratio of carrying causal variants of 20.6), low-risk haplotypes (15\% frequency, posterior mean odds ratio of 0.60 ), and neutral haplotypes (59\% frequency). The three clusters are separated by two sets of SNPs in the region that are not in linkage disequilibrium with each other, but that tag rs10811661 and rs10757283, respectively. Conclusion: Our results demonstrate that T2D risk in this region is most likely to be due to (at least) two independent causative mutations, although further work is required to determine which, if any, of the SNPs discovered by sequencing are functional.

Supported by: Wellcome Trust

\section{2}

CHOP T/C and C/T haplotypes contribute to early-onset type 2 diabetes in Italians

C. Gragnoli ${ }^{1,2}$, F. Chiaramonte ${ }^{3}$

${ }^{1}$ Lab. of Molecular Genetics of Complex \& Monogenic Disorders, Penn State University, Medicine \& Physiology, Hershey, United States, ${ }^{2}$ Center for Biotechnology \& Dept of Biology,Temple University, Sharro Institute for Cancer Research \& Molec. Med, Philadelphia, United States, ${ }^{3}$ Diabetology Unit, Santo Spirito Hospital, Rome, Italy

Type 2 diabetes (T2D) is characterized by impaired insulin secretion, insulin insensitivity and decreased beta cell mass. Multiple genes contribute to T2D. The chromosome 12q13.1 region is in linkage to T2D in different populations, including our Italians dataset. $\mathrm{CHOP}$ is a candidate gene for the linkage, as it is located in the chromosome 12q13.1 region, and may contribute to T2D by increasing beta cell apoptosis susceptibility and by impairing insulin sensitivity. Our goal was to identify any potential $C H O P$ gene variants contributing to T2D in our Italian early-onset T2D families, which show linkage to the $C H O P$ region. We directly sequenced the $C H O P$ gene in 28 Italian probands of the linked T2D families and in 115 control subjects. We performed genotype and haplotype association tests with T2D of the identified SNPs. We performed model-free and parametric association haplotype tests with T2D. We identified three SNPs (5'UTR-c.279T $>$ C, 5'UTR-c.120A $>$ G and + nt $30 \mathrm{C}>\mathrm{T}$ (F10F) in CHOP. These SNPs are in LD. The association test showed an association trend with T2D of the TT (F10F) and AG (-c.120A>G) genotypes. The haplotype association test resulted significant for the haplotypes $\mathrm{T} / \mathrm{C}$ (frequency=0.33) and C/T (frequency=0.01) (at 5'UTR-c.279T $>\mathrm{C}$ and $+n t 30 \mathrm{C}>\mathrm{T}$, respectively) under non-parametric analysis ( $\mathrm{p}$-value $=0.000)$, the recessive model $(\mathrm{p}$-value $=0.0000)$ and the additive model $(\mathrm{p}$-value $=0.0014)$. Our data show that $\mathrm{CHOP}$ described haplotypes $\mathrm{T} / \mathrm{C}$ and $\mathrm{C} / \mathrm{T}$, as a single additive and as a homozygous variant, contribute significantly to T2D in our Italian early-onset group. We conclude that the CHOP T/C and C/T haplotype contributes to our T2D linkage signal on chromosome 12q13.1

\section{3}

Genetic risk of development of posttransplantation diabetes mellitus (PTDM) in renal allograft recipients

E. Kang ${ }^{1}$, M. Kim ${ }^{2}$, H. Kim ${ }^{3}$, C. Nam ${ }^{4}$, C. Ahn ${ }^{1}$, B. Cha ${ }^{1}$, S. Kim², H. Lee ${ }^{1}$ ${ }^{1}$ Internal Medicine, Yonsei University College of Medicine, Seoul, ${ }^{2}$ Surgery, Yonsei University College of Medicine, Seoul, ${ }^{3}$ Internal Medicine, Kwandong University College of Medicine, Gangneung, ${ }^{4}$ Preventive Medicine and Public Health, Yonsei University College of Medicine, Seoul, Republic of Korea

Background and aims: Posttransplantation diabetes mellitus (PTDM) is a major metabolic complication in renal transplant recipients. Recent genome wide association studies have revealed several genes associated with type 2 diabetes in Caucasian populations. We examined the association of 13 single nucleotide polymorphisms (SNPs) within 6 genes in renal allograft recipient cohort. Materials and methods: 624 kidney recipients transplantation from 1989 to 2007, without a history of diabetes, and with a pretransplant fasting plasma glucose $<126 \mathrm{mg} / \mathrm{dL}$ were Included in the study. We analyzed 13 SNPs (rs4402960 in IGF2BP2, rs10811661 in CDKN2A/B, rs1111875, rs7923837, rs5015480 in HHEX, rs13266634 in SLC30A8, rs11196205, rs4506565, rs12243326, rs7903146, rs12255372, rs7901695 in TCF7L2, and rs734312 in WFS1) in 174 patients with PTDM and 450 patients with non-PTDM by using TaqMan real-time PCR.

Results: Among 13 SNPs examined, 5 loci were significantly associated with PTDM (rs1111875 in HHEX, $\mathrm{p}<0.001$; rs7923837 in HHEX, $\mathrm{p}=0.009$; rs5015480 in HHEX, $\mathrm{p}=0.005$; rs13266634 in SLC30A8, $\mathrm{p}=0.007$; rs7903146 in TCF7L2, $\mathrm{p}=0.029$ ). Four SNPs remain significant after adjustment for age, gender, body weight gain, and type of immunosuppressant (rs1111875 in HHEX, hazard ratio (HR) $=1.35, \mathrm{p}=0.006$; rs5015480 in HHEX, $\mathrm{HR}=1.13$, $\mathrm{p}=0.026$; rs 13266634 in SLC30A8, HR=1.32, $\mathrm{p}=0.013$; rs7903146 in TCF7L2, $\mathrm{HR}=1.45, \mathrm{p}=0.039$ ). Rs4402960 in IGF2BP2, rs10811661 in $C D K N 2 A / B$, and rs734312 in WFS1 were not associated with PTDM ( $\mathrm{p}=0.075, \mathrm{p}=0.115$, $\mathrm{p}=0.810$ respectively).

Conclusion: These data provide evidence that the SLC30A8, HHEX, TCF7L2 genetic variations are associated with PTDM and rs1111875 in HHEX was the most powerful genetic predictor for PTDM development in renal allograft recipients.

Supported by: Global 5-5-10 Project, Yonsei University 


\section{4}

Analyses of $S 100 B$ rs $2300403 \mathrm{~T} / \mathrm{C}$ contribution to T2D in a large population based-study - the HUNT study

P.M. Thorsby ${ }^{1,2}$, J.-C. Lambert ${ }^{3}$, K. Midthjell ${ }^{4}$, N. Gjerlaugsen ${ }^{1}$, J. Holmen ${ }^{4}$, K.I. Birkeland ${ }^{5,2}$, A. Meirhaeghe ${ }^{3}$

${ }^{1}$ Aker University Hospital, Hormone Laboratory, Oslo, Norway, ${ }^{2}$ Aker University Hospital, Aker- Ullevål Diabetes Research Centre, Oslo, Norway, ${ }^{3}$ Institut Pasteur de Lille, INSERM U744, Lille, France, ${ }^{4}$ Norwegian University of Science and Technology, HUNT Research Centre, Verdal, Norway, ${ }^{5}$ Aker University Hospital, Department of Endocrinology, Oslo, Norway

Aims: The $S 100 B$ gene, a glial-derived protein, is associated with beta-amyloid and phospho-tau accumulation and Alzheimer's disease. It also influences $\beta$ cell destruction. We wanted to study whether the polymorphism rs 2300403 in $S 100 B$, shown to be associated with the risk of Alzheimer's disease, could be associated with type 2 diabetes or related clinical parameters.

Methods: 65,905 individuals above 20 years of age participated in a health survey in Nord-Trøndelag county in Norway (HUNT 2) in 1995-97. We studied a population-based randomly selected group of 869 individuals with self-reported diabetes or non-fasting serum glucose above $11.1 \mathrm{mmol} / \mathrm{l}$, and 2,080 non-diabetic control subjects with serum glucose below $5.5 \mathrm{mmol} / \mathrm{l}$. The study was powered to detect differences in allele frequencies as low as $5 \%$. The S100B rs 2300403 T/C polymorphism was studied (TaqMan ${ }^{\circ}$ SNP genotyping assays in a two color real-time PCR), and traditional clinical risk factors were investigated cross-sectionaly.

Results: The cases were slightly older than the controls ( $66.8 \pm 13.7$ vs. 65.6 \pm 14.5 years, $\mathrm{p}=0.031$ ). The clinical characteristics of cases and controls from HUNT 2 are displayed in the table below.

\begin{tabular}{llll}
\hline & Type 2 diabetes & Control subjects & p \\
\hline${\text { Gender }(\mathrm{female} / \mathrm{male})^{*}}^{*}$ & $430 / 439$ & $1056 / 1024$ & $\mathrm{~ns}$ \\
\hline BMI $\left(\mathrm{kg} / \mathrm{m}^{2}\right)$ & $29.1(5.0)$ & $26.4(3.9)$ & $<\mathbf{0 . 0 0 1}$ \\
\hline Waist $(\mathrm{cm})$ & $96.2(12.5)$ & $87.9(11.2)$ & $<\mathbf{0 . 0 0 1}$ \\
\hline W/H ratio & $0.90(0.08)$ & $0.86(0.08)$ & $<\mathbf{0 . 0 0 1}$ \\
\hline SBP $(\mathrm{mm} \mathrm{Hg})$ & $152.8(25.5)$ & $144.8(24.2)$ & $<\mathbf{0 . 0 0 1}$ \\
\hline DBP $(\mathrm{mm} \mathrm{Hg})$ & $83.8(13.7)$ & $82.3(12.7)$ & $\mathbf{0 . 0 0 4}$ \\
\hline S-Cholesterol $(\mathrm{mmol} / \mathrm{l})$ & $6.1(1.3)$ & $6.3(1.3)$ & $\mathbf{0 . 0 0 6}$ \\
\hline S-HDL $(\mathrm{mmol} / \mathrm{l})$ & $1.2(0.4)$ & $1.4(0.4)$ & $<\mathbf{0 . 0 0 1}$ \\
\hline S-TG $(\mathrm{mmol} / \mathrm{l})$ & $2.5(1.8)$ & $1.7(0.9)$ & $<\mathbf{0 . 0 0 1}$ \\
\hline
\end{tabular}

The allele frequencies of the $S 100 B$ rs 2300403 polymorphism was 0.69 for the $\mathrm{T}$ allele and 0.31 for the $\mathrm{C}$ allele. There was no difference in frequencies between T2D and controls $\left(\chi^{2}=0.79\right)$, even when testing cases against the oldest third of the controls (age $>74$ years, $\chi^{2}=0.86$ ). The T allele was significantly associated with increased glucose in the control group compared with CC subjects $(4.99(0.42) \mathrm{mmol} / \mathrm{l}$ vs $4.91(0.48)$, respectively, $\mathrm{p}=0.014)$. No other clinical parameter (SBP, DBP, BMI, W/H ratio, Cholesterol, HDL or TG) showed association with this polymorphism.

Conclusion: The $S 100 B$ rs 2300403 polymorphism was not associated with T2D but it might influence glucose metabolism.

Supported by: Hormone Laboratory and Aker University Hospital

\section{5}

Impact of transcription-factor 7-like 2 (TCF7L2) gene-variants on the response to anti-hyperglycaemic treatment in non-obese patients with type 2 diabetes

S.S. Lund $^{1}$, L. Tarnow ${ }^{1}$, C.D.A. Stehouwer ${ }^{2}$, C.G. Schalkwijk ${ }^{3,2}$,

M. Frandsen ${ }^{1}$, U.M. Smidt ${ }^{1}$, T. Hansen ${ }^{1}$, O. Pedersen ${ }^{1}$, H.-H. Parving ${ }^{4}$, A.A. Vaag $^{1}$

${ }^{1}$ Steno Diabetes Center, Gentofte, Denmark, ${ }^{2}$ Department of Internal Medicine, University Hospital Maastricht, Netherlands, ${ }^{3}$ Department of Clinical Chemistry, University Medical Centre, Amsterdam, Netherlands, ${ }^{4}$ Department of Medical Endocrinology, Rigshospitalet, Copenhagen, Denmark

Background and aims: Common TCF7L2 gene-variants have been associated with type-2-diabetes (T2DM) (rs7903146 and rs12255372) and obesity (rs10885406). In non-obese T2DM-patients, we studied the impact of TCF7L2 gene-variants on anti-hyperglycaemic (Haemoglobin $\left.\mathrm{A}_{1 \mathrm{c}}\left(\mathrm{HbA}_{1 \mathrm{c}}\right)\right)$ and anti-inflammatory (high-sensitivity C-reactive-protein (hsCRP)) efficacy of metformin or repaglinide targeting insulin-resistance and insulin-secretion, respectively.

Materials and methods: Randomized, double-masked, $2 \times 4$ months crossover-study of metformin $1 \mathrm{~g}$ twice-daily versus repaglinide $2 \mathrm{mg}$ thrice-daily in 96 non-obese (BMI $\left.\leq 27 \mathrm{~kg} / \mathrm{m}^{2}\right)$ insulin-naïve Caucasian T2DM-patients (rs10885406: AA: $n=17$; AG: $n=60$; GG: $n=19$; rs7903146: TT: $n=8$; TC: $n=58$; CC: $n=30$; rs12255372: GG: $n=36$; GT: $n=55$; TT: $n=5$ ).

Results: Compared with AA-homozygotes, rs 10885406 G-allele carriers had lower $\mathrm{HbA}_{1 \mathrm{c}}$ during metformin treatment (Mean [95\%CI] $\mathrm{HbA}_{1 \mathrm{c}}$-difference during metformin: G-allele carriers versus AA-homozygotes: $-0.52 \%$ [-0.97; -0.06], $\mathrm{p}=0.026)$. In rs10885406 G-allele carriers, rs12255372 T-allele carriers and rs7903146 TT-homozygotes, hsCRP levels were lower during metformin versus repaglinide treatments (Mean [95\%CI] relative hsCRP difference metformin versus repaglinide, rs10885406 G-allel-carriers: $-26 \%$ $[-42 ;-4] ; \mathrm{p}=0.024 ;$ rs 12255372 T-allele carriers: $-31 \%[-49 ;-6] ; \mathrm{p}=0.017$; and rs7903146 TT-homozygotes: $-58 \%[-81 ;-7] ; \mathrm{p}=0.033)$. Compared with AA-homozygotes, rs10885406 G-allele carriers had increased hsCRP levels during repaglinide treatment (Mean $[95 \% \mathrm{CI}]$ relative hsCRP difference during repaglinide, rs 10885406 G-allele carriers versus AA-homozygotes: $76 \%$ $[9 ; 185], \mathrm{p}=0.021)$. Treatment-by-TCF7L2-genotype interactions were significant for hsCRP ( $r$ 10885406: $\mathrm{p}=0.029$; $r$ 12255372: $\mathrm{p}=0.046$; and rs7903146: $\mathrm{p}=0.027$ ), but not for $\mathrm{HbA}_{1 c}$.

Conclusion: In non-obese T2DM-patients, common TCF7L2 gene-variants might influence anti-hyperglycaemic treatments responses. Metformin may have improved glycaemic and/or anti-inflammatory effects in rs 10885406 G-allele carriers, rs12255372 T-allele carriers or rs7903146 TT-homozygotes, whereas repaglinide may have improved anti-inflammatory effects in rs10885406 AA-homozygotes.

Supported by: Novo Nordisk A/S; Danish Diabetes Association; Clinical Development Foundation at Steno Diabetes Center, Denmark

\section{6}

Protective role of the rare genetic variant of the hypoxia inducible factor1 alpha (HIF1a) in type 2 and type 1 diabetes

G. Nagy ${ }^{1}$, R. Nagy ${ }^{2}$, A. Székely ${ }^{3}$, N. Németh ${ }^{2}$, M. Sasvári-Székely², A. Somigyi ${ }^{1}$

${ }^{1}$ II. Department of Internal Medicine, Semmelweis University, ${ }^{2}$ Department of of Medical Chemistry, Molecular Biology and Pathobiochemistry, Semmelweis University, ${ }^{3}$ Department of Psychiatry, Eötvös Loránd University, Budapest, Hungary

Background: Hypoxia inducible factor-1 alpha (HIF1a) is a transcription factor known to increase the synthesis of the vascular endothelial growth factor (VEGF) under hypoxic conditions. By influencing VEGF levels, HIF1a is thought to play an important role in neo-vascularisation, embryonic pancreas beta-cell mass development, and beta cell function. A genetic association analysis of the HIF1a gene polymorphism performed on a Japanese sample raised the possibility that the P582S (a C $\rightarrow \mathrm{T}$ change) functional polymorphism found in the coding region HIFla gene could be a protective factor against type 2 diabetes mellitus (T2DM).

Aims: Distribution of different genetic variants may significantly vary between different populations. Thus our aim was to determine the frequency of the P582S $(\mathrm{C} \rightarrow \mathrm{T})$ mutation in a control $(\mathrm{K})$, a T2DM and a type 1 diabetic (T1DM) sample in a Caucasian (Hungarian) sample.

Materials and methods: 367 T2DM and 71 T1DM and 341 sex matched $\mathrm{K}$ subjects were recruited. Using a non-invasive sampling technique, buccal cells were collected for DNA isolation. Genotyping was performed by real time PCR using TaqMan probes.

Results: Frequency of the C allele was more pronounced in both T1DM (90.8\%) and T2DM (90.9\%) as compared to controls (86.8\%). Statistical analysis of allele-wise distribution in the three groups yielded significant results: $\chi^{2}(2)=6.515, p=0.038$. The CC genotype was more frequent in both patient groups ( $81.7 \%$ in T1DM and 82.3 in T2DM) than in the control group (75.7\%). The CT genotype was less frequent in the patient groups (18.3\% in T1DM and 17.2 in T2DM) as compared to the $22.3 \%$ in controls. The TT genotype was extremely rare in the population $(0.0 \%$ in T1DM, $0.5 \%$ in T2DM and $2 . \%$ in controls) therefore the genotypes were grouped as T absent (CC) and $\mathrm{T}$ present (CT and TT) for genotype-wise statistical analysis. Frequency of the $\mathrm{T}$ absent and $\mathrm{T}$ present groups were compared in the patient (including T1DM and T2DM) and control groups. The T absent genotype was significantly more frequent in the patient group $(82.2 \%)$ as compared to the controls $(75.7 \%) \chi^{2}(1)=4.989, p=0.026$. 
Conclusion: Genotype and allele frequency analysis confirmed the possible protective role of the HIF1 $\alpha \mathrm{T}$ allele against T2DM in a Caucasian population. Allele frequency analysis also suggested that the $\mathrm{T}$ allele might also be a protective factor in T1DM. Our results confirm the possible role of the HIF1a gene in the pathomechanism of diabetes.

Supported by: Hungarian funds ETT - 448/2006 (Somogyi A)

\section{7}

Glucose 6 phosphatase beta cell specific subtype/IGRP gene variation controls fasting plasma glucose levels in humans

N. Bouatia-Naji ${ }^{1}$, G. Rocheleau ${ }^{2}$, L. Van Lommel ${ }^{3}$, K. Lemaire ${ }^{3}$, F. Schuit ${ }^{3}$, M. Marre ${ }^{4}$, B. Balkau ${ }^{5}$, M.-R. Jarvelin 6 , R. Sladek ${ }^{2}$, P. Froguel ${ }^{1}$

${ }^{1}$ Genomics and Molecular Physiology of Metabolic Diseases, Lille, France, ${ }^{2}$ Department of Human Genetics, Faculty of Medicine, McGill University and Génome Québec Innovation Centre, Montreal, Canada, ${ }^{3} \mathrm{Gene}$ Expression unit Dept. Molecular Cell Biology, Katholieke Universiteit Leuven, Leuven, Belgium, ${ }^{4}$ Bichat Hospital, Paris, France, ${ }^{5}$ Paris Sud University, Villejuif, France, ${ }^{6}$ Department of Epidemiology and Public Health, Imperial College London, United Kingdom

Background and aims: Non-diabetic individuals with fasting plasma glucose (FPG) levels at the high end of the normal range have a higher risk of cardiovascular mortality.

Materials and methods: To identify genetic determinants that contribute to inter-individual variation in FPG, we first tested 392,935 Single Nucleotide Polymorphisms (SNPs) in 654 normoglycemic subjects for association with FPG.

Results: We replicated the most strongly associated SNP $\left(\mathrm{rs} 560887, \mathrm{p}=4 \times 10^{-7}\right)$ in an additional 9,353 subjects. SNP rs560887 maps to intron 3 of the G6PC2 (glucose-6-phosphatase catalytic subunit-related protein 2) gene which is selectively expressed in pancreatic islets (also known as IGRP, an auto antigen in T1D). This SNP is associated with FPG ( $\beta=-0.06 \mathrm{mmol} / \mathrm{l}$ per A-allele, combined $\mathrm{p}=4 \times 10^{-23}$ ) and with pancreatic beta-cell function (Homa-B model; combined $p=3 \times 10^{-13}$ ) in three European cohorts, although it is not associated with T2D risk. However, rs560887 A allele carriers have a lower risk of developing $\mathrm{FPG} \geq 6.1 \mathrm{mmol} / \mathrm{l}$ over a 9-year follow-up period (Hazard ratio $=0.83$, $\mathrm{p}=0.009$ ) suggesting that $66 P C 2 /$ IGRP may regulate FPG by modulating the set-point for glucose-stimulated insulin secretion in beta-cells. Interaction analysis of rs560887 with the glucokinase regulatory (GCKR) variant rs1260326-P446L and the glucokinase $(G C K)$ variant rs1799884/-30G/A in the D.E.S.I.R cohort showed an additive effect of the three SNPs on FPG $\left(\mathrm{p}=5 \times 10^{-21}\right)$. Individuals carrying more than four alleles associated with low FPG (10.7\% of the D.E.S.I.R population), show a mean $0.24 \mathrm{mmol} / \mathrm{l}(4.5 \%)$ decrease of FPG compared to subjects carrying only one allele.

Conclusion: In conclusion we identified the G6PC2/IGRP gene as a major regulator of glucose levels which supports further the role of various components of the glucose phosphorylation pathway in glucose homeostasis Supported by: ALFEDIAM-Servier, EURODIA, Genome Canada/Genome Quebec, KU-Leuven, JDRF, Imperial College London. and the Academy of Finland

\section{PS 005 Multiple gene influence on type 2 diabetes risk}

\section{8}

Role of TCF7L2 and HHEX/IDE risk alleles in the pathophysiological and clinical phenotype of newly diagnosed type 2 diabetic patients S. Bonetti ${ }^{1}$, E. Trabetti ${ }^{2}$, M. Trombetta ${ }^{1}$, G. Malerba ${ }^{2}$, L. Boselli ${ }^{1}$, M. Muggeo ${ }^{1}$, P. Pignatti ${ }^{2}$, E. Bonora ${ }^{1}$, R.C. Bonadonna ${ }^{1}$ ${ }^{1}$ Department of Biomedical and Surgical Sciences, ${ }^{2}$ Department of Mother, Child, Genetics, University of Verona School of Medicine, Verona, Italy

Background: Several SNP variants which carry an increased risk for type 2 diabeets (T2DM) have been identified by recent GWA studies. Among them, the risk alleles T of rs7903146 and G of rs1111875 are related to TCF7L2 and HHEX genes, respectively, . and these, in turn, are both involved in the Wnt pathway which plays a role in pancreas organogenesis. Some studies have reported a role of these risk alleles in determining beta-cell function $(\beta F)$ in nondiabetic people.

Aims: We tried to assess the role played by rs7903146 and rs1111875 in the pathophysiological and clinical phenotype of patients with newly diagnosed T2DM.

Research methods: In 345 drug-naive GAD-antibody negative patients with newly diagnosed T2DM (age: $58 \pm 0.5$ yrs; BMI: $30.4 \pm 0.3 \mathrm{~kg} / \mathrm{m}^{2}$; HbAlc: $6.9 \pm 0.1 \%$; mean \pm SEM) we performed an OGTT for $\beta \mathrm{F}$ and a standard euglycemic insulin clamp to assess insulin sensitivity (SI) (M value; $605 \pm 19 \mu \mathrm{mol}$ $\mathrm{min}^{-1} \cdot \mathrm{m}^{-2} \mathrm{BSA}$ ), on separate days. OGTT plasma glucose (G) and C-peptide concentration curves were analyzed by state-of-art mathematical modeling to quantify basal insulin secretion rate (B-ISR: $1.06 \pm 0.02 \mathrm{Uhr}^{-1} \cdot \mathrm{m}^{-2} \mathrm{BSA}$ ), and the sensitivity of $\beta$-cells to both the rate of increase of $\mathrm{G}$ (derivative or dynamic control; $583 \pm 34\left[\mathrm{pmolm}^{-2}\right]$ per $\left.[\mathrm{mM} / \mathrm{min}]\right)$ and to $\mathrm{G}$ concentration (proportional or static control; $51.3 \pm 2.6\left[\mathrm{pmol} \cdot \mathrm{min}^{-1} \cdot \mathrm{m}^{-2} \mathrm{BSA}\right]$ per $[\mathrm{mmol} / \mathrm{l}]$ ). Rs7903146 and rs1111875 were genotyped and found in H-W equilibrium.

Results: The T allele of rs7903146 in homozygosity was associated to significant reductions in B-ISR $\left(\mathrm{C} / \mathrm{C}, \mathrm{n}=123: 1.12 \pm 0.04 \mathrm{Uhr}^{-1} \mathrm{~m}^{-2} \mathrm{BSA} ; \mathrm{C} / \mathrm{T}\right.$, $\mathrm{n}=164: 1.06 \pm 0.03 ; \mathrm{T} / \mathrm{T}, \mathrm{n}=59: 0.95 \pm 0.04 ; \mathrm{p}<0.05$ by MANOVA) and in fasting triglycerides (C/C: $1.8 \pm 0.1 \mathrm{mmol} / \mathrm{l} ; \mathrm{C} / \mathrm{T}: 1.8 \pm 0.1 ; \mathrm{T} / \mathrm{T}: 1.3 \pm 0.1 ; \mathrm{p}<0.05$ by MANOVA). Rs111875 genotype showed significant associations $(\mathrm{p}<0.05$ by MANOVA) with waist circumference and SI, and suggestive trends for $\beta \mathrm{F}$, but all these associations disappeared when covariates were taken into account.

Conclusion: In newly diagnosed T2DM patients: 1. rs7903146 risk allele T of TCF7L2 is associated with reduced basal insulinization, but with less burden of atherogenic dyslipidemia; 2. definition of a role, if any, of rs 1111875 genetic variability in determining the pathophysiologic phenotype requires more extensive and detailed studies.

Supported by: University of Verona Italian Ministry of University and Research

\section{9}

Direct evidence to support the fetal insulin hypothesis as the type 2 diabetes risk alleles at the CDKAL1 and HHEX-IDE gene loci reduce birth weight

A.T. Hattersley ${ }^{1}$, R.M. Freathy ${ }^{1}$, A.J. Bennett ${ }^{2}$, S.M. Ring ${ }^{3}$, N.J. Timpson ${ }^{4,5}$, A. Pouta ${ }^{6}$, A. Ruokonen ${ }^{6}$, E. Hypponen ${ }^{7}$, C. Power ${ }^{7}$, P. Elliott ${ }^{8}$, D.P. Strachan ${ }^{9}$, M.-R. Jarvelin ${ }^{6,8}$, G. Davey Smith ${ }^{3,5}$, M.I. McCarthy ${ }^{2,4}$, T.M. Frayling ${ }^{1}$ ${ }^{1}$ Diabetes Genetics and Genetics of Complex Traits, Peninsula Medical School, Exeter, United Kingdom, ${ }^{2}$ Oxford Centre for Diabetes, Endocrinology and Metabolism, University of Oxford, United Kingdom, ${ }^{3}$ Department of Social Medicine, University of Bristol, United Kingdom, ${ }^{4}$ Wellcome Trust Centre for Human Genetics, University of Oxford, United Kingdom, ${ }^{5} \mathrm{MRC}$ Centre for Causal Analyses in Translational Epidemiology, University of Bristol, United Kingdom, ${ }^{6}$ National Public Health Institute, University of Oulu, Finland, ${ }^{7}$ MRC Centre of Epidemiology for Child Health, UCL Institute of Child Health, London, United Kingdom, ${ }^{8}$ Department of Epidemiology and Public Health, Imperial College, London, United Kingdom, ${ }^{9}$ Division of Community Health Sciences, St. George's, University of London, United Kingdom

Background and aims: The fetal insulin hypothesis proposed that the association between low birth weight and type 2 diabetes (T2D) could be explained by T2D risk alleles in the fetus reducing insulin-mediated fetal 
growth. This has been supported by epidemiological data but there has been no direct molecular genetic support. Type 2 genes may also increase birth weight by increasing maternal glycaemia. Recent genome-wide association studies have identified novel T2D risk variants but their role in fetal growth is not known.

Materials and methods: We examined five recently identified T2D loci (CD$K A L 1, C D K N 2 A / B, H H E X-I D E, I G F 2 B P 2$ and SLC30A8) for their impact on fetal growth, either through fetal genotype or maternal genotype, or a combination. We used data from 7986 mothers and 19200 offspring from four studies of white Europeans.

Results: We found strong evidence that fetal CDKAL1 and HHEX-IDE T2D risk genotypes influence birth weight. Each fetal copy of the CDKAL1 and HHEX-IDE T2D risk alleles reduced birth weight by $21 \mathrm{~g}$ ([95\%CI: 11-31g]; $\left.\mathrm{P}=2 \times 10^{-5}\right)$ and $14 \mathrm{~g}$ ([95\%CI: 4-23g]; $\left.\mathrm{P}=0.004\right)$, respectively. Adjustment for maternal genotype $(n=5,712)$, resulted in an increase in effect size (CDKAL1: birth weight reduced by $36 \mathrm{~g}$ [16-56g]; $\mathrm{P}=0.0005$. HHEX-IDE: $29 \mathrm{~g}$ [10-48g]; $\mathrm{P}=0.003$ ), consistent with an opposing effect of maternal genotype on birth weight. There were no associations between the other variants and fetal growth $(P>0.05)$

Conclusion: Our data provide the first direct evidence for the fetal insulin hypothesis by showing that common T2D risk alleles in CDKAL1 and HHEX-IDE can reduce birth weight through a predominant effect of fetal genotype. This contrasts with the predominantly maternal effect of TCF7L2 and suggests that T2D risk loci have heterogeneous effects on fetal insulin secretion.

Supported by: Diabetes UK, Medical Research Council, Wellcome Trust, University of Bristol, Academy of Finland, National Health Service Executive

\section{0}

Extended analysis of genome-wide scans provides clues about novel common and rare susceptibility loci for type 2 diabetes

E. Zeggini ${ }^{1}$, R. Lawrence ${ }^{1}$, A.T. Hattersley ${ }^{2}$, T.M. Frayling ${ }^{2}$, the Wellcome Trust Case Control Consortium, K.S. Elliott ${ }^{1}$, M.I. McCarthy ${ }^{1}$

${ }^{1}$ WTCHG, University of Oxford, United Kingdom, ${ }^{2}$ Institute of Biomedical and Clinical Science, Peninsula Medical School, Exeter, United Kingdom

Background and aims: Multiple new type 2 diabetes (T2D) susceptibility loci have been identified through genome-wide association (GWA) scans, but established associations account for only a small proportion of the genetic component of the disease. The established T2D loci PPARG, KCNJ11, TCF7L2, HHEX/IDE, CDKN2A/B, CDKAL1, FTO, SLC30A8, IGF2BP2, TCF2 and WFS1 have a cumulative sibling recurrence risk ratio (lambda-s) of 1.06. In European populations, the overall lambda-s for T2D has been estimated to be approximately 3 . We have carried out a meta-analysis of 3 T2D GWA scans across 10,128 individuals of European descent and $~ 2.2$ million SNPs (directly genotyped and imputed). Through replication testing in an independent set of samples (effective sample size of up to 53,975) we have detected at least six new loci with robust evidence for association $\left(\mathrm{p}<5 \times 10^{-8}\right)$, and modest effect sizes (largest allelic odds ratio was 1.15).

Materials and methods: On the basis of replication sample allele frequencies and effect sizes, we calculated the individual lambda-s values for these novel loci to investigate the proportion of the genetic component of T2D that they explain. To assess the role of rare variants in T2D susceptibility, we have used a super-locus approach (explained below) to analyse markers with frequency $<0.05$ from the Wellcome Trust Case Control Consortium T2D GWA scan. To do this, we looked across all gene regions genome-wide (defined as $50 \mathrm{~kb}$ either side of the gene transcriptional start and end sites). For each gene, we constructed a $2 \times 2$ table of the number of cases carrying at least one and the number of cases not carrying any rare variant minor alleles in the region, versus the number of controls carrying at least one or not carrying any rare variant alleles in the region. We tested for differences in the frequency with which cases carry at least one rare variant allele versus controls by the chisquared test (or Fisher's exact test where required).

Results: The individual lambda-s values for the novel loci detected through meta-analysis are: 1.001 for JAZF1, 1.002 for CDC123/CAMK1D, 1.001 for TSPAN8/LGR5, 1.002 for THADA, 1.002 for ADAMTS9 and 1.001 for NOTCH2. Together with the previously established 11 loci, their cumulative lambda-s is 1.07 , assuming a log-additive model. Using the rare variant superlocus approach in the WTCCC data, we detected several loci with promising evidence for association $(\mathrm{p}<0.00001)$, for example within the LOC391845 ( $1.4 \%$ of cases with at least one rare allele v. $0.2 \%$ of controls, $\left.\mathrm{p}=4 \times 10^{-6}\right)$ gene. They are being followed up by large-scale replication and deep resequencing studies.
Conclusion: The lambda-s values indicate that the known susceptibility variants account for only a small proportion of observed familial aggregation. Part of the explanation may relate to the fact that the variants causal for the observed associations have yet to be defined. However, it is also probable that there are many novel loci to be found, and that studies of the role of rare variants may prove a powerful approach for their discovery. Supported by: Wellcome Trust

\section{1}

Large-scale follow-up study does not confirm putative association signals in the NOS1AP and PKLR gene regions on chromosome 1q with type 2 diabetes

N.W. Rayner ${ }^{1}$, I. Prokopenko ${ }^{1}$, C.J. Groves ${ }^{1}$, E. Zeggini ${ }^{1}$, R.L. Hanson ${ }^{2}$, B.D. Mitchell', W. Jia ${ }^{4}$ M. Ng 5 , P. Froguel ${ }^{6}$, J. Chan ${ }^{5}$, C. Bogardus², S.C. Elbein ${ }^{7}$, A.R. Shuldiner ${ }^{3}$, M.I. McCarthy ${ }^{1}$, The International Type 2 Diabetes 1q Consortium

${ }^{1}$ WTCHG, University of Oxford, United Kingdom, ${ }^{2}$ NIH/NIDDK, Phoenix, United States, ${ }^{3}$ School of Medicine, University of Maryland, Baltimore, United States, ${ }^{4}$ Dept of Endocrinology \& Metabolism, Shanghai Diabetes Institute, Shanghai, China, ${ }^{5}$ Dept of Medicine \& Therapeutics, Chinese University of Hong Kong, ${ }^{6}$ Institut de Biologie de Lille Génomique et Physiologie Moléculaire des Maladies Métaboliques, Institut Pasteur, France, ${ }^{7}$ Division of Endocrinology and Metabolism, University of Arkansas, Little Rock, United States

Background and aims: Dense-mapping (5290 SNPs) of the well-replicated chrlq type 2 diabetes (T2D) linkage region (147.0-169.7Mb) identified two promising association signals in samples of European descent. The first resides within the NOS1AP (CAPON) gene (e.g. rs7538490, OR 1.53 [95\%CI $\left.1.28-1.81], \mathrm{p}=1.2 \times 10^{-6}\right)$, and the second within an extensive linkage disequilibrium (LD) block that includes the ASH1L and PKLR genes (e.g. rs11264372, OR 1.35 [1.17-1.56], p $\left.5.1 \times 10^{-5}\right)$. The aim of this study was to explore these putative associations in further samples.

Materials and methods: We first examined genome-wide association data from the Wellcome Trust Case Control Consortium (WTCCC) T2D sample containing 1495 T2D cases (independent of those already typed by the $1 \mathrm{q}$ consortium) and 2938 controls of UK origin. We also genotyped rs7538490 in 2 further independent sets containing 2955 T2D cases and 3336 controls from the UK. We typed rs11264371 ( $\mathrm{r}^{2} 1.0$ with $\left.\mathrm{rs} 11264372\right)$ in $3441 \mathrm{~T} 2 \mathrm{D}$ cases and 5334 controls.

Results: We found no evidence of association within the independent WTCCC sample for the NOS1AP signal (rs7538490, OR 0.98 [0.88-1.09] or the ASH1L/PKLR region (rs11264371, 1.04 [0.93-1.18]). The extended replication efforts in additional UK case-control sets also failed to demonstrate any detectable effect at either SNP. Meta-analysis of the combined replication sample showed no association at these two loci: rs7538490 OR 1.01 [0.951.08] for total 4934 T2D cases and 6789 controls; rs11264371 OR 1.03 [0.971.10] for $4351 \mathrm{~T} 2 \mathrm{D}$ cases and 6100 controls.

Conclusion: Notwithstanding differences in ascertainment between the highly-selected cases used for the original high density association screen and those used for replication, we conclude that no convincing role in T2D susceptibility was observed for these SNPs in NOSA1P and around ASH1L/ $P K L R$ region. All in all, dense association mapping across the region, in large, well-powered samples has failed to identify common SNP variants that may be contributing to the replicated linkage signal. We are now testing the hypothesis that structural variants, and low frequency, intermediate penetrance SNPs may be causal for the observed linkage.

Supported by: NIDDK Wellcome Trust Diabetes UK MRC

\section{2}

Studies of genome-wide association data support a genetic overlap between type 2 diabetes and prostate cancer

K.S. Elliott ${ }^{1}$, E. Zeggini ${ }^{1}$, N. Rayner ${ }^{1}$, C. Groves ${ }^{1,2}$, T. Frayling ${ }^{3}$, A.

Hattersley ${ }^{3}$, Diabetes Genetics Replication and Meta-analysis (DIAGRAM) Consortium, M.I. McCarthy ${ }^{2}$

${ }^{1}$ Wellcome Trust Centre for Human Genetics, University of Oxford, United Kingdom, ${ }^{2} \mathrm{Oxford}$ Centre for Diabetes, Endocrinology and Metabolism, University of Oxford, United Kingdom, ${ }^{3}$ Institute of Biomedical and Clinical Science, Peninsular Medical School, Exeter, United Kingdom

Background and aims: Recent genome- wide association (GWA) studies have identified and confirmed many type 2 diabetes (T2D) susceptibility 
genes. Several of these T2D loci (PPARG, TCF7L2, CDKN2A, HHEX and IGF2BP2) as well as some genes causal for monogenic diabetes have known roles in cancer pathogenesis. Association of T2D and prostate cancer at alternate alleles of the same variant in the HNF1B gene support a shared genetic aetiology between the two diseases. This is further supported by recent publications showing association with T2D and prostate cancer at separate unlinked variants in the JAZF1 gene.

Materials and methods: To formally assess this genetic relationship we compared meta-analysed T2D GWA data from the Diabetes Genetics Replication And Meta-analysis (DIAGRAM) Consortium with publicly available GWA prostate cancer data from the Cancer Genetics Markers of Susceptibility (CGEMS) project. For SNPs where there was data available for both diseases $(\mathrm{n}=393,763)$, we looked SNP-by-SNP at the distribution of $\mathrm{p}$-values above and below $\mathrm{p}=0.01$ for T2D and prostate cancer. Second, we adopted a more targeted approach by looking for $\mathrm{T} 2 \mathrm{D}$ and prostate cancer signals (defined by $\mathrm{p}<0.001$ ) in gene regions containing confirmed T2D and prostate cancer variants.

Results: When we looked SNP-by-SNP, genome-wide, for evidence of association signals (defined by $\mathrm{p}<0.01$ ) shared between $\mathrm{T} 2 \mathrm{D}$ and prostate cancer, we found 73 instances representing 42 independent signals compared to an expectation of 8.3 independent signals under the null (binomial test $\mathrm{p}<10^{-6}$ ). Of these 42 signals, 22 showed discordant and 20 showed concordant directions of association between the two diseases. When we focussed attention on regions already known to be implicated in disease risk (to improve power), we found a strong over-representation of prostate cancer association signals (defined by $\mathrm{p}<0.001$ ) in the 17 regions known to harbour T2D susceptibility variants compared to all other autosomal genes for which data was available $(\mathrm{n}=18,719)$ (chi-squared test $\left.\mathrm{p}=3 \times 10^{-8}\right)$ : in all, six of these regions (CDKAL1, NOTCH2, CAMK1D, JAZF1, HNF1B and KCNJ11) contained such a prostate cancer signal. In the reciprocal analysis, there were just the two known T2D signals $(H N F 1 B$ and $J A Z F 1)$ (defined by $\mathrm{p}<0.001$ ) in the 10 confirmed prostate cancer gene regions (chi-squared test $p=0.102$ ). Since there is a greater chance of finding signals in large gene regions, we tested the relationship between confirmed disease genes and gene region size and found that the mean of confirmed disease gene region sizes fell between the 15th and 85th quantiles of 10,000 random permutations.

Conclusion: The non-random co-localisation of T2D and prostate cancer signals at the genome-wide level indicates undefined overlaps in aetiological mechanisms. When false positive associations are reduced by studying gene regions harbouring confirmed T2D or prostate cancer disease variants, the over-representation of prostate cancer signals in T2D gene regions strengthens the evidence of an overlapping genetic basis for T2D and prostate cancer.

Supported by: Wellcome Trust

\section{3}

The role of confirmed type 2 diabetes risk alleles on age-of-diagnosis in a Norwegian population based sample: the HUNT Study

S. Johansson ${ }^{1}$, J.K. Hertel ${ }^{1}$, K. Midthjell' ${ }^{2}$, A. Molven ${ }^{1}$, P.R. Njølstad ${ }^{1}$

${ }^{1}$ Department of Clinical Medicine, University of Bergen, ${ }^{2}$ Department of

Public Health and General Practise, Norwegian University of Science and

Technology, Verdal, Norway

Background and aims: We and others have recently shown that the novel associated SNPs of the first wave of type 2 diabetes (T2D) whole-genome association scans are consistently replicated in many populations and under different study designs. It could be speculated that these risk alleles are associated with younger onset of diabetes due to a higher genetic load. Here, we explore the relationship between age-of-diagnosis and confirmed risk-associated SNPs close to the genes IGFBP2, CDKAL1, SLC30A8, CDKN2B, HHEX and FTO.

Materials and methods: 1449 patients with T2D and 1551 controls from the population-based HUNT-study in Norway were analysed for the six most consistently associated SNPs near IGFBP2, CDKAL1, SLC30A8, CDKN2B, HHEX and FTO. Logistic regression was used to compare the allele distribution between cases diagnosed at 35-59 years, cases diagnosed at the age of 60 and above, and all controls. Age-of-diagnosis was also studied as a quantitative trait. All analyses included BMI and gender as covariates.

Results: The risk allele at HHEX was associated with younger age of diagnosis $(\mathrm{p}=0.06$ ), with a similar but weaker trend at CDKN2B and FTO. SNPs close to CDKAL1 and SLC30A8 were not differentially distributed in the two age groups. Interestingly, the IGFBP2 risk allele at SNP rs4402960 may be associated with older age of diagnosis $(\mathrm{p}=0.08)$.
Conclusion: For three of the examined SNPs, our results are in line with the hypothesis of a higher genetic load in cases with younger onset of diabetes. However, our data on IGFBP2 suggest that certain risk alleles may be more pronounced in cases with late-onset diabetes.

Supported by: Translational Research Fund of the University of Bergen, Haukeland University Hospital, Innovest, Norwegian Research Council (FUGE)

\section{4}

Genetic analysis of "European" type 2 diabetes SNPs from whole genome association studies in African Americans

D.W. Bowden ${ }^{1}$, J.P. Lewis ${ }^{1}$, N.D. Allred ${ }^{1}$, C.D. Langefeld ${ }^{2}$, J. Divers ${ }^{2}$, B.I. Freedman ${ }^{3}$

${ }^{1}$ Center for Human Genomics, ${ }^{2}$ Public Health Sciences, ${ }^{3}$ Internal Medicine, Wake Forest University School of Medicine, Winston-Salem, United States

Several whole genome association (WGA) studies have identified type 2 diabetes mellitus (T2DM) susceptibility genes in European-derived populations. Little investigation of these loci has been reported in other ethnic groups, specifically African Americans (AAs). Differences exist between these populations for T2DM risk, suggesting they may not share identical genetic risk factors. Our objective was to examine the influence of T2DM genes identified in WGA studies in a large AA case-control population. SNPs in 12 loci (e.g. TCF7L2, IDE/KIF11/HHEX, SLC30A8, CDKAL1, PKN2, IGF2BP2, FLJ39370, and EXT2/ALX4) associated with T2DM in European-derived populations were genotyped in 993 T2DM AA cases and 1054 AA controls. In addition, 68 ancestry-informative markers (AIMs) were genotyped to account for the impact of admixture on association results. While power to detect association differed based on allele frequency and reported odds ratios, power analyses suggested evidence for association would be observed in 4-5 loci. With the exception of TCF7L2, little evidence of association with T2DM in AAs was observed. One TCF7L2 SNP (rs7903146) showed compelling evidence of association with T2DM [admixture adjusted $\mathrm{P}=1.59 \times 10$ ${ }^{6}$, additive model, $\mathrm{OR}=1.37$ (1.31-1.43)]. The only other SNP with nominal evidence of association after admixture adjustment was the intragenic SNP rs9300039 [ $\mathrm{P}=0.029$, dominant model, $\mathrm{OR}=1.48$ (1.28-1.71)]. Interestingly, 4 of the SNPs are monomorphic in the Yoruba population of the HAPMAP project with only the "risk" allele from the populations of European descent observed. We conclude that, except for TCF7L2, "European" T2DM variants do not significantly contribute to inter-individual susceptibility to T2DM in AAs. Consequently, genes contributing to T2DM in AAs and other peoples of African origin may, in part, be different from those in European-derived populations. The high frequency of risk alleles in several of these genes may, however, contribute to the increased prevalence of T2DM in AAs. Supported by: National Institutes of Health USA 


\section{PS 006 Genetic regulation of insulin action}

\section{5}

HNF4 $\alpha$ directs histone methylation to silence PED/PEA-15 expression in human hepatocytes

R. Teperino, R. Valentino, M. Ciccarelli, A. Perfetti, A.T. Alberobello, F. Giacco, R. Buonomo, P. Formisano, F. Beguinot, P. Ungaro

Dipartimento di Biologia e Patologia Cellulare e Molecolare \& IEOS-CNR, Universita Di Napoli, Italy

Background and aims: $P E D / P E A-15$ is a gene commonly overexpressed in tissues from type 2 diabetic individuals and healthy subjects at high risk of developing diabetes (such as first degree relatives). Indeed, overexpression of the PED/PEA-15 gene in mice, impairs glucose tolerance and leads to diabetes in conjunction with high-fat diet treatment. The Hepatocyte Nuclear Factor 4alpha (HNF-4alpha) is a liver-enriched nuclear receptor involved in the control of glucose homeostasis. Point mutations in HNF-4alpha impair liver and pancreatic regulation of glucose homeostasis and cause Maturity Onset Diabetes of the Young Type 1 (MODY 1). More recently, genetic and biochemical evidences indicate that HNF-4alpha may also play a role in the development of Type 2 Diabetes.

Recent evidences in our lab, indicate that HNF-4alpha inhibits PED/PEA-15 expression in liver by binding its responsive element on PED/PEA-15 promoter. The aim of our work is to understand the molecular mechanism by which HNF-4alpha exerts its action on PED/PEA-15 expression and test the hypothesis that HNF-4alpha might induce a packaging of chromatin in the region of PED/PEA-15 promoter.

Materials and methods: Hela and HepG2 cells are used in this study. A bioinformatic analysis has been performed using the software RECON to map potential nucleosomes on the core-promoter of PED/PEA-15, and Micrococcal Nuclease (MNase) Protection Assay has been used to further confirm "in silico" data. Chromatin Immunoprecipitation (ChIP) and ReChIP Assays have been performed to identify histone marks and histone-associated proteins.

Results: Our work show the fundamental role of HNF-4alpha in directing nucleosome assembly and histone methylation to maintain PED/PEA-15 gene repression in HepG2 cells. This data has been further confirmed in Hela cells overexpressing HNF-4alpha (Hela-HNF). Indeed, both in Hela-HNF and HepG2 cells HNF-4alpha expression promotes the assembly of histone deacetylase (HDAC), as well as histone methyl transferase (HMT) complexes on the PED/PEA-15 promoter and leads to the di-methylation of Lysine 9 on histone H3. Furthermore, HNF-4alpha interacts with and recruits SMRT (Silencing Mediator of Retinoic Acid and Thyroid Hormone Receptor) corepressor to $P E D / P E A-15$ promoter leading the associated chromatin to condense. These modifications are barely undetectable in both Hela and HepG2 cells transfected with a HNF-4alpha specific shRNA plasmid where HNF4alpha is expressed at low levels.

Conclusion: These results suggest that HNF-4alpha functions as a scaffold protein for both histone deacetylase and histone methyl transferase activities to inhibit $P E D / P E A-15$ transcription, thus representing a new potential molecular tool to target $P E D / P E A-15$ expression. Further in vivo studies should be done to determine whether HNF-4alpha may act via chromatin remodelling even in vivo and whether alterations of this mechanism might play a role in the overexpression of $P E D / P E A-15$ gene observed in type 2 diabetic patients and their healthy first degree relatives.

\section{6}

$P G C-1 \alpha$ and $P G C-1 \beta$ are related to markers for fibre type composition in human skeletal muscle

A.H. Olsson ${ }^{1}$, T. Rönn ${ }^{1}$, A. Vaag 2 , L. Groop ${ }^{1}$, P. Poulsen ${ }^{2}$, C. Ling ${ }^{1}$

${ }^{1}$ Clinical Sciences, Diabetes and Endocrinology, Lund University, Malmo, Sweden, ${ }^{2}$ Steno Diabetes Center, Gentofte, Denmark

Background and aims: Human skeletal muscle contains three major fibre types distinguished by their myosin heavy chain (MHC) isoforms. The mitochondria content and oxidative capacity is the highest in slow oxidative type I fibres, lower in fast oxidative type IIa fibres, and lowest in fast glycolytic type IIx/d fibres. The amount of oxidative type I fibres is reduced and glycolytic type IIx/d fibres is increased in muscle from patients with type 2 diabetes (T2D) and subjects with a family history of T2D. The transcriptional co-acti- vators $P G C-1 \alpha$ and $P G C-1 \beta$ are both implicated in the formation of oxidative fibres in skeletal muscle and are down-regulated in diabetic muscle. The aim of the present study was to investigate factors influencing the expression of markers for fibre type composition in human skeletal muscle of twins.

Materials and methods: mRNA expression of three fibre type markers; MHC7 (slow oxidative), MHCIIa (fast oxidative) and MHCIIx/d (fast glycolytic), were analysed in muscle biopsies from young $(\mathrm{n}=102)$ and elderly $(\mathrm{n}=81)$ non-diabetic twins and related to the level of $P G C-1 \alpha$ and $P G C-1 \beta$ mRNA. Single nucleotide polymorphisms (SNPs) in the three MHC gene regions (10 SNPs in MHC7, 5 SNPs in MHCIIa and 5 SNPs in MHCIIx/d) were genotyped and related to gene expression.

Results: While MHCIIa mRNA expression was reduced in skeletal muscle from elderly compared with young twins $(1.07 \pm 0.07$ versus $1.50 \pm 0.07$; $p$ $<0.001$ ), the expression of MHC7 and MHCIIx/d did not change with age. Regression analysis showed that $P G C-1 \alpha$ was positively related to the expression of all three fibre type markers in human muscle $(p<0.01)$. However, $P G C-1 \beta$ was positively related to $M H C I I a$ and negatively related to $M H C I I x / d$ expression $(p<0.05)$. One SNP, rs17794387, showed a nominal association to MHC7 expression $(p<0.05)$ and another SNP, rs3764851, to MHCIIx/d expression $(p=0.014)$ in muscle of twins. The level of $M H C 7$ was positively related to $\mathrm{VO}_{2} \max (p=0.009)$ and MHCIIa expression was positively related to insulin-stimulated glucose uptake $(p=0.008)$. Furthermore, MHCII $x / d$ mRNA expression was negatively related to both insulin-stimulated glucose uptake $(p=0.04)$ and $\mathrm{VO}_{2} \max (p<0.0001)$.

Conclusion: Age and $P G C-1 \alpha$ and $\beta$, are associated with the expression level of markers for fibre type composition in human skeletal muscle. Furthermore, these fibre type markers are related to $\mathrm{VO}_{2} \max$ and insulin-stimulated glucose uptake.

\section{7}

Association of APLN (Encoding Apelin) variants with type 2 diabetes in Chinese Han population

C. Wang, R. Zhang, C. Hu, X. Ma, W. Jia, K. Xiang

Endocrinology \& Metabolism, Shanghai Jiaotong University affiliated No.6

People Hospital, Shanghai Diabetes Institute, Shanghai, China

Background and aims: Adipocyte-derived cytokines have been recognized to be involved in body fat distribution, insulin sensitivity and contribute to the etiology of type 2 diabetes, obesity, and metabolic syndrome. Recent studies showed that apelin is one of the newly adipokines that is highly expressed in white adipose tissue. Apelin expression was up-regulated in obese mice with hyperinsulinemia, and the plasma apeline level was dramatically high in obese subjects and diabetic patients. Thus, we hypothesized that genetic variants in the APLN gene (encoding apelin) may associated with susceptibility to type 2 diabetes.

Materials and methods: The APLN gene is located on chromosome Xq25q26.3. We examined the relationship between APLN variation and type 2 diabetes (selecting four tagging SNPs capturing $>80 \%$ common variation) in a total of 3,700 Chinese Han subjects (1,892 hospital cases [age 61.2 \pm 12.6 years, $52.2 \%$ male] and 1,808 community-based control subjects with normal glucose regulation [age $57.3 \pm 12.3$ years, $41.4 \%$ male]) using Sequenom MassARRAY system. Association was measured using either $x^{2}$ or Fisher's exact test. Furthermore, cases and controls were stratified by gender respectively and association was measured for each group.

Results: After quality control steps, allele and genotype (in females) frequencies of three SNPs (rs3115759, rs2235307 and rs2281068) were compared in 1,775 type 2 diabetes cases and 1,807 control subjects. None of the SNPs showed an association with type 2 diabetes (rs3115759: $G$ allele $32.3 \%$ in case, $32.2 \%$ in controls, $P=0.95$; rs2235307: $\mathrm{T}$ allele $18.9 \%$ in cases, $18.6 \%$ in controls, $P=0.83$; rs 2281068 : T allele $32.5 \%$ in cases, $31.8 \%$ in controls, $P=$ 0.59). Similarly, no evidence showed association of APLN variants with type 2 diabetes in both female and male groups.

Conclusion: Although apelin presents itself as a good candidate for contributing to type 2 diabetes susceptibility, this study has failed to find an association between SNPs in the APLN gene region and type 2 diabetes in Chinese. Supported by: Major Project of National Natural Science Foundation of China and National Basic Research Program of China 


\section{8}

Effect of adiponectin gene polymorphisms on circulating total and highmolecular weight adiponectin and insulin resistance in non-diabetic Greek women

L. Melistas ${ }^{1}$, C.S. Mantzoros ${ }^{2}$, M. Kontogianni' ${ }^{1}$, J.M. Ordovas ${ }^{3}$,

N. Yiannakouris ${ }^{1}$

${ }^{1}$ Nutrition-Dietetics, Harokopio University of Athens, Athens, Greece,

${ }^{2}$ Division of Endocrinology, Beth Israel Deaconess Medical Center,

Harvard Medical School, Boston, United States, ${ }^{3}$ Nutrition and Genomics

Laboratory, JM-USDA-HNRCA at Tufts University, Boston, United States

Background and aims: Adiponectin is an adipokine that modulates insulin sensitivity and whose low circulating concentration is associated with insulin resistance. Recent studies have demonstrated that the high-molecular weight (HMW) multimer of adiponectin is the active form of the hormone. In the present study, we assessed whether adiponectin gene variants contribute to circulating total and HMW adiponectin, insulin resistance (IR), or cardiovascular disease risk factors in a healthy Greek women population.

Materials and methods: A total of 349 Greek non-diabetic women (mean age: $47.0 \pm 12.1 \mathrm{y}$, mean BMI: $29.5 \pm 5.6 \mathrm{~kg} / \mathrm{m}^{2}$ ) were genotyped for two single nucleotide polymorphisms (SNPs) in the adiponectin gene $[45 \mathrm{~T}>\mathrm{G}$ (rs2241766) and 276G>T (rs1501299)]. Body composition was assessed in all subjects with dual X-ray absorptiometry (DXA) and fasting plasma glucose, lipid, lipoprotein and apolipoprotein (apo) B and A1 levels were determined. Plasma insulin and adiponectin concentrations were measured by RIA. Plasma levels of HMW adiponectin were determined in a subgroup of study participants $(\mathrm{n}=211)$ by ELISA. IR was estimated with the homeostasis model assessment (HOMA). Polymorphisms were genotyped with TaqMan SNP allelic discrimination.

Results: Multiple regression analysis showed that total and HMW adiponectin were significantly negatively correlated with BMI, body fat, waist circumference, waist-to-hip ratio, fasting triglyceride, insulin levels, as well as HOMA-IR, and positively with HDL cholesterol and apoA1 levels (all $\mathrm{P}<0.001)$. Total and HMW adiponectin concentrations were not significantly different across genotypes for the two SNPs. In addition, no association was found between the two SNPs and BMI, body composition variables and plasma lipid levels. However, in multivariate analysis controlling for potential confounders SNP 276G $>\mathrm{T}$ was associated with higher fasting insulin levels $(\mathrm{P}=0.01)$ and HOMA-IR index $(\mathrm{P}=0.009)$, and $\mathrm{SNP} 45 \mathrm{~T}>\mathrm{G}$ exhibited a tendency towards association with lower insulin levels and HOMA-IR $(\mathrm{P}=0.05$ and $\mathrm{P}=0.07$, respectively). To analyze the possible interaction between body fat and the adiponectin gene SNPs on insulin resistance the study group was divided into two subgroups according to the percentage of body fat (below or above the median of $41.0 \%$ ). We found that in the highest body fat group (> $41 \%$ ) carriers of the minor $+45 \mathrm{G}$ and $+276 \mathrm{~T}$ alleles had lower $(2.63 \pm 1.42 \mathrm{vs}$. $2.11 \pm 1.19 ; \mathrm{P}=0.005)$ and higher $(2.24 \pm 1.10$ vs. $2.84 \pm 1.60 ; \mathrm{P}=0.002)$ HOMAIR, respectively, whereas no significant differences were observed among leaner individuals ( $\mathrm{P}$ for interaction $<0.05$ ). Haplotype analysis revealed that subjects homozygous for the $45 \mathrm{~T} / 276 \mathrm{G}$ haplotype had lower total adiponectin levels than did non-carriers of this haplotype (13.71 \pm 7.12 vs. $16.40 \pm 6.68$ $\mu \mathrm{g} / \mathrm{ml} ; \mathrm{P}=0.05)$.

Conclusion: The current study shows that SNPs $45 \mathrm{~T}>\mathrm{G}$ and $276 \mathrm{G}>\mathrm{T}$ in the adiponectin gene are significantly associated with insulin resistance in nondiabetic Greek women with high body fat levels. However, no association was found between these two SNPs and body composition parameters, lipid profile, or with circulating total and HMW adiponectin concentrations.
309

A Mendelian randomisation study provides initial evidence that sex hormone binding globulin (SHBG) levels alter type 2 diabetes risk J.R.B. Perry ${ }^{1}$, D. Melzer ${ }^{2}$, M. Maggio ${ }^{3}$, D. Hernandez ${ }^{4}$, A. Singleton ${ }^{4}$, L. Ferrucci ${ }^{5}$, C. Palmer ${ }^{6}$, A. Bennett ${ }^{7}$, A. Ruokonen, ${ }^{8}$, V. Panicker ${ }^{9}$,

M.-R. Jarvelin ${ }^{10}$, A. Hattersley ${ }^{1}$, M. McCarthy ${ }^{7}$, T. Frayling ${ }^{1}$

${ }^{1}$ Genetics of Complex Traits, Peninsula Medical School, Exeter, United Kingdom, ${ }^{2}$ Epidemiology and Public Health, Peninsula Medical School, Exeter, United Kingdom, ${ }^{3}$ National Institutes of Health, National Institute on Aging, Baltimore, United States, ${ }^{4}$ Laboratory of Neurogenetics, National Institute of Aging, Bethesda, United States, ${ }^{5}$ Longitudinal Studies Section, National Institute on Aging, Baltimore, United States, ${ }^{6}$ Biomedical Research Centre, University of Dundee, United Kingdom, ${ }^{7}$ Oxford Centre for Diabetes, Endocrinology and Metabolism, University of Oxford, Oxford, United Kingdom, ${ }^{8}$ Public Health Science and General Practice, University of Oulu, Finland, ${ }^{9}$ Henry Wellcome Laboratories for Integrative Neuroscience and Endocrinology, University of Bristol, United Kingdom, ${ }^{10}$ Public Health and Primary Care, Imperial College London, London, United Kingdom

Background and aims: Sex hormone binding globulin (SHBG) levels are inversely correlated with type 2 diabetes and insulin resistance but the causal direction of this association is not known. SHBG levels may be altered because they influence type 2 diabetes risk or because they are secondary to disease processes. Genetics can help dissect the causal direction of this association using a Mendelian Randomization approach. If gene variants that increase SHBG levels also decrease type 2 diabetes risk this provides evidence of a protective role of SHBG.

Materials and methods: We performed a genome wide association study for SHBG levels using 1200 individuals from the population based InCHIANTI study. Two studies with SHBG levels measured, the WATTs study $(n=546)$ and Northern Finland 1966 Birth Cohort (N=4044), were used to replicate findings. We tested the association of variants altering SHBG levels with type 2 diabetes using inverse-variance weighted meta-analysis of a type 2 diabetes case control study of 12,643 UK individuals.

Results: In the InCHIANTI study, a one standard deviation (SD) increase in SHBG levels is correlated with type 2 diabetes: odds ratio: 0.73 (0.59-0.89), $\mathrm{p}=0.002$. We identified a variant ( $\mathrm{rs1799941)}$ near the SHBG gene associated with a per allele difference of 0.21 SDs in SHBG levels $\left(\mathrm{p}=3 \times 10^{-5}\right)$ in the InCHIANTI population. The association replicated $\left(\mathrm{p}=1.4 \times 10^{-12}\right)$ and meta-analysis of all three studies provided very strong evidence of association $\left(\mathrm{p}=1.8 \times 10^{-16}\right)$. In InCHIANTI the SNP was also associated with free testosterone levels $(\mathrm{p}=0.01)$ and ratio of total testosterone/SHBG $(\mathrm{p}=0.003)$ in males but not females. The SHBG allele associated with increasing SHBG levels was associated with a reduced risk of type 2 diabetes with an odds ratio of 0.90 (95\%CIs: $0.85-0.96, \mathrm{p}=0.0009$ ). This is consistent with the expected effect given the rs1799941-SHBG and SHBG-T2D correlations. There was a trend towards this association being stronger in males 0.88 (95\%CIs:0.81-0.95, $\mathrm{p}$ $=0.002, \mathrm{~N}=6747)$ than females $0.94(95 \%$ CIs:0.86 $-1.02, \mathrm{p}=0.16, \mathrm{~N}=5896)$ but this difference was not significant.

Conclusion: Although additional data is needed to confirm the association our study suggests that SHBG is causally influencing the risk of type 2 diabetes.

Supported by: Intramural Research Program of NIH, National Institute on Aging. DM is supported by a NHS Executive National Public Health Career Scientist Award

\section{0}

Impact of the transcription factor 7like 2 polymorphism (TCF7L2) on insulin secretion and action before and after physical inactivity A.C. Alibegovic ${ }^{1}$, L. Hojbjerre ${ }^{2}$, M.P. Sonne ${ }^{2}$, O. Pedersen ${ }^{3}$, T. Hansen ${ }^{3}$, B. Stallknecht ${ }^{2}$, F. Dela ${ }^{2}$, A. Vaag ${ }^{1}$

${ }^{1} 810$, Steno Diabetes Center, Gentofte, Denmark, ${ }^{2} 4$, University of Copenhagen, Copenhagen, Denmark, ${ }^{3} 521$, Steno Diabetes Center, Gentofte, Denmark

Background and aims: Physical inactivity is a risk factor for type 2 diabetes (T2D). However, little is known about the extent to which physical inactivity is more harmful to some persons than to others. Common genetic variants encoding the TCF7L2 polymorphism have been associated with impaired betacell function and T2D. The purpose of this study was to examine whether the diabetes risk TCF7L2 rs-7903146 T-allele modulates changes in insulin secretion and whole body insulin sensitivity following ten days of bed rest (BR). 
Materials and methods: 38 healthy young Caucasian men completed the study. Subjects were recruited from a cohort of young men with low (LBW) and normal birth weight ( $<10$ and between $50-90$ percentiles, respectively). 21 subjects were carriers of the T2D risk T allele (combined CT and TT alleles) and 17 subjects were low risk CC homozygous. No impact of - or association between - birth weight and genotype were observed, and number of LBW subjects were 10 in the CC and 11 in CT/TT group, respectively. Insulin secretion and insulin action was studied before and after ten days of BR by intra venous glucose tolerance tests combined with euglycemic hyperinsulinemic clamps ( 3 hours, insulin infusion rate $80 \mathrm{mU} / \mathrm{m} 2 / \mathrm{min}$ ). The TCF7L2 rs7903146 was genotyped using allelic discrimination performed with an ABI 7900 system (KBioscience, Herts, UK). The genotyping success rate was $98 \%$ and among 38 replicate samples no mismatches were observed. The genotype distribution obeyed Hardy-Weinberg equilibrium.

Results: No significant differences in BMI, waist-hip ratio, age, fasting plasma insulin or insulin action were observed in the two groups prior to BR. However, first phase insulin secretion (FPIS) and FPIS disposition index (FPIS $x$ $\mathrm{M}$-value; FPIS-Di) were significantly lower in CT/TT group: $1559 \pm 290$ vs. $2122 \pm 355 \mathrm{pmol} / \mathrm{L} / \mathrm{min}(\mathrm{P}<0.05)$ and $21929 \pm 4462$ vs. $30409 \pm 5386 \mathrm{mg}$ glucose $/ \mathrm{kg}$ lean body mass $(\mathrm{LBM})(\mathrm{P}<0.05)$ compared to CC group prior BR. After $\mathrm{BR}, \mathrm{CT} / \mathrm{TT}$ group demonstrated significantly lower FP insulin levels $29 \pm 2 \mathrm{vs.}$ $47 \pm 7 \mathrm{pmol} / \mathrm{L}(\mathrm{P}<0.05)$ as well as FPIS $2108 \pm 299$ vs. $3503 \pm 405 \mathrm{pmol} / \mathrm{L} / \mathrm{min}$ $(\mathrm{P}<0.001)$, and FPIS-Di $23963 \pm 3720$ vs. $34289 \pm 4574 \mathrm{mg}$ glucose $/ \mathrm{kg} \mathrm{LBM}$ $(\mathrm{P}<0.05)$ compared with CC group. The increment in FPIS in response to BR was significantly lower in CT/TT group compared with CC group $555 \pm 121$ vs. $1381 \pm 396 \mathrm{pmol} / \mathrm{L} / \mathrm{min}(\mathrm{P}<0.05)$. Although insulin action decreased in all subjects in response to $\mathrm{BR}, \mathrm{CT} / \mathrm{TT}$ group tended to be more insulin sensitive after BR as compared with CC group as determined by Homeostasis-ModelAssessment (HOMA) index, 6.1 $\pm 0.6 \mathrm{vs} .9 .6 \pm 1.4(\mathrm{P}<0.05)$, and to some extent clamp M-value $11 \pm 0.4 \mathrm{vs} .10 \pm 0.6 \mathrm{mg}$ glucose $/ \mathrm{kg} \mathrm{LBM} / \mathrm{min}(\mathrm{P}>0.05)$.

Conclusion: Young healthy carriers of the diabetes risk TCF7L2 rs-7903146 $\mathrm{T}$-allele exhibit a diminished compensatory increase in first phase insulin secretion during 10 days of $\mathrm{BR}$ indicating a greater vulnerability to physical inactivity.

Supported by: European Union Framework VI EXGENESIS project

\section{1}

Human natriuretic peptides genes are strongly associated with the risk for hyperglycaemia in a French population: the D.E.S.I.R. study S. Maimaitiming ${ }^{1,2}$, R. Roussel $^{1,2}$, F. Fumeron $^{1,2}$, N. Emery ${ }^{2}$, G. Velho ${ }^{1}$, K. Mohammedi ${ }^{1,2}$, N. Munoz ${ }^{1}$, B. Balkau ${ }^{3,4}$, J. Tichet ${ }^{5}$, M. Marre $^{1,2}$ ${ }^{1}$ Faculty of Medicine Xavier Bichat, INSERM U 695, Paris, France, ${ }^{2}$ Faculty of Medicine Xavier Bichat, University Denis Diderot, Paris, France, ${ }^{3}$ INSERM, U780-IFR69, Villejuif, France, ${ }^{4}$ Faculty of Medicine, University Paris-Sud, Orsay, France, ${ }^{5}$ Institut Régional Pour la santé, IRSA, La Riche, France

Background and aims: Natriuretic peptides induce lipolysis and may play a role in metabolic diseases. We studied the associations of several polymorphisms and haplotypes of the very close ANP and BNP genes (chr.1p36.2) with type 2 diabetes and impaired fasting glucose (T2D/IFG) in the DESIR study, a general population based cohort with a 9 year follow-up.

Materials and methods: 5,212 subjects were included. Four Hapmap selected tag-SNPs (3 in BNP gene, rs198381/rs198389/rs198388 and 1 in ANP gene, rs5065) were genotyped (Taqman). Plasma BNP levels were measured in a random sample of 369 subjects (IRMA). We tested the association between the genetic variations and the metabolic parameters with ANOVA and ANCOVA, the impact of genetic variations and the incidence of T2D/IFG (566 new cases) with logistic regression and haplotype analysis with THESIAS program. When appropriate, tests were adjusted for sex, age and BMI.

Results: Subjects with rs198381 CC genotype had higher fasting glucose levels (TT $5.35 \pm 0.01 \mathrm{mmol} / \mathrm{l}$, TC $5.38 \pm 0.02$ and CC $5.57 \pm 0.08, \mathrm{p}=0.005$ ), insulinemia (TT $47.1 \pm 0.4 \mathrm{pmol} / \mathrm{l}$, TC $46.5 \pm 0.8$ and CC $54.2 \pm 2.6$, $\mathrm{p}=0.001)$ and HOMA-IR (TT $1.67 \pm 0.02$, TC $1.65 \pm 0.03$ and CC $2.05 \pm 0.11$, $\mathrm{p}=0.0001)$. The minor allele of rs 198381, rs 198388 and rs5065 were associated with higher prevalence (co-dominant model: $\mathrm{OR}=1.31, \mathrm{p}=0.0004 ; \mathrm{OR}=1.18$, $\mathrm{p}=0.004$ and $\mathrm{OR}=1.29, \mathrm{p}=0.0003$, respectively) and incidence $(\mathrm{OR}=1.22$, $\mathrm{p}=0.02 ; \mathrm{OR}=1.18, \mathrm{p}=0.04$ and $\mathrm{OR}=1.20, \mathrm{p}=0.03$, respectively) of $\mathrm{T} 2 \mathrm{D} / \mathrm{IFG}$. Six haplotypes described $98 \%$ of genetic variation. Compared to TTGT, the most frequent haplotype (55\% frequency), CCAT, TCAC and TTAC were associated with a higher risk of T2D/IFG $(\mathrm{OR}=1.86, \mathrm{p}=0.00001$; $\mathrm{OR}=1.66$, $\mathrm{p}=0.01$ and $\mathrm{OR}=2.22, \mathrm{p}=0.0001$, respectively). The rs $198381 \mathrm{C}$ allele was associated with higher BNP concentrations (TT $5.44 \pm 0.45 \mathrm{pg} / \mathrm{ml}$, TC $6.94 \pm$
0.92 and CC $8.36 \pm 1.28, \mathrm{p}=0.03)$. The haplotype CCAC had higher plasma BNP concentrations ( $\mathrm{p}=0.04)$ compared to TTGT. In order to replicate this association, we selected the normoglycemic subjects from the DESIR study as controls $(n=3,065)$ and the T2D subjects from a cardiovascular trial in diabetes, the DIABHYCAR study $(n=3,160)$ as cases. The rare alleles of $\mathrm{rs} 198388$ and rs5065 were associated with higher risk of T2D $(\mathrm{OR}=1.19, \mathrm{p}=0.01$ and $\mathrm{OR}=1.44, \mathrm{p}=0.005$, respectively). Haplotype analysis confirmed this association. The haplotypes TCAC and TTAC were associated with a higher risk of $\mathrm{T} 2 \mathrm{D}(\mathrm{OR}=1.71, \mathrm{p}=0.02$ and $\mathrm{OR}=2.52, \mathrm{p}=0.0002$, respectively).

Conclusion: These results suggest a role of natriuretic peptides genes in the pathophysiology of T2D, possibly through BNP circulating levels and insulin resistance.

\section{2}

Genetic variation in ATP5O is associated with mRNA expression in human skeletal muscle and in vivo glucose uptake

T.S. Rönn ${ }^{1}$, P. Poulsen 2 , L. Groop ${ }^{1}$, A. Vaag' 2 , C. Ling ${ }^{1}$

${ }^{1}$ Diabetes and Endocrinology, Clinical Sciences, Lund University, Malmö, Sweden, ${ }^{2}$ Steno Diabetes Center, Gentofte, Denmark

Background and aims: Impaired oxidative capacity of the mitochondria in skeletal muscle has been suggested to contribute to insulin resistance and type 2 diabetes (T2D). Furthermore, mRNA expression of genes involved in oxidative phosphorylation, including the nuclear encoded ATP5O, is reduced in skeletal muscle from patients with T2D. However, the cause of this reduction in ATP5O mRNA level remains unknown. ATP5O is located in the stalk of the ATP synthase complex connecting the catalytic core and the membrane proton channel, thereby influencing conformational changes and proton conductance. We aimed to investigate 1) mechanisms regulating ATP5O expression in skeletal muscle and the association to in vivo measures of glucose metabolism, and 2) association between ATP5O single nucleotide polymorphisms (SNPs) and risk of T2D.

Materials and methods: ATP5O mRNA expression was analysed in skeletal muscle biopsies from young $(n=86)$ and elderly $(n=68)$ non-diabetic twins taken before and after a hyperinsulinaemic euglycaemic clamp. 11 SNPs from the ATP5O locus were genotyped in the twins and in a T2D case control cohort $(n=1466)$. DNA methylation of one region of the ATP5O promoter was analysed in a subset of twins (11 young and 11 elderly) using bisulfite sequencing.

Results: The mRNA level of ATP5O in skeletal muscle was reduced in elderly compared with young twins, both in the basal $(0.19 \pm 0.01$ versus 0.28 $\pm 0.01 ; P<0.0005)$ and insulin-stimulated $(0.21 \pm 0.01$ versus $0.30 \pm 0.01 ; P$ $<0.0005)$ state. Two SNPs influenced the expression of ATP5O of the young twins ( $\mathrm{rs} 12482697: \mathrm{T} / \mathrm{T}(\mathrm{n}=65) 0.30 \pm 0.010$ versus $\mathrm{T} / \mathrm{G}(\mathrm{n}=16) 0.25 \pm$ $0.012 ; P=0.02$ and $\mathrm{rs} 11088262: \mathrm{A} / \mathrm{A}(\mathrm{n}=70) 0.31 \pm 0.012$ versus $\mathrm{A} / \mathrm{G}(\mathrm{n}=16)$ $0.25 \pm 0.011 ; P=0.004$ ) during the clamp. A multivariate regression analysis showed that ATP5O expression in skeletal muscle was positively related to insulin-stimulated $P G C-1 \alpha$ expression $(P<0.0001)$ and inversely related to age $(P<0.0001)$ and female sex $(P<0.003)$. The degree of DNA methylation around the transcription start of ATP5O was low - and similar - in young $(0.14 \% \pm 0.07)$ and elderly $(0.73 \% \pm 0.03)$ twins, and ATP5O DNA methylation was not associated to mRNA expression $(r=0.24 ; P=0.32)$. The mRNA level of ATP5O in young and elderly twins $(n=155)$ was positively related to insulin stimulated glucose uptake (regression coefficient $=6.6 ; P=$ 0.02). In addition, the 2 SNPs influencing the expression level of ATP5O in skeletal muscle of the young twins were also associated with glucose uptake (rs12482697: T/T ( $\mathrm{n}=79) 12.1 \pm 0.36$ versus T/G $(\mathrm{n}=18) 9.8 \pm 0.75 ; P=0.005$ and rs11088262: A/A $(n=85) 12.2 \pm 0.4$ versus $A / G(n=18) 9.8 \pm 0.5 ; P=$ 0.002 , respectively). We could not detect any association between SNPs in the $A T P 5 O$ region and $\mathrm{T} 2 \mathrm{D}$.

Conclusion: $A T P 5 O \mathrm{mRNA}$ expression in human skeletal muscle is regulated by age, common polymorphisms, and PGC-1 $\alpha$ expression, and is associated to peripheral insulin sensitivity.

Supported by: Swedish Research Council, Novo Nordisk, Danish Medical Research Council, Malmö University Hospital, Swegene, Diabetes Programme at Lund University, Hedlund, Bergvall, Wiberg and Lundberg 


\section{PS 007 Genetic influence on beta cell function}

\section{3}

Decreased insulin secretion and increased risk of type 2 diabetes associated with allelic variations of the WFS1 gene encoding wolframin. The DESIR prospective study

N. Cheurfa ${ }^{1}$, G.M. Brenner ${ }^{1}$, D.A.F. Ferrarezi ${ }^{1}$, A.F. Reis ${ }^{2}$, J. Tichet ${ }^{3}$,

B. Balkau ${ }^{4}$, F. Fumeron ${ }^{1,5}$, M. Marre ${ }^{1,5}$, G. Velho ${ }^{1}$

${ }^{1}$ Unité 695, INSERM, Paris, France, ${ }^{2}$ Laboratory of Molecular

Endocrinology, Federal University of Sao Paulo, Brazil, ${ }^{3}$ IRSA, Institut Inter Régional pour la Santé, La Riche, France, ${ }^{4}$ Unité 780 - IFR69, INSERM, Villejuif, France, ${ }^{5}$ Faculté de Médecine Xavier Bichat, Université Denis Diderot, Paris, France

Background and aims: Mutations in the gene encoding wolframin (WFS1), an endoplasmic reticulum membrane protein, are responsible for Wolfram syndrome, a rare, recessive disorder characterised by diabetes mellitus, optic atrophy and other neurological and endocrinological abnormalities. Recently, common variants of WFS1 were shown to be associated with type 2 diabetes in cross-sectional studies. In the present study, we investigated associations of allelic variations in the WFS1 gene with insulin secretion, insulin sensitivity and the risk for type 2 diabetes in a 9 year prospective study.

Materials and methods: We studied 3962 unrelated French subjects from the general population who completed the 9 year protocol of the prospective DESIR study. Prevalence of impaired fasting glucose (IFG) and diabetes mellitus $(\mathrm{DM})$ at baseline was $6.7 \%(\mathrm{n}=264)$ and $3.4 \%(\mathrm{n}=134)$, respectively. During follow-up, 296 new cases of IFG and 203 new cases of DM were detected. Additional cross-sectional analyses were performed with 2980 French Caucasian type 2 diabetic patients from the DIABHYCAR cohort. Two SNPs were genotyped using Assay by Design kits from Applied Biosystems: rs7672995 (G>C, R228R, exon 6) and rs734312 (A>G, H611R, exon 8). All statistics were adjusted for sex, age and BMI.

Results: Cox proportional hazards survival regression analyses showed an association of the major G-allele of R228R variant (exon 6) with the incidence of DM (hazard ratio of 1.43, 95\% C.I. 1.09-1.48, $\mathrm{p}=0.008$ for a recessive model) or the combined incidence of IFG and DM (hazard ratio of 1.13, 95\% C.I. 1.01-1.28, $\mathrm{p}=0.05)$ in the follow-up of DESIR. An association was also observed with the total prevalence of IFG/DM at the end of the study (baseline plus incident cases) assessed by logistic regression analyses (odds ratio of $1.35,95 \%$ C.I. $1.03-1.78, p=0.03$ for a recessive model). Insulin secretion assessed at baseline in normoglycaemic subjects who developed IFG or DM during the study was decreased in carriers of the at risk G-allele: $\mathrm{HOMA} \% \mathrm{~B}$ $68 \pm 30 \%(\mathrm{GG}), 71 \pm 31 \%$ (GC) and $74 \pm 30 \%$ (CC; $\mathrm{m} \pm \mathrm{SD} ; \mathrm{p}=0.02$ for log-transformed data). Insulin sensitivity (HOMA\%S) was not different in carriers of the three genotypes. Similarly, the major A-allele of the H611R variant (exon 8 ) was associated with decreased insulin secretion at baseline and increased risk of DM (incidence and total prevalence). Cross-sectional analyses of combined DESIR and DIABHYCAR cohorts confirmed the at risk effect on diabetes of R228R G-allele (odds ratio of 1.38, 95\% C.I. 1.12-1.68, $\mathrm{p}=0.002$ ), H611R A-allele (odds ratio of 1.25, $95 \%$ C.I. 1.06-1.46, $p=0.007$ ) and the $\mathrm{G} / \mathrm{A}$ haplotype (frequency of 0.532 vs. 0.563 in normoglycaemic and diabetic subjects; odds ratio of $1.18,95 \%$ C.I. $1.05-1.29, \mathrm{p}=0.008$ ).

Conclusion: Allelic variations in the WFS1 gene modulated insulin secretion in normoglycaemic subjects at risk of developing type 2 diabetes, and increased the risk of type 2 diabetes in a 9 year prospective study. In our study, the most frequent haplotype in French Caucasians at the haplotype block containing the WFS1 gene was associated with increased risk of diabetes.

\section{4}

Relative importance of early insulin response and insulin sensitivity, corrected for measurement errors, on type 2 diabetes and $\mathrm{HbA}_{1 \mathrm{c}}$. Cross-sectional and 12 year follow-up studies

L.G.T. Berglund ${ }^{1,2}$, C. Berne ${ }^{3}$, K. Svärdsudd ${ }^{4}$, H. Garmo ${ }^{1}$, B. Zethelius ${ }^{2}$ ${ }^{1}$ Uppsala Clinical Research Center, ${ }^{2}$ Uppsala University, Department of Public Health/Geriatrics, ${ }^{3}$ Uppsala University, Department of Medical Sciences, ${ }^{4}$ Uppsala University, Department of Public Health/Family Medicine and Clinical Epidemiology, Sweden

Background and aims: In epidemiological studies the effects of impairments of insulin sensitivity and early insulin response on progression to type 2 dia- betes have not been independent of each other and the effect of the former variable has been considered the largest in bivariate models. Our hypothesis was that the effect of early insulin response has been underestimated due to low reliability.

Materials and methods: We used data from 1130 men in a population-based study and a reliability study where 17 men made a replicate measurement 4 to 6 weeks after the first measurement. The relations between response variables fasting and 2 hour glucose from an oral glucose tolerance test (OGTT) at baseline (age 70), fasting glucose and $\mathrm{HbA}_{1 \mathrm{c}}$ at a seven and twelve years follow-up, prevalent type 2 diabetes at baseline and incident type 2 diabetes over seven and twelve years of follow-up, and the predictors insulin sensitivity in$\operatorname{dex}(\mathrm{M} / \mathrm{I})$ from an euglycaemic insulin clamp, and early insulin response at an OGTT, were estimated using linear and logistic regression models, when measurement errors of both predictors were taken into account. Confidence intervals for the effects of the predictors and their difference were assessed with the bootstrap method.

Results: The effects of insulin sensitivity index and early insulin response at age 70 on fasting glucose at age 82 were strengthened by $6.1 \%$ and $24.5 \%$ $(\mathrm{p}=0.13)$, respectively, and on $\mathrm{HbA}_{1 \mathrm{c}}$ at age 82 the effects were strengthened by $1.1 \%$ and $30.4 \%(p<0.001)$, respectively, when corrected for measurement errors. In the corrected models the greater strengthening of early insulin response made the effects of insulin sensitivity index and early insulin response on the response variables to be essentially similar for fasting glucose but for $\mathrm{HbA}_{1 \mathrm{c}}$ the relative importance of early insulin response was found to be higher as compared to insulin sensitivity index $(\mathrm{p}=0.03)$. Results were similar for the other response variables.

Conclusion: The results imply that the etiological effect of early insulin response has been underestimated to a greater extent due to low reliability as compared to insulin sensitivity. Our results suggest that reliability should be considered for the design of intervention studies and preventive studies.

\section{5}

SLC2A2 gene polymorphisms and human beta cell function S. Del Guerra ${ }^{1}$, R. Lupi ${ }^{1}$, M. Bugliani ${ }^{1}$, S. Torri ${ }^{1}$, M. Galli ${ }^{1}$, V. D’Aleo ${ }^{1}$, S. Del Prato $^{1}$, S. Del Prato ${ }^{1}$, U. Boggi ${ }^{2}$, F. Filipponi ${ }^{2}$, P. Marchetti ${ }^{1}$

${ }^{1}$ Endocrinology and Metabolism - Metabolic Unit, University of Pisa, ${ }^{2}$ Department of Oncology, Transplantation and Advanced Technologies in Medicine, University of Pisa, Italy

Background and aims: An association between single nucleotide polymorphisms (SNPs) in SLC2A2, the gene encoding glucose transporter 2 (GLUT2), and type 2 diabetes has been reported.

Materials and methods: To evaluate some features of islets (HI) from subjects with the rs5393 and/or rs5394 SNPs, we prepared HI from the pancreas of non-diabetic (ND) and type 2 diabetic (T2D) organ donors, matched for age and body mass index. HI were isolated by collagenase digestion and density gradient purification; SNPs were identified by the TaqMan Allelic Discrimination Assay (Applied Biosystem); insulin secretion was measured in response to acute ( $45 \mathrm{~min}$ ) exposure to 3.3 and $16.7 \mathrm{mmol} / 1$ glucose; SLC2A2 mRNA expression was assessed by quantitative RT-PCR.

Results: Subjects carrying the rs5393 or rs5394 polymorphism were respectively 50 and $20 \%$ in ND, and 40 and $45 \%$ in T2D. Both polymorphisms were present in 5 and $30 \%$ of ND and T2D respectively ( $\mathrm{p}=0.05$ by the chi-square test). Glucose-stimulated (16.7 mmol/l) insulin release (GSIR) was lower in T2D than ND $(0.045 \pm 0.022$ vs $0.168 \pm 0.112 \mu \mathrm{U} /$ islet $/ \mathrm{min}, \mathrm{p}<0.01)$, which was accompanied by reduced stimulation index (SI, $1.54 \pm 0.62$ vs $3.77 \pm 1.95$, $\mathrm{p}<0.01$ ). Both in ND and T2DM, GSIR was $~ 40 \%$ lower when the rs 5394 SNP was present, either alone or in combination with rs5393. This lead to a statistically $(p<0.05)$ higher percentage of patients with SI below the median value in the presence of rs5394 (76.9\%) than in its absence (22.7\%). The presence of the rs5393/5394 polymorphisms was in addition associated with a $40 \%$ reduction of GLUT2 gene mRNA expression.

Conclusion: The combined presence of rs5393/5394 polymorphisms of GLUT2 gene was more frequent in T2D than ND in the present series; rs5394 appeared to be associated with decreased glucose stimulated insulin release; the SLC2A2 gene polymorphisms were accompanied by a $\sim 40 \%$ reduction of SLC2A2 mRNA. 


\section{6}

CDKAL1 is associated with increased risk for type 2 diabetes and beta cell dysfunction: joint analyses of the Singapore National Health Survey 1998 and the Singapore Diabetes Cohort Study

D.P.K. Ng ${ }^{1}$, M. Tan ${ }^{2}$, S. Ye ${ }^{1}$, S. Nurbaya ${ }^{1}$, D. Koh ${ }^{1}$, W. Kon ${ }^{3}$, H. Leong ${ }^{4}$,

E. $\mathrm{Tai}^{2}$

${ }^{1}$ Community, Occupational and Family Medicine, National University of Singapore, ${ }^{2}$ Endocrinology, Singapore General Hospital, ${ }^{3}$ Endocrinology, Tan Tock Seng Hospital, Singapore, ${ }^{4}$ Clinical Services, National Healthcare Group Polyclinics, Singapore

Background and aims: Genetic variation at the cyclin-dependent kinase 5 regulatory subunit-associated protein 1-like $1(C D K A L 1)$ locus on $6 \mathrm{p} 22.3$ has been associated with increased risk for type 2 diabetes mellitus (T2DM) and beta-cell dysfunction in a number of studies on Caucasians. However, its relevance in Asians is much less established.

Materials and methods: We examined the association between the CDKAL1 rs7756992 SNP with T2DM using a case-control study design. A total of 2098 T2DM cases (1514 Chinese, 353 Malay and 231 Asian Indian) were from the primary care-based Singapore Diabetes Cohort Study (SDCS) while 2764 controls (1965 Chinese, 442 Malay and 339 Asian Indians) were subjects with normal glucose tolerance (NGT) that formed part of the population-based Singapore National Health Survey 1998 (NHS98) collection. The association between rs7756992 and diabetes-related traits including HOMA beta-cell function was analyzed using data from 2817 Chinese, 774 Malays and 583 Asian Indian subjects from the NHS98, irrespective of their level of glucose tolerance.

Results: The G allele of rs7756992 was strongly associated with T2DM in Chinese $(\mathrm{OR}=1.24,95 \% \mathrm{CI}=1.12-1.38, \mathrm{P}<0.001)$, with nominal significance among Malays $(\mathrm{OR}=1.28,95 \% \mathrm{CI}=1.03-1.58, \mathrm{P}=0.023)$ and Asian Indians $(\mathrm{OR}=1.37,95 \% \mathrm{CI}=1.03-1.81, \mathrm{p}=0.033)$ under an additive model of inheritance. These genetic associations were independent of gender and body mass index. The $\mathrm{G}$ risk allele was also associated with lower values for HOMA betacell function after adjustment for gender, age and HOMA insulin resistance among Chinese subjects $(\mathrm{P}=0.037)$ but not in the more limited collections of Malays and Asian Indians.

Conclusion: CDKAL1 and its related pathways are likely to be important in the pathogenesis of T2DM across diverse Asian ethnic groups. Supported by: National Medical Research Council, Singapore

\section{7}

eNOS D298D variant is associated to reduced first phase of insulin secretion in first degree relatives of type 2 diabetic subjects R. D'Alfonso ${ }^{1}$, S. Rizza ${ }^{1}$, M. Cardellini ${ }^{1}$, S. Frontoni ${ }^{1}$, M.L. Hribal ${ }^{2}$, M.A. Marini ${ }^{1}$, G. Sesti ${ }^{2}$, R. Lauro ${ }^{1}$, M. Federici ${ }^{1}$

${ }^{1}$ Internal Medicine, University of Rome Tor Vergata, Italy, ${ }^{2} \mathrm{Clinical}$ and Experimental Sciences, University of Catanzaro Magna Græcia, Italy

Background and aims: Type 2 diabetes mellitus is characterized by impaired insulin secretion and reduced insulin action. Cellular studies and animal models suggested that endothelial nitric oxide syntase (eNOS) plays a role in insulin action. We and others observed that eNOS D298D variant is associated to insulin resistance in first degree relatives of type 2 diabetes subjects(FDRs). However, FDRs are also characterized by impaired insulin secretion in the first phase. eNOS plays a major role in regulating microand macrovascular function and architecture. Recently it has been suggested that that in the ZDF rat model of type 2 diabetes, an inability of the islet to maintain vascular integrity may contribute to beta-cell failure, while in the Otsuka-Long-Evans-Tokushima fatty rat, islet degeneration leading to diabetes is associated with loss of capillary number within the islet. Because of the pivotal role of eNOS in regulating vascular function we investigated if the D298D eNOS variant, which was shown to impair eNOS function, might be associated to defective beta cell function.

Materials and methods: We investigated eNOS D298 variant in 296 offspring of diabetics parents (T2DM) (109 women, 187 men), 122 diabetics patients ( 59 women, 63 men) all with $\mathrm{BMI}<35$ and age $<75$ years and 38 ( 20 women, $18 \mathrm{men}$ ) normal glucose tolerant subjects. All subjects were investigated with fasting glucose and insulin levels, lipid parameters, anthropometric and blood pressure values. All FDRs were subjected to euglycemic hyperinsulinemic clamp and oral glucose tolerance test (OGTT). Control subjects have no family history of diabetes. First-phase and second-phase insulin secretion were determined using validated indexes previously proposed by Stumvoll. E298D polymorphism was studied with polymerase chain reaction (PCR) amplification followed by enzymatic digestion with $\mathrm{MboI}$. All statistical analyses were performed using the SPSS software program.

Results: Among FDRs 46.7\% were homozygous for the wild-type E298 eNOS, 30.7\% were heterozygous and $22.6 \%$ homozygous for D298 eNOS allele. Among DM2 subjects 46.3\% were homozygous for the wild-type E298 eNOS, $25.3 \%$ were heterozygous and 28.4 homozygous for D298 eNOS allele. Among control subjects 57.9\% were homozygous for the wild-type E298 eNOS, $34.2 \%$ were heterozygous and 7.9\% homozygous for D298 eNOS allele. No significant differences were observed among carriers of the 3 genotypes for age, sex, BMI and body composition. In FDRs, multivariate analysis corrected for age, sex and BMI revealed that the presence of the eNOS D298 variant was associated to slightly but significant reduced first phase of insulin secretion as estimated by the Stumvoll index $(\mathrm{p}<0.001)$. In DM2 logistic regression analysis corrected for age, sex and BMI revealed that homozygosity for D298 compared to wild-type E298 eNOS was significantly associated to secondary failure $(\mathrm{p}<0.039)$.

Conclusion: Our preliminary study suggest that the presence of the D298 eNOS variant may impair beta cell function. Further studies in larger cohorts are necessary to establish a role for this variant in regulation of insulin secretion

\section{8}

The concurrent presence of risk alleles for TCF7L2 and HHEX/IDE potentiates the insulin secretory defect in subjects at high risk of diabetes. The GENFIEV Study

C. Bianchi ${ }^{1}$, R. Miccoli ${ }^{1}$, N. Pulizzi ${ }^{1}$, R. Bonadonna ${ }^{2}$, M. Trombetta ${ }^{2}$, F. Giorgino ${ }^{3}$, S. Frontoni ${ }^{4}$, F. Cavalot ${ }^{5}$, G. Cavallo ${ }^{6}$, E. Faloia ${ }^{7}$, G. Marchesini ${ }^{8}$, F. Leonetti ${ }^{9}$, A. Consoli ${ }^{10}$, L. Groop ${ }^{11}$, S. Del Prato ${ }^{1}$

${ }^{1}$ Department of Endocrinology and Metabolism, University of Pisa, Italy, ${ }^{2}$ Department of Endocrinology and Metabolism, University of Verona, Italy, ${ }^{3}$ Department of Endocrinology and Metabolism, University of Bari, Italy, ${ }^{4}$ Department of Internal Medicine, University Tor Vergata, Roma, Italy, ${ }^{5}$ Department of Clinical Biological Sciences, University of Turin, Italy, ${ }^{6}$ Department of Endocrinology and Metabolism, University La Sapienza, Rome, Italy, ${ }^{7}$ Division of Endocrinology, Polytechnic University of Marche, Ancona, Italy, ${ }^{8}$ Diabetology, University of Bologna, Italy, ${ }^{9}$ Department of Clinical Sciences, University La Sapienza, Rome, Italy, ${ }^{10}$ Department of Medicine and Aging Sciences, University of Chieti, Italy, ${ }^{11}$ Department of Clinical Sciences, University of Malmö, Malmö, Sweden

Background and aims: Recently, genetic variants at the transcription factor 7-like 2 gene (TCF7L2) locus have been found to confer greater risk for developing diabetes. Moreover, statistically significant association with impaired insulin secretion but not with impaired insulin action has been found. Similar effects has been reported for the HHEX/IDE (hematopoietically expressed homeobox, insulin degrading enzyme) gene so that the possibility exists for gene-to-gene interaction that may enhance beta-cell dysfunction and risk of developing Type 2 diabetes (T2DM).

Materials and methods: The GENFIEV (Genetics, Physiopatolohy and Evolution of Type 2 diabtes) study is a mutlicenter Italian trial designed to identify and characterize subjects at risk of T2DM. A total of 488 non-diabetic subjects have been identified ( $41 \%$ men $/ 59 \%$ women; age $49 \pm 11$ years; BMI $29 \pm 5 \mathrm{Kg} / \mathrm{m}^{2}$ ) within the GENFIEV cohort in whom rs7903146 of TCF7L2 and rs1111875 of HHEX/IDE SNPs have been genotyped. Effects of these genotypes on measures of insulin sensitivity (1/basal insulin) and insulin secretion have been then assessed. Beta-cell function was estimated by minimal model analysis of plasma glucose and C-peptide response to a 2-hr 75-g OGTT. This analysis allows quantification of fasting insulin secretion (B-IS), beta-cell sensitivity at glucose incremental rate, i.e. derivative control (S-CD; pmol. $\mathrm{m}^{-2}$ BSA per mM.min ${ }^{-1}$ ), and glucose - proportional control (S-CP), i.e. the curve of insulin secretion in response to glucose 4.0, 5.5, 8.0 e $11 \mathrm{mM}$ (CP; pmol. $\mathrm{min}^{-1} \cdot \mathrm{m}^{-2}$ BSA per $\mathrm{mM}$ ).

Results: Subjects homozygous for the T allele of TCF7L2, but not for the G allele of $H H E X / I D E$, had higher $1 /$ fasting insulin value (ANOVA: $\mathrm{p}=0.05$ ) but significantly lower B-IS (Kruskal-Wallis Test $\mathrm{p}=0.001)$ and C-CD $(\mathrm{p}=0.002)$ as compared to CC or CT subjects. S-CP, reflecting second-phase insulin secretion, was more impaired in TT subjects than in carriers of the CT e CC alleles of TCF7L2 (ANOVA for repeated measures, $\mathrm{p}<0.05$ ) as well as than in the GG alleles of the HHEX/IDE gene (ANOVA, $\mathrm{p}<0.006$ ). Based on relatively high frequency of risk alleles for both TCF7L2 (65\%) and HHEX/IDE (85\%) genes, we evaluated whether the two genes could exert a combination effect. Both B-IS and S-CD significantly and progressively lowered with the increase 
in the number of risk alleles (no risk alleles: 109 (IQR, 47) and 1229 (1120); 1 risk allele: 98 (53) and 1224 (1120); 2 risk alleles: 97 (63) and 1406 (1323); 3 risk alleles: 95 (52) and 1013 (857); 4 risk alleles: 69 (39) and 954 (928); $\mathrm{p}<0.01$ ), while $\mathrm{S}-\mathrm{CP}$ reduction did not reach the statistical significance.

Conclusion: This preliminary analysis of the genotype and phenotype of non-diabetic subjects from the GENFIEV cohort indicates that TCF7L2 has more pronounced effect on beta-cell function than HHEX/IDE but it also suggests that interaction of TCF7L2 with HHEX/IDE has an additive effect, resulting in further impairment of beta-cell function.

\section{9}

Common genetic variation within the NR4A3 locus associates with beta cell function in non-diabetic Germans

H. Staiger, F. Machicao, N. Stefan, A. Fritsche, H.-U. Häring

Internal Medicine IV, Medical Clinic, University Hospital Tübingen,

Germany

Background and aims: Nor-1 (neuron-derived orphan receptor 1), nuclear receptor-related protein (Nurr) 77, and Nurr-1 constitute the NR4A family of orphan nuclear receptors which were recently found to regulate hepatic glucose production, insulin signalling in adipocytes, and oxidative metabolism in skeletal muscle. In this study, we assessed whether common genetic variation within the NR4A3 locus, encoding Nor-1, contributes to the development of prediabetic phenotypes, such as glucose intolerance, insulin resistance, or beta-cell dysfunction.

Materials and methods: We genotyped 1495 non-diabetic subjects from Southern Germany for the five tagging single nucleotide polymorphisms (SNPs) rs7047636, rs1526267, rs2416879, rs12686676, and rs10819699 (minor allele frequencies $>=0.05$ ) covering $100 \%$ of genetic variation within the NR4A3 locus $\left(\mathrm{D}^{\prime}=1.0, \mathrm{r}^{2}>=0.9\right)$ and assessed their association with metabolic data derived from the fasting state, an OGTT, and a hyperinsulinemic-euglycemic clamp (subgroup, $\mathrm{N}=506$ ).

Results: All five SNPs were in Hardy-Weinberg equilibrium ( $\mathrm{p}>=0.7$, all; $\mathrm{chi}^{2}$-test). The minor allele of rs7047636 was associated with elevated plasma glucose levels at $120 \mathrm{~min}$ of OGTT ( $\mathrm{p}=0.007$; dominant model; $\mathrm{t}$-test). The minor allele of rs1526267 was associated with a higher BMI and reduced clamp-derived insulin sensitivity ( $\mathrm{p}=0.01$, both; dominant model; t-test). Most convincingly however, the minor alleles of three SNPs, i.e., rs1526267, rs12686676, and rs10819699, were consistently associated with higher insulin release as derived from the insulinogenic index and the $\mathrm{AUC}_{\mathrm{C} \text {-peptide }}$-to$\mathrm{AUC}_{\text {glucose }}$ ratio ( $<<0.05$, all; dominant model; t-test).

Conclusion: Common genetic variation within the NR4A3 locus determines insulin secretion. Thus, NR4A3 represents a novel candidate gene for betacell dysfunction and type 2 diabetes mellitus.

\section{0}

Variants in CDKAL1 and IGF2BP2 affect first phase insulin secretion during hyperglycaemic clamps

L.M. 't Hart' 1 , M.J. Groenewoud ${ }^{1}$, J.M. Dekker', A. Fritsche 3 , G. Nijpels',

E. Reiling ${ }^{1}$, R.J. Heine ${ }^{2}$, J.A. Maassen ${ }^{1,2}$, F. Machicao ${ }^{3}$, S.A. Schäfer ${ }^{3}$,

H.-U. Häring ${ }^{3}$, T.W. van Haeften ${ }^{4}$

${ }^{1}$ Molecular Cell Biology, Leiden University Medical Center, Leiden,

Netherlands, ${ }^{2}$ EMGO Institute, Vrije Universiteit Medical Center,

Amsterdam, Netherlands, ${ }^{3}$ Dept. of Internal Medicine, Eberhard-Karls

University of Tübingen, Germany, ${ }^{4}$ Internal Medicine, Utrecht University

Medical Center, Netherlands

Background and aims: Recent genome-wide association (GWA) studies have identified at least 6 novel type 2 diabetes susceptibility gene regions. We assessed the effect of these regions on insulin secretion and insulin sensitivity index as determined by a hyperglycaemic clamp.

Materials and methods: Gene variants in HHEX/IDE, CDKAL1, SLC3OA8, IGF2BP2 and CDKN2A/CDKN2B were genotyped in a cohort of 146 normal glucose tolerant (NGT) and 126 impaired glucose tolerant (IGT) subjects from the Netherlands and Germany, who all underwent a hyperglycaemic clamp at $10 \mathrm{mmol} / \mathrm{l}$ glucose.

Results: Variants in CDKAL1 and IGF2BP2 are associated with reductions in first phase insulin secretion $(34 \%$ and $28 \%$ resp., $\mathrm{P}<0.01)$ and disposition index $(\mathrm{p}<0.02)$. No effect of these variants on second phase insulin secretion was shown in our cohorts. For the gene regions near HHEX/IDE, SLC30A8 and $C D K N 2 A / C D K N 2 B$ we do not find associations with altered insulin se- cretion (Figure 1). Furthermore, the gene variants were not associated with insulin sensitivity indexes.

Conclusion: Our data show that gene variants in CDKAL1 and IGF2BP2 attenuate the first phase of glucose stimulated insulin secretion, but show no effect on the second phase of insulin secretion. Our results based on hyperglycaemic clamps provide further insight into the pathogenic mechanism behind the association of these gene variants with type 2 diabetes.

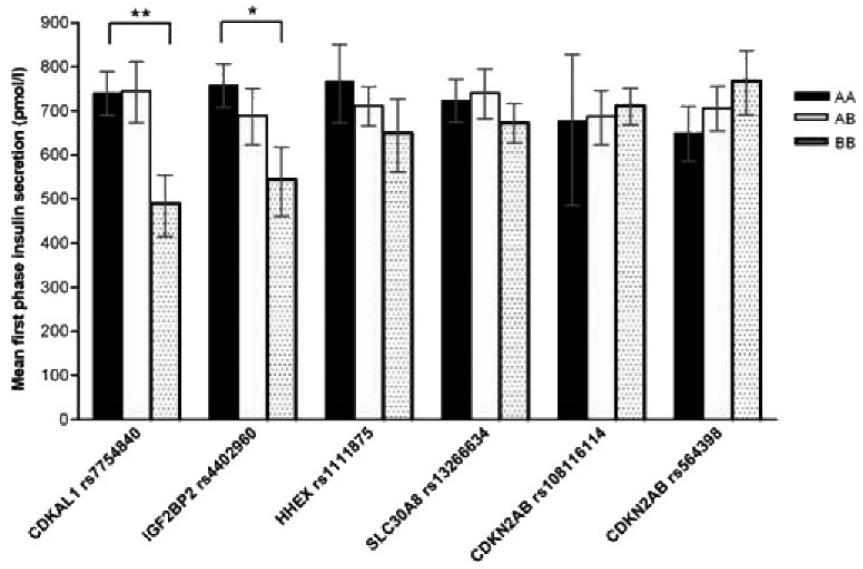

Supported by: Dutch Diabetes Research Foundation 


\section{PS 008 Genetic influence on insulin sensitivity}

\section{1}

Pro72Arg polymorphism of TP53 affects insulin sensitivity in diabetic subjects

R. Testa ${ }^{1}$, A.R. Bonfigli ${ }^{1}$, C. Sirolla ${ }^{2}$, M. Marra ${ }^{1}$, L. Spazzafumo ${ }^{2}$, M. Boemi ${ }^{1}$, F. Olivieri ${ }^{3}$, S. Salvioli ${ }^{4}$, C. Franceschi ${ }^{4}$, I. Testa ${ }^{1}$

${ }^{1}$ Diabetology Unit, IRCCS-INRCA, Ancona, ${ }^{2}$ Centre of Epidemiology and Biostatistics, IRCCS-INRCA, Ancona, ${ }^{3}$ Faculty of Medicine, Polytechnic University of Marche, Ancona, Italy, ${ }^{4}$ Department of Experimental

Pathology, University of Bologna, Italy

Background and aims: It has recently been demonstrated that p53 regulates genes implicated in energy generating metabolic pathways, suggesting its possible involvement in diabetes. The aim of this study was to evaluate whether Pro72Arg polymorphism of TP53 is associated with metabolic parameters and insulin resistance in diabetic subjects.

Materials and methods: The study design was cross-sectional and observational. We studied 303 type 2 diabetic patients (mean age \pm SD, $65.2 \pm 8.6$ years, 180 men and 123 women) and 328 non diabetic control subjects (mean age \pm SD, $65.6 \pm 8.1$ years, 190 men and 138 women) matched for age and sex. Results: The frequencies of the different genotypes were: 183 (55.8\%) Arg/ Arg, 121 (36.9\%) Arg/Pro and 24 (7.3\%) Pro/Pro genotypes in the non diabetic group and 169 (55.8\%) Arg/Arg, 109 (35.9) Arg/Pro and 25 (8.3\%) Pro/Pro genotypes in the diabetic group. This genotype distribution was not different between diabetic and non diabetic subjects $\left(\chi^{2}=0.24 ; P=0.88\right)$. The 2-way analysis of variance highlights that the increase in fasting glucose plasma levels in diabetic subjects was due to the effect of diabetes $(\mathrm{F}=313.11$, $P<0.01)$ whereas no $\mathrm{p} 53$ genotype $(\mathrm{F}=0.25 ; P=0.77)$ and $\mathrm{p} 53$ genotype-diabetes interaction effects $(\mathrm{F}=0.25 ; P=0.77)$ were found. Equally, no p53 genotype or genotype-diabetes interaction effects were detected for BMI, waist and HbAlc. Significant genotype-diabetes effect on HOMA index was found $(\mathrm{F}=4.25 ; P=0.01)$. In diabetic patients, Pro/Pro subjects showed significant lower levels of HOMA with respect to Arg/Arg and Arg/Pro carriers ( $\mathrm{F}=4.73$, $P=0.03$ and $\mathrm{F}=8.05, P<0.01$, respectively). Noteworthy, no difference between diabetic and non diabetic Pro/Pro carriers was found $(F=0.75 ; P=0.38)$. Significant p53 genotype-diabetes interaction effects were found on the levels of fasting insulin, HDL cholesterol and triglycerides. In particular, these variables showed a genotype-dependent trend in accordance with the variation of insulin resistance.

Conclusion: Our results indicate for the first time that this polymorphism may affect insulin sensitivity in diabetic subjects. The key results of our approach are the following: 1) in diabetic subjects, the Pro/Pro genotype is associated with lower values of HOMA index with respect to Arg carriers; 2) HOMA index values are similar for diabetic and non-diabetic Pro/Pro subjects. These findings could open a new field of research in the complex metabolic network regulated by $\mathrm{p} 53$.

\section{2}

The DGAT2 gene is a candidate for dissociation between fatty liver and insulin resistance in humans

K. Kantartzis' ${ }^{1}$, F. Machicao ${ }^{1}$, J. Machann² ${ }^{2}$, F. Schick² ${ }^{2}$ A. Fritsche ${ }^{1}$, H.-U. Häring ${ }^{1}$, N. Stefan ${ }^{1}$

${ }^{1}$ Department of Internal Medicine IV, ${ }^{2}$ Section on Experimental Radiology, University of Tübingen, Germany

Background and aims: The enzyme acyl-CoA:diacylglycerol acyltransferase (DGAT) catalyzes the final step of triglyceride synthesis. Mice overexpressing hepatic DGAT2, the isoform predominantly expressed in the liver, developed fatty liver, but not insulin resistance, under a high-fat diet. In the present study we hypothesized that human DGAT2 may have a similar function, and investigated whether common single nucleotide polymorphisms (SNPs) in DGAT2 contribute to dissociation between fatty liver and insulin resistance in humans.

Materials and methods: The SNPs rs10899116 C > T and rs1944438 C>T in DGAT2, which had a minor allele frequency of $>0.05$ and covered $100 \%$ of the alleles with a D' of 1 were genotyped in 187 individuals at risk for type 2 diabetes who participated in a lifestyle intervention program with diet modification and increase in physical activity. Total body fat and visceral fat were determined by magnetic resonance (MR) tomography and liver fat and intramyocellular fat by ${ }^{1} \mathrm{H}-\mathrm{MR}$ spectroscopy. Insulin sensitivity was estimated from the oral glucose tolerance test (OGTT) and in a subgroup measured by the euglycemic hyperinsulinemic clamp.

Results: After 9 months of intervention total body fat, visceral fat and intramyocellular fat significantly decreased (all $p>0.22$ ). Liver fat also decreased $(-33 \%, \mathrm{p}<0.0001)$ and insulin sensitivity increased (OGTT, 13.5\%, $\mathrm{p}<0.0001$; clamp, $13 \%, \mathrm{p}=0.04)$. There was an inverse correlation of change in liver fat with change in insulin sensitivity (OGTT, $r=-0.31, \mathrm{p}<0.0001$; clamp, $\mathrm{r}=-0.32$, $\mathrm{p}=0.03$ ). Carriers of the minor T allele of the SNP rs1944438 had less decrease in liver fat (T/T, $-24 \pm 5 \%$; $\mathrm{C} / \mathrm{T},-17 \pm 10$ ) compared to $\mathrm{C} / \mathrm{C}$ homozygotes ($39 \pm 7 \%, \mathrm{p}=0.008)$. In contrast, the SNP was not associated with change in insulin sensitivity (OGTT, $\mathrm{p}=0.79$; clamp, $\mathrm{p}=0.53$ ). Likewise, subjects with fatty liver $(n=54)$ carrying the minor T allele of SNP rs1944438 had less decrease in liver fat compared to $\mathrm{C} / \mathrm{C}$ homozygotes $(\mathrm{p}=0.02)$, but displayed similar change in insulin sensitivity calculated from the OGTT $(\mathrm{p}=0.52)$.

Conclusion: These data suggest that DGAT2 mediates dissociation between fatty liver and insulin resistance in humans. This finding may be of clinical relevance in the prevention and treatment of insulin resistance and type 2 diabetes in subjects with fatty liver.

Supported by: Deutsche Forschungsgemeinschaft

\section{3}

Insulin secretion and insulin sensitivity in first degree relatives of subjects with impaired glucose regulation

S.M. Shefin ${ }^{1}$, R.M. Hafizur ${ }^{2}$, N.M. Islam², M.M. Hossain ${ }^{2}$, Z.A. Latif ${ }^{1}$, S. Akter ${ }^{2}$, Q. Nahar ${ }^{2}$, S. Islam², K. Hasan², M.O. Faruque 2 , L. Ali ${ }^{2}$ ${ }^{1}$ Endocrinology and Diabetology, ${ }^{2}$ Dept Biochemistry \& Cell Biology, BIRDEM, Dhaka, Bangladesh

Background and aims: First degree relatives of T2 DM subjects are at increased risk for the disease, but no studies exist on the first degree relatives of subjects with impaired glucose regulation (IGR). There are also substantial genetic heterogeneity regarding the pathophysiology of diabetes and thus the basic defects of the disorder should be investigated in different populations. Present study was aimed to investigate the insulin secretory capacity and insulin sensitivity of the first degree relatives of the Bangladeshi IGR subjects. Subjects and methods: Forty one first degree relatives of 14 IFG cases (RIFG), 116 first degree relatives of 36 IGT cases (RIGT), 76 first degree relatives of 20 combined IFG-IGT cases (RIFG-IGT) and 20 age-matched healthy controls were recruited. Serum glucose was measured by glucose-oxidase method, lipid profile by enzymatic-colorimetric method and serum insulin by ELISA. Insulin secretory capacity (HOMA \% B) and insulin sensitivity (HOMA \% S) were calculated by fasting serum glucose and fasting serum insulin.

Results: BMI (M $\pm \mathrm{SD}, \mathrm{kg} / \mathrm{m}^{2}$; Control, 22 \pm 2 ; RIFG, 24 \pm 5 ; RIGT, 22 \pm 4 ; RIFGIGT, 23 \pm 4 ) was significantly higher in RIFG and RIFG-IGT subjects compared to Controls. Fasting serum glucose (FG; mmol/l; Controls, 4.8 \pm 0.4 ; RIFG, 5.4 \pm 0.8 ; RIGT, $5.8 \pm 2.2$; RIFG-IGT, $5.9 \pm 1.4$ ) was significantly higher in RIFG and RIFG-IGT ( $\mathrm{p}=0.0001)$ compared to Controls. RIGR groups had significantly ( $\mathrm{p}=0.0001$ ) higher postprandial serum glucose (Controls, $5.4 \pm 1.0$; RIFG, 7.1 \pm 2.7 ; RIGT, 8.2 \pm 5.1 ; RIFG-IGT, 8.3 \pm 4.9 ) compared to Controls. Dyslipidemia was evident in RIGR subjects compared to Controls although the values were within the normal range. HOMA\% B [Median (range); Controls, 101 (62-159); RIFG, 79 (23-185); RIGT, 76 (16-201); RIFG-IGT, 78 (13-152)] was significantly lower among the RIFG, RIGT and RIFG-IGT subjects compared to Controls. HOMA\% S [Controls, 115 (74-268); RIFG, 85(35-251); RIGT, 112 (13-288); RIFG-IGT, 84(21-288)] value in RIFG-IGT subjects were significantly lower compared to Controls. When RIGR subjects were categorized using the BMI cut-off point standardized for Bangladeshi population, all the three RIGR groups with BMI values $>23$ have shown significantly less insulin sensitivity compared to their counterparts with $\mathrm{BMI}<23$. In bivariate correlation (Spearman) analysis it was found that HOMA\% S was negatively $(\mathrm{p}<0.01)$ and HOMA\% B was positively $(\mathrm{p}<0.001)$ correlated with BMI, waist circumference and percent body fat in all the three RIGR groups. On logistic regression analysis with pooled data from all the RIGR subjects a significant positive association of the prediabetic-relative state with HOMA \%B and a significant negative association of the same state with HOMA \%S was found even when the effects of other confounding/covariates were adjusted. Multiple regression revealed that HOMA \%B in IGR is associated with BMI and WHR, and HOMA \%S in these subjects is associated with BMI and percent body fat contents when the effects of other confounding factors are adjusted. Conclusion: a) $\beta$-cell secretory dysfunction seem to have a genetic origin in all groups of IGR in Bangladeshi population, b) the genesis of the various 
phenotypes of IGR results from a complex interaction between the primary defect in insulin secretion and other primary or secondary factors contributing to insulin resistance.

Supported by: Diabetic Association of Bangladesh

\section{4}

The frequent $U C P 2-866 \mathrm{G}>\mathrm{A}$ protects against insulin resistance in Danes: large-scale studies of obesity and related metabolic traits

L. Wegner ${ }^{1}$, G. Andersen ${ }^{1}$, L.T. Dalgaard ${ }^{2}$, S. Anthonsen ${ }^{1,2}$, T. Nielsen ${ }^{1}$,

N. Grarup ${ }^{1}$, K. Borch-Johnsen ${ }^{1,2}$, T. Jørgensen ${ }^{3}$, A. Sandbæk ${ }^{4}$, T. Lauritzen ${ }^{5}$, T. Hansen ${ }^{1}$, J. Holmkvist ${ }^{1}$, O. Pedersen ${ }^{2,4}$

${ }^{1}$ Steno Diabetes Center, Gentofte, ${ }^{2}$ Department of Science, Systems and Models, Roskilde University, ${ }^{3}$ Research Centre for Prevention and Health, Glostrup University Hospital, ${ }^{4}$ Faculty of Health Science, University of Aarhus, ${ }^{5}$ Department of General Practice, University of Aarhus, Denmark

Background and aims: Uncoupling protein-2 (UCP2) is a mitochondrial transporter that uncouples oxidative phosphorylation via proton leakage from the inner mitochondrial membrane. It is involved in the regulation of reactive oxygen species production and energy metabolism and is ubiquitously expressed. The frequent rs659366 in the UCP2 promoter has been extensively studied across different populations; however, the results have not been consistent. The A-allele of rs659366 has been shown to associate with reduced risk of obesity among white Europeans, with reduced and increased risk of type 2 diabetes (T2D), and quantitative metabolic traits. The aim of this study was to investigate if the A-allele of rs659366 is associated with T2D, obesity, or quantitative metabolic traits in large-scale studies of Danes.

Materials and methods: The UCP2 rs659366 was genotyped in 13,588 Danes involving the population-based Inter99 cohort of middle-aged people $(n=6,051)$, the ADDITON study $(n=7,220)$, and a population-based sample of young healthy subjects $(n=317)$ using Taqman allelic discrimination. Results: No association of rs659366 with obesity or T2D was observed; however, in studies of 6,051 individuals of the Inter99 cohort A-allele carriers had impaired insulin release (fasting $[p=0.002]$ and 120 -min post-OGTT $[p=0.02])$ and lower fasting glycaemia $(p=0.02)$. Furthermore, young healthy A-allele carriers were less insulin-resistant (HOMA-IR, $p=0.0007$ ), and more insulin-sensitive $(p=0.02)$.

Conclusion: The A-allele of rs659366 is not associated with obesity or T2D in Danes; however, middle-aged A-allele carriers have impaired glucose-stimulated insulin release combined with increased insulin sensitivity.

Supported by: Danish Medical Research Council, Danish Diabetes Association, Gerda and Aage Haensch Foundation, A.P. Møller Foundation for the Advancement of Medical Science, University of Copenhagen, European Commission, Danish Obesity Research Centre, Danish Council for Strategic Research

\section{5}

The TRIB3 R84 variant is associated to selective insulin resistance in human umbilical vein endothelial cells in vitro and early atherosclerosis in vivo

M.L. Hribal ${ }^{1}$, G. Formoso ${ }^{2}$, F. Andreozzi ${ }^{1}$, S. Di Silvestre ${ }^{3}$, S. Prudente ${ }^{4}$, M.A. Marini ${ }^{5}$, A. Pandolfi ${ }^{3}$, V. Trischitta ${ }^{6,4}$, G. Sesti ${ }^{1}$, A. Consoli $^{2}$

${ }^{1}$ Dept of Experimental and Clinical Medicine, University of Magna Graecia, Catanzaro, ${ }^{2}$ DMSI, CeSI Institute, G. d'Annunzio University, Chieti, ${ }^{3}$ Dept of Biomedical Sciences, CeSI Institute, G. d'Annunzio University, Chieti, ${ }^{4} \mathrm{CSS}$ Mendel Institute, Rome, CSS Scientific Institute, San Giovanni Rotondo (FG), ${ }^{5}$ Dept of Internal Medicine, University of Tor Vergata, Rome, ${ }^{6}$ Dept of Clinical Sciences, Sapienza University, Rome, Italy

Background and aims: It has been proposed that PI3Kinase dependent insulin signaling pathway exerts a tonic inhibition on the MAPK dependent insulin pathway controlling, among others, potentially atherogenic insulin effects such as leukocyte adhesion molecules expression. Thus, impairment of PI3K pathway might enhance insulin atherogenic effects. An inhibitor of insulin action, TRIB3, was identified that affects insulin action by binding to and inhibiting phosphorylation of Akt, a cardinal enzyme downstream of $\mathrm{PI} 3 \mathrm{~K}$ in the insulin signaling pathway. We recently described a Q84R gain-offunction polymorphism of TRIB3 with the R84 variant being associated with insulin resistance and an earlier age at myocardial infarction. In the present study we test the hypothesis that polymorphisms associated with a loss of function in the PI3Kinase insulin pathway are correlated with upregulation of signalling pathways leading to insulin atherogenic effects.
Material and methods: We investigated insulin signaling and leukocyte adhesion molecules expression in cultured human endothelial cells (HUVECs) naturally carrying the three TRIB3 genotypes (Q84Q, Q84R, R84R).

Results: In both Q84R and R84R HUVECs, the amount of TRIB3 co-immunoprecipitated with Akt was greater than in Q84Q cells with a 50\% impairment in insulin-stimulated activation of both Akt and eNOS. In Q84Q cells, phosphorylation of ERK1/2, members of the MAPK family, was low in the basal state, and responsive to insulin. By contrast, in Q84R and R84R cells, ERK1/2 were maximally phosphorylated in the basal state without further increase in response to insulin. Moreover, in Q84Q the expression of VCAM-1, ICAM-1 and E-Selectin was induced by long term insulin stimulation. On the other hand, in Q84R and R84R cells, adhesion molecules expression was maximal in the basal state and not responsive to insulin. Both Q84R and R84R cells were not responsive to insulin-stimulated NO production. To determine whether the R84 variant was associated with early manifestation of atherosclerosis, carotid intima-media thickness (IMT) was measured by ultrasonography in 173 nondiabetic offspring of type 2 diabetics. IMT was increased in Q84R and R84R compared with Q84Q subjects $(P=0.02)$.

Conclusion: The TRIB3 R84 variant selectively impairs the vasodilatory action of insulin while enhancing MAPK activity and adhesion molecules expression. This data reinforce the hypothesis that insulin resistance, selectively affecting the PI3K pathway, might contribute to the pathogenesis of macrovascular complications of diabetes. Supported by: EFSD/Servier grant

\section{6}

ENPP1 as an insulin resistance gene

J.C. Florez ${ }^{1,2}$, E.S. Stolerman ${ }^{1,2}$, A.F. Moore ${ }^{1,2}$, A.K. Manning ${ }^{3}$,

J.B. McAteer ${ }^{1,2}$, J. Dupuis ${ }^{3}$, C.S. Fox ${ }^{3}$, L.A. Cupples ${ }^{3}$, K.A. Jablonski ${ }^{4}$, R.F. Arakaki ${ }^{4}$, B.J. Goldstein ${ }^{4}$, S.E. Kahn ${ }^{4}$, A.E. Kitabchi ${ }^{4}$, W.C. Knowler ${ }^{4}$, J.B. Meigs ${ }^{5,3}$

${ }^{1}$ Center for Human Genetic Research and Diabetes Unit, Massachusetts General Hospital, Boston, ${ }^{2}$ Program in Medical and Population Genetics, Broad Institute of Harvard and MIT, Cambridge, ${ }^{3}$ Glycemic Group, Framingham Heart Study, Framingham, ${ }^{4}$ Research Group, Diabetes Prevention Program, Rockville, ${ }^{5}$ General Medicine Division, Massachusetts General Hospital, Boston, United States

Background and aims: Functional studies suggest that the non-synonymous K121Q polymorphism (rs1044498) in the ectoenzyme nucleotide pyrophosphate phosphodiesterase ENPP1 may confer susceptibility to insulin resistance; genetic evidence quantifying its effect on type 2 diabetes, however, has been conflicting. In a comprehensive meta-analysis of 23 European population studies we recently demonstrated a modest increase in risk of type 2 diabetes for QQ homozygotes (combined odds ratio [OR] 1.38, 95\% CI 1.10$1.74, P=0.005$ ). This risk was modified by BMI, with a higher OR in studies in which the mean BMI of controls was lower. We therefore sought to 1) assess the effect of ENPP1 K121Q on quantitative gycemic traits; 2) fine-map the association signal, to establish whether it indeed stems from K121Q and not from another variant in the region; 3 ) verify its interaction with obesity; 4) examine its ability to predict diabetes onset prospectively; and 5) explore its impact on diabetes preventive strategies.

Materials and methods: We characterized the haplotype structure of ENPP1 and selected 39 tag SNPs that captured $96 \%$ of common variation in the region (minor allele frequency $\geq 5 \%$ ) with an $\mathrm{r}^{2} \geq 0.80$. We genotyped these SNPs in 2,511 Framingham Heart Study (FHS) participants and used age-sex-adjusted linear mixed effects models to test for association with quantitative metabolic traits and their interaction with BMI. We then genotyped ENPP1 K121Q in 3,548 participants in the Diabetes Prevention Program (DPP), in which lifestyle modification and metformin were compared to placebo, and performed Cox regression analyses using genotype, intervention, and interactions as predictors of diabetes incidence.

Results: In the FHS, the Q allele was associated with increased fasting plasma glucose (FPG), hemoglobin $A_{1 c}$, fasting insulin, and insulin resistance by homeostasis model assessment (HOMA-IR; all $P=0.01-0.006$ ). Linear mixed effect models indicated that the effects of all detected associations could be driven by K121Q. We found no association of K121Q with obesity, but interaction models suggested that the effect of the Q allele on FPG and HOMA-IR was stronger in those with a higher BMI $(P=0.008$ and 0.01 for interaction, respectively). There was no association of ENPP1 K121Q with diabetes incidence in FHS based on a limited sample of 241 cases. In the DPP, there was a nominally significant interaction between genotype at K121Q and intervention under a dominant genetic model $(P=0.03)$. In the placebo arm $(\mathrm{n}=996)$, 
Q allele carriers were more likely to develop diabetes than KK homozygotes (hazard ratio 1.38, 95\% CI 1.08-1.76, $P=0.009$ ). Metformin and lifestyle modification eliminated this increased risk.

Conclusion: The ENPP1 Q121 variant appears to increase risk of type 2 diabetes and is associated with hyperglycemia and insulin resistance in whites, an effect which seems to be modulated by BMI. The risk of diabetes ascribed to this polymorphism may be eliminated by preventive interventions. Supported by: NIH Research Career Award

\section{7}

Enhanced insulin sensitivity in obese patients with a rare cancer predisposition syndrome (Cowden Syndrome) due to PTEN mutations: further evidence for the Yin-Yang hypothesis

A. Pal ${ }^{1}$, T.M. Barber ${ }^{1}$, L. McCulloch ${ }^{1}$, R. Smith ${ }^{1}$, B.A. Barrow ${ }^{1}$, L. Walker ${ }^{2}$, A.L. Gloyn ${ }^{1}$

${ }^{1}$ Oxford Centre for Diabetes, Endocrinology and Metabolism, University of Oxford, ${ }^{2}$ Clinical Genetics, Churchill Hospital, Oxford, United Kingdom

Background and aims: One emerging theme from genome-wide scans for association in Type 2 diabetes (T2DM) is the frequency with which novel signals map to genes involved in cell cycle regulation. Moreover, recent studies have demonstrated that common genetic variants predisposing to cancer protect against T2DM; variants in $H N F 1 B$ have been shown to predispose to prostate cancer and also confer protection against T2DM. This yin-yang effect, whereby genetic variations at certain loci have divergent effects on cancer predisposition and the development of T2DM, is accruing substantiation. Cowden syndrome is a rare cancer predisposition syndrome (prevalence of 1 in 200000 ) caused by mutations in the phosphatase and tensin homolog tumour suppressor gene (PTEN). PTEN is a lipid phosphatase which metabolises phosphatidylinositol phospholipid-4,-5-bisphosphate (PtdIns)3,4,5-triphosphate ( $\mathrm{PIP}_{3}$ ) to PtdIns-4,-5-bisphosphate and consequently reverses a step in $\mathrm{PI}_{3}$-kinase signalling (a key pathway in insulin action). PTEN is expressed in pancreatic beta-cell, liver, muscle and adipose tissue. Studies in mice have shown that PTEN haploinsufficiency promotes insulin sensitivity, and that beta-cell specific knockout mice have increased beta-cell mass and hypoglycaemia. We hypothesised that PTEN haploinsufficient patients with Cowden syndrome could have enhanced insulin sensitivity and beta-cell function.

Materials and methods: To test our hypothesis we recruited patients with Cowden syndrome $(n=7)$ and age, BMI and gender-matched controls $(n=7)$. Subjects underwent an oral glucose tolerance test $(75 \mathrm{~g})$ and blood samples were analysed for plasma glucose and serum insulin at baseline (fasting) and at +30 minutes post-oral glucose load. Insulin sensitivity and beta-cell function were assessed with the homeostasis model of insulin resistance (HOMA2 IR) and disposition index (DI) respectively. Paired t-test analyses were implemented in SPSS.

Results: Results are shown as geometric mean [SD range]; Cowden syndrome patients vs controls. Patients with Cowden syndrome and control subjects were well-matched for gender $(\mathrm{P}=1.0)$, age $(36.5[25.8,51.6]$ vs $36.8[26.5$, 51.0], $\mathrm{P}=0.88)$ and BMI $\left(32.6 \mathrm{~kg} / \mathrm{m}^{2}[25.8,41.3] \mathrm{vs} 32.9 \mathrm{~kg} / \mathrm{m}^{2}[26.6,40.6]\right.$, $\mathrm{P}=0.79$ ). Patients with Cowden syndrome have greater insulin sensitivity (HOMA2 IR $0.51[0.19,1.38]$ vs $1.35[0.67,2.69], \mathrm{P}=0.028$ ) and although not statistically significant there was a tendency for improved beta-cell function (DI 136.2 [65.9, 281.2] vs $75.5[29.6,192.5], \mathrm{P}=0.29)$ compared to matched controls.

Conclusion: Our data support the hypothesis that in humans, a manifestation of PTEN haploinsufficiency is improved insulin sensitivity and beta-cell function. These data suggest that the beneficial affects of loss of PTEN are most marked in tissues of insulin action rather than in improved beta-cell function per se. Understanding the role of PTEN in insulin secretion/action is not only of biological importance but will also clarify the potential for adverse oncogenic events related to manipulation of these pathways to treat diabetes.

\section{8}

Impact of WFS1 polymorphisms on pre-diabetic phenotypes in a population-based sample of middle-aged people with normal and abnormal glucose regulation

T.W. Boesgaard ${ }^{1}$, T. Spars $\varnothing^{1}$, G. Andersen ${ }^{1}$, T. Jørgensen ${ }^{2}$,

K. Borch-Johnsen ${ }^{1,2}$, A. Sandbæk ${ }^{4}$, T. Lauritzen ${ }^{4}$, J. Wasson ${ }^{5}$, M.A. Permutt ${ }^{5}$, B. Glaser ${ }^{6}$, S. Madsbad ${ }^{7}$, O. Pedersen ${ }^{1,8}$, T. Hansen ${ }^{1}$

${ }^{1}$ Steno Diabetes Center, Gentofte, Denmark, ${ }^{2}$ Research Centre for Prevention and Health, Glostrup University Hospital, Denmark, ${ }^{3}$ Epidemiology, Steno Diabetes Center, Gentofte, Denmark, ${ }^{4}$ Department of General Practice, University of Aarhus, Denmark, ${ }^{5}$ Metabolism and Lipid Research Division, Wasington University School of Medicine, Saint Louis, WA, United States, ${ }^{6}$ Hadassah-Hebrew, University Medical Centre, Jerusalem, Israel, ${ }^{7}$ Department of Endocrinology, Hvidovre University Hospital, Denmark, ${ }^{8}$ Faculty of Health Science, University of Aarhus, Denmark

Background and aims: variants in WFS1 have been shown to associate with type 2 diabetes. We aimed to examine metabolic risk phenotypes of WFS 1 variants in glucose-tolerant people and in individuals with abnormal glucose regulation.

Materials and methods: The type 2 diabetes-associated WFS1 variant rs734312 (His611 Arg) was genotyped in the population-based Inter99 cohort involving 4,568 glucose-tolerant individuals and 1,471 individuals with treatment-naive abnormal glucose regulation, and in additional 3,733 treated type 2 diabetic patients from Steno Diabetes Center and the ADDITION study using TaqMan allelic discrimination.

Results: The WFS1 rs734312 A-allele showed a borderline significant association with type 2 diabetes with a relative risk consistent with previous reports. In individuals with abnormal glucose regulation the rs734312 A-allele was in an allele-dependent manner associated with a decrease in insulinogenic index $(p=0.03)$ and a decreased 30-min serum-insulin level $(p=0.05)$ after an oral glucose load while, curiously, in glucose-tolerant individuals the same allele was associated with increased fasting serum-insulin $(p=0.02)$ and insulin resistance assessed by HOMA-IR ( $p=0.03$ ), see table. We also examined for interaction with glucose tolerance status applying a linear model with glucose tolerance status as an interaction term. In this analysis we demonstrated a significant association with traits related to $\beta$-cell function (30-min seruminsulin, $p=0.009$, and insulinogenic index, $p=0.003$ ).

Table Anthropometric and metabolic characteristics of 5,384 treatment-naïve Danish individuals from the Inter99 cohort stratified according to WFS1 rs734312 genotype and glucose tolerance status

Data are means \pm standard deviation. All analyses were made using a genetic additive model

Conclusion: Type 2 diabetes-associated risk alleles of WFS1 are associated with estimates of a decreased pancreatic $\beta$-cell function among middle-aged individuals with abnormal glucose regulation. 


\begin{tabular}{|c|c|c|c|c|}
\hline \multicolumn{5}{|c|}{ Normal glucose-tolerance (NGT) } \\
\hline & A/A & $\mathrm{A} / \mathrm{G}$ & G/G & $p$ \\
\hline n (men/women) & $1,091(495 / 596)$ & $2,050(962 / 1088)$ & $958(445 / 513)$ & \\
\hline Age (years) & $45 \pm 8$ & $45 \pm 8$ & $45 \pm 8$ & \\
\hline $\mathrm{BMI}\left(\mathrm{kg} / \mathrm{m}^{2}\right)$ & $25.5 \pm 4.0$ & $25.5 \pm 4.1$ & $25.6 \pm 4.1$ & 0.56 \\
\hline \multicolumn{5}{|l|}{ Serum insulin (pmol/l) } \\
\hline Fasting & $39 \pm 24$ & $38 \pm 23$ & $37 \pm 23$ & 0.019 \\
\hline $30 \mathrm{~min}$ post-OGTT & $290 \pm 190$ & $291 \pm 178$ & $278 \pm 156$ & 0.085 \\
\hline $120 \mathrm{~min}$ post-OGTT & $174 \pm 145$ & $169 \pm 126$ & $166 \pm 130$ & 0.12 \\
\hline \multicolumn{5}{|l|}{ Plasma glucose $(\mathrm{mmol} / \mathrm{l})$} \\
\hline Fasting & $5.3 \pm 0.4$ & $5.3 \pm 0.4$ & $5.3 \pm 0.4$ & 0.77 \\
\hline 30-min post-OGTT & $8.1 \pm 1.6$ & $8.2 \pm 1.5$ & $8.2 \pm 1.5$ & 0.78 \\
\hline 120-min post-OGTT & $5.5 \pm 1.1$ & $5.5 \pm 1.1$ & $5.5 \pm 1.1$ & 0.53 \\
\hline \multicolumn{5}{|l|}{ Serum C-peptide (pmol/l) } \\
\hline Fasting & $545 \pm 208$ & $537 \pm 209$ & $534 \pm 220$ & 0.03 \\
\hline 30-min post-OGTT & $1,984 \pm 668$ & $1,988 \pm 710$ & $1,936 \pm 666$ & 0.039 \\
\hline $120-$ min post-OGTT & $2,072 \pm 804$ & $2,064 \pm 794$ & $2,025 \pm 796$ & 0.12 \\
\hline HOMA-IR (mmol/l.pmol/l) & $9.2 \pm 5.8$ & $8.9 \pm 5.7$ & $8.7 \pm 5.5$ & 0.026 \\
\hline Insulinogenic index & $31.2 \pm 20.9$ & $31.4 \pm 19.9$ & $30.0 \pm 18.1$ & 0.075 \\
\hline \multicolumn{5}{|c|}{ Abnormal glucose-tolerance (IFG, IGT, screen-detected type 2 diabetes) } \\
\hline & $\mathbf{A} / \mathbf{A}$ & $\mathrm{A} / \mathrm{G}$ & $\mathrm{G} / \mathrm{G}$ & $p$ \\
\hline $\mathrm{n}$ (men/women) & $359(224 / 135)$ & $642(385 / 257)$ & $284(166 / 118)$ & \\
\hline Age (years) & $50 \pm 8$ & $49 \pm 7$ & $49 \pm 8$ & \\
\hline $\mathrm{BMI}\left(\mathrm{kg} / \mathrm{m}^{2}\right)$ & $28.3 \pm 4.9$ & $28.3 \pm 5.3$ & $28.6 \pm 5.2$ & 0.49 \\
\hline \multicolumn{5}{|l|}{ Serum insulin (pmol/l) } \\
\hline Fasting & $54 \pm 33$ & $58 \pm 37$ & $55 \pm 33$ & 1.0 \\
\hline 30-min post-OGTT & $291 \pm 181$ & $311 \pm 224$ & $333 \pm 214$ & 0.047 \\
\hline $120 \mathrm{~min}$ post-OGTT & $370 \pm 304$ & $364 \pm 320$ & $390 \pm 354$ & 1.0 \\
\hline \multicolumn{5}{|l|}{ Plasma glucose $(\mathrm{mmol} / \mathrm{l})$} \\
\hline Fasting & $6.3 \pm 1.35$ & $6.2 \pm 1.0$ & $6.2 \pm 1.2$ & 0.38 \\
\hline 30-min post-OGTT & $10.2 \pm 1.9$ & $10.3 \pm 1.9$ & $10.2 \pm 1.7$ & 0.6 \\
\hline $120-\mathrm{min}$ post-OGTT & $8.5 \pm 2.9$ & $8.4 \pm 2.9$ & $8.2 \pm 2.3$ & 0.36 \\
\hline \multicolumn{5}{|l|}{ Serum C-peptide (pmol/l) } \\
\hline Fasting & $756 \pm 331$ & $772 \pm 348$ & $768 \pm 340$ & 0.97 \\
\hline 30-min post-OGTT & $2,039 \pm 762$ & $2,059 \pm 823$ & $2,147 \pm 751$ & 0.17 \\
\hline 120-min post-OGTT & $3,130 \pm 1,201$ & $3,054 \pm 1,223$ & $3,159 \pm 1,238$ & 0.84 \\
\hline HOMA-IR (mmol/l.pmol/l) & $15.3 \pm 10.1$ & $16.2 \pm 11.6$ & $15.3 \pm 9.9$ & 0.81 \\
\hline Insulinogenic index & $23.5 \pm 16.0$ & $25.1 \pm 19.5$ & $27.5 \pm 20.5$ & 0.025 \\
\hline
\end{tabular}

\section{9}

$C D K N 2 A / B$ polymorphism is associated with susceptibility to type 2 diabetes, but not with the gene expression in the adipose tissues in the Japanese

S. Omori ${ }^{1,2}$, Y. Tanaka ${ }^{2}$, H. Maegawa ${ }^{3}$, A. Kashiwagi ${ }^{3}$, R. Kawamori ${ }^{4}$, K. Kaku' ${ }^{5}$, Y. Nakamura ${ }^{6}$, S. Maeda ${ }^{1}$

${ }^{1}$ Laboratory for Endocrinology and Metabolism, Center for Genomic Medicine, RIKEN, Yokohama, ${ }^{2}$ Department of Internal Medicine, Division of Metabolism and Endocrinology, St. Marianna University School of Medicine, Kawasaki, ${ }^{3}$ Department of Medicine, Shiga University of Medical Science, Otsu, ${ }^{4}$ Department of Medicine, Metabolism and Endocrinology, School of Medicine, Juntendo University, Tokyo, ${ }^{5}$ Division of Endocrinology and Metabolism, Department of Internal Medicine, Kawasaki Medical School, Kurashiki, ${ }^{6}$ Laboratory of Molecular Medicine, Human Genome Center, Institute of Medical Science, University of Tokyo, Japan

Background and aims: Recently, genome-wide association studies were conducted by European and American groups, and several candidate loci were identified in populations of European descent. The aim of the present study is to further investigate the involvement of these polymorphisms in conferring susceptibility to type 2 diabetes across different ethnicities.

Materials and methods: We examined the association of 14 SNPs within 11 candidate loci (rs4402960 in IGF2BP2, rs 10811661 in CDKN2A/B, rs 1111875 and rs7923837 in HHEX, rs13266634 in SLC30A8, rs1113132 and rs11037909 in EXT2, rs9939609 and rs8050136 in FTO, rs7756992 in CDKAL1, rs1801282 in PPARG Pro12Ara, rs5219 in KCNJ11 Glu23Lys, rs7480010 in LOC387761, and rs9300039 in ch11) with type 2 diabetes in a Japanese population. We analyzed these SNPs in 1,630 Japanese subjects with type 2 diabetes and in
1,064 control subjects by using an invader assay or a TaqMan assay. In addition, we investigated the correlation between $C D K N 2 A / B$ polymorphism and its expression in the adipose tissues by using real-time RT-PCR.

Results: Among the 11 loci examined, 6 loci were significantly associated with type 2 diabetes in our population by a logistic regression analysis $(\mathrm{p}<$ 0.05 after adjusting age, gender, BMI), similar to previously reported results. We also identified an interactive effect of these 6 loci (table 1). In this population, the remaining 5 loci were not significantly associated with type 2 diabetes. In addition, we identified that the $C D K N 2 B$ is preferentially expressed in the subcutaneous adipose rather than visceral adipose (Subcutaneous: 4.78 [ $95 \%$ CI 3.00 - 7.60] vs. Visceral: 0.07 [95\% CI 0.05 - 0.10], geometric mean of arbitrary unit), whereas the expression of CDKN2A could not be detected in the adipose tissues. The $C D K N 2 B$ expressions in the adipose tissues, however, did not differ according to the $C D K N 2 A / B$ genotypes (rs10811661).

Conclusion: We have identified 6 of the 11 loci that were identified by genome-wide association studies in European descent. These loci are considered strong candidates for type 2 diabetes susceptibility across different ethnicities, although further studies are required to elucidate precise mechanisms underlying the contribution of these genes and gene polymorphisms to conferring susceptibility to type 2 diabetes.

Interactive effects of 6 SNPs on conferring susceptibility to type 2 diabetes

\begin{tabular}{lllllll}
\hline Model & $\begin{array}{l}\text { number of } \\
\text { risk genotype }\end{array}$ & 0 & 1 & 2 & 3 & 4 or $4<$ \\
\hline Recessive & Case & 286 & 578 & 473 & 188 & 36 \\
\hline & Control & 263 & 405 & 238 & 80 & 14 \\
\hline Odds ratio & 1 & 1.31 & 1.83 & 2.16 & 2.36 \\
\hline 95\% CI & - & $1.06-1.62$ & $1.45-2.30$ & $1.58-2.95$ & $1.25-4.48$ \\
\hline
\end{tabular}




\section{PS 009 Prediction of type 1 diabetes}

\section{0}

Autoantibody responses to zinc transporter 8 in children at risk for type 1 diabetes

P. Achenbach ${ }^{1}$, U. Landherr ${ }^{1}$, K. Koczwara ${ }^{1}$, V. Lampasona ${ }^{2}$, S. Krause ${ }^{1}$, A.G. Ziegler ${ }^{1,3}$, E. Bonifacio ${ }^{4}$

${ }^{1}$ Institut für Diabetesforschung, Munich, Germany, ${ }^{2}$ Dept. of Laboratory Medicine, Istituto Scientifico San Raffaele, Milan, Italy, ${ }^{3} \mathrm{Klinikum}$ Schwabing, Städt. Klinikum München GmbH, Munich, Germany, ${ }^{4}$ Center for Regenerative Therapies - Dresden, University of Technology, Germany

Background and aims: Zinc transporter 8 (ZnT8) has recently been identified as a major target antigen in the autoimmune pathogenesis of type 1 diabetes (T1DM). The aim of this study was to determine the cumulative prevalence of autoantibodies to ZnT8 in relation to other islet autoantibodies in at risk children who have been prospectively followed from birth.

Materials and methods: Antibody binding to ZnT8 was determined in sera of 1777 children, including 144 children who developed autoantibodies to insulin, GAD and/or IA-2 (78 with multiple antibodies) and 42 who progressed to T1DM, using Protein A-based radiobinding assays and 35-S labelled in vitro transcribed/translated human recombinant ZnT8 constructs. All children were screened for autoantibodies to a C-terminal (aa 268-369) portion of the A325-arginine-isoform of ZnT8 (ZnT8-C-Abs). Autoantibodies to the N-terminal (aa 1-74) portion of ZnT8 (ZnT8-N-Abs) were tested in all ZnT8C-Abs positive children and in HLA DR3 and DR4-matched ZnT8-C-Abs negative children. In addition, islet autoantibody and/or ZnT8-Abs positive children were tested for antibody binding to C-terminal constructs of the A325-tryptophan- and A325-glutamine-isoforms of ZnT8.

Results: Persistent ZnT8-C-Abs were detected in 53 children. This included 47 of 78 with multiple other islet antibodies, 2 of 35 with IAA only, 2 of 35 with GADA only, and 2 with no other islet antibodies. ZnT8-C-Abs developed at a median age of 3.2 years (IQR 2.1-5.3). The cumulative risk for developing ZnT8-C-Abs was $3.2 \%$ by age 8 years and $4.3 \%$ by age 11 years. ZnT8-C-Abs preceded autoantibodies to insulin, GAD and/or IA-2 in 3 children. ZnT8-N-Abs were often transient; at least one ZnT8-N-Abs positive sample was found in 11 children with ZnT8-C-Abs positive, 2 children who were ZnT8-C-Abs negative/islet autoantibody positive, and 8 of 633 (1.6\%) autoantibody negative children.

Seven of the islet autoantibody positive children were positive for antibodies against the ZnT8 A325-tryptophan- and/or A325-glutamine-isoforms but had no antibodies against the ZnT8 A325-arginine-isoform; 4 of these 7 developed diabetes. There were heterogenous antibody binding patterns among the ZnT8-C-Abs positive children with the majority of subjects responding equally well to all three isoforms, but some children showed an increased and few an exclusive binding to one or two of the ZnT8 isoforms. Follow-up over time revealed that these binding patterns could change suggesting epitope changes.

Thirty-one of the $60 \mathrm{ZnT} 8$-Abs positive children (51.7\%) progressed to diabetes. The 10-year cumulative risk from the time of appearance of ZnT8-Abs was $60 \%$ (95\% CI, 46-74).

Conclusion: Autoantibodies to ZnT8 can appear early in the pathogenesis of childhood T1DM and they are associated with high diabetes risk. Screening for ZnT8-Abs should consider differences in antibody binding to different ZnT8-isoforms.

Supported by: JDRF and Deutsche Forschungsgemeinschaft

\section{1}

Transplantation of adipose tissue and feeding of high fat diet protect BB rats from type 1 diabetes development

I. Klöting ${ }^{1}$, B. Wilke ${ }^{1}$, N. Klöting ${ }^{1,2}$

${ }^{1}$ Laboratory Animal Science, University of Greifswald, Karlsburg,

${ }^{2}$ Department of Internal Medicine III, University of Leipzig, Germany

Background and aims: We breed type 1 diabetic $\mathrm{BB}$ rats since more than 25 years. Several days before BB rats become diabetes their body seems to be flabby which may be attributed to loss of subcutaneous fat. This observation prompted us to search for possibilities to avoid the loss of adipose tissue. We have chosen two different ways: transplantation of adipose tissue and feeding of high fat diet to protect BB rats from type 1 diabetes.

Materials and methods: For transplantation study we used 9 complete litters of BB rats (53M:55F)) which were subcutaneously grafted with 1 or $2 \mathrm{~g}$ visceral adipose tissue of sex and MHC matched congenic, but non-diabetic $\mathrm{BB}$ rat strains at an age of 4 weeks. Sham operated and untreated BB rats $(20 \mathrm{M}: 21 \mathrm{~F}))$ served as controls. For the diet study 8 breeding pairs were fed a high fat diet (Ssniff R-Z $+10 \%$ tallow, Soest, Germany). The progeny of these breeding pairs $(n=55)$ was also given this diet up to an age of 30 weeks. 42 $\mathrm{BB}$ rats fed the normal rat diet (Ssniff R-Z) under pregnancy and during the observation period served as controls. All BB rats were observed for diabetes occurrence up to an age of 30 weeks. The diabetes frequency was compared with the Chi-square test.

Results: From the fat grafted animals in total 36 out of 53 male BB rats (68\%) and 27 out of 55 female $\mathrm{BB}$ rats (49\%) developed type 1 diabetes. In the control group 17 out of 20 male (85\%) and 20 out of 21 female BB rats (95\%) became diabetic. Therefore, significantly more grafted males than females developed diabetes up to an age of 30 weeks $(p=0.0002)$. In contrast to the transplantation study, the $\mathrm{BB}$ rats fed with high fat diet significantly more females than males were diabetic in comparison with normal fed BB controls (M $17 / 25,68 \%$ vs. $23 / 23,100 \%$ p $=0.003 ; \mathrm{F} 23 / 30,76 \%$ vs. $17 / 19,89 \%$ p $=0.23$ ). The age at onset of diabetes and the level of hyperglycaemia were largely comparable between study and control groups.

Conclusion: Both adipose tissue transplantation and high fat diet protect BB rats from type 1 diabetes development in a sex specific manner. The manipulations may influence fat accumulation and/or fat metabolism which prevent type 1 diabetes development in $\mathrm{BB}$ rats. This idea is supported by the finding that a mutation in the leptin receptor of NOD mice suppresses type 1 diabetes progression.

Supported by: Deutsche Forschungsgemeinschaft

\section{2}

An increase in C-reactive protein often precedes and predicts clinical onset of type 1 diabetes in GAD-antibody positive relatives

J. De Grijse' ${ }^{1}$, P. Couck ${ }^{2}$, I. Truyen ${ }^{1}$, I. Weets ${ }^{1}$, I. Vermeulen ${ }^{1}$, F. van Genderen $^{1}$, E. Vekens ${ }^{1}$, K. Decochez ${ }^{1}$, J. Ruige ${ }^{3}$, C. Van Schravendijk ${ }^{1}$, B. Keymeulen ${ }^{1}$, D. Pipeleers ${ }^{1}$, F. Gorus ${ }^{1,2}$

${ }^{1}$ Diabetes Research Center, Brussels Free University, ${ }^{2}$ Department of Clinical Chemistry and Radioimmunology, University Hospital Brussels Free University, ${ }^{3}$ Department of Internal Medicine, University Hospital Ghent, Belgium

Background and aims: Type 1 diabetes is a chronic inflammatory disease, characterized by insulitis, but the frequency and intensity of this process in human prediabetes is unknown. In view of previous contradictory reports we reevaluated whether circulating levels of C-reactive protein (CRP) - an established marker of inflammation - may contribute to the prediction of type 1 diabetes in a registry-based, representative and fully characterized group of first degree relatives of known patients.

Materials and methods: Random CRP levels were measured at baseline and during yearly follow-up in 211 persistently islet antibody-positive $\left(\mathrm{Ab}^{+}\right)$first degree relatives (37 with prediabetes) of type 1 diabetic patients and in 211 age- and sex-matched persistently antibody-negative relatives (median age $14.0 \pm 8.8 \mathrm{yr}$ ) with known HLA-DQ genotype (no prediabetes) followed for 81 (59-90) months and recruited by the Belgian Diabetes Registry (BDR). Serum CRP levels were measured centrally by immunoturbidimetry using Vitros high sensitivity CRP reagents on a Vitros 5,1 FS analyser (Ortho-Clinical Diagnostics, Rochester, NY, USA)

Results: Baseline CRP levels were positively correlated with female sex ( $p<$ $0.001)$, age- and sex-adjusted BMI $(p<0.001)$ and presence of GAD antibodies (GADA) $(p=0.030)$, but not with clinical outcome or overall antibody status. However, CRP levels increased more within 2 years prior to clinical onset in prediabetes (median [interquartile range] of CRP change: 0.61 [0.11 - 1.49] $\mathrm{mg} / \mathrm{L}$ ) than in individually age- and sex-matched non-diabetic relatives with or without antibodies followed equally long $(p<0.001)$. In survival analysis, an increase in CRP within one year after study entry (median [interquartile range]: $0.35[0.15-1.76] \mathrm{mg} / \mathrm{L}$ ) was associated with progression to diabetes ( $p=0.02$ versus relatives without increasing CRP levels). This association was restricted to the subgroup of persistently $\mathrm{GADA}^{+}$relatives ( $n=150 ; p<0.001$ versus $\mathrm{GADA}^{+}$relatives without CRP increase) (see figure). Multivariate Cox regression analysis confirmed CRP increase as an independent predictor of diabetes (Hazard Ratio [95\% CI]: 2.8 [1.3 - 6.1]; $p=0.01)$ besides presence of IA-2A $(p<0.001)$ and HLA-DQ2/DQ8 $(p=$ $0.002)$ in the $\mathrm{GADA}^{+}$subgroup.

Conclusion: CRP levels often increase before clinical onset of diabetes in $\mathrm{GADA}^{+}$relatives reflecting an inflammatory state and contributing to disease prediction. 


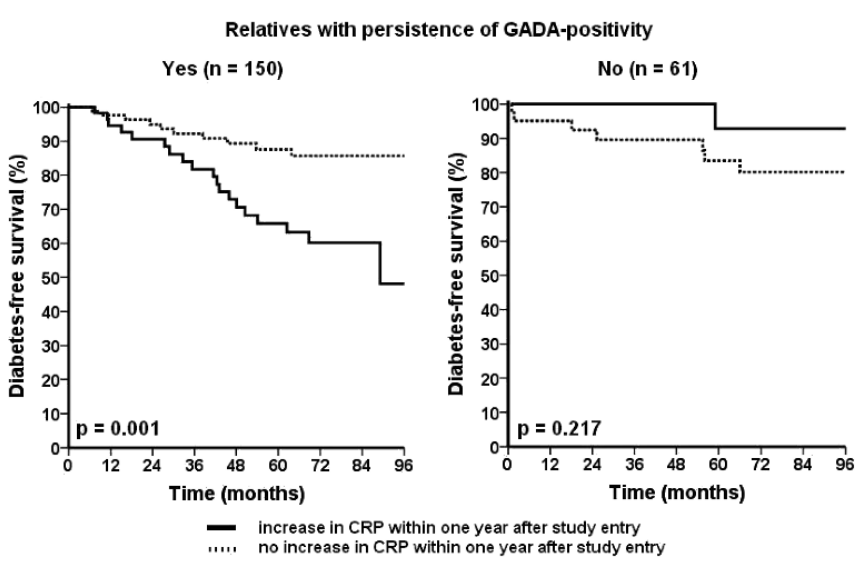

Supported by: Flemish Research Council (FWO) and JDRF Center grant

\section{3}

A platform for the identification and validation of beta cell specific membrane biomarkers

D. Flamez ${ }^{1}$, A. Berton ${ }^{2}$, I. Roland ${ }^{2}$, B. Kutlu' 3 , N. Goodman 3 , M.-C. Beckers ${ }^{4}$, D. Maréchal ${ }^{4}$, S. Goldman ${ }^{5}$, C. Van Huffel ${ }^{6}$, I. Salmon ${ }^{2}$, D.L. Eizirik ${ }^{1}$ ${ }^{1}$ Laboratory of Experimental Medicine, Brussels, Belgium, ${ }^{2}$ Laboratory of Pathology, Brussels, Belgium, ${ }^{3}$ ISB, Institute of Systems Biology, Seattle, United States, ${ }^{4}$ Eurogentec SA, Namur, Belgium, ${ }^{5}$ Laboratory of Nuclear Medicine, Brussels, Belgium, ${ }^{6}$ Keymarker SA, Namur, Belgium

Background and aims: The natural history of Type 1 diabetes is currently unknown. Onset of hyperglycemia is believed to occur when $>80 \%$ of the beta cell mass is destroyed. Detecting progressive beta cell loss and/or regeneration in pre-clinical or recent onset T1D would allow a better understanding of the natural history of diabetes and validate clinical trials to prevent the disease. The challenge for beta cell mass quantification is that islets are small micro-organs scattered in the pancreas, and none of the proposed beta cell markers are sufficiently specific for imaging. Our aim is to identify novel beta cell specific membrane proteins that can be used as biomarkers for imaging beta cell mass, purification of beta cells or for drug targeting specifically to beta cells.

Materials and methods: Bioinformatic analysis was performed on Massively Parallel Signature Sequencing (MPSS) datasets of two human pancreatic islet samples and on Affymetrix Gene Chip Rat 230.2 array datasets of purified rat beta and alpha cells using the programs TMHMM and Ingenuity Pathway Analysis (IPA). Antibodies against beta cell specific membrane proteins were tested by immunohistochemistry (IHC) on human pancreatic tissue and by Western Blotting (WB) on mouse and rat tissues and on INS-1E and AR42J cells. The sensitivity and specificity of the best candidates will be analysed using multi-tissue human and rat tissue arrays.

Results: We compared MPSS datasets obtained in human islets with publicly available MPSS data obtained in 32 human tissues and selected 848 unique islet specific genes. Using TMHMM and IPA 114, membrane proteins were selected. Genes were then classified as beta cell- or non-beta cell- specific by comparative analysis of Affymetrix microarray data obtained in primary rat beta and alpha cells. Membrane proteins that were highly changed after treatment with the cytokines IL1 $\beta$ and IFN $\gamma$ were removed from the list. We retained 44 membrane proteins that are highly enriched in beta cells. After comparison with the expression in the $\beta$-Cell Gene Atlas, 12 candidates were retained as potential beta-cell biomarkers. Specific antibodies for 7 of these membrane proteins were purchased or prepared and studied in IHC and in WB studies. Membrane and islet cell localisation was confirmed for all candidates tested. Three candidates showed low expression levels while two candidates had background staining in ductal cells. Four candidates are currently being tested in the human tissue array.

Conclusion: Using a systems biology approach we selected 12 membrane proteins that are highly enriched in pancreatic beta cells. Seven membrane proteins were validated with antibodies specifically directed against the candidate biomarkers. All seven were confirmed to be islet specific membrane proteins validating the present systems biology approach.

Supported by: Région Wallonne BioWin (Belgium) and Fonds Yvonne Boël (Brussels Belgium)

Method for high-throughput screening of HLA-B ${ }^{\star} 39$ allele to be used in the assessment of genetic risk for type 1 diabetes

M. Kiviniemi ${ }^{1}$, A.-P. Laine ${ }^{1}$, Z. Gombos ${ }^{1}$, R. Hermann ${ }^{1}$, M. Knip², J. Nurmi ${ }^{3}$, T. Lövgren ${ }^{4}$, J. Ilonen ${ }^{1}$, the Finnish Pediatric Diabetes Registry ${ }^{1}$ Immunogenetics Laboratory, University of Turku, ${ }^{2}$ The Hospital for Children and Adolescents, University of Helsinki, ${ }^{3}$ Abacus Diagnostica Ltd., Turku, ${ }^{4}$ Department of Biotechnology, University of Turku, Finland

Background and aims: An independent effect of HLA-B ${ }^{\star 39}$ allele for risk of type 1 diabetes has been established. In the Finnish population the effect of this allele is seen in HLA-DRB $1^{*} 0404-\mathrm{DQB} 1^{*} 0302$ haplotypes, the risk conferred by the haplotype is dependent on the presence of $\mathrm{B}^{\star} 39$ allele in linkage with mentioned class II alleles. A high-throughput assay is needed to exploit this information in genetic screening for natural history and prevention studies and for this purpose an assay for the detection of $\mathrm{B}^{\star} 39$ allele was developed and the effect of $\mathrm{B}^{\star} 39$ on the risk conferred by DRB1 ${ }^{\star} 0404-\mathrm{DQB1} 1^{\star} 0302$ confirmed in an expanded series of nuclear families with a diabetic child. Materials and methods: The developed assay was used to screen for the presence of HLA-B ${ }^{\star} 39$ allele in 907 Finnish nuclear families with a diabetic child. The assay uses whole blood dried on a sample collection card as sample material, and is based on an allele-specific PCR amplifying only the HLA$B * 39$ allele, followed by detection of the PCR-products by plate hybridization utilizing lanthanide-labelled probes. The chi-square function of the Epistat software was used in the statistical calculations.

Results: The developed assay was validated by screening 153 previously genotyped samples, and the results were fully concordant when compared to a formerly used standard gel detection of specifically amplified products. Since the results of the new test are produced as numbers they are easily calculated and interpreted by a computer program and integrated to databases. The effect of $\mathrm{B}^{\star} 39$ allele on the risk of type 1 diabetes conferred by HLADRB $1^{*} 0404-\mathrm{DQB} 1^{\star} 0302$ haplotype was confirmed in 907 Finnish nuclear families with a diabetic child where affected family-based artificial controls (AFBAC) controls were formed from non-transmitted haplotypes of healthy parents. The odds ratio (OR) for subjects with $\mathrm{DRB} 1^{*} 0404-\mathrm{DQB} 1{ }^{*} 0302$ $\mathrm{B}^{\star} 39$ was 8.53 (3.92-19.34) compared to 2.27 (1.54-3.34) for DRB1 ${ }^{*} 0404$ $\mathrm{DQB} 1^{\star} 0302-$ non- $\mathrm{B}^{\star} 39$. For comparison the $\mathrm{OR}$ for $\mathrm{DRB1}{ }^{\star} 0401-\mathrm{DQB1} 1^{\star} 0302$ was 7.58 (5.93-9.70), and for $\mathrm{DRB1}{ }^{\star} 0404-\mathrm{DQB1} 1^{\star} 0302$ without regarding $\mathrm{B}^{\star} 393.47$ (2.47-4.88).

Conclusion: The developed HLA-B ${ }^{\star} 39$ screening method proved to be robust, simple and fast and well suited for large-scale screening and amenable to automation. The considerably greater predisposing effect of the HLA$\mathrm{DRB1}^{\star} 0404-\mathrm{DQB1} 1^{*} 0302-\mathrm{B}^{\star} 39$ haplotype compared to that of the HLA$\mathrm{DRB}^{*}{ }^{*} 404-\mathrm{DQB} 1^{\star} 0302$-non- $\mathrm{B}^{\star} 39$ haplotype was confirmed in the study. Thus the integration of the developed method into the current screening system will increase the specificity of type 1 diabetes risk estimation.

\section{5}

First international proficiency testing of novel autoantibodies against Zn Transporter-8 and IA-2beta - Results of the Diabetes Antibody Standardisation Program

M. Schlosser ${ }^{1}$, P.W. Mueller' ${ }^{2}$, P. Achenbach ${ }^{3}$, P.J. Bingley ${ }^{4}$

${ }^{1}$ Medical Biochemistry and Molecular Biology, University of Greifswald, Karlsburg, Germany, ${ }^{2}$ Diabetes and Molecular Risk Assessment, Centers of Disease Control and Prevention, Atlanta, United States, ${ }^{3}$ Institut für Diabetesforschung, TU Munich, Germany, ${ }^{4}$ Clinical Science at North Bristol, University of Bristol, United Kingdom

Background and aims: The Diabetes Antibody Standardization Program (DASP) is a collaboration between the Immunology of Diabetes Society and US Centers for Disease Control and Prevention, set up to evaluate and improve assays for diabetes-associated autoantibodies. Autoantibodies to the novel islet antigens, ZnT8 and IA-2 $\beta$, have been associated with type 1 diabetes but their role in predictive strategies has yet to be defined. The DASP 2007 workshop set out to assess the sensitivity/specificity and concordance of assays for ZnT8 and IA- $2 \beta$ antibodies (Ab) in laboratories throughout the world, and to examine associations with established islet antibody markers. Materials and methods: Eighteen laboratories from 8 countries received coded sera from 49 patients with newly diagnosed T1DM (median age 17 years, range 5-32) and 100 controls (median age 20 years) with ZnT8 and IA-2 $\beta$ clones (donated by J Hutton, Denver and V Lampasona, Milan), and positive and negative reference standards. All labs performed in-house radiobinding 
assays (serum volume $2-10 \mu \mathrm{l}$ ) using ${ }^{35} \mathrm{~S}$-labelled in vitro transcribed/translated human recombinant ZnT8 (16 labs) and IA-2 $\beta$ (13 labs). Lab-reported results were recalculated after adjustment to the positive/negative standards (using an index for ZnT8 and multiple point standard curve for IA-2 $\beta$ ). Results were analyzed using receiver operator characteristic (ROC) curves. The sensitivity was adjusted to $95 \%$ specificity $\left(\mathrm{AS}_{95}\right)$ in controls.

Results: For ZnT8 Ab, the median lab-reported sensitivity was 51\% (interquartile range (IQR) 44-56\%), and specificity 98\% (IQR 97-98\%). ROC curve analysis showed significant differences between patients and control subjects in $15 / 16$ assays $(\mathrm{p}=0.021$ to $\mathrm{p}<0.0001)$. The median AUC of all assays was 0.71 (IQR 0.68-0.74) with $\mathrm{AS}_{95} 54 \%$ (IQR 47-59\%). Samples from 22 patients were reported positive in 12 of 16 assays, and 26 were reported positive in 8 assays. Interlaboratory concordance based on the $\mathrm{ZnT} 8 \mathrm{Ab}$ index was high $\left(\mathrm{r}^{2}=0.999\right.$; $\mathrm{p}<0.0001$ ). For IA- $2 \beta \mathrm{Ab}$, the median lab-reported sensitivity was $45 \%$ (IQR 43-50\%), and specificity $98 \%$. In all labs, ROC curve analysis showed significant differences between patients and control subjects ( $\mathrm{p}=0.004$ to $\mathrm{p}<0.0001$ ). The median AUC was 0.71 (IQR 0.68-0.74) with $\mathrm{AS}_{95}$ 53\% (IQR 48-54\%). 21 patient samples were reported positive in 11 of 12 assays, and 26 were reported positive in 6 assays. Interlaboratory concordance based on the common IA- $2 \beta$ units derived from the standard curve was high $\left(\mathrm{r}^{2}=1.0 ; \mathrm{p}<0.0001\right)$. In all labs, IA- $2 \beta$-Ab levels significantly correlated with autoantibodies to IA-2 $\left(r_{s}=0.685\right.$ to $\left.0.87 ; \mathrm{p}<0.0001\right)$.

Conclusion: The DASP evaluation of these novel autoantibodies demonstrated remarkable consistency in sensitivity and specificity with high levels of concordance between participating labs for both ZnT8 and IA-2 $\beta \mathrm{Ab}$, and confirmed their validity as diabetes-associated markers. ZnT8 and IA- $2 \beta \mathrm{Ab}$ assays perform well and are suitable for general implementation in the classification and prediction of T1DM. The DASP workshop model provides a powerful resource for rapid, comprehensive evaluation of novel autoantibody markers.

\section{6}

Genetic predictors of islet autoimmunity in early childhood. The Diabetes Autoimmunity Study in the Young (DAISY)

M.J. Rewers, J. Norris, G. Eisenbarth, K. Barriga, A. Steck, J. Hutton, W. Zhang

Barbara Davis Center for Childhood Diabetes, University of Colorado, Denver, United States

Background and aims: While multiple markers of genetic susceptibility for type 1 diabetes (T1D) are being reported from linkage and association studies, little is know concerning their independent predictive value on a population basis.

Materials and methods: DAISY is following from birth to development of islet autoimmunity (IA) and T1D 2449 high-risk children who have a highrisk HLA-DR,DQ genotype or are first-degree relatives of someone with T1D. Blood is drawn for the detection of autoantibodies to insulin, GAD65, IA-2 and ZnT8 at 9 and 15 months and annually thereafter. IA is defined as positivity for at least one of the 4 autoantibodies on two or more consecutive visits. By mean age of $7.0 \pm 4.4$ years, 163 children have developed IA and, of those, 53 have developed diabetes. Parametric models with Weilbull distribution and Cox proportional hazards models were used to examine genetic predictors of IA and diabetes, respectively.

Results: By age $12 \mathrm{yr}$, the cumulative risk (95\%CI) of IA was 36\% (19-56\%), $11 \%(3-35 \%)$, and $14 \%(8-25 \%)$ in, respectively, relatives with HLA-DR3/4, DQ8, all other relatives, and general population children with HLA-DR3/4, DQ8. The risk of T1D by age 12 in these groups was, respectively $24 \%$ (15$37 \%), 4 \%(3-7 \%)$, and $1 \%(0.4-3 \%)$. The HLA-DR,DQ genotype and, to a much lesser extend the PTPN22 and INS genotypes predicted development of IA and T1D.

Conclusion: These genotype first prospective population data including IA defined using ZnT8 as well as the classical islet autoantibodies confirms the dominant effect of genetic susceptibility conferred by the HLA-DR,DQ genotypes on the risk of IA and T1D in early childhood.

Multivariate analysis of predictors of IA and diabetes, controlling for gender, race, and family his

\begin{tabular}{lll}
\hline Covariates & HR for IA $(95 \%$ CI $)$ & HR for T1D $(95 \%$ CI $)$ \\
\hline HLA-DR3/4, DQ8 & $3.2(2.3-4.6)$ & $8.1(4.5-14.6)$ \\
\hline PTPN22 R620W & $1.5(1.1-2.1)$ & $1.7(1.0-3.0)$ \\
\hline INS -23Hph1 & $1.1(0.9-1.5)$ & $1.6(1.0-2.8)$ \\
\hline CTLA-4 T17A & $1.1(0.9-1.4)$ & $0.9(0.6-1.3)$ \\
\hline
\end{tabular}

\section{7}

The evaluation of immunological parameters, beta cell function and insulin resistance in first degree relatives of the patients with autoimmunological diabetes

K. Siewko, M. Szelachowska, A. Nikołajuk, M. Gorska

Department of Endocrinology, Diabetology and Internal Medicine, Medical University, Bialystok, Poland

Background and aims: The presence of immunological and/or metabolic markers in the blood of people predisposed to autoimmunological diabetes increases the risk of clinical onset of diabetes. Insulin resistance in type 1 diabetes has aroused a great interest recently. The aim of the study was the evaluation of the first phase of insulin secretion, C-peptide concentration, HOMA\%B index, as well as insulin resistance, measured by HOMA-IR, in relation to the occurrence of antibodies against pancreatic islets (GADA, IAA, and anti-IA2).

Materials and methods: The study was conducted in $90 \mathrm{I}^{0}$ relatives of the patients with type 1 diabetes (60 adults and 30 children), aged 9-65 years (mean age $27.1 \pm 15.5$ ), mean BMI $24.6 \pm 4.95 \mathrm{~kg} / \mathrm{m}^{2}$. Fasting C-peptide concentration was determined using ELISA method, insulin concentration during the intravenous glucose load test $(0,1,3,5 \mathrm{~min})$ - by EASIA method, and GADA, IAA, and anti-IA2 titres - by RIA method. HOMA-IR and HOMA\%B indices were calculated using computer calculator from the Website Oxford Centre for Diabetes, Endocrinology and Metabolism.

Results: The presence of a positive titre of at least one of the antibodies was found in 28 patients $(31.11 \%)$. The highest percentage concerned IAA (22.22\%). None of the persons had C-peptide concentration below normal range, however 22 people $(24.44 \%)$ showed its increased values. Significantly higher insulin concentration at $0 \mathrm{~min}(7.71 \mu \mathrm{IU} / \mathrm{ml} \pm 4.29 \mathrm{vs} 5.27 \mu \mathrm{IU} / \mathrm{ml} \pm$ $2.32 \mathrm{p}<0.002)$ and significantly lower concentrations at $1 \mathrm{~min}(46.22 \mu \mathrm{IU} / \mathrm{ml}$ \pm 33.46 vs $64.34 \mu \mathrm{IU} / \mathrm{ml} \pm 34.06 \mathrm{p}<0.008)$ and at $3 \mathrm{~min}(45.9 \mu \mathrm{IU} / \mathrm{ml} \pm 30.69$ vs $59.7 \mu \mathrm{IU} / \mathrm{ml} \pm 24.86 \mathrm{p}<0.005)$ were observed during intravenous test of glucose load in the group of the relatives as compared to the control group. The highest fasting insulin values were observed in the group of relatives with positive IAA titres. Significantly higher HOMA-IR $(1.16 \pm 0.63$ vs $0.79 \pm 0.34$, $\mathrm{p}<0.002)$ and significantly lower HOMA\%B index $(92,84 \pm 29,39$ vs $114,0 \pm$ $47,06, \mathrm{p}<0,015)$ were found in the study group as compared to the controls. Positive correlations between IAA and C-peptide concentration $(\mathrm{r}=0.282$, $\mathrm{p}<0.002)$, IAA and HOMA-IR $(\mathrm{r}=0,287, \mathrm{p}<0,002)$ and a negative correlation between IAA and HOMA\%B $(r=-0,226, p<0,015)$ were observed.

Conclusion: The increased concentrations of fasting C-peptide and insulin, with decreased beta cell function, can be referred to compensative increase in secretory capacity of beta cells in the group of $\mathrm{I}^{0}$ relatives. Higher concentrations of fasting C-peptide and insulin levels, as well as increased HOMA-IR may suggest insulin resistance in these people. The presence of significant correlations between IAA and HOMA-IR and HOMA\%B may indicate that these $\mathrm{I}^{0}$ relatives are at higher risk of the development of autoimmunological diabetes mellitus.

\section{8}

Relationship of insulin resistance to islet autoimmunity in children C. Winkler ${ }^{1}$, M. Zwilling ${ }^{1}$, A.-G. Ziegler ${ }^{1,2}$

${ }^{1}$ Institut für Diabetesforschung, Munich, ${ }^{2}$ Städt. Klinikum München $\mathrm{GmbH}$, Klinikum Schwabing, Munich, Germany

Background and aims: It has been suggested that metabolic demand and insulin resistance play a role in the development of T1D, including the onset of autoimmunity. The aim of the present study was to determine whether islet autoimmunity is associated with insulin resistance.

Materials and methods: The analysis included 779 children from the German BABYDIAB study, a prospective observational study that followed newborn offspring of mothers or fathers with T1D from birth to 17 years of age. Insulin resistance was estimated by homeostasis model assessment of insulin resistance (HOMA-IR) in 25 children at age of 5 year, in 484 children at age of 8 years, in 344 children at age of 11 years, in 85 children at age of 14 years and in 6 children at age of 17 years; 159 children had more than one HOMAIR measurement. Of the 779 children, 86 had at least one persistent islet autoantibody, median age at first islet autoantibody appearance was 5.03 (IQR, 2.12-8.10); and 15 children developed T1D, median age at diagnosis was 9.36 (IQR, 7.71-10.78).

Results: Median HOMA-IR at 5, 8, 11, and 14 years were 0.32 (IQR, 0.230.76 ), 0.76 (IQR, 0.49-1.17), 1.17 (IQR, 0.8-1.75), and 1.7 (IQR, 1.07-2.42), 
respectively. In a general linear model, increased HOMA-IR was associated with female gender $\left(P<10^{-10}\right)$, older age $\left(P=4 \times 10^{-7}\right)$ and increased BMI $(P<10$ $\left.{ }^{10}\right)$. Children with islet antibodies had in tendency lower HOMA-IR than children who were islet autoantibody negative, but this was not significant $(P=0.11)$ after correction for age of measurements, BMI and gender. Moreover, islet autoantibody positive children did not have increased BMI. Although relatively few children progressed to diabetes, an increased HOMA-IR was not associated with a faster progression rate in the antibody positive children, but rather was associated with a reduced diabetes risk $(\mathrm{P}=0.04)$. Only 5 of 15 children who developed diabetes had HOMA-IR above the median for the respective age in the last measurement prior to diabetes onset.

Conclusion: Islet autoantibody positive children are not insulin resistant soon after sero-conversion and during follow-up. These results are inconsistent with the notion that insulin resistance is a risk factor for islet autoimmunity.

Supported by: JDRF

\section{PS 010 Environmental factors and diabetes}

\section{9}

Factors associated with glucose intolerance in patients with chronic hepatitis B or chronic hepatitis C

A.N. Mavrogiannaki, B.G. Karamanos, E.K. Manesis, J.S. Koskinas, G.V. Papatheodoridis, A.C. Thanopoulou, M.M. Noutsou, A.J. Kofinis, A.J. Archimandritis

Diabetes Center, 2nd Academic Department of Internal Medicine, Athens, Greece

Background and aims: The comparison of the prevalence of glucose intolerance between patients with chronic hepatitis $\mathrm{B}(\mathrm{CHB})$ and $\mathrm{C}(\mathrm{CHC})$ is mostly based on abnormal fasting glucose or known diabetes, leading to underdiagnosis of glucose intolerance. Aim of the present study was to compare the prevalence of glucose intolerance between $\mathrm{CHB}$ and $\mathrm{CHC}$ patients, using as criterion the OGTT and furthermore to explore possible associations of glucose intolerance with biochemical abnormalities characteristic of $\mathrm{CHB}$ or $\mathrm{CHC}$ and also with the degree of the histological lesions of the liver. Materials and methods: We studied 83 consecutive CHB patients and 113 $\mathrm{CHC}$ patients examined at the outpatient liver clinic. Blood samples were obtained for laboratory investigations and OGTT was performed in all (except known diabetics). A liver biopsy was performed, according to clinical indications, in $57 \mathrm{CHB}$ and $91 \mathrm{CHC}$ patients. As glucose intolerance we considered impaired glucose tolerance (IGT), abnormal OGTT or known diabetes. All comparisons were done after adjustment for age and BMI.

Results: The prevalence of glucose intolerance was higher in $\mathrm{CHC}$ than $\mathrm{CHB}$ patients $(42.5 \%$ vs $28.9 \%, P=0.050)$. Among $\mathrm{CHB}$ patients, those with glucose intolerance compared with those with normal glucose tolerance had more frequent family history of diabetes $(58.3 \%$ vs $23.7 \%, P=0.004)$ and higher $\gamma$-GT ( $45.4 \pm 5.7$ vs $25.3 \pm 4.0 \mathrm{IU} / \mathrm{L}, P=0.008)$, while insulin resistance, by HOMA model and plasma insulin at every time point did not differ. Among CHC patients, those with glucose intolerance compared with those with normal glucose tolerance had higher insulin resistance $(5.0 \pm 0.5$ vs $2.9 \pm 0.4, P=0.000)$, higher plasma insulin at $120 \mathrm{~min}(70.2 \pm 7.0 \mathrm{vs} 39.2 \pm 4.8 \mu \mathrm{u} / \mathrm{ml}, P=0.001)$ and higher prevalence of cryoglobulinemia $(58.3 \%$ vs $32.8 \%, P=0.021)$, while family history of diabetes and fasting insulin did not differ. In both groups of $\mathrm{CHB}$ and $\mathrm{CHC}$ patients, the grade of necroinflamatory activity, the stage of fibrosis, the presence of chirrosis and the grade of steatosis, evaluated blindly, were not related with the presence of glucose intolerance. It is also noted that, the majority of patients had mild histological alterations of fibrosis.

Conclusion: Increased prevalence of glucose intolerance is documented in chronic hepatitis C compared with chronic hepatitis B, using OGTT. Factors associated with glucose intolerance are different in the two types of liver diseases, namely, family history of diabetes and higher $\gamma$-GT in CHB, while insulin resistance and cryoglobulinemia in CHC. Glucose intolerance could be another extrahepathic manifestation of $\mathrm{CHC}$, as cryoglobulinemia. Mild histological lesions are not related with glucose intolerance.

\section{0}

Specific phenotype associated with diabetes mellitus secondary to chronic hepatitis $\mathrm{C}$ infection

A. Poussier ${ }^{1}$, M. Lebouvier ${ }^{1}$, A. Penfornis ${ }^{2}$, V. Di Martino ${ }^{3}$, P. Buffier ${ }^{1}$, B. Verges ${ }^{1}$, P. Hillon ${ }^{4}$ J.-M. Petit ${ }^{1}$

${ }^{1}$ Endocrinology, CHU Bocage Dijon, Dijon, France, ${ }^{2}$ Endocrinology, CHU Besançon, Dijon, France, ${ }^{3} \mathrm{Hepatology}$, CHU Besançon, Dijon, France, ${ }^{4}$ Hepatology, CHU Bocage Dijon, Dijon, France

Background and aims: A link between chronic hepatitis $\mathrm{C}$ virus infection (HCV), type 2 diabetes mellitus and insulin resistance has been suggested by several studies. However, it seems that HCV infection is associated with insulin resistance but is not associated with metabolic syndrome. The following study, was undertaken to determine whether chronic HCV infection had an impact on the clinical characteristics of type 2 diabetes.

Materials and methods: To address this question, we studied retrospectively, a group of patients with diabetes mellitus associated with HCV infection (HCV-DM), and compared them with patients suffering from conventional type 2 diabetes (DM).

Results: The HCV-DM patients had a lower BMI ( $\mathrm{p}=0.001$ ), and systolic blood pressure $(\mathrm{p}=0.04)$ compared to patients with DM diabetes. Ten $(27.0$ 
\%) patients in the HCV-DM group and 35 (47.3\%) in the DM group had microalbuminuria $(\mathrm{p}=0.04)$. DM patients had significantly higher serum creatinine levels than HCV-DM patients $(102.9+/-62.9 \mathrm{vs} 81.9+/-32.4 \mathrm{mmol} / \mathrm{l}$, $\mathrm{p}=0.02)$. One (2.7\%) HCV-DM patients and $44(59.4 \%)$ DM patients were treated with hypolipemic therapy $(\mathrm{p}=0.0001)$. Even though nearly two-thirds of the overall DM-group were receiving cholesterol-lowering drugs, DM patients had significantly higher total cholesterol, HDL cholesterol and triglyceride levels than HCV-DM patients.

Conclusion: Our study confirms that HCV-diabetic patients had specific clinical characteristics in comparison to classical type 2 diabetic patients. These data suggest a direct effect of HCV virus on the development of insulin resistance and diabetes mellitus without the association of the metabolic syndrome.

\section{1}

Ketosis prone type $\mathbf{2}$ diabetes and human herpes virus 8 in sub-Saharan Africans

E. Sobngwi ${ }^{1}$, S.P. Choukem ${ }^{1}$, F. Agbalika ${ }^{2}$, B. Blondeau ${ }^{3}$, L.-S. Fetita ${ }^{1}$, C. Lebbe , D. Thiam², P. Cattan ${ }^{5}$, J. Larghero, , F. Foufelle ${ }^{3}$, P. Ferre ${ }^{3}$, P. Vexiau ${ }^{1}$, F. Calvo ${ }^{6}$, J.-F. Gautier ${ }^{1,3}$

${ }^{1}$ Dept. of Endocrinology, Diabetology \& Nutrition, Hopital Saint Louis, Paris, ${ }^{2}$ Department of Virology, Hopital Saint Louis, Paris, ${ }^{3}$ Inserm umrs u872, Centre de Recherche des Cordeliers, Paris, ${ }^{4}$ Department of Dermatology, Hopital Saint Louis, Paris, ${ }^{5}$ Cell Therapy Unit, Hopital Saint Louis, Paris, ${ }^{6}$ Centre d'Investigation Clinique CIC9504, INSERM, Hopital Saint Louis, Paris, France

Background and aims: An atypical form of type 2 diabetes is revealed by ketosis (Ketosis-prone type 2 diabetes- KPD), frequently in black Africans, and characterized by an acute onset requiring transient insulin therapy. Its sudden onset suggests precipitating factors. Given the geographic prevalence of KPD, we investigated in a prospective and observational cohort study, the putative role of Human Herpes Virus-8 (HHV-8) in its pathogenesis.

Materials and methods: This prospective, observational cohort study took place at Saint-Louis University Hospital. We searched antibodies against latent and lytic HHV-8 antigens, using immunofluorescence, in 187 black African consecutive diabetic patients of whom 81 had KPD, and 106 type 2 diabetes (T2D) and in 90 black African non-diabetic subjects matched for age and sex (controls). The presence of HHV-8 in genomic DNA was investigated in 22 patients at clinical onset of diabetes. We also tested whether HHV-8 was able to infect human pancreatic beta cells in culture in vitro.

Results: HHV-8 antibodies were found in $87.7 \%$ of KPD patients versus $15.1 \%$ non-ketotic type 2 diabetes (T2D), $\mathrm{OR}=39.9(17.1-93.4 ; \mathrm{p}<0.0001)$ and $40.0 \%$ of the control subjects OR=10.7 (4.9-23.4; $\mathrm{p}<0.0001)$. HHV-8 DNA was present in 6/13 KPD tested at acute onset and in 0/9 T2D. Furthermore, we showed the presence of HHV-8 proteins in human islet cells cultured for 4 days in the presence of HHV-8.

Conclusion: In KPD patients of sub-Saharan African origin, HHV-8 DNA is found in almost $50 \%$ of patients at onset and the prevalence of HHV-8 seropositivity is unexpectedly high. These data together with the capacity of HHV-8 to infect human beta cells in vitro support that HHV-8 infection may convert T2D into KPD.

Supported by: Assistance Publique - Hôpitaux de Paris, INSERM, Association Française des Diabétiques, and ALFEDIAM

\section{2}

Interaction of early cow's milk-based formula exposure and enterovirus infection in infancy in the development of type 1 diabetes-associated autoimmunity

J. Aarnisalo ${ }^{1}$, H. Honkanen ${ }^{2}$, M. Mäkelä ${ }^{1}$, S. Tauriainen ${ }^{2}$, J. Marttila ${ }^{1}$, R. Veijola ${ }^{3}$, M. Knip ${ }^{4,5}$, O. Simell ${ }^{6}$, R. Hermann ${ }^{1}$, H. Hyöty ${ }^{2}$, O. Vaarala ${ }^{7}$, J. Ilonen ${ }^{1}$

${ }^{1}$ Immunogenetics Laboratory, University of Turku, ${ }^{2}$ Department of Virology, University of Tampere, ${ }^{3}$ Department of Pediatrics, University of Oulu, ${ }^{4}$ Department of Pediatrics, University of Tampere, ${ }^{5} \mathrm{Hospital}$ for Children and Adolescents, University of Helsinki, Finland, ${ }^{6}$ Department of Pediatrics, University of Turku, ${ }^{7}$ Laboratory of Immunobiology, National Public Health Institute, Helsinki, Finland

Aims: Both enterovirus infections in childhood and cow's milk based formula feeding during infancy have been implicated in the initiation of type 1 diabetes (T1D) -associated autoimmunity. Moreover, the timing of these triggering factors seems to be crucial. In this study we aimed to analyse further the influence of these factors on autoimmunity associated with T1D.

Subjects and methods: Study subjects were participants in the Diabetes Prediction and Prevention Study (DIPP) and carried HLA-genotypes conferring risk for T1D. According to the study protocol the subjects were observed for the appearance of T1D-associated autoantibodies (ICA, IAA, GADA, IA-2A) at 3-12 month intervals. 107 case subjects positive for multiple $(\geq 2)$ autoantibodies and 447 autoantibody negative controls matched for age, gender and HLA DQB1 genotype (3-5 controls/case) were compared. Length of the follow-up was 4.5-11.9 years (median 8.0). Coxsackie B4 (CBV4) IgG and IgA, adenovirus IgG and IgA and RSV IgG antibodies were detected using EIA from serum samples collected at the age of 6,12 and 18 months. 251 of the study subjects were exposed to cow's milk-based formula (CM) before the age of 3 months and 222 subjects later in infancy.

Results: By the age of 12 months 83 of the 518 (16.0\%) subjects tested had antibodies against CBV4, $68(13.1 \%)$ against adenovirus and 152 of the 508 subjects (30.0\%) against RSV. In the Cox regression analysis, none of these virus infections detected by the age of 6,12 or 18 months were associated with the appearance of any of the diabetes-associated autoantibodies or T1D. Similarly, no association could be observed between early exposure to CM and later signs of autoimmunity. Interestingly, when the combined effect of early exposure to CM and CBV4 infection during the first 12 months of life on the appearance of autoantibodies and T1D was analysed Cox regression analysis showed a strong association between early CM exposure and CBV4 infection in infancy. The association with the appearance of autoantibodies and clinical T1D was strongest among subjects exposed to CM before 3 months of age and emerging with CBV4 antibodies between 6 and 12 months of life (HR 11.4 (95\% CI 2.9-44.9) $\mathrm{p}=0.001$ for ICA, 15.9 (3.1-81.6) $\mathrm{p}=0.001$ for IAA $16.1(3.2-80.0) p=0.005$ for GADA $7.7(1.9-32.4) p=0.005$ for IA-2A and $9.9(0.98-99.9) \mathrm{p}=0.05$ for clinical T1D) when stratified according to the HLA DQB1-DQA genotype and PTPN22 +1858C/T, insulin gene $-23 \mathrm{HphI}$ $\mathrm{A} / \mathrm{T}$ and CTLA $4+49 \mathrm{~A} / \mathrm{G}$ polymorphisms. No interplay could be observed between CM exposure and adenovirus or RSV infection.

Conclusion: Our results suggest an interplay between early insulin immunisation through cow's milk-based formula and enterovirus infection during the second half of the infancy. The needed interaction between the two hypothesised environmental factors may explain the controversial results obtained in studies observing the effect of only one of these factors.

\section{3}

Dehydroascorbate protection against dioxin-induced toxicity in the beta cell line INS-1E

V. De Tata, L. Martino, M. Novelli, M. Masini, A. Casini, P. Masiello

Experimental Pathology \& B.M.I.E., University of Pisa, Italy

Background and aims: Consistent epidemiological data have recently revealed a positive correlation between exposure to persistent organic pollutants and glucose metabolism disorders including type 2 diabetes and metabolic syndrome. These observations are particularly interesting, with reference to the continuous increase of the prevalence of type 2 diabetes in the last decades, suggesting a possible contribution of environmental factors to the pathogenesis the disease, in addition to obvious nutritional habits. We have previously demonstrated that 2,3,7,8-tetrachlorodibenzo-p-dioxin (TCDD) is highly toxic for $\beta$ cells and that it markedly impairs glucose-stimulated insulin secretion. As it is known that acute exposure to TCDD results in oxidative stress and that pancreatic $\beta$ cells are highly vulnerable to reactive oxygen species (ROS)-mediated tissue damage, in this study we aimed at investigating the protective effect of dehydroascorbic acid (DHA), a well-know antioxidant agent, against TCDD-induced toxicity in the INS-1E $\beta$-cell line.

Materials and methods: INS-1E cells were preincubated for 30 min with 0.5 or $1.0 \mathrm{mM}$ DHA and then exposed for $1 \mathrm{~h}$ to different concentrations (range 0-50 nM) of TCDD. We have then evaluated: 1) cell survival and ultrastructural alterations; 2) intracellular ROS production by fluorimetric methods; 3) mitochondrial membrane potential by cytofluorimetry; 4) glucose-stimulated insulin secretion.

Results: We have preliminarly verified that 30 -min incubation of INS-1E cells with DHA led to a rapid, significant and dose-dependent increase of the intracellular levels of ascorbic acid. $\beta$-cell viability, markedly impaired upon TCDD exposure (12-50 nM), was significantly increased following 30min preincubation with DHA. Electron microscopy showed that a peculiar ultrastructural alteration in TCDD-treated cells $(12-25 \mathrm{nM})$ was the presence of several autophagic vacuoles containing mitochondria, which also occurred 
after pre-treatment with $1 \mathrm{mM}$ DHA. However, in most cells pre-exposed to DHA, these vacuoles appeared at early stages of formation (i.e., prior to lysosome fusion), a feature that was never observed in cells treated with TCDD alone. Surprisingly, we could not detect any increase in ROS production after $1 \mathrm{~h}$ exposure of INS-1E cells to TCDD; nevertheless, we observed TCDDinduced mitochondrial membrane depolarization which was significantly prevented when the cells were pre-exposed to $1 \mathrm{mM}$ DHA. Finally, in INS-1E cells preincubated with $1 \mathrm{mM}$ DHA for $30 \mathrm{~min}$ and subsequently exposed to TCDD at a very low, non cytotoxic dose $(1 \mathrm{nM})$, the inhibitory effect of TCDD on glucose-stimulated insulin secretion was fully prevented.

Conclusion: Our data clearly show that DHA is able to protect INS-1E cells against TCDD-induced toxicity in terms of both cell function and survival. This protective effect could be related to the ability of DHA to preserve mitochondrial integrity or at least to limit TCDD-induced mitochondrial damage.

\section{4}

Insulin autoantibodies that cross-react with casein

K. Koczwara ${ }^{1}$, D. Mueller ${ }^{1}$, P. Achenbach ${ }^{1}$, S. Krause ${ }^{1}$, A.G. Ziegler ${ }^{1,2}$, E. Bonifacio ${ }^{3}$

${ }^{1}$ Institut für Diabetesforschung, Munich, ${ }^{2}$ Klinikum Schwabing, Städt. Klinikum München $\mathrm{GmbH},{ }^{3}$ Center for Regenerative Therapies - Dresden, University of Technology, Germany

Background and aims: Insulin autoantibodies (IAA) can appear early in life and precede clinical type 1 diabetes (T1DM) in children. Although the immunization events leading to IAA are unknown it has been shown that some low affinity IAA have an IgA component and a preferred binding to non-human insulin suggesting that IAA can result from immunization against molecules other than human insulin at mucosal sites. The aim of this study was to determine whether some IAA may be cross-reactive with and potentially primarily directed against exogenous antigens such as components of infant's diet.

Materials and methods: Antibody binding to ${ }^{125} \mathrm{I}$ labeled human insulin in the presence or absence of food proteins was analyzed in sera from IAA positive children with a first-degree T1DM family history from prospective follow-up studies $(\mathrm{n}=45$; median age 2.3 years, IQR 1.8-5.2). Direct binding to food proteins was performed by radiobinding assay.

Results: Initial experiments identified IAA from 3 of 9 children that were significantly inhibited by whole bovine milk powder $(98 \%, 85 \%$, and $46 \%$ inhibition of IAA binding) but not by hydrolyzed hypo-allergic milk powder, wheat flour and fowl meal, respectively. IAA of the remaining 6 children and 2 patients with T1DM were not reactive with any of these food components. Subsequent screening of further 36 IAA positive children using $0.5 \mu \mathrm{g}$ whole bovine milk powder for competition discovered 3 additional children whose IAA were partially inhibited by milk components. All of the milk-reactive IAA were of lower affinity $\left(<10^{9} \mathrm{~L} / \mathrm{mol}\right)$, and these children did not develop GAD and/or IA-2 autoantibodies or T1DM within a median follow-up of 6.1 years (IQR 2.6-7.1). The milk-reactive IAA occasionally had an IgA-IAA component. Further testing of defined milk protein components revealed that their IAA binding was completely inhibited by $0.25 \mu \mathrm{g}$ of alpha- and beta-casein, respectively, but was not affected by lactoglobulin or BSA. Moreover, antibody binding to labeled human insulin was better inhibited by casein (alpha $>$ beta) than by human insulin in these sera, indicating that the affinity of the antibodies was at least $1 \log$ higher for casein than insulin. All sera tested bound ${ }^{14} \mathrm{C}$ labeled bovine alpha-casein; binding to ${ }^{14} \mathrm{C}$-alpha-casein was not inhibited by insulin.

Conclusion: The data show that IAA can be cross-reactive to non-insulin proteins and suggest that in some cases, IAA could be secondary to immunization against food proteins. These cases do not appear to be associated with type 1 diabetes.

Supported by: JDRF and German Research Foundation

\section{5}

Islet cell antibodies associate with the presence of enteroviral RNA while glutamic acid decarboxylase antibodies do with insulin resistance in first degree relatives of type 1 diabetes

E. Cabrera-Rode ${ }^{1}$, L. Sarmiento ${ }^{2}$, I. Cuba ${ }^{1}$, G. Molina ${ }^{1}$, M. Fonseca ${ }^{2}$, L. Heng-Hung ${ }^{2}$, C. Arranz ${ }^{1}$, E. Dominguez ${ }^{1}$, P. González ${ }^{3}$, O. Diaz-Horta ${ }^{1}$

${ }^{1}$ Department of Immunology and Genetics on Diabetes, National Institute of Endocrinology, ${ }^{2}$ Department of Virology, "Pedro Kouri” Tropical Medicine Institute, ${ }^{3}$ Department of Endocrinology, Hospital "William Soler", Havana, Cuba

Background and aims: We have previously demonstrated that there is an association between the presence of enteroviral RNA and the onset of type 1 diabetes. Other groups have reported insulin resistance as a risk factor for progression of type 1 diabetes. We evaluated whether type 1 diabetes associated antibodies (ICA, GAD and IA-2 antibodies) are related to occurrence of enteroviral RNA and insulin resistance in first degree relatives of type 1 diabetic patients.

Materials and methods: Serum samples were collected from islet cell autoantibody-positive $(n=32)$ and -negative $(n=62)$ first degree relatives of type 1 diabetic patients. Plasma glucose concentrations were determined by glucose oxidase method. ICAs were determined by indirect immunofluorescence technique with prolonged incubation. Antibodies to GAD, IA-2, and insulin were determined by radioimmunoassay; and insulin resistance was estimated by the homeostasis model assessment (HOMA-IR). Enteroviral RNA was assessed using highly sensitive reverse transcriptase-polymerase chain reaction method. Results: Occurrence of enteroviral RNA was higher in ICA-positive (5/32, $15.6 \%)$ first degree relatives of type 1 diabetic patients in comparison to ICAnegative subjects $(1 / 62,1.6 \% ; \mathrm{p}=0.0164)$. In addition, the presence of enteroviral RNA was associated with high ICA titres ( $\geq 20$ JDFunits, $p=0.0064$ ). On the other hand, insulin resistance (HOMA-IR) was increased in GADApositive $(\mathrm{n}=19$, Mean $2.99 \pm 2.1)$ versus GADA-negative first degree relatives $(\mathrm{n}=75$, Mean $1.86 \pm 1.48, \mathrm{p}=0.038)$. The insulin resistance was not related to GADA titres. The presence of IA2 antibodies was associated neither with the occurrence of enteroviral RNA nor with insulin resistance.

Conclusion: These data suggest that enterovirus infections and insulin resistance are two different and independent events related to the production of type 1 diabetes associated autoantibodies (ICA and GADA). This results support the hypothesis that different factors (virus and insulin resistance) are aetiologically important as trigger and/or accelerating factors for type 1 development.

Supported by: Ministry of Health

\section{6}

Ethanol impairs GLUT4 expression in the isolated rat and human adipocytes: role of AMP-activated protein kinase alpha and myocyte enhancer factor 2

L. Feng, Q. Li, Q.B. Guan, J.J. Zhao

Endocrinology, Provincial Hospital affiliated to Shandong University, Jinan, China

Background and aims: Previous studies reported that heavy ethanol intake impaired GLUT4 expression in adipose tissues, but the precise mechanisms are not well elucidated. Recently, AMP-activated protein kinase (AMPK) is considered to be a new target of ethanol. Moreover, studies in skeletal muscle found that AMPK regulated GLUT4 via myocyte enhancer factor 2 (MEF2), a transcription regulator of GLUT4. Here, we explored whether the AMPK/ MEF2 pathway was involved in the impairment effects of ethanol on GLUT4 expression in isolated rat and human primary adipocytes.

Materials and methods: The present study was consisted of two parts. (1) To test whether an AMPK/ MEF2/ GLUT4 pathway existed in adipose tissues and observe the regulation of AMPK on MEF2 and GLUT4 in vivo, eight male Wistar rats were randomly divided into two groups, subcutaneously injected with AICAR (an AMPK activator, $0.8 \mathrm{mg} / \mathrm{g}$ body weight) or with a corresponding volume of $0.9 \% \mathrm{NaCl}$ (controls). Two hours later, epididymal adipose tissues were obtained. (2) To reconfirm the existence of this pathway in adipocytes in vitro and explore effects of ethanol on it, adipocytes isolated from normal Wistar rat and human adipose tissues were respectively incubated with and without edible ethanol, AICAR, or compound C (a selectively AMPK inhibitor) for $1 \mathrm{~h}$. Protein expression of phosphorylated-AMPK alpha (the activated AMPK), MEF2 and GLUT4 was tested by western blotting. GLUT4 mRNA levels were measured by RT-PCR. 
Results: In experiments in vivo, AICAR injection led to a marked increase in AMPK a phosphorylation $(1.9$-fold over control, $\mathrm{P}<0.01)$ in the rat adipose tissues. Following the activation of AMPK, MEF2 protein was elevated by $60.6 \%(\mathrm{P}<0.05)$. As the result, the GLUT4 mRNA levels were increased by $62.8 \%(\mathrm{P}<0.05)$ and subsequently its protein was enhanced by $58.94 \%$ $(\mathrm{P}<0.05)$. Our data showed the activated-AMPK exerted positive regulations of MEF2 and GLUT4 expression in vivo. In experiments in vitro, in AICAR treated-rat and human adipocytes, the P-AMPK $\alpha$ expression was increased by 83.37 - and $90.87 \%$ (both $\mathrm{P}<0.01$ ). Subsequently, MEF2 was enhanced by 73.31- and $53.92 \%(\mathrm{P}<0.01$ and $\mathrm{P}<0.05)$. Accordingly, for GLUT4, the mRNA levels were elevated by 51.89 - and $54.89 \%$ (both $\mathrm{P}<0.05$ ), the protein expression was increased by 48.81 - and $39.9 \%$ (both $\mathrm{P}<0.05$ ). However, if the cells were supplied with compound C prior to AICAR for $20 \mathrm{~min}$, the augmented effects of AICAR on activated-AMPK, MEF2, and GLUT4 were inhibited to nearly normal levels. These results reconfirmed the findings we observed in vivo.

To observe the action of ethanol on this pathway, the above cells were treated with edible ethanol. In relative to controls, in the rat and human adipocytes, ethanol decreased P-AMPK a protein by 38.6- and 56\% (both $\mathrm{P}<0.05$ ), then MEF2 expression was attenuated by $32-$ and $44.84 \%$ (both $\mathrm{P}<0.05$ ). As a result, the mRNA and protein levels of GLUT4 were diminished by 51.75 - and $40.03 \%$ (both $\mathrm{P}<0.05$ ) in rat adipocytes, by 39.86 - and $59.51 \%$ (both $\mathrm{P}<0.05$ ) in human adipocytes. These results suggested that the impaired GLUT4 expression by ethanol was most probably due to the decline of AMPK activity, then the inhibition of MEF2 expression.

Conclusion: In this study, we demonstrated that an AMPK/ MEF2/ GLUT4 pathway existed in both rat and human adipose tissues, the activated-AMPK positively regulated MEF2 and GLUT4 expression. The ethanol-mediated inhibition of this pathway might contribute to the impairment of GLUT4 expression.

Supported by: Natural Science Foundation of Shandong Province

\section{PS 011 Screening and prediction of type 2 diabetes}

\section{7}

Glucose challenge test two-step screening for prediabetes and early diabetes

L.S. Phillips ${ }^{1,2}$, K.M.V. Narayan ${ }^{3}$, D.C. Ziemer ${ }^{1}$, P. Kolm ${ }^{4}$, M.K. Rhee ${ }^{1}$, R. Chatterjee ${ }^{3}$, W.S. Weintraub ${ }^{4}$, V. Vaccarino ${ }^{5}$

${ }^{1}$ Division of Endocrinology and Metabolism, Emory University School of Medicine, Atlanta, ${ }^{2}$ Research Service, VA Medical Center, Decatur, ${ }^{3}$ Department of Global Health, Rollins School of Public Health, Atlanta, ${ }^{4}$ Division of Cardiology, Christiana HealthCare, Newark, ${ }^{5}$ Division of Cardiology, Emory University School of Medicine, Atlanta, United States

Background and aims: Diabetes care is limited by lack of screening. Progression from prediabetes to diabetes can be prevented or delayed, but most individuals with prediabetes are not detected, and cannot receive interventions to reduce risk. Since we lack a good screening strategy, glucose intolerance progresses for 5-10 years, many patients have early complications by the time they are recognized, and diabetes control can be difficult. We hypothesized that screening could be accomplished with a two-step "glucose challenge test" (GCT) strategy, similar to screening for gestational diabetes: a 50g oral GCT at any time of day, regardless of meal status, with a single $1 \mathrm{hr}$ sample. If the GCT exceeded a cutoff, patients would have a $75 \mathrm{~g}$ oral glucose tolerance test (OGTT) after an overnight fast.

Materials and methods: We tested our hypothesis in 1,419 adult volunteer subjects who were generally well, not pregnant, and not known to have diabetes. At a first visit, they had measurement of random plasma and capillary glucose (RPG and RCG), followed by the 50g GCT with $1 \mathrm{hr}$ samples for plasma and capillary glucose (GCTpl and GCTcap) - without prior restriction of food, and at different times during the day. All had an OGTT at a second visit - after an overnight fast, and beginning before $11 \mathrm{am}$. Glucose intolerance was defined by WHO criteria (FPG $\geq 6.1,2 \mathrm{hPG} \geq 7.8 \mathrm{mM}$ ) and screening accuracy evaluated by ROC analysis.

Results: The subjects had average age $48 \mathrm{yr}$, BMI $30.2 \mathrm{~kg} / \mathrm{m} 2$, and were $60 \%$ female and 56\% black; $5 \%$ had diabetes (DM), $21 \%$ had DM or IGT, and $24 \%$ had dysglycemia (DM or IGT or IFG). GCTpl provided area under the ROC curve (AROC) $0.89,0.82$, and 0.81 for detection of DM, DM or IGT, and dysglycemia, respectively, each higher than GCTcap (AROC 0.85, 0.77, and 0.76), $\mathrm{p}<0.05$. To detect DM or IGT, GCTpl was better than RPG (AROC $0.72)$ and GCTcap was better than RCG $(0.65)$, both $p<0.001$. GCTpl to detect DM or IGT was comparable in males and females (AROC 0.81 and 0.83 ), blacks and whites $(0.85$ and 0.80$)$, unaffected by time after meals or time of day (both $\mathrm{p}>0.2$ ), and comparable in groups with differing prevalence of glucose intolerance (AROC $0.80,0.82$, and 0.80 , for age $<40,40-55$, and $>55$ yr; AROC 0.81, 0.82, and 0.82, for BMI $<25,25-35,>35$, both $\mathrm{p}>0.6)$. Multivariate analysis showed that the $c$-statistic (equivalent to AROC) of GCTpl for detection of DM or IGT was highly significant and independent of age, sex, race, BMI, waist circumference, HDL, systolic BP, and family history $(p<0.001)$; inclusion of these variables produced an increase in AROC that was significant but small (0.85). The direct cost of GCTcap screening should be less than $\$ 5$ per person; economic modeling indicated that GCT screening and 3 years of management with metformin may be cost-saving vs. no screening in healthcare systems such as the VA in the U.S.A.

Conclusion: Two-step GCT-OGTT screening for prediabetes and unrecognized diabetes is accurate, convenient, and inexpensive. Widespread use of this strategy could help to revolutionize diabetes care, since early detection would allow preventive management which is efficacious and cost-effective. Supported by: NIDDK

\section{8}

Identification of high-risk individuals for implementation of a diabetes prevention program in an occupational setting. The DEPLAN Study S. Grammatikou, S. Liatis, K. Makrilakis, M. Katsorida,

P. Konstantopoulou, C. Pietris, C. Alexopoulos, K. Kyriakopoulos, K. Perrea, D. Perrea, N. Katsilambros

1st Internal Medicine Department, Athens University Medical School Laiko Hospital, Greece

Background and aims: DEPLAN is a European program for type 2 diabetes (T2D) prevention based on lifestyle intervention, implemented in a range of 
populations across the European Union. In this study we explored the feasibility of implementing a diabetes identification risk score questionnaire at an occupational setting.

Materials and methods: In the first step of the project, persons working at four companies were distributed a simple, validated questionnaire (FINDRISK), for identifying those at increased risk for T2D. As part of the screening procedure, the medical department of three public companies and one private company in the district of Athens, Greece, were contacted and informed about the aim of the study. A "day for diabetes prevention" was arranged, during which three doctors of the investigation team visited each company and, in collaboration with the local health professional stuff, distributed the FINDRISK questionnaire to all the employees attending their job at that specific date. The questionnaires were completed on site and the employees were given their score. A score $\geq 15$ (maximum possible 26) was considered as setting the person at high risk. The identified high-risk individuals were invited to receive an oral glucose tolerance test (oGTT) at a different day, in order to exclude persons with unknown diabetes. The remaining non-diabetic, high-risk employees were invited to attend the one-year diabetes prevention program.

Results: A total of 1001 employees (579 men and 422 women), aged 20 to 64 years completed the FINDRISK questionnaire. Fewer women than men ( $8.29 \%$ vs. $17.96 \%$ ) were included in the eldest age group (55-64 years). Mean BMI was $27.19 \pm 4.67 \mathrm{Kg} / \mathrm{m}^{2}$ (men: $28.38 \mathrm{Kg} / \mathrm{m}^{2}$, women: $25.55 \mathrm{Kg} / \mathrm{m}^{2}$ ) and mean waist circumference was $94.81 \pm 13.62 \mathrm{~cm}$. The median FINDRISK score was 9 (min: 0, max: 24). A total of 146 (14.59\%) employees [93 men $(16.43 \%)$ and 48 women $(11.76 \%)$ had a score $\geq 15$ and 83 of them $(56.84 \%)$ accepted to receive an oGTT in order to enter the lifestyle intervention program. 16 persons (19.27\%) were found to have diabetes ( 9 men and 7 women) while $36(43.37 \%)$ had impaired fasting glucose (IFG, fasting blood glucose $100-125 \mathrm{mg} / \mathrm{dl}), 8(9.64 \%)$ had impaired glucose tolerance (IGT) and 23 (27.71\%) had normal glucose metabolism. Thus, the FINDRISK questionnaire yielded a positive predictive value of $72.29 \%$ for identifying any glucose abnormality. After exclusion of the individuals with diabetes, the remaining $(n=67)$ employees were offered to enter the one-year lifestyle intervention program and 60 of them (89.55\%) accepted.

Conclusion: The FINDRISK questionnaire has a high positive predictive value $(72.29 \%)$ for identifying impaired glucose metabolism in a young and middle-aged working Greek population. A high percentage $(14.59 \%)$ of Greek employees are at high-risk for developing T2D and more than half of them $(56.84 \%)$ are willing to participate in T2D prevention programs using lifestyle modification.

\section{9}

Dietary energy density predicts the risk of type 2 diabetes: the EPICNorfolk Study, 1993-2005

J. Wang ${ }^{1}$, R. Luben ${ }^{2}$, K.-T. Khaw ${ }^{2}$, S. Bingham ${ }^{2}$, N.J. Wareham ${ }^{1}$,

N.G. Forouhi ${ }^{1}$

${ }^{1}$ Institute of Metabolic Science, Medical Research Council Epidemiology Unit, ${ }^{2}$ Department of Public Health and Primary Care, Institute of Public Health, Cambridge, United Kingdom

Background and aims: Accumulating evidence suggests that energy dense foods predispose to obesity, and there is speculation that such foods may also be associated with increased risk of type 2 diabetes (T2DM), but there is limited supportive evidence. Dietary energy density (DED) is defined as the available dietary energy per unit weight of foods. Our aim was to investigate whether there is an independent association between DED and incidence of T2DM.

Materials and methods: Population-based prospective study, the EPIC (European Prospective Investigation of Cancer)-Norfolk Cohort Study of persons aged 40-79 years at baseline. We calculated DED for overall diet (all solids and drinks) using self-reported dietary intake (from semi-quantitative food frequency questionnaire). During 12 years of follow-up, we documented 725 clinically incident cases of T2DM among 21,919 participants without diabetes, cancer or cardiovascular disease at baseline.

Results: Mean DED was 3.01 (95\% CI 3.00-3.02) kJ/g in people without diabetes, and 3.08 (95\% CI 3.03-3.13) kJ/g in those with incident T2DM after adjusting for age, sex, and baseline BMI $(P, 0.012)$. DED at baseline was positively associated with incident T2DM (odds ratio, OR, 1.21, 95\% CI 1.061.38) in analyses adjusted for age, sex, occupational status, smoking, alcohol, physical activity, family history of T2DM, total energy intake, and baseline BMI or waist circumference. There was a $60 \%$ higher risk of T2DM (OR 1.60, 95\% CI 1.19-2.16, $P$ for trend 0.002) in the highest quintile of DED (range
3.55-7.97 kJ/g) compared with the lowest quintile (range 1.04-2.43 kJ/g) in adjusted analyses. There was no significant interaction between DED and BMI or waist circumference, or DED and sex on the risk of T2DM.

Conclusion: This is the first large population-based prospective study to report that an energy dense diet may be associated with increased risk of developing type 2 diabetes, independently of obesity. The potential public health impact of a low energy dense diet on reducing the risk of diabetes deserves serious further study.

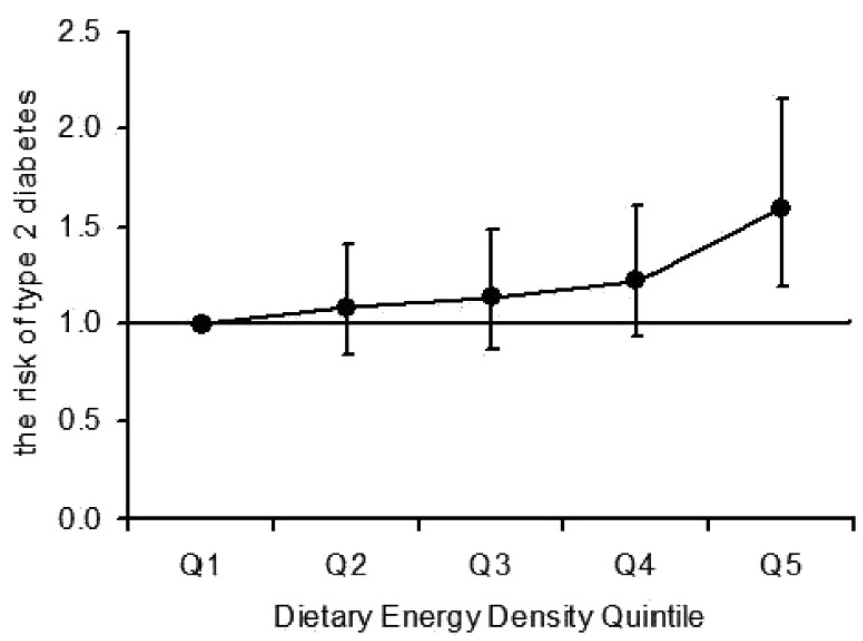

Supported by: Medical Research Council Epidemiology unit and Ellison Medical Foundation - International Nutrition Foundation

\section{0}

Low serum adiponectin predicts 10 -year incidence of type 2 diabetes and $\mathrm{HbA}_{1 \mathrm{c}}$ levels independently of obesity, lipids and inflammation -Whitehall II study

A.G. Tabák ${ }^{1}$, E.J. Brunner' ${ }^{1}$ M.A. Miller², S. Karanam², P.G. McTernan², F.P. Cappuccio ${ }^{2}$, D.R. Witte ${ }^{1}$

${ }^{1}$ Department of Epidemiology \& Public Health, University College London, ${ }^{2}$ Clinical Sciences Research Institute, Warwick Medical School, Coventry, United Kingdom

Background and aims: Several adipokines (most notably adiponectin) have emerged as possible risk factors for the development of type 2 diabetes mellitus (T2DM). While the cross-sectional association of adiponectin with obesity and insulin resistance is well-described, evidence on the prospective relationship between adiponectin and incident T2DM or glycemic control is limited. Our aim was to study the effect of adiponectin levels on the development of incident diabetes and HbAlc values, taking the major T2DM risk factors and inflammation into account.

Materials and methods: We measured baseline serum adiponectin levels in a nested case-control selection of 142 participants of the Whitehall II Cohort. All participants were Caucasian, and had no prevalent T2DM, CHD, acute or longstanding inflammatory diseases at baseline. Cases $(n=55)$ had incident T2DM according to a 75g OGTT during follow-up (mean: $11.5 \pm$ $3.0[\mathrm{SD}] \mathrm{yrs})$, controls $(\mathrm{n}=87)$ were frequency matched on age, BMI and sex. The mean age of participants was $50.9 \pm 6.3 \mathrm{yrs}$, BMI $26.8 \pm 4.2 \mathrm{~kg} / \mathrm{m}^{2}$, and $68.3 \%$ was male.

Results: Adiponectin values were lower among cases at baseline $(8.8 ; 0.4$ [SE] vs. $10.2 ; 0.4 \mu \mathrm{g} / \mathrm{mL}, \mathrm{p}=0.01)$. The risk of incident T2DM decreased by $11 \%$ (OR $0.8995 \% \mathrm{CI} 0.79-0.99, \mathrm{p}=0.03$ ) per $1 \mu \mathrm{g} / \mathrm{mL}$ increase in adiponectin. This association remained stable and significant after adjustment for potential confounders including age, sex, BMI, blood lipids (BL), systolic blood pressure (SBP), CRP, and fasting plasma glucose (FPG) at baseline. The association was independent of the effect of physical activity and family history of DM ( $\mathrm{p}<0.05$ for all). Higher adiponectin levels at baseline were associated with lower $\mathrm{HbAlc}$ values at follow-up 12 years later $(\beta-0.24,0.08 \% 95 \% \mathrm{CI}$ $0.02-0.14$ decrease in $\mathrm{HbA} 1 \mathrm{c}$ per $\mu \mathrm{g} / \mathrm{mL}$ increase in adiponectin, $\mathrm{p}=0.008$ ). This association remained stable after adjusting for age, sex, BMI, BL, SBP, and CRP. The association was independent of the effect of physical activity and family history of DM ( $\mathrm{p}<0.05$ for all), however lost significance after further adjustment for FPG. 
Conclusion: The prospective association between lower adiponectin levels and the risk of developing T2DM is independent of major T2DM risk factors and low grade inflammation, suggesting that the effect of adiponectin on T2DM may be causal. Further we showed that adiponectin predicts glycemic control 10 years later, independently of T2DM risk factors, indicating that mechanisms related to, or mediated by adiponectin may play a role in determining the severity of glycemic impairment.

Supported by: Medical Research Council; Economic and Social Research Council; British Heart Foundation; Health and Safety Executive; Department of Health; Wingate Foundation US: National Heart Lung and Blood Institute, NIH: National Institute on Aging, NIH; Agency for Health Care Policy Research; John D and Catherine T MacArthur Foundation

\section{1}

Should an oral glucose tolerance test be performed in all patients with polycystic ovary syndrome?

S.V. Fica ${ }^{1,2}$, A.I. Albu ${ }^{1}$, C.G. Barbu ${ }^{1,2}$, A.D. Reghina ${ }^{1,2}$, R. Bunghez ${ }^{1}$

${ }^{1}$ Endocrinology and Diabetes, Elias University Hospital, ${ }^{2}$ Endocrinology,

University of Medicine and Pharmacy Carol Davila, Bucharest, Romania

Background and aims: Polycystic ovary syndrome (PCOS) represents the most common endocrinopathy of reproductive age women (8-12\%). Due to association between PCOS and insulin resistance, these patients are at increased risk of developing impaired glucose tolerance (IGT) and diabetes mellitus (DM), with combined prevalence previously reported of $20-35 \%$. Nowadays there is no consensus concerning oral glucose tolerance test (OGTT) in patients with PCOS, although the majority of authors recommend it to all patients. The aim of our study was to evaluate the prevalence of glucose metabolism disturbances and OGTT utility in PCOS patients.

Materials and methods: Subjects were 258 PCOS patients with mean age $25,13 \pm 5,82$ years and mean body mass index (BMI) $29,03 \pm 7,16 \mathrm{~kg} / \mathrm{m}^{2}$, selected from our PCOS research database. Clinical (anthropometric data, family history of type $2 \mathrm{DM}$, achantosis nigricans (AN)) and paraclinical data (fasting and 2 hours glycemia during OGTT, insulinemia, lipid profile) were retrospectively obtained from patients medical records. PCOS diagnosis was based on Rotterdam Consensus criteria.

Results: Abnormal blood glucose levels were found in 18,9\% of patients (5,4\% IFG, 8,5\% IGT and 5,03\% DM). Among those patients with fasting glycemia $<126 \mathrm{mg} / \mathrm{dl}$ (247 patients) OGTT revealed in $10,1 \%$ of them the presence of IGT or DM at 2hours. Among clinical and paraclinical parameters we found that 2 hours glucose metabolism abnormalities were significantly associated with age $\geq 30$ years ( $p=0.017), B M I \geq 27 \mathrm{~kg} / \mathrm{m}^{2}(\mathrm{p}=0.018)$, waist-hip ratio $\geq 0,8$ ( $\mathrm{p}=0.036$ ), fasting blood glucose $\geq 100 \mathrm{mg} / \mathrm{dl}$ ( $\mathrm{p}<0.0001)$, HOMA inde $\geq 3(p=0.046)$, total cholesterol $>200 \mathrm{mg} / \mathrm{dl}(\mathrm{p}=0.019)$, triglycerides levels $\geq 150 \mathrm{mg} / \mathrm{dl} \quad(\mathrm{p}=0.002)$. The positive predictive value of these parameters was: $18,86 \%, 13,9 \%, 13,39 \%, 42,85 \%, 14,13 \%, 15,13 \%, 23,68 \%$ respectively. We found no association with achantosis nigricans (AN) or family history of type $2 \mathrm{DM}$ in first degree relatives (FHDM).

Conclusion: OGTT had a significant contribution to accurate characterisation of abnormal glucose metabolism in our PCOS patients. Clinical data known to be associated with increased risk for DM, as AN and FHDM were not useful markers of $2 \mathrm{~h}$ impaired glycemias. Due to low value of clinical and paraclinical data to predict $2 \mathrm{~h}$ abnormal glucose levels we suggest that all patients with PCOS should be screened with OGTT in order to improve the therapeutic approach and prognosis.

\section{2}

Adipokines in the prevention of type 2 diabetes: the effect of a long-term lifestyle intervention

E.E. Blaak ${ }^{1}$, C. Roumen ${ }^{1}$, E. Corpeleijn ${ }^{1}$, W.H.M. Saris ${ }^{1}$, E.J.M. Feskens ${ }^{2}$ ${ }^{1}$ Human Biology, Maastricht University, ${ }^{2}$ Division of Human Nutrition, Wageningen University, Netherlands

Background and aims: We investigated the effect of a 4.2 year lifestyle intervention according to general recommendations on circulating adipokine concentrations and inflammation markers in subjects at risk for diabetes as well as the potential of leptin, adiponectin, resistin, plasminogen activator inhibitor 1 (PAI-1) and Complement factor 3 (C3) as biomarkers for lifestyleinduced improvements in glucose metabolism and insulin resistance.

Materials and methods: In the Study on Lifestyle intervention and Impaired glucose tolerance Maastricht, 147 men and women with impaired glucose tolerance (IGT) were randomized to either a combined diet-and-exercise intervention (INT) or a control program (CON). At baseline and at the end of the study (after 4 or 6 years) adipokines and inflammation were measured in addition to performance of an oral glucose tolerance test, an exercise test, and anthropometric measurements. At the end of the study, complete data of 92 subjects (49 INT,43 CON) were obtained.

Results: Lifestyle intervention increased carbohydrate intake, improved maximal aerobic capacity $\left(\mathrm{VO}_{2} \max \right)$ and reduced diabetes incidence by 31 $\%\left(\mathrm{p}_{\text {all }}<0.05\right)$. Body weight first decreased in INT $(-2.47 \mathrm{~kg})$, but increased during the last part of the study $(+1.31 \mathrm{~kg})$, whereas body weight did not change in CON. 2-hr glucose levels increased less in INT compared to CON $(+0.24 \pm 0.39 \mathrm{mM}$ vs $0.95 \pm 0.38 \mathrm{mM}$, respectively, $\mathrm{p}=0.04)$. HOMA-IR levels did not change between groups $(\mathrm{p}=0.54)$. Leptin levels increased more in the control group compared to the intervention group $(+0.34 \pm 0.09 \mathrm{ng} / \mathrm{ml}$ vs $0.09 \pm 0.44 \mathrm{ng} / \mathrm{ml} ; \mathrm{p}=0.02$ ), whereas no differences were observed in adiponectin, resistin, PAI-1 and C3 concentrations. In the total population $(n=92)$ changes in leptin level were correlated with changes in BMI, waist, body fat percentage, $\mathrm{VO}_{2}$ max. $\Delta$ Leptin was related to $\Delta \mathrm{PAI}-1$ and $\Delta \mathrm{C} 3\left(\mathrm{p}_{\mathrm{all}}<0.05\right)$, but not with $\Delta$ adiponectin or $\Delta$ resistin. $\Delta$ Leptin, $\Delta \mathrm{PAI}-1$ and $\Delta \mathrm{C} 3$ were related to improvements in estimated insulin sensitivity (HOMA-IR). The relationship between $\triangle$ leptin and HOMA-IR was independent of age, sex and changes in PAI-1, C3, BMI, body fat percentage and waist circumference (Multivariate regression $\mathrm{p}=0.04)$. $\Delta$ Leptin was the only marker related to 2 -hr glucose, but not independent of $\triangle \mathrm{BMI}$.

Conclusion: A decrease in leptin was strongly associated with a decrease in insulin resistance, only partially explained by a reduction in BMI. During a long-term lifestyle intervention leptin can be regarded a biomarker for improvements in insulin sensitivity, independent of body weight loss and changes in body composition.

Supported by: Dutch Diabetes Research Foundation, Netherlands Organization for Scientific Research

\section{3}

Lifestyle and socioeconomic status do not contribute to optimising screening strategies for type 2 diabetes. The ADDITION Netherlands Study

P.G.H. Janssen ${ }^{1}$, K.J. Gorter ${ }^{1}$, R.P. Stolk ${ }^{2}$, G.E.H. Rutten ${ }^{1}$

${ }^{1}$ Julius Center for Health Sciences and Primary Care, University Medical Center, Utrecht, ${ }^{2}$ Department of Epidemiology, University Medical Center, Groningen, Netherlands

Background and aims: Level of fasting glycemic control at diagnosis is an indication of severity and duration of diabetes. We investigated whether cardiovascular risk factors, lifestyle behaviors and socioeconomic status (SES) are related to the level of fasting glycemic control in screen-detected type 2 diabetic patients. Such relationships may have consequences for screening strategies.

Materials and methods: A total of 498 screen-detected type 2 diabetic patients were divided into tertiles of fasting blood glucose. Blood pressure, BMI, waist circumference, lipids, adiponectin, hsCRP, uric acid, fasting insulin, and microalbuminuria were determined. Lifestyle factors included physical activity ( $\geq 4$ days per week at least 30 minutes), smoking, consumption of alcohol (10-40 g/day), coffee ( $>2$ cups of coffee daily), and vegetables ( $\geq 5$ times per week). A composite index of socioeconomic status (high and low SES) was created by combining educational level, income and occupational status.

Results: Triglycerides, hsCRP (especially among people with low SES), and urinary albumin excretion were positively, adiponectin (only among those with high SES), and uric acid inversely related to fasting glucose levels. Better fasting glycemic control was associated with more favorable alcohol consumption, especially among men, and higher level of physical activity. Generally, cardiovascular risk markers and lifestyle behaviors did not differ substantially between tertiles.

Conclusion: Our data indicate that lifestyle factors and SES are not helpful to identify specific categories of patients to be screened for diabetes. Supported by: NovoNordisk, GlaxoSmith Kline, Merck 
CVD risk markers in screen-detected type 2 diabetic patients compared by tertiles of FBG

\begin{tabular}{|c|c|c|c|c|}
\hline & Lowest tertile $n=169$ & Intermediate tertile $n=163$ & Highest tertile $n=165$ & $\begin{array}{l}\mathrm{P} \text { for } \\
\text { trend }\end{array}$ \\
\hline SBP $(\mathrm{mmHg})$ & $166 \pm 22$ & $166 \pm 23$ & $162 \pm 24$ & 0.316 \\
\hline$\underline{\mathrm{DBP}}(\mathrm{mmHg})$ & $89 \pm 10$ & $89 \pm 11$ & $90 \pm 11$ & 0.890 \\
\hline Waist circumfernce $(\mathrm{cm})$ & $106 \pm 12$ & $108 \pm 12$ & $106 \pm 13$ & 0.365 \\
\hline Cholesterol $(\mathrm{mmol} / \mathrm{l})$ & $5.8 \pm 1.2$ & $5.6 \pm 1.0$ & $5.7 \pm 1.2$ & 0.685 \\
\hline LDL-cholesterol $(\mathrm{mmol} / \mathrm{l})$ & $3.7 \pm 1.0$ & $3.7 \pm 0.9$ & $3.7 \pm 1.0$ & 0.613 \\
\hline Triglycerides $(\mathrm{mmol} / \mathrm{l})$ & $1.8 \pm 1.7$ & $1.9 \pm 1.1$ & $2.1 \pm 1.2 \mathrm{a}$ & 0.152 \\
\hline Adiponectin (ng/ml x 100) & $6479 \pm 3611$ & $6416 \pm 3764$ & $5967 \pm 3179$ & 0.651 \\
\hline $\operatorname{HrCRP}(\mathrm{mg} / \mathrm{ml})$ & $7.3 \pm 11.6$ & $6.1 \pm 5.4$ & $8.0 \pm 9.9 \mathrm{~b}$ & 0.117 \\
\hline Uric acid $(\mu \mathrm{mol} / \mathrm{l})$ & $390.0 \pm 99.2$ & $382.7 \pm 88.6$ & $351.8 \pm 96.8 \mathrm{c}$ & $<0.001$ \\
\hline Urinary albumin excretion $(\mathrm{mg} / \mathrm{l})$ & $16.3 \pm 27.3$ & $41.1 \pm 139.9$ & $26.8 \pm 46.3 \mathrm{a}$ & 0.090 \\
\hline $\begin{array}{l}\text { Data are presented as mean } \pm \text { SD except where otherwise } \\
\text { specified. Cardiovascular risk markers were compared with } \\
\text { adjustment for age, gender, and, where appropriate, use of } \\
\text { antihypertensive or lipid lowering medications. }\end{array}$ & $\begin{array}{l}\text { a highest tertile } \\
\text { significantly different } \\
\text { from lowest tertile }\end{array}$ & $\begin{array}{l}\text { b highest tertile } \\
\text { significantly different from } \\
\text { intermediate tertile }\end{array}$ & $\begin{array}{l}\text { c highest tertile } \\
\text { significantly different } \\
\text { from other tertiles }\end{array}$ & \\
\hline
\end{tabular}

\section{4}

Trends of diabetes mellitus in Spain from 1987 to 2003

F.J. Basterra-Gortari ${ }^{1,2}$, M. Bes-Rastrollo ${ }^{2}$, L. Forga ${ }^{1}$, J.A. Martínez ${ }^{3}$,

C. Causso-Lariena ${ }^{1}$, M.A. Martínez-González ${ }^{2}$

${ }^{1}$ Endocrinology, Hospital of Navarra, ${ }^{2}$ Preventive Medicine and Public

Health, University of Navarra, ${ }^{3}$ Physiology and Nutrition, University of

Navarra, Pamplona, Spain

Background and aims: Diabetes prevalence is increasing in Western countries. We evaluated the trend of the prevalence of diabetes mellitus in Spain from 1987 to 2003 .

Materials and methods: The Spanish National Health Surveys (NHS) of $1987(n=29,647), 1993(n=21,061), 1995 \quad(n=6,396), 1997 \quad(n=6,396), 2001$ $(n=21,067)$ and $2003(n=21,650)$ which are representative samples of the adult Spanish general population were used. Participants were classified as diabetics if they answered affirmatively to the following question "Has your doctor told you that you suffer currently from diabetes?" Linear trend chi squared test and multivariate logistic regression models (the year of the survey was used as an independent continuous variable) adjusted for age, sex and educational levels, were used. 95\% confidence intervals were calculated. Results: A significant increasing temporal trend for the prevalence of diabetes was observed in the univariate analysis $(\mathrm{p}<0.001)$ (Figure).

After adjusting for age, sex and educational level a significant increasing temporal trend was also observed $(\mathrm{p}<0.001)$. A statistically significant increasing trend was observed in males $(\mathrm{p}<0.001)$ and females $(\mathrm{p}<0.001)$ after adjusting for age and educational level. This significant increasing trend was present for men of most ages (except for those between 30-39 years) (table), however it was apparent only for women over 50 years, whereas, women between 30 39 years old had a significant decreasing trend in the prevalence of diabe- tes $(\mathrm{p}<0,001)$ and women between $40-49$ years had also a decreasing trend $(\mathrm{P}=0.06)$.

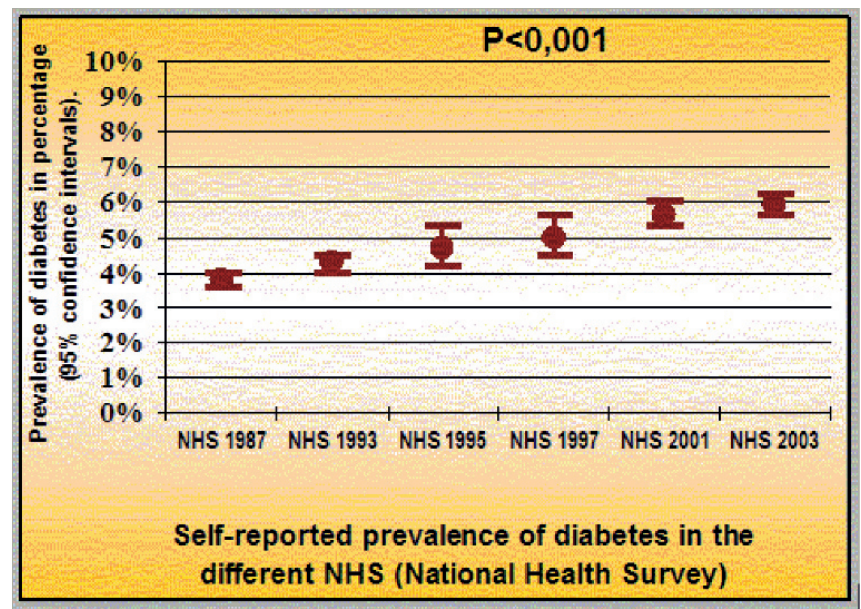

Conclusion: The prevalence of diabetes mellitus has increased in Spain between 1987 and 2003 in both males and females. Although self-reported data under-estimate the actual prevalence of diabetes, this does not necessarily invalidate this study about the prevalence trend of diabetes mellitus, because there is no reason to think that accuracy of self-reported data may have changed across surveys.

Prevalence (\%) of self-reported diabetes in the different NHS. According to sex and ages categories.

\begin{tabular}{|c|c|c|c|c|c|c|c|c|}
\hline & AGE & 1987 NHS & 1993 NHS & 1995 NHS & 1997 NHS & 2001 NHS & 2003 NHS & $\mathrm{P}$ (for trend) \\
\hline \multicolumn{9}{|l|}{ MALES } \\
\hline & $16-29$ & 0.3 & 0.3 & 0.3 & 0.6 & 0.7 & 0.5 & 0.03 \\
\hline & $30-39$ & 0.9 & 0.8 & 0.6 & 0.2 & 0.7 & 1.1 & 0.69 \\
\hline & $40-49$ & 1.7 & 2.3 & 2.0 & 2.0 & 2.9 & 2.9 & 0.005 \\
\hline & $50-64$ & 6.1 & 7.0 & 8.0 & 8.1 & 8.5 & 9.6 & $<0.001$ \\
\hline & $>65$ & 8.6 & 8.9 & 11.9 & 12.4 & 16.1 & 17.0 & $<0.001$ \\
\hline & TOTAL & 3.0 & 3.3 & 4.0 & 4.1 & 5.3 & 5.6 & $<0.001$ \\
\hline \multicolumn{9}{|c|}{ FEMALES } \\
\hline & $16-29$ & 0.6 & 0.4 & 0.1 & 0.1 & 0.5 & 0.8 & 0.69 \\
\hline & $30-39$ & 1.7 & 0.7 & 1.5 & 0.8 & 0.8 & 0.4 & $<0.001$ \\
\hline & $40-49$ & 3.0 & 1.7 & 2.8 & 3.0 & 2.5 & 1.9 & 0.06 \\
\hline & $50-64$ & 6.7 & 8.2 & 7.9 & 10.1 & 8.9 & 8.4 & 0.003 \\
\hline & $>65$ & 12.9 & 15.9 & 15.1 & 15.7 & 16.2 & 18.4 & $<0.001$ \\
\hline & TOTAL & 4.6 & 5.1 & 5.4 & 5.8 & 6.0 & 6.2 & $<0.001$ \\
\hline
\end{tabular}




\section{5}

Eight models of diabetes screening - impact on epidemiologic results A.V. Dreval, I.V. Misnikova, I.A. Barsukov

Endocrinology, Moscow Regional Resaerch Clinical Institute, Russian Federation

Background and aims: Leading international organizations (WHO, American diabetes association, IDF) offer different models of screening of glucose metabolism abnormalities, which have certain impact on screening results. The aim of the study was to compare different screening models.

Materials and methods: Population-based screening for IFG, IGT and T2D in 2508 individuals of Moscow County. FPG was measured in all participants. Standard 2-h $75 \mathrm{~g}$ OGTT was performed using HemoCue analyzers in subjects previously undiagnosed with diabetes. Depending on diagnostic criteria (American diabetes association or WHO) and research method (only FBG, OGTT) 8 screening models were considered. Sensitivity(Se) and specificity(Sp) of models is compared based on definition of T2DM, IGT, IFG on formula: $\mathrm{Se}=\mathrm{TP} /(\mathrm{TP}+\mathrm{FN}), \mathrm{Sp}=\mathrm{TN} /(\mathrm{TN}+\mathrm{FP})$, TP-True Positive, TN-True Negative, FN-False Negative, FP-False Positive.

Results: Table 1

Prevalence (Pr) of T2DM, IFG, IGT, sensitivity (Se) and specificity(Sp) of different screening models

Conclusion: Prevalence of T2DM, IFG and IGT according to diagnostic criteria (American diabetes association or WHO) and research method (FBG only, OGTT) is changed due to different sensitivity of screening models. Specificity remains high in spite of screening model choice.

\section{PS 012 Animal models for type 2 diabetes}

\section{6}

Liver transcription factor ChREBP is modified by O'glycosylation ( $\mathrm{O}$ GlcNAc) in liver of control and insulin resistant mice

C. Guinez-Lelong, V. Fauveau, A.-F. Burnol, J. Girard, C. Postic

Endocrinologie, Métabolisme et cancer, Institut Cochin, Paris, France

Background and aims: Carbohydrate Responsive Element Binding protein (ChREBP) is a key transcription factor that mediates the transcriptional effects of glucose on glycolytic and lipogenic genes in liver. The elevated ChREBP protein levels observed in liver of obese ob/ob mice strongly correlate with their state of hepatic steatosis and insulin-resistance. Under low glucose concentrations, ChREBP is an inactive phosphorylated cytosolic protein while after a raise in glucose metabolism ChREBP undergoes dephosphorylation and is translocated into the nucleus to activate its target genes. However, controversy has recently emerged concerning ChREBP regulation by phosphorylation, suggesting that additional post-traductional mechanisms may be required. O-linked beta- $\mathrm{N}$-acetylglucosamine (O-GlcNAc) modification of proteins plays an important role in transcription, translation, nuclear transport and signal transduction. Recently, increased O-GlcNAc modification of proteins was linked to the development of insulin resistance in liver. Therefore, the aim of our study was to determine whether ChREBP is regulated by O-GlcNAc in liver of control and/or insulin-resistant mice.

Materials and methods: $\mathrm{C} 57 \mathrm{Bl} / 6 \mathrm{~J}$ mice that were either fasted for 24 hours or refed on a high carbohydrate (HCHO) diet for 18 hours, were used as controls. To dissociate the effects of O-GlcNAc modification from those of glucose metabolism, glucosamine $(2,5 \mathrm{~g} / \mathrm{kg})$ was administrated orally to $18 \mathrm{~h}$ fasted C57Bl/6J mice. Glucosamine-treated mice were sacrificed 4, 8, 10 or 12 hours after the beginning of the gavage. In parallel, a group of mice receiving a glucose gavage $(5 \mathrm{~g} / \mathrm{Kg})$ was studied at similar time-points.

Results: Treatment with glucosamine resulted in O-GlcNAc modification of ChREBP and to an increase in ChREBP total protein content to similar levels than in liver of glucose and $\mathrm{HCHO}$ diet fed mice. Interestingly, in contrast to glucose gavage and to $\mathrm{HCHO}$ diet conditions, glucosamine-mediated induction of ChREBP occurred independently of a raise in hepatic glucose metabolism and was not linked to enhanced ChREBP transcription rates. In addition, while ChREBP was rapidly translocated into the nucleus after a glucose gavage (as soon as 4 hours post gavage), ChREBP remained cytosolic and as a consequence, did not activate its target genes under glucosamine treatment (all time tested). Additional experiments performed in mouse hepatocytes showed that glucosamine treatment $(5 \mathrm{mM})$ stabilized the endogenous ChREBP protein and protected it against proteasomal degradation. Finally, the elevated expression of ChREBP in liver of ob/ob mice strongly correlated with high O-GlcNAc levels.

Conclusion: Taken together, our results suggest that O-GlcNAc modification may represent an important regulation of ChREBP protein content in liver under both physiological and physiopathological conditions.

\begin{tabular}{|c|c|c|c|c|c|c|c|c|c|}
\hline \multirow[t]{2}{*}{ Screening models } & \multicolumn{3}{|l|}{ T2DM } & \multicolumn{3}{|c|}{ IFG (\%) } & \multicolumn{3}{|c|}{ IGT(\%) } \\
\hline & $\operatorname{Pr}(\%)$ & $\mathrm{Se}$ & Sp & $\operatorname{Pr}(\%)$ & $\mathrm{Se}$ & $\mathrm{Sp}$ & $\operatorname{Pr}(\%)$ & $\mathrm{Se}$ & Sp \\
\hline WHO,1999 OGTT & 3.9 & 1 & 1 & 4,06 & 1 & 1 & 6,53 & 1 & 1 \\
\hline WHO,1999 FPG only & 3.15 & 0.84 & 1 & 4.47 & 1 & 1 & - & - & - \\
\hline WHO,1999 2-hr PG only & 2.9 & 0.80 & 1 & - & - & - & 7.18 & 1 & 0.99 \\
\hline ADA,2003 FPG only & 3.15 & 0.84 & 1 & 13.08 & 1 & 0.91 & - & - & - \\
\hline IDF+ADA FPG + OGTT if FPG $>5.5 \mathrm{mmol} / 1$ & 3.75 & 0.96 & 1 & 12,48 & 1 & 0.92 & 3,59 & 0.69 & 1 \\
\hline$\overline{\mathrm{IDF}} 2007 \mathrm{FPG}+\mathrm{OGTT}$ if FPG $>6 \mathrm{mmol} / \mathrm{l}$ & 3.55 & 0.92 & 1 & 4,06 & 1 & 1 & 1,75 & 0.58 & 1 \\
\hline FPG+OGTT if FPG<6.1 mmol/l & 3.55 & 0.92 & 1 & 4.41 & 1 & 1 & 4.78 & 0.79 & 1 \\
\hline FPG+OGTT if FPG $<5.6 \mathrm{mmol} / \mathrm{l}$ & 3.31 & 0.87 & 1 & 13.08 & 1 & 0.91 & 2.95 & 0.65 & 1 \\
\hline
\end{tabular}

Supported by: Alfediam 


\section{7}

Diet-induced abdominally obese rats with normal weight developed insulin resistance and muscular mitochondrial dysfunction

L. Cao, N.W. Tong, X.H. Liu

Dept. of Endocrinology, West China hospital of Sichuan University, Chengdu, China

Background and aims: Abdominal obesity is associated with reduced insulin sensitivity. Mitochondrial dysfunctions have been found involved in mechanisms of insulin resistance in muscle. The study are designed to investigate muscular ATP production in diet-induced abdominally obese SD rats with normal weight(AONW) and its possible mechanisms.

Materials and methods: The model of rats with normal body weight(BW) and increased visceral adipose tissue(VAT) were made by self-designed diet for 20 weeks. The control group (CG) were fed by common diet. Glucose tolerance was measured by intraperitoneal injection of glucose $(2 \mathrm{~g} / \mathrm{kg}$ weight) after an overnight fast. Hyperinsulinemic-euglycemic clamp combined with intravenous infusion of $3-{ }^{3} \mathrm{H}$-glucose technique was administered to evaluate the insulin sensitivity of rats in both groups. Plasma glucose, free fatty acids, insulin were measured and compared with those in control to find metabolic disorders in model rats. Body and visceral adipose tissue were weighted just before and after sacrifice respectively. Light microscopy and electronic microscopy were used to detect changes in muscle. ATP ADP ratio(ATP/ADP) in muscle were measured by HPLC. Expression of genes in muscle involved in mitochondrial function were detected, including 1.mRNA of mitochondrial transcription factor $\mathrm{A}(\mathrm{TFAM})$, peroxisome proliferators activated receptor gamma co-activator-1 $\alpha(\mathrm{PGC}-1 \alpha)$, uncoupling protein $3(\mathrm{UCP} 3)$ and insulin receptor substrate-1(IRS-1) by RT-PCR;2.protein level of TFAM, PGC-1a and IRS- 1 by western blot.

Results: Comparing to CG, BW of AONW was normal but VAT increased significantly $(\mathrm{p}=0.01)$, while the percentage of VAT/BW(\%) 5.80(AONW) vs 3.45(CG). Glucose disposal rates $(\mathrm{mg} / \mathrm{min} \cdot \mathrm{kg}$ ) were $16.57 \pm 5.41(\mathrm{AONW})$ vs $10.74 \pm 5.16(\mathrm{CG}), \mathrm{p}=0.03$. Hepatic glucose output $(\mathrm{mg} / \mathrm{min} \cdot \mathrm{kg})$ was not suppressed by insulin infusion in $\operatorname{AONW}(7.28 \pm 6.01$ vs $8.48 \pm 3.50$,before vs after infusion, $\mathrm{p}=0.61)$. Glucose intolerance during IPGTT and high plasma FFA(836.1 \pm 142.1 vs $673.9 \pm 177.2 \mu \mathrm{mol} / \mathrm{L}$ in $\mathrm{AONW}$ vs $\mathrm{CG}, \mathrm{p}=0.048)$ were found in AONW. Adipose increment, capillary necrosis and mitochondria swelling were found in AONW muscles by microscopy. ATP/ADP in muscle of AONW was $62.1 \%$ of control, although the difference was not significantly $(\mathrm{p}=0.06)$. PGC- $1 \alpha$ mRNA level increased by 2.24 fold in AONW than in CG(p=0.07), and UCP3 mRNA level increased by 6.38 fold in AONW than in $C G(p=0.007)$. No significant differences in TFAM and IRS-1 mRNA expression were found between groups. TFAM protein level in AONW was $13.5 \%$ of that in CG(p<0.005).PGC-1 $\alpha$ level in AONW was $148.2 \%$ of that in $\mathrm{CG}(\mathrm{p}<0.005)$. IRS-1 protein expression didn't change significantly.

Conclusion: The self-designed diet-induced AONW SD rats were abdominal obese and muscular insulin resistant. High plasma FFA was the most likely one among the reasons of compensatory increased expression of PGC-1a and UCP3 in muscle of AONW. The results support a hypothesis that ATP production were probably impaired by increased plasma FFA. The disparity between PGC-1a, UCP3 and TFAM expression in AONW muscle suggests low TFAM protein might be related to the mitochondrial dysfunction, and accompanying with IRS-1 non-upregulation, the mitochondrial dysfunction might be play some role in the development of insulin resistance.

\section{8}

Intrauterine programming of beta cell development and function by maternal diabetes. What does embryo-transfer experiments in GK/Par rats tell us?

A. Chavey ${ }^{1}$, M.-N. Gangnerau ${ }^{1}$, L. Maulny ${ }^{2}$, D. Bailbé ${ }^{1}$, J. Movassat ${ }^{1}$,

J.-P. Renard ${ }^{2}$, B. Portha ${ }^{1}$

${ }^{1}$ Laboratoire de physiopathologie de la nutrition, CNRS UMR 7059, Paris,

France, ${ }^{2}$ Biologie du développement et reproduction, INRA UMR 1198,

Jouy-en-Josas Cedex, France

Background and aims: Epidemiological findings strongly suggest that in utero exposure to diabetes is associated with abnormal insulin secretion and glucose homeostasis in offspring of diabetic mothers and may participate in the excess of maternal transmission in T2D. From human studies however, isolation of the respective contribution of genetic vs. intrauterine environmental factors is hardly attainable. We have therefore used the GK/Par rat, a spontaneous model of type 2 diabetes, to determine whether the altered metabolic intrauterine/postnatal milieu of the GK mother may cause malprogramming of the beta-cell mass and adversely alter beta-cell function later in adult life. To that aim we have used an embryo transfer technology. Materials and methods: Fertilised GK or Wistar (W) oocytes (oW and oGK) were transferred into pseudo-pregnant W or GK females. Pregnancies were allowed to go to term. In some experiments, $\mathrm{W}$ newborn pups reared by gestating GK mothers (gGK) were cross-fostered to W mothers for suckling $(\mathrm{sW})$. Male offspring were tested at 8 weeks of age for phenotyping (intravenous glucose tolerance test, insulin release in response to glucose in vivo (GSIS), pancreatic insulin stores, pancreatic beta-cell mass).

Results: We found that: 1/ Offspring from GK embryo transfers into GK mothers $[\mathrm{oGK} / \mathrm{gGK} / \mathrm{sGK}$ ] were similarly hyperglycaemic compared to the non-manipulated GK/Par stock colony. 2/ Offspring from W embryo reared in hyperglycaemic GK mothers [oW/gGK/sGK] were normoglycaemic, normotolerant to glucose and normal insulin releaser in response to glucose. However, their pancreatic insulin stores (ng/mg pancreas) and beta-cell mass ( $\mu \mathrm{g} / \mathrm{mg}$ pancreas) were significantly reduced (by $40 \%$ and $31 \%$ respectively) as compared to the non-manipulated $\mathrm{W}$ offspring from our stock colony. 3/ Offspring from $\mathrm{W}$ embryo reared in hyperglycaemic GK mothers and then milked by W mothers [oW/gGK/sW] were normoglycaemic, normotolerant to glucose and normal insulin releaser in response to glucose. Again, their pancreatic insulin stores and total beta-cell mass were reduced (by $40 \%$ and $30 \%$ respectively) as compared to the non-manipulated $\mathrm{W}$ offspring from our stock colony. 4/ Offspring from GK embryo reared in normoglycaemic W mothers $[\mathrm{oGK} / \mathrm{gW} / \mathrm{sW}]$ exhibit basal hyperglycaemia, glucose intolerance and low GSIS, to the same extent as the oGK/gGK/sGK offspring. This was associated with reduced pancreatic insulin stores (by $65 \%$ vs $\mathrm{W}$ ) and beta-cell mass (by $69 \%$ vs W), to a more severe extend than that in non-manipulated GK/Par rats from the stock colony.

Conclusion: We conclude that: 1 / in W rats with no risk of diabetes, exposure to hyperglycaemia in utero only or in utero and during suckling, significantly decreases the total beta cell mass and insulin stores in their pancreas. However this does not cause long-term damages to glucose homeostasis, despite discrete alteration of GSIS. Whether or not this reflects an early (foetal) alteration of beta-cell mass development is under examination. 2/ Offspring from GK embryos transferred in the uterus of euglycaemic W mother, still develop low GSIS in vivo, low pancreatic insulin stores and low beta-cell mass when adults, therefore highlighting a major role for morbid genes. Supported by: Prograbeta granted by the French ANR

\section{9}

Non-invasive imaging of pancreatic islets targeting glucagon-like peptide- 1 receptors

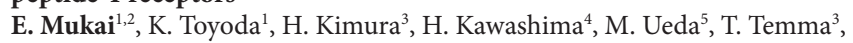
K. Hirao ${ }^{6}$, K. Nagakawa ${ }^{6}$, Y. Seino ${ }^{7}$, H. Saji ${ }^{3}$, N. Inagaki ${ }^{1}$

${ }^{1}$ Department of Diabetes and Clinical Nutrition, Graduate School of Medicine, Kyoto University, Kyoto, ${ }^{2} J a p a n$ Association for the Advancement of Medical Equipment, Tokyo, ${ }^{3}$ Department of Patho-Functional Bioanalysis, Graduate School of Pharmaceutical Sciences, Kyoto University, ${ }^{4}$ Department of Diagnostic Imaging and Nuclear Medicine, Graduate School of Medicine, Kyoto University, ${ }^{5}$ Radioisotopes Research Laboratoty, Kyoto University Hospital, Faculty of Medicine, Kyoto University, ${ }^{6}$ Arkray, Inc., Kyoto, ${ }^{7}$ Division of Diabetes and Clinical Nutrition, Kansai Denryoku Hospital, Kyoto, Japan

Background and aims: The volume of pancreatic $\beta$-cells is known to decrease during diabetes development and progression. A technique for measurement of $\beta$-cell volume in vivo is required, but a non-invasive method to detect pancreatic $\beta$-cells has not been developed. In the present study, specific imaging of pancreatic $\beta$-cells targeting glucagon-like peptide-1 receptors (GLP-1R) was evaluated using its antagonist, exendin(9-39).

Materials and methods: For evaluating receptor specificity, binding assay in vitro was performed using dispersed cells of islets isolated from 6-week-old male ddY mice. For assessment of in vivo selectivity, biodistribution studies were performed by intravenous administration of [ $\left.{ }^{125} \mathrm{I}\right]$-Exendin(9-39) (1 $\mu \mathrm{Ci}$ ) to 6-week-old male ddY mice. At appropriate time points after administration, selected organs and blood were harvested and their radioactivities were measured. In a blocking study, excess nonradioactive Exendin(9-39) (50 $\mu \mathrm{g})$ was administered before the $\left[{ }^{125} \mathrm{I}\right]$-Exendin(9-39) injection. After $\left[{ }^{125} \mathrm{I}\right]-$ Exendin(9-39) injection into transgenic mice with pancreatic $\beta$-cells expressing GFP (kindly provided by Dr. Hara, University of Chicago), fluorescent and radioactive signals of sections of pancreas were evaluated with an image analyzer. 
Results: Binding assay analysis using mouse pancreatic islet cells showed competitive inhibition of specific [125I]-Exendin(9-39) binding by nonradioactive Exendin(9-39). Biodistribution study by intravenous administration of [ $\left.{ }^{125} \mathrm{I}\right]$-Exendin(9-39) showed that radioactivity of pancreas was high at 60 and 120 min compared to other organs, while radioactivity of lung was highest. Pre-administration of excess nonradioactive Exendin(9-39) considerably and specifically blocked the radioactivity of pancreas and lung two hours after [125I]-Exendin(9-39) injection. Image analysis using sections of pancreas of $\left[{ }^{125} \mathrm{I}\right]$-Exendin(9-39)-administered mice expressing GFP in pancreatic $\beta$-cells showed that the fluorescent GFP signal and the radioactive signal were similarly located, indicating accumulation of Exendin(9-39) specifically in pancreatic $\beta$-cells. The intensity of the fluorescent signal was also correlative with that of the radioactive signal.

Conclusion: For imaging of pancreatic $\beta$-cells, Exendin(9-39) and GLP-1R can serve as a useful probe and target molecule, respectively.

Supported by: Research on Nanotechnical Medicine from the Ministry of Health, Labour, and Welfare of Japan

\section{0}

\section{Novel potential biomarkers for detection of type 2 diabetes based on transcriptome analyses of Cohen rat models}

P. Ikonomi ${ }^{1}$, S. Zangen ${ }^{2}$, S. King ${ }^{1}$, I. Raz ${ }^{2}$, C. Gelber ${ }^{1}$

${ }^{1}$ Translational Research, American Type Culture Collection, Manassas, United States, ${ }^{2}$ Diabetes Unit, Hadassah University Hospital, Jerusalem, Israel

Background and aims: The Cohen diabetic (CD) rat is a versatile animal model comprised of 2 strains that manifest many of the common features of type 2 diabetes (T2D) in humans. The sensitive strain (CDs) develops diabetes within 30 days when maintained on a high sucrose/copper poor diet (HSD), whereas the resistant strain (CDr) retains normal blood glucose levels. Neither strain shows any signs of diabetes when provided regular rodent diet (RD). Thus, incidence of $\mathrm{T} 2 \mathrm{D}$ in the $\mathrm{CD}$ rat model results from synergistic effects of genetic susceptibility and dietary composition. Pair-wise comparisons of gene expression profiles are indicative of; predisposition, resistance, progression or protection against $\mathrm{T} 2 \mathrm{D}$.

To identify specific markers that can potentially characterize resistance, progression or predisposition to $\mathrm{T} 2 \mathrm{D}$, transcriptome changes of epididymal fat and pancreatic tissue of these two strains were studied by microarray analyses using the rat expression arrays.

Materials and methods: Total RNA was extracted from epididymal fat and pancreatic tissue for each of the strains and analyzed using the Rat Expression Arrays (Affymetrix, CA). The data was analyzed using GeneSpring GX (Agilent, CA) and expression of selected transcripts was confirmed by Real Time PCR

Results: Microarray transcriptome profiling of epididymal fat tissue from $\mathrm{CD}$ rats revealed numerous biomarkers that may correlate with resistance, progression and predisposition to disease. Our analyses reveal 48 transcripts that show inverse expression trends in relevance to resistance or progression of the disease. Thus, in the model indicative of genetic resistance, we found that T2D-related genes such as Gyk, Scd2 and Nr1h3 were upregulated; interestingly, progression of T2D is characterized by downregulation of the same transcripts. In contrast, expression levels of other markers such as Lypla3, Acaa2 and Anxal increase with progression of disease but are expressed at lower levels in the resistance model. The expression pattern of the above transcripts was confirmed by quantitative PCR, and was followed throughout the development of the disease. Results show that specific quantitative changes of transcript levels are observed as early as 10 days after exposure in high sucrose diet and become more pronounced as the duration of high sucrose diet is extended to 30 days.

Similarly, our microarray and quantitative PCR analyses in pancreatic tissue resulted on selection of 14 transcripts with the quantitative changes indicative of development and resistance of Type 2 diabetes. Eight of these transcripts show quantitative expression changes associated to the disease in both epididymal fat and pancreatic tissue.

Conclusion: In conclusion, our analyses using epididymal fat and pancreatic tissue from Cohen rat models could indicate novel modification mechanism involved in T2D. We are currently profiling the expression of selected markers in whole blood cells. Such analyses will result in identification of a combination of transcripts whose monitored expression could be used not only as biomarkers for predisposition or development of the disease, but also as the basis for therapeutics for Type 2 Diabetes.

Supported by: American Type Culture Collection

\section{1}

Differences between global miRNA expression profiles in diabetic and normoglycaemic rats

B.M. Herrera ${ }^{1,2}$, H.E. Lockstone ${ }^{1}$, S. Collins ${ }^{2}$, P.J. Kaisaki ${ }^{1}$, A. Barrett ${ }^{2}$, J.C. Randall1 ${ }^{1}$, K. Argoud ${ }^{1}$, D. Gauguier ${ }^{1}$, C. Fernandez ${ }^{1}$, J.M. Taylor ${ }^{1}$,

M.I. McCarthy ${ }^{1}$, C.M. Lindgren ${ }^{1}$

${ }^{1}$ Wellcome Trust Centre for Human Genetics, University of Oxford, Oxford, United Kingdom, ${ }^{2} \mathrm{OCDEM}$, Oxford Centre for Diabetes, Endocrinology and Metabolism, Oxford, United Kingdom

Background and aims: MicroRNAs (miRNAs) are recently discovered noncoding RNA molecules ( 22 nucleotides in length) that play an important role in post-translational regulation of gene expression. MiRNA-mediated expression regulation relies on sequence-specific alignment to target mRNA molecules, and thus a single miRNA can potentially bind to a large number of target mRNAs. MiRNAs have been shown to be involved in a broad range of biological mechanisms including cell differentiation and development and more recently have been implicated in energy homeostasis. To investigate the potential involvement of miRNAs in T2D, we assessed expression of miRNAs in fat, skeletal muscle and liver samples from T2D rats and compared it to expression in genetic and physiological controls.

Materials and methods: Total RNA was extracted from skeletal muscle, fat and liver from three-month-old male rats representing: spontaneous T2D (Goto-Kakizaki [GK], n=5), normoglycaemic (Wistar-Kyoto [WKY], n=5) that share genetic background with GK rats and normoglycaemic genetically distant controls (Brown-Norway [BN], n=5). Expression levels of 239 rat miRNAs were quantified using the EXIQON microarray platform. After background correction and normalization, we looked for differences between tissues and then within tissues across strains. Validation of expression results for a selected number of miRNAs was carried out using q-RT-PCR on the TaqMan platform. In-silico prediction of miRNAs targets was carried using miRBase and Target-scan 4.1. The involvement of target genes in biological pathways was assessed using g:Profiler software.

Results: We observe considerable differences in miRNA expression profiles in our tissue specific comparisons between strains. Differential expression was detected for 20 miRNAs; 2 in muscle, 7 in fat and 11 in liver $(\mathrm{p}<0.05$, corrected for multiple testing) suggesting that these differences provide signatures of the T2D phenotype modeled by the GK strain. Notably, in fat, the GK rat showed over-expression of miR-222 and miR-27a (Fold change [FC] $=2.4$ and $1.74, \mathrm{p}<0.005$ ) which target genes involved in apoptosis ( $\mathrm{g}$ :Profiler $\mathrm{p}$-value $=7.6 \times 10-7)$. In liver, under-expression of miR-100 and miR-125b $(\mathrm{FC}=-1.2$ and $-1.44, \mathrm{p}<0.003)$ and in muscle under-expression of miR-let-7e and miR-10b $(\mathrm{FC}=-1.31$ and $-1.58, \mathrm{p}<0.02)$ which target a number of genes involved in the insulin signaling pathway (g:Profiler $\mathrm{p}$-value $=8.4 \times 10-4)$. Nine differentially expressed miRNAs were selected for validation using q-RT-PCR and of these, results were replicated for miR-140*, miR-222, miR-487b, miR103, miR-195 and miR-10b.

Conclusion: We have assessed and compared global miRNA expression profiles in three insulin target tissues from three different rat strains (GK, WKY and $\mathrm{BN}$ ) and identified tissue and strain specific profiles, further supporting evidence for miRNA involvement in the T2D phenotype. Our results suggest that altered miRNA expression levels are associated with disturbances in glucose homeostasis, likely to arise through their role in the regulation of genes involved in insulin signaling mechanisms in liver and muscle. Supported by: Diabetes UK

\section{2}

A novel genetic mouse model of diabetes, Buttermouse, has been identified

N.L. Absalom ${ }^{1}$, M. Goldsworthy ${ }^{1}$, H.C. Matthews ${ }^{2}$, D. Bogani ${ }^{1}$, C. Church ${ }^{1}$, R.D. $\operatorname{Cox}^{1}$

${ }^{1}$ Mammalian Genetics Unit, Medical Research Council, Oxfordshire, ${ }^{2}$ Department of Hepatology, Imperial College, London, United Kingdom

Background and aims: Type 2 diabetes is a complex disease that is caused by genetic and environmental factors, of which the genetic factors are not all understood. Population studies and murine models are complimentary approaches to identifying such genetic factors. Our approach was to identify novel genetic mutations causative for diabetes in a murine model. Initially, $\mathrm{BALB} / \mathrm{c}$ male mice were treated with the chemical mutagen ENU to create random mutations throughout the genome. These mice were mated to female $\mathrm{C} 3 \mathrm{H} / \mathrm{HeH}$ mice and the progeny screened for high free fed plasma glucose. 
Here we describe the genetic and physiological characterization of one mutant where male mice were identified with high plasma glucose, buttermouse. Materials and methods: After identification of the mutant mouse, the F1 male was successively backcrossed onto a $\mathrm{C} 3 \mathrm{H} / \mathrm{HeH}$ background. Genotyping analysis was performed using microsatellite markers and pyrosequencing of single nucleotide polymorphisms between $\mathrm{C} 3 \mathrm{H}$ and $\mathrm{Balb} / \mathrm{c}$ mouse strains. Candidate genes were sequenced to identify the mutation. Phenotyping was done with an intraperitoneal glucose tolerance test measuring plasma glucose at 12 weeks and plasma insulin at 16 weeks. At 19 weeks blood plasma and tissues were collected and analyzed.

Results: Genotyping analysis identified a region of 350kbp of DNA that contained the mutation. Candidate gene sequencing revealed a putative missense mutation in a chromatin remodeling enzyme. During an IPGTT plasma glucose concentrations prior to, 60 and 120 minutes after injection were significantly higher for male buttermouse than wild-type controls $(10.2 \pm 2.7$, $25.6 \pm 5.9,20.5 \pm 6.1$ compared to $7.7 \pm 1.9,15.0 \pm 4.1,10.2 \pm 2.8, \mathrm{p}<0.01, \mathrm{n}>60)$. Liver sections at 19 weeks of age indicated that male buttermouse developed hepatic steatosis and hepatocyte ballooning consistent with non-alcoholic fatty liver disease. The plasma cholesterol ( $5.1 \pm 0.5$ vs $4.3 \pm 0.4, \mathrm{p}<0.05, \mathrm{n}=10$ )and triglyceride concentrations $(6.1 \pm 1.5$ vs $4.4 \pm 1.5, \mathrm{p}<0.05, \mathrm{n}=10)$ were significantly higher for male buttermouse compared to wild-type.

Conclusion: We have identified a novel genetic mouse of diabetes and plan to continue to study buttermouse to understand how alterations in gene expression can lead to type 2 diabetes and its complications. We aim to confirm the mutation is causative for the mouse model by using complimentary techniques of knockout murine models and functional assays.

Supported by: Diabetes UK, Medical Research Council and Wellcome Trust

\section{PS 013 Classification of type 1 diabetes}

\section{3}

Two subtypes of type 1 diabetes of Japanese adult patients with low and high titers of GAD autoantibody

T. Aoyama ${ }^{1}$, H. Ikeda ${ }^{1}$, S. Honjo ${ }^{1}$, K. Nabe ${ }^{1}$, T. Kimura ${ }^{1}$, K. Nomura $^{1}$, Y. Wada ${ }^{1}$, Y. Hamamoto ${ }^{2}$, J. Fujikawa ${ }^{3}$, H. Koshiyama ${ }^{1}$

${ }^{1}$ Center for Diabetes \& Endocrinology, The Tazuke Kofukai Foundation Medical Research Institute Kitano Hospital, Osaka, ${ }^{2}$ Division of Diabetes \& Clinical Nutrition, Kyoto University, ${ }^{3}$ Department of Laboratory Medicine, The Tazuke Kofukai Foundation Medical Research Institute Kitano Hospital, Osaka, Japan

Background and aims: About 5-10\% of patients with clinically diagnosed type 2 diabetes were reported to have markers of islet autoantibodies, such as GAD autoantibodies (GADAb). They are variably referred to as latent autoimmune diabetes in adults, slowly progressing insulin-dependent diabetes, noninsulin-requiring autoimmune diabetes or type 1.5 diabetes, and it has been reported that their $\beta$-cell function then slowly declines to insulin-dependent state in several years. A recent study from Italy has reported that GADAb could define heterogeneity of adult "type 1 diabetes" and their titration might predict future risk of progression to insulin dependence. Nevertheless, there has been no such a study in Japanese patients. The aim of this study is to investigate whether GADAb can define adult "type 1 diabetes" of Japanese patients.

Materials and methods: Between December 2000 and July 2007, the serum GADAb levels were measured in a total of 1857 consecutive diabetic patients with age over 20 years in our center. GADAb were tentatively defined to be positive with a level $\geq 1.5 \mathrm{U} / \mathrm{ml}$. Diabetic patients with GADAb were then divided into two subgroups; those with low $(<10 \mathrm{U} / \mathrm{ml}$ :L group) and high ( $\geq 10 \mathrm{U} / \mathrm{ml}: \mathrm{H}$ group) titers of GADAb. Their clinical characteristics, including autoantibodies to thyroid peroxidase (TPO), were investigated. Diabetic patients without GADAb who were matched for age and gender with those with GADAb were selected as control group.

Results: A total of $103(5.5 \%)$ diabetic patients (male/female:48/55, mean age: $57 \pm 16$ years) had GADAb. Clinical profiles of diabetic patients with GADAb were significantly different from those without GADAb, showing higher $\mathrm{HbA}_{1 \mathrm{C}}$ level $(7.9 \pm 2.5 \%$ vs. $9.2 \pm 2.7 \%, \mathrm{P}<0.005)$, lower $\mathrm{BMI}(25.3 \pm 3.9 \mathrm{~kg} /$ $\mathrm{m}^{2}$ vs. $\left.23.1 \pm 4.6 \mathrm{~kg} / \mathrm{m}^{2}, \mathrm{P}<0.005\right)$, serum triglycerides $(179 \pm 124 \mathrm{mg} / \mathrm{dL}$ vs. $141 \pm 111 \mathrm{mg} / \mathrm{dL}, \mathrm{P}<0.005)$ and serum uric acid level $(5.5 \pm 1.6 \mathrm{mg} / \mathrm{dL}$ vs. $4.8 \pm 2.3 \mathrm{mg} / \mathrm{dL}, \mathrm{P}<0.001)$. Of all 103 diabetic patients with GADAb, $65(63 \%)$ were classified as L group (male/female:36/29), and 38 (37\%) were classified as $\mathrm{H}$ group (male/female:12/26). Compared with $\mathrm{L}$ group, $\mathrm{H}$ group were younger at age $(61 \pm 14$ years vs. $52 \pm 17$ years, $\mathrm{P}<0.05)$ and showed higher $\mathrm{HbA}_{1 \mathrm{C}}$ level $(8.4 \pm 2.3 \%$ vs. $10.2 \pm 2.7 \%, \mathrm{P}<0.005)$, lower serum triglycerides $(152 \pm 110 \mathrm{mg} / \mathrm{dL}$ vs. $119 \pm 109 \mathrm{mg} / \mathrm{dL}, \mathrm{P}<0.05)$ and serum uric acid level $(5.4 \pm 2.6 \mathrm{mg} / \mathrm{dL}$ vs. $3.9 \pm 1.1 \mathrm{mg} / \mathrm{dL}, \mathrm{P}<0.001)$, and higher prevalence of TPO autoantibody ( $15 \%$ vs. $48 \%, \mathrm{P}<0.05)$. Clinical profiles of $\mathrm{L}$ group showed no significant differences except for lower BMI $\left(25.3 \pm 3.9 \mathrm{~kg} / \mathrm{m}^{2}\right.$ vs. $23.1 \pm 4.8 \mathrm{~kg} /$ $\left.\mathrm{m}^{2}, \mathrm{P}<0.05\right)$ from control group.

Conclusion: This study suggests that GADAb level of $10 \mathrm{U} / \mathrm{ml}$ discriminates two subtypes of type 1 diabetes of Japanese adult patients.

\section{4}

Are GAD antibodies helpful in classification of patients with diabetes? Evidence from studies of a random population of middle-aged Danes. The Inter99 study

S. Engberg ${ }^{1}$, C. Glümer ${ }^{1}$, T. Jørgensen ${ }^{2}$, O. Pedersen ${ }^{1}$, K. Borch-Johnsen ${ }^{1}$ ${ }^{1}$ Steno Diabetes Center, Gentofte, ${ }^{2}$ Research Centre for Prevention and Health, Research Centre for Prevention and Health, Glostrup, Denmark

Background and aims: The aim of this cross-sectional study was to asses the prevalence of circulating GAD antibodies in a general Danish population according to glucose tolerance class and consequently to evaluate whether GAD antibodies are helpful in classification of patients with diabetes.

Materials and methods: In the population based Inter99 study, serum from individuals with IFG $(n=520)$, IGT $(n=751)$, screen-detected diabetes $(n=265)$, known diabetes $(n=139)$ and from a random sample of individuals with NGT ( $n=1490)$ were analysed for GAD antibodies. Individuals with known diabetes were divided into clinical type 1 and type 2 diabetes mellitus according to clinical characteristics. 
Results: No significant difference in the prevalence of positive and weak positive GAD antibodies was found between individuals with NGT (4.4\%), IFG $(2.8 \%)$, IGT $(3.5 \%)$ or screen-detected diabetes $(4.3 \%)$, whereas it was higher in individuals with known diabetes (14.3\%) compared with NGT $(p<0.0001)$. Among individuals with known diabetes, $8.5 \%$ in the clinical type 2 diabetes subgroup were GAD antibody positive and weak positive compared to $60 \%$ in the clinical type 1 diabetes subgroup $(p<0.0001)$. The prevalence of positive and weak positive GAD antibodies was not significantly different when comparing the clinical type 2 diabetes subgroup with NGT.

Conclusion: In a general Danish population, the prevalence of circulating GAD antibodies is surprisingly high in individuals with NGT. Therefore, there is a considerable risk of misclassification if GAD antibody positivity is used to reclassify individuals with clinical type 2 diabetes to aetiological type 1 diabetes according to WHO's recommendations.

Supported by: Danish Medical Research Council, Danish Center for Evaluation and Health Technology Assessment, Novo Nordisk, Copenhagen County, Danish Heart Foundation, Danish Diabetes Association, Danish Pharmaceutical Association

\section{5}

Classification of childhood-onset diabetes in Norway - The Norwegian Childhood Diabetes Registry

T. Skrivarhaug ${ }^{1}$, M. Bjørnvold ${ }^{2}$, D.E. Undlien², P.R. Njølstad ${ }^{3}$, G. Joner ${ }^{1}$ ${ }^{1}$ Dept. of Pediatric, Ullevål University Hospital, Oslo, Norway, ${ }^{2}$ Institute of Medical Genetics Faculty Division, Ullevål University Hospital, Oslo, Norway, ${ }^{3}$ Dep. of Clinical Medicine, University of Bergen, Norway

Background and aims: The Norwegian Childhood Diabetes Registry has registered newly diagnosed cases of childhood-onset diabetes nationwide with biological samples in the five-year period 2002-2007. The aim was to study the epidemiology of different forms of diabetes and classify incident cases on the basis of family history, clinical data, C-peptide, autoantibodies and HLA- genotypes.

Materials and methods: In the period 2002-2007,1551 new cases have been diagnosed below 15 years. In addition to family history and clinical data, blood samples were collected and analyzed for autoantibodies (anti-GAD, insulin-antibodies, anti-IA-2), HLA-genotypes and C-peptide. HLA genotypes were grouped into four risk categories (high, moderate, neutral and low) based on DQB1, DQA1and DRB1 genotypes including DRB1*04 sub typing. Due to the following criteria patients were classified as: 1) Type 1 diabetes (T1D): Antibodies pos. + neutral/moderate/ high risk HLA-genotype. 2) Probably T1D: Antibodies neg. + high risk HLA- genotype. 3) Unclassified: Antibodies neg. + low/moderate risk HLA-genotype. The patients were classified as autoantibodies positive $(\mathrm{ab}+)$ if one or more of the three tested was positive.

Results: Autoantibodies and HLA- genotypes were analyzed in 995 patients; $932(94 \%)$ were ab+ positive. HLA-genotype risk categories: 9 (1\%) patients were low risk, 259 (26\%) neutral risk, 473 (48 \%) moderate risk and 254 (25 $\%)$ high risk. On this basis of this 925 (93\%) of the patients were classified as T1D, $21(2 \%)$ were classified as probably T1D and $49(5 \%)$ patients were unclassified. A first degree relative with T1D were registered in 126 (12\%) of the patients and a first degree relative with Type 2 Diabetes (T2D) were registered in $21(2 \%)$ of the patients. A multiplex family (1 parent and 1 sibling with T1D) were registered in $9(1 \%)$ of the patients. In patients classified as T1D; 118 out of 925 patients (13\%) had a first degree relative with T1D, 9 out of $925(1 \%)$ had a multiplex family and 15 out of 925 (2\%) had a first degree relative with T2D.

In patients classified as probably T1D; 2 out of $21(10 \%)$ had a first degree relative with T1D. In patients registered as unclassified; 3 out of $49(7 \%)$ had a first degree relative with T1D. 21 out of 1089 patients (19\%) had diabetic ketoacidosis at diabetes onset $(\mathrm{ph}<7.3)$. Eight patients were discharged without insulin, none had high risk HLA-genotype and one of them was autoantibodies positive. This patient may represent $\mathrm{T} 2 \mathrm{D}$.

Conclusion: National diabetes registries are valuable tools in studying the epidemiology of different types of diabetes. The majority of childhood-onset diabetes was classified as T1D due to clinical data, HLA- genotypes and autoantibodies. $93 \%$ of the patients had T1D diabetes. In at least $5 \%$ of the cases further data is needed before a final diagnosis. Few children and adolescents below the age of 18 years get T2D in Norway, max 1-2 \% of new onset diabetes cases.

\section{6}

Diabetes in Down's syndrome: is it heterogeneous?

K.M. Gillespie, R.J. Aitken, J.P.H. Shield

University of Bristol, United Kingdom

Background and aims: There is an increased frequency of autoimmune thyroid disease, coeliac disease and diabetes in Down's syndrome. Understanding how an extra chromosome 21 increases the risk of autoimmune disease could provide vital insights into the mechanisms underlying autoimmunity in the general population. Diabetes in Down's syndrome in particular, is understudied. In a previous study of 40 children with Down's syndrome and diabetes in our laboratory, it was shown that children with Down's syndrome diagnosed with diabetes under the age of 21 share the same HLA characteristics as type 1 diabetes but have fewer "high risk" HLA class II genotypes than age matched children from the general population with type 1 diabetes. We therefore postulated that increased expression of genes on chromosome 21 increase the penetrance of autoimmune diabetes in children with Down's syndrome.

Materials and methods: A further 16 individuals in the UK with Down's syndrome and diabetes were identified. Clinical data and, where possible, genetic samples were collected. The frequency of diabetes according to age at diagnosis was calculated. HLA class II genotyping was carried out using DYNAL Autoreli PCR-SSOP.

Results: An unusual pattern emerged in the age at diagnosis of children with Down's syndrome and diabetes: $18 \%$ were diagnosed at, or under the age of 2 years (Fig 1) compared to an expected frequency of $7 \%$ for type 1 diabetes in the same age group in the general population. Of 9 children diagnosed under 2 years, 5 (55\%) were negative for high risk HLA class II genotypes, approximately the same frequency as the general population. Peaks in age at onset possibly consistent with type 1 and type 2 diabetes were also observed.

Conclusion: These data indicate that diabetes in very young children with Down's syndrome may not be of autoimmune origin and diabetes diagnosed in older individuals with Down's syndrome may be either type 1 or type 2 diabetes. This is consistent with the observed reduction of high risk HLA class II genotypes in individuals with Down's syndrome and diabetes, diagnosed under the age of 21 . There is therefore an urgent requirement for improved phenotyping, classification and diagnosis of diabetes in individuals with Down's syndrome.

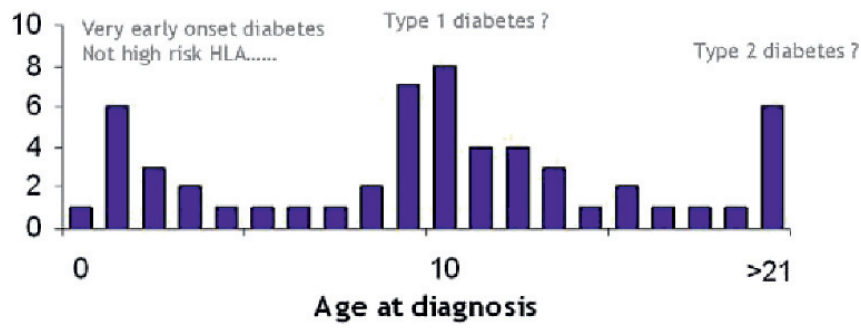

Figure 1. Age at onset of diabetes in Down's syndrome

\section{7}

Post transplant diabetes mellitus: role of insulin-resistance and pro-inflammatory cytokines in first year post-transplant V. Bonato ${ }^{1,2}$, D. Cataldo $^{1}$, M. Calabrese ${ }^{1}$, C. Tondi $^{1}$, M. Carmellini ${ }^{3}$, F. Dotta ${ }^{1,2}$

${ }^{1}$ Depatment of Internal Medicine and Endocrine and Metabolic Sciences, University of Siena, ${ }^{2}$ Fondazione Umberto Di Mario, Toscana Life Sciences, ${ }^{3}$ Transplant Unit, AOU Senese, Siena, Italy

Background and aims: Post Transplant Diabetes Mellitus (PTDM), a common complication of solid organ transplant, has a negative impact on graft and patient prognosis. Age, ethnicity, type 2 diabetes family history, and Hepatitis $\mathrm{C}$ were identified as non modifiable risk factors of PTDM. In addition type of immune-suppressive therapy, and overweight are modifiable risk factors for PTDM. Several studies demonstrated that pro-inflammatory molecules, such as MCP-1 and IL-6, are involved in the pathogenesis of type 2 diabetes. In a retrospective analysis, we found that $17 \%$ of 250 kidney transplant recipients developed an altered fasting glucose (PTDM 12.2\% and impaired fasting glucose, IFG, 4.8\%), with the highest incidence in first year post transplant. In the present study we aimed to evaluate the role of insulinresistance and proinflammatory molecules in PTDM and IFG. 
Materials and methods: At the day of kidney transplantation 53 non diabetic consecutive patients were recruited, and followed up to 1 year. At baseline the following data were collected: age, sex, body mass index (BMI), prevalence of overweight (BMI $>25 \mathrm{Kg} / \mathrm{m} 2)$, hepatitis C status, prevalence of diabetes mellitus family history, prevalence of hypertension, dyslipidemia and smoke. At follow up a blood sample was collected to determine glycaemia, lipid profile, basal insulin, creatinine, serum MCP-1 and IL-6. HOMA-IR and QUICKI were calculated to determine insulin-resistance and insulin-sensitivity. Type of immune-suppression (cyclosporine vs tacrolimus) and occurrence of acute rejection was evaluated at follow up. According with ADA guidelines patients were classified as PTDM, IFG or normoglycemic (NG).

Results: During the first year 9\% of patients developed PTDM, while $28.3 \%$ developed IFG. PTDM and IFG patients were older than NG ( $p<0.05$ and $\mathrm{p}<0.03$ ). In IFG patients HOMA-IR resulted higher than in NG group (3.10 \pm 2.72 and $1.96 \pm 2.37 \mathrm{p}=0.0082)$, while QUICKI was lower $(0.34 \pm 0.03$ and $0,36 \pm 0,03 \mathrm{p}=0.0113)$. PTDM and IFG prevalence was higher in tacrolimus-treated recipients than in cyclosporine-treated $(\mathrm{p}=0.002$ and $\mathrm{p}=0.07)$. No differences were observed in pro-inflammatory molecules, MCP-1 and IL-6. Multivariate analysis confirmed tacrolimus as independent risk factors for PTDM $(\mathrm{p}<0.02)$.

Conclusion: Our data confirm the role, in PTDM development, of known risk factors, in particular age and tacrolimus treatment, during the first year post transplant. In our work PTDM is only in part associated with insulin resistance, which is observed mainly in IFG patients. In the general population IFG is considered a "pre-diabetic" state and here we speculate that it precedes the onset of PTDM and may progress to PTDM in the presence of failure of beta cell function.

\section{8}

Heterogeneity of patients with LADA: linkage to autoimmunity is apparent only in those with perceived need for insulin treatment. Results from the Nord-Trøndelag Health Study (HUNT 2)

M. Radtke ${ }^{1}$, T.I. Lund Nilsen ${ }^{2}$, K. Midthjell' ${ }^{2}$, V. Grill ${ }^{1}$

${ }^{1}$ Department of Cancer Research and Molecular Medicine, ${ }^{2}$ Department of Public Health, Trondheim, Norway

Background and aims: Heterogeneity of Latent Autoimmune Diabetes in the Adult (LADA) has not been examined in detail. We compared phenotypes of LADA with and without perceived need for insulin treatment and contrasted these data with type 2 diabetes (T2D).

Materials and methods: We used data from the all-population-based NordTrøndelag Health Survey $(\mathrm{n}=64931)$, performed 1995-97. Data were assembled for LADA $(n=106)$ and T2D $(n=943)$ during times when diagnosis of LADA was not investigated.

Results: Comparing LADA with and without insulin: Markers of metabolic syndrome, such as BMI and blood pressure were similar. Insulin-treated subjects had higher titers of anti-GAD $(\mathrm{p}<0.001)$ and lower fasting $\mathrm{C}$-peptide $(p<0.001)$. Titre of anti-GAD and level of $C$-peptide in the insulin treated LADA group correlated negatively $(\mathrm{r}=-0.40 ; \mathrm{p}=0.009)$. Comparing LADA and T2D both without insulin: Groups were similar in all tested respects. Comparing insulin-treated LADA and insulin-treated type 2 diabetes: LADA patients were leaner, but still overweight, (BMI 28.7(27.4-30.1) vs. 30.9(CI: $30.2-31.5)$ in $T 2 D, p<0.001)$. More LADA patients received insulin $(40 \%$ vs. $22 \%, \mathrm{p}<0.001)$ Diabetes duration before start of insulin tended to be shorter $(\mathrm{p}=0.157)$, and insulin dose lower $(\mathrm{p}=0.190)$ despite lower C-peptide $(\mathrm{p}<0.001)$. Comparison between T2D with and without insulin: Insulin treated subjects were more obese, had higher $\mathrm{HbAlc}$ and lower C-peptide ( $\mathrm{p}<0.001$ for differences between groups).

Conclusion: Perceived need for insulin in LADA is dictated to a major extent by autoimmunity, despite presence of a metabolic syndrome. In contrast, in T2D a combination of insulin resistance and beta cell insufficiency prompts the decision to start insulin treatment.

Supported by: Research fellowship from the Central Norway Regional Health Authority and Norwegian University of Science and Technology

\section{9}

The prevalence of metabolic syndrome is much less in LADA and type 1 diabetes than type 2 diabetes: ACTION LADA 3

M.I. Hawa ${ }^{1}$, E. Cipponeri ${ }^{1}$, D. Collier ${ }^{1}$, Y. Loh ${ }^{1}$, P. Pozzilli ${ }^{1}$, S. Brophy ${ }^{2}$, S. Hunter ${ }^{3}$, C. Thivolet ${ }^{4}$, M. Didac ${ }^{5}$, R.D.G. Leslie ${ }^{1}$

${ }^{1}$ Department of Diabetes \& Metabolic Medicine, Barts and The London School of Medicine and Dentistry, London, United Kingdom, ${ }^{2}$ The Clinical School, University of Wales Swansea, United Kingdom, ${ }^{3}$ Department of Diabetes, Royal Victoria Hospital, Belfast, Ireland, ${ }^{4}$ Dept. of Endocrinology, Hospital Edouard Herriot, Lyon, France, ${ }^{5}$ Unit of Endocrinology, Hospital de Sabadell, Spain

Background and aims: The aim of this study was to estimate the frequency of the Metabolic Syndrome in adult European patients with diabetes and to assess whether it is associated with the type or age at onset of autoimmune diabetes.

Materials and methods: A consecutive series of patients attending hospitalbased diabetes clinics in the Europe were assessed for the frequency of Metabolic Syndrome and compared with population-based controls as part of the Action LADA study. In total, 2079 subjects (age range 30 - 70 years) were studied, including 1289 recent-onset type 2 diabetes patients without glutamic acid decarboxylase autoantibodies (GADA), 140 non-insulin requiring patients with GADA who remained off insulin therapy at least 6 months postdiagnosis and were designated latent autoimmune diabetes of adults (LADA), 291 type 1 diabetes patients and 359 control subjects. Metabolic Syndrome was defined according to both the National Cholesterol Education Program diagnostic criteria and the International Diabetes Federation.

Results: In both type 1 diabetes and LADA, levels of waist circumference and HDL cholesterol were similar, but in each group lower than in patients with type 2 diabetes ( $\mathrm{p}<0.0001$ for both comparisons). The frequency of Metabolic Syndrome was similar in type 1 diabetes (55.0\%) and LADA (54\%), but less than in type 2 diabetes patients $(81.5 \%)$ and more than controls $(36.5 \%)(\mathrm{p}<0.0001$ for all comparisons). However when glucose was removed as a variable Metabolic Syndrome frequency was similar in type 1 diabetes (27.5\%), LADA (27.0\%) and controls (35.0\%), but still less frequent in all groups than in type 2 diabetes $(47.5 \%)(\mathrm{p}<0.0001)$. Factors associated with Metabolic Syndrome included diabetes, older age and male gender. The hierarchy of frequency of features of Metabolic Syndrome in all groups, including controls, was similar.

Conclusion: Metabolic Syndrome is as prevalent in adults with LADA as in type 1 diabetes, but the frequency is nearly half that seen in type 2 diabetes. When glucose is excluded as a variable, Metabolic Syndrome is as prevalent in patients with autoimmune diabetes as controls. In terms of Metabolic Syndrome, autoimmune diabetes is distinct from type 2 diabetes. Supported by: European Union and Develogen

\section{0}

Similarities and differences between Caucasian and Asian LADA A. Petrone ${ }^{1}$, Y. Park ${ }^{2}$, S. Genovese ${ }^{3}$, J.T. Woo ${ }^{4}$, C. Giordano ${ }^{5}$, K.W. Lee ${ }^{6}$, A.E. Pontiroli ${ }^{7}$, S.H. Paik ${ }^{8}$, M.S. Nam ${ }^{6}$, P. Pozzilli ${ }^{9}$, Y.S. Kim $^{10}$, R. Buzzetti ${ }^{1}$, NIRAD Study Group, Korean National Diabetes Program ${ }^{1}$ Clinical Sciences, "Sapienza" University, Rome, Italy, ${ }^{2}$ Endocrinology, Hanyang University, Seoul, Republic of Korea, ${ }^{3}$ Endocrinology, Istituto Clinico Humanitas IRCCS, Milan, Italy, ${ }^{4}$ Endocrinology and Metabolism, Kyung Hee University School of Medicine, Seoul, Republic of Korea, ${ }^{5}$ Endocrinology, University of Palermo, Italy, ${ }^{6}$ Endocrinology and Metabolism, Ajou University, Suwon, Republic of Korea, ${ }^{7}$ San Paolo Hospital, Milan, Italy, ${ }^{8}$ Endocrinology, Korea University, Seoul, Republic of Korea, ${ }^{9}$ Endocrinology, University Campus Bio-Medico, Rome, Italy, ${ }^{10}$ Endocrinology, Inha University, Seoul, Republic of Korea

Background and aims: Latent autoimmune diabetes in adults (LADA) is an autoimmune form of diabetes in adults. Patients with LADA show more preserved beta-cell function than classic T1D, but have also clinical features of type 2 diabetes (T2D). T1D and T2D vary in epidemiology, clinical and metabolic features between Caucasian and Asian patients. Prevalence of T1D is at least ten fold lower in Asians compared to Caucasians, whereas few epidemiological studies on LADA have shown that this form of diabetes is only slightly less prevalent in Asians vs. Caucasians. No studies however, have compared the features of LADA in these ethnic groups. In a joint collaborative project between Italy and South Korea, we compared anthropometric, clinical and metabolic data of Caucasian vs. Asian LADA patients. 
Materials and methods: Two large studies on prevalence of LADA are carried out in Italy and South Korea whose results are reported elsewhere. A cross sectional analysis was performed in the 193 LADA patients diagnosed in Italy and in the 39 LADA patients diagnosed in South Korea. The same ascertainment criteria were applied for diagnosis of LADA in both groups as published in Diabetes Care in 2007. Biochemical parameters were measured centrally in both countries, respectively, GAD antibodies were evaluated in two centralized laboratories, one in Italy and one in Korea, and both participated in the DASP autoantibody Workshop. Data were analyzed with one way ANOVA test.

Results: Prevalence of LADA (reported elsewhere) was very similar $(4.4 \%$ in Italy and $4.4 \%$ in South Korea). Mean age at diagnosis was nearly identical between Caucasian and Asian LADA (50.3 yrs. \pm 12 vs. 49.6 yrs. \pm 8.3 ), as well as $\mathrm{HbAlc}$ values $(7.5 \% \pm 1.7$ vs. $7.4 \% \pm 1.5)$. Despite similar HbAlc levels, fasting blood glucose (BG) levels were significantly higher in Caucasian vs. Asians $(168.1 \mathrm{mg} / \mathrm{dl} \pm 58 \mathrm{mg} / \mathrm{dl}$ vs. $134 \mathrm{mg} / \mathrm{dl} \pm 34 \mathrm{mg} / \mathrm{dl}, \mathrm{p}<0.001)$ implying that post-prandial glucose values were higher in Asian LADA. There was a significant higher BMI in Caucasian vs. Asian LADA (27. \pm 5.1 vs. $25.3 \pm$ $3.3, \mathrm{p}<0.01)$ and higher HDL (but not total) cholesterol levels $(50 \mathrm{mg} / \mathrm{dl} . \pm 3$ $\mathrm{mg} / \mathrm{dl} \mathrm{vs} .44 .7 \mathrm{mg} / \mathrm{dl} \pm 11 \mathrm{mg} / \mathrm{dl}, \mathrm{p}<0.03)$. By contrast, triglyceride levels were significantly higher in Asians vs. Caucasian $(201 \mathrm{mg} / \mathrm{dl} \pm 169 \mathrm{mg} / \mathrm{dl}$ vs. 144 $\mathrm{mg} / \mathrm{dl} \pm 104 \mathrm{mg} / \mathrm{dl}, \mathrm{p}<0.01$ ) probably reflecting differences in diet habits.

Conclusion: Asian LADA patients are leaner than Caucasians, have similar both age at diagnosis and overall metabolic control, although fasting BG appears higher in Caucasians. LADA in these two ethnic groups has similar prevalence, clinical and some metabolic features in common, but also some relevant differences in lipid profile which may help in designing strategies for intervention according to the ethnic group.

Supported by: FoRiSID (Research Foundation of the Italian Society of diabetes) with an unrestricted grant from NOVO Nordisk, (Italy)

\section{PS 014 Incidence of type 1 diabetes}

\section{1}

The trend curve of type 1 diabetes incidence in Czech children has flattened

O. Cinek ${ }^{1}$, M. Kulich ${ }^{2}$, Z. Sumnik ${ }^{1}$

${ }^{1}$ Department of Pediatrics, ${ }^{2}$ Department of Probability and Mathematical Statistics, Charles University in Prague, Czech Republic

Background and aims: The incidence of childhood type 1 diabetes (T1D) is increasing in most populations, and this increase often forms a basis for long-term forecasts. Using data from one of the largest population-based paediatric diabetes registers, we show how unpredictably the incidence can change: a sudden stagnation can follow immediately after a very rapid relative increase.

Materials and methods: The population-bases Czech Childhood Diabetes Register records new cases of childhood-onset T1D (using EURODIAB criteria), manifested at the age of $0-14$ completed years, as reported by a network of paediatric diabetologists. The completeness of the ascertainment was calculated to $99 \%$ using data from parent organization as the secondary source. The observed numbers of cases diagnosed over 1989 - 2006 were modelled by Poison log-linear regression with logarithm of the total follow-up time as an offset. The predictors were age, gender, calendar time, and their interactions. Effect modification of calendar time by age was modelled by interacting calendar time with age group; this means the effect of calendar time was allowed to vary with age group. The analyses were adjusted for varying completeness of ascertainment. The time trends in T1D incidence were modelled by piecewise linear function of calendar time with breaks at years 1996 and 2002. The breaks were suggested by descriptive analyses.

Results: A total of 4296 cases was ascertained over 1989 - 2006 from an average paediatric population of 1.8 million. The observation period can be divided into three parts: a moderate increase in incidence over 1989-1995, followed by a steep increase between 1996-2001, and a stagnation 2002-2006. While the upward inflection of the incidence curve between the first and the second period is statistically significant only for the youngest age group (0-4 years at onset, $\mathrm{p}=0.02)$, the flattening of the incidence after 2002 is highly significant in the whole group $0-14$ years of age $(\mathrm{p}<0.001)$, as well as the two younger subgroups $0-4(\mathrm{p}<0.001)$ and $5-9$ years $(\mathrm{p}=0.005)$.

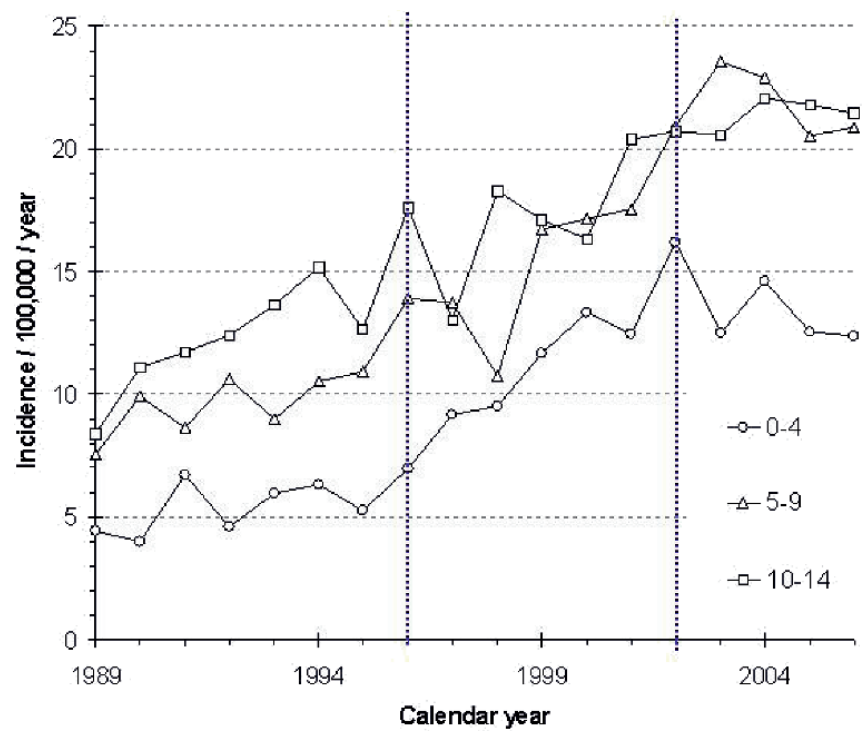

Conclusion: Stagnations in populations with previously rising T1D incidence exist and have been documented in sufficiently large datasets (e.g. from Norway with a stable incidence over 1989-1998 that was followed again by an increase). Further reports on lack of significance for the increase in incidence come from smaller registers, being related mostly to low number of cases; this is however not the case for our dataset from one of the largest operative paediatric diabetes registers. The environmental exposure (or its change) responsible for this recent inflection is unknown, but is most likely populationspecific: Austria, our close neighbour both geographically and genetically, undergoes an uninterrupted increase in incidence lasting now about two 
decades. Our data seem to contribute to the notion that no simple long- and intermediate-term trends in incidence can be reliably predicted. Supported by: Ministry of Education of the Czech Republic

\section{2}

\section{Seasonal variation of the onset of type 1 diabetes among children worldwide \\ E.V. Moltchanova, N. Schreier, N. Lammi, M. Karvonen}

Dept of Health Promotion and Chronic Disease Prevention, National Public Health Institute, Helsinki, Finland

Background and aims: Type 1 diabetes mellitus (T1DM) is thought to result from a combination of genetic and environmental factors. Among the latter, e.g., viral infections and the intake of vitamin D may be subject to seasonal variations. Several studies worldwide have found evidence of seasonality of birth and diagnosis of T1DM. However, the generalisation of the results is usually difficult due to different methods of data collection and statistical analysis. The WHO Diabetes Mondiale (DIAMOND) project provides unified data collection framework. Our aim was to study the seasonal patterns exhibited by these data.

Materials and methods: During the period 1990-1999, population-based registries were used to collect standardized data on incidence of T1DM among 0-14 year-olds worldwide within the framework of the WHO DIAMOND project. A total of 104 centers from 52 countries have provided incidence and population data sufficient to analyse seasonal patterns. Geographical coordinates for each center were also obtained. A regression model with linear trend term and seasonal Fourier terms was fitted, adjusted by age and sex.

Results: Of the 104 studied centers 42 exhibited significant seasonality (p $<=.05$ ) in the incidence of TIDM. Of these 28 had peaks in winter months (October - January) and 33 had troughs in summer months (June - August). However, some of the centers in Southern hemisphere demonstrated different pattern with peak in July-September and trough in January-March. The existence of significant seasonal pattern correlated with the higher level of incidence ( $\mathrm{p}<=.001$ ). This effect disappeared when adjusted for the geographical location, namely the latitude. Centers further away from the equator were on average more likely to exhibit seasonality $(\mathrm{p}<=.001)$. The longitude, on the other hand, had no effect. Incidence for boys exhibited significant seasonality more often than that for girls ( 33 vs 26 centers). When divided into three age groups, $0-4$ years of age, 5-9 years of age, and 10-14 years of age, the incidence in older age groups showed significant seasonality more often than that in younger age groups.

Conclusion: The analysis of worldwide data onT1DM, provided by the WHO DIAMOND Project, largely confirms the findings of smaller studies reported so far: existence of seasonal pattern of T1DM incidence with peak in winter and trough in summer in the Northern hemisphere, more often observed among boys than among girls, and among older age-groups than among the younger. In addition, the Southern hemisphere centers have shown seasonality patterns different from those in the Northern hemisphere.

\section{3}

Infant feeding patterns during the first year of life in offspring of mothers with and without type 1 diabetes: results from the TEDDY (The Environmental Determinants of Diabetes in the Young) Study J.M. Norris ${ }^{1}$, S. Hummel ${ }^{2}$, S. Schoen ${ }^{3}$, S.M. Virtanen ${ }^{4}$, W. McLeod ${ }^{5}$, C. Andren Aronsson 6 , P. Gesualdo ${ }^{7}$ for the TEDDY Study Group ${ }^{1}$ Preventive Medicine and Biometrics, University of Colorado Denver, United States, ${ }^{2}$ Immunology, Diabetes Research Institute, Munich, Germany, ${ }^{3}$ Research Institute of Child Nutrition, Dortmund, Germany, ${ }^{4}$ Epidemiology, University of Tampere, Finland, ${ }^{5}$ Pediatrics Epidemiology Center, University of South Florida, Tampa, United States, ${ }^{6}$ Diabetes and Celiac Disease Unit, Lund University, Malmo, Sweden, ${ }^{7}$ Barbara Davis Center, University of Colorado Denver, Aurora, United States

Background and aims: Previous studies have suggested that infant feeding patterns may influence the risk of islet autoimmunity and type 1 diabetes (T1D). Pediatric authorities have recommended exclusively breast-feeding newborns during the first 6 months of life. Recently the German BABYDIAB study showed that mothers with T1D breast-feed their babies less and for a shorter duration compared to mothers without T1D. The international TEDDY study has assessed the effect of maternal T1D on breast-feeding behaviour and the introduction of various foods during the first year of life.
Materials and methods: The TEDDY study is conducted in three U.S. centers (Denver, Seattle, Georgia/Florida) and three European centers (Finland, Sweden, Germany) with the aim of identifying environmental factors that may trigger islet autoimmunity and T1D. A total of 4,207 children at genetically high risk for T1D have been enrolled into the study. The 2,343 children followed for more than one year were included in the present analyses. Of these, 96 have a mother with T1D, 151 have a non-diabetic mother but a father or siblingwith T1D and 2,096 have no first-degree relative with T1D. Data on early infant diet were collected at 3-month intervals.

Results: Mothers with T1D breast-fed their children less than non-diabetic mothers. During the first 4 weeks of life only $28.1 \%$ (SE 2.4) of the children with a T1D mother were exclusively breast-fed compared to $47.7 \%$ (SE 2.8 ) of the children with a father/sibling with T1D and $43.3 \%$ (SE 0.7) of the children from the general population $(\mathrm{p}<0.001)$. Also the total breast-feeding duration was shorter in the offspring of mothers with T1D $(\mathrm{p}<0.01)$. During the first 4 weeks of life, $64.9 \%$ (SE 2.9) of the children with a mother with T1D were introduced to cow's milk vs. $49.0 \%$ (SE 2.9) of the children with a father/sibling with T1D and vs. 54.5\% (SE 0.7) of the children from the general population $(\mathrm{p}<0.001)$. In contrast, $65.5 \%$ (SE 0.8 ) of the children of the general population were introduced to gluten by 5 months of age, whereas only $37.6 \%$ (SE 4.3 ) of the children with a mother with T1D and $42.3 \%$ (SE 3.6) of the children with a father/sibling with T1D were exposed to gluten by that age $(\mathrm{p}<0.001)$.

Conclusion: This study confirms earlier findings that mothers with T1D breast-feed less and for a shorter duration compared to mothers without T1D. Furthermore offspring of mothers with T1D were exposed to cow's milk earlier, and children with a mother or other first-degree relative with T1D were exposed to gluten later compared to children from the general population. Supported by: NIDDK, NIAID, NIEHS, NICHD, JDRF and CDC

\section{4}

Increasing temporal trend in incidence of type 1 diabetes in children in Piedmont Region, Italy, period 1990-2004

G. Novelli ${ }^{1}$, F. Cerutti ${ }^{2}$, F. Cadario ${ }^{3}$, A. Perino ${ }^{3}$, G. Bona ${ }^{3}$, C. Zucco ${ }^{1}$, M. Perotto ${ }^{1}$, F. Panero ${ }^{1}$, G. Bruno ${ }^{1}$

${ }^{1}$ Internal Medicine, University of Turin, ${ }^{2}$ Dept of Pediatrics, University of Turin, ${ }^{3}$ Dept of Pediatrics, University of Novara, Italy

Background and aims: Incidence of type 1 diabetes is increasing in many countries. In Northern Italy, the incidence rates among persons up to 29 years of age have been estimated by the Registry of the Province of Turin, which has achieved high estimated completeness of ascertainment for both children and young dults. In this report, we aimed to extend the study base of the registry to all children aged 0-14 years in the whole Piedmont Region in the period 1990-2004.

Materials and methods: The study-base is the population 0-14 years of age of the Piedmont Region (500,000 children), period 1990-2004, identified through two independent sources of ascertainment. Diagnosis of type 1 diabetes was based on permanent insulin treatment. The two sample capture-recapture method was employed to estimate completeness of ascertainment Results: 948 incident cases of diabetes have been identified (completeness of ascertainment 99\%). Incidence was $12.1 / 100,000$ person-years (95\% CI 11.4-12.9) in the age group 0-14 yrs, similar in both sexes. The highest risk in both sexes was found in the age-group 10-14 yrs (0-4: 9.2/100,000 personyears, 5-9: 12.7/100,000, 10-14: 14.3/100,00). Large fluctuations in incidence rates/year were evident; moreover, comparing 5 -years time periods incidence rate, a significant increase in risk was evident: 1990-94: 10.9/100,000 personyears; 2000-2004: 14.4/100,000 person-years (RR 1.32, 95\% CI 1.13-1.53, $\mathrm{p}<0.0001)$. The highest risk was found in males in $2001(18.0 / 100,000,95 \%$ CI 13.5-23.9). Large geographical variations within the Region area were also found, not related to urban/rural characteristic, and therefore mostly unexplained at present.

Conclusion: This population-based study shows: 1) large geographical variations in risk of childhood type 1 diabetes within a single Italian Region; 2) an increasing temporal trend in risk, similar to that found in other European countries. Monitoring of the disease over time and geographical area through registries is essential to suggest hypothesis on the etiopathogenesis of the disease. 


\section{5}

Incidence of childhood type 1 diabetes (0-14 yrs) in La Palma Island: 1993-2007

B.M. Belinchón Sz.-Somoza ${ }^{1}$, J.A. Hernández Bayo²,

R. Cabrera Rodríguez ${ }^{3}$

${ }^{1}$ Family Care, Primary Care Center, San Andrés y Sauces, ${ }^{2}$ Endocrinology, General Hospital of La Palma, Breña Alta, ${ }^{3}$ Pediatrics, General Hospital of La Palma, Breña Alta, Spain

Background and aims: The incidence of type 1 diabetes shows wide geographical variability and heterogeneity. The aim of this study was to determine the incidence of type 1 diabetes in children and adolescents younger than $15 \mathrm{yr}$ in La Palma Island (the most northwest of Canary Islands, Spain: $730 \mathrm{Km}^{2}, 85000$ habitants and subtropical climate).

Materials and methods: All subjects with type 1 diabetes (according WHO and/ or ADA criteria) diagnosed between January 1993 and December 2007 (prospectively 1995-2007) were included. The population at risk (0-14 yr) fluctuated between 15711 habitants -1991 General Census- and 11899 habitants -2001 General Census-. All the reported cases were on insulin treatment. All subjects were living in La Palma Island at least six months before diagnosis of type 1 diabetes. Autoimmunity was checked measuring tyrosine phosphatase $\left(\mathrm{IA}_{2}\right)$ and glutamic acid decarboxylase (GAD) auto-antibodies. Using the capture-recapture method (primary source was hospital records, while secondary sources were membership files of La Palma Diabetic Association and Primary Care Physicians), the ascertainment was $100 \%$. The incidence rates were expressed as number of cases per $10^{5}$ habitants per year. The $95 \%$ Confidence Intervals were estimated assuming the Poisson distribution of the cases. The age adjustment for the rates was done using the direct method with a World and European Standard Population.

Results: Sixty eight subjects younger than $15 \mathrm{yr}$ had presented type 1 diabetes at the last $15 \mathrm{yr}$ [ 37 boys, 31 girls; sex ratio 1.2 ; medium age: $8.5 \pm 3.8$ yr (95\% CI: 7.6-9.4)]. In 8 subjects (11.8\%) type 1 diabetes was present in other first degree families (brothers, not twins). Autoimmunity (positive $\mathrm{IA}_{2}$ and/or GAD auto-antibodies) was present in 79.7\% (51/64): $89.2 \%$ at diagnosis (33/37), 66.7\% after diagnosis (18/27). The annual incidence fluctuates between 12.7 and $67.2 / 10^{5}$, being the average annual incidence $33.2 / 10^{5}(95 \%$ CI: 30.3-36.1). No seasonal differences were observed (spring 23.5\%, summer $26.5 \%$, autumn $26.5 \%$, and winter $23.5 \%$ ). The incidence in males was higher than in females [35/10 $0^{5}$ per yr (95\% CI: $\left.32-38\right)$ versus $31.2 / 10^{5}$ per yr $(95 \%$ CI: 28.4-34)]. The incidence was higher in the $10-14 \mathrm{yr}$ age-group $\left(38.3 / 10^{5}\right.$ per yr, 95\% CI: 35.2-41.4), followed by the $5-9$ yr age-group (37.6/10 $0^{5}$ per yr, 95\% CI: $34.5-40.7)$ and the $0-4$ yr age-group $\left(21.6 / 10^{5}\right.$ per yr, $95 \%$ CI: $19.2-$ 24). The age-adjusted incidence to World Standard Population was 31.6/10 per yr (95\% CI: 28.8-34.4). The age-adjusted incidence to European Standard Population was $32 / 10^{5}$ per yr (95\% CI: 29.1-34.9).

Conclusion: The incidence of type 1 diabetes in La Palma Island is the highest reported up to date in a Spanish community, and is close to the highest of the world. It is inconsistent with the hypothesis of a north-south gradient in diabetes risk. The knowledge of the incidence rates in La Palma Island can contribute to study the role that genetics and environmental factors may play in these differences.

\section{6}

Incidence of insulin-dependent diabetes mellitus among Italian-heritage children living in Germany

S. Muntoni ${ }^{1,2}$, S. Ehehalt ${ }^{3}$, P. Popovic ${ }^{3}$, A. Willasch ${ }^{3}$, R. Hub ${ }^{3}$, M.B. Ranke ${ }^{3}$, S. Muntoni ${ }^{2}$, A. Neu for the DIARY Group Baden-Wuerttemberg ${ }^{3}$ ${ }^{1}$ Department of Toxicology, Oncology and Molecular Pathology Unit, University School of Cagliari, Cagliari, Italy, ${ }^{2}$ Centre For Metabolic Diseases and Atherosclerosis, The ME.DI.CO. Association, Cagliari, Italy, ${ }^{3}$ University Hospital for Children and Adolescents, University School of Tuebingen, Germany

Background and aims: In order to investigate the role of genetic and environmental factors in the pathogenesis of Type 1 diabetes, we set up in Germany a migrant study with a comparative study of disease prevalence and incidence in (A) children of migrant Italians from high-risk (Sardinia) and low-risk (continental Italy) regions versus (B) German children.

Materials and methods: Children from Italy were identified by the "BadenWuerttemberg (BW) Diabetes Incidence Registry" (DIARY), in which 4,017 newly-diagnosed type 1 diabetic patients, aged 0-14 years, were registered from 1987 to 2003. In addition, data relating to type 1 diabetic children from Sardinia were elicited from more than 2,000 questionnaires.
Results: (1) Type 1 diabetes is more frequent in German children than in children of Italian migrants (IR 14.8/100,000/year, 95\% CI 14.4-15.4 vs IR $10.8 / 100,000 /$ year, $95 \%$ CI 8.2-13.6). (2) The incidence of type 1 diabetes among Italian children residing in Germany is similar to that of Italian children in the home country (IR 10.8/100,000/year, 95\% CI 8.2-13.6 vs $8.4 / 100,000 /$ year, $95 \%$ CI 7.9-8.9). (3) The prevalence of type 1 diabetes among Sardinian children is higher than that of German children $(0.11 \%$, 95\% CI 0.11-0.12) independently of the place where Sardinian children are living (Sardinian children in Germany 2.3\%, 95\% CI 0.5-6.5 vs Sardinian children in Sardinia 0.30\%, 95\% CI 0.27-0.32).

Conclusion: Children from high- and low-risk areas of Italy have incidence rates closer to the rates of their native regions than to those of German children. This indicates that genetic factors play a predominant role in the pathogenesis of type 1 diabetes.

\section{7}

Menopause in women with type 1 diabetes

L. Sjöberg' ${ }^{1}$ L. Haapala ${ }^{2}$, V. Harjutsalo ${ }^{3}$, J. Pitkäniemi² ${ }^{2}$ J. Tuomilehto ${ }^{2}$, R. Kaaja ${ }^{4}$

${ }^{1}$ Department of General Practice and Primary Health Care, University of Helsinki, ${ }^{2}$ Department of Public Health, University of Helsinki, ${ }^{3}$ Department of Health Promotion and Chronic Disease Prevention, National Public Health Institute, Helsinki, ${ }^{4}$ Department of Obstetrics and Gynaecology, Helsinki University Central Hospital, Finland

Background and aims: Earlier studies have suggested a significantly earlier menopause in type 1 diabetic women. This could be related to ovarian dysfunction due to microangiopathy and/or to autoimmune processes related to diabetes. The aim of this study was to study the timing of menopause and the factors influencing it in a large cohort of Finnish women with type 1 diabetes.

Materials and methods: The subjects of the DERI (Diabetes Research International Group) study were diagnosed with type 1 diabetes at 17 years of age or under during the years 1965-1979, placed on insulin treatment at diagnosis and residing in Finland. The cases were identified from the National Diabetes Registry and the drug reimbursement registry with an ascertainment rate approaching $100 \%$. From this cohort, we selected all women who were born in 1962 or before that and thus were in the perimenopausal age (40-55) in 2002, when the study started. Of the 1004 subjects who were alive at the start of the study, 980 could be contacted. A questionnaire on their gynaecological and reproductive history, diabetes-related facts, other diseases and life-style factors was sent . The response rate was $65 \%(635 / 980)$. To study the transition from premenopause to postmenopause a similar questionnaire was sent three years later. The response rate was $81 \%$ (513/635).

The probability of reaching menopause was evaluated by using Kaplan-Meier method and associated factors (number of deliveries, retinopathy, nephropathy, autoimmune diseases, smoking) were evaluated using Cox's proportional hazards regression modelling.

Results: Of the 635 respondents, a total of 78 had undergone hysterectomy, 4 had experienced premature menopause because of chemotherapy and 41 used hormonal contraception causing amenorrhea. These women were excluded from the analyses. Those who could report their age at the cessation of menstruation had a menopausal age of 47.8 years (median; $95 \%$ CI 46.8 48.8 ). In the study population there were, however, over one hundred women over 50 years of age who had not yet reached menopause.

We found a significant association between the age at menopause and the number of deliveries $(\mathrm{p}=0.02)$. The presence of diabetic retinopathy or nephropathy did not have a significant influence on the age at menopause, neither did the presence of other autoimmune diseases.

Conclusion: The results of our study do not indicate a significantly earlier menopause in type 1 diabetic patients in Finland. This is in contradiction to earlier study results on this subject.

Supported by: Kyllikki and Uolevi Lehikoinen foundation, Else and Wilhelm Stockmann foundation 


\section{PS 015 Cardiovascular risk}

\section{8}

Predictive value and risk of the metabolic syndrome defined by NCEP III and IDF for the development of type 2 diabetes

C. Temml ${ }^{1}$, R. Obermayer ${ }^{2}$, G. Gutjahr ${ }^{3}$, B. Ludvik ${ }^{3}$

${ }^{1}$ Health care, MA15 Dez.IV, Vienna, ${ }^{2}$ Internal Medicine III, Donauspital

SMZ-OST, Vienna, ${ }^{3}$ Internal Medicine III, University of Vienna, Austria

Background and aims: The metabolic syndrome (MS) is associated with an increases risk of type 2 diabetes (DM2). Among others, the criteria of the National Cholesterol Education Programs (NCEP- ATP III panel) or International Diabetes Federation (IDF) are used for diagnosis. The aim of the present study is to evaluate the predictive value of these criteria as regards to the development of DM2.

Materials and methods: In this longitudinal study, parameters relevant to the diagnosis of MS were assessed in 13.852 non-diabetic subjects (7.270 males (56,6\%), age $44 \pm 3 \mathrm{y}$ and 5.582 females (43,3\%), age $46 \pm 14 \mathrm{y}$, means \pm SEM). The subjects were followed up to an average of 6 years.

Results: According to IDF, $13.3 \%$ and to NCEP III $10.4 \%$ (NCEP vs. IDF, p> 0.05 ) of all subjects presented with MS at study entry. In all age groups, the prevalence of MS was higher by IDF than by NCEP definition, between 50 to 59 years of age it was 24.4 vs. $16.5 \%$ in males, and 19.1 vs. $13.8 \%$ in females. The prevalence was higher in men compared to women in both definitions for all age categories, except for female subjects over 60 years. Concordance between both definitions was modest as expressed by a kappa value of 0.63 (95\% CI 0.61-0.65). The diagnosis of MS increased the relative risk to develop DM2 over a period of 6 years according to IDF vs. NCEP in males by 7.6 vs. 7.0 in females by 13.7 vs. 16.6 ( $p>0.05$ ).

Conclusion: The results of this study reveal a higher prevalence for MS in a non-diabetic population when the IDF vs. NCEP criteria are used. The presence of MS imposes a considerably increased risk for the subsequent development of DM2. In female subjects, NCEP criteria allow a better assessment of the risk for DM2.

\section{9}

Comparison of the population specific clinical model and the IDF definition of metabolic syndrome for the prediction of type $\mathbf{2}$ diabetes in a Japanese population

T. Nakagami ${ }^{1}$, M. Tominaga ${ }^{2}$, R. Nishimura ${ }^{3}$, M. Daimon ${ }^{4}$, T. Oizumi ${ }^{4}$, N. Yoshiike ${ }^{5}$, N. Tajima ${ }^{3}$

${ }^{1}$ Diabetes Center, Tokyo Women's Medical University, ${ }^{2}$ Department of Laboratory Medicine, Yamagata University School of Medicine, ${ }^{3}$ Division of Diabetes, Metabolism and Endocrinology, Department of Internal Medicine, Jikei University School of Medicine, Tokyo, ${ }^{4}$ Department of Neurology, Hematology, Metabolism, Yamagata University School of Medicine, ${ }^{5}$ International Business-Academia Collaboration Center, National Institute of Health and Nutrition, Tokyo, Japan

Background and aims: The metabolic syndrome (MetS) has been highlighted as high-risk for type 2 diabetes (T2DM) and cardiovascular disease (CVD). Thus, we sought to compare the MetS to the clinical prediction model for DM, which is developed in the Funagata Diabetes Study, as predictors of T2DM.

Subjects and methods: A total of 1,189 non DM in the Funagata-town inhabitants were followed for 5 years, with 4,720 person years accumulated. The incident DM was identified by the 1999-World Health Organization criteria. MetS was identified by the definition according to the International Diabetes Federation (IDF). Baseline measurements included age, sex, smoking status, alcohol habits, physical activity, family history of DM, body mass index (BMI), waist circumference (Wc), systolic and diastolic blood pressures (BPs), fasting plasma glucose (FPG) and 2-hour plasma glucose, HbAlc, fasting insulin, total and high density lipoprotein cholesterol (HDL-C) and triglyceride (TG). The multivariate logistic regression model was used to develop DM prediction model in the Funagata-town inhabitants.

Results: During 5 year of follow-up, 57 subjects developed DM. The prevalence of the MetS at baseline was $11.1 \%$.The multivariate logistic regression model showed that age (odds ratio [OR] per 10 years, 1.60 [95\% CI: 1.19$2.16]$ ), Wc (OR per 1SD, 1.69 [1.26-2.28]), family history of DM (2.97 [1.227.22]), and log transformed FPG (OR per 1SD, 3.44 [2.44-4.84]) were associated with incident DM. The presence of high BP, high TG and low HDL-C by the IDF definition of MetS increased the risk of development of DM by $55 \%$, $62 \%$, and $68 \%$, respectively, in comparison to the absence of these conditions. However they were not statistically significant. Then, the population specific prediction model for $\mathrm{DM}$ was calculated as following regression parameters: $\mathrm{P}=1 /\left(1+\mathrm{e}^{-\mathrm{x}}\right)$, where $\mathrm{x}=-30.463+\left(0.472^{\star}\right.$ Age $/ 10+0.526^{\star} \mathrm{Wc} / \mathrm{SD}+1.234^{\star} \log$ FPG/SD) +1.088 (if $1^{\text {st }}$ degree relative has DM). The area under the receiver operating characteristic curve for the prediction model was 0.84 (95\% CI: 0.79-0.89) and the optimal cut-off point for the prediction of incident DM was defined as a probability value $\geq 0.04$. The model's sensitivity was $87.7 \%$, specificity $68.3 \%$, and false-positive test $26.6 \%$. While the sensitivity, specificity and false-positive test for predicting DM with MetS were 31.6\%, 89.9\%, and $86.4 \%$. At corresponding sensitivity, the prediction model had a significantly lower false positive rate $(\mathrm{p}<0.001)$ and, at corresponding false positive rate, significantly higher sensitivity $(\mathrm{p}<0.001)$ than the MetS for predicting DM. Combining the MetS with DM prediction model did not improve the prediction of DM.

Conclusion: The MetS is inferior to the population specific prediction model for T2DM.

Supported by: Ministry of Health, Labour and Warfare, Japan; Japanese Ministry of Education, Culture, Sports, Science and Technology; Japan Medical Women's Association and the Tokyo Women's Medical University Association

\section{0}

Association of endothelial function with components of the metabolic syndrome and lifestyle factors in subjects without previously diagnosed diabetes. Results from the KORA F4 Study

B. Thorand ${ }^{1}$, A. Schneider ${ }^{1}$, A. Peters ${ }^{1}$, W. Rathmann ${ }^{2}$, H.-E. Wichmann ${ }^{1}$, C. Meisinger ${ }^{1}$

${ }^{1}$ Department of Epidemiology, Helmholtz Zentrum München, Neuherberg, ${ }^{2}$ Institute of Biometrics and Epidemiology, German Diabetes Center, Düsseldorf, Germany

Background and aims: Endothelial dysfunction plays an important role in the pathogenesis of atherosclerosis and may also be of particular importance for the development of type 2 diabetes. Therefore, the aim of the present study was to investigate associations between endothelial function and different components of the metabolic syndrome, including glucose concentrations, as well as lifestyle factors related to the development of atherosclerosis and type 2 diabetes.

Materials and methods: This abstract includes preliminary data from 364 men and 374 women aged 52-71 years without previously diagnosed diabetes who participated in the ongoing prospective KORA (Cooperative Research in the Region of Augsburg) F4 study. This study is a follow-up examination of the KORA S4 study conducted between 1999 and 2001. Endothelial function was measured using reactive hyperemia-peripheral artery tonometry (EndoPAT, Itamar, Israel). Changes in pulse wave amplitude were observed at the fingertips using fingertip plethysmography probes before and after 5-minute forearm cuff inflation. The hyperemic response was determined as the log of the ratio of the post-deflation (measured 90-120 seconds following cuff deflation) to baseline pulse amplitude in the hyperemic finger normalized to the contralateral finger (log PAT ratio). A standardized oral glucose tolerance test (OGTT) was performed in all participants after an overnight fast. Endothelial function was measured approximately 50 minutes after the OGTT had been terminated and after the subjects had received a standardized breakfast. The association between sex, age, metabolic (body mass index (BMI), actual hypertension, ratio of total cholesterol/HDL-cholesterol, baseline and 2 hour glucose concentrations) as well as lifestyle factors (smoking status, physical activity, alcohol consumption) and endothelial function was assessed using linear regression analysis with log PAT ratio as the dependent variable.

Results: The mean $( \pm$ SD) $\log$ PAT ratio was $0.49 \pm 0.42$ in the total study population and $0.39 \pm 0.36$ in men and $0.58 \pm 0.45$ in women. In a multivariable linear regression model which included all covariables mentioned above except the 2 hour glucose concentration, male sex, age, BMI and fasting glucose were significantly inversely associated with log PAT ratio (all p-values $<0.002$ ). The $\mathrm{p}$-value for glucose was slightly higher when the 2 hour glucose concentration was used instead of fasting glucose $(\mathrm{p}=0.009)$ and the association was only significant for fasting glucose $(\mathrm{p}=0.03)$ when both glucose concentrations were included in the model simultaneously. None of the examined lifestyle factors was significantly associated with the log PAT ratio. Conclusion: Male sex, age, BMI and fasting glucose concentrations as well as glucose levels measured 2 hours after an OGTT were strongly inversely associated with endothelial function. Future prospective studies are needed to 
determine the temporal relationship between derangements in glucose metabolism and endothelial dysfunction.

\section{1}

The association of serum cystatin $\mathrm{C}$ with metabolic syndrome and its related components

Y. Kang, S. Kim, S. Kim, S. Lee, S. Son, I. Kim, Y. Kim

Internal Medicine, Pusan National University School of Medicine, Busan, Republic of Korea

Background and aims: Serum cystatin $\mathrm{C}$ has been proposed as a better marker than serum creatinine for estimation of kidney function and associated with cardiovascular disease (CVD), especially in the elderly. The aim of this cross-sectional study was to investigate the association of serum cystatin $\mathrm{C}$ with metabolic syndrome (MS), a gathering of cardiovascular risk factors, and its related components.

Materials and methods: Total 1,468 adults (20-70 years of age, 813 men and 655 women) who visited Center for Health Promotion in Pusan National University Hospital for routine checkup were studied. We checked metabolic parameters and biochemical markers including serum cystatin C, hs-CRP, gamma glutamyltransferase, and uric acid. We defined metabolic syndrome using modified, revised ATP III criteria.

Results: Serum cystatin C was more increased in old ages ( $\geq 50$ years, $0.91 \pm 0.18 \mathrm{mg} / \mathrm{L}$ vs. $<50$ years, $0.83 \pm 0.14 \mathrm{mg} / \mathrm{L}$ ). After adjustment for age and sex, cystatin $\mathrm{C}$ was positively correlated with waist circumference $(\mathrm{r}=0.222$, $\mathrm{p}<0.001)$, BMI $(\mathrm{r}=0.115, \mathrm{p}<0.001)$, SBP $(\mathrm{r}=0.110, \mathrm{p}<0.001)$, DBP $(\mathrm{r}=0.273$, $\mathrm{p}<0.001)$, and TG $(\mathrm{r}=0.166, \mathrm{p}<0.001)$, GGT $(\mathrm{R}=0.198, \mathrm{P}<0.001)$, hs-CRP $(\mathrm{r}=0.101, \mathrm{p}<0.001)$, and uric acid $(\mathrm{r}=0.377, \mathrm{p}<0.001)$ and inversely related with HDL cholesterol $(\mathrm{r}=-0.181, \mathrm{p}<0.001)$. Age, waist circumference, DBP, and HDL cholesterol were major independent determinant of cystatin $\mathrm{C}$ in multivariate regression analyses. Serum cystatin $\mathrm{C}$ was increased according to the increase of the number of metabolic syndrome component disorders and significantly increased in subjects with metabolic syndrome (MS, $0.90 \pm 0.19$ $\mathrm{mg} / \mathrm{L}$ vs. non-MS, $0.86 \pm 0.16 \mathrm{mg} / \mathrm{L}$ ).

Conclusion: These findings raise the possibility that increased serum cystatin $\mathrm{C}$ level might be associated CVD risk factors and inflammatory markers and would be a useful tool for cardiovascular assessment.

\section{2}

\section{Adverse cardiometabolic profiles amongst construction workers in \\ Ireland \\ S. Shah, H. Thabit, N. Burns, J. Nolan, RISC Investigators \\ Metabolic Research Unit, St James Hospital, Dublin, Ireland}

Background: Previous epidemiological studies have shown that construction workers are at increased risk for a range of chronic diseases.

Methods: The cardiometabolic risk factors of 433 male Irish construction workers (CW) were compared to 672 age- and sex-matched healthy non-diabetic European (HS) subjects from the EGIR-RISC study. Anthropometric data were collected as well as fasting blood samples for glucose, and lipid profiles. Questionnaires pertaining to physical activity were also collected from the CW. The baseline characteristics of the two cohorts are outlined in Table 1.

Results: The prevalence of the Metabolic Syndrome (MS) by the International Diabetes Federation (IDF) criteria in the CW was twice that of the HS (28.2\% vs $14.2 \%, \mathrm{p}=<0.0005)$. The CW had more adverse components of the MS compared to HS. The CW were significantly more overweight with central obesity. They had significantly higher LDL cholesterol, systolic and diastolic blood pressure. Those with abnormal plasma glucose (fasting plasma glucose $\geq 5.6 \mathrm{mmol} / \mathrm{l})$ in both groups, $(\mathrm{CW}=61$, HS $=133)$ were of similar age ( 46.6 vs 45.8 years, $\mathrm{p}=0.58$ ). However when compared to HS, CW still had a higher BMI (29.1 vs $27.8 \mathrm{~kg} / \mathrm{m} 2, \mathrm{p}=0.021)$, waist circumference (102.7 vs $97.7 \mathrm{~cm}, \mathrm{p}=0.002)$ systolic $\mathrm{BP}(140 \mathrm{vs} 127 \mathrm{~mm} / \mathrm{Hg}, \mathrm{p}<0.0005)$ and diastolic $\mathrm{BP}(86.5$ vs $78.5 \mathrm{~mm} / \mathrm{Hg}, \mathrm{p}<0.0005)$.

Conclusion: In the CW cohort, there were no differences in the BMI or metabolic profile in those who reported being physically active at work and those who reported a sedentary work pattern. However, those who reported exercising 3 or more times a week had significantly lower BMI (26.6 vs 27.8 $\mathrm{kg} / \mathrm{m} 2, \mathrm{p}=0.006$ ), waist circumference ( 95.4 vs $98 \mathrm{~cm}, \mathrm{p}=0.037$ ) and triglycerides ( 1.1 vs $1.4 \mathrm{mmol} / \mathrm{l}, \mathrm{p}=0.02$ ) than those who exercised at most once a week.
Thus, despite a physically demanding occupation, CW demonstrate higher cardiometabolic risk, probably as a result of lifestyle and other factors outside the workplace.

Table1. Data are expressed as mean (SEM). P value $<0.05$ are significant

\begin{tabular}{llll}
\hline & CW & HS & p value \\
\hline Age $($ years $)$ & $42.5(0.46)$ & $43.4(0.33)$ & 0.13 \\
\hline Weight $(\mathrm{kg})$ & $86.4(0.55)$ & $84.4(0.49)$ & 0.006 \\
\hline BMI $(\mathrm{kg} / \mathrm{m} 2)$ & $27.8(0.16)$ & $26.5(0.13)$ & $<0.0005$ \\
\hline Waist $(\mathrm{cm})$ & $99.4(0.50)$ & $93.8(0.4)$ & $<0.0005$ \\
\hline Waist:hip ratio & $1.0(0.01)$ & $0.9(0.003)$ & $<0.0005$ \\
\hline Systolic BP $(\mathrm{mm} / \mathrm{Hg})$ & $135.1(0.71)$ & $123.2(0.44)$ & $<0.0005$ \\
\hline Diastolic BP $(\mathrm{mm} / \mathrm{Hg})$ & $82.5(0.48)$ & $77.1(0.31)$ & $<0.0005$ \\
\hline Cholesterol $(\mathrm{mmol} / \mathrm{l})$ & $5.12(0.04)$ & $5.24(0.03)$ & 0.04 \\
\hline LDL-C $(\mathrm{mmol} / \mathrm{l})$ & $3.27(0.04)$ & $3.08(0.03)$ & $<0.0005$ \\
\hline HDL-C $(\mathrm{mmol} / \mathrm{l})$ & $1.23(0.01)$ & $1.24(0.01)$ & 0.8 \\
\hline Triglycerides $(\mathrm{mmol} / \mathrm{l})$ & $1.36(0.05)$ & $1.34(0.03)$ & 0.67 \\
\hline
\end{tabular}

Supported by: EU; additional support has been provided by AstraZeneca. The EGIR group activities are supported by Merck Serono, France

\section{3}

Validity of Indian anthropometric reference limits versus standard definitions in determining the metabolic syndrome in young South Indians

S. Vasan ${ }^{1}$, S. Christopher ${ }^{2}$, N. Thomas ${ }^{1}$, S.B. Carani ${ }^{3}$

${ }^{1}$ Endocrinology,Diabetes \& Metabolism, Christian Medical College, Vellore, India, ${ }^{2}$ Biostatistics, Christian Medical College, Vellore, India, ${ }^{3}$ Molecular Medicine and Surgery, Karolinska Instituet, Stockholm, Sweden

Background and aims: The prevalence of the metabolic syndrome has never been studied using Asian Indian reference intervals in young Indians. The purpose of this study is to validate standard definitions for metabolic syndrome in young Indians using Indian reference limits and to determine the prevalence rates.

Materials and methods: This was a prospective cross sectional study which involved screening for Metabolic syndrome indices in young postpubertal adults $(\mathrm{n}=1378)$ between the age of $12-28$ (Mean age 17.1 \pm 2.4 ) years in a suburban Indian region with a population of 400,000. Informed consent was obtained from all participants. Anthropometric measurements included height, weight, waist circumference, Waist-Hip ratio (WHR), Skin fold thickness in the subscapular, triceps and abdominal regions. Blood was collected for fasting lipid profile and blood sugar was measured at fasting and 2 hours postprandial intervals. The prevalence was estimated by incorporating standard Asian reference limits for anthropometry (Waist circumference - 85 Men / 80 Women, Waist-Hip ratio -0.88 Men / 0.81 Women and BMI cut off $>23$ $\mathrm{kg} / \mathrm{m} 2)$ in standard definitions for metabolic syndrome using World Health Organisation (WHO), Adult Treatment Panel III (ATPIII) and International Diabetes Federation (IDF) criteria.

Results: The overall prevalence of the Metabolic syndrome in this group of young Asian Indians was -112 (8.1\%) [Men -74(7.3\%), women -38 (10.4\%)] as defined by any criteria using Asian reference limits for anthropometry, while, the prevalence rate is $5.3 \%$ using standard definitions. The prevalence rates were significantly higher with inclusion of modified reference limits of waist circumference and BMI in place of the existing reference limits of waist circumference in the NCEP ATPIII (5.7\%), WHO (6.5\%) or IDF(4.6\%) criteria. Age-specific group analysis has shown higher prevalence among age groups $14-17$ years (50.8\%) as compared to other groups $18-21(43.5 \%)$ and $22-25$ years $(5.7 \%)$. There were significant gender differences in the mean values of several components of the metabolic syndrome like blood pressure $(\mathrm{p}=<0.001)$, HDL cholesterol $(\mathrm{p}=<0.001)$, Fasting glucose $(\mathrm{p}=<0.001)$ and triglycerides $(p=0.002)$.The $95^{\text {th }}$ centile for Skin fold thickness at three regions correlated significantly with body mass index (Subscapular $\rho=0.51$, Triceps $\rho=0.39$ and Biceps $\rho=0.45$ ). Impaired fasting glucose (IFG) was present in $27 \%$ of males and $40 \%$ of females and impaired glucose tolerance was seen in $3.2 \%$ of males and $2.7 \%$ of females. Dyslipidaemia was evidenced by elevated triglycerides ( $9.3 \%$ in males \& $5.7 \%$ in females) and low HDL cholesterol (62.2\% in males \& $65.4 \%$ in females) in this post pubertal age group and was diagnosed based on the $95^{\text {th }}$ percentile adjusted for age and sex. 
Conclusion: Our data has shown a high prevalence of the metabolic syndrome among young South Indians which is a potential early onset risk factor for macrovascular disease. Skin fold thickness, low HDL cholesterol levels and impaired fasting glycaemia are the most important risk factors that seemed to occur in this age group.

Indian anthropometric reference limits in standard definitions improve the sensitivity of defining metabolic syndrome in such a setting, when describing an Indian population.

\section{4}

Resting energy expenditure in overweight-obese non diabetic patients with metabolic syndrome

G. Seghieri ${ }^{1}$, R. Anichini ${ }^{1}$, A. De Bellis ${ }^{1}$, G. Fabbri ${ }^{1}$, R. Malagoli ${ }^{1}$, L. Alviggi ${ }^{1}$, F. Franconi ${ }^{2}$

${ }^{1}$ Internal Medicine, Spedali Riuniti, Pistoia, Italy, ${ }^{2}$ Pharmacology, University of Sassari, Italy

Background and aims: Increase in resting energy expenditure (REE) has been shown significantly associated with obesity, while insulin resistance has been found related with the decrease in REE. In addition diabetes and hypertension have been associated with higher REE levels, whereas conflicting results exist about REE in subjects with altered glucose metabolism or dyslipidemia. As a consequence of this, there are no clear-cut data about the relation, if any, between metabolic syndrome (MS) and REE.

Materials and methods: To study this aspect we evaluated $334(118 \mathrm{M} / 216 \mathrm{~F})$ non diabetic overweight-obese subjects $\left(\mathrm{BMI} \geq 25 \mathrm{Kg} / \mathrm{m}^{2}\right)$, who consecutively attended the Obesity Outpatient Clinic of our hospital to obtain dietetic advice. In all patients, divided into two groups [those with MS (MS+, n=173) and those without it (MS-, $\mathrm{n}=161$ ) according to ATP-III criteria], we measured body weight composition and REE by electrical bioimpedance, validated in a consistent sample by indirect calorimetry.

Results: Mean REE, expressed as $\mathrm{kJ} / \mathrm{d} / \mathrm{Kg}$ fat-free mass (FFM) $\pm \mathrm{SD}$ was similar in MS+ and in MS- patients (116.1 $\pm 21.4 v s 115.7 \pm 21.8 \mathrm{~kJ} / \mathrm{d} / \mathrm{Kg} ; \mathrm{p}=\mathrm{NS})$. The relative MS risk adjusted for age, sex, fat mass, and waist girth, expressed as odds ratio (OR; 95\% CI) ), was not significantly related with increase in each 1 REE SD [OR:1.167 (0.913-1.525)], while single MS components behaved differently. For impaired fasting glucose, as well as for dyslipidaemia the adjusted ORs were not significantly associated with REE, while, on the contrary, each increase in 1 REE SD $(21.628 \mathrm{~kJ} / \mathrm{d} / \mathrm{Kg}$ FFM $)$ augmented the risk of arterial hypertension of $\sim$ the 50\%: [OR:1.458; (1.100-1.995)]. Likewise, after adjusting for age, sex, waist circumference and fat mass, fasting plasma glucose was unrelated with REE, whereas mean blood pressure was significantly and independently related to REE $(\mathrm{p}=0.011)$.

Conclusion: In conclusion in overweight-obese non diabetic patients MS is unrelated to changes in REE, while, examining any single MS component, rise in REE seems to be significantly related to blood pressure, and, at the same time, to be an independent predictor of arterial hypertension in these patients.

Supported by: Fondazione Cassa di Risparmio di Pistoia e Pescia

\section{5}

Prevalence, treatment and control of cardiometabolic risk factors in subjects with high waist circumference - $5 \mathrm{~A}$ study

J.C.N. Chan 1 , C.A. Aguilar-Salinas ${ }^{2}$, A. Avezum ${ }^{3}$, E. Bruckert ${ }^{4}$, A. Misra ${ }^{5}$, I. Raz ${ }^{6}$, A.M. Sharma ${ }^{7}$, M.-T. Van der Merwe ${ }^{8}$

${ }^{1}$ Department of Medicine and Therapeutics, The Chinese University of Hong Kong, Shatin, Hong Kong, ${ }^{2}$ Department of Endocrinology and Metabolism, INCMNSZ, Mexico City, Mexico, ${ }^{3}$ Research Division, Dante Pazzanese Institute of Cardiology, Sao Paulo, Brazil, ${ }^{4}$ Departement d'Endocrinologie, Hopital Pitie Salpetriere, Paris, France, ${ }^{5}$ Department of Diabetes and Metabolism, Fortis Group of Hospital, New Delhi, India, ${ }^{6}$ Hadassah Diabetes Center, Hadassah University Hospital, Jerusalem, Israel, ${ }^{7}$ Obesity Research \& Management, University of Alberta, Edmonton, Canada, ${ }^{8}$ Unitas Hospital, Pretoria, South Africa

Background and aims: In this pandemic of overweight and obesity, there is an urgent need to document the pattern of medical care in these high risk subjects although data in this regard is generally scarce especially in developing countries. The aim of the $5 \mathrm{~A}$ Study is to assess the prevalence and control of cardiometabolic risk factors (CMRF) in a cohort of subjects with high waist circumference (WC) of $90 \mathrm{~cm}$ (35.5 inches) or more for males and 80 $\mathrm{cm}$ (31.5 inches) or more for females.

Materials and methods: Data on diagnosis and treatment of CMRF (dyslipidemia [DL], type 2 diabetes [T2D], hypertension [HT] and cardiovascular diseases [CVD]) were collected from 6291 adults in 8 countries by 3 physician specialties (17.3\% GP, 52.7\% cardiologist and 30.1\% Diabetologist). For each CMRF, we assessed its prevalence (as diagnosed by physician and/or abnormal clinical/laboratory value), the percentage diagnosed by physician, the percentage of subjects treated, and the percentage of subjects reaching internationally recommended targets $\left(\mathrm{HbA}_{1 c}<7 \%, \mathrm{BP}<130 / 80 \mathrm{mmHg}\right.$, triglyceride (TG) $<1.7 \mathrm{mmol} / \mathrm{L}, \mathrm{LDL}-\mathrm{C}<2.6 \mathrm{mmol} / \mathrm{L}, \mathrm{HDL}-\mathrm{C}>1 \mathrm{mmol} / \mathrm{L}$ ).

Results: We analyzed subjects with DL (high LDL-C and/or TG and/or low HDL-C; $n=4162 ., 66.2 \%)$, T2D ( $n=1895,30.1 \%)$, HT $(n=4128,65.6 \%)$ and $\operatorname{CVD}(\mathrm{n}=1566,24.9 \%)$. The management of each CMRF is shown in the table below.

Overall, in subjects with 1 CMRF (excluding CVD), 8.3\% were at target; in those with 2 CMRF, $0.9 \%$ were at target for both parameters; and for subjects with 3 CMRF, $1.3 \%$ were at target for 3 parameters. In subjects with CVD: percentages of patients controlled with DL or T2D or HT were $42.8 \%$ $(\mathrm{N}=328), 32.8 \%(\mathrm{~N}=115)$ and $10.7 \%(\mathrm{~N}=58)$ respectively.

Conclusion: In subjects with high WC, there were high prevalences of CMRF clustering which were often not diagnosed, treated or controlled, even in the presence of clinical CVD.

$5 \mathrm{~A}$ study $=$ Awareness of Abdominal Adiposity As A marker of cardiometabolic risk study

\begin{tabular}{|c|c|c|c|c|}
\hline & Type 2 Diabetes & Dyslipidemia & Hypertension & CVD \\
\hline Prevalence & $\begin{array}{c}30.1 \% \\
(\mathrm{~N}=1895 / 6291)\end{array}$ & $\begin{array}{c}66.2 \% \\
(\mathrm{~N}=4162 / 6291)\end{array}$ & $\begin{array}{c}65.6 \% \\
(\mathrm{~N}=4128 / 6291)\end{array}$ & $\begin{array}{c}24.9 \% \\
(\mathrm{~N}=1566 / 6291)\end{array}$ \\
\hline Diagnosed & $\begin{array}{c}31.0 \% \\
\left(\mathrm{~N}=1895 / 6109^{*}\right)\end{array}$ & $\begin{array}{c}51.4 \% \\
(\mathrm{~N}=3233 / 6291)\end{array}$ & $\begin{array}{c}54.6 \% \\
(\mathrm{~N}=3434 / 6291)\end{array}$ & $\begin{array}{c}24.9 \% \\
\left(\mathrm{~N}=1566 / 6288^{*}\right)\end{array}$ \\
\hline Treated & $92.8 \%(\mathrm{~N}=1755)$ & $57.7 \%(\mathrm{~N}=2366)$ & $80.5 \%(\mathrm{~N}=3322)$ & $74.6 \%(\mathrm{~N}=1168)$ \\
\hline At target /controlled & $34.5 \%(\mathrm{~N}=409)$ & $34.2 \%(\mathrm{~N}=618)$ & $11.8 \%(\mathrm{~N}=1404)$ & \multicolumn{1}{|c|}{} \\
& & &
\end{tabular}

Supported by: sanofi-aventis 


\section{6}

Low-grade inflammation mediates the association between the metabolic syndrome and the ankle-arm index (The CODAM Study)

M. Jacobs $\mathbf{s}^{1,2}$, M.M.J. van Greevenbroek ${ }^{1,2}$, C.J.H. van der Kallen ${ }^{1,2}$,

I. Ferreira ${ }^{2,3}$, E.E. Blaak ${ }^{4,5}$, E.J.M. Feskens ${ }^{6}$, C.G. Schalkwijk ${ }^{1,2}$,

C.D.A. Stehouwer ${ }^{1,2}$

${ }^{1}$ Cardiovascular Research Institute Maastricht (CARIM), Maastricht

University, Netherlands, ${ }^{2}$ Department of Internal Medicine, University

Hospital Maastricht, Netherlands, ${ }^{3}$ Department of Clinical Epidemiology

and Medical Technology Assessment (KEMTA), University Hospital

Maastricht, Netherlands, ${ }^{4}$ Department of Human Biology, Maastricht

University, Netherlands, ${ }^{5}$ Nutrition and Toxicology Research Institute

Maastricht (NUTRIM), Netherlands, ${ }^{6}$ Division of Human Nutrition,

Wageningen University, Wageningen, Netherlands

Background: The Metabolic Syndrome (MetS) is associated with CHD and this association may be related to low-grade inflammation. The metabolic syndrome may also be related to peripheral arterial dysfunction although this relation has not been described extensively. Low-grade inflammation has been implicated in peripheral arterial dysfunction as well.

Aims: To determine associations between the metabolic syndrome and ankle-arm index (AAIx; an available and reproducible method for the detection of peripheral arterial dysfunction) and to address the role of low-grade inflammation therein.

Materials and methods: The study population consisted of 574 subjects (age: $59.6 \pm 7.0$ years, $61.3 \%$ men, $54.7 \%$ MetS; NCEP-ATP 2005 criteria) from the Cohort Study Diabetes and Atherosclerosis (CODAM) cohort, who were invited from a large population based cohort on basis of an elevated risk for type 2 diabetes and cardiovascular diseases. The AAIx was calculated by dividing the highest of the ankle pressures (a. dorsalis pedis or a. tibialis) in either leg by the highest pressure at the brachial artery. The following inflammatory markers were measured in fasting plasma: C-reactive protein (CRP), IL6, soluble vascular cell adhesion molecule, soluble intercellular adhesion molecule (sICAM) and serum amyloid A. For each inflammation marker the Z-score was calculated and an inflammation score was then calculated as the average of the Z-scores of the five inflammation markers listed above. Multiple linear regression analyses were used to determine associations between the metabolic syndrome and low-grade inflammation on the one hand, and the AAIx on the other.

Results: The metabolic syndrome was associated with the AAIx (Beta $[\beta]=$ $-0.02,95 \% \mathrm{CI}:-0.04 ;-0.002, \mathrm{P}=0.03$ ). Adjustment for age, sex and smoking did not change this association. Inflammation markers that were associated with the AAIx were CRP ( $\beta=-0.04,95 \%$ CI: $-0.06 ;-0.01, P=0.002)$, IL6 ( $\beta=$ $-0.05,95 \%$ CI: $-0.08 ;-0.02, \mathrm{P}=0.002)$, and sICAM $(\beta=-0.10,95 \%$ CI: -0.17 ; $-0.02, \mathrm{P}=0.017)$, all adjusted for age, sex and smoking. When both the metabolic syndrome and the inflammation score were included in the linear regression model, the metabolic syndrome was no longer associated with AAIx, but the association between inflammation and the AAIx remained $\left(\beta_{\mathrm{Mets}}=\right.$ $-0.01,95 \% \mathrm{CI}:-0.03 ; 0.01 \mathrm{P}=0.19 ; \beta_{\text {in }}$ 0.002 , adjusted for age, sex, smoking).

Conclusion: Subjects with the metabolic syndrome show greater levels of peripheral arterial dysfunction (as expressed by AAIx). Importantly, chronic low-grade inflammation mediated, in a great deal, that association, and was independently associated with AAIx.

Supported by: grants of the Netherlands Organisation for Scientific Research and the Dutch Diabetes Research Foundation

\section{PS 016 Glycated haemoglobin and diabetes management}

\section{7}

The heritability of $\mathrm{HbA}_{1 \mathrm{c}}$ and fasting glucose is caused by different genetic factors

A.M.C. Simonis-Bik ${ }^{1}$, E.M.W. Eekhoff ${ }^{1}$, R.J. Heine ${ }^{1}$, E.J.C. de Geus ${ }^{2}$, M. Diamant ${ }^{1}$, J.M. Dekker ${ }^{3}$, M. van Leeuwen ${ }^{2}$, D.I. Boomsma ${ }^{2}$ ${ }^{1}$ Diabetes Centre, VU University Medical Centre, ${ }^{2}$ Biological Psychology, VU University, ${ }^{3} \mathrm{E} M G O$ institute, VU University Medical Centre, Amsterdam, Netherlands

Background and aims: The proportion of glycated haemoglobin, HbAlc, reflects the mean blood glucose level of the preceding 8-10 weeks. Although the relation between $\mathrm{HbAlc}$ and complications, including nephropathy and retinopathy, is established, the genetic contribution to variation in $\mathrm{HbAlc}$ has not been examined in detail. Specifically, it is unclear whether the genetic factors that influence the variability of HbAlc are the same as those that influence fasting blood glucose.

Materials and methods: We included 77 families of the Nederland's Twin Register in an extended twin study of different aspects of beta-cell function. All participants were healthy, age 20-45 years, with siblings of the same sex. Fasting blood glucose was measured in three different settings: at home before an OGTT, and at 2 clinic visits (2-3 samples/visit). HbAlc was measured in one of the clinic samples.

Results: The present analysis is based on 180 persons, consisting of 51 monozygotic pairs ( 21 male), 21 dizygotic pairs ( 8 male), 5 incomplete twin pairs ( 3 male) and 31 siblings ( 15 male). The mean age was $30.6 \pm 4.6$ years, mean fasting blood glucose at home $4.63 \pm 0.46 \mathrm{mmol} / \mathrm{l}$, and at the 2 clinic visits $4.37 \pm 0.40$ and $4.44 \pm 0.35 \mathrm{mmol} / \mathrm{l}$ respectively. Men had a significant higher mean fasting blood glucose level than women: 4.63 vs 4.40 respectively. and a higher HbAlc (men $5.30 \pm 0.26 \%$, women $5.21 \pm 0.22 \%$, p < 0.001 ). Raw data were used in a 4 -variate structural equation model in the $\mathrm{mx}$ software package. This model incorporated sex and age differences and estimated heritability of each parameter as well as their genetic correlations. Additive genetic effects (heritability) explained $75 \%$ of the population variance in $\mathrm{HbAlc}$, the remainder was attributable to unique environment. The heritability of fasting blood glucose was prominent in all settings (66\% in the OGTT, and $57 \%$ and $40 \%$ in the clinic visits samples respectively). There was a significant correlation between the fasting blood glucose levels in all three settings (r $0.34-0.55)$ which was mostly due to a common genetic factor influencing individual differences in glucose level in all three settings. The correlation between $\mathrm{HbAlc}$ and fasting blood glucoses was low ( $\mathrm{r} 0.11$ - 0.23) and the genetic factors influencing $\mathrm{HbAlc}$ and fasting glucose were non-overlapping.

Conclusion: The present results suggest a high heritability of $\mathrm{HbAlc}(75 \%)$ and a consistent substantial heritability for fasting glucose levels in various settings (40 - 63\%) in healthy, normoglycaemic adults. Genetic factors influencing $\mathrm{HbAlc}$ and fasting blood glucose levels were independent, suggesting that heritability of $\mathrm{HbAlc}$ largely reflects genes responsible for variability in the glycation rate of haemoglobin, independent of glycaemia.

Supported by: Dutch Diabetes Research Foundation

\section{8}

Can $\mathrm{HbA}_{1 \mathrm{c}}$ replace glucose as a diagnostic test for diabetes? D. Vistisen, R. Borg, K. Borch-Johnsen Steno Diabetes Center, Gentofte, Denmark

Background and aims: Diabetes is a condition defined by hyperglycaemia giving specific microvascular complications and increased risk of macrovascular complications. Since 1980 the WHO has defined diabetes on the basis of the oral glucose tolerance test (OGTT), but this test is time consuming, has low reproducibility, and is based on glucose-measurements sensitive to handling and transportation. Glycosylated haemoglobin (HbAlc) can be measured non-fasting, is easily and reliably measured and relatively cheap. With the recent world-wide standardisation of $\mathrm{HbAlc}$ measurements to the IFCC reference method a global standardisation is possible. This reopens the question whether $\mathrm{HbAlc}$ can replace glucose as a diagnostic test for diabetes. The aim of this project was to evaluate the implications of using $\mathrm{HbAlc}$ as a diagnostic test for Type 2 diabetes in a Danish population. 
Materials and methods: This analysis is based on the population based Inter99 study (age range 30-60 years, $\mathrm{N}=6,258$ ), excluding individuals with known diabetes or incomplete data on fasting (FPG), 2-hour plasma glucose (2hPG) or HbAlc (HCLC BioRad variant 1). Micro- and macroalbuminuria were defined as a urine albumin concentration $\geq 30 \mathrm{mg} / \mathrm{l}$ and $>300 \mathrm{mg} / \mathrm{l}$ respectively. According to the WHO 1999 classification, 259 (4.1\%) had undiagnosed diabetes and 1,271 (20.3\%) had impaired glucose metabolism (IGM). Consequently, we defined diabetes by $\mathrm{HbA1c}$ as the 259 individuals with the highest $\mathrm{HbAlc}$ levels and IGM by HbAlc as the next 1,271 individuals to retain unchanged prevalence of DM and IGM. The two definitions were compared based on the micro- and macrovascular risk profile. Furthermore, the 10 year risk of developing ischaemic heart disease (IHD) was compared using the PRECARD program. ROC analysis was used to assess the ability of FPG, 2HPG and HbA1c to distinguish between individuals in high- and low risk of developing IHD.

Results: Individuals defined by $\mathrm{HbAlc}$ were older, had male predominance, higher levels of smokers, total- and HDL cholesterol levels. Furthermore they were leaner and had lower BP levels. The prevalence of micro- or macroalbuminuria and retinopathy is higher.

\begin{tabular}{|c|c|c|c|c|}
\hline & \multicolumn{2}{|c|}{ DM } & \multicolumn{2}{|c|}{ IGM } \\
\hline & OGTT & HbAIC & OGTT & HbA1E \\
\hline \multicolumn{5}{|l|}{ Macrovascular risk profile } \\
\hline Age (years) & $50.7(6.9)$ & $51.5(6.2)$ & $48.5(7.4)$ & $48.9(7.3)$ \\
\hline Males (\%) & $63.3(57.1-69.2)$ & $64.9(58.7-70.7)$ & $58.9(56.2-61.7)$ & $60.6(57.8-63.3)$ \\
\hline BMI $(\mathrm{kg} / \mathrm{m} \mathrm{2})$ & $30.2(5.8)$ & $29.2(5.5)$ & $28.1(4.9)$ & $27.0(5.0)$ \\
\hline Waist (cm) & $98.6(14.2)$ & $96.3(14.9)$ & $92.3(13.2)$ & $89.7(13.3)$ \\
\hline BP Diastolic $(\mathrm{mmHg})$ & $90.7(11.7)$ & 87.4 (12.5) & $87.0(11.6)$ & $83.7(11.1)$ \\
\hline BP Systolic (mmHg) & $146.5(20.2)$ & $139.7(20.7)$ & 137.7 (17.9) & $132.3(17.7)$ \\
\hline Total Cholesteral $(\mathrm{mma} / \mathrm{h})$ & $5.5(1.0)$ & $5.6(1.1)$ & $5.4(1.0)$ & $5.4(1.0)$ \\
\hline HDL-cholesterol (mmol/) & $1.3(0.4)$ & $1.2(0.4)$ & $1.4(0.4)$ & $1.4(0.4)$ \\
\hline Triglycerides $(\mathrm{mma} /)$ & $2.2(1.6)$ & $1.9(1.3)$ & $1.7(2.1)$ & $1.4(1.0)$ \\
\hline Antihypertensive treatment (\%) & $20.5(15.7-25.9)$ & $15.4(11.3-20.4)$ & $11.5(9.8-13.4)$ & $7.1(5.7-8.6)$ \\
\hline Smoking (\%) & $35.9(30.1-42.1)$ & $57.9(51.6-64)$ & $35.3(32.7-38.0)$ & $49.3(46.5-52.0)$ \\
\hline Fam ily disposition to DM (\%) & $27.4(22.1-33.3)$ & $25.1(19.9-30.8)$ & $21.4(19.2-23.8)$ & $20.1(18.0-22.5)$ \\
\hline \multicolumn{5}{|l|}{ Mierovascular risk profile } \\
\hline Microalbuminuria (\%) & $8.5(5.4-12.6)$ & $9.7(6.3-13.9)$ & $3.9(2.9-5.2)$ & $3.2(2.3-4.4)$ \\
\hline Macroalbuminuria (\%) & $0.4(0.0-2.1)$ & $1.2(0.2-3.3)$ & $0.6(0.3-1.2)$ & $0.3(0.1-0.8)$ \\
\hline Retinopathy $(\%)^{*}$ & $2.4(0.6-5.9)$ & $3.1(0.6-8.7)$ & $2.5(1.0-5.2)$ & $2.7(0.9-6.1)$ \\
\hline
\end{tabular}

Maans (SD) and proportions $(95 \%-\mathrm{Cl})$. . Retinopathy assessed in a sub-sample of 970 individuals.

$\mathrm{HbAlc}$ distinguishes between individuals in high- and low risk of developing IHD over a 10 year period better than FPG and 2hPG.
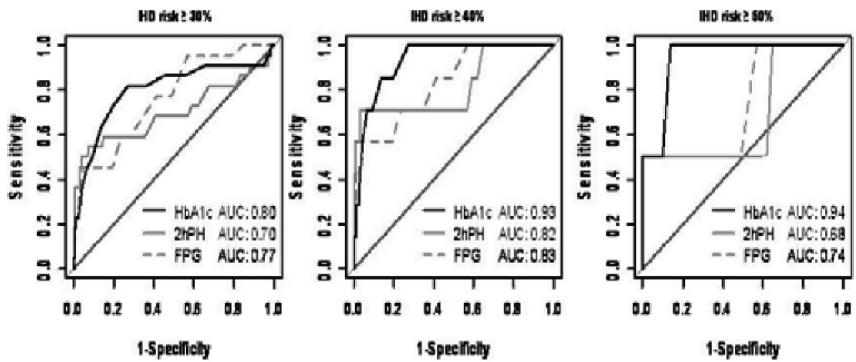

Figure 1. ROC curves for identifying individuals at high rist for developing IHD
Conclusion: Individuals with diabetes defined by HbAlc have a worse microvascular risk profile compared with those identified by the glucose based on the WHO definition. The macrovascular risk profile is not as conclusive. $\mathrm{HbAlc}$ is better at distinguishing between individuals in high- and low risk of developing IHD over a 10 year period compared with FPG and 2hPG. Supported by: Danish Medical Research Council, Danish Centre for Evaluation and Health Technology Assessment, Novo Nordisk, Copenhagen County, Danish Heart Foundation, Danish Diabetes Association, Danish Pharmaceutical Association

\section{9}

Reference values for deoxyfructosyl haemoglobin (beta) in healthy paediatric and adult population

A. Gerasimidi-Vaseou ${ }^{1}$, C. Maragou ${ }^{1}$, T. Bachou ${ }^{1}$, D. Papadatos ${ }^{1}$,

L. Spanou ${ }^{2}$, I. Drakopoulos 2 , L. Stamoyannou ${ }^{1}$, K. Makris ${ }^{2}$

${ }^{1}$ A Department of Pediatrics, P\&A Kyriakou Childrens Hospital, ${ }^{2}$ Clinical

Biochemistry, KAT General Hospital, Athens, Greece

Background and aims: There are at least 30 different laboratory assays commercially available to measure the proportion of $\mathrm{HbAlc}$ in blood all producing different results. Harmonization of the HbAlc reported results from different laboratory assays until now was achieved through national initiatives like the National Glycohemoglobin Standardisation Program (NGSP). Based on the work of NGSP the current HbAlc assay has been harmonised on reference methods that measure a mixture of glycated haemoglobins. The International Federation of Clinical Chemistry (IFCC) has recently approved a reference measurement procedure for $\mathrm{HbAlc}$ that specifically measures the concentration of only one molecular species of HbAlc. A new name, deoxyfrucosyl haemoglobin (beta) (DOFH), and measurement units, $\mathrm{mmol} / \mathrm{mol}$, were proposed to replace $\mathrm{HbAlc}$ and $\%$. DOFH represents the fraction of the b-chains of hemoglobin that has a stable hexose adduct on the N-terminal amino acid valine. This study examines the impact of these changes on laboratory result reporting and clinical practise by trying to establish reference ranges for deoxyfrucosyl haemoglobin (beta) for pediatric and adult population and compare it to the traditional $\mathrm{HbAlc}$ measurement.

Materials and methods: Blood samples were collected from 106 healthy non-diabetic children (59 boys, 47 girls) and 264 adults (112 men, 151 women). DOFH was measured in addition to HbAlc using an HPLC (HA8160 Menarini-Arkray) which is calibrated and traceable to the IFCC reference method. Our population was divided in 11 age groups: infants(<2years), pre-school age children (2-6years), school age children (6-11years), adolescents(11-16) and 7 adult groups. All adults had normal glucose tolerance in a standard 0-2hrs OGTT.

Results: In the paediatric population (table) DOFH values were not significantly different among the five age groups and between boys and girls (30.4 vs $32.79 \mathrm{mmol} / \mathrm{mol}$ ). In the adult population (table) the differences observed in the mean DOFH were statistically significant. Mean DOFH seems to increase with age. There was a positive correlation between age and DOFH in the adult population $(\mathrm{r}=0.351, \mathrm{p}<0,0001)$. No differences were observed between men and women ( $35.1 \mathrm{vs} 34,4 \mathrm{mmol} / \mathrm{mol})$

Conclusion: A single reference range can be used for the entire pediatric population $(25.7-39.7 \mathrm{mmol} / \mathrm{mol})$. Age related reference values should be used in the adult population

\begin{tabular}{lllllllll}
\hline $\begin{array}{l}\text { age group } \\
\text { (years) }\end{array}$ & $\mathrm{n}$ & $\begin{array}{l}\text { mean } \\
\text { age }\end{array}$ & $\begin{array}{l}\text { DOFH (mmol/ } \\
\text { mol) }- \text { mean(SD) }\end{array}$ & 2.5percentile & 97.5percentile & $\begin{array}{l}\text { HbA1c(\%)- } \\
\text { mean(SD) }\end{array}$ & 2.5percentile & 97.5percentile \\
\hline$<2$ & 25 & 1.0 & $33.9(2.9)$ & 30.1 & 39.7 & $5.3(0.3)$ & 4.9 & 5.8 \\
\hline $2-5$ & 23 & 3.4 & $32.2(2.4)$ & 28.0 & 36.6 & $5.1(0.2)$ & 4.7 & 5.5 \\
\hline $6-11$ & 36 & 8.8 & $34.3(3.1)$ & 29.0 & 39.2 & $5.3(0.3)$ & 4.8 & 5.7 \\
\hline $12-16$ & 22 & 13.6 & $32.1(4.5)$ & 25.7 & 39.3 & $5.1(0.4)$ & 4.5 & 5.7 \\
\hline $17-30$ & 32 & 23.0 & $30.6(5.2)$ & 22.2 & 40.4 & $5.0(0.5)$ & 4.2 & 5.8 \\
\hline $31-40$ & 25 & 36.2 & $31.9(4.7)$ & 25.7 & 41.4 & $5.1(0.4)$ & 4.5 & 5.9 \\
\hline $41-50$ & 45 & 46.0 & $32.8(5.8)$ & 23.6 & 43.1 & $5.1(0.5)$ & 4.3 & 6.1 \\
\hline $51-60$ & 46 & 55.6 & $34.2(5.7)$ & 24.6 & 43.2 & $5.2(0.5)$ & 4.4 & 6.1 \\
\hline $71-70$ & 49 & 67.0 & $35.5(5.3)$ & 27.0 & 45.1 & $5.4(0.5)$ & 4.6 & 6.3 \\
\hline$>81-80$ & 35 & 74.9 & $36.4(5.7)$ & 25.7 & 44.6 & $5.6(0.5)$ & 4.5 & 6.2 \\
\hline 80 & 32 & 84.7 & $36.0(5.0)$ & 27.9 & 44.3 & $5.4(0.5)$ & 4.7 & 6.2 \\
\hline
\end{tabular}




\section{0}

Diagnostic value of $\mathrm{HbA}_{1 \mathrm{c}}$ in different clinical settings with different prevalence of diabetes mellitus

Y. Ku, Y. Cho, S. Yoo, H. Jung, S. Choi, S. Lim, M. Moon, H. Jang, C. Shin, K. Park, S. Kim, H. Lee

Internal Medicine, Seoul National University College of Medicine, Seoul, Republic of Korea

Background and aims: In general, $\mathrm{HbA}_{1 \mathrm{c}}$ is currently not recommended as a diagnostic test for diabetes. However, its utility as a screening tool of diabetes has recently been revisited. In the 1999-2004 National Health and Nutrition Examination Survey (the prevalence of previously undiagnosed diabetes was $3.4 \%$ ), an $\mathrm{HbA}_{1}$ level of $5.8 \%$ showed the highest combination of sensitivity $(86 \%)$ and specificity (92\%). Since the diagnostic value of a particular test varies depending on the prevalence of the disease of interest, we addressed this issue in two different clinical settings with different prevalence of previously undiagnosed diabetes in Korea.

Materials and methods: We analyzed data from 17,739 subjects for routine health check-ups (9,836 men and 7,903 women, 49.4 $\pm 11.2 \mathrm{yrs})$, who had not been diagnosed with diabetes, at Kangnam Healthcare Center (KHC); and 456 subjects ( 184 men and 267 women, $52.7 \pm 9.5 \mathrm{yrs}$ ), who had not been diagnosed with diabetes but were concerned with diabetes due to various reasons, from Diabetes Clinic of Seoul National University Hospital (SNUH). $\mathrm{HbA}_{1 \mathrm{c}}$ was measured by HPLC (Bio-Rad VARIANT TM II TURBO, CA, USA). Diabetes was diagnosed by fasting plasma glucose in KHC cohort and by oral glucose tolerance test in SNUH cohort.

Results: The receiver operating characteristics curve analysis showed that an $\mathrm{HbA}_{1 \mathrm{c}}$ level of $5.9 \%$ was the best for the diagnosis of diabetes in KHC cohort while an $\mathrm{HbA}_{1 \mathrm{c}}$ level of $6.1 \%$ for $\mathrm{SNUH}$ cohort. The $\mathrm{HbA}_{1 \mathrm{c}}$ level of $5.9 \%$ had a sensitivity of $84.6 \%$ and a specificity of $86.3 \%$ in $\mathrm{KHC}$ cohort, whereas the $\mathrm{HbA}_{1 \mathrm{c}}$ level of $6.1 \%$ had a sensitivity of $87.2 \%$ and a specificity of 82.2 in SNUH cohort. Of interest, there was a marked difference in positive and negative predictive values (PV). In KHC cohort (the prevalence of previously undiagnosed diabetes was $2.8 \%$ ), the $\mathrm{HbA}$ level of $5.9 \%$ had a very low positive PV (14.9\%) but had a very high negative PV $(99.5 \%)$. Therefore, in routine health check-up condition, an $\mathrm{HbA}_{1 c}$ level below $5.9 \%$ could rule out diabetes. However, an $\mathrm{HbA}_{1 \mathrm{c}}$ level $\geq 5.9 \%$ should be re-evaluated with other methods. In SNUH cohort (the prevalence of previously undiagnosed diabetes was $25.9 \%$ ), the $\mathrm{HbA}_{1 \mathrm{c}}$ level of $6.1 \%$ had a moderate positive PV (63.1\%) but had a high negative PV $(94.8 \%)$. Therefore, in the Diabetes Clinic setting, an $\mathrm{HbA}_{1 \mathrm{c}}<6.1 \%$ could also rule out diabetes. However, an $\mathrm{HbA}_{1 \mathrm{c}}$ level $\geq 6.1 \%$ needs to be re-evaluated with other methods, although it might be useful for the diagnosis of diabetes in part.

Diagnostic value of HbA1c (Data are shown as percent (95\% CI))

\begin{tabular}{llll}
\hline & Healthcare center & Diabetes clinic & Diabetes clinic \\
\hline Alc cutoff & 5.9 & 5.9 & 6.1 \\
\hline Sensitivity & 84.6 & 92.3 & 96.5 \\
& $(81.2-87.7)$ & $(86.0-96.4)$ & $(93.5-98.4)$ \\
\hline Specificity & 86.3 & 74.2 & 82.2 \\
& $(85.8-86.8)$ & $(69.2-78.8)$ & $(77.8-86.1)$ \\
\hline Positive predictive & 86.3 & 55.6 & 63.1 \\
value & $(85.8-86.8)$ & $(48.3-62.7)$ & $(55.3-70.6)$ \\
\hline Negative predictive & 99.5 & 96.5 & 94.8 \\
value & $(99.3-99.6)$ & $(93.5-98.4)$ & $(91.6-97.1)$ \\
\hline
\end{tabular}

Conclusion: $\mathrm{HbA}_{1 \mathrm{c}}$ could be used as a screening tool for the diagnosis of diabetes. However, caution should be exercised in its interpretation according to the prevalence of diabetes in the population of interest.

Supported by: MIC \& IITA through IT-Leading R\&D Support Project

\section{1}

Limited agreement between $\mathrm{HbA}_{1 \mathrm{c}}$ and glucose levels in the general Dutch population: The New Hoorn Study

E. van 't Riet, J.M. Rijkelijkhuizen, G. Nijpels, J.M. Dekker

EMGO Institute, VU University Medical Center Amsterdam, Netherlands

Background and aims: Recent research suggests HbAlc can be used to estimate average blood glucose levels in subjects with type 2 diabetes. It may be questioned whether HbAlc could be a good indicator of mean blood glu- cose in people without diabetes. To our knowledge, information on the correlations between $\mathrm{HbAlc}$ and glucose in the general population, including subjects without diabetes, is lacking. Therefore, we investigated the correlations of HbAlc, fasting plasma glucose (FPG) and 2 hour postload plasma glucose (2hrPG) in subjects with normal glucose tolerance (NGT), impaired hyperglycaemia (IH), known diabetes (KDM2) and screen-detected diabetes (NDM2) to investigate whether harmonisation of these markers of hyperglycaemia can be applied in different categories of glucose status.

Materials and methods: For the analysis we used data of 2748 subjects 40-65 years old, randomly selected from the population register of Hoorn, The Netherlands, who underwent an OGTT in 2006/2007 (The New Hoorn Study). To define glucose status we used the WHO '06 criteria. Spearman correlations were used to investigate the correlations between markers of hyperglycaemia. To investigate whether $\mathrm{HbAlc}$ could be used to identify people with diabetes, we excluded subjects with known diabetes $(\mathrm{n}=90)$ and calculated ROC curves for $\mathrm{HbAlc}$ as a predictor of fasting glucose $\geq 7.0$ and/or postload glucose $\geq 11.1$.

Results: In subjects with NGT, IH and newly detected DM2, the correlations between HbAlc and FPG were higher than between HbAlc and 2hrPG, but all correlations were rather low (table 1). In subjects with KDM2, the correlations were higher and reached levels between 0.74 and 0.92 . The optimal cutoff point for identifying diabetes with $\mathrm{HbA1c}$, was a HbAlc level of 5.65, with a sensitivity and specificity of $78.5 \%$ and $83.5 \%$ respectively. Table 1 shows that using this $\mathrm{HbAlc}$ cut-off point for the diagnosis of diabetes compared to using the WHO ' 06 criteria would lead to inclusion of $20 \%$ of the nondiabetic and non-IH subjects and to not identifying $22 \%$ of the cases with diabetic glucose levels.

Conclusion: In subjects with known diabetes correlations are high, which supports the ongoing research into the use of HbAlc to estimate mean glucose in subjects with known diabetes. Using HbAlc as an indicator of mean glucose in subjects with NGT, IH or screen-detected diabetes should be considered with caution based on the low correlations. In addition, using HbAlc levels to identify diabetes in the general population without known diabetes at present is not recommendable. First question to address would be whether a HbAlc of $\geq 5.65$ predicts future diabetes complications in subjects with normal glucose levels.

Table 1. Spearman correlations between markers of hyperglycaemia and numbers of subjects with NGT, IH and DM2 with HbA1c levels below or above 5.65

\begin{tabular}{llllll}
\hline Correlations & \multicolumn{7}{l}{} \\
\hline & Total & NGT & IH & KDM2 & NDM2 \\
\hline $\mathrm{N}(\%)$ & $2748(100)$ & $2114(77)$ & $435(16)$ & $90(3)$ & $109(4)$ \\
\hline HbA1c vs FPG & $0.46^{*}$ & $0.26^{*}$ & $0.25^{*}$ & $0.78^{*}$ & $0.51^{*}$ \\
\hline HbA1c vs 2hrPG & $0.34^{*}$ & $0.14^{*}$ & -0.05 & $0.84^{*}$ & $0.45^{*}$ \\
\hline Cut-off point Hba1c 5.65 & & & & \\
\hline$<5.65(\mathrm{~N}(\%))$ & $2151(81)$ & $1875(89)$ & $253(58)$ & - & $23(22)$ \\
\hline$>=5.65(\mathrm{~N}(\%))$ & $503(19)$ & $237(11)$ & $182(42)$ & - & $84(88)$ \\
\hline$*=\mathrm{p}<0.05$ & & & & \\
\hline
\end{tabular}

Supported by: Novartis Pharma

\section{2}

Treatment approach and $\mathrm{HbA}_{1 \mathrm{c}}$ control among U.S. adults with type 2 diabetes

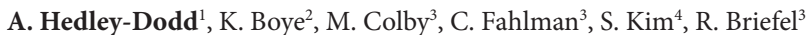
${ }^{1}$ Health Research, Mathematica Policy Research, Washington, ${ }^{2}$ Global Health Outcomes, Eli Lilly and Company, Indianapolis, ${ }^{3} \mathrm{Health}$ Research, Mathematica Policy Research, Inc., Washington, ${ }^{4}$ Stempel School of Public Health, Florida International University, Miami, United States

Background and aims: Long-term blood glucose control can substantially reduce the risk of complications from diabetes. Concurrent with the development of new diabetic therapies, glucose control in the U.S. has gradually improved in recent years, but it remains suboptimal. By supplementing the self-reported treatment approach with prescription medication data, we will show the changing pattern of diabetic therapies and the association between population level glucose control and treatment approach.

Materials and methods: Interview and prescription medication data from the National Health and Nutrition Examination Survey (NHANES) 1999-2000, 
2001-2002, and 2003-2004 were used to determine the treatment approach for adults with type 2 diabetes in the U.S. Individuals diagnosed before age 30 who reported insulin-only therapy were classified as having type 1 diabetes and excluded; all other adults with the diagnosis of diabetes were assumed to have type 2 diabetes. Oral agents were classified by primary generic ingredient (sulfonylurea, metformin, thiazolidinedione (TZD)). We calculated the proportion of adults with type 2 diabetes utilizing each major therapeutic and oral agent approach in 1999-2004. Within each treatment category, we computed the mean level of blood glucose $\left(\mathrm{HbA}_{1 \mathrm{c}}\right)$ and proportion of adults whose $\mathrm{HbA}_{1 \mathrm{c}}$ levels met recommended guidelines for control. Differences between groups were tested at the 0.05 significance level, with an adjustment for multiple comparisons.

Results: In 1999-2004, approximately $60 \%$ of adults with type 2 diabetes used only oral agents to manage their diabetes. As the distribution of oral treatment therapies changed over time, with the most prevalent treatment shifting from sulfonylurea monotherapy in 1999-2000 (23.0\%) to any oral agent regimen containing TZD in 2003-2004 (21.4\%), the mean $\mathrm{HbA}_{1 \mathrm{c}}$ level decreased from 7.8 to 7.2 . Only $38 \%$ of adults with type 2 diabetes met the International Diabetes Federation (IDF) goal for $\mathrm{HbA}_{1 \mathrm{c}}$ control $\left(\mathrm{HbA}_{1 \mathrm{c}}\right.$ less than or equal to $6.5 \%)$ in $1999-2004$. The proportion meeting the IDF goal differed across treatment therapies, ranging from $10.6 \%$ of those who used both insulin and oral agents to $70.0 \%$ of those who used no diabetic agents. Nearly $37 \%$ of the group using oral agents only met the IDF goal. Across the oral agent treatment categories, the proportion with $\mathrm{HbA}_{1 c}$ controlled at the $7.0 \%$ level was significantly lower for those on a therapy consisting of TZD, sulfonylurea, and metformin $(31.9 \%)$ than those on metformin alone $(62.2 \%)$, likely reflecting a progressive treatment approach of prescribing additional medications for those whose $\mathrm{HbA}_{1 c}$ level is not controlled. Analyses of $\mathrm{HbA}_{1 c}$ control by sex, race/ethnicity, obesity status, and length of diagnoses revealed no clear patterns of differences by treatment therapy.

Conclusion: Oral treatment regimens for adults with type 2 diabetes continue to change over time, with more adults using multiple oral agent therapies in an effort to improve $\mathrm{HbA}_{1 c}$ control. However, more than $60 \%$ of adults with type 2 diabetes have an $\mathrm{HbA}_{1 \mathrm{c}}$ level that remains above the IDF guideline, an indication that currently available treatments could be used more optimally and new agents to improve $\mathrm{HbA}_{1 \mathrm{c}}$ control may be needed.

Supported by: Eli Lilly

\section{PS 017 Epidemiology and genetics of body weight}

\section{3}

Association of FTO gene with morbid obesity and relation with insulin resistance in a women population from Southern France

R. Attaoua ${ }^{1}$, S. Ait El Mkadem¹, S. Kaouache ${ }^{1}$, J.-F. Brun², J.-C. Gris ${ }^{3}$,

E. Renard ${ }^{4}$, F. Grigorescu';

${ }^{1}$ Molecular Endocrinology Laboratory (EA 4188), University of Montpellier 1 (IURC), France, ${ }^{2}$ Centre d'Exploration et de Réadaptation des Anomalies du Métabolisme Musculaire (CERAMM), Lapeyronie Hospital, Montpellier, France, ${ }^{3}$ Haematology Laboratory, Doumergue University Hospital, Nîmes, France, ${ }^{4}$ Department of Endocrinology, Lapeyronie Hospital, Montpellier, France

Background and aims: FTO (fat mass and obesity associated) gene (Chr 16q12.2) was recently discovered by genome wide scanning as being associated with type 2 diabetes through the effect of body mass index (BMI). Association was replicated in many populations. This study was aimed to explore FTO gene association to obesity in women from Southern France in relation to in vivo insulin sensitivity.

Materials and methods: Case-control study was performed in women with class II (simple) obesity $(n=128)$, class III (morbid) obesity $(n=65)$ and lean control women $(n=42)$. Insulin sensitivity was measured by HOMA-IR and Minimal Model (IVGTT). FTO gene was genotyped through SNP rs1421085 (T/C) by sequence specific oligonucleotide (SSO)-PCR and confirmed by sequencing.

Results: Allelic frequencies (q) of rs 1421085 were $0.41,0.50$ and 0.61 for controls, simple and morbid obesity, respectively $(\mathrm{P}<0.02)$. Prevalence of homozygous $\mathrm{C} / \mathrm{C}$ were 23.1 and $40.5 \%$ in simple and morbid obesity compared to only $18.7 \%$ in controls $(\mathrm{P}<0.01)$. A gene dosage effect was revealed on BMI $\left(29.0 \pm 1.0,31.0 \pm 0.8\right.$ and $\left.33.7 \pm 1.5 \mathrm{~kg} / \mathrm{m}^{2}\right)$ between $\mathrm{T} / \mathrm{T}, \mathrm{T} / \mathrm{C}$ and $\mathrm{C} / \mathrm{C}$ carriers $(\mathrm{P}<0.01)$. The best association of $\mathrm{C} / \mathrm{C}$ was found between controls and morbid obesity with $\mathrm{P}<0.02$, OR $4.2,95 \% \mathrm{CI}$ [1.6-11.0], but it remained non-significant for simple obesity. No correlation was found between $\mathrm{C} / \mathrm{C}$ genotypes and impaired glucose tolerance (IGT) or impaired fasting glucose (IFG). By contrast, FTO genotypes were well correlated with insulin resistance (mean HOMA-IR was $1.7 \pm 0.1,2.0 \pm, 0.1,2.6 \pm 0.3$ in T/T, T/C and C/C genotypes, respectively, $\mathrm{P}<0.0035$ ). In logistic regression, $\mathrm{C} / \mathrm{C}$ was associated with insulin resistant patients (HOMA-IR $>2.49$ ) with $\mathrm{P}<0.0023$, OR 4.16, 95\%CI [1.66-10.43]. In ANOVA, HOMA-IR index was well explained by $\mathrm{C} / \mathrm{C}$ genotype with $\mathrm{P}<0.0035$, interaction factor $\alpha=0.9$ ), values which were abolished after adjustment for BMI. Although $\mathrm{C} / \mathrm{C}$ carriers with morbid obesity had lower sensitivity index ( $\mathrm{Si}$ ) than lean women $(3.0 \pm 0.5$ versus 7.8 \pm 2.6 , respectively) with a parallel increased of $\operatorname{AIRg}(28.2 \pm 19.8$ versus 12.2 \pm 3.5 ), no gene dosage effect was detected on IVGTT parameters. Analysis of other features composing the metabolic syndrome indicated good correlation between $\mathrm{C} / \mathrm{C}$ genotype and the triglycerides levels $(\mathrm{P}<0.04)$.

Conclusion: These data confirmed in morbidely obese women from Southern France population the previous relationship between FTO gene and BMI and indicate that relation between FTO genotypes and insulin resistance is more subtle and indistinguishable from the effect of BMI. In the context of our recent results on this gene in obese and infertile women, these data suggest that FTO may provide useful genetic markers for assessing the effect of obesity in metabolic complications.

\section{4}

Polymorphisms in the adiponectin encoding gene and susceptibility to type 2 diabetes in the Polish population

M. Szopa ${ }^{1}$, M. Malecki ${ }^{1}$, P. Wolkow ${ }^{2}$, J. Skupien ${ }^{1}$, A. Dembinska-Kiec ${ }^{3}$, J. Sieradzki ${ }^{1}$

${ }^{1}$ Department of Metabolic Diseases, ${ }^{2}$ Department of Pharmacology, ${ }^{3}$ Department of Clinical Biochemistry, Jagiellonian University Medical College, Krakow, Poland

Background and aims: Recently, several association studies of type 2 diabetes mellitus (T2DM) and adiponectin gene polymorphisms have been reported with conflicting results. Our aim was to search for associations between three polymorphisms $(-11.391 \mathrm{G}>\mathrm{A},+45 \mathrm{~T}>\mathrm{G},+276 \mathrm{G}>\mathrm{T})$ of adiponectin gene with T2DM and related quantitative traits in Polish Caucasians. 
Materials and methods: The study groups comprised of unrelated 495 T2DM cases and 435 controls. We compared the distribution of alleles, genotypes and haplotypes between study groups. Genotype-quantitative trait analysis in the controls included BMI as well as glucose and insulin data from the oral glucose tolerance test (OGTT). All the study subjects were genotyped for three adiponectin gene polymorphisms using the restriction fragment length polymorphism (RFLP) technique.

Results: Frequencies of minor alleles were as follows: $15.5 \%$ in T2DM vs. $19.8 \%$ in controls $(\mathrm{p}=0.0145)$ for $+276 \mathrm{G}>\mathrm{T} ; 10.6 \%$ vs. $8.2 \%$ for $-11.391 \mathrm{G}>\mathrm{A}$ $(\mathrm{p}=0.0722)$ and $7.0 \%$ vs. $8.0 \%$ for $+45 \mathrm{~T}>\mathrm{G}(\mathrm{p}=0.48)$, respectively. The difference of genotype distribution between the T2DM group and the control group was statistically significant ( $\mathrm{p}=0.0247$ ) for the $276 \mathrm{G}>\mathrm{T}$ variant: $71.31 \%$ vs. $62.99 \%, 26.46 \%$ vs. $34.48 \%$ and $2.22 \%$ vs. $2.53 \%$, respectively for the GG, $\mathrm{GT}$ and TT. Alleles of $+45 \mathrm{~T}>\mathrm{G}$ and $+276 \mathrm{G}>\mathrm{T}$ were in linkage disequillibrium $\left(D^{\prime}=0.81, r^{2}=0.011\right)$ and they formed two frequent haplotypes: T-G and T-T. Their frequencies were as follow: $77.7 \%$ in T2DM vs. $72.5 \%$ in controls for T$\mathrm{G}(\mathrm{p}=0.055)$; and $15.1 \%$ vs. $20.1 \%$ for $\mathrm{T}-\mathrm{T}$ ( $\mathrm{p}=0.0024)$, respectively. However, logistic regression analysis revealed that the effect of the T-T diplotype could be explained entirely by the $\mathrm{SNP}+276 \mathrm{G}>\mathrm{T}$. In quantitative traits analysis, the $\mathrm{T}$ allele of $+276 \mathrm{G}>\mathrm{T}$ was significantly associated $(\mathrm{p}<0.05)$ with lower insulin resistance (HOMA-IR, fasting insulin) among non-diabetic subjects. In addition, the A allele at position -11.391 was significantly associated $(\mathrm{p}<0.05)$ with higher insulin resistance (HOMA-IR, fasting insulin). In multiple regression analysis all identified associations remained significant after gender, BMI and age were included in the multiple regression model. In addition, in this model $-11.391 \mathrm{G}>\mathrm{A}$ and $+276 \mathrm{G}>\mathrm{T}$ were independently associated with T2DM.

Conclusion: We conclude that adiponectin gene polymorphisms are associated with T2DM and diabetes related phenotypes in Caucasians of Polish origin.

Supported by: Polish Governmental grant

\section{5}

Common genetic variation in the retinoic acid receptor alpha gene is associated with body fat content in subjects at increased risk for type 2 diabetes

K. Müssig' , H. Staiger ${ }^{1}$, F. Machicao ${ }^{1}$, C. Thamer ${ }^{1}$, J. Machann ${ }^{2}$, F. Schick ${ }^{2}$, C.D. Claussen ${ }^{2}$, N. Stefan ${ }^{1}$, A. Fritsche ${ }^{1}$, H.-U. Häring ${ }^{1}$

${ }^{1}$ Division of Endocrinology, Diabetology, Angiology, Nephrology, and

Clinical Chemistry, Germany, ${ }^{2}$ Section on Experimental Radiology,

University Hospital of Tübingen, Germany

Background and aims: Modulating effects of retinoic acid on adipocyte differentiation are mediated by the nuclear transcription factor retinoic acid receptor-alpha (RAR-alpha). We studied whether genetic variation within the RARA gene encoding RAR-alpha contributes to the development of obesity and obesity-related traits, such as insulin resistance and beta-cell dysfunction.

Materials and methods: We genotyped 943 subjects with an increased risk for type 2 diabetes for the four single nucleotide polymorphisms (SNPs) rs2715554, rs2715553, rs9303286, and rs482284 and performed correlational analyses with anthropometric data and parameters of insulin sensitivity and beta-cell function. For metabolic characterization, all subjects underwent an oral glucose tolerance test (OGTT), a subset additionally a hyperinsulinemiceuglycemic clamp. In a subgroup of 314 subjects, whole body fat distribution was assessed by nuclear magnetic resonance imaging. In 312 subjects, metabolic traits and anthropometric data were reevaluated after a 9-months lifestyle intervention.

Results: The four chosen SNPs cover $100 \%$ of common genetic variation (minor allele frequency $>0.10)$ within the RARA gene $\left(r^{2}>0.8\right)$. We found significant associations of the SNP rs9303286 with whole body adiposity, with carriers of the minor allele displaying lower values for body mass index (BMI), waist circumference, and body fat content ( $\mathrm{p}=0.0151, \mathrm{p}=0.0041$, and $\mathrm{p}=0.0002$, respectively). Moreover, the minor allele of SNP rs2715554 was significantly associated with a better improvement of BMI, waist circumference, and body fat content after a 9-months lifestyle intervention ( $\mathrm{p}=0.0476$, $\mathrm{p}=0.0009$, and $\mathrm{p}=0.0073$, respectively).

Conclusion: We conclude that common genetic variation within the RARA gene might affect regulation of adipose tissue mass.

Supported by: grants from the German Research Foundation and the European Community's frame program FP6

\section{6}

Expression of the bone morphogenetic protein receptor 1A gene $(B M P R 1 A)$ in adipose tissue and BMPR1A genetic variation are associated with human obesity

Y. Böttcher ${ }^{1}$, D. Schleinitz ${ }^{2}$, H. Unbehauen ${ }^{1}$, N. Klöting ${ }^{1}, K^{2}$. Ruschke ${ }^{1}$, B. Enigk ${ }^{2}$, A. Tönjes ${ }^{1}$, S. Wolf ${ }^{1}$, Y.-H. Tseng ${ }^{3}$, G.H. Scholz ${ }^{4}$, M. Blüher ${ }^{1}$, M. Stumvoll ${ }^{1}$, P. Kovacs ${ }^{2}$

${ }^{1}$ III. Medical Department, University of Leipzig, Germany, ${ }^{2}$ Interdisciplinary Center for Clinical Research IZKF, University of Leipzig, Germany, ${ }^{3}$ Harvard Medical School, Joslin Diabetes Center, Boston, United States, ${ }^{4}$ Medical Department, St. Elisabeth Hospital of Leipzig, Germany

Background and aims: Family of bone morphogenetic proteins (BMP) is involved in regulation of adipogenesis. We measured the mRNA expression of BMP members in paired samples of visceral and subcutaneous adipose tissue from 198 subjects and examined its relation to obesity. Amongst others, mRNA expression of the human bone morphogenetic protein receptor 1A (BMPR1A) was significantly increased in both visceral and subcutaneous adipose tissue of 51 overweight (BMI $>25$ and $<30 \mathrm{~kg} / \mathrm{m}^{2}$ ) and 91 obese $\left(\mathrm{BMI}>30 \mathrm{~kg} / \mathrm{m}^{2}\right)$ subjects compared with 56 lean subjects $\left(\mathrm{BMI}<25 \mathrm{~kg} / \mathrm{m}^{2}\right)$ $(P<0.05$ and $P<0.001$, respectively). We therefore investigated whether genetic variants within the BMPR1A are associated with mRNA expression in fat and whether these variants affect the pathophysiology of human obesity and type 2 diabetes (T2D)

Materials and methods: We sequenced the BMPR1A (13 exons, exon-intron boundaries, 5' and 3' UTRs) in DNA samples from 48 non-related Caucasian subjects to identify genetic variants. Twenty representative variants including HapMap tagging SNPs (single nucleotide polymorphisms) (www.hapmap. org) were initially genotyped for subsequent association studies in German Caucasian $(\mathrm{N}=1808)$ with detailed metabolic testing. For replication analyses we used an isolated population of Sorbs from Germany ( $\mathrm{N}=933)$.

Results: In a case control study including 461 lean $\left(\mathrm{BMI}<25 \mathrm{~kg} / \mathrm{m}^{2}\right)$ and 678 obese $\left(\mathrm{BMI}>30 \mathrm{~kg} / \mathrm{m}^{2}\right)$ subjects, four SNPs (rs7095025, rs7922846, rs 11202222 and rs10788528) were significantly associated with obesity (all $P<0.05$, adjusted for age and sex). Consistent with this, we found significant associations between BMPR1A SNPs and obesity/diabetes related quantitative traits (body mass index (BMI), circulating serum leptin, fasting plasma glucose, 2-h plasma glucose in oral glucose tolerance test; all $P<0.05$, adjusted for age, sex and BMI) in 896 subjects without T2D. Finally, the rs17426348 was moderately associated with T2D in a case-control study including 912 cases with T2D and 718 healthy controls with normal glucose tolerance (adjusted $P=0.05$ ). For 3 SNPs (rs7095025, rs11202222 and rs10788528), the association with measures of obesity and related traits could be replicated in the independent cohort of Sorbs (all adjusted $P<0.005$ ). In addition, in a subgroup of German Caucasian subjects with measured BMPR1A mRNA expression in fat, several variants were associated with the visceral gene expression levels (all adjusted $P<0.05)$.

Conclusion: Our data suggest that genetic variation in the BMPR1A may play a role in the pathophysiology of human obesity and T2D, possibly mediated through effects on mRNA expression.

Supported by: Medical Faculty at the University of Leipzig, Deutsche Forschungsgemeinschaft and Interdisciplinary Centre of Clinical Research Leipzig

\section{7}

Fasting and 2-hour plasma glucose levels are affected by food patterns. Longitudinal data from the Inter99 Study

C. Lau ${ }^{1}$, U. Toft ${ }^{2}$, I. Tetens ${ }^{3}$, B. Carstensen ${ }^{1}$, T. Jørgensen ${ }^{2}$, O. Pedersen ${ }^{1,4}$, K. Borch-Johnsen ${ }^{1,4}$

${ }^{1}$ Steno Diabetes Center, Gentofte, Denmark, ${ }^{2}$ Research Centre for Prevention and Health, Glostrup, Denmark, ${ }^{3}$ Technical Univesity of Denmark, National Food Institute, Søborg, Denmark, ${ }^{4}$ Faculty of Health Sciences, University of Aarhus, Denmark

Background and aims: The effect of food patterns on long term changes in continuous plasma glucose levels needs to be determined. An advantage using food patterns is the potential to detect the combined effect of foods, especially if the individual components of a pattern contribute to only a small amount of risk. We therefore examined whether food patterns previously identified at baseline in the Inter99 study were predictive of changes in plasma glucose and whether the association differed between individuals with different glucose tolerance status. 
Materials and methods: At baseline 5,824 men and women (30-60y) without diabetes were included and followed up after 1, 3 and 5 years. A traditional food pattern (primarily characterised by high-fat meat, mayonnaise salads, potatoes, butter and lard) and a modern food pattern (primarily characterised by vegetables, fruit, vegetable oil/vinegar dressing, poultry, pasta, rice and cereals) have previously been identified at baseline. A score for each of the two patterns was estimated for each person. Fasting plasma glucose (FPG) and post-challenge 2-hour plasma glucose (2h-PG) were used as continuous outcomes. Individuals were classified into normal-glucose-tolerance (NGT), isolated impaired-fasting-glycemia (i-IFG), isolated impaired-glucose-tolerance (i-IGT) and a combined group of IFG and IGT (IFG/IGT) at baseline. The effect of the baseline food patterns on changes in plasma glucose during follow-up was examined with and without taking account of glucose tolerance status using random effect models adjusting for relevant confounders. Results: Considering the modern pattern a higher score predicted a significant annual fall in $2 \mathrm{~h}-\mathrm{PG}$ between $0.010-0.017 \mathrm{mmol} / \mathrm{l}(\mathrm{p}<0.05)$, but no significant change was observed for FPG. The traditional pattern did not predict general changes for FPG or $2 \mathrm{~h}-\mathrm{PG}$ values. However, clear interactions between groups of glucose tolerance status for the modern pattern and FPG and for the traditional pattern and both FPG and 2h-PG were observed ( $\mathrm{p}$ interaction<0.05).

Conclusion: All significant effects observed for a higher modern score indicated that the modern pattern had a protective effect on changes in plasma glucose, whereas all significant effects observed for a higher traditional score indicated a detrimental effect for changes in plasma glucose. The food patterns may be more likely to affect plasma glucose levels in individuals with i-IFG, i-IGT or IFG/IGT than individuals with NGT. We suggest that the risk of worsening the plasma glucose levels may be reduced by shifting from a traditional food pattern, consisting of especially fatty meats, potatoes and butter, to a modern food pattern primarily characterised by vegetables, fruit, oil and poultry.

Supported by: Danish Research Agency, Ministry of Science Technology and Innovation

\section{8}

Body weight change between pregnancies is associated with the risk of gestational diabetes (GDM)

A. Ferrara, S.F. Ehrlich, M.M. Hedderson, T.Y. Peng

Division of Research, Kaiser Permanente Northern California, Oakland,

United States

Background and aims: The prevalence of both GDM and obesity have increased in women of reproductive age during the last decade. Obesity and being overweight are among the major risk factors for GDM. However, it is unclear whether the risk of GDM varies by weight gain or loss before pregnancy. We examined the associations between change in body weight from the first to the second pregnancies and the risk of GDM during the second pregnancy in a multiethnic population of 25,880 women who had their first two consecutive births between 1996 and 2005 at Kaiser Permanente of Northern California (USA).

Materials and methods: Electronic medical records were used to obtain data on age, race/ethnicity, body weight measured during the first trimester, gestational age at body weight measurements and GDM. GDM was defined according to the American Diabetes Association plasma glucose thresholds obtained from a $100-\mathrm{g}$ OGTT.

Results: Mean age at the first pregnancy was $26.4+/-4.8$ years, and the race/ ethnicity distribution was as follows: $46 \%$ Caucasians, $5 \%$ AA, $18 \%$ Asian, $22 \%$ Hispanic and $9 \%$ other. On average, the interval between the first and second pregnancy was 34 months and women gained $3.7 \mathrm{~kg}$ between the first and second pregnancy. 5.1\% women had GDM at the first pregnancy, 5.6\% had GDM at the second pregnancy and $1.9 \%$ had GDM at both pregnancies. The risk of GDM increased linearly with weight change between pregnancies, after adjustment for age, body weight and GDM at the first pregnancy, race/ ethnicity, gestational age at weight measurements and time between pregnancies. Compared with women whose weight remained stable $(+/-3.0 \mathrm{~kg})$, the adjusted odds ratios for GDM at the second pregnancy for those who gained 3.1 to $5.9 \mathrm{~kg}$ and for those who gained $6.0 \mathrm{~kg}$ or more were: 1.67 (1.44-1.95) and 2.45 (2.11-2.84), respectively. The adjusted odds ratios for GDM for those who lost 3.1 to $5.9 \mathrm{~kg}$ and those who lost $6.0 \mathrm{~kg}$ or more were: $0.63(0.46-0.87)$ and $0.42(0.25-0.68)$, respectively. In the same model, the odds ratio for GDM at the second pregnancy for those who had GDM at the first pregnancy was 13.84 (12.04-15.90). When women were grouped according to their GDM status at the first and second pregnancy, on average those who had GDM at the first but not at the second pregnancy gained the least amount of weight, while women who did not have GDM at the first pregnancy and developed GDM at the second pregnancy gained the greatest amount of weight between pregnancies (Figure). Similar results were obtained when women were stratified by race/ethnicity.

Conclusion: Our results provide epidemiological evidence for implementing postpartum weight loss programs in women with GDM and to prevent postpartum weight gain in women without a history GDM.

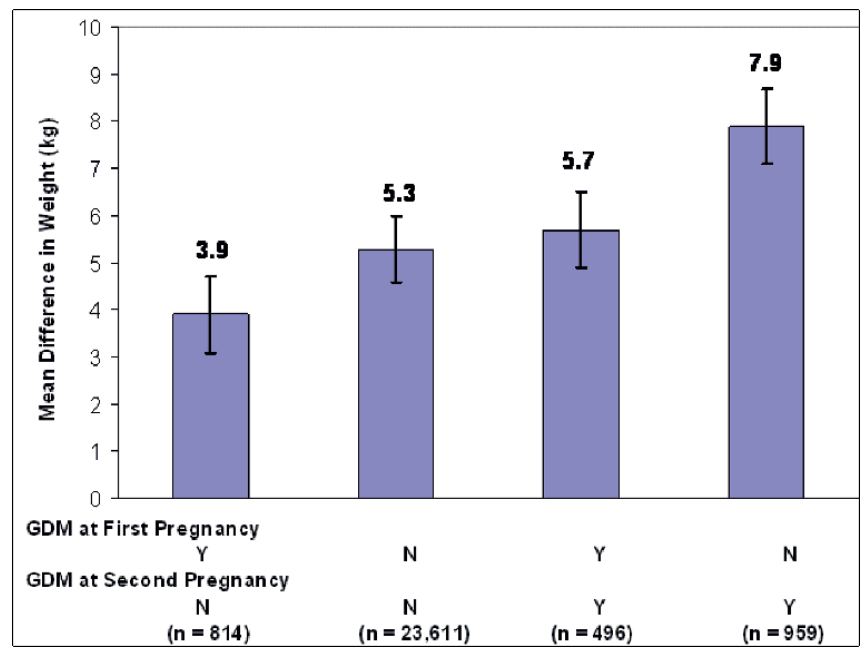

\section{9}

Only regional adiposity, but not general adiposity, is associated with type 2 diabetes in Korea

S.-W. Park ${ }^{1}$, D.-J. Kim², Y.-K. Lee ${ }^{1}$, S.-K. Kim¹, Y.-L. Kim, W.-K. Park ${ }^{1}$,

Y.-K. Choi ${ }^{1}$, S.-J. Lee ${ }^{1}$, Y.-W. Cho ${ }^{1}$

${ }^{1}$ Department of Medicine, Pochon CHA University, Sungnam, ${ }^{2}$ Department of Medicine, Ajou University School of Medicine, Suwon, Republic of Korea

Background and aims: Although general adiposity, usually assessed by BMI, is a strong risk factor for type 2 diabetes (T2DM), some studies have suggested that the pattern of body fat distribution might be more important predictor of T2DM. The purpose of the study was to investigate the associations between different measures of adiposity (BMI, waist circumference) and T2DM in ethnically non-obese population. We hypothesized that reginal adiposity measured by waist circumference (WC) is a key factor associated with T2DM in Korea, where majority of subjects with T2DM is non-obese even though obesity is defined by BMI $\geq 25 \mathrm{~kg} / \mathrm{m}^{2}$.

Materials and methods: We conducted a cross-sectional analysis of a nationally representative sample of 5,294 adults aged $\geq 20$ years, from the Third Korean National Health and Nutrition Examination Survey (KNHANES) performed in 2005. All subjects were examined and fasting plasma glucose

The odds ratio of type 2 diabetes in Koreans, stratified by BMI and waist circumference (WC).

\begin{tabular}{lllllll}
\hline & All & $(\mathrm{n}, 5294)$ & Men & $(\mathrm{n}, 2244)$ & Women & $(\mathrm{n}, 3050)$ \\
\hline & OR & $(95 \% \mathrm{CI})$ & OR & $(95 \% \mathrm{CI})$ & OR & $(95 \% \mathrm{CI})$ \\
\hline Normal BMI, Normal WC & 1 & Reference & 1 & Reference & 1 & Reference \\
\hline Normal BMI, High WC & 2.64 & $1.81-3.85$ & 2.29 & $1.31-3.99$ & 2.89 & $1.71-4.88$ \\
\hline High BMI, Normal WC & 1.33 & $0.93-1.92$ & 1.50 & $0.94-2.39$ & 1.11 & $0.62-1.99$ \\
\hline High BMI, High WC & 2.16 & $1.71-2.73$ & 2.14 & $1.54-3.00$ & 2.18 & $1.55-3.06$ \\
\hline
\end{tabular}


(FPG) was measured. General obesity was defined by BMI $\geq 25 \mathrm{~kg} / \mathrm{m}^{2}$ and central obesity was defined by the ethnicity specific cutoff for Koreans (WC $\geq 90 \mathrm{~cm}$ in men and $\geq 85 \mathrm{~cm}$ in women). Diabetes mellitus was defined by previous diagnosis of diabetes or use of anti-diabetic medications or FPG $\geq$ $7.0 \mathrm{mmol} / \mathrm{l}$. Multiple logistic regression analyses were used to identify independent factors associated with T2DM.

Results: Among various clinical parameters, age, male sex, BMI, waist circumference, family history of diabetes, and smoking status were associated with T2DM in bivariate analyses. In multiple logistic regression models, WC but not BMI was associated with diabetes controlling for age, sex, family history and smoking status. When the subjects were divided into four groups by the presence of general obesity and/or central obesity, the odds ratio of T2DM was the highest in subjects with normal BMI and high WC in both men and women. But the risk of T2DM was not increased in subjects with high BMI and normal WC (Table). The results were similar even if the outcome was restricted to newly diagnosed diabetes excluding those with known and/or treated cases.

Conclusion: In conclusion, only regional adiposity assessed by waist circumference, but not general adiposity measured by BMI, is independently associated with type 2 diabetes in Korea.

\section{0}

Associations between abdominal fat distribution and glucose intolerance and fasting insulin. The Inuit Health in Transition Study

M.E. Jørgensen ${ }^{1}$, K. Borch-Johnsen ${ }^{1}$, P. Bjerregaard ${ }^{2}$

${ }^{1}$ Steno Diabetes Centre, Gentofte, Denmark, ${ }^{2}$ Centre for Health Research in Greenland, National Institute of Public Health, University of Southern Denmark, Copenhagen, Denmark

Background and aims: Inuit populations have lower mean values of various metabolic risk factors for a given level of obesity compared to western populations. The Inuit are suggested to be protected from a high amount of subcutaneous adipose tissue (SAT) which is less metabolically active than visceral adipose tissue (VAT). We aimed to study associations between ultrasound measurements of intraabdominal fat and glucose tolerance status and fasting insulin.

Materials and methods: Data were collected from 2005 to 2007 among adult Inuit on the west coast of Greenland. Diabetes, IGT and IFG were diagnosed using the OGTT. Glucose and insulin were measured. VAT and SAT were measured by ultrasound.

Results: A total of 1633 Inuit participated. The median age of the sample was 44 years (range 18-89), and 57\% were women. The prevalence of IFG, IGT, and diabetes was $10 \%, 7 \%$, and $7.6 \%$. Mean values of abdominal fat according to glucose tolerance group are shown in the table. While SAT and VAT increased with glucose intolerance, the ratio between SAT and VAT decreased. Both SAT and VAT were positively associated with fasting insulin. SAT and VAT were highly correlated; therefore each of the two variables were modelled as a linear spline with three knots in a mixed model with fasting insulin as dependent variable. For a given level of VAT, an increase in SAT was associated with a decrease in fasting insulin.

Conclusion: The data indicate for a given level of VAT an inverse association between SAT and glucose intolerance and fasting insulin, respectively.

Association between glucose tolerance status and ultrasound measurements of fat distribution

\begin{tabular}{|c|c|c|c|c|c|c|c|}
\hline \multirow{2}{*}{$\overline{\mathrm{SAT}(\mathrm{cm})}$} & \multirow{3}{*}{$\begin{array}{l}\text { NGT } \\
\text { IFG }\end{array}$} & \multirow{2}{*}{$\frac{N}{702}$} & \multicolumn{2}{|c|}{ Women } & \multirow{2}{*}{$\begin{array}{l}\mathrm{N} \\
522\end{array}$} & \multicolumn{2}{|l|}{ Men } \\
\hline & & & 3.71 & & & 2.29 & \\
\hline & & 78 & 4.07 & & 90 & 2.33 & \\
\hline & IGT & 85 & 3.81 & & 32 & 2.50 & \\
\hline & $\mathrm{DM}$ & 67 & 3.49 & $\mathrm{p}=0.38$ & 57 & 2.73 & $\mathrm{p}<0.001$ \\
\hline \multirow{4}{*}{ VAT $(\mathrm{cm})$} & NGT & 702 & 6.50 & & 522 & 7.12 & \\
\hline & IFG & 78 & 7.59 & & 90 & 8.14 & \\
\hline & IGT & 85 & 7.63 & & 32 & 8.98 & \\
\hline & $\mathrm{DM}$ & 67 & 7.24 & $\mathrm{p}=0.09$ & 57 & 9.34 & $\mathrm{p}<0.001$ \\
\hline \multirow{4}{*}{$\begin{array}{l}\text { Ratio SAT/VAT } \\
(\%)\end{array}$} & NGT & 702 & 0.58 & & 522 & 0.32 & \\
\hline & IFG & 78 & 0.57 & & 90 & 0.30 & \\
\hline & IGT & 85 & 0.51 & & 32 & 0.30 & \\
\hline & DM & 67 & 0.46 & $\mathrm{p}<0.001$ & 57 & 0.29 & $\mathrm{p}=0.05$ \\
\hline
\end{tabular}

Supported by: Karen Elise Jensen Foundation

\section{PS 018 Epidemiology of type 2 diabetes complications}

\section{1}

Increased prevalence of retinopathy in patients of South Asian ethnicity persists, even after adjustment for potentially confounding risk factors K. Bush ${ }^{1,2}$, L. Varadhan ${ }^{1}$, N.T. Raymond ${ }^{1}$, D. Reynold ${ }^{1}$, S. Sankaran ${ }^{1}$, S. Bellary ${ }^{3}$, A.H. Barnett ${ }^{3}$, S. Kumar ${ }^{4}$, J.P. O’Hare ${ }^{1}$

${ }^{1}$ Health Sciences Research Institute, The University of Warwick, Coventry, ${ }^{2}$ Ophthalmology, Southampton General Hospital, Southampton, ${ }^{3}$ Diabetes and Endocrinology, Birmingham Heartlands Hospital, Birmingham,

${ }^{4}$ Clinical Sciences Research Institute, The University of Warwick, Coventry, United Kingdom

Background and aims: Diabetes is four times more common in Asians but the prevalence of microvascular complications is disputed. The aim of this study was to estimate and compare retinopathy prevalence and evaluate relative risk for patients of South Asian (SA) and White European (WH) ethnic background with type 2 diabetes.

Materials and methods: We used data from the Coventry Diabetes Retinal Screening service, with baseline data from the United Kingdom Asian Diabetes Study (UKADS) and Primary care practices to establish the prevalence of diabetic retinopathy in a community setting. 4316 retinal photographs ( 2 eyes $\mathrm{x} 2$ fields per patient) were graded by the retinal screening team according to National screening committee guidelines and staged based on the worst eye. Any retinopathy, sight threatening retinopathy (STR), maculopathy and previous photocoagulation prevalences were estimated and compared between ethnicity defined groups. Demographic and clinical risk factors data were collected and to allow for confounding effects, multivariable analyses were performed using conditional logistic regression matched for duration of diabetes.

Results: A total of 1079 patients were included in the study, comprising 430 SA and $649 \mathrm{WH}$. The SA group comprised more females, were younger and had shorter duration of diabetes, all factors likely to confound comparison of crude prevalence estimates. Prevalence of diabetic retinopathy comparing the SA and WH groups were: any retinopathy $45 \%$ vs. $39 \%, \mathrm{P}=0.06$, STR $16 \%$ vs. $12 \%, \mathrm{P}=0.054$, maculopathy $13 \%$ vs. $9 \%, \mathrm{P}=0.08$ and previous photocoagulation $4 \%$ vs. $5 \%, \mathrm{P}=0.28$. Conditional logistic regression showed a significant effect for ethnicity, with any retinopathy unadjusted OR SA:WH $=1.66(1.22$ to 2.25), $\mathrm{P}=0.001$ and adjusted for gender, age at diagnosis, systolic blood pressure, $\mathrm{HbA1c}$ and total cholesterol $=1.41$ (1.01 to 1.97), $\mathrm{P}=0.045$. Similar analysis for STR gave unadjusted $\mathrm{OR}=1.61$ (1.04 to 1.68) and adjusted $\mathrm{OR}=$ 1.44 (0.90 to 2.28), $\mathrm{P}=0.13$.

Conclusion: Crude prevalences of any retinopathy and STR were similar comparing the two ethnicity defined groups, but the risk profiles were quite different, with potential for confounding effects. Adjustment for confounding factors showed higher OR for any retinopathy for the SA group. Risk of STR was raised in the SA, but not statistically significant. The SA group may need more aggressive management of established risk factors to reduce development of retinopathy.

Supported by: Pfizer, sanofi-aventis, Servier Laboratories UK, Merck Sharp \& Dohme/Schering-Plough, Takeda UK, Roche, Merck Pharma, Daiichi-Sankyo UK, Boehringer Ingleheim, Eli Lilly, NovoNordisk, Bristol Myers Squibb, Solvay Health Care and Assurance Medical Society, UK

\section{2}

CRP, microalbuminuria and 5-year survival in type 2 diabetes: the Casale Monferrato Study

G. Bruno ${ }^{1}$, P. Fornengo ${ }^{1}$, O. Segre ${ }^{1}$, G. Novelli ${ }^{1}$, F. Panero ${ }^{1}$, M. Perotto ${ }^{1}$, C. Zucco', G. Bargero ${ }^{2}$, P. De Ambrogio ${ }^{3}$, P. Cavallo-Perin ${ }^{1}$

${ }^{1}$ Dept. Internal Medicine, University of Torino, ${ }^{2}$ Diabetology, Ospedale S.Spirito, Casale Monferrato, ${ }^{3}$ Laboratory, Ospedale S.Spirito, Casale Monferrato, Italy

Objective: Recent studies have focused on the measurement of high sensitivity CRP as a predictor of subsequent cardiovascular outcomes. Patients with type 2 diabetes mellitus tend to have higher CRP concentrations than do those without it, suggesting an increased role of inflammation in the accelerated atherosclerosis seen in these patients. The aim of this study was to determine to what extent a CRP value in type 2 diabetic people influences 
5-year all causes and cardiovascular mortality, independently on AER and other cardiovascular risk factors.

Research design and methods: In the Casale Monferrato Study centralized measurements of CRP-hs values were available in 2381 (73.3\%) persons with type 2 diabetes out of a population-based cohort of 3249 persons with diabetes in 2000. They were identified using independent sources of identification, obtaining an estimated completeness of ascertainment $80 \%$.All measurements were centralized. The independent role of CRP-hs as predictor of all-cause and cardiovascular mortality in the follow-up period 2000-2006 was assessed with multivariate Cox proportional hazards modeling.

Results: Results are based on 496 deaths in 11.717 person-years of observations. With respect to subjects with CRP $\leq 3 \mathrm{mg} / \mathrm{l}$, those with CRP values $>3$ $\mathrm{mg} / \mathrm{l}$ had an hazard ratio (HR) $=1.52$ (95\% CI 1.20-1.94) for all-cause mortality and $\mathrm{HR}=1.42$ (95\% CI $0.98-2.06)$ for cardiovascular mortality, after adjustment for age, sex, smoking, hypertension, apoB/apoA1, HbAlc, CVD, AER, eGFR, antidiabetic treatment. When analyses were limited to diabetic persons without CVD, respective HRs were 1.66 (95\% CI 1.23-2.23) for allcause mortality and $1.32(0.82-2.14)$ for cardiovascular mortality.

Conclusion: our population based study indicates that CRP-hs measurement is an independent predictor of short-term mortality risk in type 2 diabetic people, even in those in primary prevention of the disease. These findings would suggest the usefulness in clinical practice of CRP measurement for better stratification of risk

Supported by: Piedmont Region, Ricerca Sanitaria Finalizzata 2006

\section{3}

Reduced plasma levels of glucagon-like peptide-1 predict impaired glucose tolerance, but not cardiovascular disease, in elderly men D. Nathanson ${ }^{1}$, B. Zethelius ${ }^{2}$, Å. Sjöholm ${ }^{1}$, C. Berne ${ }^{3}$, J.J. Holst ${ }^{4}$, T. Nyström ${ }^{1}$

${ }^{1}$ Department of Internal Medicine, Stockholm South Hospital, Karolinska Institutet, Stockholm, Sweden, ${ }^{2}$ Outpatient Clinic for Obesity Care and Metabolic Diseases, Uppsala University Hospital, Uppsala, Sweden, ${ }^{3}$ Department of Medical Sciences, Uppsala University Hospital, Sweden, ${ }^{4}$ Department of Biomedical Sciences, The Panum Institute, University of Copenhagen, Denmark

Background and aims: Glucagon-like peptide-1 (GLP-1) is a promising new drug for treatment of type 2 diabetes. Its extensively documented extra-pancreatic effects, for example beneficial influence in connection with endothelial dysfunction and myocardial ischemia, are extremely interesting in this context. Also, insulin resistance is of major importance in terms of type 2 diabetes and coronary heart disease (CHD). Earlier studies demonstrated reduced plasma levels of GLP-1 in type 2 diabetes patients. However, no previous longitudinal study has investigated the association between plasma levels of GLP-1 and CHD. Therefore, we wanted to study cross-sectional and longitudinal relationships between oral glucose-stimulated GLP-1 plasma levels and: development of CHD, insulin sensitivity index (M/I) assessed by euglycemic clamp, early insulin response to an oral glucose tolerance test (OGTT), and type 2 diabetes mellitus.

Materials and methods: A population-based cohort study was conducted from August 1991 to May 1995 among 510 men in Uppsala, Sweden, aged 70 years at baseline with a follow-up of up to 12 years using registry data obtained from the National Board of Health and Welfare in Sweden. At baseline, insulin sensitivity, assessed by euglycemic insulin clamp, and early insulin response to a $75 \mathrm{~g}$ oral glucose tolerance test (OGTT) were determined. Fasting GLP-1 and the dynamic response of $\triangle$ GLP-1 (i.e., the difference between 60 min OGTT-stimulated GLP-1 and fasting GLP-1 levels), measured in frozen plasma samples with a radioimmunoassay using antiserum 89390 , were the predicting variables in the subsequent analyses. CHD was defined as death, as recorded in the Cause of Death Registry, or the first time hospitalisation for $\mathrm{CHD}$ as recorded in the In-Patient Registry (International Classification of Disease [ $9^{\text {th }}$ revision] codes 410 to 414 ). Potential associations were analyzed using logistic regression (Wald test), presented as odds ratios (OR) with their $95 \%$ confidence intervals (CIs).To adjust for possible confounders, we used a multivariable model.

Results: In multivariate analyses, $\Delta$ GLP-1 (OR 0.70, CI 0.96-0.99) predicted impaired glucose tolerance (IGT). In contrast, $\triangle$ GLP-1 did not predict type 2 diabetes (OR 1.0, CI 0.98-1.0). There were no associations between $\Delta$ GLP1 and CHD (OR 0.99, CI 0.98-1.0) or risk factors i.e. hypertension, lipids, glucose, BMI, M/I or urine albumin. Finally, using both fasting GLP-1 and the OGTT-stimulated GLP-1 level at 60 minutes as predictors in the above models, did not reveal any further associations.
Conclusion: Impaired secretion of GLP-1 after an OGTT seems to predict for IGT in elderly men. In contrast, no association was seen between GLP1 and type 2 diabetes, which may be confounded by oral treatment in that group (e.g. metformin has been shown to increase GLP-1 plasma levels). More importantly, no association between $\triangle \mathrm{GLP}-1$ and CHD, and potential risk factors for $\mathrm{CHD}$, were discerned in this cohort study, suggesting that plasma levels of GLP-1 may not reflect cardiovascular disease in this group of elderly men.

\section{4}

High triglycerides, low HDL cholesterol, and small LDL particles predict incident type 2 diabetes in non-diabetic coronary patients C.H. Saely ${ }^{1,2}$, A. Vonbank ${ }^{1}$, S. Beer ${ }^{1}$, P. Rein ${ }^{1}$, S. Aczel ${ }^{1}$, T. Marte ${ }^{1}$, H. Drexel ${ }^{1,2}$ ${ }^{1}$ VIVIT Institute, Feldkirch, Austria, ${ }^{2}$ University for Human Sciences, Triesen, Liechtenstein

Background and aims: Patients with type 2 diabetes mellitus (T2DM) exhibit a typical pattern of dyslipidemia with high triglycerides, low HDL cholester$\mathrm{ol}$, and small LDL particles, which is also frequently observed in pre-diabetic patients. We therefore aimed at testing the hypothesis that these lipid abnormalities predict incident T2DM in the high-risk population of angiographied coronary patients.

Material and methods: The incidence of T2DM was recorded over 6 years in a population of 503 consecutive non-diabetic patients undergoing coronary angiography for the evaluation of stable coronary artery disease.

Results: During follow-up, T2DM was newly diagnosed in 86 (17.1\%) of our patients. Impaired fasting glucose (IFG) $\geq 100 \mathrm{mg} / \mathrm{dl}$ at baseline was present in $53.4 \%$ of the study population, and IFG patients were at a significantly higher risk of incident T2DM than patients with normal fasting glucose $(24.3 \%$ vs. 8.9\%; $\mathrm{p}<0.001$ ); in logistic regression analysis adjusting for age, gender, BMI, hypertension, and smoking the standardized $\mathrm{OR}$ for fasting glucose as a predictor of T2DM was 1.72 [95\% CI 1.36-2.18]; $\mathrm{p}<0.001$. In line with our hypothesis, also the serum levels of triglycerides $(\mathrm{OR}=1.57$ [1.25-1.98]; $\mathrm{p}<0.001)$ and HDL cholesterol $(\mathrm{OR}=0.62[0.45-0.86] ; \mathrm{p}=0.004)$ as well as the LDL particle diameter $(\mathrm{OR}=0.57[0.44-0.75] ; \mathrm{p}<0.001)$ proved significantly predictive of incident T2DM. Importantly, triglycerides $(\mathrm{OR}=1.49$ [1.18-1.88], $\mathrm{p}=0.001)$, HDL cholesterol $(\mathrm{OR}=0.66[0.47-0.91], \mathrm{p}=0.012)$ and the LDL particle diameter $(\mathrm{OR}=0.61[0.45-0.80], \mathrm{p}<0.001)$ still significantly predicted T2DM after additional adjustment for the baseline fasting glucose values.

Conclusion: High triglycerides, low HDL cholesterol, and a small LDL particle diameter significantly and independently predict the 6-year incidence of T2DM among non-diabetic coronary patients.

Supported by: Liechtenstein Global Trust Bank; Peter Goop Stiftung; Vorarlberger Landesregierung; Medizinisches Zentrallabor Feldkirch; Vorarlberger Industriellenvereinigung; Vorarlberger Aerztekammer; Vorarlberger Landeskrankenhaus-Betriebsgesellschaft

\section{5}

ICAM-1 is independently associated with incident diabetes but not incident coronary events or stroke in the elderly at risk

N. Sattar 1 , P. Group ${ }^{2}$

${ }^{1}$ Vascular Biochemistry, Glasgow Royal Infirmary, ${ }^{2}$ Prosper, Glasgow, Leiden, Cork, United Kingdom

Background and aims: To determine whether circulating ICAM-1 as a surrogate of 'endothelial activation' was associated with risk for vascular events and /or incident diabetes in the elderly.

Materials and methods: We related baseline ICAM-1 levels to vascular events (866 combined incident fatal and non-fatal CHD and stroke from 5685 participants) and to incident diabetes ( 292 from 4945 without baseline diabetes) in the elderly at risk over 3.2 years follow up.

Results: At baseline, ICAM-1 levels correlated positively to triglyceride but negatively to LDL- and HDL-cholesterol. ICAM-1 levels were higher in smokers, and those with prior diabetes or peripheral arterial disease. ICAM1 level was higher in those who developed diabetes [388.6 (1.42) ng $/ \mathrm{ml}$ versus $369.4(1.39) \mathrm{ng} / \mathrm{ml}, \mathrm{p}=0.011]$. Furthermore, higher ICAM-1 was associated with new onset diabetes, HR 1.84 (1.26, 2.69), $\mathrm{p}=0.0015$ per unit logICAM-1 with adjustment for risk factors including age, gender, smoking, BMI, lipids, blood pressure, fasting glucose and CRP. By contrast, ICAM-1 levels were not elevated $(374.7(1.4) \mathrm{ng} / \mathrm{ml}$ versus $370.8(1.4) \mathrm{ng} / \mathrm{ml}, \mathrm{p}=0.40)$ in those who had an incident vascular event compared to those who remained event 
free. The hazard ratio (HR) (95\% Confidence Interval) and p-value for 1 unit $\log$ ICAM increment was $1.13(0.93,1.38)$; $\mathrm{p}=0.23$ falling to $0.98(0.80,1.22)$; $\mathrm{p}=0.89$ in analyses adjusted for other risk factors in the whole cohort.

Conclusion: We show for the first time the elevated ICAM-1 levels are associated with incident diabetes risk in the elderly, despite lack of association with risk for vascular (fatal or non-fatal coronary or stroke) events. ICAM-1 appears to be more closely aligned to diabetes rather than CVD risk in the elderly, a finding which significantly extends relevant data in younger populations. Supported by: Stroke Association, UK

\section{6}

Non-invasive skin fluorescence indicates cumulative glycaemic exposure J.D. Maynard ${ }^{1}$, T. Orchard ${ }^{2}$, B. Conway ${ }^{2}$, N. Matter ${ }^{1}$

${ }^{1}$ VeraLight, Inc., Albuquerque, United States, ${ }^{2}$ Epidemiology, University of Pittsburgh, Pittsburgh, United States

Background and aims: Skin advanced glycation endproducts (AGEs) are biomarkers for tissue damage related to hyperglycemia. Skin AGEs levels may also be influenced by renal function. This study evaluated the association between glycemic exposure, renal function and skin AGEs measured by noninvasive fluorescence spectroscopy.

Materials and methods: Noninvasive measurements were made on the volar forearm of 29 individuals with type 1 diabetes enrolled in the Pittsburgh Epidemiology of Diabetes Complications (EDC) Study. Fluorescence was excited with a $375 \mathrm{~nm}$ LED and detected over the 400 to $496 \mathrm{~nm}$ emission window. Reflectance was measured with $375 \mathrm{~nm}$ and white LEDs. The reflectance measurements enable compensation for skin pigmentation, hemoglobin content and light scattering. The resulting intrinsic fluorescence (IF) was agecorrected to account for the accumulation of skin AGEs in normal health and then integrated to yield a single value.

Results: The mean subject age was 52 and the mean duration of diabetes was 40 years. 4 subjects had overt nephropathy (ON, albumin excretion $>200$ $\mu \mathrm{g} / \mathrm{min}$ or history of dialysis/transplant). Skin IF was correlated $(\mathrm{r}=0.66$, $\mathrm{p}=0.0004$ ) with mean A1c (9 measurements over $\sim 20$ years) in the $25 \mathrm{ON}$ free subjects, but was not correlated $(\mathrm{r}=0.16, \mathrm{p}=0.41)$ when assessed in all 29 . Correcting the IF by the natural log of the subject's renal function (MDRD formula) improved correlation ( $\mathrm{r}=0.37, \mathrm{p}=0.051)$ with mean Alc for the 29 . In the ON free group, skin IF was also correlated with Almonths $(\mathrm{r}=0.55$, $\mathrm{p}=0.005$, calculated by multiplying the number of $\mathrm{HbA} 1 \mathrm{c}$ units above normal at each cycle by the number of months between the midpoints of the preceding and succeeding cycle intervals), mean of Alc's taken between 10 and 20 years ago $(\mathrm{r}=0.66, \mathrm{p}=0.0003)$, mean of $\mathrm{A} 1 \mathrm{c}$ 's taken between 1 and 10 years ago $(\mathrm{r}=0.57, \mathrm{p}=0.003)$, and most recent $\mathrm{Alc}(\mathrm{r}=0.40, \mathrm{p}=0.047)$.

Conclusion: Both skin AGEs and mean Alc have been shown to be better predictors of diabetic complications than most recent Alc. Since physicians typically do not have access to a patient's multi-year mean Alc, the skin IF measurement may provide a simple means of assessing the historic glycemia of a person with diabetes that does not have overt nephropathy.

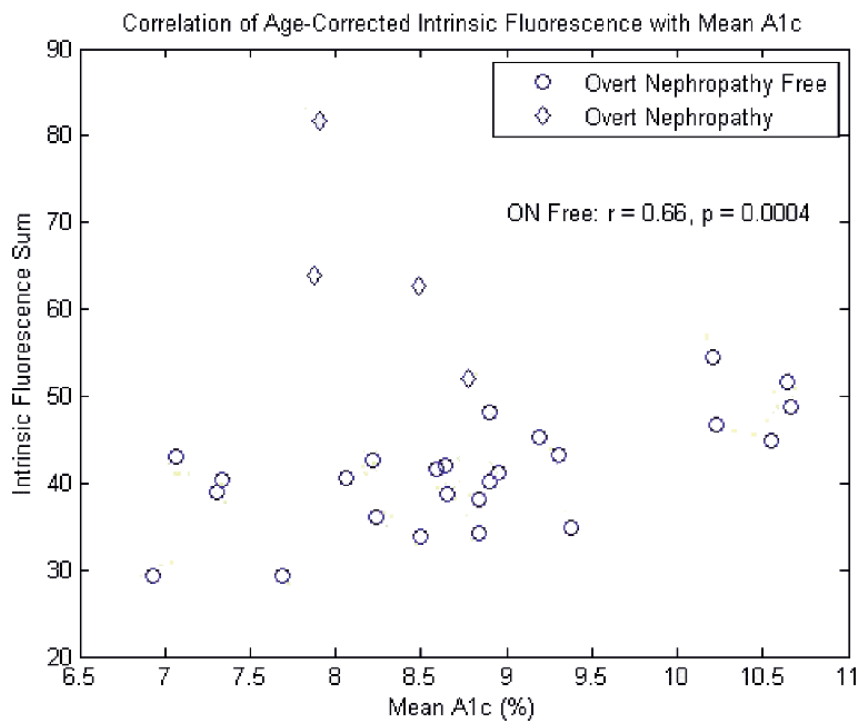

Supported by: VeraLight, Inc.

\section{7}

Changes in triglyceride levels over time and risk of type 2 diabetes. The Metabolic Life-style and Nutrition Assessment in Young Adults (MELANY) Study

A. Tirosh ${ }^{1}$, I. Shai ${ }^{2}$, R. Bitzur ${ }^{3}$, T. Shochat ${ }^{4}$, A. Rudich $^{5}$

${ }^{1}$ Department of Internal Medicine A, Sheba Medical Center, Tel-

Hashomer, ${ }^{2}$ The S. Daniel Abraham Center for Health and Nutrition, Ben-

Gurion University of the Negev, Beer-Sheva, ${ }^{3}$ The Institute of Lipid and Atherosclerosis Research, Sheba Medical Center, Tel-Hashomer, ${ }^{4}$ Medical Corps Headquarters, Israel Defense Forces (IDF), ${ }^{5}$ Department of Clinical Biochemistry, Ben-Gurion University of the Negev, Beer-Sheva, Israel

Background: The association between changes in triglycerides (TG) levels over time and diabetes is unknown.

Methods: For 13,953 26-45 years old apparently healthy men, with TG $<300$ $\mathrm{mg} / \mathrm{dL}(3.39 \mathrm{mmol} / \mathrm{L})$, we obtained TG measurements at baseline (Time1) and 3-5 years later (Time2), and followed for a mean of 5.5 years for incident type 2 diabetes.

Results: 322 type 2 diabetes cases occurred during 76,742 person-years. A multivariate model adjusted for age, body mass index (BMI), total cholesterol:HDL-cholesterol ratio, family history of diabetes, fasting glucose, blood pressure, physical activity, and smoking status, revealed a continuous independent rise in incident diabetes with increasing baseline TG levels, $(\mathrm{P}$ trend $<0.001$ ). Men in the lowest tertile of Time1 TG who progressed to the highest tertile over follow-up (Low-High), exhibited hazard ratio (HR) of 12.62 (95\%CI;3.52-31.34) compared to those remaining in the lowest tertile at both time points (reference group: Low-Low). While men who were at the top TG tertile throughout follow-up (High-High) had a HR for diabetes of 7.08 (95\%CI;2.52-14.45), those whose TG decreased to the lowest tertile (High-Low) exhibited HR of 1.97(95\%CI;0.67-6.13). Alterations in TG during follow-up were associated with changes in BMI, physical activity, and breakfast-eating habits $(\mathrm{p}<0.05)$, but remained an independent modifier of diabetes risk even after adjustment for such changes.

Conclusion: Two measurements of fasting TG obtained 3-5 years apart can assist in identifying apparently healthy young men at increased risk for diabetes, independent of traditional risk factors and of associated changes in BMI and life-style parameters.

Supported by: Ben-Gurion University of the Negev, Israel Defense Forces National Budget

\section{8}

IGF-I/IGF-binding protein 3 ratio: a mechanistic insight into cardiometabolic risk

J. Sierra-Johnson ${ }^{1}$, R.M. Fisher ${ }^{1}$, A. Romero-Corral ${ }^{2}$, V.K. Somers ${ }^{2}$, F. Lopez-Jimenez ${ }^{2}$, A. Malarstig ${ }^{1}$, A. Hamsten ${ }^{1}$, K. Brismar' ${ }^{1}$,

M.-L. Hellenius ${ }^{1}$

${ }^{1}$ Medicine, Karolinska Institutet, Stockholm, Sweden, ${ }^{2}$ Internal Medicine, Mayo Clinic, Rochester, United States

Background and aims: Recent reports suggest that IGF-I and IGFBP-3 have independent and opposing mechanistic effects on insulin, which lead us to hypothesize that by calculating the ratio of these two proteins we could detect subjects at particular high cardiometabolic risk. To assess the relationship between the insulin-like growth factor 1/ insulin-like growth factor binding protein 3 (IGF-I/IGFBP-3) ratio and the metabolic syndrome/insulin resistance.

Materials and methods: We examined 3,281 subjects (1,463 men; 1,818 women aged 20 to 49 years), otherwise healthy adults, who participated in the Third National Health and Nutrition Examination Survey (NHANES III) and had the recently released measurements of IGF-I and IGFBP-3. Insulin resistance was estimated using the computer homeostatic model assessment (HOMA-2). The updated ATP-III definition of the metabolic syndrome was used. We applied adjusted logistic and linear regression models.

Results: After adjusting for age and race, men and women in the lowest quartile of the IGF-I/IGFBP-3 ratio were 3 times more likely to meet the ATP-III definition of the metabolic syndrome and twice as likely to be insulin resistant. Mean values of the IGF-I/IGFBP-3 ratio decreased significantly as the number of metabolic syndrome components increased (ANOVA $p<0.0001$ ). Area under the curve for detecting metabolic syndrome/insulin resistance using the IGF-I/IGFBP-3 ratio was 0.760 , significantly improving upon either protein alone $(\mathrm{p}=0.01)$. 
Conclusion: The IGF-I/IGFBP-3 ratio is significantly associated with the metabolic syndrome and insulin resistance. A low IGF-I/IGFBP-3 ratio may provide a mechanistic insight into development of the different cardiometabolic entities and its clustering phenomenon. Supported by: EFSD/Lilly Research award

\section{9}

Quality of care of the risk factors in patients with and without diabetic foot syndrome in a German diabetic population The Diabetes in Germany (DIG) Study: 4 years follow-up data

P. Ott, J. Stelzer, I. Benke, C. Koehler, M. Hanefeld

Zentrum für Klinische Studien, GWT-TUD GmbH, Dresden, Germany

Background and aims: The prevention of diabetic foot syndrome requires an optimal control of blood glucose/HbAlc and of the other risk factors. The DIG study presents population based data on the quality of type 2 diabetes treatment and of major coronary risk factors in Germany for a four years period. This report analyses the data of the patients with and without diabetic foot syndrome (DFS) with the question: Is an optimal treatment of the cardiovascular risk factors connected with a lower rate of diabetic foot syndrome in the clinical practice?

Materials and methods: 4020 patients with type 2 diabetes between 35 and 80 years old were recruited by 238 physicians from general and specialised practices across Germany. Medical history, risk factor profile and clinical data were registered. We analysed $\mathrm{HbAlc}$, fasting blood glucose (FBG), postprandial blood glucose (ppBG), blood pressure (BP), triglycerides (TG), HDLand LDL-Cholesterol at baseline and the influence of concomitant disease on the development of diabetic foot syndrome. DFS was diagnosed if typical ulcer and/or amputation). 2959 patients were seen at 4 years follow-up. 91 patients have a DFS. Polyneuropathy was diagnosed if typical symptoms and/or vibration $<4 / 8$ and/or drugs for polyneuropathy was given. Diagnosis of peripheral arterial disease (PAD) was diagnosed if claudication, peripher revascularisation procedure or missing of pulses of foot were reported.

Results: The patients with DFS were significantly older (63.2 vs. 61.4 years, $\mathrm{p}<0.05)$, had a longer diabetes duration $(12.8$ vs. 8.3 years $\mathrm{p}<0.001)$ and had a larger abdominal circumference ( 111.7 vs. $104.7 \mathrm{~cm} \mathrm{p}<0.001)$. The glycaemic control was worse in patient with DFS (HbAlc -7.4 vs. $7 \% \mathrm{p}<0.01 / \mathrm{pp}$ blood glucose -9.9 vs. $9.0 \mathrm{mmol} / \mathrm{l} \mathrm{p}<0.05$ ). Puls pressure (62.6 vs. $57.4 \mathrm{~mm}$ $\mathrm{Hg} \mathrm{p}<0.01)$ and Systolic blood pressure (144.8 vs. $138.9 \mathrm{mmHg} \mathrm{p}<0.01)$ were higher too. No significant differences were found for lipids, FBG and diastolic blood pressure.

Multivariate analysis reveals polyneuropathy, PAD in combination with polyneuropathy and abdominal circumference as significant predictor for development DFS.

Conclusion: Patients with diabetic foot syndrome show a significantly worse control of HbAlc, BP and central obesity. Therefore a better control of these three major risk factors seems to be essential. This is especially necessary for patients with polyneuropathy and/or PAD.

Supported by: Pfizer GmbH

\section{0}

The impact of age on lung function in U.S. patients with diabetes: data from the National Health and Nutrition Examination Survey III (NHANES III, 1988-1994)

P.-Y. Berclaz ${ }^{1}$, H. Gao ${ }^{1}$, D. Swanson ${ }^{2}$, D. Webb ${ }^{1}$, J. Tobian' ${ }^{1}$, J. Li ${ }^{1}$, R. Crapo ${ }^{3}$, R. Jensen ${ }^{3}$

${ }^{1}$ Eli Lilly \& Co., Indianapolis, United States, ${ }^{2}$ Medical Writing, i3 Statprobe, Ann Arbor, United States, ${ }^{3}$ Pulmonary Division, LDS Hospital, Salt Lake City, UT, United States

Background and aims: Patients with diabetes have lower average forced vital capacity (FVC) and forced expiratory volume $\left(\mathrm{FEV}_{1}\right)$ than individuals without diabetes. Since lung function eventually declines with age in adults, it is important to know how the rate of decline in patients with diabetes compares to that of subjects with normal glycaemic control. We conducted a retrospective analysis of the NHANES III data set to determine whether patients with diabetes have accelerated age-related decline in lung function.

Material and methods: In the NHANES III study, spirometry was performed on 20,624 subjects who were randomly selected from across the United States. For our analysis, the Diabetes group was defined as a subset of participants who answered "yes" to the question, "Has a doctor ever told you that you had diabetes (sugar in the blood)?" The No Diabetes group consisted of subjects who answered "no" to having diabetes and had glycosylated haemoglobin $\mathrm{A}_{1 \mathrm{C}}\left(\mathrm{HbA}_{1 \mathrm{C}}\right)<6.0 \%$. Additional exclusion criteria for both groups included age $>90$, smoking, presence of asthma or chronic lung disease, or presence of pulmonary symptoms (ie, cough, wheezing or whistle in the chest, shortness of breath, or mucus). Subjects were divided into younger (18-29 y) and older ( $\geq 30 \mathrm{y}$ ) age groups to control for normal age-related lung development and decline. The effect of age on spirometry was assessed using an ANCOVA while controlling for sex, height, ethnicity and body mass index.

Results: Compared to the No Diabetes group $(n=6,678)$, the Diabetes group $(\mathrm{n}=496)$ had fewer males ( $46 \%$ vs. $38 \%)$, was older ( 41.2 vs. $60.1 \mathrm{y})$ and had higher mean $\mathrm{HbA}_{1 \mathrm{C}}$ values (5.15\% vs. $7.91 \%$ ). The ethnic distribution of the No Diabetes and Diabetes groups was: Caucasian (39.2\% vs. $30.0 \%)$, NonHispanic black (25.6\% vs. $27.4 \%$ ), Hispanic (30.7\% vs. $40.1 \%$ ), and other ( $4.5 \%$ vs. $2.4 \%$ ). For the $18-29$ y old participants, the mean values for the Diabetes group were lower but not significantly different for $\mathrm{FEV}_{1}(-.096 \mathrm{~L}$, $\mathrm{p}=.29)$ and FVC $(-.130 \mathrm{~L}, \mathrm{p}=.21)$ and there were no significant differences in the rates of change between the groups. There was increased variation within the $18-29$ y old Diabetes group due to the small sample size $(n=25)$. For the $\geq 30$ y old participants, the mean values for the Diabetes group were significantly lower for FEV $(-.094 \mathrm{~L}, \mathrm{p}<.001)$ and FVC $(-.166 \mathrm{~L}, \mathrm{p}<.001)$ and the rate of change for the Diabetes group differed from the No Diabetes group by $.0004 \mathrm{~L} / \mathrm{y}(\mathrm{p}=.78)$ for $\mathrm{FEV}$ and $-.0005 \mathrm{~L} / \mathrm{y}(\mathrm{p}=.77)$ for FVC.

Conclusion: In the NHANES III population, patients with diabetes had small reductions in pulmonary function that were statistically significant in the older $(\geq 30 \mathrm{y})$ group. There were no significant differences in the rate of change between the Diabetes and No Diabetes groups.

\begin{tabular}{|c|c|c|c|c|c|c|c|c|c|c|}
\hline \multicolumn{11}{|c|}{ Mean Pulmonary Function Adjusted for Sex, Height, Ethnicity and Body Mass Index. } \\
\hline \multirow[t]{2}{*}{ Group } & \multicolumn{5}{|c|}{ Ages 18-29y } & \multicolumn{5}{|c|}{ Ages $\geq 30 \mathrm{y}$} \\
\hline & $\mathrm{n}$ & $\begin{array}{l}\text { Pulmonary } \\
\text { Function (L) }\end{array}$ & P Value & $\begin{array}{l}\text { Rate of Change } \\
(\mathrm{L} / \mathrm{y})\end{array}$ & P Value & $\bar{n}$ & $\begin{array}{l}\text { Pulmonary } \\
\text { Function (L) }\end{array}$ & P Value & $\begin{array}{l}\text { Rate of Change } \\
\text { (L/y) }\end{array}$ & P Value \\
\hline \multicolumn{11}{|l|}{$\mathrm{FEV}_{1}$} \\
\hline No Diabetes & 2361 & $3.586(.012)$ & & $.0104(.0114)$ & & 4317 & $2.891(.010)$ & & $-.0313(.0023)$ & \\
\hline Diabetes & 25 & $3.490(.091)$ & & $.0334(.0276)$ & & 471 & $2.797(.022)$ & & $-.0309(.0027)$ & \\
\hline Difference & & $-.096(.090)$ & .29 & $.0230(.0250)$ & .36 & & $-.094(.022)$ & $<.001$ & $.0004(.0015)$ & .78 \\
\hline \multicolumn{11}{|l|}{ FVC } \\
\hline No Diabetes & 2361 & $4.197(.014)$ & & $.0327(.0131)$ & & 4317 & $3.675(.012)$ & & $-.0242(.0028)$ & \\
\hline Diabetes & 25 & $4.067(.104)$ & & $.0682(.0316)$ & & 471 & $3.509(.027)$ & & $-.0247(.0033)$ & \\
\hline Difference & & $-.130(.103)$ & .21 & $.0353(.0286)$ & .22 & & $-.166(.027)$ & $<.001$ & $-.0005(.0018)$ & .77 \\
\hline
\end{tabular}

Values are least square mean (SE). 


\section{PS 019 CVD and mortality in diabetes}

\section{1}

Antidiabetic treatments and the risk of hospitalisation with myocardial infarction: a nationwide case-control study

H.T. Horsdal ${ }^{1,2}$, J. Rungby ${ }^{2,3}$, F. Søndergaard ${ }^{1}$, S.P. Johnsen ${ }^{1,4}$

${ }^{1}$ Department of Clinical Epidemiology, ${ }^{2}$ Department of Pharmacology,

${ }^{3}$ Department of Endocrinology C, ${ }^{4}$ Center of Cardiovascular Research,

Aarhus University Hospital, Denmark

Background and aims: Data on cardiovascular risk of different types of antidiabetic treatment are sparse and conflicting. Here, we examined the risk of myocardial infarction (MI) among patients treated with sulphonylureas, metformin, insulin, any combination and no antidiabetic pharmacotherapy. Materials and methods: We conducted a population-based case-control study among all patients with type 2 diabetes mellitus in Denmark with a first-time MI and age - and gender-matched non-MI controls in the 19962004 using data from computerized registries. Using conditional logistic regression with adjustment for multiple covariates, we estimated odds ratios (ORs) of MI associated with antidiabetic treatment, using patients treated with sulphonylureas as the reference group.

Results: We identified a total of 10,616 cases hospitalized with MI and 90,697 controls, of which 7,134 (67.2\%) and 53,821 (59.3\%) had filled a prescription for an antidiabetic drug, respectively. We found lower risk of MI among users of metformin (adjusted OR=0.86, 95\% CI: 0.78-0.95), insulin (adjusted $\mathrm{OR}=0.92,95 \% \mathrm{CI}$ : 0.86-0.99), and among patients not receiving any antidiabetic pharmacotherapy (adjusted OR=0.75, 95\% CI: 0.71-0.79) compared with users of sulphonylureas. Users of any combination had similar risk of MI as users of sulphonylueas (adjusted OR=0.99, 95\% CI: 0.92-1.06). We found no differences between individual sulphonylureas, and glycaemic control had only minor impact on the risk estimates in a subanalysis including $\mathrm{HbA}_{1 c^{\circ}}$ Conclusion: Our findings provide some support for the hypothesis that sulphonylureas may be associated with increased risk of hospitalization with MI. Supported by: Research Initiative of Aarhus University Hospital, University of Aarhus, Diabetes Association, and Danish Medical Research Council

\section{2}

Cardiovascular disease risk in type 2 diabetes patients, non-obese that meet and obese who do not meet goals for glucose and blood pressure control. A Swedish National Diabetes Registry report

B. Zethelius ${ }^{1}$, J. Cederholm² , B. Eliasson ${ }^{3}$, K. Eeg-Olofsson ${ }^{3}$, P.M. Nilsson ${ }^{4}$, S. Gudbjörnsdottir ${ }^{3}$

${ }^{1}$ Dept. of Public Health and Caring Sciences/Geriatrics, Uppsala University, ${ }^{2}$ Dept. of Public Health and Caring Sciences/Family Medicine and Clinical Epidemiology, Uppsala University, ${ }^{3}$ Dept. of Medicine, Göteborg University, ${ }^{4}$ Dept. of Clinical Sciences Medicine, Lund University, Sweden

Background and aims: Glycaemic and blood pressure control are considered two important parts of multi factorial risk factor control in type 2 diabetes patients for reducing cardiovascular disease (CVD) morbidity and mortality. The aim of the present study was to estimate the relative risk for fatal and non-fatal CVD and all cause mortality in patients who meet and do not meet goals for glycaemic (HbAlc $<7 \%$, DCCT-standard, adopted by the ADA) and blood pressure control $(\mathrm{SBP} / \mathrm{DBP} \leq 130 / 80 \mathrm{mmHg})$ considering if patients were obese or not.

Materials and methods: Data from type 2 diabetes patients in the Swedish National Diabetes Register (NDR), age $\leq 85$ years, followed-up 6 years from 1998 to 2003 was used. Non-obese patients $(n=744)$ with excellent baseline control (HbAlc $<7 \%, \mathrm{BP} \leq 130 / 80 \mathrm{mmHg}$ and $\mathrm{BMI}<30 \mathrm{~kg} / \mathrm{m}^{2}$ ) and obese patients $(\mathrm{n}=1857)$ with adverse baseline control (HbAlc $\geq 7 \%$, BP $>130 / 80$ $\mathrm{mmHg}$ and $\mathrm{BMI} \geq 30 \mathrm{~kg} / \mathrm{m}^{2}$ ) were studied.

Results: Comparison of type 2 diabetes patients, non-obese with excellent and obese with adverse control showed that risk for first incident fatal/nonfatal CVD, was $40 \%$ lower with excellent control (RR, 0.60; 95\%CI, 0.47 $0.76 ; \mathrm{p}<0.001)$, after adjustment for age, sex, diabetes duration, smoking and microalbuminuria $>20 \mu \mathrm{g} / \mathrm{min}$. Corresponding risks for myocardial infarction was $39 \%$ lower (RR, $0.61 ; 95 \% \mathrm{CI}, 0.43-0.85 ; \mathrm{p}=0.004$ ), for coronary heart disease $26 \%$ lower (RR, $0.74 ; 95 \% \mathrm{CI}, 0.56-0.97 ; \mathrm{p}=0.03$ ), for stroke $61 \%$ lower (RR, $0.39 ; 95 \% \mathrm{CI}, 0.26-0.60 ; \mathrm{p}<0.001$ ), and for all cause mortality $21 \%$ lower (RR, $0.79 ; 95 \% \mathrm{CI}, 0.56-1.12$; n.s.).
Conclusion: In this population of type 2 diabetes patients from the Swedish NDR, a reduced risk for fatal and non-fatal CVDs was observed among nonobese patients who met the goals for glycaemic and blood pressure control as compared to obese patients who did not. These results support the importance of glucose and blood pressure control obtained in randomized clinical trials on multifactorial risk factor control, in order to reduce CVD. Results observed emphasize on applying target oriented treatment in type 2 diabetes as well as a need for further clinical studies on effective weight reducing treatments to be applied in type 2 diabetes patients.

\section{3}

Cardiovascular risk increases sharply in hypercholesterolaemic patients with fasting plasma glucose of $95-110 \mathrm{mg} / \mathrm{dL}$, but not in the patients treated by low-dose pravastatin

H. Kurata, N. Tajima

Internal Medicine, Jikei University School of Medicine, Tokyo, Japan

Background and aims: Accumulated research evidence demonstrated that impaired glucose tolerance is the risk factor for developing cardiovascular (CVD) risk, but it is still controversial in impaired fasting glucose (IFG). The aim of the current study is to investigate the relationship between the hypercholesterolemia and future development of CVD in patients with hypercholesterolemia, and additionally evaluated effect of the pravastatin in post-hoc analysis of the large scale primary prevention study with pravastatin (MEGA Study).

Materials and methods: The MEGA Study aimed to evaluate the effect of low dose pravastatin (10-20mg/day) on primary prevention of cardiovascular disease in a total of 7,832 Japanese patients (male, 40 to 70 years; female, post-menopause to 70 years) with mild to moderate hyperlipidemia without cardiovascular disease. Patients were randomized to diet alone or diet + pravastatin and followed up more than 5-years. A total of 6,673 patients with baseline and follow-up fasting glucose level were analyzed, and which included $68 \%$ of female and $42 \%$ of hypertension, and its fasting plasma glucose (FPG) level was $109 \mathrm{mg} / \mathrm{dL}$. The relationship between FPG and risk of CVD events was evaluated using multivariate Cox proportional hazards model with restricted quadratic spline based on three knots for FPG quartiles, adjusted by sex, age, low-density lipoprotein cholesterol, high-density lipoprotein cholesterol, hypertension, and smoking.

Results: A total of $231 \mathrm{CVD}$ events were observed in 6,673 patients with FPG value. The spline curve in diet group drew the sharp ascending curve in around 95 to $110 \mathrm{mg} / \mathrm{dL}$ in FPG, and it got to gentle in $110 \mathrm{mg} / \mathrm{dL}$ or more. In contrast, the curves in the diet plus pravastatin group drew the liner ascending curves from around $95 \mathrm{mg} / \mathrm{dL}$. The hazard ratio was constantly lower in diet plus pravastatin group than in diet group irrespective of FPG level. Conclusion: The IFG is associated with the risk for CVD in hypercholesterolemia, but it is not under the pravastatin treatment.

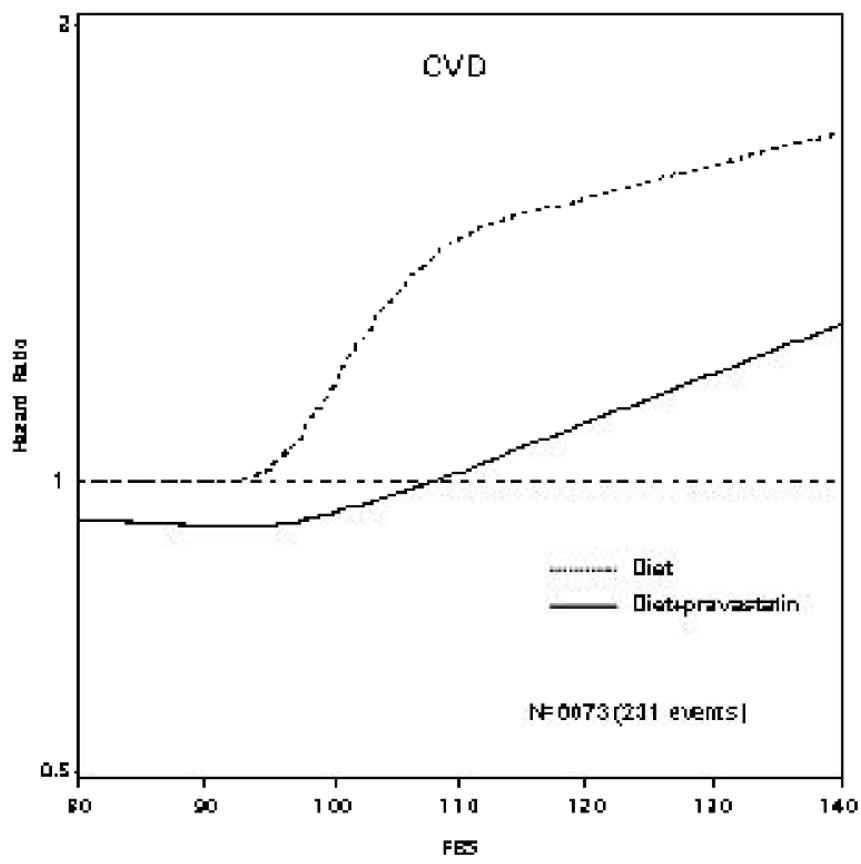

Supported by: Daiichi Sankyo 


\section{4}

The change in serum Gamma-glutamyltransferase is associated with those in triglyceride and blood pressure and with worsening of glucose tolerance in Japanese male subjects

Y. Wada ${ }^{1}$, Y. Hamamoto ${ }^{2,1}$, S. Honjo ${ }^{1}$, J. Fujikawa ${ }^{3}$, R. Hosokawa ${ }^{4}$, H. Ikeda ${ }^{1}$, K. Nabe ${ }^{1}$, T. Kimura ${ }^{1}$, T. Aoyama ${ }^{1}$, K. Nomura ${ }^{1}$, H. Koshiyama ${ }^{1,2}$

${ }^{1}$ Center for Diabetes and Endocrinology, The Tazuke Kofukai Foundation Medical Research Institute Kitano Hospital, Osaka, ${ }^{2}$ Department of Diabetes and Clinical Nutrition, Kyoto University Graduate School of Medicine, Kyoto, ${ }^{3}$ Department of Clinical Laboratory, The Tazuke Kofukai Foundation Medical Research Institute Kitano Hospital, Osaka, ${ }^{4}$ Department of Preventive Medicine, The Tazuke Kofukai Foundation Medical Research Institute Kitano Hospital, Osaka, Japan

Background and aims: While the prevalence of metabolic syndrome is explosively increasing in Japan, it is of great interest which factors can be markers to predict the progress into the metabolic syndrome. In the present study, we investigated the possibility of gamma-glutamyltransferase (GGT) as a marker for aggravation of blood pressure, dyslipidemia and glucose tolerance in subjects who received the oral glucose tolerance tests (OGTT) annually in clinical check-up in our Institute.

Subjects and methods: The study included a total of 198 male subjects (age 30 to 80 years). They received OGTT, routine blood chemistry tests as well as physical examinations in the annual regular check-up of Department of Preventive Medicine of our Institute during 2004 to 2006. The same examinations were performed one year after the first examinations. Their age was $60.3 \pm 0.8 \mathrm{SE}$ years. The subjects were divided into two groups depending on the presence $(n=124)$ or absence $(\mathrm{n}=74)$ of fatty liver, which was based on the diagnosis by ultrasonography. The correlation between the changes in GGT and those in other parameters during one year follow up were studied by linear regression analyses.

Results: First, we investigated the relationship between the changes in all parameters in the subjects as a whole. There was a significantly positive correaltion between the changes in GGT and those in 2-hour OGTT post-challenge plasma glucose levels in all subjects $(r=0.24, P=0.001)$. Furthermore, there was a singinicantly positive correaltion between the changes in GGT and those in triglyceride levels $(r=0.19, P=0.02)$.

Second, we investigated the relationship between various parameters in a subgroup of subjects who showed NGT on the first examination. There was no correlation between the changes in any parameters. Third, we investigated the relationship between the changes of parameters in a subgroup of subjects who showed IGT or DM pattern on the first examination. There was a weak but significanly positive cooreleation between the changes in GGT and those in both systolic and diastolic blood pressure (systolic pressure: $r=0.19, P=$ 0.03 ; diastolic pressure: $r=0.23, P=0.01$ ). Finally, the relationships between the changes in parameters were examined in a subgroup of subjects with fatty liver. The changes in GGT showed a significant positive correlation with those in post-challenge plasma glucose levels $(r=0.24, P=0.008)$, triglyceride $(r=$ $0.18, P=0.04)$, systolic blood pressure $(r=0.23, P=0.008)$, and diastolic blood pressure $(r=0.26, P=0.004)$. In contrast, among the subjects without fatty liver, the change in GGT showed no correlation with those in other parameters. Conclusion: The change in serum GGT levels for one year can be a predictor of aggravation of hypertriglyceridemia, hypertension and glucose intolerance in Japanese male subjects, especially in those with fatty liver.

\section{5}

The association of dyslipidaemia with cardiovascular mortality in individuals without a prior history of diabetes in the DECODE Study L. Zhang ${ }^{1,2}$, Q. Qiao ${ }^{1,2}$, J. Tuomilehto ${ }^{1,2}$, N. Hammar ${ }^{3,4}$, G. Ruotolo ${ }^{5,4}$, C.D.A. Stehouwer ${ }^{6}$, R.J. Heine ${ }^{7}$, M. Eliasson ${ }^{8}$, B. Zethelius ${ }^{9}$

${ }^{1}$ Department of Public Health, University of Helsinki, Finland, ${ }^{2}$ Department of Health Promotion and Chronic Disease Prevention, National Public Health Institute, Helsinki, Finland, ${ }^{3}$ Department of Epidemiology, Institute of Environmental Medicine, Karolinska Institute, Stockholm, Sweden, ${ }^{4}$ AstraZeneca, Mölndal, Sweden, ${ }^{5}$ Cardiovascular Biology Research Centre, San Raffaele Scientifc Institute, Milan, Italy, ${ }^{6}$ Department of Medicine, University Hospital Maastricht, AZ Maastricht, Netherlands, ${ }^{7} \mathrm{VU}$ University Medical Center, Amsterdam, Netherlands, ${ }^{8}$ Department of Public Health and Clinical Medicine, Umeå University, Sweden, ${ }^{9}$ Department of Public Health/Geriatrics, Uppsala University Hospital, Sweden

Background and aims: Dyslipidaemia is associated with an increased risk of cardiovascular disease (CVD), particularly in diabetic individuals. However, whether lipid levels predict CVD death to the same extent in different prediabetic states is not clear yet. Therefore, we evaluated the role of dyslipidaemia on the risk of CVD mortality in individuals without a prior history of diabetes by categories of glucose tolerance.

Materials and methods: Data from population-based prospective studies of 9,043 men and 8,594 women aged 25-89 years representing 14 studies in 6 European countries were jointly analysed. A total of 732 CVD deaths occurred during the average 9 years of follow-up. Multivariate-adjusted hazard ratios (HRs) and $95 \%$ confidence intervals (CIs) for CVD mortality were estimated using Cox proportional hazard analysis.

Results: For HDL cholesterol (HDL-C), multivariate adjusted HR for CVD mortality was $0.69(0.49-0.96)$ in individuals with isolated fasting hyperglycaemia (fasting plasma glucose $[\mathrm{FPG}] \geq 6.10 \mathrm{mmol} / \mathrm{l}$ and 2-hour plasma glucose $[2 \mathrm{hPG}]<7.80 \mathrm{mmol} / \mathrm{l}), 0.64(0.48-0.86)$ in those with combined glucose intolerance (CGI, FPG $\geq 6.10 \mathrm{mmol} / \mathrm{l}$ and $2 \mathrm{hPG} \geq 7.80 \mathrm{mmol} / \mathrm{l}$ ), and $0.66(0.48-0.90)$ in patients with newly diagnosed diabetes, but not in those with isolated post-load hyperglycaemia ( FPG $<6.10 \mathrm{mmol} / \mathrm{l}$ and $2 \mathrm{hPG} \geq 7.80$ $\mathrm{mmol} / \mathrm{l})$. As a consequence, total cholesterol to HDL-C ratio (TC/HDL-C) was also positively associated with CVD death in the same groups. Furthermore, non-HDL-C was positively associated with CVD death in individuals with IFG, even though was significant only for isolated fasting hyperglycaemia (1.39 [1.06-1.82]). Finally, triglycerides (TG) were positively associated with CVD death only in individuals with CGI (HR=1.20 [1.04-1.39]) or newly diagnosed diabetes $(1.20[1.02-1.40])$.

Conclusion: Distinct patterns of dyslipidaemia are associated with increased CVD mortality in individuals with isolated fasting hyperglycaemia or IFG, but not in those with isolated post-load hyperglycaemia or IGT, suggesting that a different pathophysiology underlies these two prediabetic states. Supported by: Academy of Finland, Future Forum Research Grant and Novo Nordisk Foundation. The DECODE Study has been financially supported by unrestricted grants from Novo Nordisk, Novartis Pharma AG; AstraZeneca

\section{6}

Mortality in a representative sample of diabetic patients in France - the ENTRED Study

D. Simon ${ }^{1,2}$, I. Romon ${ }^{3}$, A. Weill ${ }^{4}$, M. Varroud-Vial ${ }^{5}$, E. Eschwege ${ }^{2}$,

E. Jougla ${ }^{6}$, A. Fagot-Campagna ${ }^{3}$

${ }^{1}$ Service de Diabétologie, Hôpital de la Pitié-APHP, Paris, ${ }^{2} \mathrm{U}-780$, INSERM, Villejuif, ${ }^{3}$ Département des Maladies Chroniques, Institut National de Veille Sanitaire, Saint-Maurice, ${ }^{4}$ Direction de la stratégie, des études et des statistiques, CNAMTS, Paris, ${ }^{5}$ Service de Diabétologie, ANCRED, Corbeil, ${ }^{6}$ Cepi-DC, INSERM, Le Vésinet, France

Background and aims: Very few data are available concerning mortality rates and causes of death in national representative samples of diabetic patients, and use of routine data mortality statistics to measure the Public Health impact of diabetes in a country is debated. Based on a random sample of pharmacologically-treated diabetic patients living in France, death rates, causes of death and the proportion of death certificates mentioning diabetes were assessed from 2001 to 2006.

Materials and methods: In 2001, 9,446 adults living in France who claimed refund of oral antidiabetic agents or insulin were randomly selected from the database of the major National Health Insurance System which covers more than $70 \%$ of the French population. In 2006, the vital status was successfully extracted from the national registry for 9,101 subjects (96\%). Among the 345 cases with unknown vital status, around the $3 / 4$ were born out of France. Death certificates were available for $99 \%$ of the deceased patients.

Results: At baseline, these 9,101 diabetic patients were men for 54\%, had a mean age of 65 years and a mean diabetes duration of 12 years. Between October $1^{\text {st }} 2001$ and September $14^{\text {th }} 2006,1,388$ subjects died (15.3\%), i.e. $32.4 / 1,000$ person-years. Death rates by age and sex are shown in the Figure. Mean age at death was 74 years in men and 80 years in women. Causes of deaths were: circulatory diseases in $60 \%$ in multiple-cause (MC) analysis [32\% as underlying cause(UC)], including ischaemic heart disease in $18 \%$ MC (11\% UC) and stroke in 14\% MC ( $8 \%$ UC); all neoplasms in $32 \%$ MC ( $29 \%$ UC); all respiratory diseases in $18 \%$ MC (5\% UC); diseases of the digestive system in $11 \% \mathrm{MC}(4 \% \mathrm{UC})$ and diseases of the genito-urinary system in $10 \% \mathrm{MC}(2 \% \mathrm{UC})$, including renal failure in $9 \% \mathrm{MC}$ ( $2 \% \mathrm{UC})$. Diabetes was mentioned in $35 \%$ of death certificates including $12 \%$ as UC of death. In the 253 subjects who died with ischaemic heart disease, diabetes was mentioned in only 136 death certificates (54\%).

Conclusion: This study relies upon a national sample of diabetic patients quite representative of the French diabetic population, who could be traced 
over 5 years for vital status and causes of death with a high degree of exhaustiveness. It shows a high mortality rate, higher in men in all age classes. Circulatory diseases are the most frequent cause of deaths. Diabetes is mentioned in about one third of death certificates only, and is obviously underreported in death certificates even when taking into account multiple causes of death.

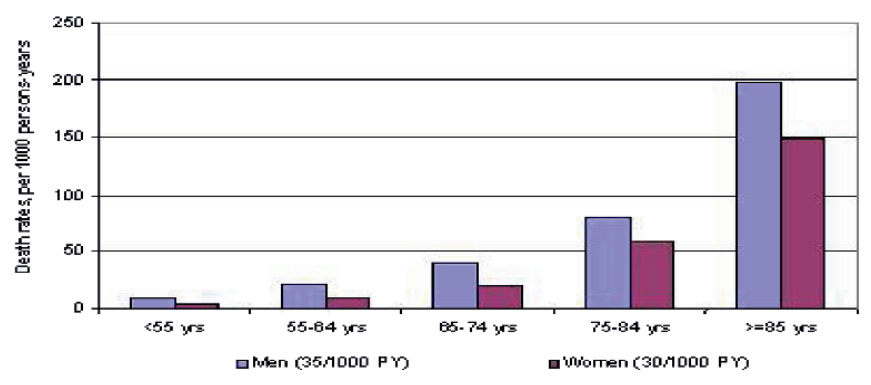

\section{7}

UKPDS outcomes model predicted total mortality in U.S. adults with type 2 diabetes

Q. Zhang ${ }^{1}$, N.D. Wong ${ }^{2}$, M. Song 2 , S. Malik 2 , V.A. Lopez 2 , H.M. Phatak ${ }^{3}$, M. Panagiotis ${ }^{3}$, C.M. Alexander ${ }^{3}$

${ }^{1}$ Global Outcomes Research, Merck \& Co, Inc, Whitehouse Station, NJ, United States, ${ }^{2}$ University of California, Irvine, United States, ${ }^{3}$ Merck \& Co., Inc., Whitehouse Station, United States

Background and aims: The validity of the United Kingdom Prospective Diabetes Study (UKPDS) Outcomes Model is not established in populations with type 2 diabetes outside the United Kingdom. We compared the total mortality predicted from the UKPDS model with the observed mortality seen among U.S. adults with type 2 diabetes who were comparable to the UKPDS study cohort at entry.

Materials and methods: We studied participants with diabetes from the National Health and Nutrition Examination Survey 1988-1994 (NHANES III) with characteristics comparable to the UKPDS cohort, including age 25-64 years, age at diagnosis of diabetes $\geq 30$ years, diabetes duration $0-5$ years, and no evidence of cardiovascular disease (CVD). The 10-year observed total mortality was compared to the UKPDS model-predicted mortality based on age, gender, height, weight, diabetes duration, glycated hemoglobin (HbAlc), total and HDL-cholesterol, systolic blood pressure, and smoking status.

Results: Participants $(n=156)$ were on average aged $49.6 \pm 1.0$ years at baseline, aged $47.1 \pm 1.0$ years at diagnosis of diabetes, with a mean duration of diabetes of $2.6 \pm 0.2$ years and were followed for an average of $10.4 \pm 2.0$ years. The UKPDS model-predicted 10-year total mortality was $15.0 \%$, similar to the observed total mortality of $13.7 \%$ ( $42.6 \%$ due to CVD) (Figure). The average annual difference between predicted and observed mortality was 0.6 years. Age and duration of diabetes were the most important variables that affected the ability of the UKPDS model to predict total mortality among diabetic patients with no CVD at baseline and age of diagnosis $\geq 30$ years.

Conclusion: The UKPDS Outcomes Model accurately predicted observed mortality in the subgroup of NHANES III participants with characteristics similar to the UKPDS.

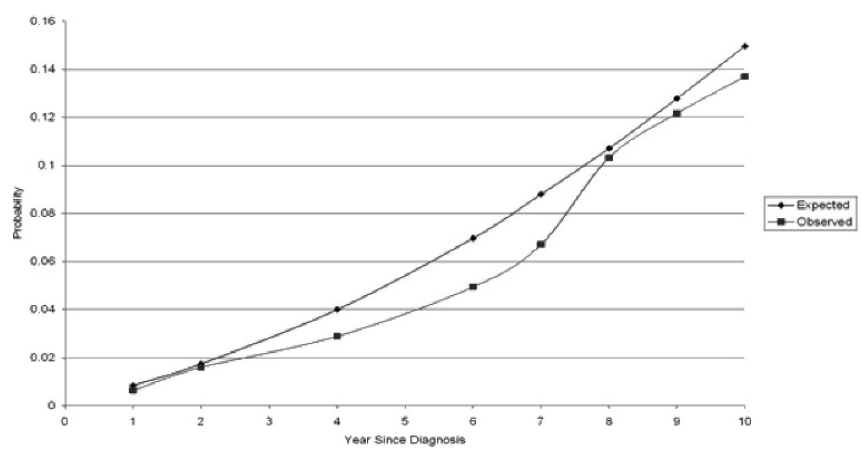

Figure 1. Expected vs. Observed Risk Rates per Year for Any Cause Mortality Aged 25-64 years with Diabetes Duration $<6$ years $(\mathrm{N}=156)$

Support by: Merck \& Co., Inc.

\section{8}

Does fasting plasma glucose predict mortality or coronary heart disease in senior citizens without diabetes or in those with undiagnosed diabetes? The Dubbo Study of Australian elderly

L.A. Simons ${ }^{1}$, J. Simons ${ }^{1}$, Y. Friedlander ${ }^{2}$, J. McCallum ${ }^{3}$

${ }^{1}$ Lipid Research Dept, University of NSW, St Vincent's Hospital, Darlinghurst NSW, Australia, ${ }^{2}$ Epidemiology Unit, Hebrew UniversityHadassah School of Public Health, Jerusalem, Israel, ${ }^{3}$ Vice-Chancellor's Dept, Victoria University, Melbourne, Australia

Background and aims: The burden of cardiovascular disease (CVD) in diabetes is well known, falling heavily on females. But fasting plasma glucose (FPG) levels below diabetes "thresholds" may also be important, although current data are meagre and controversial. This study aims to confirm whether FPG predicts death or coronary heart disease (CHD) (a) in non-diabetic subjects in the presence or absence of prior CVD (i.e. CVD at study entry), and (b) in the presence of undiagnosed diabetes.

Materials and methods: We have analysed 16 year follow-up data in a cohort of Australian senior citizens, born before 1930 and first examined in 198889 (www.dubbostudy.org). Key outcomes were all-causes mortality and CHD (ICD-9 \& 10). Diabetes was defined on historical grounds (previous diagnosis or use of medication), undiagnosed diabetics were those without history but with FPG $>6.9 \mathrm{mmol} / \mathrm{L}$. Cox models were used to calculate point estimates and $95 \%$ ci's for the Hazard Ratio of the specified outcomes, FPG being entered as a continuous variable (unless otherwise specified).

Results: Death rates in males and females with prior CVD but no diabetes were 49 and 32/1000 person-years respectively (corresponding rates in the presence of diabetes were 86 and 67). CHD event rates were 39 and 27/1000 person-years respectively (76 and 85 in diabetes).

Table 1 presents Cox models for each $1 \mathrm{mmol} / \mathrm{L}$ increment in FPG (other standard variables in the models not shown). Historical diabetes was excluded from all 4 models, but models 3-4 include those with undiagnosed diabetes or lesser degrees of glucose intolerance.

FPG did not significantly predict any outcome in any model in males!

In females, FPG was a significant predictor of death or CHD in the cohort which included prior CVD but excluded all diabetes (model 1). In this model FPG levels 5.3-6.0 predicted a $41 \%$ greater risk of death and a 33\% greater risk of $\mathrm{CHD}$ than with $\mathrm{FPG}<4.6 \mathrm{mmol} / \mathrm{L}$.

In females with undiagnosed diabetes, FPG predicted death independently of prior CVD presence, but did not significantly predict CHD (models 3-4).

Conclusion: FPG in female senior citizens without diabetes significantly predicts all-causes mortality and CHD in those with prior CVD. FPG predicts

TABLE 1. COX MODELS, HAZARD RATIOS (95\% CI”S) OVER 16 YEARS FOLLOW-UP ( $n=$ number of males, females)

\begin{tabular}{lllll}
\hline & $\begin{array}{l}\text { Model 1 } \\
\text { (incl CVD \& excl FPG }>6.0) \\
(\mathrm{n}=1048,1371)\end{array}$ & $\begin{array}{l}\text { Model 2 } \\
(\text { excl CVD \& excl FPG }>6.0) \\
(\mathrm{n}=768,1079))\end{array}$ & $\begin{array}{l}\text { Model 3 } \\
\text { (incl CVD \& incl FPG }>6.0 \\
(\mathrm{n}=1145,1470)\end{array}$ & $\begin{array}{l}\text { Model 4 } \\
(\text { excl CVD \& incl FPG }>6.0 \\
(\mathrm{n}=831,1160)\end{array}$ \\
\hline Deaths, male & $1.07(0.91-1.27)$ & $1.02(0.82-1.25)$ & $1.00(0.92-1.09)$ & $1.00(0.89-1.12)$ \\
\hline Deaths, female & $1.30(1.09-1.56)$ & $1.17(0.95-1.44)$ & $1.18(1.10-1.26)$ & $1.18(1.04-1.35)$ \\
\hline CHD, male & $1.13(0.93-1.38)$ & $1.24(0.95-1.63)$ & $0.95(0.86-1.05)$ & $0.98(0.86-1.12)$ \\
\hline CHD, female & $1.24(1.02-1.51)$ & $1.07(0.84-1.37)$ & $1.05(0.98-1.12)$ & $1.11(0.94-1.30)$ \\
\hline
\end{tabular}


all-causes mortality (but not CHD) in females with undiagnosed diabetes. FPG does not predict any outcome in males in the present study design.

\section{9}

Mortality in a cohort of individuals with and without diabetes mellitus in a Swedish community during $\mathbf{3 0}$ years of follow up S.P.O. Jansson, D.K.G. Andersson, K. Svärdsudd

Department of Public Health and Caring Sciences, Family Medicine and Clinical Epidemiology Section, Uppsala University Sweden, Sweden

Background and aims: An excess mortality in people with diabetes compared to those without diabetes is well documented. This higher mortality is in the vast majority of cases due to cardiovascular disease (CVD). Studies of mortality rates concerning sex differences are inconsistent. In Sweden, the population have experienced a substantial decline in CVD the past 25 years but if this holds true for the diabetic population is unclear. Our aim in this prospective cohort study was to compare mortality (all causes) in individuals with and without diabetes during the time period 1972-2004.

Materials and methods: Since 1972 all patients with diabetes are registered in a diabetes register at Laxå primary healthcare centre. The register has been complemented with a reference population consisting of 5 age- and gender matched nondiabetic referents per diabetic subject. During 1972-2001, a total of 776 new cases of diabetes were found. The "Laxå register" (cases and controls) has further been updated from 1972-2004 with data from the national Cause of Death Register.

Results: During the follow-up period, 473 diabetic subjects died, 233 women ( 3 type 1 - and 230 type 2 diabetes individuals) and 240 men (1 type 1 - and 239 type 2 diabetes individuals) and 2077 nondiabetic referents ( 995 women and $1082 \mathrm{men}$ ). The 10-year mortality rate for diabetic men was $40 \%$ among those diagnosed 1972-1981, 35.4\% among those diagnosed 1982-1991 and 29.7\% among those diagnosed 1992-2001. Corresponding rates for male referents were $45.6 \%, 35.1 \%$ and $19.8 \%$. Among diabetic women the mortality rates were $30.1 \%, 26.6 \%$ and $20.9 \%$ and among female referents $34.8 \%, 26.3 \%$ and $13.7 \%$.

The referents on average reduced their mortality by $5 \%$ per five-year period throughout the whole study period as compared with the diabetic subjects who reduced their mortality by $0.1 \%$ per 5 -year period.

Conclusion: Individuals with diabetes showed only a marginally reduction in mortality during the three time periods compared with the referents, a significant trend difference. Women with diabetes had a lower mortality than diabetic men.

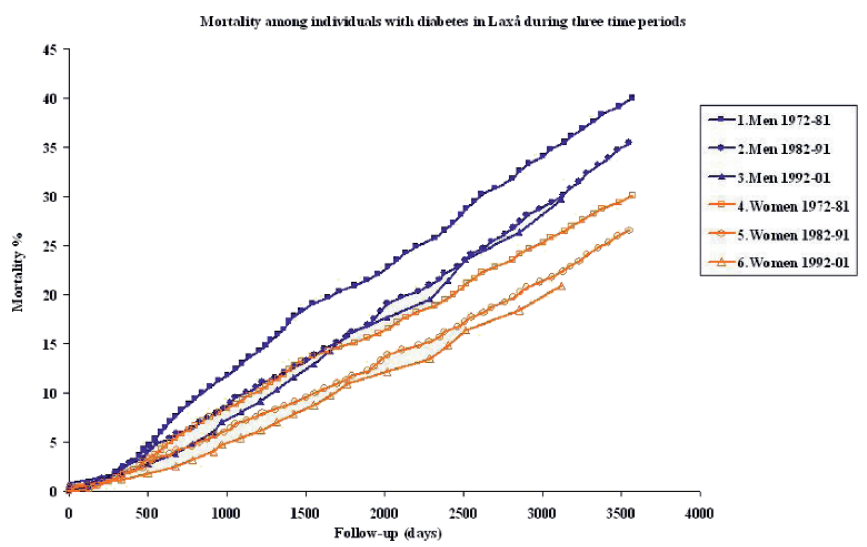

Supported by: Primary Health Care Research Unit, Örebro, Örebro County Council Research Unit, Family Medicine Research Centre, Örebro University, and Uppsala University

\section{PS 020 Diabetes and ethnicity}

\section{0}

Type 2 diabetes in the non-obese children and adolescents in Bangladesh M.A. Sayeed ${ }^{1}$, H. Mahtab ${ }^{2}$, A. Banu' ${ }^{2}$ P.A. Khanam², Z.A. Latif', A.U. Ahmed ${ }^{4}$ J.S. Nahar ${ }^{2}$, K. Khatun 2 , A. Azad Khan ${ }^{2}$ ${ }^{1}$ Community Medicine, Ibrahim Medical College, Dhaka, ${ }^{2}$ Epidemiology, BIRDEM, Dhaka, Bangladesh, ${ }^{3}$ Epidemiolgy, BIRDEM, Dhaka, ${ }^{4}$ Biochemistry, BIRDEM, Dhaka, Bangladesh

Background and aims: Burdened with the highest population density (1007 per sq.km) and frequent natural disasters Bangladesh has been striving against poverty, hunger and diseases. Inequitable distribution of food, education and healthcare has lead to undernutrition in one and obesity in the other. Additionally, rapidly urbanized environment influenced the traditional life. Childhood obesity is a well-known risk, while under-nutrition in pregnancy and low birth weight (LBW) was also attributed to T2DM and cardiovascular diseases. It is not known, however, how many of our children are underweight and how many of them are obese; and how many of them started developing diabetes. This is the first study to address the prevalence of diabetes among the obese and non-obese children and adolescents in Bangladesh.

Materials and methods: We investigated school children of age group 10 - 18 years in rural and urban communities in 2007. Investigations included socio-demographic information, height, weight, mid-upper-arm circumference (MUAC), blood pressure (SBP, DBP), fasting blood glucose (FBG), triglycerides (TG), and HDL-c. We used ADA criteria (1997) for impaired fasting glucose (IFG) and diabetes. For obesity and overweight, we used sex- and age-specific body mass index (BMI) criteria for the Asians recommended by the International Obesity Task Force.

Results: Overall, 2152 students (m / f: 1064 / 1088) volunteered the study. Their mean (SD) age was 13.3 (2.0) y, BMI was 18.5 (3.1) and MUAC was $21.2(3.4) \mathrm{cm}$. The mean (SD) FBG, TG and HDL were $4.6(0.87) \mathrm{mmol} / \mathrm{l}$, $107(44) \mathrm{mg} / \mathrm{dl}$ and $45.0(10.6) \mathrm{mg} / \mathrm{dl}$, respectively. The prevalence of normal weight was only $35.8 \%$ (m / f: $31.8 / 39.8 \%$, p $>0.05$ ), whereas, underweight was in excess ( $\mathrm{m} / \mathrm{f:} 60 / 56.1 \%, \mathrm{p}>0.05)$. In contrast, overweight and obesity were only 5.1 and $3.0 \%$, respectively. The prevalence of IFG (95\% CI) was $3.4 \%(2.63-4.17)$ and T2DM was $1.5 \%(0.99-2.01)$. FBG showed significant positive correlation with HDL in all age groups $(10-12,-14,-16,-18 y)$ and significant inverse correlation with MUAC but limited to age $<14 \mathrm{y}$. In either sex for all ages, BMI showed no association with FBG. In assessing risk for hyperglycemia $(\mathrm{FBG}=>5.6 \mathrm{mmol} / \mathrm{l})$, logistic regression showed [odds ratio (OR) with $95 \% \mathrm{CI}]$ that higher age (>14y, OR 2.04, 1.27 - 3.26), urban area (OR 11.60, 5.57 - 24.17) and higher family income (taka >10,000: OR 2.03, $1.30-3.18$ ) were found to be significant; whereas, obesity related variables (BMI, MUAC) were proved not significant.

Conclusion: Despite lower obesity prevalence among these children the development of IFG and T2DM were found not negligible. The cut-offs for obesity measures we used might be inappropriate. The lower cut-offs or waist-girth could have been proved more useful. Significant positive correlation of FBG with HDL and significant negative correlation with MUAC (not BMI) indicate a complex interrelationship among obesity indices, glycemia and dyslipidemia. A prospective cohort including other variables like waistgirth, insulin or macro- / micro-nutrients may explain how diabetes starts developing in the non-obese young children.

Supported by: WHO

\section{1}

Can ethnic group differences in insulin resistance and dyslipidaemia be explained by differences in muscle mass and visceral fat?

T. Tillin ${ }^{1}$, A. Hughes ${ }^{1}$, I. Godsland ${ }^{2}$, N. Forouhi ${ }^{3}$, P. McKeigue ${ }^{4}$,

N. Chaturvedi ${ }^{1}$

${ }^{1}$ International Centre for Circulatory Health, Imperial College, London,

${ }^{2}$ Metabolic Medicine, Imperial College, London, ${ }^{3}$ Epidemiology Unit, MRC, Cambridge, ${ }^{4}$ MRC Human Genetics Unit, University of Edinburgh, United Kingdom

Background and aims: People of Indian Asian and African Caribbean descent have high rates of diabetes and insulin resistance (IR). However the consequences of diabetes and IR differ, with Indian Asians displaying the classical pattern of dyslipidaemia and elevated rates of coronary heart disease (CHD), whilst people of African Caribbean descent have favourable lipid profiles and, in the UK, low rates of CHD. 
We studied whether differences in fat and lean tissue could explain elevated levels of IR and differing lipid profiles in British African Caribbeans and Indian Asians.

Method: Population based study of 1732 European White, 1234 Indian Asian and 405 African Caribbean non-diabetic men aged 40-69 years in West London. Measurements included fasting bloods, skinfold thicknesses and waist, hip, mid-arm and thigh circumferences. Visceral adipose tissue (VAT) and whole body skeletal muscle mass (SMM) were estimated using ethnicity-specific formulae validated against whole-body MRI and CT.

Results: Compared with European men, African Caribbeans were significantly more insulin resistant but less dyslipidaemic. They had more SMM and less VAT (table). Conversely, Indian Asians were significantly more insulin resistant and more dyslipidaemic, with more VAT and less SMM than Europeans. Adjustment for SMM abolished the ethnic difference in HOMA IR for African Caribbeans. Adjustment for VAT abolished the ethnic difference in HOMA IR for Indian Asians and obliterated ethnic differences in triglycerides in both African Caribbeans and Indian Asians compared with Europeans (table and figure).

Conclusion: Reduced visceral fat explained the favourable lipid profiles of British African Caribbean men, whilst their increased IR relative to European men appeared to be explained by increased muscle mass, suggesting abnormalities of skeletal muscle metabolism in African Caribbeans.

In contrast, increased IR and dyslipidaemia in Indian Asian men was explained by increased visceral fat. Marked ethnic differences in body fat distribution contribute to ethnic differences in IR consequences.

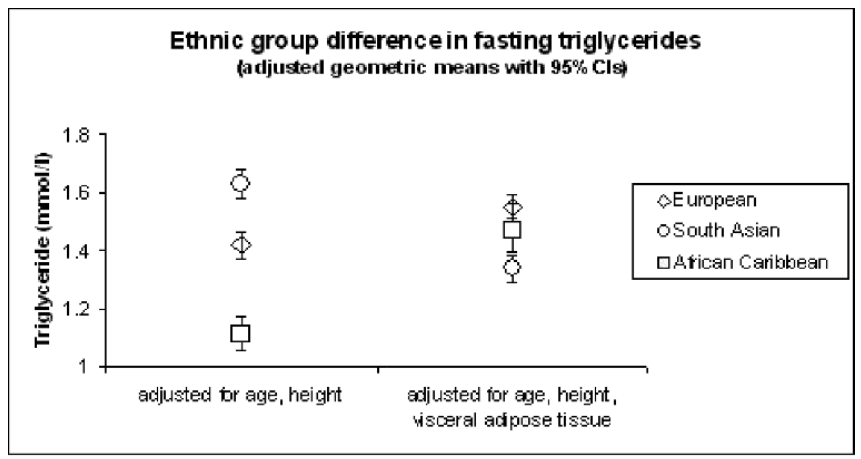

Visceral adipose tissue, skeletal muscle mass, HOMA and fasting glucose by ethnicity

\begin{tabular}{llll}
\hline & $\begin{array}{l}\text { Europeans } \\
\text { (reference) }\end{array}$ & $\begin{array}{l}\text { South } \\
\text { Asians }\end{array}$ & $\begin{array}{l}\text { African } \\
\text { Caribbeans }\end{array}$ \\
\hline Number & 1732 & 1234 & 405 \\
\hline Visceral adipose tissue (VAT), $\mathrm{cm}^{2}$ & 130 & $190^{* *}$ & $82^{* *}$ \\
\hline Skeletal muscle mass (SMM), kg & 33 & $29^{* *}$ & $36^{* *}$ \\
\hline HOMA-IR, adjusted for age, height: & 1.6 & $2.3^{* *}$ & $1.8^{* *}$ \\
\hline $\begin{array}{l}\text { HOMA-IR, adjusted for age, height, } \\
\text { VAT }\end{array}$ & 1.9 & $\mathbf{1 . 8 ^ { * * }}$ & $2.9^{* *}$ \\
\hline $\begin{array}{l}\text { HOMA-IR, adjusted for age, height, } \\
\text { SMM }\end{array}$ & 1.6 & $2.7^{* *}$ & $\mathbf{1 . 5}$ \\
\hline $\begin{array}{l}\text { Fasting glucose (mmol/l), adjusted for } \\
\text { age, height }\end{array}$ & 5.5 & $5.7^{*}$ & $5.7^{* *}$ \\
\hline $\begin{array}{l}\text { Fasting glucose (mmol/l), adjusted for } \\
\text { age, height, VAT }\end{array}$ & 5.6 & $\mathbf{5 . 5 ^ { * * }}$ & $6.0^{* *}$ \\
$\begin{array}{l}\text { Fasting glucose (mmol/l), adjusted for } \\
\text { age, height, SMM }\end{array}$ & 5.5 & $5.7^{* *}$ & $\mathbf{5 . 6}$ \\
\hline
\end{tabular}

${ }^{*} \mathrm{p}<0.05,{ }^{* *} \mathrm{p}<0.01$. Bold text indicates

attenuated/reversed associations

Supported by: Medical Research Council, British Heart Foundation

\section{2}

Questioning the appropriateness of recommended waist cut-points for Caucasians and Asian Indians based on relationships with incident diabetes A.J. Cameron ${ }^{1}$, P.Z. Zimmet ${ }^{1}$, G. Alberti ${ }^{2}$, R.A. Sicree ${ }^{1}$, J. Tuomilehto ${ }^{3}$, J.E. Shaw ${ }^{1}$

${ }^{1}$ Epidemiology Department, International Diabetes Institute, Caulfield, Australia, ${ }^{2}$ Department of Medicine, University of Newcastle, United Kingdom, ${ }^{3}$ Department of Public Heatlh, University of Helsinki, Helsinki, Finland

Background and aims: Cut-points for waist circumference, as currently used in the IDF definition of the Metabolic Syndrome, are based on concordance with BMI cut-points for overweight and obesity (Europids) and cross-sectional associations with components of the metabolic syndrome (Asians). Minimal evidence exists as to whether these bear any relation to important outcomes such as diabetes, particularly for non-Europid populations. The aim of this work is to use data from prospective cohort studies conducted in Asian Indian and Europid populations to determine whether the currently recommended waist cut-points are appropriate for these populations.

Materials and methods: Data are from 5759 non-pregnant Europid participants (45.0\% male) in the Australian Diabetes, Obesity, and Lifestyle (AusDiab) study (1999-2004) and 2233 non-pregnant ethnically Asian Indians (45.5\% male) from the Mauritius Non-Communicable Disease Study (19871992). An oral glucose tolerance test was used to obtain fasting and 2 -hr plasma glucose at both baseline and follow-up, and diabetes diagnosed according to 1999 WHO recommendations. Waist circumference (WC) was measured at the testing site. Ethical approval to conduct the studies was obtained, and informed consent was obtained from participants in both studies.

Results: Mean WC was $97.1 \mathrm{~cm}$ and $84.9 \mathrm{~cm}$ for Europid men and women, and $77.6 \mathrm{~cm}$ and $72.9 \mathrm{~cm}$ for Asian Indian men and women. A total of 240 Europid and 187 Asian Indian participants developed diabetes over five years. For a 50 year old Europid with WC of $94 \mathrm{~cm}$ (male) or $80 \mathrm{~cm}$ (female) (note - recommended cut-points for Europids), annual diabetes incidence is estimated as $0.6 \%$ for both sexes. For 50 year old Asian Indians with WC of $90 \mathrm{~cm}$ (male) or $80 \mathrm{~cm}$ (female) (note - recommended cut-points for Asians), annual diabetes incidence is estimated as $5.3 \%$ and $2.2 \%$ for men and women respectively. The WC at which a 50 year old is estimated to have a $1 \%$ annual diabetes incidence is $105 \mathrm{~cm}$ (male) and $93 \mathrm{~cm}$ (female) in Europids and $67 \mathrm{~cm}$ (male) and $63 \mathrm{~cm}$ (female) in Asian Indians. Compared to a WC at the 10th percentile $(84 \mathrm{~cm}$ and $70 \mathrm{~cm}$ in Europid men and women; $66 \mathrm{~cm}$ and $60 \mathrm{~cm}$ in Asian Indian men and women), age-adjusted annual diabetes incidence doubles at $W C$ of $97 \mathrm{~cm}$ and $85 \mathrm{~cm}$ in Europid men and women, and $76 \mathrm{~cm}$ and $75 \mathrm{~cm}$ for Asian Indian men and women.

Conclusion: We have demonstrated here that the difference between currently recommended WC cut-points for Europids and Asians ( $4 \mathrm{~cm}$ for men, identical for women) appear to underestimate the difference in WC at which risk for diabetes increases in these populations. This is true for men and particularly so for women (where current WC cut-points are identical for all ethnic groups). Similar studies in other Asian populations are needed to determine whether this is a finding common to all Asian populations. The results of prospective, outcome based studies such as these should be considered when revising ethnicity-specific waist circumference cut-points, which are vitally important as a global public health tool.

Supported by: National Heart Foundation of Australia, National Health and Medical Research Council of Australia, Mauritius Ministry of Health, WHO, University of Newcastle upon Tyne, National Public Health Institute (Helsinki, Finland), U.S. National Institutes of Health

\section{3}

Diabetes susceptibility in five ethnic minority groups compared to Norwegians - the impact of adiposity measures and other risk factors A.K. Jenum ${ }^{1}$, L.M. Diep ${ }^{2}$, G. Holmboe-Ottesen ${ }^{3}$, B.N. Kumar ${ }^{3}$,

K.I. Birkeland ${ }^{1}$

${ }^{1}$ Aker/Ullevaal Diabetes Research Center, Aker University Hospital, ${ }^{2}$ Aker Research Center, Aker University Hospital, ${ }^{3}$ Institute of General Practice and Community Medicine, University of Oslo, Norway

Background and aims: Ethnicity exerts a strong influence on diabetes susceptibility. Europeans seem to be the least affected by the increasing rates of obesity and sedentary behaviours. Immigrants from non-Western countries to Europe may have even higher diabetes prevalence rates than found in their countries of origin. The aim of this study was to assess diabetes prevalence in 
Oslo in five ethnic minority groups compared to Norwegians, and the differential impact of adiposity measures and environmental factors.

Materials and methods: Data from questionnaires, physical examinations and serum samples from two population-based health surveys in Oslo, conducted by the Norwegian Institute of Public Health according to established standards in 2000-2002, were analysed. In the Romsås in Motion Study all 31-67 year old subjects in two districts were invited, whereas in the Oslo Immigrant Health Study all 31-60 years olds born in Sri Lanka, Pakistan, Vietnam, Turkey and Iran. Subjects with non-fasting serum glucose (NFSG) levels $>6.0 \mathrm{mmol} / \mathrm{l}$ were asked to return for a fasting serum glucose (FSG) and $\mathrm{HbA}_{1} \mathrm{c}$ (normal reference range of 4.1-6.4\%). Subjects not reporting diabetes, but with FSG $\geq 7.0$ $\mathrm{mmol} / \mathrm{l}$, or $\mathrm{HbA}, \mathrm{c}>6.4 \%$, or when not attending for fasting samples, NFSG $\geq 11.1 \mathrm{mmol} / \mathrm{l}$, were categorised as having undiagnosed diabetes. Of the 4929 subjects who met, 2212 were from Norway, 459 from Iran, 563 from Vietnam, 390 from Turkey, 880 from Sri Lanka and 425 from Pakistan.

Results: 196 women and 272 men had diabetes $(\mathrm{N}=468)$, of which 277 cases were known and 191 (41\%) screening-diagnosed. Diabetes prevalence differed significantly between ethnic groups (Figure 1). Based on preliminary sex specific logistic regression analyses with Norwegians as a reference, the OR for diabetes for Sri Lankaan women and men were 9.2 and 3.0, for Pakistanis 8.4 and 2.3, for Vietnamese 4.8 and 2 and for women from Turkey 3.1 $(\mathrm{p}<0.001$ for all) adjusted for age, waist/hip- ratio, physical activity, smoking, education, full time work, body height, and parity in women. The age-adjusted impact of BMI, waist/hip ratio and waist on diabetes prevalence varied significantly by ethnicity, with higher estimated age-adjusted prevalence rates for all BMI values in the range 18-35 for all ethnic groups compared to Norwegians except for Iranians.

Conclusion: Vietnamese, Turkish, Sri Lankaan and Pakistani women had 3 10 times higher age-standardized prevalence rates than Norwegian and Iranian women. Higher diabetes susceptibility for three measures of adiposity was found for Sri Lankans, Pakistanis, Vietnamese and Turkish immigrant groups compared to Norwegians and Iranis.

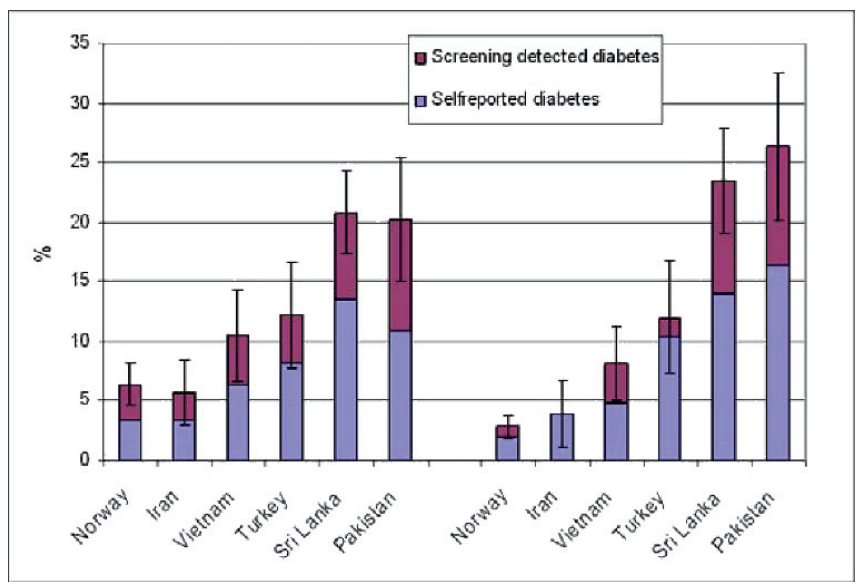

Figure 1: Diabetes prevalence $(95 \% \mathrm{CI})$ in 31-60 year olds according to sex and ethnicity, age standardized with the Norwegian population in 2000 as reference.

Supported by: Post doctoral research grant from Health Region East

\section{4}

Higher leptin levels in Asian Indians than in Creole and Caucasians a potential explanation to increased metabolic risk; data from Mauritius and Northern Sweden

M. Lilja ${ }^{1}$, M. Eliasson ${ }^{2}$, B. Stegmayr ${ }^{2}$, T. Olsson ${ }^{2}$, P. Zimmet ${ }^{3}$, J.E. Shaw ${ }^{3}$, A. Cameron ${ }^{3}$, K. Pauvaday ${ }^{4}$, J. Tuomilehto ${ }^{5}$, G. Alberti ${ }^{6}$, S. Soderberg ${ }^{2}$

${ }^{1}$ The Research and Development Unit, Jamtland County council, Ostersund, Sweden, ${ }^{2}$ Department of Public Health and Clinical Medicine, Umea University, Sweden, ${ }^{3}$ International Diabetes Institute, Melbourne, Australia, ${ }^{4}$ Ministry of Health and Quality of Life, Port Louis, Mauritius, ${ }^{5}$ Department of Public Health, University of Helsinki, Finland, ${ }^{6}$ Department of Endocrinology and Metabolic Medicine, Imperial College, London, United Kingdom

Background and aims: Leptin is an independent predictor of type 2 diabetes, cardiovascular disease and it predicts worsening of the metabolic syndrome independently of obesity. Experimental data also show that leptin may have detrimental effects on the cardiovascular system and on the insulin producing beta cell. Asian Indians have been shown to be highly suceptible to type 2 diabetes and other features of the metabolic syndrome. We hypothesised that leptin may explain this difference and the aim of this study was to explore if levels of leptin, leptin per BMI unit (leptin/BMI) and leptin per $\mathrm{cm}$ in waist circumference (leptin/waist) differed between different ethnicities (Caucacians, Asian Indians and Creoles [mainly African ancestry]).

Materials and methods: In the Northern Sweden MONICA study cross-sectional population surveys were performed in 1986, 1990 and 1994. In 1999 all survey participants were offered a follow-up with 1720 men and 1793 women participating (all Caucacians). In Mauritius, population based surveys were undertaken 1987 with a follow up in 1992. 1075 men and 1341 women of Indian $(\mathrm{n}=1815)$ and Creole $(\mathrm{n}=602)$ ethnicity participated both studies. All subjects were 25-74 years and leptin samples from the initial surveys were used. Leptin levels were determined with the same method (Linco, RIA) on samples that had been stored for approximately 10 years. Data were explored with an ANCOVA with post hoc Bonferroni analysis. Adjustment was made for BMI, smoking habits and actual age. Pregnant women were excluded. Results: Asian Indian men and women had higher leptin, leptin/BMI and leptin/waist then Creole and Caucacian men and women (all $\mathrm{p}<0.0005$ ). For men the difference between Creole (in the middle) and Caucacians (lowest values) for leptin, leptin/BMI and leptin/waist were also significant (all $\mathrm{p}<0.0005)$.

The association between age and each of leptin, leptin/waist and leptin/BMI did not differ between ethnicities.

Conclusion: Asian Indian men and women have higher levels of leptin, leptin/BMI and leptin/waist then Creole and Caucacian men and women. This could partly explain the increased vulnerability for features of the metabolic syndrome in Indians.

Supported by: County councils in northern Sweden ("Visare Norr"), Swedish National Institute of Public Health, the Swedish Heart and Lung Foundation, Ministry of Health on Mauritius

\section{5}

Diabetes, IGT and IFG: risk factors for all-cause mortality over 15 years in the multi-ethnic nation of Mauritius

D.J. Magliano ${ }^{1}$, S. Söderberg ${ }^{1,2}$, K. Pauvaday ${ }^{3}$, S. Kowlessur ${ }^{3}$, J. Tuomilehto ${ }^{4}$, G. Alberti ${ }^{5}$, J.E. Shaw ${ }^{1}$, P.Z. Zimmet ${ }^{1}$

${ }^{1}$ Epidemiology, The Baker IDI Heart and Diabetes Institute, Melbourne, Australia, ${ }^{2}$ Department of Public Health and Clinical Medicine, Cardiology, Umeå University, Sweden, ${ }^{3}$ Non Communicable Diseases Unit, Ministry for Health and Quality of Life, Port Louis, Mauritius, ${ }^{4}$ Department of Public Health, University of Helsinki, Helsinki, Finland, ${ }^{5}$ Department of Endocrinology and Metabolism, St Mary's Hospital and Imperial College, London, United Kingdom

Background and aims: To explore the relationship between diabetes, impaired glucose tolerance (IGT) and impaired fasting glycaemia (IFG) and allcause mortality adjusted for conventional risk factors in a large multi-ethnic cohort living in the developing nation of Mauritius.

Methods: Population-based surveys were undertaken in Mauritius in 1987, 1992 and $1998(n=9559)$. Participants were aged 18 years or greater and 66\%, $27 \%$, and $7 \%$ were of Indian, Creole, and Chinese descent, respectively. Response rates for all surveys were greater than $85 \%$. Questionnaires, anthropometric measurements, and a 2 hour $75-\mathrm{g}$ oral glucose tolerance test were undertaken at each survey. Glucose tolerance was classified according to World Health Organisation 1999 criteria. In 2007, a mortality follow-up was undertaken. Cox's proportional hazards model was used to obtain hazard ratios (HRs) for risk of mortality, adjusted for age, sex, prior cardiovascular disease, hypertension, smoking, total cholesterol, education, body mass index, waist and hip circumference.

Results: Over a median follow-up of 15.1 years, there were 1559 deaths, 7168 survivors and 832 participants were lost to follow-up. Compared with those with normal glucose tolerance (NGT), the all-cause mortality HRs and 95\% confidence intervals (CIs) for known diabetes mellitus (KDM), newly diagnosed diabetes mellitus (NDM) and IGT were 3.7 (2.9 to 4.6), 2.2 (1.8 to 2.7), 1.3 (1.1 to 1.6 ) in males and 3.1 (2.4 to 3.8 ), 2.0 (1.6 to 2.5 ), 1.1 ( 0.9 to 1.4 ) in females, respectively. Those with IFG were not at increased risk of mortality in either males (1.2 [0.9 to 1.6$])$ or females (0.7 [0.4 to 1.3$])$. The HRs of mortality from all diabetes fell from 6.4 (3.7 to 11.3) in those aged 18 - 34 years to 1.7 (1.4 to 2.2.) in those aged 65 years or greater. Among the Indians, compared to those with NGT, those with KDM (3.6 [3.0 to 4.4]), NDM 
(2.4, [2.0 to 2.8$])$ and IGT (1.3 [1.1 to 1.5$])$ were at increased risk of all-cause mortality, but in the Creole population, only those with KDM (2.6 [1.9 to 3.5]) and NDM (1.8 [1.4 to 2.4]) were at increased risk of death; IGT (1.1 [0.9 to1.4]) and IFG (0.9 [0.6 to 1.4]) were not associated with an increased risk of all-cause mortality. Among those of Chinese descent, only KDM were at increased risk of mortality (3.7 [1.9 to 7.2$]$ ).

Conclusion: This is one of the largest ever studies of diabetes and mortality in a developing country. The ethnic mix of Mauritians is said be a microcosm of the world's population as it represents over $60 \%$ of the world's peoples. This study shows that mortality risk from diabetes deceases with age and that KDM and NDM were independent risk factors for all-cause mortality in the three ethnic groups. IGT was only associated with mortality in the Indians, while IFG was not associated with an increased risk of death in any ethnic subgroup.

\section{6}

Insulin resistance and secretion in different ethnic populations of Kenya D.L. Christensen ${ }^{1}$, D.L. Mwaniki ${ }^{2}$, M.K. Boit ${ }^{3}$, B. Kilonzo ${ }^{4}$, H. Friis ${ }^{5}$, I. Tetens ${ }^{6}$, L. Kaduka 2 , B.O. Omondi², K. Borch-Johnsen ${ }^{1}$

${ }^{1}$ Steno Diabetes Center, Copenhagen, Denmark, ${ }^{2} \mathrm{CPHR}$, KEMRI, Nairobi, Kenya, ${ }^{3}$ Department of Exercise, Recreation and Sport Science, Kenyatta University, Nairobi, Kenya, ${ }^{4} \mathrm{CPHR}$, Kenyatta University, Nairobi, Kenya, ${ }^{5}$ Department of Human Nutrition, University of Copenhagen, Denmark, ${ }^{6}$ Department of Nutrition, Technical University of Denmark, Copenhagen, Denmark

In Africa, the aetiology of glucose intolerance has not been systematically investigated in representative populations. Differences in lifestyle are still apparent in the rural areas, and urbanisation occurs at a high rate, but their association with insulin secretion and insulin resistance is unknown. Therefore, the aim of this study was to estimate insulin secretion and action in different ethnic groups of Kenya.

In a cross-sectional study, the Luo, Kamba and Maasai were chosen as study populations to represent a fishing-agricultural, an agricultural, and an agropastoralist rural society, respectively. In total, 1,306 men and women (age range 17-68 yrs) had an OGTT. Of these, 58 individuals had diabetes and were subsequently excluded from further analysis. Insulin and glucose concentrations from venous blood samples at $0 \mathrm{~min}$ and $30 \mathrm{~min}$ from the remaining 1,248 individuals were analysed. Estimates of insulin sensitivity (HOMAIR), absolute insulin secretion (insulinogenic index) and beta cell function (Disposition Index, DI=HOMA*insulinogenic index) were calculated.

In the rural and urban populations, geometric mean HOMA-IR was 0.59 (95\% CI: $0.56 ; 0.61)$ and $0.85(0.76 ; 0.95)$, respectively, geometric mean insulinogenic index was $88.9(84.0 ; 94.1)$ and $127.3(107.7 ; 150.5)$, respectively, and geometric mean DI was $153.3(144.9 ; 162.3)$ and $153.4(127.9 ; 183.8)$. Results on insulin resistance and secretion as well as biological background of study population stratified by ethnic groups are shown in Table 1.

HOMA-IR and insulinogenic index were higher in the Maasai and Kamba compared to the Luo. This indicates a higher absolute insulin secretion in order to compensate for insulin resistance among the Maasai and Kamba. Consequently, this was reflected in the estimated beta cell function were no difference could be found between the ethnic groups. A higher HOMA-IR in the urban population was also compensated for by a higher insulinogenic index and thus there was no difference in DI between urban and rural populations. This study indicates that lifestyle differences with or without urbanisation have an effect on insulin resistance and secretion in African populations.

\section{7}

Diabetes prevalence among migrant populations in Australia: prevalence and risk factor associations

R.A. Sicree, P.Z. Zimmet, A.J. Cameron, D.J. Magliano, J.E. Shaw International Diabetes Institute, Melbourne, Australia

Background and aims: Ethnicity is a key factor affecting diabetes prevalence, with obesity linked to diabetes amongst all ethnic groups. However, there are little data on the choice of cut-points for obesity for different ethnic groups. Our objectives were to examine the relationship between ethnicity and diabetes prevalence in the Australian population, and to assess whether the relationship between obesity and diabetes is consistent across ethnicities.

Materials and methods: The AusDiab Study was a population-based cluster survey of 11,247 adults conducted in Australia during 1999-2000, in which fasting glucose and glucose tolerance were assessed for 11,078 participants. Ethnic background was coded according to country of birth, with modification for main language used at home. Waist circumference and BMI were used as indices of obesity. Diabetes was classified according to 1999 WHO criteria.

Results: Diabetes prevalence (age-and sex-standardised to 50 years) was significantly higher for those from South European (9.9\%), and Asian background (11.3\%), compared to $5.8 \%$ for the Australian/New Zealand born (both at $\mathrm{p}<0.001)$. Compared to the Australian/ New Zealand born, mean age- and sex-adjusted waist circumference was $\sim 2 \mathrm{~cm}$ greater for South European participants $(\mathrm{p}=0.001)$ and $\sim 5 \mathrm{~cm}$ less for the Asian born $(\mathrm{p}<0.001)$; similarly mean BMI was $1.5 \mathrm{~kg} / \mathrm{m}^{2}$ more for the South Europeans and 2.0 $\mathrm{kg} / \mathrm{m}^{2}$ less for Asian participants (both at $\mathrm{p}<0.001$ ), with the Australian/ New Zealand born adjusted means being 98.8 and $86.4 \mathrm{~cm}$, and 27.7 and $27.4 \mathrm{~kg} /$ $\mathrm{m}^{2}$ for males and females respectively. The Asian born group had lower rates of central obesity based on current World Health Organization and International Diabetes Federation criteria. The association of obesity measures with diabetes prevalence were the same across ethnicity groups, with interaction terms for ethnicity and waist, and ethnicity and BMI having $\mathrm{p}$ values of 0.4 and 0.8 respectively. Overall differences of about $5 \mathrm{~kg} / \mathrm{m}^{2}$ in BMI, or $11 \mathrm{~cm}$ in waist circumference were associated with twice the diabetes prevalence irrespective of ethnicities. Adjustment for obesity did not explain differences in diabetes prevalence between ethnicities; the parameter estimates suggest that to have similar prevalence to the Australian born the Asian migrant populations would need waist circumferences at least $10 \mathrm{~cm}$ lower or BMI $5 \mathrm{~kg} / \mathrm{m}^{2}$ than those of the Australian/ New Zealand born participants. Migrants from other regions (other English speaking countries, and elsewhere in Europe) had similar rates of obesity and diabetes to the Australian/New Zealand born. Among the Australian born, those of Indigenous background had a 4 -fold risk of diabetes ( $\mathrm{p}<0.001$ ), and mean waist $7 \mathrm{~cm}$ and BMI $2.8 \mathrm{~kg} / \mathrm{m}^{2}$ (both at $\mathrm{p}<0.001)$ greater than other Australian born.

Conclusion: There are marked differences in measures of obesity and diabetes prevalence among the Australian population related to ethnic background. The greater level of obesity among South Europeans partly explained their higher diabetes prevalence, but the higher diabetes rate among Asians, despite less central obesity suggests current waist cut-offs for Asian populations may be too high.

Table 1. Biological background characteristics and derived variables of OGTT. Results are presented as mean or geometric mean values $(95 \% \mathrm{CI})$.

\begin{tabular}{llllllll}
\hline & Luo & $(95 \% \mathrm{CI})$ & Kamba & $(95 \% \mathrm{CI})$ & Maasai & $(95 \% \mathrm{CI})$ & P-value \\
\hline $\mathrm{n}$ & 429 & & 459 & & 360 & & \\
\hline Females $(\%)$ & 51.5 & & 68.2 & & 51.7 & & \\
\hline Age $(\mathrm{yrs})$ & $38.1^{\mathrm{a}}$ & $(37.1 ; 39.1)$ & $40.3^{\mathrm{b}}$ & $(39.4 ; 41.2)$ & $36.2^{\mathrm{c}}$ & $(35.1 ; 37.3)$ & $<0.001^{1}$ \\
\hline BMI $\left(\mathrm{kg} / \mathrm{m}^{2}\right)$ & 21.6 & $(21.3 ; 21.9)$ & 22.2 & $(21.8 ; 22.6)$ & 21.6 & $(21.1 ; 22.1)$ & 0.058 \\
\hline HOMA-IR & $0.52^{\mathrm{a}}$ & $(0.48 ; 0.55)$ & $0.64^{\mathrm{bc}}$ & $(0.60 ; 0.69)$ & $0.70^{\mathrm{c}}$ & $(0.65 ; 0.75)$ & $<0.001^{2}$ \\
\hline Insulinogenic index & $76.9^{\mathrm{ab}}$ & $(69.8 ; 84.7)$ & $98.6^{\mathrm{bc}}$ & $(90.6 ; 107.4)$ & $105.3^{\mathrm{c}}$ & $(95.7 ; 115.9)$ & $<0.001^{2}$ \\
\hline Disposition index & 152.2 & $(138.3 ; 167.5)$ & 155.3 & $(142.4 ; 169.5)$ & 152.0 & $(137.5 ; 168.1)$ & $0.975^{2}$ \\
\hline
\end{tabular}

${ }^{1} \mathrm{P}$-value is $\mathrm{chi}^{2}$-test, and abc denotes between-group differences $(\mathrm{P}<0.05)$

${ }^{2} \mathrm{P}$-value is chi ${ }^{2}$-test. ${ }^{\text {abc }}$ denotes ethnic differences $(\mathrm{P}<0.05)$ with the Luo as reference group controlled for sex, age, and BMI.

Supported by: Danida, University of Copenhagen and Steno Diabetes Center 


\section{8}

Incidence and prevalence of diabetes among Inuit in Alberta, Canada, 1995 to 2006

L.W. Svenson ${ }^{1}$, R.L. Campbell ${ }^{2}$, J.A. Johnson ${ }^{3}$

${ }^{1}$ Community Health Sciences, University of Calgary, ${ }^{2}$ Continuing Education, Concordia University College of Alberta, ${ }^{3} \mathrm{Public}$ Health Sciences, University of Alberta, Edmonton, AB, Canada

Background and aims: It is generally known that the prevalence of diabetes is significantly higher among indigenous populations. In Alberta, there is solid evidence of this disparity for Aboriginals holding treaty status under the Canadian Indian Act. However, little is known about the epidemiology of diabetes among the Inuit in Canada. The purpose of this analysis was to examine the incidence and prevalence of diabetes among Inuit compared with status Aboriginals and the non-Aboriginal population.

Methods: The Alberta Diabetes Surveillance System (ADSS) tracks population trends in diabetes based on International Classification of Disease (ICD) diagnostic codes in physician (ICD-9-CM) and hospital claims (ICD-9 and ICD-10-CA) from administrative records collected by Alberta Health and Wellness. We applied the diabetes case definition based on the Canadian National Diabetes Surveillance System for adults aged 20 years and older. Incidence and Prevalence were tracked from 1995 to 2006. All rates were age-standardized to the 2001 Alberta population using the direct method. The federal government pays health insurance premiums on behalf of status Aboriginals in Canada. As a result the Alberta Health Care Insurance Plan identifies these individuals. Among the groups included are the Inuit.

Results: In 2006, the prevalence of diabetes was 4.3\% (95\% C.I. 4.28, 4.32), 6.2\% (95\% C.I., 4.8, 7.7) and 11\% (95\% C.I., 10.6, 11.3) for non Aboriginal, Inuit, and Status Aboriginals respectively. From 1995 to 2006, prevalence increased 51.8\% for non Aboriginals, 24.5\% for status Aboriginals, and $115.5 \%$ for Inuit. The incidence of diabetes was highest among status Aboriginals (6.5 per 1,000$)$ followed by the Inuit $(6.2$ per 1,000$)$ and the non Aboriginal population (3.7 per 1,000). From 1995 to 2006, incidence increased 44.1\%, 1.7\% for non Aboriginal and status Aboriginals respectively. The incidence fluctuated from year to year among the Inuit, due to their relatively small population, ranging from a low of 1.1 per 1,000 in 1999 to a high of 9.5 in 2005. In 2006 , the incidence was 3.6 per 1,000 .

Conclusion: The prevalence of diabetes among Inuit in Alberta is higher than the general population, but lower than status Aboriginals. However, the rate of increase in prevalence among the Inuit is greater.

Supported by: Alberta Health and Wellness and Public Health Agency of Canada

\section{PS 021 Quality of life and diabetic complications}

\section{9}

Risk of non-trauma lower extremity amputation in patients with type 1 diabetes - a population-based cohort study in Sweden

J. Miao Jonasson ${ }^{1}$, W. Ye ${ }^{2}$, P. Sparén 2 , J. Apelqvist ${ }^{3}$, O. Nyrén ${ }^{2}$, K. Brismar ${ }^{1}$ ${ }^{1}$ Molecular Medicine and Surgery, Karolinska Institutet, Stockholm,

${ }^{2}$ Medical Epidemiology and Biostatistics, Karolinska Institutet, Stockholm, ${ }^{3}$ Medicine, Lund University Hospital, Lund, Sweden

Background and aims: To estimate the risk of non-trauma lower extremity amputations (LEAs) in patients with type 1 diabetes mellitus (T1DM).

Materials and methods: We identified, in the Swedish Inpatient Register between 1975 and 2004, 31354 patients (15001 women and 16353 men) who had been hospitalized for diabetes before age 31 years. They were followed for non-trauma LEA through cross-linkage in the Inpatient Register until December 31, 2004. Information on censoring was obtained through linkages to the Death and Migration registers. Poisson regression modeling was used to compare the risk of LEAs in different calendar periods of follow-up, simultaneously adjusting for gender and attained age at follow-up. Standardized incidence ratio (SIR) was used to estimate the relative risk with the age-, sex-, and calendar-period-matched Swedish general population as reference. The cumulative probability of LEA was calculated using the Kaplan-Meier method.

Results: In total, 465 T1DM patients underwent LEAs. The risk had decreased in the most recent calendar period (2000-2004) compared to the period before 2000 (relative risk $=0.6,95 \%$ confidence interval [CI] 0.5-0.8). However, even in the most recent period, these fairly young patients had 86-fold risk of LEAs (SIR $=85.8,95 \%$ CI 72.9-100.3) compared with the matching general population. By the age of 65 years, the cumulative probability of a LEA was $11.0 \%$ for women, and $20.7 \%$ for men.

Conclusion: Despite of a tendency of decreased risk in recent calendar period, patients with type 1 diabetes are still at very high risk for LEAs.

Supported by: Family Erling-Persson Foundation, through the regional agreement on medical training and clinical research between Stockholm County Council and Karolinska Institutet Stockholm County Council, Swedish Diabetes Association and StorStockholm Diabetes Association

\section{0}

Glycaemic goal and comorbidities of elderly patients with type 2 diabetes mellitus in the US

M. Bron ${ }^{1}$, F.T. Shaya ${ }^{2}$, X. Yan ${ }^{2}$, B.J. Pandya ${ }^{1}$, R.W. Baran ${ }^{1}$

${ }^{1}$ Global Health Economics and Outcomes Research, Takeda Global Research \& Development Center, Inc., Deerfield, United States, ${ }^{2}$ Pharmaceutical Health Services Research, University of Maryland, Baltimore, United States

Background and aims: Diabetes affects a substantial proportion of the US population. Among US adults 65 years or older, the prevalence of diagnosed diabetes was $15.3 \%$ in the National Health and Wellness Survey (NHANES) data sample (1999-2002); when applied to the 2000 US census population, this represented 5.4 million individuals. Effective treatment of type 2 diabetes mellitus (T2DM) in elderly patients is especially challenging for several reasons, including an increased prevalence of comorbidities such as hypertension, heart failure, renal insufficiency, and diabetic nephropathy. This study aimed to determine the estimated prevalence of T2DM, level of glycemic control, and comorbidities in elderly patients in the US.

Materials and methods: A cross-sectional study methodology was used to examine 6 years of patient data. Elderly patients ( $\geq 65$ years of age) with T2DM were identified from the US NHANES from 1999 to 2004. T2DM, hypertension, renal insufficiency, and heart failure were identified by physician diagnosis. Nephropathy was defined as the presence of microalbuminuria (albumin/creatinine ratios 30-299 $\mu \mathrm{g} / \mathrm{mg}$ ) or macroalbuminuria (albumin/ creatinine ratios $\geq 300 \mu \mathrm{g} / \mathrm{mg}$ ). Renal insufficiency was defined further by the question "Had the doctor ever told you had weak/failing kidneys?" Current use of insulin and oral antidiabetic drugs (OADs) were based on self reporting. Targets for glycemic control were defined as $\mathrm{HbAlc}<7.0 \%$ and $<6.5 \%$, as proposed by the ADA and the EASD, respectively. Data were analyzed using SAS version 9.1.

Results: Results of our analysis found the prevalence of T2DM in the elderly over the years 1999 to 2004 was $16.6 \%$. Of these patients, most were women 
(56.6\%) 65 to 74 years of age (58.9\%); $34.1 \%$ were 75 to 84 years of age, and $7.1 \%$ were 85 years or older. Mean (SD) HbAlc in these elderly patients was $7.0 \% \pm 1.2 \%$ (median [range], $6.8 \%$ [ $4.3 \%$ to $14.3 \%$ ]); $43.8 \pm 2.6 \%$ and 60.0 $\pm 2.4 \%$ of patients did not achieve the HbAlc target of $<7.0 \%$ and $<6.5 \%$, respectively. Approximately $14.5 \%$ of elderly patients relied on diet and exercise alone as a treatment option. Most elderly patients were treated with $\mathrm{OAD}$ alone (56.4\%); the remainder was treated with insulin alone (18.7\%) or a combination of OADs and insulin (10.5\%). Hypertension, present in $73.1 \%$ of patients, was the most prevalent comorbid disease. Nephropathy, represented by the presence of micro- or macroalbuminuria, was present in $38.1 \%$ of patients and was the second most prevalent comorbid disease. Heart failure, renal insufficiency, microalbuminuria, and macroalbuminuria were present in $19.0 \%, 10.6 \%, 27.1 \%$, and $11.0 \%$ of patients, respectively.

Conclusion: Results of this study suggest that the prevalence of T2DM has increased in the elderly and that the majority of these patients are treated by OADs alone. Additionally, a large percentage of elderly patients with T2DM have substantial comorbidity burden. Thus, more effective therapeutic management is needed for elderly patients with T2DM. Therapy selection demands careful consideration of prevalent comorbidities such as hypertension, nephropathy, renal insufficiency, and heart failure.

Supported by: Takeda Global Research \& Development Center, Inc.

\section{1}

Natural course of health-related quality of life in screen-detected type 2 diabetes patients and effects of a multifactorial, intensified treatment. The ADDITION Netherlands Study

M. Van den Donk, K.J. Gorter, G.E.H. Rutten

Julius Center for Health Sciences and General Practice, University Medical Center Utrecht, Netherlands

Background and aims: Type 2 diabetes mellitus is related to worsened health-related quality of life (HRQOL). However, not much is known about the course of HRQOL after development of type 2 diabetes and the influence of intensified treatment. In this study, we examine the effects of a multifactorial, intensified treatment on HRQOL, and the course of HRQOL in screendetected type 2 diabetes patients until 3.5 years after diagnosis.

Materials and methods: This study is the Dutch part of the ongoing ADDITION Study (Anglo-Danish-Dutch Study of Intensive Treatment in People

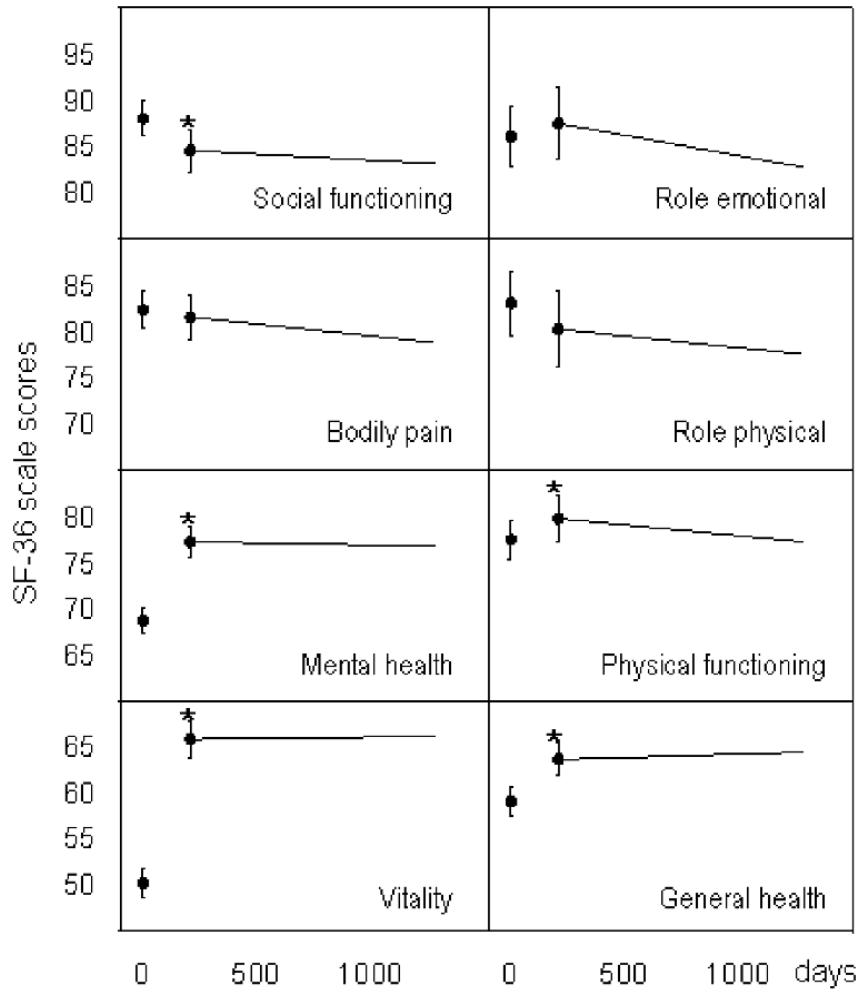

* $\mathrm{P}<0.05$ compared with baseline with Screen-detected Diabetes in Primary Care). In this cluster randomised controlled trial, 498 screen-detected type 2 diabetes patients from 77 general practices are assigned to intensified $(n=255)$ or routine treatment according to Dutch guidelines $(n=243)$. At baseline and yearly thereafter, patients filled out the Euroqol-5D and Short-Form(SF)-36 questionnaires. We analysed the effects of intensified treatment on HRQOL, and the course of HRQOL using random effects models to account for clustering and time effects.

Results: There were no differences between intensified and routine treatment on HRQOL. Therefore, we analysed the course of HRQOL in the total study population. At follow-up, patients scored lower on the SF-36 scale Social Functioning, and higher on the scales Mental Health, Physical Functioning, Vitality, and General Health, compared with baseline. During 0.5 and 3.5 years of follow-up, the scores did not significantly change.

Conclusion: Multifactorial, intensified treatment does not influence HRQOL in screen-detected DM2 patients. Therefore, healthcare professionals do not have to fear negative effects of an intensified treatment on HRQOL. After screen-detection of DM2, HRQOL improves on the SF-36 scales Mental Health, Physical Functioning, Vitality, and General Health, and worsens on the scale Social Functioning. During the next three years, HRQOL remains stable. This may be the effect of treating the disease.

Supported by: Dutch Diabetes Foundation

\section{2}

Time to anti-hyperglycaemic treatment increases with age in patients with type 2 diabetes mellitus (T2DM) in the UK

P. Mavros, C. Zhao, C.M. Alexander

Global Outcomes Research, Merck \& Co. Inc., Whitehouse Station, United States

Background and aims: T2DM is a chronic metabolic disease with increasing incidence and prevalence with age. Adequate glycaemic control is essential in reducing the risk of various micro and macro vascular complications associated with T2DM. This study was undertaken to examine whether there have been differences in treatment rates or changes in treatment trends across different age groups in the UK from 1995 to 2005.

Materials and methods: This retrospective cohort study used a sample from the MediPlus (UK) database that covers 1.8 million patients from general practitioners throughout the UK. Included in this study were newly diagnosed T2DM patients from 1995-2005 (index period). At the date (index date) of the first observed diagnosis (ICD10-CM code: E10-E14) patients were at least 30 years old with at least one year of recorded prior medical history and 2 subsequent years of recorded medical history. There were neither prior diagnoses of T2DM nor anti-hyperglycaemic treatments for at least 12 months prior to initial diagnosis. We examined the effect of age on the time to treatment and differences in treatment trends over time.

Results: 20,983 newly diagnosed T2DM patients were identified, $46.3 \%$ of which were female. The mean (standard deviation) age at time of diagnosis was 61.0 (12.6) years. The percent of patients in the age groups of 30-45, 45$65,65-75,75+$ were $11.0 \%, 47.3 \%, 27.0 \%$, and $14.7 \%$ respectively. The percentages of patients in these age groups without diabetic treatment 1 year after initial diagnosis are 49.0, 57.6, 62.6 and 64.7 respectively; at 2 years after initial diagnosis are 40.1, 47.1, 53.3 and 58.0 respectively. The hazard (to treatment) ratio (HR) associated with one additional year of age was .988 $(\mathrm{p}<0.0001)$. There is a time trend towards faster treatment times from 1995 to 2005 (HR 1.021; $\mathrm{p}<0.0001$ ). These trends were mostly evident among the 30-45 and 45-65 age groups (HR: 1.069 and 1.036 respectively; $\mathrm{p}<0.0001$ ) than in the $65-75$ or $75+$ age groups (HR 1.01; $\mathrm{p}=0.1703$ and $0.982 ; \mathrm{p}=0.0856$ respectively).

Conclusion: In this cohort of newly diagnosed T2DM patients, we observed consistently lower treatment rates with antihyperglycemic medications for older individuals. Differences in the treatment rates by age are attributed to increasing treatment rates for younger patients over time. Treatment rates for elderly patients have remained constant and lower than younger patients. 


\section{3}

Diabetes in the UK: trends in the prevalence, incidence and treatment 1996-2005

E.L. Massó González ${ }^{1}$, S. Johansson ${ }^{2,3}$, M.-A. Wallander ${ }^{2,4}$, L.A. GarcíaRodríguez ${ }^{1}$

${ }^{1}$ CEIFE, Centro Español de Investigación Farmacoepidemiológica, Madrid, Spain, ${ }^{2}$ AstraZeneca, Mölndal, Sweden, ${ }^{3}$ Institute of Medicine, Sahlgrenska Academy, Göteborg University, Sweden, ${ }^{4}$ Department of Public Health and Caring Science, Uppsala University, Sweden

Background and aims: There are limited data from longitudinal studies on time trends and treatment patterns of diabetes in the UK. The aim of this study was to estimate the incidence, prevalence, and changes in treatment of type 1 and type 2 diabetes in the UK general population from 1996 to 2005.

Materials and methods: Using The Health Improvement Network database, we identified patients with type 1 or type 2 diabetes who were aged 10-79 between 1996 and 2005. Prevalent cases $(n=49999)$ were separated from incident cases $(\mathrm{n}=42642$; type $1=1256$, type $2=41386)$. Data were collected on treatment patterns in incident cases, and on BMI in prevalent and incident cases.

Results: Incidence of diabetes in the UK increased from 2.7 per 1000 personyears in 1996 to 4.4 per 1000 person-years in 2005. Incidence of type 1 diabetes remained relatively constant throughout the study period; however, incidence of type 2 diabetes increased from 2.6 per 1000 person-years in 1996 to 4.3 per 1000 person-years in 2005. Between 1996 and 2005, the proportion of individuals newly diagnosed with type 2 diabetes who were obese increased from $46 \%$ to $56 \%$. Diabetes prevalence increased by $54 \%$ over the course of the study, from $2.8 \%$ in 1996 to $4.3 \%$ in 2005 . Treatment of both type 1 and type 2 diabetes with metformin increased across the study period, while treatment with sulfonylureas decreased. In 1996, no patients with type 1 diabetes were treated with glitazone; however, by 2005 it was being prescribed to a small number of patients. We stratified the different treatments by BMI and noted that in 2005 metformin was the most prescribed diabetes treatment among obese diabetic type 2 patients, while sulfonylureas was in 1996.

Conclusion: The prevalence and incidence of diabetes have increased in the UK over the past decade. This increase might be primarily explained by the changes in obesity prevalence. Changing diagnostic criteria over time could have influenced our findings. Finally, in the last ten years there was also a change in the treatment pattern from sulfonylureas to metformin.

Supported by: AstraZeneca RéD Mölndal, Sweden

\section{4}

Relation between glycaemic control and mortality in patients with type 2 diabetes in daily practice (Zodiac-11)

G.W.D. Landman ${ }^{1,2}$, N. Kleefstra ${ }^{2,3}$, S.J.J. Logtenberg ${ }^{2}$, K.H. Groenier ${ }^{4}$, S.T. Houweling ${ }^{3,2}$, H.J.G. Bilo ${ }^{2,5}$

${ }^{1}$ Internal Medicine, Isala clinics, Zwolle, Netherlands, ${ }^{2}$ Diabetes Centre, Isala clinics, Zwolle, ${ }^{3}$ Medical Research Group, Langerhans, Zwolle, ${ }^{4}$ General Practice, UMCG, Groningen, ${ }^{5}$ Internal Medicine, UMCG, Groningen, Netherlands

Background: Recently, the relationship between the degree of glycemic control and all-cause mortality in type 2 diabetes (T2DM) has been questioned. Therefore we analyzed mortality risk in a large group T2DM patients, stratified in different $\mathrm{HbAlc}$ ranges.

Material and Methods: The ZODIAC study started in 1998 as part of a shared care diabetes project in the Netherlands. A group of 1143 patients with T2DM was enrolled that year and seen annually. Data on mortality were assessed after an average period of 5.8 years. Mortality rates were evaluated using standardized mortality ratios (SMR).

Cox Proportional Hazard Modeling was used to assess the association between $\mathrm{HbAlc}$ and (cardiovascular and all-cause) mortality. Separate analyses were made for $\mathrm{HbAlc}$ as a continuous variable and as a grouped variable $(<6,6-7,7-8,8-9, \geq 9)$. For visualization of the results, a Kaplan-Meier curve was made. The following variables were included in the model as possible confounders: Age, gender, smoking (never, previous and current), duration of diabetes, Cockcroft, body mass index, systolic blood pressure, Chol/HDL, macrovascular complications (yes or no), use of statins (yes or no), insulin use (yes or no), and albuminuria (yes or no).

Results: The study population consisted of 653 women and 490 men. Median $\mathrm{Hba} 1 \mathrm{c}$ at baseline was $7.3 \%$ (interquartile range: 6.6-8.3), and 335 (31\%) patients died. SMR for all cause mortality was 1.86 (CI 1.66-2.06), and for cardiovascular mortality 2.24 (CI 1.91-2.61).
The hazard ratios (HRs) of HbAlc as a continuous variable was 1.19 (95\%CI: 1.08-1.31) for all-cause mortality.

HRs for the HbAlc groups 6-7, 7-8, 8-9 and $\geq 9$ were 0.92 (CI 0.55-1.55), 1.06 (CI 0.63-1.79), 1.43 (CI 0.84-2.46), 1.80(CI 1.02-3.17) (figure 1; survival curve) compared to HbA1c $<6(\mathrm{n}=100)$ for all-cause mortality.

Conclusion: Poorer glycemic control is associated with increased all-cause mortality; this is in confirmation with the results of the UKPDS-study. A recent trial concluded that intensive therapy, resulting in $\mathrm{HbAlc}$ values below $6 \%$, is associated with increased mortality. The very small differences in mortality in the lower HbAlc cohorts in our study, which was not an intervention study, but an example of daily diabetes practice, indeed suggested that there is no basis for an ever decreasing target level of $\mathrm{HbAlc}$ in type 2 diabetes mellitus, at least not with the current therapeutical arsenal.

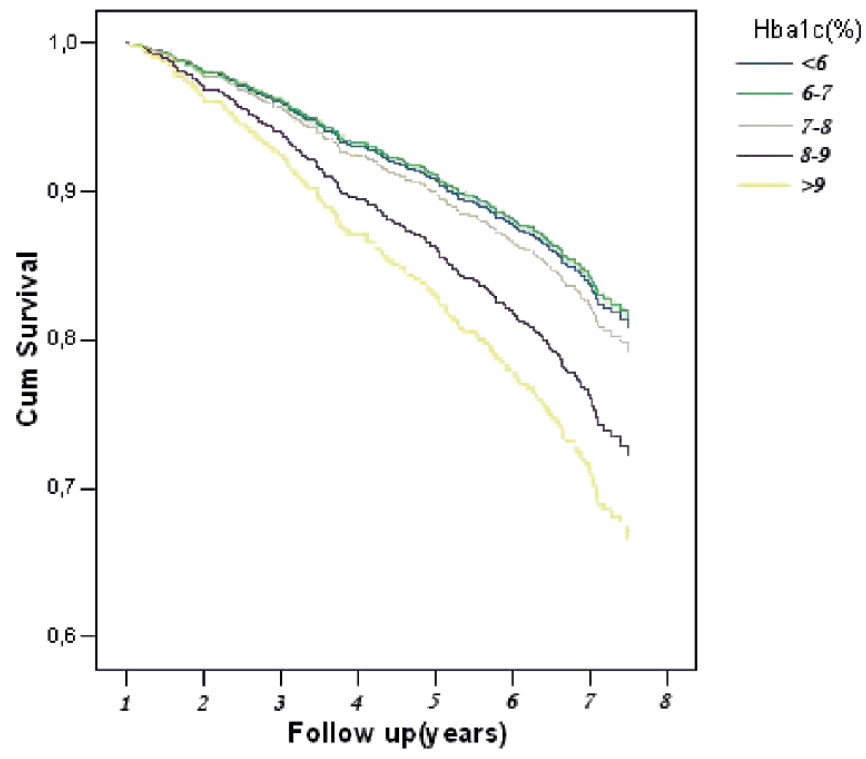




\section{PS 022 Beta cells from stem cells}

\section{5}

Functional characterisation in vivo and in vitro of encapsulated insulinproducing cells derived from mouse embryo progenitor

S. Shao, F. Xie, G. Li

National University Medical Institutes, Yong Loo Lin School of Medicine,

National University of Singapore, Singapore

Background and aims: Type 1 diabetes is an autoimmune disease caused by the destruction of pancreatic $\beta$-cells. It is traditionally treated with insulin replacement therapy by injection or insulin pump. However, such treatment involves complicated administration and is unable to cure the disease. Consequently, alternative approaches are being explored for developing better therapy.

Materials and methods: We generated lines of insulin-producing cells, named MEPI (Mouse Embryo Progenitor-derived Insulin-producing) cells, from differentiating mouse early embryo-derived progenitor cells. Detached MEPI-1 cells were cultured overnight in suspensions before mixed up with barium alginate (5 million cells in $1 \mathrm{ml}$ alginate) to generate microcapsules $(500 \mu \mathrm{m})$ using an electrostatic encapsulator. Insulin secretion capability of encapsulated cells was assessed before and after transplantation. An aliquot of capsules containing 2.5 million MEPI- 1 cells were peritoneally transplanted into each immune-competent, streptozotocin-induced diabetic mouse (C57J and Balb/c) with blood glucose levels $>350 \mathrm{mg} / \mathrm{dL}$. The transplanted capsules were washed out at different intervals for assessments of insulin fluorescence staining and cell growth rates.

Results: Dose-response study revealed that glucose-stimulated insulin secretion from attached MEPI-1 cell monolayer reached a maximum at $11.2 \mathrm{mM}$ glucose. However, insulin secretion was further increased by 1.8 folds when glucose elevated from 8.4 to $16.7 \mathrm{mM}$ in freshly made capsules. After transplantation of encapsulated cells, the diabetic mice become normoglycemia in $1-3$ days and then developed hypoglycemia at various degrees $(40 \pm 6 \mathrm{mg} / \mathrm{dl}$, $\mathrm{n}=10$ ). Despite that, these mice exhibited similar physical activity and body weight to the non-diabetic controls. When capsules were washed out during this period, a relapse of hyperglycemia occurred immediately, and positive insulin staining was found within the capsules. Separate experiments showed that about 2 months after implantation, all the mice regained hyperglycemia; the days between capsule transplantation and relapse of hyperglycemia were $52.2 \pm 4.5(\mathrm{n}=10)$. The number of transplanted MEPI- 1 cells per capsule increased initially (5-9 folds) and then decreased after 90 days transplantation on average. Additionally, the doubling time of cell population in vivo varied from 7-14 days within 42 days after transplantation, longer than that $(\sim 3.2$ days) of attached MEPI- 1 cells cultured in vitro . Moreover, the blood glucose level started to increase when the cell number in capsules decreased below that in the originally transplanted ones. Similar results were obtained in both strains of C57J and Balb/c mice.

Conclusion: These results indicated that encapsulated MEPI-1 cells displayed an improved dose-response of glucose-stimulated insulin secretion over the attached cells. Transplantation of encapsulated MEPI-1 cells could correct hyperglycemia in immune-competent diabetic mice. Cells in capsules were still able to grow in vivo but in a slower rate, while maintaining the function of insulin secretion for a considerable period. However, the capsule-transplanted mice eventually returned back to hyperglycemic apparently due to the failure of implanted cells during the protracted term.

Supported by: Biomedical Research Council of Singapore

\section{6}

Proliferation of dedifferentiated adult human pancreatic beta cells in vitro revealed by cell-lineage tracing

H.A. Russ ${ }^{1}$, Y. Bar ${ }^{1}$, P. Ravassard ${ }^{2}$, S. Efrat ${ }^{1}$

${ }^{1}$ Human Genetics and Biochemistry, Tel Aviv University, Israel, ${ }^{2}$ National Center for Scientific Research, Pierre and Marie Curie University, Paris, France

Background and aims: Expansion of insulin-producing beta cells from adult human cadaver islets could alleviate donor shortage for cell-replacement therapy of diabetes. A major obstacle to development of effective expansion protocols is the rapid loss of beta-cell markers in the cultured cells. We developed a genetic cell-lineage tracing approach for following the fate of cultured beta cells.
Materials and methods: Cells dissociated from isolated human islets were infected with 2 lentiviruses, the first expressing Cre recombinase under control of the insulin promoter, and the second a reporter cassette with the structure CMV promoter-loxP-DsRed2-loxP-eGFP.

Results: Beta cells were efficiently labeled by the dual virus system. Dedifferentiated cells derived from beta cells were shown to proliferate for a maximum of 16 population doublings. Labeled cells could be isolated and expanded in the absence of other pancreas cell types, if provided with medium conditioned by non-beta pancreatic cells.

Conclusion: Our findings provide for the first time direct evidence for dedifferentiation, survival, and replication of cultured adult human beta cells, unlike mouse beta cells. The tracing system will allow optimization of beta-cell expansion for transplantation. These findings demonstrate the feasibility of cell-specific labeling of cultured primary human cells, using a genetic recombination approach that was previously restricted to transgenic animals.

\section{7}

Expression of pluripotent stem cell markers within intact adult human pancreas and proliferative adherent cell cultures derived from digested pancreas

H.R.H. Al-Turaifi ${ }^{1}$, A.I.M. AldibbiaT ${ }^{1}$, G.N. Holliman ${ }^{1}$, S.C. Campbell', J.A.M. Shaw ${ }^{1}$, G.G.C. Huang ${ }^{2}$, S.G.C. Amiel ${ }^{2}$

${ }^{1}$ Clinical Medical Sciences, North East England Stem Cell, Newcastle Upon Tyne, United Kingdom, ${ }^{2}$ School of Medicine, Department of Diabetes, King's College London, United Kingdom

Background and aims: Pancreatic $\beta$-cell replacement offers the potential of a 'cure' for Type 1 diabetes. Shortage of suitable donors limits widespread implementation of this approach. Recent research has been focussed on potential new sources of $\beta$-cells. The existence of true stem cells within pancreas remains contentious. The aim of this study was to investigate expression of the classical stem cell markers Oct4, Sox2, Nanog and ABCG2 in adult human pancreas and evaluate potential for isolation and maintenance of stem cell marker +ve cells in proliferative culture.

Materials and methods: Human pancreatic tissue was obtained from deceased donors following ethical approval and relative consent. Unmanipulated pancreas was snap frozen for sectioning and immunofluorescence staining for differentiated / stem cell markers. Islet and ductal / exocrine-enriched fractions from fresh pancreas were separated by collagenase digestion and density gradient centrifugation. Purified cell fractions were seeded in adherent culture with proliferative 'islet survivor cell (ISC)' and 'ductal /exocrine survivor cell (DESC)' cultures established and characterised by RT-PCR / immunocytochemical staining.

Results: Pancreatic expression of Oct 4 was confirmed by positive staining localised in close proximity to ducts and within islets. There was no co-localisation with CK19-expressing differentiated duct cells or insulin-expressing mature $\beta$-cells. Expression of Oct4, Sox 2, Nanog and ABCG2 in addition to differentiated markers for endocrine, exocrine, ductal and mesenchymal phenotype was confirmed by RT-PCR in isolated islets and ductal / exocrineenriched cell fractions. ISCs were maintained in proliferative culture for up to 12 passages with maintained expression of Oct- 4 +ve cells in addition to proliferative Ki67 +ve ductal cells. DESCs were maintained in proliferative culture for up to 10 passages with persistence of a single ductal $(\mathrm{CK} 19+\mathrm{ve}) /$ mesenchymal (vimentin +ve) / $\beta$-cell precursor PDX1 +ve) phenotype. Oct4 expression was lost by passage 3 , while Sox-2 and ABCG 2 mRNA expression were maintained at late passage.

Conclusion: Expression of classical stem cell markers has been confirmed in unmanipulated adult human pancreas with maintained expression in proliferative cell cultures. Further studies are required to elucidate the potential for $\beta$-cell neogenesis from these survivor cells. Supported by: Ministry of Health, Kingdom of Saudia Arabia

\section{8}

Retinoid acid attenuates amylase expression in human embryonic stem cells-derived endoderm and improves hepatic fate J.K. Mfopou ${ }^{1}$, B. Chen ${ }^{2}$, L. Sui ${ }^{1}$, K. Sermon ${ }^{2}$, J. Van der Elst ${ }^{2}$, L. Bouwens ${ }^{1}$ ${ }^{1}$ Cell Differentiation Unit, Vrije Universiteit Brussel, ${ }^{2}$ Stem Cell Lab - Centre for Reproductive Gynecology, Vrije Universiteit Brussel, Belgium

Background and aims: Human embryonic stem cell (hESC) derivatives are now regarded as potential surrogates for cell therapy. Many patients suffering 
from various diseases would benefit from it provided the in vitro differentiation of functional cells is well mastered. As far as diabetes is concerned, hESC have been coaxed towards insulin-producing cells, but the differentiation efficiency and functional status of these cells remain to be improved. In an attempt to design a better protocol, we sequentially cultured hESC in the presence of several growth factors and chemicals previously described as improving pancreas differentiation.

Materials and methods: The hESC line VUB07 generated and characterized in our institute was maintained undifferentiated on inactivated mouse embryonic fibroblasts and differentiated into definitive endoderm by treatment with ActA (50 ng/ml) and Wnt3a $(25 \mathrm{ng} / \mathrm{ml})$, with or without BMP4 (8 ng/ $\mathrm{ml})$ and ITS supplement $(1 \mathrm{x})$ for 4 days. Retinoic acid (2.5-10 $\mu \mathrm{mol} / \mathrm{l})$ and ITS (1x) were added for 2 days and the cells further maintained in low FCS $(2 \%)$, FGF2 $(5 \mathrm{ng} / \mathrm{ml}), \mathrm{ActB}(10 \mathrm{ng} / \mathrm{ml})$ and KAAD-Cyclopamine $(250 \mathrm{nmol} /$ 1) for 4 days. During the last 5 days, Cyclopamine and ActB were replaced by Nicotinamide $(10 \mathrm{mmol} / \mathrm{l})$. Pancreatic and hepatic markers were analyzed at each stage by immunocytochemistry and PCR.

Results: Definitive endoderm was induced in this system as evaluated by Sox 17 and Foxa2 co-expression (up to $60 \%$ ) on day 4 . Analysis of pancreatic markers indicated that in the absence of retinoic acid treatment, a large majority of differentiated cells (50-60\%) strongly express amylase and low levels of Pdx1. On the contrary, addition of retinoic acid (days 4 to 6) attenuated amylase expression in the progenies, but did not result in a significant increase in Pdx1 expression. Furthermore, retinoic acid considerably improved the generation of albumin-expressing hepatocyte-like cells organized in colonies that co-expressed Foxa2, in contrast to the rare albumin-positive cells observed in its absence. The role of retinoic acid in this process and in hepato-pancreas development is further discussed.

Conclusion: Sequential exposure of hESC to growth factors and chemicals would allow controlling their differentiation towards desired progenies such as pancreatic cells needed in diabetes cell therapy. hESC-derived definitive endoderm was easily converted to amylase-expressing cells within 2 weeks, whereas its early treatment with retinoic acid shifted the progenies to a hepatic fate. This negative effect of retinoic acid on exocrine pancreas differentiation is concordant with data from mouse studies, but does not support the established requirement of retinoic acid for pancreas induction from the endoderm.
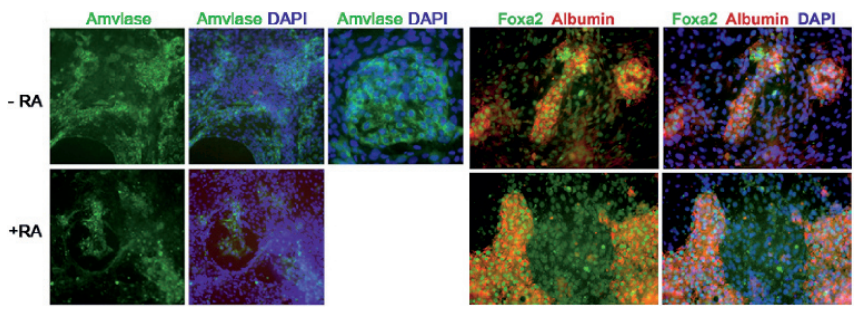

Supported by: Vrije Universiteit Brussel

\section{9}

Generation of insulin-synthesising pseudo-islets from adult stem cell cultures derived from the human pancreas

A. Aldibbiat ${ }^{1}$, G.N. Holliman ${ }^{1}$, H. Al-Turaifi ${ }^{1}$, S.C. Campbell ${ }^{1}$, G. Huang ${ }^{2}$, J. Shaw ${ }^{1}$

${ }^{1}$ Diabetes Research Group, Newcastle University, ${ }^{2}$ Diabetes Research Group, King's College London, United Kingdom

Background and aims: Despite nearly 90 years of insulin therapy, diabetes remains a leading cause of blindness, kidney failure and amputation. Intensive conventional treatment can reduce risk of complications but is associated with increased risk of hypoglycaemic collapse. True sustainable normalisation of blood glucose in Type 1 diabetes can only be achieved through replacement or regeneration of functional beta cells with insulin release in response to glucose and other stimuli. While this is possible through transplantation of whole pancreas or isolated islets retrieved from deceased donors, organ availability is sufficient for $<1 \%$ of potential recipients. The aim of the current study was to derive and characterise proliferative progenitor cells from adult human pancreas and to explore potential for generation of new insulin-synthesising cells.

Materials and methods: A proliferative population of adherent cells, termed islet survivor cells (ISCs), was derived from isolated human islets following 3 day culture in serum-supplemented CMRL medium. Cellular aggregates, termed pseudo-islets, were generated following culture of ISCs in serum-free CMRL medium. Expression of stem cell and pancreatic markers was determined by RT-PCR and ICC in ISCs and differentiated pseudo-islets. Insulin and C-peptide content was quantified by ELISA.

Results: Maintained proliferative capacity was demonstrated up to passage 10 with preserved expression of Oct4, Vimentin and cytokeratin-19 in addition to insulin, PDX1, Pax4, Glut2, Glut1, Sur1and glucokinase. Stem cell phenotype was confirmed by positive Oct 4 in addition to cytokeratin-19 and vimentin immunostaining. Endocrine phenotype was confirmed by insulin, C-peptide, glucagon, pancreatic polypeptide and somatostatin immunostaining. Differentiated pseudo-islets demonstrated down-regulation of stem cell markers in addition to positive fluorescence with the Newport Green zinc indicator, characteristic of insulin-rich beta cells. C-peptide storage and release was significantly higher in differentiated pseudo-islets in comparison to ISCs (C-peptide storage: $3.7 \pm 0.03$ vs $1.8 \pm 0.009 \mathrm{pmol} / \mathrm{million}$ cells (mean $\pm \mathrm{SD}$ ), $\mathrm{p}=0.02$. Secretion: $5.1 \pm 0.02$ vs $3.4 \pm 0.009 \mathrm{pmol} / \mathrm{million}$ cells $/ 24$ hours, $\mathrm{p}=$ 0.04 .

Conclusion: Presence of stem cells within proliferative adult human pancreas cultures has been confirmed with continued expression of end-differentiated islet phenotypic markers. Generation of new islet-like clusters with enhanced ability to store and secrete human C-peptide has been attained in vitro. Supported by: Medical Research Council; Diabetes Foundation; Diabetes Research and Wellness Foundation; and Diabetes UK

\section{0}

Pancreatic duodenal homeobox 1 expression is not essential for differentiation of mouse bone marrow mesenchymal stem cells into insulin-producing cells

Y. Hamamoto, N. Inagaki

Department of Diabetes and Clinical Nutrition, Kyoto University Graduate School of Medicine, Japan

Background and aims: Several published papers have reported that bone marrow mesenchymal stem cells (BM-MSCs) have the capacity to differentiate into insulin-producing cells (IPCs). Pancreatic duodenal homeobox-1 (Pdx1) plays a key role in the development of pancreas, and is reported that its ectopic expression induces transdifferentiation into pancreatic endocrine cells from pancreatic duct cells, hepatocytes, or BM-MSCs. Glucagon-like peptide-1 (GLP-1) is reported to promote islet neogenesis and reduce $\beta$-cell apoptosis. Here we examined the effect of constitutive Pdx1 expression and exendin-4 (Ex4) stimulation in cultured mouse BM-MSCs on differentiation into IPCs, and evaluated the expression of genes of $\beta$-cell specific transcription factors and hormones.

Materials and methods: BM-MSCs were isolated from femurs and tibias of C57BL/6 mice, and cultured under defined conditions. Pdx1 expression vector was made by insertion of cloned Pdxl gene into the downstream of CMV promoter, and the vector for control was made by inserting green fluorescence protein (GFP) gene instead of Pdx1 gene. Transfection of the vectors was carried out by lipofection on day 10-14. Ex4 (10nM) was added into culture medium at multiple time points to evaluate the effect. Four experimental groups were made by the combination of Pdx1 or GFP vector transfection and the addition of Ex4 or PBS in culture medium. On day 7,21 and 42, cells were collected and expression of $\beta$ cell specific genes such as insulin 1 (INS1), insulin 2 (INS2), Pdx1, glucose transporter 2 (GLUT2), glucokinase (GK), Neurogenin 3 (Ngn3), and GLP-1 receptor was assessed using RT-PCR. Insulin content and pdx1 expression was examined using ELISA assay and immuno-staining, respectively.

Results: After plastic adherence selection, BM-MSCs were cultured until 42 days. At first, MSCs showed spindle and fibrocyte-like appearance, but gradually some of the cells started to show morphological change into spherical appearance. Around day 14 to 21, a part of the cells aggregated and formed islet-like clusters. In order to determine whether the cells had differentiated into IPCs, mRNA expression profiles were assessed. Not only Pdx1 transfected group but also GFP transfected group, insulin expressions were observed. The probability of insulin mRNA expressions detected by RT-PCR in the two groups was about a half of total cultured dishes, and was not significantly different. Analysis of $\beta$-cell specific gene expression profiles in IPCs revealed that IPCs are expressing GK, GLUT2, and GLP-1 receptor, suggesting that the cells are equipped with machineries essential for insulin secretion. However, in the analysis of IPCs in GFP transfected group, although the expressions of $\beta$-cell specific genes were detected, Pdx1 expression was found to be absent or quite low level, and it was not different even at the early phase of differ- 
entiation evaluated on day 7. Ex4 stimulation increased probabilities of IPC differentiation by 1.5 fold without increasing endogenous $\mathrm{Pdx} 1$ expression. Conclusion: Although Pdx1 is an essential factor in the development of pancreas, our data suggest that $\mathrm{pdx} 1$ expression is not required for differentiation of mouse BM-MSCs into IPCs, and enhanced expression of Pdx1 is not enough to generate IPCs. Ex4 could increase differentiation into IPCs, suggesting that GLP-1 signal is more favorable to generate IPCs from BMMSCs.

\section{1}

Human placental lactogen- $A$ and human fibroblast growth factor-2b induce ductal PANC-1 cells to form beta cell like aggregates

M.F. Lombardo ${ }^{1}$, K. Basello ${ }^{1}$, D. Pastore ${ }^{1}$, F. Ferrelli ${ }^{2}$, B. Capuani ${ }^{1}$, A. Coppola $^{1}$, M. Tesauro ${ }^{1}$, P. Sbraccia ${ }^{1}$, M. Federici ${ }^{1}$, G. Donadel ${ }^{1}$, D. Lauro ${ }^{1}$ ${ }^{1}$ Department of Internal Medicine, University of Rome Tor Vergata, Rome, ${ }^{2}$ IRCCS, San Raffaele, Pisana, Rome, Italy

Background and aims: The increasing number of Diabetic patients (Type 1 and $2 \mathrm{DM}$ ) and the encouraging results obtained from islet transplantation has driven many scientists to deeply analyze the process of beta-cell development from precursor/staminal cells. Several evidences suggest that ductal cells or precursor/staminal cells present in the ductal layer could originate beta cells during adult life. It has been shown that mouse Placental Lactogen (PL) expression in pancreatic beta cells result in hyperplasia and hypertrophy that could be mediated by prolonged beta cell survival or by increased beta cell proliferation. There are also evidences from mice studies that attenuation of Fibroblast Growth Factor (FGF)-2b Receptor 1c signaling in the pancreas leads to beta-cell defects that are characteristic of Type $2 \mathrm{DM}$. This is characterized by reduced beta cells mass, impaired glucose sensing and perturbed pro-insulin processing. It has also been reported that FGF-2b can act in an autocrine/paracrine manner to stimulate the initial process of cell aggregation and cluster formation, an important step in the development and differentiation of endocrine pancreas. The aim of the present study is to investigate the ability of hPL-A and hFGF-2b to induce differentiation from ductal cells (PANC-1) into beta-like cells.

Materials and methods: PANC-1 cells have been treated with hPL-A, hFGF2b and hPL-A plus hFGF-2b for 96h, in order to induce islet-like cell aggregates. Then cell clusters have been analyzed by immunofluorescence and FACS to detect the expression of beta cell antigens (Insulin, PDX-1, Glut-2, Nkx6.1, Nkx2.2, and PAX-4) and ductal markers (Cytokeratin 19 and CA 19.9). Insulin concentration has been measured by RIA analysis in islet-like cell aggregates in basal condition (intracellular) and in response to glucose and potassium challenge. FACS analysis was performed to determine before and after cell differentiation the replication time of PANC-1 using Bromodeoxyuridine (BrdU) incorporation and apoptosis/necrosis using PI/Annexin $\mathrm{V}$.

Results: Treatment with hPL-A, hFGF2b and hPL-A/hFGF-2b for $96 \mathrm{~h}$ induced PANC-1 differentiation to islet-like cell aggregates. The newly cell aggregates showed an increased expression of Insulin, PDX-1, Glut-2 and $\mathrm{C}$-peptide. In particular the co-treatment with hPL-A/hFGF-2b improved expression of Insulin $(+20 \%$; $\mathrm{p}<0.05)$, PDX-1 $(+40 \%$; $<<0.05)$, Nkx2.2 $(+45 \%$; $\mathrm{p}<0.01)$, Nkx6.1 (+35\%; $\mathrm{p}<0.05)$ and, interestingly, hPL-A/hFGF-2b co-treatment reduced the expression of the typical ductal cell marker Cytokeratin 19 $(-35 \% ; \mathrm{p}<0.01)$. We also found that islet-like cell aggregates induced by hPL$\mathrm{A} / \mathrm{hFGF}-2 \mathrm{~b}$ treatment were able to secrete insulin after glucose and potassium stimulation. BrdU incorporation after $48 \mathrm{~h}$ and $96 \mathrm{~h}$ of treatment showed a reduction of cells in S-phase $(-20 \%$ and $-18 \%$, respectively; $\mathrm{p}<0.01)$, as well as a reduction in the number of apoptotic and necrotic cells, confirming the idea that hPL-A/hFGF- $2 \mathrm{~b}$ can induce cell differentiation.

Conclusion: hPL-A/hFGF- $2 \mathrm{~b}$ tratment induce PANC-1 differentiation to islet-like aggregates that are able to secrete insulin in response to glucose, suggesting a role for these two growth factors/hormones in inducing beta cell neogenesis and differentiation towards an endocrine phenotype.

Supported by: MIUR 2004 grant and Pubblic Health Department 2004 grant

\section{2}

Selective removal of residual undifferentiated embryonic stem cells from differentiation cultures

O. Naujok, S. Lenzen

Institute of Clinical Biochemistry, Hannover Medical School, Germany

Background and aims: Embryonic stem (ES) cells have the potential to produce unlimited numbers of surrogate insulin-producing cells for cell replacement therapy of type 1 diabetes mellitus. However, the transplantation of differentiated ES cells harbors the risk of uncontrolled proliferation and onset of tumors such as teratoma formation. In order to control teratoma formation and to minimize cellular overproliferation, ES cells must be generated with a negative selection marker such as a suicide gene. Here we report the generation of a mouse ES cell line, which expresses a splice-corrected variant of the HSV1 thymidine kinase and eGFP under the control of the Oct4 promoter for negative selection and ablation of undifferentiated ES cells.

Materials and methods: A $3 \mathrm{~kb}$ promoter fragment of the human Pou5fl gene (Oct4) was cloned to drive the expression of a splice-corrected variant of the HSV1 thymidine kinase and eGFP in a bicistronic gene cassette. Mouse ES-D3 cells were stably transfected and eGFP positive cells were selected and characterized. Clonal HSVtk ${ }^{+}$cells were subjected to a short differentiation protocol and the cultures were treated with ganciclovir for negative selection of residual undifferentiated cells. Treated HSVtk ${ }^{+}$and untreated $\mathrm{HSVtk}^{+}$cells were analyzed using quantitative RT-PCR, FACS and immunohistochemistry.

Results: The pOct4-scHSVtk-eGFP construct was successfully integrated into ES cells, called HSVtk ${ }^{+}$cells. These cells remained pluripotent and stained positive for the embryonic markers SSEA-1, alkaline phosphatase, Oct4 and Nanog. Treatment with varying concentrations of ganciclovir revealed sensitivity to ganciclovir with an $\mathrm{EC}_{50}$ value of $0.17 \mu \mathrm{M}$. When $\mathrm{HSVtk}^{+}$ cells were differentiated for 19 days comprising a seven day treatment with $1 \mu \mathrm{M}$ ganciclovir the cells lost very quickly eGFP fluorescence while in the untreated group up to $40 \%$ of the cells remained eGFP-positive. Staining for SSEA-1 showed negligible populations of SSEA1-positive cells in the treated group while in the untreated group the residual population of eGFP-positive cells remained also positive for SSEA-1. Staining for alkaline phosphatase showed undifferentiated clusters in the group of untreated $\mathrm{HSVtk}^{+}$cells while in treated $\mathrm{HSVtk}^{+}$cells alkaline phosphatase staining was very faint. Analysis of the gene expression of Oct4 revealed an 80 fold higher Oct 4 expression in untreated HSVtk ${ }^{+}$cells when compared to treated $\mathrm{HSVtk}^{+}$cells. Nanog, as a second marker for ES cells, showed a 27 fold decrease in treated cells. Quantitative analysis of gene expression revealed a significant 25 fold lower expression of embryonic RAS (eRAS) in treated cells. Another embryonic marker, namely Sox 2 , showed also a decrease in treated cells. Immunohistochemical staining of Oct4 and Nanog revealed that cells with embryonic properties could be easily detected if the cells were not treated with ganciclovir. In treated cells Oct 4 and Nanog was barely detectable.

Conclusion: ES cells offer great potential for cell replacement therapy. Besides the need for efficient differentiation protocols for generation of insulinproducing cells or other cell types, efforts must be undertaken to minimize the risk of tumor formation after transplantation of differentiated ES cells. Oct4 together with Nanog is a master regulator for pluripoteny in embryonic stem cells. Our results show that the Pou5f1 gene (Oct4) can be utilised to specifically target undifferentiated cells and to effectively remove these unwanted cells.

Supported by: Deutsche Forschungsgemeinschaft in the Framework of the Excellence Cluster REBIRTH 


\section{PS 023 Beta cell regeneration}

\section{3}

Intermediate filament expression in the adult and developing rat pancreas

E. Bertelli ${ }^{1}$, A. Di Bella ${ }^{1}$, M. Regoli ${ }^{1}$, C. Nicoletti ${ }^{2}$

${ }^{1}$ Dept. of Pharmacology, Sect. of anatomy, University of Siena, Italy, ${ }^{2} \mathrm{GI}$ Biology and Health Programme, Institute for Food Research, Norwich, United Kingdom

Background and aims: Intermediate filaments are frequently used as markers of cell differentiation. In order to assess if they can be useful to identify differentiating pancreatic cells, we have examined the pattern of expression of nestin, cytokeratin 20 (CK20) and vimentin in rat adult and developing pancreas

Materials and methods: Embryos from E12.5 up to E21.5 as well as pancreas from newborn, 3-days old, and adult rats were sampled and immediately frozen. Cryosections were fixed in cold acetone and laser scanning confocal microscope analysis was carried out on sections double-labelled with antibodies against nestin, vimentin or CK20 and markers of endocrine or exocrine cells. Double-labelling experiments were also carried out with antibodies anti-vimentin and anti-CK20

Results: In the developing gland CK20 was strongly expressed by duct cells, whereas endocrine cells showed a less intense staining and acinar cells were provided with a weak labelling that outlined a fine network of filaments. Since the beginning of the development of the dorsal and ventral pancreatic buds, some cells located within the dorsal pancreatic rudiment also displayed vimentin immunofluorescence. The number of these cells progressively increased with time, reaching its highest peak in newborn rats when most of the duct cells contained vimentin, and rapidly decreasing soon after. Acinar cells were always CK20-immunoreactive since the beginning of their cytodifferentiation but they never showed vimentin fluorescence. Since endocrine cells differentiate from precursor cells located in the epithelial lining of the primitive ducts we decided to verify whether vimentin-immunoreactive duct-like cells could be transitional cells in the process of differentiation into endocrine cells. Delta, beta and PP cells, though always containing a welldeveloped network of CK20 intermediate filaments, never showed vimentinimmunoreactivity at any time-point even in newborn animals when vimentin-containing duct cells were at their highest number. On the contrary, in addition to a network of CK20 filaments that was present throughout the entire development of the pancreas, alpha cells also displayed vimentin-immunoreactivity starting from the earliest gestational age studied (E12.5). At this stage of development almost all vimentin-containing cells within the dorsal pancreatic bud were alpha cells and even the reverse (i.e. alpha cells displaying a vimentin network) applied almost constantly (fig 5d-f). At E13.5 even the ventral pancreatic bud displayed the same pattern of staining. With time, however, alpha cells seemed to progressively loose vimentin filaments. Still, in newborn animals several vimentin-expressing glucagon cells could be easily found, but the number of these cells declined very quickly and after a few days from birth they were virtually absent. Nestin was observed exclusively in mesenchymal cells and in particular in endothelial cells of blood vessels Conclusion: Under our experimental conditions CK20 is not an exclusive marker of duct cells. However, the pattern of its labelling still allows the identification of the three major cell types (duct, acinar, endocrine cells). Vimentin is expressed in cells located within pancreatic ducts from the beginning of pancreatic development. Its presence in glucagon-secreting cells likely identify immature or recently differentiated alpha cells

\section{4}

Expression and function of lutheran, a laminin alpha5 binding glycoprotein receptor on human pancreatic islet cells M. Banerjee ${ }^{1}$, I. Virtanen ${ }^{2}$, O. Korsgren ${ }^{3}$, T. Otonkoski ${ }^{1}$

${ }^{1}$ Biomedicum Stem Cell Center, University of Helsinki, Finland, ${ }^{2}$ Institute of Biomedicine/Anatomy, University of Helsinki, Finland, ${ }^{3}$ Department of Clinical Immunology, University of Uppsala, Sweden

Background and aims: Lutheran blood group glycoprotein (Lu), and its truncated form known as BCAM, is a transmembrane protein of the immunoglobulin superfamily, which serves as a receptor for alpha5 chain of laminins (Lm), constituent of Lms-511 and -521. Lm-511 is found in many epithelial basement membranes (BMs) and recently we have identified it as the sole laminin isoform of the BM surrounding human pancreatic islets. The high affinity of Lu/BCAM for laminin is thought to contribute to various developmental processes including organogenesis and vascular development. In red blood cells, $\mathrm{Lu} / \mathrm{BCAM}$ is activated through cAMP signaling. The aims of the present study were (1) to examine the expression of Lu/BCAM in adult and fetal human pancreas and (2) to investigate its possible role in islet cell adhesion dependent or independent of cAMP.

Materials and methods: Frozen human pancreatic sections, freshly isolated islet cells (dispersed into single cells) and cultured endocrine cells (48 hours cultured) were used to examine the expression of Lu. Purified Lm511 and soluble recombinant $\mathrm{Lu}$ (sol-Lu) proteins were used for short-term adhesion experiments. Forskolin (activator of adenylate cyclase) and PKA-i (specific inhibitor of c-AMP dependent protein kinase A) were employed to examine cAMP dependency.

Results: Immunofluorescent staining of human pancreatic frozen sections showed bright immunoractivity for Lu in a polarized fashion co-aligned with the BM Lm alpha5 chain surrounding islets. Faint immunoreactivity was recorded in the more centrally located islet cells. Human fetal pancreas at 17-22 gestational weeks showed an essentially similar pattern of Lu immunoreactivity. Competitive inhibition by sol-Lu in short-term adhesion experiments (90 $\mathrm{min}$ ) resulted in severe impairment of islet cell adhesion (insulin and chromogranin-A positive cells) on Lm511 matrix, suggesting a central role of Lu in islet cell adhesion to Lm511. The inhibitory effect of sol-Lu was equal to that achieved with monoclonal antibodies against $\alpha 3$ and $\beta 1$ integrin subunits. To examine the role of cAMP signaling on Lu expression and islet cell adhesion, dispersed islets were pretreated with forskolin and/or PKA-i prior to adhesion to Lm511 matrix (overnight incubation). Forskolin treatment clearly enhanced islet-cell membrane expression of Lu. Quantitative analysis of cell adhesion showed significant enhancement of beta-cell adhesion by forskolin while PKA-i treated cells adhered poorly to LM511.

Conclusion: The present study discloses $\mathrm{Lu}$ as a previously unrecognized laminin binding receptor on human pancreatic endocrine cells. Islet cell adhesion to Lm511 is at least partially mediated via Lu and is cAMP dependent. Further studies are required to undertake in order to establish a relationship between $\mathrm{Lu}$ and functional status of human islets. Supported by: EU

\section{5}

Long lifespan and very low neogenesis and proliferation of human islet beta cells estimated by mathematical modelling of beta cell lipofuscin accumulation

M. Cnop ${ }^{1,2}$, S.J. Hughes ${ }^{3}$, F. Sayeed ${ }^{4}$, L. van de Laar ${ }^{4}$, J.H. Gunter ${ }^{4}$,

D.W.R. Gray ${ }^{3}$, P.R.V. Johnson ${ }^{3}$, B.C. Hansen ${ }^{5}$, J.F. Morris ${ }^{6}$,

M. Pipeleers-Marichal ${ }^{7}$, I. Cnop ${ }^{8}$, A. Clark ${ }^{4}$

${ }^{1}$ Laboratory of Experimental Medicine, Universite Libre de Bruxelles, Belgium, ${ }^{2}$ Division of Endocrinology, Erasmus Hospital, Brussels, Belgium, ${ }^{3}$ DRWF Islet Isolation Facility, Churchill Hospital, Oxford, United Kingdom, ${ }^{4}$ Oxford Centre for Diabetes, Endocrinology and Metabolism, Churchill Hospital, Oxford, United Kingdom, ${ }^{5}$ Obesity, Diabetes, and Aging Research Center, University of South Florida, Tampa, United States, ${ }^{6}$ Department of Human Anatomy, Genetics and Physiology, University of Oxford, United Kingdom, ${ }^{7}$ Diabetes Research Centre, Vrije Universiteit Brussel, Brussels, Belgium, ${ }^{8}$ Department of Mathematics, Vrije Universiteit Brussel, Brussels, Belgium

Background and aims: The longevity of pancreatic islet $\beta$-cells in man is largely unknown. A decreased $\beta$-cell mass resulting from an imbalance in turnover of insulin-secreting pancreatic islet $\beta$-cells has been proposed as a contributory factor for type 2 diabetes. Lipofuscin body (LB) accumulation is a hallmark of ageing in post-mitotic cells. Using this marker, we estimated the proportion of newly formed human $\beta$-cells.

Materials and methods: Using electron microscopical morphometry, we examined the relationship of LB accumulation in $\beta$-cell sections (two dimensions) to age in islets of human donors (aged 1-81 years, $n=50$ ), human insulinoma cells and non-human primate islets $(n=10)$. To estimate the proportion of lipofuscin-positive $\beta$-cells and the number and size of LBs in three dimensions, a three dimensional mathematical model was constructed using Mathematica software.

Results: The $\beta$-cell content of LBs increased with age. $\beta$-cell area of lipofuscin (\% of cytoplasmic area) was highly correlated with donor age ( $\mathrm{r}=0.87$, $\mathrm{p}<0.0001$ ), increasing from $0.03 \%$ of cytoplasm for the youngest donor to $14 \%$ in the oldest, and not different in type 2 diabetes $(n=6)$. By multiple regression analysis, LB content was unrelated to body mass index. Modelling 
of the total cellular lipofuscin content in 3D from the 2D data showed that, in the oldest individuals, total LB volume reached $35 \%$ of the cytoplasmic volume. LBs also accumulated in non-human primate $\beta$-cells $(r=0.88, p<0.001)$. A low LB content, unrelated to patient age, was observed in human proliferative insulinoma cells. The percentage of human $\beta$-cell sections imaged which contained a cross-section of at least one LB positively correlated with age $(r=0.82, p<0.0001)$. The percentage of LB-positive cell sections increased logarithmically from $7 \%$ at age 1 year to $100 \%$ at age 52 , whereas it remained low in insulinoma cells (10-40\%). The model indicated that the 3D LB-positive $\beta$-cell population (ageing cells) was $>90 \%$ in the first decade of life and rising to close to $100 \%$ by age 20 .

Conclusion: Our data suggest that in vivo in humans the proportions of LBnegative, newly formed $\beta$-cells are very low and that $\beta$-cells progressively accumulate LBs with time. Unlike the proposed scenario in rodents, neogenesis and/or replication are not major contributors to the islet $\beta$-cell population in humans. Ageing of long-living human $\beta$-cells could contribute to the decline of insulin secretion with age and to the onset of type 2 diabetes.

Supported by: Wellcome Trust, Diabetes UK, European Union FP6 IP EuroDia, Fonds National de la Recherche Scientifique, Belgium

\section{6}

Beta cell regeneration in type 1 diabetes: the role of Reg genes in NOD mice

E. Astorri ${ }^{1,2}$, M. Bombardieri ${ }^{2}$, C. Guglielmi ${ }^{1}$, C. Pitzalis ${ }^{2}$, P. Pozzilli ${ }^{1,3}$ ${ }^{1}$ Area Endocrinologia e Diabetologia, University Campus Bio Medico, Rome, Italy, ${ }^{2}$ Experimental Medicine \& Rheumatology, Queen Mary University, Barts \& the London, United Kingdom, ${ }^{3}$ Centre of Diabetes \& Metabolic Diseases, Queen Mary University, Barts \& the London, United Kingdom

Background and aims: Type 1 Diabetes (T1D) is an autoimmune disease characterised by pancreatic islet destruction. There is evidence that the betacell mass is not static but in a constant process of death and renewal. Reg genes, a multigene family discovered by screening the cDNA library derived from rat regenerating islets, may represent an important therapeutic target for islet regeneration.

The specific aim of this study was to evaluate the expression of Reg genes in the pancreas of NOD mice, the animal model of T1D, and to correlate gene expression with different stages of life/disease and different degrees of islets inflammatory infiltrate.

Materials and methods: 68 female pre-diabetic and diabetic NOD mice and $20 \mathrm{BALB} / \mathrm{c}$ mice as controls were culled at different time points $(4,8,12,16$, 2024,30 and $>30$ weeks). RNA was extracted from pancreas and reversetranscribed to cDNA. Quantitative real-time PCR was performed in order to investigate the mRNA expression of all Reg genes (Reg1, Reg2, Reg3 $\alpha, \operatorname{Reg} 3 \beta$, $\operatorname{Reg} 3 \gamma$, Reg $3 \delta$ ). Results were expressed as relative quantification using two endogenous controls, $\beta$-actin and 18S. Paired snap-frozen, OCT-embedded samples were analysed by Haematoxylin and Eosin staining and immunohistochemistry for $\mathrm{T}$ and $\mathrm{B}$ lymphocytes in order to evaluate immune cell infiltration as peri-islet or intra-islet infiltrates and the degree of lymphoid organization using a semiquantitative score.

Results: NOD mice showed high pancreatic expression of all Reg genes, except for Reg 4 . The expression profile of all Reg genes was similar with high expression in very young mice (4 weeks) and progressive decrease almost to zero at 10 weeks. This was followed by a dramatic peak in all Reg genes in NOD mice at disease onset (12 weeks) which lasted until around 18-20 weeks and was followed by a progressive and rapid decline. The peak in Reg genes expression was associated with the maximum rate and organization of islet inflammatory infiltrate. In control $\mathrm{BALB} / \mathrm{c}$ mice, we observed a strong constitutive expression of Reg genes at 4 weeks which decreased dramatically from high expression to undetectable at 10 weeks (similar to NOD mice). However, differently from NOD mice, this was not followed by a later peak with no residual expression up to 20 weeks of age.

Conclusion: Here we report the first quantitative analysis of Reg genes expression profile in NOD and control mice at different stages of life/disease. These results suggest that Reg genes might have an active role in the physiological expanding process during the growth of the pancreas in early life. However, once the organ has reached its adult volume, Reg genes gradually decrease their expression levels unitl undetectable from 10 weeks onwards. Conversely, in NOD mice Reg genes are induced at disease onset (12-15 weeks) most likely as an attempt to regenerate the $\beta$-cells mass following the autoimmune destructive process. Evidence that Reg gene expression peaks in parallel with the maximum inflammatory infiltrate also suggests a role of local inflammation in inducing Reg genes expression. Overall, our data contribute to clarify the role of Reg genes in the pancreas during normal and pathologic conditions. The degree of expression of Reg genes may constitute a new tool in the evaluation of beta-cell regeneration in T1D.

\section{7}

Pancreatic regenerating protein $(\mathrm{reg})$ inhibits human pancreatic stellate cell proliferation

L. Li ${ }^{1}$, M.G. Bachem ${ }^{2}$, S. Zhou ${ }^{2}$, D. Bimmler ${ }^{3}$, R. Graf ${ }^{3}$, Z. Sun ${ }^{1}$

${ }^{1}$ Depatment of Endocrinology, Southeast University, Nanjing, China,

${ }^{2}$ Department of Clinical Chemistry, Ulm University, Germany, ${ }^{3}$ Department of visceral and transplantation surgery, Zurich University, Switzerland

Background and aims: Pancreatic regenerating protein ( $r e g)$ has been discovered in the fields of diabetes and pancreatitis. In diabetes, reg is up-regulated and plays a key role in islet neogenesis/regeneration by induction of cellular proliferation. During acute and chronic pancreatitis, reg is also dramatically increased. However, the functional role of reg in pancreatitis remains unclear. Pancreatic stellate cells (PSC) play a central role in fibrogenesis associated with pancreatitis. To develop an integrated concept regarding reg protein in endocrine and exocrine pancreas we investigated its effect on PSC.

Materials and methods: reg was expressed in the yeast Pichia pastoris and purified from medium supernatants. Human PSC were obtained by outgrowth from fibrotic human pancreas tissue. reg was added at concentrations of $10 \mathrm{ng} / \mathrm{ml}$ and $100 \mathrm{ng} / \mathrm{ml}$ to cultured PSC (passage 3 - 8). Cell proliferation was determined by BrdU incorporation. PSC migration was assessed by the wound-assay. Extracellular matrix (collagen type I and fibronectin), matrix metalloproteinases (MMPs), tissue inhibitors of matrix metalloproteinases (TIMPs) and reversion- inducing cysteine-rich protein with Kazal motifs (RECK) were demonstrated on protein and mRNA level.

Results: reg inhibited PSC proliferation and migration in a dose-dependent manner. Soluble collagen I and fibronectin were reduced. reg slightly decreased the synthesis of MMP-1 and -2 and strongly decreased TIMP- 1 and 2 concentrations in PSC supernatants. RECK expression was slightly induced after reg application.

Conclusion: While reg promotes pancreatic islet $\beta$-cell growth and regeneration, our results provide novel insights that reg inhibits PSC proliferation and TIMP $1 / 2$ secretion. These data endorse our view that reg plays an important role in the reorganization of the injured pancreas. The findings suggest that reg may have a protective function during the repair phase of pancreatic injury by promoting fibrosis resolution and boosting pancreatic islet $\beta$-cell regeneration.

Supported by: Deutsche Forschungsgemeinschaft; Swiss National Science Foundation

\section{8}

Epithelial cells within the adult pancreas do not co-express mesenchymal antigens in vivo; evidence that epithelial-mesenchymal transition is an artifact of cell culture K.L. Seeberger, A. Eshpeter, R.V. Rajotte, G.S. Korbutt

Surgery, University of Alberta, Edmonton, Canada

Background and aims: Currently there is an inherent shortage of cadaveric donors necessary to meet the transplantation requirements of type-1 diabetics. Identification of an islet progenitor that could generate an unlimited supply of transplantable islets could potentially alleviate the shortage of donor islets. Previously we have demonstrated that mesenchymal stem cells (MSCs) can be expanded from the non-endocrine pancreas (ductal epithelium) that is normally discarded following islet isolation. Although pancreatic MSCs can be partially differentiated into islet-like cells that express endocrine hormone mRNA and transcription factors critical to islet development they do not express insulin protein. It has been proposed that these MSC progenitors are actually derived from human $B$-cells undergoing reversible epithelialmesenchymal transition (EMT), suggesting that these cells could be a potential source of new $B$-cells. In this study we sought to identify a population of epithelial cells in the non-endocrine pancreas that give rise to multipotent MSCs.

Materials and methods: Double immunofluorescent (IF) staining and flow cytometry were used to assess the cell phenotype of non-endocrine pancreas tissue following islet procurement and to follow changes to cell phenotype during in vitro expansion (24 hours to 21 days) and differentiation of MSCs. 
IF staining of paraffin embedded pancreatic biopsy sections was used to assess cell phenotype in vivo. Several antibodies against MSC and epithelial cell antigens along with antibodies to pancreatic endocrine and exocrine hormones were used in both methods. Flow cytometry analysis data are expressed as mean percent \pm SEM.

Results: In this study we demonstrate that: 1) pancreatic epithelial cells do not express MSC antigens in vivo; 2) following islet isolation epithelial cells (EpCAM and CK19 positive) express the MSC antigens CD44 (32 \pm 8 and $38 \pm 10)$ and $\mathrm{CD} 29(85 \pm 4$ and $64 \pm 4) ; 3)$ during in vitro expansion the number of single positive epithelial and double positive epithelial/MSCs declines while the number of single positive MSCs increases and; 4) differentiated MSCs do not revert to a true epithelial cell phenotype in our culture conditions, as epithelial cell surface markers (EpCAM, CK19 and E-Cadherin) are not re-expressed, although the MSC phenotype is altered.

Conclusion: This study demonstrates that MSCs may be derived in vitro via a pancreatic epithelial cell undergoing EMT; however it is likely that a small percentage of MSCs that reside in the adult pancreas are proliferating while the epithelial cells are negatively selected for by the experimental culture conditions.

Supported by: JDRF, CIHR and ADI

\section{9}

The induction of pancreatic islet regeneration in vitro with islet neogenesis associated protein-related pentadecapeptide

\section{T. Yang}

Dept. Endocrinology, First Affiliated Hospital of Nanjing Medical University, Nanjing, Jiangsu, China

Background and aims: To establish protocol for the isolation of mesenchymal stem cells (MSCs) from adult rat pancreatic duct and differentiation in vitro with islet neogensis associated protein-related pentadecapeptide (INGAP-PP). The insulin secreting cells produced with the novel multi-step differentiation method are the source for islet transplantation for diabetic rat model.

Materials and methods: The pancreas from adult SD rat was digested with type V collagenase solution $(0.5 \mathrm{mg} / \mathrm{ml})$ and ductal epithelial cells were isolated from the digests by centrifugation of Ficoll density gradient to delete the islet tissue. The islet-depleted ductal-epithelial cells were cultured in PRMI $1640(\mathrm{pH} 7.4)$ medium with $10 \%$ fetal calf serum at $37^{\circ} \mathrm{C}$ in $5 \% \mathrm{CO}$ and the epidermal growth factor (EGF) and basic fibroblast growth factor (bFGF) were supplemented 4 days later. The medium were switched to serum-free medium supplemented with EGF and bFGF after continuous single cell layer appeared in about 7 th day. At the last step of the differentiation the cells were divided into two groups, one cultured in the medium supplemented with islet INGAP-PP $(1.0 \mu \mathrm{g} / \mathrm{ml})$ and the others with the control peptide $(1.0 \mu \mathrm{g} /$ $\mathrm{ml}$ ), with which the same amino acids but arranged in the completely difference sequence. After the differentiation, the insulin producing test were performed for the new generated islet-like clusters (ILCs), and the expression of insulin, glucagons, Pdx-1, CK-19 and nestin in the ILCs were tested with immunohistochemistry and reverse transcription-polymerase chain reaction (RT-PCR). The insulin content of the ILCs cells in the supernatant after glucose stimulation was assayed with ELISA. The regenerated ILCs were harvested and implanted into the subcapsular region of one kidney for the streptozotocin-induced diabetic rat, and the blood glucose levels were observed after transplantation.

Results: The expression of CK-19, Pdx-1 and nestin in the ductal-epithelial cells before differentiation were detected with immunofluorescence stain and RT-PCR, and the expression of insulin and glucagons are negative. After induction with EGF and bFGF, spherical-like aggregates appeared and islet-like cells in different sizes were formed after 12 days. The expression of insulin and glucagon of the ILCs from INGAP-PP group were significantly higher than that of control group $(\mathrm{p}<0.01)$. Insulin release experiment indicated that insulin secretion ratio of ILCs from INGAP-PP group under glucose stimulation were significantly higher than that of control group $(p<0.01)$. The blood glucose level of the streptozotocin-induced diabetic rat were notably decreased than that of control group, after the kidney subcapsular implantation with of ILCs harvested from the multi-step differentiation procedure with INGAP-PP.

Conclusion: The novel INGAP-PP involved differentiation protocol for the islet-like clusters from the rat pancreatic mesenchymal stem cells was established. The insulin producing capability of the ILCs were sufficient to reverse the blood glucose level for the streptozotocin-induced diabetic rat after kidney subcapsular transplantation.

Supported by: National Nature Science Foundation of China
450

PGC-1alpha, potential mediator of the effects exerted by the glucocorticoids on pancreatic beta cell function and development B. Valtat, A. Singh-Estivalet, B. Bréant, B. Blondeau Team 8, INSERM UMRS 872, Paris, France

Background and aims: The pancreatic development is a complex process controlled by transcription factors and extrinsic signals. In this context, we have shown that glucocorticoids (GC) are negative regulators of pancreatic beta-cell development. To investigate by which mechanisms GC exert their effects, we aim at defining the GC-regulated genes and the potential implication of the transcriptional coactivator PGC-1alpha whose expression is controlled by GC and which binds to the glucocorticoid receptor.

Materials and methods: In vitro studies were performed on a beta cell line, the MIN6 cells that were treated with Dexamethasone (Dex, a synthetic glucocorticoid), infected with an adenovirus expressing PGC-1alpha or transfected with a small interferent RNA (siRNA) specific to PGC-1alpha. In vivo studies were performed on CD1 pregnant mice submitted or not to $50 \%$ food restriction and foetal pancreas were analysed at stages E13.5 to E18.5. Protein and RNA were extracted from cells or pancreata. Gene expression was measured by Western Blot and real-time quantitative PCR.

Results: First, we showed on the MIN6 cells that Dex treatment increased the expression of PGC-1alpha and decreased the expression of key beta-cell transcription factors, Pdx-1, MafA, Pax4, Pax6, Nkx2.2 and Isl1. Next, we determined the implication of PGC-1alpha in these gene regulations by overexpressing PGC-1alpha in MIN6 cells using an adenovirus and showed that it reduced the expression of the same transcription factors, suggesting that PGC-1alpha is involved in the glucocorticoid effects. We partially blocked the Dex-induced increase of the PGC-1alpha expression using a siRNA and found that Pdx-1 was also partially reduced by the Dex treatment, suggesting that PGC-1alpha is involved in the negative regulation of Pdx-1. Finally, we showed that PGC-1alpha is expressed in the foetal mouse pancreas at E13.5 with a peak of expression at E14.5-E15.5 when the pancreas is mainly composed of undifferentiated cells. When fetuses are exposed to undernutrition and thereby excess GC, the expression of PGC-1alpha is increased at the late stage E18.5 while the expression of $\mathrm{Pdx}-1$ is decreased.

Conclusion: Our work showed that 1) GC regulate PGC-1alpha expression in beta cells both in vitro and in vivo, 2) GC modulate the expression of key transcription factors for the development and the function of the beta-cells and 3) the transcriptional coactivator PGC-1alpha is involved the majority of the effects of the GC on the beta-cell transcription factors, especially the regulation of the expression of Pdx-1.

Supported by: National Research Association (ANR-Gramsy) and French Research Ministry

\section{1}

Raf-1 activation by insulin requires calcium signalling in pancreatic beta cells

E.U. Alejandro, K.J. Jacob, J.D. Johnson

Department of Cellular and Physiological Sciences, University of British Columbia, Vancouver, Canada

Background and hypothesis: The metabolic hormone insulin enhances growth and survival in many cell types including pancreatic $\beta$-cells. We previously reported that physiological doses of insulin protect $\beta$-cells from death and promote proliferation mediated by Raf- 1 signalling. We hypothesized that insulin directly stimulates the activation of Raf-1, a pro-survival serine/ threonine kinase upstream of Mek and Erk.

Materials and methods: Human and mouse islets were isolated via filtration and treated with insulin with or without BAPTA-AM, KN-62, KN93, or thapsigargin. Phosphorylated and total protein expression of Raf-1, Erk, and Bad were examined by western blotting. Primary islet cells and MIN6 cells were stained for propidium iodide and BrdU to estimate apoptosis and proliferation.

Results: First, we showed that critical protein components of the insulin/Raf1/Erk cascades are present in both human and mouse islets and MIN6-cells. Next, we demonstrated that insulin promoted dephosphorylation of the inhibitory site of Raf-1 at serine 259, and phosphorylation of the activation site of Raf- 1 at serine 338 , key events that are likely to fully activate the kinase. Over-expression of Raf-1 increased Erk phosphorylation. Indeed, insulin-induced Raf-1 activation also led to an increase in Erk phosphorylation. In addition to activation of the Raf-1/Mek/Erk pathway, we observed that insulin 
induced the phosphorylation of Bad, a novel target of Raf- 1 in the mitochondria. We have previously shown that insulin generated rapid cytosolic calcium signals in dispersed human and mouse $\beta$-cells. Thus, we tested the role of calcium signalling in Raf-1/Erk activation by insulin. The effect of insulin on Erk phosphorylation was abrogated by inhibitors of calcium signalling, such as BAPTA-AM, an intracellular calcium chelator, $\mathrm{KN}-62$ and $\mathrm{KN}-93$, specific inhibitors of the calcium/calmodulin-dependent protein kinase. These data suggest that calcium may be required for the activation of Raf- 1 and its downstream targets such as Erk and Bad. Blocking Raf-1 and calcium signalling individually resulted in Erk and Bad dephosphorylation and cell death. Moreover, dual inhibition of Raf-1 and calcium signalling did not have an additive effect, suggesting that they might be acting through the same pathways. The proliferative effects of insulin were blocked by thapsigargin, an inhibitor of the sarco/endoplasmic reticulum calcium-ATPase.

Conclusion: Our data suggest that insulin activates Raf-1/Erk and Raf-1/Bad pathways and calcium signalling may be required in the growth and survival effect of insulin in pancreatic $\beta$-cells.

Supported by: JDRF and NIH

\section{PS 024 Beta cell proliferation}

\section{2}

Protein-caloric maternal malnutrition affects pancreatic levels of Insulin-Like Growth Factor-1 and -2 during neonatal period: repercussions on beta cell mass

E. Fernández ${ }^{1}$, L. de Miguel ${ }^{1}$, M.A. Martín ${ }^{2}$, F. Escrivá ${ }^{1}$, C. Álvarez ${ }^{1}$ ${ }^{1}$ Bioq. y Biol. Mol. II, Fac. Farmacia, UCM, Madrid, ${ }^{2}$ Instituto del Frío, CSIC, Madrid, Spain

Background and aims: Developing endocrine pancreas undergoes substantial remodelling along the neonatal period and replication, neogenesis and apoptosis play a main role in the process. Insulin-like growth factors (IGFs) are major contributors to beta-cell growth, maturation and function, and are highly sensitive to nutritional status. We have previously shown that proteincaloric food restriction applied during the last third of gestation caused, in fetuses at term, an increase of beta-cell mass related to the stimulation of betacell proliferation due to locally increased pancreatic IGF-1. Nevertheless, at 4 days of life the beta-cell mass was decreased in undernourished $(U)$ neonates and this effect persisted until adult age. In order to clarify whether remodelling of endocrine pancreas occurs by the same mechanisms in control (C) and $\mathrm{U}$ neonates we quantified beta-cell mass, neogenesis and replication at 4,14 and 23 days of age. To determine the impact of food-restriction on the protein expression profile of IGFs and the consequences for the growth of beta-cells we measured serum GH, IGF-1/-2 levels and IGF-1/-2 protein content in pancreas and liver at the same age.

Materials and methods: Food-restricted pregnant rats received 35\% of control diet since the last week of gestation until the time of sacrifice of neonates. Beta-cell mass, neogenesis and replication were evaluated by immunocytochemistry and morphometry. Serum concentrations of GH and IGF-1 were measured by radioimmunoassay and of IGF- 2 by radioreceptorassay. The protein content of IGF- 1 and -2 was analyzed in pancreas and liver by Western blot.

Results: Beta-cell mass increased in $\mathrm{C}$ neonates from day 4-14 but it remained stable from 14-23 days of age. In $U$ group we did not observe any difference along the neonatal period but the beta-cell mass was significantly lower at each time point as compared to C. Beta-cell neogenesis progressively decreased with time in $\mathrm{C}$ whereas in $\mathrm{U}$ it remained stable between 4-14 days of age and then declined on day 23 staying higher than the $\mathrm{C}$ value at the same time. Beta-cell replication peaked on day 14 in $\mathrm{C}$ and decreased on day 23. In $\mathrm{U}$ rats beta-cell replication remained stable from 4-14 days of age and then decreased on day 23. Differences between $\mathrm{C}$ and $\mathrm{U}$ groups were only found at 14 days of age.

Pancreatic and hepatic IGF-2 protein levels decreased progressively with time in both groups but pancreatic IGF- 2 concentration was significantly higher at each time point in U animals. Both groups showed the same IGF-1 protein expression profile in pancreas and liver with a peak of concentration in pancreas at 14 days of age. The protein levels of hepatic IGF-1 increased linearly with time in both groups. Serum GH levels decreased progressively throughout the time of study in both populations. Serum IGF-1 levels increased progressively in both groups but these values were higher in $\mathrm{U}$ rats as compared to $\mathrm{C}$ neonates on day 14 and 23 .

Conclusion: 1) Despite beta-cell mass is decreased in $\mathrm{U}$ animals along neonatal period, the endocrine pancreas still has the capacity to regenerate; 2 ) The enhancement of pancreatic IGF-1 levels at 14 days of age could favour both stimulation of beta-cell replication in $\mathrm{C}$ neonates and beta-cell neogenesis in $U$ animals; 3 ) In the same manner, the pancreatic IGF-2 overexpression in $U$ rats may contribute to maintain an elevated rate of beta-cell neogenesis during a longer period of time.

Supported by: Ministerio de Ciencia y Tecnología and CIBER de Diabetes y Enfermedades Metabólicas, Spain 


\section{3}

Reversal in beta cell mass and glucose tolerance of diabetic mice by reactivation of Cyclin-dependent kinases with in vivo gene transfer Y. Hiki ${ }^{1,2}$, T. Sasaki ${ }^{1,3}$, M. Nemoto ${ }^{1,3}$, Y. Hirai ${ }^{4}$, T. Ohashi ${ }^{3}$, N. Tajima ${ }^{1}$ ${ }^{1}$ Division of Diabetes, Metabolism and Endocrinology, Department of Internal Medicine, Jikei University School of Medicine, Tokyo, ${ }^{2}$ Division of Metabolism, Department of Internal Medicine, Nishisaitama-chuo National Hospital, Saitama, ${ }^{3}$ Department of Gene therapy, Institute of DNA Medicine, Jikei University School of Medicine, Tokyo, ${ }^{4}$ Department of Biochemistry and Molecular Biology, Division of Gene Therapy Research, Center for Advanced Medical Technology, Nippon Medical School, Tokyo, Japan

Background and aims: Impairment of compensatory proliferation of $\beta$ cells is assumed to be critical for development of diabetes. In this process, inactivation of Cyclin-dependent kinases (CDK4/6) caused by increased expression of negative regulator, $\mathrm{p}^{16^{\mathrm{INK}}{ }^{\mathrm{Na}}}$ is essential. Therefore molecular intervention to cell cycle regulation of residual islet cells has therapeutic potential. In the present study, we aimed to restore $\beta$ cell mass by reactivating CDK 4 with in vivo gene transfer technique in adult diabetic mice.

Materials and methods: Adeno-associated virus (AAV) 8 vector was concentrated by ultracentrifugation with iodixanol gradient. For the transient in vivo gene expression, $10 \mathrm{w}$-old STZ mice were randomized for the treatment with $\mathrm{CDK} 4^{\mathrm{R} 24 \mathrm{C}}$ gene, a variant that promotes $\mathrm{G} 1 / \mathrm{S}$ transition with least suppression by p16 ${ }^{\text {INK4a }}$ (R24C treated), or with GFP gene (mock treated). The $8 \times 10^{12} \mathrm{vg} /$ body virus was directly injected to pancreas by an open surgery. At $16 \mathrm{w}$ after the treatment, paraffin-embedded tissues were analyzed by immunohistochemistry (IHC). In order to know changes in $\beta$ cell mass, total islet area of whole pancreas (total islet area) of 210 slices and $\beta$ cell area within each islet ( $\beta$ cell mass) of 360 islets for each treatment were measured. For evaluation of cellular proliferation, staining with PCNA and CDK were also performed. For evaluation of $\beta$ cell differentiation, staining with insulin, glucagons, MafA, and MafB were performed.

Results: Observation of GFP indicated about $30 \%$ of islet cells expressed the transgene as long as $16 \mathrm{w}$. IHC of CDK staining revealed that CDK4 was localized exclusively at cytoplasm of $\beta$ cells in the mock treated mice, while it localized at nucleus in addition to cytoplasm in most of $\beta$ cells in the $\mathrm{R} 24 \mathrm{C}$ treated islets, suggesting reactivation and nuclear translocation of Cyclin D/ CDK4 complex. Both total islet area and $\beta$ cell mass of the R24C treated mice turned out to be 2.5 fold greater than those of mock treated mice. Histogram analysis revealed that distribution of $\beta$ cell mass of the R24C treated mice almost recovered to that of normal mice. PCNA and TUNEL staining of the R24C treated mice showed that proliferating $\beta$ cells were significantly increased while apoptotic cells did not changed. All of the insulin-expressing cells including PCNA $(+)$ cells in islets of the R24C treated mice were shown to be $\mathrm{MafA}(+) \mathrm{MafB}(-)$, indicating the proliferated islet cells should be terminally differentiated $\beta$ cell. Plasma glucose level of the R24C treated mice after glucose load until $120 \mathrm{~min}$ were significantly lower than that of mock treated mice; $12.5 \pm 0.6$ vs. $17.9 \pm 0.9 \mathrm{mM}, \mathrm{p}=0.0002$ at 120 .

Conclusion: Our data demonstrate that the aberrant islet structure and glucose intolerance in diabetic mice was restored with improved growth regulation of $\beta$ cells when $\mathrm{CDK} 4^{\mathrm{R} 24 \mathrm{C}}$ gene was expressed in adult islets in vivo.

\section{4}

PTB1 regulates the cell cycle supressor p27Kip1 in insulinoma cells F. Ehehalt ${ }^{1}$, K.-P. Knoch ${ }^{2}$, E. Wuendrich ${ }^{2}$, R. Meisterfeld ${ }^{1}$, C. Wegbrod ${ }^{2}$, A. Altkrueger ${ }^{2}$, H.-D. Saeger ${ }^{1}$, M. Solimena ${ }^{2}$

${ }^{1}$ Dept. of Surgery, Dresden University of Technology, Germany,

${ }^{2}$ Experimental Diabetology, Dresden University of Technology, Germany

Background and aims: After glucose entry in the pancreatic beta-cell the rapid biosynthesis of insulin secretory granules (SG) starts parallel to the activation of the secretory machinery. We have shown that glucose promotes the nucleocytoplasmic translocation of polypyrimidine tract-binding protein 1 (PTB1) in beta-cells. Cytosolic PTB1 binds mRNAs encoding SG proteins, thus enhancing their stability and translation, while knockdown of PTB1 by RNA interference depletes SG stores. In this study we have focussed on the role of PTB1 for proliferation of beta cells.

Materials and methods: We investigated whether the knockdown of PTB1 is affecting the proliferation rate (BrdU incorporation) of INS-1 cells and searched for possible PTB1 targets involved in cells cycle regulation by quantitative PCR and western blot.
Results: Parallel to the depletion of SGs, PTB1 knockdown correlated with decreased expression of the proliferation repressor p27Kip1 and increased replication of INS-1 cells. Knockdown of p27Kip1 was associated with increased proliferation of INS- 1 cells, due to its reduced binding to the CdK2/ cyclinE complex, and with reduced expression of several beta-cell specific marker proteins. The similar phenotypes observed following the knockdown of PTB1 and p27Kip1 could be rescued by the independent overexpression of either protein fused to an epitope tag.

Conclusion: These data suggest that PTB1 promotes the expression of the proliferation repressor p27Kip1 in INS-1 cells, thereby inhibiting their proliferation while promoting granule biogenesis. Accordingly, the PTB1/p27Kip1 pathway may act as a switch between differentiation and proliferation of pancreatic beta-cells. Supported by: JDRF, SFB655

\section{5}

Expression of the master repressor RE-1 silencing transcription factor (REST) in beta cells leads to diabetes

D. Martin ${ }^{1}$, D. Caille ${ }^{2}$, P. Meda ${ }^{2}$, J.-A. Haefliger ${ }^{1}$

${ }^{1}$ Department of medicine, University Hospital, Lausanne, ${ }^{2}$ Department of Cell Physiology and Metabolism, University Medical Center, Geneva, Switzerland

Background and aims: The repressor RE-1 silencing transcription factor (REST) is a master regulator of neuroendocrine genes. The generation of transgenic mice expressing REST specifically in beta cells (RIP-REST mice) represents a reliable system to identify the importance of REST target genes in insulin producing cells. The observation that RIP-REST mice feature intolerance to glucose, together with the identification of several REST target genes that are key to exocytosis, such as Snap25 or synaptotagmin isoforms, attested for the essential role of these genes in beta cell function. Evidences suggest that a number of additional REST target genes are crucial for insulin secretion, beta cell survival and overall beta cell identity.

Materials and methods: Transgenic mice were characterized by Southern blot analyses of genomic DNA, and by immunohistochemical staining of pancreatic sections. The secretory response of these mice was assessed by pancreas perfusion and by measuring glycemia. Transcriptional regulation of target genes was assessed by quantitative PCR.

Results: Glucose intolerant RIP-REST animals show a decrease in beta cell mass, arguing that REST target genes are also involved in cell proliferation/ survival. This was further confirmed with a particular RIP-REST mice line that display a profound hyperglycemia leading to death after few months. This frank diabetes, observed from weaning onward, was accounted for by a major loss of beta cell mass, as assessed by immunohistochemistry and pancreatic insulin content measurements. The morphological analyses of pancreas from mice at postnatal day 2 (P2), revealed a significant insulin immunolabelling in RIP-REST diabetic mice, as compared with control animals, suggesting that the loss of beta cells occurred after birth, and did not arise from defects in embryonic development. In contrast, the islet morphology in these P2 transgenic mice was strongly affected, with glucagon and insulin positive cells scattered randomly among the islets. This phenotype, more severe than that observed with the glucose intolerant RIP-REST mice, is thought to be accounted for by a higher level of transgene expression. RE-1 sequence databases searches revealed a number of substantial putative target genes, either involved in growth signal transduction pathways, or in mechanisms of mitogenesis, that could be responsible for the observed beta cell loss. Preliminary results point out two known regulator of cell proliferation/ survival, Ica512 and islet-brain 1, as well as an uncharacterized neuroendocrine-specific regulatory subunit of cdk5, Cdk5r2 (p39), as bona fide REST target genes.

Conclusion: We point out our RIP-REST mice as a pertinent model underlining the functional importance of REST target genes in pancreatic beta cells. Specifically, we show that the expression in beta cells of a "forbidden" key master repressor, REST, can lead to diabetes, by a mechanism that may rely on beta cell dysfunction, survival or differentiation. Further comprehensive analyses of these mice will help to build up a list of essential genes of pancreatic beta cell fate, that may play a critical role in the pathophysiology of diabetes.

Supported by: FNRS 31-109530 FNRS 31-109402 


\section{6}

ARNT is required for normal glucose tolerance and beta cell proliferation in pregnancy

S.M. Lau, R. Stokes, K. Cheng, J.E. Gunton

Immunology and Inflammation, Garvan Institute of Medical Research, Sydney, Australia

Background and aims: We demonstrated that the ubiquitous and highly conserved transcription factor ARNT is reduced in expression by $>90 \%$ in the islets of people with Type 2 diabetes and is thus an important candidate gene for beta cell dysfunction. 3 of the 5 women with Type 2 diabetes also had a known history of gestational diabetes, suggesting that ARNT may also play a role in the pathogenesis of this condition. We hypothesise that ARNT may be important in regulation of beta cell proliferation during pregnancy. ARNT forms an obligate heterodimer with partners such as hypoxia inducible factor (HIF) -1 alpha, HIF-2 alpha and the aryl-hydrocarbon receptor. In beta cells, the ARNT-HIF-1 alpha complex is important in the regulation of glucose sensing, glycolysis and insulin signalling. Knockdown of ARNT in cultured Min6 islets results in decreased expression of antiapoptotic genes BCL-2 and BCL-xL. Thus ARNT may play a particularly important role in the adaptation of beta cells to stressors such as the increased insulin secretory demands of pregnancy. Beta cell dysfunction is an important component of gestational diabetes, but the cause of this defect has not been fully elucidated. We hypothesise that ARNT is required for adequate beta-cell proliferation in pregnancy, and that loss of ARNT will result in impaired glucose tolerance and decreased beta-cell proliferation. We studied mice with a beta-cell specific deletion of ARNT (beta-ARNT mice). Females have mild glucose intolerance by 9 weeks of age due to beta cell dysfunction. They have a normal body weight, insulin sensitivity and fertility.

Materials and methods: 12-14 week old nulliparous beta-ARNT and floxed control female mice underwent timed mating. An intraperitoneal glucose tolerance test was performed on gestational day 16.5. In a separate group of mated females, 5-bromo-2-deoxyuridine (BrdU) ( $30 \mathrm{mg} / \mathrm{kg}$ body weight) was injected intraperitoneally on day $14.5,16$ hours prior to removal of the pancreas for formalin fixation and paraffin embedding. Pancreatic sections were stained (immunoflurescence) for insulin, BrdU and DAPI. 3 sections per mouse were analysed for islet number, beta cell area and number of BrdUpositive nuclei per islet. The BrdU labelling index (total number BrdU positive nuclei ${ }^{\star} 100000 /$ total beta cell area) was calculated for each mouse.

Results: Beta-ARNT mice have impaired glucose tolerance in pregnancy (Fig 1). Beta cells from beta-ARNT dams have a significantly decreased BrdU labelling index and fewer BrdU- positive cells per islet.

Conclusion: Loss of beta cell ARNT results in a greater deterioration of glucose tolerance during late pregnancy. This is associated with decreased BrdU staining in beta cells. These data suggest that impaired glucose tolerance in pregnancy in beta-ARNT mice is secondary to a combination of decreased glucose sensing and reduced beta cell proliferation.

\section{B-ARNT mice have greater deterioration of glucose tolerance during pregnancy}

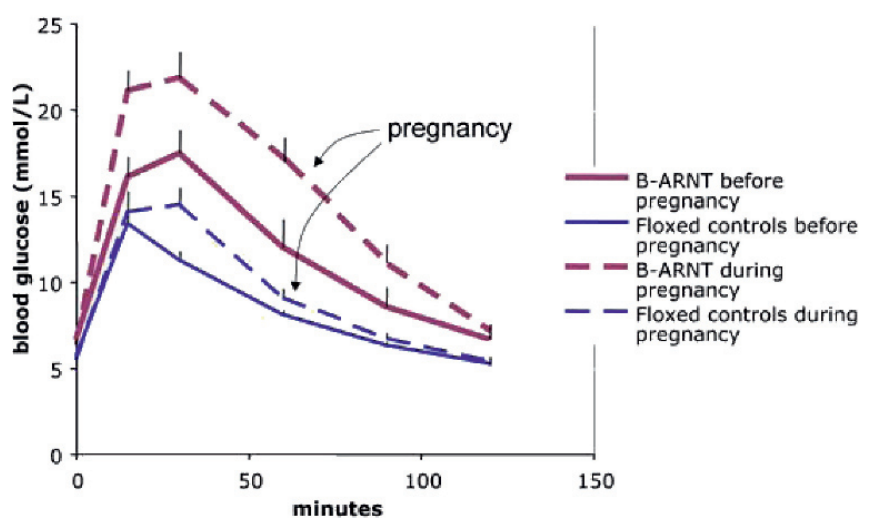

Supported by: Australasian Diabetes in Pregnancy Society Research grant
457

Glucose metabolism and insulin secretion in islets from pregnant protein-restricted rats

D.F.I. Souza ${ }^{1}$, L.M.I. Souza ${ }^{2}$, S.R.L. Reis ${ }^{2}$, R.V. Veloso ${ }^{2}$, M.A.B. Reis ${ }^{3}$, A.C. Boschero ${ }^{4}$, E.M. Carneiro ${ }^{5}$, V.C. Arantes ${ }^{2}$, M.Q. Latorraca ${ }^{2}$

${ }^{1}$ Pós-Graduação em Ciências da Saúde, Universidade Federal de Mato Grosso, Cuiabá-MT, ${ }^{2}$ Departamento de Alimentos e Nutrição, Universidade Federal de Mato Grosso, Cuiabá-MT, ${ }^{3}$ Departamento de Ciências Básicas em Saúde, Universidade Federal de Mato Grosso, Cuiabá-MT, ${ }^{4}$ Departamento de Fisiologia e Biofísica, Universidade Estadual de Campinas, CampinasSP, ${ }^{5}$ Departamento de Fisiologia e Biofísica, Universidade Estadual de Campinas, Cuiabá-MT, Brazil

Background and aims: Protein restriction reduces glucose-induced insulin secretion, due, in part, to decrease glucose transporter 2 expression and glucose metabolism. In contrast, pregnancy increases glucose-induced insulin release and reduces glucose-stimulation threshold through enhancing glucose transporter 2, glucokinase and hexokinase expressions and activities. We investigated the effect of low protein diet during pregnancy on expression levels of these proteins, the rate of glucose oxidation and the insulin secretion. Materials and methods: Adult control non-pregnant (CNP) and control pregnant $(\mathrm{CP})$ rats were fed a normal-protein diet $(17 \%)$, whereas low-protein non-pregnant (LPNP) and low-protein pregnant (LPP) rats were fed a low-protein diet $(6 \%)$ for $15 \mathrm{~d}$. Islets were isolated to determination of insulin secretion, rate of glucose oxidation and expression of glucokinase, hexokinase, and glucose transporter 2 by Western blot.

Results: Islets from pregnant rats exhibited the dose-response curves to glucose shifted to the left compared to islets from non-pregnant rats. The half-maximal response $\left(\mathrm{EC}_{50}\right)$ from LPP group was lower in relation to other groups (CNP: $8.6 \pm 0.3 \times 10^{-3} \mathrm{mM}$; CP: $7.4 \pm 0.2 \times 10^{-3} \mathrm{mM}$; LPNP: $9.9 \pm 0.6 \times 10^{-}$ ${ }^{3} \mathrm{mM}$; LPP: $6.5 \pm 0.1 \times 10^{-3} \mathrm{mM}$ de glucose). In the presence of $2.8 \mathrm{mmol}$ glucose/L, insulin secretion (ng/islet/90min) by islets of CNP and LPNP was similar and lower than that by islets of CP and of LPP rats. However, the insulin secretion by islets of LPP rats was higher than all groups (CNP: 0.19 \pm 0.02 ; CP: $0.50 \pm 0.03$; LPNP: $0.21 \pm 0.01$; LPP: $0.80 \pm 0.02$ ). In $2.8 \mathrm{mmol}$ glucose/L, pregnancy produced increase in the islet glucose oxidation rates (pmol/islet/h), but in greater degree in CP than in LPP group (CNP: 1.37 \pm 0.33 ; CP:7.23 \pm 1.59 ; LPNP:4.33 \pm 0.75 ; LPP:5.86 \pm 0.68 ). Glucokinase (CNP:12270 \pm 1037 ; CP:12282 $\pm 1040 ; \quad$ LPNP:10894 $\pm 611 ; \quad$ DP:7054 \pm 1330 ) and glucose transporter 2 expression levels (CNP:16978 \pm 1676 ;CP:15963 \pm 528 ; LPNP:16143 \pm 2586 ; LPP:9714 \pm 1973 ) were lower in islets from LPP group compared the other groups. Hexokinase levels were lower in malnourished than in the control islets and pregnancy increased its expression only in CP islets (CNP:14257 \pm 1679 ; CP:17245 \pm 1157 ; LPNP:12013 \pm 2198 ; LPP:10057 \pm 677 ).

Conclusion: Protein restriction during pregnancy improved islets glucose sensitivity despite decreased expression of proteins involved in the glucose metabolism.

Supported by: CNPq/Fapemat

\section{8}

The role of R-spondin-1/canonical Wnt signalling in glucagon-like peptide-1 regulation of beta cell growth

V.S.C. Wong, W.J. Schultz, P.L. Brubaker

Physiology, University of Toronto, Canada

Background and aims: Glucagon-like peptide-1 (GLP-1) functions as an incretin hormone, as well as a $\beta$-cell growth factor stimulating $\beta$-cell proliferation. Canonical Wnt (cWnt) signaling is an important component of pancreatic development and plays a role in $\beta$-cell proliferation. We have recently demonstrated the presence of R-spondin-1 (Rspo1), a gut growth factor and secreted activator of cWnt signaling, in murine MIN6 $\beta$-cells. These cells have also been shown to express multiple Wnt ligands, their receptors, and downstream molecules of the intracellular cWnt signaling pathway. Furthermore, we found that the GLP-1 receptor agonist, exendin-4 (Ex4) enhances Rspo1 mRNA levels in a time- (up to $8 \mathrm{hr}$ ), dose- (up to $10 \mathrm{nM}$ ) and high glucosedependent manner. We have therefore now investigated the role of Rspo1 in GLP-1 regulation of $\beta$-cell growth.

Materials and methods: MIN6 or $\beta$ TC cells or mouse islets were treated with EX4 (10 nM) in the absence or presence of inhibitors of PI3K (LY294002; $10 \mathrm{nM})$, PKA (H89; $10 \mu \mathrm{M})$, MEK1 (PD98059; $20 \mu \mathrm{M})$, or p38 MAPK (SB203580; $10 \mu \mathrm{M})$, or with immuno-purified FLAG-Rspol protein. Changes 
in mRNA transcript levels for Rspol and the cWnt target gene, c-myc, were assessed via qRT-PCR, $\beta$-catenin nuclear localization was determined by immunoblot of cell nuclear fractions, and proliferation was examined by thymidine incorporation assay.

Results: Consistent with our findings in MIN6 cells, Ex4 increased Rspo1 and c-myc mRNA levels in $\beta$ TC cells, peaking at 8 and $24 \mathrm{hr}$, respectively $(\mathrm{p}<0.05)$. A preliminary study showed that Rspol expression was also stimulated by approximately 80 -fold in isolated mouse islets after $4 \mathrm{hr}$ incubation with Ex4. In MIN6 cells, treatment with FLAG-Rspo1 alone ( $8 \mathrm{hr}, 0.001$ and $10 \mathrm{ug} / \mathrm{ml}$ ) also stimulated c-myc expression by approximately 3 -fold. Cotreatment of the cells with Ex4 and LY294002 abolished the effect of Ex4 on Rspo1 mRNA. In contrast, the stimulatory effect of Ex 4 to increase $\beta$-catenin nuclear localization (by 2-fold, $\mathrm{p}<0.05$ ), was found to be MEK1- $(\mathrm{p}<0.01)$ but not PI3K-, PKA-, or p38-dependent. Furthermore, analysis of MIN6 cell proliferation revealed that, while Ex4 stimulated cell growth by 1.2 -fold ( $\mathrm{p}<0.01$ ), FLAG-Rspol was inhibitory, decreasing thymidine incorporation by $60 \%$ ( 1 $\mathrm{ug} / \mathrm{ml} ; \mathrm{p}<0.01)$. An MTT cell survival assay did not reveal any detrimental effect of FLAG-Rspol on overall MIN6 cell viability.

Conclusion: Ex4 stimulation of Rspo1 expression is not limited to the MIN6 $\beta$-cell line, but is also found in $\beta$ TC and primary islet cells. While the effects of Ex4 to increase Rspol mRNA are PI3K-dependent, Ex4 stimulation of nuclear $\beta$-catenin is ERK-1-dependent, suggesting that Ex4 regulates Rspo1 and cWnt signaling through alternative pathways. Moreover, the inhibitory effects of Rspol on $\beta$-cell proliferation suggests that the role of Rspol in the $\beta$-cell may be to modulate the balance between proliferative behaviour and differentiated function.

Supported by: Banting and Best Diabetes Graduate Scholarship, Institute of Biomaterials and Biomedical Engineering Sally and Paul Wang Graduate Scholarship and Canadian Institutes of Health Research Doctoral Award. P.L.B. is supported by the Canada Research Chairs Program and operating grant from the Canadian Diabetes Association

\section{9}

Calcium-dependent transcription pathways are required for stimulation of beta cell proliferation by extracellular matrix

G. Parnaud ${ }^{1,2}$, E. Hammar' ${ }^{1}$ M.Y. Donath ${ }^{3}$, P.A. Halban ${ }^{1}$

${ }^{1}$ Department of Genetic Medicine and Development, University Medical Center, ${ }^{2}$ Cell Isolation and Transplantation Center, Division of Surgical Research, Department of Surgery, University Hospital, ${ }^{3}$ Division of Endocrinology and Diabetes, University Hospital, Geneva, Switzerland

Background and aims: Rat beta-cell proliferation is increased on extracellular matrix $(E C M)$ and this requires an elevation of $\left[\mathrm{Ca}^{2+}\right]_{i}$. The aim of this study was to determine $\mathrm{Ca}^{2+}$-dependent growth signalling pathways activated by ECM with a particular focus on nuclear-factor B (NF-kB) and Nuclear Factor of activated T cells (NFAT).

Materials and methods: Autofluorescence FACS-sorted rat pancreatic betacells were plated on poly-L-lysine (PLL) or on 804G-ECM (derived from rat bladder carcinoma cells) with(out) inhibitors for $48 \mathrm{~h}$ with addition of BrdU for the last $24 \mathrm{~h}$. Cell replication was determined by BrdU incorporation and the identity of beta-cells confirmed by insulin immunofluorescence. NF-kB nuclear translocation was assessed by immunofluorescence. NF-kB transcriptional activity was analyzed by quantification of mRNA levels of IkBa, (established NF-kB target gene) vs. L3 (housekeeping gene) by real-time quantitative RT-PCR. Data are mean \pm SEM for 3 or more independent experiments. Results: Treatment of beta-cells with diazoxide (opens $\mathrm{K}_{\text {атр }}$ channels, thereby preventing depolarization and $\mathrm{Ca}^{2+}$ entry) reduced $804 \mathrm{G}-\mathrm{ECM}$ induced NF$\mathrm{kB}$ nuclear translocation (by $51.84 \pm 11.93 \%$; $\mathrm{p}<0.02$ ) as well as IkBa gene expression ( $3.3 \pm 0.49$ vs. $1.5 \pm 0.16 ; 804 \mathrm{G}-\mathrm{ECM}$ - vs. + diazoxide; $\mathrm{p}<0.01)$. NF-kB inhibitor Bay 11-7082 $(5 \mu \mathrm{M})$ blocked almost completely beta-cell proliferation $\left(2.07 \pm 0.43\right.$ vs. $0.17 \pm 0.07 \% \mathrm{BrdU}^{+}$cells; $804 \mathrm{G}-\mathrm{ECM}$ - vs. + Bay 11-7082; $\mathrm{p}<0.02)$. Since IL-1beta is a known target of NF-kB that is secreted from rat beta-cells on ECM, we blocked the IL-1 receptor with IL-1Ra $(2 \mu \mathrm{g} / \mathrm{ml})$ and this resulted in $57 \pm 9 \%$ decrease in beta-cell proliferation on $804 \mathrm{G}$-ECM $(p<0.001)$. Having shown previously an inhibitory effect of cyclosporin A (inhibitor of calcineurin/NFAT), we now show that direct inhibition of NFAT by VIVIT peptide inhibits partially 804G-ECM-induced beta-cell proliferation ( $4.5 \pm 0.3$ vs. $3.1 \pm 0.2 \% \mathrm{BrdU}^{+}$cells $804 \mathrm{G}$-ECM - vs. + VIVIT; $\mathrm{p}=0.02$ ). NFAT is also modulated by non-calcineurin-dependent pathways, including MAPK, PKA and GSK3beta. Compatible with these known mechanisms, the p38 and c-Jun N terminal Kinase (JNK) MAPK inhibitors SB20358 and SP600125 $(10 \mu \mathrm{M})$ increased beta-cell proliferation on 804G-ECM by 4.3 and 1.8 fold respectively ( $\mathrm{p}=0.02$ vs. control) as did the GSK3 inhibitor BIO ( 1 $\mu \mathrm{M})\left(3.3 \pm 0.3\right.$ vs. $5.7 \pm 0.8 \% \mathrm{BrdU}^{+}$cells, $804 \mathrm{G}-\mathrm{ECM}$ - vs. $\left.+\mathrm{BIO} ; \mathrm{p}=0.04\right)$ while the PKA inhibitor H89 $(10 \mu \mathrm{M})$ decreased it $\left(2.3 \pm 0.4\right.$ vs. $0.4 \pm 0.3 \% \mathrm{BrdU}^{+}$ cells, 804G-ECM - vs. + H89; $\mathrm{p}=0.004)$.

Conclusion: Our results suggest that calcium entry which is necessary for 804G-ECM increased beta-cell proliferation is also involved in 804G-ECM induced NF-kB activity that is required for beta-cell proliferation. IL-1beta may amplify this process by feed-forward activation of NF-kB. These results also indicate a role for NFAT signalling in 804G-ECM induced beta-cell proliferation, with possible modulation via both calcineurin and non-calcineurin-dependent pathways.

Supported by: JDRF

\section{0}

The protein tyrosine phosphatase, $\mathrm{PTP}-\mathrm{BL}$, regulates pancreatic beta cell proliferation by interacting with components of the Wnt signalling pathway

A.R. Oknianska ${ }^{1}$, H.J. Welters ${ }^{1}$, K.S. Erdmann ${ }^{2}$, G.U. Ryffel ${ }^{3}$, N.G. Morgan ${ }^{1}$ ${ }^{1}$ Institute of Biomedical and Clinical Science, University of Plymouth, United Kingdom, ${ }^{2}$ Department of Biochemistry II, Ruhr-University Bochum, Germany, ${ }^{3}$ Institut fuer Zellbiologie, University of Duisburg-Essen, Germany

Introduction: In pancreatic beta-cells, increased expression of the MODY5 gene product, $H N F 1 \beta$, leads to enhanced rates of apoptosis and altered regulation of the cell cycle, suggesting that control of HNF1 $\beta$ expression may be important for the control of $\beta$-cell proliferation and viability. It is unclear how these effects of HNF1 $\beta$ are mediated but previously we identified a protein tyrosine phosphatase, PTP-BL, as an HNF1 $\beta$-regulated protein in $\beta$-cells and we have now studied the role of this protein in INS-1 $\beta$-cells.

Methods: The rat $\beta$ cell line INS- 1 was used to generate clones stably expressing either wild type (WT) PTP-BL or a tyrosine phosphatase deficient mutant (PTP-BL-CS) under the control of an inducible promoter. Cell proliferation was measured with the CellTiter $96^{\circ} \mathrm{AQ}$ uenous One Solution Cell Proliferation Assay (Promega). For routine determination of cell viability, trypan blue staining was used. Insulin secretion was measured by radioimmunoassay. Whole cell protein extracts were analysed by immunoprecipitation and Western blotting.

Results: Stably transfected INS-1 cells were generated that express either wild type (WT) or the phosphatase-deficient mutant (PTP-BL-CS) of PTPBL conditionally under the control of a tetracycline-regulated promoter. Enhanced expression of WT PTP-BL inhibited INS-1 cell growth dose-dependently (uninduced: $0.30 \pm 0.02 \mathrm{~A}$ units at $72 \mathrm{~h} ; 20 \mathrm{ng} / \mathrm{ml}$ tetracycline: $0.18 \pm 0.01$ $(\mathrm{p}<0.001) ; 1 \mu \mathrm{g} / \mathrm{ml}$ tetracycline: $0.14 \pm 0.01$ units; $(\mathrm{p}<0.001))$ but this effect was not observed when PTP-BL-CS was expressed. Neither construct altered the rate of apoptosis. PTP-BL has been reported to interact with components of the Wnt signalling pathway and addition of exogenous Wnt3a resulted in an increase in cell proliferation $(120 \pm 5 \%$ vs control; $\mathrm{P}<0.01)$ and a rise in $\beta$-catenin levels, consistent with the operation of this pathway in INS-1 cells. Upregulation of WT PTP-BL antagonised Wnt3a-induced cell growth $(85 \pm 5 \%$ vs control; $\mathrm{p}<0.001)$ and the rise in total $\beta$-catenin levels. By contrast, PTPBL-CS failed to inhibit Wnt3a-induced proliferation. Induction of PTP-BL caused a decrease in the expression of active (dephosphorylated) $\beta$-catenin in INS-1 cells consistent with the involvement of the phosphatase in the direct regulation of $\beta$-catenin functionality. The rise in $\beta$-catenin caused by Wnt3a was also suppressed by over-expression of HNF1 $\beta$, suggesting that HNF1 $\beta$ may regulate the Wnt signalling pathway via an increase in PTP-BL levels. Conclusion: We conclude that PTP-BL plays an important role in regulation of cell cycle progression in pancreatic $\beta$-cells and that it interacts functionally with components of the Wnt signalling pathway.

Supported by: EFSD/AstraZeneca grant and Diabetes UK 


\section{PS 025 Transcriptional regulation in beta cells}

\section{1}

PTB1 regulates the expression of VEGF-A in rat insulinoma INS1 cells K.-P. Knoch ${ }^{1}$, K. Kratz ${ }^{1}$, F. Ehehalt ${ }^{2}$, C. Wegbrod ${ }^{1}$, A. Altkrueger ${ }^{1}$, M. Solimena ${ }^{1}$

${ }^{1}$ Experimental Diabetology, ${ }^{2}$ Dept. of Surgery, Dresden University of Technology, Dresden, Germany

Background and aims: PTB1 plays a key role in the biogenesis of insulin secretory granules (ISGs) induced by glucose and GLP-1 shortly after their stimulation of pancreatic beta-cells. Stimulation by these agents results in the nucleocytoplasmic translocation of PTB1 through independent mechanisms and its binding to polypyrimidine-rich motifs in the 3'- and/or 5'-untranslated region (UTR) of mRNAs encoding ISG components. This, in turn, increases the stability of these mRNAs and promotes their translation in a concerted fashion, thereby enhancing ISG biogenesis. In this study we focused on the potential role of PTB1 in the expression of VEGF-A. Release of VEGF-A by beta-cells promotes islet angiogenesis, stimulates endothelial cells to produce extracellular matrix components and favors the vascularization of transplanted islets. The 5'-UTR of the VEGF-A mRNA is unusually long (>900 bp) and contains several PTB1 binding sites.

Materials and methods: We investigated therefore whether the expression of VEGF-A in rat insulinoma INS-1 cells is regulated by PTB1 by RT/PCR and reporter gene assays.

Results: We found that INS1 cells express primarily VEGF-A and that glucose stimulation induces a rapid increase of VEGF- $\mathrm{A}_{164}$ mRNA levels because of post-transcriptional mechanisms. This up-regulation was prevented upon knockdown of PTB1 by RNA interference, while over-expression of PTB1 increased the levels of VEGF-A ${ }_{164}$ mRNA. Sequential truncation of VEGF-A 5'-UTR progressively reduced its in-vitro binding to PTB1, while inclusion of PTB1 binding sites found in the VEGF-A 5'-UTR enhanced the expression of luciferase reporter constructs in glucose-stimulated INS-1 cells. This enhanced expression, however, was abolished by knockdown of PTB1.

Conclusion: Taken together, these data suggest that in addition to promoting ISG biogenesis, PTB1 is required for the rapid up-regulation of VEGF- $\mathrm{A}_{164}$ in glucose-stimulated beta cells.

Supported by: JDRF

\section{2}

Ufm1, a new ubiquitin-like protein, is required for glucose-induced insulin secretion from pancreatic beta cells

K. Lemaire ${ }^{1}$, M. Granvik ${ }^{1}$, T. Becker ${ }^{2}$, C. Newgard ${ }^{2}$, E. Waelkens ${ }^{3}$, F. Schuit ${ }^{1}$ ${ }^{1}$ Molecular Cell Biology, Gene Expression Unit, KULeuven, Belgium, ${ }^{2}$ Department of Pharmacology and Cancer Biology, Duke University Medical Center and Sarah W. Stedman Nutrition and Metabolism Center, Durham, United States, ${ }^{3}$ Molecular Cell Biology, Promega, KULeuven, Belgium

Background and aims: Ufm1 is a ubiquitin-like protein with an unknown function. Generally, ubiquitin-like proteins affect very diverse cellular processes via post-translational modification of their targets. We studied the expression and function of Ufm1 in pancreatic islets.

Materials and methods: Ufm1 mRNA levels were measured via quantitative RT-PCR. Insulin release and content were measured in Ufm1-silenced INS1832/13 cells via ELISA. Identification of Ufm1 targets was performed using affinity purification and mass spectrometry analysis.

Results: Knockdown of Ufm1 in INS1-832/13 cells resulted in a drastic decrease in glucose-induced insulin release and in insulin content. Further, we identified the first two target proteins of Ufm1, namely Ufbp1 and Cdk5Rap3. Ufbp1 is an uncharacterised protein with an unknown function. Via detailed computational protein analysis and fluorescence microscopy, we showed that Ufbp1 is a component of the secretory pathway and that it influences the distribution of Ufm1 in the cell between the nucleus and the ER.

Conclusion: Together, our data shows that Ufm 1 specifically plays a role in glucose stimulated insulin release in the pancreatic beta cell and we propose that it plays a role in regulating the protein secretory pathway via Ufbpl.

\section{3}

Pax6 and Pax6(5a) in pancreatic beta cells G. Wolf, A. Karkour, U. Henrion, R. Walther

Department of Medical Biochemistry and Molecular Biology, University of Greifswald, Germany

Background and aims: The transcription factor Pax6 is known as an important insulin-dependent regulator of the glucagon gene expression in pancreatic alpha cells, while in beta cells the influence of this transcription factor on insulin gene expression is discussed controversially. Moreover the impact of Pax6(5a), an alternative splice product, has not been investigated so far. We already identified Pax6 as an inhibitor of the rat insulin gene 2. Using starved and high glucose conditions we studied posttranslational modifications by kinases and glycosyl-transferases of both isoforms in pancreatic beta cells.

Materials and methods: Microscopical analyses of overexpressed fluorescent fusion proteins or immunostained endogenous transcription factors were done to study its intracellular localization in dependence on glucose. Glycosylation of these proteins was investigated using specific antibodies after immunoprecipitations and Western Blots. Several mutant GST fusion proteins were generated and subjected to in vitro kinase assays to detect phosphorylation sites.

Results: Under low glucose conditions Pax6 is localized in the nucleus, whereas high glucose leads to cytoplasmic localization. Translocation was conducted by phosphorylation of Pax6. In vitro both Pax6 isoforms are phosphorylated by CaMK2 and CK2, but not by PKB. The mutation of Threonin 48, which is phosphorylated by CK2 in Pax6(5a), but is absent in Pax6, caused nuclear remaining of Pax6(5a) even under high glucose. Although glycosylation has been reported to be involved in translocation, Pax6 has not been found to be glycosylated.

Conclusion: Pax6 and Pax6(5a) are posttranslational modified by phosphorylation through CaMK2 and CK2, but not $\mathrm{PKB}$, which may influence its functional activities.

\section{4}

Q268X, truncated mutant of HNF4a causing MODY1, has nucleolustargeting ability and can bind with wild-type HNF4a or its repressor SHP and accumulate them to the nucleolus in cultured cells M. Ogata ${ }^{1}$, T. Awaji ${ }^{2}$, N. Iwasaki ${ }^{1}$, G.I. Bell ${ }^{3}$, S. Miyazaki ${ }^{4}$, Y. Iwamoto ${ }^{1}$ ${ }^{1}$ Tokyo Women's Medical University, Diabetes Center, Japan, ${ }^{2}$ Saitama University, Department of Pharmacology, Tokyo, Japan, ${ }^{3}$ The University of Chicago, Medicine and Human Genetics, Chicago, United States, ${ }^{4}$ Tokyo Women's Medical University, Department of Physiology, Japan

Background and aims: Hepatocyte nuclear factor $4 \alpha(\mathrm{HNF} 4 \alpha)$ is a key promoter of gene expression leading to insulin secretion in pancreatic $\beta$ cells. A number of heterozygous HNF4a mutations causing MODY type 1 (MODY1) have been reported. HNF4a protein functions only as homodimers and usually locates in the nucleus. We focused Q268X, the first reported HNF4a mutation responsible for. Q268X is a C-terminal truncated mutant up to the 269th amino acid, lacks transactivation function, and causes diabetes phenotype with remarkable penetration, unlike point mutations such as R127W HNF4a. Q268X has been shown to bind with wild-type HNF4a in biochemical assay in vitro. However, since the nuclear distribution pattern differs between Q268X and wild-type, they might be not associated in living cells. Results: Expressed wild-type HNF4a tagged with EYFP was predominantly located in the nucleus except the nucleoli in COS7. The localization in the nucleoplasm was confirmed in CHO cells and MIN6 cells. The similar distribution was observed for point mutation R127W which has been found as a heterozygous mutation in MODY1 patients . In contrast, a truncated mutant Q268X tagged with EYFP was predominantly localized in the nucleoli in COS7 and CHO cells. In MIN6 cells, however, expressed Q268X-YFP was located in the nucleoplasm as well as the nucleolus. We thought that Q268X should be precisely examined in nuclear localization and dimerization with wild-type HNF4a in living cells. We found that Q268X binds with wild-type HNF4a in living COS7 and CHO cells, whose cells are not express HNF4a naturally, as visualized by co-transfecting the cells with genes encoding Q268X or HNF4a tagged with yellow or cyan variants of green fluorescent proteins (GFPs), respectively, and by utilizing fluorescence resonance energy transfer (FRET) between the two GFP variants. Q268X was also found to interact with small heterodimer partner (SHP), a negative regulator of nuclear receptors including HNF4a. Mutational analysis by deletion from the $\mathrm{C}$ terminus of HNF4a showed that the predominant nucleoplasm localization 
of HNF4a was conserved up to I338X mutant, and the nucleolus-targeting activity appeared from S337X. The even smaller mutant such as R154X seems to be accessible to the nucleolus but not anchored there. R154 distributed in the whole nucleus without preferential localization to the nucleoli.

Conclusion: When expressed simultaneously, Q268X was capable of accumulating HNF4a or SHP in the nucleoli, exhibiting enhanced FRET there. Altered intra-nuclear localization to nucleoli as heterodimers with Q268X could perturb normal function of HNF4a and/or SHP. HNF4a has been shown to bind with SHP at AF-2 domain. The present results indicate that there is another binding site than AF-2 domain, as suggested by previous study. The binding site for SHP is thought to be located between A250 and R150. A variety of phenotypes of MODY1 may have another possible functional modification; that is, intra-nuclear localization change of HNF4a and its repressor SHP caused by binding of the mutant.

Supported by: Grant-in-Aids for General Scientific Research

\section{5}

CREBP is involved in glucagon-stimulated glucagon gene transcription T. Moede, D. Kaiser, I.B. Leibiger, P. Vaca-Sanchez, P.-O. Berggren,

B. Leibiger

The Rolf Luft Research Center for Diabetes and Endocrinology, Karolinska Institutet, Stockholm, Sweden

Background and aims: We have previously demonstrated that glucagon gene transcription can be up-regulated by secreted glucagon through a molecular mechanism involving the glucagon receptor and multiple protein kinases, namely protein kinase $\mathrm{A}$, protein kinase $\mathrm{C}$ and CaM-kinase II. It has been shown that these signalling pathways can converge at the level of the transcription factor CRE-binding protein (CREBP). Because the glucagon promoter is known to contain a CREBP-binding site at -308 bp to $-280 \mathrm{bp}$, the aim of this study was to investigate the involvement of transcription factor CREBP binding in the positive feedback regulation of glucagon gene transcription.

Materials and methods: Glucagon mRNA levels were analysed by QPCR and normalized to cyclophilinA. Online monitoring of glucagon promoter $(-775$ to $+7 \mathrm{bp})$ driven DsRed expression was used as a readout for glucagonstimulated gene transcription, while glucagon promoter-driven firefly luciferase expression was used to assess basal promoter activity. Electrophoretic mobility shift assay (EMSA) in combination with supershift using a CREBPspecific antibody and wild type and mutant variants of the CRE cis-elements, were employed to study the in vitro binding of CREBP to the glucagon promoter CRE. Functional expression studies using wild type and mutant glucagon promoter variants in combination with pharmacological inhibitors of PKC, PKA and CaMKII were performed to test the involvement of CREBP in glucagon-mediated glucagon transcription.

Results: Stimulation of alphaTC1-9 cells with $200 \mathrm{pmol} / \mathrm{ml}$ glucagon led to a $1.56+/-0.1(\mathrm{n}=10)$ fold increase in glucagon-promoter driven DsRed fluorescence in alphaC1-9 cells. This treatment also resulted in $2.2+/-0.1 \quad(n=3)$ and $2.5+/-0.2$ fold $(\mathrm{n}=3)$ increased mRNA levels in alphaTC1-9 and isolated islets respectively. The increase in either mRNA levels or DsRed fluoresccence were abolished by treatment with inhibitors of protein kinase A (100 $\mu \mathrm{M}$ RPcAMPs), protein kinase C (150 nM bisindolylmaleimide) and CaM-kinase II (400 nM autocamtideII). Since these signalling pathways can all converge at the CREBP-CRE system, we studied the effect of a CRE-mutation, which does not allow CREBP binding, on rat glucagon promoter activity. Electrophoretic mobility shift assay in combination with a supershift assay, utilizing a CREBP-specific antibody revealed that CREBP is present in nuclear extracts of alphaTC1-9 cells and that CREBP binds to the previously identified CREelement of the glucagon promoter at -308 to $-280 \mathrm{bp}$. Mutation in the core of the CRE-element (TGACGTCA to TGtgGTCA) abolished the in vitro binding of CREBP as observed in the EMSA. When this mutation was introduced into the expression constructs for the luciferase reporter system, it resulted in $75+/-5 \%$ reduction of luciferase expression $(n=5)$, indicating a reduced basal promoter activity. Finally, the mutation in the CRE-element completely abolished the stimulatory effect of $200 \mathrm{pmol} / \mathrm{ml}$ glucagon on glucagon promoter driven DsRed expression.

Conclusion: Our findings suggest that CREBP binding to the CRE element of the glucagon promoter is critically important for both basal and glucagon stimulated glucagon gene transcription. The transcription factor CREBP appears to be the target of the signalling events underlying the positive feedback regulation of glucagon on the transcription of its own gene in the pancreatic alpha-cell.

Supported by: Novo Nordisk Fonden, Swedish Research Council Family and Stefan Persson Foundation

\section{6}

Gene expression profiling of various sulfonylureas in insulin producing INS-1E cells

N.E. Magnusson, A.B. Petersen, K. Smidt, B. Brock, O. Schmitz, J. Rungby Pharmacology, University of Aarhus, Denmark

Background and aims: Sulfonylurea derivatives (SUs) are used in the management of type 2 diabetes. They act by stimulating insulin secretion by binding to the ATP-sensitive potassium channel (K-ATP) subunit SUR1 in beta-cells. SU-receptors are found in other cell types where K-ATP channels are thought to be involved in protective physiological responses to metabolic stress. Previous studies have shown that glimepiride and glibenclamide interact with SUR subunits in other cell types. To identify SU responsive genes in the beta-cell we investigated the transcriptional regulation of gliclazide, glimepiride, and glibenclamide by microarray analyses in INS-1E cells.

Materials and methods: INS-1E cells were exposed to $10 \mu \mathrm{M}$ of each compound for $24 \mathrm{~h}$. Control groups received vehicle. Each experiment was performed as three independent replicates. Gene expression profiles were analyzed using Affymetrix GeneChip arrays. Low intensity data was reduced using the following criteria: Genes with log fold changes $>0$ and absolute signal values $<100$ for the test sample were omitted. Similarly log fold changes $<0$ and absolute signal intensities $<100$ for the control sample were omitted. Pathways were analyzed with Ingenuity Pathway analysis software using a cut-off limit of fold change $>1.5$ and $p<0.05$ (test vs control). Significant biological functions and canonical pathways were identified using Fishers exact test.

Results: A number of networks were identified for glibenclamide and glimepiride and none for gliclazide. 5 and 4 networks at various levels of significance were found for glimepiride $(\mathrm{p}=0.2-1 \mathrm{E}-26)$ and glibenclamide ( $\mathrm{p}=0.2-1 \mathrm{E}-22)$, respectively. The functional categories of these networks primarily related to cancer, immunological disease, cell death, and cell cycle. Toxicological analyses identified signalling pathways for glimepiride relating to oxidative stress and hypoxia via the $\mathrm{NAD}(\mathrm{P}) \mathrm{H}$ dehydrogenase system involved in free radical production. Also, down-regulation of AMP-activated protein kinase was identified as a function for cardiac damage. AMPK lacking the kinase activity increases the heart injury via alteration of the myocardial ischemia-reperfusion protective mechanism. Cell death signalling pathways comprised the second largest functional group for glimepiride and glibenclamide including pro-apoptotic IFN-gamma, MYC, TNF, and leptin pathways. The effect of gliclazide was analysed with reduced stringency allowing genes varying by 1.4 fold. A single network involving the beta-catenin pathway was identified. Activated beta-catenin has been shown to increase beta-cell expansion in mouse islets. In contrast to glimepiride and glibenclamide, no evidence of genes regulating beta cell apoptosis was found for gliclazide.

Conclusion: This study demonstrates that SU's affect the gene expression in INS-1E cells. Glibenclamide and glimepiride altered several transcription factors and signalling pathways involved in cell homeostasis and cell death. In contrast, a lower level of gene-regulation was found for gliclazide which may correspond to a higher specificity for SUR1 subunits of this compound. Regulation by gliclazide revealed changes compatible with beta-cell preserving networks (beta-catenin). This is in agreement with previous findings suggesting gliclazide as a general scavenger of radicals. SUs affect both beta-cell function and survival. This genome-wide scan suggests a differential profile among SUs on beta cell function and survival.

\section{7}

Effects of AMP-activated protein kinase on peroxisome proliferatorsactivated receptors $\alpha / \gamma$ and pancreatic duodenal homeobox-1 in INS-1 cells

H. Guo ${ }^{1,2}$, X.X. Lv ${ }^{1}$, X.J. Zhang', Q.B. Guan ${ }^{1}$, L. Gao ${ }^{1}$, J.J. Zhao ${ }^{1}$

${ }^{1}$ Scientific Centre, Provincial Hospital affiliated to Shandong University, ${ }^{2}$ Department of Pharmacology, Shandong University School of Medicine, Jinan, China

Background and aims: AMP-activated protein kinase (AMPK) is an energy sensor that regulates cellular metabolism and controls whole-body glucose homeostasis in multiple tissues. Although large numbers of studies have reported AMPK exerts action in several tissues, effects of AMPK on its target molecules in pancreatic $\beta$ cells remain largely elusive. One of the main purposes here was to detect the PDX-1 expression when AMPK activity changes. Pancreatic duodenal homeobox-1 (PDX-1) plays a pivotal part both in embryonic Pancreas development and in regulating pancreatic islet $\beta$-cell-spe- 
cific gene expression. Whether AMPK can affect PDX-1 expression has not previously been described. If so, accompanying with the changes of PDX-1, whether the expression changes also occurred simultaneously in PPAR $\alpha$ and PPAR $\gamma$, which are two members of the peroxisome proliferators-activated receptors (PPARs) subfamily. Owing to the more beneficial and effective function in antidiabetes of dual PPARa $/ \gamma$ agonist. Another emphasis, thus, of our aims here was to investigate whether AMPK could affect synchronously PPARa / $\gamma$ expression in $\beta$ cells.

Materials and methods: INS- 1 cells, a rat insulin-secreting cell line, were cultured in RPMI 1640 medium, and treated with or without $0.5 \mathrm{mM}$ AIC$\mathrm{AR}$ (an AMPK agonist) in the presence or absence of $10 \mu \mathrm{M}$ Compound $\mathrm{C}$ (an AMPK inhibitor). Treatment for 8 hours, total RNA was extracted for determining the contents of PPAR $\alpha / \gamma$ and PDX-1 mRNA by real-time PCR (normalized to GAPDH), total proteins were extracted for analysis of PPAR $\alpha$ protein expression by immunoprecipitation, while nuclear and cytoplasmic proteins were respectively gained for detecting PDX-1 by western blot (normalized to $\beta$ actin)

Results: Treatment with AICAR increased the PDX-1 mRNA levels approximately 2 -fold $(\mathrm{p}<0.05)$ compared to control. But, the AICAR-mediated increase was restored to the normal levels when Compound C prior to AICAR added. There are no significant difference between control and Compound $\mathrm{C}$ alone groups. Accordingly, compared to control, the nuclear PDX-1 proteins of the cells treated with AICAR alone were increased 1.8-fold $(\mathrm{p}<0.05)$. When cells were treated with AICAR and Compound $\mathrm{C}$ together, the nuclear PDX-1 protein expression was reduced by $33.3 \%$ ( $\mathrm{p}<0.05$ vs AICAR alone). However, in cytoplasmic proteins, PDX-1 expression in both AICAR group and Compound C/AICAR co-treated group was obviously higher than that in the control $(\mathrm{p}<0.05)$. The PPAR $\alpha$ and PPAR $\gamma$ mRNA contents were respectively increased about 1.68 -fold $(\mathrm{p}<0.05)$ and 2.66 -fold $(\mathrm{p}<0.05)$ in the cells treated with AICAR alone compare to control, but this increase was oppressed and was respectively reduced by $33.88 \%$ ( $\mathrm{p}>0.05$ ) and by $34.89 \%$ $(\mathrm{p}<0.05)$ in the cells treated with AICAR and Compound C together. Consistent with the changes of PPARa mRNA contents, IP experiment showed a strong stained band $(\mathrm{p}<0.05)$ representing PPAR $\alpha$ protein in the cells treated with AICAR alone, while the weakest band $(\mathrm{p}<0.05)$ was obtained in the presence of AICAR and compound $\mathrm{C}$ together.

Conclusion: AMPK activation increases PPARa, PPAR $\gamma$ and PDX-1 expression both in gene transcriptional level and in protein level in INS-1 cells under "physiological" conditions. Our knowledge concerning the effects of AMPK on its downstream targets may be useful for its involvement in islet $\beta$ cells protection.

Supported by: National Natural Sciene Foundation of China and Foundation of Weisheng Ting Shandong Province

\section{8}

Promoter activity of microsomal triglyceride transfer protein (MTTP) affected by lipids

I. Kraus-Stojanowic ${ }^{1}$, F.-A. Heinsen ${ }^{1}$, A. Schneider-Muntau ${ }^{1}$, F. Döring ${ }^{2}$, D. Rubin ${ }^{1}$, J. Schrezenmeir ${ }^{1}$

${ }^{1}$ Department of Physiology and Biochemistry of Nutrition, Max-Rubner Institute, ${ }^{2}$ Institute of Human Nutrition and Food Science, ChristianAlbrechts-University, Kiel, Germany

Background and aims: MTTP is involved in the assembly and secretion of VLDL by liver and of chylomicrons by intestine. Subjects homocygous for -493T polymorphism have fewer but more lipid-rich VLDL particles, lower cholesterol levels and therefore a less atherogenic lipoprotein profile. There is a nearly complete linkage disequilibrium in the common -493G/-400A/-164T and the rare allele -493T/-400T/-164C. The common haplotype has an almost twofold lower promoter activity than the rare haplotype. Mutagenesis experiments of our group revealed that instead of $-493 \mathrm{G}>\mathrm{T}$ as described in the literature, polymorphism at position -164 is responsible for different activity of MTTP promoter haplotypes, which seems to be mediated via SREBP1a. Further on allele specific binding sites for the SREBP cofactor NFY at -164T and for HNF1 at $-400 \mathrm{~A}$ are indicated in silico. To verify the putative transcription factor binding sites mobility shift assays (EMSA) were performed. Since there transcription factors are known to bind lipid ligands the effect of lipids on the allele specific promoter activity was studied in reporter gene assays and by qRT-PCR.

Materials and methods: For EMSA biotinylated complimentary oligos of allelic variants at position -400 and -164 were incubated with nuclear extracts of HeLa cells transfected with either NFY or HNF1 $\alpha$. Unlabled oligos were used as competitors, for super shift antibodies against NFY-A and HNF1a were added. For reporter gene assay HuH7 liver carcinoma cells were transfected with pGL4.10 vector containing different functional 966-bp segments of the MTTP promoter. Cells were incubated with $50 \mu \mathrm{M}$ palmitic, oleic, linoleic, eicosapentaenoic acid or cholesterol for $24 \mathrm{~h}$ before analysis of luminescence. MTP expression of untransfected $\mathrm{HuH} 7$ cells was determined by qRT-PCR after treatment.

Results: EMSA indicated specific binding of NFY to -164T. Binding was competed by unlabeled $-164 \mathrm{~T}$ but not $-164 \mathrm{C}$ oligos. Antibody against NFY lead to a supershift only of the $-164 \mathrm{~T}$ variant. Reporter gene assay demonstrated a nearly twofold decrease in promoter activity of $-164 \mathrm{~T}$ compared to $-164 \mathrm{C}$, indicating an inhibitory effect of SREBP1a or NFY. EMSA identified a specific binding of HNF1 $\alpha$ to the $-400 \mathrm{~T}$ allele, that is reduced by displacement reaction and shows a supershift with an antibody against HNF1a. In reporter gene assay there was no significant difference between the activity of promoter constructs $-400 \mathrm{~T}$ and $-400 \mathrm{~A}$. Palmitic acid slightly increased and oleic, linoleic and eicosapentaenoic acid decreased MTTP promoter activity, irrespective of the genotype. Multifactor ANOVA indicates a lacking interaction between fatty acids and promoter constructs. Cholesterol had no significant influence on the promoter activity of the different constructs. MTTP expression in $\mathrm{HuH} 7$ cells after incubation with fatty acids or cholesterol showed a ranking: eicosapentaenoic acid $>$ palmitic acid $>$ cholesterol $>$ untreated control $>$ oleic acid $>$ linoleic acid.

Conclusion: Binding of NFY to -164T could reduce MTTP promoter activity as a cofactor for SREBP. Binding of HNF1 $\alpha$ to $-400 \mathrm{~T}$ is of minor relevance. The impact of fatty acids is not dependent on the influence of the examined genotypes.

\section{9}

New strategy for generation of a mouse inducible model of protein expression in pancreatic beta cells

N. Bulat, C. Widmann

Physiology, University of Lausanne, Switzerland

Background and aims: RasGAP is a GTPase-activating protein specific for Ras. RasGAP is also a substrate of the caspase family of proteases. Under mild stress conditions RasGAP is cleaved by caspases into two fragments, fragment $\mathrm{C}$ (C-terminal part) and fragment $\mathrm{N}$ (N-terminal part). Fragment $\mathrm{N}$ expressed in cells, including insulinoma cell lines, induces potent survival signals that depend on the activation of Akt and the repression of NFkB. Transgenic mice expressing fragment $\mathrm{N}$ under the control of the rat insulin promoter (RIP-N mice) are more resistant to streptozotocin-induced diabetes. Islet cells isolated from this transgenic mouse are also more resistant to a series of pro-apoptotic stimuli, including inflammatory cytokines, palmitate, hypoxia and high glucose concentrations. The RIP-N mice however constitutively express fragment $\mathrm{N}$. An inducible model of fragment $\mathrm{N}$ expression in beta cells would represent a better system to assess the therapeutical potential of fragment $\mathrm{N}$ at the onset of diabetes. Generation of such a model requires that a targeting DNA sequence allowing a tight and specific regulation of fragment $\mathrm{N}$ expression in beta cells be constructed.

Materials and methods: We developed a strategy based on the ability of a fusion protein (called tTRKRAB) between KRAB (Krüppel-associated box protein) and the tetracycline repressor to block gene expression. Upon addition of tetracycline (or the doxycycline analogue), the fusion protein detaches from TREs allowing transcription to resume. We have constructed a targeting vector containing fragment $\mathrm{N}$, RIP, tTRKRAB, and TRE sequences that allows specific expression in beta cells upon doxycycline addition in vitro.

Results: This vector was used to generate mice that can be turned on and off at will for the expression of fragment $\mathrm{N}$ specifically in pancreatic beta cells. The transgenic mice line is created and is under the process of testing and will be used for possible ameliorating transplantation procedure.

Conclusion: Inducible expression of proteins in tissue specific manner by using one vector system is a much needed tool in molecular biology research. With this newly developed system we hope to generate such a mice line that will need no further backcrossing since it contain all the needed elements in one vector. This will hopefully bring us closer to the improvement of treatments for diabetic patients of both type I and type II.

Supported by: JDRF and Swiss National Science Foundation 


\section{0}

Beta cell specific silencing of the plasma membrane monocarboxylate transporter MCT1 (SLC16A1) is not effected through methylation of its promoter

T.J. Pullen ${ }^{1}$, G. da Silva Xavier ${ }^{1}$, G. Kelsey ${ }^{2}$, G.A. Rutter ${ }^{1}$

${ }^{1}$ Cell Biology, Imperial College, London, ${ }^{2}$ Developmental Genetics and

Imprinting, Babraham Institute, Cambridge, United Kingdom

Background and aims: Glucose metabolism in pancreatic $\beta$-cells is specialised to efficiently couple glucose oxidation to an increase in ATP:ADP ratio, critical for stimulating insulin secretion. Alternative metabolic pathways that could interfere with glucose sensing are suppressed by specifically "disallowing" expression of certain genes in $\beta$-cells. For example, MCT1 (SLC16A1) encodes a plasma membrane monocarboxylate (pyruvate/lactate) transporter and is widely expressed in other tissues but not in $\beta$-cells. The effects of inappropriate expression of $M C T 1$ are shown in the rare genetic disorder, Physical Exercise-Induced Hypoglycaemia, caused by mutations within the MCT1 promoter. In affected patients, during strenuous physical exercise the presence of the transporter allows circulating pyruvate/lactate to enter $\beta$-cells, resulting in inappropriate insulin release, and consequent hypoglycaemia. We aimed here to identify the mechanisms by which expression of MCT1 is specifically disallowed in $\beta$-cells. Methylation of $\mathrm{CpG}$ islands associated with promoters is an epigenetic modification associated with gene silencing in development and disease. Both human and mouse MCT1 promoters contain CpG islands. We therefore investigated whether methylation of the MCT1 promoter may contribute to $\beta$-cell-specific silencing of this gene.

Materials and methods: The mouse pancreatic $\beta$-cell line, MIN6, was treated with a methylation inhibitor, 5-azacytidine, then $M C T 1$ expression was quantified by RT-PCR. The methylation status of the MCT1 promoter was analysed by bisulphite sequencing in MIN6 cells, a mouse liver cell line mhAT3F which expresses high levels of MCT1, and isolated mouse islets. Methylated DNA immunoprecipitation was used as an independent technique to analyse the methylation status of the MCT1 promoter. Sheared genomic DNA prepared from MIN6 and mhAT3F cells was immunoprecipiated with an antibody against methylated cytosine. Pulled-down DNA fragments were detected by PCR.

Results: Treatment of MIN6 cells with 5-azacytidine led to a 1.7-fold increase in the level MCT1 mRNA ( $\mathrm{p}<0.01)$. However, bisulphite sequencing of the entire MCT1 promoter CpG island in MIN6 and mhAT3F cells revealed very low levels of methylation ( $<5 \%)$ in both cell types. The $M C T 1$ promoter was also found to be hypomethylated $(<5 \%)$ in isolated mouse islets. The quality of the bisulphite sequencing was verified by successfully analysing a normally methylated region. The hypomethylation of the MCT1 promoter was confirmed as it was not pulled-down in a methylated DNA immunoprecipitation from MIN6 or mhAT3F cells. Selective pull-down of methylated promoters by this technique was confirmed using FTMT and GAPDH as positive and negative controls respectively.

Conclusion: Although global inhibition of DNA methylation in $\beta$-cells increased expression of $M C T 1$, we have discovered that the MCT1 promoter has very low levels of methylation in both MIN6 cells and in isolated islets. Therefore while methylation may be involved in upstream events, we show that $M C T 1$ is not transcriptionally regulated through methylation of its promoter in $\beta$-cells. We are currently investigating alternative mechanisms by which MCT1 expression is specifically disallowed in $\beta$-cells, including posttranscriptional regulation by microRNAs. Supported by: Wellcome Trust and EU ("Savebeta")

\section{PS 026 Beta cell exocytosis}

\section{1}

Adenosine $\mathrm{A}_{1}$ receptors modulate glucose-induced pulses of islet hormone release

A. Salehi ${ }^{1}$, F. Parandeh ${ }^{1}$, B. Fredholm ${ }^{2}$, E. Grapengiesser ${ }^{3}$, B. Hellman ${ }^{3}$ ${ }^{1}$ Clinical Sciences, University of Lund, Malmö, ${ }^{2}$ Physiology \& Pharmacology, Karolinska Institute, Stockholm, ${ }^{3}$ Medical Cell Biology, University of Uppsala, Sweden

Background and aims: Evidence has been provided that extracellular ATP stimulates the pulsatile component of insulin, glucagon and somatostatin release by activating $\mathrm{P} 2 \mathrm{Y}_{1}$ receptors. The present investigation examines if the purine adenosine, via $A 1$ receptors $\left(A_{1} R\right)$ interferes with the pulsatile islet hormone release.

Materials and methods: Ratiometeric Fura- 2 technique was used for measuring cytoplasmic $\mathrm{Ca}^{2+}$ in individual mouse $\beta$-cells. Insulin, glucagon and somatostatin were measured by radioimmunoassay in perfusate from pancreas.

Results: Addition of $10 \mu \mathrm{M}$ adenosine to isolated mouse $\beta$-cells rapidly suppressed $\mathrm{IP}_{3}$-induced $\mathrm{Ca}^{2+}$ transients supposed to co-ordinate the insulin release pulses. This process was reversed by $100 \mathrm{nM}$ of the $\mathrm{A}_{1} \mathrm{R}$ antagonist DPCPX. In situ perfusion of mouse pancreas indicated two phases of islet hormone response when glucose was raised from 3.3 to $16.7 \mathrm{mM}$. Typically, the first phase response was characterized by a small initial dip followed by a peak, which was more pronounced for insulin and somatostatin than for glucagon. The second phase was markedly affected by knockout of $A_{1} R$. Wild type of mice $\left(A_{1} R+/+\right)$ usually lacked statistically verified insulin pulses but generated 8-9 min pulses of glucagon and somatostatin appearing in antisynchrony. In the $\mathrm{A}_{1} \mathrm{R}(-/-)$ mice the secretory rate of insulin was almost three times higher due to distinct pulses, which appeared in periods of $8 \mathrm{~min}$. There was no corresponding increase of average glucagon and somatostatin secretory rate, but a prolongation of the pulses of both hormones with $50 \%$.

Conclusion: The results indicate that $\mathrm{A}_{1} \mathrm{R}$ receptor is important both for the amplitude (insulin) and duration (glucagon and somatostatin) of islet hormone pulses.

Supported by: Swedish Research Council and Swedish Diabetes Fundation

\section{2}

Molecular mechanisms controlling the activation and function of the RalA GTPase in pancreatic beta cells

S. Ljubicic, P. Bezzi, R. Regazzi

Department of Cellular Biology and Morphology, University of Lausanne, Switzerland

Background and aims: RalA is a Ras-like monomeric GTPase implicated in the modulation of a variety of cellular processes including exocytosis and endocytosis. We previously found that RalA GTPase is expressed in pancreatic beta cells and is activated by insulin secretagogues. In addition, we discovered that silencing of RalA leads to impaired insulin secretion. In the present study, we investigated the pathways leading to RalA activation as well as the molecular mechanisms through which the GTPase controls insulin release from pancreatic beta cells.

Materials and methods: The experiments were performed in the insulin-secreting cell line INS-1E. Activation of RalA in living cells was assessed by Fluorescence Resonance Energy Transfer (FRET) using a construct including a YFP-labeled RalA and a CFP-labeled Ral effector. The role of RalA in the docking step was assessed by TIRF microscopy. The implication of the GTPase in phospholipase $\mathrm{D}$ activation was determined by measuring the generation of phosphatidic acid in cells lacking RalA.

Results: We previously demonstrated that insulin secretagogues activate $\mathrm{RalA}$. Here, we found that activation of RalA requires RalGDS, a specific Ral exchange factor. Indeed, RalGDS silencing by RNA interference reduced hormone release induced by secretagogues by more than $50 \%$, whereas overexpression of RalGDS did not alter hormone secretion. To precisely define the molecular mechanisms through which RalA controls the secretory process of beta cells we analyzed the impact of RalA silencing on docking and fusion on insulin-containing granules. We found that the reduction in RalA expression is associated with a small but significant decrease (20\%) in the number of granules recruited to the plasma membrane. Moreover, RalA silencing in INS-1E cells led to a strong decrease in secretagogues-evoked phospholipase 
D activity, indicating that this small GTPase is not only involved in the docking process but participates also in the regulation of late events leading to insulin exocytosis.

Conclusion: Taken together our data indicate that the exchange factor RalGDS plays an important role in secretagogue-induced RalA activation and insulin secretion. We also demonstrate that once activated RalA can regulate two key events in the insulin secretory pathway: the docking of secretory granules at the plasma membrane and their subsequent fusion. Supported by: Swiss National Foundation

\section{3}

Presence and role of an electrogenic $\mathrm{Na}-\mathrm{HCO}_{3}$-cotransporter in insulinproducing cells

N. Bulur ${ }^{1}$, P. Lybaert ${ }^{1}$, E. Hupkens ${ }^{1}$, K. Louchami ${ }^{1}$, Y. Zhang ${ }^{1}$, A.P. Yates ${ }^{2}$, L.C. Best ${ }^{2}$, R. Beauwens ${ }^{3}$, W.J. Malaisse ${ }^{1}$, A. Sener ${ }^{1}$

${ }^{1}$ Laboratory of Experimental Hormonology, Université Libre de Bruxelles, Belgium, ${ }^{2}$ Departements of Clinical Biochemistry and Medicine, University of Manchester, United Kingdom, ${ }^{3}$ Laboratory of Cell and Molecular Physiology, Université Libre de Bruxelles, Belgium

Background and aims: We have recently documented the presence of the electrogenic Na-HCO -cotranspoter NBCe1-A in rat islet beta cells by immunohistochemistry, western blotting and RT-PCR. The participation of this cotransporter in sodium handling by insulin-producing cells was also recently examined. The present study deals mainly with the possible role of this cotransporter in insulin release.

Materials and methods: Rat pancreatic islets, isolated by the collagenase procedure, were incubated for $90 \mathrm{~min}$ in the absence or presence of tenidap, an inhibitor of NBCe1-A in kidney proximal tubule cells. Intracellular $\mathrm{pH}$ measurements were carried out in dispersed rat islet cells. Beta-cell membrane potential was recorded using the perforated patch technique.

Results: In the 3 to $100 \mu \mathrm{mol} / \mathrm{l}$ range, tenidap cause a concentration-related inhibition of insulin release from islets exposed to $16.7 \mathrm{mmol} / \mathrm{l}$ glucose, with a half-maximal suppressing effect at about $50 \mu \mathrm{mol} / \mathrm{l}$. Further experiments conducted in the absence or presence of $100 \mu \mathrm{mol} / \mathrm{l}$ tenidap indicated that it failed to affect significantly the basal release of insulin release recorded at 5.6 $\mathrm{mmol} / \mathrm{l}$ glucose, abolished the secretory response to $8.3 \mathrm{mmol} / \mathrm{l}$ glucose and severely decreased the insulinotropic action of $16.7 \mathrm{mmol} / \mathrm{l}$ glucose. Tenidap also decreased insulin secretion evoked by $10.0 \mathrm{mmol} / \mathrm{l} 2$ - ketoisocaproate, the relative extent of such an inhibition $(42 \pm 8 \%)$ not being significantly different from that found a $8.3 \mathrm{mmol} / \mathrm{l}$ glucose $(57 \pm 10 \%)$. The secretory response to $20.0 \mathrm{mmol} / \mathrm{l} \mathrm{L}$-leucine, which was comparable to that evoked by 8.3 $\mathrm{mmol} / \mathrm{l}$ glucose was decreased by no more $24 \pm 10 \%$, however, in the presence of tenidap. Last, the enhancing action of glibenclamide $(5 \mu \mathrm{mol} / \mathrm{l})$, theophylline $(1.4 \mathrm{mmol} / \mathrm{l})$, forskolin $(5 \mu \mathrm{mol} / \mathrm{l})$ and cytochalasin $\mathrm{B}(0.2 \mathrm{mmol} / \mathrm{l})$ upon insulin release evoked by $8.3 \mathrm{mmol} / \mathrm{l}$ glucose was also abolished by tenidap. At all glucose concentrations ( 4 to $16 \mathrm{mmol} / \mathrm{l})$, tenidap $(50-100 \mu \mathrm{mol} / \mathrm{l})$ provoked an immediate lowering of intracellular $\mathrm{pH}$, this effect slowly fading out over the ensuing 7-8 min, and promoted pronounced hyperpolarisation of the plasma membrane potential.

Conclusion: The concentration-response relationship for the inhibitory action of tenidap on glucose-stimulated insulin release and its effect upon intracellular $\mathrm{pH}$ in dispersed islet cells are comparable to observations made in other cell types. The latter effect may account for the lower sensitivity of leucine-stimulated islets to the inhibitory action of tenidap. With due reservation on the specificity of tenidap inhibition of NBCe1-A, the present results are compatible with a key role for bicarbonate efflux via this cotransporter in the insulinotropic action of nutrient secretagogues.

\section{4}

NADPH as a putative regulator of voltage-gated $\mathrm{Ca}^{2+}$ channels in pancreatic beta cells

T.M. Reinbothe, U. Fransson, E. Renström

Islet Pathophysiology, Lund University, Malmö, Sweden

Background and aims: The pyridine nucleotide NADPH is formed by mitochondrial shuttles during glucose metabolism and we have previously demonstrated that it influences $\mathrm{Ca}^{2+}$-induced insulin release. NADPH also serves as a reducing equivalent in many cellular processes instrumental in the regulation of beta-cell's redox state. The aim of the present study was to further investigate the role of NADPH in stimulus-secretion coupling focusing on voltage-gated $\mathrm{Ca}^{2+}$ channels in individual beta-cells.
Materials and methods: Islets were isolated from adult Wistar rats by collagenase digestion and dissociated into single cells. Whole-cell $\mathrm{Ca}^{2+}$ currents were recorded using the patch-clamp technique in standard whole cell configuration. In order to investigate the effect of NADPH on $\mathrm{Ca}^{2+}$ channel subtypes, $\mathrm{Ca}^{2+}$ channel blockers were used as specified below. RNA interference with siRNA targeting the NADPH effector glutaredoxin (GRX) was used to determine its effect on insulin secretion which was detected using an insulin radioimmunoassay (RIA). Furthermore, cellular thiol concentration was measured after knockdown of GRX with an Ellman's assay.

Results: After the addition of $100 \mu \mathrm{M}$ NADPH to the pipette solution we could observe an inhibition of averaged $\mathrm{Ca}^{2+}$ current amplitudes at $0 \mathrm{mV}$ by $36 \%$ ( 50 $\mathrm{pA} ; \mathrm{SE}=4.6 \mathrm{pA} ; \mathrm{n}=15)$ compared to patches containing no NADPH $(78.8 \mathrm{pA}$; $\mathrm{SE}=13.4 \mathrm{pA} ; \mathrm{n}=11)$. Isradipine $(2 \mu \mathrm{M})$ was then used in the bath solution to determine the effect of NADPH on the non L-type current component of measured $\mathrm{Ca}^{2+}$ currents. In control cells without NADPH, isradipine reduced whole cell $\mathrm{Ca}^{2+}$ current amplitudes by $52 \%(38 \mathrm{pA} ; \mathrm{SE}=10 \mathrm{pA} ; \mathrm{n}=8)$ as compared with non-treated cells (78.8 pA; $\mathrm{SE}=13.4 \mathrm{pA} ; \mathrm{n}=11)$. Interestingly, NADPH stimulated the non L-type $\mathrm{Ca}^{2+}$ current component by $61.5 \%$ (61.7 pA; $\mathrm{SE}=10.3 \mathrm{pA}$, $\mathrm{n}=8$ ). By contrast, in the presence of the R-type $\mathrm{Ca}^{2+}$ channel blocker SNX482 (100 nM), NADPH was without effect. The down-regulation of GRX (KO) suppressed insulin secretion by $62 \%$ in high glucose $(16.7 \mathrm{mM}$ Glucose; $7.09 \mathrm{ng} /$ $\mathrm{mg} / \mathrm{h}$ vs. $18.65 \mathrm{ng} / \mathrm{mg} / \mathrm{h} \mathrm{KO}$ vs. control; $\mathrm{p}<0,04 ; \mathrm{n}=3$ ) and had no effect on basal insulin secretion ( $2.8 \mathrm{mM}$ Glucose; $5.69 \mathrm{ng} / \mathrm{mg} / \mathrm{h}$ vs. $5.14 \mathrm{ng} / \mathrm{mg} / \mathrm{h} \mathrm{KO}$ vs. control; $p>0.6 ; n=3)$. We could not detect an influence of GRX down-regulation on cellular thiol concentration $(0.59 \mu \mathrm{M}$ vs. $0.51 \mu \mathrm{M} \mathrm{KO}$ vs. control; $\mathrm{p}>0.4 ; \mathrm{n}=3)$. Conclusion: Our results demonstrate a $\mathrm{Ca}^{2+}$ channel subtype specific effect of the pyridine nucleotide NADPH. It inhibits L-type $\mathrm{Ca}^{2+}$ current components whereas non L-type components are stimulated. The NADPH effector glutaredoxin can also influence insulin secretion. We hypothesize that this mechanism is involved in differential regulation of the attributed roles of $\mathrm{Ca}^{2+}$ subtypes in biphasic insulin secretion.

Supported by: Marie Curie Research Training Network Cavnet, Swedish Diabetes Association and the Faculty of Medicine in Lund

\section{5}

Kv2.1 channel blockers potentiate glucose-induced insulin release in islets of type 2 diabetic rats K. Dezaki ${ }^{1}$, B. Damdindorj ${ }^{1}$, M. Kakei ${ }^{2}$, T. Yada

${ }^{1}$ Physiology, Jichi Medical University, Tochigi, ${ }^{2}$ Saitama Medical Center, Jichi Medical University, Saitama, Japan

Background and aims: Voltage-dependent potassium channels are involved in repolarization of excitable cells. In pancreatic $\beta$-cells, activation of delayed rectifier $\mathrm{K}^{+}(\mathrm{Kv})$ channels might repolarize cells and attenuate glucose-stimulated action potentials to suppress insulin secretion. Inhibition of the $\beta$-cell $\mathrm{Kv}$ current would be expected to prolong action potentials and enhance glucosestimulated insulin secretion. Among Kv channel families, Kv2.1 is reportedly expressed in islet $\beta$-cells as the major component of $\mathrm{Kv}$ currents in rodents. This study aimed to determine the effects of specific blockers of Kv2.1 channels on the glucose-induced insulin release in islet $\beta$-cells of diabetic rats. Materials and methods: Islets were isolated from male Wistar and type 2 diabetic Goto-Kakizaki (GK) rats by collagenase digestion, and insulin release was determined by ELISA. In rat single $\beta$-cells, cytosolic $\mathrm{Ca}^{2+}$ concentration $\left(\left[\mathrm{Ca}^{2+}\right]_{\mathrm{i}}\right)$ was measured by fura- 2 microfluorometry, while whole cell currents were measured in the presence of tolbutamide by nystatin-perforated patchclamp technique.

Results: In isolated islets of Wistar rats, tetraethylammonium (TEA), a nonselective blocker of $\mathrm{Kv}$ channels, increased glucose $(8.3 \mathrm{mM})$-induced insulin release. Moreover, Kv2.1 channel blockers, stromatoxin (ScTx) and guangxitoxin-1E (GxTx), significantly increased glucose-induced insulin release. The basal insulin release at $2.8 \mathrm{mM}$ glucose was not altered by these Kv channel blockers. In contrast, margatoxin, a Kv1.3 channel blocker, affected insulin release neither at stimulated nor at basal glucose. TEA and ScTx markedly attenuated $\mathrm{Kv}$ currents in $\beta$-cells. ScTx and GxTx concentration-dependently potentiated glucose $(8.3 \mathrm{mM})$-induced $\left[\mathrm{Ca}^{2+}\right]_{i}$ increases in rat $\beta$-cells, whereas basal $\left[\mathrm{Ca}^{2+}\right]_{\mathrm{i}}$ levels at $2.8 \mathrm{mM}$ glucose were not affected by these Kv2.1 channel blockers. In type 2 diabetic GK rats, Kv2.1 channel blockers increased glucose $(8.3 \mathrm{mM})$-induced insulin release from islets and potentiated $\left[\mathrm{Ca}^{2+}\right]_{\mathrm{i}}$ increases in $\beta$-cells, to similar extents to those observed in Wistar rats. Conclusion: Kv2.1 channels may physiologically limit glucose-induced $\mathrm{Ca}^{2+}$ entry to suppress insulin secretion in $\beta$-cells. Blockade of Kv2.1 channels can promote glucose-induced insulin release in diabetic GK rats as well as normal rats, providing a potential therapeutic tool to treat type 2 diabetes. 


\section{6}

ADP mediates inhibition of insulin secretion by activation of P2Y $\mathrm{Y}_{13}$ receptors

S. Amisten ${ }^{1}$, S. Meidute-Abaravecien ${ }^{2}$, A.S. Salehi ${ }^{2}$, D. Erlinge ${ }^{1}$

${ }^{1}$ Cardiology, Lund University, ${ }^{2}$ Clinical Science, Lund University, Malmö, Sweden

Background and aims: Several studies have previously suggested that extracellular nucleotides such as ATP and ADP play an important role in the regulation of insulin secretion from pancreatic beta-cells. The aim of this study was to determine which ADP sensitive P2Y receptors are present in mouse islets and pancreatic beta-cells and to investigate their role in insulin and glucagon secretion.

Materials and methods: Quantitative real-time PCR (qPCR) was used to quantify the expression of the ADP receptors $\mathrm{P} 2 \mathrm{Y}_{1}, \mathrm{P}_{2} \mathrm{Y}_{12}$ and $\mathrm{P} 2 \mathrm{Y}_{13}$ in mouse islets and isolated beta-cells. The non-biodegradable ADP-analogue 2-MeSADP was used in combination with either the P2Y antagonist MRS2179 or the $\mathrm{P} 2 \mathrm{Y}_{13}$ antagonist MRS2211 to study the influence of $\mathrm{P} 2 \mathrm{Y}_{1}$ and $\mathrm{P} 2 \mathrm{Y}_{13}$ receptors on insulin and glucagon release. The effects of endogenously released ADP on insulin and glucagon secretion were also studied by co-incubation of islets with the nucleotide degrading enzyme apyrase at different glucose concentrations.

Results: Both P2Y and $\mathrm{P}_{13} \mathrm{Y}_{13}$ were found to be expressed in mouse islets and beta-cells, but only trace amounts of $\mathrm{P} 2 \mathrm{Y}_{12}$ could be detected. The relative expression of $\mathrm{P} 2 \mathrm{Y}_{13}$ was $19 \%$ of the $\mathrm{P} 2 \mathrm{Y}_{1}$ expression in islets and $45 \%$ of the $\mathrm{P} 2 \mathrm{Y}_{1}$ expression in beta cells. Incubation of mouse islets with 2-MeS-ADP $(10 \mu \mathrm{M})$ and the selective $\mathrm{P}_{2} \mathrm{Y}_{1}$ antagonist MRS2179 $(10 \mu \mathrm{M})$ in the presence of $8.3 \mathrm{mM}$ glucose resulted in a marked decrease in insulin secretion $(-70 \%$, $\mathrm{p}<0.001)$. This indicates an inhibitory effect on insulin secretion mediated by the unblocked $\mathrm{P} 2 \mathrm{Y}_{13}$ receptor. In contrast, pre-treatment of islets with the selective $\mathrm{P}_{2} \mathrm{Y}_{13}$ receptor antagonist MRS2211 strongly increased insulin secretion $(+112 \%, \mathrm{p}<0.001)$, indicating a stimulatory effect on insulin secretion by the unblocked $\mathrm{P} 2 \mathrm{Y}_{1}$ receptor. The effect of $\mathrm{P} 2 \mathrm{Y}_{13}$ receptor blockade by MRS2211 on insulin and glucagon secretion was tested at both sub-stimulatory $(8.3 \mathrm{mM})$ and high $(20 \mathrm{mM})$ glucose concentrations. Inhibition of P2Y mediated signaling resulted in elevated secretion of both insulin $(8.3 \mathrm{mM}$ glucose: $+92 \%, \mathrm{p}<0.001 ; 20 \mathrm{mM}$ glucose: $+38 \%, \mathrm{p}=0.005)$ and glucagon $(8.3$ $\mathrm{mM}$ glucose: $+76 \%, \mathrm{p}<0.05 ; 20 \mathrm{mM}$ glucose: $+69 \%, \mathrm{p}=0.005)$. The ADP degrading enzyme apyrase was found to increase both insulin $(+122 \%, \mathrm{p}<0.001)$ and glucagon $(+45 \%, \mathrm{p}<0.001)$ secretion at low $(1 \mathrm{mM})$ glucose, whereas at high glucose $(20 \mathrm{mM})$, insulin secretion was inhibited $(-45 \%, \mathrm{p}=0.003)$ and glucagon secretion was promoted $(+118 \%, \mathrm{p}<0.001)$.

Conclusion: In this study we have found that the ADP receptors P2Y and $\mathrm{P} 2 \mathrm{Y}_{13}$ are expressed in islets and isolated beta-cells from the mouse and that P2 $\mathrm{Y}_{13}^{13}$ mediated signaling has an inhibitory effect on both insulin and glucagon secretion in the mouse. We have also shown that inhibition of the P2Y receptor results in an increased secretion of both insulin and glucagon, and that administration of the ADP degrading enzyme apyrase affects islet hormone release, suggesting that endogenous ADP is a mediator of both insulin and glucagon secretion in mouse islets. We conclude that inhibition of the $\mathrm{P} 2 \mathrm{Y}_{13}$ receptor might be an interesting novel therapeutic approach aimed at increasing the natural insulin secretion from pancreatic islets.

Supported by: Swedish Scientific Research Council, Swedish Heart and Lung Foundation, Lund Vascular Wall program, Lund University, Lund University Hospital funds, Söderberg Foundation

\section{7}

Induction of cyclic AMP responsiveness in pseudoislets constituted from beta cell-beta cell interaction

M. Iwasaki ${ }^{1}$, K. Minami ${ }^{1}$, T. Shibasaki ${ }^{1}$, T. Miki², J.-I. Miyazaki ${ }^{3}$, S. Seino ${ }^{1,4}$ ${ }^{1}$ Divisions of Cellular and Molecular Medicine, Kobe University Graduate School of Medicine, Kobe, ${ }^{2}$ Department of Autonomic Physiology, Chiba University Graduate School of Medicine, ${ }^{3}$ Department of Nutrition and Physiological Chemistry, Osaka University Graduate School of Medicine, ${ }^{4}$ Diabetes, Metabolism and Endocrinology, Kobe University Graduate School of Medicine, Japan

Background and aims: Cell-cell interactions are involved in the regulation of cellular functions. It has been shown that insulin secretion from intact pancreatic islets is greater than that from dispersed $\beta$-cells, and that reaggregation of the dispersed $\beta$-cells improves the secretory response. Thus, cell-cell interactions may well have a role in pancreatic $\beta$-cell function. However, the effect of cell-cell interaction on insulin secretion is little understood. We have addressed the role of $\beta$-cell- $\beta$-cell interaction on insulin secretory properties and intracellular signals by comparing monolayer-cultured clonal $\beta$-cells (MCCs) and three-dimensional islet-like structure or pseudoislets (PIs) constituted from clonal $\beta$-cells.

Materials and methods: PIs were formed from clonal $\beta$-cells (termed MIN6K20 cells) by culture on gelatin-coated dishes for seven days. Insulin secretion was measured by batch incubation method with stimulation of glucose, glucagons-like peptide-1 (GLP-1), or 8-Br-cyclic AMP, and cellular insulin was extracted with acid ethanol. Cellular cyclic AMP was also measured. Data were normalized by DNA content.

Results: The newly established $\beta$-cell line MIN6-K20 secretes insulin in a regulated manner, but the cells do not respond to GLP-1, an incretin hormone that potentiates insulin secretion through cyclic AMP signaling. We found that insulin content in MCCs of MIN6-K20 cells was much lower than that in native islets, formation of PIs increased insulin content $(0.95 \pm 0.02 \mathrm{ng} / \mathrm{ng}$ DNA in MCCs vs. $6.88 \pm 0.57 \mathrm{ng} / \mathrm{ng}$ DNA in PIs) in MIN6-K20 cells to a the level comparable to that in native mouse islets $(6.81 \pm 0.47 \mathrm{ng} / \mathrm{ng} D N A)$. In contrast, mRNA expression levels of insulin 1 and insulin 2 were not changed by formation of PIs. In addition, formation of PIs in the cells induced GLP1 responsiveness of insulin secretion. While cyclic AMP content was only slightly increased by GLP-1 in MCCs (2.6-fold), it was drastically increased in PIs (11.2-fold). Even in the presence of phosphodiesterase inhibitor, cyclic AMP content in PIs $(15.16 \pm 3.06 \mathrm{fmol} / \mathrm{ng} \mathrm{DNA})$ was significantly higher than that in MCCs $(0.69 \pm 0.09 \mathrm{fmol} / \mathrm{ng}$ DNA), indicating that cyclic AMP production is increased in PIs. Moreover, potentiation of insulin secretion by 8 -Br-cyclic AMP was significantly higher in PIs $(0.09 \pm 0.01 \mathrm{ng} / \mathrm{ng}$ DNA) than in MCCs $(0.06 \pm 0.01 \mathrm{ng} / \mathrm{ng} \mathrm{DNA})$, suggesting that signals downstream of cyclic AMP are enhanced in PIs.

Conclusion: Our data show that cyclic AMP signaling and insulin secretory response are induced by constitution of PIs. Thus, the three dimensional structure formed by $\beta$-cell- $\beta$-cell interaction is critical for normal regulation of insulin secretion.

\section{8}

$\mathrm{K}_{\mathrm{ATP}}$-channel independent insulinotropic effect of the imidazolines: an exception to the rule?

K. Hatlapatka ${ }^{1}$, A. Wienbergen ${ }^{1}$, A. Jörns ${ }^{2}$, M. Willenborg ${ }^{1}$, I. Rustenbeck ${ }^{1}$

${ }^{1}$ Institute of Pharmacology and Toxicology, University of Braunschweig,

${ }^{2}$ Institute of Anatomy, Hannover Medical School, Germany

Background and aims: The marked glucose-dependency of the imidazolineinduced insulin secretion has been ascribed to $\mathrm{K}_{\mathrm{ATP}}$ channel-independent effects. This hypothesis was tested using islets from SUR1 KO mice which have no functional $\mathrm{K}_{\text {ATP }}$ channels.

Materials and methods: Insulin secretion was measured by perifusion of NMRI and SUR1 KO mouse islets, $\mathrm{K}_{\text {ATP }}$ channel activity and plasma membrane potential by patch clamping and cytosolic free calcium concentration $\left[\mathrm{Ca}^{2+}\right]_{\mathrm{c}}$ by microfluorimetry.

Results: $100 \mu \mathrm{mol} / \mathrm{l}$ efaroxan enhanced insulin secretion when the glucose concentration was stimulatory $(10 \mathrm{mmol} / \mathrm{l})$ but not when it was basal $(5$ $\mathrm{mmol} / \mathrm{l})$. The secretory pattern elicited by $10 \mu \mathrm{mol} / \mathrm{l} \mathrm{RX} 871024$ closely resembled that of $100 \mu \mathrm{mol} / \mathrm{l}$ efaroxan, consistent with a $\mathrm{K}_{\mathrm{ATP}}$ channel block of comparable magnitude (reduction by $85 \%$ ). Consequently, all of the named imidazoline compounds induced a rise in $\left[\mathrm{Ca}^{2+}\right]_{c}$ in NMRI mouse islets, even in the presence of a basal glucose concentration. In contrast to $10 \mu \mathrm{mol} / \mathrm{l}$ RX871024, $100 \mu \mathrm{mol} / \mathrm{l} \mathrm{RX}$ massively stimulated insulin secretion at basal glucose. The insulin secretion by SUR1 KO islets was neither increased by efaroxan, nor by alinidine nor by phentolamine, Again, RX871024 was different in that 100 but not $10 \mu \mathrm{mol} / \mathrm{l}$ RX871024 stimulated secretion by SUR1 $\mathrm{KO}$ islets both at 5 and $10 \mathrm{mmol} / \mathrm{l}$ glucose. This was confirmed by electron microscopy of the granule content.

Conclusion: Only RX871024 at a high concentration, but not the other $\mathrm{K}_{\text {ATP }}$ channel blocking imidazolines, exerts a $\mathrm{K}_{\text {ATP }}$ channel-independent insulinotropic effect. This effect, however, does not seem to be responsible for the marked glucose dependency. 


\section{9}

Employing genetically encoded $\mathrm{Ca}^{2+}$ indicators to assess beta cell $\mathrm{Ca}^{2+}$ homeostasis

S. Speier, T. Moede, B. Leibiger, D. Kaiser, I.B. Leibiger, P.-O. Berggren The Rolf Luft Research Center for Diabetes and Endocrinology, Karolinska Institutet, Stockholm, Sweden

Background and aims: Changes in cytoplasmic free $\mathrm{Ca}^{2+}$ concentration $\left(\left[\mathrm{Ca}^{2+}\right]_{\mathrm{i}}\right)$ are a key mechanism in beta-cell signal transduction, being involved in the regulation of insulin secretion, gene transcription and cell death, respectively. To investigate these mechanisms, $\mathrm{Ca}^{2+}$ imaging experiments in beta-cells have so far mainly employed synthetic $\mathrm{Ca}^{2+}$ indicators. However, genetically-encoded $\mathrm{Ca}^{2+}$ indicators (GECIs) serve several advantages over synthetic sensors. Employing GECIs enables monitoring $\mathrm{Ca}^{2+}$ in a genetically-defined cell population or specific subcellular compartments. Futhermore, the use of GECIs would allow long-term $\mathrm{Ca}^{2+}$ imaging of beta-cells in vivo. We tested several GECIs to evaluate suitable GECIs for assessing beta-cell $\mathrm{Ca}^{2+}$ homeostasis with the aim to establish a system that enables assessment of beta-cell physiology non-invasively in vivo.

Materials and methods: Three different GECIs, YCAM2.3, Design3 and GCAMP2, were first tested in MIN6 cells and isolated islets in vitro. For the transfection of beta-cells in islets the constructs were incorporated into adenoviral vectors. Islets transduced with the GECI containing adenovirus were tested in vitro or transplanted into the anterior chamber of the eye of mice via injection through the cornea to enable in vivo studies. For measurements of changes in fluorescence of the GECIs widefield fluorescence microscopy, confocal and two-photon laser scanning microscopy were used.

Results: For stimulation, transfected MIN6 cells were perfused with $16 \mathrm{mM}$ glucose, $30 \mathrm{mM} \mathrm{KCl}$ or $100 \mu \mathrm{M}$ glibenclamide. The Fluorescence resonance energy transfer (FRET) based GECIs YCAM2.3 and Design3 showed an increase in the yellow fluorescent protein (YFP) / cyan fluorescent protein (CFP) ratio of at least $50 \%$ after stimulation. The green fluorescent protein (GFP) based GECI GCAMP2 showed an increase in single wavelength fluorescence of more than 2 -fold. Stimulation of changes in $\left[\mathrm{Ca}^{2+}\right]_{\mathrm{i}}$ by perfusion of transduced islets with $30 \mathrm{mM} \mathrm{KCl}$ and $100 \mu \mathrm{M}$ glibenclamide lead to increases in GFP fluorescence or the YFP / CFP ratio comparable to the results obtained in MIN6 cells. In response to elevated glucose levels all three GECIs showed oscillatory changes in fluorescence. First transplantations of transduced islets into the anterior chamber of the eye displayed no changes in engraftment compared to non-transduced islets and the fluorescent constructs were detectable for several months.

Conclusion: We showed that the GECIs YCAM2.3, Design3 and GCAMP2 are useful indicators to assess beta-cell physiology in vitro by measuring changes in $\left[\mathrm{Ca}^{2+}\right]_{i}$. Future experiments will evaluate the use of these GECIs in an in vivo system by transplanting islets transduced with GECIs into the anterior chamber of the eye. This will enable non-invasive long-term studies of beta-cell signal transduction in vascularized and innervated islets and will help to reveal function of the endocrine pancreas in health and disease. Supported by: JDRF, EFSD/MSD grant and Family Erling-Persson Foundation

\section{PS 027 Beta cells in type 2 diabetes}

\section{0}

Expression analyses of type 2 diabetes mellitus associated genes in clonal and primary beta cells and functional characterisation of INS-1E cells overexpressing the beta cell specific zinc transporter ZnT-8 M. Elsner, E. Knoche, S. Lenzen

Institute of Clinical Biochemistry, Hannover Medical School, Germany

Background and aims: The recent identification of new gene loci, which are associated with T2DM, opens new perspectives in the analyses of the pathomechanisms leading to the manifestation of the disease. In genome wide association studies (GWA) SNPs were discovered in the non-coding regions of several genes with so far unknown consequences for the expression of the respective gene. In the present study the gene expression of the most significant T2DM associated genes TCF7L2, SLC30A8 (ZnT-8), IGF2BP2, CDKAL1, CDKN2A and CDKN2B were analysed by quantitative real time PCR in the $\beta$-cell lines RINm5F, INS- 1 and MIN- 6 compared with primary islets and pancreas tissue.

Materials and methods: The expression of the genes was determined by absolute quantitative real time RT-PCR using a SYBR Green I assay. RNA was isolated from the cells and reverse transcribed into cDNA. For preparation of specific DNA standard cDNA fragments of the analysed genes were cloned into the pCR2.1 plasmid. The amount of specific mRNA per cell was calculated with the determined efficiency of the PCR reaction (1.8 to 1.96) using the software qPCR. For the expression study the coding region of the ZnT-8 was cloned into a lentiviral gene expression system. ZnT-8 overexpressing INS-1E cells were selected after viral transduction with the antibiotic blasticidin. The viability of the cells in dependence on the zinc content of the medium was determined by the MTT assay. Insulin secretion and content of the INS-1EZnT- 8 cells was measured after static incubation by RIA.

Results: TCF7L2 was expressed in all cell types without significant differences. The expression of IGF2BP2 was significantly higher in human pancreas than in rat and mouse cells, which indicates a particular impact for the human system. In contrast the expression of CDKAL1 at relatively low levels in comparison to rodent cells seems to be of less importance for the examined human cells. An expression of the genes CDKN2A/B which were probably involved in the regulation of cell cycle and $\beta$-cell mass could not detected in the human pancreas. The highest expression was measured in the MIN- 6 cell line. The highest expression level for the SLC30A8 gene, which encodes for the $\beta$-cell specific zinc transporter ZnT-8, was detectable in MIN-6, INS-1E cells and human pancreas. An overexpression of the ZnT-8 transporter in INS-1E cells led to a more the 2 fold increase in glucose-stimulated insulin secretion (GSIS) at glucose concentrations $>3 \mathrm{mmol} / \mathrm{l}$ in comparison to nontransduced control cells. Interestingly, this increase of GSIS was independent from the zinc concentration of the culture medium indicating that the enhancement of the GSIS might be independent on the accumulation of zinc in the insulin granules.

Conclusion: The present study provides important data for further in vitro investigations on established beta cell lines to clarify the role of T2DM candidate genes for the physiology of $\beta$-cells. The $\beta$-cell specific zinc transporter ZnT-8 has the ability to enhance glucose-stimulated insulin secretion and may thereby represent a novel pharmacological target for the treatment of diabetes.

Supported by: EU in the Framework Programme 6

\section{1}

Glucokinase activators modulate glucose metabolism and glucoseinduced insulin secretion in islets from GK/Par rats M. Fradet ${ }^{1}$, M.-H. Giroix ${ }^{1}$, D. Bailbe ${ }^{1}$, S. El Bawab ${ }^{2}$, V. Autier ${ }^{2}$, M. Kergoat ${ }^{2}$, B. Portha ${ }^{1}$

${ }^{1}$ Physiopathol. Nutrition, CNRS UMR7059, Paris, ${ }^{2}$ Biochemistry Group, Merck-Serono, Chilly-Mazarin, France

Background and aims: Recently, the discovery and development of smallmolecule glucokinase activators (GKAs) have been reported. The activators stimulate glucokinase activity via binding to an allosteric site on the enzyme and have been shown to decrease plasma glucose levels in healthy and diabetic animals. GKAs stimulate glucose phosphorylation in isolated hepatocytes and pancreatic beta-cells and it is considered that the increased glucose metabolism in these in vitro systems is accompanied by the expected 
downstream effects. Thus, in rat hepatocytes GKAs stimulate glycolysis and glycogen synthesis whilst inhibiting gluconeogenesis and glycogenolysis. In beta-cell lines and in pancreatic islets isolated from normal rodents or human, GKAs amplifie glucose-stimulated insulin secretion (GSIS). However, it has never been shown so far that this is also the case in diabetic islets, as it is unknown to what extend the beta-cell glucose metabolism is modulated by GKAs. In this respect, it was considered of interest to compare the metabolic and secretory responses to GKAs in islets freshly isolated from either normal Wistar (W) or spontaneously diabetic GK/Par rats.

Materials and methods: The two compounds Ro-28-1675 (C1) and GKA50 (C2) were tested at maximally effective dosage in vitro $(10 \mu \mathrm{M})$. The rates of appearance of ${ }^{3} \mathrm{H}_{2} \mathrm{O}$ and ${ }^{14} \mathrm{CO}_{2}$ formed from $\left[5-{ }^{3} \mathrm{H}\right]$ glucose and $\left[6-{ }^{14} \mathrm{C}\right]$ glucose during $120 \mathrm{~min}$ islet incubations at $37^{\circ} \mathrm{C}$, were measured and taken as evaluations of the islet glucose utilisation and glucose oxidation respectively. Insulin release was determined under similar conditions in parallel experiments. Results: In normal W islets, both $\mathrm{C} 1$ and $\mathrm{C} 2$ markedly increased the glycolytic flux and the glucose oxydation rate, at low $(\mathrm{G} 2.8 \mathrm{mM})$ and intermediate glucose (G $8 \mathrm{mM}$ ) concentrations, while at $\mathrm{G} 16 \mathrm{mM}$, they were far less efficient. Concerning stimulation of insulin release, both activators were more efficient at low and intermediate glucose concentrations shifting the doseresponse curve for glucose to the left with no effect on the maximal increase in GSIS. The fact that the activators did not further increase insulin secretion stimulated by $\mathrm{G} 16 \mathrm{mM}$ is consistent with the observation that at high $\mathrm{G}$ concentrations, other metabolic enzymes downstream of glucokinase become rate limiting and insulin secretion no longer parallels glucokinase activity. In the absence of G, C1 and C2 did not affect insulin release. In diabetic GK/Par islets, both compounds promoted a pattern of activation of glucose metabolism and insulin secretion very similar to that reported in $\mathrm{W}$ islets, resulting in a notable potentiation of $\mathrm{G} 8 \mathrm{mM}$-induced insulin secretion (1.6 fold and 1.8 fold, in response to $\mathrm{C} 1$ and $\mathrm{C} 2$ respectively).

Conclusion: We have shown for the first time that GKAs cause in diabetic islets (as in normal islets) an increase in glucose-induced insulin secretion correlated with an increased glucose oxidation rate. This associated with a leftward shift of the glucose dose-response profile seen in these islets, while the maximal response remains unaffected. These data further document a role for GKAs as novel utilities in the therapeutic management of mild diabetes.

\section{2}

Adaptative secretory changes to a fructose-induced insulin resistance state: role of glucokinase and glucose metabolism in the islets

B. Maiztegui, M.I. Borelli, J.J. Gagliardino

Cenexa (UNLP-CONICET), National University of La Plata School of Medicine, Argentina

Background and aims: The role of glucokinase (GK) in the compensatory increased secretion of insulin observed in rats with normoglycemia and dietary-induced insulin resistance (IR) was studied.

Materials and methods: Normal male Wistar rats received a standard commercial diet and tap water without (control, C) or with (FRD) $10 \%$ fructose for 3 weeks. Blood glucose (strips), triglyceride (commercial kit) and insulin (radioimmunoassay) levels were measured at the time of sacrifice. Islets were isolated by collagenase digestion to study glucose-induced insulin release, glucose metabolism $\left({ }^{14} \mathrm{CO}_{2}\right.$ and ${ }^{3} \mathrm{H}_{2} \mathrm{O}$ production from labeled glucose), hexokinase (HK) and GK transcription (RT-PCR), protein expression (Western blot), compartmentalization (cytosol and particulate fractions) and activity (bioassay).

Results: C and FRD rats had similar body weights. FRD rats presented normoglycemia, hypertriglyceridemia, hyperinsulinemia and increased HOMA$\mathrm{R}$ index. FRD islets released significantly more insulin and produced higher amounts of ${ }^{14} \mathrm{CO}_{2}$ and ${ }^{3} \mathrm{H}_{2} \mathrm{O}$ in response to high glucose. While no differences were found in $\mathrm{HK}$ transcription, protein expression and activity between groups, GK total and cytosolic fraction activity and protein expression were significantly higher in FRD rat islets. FRD did not induce significant changes in GK transcription rate.

Conclusion: Our results demonstrate that in rats with sustained induced IR and normoglycemia, GK activity increased due to a combination of posttranslational mechanisms of increased GK protein amount and compartmentalization, which resulted in increased glucose metabolism and insulin secretion. These data demonstrate that GK plays a pivotal role in functional islet adaptation to maintain normal glucose homeostasis.

Supported by: grants from FONCYT and CONICET

\section{3}

Insulin resistance modifies plasma membrane calcium-ATPase activity and isoform expression in rat pancreatic islets

M.E. Alzugaray ${ }^{1}$, M.E. García ${ }^{1}$, H.H. Del Zotto ${ }^{1}$, J.P.F. Rossi ${ }^{2}$,

J.J. Gagliardino ${ }^{1}$, L.E. Flores ${ }^{1}$

${ }^{1}$ Endocrinology, Cenexa (UNLP-CONICET), La Plata, Argentina, ${ }^{2}$ IQUIFIB, UBA-CONICET, Buenos Aires, Argentina

Background and aims: Despite the extensive knowledge about the patterns of Plasma Membrane Calcium-ATPase (PMCA) and their regulatory role upon insulin secretion under normal conditions, there are scarce reports on their possible changes and their participation in the pathogenesis of diseases that affect islet function, such as type 2 diabetes. Insulin resistance (IR) is an early abnormality present in such disease, so the knowledge of PMCAs state in that condition could help to understand their possible participation in the pathogenesis of type 2 diabetes. The aim of the present study is to study the effects of IR induced by dietary manipulation upon islet PMCA activity and expression, insulin secretion, glucose metabolism and morphometric parameters of $\beta$-cells.

Materials and methods: Normal male Wistar rats were fed for 21 days with a commercial diet and water without (control) or with $10 \%$ fructose (FRD). After 21 days the animals were sacrificed and blood samples were used to determine plasma glucose, triglyceride and insulin levels, and whole pancreas were removed for immunocytochemical studies and islets isolation to measure insulin release, glucose metabolism, PMCA activity and transcription/expression of PMCA isoforms.

Results: Body weight and plasma glucose levels were similar in both groups, but FRD rats have significantly higher levels of triglyceride and insulin, insulin:glucose ratio and HOMA-R index as shown in the table. FRD islets released in vitro more insulin in response to glucose and metabolized more glucose than control islets. On the other hand, they showed a significant decrease in protein content of PMCA2 ( $<<0.001)$, an increase of PMCA3 ( $\mathrm{p}<0.01)$, and no significant changes in PMCAs 1 and 4. PMCA activity was significantly lower in FRD islets ( $1.22 \pm 0.09$ vs. $1.57 \pm 0.11 \mathrm{pmol} \mathrm{Pi/ug} / \mathrm{h} ; \mathrm{p}<0.02)$, but this difference disappeared when calmodulin activity was blocked with calmidazolium. $\beta$-cell mass decreased significantly in FRD rats (6.22 \pm 1.00 vs. 3.89 $\pm 0.40 \mathrm{mg}, \mathrm{p}<0.05$ ), with no significant differences in the pancreatic PMCA cellular distribution pattern.

Conclusion: FRD simultaneously induced IR, a decrease in $\beta$-cell mass, a decrease in PMCA activity, and an increase in glucose metabolism of the islets. Functional changes favor the compensatory greater release of insulin necessary to cope with the IR state, maintaining glucose homeostasis within normal range. Thus, changes in PMCA activity and in its isoforms expression play a modulatory role upon insulin secretion during long-term adaptation to an increased hormone demand.

\begin{tabular}{llll}
\hline & Control & FRD & P Value \\
\hline $\begin{array}{l}\text { Body weight gained during } \\
\text { treatment }(\mathrm{g})\end{array}$ & $69.2 \pm 0.71$ & $72.3 \pm 0.85$ & NS \\
\hline Glucose $(\mathrm{mg} / \mathrm{dl})$ & $130.1 \pm 4.4$ & $131.6 \pm 3.2$ & NS \\
\hline Trigliceryde $(\mathrm{mg} / \mathrm{dl})$ & $98.5 \pm 3.5$ & $158.9 \pm 5.5$ & $<0.0001$ \\
\hline Insulin $(\mathrm{ng} / \mathrm{ml})$ & $0.77 \pm 0.05$ & $1.16 \pm 0.07$ & $<0.02$ \\
\hline Insulin:Glucose ratio & $0.017 \pm 0.001$ & $0.027 \pm 0.002$ & $<0.0001$ \\
\hline HOMA-R Index & $9.7 \pm 1.4$ & $22.1 \pm 3.2$ & $<0.0001$ \\
\hline
\end{tabular}

Supported by: Agencia Nacional de Promoción Científica y Tecnológica - CONICET-Argentina

\section{4}

Changes in neuronal NO synthase dimerisation state but not catalytic activity are involved in the hypersecretion of insulin in obese Zucker fa/fa rats

K. Mezghenna, M. Manteghetti, C. Jahannault, P. Petit, R. Gross,

A.-D. Lajoix

UMR 5232, CNRS, Montpellier, France

Background and aims: In rat pancreatic $\beta$-cell, we have previously shown the expression of an isoform of neuronal NO synthase (nNOS), that controls insulin secretion through metabolic and non-metabolic activities. This latter activity is related to the interaction of nNOS with its protein inhibitor 
PIN at the level of insulin secretory granules. PIN is also the light chain of cytoskeletal motors like myosin V. Zucker fa/fa rats are hyperphagic, obese, insulino-resistant and hyperinsulinic animals, due to a mutation in the leptin receptor. In these animals, the hypersecretion of insulin is persistent even if islets are maintained in culture for a long period, suggesting a constitutive defect of pancreatic $\beta$-cell. Our aim is thus to search if nNOS abnormalities could be involved in $\beta$-cell dysfunction observed at early stages of diabetes, using the Zucker fa/fa rat model.

Materials and methods: nNOS and PIN expression was studied by quantitative RT-PCR and Western blotting using monoclonal antibodies. Dimerization of nNOS was analyzed by low temperature SDS-PAGE without boiling the samples, followed by a Western blotting. nNOS catalytic activity was measured by the production of citrulline from radiolabeled arginine. Immunofluorescence studies were performed on isolated $\beta$-cells with specific polyclonal antibodies against nNOS, PIN and myosin V.

Results: In islets of Zucker fa/fa rats, we first observed a 7 -fold increase in nNOS proteic expression. We also analyzed the monomer/dimer equilibrium of nNOS and found higher amounts of nNOS dimers in fa/fa rats as compared to the fa/+ rats. Surprisingly, nNOS catalytic activity was diminished by $25 \%$ in the fa/fa rats, suggesting the presence of inactive nNOS dimers. We analyzed the presence of nNOS phosphorylation at Ser 847, known to inactivate the enzyme through inhibition of calmodulin binding. An enhanced phosphorylation of both nNOS monomers and dimers could be observed in the fa/fa rats. Using confocal microscopy, nNOS displayed a greater colocalization with insulin secretory granules and its protein inhibitor PIN, which is also overexpressed in fa/fa rats and strongly associated with myosin V.

Conclusion: As pharmacologically-induced nNOS dimers account for a monophasic enhanced insulin response to glucose, the increased nNOS dimerization state observed could be involved in the hypersecretion of insulin present in the Zucker fa/fa rats, through interactions of nNOS with PIN and myosin $\mathrm{V}$ at the insulin secretory granules rather than through an increased catalytic activity.

\section{5}

Roles of islet glucose 6 phosphatase related protein in islet function in vivo and in vitro

P.G. Pirot ${ }^{1}$, S.I. Hackl ${ }^{1}$, S.A. Sarkar ${ }^{1}$, R.M. O’Brien ${ }^{2}$, H.W. Davidson ${ }^{1}$, J.C. Hutton ${ }^{1}$

${ }^{1}$ Barbara Davis Center for Childhood Diabetes, University of Colorado Denver, Aurora, ${ }^{2}$ Barbara Davis Center for Childhood Diabetes, Vanderbilt University, Nashville, United States

Background and aims: Islet glucose 6-phosphatase catalytic subunit related protein (IGRP) is a major autoantigen in type 1 diabetes and a candidate gene in type 2 diabetes. It was originally identified on the basis of its sequence similarity to the catalytic subunit of liver glucose 6-phosphatase (G6Pase), and like G6Pase is localized to the endoplasmic reticulum. However, it is a pseudogene in the rat, and its precise role in pancreatic beta cell function in humans and mice remains unclear. The aim of this study was to address this issue by determining the catalytic properties of IGRP.

Materials and methods: G6P assays were conducted using isolated islets from Balb/c mice, and Cos-7 cells transfected with mouse G6Pase, IGRP, or G6Pase/IGRP chimeras. Cell lysates were prepared by freeze-thawing and/ or sonication and incubated for $2 \mathrm{~h}$ at $30 \mathrm{C}$ with $0.5 \hat{\mathrm{A}} \mu \mathrm{Ci}$ [14-C] G6P. After precipitation with a mixture of zinc sulfate and barium hydroxide to remove unreacted substrate the product was measured by liquid scintillation counting. Protein concentrations were measured by BCA assay, and IGRP expression analyzed by western blotting using a rabbit polyclonal antibody. In some experiments islets were cultured overnight prior to analysis.

Results: Freshly isolated islets hydrolyzed G6P at $21 \hat{\mathrm{A}} \pm 3 \mathrm{nmol} / \mathrm{min} / \mathrm{mg}$. This activity was maintained after overnight culture in $11.1 \mathrm{mM}$ glucose. In contrast, culture in low glucose $(5.6 \mathrm{mM})$ resulted in an $\sim 66 \%$ reduction in G6Pase activity $(7 \hat{\mathrm{A}} \pm 2 \mathrm{nmol} / \mathrm{min} / \mathrm{mg})$, suggesting that IGRP may be involved in the regulation of insulin secretion. The decreased activity resulted from turnover of the protein as evidenced by semi-quantitative western blotting. Cos-7 cells transfected with vector alone had an apparent basal G6Pase activity of $2 \mathrm{nmol} / \mathrm{min} / \mathrm{mg}$. A 4.3 -fold increase $(8.6 \mathrm{nmol} / \mathrm{min} / \mathrm{mg})$ was observed after transfection with wild-type IGRP, which was not seen with a mutant (R79A) in the presumptive catalytic domain. In contrast, transfection with liver G6Pase resulted in an activity of $2900 \hat{\mathrm{A}} \pm 150 \mathrm{nmol} / \mathrm{min} / \mathrm{mg}$. To investigate the structural features that resulted in the $\sim 330$ fold greater activity of the liver enzyme we created a series of chimeras. Substitution of the final 3 transmembrane domains (TMDs) of G6Pase with the equivalent regions of IGRP had no significant effect on activity. In contrast, chimeras containing only the first 3 TMDs of G6Pase had a similar activity to IGRP, which was also true of chimeras containing the first IGRP TMD and the final 8 TMDs of G6Pase. Conclusion: IGRP appears to be the principal enzyme responsible for the low level G6Pase activity in mouse islets. It undergoes rapid changes in expression in response to glucose, consistent with a role in regulating insulin secretion. Chimeras with liver G6Pase that do not involve regions predicted to form the catalytic site have no effect, suggesting that the overall topology of the 2 proteins are equivalent. In contrast, introduction of elements of the IGRP catalytic site into G6Pase significantly decreases activity. Although IGRP conceivably forms a substrate cycle with glucokinase, it appears not to be simply a regulator of glycolysis.

Supported by: NIH, JDRF, ADA and CDF

\section{6}

Rosiglitazone prevents fatty acid induced increases in uncoupling protein 2 gene expression and protects pancreatic beta cells from lipotoxicity

T. Ali $^{1}$, C. Landy ${ }^{1}$, C.E. Marriott ${ }^{1}$, R. Crawford ${ }^{2}$, A. Woollhead ${ }^{1}$,

M. Harrison ${ }^{1}$, W.M. Macfarlane ${ }^{1}$

${ }^{1}$ School of Pharmacy \& Biomolecular Sciences, University of Brighton, ${ }^{2}$ Bute Medical School, University of St Andrews, United Kingdom

Background and aims: A wealth of recent evidence implicates increasing obesity levels in the rising global epidemic of type 2 diabetes. At a cellular level, high fatty acid concentrations contribute to increasing beta-cell dysfunction and the eventual loss of beta-cell viability. The free fatty acid palmitate has been shown to act in part through elevation of uncoupling protein 2 (UCP2) mRNA levels within the beta-cells. We have recently shown that the thiazolidinedione rosiglitazone has direct effects on pancreatic beta-cell gene expression. The aim of the present study was to test the hypothesis that rosiglitazone can protect beta cells from lipotoxicity, and to determine the specific effect of this drug on UCP2 gene expression.

Materials and methods: Utilizing the mouse beta cell line Min6, real time PCR was employed to determine the effects of palmitate and rosiglitazone on UCP2 gene expression. MTT viability assays were used to determine the effects of these agents on Min6 cell viability. These methods were combined with selected inhibitors of AMPK, CK2 and PPAR $\gamma$ activity to investigate the signaling events mediating these effects.

Results: MTT assays showed that addition of $0.5 \mathrm{mM}$ palmitate for 72 hours resulted in a $70 \%$ loss of Min6 cell viability $(\mathrm{P}<0.01)$. However, the presence of $10 \mu \mathrm{M}$ rosiglitazone completely abolished the detrimental effect of palmitate, protecting beta cell viability. At a gene expression level, palmitate exposure significantly increased UCP2 mRNA levels $(\mathrm{p}<0.05)$. In contrast, rosiglitazone alone had no significant effect on UCP2 gene expression. However, addition of rosiglitazone prior to palmitate completely abolished the effect of palmitate on UCP2 expression $(\mathrm{p}<0.05)$. The ability of rosiglitazone to block palmitate-induced increases in UCP2 gene expression was abolished by addition of the PPAR $\gamma$ antagonist GW9662, suggesting that the effects of rosiglitazone on UCP2 expression under these conditions are mediated through the modulation of PPAR $\gamma$ activity. At the level of individual kinase activities, addition of $10 \mu \mathrm{M}$ rosiglitazone significantly increased the activity of both AMPK $(\mathrm{p}<0.05)$ and casein kinase $2(\mathrm{CK} 2)(\mathrm{p}<0.01)$ in Min6 cells. The AMPK inhibitor compound $\mathrm{C}$ was able to completely block the protective effect of rosiglitazone on cell viability $(\mathrm{p}<0.01)$. CK2 inhibitor TBB was able to partially block the effects of rosiglitazone on cell viability $(\mathrm{p}<0.05)$, suggesting that AMPK lies upstream of CK2 in the cell signaling events being stimulated by rosiglitazone. Neither inhibitor was able to block the ability of rosiglitazone to suppress palmitate-induced increases in UCP2 expression.

Conclusion and interpretation: The results of the present study indicate that rosiglitazone modulates multiple signaling pathways in pancreatic beta cells, protecting Min 6 cells from the detrimental effects of palmitate and blocking palmitate-induced increases in UCP2 gene expression. The present study adds to a growing body of evidence indicating that rosiglitazone can have direct protective effects on beta-cell viability and function, and that these effects occur through both PPAR $\gamma$-dependent and PPAR $\gamma$-independent signaling pathways.

Supported by: Diabetes UK 


\section{7}

Pyruvate dehydrogenase kinase 1 knock down in INS-1 832/13 Cells enhances insulin secretion and the cellular NADPH/NADP ${ }^{+}$ratio

O.V. Kotova ${ }^{1}$, U. Fransson ${ }^{1}$, M.C. Sugden ${ }^{2}$, H. Mulder ${ }^{1}$

${ }^{1}$ Clinical Science, Unit of Molecular Metabolism, Lund University, UMAS,

Malmö, Sweden, ${ }^{2}$ Centre for Diabetes and Metabolic Medicine, Barts and

London School of Medicine and Dentistry, United Kingdom

Background and aims: While it is widely agreed that fuel metabolism in the pancreatic $\beta$-cell controls glucose-stimulated insulin secretion (GSIS), it has become increasingly clear that the precise ways in which carbons enter and exit the TCA-cycle are key regulatory events. Two major pathways for entry of carbons into the TCA-cycle are the reactions catalyzed by pyruvate dehydrogenase $(\mathrm{PDH})$ and pyruvate carboxylase $(\mathrm{PC})$, respectively; the latter has received a great deal of attention because it is remarkably prominent in the pancreatic $\beta$-cell. PC controls the major anaplerotic pathway in $\beta$-cells, and we and others have shown that its inhibition results in impaired GSIS. Here, we took an alternative approach and examined the effect of silencing PDH kinase 1 (PDK1) on GSIS and metabolism in INS-1 832/13 clonal $\beta$-cells. PDK1 is known to inhibit activity of PDH via phosphorylation, and the manipulation is envisaged to enhance TCA-cycle flux through PDH.

Materials and methods: PDK1 was knocked down in INS-1 832/13 cells by RNA interference (RNAi). After $72 \mathrm{~h}$, insulin secretion, glucose oxidation, ATP/ADP ratio, $\mathrm{PDH}$ activity and the $\mathrm{NADPH} / \mathrm{NADP}^{+}$ratio were determined.

Results: PDK1 mRNA was reduced by $80 \%(\mathrm{P}<0.001)$, and PDK1 protein expression was decreased, as assessed by Western Blot. Knock down of PDK1 did not affect basal insulin secretion. Remarkably, cells treated with siRNA to PDK1 increased insulin secretion 5-fold $(\mathrm{P}<0.01)$ in response to $16.7 \mathrm{mM}$ glucose, while cells transfected with the negative control increased insulin secretion by only 3.1 -fold. Insulin secretion provoked by two other metabolic fuels, leucine and succinate, was not influenced by knock down of PDK1. Knock down of PDK1 led to a 1.7-fold increase in PDH activity, but surprisingly, did not affect either glucose oxidation or the glucose-induced rise in ATP/ADP ratio. After stimulation with $16.7 \mathrm{mM}$ glucose for $3 \mathrm{~min}$, the $\mathrm{NADPH} / \mathrm{NADP}^{+}$ratio rose in both silenced and control cells. However, the ratio was 1.8 -fold higher in 832/13 cells where PDK1 was silenced compared to control cells.

Conclusion: We suggest that maintenance of low PDK1 expression, keeping $\mathrm{PDH}$ in a dephosphorylated and active state, is an important factor in GSIS in pancreatic $\beta$-cells. Since secretion in response to leucine and succinate, which act distally to the exit of citrate and/or isocitrate from the TCA-cycle, was unaffected, it is possible that cycling of citrate and/or isocitrate to pyruvate accounts for enhanced GSIS. This agrees both with the observed exaggeration of the NADPH/NADP ${ }^{+}$ratio in the cytosol, while neither glucose oxidation nor the ATP/ADP ratio was increased.

Supported by: Swedish Research Council

\section{8}

Assessment of the AstraZeneca glucokinase activator GKA50 on pancreatic beta cell function and viability in the clonal beta cell line BRIN-BD11

N. Mullooly ${ }^{1}$, P. Newsholme ${ }^{1}$, D. Smith ${ }^{2}$

${ }^{1}$ School of Biomolecular and Biomedical Science, University College

Dublin, Ireland, ${ }^{2}$ AstraZeneca, Alderly Park, Macclesfield, Cheshire, United

Kingdom

Background and aims: Pancreatic beta cells fail to secrete appropriate amounts of insulin for glycaemic control in type 2 diabetes. Therapeutic strategy for type 2 diabetes includes increasing the insulin secretory capacity of the beta cells. Glucokinase has emerged as a promising target for an anti-diabetic agent. This enzyme has been designated the "glucose sensor" due to its concentration dependent control over glucose metabolism and thus insulin secretion. Genetic analysis and structural studies reveal the enyme posesses an allosteric activation site. Molecular compounds which bind to this site stabalize glucokinase in its closed confirmation increasing its affinity for glucose and should enhance glucose stimulated insulin secretion in the pancreatic beta cell. We have tested the glucokinase activator GKA50 with respect to clonal beta cell (BRIN-BD11) metabolism, insulin secretion and cellular integrity. This bioengineered cell line has a high glucokinase-hexokinase ratio compared to other beta cell lines, and exhibits bi-phasic glucose stimulated insulin secretion, consistent with normal beta cells.
Materials and methods: The metabolic and insulin secretory action of GKA50 was determined using basal $(1.1 \mathrm{mM})$ and stimulatory $(16.7 \mathrm{mM})$ glucose + alanine $(10 \mathrm{mM})$ static incubation conditions. Insulin secretion was determined by ELISA. The effects of GKA50 on cell growth and viability was assessed using the WST-1 cell viability assay.

Results: We have shown that micromolar concentrations of GKA50 are potent activators of glucokinase and increase glucose stimulated insulin secretion in BRIN-BD11 cells in a dose dependent manner, becoming significant at $10 \mu \mathrm{M}$ GKA50 ( $<<0.05)$. GKA50 stimulated insulin secretion was further enhanced by the presence of $10 \mathrm{mM}$ alanine. No significant difference in viability was found between cells incubated in the absence or presence of GKA50, even at concentrations of $100 \mu \mathrm{M}$.

Conclusion: Increased activation of glucokinase increases cellular glucose metabolism and insulin secretion. Our results highlight the importance of glucokinase in metabolic regulation and stimulus secretion coupling in pancreatic beta cells. Glucokinase activator drugs offer the possibility of effective pharmacological control over glycaemia and may be a novel therapeutic approach to the treatment of defective glucose stimulated insulin secretion in type 2 diabetes mellitus.

Supported by: AstraZeneca 


\section{PS 028 Metabolic signals in the beta cell}

\section{9}

Over-expression of constitutively-activated or dominant-negative forms of AMP-activated protein kinase (AMPK) selectively in the beta cells of transgenic mice exerts opposing effects on glucose tolerance

G. Sun, G. da Silva Xavier, A. Riboulet-Chavey, T. Taneja, I. Leclerc, G.A. Rutter

Cell biology, Imperial college London, United Kingdom

Background and aims: Defective insulin secretion from pancreatic $\beta$ cells underlies the progression towards type 2 diabetes. Whilst stimulation of AMP-activated protein kinase (AMPK) in insulin-sensitive tissues is an important mode of action of anti-hyperglycemic agents including metformin, the role of this enzyme in the $\beta$-cell is less clear. In order to address this question we have generated transgenic mice over-expressing constitutively-active (CA) or dominant-negative (DN) forms of AMPK under the rat insulin II (RIP) promoter.

Materials and methods: $\mathrm{cDNAs}$ encoding the truncated $\mathrm{N}$-terminal domain common to AMPKa1 and a2 (residues 1-312) and carrying an activating mutation (T172D; AMPK CA) was fused downstream of a $0.6 \mathrm{~kb}$ proximal fragment of the RIP promoter. AMPK DN was generated similarly using full length AMPKa1 cDNA (D157A), and the purified fragments were injected into oocytes from C57BL6 mice before re-implantation. The offspring of founders carrying 10 copies of the AMPK CA, or 2-5 copies of the AMPK DN transgenes, were characterized. Both RIP-AMPK CA C57BL6 mice and their wild type littermates were kept on normal chow diet, while DN mice and their wild type littermates were kept on high fat $(60 \%)$ diet from six weeks of age. Transgenic mice or littermates were fasted $16 \mathrm{~h}$ prior to intraperitoneal injection of glucose $(1 \mathrm{~g} / \mathrm{kg}$ body weight) for measurement of blood glucose (Roche, Accuchek glucometer), or $3 \mathrm{~g} / \mathrm{kg}$ body weight for measurement of plasma insulin (Merchodia, ultrasensitive rat insulin ELISA). Insulin secretion from isolated islets was measured after batch incubation with 3 or $16.7 \mathrm{mmol} / \mathrm{l}$ glucose. Secreted and total insulin were quantified by radioimmunoassay using $\mathrm{I}^{125}$-labeled insulin. All experiments were approved by UK Home Office according to Animals (Scientific Procedures) Act, 1986.

Results: RIP-AMPK CA (10 copies) female transgenic mice developed glucose intolerance at 24 weeks (peak glucose \pm SEM: transgenic $=14.76 \pm 0.3$ $\mathrm{mmol} / \mathrm{l}$, littermate controls $=12.45 \pm 0.65 \mathrm{mmol} / \mathrm{l} ; \mathrm{n}=4 ; p=0.039$ ) and male mice at 12 weeks (Peak glucose \pm SEM: transgenic $=16.78 \pm 0.27 \mathrm{mmol} / \mathrm{l}$, littermate controls $=15.33 \pm 0.037 \mathrm{mmol} / \mathrm{l} ; \mathrm{n}=2)$. Both male and female transgenic mice showed a small decrease of plasma insulin levels (Male: transgenic $=0.38 \mu \mathrm{g} / \mathrm{l}$, littermate controls $=0.72 \mu \mathrm{g} / \mathrm{l}$; Female: transgenic $=0.53 \mu \mathrm{g} / \mathrm{l}$, littermate controls $=0.55 \mu \mathrm{g} / \mathrm{l}) 15 \mathrm{~min}$ after glucose challenge, and reduced insulin secretion in islets from RIP-AMPK CA mice (transgenic $=0.25 \pm 0.05$ $\% / 30$ min., littermate controls $=0.57 \pm 0.16 \% / 30 \mathrm{~min} ; \mathrm{n}=2, p=0.044)$ when stimulated by 16.7 (vs 3.0) mmol/l glucose. Male RIP-AMPK DN ( $2-5$ copies) displayed unaltered glucose tolerance on a normal chow diet but significantly improved tolerance (Peak glucose \pm SEM: transgenic $=19.09 \pm 0.8 \mathrm{mmol} / \mathrm{l}$, littermate controls $=21.12 \pm 0.97 \mathrm{mmol} / \mathrm{l} ; \mathrm{n}=8 ; p=0.024)$ after 12 weeks exposure to a high fat $(60 \%)$ diet.

Conclusion: Over-expression of AMPK in $\beta$ cells impairs insulin secretory function in vivo, whereas this parameter is improved under conditions of $\beta$ cell stress when AMPK activity is suppressed. The development of agents selective for AMPK complexes in $\beta$ cells may provide a new therapeutic approach towards type 2 diabetes.

Supported by: Wellcome Trust, Medical Research Council UK, National Institutes of Health and EU

\section{0}

Deletion of protein kinase $\mathrm{C}$ epsilon in mice enhances glucose stimulated insulin secretion following lipid exposure by up-regulation of the amplifying pathways of insulin secretion

J. Cantley, G.L. Pearson, J.G. Burchfield, C. Schmitz-Peiffer, T.J. Biden Diabetes and Obesity Research Program, Garvan Institute of Medical Research, Sydney, Australia

Background and aims: Deletion of protein kinase C epsilon (PKCE) has been shown to protect mice from high fat diet induced glucose intolerance by enhancing glucose stimulated insulin secretion (GSIS). Islets isolated from PKC $\varepsilon$ knock-out (PKCeKO) mice showed enhanced GSIS, relative to wild type (wt) islets, following lipid pre-treatment, although the mechanisms responsible for this are unknown. The amplifying pathways of GSIS allow glucose to dose dependently increase insulin secretion in a depolarised $\beta$-cell, independently of the canonical triggering pathway of GSIS. It has been demonstrated that the amplifying pathways function, in part, by increased partitioning of longchain fatty acyl CoAs (LC-CoAs) into esterification pathways by inhibition of $\beta$-oxidation by glucose. Malonyl CoA production by acetyl CoA carboxylase (ACC) has been shown to regulate this partitioning in $\beta$-cells. Therefore, the aim of this study was to investigate the mechanisms of lipid dependent upregulation of GSIS in PKCeKO islets.

Materials and methods: Islets were isolated from 12 week old male mice and cultured for 48 hours in DMEM containing either $0.92 \%$ BSA alone (control), or $0.92 \%$ BSA coupled to $0.4 \mathrm{mmol} / 1$ palmitate (lipid). Secretion experiments were performed using KRB with $10 \mathrm{mmol} / 1$ HEPES and $0.1 \%$ BSA, containing either $2 \mathrm{mmol} / \mathrm{l}$ or $20 \mathrm{mmol} / \mathrm{l} \mathrm{D}$-glucose. Diazoxide (DZX $100 \mu \mathrm{mol} / \mathrm{l}$ ) was used to maintain open KATP channels and $\mathrm{KCl}(25 \mathrm{mmol} / \mathrm{l})$ to depolarise cell membranes. Insulin was assayed by RIA. Intracellular free calcium was measured by fluorescence microscopy. Phosphorylation of ACC and AMP activated protein kinase (AMPK) was determined by immunoblot. Data is presented as mean \pm SEM with statistical analysis using ANOVA and bonferroni post test.

Results: GSIS in response to $20 \mathrm{mmol} / \mathrm{l}$ glucose was diazoxide sensitive in wt and PKCEKO islets pre-treated with control BSA or lipid. To study amplifying GSIS, DZX and $\mathrm{KCl}$ were combined to initiate insulin secretion by depolarising cell membranes independently of KATP channel closure. Under these conditions $(\mathrm{DZX}+\mathrm{KCl}), \mathrm{PKC} \mathrm{KKO}$ lipid pre-treated islets showed a significant enhancement of amplifying GSIS in response to $20 \mathrm{mmol} / \mathrm{l}$ glucose (wt control $1.39 \pm 0.16$, wt lipid $1.76 \pm 0.22$, PKCeKO control $2.12 \pm 0.23$, PKCeKO lipid $3.55 \pm 0.40$; ng insulin secreted/islet/hour; $\mathrm{n}=3$ experiments; $\mathrm{PKC \varepsilon KO} \mathrm{lipid} \mathrm{vs}$ other groups $\mathrm{P}<0.01)$. Glucose and $\mathrm{KCl}$ induced calcium influx was normal in PKCeKO $\beta$-cells (wt control $17.4 \pm 2.0$; wt lipid $16.15 \pm 1.89$; PKCeKO control $14.84 \pm 1.3$; PKCeKO lipid $15.73 \pm 1.92$; AUC of calcium trace, $\mathrm{n}=4$ experiments, NS) indicating that enhanced secretion was not due to altered calcium influx. Immunoblots showed decreased phosphorylation of AMPK threonine 172 and ACC serine 79 in PKCeKO islets during culture with lipid.

Conclusion: These data show up-regulation of the amplifying pathways of GSIS in lipid pre-treated PKCeKO islets, following a depolarising stimulus. Reduced phosphorylation of ACC and AMPK suggests altered lipid partitioning. PKC\&KO islets are therefore a model of enhanced amplifying GSIS, and may allow further elucidation of signalling mechanisms governing the amplification pathways. As PKC\&KO mice are protected from high fat diet induced glucose intolerance, up-regulation of amplifying GSIS by inhibition of PKC $\varepsilon$ in humans represents a potential novel treatment for type 2 diabetes. Supported by: National Health and Medical Research Council of Australia

\section{1}

Insulin-stimulated $\mathrm{PI}(3,4) \mathrm{P} 2$ production by $\mathrm{PI} 3 \mathrm{~K}-\mathrm{C} 2 \alpha$ and activation of glucokinase gene transcription in pancreatic beta cells

B. Leibiger ${ }^{1}$, T. Moede ${ }^{1}$, C.J. Barker ${ }^{1}$, S. Uhles ${ }^{1}$, J. Domin ${ }^{2}$, P.-O. Berggren ${ }^{1}$, I.B. Leibiger ${ }^{1}$

${ }^{1}$ The Rolf Luft Research Center for Diabetes and Endocrinology, Karolinska Institutet, Stockholm, Sweden, ${ }^{2}$ Renal Section, Imperial College School of Medicine, London, United Kingdom

Background and aims: Pancreatic $\beta$-cells are targets for positive feedback action by secreted insulin. We have shown that insulin activates the transcription of the $\beta$-cell glucokinase transcription unit (BGK) via the insulin receptor B-type (IR-B) and signalling via a class II PI3K-C2a-like activity and PKB. The present study aimed at identifying the actual PI3K activity involved as well as the molecular mechanisms mediating this activation.

Materials and methods: BGK promoter-driven GFP expression in transfected HIT-T15, MIN6 and primary mouse $\beta$-cells served as the readout for BGK transcription. By combining the use of pharmacological inhibitors with transient over-expression of PI3K-C2 $\alpha$ and PKB variants, siRNA based knockdown of PI3Ks and PKB isoforms, PI-lipid profiling and co-localization analysis using life-cell confocal and TIRF microscopy, we now show that IR-B-mediated activation of BGK transcription involves the generation of PI $(3,4) P 2$ by PI3K-C2 $\alpha$ and the subsequent activation of the $\alpha$-isoform of PKB.

Results: Because $100 \mu \mathrm{M}$ LY294002 or $150 \mathrm{nM}$ Wortmannin was needed to block insulin-stimulated BGK promoter activity, i.e. concentrations similar to those that inhibit PI3K class II C2 $\alpha$, we studied the involvement of PI3K-C2 $\alpha$ 
in BGK transcription. Over-expression of both wild type PI3K-C2 $\alpha$ as well as plasma-membrane-targeted PI3K-C2 $\alpha$-CAAX increased BGK promoter activity, while expression of the kinase-dead mutant PI3K-C2a-R1251P had no stimulatory effect. Vice versa, siRNA-mediated knockdown of PI3K-C2 $\alpha$ abolished insulin-stimulated BGK activation without any effect on IR-A/PI3K class Ia-mediated insulin promoter activity. siRNA-mediated knockdown of PKB isoforms identified PKB $a$ to be involved in insulin-stimulated BGK transcription. siRNA-mediated knockdown of PI3K-C2 $\alpha$ abolished insulinstimulated PKB $\alpha$ activation. PI-lipid profiling, combining LY294002-mediated inhibition of class Ia PI3K and siRNA-mediated knockdown of PI3K-C2a, identified PI $(3,4) \mathrm{P} 2$ as the lipid product of PI3K-C2 $\alpha$ in insulin-stimulated MIN6 cells. Life-cell confocal and TIRF microscopy demonstrated the colocalization of both PI3K-C2 $\alpha$ and PKB $\alpha$ with IR-B at the plasma membrane of HIT and MIN6 cells.

Conclusion: Our data suggest that IR-B-mediated activation of PI3K-C2 $\alpha$ results in the generation of $\mathrm{PI}(3,4) \mathrm{P} 2$, which activates $\mathrm{PKBa}$ and subsequently the transcription of BGK in the pancreatic $\beta$-cell.

Supported by: Novo Nordisk Foundation, Family Erling-Persson Foundation, EuroDia, EFSD/Lilly grant, Swedish Research Council

\section{2}

Mediators of cyclic AMP action in glucose-stimulated pulsatile insulin secretion from single beta cells

O. Idevall-Hagren, E. Gylfe, A. Tengholm

Medical Cell Biology, Uppsala University, Sweden

Background and aims: Glucose stimulation of $\beta$-cells evokes synchronized oscillations of the cytoplasmic $\mathrm{Ca}^{2+}\left(\left[\mathrm{Ca}^{2+}\right]_{\mathrm{i}}\right)$ and sub-membrane cAMP $\left([\mathrm{cAMP}]_{\mathrm{m}}\right)$ concentrations, which underlie pulsatile insulin release. However, it is not known how cAMP oscillations contribute to pulsatile insulin release. In the present study, we investigated the involvement of protein kinase A (PKA) and exchange protein directly activated by cAMP (Epac) for glucoseinduced pulsatile insulin release from individual $\beta$-cells.

Materials and methods: Evanescent wave fluorescence imaging was used to record $[\mathrm{cAMP}]_{\mathrm{m}}$ or $\left[\mathrm{Ca}^{2+}\right]_{\mathrm{i}}$ in parallel with phosphatidylinositol-3,4,5-trisphosphate (PIP $)_{3}$. This membrane lipid is formed by autocrine insulin receptor activation and was used as readout for insulin secretion from single MIN6 $\beta$-cells.

Results: Elevation of the glucose concentration from 3 to $11 \mathrm{mM}$ induced oscillations of $[\mathrm{cAMP}]_{\mathrm{m}}$, followed after a $14 \mathrm{~s}$ delay by PIP ${ }_{3}$ oscillations reflecting pulsatile insulin release. Inhibition of cAMP formation with the adenylyl cyclase inhibitor 2'5'-dideoxyadenosine markedly reduced this PIP, response. Also the PKA inhibitors Rp-8-CPT-cAMPS $(100 \mu \mathrm{M})$ or KT5720 $(2 \mu \mathrm{M})$ diminished the time-average $\mathrm{PIP}_{3}$ response to $72 \pm 7 \%(\mathrm{n}=35, \mathrm{P}<0.02)$ and $41 \pm 10 \%(\mathrm{n}=12, \mathrm{P}<0.001)$ of control, respectively, when applied before glucose stimulation. In contrast, the PKA inhibitors had only a modest effect on already manifested $\mathrm{PIP}_{3}$ oscillations. The reduction of the early $\mathrm{PIP}_{3}$ response was accompanied by a temporal dissociation between the initial $\left[\mathrm{Ca}^{2+}\right]_{\mathrm{i}}$ and $[\mathrm{cAMP}]_{\mathrm{m}}$ elevations due to a selectively shortened time lag between glucose stimulation and elevation of $\left[\mathrm{Ca}^{2+}\right]_{\text {, from }} 164 \pm 5 \mathrm{~s}(\mathrm{n}=11)$ to $134 \pm 7 \mathrm{~s}(\mathrm{n}=20 ; \mathrm{P}<0.01)$. Although insensitive to PKA inhibition, manifested $\mathrm{PIP}_{3}$ oscillations in response to glucose were amplified by specific activators of both PKA and Epac.

Conclusion: The data indicate that coordination of $\left[\mathrm{Ca}^{2+}\right]_{\mathrm{i}}$ and $[\mathrm{cAMP}]_{\mathrm{m}}$ signals are important for glucose-induced insulin secretion. Whereas PKA is important for the establishment of proper pulsatile insulin release, maintenance of secretion seems to involve a PKA-independent cAMP effector.

\section{3}

Zinc mediates feedback inhibition of cyclic AMP signalling and insulin secretion in beta cells

O. Dyachok, O. Idevall, A. Tengholm, E. Gylfe

Department of Medical Cell Biology, Uppsala University, Sweden

Background and aims: Glucose is the principal regulator of insulin secretion from pancreatic $\beta$-cells. Glucose increases the cytoplasmic $\mathrm{Ca}^{2+}$ and cAMP concentrations in $\beta$-cells, and it is well documented that cAMP is an important amplifier of $\mathrm{Ca}^{2+}$-triggered insulin release. Insulin is stored in secretory vesicles as a crystalline zinc complex and $\mathrm{Zn}^{2+}$ co-released with insulin has been proposed to exert a negative feedback on secretion by inhibiting $\mathrm{Ca}^{2+}$ influx after activation of hyperpolarizing $\mathrm{K}^{+}$channels. Since $\mathrm{Zn}^{2+}$ has been found to interfere with cAMP turnover in some cells, we tested the effects of $\mathrm{Zn}^{2+}$ on the kinetics of cAMP and $\mathrm{Ca}^{2+}$ signaling as well as insulin secretion from individual glucose-stimulated $\beta$-cells.

Materials and methods: MIN6 $\beta$-cells were transfected with a fluorescent translocation biosensor reporting phosphatidylinositol-3,4,5-trisphosphate $\left(\mathrm{PIP}_{3}\right)$. This membrane lipid is formed by autocrine insulin receptor activation and was used as readout for insulin secretion from single cells. The cytoplasmic cAMP concentration beneath the plasma membrane ([cAMP $\left.]_{i}\right)$ was detected by another translocation biosensor based on fluorescence-labeled protein kinase A subunits. Measurements of intracellular $\mathrm{Ca}^{2+}\left(\left[\mathrm{Ca}^{2+}\right]_{i}\right)$ and $\mathrm{Zn}^{2+}\left(\left[\mathrm{Zn}^{2+}\right]_{\mathrm{i}}\right)$ concentrations were performed in cells loaded with the fluorescent indicators fura-2 and FluoZin-3, respectively. Changes in $\mathrm{PIP}_{3}$ in the plasma membrane and $[\mathrm{cAMP}]_{i}$ and $\left[\mathrm{Zn}^{2+}\right]_{\mathrm{i}}$ in the sub-membrane space were recorded with evanescent wave microscopy.

Results: MIN6 $\beta$-cells reacted to glucose stimulation with pronounced oscillations of $\left[\mathrm{Ca}^{2+}\right]_{i}$ and $[\mathrm{cAMP}]_{\text {, }}$ as well as with pulsatile insulin release. The oscillatory patterns varied among cells and the responses sometimes faded with time. Glucose also induced elevation of $\left[\mathrm{Zn}^{2+}\right]$, and oscillations were ap-

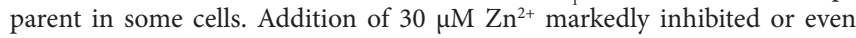
abolished the glucose-induced [cAMP], and insulin responses, but had a less pronounced effect on $\left[\mathrm{Ca}^{2+}\right]_{\mathrm{i}}$ oscillations. The inhibitory effects of added $\mathrm{Zn}^{2+}$ were not reversed by omission of the cation and chelation of residual extracellular $\mathrm{Zn}^{2+}$ with Ca-EDTA. In contrast, the membrane permeable heavy metal chelator TPEN $(50 \mu \mathrm{M})$ readily restored the $\mathrm{Zn}^{2+}$-inhibited [cAMP] oscillations and insulin release. Moreover, TPEN immediately rescued spontaneously faded $[\mathrm{cAMP}]_{\mathrm{i}}$ oscillations and pulsatile insulin release. Extracellular $\mathrm{Zn}^{2+}$ concentration-dependently suppressed basal cAMP production in the presence of the phosphodiesterase inhibitor IBMX and this effect was reversed by TPEN.

Conclusion: These data reveal a novel regulatory role for $\mathrm{Zn}^{2+}$ in $\beta$-cells by showing that $\mathrm{Zn}^{2+}$ co-released with insulin feedback inhibits cAMP formation and insulin secretion.

\section{4}

A metabolic comparison of glucose-responsive INS-1 832/13 and glucose-nonresponsive INS-1 832/2 cells

V.V. Sharoyko' ${ }^{1}$, K. Bacos ${ }^{1}$, A. Abhyankar ${ }^{2}$, C.L.F. Nagorny ${ }^{1}$, H. Mulder ${ }^{1}$, H. Luthman ${ }^{2}$

${ }^{1}$ Unit of Molecular Metabolism, Department of Clinical Sciences in Malmö, Lund University, ${ }^{2}$ Unit of Medical Genetics, Department of Clinical Sciences in Malmö, Lund University, Malmö, Sweden

Background and aims: The biochemical mechanisms underlying glucosestimulated insulin secretion (GSIS) from pancreatic beta-cells are not completely understood. Specifically, mitochondrial metabolism plays a key role in GSIS. In fact, several different mutations in mitochondrial DNA (mtDNA) cause a maturity-onset form of diabetes. In order to investigate putative mitochondrial disturbances in beta-cells that impact GSIS, we employed the two clonal isolates of INS- 1 cells the glucose-responsive 832/13 and glucosenonresponsive $832 / 2$ cells. These clones are characterized by similar insulin content but exhibit specific differences in TCA-cycle fluxes. The anaplerotic pyruvate cycling pathway is more active in the glucose-responsive 832/13 cells. We examined the rate of glucose oxidation, which reflects TCA-cycle activity. We also sequenced mtDNA, which encodes transfer and ribosomal RNAs (rRNA) as well as 13 of the proteins in the multi-subunit enzyme complexes responsible for oxidative phosphorylation in mitochondria.

Materials and methods: Glucose oxidation and mitochondrial DNA sequencing in INS-1 832/13 and INS-1 832/2 cells were performed.

Results: The rate of glucose oxidation under basal conditions $(2.8 \mathrm{mM}$ glucose) was significantly higher in $832 / 2$ cells than in $832 / 13$ cells $(2.7 \pm 0.5$ versus $0.7 \pm 0.1 \mathrm{nmol}$ glucose $/ \mathrm{mg}$ protein $/ \mathrm{h}$, respectively; $\mathrm{P}<0.01)$. Upon stimulation with $16.7 \mathrm{mM}$ glucose, the rate of glucose oxidation in $832 / 2$ and $832 / 13$ cells increased to $10.3 \pm 0.8$ and $2.5 \pm 0.3 \mathrm{nmol}$ glucose $/ \mathrm{mg}$ protein $/ \mathrm{h}$, respectively $(\mathrm{P}<0.01)$. However, whereas the absolute rates of glucose oxidation were different in the two cell lines, the increment of glucose oxidation was similar in both clones (3.6-fold versus 3.8 -fold in $832 / 13$ and $832 / 2$ cells, respectively). Moreover, mitochondrial DNA sequencing revealed a DNA sequence variant $(1242 \mathrm{~T} / \mathrm{C})$ in the gene encoding $16 \mathrm{~S}$ rRNA. T is the common allele in the rat and is found in the superior INS-1 832/13 cell line.

Conclusion: Glucose-nonresponsive INS-1 832/2 cells are characterized by a higher absolute rate of glucose oxidation compared to glucose responsive INS-1 832/13 cells, while the increment due to an increase in glucose concentration is similar. 16S rRNA is involved in the synthesis of mitochondrial 
proteins found in the respiratory chain, and we suggest that the mutation in 16S rRNA may be one of the factors leading to impaired GSIS in INS-1 $832 / 2$ cells.

Supported by: Swedish Research Council

\section{5}

Islet GABA plays a dual role in the mechanism of stimulation of insulin secretion by $a$-ketoisocaproic acid

J. Pizarro-Delgado ${ }^{1}$, I. Hernández-Fisac ${ }^{1}$, R. Martín-del-Río²,

J. Tamarit-Rodriguez ${ }^{1}$

${ }^{1}$ Biochemistry, Complutense University, ${ }^{2}$ Research Department, Hospital

"Ramón y Cajal", Madrid, Spain

Background and aims: We have previously found that $\alpha$-ketoisocaproic acid (KIC) decreases strongly islet GABA content by promoting its metabolism in the "GABA shunt". Now, we have investigated whether GABA-decrease is necessary for the stimulation of insulin secretion.

Materials and methods: Hormone release was studied in rat perifused islets and insulin radioimmunologically measured every minute in the perifusate. Islet amino acids'contents were quantified measuring their fluorescence after being derivatized with o-phtalaldialdehyde and separated by reverse-phase HPLC.

Results: $10 \mathrm{mM}$ KIC stimulated a biphasic secretion of insulin whose magnitude was approximately one third lower than the one triggered by $20 \mathrm{mM}$ glucose. Addition of a plasma membrane permeable precursor of GABA (ethyl-GABA = eGABA) at $1 \mathrm{mM}$ clamps the intracellular GABA content at the values recorded at the plasma concentration of glutamine (close to 1 $\mathrm{mM})$ : it did not modify significantly first phase $(13.1 \pm 3.8, \mathrm{n}=4$ vs. $23.8 \pm$ $6.2 \mathrm{ng} / 10 \mathrm{~min} . \mathrm{x} 40$ islets, $\mathrm{n}=6$; N.S.) but suppressed second phase of KICinduced release within $52 \%(16.2 \pm 3.0, \mathrm{n}=4$ vs. $33.5 \pm 3.0 \mathrm{ng} / 20 \mathrm{~min} . \mathrm{x} 40$ islets, $\mathrm{n}=6$; $\mathrm{p}<0.005) .30 \mathrm{mM} \mathrm{KCl}$ depolarization did not prevent eGABAinduced decrease. Surprisingly, a further reduction of the $\mathrm{K}^{+}$-electrochemical potential with $70 \mathrm{mM} \mathrm{KCl}$ induced per se a non significant decrease of the first phase $(14.2 \pm 2.2, \mathrm{n}=6$ vs. $23.8 \pm 6.2 \mathrm{ng} / 10 \mathrm{~min}$. $\mathrm{x} 40$ islets, $\mathrm{n}=6$; N.S. $)$ and markedly suppressed by $52.5 \%(15.9 \pm 2.4, \mathrm{n}=6$ vs. $33.5 \pm 3.0 \mathrm{ng} / 20$ min. $x 40$ islets, $n=6 ; \mathrm{p}<0.001$ ) the 2 nd phase of KIC-induced release to a similar extent than $1 \mathrm{mM}$ eGABA. No further reduction of 2 nd phase insulin secretion was obtained by eGABA ( 5 or $10 \mathrm{mM}$ ) addition to $70 \mathrm{mM} \mathrm{KCl}$ but it was prevented by $10 \mathrm{mM}$ glutamine $(14.6 \pm 2.3, \mathrm{n}=6$ vs. $6.3 \pm 1.1 \mathrm{ng} / 20$ min. $x 40$ islets, $n=6 ; p<0.008$ ). Amino acid analysis showed that $70 \mathrm{mM} \mathrm{KCl}$ decreased islet GABA content $(752.5 \pm 77.4, \mathrm{n}=7$ vs. $1,109.1 \pm 74.2 \mathrm{pmol} / 25$ islets, $\mathrm{n}=7 ; \mathrm{p}<0.006)$ to the same extent as $10 \mathrm{mM} \mathrm{KIC}(644.4 \pm 65.6, \mathrm{n}=7$ vs. $1,109.1 \pm 74.2 \mathrm{pmol} / 25$ islets, $\mathrm{n}=7 ; \mathrm{p}<0.0005)$. However, at variance with KIC, $70 \mathrm{mM} \mathrm{KCl}$ induced a strong release of GABA $(619.6 \pm 106.5, \mathrm{n}=8$ vs. 305.0 $\pm 22.9 \mathrm{pmol} / 25$ islets, $\mathrm{n}=6 ; \mathrm{p}<0.01)$ and taurine $(227.0 \pm 41.5, \mathrm{n}=8$ vs. $110.4 \pm$ $11.2 \mathrm{pmol} / 25$ islets, $\mathrm{n}=8 ; \mathrm{p}<0.017)$ and this pattern was not modified by the simultaneous application of $\mathrm{KIC}$ and $\mathrm{KCl}$. Taurine content was not modified by KIC but it was decreased by $70 \mathrm{mM} \mathrm{KCl}(197.8 \pm 20.7, \mathrm{n}=8$ vs. $327.8 \pm 24.9$ $\mathrm{pmol} / 25$ islets, $\mathrm{n}=8 ; \mathrm{p}<0.001$ ).

Conclusion: KIC-induced reduction of the intracellular GABA concentration is necessary for the stimulation of insulin secretion because clamping it at "physiological" levels suppressed the second phase of release. Intracellular GABA depletion induced by its increased efflux at $70 \mathrm{mM} \mathrm{KCl}$ did not allow a normal stimulation of insulin secretion by KIC. GABA seems to play a dual role in the regulation of KIC-induced insulin release: maintaining its resting concentration suppresses secretion stimulation but the latter is favoured when GABA is lowered by increasing its metabolism.

Supported by: Instituto de Salud Carlos III

\section{6}

Molecular mechanisms of nutrient stimulated insulin-containing vesicle transport in pancreatic beta cells - identification of secretory vesiclespecific motor protein receptors

S. Lajus, A. Varadi

Faculty of Applied Sciences, University of the West of England, Bristol, United Kingdom

Background and aims: Pancreatic beta-cells synthesize insulin and store it in large dense core vesicles (LDCVs). When the blood-glucose concentration increases, a biphasic secretion of insulin occurs. The first phase is rapid and transient whereas the second phase is slower and more sustained. Our pre- vious studies have demonstrated that transport of LDCVs driven by motor proteins, kinesin 1 and myosin $\mathrm{Va}$, from reserve pool to plasma membrane is important for the second phase of secretion. The recruitment of these motor proteins to LDCVs requires specific receptors. However, the molecular components of the motor protein - LDCV complex and their direct regulators have not been identified in pancreatic beta-cells.

Materials and methods: We used reverse transcription polymerase chain reaction (RT-PCR) to identify the beta-cell specific splice variants of the globular tail domain of myosin Va heavy chain which is known to be involved in cargo binding. We used immunoprecipitation with a myosin Va and a kinesin 1 specific antibody, GST pull-down experiments, followed by MALDI-TOF method in order to identify kinesin 1 and myosin Va-specific receptors and interacting partners on LDCVs.

Results: Myosin Va-organelle receptor is well characterised in melanocytes where exons $\mathrm{ABCDEF}$ are co-expressed. Exons $\mathrm{F}$ and $\mathrm{D}$ are essential for cargo binding and interaction with MyRIP. However, in beta-cells these exons are absent suggesting that the putative interaction partners of myosin Va in this cell type are different than that of in melanocytes. Immunoprecipitation experiments revealed that myosin Va and kinesin 1 are part of the same protein complex in beta-cells. To confirm the specific interaction as expected neither of these proteins form complex with the mitochondrial resident cytochrome C.

Conclusion: We now use the natural splicing variants of myosin Va and kinesin 1 to identify the novel secretory vesicle-specific motor protein receptors in beta-cells. Furthermore, the nutrient-induced signalling pathway implicated in the recruitment of these motors to LDCVs will be addressed. This study provides fundamental information on the molecular basis and regulation of insulin-granule mobilisation which is essential for the sustained phase of insulin release.

Supported by: BBSRC

\section{7}

Down regulation of the mitochondrial malate-asparte shuttle by knockdown of its key component Aralar1 in INS-1E beta cells M. Casimir, P. Maechler

Department of Cell Physiology and Metabolism, University of Geneva, Switzerland

Background and aims: The NADH shuttle system transports reducing equivalents from the cytosol to the mithochondrial matrix and is essential for efficient coupling of glucose metabolism to insulin secretion. Among the NADH shuttles identified, the malate-aspartate has been shown to play the major role in pancreatic beta-cells. A key component of the malate-aspartate shuttle is Aralar1, also referred to as aspartate-glutamate carrier 1. We previously demonstrated that over-expression of Aralar1 increases glucose derived products into mitochondria and results in potentiated metabolism secretion coupling. In the present study, we addressed the reverse question by decreasing expression of Aralar1 by means of siRNA approach. This way, we aimed at evaluating the contribution of the malate-aspartate shuttle in insulin secreting cells along with possible compensatory mechanisms.

Materials and methods: We first identified siRNA construct down-regulating Aralar1. The selected construct was cloned into a recombinant adenovirus. Expression levels were assessed by immunoblotting. The most efficient titer $(0.1 \mu \mathrm{l}$ per well of 24 -well plates) was used to transduce INS-1E beta-cells for 1 hour and cells were subsequently cultured for 4 days before analyses. An empty adenovirus construct was used in control cells. Then, we measured parameters of the metabolism secretion coupling: cellular ATP concentrations, glucose oxidation, glucose utilization, lactate production, and NADH levels. Insulin secretory responses (analysed by RIA) to stimulatory glucose concentrations were also tested.

Results: Immunoblotting revealed that 4 days after transduction with antiAralar1 siRNA adenovirus, expression of Aralar1 was decreased by about $50 \%$. The reduction in Aralarl expression dramatically decreased glucose induced NADH generation. In parallel, depletion of Aralar1 decreased glucose oxidation by $20 \%$ compared to control cells $(p<0.01)$. ATP measurements showed a typical $20 \%$ increase in control cells stimulated with $15 \mathrm{mM}$ versus basal $2.5 \mathrm{mM}$ glucose $(\mathrm{p}<0.01)$. Glucose-evoked ATP generation was only $50 \%$ of controls in Aralar1 knockdown INS-1E cells $(\mathrm{p}<0.05)$. Parameters reflecting pathways up-stream of mitochondrial metabolism, such as glucose utilization and lactate production, were not modified by Aralarl knockdown. Despite the observed impairment in glucose induced mitochondrial activation, glucose stimulated insulin secretion was not significantly affected in Aralar1 knockdown INS-1E cells compared to controls. 
Conclusion: Down regulation of the malate-aspartate shuttle by knockdown of Aralar1 resulted in marked reduction in glucose induced mitochondrial activation. Unexpectedly, such impairment was not accompanied by decreased glucose stimulated insulin secretion. Ongoing experiments investigate possible compensatory mechanisms.

Supported by: Swiss National Science Foundation

\section{8}

Effects of diazoxide on beta cell mitochondrial function and mass are glucose-dependent

G. Larsson-Nyrén ${ }^{1}$, Z. Ma ${ }^{2}$, V. Grill ${ }^{3,2}$, A. Björklund ${ }^{2}$

${ }^{1}$ Integrative Medical Biology, Histology and Cell Biology, Umeå, Sweden, ${ }^{2}$ Dept. of Molecular Medicine and Surgery, Karolinska Institutet, Karolinska Hospital, Sweden, Rolf Luft Center of Diabetes Research, Stockholm, Sweden, ${ }^{3}$ Dept. of Cancer Research and Molecular Medicine, Trondheim, Norway, Norwegian University of Science and Technology, Trondheim, Norway

Background and aims: Diazoxide protects insulin secretion during chronic hyperglycemia and the drug has been considered for therapy. Beneficial effects could be secondary to opening effects of the drug on K-ATP channels in the plasma membrane. Also effects on beta cell mitochondria could potentially be important but are insufficiently clarified. Here we tested for effects of diazoxide on beta cell mitochondrial function and mass during culture conditions

Materials and methods: Rat pancreatic islets were used to investigate effects of diazoxide on mitochondrial membrane potential (MMP). Islets were cultured for $48 \mathrm{~h}$ at 5.5 or $27 \mathrm{mM}$ glucose with and without diazoxide $(325 \mu \mathrm{M})$. Islets were then loaded with Rhodamine $123(32 \mu \mathrm{M})$ for $10 \mathrm{~min}$ at $37^{\circ} \mathrm{C}$. Measurements were carried out with a SPEX fluorolog-2 system coupled to an inverted microscope. Results were standardized by reference to the fluorescence obtained after the addition of $10 \mu \mathrm{M}$ FCCP during the process of measurement.

Mitochondrial mass was assessed in isolated beta cells from $o b / o b$ islets. Cells were cultured for $48 \mathrm{~h}$ in 5.5 or $27 \mathrm{mM}$ glucose with or without diazoxide. After culture the distribution of the probe MitoTracker Red, which accumulates in active mitochondria, was measured using a Leica SP2 spectral laser scanning confocal microscopy equipped with a $250 \mathrm{~mW}$ argon/krypton laser.

Results: Culture of rat islets in $27 \mathrm{mM}$ vs. $5.5 \mathrm{mM}$ glucose expectedly increased (hyperpolarized) MMP ( $62 \pm 2.9$ vs. $68 \pm 2.3 \%$ of max FCCP, $p<0.001$, $\mathrm{n}=23)$. Pre-exposure of rat islets to diazoxide during the culture period $(48 \mathrm{~h})$ affected MMP in an opposite fashion when co-cultured with the high compared with the low glucose concentration. Thus, hyperpolarization was seen after co-culture with $27 \mathrm{mM}$ glucose $(-6 \pm 4.2 \%$ of $\max$ FCCP, $\mathrm{n}=7)$ but depolarization after co-culture at $5.5 \mathrm{mM}$ glucose $(+4 \pm 1.4 \%$ of $\max$ FCCP, $\mathrm{n}=10)$. The difference between the MMP effect of diazoxide when co-cultured with $27 \mathrm{mM}$ vs. when co-cultured with $5.5 \mathrm{mM}$ glucose was highly significant, $\mathrm{p}<0.001$. Culturing beta cells from islets of $o b / o b$ mice at elevated glucose $(27$ $\mathrm{mM}$ ) increased the total area of each beta cell (from $132 \pm 4, \mathrm{n}=29$ to $160 \pm 6$ arbitrary units, $\mathrm{n}=34, \mathrm{p}<0.005)$. The mitochondrial area increased in proportion to the total cell area (from $95 \pm 5, n=29$ to $110 \pm 6$ arbitrary units, $n=34$, $\mathrm{p}<0.1)$. Both total cell and mitochondrial areas were significantly reduced by previous diazoxide (to $132 \pm 6, \mathrm{n}=26, \mathrm{p}<0.001$ and $88 \pm 4, \mathrm{n}=26, \mathrm{p}<0.019$ vs. 27 $\mathrm{mM}$ glucose only). In contrast, pre-exposure to diazoxide during co-culture with $5.5 \mathrm{mM}$ glucose neither affected the total, nor the mitochondrial area.

Conclusion: The glucose concentration during culture determines the impact of diazoxide on MMP, indicating that diazoxide effects are governed by the degree to which mitochondria are energized. The glucose-dependent inhibitory effects by diazoxide on total and mitochondrial mass could reflect reduced demands for insulin biosynthesis and secretion. The glucose dependencies demonstrated here are in line with, and may be linked to the beneficial effects of diazoxide on insulin secretion which are restricted to sustained hyperglycemia.

\section{PS 029 Modulators of insulin secretion}

\section{9}

On the urotensin II inhibition of insulin release, study in the perfused rat pancreas

R.A. Silvestre, E.M. Egido, R. Hernández, J. Marco

Exp. Endocrinology, Hospital Universitario Puerta de Hierro, Madrid, Spain

Background and aims: Urotensin-II (UII), a somatostatin-like peptide, has been found to be a highly potent vasoactive peptide in some vascular beds. We have previously demonstrated that UII is present in the pancreas and, in the perfused rat pancreas, it exerts a dose-response inhibition of glucose-induced insulin secretion. Furthermore, specific UII antagonists (urantide and palosuran) potentiate glucose-induced insulin secretion, thus supporting the concept that endogenous UII is an insulinostatic peptide. To gain further insight into the insulinostatic effect of UII, we have investigated the effect of this peptide on glucose-induced insulin secretion in the rat pancreas perfused at normal and high glucose concentrations, as well as its effect on the insulin release elicited by non-glucose secretagogues (tolbutamide, $\mathrm{KCl}$ and exendin-4).

Materials and methods: The study was performed in the isolated perfused rat pancreas. Rat UII (Peptide Institute Inc., Japan) was infused at 1 and 10 $\mathrm{nM}$. Insulin secretion was stimulated by increasing perfusate glucose concentration (from 5.5 to $9 \mathrm{mM}$ and from 9 to $13 \mathrm{mM}$ ), by increasing perfusate $\mathrm{K}^{+}$ concentration (from 5 to $11 \mathrm{mM}$ ), and by infusing tolbutamide $(100 \mathrm{mg} / \mathrm{l})$ or exendin-4 $(1 \mathrm{nM})$. The effect of UII on exendin-4-induced insulin secretion was also studied in rats pretreated with pertussis toxin (Bordetella pertussis; Sigma-Aldrich, St. Louis, MO, USA; $3 \mu \mathrm{g} / 100$ g b.w., i.p., 3 days before experiments). Insulin was analyzed by RIA.

Results: $1 \mathrm{nM}$ UII significantly reduced the insulin secretion elicited by an increase in perfused glucose level from 5.5 to $9 \mathrm{mM}$, but not the insulin response induced by increasing glucose concentration from 9 to $13 \mathrm{mM}$. The insulin response to the latter glucose stimulus was reduced by a higher UII concentration $(10 \mathrm{nM})(\mathrm{)})$ UII, at $1 \mathrm{nM}$, significantly blocked the insulin response elicited by tolbutamide (incremental area: $377 \pm 73$ vs. $665 \pm 72 \mathrm{ng} / 15$ min in controls; $\mathrm{p}<0.05)$ but not by $\mathrm{KCl}(44.8 \pm 10.7$ vs. $55.5 \pm 9.8 \mathrm{ng} / 15 \mathrm{~min}$ in controls; $\mathrm{p}=0.486$ ). The insulin response to exendin- 4 , a GLP-1 analogue, was also reduced by $1 \mathrm{nM}$ UII $(34 \pm 6 \mathrm{vs}$. $76 \pm 9 \mathrm{ng} / 15 \mathrm{~min} ; \mathrm{p}<0.01)$. The insulinostatic effect of UII on exendin-4-induced insulin secretion, observed in control rats, was not observed in Pertussis toxin-pretreated rats (exendin-4+UII: $242 \pm 79$ vs. $198 \pm 75 \mathrm{ng} / 15 \mathrm{~min}$ in exendin- 4 experiments; $\mathrm{p}=0.697$ ).

Conclusion: Our results confirm the insulinostatic effect of UII on glucoseinduced insulin release at low glucose levels, and indicate that, at a high glucose concentration, the B cell is less sensitive to UII than at a low glucose level. Furthermore, UII inhibits tolbutamide-induced insulin secretion and does not affect the insulin response elicited by $\mathrm{K}^{+}$depolarization, thus suggesting that ATP-dependent $\mathrm{K}^{+}$channels might be implicated in its insulinostatic effect. Finally, UII also reduced exendin-4-induced insulin secretion, an effect not observed in Pertussis toxin-treated rats. Thus, the insulinostatic effect of UII can be accounted for, at least in part, by its inhibitory action on the adenylate cyclase/cAMP system via a Pertussis toxin-sensitive Gi protein. Supported by: Ministerio de Educación y Ciencia, FIS and Fundación Médica $M M A$, Spain

\section{0}

Role of cb1 and cb2 cannabinoid receptors on insulin secretion and glucose metabolism

R. Maldonado, C. Touriño, M.A. Martín, B. Rubi

Neurophar, Universitat Pompeu Fabra, Barcelona, Spain

Background and aims: Overactivation of the endocannaboid system is linked to the development of abdominal obesity and associated metabolic disorders, contributing to a high risk of cardiovascular disease and type II diabetes. However, the specific role of $\mathrm{CB} 1$ and $\mathrm{CB} 2$ receptors on glucose homeostasis and pancreatic endocrine function is still controversial. Here, we analyzed the role of cannabinoid receptors on glucose homeostasis in vivo by using the mixed CB1-CB2 agonist CP55,490, the CB1 antagonist rimonabant and the CB2 antagonist SR144528 in C57BL/6J mice. In addition, we tested the effects of CB1-CB2 receptor activation on glucose-stimulated insulin secretion ex vivo in isolated mouse pancreatic islets. 
Materials and methods: $\underline{I n}$ vivo experiments: Overnight $(15 \mathrm{~h})$ fasted C57BL/ 6J mice were treated i.p. with $3 \mathrm{mg} / \mathrm{kg}$ rimonabant, $3 \mathrm{mg} / \mathrm{kg} \mathrm{SR} 144528$ and $/$ or $0.1 \mathrm{mg} / \mathrm{kg} \mathrm{CP} 55,490$, at $\mathrm{t}=-25,-20$ and $-15 \mathrm{~min}$ respectively. At $\mathrm{t}=0$ mice were administered glucose $(2 \mathrm{~g} / \mathrm{kg})$. Blood samples were collected from the tail vein at $\mathrm{t}=0,15,30,45,60$ and $120 \mathrm{~min}$. Blood glucose was measured with Glucometer Elite device (Bayer, Leverkusen, Germany). Ex vivo experiments: Mouse pancreatic islets were isolated by collagenase digestion and cultured free-floating for $24 \mathrm{~h}$ in RPMI-1640 medium before use. For static incubations, islets were washed and preincubated in $2.8 \mathrm{mM}$ glucose Krebs-Ringer bicarbonate HEPES buffer ( $0.1 \%$ bovine serum albumin). After 30 min preincubation, islets were washed in the same buffer. Batches of 3 islets were hand-picked and incubated for $60 \mathrm{~min}$ at $37^{\circ} \mathrm{C}$ in $200 \mu \mathrm{l}$ at basal $(2.8 \mathrm{mM})$ or stimulatory $(11 \mathrm{mM})$ glucose concentrations in the presence or absence of CP55,490 (5 nM, $50 \mathrm{nM}, 500 \mathrm{nM}, 5$ microM). Insulin was measured with Ultrasensitive mouse insulin ELISA (Mercodia AB, Uppsala, Sweden).

Results: Simultaneous activation of CB1 and CB2 receptor by CP55,940 did not affect glucose tolerance in vivo. Accordingly, insulin secretion in mouse islets stimulated with $11 \mathrm{mM}$ glucose (5.2 fold vs. $2.8 \mathrm{mM}$ glucose, $\mathrm{p}<0.05$ ) was not modified by the administration of CP55,940 at concentrations up to 500. A higher concentrations of CP 55,940, 5 microM, was required to inhibit glucose stimulated insulin secretion $(-38 \% \mathrm{p}<0.05)$. The selective activation of CB1 receptor in vivo by the co-administration of CP55,490 and the CB2 antagonist SR144528 induced a right shift on the glucose tolerance curve, indicating glucose intolerance. This effect was suppressed by the CB1 antagonist rimonabant.

Conclusion: $\mathrm{CB} 1 \mathrm{AND}$ CB2 receptor modulate glucose metabolism in vivo and insulin release in pancreatic islets ex vivo. $\mathrm{CB} 1$ activation in vivo impairs pancreatic function and glucose homeostasis in mice and this effect is reverted by the $\mathrm{CB} 1$ antagonist rimonabant.

Supported by: sanofi-aventis

\section{1}

Zinc transporter ZnT-8 regulates insulin and glucagon secretion in Min6 and $\alpha$ TC1-9 pancreatic cell lines

S.C. Souza, L. Qiu, K.E. Inouye, J.J. Roix, H. Chen

Cardiovascular and Metabolic Diseases, Novartis Institutes for BioMedical

Research, Cambridge, United States

Background and aims: Alterations in zinc homeostasis are involved in the pathology of diabetes. In pancreatic islets, zinc plays an important role in insulin processing, storage, and secretion. Specifically, zinc is required for the formation of zinc-insulin hexamers within the secretory granules and packaging of insulin in this format is necessary for its efficient secretion. Control of zinc uptake and efflux is achieved through the action of specialized zinc transporter proteins. Recently, ZnT-8 (SLC30A8) was cloned and found to be, predominantly expressed in pancreatic $\beta$ cells. Over-expression of human ZnT-8 in INS1 cells increased the intracellular zinc content and glucosestimulated insulin secretion (GSIS) (J.Cell.Sci., 2006,119:4199). Of particular interest, whole-genome association studies have linked a non-synonymous variation of the ZnT-8 gene, R325W, with an increased risk of human type 2 diabetes (T2D) (Nature, 2007, 445:881). This study aimed to further explore the role of ZnT-8 in pancreatic clonal cells, and to understand how genetic variations in ZnT-8 affect the disease risk at cellular levels.

Materials and methods: Adenoviruses expressing human ZnT- 8 variants W325 and R325 (the risk allele) were used to transduce Min6 insulinoma and aTC1-9 glucagonoma cells. Mouse ZnT- 8 shRNAs were used to generate Min6 or aTC1-9 pancreatic stable cell lines with reduced ZnT-8 expression. Concentration of insulin and glucagon was determined using an Elisa assay. Sub-cellular localization of ZnT-8 variants proteins were assessed by confocal microscopy.

Results: In Min6 cells, over-expressed ZnT-8 W325 or R325 were co-localized with insulin. Moreover, over-expression of both variants resulted in a glucose dose-dependent stimulation of insulin secretion (up 80\% at $20 \mathrm{mM}$ of glucose). There was no change in cellular insulin content and mRNA levels. Conversely, 50\% knockdown of ZnT-8 in Min6 cells reduced basal insulin secretion by $\sim 40 \%$. This reduction was accompanied by a $\sim 1.7$ fold increase in intracellular insulin content. aTC1-9 cells expressed high levels of endogenous ZnT-8. Over-expression of ZnT-8 W325 or R325 in aTC1-9 cells decreased glucagon secretion by $\sim 50 \%$ and its cellular content by $\sim 30 \%$. mZnT-8 knockdown in aTC1-9 cells, on the other hand, led to an increase of glucagon mRNA $(\sim 50 \%)$ and secretion $(\sim 69 \%)$.

Conclusion: The present study demonstrates that ZnT-8 is not only critical for insulin secretion in $\beta$ cells, but also plays a complementary role in inhibit- ing glucagon secretion directly in a cells. Although we have yet to determine the differential effects of the wild type and risk allele of ZnT-8 on islet secretory functions, our data provides evidence that ZnT- 8 deficiency could adversely affect both insulin and glucagon metabolism, potentially contributing to an increased risk of T2D.

\section{2}

ZnT-3 knockout effects expression and glucose-mediated secretion of insulin in INS-1E cells

A.B. Petersen, K. Smidt, N.E. Magnusson, L. Egefjord, B. Brock, O. Schmitz, J. Rungby

Department of Pharmacology, University of Aarhus, Denmark

Background and aims: Insulin secreting $\beta$-cells contain large amounts of zinc which is necessary for the formation of stable insulin hexamers in secretory vesicles. Rising evidence points to a connection between zinc transporters (ZnTs) and diabetes. It has recently been shown that ZnT-8 functions as an antigen in the development of Type 1 diabetes and that mutations in the same gene is associated with Type II diabetes. Transporters of the Slc30Afamily (ZnT-1 to 10) regulate zinc efflux from the cytoplasm. ZnT-3 has been identified in $\beta$-cells, the expression of ZnT-3 is glucose sensitive and ZnT-3 KO mice have decreased glucose tolerance. In this study we used siRNA-mediated knockdown to further investigate the role of ZnT-3 in insulin secretion from $\beta$-cells.

Materials and methods: $100 \mathrm{nM}$ siRNA concentration was used to perform ZnT-3 knockdown in INS-1E cells. Knockdown of ZnT-3 and insulin expression at mRNA level was detected using Q-PCR. Following transfection and 48 hours of recovery the knockout and control cells were stimulated with various concentrations of glucose $(2,0 ; 6,6 ; 16,7$ and 24,6 mM) for 2 hours. Intracellular and secreted insulin following stimulation was then measured. Results: Following transfection the expression of ZnT-3 was reduced to 57,86 $\%(\mathrm{p}<0,05)$ compared to mock-transfected controls. Insulin expression in cells transfected with ZnT-3 siRNA showed a fold change of $-1,796$ ( $\mathrm{p}<0,05)$ compared to mock-transfected samples. Insulin secretion data was analysed using 2-way ANOVA. ZnT-3 knockdown had a negative effect on the secretion of insulin at $16,7 \mathrm{mM}$ glucose (figure) . At $25 \mathrm{mM}$ the difference was abolished. The intracellular insulin content was unchanged in ZnT-3 transfected cells compared to the mock-transfected after the 2 hour glucose stimulaton.

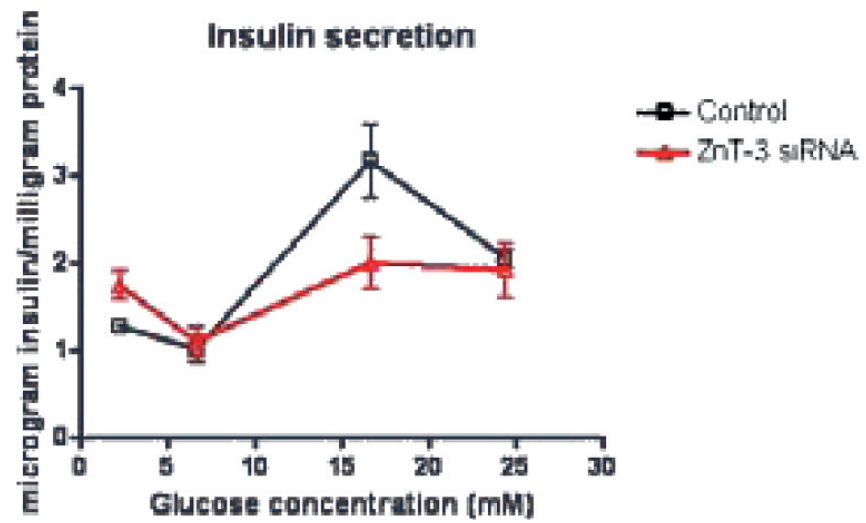

Conclusion: The influence of ZnT-3 on insulin expression and secretion has until now not been described. It has been shown that ZnT-3 is glucose-sensitive. The gene is up-regulated at high glucose levels when increased amounts of secreted insulin are needed. In this study we have shown that siRNA mediated knockdown of the ZnT-3 zinc-transporter in INS-1E cells results in decreased expression and glucose-mediated secretion of insulin at high glucose levels, although at $24,6 \mathrm{mM}$ we found no difference, most likely due to glucose-toxicity. These findings add to the rising evidence demonstrating a connection between ZnTs and insulin secretion.

Supported by: Generalkonsul Friedrich Bøhm og datter Else Bøhms Fond and Kornings Fond 


\section{3}

Dopamine transporter modulates glucose-stimulated insulin secretion in mouse pancreatic islets

B. Rubí, R. Maldonado

Departament de Ciències Experimentals i de la Salut, Laboratori de

Neurofarmacologia, Barcelona, Spain

Background and aims: Recent studies highlight the relevance of widely known neurotransmitters, such as dopamine, in the modulation of various physiological functions outside of the CNS. In pancreatic islets, dopamine inhibits insulin secretion. Dopamine receptors are present in insulin granules of rodent and human beta cells. Dopamine D2 receptor polymorphisms are associated with obesity and type 2 diabetes and are predictive of body mass index categories. However, the origin and mechanism of action of dopamine acting in pancreatic islets is not yet fully understood. Here, we investigated whether dopamine transport into pancreatic islets is necessary for the inhibitory actions of dopamine. For this purpose, we analyzed the effects of the dopamine transporter inhibitor nomifensine on glucose stimulated insulin secretion in isolated mouse islets.

Materials and methods: Mouse pancreatic islets were isolated by collagenase digestion and cultured free-floating for $24 \mathrm{~h}$ in RPMI-1640 medium before use. For static incubations, islets were washed and preincubated in $2.8 \mathrm{mM}$ glucose Krebs-Ringer bicarbonate HEPES buffer $(0.1 \%$ bovine serum albu$\mathrm{min})$. After the $30 \mathrm{~min}$ preincubation, islets were washed in the same buffer. Batches of 3 islets were hand-picked and incubated for $60 \mathrm{~min}$ at $37^{\circ} \mathrm{C}$ in 200 microl at basal $(2.8 \mathrm{mM})$ or stimulatory $(11 \mathrm{mM})$ glucose concentrations in the presence or absence of 10 microM dopamine and/or 10 microM nomifensine. Insulin secretion was determined using an ultrasensitive mouse insulin ELISA (Mercodia AB, Uppsala, Sweden).

Results: Mouse islets were washed after the $30 \mathrm{~min}$ low glucose preincubation and insulin secretion was stimulated with $11 \mathrm{mM}$ glucose ( 7.5 fold vs. 2.8 $\mathrm{mM}$ glucose, $\mathrm{p}<0.05)$. Ten microM dopamine resulted in inhibition of glucose-stimulated insulin secretion (56\% inhibition vs. $11 \mathrm{mM}$ glucose control, $\mathrm{p}<0.05)$. Blockade of dopamine uptake by 10 microM nomifensine rescued the response to glucose $(132 \%$ increase vs. dopamine alone, $\mathrm{p}<0.05)$, whereas 10 microM nomifensine alone had no effect on glucose-stimulated insulin secretion in mouse islets. Intriguingly, we observed that islets that were not washed after the $30 \mathrm{~min}$ low glucose preincubation were much less responsive to glucose (3.6 fold increase vs. $2.8 \mathrm{mM}$ glucose, $\mathrm{p}<0.05$ ). The same islets responded potently to glucose in the presence of 10 microM nomifensine (11.5 fold increase vs. $2.8 \mathrm{mM}$ glucose, $\mathrm{p}<0.05)$. Therefore nomifensine was able to rescue the basal dopamine dependent inhibition of glucose response.

Conclusion: Our results show that dopamine requires to be taken up by pancreatic islet cells in order to inhibit insulin secretion. Additionally, our data suggest that uptake of pancreatic tissue-derived dopamine decreases islet glucose-response. The drug of abuse cocaine and several pharmacological compounds, such as methylphenidate and bupropion, are inhibitors of DAT. Knowledge of the role of DAT on pancreatic endocrine function might help to understand some of the metabolic effects of these substances. Supported by: EFSD/AstraZeneca research Award and (Ministerio de Educación y Ciencia)

\section{4}

SK4 channel knock-out improves glucose homeostasis by influencing the function of pancreatic beta cells

M. Düfer, B. Gier, D. Wolpers, P. Ruth, P. Krippeit-Drews, G. Drews

Pharmazeutisches Institut, Universität Tübingen, Germany

Background and aims: Besides ATP-dependent $\mathrm{K}^{+}$channels, the key regulators of glucose-stimulated insulin secretion, pancreatic beta-cells express voltage- and $\mathrm{Ca}^{2+}$-regulated $\mathrm{K}^{+}$channels. To elucidate the significance of $\mathrm{Ca}^{2+}$ activated $\mathrm{K}^{+}$channels of intermediate conductance (SK4, IK1) for stimulussecretion coupling we investigated whether SK4 channel knock-out mice (SK4-KO) show alterations in beta-cell function and/or glycemic control.

Materials and methods: Glucose tolerance and insulin sensitivity were measured in male wildtype (WT) and SK4-KO mice at an age of 12 and 24 weeks by intraperitoneal (i.p.) injection of $2 \mathrm{~g} / \mathrm{kg} \mathrm{BW}$ glucose and 0.7 I.U./kg BW insulin, respectively. Membrane potential and ion current recordings were performed in the perforated-patch configuration and $\left[\mathrm{Ca}^{2+}\right]_{\mathrm{c}}$ was determined using fluorescence techniques.

Results: Blood glucose concentrations in the fed and fasted state were similar in SK4-KO and WT mice at an age of 12 and 24 weeks (fasted: $6.6 \pm 0.5$ vs.
$5.8 \pm 0.6 \mathrm{mM}$, fed: $10.4 \pm 0.8$ vs. $11.1 \pm 1.1 \mathrm{mM}, \mathrm{n}=5-6,12$ weeks, respectively). However, SK4-KO animals showed a significantly lower increase in blood glucose concentration after i.p. glucose challenge (2-hour plasma glucose concentration of $6.2 \pm 0.5 \mathrm{mM}$ in SK4-KO vs. $9.5 \pm 1.2 \mathrm{mM}$ in WT animals, 12 weeks, $\mathrm{n}=5, \mathrm{p}<0.05)$. As the improved glucose tolerance was not accompanied by an increased insulin sensitivity $(n=5-6)$ we investigated whether SK4$\mathrm{KO}$ influences beta-cell function. SK4-KO did not alter the resting membrane potential in $0.5 \mathrm{mM}$ glucose (SK4-KO: $-66 \pm 2 \mathrm{mV}, \mathrm{n}=6$, WT: $-68 \pm 1 \mathrm{mV}, \mathrm{n}=3$ ). In the presence of $15 \mathrm{mM}$ glucose the frequency of $\mathrm{Ca}^{2+}$ action potentials was significantly elevated in SK4-KO beta-cells (SK4-KO: 97 $\pm 5 / \mathrm{min}, \mathrm{n}=53$ vs. WT: $74 \pm 12 / \mathrm{min}, \mathrm{n}=18, \mathrm{p}<0.05)$. In addition, the threshold for glucose-induced membrane depolarization and $\mathrm{Ca}^{2+}$-influx was shifted to lower glucose concentrations. The glucose concentration at which $50 \%$ of all cells tested showed a rise in $\left[\mathrm{Ca}^{2+}\right]$ was $6.37 \mathrm{mM}$ (95\% confidence interval CI: 6.09 to $6.68 \mathrm{mM}$ ) for WT beta-cells and was reduced to $5.67 \mathrm{mM}$ (95\% CI: 5.29 to $6.05 \mathrm{mM}$ ) for SK4-KO beta-cells. Importantly, in WT beta-cells that were stimulated with $15 \mathrm{mM}$ glucose $\left[\mathrm{Ca}^{2+}\right]_{c}$ was increased by addition of the SK4 channel blocker TRAM-34 (1 $\mu \mathrm{M}, \mathrm{n}=5)$.

Conclusion: These data implicate that SK4 channels participate in the regulation of beta-cell stimulus-secretion coupling. The ablation of SK4 channels increases beta-cell responsiveness to glucose stimulation and improves glucose tolerance in vivo without alterations in insulin sensitivity. As $\left[\mathrm{Ca}^{2+}\right]_{c}$ of WT beta-cells could be augmented by blocking SK4 channels pharmacologically, SK4 channels might provide a target to improve beta-cell function in patients with impaired glucose homeostasis. Supported by: Deutsche Forschungsgemeinschaft

\section{5}

Chronic exposure to high leucine decreases glucose-stimulated insulin secretion probably by AMP-activated protein kinase mediating pathway in INS-1 cells

X.J. Zhang ${ }^{1}$, Y.T. Li $^{2}$, W. Xin ${ }^{3}$, L. Gao ${ }^{3}$, J.J. Zhao

${ }^{1}$ Endocrinology, ${ }^{2}$ Clinical Laboratory, Provincial Hospital affiliated to Shandong University, Jinan, China, ${ }^{3}$ Scientific Center, Jinan, China

Background and aims: AMP-activated protein kinase (AMPK) reacts in modulating insulin secretion.Glucokinase( GCK) plays a key role in glucose metabolism, particularly glucose-stimulated insulin secretion(GSIS). Studies have shown AMPK regulates GCK at high glucose circumstance. Based on that, we supposed that there existed an regulatory effect of AMPK on GCK under physiological condition. Furthermore, how AMPK regulating GCK exposed to high leucine was our interest as well. Pancreatic duodenal homeobox (PDX) - 1 can transactivate the expression of $\beta$-cell-specific genes, such as GCK, GLUT2. We assumed that AMPK regulated GCK / GLUT2 via PDX-1. It is reported that chronic exposure of pancreatic $\beta$-cells to high concentration of leucine impairs GSIS in vitro. However, the exact mechanism is unclear. Some studies showed that exposure to leucine related with the expression of AMPK and GCK/GLUT2. Hence, we hypothesized that chronic exposure to leucine decreased GSIS associated with the assumed pathway above.In the present study, we firstly tested if AMPK regulated GCK / GLUT2 via PDX-1 in beta cells under physiological condition, then studied if chronic exposure to leucine decreased GSIS associated with the pathway.

Materials and methods: INS-1 cells were cultured in RPMI 1640 with and without $0.5 \mathrm{mM}$ AICAR (an AMPK activator) or $10 \mu \mathrm{M}$ Compound C (an AMPK inhibitor) for 48 hours. In addition, DN-Pdx-1 28 cells, another INS1 stable cell line, allowing the absent expression of PDX-1 inducing with 500 $\mathrm{ng} / \mathrm{ml}$ doxycycline, were treated with and without $0.5 \mathrm{mM}$ AICAR or $10 \mu \mathrm{M}$ Compound C for 48 hours as well. Then INS- 1 cells were treated with and without the elevated concentrations of leucine (10 mM, $20 \mathrm{mM}$ or $40 \mathrm{mM})$ for 48 hours. After that, the GSIS and intracellular insulin contents were determined by radioimmunoassay. AMPK, PDX-1, GCK and GLUT2 protein expression was detected by Western blotting.

Results: (1) In INS-1 cells, relative to the control, AICAR treatment decreased the expression of PDX-1 and its downstream targets, GCK and GLUT2 $(\mathrm{p}<0.05)$. While Compound C treatment had the opposite effects. (2) In DN-Pdx-1 $1^{\sharp} 8$ cells, we observed that there was nearly no PDX-1 protein band occurence under induced condition. In agreenmet with this, weaker bands $(\mathrm{p}<0.05)$ were seen in GLUT2 and GCK expression compared with those under non-induced condition. Under non-induced condition, AICAR or Compound C had the same effects as those observed in the INS-1 cells above, however, under induced condition, whatever AICAR or Compound C could not affect the expression of GCK and GLUT2.(3) When the INS-1 cells were exposed to leucine, there was not difference of the GSIS levels and insu- 
lin content between control and $10 \mathrm{mM}$ or $20 \mathrm{mM}$ leucine. However, exposed to $40 \mathrm{mM}$ leucine decreased the GSIS levels by $45 \%(\mathrm{p}<0.01)$ at high glucose stimulation and dimished the intracellular insulin contents by $32 \%(\mathrm{p}<0.01)$. It also increased $\mathrm{p}-\mathrm{AMPK}$ activation $(\mathrm{p}<0.05)$ and decreased PDX-1, GLUT2 and GCK expression $(\mathrm{p}<0.05)$.

Conclusion: In conclusion, the present study demonstrates that in pancreatic $\beta$-cells, AMPK regulates GCK / GLUT2 via PDX-1 and chronic exposure to leucine for $48 \mathrm{~h}$ decreases GSIS and insulin content probably associated with the changes of the signaling pathway.

\section{6}

STAT3 is the primary signalling pathway used by CNTF in rat pancreatic islets

L.F. Rezende ${ }^{1}$, A. Negro ${ }^{2}$, F. Langone ${ }^{1}$, A.C. Boschero ${ }^{1}$

${ }^{1}$ Physiology and Biophysics, UNICAMP, Campinas, Brazil, ${ }^{2}$ Department of Biochemistry, Universitá di Padova, Padova, Italy

Background and aims: CNTF is a cytokine that promotes survival and/or differentiation in many cell types, including pancreatic islet-cells, but until now, the signaling mechanisms of CNTF effects in pancreatic islets are not known. In this work, we elucidated the underlying mechanisms of CNTF signaling in cultured neonatal pancreatic islets.

Materials and methods: Pancreatic islets from neonatal rats were isolated by the collagenase method and cultured for 3 days in RPMI medium without (CTL) or with $1 \mathrm{nM}$ of CNTF (CNTF). The medium also contained, when required, specific inhibitors of the PI3K, MAPK and JAK/STAT3 pathways. mRNA expression (RT-PCR) and protein phosphorylation (Western blot) of Akt, ERK1/2 and STAT3, and SOCS-3 (RT-PCR and Western-blot), as well as glucose-stimulated insulin secretion (GSIS) (Radioimmunoassay), were analysed.

Results: Results: Akt, ERK1 and STAT3 mRNA expression, as well as phosphorylated Akt and ERK1/2, was not affected by CNTF treatment. CNTF increased cytoplasmatic and nuclear phosphorylated STAT3, and the mRNA and protein expression of SOCS3 (target-gene of STAT3 activation). These effects were blocked by the STAT3 inhibitor, curcumin. Finally, GSIS was lower in CNTF-treated islets and this inhibitory effect was counteracted by curcumin, but not by the MAPK inhibitor, PD98059, nor by the PI3K inhibitor, wortmannin.

Conclusion: CNTF chronically affects pancreatic islets, activating STAT3, but not, MAPK and PI3K pathways, increasing SOCS3 expression and impairing GSIS. These findings demonstrate that STAT3 is the primary signaling pathway activated by CNTF in rat pancreatic islets.

Supported by: FAPESP/CNPq

\section{7}

Identification of putative prion protein interacting proteins in islet beta cells

A. Strom ${ }^{1}$, G.-S. Wang ${ }^{1}$, A.W. Stuke ${ }^{2}$, F.W. Scott ${ }^{1}$;

${ }^{1}$ Chronic Disease Program, Ottawa Health Research Institute,

Ottawa,Canada, ${ }^{2}$ Virology and Immunology, German Primate Centre,

Göttingen, Germany

Background and aims: Cellular prion protein $\left(\operatorname{PrP}^{\mathrm{C}}\right)$ is a glycoprotein ubiquitously expressed in neuronal and neuroendocrine cells. In the pancreas, $\operatorname{PrP}^{\mathrm{C}}$ is specifically and abundantly expressed in islets. However, the function of $\operatorname{PrP}^{\mathrm{C}}$ in pancreatic islets and specifically in $\beta$-cells is not known. We recently investigated expression of $\operatorname{PrP}^{\mathrm{C}}$ in the pancreas of diabetes-prone BioBreeding (BBdp) rats compared with control strains. There was strong $\operatorname{PrP}^{\mathrm{C}}$ expression in all hormone secreting cells within the islets of Langerhans and cytosolic $\mathrm{PrP}^{\mathrm{C}}$ aggregates, found exclusively in $\mathrm{B}$-cells, were more frequent in BBdp rats compared with the BB control strain $(P<0.0001$, at 100 days of age). Furthermore, $\operatorname{PrP}^{\mathrm{C}}$ expression was suppressed in $\mathrm{B}$-cells of Sprague Dawley rats under hyperglycemic conditions and a pronounced $\operatorname{PrP}^{\mathrm{C}}$ aggregation was observed while the blood glucose returned to physiological level. These results suggested that the function of $\mathrm{PrP}^{\mathrm{C}}$ in $\mathrm{B}$-cells is closely related to glucoregulation and/or insulin secretion. The aim of the present study was to identify potential $\operatorname{PrP}^{\mathrm{C}}$ binding proteins in $B$-cells that might reveal $\mathrm{PrP}^{\mathrm{C}}$ related pathways.

Materials and methods: Extracts of soluble (cytosolic) and insoluble (membrane) proteins from rat insulinoma INS-1E cells (kind gift of Dr. P. Maechler; Geneva, Switzerland) were separated by SDS-PAGE and immunoblotted onto nitrocellulose membranes. Far-Western immunoblotting, also referred to as protein overlay assay, was performed in which membranes were blocked, then probed with $200 \mathrm{ng} / \mathrm{ml}$ of recombinant $\operatorname{PrP}^{\mathrm{C}}\left(\mathrm{rPrP}^{\mathrm{C}}\right)$ (Prionics, Switzerland), followed by incubation with primary anti- $\operatorname{PrP}^{\mathrm{C}} 4 \mathrm{~F} 2$ antibody and secondary anti-mouse/HRP antibody. Candidate $\mathrm{rPrP}^{\mathrm{C}}$ interacting proteins were excised and analyzed by MALDI-TOF mass spectrometry. Full length and Cterminally truncated constructs of $\mathrm{H}^{\circ}$ (kind gift of Dr. J. Hansen; Colorado State University) were expressed in BL21(DE3)LysS E.coli strain.

Results: Probing protein extracts of INS-1E cells with $\mathrm{rPrP}^{\mathrm{C}}$ revealed several candidate interacting proteins. One of the candidate $\operatorname{PrP}^{\mathrm{C}}$ interacting proteins was identified by mass spectrometry as histone H3. To confirm the specificity of $\mathrm{rPrP}^{\mathrm{C}}$ binding to $\mathrm{H} 3$ we immunoblotted purified recombinant histones $\mathrm{H} 1^{\circ}, \mathrm{H} 2 \mathrm{~A}, \mathrm{H} 2 \mathrm{~B}, \mathrm{H} 3$ and $\mathrm{H} 4$ (New England BioLabs) and probed the blot with $\mathrm{rPrP}^{\mathrm{C}}$. Recombinant $\operatorname{Pr}^{\mathrm{C}}$ showed strong binding to the histone $\mathrm{H} 3$ and also to the linker histone $\mathrm{H} 1^{0}$. Using different $\mathrm{C}$-terminal truncation mutants of histone $\mathrm{H}^{0}$ expressed in E.coli we demonstrated by far-Western immunoassay that $\mathrm{rPrP}^{\mathrm{C}}$ binds to the $\mathrm{C}$-terminal domain of $\mathrm{H}^{0}$ (aa 163-193). Conclusion: Recombinant $\operatorname{PrP}^{\mathrm{C}}$ interacts in vitro with two different structural proteins from chromatin. These findings raise the possibility that $\operatorname{Pr}^{\mathrm{C}}$ participates in epigenetic gene silencing or activation in B-cells. Further investigations must confirm these interactions and evaluate their physiological effects. The elucidation of the function of $\mathrm{PrP}^{\mathrm{C}}$ in $\beta$-cells may provide new mechanisms through which this unique cell type responds to and maintains blood glucose levels.

Supported by: JDRF, CIHR and CDA 


\section{PS 030 Lipid effects in the beta cell}

\section{8}

The monounsaturated fatty acid, palmitoleate, attenuates the PERK signalling pathway of ER stress by regulating the phosphorylation of eIF2 $\alpha$

E. Diakogiannaki, N.G. Morgan

Endocrine pharmacology, Peninsula Medical School, Plymouth, United Kingdom

Background and aims: Among the various factors which have been implicated in the loss of beta-cell mass in type 2 diabetes are the elevated levels of free fatty acids (FA). FA species differ considerably in their ability to regulate beta-cell viability, with long chain saturated FA (such as palmitate (C16:0)) being cytotoxic whereas long chain monounsaturated species (e.g. palmitoleate $(\mathrm{C} 16: 1))$ are well tolerated. The most striking effect occurs during exposure to combinations of palmitate and palmitoleate. Under these conditions, the cytotoxicity of palmitate is prevented and the beta-cells remain viable and proliferate normally. Studies of cell morphology have revealed that palmitate causes alterations to the cellular architecture and, in particular, to the morphology of the endoplasmic reticulum (ER). By contrast, palmitoleate is much less disruptive. The combination of the two FA results in changes that are less extensive than those seen with palmitate alone. These results suggest that alterations to ER homeostasis could be involved in the differential effects of FA on cell viability. Therefore, the aim of this study was to investigate whether the cytoprotective effect of palmitoleate is associated with an amelioration of the beta-cell ER stress response.

Materials and methods: The rat pancreatic beta-cell line BRIN-BD11 was employed and the expression of markers associated with ER stress was detected by Western blotting following exposure to fatty acids.

Results: Palmitate dose-dependently $(0.05-0.25 \mathrm{mM})$ induced apoptosis of BRIN-BD11 cells and it augmented the expression of the ER associated proapoptotic factor CHOP-10 over the same concentration range. By contrast, palmitoleate (either alone or in combination with palmitate) was not toxic and did not up-regulate CHOP-10. Markers of the PERK signalling pathway, such as the transcription factor ATF4 and the phosphorylated form of eIF2 $\alpha$, were highly expressed in palmitate treated cells while the addition of palmitoleate minimised this response. Surprisingly, the major ER chaperone GRP78 and the phosphorylated form of PERK were not induced in FA treated cells although both proteins were increased after exposure to the established ER stress inducer tunicamycin. Similar to its actions in palmitate-treated cells, palmitoleate attenuated the induction of CHOP-10, ATF4 and phosphoeIF2 $\alpha$ in cells exposed to tunicamycin. However, it failed to alter the levels of GRP78 in response to tunicamycin. Palmitoleate also significantly improved the viability of cells treated with tunicamycin. Based on these observations it was hypothesised that the protective effect of palmitoleate may derive from its ability to regulate the level of eIF2 $\alpha$ phosphorylation either by inhibiting a relevant kinase or by enhancing the activity of an eIF2 $\alpha$ phosphatase. When cells were exposed to the phosphatase inhibitor salubrinal, the extent of eIF2 $\alpha$ phosphorylation was increased. Palmitoleate minimised this effect, consistent with the possibility that it may regulate the activity of an eIF2 $\alpha$ kinase. Conclusion: Palmitoleate prevents the expression of markers of ER stress stimulated by palmitate and tunicamycin in beta-cells. This cytoprotection may be attributable to the inhibition of an eIF2a kinase(s).

Supported by: Boehringer Ingelheim, Germany

\section{9}

Effects of exendin-4 on free fatty acid-induced endoplasmic reticulum stress and apoptosis in pancreatic beta cells

D.A. Cunha, L. Ladrière, F. Ortis, M. Igoillo-Esteve, D.L. Eizirik, M. Cnop Laboratory of Experimental Medicine, Université Libre de Bruxelles, Belgium

Background and aims: Chronic exposure of pancreatic beta cells to saturated free fatty acids (FFA) causes endoplasmic reticulum (ER) stress and apoptosis, and may contribute to beta cell loss in type 2 diabetes. It has been suggested that GLP-1 receptor agonists, such as exendin-4, preserve beta cell function and mass in part by protecting beta cells against ER stress via upregulation of ATF4-CHOP-GADD34 and consequent dephosphorylation of eIF2 $\alpha$. We have previously shown that salubrinal (sal), a selective inhibitor of eIF2 $\alpha$ dephosphorylation, is deleterious to beta cells by potentiating FFA in- duction of ATF4 and CHOP. The aim of this study was to evaluate the effects of exendin -4 on FFA \pm sal-induced ER stress in beta cells.

Materials and methods: INS-1E or FACS-purified rat beta cells were exposed to the chemical ER stressors thapsigargin (thap, $1 \mu \mathrm{M}$ ) and the FFA oleate or palmitate $(0.5 \mathrm{mM}, 1 \% \mathrm{BSA})$ in the presence or absence of exendin-4 (Ex, 10$50 \mathrm{nM}$ ) or the cAMP generator forskolin $(\mathrm{Fo}, 20 \mu \mathrm{M})$. Apoptosis was detected by Hoechst/propidium iodide staining and by Cell Death Detection ELISA${ }_{\text {PLUS }}$ assay. mRNA expression was analyzed by real time RT-PCR, and protein expression and eIF $2 \alpha$ phosphorylation by Western blot. CHOP knockdown was achieved by siRNA transfection.

Results: Ex or Fo protected primary beta cells against thapsigargin (thap $26 \pm 1 \%$, thap + Ex $17 \pm 1 \%$, thap + Fo $13 \pm 3 \%$ apoptotic cells after $24 \mathrm{~h}, \mathrm{n}=3$ $4, \mathrm{p}<0.05$ vs thap alone; apoptosis in control condition $9 \pm 2 \%$ ), and against oleate (oleate $23 \pm 2 \%$, oleate $+\mathrm{Ex} 15 \pm 3 \%$, oleate + Fo $15 \pm 3 \%$ apoptosis after 3 days, $\mathrm{n}=10, \mathrm{p}<0.05$ ) but not palmitate. Ex or Fo also protected primary beta cells against the combination of oleate + sal (oleate + sal $55 \pm 6 \%$, oleate + sal + Ex $43 \pm 6 \%$, oleate $+\mathrm{sal}+$ Fo $30 \pm 5 \%, \mathrm{n}=7-9, \mathrm{p}<0.05)$ or palmitate + sal (palmitate + sal $65 \pm 7 \%$, palmitate + sal + Ex $43 \pm 6 \%$, palmitate + sal + Fo $43 \pm 8 \%$, $\mathrm{p}<0.05)$. Findings were similar in INS-1E cells $(24 \mathrm{~h})$ and also when evaluating apoptosis by ELISA. Fo tended to increase ATF4 and CHOP mRNA and protein expression in INS-1E cells exposed to FFA + sal for 7-14 h, and it increased GADD34 mRNA after $14 \mathrm{~h}$ (respectively 2.2- and 2.4-fold for oleate + sal and palmitate + sal compared to FFA + sal alone, $\mathrm{n}=5-6, \mathrm{p}<0.05)$ whereas the increase by Ex did not reach significance. Sal augmented eIF2 $\alpha$ phosphorylation (oleate + sal 1.6-fold of control compared to 0.9 -fold for oleate, palmitate + sal 3.6-fold of control compared to 1.5-fold for palmitate at $6 \mathrm{~h}, \mathrm{n}=4, \mathrm{p}<0.05$ ) and this was decreased by Ex or Fo. To examine whether Ex or Fo dephosphorylated eIF2 $\alpha$ via enhanced ATF4-CHOP-GADD34 signaling, we knocked down CHOP. CHOP inhibition decreased palmitate + sal-induced apoptosis (from $30 \pm 3 \%$ to $17 \pm 1 \%$ apoptotic INS-1E cells after $14 \mathrm{~h}, \mathrm{n}=5, \mathrm{p}<0.05)$, but it did not alter the protective effects of Fo against palmitate + sal. Fo prevented sal-mediated eIF2 $\alpha$ phosphorylation very early on (20 min-6 h), confirming that Ex and Fo do not protect beta cells against lipotoxic ER stress via enhanced negative feedback through CHOP.

Conclusion: Ex and Fo partially decrease FFA \pm sal-induced apoptosis in beta cells. This anti-apoptotic effect is not due to enhanced CHOP signaling, as previously proposed. Activation of the GLP-1 receptor may instead directly modulate eIF2 $\alpha$ phosphorylation and thereby improve beta cell viability. Supported by: EU, Belgian Lipid Club research fellowship, Octave, Dupont Foundation (Royal Academy of Science of Belgium), Fonds National de la Recherche Scientifique, Fonds de la Recherche Scientifique Médicale and Actions de Recherche Concertées de la Communauté Française, Belgium

\section{0}

Mono-oleoyl-glycerol acutely stimulates basal insulin secretion and rapidly inhibits glucose-induced insulin secretion from rat pancreatic islets

J.T. Deeney ${ }^{1}$, M. Saadeh ${ }^{2}$, T.C. Ferrante ${ }^{2}$, A. Kane ${ }^{2}$, B.E. Corkey ${ }^{1}$

${ }^{1}$ Department of Medicine, Boston University School of Medicine, United States, ${ }^{2}$ Department of Medicine, Boston Medical Center, United States

Background and aims: Chronic exposure (24-72 hrs) of pancreatic islets to elevated glucose and fatty acid (FA) leads to glucolipotoxicity (GLT) characterized by basal insulin hypersecretion and impaired glucose-stimulated insulin secretion (GSIS) mimicking type 2 diabetes. The aim of this study was to study the effects of an acute increase in lipids on insulin secretion from pancreatic $\beta$-cells.

Materials and methods: Insulin secretion was measured from batch incubated and perifused rat pancreatic islets as well as batch incubated INS-1 832/13 cells. Glycerol and long-chain acyl-CoA (LC-CoA) were measured enzymatically, intracellular $\mathrm{Ca}^{2+}$ was measured in single cells loaded with fura 2-AM and $\mathrm{O}_{2}$ consumption was measured using a multi-well plate $\mathrm{O}_{2}$ sensor.

Results: Mono-oleoyl-glycerol (MOG) (0.1-1 mM) acutely stimulated basal insulin secretion from rat pancreatic islets and INS-1 832/13 cells in a dose dependent manner without increasing intracellular $\mathrm{Ca}^{2+}$. Diazoxide $(0.4$ $\mathrm{mM}$ ) did not prevent the acute stimulatory effect of MOG confirming that the effect was independent of the $\mathrm{K}_{\text {ATP }}$-dependent (triggering) pathway of secretion. How MOG enhances insulin secretion is currently under investigation. MOG has been reported to increase diacylglycerol (DG) through inhibition of DG kinase (DGK). The DGK inhibitor R59949 (1-5 $\mu \mathrm{M})$ did not increase basal insulin secretion in INS-1 cells indicating that increasing DG through this pathway does not stimulate insulin secretion. MOG was however metabolized by INS- 1 cells, which was indicated by increased glycerol 
production and a 6-fold increase in LC-CoA within $30 \mathrm{~min}$. The metabolism of MOG did not result in an increase in basal respiration in INS-1 cells, which was consistent with a non-triggering pathway for increased insulin secretion. Rat islets perifused with MOG $(0.2 \mathrm{mM})$ exhibited a large transient increase in basal insulin secretion (peak value increased 10-fold over basal), which remained 2-fold elevated compared to the pre-MOG basal level. Glucose (11 $\mathrm{mM}$ ) failed to stimulate insulin release from the same islets pre-exposed to MOG for $30 \mathrm{~min}$. $\mathrm{KCl}(40 \mathrm{mM})$ stimulated insulin secretion was reduced by $90 \%$ after MOG exposure. Consistent with this inhibition of secretion $30 \mathrm{~min}$ of MOG exposure inhibited the glucose stimulated $\mathrm{Ca}^{2+}$ increase in dissociated rat pancreatic islets by $75 \%$ (average of 30 cells) and the $\mathrm{KCl}$ stimulated $\mathrm{Ca}^{2+}$ increases in dissociated rat pancreatic islets and INS- 1 cells. The effects of MOG to inhibit insulin secretion were chain length dependent with monodecanoyl-glycerol having little inhibitory effect after 30 min preincubation. Conclusion: B-cell glucolipotoxicity characterized by basal hypersecretion and impaired GSIS can be rapidly mimicked by MOG exposure in the absence of elevated glucose and FA. The rapidity of the affects provide a model to examine the effects of elevated lipids on insulin secretion independent of genetic alterations that occur over the time required to achieve GLT by more conventional means. An acute increase in LC-CoA may lead to both increased basal insulin secretion and subsequent decreased GSIS in rat pancreatic islets.

Supported by: NIH

\section{1}

The once-daily human GLP-1 analogue liraglutide preserves pancreatic beta cells in diabetic $d b / d b$ mice through both acute and chronic action mechanisms

M. Shimoda, Y. Kanda, K. Tawaramoto, M. Hashiramoto, M. Matsuki, K. Kaku

Diabetes and Endocrine Division, Kawasaki Medical School, Okayama, Japan

Background and aims: GLP-1 increases the pancreatic beta-cell mass by stimulating cell proliferation and inhibiting apoptosis. In this study, we investigated the molecular mechanism for the proliferative effect of liraglutide on beta-cell mass in the obese diabetic animal model $d b / d b$ mice.

Materials and methods: Ten week-old male $d b / d b$ mice recieved liraglutide $(0.2 \mathrm{mg} / \mathrm{kg}$ twice daily s.c.) or vehicle (control) for 2 days (Study 1$)$ or 2 weeks (Study 2). Body weight (BW), fasted blood glucose (FBG), insulin (FIRI), and triglyceride (TG) were measured at before and after liraglutide intervention, and intraperitoneal insulin tolerance test (ipITT:1 IU/kg BW) was performed at 12 weeks of age. The beta-cell mass and cell proliferation/apoptosis were assessed by histological analysis including proliferating cell number antigen (PCNA), 4-hydroxynonenal modified protein (4-HNE) immunostaining, and TUNEL assay of the islet tissue. Gene expressions specific for the core area of pancreatic islet were analyzed by Laser Capture Microdissection method and real time reverse transcriptase-polymerase chain reaction (RTPCR). Primer pairs encoding genes associated with pancreatic hormones, cell proliferation, apoptosis, cell cycle, and oxidative stress were prepared, and real-time RT-PCR with SYBR Green was applied. Each gene expression was relatively quantified by the comparative CT method with each result in the 18 SrRNA as a control.

Results: Study 1: After 2 days' intervention with liraglutide, FBG and FIRI were readily lowered in liraglutide-treated mice. The pancreatic beta-cell mass was not affected by liraglutide treatment, but islet cell gene expressions of Cycline D and Hlxb-9, a marker of cell differentiation, were significantly up-regulated, and Hes-1, a marker of anti-cell differentiation, was down-regulated in mice treated with liraglutide. Gene expressions of catalase, GSHPx and XBP-1 related with oxidative stress and endoplasmic reticulum (ER) stress did not differ between groups. Immunohistochemical findings and TUNEL assay did not show any significant difference between groups. Study 2: After 2 weeks' intervention, BW was significantly lower in the liraglutide group than in the control $(42.1 \pm 0.5 \mathrm{~g}$ vs. $44.6 \pm 0.6 \mathrm{~g}$, mean $\pm \mathrm{SE}, p<0.01, \mathrm{n}=10$ for each). FBG, FIRI, and TG levels were significantly lower in the liraglutide group than in the control (glucose: $189 \pm 10.3$ vs. $272 \pm 26.0 \mathrm{mg} / \mathrm{dl}, p<0.05$, insulin: $4.3 \pm 0.5$ vs. $6.4 \pm 0.7 \mathrm{ng} / \mathrm{ml}, p<0.05$, TG: $113 \pm 2.4$ vs. $133 \pm 6.5 \mathrm{mg} / \mathrm{dl}$, respectively, $p<0.01)$. The pancreatic beta-cell mass was higher in liraglutide-treated mice than in the control $(7.7 \pm 0.7$ vs. $4.9 \pm 0.5 \mathrm{mg}, p<0.01, \mathrm{n}=5$ for each). The islet TG content was reduced by liraglutide. PCNA-positive cells were increased and 4-HNE-positive cells were decreased by liraglutide treatment. TUNEL assay showed the less beta-cell apoptosis in liraglutidetreated mice. NeuroD, ERK-1 and cycline D mRNA levels were significantly higher in liraglutide mice than in the control. SREBP-1c, FAS, and XBP-1 gene expressions were down-regulated, and catalase and GSHPx genes were up-regulated by 2 weeks' intervention with liraglutide.

Conclusion: Liraglutide increased pancreatic beta-cell mass in diabetic $d b / d b$ mice. The mechanism was both regulation of cell kinetics as an acute effect but also suppression of oxidative stress and ER stress by ameliorating glucolipotoxicity as a chronic effect. Supported by: Novo Nordisk

\section{2}

High density lipoproteins protect pancreatic beta cells by targeting $4 \mathrm{E}-$ BP1

J. Petremand ${ }^{1}$, A.-C. Butty ${ }^{1}$, J.-Y. Yang ${ }^{1}$, B. Natasa ${ }^{1}$, C. Poussin ${ }^{1}$, B. Thorens ${ }^{1}$, G. Waeber ${ }^{2}$, C. Widmann ${ }^{1}$

${ }^{1}$ Physiology, University of Lausanne, ${ }^{2}$ Medecine Interne, CHUV, Lausanne, Switzerland

Background and aims: Low level of HDL cholesterol is one of the lipid phenotype observed in diabetic patients. HDLs exert numerous protective effects on endothelial cells such as prevention of LDL oxidation, endothelial dysfunction and apoptosis. In the case of pancreatic beta cells, HDLs have been shown to protect against apoptosis elicited by various stress stimulus such as serum starvation, cytokines, or oxidized LDL. However, the molecular mechanisms of HDL-induced beta cells protection are not entirely identified and represent a promising way to discover new potential target for the treatment of diabetes.

Materials and methods: Affymetrix gene chip technology was used in stress or normal conditions to identify the target genes by which HDL exert their anti-apoptotic activities in insulin-secreting cells.

Results: One interesting target identified was the translational repressor 4Ebinding protein (4E-BP1). This small protein regulates protein synthesis by sequestering eIF4E which is necessary for initiation of translation. 4E-BP1 expression is induced by starvation or hypoxia and has been demonstrated to induce apoptosis. In this study, stresses such as serum starvation or IL-1 beta treatment induced 4E-BP1 expression in insulin-secreting beta cell lines and in human islets. The induction of $4 \mathrm{E}-\mathrm{BP} 1$ expression was significantly reduced by HDL, and this effect was correlated with prevention of apoptosis. Moreover, overexpression or lentiviral infection with 4E-BP1 constructs induced apoptosis, mimicking the starvation effect. Finally, the use of 4E-BP1 shRNA reduced the induction of $4 \mathrm{E}-\mathrm{BP} 1$ expression by the stress and the stress-induced apoptotic response was also reduced showing that there is a direct link between stress conditions, increased 4E-BP1 levels and apoptosis in beta cells.

Conclusion: These data indicate that HDL block stress-induced expression of $4 \mathrm{E}-\mathrm{BP} 1$ and prevent beta cell apoptosis, showing that $4 \mathrm{E}-\mathrm{BP} 1$ is a possible target to prevent development of diabetes.

Supported by: Swiss National Fondation

\section{3}

The importance of free fatty acid binding receptors and ER stress for lipotoxicity in insulin-producing cells W. Gehrmann ${ }^{1}$, M. Elsner ${ }^{1}$, A. Jörns ${ }^{1,2}$, S. Lenzen ${ }^{1}$

${ }^{1}$ Institute of Clinical Biochemistry, ${ }^{2}$ Centre of Anatomy, Hannover Medical School, Hannover, Germany

Background and aims: Elevated levels of free fatty acids (FFA) can cause $\beta$ cell dysfunction and apoptosis in type 2 diabetes. Only saturated FFAs have toxic effects upon insulin-producing cells whereas unsaturated FFAs are protective. However, the molecular mechanisms of lipotoxicity are only partially understood. It is still under discussion if the FFA binding receptors GPR40 and GPR120 are involved and if ER stress is a crucial mechanism of lipotoxicity. Disturbed ER function lead to an accumulation of unfolded or misfolded proteins which activates a specific ER stress response. Therefore the aim of the present study was to analyse the gene expression of the GPR-receptors and different ER stress marker genes.

Materials and methods: To investigate the cytotoxic effects of palmitic acid (PA) upon different insulin-producing cell lines viability was measured by MTT assay after $24 \mathrm{~h}$ exposure. The effect of PA and oleic acid (OA) on the mRNA expression levels of the ER stress marker genes Bip, CHOP, XBP-1 and XBP-1 spliced and the expression levels of GPR40 and GPR120 were determined by quantitative RT-PCR. The receptor mediated enhanced glucose 
stimulated insulin secretion (GSIS) due to FFA treatment was measured by a static incubation. Electron microscopy analyses were performed to detect morphological changes due to FFA treatment in RINm5F and INS-1E cells. Results: The cytotoxic effect of PA was much more pronounced in RINm5F $\left(\mathrm{EC}_{50}=113 \mu \mathrm{M} \pm 6\right)$ cells than in INS-1E $\left(\mathrm{EC}_{50}=1860 \mu \mathrm{M} \pm 540\right)$ and MIN6 $\left(\mathrm{EC}_{50}=993 \mu \mathrm{M} \pm 123\right)$ cells. Interestingly, in RINm5F cells, which showed the highest sensitivity for PA, the GPR40 and GPR120 receptors were not detectable by quantitative RT-PCR. By contrast the less sensitive cell lines INS-1E and MIN6 expressed both receptors. An enhanced GSIS due to FFA treatment was only detected in MIN6 and INS-1E cells. Palmitic acid led to a 5 fold increased insulin secretion at $10 \mathrm{mM}$ and $30 \mathrm{mM}$ glucose in MIN6 cells and to 2 fold increase in INS-1E cells. By contrast OA had a minor effect. The induction was only 3 fold in MIN6 cells and 1.5fold in INS-1E cells. In electron microscopy analyses we found damage to the endoplasmic reticulum induced by PA in RINm5F cells whereas the endoplasmic reticulum was less damaged in INS-1E cells. The expression level of the ER stress marker gene CHOP was increased 21 fold in RINm5F cells and 6 fold in INS-1E cells after $12 \mathrm{~h}$ incubation with PA. Furthermore the expression level of XBP-1 spliced was increased 10 fold in RINm5F cells, whereas the expression level in INS$1 \mathrm{E}$ cells was unaltered. In both cell lines the expression level of Bip was only slightly increased and of XBP-1 unchanged after PA treatment. Incubation with OA did not affect the expression level of all analysed ER stress marker genes. Moreover, OA was able to prevent induction of CHOP and XBP-1 spliced due to PA treatment.

Conclusion: The presented data indicate that the toxicity of PA is probably not mediated through the FFA binding receptors GPR40 and GPR120, because the most sensitive cell line RINm5F did not express both receptors. The enhanced GSIS due to FFA in MIN6 and INS-1E cells showed the functionality of the GPR40 receptor.The different induction of ER stress marker genes in the analysed beta cell lines RINm5F and INS-1E cells after PA treatment which is also reflected by the results of the viability assay indicates that ER stress plays an important role for lipotoxicity.

\section{4}

Visfatin decreases cell viability, activates insulin receptor and alters expression of key genes in a clonal beta cell line

S.J. Dunmore ${ }^{1}$, D. Onyango ${ }^{1}$, J.E.P. Brown ${ }^{2}$;

${ }^{1}$ Diabetes Research Group, University of Wolverhampton, ${ }^{2}$ Endocrinology and Metabolism Research Group, University of Warwick, Coventry, United Kingdom

Background and aims: The novel adipokine visfatin is reported to have insulin-like properties and to activate insulin signalling although there is some controversy as to whether this effect is genuine or whether the protein produces its effects through its nicotinamide phosphoribosyltransferase enzymic activity, the first step of the NAD synthetic pathway. Due to its high expression in visceral fat tissue and its insulin-like effects it has been suggested to be involved in the pathogenesis of type 2 diabetes, although this link has yet to be well established. Since insulin receptors are expressed in beta-cells, and a recent report has demonstrated progressive beta-cell failure with increased visfatin serum concentrations, we sought to evaluate the effects of visfatin on beta-cell viability and on the insulin signalling (insulin receptor (IR) phosphorylation) pathway. We also investigated the effect of incubation with visfatin on mRNA expression of a range of genes in the pancreatic beta-cell using a diabetes gene pathway specific PCR array.

Materials and methods: Beta-TC6 clonal mouse pancreatic beta-cells were cultured in RPMI-1640 before being treated for 24 hours with a range of recombinant visfatin concentrations ( 0 \& $0.5 \mathrm{nmol} / 1$ for gene expression; 0,1 \& $10 \mathrm{nmol} / \mathrm{l}$ for IR phosphorylation; $0,2,4,6,8 \& 10 \mathrm{nmol} / \mathrm{l}$ for cell viability), a positive control of $1 \mathrm{nmol} / \mathrm{l}$ insulin was used for the IR phosphorylation assay. Cell viability was measured by a modified MTS method and insulin signalling by Western blotting of protein extracts using an anti-IR phosphotyrosine (phospho Y972) antibody antibody. Total RNA was extracted and PCR array technology was used to compare mRNA expression in treatment sample to control sample.

Results: Incubation with visfatin dose-dependently decreased beta cell viability with significant $(\mathrm{p}<0.05)$ decreases of $24 \%$ and $40 \%$ at 8 and $10 \mathrm{nmol} / \mathrm{l}$ respectively. Visfatin increased insulin receptor phosphorylation by 5 -fold compared to at $1 \mathrm{nmol} / \mathrm{l}$ (similar to the effect of insulin) and by 10 fold at 10 $\mathrm{nmol} / \mathrm{l}$. Visfatin caused changes in expression of a number of genes relevant to beta-cell function including greater than ten-fold increases in glucagon receptor, interleukin $12 \mathrm{~b}$, and transforming growth factor beta 1 . Decreases of more than 50\% were seen in FOXg1 and G6PD.
Conclusion: Pathologically relevant concentrations of visfatin decrease betacell viability and can activate early-stage insulin signalling in these cells as well as altering expression of a number of genes which may affect the function in these cells. This supports the suggestion that visfatin may have a role in loss of beta-cell function in obesity-related diabetes.

\section{5}

Loss of insulin synthesis and beta cell survival induced by oxidized LDL require activation of reticulum endoplasmic stress: a mechanism countered by HDL

D. Favre ${ }^{1}$, S. Brajkovic ${ }^{1}$, G. Niederhauser ${ }^{1}$, G. Waeber ${ }^{2}$, A. Abderrahmani ${ }^{1}$ ${ }^{1}$ Dpt of Cell biology and Morphology, ${ }^{2}$ Dpt of Internal Medicine, Centre Hospitalier Universitaire Vaudois, Lausanne, Switzerland

Elevated circulating concentrations in modified LDL-cholesterol particles (e.g. oxidised LDL) and low levels in HDL increase not only the risk for diabetic patients to develop cardiovascular diseases but also may contribute to development and progression of diabetes by directly having adverse effects on $\beta$-cells. Chronic exposure of $\beta$-cells to $2 \mathrm{mM}$ human oxidised LDL-cholesterol (oxLDL) increases the rate of apoptosis, reduce insulin biosynthesis and the secretory capacity of the cells in response to nutrients. In line with the protective role, HDL efficiently antagonised the harmful effects of oxLDL, suggesting that low levels of HDL would be inefficient to protect $\beta$-cells against oxLDL attack in patients. Activation of endoplasmic reticulum (ER) stress is pointed out to contribute to $\beta$-cell dysfunction elicited by environmental stressors. In this study we investigated whether activation of ER stress is required for oxLDL to mediate detrimental effects on $\beta$-cells and we tested the potential antagonist properties of HDL: The mouse MIN6 insulin-secreting cells were cultured with $2 \mathrm{mM}$ of LDL-cholesterol preparation (native or in vitro oxidized) in the presence or absence of $1 \mathrm{mM}$ of HDL-cholesterol or the ER stress inhibitor 4-phenylbutyrate (4-PBA): Prolonged exposure of MIN6 cells to $2 \mathrm{mM}$ oxLDL-cholesterol for 48 hours led to an increase in expression of ER stress markers such as ATF4, CHOP and p58 and stimulated the splicing of XBP-1 whereas, induction of these markers was not observable in the cells cultured with native LDL. Treatment of the cells with the 4-PBA chemical chaperone molecule efficiently blocked activation of the ER stress markers induced by oxLDL. The latter mediates $\beta$-cell dysfunction and apoptosis by diminishing the expression of islet brain 1 (IB1) and Bcl2. The levels of these two proteins were preserved in the cells that were co-treated with oxLDL and the 4-PBA. Consistent with this result we found that blockade of ER stress activation alleviated the loss of insulin synthesis and abolished apoptosis evoked by oxLDL. However incubation of the cells with 4-PBA did not prevent impairment of insulin secretion elicited by oxLDL, indicating that ER stress is not responsible for the oxLDL-mediated defect of insulin secretion. Co-incubation of the cells with HDL mimicked the effects of 4-PBA on the expression of IB1 and Blc2 and thereby counteracted oxLDL attacks on insulin synthesis and cell survivals. We found that HDL efficiently inhibited activation of the ER stress mediated by oxLDL: These data highlight the contribution of the ER stress in the defects of insulin synthesis and cell survivals induced by oxLDL and emphasize the potent role of HDL to counter activation of the oxLDL-mediated ER-stress activation:

Supported by: EFSD/MSD grant 


\section{PS 031 Cytokine toxicity in beta cells}

\section{6}

Influence of antioxidative enzyme overexpression and proinflammatory cytokines on Bcl-2 promoter activity and activation of caspase-9 in insulin-producing cells

I. Mehmeti, S. Lortz, S. Lenzen

Institute of Clinical Biochemistry, Hannover Medical School, Germany

Background and aims: Type 1 diabetes is caused by autoimmune destruction of pancreatic B-cells. This ß-cell destruction process appears to result from proinflammatory cytokines, such as IL-1beta, IFN-gamma and TNF-alpha, mainly by activation of intracellular signal cascades leading finally to apoptosis. One of the preferential target structures of these apoptosis pathways are mitochondria. Integrity and stability of mitochondria are critical for $\beta$ cell viability or initiation of apoptosis. In previous studies we have shown that overexpression of a mitochondrially located catalase (MitoCat) induced an increased gene and protein expression of the antiapoptotic acting Bcl-2 molecule. Therefore, the aim of this study was to characterise the influence of catalase overexpression and proinflammatory cytokines on the transcriptional regulation of $\mathrm{Bcl}-2$ expression and to quantify caspase- 9 activity before and after cytokine exposure.

Materials and methods: Quantification of Bcl-2 promoter activity was performed after transient transfection with firefly luciferase reporter gene constructs under the control of the Bcl-2 promoter (with intact or deleted cAMPresponse element (CRE)) and exposure to IL-1beta or to a cytokine mixture (IL-1beta, TNF-alpha und IFN-gamma) for $24 \mathrm{~h}$. To examine the importance of the CRE site for the cytokine-mediated transcriptional regulation of $\mathrm{Bcl}-2$ expression, we additionally transfected insulin-producing RINm5F cells with a reporter gene construct containing the luciferase reporter gene under the control of four tandem repeats of CRE. Caspase- 9 activity of insulin-producing control cells and cells overexpressing either cytosolic catalase (Cat) or mitochondrially located catalase (MitoCat) was measured by a flow cytometry-based caspase- 9 assay after $24 \mathrm{~h}$ cytokine exposure.

Results: RINm5F control cells transfected with a Bcl-2 promoter construct containing the intact CRE site showed a significantly decreased Bcl-2 promoter activity after cytokine treatment, whereas the CRE site deleted Bcl2 promoter showed no significant changes of Bcl-2 promoter activity. This cytokine-mediated decrease of reporter gene activity was also seen with the CRE construct in RINm5F control cells. In MitoCat cells but not in CytoCat cells the CRE and Bcl-2 promoter activity was well preserved after cytokine exposure. Cytokine stimulation of control cells and cells overexpressing cytosolically localised catalase led to a significantly increased caspase- 9 activity, whereas MitoCat overexpression prevented the increase of caspase- 9 activity after cytokine treatment.

Conclusion: Cytokine-induced reduction of CRE and Bcl-2 promoter activity indicates that the CRE site within the Bcl-2 promoter is essential for the down-regulation in response to cytokines. These data correspond to the $\mathrm{Bcl}-2$ expression in these cells. Cytokines significantly increased caspase- 9 activity in control cells, suggesting that the mitochondrial apoptosis pathway is crucial for B-cell death. Overexpression of MitoCat could successfully avoid downregulation of $\mathrm{Bcl}-2$ promoter activity and therefore maintain higher expression of Bcl-2 compared with control cells, resulting in a higher resistance against proinflammatory cytokines.

\section{7}

Identification of gene networks regulated by the cytokines IL-1 $\beta$ and TNFa in primary rat beta cells

F. Ortis, N. Naamane, D. Flamez, F. Moore, D.L. Eizirik

Laboratory of Experimental Medicine, Université Libre de Bruxelles,

Brussel, Belgium

Background and aims: Cytokines contribute to pancreatic beta cell death in type 1 diabetes. Cytokine induced beta cell apoptosis depends on the expression of hundreds of genes. To understand the regulation of these gene networks at its real level of complexity, we have performed array analysis of primary beta cells exposed to IL- $1 \beta+$ IFN $\gamma$ or TNF $\alpha+$ IFN $\gamma$ using the new Affymetrix GeneChip containing more than 31.000 probe sets. This was followed by global analysis of gene expression using Ingenuity Pathway Analysis (IPA 5.5).

Material and methods: Primary rat beta cells were FACS-purified and exposed for 6 or $24 \mathrm{~h}$ to control condition, IL-1 $\beta(50 \mathrm{U} / \mathrm{ml})+\mathrm{IFN} \gamma(100 \mathrm{U} / \mathrm{ml})$ or TNFa $(1000 \mathrm{U} / \mathrm{ml})+\mathrm{IFN} \gamma(100 \mathrm{U} / \mathrm{ml})$ before array analysis (GeneChip ${ }^{\circ}$ Rat Genome 2302.0 Array). Results of 3 independent biological/array experiments were analyzed and mRNA expression considered modified by cytokines when $\mathrm{q} \leq 0.025$ compared to control condition. These results were analyzed by the IPA 5.5 software to identify networks and pathways regulated by cytokines. Cell viability was evaluated by Hoechst 342 and propidium iodide and nitrite determined by the Griess method. NF- $\kappa B$ translocation to the nucleus analyzed by immunostaining for $\mathrm{p} 65$.

Results: Cytokines induced beta cell death $(\mathrm{P} \leq 0.001$ for cytokines vs control; $72 \mathrm{~h}$ ), with $17 \pm 2 \%$ apoptotic cells in the control, $44 \pm 2 \%$ in IL- $1 \beta+$ IFN $\gamma$ and $41 \pm 2 \%$ in TNF $\alpha+$ IFN $\gamma$ treated cells. IL- $1 \beta+$ IFN $\gamma$-induced higher NO production than TNF $\alpha+\operatorname{IFN} \gamma\left(21 \pm 4\right.$ vs $13 \pm 3 \mathrm{pM} / 10^{3}$ cells, respectively). The microarray analysis detected nearly 16.000 transcripts in control and cytokine-treated cells. Of these, 92 and 3.655 displayed significant changes in expression after respectively 6 and $24 \mathrm{~h}$ treatment with IL- $1 \beta+$ IFN $\gamma$. After TNF- $\alpha+$ IFN $\gamma$ exposure, 376 and 991 transcripts were modified after 6 and $24 \mathrm{~h}$, respectively. Among the top 5 canonical pathways significantly changed by cytokines, 2 were common for IL-1 $\beta+$ IFN $\gamma$ and TNF $\alpha+$ IFN $\gamma$, namely: "Antigen presentation" ( $\mathrm{p}$-value $\leq 5 \mathrm{E}-03$ ) and "Acute phase response" (pvalue $\leq 2 \mathrm{E}-02$ ). The "Interferon signalling" pathway was identified as significantly changed only at $24 \mathrm{~h}$ TNF- $\alpha+$ IFN $\gamma$ treatment. A total of 2 and 100 networks (representing key transcription factors and their interactions with more than 12 focus genes) were modified by IL- $1 \beta+$ IFN $\gamma$ after respectively 6 and $24 \mathrm{~h}$. Using the same criteria, we found 11 and 50 networks modified by the TNF $\alpha+\operatorname{IFN} \gamma$ treatment after respectively 6 and $24 \mathrm{~h}$. The networks with higher scores for both treatments were regulated by Myc, NF-kB and Jun. These networks, however, had a low agreement in the identity of the genes induced by IL- $1 \beta$ or TNF- $\alpha$ : $21 \%$ (Myc), $17 \%$ (NF-kB) and $11 \%$ (Jun). Additional experiments indicated that IL- $1 \beta$ induced an earlier $\mathrm{p} 65$ translocation to the nucleus as compared to TNF- $\alpha(84 \pm 2 \%$ vs $10 \pm 3 \%$ nuclear positive cells for respectively IL- $1 \beta$ and TNF $\alpha ; \mathrm{n}=4, \mathrm{p} \leq 0.001$ at $10^{\prime}$ ). There was also a more sustained p 65 nuclear localization following IL-1 $\beta$ exposure, with higher values at 1, 2, 8, 12, 24 and $48 \mathrm{~h}$ as compared to TNFa.

Conclusion: There are major temporal differences between the effects of IL$1 \beta$ and TNFa in beta cells, with higher induction of gene networks by TNF $\alpha$ at $6 \mathrm{~h}$ and by IL-1 $\beta$ at $24 \mathrm{~h}$. IL-1 $\beta$ and TNFa induce similar key transcription factor and gene networks, but there is a differential usage of genes involved in these networks. These different effects may be in part explained by a different temporal induction of key regulatory transcription factors, as presently shown for NF-kB.

Supported by: Communauté Française de Belgique - Actions de Recherche Concertées, Fonds National de la Recherche Scientifique Belgium, the Belgium Program on Interuniversity Poles of Attraction initiated by the Belgium State and European Union

\section{8}

Liraglutide induces cell proliferation and protects from interleukin-1beta induced beta cell apoptosis in human islets

R. Prazak ${ }^{1}$, S. Rütti ${ }^{1}$, H. Ellingsgaard ${ }^{1}$, L. Knudsen ${ }^{2}$, M.Y. Donath ${ }^{1}$ ${ }^{1}$ Endocrinology, UniSpital Zürich, Switzerland, ${ }^{2}$ Novonordisk, Malov, Denmark

Background and aims: Glucagon-Like Peptide-1 (GLP-1) analogs induce beta-cell proliferation and have anti-apoptotic effects in rodent islets. However the effects in human islets are less studied. Interleukin-1beta (IL-1beta) production is increased in islets of patients with type 2 diabetes and induces beta-cell apoptosis. The aim of the study was to determine whether the oncedaily human GLP-1 analog liraglutide affects proliferation and apoptosis in human islets.

Materials and methods: Mouse, and human islets from six organ donors were cultured on extracellular matrix-coated plates at $11 \mathrm{mM}$, and $5.5 \mathrm{mM}$ glucose respectively, and exposed to liraglutide in the presence and absence of IL-1beta and of LDL particles.

Results: As previously shown, exposure to $300 \mathrm{nM}$ liraglutide for 4 days induced a 2.1-fold cell proliferation in mouse islets while human beta-cell proliferation remained unchanged as assessed by Ki-67 staining. However, detection of BrdU added throughout the culture period displayed an $1.74+/$ 0.31 -fold increase in the number of positive endocrine cells in the liraglutide treated human islets $(n=5)$. The quality of the insulin double staining did not allow for a clear characterization of the endocrine positive BrdU cells. Furthermore, liraglutide prevented beta-cell apoptosis induced by $2 \mathrm{ng} / \mathrm{ml} \mathrm{IL}-$ lbeta $(2.77+/-0.7$ versus $0.81+/-0.32$ fold increase in TUNEL positive beta cells in IL-1beta alone versus IL-1beta and liraglutide, respectively, compared 
to baseline; $n=3)$. Moreover, liraglutide prevented the decrease in mouse $\beta$ cell proliferation ( 0.5 fold of control) caused by $3.1 \mathrm{mM}$ LDL particles $(n=6)$. Conclusion: Liraglutide induces endocrine cell proliferation in human islets detected by continuous BrdU exposure during prolonged incubation, but not by Ki-67 staining reflecting only the last 12 hours of proliferative activity. In analogy to the proliferative effect of liraglutide on rodent islets, it is likely that the BrdU positive human endocrine cells are replicating islet beta-cells and not non-beta-endocrine-cells. Finally, liraglutide prevents IL-1beta induced beta-cell apoptosis

\section{9}

Withdrawn

\section{0}

The contribution of different cytokines and nitric oxide to ER stress and cell death in insulin-producing cells

S.A. Kacheva, S. Lenzen, E. Gurgul-Convey

Institute of Clinical Biochemistry, Hannover Medical School, Germany

Background and aims: Proinflammatory cytokines and especially IL- $1 \beta$ play a crucial role in beta cell damage and death during the development of type 1 diabetes mellitus (T1DM). But the underlying mechanisms are not fully understood. ER stress has been considered to play an important role in cytokine-meditated beta cell toxicity. Little is known, however, about the importance of the different cytokines as well as nitric oxide in this process. Therefore, we analyzed the role of various cytokine combinations and the contribution of nitric oxide in ER stress in insulin-producing cells.

Materials and methods: Insulin-producing INS1E cells were incubated for $72 \mathrm{~h}$ with IL- $1 \beta$, TNF $\alpha$ and IFN $\gamma$ alone or in different combinations in the presence or absence of an iNOS blocker (5 mM L-nitro-arginine) and thereafter cell viability was measured (MTT assay). The expression of different ER stress markers was analyzed by Real-Time PCR. Caspase- 3 activity was measured by a chemiluminescent method, NFkB by a SEAP reporter gene assay and nitrite production by a Griess method.

Results: IL- $1 \beta$, TNF $\alpha$ and IFN $\gamma$ showed a concentration-dependent toxicity in the MTT assay. The high concentrations of these cytokines (IL-1 $\beta 600$ $\mathrm{U} / \mathrm{ml}$, TNF $\alpha 1850 \mathrm{U} / \mathrm{ml}, \mathrm{IFN} \gamma 140 \mathrm{U} / \mathrm{ml}$ ) after $72 \mathrm{~h}$ decreased the viability to $40 \%, 76 \%$ and $43 \%$, vs. untreated controls, respectively. The combinations of lower concentrations of cytokines were more toxic than any cytokine used alone (viability after $72 \mathrm{~h}$ : IL-1+TNF $32 \%$, IL-1+IFN $13 \%$, TNF+IFN $33 \%$ ). The mixture of all three cytokines was the most toxic combination with $5 \%$ viable cells after $72 \mathrm{~h}$. The iNOS blocker prevented nitrite formation and this was accompanied by a partial protection against cytokine toxicity (viability after 72 h: IL-1ß $73 \%$, Mix $12 \%$, IL-1+TNF $73 \%$, IL-1+IFN $40 \%$, TNF+IFN $35 \%)$. Cytokines affected the expression of ER stress markers with the maximal effects on CHOP and Bip after a $12 \mathrm{~h}$ incubation (CHOP: IL-1 $630 \%$, Mix 733 \%, IL-1+TNF 827 \%, IL-1+IFN 593 \%, TNF+IFN 362 \%; Bip: IL-1 45 $\%$, Mix, $40 \%$ IL-1+TNF $60 \%$, IL-1+IFN $42 \%$, TNF+IFN $45 \%$ ). These effects were strongly counteracted in the case of CHOP ( $12 \mathrm{~h}$ iNOS blocker: IL-1 89 $\%$, Mix $129 \%$, IL-1+TNF $62 \%$, IL-1+IFN $54 \%$, TNF+IFN $162 \%$, p<0.05) and to some extend in the case of Bip expression (12 h iNOS blocker: IL-1 83 $\%$, Mix $54 \%$, IL-1+TNF $75 \%$, IL-1+IFN $67 \%$, TNF+IFN $92 \%$, p $<0.05$ ) by a use of the iNOS blocker. On the other hand, cytokines only slightly affected the expression of XBP-1 and XBP-1 spliced and this effect was NO-independent. IL- $1 \beta$ and the three cytokine mixture induced caspase- 3 activity (after 24 h: IL-1 $140 \%$, Mix $163 \%$ ) and this was prevented by the iNOS blocker (IL-1 $108 \%$, Mix $78 \%, \mathrm{p}<0.05)$. Cytokines induced the NFkB activation after $6 \mathrm{~h}$ also in a NO-dependent manner (cytokines vs. cytokines with iNOS blocker: IL-1 269 vs. $126 \%$, Mix 317 vs. $148 \%$, IL-1+TNF 314 vs. $108 \%$, IL-1+IFN 195 vs. $150 \%$, TNF+IFN 121 vs. $92 \%$ ).

Conclusion: IL- $1 \beta$ and the cytokine mixture, which show the highest toxicity in the MTT assay and caspase- 3 activation have also the strongest effects on the expression of ER stress markers and this can be related to an increased production of nitric oxide. We postulate that the cytokine-induced NO production changes the unfolded protein response, which in turn leads to beta cell destruction and death.

\section{1}

Toxic cytokines interleukin- $1 \beta$, tumor necrosis factor- $\alpha$ and interferon$\gamma$ regulate zinc transporter gene expression in beta cells and Slc30A8 protects against cytokine-induced cell death

L. Egefjord ${ }^{1}$, A.B. Petersen ${ }^{1}$, C.H. Bang-Berthelsen ${ }^{2}$, F. Pociot ${ }^{2}$, A.E.

Karlsen ${ }^{3}$, K. Schmidt ${ }^{1}$, F. Chimienti ${ }^{4}$, B. Brock ${ }^{1}$, O. Schmitz ${ }^{1}$, J. Rungby ${ }^{1}$, N.E. Magnusson ${ }^{1}$

${ }^{1}$ Pharmacology, University of Aarhus, Denmark, ${ }^{2}$ Steno Diabetes Center, Gentofte, Denmark, ${ }^{3}$ NovoNordisk, Copenhagen, Denmark, ${ }^{4}$ CEA, MELLITECH, Grenoble, France

$\beta$-cells depend on zinc influx to secretory vesicles for the crystallization of insulin since insulin is stored as a hexamer complexed with two zinc ions. Tight regulation of zinc homeostasis is ensured by zinc transporters (ZnTs and ZIPs). $\beta$-cell function and mass is severely affected by the presence of a number of cytokines, in particular interleukin-1beta (IL-1 $\beta$ ), in both type-1 and type-2 diabetes. Over-expression of the ZnT8, zinc transporter protein encoded by Slc30A8, which has been identified as a major risk gene for type-2 diabetes and an antigen associated with the development of type-1 diabetes, seems to protect $\beta$-cells from oxidative stress.

We therefore examined the expression profiles of ZnTs and ZIPs in cultured $\beta$-cells and islets after cytokine exposure and the survival of Slc30A8 overexpressing cells after cytokine treatment.

For the cell experiments, INS 1 cells were stimulated for 1 or 24 hours with 60-180 U IL- $1 \beta / \mathrm{mL}, 200$ U TNF- $\alpha / \mathrm{mL}, 200$ U INF- $\gamma / \mathrm{mL}$, or a mixture comprising $180 \mathrm{U}$ IL- $1 \beta / \mathrm{mL}, 200 \mathrm{U}$ TNF- $\alpha / \mathrm{mL}$ and $200 \mathrm{U}$ INF- $\gamma / \mathrm{mL}$ (PharMingen International, San Diego, CA, USA). Controls were stimulated with RPMI 1640 medium. Gene-expression was measured by Q-PCR. For islet experiments, rat neonatal islets were cultured at $11 \mathrm{mM}$ glucose. Stimulations were performed for $1,2,4,6$ and $24 \mathrm{~h}$ with $250 \mathrm{pg} / \mathrm{mL}$ IL- $1 \beta / \mathrm{mL}, 10 \mathrm{ng} /$ $\mathrm{mL}$ INF- $\gamma$, or a mixture comprising $250 \mathrm{pg} / \mathrm{mL}$ IL- $1 \beta$ and $10 \mathrm{ng} / \mathrm{mL}$ INF$\gamma$. Controls were stimulated with RPMI 1640 medium. Gene-expression was measured by Q-PCR. For survival, INS 1E cells over-expressing Slc30A8 were compared to wild-type after exposure to a mixture of cytokines as above and survival was measured by MTT for cell death.

A number of cytokine-induced changes in $\mathrm{Zn}$-transporter gene-expression were found. The figure shows significant changes in isolated INS 1 cells. Similar results were found in islets. Over-expression of Slc30A8 significantly reduced cytokine-induced apoptosis ( $<<0.05$ when MTT activity was measured against wild type).

Our results indicate a dynamic response of genes responsible for $\beta$-cell zinc homeostasis to cytokines and provide clues for further exploration of the role of these proteins in both the development of diabetes and $\beta$-cell dysfunction during cytokine stress which is high in diabetes. Further, we demonstrate that high levels of Slc30A8 confer a protection against cytokines.

\section{Test vs control, $\mathrm{p}<0.05,{ }^{*} \mathrm{p}<0.01$}

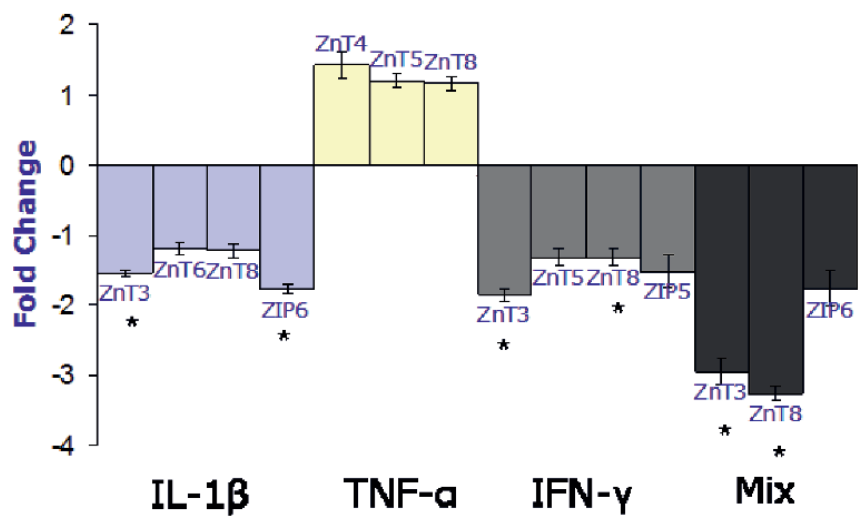

Supported by: The Danish Diabetes Association and Novo Scholarship Programme 


\section{2}

An IKK $\beta$ inhibitor protects against IL-1 $\beta$ induced production of reactive oxygen species and beta cell death

J. Friberg ${ }^{1}$, F. Pociot ${ }^{2}$, M. Tonnesen ${ }^{2}$, A.E. Karlsen ${ }^{1}$

${ }^{1}$ Diabetes biology, Novo Nordisk A/S, Maaloev, Denmark, Steno Diabetes

Center, Gentofte, Denmark

Background and aims: Activation of inflammatory pathways has been reported in obesity, insulin resistance and both type 1 and type 2 diabetes. The notion that excess circulating nutrients stimulate an inflammatory response in the islets of Langerhans and its effect on insulin secretion from the $\beta$-cells has until now attracted little attention. The aim of the present work was to investigate the relationship between inflammatory pathways, induction of reactive oxygen species (ROS) and $\beta$-cell failure.

Materials and methods: ROS was measured with a fluorescent probe CMH2DCFDA. Expression levels of genes were quantified using RT-PCR. NFkB activity was defined by EMSA using a radioactively labeled NFKB-binding oligonucleotide as probe. Reduced caspase-3 cleavage caused by DMT1 specific siRNA was detected by western blot. Mitochondrial activity was assessed by MTT assay and cell death was assessed as caspase 3/7 activity. Insulin concentrations were determined by LOCI assay.

Results: In newborn rat islets Interleukin- $1 \beta$ (IL- $1 \beta$ ) induces ROS production with a maximal production after $24 \mathrm{hrs} \mathrm{IL-} 1 \beta$ exposure ( $>200 \%$ of controls). IL- $1 \beta$ exposure was accompanied by increased gene-expression of inflammatory markers like TNFa, IL6, iNOS and the macrophage specific marker CD68. Pre-treatment with an IKK $\beta$-inhibitor reduced IL- $1 \beta$ mediated NFKB activity, reversed the ROS production and normalized the expression levels of TNFa, IL6, iNOS and CD68. This could indicate that one beneficial effect of anti-inflammatory drugs is mediated through inhibition of the activity of passenger macrophages within de islets. Furthermore, in beta-cells IL-1 $\beta$ exposure caused an even greater induction of ROS. This was time- and dose-dependent, with a maximal ROS production after $24 \mathrm{hrs} \mathrm{IL-1} \beta$ exposure ( $>400 \%$ of controls) and accompanied by reduced insulin secretion, glucose intolerance and apoptosis. Pre-treatment with the IKK $\beta$-inhibitor reduced IL- $1 \beta$ mediated NFKB activity, reversed the ROS production and rescued cells from apoptosis. In addition IKK $\beta$-inhibition prevented IL- $1 \beta$ mediated loss of accumulated and glucose stimulated insulin secretion.

To explore the mechanism behind IL- $1 \beta$ induced ROS production, expression of NFKB regulated genes was addressed. Expression of two NFKB regulated genes, iNOS and Divalent Metal Transporter 1 (DMT1), were increased by IL- $1 \beta$ and normalized by pre-treatment with the IKK $\beta$ inhibitor. The production of ROS was reduced by an inhibitor of iNOS induced NO production (L-NAME), and by an iron chelator (DFO). Furthermore, siRNA-mediated DMT1 knockdown completely protected against IL-1-induced caspase-3 cleavage. This indicates that ROS is partly NO generated and in part depends on the reaction between hydrogen peroxide and iron, generating highly reactive hydroxyl radicals - known as the Fenton reaction.

Conclusion: IL-1 $\beta$ exposure activates inflammatory pathways, including a significant increase in the production of ROS. In INS-1 cells the inflammatory response is followed by glucose intolerance and $\beta$-cell apoptosis. Glucose intolerance and $\beta$-cell apoptosis can be prevented by inhibition of IKK $\beta$ activity. Our data suggest that this process depends upon the expression of the NFKB-regulated genes DMT1 and iNOS, and thus the increased ROS production involve both NO and hydroxyl, resulting from the iron dependent Fenton reaction.

\section{3}

Alpha 1anti trypsin, modulates insulin secretion and prevents from cytokine mediated apoptosis

R. Kumar, M. Kallis, C. Cilio, S. Janciauskiene, A. Salehi

Clinical Sciences, Lund University, Malmo, Sweden

Background and aims: Alpha 1-anti trypsin (A1AT) is the prototypic member of the serine protease inhibitor (serpin) super family of proteins, which has a major role in inactivating neutrophil elastases and other proteases to maintain protease-antiprotease balance. Alpha 1-anti trypsin (A1AT) has been used as key suppressor of inflammatory process in different tissues. In the present study we investigated the effect of A1AT treatment on the beta cell function and survival.

Materials and methods: INS- 1 cells and isolated murine pancreatic islets were used to study the dose dependent response of A1AT on the insulin secretion at different glucose levels. The modulating effects of cyclic AMP in- hibiting/stimulating agents e.g clonidine, GLP-1 and forskolin in the absence and presence of A1AT were investigated and cytokine induced apoptosis was also measured.

Results: A1AT protected the apoptotic cell death induced by cytokines (TNF-alpha, IL-1-beta, and IFN-gamma ) in INS-1 cells ( $<<0.001)$. A1AT increased insulin secretion in dose dependent manner in response to a sub stimulatory glucose concentration $(8.3 \mathrm{mmol} / 1)$ from both INS- 1 cells and pancreatic islets. The insulin secretory response of pancreatic islets at different glucose concentrations was markedly potentiated in the presence of A1AT $(0.5 \mathrm{mg} / \mathrm{ml})$. The inhibitory effect of clonidine on insulin release was reversed by A1AT $(\mathrm{p}<0.001)$ and the stimulatory effect of GLP-1 or forskolin was further increased in the presence of A1AT $(p<0.001)$. Preliminary data from INS-1 cells showed that A1AT has stimulatory effects on cyclic AMP generation. $(\mathrm{p}<0.01)$.

Conclusion: Our data suggest that A1AT in addition to its anti inflammatory properties, has beneficial effects on potentiating insulin release by elevating cyclic AMP production which is a key player in different intracellular pathways. 


\section{PS 032 Glucotoxicity in beta cells}

\section{4}

High glucose-induced antiangiogenic environment impairs beta cells and microcapillary endothelial cells interactions

G. Cohen ${ }^{1}$, N. Kaiser ${ }^{2}$, S. Sasson

${ }^{1}$ Pharmacology, Hebrew University School of Medicine, ${ }^{2}$ Endocrionology \& Metabolism Service, Hadassah-Hebrew University Medical Center, Jerusalem, Israel

Background and aims: An intact and functional vascular microcapillary network in islets of Langerhans supports normal islet viability and function. Transplantation of pancreatic islets is emerging as a therapeutic option for type-1 diabetes. Upon isolation of islets from donors' pancreases their vasculature is severed. Thus, implanted islets must re-establish their vascular connection in the host. This revascularization process requires pro-angiogenic factors to induce the formation of new microvessels. Research efforts have been directed toward improving neovascularization of transplanted islets for developing better methods for successful engraftment and preservation of $\beta$ cell mass after implantation. However, it has not always been acknowledged that the efficiency of such approach is complicated by the presence in the graft and host of (i) anti-angiogenic factors and (ii) altered $\beta$-cell and endothelial cell-extracellular matrix (ECM) interactions within the islets. We aim at investigating these processes in a novel in vitro co-culture system.

Materials and methods: The newly developed co-culture system allows for a highly organised $\beta$-cell and capillary endothelial cell multicellular interaction: When plated on Matrigel- or 804G cell ECM-coated surfaces, capillary endothelial cells (bEnd 3 of murine brain or MS-1 of murine islets of Langerhans) readily form distinct microcapilary-like network within a few hours (Panel A, Matrigel). The striking finding is that when $\beta$-cells (INS-IE) are added to such networks, they migrate, cluster and aggregate around and along the network, forming a unique three dimensional organization (Panel C), while in the absence of the network they remain uniformaly spread (Panel B).

Results: Using this system we have found that high glucose levels impair the capacity of endothelial cells to form such well-organised micrcapillary-like network. Moreover, it alters both $\beta$-cell and endothelial cell integrin-mediated interactions with Matrigel or 804G ECM. We have also discovered that high glucose levels augment the expression of the protein calreticulin and of its proteolytic product, vasostatin, in both cultured INS-1E and in microcapillary endothelial cells.Vasostatin is a potent endogenous anti-angiogenic factor. In addition to its anti-angiogenic potential, calreticulin also interferes with normal cell-matrix interactions by inhibiting integrin receptor-mediated cell adhesion. When added directly to microcapillary endothelial cell cultures calreticulin impeded their capacity to form organized microcapilary-like structures. It also blocked the migration and aggregation of INS-1E cells with pre-formed networks.

Conclusion: These data suggest that hyperglycaemia can induce antiangiogenic environment both in the host and in the graft and may contribute to a delayed revascularization of the transplanted islets in type 1 diabetic patients.

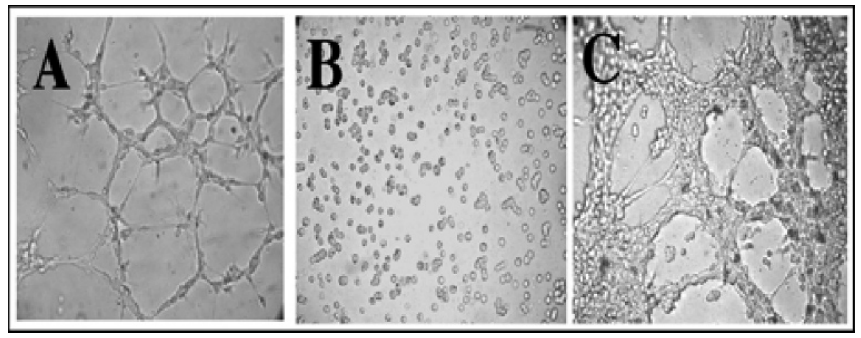

Supported by: JDRF International and the David R. Bloom Center for Pharmacy, The Hebrew University of Jerusalem

\section{5}

Proteomic profile of insulin producing INS-1E cells upon exposure to high glucose

M. Maris ${ }^{1}$, L. Overbergh ${ }^{1}$, W. D'Hertog ${ }^{1}$, E. Waelkens ${ }^{2}$, C. Mathieu ${ }^{1}$ ${ }^{1}$ Catholic University of Leuven, Laboratory of Experimental Medicine and Endocrinology, ${ }^{2}$ Catholic University of Leuven, Laboratory of Protein Phosphorylation and Proteomics, Belgium

Background and aims: Type 2 diabetes is a dual disease that develops when the pancreatic $\beta$-cells can no longer compensate for insulin resistance in the target organs, mainly skeletal muscles, but also liver and adipocytes. This relative insulin deficiency is the consequence of $\beta$-cell dysfunction and/or $\beta$-cell death. The cause of this progressive $\beta$-cell failure remains elusive, but it has repeatedly been demonstrated in vivo that hyperglycemia is a prerequisite for $\beta$-cell dysfunction. It has been observed, in whole islets, as well as in different $\beta$-cell lines, such as INS-1E and MIN6, that insulin gene expression, insulin content and glucose stimulated insulin secretion, were drastically compromised over time upon exposure to high glucose concentrations. To date, the exact mechanisms and pathways involved in $\beta$-cell dysfunction by high glucose treatment have not been unraveled. The aim of the present study was to analyze protein changes in insulin-producing INS-1E cells exposed to high glucose.

Materials and methods: INS-1E cells were exposed to $25 \mathrm{mM}$ glucose for 24 hrs (as compared to $11 \mathrm{mM}$ for control cells) and alterations in protein profile were analysed by two-dimensional Difference gel electrophoresis (DIGE) (covering 2 different $\mathrm{pH}$ ranges, $\mathrm{pH} 4-7$ and $\mathrm{pH} 6-9$ ). Proteins altered in expression $(n=4, p<0,05)$ were identified by MALDI-TOF/TOF. The proportion of apoptotic and necrotic cells was evaluated by microscopic counting after Hoechst/Propidium Iodide staining.

Results: Exposure of the cells to high glucose, increased the proportion of apoptotic cells $(14,40 \pm 1,71$ versus $2,87 \pm 0,19$ for control $(p<0.001))$, as well as the necrotic rate $(6,94 \pm 2,2$ versus $1,15 \pm 0,15$ for control $(p<0.05))$. 2D-DIGE analysis revealed within two different $\mathrm{pH}$ ranges a total of $2189 \pm$ $16(\mathrm{pH} \mathrm{4-7)}$ and $1467 \pm 97$ ( $\mathrm{pH} 6-9)$ spots. Of these, 124 protein spots were altered in expression $(\mathrm{n}=4, p<0.05)$, of which 102 spots $(82.3 \%)$ could unambiguously be identified by MALDI-TOF/TOF. The identified proteins were involved in different pathways of which the glycolysis/TCA cycle, endoplasmic reticulum (ER) stress response/protein folding, cellular transport, protein synthesis and degradation were most abundant. More specific, many chaperone proteins were downregulated by high glucose treatment. As these proteins have previously been associated with protective and anti-apoptotic functions, we conclude that downregulation of these proteins may hamper $\beta$-cell defenses, making them more vulnerable to apoptosis. In addition, also proteins involved in intracellular transport were downregulated by high glucose. These included proteins involved in nucleo-cytoplasmatic transport, trafficking to the ER, transport between ER and Golgi apparatus and exocytosis-related proteins. We conclude that perturbations in this intracellular trafficking may hamper correct protein synthesis and formation of post-translational modifications, leading to $\beta$-cell dysfunction and death.

Conclusion: In this study we identified new molecular pathways that were not described before as being involved in high glucose-induced $\beta$-cell dysfunction and death. In addition, the present findings may provide new key target proteins for the prevention of $\beta$-cell dysfunction and death in type 2 diabetes.

\section{6}

Effects of deglycating enzyme Fructosamine-3-kinase gene knockout on pancreatic beta cell glucotoxicity

S.M.A. Pascal ${ }^{1}$, M. Veiga-da-Cunha ${ }^{2}$, E. Van Schaftingen ${ }^{2}$, J.-C. Jonas ${ }^{1}$ ${ }^{1}$ Unit of Endocrinology and Metabolism, Université catholique de Louvain, ${ }^{2}$ Laboratory of Physiological Chemistry, de Duve Institute and Université catholique de Louvain, Brussels, Belgium

Background and aims: Non-enzymatic protein glycation and the formation of Advanced Glycation End Products may play a role in the toxic effects of chronic hyperglycemia in various cell types. We have recently shown that the intracellular enzyme Fructosamine-3-kinase (FN3K), which initiates protein deglycation by phosphorylating fructosamines on the third carbon, is expressed in mouse pancreatic islets together with FN3K-related protein (FN3K-RP), a closely related enzyme that phosphorylates mainly ribulosamines and erythrulosamines. To determine to what extent protein glycation contributes to beta-cell glucotoxicity, we tested the effect of Fn3k gene 
ablation on the deleterious effects of high glucose and ribose on beta-cell function and survival.

Materials and methods: Islets isolated from Fn3k deficient mice $\left(F n 3 k^{-1-}\right)$ and their wild-type littermates (WT) were cultured for 1 week in serum-free RPMI medium containing 5g/l BSA and 10-30 mmol/l glucose (G10-G30) or $\mathrm{G} 10+25 \mathrm{mmol} / \mathrm{l}$ ribose. After culture, glucose-induced changes in cytosolic $\mathrm{Ca}^{2+}$ concentration $\left(\left[\mathrm{Ca}^{2+}\right]_{\mathrm{c}}\right.$ ) and insulin secretion (normalized for differences in islet DNA content) were measured in perifused islets. Islet caspase activation was measured with FAM-VAD-fluoromethylketone. The mRNA levels of Fn $3 k$, Fn $3 k-r p, c-M y c$ and Preproinsulin were determined by real-time RTPCR. Results are means \pm S.E. for at least 7 islets from 3 experiments.

Results: The $F n 3 k$ and $F n 3 k-r p$ to $T b p$ mRNA ratios were similar in islets from WT mice $(1.24 \pm 0.21$ and $1.01 \pm 0.18, \mathrm{n}=4)$. Fn $3 k-r p$ islet mRNA levels were not affected by Fn $3 k$ gene ablation. After 1wk culture in G10, stepwise glucose stimulation (from 0.5 to 7,15 and $30 \mathrm{mmol} / \mathrm{l}$ ) induced an initial decrease in $\left[\mathrm{Ca}^{2+}\right]$ without changes in insulin secretion in $\mathrm{G}$, followed by concentration-dependent rises in $\left[\mathrm{Ca}^{2+}\right]_{\mathrm{c}}$ and insulin secretion in G15 and G30 that were fully abrogated by $250 \mu \mathrm{mol} / \mathrm{l}$ diazoxide. In comparison, culture in G30 increased the glucose sensitivity for the rise in $\left[\mathrm{Ca}^{2+}\right]_{c}$ and the stimulation of insulin secretion and reduced by $\sim 40 \%$ the maximal rate of insulin secretion in G30 and by 25\% the islet insulin to DNA content ratio. These changes, which occurred without significant changes in Preproinsulin and $c$ $M y c$ mRNA levels nor in caspase activity, were similar in Fn3k ${ }^{-1}$ and WT islets. After culture in $\mathrm{G} 10+25 \mathrm{mmol} / \mathrm{l}$ ribose, the glucose-induced changes in $\left[\mathrm{Ca}^{2+}\right]_{\mathrm{c}}$ were similar to those observed after culture in G10, but the amplitude of glucose-stimulated insulin secretion was significantly reduced by $\sim 70 \%$, in parallel with a $\sim 20 \%$ decrease in the islet insulin to DNA content ratio. These changes were accompanied by a $\sim 40 \%$ reduction in Preproinsulin mRNA levels and a $\sim 3$-fold increase in $c-M y c$ mRNA levels without detectable changes in caspase activity. As observed after culture in high glucose, the effects of ribose were similar in $\mathrm{Fn} 3 \mathrm{k}^{-1}$ and WT islets.

Conclusion: A lack of FN3K deglycating enzyme does neither increase nor decrease the sensitivity of mouse islets to the deleterious effects of a 1 week culture in the presence of $30 \mathrm{mmol} / \mathrm{l}$ glucose or $25 \mathrm{mmol} / \mathrm{l}$ ribose, two conditions that increase protein glycation and oxidative stress in beta-cells. The possible role of other deglycating enzymes, such as FN3K-RP, in beta-cell glucotoxicity deserves further investigation.

\section{7}

Effect of RAGE ligands on pancreatic beta cell

S.-H. Ihm ${ }^{1}$, B.-W. Lee ${ }^{1}$, J.-G. Kang ${ }^{1}$, M.-G. Choi ${ }^{1}$, H.-J. Yoo ${ }^{1}$, J. Ihm² ${ }^{1}$ Internal Medicine, Hallym University, Anyang, ${ }^{2}$ Chemistry, Kyonggi University, Suwon, Republic of Korea

Background and aims: Type 2 diabetes is characterized by progressive betacell failure. AGEs formed under chronic hyperglycemia act via receptors such as RAGE and lead to cellular damage underlying the microvascular and macrovascular complications of diabetes. A recent study has shown that a RAGE ligand (S100b) in vitro directly upregulated the expression of inflammatory COX2 gene in human islets via RAGE activation. RAGE and a RAGE ligand (S100b) have been observed on islet cells with an inflammatory infiltrate in diabetic NOD mouse by immunohistochemistry (IHC). However, it is not clearly known to what extent AGE directly influence pancreatic beta cell function. Thus, the objective of this study was to investigate the direct effect of RAGE ligands (S100b \& HMG-1) on islet cells in vitro and to see whether a RAGE ligand (S100b) and RAGE can be detected in islets of OLETF rats.

Materials and methods: RAGE expression in INS-1 cells and isolated islets was studied with RT-PCR and Western blot. S100b and RAGE expression in pancreatic section of OLETF rats was studied by IHC. INS-1 cell apoptosis was detected by FACS with Annexin-V and PI and ELISA of cytoplasmic fragmented DNA. Apoptosis of isolated islet cells was detected by APOPercentage assay. Intracellular (IC) ROS and MMP (mitochondrial membrane potential) were measured with specific fluorescent dyes.

Results: RAGE was detected in INS-1 cells, isolated rat islets and human islets at mRNA and protein levels. S100b and RAGE were observed on islet cells in 28 week-old OLETF rats by IHC. RAGE ligands (S100b, 3, $6 \mathrm{ug} / \mathrm{mL}$; HMG-1, 0.1, $1 \mathrm{ug} / \mathrm{mL} ; 4-48 \mathrm{hr}$ ) induced apoptotic cell death of INS-1 cells (Fig.), isolated rat and pig islet cells in a dose- and time-dependent manner. RAGE ligands-induced INS-1 cell apoptosis was inhibited by antioxidants (NAC $1 \mathrm{mM}$, a-lipoic acid $150 \mathrm{uM}$ ) and accompanied by increased IC ROS and decreased MMP. This increase of IC ROS by RAGE ligands was inhibited by NADPH oxidase inhibitor, AEBSF (20 uM).
Conclusion: Our results suggest that RAGE ligands and RAGE interaction which increases in diabetes may contribute not only to the development of chronic diabetic complications but also to the progressive pancreatic beta cell loss in type 2 diabetes by inducing IC oxidative stress.

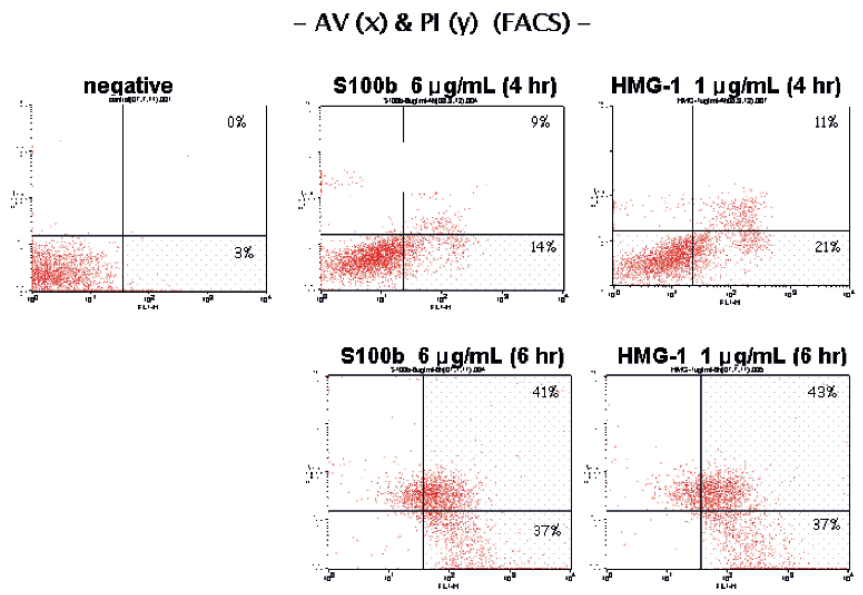

Fig. Effect of S100b \& HMG-1 (6hr) on INS-1 Cell

Supported by: Innovative Research Institute for Cell Therapy, Republic of Korea

\section{8}

$\mathrm{Ca}^{2+} /$ calmodulin-dependent protein kinase II $\delta_{2}$ (CamK II) is necessary for the expression of beta cell phenotypic genes and may link to reversible glucotoxicity

M.A. Osterhoff' ${ }^{1,2}$, S. Bessenyei ${ }^{1}$, A. Sharma ${ }^{1}$, Ö. Gögebakan ${ }^{1,2}$, M. Möhlig $^{1,2}$, A.F.H. Pfeiffer ${ }^{1,2}$

${ }^{1}$ Clinical Nutrition, German Institute of Human Nutrition, Nuthetal, ${ }^{2}$ Department for Endocrinology, Diabetes and Nutrition, Charité University Medicine, Berlin, Germany

Background and aims: The expression of CamK II $\delta_{2}$ is necessary for insulin gene-expression in the rat insulinoma cell line INS-1. Prolonged activation of CamK II by chronically elevated [Ca2+], e.g. after chronic glucose-stimulation, leads to deactivation of CamK II and decreased expression of typical $\beta$ cell genes. In the present study we show that CamK II mediates the regulation of expression of PDX- 1 controlled typical $\beta$-cell genes in INS- 1 cells.

Materials and methods: INS- 1 and, by an antisense RNA approach, CamK II $\delta_{2}$ suppressed INS $\delta$-W12 cells were cultured in RPMI 1640 media. CamK II was re-expressed by transfecting a construct coding for the human hCamK II $\beta_{1}$ into INS $\delta$-W12 cells by use of FuGene 6 (Roche). The gene-expression of b-cell genes was determined by quantitative real-time PCR (RotorGene, Corbett Research) using $\alpha$-tubulin as a standard. For suppression of CamK II $\delta$ expression by an independent approach in unmodified INS-1 cells CamK II $\delta$ specific siRNA (Qiagen) was used and protein concentration detected in western-blots by use of a CamK II $\delta_{2}$ specific antibody.

Results: The reduction of the CaMK II $\delta_{2}$-expression in INS- 1 cells by an antisense RNA approach (INS $\delta$-W12) led to a markedly decreased expression of the $\beta$-cell genes insulin, IAPP, GLUT- 2 and glucokinase while the expression of hexokinase I was increased. Metabolic assays showed a right-shift of the half-maximum glucose-induced metabolic activity from $5 \mathrm{mmol} / \mathrm{l}\left(\mathrm{K}_{\mathrm{m}}\right.$ of glucokinase) to $0.5 \mathrm{mmol} / \mathrm{l}\left(\mathrm{K}_{\mathrm{m}}\right.$ of hexokinase). The re-transfection of hCaMK II $\beta_{1}$ into INS $\delta$-W12 cells was able to restore - at least in part - the $\beta$-cell phenotype of INS- 1 cells, represented by a doubling of the gene-expression of insulin, glucokinase and GLUT-2 while the expression of hexokinase I was reduced to $50 \%$. Ongoing experiments demonstrated that CamK II $\delta$ expression can also be suppressed by transfection of CamKII $\delta$ specific siRNA into INS-1 cells. Conclusion: The rescue of the $\beta$-cell phenotype of INS- 1 cells after CamK II $\delta_{2}$ suppression by re-transfection of human CaMK II $\beta 1$ not only proves the specifity of the retroviral antisense mRNA approach to selectively reduce the CaMK II $\delta_{2}$ expression. It also shows clearly that CamK II is not deactivated in parallel to but controls the expression of $\beta$-cell genes. Possibly, CaMK II is a mediator of reversible glucotoxicity since high intracellular $\mathrm{Ca}^{2+}$-levels led to deactivation of CaMK II and by this to a decreased expression of $\beta$-cell genes and finally to dedifferentiation of $\beta$-cells. 


\section{9}

Lipotoxicity in insulin-producing cells is neither amplified by high glucose nor mediated by glucokinase

H. Schmitt, S. Lenzen, S. Baltrusch

Institute of Clinical Biochemistry, Hannover Medical School, Germany

Background and aims: In pancreatic beta cells, glucokinase (GK) acts as a glucose sensor and catalyzes the rate-limiting step for initiation of glucoseinduced insulin secretion. Using the recently developed RheoSwitch Mammalian Inducible Expression System, based on the synthetic inducer RSL1, it was shown that precise up-regulation of GK in the presence of high glucose did not cause cellular toxicity. In contrast, GK overexpression resulted in an increase in glucose-induced insulin secretion. However, high glucose as well as enhanced glucose metabolism has been considered to potentiate toxic effects of free fatty acids (FFA) in beta cells. The aim of this study was to elucidate whether high glucose or GK expression amplify lipotoxicity in insulin-producing cells.

Materials and methods: Previously generated RINm5F-R-EYFP-GK cells, in which GK can be adjustably expressed in response to the synthetic inducer RSL1 were incubated at different glucose concentrations with or without the FFA palmitic acid (PA). Cell viability was measured by MTT assay and GK enzyme activity by a photometric assay. The mitochondrial membrane potential was measured using the potentiometric dye JC-1 or TMRE in combination with MitoTracker Green ${ }^{\circ}$. The change of mitochondrial membrane potential induced by $10 \mathrm{mmol} / \mathrm{l}$ glucose was analyzed every minute over $60 \mathrm{~min}$ by fluorescence microscopy. The slope of the JC-1 ratio change and TMRE/ MitoTracker Green ratio change of the individual cells was calculated offline.

Results: RINm5F-R-EYFP-GK cells were cultured for $48 \mathrm{~h}$ in the absence or presence of $0.5,62.5,125,250$ and $500 \mathrm{nmol} / 1$ of the inducer RSL1 in medium supplemented with 1,10 or $30 \mathrm{mmol} / \mathrm{l}$ glucose with or without PA. The cell viability decreased consistently at all glucose concentrations and GK expression levels by $40 \%$ and $70 \%$ in the presence of 100 and $200 \mu \mathrm{mol} / \mathrm{l}$ palmitic acid, respectively. The toxic effect of PA was comparable to that observed in RINm5F control cells. The glucokinase enzyme activity in RINm5F-REYFP-GK cells was only slightly affected by PA. An extended incubation time of 14 days at $30 \mathrm{mmol} / \mathrm{l}$ glucose in the presence of 0 or $500 \mathrm{nmol} / \mathrm{l}$ of the inducer RSL1 did not decrease cell viability of RINm5F-R-EYFP-GK cells. Furthermore, these cells pre-cultured at high glucose did not show a higher sensitivity in response to a $48 \mathrm{~h}$ incubation at $200 \mu \mathrm{mol} / \mathrm{l} \mathrm{PA}$. In addition, the mitochondrial membrane potential change was measured in RINm5F-REYFP-GK cells pre-cultured at 10 or $30 \mathrm{mmol} / \mathrm{l}$ glucose. After starvation for $90 \mathrm{~min}$ in the absence of glucose, RINm5F-R-EYFP-GK cells with GK expression showed in response to $10 \mathrm{mmol} / \mathrm{l}$ glucose a significantly faster increase in the mitochondrial membrane potential than RINm5F-R-EYFP-GK cells without GK expression.

Conclusion: Our studies show that culture at high glucose does not amplify lipotoxicity in insulin-producing cells. Furthermore, glucose metabolism in beta cells is apparently not involved in FFA induced toxicity. Increased expression of the glucose phosphorylating enzyme glucokinase accelerates metabolism and transmits the metabolic signal faster to the mitochondria.

\section{0}

Overexpression of anti-oxidative $\mathrm{K}_{\mathrm{ATP}}$ channels protects pancreatic beta cell functions from glucose toxicity

T. $\mathbf{L u}^{1}$, T. He${ }^{2}$, H.-C. Lee ${ }^{1}$

${ }^{1}$ Internal Medicine, ${ }^{2}$ Anesthesiology, Mayo Clinic, Rochester, United States

Background and aims: ATP-sensitive $\mathrm{K}^{+}\left(\mathrm{K}_{\mathrm{ATP}}\right)$ channels, encoded by Kir6.2/ SUR, are crucial for controlling pancreatic $\beta$-cell insulin secretion. Over produced reactive oxygen species (ROS) are known to be the key mechanism of $\beta$-cell glucose toxicity and impairs $\beta$-cell insulin secretion. However, whether ROS-mediated $\mathrm{K}_{\text {ATP }}$ channel modulation would affect $\beta$-cell function has not been determined.

Methods: Using patch clamp techniques and molecular biological approaches, we compared the $\mathrm{K}_{\text {ATP }}$ channel property and insulin secretion from HIT-T15 cell lines in $5 \mathrm{mM}$ glucose $(\mathrm{NG}$ ) and $22 \mathrm{mM}$ glucose (HG) culture conditions. We found that the $\mathrm{K}_{\text {ATP }}$ current of HIT-T15 cells has a strong inward component and relative small outward component of currents in NG, appearing a weak inwardly rectifying property (Fig. A). In contrast, 2-week exposure to $\mathrm{HG}$ reduced the inward component of $\mathrm{K}_{\mathrm{ATP}}$ currents from $-21.9 \pm 4.3 \mathrm{pA} / \mathrm{pF}$ in $\mathrm{NG}(\mathrm{n}=6)$ to $-5.9 \pm 3.2 \mathrm{pA} / \mathrm{pF}$ in $\mathrm{HG}\left(\mathrm{n}=6, \mathrm{p}<0.05^{*}\right)$ at $-100 \mathrm{mV}$, and enhanced the outward component of currents from $1.2 \pm 2.4 \mathrm{pA} / \mathrm{pF}$ in $\mathrm{NG}(\mathrm{n}=6)$ to $31.4 \pm 2.3 \mathrm{pA} / \mathrm{pF}^{*}$ in $\mathrm{HG}(\mathrm{n}=6)$ at $+100 \mathrm{mV}$ (Fig. B). Thus, culture with HG converted the channel property from an inward rectifier into an outward rectifier (Fig. C). The $\mathrm{HG}$ effects on $\mathrm{K}_{\mathrm{ATP}}$ channel property were mimicked by $\mathrm{H}_{2} \mathrm{O}_{2}$ applied to the cytoplasmic surface of cells, which were reversible by DDT, suggesting that the mechanism of cysteine oxidation was involved (Fig. D). Molecular determinant studies confirmed that the cysteine residue at 166 (C166) of Kir6.2 subunit were responsible for the $\mathrm{HG}$ and $\mathrm{H}_{2} \mathrm{O}_{2}$ effects. Replacement of $\mathrm{C} 166$ by alanine (C166A) on Kir6.2 eliminated $95 \%$ the $\mathrm{H}_{2} \mathrm{O}_{2}$ effects (Fig. E) and completely abolished the HG effects. 2 weeks culture with HG reduced the HIT-T15 cell insulin secretion in response to $22 \mathrm{mM}$ glucose stimulation (from $1.08 \pm 0.06 \mathrm{ng} / \mathrm{ml}$ of baseline to $1.82 \pm 0.04 \mathrm{ng} / \mathrm{ml}$ at $60 \mathrm{~min}$ exposure to $22 \mathrm{mM}$ glucose, $\mathrm{n}=3$ ), compared to those cells cultured with NG (from $1.52 \pm 0.05 \mathrm{ng} / \mathrm{ml}$ of baseline to $3.05 \pm 0.11 \mathrm{ng} / \mathrm{ml}{ }^{*}$ at $60 \mathrm{~min}$ exposure to $22 \mathrm{mM}$ glucose, $\mathrm{n}=3$ ) (Fig. F). Importantly, over expression of Kir6.2C166A in HIT-T15 $\beta$-cells significantly increased insulin secretion by $50 \%$ in HG condition (Fig. F).

Conclusion: Our results indicated that redox modulation of $\mathrm{K}_{\text {ATP }}$ channels impaired pancreatic $\beta$-cell insulin secretion. The C166 residue of Kir6.2 is the molecular target of ROS. Prevention of $\mathrm{K}_{\text {ATP }}$ channel oxidation would enhance pancreatic $\beta$-cell functions in chronic hyperglycemia.
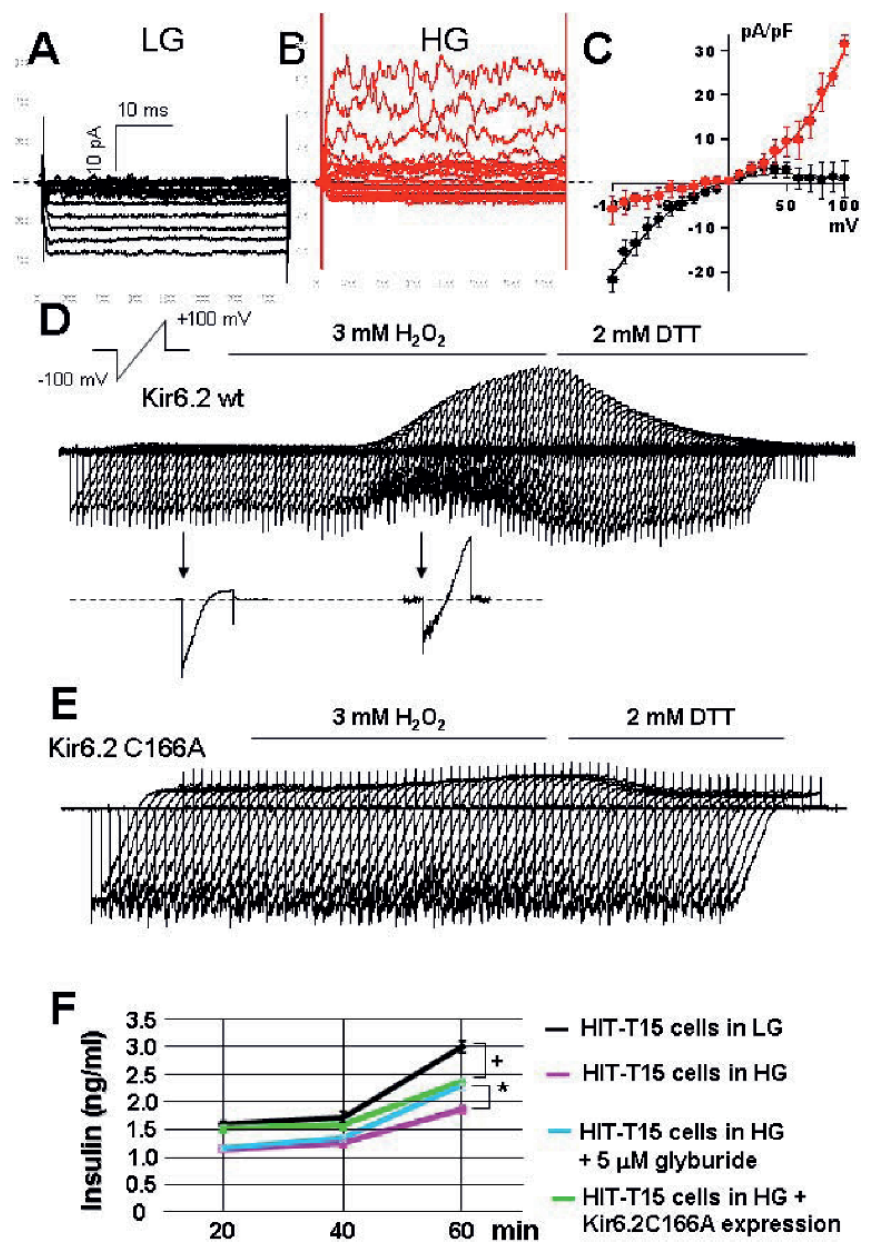

Supported by: Junior Faculty Award from American Diabetes Association 


\section{1}

Development of a predictive model for pancreatic islet amyloidosis in the baboon based on fasting plasma glucose and age. Implication for the study of beta cell failure in human type 2 diabetes

F.B. Folli ${ }^{1}$, A. Davalli ${ }^{2}$, A.O. Chavez ${ }^{1}$, A. Comuzzie ${ }^{3}$, E. Tejero ${ }^{4}$, P. Zuo ${ }^{1}$, Z. Chang ${ }^{1}$, E. Dick, Jr. ${ }^{4}$, G. Hubbard ${ }^{4}$, A. Cruz ${ }^{5}$, C. Perez ${ }^{5}$, G. Halff', A. Gastaldelli ${ }^{2}$, R.A. DeFronzo ${ }^{1}$, R. Guardardo Mendoza ${ }^{1}$

${ }^{1}$ Medicine/Diabetes, UTHSCSA, San Antonio, United States, ${ }^{2}$ Dept of Medicine, Istituto Scientifico San Raffaele, Milano, Italy, ${ }^{3}$ Southwest

National Primate Center, Southwest Foundation for Biomedical Research, San Antonio, United States, ${ }^{4}$ Diabetes Research, Southwest Foundation for Biomedical Research, San Antonio, United States, ${ }^{5}$ Diabetes Research, Universidad de Colima, Mexico

Background and aims: Pancreatic islet amyloidosis (PIA) has been implicated in the pathogenesis of beta cell failure in human type 2 diabetes. The baboon genome shares $98 \%$ overlap with the human. Thus, the baboon represents an ideal model to understand the pathophysiology of PIA and beta cellfailure in humans. Thus, the aim of this work was to identify the predictors of PIA in baboon model of insulin resistance and T2DM.

Materials and methods: We studied 150 baboons in which glucose, insulin and glucagon concentrations were measured prior to necropsy. PIA, islet size, islets and amyloid volumes were quantified at necropsy using a stereological approach. Genetic characterization of the baboon islet amyloid polypeptide amylin (IAPP) showed that the sequence was $90 \%$ identical to that of humans at the cDNA level and $97 \%$ identical at the protein level.

Results: Severity of amyloid deposition was stratified into quintiles $(\mathrm{Q} 1=5 \%$ to $\mathrm{Q} 5=51 \%$ ). Progressive increases in age (from 16 to 22), FPG (from 75 to $187 \mathrm{mg} / \mathrm{dl}$ ), $\mathrm{HbA}_{1 \mathrm{C}}$ (from 4.6 to $9.3 \%$ ), FFA (from 0.53 to $1.15 \mathrm{mmo} / \mathrm{l}$ ), glucagon (from 60 to $112 \mathrm{ng} / \mathrm{l}$ ), islet size (from 9 to $13 \mathrm{~mm}^{2}$ ) and islets volume (from 2.6 to $5.4 \%$ ) were observed from quintile I to quintile V. (all $\mathrm{p}<0.05$ ). After adjusting for gender, age and weight, the differences in FPG and HbAlc persisted $(\mathrm{p}<0.05)$. We used HOMA-B (log transformed due to the skewed distribution) as an index of beta cell function. $\operatorname{Ln}($ HOMA-B) decreased progressively with increasing amyloid severity $(r=-0.509, p<0.0001)$ and increasing FPG $(r=-0.589, \mathrm{p}<0.0001)$. In a multivariate analysis, age, gender, obesity, FFA, $\operatorname{Ln}$ (glucose), $\operatorname{Ln}$ (insulin) and $\operatorname{Ln}$ (glucagon) were the most important predictors of PIA (adjusted $\mathrm{R}^{2}=0.535, \mathrm{p}<0.0001$ ). Using only glucose and age, the adjusted $\mathrm{R}^{2}$ was $0.519, \mathrm{p}<0.0001$. Thus, amyloid severity (\%) was predict by the following equation $=1.118 \times$ Age (years) $+37.57 \times \operatorname{Ln}$ (glucose $\mathrm{mg} / \mathrm{dl})-164$.

Conclusion: (1) FPG directly correlates with PIA severity in baboons, over the range of the metabolic abnormalities typically observed in human T2DM; (2) The main predictors of PIA in baboons are FPG and age. These data support the concept that the baboon is a novel and important non-human primate model for the study of pathophysiology, including beta cell failure, and novel therapies for preventing PIA in T2DM.

\section{PS 033 Apoptotic mechanisms in beta cells}

\section{2}

Impaired glucose tolerance in calbindin D28k-ablated mice in vivo is associated with impaired calcium response and insulin secretion in islets in vitro

S. Rohleder ${ }^{1,2}$, U. Zeitz ${ }^{1}$, R.G. Erben ${ }^{1,2}$

${ }^{1}$ Institute of Pathophysiology, Dept. of Biomedical Sciences, University of Veterinary Medicine Vienna, Austria, ${ }^{2}$ Institute of Animal Physiology, Ludwig Maximilians University, Munich, Germany

Background and aims: The calcium binding protein calbindin D28k (D28k) has been identified in various tissues including brain, kidney, bone, and pancreas. D28k is thought to enhance renal tubular calcium reabsorption, and may protect cells from apoptosis in the brain. Although it has been suggested the D28k may act as a calcium buffer in pancreatic alpha and beta cells, the role of D28k in islet cells in vivo is still unclear. It was the aim of the present study to explore further the functional role of D28k for insulin secretion and intracellular calcium handling in islets of Langerhans, using D28k knockout (D28k-KO) mice.

Materials and methods: All in vivo experiments were performed in 12-weekold male wild-type (WT) or homozygous D28k-KO mice. After an overnight fast, $1.5 \mathrm{mg}$ glucose per g body weight was administered at time 0 by subcutaneous injection or gavage. Blood glucose levels were determined at 0,10 , 20, 30, 60 and $120 \mathrm{~min}$ in whole blood samples from the tail tip by a standard test system. At baseline and 10 minutes, additional blood samples were taken for measurement of serum insulin. Islet and beta cell area were quantified histomorphometrically in paraffin sections after anti-insulin immunostaining. For in vitro studies, islets were isolated from WT and D28k-KO mice by collagenase digestion. Static glucose stimulation tests were performed directly after isolation. Subsequently, the same islets were cultured in RPMI 1640 and $10 \%$ FCS; insulin secretion tests were repeated on day 2 of culture. For quantification of intracellular calcium peaks, freshly isolated islets were incubated with Fura2-AM and fastened in a quartz cuvette in modified KRBH buffer containing $5 \mathrm{mM}$ glucose after adherence to APES/polylysin-treated glass cover slips. Changes in intracellular calcium in response to a $30 \mathrm{mM}$ glucose stimulus were quantified using a Jasco FP 6300 spectrophotometer. Whole intracellular calcium was quantified after dissolution of 20 islets in a small volume quartz cuvette. All insulin samples were quantified by a mouse specific ELISA.

Results: In subcutaneous, but not oral, glucose tolerance tests we found slightly, but significantly impaired glucose tolerance (AUC increased by $8 \%$ from $28914 \pm 346$ to $31403 \pm 1132, \mathrm{P}<0.05$ ) and a trend for lower insulin secretory capacity (reduction from $0.1649 \pm 0.02320$ to $0.1101 \pm 0.013 \mathrm{ng} / \mathrm{ml}$, $\mathrm{P}<0.051)$ in D28k-KO mice (data are means \pm SEM). Freshly isolated islets of knockout mice displayed impaired insulin secretion, a 3-fold reduction in the intracellular calcium peak following glucose stimulation, and $80 \%$ lower islet calcium content compared with WT. However, insulin secretion returned to normal in islets of D28k-KO mice after 2 days of culture with 10\% FCS. Histomorphometry revealed a $6 \%$ higher beta cell area per islet area in D28k-KO mice.

Conclusion: Our data show that D28k has a functional role in beta cells in vivo. The impaired insulin secretion in D28k-ablated mice may be caused by deteriorated calcium handling within beta cells. We hypothesize that this cellular defect is compensated for by an increase in beta cell mass in vivo, which might explain why it remains subclinical, and is only detectable after a glucose challenge. Further experimentation is required to dissect the intracellular function of D28k in the beta cell.

Supported by: Deutsche Forschungsgemeinschaft

\section{3}

Subcellular distribution and function of Bcl-2 and Bcl-xL in the beta cell D.S. Luciani, S. Widenmaier, J. Yeung, C. McIntosh, J.D. Johnson Department of Cellular and Physiological Sciences, University of British Columbia, Vancouver, Canada

Background and aims: Bcl-2 family proteins are integral in the control of $\beta$ cell apoptosis and emerging evidence also suggests important roles in $\beta$-cell physiology. Both pro- and anti-apoptotic members of this family interact to regulate programmed cell death, and this control can be exerted at several 
cellular organelles. Bcl-2 and Bcl-xL are both believed to be important for $\beta$-cell survival, but their relative abundance and localization within the $\beta$-cell are not well characterized. Moreover, much of the current knowledge regarding Bcl-2 family members stems from overexpression studies which do not necessarily recapitulate the state of the endogenous proteins. The aims of the present study were to determine the subcellular distribution of endogenous and overexpressed Bcl-2 and Bcl-xL in $\beta$-cells and to further clarify the organelle-specific functions of these important pro-survival proteins.

Materials and methods: Immunofluorescent staining of primary mouse $\beta$ cells, MIN6 cells and mouse pancreas sections was employed to visualize the localization of $\mathrm{Bcl}-2$ and $\mathrm{Bcl}-\mathrm{xL}$ relative to intracellular organelles. Association of Bcl-2 and Bcl-xL with organelles was further probed by subcellular fractionation and western blot. Liposome-based transfection was used to transiently overexpress fluorescent protein-tagged Bcl-2 and Bcl-xL. Small molecule inhibitors were used in combination with live cell microscopy and western blotting to study the impact of Bcl-2/Bcl-xL antagonism on mitochondrial function, glucose-stimulated insulin secretion and $\beta$-cell viability. Results: In both cultured $\beta$-cells and pancreatic sections immunofluorescent staining with monoclonal and polyclonal antibodies to Bcl-2 revealed clear nuclear localization with no detectable signal in the cytosolic compartment. This differed significantly from the distribution of exogenous GFP-tagged Bcl-2 in transfected cells. Bcl-2:GFP was recognized by the antibodies, but localized exclusively to the cytoplasm with prominent mitochondrial co-localization. In contrast to endogenous $\mathrm{Bcl}-2$, endogenous $\mathrm{Bcl}-\mathrm{xL}$ was found by both immunostaining and cell fractionation to be primarily mitochondrial and non-nuclear. Indeed, a small molecule inhibitor of $\mathrm{Bcl}-\mathrm{xL} / \mathrm{Bcl}-2$ caused immediate mitochondrial hyperpolarization and KATP-channel-dependent cytosolic $\mathrm{Ca}^{2+}$ signals. These events occurred hours before the onset of caspase-3-dependent apoptosis. We also observed an immediate effect on glucose-stimulated insulin secretion using Bcl-xL/Bcl-2 inhibitors. Together these data suggest links between $\mathrm{Bcl}$ family members and $\beta$-cell function.

Conclusion: Endogenous $\mathrm{Bcl}-2$ and $\mathrm{Bcl}-\mathrm{xL}$ differ markedly in their localization within the pancreatic $\beta$-cell. While Bcl-2 is found in the nucleus, Bcl-xL shows strong association with mitochondria. Prominent mitochondrial effects of Bcl-2/Bcl-xL inhibitors thus suggest that of the two proteins, Bcl-xL may be the primary regulator of $\beta$-cell mitochondrial apoptosis and physiology. Further studies on the roles of nuclear-targeted endogenous Bcl-2 are warranted. Moreover, differences in the intracellular partitioning of exogenous and endogenous Bcl-2 advocate caution when using overexpression to study Bcl-2 function in $\beta$-cells.

Supported by: JDRF, CIHR and CDA

\section{4}

Insulin induces apoptosis at long-term in pancreatic beta cells

M. Benito, A. Bartolome, C. Guillen

Bioquimica y Biologia molecular, Facultad De Farmacia, Universidad

Complutense, Madrid, Spain

Background and aims:Type 2 diabetes results from a combination of insulin resistance and impaired insulin secretion Insulin resistant states are associated with an increase in the beta cell mass and also high levels of circulating insulin. Ultimately the beta cells undergo a failure in the insulin secretion that leads to uncontrolled diabetes. At this stage, a question arises if those persistent high levels of circulating insulin may contribute to beta-cell damage. To address this important issue, we have submitted beta cells to a prolonged effect of increasing concentrations of insulin.

Materials and methods: We have previously established mouse beta cell lines lacking or not insulin receptors (IR) in culture from the LoxP/LoxP IR mice. Thus, immotalized loxP/LoxP IR beta cells were infected with adenoviruses bearing or not Cre and subsequently subcloned to assure cells lacking IR. These beta cells proliferate independently in response to insulin or glucose through the convergent signaling pathway mTOR/p70 S6 kinase.

Results: Insulin $10 \mathrm{nM}$ induces at short term Akt and also p70 S6 kinase phosphorylation in beta cells in the absence of glucose (from 2 to $8 \mathrm{~h}$ ). However, insulin effect on survival signaling attenuated at $15 \mathrm{~h}$ and was totally impaired at $24 \mathrm{~h}$. In parallel, glucose-deprived beta cells enhanced caspase- 3 in its active form (cleaved caspase-3). Under the same experimental conditions, insulin inhibited cleaved caspase- 3 expression at short term, but increased its expression at long term. At $24 \mathrm{~h}$, glucose-deprived beta cells increased caspase-3 activity and also the number of haplodiploid cells but decreased the cell number. Under these conditions, there was a shift from inhibitory to stimulatory effects of insulin on caspase 3 activity and also haplodiploids cell number in a dose-dependent manner. Opposite effects were observed regard- ing beta cells number. The stimulatory effect at $24 \mathrm{~h}$ of high levels of insulin (from 10 to $100 \mathrm{nM}$ ) on cleaved caspase-3 expression, as confirmed by twodimensional electrophoresis immunoblotting, was not observed in beta cells lacking IR or in beta cells bearing IR in the presence of glucose $5 \mathrm{mM}$. Finally, the long-term effect of insulin on the above parameters was cycloheximidedependent, suggesting that a endogenous protein factor was required for the deleterious effect of insulin.

Conclusion: All these results seem to suggest that insulin, beside its survival short-term effect, was able to induce beta-cell death owing to apoptosis in a dose- a time-dependent manner. More importantly, this long-term effect of insulin was entirely dependent on endogenous protein synthesis. Thus persistent supra physilogical levels of insulin observed in several insulin resistant states might contribute to beta-cell damage and subsequently to the progression to type 2 diabetes.

Supported by: M.E.C, Spain

\section{5}

Serine/threonine protein phosphatase 5 mediates dexamethasoneinduced beta cell death through its modulation of p38 MAPK phosphorylation

H. Ortsäter, L. Fransson, N. Grankvist, R.E. Honkanen, Å. Sjöholm Dept. of Clinical Science and Education, Karolinska Institutet, Stockholm, Sweden

Background and aims: Serine/threonine protein phosphatase 5 (PP5) is a single polypeptide chain containing a catalytic domain near its C-terminus and a regulatory domain at the $\mathrm{N}$-terminus end. Through tetratricopeptide repeat domains the phosphatase interacts with heat-shock protein 90. By this interaction PP5 has been shown to coimmunoprecipitate with the glucocorticoid receptor (GR). A reduction in cellular levels of PP5 affects the pattern of genes that are activated after glucocorticoid treatment. Since glucocorticoids can evoke both insulin secretory dysfunction and pancreatic $\beta$-cell death when given to islets of Langerhans in vitro, and steroid diabetes in patients, we wanted to investigate whether PP5 influences dexamethasone-induced cell death in the insulin-producing cell line MIN6.

Materials and methods: Clonal pancreatic MIN6 cells were treated with a siRNA directed against PP5 to reduce the cellular levels of PP5. After siRNA treatment cells were exposed to $100 \mathrm{nM}$ of dexamethasone with/out either $1 \mu \mathrm{M}$ of the GR antagonist RU486 or $20 \mu \mathrm{M}$ of the p38 MAPK inhibitor SB203580. After culture we assessed apoptosis induction by measuring the levels of cytoplasmic DNA-histone complexes. We also analyzed PP5 mRNA levels by quantitative PCR. Finally, we measured PP5 protein levels and the degree of p38 MAPK phosphorylation by Western blotting.

Results: In MIN6 cells, PP5 mRNA and protein levels were reduced by 50 $60 \%$ after 24-48 hours of siRNA treatment. Treating control MIN6 cells with dexamethasone for 30 hours caused a $4.5 \pm 0.6(n=9)$ fold induction of apoptosis compared to untreated cells. Such an effect was fully blocked by co-treating the cells with RU486. The ability of dexamethasone to induce apoptosis was attenuated by $25 \pm 4 \%(n=9, p<0.05)$ in cells with reduced levels of PP5. We next investigated the ability of dexamethasone to activate p38 MAPK signaling. p38 MAPK phosphorylation was 5.1 $\pm 1.0(n=5)$ fold increased after a 30-hour dexamethasone exposure. Again, such an effect was fully blocked by co-treating the cells with RU486. In cells with reduced levels of PP5, dexamethasone only managed to cause a $3.0 \pm 0.7$ fold $(n=5)$ increase in p38 MAPK phosphorylation. Finally, we investigated whether inhibition of p38 MAPK signaling could protect MIN6 cells from dexamethasone-induced apoptosis. Co-culturing cells with dexamethasone and the p38 MAPK inhibitor SB203580 reduced apoptosis by $50 \%$ compared to cells treated with dexamethasone alone.

Conclusion: In the present study, we show that dexamethasone induces phosphorylation of p38 MAPK and cell death in insulin-producing MIN6 cells. Both of these effects seem to be mediated via the GR since RU486 inhibited them fully. It is also evident that they are connected because disruption of p38 MAPK signaling reduced dexamethasone-induced apoptosis. Furthermore, it is shown that PP5 is a key player in regulating glucocorticoid signaling in these cells. When cellular levels of PP5 were reduced by means of siRNA treatment, both induction of p38 MAPK phosphorylation and dexamethasone-induced apoptosis were attenuated. We hypothesize that reduced levels of PP5 change the GR transcriptional activity that subsequently reduces p38 MAPK phosphorylation and apoptosis. Inhibition of PP5 may prove valuable in efforts aimed at $\beta$-cell protection against apoptosis in steroid diabetes. Supported by: Swedish Society for Medical Research 


\section{6}

Transient oxidative stress in INS-1E cells and rat islets induces mitochondrial damages promoting prolonged beta cell dysfunction N. Li, P. Maechler

Department of Cell Physiology and Metabolism, University of Geneva, Switzerland

Background and aims: Mitochondrial injuries caused by oxidative stress are putative mechanism of pancreatic beta-cell failure in the course of diabetes. We and others have previously shown that transient exposure of beta-cells to oxidant interrupts the transduction signals normally coupling glucose metabolism to insulin secretion. Here, we analysed beta-cell function after a 3day recovery period following single exposure to oxidative stress.

Materials and methods: Using INS-1E beta-cell and isolated rat islets, we studied long-term impact of a single transient oxidative stress $(200 \mu \mathrm{M} \mathrm{H} 2 \mathrm{O} 2$ for $10 \mathrm{~min}$ ) applied 3 days before analyses. Then, key parameters for metabolism secretion coupling were measured: mitochondrial membrane potential (rhodamine-123 fluorescence), cytosolic ATP production (luciferase bioluminescence), oxygen consumption (on isolated mitochondria in an oxymeter), and glucose-stimulated insulin secretion (RIA). In order to detect putative mechanisms underlying prolonged dysfunction, mitochondrial ROS generation (Amplex Red), integrity of respiratory chain complex subunits (western blot), and mitochondrion associated gene expression (qRT-PCR) were analysed.

Results: Three days after the single transient oxidative stress INS-1E betacells exhibited markedly reduced mitochondrial function and secretory responses to glucose. Compared to non-stressed INS-1E cells, we observed impaired mitochondrial hyperpolarization (-55\% upon glucose stimulation), reduced ATP production (-59\%), decreased oxygen consumption $(-43 \%$ and $-60 \%$ upon $\mathrm{NADH}$ and succinate+ADP stimulations, respectively), and blunted glucose-stimulated insulin secretion $(-51 \%, \mathrm{P}<0.01)$. Similar effects were observed in isolated rat islets. These parameters correlated with increased mitochondrial ROS formation $(+60 \%$ and $+40 \%$ upon NADH and succinate stimulations, respectively) accompanied by down-regulation of the respiratory chain subunits complex I (-24\%), complex III (-21\%) and complex IV (-29\%). We also measured decreased expression of genes responsible for mitochondrial biogenesis, such as TFAM (-74\%) and PGC-1alpha (-84\%). Three weeks after single oxidative stress, both mitochondrial respiration and secretory responses of INS-1E cells were totally recovered. Moreover, such recovered INS-1E cells exhibited resistance to a second transient oxidative stress.

Conclusion: INS-1E beta-cells and rat islets memorized one single transient oxidative stress as revealed by impairment of their mitochondrial respiratory chain and blunted glucose-stimulated insulin secretion 3 days after stress. This memory was induced by transient ROS exposure that in turn promoted molecular changes maintaining subsequent endogenous ROS generation. The cells could recover metabolism-secretion coupling after 3 weeks and even became more resistant to the secondary oxidative attack. Supported by: EFSD research award supported by BMS

\section{7}

\section{Serine/threonine protein phosphatase 5 regulates the phosphorylation} status of JNK after hydrogen peroxide exposure in pancreatic beta cells N. Grankvist, J. Pettersson, R. Honkanen, ̊. Sjöholm, H. Ortsäter Department of Clinical Science and Education, Karolinska Institutet, Stockholm, Sweden

Background and aims: In type 2 diabetes, where pancreatic $\beta$-cells are exposed to elevated glucose concentrations, a reduced $\beta$-cell mass is seen. This could be the result from an increased production of reactive oxygen species (ROS) that cause cellular damage and initiate apoptosis. An important positive regulator of apoptosis is the mitogen-activated protein kinase (MAPK) signaling pathway. One mammalian MAPK is c-Jun N-terminal kinase (JNK) which can be activated by an apoptosis signal-regulating kinase (ASK1). Serine/threonine protein phosphatase 5 (PP5) is a single polypeptide chain that can be present both in the cytosol and the nucleus. It has been suggested that PP5 can act as a negative regulator of ASK1/JNK signaling. The aim of this study was to investigate the role of PP5 in the regulation of JNK signaling in MIN6 cells exposed to hydrogen peroxide $\left(\mathrm{H}_{2} \mathrm{O}_{2}\right)$.

Materials and methods: Clonal pancreatic MIN6 cells were exposed to $0.5 \mathrm{mM} \mathrm{H}_{2} \mathrm{O}_{2}$ to induce cell death, for 4,8 and 16 hours. Cells were also cotreated with the $\mathrm{H}_{2} \mathrm{O}_{2}$ and ROS scavengers catalase and tempol. To analyze if cells were able to recover from the $\mathrm{H}_{2} \mathrm{O}_{2}$ treatment, cells were exposed to $\mathrm{H}_{2} \mathrm{O}_{2}$ for $30 \mathrm{~min}$ and were then allowed to recover. Cell viability was then analyzed by lactate dehydrogenase activity assay. In order to reduce the PP5 expression level, MIN6 cells were treated with siRNA directed against PP5. The cells were then exposed to $\mathrm{H}_{2} \mathrm{O}_{2}$ for $0,5,15$ and $30 \mathrm{~min}$, together with control cells without reduced levels of PP5. In MIN6 cells with reduced levels of PP5 the scavengers catalase, MnSOD, CuZnSOD and thioredoxin peroxidase were measured. The PP5 and the different scavengers mRNA levels were analyzed by quantitative PCR. The protein levels and degree of JNK phosphorylation were measured by Western blotting.

Results: MIN6 cells treated with $0.5 \mathrm{mM} \mathrm{H}_{2} \mathrm{O}_{2}$ for 4,8 and 16 hours showed a $60-70 \%$ decrease in cell viability. This decrease was fully and partly prevented by the ROS scavengers catalase and tempol, respectively. A 30 min exposure to $0.5 \mathrm{mM} \mathrm{H}_{2} \mathrm{O}_{2}$ did not induce a decrease in cell viability. However, if the cells were left in the absence of $\mathrm{H}_{2} \mathrm{O}_{2}$ for an additional time period $(3.5,7.5$ or 15.5 hours) reduced cell viability was as marked as in cells continuously exposed to $\mathrm{H}_{2} \mathrm{O}_{2}$. These results indicate that the cells were not able to recover from the $\mathrm{H}_{2} \mathrm{O}_{2}$ treatment. The degree of JNK phosphorylation was rapidly induced by $\mathrm{H}_{2} \mathrm{O}_{2}$ exposure. Phosphorylation of both JNK1 and 2 was enhanced 20 -fold after 15 min of exposure. In cells treated with siRNA against PP5 mRNA levels of the phosphatase were reduced by $50 \%$ after 24 hours. The ability of $\mathrm{H}_{2} \mathrm{O}_{2}$ to increase the phosphorylation levels of JNK was doubled in cells with reduced PP5 levels. Down-regulation of PP5 as such did not affect catalase, MnSOD, CuZnSOD and thioredoxin peroxidase mRNA levels.

Conclusion: These data show that $\mathrm{H}_{2} \mathrm{O}_{2}$-induced $\beta$-cell damage is a rapid event that is hard for the cells to recover from. A cellular event that rapidly occurs after $\mathrm{H}_{2} \mathrm{O}_{2}$ exposure is phosphorylation of JNK1 and 2. The data presented here suggests that PP5 is a negative regulator of JNK signaling since MIN6 cells treated with siRNA against PP5 had much higher levels of phosphorylated JNK after exposure to $\mathrm{H}_{2} \mathrm{O}_{2}$ as compared to control cells. These findings suggest that enhancement of PP5 activity might be a way to reduce cellular damage induced by ROS and may prove valuable in efforts aimed at $\beta$-cell protection against apoptosis in diabetes.

\section{8}

Effects of exendin-4 and interferon-gamma antibody on beta cell apoptosis and proliferation in BB rat

C. Segundo ${ }^{1}$, G. Pérez-Arana ${ }^{1}$, M. Blandino-Rosano ${ }^{1}$, A. Cebada-Aleu ${ }^{1}$, M. Aguilar-Diosdado ${ }^{2}$

${ }^{1}$ Investigation Unit, ${ }^{2}$ Endocrinology and Nutrition Service, Puerta del Mar Hospital, Cadiz, Spain

Background and aims: The autoimmune Diabetes Mellitus type 1 (DM1) is characterised by a loss of beta cell mass due to an autoimmune process (insulitis). Proinflammatory cytokines and free radicals mediated apoptosis has been considered the main mechanism of beta cell destruction by altering the natural death/proliferation balance. Exendin-4, a GLP-1 analog, and interferon-gamma antibody (IFN- $\gamma \mathrm{Ab}$ ) have been shown to make a benefit effect on beta cell mass. The aim of this work is to study "in vivo" changes in proliferation and apoptosis over the insulitis period after exendin-4 and IFN$\gamma \mathrm{Ab}$ administration.

Materials and methods: Biobredding (BB) rat strain was used as DM1 model. Animals were killed at 5, 7, 9 and 11 weeks of age and beta cell apoptosis and proliferation were quantified in pancreatic tissue by TUNEL and BrdU staining, respectively. A daily treatment with intraperitoneal Exendin-4 $(4186,6 \mathrm{ng} / \mathrm{kg})$, alone or in combination with weekly IFN- $\gamma \mathrm{Ab}$ administration $(100 \mathrm{mg} / \mathrm{Kg})$ was initiated at 5 weeks of age. Normal saline treated rats were used as control and proliferation, apoptosis and lymphocytic infiltration score were compared in both groups.

Results: A decrease in proliferating beta cell percentage at 7, 9 and 11 weeks of age in $\mathrm{BB}$ rat was observed. The start point of the loss of proliferation was previous to the apoptosis enhancement which reached statistical significance at 9 weeks of age. Treatment of BB rat with Exendin-4 alone recovered beta cell proliferative capacity at 7 and 9 but not at 11 weeks of age, and decreased beta cell apoptosis at 11 weeks of age. Combination of Exendin- 4 and IFN- $\gamma$ $\mathrm{Ab}$ improved Exendin- 4 effects alone by maintaining beta cell proliferation values higher than those in untreated rats all over the study. These effects were not related with the severity of insulitis.

Conclusion: The BB murine DM1 model presents a diminished beta cell proliferation from early stages of insulitis. Exendin- $4+$ anti IFN- $\gamma$, and to a lesser extent Exendin-4 alone, partially restores proliferative capacity and inhibits apoptosis in beta cells during insulitis without affecting islet infiltration. These results support a role of regulatory events of proliferation in DM1 
pathogenesis and suggest that either beta cell apoptosis and proliferation control could be important in preventive strategies against DM1.

Supported by: Diabetes Group Network of the Spanish Ministry of Health and the Andalusia Department of Health

\section{9}

Oleate prevents apoptosis but not UPR activation in palmitate-treated

INS-1E beta cells

E. Sargsyan, E.M. Sol, P. Bergsten

Medical Cell Biology, Uppsala University, Sweden

Background and aims: Saturated fatty acid palmitate (PA) and unsaturated fatty acid oleate $(\mathrm{OA})$ are two of the most abundant fatty acids in the circulation. Whereas long-term incubation of $\beta$-cells with PA induces apoptosis, incubation with OA has no such effect. Interestingly, the negative effect of PA on $\beta$-cell apoptosis can be prevented by co-culturing the cells with PA and $\mathrm{OA}$. Mechanisms for this protective effect of OA on $\beta$-cells exposed to PA are unclear, however. One of the potential mechanisms of how PA induces apoptosis is the unfolded protein response (UPR), which is not initiated in $\beta$-cells cultured in the presence of OA. Taking into consideration that OA reverses PA-induced apoptosis, we examined if the mechanism of such OA-induced reduction in apoptosis is via inhibiting development of the UPR.

Materials and methods: INS-1E cells were cultured for 24 hours in the presence of $0.5 \mathrm{mM}$ PA, $0.5 \mathrm{mM}$ OA complexed with $0.5 \%$ BSA or a combination of $0.5 \mathrm{mM}$ PA and $0.5 \mathrm{mM}$ OA complexed with $1 \%$ BSA. After culture, cells were lysed and cellular protein was isolated and used for measurements of phosphorylated (p)-PERK, p-eIF2 $\alpha$, ATF4, CHOP, Bcl2 and caspase 3. Apoptosis was determined by measuring cytoplasmic oligonucleosomes and related to DNA content. Total protein concentration was also determined. Results: INS-1E cells cultured in the presence of OA for 24 hours showed no induction of apoptosis. In contrast, when OA was replaced by PA seven-fold increase of apoptosis was observed. This rise in apoptosis was reverted when $\mathrm{OA}$ was also included in the culture medium. Protein content was reduced by $40 \%$ in cells treated with PA but remained unchanged in cells treated with combination of PA and OA, consistent with measurements of apoptosis. The PERK pathway plays a major role in the development of the UPR in PA-treated INS-1E cells. Therefore we measured targets of this pathway. We found that p-PERK, p-eIF2 $\alpha$, ATF4 and CHOP were strongly up-regulated in cells cultured in the presence of PA but not induced when cells were cultured in the presence of OA. When both OA and PA were present in the culture medium, expression levels of the UPR targets were not lowered but remained at levels observed in cells cultured in the presence of PA alone. In support of other mechanisms than UPR activation responsible for PA-induced apoptosis, we found that neither pro-apoptotic protein $\mathrm{Bcl}-2$, a downstream target of CHOP, nor caspase-3, which is essential for ER-stress induced apoptosis were affected in INS-1E cells exposed to palmitate.

Conclusion: The protective effect of OA on PA-treated $\beta$-cells is not mediated via attenuation of ER stress. Absence of PA effect on Bcl-2 and caspase-3 expression levels makes it unlikely that activation of UPR plays a major role in PA-induced apoptosis.

Supported by: EFSD/MSD grant, Swedish Medical Research Council and Swedish Diabetes Association

\section{PS 034 Inflammation and ROS in beta cells}

\section{0}

Communication between insulin-resistant skeletal muscle and the beta cell

K. Bouzakri' ${ }^{1}$, P. Ribaux ${ }^{1}$, T. Berney ${ }^{2}$, M. Donath ${ }^{3}$, P.A. Halban ${ }^{1}$

${ }^{1}$ Genetic and development, Centre Medical Universitaire, Geneva, ${ }^{2}$ Surgery, Cell Isolation and Transplantation Center, Division of Surgical Research, Geneva, ${ }^{3}$ Internal Medicine, Division of Endocrinology and Diabetes, Zurich, Switzerland

Background and aims: Obesity and type 2 diabetes mellitus are widespread metabolic disorders characterized by an overlapping phenotype of insulin resistance with a deficiency in insulin secretion underlying hyperglycemia. Systemic inflammation is also a feature of obesity and type 2 diabetes, raising the hypothesis that elevated cytokine levels may contribute to peripheral insulin resistance and decreased beta cell functional mass. In healthy humans, TNF- $\alpha$ infusion induces skeletal muscle insulin resistance. We now explore the potential communication between insulin-resistant skeletal muscle and the beta cell.

Materials and methods: Human skeletal muscle cells were cultured for up to $24 \mathrm{~h}+/-20 \mathrm{ng} / \mathrm{ml}$ TNF- $\alpha$ to induce insulin resistance. mRNA expression for cytokines was analysed after $8 \mathrm{~h}$ by hybridising cDNA to low-density oligo-nucleotide arrays. Conditioned media were collected and the concentration of a small number of cytokines measured by Luminex multiplex assay. MIN6B1 cells (well-differentiated transformed mouse beta cell line) were used to explore the impact of exposure to these conditioned media for $24 \mathrm{~h}$ on apoptosis (TUNEL assay), proliferation (BrdU incorporation) and shortterm insulin secretion (1h $2.8 \mathrm{mM}$ glucose followed by $1 \mathrm{~h} 16.7 \mathrm{mM}$ glucose). Data are mean \pm SE, for $n=4$ (muscle cells) and $n=3$ (MIN6B1 cells using conditioned media from 3 individual human muscle cells each time) independent experiments.

Results: Muscle cells treated for $8 \mathrm{~h}$ show an increase of 19 chemokine genes on the oligo-nucleotide arrays. These results were confirmed and quantified by qRT-PCR for the following: CCL5; CX3CL1; CXCL10; CXCL2; IL1b; IL6; IL8; C3; CCL11; CCL2; CCL7; CXCL11; CXCL6; CXCL3; CXCL9; IL15; CXCL1; TNFa; IL-1Ra. The concentration of most candidate cytokines in the conditioned medium after $24 \mathrm{~h}$ of TNF- $\alpha$ treatment was too low for reliable measurement but IL- 8 was clearly increased to $>1.5 \mathrm{ng} / \mathrm{ml}$. TNF- $\alpha$ conditioned medium increased MIN6B1 cell apoptosis by 13-fold compared to control cells ( $13.4 \pm 0.5$ vs. $1.1 \pm 0.3 \%$ TUNEL positive cells; $\mathrm{p}<0.001)$ and decreased proliferation compared to control cells at both low $(2.8 \mathrm{mM})$ (9.8 \pm 0.3 vs. $5.9 \pm 0.3 \%$ BrdU positive cells; $\mathrm{p}<0.01)$ and high $(16.7 \mathrm{mM})$ glucose $(26.7 \pm 0.7$ vs. $8.7 \pm 1.3 \%$ BrdU positive cells; $p<0.01)$. There was also inhibition of glucose-stimulated insulin secretion after 24h culture of MIN6B1 cells in TNF- $\alpha$ conditioned medium compared to control cells $(27.3 \pm 1.7$ vs. $14.0 \pm 2.6 \%$ total insulin content $/ \mathrm{h} ; \mathrm{p}<0.01)$ and a small but significant increase in basal secretion $(8.6 \pm 1.7$ vs. $3.3 \pm 0.2 \%$ total insulin content $/ \mathrm{h}$; $\mathrm{p}<0.01)$. These changes were not due to the direct effect of TNF- $\alpha$ itself on the beta cells but rather to the presence of as yet uncharacterised factors in the conditioned medium of muscle cells cultured with TNF- $\alpha$. These results were confirmed using primary rat beta cells and in preliminary studies with human beta cells.

Conclusion: Taken together these results showed that induction of insulinresistance skeletal muscle by TNF- $\alpha$ leads to secretion of myokines which impact negatively on beta cell function and survival. The identification of these myokines and their molecular targets on beta cells opens the possibility for new therapeutic strategies for preservation of functional beta cell mass in type 2 diabetes.

\section{1}

Islet cell mass and turnover in patients with chronic pancreatitis B.A. Menge ${ }^{1}$, H. Schrader ${ }^{1}$, S. Schneider ${ }^{1}$, A. Tannapfel ${ }^{2}$, W. Uhl ${ }^{1}$, W.E. Schmidt ${ }^{1}$, J.J. Meier ${ }^{1}$

${ }^{1}$ Ruhr-University Bochum, St. Josef-Hospital, ${ }^{2}$ Ruhr-University Bochum, Institute for Pathology, Bochum, Germany

Background and aims: Chronic pancreatitis (CP) often leads to the subsequent development of diabetes, but the changes in islet cell mass and turnover in such patients have not yet been analysed in detail. We addressed the ques- 
tions (1) Is beta-cell mass reduced in patients with CP? (2) Is beta-cell turnover increased in CP patients? (3) Are beta-cells less vulnerable to apoptosis than acinar cells in patients with $\mathrm{CP}$ ?

Materials and methods: Pancreatic tissue from 29 patients with CP and 14 control subjects was stained for insulin, glucagon, Ki67, and TUNEL and morphometric analyses were performed. Pancreas volume was determined based on abdominal computed tomographies in 13 patients with CP and 11 control subjects. Islet cell mass was estimated from the product of the fractional islet cell areas and the mean pancreas volume in each group.

Results: The mean pancreatic volume was $64.9 \pm 4.3 \mathrm{~cm}^{3}$ in CP patients and $82.3 \pm 6.7 \mathrm{~cm}^{3}$ in control subjects $(\mathrm{p}=0.035)$. Fractional beta-cell area was $0.64 \pm 0.09 \%$ in CP patients and $0.78 \pm 0.07 \%$ in controls $(\mathrm{p}=0.30)$. Thus, beta-cell mass was estimated to be $0.42 \pm 0.06 \mathrm{~g}$ in $\mathrm{CP}$ patients and $0.64 \pm$ $0.05 \mathrm{~g}$ in controls $(\sim 34 \%$ reduction; $\mathrm{p}=0.016)$. There was a significant reduction in islets number in $\mathrm{CP}$ patients $(\mathrm{p}=0.0017)$, whereas islet size was even slightly increased $(p=0.10)$. Alpha-cell mass was not different between the groups $(p=0.64)$. There were no differences in the frequencies of replication in beta-cells, duct cells and acinar cells between the groups. The frequency of apoptosis was similar in beta-cells ( $\mathrm{p}=048$ ), but $\sim 10$ fold increased in acinar cells in CP patients $(\mathrm{p}<0.0001)$.

Conclusion: Beta-cell mass is reduced by only $\sim 35 \%$ in patients with CP, largely because of a reduction in the total number of islets. The similar frequencies of beta-cell replication in CP patients and controls despite an $\sim 10$ fold increase in acinar cell apoptosis suggest that the self-destruction of the pancreas in patients with chronic pancreatitis is highly specific for the exocrine compartment and affects the endocrine islets to a much lesser extent. These findings serve to explain the late onset of overt diabetes in patients with CP.

Supported by: Deutsche Forschungsgemeinschaft, Ruhr-University Bochum

\section{2}

Bone marrow-derived dendritic cells from NOD mice display elevated expression of CCR2 ligands

K. Takahashi' ${ }^{1}$, Y. Oka ${ }^{2}$, J. Satoh

${ }^{1}$ Department of Diabetes and Metabolism, Iwate Medical University, Morioka, ${ }^{2}$ Department of Molecular Metabolism and Diabetes, Tohoku Uinversity, Sendai, Japan

Background and aims: Dendritic cells (DC) are central to the initiation of immune response and maintenance of self-tolerance. In type 1 diabetes, DC display defective phenotype and function in type 1 diabetes, and possibly play crucial roles in the pathogenesis of this disease. To characterise molecular changes in $\mathrm{CD} 11 \mathrm{c}+$ bone marrow (BM)-derived DC from NOD mice, a model of type 1 diabetes, we compared transcript profiles of BMDC with those from the sister strain, NON mice. In the present study, we analaysed our microarray data focusing on the chemokine (C-C motif) ligands (CCLs), which have roles in directing cell movements necessary for the initiation of Tcell immune response, and influence the direction of T-cell differentiation.

Materials and methods: BM cells from 4-week-old female NOD and NON mice were cultivated in the presence of GM-CSF and IL- 4 over 5 days, and with LPS for following 2 days. The CD11c+ DC were then sorted by magnetic beads-conjugated anti-CD11c+ antibodies (MACSTM, Miltenyi Biotech Inc, Bergisch Gladbach, Germany). RNA extracted from the CD11c+ DC from 3 independent mice $(10 \mu \mathrm{g} /$ mouse) was pooled, and used to prepare targets for hybridization to the GeneChip Mouse Genome 430A Arrays (Affymetrix, Santa Clara, CA).

Results: Among all known C-C motif ligands, mouse mature BMDC expressed CCL2-9, 12, 17 and 24, whereas CCL1, 11, 19-21, 25, 27 and 28 were absent. CCL2 (macrophage chemotactic protein 1; MCP1), CCL7/MCP3 and CCL12/MCP5 were noted to be more strongly expressed in the BMDC from NOD mice than those from NON mice $(462 \%, 531 \%$ and $1417 \%$ vs NON, respectively). The other chemokines detected showed about equal intensity of expression between these strains.

Conclusion: Expression of CCL2, 7 and 12 was found in BMDC from 4week-old female NOD mice, which are known to be CCR2 ligands. The early inflammatory changes associated with insulitis are heralded by infiltration of macrophages and DC. CCR2 on macrophages and immature DC are involoved in trafficking to lymph nodes and site of inflammation. Dysregulated expression of CCR2 ligands possibly lead to developing type 1 diabetes in NOD mice by affecting recruitment of antigen presenting cells into islets in a paracrine manner.

\section{3}

The role of oxidants and anti-oxidants in beta cell function and integrity M. Michalska ${ }^{1,2}$, P. Newsholme ${ }^{1}$, G. Wolf ${ }^{2}$, R. Walther ${ }^{2}$

${ }^{1}$ School of Biomolecular and Biomedical Science, University College Dublin, Ireland, ${ }^{2}$ Department of Medical Biochemistry and Molecular Biology, Ernst-Moritz-Arndt-Universität, Greifswald, Germany

Background and aims: Type 1 diabetes mellitus is an autoimmune disease that results from selective destruction of pancreatic $\beta$-cells within the islets of Langerhans. Damage of the insulin-producing $\beta$-cells occurs via T-cell and macrophage infiltration of the islet. Immune cells can produce interleukin (IL)- $1 \beta$, tumor necrosis factor (TNF- $\alpha$ ) and $\gamma$-interferon (IFN- $\gamma$ ). These cytokines induce the synthesis of reactive oxygen (ROS) and nitrogen (NO) species, in the beta cell which prompt dysfunction and apoptosis. The contribution of reactive oxygen species and NO production to glucolipotoxic bcell dysfunction and death are unknown. Insulin-producing $\beta$-cells are very sensitive to oxidative stress due to their low antioxidant defense system e.g. superoxide dismutase (SOD) or catalase. For this reason, there is a significant interest in the regulation of expression of antioxidants such as peroxiredoxin III, superoxide dismutase or catalase in pancreatic islets. We have recently hypothesized that inhibition of ROS and/or NO generation at source would lead to better protection from insult. In addition to testing this hypothesis, we have investigated a possible novel role of Prx III the functionality of the insulin-secreting cells.

Materials and methods: Clonal BRIN BD 11 beta cells or mouse islets were treated with various stressors (cytokines: $0.3125 \mathrm{U} / \mathrm{ml} \mathrm{IL}-1 \beta, 31.25 \mathrm{U} / \mathrm{ml}$ TNF$\alpha, 15.625 \mathrm{U} / \mathrm{ml}$ IFN- $\gamma$, or hydrogen peroxide $0.3 \%$, or high glucose $25 \mathrm{mM}$, or palmitic acid $0.1 \mathrm{mM}$ ) in the presence or absence of antioxidants such as superoxide dismutase $(20 \mathrm{U} / \mathrm{ml})$, catalase $(100 \mathrm{U} / \mathrm{ml}), \mathrm{NAC}(0.2 \mathrm{mM})$, the NADPH oxidase inhibitor Apocynin (0.2mM), or the iNOS inhibitor 1400W $(0.2 \mathrm{mM})$. Using Western blot analyses the expression of Prx III or p47phox was determined in BRIN BD 11 cells. To examine beta cell functionality after stress or protection (exposure to various antioxidants), insulin secretion was determined by ELISA.

Results: Treatment with either cytokines, hydrogen peroxide, or palmitic acid resulted in a 2 - fold decrease in chronic (24hr) stimulated insulin secretion from the BRIN BD11 cell line, and a 3-fold decrease after exposure to cytokines or palmitic acid in mouse islets. Acute exposure to cytokines also resulted in decrease in insulin secretion, but to a lesser extent. Furthermore, antioxidants such as catalase, superoxide dismutase and $\mathrm{N}$-acetylcysteine protected the secretory response against cytokines $(\mathrm{p}<0.01)$, hydrogen peroxide $(\mathrm{p}<0.01)$, or high glucose $(\mathrm{p}<0.01)$ in the BRIN BD11 cell line, and against cytokines $(\mathrm{p}<0.01)$, or palmitic acid $(\mathrm{p}<0.01)$ in mouse islets. The expression of $\mathrm{p} 47 \mathrm{phox}$ was upregulated at the protein level after treatment with cytokines or $\mathrm{H} 2 \mathrm{O} 2$ in the cell line. The addition of Apocyanin and 1400W fully protected chronic $(24 \mathrm{hr})$ insulin secretion in mouse islets $(\mathrm{p}<0.01)$.

Conclusion: The results demonstrate that various antioxidants protect pancreatic beta cells against immune, nutrient or hydrogen peroxide inducers of oxidative stress thus maintaining insulin secretion and preventing beta cell death.

Supported by: Toneca

\section{4}

The $K_{\text {ATP }}$ channel subunit SUR1 influences cell viability of pancreatic beta cells exposed to oxidative stress

B. Gier ${ }^{1}$, P. Krippeit-Drews ${ }^{1}$, L. Aguilar-Bryan ${ }^{2}$, J. Bryan², M. Düfer ${ }^{1}$

${ }^{1}$ Pharmazeutisches Institut, Universität Tübingen, Germany, ${ }^{2}$ Pacific Northwest Research Institute, University of Washington, Seattle, United States

Background and aims: In stimulated wildtype (WT) islets we observed that insulin secretion is impaired by low concentrations of $\mathrm{H}_{2} \mathrm{O}_{2}$, whereas hormone release from islets of SUR1 knockout (SUR1-KO) mice was not affected. To elucidate whether the protection of $\mathrm{K}_{\text {ATP }}$ channel-deficient pancreatic beta-cells against ROS-induced cell damage is coupled to cell viability, we determined apoptosis of WT and SUR1-KO beta-cells exposed to oxidative stress.

Materials and methods: Apoptotic beta-cells were identified by TUNEL and NucView $^{\mathrm{TM}}$ (determination of active caspase3) labeling, respectively.

Results: After one hour of incubation, $10 \mu \mathrm{M} \mathrm{H}_{2} \mathrm{O}_{2}$ increased the number of caspase3-positive WT beta-cells from $9.9 \pm 2.0 \%$ to $28.2 \pm 4.1 \%(\mathrm{n}=3, \mathrm{P}<0.01)$. In SUR1-KO beta-cells basal apoptosis was increased compared to WT, but 
cell viability was not affected by the addition of $10 \mu \mathrm{M} \mathrm{H}_{2} \mathrm{O}_{2}$. Furthermore, 25 $\mu \mathrm{M} \mathrm{H}_{2} \mathrm{O}_{2}$ augmented apoptosis of WT beta-cells from $9.9 \pm 2.0 \%$ to $38.8 \pm 6.8 \%$ $(\mathrm{n}=3, \mathrm{P}<0.01)$ compared to an elevation from $17.52 \pm 0.5 \%$ to only $24.9 \pm 1.0 \%$ $(\mathrm{n}=3, \mathrm{P}<0.01)$ in SUR1-KO beta-cells. Similar results were obtained after six hours of incubation with both concentrations of $\mathrm{H}_{2} \mathrm{O}_{2}$. In order to elucidate if the effect is restricted to $\mathrm{H}_{2} \mathrm{O}_{2}$, we analyzed the influence of the NO-donor SNOC $(100 \mu \mathrm{M})$ on cell survival. The rate of apoptotic cells was increased from $9.9 \pm 2.0 \%$ to $25.8 \pm 2.5 \%(\mathrm{n}=3, \mathrm{P}<0.001)$ in WT beta-cells, whereas cell viability remained unchanged in SUR1-KO beta-cells. To investigate whether pharmacological closure of $\mathrm{K}_{\text {ATP }}$ channels induces similar protective effects against oxidative stress in WT beta-cells, they were preincubated with $25 \mu \mathrm{M}$ tolbutamide for four hours. This maneuver prevented WT beta-cells from the destructive effect of $10 \mu \mathrm{M} \mathrm{H}_{2} \mathrm{O}_{2}$ and decreased the extent of apoptotic cells provoked by $25 \mu \mathrm{M} \mathrm{H}_{2} \mathrm{O}_{2}$.

Conclusion: Modulation of $\mathrm{K}_{\mathrm{ATP}}$ channel activity rendered pancreatic betacells less sensitive to oxidative stress. Cell function as well as cell viability were preserved in beta-cells from SUR1-KO mice during an oxidative insult with low concentrations of $\mathrm{H}_{2} \mathrm{O}_{2}$. Influencing $\mathrm{K}_{\text {Tp }}$ channels in WT beta-cells significantly reduced ROS-induced apoptotic cell death. Thus, changing $K_{\text {ATP }}$ channel activity may be an interesting approach to prevent beta-cell death mediated by oxidative stress during the development of diabetes mellitus as well as before islet transplantation.

Supported by: Deutsche Forschungsgemeinschaft, the U.S. National Institute of Health and the JDRF

\section{5}

Role of exogenous sphingosine-1-phosphate and over-expressed sphingosine kinase 1 in glucolipotoxicity-induced pancreatic beta cell death

J. Veret, J. Movassat, B. Portha, H. Le Stunff

Laboratoire de physiopathologie de la nutrition, UMR CNRS 7059, Paris, France

Background and aims: Chronic hyperglycaemia as seen in type 2 diabetes is often associated with excessive circulating lipid levels. Both abnormalities have been reported to exert adverse effects on beta cell function and survival, a phenomenon referred as glucolipotoxicity. The excessive delivering of lipids in islets of Langherans influences diabetes progression through the accumulation of the bioactive sphingolipid ceramide, which has been involved in the induction of apoptosis in mammals. In contrast to ceramide, sphingosine-1phosphate (S1P) another sphingolipid, stimulates vital biological processes such as cell proliferation and survival. Inside the cells, S1P is produced by the phosphorylation of sphingosine catalyzed by sphingosine kinases (SphKs). Sphingosine is also a precursor of ceramide synthesis. Because of the interconvertibility of ceramide and S1P and their opposing effects on cell fate, the dynamic balance between S1P and ceramide has been proposed to form a "sphingolipid biostat" that determines cell survival or death. Therefore, in the present study, we sought to determine the role of the SphK/S1P axis in glucolipotoxicity-induced beta cell death.

Materials and methods: INS- 1 cells were transfected either by an empty plasmid (vector) or a SphK1 plasmid. INS-1 cells were cultured with 1\% FCS, 0.4 $\mathrm{mM}$ palmitate, various concentrations of glucose in the absence or the presence of S1P for $48 \mathrm{~h}$. INS- 1 cell viability was measured by MTT assay. INS- 1 cell apoptosis was determined by chromatin condensation assessed by DAPI staining. SphK activity was measured in INS- 1 cells using an in vitro kinase assay.

Results: Palmitate and glucose plus palmitate treatments induced a 3 foldincrease in chromatin condensation in INS-1 cells within $48 \mathrm{~h}$, indicative of apoptosis. Millimolar concentrations of exogenous S1P were unable to prevent beta cell apoptosis induced by palmitate. In contrast, nanomolar concentrations of S1P completely inhibit the synergic apoptotic action of $0.4 \mathrm{mM}$ palmitate in the presence of $30 \mathrm{mM}$ glucose. Both mammalian sphingosine kinase isoforms are expressed in INS-1 cells. In order to determine the role of SphK1 in glucolipotoxicity-induced beta cell death, we over-expressed SphK1 in INS-1 cells. Surprisingly, the over-expressed SphK1 was unable to reduces beta cell viability induced either by palmitate alone or combined with high glucose concentrations. Moreover, we found that glucolipotoxicity-induced beta cell death was associated with a reduction of SphK1 activity. Replacing D-glucose by the non-metabolisable L-glucose neither potentiated palmitate-induced beta cell death nor decreased SphK1 activity. Similarly, palmitate methyl ester, which is not metabolized, did not affect beta cell survival and SphK1 activity. In contrast, 2-bromopalmitate, an inhibitor of fatty acid mitochondria beta oxidation, reproduced the effect of palmitate on beta cell survival.
Conclusion: Collectively, our results suggest that S1P prevents glucolipotoxicity-induced apoptosis. However, exogenous S1P and SphK1, which produces intracellular S1P, have diverging role in glucolipotoxicity-induced beta cell death. These results also suggest that palmitate-induced SphK1 inhibition is associated with a glucose-dependent esterification of free fatty acid. Supported by: CNRS and ANR

\section{6}

Autophagy: an age-related change in beta cells

S. Wang ${ }^{1}$, X.J. $\mathrm{Li}^{2}$, J. Li ${ }^{1}$, L. Chao ${ }^{1}$, B.R. Dong ${ }^{1}$

${ }^{1}$ Geriatrics, ${ }^{2}$ Endocrinology and Metabolism, West China Hospital, Chengdu, China

Background and aims: Autophagy is a highly regulated cellular mechanism for the bulk degradation of cytoplasmic contents. It has been implicated in a variety of physiological and pathological conditions such as cancer, neurodegeneration disease, as well as aging. However, whether autopahgy involved in the age-related $\beta$-cell change and the high prevalence of glucose intolerance and type 2 diabetes in older population are not known. Thus, the present study was designed to observe the phenomena of autophagy, and its relation, if any, to apoptosis, which might be associated with the reduction of $\beta$-cell mass, and age-related ultrastructural changes in $\beta$-cell of different age rats. Materials and methods: Natural aging SD rats with different age $(6,12,20$ $24 \mathrm{mon}$ ) was used in this experiment. After the BW were measured, rats were anesthetized with sodium pentobarbital $(30 \mathrm{mg} / \mathrm{kg}$, ip), blood were drawn from heart for detecting fasting blood glucose, lipid profile and FFA as basal metabolic parameters. Pancreases were isolated quickly, fixated and embed. Islets were observed for: 1 . Autophagolysosme and apoptotic bodies: observed by EM (H-600IV, HITACHI, Japan); 2. Apoptosis rate : tested by the method of Tunel immunohistochemical staining (Tunel-POD assay, Roche) and EM.

Results: 1 . The significantly higher concentration of FFA was found in 12, 20 - 24 months groups $(386.02 \pm 88.08 ; 349.43 \pm 93.47$ vs. $263.93 \pm 68.77 \mathrm{mmol} /$ 1 , respectively. $\mathrm{p}<0.05$ ), with no difference in WT, FBG, lipid profile among three groups; 2 . Lysomic vacuoles were first found in the $\beta$-cell of $12 \mathrm{mon}$. age rats (Fig. A); 3. Autophagic vacuoles (AV) appeared in $\beta$-cells of $20-24$ mon. age rats (Fig. B), with no any similar change in $6 \mathrm{mon}$. rats (Fig. C). 4 . There was no any evidence of apoptosis by both ways of EM and Tunel immunohistochemical staining in $\beta$-cellsin different stages of aged rats.. Furthermore, swelling and vacuolar degeneration were found in both mitochondrion and endoplasmic reticulum in $\beta$-cell of the oldest rats.

Conclusion: Our results showed for the first time that it is autophagy not apoptosis involved in age-related $\beta$-cell change, which firstly occurred in middle aged (12 month) rats. The mechanism within it is not yet understood. The relationship between activated autophagy and insulin secretion impairment in $\beta$-cell of aged rats needed to be further explored.

Fig: Autophagy in $\beta$-cells of different month SD rats. A: 12 month, lysomic vacuoles (thick arrow); B: 20-24 month, autophagic vacuoles (thin arrow), atrophic mitochondria (triangle); C: 6 month.
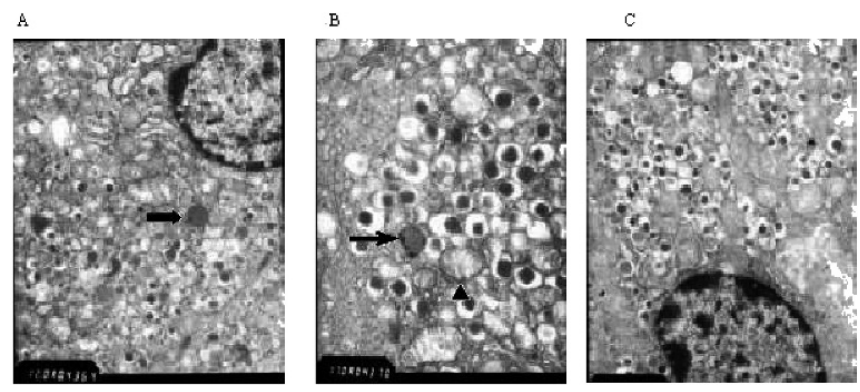

Supported by: National Natural Science Foundation of China 


\section{7}

Serum glucocorticoid inducible kinase 1 inhibits degradation of insulin receptor substrate- 2 in insulin secreting cells

S.J. Ullrich ${ }^{1}$, D. Avram ${ }^{1}$, F. Ranta ${ }^{1}$, J. Leveringhaus ${ }^{1}$, A.M. Hennige ${ }^{1}$, S. Hopp ${ }^{1}$, F. Lang ${ }^{2}$, H.-U. Häring ${ }^{1}$

${ }^{1}$ Internal Medicine IV, University Hospital of Tuebingen, ${ }^{2}$ Institute of

Physiology, University of Tuebingen, Germany

Background and aims: In insulin secreting cells, insulin receptor substrate-2 (IRS-2) transmits insulin and IGF-1 receptor signalling. Indeed, IRS-2 expression and stimulation has been found to guarantee survival and proper functioning of these cells. In addition to modifications of IRS-2 by stimulatory and inhibitory phosphorylations, IRS-2 has a fast turnover rate and thus signalling largely depends on protein degradation and synthesis. The serumglucocorticoid-inducible kinase 1 (SGK1) regulates proteasomal degradation of membrane proteins. Previously, we observed that the synthetic glucocorticoid dexamethasone (dex) induces the expression of SGK1 in insulin secreting cells. While dex treatment inhibited tyrosine phosphorylation of IRS-2, the protein was increased 2-fold without changes in mRNA levels. This study was performed to analyze the role of SGK1 during glucocorticoid induced cell death and to examine whether SGK1 is responsible for increased expression of IRS-2 observed in insulin secreting cells under glucocorticoid excess. Materials and methods: INS- 1 cells were stably transfected with hSGK1. Apoptotic cell death was assessed using TUNEL staining of nuclear DNA. Mitochondrial potential was measured by fluorescence activated cell sorting (FACS) after staining of the cells with tetramethylrhodamine ethylester (TMRE). Expression and phosphorylation of proteins were analyzed using Western blot analysis. The rate of protein degradation was estimated in the presence of inhibition of protein synthesis by cycloheximide.

Results: In contrast to dex, stable transfection of INS-1 cells with hSGK1 did not affect cell growth, apoptotic cell death or glucose-induced insulin secretion. In accordance, cells in stimulatory high glucose developed a more negative mitochondrial potential than cells kept at $2.8 \mathrm{mM}$ glucose. While in control cells incubated with cycloheximide, $60 \%$ of IRS-2 protein was degraded after $8 \mathrm{~h}$, in hSGK1 transfected cells no significant degradation was observed. The PI3K inhibitor LY294002 inhibited degradation of IRS-2 also in control cells. This inhibition of degradation was accompanied by an inhibition of an increase in apparent molecular weight of IRS-2. In contrast, SGK1 did not inhibit the shift in molecular weight of IRS-2.

Conclusion: These observations suggest that SGK1 does not contribute to dex-mediated apoptotic cell death but the kinase inhibits degradation of IRS2 in insulin secreting cells.

\section{PS 035 ER stress in the beta cell}

\section{8}

JunB inhibits ER stress and apoptosis in pancreatic beta cells E.N. Gurzov, F. Ortis, F. Moore, D.L. Eizirik

Laboratory of Experimental Medicine, Universite Libre de Bruxelles, Belgium

Background and aims: Cytokines contribute for pancreatic beta cell apoptosis in type 1 diabetes but the mechanisms involved are not well understood. The transcription factor Activator Protein-1 (AP-1) is a key player in inflammation and apoptosis. Based on microarray analysis of cytokine-treated insulin-producing INS-1E and primary beta cells we have previously identified modifications in expression of the AP- 1 components. We presently evaluated the role of the AP-1 subunit JunB for cytokine-induced beta cell dysfunction and death.

Materials and methods: INS-1E and primary FACS-purified beta cells were transfected with control or siRNAs targeting JunB and treated with cytokines (IL-1 $\beta$ 10-50 U/ml+ IFN- $\gamma$ 100-1000 U/ml). The siRNA-induced JunB knockdown (KD) phenotype was compared to fibroblasts constitutively lacking JunB expression. Upregulation of JunB was achieved by transfection with an adenoviral vector. Real time RT-PCR, Western Blot, and viability assays (Hoechst/PI) were performed to identify proteins involved in JunB-mediated responses.

Results: Cytokines induced an early increase in JunB expression in INS-1E cells ( 3 -fold protein increase by $8 \mathrm{~h}, \mathrm{n}=3, \mathrm{P}<0.05$ ). This protein upregulation was prevented by an NF- $\kappa$ B repressor. JunB KD by RNA interference $(>80 \%$ inhibition) in INS-1E cells augmented cytokine-induced iNOS (33-fold increase, $\mathrm{n}=3, \mathrm{P}<0.05$ ) and Chop expression ( 15 -fold increase, $\mathrm{n}=3, \mathrm{P}<0.05$ ), leading to increased cell death (44\% apoptosis in cytokine-treated JunB KD cells vs $19 \%$ apoptosis in cells treated with cytokines and control siRNA, $\mathrm{n}=5$, $\mathrm{P}<0.001)$. Similar findings were observed in FACS-purified beta cells. Other ER stress markers (XBP-1s and ATF-4) were also upregulated following JunB $\mathrm{KD}$ and cytokine treatment. Chop expression and ER stress markers were similarly increased in JunB KD INS-1E cells after addition of cyclopiazonic acid, an endoplasmic reticulum stressor. In line with this, cytokines induced iNOS and apoptosis in mouse JunB knockout fibroblasts but not in wild-type cells. Conversely, adenovirus-mediated overexpression of JunB diminished cytokine-induced iNOS and Chop expression and protected INS-1E and primary beta cells against cytokines (10-14\% apoptosis in JunB overexpressing and cytokine-treated INS-1E and primary beta cells respectively, vs $>25 \%$ apoptosis in cytokine-treated control cells; $\mathrm{n}=3-5 ; \mathrm{P}<0,05)$.

Conclusion: Our findings indicate that JunB has a protective role against cytokine-induced ER stress and apoptosis in beta cells. This protein is probably part of the defense mechanisms triggered by beta cells during exposure to pro-inflammatory cytokines. Therefore, overexpression of JunB may be an interesting strategy for beta cell protection in early diabetes.

Supported by: Communauté Française de Belgique - Actions de Recherche Concertées, Fonds National de la Recherche Scientifique Belgium, the Belgium Program on Interuniversity Poles of Attraction initiated by the Belgium State and European Union

\section{9}

Involvement of endoplasmic reticulum stress in pancreatic beta cell death: a proteomic approach

L. Overbergh ${ }^{1}$, W. D’Hertog 1 , A. Cardozo ${ }^{2}$, D. Eizirik ${ }^{2}$, E. Waelkens ${ }^{3}$, C. Mathieu ${ }^{1}$

${ }^{1}$ Catholic University of Leuven, Laboratory of Experimental Medicine and Endocrinology, Leuven, ${ }^{2}$ Université Libre de Bruxelles, Laboratory for Experimental Medicine, Brussels, ${ }^{3}$ Catholic University of Leuven, Laboratory of Protein Phosphorylation and Proteomics, Leuven, Belgium

Background and aims: Pancreatic $\beta$-cells are the crucial cells responsible for synthesizing, processing and secreting insulin. Because of this high demand on insulin processing, $\beta$-cells have a highly developed endoplasmic reticulum (ER) and are thus very sensitive to ER stress, in particular to agents that deplete the $\mathrm{ER} \mathrm{Ca}^{2+}$, such as cyclopiazonic acid (CPA). In previous studies we have investigated, at the mRNA level, the global alterations induced by CPA, in rat insulin-producing INS-1E cells. The aim of this study was to investigate the changes induced by CPA at the protein level.

Materials and methods: INS- $1 \mathrm{E}$ cells were treated with $25 \mu \mathrm{M}$ CPA dissolved in DMSO or with DMSO alone during 6 or 12 hours. Cell viability was as- 
sessed by microscopic cell counting (Hoechst/Propidium Iodide staining). Differential protein expression was analysed by two-dimensional difference gel electrophoresis (2D-DIGE), covering 2 different $\mathrm{pH}$ ranges ( $\mathrm{pH} 4-7$ and pH6-9). Proteins altered in expression $(n=4, p<0,05)$ were identified after ingel trypsin digestion and MALDI-TOF/TOF analysis. Altered expression of some of the identified proteins was confirmed by western blotting and analyzed at the mRNA level by real-time RT-PCR.

Results: Culturing of the INS-1E cells in the presence of $25 \mu \mathrm{M}$ CPA resulted in an increase in the proportion of apoptotic cells from $1,12 \%+/-0.84 \%$ to $11,45 \%+/-3.10 \%$ after 6 hours $(n=4, p<0.01)$ and to $18,24 \%+/-6.39 \%(n=4$, $\mathrm{p}<0.01$ ) after 12 hours of treatment. mRNA levels for the pro-apoptotic transcription factor CHOP increased 26-fold to 31-fold after 6 and 12 hours of CPA treatment, respectively $(\mathrm{p}<0.01)$. For the $2 \mathrm{D}-\mathrm{DIGE}$ proteomic study, $1841 \pm 294$ spots could be matched for the 6 and 12 hour time point in the pH 4-7 range. Of these, 48 and 90 spots were differentially expressed between control and CPA-treated cells for the 6 and 12 hour time point respectively $(\mathrm{p}<0,05)$. No differentially expressed spots (with $\mathrm{p}<0,05)$ were observed in the $\mathrm{pH}$ 6-9 range. Of these, 91 spots were unambiguously identified, resulting in 60 distinct proteins. We grouped these proteins according to their function in 9 groups. The results point to a marked decrease in multiple proteins involved in the ubiquitin-proteasome pathway. Also several chaperones were downregulated, a finding that was not observed at the mRNA level.

Conclusion: Discrepancies between mRNA and protein levels of several molecular chaperones point to the importance of post-transcriptional regulation in this pathway. Since chaperones are known to play a role in $\beta$-cell defenses, the observed downregulation may contribute to the high vulnerability of $\beta$ cells to ER stress.

\section{0}

Carboxypeptidase E deficiency causes beta cell dysfunction and apoptosis in vitro and in vivo

J.D. Johnson, K.D. Jeffrey, D.S. Luciani, E.U. Alejandro, T.B. Kalynyak

Cellular and Physiological Sciences, Diabetes Research Group, University of British Columbia, Vancouver, Canada

Background and aims: Diabetes is associated with high levels of circulating lipids. Many studies have shown that free fatty acids, such as palmitate, can cause beta-cell dysfunction and death. In an unbiased proteomics screen, we identified carboxypeptidase $\mathrm{E}$ as a major protein target of palmitate. In the present study, we investigated the islet phenotype of mice lacking carboxypeptidase $\mathrm{E}$.

Materials and methods: Glucose homeostasis in CPE null mice was examined with intraperitoneal glucose tolerance tests. Pancreas sections were analyzed for beta-cell area, alpha-cell area, islet architecture, average islet size and islet density. Pancreatic beta-cell TUNEL and BrdU staining were employed to estimate apoptosis and proliferation in vivo. Islets were isolated via filtration. Apoptosis-related protein expression was examined by western blot using antibodies to cleaved caspase- 3 and CHOP. The function of islets was assessed using islet perifusion. Proinsulin maturation was assessed using immunofluorescent staining. Fully processed insulin (i.e. CPE-cleaved) was identified using the $\mathrm{mAB} 1$ monoclonal antibody, whereas total insulin was identified using a polyclonal antibody. CPE levels were modulated in MIN6 cells using shRNA (combined with FACS sorting) or transient transfection.

Results: As noted by others, CPE null mice had impaired fasting glucose and significant glucose intolerance when compared to wildtype littermate controls. Pancreas sections from these mice had larger islets and $>2$ fold increased beta-cell mass, although the beta-cells had much weaker insulin staining. CPE null beta-cells exhibited a markedly higher rate of both apoptosis and proliferation in vivo. Similarly, islets isolated from CPE null mice had increased cleaved caspase- 3 and CHOP protein levels. This was recapitulated in MIN6 cells with shRNA-mediated knockdown of CPE. CPE nulls islets were also displayed evidence of functional defects. For example, co-staining with antibodies to CPE-cleaved insulin and total insulin showed that fully mature insulin was virtually absent in pancreas sections from CPE null mice. Interestingly, this was also observed in islets from high fat fed mice and palmitate-treated MIN6 cells, corresponding to a reduction in CPE seen in both models. Perifusion experiments indicated that islets isolated from CPE null islets had elevated basal insulin secretion whereas the fold insulin responses to glucose and $\mathrm{KCl}$ were both impaired relative to control mice.

Conclusion: In the present study, we characterized key aspects of the islet phenotype of CPE null mice and of MIN6 cells with a 30\% reduction in CPE. Together with our previous work demonstrating that palmitate and high fat feeding decrease beta-cell CPE levels, these new results suggest that some aspects of type 2 diabetes pathology can be explained by lipid-induced suppression of CPE.

Supported by: Canadian Diabetes Association, Canadian Institute for Health Research

\section{1}

Diazoxide protects against hypoxia-induced beta cell damage

A. Björklund ${ }^{1}, \mathrm{Z} . \mathrm{Ma}^{1}, \mathrm{~K}$. Brismar ${ }^{1}$, V. Grill ${ }^{1,2}$

${ }^{1}$ Molecular Medicine and Surgery, Rolf Luft Center of Diabetes Research, Karolinska Institutet, Sweden, ${ }^{2}$ Norwegian University of Science and Technology, Dept of Cancer Research and Molecular Medicine, Trondheim, Norway

Background and aims: Islet transplantation is hampered by relative hypoxia after transplantation. Means to improve resilience toward hypoxia-induced beta cell damage could potentially improve the success of transplantation. To survey the impact of hypoxia and ways of promoting resilience we tested for effects of hypoxia in vitro on beta cell function and for a modulating effect of diazoxide, known from other tissues to protect against hypoxia-induced damage.

Materials and methods: Isolated rat islets were cultured in $11 \mathrm{mM}$ glucose (G) over night (22h). The following day islets were exposed for $6 \mathrm{~h}$ to hypoxia $\left(<1 \% 0_{2}\right)$. Glucose-(16.7 mM G) -induced release was tested 30 min later in $60 \mathrm{~min}$ batch incubations as well as after continued culture over night (22h) at normoxia (=re-oxygenation). Effects of diazoxide $(325 \mu \mathrm{M})$ were tested either by pre-exposure to the drug for $22 \mathrm{~h}$ preceding hypoxia or by addition of the drug during the $6 \mathrm{~h}$ of hypoxia.

Results: $6 \mathrm{~h}$ of hypoxia attenuated insulin responses to $16.7 \mathrm{mM} \mathrm{G}$ when measured $30 \mathrm{~min}$ after hypoxia $(8 \pm 1.2$ vs. $65 \pm 15.7 \mu \mathrm{U} / \mathrm{islet} / \mathrm{h}(\mathrm{p}=0.012$, $\mathrm{n}=7$ ). Hypoxia-inducible protein (HIF)-1 alpha was up-regulated immediately following hypoxia (Western blotting). Re-oxygenation improved insulin response to glucose in previously hypoxia- cultured islets ( $8 \pm 1.2$ vs. $22 \pm 2.8$ $\mu \mathrm{U} /$ islet, $\mathrm{p}<0.001, \mathrm{n}=7)$. However, the response was still much decreased vs. islets that had been continuously cultured in normoxia $(22 \pm 2.8$ vs. $78 \pm 17.2$, $\mathrm{p}=0.01, \mathrm{n}=7$ ). Hypoxia also decreased islet insulin content measured both directly after the $6 \mathrm{~h}$ of hypoxia (from $954 \pm 84$ to $735 \pm 95 \mu \mathrm{U} /$ islet, $\mathrm{p}<0.001, \mathrm{n}=7$ ) and after re-oxygenation (from $1044 \pm 77$ to $409 \pm 44 \mu \mathrm{U} /$ islet, $\mathrm{p}<0.001, \mathrm{n}=7$ ). The $\mathrm{pdx}-1$ protein was down-regulated after re-oxygenation vs. continuously normoxic controls. Previous diazoxide did not rescue the glucose-induced insulin response when tested immediately after $6 \mathrm{~h}$ of hypoxia. However, previous diazoxide improved the response to $16.7 \mathrm{mM} \mathrm{G}$ in hypoxia-exposed islets when measured after re-oxygenation $(47 \pm 5$ vs. $26 \pm 3 \mu \mathrm{U} /$ islet $/ \mathrm{h}, \mathrm{p}=0.027$, $\mathrm{n}=4$ ). Furthermore, previous diazoxide increased islet insulin content when measured after re-oxygenation $(926 \pm 208 \mu \mathrm{U} /$ islet vs. $388 \pm 58 \mu \mathrm{U} /$ islet, $\mathrm{p}=0.01$, $\mathrm{n}=4$ ). Diazoxide present during hypoxia failed to affect insulin secretion or content following re-oxygenation (results not shown). Thus diazoxide was beneficial only when present prior to hypoxia.

Conclusion: 1) hypoxia can induce permanent functional beta cell damage which affects both insulin secretion and biosynthesis 2) pre-exposure to diazoxide lessens these damaging effects. These findings may have implications for islet transplantation procedures.

Supported by: Swedish Society of Medicine, Swedish Medical Research Council, EFSD/MSD grant

\section{2}

Proteomically identified elevated serum levels of apolipoprotein CIII in individuals with type 2 diabetes mellitus may induce MAPK-mediated beta cell apoptosis

E.M. Sol, T. Sundsten, P. Bergsten

Department of Medical Cell Biology, Uppsala University, Sweden

Background and aims: Type 2 diabetes (T2DM) results from complex interplay between genetic predisposition and environmental factors that together promote impairment of the $\beta$-cell. To identify blood-borne proteins involved in the detrimental process, we have previously protein profiled serum from control individuals and T2DM individuals with impaired $\beta$-cell function. Among the differentially displayed proteins apolipoprotein CIII (apoCIII) was significantly up-regulated in T2DM individuals. ApoCIII plays an important role for plasma triglyceride levels and elevated levels of the lipoprotein have been connected with dyslipidemia in T2DM subjects. The present study was undertaken to investigate if the elevated levels of apoCIII could be caus- 
ally linked to impaired $\beta$-cell function. In support of such a role increased $\beta$-cell cytoplasmic calcium concentration and apoptosis has been reported in the presence of the protein. We therefore hypothesized that the elevated intracellular levels of calcium might be related to induction of endoplasmic reticulum (ER) stress. In addition, activation of mitogen-activated protein kinases (MAPKs) was suggested to be a mechanism by which the lipoprotein could negatively affect the $\beta$-cell. The hypotheses were addressed by measuring the expression of ER stress markers and MAPKs in $\beta$-cells exposed or not to apoCIII.

Materials and methods: INS-1E cells were cultured in the absence or presence of apoCIII $(10 \mu \mathrm{g} / \mathrm{ml})$ for 24 hours. In cells examined for expression of ERK and p38, ERK inhibitor PD98059 (100 $\mu \mathrm{M})$, p38 inhibitor SB203580 (10 $\mu \mathrm{M})$ or L-type calcium-channel inhibitor verapamil $(100 \mu \mathrm{M})$ were added or not to the culture medium prior to introducing apoCIII. After culture, apoptosis was determined by measuring the amount of cytoplasmic oligonucleosomes and related to DNA content. Protein expression of ER-stress markers phosphorylated (p)-eIF2 $\alpha$ and CHOP, and phosphorylated and unphosphorylated forms of the MAPKs ERK, p38 and JNK were determined with western blot analysis at different time points.

Results: INS-1E cells cultured for 24 hours in the presence of apoCIII had 2.5 -fold increased apoptosis. ER-stress markers p-eIF2 $\alpha$ and CHOP were not changed at the end of the culture period. The expression of p-ERK was rapidly induced 3-fold 0.5 hour after introduction of apoCIII and declined to reach basal level after 12 hours culture. Inhibition of the L-type calcium channel with verapamil blocked the ERK induction. JNK was not altered by apoCIII. In contrast, $\mathrm{p} 38$ was induced by the lipoprotein after 18 hours and manifests as 2 -fold rise after 24 hours. When inhibiting ERK and/or p38 activation, apoptosis in apoCIII-treated cells was modulated.

Conclusion: The observed elevated circulating apoCIII levels may induce $\beta$ cell apoptosis via activation of MAPKs ERK and/or p38.

Supported by: EFSD/MSD grant, Swedish Medical Research Council, and Swedish Diabetes Association

\section{3}

Direct effects of palmitate on ER calcium, cytosolic calcium, and beta cell death, measured with real-time assays and fluorescent protein-based indicators

K.S. Gwiazda, T.-L.B. Yang, Y. Lin, D.S. Luciani, J.D. Johnson

Cellular and Physiological Sciences, University of British Columbia,

Vancouver, Canada

Background and aims: There is a strong link between obesity, elevated free fatty acids, and type 2 diabetes. It has been shown that palmitate has pleiotropic effects on beta-cell function and survival. Acute administration of the fatty acid mildly augments glucose-stimulated insulin secretion, but chronically elevated levels inhibit insulin secretion and promote beta-cell apoptosis. Palmitate-induced signalling is thought to involve altered calcium homeostasis. Exposure to the fatty acid induces intracellular calcium signalling in INS-1 and HIT-T15 cells, as well as in primary mouse islets. However, more detailed studies regarding the role of these calcium signals are needed.

Materials and methods: We used both human beta-cells and the MIN6 betacell line. Calcium imaging in primary cells was performed using Fura-2-AM dye. Calcium dynamics in specific cellular locations were measured using the fluorescent protein-based genetically encoded calcium sensors D3cpv and D1ER for the cytosol and ER, respectively. Western blotting was used to assess the time-course of CHOP activation and propidium iodide incorporation was used to assay cell death in real-time. The in vivo effects of hyperlipidemia were assessed in C57B6/J mice fed a high fat diet.

Results: Acute palmitate treatment of MIN6 cells and human islet cells increased cytosolic calcium through intracellular calcium store release as well as extra-cellular calcium influx. The non-metabolizable analogue 2bromopalmitate, which is not thought to activate ATP-dependent extra-cellular calcium influx, also increased cytosolic calcium, but to a much lesser extent. Both palmitate and 2-bromopalmitate induced cell death, although propidium iodide incorporation induced by 2 -bromopalmitate was significantly slower. Palmitate also induced the expression of the pro-apoptotic transcription factor $\mathrm{CHOP}$, indicating an increase in ER stress and suggesting a possible depletion of ER calcium. Indeed, acute palmitate treatment in MIN6 cells caused reversible calcium depletion from the ER lumen in a subpopulation of cells. We observed sustained oscillations of cytosolic calcium in cells treated with palmitate for two hours. However, we detected little palmitate-induced insulin secretion, suggesting these calcium signals are poorly coupled to exocytosis. We fed wild type mice a high fat diet to determine the chronic effects of elevated lipid exposure. Islets from these mice exhibited basal hyperinsulinemia and a reduced relative glucose-stimulated insulin response, a phenotype similar to human type 2 diabetes.

Conclusion: We have characterized calcium-dependent mechanisms involved in altered beta-cell function and survival induced by the free fatty acid palmitate. We present the first direct evidence that palmitate depletes calcium from the beta-cell ER. Together, these data shed light on one of the many pathways that may be involved in the pathogenesis of lipotoxicity and type 2 diabetes.

Supported by: Canadian Diabetes Association and the Canadian Institute for Health Research

\section{4}

Exposure to persistant organic pollutants, polychlorinated biphenyls, potentiates fatty acid-mediated beta cell death: role of ER-stress

L. Okafor ${ }^{1}$, I. Chadbourn ${ }^{2}$, A. Wale ${ }^{1}$, E. Katsoulieris ${ }^{2}$, J. Cunningham², P. Chatterjee ${ }^{2}$ J.G. Mabley ${ }^{2}$

${ }^{1}$ Pharmacology, Brighton \& Sussex Medical School, ${ }^{2}$ School of Pharmacy \& Biomolecular Sciences, University of Brighton, United Kingdom

Background and aims: Exposure to polychlorinated biphenyls (PCB) -environmental contaminants - has been linked to increased incidence of Type II diabetes. Although exposure, particularly to PCB 126, has been shown to be a risk factor for development of Type II diabetes independent of obesity it has been proposed that environmental contaminant exposure coupled with a high fat diet synergistically increases the risk of developing Type II diabetes. The aim of this study was to determine the in vitro effect of PCB 126 alone and in combination with palmitic acid on $\beta$-cell viability and death.

Materials and methods: BRIN-BD11 cells were exposed to PCB $126(1,3,10$ or $20 \mu \mathrm{M}$ ) for 24 or $48 \mathrm{~h}$. Cell viability was determined using the MTT assay and cell death assessed by propidium iodide/Hoechst staining. BRIN-BD11 cells were also exposed to PCB 126, 1 or $3 \mu \mathrm{M}$, in combination with palmitic acid $(10$ or $30 \mu \mathrm{M})$ for $24 \mathrm{~h}$ before levels of cell viability and death were determined. Endoplasmic reticulum (ER) stress was measured in BRIN-BD11 cells exposed to palmitic acid alone or in combination with PCB 126 by Western blotting for the ER stress proteins GRP78 and CHOP.

Results: PCB 126 exposure for $24 \mathrm{~h}$ decreased BRIN-BD11 cell viability only at $20 \mu \mathrm{M}$ (reduced to $77 \% \pm 4 ; \mathrm{p}<0.05$ vs. untreated cells). However, $48 \mathrm{~h}$ exposure to PCB 126 reduced BRIN-BD11 cell viability to $95 \% \pm 4,81 \% \pm 6,68 \% \pm 4$ and $52 \% \pm 5$ ( $\mathrm{p}<0.05$ vs. untreated cells) for $1,3,10$ and $20 \mu \mathrm{M}$ respectively. Exposure to PCB 126 for $24 \mathrm{~h}$ increased BRIN-BD11 cell necrosis (from $2.6 \%$ to $7.4 \%, 8.5 \%$ and $15.5 \%$ for 1,3 or $10 \mu \mathrm{M}$ PCB 126 ; $\mathrm{p}<0.05$ vs. untreated cells) but apoptosis levels were unaffected. Exposure to PCB $126(1$ or $3 \mu \mathrm{M})$ or palmitic acid $(10$ or $30 \mu \mathrm{M})$ alone for $24 \mathrm{~h}$ had either no or minimal $(<10 \%$ decrease) effect on cell viability. However, simultaneous treatment of BRINBD11 cells with $10 \mu \mathrm{M}$ palmitic acid plus 1 or $3 \mu \mathrm{M}$ PCB 126 synergistically reduced cell viability to $92 \% \pm 3$ and $82 \% \pm 3$ ( $\mathrm{p}<0.01$ vs. PCB 126 or palmitic acid alone) and $30 \mu \mathrm{M}$ palmitic acid combined with 1 or $3 \mu \mathrm{M}$ PCB 126 reduced cell viability to $71 \% \pm 4$ and $58 \% \pm 5$ respectively ( $<<0.01$ vs. PCB 126 or palmitic acid alone).Combination of PCB $126(1$ or $3 \mu \mathrm{M})$ with $10 \mu \mathrm{M}$ palmitic acid increased BRIN-BD11 apoptosis levels but had no effect on cell necrosis. PCB 126 at 1 or $3 \mu \mathrm{M}$ had no effect on $B$-cell apoptosis levels whereas palmitic acid $(10 \mu \mathrm{M})$ increased it from $2.6 \pm 0.7$ to $12 \% \pm 4(\mathrm{p}<0.01$ vs. untreated cells). Simultaneous treatment of BRIN-BD11 cells with $10 \mu \mathrm{M}$ palmitic acid and 1 or $3 \mu \mathrm{M}$ PCB 126 significantly increased apoptosis levels to $31 \% \pm 6$ and $36 \% \pm 7$ respectively. ER stress protein levels of GRP78 and CHOP were increased by $10 \mu \mathrm{M}$ palmitic acid but unaffected by PCB 126 . Simultaneous treatment with both palmitic acid and PCB 126 synergistically increased B-cell expression of both GRP78 and CHOP above that observed in untreated, palmitic acid or PCB treated cells.

Conclusion: PCB 126-mediated loss of B-cell viability and increased cell death is observed only with high concentrations and long exposure times. However, exposure of $\beta$-cells to PCB 126 in combination with palmitic acid synergistically increased $\beta$-cell apoptosis possibly via potentiated ER stress. This data suggests that environmental contaminants may synergise with dietary fatty acids leading to increased $ß$-cell loss and susceptibility to development of Type II diabetes. 


\section{5}

Endoplasmic reticulum stress in INS-1 cells and human islets induced by glucose, TNF- $\alpha$ und IL-1ß

S. Erbel, C. Reers, G.L. Jansky, P.P. Nawroth, R.A. Ritzel

Department of Internal Medicine I and Clinical Chemistry, University of

Heidelberg, Germany

Background and aims: Type 2 diabetes mellitus is characterized by insulin resistance, beta-cell dysfunction and beta-cell deficit due to apoptosis. One important mechanism is ongoing beta-cell stress, especially stress in the endoplasmic reticulum (ER). Chronic beta-cell stress is triggered by insulin resistance, hyperglycemia and as recently suggested by adipocytokines. Therefore we posed the following questions: 1) Which pathways of ER stress are activated in beta-cells by glucose and/or adipocytokines? 2) Does beta-cell stress impair beta-cell function and survival? 3) Does the incretin mimetic exenatide reduce beta-cell stress?

Materials and methods: INS- 1 cells were incubated with 11 and $30 \mathrm{mM}$ glucose and TNF- $\alpha$ (100ng/ml) or IL-1ß (50U/ml) for 1 hour ( $\mathrm{n}=3$ experiments). The expression of ER stress markers were measured by immunoblotting ( $\mathrm{p}-$ eIF2 $\alpha, p-J N K)$. Isolated human islets ( $n=3$ donors) were incubated with glucose $(5$ and $30 \mathrm{mM})$ and TNF- $\alpha(10 \mathrm{ng} / \mathrm{ml})$ or IL- $1 ß(50 \mathrm{U} / \mathrm{ml})$ for 48 hours. The function of human islets was determined by insulin secretion analysis in static incubation ( $2 \mathrm{~h}$ at 5 and $20 \mathrm{mM}$ glucose). ER stress and apoptosis were measured by immunofluorescence (CHOP, TUNEL). In addition islets were incubated with exenatide $(50 \mathrm{nM})$ at identical conditions.

Results: In INS- 1 cells different ER stress pathways were activated. The expression of p-eIF2 $\alpha$ was increased by $30 \mathrm{mM}$ glucose $(+32 \pm 14 \%, \mathrm{p}=0.07)$, TNF- $\alpha(+64 \pm 14 \%, \mathrm{p}=0.02)$ and IL- $1 ß(+129 \pm 15 \%, \mathrm{p}=0.007$, each versus control). Similar results were obtained for $\mathrm{p}-\mathrm{JNK}$ (glucose $+27 \pm 12 \%, \mathrm{p}=0.08$; TNF- $\alpha+53 \pm 9 \%, p=0.01$; IL- $1 \beta+163 \pm 64 \%, p=0.06$ ). In human islets CHOP expression (perinuclear and nuclear) was elevated 5 and 8 -fold by $30 \mathrm{mM}$ glucose und TNF- $\alpha$, respectively ( $<<0.01$ in all groups). IL- $1 \beta$ increased the CHOP expression 6- and 15 -fold $(\mathrm{p}<0.001)$. Islet function was impaired after $48 \mathrm{~h}$ incubation with TNF- $\alpha(-55 \pm 3 \%, \mathrm{p}<0.0001)$, while $30 \mathrm{mM}$ glucose or IL$1 ß$ did not alter insulin secretion ( $\mathrm{p}=\mathrm{n} . \mathrm{s}$.). The number of apoptotic cells was increased with $30 \mathrm{mM}$ glucose or TNF- $\alpha$ (Control: $1.2 \pm 0.3 \%$ TUNEL-positive cells/islet; $30 \mathrm{mM}$ glucose: $2.9 \pm 0.4 \%, \mathrm{p}=0.003$; TNF- $\alpha$ : $2.6 \pm 0.4 \%, \mathrm{p}=0.01$ ), but not after incubation with IL- $1 ß(1.9 \pm 0.4 \%, \mathrm{p}=0.15)$. ER stress in human islets $(\mathrm{CHOP})$ was reduced by incubation with exenatide $(\mathrm{p}<0.05)$ in all groups, while after $48 \mathrm{~h}$ incubation there was no change in apoptosis.

Conclusion: Glucose, TNF- $\alpha$ and IL-1ß activate different ER stress pathways in beta-cells. Exenatide reduced ER stress in human islets. Yet, ER stress, beta-cell function and beta-cell apoptosis are not synchronically regulated. Supported by: DFG and a project grant by the DDG

\section{PS 036 Experimental immunology}

\section{6}

The murine pro-insulin II molecule contains strong-binding motifs for several H2-A and H2-E alleles that protect NOD mice from diabetes G.K. Papadopoulos ${ }^{1}$, G. Bondinas ${ }^{1}$, A.K. Moustakas ${ }^{2}$ ${ }^{1}$ Floriculture and Landscape Architecture, Epirus Institute of Technology, Arta, ${ }^{2}$ Organic Farming, Technological Educational Institute of Ionian Islands, Argostoli, Greece

Background and aims: The incidence of type 1 diabetes in NOD mice can be markedly reduced by the transgenic expression of several mouse MHC II alleles, both of the H2-A and H2-E loci. We wished to investigate if this introduction in any way affects the antigenic presentation of the mouse proinsulin II molecule, especially the peptide B9-23, via tighter binding of overlapping epitopes.

Materials and methods: As a first step, we scanned the the entire mouse proinsulin II molecule for possible epitopes of $\mathrm{H} 2-\mathrm{A}^{\mathrm{b} / \mathrm{d} / \mathrm{k}}$ and $\mathrm{H} 2-\mathrm{E}$ allele molecules. The presumed epitopes fulfilling estsablished motifs were then modelled onto the respective structures of the MHC II alleles by energy minimisation, using the programme Discover of Accelrys. The various MHC II structures were obtained from the Protein Data Bank. Well-fitting epitopes were then compared for vicinity to known reactivities restricted to $\mathrm{H} 2-\mathrm{A}^{\mathrm{g} 7}$ of NOD mice.

Results: A number of epitopes (core nonamers, anchors in bold) were identified for each of the alleles in the following descending order of affinity: For H2-A ${ }^{\text {b }}$ InsB1-9 (FVKQHLCGS), InsB6-14 (LCGSHLVEA), InsC53-61 (LQTLALEVA), InsA2-10 (VDQCCTSIC), InsC34-42 (VEDPQVAQL), InsB12-20 (VEALYLVCG), and InsC58-A1 (LEVAQQKRG). For H2-A $\mathrm{A}^{\mathrm{d}}$, InsB6-14, InsC53-61, InsC34-42, InsB12-20, InsB58-66, InsB1-9, and InsA210. For H2-A ${ }^{\mathrm{k}}$, InsA4-12 (DQCCTSICS), InsC52-60 (DLQTLALEV), and InsB4-12 (QHLCGSHLV). Remarkably, all H2-E alleles with known motifs can bind to the epitope B24-C32 (FFYTPMSRR). Furthermore, all epitopes identified are overlapping with known major NOD mouse epitopes, either for $\mathrm{H} 2-\mathrm{A}^{\mathrm{g} 7}$ (immunogenic epitope InsB9-23 with most probable core nonamer InsB12-20, regulatory epitope InsB24-C32), or for H2-K $\mathrm{K}^{\mathrm{d}}$ (InsB15-23 and InsB25-C34).

Conclusion: The results of modeling reveal that the above mouse MHC II alleles confering protection to NOD mice from type 1 diabetes when transgenically expressed, probably do so by tightly binding to overlapping epitopes of known peptide fragments that are diabetogenic in NOD mouse, and do not bind so tightly to $\mathrm{H} 2-\mathrm{A}^{\mathrm{g} 7}$. The possible biological results of such tight binding (epitope stealing, induction of Tregs, or other unknown mechanisms) must be investigated.

\section{7}

A pivotal role of proinflammatory cytokines in diabetes development and after recurrence of diabetes after pancreas transplantation in type 1 animal models

A. Jörns ${ }^{1}$, A. Meyer zu Vilsendorf ${ }^{2}$, T. Taivankhuu ${ }^{1}$, L. Overbergh ${ }^{3}$,

C. Mathieu ${ }^{3}$, S. Lenzen ${ }^{1}$

${ }^{1}$ Institute of Clinical Biochemistry, Hannover Medical School, Germany,

${ }^{2}$ Visceral- and Transplantation Surgery, Hannover Medical School, Germany, ${ }^{3}$ Department of Experimental Medicine, Laboratory of Experimental Medicine and Endocrinology, Katholieke Universiteit Leuven, Belgium

Background and aims: The IDDM (LEW.1AR1-iddm) rat and the NOD mouse are two animal models of human type 1 diabetes mellitus with either an early invasive islet infiltration without signs of peri-insulitis or with a long pre-diabetic period with signs of peri-insulitis resulting in an invasive infiltration. The aim of the study was to identify the pro-inflammatory cytokine pattern in the infiltrated islets and in parallel to identify changes in the cytokine profile in serum samples.

Materials and methods: For the purpose of identification of the different anti- and pro-inflammatory cytokines during spontaneous diabetes development as well as after isogeneic pancreas transplantation gene expression (in situ and real time RT-PCR) and protein expression (immunohistochemistry and bead-based Multiplex assay) of Th1- and Th2-cytokines were performed in pancreas organs at different stages during the development of insulitis/ diabetes manifestation and after recurrence of diabetes following pancreas transplantation. 
Results: NOD mouse islets with signs of peri-insulitis showed no cytokine expression. Only after the invasive immune infiltration into the islet organ the pro-inflammatory cytokines IL- $1 \beta$ and TNF- $\alpha$ as well as the anti-inflammatory cytokines IL- 4 and IL- 10 became evident in the immune cell infiltrate leading to diabetes manifestation over a period of two weeks. In the IDDM rat concomitant with the first appearance of immune cells pro- and antiinflammatory cytokines became evident, initially IL- $1 \beta$ followed by TNF- $\alpha$ leading to diabetes manifestation within 3-7 days. In both animal models the pro-inflammatory cytokine IFN- $\gamma$ was detectable to a negligible extent only in the infiltrated islets as well as in the in serum. After a whole pancreas transplantation in the IDDM rat half of the transplanted recipient rats remained normoglycaemic over 6 months after transplantation whereas the other half showed a recurrence of the disease with a very rapid onset. The immune cells in the grafted pancreas now revealed a massive IFN- $\gamma$ expression both at the gene and protein level which was accompanied by an increase of IFN- $\gamma$ in serum. This confirms similar observations after islet transplantation in the NOD mouse.

Conclusion: Primary diabetes manifestation in both type 1 diabetes animal models took place upon significant expression of the pro-inflammatory cytokines IL- $1 \beta$ and TNF- $\alpha$, but without a significant expression of IFN- $\gamma$ in the islet infiltrate. Pancreas transplantation in an isograft manner permanently cured diabetes in half of the recipients whereas the other half showed a recurrence of diabetes in a fudroayant manner, characterized by a very significant involvement of IFN- $\gamma$.

\section{8}

Granulocyte macrophage-colony stimulating factor (GM-CSF) induces precursors of bone marrow-derived dendritic cells in vivo to acquire a tolerogenic phenotype in NOD mice

S. Gaudreau, C. Guindi, G. Besin, G. Dupuis, A. Amrani

Pedatrics, Immunology division, Université de Sherbrooke, Sherbrooke, Canada

Background and aims: The induction of tolerance is critical for the maintenance of immune homeostasis. In autoimmune diseases such as Insulindependent diabetes (IDDM), the immune system is defective in maintaining $\mathrm{CD} 4^{+}$and $\mathrm{CD} 8^{+} \mathrm{T}$ cell peripheral tolerance. IDDM is the result of a complex $\mathrm{CD}^{+}$and $\mathrm{CD}^{+} \mathrm{T}$ cell-dependent autoimmune process directed against pancreatic $\beta$ cells. Professional antigen presenting cells, such as macrophages and dendritic cell (DCs) also play an important role in the pathogenesis of IDDM. In diabetes and particularly in NOD mice, it is well established that there is an accumulation of DCs and macrophages previous to the arrival of CD4+ and $\mathrm{CD} 8+\mathrm{T}$ cells in the islet. These DCs capture islets antigens and then initiate the recruitment and activation of autoreactive $\mathrm{CD} 4^{+}$and $\mathrm{CD} 8^{+} \mathrm{T}$ cells in the islet. In vivo and in vitro, the development of DCs cells requires stimulation of bone marrow haematopoietic precursors with GM-CSF. We have recently reported that treatment with GM-CSF suppresses diabetes in NOD mice by inducing tolerogenic DC in the periphery. In present study, we investigated whether GM-CSF had a direct influence on BMDC stem cell precursor's commitment into a tolerogenic state in vivo.

Materials and methods: Three weeks old prediabetic female NOD mice were injected with rmGM-CSF or PBS twice a week beginning at 3 weeks of age until 40 weeks. Thereafter, DCs were generated from the bone marrow cells of GM-CSF-treated (GM-CSF.BMDC) and PBS-treated (PBS.BMDC) mice using a GM-CSF and IL-4 and assess their ability to induce tolerance of diabetogenic $T$ cells in vitro and in vivo.

Results: We found that GM-CSF.BMDC produced more IL-10, less IL-12p70 and IFN $\gamma$ as compared to PBS.BMDC when stimulated with pro-inflammatory agents such as liposaccharide (LPS). Furthermore, LPS-activated GM-CSF. BMDC expressed high levels of PD-L1, a cell surface molecule important to maintain T cell tolerance. GM-CSF.BMDC were also poor activators of diabetogenic $\mathrm{T}$ cells and induces an increased production of $\mathrm{IL}-10$ by $\mathrm{CD} 4^{+} \mathrm{CD} 25^{+}$ and $\mathrm{CD} 4^{+} \mathrm{CD} 25^{-} \mathrm{T}$ cells. In addition, only GM-CSF.BMDC can promote conversion of $\mathrm{CD} 4+\mathrm{CD} 25$ - naïve $\mathrm{T}$ cells in $\mathrm{CD} 4+\mathrm{CD} 25+\mathrm{Foxp} 3+$ regulatory $\mathrm{T}$ cells. Finally, a single injection of $2 \times 10^{6}$ LPS conditioned GM-CSF.BMDC into pre diabetic mice delayed significantly development of diabetes as compared to recipients injected with LPS conditioned PBS.BMDC.

Conclusion: Our results showed that GM-CSF injection into young pre-diabetic NOD mice induced BMDC precursors to differentiate into a tolerogenic DCs that promote tolerance of diabetogenic $\mathrm{T}$ cells. Therefore, these work may contribute to design novel therapeutic interventions that target BMDC to prevent development of diabetes in humans. Supported by: JDRF

\section{9}

Priming monospecific, diabetes-inducing CD8+ T cell responses to insulin epitopes and mimotopes

A. Spyrantis ${ }^{1}$, T. Rajasalu ${ }^{2}$, H. Brosi ${ }^{1}$, R. Schirmbeck ${ }^{1}$, B.O. Boehm ${ }^{1}$

${ }^{1}$ Department of Internal Medicine I, University Medical Center of Ulm,

Germany, ${ }^{2}$ Department of Internal Medicine and Immunology, University of Tartu, Estonia

Background and aims: The role of insulin as a major autoantigen for T celldependent autoimmunity in type 1 diabetes (T1D) has been well characterized. We have previously shown that genetic vaccination with insulin can induce diabetes in RIP-B7.1 (CD80) transgenic mice. Single epitope immunisations are also an efficient method to induce experimental autoimmune diabetes in RIP-B7.1 (CD80) mice. Here we have used single epitope immunisation to define both $\mathrm{H}-2 \mathrm{~K}^{\mathrm{b}}$ and $\mathrm{H}-2 \mathrm{~K}^{\mathrm{d}}$ restricted insulin $\mathrm{A}$ - and $\mathrm{B}$-chain epitopes.

Materials and methods: RIP-B7.1 $1^{+}$C57/BL6 mice and RIP-B7.1 ${ }^{+}$BALB/C mice were immunised with A- and B-chain derived epitopes. The immunisation method involves the mixture of the epitope sequence extended by the cationic cell penetrating sequence derived from the HIV-tat ${ }_{49-57}$ motif RKKRRQRRR, complexed with short TLR-9-stimulating oligonucleotides (ODN1826), in a 6:1 molar ratio. Diabetes was defined by blood glucose $>250$ $\mathrm{mg} / \mathrm{dl}$. Epitope specific T cell activation and cytokine profiles were analyzed by multiparameter FACS.

Results: Hyperglycemia is observed in RIP-B7.1 C57/BL6 mice when immunised with insulin A-chain $\mathrm{H}-2 \mathrm{~K}^{\mathrm{b}}$-restricted $\mathrm{A}_{12-21}$-tat/ODN and in RIP$\mathrm{B} 7.1 \mathrm{BALB} / \mathrm{c}$ mice when immunised with the extended $\mathrm{H}-2 \mathrm{~K}^{\mathrm{d}}$-restricted $B_{9-23}$-tat/ODN, both with a median onset of 7-8 weeks after immunization and a cumulative diabetes incidence of $>95 \%$ at week 9-10. In vitro IFN $\gamma^{+}$ $\mathrm{CD} 8^{+} \mathrm{T}$ cell frequencies were usually low $\left(\leq 0.05 \%\right.$ of total $\mathrm{CD} 8^{+} \mathrm{T}$ cell population). To check, if antigenicity of the peptide can be improved by changing its sequence, we replaced the $\mathrm{C}$-terminal asparagine $(\mathrm{N})$ at position $\mathrm{A} 21$ with alanine $(\mathrm{A})$ of the A-chain cationic peptide $\left(\mathrm{A}_{12-21 / \mathrm{N} 21 \mathrm{~A}}\right.$-tat/ODN). This insulin mimotope accelerated diabetes onset (week 5-6 post-immunization; diabetes incidence $>95 \%$ by week 7-8). In vitro IFN $\gamma^{+} \mathrm{CD} 8 \mathrm{~T}$ cell frequencies for the modified peptide $\mathrm{A}_{12-21 \mathrm{~N} 21 \mathrm{~A}}$ were found to be largely increased (up to $5,5 \%$ of total CD8 $8^{+} \mathrm{T}$ cells).

Conclusion: The approach taken confirms the central role of insulin-specific $\mathrm{CD}^{+} \mathrm{T}$ cell reactivity in T1D and outlines the efficiency of a single epitope immunization to induce T1D. In addition, it provides a framework for preclinical assessment of novel peptide variants that may either augment $\mathrm{T}$ cell responses or may lead to $\mathrm{T}$ cell anergy or tolerance. Supported by: Deutsche Forschungsgemeinschaft

\section{0}

Intracellular inhibition of JNK kinase reduces lymphocyte homing into endocrine pancreas of non obese diabetic (NOD) mouse C.P. Khoo ${ }^{1,2}$, N. Hill ${ }^{1}$, M.G. Valorani ${ }^{1,3}$, A. Signore ${ }^{4}$, P. Pozzilli ${ }^{1,2}$

${ }^{1}$ Centre for Diabetes and Metabolic Medicine, Queen Mary University of London, United Kingdom, ${ }^{2}$ Area di Endocrinologia, Universita' Campus Bio-Medico, Rome, Italy, ${ }^{3}$ Stem Cell Research Department, Livio Patrizi Foundation, Research Group BIOS, Rome, Italy, ${ }^{4}$ Medicina Nucleare, University La Sapienza, Rome, Italy

Background and aims: Members of JNK family, JNK2 and JNK1 have often been implicated in type 2 and type 1 diabetes progression by causing $\beta$-cell apoptosis. XG-102 is a neuroprotectant acting by intracellular-inhibition of JNK kinase and is a protease resistant peptidic derivative of the human protein IB-1 selectively blocking the access of JNK to c-Jun and other substrates by a competitive mechanism. The role of JNK1 in type 1 diabetes however is not known. Since apoptosis of $\beta$-cells is one of the putative mechanisms involved in the cascade of events leading to type 1 diabetes, we intend to test XG-102 for $\beta$-cells protection and prevention of $\beta$-cells loss in the NOD mouse.

Materials and methods: Two groups of newly diabetic (0-3 days) mice (1520 weeks of age) were used in this study: Group A- mice injected with XG-102 for once every week for 4 weeks and mice lasting past $7^{\text {th }}$ week of follow-up re-administered with XG-102. Group B-control mice injected with PBS injected for once every week for 4 weeks. In order to investigate the influence of XG-102 on the homing of committed T-cells in the endocrine pancreas, insulitis was rated using $\mathrm{H} \& \mathrm{E}$ stained sections. Insulitis was quantified in all islets present in two H\&E-stained sections from each animal, each section being 
separated from the next by at least $200 \mu \mathrm{m}$. Blood glucose level of mice before culling was taken to compare the effect of XG-102 to delay $\beta$-cell death.

Results: Insulitis of both treated and non-treated mice was evaluated in "blind". After 4 weeks of injection with XG-102, insulitis in group A mice was less prevalent compared to the control mice. The majority ( $45 \%$ ) of islets from Group A mice showed little infiltration and destruction of pancreatic $\beta$-cells (grade 0 insulitis) whereas only $18 \%$ of islets from Group B mice had no insulitis (P-value : 0.002). Group A mice have twice the number of islets in treated mice (Group A, Average number of islets, Av $=20$ ) compared to the control mice (Group B, Av = 13)(P-value: 0.047).

Conclusion: These preliminary data suggest that XG-102 may be acting by reducing lymphocyte homing into the islets as well as blocking apoptosis, which in turn may promote islet survival.

Supported by: Queen Mary University, London and Xigen, Switzerland

\section{1}

A diet rich in protein and poor in starch with reduced food intake prevents diabetes in the NOD mouse: the significance of Reg gene expression in the pancreas

C. Guglielmi ${ }^{1}$, E. Astorri ${ }^{2}$, R. Portuesi ${ }^{1}$, M. Bombardieri ${ }^{2}$, M. Valorani ${ }^{3,4}$, P. Pozzilli ${ }^{1}$

${ }^{1}$ Endocrinology and Diabetes, Università Campus Bio-Medico, Roma, Italy, ${ }^{2}$ Centre for Experimental Medicine \& Rheumatology, Barts and The London, London, United Kingdom, ${ }^{3}$ Diabetes \& Metabolic Medicine, ICMS, Queen Mary University of London, United Kingdom, ${ }^{4}$ Stem Cell Research Department, Livio Patrizi Foundation, Research Group BIOS, Rome, Italy

Background and aims: The prevalence of diabetes is increasing rapidly in industrialized countries. Although most attention has focused on the increase in type 2 diabetes (T2D), there has been a parallel increase in type 1 diabetes (T1D). Hyperglycaemia might result from failure of the beta cells to secrete insulin, resistance of tissues to its action, or a combination of both. Increase of body weight can accelerate the loss of beta cells through apoptosis, i.e. constitution, insulin resistance and autoimmunity. The overall aim of this study was to evaluate the effects of a reduced amount of food (4 gr. per day) instead of the "ad libitum" diet on the development of diabetes in the NOD mouse model. The relationship between body weight and onset of diabetes has been examined in the context of weight at birth, weight changes since birth and weight at disease onset. Furthermore we tested the hypothesis that a diet with a lower protein and a greater starch amount could also impact disease development.

Materials and methods: 48 female NOD mice were randomly allocated in two different groups: group A) reduced food intake with 4 gr. per day using a high nutrient diet (RM3) and group B) reduced food intake with $4 \mathrm{gr}$. per day using a diet poor in protein and rich in starch (RM1). Mice were weighed regularly throughout their life span and tested weekly for urinary glucose ( $>56 \mathrm{mmol} / \mathrm{l})$, as a sign of development of diabetes. At diabetes onset mice were removed from the experiment and their diabetic status confirmed by a blood-glucose reading of $11.5 \mathrm{mmol} / \mathrm{l}$ or greater. Culled mice at diabetes diagnosis were dissected and pancreas retained for studies aimed at expression of Reg genes (Reg1, Reg2, Reg $3 \alpha$, Reg3 $\beta$, Reg $3 \gamma$, and Reg $3 \delta$ ) associated with beta cell regeneration. RNA from pancreas was extracted and than reverse-transcribed into cDNA using the Thermoscript RT-PCR System. The real-time PCRs were than run in duplicate and all Reg genes pancreatic expression was evaluated.

Results: No significant difference was observed between group A and group $B$ in terms of weight at birth (10.3 \pm 2 vs. $10.6 \pm 1.9)$, weight changes since birth considering the pre-diabetes phase $(24.9 \pm 2.1$ vs. $23.6 \pm 2.1)$ and weight at disease onset $(23.7 \pm 2.1$ vs. $21.5 \pm 3.5)$. Incidence of diabetes was significantly reduced in group A $(20.8 \%)$ compared to group B $(45.8 \%, \mathrm{p}=0.001)$. All Reg genes were expressed in pancreas but a different trend in their expression between the two groups was observed. Expression of all Reg genes increased until 30 weeks of age and then decreased in group A; on the contrary, in group B Reg gene expression decreased first and then peaked up again at 30 weeks of age suggesting active beta cell regeneration in animals that develop diabetes but which was not capable to reverse hyperglycaemia.

Conclusion: NOD mice exposed to a reduced food intake and consuming $33 \%$ vs. $44 \%$ of starch develop less diabetes. Both groups of mice showed different trends of Reg genes expression suggesting a relation between beta cell regeneration and disease onset, a finding which indicates that after onset of hyperglycaemia beta cell regeneration is an active, although unsuccessful, process.

\section{2}

Immunomodulation action of an agonistic anti CTLA-4 and a modulating anti CD4 antibody in the LEW.1AR1-iddm rat, a model for type 1 diabetes mellitus

H. Weiss ${ }^{1}$, A. Siepert ${ }^{1}$, R. Pagel ${ }^{1}$, H. Nizze ${ }^{2}$, M. Lehmann ${ }^{1}$, M. Tiedge ${ }^{1}$

${ }^{1}$ University of Rostock, Institute of Medical Biochemistry and Molecular Biology, ${ }^{2}$ University of Rostock, Institute of Pathology, Rostock, Germany

Background and aims: The LEW.1AR1-iddm rat is an animal model of type 1 diabetes mellitus (T1DM) with a strong genetic component and an incidence of $60 \%$. Modulation of the T-cell repertoire using monoclonal antibodies (mabs) may induce immune tolerance. It was the aim of this study to test whether (1) an agonistic anti CTLA-4 antibody (H4) and (2) a modulating anti $\mathrm{CD}^{+}$antibody (RIB5/2) may reverse autoimmune diabetes after onset of the disease.

Materials and methods: Diabetic LEW.1AR1-iddm rats (blood glucose $>7.8$ $\mathrm{mmol} / \mathrm{l})$ were treated for 10 days with mabs $(5 \times 5 \mathrm{mg} / \mathrm{kg}$ b.w.) immediately after diabetes diagnosis. 20 days after diabetes manifestation the animals were killed and blood samples were analyzed for immune status via flow cytometry (FACS) for the T-cell surface markers T-cell receptor (R73), CD4 (Ox35), CD8 $\beta$-chain (341), CD25 (Ox39), CTLA-4/CD152 (WKH1203). Pancreas samples were examined for beta cell destruction and immune cell infiltration.

Results: From our cohort 15 out of 24 animals got diabetes (60\%). Seven animals were treated with $\mathrm{H} 4$, eight animals were treated with RIB5/2. Six out of seven animals from the $\mathrm{H} 4$ cohort required insulin substitution starting 5 to 10 days after diabetes onset. Seven out of eight animals of the RIB5/2 cohort required insulin starting between day 4 and 16 after diabetes onset. One animal of the RIB5/2 cohort showed a complete remission of the diabetic state. FACS analysis of peripheral blood lymphocytes did not show significant differences in T-cell content between control, H4 and RIB5/2 treated animals. The content of $\mathrm{CD} 4^{+}$cells was significantly $(\mathrm{p}<0.05)$ different between all groups. The RIB5/2 cohort showed a low $\mathrm{CD} 4^{+}$cell content indicating a changed distribution in lymphoid compartments. In contrast a higher $\mathrm{CD} 4^{+}$ cell content was observed in the $\mathrm{H} 4$ group compared to the control and the $\mathrm{RIB5} / 2$ group. The $\mathrm{CD} 8^{+} \mathrm{T}$-cell content was significantly higher $(\mathrm{p}<0.001)$ in the RIB5/2 group compared to the control and to the H4 group $(\mathrm{p}<0.001)$. Animals of the RIB5/2 group additionally showed significantly decreased levels of $\mathrm{CD}_{2} 5^{+}(\mathrm{p}<0.01)$ and $\mathrm{CD} 152^{+}(\mathrm{p}<0.01)$ T-cells compared to the controls. Neither treatment with $\mathrm{H} 4$ nor RIB5/2 did reverse massive infiltration of islets by immune cells. Also beta cell mass was not different between the antibody-treated cohorts and control.

Conclusion: The therapeutic application of immunomodulating antibodies did significantly change the composition of the peripheral blood lymphocytes but did not lead to a regeneration of the beta cell mass or a slow down in disease progression. However, the reversion of the diabetic state after the administration of RIB5/2 shows that the modulation of CD4 on T-cells and the reallocation of $\mathrm{CD} 4^{+} \mathrm{T}$-cells could have a positive influence on beta cell mass. In our model the inhibition of T-cell response by stimulation of the CTLA-4 signalling pathway could not stop the fulminant destruction of the beta cells. In future experiments we will characterize the prophylactic therapeutic efficiency of the antibodies when applied in the prediabetic state. Supported by: Deutsche Diabetes Gesellschaft; FORUN

\section{3}

New approach for free rat C-peptide immunodetection

A.V. Kharitonov, A.G. Katrukha

R\&D Department, HyTest Ltd, Turku, Finland

Background and aims: C-peptide - the product of enzymatic cleavage of proinsulin - is a peptide linking A and B chains of insulin in proinsulin molecule. While C-peptide is released into the blood stream in amounts equimolar to insulin and its half-life in blood is much longer than that of insulin (30 min vs. $4 \mathrm{~min}$ for insulin) C-peptide was accepted as a reliable marker for proinsulin metabolism disturbances during proinsulin processing. Measurement of C-peptide levels in the blood or urine is widely used for diagnosis of hypoglycemia and for investigation of pathogenesis of diabetes mellitus type I.

A rat is widely used animal model for the studying of pathophysiological mechanisms underlying diabetes mellitus. Unlike other animals, rats and mice have two isoforms of C-peptide - C-peptides I and II. A number of assays for rat $\mathrm{C}$-peptide immunodetection are available. Most of commer- 
cially available assays are radioimmunoassays or assays based on competition principle. However all of them have considerable disadvantages. The major problem of commercially available immunoassays is cross-reaction with intact and partially processed proinsulin (32,33 split; des 32 split; des 31,32 split; 65,66 split; des64 split and des64,65 split forms). Cross-reaction can be diminished by removal of cross-reactants by gel filtration chromatography but it is not suitable for routine diagnostics procedures.

The aim of our study was to develop monoclonal antibodies (MAbs) for sensitive and specific rat C-peptide immunoassay that would not cross-interact with proinsulin and all known forms of partially processed proinsulin.

Materials and methods: Special strategy of MAbs selection was applied.

Results: We have got a panel of unique monoclonal antibodies that recognize free rat C-peptide with high sensitivity and do not bind to rat proinsulin. MAbs CII-11, CII-29 (specific to N-terminal part of C-peptide molecule) and CC18, CC20, CC34 (specific to C-terminal part of C-peptide) detect both rat C-peptides I and II. Combination of $\mathrm{N}$ - and $\mathrm{C}$-terminal specific MAbs can be used for the creation of highly specific C-peptide immunodetection systems of sandwich type. The assays utilizing pairs of MAbs described above have demonstrated high sensitivity (less than $30 \mathrm{pg} / \mathrm{ml}$ of free rat C-peptide in the sample). Moreover we have developed MAbs that could be also used for the differentiation between rat C-peptides I and II: MAbs CI-0 and 6H1 specifically recognize C-peptide I while MAbs CII-106 and CII-138 bind to C-peptide II and do not interact with C-peptide I.

Conclusion: We obtained a new generation of anti-rat C-peptide monoclonal antibodies that specifically recognize free rat C-peptides I and II with high sensitivity and having no cross-reactivity with rat proinsulin. These MAbs are recommended to be used in different immunoassays for quantitative determination of free rat C-peptides in biological samples.

\section{4}

Cellular therapy of type 1 diabetes: analysis of the immunomodulatory capacity of mesenchymal stem cells

M. Steinsdoerfer, J. Seissler, C. Sattler

Diabetes Center, Medical Clinic Innenstadt, Munich, Germany

Background and aims: Until now the transplantation of the whole pancreas organ or isolated Langerhans-islets remains the only method to cure type 1 diabetes. Transplantation is largely limited by the lack of organ donors and the considerable side effects of the immunosuppressive therapy. The aim of this study was to develop a new strategy for tolerance induction after islet transplantation using the pleiotropic effects of mesenchymal stem cells (MSCs). Materials and methods: From bone marrow of Balb/c mice MSCs were isolated and cultured for several passages. MSCs were characterised by FACS and by their capacity to differentiate into adipocytes, osteoblasts and chondrocytes using specific supplements. The immunosuppressive effect of unstimulated or IFN $\gamma+/$ - TNFa pretreated MSCs was analysed by mixed lymphocyte reaction (MLR). T cell proliferation was determined by $\left[{ }^{3} \mathrm{H}\right]$-thymidine incorporation. To identify the mechanisms of MSC-mediated immunosuppression, we investigated the role of indoleamin-2,3-dioxygenase (IDO), hepatocyte growth factor (HGF) and cyclooxygenase-2 (Cox2) by semiquantitative RT-PCR, Western blot and the measurement of the tryptophane catabolite kynurenine, respectively.

Results: FACS analysis revealed the expression of characteristical MSC markers: CD105, CD73, CD140b, CD29, Sca1 and VCAM. Expression of CD90 and CD44 was weak. By using specific supplements the cells could be differentiated into adipocytes, osteoblasts and chondrocytes. MSCs stimulated with IFN $\gamma$ reduced $T$ cell proliferation significantly in the MLR experiments $(p<0.01)$. This effect was enhanced by prestimulation of MSCs with IFN $\gamma$ and TNFa $(p<0.01)$. Unstimulated MSCs expressed IDO only at a low level. After stimulation with IFN $\gamma$ or IFN $\gamma+$ TNFa the expression of IDO mRNA significantly increased. However, no functional IDO protein was observed. HGF was found to be constitutively expressed in our MSCs. After stimulation with proinflammatory cytokines Cox2 expression was detected.

Conclusion: The murine MSCs described by our group show a typical expression of surface markers and distinct multipotency. Although no expression of active IDO protein after stimulation could be shown, the cells inhibited T cell proliferation by an IDO-independent mechanism. Our findings on the induction of Cox2 suggest that the immunomodulatory effect of MSCs may be associated with PGE2 production. In addition, HGF could be involved in the downregulation of $\mathrm{T}$ cell activity. Further experiments are being carried out to assess the molecular pathways of the immunosuppressive properties of MSCs. These data suggest that the cotransplantation of Langerhans-islets and MSCs could be a promising alternative to the standard therapy.

\section{PS 037 Autoimmunity against GAD}

\section{5}

GAD65 vaccination significantly reduces insulin dependence at five years follow-up in a dose escalating study in adult-onset autoimmune diabetes patients

C.-D. Agardh, K. Lynch, M. Palmér, K. Link, Å. Lernmark

Lund University, Malmö University Hospital, Sweden

Background and aims: Treatment of GAD65-autoantibody positive diabetes patients with alum-formulated recombinant GAD65 (Diamyd ${ }^{\mathrm{TM}}$ ) has previously been reported to be safe and not to compromise beta-cell function. The aim was to test the hypothesis that Diamyd ${ }^{\mathrm{TM}}$ is still safe after five years follow-up and that the dose level affects the beta cell function.

Materials and methods: The study was a Phase II randomised, double blind, placebo-controlled, dose-escalation clinical trial including 47 GADA positive non-insulin dependent patients who at weeks one and four received subcutaneous injections of placebo, 4, 20, 100 or $500 \mu \mathrm{g}$ Diamyd ${ }^{\mathrm{TM}}$. Data on safety evaluations and insulin treatment during follow-up were obtained for all patients. Fasting C-peptide levels were measured at baseline and 5 years and used to confirm insulin dependency and change in beta cell function. Metabolic control was examined among patients who had not started insulin treatment.

Results: No significant study-related adverse events occurred during the five year follow-up. Six patients (three started insulin) dropped out in the first 13 months of the study, and another died (unrelated to the study) within the third year without starting insulin treatment. These patients were excluded from the rest of the analyses.Of the remaining 40 patients followed for five years, dose level was significantly associated with number of patients starting insulin treatment (placebo 7/11 (64\%); $4 \mu \mathrm{g}$ group 5/7(71\%); $20 \mu \mathrm{g} 1 / 7$ (14\%); $100 \mu \mathrm{g}$ 1/7(14\%); $500 \mu \mathrm{g}$ 5/8 (63\%); p=0.046). Fasting C-peptide levels had declined significantly in the placebo (mean decline $=0.18 ; 95 \% \mathrm{CI}=0.02$ $-0.34 \mathrm{nmol} / \mathrm{L} ; \mathrm{p}=0.03$ ) and $500 \mu \mathrm{g}$ group (mean decline $=0.33 ; 95 \% \mathrm{CI}=0.07$ $-0.57 \mathrm{nmol} / \mathrm{L} ; \mathrm{p}=0.02$ ), but no change was observed in the 4,20 and $100 \mu \mathrm{g}$ dose groups. Compared to placebo, fasting C-peptide had increased significantly only in the $20 \mu \mathrm{g}$ dose group $(0.34 ; 0.09-0.60 \mathrm{nmol} / \mathrm{L}, p=0.01)$. Of the patients who had not started insulin treatment, mean change in HbAlc was observed (although not statistically significantly) in the placebo $(\mathrm{n}=4,0.7$ percentage), $4 \mu \mathrm{g}$ ( $\mathrm{n}=2,1.8$ percentage), $100 \mu \mathrm{g}(\mathrm{n}=6,1.3$ percentage) and $500 \mu \mathrm{g}(\mathrm{n}=2,1.0$ percentage) dose group but not in the $20 \mu \mathrm{g}$ dose group $(\mathrm{n}=6,-0.0)$.

Conclusion: These findings suggest that a prime and boost injection with $\mathrm{Di}$ amyd $^{\mathrm{TM}}$ appears safe long term. The trial design allowed testing of doses to be potentially used in future efficacy studies. The $20 \mu \mathrm{g}$ group dose continues to show preserved beta cell function and no adverse events. The rationale to test the $20 \mu \mathrm{g}$ dose for safety and efficacy in larger clinical trials is ongoing. Supported by: Diamyd Therapeutics AB

\section{6}

The clinical implications of GAD antibody positivity in older patients with established non-insulin requiring diabetes

G.A. Hitman ${ }^{1}$, M.I. Hawa ${ }^{1}$, R.D.G. Leslie ${ }^{1}$, T. Ola ${ }^{1}$, C.-C. Wun ${ }^{2}$,

D. DeMicco ${ }^{2}$, D.J. Betteridge ${ }^{3}$, P.N. Durrington ${ }^{4}$, J.H. Fuller ${ }^{5}$, H.A.W. Neil ${ }^{6}$, H.M. Colhoun ${ }^{7}$

${ }^{1}$ Centre for Diabetes and Metabolic Medicine, Barts and The London School of Medicine and Dentistry, London, United Kingdom, ${ }^{2}$ Pfizer, New York, United States, ${ }^{3}$ Department of Diabetes, University College London, United Kingdom, ${ }^{4}$ Department of Medicine, University of Manchester, United Kingdom, ${ }^{5}$ Department of Epidemiology and Public Health, University College London, United Kingdom, ${ }^{6}$ Centre for Diabetes, Endocrinolgy and Metabolism, University of Oxford, United Kingdom, ${ }^{7}$ Public Health, University of Dundee, United Kingdom

Background and aims: Whilst the importance of screening using antibodies to glutamic acid decarboxylase (GADA) in younger patients with apparent type 2 diabetes (T2D) is established, its relevance in older patients in predicting the requirement for insulin treatment and susceptibility to microvascular complications is unknown.

Materials and methods: GADA were determined by a radio-immunoprecipitation method in 2425 patients participating in the Collaborative Atorvastatin Diabetes Study (CARDS); a randomised placebo controlled trial of 2838 
patients with presumed T2D (defined by WHO criteria, median age 62yrs and duration of diabetes 8yrs) who received atorvastatin or placebo and were evaluated for diabetes outcomes over a median follow up of $4 \mathrm{yrs}$.

Results: Of the diabetes patients, $7.1 \%(n=173)$ were GADA positive (GAD>40units). On multivariate analysis at baseline, GADA positive patients compared to GADA negative patients were younger (51.5 vs 55.0 yrs), leaner (BMI 27.3 vs $28.9 \mathrm{~kg} / \mathrm{m} 2$ ), had higher HbAlc ( 8.5 vs $7.8 \%$ ), less metabolic syndrome (64 vs $80 \%$ ) and were more likely to be on insulin both initially ( $56 \%$ vs 17\%; OR 5.8, 95\%CI 4.1-8.2), and after 4yrs follow-up: cumulative incidence of insulin treatment in those not on insulin at baseline was 16 vs $5 \%$ (OR 3.1, 95\% CI 2.0-4.9); all $\mathrm{p}<0.0001$ apart from age of diagnosis ( $\mathrm{p}=0.01$ ). GADA positivity did not predict microvascular complications at baseline or follow-up.

Conclusion: Adult-onset autoimmune diabetes is not uncommon in these established patients with clinically defined type 2 diabetes. However, in such GADA positive patients, the rate of progression towards insulin treatment was slow (at 8 yrs post-diagnosis $44 \%$ were not on insulin) and was not associated with an increased risk of microvascular complications. Supported by: Diabetes UK, UK Department of Health and Pfizer

\section{7}

$\mathrm{GAD}_{65}$ treatment induces high GADA levels in type 1 diabetic children - a relation to $\mathrm{C}$-peptide response, but not to adverse events

M. Chéramy, J. Ludvigsson, R. Casas

Clinical and Experimental Medicine, Division of Pediatrics, Linköping, Sweden

Background and aims: In mice the protective effect of $\mathrm{GAD}_{65}$ has been associated with the induction of high levels of GAD-specific IgG1 antibodies (GADA). In a previous study in LADA patients, GADA levels were not affected by GAD injection except at a very high dose $(500 \mu \mathrm{g})$. We investigated the effect of treatment with $\mathrm{GAD}_{65}\left(\right.$ Diamyd $\left.^{\mathrm{TM}}\right)$ on the antibody response in type 1 diabetic children and its relation to C-peptide preservation and adverse events.

Materials and methods: 70 type 1 diabetic patients (10-18 years old) diagnosed within the previous 18 months, were included in a double-blind, randomised, placebo-controlled trial receiving a primary injection on day 1 of $20 \mu \mathrm{g}$ recombinant human $\mathrm{GAD}_{65}(\mathrm{n}=35)$ or placebo $(\mathrm{n}=35)$ and a booster dose after 4 weeks. Blood samples were collected before the first injection and after 1, 3, 9, 15, 21 and 30 months. The serum levels of GADA, and antibodies to the islet cell antigen IA-2 (IA-2A), tetanus toxoid antibodies and total IgE were analysed, as well as C-peptide (AUC) after a Mixed Meal Tolerance Test. Adverse events were registered.

Results: Patients receiving $\mathrm{GAD}_{65}$ or placebo had similar levels of the autoantibodies at baseline. GAD treatment significantly increased GADA levels in the treated patients in comparison to the placebo group after 3 months $(\mathrm{p}=0.001)$. The levels remained higher in $\mathrm{GAD}_{65}$ treated children still after 30 months $(\mathrm{p}<0.05)$. The $10 \%$ patients with best $\mathrm{C}$-peptide preservation had higher levels of GADA pre treatment compared to intermediate responders $(\mathrm{p}<0.05)$ and to non-responders (loss $>65 \%$ of $\mathrm{C}$-peptide) ( $\mathrm{p}=0.07)$. No differences in the levels of IA-2A, tetanus antibody or IgE post treatment were observed indicating a treatment-specific effect. High GADA titers, seen in both groups, were not related to any signs of neurological or other adverse events.

Conclusion: The effect of $\mathrm{GAD}_{65}$ treatment on the humoral response was antigen specific and induced a long lasting specific B cell memory, without adverse clinical events. The best C-peptide preservation was observed in patients with high GADA levels at baseline. Our data constitute novel information in the understanding of the effect of treatment with $\mathrm{GAD}_{65}$ in children with type 1 diabetes.

Supported by: Swedish Child Diabetes Foundation, Medical Research Council of Southeast Sweden and Diamyd Medical AB

\section{8}

$\mathrm{GAD}_{65}$ specific monocyte activation in type 1 diabetic children treated with alum-formulated $\mathrm{GAD}_{65}$

S. Axelsson, J. Ludvigsson, R. Casas

Clinical and Experimental Medicine, Division of Pediatrics, Linköping, Sweden

Background and aims: We have previously demonstrated that injection of alum-formulated $\mathrm{GAD}_{65}\left(\right.$ Diamyd $\left.^{\mathrm{Tm}}\right)$ to children with recent onset of type 1 diabetes (T1D) resulted in long lasting specific immune responses, which accompany a significant preservation of C-peptide. The immunomodulatory effect of $\mathrm{GAD}_{65}$ was antigen specific and observed both in $\mathrm{T}$ and $\mathrm{B}$ cell immune response in treated individuals. However, memory specific immune responses of the adaptive immune system need to interact with the innate immune system for an effective activation, as the two systems complement each other and act in parallel. Among other components of the innate immune system, monocytes act to initiate and reinforce immune responses, permitting the establishment of the immunological memory. Thus, this study aimed to investigate the effect of $\mathrm{GAD}_{65}$ treatment in the activation and maturation of monocytes.

Materials and methods: The phase II Diamyd ${ }^{\mathrm{m}}$ study was designed as a randomised, double-blind, placebo-controlled, multi-centre trial including 70 T1D children, with a primary injection day one and a booster injection after 4 weeks. Peripheral blood mononuclear cells (PBMC) were collected 30 months after receiving $20 \mu \mathrm{g}$ alum-formulated $\mathrm{GAD}_{65}(\mathrm{n}=21)$ or placebo $(\mathrm{n}=24)$, and stimulated with $\mathrm{GAD}_{65}$ and IA-2 for $18 \mathrm{hrs}$, in parallel to cells cultured only in medium. Expression of CD14, CD80, CD86 and CD58 was analysed by flow cytometry. The chemokines IP-10, MCP-1, MIP-1a, MIP$1 \beta$ and RANTES were measured in cell supernatants using multiplex fluorochrome technique (Luminex).

Results: After $\mathrm{GAD}_{65}$ stimulation, the percentage of $\mathrm{CD} 14+$ cells expressing CD80/CD58 $(\mathrm{p}<0.05)$ and CD80/CD86 $(\mathrm{p}<0.05)$ was higher among the $\mathrm{GAD}_{65}$ treated children. Additionally, stimulation with $\mathrm{GAD}_{65}$ resulted in a reduction of the percentage of CD14 expressing cells $(p<0.05)$ as well as the $\mathrm{CD} 14$ median intensity $(\mathrm{p}<0.05)$ in the same group. In contrast, no difference between the two groups was observed when cells were cultured in medium alone or in presence of IA-2. Further, after $\mathrm{GAD}_{65}$ stimulation the levels of MCP-1, MIP- $1 \alpha$ and MIP- $1 \beta$ increased in the cell supernatants from children who received $\mathrm{GAD}_{65}(\mathrm{p}<0.01)$.

Conclusion: Up-regulation of co-stimulatory molecules and decreased expression of CD14, in parallel with increased chemokine secretion after $\mathrm{GAD}_{65}$ stimulation, suggests that recall responses in T1D children treated with $\mathrm{GAD}_{65}$ involve activation of the innate immune system. This could in turn lead to a more efficient antigen presentation and antigen-specific $\mathrm{T}$ and B lymphocyte activation.

Suported by: Swedish Child Diabetes Foundation the Medical Research Council of Southeast Sweden and Diamyd Medical AB

\section{9}

A low peptide concentration selectively promotes expansion of high avidity $\mathrm{T}$ cell clones: study of DRB1 ${ }^{\star} 0404$-restricted human $\mathrm{T}$ cell clones specific to the GAD65 555-567 epitope

V. Öling ${ }^{1}$, K. Geubtner ${ }^{2}$, J. Ilonen ${ }^{1}$, H. Reijonen ${ }^{2}$

${ }^{1}$ Immunogenetics Laboratory, University of Turku, Finland, ${ }^{2}$ Benaroya Research Institute, Virgina Mason, Seattle, United States

Background and aims: $T$ cells specific for islet proteins can be detected in the peripheral blood of T1D patients and at-risk individuals, suggesting failure of the central tolerance and the negative selection. Cells with low-avidity to self-antigens may escape the selection and later contribute to autoimmunity. We addressed a question how the antigen dose affects the diversity of autoreactive T cells. Our hypothesis was that low antigen dose will preferentially stimulate high avidity T cells. T cell clones specific for GAD65, a major autoantigen in T1D, were isolated and the T cell clones avidity and phenotype were investigated.

Materials and methods: CD4+ T cells isolated from a DR404-positive subject with a known response to GAD65 555-567 were stimulated with 10,1 or 0.1 $\mu \mathrm{g} / \mathrm{ml}$ of GAD65 557I peptide in the presence of adherent CD4 negative cells for 14 days. GAD65 557I is an agonist peptide previously described to induce a stronger proliferative response of GAD65 555-567 specific T cell clones. After 14 days the cells were stained with GAD65 and control tetramers. GAD65 tetramer binding CD4+ cells were single cell sorted and expanded. Peptide specificity of the $\mathrm{T}$ cell clones was confirmed by tetramer staining and antigen specific proliferation. Avidity was determined by proliferation in decreasing concentrations of the GAD65 557I peptide $(0.001-10 \mu \mathrm{g} / \mathrm{ml})$ and cytokine secretion was analysed in Cytometric Beads Array assay. T cell receptor usage was tested with $\mathrm{V} \beta$ chain specific monoclonal antibodies using FACS.

Results: Avidity of the T cell clones was determined by $50 \%$ of maximal proliferation response and the level of tetramer binding. An inverse correlation between the antigen dose used in the original cultures and the overall avidity of the isolated T cell clones was observed: $\mathrm{T}$ cell clones isolated from cultures originally stimulated by a low antigen dose $(0.1$ and $1 \mu \mathrm{g} / \mathrm{ml}$ GAD65 557I peptide), displayed a higher proliferative response in decreasing amounts 
of the specific peptide in comparison to $\mathrm{T}$ cell clones originally cultured in presence of the highest antigen dose $(10 \mu \mathrm{g} / \mathrm{ml})$ (Mann-Whitney $\mathrm{U}$ test, tied $\mathrm{p}=0.008$ and $\mathrm{p}=0.023$, respectively). $\mathrm{T}$ cells clones derived from cells stimulated with the lowest peptide dose also displayed the highest tetramer binding affinity in comparison to the other $\mathrm{T}$ cell clones (tied $\mathrm{p}=0.058$ ). We did not detect any significant differences in the release of IFN- $\gamma$, TNF- $\alpha$, IL-10, IL-5, IL-4 and IL-2 between the T cell groups. Majority (75\%) of the T cell clones induced by the low antigen dose $(0.1 \mu \mathrm{g} / \mathrm{ml}$ GAD65 557I) expressed the V $\beta 5.1$ chain whereas only two $(40 \%)$ of the $\mathrm{T}$ cell clones induced by the intermediate antigen dose $(1 \mu \mathrm{g} / \mathrm{ml})$ and only one $(14 \%) \mathrm{T}$ cell clone from the high antigen dose cultures $(10 \mu \mathrm{g} / \mathrm{ml})$ was V $\beta 5.1$ positive.

Conclusion: Our results suggest that antigen dose modulates diversity of the peripheral $\mathrm{T}$ cell repertoire observed subsequent to in vitro $\mathrm{T}$ cell expansion, both avidity and TCR usage of the T cell clones are dependent on the dose of peptide used for stimulation. Therefore, factors influencing the overall functional avidity should be taken into consideration when designing antigen specific assays to evaluate the diversity of autoreactive $\mathrm{T}$ cell populations and changes induced by immunomodulatory therapies in T1D.

\section{0}

Study of T cell response to Ii-key/MHC class II epitope hybrides in human type 1 diabetes

H. Beyan ${ }^{1}$, E. Von Hofe ${ }^{2}$, R.D. Leslie ${ }^{1}$

${ }^{1}$ Centre for Diabetes and Metabolic Medicine, Institute of Cell and

Molecular Science, London, United Kingdom, ${ }^{2}$ Antigen Express, Biotech 3, Worcester, United States

Background and aims: Type 1 diabetes (T1DM) is a chronic, T cell-mediated autoimmune disease mediated by an adaptive immune response to antigen including glutamic acid decarboxylase65 (GAD65). A peptide of the Ii protein (Ii-Key) enhances in vitro presentation of antigenic peptides, thus creating a therapeutic opportunity to regulate the antigenic peptide binding to MHC Class II molecules. The aim of our study was to apply this technique to testing the use of Ii-Key/MHC class II GAD65 epitope hybrids in characterizing anti-GAD65 $\mathrm{T}$ cell responses in patients with T1DM.

Materials and methods: GAD65 peptides predicted to be recognized by DRB $1^{*} 0301$ and $\mathrm{DRB} 1^{*} 0401$ were identified with the Rammensee SYFPEITH program. Peripheral blood mononuclear cells (PBMC) were tested from 21 established T1DM patients (age mean 43 years range 24 - 46 years, 11 male; mean disease duration 22 years) and 24 normal subjects (age mean 49 years range 25 - 58 years, 12 male). Patients and controls were selected for HLA-DR3 or 4 and tested for GAD65 autoantibodies. In an optimized assay PBMCs were cultured with positive and negative control peptides, whole GAD65 and GAD65 peptides, then analysed for interferon gamma (IFNg) and interleukin-10 (IL-10) by U-Cytech ELISPOT.

Results: Patients with T1DM had significantly greater IFNg responses to whole GAD65 compared to controls $(\mathrm{p}<0.01)$, but there was no difference in IL-10 responses between them. Patients with T1DM also showed increased IFNg responses to any given GAD65 peptide compared with controls $(\mathrm{p}<0.03)$, again without differences in IL-10 responses. There was no dominant GAD65 peptides and the responses to GAD65 peptides were not influenced by the li-key.

Conclusion: We found out that patients with established T1DM have greater IFN gamma responses to both whole GAD65 and GAD65 peptides compared to controls. IL-10 responses, on the other hand, were similar between T1DM and controls. The results are consistent with a polarized pro-inflammatory response to GAD65 peptides in T1DM independent of the li-key protein. We conclude that even after many years of T1DM there is a detectable cellular and humoral immune response to GAD65.

Supported by: Antigen Express Inc.

\section{1}

GAD and IA-2 immunoreactivity in type 1 diabetic patient saliva C. Tiberti ${ }^{1}$, B. Shashaj ${ }^{2}$, A. Verrienti ${ }^{1}$, E.G. Vecci ${ }^{1}$, F. Lucantoni ${ }^{1}$, M. Suma ${ }^{1}$, D. Masotti ${ }^{1}$, S. Morano ${ }^{1}$, N. Sulli ${ }^{2}$, F. Dotta ${ }^{3}$

${ }^{1}$ Clinical Sciences, University of Rome "Sapienza", ${ }^{2}$ Pediatrics, University of Rome "Sapienza", ${ }^{3}$ Internal Medicine, Endocrine and Metabolic Sciences, University of Siena, Italy

Background and aims: Type 1 diabetes is characterized by the presence of autoantibodies directed against various islet autoantigens, including glutamic acid decarboxylase (GAD) and tyrosine phosphatase 2 (IA-2) proteins. Some attempts have been made in assaying GAD autoantibodies (GADA) in human saliva, a body fluid which can be easily obtained by non-invasive techniques, bypassing blood sample collection. However, these salivary assays were not sensitive, specific and accurate enough in comparison to serum assays. The poor performances of GADA assays probably dissuaded the investigators to search for other diabetic-specific autoantibodies in human saliva, thus to our knowledge, no attempts were made to detect other diabetes specific autoantibodies in human saliva. Aim of this study was to evaluate the ability of a highly sensitive fluid-phase ${ }^{35} \mathrm{~S}$-radioimmunoassay method to detect salivary GAD and IA-2 immunoreactivity in newly diagnosed (ND) and long-standing (LS) type 1 diabetic patients.

Materials and methods: Human recombinant full-length GAD65 and IA2(605-979) autoantibodies were detected in paired saliva and serum samples collected from: $39 \mathrm{ND}$ (21m, 18f), $31 \mathrm{LS}$ patients (15m, 16f), 24 GADA and IA-2A negative first degree relatives of patients with autoimmune disease (FDR) $(13 \mathrm{~m}, 11 \mathrm{f}), 76$ healthy subjects $(32 \mathrm{~m}, 44 \mathrm{f})$. To detect salivary autoantibodies were utilized the supernatants of unstimolated whole saliva samples previously spinned at $10000 \mathrm{rpm}$.

Results: GADA were detected in the serum of $25 / 39(64.1 \%)$ ND, 20/31(64.5\%) LS and in the saliva of $24 / 39(61.5 \%)$ ND and $19 / 31(61.3 \%)$ LS patients. IA$2 \mathrm{~A}$ were detected in the serum of 19/39(48.7\%) ND, 14/31(46.2\%) LS and in the saliva of 18/39(46.2\%) ND and 12/31(38.7\%) LS patients. No presence of GADA and IA-2A was found in serum and saliva of GADA and IA-2A negative FDR subjects. A significant correlation was found between serum and saliva GADA titers $(\mathrm{r}=0.739, \mathrm{p}<0.0001)$ and between serum and saliva IA-2A titers $(\mathrm{r}=0.687, \mathrm{p}<0.0001)$. Altogether, salivary and serum GAD and IA- $2 \mathrm{im}$ mune responses were concordant in $94.3 \%$ and $93.3 \%$ of subjects analyzed, respectively.

Conclusion: The present study demonstrates that immune changes typical of type 1 diabetes may be reflected in oral secretions. The use of saliva supernatant, the accurate choice of its dilution and the use of a fluid-phase radioimmunoassay method make possible the detection of salivary GAD and IA-2A with high sensibility and specificity. Human saliva, a speciman that can be easily collected, also repeatedly in the time by non-invasive procedures, may therefore represent a reliable, powerful tool for the study of humoral autoimmunity in type 1 diabetes.

\section{2}

Decreasing humoral activity in siblings of type 1 diabetes children over a ten year period with increasing incidence of the disease J. Svensson ${ }^{1}$, A. Nilsson ${ }^{2}$, S. Eising ${ }^{3}$, H. Mortensen ${ }^{1}$, A. Lernmark ${ }^{2}$, F. Pociot ${ }^{3}$, J. Johannesen ${ }^{1}$

${ }^{1}$ Paediatric Department, Glostrup University Hospital, Glostrup, Denmark, ${ }^{2}$ Department of Clinical Sciences, Lund University/CRC, Malmö, Sweden, ${ }^{3}$ Steno Diabetes Center, Gentofte, Denmark

Background and aims: Type 1 diabetes (T1D) is an immune-mediated disease, which develops on the basis of complex gene-environment interactions. Autoantibodies are important markers of the autoimmunity against the pancreatic islet beta cells. Despite the number of autoantibodies predict T1D, their possible pathogenic role remains unclear. High concentrations of GAD antibodies are associated with slower progression to clinical disease and Harrison et al. found in 1993 an inverse relation between humoral and cellular immunity to GAD in relatives of T1D patients. The objective of the present study was to test the hypothesis that levels of autoantibodies (GAD65 and IA2A) may decrease over a ten year period in high risk subjects (siblings of T1D patients) and at onset in T1D children as an explanation of a more accelerated disease process which would lead to an increasing trend in incidence rate.

Materials and methods: Data for the study are derived from a large population-based register of diabetic children with an attached bio bank. It is a random sample in newly diagnosed children $(n=481)$ with blood sampling less than 3 months after onset collected 1997-2005. There were 479 siblings samples from the same time period. Blood samples are stored at minus 80 degrees until analysis. Autoantibodies (GAD65A, IA-2A) were measured using radioimmunoassay (RIA). The 97.5 percentile cut-off limits GAD65A and IA2A were 32 and $6 \mathrm{U} / \mathrm{ml}$, respectively

Results: Six out of 479 siblings developed T1D 0.5-3.4 years after blood sampling. Among the siblings eight (1.7\%) had both IA2A and GAD65A above the cut-off limit, three of those developed T1D. Two $(2 / 479,0.4 \%)$ had IA2A levels above $6 \mathrm{U} / \mathrm{ml}$ but GAD65A below cut-off, 19 (19/479. 4.0\%) had GAD65A above $32 \mathrm{U} / \mathrm{ml}$ but IA2A below cut-off, one from each of these 
groups developed diabetes. There was a significant negative correlation between year of measure and GAD65A and IA2A levels observed only in healthy siblings, not in the newly diagnosed T1D children. The IA2A level was 0 in 427 siblings, whereas GAD65A was only 0 in one sibling. GAD65A was positively correlated with age in patients, whereas IA2A showed no association with age. The GAD65A were significantly higher in female patients. Conclusion: The levels of GAD65A and IA-2A was unchanged in newly diagnosed T1D children 1997-2005 while a decreasing level of GAD65A and IA2A were observed in their healthy siblings. The IA2A level was only above 0 in 52 cases, therefore the correlation with sample year and IA2A should be taken with this in mind. If lower levels of autoantibodies reflects less humoral activity, then siblings of T1D children seems over this ten year period to move towards a higher risk of developing diabetes in accordance with the increasing trend in incidence.

Supported by: Danish Diabetes Association, Danielsen Fonden, Copenhagen County

\section{3}

Latent autoimmune diabetes in adults vs recent-onset type 1 diabetes: differences in autoantibody levels, clinical course and residual insulin secretion capacity

L. Lukic, N.M. Lalic, A. Jotic, T. Milicic, M. Zamaklar, K. Lalic, N. Rajkovic Institute for Endocrinology, Clinical Center of Serbia, Belgrade, Serbia

Background and aims: Previous studies have suggested that Latent Autoimmune Diabetes in Adults (LADA) exhibits similar immunological, metabolic and clinical characteristics compared to recent-onset Type 1 diabetes (T1D), but the similarities and differences between the two disorders have not yet been clarified. Therefore the aim of this study was to compare: (a) the incidence of glutamic acid decarboxilase (GAD) and thyrosin phosphatase (IA2) autoantibody positivity, (b) clinical course and (c) insulin secretory capacity during the first year of the disease in 30 patients with LADA (age $>35 \mathrm{yrs}$ ) (group A) and in 30 patients with recent-onset T1D (age >26 yrs) (group B) Materials and methods: All patients were analysed in insulin requiring state. The GADA and IA2 positivity was determined by RIA. The clinical course was evaluated using the following parameters: duration of simptoms before diagnosis, frequency of ketosis, weight loss and incidence of clinical remission (CR). The CR was defined as optimal metabolic control without insulin lasting $>30$ days. The insulin secretion capacity was evaluated by determining C-peptide levels (RIA) before and after $1 \mathrm{mg}$ intravenous glucagon stimulation $(0 / 6 \mathrm{~min})$

Results: We found that percentage both of GADA and IA2 positive patients was lower in group A compared to the group B (A:3.7\% vs B:14.3\%, $<<0.01$ ). However, when GADA positivity was analysed separately, the percentage was higher in group A in comparison to the group B (GADA A: $92.6 \%$ vs B: $65.3 \%, \mathrm{p}<0.01$ ). On the other hand, when IA2 positivity was separately analysed we did not find significant difference between groups (IA2 A: $2.5 \%$ vs $B: 2.8 \% ; p=N S)$. Simultaneously, the incidence of $C R$ was significantly lower in group A than in the group B (A:13.8\% vs B: $41.4 \%, \mathrm{p}<0.05)$. Also, in group A compared to group B we detected significantly shorter duration of simptoms (A: $2.83 \pm 1.97$ vs B $7.57 \pm 0.87$ months, $\mathrm{p}<0.01$ ), lower frequency of ketosis (A: $19.2 \%$ vs $B: 50 \%, p<0.01$ ), and smaller weight loss (A: $4.03 \pm 2.18$ vs B: $6.73 \pm 3.64 \mathrm{~kg}, \mathrm{p}<0.05)$. In addition, we found that basal and stimulated $\mathrm{C}$ peptide levels were significantly higher in group A than in group B (C-pep. 0 min. A: 0.37 vs B: $0.20 \mathrm{nmol} / 1$; C-pep. 6 min. A: 0.53 vs B: $0.35 \mathrm{nmol} / \mathrm{l}$, $\mathrm{p}<0.01$, respectively).

Conclusion: Our data have shown that patients with LADA exhibited lower autoantibody response, together with milder clinical course and better preserved insulin secretion capacity. In contrast, our results have demonstrated that the LADA patients exhibited lower incidence of CR in comparison to the adult recent-onset T1D.

\section{PS 038 Clinical immunology}

\section{4}

Interim results of a phase I/II clinical trial of a DNA plasmid (BHT-3021) for type 1 diabetes

P. Gottlieb ${ }^{1}$, G.S. Eisenbarth ${ }^{1}$, M. Kipnes ${ }^{2}$, R. Ratner ${ }^{3}$, J. Rockell ${ }^{1}$,

R. Wagner ${ }^{1}$, F. Valone ${ }^{4}$, H. Garren ${ }^{4}$

${ }^{1}$ Barbara Davis Center, University of Colorado Health Sciences Center, Aurora, ${ }^{2}$ Diabetes and Glandular Disease Clinic, San Antonio, ${ }^{3}$ Diabetes, Medstar Research Institute, Washington, ${ }^{4}$ Bayhill Therapeutics, Palo Alto, United States

Background and aims: Current therapies approved and in development for autoimmune diseases are only partially effective and largely non-antigen specific. Recently, DNA plasmids have proven to be a very effective antigen-specific method of autoimmune disease treatment in various animal models and in a clinical trial in multiple sclerosis. We have previously demonstrated that a proinsulin encoding DNA vaccine is effective in treating the NOD animal model of T1D, and that it causes antigen-specific immune tolerance. We have initiated a phase I/II clinical trial of BHT-3021, a DNA plasmid that encodes full-length human proinsulin, in type 1 diabetes (T1D) patients.

Materials and methods: This is a randomized, blinded, placebo-controlled trial in T1D patients. Key eligibility criteria are an age of 18 to 40 years at the time of diagnosis of TID diabetes and the presence of antibodies to at least one diabetes-related antigen (insulin, GAD-65, or IA-2). Both C-peptide + and - patients are enrolled in two separate groups. Within each group, subjects are randomized to BHT-3021 or saline placebo in a 2:1 ratio. BHT3021 or placebo is injected intramuscularly weekly for 12 weeks after which subjects are followed in a blinded manner for 40 weeks. Four dose levels of BHT-3021 are being tested $(0.3 \mathrm{mg}, 1 \mathrm{mg}, 3 \mathrm{mg}$ and $6 \mathrm{mg})$. The primary outcome measures are safety and changes in antigen-specific immune response. Immune response measures include antibody and $\mathrm{T}$ cell reactivity to insulin, GAD-65 and IA-2 and T cell frequency assessed by tetramer assays.

Results: To date, 14 patients (9 C peptide positive; $5 \mathrm{C}$ peptide negative) have been randomize to the initial $1.0 \mathrm{mg}$ dose level. Safety data on these patients demonstrate that BHT-3021 is safe with no increase in adverse events relative to placebo. No subjects have discontinued therapy and no serious adverse events have been reported. Adverse events (AEs) occurred with equal frequency in the placebo and BHT-3021 treatment arms. All AEs were mild or moderate in severity. There were no clinically significant laboratory abnormalities. There was also no evidence of accelerated loss of beta cell function as measured by C-peptide levels. In fact, in the three BHT-3021 treated patients where we have data to week 15 , the mean change in peak stimulated C-peptide is an increase by $19 \%$ compared to baseline.

Preliminary data on the first several patients treated with BHT-3021 demonstrate that in both patients treated with $1 \mathrm{mg}$ BHT-3021, there was an approximately $50 \%$ reduction in titers of anti-insulin antibodies rapidly after the initiation of dosing that was sustained throughout the dosing period. In contrast, in the one placebo treated patient from whom we have data, the anti-insulin antibody titers were unchanged with placebo dosing. Autoimmune $\mathrm{T}$ cell data from these patients are pending at this time.

Conclusion: The preliminary data from this phase I/II trial indicate that BHT-3021 is safe and may suppress immune responses in an antigen-specific manner. Additional patient data are expected by the middle of 2008 . Supported by: Bayhill Therapeutics, Inc.

\section{5}

High prevalence of autoimmunity in adult onset diabetes subjects with high GADA titre

M. Capizzi ${ }^{1}$, A. Petrone ${ }^{1}$, C. Tiberti ${ }^{1}$, M. Spoletini ${ }^{1}$, S. Di Pietro ${ }^{2}$,

M. Locatelli ${ }^{3}$, F. Dotta ${ }^{4}$, A. Falorni ${ }^{5}$, E. Bosi ${ }^{6}$, R. Buzzetti ${ }^{1}$

${ }^{1}$ Clinical Sciences, "Sapienza" University, Rome, ${ }^{2}$ Diabetes Unit, Instituto

Nazionale Riposo e Cura Anziani (INRCA), Rome, ${ }^{3}$ Endocrinology, Bambino Gesu` Hospital, Rome, ${ }^{4}$ Internal Medicine, Siena University, ${ }^{5}$ Internal Medicine, University of Perugia, ${ }^{6}$ General Medicine, Diabetes \& Endocrinology, San Raffaele Scientific Institute and Vita Salute University, Milan, Italy

Background and aims: Previous studies showed that LADA is not one clearcut disease entity, but a heterogeneous population of diabetes subjects wherein subgroups can be identified based on their autoimmune status: the titre of 
autoantibodies to GAD. Compared to low GADA, subjects with high GADA titre have more prominent traits of insulin deficiency and a higher prevalence of the genotypes known to increase the risk of type 1 diabetes. The aim of the present study was to correlate GADA titre, in LADA subjects, with the presence of other organ and non organ specific autoantibodies.

Materials and methods: Adult onset GADA positive autoimmune diabetes subjects $(\mathrm{n}=191)$ and a twofold number of age and sex matched GADA negative type 2 diabetes subjects $(n=382)$ were selected from the Non Insulin Requiring Autoimmune Diabetes (NIRAD) study cohort of 4,250 type 2 diabetes subjects. The analysis of GADA titre showed a bimodal distribution which identified two subgroups of patients with high ( $>32$ GADA U) and low GADA titre ( $\leq 32$ GADA U). All subjects were further characterized for other autoantibodies as protein tyrosine phosphatase IA-2 (IA-2A), thyroid peroxidase antibodies, (TPO), steroid-21-hydroxylase (21OHAb), anti-parietal cell antibodies (APCA), tissue transglutaminase antibody IgA (tTGA). IA-2As were measured using a radiobinding assay with in vitro-translated IA- 2 (amino acids 605-979). Thyroid peroxidase (TPO) antibodies were measured by a radioimmunoassay using a commercial kit. 21-hydroxylase were measured using a radiobinding assay. IgG anti-tyrosine phosphatase 2 (IA-2 $\mathrm{A}_{\mathrm{IC}}$ ) and IgA antitransglutaminase autoantibodies (tTGA) were detected by a radioimmunoprecipitation assay using the ${ }^{35} \mathrm{~S}$-methionine labeled human antigen. Anti-parietal cell antibodies (APCA) were detected by using a commercial ELISA method. Results: Compared with those with low GADA titer, LADA subjects with high GADA titer showed a profile of more severe autoimmunity resulting in higher prevalence of several types of autoantibodies. Patients with high GADA compared to low GADA titer showed a significantly higher prevalence of IA- $2 \mathrm{~A}_{\mathrm{IC}}$ ( $25.5 \%$ vs $8.2 \%$; $\left.\mathrm{p}<0.002\right)$, TPOAb ( $37.2 \%$ vs $16.5 \%$; $\left.\mathrm{p}=0.002\right)$ reported elsewhere, and APCA (23.3\% vs 9.3\%; $\mathrm{p}<0.01)$. 21OHAb showed a prevalence of $3.2 \%$ (3/94) in high GADA titer and were not present in low GADA titer (0/97) ( $\mathrm{p}=\mathrm{ns})$ and in type 2 diabetes subjects (0/382; high GADA titre vs type 2 diabetes $\mathrm{p}=0.03)$. tTGA showed a prevalence of $4 \%(3 / 94)$ in high GADA, $1.2 \%(1 / 97)$ in low GADA and $0.4 \%$ in type 2 diabetes ( $\mathrm{p}$ for trend $=0.007$, high GADA vs type 2 diabetes $\mathrm{p}=0.03$ ).

Conclusion: High GADA titre, in LADA subjects, is associated with a profile of more severe autoimmunity consisting in higher prevalence of organ and non organ specific autoantibodies. Previous longitudinal studies indicate that the prevalence of multiple autoantibodies is a better index of disease progression than prevalence of antibodies directed at individual antigens. This may indicate intermolecular epitope spreading and, hence, amplifying the autoimmune response.

Supported by: FoRiSID (Research Foundation of the Italian Society of diabetes) and NOVO Nordisk, Italy

\section{6}

Innate immunity and human insulitis during beta cell coxsackie B4 infection

F. Dotta ${ }^{1,2}$, V. Bonato ${ }^{1,2}$, R. Gallo ${ }^{1,2}$, F.A. Grieco ${ }^{1,2}$, G. Sebastiani ${ }^{1,2}$,

P. Marchetti ${ }^{3}$, G. Gambelunghe ${ }^{4}$, A. Falorni ${ }^{4}$

${ }^{1}$ Department of Internal Medicine and Endocrine and Metabolic sciences, University of Siena, ${ }^{2}$ Fondazione Umberto Di Mario, Toscana Life Sciences, Siena, ${ }^{3}$ Department of Endocrinology and Metabolism, University of Pisa, ${ }^{4}$ Department of Internal Medicine, Section of Internal Medicine and Endocrine and Metabolic Sciences, University of Perugia, Italy

Background and aims: Type 1 diabetes mellitus (TlDM) is a multifactorial disease in which multiple genes interplay with environmental factors leading to autoimmunity against self-antigens in T-cells. Viral infections are associated epidemiologically with TIDM. Recently we reported that in a subset of TIDM patients signs of enteroviral infection were detected. Infecting virus was identified as a novel Coxsackie B4 (CVB4) strain, named 'Tuscany Strain". Viral infection, was beta-cell specific and caused beta-cell dysfunction as shown by impaired glucose-stimulated insulin secretion. Several studies demonstrated that during stress, such as viral infections, cells synthesize factors, such as FAS, IFN-alfa, MICA, which interact with innate immunity. In particular MICA (MHC class I chain-related) molecules are stress induced, and recognized by NKG2D receptors on natural killer (NK) and CD8-positive T-cells. In addition studies on animal models demonstrated the pathogenetic role of CXCR3+ autoreactive lymphocytes, recruited in the pancreatic islet by CXCL10 and CXCL9. We aimed at characterizing the occurrence of innate immunity-related phenomena and chemokine expression in human insulitis during CVB4 infection.

Materials and methods: Pancreas and collagenase purified islets were obtained from organ donors ( 2 recently diagnosed TIDM found positive at islet levels for CVB4 strain, and 5 controls). Pancreatic specimens were formalinfixed and paraffin-embedded for immunohistochemical studies, while pancreatic islets were employed for mRNA expression studies.

Results: In pancreas of CVB4 positive T1DM patients, insulitis was present and was characterized mainly by NK cells (CD94+) and by a small amount of CD3 expressing T-cells. Strong positivity for IFN-alfa and FAS was detected in the majority of pancreatic islets as shown by immunohistochemestry. In addition insulitic lesions were characterized by presence of elevated levels of the chemokine CXCL10 and infiltration of lymphocytes expressing the corresponding chemokine receptor CXCR3. This result was paralleled by real time PCR analyses on isolated TIDM islets. Both in islet and endothelial cells we observed an over-expression of class I MHC molecules. Interestingly, MICApositive cells were detected in most of the islets and in scattered exocrine cells. MICA expression co-localized with insulin producing beta-cells in the islets as shown by confocal microscopy experiments.

Conclusion: We have demonstrated that: during beta-cell viral infection, innate immunity is indeed involved in human TlDM, thus suggesting that strategies aimed at modulating NK cell function may interfere with the natural history of beta-cell destruction. In addition, our study suggested a relevance of the CXCL10-CXCR3 pathway in facilitating human leucocyte migration to inflamed pancreatic islets, which may lay the basis for the development of a novel therapeutic target.

\section{7}

Polyclonal anti-T-lymphocyte globulin preserves C-peptide production in type 1 diabetes of recent onset

F. Saudek ${ }^{1}$, T. Havrdova ${ }^{1}$, P. Boucek ${ }^{1}$, H.H. Hirsch ${ }^{2}$, K. Zacharovova ${ }^{1}$, V. Lanska ${ }^{1}$

${ }^{1}$ Diabetes Center, Institute for Clinical and Experimental Medicine, Praha 4, Czech Republic, ${ }^{2}$ Institute for Medical Microbiology, University of Basel, Switzerland

Background and aims: The destruction of pancreatic B-cells typical for type 1 diabetes (T1DM) is mediated by autoreactive T-lymphocytes. Their identifications and specific targeting has been so far not successful. Recently, studies with 2 different monoclonal antibodies directed against the CD3 molecule showed encourraging results in delaying the loss of C-peptide production and improving metabolic control in T1DM of recent onset. We report on the results of an immune-intervention study in new onset T1DM (within 6 weeks of diagnosis) using polyclonal anti-T-lymphocyte globulin which in contrast to anti-CD3 antibodies displays multiple anti-T-cell specificities and might stop the autoimmune process by deletion of different activated T-cells clones and restore self-tolerance.

Materials and methods: Twenty four adult subjects fullfilling the ADA criteria of T1DM 18-35 years of age with C-peptide level following glucagone stimulation $\geq 0.3 \mathrm{nmol} / 1$ and positivity of 1 or more islet autoantibody tests were randomized for treatment with 4 doses of ATG-Fresenius $(9 \mathrm{mg} / \mathrm{kg}$ with subsequent 3 doses of $3 \mathrm{mg} / \mathrm{kg}$ ) in addition to intensive insulin treatment (ATG-group, $\mathrm{n}=13$ ) or for intensive insulin therapy only (I-Group, $\mathrm{n}=11$ ). The insulin doses, glucagon-stimulated C-peptide levels and metabolic control were compared at 6,12 and 24 months after the study start.

Results: While the basal and stimulated C-peptide levels did not differ between the groups, the area under the C-peptide curves were different (basal C-peptide 0 - 12 months $\mathrm{p}=0.016,0-24$ months $\mathrm{p}=0.021$, stimulated C-peptide $0-12$ months $p=0.016,0-24$ months $p=0.036$; Mann-Whitney test). ANOVA with repeated measures and grouping factor showed different trends in Cpeptide levels and insulin doses between th groups (all $\mathrm{p}<0.05$ ). Mean fasting C-peptide levels in the ATG and Placebo groups at 12 months were $0.52 \pm 0.40$ and $0.23 \pm 0.19 \mathrm{pmol} / \mathrm{ml}$, respectively; $\mathrm{p}=0.03$ ) and stimulated levels were $0.90 \pm 0.66$ and $0.36 \pm 0.32 \mathrm{pmol} / \mathrm{ml}$, respectively; $\mathrm{p}=0.026$ (Mann-Whitney test) and the differences remained significant for 24 months. Complete clinical remission occurred in 2 subjects in the ATG group and in none in the I Group. The ATG treament resulted in long-lastig inversion of the CD4/CD8 T-cell ratio suggesting immunoregulatory effect of the therapeutic intervention. Adverse events were observed only in the $1^{\text {st }}$ month of therapy and included cytokine release syndrome and transient symptoms of serum sickness both in 7 subjects in the ATG-group. For comparison, symptoms of the serum-sickness were retrospectively not found in any of the last 110 consecutive pancreas and kidney recipients and 7 islet recipients treated with similal doses of ATG in combination with other immunosuppressive drugs. Specific virological examination did not show any activation of the herpes virus type 1 and 2, cytomegalovirus or EB-virus in any of the ATG treated stubjects during a 1-year follow-up. 
Conclusion: Short-term ATG therapy in T1DM of recent onset results in lower insulin need due to preserved C-peptide production. Safety of this therapeutic approach could be possibly improved by prevention of the febrile serum-sickness-like disease. Combination therapy with other drugs modulating the immune system or stimulating beta-cell regeneration could further improve the efficacy of this approach.

Supported by: grant No. MZO 00023001 MZ CR

\section{8}

Prospective study of latent pernicious anaemia markers in patients with type 1 diabetes mellitus

E. Colomé ${ }^{1}$, N. Alonso ${ }^{1}$, M. Granada ${ }^{2}$, J. Juncà 3 , E. Martínez-Cáceres ${ }^{4}$, B. Soldevila ${ }^{1}$, I. Salinas ${ }^{1}$, A. Sanmartí ${ }^{1}$

${ }^{1}$ Endocrinology and Nutrition Service, ${ }^{2}$ Biochemistry Service,

${ }^{3}$ Hemathology Service, ${ }^{4}$ Immunology Service (LIRAD-BST), Hospital

Universitari Germans Trias i Pujol, Badalona, Spain

Background and aims: Patients with type 1 diabetes mellitus (DM1) present a high prevalence of autoimmune associated diseases such as pernicious anaemia that has a long pre-clinical or latent phase. Recently, we described a prevalence of latent pernicious anaemia (LPA) of $12.4 \%$ in DM1. A five year follow-up study of a cohort of DM1 patients in order to evaluate the evolution of biochemical markers of LPA, defined as a serum pepsinogen I (PI) concentration below normal limits, as well as several hemathological parameters.

Materials and methods: Prospective study of 186 DM1 patients (93 men, aged $30 \pm 9.4$ yrs). All patients underwent in 2001 and 2006 a measurement of: serum PI (2001: RIA; 2006: ELISA), gastrin (RIA), HbAlc, cobalamin and parietal cell antibodies (PCA) (indirect immunofluorescence, positive title $\leq$ $1 / 40)$.

Results: In 2001, 23 out of 186 DM1 patients presented LPA. Of these, 17 (77.3\%) persisted with low PI in 2006 and in 5 PI concentrations were normalized. One patient was lost at follow-up. $100 \%$ of patients with positive PCA and low PI in 2001 continued with low PI in 2006. Nevertheless, only $33.3 \%$ (3/9) of patients with low PI and negative PCA in 2001 persisted with low PI in 2006. All those patients whose PI normalized in 2006 had normal gastrin concentrations and negative PCA in 2001. In 2006, PI was low in 6 new patients who presented normal PI in 2001. Of these, 50\% presented positive PCA and another $33 \%$ high gastrin concentrations in 2001 . However, only $8.8 \%(3 / 34)$ of patients with positive PCA and normal PI in 2001 evolved to LPA in 2006. The cobalamin concentration in 2001 was similar in patients with or without LPA, but in 2006 it was significantly lower in the group with LPA diagnosed in $2001(\mathrm{p}=0.007)$.

Conclusion: The best markers of LPA in DM1 patients were the presence of low concentrations of PI together with positive PCA. The prevalence of LPA in DM1 patients increases with the temporal evolution of diabetes. This combination would allow to identify and treat those patients with low cobalamin concentrations and therefore avoid the possible clinical complications derived from its deficit.

\section{9}

Prevalence of atopy in children with type 1 diabetes, hepatitis B virus carriers and healthy children: role of Th1 type immune response G. Karagüzel ${ }^{1}$, F. Orhan ${ }^{2}$, M. Cakır ${ }^{3}$, A. Ökten ${ }^{1}$

${ }^{1}$ Pediatric Endocrinology, Karadeniz Technical University, School of Medicine, Trabzon, ${ }^{2}$ Pediatric Allergy, Karadeniz Technical University, School of Medicine, Trabzon, Turkey, ${ }^{3}$ Pediatrics, Karadeniz Technical University, School of Medicine, Trabzon, Turkey

Background and aims: Type 1 diabetes (DM1) and a chronic viral infection, hepatitis B virus (HBV) carriers - are associated with a Th1-dominant and Th1-insufficient cytokine profile, respectively. Our aim was to analyze the frequency of allergic disease in patients with DM1 and in HBV carriers in order to evaluate the role of Th1 type immune response in atopy and allergic disease.

Materials and methods: The study included the patients with DM1 (group I, n: 52), HBV carriers (group II, n: 47); and healthy control group (group III, n: 209). Participants were screened for allergic disease and atopic sensitization. Results: There was no significant difference between the groups in terms of age and gender. The duration of disease was $4.3 \pm 1.2$ and $3.1 \pm 2.1$ years in patients with DM1 and HBV carriers, respectively. The prevalence of atopy in HBV carriers (36.1\%) was significantly higher than in both patients with DM
$(13.4 \%)$ and the children in the control group $(18 \%)(p<0.05)$. Comparison of the frequency of the allergic symptoms and allergic diseases showed that the prevalence of history of ever wheezing, current wheezing, and doctor-diagnosed asthma or eczema were significantly higher in chronic HBV carriers than in patients with DM1 ( $<<0.05$ for all parameters). The IgE levels were $193.7 \pm 201.2,208 \pm 347.4$ and $166.1 \pm 231.8 \mathrm{IU} / \mathrm{mL}$ in DM, chronic HBV carriers and the control group, respectively and there were no statistically significant differences between the groups. No significant difference was found between the groups in terms of family history of atopic diseases.

Conclusion: Our study supports the Th1/Th2 model. The prevalence of allergic disease and atopy is decreased in Th1-mediated autoimmune disease, DM1 and conversely is increased in insufficient Th1 response, chronic HBV carriers.

Supported by: Karadeniz Technical University Scientific Research Projects Unit

\section{0}

Organ specific autoantibodies in patients with type 1 diabetes, LADA and type 2 diabetes

N.C. Schloot ${ }^{1}$, M.I. Hawa ${ }^{2}$, W.A. Scherbaum ${ }^{3}$, P. Pozzilli ${ }^{4}$, R. Leslie ${ }^{2}$, J. Seissler ${ }^{5}$ for the Action LADA consortium

${ }^{1}$ German Diabetes Center, Heinrich-Heine University, Düsseldorf, Germany, ${ }^{2}$ Queen Mary's College, Institute of Cell and Molecular Science, London, United Kingdom, ${ }^{3}$ University Hospital Duesseldorf, Heinrich-Heine University, Dept of Endocrinology, Diabetes and Rheumatology, Germany, ${ }^{4}$ University Campus Bio-Medico, University Campus Bio-Medico, Rome, Italy, ${ }^{5}$ Medical Clinic, Diabetes Center, Muenchen, Germany

Background and aims: LADA (latent autoimmune diabetes of the adult) presents clinically similar to type 2 diabetes but is characterized by type 1 diabetes associated autoantibodies. Objective of the study was the investigation of patients with diabetes for organ-specific autoantibodies including type 1 associated autoantibodies.

Materials and methods: We investigated 998 patients (mean age 52.8years, 30-69 years) with diabetes duration less than five years. Patients were recruited from the Action LADA study. Serum samples of patients from Germany, Austria, Belfast, Rome and London were investigated in a blinded fashion for antibodies: glutamic acid decarboxylase (GAD)65, thyrosine phosphates like antigen (IA2A), islet cells (ICA), thyroid peroxidase (TPO), parietal gastric cells (PCA) and tissue transglutaminase (tTG) antibodies.

Results: Adult patients with diabetes showed positive antibodies in $10.7 \%$ for GADA, 2.6\% for IA2A, 4.0\% for ICA, 14.9\% for TPO, 10.8\% for PCA and $0.9 \%$ for tTGAb. Further analysis showed, that $6.5 \%$ of all patients with diabetes were positive for only GADA, $0.3 \%$ positive for only ICA, $0.2 \%$ positive for only IA2A. 3.5\% of diabetes patients were positive for GADA and ICA, $2.6 \%$ were positive for GADA and IA2A, $1.6 \%$ were positive for GADA,IA2A and ICA. $11.29 \%$ of the patients were positive for GADA or ICA or IA2A. In GADA positive patients we found: $23.8 \%$ positive for IA2A, $32 \%$ positive for ICA, $14.8 \%$ positive for IA2A and ICA, $19.7 \%$ positive for TPO, $17.2 \%$ positive for PCA and $1.6 \%$ positive for tTGA.

Conclusion: Measurement of GADA identifies $94 \%$ of patients positive for type 1 diabetes associated autoantibodies. The prevalence of organ-specific autoantibodies is increased in patients with GADA compared to patients negative for GADA.

Supported by: $E U$

\section{1}

Antibodies against intestinal $\mathrm{CD}_{163^{+}}$macrophages in a patient with type 1 diabetes and celiac disease

B. Sonier, G.-S. Wang, A. Strom, F.W. Scott

Chronic Disease Program, Ottawa Health Research Institute, Ottawa, Canada

Background and aims: Recent studies have described histological and immunological abnormalities in the gastrointestinal tract of animal models and humans with type 1 diabetes (T1D). Oral exposure to dietary antigens normally leads to the induction of tolerance in the mucosal immune system, a process that is facilitated by resident antigen presenting cells. Our group has described the case of a highly wheat sensitive index patient with both T1D and celiac disease $(\mathrm{CD})$ who displayed antibody and $\mathrm{T}$ cell reactivity to wheat proteins, including a homologue of wheat storage globulin-1 (Glb1). Antibody and $\mathrm{T}$ cell reactivity to Glb1 was also observed in a subset of patients 
with T1D. In the present study, we purified Glb1 antibodies from this index patient to identify possible autoimmune targets in the gastrointestinal tract. Materials and methods: Recombinant His-tagged Glb1 (His-rGlb1) was produced using the Bac-to-Bac Baculovirus Expression System in SF21 insect cells. His-rGlb1 was then used to enrich Glb1-specific antibodies from plasma of the index patient by batch affinity chromatography. The presence of antibodies in the enriched sample was confirmed by ELISA. This antibody mixture was used to probe Bouin's fixed intestinal sections from 11, 30, 45, 70 and 100 day old Wistar Furth rats. The antibody preparation was also used to probe fixed human monocytes which were enriched from peripheral blood mononuclear cells (PBMC).

Results: Probing rat intestinal sections with the enriched Glb1 antibody preparation revealed binding to a subset of cells in the lamina propria. This labeling was only present on sections from animals that were past the age of weaning. Binding was not blocked by $\mathrm{rGlb} 1$ and was therefore attributable to non-Glb1 antibodies that were co-purified during the enrichment process. Additional experiments indicated that this labeling predominantly co-localized with cells that express CD163, a marker of alternatively activated, mature tissue-resident macrophages. A subset of PBMC-derived human monocytes was also labeled, suggesting that these anti-macrophage antibodies could represent novel T1D- and/or CD-related autoantibodies. Additional preliminary data suggested that macrophage autoreactivity was also present in other autoimmune patients with T1D, CD or both T1D/CD.

Conclusion: These results suggest that intestinal macrophages are an autoimmune target in this index patient. Although this immune reactivity was not Glb1 antibody specific, putative anti-macrophage autoantibodies were co-purified during the batch affinity chromatography. Resident intestinal macrophages are thought to be important for maintaining immune tolerance in the gut. Immune reactivity was only detected on rat gut sections of animals $\geq 30$ $\mathrm{d}$ of age, past the age of weaning. This is consistent with an immune response that targets a population of $\mathrm{CD} 163^{+}$mature intestinal macrophages that are only present in animals that have been exposed to complex dietary antigens. This study raises the novel possibility that an autoimmune response against mucosal immune cells is present in some patients with T1D and/or CD. Supported by: CIHR, JDRF, and FRSQ

\section{PS 039 Autoantibodies and regulatory T-cells in type 1 diabetes}

\section{2}

Association between anti-ZnT8 autoantibodies and SLC30A8 Arg325Trp variant

E. Kawasaki ${ }^{1}$, M. Uga ${ }^{1}$, G. Kuriya ${ }^{1}$, T. Satoh ${ }^{2}$, K. Fujishima ${ }^{2}$, A. Moriuchi ${ }^{1}$, K. Fukushima ${ }^{1}$, M. Ozaki ${ }^{3}$, N. Abiru' ${ }^{2}$, H. Yamasaki ${ }^{3}$, K. Eguchi ${ }^{1}$

${ }^{1}$ Department of Metabolism/Diabetes and Clinical Nutrition, Nagasaki University Hospital of Medicine and Dentistry, ${ }^{2}$ Department of Endocrinology and Metabolism, Graduate School of Biomedical Science, Nagasaki University, ${ }^{3}$ Health Center, Nagasaki University, Nagasaki, Japan

Background and aims: Recently, the zinc transporter protein member 8 (ZnT8) has been reported as a new autoantigen in type 1 diabetes. Furthermore, a recent genome-wide association study identified the SLC30A8 rs13266634 polymorphism encoding an Arg325Trp polymorphism in the ZnT8 to be associated with type 2 diabetes. The facts that major epitope(s) for ZnT8 antibodies lies within the cytoplasmic domain of the molecule (aa268-369) and the variant residue at aa325 is located at the most distal loop between $\beta$-strand and $\alpha$-helix prompt us to analyze the association between humoral autoreactivity to ZnT8 and SLC30A8 polymorphism.

Materials and methods: Sera from total 286 patients with type 1 diabetes were screened for ZnT8 antibodies using radioligand binding assay and a hybrid ZnT8 cytoplasmic cDNA construct generated by ligation of the cDNA with 325Arg (CR) and 325Trp (CW). Autoreactivity to CR or CW construct was also determined separately using each cDNA.

Results: Of 286 patients with type 1 diabetes 75 (26\%) were ranked as positive for $\mathrm{ZnT} 8(\mathrm{CRCW})$ antibodies. Among them 44 patients whose genomic DNA were available were used for further analysis. Of these $15(34 \%)$ patients were homozygotes of the $325 \operatorname{Trp}(\mathrm{WW})$ in SLC30A8, 19 (43\%) were RR homozygotes, and 10 (23\%) were RW heterozygotes, respectively. The autoreactivity to ZnT8 was closely associated with SLC30A8 genotype, and the level of ZnT8(CR) antibodies in patients with R allele (RR+RW) was significantly higher than that in WW homozygotes $(0.139 \pm 0.158$ vs. $0.002 \pm 0.014$, $\mathrm{P}<0.0001)$. Furthermore, the level of ZnT8(CW) antibodies in patients with $\mathrm{W}$ allele $(\mathrm{WW}+\mathrm{RW})$ was significantly higher than that in RR homozygotes $(0.093 \pm 0.103$ vs. $0.029 \pm 0.053, \mathrm{P}<0.005)$. Among 19 patients with RR genotype CW-specific antibodies were found in only 1 patient (5\%), CR-specific antibodies in 7 patients (37\%), and CR/CW cross-reactive antibodies in 7 patients (37\%), respectively. In contrast, 11 (73\%) of 15 patients with WW genotype had CW-specific antibodies and no one had CR-specific antibodies. Nine (90\%) of 10 patients with RW genotype reacted with either CR or CW constructs.

Conclusion: These results indicate that variant residue at aa325 is a key determinant of humoral autoreactivity to ZnT8 and SLC30A8 genotype is an important determinant of autoantibody specificity.

Supported by: Ministry of Education, Culture, Science, Sports and Technology of Japan

\section{3}

The common non-synonymous SNP in the Slc30A8 gene at aa325 determines $\mathrm{ZnT} 8$ autoantibody specificity in type 1 diabetes J.M. Wenzlau, O. Moua, Y. Liu, L. Yu, L.M. Frisch, K.T. Fowler, S. Rangasamy, G.S. Eisenbarth, H.W. Davidson, J.C. Hutton Barbara Davis Center for Childhood Diabetes, University of Colorado Denver, Aurora, United States

Background and aims: Zinc transporter 8 (Slc30A8) is a major target of autoimmunity in human type1A diabetes (T1D). Genome wide SNP association studies have shown that it is also associated with type 2 diabetes susceptibility, which may suggest that there are common mechanisms in the pathogenesis of T1D and T2D. The common non-synonymous polymorphism at aa325 is located in a region where more than $60 \%$ of new onset T1D patients exhibit antibodies. The aim of this study was to examine the relationship between subject genotype and autoreactivity in this region and its association with the prevalence of autoantibodies, their levels and the age of onset of clinical disease.

Materials and methods: T1D new onset individuals $(n=348$; median age at onset 8.6yr; range 1-38yr) were assayed for $\mathrm{ZnT} 8$ autoantibodies (ZnT8A) by radioimmunoprecipitation assays using $\mathrm{C}$-terminal 102aa constructs bearing 
the common polymorphic aa325 variants encoding Arg and Trp as well as the rare Gln variant. Assay specificity was confirmed by preabsorption with recombinant versions of the corresponding variant constructs. Genotypes were determined by PCR-based SNP analysis using quantitative RTPCR (ABI assay on demand) and direct nucleotide sequencing.

Results: ZnT8 autoantibodies were found in 63\% of subjects. Individually, $56 \%$ were reactive to $\operatorname{Arg} 325$ constructs, $44 \%$ to $\operatorname{Trp} 325$, and $36 \%$ with Gln325. Of the total, 15-20\% were Arg325-restricted (Arg but neither Trp nor Gln reactive), 6-10\% Trp325-restricted (Trp but neither Arg nor Gln reactive), and $0.3 \%$ Gln-restricted. Restriction to Arg325 and Trp325 corresponded to the inheritance of the corresponding $\mathrm{C}$ or $\mathrm{T}$ allele. A strong gene dosage effect was also evident such that the frequency of Arg or Trp-restricted ZnT8A levels were much lower in heterozygous than in homozygous individuals. Gln variant reactivity in the majority of cases was equivalent to Arg and Trp construct reactivity and not affected by genotype. The SLC30A8 SNP allele frequency in our group $(75 \% \mathrm{C} ; 25 \% \mathrm{~T})$ was typical of non-diabetic Caucasian populations, and the SLC30A8 genotype neither affected the levels and prevalence of insulin, GAD65 and IA-2 autoantibodies, nor correlated with age of T1D onset. The median age of onset of type 1 diabetes did not vary as a function of genotype or allele frequency however in the 0-4yr age group the CC genotype was more frequent ( 65 vs $50 \%$ in the $10-15$ age group) accompanied by a lower prevalence of Trp-restricted autoreactivity.

Conclusion: Humoral autoreactivity to $\mathrm{ZnT} 8$ shows a remarkable restriction depending on variation of a single amino acid in the molecule. Although the autoantibody response is polyclonal, it is primarily focused on a limited number of epitopes, two of which are sensitive to residue 325 (Arg or Trp), and at least one other that is independent of the residue at this position. The strict linkage between genotype and Arg- or Trp-restricted antibodies indicates that autoimmunity to ZnT8 is indeed reactivity to self rather than arising from immunity to a foreign antigen through a process of molecular mimicry. Moreover, the existence of "isoepitopes" in autoantigens has both implications for diagnostic strategies and for antigen-based therapeutic interventions. In particular, it raises the question as to whether immunoregulation should be tailored to self or non-self molecular variants in homozygous individuals.

Supported by: JDRF

\section{4}

Zinc transporter 8 antibodies complement GAD and IA-2 antibodies in the identification of adult-onset autoimmune diabetes

V. Lampasona ${ }^{1}$, R. Buzzetti ${ }^{2}$, E. Bazzigaluppi ${ }^{1}$, A. Petrone ${ }^{2}$, M. Capizzi ${ }^{2}$, C. Tiberti ${ }^{2}$, M. Songini ${ }^{3}$, F. Giorgino ${ }^{4}$, E. Bonifacio ${ }^{5}$, E. Bosi ${ }^{1}$

${ }^{1}$ Internal Medicine, San Raffaele Scientific Institute, Milan, Italy, ${ }^{2}$ Clinical Sciences, La Sapienza University, Rome, Italy, ${ }^{3}$ Diabetes, San Michele Hospital, Cagliari, Italy, ${ }^{4}$ Endocrinology \& Metabolism, Bari University, Italy, ${ }^{5}$ Center for Regenerative Therapies, Dresden University of Technology, Germany

Background and aims: Zinc Transporter 8 (ZnT8) is an islet beta-cell secretory granule membrane protein that has recently been reported as autoantigen in type 1 diabetes. ZnT8 antibodies (ZnT8A) are directed against epitopes expressed in the cytosolic domains in the $\mathrm{COOH}$-terminal and, less frequently, within the $\mathrm{NH}_{2}$-terminal of the protein. The aim of this study was to determine the prevalence of ZnT8A in adult-onset diabetes.

Materials and methods: Overall, 541 patients with initial non insulin-requiring diabetes participating in the NIRAD study were studied: of these, 193 (age $50.3 \pm 12.8 \mathrm{yr}$ ) had autoimmune diabetes defined as having antibodies to either GAD(A) or IA-2(A) (4.5\% overall prevalence) and 348 were age and sex matched patients with antibody-negative type 2 diabetes (age 51.1 $\pm 10.8 \mathrm{yr}$ ). ZnT8A were measured by a radiobinding and immunoprecipitation assay using in vitro translated and transcribed ${ }^{35} \mathrm{~S}$-labelled $\mathrm{COOH}$-terminal (aa 268369) and $\mathrm{NH}_{2}$-terminal (aa 1-74) ZnT8 cloned constructs. Sensitivity of the assay in juvenile type 1 diabetes was $64 \%$ for $\mathrm{ZnT} 8 \mathrm{~A}-\mathrm{COOH}$ and $11 \%$ for ZnT8A-NH, with $99 \%$ specificity.

Results: ZnT8A-COOH were detected in 36/193 (18.6\%) autoimmune (all 36 with GADA, 2 with both GADA and IA-2A) and 6/348 (1.7\%) patients with type 2 diabetes $(p<0.0001)$; within the GADA positive patients, GADA titre was higher in the ZnT8A-COOH positive group (median 100 vs 19.4 units, respectively; $\mathrm{p}=0.001 \mathrm{Mann}$-Whitney test). $\mathrm{ZnT} 8 \mathrm{~A}-\mathrm{NH}_{2}$ were rare and found in 4/193 (2.1\%) autoimmune (3 having also ZnT8A-COOH) and 1/348 (0.3\%) patients with type 2 diabetes.

Conclusion: ZnT8A-COOH appear to be a valuable additional marker of adult-onset autoimmune diabetes since they confirm autoimmunity in a sub- group of single GADA positive patients, and identify additional patients who had none of the previous markers of autoimmune diabetes. It remains to be established whether the single $\mathrm{ZnT} 8 \mathrm{~A}-\mathrm{COOH}$ positive patients have clinical features associated with autoimmune diabetes.

Supported by: Research Foundation of the Italian Society of Diabetes based on a grant from NovoNordisk Italy

\section{5}

Engineering single chain multi-component autoantigens for enhanced detection of autoantibodies in type 1 diabetes

J.C. Hutton, Y. Liu, C. Patel, L. Yu, G.S. Eisenbarth, J.M. Wenzlau, H.W. Davidson

Barbara Davis Center for Childhood Diabetes, University of Colorado Denver, Aurora, United States

Background and aims: Autoantibodies provide highly sensitive and specific measures of autoimmunity prior to clinical diabetes in genetically-at-risk populations. However, the currently available assays fall short of the required level of $>99.5 \%$ specificity required to make neonatal screening in the general population a realistic possibility. To reach this level it is estimated that up to 10 single target assays might have to be run concurrently. We therefore investigated the potential of multimerizing epitopes and combining epitopes from different autoantigens into single vectors for improving specificity and increasing throughput of autoantibody assays.

Materials and methods: Model systems were developed using ZnT8, an autoantigen that has has 2 major polymorphic variants with differential reactivity, and IA2 and phogrin that represent related autoantigens encoded by different genes. Molecular modeling of ZnT8 using the bacterial Ziip gene product as template was used to construct free energy minimized homo- and heterodimers of ZnT8. The actual crystal structure of IA2 and deduced structure of phogrin (IA2ß) were similarly used to design dimeric structures of these proteins. Multidomain ZnT8 and IA2/IA2ß fusion proteins were fused into a single chain analyte using a $\mathrm{Fc}$ hinge to connect to the 2 molecule flanked by Gly triplets. All constructs were tested against a panel of 145 sera derived from new onset T1D patients and a blinded panel of 50 diabetic and 100 control sera provided by the Diabetes Autoantigen Standardization Program (DASP).

Results: Antibodies to the ZnT8 Arg325 variant was detected in 53\% of new onset sera compared to $48 \%$ that bound the ZnT8 Trp variant. However, the same sera analyzed with a heterodimer of ZnT8 Trp variant fused to ZnT8 Arg variant showed $78 \%$ sensitivity without loss in specificity (>99\%). The improved sensitivity was both the result of combining the 2 major epitopes in one molecule (nominally 63\%) and the higher avidity resulting from duplication of other epitopes within the monomers. The latter was evidenced by the increase sensitivity of assays to homodimers versus monomers that was achieved without any loss in the detection rate or change in the background signal in the assay $(<1 \%)$. An additional $2.5 \%$ increase in detection was attributable to neoepitopes present in the dimeric but not the monomeric construct. Comparison of responses to homodimeric and heterodimeric constructs of IA2 and phogrin showed increased sensitivity of the assays both as a result of the duplication of the epitopes and the incorporation of multiple epitopes within the same molecule. A heterodimer of IA2 with ZnT8 could be used to detect both antigenic specificities in a single molecule without changes in the background.

Conclusion: Our data indicate that there is a substantial advantage in assaying multimeric autoantigen constructs, both in terms of increasing the number of epitopes within the one molecule and increasing the sensitivity of the assays as a result of higher avidity. It should be noted that the increased sensitivity and specificity is accompanied by the loss of specific information regarding the molecular form of the autoantigen and isoepitopes involved, although this is unlikely to be a concern for general screening. Our strategies could presumably be extended to trimeric and higher order constructs, provided that the background signal in such assays are not compromised. Supported by: JDRF 


\section{6}

Long term $v s$. short-term clinical remission in type 1 diabetes: an analysis of $\mathrm{CD} 4+\mathrm{CD} 25+$ and $\mathrm{CD} 4+\mathrm{CD} 161+\mathrm{T}$ cell subsets

A. Jotic ${ }^{1}$, N.M. Lalic ${ }^{1}$, T. Milicic ${ }^{1}$, I. Markovic ${ }^{2}$, B. Djuricic ${ }^{2}$, M. Zamaklar ${ }^{1}$, K. Lalic ${ }^{1}$, L. Lukic ${ }^{1}$, N. Rajkovic ${ }^{1}$, M. Macesic ${ }^{1}$

${ }^{1}$ Institute for Endocrinology , Diabetes and Metabolic Diseases, Clinical Centre of Serbia, ${ }^{2}$ Institute for Biochemistry, School of Medicine, Belgrade, Serbia

Background and aims: It has been previously suggested that changes in $\mathrm{CD}^{+} \mathrm{T}$ lymphocyte subsets might correlate with the clinical course of the disease in patients with recent-onset Type 1 diabetes (T1D). However, the relevance of the changes in $\mathrm{CD} 25+$ and $\mathrm{CD} 161+$ subsets of the regulatory $\mathrm{T}$ cells, potentially exerting suppressive effect on the ongoing autoreactive response, on the duration of clinical remission (CR) has not been clarified. Therefore, the aim of this study was to compare the changes in $\mathrm{CD}^{+} \mathrm{T}$ cell subsets in peripheral blood between two groups of recent-onset T1D patients exhibiting difference in duration of CR. We included 64 recent-onset T1D patients grouped as follows: group $\mathrm{A}(\mathrm{N}=34)$ long-term remission ( $>180$ days) (392+/-62 days), group B ( $\mathrm{N}=30)$, short-term remission (< 180 days) $(135+/-$ 37 days). In each patient, the analysis of the percentage of regulatory CD25+ and CD161+ subsets of CD4+ T lymphocytes was done sequentially: (a) in insulin-requiring state (IRS) and (b) at day 30 of CR.

Materials and methods: The CR was defined as optimal metabolic control without insulin lasting $>30$ days. The percentages of $\mathrm{CD} 3+$, total $\mathrm{CD} 4+$, $\mathrm{CD} 4+\mathrm{CD} 25+$ and $\mathrm{CD} 4+\mathrm{CD} 161+\mathrm{T}$ cell subsets were analyzed in peripheral blood by using two-color immunofluorescence staining and flowcytometry Results: The percentages of $\mathrm{CD} 3+$, and total CD4+ T cells did not differ significantly neither between IRS and CR in the group A, nor between the groups $A$ and B. However, when the percentage of CD4+CD25+ T lymphocytes was analyzed at onset of T1D, in IRS, it was found to be higher in group A vs group B (A/IRS: $2.78 \pm 0.51$ vs B: $2.01 \pm 0.65 \%, \mathrm{p}<0.05$ ). Moreover, in the state of $\mathrm{CR}$, in both groups the percentage of CD4+CD25+ T cells increased but remain higher in group A than in group B (A/CR: $5.52 \pm 0.17$ B: $3.41 \pm 0.55 \%$, $\mathrm{p}<0.05)$, while the increase was significantly higher in group $A$ vs group $B(p<0.05)$. Simultaneously, the percentage of CD4+CD161+ T cells was found to be higher at IRS in group A vs group B (A/IRS: $4.28 \pm 0.63$ vs B/IRS: $3.18 \pm 1.01, \mathrm{p}<0.05)$, while in the state of $\mathrm{CR}$ the percentage of $\mathrm{CD} 4+\mathrm{CD} 161+$ $T$ increased and remained higher compared to the group B (A/CR: 6.23 \pm 0.86 , vs $B / C R 4.28 \pm 0.98, p<0.05)$ and the increase was also higher in group $A$ vs group $\mathrm{B}(\mathrm{p}<0.05)$.

Conclusion: Our results have demonstrated that in recent-onset T1D patients, the duration of CR was strongly associated with an increase in both CD25+ and CD161+ regulatory CD4+ T cell subsets. The results imply that the clinical course of T1D might be strongly influenced on the level of the activity of these suppressor subsets of the regulatory CD4+ T lymphocytes.

\section{7}

Distinctive differences in the phenotypic characteristics of Tregs of newly-diagnosed type 1 diabetics, long-standing patients, relatives and controls

S.A. Paschou ${ }^{1}$, G. Vartholomatos ${ }^{2}$, L. Dova², N. Kolaitis ${ }^{3}$, E. Giotaki ${ }^{4}$, A. Tsatsoulis ${ }^{1}$, G.K. Papadopoulos ${ }^{5}$

${ }^{1}$ Endocrinology Clinic, University of Ioannina Regional Hospital, Ioannina, ${ }^{2}$ Haematology Laboratory, Molecular Biology Unit, University of Ioannina Regional Hospital, Ioannina, ${ }^{3}$ Haematology Laboratory, University of Ioannina Regional Hospital, Ioannina, ${ }^{4}$ Department of Nursing, Epirus Institute of Technology, Ioannina, ${ }^{5}$ Laboratory of Biochemistry and Biophysics, Faculty of Agricultural Technology, Epirus Institute of Technology, Arta, Greece

Background and aims: In order to gain better understanding of the mechanisms leading to the autoimmune destruction of beta-cells and consequent type 1 diabetes ( $\mathrm{t} 1 \mathrm{~d}$ ), we have documented by flow cytometry the proportion of T regulatory lymphocytes (Tregs, $\mathrm{CD} 4{ }^{+} \mathrm{CD} 25^{\text {high }}$ ) as well as the phenotypic characteristics of Tregs from newly-diagnosed and long-standing t1d patients as well as first and second-degree relatives and controls.

Materials and methods: Peripheral blood from 9 newly-diagnosed patients (ages $14.9+/-10.3,6 \mathrm{M} / 3 \mathrm{~F}$ ), 17 tld patients with long-term disease (ages 29.2 $+/-9.2$, mean disease duration 13.8 years, $8 \mathrm{M} / 9 \mathrm{~F}$ ), 29 controls with no first or second degree relatives suffering from any autoimmune disesse (ages 25.3 $+/-11.5,11 \mathrm{M} / 18 \mathrm{~F})$, and 13 first and second degree relatives of t1d patients (ages $26.2+/-5.8,5 \mathrm{M} / 8 \mathrm{~F}$ ), was analysed by triple colour flow cytometry for various phenotypic markers (CD27, CD28, CD45RO, CD95, CD122 (IL2R $\beta$ ), CD127, CD152 (CTLA-4, membrane and intracellular), CD220 (InsR), TGF $\beta$, TGF $\beta$ RII, and the FoxP3 transcription factor, characteristic of Tregs. The Tregs were distinguished from the Teffector population in CD4-CD25 dot and contour plots, as well as by the distinct expression of FoxP3 (high in Tregs) and CD127 (low in Tregs).

Results: There is a highly statistically significant difference in the Tregs of newly-diagnosed, long standing t1d patients and relatives of t1d patients, all compared to controls, expressed as percent of CD4+ T cells $(1.046+/-0.240$ $(\mathrm{p}<0.001), 1.330+/-0.229(\mathrm{p}<0.001), 1.842+/-0.243(\mathrm{p}=0.001), 2.297+/-$ 0.527 , respectively). This difference is also significant between newly-diagnosed and long term patients $(\mathrm{p}=0.007)$, and between each these two groups and relatives $(\mathrm{p}<0.001$, and $\mathrm{p}<0.001)$. Besides the previously reported lower frequency of TGF $\beta^{+}$and CD220 $0^{+}$Tregs but at higher intensity per cell in newly-diagnosed tld patients, we note a remarkable difference in the level per cell of mCD152 in the Tregs of long standing diabetics versus controls (368 +/- 272 versus $199+/-86, \mathrm{p}=0.027$ ), and a similar but not statistically significant difference in the Tregs of newly-diagnosed t1d patients. Yet, newlydiagnosed and long standing t1d patients have a lower percent of membrane $\mathrm{CD} 152^{+}$Tregs compared to controls. The vast majority of Tregs from newlydiagnosed t1d patients express icCD152 at significantly higher proportion compared to long-term patients $(\mathrm{p}=0.039)$, relatives $(\mathrm{p}<0.001)$ and controls $(\mathrm{p}=0.01)$ (values at $85.96+/-13.9 \%, 59.1+/-39.2,38.9+/-23.4$, and 61.9 +/- 36.2 respectively).

Conclusion: The significally lower proportion of Tregs in tld extends to long-term patients and relatives, in addition to newly-diagnosed patients. There are several defects in $\mathrm{m} / \mathrm{icCD} 152$ expression in patients and relatives which point to a possible role of this molecule, an already known genetic factor for the disease, in t1d pathogenesis.

Supported by: A grant of the programme ARCHIMEDES

\section{8}

Recent-onset type 1 diabetes: clinical remission is associated with increases of CD 25+ and CD 45RA+ regulatory subsets of CD 4+ T lymphocytes and improvements in insulin sensitivity level

T. Milicic ${ }^{1}$, N.M. Lalic ${ }^{1}$, A. Jotic ${ }^{1}$, I. Markovic ${ }^{2}$, B. Djuricic ${ }^{2}$, M. Zamaklar ${ }^{1}$, K. Lalic ${ }^{1}$, L. Lukic ${ }^{1}$, N. Rajkovic ${ }^{1}$, M. Macesic ${ }^{1}$

${ }^{1}$ Institute for Endocrinology, Diabetes and Metabolic Diseases, Clinical Centre of Serbia, ${ }^{2}$ Institute for Biochemistry, School of Medicine, Belgrade, Serbia

Background and aims: Previous studies have reported an important role of the changes in $\mathrm{CD} 4+\mathrm{T}$ cell subsets in the initial phase of Type 1 diabetes (T1D). However, the relevance of the changes in CD4+CD25+ and $\mathrm{CD} 4+\mathrm{CD} 45 \mathrm{RA}+\mathrm{T}$ subset of the regulatory suppressive $\mathrm{T}$ cells and insulin sensitivity level, during the initial phase of T1D has not been clarified. Therefore, the aim of this study was to analyze (a) the percentage of regulatory suppressor CD4+CD25+ and CD4+CD45RA+ T cell subsets; (b) insulin sensitivity level during the first year of the clinical course of the disease comprising (1) insulin-requiring state (IRS) at the onset and (2) the state of clinical remission (CR) in 37 recent-onset T1D patients (group A) as well as in 20 healthy, age-matched control subjects (group B).

Materials and methods: The CR was defined as optimal metabolic control without insulin lasting $>30$ days. The percentage of CD4+CD25+ and CD4+ CD45RA+ T cell subsets was analysed by using two-color immunofluorescence staining and flowcytometry. Euglycemic hyperinsulinemic clamp was performed and insulin was infused at the rate of $1 \mathrm{mU} / \mathrm{kgbw} / \mathrm{min}$ during 180 min and glucose infusion was adjusted manually, at 5 minutes intervals, to maintain target glycemia at $90 \mathrm{mg} / \mathrm{dl}$. Total glucose uptake was calculated on the basis of the amount of glucose infused during steady state period (20-180 minutes).

Results: When the percentage of CD4+CD25+ T lymphocytes was analyzed, we found that at onset of T1D, in IRS, it was lower in group A vs group B (A: $2.33 \pm 0.30$ vs $B$ : $5.17 \pm 0.74 \%, p<0.01)$. However, in the state of $C R$, in group A the percentage of $\mathrm{CD} 4+\mathrm{CD} 25+\mathrm{T}$ cells increased and it became higher than at onset (A: $4.17 \pm 0.11$ vs B: $5.19 \pm 0.72 \%, p=N S$ ), reaching the values similar to those in group B. Moreover, the percentage of CD4+CD45RA+T cells was found to be lower at IRS in group A vs group B (A/IRS: $23.0 \pm 1.1$ vs $\mathrm{B}: 28.4 \pm 2.4, \mathrm{p}<0.05)$, while in the state of $\mathrm{CR}$ it also increased and became similar to those in the group B (A/CR: $26.9 \pm 1.1, p=N S)$. In addition, total glucose uptake was significantly lower in group $A$ at onset of T1D in comparison to group B (A: $3.83 \pm 0.12$ vs B: $6.78 \pm 0.25 \mathrm{mg} / \mathrm{min} / \mathrm{kg}$, $\mathrm{p}<0.01$ ). However, 
in the state of CR, total glucose uptake significantly increased in group A, reaching the levels detected in healthy controls (A: $5.95 \pm 0.10$ vs B: $6.78 \pm 0.25$ $\mathrm{mg} / \mathrm{min} / \mathrm{kg}$, $\mathrm{p}=\mathrm{NS}$ ).

Conclusion: Our results have demonstrated that in recent-onset T1D patients, the state of CR was associated with an increase in regulatory CD4+CD25+ and CD4+ CD45RA+ T cell subsets and in insulin sensitivity levels. The results imply that the appearance of the CR might be influenced by both immunological and metabolic phenomena, i.e. by the increase in the number of the suppressor subsets of the CD4+ T lymphocytes as well as by the improvements in insulin sensitivity.

\section{9}

Higher serum dipeptidyl peptidase- 4 activity in type 1 diabetes mellitus than in type 2: a direct comparison

T. Varga, A. Somogyi, G. Nagy, Z. Tulassay, L. Selmeci, G. Firneisz 2nd Department of Internal Medicine, Semmelweis University, Budapest, Hungary

Background and aims: Serum dipeptidyl peptidase-4 (DPP-4, CD26) has an important role in the carbohydrate metabolism with the degradation of the entero-hormones (gastric inhibitory polypeptide/GIP/, glucagon-like peptide-1/GLP-1/). Certain studies implicate that type 2 diabetic (T2DM) patients have impaired incretin effect. The function of the entero-insular axis might be influenced by the enzymatic activity of the DPP-4 through the degradation of both incretin hormones. Soluble CD26 has been identified as an activation marker in immune cells which can modulate in vitro T-cell proliferation. Serum DPP-4 levels are altered in different autoimmune diseases. The present study was designed to assess serum both the fasting and postprandial DPP-4 activity in patients with type 1-(T1DM), type 2(T2DM) diabetes and healthy controls.

Materials and methods: A cross sectional study of $87(\mathrm{~F} / \mathrm{M}=47 / 40)$ patients with T2DM, 41(F/M=17/24) with T1DM and for comparison sex- age- and BMI-matched $25(\mathrm{~F} / \mathrm{M}=15 / 10)$ healthy volunteers was performed. Fasting serum DPP-4 activity was assessed in all the 153 subjects. Postprandial serum DPP-4 enzyme activities were also determined at $60 \mathrm{~min}$ and $180 \mathrm{~min}$ periods after test meal $(50 \mathrm{~g}$ carbohydrate $+24 \mathrm{~g}$ protein $+12 \mathrm{~g}$ fat $=410 \mathrm{kcal})$ in 50 patients( 15 type $1-17$ type 2 diabetic and and 18 healthy individuals) out of 153 subjects. Serum DPP-4 activity was measured by microplate-based (Multiskan-Plus-MKII, Labsystem at $405 \mathrm{~nm}, 25^{\circ} \mathrm{C}$ for $30 \mathrm{~min}$ ) kinetic assay. Gly-Pro-pNA (Bachem, Bubendorf, Switzerland) was used as substrate. Results are expressed in $\mathrm{nmol} / \mathrm{ml} / \mathrm{min}(\mathrm{U} / \mathrm{L})$ pNA hydrolysis.

Results: There was no increase after test meal in serum DPP-4 activities in any of the groups studied (type 1-, type 2 diabetes, healthy control(CNTRL)). However we experienced significant increase(T1DM vs CNTRL $p<0.0075$; T1DM vs T2DM $\mathrm{p}<0.0001)$ in the fasting serum DPP-4 enzyme activity in type 1 diabetes (29.065 (95\%CI:27.30-30.826)) compared not only to healthy individuals (25.64 (95\%CI:24.348-27.430)) but also in comparison with type 2 diabetes( 24.10 (95\%CI:22.75-25.46)). There was neither difference in the fasting plasma glucose $(\mathrm{T} 1 \mathrm{DM}$ : mean $=\mathbf{9 . 2} \mathrm{mmol} / \mathrm{L} \pm 3.91$, T2DM: mean= 8.1 mmol/L \pm 3.037 ) nor in the HbAlC mean values (T1DM: mean $=\mathbf{7 . 8 0} \%$ \pm 1.574 ); (T2DM: mean=7.47 \pm 1.569 ) between the two diabetic groups.

Conclusion: We concluded that not the hyperglycaemia, rather the type of diabetes determinates the the serum DPP- 4 enzymatic activity. The increased serum DPP-4 enzyme activity in type 1 diabetes, may be the cosequence of the autoimmune process itself, hormonal feed back mechanism or the endothelial damage. Serum DPP-4 as an activation marker of the immune cells (T-lymphocytes) may be an indicator of the autoimmune inflammation in the pancreas. Furthermore the increase in the serum DPP-4 activity may even be a result of a concomitant autoimmune disorder such as the Basedow-Graves disease. The diagnostic accuracy of the serum DPP-4 activity determination is limited by its highly aspecific alterations, therefore in T1DM the enzyme activity alone maybe insufficient for the discrimination from the T2DM. However the increase in fasting serum DPP- 4 enzyme activity presented here in patients with T1DM may call for further studies on the potential extension of the indications of the DPP-4 inhibitor treatment, especially because these drugs demonstrated beta-cell protective properties in certain experimental animal studies in diabetes.

Supported by: Hungarian Metabolism and Diabetes Foundation and the OTKA PD73606 grant

\section{PS 040 Clinical islet and pancreas transplantation}

\section{0}

Supplemental islet infusions restore insulin independence after graft dysfunction in islet transplant recipients

A. Koh, S. Imes, J. Shapiro, P. Senior

Clinical Islet Transplant Program, University of Alberta, Edmonton, Canada

Background: Previous reports suggest that the success of supplemental islet infusions (SII) to restore insulin independence in islet transplant recipients with graft dysfunction was due to the use of exenatide. However, recent use of more potent induction therapies and intensive peri-transplant anticoagulation and metabolic control has led to improved islet transplant outcomes. We sought to determine whether SII alone using current protocols is effective to restore insulin independence in islet transplant recipients with graft dysfunction.

Methods: Ten previously insulin-independent subjects who had resumed insulin therapy because of graft dysfunction (fasting glucose $>7 \mathrm{mmol} / \mathrm{L}$ and/or post-prandial glucose $>10 \mathrm{mmol} / \mathrm{L}$; and/or C-peptide : glucose < $0.06 \mathrm{nmol} / \mathrm{mmol}$ ) received a single SII. One subject received a second SII. Induction therapy was with thymoglobulin plus etanercept $(n=7)$ or daclizumab (Edmonton protocol, $n=3$ ). All subjects received intensive peri-transplant management with intravenous heparin and insulin infusions for 48 hours. Maintenance immunosuppression consisted of tacrolimus plus either sirolimus or cellcept.

Results: SII was performed 49.3 (34.5 - 57.8) months (median with interquartile range) after the preceding islet transplant. Subject characteristics are summarized in Table 1.

\section{Table 1: Subject Characteristics}

\begin{tabular}{ll}
\hline Gender (M/F) & $7 / 3$ \\
\hline Duration of insulin independence prior to SII (months) & $15.9(14.0-23.3)$ \\
\hline Pre-SII Fasting glucose (mmol/L) & $8.1(7.6-9.8)$ \\
\hline Pre-SII 90-min glucose after mixed meal (mmol/L) & $15.2(11.7-19.8)$ \\
\hline Pre-SII Fasting C-Peptide/Glucose (nmol/mmol) & $0.04(0-0.09)$ \\
\hline $\begin{array}{l}\text { Pre-SII Mixed meal Stimulated C-Peptide/Glucose } \\
\text { (nmol/mmol) }\end{array}$ & $0.05(0.03-0.09)$ \\
\hline Pre-SII Insulin use (units/kg/day) & $0.38(0.27-0.48)$ \\
\hline Pre-SII HbA1c(\%) & $7.0(5.9-7.6)$ \\
\hline
\end{tabular}

Subjects received an islet mass of 5493 (4951 - 6134) IEQ/kg for the SII vs. 8787 (6661 - 12427) IEQ/kg with their initial transplant(s) $(\mathrm{p}=0.059)$. Nine of the 10 subjects became insulin independent $2.7(1.5$ - 4.3) months after SII. After a follow-up period of $17.3(10.1-20.8)$ months, insulin independence has been maintained in 8 of the 9 subjects. Only 1 subject has returned to insulin use to date, after 14.7 months of insulin independence. The duration of insulin independence following SII has already exceeded that of the initial transplant(s) in 2 subjects. All subjects have maintained good glycemic control after SII as indicated by current fasting glucose $6.1(5.7-6.6) \mathrm{mmol} / \mathrm{L}$, and 90-min glucose after mixed meal $7.4(6.1-8.3) \mathrm{mmol} / \mathrm{L}$. The peak mix meal stimulated C-peptide:glucose ratio after SII was $0.31(0.25-0.37) \mathrm{nmol} /$ $\mathrm{mmol}$, indicating excellent islet graft function.

Conclusion: Using current potent induction and intensive peri-transplant therapies, SII alone with a relatively small islet mass is effective to achieve impressive insulin independence rates. Longer follow-up will be required to confirm the durability of insulin independence achieved in this study.

\section{1}

Perfluorocarbons, a new tool for the preservation of pancreatic islets in vitro

E. Maillard ${ }^{1}$, M. Sanchez Dominguez ${ }^{2}$, C. Kleiss ${ }^{1}$, M.-C. Sencier ${ }^{1}$,

A. Langlois ${ }^{1}$, W. Beitiger ${ }^{1}$, C. Vodouhe ${ }^{1}$, M.-P. Krafft ${ }^{2}$, M. Pinget ${ }^{1}$,

A. Belcourt ${ }^{1}$, S. Sigrist ${ }^{1}$

${ }^{1}$ Laboratoire de recherche, Centre Européen d'Etude du Diabète, ${ }^{2} \mathrm{CNRS}$ UPR22, Institut Charles Sadron, Strasbourg, France

Background and aims: Pancreatic islets transplanted to treat type 1 diabetes often fail to function likely because of islets hypoxia. Perfluorocarbons 
(PFCs) have a high oxygen solubility coefficient and maintain high oxygen partial pressures for extended time. They serve also as oxygen "reservoirs" for harvested organs in pancreas organ transplantation. The use of oxygen carriers as perfluorocarbons could reduce islets loss. The aim of this study was to test whether the use of PFCs could be beneficial for the secretory activity and overall viability of cultured purified islets before transplantation.

Materials and methods: Purified rat islets were cultured in static conditions for 1,3 or 5 days with or without emulsion of PFCs $10 \%(\mathrm{v} / \mathrm{v})$ saturated or not with oxygen. Cell death and apoptosis were assessed by Fluorescein Diacetate/Propidium Iodure and Hoechst staining and by the measure of Bax, Bcl-2 mRNA expression using RT-PCR. Insulin secretion was measured by ELISA and expressed as stimulation index. Hypoxia was evaluated by the study of VEGF, Matrix Metalloproteinase-2 and Matrix Metalloproteinase9 expression using RT-PCR, western blotting, immunohistochemistry and ELISA.

Results: Study of islet viability demonstrated no toxicity of the treatment during the experiment, whereas there was a significant increase of Bcl-2 mRNA expression in islets incubated in PFCs emulsions $(1.28 \pm 0.3$ vs $0.36 \pm 0.1$; $\mathrm{p}<0.001 ; \mathrm{n}=3)$ and a significant decrease of Bax mRNA expression $(0.5 \pm 0.2$ vs $0.9 \pm 0.07 ; \mathrm{p}<0.05 ; \mathrm{n}=3$ ) after 24 hours of culture. Moreover, there was a preservation of islets functionality in the presence of PFCs during the experiment, while a decrease of this functionality was observed in culture medium alone $(2.78 \pm 0.54 ; 1.46 \pm 0.07$ and $1.15 \pm 0.56(\mathrm{p}<0.01)$ after 1,3 and 5 days respectively). The study of hypoxia showed a decrease of VEGF mRNA expression with oxygenated PFCs $(0.49 \pm 0.15)$ as compared to control $(0.75 \pm 0.19$; $\mathrm{p}<0.05 ; \mathrm{n}=5)$. The analysis of protein expression showed a decrease of VEGF secretion with oxygenated PFCs after 2 and 3 days of culture $(0.05 \pm 0.03 \mu \mathrm{g}$ of $\mathrm{VEGF} / \mathrm{mg}$ of protein vs $0.44 \pm 0.12 \mu \mathrm{g}$ of $\mathrm{VEGF} / \mathrm{mg}$ of protein for control; $\mathrm{p}<0.001 ; \mathrm{n}=3$ ). In agreement with these results, MMP-2 secretion of PFCs incubated islets was decreased in comparison to control $(0.05 \pm 0.03$ versus $0.13 \pm 0.06 \mathrm{pg}$ of MMP- $2 / \mathrm{mL}$ ) after 1 and 3 days of incubation. Finally immunohistochemistry studies showed that this decrease of hypoxia was linked to a preservation of islet structure in the presence of PFC. In contrast, without PFCs, a disruption of islets and fibrosis was observed.

Conclusion: Our results suggested that PFCs are very useful to fight against hypoxia. Thus, the use of PFC emulsions in the culture may provide advantage over conventional protocols to preserve islets before transplantation. Supported by: EC

\section{2}

Metabolic effect of sirolimus versus mycophenolate mofetil on pancreatic graft function - a prospective randomised study

T. Havrdova ${ }^{1}$, F. Saudek ${ }^{1}$, P. Boucek ${ }^{1}$, K. Lipar ${ }^{2}$, R. Koznarova ${ }^{1}$,

T. Jedinakova ${ }^{1}$, J. Skibova ${ }^{3}$

${ }^{1}$ Diabetes Center, ${ }^{2}$ Transplant Center, ${ }^{3}$ Statistical Department, Inst Clin Exp Medicine, Prague, Czech Republic

Background and aims: Metabolic effects of immunosuppressive agents are of great importance in pancreas or islet transplantation. The aim of our study was to compare glucose metabolism in Type 1 diabetic recipients of kidney and pancreatic grafts on tacrolimus-based immunosuppression in conjunction with sirolimus (RAPA) or mycophenolate mofetil (MMF) in a prospective randomised study.
Materials and methods: The investigation was performed in 32 insulin-independent rejection-free patients after simultaneous pancreas and kidney transplantation (with systemic venous drainage of pancreatic graft) at 2 occasions: at $0.6 \pm 0.2$ [mean $\pm \mathrm{SD}$ ] month post-transplant (with steroid dose 11.6 $\pm 3.91 \mathrm{mg} /$ day) and subsequently at $22.1 \pm 10.67$ months (steroid-free). All recipients had a good function of the kidney graft. Fasting glycemia, insulin and C-peptide levels, $\mathrm{HbA}_{1 \mathrm{c}}$, IVGTT with $\mathrm{K}_{\mathrm{G}}$-calculation were assessed in both groups. Insulin sensitivity was evaluated by HOMA-IR. Areas under the insulin/C-peptide curves during the IVGTT (AUC-IRI, AUC-CP) were used as the parameters of insulin/C-peptide secretion.

Results: The RAPA and MMF groups did not differ in age, BMI, post-transplant period and steroid daily dose. Trough levels of tacrolimus had no significant impact on any of examined parameters. $\mathrm{K}_{\mathrm{G}}$ and HOMA-IR of whole study group significantly improved between the exams $(1.03 \pm 0.4$ vs. $1.35 \pm$ $0.54, \mathrm{p}<0.01$ and $3.57 \pm 2.32$ vs. $2.42 \pm 1.7, \mathrm{p}<0.05$, respectively). We found the significant differences between the groups in AUC-CP and $\mathrm{K}_{\mathrm{G}}$ values after steroid withdrawal.

Conclusion: Glucose tolerance measured with the use of IVGTT significantly improved due to better insulin sensitivity after steroid withdrawal in whole study group. Stimulated insulin production did not changed during our study. Parameters of glucose metabolism in steroid-free period $\left(\mathrm{K}_{\mathrm{G}}\right.$ and AUC-CP) were more favourable in patients treated with MMF than in recipients treated with RAPA.

Supported by: MZO 00023001

\section{3}

Successful combined pancreas kidney transplantation in type 1 diabetic patients: normal intrahepatocellular and -myocellular lipid content despite systemic venous drainage

M. Anderwald-Stadler ${ }^{1,2}$, C. Anderwald ${ }^{3}$, S. Zbyn ${ }^{4}$, G. Pacini ${ }^{5}$,

M. Promintzer, , M. Mandl', M. Bischof ${ }^{3}$, S. Gruber ${ }^{4}$, P. Nowotny ${ }^{3}$,

A. Luger ${ }^{3}$, R. Prager ${ }^{1,2}$, M. Krebs ${ }^{3}$

${ }^{1} 3$ rd Medical Department of Metabolic Diseases and Nephrology, Hietzing Hospital Vienna, Austria, ${ }^{2}$ Metabolic Diseases and Nephrology, Karl

Landsteiner Institut, Vienna, Austria, ${ }^{3}$ Department of Internal Medicine III, Division of Endocrinology and Metabolism, Medical University Vienna, Austria, ${ }^{4}$ NMR Center of Excellence, Medical University Vienna, Austria, ${ }^{5}$ Metabolic Unit, ISIB-CNR, Padova, Italy

Background and aims: Insulin replacement in Type 1 diabetes mellitus (T1DM) is usually performed via a systemic (subcutaneous) instead of the physiologic portal route. So far it is unclear whether this systemic route of insulin delivery might contribute to metabolic defects in these patients. This hypothesis is supported by the finding that T1DM is associated with decreased intrahepatocellular and increased intramyocellular lipid content. Successful pancreas kidney transplantation (PKT) with systemic venous drainage is an ideal model of optimized systemic insulin therapy resulting in systemic hyperinsulinemia and relative portal hypoinsulinemia. Therefore, the aim of the present study was to investigate the effects of PKT on intracellular lipid distribution and insulin sensitivity.

Materials and methods: A 3hour 75g-oGTT was performed in 9 T1DM patients after successful PKT $\left(26 \pm 1 \mathrm{~kg} / \mathrm{m}^{2}, 50 \pm 3 \mathrm{yrs}, 4 \mathrm{f} / 5 \mathrm{~m}\right)$ with systemic venous drainage, thus reversed to nondiabetic state (basal glucose $99 \pm 3 \mathrm{mg} / \mathrm{dl}$,

\begin{tabular}{|c|c|c|c|c|}
\hline & \multicolumn{2}{|l|}{$\begin{array}{l}\text { RAPA group } \\
(\mathrm{n}=16)\end{array}$} & \multicolumn{2}{|l|}{$\begin{array}{l}\text { MMF group } \\
(\mathrm{n}=16)\end{array}$} \\
\hline & Examination 1(a) & Examination 2 (b) & Examination 1 (c) & Examination $2(\mathrm{~d})$ \\
\hline HbAlc (\%) & $6.3 \pm 0.9$ & $\begin{array}{l}5.7 \pm 0.5 \\
(\mathrm{p}<0.05 \text { vs. a) }\end{array}$ & $5.8 \pm 0.5$ & $5.5 \pm 0.5$ \\
\hline Fasting glycemia $(\mathrm{mmol} / \mathrm{L})$ & $5.3 \pm 0.7$ & $4.7 \pm 0.5$ & $5.5 \pm 0.8$ & $\begin{array}{l}4.7 \pm 0.8 \\
(p<0.05 \text { vs. } c)\end{array}$ \\
\hline KG (\%/min.) & $1.0 \pm 0.5$ & $1.16 \pm 0.43$ & $1.0 \pm 0.3$ & $\begin{array}{l}1.55 \pm 0.6 \\
(\mathrm{p}<0.05 \text { vs. b, c) }\end{array}$ \\
\hline AUC-IRI (mIU/L/60min.) & $1863 \pm 1002$ & $1837 \pm 894$ & $\begin{array}{l}2765 \pm 1205 \\
(\mathrm{p}<0.05 \text { vs. a) }\end{array}$ & $2358 \pm 1300$ \\
\hline AUC-CP(pmol/mL/60min.) & $140.3 \pm 62.7$ & $\begin{array}{l}86.9 \pm 28.9 \\
(\mathrm{p}<0.05 \text { vs. a) }\end{array}$ & $171.1 \pm 75.4$ & $\begin{array}{l}112.6 \pm 31.8 \\
(\mathrm{p}<0.05 \text { vs. b, c })\end{array}$ \\
\hline $\begin{array}{l}\text { Stimulated AUC-CP } \\
\text { (pmol/mL/60min.) }\end{array}$ & $50.2 \pm 25.3$ & $43.7 \pm 20$ & $70.6 \pm 31.4$ & $\begin{array}{l}59.5 \pm 20.0 \\
(\mathrm{p}<0.05 \text { vs. b) }\end{array}$ \\
\hline HOMA-IR & $2.8 \pm 1.8$ & $2.2 \pm 1.2$ & $4.2 \pm 2.5$ & $2.7 \pm 2.0$ \\
\hline
\end{tabular}


HbAlc $5.4 \pm 0.1 \%$ ) and in 9 matching nondiabetic controls (CON) $(26 \pm 1 \mathrm{~kg} /$ $\mathrm{m}^{2}, 53 \pm 3 \mathrm{yrs}, 3 \mathrm{f} / 6 \mathrm{~m}, 92 \pm 3 \mathrm{mg} / \mathrm{dl}, 5.6 \pm 0.1 \%$ ) to assess plasma concentrations of glucose, free fatty acids (FFA), insulin and C-peptide. Whole body insulin sensitivity was calculated from oGTT using the clamp-like insulin sensitivity index (CLIX). Fasting endogenous glucose production (EGP) was measured using DD-glucose tracer dilution. Intracellular lipid content of the liver (IHCL) and the soleus muscle (IMCL) was non-invasively measured using ${ }^{1} \mathrm{H}$-nuclear-magnetic-resonance spectroscopy.

Results: Fasting concentrations of insulin and C-peptide were markedly higher in PKT vs. CON (insulin: $17 \pm 3$ vs. $9 \pm 2 \mu \mathrm{U} / \mathrm{ml}$; C-peptide: $3.0 \pm 0.4$ vs. $2.2 \pm 0.3 \mathrm{nmol} / 1$; both $\mathrm{p}<0.02$ ), while fasting glucose and FFA and areas under concentration curves of insulin, C-peptide, glucose and FFA were similar. IHCL (PKT:2.9 \pm 1.0 ; CON:4.4 $\pm 2.5 \%$ ) and IMCL (PKT:1.0 \pm 0.2 ; CON:1.0 $\pm 0.2 \%$ ), CLIX and EGP were comparable between in PKT and CON.

Conclusion: Despite systemic hyperinsulinemia, T1DM after successful PKT exhibit unchanged intracellular lipid content of liver and skeletal muscle, whole body insulin sensitivity and EGP in comparison with age-and BMImatched non-diabetic controls. Thus, this study (which for the first time evaluated IHCL and IMCL in PKT) indicates that systemic insulin substitution does not cause alterations or intracellular lipid distribution or of insulin sensitivity.

Supported by: grant of the Austrian Diabetes Association and of the Austrian National Bank

\section{4}

\section{Neuropathy assessment in type 1 diabetic patients after islet transplantation}

M.-C. Vantyghem ${ }^{1,2}$, J.-F. Hurtevent ${ }^{3}$, F. Faivre-Defrance ${ }^{4}$, V. Raverdy ${ }^{1}$,

T. Hubert ${ }^{1}$, M. Hazzan ${ }^{5}$, J. Kerr-Conte ${ }^{1}$, C. Noel ${ }^{5}$, F. Pattou ${ }^{1}$

${ }^{1}$ INSERM U859, ${ }^{2}$ Endocrinology and Metabolism Department,

${ }^{3}$ Neurophysiology Department, Endocrinology and Metabolism

Department, ${ }^{5}$ Lille University Hospital, Nephrology Department, Lille, France

Background and aims: The benefits of islet transplantation in type 1 diabetic patients should be weighed against the potential side effects of immunosuppressive drugs. The aim of this study was to assess whether islet transplantation $(\mathrm{Tx})$ was able to stabilize neuropathy $(\mathrm{NP})$ in type 1 diabetic patients with or without kidney graft.

Patients and methods: From 2003 to 2007, 24 type 1 diabetic patients aged $44 \pm 7$ years, weighing $72 \pm 9 \mathrm{~kg}$ received 2 to 3 islet infusions during a period of $3(n=20)$ to 6 months $(n=4)$. The 24 patients were divided in 2 groups: 14 having received islet transplantation alone (ITA) and 10 islet-after-kidney transplantation (IAK) with a mean time between kidney and islet graft of $22 \pm 9$ months. The immunosuppressive regimen included daclizumab, tacrolimus, and sirolimus. An electromyogram with study of sensory and motor potentials of the 4 limbs was performed before islet transplantation (and after kidney graft for IAK), and then yearly in the 23/24 patients having a distance of at least 1 year from first islet transplantation (14 ITA + 9 IAK), whatever blood C-peptide level. At the last assessment, 20/23 patients had a detectable blood C-peptide level, $10 / 23$ received insulin, and 16/23 patients had HbAlc level below $6.5 \%$.

Results: Before transplantation, 6/14 ITA and 8/9 IAK patients showed a NP and 8/14 ITA and 1/9 IAK no neuropathy. One to 4 years after transplantation, an improvement of NP was observed in 3/6 ITA and 4/8 IAK patients suffering from NP before transplantation. A steadiness was noticed in the 7 other patients suffering from NP (3 ITA and 4 IAK) before transplantation. No deterioration was noted in the 8 ITA and in the unique IAK patient without NP before transplantation. A significant improvement of the mean sensitive nerve conduction velocity of the upper (pre Tx $46.7 \pm 9.8$ vs post Tx: $51.2 \pm 7.9 \mathrm{~m} / \mathrm{s} ; \mathrm{p}<0.02$ ) and lower (pre Tx: $41.6 \pm 5.4$ vs post Tx: $46.3 \pm 7.8$ $\mathrm{m} / \mathrm{s} ; \mathrm{p}<0.01)$ limbs was observed. Nevertheless it did not significantly correlate with one-year blood C-peptide, anti-GAD auto-antibodies, tacrolimus, sirolimus, and $\mathrm{HbAlc}$ levels despite a trend toward a positive correlation between HBAlc and sensitive nerve conduction velocity. Discussion : DCCT study has demonstrated that optimized insulin therapy could prevent occurrence of NP (1995) and improve subclinical forms (2007). An improvement of electrophysiologic scores in IAK patients has also been shown (Del Carro, Diabetes Care 2007).

Conclusion: Our results suggest that islet transplantation allows not only to prevent, but also to improve established NP. Indeed 1) An improvement of the NP shown by electromyogram was demonstrated in $7 / 14$ patients $(50 \%)$ initially suffering from NP one to 4 years after islet transplantation; 2) The same efficiency was observed in ITA and IAK patients; 3) Despite the potential neurotoxicity of immunosuppressive drugs, no deterioration was noticed in patients with no neuropathy before transplantation. Suported by: PHRC 2001

\section{5}

Does pancreas transplantation in type 1 diabetes reduce the progression of foot pathology leading to amputation?

J. Aron-Wisnewsky ${ }^{1}$, M.-L. Tanguy ${ }^{2}$, B. Barrou ${ }^{3}$, A. Harteman-Heurtier ${ }^{1}$ ${ }^{1}$ Diabetology, ${ }^{2}$ Biostatistic, ${ }^{3}$ Transplant Surgery, Pitie-Salpetriere hospital, Paris, France

Background and aims: Simultaneous pancreas-kidney transplantation (SPKT) has become the therapy of choice in patients with type 1 diabetes mellitus with end stage renal disease as it improves patient survival and quality of life. Moreover stabilization or even reversion of diabetic microvascular complications is known to be achieved after SPKT. But little is known about its effect on foot complications. The aim of our study was to evaluate the outcome of successful SPKT on foot pathology.

Materials and methods: A retrospective single center review was made of all the simultaneous pancreas kidney transplants performed in type 1 diabetes patients between January 1989 and April 2006 with a follow up of at least one year. Mono and multivariate statistical analysis were conducted to identify foot complication's risk factors. Differences between the group of patients with a functioning graft and those who rejected it were tested with a chisquared test.

Results: 69 patients, mean age at transplantation $37 \pm 7$ years, mean duration of diabetes $24 \pm 7$ years, mean $\mathrm{HbAlc} 9.2 \pm 2.4 \%$, mean duration of dialysis prior to the transplant of $18 \pm 14$ months, were followed for a mean period of $9.3 \pm 4.1$ years. At inclusion, $100 \%$ of our cohort suffered from peripheral neuropathy complication and $20 \%$ had peripheral vascular disease. At the end of the follow up period, 30 patients developed at least one foot lesion (annual incidence of 5\%) and 16 patients suffered from either a lower limb or toe amputation (amputation rate 23\%). Peripheral disease prior to transplantation was the only significant risk factor of foot lesions after SPKT $(\mathrm{p}<0.04)$ and pretransplant history of foot lesions was the only significant risk factor of amputation after SPKT $(\mathrm{p}=0.008)$.

Conclusion: From our results, transplanted patients have the same risks than diabetic patients with peripheral neuropathy (grade 1) with regards to foot problems. We conclude that simultaneous pancreas kidney transplantation fails to halt or reduce the progression of foot complications. We suggest that even after successful pancreas transplantation, patients should continue to benefit from close lower limb medical supervision.

\section{6}

Islet and pancreas transplantation alone: one center short-term results P. Girman ${ }^{1}$, M. Mindlova ${ }^{1}$, Z. Berkova ${ }^{1}$, T. Koblas ${ }^{1}$, K. Zacharovova ${ }^{1}$, L. Pektorova ${ }^{1}$, E. Dovolilova ${ }^{1}$, E. Vavrova ${ }^{1}$, R. Koznarova ${ }^{1}$, V. Bobek ${ }^{1}$, M. Adamec ${ }^{2}$, K. Lipar' ${ }^{2}$, F. Saudek ${ }^{1}$;

${ }^{1}$ Diabetes Center, ${ }^{2}$ Transplantation Surgery, IKEM, Prague, Czech Republic

Background and aims: Pancreas transplantation alone (PTA) is an established approach in the treatment of selected non-uremic Type-1 diabetic patients with hypoglycemia unawareness syndrome. Recently, transplantation of pancreatic islet (ITx) was introduced in our center as an alternative method for the same indication. The aim of the study was to compare the short-term safety and efficacy of both procedures in terms of patient and graft survival rates metabolic control and the rate of complications.

Materials and methods: From 1998 to 200727 PTA (and 4 re-transplantations) and 14 ITx were performed in 31 and 8 type- 1 diabetic recipients with hypoglycemia unawareness syndrome, respectively. Pancreas was placed extraperitoneally with systemic venous anastomosis and either with enteral or bladder pancreatic duct drainage. Islets were isolated from pancreases distended by Liberase and purificated in COBE 2991. Patient survival, graft function, complications and metabolic control were compared at 6 month after transplantation and after the last infusion of islets.

Results: Survival of patients was $100 \%$ at $6 \mathrm{~m}$ in both groups. At the time of follow-up 70\% (19/27) patients with PTA and 13\% (1/8) after ITX were insulin-free. 29\% (8/27) patients after PTA lost their grafts early after transplantation due to thrombosis (5) rejection (2) and bleeding (1). In ITx group 1 patient had primary non-function, in the others insulin daily dose $( \pm S D)$ 
decreased significantly from $31 \pm 5$ to $13 \pm 6$ IU/day. In comparison with the ITx group (21\%), the PTA group had a high rate of serious complications (62\%) The mean values $( \pm \mathrm{SD}$ ) of $\mathrm{HbAlc}$ were $3.3 \pm 0.7$ and $5.4 \pm 0.6 \%$ in PTA and ITx recipients, respectively (normal value $<4.2 \%$ ).

Conclusion: PTA immediately restored normal glucose metabolism in the majority of diabetic recipients. More than a half of the recipients experienced surgical or other serious adverse event. ITx itself did not lead to insulin independence in most of patients. However, it stabilized glucose control close to the normal range and eliminated the episodes of severe hypoglycemia. Here we present our first clinical results with islet transplantation which, of course, need further improvement.

Supported by: grant VZ 00023001

\section{7}

The direct effects of immunosuppressive drugs on isolated human islets M. Bugliani, M. Masini, R. Lupi, S. Del Guerra, M. Galli, V. D’Aleo, F. Filipponi, S. Del Prato, U. Boggi, F. Mosca, P. Marchetti Dept. Endocrinology and Metabolism, Metabolic Unit, University of Pisa, Italy

Background and aims: The use of immunosuppressive drugs is associated with the development of diabetes, possibly due to beta-cell toxicity. To better understand the mechanisms responsible for these effects, we investigated the actions of prolonged exposure of isolated human islets to therapeutical levels of tacrolimus (Tac) or cyclosporin A (CsA).

Materials and methods: Islets were isolated from the pancreas of $16 \mathrm{mul}-$ tiorgan donors (age: $63 \pm 14$ yrs; gender: $10 \mathrm{M} / 6 \mathrm{~F} ; \mathrm{BMI}: 25.1 \pm 3.7 \mathrm{~kg} / \mathrm{m}^{2}$ ) by enzymatic digestion and density gradient centrifugation. Functional, survival and molecular studies were then performed after $96 \mathrm{hr}$ incubation with 10 $\mathrm{ng} / \mathrm{ml} \mathrm{Tac}$ or $150 \mathrm{ng} / \mathrm{ml}$ CsA.

Results: Glucose-induced insulin secretion was significantly decreased in Tac $(p<0.05)$, but not in CsA exposed islets, (stimulation index, i.e. the ratio of insulin release at 16.7 vs $3.3 \mathrm{mM}$ glucose: control islets, Ctrl, $2.3 \pm 0.5$; Tac 1.6 \pm 0.2 ; CsA, $2.0 \pm 0.4$ ). Electron microscopy (EM) showed a significant $(\mathrm{p}<0.05)$ decrease of insulin granules in Tac exposed beta-cells (volume density, ml\%: $0.63 \pm 0.39$ ), but not in the CsA group (1.55 \pm 0.01 ), as compared to $\mathrm{Ctrl}(1.54 \pm 0.09)$. The percentage of apoptotic beta-cells was higher in Tac (11.7 \pm 4.7$)$ than Ctrl $(2.7 \pm 1.2)$ (the respective value in CsA was 6.31.2). Microarray analysis (Affymetrix) showed that of the 22,215 probe sets present on the HG U133A chip, 94 and 60 were significantly $(\mathrm{p}<0.05)$ altered in Tac and CsA, respectively. In the Tac group, 23 genes were upregulated and 71 downregulated: among them, 5 were associated with apoptosis, 4 with cell proliferation, and 6 with transcription pathways. In the CsA group, 23 genes were upregulated and 37 were downregulated, with 2 regarding apoptosis and 7 transcription phenomena. Compared to Tac, microarray of CsA treated islets showed 78 upregulated and 51 downregulated genes, of which 6 regarded apoptosis, 8 cell proliferation and 12 transcription pathways. FKBP 12.6, a key gene involved in calcineurin inhibition, was significantly less expressed with CsA than Tac, as shown by quantitative RT-PCR.

Conclusion: In conclusion, CsA had less deleterious effects on isolated human islets, possibly due to a lower influence on gene expression.

\section{PS 041 Experimental islet transplantation}

\section{8}

Effectiveness of exenatide as a potent anti-rejection therapy for islet cell allo-transplantation in the non-human primate

J.L. Buss ${ }^{1}$, A. Rajab ${ }^{1}$, E. Diakoff 2 , K. Osei ${ }^{2}$

${ }^{1}$ Surgery, The Ohio State University, Columbus, ${ }^{2}$ Endocrinology, Diabetes and Metabolism, The Ohio State University, Columbus, United States

Background and aims: The use of islet cell transplantation in the treatment of type 1 diabetes has shown promising results. However, numerous limiting factors such as islet availability, early death of islets post-transplant and ineffective immunosuppressive regimens have continued to prevent islet cell transplantation from becoming more widely used clinically. Exenatide is an incretin mimetic and has demonstrated a wide range of beneficial effects on islet beta cells. Among these are protection from apoptosis, improved insulin production in response to glucose and promotion of beta cell regeneration. We propose that the beneficial effects of Exenatide will make it an effective in vivo treatment for islet cell allo-transplantation and that Exenatide alone may even be sufficient to protect islet allografts from rejection.

Materials and methods: Diabetes was induced in the non-human primate, Macaca fascicularis by total pancreatectomy. Islets were isolated following the procedure for human islet isolation and transplanted into the portal vein $(11,096 \pm 6996 \mathrm{IEq} / \mathrm{kg})$. Group $1(\mathrm{~N}=3)$ received islet allografts and were

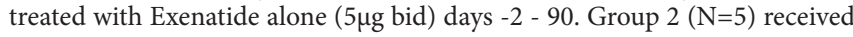
islet allografts and were treated with rabbit anti-thymocyte globulin $(1.5 \mathrm{mg} /$ $\mathrm{kg}$ ) and prednisone on days $0-3$ and cyclosporine $(25 \mathrm{mg})$ and mycophenolate mofetil $(250 \mathrm{mg})$ days $0-90$. Group $3(\mathrm{~N}=5)$ received islet allografts and were treated with the same regimen as Group 2 plus Exenatide ( $5 \mu \mathrm{g}$ bid) on days -2 - 90. Finally, Group $4(\mathrm{~N}=2)$ received islet allografts with no immunosuppressive treatment. Blood glucose was monitored daily for the first month posttransplant and weekly thereafter. Intravenous glucose tolerance tests were performed on days 0,28 and 90 , and glucose disappearance rate constants $\left(\mathrm{k}_{\mathrm{C}}\right)$ at 10-30 min were determined.

Results: Blood glucose monitoring indicated that all animals in Groups 1-3 (islet allografts with Exenatide and/or immunosuppression treatment) remained normoglycemic for at least 90 days post-transplant with an average blood glucose of $51.2 \pm 26.0 \mathrm{mg} / \mathrm{dl}$. Blood glucose for animals receiving islet allo-grafts and Exenatide treatment alone (Group 1) at 90 days post-transplant was $47.7 \pm 7.23 \mathrm{mg} / \mathrm{dl}$, indicating functional islet grafts despite a lack of conventional immunosuppression in these animals. One animal in Group 4 (allograft + no treatment) showed immediate rejection of the islet graft with blood glucose levels rising to $>200 \mathrm{mg} / \mathrm{dl}$ by day 2 post-transplant.

ivGTTs post-transplant showed normal responses in animals from Groups 1-3 in both blood glucose and serum insulin levels. In fact, serum insulin levels were notably higher in Group 1 than in any other group (Figure 1). Additionally, there was not a significant difference in the $\mathrm{k}_{\mathrm{G}}(\% / \mathrm{min})$ for allograft recipients plus Exenatide alone before and after transplant $(6.73 \pm 3.49$ vs. $4.48 \pm 3.68, \mathrm{p}=0.34$ ).

Conclusion: We conclude that Exenatide appears to be an effective in vivo anti-rejection treatment in islet allo-grafts in the non-human primate. Despite a lack of conventional immunosuppressive treatment in animals treated with Exenatide alone, islet allografts showed no signs of rejection and, in fact, demonstrated increased insulin production compared to animals not receiving Exenatide. The mechanisms of the anti-rejection properties of Exenatide remain to be elucidated.

Supported by: Amylin Pharmaceuticals, Inc.

\section{9}

Effects of IL-1 on the revascularization of islet grafts

O. Castaner, N. Tellez, E. Estil.les, G. Joanny, J. Escoriza, E. Montanya Lab. Diabetes and Experimental Endocrinology. University of Barcelona, Endocrine Unit, Hospital Universitari de Bellvitge; IDIBELL; CIBERDEM, L'Hospitalet de Llobregat, Spain

Background and aims: In the initial days after islet transplantation, a nonspecific inflammatory process takes place at the grafted site, and contributes to the initial damage of transplanted islets and to early graft failure. The inflammation involves the expression of pro-inflammatory cytokines, among 
them IL-1 $\beta$. We have recently shown that the blockade of IL- 1 action by IL-1receptor antagonist protein (IL1RN) improved the metabolic outcome of the recipients, that achieved consistent normoglycaemia despite the transplantation of a borderline beta cell mass (800 islets). These results supported the deleterious role of IL-1 expression on islet grafts and the potential therapeutic value of IL1RN. In experimental tumor models, IL-1 $\beta$ has increased endothelial migration and proliferation, and modulated angiogenesis, and IL-1 receptor antagonism has shown anti-angiogenic effects. IL-1 could therefore play a role on the revascularization of islet grafts. We aimed to determine whether the over expression of IL1RN in transplanted islets, modifies the revascularization of islet grafts.

Materials and methods: Islets from Lewis rats were infected ex vivo with an adenovirus encoding for IL-1-receptor antagonist (Ad-Ill rn). Streptozotocin-diabetic Lewis rats were syngenically transplanted with 500 or 1200 AdIll $r n$ infected (Ad-Illrn group) or uninfected islets (control group) under the kidney capsule. 500 islets are a clearly insufficient mass to restore normoglycaemia, and we expected that all animals would remain hyperglycaemic, after transplantation. In contrast, 1200 islets are a sufficient mass to restore normoglycaemia. The islet grafts, harvested on days 10 and 28 after transplantation, were double stained with the lectin Griffonia Simplicifolia, which binds to $\alpha$ - galactosyl and N-acetylgalactosaminyl terminations, expressed on the surface of the rat's endothelial cells, and with an insulin antibody. Vascular density was determined by point counting morphometry.

Results: As expected, rats transplanted with 500 islets remained hyperglycaemic throughout the study (Ad-Il1rn group: 20.1 \pm 1.83 ; control group: $21.03 \pm 1.19 \mathrm{mmol} / \mathrm{L}$ ). All animals showed similar hyperglycemia ensuring comparable metabolic conditions among groups. Normoglycaemia was achieved in the recipients of 1200 islets (day 30: Ad-Illrn group: 5.47 \pm 0.11 ; control group: $5.81 \pm 0.18 \mathrm{mmol} / \mathrm{L}$ ). On day 10 after transplantation, the vascular density was lower in the 500-islet Ad-Illrn group than in the control group (5.96 \pm 0.7 vs $11.8 \pm 3.0 \%, \mathrm{p}<0.05)$. On day 28 after transplantation, vascular density in the Ad-Illrn group was higher than on day $10(13.3 \pm 0.9 \%$ $\mathrm{p}<0.01$ ), and similar to the control group $(13.2 \pm 2.6 \%)$. In normoglycaemic 1200 -islet grafts, the vascular density was determined on day 30 after transplantation, and was also similar in Ad-Illrn and control groups (15.4 $\pm 0.9 \mathrm{vs}$ $12.5 \pm 2.4 \%)$.

Conclusion: The over expression of IL-1 receptor antagonist protein in transplanted islet grafts inhibited the initial revascularization of the graft. The inhibition of angiogenesis was observed as a delay, as vascular density increased subsequently and reached similar values to those of control islet grafts, both in normoglycaemic and in hyperglycaemic recipients. Our results suggest that, the initial expression of IL-1 may enhance the revascularitzation of the graft in islet transplantation.

Supported by: FIS PI-060891

\section{0}

Muscle-targeted gene therapy for the treatment of diabetes mellitus: investigating the role of the immune system when employing mutant (pro)insulin plasmids

S.J.M. Niessen ${ }^{1,2}$, A. Mahmoud ${ }^{2}$, A. Aldibbiat ${ }^{2}$, C. Huggins ${ }^{3}$, D.B. Church ${ }^{1}$, J.A.M. Shaw ${ }^{2}$

${ }^{1}$ Department of Veterinary Clinical Sciences, Royal Veterinary College, University of London, Hatfield, ${ }^{2}$ Diabetes Research Group, Newcastle University, ${ }^{3}$ Comparative Biology Centre, Newcastle University, United Kingdom

Background and aims: The tight glycaemic control required to prevent microvascular complications without limiting hypoglycaemia in Type 1 and 2 diabetes is difficult to attain using conventional subcutaneous insulin therapy. Alternative approaches have therefore been pursued. Muscle-targeted gene therapy (MTGT) employing (pro)insulin constructs is promising and provides possible cost-effective off-the-shelf treatment for diabetes. However, uncertainty still remains over the influence of the immune system on this type of treatment, especially in view of currently encountered 10-fold decreased levels of efficiency with mutant proinsulin constructs designed to yield fully processed insulin, compared to wild-type constructs. This study aimed to assess the influence of immune system on efficacy of MTGT with wild-type (hppI1) and mutant furin-cleavable (hppI4) human proinsulin constructs or $\beta$-galactosidase reporter gene ( $\beta$-gal) (all subcloned in pVR1012 plasmid). Materials and methods: After injection of $10 \mathrm{U}$ hyaluronidase, tibialis anterior muscles in 9 male CD-1 mice and 9 male SCID (immunodeficient) mice

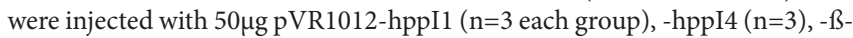
gal $(n=2)$ or $50 \mu$ saline $(n=1)$, followed by electroporation ( 8 pulses, $200 \mathrm{~V}$ / $\mathrm{cm}, 20 \mathrm{~ms}, 2 \mathrm{~Hz}$ ). Blood samples for glucose, human (pro)insulin and C-peptide estimation were obtained at set time points, as were muscles for histology (with insulin and C-peptide immunostaining), human (pro)insulin, C-peptide and $\beta$-gal estimation. The same plasmid preparations were used for in vitro transfections in the $\mathrm{C} 2 \mathrm{C} 12$ murine muscle cell line to ensure adequate functioning of these plasmids, with subsequent RT-PCR to prove preproinsulin expression.

Results: Blood glucose and body weight was not affected in any animal. Serum human proinsulin was $35.1 \pm 10.2$ and $35.3 \pm 21.2 \mathrm{pmol} / \mathrm{l}(\mathrm{mean} \pm \mathrm{SD})$ at 2 and 7 days post gene-transfer in hppI1-injected CD-1 mice and $29.8 \pm 15$ and $37 \pm 7.4 \mathrm{pmol} / \mathrm{l}$ in hppIl-treated SCID mice. Human insulin was undetectable in either group. Although expression was confirmed by insulin and C-peptide immunostaining of muscle sections, no significant serum human proinsulin, insulin or C-peptide concentrations were detected following hppI4 gene transfer in CD-1 or SCID mice. $\beta$-gal activities in muscle homogenates of $\beta$ gal transfected mice were $3.3 \pm 0.3(\mathrm{CD}-1)$ and $0.34 \pm 0.05$ (SCID) $\mathrm{U} / \mu \mathrm{l}$. In vitro transfection studies revealed adequate functioning of the employed plasmids with significant proinsulin in hppI1 (medium: 328 \pm 52 , lysate: $18.2 \pm 6.9 \mathrm{pmol} / \mathrm{l}$ ), insulin in hppI4 (medium: $16.4 \pm 4.4$, lysate: $8.7 \pm 6.8 \mathrm{pmol} /$ l) and $\beta$-gal activity $(52.8 \pm 2.0 \mathrm{U} / \mu \mathrm{l})$ in $\mathrm{B}$-gal transfected cells. RT-PCR confirmed preproinsulin expression in hppIl and hppI4 transfected cells. Conclusion: Immune responses do not appear to play a significant role in the low insulin levels encountered when employing mutant furin-cleavable proinsulin plamids for MTGT. Absence of significant C-peptide demonstrates that increased metabolism of mature insulin is also not the sole cause. Successful clinical translation will require plasmid refinement and enhancement of gene transfer efficiency.

\section{1}

Optimal liraglutide therapy improves engraftment and function of a marginal mass islet transplant in mice

S. Merani ${ }^{1}$, W. Truong ${ }^{1}$, J.A. Emamaullee ${ }^{1}$, C. Toso ${ }^{1}$, L.B. Knudsen ${ }^{2}$,

A.M.J. Shapiro ${ }^{1}$

${ }^{1}$ Surgical Medical Research Institute, University of Alberta, Edmonton, Canada, ${ }^{2}$ Diabetes Research Unit, Novo Nordisk, Måløv, Denmark

Background and aims: The scarcity of high quality deceased pancreas donors prevents widespread application of islet transplantation for treatment of labile type 1 diabetes mellitus. Opportunities for the improvement of current techniques includes optimization of islet isolation and purification, use of culture with pharmacologic insulinotropic agents, strategies to reduce graft rejection and inflammation, and the search for alternative insulin producing tissue. Here we report the efficacy of the once-daily human glucagonlike peptide 1 analog, liraglutide, in a mouse model of marginal mass islet transplantation.

Materials and methods: Liraglutide was administered (200 ug/kg sc) either once or twice daily because it is metabolized more rapidly in mice than in humans. The model used was a marginal mass (250 syngeneic islets/mouse) syngeneic islet transplant in streptozotocin-induced diabetic BALB/c mice. Results: Time-to-normoglycemia, defined as the first day of sustained posttransplantation normoglycemia was significantly shorter in twice daily liraglutide treated animals versus control (median 1 day vs. 7 days, $p=0.0003$ ), even if animals were concurrently administered a daily dose of sirolimus (median 1 day vs. 72.5 days, $\mathrm{p}<0.0001$ ). However, animals given only a single dose of liraglutide daily, or given a once daily liraglutide plus pantoprazole (to increase serum gastrin levels, administered $50 \mathrm{mg} / \mathrm{kg} /$ day ip) did not show improved islet engraftment. Twice daily liraglutide treated animals also demonstrated improved glucose tolerance during intraperitoneal glucose tolerance test (IPGTT).

To investigate the mechanism of action of twice daily liraglutide treatment on islet engraftment, animals were discontinued of liraglutide on post-transplant day 90, which resulted in diminished glucose tolerance during IPGTT. Late start liraglutide therapy at 90 days prost-transplant resulted in no improvement in engraftment. These findings suggest that liraglutide therapy mediates both early and late insulinotropic effects. Insulin/TUNEL fluorescence microscopy showed reduced transplanted beta-cell apoptosis in liraglutide treated recipients 48 hours post-transplant.

Conclusion: Overall, our data shows that liraglutide has beneficial impact on the engraftment and function of syngeneic islet transplants in mice, when administered continuously starting on the day of transplant. 


\section{2}

Improved glucose metabolism by islet transplant at periportal site than intraportal site

K. Toyoda ${ }^{1}$, T. Okitsu ${ }^{2}$, T. Uonaga ${ }^{1}$, X. Liu ${ }^{1}$, S. Yamane ${ }^{1}$, Y. Seino ${ }^{3}$,

N. Inagaki ${ }^{1}$

${ }^{1}$ Diabetes and Clinical Nutrition, Kyoto University, ${ }^{2}$ Transplantation Unit,

Kyoto University Hospital, ${ }^{3}$ Diabetes and Clinical Nutrition, Kansai-

Denryoku Hospital, Osaka, Japan

Background and aims: Intraportal site is common in clinical islet transplantation. However, its superiority is not elucidated yet. In terms of glucose metabolism, it should have a drawback because islets are located at the peripheral end of portal vein and insulin acts only on the restricted area of the liver. We, therefore, consider periportal site as another site for islet transplantation, which allows whole liver to be exposed to secreted insulin. Then, we tested the efficacy of periportal site by comparing with intraportal site using mouse islet transplantation model.

Materials and methods: Syngeneic mouse islet transplantation was performed using male C57BL/6 mice. Recipient animals were rendered diabetic by streptozotocin (STZ) and received four hundred islets. Body weight and blood glucose were monitored in the mice of the following four groups; STZ induced diabetic mice receiving islets at periportal site (PP group), those at intraportal site (IP group), those without any islet transplant (STZ group) and sham-operated wild mice (Control group). Oral glucose tolerance test (OGTT) was performed 30 days and 60 days after transplantation and HOMA-IR was calculated.

Results: In both PP and IP group, random non-fasting glucose levels were significantly lower than STZ group after 400 islets transplantation, but it took significantly longer time to get normoglycemia in PP group than IP group (PP $11.2 \pm 1.5$, IP $3.5 \pm 1.2$ (days), $\mathrm{p}<0.01$ ) (Figure.1). OGTT in PP group showed higher levels of post-load blood glucose than IP and Control group on Day30 and showed similar normal glucose tolerance as IP and Control group on Day60. HOMA-IR on Day60 in IP group was also significantly higher than PP and Control group (both, $\mathrm{p}<0.05$ ) (Control 1.53 \pm 0.15 , PP $1.35 \pm 0.10$, IP 2.46 \pm 0.35 ) (Figure.2).

Conclusion: These results indicated that islet transplantation at periportal site was superior in terms of glucose metabolism to intraportal site, although this superiority is limited only after islet transplant became engrafted. The periportal islet transplantation site might enable islet grafts to survive for longer term due to its less stressful condition than intraportal site, and might serve as a possible strategy to increase feasibility of clinical islet transplantation.

\section{Percentage of normoglycemic recipient}

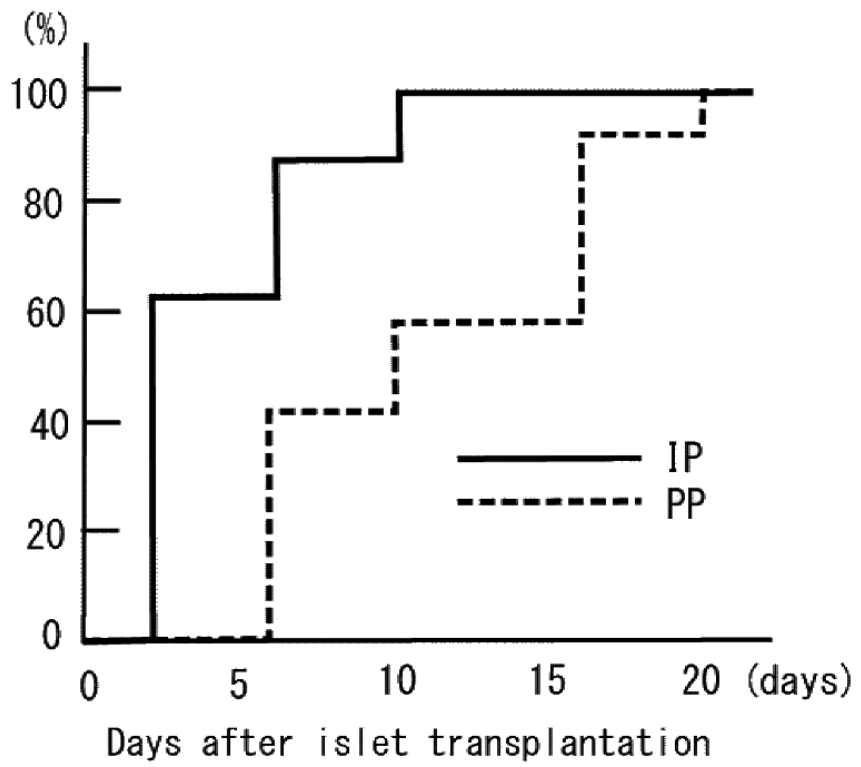

Figure 1.

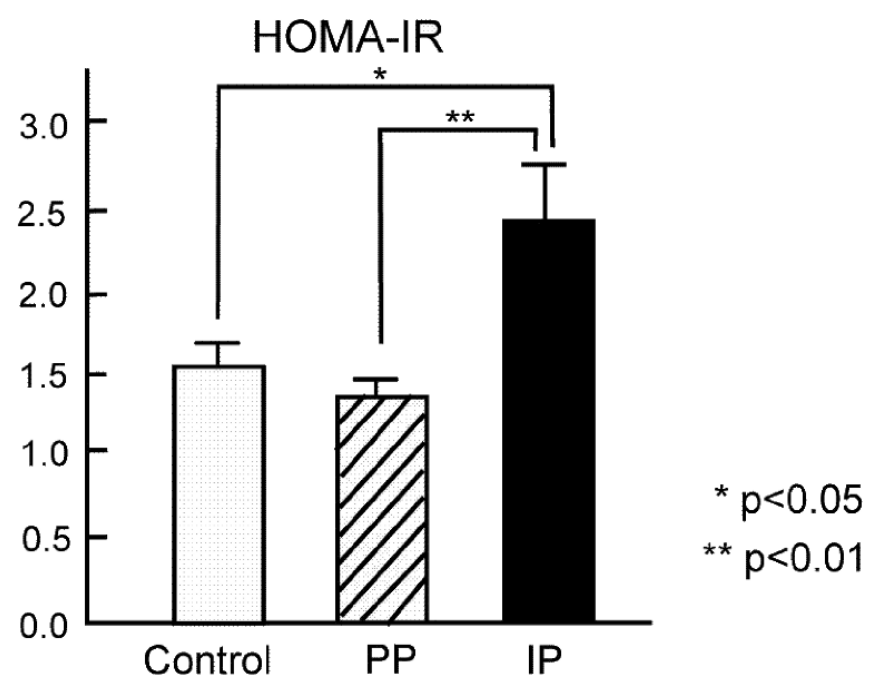

Figure 2.

Supported by: Ministry of Education, Culture, Sports, Science and Technology of Japan, and by Research on Nanotechnical Medicine from the Ministry of Health, Labour and Welfare of Japan

\section{3}

IBMIR reaction and macrophage migration: role of the exocrine tissue S. Sigrist ${ }^{1}$, N. Ebel ${ }^{1}$, W. Bietiger ${ }^{1}$, C. Vodouhe ${ }^{1}$, A. Langlois ${ }^{1}$, A. Belcourt ${ }^{1}$, L. Kessler ${ }^{1}$, J. Oberholzer ${ }^{2}$, M. Pinget ${ }^{1}$

${ }^{1}$ Research laboratory, CeeD, Strasbourg France, ${ }^{2}$ Division of Transplantation, University of Chicago, United States

Background and aims: After infusion into the portal vein, a substantial percentage of islets are lost in the immediate post-transplant period through an inflammatory response termed the instant blood-mediated inflammatory reaction (IBMIR). Coagulation, platelet aggregation, complement activation, and neutrophil and monocyte infiltration play roles in this reaction. There is also evidence that exocrine contamination may impair the implantation of islet tissue. Our objective was to identify the role of exocrine tissue in the IBMIR reaction and to prevent these reactions using several anti-inflammatory strategies.

Materials and methods: The culture mediums conditioned for 48 hours by rat pancreatic islets were incubated with peritoneal murine macrophages to study their migration. Chemotaxis was evaluated using the Boyden chamber and expressed as the chemotactic index. To validate that the Boyden chamber mimic the IBMIR, we developed 3 models: syngenic model was realized with Lewis rat, allogenic with Lewis rat and xenogenic with human pancreatic islet and Lewis rat macrophages To understand the role of exocrine tissue on IBMIR, we have exposed macrophages to several purity of islet supernatant from $90 \%$ to $1 \%$. Finally, to modulate inflammation, macrophages were preincubated with PSC-RANTES $(1,10,100 \mathrm{nmol} / \mathrm{L})$, a potent antagonist of CCR5 or added heparin or pentosan $(1 \mu \mathrm{mol} / \mathrm{L})$.

Results: Study on macrophages migration using the syngenic, allogenic et xenogenic models showed that the Boyden chamber mimics the IBMIR reaction because macrophage migration was comparable in all the conditions tested. Moreover, this migration was increased in the presence of exocrine tissue from $1,41 \pm 0.12$ with $90 \%$ of purity to $1.92 \pm 0.19$ with $50 \%(n=6 ; p<0.001)$ indicating that exocrine tissue play a crucial role in IBMIR. Finally, PSCRANTES significantly reduced, in a dose-dependent manner, macrophage chemotaxis by islets supernatant from $1.92 \pm 0.19$ to $0.67 \pm 0.18(\mathrm{n}=6 ; \mathrm{p}<0.01)$. The trapping of chemokines by heparin or pentosan also reduced the chemotactic activity of islets supernatant with heparin or pentosan from $1.92 \pm 0.19$ to $1.1 \pm 0.12$ and $0.95 \pm 0.19$ respectively.

Conclusion: The quality of human islets is one of the factors decisive for the success of human islet transplantation. Exocrine tissue may be more immunogenic than islet tissue, and complete purification may have advantages for the prevention of IBMIR. Furthermore, modulation of macrophages migration using PSC-RANTES or heparinoids could be a strategy to limit IBMIR reaction and merits further evaluation in vivo. 


\section{4}

Magnetic iron nanoparticles in rat pancreatic islets: post-transplantation tracking

K. Zacharovova ${ }^{1}$, Z. Berkova ${ }^{1}$, V. Herynek ${ }^{2}$, P. Girman ${ }^{1}$, T. Koblas ${ }^{1}$,

L. Pektorova ${ }^{1}$, E. Dovolilova ${ }^{1}$, M. Vancova ${ }^{3}$, J. Nebesarova ${ }^{3}$, F. Saudek ${ }^{1}$

${ }^{1}$ Laboratoty of Langerhans Islets, IKEM, Prague, ${ }^{2}$ Department of Magnetic

Resonance, IKEM, ${ }^{3}$ Institute of Parasitology, Biology Centre, Ceske

Budejovice, Czech Republic

Background and aims: The pancreatic islets in vitro cultivated with iron paramagnetic ferucarbotran nanoparticles - Resovist ${ }^{\bullet}$ - incorporate the particles into the cells including endocrine cells. The integrated iron particles allow the islets detection for several months by magnetic resonance after transplantation. We used transmission electron microscopy and immunohistochemical methods for post-transplantation tracking of the iron localization and the way of particles storage and retention.

Materials and methods: Isolated rat islets were incubated with ferucarbotran for 48 hours. Iron labeled islets were transplanted into the portal vein and/or under the kidney capsule of syngeneic animals. The livers and kidneys were removed 7, 30 days and 3 months after transplantation. For transmission electron microscopy samples were fixed with mixture of $4 \%$ formaldehyde and $2 \%$ glutaraldehyde, postfixed in $1 \% \mathrm{OsO}_{4}$, dehydrated and embedded in Epon resin. For immunohistochemistry the tissue was fixed in Bouin's solution, embedded in resin Tissue Tek and frozen. Immunodetection of endocrine cells and macrophages was combined with $\mathrm{Fe}^{3+}$ iron detection.

Results: After the islet transplantation, the iron particles changed the localization. After 1 week post-transplantation, ferucarbotran was rarely found in the endocrine cells however it was detected in huge amounts inside the macrophages and fibroblasts surrounding the islets. The particles were found surprisingly also inside the endothelium of newly formed vessels. 1 month after transplantation, the iron wasn't detectable in the endocrine cells however it was even more accumulated in islet surrounding cells. After 3 month post-transplantation period, the localization of iron particles was the same as after the 1-month period, only the extracellular matrix fibers surrounding the islets were multiplied. The immunodetection combined with iron detection excluded the localization of iron in endocrine cells after the transplantation. The ultrastructure of the islets and surrounding tissue arrangement was very similar in liver and under kidney capsule.

Conclusion: After the islet transplantation, the ferucarbotran particles translocated from the endocrine cells and iron was concentrated inside the macrophages and other islet surrounding cells. The iron particles were stored in these cells without changes for months. The process of posttransplant particle transfer needs to be further investigated.

Supported by: grant No. 2B06175.MSMT, Czech Republic

\section{5}

Labelling of insulin-secreting INS-1E cells and rodent islets of Langerhans with ferucarbotran for non-invasive MR imaging V.J. Auer ${ }^{1,2}$, E. Schremmer-Danninger ${ }^{1,2}$, J.N. Bucher ${ }^{1,2}$, N. Schantz ${ }^{1,2}$, M.C. Raggi ${ }^{2}$, M. Reiser ${ }^{3}$, M.J. Stangl ${ }^{2}$, F. Berger ${ }^{3}$

${ }^{1}$ IMETUM, Technical University Munich, Garching, ${ }^{2}$ Transplant Surgery, Technical University Munich, ${ }^{3}$ Clinical Radiology, Ludwig-MaximiliansUniversity Munich, Germany

Background and aims: Transplantation of purified pancreatic islets is a promising method of restoring insulin secretion in selected patients with type 1 diabetes mellitus. To monitor the fate of transplanted islets, transplantation efficiency and graft survival, a method to evaluate the location, distribution and long term viability in a noninvasive manner in vivo is needed over a period of time. MRI of islets labeled with magnetically visible contrast agents after transplantation has the potential to fulfill this goal as MRI provides high spatial and high contrast resolution images. The aim of the study is to set up viability assays and to establish protocols for labeling and visualizing. INS-1E cells, a stable differentiated, insulin secreting cell line, and rodent islets in vitro were used as our models for the potential human clinical setting using FDA-approved SPIOs and clinical scanners.

Materials and methods: For labeling INS-1E cells in vitro, concentrations of ferucarbotran range from $12,5 \mu \mathrm{g} / \mathrm{ml}$ to $1000 \mu \mathrm{g} / \mathrm{ml}$ in the culture medium. The INS-1E cells were incubated for 3 days. Labeling efficiency was measured by separating magnetically labeled cells from unlabeled by incubation in a special magnetic tool. Rat islets were labeled with $5,4 \mathrm{mg} / \mathrm{ml}$ ferucarbotran in the culture medium. For assessment of magnetic resonance contrast in a clinical 3T scanner, we placed both a layer of labeled INS-1E cells and 5-10 SPIO-labeled, handpicked rodent islets between two layers of $4 \%$ gelatine. Viability assay was performed by double staining the cells with ethidium bromide as an exclusion dye and calcein AM as an inclusion dye for living cells, and by WST-1 test. Cell function was evaluated by static incubation assay to assess insulin secretion. Using the calcium-indicator Fluo-3AM, intracellular calcium concentration was studied in response to glucose. Fluorescence was measured in a multiwell spectrophotometer.

Results: Iron labeled insulin secreting INS-1E cells did not show altered viability or reduced glucose stimulated insulin secretion in vitro. Double staining of INS-1E cells showed no difference between labeled and non labeled INS-1E cells. This result was confirmed by WST-1 test. We visualized SPIOlabeled INS-1E cells and rat islets in vitro using a clinical $3 \mathrm{~T}$ scanner. Diabetic rats transplanted with 500-1000 SPIO-labeled islets became normoglicemic. $\mathrm{T} 2^{*}$-weighted MRI protocols for visualizing transplanted islets in rats, are under way.

Conclusion: Labeling the insulin secreting INS-1E cell line and rat islets with SPIOs does not alter their viability, while enabling MR imaging contrast. Supported by: Bayerische Forschungsstiftung

\section{6}

Optimization of pancreatic islets revascularization after transplantation: pharmacological approach or gene therapy?

A. Langlois, W. Bietiger, E. Seyfritz, K. Mandes, E. Maillard, A. Belcourt, M. Pinget, L. Kessler, S. Sigrist

Laboratoire de recherche, Centre européen d'etude du Diabète, Strasbourg, France

Background and aims: The formation of new microvasculature by capillary sprouting at the site of islet transplantation is crucial for the long-term survival and function of the graft. Vascular endothelial growth factor (VEGF), an endothelial cell-specific mitogen with potent angiogenic and vascular permeability-inducing properties, may be a key factor in modulating the revascularization of islets after transplantation. Delivery of human vascular endothelial growth factor (hVEGF) gene to the islets is likely to promote islet revascularization and survival. Some of the gene therapy enhancement presents significant ethical/societal questions. Then development of pharmacological approach to enhance VEGF expression could be an alternative to gene therapy. The aim of this work was to study the effect of adenoviral infection or Deferoxamine (DFO), an iron chelator, on islets revascularization and metabolic control of diabetic rat after transplantation.

Materials and methods: In vivo streptozotocin-treated diabetic Lewis rats received 1000 Lewis islets beneath the liver via the portal vein. Transplanted islets were not treated or infected in vivo using an adenovirus encoding human VEGF gene $\left(4.10^{8} \mathrm{pfu} /\right.$ pancreas $)$ or incubated with DFO $(10 \mu \mathrm{mol} / \mathrm{L})$ during 1 and 3 days. Islets revascularization was studied by immunohistochemistry using lectine, VEGF and insulin staining. Metabolic control of diabetic rat was evaluated by the measures of body weight gain, Fasting blood glucose and the secretion of C-peptide during two months.

Results: Immunostaining study of vascularisation surrounding the islets showed a stimulation of islet revascularization using DFO which was maintained during the experiment. Moreover, this revascularization was associated to a preservation of islets viability. These results suggested that overexpression of VEGF promoted islet survival by the inhibition of necrosis and apoptosis. The metabolic study has shown a gain of body weight using DFO and infection 2 months after transplantation (DFO: $28.5 \pm 34.45$ g; Infection: $30.67 \pm 57.33 \mathrm{~g} v s-12.70 \pm 17.03 \mathrm{~g} ; \mathrm{n}=3$ ), a syngeneic recipients were euglycemic for all conditions during the fast and a better secretion of C-peptide after fasting and refeeding using DFO (1 month after fasting: DFO: 533.38 \pm 44.61 pmol/L; Infection: $256.88 \pm 126.40 \mathrm{pmol} / \mathrm{L}$ vs $270.33 \pm 44.61 \mathrm{pmol} / \mathrm{L} ; \mathrm{n}=3$ ).

Conclusion: These results demonstrate that VEGF production in islets stimulates graft angiogenesis and enhances islet revascularization. This mechanism might be explored as a novel strategy to accelerate islet revascularization and improve long-term survival of functional islet mass posttransplantation. In conclusion, the pharmacological approach of VEGF overexpression using DFO seemed more promising to improve islets vascularization after transplantation than adenoviral infection with human VEGF 165 gene. 


\section{PS 042 Intestinal glucose absorption and metabolism}

\section{7}

Intestinal gluconeogenesis is a key factor in the early improvement of insulin sensitivity after gastric by-pass in mice fed on a high fat-diet G. Mithieux ${ }^{1}$, S. Troy ${ }^{2}$, M. Soty ${ }^{1}$, L. Ribeiro ${ }^{2}$, S. Migrenne ${ }^{3}$, X. Fioramonti ${ }^{3}$, V. Fauveau ${ }^{4}$, R. Aubert ${ }^{2}$, B. Viollet ${ }^{5}$, M. Foretz ${ }^{5}$, J. Leclerc ${ }^{5}$, C. Zitoun ${ }^{1}$, B. Thorens ${ }^{6}$, C. Magnan ${ }^{3}$, F. Andreelli ${ }^{2}$

${ }^{1}$ Unité 855, Inserm, Lyon, France, ${ }^{2}$ Unité 695, Inserm, Paris, France, ${ }^{3}$ Unité 7059, CNRS, Paris, France, ${ }^{4}$ Institut Cochin, IFR Alfred Jost, Plateforme de microchirurgie, Paris, France, ${ }^{5}$ Institut Cochin, Université Paris Descartes, France, ${ }^{6}$ Department of Physiology and Center for Integrative Genomics, University of Lausanne, Switzerland

Background and aims: Gastric by-pass (GBP) surgery promotes a rapid improvement of insulin sensitivity and insulin secretion in obese diabetic patients, before a significant weight loss has occurred. On the contrary, the improvements observed in the patients after gastric lap-band (GLB) surgery take place lately, after weight loss. It is generally thought that GLP-1 could be a possible effector of this improvement. The aim of this work was to document the role of GLP-1 and of other possible mechanisms involved in the amelioration of glucose homeostasis after GLB in mouse models

Materials and methods: we studied food intake, weight gain, insulin sensitivity (glucose and insulin tolerance tests, hyperinsulinic euglycemic clamp), the role of GLP-1 (effect of the exendin [9-39] amide antagonist), the expression of intestinal genes of gluconeogenesis (glucose- 6 phosphatase and phosphoenolpyruvate carboxykinase), intestinal glucose fluxes (arterio-venous glucose balance and $3[3 \mathrm{H}]$ glucose tracer dilution determinations), in mice fed on a high-fat diet, after GBP or GLB surgery, compared to sham pair-fed mice. Results: GBP mice exhibited a strong and lasting decrease of food intake. In addition, a marked restoration of insulin sensitivity, of glucose tolerance, and of insulin secretion, was observed by 10 days only after surgery. Hyperinsulinic euglycemic clamp revealed that the improvement took place at the level of hepatic glucose production. In contrast, no restoration of these parameters was observed in pair-fed sham- and GLB mice. In GBP mice treated with the GLP-1 antagonist, the increase of insulin secretion was cancelled, whereas the decreased food intake and the restored insulin sensitivity and glucose tolerance were still present. Glucose- 6 phosphatase and phosphoenolpyruvate carboxykinase enzymes were markedly increased along the whole small intestine of GBP mice, but not in sham- and GLB mice. The intestinal glucose release, measured in postabsorptive GBP mice, was sufficient to counterbalance the intestinal glucose uptake, so that the portal glucose concentration was equivalent to the arterial glucose concentration. No portal glucose release took place in pair-fed sham- mice. The beneficial effects of GBP were not observable in mice with invalidation of the glucose transporter Glut-2, or in mice with inactivation of portal nervous afferences.

Conclusion: Portal glucose appearance per se has been shown to decrease food intake, suppress hepatic glucose production, and increase peripheral glucose uptake. These effects do not take place in Glut-2 invalidated mice or after inactivation of portal nervous afferences. Our data thus strongly suggest that: 1) GLP-1 might play a key role in the restoration of insulin secretion in GBP mice; 2) a "portal glucose signal", resulting from intestinal gluconeogenesis, is a crucial factor for the decreased food intake and the rapid improvement of insulin sensitivity occurring after gastric by pass in mice.

\section{8}

Impact of weight loss on the incretin effect in obese non-diabetic subjects E. Muscelli ${ }^{1}$, S. Camastra ${ }^{1}$, M. Anselmino ${ }^{2}$, F. Santini ${ }^{3}$, A. Mari ${ }^{4}$, J.J. Holst ${ }^{5}$, E. Ferrannini ${ }^{1}$

${ }^{1}$ Department of Internal Medicine, University of Pisa, Italy, ${ }^{2}$ IV Surgery Unit-Bariatric Surgery Division, Santa Chiara Hospital, Pisa, Italy, ${ }^{3}$ Department of Endocrinology, University of Pisa, Italy, ${ }^{4}$ Institute of Biomedical Engineering, Consiglio Nazionale delle Ricerche, Padua, Italy, ${ }^{5}$ Department of Medical Physiology, Panum Institute, Copenhagen, Denmark

Background and aims: The incretin effect is commonly defined as the potentiation of insulin release after oral glucose (OS) vs intravenous glucose (IV) administration at matched plasma glucose levels. Both diabetes and obesity are associated with an impaired incretin effect independently of one another, but whether weight loss (WL) restores the incretin effect is not known. Materials and methods: Oral (75 g glucose, 3 hours) and isoglycaemic intravenous IV glucose administrations were performed on separate days in 15 obese non-diabetic subjects ( $42 \pm 3$ years; BMI $\left.=40.3 \pm 2.2 \mathrm{~kg} . \mathrm{m}^{-2}\right)$ before and after weight loss (induced by caloric restriction in 6 patients, laparoscopic gastric banding in 7 patients and gastric bypass in 2 patients). C-peptide deconvolution was used to calculate insulin secretion rates, and a mathematical model was used to measure $B$-cell glucose sensitivity ( $B G S$ ), defined as the slope of the dose-response curve of insulin secretion rate and plasma glucose concentration. Incretin effect was taken to be the ratio of OS to IV responses. Insulin sensitivity was also estimated by the oral glucose sensitivity index, OGIS.

Results: Over a period of 1 year, subjects lost $21 \pm 2 \%$ of their initial weight and $63 \pm 6 \%$ of their weight excess. Plasma glucose responses were similar with oral and isoglycemic IV glucose both before and after WL, and were somewhat lower on the OGTT after WL $(\%, \mathrm{p}=0.06)$. The total amount of insulin secreted with oral glucose (TIS) was higher than with IV glucose both before ( $75 \pm 6$ vs $61 \pm 6$ nmol.m $\left.{ }^{-2}, \mathrm{p}=0.004\right)$ and after WL $(63 \pm 5$ vs $43 \pm 4$ $\left.\mathrm{nmol} \cdot \mathrm{m}^{-2}, \mathrm{p}=0.0007\right)$. The incretin effect on TIS was significantly improved by weight loss $(\mathrm{OS} / \mathrm{IV}=1.3 \pm 0.1$ before $\mathrm{WL}$ vs $1.5 \pm 0.1$ after $\mathrm{WL}, \mathrm{p}=0.03)$. ßGS was higher during oral than IV glucose both before $(113 \pm 11$ vs $84 \pm 13$ pmol.min $\left.{ }^{1} \cdot \mathrm{m}^{-2} \mathrm{mM}^{-1}, \mathrm{p}<0.0001\right)$ and after WL $\left(107 \pm 10\right.$ vs $66 \pm 9$ pmol. $\mathrm{min}^{-1} \mathrm{~m}^{-2} \cdot \mathrm{mM}^{-1}$, $\mathrm{p}=0.002)$. The incretin effect on $B G S$ was only marginally improved $(1.6 \pm 0.2$ vs $1.9 \pm 0.2, \mathrm{p}=\mathrm{ns})$. Both, insulin sensitivity $\left(325 \pm 20\right.$ vs $410 \pm 21 \mathrm{ml} \cdot \mathrm{min}^{-1} \cdot \mathrm{m}$ $\left.{ }^{2}, \mathrm{p}=0.002\right)$ and insulin clearance $\left(1.76 \pm 0.18\right.$ vs $\left.2.28 \pm 0.241 . \mathrm{m}^{-2}, \mathrm{p}=0.01\right)$ increased after WL. The WL-induced change in the incretin effect on IS was directly related to corresponding change in $\mathrm{BMI}(\mathrm{r}=0.61 \mathrm{p}<0.02)$. In contrast, neither the GLP-1 nor the GIP response to oral glucose changed significantly after WL (GLP-1 $=2.97 \pm 0.40$ vs $3.74 \pm 1.12 \mathrm{nmoll}^{-1} \cdot 3 \mathrm{~h}$, $\mathrm{p}=\mathrm{ns}$; $\mathrm{GIP}=5.85 \pm 1.32$ vs $\left.4.56 \pm 0.58 \mathrm{nmoll}^{-1} \cdot 3 \mathrm{~h}, \mathrm{p}=\mathrm{ns}\right)$.

Conclusion: In obese non-diabetic subjects, substantial weight loss proportionally improves the incretin effect on insulin secretion - but not the incretin effect on B-cell glucose sensitivity - largely independently of changes in GLP1 or GIP responses.

\section{9}

Resistin-like molecule beta regulates intestinal glucose absorption J.-C. Marie, P. Lettéron, C. Nazaret, R. Belharbi Krimi U773, CRB3, INSERM, Paris, France

Background and aims: Gastrointestinal peptides can modulate intestinal absorption of alimentary glucose, while the primary regulator is glucose itself. We investigated the effect of gut derived resistin-like molecule $\beta$ (RELM $\beta$ ) on intestinal sugar absorption.

Materials and methods: Oral (OGTT) and intraperitoneal (IGTT) glucose tolerance tests $(1 \mathrm{~g} / \mathrm{Kg})$ were performed in wild type Wistar rats and C57BL/6 mice. Studies of rat Jejunal transmural transport and uptake of $\mathrm{D}-\left({ }^{14} \mathrm{C}\right)$-glucose were performed as well as Western blotting.

Results: After a bolus of glucose without (control) or with 1nM RELM $\beta$, blood glucose level of mice or rats was significantly $(\mathrm{p}<0.05)$ higher with RELM $\beta$ versus control. Mice receiving an intraperitoneal injection (IP) of $55 \mu \mathrm{g} / \mathrm{Kg}$ RELM $\beta$ or not (control) had similar glucose level during OGTT or IGTT. The addition of $1 \mathrm{nM}$ RELM $\beta$ increased $30 \mathrm{mM}$ glucose transmural transfer by approximately 2 -fold as compared to control. Such a potentiating effect of RELM $\beta$ on sugar transfer was significantly inhibited by 50 to $60 \%$ in the presence of either an intracellular traffic inhibitor, cytochalasin $\mathrm{B}(20 \mu \mathrm{M})$ or an AMPK inhibitor, compound C $(10 \mu \mathrm{M})$ or a PKC inhibitor; chelerythrine chloride $(1 \mu \mathrm{M})$. In agreement with these results, stimulation of rat intestinal glucose uptake by luminal RELM $\beta$ was respectively associated with recruitment of GLUT-2 to the brush-border membrane and phosphorylation of PKC-BII, AMPK.

Conclusion: The gut hormone RELM $\beta$ can directly affect intestinal sugar transport at the luminal site with a likely involvement of GLUT-2, and AMPK, PKC activation.

Supported by: INSERM and Nestlé France 


\section{0}

Regulation of intestinal sugar absorption by GLUT2 in healthy and insulin resistant mice

E. Brot-Laroche ${ }^{1}$, M. LeGall ${ }^{1}$, V. Tobin ${ }^{1}$, X. Fioramonti ${ }^{1}$, E. Stolarczyk ${ }^{1}$, A.G. Blazquez ${ }^{2}$, P. Serradas ${ }^{1}$, C. Magnan ${ }^{3}$, A. Leturque ${ }^{1}$

${ }^{1}$ Centre de Recherche des Cordeliers, INSERM UMRS 872, Paris, France,

${ }^{2}$ CIBERehd, University Salamanca, Spain, ${ }^{3}$ Université Paris 7, UMR 7059

CNRS, France

Background and aims: Dietary manipulations in mice revealed regulations of intestinal sugar absorption at the level of transporter gene expression. Intestinal sugar transport is mediated by SGLT1 and by GLUT5 in the brush border membrane (BBM) of enterocytes and by basolateral GLUT2. A transient translocation of GLUT2 into the BBM in response to sugar ingestion occurs to complement SGLT1 and GLUT5 transport. Sugar absorption then promotes insulin secretion. The aim of the study was to investigate the role of insulin on GLUT2 trafficking in enterocytes. Insulin effects were analysed in healthy and insulin resistant mice.

Materials and methods: Mice fed a standard chow diet were compared to mice fed high fructose (65\%) or high fat (72\%), both diets known to induce insulin resistance. Insulin sensitivities were measured in hyperinsulinemiceuglycaemic (HI-EG) clamp conditions (glucose infusion rates: control $=67$ \pm 4 ; high-fructose $=36 \pm 6$; high-fat $=7 \pm 4$ mg.kg-1.min-1). Compared to control, GLUT2 protein was increased by high-fructose but not by low-sugar high-fat diet. Enterocyte membranes were sorted on density gradients (iodixanol; $10-30 \%$ ) and analysed by western blotting for GLUT2 content and appropriate BBM, basolateral and intracellular membrane markers.

Results: Insulin decreased fructose uptake in jejunal rings of control mice. This was associated with a decrease in BBM GLUT2 and a mirror increase in internal membranes. Interestingly, the injection of insulin to control mice prior an oral fructose or glucose load prevented GLUT2 from inserting into the BMM as expected in response to dietary sugars. Consequently sugar absorption was reduced. Total GLUT2 was unaltered by insulin perfusion. In both type of insulin resistant mice, BBM GLUT2 was abnormally high in the basal state and could not be removed by HI-EG clamp. In addition, GLUT2 levels in basolateral membranes were reduced in high-fat fed mice in both basal and clamp conditions. This could explain why initial rates of glucose absorption in mice fed a high-fat diet were lower than that measured in fructose-fed mice despite increased apical GLUT2 location in both groups.

Conclusion: Insulin resistance in mice was characterized by a permanently high amount of GLUT2 in the BBM of enterocytes, then basolateral GLUT2 levels will determine the efficiency of dietary sugar absorption. Taken together, our results indicate that the rapid acceleration of sugar absorption by the transient translocation of GLUT2 into the BBM in response to dietary sugar is physiologically controlled down by insulin. The intestinal action of insulin by delayed sugar absorption might thus constitute a mechanism to limit the magnitude of glucose excursions in blood after a meal.

Supported by: INSERM, UPMC Paris 6 University, ATC Nutrition, ALFEDIAM/Merk-Lipha, Spanish Ministerio de Educacion y Ciencia, French Ministère de l'Enseignement Supérieur et de la Recherche

\section{1}

Diurnal variation in the incretin response to a meal challenge in humans O. Lindgren ${ }^{1}$, M. Sörhede Winzell ${ }^{1}$, C. Deacon ${ }^{2}$, B. Ahrén ${ }^{1}$

${ }^{1}$ Department of clinical sciences, Lunds University, Sweden, ${ }^{2}$ Biomedical Sciences, Copenhagen Univeristy, Denmark

Background and aims: Glucose tolerance is better in the morning than in the evening. As the incretin effect is responsible for a substantial portion of insulin secretion after a meal, we investigated whether the diurnal variation in glucose tolerance may be explained by a similar diurnal variation in the release of incretins. To accomplish this we compared the incretin response to a standardized meal consumed in the morning $(8 \mathrm{am})$ and in the late afternoon $(5 \mathrm{pm})$ in young healthy males.

Materials and methods: The study was undertaken in healthy lean males $\left(\mathrm{n}=12\right.$; BMI $\left.20-25 \mathrm{~kg} / \mathrm{m}^{2}\right)$. After an overnight fast the subjects consumed a standard breakfast at 8.00 am and 5.00 pm respectively. The subjects were fasted between the test meals. Blood samples were collected before and up to 5 hours after the meal for measurement of glucose, insulin and total and intact glucagon-like peptide-1 (GLP-1) and glucose-dependent insulinotropic polypeptide (GIP).
Results: Baseline glucose was slightly lower in the afternoon compared to in the morning ( $4.3 \pm 0.1$ vs. $4.6 \pm 0.1 \mathrm{mM}, \mathrm{P}=0.0008$ ). Plasma glucose increased similarly after meal ingestion during the first $30 \mathrm{~min}$ with no difference between morning and afternoon. However, following the first $30 \mathrm{~min}$, glucose levels declined after the morning meal, whereas glucose continued to rise throughout a $60 \mathrm{~min}$ period in the afternoon meal. Thus, the mean glucose peak was reached earlier in the morning $(26 \pm 3 \mathrm{~min})$ than in the afternoon $(56 \pm 2, \mathrm{P}<0.0001)$ and the peak level was higher in the afternoon than in the morning ( $7.4 \pm 0.3$ vs. $6.1 \pm 0.2 \mathrm{mM}, \mathrm{P}=0.0015)$. Baseline insulin did not differ between morning and afternoon. There was however, a marked delay in the insulin response in the afternoon and insulin levels peaked later compared to the morning meal $(61 \pm 5$ vs. $30 \pm 2$ minutes, $\mathrm{P}=0.0005)$. The early $(0-30$ $\mathrm{min}$ ) insulin response was higher in the morningas evident by a higher insulinogenic index $(530 \pm 201$ vs. $128 \pm 31 \mathrm{pmol}$ insulin/mmol glucose at $5 \mathrm{pm}$, $\mathrm{P}=0.017$ ), whereas the total response was higher in the afternoon than in the morning(AUCins: $34.4 \pm 4.1$ vs. $24.8 \pm 3.3 \mathrm{nM}^{\star} \min , \mathrm{P}=0.011$ ), which was due to a prolonged increase throughout a $60 \mathrm{~min}$ period. Baseline iGLP-1 was significantly higher at 8 am compared to $5 \mathrm{pm}(6.7 \pm 2.8$ vs. $5.5 \pm 2.2 \mathrm{pM}$, $\mathrm{P}=0.0322$ ), while there was no difference in tGLP-1. There was an increase in GLP-1 levels after meal ingestion both in the morning and in the afternoon. The peak of tGLP- 1 after meal was higher in the morning ( $35 \pm 4$ vs $28 \pm 2 \mathrm{pmol} / \mathrm{l} ; \mathrm{P}=0.03)$ and the peak was reached faster $(24 \pm 3$ vs $46 \pm 3 \mathrm{~min}$; $\mathrm{P}=0.0005)$. There was however no statistical difference for iGLP-1, although at 20 minutes iGLP-1 was significantly higher in the morning $(12.2 \pm 2.7$ vs $9.2 \pm 2.1 \mathrm{pM}, \mathrm{P}=0.0342$ ). There was no difference in baseline values for neither tGIP nor iGIP between the morning and the afternoon meal.

tGIP peaked later in the afternoon ( $51 \pm 4$ vs. $34 \pm 2 \mathrm{~min}$ at $8 \mathrm{am}, \mathrm{P}=0.0020)$ but with no difference in peak height. iGIP also peaked later in the afternoon ( $43 \pm 4$ vs. $23 \pm 2 \mathrm{~min}$ at $8 \mathrm{am}, \mathrm{P}=0.0039$ ).

Conclusion: Glucose tolerance after a standardized meal is better in the morning than in the afternoon. This is associated with an increased early insulin response and increased GLP-1 secretion in the morning than in the afternoon. The results suggest that the diurnal variation in glucose tolerance can, at least partly, be explained by a similar diurnal variation in incretin release with a more abundant GLP-1 secretion in the morning than in the afternoon as the predominant mechanism.

Supported by: Swedish research Council; Faculty of Medicine, Lund University, Sweden; Region Skåne, Sweden 


\section{PS 043 Insulin action: liver and skeletal muscle}

\section{2}

Regulation of glucose metabolism in rat skeletal muscle cells by the nuclear orphan receptor Nur77

T. Kanzleiter, D. Wilks, E. Preston, N. Turner, G.J. Cooney

Diabetes and Obesity Research Program, Garvan Institute of Medical

Research, Darlinghurst, Sydney, Australia

Background and aims: Skeletal muscle accounts for $~ 50 \%$ of energy expenditure and is a major tissue for nutrient oxidation. Muscle plays a key role in insulin-stimulated postprandial glucose disposal, a process known to be impaired in Type 2 Diabetes. Therefore a detailed knowledge of the molecular mechanisms regulating substrate metabolism in skeletal muscle is of the utmost importance for the understanding of this disease. Recently it was suggested that the nuclear orphan receptor Nur77 is involved in the transcriptional regulation of several genes important for glucose and lipid metabolism in skeletal muscle. The aim of our study was therefore to investigate the functional consequences of Nur77 regulated changes in gene expression to glucose and lipid metabolism in skeletal muscle cells.

Materials and methods: L6 myotubes were used 7 days post differentiation. The myotubes were transfected with a Nur77 coding adenovirus or a GFP control virus. Two days post transfection the cells were incubated in the presence of ${ }^{14} \mathrm{C}$-glucose or ${ }^{14} \mathrm{C}$-oleate for 1 hour at $37^{\circ} \mathrm{C}$ to determine oxidation of these substrates. Medium was transferred to a sealed container and acidified to release ${ }^{14} \mathrm{CO} 2$. For ${ }^{14} \mathrm{C}$-glucose treated cells glycogen was precipitated and ${ }^{14} \mathrm{C}$-glucose incorporation into glycogen and total glycogen content were also measured. Statistical significance of differences between treatments was assessed by unpaired Student's t-test.

Results: Nur77mRNA expression was increased 77-fold two days after adenoviral transfection compared to control cells. There was no significant difference in fatty acid oxidation between Nur77 over-expressing cells (L6-Nur77) and control cells (L6-Con) ( $3.0 \pm 0.2$ vs. $3.4 \pm 0.2 \mathrm{nmoles}$ oleate/well/hour, respectively). However, basal glucose oxidation was significantly increased in L6-Nur77 cells ( $2.0 \pm 0.1$ vs. $1.5 \pm 0.2 \mathrm{nmoles}$ glucose/well/hour, $\mathrm{p}=0.014)$. An increase of a similar magnitude was also observed when the cells were insulin-stimulated ( $2.3 \pm 0.1$ vs. $1.7 \pm 0.1 \mathrm{nmoles}$ glucose/well/hour, $\mathrm{p}=0.005)$. The incorporation of $14 \mathrm{C}$-glucose into glycogen was also increased in L6-Nur77 cells in the presence and absence of insulin as compared to control cells (+Ins: $1.5 \pm 0.1$ vs. $1.1 \pm 0.03, \mathrm{p}=0.003$ and - Ins: $1.0 \pm 0.1$ vs. $0.8 \pm 0.06$ pmoles glucose/well/hour, $\mathrm{p}=0.003$, respectively). The total glycogen content was not significantly different between L6-Nur77 and control cells (+Ins: $2.4 \pm 0.03$ vs. $2.3 \pm 0.04$ and -Ins: $2.3 \pm 0.05$ vs. $2.5 \pm 0.04 \mu$ moles glucose).

Conclusion: Our data provide compelling evidence that the nuclear orphan receptor Nur77 is a regulator of skeletal muscle glucose metabolism. Nur77 overexpression augments two functional endpoints of glucose metabolism (glucose oxidation and glycogen synthesis) but has no effect on lipid oxidation. Interestingly, the observed increases in glucose metabolism were independent of the stimulatory effects of insulin, indicating that Nur77 may regulate pathways of glucose metabolism directly rather then insulin signalling mechanisms.

Suported by: Deutsche Forschungsgemeinschaft, Diabetes Australia Research Trust and NHMRC Australia

\section{3}

Regulatory mechanisms of AMP-activated protein kinase gene expression and protein level in young and old twins

B. Mortensen ${ }^{1}$, P. Poulsen ${ }^{1}$, R. Ribel-Madsen ${ }^{1}$, M. Friedrichsen ${ }^{1}$, J.B. Birk ${ }^{2}$, A. Vaag ${ }^{1}$, J. Wojtaszewski ${ }^{2}$

${ }^{1}$ Steno Diabetes centre, Gentofte, Denmark, ${ }^{2}$ Department of Exercise and Sport Sciences, University of Copenhagen, Molecular physiology group, Denmark

Background and aims: Some of the beneficial effects of exercise on insulin action and prevention of type 2 diabetes are currently suggested to be mediated by activation of AMP-activated protein kinase (AMPK). Recent studies suggest that antidiabetic drugs such as metformin acts through AMPK. These findings indicate that AMPK might be involved in development of insulin resistance, and might be a target candidate for treatment of type 2 diabetes.
We aimed to identify mechanisms involved in regulation of AMPK gene and protein expression and to identify the impact of AMPK protein levels on glucose metabolism.

Materials and methods: This cross-sectional study included young $(\mathrm{n}=100)$ and old $(n=82)$, non-diabetic twins. AMPK $\alpha 1, \alpha 2$ and $\gamma 3$ mRNA and protein levels were analyzed in skeletal muscle biopsies excised during the basal and insulin-stimulated state of a physiological hyperinsulinemic euglycemic clamp $\left(40 \mathrm{mU} / \mathrm{m}^{2} / \mathrm{min}\right)$. Gene expression levels were investigated using real time PCR with Cyclophilin-A as endogenus control. Protein levels were measured by Western blot technique.

Results: Males had higher expression of AMPK $\alpha 2$ mRNA (1.43 \pm 0.07 vs. $1.01 \pm 0.06 ; \mathrm{p}<0.0001)$, and AMPK $\gamma 3$ mRNA $(1.58 \pm 0.09$ vs. $0.81 \pm 0.06$; $\mathrm{p}<0.0001)$ and protein $(1374 \pm 44$ vs. $928 \pm 40$; $<<0.0001)$ compared to females. No influence of sex was demonstrated on AMPK $\alpha 1$ mRNA or protein levels. AMPK $\alpha 1$ or $\alpha 2 \mathrm{mRNA} /$ protein levels were similar in young and old subjects, whereas AMPK $\gamma 3$ expression was higher in young compared to elderly subjects, both on mRNA $(1.38 \pm 0.08$ vs. $0.92 \pm 0.05$; $\mathrm{p}<0.0001)$ and protein level $(1182 \pm 47$ vs. $1113 \pm 49 ; \mathrm{p}=0.0451)$. Comparison of intra-pair correlations on AMPK measures from monozygotic and dizygotic twins demonstrated that AMPK mRNA and protein levels were not under genetic influence. Multivariate regression analysis demonstrated that wholebody $\mathrm{VO}_{2 \max }$ was negatively related to AMPK $\gamma 3 \mathrm{mRNA}(\mathrm{p}=0.004)$ and protein expression ( $\mathrm{p}=0.001$ ), and total body fat was negatively related to AMPK $\alpha 2$ mRNA ( $p=0.03$ ) and AMPK $\gamma 3$ protein expression $(p=0.04)$. AMPK $\gamma 3$ protein level was negatively associated to insulin-stimulated glucose uptake $(\mathrm{p}=0.048)$ through the non-oxidative glucose metabolic pathway $(\mathrm{p}=0.0034)$, the latter association being independent of aerobic capacity and obesity. Conclusion: Skeletal muscle AMPK mRNA and protein levels are regulated by sex, age, obesity and aerobic capacity. AMPK $\gamma 3$ is associated with glucose metabolism through the non-oxidative glucose metabolic pathway. This association may mechanistically be linked through the ability of AMPK to down regulate glycogen synthase activity.

\section{4}

Regulation of gluconeogenic genes expression via resveratrol J.-M. Park ${ }^{1,2}$, T.-H. Kim ${ }^{1,2}$, J.-S. Bae ${ }^{2,3}$, M.-Y. Kim ${ }^{2,3}$, Y.-S. Park ${ }^{1,2}$, Y.-H. Ahn ${ }^{1,2}$

${ }^{1}$ Brain Korea 21 Project for Medical Sciences, ${ }^{2}$ Center for Chronic Metabolic Disease Research, ${ }^{3}$ Dept. of Biochemistry and Molecular Biology, Yonsei University College of Medicine, Seoul, Republic of Korea

Background and aims: Resveratrol (RSV), a naturally occurring polyphenol found in grape and red wine, is known as an activator of silent information regulator 2 (Sir2) which is a $\mathrm{NAD}^{+}$-dependent histone deacetylase. Recently, it has reported that expression of hepatic gluconeogenic genes, such as phosphoenolpyruvate carboxykinase (PEPCK) and glucose-6-phosphatase (G6Pase), are inhibited by insulin and the inhibition was relieved by RSV treatment. However the molecular mechanism of RSV action on the insulin-inhibition of gene expression is not well established yet. The forkhead transcription factor (FOXO1) is known as a transcriptional activator of genes involved in the gluconeogenesis. In the presence of insulin, FOXO1 is phosphorylated and exported from nucleus to cytosol, losing the ability to activate gene transcription. In this study, we demonstrate that RSV up-regulates the expression of gluconeogenic genes through deacetylation of FOXO1 by activating SIRT1.

Materials and methods: The mRNA level of gluconeogenic genes was measured in primary cultured hepatocytes of rat and mouse by real-time PCR. In the presence of insulin, the effect of activator or inhibitor of SIRT1 was studied using Western-blot analysis using primary cultured hepatocytes of rat. Also its effect was identified by observing the localization of FOXO1 and SIRT1 in Alexander cell lines by using immunofluorescence microscopy. The binding of FoxO1 to the promoter regions of gluconeogenic genes are tested by chromatin immunoprecipitation (CHIP) assay using primary cultured rat hepatocytes.

Results: Down-regulation of gluconeogenic gene by insulin was reversed by RSV in a dose-dependent manner and its effect was counteracted by Sirtinol. Insulin signaling pathway was affected by activator or inhibitor of SIRT1. RSV induced the localization of FOXO1 to nucleus, presumably by increasing its deacetylated form. As a result, FOXO1 may be able to bind to the promoter regions of gluconeogenic gene Indeed, CHIP assay showed that nuclear FOXO1 directly binds to the promoter and increases the mRNA level of gluconeogenic gene in the presence of insulin. 
Conclusion: SIRT1 is known to deacetylate the FOXO1 through direct interaction. In this study, we showed that SIRT1 activator, RSV, may increase the deacetylated form of FOXO1, a form which may be less phosphorylated by insulin and thereby localized to nucleus. As a result, the deacetylated FOXO1 binds to the promoter regions of gluconeogenic genes. This observation may help explain how RSV increases gluconeogenic gene expression which is suppressed by insulin.

Supported by: KOREA Science and Engineering Foundation grant funded by the KOREA government and Brain KOREA 21 project for Medical Sciences of Yonsei University College of Medicine

\section{5}

Tfe3 activates hepatic glucokinase gene expression at transcriptional level

M.-Y. Kim ${ }^{1,2}$, S.-S. Im ${ }^{3}$ T.-H. Kim², ${ }^{2,4}$ J.-S. Bae ${ }^{1,2}$, J.-M. Park ${ }^{2,4}$, Y.-S. Park ${ }^{2,4}$, Y.-H. Ahn ${ }^{2,4}$

${ }^{1}$ Biochemistry and Molecular Biology, Yonsei University College of Medicine, Seoul, Republic of Korea, ${ }^{2}$ Center for Chronic Metabolic Disease Research, Yonsei University College of Medicine, Seoul, Republic of Korea, ${ }^{3}$ Molecular Biology and Biochemistry, University of California, Irvine, United States, ${ }^{4}$ Brain Korea 21 Project for Medical Sciences, Yonsei University College of Medicine, Seoul, Republic of Korea

Background and aims: TFE3 (transcription factor E3) is a bHLH protein that is first identified and characterized in enhancer element in the immunoglobulin heavy-chain and $\mathrm{T}$ cell receptor gene. E-boxes, the cis-elements for bHLH protein binding, are located in the promoters of numerous genes involved in metabolism. Most bHLH proteins have been shown to be responsible for the gene expression involved in the metabolism. Previously it was reported that TFE3 activates hepatic IRS-2, an important component of insulin signaling. In this study, we demonstrate that glucokinase (Gck) gene, which is a key regulator of glycolysis in liver and pancreas is upregulated by TFE3 at the transcriptional level.

Materials and methods: Transcriptional activity of hGck promoter by TFE3 was measured by luciferase assay in HepG2 cells. In vitro translated TFE3 protein was used in EMSA to show the binding of TFE3 in hGck gene promoter. Chromatin immunoprecipitation (ChIP) was performed to show the binding of TFE3 to hGck promoter in vivo. mRNA level of Gck was measured by real time PCR in the primary cultured rat hepatocyte which is infected with TFE3 adenovirus.

Results: In silico search suggested that there are several E-boxed in the human Gck (hGck) promoter. Luciferase assay showed that TFE3 activates the hGck promoter activity by 20 fold. Introduction of mutation at the E-boxes resulted in the decrease the transcriptional activity. EMSA and ChIP assay showed that TFE3 binds directly to hGck promoter. Infection of TFE3-adenovirus to primary cultured hepatocytes increased endogeneous hGck mRNA level. The transcriptional activation of hGck by TFE3 was greater in hepatocyte maintained at low glucose media than in high glucose media.

Conclusion: We demonstrated that TFE3 activates the gene expression of Gck by direct binding on Gck promoter.

Supported by: Korea Science and Engineering Foundation grant funded by the Korea government and Brain Korea 21 project for Medical Sciences of Yonsei University College of Medicine

\section{6}

PET measurements of glucose uptake in the skeletal muscle and myocardium of Wistar and diabetic ZDF rats

C. Schölch, C. Hecker, P. Thomanek, C. Hertenberger, H.G. Niessen, D. Stiller

Boehringer Ingelheim Pharma GmbH \& Co. KG, Biberach an der Riss, German

Background and aims: Type 2 diabetes is a metabolic disorder characterized by hyperglycaemia, relative insulin deficiency and insulin resistance with abnormal glucose uptake and utilization. Here, the skeletal muscle is the major locus for insulin resistance, playing a key role in whole-body glucose homeostasis. Additionally, it has been demonstrated in rodents that the diabetic heart exhibits increased fatty acid oxidation that preceded reductions in carbohydrate oxidation. Rosiglitazone (RSG), a peroxisome proliferatoractivated-receptor- $\gamma$ (PPAR- $\gamma$ ) agonist, improves glucose tolerance and the sensitivity of peripheral tissues to insulin in various insulin-resistant states.
The aim of the study was to determine whether small-animal PET can be used to non-invasively measure substrate metabolism in skeletal muscle and myocardium and to quantify changes after RSG treatment.

Materials and methods: In our study, we used non-invasive small animal PET as a method to quantify insulin-stimulated $2-\left[{ }^{18} \mathrm{~F}\right]$ fluoro-2-deoxy-Dglucose (FDG) uptake in forearm skeletal muscle and myocardium of fed rats under Pentobarbital anaesthesia. Immediately after iv. injection of FDG into the tail vein, male Wistar and male diabetic ZDF rats had been treated iv. with different doses of insulin (Wistar rats: 0.01 and $0.05 \mathrm{IE} / \mathrm{kg}$ BW; ZDF rats: $0.05,1$, and $2 \mathrm{IE} / \mathrm{kg} \mathrm{BW}$ ) to stimulate muscle glucose uptake. In a subchronic experiment male obese ZDF rats have been treated for 14 days with RSG (15 $\mathrm{mg} / \mathrm{kg} /$ day). FDG uptake had been measured after stimulation with $1 \mathrm{IE} / \mathrm{kg}$ BW insulin using small animal PET on day 15. In all studies the glucose utilization was determined by Patlak analyses of PET data.

Results: In Wistar and ZDF rats an insulin-dose dependent increase in FDG uptake in the skeletal muscle tissue was observed. In ZDF rats higher insulin doses had to be used, reflecting their severe insulin resistance. However, despite using higher insulin concentrations, insulin stimulated myocardial FDG uptake was much higher in Wistar rats compared to ZDF rats. Treatment with RSG significantly decreased fasting blood glucose (-52\%) and HbAlc $(-0.7 \%)$ by the end of the treatment period in ZDF rats. This improvement of the diabetic state was accompanied by a significant increase in insulin stimulated FDG uptake in the skeletal muscle and also in the myocardium compared to untreated ZDF rats.

Conclusion: We show that non-invasive PET measurements of skeletal muscle substrate metabolism in ZDF rats are consistent with expected metabolic abnormalities that occur in this well characterized model of type 2 diabetes compared to normal Wistar rats. Furthermore, the results of our study are consistent with the well established early onset of cardiac substrate metabolic derangement in the ZDF rat. Treatment with RSG improved the diabetic situation, with an increased FDG uptake in skeletal muscle and interestingly also increased myocardial FDG uptake, therefore reflecting an overall improvement of the diabetic situation in the RSG treated rats. In summary, small animal PET is a suitable method to measure basal or treatment-induced changes in muscle and myocardium glucose utilisation with the advantage of repeated non-invasive measurements in one individual rat.

\section{7}

Regulation of insulin signalling by insulin degrading enzyme in HepG2 cell line

O. Pivovarova ${ }^{1,2}$, N.N. Rudovich ${ }^{1,2}$, A.F.H. Pfeiffer ${ }^{1,2}$

${ }^{1}$ Department of Clinical Nutrition, German Institute of Human Nutrition Potsdam-Rehbruecke, Nuthetal, ${ }^{2}$ Department of Endocrinology, Diabetes and Nutrition, Charité Unversity Medical School Berlin, Germany

Background and aims: Insulin-degrading enzyme (IDE) is thought to be the major enzyme responsible for insulin degradation. Evidence for a role of IDE in the pathogenesis of type 2 diabetes mellitus (T2DM) was obtained in animal models and in associative studies in some human populations, but the mechanism of this influence is poorly understood. The aim of present study was to investigate the role of IDE in insulin signaling in HepG2 human hepatoma cells.

Materials and methods: Inhibition of IDE (by bacitracin and specific siRNA) and IDE overexpression was used to study its function in insulin signaling. HepG2 cells were exposed to short-term (30,60 min, $100 \mathrm{nM})$ or long-term $(24 \mathrm{~h}, 10 \mathrm{nM})$ insulin treatment. For IDE inhibition experiments, bacitracin $(1 \mathrm{mg} / \mathrm{ml})$ was added to the cell medium $1 \mathrm{~h}$ before insulin treatment. For IDE silencing, HepG2 cells were transfected with two specific human IDE siRNAs $(80 \mathrm{nM})$. For IDE overexpression, cells were transfected with pCMV-hIDE vector. Insulin signaling activation after insulin treatment was assessed by western blotting (phospho-Akt, FOXO1), expression of insulin target genes - by real-time PCR (G6PC, PEPCK, FAS, ACC).

Results: In comparison to control cells, IDE protein level was decreased by up to $30 \%$ in IDE knockout cells and increased 1,5-2 fold by IDE overexpression. Inhibition of IDE, both with bacitracin and IDE siRNA, increased Akt phosphorylation by $15-25 \%$ and decreased FOXO1 levels by $20-35 \%$ after insulin treatment. Inhibition of IDE also elevated basal activity of Akt by $25 \%$ in cells without insulin stimulation. Moreover, in insulin-untreated IDE knockout cells, gluconeogenic gene expression (G6PC, PEPCK) was decreased by $35 \%$, whereas expression of lipogenic genes FAS and ACC was not significantly affected. IDE overexpression led to opposite effects that confirmed received data. The effects of IDE suppression or overexpression in insulin-untreated 
cells may be associated with recently described regulation of proteasome activity by IDE or other, as yet unknown, mechanism.

Conclusion: Alterations of IDE levels influence insulin signaling and gluconeogenic gene expression in HepG2 hepatoma cells. IDE appears to inhibit insulin signaling not only through the mechanism of insulin degradation, but also through some other mechanism, possibly through the regulation of proteasome activity. Thus, disturbances in the regulation of IDE expression and activity in liver may contribute in T2DM pathogenesis.

Supported by: grant from the German Ministry of Education and Science

\section{8}

Sex differences in human skeletal muscle metabolism

A. Rune, F. Salehzadeh, L. Al-Khalili

Molecular Medicine and Surgery, Karolinska Institutet, Stockholm, Sweden

Background and aims: Men and women appear to metabolize lipids differently. While men store fat in the abdominal area, women preferably store fat subcutaneously and around the hip area. Increased abdominal obesity is positively correlated with an increased incidence of type 2 diabetes. Resting fat oxidation is lower and intramuscular triglyceride storage is higher in women compared to men. During exercise women metabolize lipids to a higher degree than men. The aim of this study was to determine whether intrinsic sex differences influence glucose and lipid metabolism in human skeletal muscle.

Materials and methods: Human skeletal muscle cell cultures were established from vastus lateralis muscle biopsies obtained from 9 male and 9 female donors. The women were post-menopausal ( $60 \pm 1$ year) and age-matched to the men ( $61 \pm 2$ year). Subjects reported no known metabolic disorders. Cultures were grown in steroid-free serum, and differentiated to form myotubes. Glucose and lipid metabolism and mRNA expression of candidate genes was determined.

Results: Basal and insulin-stimulated glucose incorporation to glycogen was similar between cultures established from women and men. Basal glycogen synthesis was $8.0 \pm 1.4$ vs. $7.4 \pm 0.8 \mathrm{nmol} / \mathrm{mg}$ protein/h, for men vs. women, respectively. Insulin-stimulated glycogen synthesis was $18.7 \pm 3.1$ vs. $16.0 \pm 3.8$ $\mathrm{nmol} / \mathrm{mg}$ protein/h for men vs. women, respectively. Interestingly, cultures established from men had a higher basal rate of lipid oxidation, as compared to cultures established from women $(675 \pm 175$ vs. $361 \pm 70 \mathrm{CPM} / \mathrm{mg}$ protein $/ 120 \mathrm{~min}$, for men vs. women, respectively; $\mathrm{p}<0.05)$. Moreover, the magnitude of the insulin-induced suppression of lipid oxidation was greater in men compared to women. mRNA of expression of uncoupling protein (UCP) 2, UCP3, fatty acid binding protein 3, sterol regulatory element binding protein, carnitine palmitoyltransferase 1 , peroxisome proliferator-activated receptor (PPAR) $\gamma$ was similar between the sexes. Paradoxically, mRNA expression of PPAR $\delta$, a gene implicated in regulation of lipid oxidation, was 1.6-fold higher in muscle cultures established from women vs. men $(1.90 \pm 0.3$ vs. $1.18 \pm 0.1 \mathrm{AU}$ for women vs men, respectively; $\mathrm{p}=0.04$ ).

Conclusion: Glucose metabolism in primary human skeletal muscle cultures does not appear to be influenced by intrinsic sex differences. In contrast, basal lipid oxidation was higher in muscle cultures derived from male donors compared to female donors indicating lipid oxidation is influenced by sex differences between men and women. Intrinsic sex differences in skeletal muscle metabolism may account for alterations in whole body lipid metabolism between men and women

Supported by: Swedish Research Council, Novo-Nordisk Foundation, Karolinska Institute Centre for Gender Medicine, and EU

\section{PS 044 ROS and mitochondria: effects on insulin secretion and action}

\section{9}

Absence of CuZn superoxide dismutase (SOD 1) leads to glucose intolerance

G. Muscogiuri ${ }^{1}$, B. Balas ${ }^{1}$, S. Reyna ${ }^{1}$, A. Giaccari ${ }^{2}$, H. Van Remmen ${ }^{1}$, R. Defronzo ${ }^{1}, \mathrm{~N}$. Musi ${ }^{1}$

${ }^{1}$ Diabetes, University of Texas Health Science Center, San Antonio, United States, ${ }^{2}$ Endocrinology, Catholic University, Rome, Italy

Background and aims: Oxidative stress is thought to be involved in the pathogenesis of insulin resistance and type 2 diabetes mellitus (T2DM). CuZn superoxide dismutase (SOD 1) plays a critical role in scavenging superoxide anion production in the cytosol. We studied mice with whole body deletion of SOD 1 to test the hypothesis that increased oxidative stress (due to SOD 1 ablation) would impair insulin action.

Materials and methods: We performed $2 \mathrm{mg} / \mathrm{hg}$ I.P. glucose tolerance test in SOD 1 knock out $(\mathrm{KO})$ mice $(\mathrm{n}=11)$ and wild type (WT) mice $(\mathrm{n}=10)$. To determine wheter the glucose intolerance resulted from insulin resistance, whole body insulin sensitivity was measured with a $2 \mathrm{~h}$ euglycemic hyperinsulinemic $(18 \mathrm{mU} / \mathrm{kg} / \mathrm{min})$ with tritiated glucose $(\mathrm{n}=6$ per group). We determined also insulin secretion during a hyperglicemic clamp (+350 $\mathrm{mg} / \mathrm{dl})$ for 90 minutes.

Results: The body weight in KO mice was lower compared with the WT ( $20.3 \pm 4.2$ vs. $24.8 \pm 2.7 \mathrm{~g} ; \mathrm{p}<0.05)$. Fasting plasma glucose and insulin concentrations were similar in both groups. Ablation of SOD1 resulted in glucose intolerance during an intraperitoneal $(2 \mathrm{mg} / \mathrm{hg})$ glucose tolerance test $(\mathrm{AUC}=$ $28853 \pm 5831$ in WT vs. $36235 \pm 5065$ in KO mice; $\mathrm{p}=0.05$ ). No differences in peripheral (muscle) and hepatic insulin sensitivity were observed between $\mathrm{WT}$ and KO mice during $2 \mathrm{~h}$ euglicemic hyperinsulinemic clamp. During the hyperglicemic clamp, first phase (0-10 $\mathrm{min}$ ) insulin secretion was significantly reduced in $\mathrm{KO}$ mice (plasma insulin $\mathrm{AUC}=7566 \pm 2146$ in WT vs. $4324 \pm$ $410 \mathrm{mU} / \mathrm{ml} / \mathrm{min}$ in $\mathrm{KO} ; \mathrm{p}<0.05, \mathrm{n}=3$ per group). Second phase (10-90 min) insulin secretion tended to be reduced in KO mice (16238.82 2900.18 in KO vs. $20468.53 \pm 8805.628$ in WT; $\mathrm{p}=\mathrm{NS}$ ).

Conclusion: In summary increased oxidative stress due to SOD 1 ablation leads to glucose intolerance due to impaired beta cell insulin secretion without change in muscle or hepatic sensitivity to insulin. Oxidative stress in beta cells could be involved in the defective first phase insulin secretion observed in the early stages of T2DM.

Supported by: Nathan Shock Center of Excellence American Diabetes Association National Institutes of Health

\section{0}

Mitochondrial reactive oxygen species generation in obese non-diabetic and T2DM subjects

M. Abdul-Ghani, R. Jani, A. Chaves, M. Molina, D. Tripathy, R. DeFronzo University of Texas Health Science Center at San Antonio, San Antonio, United States

Background and aims: Increased oxidative stress has long been implicated in the pathogenesis of type 2 diabetes mellitus (T2DM) and its complications. However, no previous study has directly measured the rate of reactive oxygen species (ROS) production in insulin resistant individuals. Since insulin resistance in skeletal muscle is a central feature in T2DM, the aim of this study was to quantitate directly mitochondrial ROS production in skeletal muscle of obese, insulin resistant, normal glucose tolerant (NGT), and T2DM subjects and compared the result with those in lean healthy NGT individuals.

Materials and methods: Ten lean healthy NGT subjects (BMI $=26.0 \pm 0.9$, age $=40 \pm 2), 10$ obese NGT subjects $(\mathrm{BMI}=37.3 \pm 2.5$, age $40 \pm 3)$ and $9 \mathrm{~T} 2 \mathrm{DM}$ drug naïve subjects $(\mathrm{BMI}=38.8 \pm 2.4$, age $=43 \pm 3$ ) were studied with insulin clamp and vastus lateralis muscle biopsy. Mitochondrial ATP synthesis rate and ROS production were measured ex vivo in isolated muscle mitochondria with chemiluminescence and fluorescent techniques, respectively.

Results: Compared to lean NGT subjects, both obese and T2DM subjects had a reduced rate of ATP synthesis ( $58 \pm 4$ vs $39 \pm$ and $44 \pm 5 \mathrm{nmole} / \mathrm{mg}$ protein. min with glutamate $[\mathrm{p}<0.01]$, and $62 \pm 9$ vs $39 \pm 4$ and $42 \pm 6 \mathrm{nmole} / \mathrm{mg}$ 
protein.min with succinate, respectively $[\mathrm{P}<0.05]$ with ANOVA). However, the rate of ROS production with complex I and complex II substrates was decreased in obese vs lean subjects, $(85 \pm 21$ vs $247 \pm 58$ pmole $/ \mathrm{mg}$ protein.min with glutamate $[\mathrm{p}<0.002)]$ and $31 \pm 7$ vs $80 \pm 23$ with succinate $[\mathrm{p}<0.05])$. In contrast, in T2DM, ROS production from glutamate $(247 \pm 58)$ and succinate $(85 \pm 21)$ was similar to lean controls. ROS production in non-diabetic (lean and obese) subjects, with both substrates, strongly correlated with the rate of mitochondrial ATP production $(\mathrm{r}=0.71, \mathrm{p}<0.0009$ for glutamate and $\mathrm{r}=0.83$, $\mathrm{p}<0.0001$ for succinate) and with total glucose disposal measured with the euglycemic hyperinsulinemic clamp $(\mathrm{r}=0.74, \mathrm{p}<0.0004$ for glutamate and $\mathrm{r}=0.51, \mathrm{p}<0.02$ for succinate). The ATP/ROS ratio was 470,498 and 233 with glutamate and 1611, 1623 and 703 with succinate in lean, obese and T2DM subjects, respectively $(\mathrm{P}<0.02$ for glutamate and $\mathrm{P}<0.05$ with succinate with ANOVA).

Conclusion: (i) mitochondrial ATP production rate is reduced in both obese insulin resistant and T2DM subjects compared to lean NGT individuals; (ii) the decrease in ROS production in obese NGT subjects and "normal" ROS production in T2DM compared to lean NGT subjects argue against a significant role for excessive mitochondrial ROS production in the pathogenesis of insulin resistance in human skeletal muscle.

Supported by: NIH

\section{1}

Increased skeletal muscle phosphodiester contents of patients with type 2 diabetes mellitus relate to insulin resistance and impaired mitochondrial function

J.M. Szendroedi ${ }^{1}$, A.I. Schmid ${ }^{2}$, M. Chmelik ${ }^{2}$, P. Nowotny ${ }^{3}$, M. Wolzt ${ }^{3}$, M. Roden ${ }^{1}$

${ }^{1} 1$ st Medical Department, Hanusch Hospital, Karl-Landsteiner Institute for Endocrinology and Metabolism, ${ }^{2}$ Medical University of Vienna, MR centre of excellence, Vienna, ${ }^{3}$ University of Vienna, Department of Internal Medicine 3, Vienna, Austria

Background and aims: The membrane composition of skeletal muscle has been related to insulin action. Possible mechanisms suggested include changed membrane fluidity, which could affect insulin receptor activation, release of different second messenger molecules influencing cellular processes, and altered cellular energy expenditure influencing lipid stores. However, the factors determining muscle phosphodiester concentrations are yet unclear.

Materials and methods: Glucose metabolism, ectopic lipid deposition in muscle (IMCL) and mitochondrial function were measured in 10 overweight type- 2 diabetic patients (T2DM: age: $59 \pm 6$ a, BMI: $27 \pm 3 \mathrm{~kg} / \mathrm{m}^{2}$ ), 10 sex-/age/body mass-matched elderly (CONo: $57 \pm 6 \mathrm{a}, 26 \pm 3 \mathrm{~kg} / \mathrm{m}^{2}$ ) and 11 younger controls (CONy: $27 \pm 2$ a, $23 \pm 2 \mathrm{~kg} / \mathrm{m}^{2}$ ). Phosphodiester content (PDE), ATP synthetic flux (fATP) and IMCL were assessed with ${ }^{31} \mathrm{P} /{ }^{1} \mathrm{H}$ magnetic resonance spectroscopy, insulin sensitivity was determined via hyperinsulinemiceuglycemic clamp tests (M-value).

Results: PDE was $\sim 31 \%$ lower in CONy than in overweight CONo and T2DM (T2DM: $2.8+/-0.2$, CONo: $2.5+/-0.2$, CONy: $1.8+/-0.1 \mathrm{mmol} / 1, \mathrm{P}=0.002$ ) despite comparable muscle lipid contents. $\mathrm{M}$ was lower in diabetic patients ($35 \%$ vs. CONo, $-56 \%$ vs. CONy; $\mathrm{P}<0.05)$. T2DM had $~ 37 \%$ lower fATP than CONy (8.6 \pm 2 vs. $11.8 \pm 3 \mu$ mol. ${ }^{-1}$ muscle. $\mathrm{min}^{-1} ; \mathrm{P}<0.05$, CONo (10.4 \pm 3$)$. PDE related negatively to $\mathrm{M}(\mathrm{r}=-0.620, \mathrm{P}<0.001)$ and fATP $(\mathrm{r}=-0.371, \mathrm{P}<0.05)$, and positively to age $(\mathrm{r}=0.610, \mathrm{P}<0.001)$ and $\mathrm{BMI}(\mathrm{r}=0.672, \mathrm{P}<0.001)$ but did not relate to IMCL. HbAlc $(\mathrm{r}=0.672, \mathrm{P}<0.001)$ and fasting plasma glucose $(\mathrm{r}=0.614, \mathrm{P}<0.001)$ related positively to $\mathrm{PDE}$ over the entire study group and also within the group of T2DM patients.

Conclusion: PDE content of skeletal muscle strongly relates to insulin resistance, impairment of mitochondrial function and parameters of metabolic control, is increased in elderly and obese and does not depend on intramyocellular lipid contents.

Supported by: Austrian Science Foundation, Austrian National Bank, EFSD/ Novo Nordisk Type 2 Programme Grant, Herzfelder Family Trust, Hochschuljubiläumsstiftung Vienna and Novo Nordisk and Baxter to M.R.

\section{2}

Impaired macroautophagy as a cause of insulin resistance and mitochondrial dysfunction associated with aging

H. Jung ${ }^{1}$, S. Kim², N. Cho' ${ }^{2}$ J. Kim², M.-K. Lee², K.-W. Kim², H.-K. Lee, M.-S. Lee ${ }^{2}$

${ }^{1}$ Samsung Medical Center, ${ }^{2}$ Seoul National University Hospital, Seoul, Republic of Korea

Background and aims: Macroautophagy is a cellular process whereby cellular organelles including mitochondria are degraded. Macroautophagy has been reported to decline with aging, which may lead to decreased mitochondrial function. In the present study, we studied the role of macroautophagy in age-associated insulin resistance and mitochondrial dysfunction.

Materials and methods: Glucose tolerance test and insulin tolerance test were performed in young and old C57BL/6 mice. Insulin signaling in vivo was examined in the skeletal muscle and liver tissues by immunoblotting. Resting $\mathrm{O}_{2}$ consumption and mitochondrial complex activities were measured. Steady-state macroautophagy level was assessed by LC3 immunoblotting and electron microscopy. The relationship between macroautophagy, mitochondrial complex activity and insulin resistance was investigated using differentiated L6 myotubes.

Results: Old mice (> 18 months) showed glucose intolerance, hyperinsulinemia and increased HOMA index, suggesting insulin resistance. Insulin signaling, e.g., IRS1 and AKT phosphorylation after insulin administration in vivo was impaired in old mice. Mitochondrial complex I and II/III activities of muscle and liver tissues were decreased, potentially explaining insulin resistance in aged mice. Resting $\mathrm{O}_{2}$ consumption was also lower in old mice (Fig.1). Electron microscopy and LC3 immunoblotting showed that macroautophagy was impaired in old mice (Fig.2). The inhibition of macroautophagy in L6 myotubes induced mitochondrial dysfunction and impaired insulin signaling (Fig.3).

Conclusion: Mitochondrial complex activities and macroautophagy were decreased in the skeletal muscle and liver tissues of insulin-resistant old mice. As inhibition of macroautophagy in vitro induced mitochondrial dysfunction and impaired insulin signaling, decline of macroautophagy may be one of the important causes of age-associated mitochondrial dysfunction and insulin resistance.

\section{(A) Muscle}

Complex I

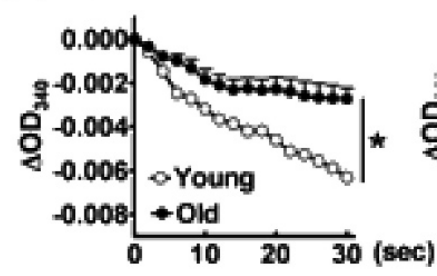

(B) Liver
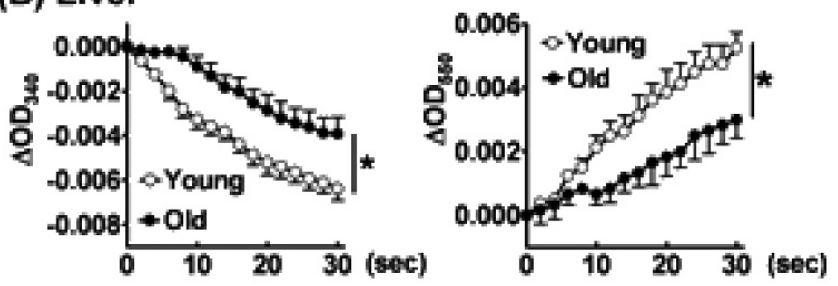

\section{(C) $\mathrm{O}_{2}$ consumption}

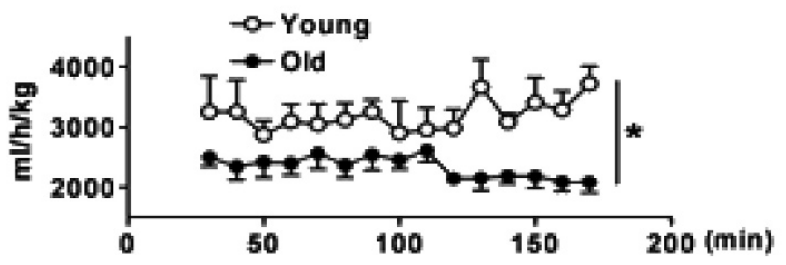

Figure 1. 


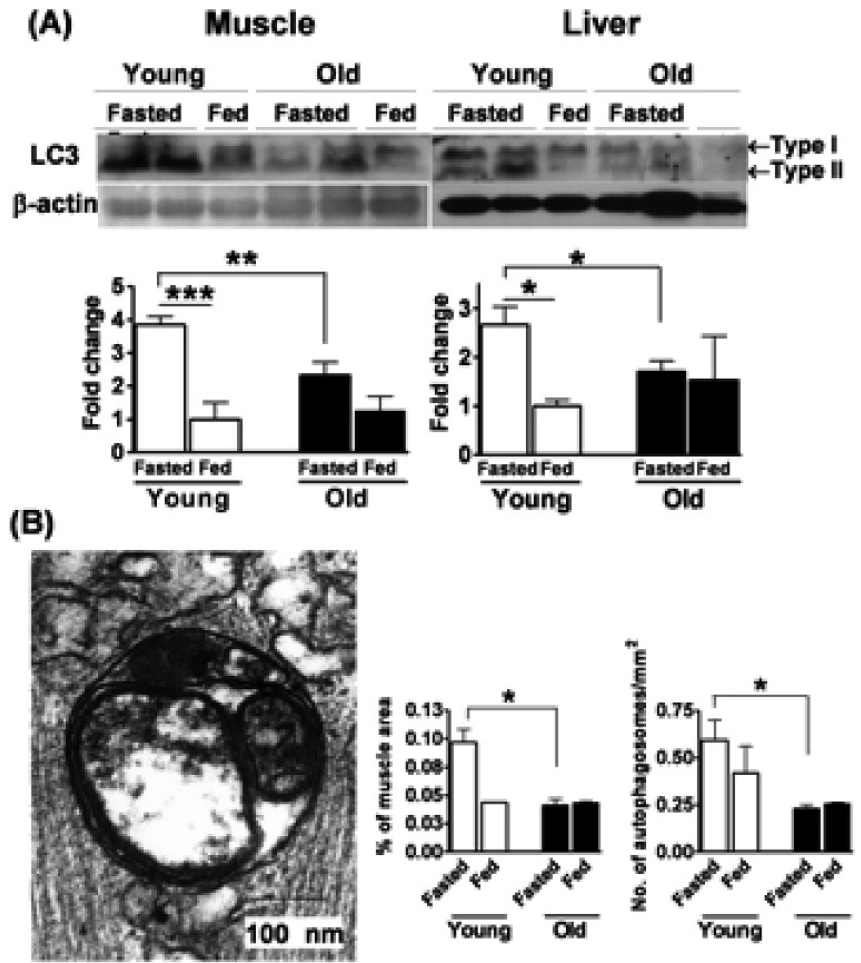

Figure 2.

\section{3}

A new type of insulin sensitiser, BGP-15 regulates mitochondrial function

A. Kolonics ${ }^{1}$, A. Cselenyak ${ }^{2}$, E. Kiss ${ }^{3}$, P.N. Literati ${ }^{4}, K^{2}$ Tory ${ }^{1}$

${ }^{1}$ Molecular pharmacology, N-Gene Research Laboratories, ${ }^{2}$ Human

Physiology and Clinical Experimental Research,, Semmelweis University, ${ }^{3}$ Immunology, Eotvos Lorand University, ${ }^{4}$ Molecular phamacology, N-Gene Research Laboratories, Budapest, Hungary

Background and aims: The insulin-sensitizing property of BGP-15 (R,SO-(3-piperidino-2-hydroxy-1-propyl)-nicotinic-acid-amidoxime) has been demonstrated in diabetic animal models and in insulin-resistant patients by hyperinsulinemic euglycemic clamp method (Diabetes 55 Suppl. 1. A483, 2006). JUN kinase mediated inhibition of insulin signaling is a common pathogenic mechanism in insulin resistance associated with obesity, inflammation and mitochondrial dysfunction. Inhibition of JUN kinase activation by the stimulation of stress protein (HSP72) expression has been demonstrated as a novel insulin sensitizing mechanism of BGP-15 (PNAS 105: 1739, 2008). We aimed to study the cellular mechanisms of insulin sensitizing effect of BGP-15 in insulin sensitive cell culture.

Materials and methods: The effect of BGP-15 was evaluated in insulin responsive human keratinocyte cell line (HaCaT) in hyperglycaemic condition. Glucose transport was measured by 2-deoxy-D-glucose uptake (2-DOG). Insulin signaling was characterized by phosphorylation level of IRS-1 (Tyr989) and Akt (Ser473). Laser Confocal Microscopy was applied for localization of actin, PIP-2 and BGP-15-Biotine conjugate. The effects of a 5-day BGP15 treatment were examined by measuring mitochondrial mass and insulin signaling in soleus of diabetic Gotoh-Kakizaki (GK) rats.

Results: Hyperglycaemia reduced insulin induced 2-DOG uptake and IRS-1 and Akt phosphorylation. BGP-15 treatment $\left(30^{\prime}, 24 \mathrm{~h}\right)$ significantly stimulated 2-DOG uptake but in contrast to insulin it resulted in marginal increase in IRS-1 and Akt phosphorylation in hyperglycaemic HaCaT cells. BGP-15 induced waves of PIP-2 formation and actin polymerization in 1 hour. Temporary elevation of mitochondrial 3-nitro-tyrosine level and phosphorylation of AMPK were also observed. 3-nitro-tyrosine derived partly from increased nitric oxide production. It was accompanied by increased citosolic appearance of constitutive nitric oxide synthase and HSP72. A 3-day BGP-15 treatment restored insulin induced phosphorylation of IRS-1 and improved mitochondrial mass in hyperglycaemic HaCaT cells. A 5-day BGP-15 treatment resulted in improved insulin sensitivity, insulin signaling and increased mitochondrial mass in soleus of Goto-Kakizaki diabetic rats. Biotine-BGP15/Streptavidine-FITC staining on fixed cells revealed the accumulation of the conjugated drug in mitochondria. Precipitation study with Biotine-BGP15/CaptAvidin agarose exposed mitochondrial Complex I (75 kDa subunit) binding.
(A)

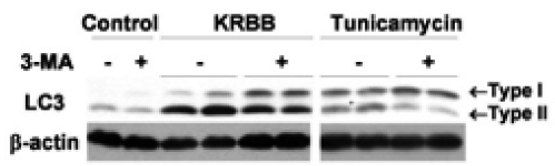

(B)
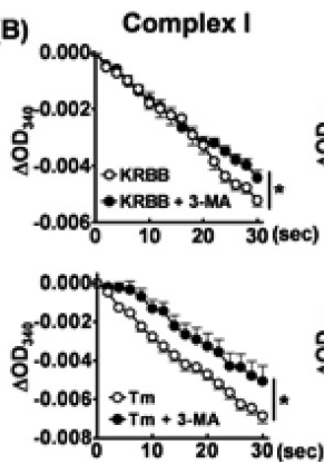

Complex II/III
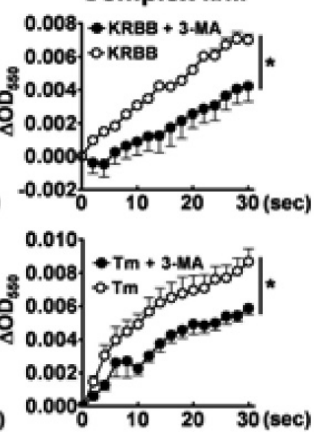

(C)
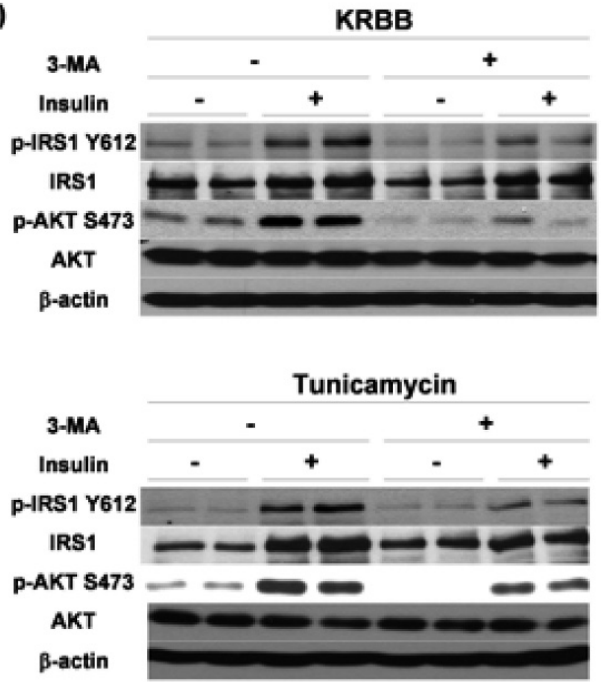

Figure 3.

Supported by: Nano/Bio Science Program Grant and 21C Frontier Functional

Proteomics Project 
Conclusion: These observations suggest that BGP-15 regulates mitochondrial function through nitric oxide production and binding to Complex I. BGP-15 induced cytoskeletal rearrangement and rapid chaperone release may be a secondary consequence. These changes may contribute to improved insulin sensitivity.

\section{4}

Mt2a is involved in TNFa induced insulin resistance

S. Dubois ${ }^{1}$, T. Connor ${ }^{2}$, N. Konstantopoulos ${ }^{2}$, C. Swinton ${ }^{2}$, K. Walder ${ }^{2}$

${ }^{1}$ Faculty of Medicine Lyon Sud, Inserm870/INRA1235, Oullins, France,

${ }^{2}$ Metabolic Research Unit, Deakin University, Geelong, Australia

Background and aims: The Adipose Tissue is now recognised as an endocrine/paracrine organ which can influence whole body metabolism not only through the release of non-esterified free fatty acids but also via many adipokines such as Tumor Necrosis Factor $a(\mathrm{TNF} \alpha)$. TNFa is a proinflammatory cytokine that has been implicated as a contributing cause of insulin resistance-eg reduced proteins levels of GLUT4 and several insulin signaling proteins; but although the effects of TNFa are well known, the mechanisms by which TNFa induces insulin resistance are not well understood. Our goal was to investigate potential new targets of TNF $\alpha$. One of them could be the Metallothionein 2a (Mt2a) which is part of the heavy metal-binding proteins family and has been shown to be regulated by cytokines such as TNFa. Mt2a polymorphims have been recently described and associated to type 2 diabetes and its complications. On the other hand, in type 2 diabetic patients, key factors regulating mitochondrial metabolism and biogenesis are reduced, notably the Complex I of the Electron Transport Chain (ETC). Our aim was to investigate the hypothesis that Mt2a and/or Complex I might be involved in TNFa induced insulin resistance.

Materials and methods: We used 3T3-L1 as an in vitro model of adipocytes in which insulin resistance (impaired insulin action) was induced by treating mature adipocytes with TNFa $(10 \mathrm{ng} / \mathrm{ml}, 72 \mathrm{~h})$. In order to further explore the potential role of Mt2a or Complex I in insulin resistance; we treated 3T3-L1 mature adipocytes with Mt2a protein (500ng, 24h) or the Complex I inhibitor (Rotenone, $200 \mathrm{nM}, 24 \mathrm{~h}$ ). Following treatments, insulin sensitivity was determined by 2-deoxyglucose uptake and RNA and proteins were extracted. Real-Time PCR of specific target genes was performed.

Results: Preliminary results show that insulin-stimulated glucose uptake was inhibited by $55 \%(\mathrm{p}<0.0001$ compared to vehicle, $\mathrm{n}=5)$ and by $53 \%(\mathrm{p}<0.001$ compared to vehicle, $n=5$ ) when 3T3-L1 adipocytes were treated with TNFa or Mt2a, respectively. Results of gene expression analysis show that Mt2a and Complex I (NduFa9) mRNA levels were significantly increase $(\mathrm{p}<0.001, \mathrm{n}=3)$ and decrease $(\mathrm{p}=0.01, \mathrm{n}=3)$, respectively following TNFa treatment. Key proteins involved in insulin signaling pathway such as AKT gene expression was unchanged with Mt2a treatment, but significantly decrease with Rotenone treatment $(p<0.01, n=3)$. Similarly, opposite results between treatments were observed for Protein Tyrosine Phosphatase 1B (PTP1B) gene expression that was increase with Mt2a treatment $(\mathrm{p}<0.01, \mathrm{n}=3)$ and unchanged with Rotenone treatment.

Conclusion: We conclude that Mt2a and ETC/Complex I might be involved in TNFa induced insulin resistance via an up/down regulation of several and different insulin signaling key proteins. These results raise the concept of considering metallothioneins proteins and notably Mt2a or Complex I (NduFa9) as potential therapeutic targets for type 2 diabetes. Future directions involved to measure insulin signaling specific genes protein level following Mt2a or Rotenone treatment.

\section{PS 045 Vascular effects of insulin}

\section{5}

High fat feeding induced loss of insulin-mediated vascular sensitivity in muscle

S. Rattigan, P. St-Pierre, A.J. Genders, L. Keith, M.A. Vincent,

S.M. Richards

Menzies Research Institute, University of Tasmania, Hobart, Australia

Background and aims: It has been widely reported that skeletal muscle glucose uptake is blunted in rats fed a high fat diet. We have demonstrated that Obese Zucker rats (a genetic model of obesity and insulin-resistance), have impaired insulin-mediated muscle microvascular recruitment, even at high doses of insulin $\left(20 \mathrm{mU} \cdot \mathrm{min}^{-1} \cdot \mathrm{kg}^{-1}\right)$. The effect high fat feeding has on insulin's effect to increase total blood flow and microvascular recruitment is unknown. Thus the aim of this study was to determine the effect of high fat feeding on the haemodynamic actions of insulin.

Materials and methods: Hooded Wistar rats were placed on a high fat diet ( $60 \%$ of calorie intake derived from fat) for 4 weeks or a normal chow diet. All rats were fasted overnight prior to euglycaemic hyperinsulinaemic clamps performed at two doses of insulin $\left(3 \mathrm{mU} \cdot \mathrm{min}^{-1} \cdot \mathrm{kg}^{-1} \mathrm{x} 2 \mathrm{~h}\right.$ and $10 \mathrm{mU} \cdot \mathrm{min}^{-1} \cdot \mathrm{kg}^{-1}$ $\mathrm{x} 2 \mathrm{~h}$ ). The jugular veins and the carotid artery were cannulated for intravenous infusions, arterial blood sampling and monitoring of arterial blood pressure. Femoral blood flow (FBF) was monitored by a probe positioned around the femoral artery. Microvascular recruitment was determined by the metabolism of infused 1-methyl xanthine.

Results: Compared to rats fed a normal diet (NF), high-fat fed animals (HFF) showed an increase in total body mass $(\mathrm{NF}=232.50 \pm 1.71 \mathrm{~g}$ vs $\mathrm{HFF}=248.21$ $\pm 2.60 \mathrm{~g}, \mathrm{p}<0.01)$ and in epididymal adipose tissue mass $(\mathrm{NF}=1376 \pm 66 \mathrm{mg}$ vs $\mathrm{HFF}=2755 \pm 146 \mathrm{mg} \mathrm{p}<0.01)$. High fat feeding induced a state of insulin resistance as seen by an increase in fasting blood glucose $(\mathrm{NF}=2.82 \pm 0.06 \mathrm{mM}$ vs $\mathrm{HFF}=3.52 \pm 0.09 \mathrm{mM}, \mathrm{p}<0.05)$ and plasma insulin $(\mathrm{NF}=73 \pm 11 \mathrm{pmol} / \mathrm{L}$ vs $\mathrm{HFF}=147 \pm 12 \mathrm{pmol} / \mathrm{L}, \mathrm{p}<0.05)$. High fat feeding had no effect on blood pressure or heart rate. Whole body insulin sensitivity reflected by the glucose infusion rate during the clamps was significantly lower in HFF compared to NF. The loss of insulin sensitivity was greater at $3 \mathrm{mU} \cdot \mathrm{min}^{-1} \cdot \mathrm{kg}^{-1}(\Delta-70 \%)$ compared to $10 \mathrm{mU} \cdot \mathrm{min}^{-1} \cdot \mathrm{kg}^{-1}(\Delta-20 \%)$. In HFF there was no change in FBF at $3 \mathrm{mU} \cdot \mathrm{min}^{-1} \cdot \mathrm{kg}^{-1}$ insulin $(\Delta+12 \%$, NS) but a significant increase with $10 \mathrm{mU}$. $\mathrm{min}^{-1} \cdot \mathrm{kg}^{-1}$ insulin $(\Delta+67 \%, \mathrm{p}<0.01)$, although this was blunted compared to the increase observed in the NF animal $(\Delta+142 \%$, NF vs HFF $p=0.11)$. HFF animals had impaired insulin-mediated microvascular recruitment but the effect was greater at the lower dose of insulin $\left(3 \mathrm{mU} \cdot \mathrm{min}^{-1} \cdot \mathrm{kg}^{-1} \mathrm{NF}=\Delta+48 \%\right.$ vs $\mathrm{HFF}=\Delta+15 \%, 10 \mathrm{mU} \cdot \mathrm{min}^{-1} \cdot \mathrm{kg}^{-1} \mathrm{NF}=\Delta+42 \%$ vs $\left.\mathrm{HFF}=\Delta+29 \%\right)$. Preliminary results examining the effect of muscle contraction using field stimulation indicated that at low intensity contraction, HFF also decreased the microvascular responses to contraction.

Conclusion: We conclude that high fat feeding causes marked impairment in microvascular responses in skeletal muscle, and is likely to increase the metabolic impact of high fat feeding and cause greater whole body insulin resistance by limiting the delivery of hormones and nutrients to muscle. However, in contrast to the complete lack of vascular responsiveness of the Zucker obese rat to insulin, the HFF rat showed a reduced sensitivity of both microvascular recruitment and muscle glucose uptake to insulin. This situation is akin to the inhibition of insulin-mediated microvascular recruitment by tumour necrosis factor-alpha, which can be overcome at higher doses of insulin.

Supported by: NHMRC Australia

\section{6}

Insulin increases matrix metalloproteinase-2 synthesis, secretion and activity in aortic vascular smooth muscle cells through the PI3-K pathway: effect reduced by insulin resistance

G. Doronzo, M. Viretto, I. Russo, P. Del Mese, L. Mattiello, G. Anfossi, M. Trovati

Department of Clinical and Biological Sciences, Diabetes Unit, San Luigi Gonzaga Hospital, Orbassano-Turin, Italy

Background and aims: Matrix metalloproteinases (MMPs) are zinc-dependent endopeptidases secreted as pro-enzymes, activated after specific cleavage and inhibited by specific tissue inhibitors (TIMPs). They degrade extracellular matrix and play a role in physiological and pathological angiogenesis, in the 
vascular smooth muscle cell (VSMC) migration from media to intima occurring in the formation of atherosclerotic plaques, in plaque instabilization via fibrous cup rupture and in the pathogenesis of aortic aneurysms. It has been described that insulin exerts pro-atherogenic effects via the MAPK-pathway and anti-atherogenic effects via the PI3-K pathway, which is less activated by insulin in the insulin-resistant states. Aim of the study is to clarify whether insulin influences in VSMC synthesis and activation of MMP-2, which is constitutively produced in these cells, and whether this putative effect is modified by the presence of insulin resistance.

Materials and methods: The study has been carried out in VSMC obtained as primary culture in our laboratory from aortas of insulin sensitive lean Zucker rats $(\mathrm{fa} /+)$ and insulin resistant obese Zucker rats $(\mathrm{fa} / \mathrm{fa})$, by measuring the influence of a 24-hour incubation with $2 \mathrm{nM}$ insulin on MMP-2 protein synthesis and secretion (western immunoblotting) $(\mathrm{n}=6)$, on TIMP-2 synthesis (western immunoblotting) ( $\mathrm{n}=6$ ) and on MMP-2 activity (gelatin zimography) $(\mathrm{n}=6)$, in the absence and in the presence of a 1-hour pre-incubation with the specific PI3-K inhibitor LY294002 $(50 \mu \mathrm{M})(\mathrm{n}=4)$. Results obtained after densitometric assays are expressed as mean \pm SEM.

Results: In aortic VSMC from lean Zucker rats, insulin 2nM: i)increased MMP-2 synthesis, secretion and activity $(\mathrm{p}=0.002-0.0001)$ by a PI3-K-dependent mechanism blunted by LY294002; ii)did not modify TIMP-2 synthesis. In aortic VSMC from obese Zucker rats, insulin increased synthesis, secretion and activity of MMP-2 ( $\mathrm{p}=0.05-0.004)$, but at an extent significantly lower than in VSMC from lean Zucker rats. In particular: i)synthesis of MMP-2 was $216.53 \pm 31.06 \%$ of baseline in lean vs $127.8 \pm 11.51 \%$ in obese Zucker rats ( $\mathrm{p}=0.002)$; ii)secretion of MMP- 2 was $166 \pm 11.62 \%$ of baseline in lean vs $109.4 \pm 2.82 \%$ in obese Zucker rats ( $\mathrm{p}=0.0001$ ); iii)activity of MMP2 was $271.8 \pm 40.6 \%$ of baseline in lean vs $123.8 \pm 10.6 \%$ in obese Zucker rats $(\mathrm{p}=0.013)$.

Conclusion: We observed that insulin increases MMP-2 synthesis, secretion and activity in aortic VSMC from lean, insulin-sensitive Zucker rats with a mechanism mediated by the PI3-K pathway and that this effect is attenuated in VSMC from obese, insulin resistant Zucker rats. MMPs are involved both in reparative vascular processes and in vascular damage: thus, an increase of their production and activity is not "good" or "bad" per se, but depends on the different settings. However, if we consider the role of MMPs in plaque formation and instabilization, our data support the conclusion that insulin exerts a pro-atherogenic effect via the signalling pathway usually considered anti-atherogenic, and that this pro-atherogenic insulin action is impaired in a classical animal model of insulin resistance. Thus, vascular insulin actions mediated by the PI3-K pathway are not necessarily "good", and their impairment in the insulin-resistant states is not necessarily "bad".

Supported by: grant of the Italian Ministry for University and Research

\section{7}

Insulin-mediated capillary recruitment in muscle is sensitive to intracerebroventricular NOS inhibitor

M.G. Clark, E.A. Bradley, K. Willson, D. Choi-Lundberg, S.M. Richards, S. Rattigan

Menzies Research Institute, University of Tasmania, Hobart, Australia

Background and aims: We have previously reported that insulin acts in vivo to increase capillary recruitment in hindlimb muscle of rats to enhance delivery and thus increase glucose uptake. In normally responsive rats these events are NO-dependent such that systemic administration of nitric oxide synthase (NOS) inhibitor increases blood pressure, blocks systemic insulin-mediated capillary recruitment and glucose uptake in muscle. In addition, others have reported that intra-cerebroventricular (ICV) administration of NOS inhibitor to conscious rats raises blood pressure, increases plasma glucose levels and reduces whole body glucose disposal during insulin clamp. Accordingly, the present study was conducted to test whether there was a NO-dependent pathway in the central nervous system involved in insulin-mediated muscle capillary recruitment and glucose uptake.

Materials and methods: Anaesthetized rats underwent standard surgery for a hyperinsulinaemic euglycaemic clamp and then were placed in a Kopf stereotactic apparatus for insertion of an ICV cannula into the lateral ventricle. Animals were allowed to stabilize for $60 \mathrm{~min}$ and then underwent an insulin clamp $\left(10 \mathrm{mU} \cdot \mathrm{min}^{-1} \cdot \mathrm{kg}^{-1}\right)$ for $70 \mathrm{~min}$ while still under anaesthesia. L-NMMA $(167 \mu \mathrm{g} / \mu \mathrm{l}$ in CSF) was infused ICV at $0.5 \mu \mathrm{l} / \mathrm{min}$ for two periods of $3 \mathrm{~min}$ at 0 and $30 \mathrm{~min}$ of the clamp, or infused systemically (to simulate spillover from ICV) with insulin for 70 min so that the total amount infused by either route was $500 \mu \mathrm{g}$. Blood pressure (BP), heart rate (HR), femoral blood flow (FBF), glucose infusion rate to maintain euglycaemia (GIR), muscle glucose uptake (2-deoxyglucose, 2DG), capillary recruitment by 1-methylxanthine metabolism and plasma insulin were determined. The position of the ICV needle was confirmed by dye infusion and sectioning post-mortem.

Results: Systemic infusion of $500 \mu \mathrm{g}$ of L-NMMA with insulin was insufficient to affect BP, HR GIR, FBF, 2DG, capillary recruitment or plasma insulin when compared to insulin alone. However, ICV infusion of $500 \mu \mathrm{g}$ L-NMMA blocked insulin-mediated increases in FBF $(2.08 \pm 0.09$ to $1.57 \pm 0.16 \mathrm{ml} /$ $\mathrm{min}), 2 \mathrm{DG}\left(16.5 \pm 1.4\right.$ to $\left.9.8 \pm 0.4 \mu \mathrm{g} \cdot \mathrm{g}^{-1} \cdot \mathrm{min}^{-1}\right)$ and capillary recruitment $(10.3 \pm 0.4$ to $6.6 \pm 0.9 \mathrm{nmol} / \mathrm{min}$ ) (data are means $\pm \mathrm{SE}$ and for each change $\mathrm{P}<0.05, \mathrm{n}=5-8$ ). Plasma insulin was $2430 \pm 240$ (ICV L-NMMA) and 2060 $\pm 150 \mathrm{pM}$ (systemic L-NMMA). HR and BP were unaffected at this dose of L-NMMA given ICV but higher doses increased BP.

Conclusion: The present findings indicate that the effects of systemic insulin to increase femoral arterial blood flow and capillary recruitment may be regulated by a central pathway that is sensitive to the nitric oxide synthase inhibitor L-NMMA. We propose that a central NO-dependent vasomotor neuronal pathway is involved in insulin action to increase muscle capillary recruitment and thus glucose uptake.

Supported by: NHMRC Australia and NIH USA

\section{8}

Zaprinast acutely augments insulin-mediated capillary recruitment and glucose disposal in rats in vivo

A.J. Genders, E.A. Bradley, S.M. Richards, M.G. Clark, S. Rattigan Menzies Research Institute, University of Tasmania, Hobart, Australia

Background and aims: Insulin increases glucose uptake into muscle in vivo in rats and humans by increasing delivery of hormones and nutrients through capillary recruitment. In addition, insulin-mediated capillary recruitment and vasodilatation has been thought to involve endothelial production of nitric oxide which then migrates to neighbouring smooth muscle cells to activate guanylate cyclase and produce cyclic GMP (cGMP). However, cGMP is degraded by a class of enzymes, the phosphodiesterases (PDEs) and inhibitors of these can amplify nitric oxide signalling by decreasing the degradation of cGMP. Thus PDE inhibitors have the potential for increasing capillary recruitment and enhancing insulin action. Accordingly, the aim of this study was to investigate the effect of the cGMP PDE inhibitor Zaprinast on insulinmediated capillary recruitment and skeletal muscle glucose uptake.

Materials and methods: Hooded-Wistar rats were anesthetized and had cannulas surgically implanted into their carotid artery, for arterial blood glucose (BG) sampling and measurement of blood pressure (BP), and the jugular vein for intravenous infusions. A flow probe was positioned around the femoral artery for continual measurement of femoral blood flow (FBF) and heart rate (HR). The euglycaemic insulin-clamp $\left(3 \mathrm{mU} \cdot \mathrm{min}^{-1} \cdot \mathrm{kg}^{-1}\right)$ was used to assess whether a dose of Zaprinast (1.6mg bolus followed by a $10 \mu \mathrm{g} \cdot \mathrm{min}^{-1} \cdot \mathrm{kg}^{-1}$ infusion for the duration of the experiment) enhances insulin-mediated glucose uptake. Four experimental groups were studied; saline, insulin, saline + Zaprinast, insulin + Zaprinast. Arterial glucose and lactate, glucose infusion rate (GIR), whole body glucose appearance and disappearance (Ra and Rd by ${ }^{3} \mathrm{H}$-glucose tracer techniques), capillary recruitment by metabolism of infused 1-methylxanthine(1-MX), plasma levels of Zaprinast and 1-MX were determined.

Results: BG, BP and HR were not significantly different between the four treatment groups. Plasma Zaprinast concentration was $30 \pm 2.7 \mu \mathrm{M}$ (Zaprinast) and $25 \pm 1.6 \mu \mathrm{M}$ (Zaprinast + insulin). Zaprinast treatment alone resulted in a significant $(P<0.05, \mathrm{n}=8)$ increase in capillary recruitment, $\mathrm{Rd}$ and arterial lactate concentrations. Zaprinast treatment during the insulinclamp significantly $(\mathrm{P}<0.05)$ increased capillary recruitment, Rd, GIR and plasma lactate.

Conclusion: The main findings indicate that effects of acute infusion of Zaprinast in vivo are to potentiate insulin-mediated capillary recruitment, $\mathrm{Rd}$ and GIR. We propose that a Zaprinast-sensitive cGMP PDE specifically expressed in arterioles controlling blood flow entry to capillaries can be targeted to increase insulin action to recruit capillaries in muscle and therefore improve whole body insulin-mediated glucose disposal in vivo. Supported by: NHMRC Australia 


\section{9}

In vivo chronic hyperglycaemia induces endothelial insulin-resistance P. Di Fulvio' ${ }^{1}$, A. Pandolfi', S. Di Silvestre ${ }^{2}$, P. Di Tomo², A. Giardinelli², N. Di Pietro ${ }^{2}$, G. Formoso ${ }^{1}$, M. Marchisio ${ }^{3}$, A. Consoli ${ }^{1}$

${ }^{1}$ Medicine and Aging Sciences, ${ }^{2}$ Biomedical Sciences, ${ }^{3}$ Biomorphology,

G.d'Annunzio University, Chieti, Italy

Background and aims: Endothelium is an insulin target organ and endothelial insulin-resistance is thought to play a pivotal role for the increased cardiovascular risk associated to metabolic syndrome and type 2 diabetes. Hyperglycaemia, through glucotoxicity, has been shown to induce insulinresistance at liver, skeletal muscle and adipose tissue site. Aim of our study was to determine whether chronic in vivo exposure to hyperglycaemia can induce insulin-resistance also in endothelial cells.

Materials and methods: We studied human tissues and endothelial cells (HUVEC) obtained from umbilical cords of women with Gestational Diabetes (H-GD) and women with normal carbohydrates tolerance (H-CT). In HUVEC cultured at low number of passages we studied NO production (conversion of $3 \mathrm{H}$-arginin in $3 \mathrm{H}$-citrulline) and Akt phosphorylation (Western blot) in the basal state and after 10 minutes stimulation with insulin (100 $\mathrm{nM}$ ); eNOS expression (Western blot) in the basal state and after $24 \mathrm{hrs}$ of stimulation with insulin $(100 \mathrm{nM})$; and, only in the basal state, phosphorylation levels of Protein Kinase-C alpha (PKCa, Western blot), an enzyme potentially implicated in insulin-resistance. PKCa phosphorylation levels were also evaluated ex vivo on umbilical cords sections (immunohistochemistry). Results: In H-CT, as expected, insulin stimulated NO production (+ $40 \%$ vs basal levels after 10 minutes stimulation), Akt phosphorylation ( $+35 \%$ vs basal levels after 10 minutes stimulation) and eNOS expression ( $+53 \%$ vs basal after a 24 hrs stimulation), in H-GD, instead, insulin failed to affect these parameters, which were not different from basal levels after insulin stimulation. Moreover, in H-GD, PKCa phosphorylation was increased up to $25 \%$ vs $\mathrm{H}$ CT. Immunohistochemical analysis of tissue samples confirmed an increase in PKCa phosphorylation levels in umbilical cords from gestational diabetic women vs control.

Conclusion: Our data demonstrate that chronic in vivo hyperglycaemia, probably via a chronic activation of PKC isoforms, induces insulin-resistance at the IRS1/PI-3-Kinase/Akt/eNOS pathway in endothelial cells. This allows to speculate that hyperglycaemia induced endothelial insulin-resistance could contribute to mechanisms by which hyperglycaemia causes vascular damage.

Supported by: CARONUT AP 2006-2008 to A. Pandolfi from MIPAF (Ministero delle Politiche Agricole e Forestali), EFSD/SERVIER Research Programme and by Italian Government Grant PRIN $2006 n^{\circ} 2006069102$ and Ministero della Istruzione, Università e Ricerca

\section{0}

Effect of ten days of bed rest on insulin action and endothelial function in subjects with and without increased risk of type 2 diabetes

M.P. Sonne ${ }^{1}$, L. Højbjerre ${ }^{1}$, A.C. Alibegovic ${ }^{2}$, B. Stallknecht ${ }^{1}$, A. Vaag ${ }^{2}$,

F. Dela ${ }^{1}$

${ }^{1}$ Department of Biomedical Sciences, Sections of Systems Biology Research, University of Copenhagen, ${ }^{2}$ Steno, Diabetes Center, Gentofte, Denmark

Background and aims: Sedentary lifestyle is a major and growing problem in the Western societies, contributing to lifestyle- related diseases such as type 2 diabetes and cardiovascular disease. We used bed rest as a model of physical inactivity to study the influence of physical inactivity on insulin action and endothelial function in a group of young healthy men with first degree relatives with type 2 diabetes (FDR) compared with matched controls (CON). Materials and methods: 20 CON (25 \pm 1 years, BMI $\left.24 \pm 0.5 \mathrm{~kg} \mathrm{~m}^{2-1}\right)$ and 13 FDR $\left(26 \pm\right.$ lyears, BMI $\left.25 \pm 1 \mathrm{~kg} \mathrm{~m}^{2-1}\right)$ participated in the study. Whole body insulin-mediated glucose uptake and forearm endothelial function was studied before and after 10 days of strict bed rest by intra arterial infusions of adenosine (Adn) and acetylcholine (Ach) in one arm while the other served as control, before and during a 3-hour hyperinsulinemic $\left(40 \mathrm{mU} / \mathrm{min} / \mathrm{m}^{2}\right)$ isoglycemic clamp. Forearm blood flow was measured by venous occlusion plethysmography simultaneously in both arms. Diet was controlled before and under the intervention.

Results: Both before and after bed rest the FDR were more insulin resistant compared with the CON (both $\mathrm{P}<0.05$ ). Insulin-stimulated glucose uptake decreased significantly in both groups in response to 10 days of bed rest but the decrease did not differ between the two groups $(\mathrm{P}>0.05)$. CON decreased from $7.2 \pm 0.5$ to $4.9 \pm 0.4 \mathrm{mg} \mathrm{min}^{-1} \mathrm{~kg}^{-1}(\mathrm{P}<0.001)$ and FDR decreased from $5.0 \pm 0.7$ to $3.6 \pm 0.3 \mathrm{mg} \mathrm{min}^{-1} \mathrm{~kg}^{-1}$. Before bed rest systemic hyperinsulinemia

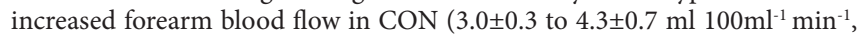
$\mathrm{P}<0.05)$, whereas in FDR no increase was observed. After bed rest hyperinsulinemia did not significantly increase forearm blood flow in either group. Before bed rest FDR demonstrated a significantly lower forearm blood flow response to Adn and Ach compared to CON. In both FDR and CON the ten days of bed rest did not change the forearm blood flow response to Adn and Ach + - - insulin-stimulation.

Conclusion: Short time physical inactivity induced whole body insulin resistance in both FDR and CON. The CON demonstrated impaired vascular insulin response after bed rest. The FDR demonstrated metabolic and vascular insulin resistance and endothelial dysfunction before bed rest compared with CON. Surprisingly the vascular responsiveness to Adn and Ach was unaffected after bed rest in both groups. It could be speculated that the FDR demonstrated a greater vulnerability to physical inactivity than CON since they after bed rest demonstrated a more severe degree of insulin resistance even though they were insulin resistant before hand.

Supported by: EU Framework VI EXGENESIS 


\section{PS 046 Regulation of incretin secretion and action}

\section{1}

GLP-1 inhibits glucagon secretion by affecting several cellular mechanisms

Y.Z. De Marinis ${ }^{1}$, Q. Zhang ${ }^{2}$, A. Salehi ${ }^{1}$, L. Eliasson ${ }^{1}$, P. Rorsman ${ }^{2}$ ${ }^{1}$ Lund University Diabetes Centre, Dept of Clincal Sciences Malmö, Sweden, ${ }^{2}$ Oxford Centre for study of Diabetes, Endocrinology, and Metabolism, University of Oxford, United Kingdom

Background and aims: Glucagon secretion is regulated by several agonists and antagonists and is dependent on the inactivation of voltage-dependent $\mathrm{Na}^{+}$-channels and influx of $\mathrm{Ca}^{2+}$ through $\mathrm{N}$-and L-type $\mathrm{Ca}^{2+}$-channels, The incretin hormone GLP-1 inhibits glucagon secretion whereas it stimulates insulin secretion, making it an interesting candidate for new treatment of type 2 diabetes mellitus. The aim of this study was to investigate the cellular mechanism whereby GLP-1 inhibits whereas other cAMP-increasing agents, such as adrenaline, stimulate glucagon secretion.

Materials and methods: Glucagon secretion was measured on freshly isolated mouse islets and analysed using radioimmunoassay (RIA). The electrical properties of the A-cells such as ion channel currents, electrical activity and changes in membrane capacitance (to determine the exocytotic response) was measured on single A-cells using the perforated patch configuration of the patch clamp technique.

Results: We first investigated how the ability of GLP-1 to inhibit glucagon secretion is associated with membrane potential by measuring glucagon secretion at $1 \mathrm{mM}$ glucose and increasing $\mathrm{K}^{+}$-concentration. At $4.5 \mathrm{mM} \mathrm{K}$ GLP-1 significantly inhibited glucagon secretion $(\mathrm{P}<0.001 ; \mathrm{n}=8)$. At $15 \mathrm{mM}$ K+ GLP-1 had no effect and at strongly depolarizing levels $(70 \mathrm{mM})$ a small stimulation $(\mathrm{P}<0.05 ; \mathrm{n}=8)$ was observed. However, at all $\mathrm{K}^{+}$concentrations, glucagon secretion was significantly stimulated $(\mathrm{P}<0.001)$ by other cAMPincreasing agents such as adrenaline $(5 \mu \mathrm{M})$ or forskolin $(10 \mu \mathrm{M})$. The inhibitory effect of GLP-1 was prevented by lowering extracellular $\mathrm{K}^{+}$to $1.5 \mathrm{mM}$. Low concentrations of forskolin $(10 \mathrm{nM})$ mimicked the inhibitory effect of GLP-1, suggesting that the inhibitory effect may be mediated by low cAMP levels whereas high concentrations stimulate release. In electrophysiological measurements on single A-cells, GLP-1 was found to reduce action potential amplitude from $37 \pm 2 \mathrm{mV}(\mathrm{n}=4)$ under control conditions to $18 \pm 4 \mathrm{mV}(\mathrm{n}=4$; $\mathrm{P}<0.017)$ but not inter spike time between the action potentials. Forskolin $(10$ $\mu \mathrm{M})$ did not affect the spike amplitude. At the level of exocytosis it was demonstrated, by measuring voltage-dependent changes in membrane capacitance, that forskolin, adrenaline and the CAMP-GEF2 agonist 8-Br-cAMPs all shift the voltage-dependence of exocytosis to a more negative potential, which do not occur in the presence of GLP-1. Thus, exocytosis occurs at much lower membrane potential than under control conditions using cAMP-increasing agents except for GLP-1.

Conclusion: We suggest that GLP-1 is unable to increase the cAMP-concentration in the A-cell to the same extent that other cAMP-increasing agents leading to inhibition of action potential amplitude, possibly via changing the inactivation properties of currents involved in A-cell action potential firing, and the inability to activate CAMP-GEF2 resulting in the observed failure to shift the voltage-dependence of exocytosis and reduced glucagon secretion. Supported by: Crafoord Foundation, the Swedish MRC and the Wellcome Trust

\section{2}

Near normalisation of blood glucose improves the secretion of amylin during GLP-1 potentiation of glucose-induced insulin secretion in patients with type 2 diabetes

M. Asmar ${ }^{1,2}$, P. Højberg ${ }^{2}$, J. Holst ${ }^{1}$, S. Madsbad ${ }^{2}$

${ }^{1}$ Department of Biomedical Sciences, University of Copenhagen,

${ }^{2}$ Department of Endocrinology, Hvidovre Hospital, Denmark

Background and aims: Amylin is a 37-amino acid polypeptide produced in the pancreatic beta-cells, co-stored with insulin in the secretory granules and co-secreted with insulin on glucose stimulation. In type 2 diabetes mellitus, circulating levels of amylin decrease as amyloid deposits accumulate and $\beta$ cell function deteriorates. This study was undertaken to determine whether 4 weeks of near normalisation of blood glucose (BG) improves the secretion of amylin during GLP-1 potentiation of glucose induced insulin secretion in type 2 diabetes mellitus.

Materials and methods: Nine obese patients with type 2 diabetes (T2DM) and poor glycaemic control ( $\mathrm{HbAlc} 8.0 \pm 0.4 \%$ ) were investigated before and after 4 weeks of near normalisation of BG using insulin treatment (mean BG $6.4 \pm 0.3 \mathrm{mmol} / \mathrm{l}, \mathrm{HbAlc} 6.6 \pm 0.3 \%$ ). Nine matched healthy subjects were also studied. Amylin and C-peptide secretion were investigated before and after insulin treatment on two days using stepwise glucose infusions and infusion of saline or GLP-1 (1.0 pmol/ $\mathrm{kg} / \mathrm{min})$, resulting in supraphysiological total GLP-1 concentrations of approximately $200 \mathrm{pmol} / \mathrm{l}$.

Results: In T2DM, the AUC under the amylin curves during saline infusion, before and after near normalisation of BG, were $638 \pm 103$ and $726 \pm 142 \mathrm{pM} \mathrm{x}$ min, p: NS. During GLP-1 infusion, the corresponding AUCs were 2450 and $4295 \mathrm{pM} x \mathrm{~min}, \mathrm{p}=0,004$. In the healthy subjects, the AUC was $4205 \pm 352 \mathrm{pM}$ $\mathrm{x}$ min during saline infusion and $7393 \pm 439 \mathrm{pM} x$ min during GLP-1 infusion, $\mathrm{p}=0.004$. The corresponding AUC under the C-peptide curves during the saline infusion in the T2DM patients before and after near-normalisation of BG were $124 \pm 19$ and $134 \pm 16 \mathrm{nM}$ x min, p: NS, and $268 \pm 48$ and $329 \pm 47$ $\mathrm{nMx} / \mathrm{min}, \mathrm{p}=0.02$ during GLP- 1 infusion. In the healthy subjects, the AUC was $267 \pm 33 / \mathrm{min}$ during saline infusion and $454 \pm 50 \mathrm{nM} \mathrm{x}$ min during GLP-1 infusion, $\mathrm{p}<0,001$. The ratios between TAUC amylin/TAUC C-peptide did not differ before and after insulin treatment in T2DM during saline infusion $(0.51 \%$ and $0.54 \%$, respectively; $\mathrm{p}=$ NS.) but a significant difference was seen during the GLP-1 days, before and after insulin treatment $(0.91 \%$ before and $1.3 \%$ after, $p=0.004$ ). The ratios were higher on both GLP-1 days than on the saline days. In the healthy subjects, the corresponding ratios were $0.93 \%$ during saline infusion and $2.7 \%$ during GLP-1 infusion, p: NS. No significant difference was seen in the ratio during the saline and GLP-1 days in the healthy participants and during the GLP-1 day after insulin treatment in the T2DM.

Conclusion: These data imply that 4 weeks of near normalisation of blood glucose and a supraphysiological dose of GLP-1 enhances beta cell function, as estimated from the high circulating levels of amylin.

Supported by: Novo Nordisk Foundation, the Danish Diabetes Association and the Danish Reasearch Agency

\section{3}

Insulin and exendin-4 stimulate proliferation of GLP-1-secreting cells C.B. Miller ${ }^{1}$, Q. Zhang ${ }^{1}$, Å. Sjöholm ${ }^{2}$

${ }^{1}$ Karolinska Institutet, Research center, ${ }^{2}$ Karolinska Institutet, Internal medicine, Stockholm South Hospital, Stockholm, Sweden

Background and aims: Glucagon-like peptide-1 (GLP-1) is an incretin hormone, secreted from gut L-cells upon nutrient intake, and forms the basis for novel drugs against type 2 diabetes. Secretion of GLP-1 is impaired in type 2 diabetes. Glucagon, secreted from pancreatic $\alpha$-cells, acts as the counterpart of insulin. Abnormalities in a-cell function contribute to hyperglycemia and insulin resistance in diabetes. Little is known about the influence of antidiabetic drugs on $\alpha$ - and L-cell function and regeneration. The aim of this study was to investigate the long term impact of anti-diabetic agents on the proliferation of GLP-1- and glucagon-secreting cells and mechanisms underlying such effects.

Materials and methods: The GLP-1-secreting cell line GLUTag and glucagon-secreting aTC1-9 cells were cultured in DMEM media. Cell proliferation was evaluated by ${ }^{3} \mathrm{H}$-thymidine incorporation into DNA and by MTT assay after a $48 \mathrm{~h}$ incubation with the anti-diabetic agents.

Results: At a basal concentration of glucose $(3 \mathrm{mM})$, insulin and the stable GLP-1 analogue Exendin-4 significantly increased proliferation of the GLP1-secreting GLUTag cells, while not influencing proliferation of the glucagon-producing aTC1-9 cells. In contrast, metformin significantly inhibited proliferation of both GLUTag and aTC1-9 cells. Effects of the drugs on hormone secretion by these cells are now being investigated.

Conclusion: The present study shows that insulin and Exendin- 4 significantly stimulate the proliferation of GLP-1-producing cells, whereas metformin does the opposite and also suppresses proliferation of glucagon-producing cells. The enhanced proliferation of GLUTag cells by Exendin- 4 also suggests that GLP-1, secreted from these cells, may stimulate cell growth through a positive autocrine/paracrine feedback loop. Since serum GLP-1 levels are decreased in type 2 diabetic patients, the mitogenic stimulation of GLP-1-producing cells by insulin and Exendin- 4 noted here suggests a novel beneficial long term effect of these antidiabetic drugs in clinical practice, i.e. by augmenting the incretin effect. Likewise, the inhibitory influence of metformin on the proliferation of glucagon-secreting cells may benefit diabetic patients 
treated with this drug, as relative hyperglucagonemia contributes to impaired glycemic control in type 2 diabetes.

\section{4}

Down regulation of GIP receptor by hyperlipidaemia: possible contribution to impaired incretin effects in type 2 diabetes

G. Xu' ${ }^{1}$, R. Fan ${ }^{1}$, X. Gu${ }^{1}$, X. $\mathrm{Li}^{1}$, K. Li ${ }^{1}$, R. Ma ${ }^{1}$, H. Zhao ${ }^{1}$, P. Tong ${ }^{1}$, J. Chan ${ }^{1}$, R. Laybutt ${ }^{2}$

${ }^{1}$ Medicine and Therapeutics, The Prince of Wales Hospital, The Chinese University of Hong Kong, Hong Kong SAR, China, ${ }^{2}$ Diabetes and Obesity Research Program, Garvan Institute of Medical Research, St Vincent's Hospital, Sydney, Australia

Background and aims: Stimulation of insulin secretion by the incretin hormone, glucose-dependent insulinotropic peptide (GIP) has been found to be diminished in type 2 diabetes. We hypothesized that this impairment is due to a defect at the receptor level induced by the diabetic state. We have shown that hyperglycemia decreased expression of incretin receptors in $\beta$-cells associated with impairments of insulin secretion. In the present study we aim to examine the role of hyperlipidemia in the regulation of GIP receptor (GIP R) expression in pancreatic $\beta$-cells.

Materials and methods: Two insulin-secreting $\beta$-cell lines, MIN6 and INS$1 \mathrm{E}$, were cultured in standard media supplemented with palmitate or oleate (both $0.4 \mathrm{mM}$ coupled to $0.92 \% \mathrm{BSA}$ ) and RNA were extracted for analysis of GIP receptor expression. For in vivo experiments, islets isolated from $d b / d b$ mice and pancreas from Zucker fatty rats were used to evaluate the expression of the incretin receptor.

Results: Gene expression levels of GIP R were significantly decreased in both insulin secreting cell lines after palmitate treatment for 24 hours, but were not changed with oleate treatment. $d b / d b$ mice displayed higher blood glucose, plasma NEFA, and plasma triglyceride levels at 16 weeks of age compared with age-matched $d b /+$ (control) mice. Islets from hyperlipidemic $d b / d b$ mice displayed a decrease in GIP R expression, with recovery when lipid levels were normalized by benzafibrate (Sigma), which selectively lowered plasma lipids. Hyperlipidemic Zucker fatty rats also displayed a decrease in GIP R protein expression in pancreatic $\beta$-cells by immunofluorescence staining with a GIP R specific antibody.

Conclusion: These data demonstrate that hyperlipidemia is associated with decreased GIP receptor expression and this decrease likely contributes to the impaired incretin effects found in diabetes.

\section{5}

GLP-1 is not a physiological mediator of glucose homeostasis in dogs B.A. Aulinger ${ }^{1}$, J. Heyl ${ }^{1}$, C.T. Elfers ${ }^{1}$, H.L. Rilo ${ }^{2}$, D.A. D’Alessio ${ }^{1}$

${ }^{1}$ Division of Endocrinology and Metabolism, ${ }^{2}$ Department of Surgery, University of Cincinnati, United States

Background and aims: Glucagon-like-peptide 1 (GLP-1) is a gut peptide that plays a key role in the regulation of glucose homeostasis. GLP-1 is highly conserved across mammalian species and interference with the action of this peptide causes glucose intolerance in mice, rats and humans. Exendin-4 (Ex4), an agonist of the GLP-1 receptor (GLP-1r), has become an effective drug for treatment of diabetes and acts primarily by enhanced insulin secretion, suppressed glucagon release and delayed gastric emptying in humans. Recent studies using canine models suggest that Ex-4 and GLP-1 maintain glucose homeostasis with only minimal changes of pancreatic hormone levels. The aim of this study was to determine the role of endogenous GLP-1 in postprandial glucose regulation in dogs.

Materials and methods: To test the effect of pharmacological levels of GLP$1 \mathrm{r}$ agonism Mongrel dogs weighing 11-14 kg underwent a mixed meal test (70 g sour cream with $25 \mathrm{~g}$ dextrose) after s.c. injection of either Ex-4 (10 $\mu \mathrm{g})$ or saline. For determination of gastric emptying $\mathrm{D}$-xylose was added to the meal. On the morning of the test, fasted dogs were brought to the procedure room, and peripheral catheters were placed for infusions and blood draws. Fifteen min after giving either one of the treatments the dogs were fed and samples were taken for determination of glucose, insulin and D-xylose for $180 \mathrm{~min}$ thereafter. To investigate the role of endogenous GLP-1 the same meal test was done during infusion of the GLP-1r antagonist Exendin-9 (Ex9) $(750 \mathrm{pmol} / \mathrm{kg} / \mathrm{min})$ or saline. In another set of experiments insulin secretion during a $20 \mathrm{~min}$ hyperglycemic squarewave was compared when either GLP-1 (3 pmol/ $\mathrm{kg} / \mathrm{min})$ or saline was given iv.
Results: Administration of Ex-4 before the meal resulted in profound blockade of gastric emptying measured by D-xylose appearance (AUC $22.0 \pm 4.3$ vs. $\left.77.9 \pm 3.2 \mathrm{mM}^{*} \min , \mathrm{p}<0.001\right)$. Postprandial blood glucose increased only moderately after Ex-4 treatment (basal 91, peak $103 \mathrm{mg} / \mathrm{dl}$ at $180 \mathrm{~min}$ ), and there was no rise in plasma insulin. Saline treated dogs had typical postprandial glucose (95 to $122 \mathrm{mg} / \mathrm{dl}$ at $90 \mathrm{~min}$ ) and insulin (35 to $131 \mathrm{pM}$ at 90 $\mathrm{min}$ ) profiles. Postprandial D-xylose, glucose or insulin levels did not differ between saline and Ex-9 treated animals, indicating that blockade of endogenous GLP-1 had no effect under these conditions. During fixed hyperglycemic conditions and either saline or GLP-1 infusion insulin levels did not differ, confirming a weak insulinotropic action of GLP-1 in dogs.

Conclusion: These findings suggest that dogs have a distinct pattern of GLP1 actions. Pharmacologic GLP-1r agonism with Ex-4 had a marked effect to reduce gastric emptying and prevent a glycemic excursion. The failure of Ex-4 to stimulate insulin secretion in this setting was likely due to near normal glycemia, but limited insulinotropism mediated through the GLP-1r of dogs was evident during application of a dose of GLP-1 that is that is highly potent in humans. Importantly, blockade of the GLP-1r with Ex-9 suggests that GLP-1 secreted after our test meal did not affect gastric emptying, insulin secretion or glucose tolerance. These findings raise questions as to the physiological role of GLP-1 in dogs and suggest considerable variations of the incretin system across different species.

Supported by: NIH - NIDDK

\section{6}

The twenty-four hour GIP responses are impaired in normoglycaemic men with the metabolic syndrome but not in men with type 2 diabetes D.L. Goede ${ }^{1}$, M.E. Tushuizen ${ }^{1}$, J.J. Holst ${ }^{2}$, R.J. Heine ${ }^{1}$, M. Diamant ${ }^{1}$ ${ }^{1}$ Endocrinology / Diabetes Centre, VU University Medical Centre, Amsterdam, Netherlands, ${ }^{2}$ Panum Institute, University of Copenhagen, Denmark

Background and aims: The incretins glucagon-like peptide (GLP)-1 and glucose-dependent insulinotropic polypeptide (GIP) are important in the regulation of the postprandial insulin response. Previous studies suggest that an impaired meal-induced GLP-1 response may contribute to the abnormal beta-cell secretion function as observed in patients with impaired glucose metabolism and type 2 diabetes mellitus (T2DM). However, the findings regarding GIP secretion in relation to T2DM and pre-diabetic states are as yet non conclusive. We studied the 24-h profiles of GLP-1 and GIP in males with the metabolic syndrome (MetS), in whom glucometabolic abnormalities were excluded by an oral glucose tolerance test (OGTT), in age- and BMImatched males with T2DM and healthy males.

Materials and methods: We monitored the 24-h profiles of plasma glucose, insulin, triglycerides (TG), GIP and GLP-1 in 18 T2DM (mean \pm SD age $56 \pm 5$ yrs, BMI $33 \pm 4 \mathrm{~kg} / \mathrm{m}^{2}$, HbAlc $7.0 \pm 1.0 \%$ ), 14 MetS (age $56 \pm 6$ yrs, BMI $31 \pm 3$ $\mathrm{kg} / \mathrm{m}^{2}, \mathrm{HbAlc} 5.9 \pm 0.4 \%$, fasting plasma glucose $5.5 \pm 0.4 \mathrm{mmol} / \mathrm{l}$, 2h-OGTT glucose $6.1 \pm 0.8 \mathrm{mmol} / \mathrm{l}$ ) and 18 healthy males. During day-time, subjects received three identical standardized high-fat mixed meals $(900 \mathrm{kcal} ; 50 \mathrm{~g}$ fat, $75 \mathrm{~g}$ carbohydrate, $35 \mathrm{~g}$ protein) as breakfast $(\mathrm{t}=0)$, lunch $(\mathrm{t}=4 \mathrm{~h})$ and dinner $(\mathrm{t}=8 \mathrm{~h})$, thus mimicking real-life situation. Blood samples were collected at 9 time-points throughout the 24 -h period, at preset intervals. Patients were treated with diet and/or metformin and/or sulphonylurea drugs.

Results: T2DM versus MetS males had higher HbA1c, FPG and HOMA-IR, but similar BMI, waist, blood pressure, fasting TG and insulin. As expected, plasma 24-h AUC glucose, insulin and TG were increased in T2DM relative to controls $(P<0.05)$, but 24 -h AUC TG and insulin were comparable with MetS ( $P=0.93$ and $P=0.56$, respectively). Twenty-four hour AUC glucose was similar for the MetS and control subjects $(P=0.38)$. Fasting incretins and 24h AUC GLP-1 did not differ among the 3 groups, whereas 24-h AUC GIP was significantly lower in MetS compared to T2DM and controls (1039 \pm 600 vs $1689 \pm 664$ and $1420 \pm 113$, respectively, $P=0.01$ ). No significant differences were detected for 24-h AUC GIP between T2DM and controls ( $P=0.17)$.

Conclusion: In well-controlled T2DM males, 24-h postprandial GLP-1 and GIP responses were comparable with healthy subjects, whereas in MetS without glucometabolic abnormalities, 24-h GIP secretion was significantly impaired. Since disruption of GIP signalling reportedly prevented diet-induced obesity in mice, the lower GIP secretion in men with the MetS may be an adaptation to insulin resistance and obesity. This hypothesis, however, needs to be confirmed in humans.

Supported by: Dutch Diabetes Foundation 


\section{7}

Reduced incretin effect in obese subjects with normal glucose tolerance as compared to lean control subjects

F.K. Knop ${ }^{1,2}$, K. Aaboe ${ }^{1}$, T. Vilsbøll ${ }^{1}$, S. Madsbad ${ }^{3}$, T. Krarup ${ }^{1}$, J.J. Holst ${ }^{2}$ ${ }^{1}$ Department of Internal Medicine F, Gentofte Hospital, ${ }^{2}$ Department of Biomedical Sciences, University of Copenhagen, ${ }^{3}$ Department of Endocrinology, Hvidovre Hospital, Copenhagen, Denmark

Background and aims: The incretin effect (the enhancement of glucose-induced insulin secretion after oral glucose tolerance test (OGTT) compared to isoglycaemic intravenous (iv) glucose infusion) is markedly reduced in patients with type 2 diabetes mellitus (T2DM). Data support the notion that this deficiency is a consequence of the diabetic state. However, the impact of insulin resistance on the incretin effect in obese individuals who uphold a normal glucose tolerance (NGT) despite their insulin resistant state remains to be elucidated.

Materials and methods: The incretin effect was measured over 2 days by means of 4-hour OGTT (50 g of glucose) and isoglycaemic iv glucose infusion in four groups of volunteers: 1$)$ Eight obese patients with T2DM $\left(\mathrm{HbA}_{1} \mathrm{c}\right.$ : $7.2(5.9-10.2) \%$ (mean(range)); BMI: $\left.37(35-41) \mathrm{kg} / \mathrm{m}^{2}\right) ; 2$ ) Eight lean patients with T2DM (HbA c: 6.8 (5.7-8.7)\%; BMI: $\left.\left.24(22-25) \mathrm{kg} / \mathrm{m}^{2}\right) ; 3\right)$ Eight obese subjects with NGT (HbA c: 5.6 (5.4-5.8)\%; BMI: $\left.33(35-38) \mathrm{kg} / \mathrm{m}^{2}\right)$; and 4) Eight lean healthy subjects ( $\mathrm{HbA}_{1} \mathrm{c}: 5.6$ (5.1-5.8)\%; BMI: $\left.23(20-25) \mathrm{kg} / \mathrm{m}^{2}\right)$. Results: Isoglycaemia during the two glucose administration forms was obtained in all 4 groups. In patients with T2DM insulin resistance according to the homeostasis model assessment (HOMA) amounted to $7.7 \pm 1.0$ (mean \pm SEM) (obese) and 2.2 \pm 0.6 (lean), $P<0.05$; and in subjects with NGT HOMA amounted to $3.2 \pm 0.6$ (obese) and $1.1 \pm 0.2$ (lean), $P<0.05$. As expected the incretin effect $\left(100 \% \times\left(\mathrm{AUC}_{\text {ins, OGTT }}-\mathrm{AUC}_{\text {ins }, \mathrm{i}}\right) / \mathrm{AUC}_{\text {ins,OGTT }}\right)$ was significantly $(P<0.05)$ reduced in patients with T2DM (obese: $7 \pm 7 \%$ (mean \pm SEM); lean: $29 \pm 8 \% ; P=\mathrm{NS}$ ) as compared to subjects with NGT (obese: $41 \pm 4 \%$; lean: $53 \pm 4 \%$; $P<0.05$ ).

Conclusion: Interestingly, as noted above, the incretin effect was significantly higher among lean insulin sensitive subjects with NGT as compared to obese insulin resistant subjects with equivalent glucose tolerance. This suggests that the incretin effect attenuates alongside the development of obesity-related insulin resistance thereby reflecting a very early pathophysiological trait in the development of T2DM.

Supported by: Danish Diabetes Association

\section{8}

Serum active ghrelin levels in hypothyroidism

N.B. Tutuncu ${ }^{1}$, M. Kulaksizoglu ${ }^{2}$, S. Kulaksizoglu ${ }^{3}$

${ }^{1}$ Endocrinology and Metabolism, Baskent University Faculty of Medicine, Ankara, ${ }^{2}$ Endocrinology and Metabolism, Baskent University Faculty of Medicine, Konya, ${ }^{3}$ Biochemistry, Baskent University Faculty of Medicine, Konya, Turkey

Background and aims: Ghrelin is a 28 aminoacid peptide predominantly produced by the stomach oxyntic-XA like cells. Ghrelin has been discovered as a natural ligand of the growth hormone receptor but has effects on energy metabolism, food intake, gastric acidity and motility also on pancreas function. Alterations in thyroid function are associated with a large variety of symptoms, including changes in body weight and food intake. Unexpectively, ghrelin levels in hyperthyroidism is found to be decreased. In the case of hypotyhroidism controversial results have been reported. The aim of this study is to evaluate ghrelin levels in hypothyroidism.

Materials and methods: 30 hypothyroid patients were evaluated with 23 age, sex, body mass index matched controls. Fasting plasma TSH, fT4, fT3, anti-thyroglobulin, anti-thyroidperoxidase, total-LDL-HDL cholesterol, triglyseride, c-reactive protein, gastrin, insulin, glucose and ghrelin levels were studied. Ghrelin was eveluated with Linco active ELISA KIT. Aprotinin SIGMA was added to samples. Samples were centrifugated at $+4{ }^{0} \mathrm{C} 1500 \mathrm{~g}$ for 15 minutes. They were kept $-40^{\circ} \mathrm{C}$ till the time for eveluation

Results: Ghrelin levels vere significantly elevated in the hypothyroid patients compared to the control group. After euthyroidism was achieved, ghrelin levels were found to be similar with the healthy controls. Ghrelin levels correlated negatively with fT3 and fT4 and positively with HDL levels.

Conclusion: Ghrelin could be the reason for weight gain and increased adiposity in hypothyroidism. Whether circulating ghrelin plays an important role in feeding behaviour and energy homeostasis is not still known. However, our results indicate that active ghrelin may have some critical actions in hypothyroidism induced weight gain. Further studies are needed to clarify the exact mechanism.

\section{PS 047 Animal models: altered glycaemia and insulin resistance}

\section{9}

Regulation of glycaemia in the absence of glucose production by the liver F. Rajas ${ }^{1,2}$, E. Mutel ${ }^{1,2}$, A. Stefanutti ${ }^{1,2}$, G. Mithieux ${ }^{1,2}$

${ }^{1}$ Unité 855, Inserm, ${ }^{2}$ Université Claude Bernard Lyon 1, IRF62, Lyon, France

Background and aims: Only three organs of the body - the liver, kidney and small intestine - have the capacity to release endogenous glucose, because they are the only tissues to express glucose-6-phosphatase (G6Pase). This enzyme catalyzes the last step common to glycogenolysis and gluconeogenesis, i.e., the hydrolysis of glucose-6-phosphate into glucose and inorganic phosphate. The inherited deficiency of the catalytic subunit (G6PC) of this enzyme (glycogen storage disease type 1a) underlies serious hypoglycaemic episodes in the post-absorptive period. The patients cannot use hepatic glycogen stores to produce glucose and develop hepatomegaly and steatosis. Up to now, hepatic glucose production is very often assimilated to endogenous glucose production. However, in some physiological states, renal and intestinal production of glucose might represent more than half of total production. In this work, we raised the question of the regulation of glycaemia in the absence of glucose production by the liver.

Materials and methods: We generated liver-specific G6Pase knockout mice (g6pc -/-) using an inducible CRE-lox strategy. G6Pase expression was analysed in the liver, kidney and intestine of wt $(\mathrm{g} 6 \mathrm{pc}+/+)$, heterozygote (g6pc $+/-)$ and g6pc -/- mice, ten days after induction of the liver g6pc deletion. Glycogen, G6P and triglyceride levels were determined from liver homogenates. The glycaemia of mice was followed during prolonged fasting (45h).

Results: Ten days after hepatic g6pc deficiency, G6Pase activity was inhibited by more than $95 \%$ in the liver of g6pc -/- mice compared to wt mice $(0.3 \pm 0.1$ vs $10.7 \pm 0.7 \mathrm{U} / \mathrm{g}$ of tissue, $\mathrm{p}<0.01$ ). The livers of $\mathrm{g} 6 \mathrm{pc}-/$ - mice rapidly accumulated glycogen $(23.9 \pm 1.2 \mathrm{vs} 10.1 \pm 0.8 \mathrm{mg} / \mathrm{g}$ of tissue in wt mice, $\mathrm{p}<0.01)$ and glucose-6-phosphate $(1.4 \pm 0.01$ vs $0.4 \pm 0.03 \mu \mathrm{mol} / \mathrm{g}$ in wt mice, $\mathrm{p}<0.01)$. These mice also developed hepatomegaly and liver steatosis, accumulating triglycerides $(23.4 \pm 1.4$ versus $8.4 \pm 1.1 \mathrm{mg} / \mathrm{g}$ of tissue in wt mice, $\mathrm{p}<0.01)$, after 5 weeks of hepatic G6Pase deficiency. It is worth noting that hepatic g6pc-/- mice were viable while mice with total invalidation of Glc6Pase die if not supplemented with glucose injection. Despite the absence of hepatic glucose production, the glycaemia of $\mathrm{g} 6 \mathrm{pc}-/$ - mice was similar to that of wild type mice in the fed state. During fasting ( 6 to $45 \mathrm{~h}$ ), the lowering of glycemia was slightly more rapid in g6pc-/- mice than in wt mice $(90 \pm 10 \mathrm{mg} / \mathrm{dL}$ vs $150 \pm 13 \mathrm{mg} / \mathrm{dL}$ at $6 \mathrm{~h}$ ), likely because of the lack of liver glycogenolysis. However, no significant difference was observed at a later time, between 12 and 45 $\mathrm{h}$ of fasting. This might be a consequence of the induction of an extra-hepatic glucose production. It was noteworthy that two groups of g6pc -/- mice could be distinguished. In the first group, mice exhibited an increase of intestinal G6Pase activity, by two fold ( $3.8 \pm 0.8$ vs $1.9 \pm 0.3 \mathrm{U} / \mathrm{g}$ of tissue in wt mice, $\mathrm{p}<0.01$ ), whereas renal G6Pase activity was unchanged (11.6 \pm 0.5 vs $12.2 \pm$ $0.5 \mathrm{U} / \mathrm{g}$ of tissue in wt mice). In the second group, mice exhibited an induction of renal G6Pase activity ( $17.3 \pm 1.1$ vs $12.2 \pm 0.5 \mathrm{U} / \mathrm{g}$ of tissue in wt mice, $\mathrm{p}<0.01$ ), whilst intestinal G6Pase activity was unchanged ( $1.4 \pm 0.2$ vs $1.9 \pm$ $0.3 \mathrm{U} / \mathrm{g}$ of tissue in wt mice).

Conclusion: These data suggest that: 1) glucose production by the liver is essential for the regulation of glycemia during the transition from the postprandial to the post-absorptive period; 2) glucose production by the kidney and the small intestine are sufficient for the maintenance of glycemia during fasting.

Supported by: Association Francophone des Glycogenoses and Agence Nationale de la Recherche-MRAR

\section{0}

ERK1 deficiency improved the insulin resistance of the obese $o b / o b$ mice J.-F. Tanti ${ }^{1}$, J. Jager ${ }^{1}$, V. Corcelle ${ }^{1}$, T. Grémeaux ${ }^{1}$, K. Laurent ${ }^{1}$, G. Pages ${ }^{2}$, J. Pouyssegur ${ }^{2}$, Y. Le Marchand-Brustel ${ }^{1}$, R. Burcelin ${ }^{3}$, F. Bost ${ }^{1}$ ${ }^{1}$ Inserm, INSERM U895, Nice, ${ }^{2}$ Cnrs, CNRS UMR 6543, Nice, ${ }^{3}$ Inserm, INSERM U 858, Toulouse, France

Background and aims: Insulin resistance is a main feature of obesity and type II diabetes. Activity of MAP Kinase family members, including ERK, is increased in adipose tissue from obese and type II diabetic animals and patients. Strong evidences suggest that activation of MAPK is implicated in the 
down-regulation of insulin signaling and action in insulin resistance state. We have shown that mice lacking ERK1 are resistant to high fat diet induced obesity and protected from insulin resistance due to a decrease in adipogenesis and a higher post-prandial metabolic rate. To investigate the role of ERK1 in the development of insulin resistance in obese mice, we generated leptindeficient mice (ob/ob) invalidated for ERK1 (ob/ob-erk1-/-).

Materials and methods: Ob/ob mice lacking erk1 (ob/ob erk1-/-) was generated by crossing ob/+erk1-/-. The mice were studied at 12 weeks of age. Insulin sensitivity was investigated by glucose tolerance test and hyperinsulinemic euglycemic clamp. Genes expression was measured by real-time PCR.

Results: We found that $o b / o b$-erk1-/- mice remained as obese as $o b / o b$ mice but their fed glycemia is markedly reduced ( ob/+ $160 \mathrm{mg} / \mathrm{dl}$, ob/ob $326 \mathrm{mg} /$ $\mathrm{dl}$, ob/ob erk1-/- 200mg/dl). Glucose tolerance test showed an improved insulin sensitivity of ob/ob-erk1-/- mice. Using the hyperinsulinemic euglycemic clamp, we showed that glucose infusion rate (GIR) is increased by $50 \%$ in ob/ob erk1-/- mice compared to ob/ob mice (26.29 vs $17.47 \mathrm{mg} / \mathrm{kg} / \mathrm{min}$ ) and that glucose uptake in muscles of ob/ob erk1-/- mice is increased and more specifically in glycolitic muscles. We found that expression of inflammatory markers (TNFalpha, PAI-1 and IL-1beta) and of lipogenic genes are decreased in visceral adipose tissue of ob/ob-erk1-/- mice. Further, the level of circulating free fatty acids measured at the end of the clamp is $35 \%$ lower in the ob/ob erk1-/- mice compared to the ob/ob mice (12.53 vs19.41 mg/ dl) suggesting that the anti-lipolytic effect of insulin in adipose tissue is improved. We also demonstrated that $o b / o b-e r k 1-/-$ are partially protected from liver steatosis with a reduction in liver triglycerides content $(\mathrm{ob} /+2.10, \mathrm{ob} / \mathrm{ob}$ 119.92 , ob/ob erk1-/- $64.04 \mathrm{mg} / \mathrm{g}$ of liver) and a reduction in acetyl-CoA carboxylase expression (ob/+ 1.16 AU, ob/ob 5.05 AU, ob/ob erk1-/- $3.75 \mathrm{AU})$. Conclusion: Our data suggest that the specific targeting of erk 1 could protect obese mice against insulin resistance and liver steatosis by improving adipose tissue inflammation and by an increase in muscles glucose uptake. New pharmacological strategy that target ERK pathway and more specifically ERK1 could thus be of particular interest to fight obesity and insulin resistance. Supported by: European Grant HEPADIP

\section{1}

High fat diet modifies the protective effects of adiponectin overexpression on streptozotocin-induced diabetes in transgenic mice S. Ait El Mkadem ${ }^{1}$, R. Attaoua ${ }^{1}$, S. Zouaoui ${ }^{1}$, L. Monnier ${ }^{1}$, S.M. Brichard ${ }^{2}$, F. Grigorescu ${ }^{1}$

${ }^{1}$ Molecular Endocrinology (EA4188), University of Montpellier 1, IURC, Montpellier, France, ${ }^{2}$ Endocrinology and Metabolism Unit, University of Louvain, Brussels, Belgium

Background and aims: Adiponectin $(\mathrm{ApN})$ is an adipokine which plays a crucial role in glucose homeostasis and activation of vascular endothelial cells. Indeed, its levels are decreased in insulin-resistant states such as type 2 diabetes and metabolic syndrome. We recently generated a transgenic (Tg) mouse model overexpressing full-length $\mathrm{ApN}$ and discovered that $\mathrm{Tg}$ mice on standard diet (STD) are protected from streptozotocin (STZ)-induced diabetes. In the hypothesis that this protection was linked to the insulin-sensitizing effects of ApN, this study was aimed to explore the effects of ApN on diabetes under conditions of high fat diet (HFD).

Materials and methods: $\mathrm{Tg}$ and wild type (WT) mice ( $\mathrm{n}=5-9 /$ group) were fed either a STD or a HFD and received multiple ip injections of low doses of STZ. Induction and duration of diabetes were followed until 8 weeks by measurements of glucose levels. Glucose intolerance was determined by ip glucose tolerance test (ipGTT) while insulin sensitivity was determined by insulin tolerance test (ITT). For molecular analysis, mice were sacrificed, tissues removed $5 \mathrm{~min}$ after insulin stimulation (5U) and proteins activation analyzed by immunoblotting using phospho-specifics antibodies.

Results: Under STD conditions, STZ treatment induced progressive hyperglycemia in WT mice reaching in the fed state $350 \mathrm{mg} / \mathrm{dl}$ at 8 weeks, while glycemia of Tg mice remained significantly lower $(<200 \mathrm{mg} / \mathrm{dl}, \mathrm{P}<0.001)$. Similar differences were observed for fasting glycemia $(217 \pm 30.7$ for WT vs $126.4 \pm 12.5 \mathrm{mg} / \mathrm{dl}$ for Tg, $\mathrm{P}<0.02)$. The ITT showed that WT mice developed severe insulin-resistance after STZ treatment while Tg mice showed extreme insulin-sensitivity. Under HFD, the protective effect of ApN on STZinduced diabetes persisted on fasting glycemia with significant differences at 7 weeks between WT and Tg mice $(266.8 \pm 21$ vs $162.6 \pm 8.1 \mathrm{mg} / \mathrm{dl}$, respectively, $\mathrm{P}<0.0001)$. However, no difference was observed on glycemia in the fed state, which increased similarly $(\sim 230 \mathrm{mg} / \mathrm{dl})$ in WT and Tg mice after STZ treatment. The ipGTT revealed that HFD induced glucose intolerance in WT animals. STZ treatment aggravated the glucose intolerance of WT and
$\mathrm{Tg}$ mice. However, glycemia at $2 \mathrm{~h}$ of $\mathrm{Tg}$ mice dropped to $320 \mathrm{mg} / \mathrm{dl}$ while that of WT remained $>440 \mathrm{mg} / \mathrm{dl}$. Molecular mechanisms involved in the protective effect of ApN under HFD are still unknown, but data obtained on hepatic tissue of mice under STD showed that in parallel to a $\sim 2$-fold increase of AMPK activity (Thr172), phosphorylation of Ser 632 of IRS-1 (a known negative regulatory mechanism of insulin action) was decreased after insulin stimulation in Tg mice when compared to WT diabetic animals. This potentialization of insulin signaling manifested by the $\sim 3$-fold enhanced Akt activity (Thr308) was explained by major reduction of phosphorylation on Thr389 of p70S6K in Tg mice.

Conclusion: Chronic over-expression of ApN exerts protective effects against experimental induced diabetes by modulating peripheral insulin resistance, although distinct effects were observed pending on fat content of the diet. These data will give a new insight into the molecular mechanism of crosstalk between ApN and insulin pathways in vivo and potential use of ApN as therapeutic agent for diabetes and related complications. Supported by: ALFEDIAM/Roche Pharma

\section{2}

Effect of food protein on the repartition of endogenous glucose production among gluconeogenic organs, and on its sensitivity to insulin B. Pillot, M. Soty, A. Gautier-Stein, C. Zitoun, G. Mithieux Faculté de Médecine Laennec, Inserm U855 / UCB Lyon1, Lyon, France

Background and aims: Food proteins induce the gene expression of intestinal and renal gluconeogenic enzymes in rats. However, a normal glycemia is maintained in protein-fed rats. Furthermore, some parameters of glucose homeostasis are improved in type 2 diabetic patients fed with protein-enriched diet (PED). The aim of our study was to determine the impact of PED on the repartition of endogenous glucose production (EGP) among gluconeogenic organs and to test the effects of this diet on insulin sensitivity of EGP in rats.

Materials and methods: In rats fed on PED for 3 days (50\% proteins vs. $17 \%$ in normal starch-enriched diet (SED)), we: 1) studied the expression of hepatic and renal gluconeogenic enzymes (glucose- 6 phosphatase and phosphoenolpyruvate carboxykinase); 2) quantified renal glucose release by a combination of glucose tracer dilution and arterio-venous glucose balance, 3) estimated insulin action on EGP (insulin perfusion at $240 \mathrm{pmol} / \mathrm{h}$ ) and on peripheral glucose utilization (insulin perfusion at $2880 \mathrm{pmol} / \mathrm{h}$ ), using hyperinsulinemic euglycemic clamps, 4) quantified the hepatic glycogen content after the clamp.

Results: Glucose- 6 phosphatase and phosphoenolpyruvate carboxykinase were markedly increased in the kidney, but not in the liver in PED-fed rats. The renal glucose release was strongly increased in PED-fed rats $(26.0+/$ $4.7 \mu \mathrm{mol} / \mathrm{kg} / \mathrm{min}$ vs. $12.5+/-1.9 \mu \mathrm{mol} / \mathrm{kg} / \mathrm{min}$ in SED-fed rats) and represented about $40 \%$ of EGP, compared to $17 \%$ in SED-fed rats. However, EGP was similar in both groups. Hyperinsulinemic euglycemic clamps showed a stronger EGP suppression with PED (about $-50 \%$ vs. - $30 \%$ in SED-fed rats). There was no difference in glucose utilization at either dose of insulin infused. Moreover, hepatic glycogen content was markedly higher in PED-fed rats than in SED-fed rats ( $18+/-3.5 \mathrm{vs} .4 .7+/-3.2 \mathrm{mg} / \mathrm{g}$ of tissues) at the end of the clamp.

Conclusion: Added to previous data relating to glucose production by the small intestine, we conclude that protein feeding induces a redistribution of glucose fluxes among gluconeogenic organs. This is accompanied by an improvement of insulin sensitivity of suppression of EGP, likely dependent on suppression of hepatic glucose production, linked to a sparing of hepatic glycogen storage. These results may explain at least in part the spectacular improvement of glucose homeostasis observed in type 2 diabetic patients, upon increasing the proportion of protein in their diet.

Supported by: Société Française de Nutrition and Institut Appert

\section{3}

The chronological difference in the evolution of tissue insulin resistance X.J. Li ${ }^{1}$, J.L. Wang ${ }^{1}$, Y.H. Wu ${ }^{1}$, J. Li ${ }^{1}$, X.W. Ran ${ }^{1}$, X.X. Zhang ${ }^{1}$, Y.C. Wang ${ }^{2}$ ${ }^{1}$ Endocrinology, ${ }^{2}$ Psychology, West China Hospital, Chengdu, China

Background and aims: It has well been documented that insulin resistance (IR) features notable tissue selectivity and heterogeneity. Less attention, however, has been paid to the chronological related events of different tissues in the evolution of IR. Recently, we and others showed that IR can be induced 
Table. The dynamic changes of IRC in rat islets in both groups over 12 wks.

\begin{tabular}{lllllll}
\hline & $1 \mathrm{~W}$ & $2 \mathrm{~W}$ & $4 \mathrm{~W}$ & $6 \mathrm{~W}$ & $8 \mathrm{~W}$ & $12 \mathrm{~W}$ \\
\hline $\mathrm{NC}$ & $1.37 \pm 0.53$ & $1.25 \pm 0.46$ & $1.20 \pm 0.49$ & $1.44 \pm 0.70$ & $1.46 \pm 0.37$ & $1.47 \pm 0.44$ \\
\hline $\mathrm{HF}$ & $0.95 \pm 0.28^{*}$ & $0.13 \pm 0.13^{* *}$ & $0.57 \pm 0.36^{* *}$ & $0.85 \pm 0.26^{* *}$ & $0.48 \pm 0.33^{* *}$ & $0.66 \pm 0.34^{* *}$ \\
\hline
\end{tabular}

in pancreatic aand $\beta$ cells i.e. "islet cell insulin resistance" in high-fat-fed rats. This study is designated to observe the chronological difference of the appearance of IR in diverse tissues, with pancreatic islet, in particular.

Materials and methods: 60 Wistar rats were randomly allocated into highfat fed (HF) and regular chaw fed control groups (NC). Rats in both groups were sacrificed and tissues i.e., pancreas, liver, $\mathrm{m}$. seleus and epidedymis fat pads were obtained at 1,2, 4, 6, 8 and 12 wk. respectively, for detecting insulin receptor (IRC) and IRS-1 protein and phosphorylation of their protein tyrosine kinase (PTK-P). (Western blotting and immunoprecipitation.).FPG, insulin(FIn), TG., FFA and Ch. were also measured at each occasion. HOMAIR, ISI, were calculated by Fin and FPG.

Results: Significant increase of TG $(2.34 \pm 0.62$ vs. $1.74 \pm 0.4 \mathrm{mmol} / \mathrm{L}, \mathrm{P}<0.05)$ at $6 \mathrm{wk}$, and of FIn $(1.68 \pm 0.35 \mathrm{vs} .1 .16 \pm 0.08 \mu \mathrm{u} / \mathrm{ml}, \mathrm{P}<0.05)$, FFA $(785.5 \pm 207.9$ vs., $573.8 \pm 148.5 \mu \mathrm{mol} / \mathrm{L}, \mathrm{P}<0.05)$, and $\mathrm{Ch}$ at 8 wk in $\mathrm{HF}$ rats vs. NC was found. FPG remained unchanged over $12 \mathrm{wk}(4.88 \pm 0.54$ vs. $5.10 \pm 0.45 \mathrm{mmol} / \mathrm{L})$, ISI remarkably impaired at $12 \mathrm{wk}$ in $\mathrm{HF}$ vs. $\mathrm{NC}(1.09 \pm 0.02$ vs. $0.17 \pm 0.02, \mathrm{P}<0.05)$, whereas HOMA-IR was significant higher in HF than in NC $(0.46 \pm 0.19$ vs. $0.21 \pm 0.02, \mathrm{P}<0.05)$ at the same time. The following table shows the sequential changes of IRC in islets in both groups over the $12 \mathrm{wks}$.

${ }^{*}$ vs. NC, $\mathrm{P}<0.05$, ${ }^{* *}$ vs. NC, $\mathrm{P}<0.01$. Similar changes of IRC PTK-P and IRS- 1 in islets were found in which both variables were notable lower in HF vs. NC, occurring in the early stage ( $2 \mathrm{wk}$ ), and remained low during the course of IR ( $123989 \pm 26010$ vs., $684142 \pm 86844$ and $0.34 \pm 0.32$ vs. $1.24 \pm 0.7, \mathrm{P}<0.01,2$ wk; and $118049 \pm 40613$ vs. $799344 \pm 281085$, and $0.33 \pm 0.29$ vs. $1.33 \pm 0.49, \mathrm{P}<0.01$, $12 \mathrm{wk})$. However, significant decline of IRS-1-P did not occur until $12 \mathrm{wk}$. Similar changes of IRC were also found in liver $(1.11 \pm 0.22$ vs. $1.59 \pm 0.17$, $\mathrm{P}<0.05,1 \mathrm{wk}$, and $1.05 \pm 0.32$ vs. $1.63 \pm 0.49, \mathrm{P}<0.01,12 \mathrm{wk})$ with parallel changes of IRC PTK-P, IRS-1, and IRS-1-P. The appearance of muscle IRC reduction was delayed compared with islet and liver $(0.68 \pm 0.43$ vs. $1.30 \pm 0.44$, at $6 \mathrm{wk})$, although changes of IRC-P, IRS-1, and IRS-1-P. were similar to the former tissues. Fat IRC decline occurred at $12 \mathrm{wK}(0.57 \pm 0.02$ vs. $0.80 \pm 0.19$, $\mathrm{P}<0.05$ ), which was much more delayed than the above 3 tissues with no any meaningful change of IRC-PTK-P at $12 \mathrm{wk}$.

Conclusion: Our dynamic observations showed that chronological differences exist in the genesis of IR in diverse tissues, and that islet cell IR and hepatic IR may precede peripheral IR. Further studies are needed to elucidate the implication of the phenomenon.

Supported by: National Natural Science Foundation of China

\section{4}

Regulation of liver glucokinase activity in intact normal rats and in rats with dietary-induced insulin resistance

L.M. Massa, J.J. Gagliardino, F. Francini

CENEXA Center of Experimental and Applied Endocrinology, National

University of La Plata, Argentina

Background and aims: To study the regulation of liver glucokinase activity by its gene transcription, protein expression, cellular translocation and 6-phosphofructo-2-kinase/fructose-2,6-biphosphatase (PFK2), in rats with insulin resistance induced by a fructose-rich diet (FRD).

Materials and methods: Glycemia (GOD-PAP), triglyceridemia (enzymatic assay), insulinemia (RIA), GK activity (spectrophotometry), GK and PFK2 protein and gene expression (Western blot and real- time PCR) were measured in liver of control and FRD rats.

Results: FRD rats had significantly higher values of glycemia, insulinemia, and triglyceridemia. GK activity in the cytosolic fraction (CF) of FRD animals was also higher than in the control group $(360 \pm 9.9$ vs. $100 \pm 3.8 \%$; $\mathrm{P}$ $<0.001)$. GK protein level in CF was higher, though not significantly, in FRD animals (116 vs. $100 \%)$. On the other hand, GK activity was higher in the digitonin nuclear fraction (DNF) of C rats [69 \pm 3.4 vs. $31 \pm 1.2 \%$ of total activity $(\mathrm{DNF}+\mathrm{CF}) ; \mathrm{P}<0.001]$. Opposite results were obtained in FRD animals, i.e., GK activity was lower in DNF than in CF [ $14 \pm 1.1$ vs. $82 \pm 2.3 \%$ of total activity $(\mathrm{DNF}+\mathrm{CF}) ; \mathrm{P}<0.001]$. GK transcription level was similar in both groups, while PFK2 gene expression was 4-fold higher in FRD animals. PFK2 protein expression in CF was 5 -fold higher in FRD and its immunological blockage decreased significantly GK activity ( $70 \%$ decrease).
Conclusion: The regulatory effect of GK compartmentalization and PFK2 role upon liver GK activity reported in vitro fully operate in normal intact animals. According to the results of the immunological blockade of PFK2, in this model this enzyme would be the main responsible for the increase in GK activity. Thus, these two mechanisms (cellular translocation and PFK2 interaction) would participate in the adaptative response of liver metabolism to insulin resistance to maintain normal glucose homeostasis. These results provide a new potential site for therapeutic interventions in type 2 diabetes. Supported by: Fondo Nacional para la Investigación Científica y Tecnológica and Consejo Nacional de Investigaciones Científicas y Técnicas (CONICET)

\section{5}

A high cholesterol diet increases the association between caveolae and insulin receptor in rat liver

M. Hahn-Obercyger ${ }^{1}$, L. Graeve ${ }^{2}$, Z. Madar ${ }^{1}$

${ }^{1}$ Institute of Biochemistry, Food Science and Nutrition, The Hebrew University of Jerusalem, Rehovot, Israel, ${ }^{2}$ Institute of Biological Chemistry and Nutrition, University of Hohenheim, Stuttgart, Germany

Background and aims: Caveolin-1, a component of caveolae, regulates signaling pathway compartmentalization by interacting with tyrosine kinase receptors and their substrates. Cholesterol is an essential structural component of lipid rafts and caveolae. Perturbations in caveolae lipid composition have been shown in vitro to displace proteins from lipid microdomains, thereby altering their functionality and subsequent downstream signaling. The role of caveolin-1 in insulin receptor (IR) signaling has been widely investigated invitro in 3T3-L1 adipocyte cells. However, in-vivo experiments investigating this connection in liver tissue have not been carried out. This may be due to the fact that the liver contains a lower level of caveolin-1. Nevertheless, it has been shown that caveolin-1 is located in liver parenchymal cells, with negligible levels detected in endothelial cells. The discovery that the IR is compartmentalized into caveolae introduced a new framework for the understanding of the molecular mechanisms whereby exposure to a high cholesterol diet may alter insulin signaling. Therefore, the aim of this study was to determine whether a high cholesterol diet modulates the hepatic caveolar lipid environment and IR microdomain localization and function in vivo.

Materials and methods: Twenty-four Sprague-Dawley rats were fed with a standard diet or a high cholesterol diet (1\%) for 21 days. Half animals in each group received insulin (10U, i.p.) while the remainder received a saline injection. After 30 min liver tissue was removed and mRNA (RT-PCR) and protein (Western blot and Immunoprecipitation) analysis in total homogenate and lipid rafts fractions were performed.

Results: In comparison to a standard diet, a diet rich in cholesterol significantly altered liver caveolae by increasing both caveolin-1 $(66 \%, \mathrm{p}<0.05)$ and caveolin-2 $(55 \%, \mathrm{p}<0.05)$ expression while caveolin-1 mRNA levels were reduced. Concomitantly, a 25\% increase in localization of the caveolae-resident signaling protein IR was observed. The distribution (see figure) of caveolar and non-caveolar phosphorylated IR was unaffected but insulin-induced IR activation was significantly enhanced following consumption of the high cholesterol diet $(120 \%, \mathrm{p}<0.001)$. Insulin stimulation failed to induce tyrosine phosphorylation of caveolin-1 in hypercholesterolemic rats.

Conclusion: It has been demonstrated, for the first time, that a high cholesterol diet can markedly alter protein composition of liver caveolae microdomains. These findings suggest a mechanism by which a high cholesterol diet altered caveolin-1 expression in-vivo accompanied by altered IR localization and activity. 


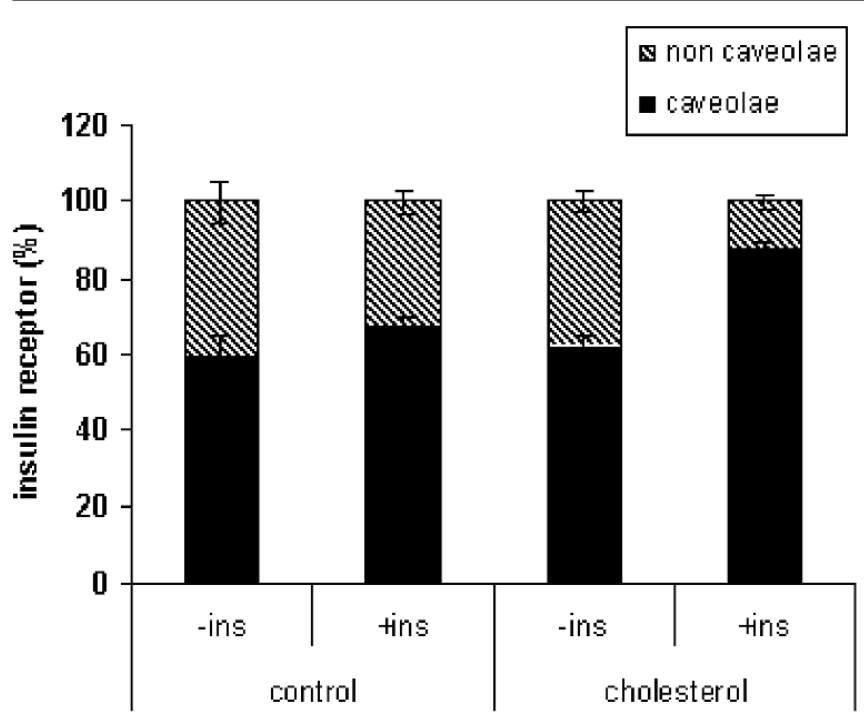

Insulin receptor localization in liver caveolae of hypercholesterolemic rats. Distribution of IR in caveolar and non-caveolar fractions in liver tissue of hypercholesteroliemic rats were compared with control and rats treated with insulin prior to the liver removal. The results $(n=3)$ were expressed as percents of total protein. Data represent Mean + SE

Supported by: Hohenheim foundation

\section{6}

Effects of aging and calorie restriction on rat skeletal muscle glycogen synthase and glycogen phosphorylase

M. Montori-Grau', R. Minor ${ }^{2}$, C. Lerin ${ }^{3}$, J. Allard², C. García-Martínez ${ }^{1}$, R. de $\mathrm{Cabo}^{2}$, A.M. Gómez-Foix ${ }^{1}$

${ }^{1}$ Bioquímica i Biologia Molecular, Universitat de Barcelona, Spain, ${ }^{2}$ Laboratory of Experimental Gerontology, National Institute on Aging, Baltimore, United States, ${ }^{3}$ Research Division, Joslin Diabetes Center, Boston, United States

Background and aims: Aging in skeletal muscle is associated with loss of metabolic competences and glucose tolerance. Calorie restriction (CR) has been shown to prevent the age-associated impairment of glucose tolerance and metabolic functional decline of muscle tissue. Here we studied age-associated changes in glycogen metabolizing enzymes and the effects of CR, in oxidative soleus (SOL) and glycolytic tibialis anterior (TA) rat muscle.

Materials and methods: Muscle glycogen synthase (GS) and glycogen phosphorylase (GP) total enzyme activity, activity ratio, immunoblotted protein and mRNA levels have been examined as well as mRNA levels of glycogentargeting protein phosphatase 1 subunits PTG and $\mathrm{G}_{\mathrm{M}} / \mathrm{R}_{\mathrm{GL}}$ in old (24-month) compared to young (6-month) rats maintained in a normal or CR diet.

Results: GS activity and immunoblotted protein levels, but not muscle GS mRNA, were lower in SOL and TA in 24-month-compared to 6-month-old rats, maintained in a normal diet. GS activity ratio was also reduced, more in SOL than in TA, and this was correlated to a decrease in PTG mRNA levels. CR prevented the decline in GS activity and protein levels, in both SOL and TA and prevented the inactivation of GS and raised PTG mRNA levels, more in SOL than in TA. GP activity, but not muscle GP mRNA levels, was reduced in SOL of 24-month- compared to 6-month-old rats, but preserved in TA. Immunoblotted GP protein content changed inversely to GP activity in response to aging and muscle-type. GP activity ratio was considerably reduced in 24-month-old rats in TA only. CR did not prevent the decline in GP activity or the rise in GP protein in SOL, but it did prevent the inactivation of GP in TA.

Conclusion: A reduction in both GS and GP activity was observed in SOL in old (24-month) compared to young (6-month) rats, whereas in TA GS also was decreased, but GP was maintained. GS was inactivated in SOL and TA and GP was inactivated in TA alone. CR prevented the age-associated reduction in muscle GS activity/protein and the inactivation of GS and GP. Supported by: Ministerio de Ciencia y Tecnología and CIBERDEM from the Ministerio de Sanidad y Consumo, Instituto de Salud Carlos III, Spain

\section{PS 048 Regulation of insulin secretion}

\section{7}

Induction of acute beta cell lipotoxicity in non-diabetic subjects genetically predisposed to type 2 diabetes mellitus M. Mathew, P. Kumar, R. Ali, A. Gastaldelli, S. Wang, K. Cusi Diabetes/Medicie, UTHSCSA, San Antonio, United States

Background and aims: Loss of first phase (0-10 min.) insulin secretion in response to intravenous glucose precedes the development of type 2 diabetes mellitus. We have previously shown a similar loss in first phase insulin secretion by a chronic low-dose lipid infusion in $\mathrm{FH}+$ subjects, suggesting that it may be a marker for susceptibility to type 2 diabetes mellitus. However, the effect of an acute lipid infusion on insulin secretion has not been carefully studied in non-diabetic genetically predisposed subjects. To this end, we studied the effect/time-course of a 4-hour lipid infusion on acute glucosestimulated insulin release (AIR 0-10 min.) in 20 lean subjects, divided into 3 groups well matched for age/BMI/ethnicity based on a positive $(\mathrm{FH}+)$ or negative (controls) family history of type 2 diabetes mellitus in first degree relatives, as well as on a screening OGTT: 1) $7 \mathrm{FH}+$ with IGT (FH+IGT) (age $=51 \hat{\mathrm{A}} \pm 3 \mathrm{yr}$, body mass index $(\mathrm{BMI})=27.3 \hat{\mathrm{A}} \pm 0.9 \mathrm{~kg} / \mathrm{m} 2$, fasting plasma glucose $=108 \hat{\mathrm{A}} \pm 2 \mathrm{mg} / \mathrm{dl}) ; 2) 7 \mathrm{FH}+$ with normal glucose tolerance $(\mathrm{FH}+\mathrm{NGT})$ $($ age $=36 \hat{\mathrm{A}} \pm 8 \mathrm{yr}, \mathrm{BMI}=26.3 \hat{\mathrm{A}} \pm 1.1 \mathrm{~kg} / \mathrm{m} 2$, fasting plasma glucose $=93 \hat{\mathrm{A}} \pm 2$ $\mathrm{mg} / \mathrm{dl})$ and 3) 6 controls with NGT (age $=47 \hat{\mathrm{A}} \pm 4 \mathrm{yr}, \mathrm{BMI}=27.1 \hat{\mathrm{A}} \pm 1.9 \mathrm{~kg} / \mathrm{m} 2$, fasting plasma glucose $=95 \hat{\mathrm{A}} \pm 2 \mathrm{mg} / \mathrm{dl}$ ).

Materials and methods: Patients were admitted twice to the research unit (GCRC). After an overnight fast, they received an infusion of either normal saline (SAL) or lipid (Liposyn III at $90 \mathrm{ml} / \mathrm{h}$ ) for 4 hours and the time-course of AIR (0-10 min. C-peptide AUC) was assessed at $60 \mathrm{~min}$. (AIR1), $120 \mathrm{~min}$. (AIR2) and 240 (AIR3) min. following a 0.3 gram per kilogram glucose bolus. Glucose, insulin and FFA were also measured.

Results: Fasting plasma glucose was higher in FH+IGT vs. FH+NGT and controls $(\mathrm{p}<0.03)$, but post-bolus glucose levels were similar among the 3 groups (AIR1, 2 and 3). Compared to controls, FH+IGT and FH+NGT were insulin resistant as calculated by the Matsuda index derived from the screening oral glucose tolerance test. During SAL, compared to controls, AIR in FH+IGT was significantly reduced by $40 \%, 47 \%$ and $25 \%$ for AIR 1 , AIR 2 and AIR3, respectively $(\mathrm{p}<0.1-0.02)$ and also in FH+NGT $(25 \%, 21 \%$ and $34 \%$ for AIR1, AIR2 and AIR3, respectively ( $<<0.1-0.001)$. Lipid infusion did not impair insulin secretion in controls, but markedly reduced AIR1, 2 and 3 in FH+IGT by $75 \%, 54 \%$ and $33 \%$ ( $<<0.02-0.04$ vs. SAL and $\mathrm{p}<0.05-0.001$ vs. controls) and to a lesser extent in FH+NGT, with a more modest decrease in AIR2 and 3 compared controls.

Conclusion: Subjects with a strong genetic predisposition to type 2 diabetes mellitus have early subtle defects in first phase insulin secretion. An acute lipid infusion significantly impairs insulin secretion in $\mathrm{FH}+$ subjects with impaired glucose tolerance, but not as significantly in normal glucose tolerant $\mathrm{FH}+$ subjects. This suggests that in more advanced stages of the "pre-diabetic" state (FH+ with IGT), lipotoxicity is an extremely relevant contributing factor in the progression to T2DM. This may explain, at least in part, the role of obesity in the development to type 2 diabetes mellitus. We speculate that an acute lipid infusion may potentially be a useful test to identify subjects at the highest risk of developing type 2 diabetes mellitus.

\section{8}

Association of serum free IGF-1 and IGFBP-1 with insulin sensitivity in impaired glucose tolerance (IGT) and type 2 diabetic subjects M.G. Kabir ${ }^{1}$, M.H. Mosaraf ${ }^{1,2}$, S.N. Hassan ${ }^{1,2}$, Z. Hassan ${ }^{3}$, I. Khan $^{2}$, Q. Nahar ${ }^{2}$, M. Alauddin ${ }^{1}$, M.O. Faruque ${ }^{4}$, L. Ali ${ }^{2}$

${ }^{1}$ Biochemistry \& Molecular Biology, University of Chittagong, ${ }^{2}$ Biochemistry \& Cell Biology, BIRDEM, Dhaka, ${ }^{3}$ Physiology \& Molecular Biology, BIRDEM, Dhaka, ${ }^{4}$ Biochemistry \& Molecular Biology, BIRDEM, Dhaka, Bangladesh

Background and aims: Free IGF-1 and IGFBP-1 are associated with obesity which is one of the major features of insulin resistance. Although both the obesity and insulin resistance are involved in the development of diabetes, but very few studies exist on free IGF-1 and IGFBP-1 in diabetes and IGT subjects. The present study was undertaken to investigate the association of free IGF-1 and IGFBP-1 with insulin sensitivity in IGT and type 2 diabetic subjects. 
Subjects and methods: Ninety one subjects with impaired glucose tolerance (IGT) and 68 with type 2 diabetes mellitus (DM) were studied along with age- and BMI-matched 61 healthy Controls without family history of diabetes or prediabetes.. Serum glucose was measured by glucose-oxidase method, lipid profile by enzymatic-colorimetric method, and insulin, free IGF-1 and IGFBP- 1 by standard ELISA method. Insulin secretory capacity (HOMA-B\%) and insulin sensitivity (HOMA-S\%) were calculated using fasting glucose and fasting insulin by HOMA-CIGMA software.

Results: Waist-hip ratio (WHR) [Control, 0.91 \pm 0.05 ; IGT, 0.94 \pm 0.05 ; DM, $0.93 \pm 0.04]$ was significantly higher in $\mathrm{DM}(\mathrm{p}=0.01)$ subjects compared to Controls. Median (range) of fasting serum insulin [pmol/1; Control, 51.7 (7.8-155.9); IGT, 67.7 (6.9-237.6); DM, 69.3 (17.4-525.3)] was significantly higher in IGT $(\mathrm{p}=0.004)$ and DM $(\mathrm{p}=0.014)$ group compared to Controls. HOMA-B\% [Control, IGT and DM subjects were 99 (21-187), 99 (26-278) and $62(14-296)$ ] was significantly $(\mathrm{p}=0.001)$ lower in DM subjects compared to Controls. HOMA-S\% [Control, IGT and DM subjects were 86 (29-554), 66 (20-661) and 59 (9-256)] was significantly lower in IGT $(\mathrm{p}=0.001)$ and DM subjects $(\mathrm{p}=0.001)$ compared to Controls. Fasting serum free IGF-1 level [pg/ $\mathrm{ml}$; Control, IGT and DM were 118.2 (39.4-486.1), 118.2 (19.9-465.9) and 140.1 (19.9-803.5)] was not significantly different among the study groups. Similarly fasting serum IGFBP-1 level [ng/ml; Control, IGT and DM were 11.5 (1.1-83.97), 13.8 (1.6-68.1) and $11.9(0.68-146)]$ was not significantly different among the groups. When the study subjects were categorized on the basis of BMI cut-off point standarderized for Bangladeshi population then the level of free IGF-1 was significantly $(\mathrm{p}=0.03)$ higher in type $2 \mathrm{DM}$ [182.62 (72.32-365.45)] subjects with BMI $\leq 23$ compared to Controls [ 95.70 (39.4-161.53)] with similar BMI range. Increased level $(\mathrm{p}=0.05)$ of free IGF1 was also observed in healthy Controls with BMI $>23$ than Controls with $\mathrm{BMI} \leq 23$. Binary logistic regression analysis with Control group have shown that both IGT and DM were associated with IGFBP-1 (IGT: $\beta=0.377, p=0.03$; DM: $\beta=0.075, p=0.028$ ) but not with free IGF-1. On multiple linear regression analysis of free IGFBP- 1 concentration, after adjusting the confounding variables, showed that IGFBP- 1 was positively $(b=0.152, p=0.052)$ associated with HOMA $S$ in the combined group of IGT and DM subjects.

Conclusion: Free IGF-1 is associated with body weight and it may not be directly associated with diabetes. On the other hand IGFBP-1 seems to be associated with insulin resistance in prediabetic and diabetic states.

Supported by: Diabetic Association of Bangladesh; IPICS, Sweden

\section{9}

Proteomic analysis identified novel signalling pathways activated in human pancreatic islets of type 2 diabetes patients

H.K. Nyblom ${ }^{1}$, M. Bugliani ${ }^{2}$, Y.M.E. Fung ${ }^{3}$, R.A. Zubarev ${ }^{3}$, P. Marchetti' ${ }^{2}$, P. Bergsten ${ }^{1}$

${ }^{1}$ Department of Medical Cell Biology, Uppsala University, Sweden, ${ }^{2}$ Department of Endocrinology and Metabolism, Pisa University, Italy, ${ }^{3}$ Department of Cellular and Molecular Biology, Uppsala University, Sweden

Background and aims: Islet dysfunction in type 2 diabetes mellitus (T2DM) is caused by genetic and environmental factors, which alter expressions of multiple genes. Given the complexity it is not surprising that, although specific $\beta$-cell genes that contribute to impaired function have been identified, the mechanisms for islet dysfunction remain unclear. The aims of the present study were to optimize the proteomic approach liquid chromatography Fourier transform ion cyclotron resonance mass spectrometry (LC FT-ICR MS) so that expression profiles can be determined from a small number of islets, generate protein expression data sets from freshly isolated human islets obtained from T2DM and control donors and analyze the results to reveal signaling pathways involved in destruction of islet function.

Materials and methods: Islets of Langerhans were isolated from five T2DM and ten control donors. The two groups were age and weight matched. Islets were either used for determination of insulin release in response to glucose or glibenclamide or for expression measurements. For the latter purpose islets were lyzed and pooled resulting in one sample containing islet lysates from the T2DM individuals and one from the control individuals. The two samples were analyzed by LC FT-ICR MS and proteomic expression data sets generated. Subsequent analysis of the data sets determined differential signaling pathway activation between islets obtained from T2DM and control donors. In addition, key-node molecules of dominant signaling pathways were determined.

Results: Insulin release from control islets increased 4-fold when the glucose concentration was increased from 3 to $17 \mathrm{mM}$ glucose. Similar rises were observed when glibenclamide was added. In contrast, insulin release only increased 2-fold from islets isolated from T2DM donors when either the glucose concentration was raised or glibenclamide was added. Protein profiles of islet lysates from control and T2DM donors contained expression levels of approximately 400 identified proteins. When the data sets were used for signaling pathway analysis, a number of pathways were activated in islets of T2DM individuals compared to islets obtained from control individuals $(\mathrm{p}<0.05)$. Among the significantly activated pathways several associated with apoptosis were identified. Activation of these signaling pathways in T2DM was confirmed when the identified key-nodes were mapped onto known pathways.

Conclusion: The study is the first account of protein profiles of islets obtained from individuals with T2DM and demonstrates the feasibility of performing proteomic studies on as little as 100 freshly isolated human islets when using the LC FT-ICR MS approach. Mechanisms of islet dysfunction were delineated by subjecting the expression data sets to signaling pathway and key-node analyses, which revealed activation of apoptotic and other pathways in T2DM donors compared to control. Verification and validation of the discovered pathways as well as performing expression measurements on more individuals will determine the importance of the identified pathways for islet dysfunction in T2DM.

Supported by: EFSD/MSD Grant, Swedish Medical Research Council, and Swedish Diabetes Association

\section{0}

Reduced insulin secretion/insulin resistance (disposition) index is the primary determinant of glucose intolerance in the pre-diabetic state: results from the ACT NOW Study

R.A. DeFronzo ${ }^{1}$, M.A. Banerji ${ }^{2}$, G.A. Bray ${ }^{2}$, T.A. Buchanan ${ }^{2}$, S. Clement ${ }^{2}$,

R.R. Henry ${ }^{2}$, A. Kitabchi' ${ }^{2}$, S. Mudaliar', N. Musi ${ }^{1}$, R. Ratner ${ }^{2}$, P. Reaven ${ }^{2}$, D. Schwenke ${ }^{2}$, D. Tripathy ${ }^{1}$

${ }^{1}$ Medicine/Diabetes, UTHSCSA, United States, ${ }^{2}$ Diabetes, ACT NOW Investigators, San Antonio, United States

Aims: To evaluate the relationship between insulin sensitivity, $\beta$-cell function and glucose tolerance at baseline in subjects with varying degrees of glucose tolerance in the ACT NOW study.

Methods: ACT NOW is multicenter study for the prevention (pioglitazone vs. placebo) of diabetes in subjects with impaired glucose tolerance (IGT). We present the baseline data from 602 IGT subjects from 8 US centers. During the screening process, 115 subjects with normal glucose tolerance (NGT) and 50 subjects with impaired fasting glucose (IFG $=110-125 \mathrm{mg} / \mathrm{dl})$ were identified. Of 602 IGT subjects, 174 had isolated IGT (FPG $<100 \mathrm{mg} / \mathrm{dl} ; 2$ $\mathrm{h}$ glucose $=140-199 \mathrm{mg} / \mathrm{dl})$ and $428 \mathrm{had}$ combined IGT plus IFG $(\mathrm{FPG}=$ $100-125 \mathrm{mg} / \mathrm{dl}$; 2 -h glucose $=140-199 \mathrm{mg} / \mathrm{dl}$ ). Indices of insulin secretion and insulin sensitivity were derived from the plasma glucose, insulin, and $\mathrm{C}$ peptide concentrations during the OGTT. The acute insulin response (AIR $\mathrm{min}$ ) and insulin sensitivity $\left(\mathrm{S}_{\mathrm{I}}\right)$ were measured with the frequently sampled intravenous glucose tolerance test (FSIVGTT) in IGT and IGT/IFG subjects. Results: Matsuda index of insulin sensitivity from OGTT was similar in NGT and IFG subjects ( $6.5 \pm 0.6$ vs $5.8 \pm 0.7, \mathrm{p}=\mathrm{ns})$, but worsened progressively moving form NGT to IGT ( $6.5 \pm 0.6$ vs $5.0 \pm 0.2, \mathrm{p}=0.03$ ) to combined IGT/IFG (3.9 $\pm 0.2, \mathrm{p}<0.0005)$. The insulin secretion/insulin resistance index $\left(\Delta \mathrm{I}_{0-120} / \Delta \mathrm{G}_{0-120} \mathrm{x}\right.$ Matsuda index) was similar in NGT and IFG subjects but was markedly reduced in IGT versus NGT $(4.5 \pm 0.2$ vs $11.5 \pm 1.1, \mathrm{p}<0.0001)$ and was further decreased in subjects with combined IGT/IFG $(3.2 \pm 0.1$, $\mathrm{p}<0.0001)$. Late phase insulin secretion $\left(\Delta \mathrm{I}_{60-120} / \Delta \mathrm{G}_{60-120} \mathrm{x}\right.$ Matsuda index and $\Delta \mathrm{ISR}_{60-120} / \Delta \mathrm{G}_{60-120} \times$ Matsuda index; ISR = insulin secretory rate by C-peptide deconvolution) were similar in NGT and IFG, but significantly decreased in IGT $(\mathrm{p}<0.0001)$ and combined IGT/IFG $(\mathrm{p}<0.00001)$. Subjects with combined IGT/IFG had a lower $\operatorname{AIR}_{0-10}(329 \pm 18$ vs $429 \pm 26$, $\mathrm{p}=0.002)$ and disposition index $\left(\operatorname{AIR}_{0-10} \times \mathrm{S}_{\mathrm{i}}\right)(751 \pm 43$ vs $991 \pm 86, \mathrm{p}=0.01)$ compared to subjects with isolated IGT. Si correlated with the Matsuda index $(r=0.518, \mathrm{p}<0.0001)$. When subjects were stratified according to FPG, the insulin AUC during OGTT progressively increased with increasing FPG; however, both $\Delta \mathrm{I}_{0-120}$ / $\Delta \mathrm{G}_{0-120} \times$ Matsuda index and $\Delta \mathrm{ISR}_{0-120} / \Delta \mathrm{G}_{0-120} \times$ Matsuda index declined progressively with rising FPG concentrations. Subjects with FPG $=115-125 \mathrm{mg} /$ dl had an $80 \%$ decrease in beta cell function compared to subjects with FPG $=70-90 \mathrm{mg} / \mathrm{dl}$. Insulin sensitivity also decreased progressively by $40 \%$ over the same range of FPG.

Conclusion: (1) The decline in $\beta$-cell function (regardless of method of estimation: insulin and C-peptide levels during OGTT or IVGTT) is the primary determinant of worsening glucose tolerance in "pre-diabetic" subjects; (2) Impaired early insulin secretion (with normal/near normal insulin sensitivi- 
ty) is the major defect hyperglycemia in subjects with isolated IFG; (3) insulin resistance and impaired early and late phase insulin secretion are responsible for post-glucose load hyperglycemia in IGT.

Supported by: Takeda Pharmaceuticals

\section{1}

Progression of beta cell dysfunction in subjects at risk for type 1 diabetes A. Mari ${ }^{1}$, V. Nofrate ${ }^{1}$, J. Skyler ${ }^{2}$, E. Ferrannini ${ }^{3}$

${ }^{1}$ Institute of Biomedical Engineering, National Research Council, Padova, Italy, ${ }^{2}$ Division of Endocrinology, Diabetes, \& Metabolism, University of Miami Leonard M. Miller School of Medicine, Miami, United States, ${ }^{3}$ Department of Internal Medicine, University of Pisa School of Medicine, Italy

Background and aims: First-degree relatives of type 1 diabetic patients $\left(\mathrm{FDR}_{1}\right)$ have increased risk of developing diabetes. The pathophysiological mechanisms and mode of onset of diabetes in this high-risk group have not been investigated.

Materials and methods: In 325 islet-cell autoantibody-positive, non-diabetic FDR from the DPT-1 Study (age=11 [9] years, median [interquartile range]), we analyzed 1,898 OGTTs (6 [6] per subject) performed between baseline and last follow-up (2.7 [2.8] years), when 113 subjects had developed diabetes (progressors, at 2.1 [2.0] years). The insulin-secretion/plasma glucose doseresponse function was resolved by mathematical modelling of the OGTT glucose and C-peptide and beta-cell glucose sensitivity ( $\beta$-GS, slope of the dose-response) and insulin secretion at $5 \mathrm{mM}$ glucose (IS ${ }_{5}$ ) were calculated. In the subjects with $\geq 4$ OGTTs ( $\mathrm{n}=52$ ), time-series of individual parameters were fitted using a biphasic function (with an initial slope, a transition time and a final slope) to determine the temporal pattern.

Results: Transition to hyperglycaemia was clearly biphasic in $67 \%$ of subjects, monophasic in $8 \%$ and ill-defined in $25 \%$. In the subjects with biphasic transition ( $\mathrm{n}=48)$, 2-hour glucose sharply increased to diabetic levels (rate of change 12.9 [20.1] mM/year) following an initial stable phase (0.0 [1.3] $\mathrm{mM} /$ year). The time at which the transition occurred was $0.76[1.04]$ years before diagnosis. In the non-progressors $(n=157)$, only a slow drift $(0.07$ [0.4] $\mathrm{mM} /$ year) was observed in most subjects ( $90 \%)$. Two-hour glucose levels were higher in the progressors during the entire observation period (6.7 [2.1] vs 5.9 [1.7], $\mathrm{p}<0.01$ at baseline). Baseline $\beta$-GS was lower in progressors than non-progressors (46 [34] vs 87 [65] pmolmin ${ }^{-1} \mathrm{~m}^{-2} \mathrm{mM}^{-1}, \mathrm{p}<0.0001$ ). The temporal pattern of $\beta$-GS was less clearly defined than that of 2 -hour glucose levels, in part due to noisier data. Using a linear approximation, the rate of decline of $\beta$-GS in progressors $\left(-9.9[12.6]\right.$ pmol $\mathrm{min}^{-1} \cdot \mathrm{m}^{-2} \cdot \mathrm{mM}^{-1}$ year-1) was faster than in non-progressors (-4.2 [12.9], $\mathrm{p}<0.0001)$. In the 35 subjects in which 2-hour glucose was markedly biphasic, $\beta$-GS was initially flat ( 4.3 [25.5] pmol $\mathrm{min}^{-1} \mathrm{~m}^{-2} \cdot \mathrm{mM}^{-1}$ year ${ }^{-1}$ ) and then rapidly declined (at a rate of -40.9 [33.7] pmol $\mathrm{min}^{-1} \mathrm{~m}^{-2} \mathrm{mM}^{-1}$ year ${ }^{-1}$ ) starting 1.4 [1.2] years before diagnosis. In this subgroup, the time of the $\beta$-GS transition was correlated with the time of the 2-hour glucose transition $(r=0.42, \mathrm{p}<0.02)$ and anticipated the latter by

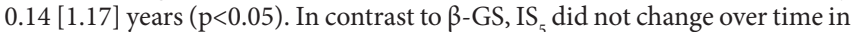
progressors (rate of change $-0.1[15.3] \mathrm{pmol}^{-1} \mathrm{~min}^{-1} \mathrm{~m}^{-2}$. $\mathrm{year}^{-1}, \mathrm{p}=\mathrm{ns}$ ), though at baseline it was lower in progressors (53 [37] vs 69 [48] pmolmin ${ }^{-1} \cdot \mathrm{m}^{-2}$ year ${ }^{-1}$, $\mathrm{p}<0.05$ ). Insulin sensitivity (OGIS) showed an average decline similar to $\beta$ GS, though its phasic characteristics were less defined. In all progressors (660 OGTTs), 2 -hour glucose was simultaneously related to both $\beta$-GS and insulin sensitivity $\left(r^{2}=0.41, \mathrm{p}<0.0001\right)$.

Conclusion: In $\mathrm{FDR}_{1}$, (a) transition to diabetic hyperglycaemia occurs mostly in a biphasic time-course and is preceded by a similar biphasic change in $\beta$-GS; (b) subjects who develop diabetes have a reduced $\beta$-GS; and (c) both $\beta$-GS and insulin sensitivity are strong determinants of glucose levels.

\section{2}

Frequency of insulin resistance and beta cell dysfunction and their relations with clinical and biochemical features in newly-diagnosed type 2 diabetes

E. Bonora, V. Stoico, C. Negri, M. Trombetta, C. Tommasi, F. Grippaldi, C. Brangani, I. Pichiri, F. Tecilazich, M. Muggeo, R.C. Bonadonna Endocrinologia e Malattie del Metabolismo, University of Verona, Italy

Background and aims: To evaluate the frequency of insulin resistance and beta-cell dysfunction and their relations with several clinical and biochemical features in newly-diagnosed type 2 diabetes.
Materials and methods: Insulin resistance and beta-cell dysfunction were assessed by hyperinsulinemic euglycemic clamp and minimal model analysis of C-peptide and glucose levels during OGTT with in 377 newly-diagnosed untreated type 2 diabetic patients. In these subjects the presence of chronic complications was explored by medical history, opthalmoscopy, ECG, carotid and femoral-popliteal echo-doppler and albuminuria (micro and macro) assessment. Several biomarkers were measured, including FFA and adiponectin (markers of adipose tissue dysregulation), CRP, IL-6 and TNF- $\alpha$ (markers of inflammation), P-selectin, E-selectin, and ICAM-1 (markers of endothelial dysfunction), and oxidized LDL (marker of oxidant stress). Also standard clinical (e.g, waist circumference) and biochemical parameters (e.g., HbA1c) were assessed. Mean pre- and post-prandial glucose levels were computed based upon six time-points home blood glucose monitoring on 5 different days.

Results: Most subjects (86\%) had both insulin resistance (glucose disposal during clamp, M-clamp, within the bottom quartile of 500 nonobese healthy subjects) and beta-cell dysfunction (beta-index computed with minimal model applied to OGTT within the bottom quartile of 125 nonobese healthy subjects), whereas $6 \%$ had isolated insulin resistance and $8 \%$ had isolated beta-cell dysfunction. The prevalence of chronic complications was as follows: background retinopathy $6.3 \%$, proliferative retinopathy $0 \%$, microalbuminuria $11.8 \%$, macroalbuminuria $1.4 \%$, CHD $4.9 \%$, increased carotid IMT $55.4 \%$, carotid plaques $5.9 \%$, increased femoral IMT $48.8 \%$, femoral-popliteal plaques 5.2\%. After adjusting for sex, age, smoking, HbAlc, LDL cholesterol and blood pressure, no difference in insulin resistance or beta-cell dysfunction was found between subjects with or without microangiopathy or with or without macroangiopathy in any site. In multiple regression analyses which included also sex, age and smoking, M-clamp was independently associated with waist circumference, FFA, adiponectin, oxidized LDL and E-selectin, whereas beta-index was independently associated with waist circumference, FFA and HbAlc. In multiple regression analysis both M-clamp and beta-index were independently associated to HbAlc and blood glucose (fasting, 2-h OGTT, mean pre-prandial, mean post-prandial) but the strength of association with beta-index was two-fold higher.

Conclusion: Most subjects with newly-diagnosed type 2 diabetes have both insulin resistance and beta-cell dysfunction. On a cross-sectional basis, none of these pathogenetic features of type 2 diabetes seems to be associated with chronic complications. Adipose tissue dysregulation is associated, perhaps causally, with both insulin resistance and beta-cell dysfunction, whereas endothelial dysfunction and oxidant stress might contribute to (or are caused by) insulin resistance. Beta-cell dysfunction explains the degree of glucose control to a greater extent than insulin resistance.

Supported by: Italian Ministry of University and Research

\section{3}

TLR agonist induced changes in BRIN-BD11 clonal beta cell metabolism, signal transduction and insulin secretion

A.M. Kiely, P. Newsholme

Biochemistry Department, Conway Institute of Biomolecular and Biomedical Research, Dublin, Ireland

Background and aims: Insulin resistance associated with obesity and type 2 diabetes is commonly associated with changes in muscle, adipose tissue and liver signal transduction pathways. Pro-inflammatory cytokines (such as TNF- $\alpha$ and IL-1 $\beta$ ) can alter patterns of insulin signalling in these target tissues, resulting in resistance to the action of insulin. More recently evidence for involvement of innate immune product receptors, e.g. Toll like receptors, in insulin resistance has been published. TLR4 and TLR2, whose agonists include lipopolysaccharides and saturated fatty acids, are implicated in altered patterns of signalling in animal models of insulin resistance and obesity. Indeed loss of function mutations in the TLR4 receptor had a beneficial effect on the insulin signalling pathways in adipose, muscle and liver tissues in mice and improved insulin action.

Materials and methods: Clonal BRIN BD11 $\beta$-cells were treated for 24 hours with increasing concentrations of LPS. The media was removed and stored to determine chronic $(24 \mathrm{~h})$ insulin secretion and nutrient consumption. Acute $(20 \mathrm{~min})$ stimulated insulin secretion was subsequently determined. Glucose and glutamine concentrations were determined by enzymatic assays using the YSI 7100 MBS Multiparameter Bioanalytical System. Protein was extracted using RIPA buffer, subjected to SDS-PAGE and electrophoretically transferred onto a nitrocellulose sheet. The sheet was blocked in 5\% milk protein, incubated with polyclonal anti IRS-1, p-IRS-1, Akt, p-Akt or IR $\beta$ 
(Isis), washed and probed with horseradish peroxidase and visualised with SuperSignal West Pico (Pierce).

Results: Incubation of BRIN-BD11 cells for 24 hours in the presence of increasing concentrations of the TLR4 ligand LPS significantly decreased chronic ( 24 hour) insulin secretion from $1.09 \pm 0.19$ to $0.76 \pm 0.18 \mu \mathrm{g}$ insulin / $\mathrm{mg}$ protein in the presence of $100 \mathrm{ng} / \mathrm{ml}$ LPS $(\mathrm{p}<0.05)$. There was no change in acute $(20 \mathrm{~min})$ stimulated insulin secretion, or insulin content. The reduction in chronic insulin secretion was not accompanied by changes in nutrient (glucose or glutamine) consumption. IR $\beta$ expression levels were increased significantly from $1 \pm 0.52$ to $8.6 \pm 1.83$ units $(\mathrm{p}<0.01$ ), and Akt phosphorylation was slightly reduced. There was no change in IRS-1 protein expression or phosphorylation after 24 hours. Incubation of treated cells for a further 24 hours in the absence of LPS demonstrated a recovery of chronic (24hr) insulin secretion.

Conclusion: We have demonstrated a small but significant reduction in insulin secretion associated with $24 \mathrm{hr}$ exposure to the TLR4 agonist LPS. It is known that glucose and insulin induce expression of IR $\beta$ in pancreatic $\beta$-cells. Here we have demonstrated that LPS increased IR $\beta$ expression and reduced insulin secretion. Insulin content was not altered. We also found exposure to LPS resulted in a reduction in the level of activated Akt which could potentially impair the viability of the $\beta$-cell after longer periods of incubation. However, the concentrations of LPS used in this study did not impair cell viability during the period of our experiments. Importantly, defects in insulin secretion associated with initial $24 \mathrm{hr}$ exposure to LPS were reversed after a further 24 hour incubation in the absence of LPS.

Supported by: Health Research Board of Ireland

\section{4}

BLX-1002, a novel thiazolidinedione with no PPAR affinity, stimulates AMP-activated protein kinase activity, raises cytosolic $\mathrm{Ca}^{2+}$ and enhances glucose-stimulated insulin secretion in a PI3K-dependent manner F. Zhang ${ }^{1}$, D. Dey ${ }^{2}$, R. Bränström ${ }^{3}$, L. Forsberg ${ }^{3}$, M. Lu ${ }^{3}$, Q. Zhang ${ }^{1}$,

Å. Sjöholm ${ }^{1}$

${ }^{1}$ Department of Clinical Science and Education, Karolinska Institutet, Stockholm, Sweden, ${ }^{2}$ Bexel Pharmaceuticals, Inc., Union City, United States, ${ }^{3}$ Department of Molecular Medicine and Surgery, Unit of Endocrine Surgery, Karolinska Institutet, Stockholm, Sweden

Background and aims: BLX-1002 is an amino acid conjugated, novel small molecule $(M W<500)$ with no affinity to PPAR receptors. It has been shown to lower blood glucose in type 2 diabetes without adipogenic effects in vivo. The function of the drug on pancreatic $\beta$-cells remains unkown. We have investigated effects of the drug on $\beta$-cell function in islet cells from $o b / o b$ mice.

Materials and methods: Pancreatic islets were isolated from $o b / o b$ mice by enzyme digestion and disrupted into cells in a $\mathrm{Ca}^{2+}$-free buffer with EGTA. Insulin secretion was investigated in batch-incubation during 20 minutes. $\mathrm{Cy}$ tosolic free $\mathrm{Ca}^{2+}$ concentration $\left(\left[\mathrm{Ca}^{2+}\right]_{\mathrm{i}}\right)$ was measured in Fura-2 pre-loaded cells using a time-sharing spectrofluorometer. The activities of voltage-gated $\mathrm{Ca}^{2+}$ channels and ATP-dependent $\mathrm{K}^{+}\left(\mathrm{K}_{\text {ATP }}\right)$ channels were monitored using patch-clamp technique. Activation of AMPK was evaluated by Western blotting using anti-phospho-AMP-activated protein kinase (AMPK) $\alpha$ (Thr172). Results: Incubation of the $\beta$-cells with BLX-1002 enhanced high glucosestimulated insulin secretion without affecting release of the hormone at glucose concentrations of 3 or $8 \mathrm{mM}$ during a 20 min-incubation, while its major matabolite BLX-1015 had no effect. BLX-1002 also augmented $\left[\mathrm{Ca}^{2+}\right]_{i}$ at high glucose, an effect that was abolished by pre-treatment of the cells with the $\mathrm{Ca}^{2+}$-ATPase inhibitor thapsigargin. In contrast, BLX-1002 interfered neither with voltage-gated $\mathrm{Ca}^{2+}$ channel nor with $\mathrm{K}_{\text {ATP }}$ channel activities. Stimulation of the $\beta$-cells with BLX-1002 induced activation of AMPK at high glucose. The stimulatory effect of BLX-1002 on insulin secretion at high glucose was abolished by treatment of the cells with the selective $\mathrm{PI}_{3} \mathrm{~K}$ inhibitors wortmannin or LY294002.

Conclusion: This study suggests that BLX-1002 potentiates glucose-stimulated insulin secretion in pancreatic $\beta$-cells in a $\mathrm{PI}_{3} \mathrm{~K}$-dependent manner. This stimulatory effect of BLX-1002 on insulin release occurs only in the presence of high glucose and is associated with AMPK phosphorylation and an enhanced $\left[\mathrm{Ca}^{2+}\right]_{i}$ mediated through mobilization of $\mathrm{Ca}^{2+}$ from intracellular $\mathrm{Ca}^{2+}$ stores. Changes in voltage-gated $\mathrm{Ca}^{2+}$ channel or $\mathrm{K}_{\mathrm{ATP}}$ channel activities do not seem to be involved in the increase in $\left[\mathrm{Ca}^{2+}\right]_{\mathrm{i}}$ induced by the drug. The glucose-sensitive stimulatory impact of BLX-1002 on $\beta$-cell function may translate into substantial clinical benefits of the drug in the management of type 2 diabetes, by avoidance of hypoglycemia.

Supported by: Research Center at Södersjukhuset, Stockholm

\section{5}

Bisperoxovanadate prevents fat-induced beta cell dysfunction in vitro and in vivo

A.I. Oprescu' ${ }^{1}$, P. $\mathrm{Han}^{2}$, S.A. Constantin-Marino ${ }^{2}$, L. Lam ${ }^{2}$, G.F. Lewis ${ }^{1,2}$, I.G. Fantus ${ }^{1,2}$, M.B. Wheeler ${ }^{2}$, A. Giacca ${ }^{1,2}$

${ }^{1}$ Institute of Medical Science, ${ }^{2}$ Physiology, University of Toronto, Toronto, Canada

Background and aims: Fatty acids induce insulin resistance and chronically decrease $\beta$-cell function. The insulin signaling pathway plays a role in insulin secretion, however it is still unclear whether the FFA-induced decrease in $\beta$ cell function involves $\beta$-cell insulin resistance.

Materials and methods: To address the role of generalized and $\beta$-cell insulin resistance in FFA-induced $\beta$-cell dysfunction we coinfused with fat a peroxovanadium compound, bisperoxovanadate $(\mathrm{bpV})$, in normal Wistar rats. $\mathrm{BpV}$, a tyrosine phosphatase inhibitor, acts as insulin mimetic and is devoid of any antioxidant effect that could prevent $\beta$-cell dysfunction, unlike most insulin sensitizers. Two fat infusions were given iv to elevate plasma FFA by approximately 2-fold: a direct infusion of oleate in bovine serum albumin, or an emulsion of $20 \%$ olive oil $\left(5 \mu \mathrm{l} \cdot \mathrm{min}^{-1}\right)+$ heparin $\left(50 \mathrm{U} \cdot \mathrm{ml}^{-1}\right)$ for $48 \mathrm{~h}$ in Wistar rats, with or without $\mathrm{bpV}$ at $0.0025 \mathrm{pmol} \cdot \mathrm{kg}^{-1} \mathrm{~min}^{-1}$. At the end of the $48 \mathrm{~h}$ infusions we performed two-step hyperglycemic clamps to evaluate glucose stimulated insulin secretion (GSIS) in vivo, or we isolated islets to evaluate GSIS ex vivo. We also exposed the islets in culture conditions to $0.4 \mathrm{mM}$ oleate in $0.5 \% \mathrm{BSA} \pm 4 \mu \mathrm{M} \mathrm{bpV}$ for $48 \mathrm{~h}$ to evaluate GSIS in vitro.

Results: $48 \mathrm{~h}$ infusion of oleate or olive oil impaired $\beta$-cell function in vivo $(\mathrm{p}<0.01)$ as measured with the disposition index during a two-step hyperglycemic clamp (13 and $22 \mathrm{mM}$ glucose), whereas coinfusion of bpV prevented the effect of oleate or olive oil. GSIS ex vivo in isolated islets of oleate or olive oil-treated rats was impaired (both oleate and olive oil $p<0.001$ vs. control, at 13 and $22 \mathrm{mM}$ ), but was restored by coinfusion of bpV (both oleate $+\mathrm{bpV}$ and olive oil $+\mathrm{bpV} p=\mathrm{NS}$ vs control), while $\mathrm{bpV}$ alone had no effect. Similar results were obtained in cultured islets, where $48 \mathrm{~h}$ exposure to oleate impaired the GSIS at $13 \mathrm{mM}$ (oleate: $0.125 \pm 0.023 \mathrm{pmol} /$ islet $/ \mathrm{h}, \mathrm{n}=10, \mathrm{p}<0.001$ vs. control: $0.248 \pm 0.022 \mathrm{pmol} /$ islet $/ \mathrm{h}, \mathrm{n}=9$ ), and $22 \mathrm{mM}$ (oleate: $0.212 \pm 0.026$ $\mathrm{pmol} /$ islet/h, $\mathrm{n}=10, \mathrm{p}<0.001$ vs. control: $0.396 \pm 0.033 \mathrm{pmol} / \mathrm{islet} / \mathrm{h}, \mathrm{n}=9)$, an effect prevented by bpV (oleate $+\mathrm{bpV}: 0.367 \pm 0.111 \mathrm{pmol} /$ islet $/ \mathrm{h}$ at $13 \mathrm{mM}$, $\mathrm{n}=7$, oleate $+\mathrm{bpV}: 0.539 \pm 0.147 \mathrm{pmol} / \mathrm{islet} / \mathrm{h}$ at $22 \mathrm{mM}, \mathrm{n}=7$, both $\mathrm{p}=\mathrm{NS}$ vs control), while $\mathrm{bpV}$ alone had no effect $(\mathrm{n}=4)$.

Conclusion: Our results suggest a role of FFA in decreasing $\beta$-cell function by impairing tyrosine phosphorylation at the $\beta$-cell level, consistent with the induction of $\beta$-cell insulin resistance.

Supported by: Canadian Institutes of Health Research

\section{6}

Direct effects of rapid acting insulin analogues on insulin signalling in human pancreatic islets

V. D'Aleo ${ }^{1}$, R. Lupi ${ }^{1}$, R. Mancarella ${ }^{1}$, S. Del Guerra ${ }^{1}$, U. Boggi ${ }^{2}$, F. Filipponi ${ }^{2}$, P. Marchetti ${ }^{1}$

${ }^{1}$ Endocrinology and Metabolism, ${ }^{2}$ Department of Oncology, Transplantation and Advanced Technologies in Medicine, University of Pisa, Pisa, Italy

Background and aims: In recent years, rapid acting insulin analogs (RAIA: Lispro, Aspart and Glulisine) have been introduced to optimize insulin treatment in diabetes. These molecules have similar, but not identical, pharmacodynamic and pharmacokinetic characteristics. In particular, work with cell lines and rodents have suggested that RAIA may have different insulin signaling system (SigSys) properties. Since SigSys is present in pancreatic islets and can modulate beta-cell function and survival, we studied the effects of Aspart, Glulisine and regular insulin (RI) on isolated human islets (IHI).

Materials and methods: IHI were prepared by collagenase digestion and density gradient purification by the pancreas of multiorgan donors (age: $70 \pm 13$ yrs; gender; 1 male and 3 females; body mass index: $24.7 \pm 1.9 \mathrm{~kg} / \mathrm{m} 2)$, and then cultured for $48 \mathrm{~h}$ in the presence of $5.5(\mathrm{~g})$ or $22.2(\mathrm{G}) \mathrm{mmol} / \mathrm{l}$ glucose, either with or without the addition of Aspart, Glulisine or RI $(10 \mathrm{nmol} / \mathrm{l})$. Functional and molecular studies were performed at the end of the different incubation conditions.

Results: Endogenous insulin secretion (C-peptide, pmol/l) during the $48 \mathrm{~h}$ culture was $1287 \pm 540$ at $g$ and increased to $3130 \pm 1666$ at $G(p<0.05)$. The presence of RAIA or RI did not affect this feature significantly. IHI pre-cultured at $\mathrm{g}$ and acutely challenged with 3.3 and $16.7 \mathrm{mmol} / \mathrm{l}$ glucose released $26.6 \pm 3.3$ and $56.7 \pm 5.7 \mu \mathrm{U} / \mathrm{ml}$ insulin, respectively $(\mathrm{p}<0.01)$. Pre-exposure 
to $G$ caused a significant $(\mathrm{p}<0.05)$ increase of insulin secretion (IS) at 3.3 $\mathrm{mmol} / \mathrm{l}$ glucose $(35.2 \pm 2.43 \mu \mathrm{U} / \mathrm{ml})$ and a reduction $(\mathrm{p}<0.05)$ of IS in response to $16.7 \mathrm{mmol} / \mathrm{l}$ glucose $(44.2 \pm 6.4 \mu \mathrm{U} / \mathrm{ml})$. These corresponded to stimulation index (SI, ratio or stimulated over basal insulin release) values of $2.2 \pm 0.5$ and $1.2 \pm 0.1(p<0.01)$, which were not further affected by the added insulins. Several changes at the level of molecules involved in the early steps of SigSys occurred at the end of the different incubation conditions. Compared to g, pre-exposure to $\mathrm{G}$ determined increased transcription of IRS-1 and IRS-2, and reduced expression of insulin receptor (IR), whereas phosphatidyl-inositol-3 kinase (PI3K, p110a subunit) did not change significantly. Glulisine did not affect IR expression, which however was inhibited by RI in the islets kept at $\mathrm{g}$ and induced by Aspart and RI in the islets pre-cultured at G. Modest changes (within 10\%) were observed as for the expression of IRS-1 and IRS-2, whatever the incubation condition. PI3K expression was significantly induced by Glulisine and Aspart at g, but not at G.

Conclusion: In summary, the results of the present study suggest that the tested RAIA and RI have different effects on the expression of molecules involved in the early steps of the insulin signaling system in human islets, with the overall action also depending on the degree of glucose exposure.

Supported by: sanofi-aventis

\section{PS 049 Other hormones}

\section{7}

Growth hormone increases intrahepatic rates of glycogen synthesis in growth hormone deficient adults

W. Schwenk ${ }^{1}$, S. Kirmani ${ }^{2}$

${ }^{1}$ Pediatrics and Adolescent Medicine, ${ }^{2}$ Medical Genetics, Mayo Clinic, Rochester, United States

Background and aims: We have previously shown that growth hormone replacement is associated with a $40 \%$ increase in intrahepatic rates of glycogen synthesis during refeeding in children with idiopathic growth hormone deficiency. Our aims were to see whether growth hormone replacement in adults with growth hormone deficiency would be associated with a similar increase in the rates of intrahepatic glycogen synthesis and to determine whether such an increase in the rates of intrahepatic glycogen synthesis was due to increased direct uptake of glucose by the liver (direct pathway) and/or increased rates of gluconeogenesis (indirect pathway).

Materials and methods: 5 adults with growth hormone deficiency were randomly studied twice, off and on growth hormone replacement (OFF and ON, respectively). Patients were fasted overnight and then infused with glucose $(15.7 \pm 0.4 \mu \mathrm{mol} / \mathrm{kg}$ per $\mathrm{min})$. Intrahepatic rates of glycogen synthesis were estimated by infusing $\left[1-{ }^{14} \mathrm{C}\right]$ galactose to steady state, administering acetaminophen, and measuring the specific activity $(\mathrm{SA})$ of $\left[{ }^{14} \mathrm{C}\right]$ acetaminophen glucuronide in the urine in order to calculate steady state intrahepatic uridine diphosphate glucose (UDP-glucose) flux. The percentage of UDP-glucose coming from direct uptake of glucose was estimated by infusing $\left[3-{ }^{3} \mathrm{H}\right]$ glucose, and measuring the SA of $\left[{ }^{3} \mathrm{H}\right\}$ acetaminophen glucuronide in the urine.

Results: Growth hormone replacement was associated with a $34 \%$ increase in estimated rates of intrahepatic glycogen synthesis (4.42 \pm 0.76 vs $5.90 \pm$ $0.81 \mu \mathrm{mol} / \mathrm{kg}$ per minute for OFF and ON, respectively; $\mathrm{p}<0.05)$ The percentage of glucose coming from direct uptake of glucose was not affected by growth hormone treatment ( $44.6 \pm 5.9 \%$ vs $47.4 \pm 5.8 \%$ for OFF and ON, respectively). In summary, growth hormone treatment in growth hormone deficiency adults increased estimated rates of intrahepatic glycogen synthesis by increasing the amount of glucose entering UDP-glucose via the direct and indirect pathways equally.

Conclusion: Growth hormone appears to increase intrahepatic rates of glycogen synthesis not only by stimulating gluconeogenesis, but also by increasing direct uptake of glucose.

Supported by: Genetech Foundation

\section{8}

Liver is the primary site for $11 \beta H S D 1$ gene expression in splanchnic tissues in humans

A. Basu' ${ }^{1}$, R. Basu' 1 , M. Grudzien², P. Jung², P. Jacobson ${ }^{2}$, M.G. Sarr

R.A. Rizza ${ }^{1}$

${ }^{1}$ Endocrinology,Metabolism \& Nutrition, Mayo Clinic College Of Medicine, Rochester, ${ }^{2}$ Global Pharmaceutical Research \& Development, Abbott, Abbott Park, ${ }^{3}$ Surgery, Mayo Clinic College Of Medicine, Rochester, United States

Background and aims: Experiments in the awake dog model suggest that the liver is the primary site for splanchnic cortisol production with minimal cortisol production occurring in the viscera. The purpose of this study was to compare gene expression between liver and omental fat in obese humans for genes regulating the glucocorticoid receptor (NR3C1), and 11 beta hydroxysteroid dehydrogenase enzymes (11ßHSD1 and 2). 11ßHSD1 converts inactive cortisone to active cortisol while 11ßHSD2 converts cortisol to cortisone. These enzymes modulate the availability of cortisol to bind to its receptor (NR3C1). Since splanchnic cortisol production has been implicated in obesity and type 2 diabetes, this information would be key for future development of tissue-specific therapeutic targets.

Materials and methods: Liver and omental fat tissue from subjects ( $\mathrm{n}=7$; age $53 \pm 3 \mathrm{yrs}$; BMI $46 \pm 3 \mathrm{~kg} / \mathrm{m}^{2}$ ) were collected during routine abdominal surgery. Samples from each subject were quick frozen with liquid nitrogen and stored in dry ice. $150 \mathrm{mg}$ (liver) and $200 \mathrm{mg}$ (fat) sections were weighed and lysed using an appropriate amount of Qiazol Lysis Reagent and mechanical homogenization. Qiazol homogenates were used to isolate total RNA using automated Qiagen RNeasy technology on the Qiagen BioRobot 3000. Reverse transcription and PCR were performed at standard temperatures using the 
Invitrogen SuperScript III Platinum One-Step Quantitative RT-PCR System. All samples were analyzed for RNA integrity using the Agilent 2100 Bioanalyzer. The samples were run in triplicates. Data analysis was performed by first normalizing all data to the Total Human Reference RNA standard curve for each gene to achieve a relative amount of target RNA (ng). Then, each sample was normalized to the average amount of RNA (ng) for the three control genes (PPIA, RPLPO, and RPL19). This approach permitted assessment of relative expression between samples of interest for a specific target gene. However comparison of absolute amounts of expression between genes was not possible.

Results: NR3C1 gene expression was detected in all tissues tested, with no difference in expression between liver and omental fat. 11ßHSD1 gene expression was highly abundant in liver.11ßHSD1 expression in liver and fat was variable between subjects, but expression was $\sim 9$ fold higher in liver tissue versus omental fat. In contrast, although detected, $11 \beta \mathrm{HSD} 2$ gene expression was very low $(<0.5$ relative ng of human reference RNA standard curve) in both liver and omental fat and neither tissue type had elevated expression over the other.

Conclusion: These data shows that in obese humans, gene expression of $11 \beta H S D 1$ occurs predominantly in the liver compared to omental fat. This implies that the liver is likely to be the primary site of splanchnic cortisol production in humans.

Supported by: NIH

\section{9}

Combined treatment with epoetin- $\beta$ and testosterone (mix of esters) of males suffering with diabetes of type 1 and receiving haemodialysis A. Lepetukhin ${ }^{1}$, N. Goncharov ${ }^{1}$, M. Shestakova ${ }^{1}$, A. Mordik ${ }^{2}$, N. Biskaeva ${ }^{2}$ ${ }^{1}$ Diabethic nephropathy, Research Center of Endocrinology, ${ }^{2}$ Diabethic nephropathy, Extracorporeal therapy center "Fespharm", Moscow, Russian Federation

Background and aims: investigation of androgenic deficit impact on erythropoietin and correction of anemia among males who suffer from with diabetes mellitus of type 1 (T1 DM) and receive haemodialysis by virtue of combined therapy with epoetin- $\beta$ (EPO- $\beta$ ) and testosterone (mix of esters, TME).

Materials and methods: 43 males suffering with type1 (T1DM) and receiving haemodialysis were included in investigation. Males younger that 40 years old (median age is $34.0 \pm 3.9$ years) comprised group 1. Males of 40 years old and older (median age is $47.3 \pm 3.5$ years) comprised group 2. Level of total testosterone (tT) was $21.2 \pm 6.6 \mathrm{nmol} / \mathrm{l}$. tT level in group 2 was $9.8 \pm 3.9 \mathrm{nmol} / \mathrm{l}$. Subgroups for treatment (combined treatment with EPO- $\beta$ and TME) and control subgroup (treatment only with EPO- $\beta$ ) were organized: $\mathrm{A}(\mathrm{n}=14)$ and $B(n=6)$ in group 1 and $C(n=14)$ and $D(n=9)$ in group 2 , respectively. Subgroups for treatment and control didn't differ by age, duration of DM and haemodialysis therapy, by level of HbAlc (\%), $\mathrm{Hb}(\mathrm{g} / \mathrm{l}), \mathrm{tT}(\mathrm{nmol} / \mathrm{l})$. Duration of investigation comprised 6 months. EPO- $\beta$ was prescribed in according to $\mathrm{Hb}$ level , 1/0 $\mathrm{ml}$ of TME was prescribed once in three weeks. $\mathrm{tT}$ level after injection of TME was measured 24 hours, 1,2 , and 3 weeks after the injection and was equal to $60.4 \pm 12.9,26.7 \pm 6.8,17.2 \pm 3.4$, and $11.9 \pm 3.9 \mathrm{nmol} / 1$, respectively. Statistic processing of data was performed with use of non-parametric methods ( $\mathrm{p}$ ), data are presented as $\mathrm{M} \pm \mathrm{SD}$.

Results: no authentic and reliable increase of $\mathrm{Hb}$ level was observed in group 1. Increase of $\mathrm{Hb}$ level by $16.2 \%$ was observed in subgroup C. Decrease of EPO- $\beta$ dose by $32.1 \%$ and $41.1 \%$ a week was observed in subgroups A and C, respectively. Authentic and reliable decrease of EPO- $\beta$ dose in a week was not observed in control groups (see Table).

Indicants before and after treatment $(\mathrm{M}+\mathrm{SD})$

\begin{tabular}{lllll}
\hline Groups & Indicants & Before treatment & $\begin{array}{l}6 \text { months after } \\
\text { treatment }\end{array}$ & p-value \\
\hline Group1 & $\mathrm{A} \mathrm{Hb}(\mathrm{g} / \mathrm{l})$ & $118.1 \pm 9.6$ & $118.2 \pm 6.7$ & 0.8 \\
& $\mathrm{~B}$ & $113.0 \pm 5.7$ & $109.6 \pm 9.4$ & 0.17 \\
\cline { 2 - 5 } & $\mathrm{A} \mathrm{EPO}-\beta(\mathrm{ME} /$ week $)$ & $3857.1 \pm 1231.4$ & $2428.5 \pm 851.6$ & 0.007 \\
$\mathrm{~B}$ & $3334.3 \pm 1032.8$ & $3666.6 \pm 1505.5$ & 0.5 \\
\hline Group2 & $\mathrm{C} \mathrm{Hb}(\mathrm{g} / \mathrm{l})$ & $94.9 \pm 5.7$ & $113.5 \pm 6.3$ & 0.009 \\
& $\mathrm{D}$ & $98.4 \pm 7.0$ & $99.4 \pm 6.8$ & 0.5 \\
\cline { 2 - 5 } & $\mathrm{C}$ EPO- $\beta(\mathrm{ME} /$ week $)$ & $7428.5 \pm 1452.5$ & $4285.7 \pm 1325.9$ & 0.001 \\
$\mathrm{D}$ & $7111.2 \pm 1242.9$ & $7233.3 \pm 1414.2$ & 0.7 \\
\hline
\end{tabular}

Conclusion: reduction of testosterone level in blood of males with type $1 \mathrm{DM}$ receiving haemodialysis is of age-dependent nature. Combined treatment with EPO- $\beta$ and TME contributes to increase of $\mathrm{Hb}$ level in blood of senior age group and to reduction of need in EPO- $\beta$ irrespective of patient age.

\section{0}

Dehydroepiandrosterone (DHEA) reduced preadipocyte proliferation via androgen receptor

T. Ishizuka, K. Kajiata, T. Ikeda, I. Mori, Y. Uno, K. Fujioka, H. Morita Department of General Internal Medicine, Gifu Graduate School of Medicine, Japan

Background and aims: A great number of evidences indicate that DHEA administration results in reduced adiposity in men and animals. On the other hand, recent researches revealed that low testosterone (TEST) levels are associated with type 2 diabetes and obesity in men. Considering the fact that androgen receptor (AR) deficient mice represent obesity, AR acts to prevent fat accumulation in whole body. We have previously shown that senescence associated $\beta$-galactosidase (SA- $\beta$-Gal) was detected in adipose tissue isolated from obese animal was prevented with DHEA administration. In this study, we have further examined the effect of DHEA on telomere length and preadipocyte distribution in adipose tissue. Moreover, we assessed the role of AR in DHEA-induced growth suppression in preadipocyte.

Materials and methods: Male Otsuka Long Evans Fatty (OLETF) rats, hereditary obese type 2 diabetic animals derived from Long Evans Tokushima (LETO) rats, LETO rats were fed with or without (control) $0.4 \%$ DHEA containing food for $52 \mathrm{wk}$. Two $\mu \mathrm{g}$ genomic DNA isolated from adipose tissue was digested with restriction enzyme, Hinf 1/Rsal, and then Southern analysis was performed to measure telomere length. Moreover, 3T3-L1 preadipocytes were treated with DHEA and TEST for $24 \mathrm{hr}$, and then cell proliferation was assayed with BrdU uptake. We also assessed the effect of flutamide, AR inhibitor, and fulvestrant, estrogen receptor (ER) inhibitor, on DHEA-induced reduction of cell growth utilizing siRNA.

Results: Treatment with DHEA for 52 wk reduced body weight and fat weight, but not food consumption, in LETO and OLETF rats. These results clearly demonstrated that treatment with DHEA for $52 \mathrm{wk}$ decreased fat mass without affecting food intake. Decreased telomere length was observed in genomic DNA isolated from adipose tissue in control OLETF, which was prevented with DHEA administration. These results indicated that accelerated cell division associated with obese adipose tissue resulted in rapid telomere shorting. These results suggested that DHEA-induced growth suppression in preadipocyte may lead to attenuate subsequent differentiation, which resulted in increased preadipocyte cell number. Actually, incubation with DHEA and TEST decreased BrdU uptake to the similar extent in 3T3-L1 preadipocyte. Pretreatment with flutamide, but not fulvestrant abolished this effect. AR siRNA also inhibited DHEA-induced decreases in BrdU uptake. Moreover, we found that no difference was observed between DHEA and TEST on cell growth. Recently, three major mechanisms of DHEA action, precursor of sex steroid, neurosteroid and activator of calcium-gated potassium channels, have been postulated. Especially, researchers have revealed that inactive steroid hormones are selectively uptaken and converted into active form, of which mechanism is named intracrine, with terminal steroid transforming enzymes, such as $17 \beta-\mathrm{HSD}$ and $3 \beta$-HSD in peripheral tissue. Our results of inhibitor and siRNA study revealed that this effect was mediated via AR. Conclusion: These results suggested that DHEA-induced suppression of preadipocyte proliferation, might lead to anti-obesity and anti-senescence effect.

\section{1}

Molecular analysis of postprandial glucagon responses in patients with type 2 diabetes mellitus

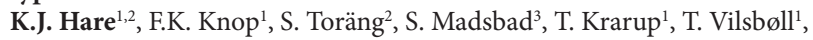
J.J. Holst ${ }^{4}$

${ }^{1}$ Department of Internal Medicin F, Gentofte Hospital, Hellerup, ${ }^{2}$ Department of Biomedical Sciences, University of Copenhagen, ${ }^{3}$ Department of Endocrinology, Hvidovre Hospital, ${ }^{4}$ Department of Biomedical Scieces, University of Copenhagen, Denmark

Background and aims: Hyperglucagonemia and lack of postprandial glucagon suppression, as measured by conventional C-terminal radioimmunoassay (RIA) is thought to contribute to the hyperglycemia in patients with type 2 
diabetes mellitus (T2DM). The proglucagon (PG) gene is expressed both in pancreatic alpha-cells and in intestinal L-cells. Processing of the gene is different in the two cell types as glucagon is only secreted from the alpha-cell. The aim of this study was to clarify the nature of the hypersecreted glucagon in patients with T2DM - is it fully processed glucagon or is it an intestinally derived PG product?

Materials and methods: Ten patients with T2DM (age: 64 [51-80] years; body mass index (BMI): 23 [21-26] kg/m²; $\mathrm{HbA}_{1} \mathrm{c}$ : 6.9 [6.2-8.7]\%; mean values [range]) and ten healthy control subjects matched for sex, age and BMI were studied. Blood was sampled following a 50-g OGTT. In the control subjects an appropriate suppression of glucagon was observed during the first 45 min of the OGTT (-36 $\pm 12 \mathrm{mM} \mathrm{x} 45 \mathrm{~min})$ whereas the patients hypersecreted glucagon in response to the OGTT ( $10 \pm 16 \mathrm{mM} \mathrm{x} 45 \mathrm{~min})$. Plasma samples (obtained at the time of the glucagon peak) were pooled, acidified and applied to a HPLC Vydac $\mathrm{C}_{18}$ column. Eluted fractions were analyzed with 3 different glucagon RIAs with antibodies directed towards the mid region and the $\mathrm{N}$ - and C-terminals of the molecule.

Results: There was high glucagon immunoreactivity in samples from patients with T2DM, but very little in samples from control subjects due to adequate suppression of glucagon. The analysis of the fractionated plasma from T2DM showed that over $80 \%$ of the immunoreactivity identified by the C-terminal RIA was due to fully processed glucagon (33-61) (coelution with glucagon standard, identification in all 3 assays).

Conclusion: We conclude that the postprandial hyperglucagonemia in T2DM represents fully processed glucagon and therefore probably results from inappropriate secretion of the pancreatic alpha-cell rather than representing intestinally derived forms.

Suported by: Novo Nordisk Foundation, Nordic Network of Endocrinology and Diabetes

\section{2}

Prednisolone-treatment increases fasting glucose and insulin levels, but does not affect hepatic and peripheral insulin sensetivity

A.J. Laskewitz ${ }^{1}$, T.H. van Dijk ${ }^{1}$, A. Grefhorst ${ }^{1}$, D.J. Reijngoud ${ }^{1}$, W. Dokter ${ }^{2}$,

F. Kuipers ${ }^{1}$

${ }^{1}$ Research lab Pediatrics, University Medical Center Groningen,

Netherlands, ${ }^{2}$ Department of Pharmacology, NV Organon, part of ScheringPlough Corp., Oss, Netherlands

Background and aims: Synthetic glucocorticoids, such as prednisolone and dexamethasone, are widely used as anti-inflammatory and immunosuppressive drugs for treatment of chronic inflammatory diseases. However, chronic use of glucocorticoids is limited by their severe side effects. Insulin resistance is commonly associated with chronic use of glucocorticoids. The underlying mechanisms of glucocorticoid-induced insulin resistance, however, are still elusive. To identify these mechanisms we characterized glucocorticoid-induced insulin resistance in $\mathrm{C} 57 \mathrm{Bl} / 6 \mathrm{~J}$ mice fed either a chow diet or a high fat diet to induce a state of compromised insulin sensitivity.

Materials and methods: C57Bl/6J mice were fed a normal chow (C) diet or a high fat (HF 36\%) diet for 6 weeks and were treated with either vehicle (V) or prednisolone $(\mathrm{P})(10 \mathrm{mg} / \mathrm{kg})$ for the last 7 days. Mice were extensively characterized for general metabolic parameters. Insulin resistance was assessed by a whole body glucose test (WBGT), a hyperinsulinemic euglycemic clamp (HIEC) and in vivo measurements of hepatic carbohydrate metabolism by employing mass isotopomer distribution analysis (MIDA) after an overnight fast. For the WBGT mice were injected with a trace amount of $\left[\mathrm{U}-{ }^{13} \mathrm{C}\right]$-glucose from which endogenous glucose production (EGP) and metabolic clearance rate (MCR) were calculated. For MIDA procedure, mice were infused with a solution containing $\left[\mathrm{U}-{ }^{13} \mathrm{C}\right]$-glucose, $\left[2-{ }^{13} \mathrm{C}\right]$-glycerol, $\left[1-{ }^{2} \mathrm{H}\right]$-galactose and paracetamol to quantify relevante fluxes of hepatic glucose metabolism. Results: Prednisolone treatment in combination with a high fat diet increased the HOMA-score (CV $0.96 \pm 0.47$ vs CP $2.32 \pm 2.59$, ns; HFV $3.59 \pm 3.44$ vs HFP $7.27 \pm 3.48 \mathrm{p}<0.05)$. In addition, plasma cholesterol (CV $2.96 \pm 0.32$ vs CP $3.38 \pm 0.61$, ns; HFV $5.85 \pm 0.41$ vs HFP $7.27 \pm 0.99 \mathrm{mmol} / \mathrm{l}, \mathrm{p}<0.05)$ and triglycerides (CV $0.74 \pm 0.19$ vs CP $1.03 \pm 0.28, \mathrm{p}<0.05$; HFV $1.29 \pm 0.18$ vs HFP $1.88 \pm 0.39 \mathrm{mmol} / \mathrm{l}, \mathrm{p}<0.05)$ were increased upon prednisolone treatment. An increased endogenous glucose production and reduced metabolic clearance rate normalized for insulin levels in the WBGT suggested glucocorticoid-induced insulin resistance in prednisolone-treated mice. However, when insulin resistance was assessed with the "gold standard", i.e., HIEC, no evidence for insulin resistance on the basis of glucose infusion rates (CV $617 \pm 28 v s$ CP $612 \pm 23$, ns; HFV $232 \pm 25$ vs HFP $253 \pm 24 \mu \mathrm{mol} / \mathrm{kg} / \mathrm{min}$, ns) was observed, although high fat diet clearly reduced the glucose infusion rate required to maintain euglycemia, establishing diet-induced insulin resistance. At hepatic level, a clearly negative glycogen balance was induced by prednisolone treatment (CV $-0.48 \pm 3.7$ vs CP $-23.68 \pm 3.22$, $\mathrm{p}<0.05$; HFV 9.8 $\pm 4.0 v s$ HFP $-11.8 \pm 7.5, \mathrm{p}<0.05)$, indicative for adapted glucose-6-phosphate flux to glycogen.

Conclusion: Our results demonstrate that prednisolone does not induce a clear insulin resistance in mice, but strongly affects hepatic glucose metabolism. In addition, this study clearly establishes that fasting glucose and insulin levels, routinely translated into a HOMA-score, do not necessarily reflect insulin sensitivity in mice.

Supported by: Dutch Top Institute Pharma

\section{3}

Acute and subacute exposure to glucocorticoids differentially impair various aspects of beta cell function in healthy males

D.H. van Raalte ${ }^{1}$, A. Mari ${ }^{2}$, M.C. Bunck ${ }^{1}$, V. Nofrate ${ }^{2}$, U. Nässander ${ }^{3}$, W.H.A. Dokter ${ }^{3}$, R.J. Heine ${ }^{1}$, M. Diamant ${ }^{1}$

${ }^{1}$ Department of Endocrinology / Diabetes Centre, VU University Medical Centre, Amsterdam, Netherlands, ${ }^{2}$ Institute of Biomedical Engineering, National Research Council, Padova, Italy, ${ }^{3} \mathrm{NV}$ Organon, part of ScheringPlough Corporation, Oss, Netherland

Background and aims: Glucocorticoids are the most frequently prescribed anti-inflammatory agents, but their use is also associated with dysmetabolic side-effects, including glucose intolerance and diabetes. Although glucocorticoid-induced insulin resistance is well-established, the effects of glucocorticoids on beta-cell function, and in particular under physiological conditions, are less well characterized.

Materials and methods: We conducted a randomized, double-blind trial in healthy men (age $25.5 \pm 4.8$ yrs; BMI $\left.24.1 \pm 1.7 \mathrm{~kg} / \mathrm{m}^{2}\right)$. Participants $(\mathrm{n}=23)$ received either prednisolone $30 \mathrm{mg}$ or placebo once daily for a period of 15 days (study 1). In study 2, a single oral dose of $75 \mathrm{mg}$ prednisolone was administered to 6 healthy men. High-fat mixed-meal tests (34g protein, $50 \mathrm{~g}$ fat, $75 \mathrm{~g}$ carbohydrates) were performed before and during glucocorticoid therapy (studies 1 and 2), and after discontinuation (study 2). Insulin secretion rate was calculated by C-peptide deconvolution and beta-cell function was evaluated with a model that describes insulin secretion rate as a function of absolute glucose levels (insulin secretory tone and glucose sensitivity), the glucose rate of change (rate sensitivity), and a potentiation factor.

Results: At day 15, prednisolone $30 \mathrm{mg}$ daily increased fasting glucose $(\mathrm{p}=$ $0.023)$, and decreased insulin sensitivity $(\mathrm{p}=0.03)$, without affecting nonesterified fatty acid- and triglyceride-levels. During the meal, prednisolone augmented the area under the glucose response curve $\left(\mathrm{AUC}_{\text {gluc }}\right)(\mathrm{p}<0.001)$, despite a marked increase in insulin secretion $(p=0.049)$. Prednisolone impaired various indices of beta-cell function, including the insulinogenic index adjusted for insulin sensitivity $(\mathrm{p}=0.08)$ and potentiation $(\mathrm{p}=0.007)$, however, glucose- and rate sensitivity were unaltered. A single dose of $75 \mathrm{mg}$ prednisolone acutely induced insulin resistance, and markedly decreased the insulin response to the meal test, resulting in an increased $\mathrm{AUC}_{\text {gluc }}(\mathrm{p}=0.003)$. Acute high-dose of prednisolone impaired glucose sensitivity $(\mathrm{p}=0.033)$ and potentiation $(\mathrm{p}=0.032)$, but did not affect rate sensitivity. One day after discontinuation of the single high prednisolone-dose, all values had normalized, except for insulin secretion which rose during the meal test $(\mathrm{p}<0.001)$.

Conclusion: Acute and short-term exposure to glucocorticoids differentially affect beta-cell function. Collectively, these abnormalities, in addition to glucocorticoid-induced insulin resistance, may account for glucocorticoid-related diabetogenic effects.

\section{4}

Characterisation of alpha cell function in Africans with ketosis-prone non-autoimmune diabetes during insulin-free remission

S. Choukem ${ }^{1}$, E. Sobngwi ${ }^{1,2}$, P. Boudou ${ }^{3}$, L.-S. Fetita ${ }^{1}$, P. Vexiau ${ }^{1}$, F. Calvo ${ }^{4}$, J.-F. Gautier ${ }^{1,5}$

${ }^{1}$ Endocrinology and Diabetes, Saint Louis Hospital, Paris, France, ${ }^{2}$ Institute of Health and Society, University of Newcastle, United Kingdom, ${ }^{3}$ Hormones Laboratory, Saint Louis Hospital, Paris, France, ${ }^{4} \mathrm{Clinical}$ Investigation Centre, Saint Louis Hospital, Paris, France, ${ }^{5} \mathrm{Umrs} 872$, INSERM, Paris, France

Background and aims: Ketosis-prone non-autoimmune diabetes (KPD), also known as idiopathic type 1 or type $1 \mathrm{~B}$ diabetes is a syndrome of un- 
clear pathogenesis found mainly in people of sub-Saharan African ancestry. It is characterised by an acute onset with ketosis or ketoacidosis that requires urgent insulin treatment. This is followed by a prolonged long-term nearnormoglycaemic remission without insulin treatment in more than $50 \%$ of patients. The aim of this study was to characterize the alpha cell function during remission.

Materials and methods: We studied 19 sub-Saharan Africans with KPD during insulin-free remission (HbAlc $\leq 7 \%$ for $\geq 3$ months without insulin) along with 19 control subjects with normal glucose tolerance, matched for age ( $44.5 \pm 2.4$ vs. $43.7 \pm 1.9$ years respectively), sex and geographical origin. Glucagon levels were measured in response to oral glucose (75g-OGTT), to IV glucose (200-min glucose ramp from 2 to $10 \mathrm{mg} \cdot \mathrm{kg}^{-1} \cdot \mathrm{min}^{-1}$ glucose infusion), to arginine (5g IV at the end of the glucose ramp) and to induced hyperinsulinaemia (euglycaemic hyperinsulinaemic clamp). The product glucagon $\mathrm{x}$ glucose $\left(10^{-3} \mathrm{pg} . \mathrm{mmol}_{\mathrm{ml}} \mathrm{l}^{-2}\right)$ was used as a surrogate of the resistance to glucagon suppression by glucose during OGTT. During the glucose ramp, the slope of glucagon decrement per glucose increment was used to measure the dose-response effect. The incremental area under the curve $\left(\mathrm{AUC}_{\mathrm{T}}\right)$ and the acute glucagon response to arginine (AGR $=$ [mean glucagon concentration from 2 to 5 min post-arginine bolus] - [glucagon concentration prior to the bolus]) were estimated in response to arginine. Results are expressed as percentage or mean \pm standard error. Comparison between groups was performed using the non-parametric Mann-Whitney $\mathrm{U}$ test.

Results: Basal glucagon level prior to each test was similar between groups. During OGTT, the resistance index was $881.4 \pm 105.5$ vs. $614.5 \pm 48.7(\mathrm{p}=$ $0.028)$ at baseline, $1576.9 \pm 138.7$ vs. $1188.4 \pm 130.2(\mathrm{p}=0.049)$ at $30 \mathrm{~min}$, and $1680.8 \pm 147.2$ vs. $652.6 \pm 39.9$ ( $\mathrm{p}<0.001)$, at $120 \mathrm{~min}$ in patients and controls respectively. During the glucose ramp, each glucose increment of $1 \mathrm{mmol} / \mathrm{l}$ lead to a $1.6 \pm 0.2 \%(1.9 \pm 0.3 \mathrm{pg} / \mathrm{ml})$ decrease in glucagon concentrations in patients vs. $4.5 \pm 0.6 \%(5.3 \pm 0.9 \mathrm{pg} / \mathrm{ml})$ in controls $(\mathrm{p}=0.001)$. During the hyperinsulinaemic clamp, the glucagon levels decreased from baseline by the same extend in both groups (patients: $119.9 \pm 10.7$ to $81.3 \pm 7.1 \mathrm{pg} / \mathrm{ml}$, controls: $124.4 \pm 9.3$ to $89.0 \pm 8.2 \mathrm{pg} / \mathrm{ml}$; p = NS). After adjustment to insulin and glucose levels prior to the arginine bolus, no difference was found between groups in response to arginine using either the $\mathrm{AUC}_{\mathrm{I}}$ or the AGR.

Conclusion: During remission, patients with KPD display alterations of glucagon suppression in response to oral and IV glucose similar to those reported in patients with type 2 diabetes. These results suggest that KPD should be considered as a form of type 2 diabetes.

Supported by: An institutional grant (PHRC) and Association Française des Diabétiques

\section{5}

A NO/sGC/cGMP dependent mechanism is involved in the regulation of net hepatic glucose uptake by hepatic nitric oxide in vivo

Z. An, J.J. Winnick, M.C. Moore, D. Neal, B. Farmer, T. Rodewald, W. Snead, A.D. Cherrington

Department of Molecular Physiology and Biophysics, Vanderbilt University School of Medicine, Nashville, United States

Background and aims: Our previous study showed that portal but not peripheral infusion of a nitric oxide donor (SIN-1) reduced net hepatic glucose uptake (NHGU) in the presence of the portal glucose delivery, hyperglycemia and hyperinsulinemia, suggesting that the increased hepatic NO resulting from portal SIN-1 infusion regulates NHGU. Because NO has been shown to activate soluble guanylate cyclase (sGC), the aim of the present study was to determine whether a sGC/cGMP dependent mechanism could be involved in the regulation of NHGU by hepatic NO.

Materials and methods: Studies were performed on two groups of 42-h-fasted conscious dogs that had undergone laparotomy for vascular cannulation $\sim 16 \mathrm{~d}$ before study. After a basal period (-30 to $0 \mathrm{~min}$ ), somatostatin was given peripherally along with $4 \mathrm{X}$ basal insulin and basal glucagon intraportally. At $0 \mathrm{~min}$, glucose was delivered at a variable rate via a leg vein to increase the hepatic glucose load to $2 \mathrm{X}$ basal. At $90 \mathrm{~min}$, an intraportal infusion of a sGC inhibitor (ODQ, $0.8 \mu \mathrm{g} / \mathrm{kg} / \mathrm{min}$ ) was started in the ODQ group ( $\mathrm{n}=10)$ while saline was given in the CON group $(n=10)$.

Results: No significant hemodynamic changes were observed in either group. The arterial insulin levels were $4 \mathrm{X}$ basal $(22 \pm 2$ and $21 \pm 2 \mu \mathrm{U} / \mathrm{mL})$ while glucagon was basal $(40 \pm 4$ and $39 \pm 4 \mathrm{pg} / \mathrm{mL})$ in the CON and ODQ groups, respectively. NHGU $(\mathrm{mg} / \mathrm{kg} / \mathrm{min})$ was $2.4 \pm 0.3$ and $2.3 \pm 0.3$ in the CON and ODQ groups, respectively prior to ODQ infusion (0-90 min). During the last half hour of the ODQ infusion period (240-270 min), NHGU was $3.2 \pm 0.3$ and $4.9 \pm 0.5(\mathrm{P}<0.05)$ in the CON and ODQ groups respectively.
Conclusion: The NO/sGC/cGMP dependent pathway has the ability to regulate net glucose uptake by the liver in vivo in the conscious dog. Supported by: National Institutes of Health and Vanderbilt University Diabetes Research and Training Center

\section{6}

The changes of plasma FGF-21 levels in type 2 diabetic mellitus, diabetic ketosis and normal subjects

G. Yang ${ }^{1}$, L. $\mathrm{Li}^{2}$, M. Yang ${ }^{1}, \mathrm{~N} . \mathrm{He}^{1}$

${ }^{1}$ Department of Endocrinology, the Second Affiliated Hospital, ${ }^{2}$ Department of Clinical Biochemistry, Chongqing Medical University, Chongqing, China

Background and aims: Fibroplast growth factor-21 (FGF-21) has been recently characterized as a potent metabolic regulator, but its pathophysiologic role in humans remains unknown. In this study we investigate whether plasma FGF-21 level is different in patients with type 2 diabetes mellitus (T2DM) and diabetic ketosis (T2DK).

Materials and methods: Sixty-eight patients with T2DM, 41 subjects with T2DK, and 52 sex-and age - matched normal controls participated in the study. Plasma FGF-21 levels were measured with a radioimmunoassay. The relationship between plasma FGF-21 levels and anthropometric and metabolic parameters was also analyzed.

Results: Plasma FGF-21 levels were higher in patients with T2DK and T2DM than in controls $(4.05 \pm 0.18$ and $2.82 \pm 0.14$ vs. $2.28 \pm 0.16 \mu \mathrm{g} / \mathrm{L}, P<0.01$ and $P<0.05$, respectively). And it was higher in patients with T2DK than T2DM $(P<0.01)$. Fasting plasma FGF-21 was found to correlate positively and significantly with SBP $(r=0.17, P<0.05), \mathrm{DBP}(r=0.18, P<0.05), \mathrm{FBG}$ $(r=0.52, P<0.01), 2 \mathrm{hPBG}(r=0.57, P<0.01), \mathrm{HbA}_{1} \mathrm{c}(r=0.43, P<0.01)$, HDL-C $(r=0.18, P<0.05)$ and FFA $(r=0.18, P<0.05)$, but negatively with fasting plasma insulin $(r=-0.38, P<0.01), 2 \mathrm{hIns}(r=-0.54, P<0.01)$ and HOMA- ${ }_{\text {IS }}(r=-0.54$, $P<0.01)$. Multiple regression analysis showed that DBP, WHR, 2hIns, $2 \mathrm{hPBG}$ and FFA were independent related factors influencing plasma FGF-21 levels $\left(\mathrm{Y}_{\mathrm{FGF}-21}=3.186+0.058 \mathrm{X}_{2 \mathrm{hPG}}-0.007 \mathrm{X}_{2 \mathrm{hIns}}+0.491 \mathrm{X}_{\mathrm{FFA}^{-}}-3.206 \mathrm{X}_{\mathrm{WHR}}+0.021 \mathrm{X}_{\mathrm{DBP}}\right)$. Multivariate logistic regression analysis showed that plasma FGF-21 level was also significantly associated withT2DM and T2DK even after controlling for anthropometric variables, blood pressure and lipid profile. Increasing levels of FGF-21 showed a significant linear trend and were independently associated with T2DM, especially when concentrations were analysised both by Mow Mean Scores and Cochran-Armitage Trend Test

Conclusion: Our results of elevated FGF-21 in T2DM and T2DK indicate that FGF-21 may play a role in the pathogenesis of T2DM and T2DK. However, further experiments need to be done to clarify the role of FGF-21. Supported by: National Natural Science Foundation of China and Chongqing Municipal Education Commission 


\section{PS 050 Exercise physiology}

\section{7}

Blood glucose homeostasis of patients with type 1 diabetes mellitus: effects of physical activity at high compared to low altitude

T. Siegmund ${ }^{1}$, B. Gutt ${ }^{1}$, A. Wiesmeth ${ }^{1}$, S. Graf ${ }^{1}$, R. Fischer ${ }^{2}$,

P.-M. Schumm-Draeger ${ }^{1}$

${ }^{1}$ Endocrinology, Diabetes and Vascular Medicine, Klinikum Munich GmbH,

${ }^{2}$ Medical Department, University Hospital Munich, Germany

Background and aims: Increasing numbers of patients with type 1 diabetes practice intense forms of physical activity at higher altitudes, including highaltitude trekking, mountain climbing, mountain biking and skiing. To date only marginal data is available comparing blood glucose (BG) homeostasis at low and high altitudes.

Materials and methods: We conducted a study with 10 patients with type 1 diabetes performing an insulin pump therapy. All participants performed two standardized endurance trainings periods ( 75 watts over 2 hours) on a bike ergometer at low altitude (L), in Munich $530 \mathrm{~m}$ above sea level and at high altitude on the Zugspitze mountain $(\mathrm{H})$, Environmental Research Station Schneefernerhaus, $2650 \mathrm{~m}$ above see level. To evaluate differences in BG homeostasis BG measurements as well as the continuous glucose monitoring system (CGMS, Medtronic) were used. Consumption of carbohydrates (C) and insulin for BG stabilisation was allowed.

Results: Data is presented as medians and ranges. Before the exercise BG was similar at L (0 min: $7.7 \mathrm{mmol} / \mathrm{l}[6.4-15.8])$ and $\mathrm{H}(0 \mathrm{~min}: 8.9 \mathrm{mmol} / \mathrm{l}$ [4.1-12.2], n.s.). Two hours after starting the endurance training BG was significantly lower in L (6.3 mmol/l [3.6-7.9]; $\mathrm{p}=0.04)$ as well as $\mathrm{H}(6.6 \mathrm{mmol} / \mathrm{l}$ [2.6-12.5]; $\mathrm{p}=0,04)$. Ingested C were comparable (L: 42 gr. [0-96]; H: 36 gr. [0-84]; n.s.). In L, 8 out of 10 participants showed a trend of initial BG rising, after $80 \mathrm{~min}$, BG levels returned to their starting level. After $120 \mathrm{~min}$ BG was significantly lower $(6.2 \mathrm{mmol}$ [3.8-7.7]; $\mathrm{p}=0.04)$. In contrast at $\mathrm{H}, 8$ out of 10 participants showed an immediate BG drop, after 25 min BG was significantly lower $(6.9 \mathrm{mmol} / 1$ [2.2-11.2]; $\mathrm{p}=0.03$ and stayed significantly lower until the end of the exercise.

Conclusion: At high altitude $(2650 \mathrm{~m})$ physical activity caused a significantly early BG drop compared to low altitude $(530 \mathrm{~m})$. Patients with type 1 diabetes should reduce insulin dose appropriately or ingest more carbohydrates when exercising at high altitude.

Supported by: German Society for Mountain Medicine

\section{8}

Moderate intensity exercise was more effective in body fat loss, but vigorous exercise improved the insulin resistance in overweight type 2 diabetes mellitus

K. Han, B. Koo, H. Kim, H.-J. Kim, K. Pak, K. Min, E. Kim

Department of Internal Medicine, Eulji university, Seoul, Republic of Korea

Clinical practice guidelines on the application of exercise intervention in Type 2 diabetes do not provide much detail regarding the intensity of exercise. The purpose of this study was to evaluate the effect of exercise of differing intensity on body fat, adipokines and insulin sensitivity, especially comparing with dietary caloric restriction. 57 overweight female subjects with type 2 diabetes were randomly assigned to each five groups and completed four different 12-week weight loss program; $\operatorname{control}(\mathrm{C})$, diet group(DG), moderate intensity exercise group(MExG), and vigorous intensity exercise group(VExG), and diet plus exercise group(DEG). Participants were encouraged to take the calorie restriction $(<1300 \mathrm{kcal} /$ day $)$ for DG. Increase of activity energy expenditure ( $>300 \mathrm{kcal} /$ day) with moderate intensity exercise for MExG, with vigorous intensity exercise for HExG, and both for DEG. We monitored the subject's diet with self reported diet diary and exercise with the accelerometer. We assessed body mass index(BMI), total fat(ATF), subcutaneous fat(ASF) and visceral fat(AVF) at abdomen, liver(LF) and spleen fat(SF), and subcutaneous(TSF), subfacial(TSF), and intramuscular fat(TIMF) at thigh level using computed tomography, insulin sensitivity by insulin tolerance test $\left(\mathrm{K}_{\mathrm{ITT}}\right)$ and fasting serum levels of several adipokines before and after intervention. At baseline, age and BMI were not different the differences across 5 groups (age:55.4 \pm 7.7 years, BMI: $27.2 \pm 2.5 \mathrm{~kg} / \mathrm{m} 2$ ). After intervention, BMI, ATF and ASF were greater reduced in DG, MExG and DEG, but not in HExG and loss of AVF was greater in DG and MExG, but not in DEG and HExG, compared with C (Table 1). The changes of LF, SF, TSF, TSF, and TIMF were not different among 5 groups. Retinol binding protein- 4 (RBP-4) increased by $42 \%$ in DG which was significantly different with other three intervention groups. Vaspin showed smaller increase in DG than in C and MExG. Adiponectin was significantly changed with ASF, leptin with AVF, RBP-4 and apoprotein B with both ASF and AVF during 12 weeks trial after adjusted with age. Compared to baseline, $\mathrm{K}_{\mathrm{ITT}}$ was improved only within HExG and DEG $(P<0.01)$ without the difference across groups. By stepwise multiple regression analysis, major determinants of the percent changes of $\mathrm{K}_{\mathrm{ITT}}$ were baseline $\mathrm{K}_{\mathrm{ITT}}$ and activity energy expenditure during trial $(P<0.01)$. The equivalent energy expenditure from moderate and vigorous activity resulted that moderate activity was more effective in body fat loss, vigorous exercise might improved the insulin resistance, although diet restriction was superior to exercise in weight reduction in overweight type 2 diabetes mellitus. Adiponectin, leptin, and RBP-4 changed with different compartments of body fat.

Table 1. Change of the body fat and adipokines during 12 week weight reduction program.

\begin{tabular}{lllll} 
Control & Diet & Moderate & Vigorous & Significance \\
$\mathrm{N}=11$ & $\mathrm{~N}=10$ & Intensity Exercise & intensity Exercise \\
$\mathrm{N}=9$ & $\mathrm{~N}=14$ & $\begin{array}{l}\text { Diet and } \\
\text { Exercise } \\
\mathrm{N}=10\end{array}$ & \\
& & & & \\
\hline
\end{tabular}

\begin{tabular}{|c|c|c|c|c|c|c|}
\hline \multirow{2}{*}{\multicolumn{7}{|c|}{ Energy intake and expenditure during 12 week intervention (cal/ $\mathrm{kg}$ of body weight) }} \\
\hline & & & & & & \\
\hline Diet intake & $27.4 \pm 3.6$ & $20.2 \pm 2.5^{\star *}$ & $26.4 \pm 1.8$ & $27.4 \pm 2.7$ & $20.8 \pm 2.4^{* *}$ & $<.01$ \\
\hline Total energy expenditure & $29.7 \pm 3.0$ & $27.7 \pm 1.7$ & $32.1 \pm 2.0^{*}$ & $33.4 \pm 2.0^{\star *}$ & $32.0 \pm 4.8^{\star *}$ & $<.01$ \\
\hline Exercise energy expenditure & $5.1 \pm 1.8$ & $4.4 \pm 1.3$ & $7.0 \pm 1.2^{\star}$ & $8.1 \pm 1.5^{\star \star}$ & $8.0 \pm 3.2^{\star \star}$ & $<.01$ \\
\hline \multicolumn{7}{|c|}{ Percent change of BMI and body fat after 12 week intervention (\%) } \\
\hline BMI & $-2.3 \pm 2.2$ & $-5.9 \pm 3.6^{* *}$ & $-5.0 \pm 2.6^{*}$ & $-3.0 \pm 2.1$ & $-6.7 \pm 1.3^{* *}$ & $<.01$ \\
\hline Total fat (\%) & $-0.9 \pm 8.7$ & $-10.2 \pm 13.2^{*}$ & $-12.6 \pm 10.3^{*}$ & $-7.3 \pm 9.8$ & $-21.3 \pm 10.4^{\star *}$ & $<.01$ \\
\hline Visceral fat $(\%)$ & $-1.2 \pm 14.7$ & $-12.5 \pm 18.9$ & $-15.9 \pm 14.1^{*}$ & $-8.4 \pm 16.1$ & $-26.3 \pm 9.8$ & $<.01$ \\
\hline Subcutaneous fat & $0.3 \pm 7.1$ & $-8.8 \pm 15.8$ & $-10.1 \pm 10.5^{\star}$ & $-5.3 \pm 9.8$ & $-17.2 \pm 11.8^{\star *}$ & 0.01 \\
\hline \multicolumn{7}{|c|}{ Percent change of adipokines after 12 week intervention (\%) } \\
\hline Retinol binding protein- 4 & $20.9 \pm 29.4$ & $41.9 \pm 66.9$ & $3.4 \pm 40.0 \dagger$ & $6.2 \pm 29.1 \dagger$ & $-4.4 \pm 20.4 \dagger$ & 0.08 \\
\hline Adiponectin leptin ratio & $15.7 \pm 51.6$ & $31.5 \pm 60.7$ & $52.3 \pm 52.6$ & $44.6 \pm 47.4$ & $88.3 \pm 65.3^{* *} \dagger$ & 0.05 \\
\hline Vaspin & $41.5 \pm 91.3$ & $3.4 \pm 37.4^{\star}$ & $148.8 \pm 206.9 \neq$ & $16.9 \pm 24.9$ & $34.0 \pm 115.8$ & 0.06 \\
\hline
\end{tabular}

** significant different compared with control $(\mathrm{p}<0.01)$

* significant different compared with control $(\mathrm{p}<0.05)$

$\ddagger$ significant different compared with diet group $(\mathrm{p}<0.01)$

$\dagger$ significant different compared with diet group $(\mathrm{p}<0.05)$ 


\section{9}

Effect of exercise on gene transcript profiles in skeletal muscle of individuals with and without a family history of diabetes

O. Hansson, T. Elgzyri, H. Parikh, K.-F. Eriksson, L. Groop

Clinical Sciences, Lund University, Malmö, Sweden

Background and aims: It is well established that the risk of developing type 2 diabetes (T2D) increases with age, obesity and reduced physical activity, but the effect of the environment seems to be exaggerated by a family history of type 2 diabetes $(\mathrm{FH}+)$. It has previously been shown that the expression of genes involved in oxidative phosphorylation is co-ordinately down regulated in human diabetic muscle and that this reduction in gene expression is correlated with total-body aerobic capacity. It is, however not known whether down-regulation of these genes is primary (genetic) or secondary to reduced physical activity. One way to test this hypothesis is to study whether there is a difference in gene transcript profiles in skeletal muscle between individuals with and without a $\mathrm{FH}+$ and whether this is reversible by a 6 month exercise period.

Materials and methods: 19 male subjects with and 20 without a FH matched for age, $\mathrm{BMI}$ and $\mathrm{VO} 2 \mathrm{max}$ were after basal muscle biopsy (from vastus lateralis), a VO2 measurement and OGTT exposed to 6 months of aerobic training (3 group-training sessions per week, $\sim 60 \mathrm{~min}$ training / session) whereafter the measurements and biopsies were repeated. Gene expression profiles were measured using Affymetrix expression arrays. The expression data have been analysed using pathway analysis tools, including Gene Set Enrichment Analysis (GSEA). Key changes in gene expression have been verified using rt-Q-PCR.

Results: $\mathrm{FH}+$ individuals had reduced expression of mitochondrial genes and of genes associated with fatty acid metabolism in skeletal muscle, including PGC1 $\alpha$, NDUFB3, CPT1, CPT2 and malonyl-CoA decarboxylase compared with $\mathrm{FH}$ - subjects. In $\mathrm{FH}+$ subjects exercise training up-regulated mitochondrial genes, as well as genes involved in electron transport, e.g. NDUFB1, NDUFB5, NDUFB9 and cytochrome $\mathrm{C}$ to the same extent as in FH- subjects, but had no effect on genes involved in fatty acid metabolism.

Conclusion: Individuals with a family history of T2D show down-regulation of mitochondrial genes in skeletal muscle, which is reversible by exercise. On the other hand, inability to upregulate genes in fatty acid metabolism could point at metabolic inflexibility.

Suported by: Novo Nordisk Fonden, Nordic Center of Excellence, UMAS fonder, Magn. Bergvall and Lund University Diabetes Center

\section{0}

Cause and effect in the relationship between body fat and physical activity in children: a longitudinal study

B. Metcalf, J. Hosking, A. Jeffery, L. Voss, T. Wilkin

Endocrinology \& Metabolism, Peninsula Medical School, Plymouth campus, United Kingdom

Background and aims: Several cross-sectional studies have concluded, based on weak inverse associations, that less activity leads to more body fat. However, such study designs cannot determine direction of causality, and the observed association may equally reflect the reverse - that more body fat leads to less activity. The aim of this study is to investigate the direction of causality between physical activity and body fat in children, applying the rule of temporality to longitudinal data.

Materials and methods: Physical activity (PA) was measured objectively by 7-day accelerometry (MTI Actigraphs) at four annual time-points - 6y, 7y, 8y and $9 \mathrm{y}$. Percent body fat (\%BF) was measured by DEXA at 7y and 8y. Timelagged correlations between $\% \mathrm{BF}$ and $\mathrm{PA}$ one year before $\left(\mathrm{PA}_{[t-1]}\right)$ and one year after $\left(\mathrm{PA}_{[t+1]}\right)$ the measurement of $\% \mathrm{BF}_{[t]}$ were performed at two timepoints $(t=7 y$ and $t=8 y)$. Analysis was performed on 185 children (104 boys, 81 girls) from the EarlyBird Diabetes Study.

Results: The correlation of $\% \mathrm{BF}_{[t]}$ with $\mathrm{PA}_{[t+1]}$ was stronger than $\% \mathrm{BF}_{[t]}$ with $\mathrm{PA}_{t \mathrm{t}-1}$ when $\mathrm{t}=7 \mathrm{y}\left(\% \mathrm{BF}_{7}-\mathrm{v}-\mathrm{PA}_{8}, \mathrm{r}=-0.21, \mathrm{p}<0.01, \% \mathrm{BF}_{7}-\mathrm{v}-\mathrm{PA}, \mathrm{r}=-0.06\right.$, $\mathrm{p}=0.40)$ and again when $\mathrm{t}=8 \mathrm{y}\left(\% \mathrm{BF}_{8}-\mathrm{v}-\mathrm{PA}_{9}, \mathrm{r}=-0.20, \mathrm{p}<0.01, \mathrm{BF}_{8}-\mathrm{v}-\mathrm{PA}_{7}, \mathrm{r}\right.$ $=-0.13, \mathrm{p}=0.08)$. On average the correlations were: $\% \mathrm{BF}_{[t]}-\mathrm{v}-\mathrm{PA}_{[t+1]} \mathrm{r}=-0.20$ and $\% \mathrm{BF}_{[t]}-\mathrm{v}-\mathrm{PA}_{[t-1]} \mathrm{r}=-0.10$ with the magnitude of the difference remaining even after controlling for $\mathrm{PA}_{[\mathrm{t}]}$.

Conclusion: Body fat is associated more strongly with PA measured one year later than with PA measured one year earlier, implying that fatness may influence activity more than activity influences fatness. The weakness of the association and its direction of causality could together be reasons why attempts to tackle childhood obesity by promoting physical activity have been largely unsuccessful. If confirmed, the findings may shift the focus of prevention to calorie intake.

Supported by: The Bright Futures Trust, the Child Growth Foundation, the EarlyBird Diabetes Trust, GSK, the Kirby Laing Foundation, Nestle, Novo Nordisk

\section{1}

Standardised depletion of intramyocellular lipids (IMCL) in subjects with different degree of insulin resistance and exercise capacity A. Egger ${ }^{1}$, P.M. Huber ${ }^{2}$, M. Ith ${ }^{2}$, I. Krull ${ }^{1}$, J.-P. Schmid ${ }^{3}$, R. Kreis ${ }^{2}$, E. Christ ${ }^{1}$, C. Boesch ${ }^{2}$

${ }^{1}$ Div. of Endocrinology, Diabetes and Clinical Nutrition, Inselspital, University Hospital, ${ }^{2}$ Department of Clinical Research, MR Spectroscopy and Methodology, University Bern, ${ }^{3}$ Department of Cardiology, Cardiovascular Prevention \& Rehabilitation, Inselspital, University Hospital, Bern, Switzerland

Background and aims: Intramyocellular lipids (IMCL) are metabolically active fuel stores that are related to individual insulin sensitivity. Diet and physical exercise are important factors influencing IMCL, which can be measured non-invasively by ${ }^{1} \mathrm{H}$ magnetic resonance spectroscopy $\left({ }^{1} \mathrm{H}-\mathrm{MRS}\right)$.

Depletion and subsequent replenishment of IMCL give insight into availability and dynamics of this energy store. The aim of the present study was to develop a standardized IMCL depletion protocol that is well tolerated by sedentary patients and sufficiently depletes IMCL in well trained athletes.

Materials and methods: 12 male subjects, including 4 patients with diabetes type 2 treated by oral antidiabetic drugs, were tested for physical performance and insulin sensitivity, by spiroergometry $\left(\mathrm{VO}_{2}\right)$ and a euglycaemic hyperinsulinaemic clamp, respectively. Using ${ }^{1} \mathrm{H}-\mathrm{MRS}$, IMCL were measured in $\mathrm{m}$. tibialis anterior and $\mathrm{m}$. vastus intermedius once before (day 1 ) and three times (days $5,6,7$ ) within a week with daily moderate exercise ( 1 hour at $60 \%$ $\mathrm{VO}_{2 \text { peak }}$ ) and moderate low-fat (5\% of energy expenditure) diet.

Results: The enrolled subjects intentionally showed wide ranges of BMI (between 21.5 and $\left.31.7 \mathrm{~kg} / \mathrm{m}^{2}\right)$, physical performance $\left(\mathrm{VO}_{2}\right.$ peak between 27.9 and $55.8 \mathrm{ml}^{\star} \mathrm{min}^{-1 *} \mathrm{~kg}^{-1}$ ), insulin sensitivity (glucose disposal rate per lean body mass between 4.7 and $15.3 \mathrm{mg}^{*} \mathrm{~min}^{-1 *} \mathrm{kgLBM}^{-1}$ ) and age (between 24 and 63 years). While IMCL levels are higher in the vastus muscle as compared to the m.tibialis anterior, both muscles show the same behavior following exercise and low fat intake (Figure): compared to baseline IMCL measurements (day 1 ) a highly significant $(\mathrm{p}<0.001$ for each muscle) IMCL reduction is achieved after 3 days of moderate exercise and diet. During the following days, IMCL levels remain constant on a minimal level (day 4 and day 5). Depleted IMCL levels were independent of insulin sensitivity in both muscles. However, subjects with higher $\mathrm{VO}_{2 \text { peak }}$ showed lower depleted IMCL levels (average over 3 days) in both muscles (correlation $\mathrm{p}=0.08$ for m.tibialis anterior and $p=0.05$ for m.vastus intermedius).

Conclusion: These findings suggest that a) the presented protocol is robust and feasible in a wide range of subjects with large differences in physical performance and insulin sensitivity b) it leads to a "minimal IMCL" after 3 days, independent of previous diet and insulin sensitivity, and c) after reaching this "minimal IMCL" level, this protocol allows studies of replenishment in very different groups of subjects, including patients and athletes. To conclude, this protocol allows comparison of IMCL dynamics in a standardized fashion.

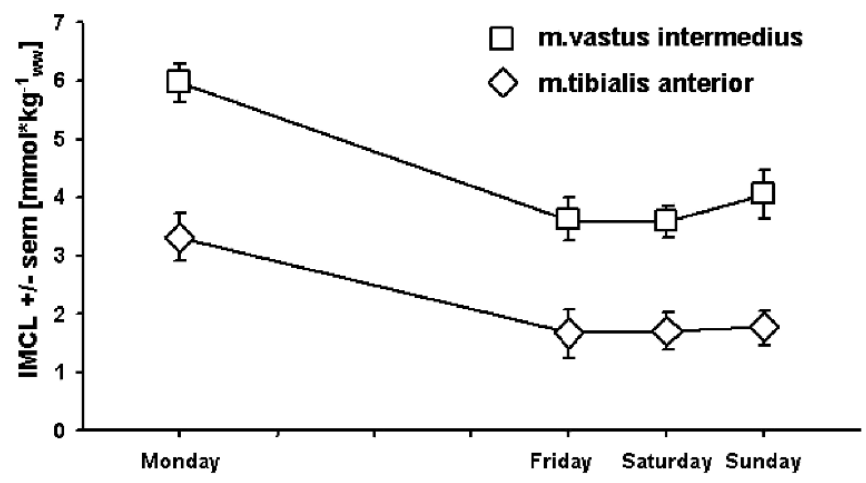

Supported by: Swiss National Science Foundation 


\section{2}

Tissue-specific regulation of adiponectin and its receptors by rosiglitazone and exercise treatment in obese diabetic OLETF rat M. Nam, Y.-J. Oh, S.-H. Kim, Y.-S. Kim

Internal Medicine, Inha University College of Medicine, Incheon, Republic of Korea

Background and aims: Aerobic exercise is recommended with or without oral hypoglycemic agents, particularly thiazolidinediones(TZDs), for the treatment of obese type 2 diabetes mellitus. Adiponectin, an abundant adipose-derived cytokine, plays an important role in modulation of insulin sensitivity, and the metabolism of glucose and lipids. It is not fully understood whether the independent and combined effects of rosiglitazone and exercise treatments are associated with improvements in adiponectin resistance.

Materials and methods: We evaluated the independent and combined effects of aerobic exercise $(15 \mathrm{~m} / \mathrm{min}$ and $30 \mathrm{~min} /$ day) and rosiglitazone $(3 \mathrm{mg} /$ $\mathrm{kg} /$ day) for 12 weeks on metabolic parameters, adiponectin and its receptors expression, glucose transport, fatty acid oxidation in an obese diabetic prone animal model, the Otsuka Long-Evans Tokushima Fatty (OLETF) rat.

Results: Increased lean body mass, decreased epididymal fat, and improved intraperitoneal glucose tolerance test were observed in both rosiglitazone plus exercise and exercise only groups (each group $\mathrm{n}=15$ ). With regard to the plasma lipid profile change, significantly decrease in triglyceride levels were observed in both exercise and rosiglitazone groups but decreased LDL levels were shown in rosiglitazone only and rosiglitazone plus exercise group but not in the exercise only group. Decreased plasma adiponectin concentration and decreased adipoR1/R2 mRNA expression both in skeletal muscle and adipose tissue were observed in diabetic control group. Exercise training, but not rosiglitazone increased adipoR1, phosphorylation of AMP-activated protein kinase(AMPK) alpha/beta1, GLUT4 and decreased Acetyl-CoA carboxylase(ACC) in skeletal muscle. In contrast, rosiglitazone, but not exercise reversed adiponectin resistance in adipose tissue. However, the rosiglitazone and exercise combined group was able to reverse adiponectin resistance in both skeletal muscle and adipose tissue.

Conclusion: exercise training and rosiglitazone may act as complementary therapies for the treatment of adiponectin resistance in obese diabetes.

\section{3}

A model for implementation and maintenance of physical activity in patients with type 2 diabetes

S.M. Sørensen ${ }^{1,2}$, S. Larsen ${ }^{1}$, K. Hove ${ }^{1}$, T. Gjørup ${ }^{1}$, A. Vaag ${ }^{1}$, B. Kiens ${ }^{2}$

${ }^{1}$ Steno Diabetes Centre, Gentofte, Denmark, ${ }^{2}$ Copenhagen Muscle Research Centre, Department of Human Physiology, Institute of Exercise and Sport

Sciences, University of Copenhagen, Denmar

Background and aims: The beneficial effects of physical activity in prevention and treatment of type 2 diabetes (T2D) are well documented. Although lifestyle intervention programs may work in some community settings and in early diabetic and/or prediabetic subjects, no models or guidelines are developed to implement training programs in a clinical diabetes setting involving patients with longer duration of disease. Therefore, the aim was to develop and test an individualized and supervised program to implement physical activity in such patients.

Materials and methods: One hundred and sixty two patients with T2D from the Steno Diabetes Centre were contacted by letter. Thirty responded positively and 21 sedentary patients (10 men and 11 women, aged 48-65 years, average duration of T2D 12,6 years) were enrolled in the study. For women and men, respectively, average values for body weight were 81,3 (SD 15,6) $\mathrm{kg}$ and 99,5 (SD 10,3) $\mathrm{kg}$, BMI 31,4 (SD 5,2) $\mathrm{kg} / \mathrm{m}^{2}$ and $32,2\left(\right.$ SD 3,1) $\mathrm{kg} / \mathrm{m}^{2}$, body fat $\%$ averaged 43,2 (SD 4,5) and 34,7 (SD 3,7). Plasma [HbAlc] in the whole group averaged 8,03 (SD 1,00)\%. The patients were randomly assigned to one of two intervention groups. Both included information about benefits of physical activity, and individual oral guidance on how to implement selfchosen physical activities in everyday life. Additionally, group 1 (5 men and 6 women) participated in group-sessions of supervised exercise twice a week in their local environments. On the remaining days of the week the patients were responsible for their own daily activity. Group 2 ( 5 men and 5 women) was on a daily basis requested to exercise individually or in self-established groups. In order to supervise the process, every second week, group 2 was contacted by their coach. Activities included Nordic walking, jogging, biking, water activities and resistance training. At baseline and after 10 weeks of intervention, all patients went through a standardised test protocol including fasting blood samplings, DXA-scanning and measurements of physical fitness level on a bicycle ergometer.

Results: Heart rate measured during submaximal exercise at the same absolute work load (a marker for physical fitness level) was decreased in the patients of group 1 to an average of 126 (SD 12,4) bpm vs. baseline values of 134 (SD 11,4) bpm; $(\mathrm{p}<0.02)$. Furthermore, they had an increase in lean body mass from 50,7 (SD 9,6) $\mathrm{kg}$ to $52,1(\mathrm{SD} 9,8) \mathrm{kg}(\mathrm{p}=0,002)$, reduction of fat $\%$ from $38,4(\mathrm{SD} 6,5)$ to $36,8(\mathrm{SD} 7,1)(\mathrm{p}=0,003)$ and a decrease in plasma HbAlc concentration from 8,14 (SD 1,1) \% to $7,74(\mathrm{SD} 1,18) \%(\mathrm{p}=0,03)$ after the intervention. In group 2, no significant changes were observed.

Conclusion: An increase in aerobic fitness level after 10 weeks exercise intervention was associated with a significantly lowered $\mathrm{HbAlc}$. This was obtained when exercise was performed even at low to moderate intensity levels. In spite of no changes in body weight, the activities lead to changes in body composition through increased muscle mass and lowered fat $\%$. Furthermore, self-reported personal wellbeing (quality of life) was increased. The present study clearly demonstrates that in order to implement and maintain physical activity in the everyday life of patients with long term T2D, individually modified exercise programs, regular personal contact combined with supervised exercise are necessary; and that physical activity is realistic and possible to implement in clinical settings.

Supported by: Steno Diabetes Center 


\section{PS 051 Hypoglycaemia}

\section{4}

Involvement of active ghrelin in glucose homeostasis: a protective role against the occurrence of hypoglycaemia

E. Disse, C. Veyrat-Durebex, N. Deblon, F. Rohner-Jeanrenaud

Departement of Internal Medecine, Endocrinology, Laboratory of

Metabolism, Geneva, Switzerland

Background and aims: Ghrelin, a 28-amino acid peptide predominantly produced by the stomach, is the most powerful orexigenic hormone in mammalian physiology. The deletion of ghrelin in ob/ob mice, a murine model of obesity, augments glucose-induced insulin secretion and markedly reduces the level of hyperglycemia exhibited by these mice. The aim of our study was to determine the influence of an acute peak of plasma ghrelin on glucose homeostasis and to determine in vivo the mechanisms of this hyperglycaemic effect . Furthermore, we wondered whether these ghrelin-elicited effects were physiologically relevant, using mice knock-out for the ghrelin receptor (GHSRla -/-).

Materials and methods: We IP administrated $10 \mathrm{nmol}$ of acylated ghrelin or $250 \mu \mathrm{l}$ of saline in Wistar rats with a chronic jugular catheter. Blood samples were taken every 30 minutes during the 4 following hours to quantify active ghrelin, glucose, insulin, glucagon, leptin, GLP-1, NEFA and adiponectin. We then performed a glucose tolerance test in these animals after an ICV injection of $250 \mathrm{pmol}$ of ghrelin in a way to determine whether the ghrelin-elicited effects were centrally mediated. The physiological relevance of these effects was determined by testing GHSR1a-/- mice during GTT, ITT and prolonged fasting. We finally worked on the ghrelin-elicited effects during insulin-induced hypoglycemia in C57Bl6 mice after the blockade of the autonomous nervous system which is implicated in the hormonal counterregulation to hypoglycaemia. We used a pharmacological blockade of the parasympathetic system (Atropine) and a genotypic blockade of the sympathetic system (mice deleted for the $\beta 1, \beta 2$ and $\beta 3$ adrenoreceptors).

Results: The glucose AUC (Area Under Curve) was significantly increased in the ghrelin-treated group ( $42.552 \pm 1.764$ vs $32.305 \pm 1.193 \mathrm{mmol} \mathrm{x} 240 \mathrm{~min}$, $\mathrm{p}<0.005)$, while the insulin AUC and the insulinosecretion index, AUC insulin-to-AUC glucose ratio, were significantly decreased (51.757 \pm 9.549 vs $80.970 \pm 9.549 \mathrm{pg} \times 240 \mathrm{~min}, \mathrm{p}<0.05$ and $2.46 \pm 0.2$ vs $1.25 \pm 0.4, \mathrm{p}<0.005$, respectively) after ghrelin injection. This hyperglycaemic and insulinostatic effect was also found after the ICV injection of $250 \mathrm{pmol}$ of active ghrelin, demonstrating that the ghrelin-elicited effect was at least partially centrally mediated. In GHSR1a -/- mice, glucose tolerance was unaltered, but mice exhibited deeper glycaemic nadir during the insulin-induced hypoglycaemia and lower glycaemic levels during prolonged fasting. The hyperglycaemic effect of ghrelin was still present in C57Bl6 mice during insulin-induced hypoglycaemia, in which endogenous insulin secretion is inhibited, but this effect was blunted in mice deleted for the three $\beta$-adrenoreceptors.

Conclusion: Altogether, these data suggest that ghrelin induces hyperglycemia. This effect is mediated through an inhibition of glucose-induced insulin secretion, partially centrally mediated and a mechanism independent of insulin secretion mediated through the sympathetic pathway. The ghrelinelicited effects on glucose homeostasis could be physiologically relevant to a protective effect against the occurrence of hypoglycaemia.

Supported by: Fondation Romande pour la Recherche sur le Diabète Fondation Endocrinologie Genève

\section{5}

The effects of acute insulin-induced hypoglycaemia on spatial ability in adults with type 1 diabetes

R.J. Wright ${ }^{1}$, I.J. Deary ${ }^{2}$, B.M. Frier ${ }^{1}$

${ }^{1}$ Department of Diabetes, Royal Infirmary of Edinburgh, ${ }^{2}$ Psychology

Department, University of Edinburgh, United Kingdom

Background and aims: The effects of acute insulin-induced hypoglycaemia have been studied on several cognitive domains, both in people with diabetes and in non-diabetic subjects. However, the effects on spatial abilities have not been studied in detail. Spatial ability is important for the conduct of cognitively demanding tasks such as navigating whilst driving, and an adverse effect of hypoglycaemia could influence several daily activities.

Materials and methods: Sixteen people with type 1 diabetes $(7$ male; median age 28 years; median HbAlc 7.8\%) underwent hyperinsulinaemic glucose clamps on two separate occasions. Arterialised blood glucose was maintained at $4.5 \mathrm{mmol} / \mathrm{l}$ for 30 minutes, and either continued at this level (euglycaemia condition) or lowered to $2.5 \mathrm{mmol} / \mathrm{l}$ (hypoglycaemia condition), for $60 \mathrm{~min}$ utes. The experimental conditions were counter-balanced. The Digit Symbol Substitution Test (DSST) and a battery of specific spatial ability tests, derived from the French and Ekstrom Kit of Factor-referenced Cognitive Tests, were applied during each experimental condition. Results were analysed using repeated measures ANOVA with condition as a within-subjects factor.

Results: Digit symbol substitution was significantly impaired during hypoglycaemia ( $<<0.001)$, as anticipated. The Hidden Patterns, Card Rotations, Paper Folding and Maze Tracing tests were all impaired significantly (all $\mathrm{p} \leq 0.001$ ) during hypoglycaemia, as was the Cube Comparisons test ( $\mathrm{p}=0.03$ ). The Map Memory test was not significantly affected by hypoglycaemia. The results are shown in Table 1 (as mean [SD]).

Conclusion: In the first detailed examination of this cognitive domain, the ability to perform several tests of spatial ability was significantly impaired during hypoglycaemia, when compared with performance during a euglycaemic control period. This has important implications for people with type 1 diabetes in the performance of everyday tasks.

Table 1: Results of spatial ability testing

\begin{tabular}{llll}
\hline Spatial Test & $\begin{array}{l}\text { Euglycaemia } \\
\text { score }\end{array}$ & $\begin{array}{l}\text { Hypoglycaemia } \\
\text { score }\end{array}$ & p-value \\
\hline Hidden Patterns & $94.5(21.8)$ & $73.7(21.0)$ & $<0.001$ \\
\hline Card Rotations & $51.9(15.5)$ & $40.4(18.7)$ & 0.001 \\
\hline Cube Comparisons & $11.7(4.1)$ & $9.4(5.7)$ & 0.03 \\
\hline Paper Folding & $6.0(1.9)$ & $4.7(2.0)$ & 0.001 \\
\hline Map Memory & $8.6(3.1)$ & $7.8(2.1)$ & 0.3 \\
\hline Maze Tracing & $11.1(3.0)$ & $9.4(2.5)$ & $<0.001$ \\
\hline
\end{tabular}

\section{6}

No effect of GLP-1 on human brain glucose delivery during

hypoglycaemia

S. Lerche ${ }^{1}$, B. Brock ${ }^{1}$, J. Rungby ${ }^{1}$, H. Boetker ${ }^{1}$, N. Moeller ${ }^{1}$, K. Vang ${ }^{2}$,

J.J. Holst ${ }^{3}$, A. Gjedde ${ }^{2}$, O. Schmitz ${ }^{1}$

${ }^{1}$ Department of Pharmacology, Aarhus University, ${ }^{2}$ PET center, Aarhus

University Hospital, ${ }^{3}$ Department of medical Physiology, Copenhagen University, Denmark

Background and aims: The brain almost exclusively depends on circulating glucose. Hypoglycaemia therefore is deleterious to brain function. The Michaelis-Menten formalism describes the magnitude of blood-brain glucose transfer across the blood-brain barrier (BBB) at glucose concentrations in arterial plasma ranging from hypo- to hyperglycaemia. Glucagon-like-peptide1 (GLP-1) inhibits blood-brain glucose exchange at normoglycaemia and predictably also at hyperglycaemia. We believe that this action may explain GLP-1's neuroprotective effect at normal or higher glucose levels. However, the Michaelis-Menten formalism predicts that hypoglycaemia minimizes the effect on glucose transport of GLP-1's action on the maximum glucose transport capacity.

Materials and methods: To test this prediction of the effect of GLP-1 in hypoglycaemia, we determined glucose transport and consumption rates in 7 healthy men in a randomized, double-blinded placebo-controlled cross-over experimental design. The acute effect (independent of insulin) of GLP-1 on glucose transfer in the brain was measured by positron emission tomography (PET) during a pituitary-pancreatic stepwise hypoglycaemic clamp with 18fluoro-deoxy-glucose (FDG) as tracer of glucose.

Results: The plasma glucose (PG) during PET averaged $3.0 \mathrm{mM}$ with an insulin infusion rate of $0.8 \mathrm{mU} / \mathrm{kg}^{*} \mathrm{~min}$. We initially maintained growth hormone (GH) and glucagon close to baseline but both increased significantly in the hypoglycaemic phase, as did epinephrine levels. PG, insulin, glucagon, GH, epinephrine levels and glucose infusion rate were similar with GLP-1 and placebo. Total and intact GLP-1 levels stayed in the pharmacological range. In total cerebral grey matter, the cerebral glucose uptake remained unchanged during hypoglycaemia with GLP-1, $0.37 \pm$ (SEM) 0.03 (GLP-1) vs. $0.40 \pm 0.02$ $\mathrm{micromol} / \mathrm{cm}^{3} / \mathrm{min}$ (placebo), $(\mathrm{P}=0.25)$. The cerebral metabolic rate for glucose remained unchanged at $0.29 \pm 0.01$ vs. $0.30 \pm 0.01 \mathrm{micromol} / \mathrm{cm}^{3} / \mathrm{min}$, $(\mathrm{P}=0.28)$ with GLP-1, as was the intracerebral glucose concentration at 0.63 \pm 0.05 vs. $0.76 \pm 0.16 \mathrm{mmol} / \mathrm{L},(\mathrm{P}=0.39)$.

Conclusion: The results complement our previous study of GLP-1 in normoglycaemia. The confirmation that the effect of GLP-1 on glucose transport 
rates is too small to measure at low glucose concentrations contributes to the understanding of GLP-1's generally beneficial action also in hypoglycaemia.

\section{7}

Insulin VIAject ${ }^{\text {tw }}$ and regular human insulin in patients with type 1 diabetes: efficacy and safety in an open-label multicenter clinical trial F.M. Flacke ${ }^{1}$, D. Klonoff ${ }^{2}$, B. Sethi ${ }^{3}$, T. Forst ${ }^{4}$, P. Simms ${ }^{1}$, L. Heinemann ${ }^{5}$, A. Pfuetzner ${ }^{4}$, S.S. Steiner ${ }^{1}$

${ }^{1}$ Clinical, Biodel Inc., Danbury, United States, ${ }^{2}$ Clinical Research, Mills Peninisula Health Service, San Mateo, United States, ${ }^{3}$ Clinical Research, Care Hospital, Hyderabad, India, ${ }^{4}$ Clinical Research, IKFE, Mainz, Germany, ${ }^{5}$ Clinical Research, Profil, Düsseldorf, Germany

Background and aims: VIAject ${ }^{\text {tix }}$ (VIA) is an ultra-rapid acting formulation of regular human insulin (RHI). The aim of this ongoing 6 month open-label, parallel-group, multi-center study (USA, Germany, India) is to study metabolic control and safety in $>400$ patients with type 1 diabetes using VIA compared to RHI for prandial insulin therapy.

Materials and methods: Patients with type 1 diabetes (age $41 \pm 14$ years; 264 male, 184 female; BMI $26 \pm 4.9 \mathrm{~kg} / \mathrm{m}^{2}$ ) were sequentially recruited for this trial. During their first visit, body weight, BMI, and current insulin regimen were recorded. After randomization, all patients were switched to an intensified insulin treatment with insulin Lantus as basal insulin, while their prandial insulin was changed to either VIA or RHI. Subsequent visits recorded their weight and insulin dose. Adverse events were recorded as well.

Results: Weight changes could be analyzed for 210 patients for the first half of the study. For those on VIA ( $\mathrm{n}=102)$, no change in weight compared to baseline was observed, while a weight gain $(1.4 \pm 2.9 \mathrm{~kg})$ was seen in the patients $(\mathrm{n}=108)$ on RHI $(\mathrm{p}<0.001)$. With comparable glycemaemic control between the groups, prandial insulin dose was reduced in the first 6 weeks of the study with VIA $(\mathrm{n}=106)$ by $34 \%$ compared to baseline $(\mathrm{p}<0.001)$ whereas there was only a non-significant decrease of $-2.4 \pm 15.3$ units with RHI. Analysis of 8111 mild and moderate hypoglycaemic events recorded thus far showed a lower number of incidents in the VIA group (3691; $\mathrm{p}<0.001)$ than in the RHI group (4420).

Conclusion: Prandial insulin therapy with VIA showed less weight gain, lower insulin dose requirements and less hypoglycaemia in patients with type 1 diabetes compared to RHI. This confirms that the more rapid onset of action and reduced variability seen with VIA in previous short term clinical studies translates into clinically significant advantages in this long term Phase 3 pivotal trial.

\section{8}

Insulin VIAject $^{\mathrm{st}}$ and regular human insulin in patients with type 2 diabetes: efficacy and safety in an open-label multicenter clinical trial S. Steiner ${ }^{1}$, A. Pfuetzner ${ }^{2}$, B. Sethi ${ }^{3}$, T. Forst ${ }^{2}$, D. Klonoff $f^{4}$, P. Simms ${ }^{1}$, R. Pohl ${ }^{1}$, F. Flacke ${ }^{1}$

${ }^{1}$ Clinical, Biodel Inc, Danbury, United States, ${ }^{2} \mathrm{Clinical}$, IKFE, Mainz, Germany, ${ }^{3}$ Clinical, Care Hospital, Hyderabad, India, ${ }^{4}$ Diabetes, Mills Penninsula, San Mateo, United States

Background and aims: VIAject ${ }^{\text {tu }}$ (VIA) is an ultra-rapid acting formulation of regular human insulin (RHI). The aim of this ongoing 6 month open-label, parallel group, multi-center study (USA, Germany, India) is to compare metabolic control and safety in $>400$ patients with type 2 diabetes using VIA vs. RHI for prandial insulin therapy. Data presented here include safety information and first efficacy results.

Materials and methods: Patients with type 2 diabetes (age $56 \pm 9$ years; 249 male, 181 female; BMI $33.5 \pm 6.1 \mathrm{~kg} / \mathrm{m}^{2}$ (mean \pm SD)) were sequentially recruited into this trial. During their first visit, body weight, BMI, and current insulin regimen were recorded. After randomization, patients were switched to an intensified insulin treatment with insulin Lantus as basal insulin, while their prandial insulin was changed to either VIA or RHI. Patients entering the study on TZDs or Metformin stayed on their oral treatment. Subsequent visits recorded their weight and insulin dose. All adverse events were recorded. Results: Weight changes were analyzed for 353 patients for the first six weeks of the study. With VIA $(\mathrm{n}=173)$ a weight reduction $(-0.5 \pm 4.7 \mathrm{~kg})$ compared to baseline was observed. In contrast, a weight gain $(0.8 \pm 6.2 \mathrm{~kg})$ was observed in the patients $(\mathrm{n}=180)$ on RHI $(\mathrm{p}<0.05)$ for a net difference of $1.3 \mathrm{~kg}$. With comparable glycaemic control, patients on VIA reduced their dose of prandial insulin by $35.9 \%$ on average ( 16 units; $\mathrm{p}<0.001$ compared to baseline). Pa- tients on RHI increased their dose $15 \%$ ( 6 units; $\mathrm{p}<0.01$ ) for a net difference of $50.9 \%$. Analysis of the 2438 mild and moderate hypoglycaemic events reported thus far showed $47 \%$ fewer number of incidents with VIA (844 events) compared to the patients receiving RHI (1594 events, $\mathrm{p}<0.001)$.

Conclusion: Patients receiving VIA in this pivotal Phase 3 clinical trial showed weight loss, reduced insulin requirements and fewer hypoglycaemic events compared to RHI. Prandial insulin therapy with this novel regular insulin formulation showed benefits with respect to weight gain, insulin dose and hypoglycaemic events in comparison to the RHI in this large clinical study. The physiological profile of VIA leads to clinically relevant improvements in intensive insulin therapy in patients with type 2 diabetes.

\section{9}

Recurrent severe hypoglycaemias and licence to drive. A community based study

M.T. Honkasalo ${ }^{1}$, O.M. Elonheimo ${ }^{2}$, T.A. Sane ${ }^{3}$

${ }^{1}$ Diabetes care unit, Nurmijärvi Health Centre, Nurmijärvi, ${ }^{2}$ Network of Academic Health Centres, Helsinki University, ${ }^{3}$ Department of Endocrinology, Helsinki University Central Hospital, Finland

Background and aims: The knowledge of the incidence of severe hypoglycaemias ( $\mathrm{SH}$ ) is quite limited and even controversial in insulin treated patients with diabetes. In our retrospective study we analyzed the incidence and predisposing factors of $\mathrm{SH}$ in two medium sized Finnish communities, altogether with 67261 inhabitants. In the present study we evaluated whether diabetic patients with recurrent (at least 3 or more) episodes of SH during the year investigated hold a valid license to drive. According to the current medical standards for diabetes and driving the driving licence should be at least temporarily cancelled in a diabetic patient with recurrent episodes of $\mathrm{SH}$ until the driving fitness is properly maintained with adjustment of treatment. Moreover the insulin treated diabetic patients with unawareness of hypoglycaemia and occurrence of any SH should be restricted of having licence to drive commercial vehicles (lorry, minicab and taxi).

Materials and methods: From our target population we identified altogether 1776 medically treated diabetic patients, of whom 1326 (74.7\%) filled a questionnaire and reported the number of SH episodes during the preceding 12 months. Data on the occurrence of SH during the same year was also collected from electronic patient records used in primary health care centres and local hospitals offering 24 hour emergency room service and from the 24 hour ambulance service registers of these two communities. SH was defined as a hypoglycaemic episode requiring help from another person. The data of the current driving licenses was received from the local police authorities. Results: During the preceding year $27.3 \%$ of the patients with T1DM and $12.1 \%$ of insulin treated patients with T2DM reported at least one SH (the incidence rates of SH 66.7/100 and 26.5/100 pt years, respectively). Of noninsulin treated patients with T2DM $3.8 \%$ reported at least one SH with incidence of 6.0/100 pt years. Of the patients with T1DM $10.4 \%$ and of the patients with T2DM $3.2 \%$ needed ambulance care of SH (incidence rates of SH $24.3 / 100$ and 3.4/100 pt years, respectively). Of all insulin treated patients $56(7.3 \%)$ reported three or more episodes of SH during the preceding year. Thirty seven (66\%) of them still hold a licence to drive, and even $11(20 \%)$ of them hold a license to drive commercial passenger or goods carrying vehicles up to 3.5 tons (lorry, minicab and taxi).

Conclusion: Our community based data shows that the incidence of $\mathrm{SH}$ is more common than expected both in insulin treated patients with T1DM and T2DM, although the episodes of SH were clustered in a minority of patients. Recurrent episodes of SH occurred in $7.3 \%$ of insulin treated patients, which may impair their fitness to drive and increase the risk to driving mishaps. Two thirds of these patients still hold a valid license to drive and some of them were even licensed to drive commercial vehicles. Our data indicate that the current EU medical standards for diabetes and driving are not very well known among the Finnish doctors.

Supported by: Finnish Diabetes Research Foundation 


\section{0}

The clinical and economic burden of hospital encounters due to hypoglycaemia among diabetic patients

J.L. Natoli ${ }^{1}$, C.M. Alexander ${ }^{2}$, T. Haidar ${ }^{1}$, R.W. Dubois ${ }^{1}$, S.M. Curkendall ${ }^{1}$

${ }^{1}$ Cerner LifeSciences, Cerner, Beverly Hills, ${ }^{2}$ Outcomes Research, Merck and Co., Inc., Whitehouse Station, United States

Background and aims: Limited data are available describing the direct medical costs of hypoglycemia and associated economic burden. Although several economic analyses using claims data in managed care plan have been conducted, these studies are relatively old and provide little insight into the resource utilization associated with hypoglycemia. The primary objective of this retrospective study was to estimate the burden (including costs, mortality, and length of hospital stay) of a hospital visit for hypoglycemia among diabetic patients.

Materials and methods: Data from 70 hospitals participating in Cerner's Health Facts" EMR database were used to identify all encounters during 20002006 for adult diabetic patients. Of these, encounters for which hypoglycemia was the primary reason for the hospital visit were defined as those with a primary inpatient (IP) diagnosis or any emergency department (ED) diagnosis of hypoglycemia (ICD-9-CM code 251.0, 251.1, or 251.2). Total costs (2006US\$) were calculated based on the encounter type. For ED-only encounters, costs were calculated by using a 50\% cost-to-charge ratio. For encounters with an IP component (IP-only, or ED encounters with subsequent admission [ED to IP]), costs were based on the estimated US average Medicare payment in 2006 for the encounter's DRG. Other outcomes were subsequent IP admission among ED patients, IP length of stay (LOS), and in-hospital mortality.

Results: Of 519,317 hospital encounters of diabetic patients, there were 732 encounters $(0.14 \%)$ due to hypoglycemia, including $667 \mathrm{ED}$-only, $24 \mathrm{ED}$ to IP, and 41 IP-only encounters. Overall, the mean cost for these encounters was $\$ 1,089$. The mean cost was $\$ 627$ for ED-only encounters, $\$ 5,081$ for ED to IP encounters, and $\$ 6,272$ for IP-only encounters (see Table). The mean total cost for an encounter with any IP component was $\$ 5,844$. Of the 691 encounters that began in the ED, 24 (3.5\%) were subsequently admitted. Mean LOS was 4.8 days for ED to IP or IP-only encounters. In-hospital mortality was $0.5 \%$ for ED-only and $4.6 \%$ for ED to IP or IP-only encounters.

Conclusion: The average cost for diabetic patients presenting to an acute care facility with hypoglycemia was $\$ 1,089$. This mean was influenced by the fact that the majority of hypoglycemia encounters were confined to the ED with a mean cost of only $\$ 627$; however, the hospitalization cost for diabetic patients presenting to an acute care facility with hypoglycemia was $\$ 5,844$, more than 9 times higher than for an ED encounter.

Table. Hypoglycemia diagnosis (ICD-9-CM codes 251.0, 251.1, or 251.2) and outcomes

\begin{tabular}{llllll}
\hline & \multicolumn{2}{l}{$\begin{array}{l}\text { ED only } \\
(\mathbf{n}=\mathbf{6 6 7})\end{array}$} & & \multicolumn{2}{l}{$\begin{array}{l}\text { ED to IP or IP Only } \\
(\mathbf{n}=\mathbf{6 5})\end{array}$} \\
\cline { 2 - 3 } \cline { 6 - 7 } & $\mathbf{N}$ & Mean or $\%$ & & $\mathbf{N}$ & Mean or \% \\
\hline Mortality & 3 & $0.5 \%$ & & 3 & $4.6 \%$ \\
\hline Length of Stay & - & - & 65 & 4.8 days \\
\hline Total Costs (2006US\$) & 582 & $\$ 627$ & 64 & $\$ 5,844$ \\
\hline
\end{tabular}

Supported by: Merck and Co., Inc.

\section{1}

Prevalence of impaired awareness of hypoglycaemia in insulin-treated type 2 diabetes and its effect on hypoglycaemia frequency

J.E. Schopman, J. Geddes, B.M. Frier

Diabetes Department, Royal Infirmary of Edinburgh, United Kingdom

Background and aims: While impaired awareness of hypoglycaemia (IAH) is a major limitation to maintaining strict control in Type 1 diabetes, in insulin-treated Type 2 diabetes (T2DM) the prevalence of this syndrome and its influence on hypoglycaemia frequency have seldom been examined. The present study sought to ascertain the prevalence of IAH in people with insulin-treated T2DM and how it influenced their exposure to hypoglycaemia. Materials and methods: Participants completed a questionnaire documenting baseline characteristics and assessing their symptomatic awareness status using a validated method (Gold et al, Diabetes Care, 1994; 17: 697). Capillary blood glucose (BG) was then monitored prospectively four times a day, over a 4-week period; any episode of biochemical hypoglycaemia (defined as BG < $3.0 \mathrm{mmol} / \mathrm{L}$ ) was identified and any associated symptoms were recorded and classified. Severe hypoglycaemia (defined as requiring external assistance) was estimated retrospectively over the preceding year.

Results: Data were obtained from 122 people with insulin-treated T2DM (63 male; mean (SD) HbAlc $8.4 \%$ (1.5); median (inter quartile range, IQR) age, 67 (58-72) years; duration of T2DM, 15 (10-20) years; duration of insulin therapy, 6 (4-9) years). The prevalence of IAH was $9.8 \%$. The patients with IAH had a five-fold higher incidence of episodes of biochemical hypoglycaemia over the 4-week monitoring period compared to those with normal awareness of hypoglycaemia (mean (SD) 2.34 (4.39) vs 0.46 (1.21) episodes; $\mathrm{p}<0.001$ ). By extrapolation, those with normal awareness of hypoglycaemia would have an annual incidence of 6 episodes per person per year of biochemical hypoglycaemia, compared to 29 episodes per person per year in the cohort with IAH. No symptomatic differences were observed in the reported intensity of symptoms experienced between the groups during biochemical hypoglycaemia (autonomic symptoms $\mathrm{p}=0.42$; neuroglycopenic symptoms $\mathrm{p}=0.52$ ). In the year preceding the study, the incidence of severe hypoglycaemia as reported by those with IAH was 17 -fold higher than in people with normal hypoglycaemia awareness $(0.83(1.12)$ vs. $0.05(0.28)$ episodes per patient; $\mathrm{p}<0.001$ ).

Conclusion: The observed prevalence of IAH in insulin-treated T2DM $(9.8 \%)$ was consistent with a previous estimate from our centre based on clinical history alone (8\%). While IAH in insulin-treated Type 2 diabetes occurs less commonly than in Type 1 diabetes, in those affected it is associated with a greatly increased risk of biochemical and severe hypoglycaemia. The presence of IAH in people with insulin-treated T2DM should therefore be evaluated routinely at clinical review. 


\section{PS 052 Drug effects on insulin sensitivity and insulin action}

\section{2}

Stimulation of GLUT4 vesicles transport and tethering is coupled to improved insulin sensitivity in human adipose tissue in response to rosiglitazone: resolution by proteomics

M. Ahmed ${ }^{1,2}$, F. Karpe ${ }^{1}$

${ }^{1}$ Oxford Centre for Diabetes, Endocrinology and Metabolism, University of Oxford, United Kingdom, ${ }^{2}$ Department of Physiology, Ibrahim Medical College, Dhaka, Bangladesh

Background and aims: Adipose tissue plays a crucial role in regulation of lipid and carbohydrate metabolism and many of these actions are regulated by insulin. The present study aims to detect potential novel protein targets for insulin sensitization in human adipose tissue by using unbiased proteomic approaches.

Materials and methods: Ten healthy moderately obese men aged $42 \pm 2$ years were treated with rosiglitazone $4 \mathrm{mg} \mathrm{BD}$ for 14 days and adipose tissue biopsies and plasma samples were taken at day 0 (without medication), day 1 and day 14. Adipose tissue protein profiles were obtained by two-dimensional gel (2-DG) electrophoresis and label-free LC-MS/MS

Results: Fasting serum insulin was reduced by $20 \%$ after two weeks treatment with rosiglitazone. This suggested a whole body effect towards insulin sensitization. Analysis revealed distinct up- or down-regulation ( $\geq 2$-fold) in multiple proteins. On the 2-DG images, treatment with rosiglitazone increased the expression of annexin $A 1$, annexin $A 2$, calmodulin, ferritin light chain, guanine nucleotide-binding protein $\mathrm{G}(\mathrm{I}) / \mathrm{G}(\mathrm{S}) / \mathrm{G}(\mathrm{T})$ subunit $\beta 1$ (GNB1), GNB3, heat shock $27 \mathrm{kDa}$ protein, LIM and SH3 domain protein 1 , moesin, myosin-9, superoxide dismutase [Mn], transferrin, tubulin $\beta-1$ chain and vimentin. LC-MS/MS based comparative proteomics validates the data from 2 DGE and also ascertain differential expression of additional proteins, such as exocyst components, sec5, sec6, sec8, Exo84 and Exo70, involved in GLUT4 tethering and exocytosis in human adipose tissue.

Conclusion: Some of major adipose tissue proteomic changes were involved cytoskeletal rearrangement, inflammatory and redox signals, whereas coordinated up-regulation of GLUT4 granule transport and fusion in human adipose tissue was seen. Such orchestrated changes in expression of multiple proteins belonging to the same pathway provide insights into the mechanism underlying the increased uptake of glucose and improvement of insulin sensitivity in response to rosiglitazone treatment.

\section{3}

Peroxisome proliferator-activated receptor gamma ligands suppress the transcriptional activation of $P E D / P E A-15$ gene

P. Mirra, F. Oriente, S. Iovino, G. Botta, V. D’Esposito, I. Esposito,

P. Formisano, P. Ungaro, F. Beguinot

Dipartimento di Biologia e Patologia Cellulare e Molecolare \& IEOS-CNR, Universita Di Napoli, Italy

Background and aims: The phosphoprotein enriched in diabetes/phosphoprotein enriched in astrocytes (PED/PEA-15) gene is overexpressed in tissues of individuals with type 2 diabetes. In cultured cells and in vivo, ped/pea-15 overexpression impairs insulin activation of protein kinase $\mathrm{C}$ zeta (PKCzeta) and glucose disposal. Indeed, transgenic mice ubiquitously overexpressing ped/pea-15 feature impaired glucose tolerance and insulin resistance. However, it is still unknown how ped/pea-15 gene expression is regulated. Recently, we have obtained evidence that Thiazolidinediones (TZDs), a class of peroxisome proliferator-activated receptor (PPAR)gamma ligands, repress ped/pea-15 expression both at mRNA and protein levels. PPARgamma is a member of the nuclear hormone receptor superfamily that modulate gene expression upon ligand binding. Ligand-mediated activation of PPARgamma has been linked to glucose homeostasis, cellular differentiation, apoptosis and anti-inflammatory responses. The aim of this work is to study how activated PPARgamma may exert its action on PED/PEA-15 expression.

Materials and methods: ped/pea-15 protein expression was determined in tissues from C57BL/6J mice. Differentiated L6 myotubes were used as an in vitro skeletal muscle model. Luciferase assay and Electrophoretic Mobility Shift Assay (EMSA) were conducted in Hela cells.

Results: In L6 skeletal muscle cells, the TZDs rosiglitazone and troglitazone decreased ped/pea-15 protein expression leading to the activation of PKC zeta and increased 2-deoxy-glucose uptake. TZDs also reduces ped/pea-15 mRNA levels by $40 \%$. Consistent with the in vitro data, muscle tissue from control mice showed a $90 \%$ decrease of ped/pea- 15 levels upon 10 days treatment with rosiglitazone $(26 \mathrm{mg} / \mathrm{Kg} / \mathrm{die})$. Hela cells treated with TPA, the prototypical AP-1 activator showed an increase in PED/PEA-15 mRNA levels. This finding was confirmed also on the PED/PEA-15 promoter activity by luciferase assays. Interestingly, serum and TPA effect were suppressed by rosiglitazone in a concentration dependent-manner. Since PPARgamma activation by TZDs blocks AP-1-mediated gene transcription, we hypothesized that PPARgamma transrepression of the ped/pea-15 gene is due to competition for limiting amounts of coactivators present in the cell. Indeed, the suppressive effect of rosiglitazone on PED/PEA-15 was blocked by overexpressing a dominant negative form of PPARgamma that lacks the ability to recruit the coactivator $\mathrm{CBP} / \mathrm{p} 300$ (CREB-binding protein). EMSA also revealed that TPA caused an increased binding to the CRE site (a putative AP-1 binding site) on the PED/PEA-15 promoter, an effect that was suppressed by rosiglitazone. Supershift and Chip experiments are ongoing in our laboratory in order to identify the component of the DNA binding complex.

Conclusion: Taken together, these results indicate that regulation through the CRE site on PED/PEA-15 promoter induces PED/PEA-15 transcription. This effect was blocked by treatment with PPARgamma ligands. Finally, the results of this study identify PED/PEA-15 gene as a potential target for PPARgamma-based therapeutic action.

\section{4}

Thiazolidinedione enhancement of insulin action in skeletal muscle and liver cells: role of protein kinase $\mathrm{C}$ delta (PKC $\delta$ )

T. Brutman-Barazani, V. Attali, M. Horovitz-Fried, C. Brodie,

S.R. Sampson

Life Sciences, Bar-Ilan University, Ramat-Gan, Israel

Background and aims: Thiazolidinediones (TZDs) are a class of oral antidiabetic agents that improve insulin sensitivity and glucose homoestasis in type 2 diabetic patients, as well as in various animal models of diabetes. The TZDs have been in use in clinical practice but the mechanisms by which TZDs improve insulin sensitivity are not yet fully understood. An important family of proteins that has been implicated in the insulin signaling cascade is the PKC family of serine threonine kinases. PKC $\delta$. Thiazolidinediones (TZDs) are a class of oral antidiabetic agents that improve insulin sensitivity and glucose homoestasis in type 2 diabetic patients, as well as in various animal models of diabetes. The TZDs have been in use in clinical practice but the mechanisms by which TZDs improve insulin sensitivity are not yet fully understood. An important family of proteins that has been implicated in the insulin signaling cascade is the PKC family of serine threonine kinases. PKC $\delta$ was found to play an essential role in the mediation of insulin-induced glucose uptake in skeletal muscle. In addition, insulin has been shown to rapidly increase PKC $\delta$ protein levels in skeletal muscle. In this study we examined the possibility that PKC $\delta$ may also be involved in TZD enhancement of insulin effects in skeletal muscle and liver cells.

Materials and methods: Studies were performed on the AML-12 (Alpha Mouse Liver) and L6 skeletal muscle cell lines. Activation of PKB and GSK3 downstream proteins was examined by Western blotting using phospho-specific antibodies. Glucose transport was determined by the measurement of $\left[{ }^{3} \mathrm{H}\right] 2$-deoxy-D-glucose (2-DG) uptake. Inhibition of PKC $\delta$ was achieved pharmacologically with the use of Rottlerin and by infection with specific dominant negative (DN) adenovirus constructs. PKC $\delta$ was over expressed by infection with wild type (WT) adenovirus constructs.

Results: TZDs induced an increase in PKB activity and GSK phosphorylation similar to insulin. The rate of 2-DG uptake was elevated by both insulin (165\%) and TZD (138\%). Results indicate that pretreatment of the AML-12 cells with TZD followed by insulin treatment increased the effect of insulin. The effects on 2-DG uptake appeared to be additive. We examined the possibility that PKC $\delta$ may be involved in the effect of TZD on the insulin signalling pathway. Inhibition of PKC $\delta$ decreased both TZD- and insulin-induced elevation in 2-DG uptake. In contrast, over expression of WTPKC $\delta$ increased 2-DG uptake in response to TZD. Finally, TZD was found to rapidly increase PKC $\delta$ protein levels in both skeletal muscle and liver cells in a manner similar to insulin.

Conclusion: The results indicate that $\mathrm{PKC} \delta$ plays an important role in the mediation of TZD-induced effects on glucose metabolism. Moreover, TZD may increase insulin sensitivity via PKC $\delta$.

Supported by: Russel Berrie Foundation and D Cure Israel 


\section{5}

Timed dopamine agonist therapy induces a postprandial-selective insulin sensitivity

M. Ezrokhi, S. Luo, J. Cincotta, A.H. Cincotta

Research and Development, VeroScience, Tiverton, United States

Background and aims: Once daily, morning administration of Cycloset, a quick-release formulation of bromocriptine (BC) (a dopamine D2 receptor agonist), improves post-meal glycemic control in insulin resistant and type 2 diabetes subjects across the 3 standard meal periods of the day. However, Cycloset is substantially removed from the circulation before the noon meal. Pre-clinical studies have identified aberrant hypothalamic fuel-sensing pathways potentiating insulin resistance that are normalized by timed daily BC administration. Collectively, these findings suggest that timed administration of Cycloset exerts, via the hypothalamus, a postprandial-selective improvement in insulin sensitivity and this would represent a novel therapeutic mechanism for improving glycemic control in insulin resistant states. Given the impact of elevated postprandial glucose $(\mathrm{G})$ on cardiometabolic risk, it is important to delineate such a bromocriptine impact on post-meal metabolism. The aim of this study was to identify any differences in bromocriptine influence on insulin sensitivity in the euglycemic (to approximate fasting glucose) versus hyperglycemic (to approximate postprandial glucose) states and to examine the impact of bromocriptine on hepatic intermediary glucose metabolism that may affect post-meal hepatic glucose disposal.

Materials and methods: Obese, insulin resistant male 15 month old Syrian hamsters were held on 14 hour daily photoperiods from birth and treated for 14 days with either BC $(4.4 \mathrm{mg} / \mathrm{kg})$ or vehicle at 1 hour after light onset and then subjected to a euglycemic-hyperinsulinemic clamp (insulin $3 \mathrm{mU} /$ $\mathrm{kg}^{\star} \mathrm{min},{ }^{3} \mathrm{H}-3-\mathrm{G} 0.2 \mu \mathrm{Ci} / \mathrm{kg}^{\star} \mathrm{min}$, steady state glucose (SSPG) $110 \mathrm{mg} / \mathrm{dL}$ ) followed by a hyperglycemic-hyperinsulinemic clamp (insulin $3 \mathrm{mU} / \mathrm{kg}^{\star} \mathrm{min}$, ${ }^{3} \mathrm{H}-3$-Glucose infusion $0.2 \mu \mathrm{Ci} / \mathrm{kg}^{\star} \mathrm{min}$, SSPG $\left.170 \mathrm{mg} / \mathrm{dL}\right)$. Livers isolated from similarly treated animals were analyzed for G-6-phosphatase and G-6phosphate dehydrogenase activities by spectrophotometric methods.

Results: Basal plasma G and insulin (I) levels were $116 \pm 11 \mathrm{mg} / \mathrm{dL}$ and $120 \pm 6$ $\mathrm{mg} / \mathrm{dL}$ and $3.8 \pm 0.5 \mathrm{ng} / \mathrm{ml}$ and $4.8 \pm 0.5 \mathrm{ng} / \mathrm{ml}$, respectively for BC and vehicle treated animals, respectively ( $\mathrm{p}=0.06$ for I difference). During the euglycemic clamp, BC treatment increased $\mathrm{G}$ disposal rate by $21 \%$ from $20.1 \pm 0.9$ $\mathrm{mg} / \mathrm{kg}^{*} \min$ to $25.4 \pm 0.6 \mathrm{mg} / \mathrm{kg}^{*} \mathrm{~min}(\mathrm{p}=0.02)$ and decreased hepatic $\mathrm{G}$ output (HGO) by $26 \%$ from $3.4 \pm 0.4 \mathrm{mg} / \mathrm{kg}^{*} \mathrm{~min}$ to $2.6 \pm 0.1 \mathrm{mg} / \mathrm{kg}^{*} \mathrm{~min}(\mathrm{p}<0.05)$. However, during the hyperglycemic clamp period, BC increased $\mathrm{G}$ disposal rate by $50 \%$ from $28.3 \pm 1.9 \mathrm{mg} / \mathrm{kg}^{*} \mathrm{~min}$ to $42.4 \pm 2 \mathrm{mg} / \mathrm{kg}^{*} \min (\mathrm{p}<0.0001)$, and decreased HGO by $38 \%$ from $3.4 \pm 0.2 \mathrm{mg} / \mathrm{kg}^{*} \mathrm{~min}$ to $2.1 \pm 0.3 \mathrm{mg} / \mathrm{kg}^{*} \mathrm{~min}$, $(\mathrm{p}=0.005)$. Insulin levels during the clamp did not vary between the groups (BC: $5.4 \pm 0.5 \mathrm{ng} / \mathrm{ml}$, vehicle $6.5 \pm 0.6 \mathrm{ng} / \mathrm{ml}$ ). The difference in $\mathrm{BC}$ impact on $\mathrm{G}$ disposal rate during hyperglycemia versus euglycemia was also significant $(\mathrm{p}<0.01)$. BC treatment increased the Km for G-6-phosphatase activity by $27 \%$ (from $2.6+/-0.1$ to $3.3+/-0.15 \mathrm{M}, \mathrm{p}<0.05$ ) and increased G-6-phosphate dehydrogenase activity by $62 \%$ (from $2.6+/-0.4$ to $4.2+/-0.2 \mathrm{~nm} / \mathrm{min} / \mathrm{mg}$, $\mathrm{P}<0.05)$. Such a shift in $\mathrm{G}$ flux enzymatic activities can potentiate hepatic $\mathrm{G}$ utilization by reducing HGO and stimulating the pentose phosphate pathway. Conclusion: Timed BC administration exerts a postprandial-selective insulin sensitizing effect to reduce HGO and increase peripheral glucose uptake. This unique mechanism of action may in turn reduce cardiovascular risk associated with postprandial hyperglycemia.

\section{6}

CVT-3619, a partial $A_{1}$ adenosine receptor agonist, potentiates the antilipolytic effect of insulin

A.K. Dhalla, M. Santikul, L. Belardinelli

Pharmacology, CV Therapeutics, Inc., Palo Alto, United States

Background and aims: Adenosine mediates its anti-lipolytic effects via $\mathrm{A}_{1}$ adenosine receptors. In addition, adenosine also modulates insulin actions and sensitivity in skeletal muscle and adipose tissue. Previously, we have shown that CVT-3619, a partial A receptor agonist, lowers circulating free fatty acid (FFA) levels and improves insulin sensitivity in rodents. The present study was undertaken to determine the interaction between the anti-lipolytic effects of insulin and CVT-3619.

Methods: The in-vitro anti-lipolytic effects of CVT-3619 and insulin were determined by measuring the release of FFA from adipocytes from normal (SD) and diabetic (ZDF) rats, whereas the in-vivo effects on circulating FFA were determined in awake unrestrained SD and ZDF rats.
Results: In adipocytes from normal SD rats, CVT-3619 or insulin inhibited lipolysis with $\mathrm{IC}_{50 \mathrm{~s}}$ of $63 \mathrm{nM}$ and $36 \mathrm{pM}$, respectively. CVT-3619 (10 nM) caused a leftward and downward shift of the insulin concentration-response curve. Adipocytes from ZDF rats were highly resistant to the anti-lipolytic effects of insulin $\left(\mathrm{IC}_{50}=30 \mathrm{nM}\right)$ but only slightly to CVT-3619 $\left(\mathrm{IC}_{50}=130\right.$ $\mathrm{nM})$. CVT-3619 (10 nM) restored insulin's sensitivity to inhibit FFA release from $\mathrm{ZDF}$ adipocytes $\left(\mathrm{IC}_{50}=110 \mathrm{pM}\right)$. Similar to isolated adipocytes from ZDF rats, the anti-lipolytic effect of insulin was markedly reduced in ZDF rats. CVT-3619 $(0.5 \mathrm{mg} / \mathrm{kg}$, a dose associated with a very small FFA lowering effect) caused a 10-fold increase in the potency of insulin to lower plasma concentrations of FFA.

Conclusion: CVT-3619 potentiates the anti-lipolytic effect of insulin, both in-vitro and in-vivo, especially in ZDF rats which are highly insulin resistant. Thus, CVT-3619 could be useful in insulin resistant states where anti-lipolytic effect of insulin is impaired resulting in higher rate of lipolysis leading to high circulating levels of FFA.

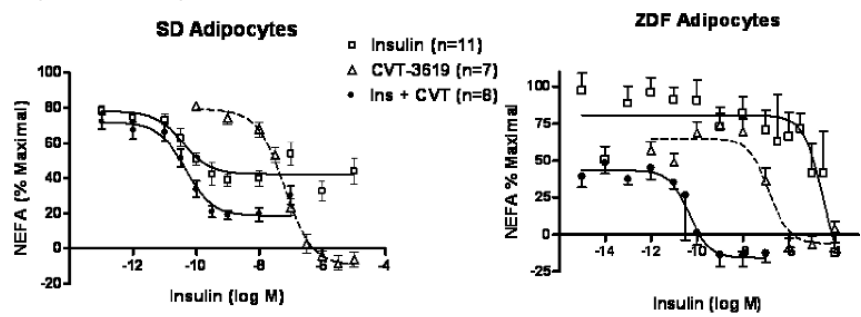

\section{7}

Testing efficient carriers for intracellular PIP3 delivery to induce insulin actions using biomimetic liposomes and cellular model systems I. Kachko - Chernetsky ${ }^{1}$, T. Traitel ${ }^{1}$, R. Jelinek ${ }^{2}$, A. Rudich ${ }^{3}$, J. Kost ${ }^{1}$ ${ }^{1}$ Department of Chemical Engineering, ${ }^{2}$ Department of Chemistry, ${ }^{3}$ Department of Clinical Biochemistry, Ben-Gurion University of the Negev, Beer-Sheva, Israel

Background and aims: Production of phosphatidylinsoitol-3,4,5-trisphosphate (PIP3), induced by insulin is a major signaling step in the induction of insulin's metabolic actions in muscle and fat, and is impaired in various states of insulin resistance. Intracellular delivery of PIP3 into cells might therefore constitute an approach for bypassing such a signaling defect. However, this approach is challenged by the need to overcome permeability barriers through the plasma membrane, and to introduce the agent in well-defined spatial-temporal coordinates corresponding to endogenous PIP3 generation. Exogenous PIP3 may be introduced into cells using polycationic carriers which should mask its negative charges. However so far, these approaches only partially mimicked insulin's effects in normal, insulin sensitive cells, and displayed poor efficiency and low reproducibility. The overall aim of this work was to assess the therapeutic potential of intracellular delivery of exogenous PIP3 to overcome cellular insulin resistance.

Materials and methods: Membrane-complex interactions and cellular localization were verified using biomimetic liposomal (lipid/polydiacetylene) membrane model and in L6 muscle cells in culture with fluorescent-PIP3 and live-cell microscopy. The ability of PIP3-carrier to induce insulin signaling and metabolic effects were evaluated in L6 myoblasts and in 3T3-L1 adipocytes electroporated with GFP-GLUT4myc plasmids.

Results: A polymeric carrier, polyethyleneimine (PEI), was utilized at different molecular weights and branched/linear backbone to generate efficient electrostaticaly bound PIP3-carrier complexes. Fluorescent liposomes surface perturbation and ESR (Electron Spin Resonance) analyses revealed that PIP3-PEI formed complexes and penetrated the lipid bilayer. Branched PEI $(25 \mathrm{kDa})$ carrier was more efficient in membrane internalization than linear PEI of the same molecular weight. Maximal liposome-complex interaction occurred at $40 \mathrm{~min}$. Live-cells kinetic studies revealed that the branched PEI-PIP3 complex enabled the retention of PIP3 at the cell periphery as compared to PIP3 alone. PIP3 delivered with PEI was found biologically available for binding to its intracellular ligand (GRP-PH) and for generating signaling effects, resulting in increased PKB phosphorylation, higher than that observed with neomycin as a carrier. Moreover, PIP3-carrier exposure increased Glut4 translocation and externalization in 3T3-L1 adipocytes.

Conclusion: We demonstrated a polymeric system for exogenous PIP3 delivery that can induce insulin signaling and metabolic effect in muscle and fat cells based on biomimetic liposomes and cellular systems. Inducing insulin 
actions by intracellular PIP3 delivery in insulin resistant cells will shed light on the potential therapeutic use of this technology.

Supported by: Israel Science Foundation

\section{8}

Nebivolol prevents insulin resistance and enhances plasma nitrates in sucrose-fed rats

M.P. Guarino ${ }^{1}$, M. Mota-Carmo ${ }^{1}$, A.I. Santos 2 , P.F. Costa ${ }^{2}$, A.B. Rendas ${ }^{1}$ ${ }^{1}$ Pathophysiology, ${ }^{2}$ Physiology, Faculdade Ciências Médicas, Lisbon, Portugal

Background and aims: Nebivolol is a $\beta-1$ selective adrenergic receptor antagonist, which also increases nitric oxide (NO) bioavailability. NO is required for physiological insulin action, suggesting that nebivolol may improve insulin sensitivity in pathologies where the NO signaling pathway is compromised. The High-Sucrose Diet Rat (HSu) is an animal model of insulin resistance with impaired NO signaling pathway. We hypothesized that HSu rats treated with nebivolol would show enhanced insulin sensitivity, correlating with an increase in plasma nitrates, a marker of endogenous NO production. These results were compared with the effect of atenolol, a $\beta$-blocker non-promoter of NO release, on insulin sensitivity.

Materials and methods: Rats were divided into six groups according to the composition of water supplied for four weeks, namely: Control - regular animal facility water; $\mathrm{S}$ - 35\% sucrose solution; $\mathrm{N}$ - nebivolol $1 \mathrm{mg} / \mathrm{kg} /$ day; $\mathrm{SN}$ - 35\% sucrose plus nebivolol $1 \mathrm{mg} / \mathrm{kg} /$ day; A - atenolol $10 \mathrm{mg} / \mathrm{kg} / \mathrm{day} ; \mathrm{SA}$ - 35\% sucrose plus atenolol $10 \mathrm{mg} / \mathrm{kg} /$ day. Insulin sensitivity was evaluated with the Insulin Tolerance Test and calculation of the rate of blood glucose decay $\left(\mathrm{K}_{\mathrm{ITT}}\right)$. NO metabolites were determined in arterial blood samples using a $\mathrm{NO} /$ ozone chemiluminescence technique

Results: $\mathrm{K}_{\mathrm{ITT}}$ was $5.02 \pm 0.42 \% / \mathrm{min}$ in the Control Group $(\mathrm{n}=8)$ and $2.52 \pm 0.33$ $\% / \mathrm{min}$ in the $\mathrm{S}$ Group $(\mathrm{n}=8, \mathrm{p}<0.001)$. The lower $\mathrm{K}_{\mathrm{ITT}}$ value found in the $\mathrm{S}$ Group was suggestive of insulin resistance.

In the $\mathrm{N}$ Group, the $\mathrm{K}_{\mathrm{ITT}}$ was $4.21 .02 \pm 0.44 \% / \mathrm{min}(\mathrm{n}=8)$, which was not significantly different from Control. Atenolol decreased insulin action as shown by a $\mathrm{K}_{\mathrm{ITT}}$ in the A group of $3.08 \pm 0.57 \% / \mathrm{min}(\mathrm{n}=7, \mathrm{p}<0.05$ from Control). In the SN Group, $\mathrm{K}_{\mathrm{ITT}}$ was $4.21 \pm 0.44 \% / \mathrm{min}(\mathrm{n}=8, \mathrm{p}<0.01$ from $\mathrm{S}$ group). In the $\mathrm{SA}$ group, $\mathrm{K}_{\mathrm{ITT}}$ was $2.67 \pm 0.64 \% / \mathrm{min}, \mathrm{n}=6$, not significant from $\mathrm{S}$ group). These results show that nebivolol prevented the development of insulin resistance induced by the sucrose diet. Atenolol, on the other hand, did not restore insulin sensitivity in $\mathrm{HSu}$ rats. Plasma $\mathrm{NO} / \mathrm{NO}_{3}{ }^{-}$was $61.7 \pm 13.5 \mu \mathrm{M}$ in the Control Group and $51.3 \pm 1.7 \mu \mathrm{M}$ in the $\mathrm{S}$ Group $(\mathrm{p}<0.05)$. In the $\mathrm{SN}$ Group, nebivolol increased plasma $\mathrm{NO} / \mathrm{NO}_{3}$ concentration to $83.0 \pm 13.5 \mu \mathrm{M}$. Plasma $\mathrm{NO} / \mathrm{NO}_{3}{ }^{-}$in the SA Group was $39.7 \pm 3.3 \mu \mathrm{M}$.

Conclusion: We concluded that nebivolol prevents the development of insulin resistance induced by a high-sucrose diet, as opposed to atenolol. Also, the enhancement of insulin sensitivity induced by nebivolol correlated with an increase in endogenous NO production, which is in agreement with the proposed hypothesis.

Supported by: A. Menarini Diagnostics

\section{PS 053 Mechanisms of insulin resistance: cellular studies}

\section{9}

The role of HSP70 on in vitro ENPP1 expression and insulin receptor activation and in vivo insulin resistance-related traits

R. Di Paola ${ }^{1}$, A. Marucci ${ }^{1}$, G. Miscio ${ }^{1}$, L. Padovano ${ }^{1}$, W. Boonyasrisawat ${ }^{1}$, J.C. Florez ${ }^{2,3}$, A. Doria ${ }^{4,2}$, V. Trischitta ${ }^{1,5}$

${ }^{1}$ Research Unit of Diabetes and Endocrine Diseases, Casa Sollievo della Sofferenza, San Giovanni Rotondo, Italy, ${ }^{2}$ Department of Medicine, Harvard Medical School, Boston, United States, ${ }^{3}$ Diabetes Unit/Center for Human Genetic Research, Massachusetts General Hospital, Boston, United States, ${ }^{4}$ Research Division, Joslin Diabetes Center, Boston, United States, ${ }^{5}$ Department of Clinical Sciences, University “Sapienza”, Rome, Italy

Background and aims: Ectonucleotide pyrophosphatase phosphodiesterase 1 (ENPP1) inhibits insulin receptor (IR) signaling and, when over-expressed, induces insulin resistance. Understanding the regulation of ENPP1 expression may unravel new molecular mechanisms of insulin resistance. We sought to identify trans-acting proteins binding the ENPP1-3'UTR and investigate their role on ENPP1 expression and insulin resistance.

Methods: We demonstrated binding of the heat shock protein 70 (HSP70) to ENPP1-3'UTR by RNA electrophoresis mobility shift analysis and tandem mass spectrometry. We used quantitative real time-PCR (QRT-PCR) and western blot analysis to investigate the effect of HSP70 on ENPP1 expression and IR-autophosphorylation. We genotyped HSP70 gene variants in 924 nondiabetic Italian subjects and measured HSP70 mRNA by QRT-PCR.

Results: By binding ENPP1-3'UTR, HSP70 stabilizes ENPP1 mRNA and increases ENPP1 transcript and protein levels. Through the positive modulation of ENPP1 expression, HSP70 inhibits insulin-induced IR autophosphorylation. The minor alleles at rs9281590 and rs2763979 in HSP70 are associated with better glucose and lipid levels in nondiabetic Europeans. Associations with lipid levels are replicated in a family-based sample, and those with glucose levels in the Diabetes Genetics Initiative sample. The minor allele at rs9281590, was also associated with reduced HSP70 expression in lymphomonocytes from nondiabetic subjects.

Conclusion/Interpretation: HSP70 increases ENPP1 expression and decreases IR activation. The HSP70 rs9281590 variant is associated with reduced HSP70 expression and lower glucose and lipids levels. Taken together, these data suggest that HSP70 may be a novel mediator of insulin resistance. Supported by: Italian Ministry of Health, Italian Ministry of University and Research Grant, Telethon, National Institutes of Health and a Research Development Career Award

\section{0}

Impaired insulin signalling is associated with decreased transformation potential of the skin

J. Russ ${ }^{1}$, G. Weingarten ${ }^{1}$, J. Takeda ${ }^{2}$, C. Kahn ${ }^{3}$, E. Wertheimer ${ }^{1}$

${ }^{1}$ Pathology, Sackler Medical School Tel Aviv University, Tel Aviv, Israel, ${ }^{2}$ Dermatology, Osaka University Medical School, Osaka, Japan, ${ }^{3}$ Joslin diabetes center, Harvard Medical School, Boston, United States

Background and aims: One of the major complications of diabetes mellitus is impaired wound healing. Traditionally, this pathology is believed to develop secondary to the neuropathy and microangiopathy associated with diabetes. However, we have previously demonstrated that insulin plays a direct role in normal skin turnover, including regulation of skin proliferation and differentiation, suggesting that insulin resistance might be directly involved in the development of diabetic skin complications. In order to further substantiate our findings on the importance of insulin signaling on skin proliferation and differentiation, in the present study we applied cellular transformation protocols on murine skin and primary keratinocytes lacking insulin signaling, aiming to demonstrate that lack of insulin signaling will result in decreased skin transformation potential.

Materials and methods: Our hypothesis was tested in skin-specific insulin receptor (IR) knockout mice (SIRKO), where disruption of IR expression is restricted to the epidermal layer of the skin. Skin tumors were induced according to a well characterized initiation-promotion carcinogenesis protocol and the kinetics of tumor formation, histopathology and expression of specific epidermal markers was followed. 
Results: We found that lack of IR expression in skin resulted in a marked decrease in tumor induction efficiency and tumor proliferation capacity. Next we analyzed which stage in the carcinogenesis was disrupted by the abrasion of the IR. There was no difference in the initiation stage, the stage when the initial mutation leading to the development of the tumor is induced chemically. However, a marked decrease was observed in the response of the IR lacking skin to the promoting agent, which is applied for further support and development of the transformed initiated cells. In order to reveal the mechanism underlying the decreased carcinogenic potential, we studied cultured primary skin epidermal cells, isolated from the SIRKO and control mice. Interestingly, the transformation of the murine primary keratinocytes in culture was inefficient in the SIRKO cells; they exhibited impaired spindle formation and appearance of multinuclear senescent cells.

Conclusion: Our data provide evidence that complete abrasion of the IR in the epidermis leads to changes in both skin turnover in vivo as well as in the intrinsic properties of the keratinocytes. Further understanding the regulation of skin turnover by insulin signaling might lead to development of new treatments for various skin pathologies associated with impaired insulin signaling.

\section{1}

Glucosamine-induced insulin-resistance in L6 skeletal muscle cells is associated with endoplasmic reticulum stress

G.A. Raciti ${ }^{1}$, L. Ulianich ${ }^{1}$, C. Iadicicco ${ }^{1}$, A. Lombardi ${ }^{2}$, F. Andreozzi ${ }^{3}$, B. Di Jeso ${ }^{2}$, C. Garbi ${ }^{1}$, P. Formisano ${ }^{1}$, F. Beguinot ${ }^{1}$, C. Miele ${ }^{1}$

${ }^{1}$ Dipartimento di Biologia e Patologia Cellulare e Molecolare \& IEOS-CNR, University of Naples, ${ }^{2}$ Dipartimento di Scienze e Tecnologie Biologiche e Ambientali, University of Lecce, ${ }^{3} \mathrm{Dip}$ di Medicina Sperimentale e Clinica, University of Catanzaro, Italy

Background and aims: Endoplasmic reticulum (ER) stress might play an important role in the pathogenesis of insulin-resistance and Type 2 diabetes. Hyperglycaemia causes insulin resistance and this process appears to be linked to ER stress. Glucosamine, generated by hexosamine pathway during hyperglycaemia, also causes insulin resistance and disturbances similar to glucose toxicity. Here, we sought to evaluate the possible role of ER stress on the insulin-resistance induced by glucosamine in skeletal muscle cells

Materials and methods: L6 skeletal muscle cells were incubated either with $10 \mathrm{mM}$ glucosamine (GlcNac) or with $0.5 \mu \mathrm{M}$ thapsigargin (Thap), a well known ER stressing agent, in the presence or in absence of insulin (100nM). Results: Upon 24 hours of GlcNac treatment, L6 cells showed a 6-fold increase of the ER stress marker BiP/GRP78 mRNA levels $(p<0.001)$ and a reduced uptake of the glucose analogue 2-deoxy-D-[3H]-glucose (2DG) in response to insulin $(\mathrm{p}<0.01)$. These effects were paralleled by a $70 \%$ decrease of both mRNA and protein levels of the insulin-sensitive glucose transporter GLUT4. GLUT4 regulation was, very likely, at the transcriptional level, as demonstrated by parallel mRNA downregulation of two transcription factors known to regulate GLUT4 expression, such as MEF2A and PGC1. Time course experiments showed that GLUT4 expression was reduced by $30 \%$ after 4 hours $(\mathrm{p}<0.001)$, and by $70 \%$ after 8 hours $(\mathrm{p}<0.001)$ following GlcNac treatment. MEF2A mRNA showed a similar kinetic. Furthermore, immunofluorescence confocal imaging experiments showed that GlcNac, besides its effect on GLUT4 transcription, impaired also the insulin induced GLUT4 translocation onto the plasma membrane. The use of the chemical chaperon 4-Phenyl Butyric Acid (PBA), known to alleviate ER stress conditions, prevented both BiP/GRP78 induction and GLUT4 downregulation. As for GLUT4, also MEF2A and PGC1 mRNA levels did not show any appreciable variation when L6 cells were treated with GlcNac in the presence of PBA. Furthermore, PBA treatment restored insulin-induced 2DG uptake to levels comparable to those of L6 control cells. Interestingly, GlcNac caused a $60 \%$ increase of both mRNA and protein levels of the glucose transporter GLUT1 $(p<0.01)$, suggesting that GLUT1 gene could be considered an early target of ER stress. Indeed, bioinformatics researches revealed the presence of four ERSE (ER stress Responsive Element) consensus sequence for ER stress activated transcription factors in the proximal region of the rat GLUT1 promoter sequence.

Conclusion: Glucosamine-induced ER stress may play a role in insulin-resistance of L6 skeletal muscle cells through the modulation of the expression and localization of the glucose transporters GLUT1 and GLUT4.

Supported by: EFSD/GSK grant

\section{2}

S-adenosyl-L-methionine inhibits IkappaB kinase- $\beta$ activity M. Moon ${ }^{1}$, M. Kim ${ }^{1}$, H. Lee ${ }^{2}$, Y. Cho ${ }^{1}$, Y. Park ${ }^{1}$, S. Choi ${ }^{1}$, H. Jang ${ }^{1}$, K. Park ${ }^{1}$, H. Lee ${ }^{1}$

${ }^{1}$ Internal Medicine, Seoul National University College of Medicine, ${ }^{2}$ Internal Medicine, Seoul National University Boramae Hospital, Seoul, Republic of Korea

Background and aims: S-adenosylmethionine (SAM) was reported to improve insulin sensitivity in OLETF rats. SAM has not only several features as a good antioxidant but also anti-inflammatory property. Both of oxidative stress and activation of I $\kappa$ B kinase $\beta(\mathrm{IKK}-\beta) / \mathrm{NF}-\kappa \mathrm{B}$ pathway by inflammation are possible mechanism in the development of insulin resistance. We investigated the effects of SAM on the activity of IKK- $\beta$, NF- $\mathrm{kB}$ binding activity, glucose transport and insulin signaling in rat L6 cells.

Materials and methods: The kinase activity of IKK- $\beta$ was determined by a colorimetric ELISA assay. NF- $\mathrm{\kappa B}$ activities were determined by Electrophoretic mobility shift assay. Phosphorylation of IRS-1(Y612) and Akt(Ser473) were determined by western blot analysis. Glucose transport was measured by the 2-deoxy- $\left[{ }^{3} \mathrm{H}\right]$-D-glucose uptake.

Results: Treatment with $1 \mathrm{mM}$ of SAM could inhibit the activity of IKK- $\beta$ by $60 \%$ compared to control material $(\mathrm{P}<0.05)$. Treatment of cells with $1 \mathrm{mM}$ of SAM could decrease NF- $\mathrm{KB}$ binding activity by about $20 \%$ compared to those activated with TNF- $\alpha$. Treatment of L6 cells with $0.5 \mathrm{mM}$ of SAM increased basal and insulin-stimulated glucose transport to $17.2 \%(\mathrm{P}<0.001)$ and $12.8 \%(\mathrm{P}=0.014)$, respectively, of that in untreated cells. $1 \mathrm{mM}$ of SAM also increased basal and insulin-stimulated glucose transport to $77.8 \%$ $(\mathrm{P}<0.001)$ and $73.5 \%(\mathrm{P}<0.001)$, respectively, of that in untreated cells. Both of $0.5 \mathrm{mM}$ and $1 \mathrm{M}$ of SAM increased the tyrosine phosphorylation of IRS-1 and Akt in L6 cells.

Conclusion: SAM inhibited the activity of IKK- $\beta$ and binding activity of NF$\kappa B$ induced by TNF- $\alpha$. SAM also improved glucose transport and insulin signaling in L6 cells. These results suggest that SAM could have a beneficial role on the treatment of type 2 diabetes.

Supported by: grant from Seoul National University Hospital Research Fund

\section{3}

Study of sam 68 implication in insulin receptor signalling by BRET C. González-Yanes ${ }^{1}$, S. Najib ${ }^{1}$, C. Blanquart ${ }^{2}$, T. Issad ${ }^{2}$,

V. Sánchez-Margalet ${ }^{1}$

${ }^{1}$ Medical Biochemistry and Molecular Biology, Virgen Macarena University Hospital, Sevilla, Spain, ${ }^{2}$ Cellular Biology, Institut Cochin, Paris, France

Background and aims: Sam68 is a RNA-binding protein belonging to the signal transduction and activation of RNA metabolism (STAR) family and it was first identified as a target of the tyrosine kinase Src. STAR proteins are characterized by a hnRNP $\mathrm{K}$ domain $(\mathrm{KH})$ embedded in a high conserved region called GSG domain, which is required for homodimerization and sequence-specific RNA binding. Sam68 has been implicated in the regulation of cell cycle progression, RNA processing and apoptosis. Self-association of Sam68 has been previously described and RNA binding is necessary for this interaction. Sam68 phosphorylation by Src disrupts this oligomerization and RNA binding. Tyr-phosphorylation of Sam68 enables the molecule to interact with SH2-domain containing proteins involving Sam68 in signal transduction. We have previously shown that Sam68 is implicated in insulin receptor (IR) signalling as an adaptor protein mediating metabolic and proliferative effect of insulin. In this work, we aimed to further investigate Sam68 interactions with IR signalling proteins by using bioluminescence resonance energy transfer (BRET).

Materials and methods: We first prepared the constructs for BRET technology: Sam68-EYFP, Sam68-phRLuc, Sam68-pcDNA3 and we have also used IR-phRLuc, IRS1-EYFP and p85a-EYFP previously employed for study of IR signalling by BRET. Transient transfections with phRluc and/or EYFP fusion or other constructs were performed in HEK293T cells. Twenty four hours post-transfection cells were detached and distributed in 96-well microplates. BRET experiments were performed on the following day. Upon the addition of $5 \mathrm{uM}$ of coelenterazine bioluminescence resulting from its degradation and the energy transferred to EYFP was detected. The BRET signal (BRET ratio) was quantified by calculating the $530 \mathrm{~nm} / 480 \mathrm{~nm}$ light-emission ratio. Results: We have tested the interaction of Sam68 with IRS-1, and the homopolymerization of Sam68. We found that Sam68 interacts with IRS-1 measuring BRET signal using Sam68-Luc/IRS1-EYFP. This association is 
potentiated by the overexpression of IR, and insulin stimulation further enhances the Sam68-IRS-1 association. On the other hand Sam68 self-association was confirmed by BRET studies.

Conclusion: In HEK293, IR overexpression and insulin stimulation positively modulates protein-protein interaction increasing the BRET signal of Sam68/IRS-1 and Sam68/Sam68 interaction. These results demonstrate the implication of Sam68 in the first steps of IR signalling.

Supported by: Fondo de Investigación Sanitaria (CP05/00273)

\section{4}

Glucose deprivation simultaneously upregulates BiP/GRP78 and downregulates pIRS1(Ser312) in human adipocytes

E.M. Murphy, G. Ceolotto, A. Avogaro

Clinical and Experimental Medicine, University of Padua, Italy

Background and aims: Adipocytes play a critical role in energy metabolism. Their ability to respond appropriately to extracellular glucose concentrations, storing energy in the form of triglycerides when glucose is abundant, and releasing FFAs into the circulation when glucose is scarse, is dependent on adequate insulin signaling. IRS1 phosphorylation at Ser 312 has been shown to decrease IRS1 tyrosine phosphorylation and to consequently impair insulin signal transduction. Impaired insulin signaling has been shown to be associated with endoplasmic reticulum (ER) stress in some tissues. In cells deprived of glucose, ER stress may be activated and therefore may modulate insulin signal transduction by molecular mechanisms not completely known. Our aim was therefore to examine the link between glucose deprivation, IRS1 phosphorylation at Ser312, and ER stress activation in adipocytes, using BiP/ GRP78 (Binding Protein/ 78 kDa Glucose-Regulated Protein) as a marker for ER stress activation.

Materials and methods: We differentiated a cell line of normal human white preadipocytes (HWP) into mature adipocytes . Cells were incubated for 18 hours in DMEM:F12 containing $2.5 \mathrm{mM}$ L-glutamine and 3\% FBS, with either $0 \mathrm{mM}$ glucose (glucose deprivation) or $5.5 \mathrm{mM}$ glucose (normal glucose), with and without insulin (100nM). Protein expression of pIRS1(Ser312), IRS1, BiP/GRP78 and GAPDH were determined by immunoblotting with specific antibodies.

Results: We found that glucose deprivation induced a decrease in protein expression of both unphosphorylated IRS1 and phosphorylated pIRS1(Ser312), both in the absence and presence of insulin, with a reduced ratio of pIRS1(Ser312) to unphosphorylated IRS1 under glucose deprivation. BiP/ GRP78 expression was increased in glucose-deprived cells in comparison to cells grown at normal glucose levels. The decrease in IRS1(Ser312) phosphorylation is associated with an increase of BiP/GRP78 protein expression.

Conclusion: The ER stress induced by glucose deprivation in human white adipocytes is associated with a downregulated phosphorylation of IRS1 at Ser312. This downregulation may provide a novel mechanism by which cells improve insulin sensitivity when glucose is scarse. IRS1(Ser312) phosphorylation is sensitive to glucose deprivation even in the absence of insulin, demonstrating an insulin-independent effect of glucose on insulin signaling.

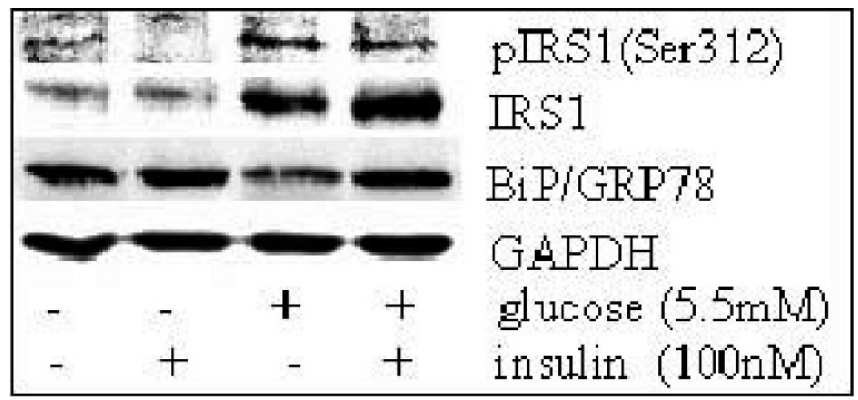

\section{5}

Insulin resistance by oxidative stress through the life-span determinant p66 $^{\text {shcA }}$ and the $\mathrm{mTOR} / \mathrm{S6K}$ cascade

G. Pani, S. Chiatamone Ranieri, S. Fusco

Institute of General Pathology, Catholic University Medical School, Rome, Italy

Background and aims: Mitochondrial oxidative stress and hyperfunction of the nutrient-sensitive signaling pathway triggered by the mammalian Target of Rapamycin (mTOR) have been independently linked to the establishment of peripheral insulin resistance. While mTOR downregulates Insulin Receptor (IR) signaling through the S6 kinase-dependent inhibitory phosphorylation of the IR Substrate-1 (IRS-1), molecular mechanisms linking generation of Reactive Oxygen Species (ROS) to impaired Insulin responses are still largely elusive. The life-span determinant p66shcA mediates cellular damage and cell death by oxidative insult in a number of pathophysiological conditions including diabetic glomerulopathy, and participates in the generation of mitochondrial ROS; however, a potential role for this molecule in oxidant-induced IR desensitization has not been investigated. Aim of the present study is to clarify the molecular mechanism underlying insulin resistance by oxidative stress, and the potential involvement of p66shcA in this phenomenon.

Materials and methods: Undifferentiated 3T3L1 pre-adipocytes were serumstarved for 24 hours in the presence of redox stimuli (Catalase, $2500 \mathrm{U} / \mathrm{ml}$; Glucose Oxidase [GOX], 5mU/ml), Rapamycin (200 ng/ml) or glucose-free medium, and stimulated with insulin $(100 \mathrm{ng} / \mathrm{ml})$ for 10' Phosphorylation of AKT/PKB, IRS-1, S6K and S6 were evaluated by phospho-specific immunoblotting of total protein extracts. P66 WT and p66KO pre-adipocytes were isolated from mice of the corresponding genotype and stimulated in vitro as described above. To induce insulin resistance cells were kept for $\geq 2$ days in the presence of TNF-alpha $(10 \mathrm{ng} / \mathrm{ml})$, GOX $(5 \mathrm{mU} / \mathrm{ml})$ or FFA $(1 \mathrm{mM})$ before insulin challenge.

Results: In the preadipocytic 3T3L1 cell line, exposure to insulin led to the robust phosphorylation of AKT and, in parallel, to activation of the mTOR/ S6K cascade, as monitored by the phosphorylation of the S6 ribosomal protein and the S6K-dependent, inhibitory phosphorylation of IRS-1 on Serine 1101. mTOR blockade by Rapamycin or by glucose withdrawal from the culture medium strongly inhibited mTOR-dependent events, while AKT phosphorylation was unaffected or slightly increased. Interestingly, a very similar response was elicited by cell treatment with the peroxide scavenger Catalase; conversely, generation of peroxide by GOX inhibited AKT responses and increased both mTOR activity and IRS-1 (Ser-1101) phosphorylation, suggesting that oxidants downregulate early insulin signaling, at least in part, through the redox-dependent activation of the $\mathrm{mTOR} / \mathrm{S} 6 \mathrm{~K}$ pathway. In adipocyte precursors lacking $\mathrm{p} 66 \mathrm{shcA}$, nutrient and insulin activation of the mTOR cascade was defective with respect to WT controls. P66 deficient cells also displayed reduced IRS-1 phosphorylation, while both basal and insulininduced phosphorylation of AKT/PKB was increased, expecially under resistance-inducing (TNF-alpha, GOX and excess free fatty acids) conditions. Conclusion: The above findings outline a model whereby oxidative stress attenuates early insulin signaling, at least in part, by activating a retro-inhibitory loop brought about by the mTOR/S6K cascade. Importantly, p66shc plays a major role, upstream of mTOR, in such inhibitory circuit. The p66-mTOR axis may therefore represent a novel target for the pharmacological control of Type II Diabetes.

Supported by: EFSD/GSK grant

\section{6}

Resveratrol ameliorates high insulin-induced insulin resistance in skeletal muscle cells by regulating mTOR and p70 S6K phosphorylation E. Tsiani, B. Faubert, T. Sanli

Community Health Sciences, Brock University, St Catharines, Canada

Background and aims: Insulin resistance, the hallmark of type 2 diabetes mellitus, is linked to hyperinsulinemia, which develops to counterbalance peripheral hormone resistance. In vitro and in vivo studies indicate that chronically elevated levels of insulin (HI) may lead to skeletal muscle insulin resistance by causing defects within the insulin-signalling cascade. Hyperinsulinemia is thus both a result and a cause of insulin resistance. Recently, the polyphenol resveratrol (RSV) has been shown to have anti-diabetic properties in vitro and in vivo. In the present study we examined the effect of RSV on $\mathrm{HI}$-induced insulin resistance in skeletal muscle cells in vitro and investigated the mechanisms involved. 
Materials and methods: L6 rat skeletal muscle cells were used. ${ }^{3} \mathrm{H}$-2-deoxyglucose (2DG) uptake was measured and total and phosphorylated levels of specific proteins were examined by western blotting.

Results: Chronic incubation (16-24 h) of L6 cells with $100 \mathrm{nM}$ insulin decreased acute insulin (INS)-stimulated 2DG uptake (INS: $179 \pm 9 \%$, HI+INS: $101 \pm 4 \%$ of control) indicating insulin resistance. However, in the presence of $25 \mathrm{uM} \mathrm{RSV} \mathrm{(16-24h)} \mathrm{insulin} \mathrm{resistance} \mathrm{(the} \mathrm{effect} \mathrm{of} \mathrm{HI}$ ) was prevented ((RHI+INS: $175 \pm 7 \%$ of control). The serine/threonine kinases mTOR and p70 S6K have been implicated to mediate insulin resistance by causing phosphorylation of serine ${ }^{307}$ of the insulin receptor substrate 1 (IRS-1) which leads to impaired downstream insulin signalling and inhibition of Akt phosphorylation /activation. HI significantly increased the phosphorylation levels of mTOR $(170 \pm 14.5 \%$ of control, $\mathrm{P}<0.01)$ and p70 S6K $(227 \pm 33.6 \%$ of control, $\mathrm{P}<0.05$ ) and RSV completely abolished these HI-induced responses (mTOR: $79 \pm 21 \%$ of control, $\mathrm{P}<0.001$, p 70 S6K: $117 \pm 19 \%$ of control, $\mathrm{P}<0.05$ ). Furthermore, HI increased ser ${ }^{307}$ phosphorylation of IRS-1 (270 $\pm 79 \%$ of control), and RSV was able to bring it back to normal ( $130 \pm 31 \%$ of control). In addition $\mathrm{HI}$ reduced insulin-stimulated Akt phosphorylation and RSV significantly blocked this inhibition.

Conclusion: Our results indicate that chronic HI induces skeletal muscle insulin resistance, and RSV can prevent it by regulating mTOR and p70 S6K phosphorylation. Resveratrol may be used towards preventing and/or treating insulin resistance and Type 2 diabetes.

Suported by: NSERC

\section{7}

Long-term mTOR inhibition exarcerbates insulin resistance and glucose intolerance induced by high fat diet

C. Veyrat-Durebex ${ }^{1}$, M. Vinciguerra ${ }^{2}$, X. Montet $^{3}$, N. Deblon ${ }^{1}$, M. Fournier ${ }^{2}$, G. Porcheron ${ }^{2}$, C. Maeder ${ }^{2}$, F. Rohner-Jeanrenaud ${ }^{1}$, M. Foti $^{2}$

${ }^{1}$ Internal Medicine, ${ }^{2}$ Cell physiology and Metabolism, ${ }^{3}$ Radiology, University of Geneva, Switzerland

Background and aims: The mammalian target of rapamycin (mTOR) has been recently shown to induce IRS1 degradation and thus to act as a negative regulator of insulin signalling. In addition, mTOR activation by fatty acids was shown to trigger hepatic steatosis by inducing PTEN downregulation in the liver. In this study we evaluate the hypothesis that blockade of mTOR activity could alleviate metabolic disorders, such as insulin resistance and hepatic steatosis.

Materials and methods: High fat diet-induced obese Wistar rats were treated with sirolimus ( $2 \mathrm{mg} / \mathrm{kg} /$ day ip) during 21 days. Body weight, food intake and the main metabolic parameters were measured. Glucose tolerance and insulin sensitivity were evaluated in vivo using glucose and insulin tolerance tests. In addition, the effects of mTOR inhibitors on insulin signalling, glucose and fatty acid metabolism were evaluated in explanted tissues from Wistar rats and in cultured cell lines.

Results: Sirolimus-treated rats showed lower body weight gain, food intake, plasma free fatty acid and triglyceride levels than control obese rats. PTEN downregulation and lipid accumulation in the liver induced by the high fat diet were decreased in sirolimus-treated rats. However, sirolimus treatment markedly worsened glucose intolerance and insulin resistance, despite a 4 fold increase in insulinaemia. Interestingly, IRS1 mRNA levels were strongly downregulated in explanted skeletal muscles and livers from sirolimus-treated rats, suggesting that long-term mTOR inhibition alters insulin signalling and glucose metabolism by reducing IRS1 expression. Consistent with these in vivo data, glucose uptake and glycogen synthesis were impaired in rat L6 myotubes exposed $24 \mathrm{~h}$ to the mTOR inhibitor, rapamycin.

Conclusion: Long-term inhibition of mTOR with rapamycin analogues prevents the development of hepatic steatosis in obese rats, but concomitantly impairs insulin sensitivity and glucose metabolism in skeletal muscles. These data call for cautiousness in the clinical use of rapamycin analogues as immune suppressors or anti-cancer therapy.

\section{PS 054 Insulin sensitivity and resistance: human subjects}

\section{8}

Prediction of the Minimal Model insulin sensitivity index through one hour intravenous glucose tolerance test data

A. Tura, S. Sbrignadello, G. Pacini

ISIB, CNR, Padova, Italy

Background and aims: Minimal Model (MM) analysis of intravenous glucose tolerance test (IVGTT) data is widely used to estimate insulin sensitivity (IS). However, this requires a computer program and relevant expertise to run the mathematical model properly. Furthermore, reliable results based on MM require many plasma insulin and glucose samples for a time interval of at least three hours after glucose injection. The aim of this study was: i) developing a predictor of MM IS index from IVGTT data limited to one hour or less, calculated with empirical parameters not requiring complex mathematical models; ii) assessing its performance in different groups of subjects with specific clinical characteristics.

Materials and methods: We studied 114 control subjects with normal glucose tolerance, CNT (age $=33.8 \pm 1.4 \mathrm{yrs}, \mathrm{BMI}=23.4 \pm 0.4 \mathrm{~kg} / \mathrm{m}^{2}$, basal glucose, $\mathrm{G}_{\mathrm{b}}=4.6 \pm 0.05 \mathrm{mmol} / \mathrm{l}($ mean $\pm \mathrm{SE})$ ), 127 subjects with impaired fasting glucose or impaired glucose tolerance, IFG/IGT $(42.8 \pm 1.4 ; 28.0 \pm 0.6), 55$ subjects with chronic renal disease, REN (age $=44.3 \pm 2.9$; $\mathrm{BMI}=25.7 \pm 1.0$ ), and 10 subjects with hyperparathyroidism, PAR, before and after surgery (age $=64.3 \pm 2.3$, $\mathrm{BMI}=25.8 \pm 1.6$ before and $25.4 \pm 1.5 \mathrm{after}$ ). All the subjects underwent a $3-\mathrm{h}$ frequently sampled IVGTT. IS index was estimated by MM analysis $\left(\mathrm{S}_{\mathrm{T}}\right)$. A predictor of $\mathrm{S}_{\mathrm{I}}$ was calculated as $\mathrm{CS}_{\mathrm{I}}=\mathrm{T} \times \mathrm{K}_{\mathrm{G}} / \Delta \mathrm{AUC}_{\mathrm{INS}}$, where $\mathrm{K}_{\mathrm{G}}$ is the rate of glucose disappearance and $\triangle \mathrm{AUC}_{\mathrm{INS}}$ is the area under the insulin curve above basal. $T$ is the time interval between 10 and 50 min when both $\mathrm{K}_{\mathrm{G}}$ and $\triangle \mathrm{AUC}_{\mathrm{INS}}$ are computed: ie, $\mathrm{T}=40 \mathrm{~min}$.

Results: In CNT $S_{I}$ and CS showed strong correlation $\left(\mathrm{R}^{2}=0.87, \mathrm{P}<0.0001\right)$. The slope of the regression line was 0.285 , and that value was used to correct the CS value in each subject of the other studied groups. Strong correlation was then found in IFG/IGT (slope $=0.97, \mathrm{R}^{2}=0.82, \mathrm{P}<0.0001$; figure) and in REN (slope $=0.92, \mathrm{R}^{2}=0.80, \mathrm{P}<0.0001$ ). Both slopes were not significantly different from unit. In PAR, $\mathrm{CS}_{\mathrm{I}}$ was again strongly correlated with $\mathrm{S}_{\mathrm{I}}$ and able to detect the increase in IS after surgery, similarly to $\mathrm{S}_{\mathrm{I}}\left(\mathrm{CS}_{\mathrm{I}}=3.84 \pm 0.5510^{-4} \mathrm{~min}\right.$ ${ }^{1}(\mu \mathrm{U} / \mathrm{ml})^{-1}$ before, $5.91 \pm 0.87$ after, $\mathrm{P}=0.006 ; \mathrm{S}_{\mathrm{l}}=3.23 \pm 0.54$ before, $4.97 \pm 0.70$ after, $\mathrm{P}=0.018$ ).

Conclusion: A few studies have developed simplified methods for IS from IVGTT data and they were based on small datasets, while the simplified approach proposed in this study has been tested in large and heterogeneous groups of patients. $\mathrm{CS}_{\mathrm{I}}$ revealed to be an excellent predictor of IS. Since the evaluation of the early part of IVGTT is a reliable method for quantifying insulin secretion, our method will allow a combined estimation of IS and beta cell function in a single individual with an easy procedure.

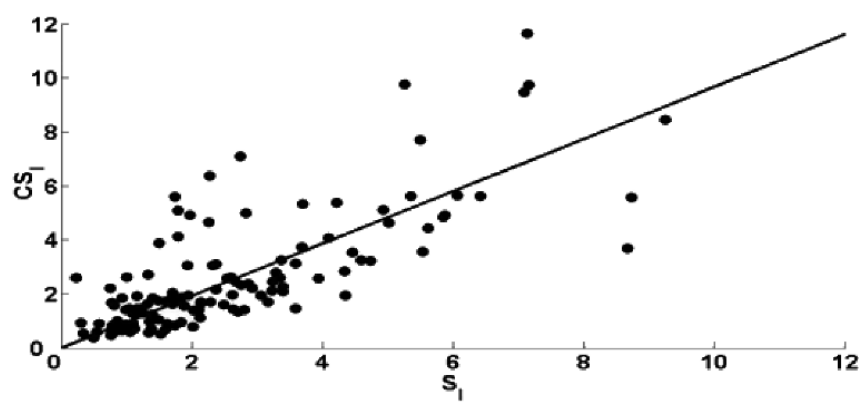

699

Application of mathematical model to evaluate insulin sensitivity and secretion function from the 3-hour OGTT

Y. Xie

Metabolic Disease Hospital, Tianjin Medical University, China

Background and aims: To establish a mathematical model to assess insulin sensitivity and secretion function from the 3-hour OGTT.

Materials and methods: The mathematical model LMM (linear minimal model) has been developed from five physiological assumptions to evalu- 
ate insulin sensitivity and secretion function from the 3-hour OGTT (oral glucose tolerance test). The equations are $d G / d t=P-a G-b I-v(G)+f(t)$ and $\mathrm{dI} / \mathrm{dt}=\mathrm{c}\left(\mathrm{G}-\mathrm{G}^{*}\right)-\mathrm{dI}$, where $\mathrm{a}$ is the coefficient of plasma glucose autogenous regulation, $b$ is the coefficient of plasma glucose reduction due to the effect of insulin, $\mathrm{c}$ is the coefficient of insulin secretion stimulated by plasma glucose, $\mathrm{d}$ is the coefficient of insulin degradation, $\mathrm{P}$ is the critical rate of plasma glucose increase caused by hepatic glucose output, $v(G)$ is the rate of plasma glucose decrease caused by urine glucose output and $f(t)$ is the rate of plasma glucose increase caused by glucose ingested from intestines and stomach, The coefficients $b$ and $c$ were defined as the insulin sensitivity index LMM-ISI and secretion function index LMM-BCI respectively after being processed by fuzzy mathematics. The insulin sensitivity index LMM-ISI and secretion function index LMM-BCI can be calculated from the plasma glucose values and insulin values at five time points $(0 \mathrm{~min}, 30 \mathrm{~min}, 60 \mathrm{~min}, 120 \mathrm{~min}$ and $180 \mathrm{~min}$ ) in the 3 -hour OGTT by a computer program developed from the model LMM. Using the data from the euglycemic clamp test, IVGTT and the 3-hour OGTT on 99 non-diabetic American Pima Indians (NGT 47 subjects and IGR 52 subjects, BMI was $35.53 \pm 8.13$ ), the feasibility of LMM-ISI and LMM-BCI was studied in this paper.

Results: The Pearson's correlation coefficient between LMM-ISI and GIR ((glucose infusion rate at steady state of the euglycemic clamp test) was 0.82 $(\mathrm{P}<0.001)$, which was larger than those corresponding values calculated from QUICKI, HOMA-ISI, IAI (insulin action index, $I A I=1 /\left(\mathrm{G}_{0} \mathrm{I}_{0}\right)$, Supamai-ISI and Composite-ISI $\left.\left(=10^{4} /\left(\mathrm{G}_{0} \times \mathrm{I}_{0} \times \mathrm{G}_{\text {mean }} \times \mathrm{I}_{\text {mean }}\right)^{0.5}\right)\right)$, the respective values of which were $0.54,0.58,0.58,0.67$ and 0.66 . After correcting the effect of GIR, the partial correlation between LMM-BCI and plasma glucose level $\left(\mathrm{G}_{120}\right.$ or $\mathrm{G}_{\text {Trapezoid }}$ the mean value of plasma glucose concentrations within 180 minutes during the OGTT calculated by the trapezoid method) was most significant $(\mathrm{r}=-0.52,-0.56$ respectively, $\mathrm{P}<0.001)$ among the six insulin secretion function indices (AIR (acute insulin response), HOMA- $\beta, \Delta \mathrm{I}_{30} / \Delta \mathrm{G}_{30}, \Delta \mathrm{I}_{60} / \Delta \mathrm{G}_{60}$, MBCI (modified beta cell index, $\mathrm{MBCI}=\mathrm{G}_{0} \times \mathrm{I}_{0} /\left(\mathrm{G}_{60}+\mathrm{G}_{120}-7\right)$ )and $\left.\mathrm{LMM}-\mathrm{BCI}\right)$. Combination of LMM-ISI and LMM-BCI could explain the change of $\mathrm{G}_{\text {Trap- }}$. better (the coefficient of determination $\mathrm{R}^{2}$ was $0.73, \mathrm{P}<0.001$ ).

Conclusion: Thus LMM-ISI is a relatively good insulin sensitivity index, while LMM-BCI is quite a reliable insulin secretion function index derived from the oral glucose tolerance test.

Key words: Mathematical model, Oral Glucose Tolerance Test, Insulin sensitivity index, Insulin secretion function index

\section{0}

Impact of postprandial or postabsorptive administration of ${ }^{2} \mathrm{H}_{2} \mathrm{O}$ on estimates of gluconeogenesis in diabetic and non-diabetic humans

R. Basu, A. Basu, R. Rizza

Endocrinology,Metabolism \& Nutrition, Mayo Clinic College Of Medicine, Rochester, United States

Background and aims: The deuterated $\left({ }^{2} \mathrm{H}_{2} \mathrm{O}\right)$ water method commonly is used to measure gluconeogenesis. The fifth carbon (C5) is labeled with deuterium during gluconeogenesis whereas the second carbon of glucose (C2) is labeled during both gluconeogenesis and glycogenolysis. The $\mathrm{C} 5 / \mathrm{C} 2$ glucose ratio is used to estimate the percent of plasma glucose derived from gluconeogenesis. However, glycogen cycling (plasma glucose to glucose-6 phosphate to glycogen to glucose- 6 phosphate to plasma glucose) is believed to occur in both the fed and fasted state. Since gluconeogenically derived C5 glucose can pass through glycogen before entering plasma, the $\mathrm{C} 5 / \mathrm{C} 2$ glucose ratio and therefore estimates of gluconeogenesis may be influenced by the time between eating and ${ }^{2} \mathrm{H}_{2} \mathrm{O}$ administration.

Materials and methods: 10 non-diabetic and 9 with type 2 diabetes ate a meal at 1800 and were randomized to receive $1.67 \mathrm{gms} / \mathrm{kgFFM}$ of ${ }^{2} \mathrm{H}_{2} \mathrm{O}$ in divided doses at either 1800,2000 and 2200 (postprandial) or 2200,2400 and 0200 (postabsorptive); plasma samples for C5 glucose, C2 glucose and plasma ${ }^{2} \mathrm{H}_{2} \mathrm{O}$ enrichment were collected at 0800 the following morning.

Results: Postprandial and postabsorptive plasma C5 glucose, C2 glucose, and ${ }^{2} \mathrm{H}_{2} \mathrm{O}$ enrichments did not differ in either the non-diabetic $(0.26 \pm 0.02$ vs. $0.24 \pm 0.02 ; 0.48 \pm 0.01$ vs. $0.40 \pm 0.03 ; 0.52 \pm 0.03$ vs. $0.44 \pm 0.03$ ) or diabetic $(0.29 \pm 0.03$ vs. $0.23 \pm 0.02 ; 0.46 \pm 0.05$ vs. $0.42 \pm 0.03 ; 0.53 \pm 0.04$ vs. 0.48 \pm 0.03 ) subjects. This resulted in a comparable estimate of the percent of plasma glucose derived from gluconeogenesis (i.e. the plasma C5/C2 glucose ratio) following postprandial and postabsorptive ${ }^{2} \mathrm{H}_{2} \mathrm{O}$ administration in the non-diabetic $(0.54 \pm 0.04$ vs. $0.59 \pm 0.03)$ but lower $(\mathrm{p}<0.02)$ estimates with postabsorptive administration in the diabetic subjects at $0800(0.64 \pm 0.01$ vs. $0.54 \pm 0.02$ ) but not when glycogen is further depleted with 4 additional hours of fasting $(0.72 \pm 0.04$ vs. $0.64 \pm 0.06)$. Plasma $\mathrm{C} 2$ glucose and ${ }^{2} \mathrm{H}_{2} \mathrm{O}$ enrichments also did not differ following postprandial and postabsorptive ${ }^{2} \mathrm{H}_{2} \mathrm{O}$ administration indicating adequate time for ${ }^{2} \mathrm{H}_{2} \mathrm{O}$ to equilibrate with the hepatic $\mathrm{C} 2$ glucose pool .

Conclusion: Administration of ${ }^{2} \mathrm{H}_{2} \mathrm{O}$ immediately following or 4 hours after the evening meal results in an equivalent estimate of gluconeogenesis by 0800 the following morning in non-diabetic subjects consistent with extensive nocturnal hepatic glycogen cycling. In contrast, the higher $\mathrm{C} 5 / \mathrm{C} 2$ glucose at 0800 but not at 1200 when ${ }^{2} \mathrm{H}_{2} \mathrm{O}$ is given immediately after meal ingestion in diabetic subjects implies increased postprandial labeling of glycogen with $\mathrm{C5}$ glucose via the indirect pathway. Therefore,the timing of ${ }^{2} \mathrm{H}_{2} \mathrm{O}$ administration relative to ingestion of the evening meal influences estimates of gluconeogenesis measured the following morning in diabetic but not non-diabetic subjects.

Supported by: NIH grant

\section{1}

Prediction of postprandial blood glucose level according to the amount of carbohydrates consumed during a meal in type 2 diabetes patients S. Franc, D. Dardari, J.-P. Riveline, G. Charpentier

Department of Diabetes, Centre Hospitalier Sud Francilien, Corbeil, France

Background and aims: Identification of the 'main meal' (i.e. the meal with the highest postprandial blood glucose [PPBG]) in patients with Type 2 diabetes mellitus (T2DM) can help optimise glycaemic control in basal-bolus insulin regimens, which may initially use a single prandial insulin injection to target the main meal as a stepwise approach towards intensifying insulin therapy. However, the main meal can vary with the dietary habits of patients and remains an unpredictable factor in the treatment of T2DM. The aim of our study was to determine the relationship between the amount of carbohydrate consumed and PPBG in T2DM patients in order to predict the main meal.

Materials and methods: Three-day blood glucose (BG) profiles were measured in $37 \mathrm{~T} 2 \mathrm{DM}$ patients treated with sulfonylurea, metformin and well titrated on insulin glargine (mean \pm SD $\mathrm{HbA}_{1}: 7.7 \pm 0.8 \%$ ), using a continuous glucose-monitoring system (CGMS) either once $(n=5)$ or twice (1 month apart; $n=32$ ). During the three CGMS days, food intake from 680 meals was accurately recorded by patients and quantified by a dietitian.

Results: Overall analysis of the 680 meals failed to show a correlation between $\mathrm{BG}$ excursion $\left(\triangle \mathrm{BG}=\mathrm{PPBG}_{\max }\right.$-[fasting blood glucose]) and carbohydrate amount, even after standardisation with body weight. In contrast, analysis of the data according to the meal showed a correlation and a simple global relationship could be established; breakfast: $\Delta \mathrm{BG}(\mathrm{mg} / \mathrm{dL})=65 \mathrm{x}$ carbohydrate/body weight +73 ; the slope was reduced by half at lunch and by a third at dinner. A quadratic polynomial equation including several parameters (body weight; C-peptide; Howorka-defined $\mathrm{K}$ insulin sensitivity index; $\mathrm{HbA}_{1}$; mean carbohydrate; and lipid and protein intake) was used to improve the $\triangle \mathrm{BG}$ model. This model was more reliable at predicting $\triangle \mathrm{BG}$ in the whole population $\left(\mathrm{R}^{2}=0.26\right)$ but had high inter-individual variability. Two groups of patients could be identified: 'responder' patients, whose $\triangle \mathrm{BG}$ was well correlated with carbohydrate amount $\left(\mathrm{R}^{2} \geq 0.30, \mathrm{n}=8\right)$, and 'non-responder' patients, who showed less correlation $\left(\mathrm{R}^{2}<0.30, \mathrm{n}=29\right)$. 'Responder' versus 'non-responder' patients were leaner (BMI, $27 \pm 3$ vs $30 \pm 4 \mathrm{~kg} / \mathrm{m}^{2} ; \mathrm{p}=0.019$ ), had higher mean PPBG excursions (mean $\triangle \mathrm{BG}, 113 \pm 23$ vs $92 \pm 21 \mathrm{mg} / \mathrm{dL}$; $\mathrm{p}=0.022)$ and consumed on average more lipids $(28 \pm 7$ vs $23 \pm 6 \mathrm{~g} ; \mathrm{p}=0.045)$. Three variables appeared to be independent and predictive factors for a 'responder' (contribution in descending order): 1) extent of the mean PPBG excursion (relative risk $[R R] 1.2 ; \mathrm{p}=0.04) ; 2$ ) mean carbohydrate load (RR $0.89 ; \mathrm{p}=0.03$ ); and 3 ) mean lipid load (RR 1.3; $\mathrm{p}=0.05$ ). For these responder patients, the quadratic polynomial equation was much more reliable at predicting $\triangle B G\left(R^{2}=0.51\right)$.

Conclusion: In conclusion, besides carbohydrate counting, other factors play a role in the extent of the PPBG excursion in T2DM patients. Characterisation of these factors and of their respective roles may improve T2DM management by providing new therapeutic targets.

Supported by: sanofi-aventis 


\section{2}

Influence of hepatic and peripheral insulin resistance on glucose tolerance in non-diabetic subjects: the RISC study

A. Gastaldelli ${ }^{1}$, G. Pacini ${ }^{2}$, A. Mari ${ }^{2}$, the RISC investigators

${ }^{1}$ Institute of Clinical Physiology, National Research Council, Pisa, ${ }^{2}$ Institute of Biomedical Engineering, National Research Council, Padova, Italy

Background and aims: Fasting hyperglycemia is prevented as long as hepatic autoregulation maintains endogenous glucose production (EGP) within the normal range. On the other hand, postprandial glycemia starts to increase long before hepatic autoregulation is lost, mainly because of insulin resistance both at the level of the muscle and of the liver (through impaired suppression of EGP). The contribution of hepatic insulin resistance (HIR) to fasting and/ or postprandial glucose concentration is still unknown. Aim of this study was to evaluate EGP and HIR in a large group of non-diabetic subjects and the relationships with peripheral insulin resistance and $B$-cell function.

Methods: The RISC study recruited 1,290 non-diabetic subjects (age 30-60 years) without hypertension or dyslipidaemia in 19 centers in Europe. Body composition, liver enzymes, lipid profile, peripheral insulin sensitivity (by hyperinsulinaemic clamp), $B$-cell function and glucose tolerance (from the OGTT) were measured. In a subgroup of subjects $(n=398), 6,6-{ }^{2} \mathrm{H}$-glucose was used to determine EGP and glucose disposal (Rd) during fasting and clamp. HIR was calculated as the product of fasting EGP and fasting plasma insulin concentration.

Results: Both fasting EGP $\left(6-43 \mu \mathrm{mol} \cdot \mathrm{min}^{-1} \mathrm{~kg}_{\text {ffim }}{ }^{-1}\right)$ and HIR $(87-3045 \mu \mathrm{mol}$ $\mathrm{min}^{-1} \mathrm{~kg}_{\text {fim }}{ }^{-1} \mathrm{pM}$ ) ranged widely. When subjects were divided into HIR quartiles (Q1 to Q4: $197 \pm 5,329 \pm 4,480 \pm 5,905 \pm 37 \mu \mathrm{molmin}^{-1} \mathrm{~kg}_{\text {ffm }}{ }^{-1} \mathrm{pM}$, respectively), graded increases in both fasting plasma glucose (from $4.8 \pm 0.1 \mathrm{mmol} / \mathrm{l}$ in $\mathrm{Q} 1$ to $5.2 \pm 0.1 \mathrm{mmol} / \mathrm{l}$ in $\mathrm{Q} 4, \mathrm{p}=0.0002)$ and 2-hour plasma glucose concentrations (from $5.3 \pm 0.1 \mathrm{mmol} / \mathrm{l}$ in Q1 to $6.1 \pm 0.2 \mathrm{mmol} / \mathrm{l}$ in $\mathrm{Q} 4, \mathrm{p}=0.001$ ) was observed. On the clamp, Rd ranged $15-151 \mu \mathrm{mol} \mathrm{min}^{-1} \mathrm{~kg}_{\mathrm{ffm}}{ }^{-1}$. Across HIR quartiles, fasting Rd increased from $13.9 \pm 0.3$ in Q1 to18.8 $\pm 0.6 \mu \mathrm{mol}$ $\mathrm{min}^{-1} \mathrm{~kg}_{\text {ffm }}{ }^{-1}$ in $\mathrm{Q} 4(\mathrm{p}=0.0001)$, while clamp Rd decreased from $64 \pm 2$ in Q1 to $46 \pm 2 \mu \mathrm{molmin}{ }^{-1} \mathrm{~kg}_{\text {ffim }}{ }^{-1}$ in $\mathrm{Q} 4$ ( $\left.\mathrm{p}=0.0001\right)$. Both fasting and clamp Rd correlated positively with adiponectin $(r=0.20$ and 0.32 respectively, $\mathrm{p}<0.0001)$. No correlation was found between fasting and clamp Rd. Residual EGP measured during clamp varied from -27 to $26 \mu \mathrm{mol} \mathrm{min}^{-1} \mathrm{~kg}_{\text {ffm }}{ }^{-1}$ (15\% of measurements with negative values). A weak, if significant, positive correlation $(r$ $=0.18, p=0.0004)$ was found between fasting and clamp EGP. HIR was associated with decreased adiponectin (partial $r=-0.21, \mathrm{p}<0.0001$, after adjusting for sex, age, BMI and centre), decreased hepatic insulin clearance (partial $r$ $=-0.24, \mathrm{p}<0.0001$ ), increased total insulin response during the OGTT (partial $r=0.31, \mathrm{p}<0.0001$ ) and decreased potentiation of insulin release (partial $r=-$ $0.15, \mathrm{p}<0.005$ ). In multivariate models adjusting for sex, age, BMI and centre, fasting glucose was predicted by HIR (partial $r=0.17, \mathrm{p}=0.0009$ ) while 2-hour glucose was predicted by clamp Rd (partial $r=-0.23 \mathrm{p}<0.0001$ ).

Conclusion: In non-diabetic subjects, hepatic insulin resistance is the main factor associated with an increase in fasting glucose concentration while peripheral insulin-mediated glucose uptake is the main factor associated with postprandial glucose concentration.

Supported by: EU, Astra Zeneca Sweden

\section{3}

Insulin-mediated ablation of nocturnal glycaemic excursion in type 2 diabetes improves daytime glycaemia and insulin sensitivity by supressing nighttime glucose production

J. Radziuk, S. Pye

Medicine, Ottawa Hospital (Civic Campus), Ottawa, Canada

Background and aims: Fasting hyperglycaemia in type 2 diabetes (T2DM) represents peak glucose levels in a circadian cycle of glucose levels and endogenous glucose production (EGP) which rise during the night and decrease in daytime. It has been suggested that the transition from a steady state glucose to such a cyclic EGP and glucose levels, is characteristic of the nonlinear nature of the glucose system. This study was designed to examine the daytime metabolic effects of insulin-mediated suppression of the nocturnal increase in EGP, and thus an abbrogation of the cyclic changes in EGP.

Materials and methods: 10 subjects with T2DM underwent 2 studies. In both they fasted from 10:00h on day 1 of the study. An infusion of [U-13C]glucose and [3-14C]lactate was initiated at 17:00h and continued until 18:00h on day 2 . During the second study (I) a variable insulin infusion was used to normalize glycemia to the extrapolated baseline (nadir) levels determined in the first study (B). Glucose fluxes (EGP), glucose removal, metabolic clearance rate
(MCR) and an index of gluconeogenesis) were determined using compartmental modelling of glucose kinetics.

Results: Glucose concentrations initially were $7.7 \pm 0.5$ and $7.8 \pm 0.5 \mathrm{mmol} / \mathrm{L}$ in $\mathrm{B}$ and I respectively, peaking at $9.1 \pm 0.6$ and $7.2 \pm 0.4 \mathrm{mmol} / \mathrm{L}(\mathrm{p}<0.05)$ at $8: 00 \mathrm{~h}$ on Day 2 and falling to $6.6 \pm 0.4$ and $5.7 \pm 0.3(\mathrm{p}<0.05) \mathrm{mmol} / \mathrm{L}$ by $18 \mathrm{~h}$. Insulin concentrations were initially $120 \pm 12$ and $114 \pm 12 \mathrm{pmol} / \mathrm{L}$ in B and I, rising to $162 \pm 18$ at $4: 30 \mathrm{~h}$ in I during its infusion (vs $102 \pm 12 \mathrm{pmol} / \mathrm{L}$ for $\mathrm{B}, \mathrm{p}<0.05$ ). Initial EGP was $8.4 \pm 0.5$ and $8.4 \pm 0.25 \mu \mathrm{mol} / \mathrm{kg}^{-1} \mathrm{~min}^{-1}$ in $\mathrm{B}$ and $\mathrm{I}$, rising by $1.71 \pm 0.30$ and $0.55 \pm 0.21 \mu \mathrm{mol} / \mathrm{kg}^{-1} \mathrm{~min}^{-1}(\mathrm{p}<0.05)$ at $7: 00 \mathrm{~h}$ on day 2 and decreasing to $6.7 \pm 0.3$ and $6.8 \pm 0.2 \mu \mathrm{mol} / \mathrm{kg}^{-1} \mathrm{~min}^{-1}$ by $18: 00 \mathrm{~h}$. The increase in EGP was explained by a peak gluconeogenic index of $3.8 \pm 0.3$ and $2.4 \pm 0.2 \mu \mathrm{mol} / \mathrm{kg}$ ${ }^{1} \mathrm{~min}^{-1}$ in B and I $(\mathrm{p}<0.05)$. Glucose removal decreased uniformly in both studies from $8.8 \pm 0.4$ to $7.2 \pm 0.3 \mu \mathrm{mol} / \mathrm{kg}^{-1} \mathrm{~min}^{-1}$. The decrease in glycemia at $18: 00 \mathrm{~h}$ (Day 2) was therefore explained by an increase in MCR from 1.11 \pm 0.07 (B) to $1.25 \pm 0.05 \mathrm{ml} / \mathrm{kg}^{-1} \mathrm{~min}^{-1}(\mathrm{I}),(\mathrm{p}<0.05)$ at this time. The increase in MCR in (I) occurs with lower insulin levels $78 \pm 6$ (I) vs $96 \pm 6 \mathrm{pmol} / \mathrm{L}$ (B).

Conclusion: The suppression of nocturnal gluconeogenesis in type 2 diabetic subjects, not only improves morning EGP and glycemia, but exerts more remote effects by improving glycaemia and increasing insulin sensitivity the following afternoon. This may be another expression of the nonlinear behaviour of the glucose system.

Supported by: Canadian Diabetes Association and Canadian Instiutes of Health Research

\section{4}

Physically active but insulin resistant subjects have features of lipotoxicity

J. Nolan, H. Thabit, N. Burns, S. Shah, the RISC investigators

Metabolic Research Unit, St James Hospital, Dublin, Ireland

Background and aims: We reported that patients with early onset type 2 diabetes mellitus had no improvement in insulin sensitivity despite aerobic exercise training for 3 months. We hypothesised that this non-response is mainly due to lipotoxicity.

Methods: In the RISC study, 807 healthy non-diabetic subjects underwent a 2-hour hyperinsulinaemic euglycemic clamp for measurement of glucose disposal rate (M/I) as well as measurement of physical activity by Actigraph accelerometers. We compared two sub-groups from this population, the active (top tertile for physical activity) but insulin resistant (bottom tertile for $\mathrm{M} / \mathrm{I}$ ) (AIR) and the inactive (bottom tertile for physical activity) but insulin sensitive (top tertile for M/I) (IIS) groups.

Results: The AIR subjects had higher Actigraph counts (561533 vs 197977 counts, $\mathrm{p}<0.005)$ and lower $\mathrm{M} / \mathrm{I}$ values $(77.1 \pm 21.2$ vs $217.5 \pm 93.5 \mu \mathrm{mol}$. min- $1 \mathrm{kgffm}-1 \mathrm{mM}-1, \mathrm{p}<0.005)$. Both groups were of similar age $(43.2 \pm 8.2 \mathrm{vs}$ $44.5 \pm 8.5$ years, $\mathrm{p}=0.36$ ). There were more males in the AIR group compared to the IIS group ( $63 \%$ vs $24 \%$ ). In comparison with the IIS group, the AIR subjects had elevated waist: hip ratio, 2-hour glucose post OGTT, fasting insulin, NEFA and reduced adiponectin and HDL-C $(1.3 \pm 0.3$ vs $1.6 \pm 0.4, \mathrm{p}<0.005)$. However, when males and females were looked at separately there were no differences in age, weight or fat mass between AIR and IIS. There were no differences in waist: hip ratio between AIR males and IIS males. However, AIR females had higher waist: hip ratio $(0.85 \pm 0.1$ vs $0.79 \pm 0.07, \mathrm{p}=0.01)$ and waist circumference (83.6 \pm 11.4 vs $77.6 \pm 8.7 \mathrm{~cm}, \mathrm{p}=0.012)$ than IIS females. NEFA was higher in AIR compared to IIS in either males or females $(0.53 \pm 0.2$ vs $0.35 \pm 0.2 \mathrm{mmol} / \mathrm{l}, \mathrm{p}=$ 0.008 in males, and $0.64 \pm 0.2$ vs $0.53 \pm 0.2, \mathrm{p}=0.03$ in females). Adiponectin and HDL-C were lower in AIR compared to IIS only in females (8.2 \pm 3.2 vs $10.8 \pm 3.8$ $\mathrm{mg} / \mathrm{l}, \mathrm{p}=0.007$, and $1.4 \pm 0.3$ vs $1.7 \pm 0.3 \mathrm{mmol} / \mathrm{l}, \mathrm{p}=0.003$ respectively.

Conclusion: Thus the key distinguishing features of physically active, but insulin resistant, subjects are consistent with abnormal lipid metabolism, which may impair skeletal muscle response to exercise.

Table1. Data are expressed as Mean (SD). P value $<0.05$ are significant

\begin{tabular}{llll}
\hline & $\begin{array}{l}\text { AIR subjects } \\
(\mathbf{N}=\mathbf{5 4})\end{array}$ & $\begin{array}{l}\text { IIS Subjects } \\
(\mathbf{N}=\mathbf{8 5})\end{array}$ & p value \\
\hline BMI $\left(\mathrm{kg} / \mathrm{m}^{2}\right)$ & $25.8(3.8)$ & $23.6(3.2)$ & $<0.005$ \\
\hline Waist:hip ratio & $0.9(0.09)$ & $0.82(0.08)$ & $<0.005$ \\
\hline Fasting Glucose $(\mathrm{mmol} / \mathrm{l})$ & $5.2(0.5)$ & $5.1(0.4)$ & 0.08 \\
\hline 2-Hour Glucose post OGTT $(\mathrm{mmol} / \mathrm{l})$ & $6.2(1.4)$ & $5.2(1.2)$ & $<0.005$ \\
\hline Fasting Insulin $(\mathrm{pmol} / \mathrm{l})$ & $47.1(22.6)$ & $28.0(12.2)$ & $<0.005$ \\
\hline Non-esterified fatty acid(mmol/l) & $0.57(0.2)$ & $0.49(0.2)$ & 0.031 \\
\hline Adiponectin $(\mathrm{mg} / \mathrm{l})$ & $6.7(3.1)$ & $9.9(3.9)$ & $<0.005$
\end{tabular}


Supported by: EU, AstraZeneca (Sweden). The EGIR group activities are supported by an unrestricted research grant from Merck Serono, France

\section{5}

Mechanism of hyperglycaemia after oral glucose ingestion in men with type 2 diabetes

V. Rigalleau ${ }^{1}$, M.-C. Beauvieux ${ }^{1}$, J.-L. Gallis ${ }^{1}$, H. Gin ${ }^{1}$, P. Schneiter ${ }^{2}$,

L. Tappy ${ }^{2}$

${ }^{1}$ Nutrition Diabetologie, Hopital Haut Leveque USN, Pessac, France, ${ }^{2}$ Institut de Physiologie, Université de Lausanne, Switzerland

Background and aims: Postprandial hyperglycaemia may be due to insufficiently suppressed Endogenous Glucose Production in Type 2 Diabetes, but this has not been demonstrated in conditions of controlled hyperglycaemia. Materials and methods: Eight men with T2D (age: $52 \pm 7$ yrs; BMI:26.6 \pm 2.3 $\mathrm{kg} / \mathrm{m} 2$; fasting glycaemia: $7.1 \pm 1.5 \mathrm{mmol} / \mathrm{L}$, without any oral antidiabetic agent) were compared to eight healthy controls (age: $51 \pm 5$; BMI: $24.6 \pm 2.9$; glycaemia: $4.9 \pm 0.4)$. Their glucose turnover rate and hepatic cycle were measured by a [2H7] glucose infusion with $\mathrm{m}+7$ and $\mathrm{m}+6$ enrichments monitoring, 3 hours before and four hours after the ingestion of [6,6-2H2] labelled glucose, while their glycaemia was maintained at $10 \mathrm{mmol} / \mathrm{L}$ by the pancreatic clamp technique.

Results: Insulin levels were similarly increased for T2D (from $84 \pm 5$ to $201 \pm 16 \mathrm{pmol} / \mathrm{L}$ ) and control subjects (from $41 \pm 9$ to $160 \pm 10 \mathrm{pmol} / \mathrm{L}$ ), with prompt C-peptide suppression after the onset of the somatostatin-insulin infusions. Glucagon levels remained slightly, but non significantly, higher in T2D $(96 \pm 25 \mathrm{ng} / \mathrm{L})$ than controls $(61 \pm 7 \mathrm{ng} / \mathrm{L})$. Seventy-one percent of the 700 $\mathrm{mg} / \mathrm{kg}$ oral glucose load appeared in the systemic circulation of the T2D subjects (63\% for controls, NS). The results are summarized in the table.

Conclusion: When T2D subjects are compared to normal subjects with similar controlled hyperglycaemia after oral glucose ingestion, they mainly differ on peripheral glucose uptake, not Endogenous Glucose Production. By contrast with the fasting situation, the main mechanism of postprandial hyperglycaemia in T2D is a reduction of glucose utilization. The low hepatic glucose cycle rate argues against any role for a putative Glucose-6-Phosphatase overactivity in the postprandial hyperglycaemia of T2D.

Rates as $\mathrm{mg} / \mathrm{kg} / \mathrm{min}$, before and after oral glucose ingestion

\begin{tabular}{lllll}
\hline & & T2D & Controls & $\mathrm{p}$ \\
\hline Peripheral Uptake & Before & $4.5 \pm 1.3$ & $7.8 \pm 2.5$ & $<0.01$ \\
& After & $5.7 \pm 2.4$ & $9.3 \pm 2.9$ & $<0.05$ \\
\hline Endogenous Production & Before & $0.48 \pm 0.87$ & $0.14 \pm 0.56$ & NS \\
& After & $0.14 \pm 0.39$ & $0.37 \pm 0.59$ & NS \\
\hline Hepatic Cycle & Before & $0.29 \pm 0.22$ & $0.51 \pm 0.21$ & NS (p=0.06) \\
& After & $0.44 \pm 0.56$ & $0.62 \pm 0.26$ & NS \\
\hline
\end{tabular}

Supported by: Alfediam-Roche and Merck

\section{PS 055 Insulin sensitivity and glucose metabolism in humans: various mechanisms}

\section{6}

Decreased glucose transport into the thalamus in type 1 diabetes O. Heikkilä ${ }^{1,2}$, N. Lundbom ${ }^{3}$, M. Timonen ${ }^{3}$, P.-H. Groop ${ }^{1,2}$, S. Heikkinen ${ }^{4}$, S. Mäkimattila ${ }^{1}$

${ }^{1}$ Institute of Genetics, Folkhälsan Research Center, ${ }^{2}$ Division of Nephrology, Department of Medicine, Helsinki University Hospital, ${ }^{3} \mathrm{Helsinki}$ Medical Imaging Center, University of Finland, ${ }^{4}$ Laboratory of Organic Chemistry, University of Helsinki, Finland

Background and aims: In type 1 diabetes (T1D), both severe hypoglycemia and recurrent hyperglycemia have been associated with cognitive impairment and regional brain volume differences. Hyperglycemia disrupts cellular homeostasis resulting in end-organ damage that may pertain also the brain. In rats, chronic hyperglycemia has been shown to down-regulate glucose transport into the brain. In humans, the effect of acute hyperglycemia has been studied in T1D patients with recurrent hypoglycemias and in occipital cortex only; no down-regulation was observed. We studied if the brain of T1D patients who are used to high plasma glucose excursions is more resistant to acute hyperglycemia compared to non-diabetic subjects. We also searched for possible regional variation in glucose transport into the brain. Materials and methods: We studied seven 22-43-year old men with T1D (duration $12 \pm 3$ yrs), and eleven age- and weight-matched men without T1D. The men with T1D had high mean amplitude of glucose excursions (11.3 \pm $2.9 \mathrm{mmol} / \mathrm{l}$ ) but no history of severe hypoglycemias. Glucose, $\mathrm{N}$-acetyl aspartate, creatine, choline, and myo-inositol (mI) in frontal cortex (FC), frontal white matter (FWM) and in thalamus (TH) were quantified with proton magnetic resonance spectroscopy during basal fasting glycemia and twice during $2 \mathrm{~h}$ hyperglycemic clamp that aimed to increase plasma glucose by 10 $-15 \mathrm{mmol} / \mathrm{l}$. To avoid endogenous insulin secretion and its possible effect on brain metabolism, the non-diabetic men received somatostatin.

Results: Plasma glucose increased $11.6 \pm 3.0$ (from $7.7 \pm 2.8$ to $19.3 \pm 2.4$ ) $\mathrm{mmol} / \mathrm{l}$ in diabetic vs. $12.3 \pm 1.0$ (from $4.7 \pm 0.4$ to $17.0 \pm 1.0$ ) $\mathrm{mmol} / \mathrm{l}$ in nondiabetic men $(\mathrm{p}=\mathrm{NS})$. Serum insulin concentrations were equal between the groups throughout the study. During basal fasting glycemia, the diabetic men had higher glucose level in the FWM $\left([39.3 \pm 10.9] \cdot 10^{-6}\right.$ vs. [ $\left.26.6 \pm 6.1\right]$ $\left.\cdot 10^{-6}, \mathrm{p}=0.03\right)$ compared to the non-diabetic men, but equal in the FC $([43.0$ $\pm 12.0] \cdot 10^{-6}$ vs. [37.4 \pm 9.2$\left.] \cdot 10^{-6}\right)$ and $\mathrm{TH}\left([34.1 \pm 14.0] \cdot 10^{-6}\right.$ vs. [28.3 \pm 12.6$]$ $\left.\cdot 10^{-6}\right)$. In the diabetic men, no regional variation of brain glucose was found. In the non-diabetic men, glucose level in FC was higher than in FWM during basal fasting glycemia $(\mathrm{p}=0.02)$ and acute hyperglycemia $(\mathrm{p}=0.04)$. During hyperglycemic clamp, the brain glucose levels increased significantly in both groups: in FC by $2.0 \pm 0.7$ vs. $2.7 \pm 0.9$ (NS), in FWM $1.5 \pm 0.7$ vs. $1.7 \pm 0.7$ (NS), in TH $1.1 \pm 0.4$ vs. $2.3 \pm 0.7 \mathrm{mmol} / \mathrm{l}(\mathrm{p}=0.01)$ for diabetic and nondiabetic men. The diabetic men had higher $\mathrm{mI}$ in the FWM at baseline and during hyperglycemia, and in the FC during hyperglycemia.

Conclusion: During basal fasting glycemia, regional variation in brain glucose levels was found in non-diabetic subjects but not in T1D patients. During hyperglycemic clamp, the glucose transport into the TH was attenuated in T1D, which is a new finding and may sign down-regulation of glucose transport. The increased $\mathrm{mI}$ signal in diabetic FC and FWM may either arise from the increased glucose level or represent osmotic adaptation of the diabetic brain. Our results suggest that in T1D, high glucose excursions result in regional changes of glucose transport that may underlie the functional and structural pathology of the brain.

Supported by: Folkhälsan Research Foundation, Finnish Medical Society, a governmental grant for health sciences research, Diabetes Research Foundation, Waldemar von Frenckell's Foundation, and Wilhelm and Else Stockmann Foundation. 


\section{7}

PTEN expression in the adipose and skeletal muscle tissue in type 2 diabetics versus healthy controls

P. Plomgaard, A.R. Nielsen, C. Broholm, O.H. Mortensen, C.P. Fischer, S. Taudorf, B.K. Pedersen

Centre of Inflammation and Metabolism, Rigshospitalet, Copenhagen, Denmark

Background and aims: Phosphatase and tensin homolog deleted on chromosome 10 (PTEN) is a lipid phosphatase, which has been proposed as a negative regulator of insulin sensitivity. PTEN dephosphorylates $\operatorname{PtdIns}(3,4,5) \mathrm{P}_{3}$ thereby inhibiting the intracellular signal transduction of insulin. In vitro studies have demonstrated that TNF- $\alpha$ increase the expression of both PTEN mRNA and protein in myotubes resulting in an impaired insulin mediated glucose uptake. This is in line with in vivo studies in mice where inhibition of PTEN has improved insulin sensitivity. In humans PTEN promoter polymorphisms in relation to diabetes have given conflicting results. The aim of the present study was to investigate if PTEN mRNA in muscle and adipose tissue were differentially expressed in healthy human subjects compared to type 2 diabetic patients.

Materials and methods: Two groups of age- gender and BMI-matched subjects were evaluated; healthy control $(n=103)$ subjects with type II diabetic patients $(n=96)$. The subjects underwent a clinical examination including having a blood sample drawn, and a DXA scanning for fat mass determination. Furthermore, a biopsy was obtained from the subcutaneous adipose tissue and the skeletal muscle tissue. In the plasma samples fasting glucose and insulin were measured as well as TNF- $\alpha$ and IL-6. The content of PTEN mRNA was quantified by use of real-time PCR. In a subpopulation of obese and non-obese diabetic and healthy controls $(n=40)$ PTEN protein expression was assessed by western blot.

Results: Type 2 diabetics were compared to the healthy controls, and a higher plasma level of fasting insulin $(26 \mathrm{pmol} / \mathrm{L}, \mathrm{P}<0.0001)$ and glucose $(3.9 \mathrm{mmol} / \mathrm{L}, \mathrm{P}<0.0001)$ was found in type 2 diabetic patients, furthermore TNF- $\alpha(\mathrm{P}<0.001)$ and IL-6 $(\mathrm{P}<0.05)$ were $0.3 \mathrm{pg} / \mathrm{ml}$ higher in type 2 diabetic patients compared to the control group. However no significant difference was observed in the fat mass, still WHR was $3.2 \mathrm{~cm}(\mathrm{P}<0.05)$ increased in the diabetic patients. PTEN mRNA expression in the adipose and muscle tissue were not different between the groups. However PTEN mRNA in the adipose tissue was negatively associated with WHR $(\mathrm{P}<0.001)$ and muscle PTEN mRNA negatively associated with plasma TNF- $\alpha$. The western blot analysis did not reveal any difference in PTEN protein expression in relation to diabetes or obesity.

Conclusion: The present study demonstrates that PTEN mRNA in muscle and adipose tissue is not associated with insulin resistance. However, an association did exist regarding PTEN mRNA content in the adipose tissue in relation to WHR. In accordance with the mRNA expression of PTEN in the skeletal muscle no difference was observed on the protein expression of PTEN. These data imply that PTEN mRNA content in muscle and adipose tissue is of limited clinical significance in relation to insulin resistance. Supported by: Commission of the European Communities, Novo Nordisk Foundation and the Danish Diabetes Association. The Centre of Inflammation and Metabolism is supported by the Danish National Research Foundation. The Copenhagen Muscle Research Centre is supported by the University of Copenhagen, the Faculties of Science and of Health Sciences at this university, and the Copenhagen Hospital Corporation and support from the Danish Medical Research Council and the Danish Natural Research Council

\section{8}

Higher iron content in liver assoicates with insulin resistance in subjects with thalassemia minor

P.C.Y. Tong ${ }^{1}$, C.Y. Yeung ${ }^{1}$, A.P.S. Kong ${ }^{1}$, R.C.W. $\mathrm{Ma}^{1}$, W.W.M. Lam ${ }^{2}$, C.W. $\mathrm{Lam}^{3}$, M. $\mathrm{Ng}^{4}$, F.C.C. Chow ${ }^{1}$, J.C.N. Chan ${ }^{1}$

${ }^{1}$ Department of Medicine \& Therapeutics, ${ }^{2}$ Department of Diagnostic Radiology and Organ Imaging, ${ }^{3}$ Department of Chemical Pathology, ${ }^{4}$ Department of Anatomical and Cellular Pathology, The Chinese University of Hong Kong, Shatin, Hong Kong

Background and aims: Chinese subjects with thalassaemia minor have raised inflammatory marker levels and are insulin resistant. In a sib-pair analysis, we examined the relationships between thalassaemia minor, insulin resistance, iron content and oxidative stress.
Materials and methods: Forty-two pairs of sibs with or without thalassemia were recruited. All subjects had a family history of diabetes in their first degree relative, and had normal glucose tolerance on oral glucose tolerance test. Insulin resistance (HOMA-IR) was derived from the homeostasis model assessment. Oxidative stress was measured by plasma (P-IsoP) and red cell membrane isoprostanes (M-IsoP) level. Iron content was determined by MRI-reciprocal T2 relaxation time $\left(\mathrm{r}-\mathrm{T} 2^{*}\right)$ in the liver and in the pancreas. Results: When compared with siblings with no hemoglobinopathy, subjects with thalassemia minor had higher fasting plasma glucose $(2.5 \pm 8.0 \%$, $\mathrm{p}=0.04)$, higher fasting plasma insulin $(37.7 \pm 83.8 \%, \mathrm{p}=0.01)$ and higher degree of insulin resistance as estimated by HOMA-IR $(42.7 \pm 89.0 \%, \mathrm{p}<0.01)$. Iron content was higher in subjects with thalassemia minor as reflected by higher reciprocal of T2 relaxation time measured by MRI liver $(12.7 \pm 30.5 \%$, $\mathrm{p}=0.01)$ and pancreas $(13.0 \pm 38.3 \%, \mathrm{p}=0.04)$. Oxidative stress, as measured by plasma isoprostane and membrane isoprostanes, was not significantly different among two groups. On linear regression analysis, HOMA-IR was associated with waist circumference, high-sensitive CRP, white cell count and $\mathrm{r}-\mathrm{T} 2^{*}$ in the liver among subjects without hemoglobinopathy. In subjects with thalassemia minor, HOMA-IR was related to waist circumference, white cell count, P-IsoP, $\mathrm{r}-\mathrm{T} 2{ }^{*}$ in the liver, age, sex and urate.

Conclusion: Results from this sib-pair study suggest that thalassemia minor causes further degree of insulin resistance in subjects that are at high risk of developing diabetes. The presence of higher hepatic iron content in thalassemia minor may induce abnormality in glucose metabolism via inflammatory and oxidative stress pathways.

Supported by: RGC Earmarked grant

\section{9}

Circulating RBP4 concentration might reflect insulin resistanceassociated iron overload

W. Ricart ${ }^{1}$, J. Moreno-Navarrete ${ }^{1}$, J. Bassols ${ }^{1}$, F. Ortega ${ }^{1}$, G. Pardo ${ }^{1}$, J. Vendrell ${ }^{2}$, J. Fernandez-Real ${ }^{1}$

${ }^{1}$ Endocrinology, Hospital of Girona, Spain, ${ }^{2}$ Endocrinology, Hospital of Tarragona, Spain

Background and aims: The mechanisms behind the association between retinol binding protein 4 (RBP4) and insulin resistance are not well understood. An interaction between iron and vitamin A status, of which RBP4 is a surrogate, has long been recognized. We hypothesized that iron-associated insulin resistance could be behind the impaired insulin action caused by RBP4.

Materials and methods: Serum ferritin and RBP4 concentration, and insulin resistance were evaluated in a sample of middle-aged men $(n=132)$, and in a replication, independent study. Serum RBP4 was also studied before and after iron depletion in patients with type 2 diabetes. Finally, the effect of iron on RBP4 release was evaluated in vitro in adipose tissue.

Results: A positive correlation between circulating RBP4 and log serum ferritin $(r=0.35$ and $r=0.61, p<0.0001)$ was observed in both independent studies. Serum RBP4 concentration was higher in men than women in parallel to increased ferritin levels. On multiple regression analyses to predict serum RBP4, log serum ferritin contributed significantly to RBP4 variance after controlling for BMI, age and HOMA value. Serum RBP4 concentration decreased after iron depletion type 2 diabetic patients (percent mean differences -13.7 ( -25.4 to $-2.04, \mathrm{p}=0.024)$. The iron donor lactoferrin led to increased dose-dependent adipose tissue release of RBP4 (2.4 fold, $\mathrm{p}=0.005$ ) and increased RBP4 expression while apotransferrin and deferoxamine led to decreased RBP4 release.

Conclusion: The relationship between circulating RBP4 and iron stores, both cross-sectional and after iron depletion, and in vitro findings suggest that iron could play a role in the RBP4-insulin resistance relationship.

Supported by: Institute for Biomedical Research; and CIBER Fisiopatología de la Obesidad 


\section{0}

Men with hyperferritinaemia and diabetes do not have a higher iron overload than those without diabetes. Results from a genetic screening programme for hereditary haemochromatosis

J.-M. Gonzalez-Clemente ${ }^{1}$, N. Freixenet ${ }^{1}$, O. Giménez-Palop ${ }^{1}$, A. Caixàs ${ }^{1}$, F. Blanco-Vaca ${ }^{2}$ À. Remacha ${ }^{3}$, C. Cabistañ ${ }^{4}$, Y. Sánchez ${ }^{4}$, V. Bach ${ }^{5}$, M. Baiget ${ }^{5}$, J. Felez ${ }^{4}$

${ }^{1}$ Diabetes, Endocrinology and Nutrition, Hospital de Sabadell, Spain, ${ }^{2}$ Biochemical Laboratory, Hospital de Sant Pau, Barcelona, Spain, ${ }^{3}$ Hematology, Hospital de Sant Pau, Barcelona, Spain, ${ }^{4}$ CAP Canaletes, Institut Català de la Salut, Cerdanyola del Vallès, Spain, ${ }^{5}$ Genetics, Hospital de Sant Pau, Barcelona, Spain

Background and aims: Recent studies showed that a decrease in beta cell function (BCF) instead of a decrease in insulin sensitivity, is the main pathogenic factor involved in the development of diabetes associated with hereditary hemochromatosis $(\mathrm{HH})$. However, most subjects with hyperferritinemia do not have $\mathrm{HH}$, although it is well-known that they have a lower insulin sensitivity and a higher prevalence of the components of the metabolic syndrome (MS). Our aim was to evaluate whether BCF and IS were different or not in diabetics as compared to the non-diabetics, in a group of men with hyperferritinemia none of them with $\mathrm{HH}$.

Materials and methods: During 1 -year period, 150 men (age: $54.0 \pm 13.1 \mathrm{yrs}$ ), were genetically screened for HH (mutations of the HFE gene: C282Y, H63D, S65C) because of hyperferritinemia (SF $>200 \mu \mathrm{g} / \mathrm{L}$, twice, one month apart). They were classified into two groups, with and without type 2 diabetes (current American Diabetes Association criteria) and were evaluated for the components of the MS (curren ATP III criteria), insulin sensitivity (HOMA\%S) and insulin secretion (HOMA\%B), high-sensitivity C-reactive protein (hsCRP), transferrin saturation index and the soluble fraction of the transferrin receptor (sTfR).

Results: Subjects with type 2 diabetes $(n=51)$ were older than those without $(\mathrm{n}=99)(60.3 \pm 9.0$ vs $55.1 \pm 12.3$ yrs, respectively, $\mathrm{p}=0.012)$. They also had a lower BCF (HOMA\%B: 54.1 (26.6-80.2) vs 101.1 (67.4-142.6)\%; p < 0.001) than those without diabetes, but no significant differences were found between both groups, regarding body mass index $(28.8 \pm 3.2$ vs $28.9 \pm 3.6 \mathrm{~kg} /$ $\mathrm{m} 2)$, waist circumference $(1.00 \pm 0.84$ vs $1.00 \pm 0.93 \mathrm{~m})$, systolic blood pressure $(138 \pm 15$ vs $141 \pm 18 \mathrm{mmHg}$ ), diastolic blood pressure ( $81 \pm 9$ vs $82 \pm 11$ $\mathrm{mmHg})$, HDL cholesterol $(1.34 \pm 0.33$ vs $1.37 \pm 0.37 \mathrm{mmol} / \mathrm{L})$, triglycerides (1.62(1.23-1.99) vs $1.59(1.13-2.48) \mathrm{mmol} / \mathrm{L})$, insulin sensitivity (HOMA\%S: 55.4(26.9-86.7) vs 79.5(27.8-146.6\%), serum hs-CRP (2.52(1.34-4.20) vs 2.02 (1.06-4.12) mg/L), ferritinemia ( $576 \pm 184$ vs $586 \pm 214 \mu \mathrm{g} / \mathrm{L})$, transferrin saturation index $(34.0(26.0-42.0)$ vs 34.7 (26.4-39.9) \%), sTfR $(2.30(2.01-2.77)$ vs $2.49(2.07-3.15) \mathrm{mg} / \mathrm{L})$ and the allelic frequency of the mutations of the HFE gen of the HH. The differences between groups in BCF persisted after adjustment for differences in age.

Conclusion: The main determining factor for type 2 diabetes in men with hiperferritinemia is BCF. No differences exist in iron overload parameters and insulin sensitivity between hyperferritinemic men with and without type 2 diabetes.

Supported by: Intensification Grant from the Ministerio de Sanidad y Consumo (JM González-Clemente), Ministerio de Sanidad y Consumo, Instituto de Salud Carlos III, Associació Catalana de Diabetis

\section{1}

Glycaemic disorders in patients with schizophrenia: The METEOR Study K. Shaw ${ }^{1}$, M. De Hert ${ }^{2}$, B. Falissard ${ }^{3}$, M. Mauri ${ }^{4}$, T. Wetterling ${ }^{5}$

${ }^{1}$ Queen Alexandra Hospital, Portsmouth Hospitals NHS Trust, Portsmouth, United Kingdom, ${ }^{2}$ UPC KUL, Campus Kortenberg, Belgium, ${ }^{3}$ Inserm u669, Maison de Solenn, Paris, France, ${ }^{4}$ Clinica Psichiatrica, Università di Pisa, Italy, ${ }^{5}$ Klinik für Psychiatrie, Vivantes Klinikum Hellersdorf, Berlin, Germany

Background and aims: An association between schizophrenia and increased risk for type 2 diabetes mellitus has been suggested for many decades and may reflect contributions from lifestyle risk factors, a risk associated with the underlying pathophysiology of schizophrenia and an effect of antipsychotic drug treatment. Atypical antipsychotic drug use has been associated with metabolic disorders such as weight gain, dyslipidaemia and hyperglycaemia. Most of the available information comes from retrospective analyses, mostly from North America, where the distribution of lifestyle risk factors differs from Europe. To complement these studies, a large, observational, cross-sec- tional pharmacoepidemiological study was initiated in 2006 in order to determine the prevalence of diabetes and other metabolic disorders in patients with schizophrenia receiving atypical antipsychotic drugs in twelve countries in Europe. Secondary objectives included identification of risk factors for metabolic disorders.

Materials and methods: The study included adult outpatients fulfilling DSM-IV-TR diagnostic criteria for schizophrenia and treated for at least three months by an antipsychotic drug. In each country, patients treated with classical or atypical antipsychotic drugs were recruited into two parallel strata in a ratio of 1:3. The study consisted of a single visit at which a fasting blood sample was taken and height, weight, waist and hip circumference and blood pressure measured. Information was collected on socio-demographic variables, lifestyle, comorbidities and co-medications, metabolic and cardiovascular risk factors, psychiatric history and antipsychotic drug history. Biochemical parameters assessed included fasting blood glucose (FBG), insulin, $\mathrm{HbA1c}$ and serum lipids. An oral glucose tolerance test was performed in certain centres (284 patients).

Results: Overall, 2463 patients (54.6\% male) were included with a median age of 41.0 years. Of these, 86 patients were treated for diabetes $(3.8 \%)$, but an additional 75 untreated patients (3.4\%) also presented an FBG $>126 \mathrm{mg} / \mathrm{dl}$ and $484(25.8 \%)$ an FBG between 100 and $126 \mathrm{mg} / \mathrm{dl}$. Multivariate logistic regression analyses identified age $>40$ years (OR: $2.00[1.48 ; 2.70]$ ), weight circumference $>95 \mathrm{~cm}$ (OR: 1.81 [1.23;2.66]), serum triglycerides $>200 \mathrm{mg}$ / $\mathrm{dl}$ (OR: 1.69 [1.23;2.33]), female gender (OR: 1.32 [1.07;1.64]) and clozapine treatment (OR: 1.90 [1.37;2.62]) as being associated with FBG $>100 \mathrm{mg} / \mathrm{dl}$. In contrast, amisulpride treatment was associated with a lower risk of FBG $>100 \mathrm{mg} / \mathrm{dl}$ (OR: 0.50 [0.33;0.75]). In addition, $9.1 \%$ of patients presented a HbAlc level $>6 \%$ and $31.6 \%$ a fasting serum insulin concentration $>12$ $\mathrm{mU} / \mathrm{l}$. Blood glucose levels were $>140 \mathrm{mg} / \mathrm{dl}$ two hours after oral glucose challenge in $16.7 \%$ of tested patients. No difference was observed in the proportion of patients with glycaemic disorders between the classical or atypical antipsychotic strata.

Conclusion: Our results indicate a relatively high prevalence of diabetes and dysglycaemia in patients with schizophrenia treated with antipsychotic drugs. Diabetes was underdiagnosed, undertreated and inadequately controlled. The findings emphasise the need for active physician follow-up of glycaemia in this patient group.

Supported by: sanofi-aventis

\section{2}

Dose seriousness of depressive symptoms influence on insulin resistance - a study using hyperinsulinaemic euglycaemic clamp

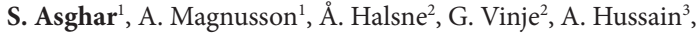
P.M. Thorsby ${ }^{2,4}$

${ }^{1}$ University of Oslo, Inst. for Psychiatric Research, ${ }^{2}$ Aker University Hospital, Hormone Laboratory, ${ }^{3}$ University of Oslo, Department of International Health, ${ }^{4}$ Aker University Hospital, Aker- Ullevål Diabetes Research Centre, Oslo, Norway

Background: Epidemiological data have shown that depression is a significant risk factor for the development of diabetes. The association between depressive symptoms and insulin resistance has been inadequately investigated.

Objective: To assess the association of elevated levels of depressive symptoms on insulin resistance.

Materials: In a depression treatment study of 24 middle aged women in Norway of immigrant origin (Pakistani), we use hyperinsulinemic euglycaemic clamp to evaluate insulin resistance. Depression is evaluated using Montgomery and Åsberg Depression Rating Scale (MADRS), and all included have depressive symptoms (MADRS score $>20$ ). Those suffered from major personality disorder or co morbid conditions or pregnant are excluded from the study. Reinvestigation for insulin resistance is performed in the same individuals following the same procedure after treatment from depression (therapy and medication), when their MADRS score is lowered to less than 10. So far we have investigated 18 women at baseline and 9 at follow up. Before inclusion all have preformed a OGTT to exclude IGT and diabetes and non received treatment for depression.

Results: At baseline $(\mathrm{n}=18)$, mean age 42.5 years $(11.2, \mathrm{SD})$, with a depression score (MADRS) of 30.1 (4.0), GDR $5.0 \mathrm{mg} / \mathrm{kg} / \mathrm{min}$ (3.0), FBG 5,8 mmol/1 (0.96), BMI $27.5 \mathrm{~kg} / \mathrm{m}^{2}$ (4.2) and waist circumference $88.7 \mathrm{~cm}$ (10.5). There was a significant negative correlation between insulin resistance and BMI $(\mathrm{r}=-0.51, \mathrm{p}<0,05)$ and waist circumference $(\mathrm{r}=-0.48, \mathrm{p}<0,05)$. Controlling for BMI, depression score (MADRS) had a significant association with insu- 
lin resistance $(\mathrm{GDR})$ at baseline $\left(\mathrm{r}^{2}=0.51, \mathrm{p}=0.005\right)$ and the contribution of each parameter to variation in GDR was equal (MADRS $\mathrm{r}^{2}=-0.53, \mathrm{p}=0.015$, BMI $r^{2}=-0.69, p=0.003$ ) following a linear regression model. The paired mean difference from the $1^{\text {st }}$ examination (depressed) to the $2^{\text {nd }}$ examination (improved from depression) $(\mathrm{n}=9)$ was MADRS $20.9(3.8, \mathrm{p}<0.001)$ and GDR 0.4 $\mathrm{mg} / \mathrm{kg} / \mathrm{min}(1.4, \mathrm{~ns})$

Conclusion: There appears to be an association between insulin resistance and seriousness of depression. Insulin resistance in turn is also significantly correlated with BMI and waist circumference. Psychiatric intervention and lifestyle change such as weight reduction in depressed patients, may improve the degree of insulin resistance.

Supported by: Aker University Hospital research grant

\section{3}

Adiponectin plasma levels in patients with schizophrenia and schizoaffective disorder: relationships with metabolic syndrome and glucose abnormalities

A.J. Scheen ${ }^{1}$, L. Hanssens ${ }^{2}$, R. van Winkel ${ }^{3}$, M. Wampers ${ }^{4}$, D. Van Eyck ${ }^{4}$, J. Collette ${ }^{5}$, J. Peuskens ${ }^{4}$, M. De Hert ${ }^{4}$

${ }^{1}$ Diabetes, Nutrition \& Metabolic Disorders, CHU Liège, University Liège, Belgium, ${ }^{2}$ Epidemiology \& Public Health, University Liège, Belgium, ${ }^{3}$ Psychiatry \& Neuropsychology, University Maastricht, Netherlands, ${ }^{4}$ Psychiatry, University KU Leuven, Kortenberg, Belgium, ${ }^{5}$ Clinical Biology, CHU Liège, Belgium

Background and aims: Schizophrenic patients have increased rates of hyperglycaemia, dyslipidaemia, metabolic syndrome, leading to a higher incidence of cardiovascular disease. The underlying mechanism, however, is poorly understood. Low levels of adiponectin are associated with metabolic abnormalities such as abdominal obesity, insulin resistance, type 2 diabetes and cardiovascular disease in the general population. The present study aims at investigating the relationships between plasma adiponectin levels and metabolic abnormalities in patients with schizophrenia and schizoaffective disorders.

Materials and methods: Fasting adiponectin levels were measured in a crosssectional sample of 386 patients with schizophrenia or schizoaffective disorder. All patients were on monotherapy of second-generation antipsychotics (SGA) and underwent an extensive metabolic screening including an oral glucose tolerance test (OGTT). Glucose abnormalities were divided into impaired fasting glucose (IFG), impaired glucose tolerance (IGT) or diabetes (based on fasting and/or 2-h post-OGTT glucose levels). Metabolic syndrome (MetS) was defined according to adapted National Cholesterol Educational Program -Adult Treatment Panel (NCEP-ATP)-III or International Diabetes Federation (IDF) criteria.

Results: The demographic characteristics were as follows - mean (STD) - : age 35.9 (11.8) years; 64\% males; 99\% Caucasians; body weight 78.7 (16.0) kg; BMI $26.1(5.0) \mathrm{kg} / \mathrm{m}^{2} ; 28 \%$ with family history of diabetes. Mean fasting adiponectin levels were $8385(6016) \mathrm{ng} / \mathrm{ml}$, being lower in men than in women $(\mathrm{p}<0.0001)$. Adiponectin plasma levels were inversely correlated with BMI $(\mathrm{p}<0.0001)$, and differed between patients with normal weight, overweight or obesity $(\mathrm{p}<0.05)$. Plasma adiponectin levels negatively correlated with waist $(\mathrm{r}=-0.341)$, triglycerides levels $(\mathrm{r}=-0.298)$, systolic blood pressure $(\mathrm{r}=-0.119)$ and fasting glucose levels $(\mathrm{r}=-0.202)$, whereas a positive correlation was observed with HDL levels $(r=0.404)$ (all $p<0.0001)$. Patients with MetS (NCEPATP-III : 29.3\%; IDF: $31.9 \%$ ) had significantly lower adiponectin levels than patients without MetS $(\mathrm{p}<0.0001)$. Patients with diabetes $(5.7 \%)$ had significantly higher adiponectin levels than patients without glucose abnormalities $(78 \%)(\mathrm{p}<0.05)$. Patients with prediabetes (IFG and/or IGT) $(16.3 \%)$ had intermediate adiponectin levels. After controlling for components of MetS and sex, antipsychotic medication independently influenced adiponectin levels, with the lowest levels in patients on clozapine and olanzapine $(p<0.0001)$.

Conclusion: Adiponectin levels in schizophrenic patients mirror what is observed in the general population, with the lowest levels in the most metabolically compromised subjects. However, antipsychotic medication may also influence adiponectin regulation independently, a finding that should be confirmed in longitudinal studies. The role of low adiponectin levels in the higher prevalence of glucose abnormalities associated with clozapine and olanzapine remains to be elucidated.

\section{4}

BGP-15, a novel type insulin sensitiser inhibits insulin resistance and body weight gain induced by atypical antipsychotic drugs

K. Tory ${ }^{1}$, L. Jaszlits ${ }^{2}$, Z. Literati-Nagy ${ }^{1}$, A. Bajza ${ }^{2}$ S. Bernath ${ }^{2}$, A. Kolonics ${ }^{1}$, B. Peitl ${ }^{3}$, B. Literati-Nagy ${ }^{3}$, Z. Szilvássy ${ }^{3}$, P. Literati-Nagy ${ }^{2}$

${ }^{1}$ Department of Molecular Pharmacology, N-Gene Research Laboratory, ${ }^{2}$ Department of Pharmacology, N-Gene Research Laboratory, ${ }^{3}$ Department of Pharmacology and Pharmacotherapy, University of Debrecen, Hungary

Background and aims: BGP-15 (R,S-O-(3-piperidino-2-hydroxy-1-propyl)nicotinic-acid-amidoxime) is an insulin sensitizer drug candidate. Its activity has been demonstrated in different diabetic animal models as well as in insulin-resistant patients by hyperinsulinemic euglycemic clamp method. JUN kinase mediated inhibition of insulin signaling is a common pathogenic mechanism in insulin resistance associated with obesity, inflammation and mitochondrial dysfunction. Inhibiting JUN kinase activation via stimulating stress protein (Hsp70) expression by BGP-15 has been demonstrated as an essential and novel insulin sensitizing mechanism. Atypical antipsychotic drugs frequently cause serious metabolic side effects such as insulin resistance, weight gain and increased risk of cardiovascular diseases. The available antidiabetic agents fail to provide sufficient protection against these side effects. Aim of the study is preclinical and clinical evaluation of the efficacy of BGP-15 on the metabolic side effects of atypical antipsychotic agents.

Materials and methods: Material and Methods: Atypical antipsychotic agents (olanzapine, clozapine, risperidone) were administered in rat, guinea pig and rabbit to provoke metabolic side effects. Insulin sensitivity of control, antipsychotic, antipsychotic + BGP-15 treated groups was determined by hyperinsulinemic euglycemic clamp method. BGP-15 treatment was applied orally. The pharmacokinetic interaction of olanzapine $(10 \mathrm{mg})$ and BGP-15 was studied in 42 healthy individuals during a 17-day treatment period. The effect of BGP-15 on drug-induced insulin resistance was evaluated by hyperinsulinemic euglycemic clamp and weight gain was measured.

Results: In preclinical experiments, five-day olanzapine treatment $(1 \mathrm{mg} / \mathrm{kg}$, b.i.d., p.o.) resulted in significant (50-65\%) decrease in insulin sensitivity in rat, guinea pig and rabbit. Simultaneously applied BGP-15 treatment (10 mg/ $\mathrm{kg}$ ) either greatly improved or normalized insulin induced glucose utilization. BGP-15 efficiently reduced olanzapine induced insulin resistance and inhibited body weight increase during a 28 -day treatment period. Insulin sensitizing activity of BGP-15 was evident on the already established insulin resistance following the 28-day clozapine treatment. In addition, BGP-15 (20 $\mathrm{mg} / \mathrm{kg}$, p.o.) prevented risperidone-induced increase in food intake and body weight gain in rats. In the human clinical study, the treatment period has been completed. Laboratory pharmacokinetic and limited efficacy data will be available by the time of the meeting.

Conclusion: Preclinical experiments suggest that BGP-15 is a new drug candidate able to control the metabolic side effects of atypical antipsychotic drugs. 


\section{PS 056 Adipokines}

\section{5}

Visfatin overexpression ameliorates insulin sensitivity and lipid profile by up-regulating PPAR $\gamma$ activities - twice euglycaemichyperinsulinaemic clamps in identical individual

C. Yulan ${ }^{1,2}$, L. Li ${ }^{1,2}$, G. Yang ${ }^{3}$, R. Li ${ }^{1,2}, \mathrm{~K}^{2} \mathrm{Li}^{3}$

${ }^{1}$ Department of Clinical Biochemistry, ${ }^{2}$ The Key Laboratory of Laboratory Medical Diagnostics in Ministry of Education, ${ }^{3}$ Department of Endocrinology, the Second Affiliated Hospital, Chongqing Medical University, Chongqing, China

Background and aims: Visfatin is an adipose-derived hormone proposed to exert insulin-mimicking effects and play a positive role in attenuating insulin resistance. However, the precise mechanisms underlying the beneficial effects of visfatin on insulin sensitivity remain largely unknown. To further characterize the physiological role of visfatin on the correction of metabolic abnormalities, we therefore examined the effects of visfatin gene overexpression on glucose-lipid metabolism and insulin sensitivity in identical individual as an own control in rats.

Materials and methods: Four-week-old male SD rats were randomly divided into three groups. Two group was fed a normal-chow diet and the another group was fed a high-fat diet for 16 weeks. On day 4 after the surgery, Euglycemic -hyperinsulinemic clamps were performed with awake and unrestrained rats. Blood samples were obtained for determination. At the end of Clamp, normal-chow rats were subdivided into two groups. One group $(\mathrm{NT}, \mathrm{n}=10)$ was given visfastin plasmid that constructed recombinant plasmids of pcDNA3.1-visfatin. Another group $(\mathrm{NC}, \mathrm{n}=5)$ was given pcDNA3.1 as control. High-fat rats $(\mathrm{HT}, \mathrm{n}=10)$ were given pcDNA3.1 - visfatin plasmid. On postinjection day 4 , insulin sensitivity was assessed using another euglycemic- hyperinsulinemic clamp in identical individual. Metabolism gene mRNA expression were measured by RT-PCR, and plasma visfatin protein by Western blot.

Results: In both normal-chow (NT)and high-fat diet (HT) animals with pcDNA3.1 - visfatin treatment, plasma visfatin protein levels were significantly increased (NT, from $0.99 \pm 0.08$ to $1.54 \pm 0.06, P<0.01$ and HT, from $0.99 \pm 0.16$ to $1.92 \pm 0.18, P<0.01)$. Total cholesterol(TC) and high-density lipoprotein cholesterol(HDL-c) were significantly decreased (TC from $1.76 \pm 0.22$ to $1.31 \pm 0.10 \mathrm{mmol} / \mathrm{L}$ for NT; from $2.36 \pm 0.22$ to $1.60 \pm 0.21 \mathrm{mmol} / \mathrm{L}$ for HT, both $\mathrm{P}<0.05$; HDL-C, from $0.95 \pm 0.15$ to $0.59 \pm 0.04$ for NT; from $1.41 \pm 0.24$ to $0.88 \pm 0.11 \mathrm{mmol} / \mathrm{L}$ for $\mathrm{HT}$, both $\mathrm{P}<0.05)$ 0n day 3 after plasmid injection. During two glucose clamp, the glucose infusion rate required to maintain euglycemia was increased by approximately $28 \%$ in the NT rats and $36 \%$ in the HT compared with before plasmid injection(both $\mathrm{P}<0.01$ ). After plasmid injection, the mRNA expression of PPAR $\gamma$ in liver tissue was significantly up-regulated in NT and HT compared with NC $(0.98 \pm 0.08$ and $1.59 \pm 0.15$ vs. $0.68 \pm 0.09$, both $\mathrm{P}<0.05)$. In addition, sterol regulatory element binding protein 2 (SREBP2) and HMG-CoA reductase mRNA expression in liver was also significantly increased in NT and HT compared with NC (SREBP2, $0.37 \pm 0.06$ and $0.36 \pm 0.07$ vs. $0.21 \pm 0.04$, both $P<0.05$; HMG-CoA reductase, $0.89 \pm 0.12$ and $0.55 \pm 0.16$ vs. $0.37 \pm 0.07, P<0.01$ and $P<0.05)$. Elevated visfatin increased the extent of IRS- 1 tyrosine phosphorylation in response to insulin in liver tissues in NT and HT than in NC $(P<0.05)$.

Conclusion: These results indicate that that visfatin could exert its increasing insulin sensitivity and hypocholesterolemic effects at least partially through regulation of the tyrosine phosphorylation of IRS-1 protein and the up-regulation of PPAR $\gamma$ and SREBP2 activation.

Supported by: National Natural Science Foundation of China, Chongqing Municipal Education Commission

\section{6}

Apelin, a new beneficial adipokine with anti-diabetic properties

C. Dray, C. Knauf, D. Daviaud, A. Waget, J. Boucher, P.D. Cani, M. Buleon, C. Guigne, C. Carpene, R. Burcelin, I. Castan-Laurell, P. Valet

U858, INSERM, Toulouse, France

Background and aims: Our team has recently identified apelin as an adipocyte-derived factor secreted and produced by adipocytes in humans and mice. Apelin is a bioactive peptide which is the endogenous ligand of APJ, a G protein-coupled receptor. Apelin and APJ mRNA are widely expressed in mammal tissues. In adipose tissue, apelin is regulated by key factors involved in diabetes like insulin and TNFalpha. In addition, the amount of apelin in blood is largely increased in obese and different mouse models of obesity. Althought a tight relation exists between insulin and apelin, the effect of apelin on glucose utilization has not been adressed so far. Thus, the effects of apelin on glucose metabolism were studied in mice.

Materials and methods: A catheter has been indwelled into the femoral vein of anaesthetized mice, sealed under the back skin, and glued on the top of the skull in order to inject apelin. Oral glucose tolerance test (OGTT), basal glucose turnover and hyperinsulinemic euglycemic clamp were performed in control and high-fat fed C57Bl6/j mice. In vitro, both glucose transport and the signalling pathway involved in apelin response were measured in soleus muscle.

Results: Apelin injection $(200 \mathrm{pmol} / \mathrm{kg})$ in normo-ponderal mice induced a significant decrease of glycemia and clearly improved glucose tolerance after oral glucose load. In basal conditions, apelin significantly increased glucose turnover by stimulating whole-body glycolysis and glycogen synthesis rate compared to saline-injected mice. Infusion of 2 deoxy- $(3 \mathrm{H})$-glucose during the last hour of hyperinsulinemic euglycemic clamp showed that apelin specifically increased glucose utilization in adipose tissue and skeletal muscles. This effect was direct since, in vitro, apelin increased in a dose-depedent manner glucose uptake in soleus muscle. Interestingly, $10 \mathrm{nM}$ apelin was able to increase maximal insulin-stimulated glucose transport suggesting an additive effect. Thus, in order to determine apelin signalling in soleus muscle, we focused on cellular targets belonging to insulin-independent pathways. Apelin stimulated muscle glucose transport both in vivo and in vitro by endothelial nitric oxide synthase (eNOS), AMP-activated protein kinase (AMPK) and Akt-dependent pathways. Finally, in obese and insulin resistant mice, apelin is still able to improve glucose tolerance and stimulated glucose utilization in skeletal muscles and white adipose tissue.

Conclusion: This work clearly demontrates for the first time that apelin is involved in glucose metabolism by using AMPK and eNOS pathways in soleus muscle. The relative contribution of apelin, besides other adipokines (leptine, adiponectin), is of major interest and should yield new insights into the physiology and physiopathology of energy metabolism. Finally, since apelin exibits a sustained effect on glucose utilzation in insulin resistant mice, it appears to be a very promising target for the treatment of type 2 diabetes.

\section{7}

Expression analysis and functional characterisation of EGFL6, a novel secreted protein of the human adipose tissue

R. Oberauer, H. Stahl, W. Rist, M. Lenter, B. Hamilton, H. Neubauer Boehringer Ingelheim Pharma GmbH \& Co. KG, Biberach, Germany

Background and aims: With increasing rates of obesity driving the incidence of type 2 diabetes and cardiovascular diseases to epidemic levels, understanding the role of adipose tissue in the pathogenesis of these morbidities is focus of current research. Identification and characterization of novel adipocytokines could provide further insights into the endocrine function of adipose tissue and potential new therapeutic targets. We recently presented the identification of the epidermal growth factor like 6 (EGFL6; EASD 2007) as a novel secreted protein of the human adipose tissue showing increased expression in obesity and decreased expression after weight loss. The aim of this work was to analyze EGFL6 expression during human in vitro adipogenesis on RNA and secreted protein level. Further, in order to identify the target cell of secreted EGFL6 in adipose tissue, the human protein was expressed, purified and analyzed for its binding capability to human preadipocytes by FACS analysis.

Materials and methods: For expression analysis, human stromal-vascular cells were isolated from adipose tissue biopsies $(n=5)$, cultured and induced for in vitro adipogenesis. During adipogenesis, monitored by Oil-Red$\mathrm{O}$ staining, total RNA and conditioned media samples were obtained and the EGFL6 expression profile was analyzed by quantitative RT-PCR, direct ELISA and Western blotting. Full-length hsEGFL6 with a C terminal His-tag was expressed in a Baculovirus insect cell system and full-length recombinant protein and defined $\mathrm{N}$ terminally truncated versions were purified from conditioned media by affinity chromatography and gel filtration. For FACS analysis, human preadipocytes were incubated with rec. hsEGFL6-His proteins and binding was measured with an anti-C terminal His-tag antibody.

Results: The weak expression of EGFL6 observed in human preadipocytes increased significantly during human in vitro adipogenesis (10-fold on RNA level). In agreement with this, both direct ELISA and Western blotting, showed significantly increased levels of secreted hsEGFL6 in the conditioned media of differentiated adipocytes (1.6 fold increased compared to non-dif- 
ferentiated preadipocytes on day $14, \mathrm{p}<0.01)$. For identification of a molecular target cell of EGFL6, human preadipocytes were pre-incubated with rec. hsEGFL6-His full-length and purified N terminally truncated proteins. Differential and domain-dependent binding of the proteins to the surface of the cells was observed by FACS analysis and will be discussed.

Conclusion: Increased expression of hsEGFL6 during human in vitro adipogenesis was shown on RNA and secreted protein level, indicating that EGFL6 is mostly secreted by adipocytes. The secreted rec. hsEGFL6 protein was shown to bind to human preadipocytes with the binding being mediated by specific protein domains. Further studies are planned to define the binding partners and function of EGFL6 in human adipose tissue physiology.

\section{8}

Lipocalin-2 expression related to obesity and pioglitazone treatment in adipose tissue of rodent and humans

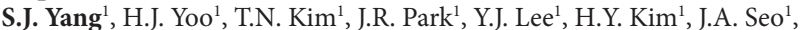
D.H. Shin ${ }^{2}$, S.G. Kim ${ }^{1}$, Y.H. Kim ${ }^{3}$, N.H. Kim ${ }^{1}$, D.S. Choi ${ }^{1}$, S.H. Baik ${ }^{1}$, K.M. Choi ${ }^{1}$

${ }^{1}$ Endocrinology, Korea University, Seoul, ${ }^{2}$ Endocrinology, Bundang Jasesang hospital, Kyoung-gi do, ${ }^{3}$ Endocrinology, Bundang Jaesang hospital, Kyounggi do, Republic of Korea

Background and aims: Adipose tissue is not just an inert storage compartment for triglyceride, but is a metabolically dynamic endocrine organ. PPAR$\gamma$ agonist plays key roles in the regulation of the function of adipose tissue, energy homeostasis and inflammation. The Otsuka Long-Evans Tokushima Fatty (OLETF) rat is a useful animal model of metabolic syndrome. To clarify the hidden pathogenesis of metabolic syndrome, the OLETF rats have been used by many investigators. Therefore, in the present study, we examined the significant overlapping gene which increased in OLETF rat and regulated by PPAR- $\gamma$ agonist simultaneously in adipose tissue using oligonucleotide microarray analyses and validated the significant gene using independent rodent and human samples.

Materials and methods: A standard diet fed $d b / d b$ mice versus a synthetic PPAR- $\gamma$ agonist treated $d b / d b$ mice and OLETF rats versus LETO rats were used for two different kinds of oligonucleotide microarray analyses. And the overlapping gene was validated using independent rodent and human samples by real time RT-PCR. Six premenopausal obese women (BMI $>25 \mathrm{~kg} /$ $\left.\mathrm{m}^{2}\right)$ and age matched six non-obese premenopausal women (BMI $<23 \mathrm{~kg} /$ $\mathrm{m}^{2}$ ) who were scheduled for gynecologic surgery were enrolled in this study. Subcutaneous and visceral adipose tissue was obtained during the gynecological surgery and serum glucose, lipid profiles, IL-6, A-FABP levels and WBC counts were measured.

Results: We examined the significant overlapping gene which increased in OLETF rats and regulated by PPAR- $\gamma$ agonist simultaneously in adipose tissue using oligonucleotide microarray analyses and as a result, we chose the lipocalin-2 as the significant overlapping gene which had to be validated by real time RT-PCR. The expression level of lipocalin- 2 in visceral adipose tissue of pioglitazone treated $d b / d b$ mice showed statistically significant decrease compared with that of normal chow fed $d b / d b$ mice $(P<0.001)$, while there was significant increase of lipocalin-2 expression in visceral adipose tissue of OLETF rats compared with that of LETO rats $(P=0.01)$ In human study, the average of BMI in obese group was $29.2 \pm 3.4 \mathrm{~kg} / \mathrm{m}^{2}$, whereas that of nonobese group was $21.4 \pm 1.2 \mathrm{~kg} / \mathrm{m}^{2}(P<0.001)$. In the obese group, there was significant increase of serum glucose $(P=0.05)$, WBC counts $(P=0.004)$, A-FABP $(P=0.004)$ and lipocalin-2 $(P=0.03)$ levels compared with those of the non-obese group. The expression level of lipocalin-2 in visceral adipose tissues of the obese group was significantly increased compared with that of the non-obese group $(P=0.01)$. However, there was no significant difference in the expression of lipocalin- 2 in subcutaneous adipose tissue between the two groups $(P=0.418)$. And the expression of lipocalin-2 in visceral adipose tissue was significantly correlated with BMI $(r=0.68, P=0.015)$, serum IL-6 $(r=0.59, P=0.044)$, A-FABP levels $(r=0.60, P=0.04)$ and WBC counts $(r$ $=0.72, P=0.008$ ).

Conclusion: This study showed that lipocalin-2 is a significant obesity-related adipokine which is controlled by PPAR- $\gamma$ agonist both in rodents and humans and further study is needed to clarify the functional role of lipocalin2 in the pathogenesis of obesity and metabolic syndrome.

\section{9}

Serum lipocalin-2 concentrations are elevated in human subjects with impaired glucose tolerance and type 2 diabetes

Z. Che ${ }^{1}$, Y. Tan ${ }^{1}$, W. Zhou ${ }^{1}$, P. Zhou ${ }^{1}$, Z. Zhou ${ }^{1}$, H. Zhang ${ }^{2}$, A. Xu ${ }^{2}$

${ }^{1}$ Diabetic Center,Metabolic Syndrome Research Center, Institute of Metabolism and Endocrinology, Central South University, Changsha, Department of Medicine and Research Centre of Heart, Brain, Hormone and Healthy Aging, University of Hong Kong, Hong Kong, China

Background and aims: Chronic low-grade inflammation is now recognized to be a key mediator in the development of type 2 diabetes. Lipocalin-2, a $25-\mathrm{kDa}$ secreted glycoprotein, is abundantly expressed in adipose tissue and liver. It was recently reported that lipocalin- 2 provide a link between obesity, insulin resistance and hyperglycemia in human. The results suggested that Lipocalin-2 can be considered a marker for monitoring obesity-related low-grade inflammation in humans. This study is aimed to evaluate whether lipocalin-2 can be an inflammatory marker in pre-diabetes.

Materials and methods: An in house enzyme-linked immunosorbent assay was developed to measure human lipocalin-2 serum concentrations, which were then compared with various parameters in subjects with normal glucose tolerance (NGT; $n=131$ ), impaired glucose tolerance (IGT; $n=81$ ), and type 2 diabetes (T2DM; $n=50$ ).

Results: No gender-associated difference in serum lipocalin-2 levels was observed. Serum lipocalin-2 concentrations were positively correlated with body mass index (BMI), waist circumference (WC), fat percentage, and high sensitivity C- reactive protein (hsCRP) after adjusted for sex and age. Serum lipocalin-2 concentrations were negatively correlated with high density lipoprotein cholesterol (HDL-C).The hsCRP and HDL-C were found to be independent determinants for serum lipocalin-2 concentrations. Serum lipocalin-2 levels were higher in the IGT and type 2 diabetic groups than in the NGT group (median 32.25 [range (9.25-92.25), 32.5 (9.75-89.5), and 27.0 (10.75-123.25)ug/L, $\mathrm{p}=0.006$ and 0.024 , respectively]. However, no difference was found between serum lipocalin- 2 concentrations in the IGT and type 2 diabetic groups.

Conclusion: Serum lipocalin-2 concentrations were found to be elevated in subjects with IGT or type 2 diabetes and to be related to various clinical parameters and inflammatory factor. Furthermore, we put forward for the first time that serum lipocalin-2 concentrations were elevated in subjects with IGT, suggesting that serum lipocalin-2 can be a useful marker for evaluating inflammatory status in pre-diabetes.

Supported by: National Basic Research Program of China, Hunan Provincial Natural Science Foundation of China

\section{0}

Visfatin induced expression of inflammatory mediators in human endothelial cells via NF-kB pathways

W.H. Sheu ${ }^{1}$, C.S. Wu ${ }^{2}$, W.J. Lee ${ }^{3}$, C.M. Wu ${ }^{1}$, L. Ho ${ }^{4}$, I.T. Lee ${ }^{1}$

${ }^{1}$ Dept of Internal Medicine, , ${ }^{2}$ Dept of Medical Research and Education, ${ }^{3}$ Dept of Medical Education and Research, ${ }^{4}$ Dept of Life Science, National Chung-Hsing University, Taichung, Taiwan

Background and aims: Visfatin is an adipokine that is highly expressed in visceral fat and whose blood levels have been reported higher in subjects with obesity and/or type 2 diabetes mellitus We studied effect of visfatin on endothelial dysfunctions and possible mechanisms.

Materials and methods: Primary human umbilical vein endothelial cells (HUVECs) pretreated with visfatin $(1,10,50 \mathrm{ng} / \mathrm{ml})$ were used to study the relationship between visfatin and endothelium dysfunction. Expression of adhesion molecules (ICAM-1, VCAM-1and E-selectin) and cytokines (IL-6 and IL-8) affected by visfatin were investigated by ELISA, flow cytometry and real-time PCR. Activity of NF- $\mathrm{kB}$ was examined by electrophoretic mobility shift assay (EMSA).

Results: At a visfatin concentration of $50 \mathrm{ng} / \mathrm{ml}$, significant increases in IL-6, IL-8, ICAM-1, VCAM-1 and E-selectin gene expression along with increased IL-6, IL-8 and sE-selectin protein levels in the conditioned medium were detected. Visfatin significantly increased ICAM-1 expression on $10 \mathrm{ng} / \mathrm{ml}$, and VCAM-1 expression on $50 \mathrm{ng} / \mathrm{ml}$ by flow cytometry. EMSA confirmed that visfatin increased DNA binding activity of NF- $\kappa B$. In addition, increased human monocyte cell line THP-1 attaching to HUVECs when pretreated with visfatin $10,50 \mathrm{ng} / \mathrm{ml}$ ) was also demonstrated.

Conclusion: We demonstrated that visfatin increased inflammatory and adhesion molecule expression, at least partly via up-regulation of NF- $\kappa$ B activ- 
ity. Our findings provide direct evidence that visfatin causes endothelial dysfunction, which may help establish a link between obesity and cardiovascular disease.

Supported by: National Science Council, Taiwan

\section{1}

Effect of obesity on growth-related oncogen factor-a, thrombopoietin and tissue inhibitor metalloproteinase-1 serum levels

E. Maury ${ }^{1}$, S.M. Brichard ${ }^{1}$, L. Noël ${ }^{1}$, Z. Pataky ${ }^{2}$, A. Carpentier ${ }^{2}$, A. Golay ${ }^{2}$, E. Bobbioni-Harsch ${ }^{2}$

${ }^{1}$ Endocrinology and Metabolism, Catholic University of Louvain, Brussels, Belgium, ${ }^{2}$ Service of Therapeutic Education for Chronic Disease, Geneva University Hospital, Switzerland

Background and aims: We have recently identified several adipokines oversecreted by omental adipose tissue of obese subjects: two chemokines [Growth-Related Oncogen factor- $\alpha$ (GRO- $\alpha$ ), Macrophage Inflammatory Protein-1ß (MIP-1ß)], a tissue inhibitor of metalloproteinase (TIMP-1), an interleukin (IL-7) and a growth factor (thrombopoietin; TPO). As these adipokines are already known to be involved in cardiovascular disease, insulin resistance and type 2 diabetes, they may link obesity to its cardiovascular or metabolic co-morbidities. The aims of the present study were: 1) to examine whether the increased production by adipose tissue in obesity was reflected by higher circulating levels and 2) to attempt to identify the potential responsible factors.

Subjects and methods: Serum levels of GRO- $\alpha$, MIP-1ß, TIMP-1, IL-7 and TPO were measured by ELISA in 32 lean, 15 overweight, 11 obese and 17 morbid obese age-matched women. Other anthropometric, metabolic and hormonal parameters were also determined: body composition, blood pressure, serum levels of lipids, glucose, insulin, leptin and adiponectin. Insulin sensitivity was evaluated by the euglycemic-hyperinsulinemic glucose clamp. Energy expenditure and substrate oxidation were determined by indirect calorimetry.

Results: Obese and morbid obese women exhibited several clinical or laboratory features of the metabolic syndrome. When compared to age-matched controls, they were characterized by higher blood pressure, hyperinsulinemia and insulin resistance, abnormal lipid profile, increased serum leptin and decreased serum adiponectin concentrations. Out of the five adipokines studied, three exhibited higher circulating levels in obese and/or morbid obese women than in lean ones $(\sim+20 \%, 30 \%$ and $55 \%$ for TIMP-1, GRO- $\alpha$ and TPO, respectively; $P<0.05$ or less). Serum levels of these three adipokines did strongly positively correlate with BMI, fat mass and waist circumference $(P<0.01)$. Serum levels of GRO- $\alpha$, TIMP- 1 and TPO did positively correlate with hyperinsulinemia and/or negatively with insulin sensitivity $(P<0.05$ or less). Serum levels of TIMP-1 did also positively correlate with leptin and blood pressure while TPO was negatively related to adiponectin but positively to circulating lipids levels (free fatty acids and triglycerides; $P<0.05$ or less). Multiple regression analysis showed that fat mass was a significant independent determinant of GRO- $\alpha$, TIMP- 1 and TPO levels. Insulinemia and glycemia were two additional independent determinants of TPO.

Conclusion: As already shown for TIMP-1, we demonstrate that circulating levels of GRO- $\alpha$ and TPO were higher in obese and/or morbid obese women and related to adiposity. These higher systemic levels may, at least in part, reflect the exacerbated secretion of these adipokines previously described in omental adipose tissue in obesity. Herein, that adiposity per se was an independent determinant of these high systemic levels reinforces the concept that hypertrophied adipocytes and surrounding recruited macrophages are fundamental contributors to this "hyperadipokinemia". Because circulating levels of these adipokines did correlate with several features of the metabolic syndrome, these levels may link obesity to its cardiovascular and metabolic disorders.

Supported by: EM has a fellowship from the FRIA (Belgium)

\section{2}

Osteoprotegerin and adiponectin are similarly related to insulin sensitivity in healthy adults

D.T. Ashley ${ }^{1}$, E.P. O’Sullivan ${ }^{2}$, N. Devlin ${ }^{1}$, N.M. Moyna ${ }^{1}$, D. Smith², D.J. O'Gorman ${ }^{1}$

${ }^{1}$ School of Health and Human Performance, Dublin City University,

${ }^{2}$ Diabetes and Endocrinology, Beaumont Hospital, Dublin, Ireland

Background and aims: Circulating osteoprotegerin (OPG), a member of the TNF receptor superfamily, correlates with severity of vascular calcification and coronary artery disease with higher serum levels in diabetic compared to non-diabetic controls. OPG is a decoy receptor for TNF-Related ApoptosisInducing Ligand (TRAIL) but little is known about the relationship between OPG and TRAIL and whether either or both factors are related to insulin sensitivity.

Materials and methods: A total of 102 subjects (38 lean, 42 overweight and 22 obese) without diabetes or cardiovascular disease volunteered for the study. Insulin sensitivity was estimated using a 3 -hr oral glucose tolerance test with OGIS analysis. OPG, TRAIL and adiponectin were analysed using commercially available ELISA assays.

Results: OPG ( $6.0 \pm 0.3$ vs $4.8 \pm 0.3$ pmoll $\left.^{-1}\right)$ and adiponectin $(10.5 \pm 0.9$ vs. $5.9 \pm 0.9 \mu \mathrm{g} \mathrm{ml}^{-1}$ ) were significantly decreased in the obese compared to lean subjects while the TRAIL-to-OPG ratio was greater ( $13.6 \pm 1.3$ vs. $20.3 \pm 2.7$, $\mathrm{p}<0.05)$. There was a strong positive relationship between adiponectin and OPG $(r=0.48, p<0.05)$ for all subjects. Both OPG and adiponectin were negatively correlated with body weight, BMI, waist circumference, and fasting plasma insulin while positively correlated with insulin sensitivity $(\mathrm{p}<0.05)$. TRAIL was positively related to body weight $(\mathrm{r}=0.24, \mathrm{p}=0.02)$ and waist circumference $(\mathrm{r}=0.26, \mathrm{p}=0.01)$ and there was a tendency for a negative relationship with adiponectin $(\mathrm{p}=0.06)$. The TRAIL-to-OPG ratio was positively related to body weight $(\mathrm{r}=0.35, \mathrm{p}<0.001)$, $\mathrm{BMI}(\mathrm{r}=0.26, \mathrm{p}=0.008)$ and waist circumference $(r=0.34, p=0.001)$ and negatively related with insulin sensitivity $(\mathrm{r}=-0.32, \mathrm{p}=0.001)$ and adiponectin $(\mathrm{r}=-0.65, \mathrm{p}<0.001)$.

Conclusion: The common relationships between OPG and adiponectin suggests a link between their release or function in healthy individuals. It is possible that the increase in OPG observed in type 2 diabetes may be a compensatory mechanism to maintain insulin sensitivity and that a change in the ratio of TRAIL-to-OPG may be a more important clinical determinant than OPG alone.

Supported by: Postgraduate studentship from the Irish Research Council for Science, Engineering and Technology 


\section{PS 057 Adiponectin}

\section{3}

Adiponectin inhibits spontaneous lipolytic rate in human isolated adipocytes

J. Polak ${ }^{1,2}$, Z. Hnevkovska ${ }^{1,2}$, J. Dietrich ${ }^{1}$, K. Kolostova ${ }^{3}$, Z. Kovacova ${ }^{1}$, E. Klimcakova ${ }^{1}$, M. Kovacikova ${ }^{1}$, M. Vitkova ${ }^{1}$, M. Bajzova ${ }^{1,2}$, M. Duskova ${ }^{4}$, J. Broz ${ }^{2}$, V. Stich ${ }^{1,5}$

${ }^{1}$ Sports Medicine Department, Third Faculty of Medicine, Charles University in Prague, ${ }^{2} 2$ nd Internal Medicine Department, Vinohrady Teaching Hospital, ${ }^{3}$ Cancer Biology Department, Third Faculty of Medicine, Charles University in Prague, ${ }^{4}$ Plastic Surgery Department, Vinohrady Teaching Hospital, ${ }^{5}$ Franco-Czech Laboratory for a Clinical Research on Obesity, Charles University in Prague, Third Faculty of Medicine, Praha, Czech Republic

Background and aims: Adiponectin is an adipose tissue derived cytokine increasing glucose and fatty acid metabolism in muscle and improving wholebody insulin sensitivity. Plasma adiponectin level is decreased in obese, insulin-resistant and Type 2 diabetic patients. The aim of this study was to investigate the role of adiponectin in the regulation of adipocyte lipolytic activity. Materials and methods: Adipocytes were isolated by collagenase digestion from abdominal adipose tissue obtained during selective surgical operations from 12 subjects (age $43.1 \pm 14.3$ years, $B M I=27.9 \pm 5.5 \mathrm{~kg} / \mathrm{m} 2,3$ men + 9 women). To obtain basal values of glycerol concentration (marker of lipolysis) in the culture media (Krebs Ringer Buffer with 10mM HEPES and $2 \%$ bovine serum albumin (KRBHA), $\mathrm{pH}$ 7.4.), cells were incubated for one hour without any treatment at $37 \mathrm{oC}$. Adipocytes were then subjected to three different conditions for the next 2 hours: 1) control incubation without any pharmacological substances (KRBHA) 2) incubation with recombinant human adiponectin $(20 \mathrm{ug} / \mathrm{ml})$ and 3$)$ incubation with $0.5 \mathrm{mM} \mathrm{AICAR} \mathrm{-} \mathrm{chemi-}$ cal activator of AMPK (adenosine monophosphate activated protein kinase) and glycerol concentrations in the adipocyte-conditioned media were determined colorimetrically.

Results: No differences in basal lipolysis were detected between tubes after 1 st hour. Following the administration of adiponectin a reduction in spontaneous lipolytic rate by $18.1 \%$ ( $95 \%$ Confidence Interval for the mean reduction $5.1-31.0 \%, \mathrm{p}<0.05)$ was observed after 2 hours of subsequent incubation. AICAR suppressed spontaneous lipolytic rate by $65.3 \%$ (CI for the mean reduction 17.6 - 51.8\%, $\mathrm{p}<0.05)$.

Conclusion: Our results show that adiponectin in physiological concentration inhibits spontaneous lipolysis in isolated human adipocytes. This mechanism might be mediated by an activation of AMPK. We suggest, that inhibition of spontaneous lipolytic rate in adipose tissue by adiponectin is an important factor limiting release of free fatty acids (FFA) into circulation and thus leading to improved glucose metabolism and insulin sensitivity. This novel finding might help to explain how hypoadiponectinemia might contribute directly to elevated FFA levels and development of type 2 diabetes mellitus.

Supported by: Grant Agency of Czech Republic, by grant of Ministry of Education of Czech Republic and by HEPADIP supported by the European Commission

\section{4}

Adiponectin is an independent marker of glucose metabolism: a crosssectional study in $\mathbf{4 0 1 3}$ middle-aged men based on detailed oral glucose tolerance testing

P. Khalili ${ }^{1}$, J. Jendle ${ }^{1}$, F. Lundin ${ }^{2}$, G. Engström ${ }^{3}$, J. Frystyk ${ }^{4}$, A. Flyvbjerg ${ }^{4}$, P.M. Nilsson ${ }^{5}$

${ }^{1}$ Internal Medicine, School of Health and Medical Sciences, Karlstad,

Sweden, ${ }^{2}$ Internal Medicine, Värmland County Research Council,

Karlstad, Sweden, ${ }^{3}$ University Hospital MAS, Department of Clinical

Sciences, Malmö, Sweden, ${ }^{4}$ Aarhus University Hospital, Medical Research

Laboratories, Denmark, ${ }^{5}$ Department of Clinical Sciences, School of Health and Medical Sciences, Malmö, Sweden

Background and aims: The adipocyte-derived plasma protein adiponectin has been implicated as a marker of insulin sensitivity and glucose metabolism. Previous studies have shown an inverse correlation between circulating adiponectin levels and measures of insulin resistance. The aim of this study was to examine whether adiponectin is an independent marker of glucose metabolism, adjusting for obesity, in middle-aged men.
Materials and methods: A preventive case-finding programme for detection of cardiovascular risk factors started in 1974 in southern Sweden, the Malmö Preventive Project (MPP). The aim was to find high-risk individuals for preventive interventions. Subjects were invited to participate in health screening, including a physical examination and laboratory tests. Blood samples have been stored and total adiponectin levels were analysed in a subgroup of 4013 middle-aged males, born in pre-specified years and living in Malmö, but not selected in any other way. The reason to select this subset of MPP was that all individuals performed an oral glucose tolerance test (OGTT) with multiple measurements of blood glucose $\left(0,10^{\prime}, 30^{\prime}, 60^{\prime}\right.$ and $\left.120^{\prime} \mathrm{min}\right)$, thus enabling us to calculate the area under the curve (AUC) of glucose. The material was divided into quintiles (Q1-Q5) from lowest (Q1) to highest adiponectin levels (Q5). Baseline characteristics were analyzed and multiple regression analyses (MRA) were performed for examining the association between adiponectin and glucose metabolism.

Results: The mean age was 47 years $(\mathrm{SD} \pm 3.3)$. Subjects in Q1 differed from Q5 in mean BMI, SBP, triglycerides, blood glucose (fasting, 2h, AUC) and HOMA-IR index. The means of these variables were significantly $(\mathrm{p}<0.05)$ higher in Q1 than in Q5. Total cholesterol did not show any difference between quintiles. Blood glucose (fasting, 2h, AUC) and HOMA-IR index were each used as independent variables in separately performed MRA. Plasma adiponectin was significantly associated with all measures of glucose metabolism (all $\mathrm{p}<0.001$ ), after adjustment for age, BMI, SBP, cholesterol and log triglycerides.

Conclusion: An inverse and independent cross-sectional correlation exists between circulating total adiponectin levels and markers of glucose metabolism and insulin sensitivity in middle-aged men. This was independent of age and general obesity (BMI). Our findings support and expand previous observations in different populations.

Supported by: Värmland County Research Council, Sweden

\section{5}

The effects of shRNA-mediated adiponectin expression inhibition on insulin signalling and glucose turnover in the 3T3-L1 adipocytes L. Jianlei ${ }^{1,2}$, L. Li ${ }^{1,2}$, G. Yang ${ }^{3}$, Y. Yang ${ }^{1,2}, \mathrm{~K} . \mathrm{Li}^{3}$

${ }^{1}$ The Key Laboratory of Laboratory Medical Diagnostics, Ministry of Education, ${ }^{2}$ Department of Clinical Biochemistry, ${ }^{3}$ Department of Endocrinology, the Second Affiliated Hospital, Chongqing Medical University, Chongqing, China

Background and aims: Adiponectin (Acrp30) is a physiologically active polypeptide hormone secreted by adipose tissue that shows insulin-sensitizing, antiinflammatory, and antiatherogenic properties. However, the underlying cellular and molecular mechanisms remain largely unknown. Therefore, we reasoned that a more limited reduction in adiponectin expression in 3T3L1 cells using vector-mediated RNAi methodology may provide additional insight into the physiologic role of adiponectin. In this study, we investigated whether posttranscriptional gene silencing by vector-mediated RNAi will inhibit adiponectin expression and its effects on glucose turnover and some metabolism gene expression in 3T3-L1 cells.

Materials and methods: Oligonucleotides coding for a short hairpin RNA (shRNA) against Acrp30 were designed and adenovirus vector plasmids, pAd-U6- ${ }_{\text {Acrp30-1 }}$ was constructed according to a two-step transformation protocol. A 3T3-L1 cell line was transfected with the Acrp30-shRNA adenovirus expression vector. Acrp30 mRNA expression and protein level were measured by RT-PCR and ELISA in these cells. Glucose turnover and gene expression were determined by LSC and RT-PCR.

Results: The Acrp30 mRNA expression and protein level in 3T3-L1 cells with adenovirus plasmids was statistically significantly lower than in controls ( $34 \%$ and $39 \%$, both $P<0.01$ ). The rate of glucose disappear $\left(\mathrm{G}_{\mathrm{Rd}}\right)$ and the uptake rates of glucose (GUR) was significantly decreased by $90 \%$ and $77 \%$ in adiponectin-deficient adipocytes compared with the controls $(P<0.05$ and $P<0.01)$. PPAR- $\alpha$ mRNA expression in adiponectin-deficient cells with insulin-stimulated was significantly down-regulated by $66 \%(P<0.05)$, and IRS-1 mRNA expression in these in both basal and insulin stimulated state was down-regulated by $52 \%$ and $72 \%$, respectively (both $P<0.05$ ). In addition, hormone-sensitive lipase (HSL) mRNA expression was down- regulated by $40 \%$ and $57 \%$ (both $P<0.05$ ), and ATGL mRNA expression was also reduced by $75 \%$ after insulin-stimulating $(P<0.05)$. Another adipocytokines, visfatin mRNA expression was reduced by $83 \%$ in the basal and insulin-stimulated states of adiponectin-deficient cells.

Conclusion: These results show that the siRNA adenoviral vector against Acrp30 mRNA has been successfully constructed, and it effectively inhibits 
the expression of Acrp30 mRNA and protein in 3T3-L1 cells. Adiponectin deficiency exerts a negative effect on glucose transport and metabolism by down-regulating GLUT-1, GLUT-4 and IRS-1 expression, and also resulted in marked alteration of visfatin, HSL and ATGL mRNA expression. Supported by: National Natural Science Foundation of China and Chongqing Municipal Education Commission

\section{6}

Effects of angiotensin II on the production of adiponectin in 3T3-L1 adipocyte

T. Ikeda, K. Kajita, K. Fujioka, I. Mori, Y. Uno, H. Morita, T. Ishizuka General Internal Medicine, Gifu University Graduate School of Medicine, Gifu, Japan

Background and aims: Various hormones, including glucagons, ACTH, parathyroid hormone and angiotensin II (AII), activate phorbol ester-sensitive PKC $(\mathrm{c} / \mathrm{nPKC})$ to obtain various hormone actions. We speculated that $\mathrm{c} / \mathrm{nPKC}$ might regulate adipocyte-specific gene and cytokine-related gene, as $\mathrm{c} / \mathrm{nPKC}$ activate NF-kappa B. Our previous research revealed that treatment with phorbol ester reduced the expression of PPAR gamma and related genes, such as aP2, lipoprotein lipase (LPL) and adiponectin in adipocyte. AII stimulation causes the inhibition of insulin signaling by serine phosphorylation of insulin receptor substrate I via activation of $\mathrm{c} / \mathrm{nPKC}$. In this study, we evaluated the effect of AII on the expression of cytokines including IL-6, IL10 and monocyte chemoattractant protein-1 (MCP-1) in order to search the association between hypertension-induced insulin resistance and adiponectin production in adipocyte.

Materials and methods: We examined the effect of high glucose, insulin and AII on the mRNA expression of these genes in adipocytes. Fully differentiated 3T3-L1 adipocytes were incubated with 10nM TNF alpha, 100nM TPA, $10 \mathrm{nM}$ insulin, $15 \mathrm{mM}$ glucose (high glucose) and $50 \mathrm{nM}$ AII for $24 \mathrm{hr}$. Total RNA was isolated to measure the expression levels of PPAR gamma, aP2, LPL, adiponectin, suppressor of cytokine signal-1 (Socs-1) and MCP-1 were measured with real-time PCR. Western blot was performed to determine activities of c/nPKC with anti-phospho MARKS antibody. NF-kappa B activity was assayed with commercially available kit. (Trans AMTM NF-kappa B, Active Motif).

Results: Treatment with $100 \mathrm{nM}$ TPA, as well as $10 \mathrm{nM}$ TNF alpha, decreased the PPAR gamma mRNA levels in 3T3-L1 adipocytes. Levels of aP2, LPL, FAS and adiponectin mRNA decreased concomitantly. TNF alpha markedly increased MCP-1 and Socs-1 mRNA level (522\% and 232\% respectively), however TPA slightly increased MCP-1 and Socs-1 mRNA levels (234\% and $125 \%$ respectively). Treatment with $50 \mathrm{nM}$ AII, but not insulin and high glucose, decreased the PPAR gamma, aP2, LPL, FAS and adiponectin mRNA levels, whereas it did not alter Socs-1 and MCP-1 levels. Go6976, c/nPKC inhibitor, prevented the AII-induced reduction in levels of PPAR gamma mRNA. Incubation with TPA and AII, but not insulin and high glucose, for $24 \mathrm{hr}$ increased c/nPKC and NF-kappa B activity.

Conclusion: These results indicate that activation of $\mathrm{c} / \mathrm{nPKC}$ negatively regulates expression level of PPAR gamma and adiponectin in adipocyte, probably mediates via NF-kappa B activation. Moreover, AII also down-regulated adiponectin mRNA level, which might contribute to insulin resistance.

\section{7}

Adiponectin gene expression is significantly higher in subcutaneous adipose tissue of diabetic ZDF fa/fa rats than in nondiabetic ZDF fa/fa, diabetic Heterozygous ZDF or lean Zucker rats

B. Seyoum, A. Fite, A. Abou-Samra

Division of Endocrinology, Diabets and Metabolism, Wayne State University, Detroit, United States

Background and aims: Zucker diabetic fatty (ZDF) rats have been used as a model for type 2 diabetes mellitus (T2DM) since the late 1980s. ZDF rats have a missense mutation in the leptin receptor gene (fa/fa), which results in decreased leptin signalling and defective receptor-mediated leptin transport into the central nervous system. Homozygous fa/fa ZDF rats naturally become hyperphagic, hyperinsulinemic, hyperlipidemic, develop substantial ectopic fat deposition in muscle cells and eventually develop T2DM. The mechanism by which ZDF rats develop metabolic syndrome and insulin resistance and the role of ectopic fat deposition in muscle tissues is not well understood. The communication between muscle and fat cells through adi- pocytokines such as adiponectin may be important pathophysiologically for the obesity and diabetes phenotype of these rats. The purpose of this study was to characterize adiponectin gene expression in adipose and muscle tissues obtained from ZDF rats.

Materials and methods: Using real-time PCR, we analyzed adiponectin mRNA levels in total RNA prepared from skeletal muscles and subcutaneous and visceral adipose tissues from 4 different ZDF rat groups, homozygous or heterozygous for the leptin receptor mutation with contrasting metabolic conditions: $\mathrm{fa} / \mathrm{fa}$ diabetic, $\mathrm{fa} / \mathrm{fa}$ nondiabetic, fa/wt diabetic and wt/wt lean non-diabetic.

Results: One-way analysis of variance was used to calculate statistical significance. The data show dramatic differences in adiponectin gene expressions between diabetic and nondiabetic ZDF rats: in subcutaneous adipose tissues adiponectin mRNA expression was 16 fold higher in fa/fa diabetic than in $\mathrm{fa} / \mathrm{fa}$ nondiabetic rat $(\mathrm{p}=0.003)$; there was no statistical difference between diabetic fa/wt and wt/wt non-diabetic lean rats. In contrast, visceral fat adiponectin mRNA expression was 4 fold lower in the diabetic than in the nondiabetic fa/fa rats $(\mathrm{p}<0.05)$; a similar trend was observed in the $\mathrm{wt} / \mathrm{fa}$ diabetic rats when compared to the wt/wt non-diabetic rats. The levels of adiponectin gene expression in abdominal, thigh and leg muscles was different in the different animal groups. In abdominal, thigh and leg muscles, adiponectin mRNA was 3-4 fold higher in nondiabetic than in diabetic $\mathrm{fa} / \mathrm{fa}$ rats $(\mathrm{p}<0.05)$. The highest adiponectin mRNA level was observed in the fa/wt diabetic rat when compared to all the other groups.

Conclusion: The data obtained in skeletal muscles and visceral adipose tissues in fa/fa ZDF rats are consistent with the literature that diabetes is associated with low adiponectin expression. In contrast, diabetes was associated with higher expression of adiponectin mRNA in subcutaneous adipose tissues in the diabetic fa/fa rats compared to the non-diabetic fa/fa rats. The higher expression of adiponectin mRNA in subcutenous adepose tissues of diabetic fa/fa ZDF rats may reflect a feedback up regulation of adiponectin gene transcription in subcutaneous fat by the diabetic state of the animal.

\section{8}

The relationship between skeletal muscle or plasma adiponectin concentration and insulin resistance in hereditary hypertriglyceridaemic rats

M. Cahova, M. Maxova, I. Markova, L. Kazdova

Department of Metabolism of Diabetes, Institute for Clinical and Experimental Medicine, Prague, Czech Republic

Background and aims: Adiponectin has been shown to have insulin-sensitising effect and it is well-documented that plasma adiponectin is lower in obese subjects and in obese diabetic patients. Although the association between adiponectin levels and adiposity is well-established, the causal relationship between age-related or dietary induced changes in insulin sensitivity and changes in adiponectin levels is still not fully understood. The aim of our study was to investigate the age-related changes in serum and tissue concentration of adiponectin and tissue insulin sensitivity in non-obese experimental model of insulin resistance - hereditary hypertriglyceridemic rats (HHTg) and control normotriglyceridemic (C) rats at age 3 - and 18- months.

Materials and methods: The experiments were carried out on adult male HHTg and Wistar rats fed 8 weeks with high-sucrose diet $(70 \mathrm{cal} \%$ as sucrose) at the age of 3- and 18- months. The adiponectin was determined by Elisa kit (B-Bridge Inc, USA). The insulin sensitivity of skeletal muscle and adipose tissue was estimated according to the insulin-stimulated ${ }^{14} \mathrm{C}$-U-glucose incorporation into glycogen and lipids.

Results: Advancing age and adiposity was associated with the decrease in serum and muscle adiponectin in both control and HHTg animals. In both groups the serum adiponectin concentration was by $100 \%$ lower in the 18 month compared to the 3-month old animals. The age-related drop in muscle adiponectin concentration was more pronounced in HHTg than in the control group (110 vs $40 \%$ ). Despite similar serum and tissue adiponectin levels in both groups, 3- month old HHTg rats exhibited an impaired glucose tolerance measured as glucose and insulin AUC during OGTT when compared with controls. This finding is in accordance with the impaired in vitro insulin sensitivity in adipose tissue and skeletal muscle in young HHTg animals. In aged animals, the glucose intolerance and in vitro insulin sensitivity was more severe in HHTg than in control animals in accordance with tissue but not serum adiponectin concentration.

Conclusion: Our data indicate that adiponectin serum concentration inversely correlates with age and thus the lower adiponectin levels may contribute to the development of age-related insulin resistance that is not associated 
with obesity. Nevertheless, the decrease in insulin sensitivity is not directly proportional to the changes in plasma adiponectin concentration and seems to be associated more tightly with tissue (skeletal muscle) adiponectin levels. Supported by: MH CR no. NR 9387-3.

\section{9}

Association of insulin, leptin and adiponectin with fetal growth in term babies of nondiabetic and diabetic mothers

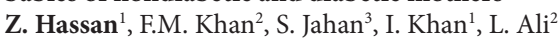

${ }^{1}$ Physiology and Molecular Biology, ${ }^{2}$ Dept of Biochemistry and Cell Biology, ${ }^{3}$ Dept of Obstetrics and Gynecology, BIRDEM, Dhaka, Bangladesh

Background and aims: Leptin and adiponectin play important roles in energy homeostasis. However, their precise role in the fetal growth and development, and their interaction with insulin still needs to be clarified in various ethnic groups due to the dependence of these hormones on genetic and environmental factors. The present study was designed to evaluate cord blood levels of insulin, leptin and adiponectin in full term newborn babies and to analyze the growth parameters of the fetus in relation to their insulinemic, leptinemic and adiponectinemic status.

Materials and methods: Blood samples from umbilical cord were taken from 128 newborn babies, out of whom 94 were of nondiabetic mothers (BNDM group) and 34 of diabetic mothers (BDM group). During analysis babies were classified as small for gestational age (BNDM-SGA and BDM-SGA) or average for gestational age (BNDM-AGA and BDM-AGA) based on WHO criteria. Weight and length of the babies as well as their anthropometric parameters (mid-arm circumference, head circumference, abdominal circumference) were measured following standard procedures. Leptin and adiponectin were measured by enzyme-linked immunosorbant assay (ELISA). Results were expressed as median (range).

Results: BDM had significantly lower total cholesterol (mg/dl) level compared to the BNDM [30 (13-80) vs $48(18-108), \mathrm{p}<0.001]$ group. This tendency was more pronounced in BDM-AGA compared to both BNDMAGA $(p=0.001)$ and BNDM-SGA $(p=0.012)$ groups. Even in the presence of normal birth weight and blood glucose values BDM group had significantly higher insulin $(\mu \mathrm{IU} / 1)$ levels compared to BNDM group [5.1 (0.5-2.2) vs 3.39 (0.5-66.6), $\mathrm{p}=0.012$ ]. Higher level was also observed in BDM-AGA compared to BNDM-AGA [5.7 (0.5-17.8) vs $3.3(0.5-66.6),(\mathrm{p}=0.021)]$. No difference was observed between BNDM-SGA and BNDM-AGA groups. Cord blood leptin level $(\mathrm{ng} / \mathrm{ml})$ in BNDM and BDM did not show statistical difference ( $\mathrm{p}=0.246)$. BNDM-AGA [9.3 (0.5-48.0)] and BDM-AGA ([11.5 (1.03-89.9)] had significantly higher leptin levels compared to BNDM-SGA [3.7 (0.6-29.6)] ( $\mathrm{p}=0.013$ and 0.012 respectively). However, leptin levels between BNDM-AGA and BDM-AGA did not show any statistical difference $(\mathrm{p}=0.432)$. Lptin levels showed positive correlation with fetal variables: birth weight $(r=0.335, p=0.001)$, blood glucose $(r=0.268, p=0.009)$ and maternal variables: $\mathrm{BMI}(\mathrm{r}=0.338, \mathrm{p}=0.001)$ and diastolic blood pressure $(\mathrm{r}=0.236$, $\mathrm{p}=0.023$ ) in absence of maternal diabetes. Multivariate analyses with leptin as dependent variable and birth weight, blood glucose, insulin and gender as independent variable revealed association with birth weight $(\mathrm{p}=0.001-<0.001)$ and blood glucose ( $\mathrm{p}=0.019-0.035)$ only in absence of maternal diabetes. But in cases of maternal variable, only BMI persistently showed significant association with leptin. Cord blood adiponectin level $(\mathrm{ng} / \mathrm{ml})$ between BNDM [68.2 (4.9-106)] and BDM [67.1 (21.4-91.8)] $(\mathrm{p}=0.650)$ did not show any significant difference and the trend was similar in the subgroups. Correlation analyses also failed to demonstrate any association with adiponectin and fetal and maternal variables either in nondiabetic and diabetic groups.

Conclusion: Leptin, in association with insulin, seems to play a crucial role in fetal growth. Adiponectin does not seem to have any association with fetal development.

Supported by: International Program in the Chemical Sciences and Diabetic Association of Bangladesh

\section{0}

Comparison of adiponectin multimeric complexes secreted from adipose tissue explants with plasma levels in obese women: impact of very low calorie diet

Z. Kovacova ${ }^{1}$, J. Polak ${ }^{1,2}$, M. Vitkova ${ }^{1,3}$, M. Kovacikova ${ }^{1}$, E. Klimcakova ${ }^{1}$, M. Bajzova ${ }^{1,2}$, Z. Hnevkovska ${ }^{1,2}$, L. Rossmeislova ${ }^{3}$, V. Stich ${ }^{1,3}$

${ }^{1}$ Department of Sport Medicine, Third Faculty of Medicine, Charles University, Prague, Czech Republic, ${ }^{2} I$ I. Internal Medicine Department, Vinohrady Teaching Hospital, Prague, Czech Republic, ${ }^{3}$ Franco Czech Laboratory for the Clinical Research on Obesity, INSERM France and Third Faculty of Medicine Charles University, Prague, Czech Republic

Background and aims: Adiponectin is adipose tissue-produced adipocytokine with important role in glucose and lipid metabolism. It circulates in plasma as high, middle and small molecular weight isoforms (HMW, MMW, LMW). Plasma adiponectin levels are decreased in obese, insulin-resistant and Type 2 diabetic patients. The aim of our study was to investigate the secretion of adiponectin polymers from adipose tissue explants in relationship to plasma and to investigate the impact of low-caloric diet-induced weight loss on metabolism of specific adiponectin isoforms in adipose tissue.

Materials and methods: A novel ELISA assay (ALPCO Diagnostics, Salem, USA) and nondenaturing Western blot analysis were used to determinate adiponectin complex secretion from adipose tissue and distribution in plasma of 14 obese subject ( 11 women and 3 men of age $39.3 \pm 6.73$ years; BMI, 31.99 $\pm 4.49 \mathrm{~kg} / \mathrm{m}^{2}$ ) before and after eight weeks' very low-calorie diet (VLCD). A needle biopsy of subcutaneous abdominal adipose tissue and blood samples were taken before and after dietary intervention. About $400 \mathrm{mg}$ of adipose tissue cut in small pieces was incubated in culture medium for four hours and the medium used for analysis.

Results: The most abundant isoform in cultured media was the HMW form which represented $88.6 \%$ of total secreted adiponectin into media (MMW was $11.4 \%$ of total adiponetin; LMW was not detected in media). We compared this distribution to plasma samples where the percentage of HMW to total adiponectin was $54.2 \%$ shifted to others isoforms (MMW $16.5 \%$; LMW $29.2 \%$ ). Western blot confirmed the results of ELISA with respect to the distribution of adiponectin polymers in both plasma and media. The subjects body weight and BMI decreased (by $11.12 \%$ and $11.14 \%$ respectively) after VLCD. We found no effect of diet on the composition of polymeric isoforms produced from adipose tissue into culture media or appearing in plasma despite of the diet-induced improvement of insulin sensitivity.

Conclusion: Adiponectin secreted from human adipose tissue is represented predominantly by HMW isoforms. The proportion of HMW in secreted medium is different from that in plasma. The secretion profile of adiponectin was not affected by the diet-induced weight reduction associated with changes of insulin sensitivity. This suggests that the diet-induced change in insulin sensitivity is not related to changes in HMW polymers of adiponectin. Supported by: Grant agency and Ministry of Health of Czech Republic and by HEPADIP project supported by EU

\section{1}

Effect of glomerular filtration rate on circulating adipokines in patients with type 2 diabetes mellitus

M. Al Arouj ${ }^{1}$, A. Ben Nakhi ${ }^{1}$, O. Mojiminiyi ${ }^{2}$, N. Abdella ${ }^{3}$

${ }^{1}$ Medicine, DASMAN Diabetes Center, ${ }^{2}$ Pathology, Faculty of Medicine, Kuwait University, ${ }^{3}$ Medicine, Faculty of Medicine, Kuwait University, Kuwait

Background and aims: Obesity and insulin resistance are known to contribute to the progression of renal disease via several potential mechanisms. We postulate that one of these mechanisms may involve retention of low molecular weight adipokines when glomerular filtration rate (GFR) decreases. Therefore, the main objective of this study was to evaluate the relationships between adiponectin $(30 \mathrm{kDa})$, resistin $(12.5 \mathrm{kDa})$ and leptin $(16 \mathrm{kDa})$ concentrations with estimated GFR (eGFR) in patients with Type 2 diabetes (T2DM) and varying degrees of diabetic nephropathy

Materials and methods: Fasting adiponectin, resistin and leptin were determined in 210 T2DM patients who were classified as normo- $(\mathrm{n}=139)$ or microalbuminuric $(\mathrm{n}=71)$. eGFR was determined by the abbreviated Modification of Diet in Renal Disease (MDRD) equation. Uni- and multi-variate regression (with inclusion of age, gender, waist circumference (WC), presence of coronary heart disease, hypertension and smoking status as potential 
confounders) analyses were used to determine the associations of eGFR with adipokine concentrations.

Results: $37 \%$ of patients had eGFR $<60 \mathrm{ml} / \mathrm{min} / 1.73 \mathrm{~m}^{2}$ and in these patients mean WC (105 vs $107 \mathrm{~cm}$ ) was lower but mean adiponectin (15.8 vs $14.3 \mathrm{ng} /$ $\mathrm{ml})$, resistin $(24.7 \mathrm{vs} 23.3 \mathrm{ng} / \mathrm{ml})$ and leptin $(39.9 \mathrm{vs} 39.2 \mathrm{ng} / \mathrm{ml})$ were higher than in patients with eGFR $>60 \mathrm{ml} / \mathrm{min} / 1.73 \mathrm{~m}^{2}$. Adiponectin $(\mathrm{r}=-0.38)$, leptin $(r=0.45)$ and resistin $(r=0.49)$ were significantly correlated with WC and these correlations remained significant after correction for eGFR and the above confounding variables. In uni- and multi-variate regression analyses, no significant associations were found between adiponectin, leptin and resistin and eGFR. Sub-group analysis of patients with eGFR $<60 \mathrm{ml} / \mathrm{min} / 1.73 \mathrm{~m}^{2}$ failed to show associations between adipokines and eGFR

Conclusion: Our results reject the hypothesis that there is GFR dependent retention of adipokines in patients with T2DM in whom the degree of adiposity appears to be the most significant determinant of circulating adipokines. Supported by: KFAS

\section{PS 058 Animal models of obesity}

\section{2}

Hepatoprotective effect of rimonabant is associated with upregulation of adiponectin in the obese, insulin resistant dog

M. Kabir, M.S. Iyer, D. Stefanovski, I.R. Hsu, O. Woolcott, D. Zheng, S.P. Kim, K.J. Catalano, J.D. Chiu, L.N. Harrison, M. Lottati, V. Ionut, R.N. Bergman, J.M. Richey

Physiology and Biophysics, USC, Keck school of medicine, Los Angeles, United States

Background and aims: Recent studies demonstrated that the CB-1 receptor antagonist Rimonabant (RIM) may have beneficial effects on liver disease. In a previous study, we showed that RIM improved hepatic insulin sensitivity and reduced both visceral and subcutaneous adiposity in the obese, insulin resistant dog model. In addition, RIM increased plasma adiponectin levels by two fold. Based on these data, we hypothesized that the effect of RIM on the liver may be mediated in part by an increase in the purported insulin sensitizer adiponectin. Hence, we undertook the current study to investigate the effect of RIM on liver morphology as well as adiponectin gene expression in subcutaneous (SQ) and visceral (VIS) fat depots.

Materials and methods: Dogs were fed a high fat diet (HF; 52\% fat) to induce mild obesity and insulin resistance. After 6 wks of fat feeding, animals were continued on HF diet for an additional 16 wks either with placebo $(P ; n=4)$ or with RIM ( $1.25 \mathrm{mg} / \mathrm{kg}$ per day; RIM; $\mathrm{n}=4$ ) treatment. Biopsies from liver and fat were obtained in RIM and P groups for histology and gene expression.

Results: After 16 weeks of high fat diet (P), histological examination of the liver revealed slight lipid accumulation, in addition to hydropic change. Administration of RIM reduced this hepatic lipid accumulation and improved hydropic change. Measurement of adiponectin expression, using real time PCR, demonstrated that RIM treatment increased adiponectin gene expression by 6 fold in the VIS depot $(\mathrm{P}<0.001)$, while there was only a slight tendency to decrease VIS adiponectin expression in $\mathrm{P}$ group $(\mathrm{P}=0.058)$. In addition, adiponectin gene expression increased 2 fold in SQ depots with both treatment groups $(\mathrm{P}<0.05)$.

Conclusion: Increased gene expression of adiponectin from visceral fat may increase the delivery of adiponectin into the liver thereby improving hepatic sensitivity. Furthermore adiponectin and/or Rimonabant itself may have beneficial effects on the liver by reducing both fat accumulation and hydropic change. Thus, Rimonabant may play a hepatoprotective role in obesity and may have an important pharmacological application for obesity associated with liver disease.

Supported by: sanofi-aventis

\section{3}

Characterisation of the insulin signalling cascade defects in the liver of obese-insulin resistant baboons

A.O. Chavez ${ }^{1}$, S. Kumath ${ }^{1}$, R. Guardardo-Mendoza ${ }^{1}$, G. Hubbard ${ }^{2}$, E. Dick ${ }^{2}$, A. Comuzzie ${ }^{2}$, A. Gastaldelli ${ }^{1}$, R.A. DeFronzo ${ }^{1}$, F. Folli ${ }^{1}$

${ }^{1}$ Medicine/Diabetes, UTHSCSA, ${ }^{2}$ Diabetes Research, Southwest Foundation for Biomedical Research and Southwest National Primate Research Center, San Antonio, United States

Background and aims: Baboons are valuable models for the study of human disease, sharing $98 \%$ genetic similarity. Recently, we have demonstrated that the baboon is a pertinent model for the study of insulin resistance. In humans, obesity and type 2 diabetes are associated with impaired hepatic insulin sensitivity. However, the molecular abnormalities present in the human insulin resistant liver are largely unknown. In the present study, we analyzed key insulin signaling molecules in liver of baboons with varying degrees of adiposity and insulin sensitivity.

Materials and methods: Eight obese insulin resistant (IR) baboons (age $=21 \pm 2$ y, BMI $=28 \pm 2^{*} \% \mathrm{BF}=15 \pm 3^{*} \mathrm{FPG}=114 \pm 7 \mathrm{mg} \%{ }^{*} \mathrm{Rd}=2 \pm 0.3 \mathrm{mg} /$ kg.min ${ }^{*}$, HOMA-IR $\left.=7.4 \pm 1.7^{*}\right)$ and eight insulin sensitive (IS) baboons $(19 \pm 2$ y, BMI $=22 \pm 1, \% B F=4.5 \pm 0.2, F P G=97 \pm 8, R d=11 \pm 1 \mathrm{mg} / \mathrm{kg} \cdot \mathrm{min}$, HOMAIR $=2.0 \pm 0.8,{ }^{*}=$ all $\mathrm{p}<0.05$ between groups) were studied. After an overnight fast, a $60 \mathrm{mU} / \mathrm{m} 2$.min euglycemic insulin clamp was performed under general anesthesia. Prior to insulin, a baseline needle liver biopsy was performed, and repeated at 30 and $120 \mathrm{~min}$ after insulin. Insulin sensitivity was calculated as the mean rate of glucose uptake (Rd) from 90 to 120 min during the clamp. Insulin signaling cascade molecules including: phosphorylation ( $\mathrm{p}$ ) of insulin 
receptor (Ins-R) at Tyr1146, Tyr1150/51 and Tyr1345, total IRS-1 and pIRS-1 (Ser612), total Akt, and pAkt (Thr308 and Ser473) were analyzed by ELISA in liver homogenates. To quantify insulin-stimulated phosphorylation, the delta between basal and 30 and 120 min after insulin stimulation were calculated. Differences between groups at different time points were analyzed by ANOVA and Pearson correlation was used to estimate the relationship between different metabolic parameters and insulin-stimulated signaling.

Results: In both IR and IS baboons hepatic pInsR-Tyr1146 increased significantly $(\mathrm{p}<0.05)$ from baseline with a maximal response at $30 \mathrm{~min}$; pInsRTyr1150 maximally increased at $120 \mathrm{~min}$ in both groups, $(\mathrm{p}<0.05 \mathrm{vs}$. baseline $)$ although to a lesser extent in the IR group. For pInsR-Tyr1345 the maximal response occurred at $120 \mathrm{~min}$ ( $\mathrm{p}<0.001 \mathrm{vs.} \mathrm{baseline)} \mathrm{similarly} \mathrm{in} \mathrm{IR} \mathrm{and} \mathrm{IS}$ baboons. There was no difference in total IRS-1 or pIRS-1 (Ser612) between groups. Aktl protein levels were significantly higher in the IR group at baseline $(\mathrm{p}<0.05)$, and the stimulation of Akt phosphorylation (pAkt1/Akt1) was impaired in IR vs. IS $(\mathrm{p}<0.05)$. pAkt-ser473 increased significantly after insulin with a peak at $30 \mathrm{~min}$ ( $\mathrm{p}<0.001$ in both groups), but the response was markedly reduced in the IR vs IS group $(25 \%, \mathrm{p}=0.02)$. pAkt1-Thr308 was maximally expressed at $120 \mathrm{~min}$ in both groups ( $\mathrm{p}<0.01 \mathrm{vs}$. basal), but in the IR group was $40 \%$ lower $(p=0.02)$. BMI was correlated directly with insulin receptor phosphorylation $(\mathrm{r}=0.60, \mathrm{p}=0.03)$ and inversely with pAkt $(\mathrm{r}=-0.65$, $\mathrm{p}=0.01)$.

Conclusion: The signaling defects in liver from obese insulin resistant baboon are characterized by modest impairment at the insulin receptor tyrosine phosphorylation, and a severe defect in pAkt. These abnormalities are tightly related to BMI and insulin resistance. Because of the strong genetic similarity between humans and baboons study of the baboon provides an important model of hepatic insulin resistance in man.

\section{4}

Effects of high fat diet on metabolic parameters in ghrelin receptor (GHS-R) knockout mice

K. Dahl, K. Raun, T. Bödvarsdottir, K. Juhl, F. Strauss, L. Kristensen, C. Nilsson

GLP1 and Obesity Pharmacology, Novo Nordisk, Måløv, Denmark

Background and aims: Ghrelin is the endogenous ligand of the ghrelin receptor (GHS-R) and is the only known orexigenic gut hormone. For this reason ghrelin has been the focus of intensive research within the therapeutic areas of appetite regulation and obesity treatment. The aim of this study was to examine if abolished ghrelin action through the GHS-R is affecting standard metabolic parameters in mice fed a high fat diet.

Materials and methods: Fifteen female and 12 male C57Bl/J6 GHS-R knockout mice (GHS-R KO), and 10 female and seven male wild type C57Bl/J6 mice (WT) were subjected to an oral glucose tolerance test (oGTT). After the initial oGTT, mice were fed a high fat (HF) diet for seven months, where after the mice were subjected to a second oGTT. Body weight was followed and body composition (NMR scan) was performed after seven months of high fat diet.

Results: The initial oGTT showed no significant difference between WT and GHS-R KO mice in blood glucose area under the curve (AUC), neither for female (GHS-R KO:263 $\pm 51 \mathrm{mM}^{*}$ min, WT:235 $\pm 25 \mathrm{mM}^{\star}$ min) nor male mice (GHS-R KO:276 $\pm 36 \mathrm{mM}^{*} \min , \mathrm{WT}: 190 \pm 35 \mathrm{mM}^{*} \mathrm{~min}$ ). After seven months of $\mathrm{HF}$ diet there were still no significant difference in glucose tolerance between female GHS-R KO mice $\left(420 \pm 48 \mathrm{mM}^{*} \mathrm{~min}\right)$ and WT mice $\left(307 \pm 27 \mathrm{mM}^{*} \mathrm{~min}\right)$. A significant effect $(\mathrm{p}<0.05)$ was seen for glucose tolerance in female GHS-R KO before HF diet as compared to after HF diet. This was not seen for female WT mice. After HF diet, male GHS-R KO mice exhibited a significantly impaired glucose tolerance as compared to male WT mice (GHS-R KO: $812 \pm 92$ $\mathrm{mM}^{\star}$ min vs. WT: $\left.399 \pm 29 \mathrm{mM}^{\star} \mathrm{min}, \mathrm{p}<0.001\right)$. There was also significantly reduced glucose tolerance after HF diet in male GHS-R KO mice when comparing with before HF diet $(\mathrm{p}<0.001)$ and this reduction was not seen in WT mice after HF diet. Basal body weight, i.e. before HF diet, did not differ between GHS-R KO and WT mice, neither for females (GHS-R KO:20.5 \pm 0.4 g vs. WT:21.7 \pm 0.3 ) nor males (GHS-R KO:30.0 \pm 0.6 g vs. WT:29.2 $\pm 0.6 \mathrm{~g}$ ). After HF diet, all mice had a significantly increased body weight as compared to before HF diet $(\mathrm{p}<0.001)$. The impact of age can with the present study design not be separated from the impact of HF diet. There were no significant effect on body weight after HF diet between male GHS-R KO mice $(44.2 \pm 1.5$ g) and male WT mice (48.2 $\pm 2.1 \mathrm{~g})$. In contrast, female GHS-R KO exhibited a significantly $(\mathrm{p}<0.001)$ lower body weight after HF diet $(40.9 \pm 1.9 \mathrm{~g})$ than female WT mice $(50.6 \pm 1.5 \mathrm{~g})$. The reduction in body weight was in female GHS-R KO accompanied by a significant reduction $(\mathrm{p}<0.01)$ in body fat as compared to WT mice (GHS-R ko:19.5 $\pm 2.4 \mathrm{~g}$, WT:29.3 $\pm 1.5 \mathrm{~g}$ ). The amount of body fat was not significantly different for male GHS-R KO mice $(16.2 \pm 1.0$ g) as compared to male WT mice (18.4 $\pm 1.1 \mathrm{~g})$.

Conclusion: In conclusion, there were no differences in body weight and glucose tolerance between young GHS-R KO mice and WT mice exposed to normal diet. However, after exposure to seven months of HF feeding, male GHS-R KO mice exhibit a reduction in glucose tolerance compared to WT mice, whereas deletion of the GHS-R did not have any impact on glucose tolerance after HF feeding in female mice. In contrast, deficiency of ghrelin action through the GHS-R, resulted in female mice being less prone to develop diet induced obesity than wild type mice, which was not seen in male GHS-R $\mathrm{KO}$ mice. Results from this study indicate a gender specific influence of the metabolic actions of ghrelin via the GHS-R in mice.

\section{5}

Chemical derivatives of DHA in the treatment of obesity and glucose intolerance associated with high-fat feeding in mice

M. Rossmeisl ${ }^{1}$, Z. Jilkova ${ }^{1}$, T. Jelenik ${ }^{1}$, M. Hensler ${ }^{1}$, V. Mohamed-Ali ${ }^{2}$, M. Bryhn ${ }^{3}$, K. Berge ${ }^{3}$, A.K. Holmeide ${ }^{3}$, J. Kopecky ${ }^{1}$

${ }^{1}$ Dept. of Adipose Tissue Biology, Institute of Physiology, Prague, Czech Republic, ${ }^{2}$ Adipokines and Metabolism Research Group, Dept. of Medicine, University College London, United Kingdom, ${ }^{3}$ Pronova BioPharma AS, Lysaker, Norway

Background and aims: $n-3$ polyunsaturated fatty acids, especially eicosapentaenoic acid and docosahexaenoic acid (DHA), exert hypolipidemic effects and prevent development of obesity and insulin resistance in animals fed high-fat diets. We sought to determine whether $\alpha$-substituted DHA-derivatives could exert a higher efficacy as anti-obesity and anti-diabetic agents, compared to DHA.

Materials and methods: $\mathrm{C} 57 \mathrm{BL} / 6$ mice were given a corn oil-based high fat (35\% weight/weight) diet (cHF), or cHF with $1.5 \%$ of lipids replaced with $\alpha$-methyl DHA ethyl ester (Substance 1), $\alpha$-ethyl DHA ethyl ester (Substance 2), $\alpha, \alpha$-di-methyl DHA ethyl ester (Substance 3), or $\alpha$-thioethyl DHA ethyl ester (Substance 4) for 4 months. We also investigated whether Substance 2 could reverse glucose intolerance in obese cHF-fed mice. Plasma markers of glucose and lipid metabolism, glucose tolerance, tissue morphology, lipid content and gene regulation were characterized.

Results: cHF induced obesity, hyperlipidemia, impairment of glucose homeostasis and adipose tissue inflammation. Except Substance 3, all other substances prevented weight gain and Substance 2 exerted the strongest effect (63\% of cHF-controls). Glucose intolerance (area under the curve) was significantly prevented ( 67\% of cHF) by both Substance 1 and Substance 2 . Moreover, Substance 2 lowered fasting glycemia, plasma insulin, triacylglycerols, and nonesterified fatty acids $(73,9,47$, and $81 \%$ of cHF-controls, respectively). Substance 2 reduced accumulation of lipids in the liver and skeletal muscle, as well as adipose tissue inflammation associated with obesity. It also reduced obesity and reverted glucose intolerance in the obese mice. Conclusion: Substance 2 represents a novel compound with a promising potential in the treatment of obesity and associated metabolic disturbances. Supported by: Czech Science Foundation, Pronova BioPharma AS

\section{6}

Antiobesity and antidiabetic effects of the melanocortin receptor modulating agent AP1030 in selective breed DIO rats (LR rat) T.E.N. Jonassen ${ }^{1}$, A. Artmann ${ }^{2}$, K.S. Madsen ${ }^{1}$, M. Tang-Christensen ${ }^{2}$ ${ }^{1}$ Action Pharma A/S, Holte, Denmark, ${ }^{2}$ Rheoscience A/S, Rødovre, Denmark

Background: AP1030, the lead compound within a novel group of phenyl pyrrol aminoguanidines (PPAGs) with melanocortin (MCr) type 1 and 4 receptor modulating effects is an orally available small molecule intended for once daily treatment. After single dose administration to normal SpragueDawley (SD) rats AP1030 dose dependently reduced food intake without concomitant effects on locomotor activity or overt sign of malaise.

Aim and methods: The aim of the present study was to examine the effects of 33 days administration of AP1030 (25 mg/kg po) on feeding and metabolic parameters in male selectively breed SD, diet-induced obesity (DIO) rats (LRrat) (18 weeks of age) on a high fat diet ( $4.41 \mathrm{kcal} / \mathrm{g}$ - Energy \%: Carbohydrate $51.4 \%$, Fat $31.8 \%$, Protein $16.8 \%$ ). Rats treated with vehicle (Veh) (20\% PEG 200) were used as controls and Sibutramine $(5 \mathrm{mg} / \mathrm{kg}$ po) treated rats were used as positive control. $\mathrm{N}=10$ in all groups (all values given as mean $\pm \mathrm{SE}$ ). 
Results: Food intake at the end of the treatment period as \% of baseline levels were as follows: Veh: $96.8 \pm 7.6 \%$; AP1030: $85.0 \pm 17.1 \%$, p $<0.01$ vs Veh; Sibutramine: $84.3 \pm 16.4 \%, \mathrm{p}<0.01$ vs Veh. Furthermore, AP1030 reduced weight gain with $\sim 10 \%$ BW from baseline (day 0) and witha $~ 15 \%$ reduction when compared to vehicle treated animals (Veh: 105.9 $\pm 2.2 \%$; AP1030: 90.4 $\pm 5.5 \%$, $\mathrm{p}<0.01$ ) (Sibutramine: $92.8 \pm 2.3 \%)$. In AP1030 animals the changes in body fat was associated with a $\sim 50 \%$ reduction in retroperitoneal and mesenteric fat depots in comparison to vehicle treated and by $~ 25 \%$ reduction in comparison to sibutramide treated rats. After 28 days of treatment with AP1030 fasting levels of glucose, insulin and cholesterol were reduced significantly in comparison to vehicle treated animals (Glucose: Veh: $7.09 \pm 0.62 \mathrm{mM}$; AP1030: $6.42 \pm 0.85 \mathrm{mM}, \mathrm{p}<0.01$ ), (Insulin: Veh: $544.8 \pm 137.6 \mathrm{pM}$; AP1030: $375.5 \pm 83.7 \mathrm{pM}, \mathrm{p}<0.01$ ), (Cholesterol: Veh: $2.72 \pm 0.82 \mathrm{mM}$; AP1030: 1.29 $\pm 0.25 \mathrm{mM}, \mathrm{p}<0.01$ ). Finally, an oral glucose tolerance test (OGTT) was conducted at study day 28. Both AP1030 and sibutramine had beneficial effects on glucose metabolism: Peak glucose $(\mathrm{t}=15 \mathrm{~min})$ : Veh: $12.03 \pm 2.02 \mathrm{mM}$; AP1030: $9.79 \pm 1.15 \mathrm{mM}, \mathrm{p}<0.01 \mathrm{vs}$ Veh; Sibutramine: $11.05 \pm 1.07 \mathrm{mM}$, NS vs Veh; Peak Insulin ( $\mathrm{t}=15 \mathrm{~min}$ ): Veh: $1063 \pm 437 \mathrm{pM}$; AP1030: 429 $\pm 212 \mathrm{pM}$, $\mathrm{p}<0.01$ vs Veh; Sibutramine: $492 \pm 207 \mathrm{pM}, \mathrm{p}<0.01$ vs Veh; Insulin sensitivity

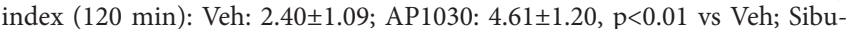
tramine: $4.44 \pm 1.65, \mathrm{p}<0.01$ vs Veh.

Conclusion: In conclusion, these results show that once daily treatment with the novel melanocortin effect AP1030 in DIO rats exerts marked antiobesity and antidiabetic effects a strong body weigth and glucose homeostasis and may prove to be feasible therapeutic route for the treatment of obesity and type 2 diabetes and related metabolic disorders.

\section{7}

Short-term high-fat feeding induces insulin resistance that is independent of adipose tissue macrophage infiltration and prevented by osteopontin deficiency

T.M. Stulnig ${ }^{1}$, F.W. Kiefer ${ }^{1}$, K. Gollinger ${ }^{1}$, J. Todoric ${ }^{1}$, T. Weichhart ${ }^{2}$, R. Geyeregger ${ }^{1}$, M. Zeyda ${ }^{1}$

${ }^{1}$ Dept. Internal Medicine III, Medical University Vienna, Clin. Division of Endocrinology and Metabolism, ${ }^{2}$ Dept. Internal Medicine III, Medical University Vienna, Clin. Division of Nephrology and Dialysis, Vienna, Austria

Background and aims: Obesity is associated with a state of chronic low-grade inflammation which is tightly linked to insulin resistance and type 2 diabetes and is associated with adipose tissue (AT) infiltration by macrophages. Osteopontin (OPN) is an inflammatory cytokine, the expression of which is strongly upregulated in AT during obesity. OPN was suggested to contribute to obesity-induced AT inflammation by promoting AT macrophage (ATM) accumulation. In the present study we examined effects of short-term (3 weeks) high-fat diet (HF) feeding on AT inflammation and insulin sensitivity in wild-type (WT) and OPN-/- mice.

Materials and methods: OPN-/- and WT control mice were placed for 3 weeks on HF and low-fat diet (LF). At the end of the feeding period an insulin tolerance test was performed. After sacrifice, blood plasma was collected and gonadal white AT was removed for immunological and gene expression analyses.

Results: Despite the absence of obesity and ATM accumulation by shortterm HF, insulin sensitivity was reduced in both genotypes compared with $\mathrm{LF}(\mathrm{P}=0.01$ for WT and OPN-/-, respectively), indicating an early onset of insulin resistance independent of ATM infiltration. However, deterioration of insulin sensitivity was significantly less in HF-fed OPN-/- mice compared with WT animals $(\mathrm{P}=0.03)$. Short-term feeding of HF was sufficient to enhance gene expression of inflammatory cytokines in AT of WT and OPN-/mice. However, gene expression of TNF- $\alpha$, MCP- 1 and iNOS markedly less increased in OPN-/- compared with WT animals indicating a role of OPN in HF-induced inflammatory alterations. Moreover, mRNA expression of insulin-sensitizing adiponectin was significantly higher in OPN-/- vs. WT mice $(\mathrm{P}=0.04)$ fed HF.

Conclusion: These data strongly indicate that short-term HF feeding impairs insulin sensitivity independent of ATM infiltration. Notably, the beneficial effects of OPN deficiency on short-term HF-induced insulin resistance could be mediated by interference with AT inflammation independent of ATM accumulation.

Supported by: Austrian Science Fund

\section{8}

Induction of muscle thermogenesis by high-fat diet in mice: association with obesity-resistance

J. Kopecky ${ }^{1}$, V. Kus ${ }^{1}$, T. Prazak ${ }^{1}$, P. Brauner ${ }^{1}$, M. Hensler' ${ }^{1}$ O. Kuda ${ }^{1}$, P. Flachs ${ }^{1}$, P. Janovska ${ }^{1}$, D. Medrikova ${ }^{1}$, M. Rossmeis ${ }^{1}$, Z. Jilkova ${ }^{1}$, B. Stefl ${ }^{2}$ ${ }^{1}$ Department of adipose tissue biology, Institute of Physiology, Academy of Sciences of the Czech Republic, ${ }^{2}$ Department od Physiology and Developmental Biology, Charles University, Prague, Czech Republic

Background and aims: Obesogenic effect of high-fat (HF) diet is counterbalanced by stimulation of energy expenditure and lipid oxidation in response to the meal. Aim of this study was to reveal whether muscle nonshivering thermogenesis could be stimulated by HF diet, especially in obesity-resistant $\mathrm{A} / \mathrm{J}$ as compared with obesity-prone $\mathrm{C} 57 \mathrm{BL} / 6 \mathrm{~J}(\mathrm{~B} / 6 \mathrm{~J})$ mice.

Materials and methods: Experiments were performed on male mice born and maintained at $30{ }^{\circ} \mathrm{C}$. Four-week-old mice were randomly weaned onto a lowfat (LF) or HF ( $60 \%$ calories from fat, mainly corn oil) diet for 2 weeks. Energy expenditure was evaluated using indirect calorimetry system (system INCA, Somedic, Horby, Sweden). Oxygen consumption in soleus or gastrocnemius muscle was followed ex vivo, using Oroboros Oxygraph (Paar KG, Graz, Austria). AMP-activated protein kinase (AMPK) activity was characterised using Western blots and antibodies against total and phosphorylated form of AMPK. Fatty acid oxidation in muscles was evaluated ex vivo using $\left[{ }^{14} \mathrm{C}(\mathrm{U})\right]$-palmitate. Plasma leptin was assessed by a Mouse Leptin RIA Kit (LINCO Research, St. Charles, MO, USA). Gene expression in muscles was quantified using qRT-PCR.

Results: In the A/J LF mice, cold exposure $\left(4^{\circ} \mathrm{C}\right)$ resulted in hypothermia, while the A/J HF, B/6J LF and B/6J HF mice were cold-tolerant. Cold-sensitivity of the A/J LF mice was associated with a relatively low whole-body energy expenditure under resting conditions, which was normalized by HF diet ( $35.6 \pm 2.6$ vs $49.2 \pm 1.9 \mathrm{ml}$ oxygen $/ \mathrm{kg} / \mathrm{min}$; measured at $30{ }^{\circ} \mathrm{C}$ during the light phase of the day; $p<0.05)$. In both strains, HF diet induced uncoupling protein 1-mediated thermogenesis (1.6- and 1.3-fold, in $\mathrm{A} / \mathrm{J}$ and $\mathrm{B} / 6 \mathrm{~J}$, respectively; measured as norepinephrine-induced respiratory rate in pentobarbital-anaesthetized mice using the INCA) in A/J mice. Only in A/J mice: (i) HF diet augmented activation of whole body lipid oxidation in cold (as revealed by indirect calorimentry); and (ii) at $30^{\circ} \mathrm{C}$, oxygen consumption in soleus muscle was increased by HF diet ( $145 \pm 27$ vs $255 \pm 30 \mathrm{ml}$ oxygen/mg protein/initial $5 \mathrm{~min} ; p<0.05$ ), as well as total content and phosphorylation of AMPK, and AICAR-stimulated palmitate oxidation. Only in A/J mice, HF diet increased leptinemia compared with the LF diet-fed mice ( $3.35 \pm 0.34$ vs $9.42 \pm 1.15 \mathrm{ng}$ leptin $/ \mathrm{ml} ; p<0.05)$. Gene expression data in soleus muscle of the A/J HF mice indicated a shift from carbohydrate to fatty acid oxidation. Conclusion: Our results suggest a role of muscle nonshivering thermogenesis and lipid oxidation in the obesity-resistant phenotype of $\mathrm{A} / \mathrm{J}$ mice and indicate that dietary fat could induce thermogenesis in oxidative muscle, possibly by the leptin-AMPK axis.

\section{9}

A central role for PI3K $\gamma$ in obesity and insulin resistance

G. Solinas ${ }^{1}$, B. Becattini ${ }^{1}$, F. Zani ${ }^{1}$, A.G. Dulloo ${ }^{1}$, R. Marone ${ }^{2}$,

M.P. Wymann ${ }^{2}$

${ }^{1}$ Medicine, Division of Physiology, University of Fribourg, ${ }^{2}$ Inst.

Biochemistry \& Genetics, Department of Biomedicine, University of Basel, Switzerland

Background and aims: Obesity is associated with a state of chronic lowgrade inflammation (metabolic inflammation) that is believed to play an important role in the development of insulin resistance. Metabolic inflammation is characterized by macrophage (MØ) infiltration into obese adipose tissue. MØs localize around dead adipocytes forming crown-like structures (CLS). The mechanisms leading to CLS formation are currently under investigation. Recent studies have identified an important role for the chemokine $\mathrm{MCP} 1$ and its receptor CCR2 in metabolic inflammation. Furthermore mice bearing a targeted mutation at the ccr2 gene show decreased food intake and weight gain when placed in high fat diet. Interestingly, both MCP1 and CCR2 were implicated in the formation of atherosclerotic lesions, suggesting that CLS formation might share some molecular mechanisms with atherosclerotic plaque formation. The lipid kinase phosphoinositide 3-kinase $\gamma(\mathrm{PI} 3 \mathrm{~K} \gamma)$ has previously been shown to be central in chemotaxis in the response of myeloid cells to different chemoactractant, including MCP1. Recently, a role for $\mathrm{PI} 3 \mathrm{~K} \gamma$ has been defined in atherosclerosis. In this study we investigated the role of PI3K $\gamma$ in diet-induced obesity and insulin resistance. 
Materials and methods: To study the role of PI3K $\gamma$ in diet-induced obesity and insulin resistance C57BL/6J mice (wt) and mice with a targeted Pi3k $\gamma$ locus (Pi3k $\gamma$-/-) were exposed to a high-fat diet ( $60 \%$ of calories from fat) for 16 weeks. Body weight was measured weekly to determine growth curves. Precise measurements of food intake was performed for 7 weeks, and lipid content of feces was determined by phenol-chloroform extraction. To test the role of PI $3 \mathrm{~K} \gamma$ in glucose homeostasis and insulin sensitivity we performed glucose and insulin tolerance tests.

Results: When placed on chow diet, no significant difference was observed between wt and Pi3k $-/$ - mice. When fed with high-fat diet, however, Pi3k $\gamma$ /- mice were resistant to obesity. The quantification of food intake did not reveal a significant difference between wt animals and Pi3k $\gamma$-/- mice. Hence, the obesity-resistant phenotype of Pi3k $\gamma$-/- mice is not due to decreased food intake. Analysis of fecal chloroform/methanol extracts also revealed no difference among the two groups, indicating that the leaner phenotype of Pi3k $\mathrm{\gamma}$ /- mice observed on high fat diet was not due to reduced lipid adsorption. Our data illustrate that Pi3k $\gamma$-/- mice are resistant to diet-induced obesity because of decreased efficiency of weigh-gain per food intake. Interestingly, glucose tolerance was also improved in Pi3k $\gamma$-/- mice placed on high fat diet for 16 weeks compared to Wt controls. Insulin tolerance was markedly improved in Pi3k $\gamma-/$ - animals versus wt mice, suggesting that the improved glucose tolerance might be due to increased insulin sensitivity.

Conclusion: In conclusion, we demonstrate here for the first time that the enzyme PI $3 \mathrm{~K} \gamma$, a pro-inflammatory kinase specifically expressed in cardiomyocytes and hematopoyetic cells, is directly involved in diet-induced obesity and glucose intolerance.

Supported by: Swiss National Science Foundation and Roche Research Foundation

\section{0}

Thyroid transcription factor-1 mediates leptin signalling pathway in the hypothalamus

I. Nam-Goong, J. Kim, E. Kim, Y. Kim

Internal Medicine, Ulsan University Hospital, Ulsan, Republic of Korea

Background and aims: Thyroid transcription factor-1 (TTF-1) is a member of the NKx family of homeodomain genes. It has been known to play an important role in the control of neuroendocrine functions by our research group. We have recently reported a novel role of TTF-1 in the regulation of feeding behavior of rats. Leptin is an adipose tissue-derived hormone that suppresses appetite by regulating activities of satiety center in the brain. In this study, we determined TTF-1 function on the leptin-induced control of feeding behavior.

Materials and methods: For the experiment of intracerebroventricular (i.c.v.) administration of leptin $(5 \mu \mathrm{g})$, we used two month-old male SpragueDawley rats with 5 animals of each group, polyethylene cannula was stereotaxically implanted into the lateral ventricle of rat. For immunohistochemical analysis, coronal sections of rat brain were hybridized with TTF-1 and STAT3 antibody.

Results: Administration of leptin via i.c.v. significantly decreased amount of food intake ( $10.1 \pm 0.8$ versus $7.4 \pm 1.2$ gram for 4 hour food intake, $\mathrm{p}<0.05$ ) and increased hypothalamic content of signal transducer and activator of transcription 3 (STAT3) $(182 \pm 22$ percent of control, $\mathrm{p}<0.001)$ that is well known to mediate leptin signaling pathway. The same treatment markedly reduced TTF-1 expression in the hypothalamus $(33 \pm 5$ percent of control, $\mathrm{p}<$ $0.001)$. Immunohistochemical analysis showed that TTF-1 is co-localized in the STAT3-positive cells in the arcuate nucleus. TTF-1 physically interacted with STAT3 in the in vivo hypothalamic extracts as well as in vitro cellular extracts from the B-35 cells transfected with TTF-1 and STAT3 expression vectors. TTF-1 attenuated STAT3-induced increase of proopiomelanocortin and decrease of agouti-related protein transcription (1080 \pm 110 percent of control and $54 \pm 2$ percent of control, respectively).

Conclusion: These data suggest that TTF-1 is involved in the regulation of central feeding behavior via leptin signaling pathway.

\section{PS 059 Ectopic fat: liver and pancreas}

\section{1}

Liver fat and type 2 diabetes: specific roles of diabetes and overweight L. Bozzetto ${ }^{1}$, G. Annuzzi ${ }^{1}$, A. Prinster ${ }^{2}$, M. Mancini ${ }^{2}$, R. Giacco ${ }^{3}$, C. De Natale ${ }^{1}$, M. Salvatore ${ }^{2}$, G. Riccardi ${ }^{1}$, A.A. Rivellese ${ }^{1}$

${ }^{1}$ Department of Clinical and Experimental Medicine, Federico II University, Naples, ${ }^{2}$ Department of Radiology, Federico II University, Naples, ${ }^{3}$ Institute of food sciences and technology, National Council of Research, Avellino, Italy

Background and aims: Fat accumulation in the liver is a common feature of conditions associated with insulin-resistance such as obesity and type 2 diabetes. Liver fat is often related to an increased abdominal visceral fat content. Nevertheless, the specific roles of insulin-resistance, obesity, and diabetes in relation to liver steatosis have not been clearly defined. To elucidate this issue hepatic fat content was determined in obese patients with and without type 2 diabetes and in normal weight subjects.

Materials and methods: Eleven diabetic obese subjects (DO), 9 non diabetic obese subjects (NDO), and 9 normal weight controls (C) were studied. All participants were male, aged 28-63 years, and with normal plasma triglyceride levels. Obese subjects with and without diabetes were matched for age, BMI and waist circumference. Diabetic patients were in good glycaemic control $(\mathrm{HbAlc}=6,8 \pm 0,7 \%)(\mathrm{M} \pm \mathrm{SD})$ under treatment with diet alone $(\mathrm{n}=7)$ or metformin $(n=4)$. Anthropometric parameters were measured by standardized methodologies, liver fat content by ${ }^{1} \mathrm{H}$-magnetic resonance (NMR) spectroscopy, abdominal fat distribution by NMR, and insulin sensitivity by hyperinsulinemic euglycaemic clamp (M/I: glucose infusion rate/plasma insulin concentration).

Results: DO had more liver fat $(13.0 \pm 7.3 \%)$ than NDO $(5.2 \pm 2.8 \%$, $\mathrm{p}<0.05)$ and $\mathrm{C}(2.0 \pm 2.4 \%, \mathrm{p}<0.001)$. Abdominal fat content was higher both in DO and NDO compared with the lean normoglycaemic controls (visceral: DO $3184 \pm 843$, NDO $2843 \pm 1378$, C $1348 \pm 742 \mathrm{~cm}^{3}, \mathrm{p}<0.001$; subcutaneous: DO $4029 \pm 362$, NDO $5197 \pm 1398$, C $1590 \pm 504 \mathrm{~cm}^{3}, \mathrm{p}<0.001$ ), being visceral fat not significantly different between the two obese groups, and subcutaneous fat significantly less in DO than in NDO $(\mathrm{p}<0.05)$. Whole-body insulin-sensitivity $(\mathrm{M} / \mathrm{I})$ was similar in $\mathrm{DO}(2.4 \pm 1.1)$ and $\mathrm{NDO}(1.8 \pm 0.8)$ in respect to controls $(6.5 \pm 2.9, \mathrm{p}<0.001)$

Conclusion: In type 2 diabetic patients with visceral adiposity and impaired insulin sensitivity, hyperglycemia plays an independent and additional role in the development of fat liver accumulation. It is likely that in diabetic obese patients increased liver fat content is part of a more general alteration of regional fat distribution, rather than the consequence of a simple increase of abdominal visceral fat.

\section{2}

Induction of the lipogenic pathway through ChREBP overexpression leads to hepatic steatosis without insulin resistance

C. Robichon, P.-D. Denechaud, F. Benhamed, M. Moldes, V. Fauveau, J. Girard, C. Postic

Endocrinologie, Métabolisme et Cancer, Institut Cochin, Paris, France

Background and aims: Nonalcoholic hepatic steatosis is an excessive accumulation of triglycerides (TG) in liver and is part of a large spectrum of hepatic disorders called NAFLD (Nonalcoholic fatty liver disease). Hepatic steatosis is now considered as the liver component of the metabolic syndrome characterized by obesity, insulin resistance, dyslipidemia and hypertension. Fatty acids utilized for hepatic TG synthesis are available from the plasma non-esterified fatty acid pool or from fatty acids newly synthesized from glucose through de novo lipogenesis. Induction of lipogenic genes is under the concerted action of transcription factors Carbohydrate Responsive Element Binding Protein (ChREBP) and Sterol Regulatory Element Binding Protein-1c (SREBP-1c) in response to glucose and insulin, respectively. Despite the emergence of ChREBP in the control of lipid synthesis, its specific contribution to hepatic lipogenesis remains to be clearly established. Therefore, the aims of this study were to determine: 1) whether the overexpression of ChREBP in liver is sufficient to stimulate lipogenesis and 2) if excessive lipogenic rates can lead to the development of hepatic steatosis and/or insulin resistance.

Materials and methods: Physiological and metabolic consequences of adenoviral ChREBP overexpression were studied 10 days (short-term) and 30 
days (long-term) after a single injection in C57BL6/J mice. Gene expression was analyzed by real time quantitative polymerase chain reaction and western blot. Insulin and glucose tolerance tests were performed to evaluate the general insulin sensitivity of mice. Moreover activation of the hepatic insulinsignaling pathway after a 3 minutes insulin pulse in the portal vein was studied. Oil red-O staining and lipid species quantification by high performance liquid and gas chromatography were performed to study the development of hepatic steatosis.

Results: Short-term and long-term overexpression of ChREBP in liver induced glycolytic (L-PK) and lipogenic (ACC, FAS, SCD1) gene expression without any induction of SREBP-1c. Under long-term ChREBP overexpression conditions, oil red-O staining of hepatic cryosections showed an important lipid accumulation confirmed by lipidomic analysis. Interestingly, in spite of a three-fold increase in hepatic TG concentrations, mice overexpressing ChREBP in liver for 30 days did not develop insulin resistance as revealed by normal insulin tolerance and by the adequate phosphorylation of key effectors of the insulin-signaling pathway.

Conclusion: Our results show that stimulation of de novo hepatic lipogenesis through ChREBP overexpression is sufficient to induce hepatic steatosis but that intra-hepatic lipid composition and/or accumulation in this mouse model is not associated with insulin resistance.

Supported by: INSERM, ANR and ALFEDIAM

\section{3}

The relationship between reactive oxygen species, insulin resistance and nonalcoholic fatty liver disease. A study using in vitro model

K.A. Lockman ${ }^{1}$, C. Filippi' ${ }^{2}$, J. Baren ${ }^{3}$, C. Pembleton ${ }^{3}$, F. Howie ${ }^{4}$, P. Lee ${ }^{3}$, P. Cowan ${ }^{3}$, A. Pryde ${ }^{3}$, A. Jaap ${ }^{1}$, P.C. Hayes ${ }^{3}$, J.N. Plevris ${ }^{3}$

${ }^{1}$ Department of Diabetes, Royal Infirmary of Edinburgh, ${ }^{2}$ Scottish Centre of Regenerative Medicine, University of Edinburgh, ${ }^{3}$ Department of Hepatology, University of Edinburgh, ${ }^{4}$ Department of Biochemistry, University of Edinburgh, United Kingdom

Background and aims: Insulin resistance plays a vital role in the pathogenesis of nonalcoholic fatty liver disease (NAFLD). However, currently available human in vitro models of steatotic hepatocytes are yet to demonstrate evidence of insulin resistance. Indeed, it has previously shown that cell exposure to oleic acid increases insulin sensitivity despite extensive steatosis. The aim of this study is to develop an in vitro model of NAFLD that demonstrate evidence of insulin resistance. Our hypothesis is that the presence of high mitochondrial reactive oxygen species (ROS) is essential for the development of in vitro model that would exhibit insulin resistance.

Materials and methods: Human C3A cell line, a clonal derivative HepG2 cell line, was selected for its better differentiated hepatic phenotype. Cells were cultured for 3 days with various combination of; Lactate (L), Pyruvate (P), Octanoate $(\mathrm{O})$ and Ammonia $(\mathrm{N})$ and Oleic acid $(\mathrm{Ol})$. ROS were detected using MitoSox Red and quantified using fluorescence activated cell sorter. Triglyceride concentrations were measured using enzymatic assay. Mitochondrial function was assessed by microscopy using fluorescent probe JC-1. Insulin sensitivity was assessed by measuring endogenous glucose production (EGP) and glycogen breakdown in the presence of increasing insulin concentrations (0-10nM). Glucose, lactate and pyruvate production were measured according to Bergmeyer methods. Cell viability was assessed using propidium iodide staining.

Results: All preconditioning media induced intracellular fat accumulation but to different degree as demonstrated by triglyceride concentrations $(\mathrm{Ol}>\mathrm{L}$ PON $>$ LPO $>$ OCT $>$ LP $>$ CONTROL; $p<0.001, n=3$ ).

The different preconditioning groups showed various ROS synthesis unrelated to the degree of fat accumulation (LPON $>\mathrm{LPO}>\mathrm{OCT}>\mathrm{LP}>\mathrm{CONTROL}$ $>\mathrm{Ol}$ ) with highest peak fluorescent intensity in LPON (LPON (mean \pm SEM) $49.67 \pm 3.38$ vs control $20.33 \pm 6.43, p<0.01$; LPON $49.67 \pm 3.38$ vs Ol $12.33 \pm$ $4.37, \mathrm{p}<0.0025 ; \mathrm{n}=3$ ). Insulin resistance was most prominent in LPON whilst OCT, like Ol, showed insulin sensitivity. In the absence of insulin, glycogen breakdown and EGP were highest in OCT but diminished with the addition of insulin in dose dependent pattern whilst EGP and glycogen breakdown increase with insulin in LPON (Preconditioning effect on EGP: $p<0.0001$; and on glycogen breakdown: $\mathrm{p}<0.0001 ; \mathrm{n}=3$ ).

JC-1 study of LPON cells showed a decrease of mitochondrial membrane potential when compared with control. Preconditioning did not affect cellular viability in all groups $(\mathrm{p}=0.23)$

Conclusion: In addition to the intracellular lipid accumulation, LPON preconditioned cells produced significant amount of ROS, insulin resistance and mitochondrial dysfunction. Despite the presence of lipid accumulation in other groups, insulin sensitivity was preserved thus suggesting other factors including ROS may have an important role in the pathogenesis of hepatic insulin resistance. Therefore, we propose that LPON preconditioned cells would be a viable in vitro model to complement the currently available in vivo model of NAFLD.

\section{4}

Small, dense LDL in metabolic syndrome and fatty liver

T. Matsumoto, I. Sugino, S. Nakano, K. Iso, K. Kuboki, H. Ueshiba, G. Yoshino

Division of Diabetes, Metabolism and Endocrinology, Department of Internal Medicine, Toho University School of Medicine, Tokyo, Japan

Background and aims: Small,dense LDL is known as an atherogenic lipoprotein and is often associated with not only patients with coronary artery disease but also metabolic syndrome (MS) subjects. High frequency of small,dense LDL (sLDL) is found in hypertriglyceridemic subjects. Also, Fatty liver is also often associated with MS. Therefore, the present study was conducted to examine plasma sLDL-cholesterol (C) level in MS patients with and without fatty liver.

Materials and methods: Two-hundred and seventy-two MS patients and 154 non-MS healthy subjects were enrolled in this study. Fatty liver was estimated by echogram and classified according to the stage of fatty liver (none, mild, moderate and severe). Presence of MS was diagnosed according to the Japanese Guidelines for the Definition of Metabolic Syndrome. Plasma sLDL-C was measured by the 2-step (precipitation and homogeneous) assay system established by Hirano et al. Plasma triglyceride and choline-esterase (CE) were measured by a standard autoanalyzer method. A one-way analysis of variance (ANOVA) was used to compare mean values among groups. Stepwise multiple linear regression analysis were performed to evaluate the relationship between sLDL-C and other parameters. All values are expressed as mean \pm SD. A significant difference was defined as $p<0.05$.

Results: SLDL-C of MS group was higher than that of non-MS healthy group ( $38.5 \pm 18.0$ vs $20.6 \pm 6.3 \mathrm{mg} / \mathrm{dl}, \mathrm{p}<0.001)$. There was slight but significant correlation between sLDL-C and fatty liver stage $(r=0.37, p<0.05)$. On the other hand, there was a significant relationship between sLDL-C and plasma triglyceride $(\mathrm{r}=0.47, \mathrm{p}<0.01)$. This became stronger in fatty liver stage (mild, moderate and severe) group $(\mathrm{r}=0.53, \mathrm{p}<0.001)$. Furthermore, there was highly significant correlation between sLDL-C/LDL-C ratio and plasma triglyceride $(\mathrm{r}=0.70, \mathrm{p}<0.0001)$. There was a weak relationship between plasma $\mathrm{CE}$ and fatty liver stage $(r=0.42, p<0.05)$. Although a weak relationship was found between CE and SLDL-C, we failed to show a significant correlation between $\mathrm{CE}$ and sLDL-C/LDL-C ratio. Multiple regression analysis revealed that the most powerful determinant of plasma sLDL-C was triglyceride, followed by fatty liver stage and CE ( $\mathrm{p}=0.0002,0.014$ and 0.028 , respectively).

Conclusion: The liver in MS may produce triglyceride from excess circulating glucose, free fatty acid and triglyceride, which may result in development of fatty liver. In order to avoid further triglyceride deposition, fatty liver may secrete large, triglyceride-enriched VLDL particles, which is recognized as a precursor of sLDL particles. In the present study, we found a significant relationship between sLDL-C and plasma triglyceride. Furthermore, this became stronger in fatty liver group, indicating that fatty liver may produce triglyceride-rich VLDL, thereby contributing to an appearance of sLDL particles in plasma of MS patients with fatty liver. Since correlation coefficient between sLDL-C/LDL-C ratio and plasma triglyceride was higher than that between LDL-C and plasma triglyceride ( $\mathrm{r}=0.70 \mathrm{vs} \mathrm{r}=0.47$ ), plasma triglyceride metabolism may have more influence on LDL peak sizes (sLDL-C/LDL-C ratio) than absolute value of sLDL-C.

\section{5}

Fatty infiltration of pancreas influences beta cell function in Korean type 2 diabetic patients

J.-Y. Yoon, J.-O. Chung, D.-H. Cho, D.-J. Chung, M.-Y. Chung

Department of Endocrinology and Metabolism, Chonnam National University Medical School,South Korea, Gwangju, Republic of Korea

Background and aims: Type 2 diabetes is characterized by progressive $\beta$-cell dysfunction as well as insulin resistance. Chronic exposure of the islet cells to nonesterified fatty acids cause $\beta$-cell damage. Some experimental studies suggest that fatty infiltration of pancreas may result in a decrease in $\beta$-cell mass and dysfunction. The majority of patients with type 2 diabetes in Korea are nonobese and $\beta$-cell dysfunction may be one of predominant features. We 
studied whether fatty infiltration of pancreas is associated with $\beta$-cell dysfunction in type 2 diabetic patients.

Materials and methods: Fifty-six type 2 diabetic patients (M: 30, F: 26; mean age: $51.86 \pm 10.08$ years) and forty control subjects (M: 28 , F: 22 ; mean age: $55.37 \pm 9.66$ years) were recruited. All subjects underwent assessment for the degree of obesity and measured fasting plasma glucose, fasting immunoreactive insulin, $\mathrm{HbA}_{1 \mathrm{C}}$, fasting plasma C-peptide, fasting free fatty acid and lipid profiles. The homeostasis model assessment of insulin resistance (HOMA-IR) and HOMA- $\beta$ were calculated. The degree of fatty infiltration of pancreas was assessed by ultrasonography and the echogenicity of the pancreas was compared with that of hepatic parenchyma and divided three different categories (lower or similar, higher, and distinctly higher).

Results: Type 2 diabetic patients had higher fasting plasma glucose (156.97 \pm 60.91 vs. $92.42 \pm 10.23 \mathrm{mg} / \mathrm{dL} ; \mathrm{P}<0.001), \mathrm{HbA}_{1 \mathrm{C}}(8.21 \pm 1.90$ vs. 5.56 $\pm 0.25 \% ; \mathrm{P}<0.001)$ and triglyceride $(193.42 \pm 178.12$ vs. $124.87 \pm 80.83 \%$; $\mathrm{P}=0.013)$ than control subjects, and they had lower logarithmic HOMA- $\beta$ $(3.98 \pm 1.15$ vs. $5.22 \pm 0.55 ; \mathrm{P}=0.016)$ than control subjects. Patients with type 2 diabetes were positively associated with pancreatic fatty infiltration $(\mathrm{r}=0.331, \mathrm{P}=0.001)$. In type 2 diabetic patients, the higher degree of fatty pancreas showed the lower levels of fasting plasma C-peptide $(2.79 \pm 1.56$ vs. $2.33 \pm 0.98$ vs. $1.73 \pm 0.71 \mathrm{ng} / \mathrm{mL}, \mathrm{P}<0.05)$. The degree of fatty pancreas negatively correlated with fasting $\mathrm{C}$-peptide $(\mathrm{r}=-0.364, \mathrm{P}=0.011)$. Gender, duration of diabetes, BMI, fasting free fatty acid, HOMA-IR, and HOMA- $\beta$ were not different according to the degree of fatty infiltration of pancreas. On a multivariate analysis, fasting plasma C-peptide concentration was independently associated with pancreatic fatty infiltration.

Conclusion: This study suggested that fatty infiltration of pancreas was associated with $\beta$-cell dysfunction in type 2 diabetic patients.

\section{6}

High lipid oxidation and incorporation in pancreatic islets of undernourished rats

M.A.B. Reis ${ }^{1}$, V.C. Arantes ${ }^{2}$, L.F. Stoppiglia ${ }^{2}$, M.Q. Latorraca ${ }^{2}$,

E.M. Carneiro ${ }^{3}$, A.C. Boschero ${ }^{3}$

${ }^{1}$ Departamento de Ciências Básicas em Saúde, Universidade Federal de Mato Grosso, Cuiabá, ${ }^{2}$ Departamento de Alimentos e Nutrição,

Universidade Federal de Mato Grosso, Cuiabá, ${ }^{3}$ Departamento de Fisiologia

e Biofísica, Universidade Estadual de Campinas, Campinas, Brazil

Background and aims: The reduced glucose-stimulated insulin secretion (GSIS) associated to intrauterine growth restriction is well established however, the mechanistic basis of the secretory defect in pancreatic islets of is poorly understood. Rodents fed a low-protein diet during neonatal life show an impaired GSIS and a chronically elevated levels of NEFA serum. Since numerous studies attribute to high NEFA level a deleterious role on the GSIS, we carry on a study with pancreatic islets of undernourished rats to evaluate the effects of palmitate on GSIS and glucose and lipid metabolism.

Materials and methods: Rats were submitted to a control (17\% protein, C group) or a low-protein diet (6\% protein, LP group) from fetal life to 40 days old. Islets from $C$ and LP rats were cultured at $5.6 \mathrm{mmol}$ glucose/l in the absence (C and LP islets) or presence of $0.6 \mathrm{mmol}$ palmitate/BSA/l (CP and LPP islets) during $72 \mathrm{~h}$. Results: Express as medium \pm SEM. At $2.8 \mathrm{mmol}$ glucose/l the insulin secretion by $\mathrm{CP}$ was higher than $\mathrm{C}$ islets $(\mathrm{p}<0.05)$ and similar to $\mathrm{CP}$ islets stimulated by $16.7 \mathrm{mmol}$ glucose/l. LP and LPP islets shown the lowest insulin secretion at $2.8 \mathrm{~mm}$ glucose $/ 1$. At $16.7 \mathrm{mmol}$ glucose/l the GSIS were similar between LP and LPP but both were significantly lower than C and CP islets $(\mathrm{p}<0.05)$ (in ng/islet.90 min, at $2.8 \mathrm{~mm}$ glucose/l: C $0.95 \pm 0.1$, CP $2.51 \pm$ 0.83 , LP $0.52 \pm 0.1$, LPP $0.32 \pm 0.05$ and at $16.7 \mathrm{mmol} /$ glucose: C $5.35 \pm 0.64$, CP $2.52 \pm 0.3$, LP $2.33 \pm 0.36$ and LPP $2.24 \pm 0.35)$. LP and LPP islets showed lower glucose oxidation rate than $\mathrm{C}$ and $\mathrm{CP}$ islets $(\mathrm{p}<0.05)$ while NS difference was seen between $\mathrm{C}$ and $\mathrm{CP}$ islets (in pmol/islet./h, C $13.5 \pm 0.8, \mathrm{CP} 12$ \pm 1.3 , LP $10.8 \pm 1.0$, LPP $9.2 \pm 0.8$ ). At $16.7 \mathrm{mmol}$ glucose/l, LP islets showed higher palmitate oxidation compared with C (in nmol/100 islets.2h, LP 1.17 \pm 0.08 and C $0.67 \pm 0.09, p<0.05$ ), but NS difference was observed among LP, LPP and CP islets. The $1-{ }^{14} \mathrm{C}$-palmitate incorporation in total lipids was higher in LP and LPP compared with C and CP islets (in pmol/islet.h, C 0.096 \pm 0.001, CP $0.093 \pm 0.003$, LP $0.123 \pm 0.007$ and LPP $0.117 \pm 0.003, \mathrm{p}<0.05$ ). Conclusion: Protein undernutrition causes an early damage in glucose metabolism, which can account for the reduced GSIS. However, a higher lipid incorporation, allowing the fatty acids to be available for exocytosis of insulin, and a high lipid oxidation, could indicate an important role of the free fatty acids in the maintenance of the GSIS in LP islets.

Supported by: Brazilian foundations CNPq and FINEP/PRONEX

\section{7}

Human pancreatic steatosis: does it exist?

I. Lingvay ${ }^{1}$, L.S. Szczepaniak ${ }^{2}$

${ }^{1}$ Endocrinology, ${ }^{2}$ Internal Medicine and Radiology, University of Texas Southwestern Medical Center, Dallas, United States

Background and aims: Pancreatic steatosis, defined as excessive accumulation of fat within pancreatic tissue, is proposed based on studies in animal models of obesity. It is hypothesized that accumulation of fat in the pancreas contributes to the pathogenesis of type 2 diabetes through beta-cell lipotoxicity. To evaluate this hypothesis in humans we studied pancreatic triglyceride content in vivo using magnetic resonance imaging and spectroscopy (MRS). Materials and methods: Fifty nine subjects of both genders and within age range from 18 to 65 years were studied. Subjects were excluded if: 1) using medications known to alter glycemic or lipid status; or 2) sustained significant change in lifestyle (diet, exercise) or weight within 6 months prior to enrollment. Glycemic status was evaluated with a standard $75 \mathrm{~g}$ oral glucose tolerance test. Metabolic risk factors were assessed through a structured interview and anthropometric measurements. Pancreatic triglyceride content was measured by localized spectroscopy using a 1.5 Tesla clinical magnetic resonance system (Philips Medical Systems) with utilization of respiratory gating and cardiac triggering during data acquisition. Results are reported as fat to water ratio $(\mathrm{f} / \mathrm{w} \%)$.

Results: MRS measurements of pancreatic triglyceride content were highly reproducible. The reproducibility of the method was assessed in 30 subjects with a wide range of pancreatic triglyceride content, by repeating the measurements 1-30 days apart. The pancreatic triglyceride content in subjects with normal weight and no family history of diabetes was up to $2 \%$. The intraclass correlation coefficient was $0.92(\mathrm{P}<0.001$, Figure 1$)$. Subjects were divided into 3 groups based on glycemic status (Figure 2). The pancreatic triglyceride content was 3 -fold higher in subjects with impaired glucose tolerance and diabetes, compared with the normal glucose tolerance group ( $\mathrm{p}=0.01$ for both comparissons). No significant correlation between the pancreatic triglyceride content and body mass index or age was observed.

Conclusion: MRS technique is a quantitative, reproducible, and non-invasive evaluation method for human pancreatic steatosis. Pancreatic steatosis is present in subjects with impaired glucose tolerance and type 2 diabetes, suggesting a possible link between ectopic fat accumulation in the pancreas and beta-cell dysfunction leading to type 2 diabetes.

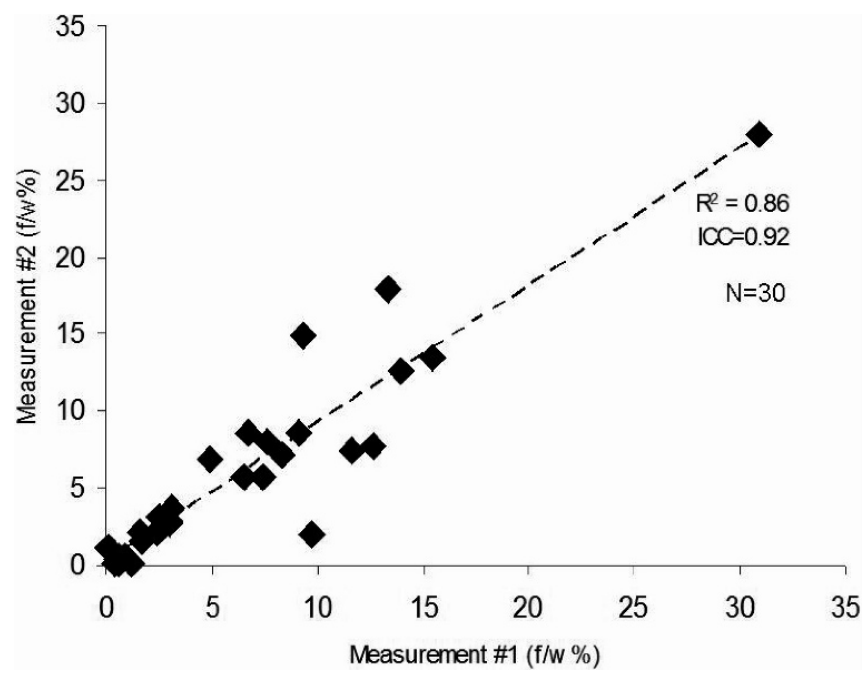

Figure 1. Reproducibility of the pancreatic MRS procedure. The intraclass correlation coefficient $($ ICC $)=0.92(p<0.001)$ 


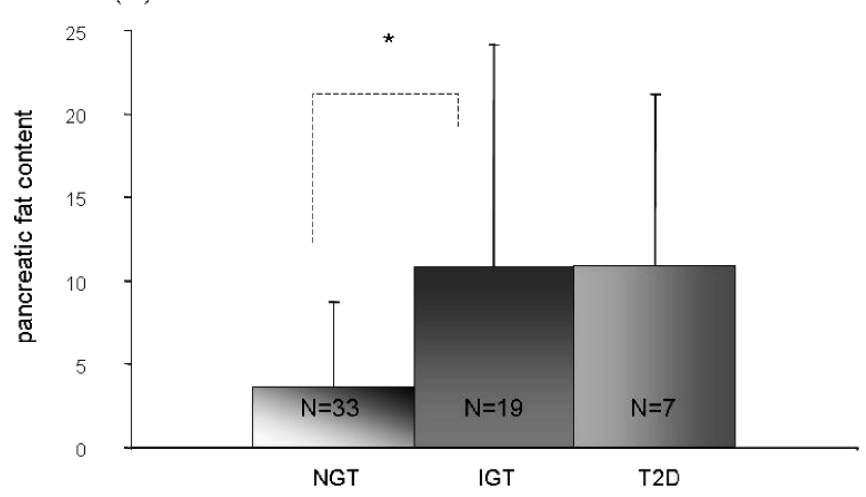

Figure 2. Comparison of pancreatic fat content in subjects with different degrees of glucose tolerance: ${ }^{*} \mathrm{p}=\mathbf{0 . 0 1}$; NGT - Normal Glucose Tolerance; IGT - Impaired Glucose Tolerance; T2D - Type 2 Diabetes

Suported by: NIH

\section{PS 060 Lipids and lipoproteins}

\section{8}

LDL glycated by methylglyoxal to physiological extent in vitro has increased binding of proteoglycan without impairment of LDL receptor binding

M. Xue, P.J. Thornalley, N. Rabbani

Clinical Sciences Research Institute, University of Warwick, Coventry,

United Kingdom

Background and aims: Glycation of low density lipoprotein (LDL) is increased in diabetes and may be linked to increased risk of atherosclerosis. The major protein of LDL, apolipoprotein B100 (apoB100), is susceptible to glycation mainly on lysine residues by glucose. Methylglyoxal (MG) is a physiological dicarbonyl glycating agent with a plasma concentration increasing disproportionately ( 3 - 5 fold) with hyperglycaemia in diabetes. Glycation by MG is directed to arginine residues and may target functionally important arginine residues in apoB100, conferring dysfunctional metabolism of LDL in diabetes. Functional activity of LDL glycated by MG in vitro has been studied previously but to high, supraphysiological extents. The aim of this study is to assess the functional activity of human LDL modified to minimal, physiological extent by MG in vitro - particularly for binding activity to the LDL receptor, proteoglycan and scavenger receptor.

Materials and methods: LDL was isolated from venous plasma, stabilised with aprotinin, collected from fasted healthy human subjects by density gradient ultracentrifugation using Iodixanol and washed with argon-purged water by ultrafiltration over a $100 \mathrm{kDa}$ microspin flter. LDL was glycated minimally by MG by incubation of LDL $(8.4 \mathrm{mg} / \mathrm{ml})$ with $100 \mu \mathrm{M} \mathrm{MG}$ in phosphate buffered saline- $0.6 \mathrm{mM}$ EDTA at $37^{\circ} \mathrm{C}$ for $24 \mathrm{~h}$. The content of advanced glycation endproduct (AGE) residues was analysed after de-lipidation and exhaustive enzymatic hydrolysis by stable isotope dilution analysis with LC-MS/MS. LDL and MGmin-LDL were modified with iodine-125 using iodogen reagent. Cultures of human hepatocyte-like HepG2 cells and human macrophage-like THP-1 cells (differentiated with phorbol ester) were prepared with $24 \mathrm{~h}$ pre-incubation with lipoprotein deficient serum. Specific and non-specific binding of LDL and MGmin-LDL, competitive binding between these two ligands and with fucoidan, and proteoglycan binding (as heparin displaceable cell binding of LDL ligands) was characterised.

Results: LDL glycated minimally by MG had increased content of MG-derived AGE residues ( $\mathrm{mol} / \mathrm{mol}$ apoB100): the major AGE was hydroimidazolone MG-H1 - MGmin-LDL $4.08 \pm 0.22$ vs LDL $0.15 \pm 0.01$. Incubation of HepG2 cells with MGmin-LDL showed similar binding kinetics and affinity to unmodified LDL for the LDL receptor and similar rates of catabolism. Binding of MGmin-LDL and unmodified LDL were competitive. Binding of MGmin-LDL to human THP-1-derived macrophages was low, competitive with LDL and non-inhibitable with the scavenger receptor ligand fucoidan. Binding of MGmin-LDL to cellular proteoglycan was increased over 2-fold compared to LDL.

Conclusion: These results suggest that the likely physiological effect of increased modification of LDL by MG in diabetes in vivo is increased proteoglycan binding and not increased binding of scavenger receptors directly. Consequenct increased residence of MG modified LDL in the extracellular millieu is likely to increase its risk of oxidation and atherogenicity in vivo. Supported by: British Heart Foundation

\section{9}

\section{WITHDRAWN}

\section{0}

Different fatty acids inhibit apoB100 secretion by both common and unique molecular pathways

T. Ota ${ }^{1}$, C. Gayet ${ }^{2}$, H.N. Ginsberg ${ }^{2}$

${ }^{1}$ Frontier Science Organization, Kanazawa University, Kanazawa, Ishikawa, Japan, ${ }^{2}$ Department of Medicine, Columbia University, New York, United States

Background and aims: Increased secretion of apolipoprotein B (apoB)-containing lipoproteins, particularly VLDL, and hepatic steatosis are characteristic concomitants of insulin resistance, the metabolic syndrome, and type 2 diabetes. Endoplasmic reticulum (ER) stress plays a central role in the devel- 
opment of hepatic insulin resistance and steatosis. Recently, we have shown that oleic acid (OA)-induced ER stress causes selective inhibition of apoB100 secretion and steatosis (J Clin Invest 118:316,2008). However, it is not known if different fatty acids (FAs) induce ER stress or inhibit apoB.

Materials and methods: We compared the effects of palmitic acid (PA) and docosahexanoic acid (DHA) to those of OA on ER stress and secretion of apoB100 by incubating McA-RH7777 (McA) cultured liver cells with PA, DHA or OA and by infusing PA, DHA or OA into C57BL/6J mice.

Results: [In vitro study] Incubation of McA cells with OA, PA, or DHA (0-1.2 $\mathrm{mM}$ ) for $16 \mathrm{hrs}$ resulted in a dose dependent increases in cell TG. PA led to less accumulation of cell TG vs.OA or DHA (PA 86.5 \pm 17.9 , OA 125.8 \pm 18.2 , DHA $128.4 \pm 5.4 \mu \mathrm{g} / \mathrm{mg}$ cell protein at $0.4 \mathrm{mM}, P<0.05$, PA vs OA or DHA). $0.4 \mathrm{mM}$ OA for $16 \mathrm{hrs}$ stimulated apoB100 secretion, this effect was lost at $0.8 \mathrm{mM} \mathrm{OA}$ and $1.2 \mathrm{mM} \mathrm{OA}$ actually inhibited apoB100 secretion. $0.4 \mathrm{mM}$ PA for $16 \mathrm{hrs}$ did not stimulate apoB100 secretion and $0.8 \mathrm{mM}$ PA actually inhibited apoB100 secretion. 0.4mM DHA for $16 \mathrm{hrs}$ inhibited apoB100 secretion. None of these doses of FA affected apoB48, apoA-I, or albumin secretion. Incubation of McA cells with OA $(0.2-1.2 \mathrm{mM})$ for 3-16hrs caused increasing ER stress as evaluated by GRP78 and phosphorylation of eIF2 $\alpha$. PA increased GRP78 and phospho-eIF2 $\alpha$ more than OA at all doses and all time points $(P<0.05$ vs OA). However, DHA did not stimulate ER stress responses at any doses and durations. Ceramide levels in McA cells were significantly increased with PA compared with control incubations (2.9-fold at $0.8 \mathrm{mM}, P<0.05)$ but were unchanged with OA or DHA. Inhibition of ceramide synthesis with myriocin reduced PA-induced ER stress to levels similar to OA-induced ER stress. Myriocin prevented PA-induced inhibition of apoB100 secretion. There was no effect of myriocin on inhibition of apoB100 secretion by either OA or DHA treatment. PA-induced ER stress responses were further enhanced by knockdown of stearoyl-CoA desaturase 1 (SCD1) by siRNA, while OA-induced ER stress was not affected by knockdown of SCD1. Knockdown of SCD1 enhanced PA-mediated inhibition of apoB100 secretion but had no effect on OA-mediated reductions of apoB100 secretion. Either vitamin $\mathrm{E}$ or desferoxamine prevented PA- or DHA- but not OA-associated inhibition of apoB100 secretion. [In vivo study] Intravenous infusions of either $6 \mathrm{mM}$ OA or PA into male C57BL/6J mice for $6 \mathrm{hrs}$ raised plasma FA levels 3-4 fold vs. saline and induced phosphorylation of eIF2 $\alpha$ and increased GRP78 protein in the liver: infusion with PA caused more ER stress than OA. Infusion of OA, but not PA, significantly increased the secretion of apoB100 compared with saline. Infusion of $6 \mathrm{mM}$ DHA for $6 \mathrm{hrs}$ did not increase apoB100 secretion and did not induce ER stress.

Conclusion: Each FA, when administered for prolonged periods of time, can, at certain doses, inhibit apoB secretion in vitro and in vivo. The effects of $\mathrm{OA}$ and PA parallel their ability to induce ER stress; PA is more potent and acts, in part, via ceramide. DHA is the most potent inhibitor but this is independent of ER stress. It is not clear how OA causes ER stress or inhibits apoB secretion.

Supported by: NIH/National Heart, Lung, and Blood Institute

\section{1}

VLDL and cholesterol remnant levels following 3 consecutive meals are associated with liver fat in men with type 2 diabetes and men with the metabolic syndrome

M.E. Tushuizen ${ }^{1}$, P.J.W. Pouwels ${ }^{2}$, S. Bontemps ${ }^{1}$, C. Rustemeijer ${ }^{3}$, N. Matikainen ${ }^{4}$, M.-R. Taskinen ${ }^{4}$, R.J. Heine1, M. Diamant ${ }^{1}$; ${ }^{1}$ Endocrinology / Diabetes Centre, VU University Medical Centre, Amsterdam, Netherlands, ${ }^{2}$ Physics \& Medical Technology, VU University Medical Centre, Amsterdam, Netherlands, ${ }^{3}$ Internal Medicine, Hospital Amstelland, Amstelveen, Netherlands, ${ }^{4}$ Department of Medicine, University of Helsinki, Helsinki, Finland

Background and aims: Abnormal postprandial metabolism, which is exaggerated and prolonged in insulin resistant states and type 2 diabetes (T2DM), is associated with increased cardiovascular disease (CVD) risk. Liver fat accumulation is related to components of the metabolic syndrome (MetS) and T2DM. We hypothesised that the severity of postprandial dyslipidaemic derangements during a $24-\mathrm{h}$ period in subjects with T2DM and those with the MetS may be related to hepatic fat content.

Materials and methods: We monitored 24-h profiles of plasma lipids in 14 T2DM, 14 MetS and 14 healthy age-matched males, following a standardised high-fat mixed breakfast $(9.00 \mathrm{am})$, lunch $(1.00 \mathrm{pm})$ and dinner $(5.00 \mathrm{pm})$, mimicking the real-life situation. Blood samples were collected before and at $\mathrm{t}=2,4,6,8,12,16,20$ and $24 \mathrm{~h}$ following breakfast. Liver fat was measured in the fasting state by localized proton MR-spectroscopy.
Results: Patients (mean \pm SD, age $55 \pm 4.2$ yrs; HbA1c $7.2 \pm 1.1 \%$ ) versus MetS subjects did not differ with respect to age, BMI, waist, blood pressure, triglycerides and HDL-cholesterol. 24h-AUC of triglycerides, apoB-100, and cholesterol remnants differed significantly between groups (all $\mathrm{P}<0.05)$, whereas AUC apoB-48 did not differ between T2DM and MetS males. Liver fat content was higher in T2DM compared to MetS and controls (median 17.8 (IQR 7.6-31.7)\%, 7.6 (2.9-10.2)\% and 3.9 (2.1-7.0)\%, respectively, $\mathrm{P}=0.001)$. Liver fat content was associated with postprandial 24-h AUC triglycerides, apoB100 and cholesterol rich remnants $(r=0.50, P=0.001, r=0.37, P=0.017 ; r=0.41$, $\mathrm{P}<0.01$, respectively), but not with 24 -h AUC apoB- 48 particles $(\mathrm{r}=0.14$, $\mathrm{P}=0.38$ ).

Conclusion: Hepatic fat content was associated with postprandial dyslipidaemic responses in subjects with T2DM and those with the MetS. These findings suggest that targeting hepatic fat accumulation in these high-risk subjects, may favourably affect postprandial metabolic derangements and ultimately reduce CVD risk.

Supported by: Dutch Diabetes Foundation

\section{2}

Measurement of postprandial triacylglycerol kinetics in both endogenous and exogenous lipoproteins using a novel immunoaffinity chromatography method

F. Sun ${ }^{1}$, M. Stolinski ${ }^{1}$, F. Shojaee-Moradie ${ }^{1}$, R. Hovorka ${ }^{2}$, A.M. Umpleby'; ${ }^{1}$ Diabetes and Endocrinology, University of Surrey, Guildford, ${ }^{2}$ Diabetes Modelling, University of Cambridge, United Kingdom

Background and aims: Abnormally elevated postprandial triacylglycerols (TAG) are a feature of metabolic syndrome and type 2 diabetes and are predictive of an increased risk of cardiovascular disease. The cause is poorly understood due to the lack of selective and sensitive methods to accurately quantify the kinetics of postprandial exogenous (chylomicrons) and endogenous (VLDL) TAG-rich lipoproteins. In this study postprandial TAG kinetics were measured in healthy subjects using a novel method to separate exogenous and endogenous lipoproteins.

Materials and methods: Six healthy volunteers $(2 \mathrm{M}, 4 \mathrm{~F})$, age $36.8 \pm 3.9 \mathrm{yr}, \mathrm{BMI}$ $24.4 \pm 1.1 \mathrm{~kg} / \mathrm{m}^{2}$ (mean \pm SEM) were studied on 2 occasions in random order 1] after an overnight fast and 2] during a continuous feeding protocol with 6 liquid meals ( $486 \mathrm{kcal}, 11 \%$ carbohydrate, $88 \%$ fat) every $2 \mathrm{~h}$. In both studies a bolus injection of $\left[{ }^{2} \mathrm{H}_{5}\right]$-glycerol was administered to label TAG. Chylomicrons and VLDL1 $\left(\mathrm{S}_{\mathrm{f}}>60\right)$ and small chylomicrons and VLDL2 $\left(\mathrm{S}_{\mathrm{f}} 20\right.$ 60 ) were separated by sequential flotation ultracentrifugation. Endogenous and exogenous particles were isolated using a novel immunoaffinity method (Protein G Sepharose coupled to three monoclonal antibodies specific for apoB100-4G3, 5E11 and Bsol16) which allowed complete separation of apoB100 and apoB48 containing lipoproteins. TAG was extracted from the purified particles and glycerol enrichment measured by gas chromatography mass spectrometry. TAG fractional catabolic rate (FCR) was calculated using a mathematical model of the exogenous and endogenous TAG pathways.

Results: Plasma TAG concentration was $0.88 \pm 0.11 \mathrm{mmol} / \mathrm{L}$ in the fasted study and $1.47 \pm 0.20 \mathrm{mmol} / \mathrm{L}$ in the fed study. There was no difference in VLDL1 (V1) and VLDL2 (V2) TAG FCR between the fasted study (V1, $43.8 \pm 15.3$ pools/day; V2, $26.8 \pm 4.4$ pools/day) and fed study (V1, $52.2 \pm 14.1$ pools/day; V2, $20.4 \pm 2.1$ pools/day). In the fed study chylomicron TAG FCR $(48.0 \pm 18.5$ pools/day) was not different from VLDL1 FCR but small chylomicron TAG FCR $(12.7 \pm 1.9$ pools/day) was significantly lower than VLDL2 $\mathrm{FCR}(\mathrm{p}=0.022)$.

Conclusion: Feeding did not change the clearance rate of VLDL1 and VLDL2 in healthy subjects. Although chylomicrons are cleared at the same rate as VLDL1 the clearance of small chylomicrons of the same density as VLDL2 is lower than VLDL2.

Supported by: Heart Research UK 


\section{3}

Nuclear magnetic resonance lipoprotein subfraction profile and early carotid atherosclerosis: the GOCADAN study

M. Masulli ${ }^{1}$, L. Patti ${ }^{1}$, G. Riccardi ${ }^{1}$, O. Vaccaro ${ }^{1}$, G. Annuzzi ${ }^{1}$, S. Ebbesson ${ }^{2}$, H. Wang ${ }^{3}$, B.V. Howard ${ }^{3}$, A.A. Rivellese ${ }^{1}$

${ }^{1}$ Department of Clinical and Experimental Medicine, "Federico II"

University of Naples, Naples, Italy, ${ }^{2}$ Norton Sound Health Corporation,

${ }^{3}$ MedStar Research Institute, United States

Background and aims: The relationship between lipoprotein subfractions and atherosclerosis is not often explored because laboratory measurement of lipoproteins is laborious and time-consuming. Proton Nuclear Magnetic Resonance (NMR) spectroscopy provides an advanced, rapid, and cost-effective method to measure directly the lipoprotein particle concentration, distribution, and size. The aim of this study was to evaluate the relationships between lipoprotein subfractions and early subclinical atherosclerosis in carotid vessels in a population based sample of Alaska Eskimos, characterized by a high cardiovascular risk.

Materials and methods: Seven hundred and sixty non diabetic Alaska Eskimos (age $\geq 35 \mathrm{yrs}$ ) were included in this analysis. Early atherosclerosis was investigated by ultrasound measurement of Intimal Media Thickness (IMT). Plaque was defined as the presence of focal protrusion of the vessel at least $50 \%$ greater than surrounding wall; plaque score was defined as the number of segments (0-7) of each carotid artery containing plaque. Subclasses (large, intermediate and small) of plasma VLDL, LDL and HDL were characterized for concentration and size by proton NMR spectroscopy, and related to tertiles of IMT (tertile $1=0.335-0.570 \mathrm{~mm}$; tertile $2=0.575-0.675 \mathrm{~mm}$; tertile $3=0.680-1.100 \mathrm{~mm}$ ) and plaque score (group $1=$ plaque score 0 ; group $2=$ plaque score 1; group 3 = plaque score 2-7).

Results: Higher IMT values were associated with a) lower concentrations of large VLDL particles $(\mathrm{p}<0.05)$, independently of other confounders, including plasma LDL cholesterol concentration, and b) higher concentrations of total $(\mathrm{p}<0.05)$, intermediate $(\mathrm{p}<0.05)$ and small $(\mathrm{p}<0.05)$ LDL particles, independently of other confounders, including plasma triglyceride concentrations. Higher plaque scores were associated with (a) lower concentrations of either large or small VLDL particles ( $\mathrm{p}<0.01$ for both), independently of other confounders, including LDL cholesterol, and (b) higher total $(\mathrm{p}<0.01)$, large $(\mathrm{p}<0.02)$ and intermediate $(\mathrm{p}<0.05)$ LDL particles, independently of other confounders, including plasma triglycerides. Neither IMT nor plaque score were associated with HDL particle concentration and size.

Conclusion: In a population at high cardiovascular risk, NMR lipoprotein particle profile discriminates sub-clinical atherosclerosis in carotid arteries. The data in our study suggest an independent inverse effect of large VLDL particles and an independent direct effect of LDL particles on several stages of atherosclerosis.

\section{4}

Low HDL-cholesterol: prevalence and associated risk factors in a large French population

P.J. Lecomte ${ }^{1}$, M. Rouvre 1 , S. Vol ${ }^{2}$, G. Gusto ${ }^{2}$, O. Lantieri ${ }^{3}$, J. Tichet ${ }^{3}$

${ }^{1}$ UCM, CHRU Bretonneau, Tours, ${ }^{2}$ Biostatistics, IRSA, La Riche, ${ }^{3}$ Directory, IRSA, La Riche, France

Background and aims: Besides LDL-cholesterol levels, HDL-cholesterol (HDLc) is routinely measured to evaluate cardio-vascular risk. However, only few studies have evaluated the prevalence of low HDLc levels in the general population and none in France. Our aims were i/ to estimate the prevalence of low HDLc levels in a French population using the definition of the NCEPATP III $[<40 / 50 \mathrm{mg} / \mathrm{dL}$ (men/women)] and ii/ to examine in this population the level of each component of the metabolic syndrome (NCEP-ATP III), other biological parameters and lifestyle conditions in subjects with regard to HDLc levels.

Materials and methods: The subjects were 21,590 men and 26,263 women aged 16 to 79 participating to a free routine medical and biological check-up. All participants completed a self-questionnaire about their socioeconomic and family status, personal and familial medical history, lifestyle behaviours (physical activity, smoking, dietary habits). Deprivation status was defined by EPICES score from 11 weighted questions related to material and social deprivation. The fatty liver index (FLI), an algorithm based on BMI, waist circumference, triglycerides and GGT estimated the prediction of fatty liver. Blood samples were collected after an overnight fast. Variables of interest were studied in 3 groups defined by age and gender-specific percentiles classes: $\leq$ $5^{\text {th }}\left(\mathrm{HDL}_{5}\right), 7$ median percentiles $\left(\mathrm{HDL}_{\mathrm{m}}\right)$ and $>95^{\text {th }}\left(\mathrm{HDL}_{95}\right)$. Data were analyzed by gender and adjustments on alcohol consumption and smoking habits were performed by logistic regression or GLM ANOVA. Gender-specific logistic regression models were built to select variables associated to $\mathrm{HDL}_{5}$ .The data were analysed using NCSS (Number Cruncher Statistical Systems) 2000.

Results: The prevalence of low HDLc levels was $12.1 \%$ in men and $28.1 \%$ in women and these figures decreased with age. A low percentage of subjects $(9.1 \%$ of men and $5.6 \%$ of women) were treated with lipid-lowering drugs. The frequency of the metabolic syndrome decreased drastically when HDLc increased $\left(\mathrm{HDL}_{5} / \mathrm{HDL}_{\mathrm{m}} / \mathrm{HDL}_{95}\right): 44 \% / 7 \% / 2 \%(\mathrm{p}<0.00001)$ in men and $42 \% / 4 \% / 1 \%(\mathrm{p}<0.00001)$ in women as for high FLI $(\mathrm{FLI} \geq 60)$ : $38 \% / 24 \% / 10 \%(\mathrm{p}<0.00001)$ in men and $31 \% / 8 \% / 3 \%(\mathrm{p}<0.00001)$ in women. When HDLc increased, sedentarity, current smoking and deprivation decreased $(p<0.00001)$ but energy intake (men only) and alcohol consumption increased $(p<0.00001)$. When data were adjusted on smoking and alcohol consumption, all differences were still significant, excepted energy intake in men. For both genders, the same variables were associated with $\mathrm{HDL}_{5}$, a positive contribution for waist circumference, smoking, triglycerides, leucocytes, diabetes and a negative contribution for alcohol consumption, family history of cholesterol, cholesterol level. Smoking and abdominal obesity were the preponderant variables.

Conclusion: The prevalence of low HDLc levels in this French population was similar to that observed in Japanese but lower than that found in American. An HDLclevel less than the age-gender specific $5^{\text {th }}$ percentile was associated with cardiovascular risk factors (sedentary, smoking habits, high triglyceride levels, diabetes, obesity), metabolic syndrome, fatty liver and with deprivation. Most of these parameters should be abated by an adequate prevention policy and first of all, on smoking and abdominal obesity. 


\section{PS 061 Ectopic fat muscle}

\section{5}

Intramyocellular triglyceride fatty acid composition implicates on muscle triglyceride turnover, insulin action and glycaemic control in man

S.B. Haugaard ${ }^{1}$, S. Madsbad ${ }^{2}, \mathrm{H} . \mathrm{Mu}^{3}$, A. $\mathrm{Vaag}^{4}$

${ }^{1}$ Clinical Research Centre, Copenhagen University Hospital, Hvidovre, ${ }^{2}$ Dept. of Endocrinology, Copenhagen University Hospital, Hvidovre, ${ }^{3}$ Dept. of Systems Biology, Technical University of Denmark, Lyngby, ${ }^{4}$ Steno Diabetes Centre, Copenhagen University, Gentofte, Denmark

Background and aims: In-vitro studies have shown that the concentration of palmitic acid (C16:0) in intramyocellular triglyceride (IMTG) correlates with insulin resistance. In the present study we investigated the fatty acid (FA) composition of IMTG and its relation to IMTG content, sex, and insulin resistance in obese and lean sedentary subjects with and without type 2 diabetes (T2DM), in whom we have reported previously that obese women possess twice as much IMTG as matched obese men and lean women.

Materials and methods: FA composition of IMTG was determined by gasliquid-chromatography in skeletal muscle biopsies harvested from sedentary subjects, 34 women ( 27 obese, 7 T2DM) and 25 men ( 20 obese, 6 T2DM). Insulin resistance was assessed by homeostasis model assessment insulin resistance index (HOMA-IR).

Results: In all subjects $(n=59)$ total saturated FA correlated inversely and total monounsaturated FA correlated positively with IMTG content. In gender specific analyses these associations were found in women only $(r=-$ $0.54, \mathrm{P}<0.002, \mathrm{r}=0.43, \mathrm{P}=0.01$, respectively) and were also significant in the group of obese women. Obese women exhibited lower total saturated FA and palmitic acid than obese men ( $13 \%$ and $9 \%$, respectively, Ps $<0.001)$, which remained significantly reduced after correction for IMTG. Total polyunsaturated FA and long-chain-polyunsaturated FA did not correlate with IMTG. Palmitic acid correlated with insulin resistance (HOMA-IR, $\mathrm{r}=0.33, \mathrm{P}=0.014$ ) and impaired glycaemic control (fasting plasma glucose, $r=0.41, \mathrm{P}<0.002$ ) after correction for gender and IMTG content.

Conclusion: Excess IMTG content associates to decreased IMTG saturated FA, which could be secondary to a low turnover rate of excess IMTG, and, thus, longer duration of exposure of IMTG to enzymatic activity by in situ desaturases. IMTG FA composition implicates on insulin sensitivity and glycaemic control independent of sex and IMTG content.

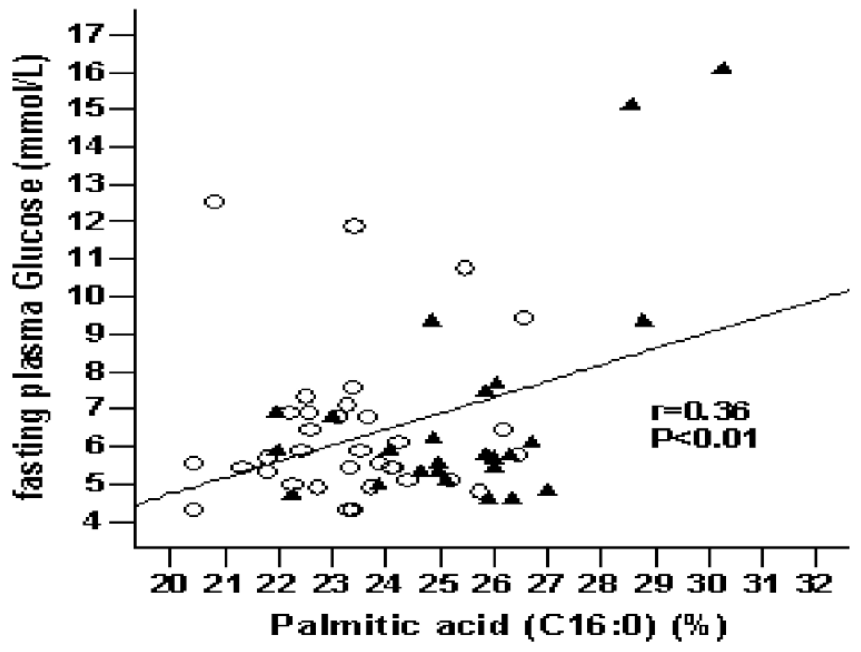

Correlation between the level of glycaemic control (fasting glucose) and intramyocellular triglyceride (IMTG) palmitic acid in men (filled triangles) and in women (open circles). P-value and correlation coefficient are given. the correlation remained significant after correction for sex and IMTG content $(\mathrm{r}=0.41, \mathrm{P}<0.002)$

Supported by: Danish Hospital Foundation for Medical Research, Region of Copenhagen, Faroe Islands and Greenland; Danish Diabetes Association; Bernhard and Marie Klein Foundation; A.P. Møller Foundation

\section{6}

Smoking cessation increases insulin action, but does not change intramuscular triglyceride concentration or turnover

B.C. Bergman, L. Perreault, M.C. Playdon, A.M. Samek, R.H. Eckel Endocrinology, University of Colorado Health Sciences Ctr, Denver, United States

Background and aims: Smoking has been shown to decrease insulin action and increase risk of type 2 diabetes in humans. Mechanisms responsible for smoking-induced insulin resistance are unclear. Smoking has been shown to increase adipocyte lipolysis, FFA concentration, and skeletal muscle LPL, but not change whole body fat oxidation suggesting muscle lipid accumulation in this group. We hypothesized smokers (SM) would have increased intramuscular triglyceride (IMTG) and diglyceride (DAG) concentration, but decreased IMTG fractional synthesis rate compared to non-smokers (CON) which would return to control levels after smoking cessation.

Materials and methods: $\operatorname{CON}(\mathrm{n}=12,20.4 \pm 0.6 \mathrm{yrs}, 22.1 \pm 0.4 \mathrm{~kg} / \mathrm{m} 2,19.7 \pm 2 \%$ fat, $0 \mathrm{cig} /$ day $)$ and $S M(n=10,21.4 \pm 0.7 \mathrm{yrs}, 22.3 \pm 0.5 \mathrm{~kg} / \mathrm{m} 2,17.9 \pm 2 \%$ fat, $17 \pm 1 \mathrm{cig} /$ day) were studied in the fasted state after 3 days of a standardized diet, before and after a smoking cessation program. [U-13C]palmitate was infused for 4 hours after which a muscle biopsy was taken, and an IVGTT performed. Muscle biopsies were dissected free of visible fat, lyophilized, lipid fractions were isolated using solid phase extraction, and analyzed by GC/MS for concentration and GC/C/IRMS for enrichment. Western blots were performed using standard techniques.

Results: SM were less insulin sensitive compared to CON (Si: $5.5 \pm 0.5$ CON vs $3.8 \pm 0.5 \mathrm{SM} \mathrm{mU} / \mathrm{L} / \mathrm{min}$ ), $\mathrm{p}=0.03$ ), which did not change in CON, but significantly increased in SM after smoking cessation $(4.3 \pm 0.4 \mathrm{SM} \mathrm{mU} / \mathrm{L} / \mathrm{min}$, $\mathrm{p}=0.02)$. We found no significant differences in basal IMTG $(19.8 \pm 2.3 \mathrm{CON}$ vs $24.3 \pm 2.7 \mathrm{SM} \mathrm{ug} / \mathrm{mg}$ dry wt) or DAG $(0.34 \pm 0.02 \mathrm{CON}$ vs $0.34 \pm 0.02 \mathrm{SM} \mathrm{ug} /$ mg dry wt) concentration, or IMTG turnover $(0.68 \pm 0.08 \mathrm{CON}$ vs $0.61 \pm 0.10$ SM \%/hr) before or after smoking cessation. IMTG and DAG composition was more saturated in SM (IMTG: $31.8 \pm 1.0 \mathrm{CON}$ vs $35.0 \pm 1.1 \mathrm{SM} \%, \mathrm{p}=0.04$ ) (DAG: $53.3 \pm 1.7$ CON vs $59.5 \pm 1.9$ SM \%, p=0.02), which did not significantly change after smoking cessation. We found increased IRS-1 ser636 phosphorylation in SM compared to $\mathrm{CON}(0.84 \pm 0.09 \mathrm{CON}$ vs $1.27 \pm 0.08 \mathrm{SM} \mathrm{AU}$, $\mathrm{p}=0.003)$ which significantly decreased after smoking cessation $(1.02 \pm 0.1$ CON vs $0.96 \pm 0.08$ SM AU, $p=0.04$ ). Significant positive relationships were found between IMTG and DAG saturation and IRS-1 ser636 phosphorylation (IMTG: $\mathrm{R} 2=0.40, \mathrm{p}=0.005$, DAG: $\mathrm{R} 2=0.25, \mathrm{p}=0.04$ ).

Conclusion: These data suggest: 1) insulin action is impaired in smokers, but increases after smoking cessation 2) differences in IMTG concentration and turnover are not related to alterations in insulin action in smokers compared to non-smokers, but may relate to 3 ) higher IMTG and DAG saturation in smokers 4) Basal inhibition of IRS-1 appears related to the saturation of IMTG and DAG, however, insulin signaling may be restored after smoking cessation independent of changes in IMTG and DAG saturation. Supported by: Colorado Tobacco Research Program

\section{7}

Elevated skeletal muscle TIP47 expression in obesity and type 2 diabetes S. Bajpeyi, C. Moro, S.R. Smith Endocrinology, Pennington Biomedical Research Center, Baton Rouge, United States

Background and aims: Proteins of the PAT family (Perilipin, Adipophilin (ADRP), Tail-interacting protein of 47kDa (TIP47), OXPAT, and S3-12), play an important role in the regulation of lipid storage/mobilization in many tissues [e.g. adipose tissue]. Recent data from our laboratory provide evidence that the dysregulation of intramyocellular lipid metabolism leads to insulin resistance; we refer to this as intramyocellular lipotoxicity. PAT proteins lie at the interface of the lipid droplet and the cytosol and therefore serve as logical candidates in the regulation of skeletal muscle intramyocellular lipid metabolism.

Materials and methods: We measured PAT protein gene expression by RTqPCR in vastus lateralis samples from lean (BMI 22.9 $\pm 1.0 ; \mathrm{n}=8$ ), obese (BMI $32.0 \pm 0.8 ; \mathrm{n}=8$ ), and type 2 diabetic subjects (BMI $38.6 \pm 2.8 ; \mathrm{n}=8$ ). Using cultured myotubes we also determined the localization of selected PAT proteins in relationship to intramyocellular lipids by confocal imaging. Results: ADRP and TIP47 were highly expressed in human muscle tissue while S3-12 and perilipin were not detected and OXPAT was at the lower 
limit of detection. TIP47 expression was increased in obese ( 2 fold) and type 2 diabetic ( 2.3 fold) subjects compared to lean $(\mathrm{p}<0.05)$ while ADRP and OXPAT were not different across the three groups. ADRP and TIP47 co-localized to lipid droplets in cultured myotubes.

Conclusion: The present data show for the first time that ADRP and TIP47 are highly expressed in human skeletal muscle and colocalize with lipid droplets. TIP47 gene expression is increased in obesity and T2DM, suggesting an important role in skeletal muscle lipid metabolism.

Supported by: grant from Health \& Performance Enhancement and Clinical Obesity Divisions, Pennington Biomedical Research Center

\section{8}

Utilization of intramyocellular lipids for oxidation in human skeletal muscle cells from lean and type 2 diabetic subjects

E. Corpeleijn ${ }^{1}$, N.P. Hessvik ${ }^{2}$, E.E. Blaak ${ }^{1}$, G.H. Thoresen ${ }^{2}$, M. Gaster ${ }^{3}$, A.C. Rustan ${ }^{2}$

${ }^{1}$ Department of Human Biology, NUTRIM, Maastricht University, Netherlands, ${ }^{2}$ Department of Pharmaceutical Biosciences, School of Pharmacy, University of Oslo, Norway, ${ }^{3} \mathrm{KMEB}$, Department of Endocrinology, Odense University Hospital, Denmark

Background and aims: It is generally accepted that insulin resistance and type 2 diabetes (T2D) are associated with lipid accumulation in skeletal muscle. How this lipid storage and oxidation are regulated is less well known. Therefore, the regulation of lipid oxidation from intracellular and extracellular sources was studied in human myotubes established from healthy lean $(\mathrm{n}=8)$ and obese T2D subjects $(\mathrm{n}=9)$.

Material and methods: Myotubes were cultured from satellite cells, differentiated and pretreated with $100 \mu \mathrm{M}$ oleic acid (OA) for 2 days to upregulate lipid metabolism. The cells were treated with enriched ${ }^{14} \mathrm{C}$-OA either during the pretreatment period or during the acute experiment, to distinguish between ${ }^{14} \mathrm{CO}_{2}$-production from intracellular or extracellular lipid sources. OA oxidation was measured by trapping the production of ${ }^{14} \mathrm{CO}_{2}$ during 4 hours of incubation. In this acute $4 \mathrm{~h}$-experiment, the cells were exposed to one of four conditions of variable glucose and OA availability: $\mathrm{gf}(0.1 \mathrm{mM}$ glucose $/ 5$ $\mu \mathrm{M}$ OA), gF (0.1 mM glucose/100 $\mu \mathrm{M} \mathrm{OA}), \mathrm{Gf}(5.0 \mathrm{mM}$ glucose $/ 5 \mu \mathrm{M} \mathrm{OA})$, or GF (5.0 mM glucose/100 $\mu \mathrm{M} \mathrm{OA})$. Group and treatment effects were analyzed with linear mixed model (SPSS 11.5).

Results: The T2D subjects were characterized in vivo by insulin resistance and metabolic inflexibility, i.e. an impaired switch from fat oxidation to carbohydrate oxidation after insulin-stimulation. The utilization of intracellular OA for oxidation $\left(\mathrm{ICL}_{\mathrm{OX}}\right)$ was significantly and consistently reduced in myotubes from T2D subjects (gf: $\mathrm{ICL}_{\mathrm{OX}}$ (lean) $=4.71 \pm 1.0 \mathrm{nmol} / \mathrm{mg}$ protein, $\mathrm{ICL}_{\mathrm{Ox}}(\mathrm{T} 2 \mathrm{D})=3.36 \pm 0.67 ; \mathrm{gF}: \mathrm{ICL}_{\mathrm{Ox}}$ (lean) $=3.64 \pm 1.0 \mathrm{nmol} / \mathrm{mg}$ protein, $\mathrm{ICL}_{\mathrm{OX}}(\mathrm{T} 2 \mathrm{D})=1.93 \pm 0.46 ; \mathrm{Gf}^{-} \mathrm{ICL}_{\mathrm{OX}}($ lean $)=4.50 \pm 1.2 \mathrm{nmol} / \mathrm{mg}$ protein, $\mathrm{ICL}_{\mathrm{OX}}(\mathrm{T} 2 \mathrm{D})=2.49 \pm 0.45 ; \mathrm{GF}: \mathrm{ICL}_{\mathrm{OX}}($ lean $)=2.92 \pm 0.8 \mathrm{nmol} / \mathrm{mg}$ protein, $\mathrm{ICL}_{\mathrm{OX}}(\mathrm{T} 2 \mathrm{D})=1.38 \pm 0.30$. Group effect $\left.\mathrm{P}=0.002\right)$. Also total $\mathrm{OA}$ oxidation was reduced (group effect $\mathrm{P}=0.003$ ). The in vivo fasting respiratory quotient (RQ) was consistently inversely associated with outcomes for in vitro lipid oxidation, including the oxidation of intracellular lipids under all conditions and the oxidation of extracellular lipids under increased $(100 \mu \mathrm{M}) \mathrm{OA}$ conditions (Spearman $\left.\mathrm{r}_{\mathrm{ALL}}>-0.59, \mathrm{P}_{\mathrm{ALL}}<0.05\right)$. Myotubes established from T2D subjects showed an impaired adaptability of total OA oxidation $(\mathrm{P}<0.001)$, which is the capacity to increase lipid oxidation in response to an increased $\mathrm{OA}$ availability. These myotubes also tended to show an increased suppressibility (gf vs Gf, $P=0.036$, gF vs GF, $P=0.136$ ), which is the sensitivity of the myocyte to the suppressive effect of glucose on lipid oxidation.

Conclusion: A low fasting fat oxidation in vivo, as exemplified by a high RQ, was reflected by a low lipid oxidation in vitro, suggesting that the characteristics of the in vivo phenotype are retained in vitro either by genetic, epigenetic or other unknown mechanisms. An impaired capacity to oxidize intracellular lipids, an impaired capacity to increase lipid oxidation and an increased sensitivity for the suppressive effect of glucose may contribute to the in vivo phenotype, characterized by insulin resistance and metabolic inflexibility, and may play a primary role in the development of insulin resistance and type 2 diabetes.

Supported by: Dutch Diabetes Foundation

\section{9}

Dysfunction of skeletal muscle lipolysis contributes to lipotoxicity and insulin resistance in obesity and type 2 diabetes

C. Moro ${ }^{1}$, S. Bajpeyi ${ }^{1}$, A. Mairal ${ }^{2}$, G. Liebisch ${ }^{3}$, S. Thomas ${ }^{1}$, G. Schmitz ${ }^{3}$, D. Langin ${ }^{2}$, S.R. Smith ${ }^{1}$

${ }^{1}$ Molecular and Experimental Endocrinology, Pennington Biomedical Research Center, Baton Rouge, United States, ${ }^{2}$ Obesity Research Laboratory, INSERM U858, Institute of Molecular Medicine, Toulouse, France, ${ }^{3}$ Institute of Clinical Chemistry, University of Regensburg, Germany

Background and aims: Insulin resistance is associated with elevated skeletal muscle lipid deposition. We hypothesized that a dysfunction of skeletal muscle lipolysis could produce per se lipotoxicity and insulin resistance.

Materials and methods: We measured triglyceride and diglyceride hydrolase activities, along with triglyceride, diglyceride and ceramide content in human vastus lateralis samples of lean $(\mathrm{n}=10)$, obese $(\mathrm{n}=16)$ and type 2 diabetes mellitus $(\mathrm{T} 2 \mathrm{DM})(\mathrm{n}=10)$ subjects. We then investigated in vitro in cultured myotubes the relationship between dysfunction of lipolysis and insulin action. Results: Across subjects, we found that the ratio diglyceride-to-triglyceride hydrolase activity was a determinant of insulin sensitivity and lipotoxicity. This ratio was reduced in obese and T2DM subjects $(25 \%, \mathrm{p}<0.01)$, in line with elevated ATGL and reduced HSL protein expression in obese and T2DM subjects. Intramyocellular triglyceride, diglyceride and ceramide were elevated in T2DM subjects, while only diglyceride levels were increased in obese compared to lean subjects. We also show that adipose triglyceride lipase (ATGL) is an important determinant of skeletal muscle triglyceride hydrolase activity and requires CGI-58 for full enzymatic activation. In cultured myotubes, overexpression of ATGL or its coactivator CGI-58 elevated diglyceride and ceramide content $(\mathrm{p}<0.05)$, and inhibited insulin-stimulated glycogen synthesis and Ser473-Akt phosphorylation $(\mathrm{p}<0.001)$.

Conclusion: Altogether, these data indicate for the first time an imbalance between ATGL and HSL in skeletal muscle that leads to incomplete triglyceride hydrolysis, and highlight a new mechanism by which defective lipolysis produces intramyocellular lipotoxicity and insulin resistance. Supported by: USDA and the Philippe Foundation Inc (CM)

\section{0}

Effects of trimetazidine on myocardial glucose and fatty acid metabolism and cardiac function in non-diabetic obese humans

M. Bucci ${ }^{1}$, K. Någren ${ }^{1}$, J. Pärkka ${ }^{1}$, R. Maggio ${ }^{1}$, R. Borra ${ }^{1}, H^{2}$. Tuunanen ${ }^{1}$, M. Taittonen ${ }^{1}$, T. Viljanen ${ }^{1}$, S. Rigazio ${ }^{1}$, M. Komu ${ }^{1}$, R. Parkkola ${ }^{2}$, J. Knuuti ${ }^{1}$, P. Nuutila ${ }^{1}$, P. Iozzo ${ }^{1,3}$

${ }^{1}$ Turku Pet Centre, Turku Central University Hospital, Finland, ${ }^{2}$ Department of Radiology, Turku Central University Hospital, Finland, ${ }^{3}$ Institute of Clinical Physiology, CNR National Research Council, Pisa, Italy

Background and aims: Trimetazidine is a metabolic agent with anti-ischemic properties used in clinical practice in patients with coronary artery diseases. Only few studies have focused on its mechanism of action in vivo, in humans. The aim of the current study was to non-invasively investigate the effects of trimetazidine treatment on the heart lipid and glucose metabolism in obese non-diabetic subjects, with no cardiac complications.

Materials and methods: Positron Emission Tomography with ${ }^{18} \mathrm{~F}$-fluorodeoxyglucose and ${ }^{11} \mathrm{C}$-palmitate was used to quantify the metabolism of glucose and fatty acids, respectively. Myocardial fatty acid oxidative (FAO) metabolism was determined by bi-exponential fitting of the heart ${ }^{11} \mathrm{C}$-palmitate wash-out curve. Magnetic Resonance Spectroscopy and Imaging were used to quantify the content of lipids in the heart and assess cardiac function and dimensions.

Results: Eight obese subjects (BMI, $31.5 \pm 3.4 \mathrm{~kg} / \mathrm{m} 2$, range 27-38) were studied in fasting conditions. The basal hemodynamic and Left Ventricular (LV) parameters in the study group were: Ejection Fraction (EF, \%) 66 \pm 6 , Cardiac Output (CO, 1/min) 6.2 $\pm 0.6, \mathrm{LV}$ mass (g) 95.0 \pm 25.1 , Stroke Volume (SV, ml) 98.1 \pm 11.8 , Heart Rate (HR, bpm) $64 \pm 9$, LV work $\left(\mathrm{mmHg}^{*} \mathrm{l}^{\star} \mathrm{min}^{-1}\right)$ 655.3 \pm 135.2 , Peripheral Vascular Resistance $\left(\mathrm{mmHg}^{\star} \min ^{*} \mathrm{ml}^{-1}\right) 16.9 \pm 2.9$. Basal metabolic parameters, as assessed by PET, were: LV glucose uptake (LV GU, mol(glucose) $)^{*} \min ^{-1}$ ) 3.4 $\pm 4.6, \mathrm{LV}$ fatty acid uptake (LV FAU, mol(palmitate) ${ }^{*}$ min-1) $10.0 \pm 3.2$. Heart lipid content (\%) was $0.8 \pm 0.7$ and LV lipid content $(\mathrm{g})$ was $0.8 \pm 0.6$. After one month of trimetazidine treatment (35 mg q.i.d.), $E F$ was increased by $6 \pm 4 \%(\mathrm{p}<0.01)$, SV was increased by $9.0 \pm 6.4 \%(\mathrm{p}<0.01)$. The heart lipid $\%$ content did not change significantly. 
The late wash-out of fatty acids from the myocardium, accounting for a significant proportion of oxidized palmitate, decreased on average by $36.3 \%$ $(\mathrm{p}=0.05$ range: from $-74 \%$ to $+0.1 \%$ ). The BMI was positively associated with LV work, Rate Pressure Product $(r>0.85, \mathrm{p}<0.01)$ and FAO $(\mathrm{r}=0.71, \mathrm{p}<0.05)$ and negatively related with SV $(\mathrm{r}=-0.70, \mathrm{p}=0.05)$. In turn, LV FAU and FAO were positively correlated with the LV work $(r=0.85$ and 0.83 , respectively, $\mathrm{p} \leq 0.01$ ). Changes in FAO positively correlated with changes in systemic triglycerides $(\mathrm{r}=0.77, \mathrm{p}=0.025)$. LV GU and the SV were positively related $(\mathrm{r}=0.76, \mathrm{p}=0.029)$. Changes in LV GU and $\mathrm{CO}$ after treatment were negatively associated $(\mathrm{r}=-0.80, \mathrm{p}=0.017)$.

Conclusion: In conclusion, the degree of obesity is associated with higher cardiac work and FAO, and reduced SV. Trimetazidine improves LV function by reducing FAO in obese subjects.

Supported by: Turku University Hospital, Finnish Diabetes Foundation, EFSD/ Lilly Research award and Novo Nordisk Foundation

\section{PS 062 Mitochondrial function and metabolism}

\section{1}

Expression of a malonyl-CoA-insensitive CPT1 to decrease palmitateinduced apoptosis in muscle cells

C. Hénique, G. Fumey, J. Girard, C. Prip-Buus, I. Cohen

Endocrinolgy, Metabolism and Cancer, Cochin Institute - INSERM U567

- CNRS UMR 8104, Paris, France

Background and aims: Obesity is a prevalent metabolic disorder that is a major risk for other alterations including insulin resistance and type 2 diabetes. While fatty acids (FA) are vital metabolic substrates for many cellular processes, lipid overload leads to inappropriate FA accumulation in nonadipose tissues such as skeletal muscle, which is responsible for cell dysfunctions and death (lipoapoptosis). Both chronic exposure of skeletal muscle to elevated concentrations of lipids and altered long-chain fatty acid (LCFA) mitochondrial oxidation contribute to the muscular dysfunctions through accumulation of deleterious FA metabolites. LCFA are oxidized in the mitochondrial matrix and their entry involves the carnitine palmitoyltransferase (CPT) system. Because of its inhibition by malonyl-CoA, an intermediate of lipogenesis, CPT1 represents the rate limiting step of mitochondrial LCFA oxidation.

Materials and methods: To determine if an increase in CPT1 activity can protect muscle cells from palmitate-induced apoptosis, we have expressed in the $\mathrm{C} 2 \mathrm{C} 12$ muscle cell line, using an adenovirus approach, a mutant form of CPT1 (CPT1mt) which is insensitive to malonyl-CoA inhibition.

Results: In C2C12 muscle cells, we demonstrate that palmitate, a saturated LCFA, induces myotube apoptosis in a time- and concentration-dependent manner. A 24 hours-incubation of the cells with $0.8 \mathrm{mM}$ palmitate causes caspase 3 cleavage (a $50 \%$ decrease in caspase 3 precursor, determined by western blot) and enhances by $60 \%$ its proteolytic activity, as measured by flow cytometry. Compared with control cells, CPT1mt expression in C2C12 myotubes induces a 2.7 -fold increase in CPT1 activity ( $1.6 \pm 0.2$ vs $0.6 \pm 0.1$ $\mathrm{nmol} / \mathrm{mg}$ protein $/ \mathrm{min}, \mathrm{p}<0.01$ ), which is maintained in the presence of $5 \mu \mathrm{M}$ malonyl-CoA ( $0.8 \pm 0.1$ vs $0.05 \pm 0.03 \mathrm{nmol} / \mathrm{mg}$ protein $/ \mathrm{min}, \mathrm{p}<0.001)$. This leads to a 2.6 -fold increase in LCFA oxidation flux ( $6.5 \pm 0.3$ vs $2.5 \pm 0.2$ $\mathrm{nmol} / \mathrm{mg}$ protein $/ 3 \mathrm{~h}, \mathrm{p}<0.001$ ) whereas oxidation of medium-chain fatty acid, which enters the mitochondria independently of the CPT system, was unaffected. In the presence of high glucose $(20 \mathrm{mM})$ and insulin $(100 \mathrm{nM})$ concentrations, CPT1mt-expressing cells maintain a high rate of LCFA oxidation whereas this pathway is more inhibited in control cells $(4.5 \pm 0.2$ vs $1.05 \pm 0.05 \mathrm{nmol} / \mathrm{mg}$ protein $/ 3 \mathrm{~h}, \mathrm{p}<0.001$ ). In myotubes exposed to $0.8 \mathrm{mM}$ palmitate for 24 hours, CPT1mt expression confers a less decrease in caspase 3 cleavage (a $10 \%$ decrease in caspase 3 precursor vs $50 \%, \mathrm{p}<0.001$ ). Thus, CPT1 exerts a protective effect against palmitate-induced apoptosis.

Conclusion: These results strongly favor the hypothesis that any strategy designed to enhance LCFA oxidation in muscle should protect cells from lipidinduced apoptosis and we propose CPT1 as a possible therapeutic target for the treatment of type 2 diabetes.

\section{2}

The differential impact of antiretroviral (ARV) therapies on mitochondrial and lipid metabolism genes in human adipose tissue K.C. McGee ${ }^{1}$, A.L. Harte ${ }^{1}$, M. Hill'1, M. Boothby ${ }^{2}$, J. Tomlinson ${ }^{3}$, L.L. Gathercole ${ }^{4}$, S. Kumar ${ }^{1}$, M. Shahmanesh ${ }^{2}$, P. McTernan ${ }^{1}$

${ }^{1}$ Unit for Diabetes and Metabolism, University of Warwick, Coventry, ${ }^{2} \mathrm{HIV}$ Medicine, University Hospital Birmingham, ${ }^{3}$ Division of Medical Sciences, University of Birmingham, ${ }^{4}$ Division of Clinical Sciences, University of Birmingham, United Kingdom

Background and aims: Our previous studies have shown that both central obesity and diabetic status impact on lipid metabolism and mitochondrial gene expression. However, less is understood on the impact of HIV infection itself and its treatments. HIV infection causes a disturbance in lipid metabolism, and some antiretroviral (ARV) regimens, such as those containing zidovidine (AZT), have been associated with lipodystrophy, diabetes and the metabolic syndrome. For this study we investigated the effect of (1) HIV status and (2) antiretroviral therapy on mitochondrial and lipid related genes. Materials and methods: Subcutaneous adipose tissue (AT) biopsies from the ileac crest were obtained from 15 controls and 32 HIV subjects pre- and 
post- 6 month ARV treatment (HIV: BMI: $25.5\left(\right.$ mean \pm SD) $\pm 5.0 \mathrm{~kg} / \mathrm{m}^{2}$; Age: $35.9 \pm 9.49 \mathrm{yrs}$; Control: BMI: $26.8 \pm 4.24 \mathrm{~kg} / \mathrm{m}^{2}$; Age: $\left.34.8 \pm 9.7 \mathrm{yrs}\right)$. Patients were randomised to either $\mathrm{AZT} / 3 \mathrm{TC} / \mathrm{EFV}$ (zidovidine/lamuvidine/efavirenz), or $\mathrm{TDF} / \mathrm{FTC} / \mathrm{EFV}$ (tenofovir/emtricitabine/efavirenz) treatment. Real time PCR examined genes related to mitochondrial metabolism and lipid metabolism including FAS, FABP4, LPL, and HSL. All PCR reactions were multiplexed with the housekeeping gene $18 \mathrm{~S}$.

Results: Mitochondrial related genes from human AT biopsies were increased in HIV patients, at baseline compared with non HIV case controls (COX3: $\mathrm{p}<0.05$; CYTB: $\mathrm{p}<0.05$; HCS: $\mathrm{p}<0.05$; NRF- 1 : $\mathrm{p}<0.05$ ). AT biopsies taken from HIV patients 6 months post-treatment showed that both AZT and TDF ARV therapy led to an overall significant increase in mitochondrial related gene expression of TFAM $(\mathrm{p}<0.01), \operatorname{HCS}(\mathrm{p}<0.05), \mathrm{ND}-4(\mathrm{p}<0.001)$ COX-4 ( $<<0.05)$, in addition to lipid metabolic genes LPL ( $\mathrm{p}<0.001)$, FABP4 $(\mathrm{p}<0.001)$, FAS $(\mathrm{p}<0.001)$ and HSL $(\mathrm{p}<0.001)$ as compared with naïve HIV patients. Individual analysis of the impact of each drug therapy highlighted that treatment of HIV patients with AZT/3TC/EFV for 6 months led to increased mitochondrial related gene expression of COX4 ( $<<0.01)$, ND-4 $(\mathrm{p}<0.01)$, whilst down-regulating CYTB $(\mathrm{p}<0.001)$ and COX-3 $(\mathrm{p}<0.05)$. TDF/FTC/EFV 6 month post treatment up-regulated TFAM (vs baseline HIV patients: $\mathrm{p}<0.01$ ) and ND-4 (vs baseline HIV patients: $\mathrm{p}<0.01$ ) but had little impact on CYTB or COX-3.

Conclusion: HIV patients, prior to ARV treatment, appear to have increased mitochondrial function compared with non-HIV case controls. In addition, short term ARV therapy increased mitochondrial related gene expression and lipid related genes, overall. Comparative analysis of the effects of the different ARV therapies on regulation of mitochondrial related gene expression highlighted that there was little difference between the AZT/3TC/EFV and TDF/FTC/EFV regimens, although there was significantly decreased expression of mitochondrially encoded CYTB and COX3 6 month post AZT/3TC/ $\mathrm{EFV}$ treatment, thus suggesting a potential mitochondrial respiratory gene dysfunction. Future studies will be required to elucidate the influence of HIV status and drug therapies on human AT, which may affect the lipodystrophyic phenotype and contribute to the reduction/progression of metabolic disease. Supported by: Department of Health

\section{3}

Stability of mitochondrial DNA against reactive oxigen species generated in diabetes

O. Savu ${ }^{1,2}$, A. Nikosjkov ${ }^{1}$, V. Sunkari ${ }^{1}$, I. Botusan ${ }^{1,3}$, J. Grünler ${ }^{1}$, K. Brismar ${ }^{1}$, S.-B. Catrina ${ }^{1,3}$

${ }^{1}$ Molecular Medicine and Surgery, Karolinska Institute, Stockholm, Sweden, ${ }^{2}$ Diabetes, "N.C. Paulescu" National Institute, Bucharest, Romania, ${ }^{3}$ Endocrinology, University of Medicine and Pharmacy "Carol Davila", Bucharest, Romania

Background and aims: The hyperglycaemia-induced overproduction of reactive oxygen species (ROS) by the mitochondria was proposed to be the unifying mechanism in the pathogenesis of diabetic complications. Local hypoxia, which is another source of ROS, has also emerged as an important factor in the development of chronic complications of diabetes. Even though mitochondrial DNA (mtDNA) is a very sensitive target for oxidative damage there is few data available on the unevenly somatic point mutations and deletions of mtDNA in diabetes and only from transversal studies. Moreover there is no direct investigation on the influence of chronic hyperglycaemia and/or hypoxia on mtDNA stability and repair. We propose to study the stability of mtDNA against ROS overproduction in diabetic condition.

Materials and methods: mtDNA mutations were evaluated both by cloning and sequencing a $600 \mathrm{bp}$ region from cytochrome B and by long fragment polymerase chain reaction (PCR) ( $10 \mathrm{kbp})$ normalized for mitochondria copy number. In vitro studies were performed in primary human dermal fibroblasts and endothelial cells exposed to different glucose concentrations and oxygen tensions. The in vivo studies were performed in diabetic $(\mathrm{db} / \mathrm{db})$ mice at different phases of the disease and compared with their heterozygotes littermates. A special strain of a mtDNA mutator mice was used as positive control. Cellular oxidative stress was measured by dichlorofluorescein assay in vitro and by nitrotyrosine immunobloting in vivo. Gene expression for manganese superoxide dismutase (MnSOD) and catalase (CAT) were analyzed by quantitative Real-Time PCR. Mitochondrial base excision repair (mtBER) activity was investigated by using abasic site-containing oligonucleotides as substrates.

Results: The combination of hyperglycaemia and hypoxia induced a higher incidence of mtDNA damage both in endothelial and in fibroblasts in vitro.
An increased incidence of point mutations was identified by sequencing in "old" (34 weeks) $\mathrm{db} / \mathrm{db}$ mice $(\mathrm{p}<0.05)$ but not in the "young" $\mathrm{db} / \mathrm{db}$ mice when compared with their age matched non diabetic littermates. Surprisingly we found a significantly decreased incidence of mtDNA damage in old diabetic mice than in controls ( $<<0,01$ for any of organs analysed). However ROS overproduction was observed in diabetic conditions both in vitro and in vivo experiments. Gene expression for $\mathrm{MnSOD}$ and CAT was significantly higher ( $\mathrm{p}=0,002$, and $\mathrm{p}=0,005$ respectively) in "old" $\mathrm{db} / \mathrm{db}$ mice but not in "young" db/db mice when compared with their aged matched controls. Conclusion: Our results reflect mtDNA instability in diabetic conditions but the mechanisms behind it are not clear yet. Gene expression studies suggest an up regulation of antioxidant defense in mitochondria which might reflect in an effective repair system in vivo.

Supported by: Family Stefan Persson Foundation, Fredrik och Ingrid Turings Stiftelse, Bert von Kantzows Stiftelse

\section{4}

Molecular and metabolic evidence for mitochondrial dysfunction associated with beta-cell failure in type 2 diabetes

H. Lu, V. Koshkin, A. Gyulkhandanyan, M.B. Wheeler

Department of Physiology, University of Toronto, Canada

Background and aims: The efficient coupling of glucose metabolism to energy production in mitochondria of $\beta$-cells is required for appropriate insulin secretion. The importance of this process is underscored by the observation that disrupting mitochondrial oxidative metabolism in $\beta$-cells blocks nutrient-mediated secretion. However, a specific relationship between mitochondrial dysfunction and $\beta$-cell failure has not been clearly established in type- 2 diabetes (T2D). To explore this probable association, we studied the MKR mouse, a genetic model of T2D caused by an IGF-1 receptor mutation in skeletal muscle. The goal of the study was first, to determine the potential contribution of mitochondrial dysfunction to $\beta$-cell failure and second, to identify the molecular events responsible for mitochondrial dysfunction.

Materials and methods: Both metabolic and proteomic approaches were used in this study. MKR and age-matched control mice were characterized by the assessment of blood glucose, plasma insulin and glucose tolerance tests (GTT). Pancreatic islet cell morphology was examined using immunohistochemistry and electron microscopy, while islet secretory function was measured by glucose-stimulated insulin secretion. Islet mitochondrial metabolism was assessed by mitochondrial membrane potential, ATP, reactive oxygen species, mitochondrial $\mathrm{Ca}^{2+}$ accumulation and oxygen consumption. Global islet protein expression profiles were measured using iTRAQ proteomics.

Results: Three week-old MKR mice were insulin resistant, whereas 10 weekold mice were insulin resistant and hyperglycaemic. The GTT revealed that 10-week-old MKR mice displayed glucose intolerance with the loss of first phase insulin secretion, whereas 3-week-old MKR mice retained firstphase insulin secretion in response to glucose. Ex vivo MKR and control islets showed similar secretory responses to glucose at 3 weeks, but secreted $51 \%$ less insulin under high glucose $(20 \mathrm{mM})$ conditions at 10 weeks of age $(p<0.001)$. Immunohistochemical analysis of islets showed no gross morphological changes in 3-week-old MKR mice, whereas 10 week-old MKR islets were hypertrophic and hyperplasic. Electron microscopy revealed the mitochondria from 10 -week-old MKR $\beta$-cells were significantly swollen with abnormal cristae. Whole islets or dispersed cells from 10-week-old MKR mice exhibited 38\% less ATP $(p<0.001), 46 \%$ reduced glucose-stimulated hyperpolarization of the mitochondrial membrane potential $(p<0.001), 50 \%$ attenuation in glucose-stimulated mitochondrial $\mathrm{Ca}^{2+}$ uptake $(p<0.001)$, a $31 \%$ decrease in the cytochrome $c$ oxidase-dependent oxygen consumption rate $(p<0.01)$, and a two fold increase in reactive oxygen species (ROS) levels $(p<0.001)$ compared to controls. In line with a defect in mitochondrial function, islet protein profiling revealed that the expression of 38 mitochondrial proteins were significantly dysregulated in MKR diabetic islets. Consistent with a defect in oxidative phosphorylation, there was a prominent downregulation of proteins (Ndufa9, Uqcrh, Cox2, Cox4i1, Cox5a and Atp5j2) of the mitochondrial respiratory chain.

Conclusion: Our results indicate that the loss of glucose-stimulated insulin secretion in the diabetic MKR mouse is likely caused by a defect in mitochondrial function as demonstrated by gross abnormalities in mitochondrial morphology, function and the expression of proteins involved in electron transport.

Supported by: Canadian Diabetes Association 


\section{5}

Inhibition of liver fatty acid oxidation by Teglicar improves the hypoglycaemic effect of metformin both "in vitro" and "in vivo" R. Conti, R. Liberati, S. Di Donna, P. Carminati, F. Giannessi Endocrinology and Metabolism, sigma-tau industrie farmaceutiche riunite SpA, Pomezia, Italy

Background and aims: Metformin action in reducing hepatic glucose production includes mitochondrial Complex I inhibition, increase in AMP level and activation of AMPK.

Teglicar, a recently identified reversible inhibitor of the liver isoform of Carnitine palmitoyltransferase1 (L-CPT1), currently in clinical phase II, reduces gluconeogenesis by decreasing pyruvate carboxylase activity through inhibition of liver fatty acid oxidation. The aim of this study was to investigate, using Teglicar, the role of liver fatty acid oxidation on the hypoglycemic effect of Metformin, both in vitro on freshly isolated hepatocytes and in vivo on $\mathrm{db} / \mathrm{db}$ mice.

Materials and methods: Sprague-Dawley rat liver cells, obtained after 24 hours of fasting, were incubated for $150 \mathrm{~min}$ at $37^{\circ} \mathrm{C}$ in $0.8 \mathrm{~mL}$ of gassed (95:5; $\mathrm{O}_{2}: \mathrm{CO}_{2}$ ) Krebs medium containing $2 \%$ defatted BSA, $1 \mathrm{mM}$ glucose, 1 $\mathrm{mM}$ pyruvate, $9 \mathrm{mM}$ lactate. The hepatocytes were preincubated for $30 \mathrm{~min}$ with the drugs prior to addition of $1 \mathrm{mM}$ oleate. The in vivo study was performed on hyperglycemic $\mathrm{db} / \mathrm{db}$ mice orally treated with a low dose of Teglicar $(60 \mathrm{mg} / \mathrm{kg} / \mathrm{die})$ for four days. On the last day, Metformin $(100 \mathrm{mg} / \mathrm{kg})$ was given orally at time 0 . Tail blood was collected at 60,120 and $270 \mathrm{~min}$ and blood glucose measured by glucometer.

Results: Control hepatocytes linearly released glucose and ketone bodies in the medium (around 120 and $55 \mu \mathrm{g} / 10^{6} \mathrm{cell} / \mathrm{s} / \mathrm{h}$, respectively). Teglicar and Metformin, when tested alone, reduced glucose production $\left(\mathrm{ED}_{50}=10 \mu \mathrm{M}\right.$ and $1.2 \mathrm{mM}$, respectively). When increasing concentrations of Teglicar $(1,2$ or $10 \mu \mathrm{m})$ were added to Metformin $(0.3,1.2$ or $1.8 \mathrm{mM})$, a further reduction of hepatocyte glucose production was measured at all studied concentrations. As Metformin does not affect the reduction of ketone bodies operated by Teglicar $\left(\mathrm{ED}_{50}=1 \mu \mathrm{M}\right)$, it is evident that inhibition of $\beta$-oxidation improves Metformin action. The mitochondrial redox state (by the $\beta$-Hydroxybutyrate/Aceto-acetate ratio), was profoundly increased by Metformin ( 8.5 at Metformin $1.8 \mathrm{mM}$ vs 0.7 control) relating to a reduction in NADH utilization on the electron transport chain. In the presence of Teglicar, the redox state progressively dropped to much lower values, suggesting that most of the NADH production, under our conditions, is $\beta$-oxidation-dependent. The increase of mitochondrial redox state, related to Complex I inhibition, is in agreement with a reduced hepatocyte ATP content $(-70 \%$ at $1.8 \mathrm{mM}$ Metformin with respect to controls). Teglicar alone did not alter the ATP nor did it affect the Metformin-induced drop in ATP content. The potentiation of Metformin effect was also shown in vivo on $\mathrm{db} / \mathrm{db}$ mice. Blood glucose, after a single low Metformin dose, following a subchronic low Teglicar dose, resulted $48 \%$ lower than with the two drugs alone.

Conclusion: Our results prove that the reduction of liver $\beta$-oxidation operated by Teglicar improves the hypoglycemic effect of Metformin.

\section{6}

Is the sub-normal insulin stimulation of muscle ATP synthesis in type 2 diabetes always a consequence of low rates of muscle glycogen synthesis? E. Lim, M.I. Trenell, K.G. Hollingsworth, F.E. Smith, P.E. Thelwell,

R. Taylor

Institute of Cellular Medicine, Newcastle Magnetic Resonance Centre, Newcastle upon Tyne, United Kingdom

Background and aims: Abnormalities in mitochondrial ATP synthesis in muscle of people with type 2 diabetes have been reported. Although these may be primary to the development of insulin resistance and type 2 diabetes, the measurement must be interpreted in the light of the energy demands of the muscle cell. In type 2 diabetes, the rate of muscle glycogen synthesis is reduced and this may lead to a lower requirement of mitochondrial ATP synthesis as a secondary phenomenon. We set out to test the hypotheses that a) the lower rates of mitochondrial ATP synthesis observed in muscle of type 2 diabetes is a consequence of subnormal rates of muscle glycogen synthesis rather than a primary defect in mitochondrial function and b) mitochondrial ATP synthesis can be restored by suppressing fatty acid oxidation.

Materials and methods: ${ }^{13} \mathrm{C}$ magnetic resonance spectroscopy (MRS) was used for the direct in vivo measurement of the rates of muscle glycogen synthesis in response to an isoglycaemic-hyperinsulinaemic clamp. The preci- sion of the measurement of muscle glycogen was improved by infusing ${ }^{13} \mathrm{C}$ enriched glucose. The rate of mitochondrial ATP synthesis was assessed by ${ }^{31} \mathrm{P}$ MRS measurement of saturation transfer between ATP and inorganic phosphate in the calf muscle. Diabetic subjects ( $>2$ years duration; BMI 28.3 $\pm 4.2 \mathrm{~kg} / \mathrm{m}^{2} ; \mathrm{HbA}_{1 \mathrm{c}} 6.6 \pm 0.6 \%$ ) were studied on 2 separate days - during an isoglycaemic-hyperinsulinaemic clamp and during the same with complete suppression of NEFA levels using acipimox. Normal controls were studied once only, without acipimox.

Results: In normal controls, after $20 \mathrm{~min}$ of insulin stimulation, the muscle glycogen signal rose by $30.0 \pm 12.5 \%$ (blood glucose of $4.8 \pm 0.4 \mathrm{mM}$ ) with a corresponding increase in ATP flux of $15 \pm 26 \%$. In the diabetic subjects, $20 \mathrm{~min}$ of insulin stimulation brought about no change in muscle glycogen signal $(-1.3 \pm 6.1 \%)$ during isoglycaemia $(6.05 \pm 0.50 \mathrm{mmol} / \mathrm{l})$ and minimal change in ATP flux $(4 \pm 16 \%)$ on placebo day. Suppression of NEFA levels were achieved with acipimox $250 \mathrm{mg}$ given at -3 hours and at the start of the insulin clamp in diabetic subjects (at $15 \mathrm{~min}=0.09 \pm 0.02$ vs. $0.62 \pm 0.10$ $\mathrm{mmol} / \mathrm{l}$ with and without acipimox; $\mathrm{p}<0.01)$. During NEFA suppression in the diabetic subjects, insulin stimulation brought about a $24 \pm 10 \%$ increase in ATP flux by $20 \mathrm{~min}$ during isoglycaemia $(5.6 \pm 0.3 \mathrm{mmol} / \mathrm{l})$. However, despite this significant increase in ATP flux, there was only minimal increase in muscle glycogen signal $(5 \pm 4 \%)$. Insulin-stimulated whole body glucose disposal was $3.79 \pm 0.71$ and $2.65 \pm 0.09 \mathrm{mg} / \mathrm{kg} / \mathrm{min}$ during isoglycaemia in controls and diabetic subjects respectively.

Conclusion: Although insulin stimulated glycogen and ATP synthesis rates are similarly sub-normal in type 2 diabetes, suppression of fatty acid oxidation created a condition in which the former remains unchanged despite increase in the latter. We conclude that that ATP synthesis rates are not primarily determined by glycogen synthesis rate and these two parameters can be separately modulated in type 2 diabetes. The subnormal muscle ATP synthesis rate of type 2 diabetes can be restored by suppressing fatty acid oxidation. Supported by: Wellcome Trust

\section{7}

Mitochondrial nitric oxide is increased in the ob-/- mice model of metabolic syndrome

P.V. Finocchietto, F.J. Barreyro, S.A. Holod, M.C. Carreras, J.J. Poderoso

Laboratory of Oxygen Metabolism, University Hospital University of Buenos Aires, Argentina

Insulin resistance (IR) is associated with the increase of visceral adipose tissue (WAT). Although the mechaism is unknown, there are several reports about mitochondrial abnormalities in metabolic tissues in IR. Nitric Oxide (NO) is a signaling molecule with many of its effects on cell function being elicited at the mitochondrial level. In different tissues, Nitric Oxide Synthase traslocates to mitochondria (mtNOS) and synthesizes $\mathrm{NO}$ vectorially directed to the matrix. It is noteworthy that NO steady-state concentration modulates electron transfer, $\mathrm{O} 2$ uptake, and the reactive oxygen species yield.

Aim: to determine the activity and expression of mtNOS in WAT, Muscle and Liver of leptin deficient Ob-/- mice.

Methods: we used Ob -/- and C57BL/6 WT mice (6-9 mo old); mice were divided in two subgroups with no intervention or receiving leptin replacement ( 6 ug bid IP 4 days). Epidydimal WAT, muscle and liver were excised and isolation and purification of mitochondria was done by differential centrifugation. Mitochondrial NO was determined by flow citometry with DAF in the presence or not of NOS inhibitor L-NMMA, the expression of mtNOS by Western Blot and RT-PCR and mitochondrial Complex I-IV activities were followed spectrophotometrically. Complexes were separated by BN-PAGE, and tyrosine nitration was detected by Western Blot. We evaluated mtNOS content and mitochondrial morphology by electronic microscopy.

Results: 1 ) Ob-/- have a significant increase of mtNOS activity and NO production in accord to 3 -fold increase of expression. 2) Complex I activity resulted markedly reduced in WAT $(-81 \% \mathrm{p}<0,05)$, muscle $(-72 \% \mathrm{p}<0,05)$ and liver $(-61 \% \mathrm{p}<0,05)$ from $\mathrm{Ob}-/-$; there were no significant changes of the activity of Complexes II-III and IV. 3)According to its low activity, Complex I exhibited 3-4 fold increased tyrosine nitration in WAT, muscle and liver mitochondria from $\mathrm{Ob}-/$-. 4) Leptin replacement completely normalized mtNOS activity and Complex I activity, with significantly decreased nitration to the same level of controls. 5) Electronic microscopy confirmed the variations of mtNOS content in mitochondria.

Conclusion: a) Mitochondria of Ob-/- mice tissues are exposed to high NO matrix concentration that conducts to Complex I nitration and subsequent reduced activity; b) Likewise, more than $60 \%$ Complex I inhibition contributes to low Ob-/- O2 uptake and obesity; c) Enhanced NO depends on high 
mtNOS expression and as well preliminary data of our group indicate on that insulin increases mtNOS activity and leptin reduces mtNOS activity; d) thus, in this rodent model of metabolic syndrome, mitochondrial hypometabolism should depend on the balance between leptin deficiency and reduced sensitivity to insulin. These evidences show that after energy replenishment, the inhibition of mitochondrial respiration in metabolic tissues via NO permits substrates to be deposited as macromolecules; at hyperinsulinemia and leptin deficiency, persistent mtNOS activation could contribute to mitochondrial dysfunction with insulin resistance and obesity and therefore to the progression of the ob -/- metabolic syndrome.

\section{8}

Role of mitochondria-derived reactive oxygen species in fat-induced insulin resistance in liver

T. Takamura, N. Nagata, S. Nakamura, H. Takayama, M. Sugimori, H. Misu, S. Kaneko

Department of Disease Control and Homeostasis, Kanazawa University Graduate School of Medical Science, Japan

Background and aims: Insulin resistance is a key pathophysiological feature of metabolic syndrome. Visceral adiposity in obesity causes excessive free fatty acids (FFAs) flux into the liver via the portal vein and may cause fatty liver disease and hepatic insulin resistance. However, the initial events triggering the development of insulin resistance and its causal relations with dysregulation of glucose and fatty acids metabolism remain unclear.

Materials and methods: We investigated biological pathways that have the potential to induce insulin resistance in male C57BL/6J mice fed a high-fat diet (HFD) containing 60\% fat. To investigate the direct and initial effects of FFAs on hepatocytes, we treated H4IIEC 3 cells, a rat hepatocyte cell line, with a monounsaturated fatty acid (oleate, $0.25 \mathrm{mM}$ ) and a saturated fatty acid (palmitate, $0.25 \mathrm{mM}$ ) in the presence of $2 \%$ FFA-free bovine serum albumin. Results: (1) Comprehensive gene expression analyses by using DNA chip technology revealed that the pathways for reactive oxygen species (ROS) production and oxidative stress are coordinately up-regulated in both the liver and adipose tissue of mice fed a HFD prior to the onset of insulin resistance and obesity through discrete mechanisms. (2) In the liver, a HFD up-regulated genes involved in sterol regulatory element binding protein (SREBP)1c-related fatty acid synthesis and peroxisome proliferator-activated receptor (PPAR)- $\alpha$-related fatty acid oxidation. (3) In the adipose tissue, however, the HFD down-regulated genes involved in fatty acid synthesis and up-regulated genes involved in forming the NADPH oxidase complex. (4) Increased ROS production preceded the elevation of TNF- $\alpha$ and FFAs in the plasma and liver. (5) Protein carbonyl was accumulated in the liver, but not in the adipose tissue of HFD mice before the onset of insulin resistance, partly due to up-regulation of gene for glutathione peroxidase, one of the redox proteins, only in adipose tissue. In cultured H4IIEC3 hepatocytes, (6) Palmitate, but not oleate, inhibited insulin-stimulated tyrosine phosphorylation of insulin receptor substrate 2 and serine phosphorylation of Akt, through c-Jun $\mathrm{NH}_{2}-$ terminal kinase (JNK) activation. (7) Among the well-established stimuli for JNK activation, ROS played a causal role in palmitate-induced JNK activation. (8) Etomoxir, an inhibitor of carnitine palmitoyltransferase-1, which is the rate-limiting enzyme in mitochondrial fatty acid $\beta$-oxidation, as well as inhibitors of the mitochondrial respiratory chain complex (thenoyltrifluoroacetone and carbonyl cyanide $m$-chlorophenylhydrazone) decreased palmitate-induced ROS production.

Conclusion: In the early stage during the development of fat-induced insulin resistance, there are compensatory alterations in the expression of genes that regulate fatty acid metabolism between the liver and adipose tissue. However, oxidative stress may be an initial key event triggering HFD-induced insulin resistance especially in the liver. Our findings in cultured hepatocytes indicate that palmitate inhibits insulin signal transduction through JNK activation, and that accelerated $\beta$-oxidation of palmitate causes excess electron flux in the mitochondrial respiratory chain, resulting in ROS overproduction. Thus, mitochondria-derived ROS induced by palmitate might be major contributors to JNK activation and insulin resistance in the liver. Supported by: Ministry of Education, Science, Sports, and Culture of Japan

\section{PS 063 Animal models of type 2 diabetes}

\section{9}

Reduced beta cell proliferation and impaired glucose tolerance in pancreatic beta cell specific Extl3 knockout mice

I. Takahashi ${ }^{1}$, K. Nata ${ }^{2}$, N. Noguchi ${ }^{1}$, T. Ikeda ${ }^{3}$, K. Sugihara ${ }^{4}$, M. Asano ${ }^{4}$,

T. Yoshikawa ${ }^{1}$, A. Yamauchi ${ }^{5}$, N.J. Shervani ${ }^{1}$, A. Uruno ${ }^{1}$, M. Unno ${ }^{6}$,

S. Takasawa ${ }^{5}$, H. Okamoto ${ }^{1}$, A. Sugawara ${ }^{1}$

${ }^{1}$ Advanced Biological Sciences for Regeneration (Kotobiken Medical Laboratories), Tohoku University, Sendai, ${ }^{2}$ Medical Biochemistry, Iwate Medical University, Yahaba-cho, ${ }^{3}$ Innovation Plaza Miyagi, JST, Sendai, ${ }^{4}$ Transgenic Animal Science, Kanazawa University, Kanazawa, ${ }^{5}$ Biochemistry, Nara Medical University, Kashihara, ${ }^{6}$ Surgery, Tohoku University, Sendai, Japan

Background and aims: EXTL3 (exostosin tumor-like 3) was originally identified as a member of the exostosin tumor (EXT) family in human. This molecule functions as a glycosyltransferase involved in heparan sulfate (HS) proteoglycans (PGs) biosynthesis. Recently, sulfated PGs including HSPGs have been reported to be involved in pancreatic endocrine cell differentiation. However, it remains unclear whether EXT family plays an important role in pancreatic $\beta$-cell development and its function in vivo. EXTL3 catalyzes both the initiation and the elongation of HS chain, and among EXT family members, its mRNA was observed to be expressed most abundantly in mouse pancreatic islet. We therefore generated pancreatic $\beta$-cell specific Extl 3 knockout $(\beta E x t l 3 K O)$ in order to clarify this issue.

Materials and methods: $\beta$ Extl3KO mice were generated through gene targeting using the rat insulin promoter-Cre/loxP recombination system. Immunohistochemical analyses of pancreatic islets were performed in 1-, 2-, 4 - and 8-week old mice using insulin and glucagon antibodies. The $\beta$-cell proliferation activity was determined by the percentage of insulin and proliferating cellular nuclear antigen (PCNA) double-positive cells against insulinpositive cells. For measurement of pancreatic insulin content, pancreata were removed from 2-, 4- and 8-week old mice and insulin was extracted by the acid-ethanol method. Glucose tolerance and insulin sensitivity were evaluated by an intraperitoneal glucose tolerance test and an insulin tolerance test, respectively, in 8-week old mice.

Results: Histological examination of pancreatic islets revealed an atypical organization (glucagon-positive cells existed in the central region of the islets) in $\beta$ Extl3KO mice at $2-8$ weeks old, and the $\beta$-cell number/islet cell number ratio was significantly diminished in $\beta$ Extl3KO islets compared to that in control islets. In $\beta$ Extl3KO mice, the $\beta$-cell proliferation activity determined by PCNA positivity was decreased to $29 \%$ (at 2 weeks) and $41 \%$ (at 4 weeks) of those in control mice $(\mathrm{p}<0.05, \mathrm{n}=3)$, although it was not different from that in control mice at 8 weeks. Pancreatic insulin content in $\beta$ Extl3KO mice was decreased to $80 \%$ of control at 2 weeks old $(p<0.05, n=5)$. However, there was no difference in pancreatic insulin content between $\beta$ Extl3KO mice and their controls at 8 weeks old. Although random blood glucose and plasma insulin concentrations were normal in $\beta$ Extl3KO mice at 8 weeks, they showed glucose intolerance coupled to impaired insulin secretion upon glucose challenge. No insulin resistance was observed in $\beta$ Extl3KO mice.

Conclusion: Our data indicate that HS plays important role(s) in $\beta$-cell proliferation and the mechanism of insulin secretion. Extl3 may therefore be a candidate gene for diabetes mellitus, the abnormality of which leads to defective $\beta$-cell proliferation and insulin secretion.

\section{0}

Causes for diabetic hyperphagia in type 2 diabetic Goto-Kakizaki(GK) rats with normal body weight

A. Ando ${ }^{1,2}$, M. Nakata ${ }^{1}$, K. Dezaki ${ }^{1}$, F. Maekawa ${ }^{1}$, K. Eto ${ }^{2}$, S. Nagasaka ${ }^{2}$, S. Ishibashi ${ }^{2}$, T. Yada ${ }^{1}$

${ }^{1}$ Integrative Physiology, ${ }^{2}$ Endocrinology and Metabolism, Jichi Medical University, Shimotsuke, Japan

Background and aims: Diabetic hyperphagia has been reported in several animal models, which include STZ treated type 1 diabetic rats showing insulin and leptin depletion and massively obese type 2 diabetic models such as $o b / o b, d b / d b$ and Ay mice that lack leptin signaling. We have previously shown that GK rats, a type 2 diabetic model, display hyperphagia without 
alteration in body weight at the young-adult ages of 6-13 weeks, in addition to hyperglycemia. This hyperphagia is associated with visceral fat accumulation at mesenteric and perirenal portions, central leptin resistance, and upregulated NPY mRNA expression in the hypothalamic arcuate nucleus. The aim of this study was to evaluate the mutual causal relationship between hyperphagia, visceral fat accumulation and hyperglycemia. For this purpose we examined the effect of restriction of food intake on adiposity and glucose homeostasis in GK rats. We also examined whether the neurons in the feeding center, the arcuate nucleus (ARC), are altered in GK rats in their ability to sense the peripheral metabolic factors, leptin, ghrelin and glucose.

Materials and methods: Wistar rats $(n=6), G K$ rats $(n=6)$ and pair-fed GK rats $(n=6)$ were fostered for 7-14 weeks, and daily food intake was measured. In pair-fed groups, GK rats were given the chow whose amount was the same as that eaten by Wistar rats every day in average (pair feeding). Fat accumulation was measured at 14 weeks of age. Cytosolic $\mathrm{Ca}^{2+}$ concentration $\left(\left[\mathrm{Ca}^{2+}\right] \mathrm{i}\right)$ in isolated arcuate neurons was measured by fura- 2 microfluorometry. Serum insulin and leptin levels were determined by EIA.

Results: Daily food consumption in GK rats was larger than that in Wistar rats during 7-14 weeks. Throughout the observation period, blood glucose levels were higher in both regularly-fed and pair-fed GK rats compared to Wistar rats. Pair feeding failed to correct the elevated blood glucose level of GK rats. Accumulations of both mesenteric and perirenal fats in GK rats were reduced by pair feeding $(\mathrm{p}<0.05)$. At 14 weeks old, GK rats showed significantly elevated concentrations of serum leptin $(\mathrm{p}<0.05)$ and insulin $(\mathrm{p}<0.01)$ compared to Wistar rats, and pair feeding substantially reduced serum leptin levels $(\mathrm{p}<0.05)$, but not serum insulin levels, in GK rats. At 13-14 weeks, to assess neuronal activities, $\left[\mathrm{Ca}^{2+}\right] \mathrm{i}$ was measured in the neurons isolated from the ARC of rats. Lowering glucose to $1 \mathrm{mM}$ increased $\left[\mathrm{Ca}^{2+}\right] \mathrm{i}$ in a subpopulation of the ARC neurons, and the amplitude of $\left[\mathrm{Ca}^{2+}\right] \mathrm{i}$ increases was significantly $(\mathrm{p}<0.05)$ larger in GK than Wistar rats at 14 weeks. By contrast, $\left[\mathrm{Ca}^{2+}\right] \mathrm{i}$ responses to ghrelin and those to leptin were not altered in GK rats. In GK rats at early ages of 3-4 weeks, blood glucose level was already increased. Furthermore, although the amount of food eaten daily was almost similar to Wistar rats, body weight was smaller, resulting in increased food intake per body weight already at 3 weeks.

Conclusion: Visceral fat accumulation in GK rats was largely corrected by pair feeding, suggesting that visceral fat accumulation primarily results from hyperphagia. Blood glucose was continuously elevated at least from 3 weeks. At 14 weeks, glucose sensing (high glucose inhibition) of the ARC neurons was abnormal. The results suggest that hyperglycemia from early stages could influence the central glucose sensing in the ARC neurons and induce hyperphagia in GK rats.

\section{1}

The prediabetic period is characterized by islet microangiopathy in the Goto-Kakizaki rat, a spontaneous model of type 2 diabetes

F. Homo-Delarche ${ }^{1}$, M.-H. Giroix ${ }^{1}$, G. Lacraz ${ }^{1}$, S. Calderari ${ }^{2}$, M. Cornut ${ }^{3}$, J.A. Ehses ${ }^{4}$, F. Schmidlin ${ }^{5}$, J. Coulaud ${ }^{1}$, M.-N. Gangnerau ${ }^{1}$, P. Serradas ${ }^{6}$, J.-C. Irminger ${ }^{3}$

${ }^{1}$ Paris-Diderot University, CNRS UMR 7059, Paris, France, ${ }^{2}$ Collège de France, INSERM U833, Paris, France, ${ }^{3} \mathrm{CMU}$, Dpt of Genetic Medecine \& Development, Geneva, Switzerland, ${ }^{4}$ University Hospital of Zürich, Division of Endocrinology and Diabetes, Switzerland, ${ }^{5}$ Merck, Serono, Chilly Mazarin, France, ${ }^{6}$ Pierre \& Marie-Curie University, INSERM U505, Paris, France

Background and aims: Endothelial signals are essential during pancreas development. Goto-Kakizaki (GK) rats have a $70 \%$ decrease in $\beta$-cell mass at E18, while it is similar at E13 to that of Wistar control rats. Because adult diabetic GK rats are hypercholesterolemic, we hypothesized that maternal hypercholesterolemia together with maternal/fetal hyperglycemia might alter perinatal pancreas development through islet microangiopathy (atherosclerosis) programming. Our aims were to: 1 ) demonstrate alterations of vascularisation in islets from 1-week-old GK rats, during the so-called prediabetic period; 2) measure systemic levels of lipids and various cytokines and chemokines around birth.

Materials and methods: 1 ) Sera were obtained from 1-week-old male Wistar and GK rats (except otherwise stated) for radioimmunoassays; 2) Islets from these rats were hand-picked after collagenase digestion; 3) Molecular approaches included Affymetrix gene array and low density arrays targeted at endothelium, angiogenesis and cardiovascular disease biomarkers (OligoGEArrayRat). Gene expression was confirmed by qRT-PCR.
Results: Affymetrix gene array (230A) showed differential expression of several genes which might be involved in inflammation in GK versus Wistar islets. Notably, the gene encoding epoxide hydrolase, the enzyme responsible for epoxyeicosatrienoic acids (EETs) conversion was decreased by $90 \%$. EETs have anti-inflammatory and protective vascular effects. We then analysed expression of genes present on the 3 targeted arrays mentioned above (128 different genes/array). We obtained 20 up- and 8 down-regulated genes. Among the up-regulated genes were those encoding: caspase 1 (IL-1-converting enzyme, x10) that cleaves IL- 1 and IL-18 into their active forms; various cytokines/chemokines involved in macrophage/granulocyte movements: CXCL2 (MIP-2 $\alpha, x 4.1)$, CXCL-1 (chemokine KC, x3.9), IL-15 (x1.4) and IL18 (x3.7); also fibronectin 1 (x2.3), ICAM-1 (x2.6), VEGFR3 (x2), carboxypeptidase 2 (TAFI, $\mathrm{x} 1.9)$ and angiotensinogen (x1.9). These 3 groups have pro-atherosclerotic effects. Among the down-regulated genes were those encoding: osteoproteogerin $(\mathrm{x} 0.4)$, neuropilin $1(\mathrm{x} 0.6)$, platelet-derived growth factor ( $\mathrm{x} 0.6$ ), coagulation factor III (tissue factor, $\mathrm{x} 0.4$ ) and the neuropeptide $\mathrm{Y}(\mathrm{x} 0.1)$ that acts as vascular mitogen. These data suggest that angiogenesis is deficient in neonatal GK islets. Concomitantly, prediabetic 1-week old-GK rats had lower leptin and higher circulating cholesterol/HDL ratio, MCP-1 and MIP-1a levels than Wistar rats. The latter data might reflect systemic vascular disturbances in GK neonates.

Conclusion: Soon after birth many pro-atherosclerotic genes were upregulated in GK islets, while pro-angiogenic genes were downregulated. These data highlight early deficient islet vascularisation that might be responsible for the GK $\beta$-cell mass defect and therefore may be of potential therapeutic value. Supported by: EFSD/MSD grant

\section{2}

Adaptive compensation of beta cells in an animal model of reduced beta cell mass subjected to high fat diet

P. Masiello ${ }^{1}$, M.-L. Peyot ${ }^{2}$, M. Latour ${ }^{2}$, M. Novelli ${ }^{1}$, V. D’Aleo ${ }^{1}$, R. Lussier ${ }^{2}$, P.-Y. Boyer', E. Joly' ${ }^{2}$, C.J. Nolan ${ }^{3}$, M. Prentki ${ }^{2}$

${ }^{1}$ Dept. of Experimental Pathology, University of Pisa, Italy, ${ }^{2}$ Montreal Diabetes Research Center, University of Montreal, Canada, ${ }^{3}$ Dept. of Endocrinology, Australian National University, Canberra, Australia

Background and aims: We have previously established a non-obese animal model of reduced $\beta$-cell mass (adult Wistar rats given streptozotocin plus nicotinamide, STZ-NA rats), characterized by mild stable hyperglycaemia, glucose intolerance, responsiveness to sulphonylureas, lack of insulin resistance. The aims of this study were: a) to develop an improved model of type 2 diabetes through induction of insulin resistance in STZ-NA rats by a high fat dietary regimen (HFD); b) to explore if a reduction in $\beta$-cell mass would result in more rapid failure of $\beta$-cell compensation in front of increased calorie/fat intake and overweight.

Materials and methods: Male Wistar rats (240 g b.w.) were given $200 \mathrm{mg} / \mathrm{kg}$ NA i.p. and $60 \mathrm{mg} / \mathrm{kg} \mathrm{STZ} \mathrm{i.v.,} 15 \mathrm{~min}$ later. Three weeks after diabetes induction, rats were subjected to HFD (19\% protein, $41 \%$ carbohydrate and $40 \%$ fat in calories, $4.5 \mathrm{kcal} / \mathrm{g}$ versus $3.4 \mathrm{kcal} / \mathrm{g}$ of chow) for 11 weeks. Food intake, body weight, plasma glucose, triglyceride (TG), free fatty acid (FFA) and insulin levels were monitored throughout the experiment. Glucose and insulin tolerance test (GTT and ITT) were also performed. At the end of the experiment, islets were isolated from a number of rats and challenged in vitro with glucose and FFA. The remaining rats were subjected to euglycaemic hyperinsulinaemic clamp to evaluate development of insulin resistance; their pancreatic $\beta$-cell mass was quantitated by immunohistochemical methods and morphometric analysis.

Results: In mildly hyperglycaemic STZ-NA rats, HFD induced a 2-fold increase in plasma FFA, TG and insulin levels, without any further augmentation of blood glucose. Of note, HFD-fed rats consumed less food than chowfed animals, so that the excess in calorie intake was $\sim 15 \%$. During IVGTT, carried out after 4.5 weeks of HFD, the glucose intolerance of STZ-NA rats, due to a reduced acute insulin response, was aggravated in HFD-fed diabetic rats, despite a clear-cut enhancement in post-loading insulinaemia indicating a noteworthy compensatory adaptation of $\beta$ cells. An ITT performed after 6 weeks of HFD showed a slightly delayed but substantially preserved insulin sensitivity in HFD-fed diabetic animals. However, after 11 weeks of HFD, both control and STZ-NA rats eventually developed a pronounced insulin resistance, as assessed by the euglycaemic hyperinsulinaemic clamp. In isolated islets from HFD-fed diabetic rats, an enhancement in glucose- and FFA-induced insulin secretion occurred, with a shift to the left of the dose-response curve. The compensatory adaptation in these animals was also testified by a 
significantly higher $\beta$-cell mass with respect to chow-fed diabetic rats $(60 \%$ versus $40 \%$ of that of healthy controls).

Conclusion: STZ-NA rats with reduced $\beta$-cell mass develop insulin resistance when fed with HFD for several weeks. Thus, they closely reproduce two major features of human type 2 diabetes. Surprisingly, in such a model, despite marked reduction in $\beta$-cell mass and chronic mild hyperglycaemia, compensatory mechanisms in residual $\beta$ cells are able to match for long time the increased insulin demand driven by excess calorie intake and development of insulin resistance. Further investigations are needed to clarify the molecular mechanisms and the duration of such adaptation.

\section{3}

Mkr mice have increased dynamic glucose disposal despite metabolic inflexibility, hepatic and peripheral insulin insensitivity

B. Vaitheesvaran ${ }^{1}$, D. LeRoith ${ }^{2}$, I.J. Kurland ${ }^{1}$

${ }^{1}$ Medicine, State University of New York, Stony Brook, ${ }^{2}$ Medicine, Mt.Sinai School of Medicine, New York, United States

Background and aims: MKR mice (dominant negative IGF-1 receptor overexpressed solely in skeletal muscle), are lean, suggesting metabolic flexibility, but demonstrate early development of Type 2 DM with severe insulin resistance, measured by EU clamp. The EU clamp assesses static glucose disposal in response to a insulin step at basal glucose. Therefore this study was done to assess the metabolic flexibility, in vivo lipolysis and static basal versus dynamic glucose disposal during an ipGTT, in MKR and FVB-wt mice.

Materials and methods: Dynamic glucose disposal was assessed by determining the disappearance rate of $\left[6,6-{ }^{2} \mathrm{H}\right]$-glucose $(\mathrm{D} 2$ glucose). Intraperitoneal glucose tolerance test was performed using $\left[6,6{ }^{2} \mathrm{H}\right]$-glucose $(1 \mathrm{mg} / \mathrm{gram}$ body weight). Static basal glucose disposal, which is equal to hepatic glucose production (HGP) in the fasted state, was determined by constant infusion of $\left[\mathrm{U}-{ }^{13} \mathrm{C}_{6}\right]$-glucose by Alzet minipump. In vivo lipolysis was assessed using 2${ }^{13} \mathrm{C}$-glycerol. Production rate PR $(\mathrm{mg} / \mathrm{kg}$ body weight $/ \mathrm{min})$ was determined using equation $P R=$ infusion rate $x\left(1 / \mathrm{e}_{\text {tracer }}-1\right)$. Infusion rate $(\mathrm{mg} / \mathrm{kg} / \mathrm{min})=$ miniosmotic pump rate $(8 \mu \mathrm{l} / \mathrm{hr}) \mathrm{x}$ plasma tracer concentration/mouse body weight $(\mathrm{kg})$. Indirect calorimetry was done using a 8-chamber Oxymax system (Columbus Instruments, Columbus, $\mathrm{OH}$ )

Results: MKR mice had an approximately $50 \%(\mathrm{p}<0.05)$,increase in fasting insulin. The AUC for the D2 glucose response for MKR mice was decreased ( $80 \pm 6$ vs $120 \pm 5$ for MKR vsFVB-wt mice, $\mathrm{p}<0.05$ ), suggesting faster glucose disposal for MKR mice,despite a similar AUC for total glucose. This suggests HGP was less suppressible in MKR mice in the fed state, in accordance with previously published EU clamp results. $\mathrm{HGP}\left[\mathrm{U}_{-}{ }^{13} \mathrm{C}_{6}\right.$. glucose) was similar in the fasted state despite higher basal insulin (hepatic insulin resistance), however the fraction of glucose molecules $(\mathrm{F})$ that recycled was decreased $(28 \%$ vs $35 \%, \mathrm{p}<0.05)$, which may indicate decreased Cori cycling, and that glucose being utilized more than fatty acids peripherally. Glycerol production using $2-{ }^{13} \mathrm{C}$ - glycerol was significantly increased in the fasted state of MKR mice, despite increased fasting insulin. This indicates insulin resistant lipolysis in MKR mice may be responsible for the decreased fat mass seen in MKR mice, despite increased FFA. Indirect calorimetery indicates metabolic inflexibility is present, in MKR mice. There is a significantly higher respiratory quotient (RQ) for MKR vs. FVB-wt mice in the 15 hour fasted state (RQ=0.884 vs $0.774, \mathrm{p}<0.0001)$, but not in the 5 hour refed state $(\mathrm{RQ}=0.95 \mathrm{vs} .0 .978)$, which supports stable isotope measurements indicating glucose, rather than fatty acids, as a preferred fuel for MKR muscle.

Conclusion: These results suggest that hepatic and adipose insulin resistance is compensatory for preservation of fuel homeostasis in MKR mice. Glucose is the preferred fuel for MKR muscle. In contrast to static EU clamp glucose disposal results, dynamic glucose disposal is increased for the same dynamic insulin response, however elevated MKR basal insulin is needed for MKR mice to match static FVB-wt basal glucose disposal. Liver and adipose insulin resistances are secondary, and may be compensatory, serving to provide glucose, as well as an increased supply of fatty acids, for metabolic needs. Supported by: NIH

\section{4}

Alterations on both hypothalamic insulin and serotonin in a type 2 diabetes model

K. Gerozissis ${ }^{1}$, E.M. Markaki ${ }^{1}$, C. Rouch ${ }^{1}$, S.M. Banas ${ }^{1}$, F. Berthou ${ }^{2}$, D. Bailbe ${ }^{1}, M$. Taouis ${ }^{2}$

${ }^{1}$ UMR 7059, University Paris 7, Paris, ${ }^{2}$ Neuroendocrinologie Moléculaire de la Prise Alimentaire,, University Paris-Sud 11, Orsay, France

Background and aims: Epidemiological studies associate depression and metabolic diseases (MD). Patients with clinical depression exhibit a reduced sensitivity to insulin, whereas the metabolic syndrome is associated with suppressed neuroendocrine responses to serotonin (5HT). However the molecular mechanisms involved are not clear. Two molecules acting in the brain seem to be of particular interest: insulin and 5HT, known to regulate in interaction, energy and glucose homeostasis. It is hypothesized that diminished efficiency or responsiveness of either molecule, contributes to the development of MD. The present study focuses on hypothalamic 5HT release and insulin gene expression in a T2D model, the Goto Kakizaki (GK) rat.

Materials and methods: Brain microdialysis was performed in awake male adult Wistar (W) and GK rats, one week after stereotaxic implantation of a guide in median hypothalamus, under anesthesia. Extracellular hypothalamic $5 \mathrm{HT}$ release in response to a laboratory chow meal (5\% fat, 59\% CHO) (LF) in rats fed either a LF or a high fat ( $40 \%$ fat, $15 \%$ CHO) (HF) diet, was measured by electrochemical detection following HPLC. Hypothalamic mRNA was assessed by real-time RT-PCR. Behavioral, endocrine and metabolic parameters were determined with appropriate methods. Statistics: One way and two way analysis of variance followed by a Fisher PLSD for 5HT, students' t-test for gene expression body weight (BW), food intake (FI), fatty acids and glucose. Significance was set at $\mathrm{p}<0.05 . \mathrm{N}=7-10$ rats per group.

Results: Compared to age matched W, glycemia and insulinemia was increased in GK rats, FI and BW were lower, free fatty acid plasma levels were identical, leptinemia was (marginally) increased. Basal 5HT levels were identical in W and GK rats. FI increased 5HT release. Compared to W, the increase of 5HT in GK rats, was significantly lower. The decreased 5HT responsiveness in GK was similar to that observed in W rats fed a HF diet for one week, a diet known to induce insulin resistance. Compared to LF fed W rats, hypothalamic insulin gene expression was reduced in LF fed GK rats, but mRNA of IR, IRS1 and IRS2 were similar in the two groups.

Conclusion: The present study indicates, in addition to peripheral changes, important modifications of hypothalamic 5HT responsiveness to FI and of insulin gene expression in a type 2 diabetes rat model. Gene expression of IR, IRS1 and IRS2, important elements of insulin signaling, are increased in $\mathrm{W}$ rats fed a HF diet, suggesting potential adaptive mechanisms, aiming to rapidly increase brain insulin efficiency and compensate altered regulatory action in the brain of peptides and neurotransmitters involved in energy and glucose homeostasis. In the diabetic rat, such a compensatory mechanism was lacking. Taken together with epidemiological studies and own previous observations indicating 1) insulin-5HT interaction in the hypothalamus 2) cross talk of the two neuroregulators in intracellular signaling pathways (STAT-3, MAPK, IRS/PI3K) in the neuronal and brain level, and 3) alteration of those signaling mechanisms in pathological situations, this study reveals an aspect of the central biological mechanisms potentially linking depressive disorders and diabetes.

\section{5}

Oral glucose tolerance test fails to detect substantial metabolic abnormalities induced by antipsychotic agents

M. Ader, J.M. Richey, K. Hucking, V. Ionut, K.J. Catalano, S.P. Kim,

M. Kabir, R.N. Bergman

Physiology and Biophysics, University of Southern California, Los Angeles, United States

Background and aims: Recent reports of metabolic abnormalities associated with atypical antipsychotic therapy have prompted calls for increased monitoring of treated patients. The oral glucose tolerance test (OGTT) is a common screening procedure for subjects at increased diabetes risk, and could potentially be applied to the psychiatric population receiving antipsychotic agents.

Materials and methods: To determine whether changes in glucose tolerance would reveal drug-induced alterations in key metabolic factors, we tested in conscious dogs the effects of 6-wk treatment with the antipsychotic olanzapine (OLZ; $15 \mathrm{mg} / \mathrm{d}, \mathrm{n}=10)$ or placebo $(\mathrm{n}=6)$ on the following: (a) glucose 
tolerance by $25 \mathrm{~g}$ OGTT, (b) insulin sensitivity $\left(\mathrm{S}_{\mathrm{T}}\right)$ by euglycemic clamp, (c) $\beta$-cell function by hyperglycemic clamp, and (d) adiposity by MRI.

Results: OLZ induced marked decrements in hepatic SI (pre: $6.1 \pm 1.0$, post: $1.5 \pm 0.9 \mathrm{dl} / \mathrm{min} / \mathrm{kg}$ per $\mu \mathrm{U} / \mathrm{ml} ; \mathrm{p}=0.009)$, with substantial increases in both visceral $(+84 \pm 22 \%)$ and subcutaneous $(+106 \pm 24 \%)$ adiposity $(\mathrm{p}<0.0001)$. Moreover, OLZ prevented $\beta$-cell upregulation of insulin secretion (pre: $1.24 \pm 0.15$, post: $1.07 \pm 0.25 \mu \mathrm{U} / \mathrm{ml}$ per $\mathrm{mg} / \mathrm{dl} ; \mathrm{p}=0.59$ ). But these defects in glucose homeostasis were not reflected in any deterioration of glucose tolerance, defined as total glucose area under the OGTT curve (AUC) (pre: $21484 \pm 616$, post: $21492 \pm 612 \mathrm{mg} / \mathrm{dl} ; \mathrm{p}=0.98$ ). With OLZ, good tolerance was maintained by elevation in $A U C_{\text {insulin }}$ (pre: $5812 \pm 643$, post: $9293 \pm 1317$ $\mu \mathrm{U} / \mathrm{ml} ; \mathrm{p}=0.009$ ). Maintenance of glucose tolerance during OLZ was similar to that observed with placebo (pre: $20934 \pm 1092$, post: $19351 \pm 1125 \mathrm{mg} / \mathrm{dl}$; $\mathrm{p}=0.98$ ), which demonstrated no defects in SI, $\beta$-cell function, and only modest $(25-30 \%)$ increase in adiposity $(\mathrm{p}<0.05)$.

Conclusion: These data demonstrate that "normal" OGTT-measured glucose tolerance may well not reflect insulin resistance or altered $\beta$-cell function. Glucose tolerance from the OGTT may underestimate significant metabolic defects caused by antipsychotic agents.

Supported by: Janssen Pharmaceutica and NIH

\section{PS 064 Cytokines: IL-1 and others}

\section{6}

Genetic-dependent differences in sensitivity and resistance of rat beta cells to interleukin-1-beta inducing inhibition of glucose stimulated insulin secretion

G. Aharon-Hananel ${ }^{1}$, S. Lenzen ${ }^{2}$, A. Jörns ${ }^{3}$, I. Raz ${ }^{1}$, S.W. Zangen ${ }^{1}$

${ }^{1}$ Diabetes Center, Hadassah University Hospital, Jerusalem, Israel, ${ }^{2}$ Institute of Clinical Biochemistry, Hannover Medical School, Germany, ${ }^{3}$ Centre of Anatomy, Hannover Medical School, Germany

Background and aims: Interleukin-1-beta (IL-1 $\beta$ ) is a potent inhibitor of $\beta$ cell function. The Cohen diabetic sensitive (CDs) rats develop diabetes when fed a diabetogenic diet (DD) but maintain normoglycemia when fed regular diet (RD). The hyperglycemic CDs rat exhibit diminished insulin secretion that was recently shown to be associated with IL1- $\beta$-positive macrophage infiltration in the exocrine pancreas. The resistant (CDr) rat maintains normoglycemia and does not exhibit these pancreatic pathologies. In contrast to the in vivo studies, islets isolated from hyperglycemic CDs rats exhibit adequate insulin secretion and proinsulin biosynthesis. We aimed to study the pathways by which IL1- $\beta$ triggers the in vivo reduction in glucose stimulated insulin secretion exhibited by the hyperglycemic CDs rat.

Materials and methods: In vivo, 5 male CDs and CDr rats were fed 30 days $\mathrm{RD}$ or DD. Blood glucose and insulin levels were assessed by IVGTT. Ultrastructural and immunohistochemical analysis of the endocrine and exocrine parenchyma was performed on pancreatic sections. In vitro, islets isolated from the $\mathrm{CDs}$ and $\mathrm{CDr}$ rats fed $\mathrm{RD}$ were pre-incubated for a period of $1 \mathrm{~h}$ with or without IL1- $\beta$ (30 or $60 \mathrm{U} / \mathrm{ml}$ ). Insulin secretion was evaluated in the presence of 1.7 and $3.3 \mathrm{mmol} / \mathrm{l}$ (basal insulin secretion) or $16.7 \mathrm{mmol} / \mathrm{l}$ glucose (stimulated insulin secretion).

Results: In vivo, the glucose AUC of CDs-fed DD was elevated $(\mathrm{P}<0.009)$ and insulin AUC was reduced $(\mathrm{P}<0.01)$ compared to the 3 other study groups. The AUC of both glucose and insulin of CDr-DD were in the normal range and were not different from CDs or CDr rats fed RD. Macrophages, showing ultrastructural signs of activation and expressing IL- $1 \beta$ immunostaining were observed in the exocrine parenchyma of the CDs-DD rats. No macrophages were found in the exocrine tissue of $\mathrm{CDr}$-DD rats or in rats fed $\mathrm{RD}$. In vitro, insulin secretion from CDs islets incubated with 30 and $60 \mathrm{U} / \mathrm{ml}$ IL1- $\beta$ was reduced by $25 \%$ compared to the same islets incubated without IL1- $\beta$. In contrast, insulin secretion of the CDr islets was not affected by exposure to IL1- $\beta$ (30 or $60 \mathrm{U} / \mathrm{ml}$ ).

Conclusion: The abnormal insulin secretion exhibited by the hyperglycemic CDs rats in vivo may be reproduced in vitro by a short exposure of isolated islets to moderate levels of IL1- $\beta$. In contrast, exposure of the CDr islets to similar levels of IL1- $\beta$ did not impair insulin secretion. This may suggest a genetic-dependent difference in the sensitivity and resistance of the CDs and $\mathrm{CDr}$ rat $\beta$-cells to IL-1 $\beta$. Using this in vitro set up, we are currently studying the pathogenic mechanism pathways providing sensitivity or protection of islets cells from the deleterious effect of IL1- $\beta$.

Supported by: Russell Berrie Foundation and D-Cure, Diabetes Care in Israel and by AM Cohen Foundation for the Advancement of Research of the Cohen Diabetic Rat

\section{7}

The anti-inflammatory cytokine interleukin-1 receptor antagonist improves beta cell function and survival by maintaining nuclear PDX-1 expression

N.S. Sauter, F.T. Schulthess, K. Maedler

Centre for Biomolecular Interactions, University of Bremen, Germany

Background and aims: Sub-clinical inflammation is a recently discovered mechanism in type 2 diabetes (T2DM). Elevated cytokines impair $\beta$-cell function and survival. A recent clinical trial shows that blocking Interleukin$1 \beta$ (IL-1 $\beta$ ) signaling by Interleukin-1 Receptor Antagonist (IL-1Ra) improves $\beta$-cell secretory function in patients with T2DM. In the present study, we provide mechanisms of this protective effect.

Materials and methods: Six-week-old IL-1Ra overexpressing mice (IL-1Ra$\mathrm{OE}$ ) and their wildtype littermates $(\mathrm{C} 57 \mathrm{Bl} / 6)$ were fed a high fat/ high sucrose diet (HFD) or a control diet for 16 weeks. Body weight and food consumption were measured weekly, intra-peritoneal glucose and insulin tolerance tests (ipGTT/ ipITT) were performed every 4 weeks. After 16 weeks, mice were 
sacrificed and their islets isolated and analyzed for insulin and PDX-1 mRNA expression, $\beta$-cell apoptosis and PDX-1 localization. Human islets were cultured for $72 \mathrm{~h}$ with increasing glucose concentrations or with $2 \mathrm{ng} / \mathrm{ml} \mathrm{IL-1} \beta$ with or without addition of recombinant human IL-1Ra, PDX-1 localization was analyzed in Bouin fixed islet sections.

Results: IL-1Ra improved glucose tolerance and insulin secretion in vivo in C57BL/6J mice fed a high fat/ high sucrose diet (HFD). Studies on isolated islets revealed that IL-1Ra specifically acted on the $\beta$-cell. IL-1Ra protected from $\beta$-cell apoptosis, induced $\beta$-cell proliferation and improved glucosestimulated insulin secretion. Insulin mRNA was reduced in islets from mice fed a HFD, but normalized in the IL-1Ra group, whereas PDX-1 mRNA levels were oppositely regulated, increased by the HFD and normalized by IL-1Ra treatment. PDX-1 protein was localized in the cytosol in the HFD animals but remained in the nucleus in the IL-1Ra group. Importantly, IL-1Ra induced a similar restoration of PDX-1 nuclear translocation in human islets. We found the JNK-pathway as a possible link in the downstream signaling from IL-1Ra to PDX-1. In isolated human islets, phosphorylated JNK-levels were increased by 11.1 and $33.3 \mathrm{mM}$ glucose or in the presence of IL-1 $\beta$, this was inhibited when IL-1Ra was added to the culture medium. Previous studies suggested that JNK-activation leads to PDX-1 export. Human islets were treated with $22.2 \mathrm{mM}$ glucose or IL-1 $\beta$, with or without the addition of a small peptide that inhibits JNK-activity. In the glucose and IL-1 $\beta$ treated cells, we found cells that co-express insulin and PDX-1 in the cytoplasm, as well as cells that showed cytoplasmic PDX-1 with almost no detectable insulin. JNK-inhibition prevented glucose- and IL-1 $\beta$-induced PDX-1 translocation and kept the transcription factor in the nucleus in most cells, indicating that the JNK-pathway is involved in regulating PDX-1 localization.

Conclusions: Our results show that IL-1Ra improves $\beta$-cell survival and function through maintaining PDX-1 expression in the nucleus and suggest a potential role for IL-1Ra in the treatment of diabetes.

Supported by: American Diabetes Foundation

\section{8}

Islet endothelial dysfunction in young adult GK rat, a spontaneous model of type 2 diabetes: reversal by IL-1 Ra treatment

M.-H. Giroix ${ }^{1}$, G. Lacraz ${ }^{1}$, N. Kassis ${ }^{1}$, J.A. Ehses ${ }^{2}$, J.-C. Irminger ${ }^{3}$, F. Schmidlin ${ }^{4}$, J. Coulaud ${ }^{1}$, B. Portha ${ }^{1}$, M.Y. Donath ${ }^{2}$, F. Homo-Delarche ${ }^{1}$ ${ }^{1}$ Laboratory of Nutrition Physiopathology-CNRS UMR 7059, University Paris-Diderot, Paris cedex 05, France, ${ }^{2}$ Division of Endocrinology and Diabetes, University Hospital of Zürich, Switzerland, ${ }^{3}$ Department of Genetic Medicine and Development, CMU, University of Geneva, Switzerland, ${ }^{4}$ Merck, Serono, Chilly-Mazarin, France

Background and aims: Inflammation is recognized to be present in obesity, insulin resistance and type 2 diabetes (T2D), both at the systemic level and in adipose tissue. Recently, we showed that inflammation is an integral component in rodent and human islets in T2D. In the Goto-Kakizaki (GK) rat, we also demonstrated that extracellular matrix deposition progresses from intra-/peri-islet vessels, as in microangiopathy. In the present study, our hypothesis was that islet inflammation might have originated from endothelial cells (EC). Using a molecular approach we investigated: 1) EC activation in islets of 2-month-old GK rats, i.e., after 1 month of mild chronic hyperglycemia associated with hyperlipidemia; 2) the in vivo effects of IL-1 receptor antagonist (IL-1Ra), an immunosuppressive agent, which has been shown to improve type 2 diabetic patient state, on GK islet inflammation and glucose homeostasis.

Materials and methods: 1) Islets were isolated from 2-month-old male GK rats and Wistar control rats and expression of selected genes was analysed by qRT-PCR; 2) Immunochemistry was performed on whole pancreata; 3 ) GK rats were treated twice daily with IL-1Ra (Kineret, Amgen) at 2 different doses (10 and $50 \mathrm{mg} / \mathrm{kg} /$ day) or saline from weaning (1 month of age, at onset of diabetes) to 2 months of age ( $n=5 /$ group). Glycemia was measured 3 times/week, throughout the treatment period and pancreata and islets were processed as above.

Results: The selected genes encoding molecules known to be involved in EC activation were the following: plasminogen activator inhibitor 1 (Pai1), integrins (like Pecam1, Icam1, Vcam1, and E-selectin (Sele)), Toll-like receptor 4 (Tlr4), endothelin-1 (Edn1), angiotensin-converting enzyme 1 (Ace1), angiotensin receptor 1 and angiotensinogen (Agt), endothelial nitric oxide synthase (eNOS), prostacyclin synthase (Ptgis), cyclo-oxygenase 2 (Cox2), NADPH-oxidase 2 (Nox2) and hypoxia-induced factor-1a (Hifla). All genes except Agt (that was decreased), were significantly up-regulated in 2-monthold GK islets. IL-1Ra treatment improved glucose homeostasis, islet mor- phology and decreased islet fibrosis in the GK rat. At the molecular level, all genes that were assessed were significantly down-regulated in a dose-response manner by IL-1Ra treatment, except Sele and Hifla. We also found a significant decrease of genes encoding the following proteins: IL-6, GRO/KC (rodent IL-8 equivalent), MCP-1 and MIP- $1 \alpha$, fibronectin, collagen I and III. Conclusion: The extensive endothelial activation that is at play during mild chronic hyperglycemia (from weaning onwards) and hyperlipidemia (from the perinatal period onwards) might alter $\beta$-cell microenvironment and consequently contribute to $\beta$-cell dysfunction in GK rats. The beneficial effect of IL-1Ra highlights the early and deleterious role of IL-1 in the GK endothelial dysfunction and offers a new therapeutic approach in T2D. Supported by: EFSD/MSD grant

\section{9}

Elevated levels of the anti-inflammatory interleukin-1 receptor antagonist (IL-1Ra) precede, but do not prevent, the onset of type 2 diabetes (Whitehall II Study)

C. Herder ${ }^{1}$, E. Brunner ${ }^{2}$, A. Tabak ${ }^{2}$, N.C. Schloot ${ }^{1}$, D.R. Witte ${ }^{2}$

${ }^{1}$ Institute for Clinical Diabetes Research, German Diabetes Centre, Duesseldorf, Germany, ${ }^{2}$ Department of Epidemiology and Public Health, University College London, United Kingdom

Background and aims: Low-grade inflammation has emerged as risk factor for the development of type 2 diabetes (T2D). Several cytokines and chemokines contribute to insulin resistance, and the pro-inflammatory cytokine interleukin-1 $\beta$ (IL-1 $\beta$ ) has been reported to inhibit beta-cell function and promote beta-cell apoptosis. A randomised controlled trial recently demonstrated that the anti-inflammatory interleukin-1 receptor antagonist (IL$1 \mathrm{Ra})$, a natural competitive inhibitor of IL- $1 \beta$, improves beta-cell function and glycaemic control in patients with type 2 diabetes. The aim of the current study was to investigate for the first time whether high systemic levels of IL1 Ra were associated with incident T2D in a prospective study.

Materials and methods: Serum levels of IL-1Ra were measured in a nested case-control study within the Whitehall II cohort. At baseline, participants were aged 50.8 years (SD: 6.2) and had an average BMI of 26.6 (SD 4.1). 366 controls with normal glucose tolerance at study baseline (75g OGTT, 19911993) and throughout follow up (up to 2002-2004) were compared with 189 individuals without T2D at baseline, with incident T2D during the follow-up period (cases). Controls were frequency-matched to cases for age, sex and BMI. Persons with prevalent or incident coronary heart disease, acute or chronic inflammatory conditions and non-white ethnicity were excluded.

Results: IL-1Ra concentrations at baseline were higher in cases (mean $(95 \% \mathrm{CI}) 296(266-326 \mathrm{pg} / \mathrm{ml})$ than in controls (mean (95\%CI) 234 (222-246) $\mathrm{pg} / \mathrm{ml})(\mathrm{p}<0.0001)$. An incremental increase in IL-1Ra of $100 \mathrm{pg} / \mathrm{ml}$ was associated with increased T2D risk (age and sex-adjusted OR (95\% CI) 1.29 (1.14-1.47); $\mathrm{p}<0.001)$. This association remained significant after additional adjustment for BMI, lipids, blood pressure, fasting glucose, fasting insulin and C-reactive protein as potential confounders.

Conclusion: Our findings indicate that individuals who will develop T2D are not only characterised by an upregulation of pro-inflammatory cytokines and chemokines, but also by the upregulation of at least one anti-inflammatory immune marker. Given the therapeutic efficacy of IL-1Ra in a clinical trial it is tempting to speculate that high IL-1Ra levels cannot be considered as genuine risk factor. Our data rather point towards the presence of an early compensatory, anti-inflammatory response that precedes, but does not prevent, the development of T2D.

Supported by: Germany: Federal Ministry of Health and Social Security; Ministry of Science and Research of the state North Rhine-Westphalia; UK: Medical Research Council, Economic and Social Research Council, British Heart Foundation, Health and Safety Executive, Department of Health; USA: National Heart Lung and Blood Institute NIH, National Institute on Aging NIH, Agency for Health Care Policy Research John and Catherine MacArthur Foundation 


\section{0}

Plasma YKL-40 - a BMI-independent marker of type 2 diabetes

A.R. Nielsen ${ }^{1}$, P. Plomgaard ${ }^{1}$, J.S. Johansen ${ }^{2}$, C.P. Fischer ${ }^{1}$,

R. Krogh-Madsen ${ }^{1}$, B.K. Pedersen ${ }^{1}$

${ }^{1}$ Centre of Inflammation and Metabolism - Rigshospitalet, Copenhagen,

${ }^{2}$ Department of Rheumatology, Herlev Hospital, Herlev, Denmark

Background and aims: YKL-40, a member of the "mammalian chitinase-like proteins," is produced by macrophages, and serum concentrations of YKL40 is elevated in patients with diseases characterized by inflammation. The possible relationship between YKL-40 and obesity, type 2 diabetes and inflammation was examined. Furthermore, using clamp technique, we assessed the effect of acute changes in blood glucose and insulin levels on circulating YKL-40.

Materials and methods: In 199 subjects divided into groups of normal glucose tolerant $(n=103)$ and type 2 diabetes $(n=96)$. These groups were subdivided on the basis of BMI (BMI $<30$ or BMI $\geq 30$ ). Blood samples and adipose tissue biopsies were obtained and fat mass was determined by DXA. Plasma YKL-40 and adipose tissue YKL-40 mRNA levels were measured using ELISA and real-time PCR, respectively. The relationship between circulating YKL-40, adipose tissue YKL-40 mRNA levels and parameters of obesity, parameters of type 2 diabetes and markers of inflammation were investigated. A second study, including healthy young male subjects $(n=7)$, investigated the acute effect of hyperglycaemia (plasma glucose: $15 \mathrm{mM}$ ) and hyperinsulineamia (plasma insulin: 900 pM) on plasma YKL-40 levels.

Results: Patients with type 2 diabetes had higher plasma YKL-40 levels ( $\mathrm{p}=$ $0.0001)$, but not higher expression of adipose tissue YKL-40 mRNA $(p=0.2)$, compared to subjects with normal glucose tolerance. Obesity had no effect on plasma YKL-40 or adipose tissue YKL-40 mRNA levels. Multivariate regression analysis revealed that plasma YKL-40 was associated with fasting plasma glucose $(p=0.0004)$ and plasma IL-6 $(p=0.0303)$. In the intervention study, no effect of hyperglycaemia or hyperinsulinaemia was observed.

Conclusion: Plasma YKL-40 has been identified as an obesity-independent marker of type 2 diabetes, which is related to fasting plasma glucose and plasma IL- 6 level. The second study suggests that the observed association between plasma glucose and YKL-40 in the circulation is not acutely regulated, but may be a consequence of a chronic dysregulated glucose homeostasis. Supported by: Danish National Research Foundation, Danish Medical Research Council, the Commission of the European Communities, Copenhagen Hospital Corporation, University of Copenhagen, "Overloege Johan Boserup and Lise Boserups Fond"

\section{1}

The chemokine IP-10 induces beta cell death through TLR4-signalling K. Maedler, N.S. Sauter, F.T. Schulthess

Centre for Biomolecular Interactions, University of Bremen, Germany

Background and aims: In both, type 1 (T1DM) and type 2 diabetes (T2DM), $\beta$-cell destruction by apoptosis results in decreased $\beta$-cell mass and progression of the disease. We hypothesize that pro-inflammatory cytokines and chemokines contribute to the etiology. In the present study, we found that the Interferon-gamma-inducible Protein-10 (IP-10) plays an important role in triggering $\beta$-cell destruction.

Materials and methods: Isolated human islets were exposed to IP-10, $\beta$-cell apoptosis (by the TUNEL assay), replication (by Ki-67 staining) and glucose stimulated insulin secretion (GSIS) were measured. Additionally, cell viability was determined using the MTT colorimetric assay. IP- 10 secretion, localization and mRNA were analyzed in isolated islets from healthy organ donors and donors with type 2 and type 1 diabetes. Akt and Pak-2 signaling were analyzed by western blot analysis. To identify the receptor which mediates the IP-10 effects in the $\beta$-cell we used the Dynabead technology. Paramagnetic beads were coated with IP-10 prior to incubation with human islet lysates and then investigated by western blot analysis. IP-10 signaling was also tested in TLR4 ${ }^{-/}$mice.

Results: Islets isolated from patients with T2DM secreted IP-10 and contained 33.5-fold more IP-10 mRNA than islets from control patients. Pancreatic sections from patients with T2DM and T1DM expressed IP-10 in $\beta$-cells. Treatment of isolated human islets with recombinant IP-10 decreased $\beta$-cell viability. This was accompanied by impaired insulin secretion and decreased insulin mRNA. IP-10 induced sustained activation of Akt and JNK and downstream cleavage of $\mathrm{p} 21$-activated protein kinase 2 (PAK-2), switching Akt signals from proliferation to apoptosis. These effects were not mediated by the commonly known IP-10 receptor CXCR3; islets from CXCR3 ${ }^{-1-}$ mice showed also decreased $\beta$-cell viability. In contrast, we identified TLR4 as the receptor for IP-10 in the $\beta$-cells. We examined the ability of IP-10 to regulate TLR4 receptor expression. $0.1 \mathrm{ng} / \mathrm{ml}$ IP- 10 caused a 2 -fold induction of TLR4 mRNA. To investigate whether IP-10 signaling is TLR4 dependent, we tested the effect of IP-10 on islets isolated from TLR $4^{-/}$mice and measured $\beta$-cell apoptosis. We used lipopolysaccharide (LPS), the major ligand for TLR4 as positive control to induce TLR4 dependent apoptosis. LPS induced a 2.9 -fold induction of $\beta$-cell apoptosis, which was prevented in isolated islets from TLR $4^{-1}$. IP-10 treatment induced a 2.2 -fold induction of $\beta$-cell apoptosis in the wildtype islets, but not in the TLR4 ${ }^{-1}$.

Conclusion: Our data suggest IP-10 as a marker for $\beta$-cell failure in diabetes. Strategies to block IP-10 signaling through blocking TLR4 signals could be a new target for maintaining $\beta$-cell survival.

Supported by: Larry Hillblom Research Foundation

\section{2}

Free fatty acids cause alterations in the insulin signalling pathway in JEG-3 cells

M. Lappas, A. Mitton, M. Permezel

Obstetrics and Gynaecology, University of Melbourne, Heidelberg, Australia

Background and aims: Free fatty acid (FFA) levels increase during normal pregnancy and have been implicated in the development of the progressive insulin resistance observed during normal pregnancy. Moreover, women with gestational diabetes mellitus (GDM) have a significant elevation in fasting FFA levels compared to normoglycaemic controls. Recent studies suggest that lipid- and fat-derived FFAs have been reported to induce insulin resistance in vitro and in vivo. Recent studies from our laboratory have shown that GDM women have defects in insulin signalling in placenta, however the effect of FFAs on insulin signalling in this tissue is not known. Thus the aim of this study was to elucidate the role of FFAs (saturated FFA palmitate (C16:0) and the monounsaturated FFA oleate $(\mathrm{C} 18: 1 \omega 9)$ on the insulin signalling pathway in human placental JEG-3 cells.

Materials and methods: Human placental JEG-3 cells ( 3 independent experiments) were then incubated in serum free DMEM for 24 hours in the presence or absence of $0.2,0.5$ and $0.75 \mathrm{mg} / \mathrm{ml}$ oleate and palmitate. Cells were stimulated with $100 \mathrm{nM}$ insulin for 1 hour. Cells were collected and lysed, and the effect of oleate and palmitate on the insulin signalling pathway (IR- $\beta$ (total and phosphorylated), IRS-1 (total and phosphorylated), PI3K p110 and PI3K p85a) was determined by Western blotting.

Results: Incubation of JEG- 3 cells with insulin caused an increase in IR- $\beta$ and IRS-1 tyrosine phosphorylation. Both oleate and palmitate inhibited insulin stimulated insulin signalling in JEG-3 cells. Specifically, oleate and palmitate decreased the protein expression of insulin stimulated phosphorylation of IR$\beta$ and IRS-1.

Conclusion: The data presented in this study demonstrate that FFAs interfere with the initial steps of the insulin signalling cascade in human placental cells. A raised level of FFA may be responsible for the insulin resistance of GDM. Supported by: National Health and Medical Research Council

\section{3}

Insulin-resistance and low-grade inflammation in type 1 diabetes mellitus

G. Llauradón ${ }^{1}$, R. Tirado 1 , A. Megia 2 , I. Simón ${ }^{2}$, L. Gallart ${ }^{2}$, A. Caixàs ${ }^{1}$, O. Giménez-Palop ${ }^{1}$, J. Vendrell2 ${ }^{2}$ J.-M. González-Clemente ${ }^{1}$

${ }^{1}$ Diabetes, Endocrinology and Nutrition, Hospital de Sabadell, Sabadell, ${ }^{2}$ Diabetes, Endocrinology and Nutrition, Hospital Universitari Joan XXIII de Tarragona. CIBERDEM, Spain

Background and aims: Subjects with type 1 diabetes (T1DM) have an increase in insulin resistance and insulin resistance is associated with a lowgrade systemic inflammatory response. However, few data exist regarding the relationships between insulin-resistance and low-grade systemic inflammation in T1DM, although both factors have been associated with the presence of micro and macrovascular complications. We aimed at evaluating the relationship between insulin resistance and some inflammation-related plasma proteins in a group of subjects with T1DM, taking into account the presence of microvascular complications.

Materials and methods: One hundred and twenty subjects (60 men and 60 women), all diagnosed with T1DM 14 years before, were evaluated for (1) 
sex, age, body mass index, waist-to-hip ratio (WHR), blood pressure, smoking, alcohol intake, insulin dose, HbAlc and lipid profile; (2) microvascular complications (neuropathy -peripheral and cardiovascular autonomic-, retinopathy and nephropathy); (3) concentrations of plasma-related inflammation proteins (soluble receptors type 1 and 2 of TNF-alpha, interleukin-6, adiponectin, leptin and high-sensitivity C-reactive protein (hs-CRP); and (4) insulin resistance (by way of the Williams' mathematical estimation of the glucose disposal rate -eGDR-). Those subjects with an eGDR less than the median of the same sex group were classified as insulin resistants and the others were classified as non-insulin resistants.

Results: Insulin resistant men, as compared to non-insulin resistant, had higher WHR $(0.89 \pm 0.08$ vs $0.83 \pm 0.05$, respectively, $\mathrm{p}<0.01)$, higher systolic ( $121 \pm 9$ vs $114 \pm 10 \mathrm{mmHg} ; \mathrm{p}=0.01)$ and diastolic blood pressure ( $72 \pm 9$ vs $70 \pm 7 \mathrm{mmHg}, \mathrm{p}=0.02)$, higher HbAlc values $(9.0 \pm 1.2$ vs $7.4 \pm 0.9 \%, \mathrm{p}<0.01)$ and higher concentrations of hsCRP $(1,16(0,61-3.10)$ vs $0.49(0.31-0.82) \mathrm{mg} /$ $\mathrm{dL}, \mathrm{p}=0.01)$. In contrast, they were no different from the other evaluated inflammation-related plasma proteins. Insulin resistant women, as compared to the non-insulin resistant, had higher WHR $(0.83 \pm 0.11$ vs $0.74 \pm 0.06$, respectively, $\mathrm{p}<0.01)$ and $\mathrm{HbAlc}$ values $(9.2 \pm 1.7$ vs $7.6 \pm 1.1 \%$, $\mathrm{p}<0.01)$, but no differences were found between both groups, regarding the other variables evaluated. After adjusting for age, sex, alcohol intake and smoking in males, hs-CRP correlated significatively with WHR $(r=0,45 ; \mathrm{p}<0.01)$ but not with blood pressure nor HbAlc.

Conclusion: Insulin resistant males with T1DM have an increase in plasma concentrations of hs-CRP, which were not confirmed in insulin resistant women. Central obesity is the main determinant of this increase in hs-CRP. Supported by: Intensification Grant from the Ministerio de Sanidad y Consumo, Instituto de Salud Carlos III and CIBERDEM (Instituto de Salud Carlos III)

\section{PS 065 AMPK and ERS}

\section{4}

Implication of AMPK and mTOR in brown adipocyte differentiation M. Lorenzo, R. Vila-Bedmar, I. Nieto-Vazquez, L. Garcia-Guerra, S. Fernandez-Veledo

Bioquimica y Biologia Molecular, Universidad Complutense, Madrid, Spain

Background and aims: Brown adipose tissue (BAT) is considered of metabolic significance in human physiology, since it plays an important role in regulating energy balance. Alterations in this tissue have been related to obesity and associated metabolic disorders, such as insulin resistance and type 2 diabetes. BAT is developed throughout fetal stage. However, the physiological signals and molecular mechanisms modulating this process are still unknown. In this study, we further examine some of the intracellular signaling pathways involved in brown adipocyte differentiation, as well as the ability of different nuclear receptor agonists to induce adipogenesis and thermogenesis.

Materials and methods: BAT differentiation was studied in a stable cell line obtained by immortalization of primary rat fetal brown adipocytes with a retroviral construct of SV40 Large T Ag. Differentiation was induced with a specific differentiation medium, suplemented with the PPARgamma agonist Rosiglitazone. Phosphorylation and expression of different kinases (MAPKs, mTOR-AMPK and Wnt pathways) were analyzed in the absence or presence of the different inhibitors and nuclear receptor agonists.

Results: Inmortalized cells were able to differentiate, expressed specific adipogenic and thermogenic markers, and reproduced metabolic functions typical of BAT. Differentiation was accomplished by a sequential activation of p38MAPK and AMPK, and by a significant attenuation in the activation of ERK1/2 and mTOR-p70 pathways. The inhibition of either the mTOR or the AMPK cascades prevented cell differentiation. In addition, a degradation of beta-catenin, a major component of Wnt pathway, was detected throughout the differentiation process. This inactivation of Wnt pathway was dependent on mTOR signaling. On the other hand, an increase in adipogenic but not in thermogenic markers was observed in cells differentiated in the presence of the LXR agonist instead of Rosiglitazone.

Conclusion: These results show a critical role for AMPK and mTOR-p70, as well as for the Wnt signaling pathways, in regulating brown adipocyte differentiation. Nuclear receptor agonists differentially regulate adipogenic and thermogenic programs in brown adipose tissue.

Supported by: Ministerio de Educacion y Ciencia, Comunidad Autonoma de Madrid, and CIBERDEM from Ministerio de Sanidad y Consumo (Spain)

\section{5}

Effects of the AMPK activators AICAR and metformin on basal and insulin-induced glucose-uptake in human adipocytes

J. Grisouard ${ }^{1}$, T. Radimerski ${ }^{1}$, D. Seboek Kinter ${ }^{1}$, H. Zulewski ${ }^{1}$, B. Kola ${ }^{2}$, M. Korbonits ${ }^{2}$, B. Müller ${ }^{3}$, U. Keller ${ }^{1}$, M. Christ-Crain ${ }^{1}$

${ }^{1}$ Department of Biomedicine, Metabolism Group, University Hospital Basel, Switzerland, ${ }^{2}$ Centre for Endocrinology, Barts and the London Medical School, University of London, United Kingdom, ${ }^{3}$ Department of Internal Medicine, Kantonsspital Aarau, Switzerland

Background: Metfomin and 5-aminoimidazole-4-carboxamide 1-beta-Dribofuranoside (AICAR) activate AMP-activated protein kinase (AMPK) in peripheral tissues. The role of AMPK in regulating glucose uptake in adipose tissue remains controversial since both, increasing and decreasing effects have been reported in rodent adipocytes. Very few data exist for primary human fat cells.

Materials and methods: Herein we have analysed the effects of AICAR and metformin on basal and insulin-mediated glucose uptake in human adipocyte culture in vitro. Human mesenchymal stem cells and human pre-adipocytederived adipocytes obtained from surgical biopsies were differentiated in vitro into adipocytes, and the effects of insulin (20 min, $100 \mathrm{nM})$, AICAR (24 h, $1 \mathrm{mM})$, metformin $(24 \mathrm{~h}, 1 \mathrm{mM})$ and their combinations on glucose uptake were determined.

Results: Both, AICAR and metformin induced AMPK activity as mirrored by increased AMPK and ACC phosphorylation. Insulin increased glucose uptake to $204 \pm 39 \%$ of control $(\mathrm{P}<0.01)$. In adipocytes treated with AICAR basal glucose uptake was not significantly altered $(118 \pm 15 \%$ of control) whereas insulin-induced glucose uptake was reduced to $82 \pm 9 \%$ of insulin alone $(\mathrm{P}<0.05)$. AICAR increased GLUT-4 protein content, and phosphorylation 
of AS160 and c-Cbl, two downstream mediators of insulin-induced glucose uptake. However, insulin-induced translocation of GLUT-4 to the plasma membrane analysed by immunodetection of GLUT-4 in plasma membrane fraction was inhibited by AICAR. Treatment of adipocytes with metformin increased basal $(226 \pm 21 \%$ of control $(\mathrm{P}<0.01))$ and insulin-induced glucose uptake $(155 \pm 19 \%$ of insulin alone $(\mathrm{P}<0.01))$. Metformin-induced glucose uptake was inhibited by phloretin, an inhibitor of glucose transporters. Metformin increased GLUT-4 protein content, GLUT-4 translocation to the plasma membrane and induced glucose-uptake even in the presence of wortmannin, an inhibitor of the classical "PI3K/Akt"-insulin signalling pathway. Conclusion: AICAR inhibits insulin-induced GLUT-4 translocation and thus prevents the increase in insulin-induced glucose uptake. In contrast, metformin appears to induce GLUT-4-dependent glucose uptake independently of the classical "PI3K/Akt"-insulin signalling pathway. In addition to the insulin signalling pathway, activation of AMPK by metformin may represent an alternative mechanism to improve glucose uptake in adipocytes.

\section{6}

AMP-activated protein kinase and transcriptional control of gluconeogenic gene expression

J.K. Rundle ${ }^{1}$, E.R. Pearson ${ }^{2}$, C. Sutherland ${ }^{1}$

${ }^{1}$ Department of Pathology and Neuroscience, ${ }^{2}$ Department of Medicine and Therapeutics, University of Dundee, Dundee, United Kingdom

Background and aims: AMP-activated protein kinase (AMPK) is activated by stimuli that raise intracellular AMP concentrations. Once activated, AMPK antagonises cellular processes which consume ATP and initiates processes that generate ATP, thereby restoring energy homeostasis. AMPK has been identified as a target of metformin, the most widely prescribed anti-diabetic agent. Indeed, animals lacking AMPK in the liver fail to respond to metformin. Previous work has suggested that AMPK activation in liver reduces the transcription of PEPCK and G6Pase genes, two rate-controlling enzymes of gluconeogenesis. A number of small molecules activate AMPK, for example 5-aminoimidazole4-carboxamide-1- $\beta$-D-ribofuranoside (AICAR) activates AMPK indirectly as it is metabolised to the AMP mimic molecule, ZMP. More recently, thienopyridone compound, A-769662, has been developed to directly target and activate AMPK. In this study, we compare AICAR and A-769662 activation of AMPK and subsequent affects on gluconeogenic gene expression.

Materials and methods: Rat hepatoma cell line HL1C was incubated with the AMPK activators AICAR or compound A-769662 for $30 \mathrm{~min}, 4 \mathrm{~h}$, or $16 \mathrm{~h}$ and changes in AMPK, ACC, and ERK 1/2 phosphorylation were measured by Western blot as surrogates for activation of AMPK and ERK. PEPCK and G6Pase gene transcription were measured by real-time PCR. HL1C cells are stably transfected with a chloramphenicol acetyltransferase (CAT) reporter gene construct under the control of the PEPCK promoter. Therefore, PEPCK promoter activity was also assessed by a CAT enzyme activity assay in response to hormones and AMPK activators.

Results: AICAR transiently activates AMPK whereas activation by A-769662 appears to be chronic. Activation of AMPK by A-769662 could still be detected $16 \mathrm{~h}$ after initial exposure.

PEPCK and G6Pase gene transcription is induced by the application of dexamethasone (synthetic glucocorticoid) and 8CPT-cAMP (mimics glucagon) to liver cells. This induction is completely inhibited by the presence of insulin. G6Pase gene expression was efficiently inhibited by AICAR (80\%) and partially by A-769662 (40\%); however, PEPCK gene expression was only repressed by AICAR ( $50 \%)$. This result was confirmed by the PEPCKCAT promoter activity assay. This suggested that AMPK activation was not sufficient to inhibit PEPCK gene transcription. AICAR has been shown to activate the ERK pathway in human muscle. ERK 1/2 phosphorylation was significantly increased when HL1C cells were stimulated with AICAR at all time points. A specific inhibitor of ERK 1/2 activation, PD184352, did not alter the repression of these genes by AICAR, suggesting this pathway is not required for the regulation of PEPCK by AICAR.

Conclusion: Our data suggests that the repression of PEPCK gene transcription by AICAR is AMPK-independent. We also show that this repression by AICAR is independent of ERK $1 / 2$ activation, despite AICAR efficiently activating ERK at all time points studied. Direct activation of AMPK by A769662 is sufficient to reduce G6Pase transcription, albeit much less potently than insulin, but this compound has no effect on PEPCK gene expression. Taken together, these data show that PEPCK and G6Pase are regulated differently from one another in response to AMPK activation, and therefore metformin. Repression of PEPCK must be independent of AMPK.

Supported by: Tenovus Scotland Diabetes UK

\section{7}

Infusion of a lipid emulsion modulates AMP-activated protein kinase (AMPK) and related proteins in rat liver, muscle and adipose tissues S. Anavi, E. Ilan, O. Tirosh, Z. Madar

Faculty of Agriculture, The Hebrew University Of Jerusalem, Rehovot, Israel

Background and aims: It has been shown that fat accumulation in liver, muscle and adipose tissue may play an important role in the development of the metabolic syndrome, but the precise mechanisms are not fully known. AMPK, a serine/theronine protein kinase, has been proposed to function as a 'fuel gauge' to monitor cellular energy status in response to nutritional variation. There is also compelling evidence that adipose tissue is an endocrine organ through secretion of "adipokines" which may mediate AMPK action. Recent studies indicate alteration/defects in AMPK signaling at the onset of the metabolic syndrome. The primary objective of this study was to examine the effects of exposure to a lipid emulsion (LE) on AMPK expression in rat liver, muscle and adipose tissues. Other proteins involved in AMPK pathway were also examined.

Materials and methods: Wistar rats were infused via the jugular vein with 10 $\mathrm{ml}$ of LE or PBS for $4 \mathrm{~h}$ per day $(2 \mathrm{ml} / \mathrm{h})$ for 6 days. Liver, muscle and adipose tissues were removed and mRNA (RT-PCR), protein (Western blot) and protein phosphatase 2C (PP2C) activity analyses were performed.

Results: Following rat treatment with LE for 6 days AMPK and acetyl-coA carboxylase (ACC) phosphorylation were significantly $(\mathrm{p}<0.007-0.02)$ decreased by $64 \%$ and $62 \%$ respectively and a significant increase $(\mathrm{p}=0.007)$ in PP2C activity of 1.5-fold were observed. Hepatic mRNA levels of AMPKa2, ACC $\alpha$, adiponectin receptor 1 (AdipoR1), AdipoR2 and PEPCK were also significantly $(\mathrm{p}<0.004-0.05)$ reduced by approximately 2 -3-fold. No significant changes in AMPKal, peroxisome-proliferator-activated receptor- $\gamma$ coactivatorl $\alpha$ (PGC1 $\alpha$ ), PGC1 $\beta$ and PP2C expression were detected. In contrast, muscles exhibited significantly increased AMPK and ACC phosphorylation of $148 \%$ and $150 \%$ respectively $(\mathrm{p}<0.01-0.02)$. Muscle mRNA levels of AMPKa2, ACC $\beta$, AdipoR1, PGC1 $\alpha$ and PGC1 $\beta$ mRNA were also increased by 3-50-folds ( $\mathrm{p}<0.001-0.05)$. No significant effect of LE on AdipoR2 mRNA levels or PP2C activity was found. In adipose tissue there was no significant alteration in AMPK or ACC phosphorylation but AMPKa1 and AMPKa2 mRNA levels were significantly $(\mathrm{p}<0.04)$ increased by 12.8 and 6.2 respectively. Adipose mRNA levels of ACC $\alpha$, PGC1 $\alpha$, PGC1 $\beta$, AdipoR1 and AdipoR2 remained unchanged.

Conclusion: These results demonstrate that LE suppresses the AMPK signaling pathway and key proteins involved in fat oxidation, mitochondrial function and biogenesis in the liver. Therefore we speculate that alterations in the liver rather than in skeletal muscle and even less in adipose tissue may be associated with the onset of the metabolic syndrome.

\section{8}

Improved glucose tolerance after long-term exercise and 5Aminoimidazole-4-carboxamide-1- $\beta$-D-ribofuranoside (AICAR) treatment in Goto-Kakizaki rats despite reduced beta cell mass R. Pold ${ }^{1}$, C. Godfredsen ${ }^{2}$, L. Solskov ${ }^{1}$, O. Schmitz' ${ }^{1}$, A. Flyvbjerg ${ }^{3}$, S. Lund ${ }^{3}$ ${ }^{1}$ Pharmacology, Aarhus University, Denmark, Novo Nordisk A/S, Måløv, Denmark, ${ }^{3}$ Aarhus University Hospital, Aarhus Sygehus (NBG). Medical Department M (Diabetes and Endocrinology), and Medical Research Laboratories, Aarhus, Denmark

Background and aims: Type 2 diabetes is characterized by insulin resistance and a compromised $\beta$-cell function. Accordingly compensatory retrieval in $\beta$-cell mass and the preservation of the $\beta$-cell function are essential in prevention of diabetes. Recent studies on an obese rat model for type 2 diabetes (ZDF-rats), characterized by severe insulin resistance combined with a $\beta$-cell defect, have shown that long-term aerobic training or 5-Aminoimidazole4-carboxamide-1- $\beta$-D-ribofuranoside (AICAR) administration can preserve islet morphology in the pancreas. To clarify if the changes are secondary to the improvements in insulin sensitivity or due to a direct effect on the $\beta$-cell the present study was undertaken to determine the $\beta$-cell morphology and the $\beta$-cell mass after 6 weeks of aerobic exercise and AICAR treatment in a lean animal model of type 2 diabetes (GK-rats) characterized primarily by a $\beta$-cell defect.

Materials and methods: Five weeks old GK-rats were allocated into 3 groups: an exercise, an AICAR treated and an untreated group. Wistar rats were used as non-diabetic controls. The exercise treated GK-group was allocated to treadmill running for one hour five days a week. AICAR treated 
animals received a daily subcutaneous injection every morning of $0.5 \mathrm{mg}$ AICAR/g body weight. After 6 weeks of treatment an oral glucose tolerance test (OGTT) was performed. After the intervention period pancreas was removed for further studies

Results: The OGTT revealed significant improvements in the glucose tolerance in the treated groups. Consistently the insulin resistance index was increased in the untreated GK-rats after the intervention period. In contrast the $\beta$-cell mass was significantly lower in exercise treated and AICAR treated animals when compared to the untreated GK-rats (AICAR; $2.5 \pm 0.3 \mathrm{mg}$, Exercise; $2.7 \pm 0.4 \mathrm{mg}$ Untreated; $4.9 \pm 0.8 \mathrm{mg}$, Wistar; $7.4 \pm 1.2 \mathrm{mg}$ ). No differences in $\beta$-cell morphology between the treated and untreated GK-rats were observed

Conclusion: In conclusion, both exercise and AICAR treated GK-rats had improved glucose tolerance despite diminished $\beta$-cell mass. This improvement can contribute to the preservation of the $\beta$-cell which is essential for delaying or preventing the onset of diabetes

\section{9}

\section{SCD1 knockdown in the beta cell protects against apoptosis and} improves ER folding capacity

K. Thörn, M. Hovsepyan, P. Bergsten

Medical Cellbiology, Uppsala Universitet, Sweden

Background and aims: Stearoyl-CoA desaturase 1 (SCD1) is an endoplasmic reticulum (ER) resident protein involved in the synthesis of mono-unsaturated fatty acids. Although it has been shown that high levels of SCD1 have a protective effect in vitro, mice lacking SCD1 have protection from dietinduced obesity. The lack of SCD1 results in higher energy expenditure, reduced fatty acid de novo synthesis, decreased expression of lipogenic genes and increased insulin sensitivity. However, these results have been obtained in insulin target tissue and no studies have so far addressed the potential beneficial effects of SCD1 disruption or reduction in the pancreatic $\beta$-cell. The aim of the present study was to investigate the molecular changes in the $\beta$-cell caused by decreasing the levels of SCD1.

Materials and methods: Insulin-producing MIN6 cells with reduced levels of SCD1 were obtained by transfecting the cells with siRNA against SCD1. Cells with normal or reduced SCD1 were cultured for 72 hours after which apoptosis was measured. Also, cells were lyzed and proteins separated by twodimensional (2D) gel electrophoresis. Subsequently, differentially expressed proteins were identified by peptide mass fingerprinting employing MALDITOF MS. Proteins of cell lysates were also separated by SDS-PAGE and immunoblotted with antibodies directed towards ER resident proteins.

Results: In MIN6 cells transfected with siRNA against SCD1 the protein level of the enzyme was reduced by 70\%. This knockdown of SCD1 in MIN6 cells resulted in reduced apoptosis 72 hours post transfection. Analysis of differentially regulated protein by $2 \mathrm{D}$ gel electrophoresis indicated regulation of proteins mainly involved in protein folding and degradation. Protein disulfide isomerase, prolyl 4-hydroxylase and glucose regulated protein 94 (GRP94), chaperones involved in protein folding in the ER, were all up-regulated by more than $50 \%$. Indications of increased function of the proteasome was also found as two of its components, proteasome subunit a type 3 and proteasome $26 \mathrm{~S}$ subunit, were up-regulated by more than $50 \%$. To investigate if the lower apoptosis and increased levels of chaperones seen after knockdown of SCD1 were caused by altering activity of the regulatory proteins in the unfolded protein response (UPR), phosphorylation of PERK and cleavage of ATF6 was investigated. Phosphorylation of PERK was decreased and cleavage of ATF6 was increased in $\beta$-cells with lowered SCD1 levels.

Conclusion: The beneficial phenotype of SCD1 knockdown in the $\beta$-cell may be caused by enhanced capacity of the ER as evidenced by increased cleavage of ATF6 and up-regulation of chaperones involved in protein folding and proteins involved in degradation. Furthermore, decreased apoptosis may in part be mediated by lower activity of the PERK pathway of the UPR. Supported by: EFSD/MSD grant, Swedish Medical Research Council, and Swedish Diabetes Association

\section{0}

Obesity is associated with activation of innate immune pathway and ER stress in human abdominal subcutaneous adipose tissue G. Tripathi, C.M. Kusminski, A.L. Harte, K.C. McGee, P.J. O’Hare, P.G. McTernan, S. Kumar

Warwick Medical School, University of Warwick, United Kingdom

Background and aims: Obesity and type 2 diabetes mellitus (T2DM) are closely associated with chronic inflammation characterised by abnormal cytokine production and activation of inflammatory signalling pathways, but the mechanisms underlying the pathogenesis of obesity induced inflammation remain unclear. Recent findings in rodent models indicate that endoplasmic reticulum (ER) stress is critical to the initiation and integration of pathways of inflammation and insulin action in obesity and T2DM. The ER represents a fundamental site for protein folding, maturation, storage and transport. Recent studies have highlighted that ER stress is caused by the accumulation of unfolded/misfolded proteins. This leads to the subsequent activation of the unfolded protein response (UPR) to restore functional integrity which, in turn, leads to upregulation of genes such as PKR-like ER kinase (PERK) and activating transcription factor 6 (ATF6). Both PERK and ATF6 have been implicated to influence JNK and NFkB pathways. However, to date, no study has examined the potential relationship between ER stress and JNK/NFKB in human adipose tissue. Therefore our aims were to 1) examine the impact of increasing adipose tissue mass on ER stress activated genes 2) and the influence of obesity on the NFKB and JNK signalling pathways in human adipose tissue.

Materials and methods: Abdominal subcutaneous adipose tissue (AbSc AT) was collected from patients undergoing elective liposuction surgery. Samples were divided into lean $(n=20)$ and obese $(n=20)$ sub-groups (lean: age, $45 \pm 3.3$ yrs; BMI, $21.9 \pm 2.4 \mathrm{~kg} / \mathrm{m}^{2}$; obese: $\left.47 \pm 4.1 \mathrm{yrs} ; 29.6 \pm 4.2 \mathrm{~kg} / \mathrm{m}^{2}\right)$. Real-time PCR and western blotting was utilised to examine ATF6, eIF2 $\alpha$ and PERK expression and also the NF- $\mathrm{\kappa B}$ and JNK pathway.

Results: ATF6, eIF2 $\alpha$ and PERK mRNA levels were 7.3-fold, 9.1-fold and 8.2fold higher in obese AbdSc AT when compared with lean (ATF6: Lean $\triangle \mathrm{Ct}$ : 10.22 \pm 0.96 ; Obese $\Delta \mathrm{Ct}$ : 7.36 \pm 1.49 ; $<<0.001$; eIF2 $\alpha$ : Lean $\Delta \mathrm{Ct}$ : $10.84 \pm 1.36$; Obese $\Delta$ Ct: $7.65 \pm 1.82 ; \mathrm{p}<0.001$; PERK: Lean $\Delta \mathrm{Ct}$ : $10.36 \pm 0.43$; Obese $\Delta \mathrm{Ct}$ : $7.42 \pm 0.95 ; \mathrm{p}<0.001)$. In parallel, expression of key components of the NFKB pathways were markedly unregulated in obese AT in comparison with lean (MyD88: 1.94-fold $\uparrow, \mathrm{p}<0.001, \mathrm{n}=6$; TRAF-6: 1.13 -fold $\uparrow, \mathrm{p}<0.05, \mathrm{n}=6$; NFkB: 1.23 -fold $\uparrow, p<0.05, n=6)$. JNK1 and JNK2 expression were also analysed by RT-PCR in lean $(n=12)$ and obese cohorts $(n=12)$. No significant difference was observed with JNK1 gene expression in relation to adiposity status (Lean: JNK1, $\Delta$ Ct 13.39 \pm 0.55 ; JNK1, Obese $\Delta$ Ct 14.17 \pm 0.68 ). However, JNK2 expression was significantly higher in the obese cohort (Lean: JNK2, $\Delta \mathrm{Ct} 10.39 \pm 0.17$; JNK2, Obese: $\Delta \mathrm{Ct} 9.6 \pm 0.23 ; \mathrm{p}<0.05$ ). We also examined the effect of adiposity for total and phosphorylated JNK1 expression. Phosphorylated JNK1 was significantly higher in obese (Lean: $1.1 \pm 0.3$, Obese: $3.2 \pm 0.3$; $\mathrm{p}<0.001)$.

Conclusion: Our findings confirm, for the first time, ER stress in human AbSc AT from obese subjects as compared with lean subjects. Our data also demonstrate upregulation of key factors of the innate immune signalling pathway; suggesting ER stress and inflammation in adipose tissue may underlie sub-clinical inflammation that may potentially contribute to pathogenesis of obesity associated T2DM. Further mechanistic studies are underway to show that ER stress may be one of the factors that induces pro-inflammatory cytokine response in human adipose tissue.

Supported by: Diabetes UK

\section{1}

Endoplasmic reticulum stress prevention improves hepatic steatosis of ob/ob mice through an inhibition of SREBP-1c proteolytic cleavage H. Kammoun, H. Chabanon, I. Hainault, P. Ferré, F. Foufelle Metabolic and nutritional pathologies : obesity and type 2 diabetes, INSERM UMRS872, Paris, France

Background and aims: Hepatic steatosis is strongly associated with the metabolic syndrome, including type 2 diabetes and obesity. In rodents, lipogenesis has been shown to be one of the major pathway implicated in the set up of steatosis. The transcription factor SREBP- $1 \mathrm{c}$ has a central role in the transcriptional control of lipogenic enzymes. SREBP-1c is regulated by insulin both at the transcriptional and post-translational level (activation of the proteolytic cleavage). Interestingly, high level of nuclear SREBP-1c are detected 
in the fatty liver of rodents characterized by a severe hepatic insulin resistance suggesting that another mechanism than insulin can trigger the activation of SREBP-1c. Endoplasmic reticulum (ER) stress has been shown to be induced in the liver of obese rodents. We hypothesized that ER stress activation in obese animals can activate SREBP-1c proteolysis leading to a stimulation of lipogenesis and finally to the development of hepatic steatosis.

Materials and methods: ob/ob mice were used as a model of fatty liver. ER stress was inhibited using an adenoviral vector allowing the overexpression of the BiP/GRP78 chaperone. The consequences of ER stress inhibition were analysed on nuclear and microsomal SREBP-1c and on SREBP-1c target genes as well as liver and serum triglycerides content.

Results: As described previously, several ER stress protein markers (ATF6 and XBP1) are induced in the liver of ob/ob mice compared to their lean control. Nuclear content of SREBP-1c as well as the expression of lipogenic enzymes are also greatly induced in the liver of ob/ob mice. Hepatic overexpression of protein BiP/GRP78 results in (1) a decrease of ER stress markers, (2) a decrease in precursor and nuclear content of SREBP-1c, (3) a decrease in lipogenic enzyme gene expression (4) a reduction in hepatic and serum triglycerides concentrations. This leads to an improvement of liver insulin sensitivity as shown by the fact that the insulin/glucose ratio and the expression of gluconeogenic enzymes are strongly decreased.

Conclusion: The ER stress pathway can contribute to the development of hepatic steatosis in obese mice by activating the transcription factor SREBP-1c and hepatic de novo lipogenesis. This pathway could then be a pharmacological target to reduce hepatic steatosis.

Supported by: French Research Agency

\section{PS 066 Obesity}

\section{2}

Rimonabant reduces body weight and adiposity independent of changes in resting metabolic rate and food intake

O.O. Woolcott, D. Stefanovski, L.N. Harrison, V. Mooradian, D. Zheng,

M. Lottati, J. Dittmann, C.M. Kolka, S.P. Kim, I.R. Hsu, K.J. Catalano,

V. Ionut, S. Yae, R.N. Bergman, J.M. Richey

Physiology \& Biophysics, Keck School of Medicine, University of Southern

California, Los Angeles, United States

Background and aims: Rimonabant (RIM), a drug used to treat obesity, is believed to reduce body weight (BW) mainly by decreasing appetite. Recently, it has been shown that RIM acutely increases energy expenditure in rodents. In this study, we longitudinally monitored fat distribution, food intake and resting metabolic rate (RMR) in dogs fed a high-fat diet and chronically treated with RIM to determine the mechanism by which RIM reduces BW.

Materials and methods: Twelve male dogs fed a hypercaloric high-fat diet (HHFD) consisted of $\sim 5400 \mathrm{kcal} /$ day (fat: $53 \%$ ), starting at week -6 . After 6 weeks of HHFD (baseline: week 0), dogs were divided in two groups: 7 dogs started RIM treatment ( $1.25 \mathrm{mg} / \mathrm{kg} / \mathrm{d}$, P.O.), and 5 dogs received placebo (PBO) during 16 weeks. HHFD was maintained until the end of the study. Fat and non-fat content changes $(\Delta)$ in the abdominal trunk were monitored by magnetic resonance imaging (MRI) at weeks $-6,0,2$, and 16 . Body composition in the trunk was divided in 5 categories: visceral adipose tissue (VAT), subcutaneous adipose tissue (SAT), total fat $(\mathrm{TF}=\mathrm{VAT}+\mathrm{SAT})$, non-fat tissue (NFT) and total tissue (TT=TF+NFT). At week -6, BW and body composition were not significantly different between groups. RIM and PBO groups had similar baseline BW $(32.5 \pm 1.2$ and $32.3 \pm 1.3 \mathrm{~kg}$, respectively, mean \pm SEM). RMR was measured by indirect calorimetry.

Results: After 16 weeks of treatment, concomitant with a HHFD, RIM dogs lost weight by $4.3 \%(\mathrm{P}=0.005)$, while $\mathrm{PBO}$ dogs increased their $\mathrm{BW}$ by $5.9 \%$ $(\mathrm{P}=0.01)$. RIM reduced food intake by $26.3 \%(79.8 \pm 5.4 \mathrm{vs} .58 .8 \pm 5.6 \mathrm{kcal} / \mathrm{kg} /$ $\mathrm{d}, \mathrm{P}=0.019$ ) only in the first two weeks of the study. Food intake in the PBO group remained unchanged. RIM consistently decreased TF and SAT contents by $18.2 \%(\mathrm{P}=0.018)$ and $24.4 \%(\mathrm{P}=0.011)$, respectively. In contrast, $\mathrm{PBO}$ group showed an average increase in SAT by $37 \%(\mathrm{P}=0.002)$ and TF by $24.2 \%$, though not significant $(\mathrm{P}=0.075)$. In the RIM group, compared with $\mathrm{PBO}$, the $\Delta$ of TF and SAT was significantly different $(81.2 \pm 5.2$ vs. $123.9 \pm 10.4 \%$ $[\mathrm{P}=0.002]$, and $76.1 \pm 4.4$ vs. $136.6 \pm 3.4 \%[\mathrm{P}<0.001]$, respectively). NFT and VAT remained unchanged in both groups. TT was correlated with BW in the RIM and PBO group $\left(\mathrm{r}^{2}=0.67[\mathrm{P}=0.025]\right.$ and $\mathrm{r}^{2}=0.77[\mathrm{P}=0.049]$, respectively). Surprisingly, compared with baseline, RIM group showed a decrease in RMR at week $16(1414.1 \pm 85.5$ vs. $1618.8 \pm 76 \mathrm{kcal} / \mathrm{d}, \mathrm{P}=0.047)$ while $\mathrm{PBO}$ group showed no changes $(1628.5 \pm 139.9$ vs. $1632.9 \pm 63.7 \mathrm{kcal} / \mathrm{d})$. However, there was no correlation between RMR and BW, TF, VAT or SAT in any group, at any week. Moreover, there was no difference in RMR between groups.

Conclusion: Chronic treatment with RIM resulted in reduction of BW and fat content in the abdominal trunk. However, these findings are not explained by either changes in food intake or RMR. Together, these results suggest that weight loss and fat reduction induced by RIM may be due to an effect on physical activity.

Supported by: sanofi-aventis

\section{3}

Weight loss improves platelet sensitivity to thiol antioxidant $\mathrm{N}$-acetylL-cysteine in obese insulin-resistant subjects: relationships with improvement of insulin sensitivity

G. Anfossi, I. Russo, M. Viretto, P. Del Mese, G. Doronzo, K. Bonomo, C. Giordanino, L. Mattiello, M. Trovati

Diabetes Unit, Department of Clinical and Biological Sciences, University of Turin, San Luigi Gonzaga Hospital, Italy

Background and aims: N-acetyl-L-cysteine (NAC) is a thiol compound with both extra- and intracellular antioxidative properties, able to reduce platelet responses to agonists by enhancing the inhibitory effects of nitric oxide (NO) via an increase of the anti-aggregating nucleotide cyclic guanosine monophosphate (cGMP). We previously observed that in central obesity platelets are resistant to the anti-aggregating effects of NAC, as a piece of a multifaceted resistance to anti-aggregating substances, deeply involved in the increased atherothrombotic risk. Lifestyle changes prevent the occurrence of cardiovascular events in obese subjects: few studies, however, evaluated so far whether weight loss reduces the obesity-related platelet dysfunction. Aim of this study is to clarify whether a diet intervention to obtain a body weight reduction of at least $10 \%$ of baseline improves platelet sensitivity to NAC.

Materials and methods: 10 subjects affected by central obesity [M/F: 5/5; age: $38.4 \pm 3.4$ years; BMI $35 \pm 1.5 \mathrm{~kg} / \mathrm{m}^{2}$; waist circumference (WC): $102.7 \pm 3.5$ $\mathrm{cm}$ ] were investigated at baseline and after a 6-month diet intervention program, by measuring: i)insulin resistance by Homeostasis Model Assessment (HOMA-IR) Index; ii)circulating markers of endothelial dysfunction (such as sVCAM) and of platelet activation (such as SCD40 Ligand); iii)platelet concentrations of cGMP in response to a 3-min incubation with $10 \mu \mathrm{mol} / \mathrm{l}$ of the NO donor Sodium Nitroprusside (SNP), with or without a 30-min pre-incubation with $3 \mathrm{mmol} / \mathrm{l}$ of NAC. The effect of NAC on the synthesis of cGMP induced by SNP was calculated also as the ratio between the cGMP levels elicited by NAC+SNP and the cGMP levels elicited by SNP alone. Data are expressed as mean \pm SEM.

Results: At the end of diet intervention, obese subjects showed: i) significant reductions of body weight $(-13.5 \pm 1.4 \%)$, BMI $(-12.8 \pm 1.5 \%)$ and WC ($9.0 \pm 1.3 \%$ ); ii) a decrease of HOMA IR from $7.7 \pm 1.2$ to $3.3 \pm 0.4$ ( $\mathrm{p}<0.002)$; iii) a decrease of sVCAM from $1093.8 \pm 77.8$ to $685.1 \pm 54.9(\mathrm{ng} / \mathrm{ml})(\mathrm{p}<0.0001)$ and of sCD40L from $5.9 \pm 0.5$ to $2.1 \pm 0.5 \mathrm{ng} / \mathrm{ml}(\mathrm{p}<0.0001)$; iv) an increase of platelet cGMP concentrations ( $\mathrm{pmol} / 10^{9} \mathrm{plts}$ ) in response to NAC from $7.57 \pm 0.54$ to $15.32 \pm 1.01(\mathrm{p}<0.0001)$, in response to SNP from $9.73 \pm 0.70$ to $33.36 \pm 4.23(\mathrm{p}<0.0001)$, in response to NAC+SNP from $26.22 \pm 2.60$ to $188.80 \pm 6.73(\mathrm{p}<0.0001) ; \mathrm{v})$ an increase of the ratio between platelet cGMP in response to $\mathrm{NAC}+\mathrm{SNP}$ and platelet cGMP in response to SNP alone, from $3.02 \pm 0.56$ to $7.06 \pm 1.46(\mathrm{p}<0.019)$. By the simple regression analysis, cGMP levels measured in the presence of NAC+SNP inversely correlated with BMI $(\mathrm{r}=-0.538, \mathrm{p}<0.014)$, WC $(\mathrm{r}=-0.528, \mathrm{p}<0.017)$, HOMA IR $(\mathrm{r}=-0.805$, $\mathrm{p}<0.007)$, sVCAM $(\mathrm{r}=-0.709, \mathrm{p}<0.0001)$ and CD40L $(\mathrm{r}=-0.718, \mathrm{p}<0.0001)$. In multiple regression analysis, HOMA-IR was the only parameter significantly correlated with the cGMP response to NAC+SNP ( $\mathrm{p}=0.0001)$.

Conclusions: Diet-induced weight loss in subjects affected by central obesity improves platelet sensitivity to NAC, with a significant relationship with the improvement of insulin sensitivity. This phenomenon could be involved in the decrease of the atherothrombotic risk induced by weight loss related to the correction of insulin resistance.

Supported by: grant from Piedmont Region 


\section{4}

Mechanism of improvement of glucose tolerance in obese subjects with type 2 diabetes following gastric bypass surgery

M. Anselmino ${ }^{1}$, M. Nannipieri ${ }^{2}$, A. Mari ${ }^{3}$, E. Mancini ${ }^{2}$, E. Barsotti ${ }^{2}$, E. Ferrannini ${ }^{2}$

${ }^{1}$ Bariatric Surgery, Azienda Ospedaliera Universitaria of Pisa, ${ }^{2}$ Internal

Medicine, University of Pisa, ${ }^{3}$ Bio-Engineery, CNR of Padua, Italy

Background and aims: $\beta$-cell dysfunction and insulin resistance are the main pathophysiological defects responsible for the development of hyperglycaemia. In morbidly obese patients with type 2 diabetes (T2DM), bilio-pancreatic-diversion (a predominantly malabsorptive surgical approach) restores euglycaemia and the acute insulin response to glucose. Aim of this study was to analyse the mechanisms underlying the improvement in glucose tolerance in morbidly obese T2DM patients undergoing Roux-en-Y gastric bypass (GBP, a predominantly restrictive operation).

Materials and methods: We evaluated glucose tolerance by a standard 75-g OGTT, insulin sensititvity by the OGTT-derived OGIS index, $\beta$-cell function by modelling analysis of the C-peptide response to oral glucose in 9 non-diabetic subjects (NGT) and 12 T2DM subjects before and 45 days after surgery.

Results: At 45 days post-GBP, body mas index (BMI) decreased to the same extent in NGT and T2DM subjects (from $43.7 \pm 4.9$ to $36.9 \pm 4.4 \mathrm{~kg} \mathrm{~m}^{-2}$ in NGT and from $47.5 \pm 7.2$ to $40.4 \pm 7.2$ in T2DM, mean $\pm S D$, $p<0.0001)$. GBP improved glucose tolerance in all subjects $(\mathrm{p}<0.0001)$ but especially in T2DM ( $\mathrm{p}<0.01$ for the interaction) (mean glucose during the OGTT: from $7.2 \pm 0.6$ to $6.3 \pm 0.7$ in NGT and from $11.6 \pm 2.5$ to $9.0 \pm 2.6 \mathrm{mM}$ in T2DM). At baseline, insulin sensitivity was reduced in T2DM as compared to NGT $(279 \pm 33$ vs $368 \pm 43, \mathrm{p}<0.0001$ ) despite a similar degree of obesity. Following surgery, insulin sensitivity improved significantly in all subjects (to $332 \pm 54 \mathrm{ml} \cdot \mathrm{min}^{-}$ ${ }^{1} \mathrm{~m}^{-2}$ in NGT and to $412 \pm 54$, in T2DM, $\left.\mathrm{p}<0.0001\right)$. At baseline, $\beta$-cell glucose sensitivity (i.e., the slope of the dose-response curve of insulin secretion rates and plasma glucose concentrations) was severely impaired in T2DM (41 \pm 23 pmolmin ${ }^{-1} \mathrm{~m}^{-2} \mathrm{mM}^{-1}$ vs $80 \pm 33$ of NGT subjects, $\left.\mathrm{p}<0.01\right)$. After GBP, $\beta$-cell glucose sensitivity improved in all subjects (to $67 \pm 43 \mathrm{pmol} \mathrm{min}^{-1} \mathrm{~m}^{-2} \mathrm{mM}^{-1}$ in T2DM and to $131 \pm 55$ in NGT, $\mathrm{p}=0.0005$ ), although it remained impaired in T2DM $v$ s NGT $(\mathrm{p}<0.01)$. The surgery-induced percent changes in $\beta$-cell glucose sensitivity were positively related to the corresponding changes in $\operatorname{BMI}(r=0.58, \mathrm{p}<0.01)$.

Conclusion: Following gastric bypass surgery, glucose tolerance improves as a result of an increase in both insulin sensitivity and $\beta$-cell glucose sensitivity. These changes are of rapid onset and roughly proportional to the weight loss regardless of initial glucose tolerance.

Supported by: grant from the Italian Ministry of Research and Education

\section{5}

Effect of weight loss on the mRNA expression of high-mobility group box 1 (HMGB1) protein in subjects with impaired glucose metabolism and the metabolic syndrome - the GENOBIN study

V.D.F. Mello ${ }^{1,2}$, M. Kolehmainen ${ }^{1}$, L. Pulkkinen ${ }^{1}$, U. Schwab ${ }^{1,3}$, U. Mager ${ }^{1}$, D.E. Laaksonen ${ }^{3}$, H. Gylling ${ }^{1,3}$, M. Uusitupa ${ }^{1}$

${ }^{1}$ School of Public Health and Clinical Nutrition/Food and Health Research Centre, University of Kuopio, Finland, ${ }^{2}$ Internal Medicine, Universidade Federal do Rio Grande do Sul, Porto Alegre, Brazil, ${ }^{3}$ Institute of Clinical Medicine, Internal Medicine, Kuopio University Hospital, Finland

Background and aims: HMGB1 is a protein that can act as anmune marker and a proinflammatory cytokine, participating in the induction of cytokine production. Previously we have shown that weight loss altered gene expression of selected cytokines and receptors of the NFKB pathway in peripheral blood mononuclear cells (PBMCs) of overweight subjects with abnormal glucose metabolism and the metabolic syndrome (MS). In this study we sought to evaluate how weight loss and the associated improvement in insulin sensitivity affected mRNA expression of HMGB1 in PBMCs in overweight individuals with the MS.

Materials and methods: Altogether 34 overweight or obese (BMI: $32.6 \pm 3.1$ $\mathrm{kg} / \mathrm{m}^{2}$ ) subjects with the MS randomized to either a weight reduction (WR) or a control group for 33 weeks were studied. Subjects in the WR group had an intensive 12 -wk weight loss period followed by a 21 -wk period where the minimum aim was weight maintenance. The mRNA expression was studied using real-time PCR and TaqMan chemistry at baseline and wk 33. Intra- venous and oral glucose tolerance tests and body composition were also assessed in the beginning and at the end of the study.

Results: In the WR group, body weight, BMI and waist circumference decreased $(\mathrm{p}<0.01)$, and the insulin sensitivity index $\left(\mathrm{S}_{\mathrm{T}}\right)$ improved $(\mathrm{p}<0.05)$. In the WR group a decrease in mRNA expression of HMGB1 occurred (median $-18 \%$ [interquartile range: $-25.0-2.0 \%$ ], $\mathrm{p}<0.001$ ). The decrease in HMGB1 mRNA expression correlated with an increase in the $\mathrm{S}_{\mathrm{I}}$ after adjustment for changes in waist circumference $(\mathrm{r}=-0.46, \mathrm{p}=0.03)$ or $\mathrm{BMI}(\mathrm{r}=-0.46, \mathrm{p}=0.06)$. Only the changes in HMGB1 expression $(\beta=-0.46, p=0.04)$ and waist circumference $(\beta=-0.58, p=0.01)$ had an independent effect on the improvement in $\mathrm{S}_{\mathrm{I}}$ in multiple regression analysis. Adjustment for changes in physical activity frequency, energy adjusted intake of total fat, saturated fat, monounsaturated fat, polyunsaturated fat or total fiber did not attenuate these independent effects. The decrease in HMGB1 expression also correlated with the change in TNF mRNA expression ( $r=0.46, \mathrm{p}=0.04)$. Moreover, baseline levels of mRNA HMGB1 expression correlated with baseline fasting insulin concentration $(\mathrm{r}=0.44, \mathrm{p}<0.01)$. Adjustments for baseline BMI and gender did not alter this result.

Conclusion: Weight loss downregulates the mRNA expression of HMGB1 in PBMCs. HMGB1 expression after WR in turn was associated with the improvement in $S_{I}$ independently of confounding factors. These results suggest, along with the association between HMGB1 expression and fasting insulin concentration, that mRNA expression of HMGB1 in obese subjects with the MS is involved in the regulation of insulin sensitivity and might reflect the local inflammatory response in the PBMCs.

Supported by: Academy of Finland, Sigrid Juselius Foundation. V.D.F. Mello was supported by CNPq, Brazil.

\section{6}

Dapagliflozin-induced glucosuria is accompanied by weight loss in type 2 diabetes patients

V. Woo ${ }^{1}$, J.F. List ${ }^{2}$, E. Morales ${ }^{3}$, W. Tang ${ }^{2}$, F.T. Fiedorek ${ }^{2}$

${ }^{1}$ Diabetes Research \& Treatment Centre, Health Sciences Centre, Winnipeg, Canada,Bristol-Myers Squibb, Princeton, United States, ${ }^{3}$ Cardiometabólica, Centro de Investigación Cardiometabólica, Aguascalientes, Mexico

Background and aims: Dapagliflozin, a novel oral antidiabetic agent that potently and selectively inhibits the renal sodium-glucose co-transporter 2, is being developed for the treatment of patients with type 2 diabetes (T2DM). By inhibiting glucose reabsorption, and with it the urinary loss of calories, the novel mechanism of dapagliflozin has the potential to drive weight loss. The effect of dapagliflozin on weight was evaluated in treatment-naive T2DM patients.

Material and methods: In this 12 -wk, double-blind, parallel-group, doseranging, placebo $(\mathrm{PBO})$-controlled trial, 389 treatment-naïve T2DM patients with inadequate blood glycemic control and low mean glucosuria at baseline were randomized in equal ratios to once-daily dapagliflozin $2.5,5,10,20$, or $50 \mathrm{mg}$, metformin (Met) XR $750 \mathrm{mg}$ titrated to $1500 \mathrm{mg}$ or PBO.

Results: Dapagliflozin treatment led to consistent and sustained increases in urinary glucose excretion, rising to mean glucosuria values between 51.8 to 85.0 grams per day $(\mathrm{g} / \mathrm{d})$ at wk 12 in the dapagliflozin study arms from baseline means between 5.8 to $10.9 \mathrm{~g} / \mathrm{d}$. Mean glucosuria with PBO and Met both remained low (5.7 and $5.6 \mathrm{~g} / \mathrm{d}$, respectively, at wk 12). Mean percent reductions for body weight and absolute changes in body mass index (BMI) over 12 wks are shown below. A higher proportion of patients in each of the dapagliflozin groups achieved a $5 \%$ weight reduction than PBO. Mean daily 24-hour urine volumes at baseline were between 1.9 and $2.2 \mathrm{~L}$ per day; small dose-related increases in 24-hour urine volumes were observed at wk 12 from $+107 \mathrm{~mL}$ (2.5-mg dose) to $+470 \mathrm{~mL}$ (50-mg dose) compared with $-112 \mathrm{~mL}$ for $\mathrm{PBO}$ and $-96 \mathrm{~mL}$ for Met. There was no apparent effect of dapagliflozin treatment on appetite, as assessed by a visual analogue scale. Adverse events of polyuria/pollakiuria were reported by 4 (1.4\%) subjects in the dapagliflozin groups and there were no reports of nocturia.

Conclusion: Overall, dapagliflozin treatment led to greater degrees of weight loss than PBO in patients with T2DM. 
Table. Mean Changes from Baseline at Wk 12.

\begin{tabular}{|c|c|c|c|c|c|c|c|}
\hline & \multicolumn{7}{|c|}{ Dapagliflozin dose (mg) } \\
\hline & $\begin{array}{l}2.5 \\
\mathrm{n}=59\end{array}$ & $\begin{array}{l}5 \\
\mathrm{n}=58\end{array}$ & $\begin{array}{l}10 \\
\mathrm{n}=47\end{array}$ & $\begin{array}{l}20 \\
\mathrm{n}=59\end{array}$ & $\begin{array}{l}50 \\
\mathrm{n}=56\end{array}$ & $\begin{array}{l}\mathrm{PBO} \\
\mathrm{n}=54\end{array}$ & $\begin{array}{l}\text { Met } \\
\mathrm{n}=56\end{array}$ \\
\hline Baseline weight - kg (SD) & $90(20)$ & $89(17)$ & $86(17)$ & $88(18)$ & $91(19)$ & $89(19)$ & $88(20)$ \\
\hline Mean reduction (LOCF) in weight - \% $[95 \% \mathrm{CI}]$ & $\begin{array}{l}-2.7 \\
{[-3.4,} \\
-1.9]\end{array}$ & $\begin{array}{l}-2.5 \\
{[-3.3} \\
-1.8]\end{array}$ & $\begin{array}{l}-2.7 \\
{[-3.5} \\
-1.8]\end{array}$ & $\begin{array}{l}-3.4 \\
{[-4.1,} \\
-2.6]\end{array}$ & $\begin{array}{l}-3.4 \\
{[-4.1,} \\
-2.6]\end{array}$ & $\begin{array}{l}-1.2 \\
{[-2.0} \\
-0.4]\end{array}$ & $\begin{array}{l}-1.7 \\
{[-2.4} \\
-0.9]\end{array}$ \\
\hline Baseline BMI - kg/m² (SD) & $31(5)$ & $31(5)$ & $30(5)$ & $31(5)$ & $32(4)$ & $32(6)$ & $32(5)$ \\
\hline Mean reduction in BMI [95\% CI] & $\begin{array}{l}-0.9 \\
{[-1.1} \\
-0.7]\end{array}$ & $\begin{array}{l}-0.8 \\
{[-1.2} \\
-0.4]\end{array}$ & $\begin{array}{l}-0.8 \\
{[-1.1} \\
-0.5]\end{array}$ & $\begin{array}{l}-1.0 \\
{[-1.2} \\
-0.8]\end{array}$ & $\begin{array}{l}-1.1 \\
{[-1.3} \\
-0.9]\end{array}$ & $\begin{array}{l}-0.3 \\
{[-0.5} \\
0.0] \\
\end{array}$ & $\begin{array}{l}-0.5 \\
{[-0.8} \\
-0.3]\end{array}$ \\
\hline
\end{tabular}

Supported by: Bristol-Myers Squibb

\section{7}

The reduction of body weight with liraglutide, a once-daily human GLP1 analogue for type 2 diabetes, primarily comes from fat tissue and the fat tissue lost is predominantly visceral fat

J. Jendle ${ }^{1}$, M.A. Nauck ${ }^{2}$, D. Matthews ${ }^{3}$, A. Frid ${ }^{4}$, K. Hermansen ${ }^{5}$, M. During, M. Zdravkovic ${ }^{6}$, B. Strauss ${ }^{7}$, A. Garber ${ }^{8}$

${ }^{1}$ Clinical Medicine, University of Orebro, Orebro, Sweden,

${ }^{2}$ Diabeteszentrum, Bad Lauterberg im Harz, Germany, ${ }^{3}$ OCDEM,

University of Oxford, United Kingdom, ${ }^{4}$ Oresund Diabetes Team, University Hospital, Lund, Sweden, ${ }^{5}$ Endocrinology and Metabolism, Aarhus University Hospital, Aarhus, Denmark, ${ }^{6}$ Novo Nordisk A/S, Bagsvaerd, Denmark, ${ }^{7}$ Endocrinology, Monash University, Clayton, Australia, ${ }^{8}$ Baylor College of Medicine, Methodist Hospital, Houston, United States

Background and aims: The majority of subjects with type 2 diabetes are overweight and most antidiabetes medications exacerbate weight gain. Therefore, therapies that minimise weight gain while improving glycaemic control are desirable.

Materials and methods: Two trials recruited subjects with type 2 diabetes [LEAD 2, baseline body weight (BW) $92.1 \pm 14.0 \mathrm{~kg}$; LEAD 3, baseline body weight (BW) $92.7 \pm 19.6 \mathrm{~kg}$ ]: LEAD 2 was a 26 -week double-blind, doubledummy, randomised, active control, parallel group trial; LEAD 3 was 52 weeks long, with treatments for both trials as indicated in the table. Measurements were taken at baseline and week 26 for LEAD 2, and baseline and week 52 for LEAD 3. Dual-energy X-ray absorptiometry (DEXA) was used to measure changes in total lean body tissue and fat as well as fat percentage in a subgroup of subjects from both studies. Visceral and sc adipose tissue in the central region of the body was measured using computerised tomography (CT) in LEAD 2 only.

Results: A 13-17\% reduction from baseline in central body visceral adipose tissue in liraglutide + metformin was demonstrated by CT. Central body sc adipose tissue was reduced by $5-9 \%$ with liraglutide + metformin, which was significantly different from glimepiride + metformin ( $3 \%$ increase) but not from placebo + metformin ( $4 \%$ reduction). An increase in liver-spleen attenuation ratio (indicating reduced hepatic steatosis) was seen in liraglutide $1.8 \mathrm{mg}+$ metformin, which was significantly different from glimepiride + metformin. The reduction in percentage of body fat (fat $\%)$ with liraglutide $1.2 \mathrm{mg}+$ metformin and liraglutide $1.8 \mathrm{mg}+$ metformin was significantly different from the increase in fat $\%$ with glimepiride + metformin. Reduction in fat $\%$ did not differ significantly between liraglutide + metformin and placebo
+ metformin. With liraglutide monotherapy, the reductions in fat mass and percentage with both doses of liraglutide were significantly different to the increases associated with glimepiride.

Conclusion: Liraglutide either as monotherapy or added to metformin significantly reduced body weight, fat mass, and fat percentage compared with glimepiride in subjects with type 2 diabetes.

\section{8}

Enhanced weight loss following co-administration of pramlintide with sibutramine or phentermine in obese subjects

A. Halseth ${ }^{1}$, L. Aronne ${ }^{2}$, C. Burns ${ }^{1}$, L. Porter ${ }^{1}$, L. Shen ${ }^{1}$

${ }^{1}$ Amylin Pharmaceuticals, Inc., San Diego, ${ }^{2}$ Medicine, Weill-Cornell Medical College, New York, United States

Background and aims: In diet-inducd obese rats, monotherapy with the anorexigenic small molecules sibutramine or phentermine or the neurohormone amylin elicited significant vehicle-corrected weight loss of up to $\sim 8 \%$, while combination treatment with amylin and sibutramine or phentermine elicited $12-13 \%$ weight loss. Similar results were seen with sibutramine, phentermine or the amylinomimetic pramlintide monotherapy in obese humans.

Materials and methods: This randomised trial was designed to assess the safety and efficacy of sibutramine or phentermine with pramlintide. Following a 1 -wk placebo lead-in, 244 overweight or obese subjects (ITT, $88 \%$ female, $41 \pm 11 \mathrm{y}$, BMI $37.7 \pm 5.4 \mathrm{~kg} / \mathrm{m}^{2}$, mean $\pm \mathrm{SD}$ ) received placebo or pramlintide (120 $\mu \mathrm{g}$ TID) alone via SC injection or pramlintide in combination with sibutramine (10 mg QAM) or phentermine (37.5 mg QAM) for $24 \mathrm{wks}$ along with diet and lifestyle intervention.

Results: At 24 wks (Evaluable), weight loss with pramlintide+sibutramine $(11.3 \pm 1.2 \mathrm{~kg}$; mean $\pm \mathrm{SE})$ and pramlintide+phentermine $(11.3 \pm 0.9 \mathrm{~kg})$ was significantly greater than with pramlintide $(3.6 \pm 0.7 \mathrm{~kg})$ or placebo $(2.1 \pm 0.9 \mathrm{~kg})$ $(\mathrm{P}<0.0001$ combination arms vs. pramlintide or placebo). More subjects achieved $\geq 5 \%$ weight loss and $\geq 10 \%$ weight loss in the pramlintide+sibutramine ( $78 \%$ and $49 \%$, respectively) and pramlintide +phentermine ( $82 \%$ and $56 \%$ ) than pramlintide (36\% and $11 \%)$ or placebo treatment groups ( $28 \%$ and $3 \%)(\mathrm{P}<0.001$ combination arms vs. pramlintide or placebo). The safety profiles of the combinations were generally consistent with those previously established for the individual agents.

Conclusion: These results are consistent with previous findings in DIO rats and suggest that targeting multiple pathways through the combination of the amylin agonist pramlintide and other centrally acting anorectic agents may result in robust weight loss.

\begin{tabular}{|c|c|c|c|c|c|}
\hline LEAD $2(n=160)$ & $\begin{array}{l}\text { Liraglutide } 0.6 \mathrm{mg}+ \\
\text { metformin }\end{array}$ & $\begin{array}{l}\text { Liraglutide } 1.2 \mathrm{mg}+ \\
\text { metformin }\end{array}$ & $\begin{array}{l}\text { Liraglutide } 1.8 \mathrm{mg}+ \\
\text { metformin }\end{array}$ & $\begin{array}{l}\text { Placebo }+ \\
\text { metformin }\end{array}$ & $\begin{array}{l}\text { Glimepiride + } \\
\text { metformin }\end{array}$ \\
\hline Change in HbAlc $\ddagger$ & $-0.69 \pm 0.07$ & $-0.97 \pm 0.07$ & $-1.0 \pm 0.07$ & $+0.09 \pm 0.09$ & $-0.98 \pm 0.07$ \\
\hline$\Delta$ fat mass, $\mathrm{kg}$ & $-0.74 \pm 0.81^{\star}$ & $-1.64 \pm 0.84^{*}$ & $-2.40 \pm 0.69^{\star}$ & $-1.13 \pm 0.94$ & $+1.13 \pm 0.84$ \\
\hline$\Delta$ lean body mass, $\mathrm{kg}$ & $-0.26 \pm 0.56^{*}$ & $-0.84 \pm 0.58^{*}$ & $-1.54 \pm 0.47^{*}$ & $-1.33 \pm 0.65$ & $+1.28 \pm 0.58$ \\
\hline$\Delta$ fat $\%$ & $-0.5 \pm 0.7$ & $-1.1 \pm 0.8^{*}$ & $-1.2 \pm 0.6^{*}$ & $-0.2 \pm 0.8$ & $+0.4 \pm 0.8$ \\
\hline LEAD $3(\mathrm{n}=45)$ & & Liraglutide $1.2 \mathrm{mg}$ & Liraglutide $1.8 \mathrm{mg}$ & & Glimepiride \\
\hline Change in HbAlc $\neq$ & & $-0.85 \pm 0.08$ & $-1.14 \pm 0.08$ & & $-0.51 \pm 0.08$ \\
\hline$\Delta$ fat mass, kg & & $-1.97 \pm 0.77^{\dagger}$ & $-0.95 \pm 0.75^{\dagger}$ & & $+2.41 \pm 0.89$ \\
\hline$\Delta$ lean body mass, $\mathrm{kg}$ & & $-1.11 \pm 0.53$ & $-1.51 \pm 0.54$ & & $-0.55 \pm 0.65$ \\
\hline$\Delta$ fat $\%$ & & $-0.88 \pm 0.53^{\dagger}$ & $-0.27 \pm 51^{\dagger}$ & & $+2.6 \pm 0.61$ \\
\hline
\end{tabular}

Least squares changes from baseline. Means \pm SEM; ${ }^{\dagger} p<0.05$ vs. Glimepiride; ${ }^{*} p<0.05$ vs. glimepiride + metformin. $\neq$ From all randomised subjects.

Supported by: Novo Nordisk A/S 


\section{9}

Effect of weight loss by very-low-calorie-diet on free fatty acid uptake in liver, pancreas, femoral adipose tissue and femoral muscle

A.C. Karmi, A. Viljanen, K. Virtanen, P. Iozzo, P. Nuutila

Turku PET Centre, University of Turku, Finland

Background and aims: The importance of free fatty acids (FFAs) in the pathogenesis of metabolic syndrome is very well known. The aim of this study was to evaluate FFA uptake in obese subjects having metabolic syndrome (MBO) in liver, pancreas, femoral adipose tissue and femoral muscle, and whether weight reduction modifies this uptake.

Materials and methods: Study population included 22 obese subjects with MBO (age $43 \pm 8$ year, BMI $33.5 \pm 4.1 \mathrm{~kg} / \mathrm{m}^{2}$ ) who were studied during fasting condition using PET with $\left[{ }^{18} \mathrm{~F}\right]$ fluoro-6-thia-heptadecanoic acid (FTHA) and with $\left[{ }^{11} \mathrm{C}\right]$-palmitate. In mitochondria, $\left[{ }^{18} \mathrm{~F}\right]$-FTHA is trapped after initial steps of oxidation and represents the total uptake of FFAs while $\left[{ }^{11} \mathrm{C}\right]$-palmitate represents the non-oxidative residual. After baseline measurements, subjects were started on a very-low-calorie-diet for 6 weeks and were restudied after 1-week recover with eucaloric diet. Fatty acid uptake rates were measured using fractional uptake rates for liver, panceas, femoral adipose tissue and muscle.

Results: Body weight decreased $11.0 \pm 2.5 \mathrm{~kg}$. Serum lipids and glycemic control improved, but fasting FFA levels were not changed. There was reduction in FFA uptake in all organs. Liver FFA uptake decreased from $10.1 \pm 3.1$ to $8.6 \pm 2.5 \mu \mathrm{mol} /\left(100 \mathrm{~g}\right.$ tissue $\left.{ }^{\star} \mathrm{min}\right)(\mathrm{p}<0.05)$ mostly due to the decreased nonoxidative FFA retention $(\mathrm{p}<0.06)$. Simultaneously with improved pancreatic beta-cell response to glucose, total uptake of FFA decreased from $1.78 \pm 0.82$ to $1.40 \pm 0.66 \mu \mathrm{mol} /\left(100 \mathrm{~g}\right.$ tissue $\left.{ }^{*} \mathrm{~min}\right)(\mathrm{p}<0.04)$. Femoral subcutaneous adipose FFA uptake decreased from $0.20 \pm 0.07$ to $0.15 \pm 0.04 \mu \mathrm{mol} /\left(100 \mathrm{~g}\right.$ tissue ${ }^{*}$ min) $(\mathrm{p}<0.006)$, but no change was observed in femoral muscle FFA uptake.

Conclusion: Weight loss decreases effectively FFA retention in the key organs involved in metabolic syndrome in the fasting state.

Supported by: Academy of Finland

\section{0}

Liraglutide, the once daily human GLP-1 analogue in combination with the $\mathrm{CB}-1$ antagonist rimonabant reduces body weight in obese rats in a synergistic manner

K. Raun, K. Dahl, L.B. Knudsen

Novo Nordisk A/S, Maaloev, Denmark

Background and aims: Obesity has reached epidemic proportions throughout most of the world and continues to increase at an alarming rate, giving rise to serious health consequences especially risk of developing type 2 diabetes. At present drug efficacy, in terms of weight loss, is modest with a relative high level of side effects. The ultimate goal for obesity drugs should be to have the effectiveness of bariatric surgery, with the safety of lifestyle intervention. A potential approach for obtaining this goal could be by combining different drugs in lower doses and that way get the advantage of reduced side effects together with elimination of the strong counter regulatory response when using single drug approach. The once-daily human GLP-1 analogue liraglutide has completed phase 3 clinical development for treatment of type 2 diabetes, and is currently in development also for the treatment of obesity. Beyond superior improvement of glycaemic control, liraglutide also reduces body weight with a mechanism that in animals has been shown to include lowering of food intake, altered food choice and maintained energy expenditure. The most recent obesity drug is the anorexigenic cannabinoid receptor- 1 antagonist rimonabant which is available in Europe. In the current study, we have investigated the effect on body weight loss in diet induced obese (DIO) rats after treatment with combinations treatment of liraglutide and rimonabant. Materials and methods: 5 groups $(n=8)$ of DIO rats were used for the study. Sub maximal efficacy doses were chosen in order to get a window for investigating combination effects. The rats were treated with either vehicle (s.c.+ p.o.), liraglutide ( $30 \mathrm{ug} / \mathrm{kg}$, s.c.), rimonabant ( $1 \mathrm{or} 3 \mathrm{mg} / \mathrm{kg}$ p.o.) or lira+rimo combinations (30 ug/ $/ \mathrm{kg}+1 \mathrm{mg} / \mathrm{kg}$ or $30 \mathrm{ug} / \mathrm{kg}+3 \mathrm{mg} / \mathrm{kg}$ for 3 weeks. Body weight was monitored daily.

Results: All data are presented as mean \pm SEM. The rats lost from 4.8 to $10.4 \%$ bodyweight over the entire treatment period with least weight loss in the vehicle treated group and the highest in the lira $30+$ rimo 3 treated group. Tachyphylaxia in body weight loss occurred in all groups over time as the bodyweight came closer to that of normal weight rats. This leads to a decline in differences between groups over time, meaning that the differences are more obvious at a time point where the slopes of the weight loss curves are still declining. After two weeks treatment the vehicle subtracted weight loss for $30 \mathrm{ug} / \mathrm{kg}$ liraglutide was $-13.5 \mathrm{~g} \pm 5.2 \mathrm{~g}$ and for 1 and $3 \mathrm{mg} / \mathrm{kg}$ rimonabant $-5.6 \mathrm{~g} \pm 5.0 \mathrm{~g}$ and $-14.0 \mathrm{~g} \pm 4.5 \mathrm{~g}$ respectively. The weight loss observed for the liraglutide+rimonabant combinations was $-29.5 \mathrm{~g} \pm 4.4 \mathrm{~g}$ for lira $30+$ rimo 1 and $-39.0 \pm 3.0$ for the lira $30+$ rimo 3 . This is a significantly greater weight loss than would be expected from adding the effects of the individual doses. A one-sample $\mathrm{t}$-test resulted in $\mathrm{p}<0.05$ (compared to $19.1 \mathrm{~g}$, the added value for lira 30 and rimo 1 ) and $\mathrm{p}<0.01$ (compared to $27.5 \mathrm{~g}$ the added value for lira 30 and rimo 3 ). At the end of the study when the bodyweight of the combination groups were approaching that of normal weight rats the synergy obtained by combinations became non significant.

Conclusion: Liraglutide can induce weight loss in combination with other anti-obesity agents in diet induced obese rats. Liraglutide lowered body weight in a synergistic manner when combined with rimonabant. Supported by: Novo Nordisk A/S 


\section{PS 067 Oxidative stress}

\section{1}

The involvement of protein S-nitrosylation in adipose tissue dysfunction H. Ovadia, N. Bashan, A. Rudich

Clinical Biochemistry, Ben Gurion University, Beer Sheva, Israel

Background and aims: Oxidative and Nitrosative stress occurs systemically in diabetes, and was suggested to be involved in the development of muscle insulin resistance in various experimental systems. $S$-nitrosylation of protein cysteinyl residues has been suggested to be an important nitric oxidedependent post-translational modification. While increased oxidative stress is well established in fat tissue, it is unknown whether increased nitrosative stress occurs in fat tissue in obesity, and if so, what is its role in the induction of adipose tissue dysfunction. Aims: i. To evaluate protein $S$-nitrosylation in omental fat tissue of High Fat fed (HFD) and ob/ob mice. ii. To compare the level of protein $S$-nitrosylation in human omental fat in obesity iii. To assess in 3T3-L1 adipocytes the ability of S-nitrosoglutathion (GSNO) to induce insulin resistance.

Materials and methods: For studying in-vivo proteins $S$-nitrosylation in obesity we utilized 2 animal models (male C57BL/6J mice after 20 weeks of high fat feeding, and ob/ob mice at the age of 16 weeks), and human omental fat biopsies. $S$-nitrosylation was evaluated in fat tissue by the Biotin switch technique. For studying the effect of nitrosylation in 3T3-L1 adipocytes the cells were exposed to $1 \mathrm{mM} \mathrm{GSNO}$ for up to $8 \mathrm{~h}$.

Results: Total S-nitrosylation of adipose tissue proteins obtained for HFD and $\mathrm{ob} / \mathrm{ob}$ mice was elevated compared to lean mice. A significant increased (2.5-11.8-fold of control) of $S$-nitrosylated proteins was documented in bands corresponding to $\mathrm{Mw}$ of: $\sim 60 \mathrm{kDa}, \sim 50 \mathrm{kDa}, \sim 40 \mathrm{kDa}$ and $\sim 37 \mathrm{kDa}$. In accordance, human omental fat tissues obtained from obese women demonstrate 2-17-fold increased S-nitrosylation in proteins with similar molecular weights. In order to evaluate the role of $S$-nitrosylation on insulin signaling, 3T3-L1 adipocytes were exposed to GSNO, conditions which enhanced proteins $S$-nitrosylation. In association with the increased S-nitrosylation, insulin stimulated $\mathrm{PKB} /$ Akt phosphorylation and the serine phosphorylation of various PKB substrates were reduced by $10-47 \%$ in the treated cells compared to control.

Conclusion: Obesity is associated with increased $S$-nitrosylation of adipose tissue proteins, which may contribute to the induction of adipocyte insulin resistance.

Supported by: Israel Science Foundation

\section{2}

Myeloperoxidase deficiency diminishes hepatic steatosis without affecting plasma glucose levels in mice

S. Rensen ${ }^{1}$, E. Loyens ${ }^{2}$, V. Bieghs ${ }^{2}$, K. Wouters ${ }^{2}$, R. Shiri-Sverdlov ${ }^{2}$, W. Buurman ${ }^{1}$

${ }^{1}$ Surgery, Maastricht University, ${ }^{2}$ Molecular Genetics, Netherlands

Background and aims: Type 2 diabetes is associated with non-alcoholic steatohepatitis (NASH). In both conditions, inflammatory processes are thought to play a defining role in pathogenesis and progression. We have investigated the role of myeloperoxidase (MPO), an important neutrophil enzyme at the crossroad of inflammation and oxidative stress, in the development of NASH and insulin resistance.

Materials and methods: Male LDLR knockout mice received bone marrow from wild-type (WT) or MPO knockout (KO) mice ( $\mathrm{n}=9$ in each group). After 10 weeks recovery, mice were fed a high fat diet (HFD) for 8 weeks. Bodyweight, lipid profile and glucose and insulin levels were monitored during the experiment. After 8 weeks, mice were sacrificed and liver and adipose tissue were analyzed using biochemical assays, immunohistochemistry and Q-PCR analyses.

Results: Bodyweight increased from $20.0 \mathrm{~g}$ in both groups to $21.8 \mathrm{~g}$ (WT) and $21.3 \mathrm{~g}(\mathrm{KO})$, respectively ( $\mathrm{p}=0.03$ for both groups, difference between groups not significant). The number of leukocytes and the relative proportion of neutrophils, macrophages, B-lymphocytes and T-lymphocytes was comparable between the groups both at baseline and at 8 weeks HFD. Interestingly, glucose levels increased from $7.5 \mathrm{mmol} / \mathrm{l}$ at baseline to $8.5 \mathrm{mmol} / \mathrm{l}$ in the WT group at 4 weeks HFD $(\mathrm{p}=0.07)$, whereas KOs only displayed an increase from 7.6 to $8.0 \mathrm{mmol} / \mathrm{l}$ ( $\mathrm{p}=0.33$ ). However, glucose levels dropped again towards comparable levels in both groups at 8 weeks HFD ( $7.6 \mathrm{vs.} 7.5 \mathrm{mmol} / \mathrm{l}$ for WT vs. KO, respectively). Liver triglyceride accumulation was similar in the $\mathrm{KO}$ group compared to the WT group ( $0.31 \mathrm{vs} 0.35 \mu \mathrm{g} / \mu \mathrm{g}$ protein; $\mathrm{p}=0.24)$. In contrast, hepatic accumulation of cholesterol was significantly higher in the WT group ( 0.090 vs. $0.072 \mu \mathrm{g} / \mu \mathrm{g}$ protein; $\mathrm{p}=0.01$ ).

Conclusion: Our preliminary results indicate that reduced inflammation caused by MPO deficiency results in attenuated hepatic steatosis without affecting plasma glucose levels.

Supported by: Senter Novem IOP Genomics

\section{3}

Inhibition of oxygen consumption in adipocytes by reactive oxygen species

Y. $\mathrm{Si}^{1}$, T. Wang ${ }^{1}$, O.S. Shirihai ${ }^{2}$, V. Schultz ${ }^{1}$, H. Si ${ }^{1}$, R.F. Corkey ${ }^{1}$, L. Hu ${ }^{1}$, J.T. Deeney ${ }^{1}$, W. Guo ${ }^{1}$, B.E. Corkey ${ }^{1}$

${ }^{1}$ Obesity Research Center, Boston University School of Medicine, ${ }^{2}$ Department of Pharmacology and Experimental Therapeutics, Tufts University School of Medicine, Boston, United States

Background and aims: The main fate of fuels (glucose and fatty acids) entering white adipocytes is utilization for storage (in the form of triglyceride, TG). Shifting the fuel distribution toward less storage and more burning could provide a strategy to reduce adiposity. To this end, it is critical to understand the regulatory mechanisms by which respiration is modulated in adipocytes. Adipose tissue is subject to inflammatory infiltration, and existing evidence supports a role for reactive oxygen species (ROS) in intracellular signaling, inflammatory processes and insulin resistance. This study was designed to test the hypothesis that adipocyte respiration is inhibited by ROS, and the removal of ROS stimulates respiration and reduces TG stores.

Material and methods: Basal and uncoupled maximal respiration, mitochondrial membrane potential, ATP/ADP ratio, and intracellular levels of ROS were measured in rat primary adipocytes treated with the ROS scavengers, pyruvate or N-acetylcysteine (NAC). Mice provided with NAC in their drinking water $(3 \mathrm{mg} / \mathrm{ml})$ were imaged by $\mathrm{NMR}$ and their rates of respiration were assessed using indirect calorimetry.

Results: We demonstrated a persistent basal rate of adipocyte oxygen consumption $(4.3 \pm 0.3 \mathrm{nmol} / \mathrm{min} / \mathrm{mg}$-protein, $\mathrm{n}=65)$ despite addition of $\mathrm{fu}$ els or FCCP (carbonyl cyanide 4- (trifluoromethoxy) phenylhydrazone), a chemical uncoupler of oxidative phosphorylation. Combination of FCCP with pyruvate uniquely stimulated adipocyte oxygen consumption up to 5fold. The stimulatory effect of pyruvate could not be replaced by other fuels, and was completely inhibited by a mitochondrial pyruvate transport inhibitor. Additionally, NAC also stimulated adipocyte oxygen consumption. Both NAC and pyruvate decreased intracellular ROS levels. In vivo, NAC-treated mice showed increased oxygen consumption (by 23\%, P<0.05) and decreased body fat (by 35\%, $\mathrm{P}<0.05$ ).

Conclusion: This study suggests for the first time that oxygen consumption in the adipocyte is inhibited by ROS. ROS inhibition of oxygen consumption may explain the difficulty in identifying effective strategies to increase fat burning and subsequent fat loss in adipocytes. Stimulating fuel oxidation in adipocytes by decreasing ROS may provide a novel means to shift the balance from fuel storage to fuel burning.

Supported by: NIH

\section{4}

The effect of combined treatment of $\alpha$-lipoic acid and insulin on skeletal muscle in OLEFT rats

S.-J. Yoo' ${ }^{1}$, O.-K. Hong ${ }^{2}$, H.-S. Kwon², S.-S. Lee ${ }^{1}$, S.-R. Kim¹, D.-S. Kim³, D.-M. Kim ${ }^{4}$, K.-H. Yoon ${ }^{2}$, M.-I. Kang', B.-Y. Cha ${ }^{2}$, S.-W. Kim ${ }^{5}$ K.-W. Lee ${ }^{2}$, H.-Y. Son' ${ }^{2}$ S.-K. Kang ${ }^{1}$

${ }^{1}$ Endocrinology and Metabolism, The Catholic University of Korea Holy Family Hospital, Bucheon, Kyunggi-do, ${ }^{2}$ Endocrinology and Metabolism, The Catholic University of Korea, Seoul, ${ }^{3}$ Endocrinology and Metabolism, The Hanyang University, Seoul, ${ }^{4}$ Endocrinology and Metabolism, The Hallym University Kangdong Sacred Heart Hospital, Seoul, ${ }^{5}$ Endocrinology and Metabolism, Sungkyunkwan University School of Medicine, Seoul, Republic of Korea

Background and aims: $\alpha$-lipoic acid (ALA) is a naturally occurring short chain fatty acid with sulfhydryl groups. It is a powerful antioxidant and is associated with improvements in skeletal muscle glucose transport activity and whole-body glucose tolerance in insulin-resistant animals and type 2 diabetic 
human. It has been reported that ALA prevents the development of diabetes in diabetes-prone obese rats by reducing triglyceride accumulation in nonadipose tissues. To investigate the basic machanism of ALA on preventing diabetes through skeletal muscle metabolism, we evaluated the effect of ALA on growth regulation and composition of skeletal muscle.

Materials and methods: Male Otsuka Long-Evans Tokushima Fatty (OLETF) and their diabetes-resistance counterpart Long-Evans Tokushima Otsuka (LETO) rats which served as normal controls, aged 24 weeks, were treated with or without ALA (100 mg/Kg body weight/day in drinking water) and / or insulin (insulin glargine, $2 \mathrm{U} / \mathrm{Kg}$ body weight/day; subcutaneous injection) for 12 weeks. Total RNA was isolated and purified from skeletal muscle using the Rneasy Fibrous Tissue Mini Kit (Qiagen Inc., Valencia, CA). RNA samples were quantified on UV spectrophotometer (NanoDrop Technologies Inc., Wilmington, DE). Quantitative real-time PCR analysis of myostatin, TGF- $\beta 1$, connective tissue growth factor (CTGF) and MHC isoform (MHC I, IIa, IIb, IId/x) mRNA expression was performed using a Bio-Rad iCycler and each mRNA expression normalized to $18 \mathrm{~S}$ ribosomal RNA expression for each sample.

Results: The treatment of ALA with or without insulin reduce body weight significantly and save relatively larger amount of skeletal muscle mass from lower leg (gastrocnemius) compared with insulin-alone treated group and control group of OLETF rats. Myostatin gene expression in skeletal muscle was increased in ALA-alone and insulin-alone treated group and decreased significantly in combined treatment group of ALA and insulin compared with control group of OLETF and LETO rats. TGF- $\beta 1$ and CTGF gene expression in skeletal muscle was decreased in combined treatment group of ALA and insulin compared with control group of OLETF rats. The isoform profile of MHC was significantly increased in MHC I, MHC IIa and MHC IId/x in ALA-alone treated group whereas decreased in MHC I, MHC IIa, MHC IIb and MHC IId/x in combined treatment group of ALA and insulin compared with the control group of OLETF and LETO rats.

Conclusion: The effect of ALA in preventing weight gain was not affected by combined insulin treatment. However the effect on growth regulation and composition of skeletal muscle was totally depend on the presense of insulin treatment. With appropriate glycemic control with insulin, it may cause less amount of glycolytic MHC fiber compositon and has an important role in preserving skeletal muscle mass by decreasing myostatin gene expression in animal model of obesity and diabetes.

\section{5}

Adaptive responses of human endothelial cells and T-lymphocytes to oxidative stress of high concentrations of glucose and palmitate, and reversal with linolenate

A.E. Kitabchi, F. Stentz, A.B. Warren, A. Aluoch, L. Razavi

Division of Endocrinology, University of Tennessee Health Science Center, Memphis, United States

Background and aims: Incubation of human T-lymphocytes (T-cells) and aortic endothelial cells (E-cells) in 15 and $30 \mathrm{mM}$ glucose (but not $5 \mathrm{mM}$ ) - results in their activation, characterized by de novo emergence of growth factor receptors for insulin (IR), IGF1 (IGFR), IL2 (IL2R), and Glut 4, with simultaneous production of lipid peroxide, as detected by thiobarbituric acid (TBA)-reacting material (MDA) and reactive oxygen species (ROS), using dichlorofluorescein-DCF as marker. These cells then metabolize glucose and become insulin sensitive tissues. Hyperglycemia and hyperlipidemia are prevalent in uncontrolled diabetes with production of MDA and ROS. As incubation of the E or T- cells with palmitate (but not linolenate) in 1, 50, and $500 \mu \mathrm{M}$ resulted in activation of these cells, we proposed that such activation might be associated with the development of fatty acid receptors, (GPR40) and transporters (FAT/CD36). Herein we report the results of our studies and responses of these cells to palmitate and linolenate.

Materials and methods: We incubated E and T-cells in various concentrations of palmiate and linolenate $(1,50$, and $300 \mu \mathrm{M})$ alone and together from 0-72 hours and measured DCF, MDA, IR, Glut4, E-selectin, as well as GPR40 and FAT/CD36. These compounds were assayed by the use of specific antibodies and quantitated by Western blot analysis.

Results and conclusion: Addition of palimtate to these cells resulted in their time and concentration-dependent activation by increased levels of E-selectin, DCF, MDA, and emergence of IR, Glut4, FAT/CD36, and GPR40 with peak levels at $300 \mu \mathrm{M}$ of palmitate, which were 2 -fold or greater above the baseline values. Linolenate up to $300 \mu \mathrm{M}$ resulted in no detectable changes in values from the baseline for the above parameters. Addition of linolenate to palmitate, however, reduced the palmitate-induced changes in all the above parameters in a dose-dependent fashion by $50-70 \%$. We conclude that the affluent environment of high palmitate and hyperglycemia in T2DM activate these cells and triggers early complications, which could be attenuated with linolenate. However, orderly development of appropriate receptors for dissipation of excess glucose (Glut4 and IR) and palmitate (GPR40 and FAT/CD36) may prevent deleterious and toxic acute effects of high glucose and palmitate as adaptive responses. The implication that unsaturated fatty acids (i.e. omega 3) can act as protective substrates may be of nutritional significance. Supported by: NIH Division of Research Resources for General Clinical Research Center through the University of Tennessee Health Science Center

\section{6}

Insulin glargine reduces carotid initmal hyperplasia after balloon catheter injury in Zucker fatty rats potentially by IGF-1 suppression of oxidative stress

P.J. Kadowitz ${ }^{1}$, S.N. Murthy ${ }^{2}$, S. Chandra' ${ }^{1}$, S. Sukhanov ${ }^{3}$, J. McGee ${ }^{4}$, P. Delafontaine ${ }^{3}$, D.B. McNamara ${ }^{1}$, V.A. Fonseca ${ }^{2}$

${ }^{1}$ Pharmacology, ${ }^{2}$ Medicine, ${ }^{3}$ Cardiology, ${ }^{4}$ Surgery, Tulane University Health Sciences Center, New Orleans, United States

Background and aims: Impaired glucose tolerance and type 2 diabetes are associated with increased cardiovascular disease morbidity and mortality particularly after vascular injury. Very little is known about the effect of insulin on the vasculature early in the course of the disease. Therefore we tested the effect of 2 insulins: glulisine (a short acting insulin) and glargine (long acting and known to increase IGF-1 levels and action) in Zucker fatty rat (insulin resistant but non-diabetic) carotid artery following balloon catheter injury. We have previously shown that IGF-1's atheroprotective effect correlates with a reduction in oxidative stress and eNOS upregulation.

Materials and methods: Zucker fatty rats were sc injected a daily dose 0.45 $\mathrm{mg} / \mathrm{kg}$ of glargine (once) or glulisine (twice) before meal (given twice for glulisine rats) for one week before and three weeks after balloon catheter mediated injury of the carotid artery. Fasting and postprandial glucose was measured in the tail vein blood twice weekly. Injured and the contra lateral arteries, liver, aorta and blood were harvested on day 22 after injury. In $\mathrm{H} \&$ E stained carotid artery sections the intima/media (I/M) ratio, an index of intimal hyperplasia was measured by microscopy in a blinded manner. The unstained sections were used for immunohistochemistry of nitro-tyrosine as a measure of oxidative stress. The serum was analyzed for IGF-1, and the aortic protein for 8 -isoprostane. The aortic eNOS was measured by Westernblot methods.

Results: Both fasting and postprandial glucose did not vary significantly between the control and insulin groups. As against controls, the I/M was reduced in the insulin treated groups, but was statistically significant in the glargine group [ $0.9 \pm 0.1$-control; $0.6 \pm 0.1$-glulisine; $0.4 \pm 0.1$-glargine, $\mathrm{p}<0.05$ ]. A trend for increased eNOS was observed in both insulin treated groups. Compared to control rats IGF-1 levels were higher in both insulin groups; however, it was significantly higher only in the glargine treated rats [567 \pm 121 (ng/ml)-control; $763 \pm 161$ (ng/ml)-glulisine; $1059 \pm 150$ (ng/ml)-glargine, $\mathrm{p}<0.05$ ]. Immunopositivity for nitrotyrosine was lower than control in the insulin treated groups and lowest in the arteries from the glargine treated animals. Aortic 8 -isoprostane was significantly reduced in the glargine group (921 $\pm 419 \mathrm{pg} / \mathrm{mg}$ protein vs. $2566 \pm 399 \mathrm{pg} / \mathrm{mg}$ protein in control; $\mathrm{p}<0.05)$. Conclusion: We conclude that insulin glargine (or to a lesser extent glulisine) reduces intimal hyperplasia following balloon catheter injury in obese non diabetic rats with an increase in IGF-1 and eNOS and a concomitant reduction in oxidative stress. These data support the concept of a beneficial effect of insulin in general and glargine in particular in vascular disease.

\section{7}

Activation of Nrf2-ARE pathway by simvastatin

P.G. Ziros ${ }^{1}$, D.V. Chartoumpekis ${ }^{1}$, A. Psyrogiannis ${ }^{1}$, V. Kyriazopoulou, A.G. Papavassiliou' ${ }^{2}$ I.G. Habeos ${ }^{1}$

${ }^{1}$ Medicine, University of Patras, Rio, Patras, Greece, ${ }^{2}$ Biochemistry, University of Athens, Greece

Background and aims: Statins inhibit the activity of the rate-limiting enzyme in cholesterol biosynthesis, HMGCoA reductase and are widely used in the treatment of cardiovascular diseases. Beyond their cholesterol reducing effect, they have also been reported to have antioxidant activities. On the 
other hand, Nrf2 is a broadly expressed transcription factor that lies central to the antioxidant defense cellular system. Nrf2 controls the expression of a number of protective genes in response to oxidative stress and electrophiles, through binding to antioxidant response elements (AREs) located on target genes. Although, the effect of statins on endogenous antioxidants, has not been systematically studied. Taking these into account, we wanted to investigate the potential role of $\mathrm{Nrf} 2$ in mediating the reported antioxidant effects of statins.

Material and methods: Subjects were male wistar rats (100-200 g) were randomized into two groups fed a standard $4 \%$ rat diet. One group was given simvastatin dissolved in methylcellulose by gavage at a dose $120 \mathrm{mg} / \mathrm{kg}$ while the other was given the same amount of methylcellulose. ST2 cells stably expressing a luciferase reporter plasmid with an ARE response element (ST2ARE) which binds Nrf2, was used to study the effect of simvastatin in vitro. Experiments with ST2-ARE cells and primary hepatocytes were performed in lipid free serum. Nuclear and total protein extracts were prepared from liver as well as primary hepatocytes Renonuclease protection assay was used to quantitate mRNA expression

Results: Wistar rats were treated with simvastatins $(120 \mathrm{mg} / \mathrm{kg})$ or vehicle for 5 days and total as well as nuclear liver extracts were prepared. Western blot experiments showed that Simvastatin induces nuclear translocation of Nrf2 in rat liver. This was confirmed in primary hepatocytes treated with simvastatin $5 \mu \mathrm{M}$ for 24 hours. Furthermore, the effect of simvastatin on primary hepatocytes was mevalonate dependent and cholesterol independent. The DNA binding activity of Nrf2 was also increased in the same nuclear extracts from the liver and primary hepatocytes treated with simvastatin. The above findings were further supported from in vitro studies using as a model the stably transfected st2 cell line (st2-ARE). Treating these cells with simvastatin enhances Nrf2 mediated transcriptional activity in a dose dependent way up to $5 \mu \mathrm{M}$, by a factor of 3, while sulforaphane, a known inducer of Nfr2 enhances the activity by a factor of 7 . Moreover pretreatment of these cells with mevalonate abolished the above result, while cholesterol had no effect. Finally two of the known targets of Nrf2 (HO-1 and GPX2) were found to be increased, when they were quantitated by RPA in rat liver RNA, after simvastatin treatment.

Conclusion: Overall our results provide in vitro as well as in vivo evidence that the antioxidant effects of simvastatin might be, at least partly, mediated by the transcription factor Nrf2.

Supported by: Hellenic Endocrine Society

\section{8}

Comparison of the effects of rosuvastatin and colestimide on metabolic parameters, adipokines and oxidative stress in type 2 diabetes patients complicated by hyperlipidaemia

K. Takebayashi, M. Suetsugu, S. Matsumoto, Y. Aso, T. Inukai

Internal Medicine, Dokkyo Medical University Koshigaya Hospital,

Koshigaya, Japan

Background and aims: Rosuvastatin, a strong statin, and colestimide, a new anion exchange resin, are both clinically useful drugs for treatment of hypercholesterolemia. This study was designed to compare the effects of rosuvastatin and colestimide on metabolic parameters including circulating lipids and glucose levels, adipokines, and markers of oxidative stress and diabetic nephropathy in patients with type 2 diabetes complicated by hyperlipidemia. Materials and methods: Design: A total of 40 patients with type 2 diabetes complicated by hyperlipidemia were prospectively recruited. The patients were assigned randomly in equal numbers to rosuvastatin $(2.5 \mathrm{mg} /$ day $)$ and colestimide ( $3.0 \mathrm{~g} /$ day) groups. Blood and urine were measured at the beginning of the study and after 12 weeks.

Results: Rosuvastatin significantly decreased the level of serum retinol binding protein-4 (RBP4: an insulin-resistant adipokine) in a subgroup of patients with poor glycemic control (HbAlc more than $8.0 \%$ ), in addition to exerting a strong low-density lipoprotein (LDL-C)-lowering effect. Colestimide significantly decreased $\mathrm{HbAlc}$, even in patients treated with a sulfonylurea at a more than a moderate dose (more than gibenclamide $2.5 \mathrm{mg}$ /day or glimepiride $3.0 \mathrm{mg} /$ day), without influencing insulin resistance or adiponectin (an insulin-sensitive adipokine) and RBP4 levels. Colestimide (but not rosuvastatin) significantly decreased the levels of urinary 8 -iso-prostaglandin (PG) F2a (a marker of oxidative stress) and urinary monocyte chemoattractant protein-1 (MCP-1) (a marker of diabetic nephropathy).

Conclusion: Our results suggest that rosuvastatin and colestimide exert different beneficial effects in type 2 diabetic patients complicated by hyperlipidemia. Accordingly, concomitant use of these drugs may be useful for prevention of progression of diabetic complications.

\section{PS 068 Cytokines: TNF alpha and related cytokines}

\section{9}

$\mathrm{N}^{\varepsilon}$-(Carboxymethyl)lysine accumulates in adipose tissue of obese patients and alters the expression of adipocytokines

K.H.J. Gaens ${ }^{1,2}$, M.P.H. van de Waarenburg ${ }^{1,2}$, J. Scheijen ${ }^{3}$, J. Nijhuis ${ }^{4}$, M.C.G. Brouwers ${ }^{1,2}$, C.D.A. Stehouwer ${ }^{1,2}$, C.G. Schalkwijk ${ }^{1,2}$

${ }^{1}$ Department of Internal Medicine, Maastricht University, ${ }^{2}$ Cardiovascular Research Institute Maastricht, Maastricht University, ${ }^{3}$ Department of Clinical Genetics, Maastricht University, ${ }^{4}$ Department of General Surgery, Maastricht University, Netherlands

Background and aims: Obesity is closely linked to a wide variety of pathophysiologic conditions. Dysregulated production of adipocytokines is associated with obesity, providing a link between obesity and inflammation, changes in glucose homeostasis and lipid metabolism. Factors by which obesity leads to a dysregulated production of adipocytokines remain largely unknown. Because of local conditions in adipose tissue of obese patients, such as increased levels of polyunsaturated fatty acids, high rate of lipid peroxidation and oxidative stress, we speculate an increased formation of the lipoxidation endproduct $\mathrm{N}^{\varepsilon}$-(Carboxymethyl)lysine (CML) in adipose tissue and we hypothesize that CML contributes to the altered expression of adipocytokines. Materials and methods: Accumulation and localization of CML in human adipose tissue was assessed by immunohistochemistry using a monoclonal antibody against CML. Colocalisation studies of CML with macrophages and endothelial cell were done using antibodies against CD68 and CD31, resp. Plasma concentrations of protein-bound CML were determined in control subjects $(n=77)$, familial combined hyperlipidemia $(F C H)$ patients $(n=42)$ and obese patients $(n=44)$ by UPLC tandem MS. To examine the biological effects of CML, human preadipocytes were incubated with CML-modified albumin and changes in gene expression of plasminogen activator inhibitor-1 (PAI-1), interleukin-6 (IL-6) and the receptor for AGE (RAGE) were analyzed by Real Time PCR.

Results: Immunohistochemistry demonstrated the presence of CML in adipocytes, macrophages and endothelial cells of adipose tissue of obese patients. Plasma CML concentrations were surprisingly lower in obese $(1.27 \pm$ $0.21 \mu \mathrm{M}, \mathrm{p}<0.001)$ and $\mathrm{FCH}$ patients $(1.31 \pm 0.28 \mu \mathrm{M}, \mathrm{p}<0.001)$ as compared with control subjects $(1.94 \pm 0.52 \mu \mathrm{M})$. A negative correlation was observed between CML plasma concentrations and BMI $(r=-0.475, \mathrm{p}<0.001)$, visceral adipose tissue $(r=-0.548, \mathrm{p}<0.001)$, but not with subcutaneous adipose tissue $(r=-0.148, \mathrm{p}=0.238)$. Incubation of human preadipocytes with CML-albumin during $24 \mathrm{~h}$ and $48 \mathrm{~h}$ had no effect on PAI-1, IL-6 and RAGE expression, while an incubation of $72 \mathrm{~h}$ with minimally-modified CML-albumin increased the expression of IL-6, PAI-1 and RAGE 2.1-fold, 1.7-fold and 3.4-fold, resp. Incubation with highly-modified CML-albumin increased the IL-6, PAI-1 and RAGE mRNA expression by 2.4 -fold, 1.8 -fold and 4.9 -fold, resp.

Conclusion: We demonstrated for the first time that CML is found in adipose tissue, namely in adipocytes, macrophages and endothelial cells of obese patients. This accumulation is a local process, since plasma CML levels are decreased in obese patients as compared to control subjects. In addition, the inverse relationship with plasma levels of CML-modified proteins and visceral adipose tissue also suggests an uptake of CML in visceral adipose tissue. A dysregulated production of adipocytokines by CML-albumin, as shown in human preadipocytes, supports our hypothesis that CML has important biological effects regarding the dysregulation of adipocytokines. Supported by: Dutch Diabetes Foundation

\section{0}

The effect of PKC $\delta$ on the IL-6 signalling and action in adipose cells E. Wallerstedt, C.X. Andersson, U. Smith

Department of molecular and clinical medicine/Diabetes, Medicine, Gothenburg, Sweden

Background and aims: We have recently shown in differentiated 3T3-L1 cells that insulin antagonizes interleukin-6 (IL-6) signaling and exerts antiinflammatory effects by significantly reducing the tyrosine phosphorylation of the intracellular signaling molecule STAT3. Treatment with the MEK inhibitor PD98059 in these cells reduced both the serine phosphorylation of STAT3 and the anti-inflammatory effect of insulin on IL-6 signaling. It has 
previously been described that serine phosphorylation of STAT3 is important for the transcriptional activity of STAT3 and that PKC $\delta$ is involved in this phosphorylation. To study the involvement of PKC $\delta$ on IL-6 signaling and the anti-inflammatory properties of insulin, we analyzed differentiated 3T3L1 cells treated with the PKC-activating phorbol ester PMA or the specific PKC $\delta$ inhibitor rottlerin. In addition, wt MEFs and cells expressing a dominant negative PKC $\delta$ (PKC $\delta \mathrm{dn}$ ) were also used.

Materials and methods: Differentiated 3T3-L1 cells were cultured and preincubated with or without rottlerin $(6 \mu \mathrm{M})$ and PMA $(100 \mathrm{nM})$ for 30 minutes before stimulation with insulin (100 nM) and/or IL-6 (20 ng/ml) for $30 \mathrm{~min}$ utes up to 24 hours. Wt MEFs and PKC $\delta$ dn MEFs were incubated with IL-6 $(30 \mathrm{ng} / \mathrm{ml})$ and soluble IL- 6 receptor $(30 \mathrm{ng} / \mathrm{ml})$ for 60 minutes and 24 hours. RNA was extracted and the gene expression was analyzed with real-time RTPCR (TaqMan) while the proteins were analyzed by immunoblotting

Results: Both insulin and PMA, but not IL-6, increased the Tyr-311 phosphorylation of PKC $\delta$ in differentiated 3T3-L1 cells while all ligands increased the Ser-727 phosphorylation of STAT3. Cells treated with rottlerin showed a reduced IL-6-induced Ser-727 phosphorylation of STAT3 whereas the insulin-induced activation of STAT3 was not effected. IL-6-induced gene expression of the negative feedback inhibitor of IL-6 signaling, SOCS3, as well as the inflammatory markers SAA3 and haptoglobin were significantly reduced in the presence of rottlerin. The importance of PKC $\delta$ for the effect of IL- 6 through the STAT3 pathway was further supported by studies in PKC $\delta$ dn MEFs which showed a significantly reduced IL-6-induced gene expression of SOCS3, haptoglobin and SAA3 compared to wt MEFs.

Conclusion: These data support the concept that PKC $\delta$ is important for the pro-inflammatory effect of IL-6 and involves Ser-727 phosphorylation of STAT3. However, PKC $\delta$ does not seem to be involved in the insulin-induced and PMA-induced Ser-727 phosphorylation of STAT3, which mainly seems to be dependent on the MAPK pathway.

Supported by: grants from Martina and Wilhelm Lundgren Foundation, Magn. Bergvalls Foundation, Thurings Foundation, Åke Wibergs Foundation, GBG Läkaresällskap and HEPADIP

\section{1}

PTEN downregulation in adipocytes promotes inflammation of adipose tissue

M. Foti ${ }^{1}$, C. Veyrat-Durebex ${ }^{2}$, C. Juge-Aubry ${ }^{2}$, C. Darimont ${ }^{3}$, V. Giusti ${ }^{4}$, C.A. Meier ${ }^{2}$, F. Rohner-Jeanrenaud ${ }^{2}$, M. Vinciguerra ${ }^{1}$

${ }^{1}$ Dept. of Cellular Physiology and Metabolism, Faculty of Medicine,

University of Geneva, Switzerland, ${ }^{2}$ Dept. of Internal Medicine, University

Hospital, Geneva, Switzerland, ${ }^{3}$ Nestec, Nestle Research Center, Lausanne,

Switzerland, ${ }^{4}$ Dept. of Medicine, University Hospital, Lausanne, Switzerland

Background and aims: The phosphoinositide phosphatase PTEN is a potent tumor suppressor and a regulator of insulin sensitivity in peripheral tissues. In adipocytes, experimental alterations of PTEN expression modulate the sensitivity of these cells to insulin. However, virtually nothing is known about the pathophysiological regulation of endogenous PTEN in adipose tissue. Herein, we investigated in vivo and in vitro whether alterations of PTEN expression in adipocytes are associated with the metabolic syndrome and what are the functional outcomes of dysregulated PTEN expression/activity. Materials and methods: PTEN expression was examined in vivo in adipose tissue of rats and human with the metabolic syndrome. Metabolic factors mediating dysregulation of PTEN expression in adipocytes and the subsequent effects on the physiology of these cells were investigated in vitro using human CHUB-S7 preadipocytes.

Results: We demonstrated that PTEN is downregulated, both at the mRNA and protein levels, in adipose tissue of diabetic/obese ZDF rats and in subcutaneous adipose tissue of obese human patients. PTEN downregulation correlated with degradation of IкBa and hyperactivation of NF- $\mathrm{kB}$, a transcription factor previously described to modulate PTEN expression. The expression of SHIP2, another PtdIns(3,4,5)P3 phosphatase involved in the control of insulin sensitivity and the development of obesity, was not altered. In vitro analyses using differentiated human CHUB-S7 preadipocytes showed that PTEN downregulation is not triggered by high concentrations of glucose or fatty acids. In contrast, the pro-inflammatory cytokines IL- $1 \alpha$ and TNFa, significantly downregulate PTEN expression. Consistent with the IL1a-dependent PTEN downregulation, long-term incubation of CHUB-S7 cells with IL-1 1 potentiates insulin-induced Akt and ERK1/2 signaling. We finally showed that PTEN downregulation in CHUB-S7 preadipocytes by PTEN siRNAs induced an increased secretion of the pro-inflammatory cytokines IL- $1 \beta$, IL- 6 and TNF $\alpha$.

Conclusion: Taken together, these data indicate that PTEN expression is downregulated in adipose tissue of obese/diabetic subjects, potentially via cytokine-mediated activation of the NF- $\mathrm{KB}$ pathway. PTEN downregulation in adipocytes might in turn worsen adipose tissue inflammation through a vicious circle by further stimulating the secretion of pro-inflammatory cytokines. Supported by: Swiss National Science Foundation and from the Carlos and Elsie de Reuter Foundation

\section{2}

Effect of 24-hour hypertriglyceridaemia on tumor necrosis factor alpha and resistin in type 2 diabetes and healthy subjects

J. Kopecky jr. ${ }^{1}$, E. Krusinova ${ }^{1}$, P. Wohl ${ }^{1}$, L. Kazdova ${ }^{1}$, P. Mlejnek ${ }^{2}$, M. Hill ${ }^{3}$, T. Pelikanova ${ }^{1}$

${ }^{1}$ Diabetes Center, Institute for Clinical and Experimental Medicine,

${ }^{2}$ Institute of Physiology, Academy of Sciences of the Czech Republic,

${ }^{3}$ Obesity Management Centre, Institute of Endocrinology, Prague, Czech Republic

Background and aims: Tumor necrosis factor alpha (TNF) and resistin are implicated in the pathogenesis of insulin resistance (IR). Its regulation during experimental lipid infusion which is known to cause IR has not been thoroughly investigated so far.

Materials and methods: The aim of the study was to assess plasma concentrations of TNF and resistin during prolonged hypertriglyceridemia in type 2 diabetes and healthy subjects. 11 patients with type 2 diabetes (D) and 11 age-matched healthy control subjects (C) underwent 24-hour infusion of lipid emulsion (Intralipid 20\%; $3 \mathrm{~g}$ of fat $/ \mathrm{kg} /$ day; HTG). Plasma concentrations of TNF and resistin were measured before ( $0 \mathrm{~min}$ ), at $30 \mathrm{~min}, 240 \mathrm{~min}$ and 24 hours of HTG. Needle biopsy of abdominal subcutaneous adipose tissue was performed before, at $240 \mathrm{~min}$ and 24 hours of infusion to asses the expression of selected cytokines by real-time PCR (expression of adipokine mRNA relative to cyclophilin mRNA).

Results: Plasma concentrations of TNF were significantly higher in D compared to $C(p<0.001)$. During HTG, plasma levels of TNF showed in D no dynamic changes, whereas in $\mathrm{C}$ significant increase at 24 hours of the infusion was detected ( $<<0.001$; ANOVA). Expressions of TNF in subcutaneous adipose tissue were significantly greater in $\mathrm{D}$ than in $\mathrm{C}(\mathrm{p}<0.001$, ANOVA), changes in time throughout the HTG were not significant. Plasma concentrations of resistin significantly rose during the HTG ( $<0.05$; ANOVA) and the difference between the two groups was not statistically significant. Differences in expressions in adipose tissue were not significant.

Conclusion: In D, plasma concentrations of TNF and it's expression in abdominal subcutaneous adipose tissue are increased. Mainly in C, prolonged lipid infusion is associated with increase in plasma TNF and resistin. Our results are in accordance with the hypothesis that TNF and resistin are implicated in lipid induced $<$ br

Table: Plasma concentrations (plasma; pg/mL) and expressions in abdominal subcutaneous adipose tissue (expressions; TNF mRNA relative to cyclophilin mRNA) of Tumor necrosis factor alpha (TNF) and Resistin. Data expressed as mean \pm SEM. (D, type 2. diabetes; C, healthy controls) IR.

\begin{tabular}{|c|c|c|c|c|c|c|c|}
\hline \multirow{3}{*}{$\overline{\text { Group }}$} & \multicolumn{7}{|l|}{ Time } \\
\hline & \multicolumn{4}{|l|}{ Plasma } & \multicolumn{3}{|l|}{ Expressions } \\
\hline & $0 \mathrm{~min}$ & $30 \mathrm{~min}$ & $240 \mathrm{~min}$ & $24 \mathrm{~h}$ & $0 \mathrm{~min}$ & $240 \mathrm{~min}$ & $24 \mathrm{~h}$ \\
\hline $\mathrm{D}$ (TNF) & $8.58 \pm 0.73$ & $7.85 \pm 0.46$ & $7.77 \pm 0.42$ & $8.06 \pm 0.45$ & $25.1 \pm 5.99$ & $33.58 \pm 7.19$ & $42.86 \pm 14.21$ \\
\hline $\mathrm{C}$ (TNF) & $0.96 \pm 0.19$ & $1.07 \pm 0.21$ & $1.52 \pm 0.29$ & $2.56 \pm 0.58$ & $6.38 \pm 1.25$ & $7.5 \pm 1.14$ & $6.53 \pm 0.96$ \\
\hline $\mathrm{D}$ (Resistin) & $4.13 \pm 0.34$ & $4.19 \pm 0.42$ & $4.53 \pm 0.56$ & $4.15 \pm 0.33$ & $24.83 \pm 9.06$ & $19.26 \pm 5.83$ & $23.24 \pm 11.16$ \\
\hline C (Resistin) & $3.5 \pm 0.29$ & $3.61 \pm 0.38$ & $4.13 \pm 0.32$ & $4.2 \pm 0.47$ & $8.7 \pm 2.34$ & $11.79 \pm 3.46$ & $10.18 \pm 2.18$ \\
\hline
\end{tabular}

Supported by: IGA MZ ČR NR 8991-3 


\section{3}

The changes of PPAR $\gamma$, ATGL and adipocytokines in TNF- $\alpha$ induced insulin resistance in mice

S. Shi ${ }^{1,2}$, L. Li ${ }^{1,2}$, G. Yang ${ }^{3}$, K. Li $^{3}$

${ }^{1}$ The Key Laboratory of Laboratory Medical Diagnostics in Ministry of Education, ${ }^{2}$ Department of Clinical Biochemistry, ${ }^{3}$ Department of Endocrinology, the Second Affiliated Hospital, Chongqing Medical University, Chongqing, China

Background and aims: Tumor necrosis factor- $\alpha$ (TNF- $\alpha$ ) has been linked to obesity-related insulin resistance and metabolic disorder, but the molecular mechanisms have not been elucidated. We undertook the present study to further investigate the in vivo molecule mechanisms that TNF- $\alpha$ contributes to the pathogenesis of insulin resistance( IR ) on the related gene of lipid metabolism in mice.

Materials and methods: C57BL/6J mice were treated by TNF- $\alpha$ for 7 days. After 7 days, we examined the in vivo effects of TNF- $\alpha$ on glucose-lipid glucose metabolism and insulin sensitivity by intravenous glucose tolerance tests (IVGTT) and euglycemic - hyperinsulinemic clamp. In addition, we also analyzed the in vivo role of TNF- $\alpha$ on some metabolism-related genes and adipocytokines in the development of insulin resistance.

Results: Plasma FFA, insulin and fasting blood glucose levels were significantly higher in TNF- $\alpha$ treatment animals than in controls $(7.86 \pm 0.40$ vs. $5.58 \pm 0.33 \mathrm{mmol} / \mathrm{L}, P<0.01 ; 32.4 \pm 1.9$ vs. $12.4 \pm 1.12 \mathrm{mU} / \mathrm{L}, P<0.05 ; 1.78 \pm 0.05$ vs. $1.54 \pm 0.11 \mathrm{mmol} / \mathrm{L}, P<0.05)$. Insulin responses to IV glucose in IVGTT were drastically reduced in TNF- $\alpha$ treatment mice compared to controls. During euglycemic - hyperinsulinemic clamp, The rate of glucose infusion needed to maintain euglycemia (GIR) in TNF- $\alpha$ treatment mice was markedly lower than in the controls $\left(39.1 \pm 2.3\right.$ vs. $\left.54.2 \pm 2.2 \mathrm{mg} \cdot \mathrm{kg}^{-1} \cdot \mathrm{min}^{-1}, P<0.01\right)$, and insulin-stimulated whole body glucose uptake and skeletal muscle glucose uptake were significantly decreased by $11 \%$ and $24 \%$, respectively. Insulin's ability to suppress HGP during clamps was significantly impaired in TNFa mice( \%), indicating severe insulin resistance in these mice. The mRNA expression of PPAR $\gamma$ in adipose tissue was significantly down-regulated in mice with TNF- $\alpha$ treatment compared with controls $(0.83 \pm 0.06$ vs. $1.07 \pm 0.07$ arbitrary units, $P<0.05)$. Adipose ATGL mRNA expression and plasma protein level were significantly lower in mice with TNF- $\alpha$ treatment compared with controls $(0.85 \pm 0.09$ vs. $1.37 \pm 0.12$ and $0.53 \pm 0.03$ vs. $0.65 \pm 0.05$ arbitrary units, $P<0.01$ and $P<0.05)$. Adiponectin mRNA content in adipose tissues and plasma protein levels were lower in the TNF- $\alpha$ treatment rats than in the controls $(0.48 \pm 0.04$ vs. $0.95 \pm 0.12$ arbitrary units, $P<0.01$ and $16.43 \pm 0.35 v s$. $21.94 \pm 0.59 \mu \mathrm{g} / \mathrm{ml}, P<0.01)$. In treated rats with TNF- $\alpha$, plasma visfatin was significantly lower than in the controls $(0.90 \pm 0.05$ vs. $1.14 \pm 0.13$ arbitrary units, $P<0.05$ ), and mRNA expression of visfatin in adipose tissue was significantly down-regulated $(0.74 \pm 0.03$ vs. $0.92 \pm 0.06$ arbitrary units, $P<0.01)$.

Conclusion: This is the first report that chronic TNF- $\alpha$ treatment in vivo not only downregulated adipose tissue PPAR $\gamma$ and ATGL mRNA expression, and ATGL plasma protein levels but also reduced adipose tissue adiponectin and visfatin mRNA expression and their plasma protein levels. These alterations may contributes to TNF- $\alpha$ - mediated elevated FFA levels and insulin resisitance.

Supported by: National Natural Science Foundation of China and Chongqing Municipal Education Commission

\section{4}

Inflammatory role of the cytokine TWEAK and its receptor Fn14 on human adipocytes. Relationship of the soluble fraction with systemic inflammatory markers in obese and type 2 diabetic patients

J.J. Vendrell' ${ }^{1}$ M.R. Chacon ${ }^{1}$, M. Miranda ${ }^{1}$, V. Ceperuelo-Mallafré ${ }^{1}$, J. Fernández-Real ${ }^{2}$, A. Megia ${ }^{1}$

${ }^{1}$ Endocrinology and Diabetes Unit, CIBERDEM. University Hospital of Tarragona Joan XXIII. Rovira i Virgili University, Tarragona,

${ }^{2}$ Endocrinology and Diabetes Unit, CIBEROBN. Josep Trueta Hospital, Girona, Spain

Background and aims: Tumor necrosis factor-like weak inducer of apoptosis (TWEAK) is a member of the tumor necrosis factor superfamily of cytokines. Soluble TWEAK can be found as biologically active form circulating in human plasma and interacting with a receptor named fibroblast growth factor-inducible 14 (Fn14). This binding activates the NF-kB signalling pathway, known to play an important role in the inflammatory processes. Type 2 diabetes and obesity are both subjected to a chronic inflammatory "low degree" condition driven by a combination of a pro-inflammatory adipose tissue expression profile with an increase in some inflammatory cytokines at bloodstream. In previous studies, we have shown that mRNA of TWEAK and Fn14 are highly expressed in human subcutaneous fat from obese and type 2 diabetes patients. Therefore, our interest is focused on investigating whether TWEAK/Fn14 has a pro-inflammatory role on human adipocytes in vitro and to determine if its circulating levels are related to human obesity and type 2 diabetes contributing to the inflammatory environment.

Research design and methods: 71 non-diabetic subjects $(n=26$ with $B M I<25$ and $\mathrm{n}=45$ with $30 \geq \mathrm{BMI}<40$, matched for age and gender) and 28 type 2 diabetic subjects (age: $65.4 \pm 8.4$ years, BMI: $29.8 \pm 3.4$ ) were included in the study. Plasma levels of sTWEAK, sMCP-1, sTNFR1, sTNFR2 and sIL6 were measured by ELISA. Glycerol levels were measured by a colorimetric assay. TWEAK/Fn14 expression was analysed by RT-PCR during SGBS preadipocyte cell line differentiation to mature adipocytes. The dose effect of TWEAK protein on human preadipocyte and differentiated adipocytes cell line was studied and compared to TNFa stimulus (from 1 to $50 \mathrm{ng} / \mathrm{mL}$ for both cytokines). The expression of MCP-1, IL-6, TNF $\alpha$. APM1, and Lep was analysed by RT-PCR.

Results: Non diabetic cohort: STWEAK in serum was found to be positively correlated with plasma glycerol and sTNFR2 ( $\mathrm{r}=0.419, \mathrm{p}<0.001$ and $\mathrm{r}=0.275$, $\mathrm{p}=0.026$ ). Type 2 diabetes cohort: $s$ TWEAK showed a strong association with plasma glycerol levels $(\mathrm{r}=0.426 \mathrm{p}<0.006)$. Multiple regression analysis showed that sTWEAK was only positively determined by glycerol levels $(B=608.75$, 95\% CI (402.7/814.7) $\mathrm{p}<0.001)$. TWEAK and Fn14 mRNA were found to decrease during adipocyte differentiation. In vitro effect of TWEAK on differentiated adipocytes up-regulated the expression of $T N F \alpha, M C P-1, I L-6$ and down regulates expression of APM1. This cytokine up-regulation was less powerful than the observed for TNF $\alpha$. TWEAK also up-regulated cytokines expression on preadipocytes in culture.

Conclusion: The circulating levels of this cytokine may be related with the lipolytic activity in adipose tissue. TWEAK is expressed by adipocytes and may have a paracrine effect over structures and cell types responsible for the inflammatory component found in the adipose tissue by increasing expression on IL-6 and TNF $\alpha$ mainly.

Supported by: CIBERDEM , Dr. Matilde Rodriguez Chacón is supported by a fellowship from Fondo de Investigación Sanitaria

\section{5}

Effect of acute hyperinsulinaemia and angiotensin receptor blockade on plasma concentrations and expressions of tumor necrosis factor $\alpha$ and resistin in diabetes mellitus

E. Krušinová ${ }^{1}$, J. Kopecký jr. ${ }^{1}$, P. Mlejnek ${ }^{2}$, P. Wohl ${ }^{1}$, L. Kazdová ${ }^{1}$, M. Pravenec ${ }^{2}$, T. Pelikánová ${ }^{1}$

${ }^{1}$ Diabetes Center, Institute for Clinical and Experimental Medicine,

${ }^{2}$ Institute of Physiology, Academy of Sciences of the Czech Republic, Prague, Czech Republic

Background and aims: Angiotensin II type 1 receptor blockade (ARB) has been suggested to have distinct antidiabetic and insulin-sensitizing properties, however the underlying mechanisms are not clear. The effect of ARB on tumor necrosis factor a (TNF) and resistin that are implicated in insulin resistance, is not fully understood. We aimed to assess plasma concentrations of resistin and TNF and their expressions in subcutaneous adipose tissue in response to acute hyperinsulinaemia and acute $A R B$ in subjects with type 2 diabetes (D) and control healthy subjects (C).

Materials and methods: Plasma concentrations of resistin and TNF were measured: 1) at $0 \mathrm{~min}, 30 \mathrm{~min}$ and $240 \mathrm{~min}$ of hyperinsulinaemic (1 mU.kg${ }^{1} \cdot \mathrm{min}^{-1}$ ) euglycaemic (5 mmol. $\mathrm{l}^{-1}$ ) clamp (HEC), and 2) during HEC after acute ARB (following administration of losartan $200 \mathrm{mg}$ ) (AT-HEC) using the same protocol, in 11D and 12 age-matched C. Needle biopsy of abdominal subcutaneous adipose tissue was performed at $0 \mathrm{~min}, 30 \mathrm{~min}$ and 240 min of both clamps to assess the relative expressions of TNF and resistin using the real-time PCR method (expression of adipokine mRNA relative to cyclophilin mRNA).

Results: Insulin sensitivity, expressed as metabolic clearence rate of glucose, was significantly higher in $\mathrm{C}$ compared to $\mathrm{D}(\mathrm{p}<0.001)$, without any changes during AT-HEC in both groups. TNF plasma concentrations and adipose tissue expressions were significantly higher in D compared to $\mathrm{C}(\mathrm{p}<0.001$; ANOVA). While plasma TNF did not change during hyperinsulinaemia or after ARB in both groups, its expressions increased during HEC but remained stable during AT-HEC ( $<<0.05$; ANOVA) in both groups. Plasma concetrations, as well as relative expressions of resistin were also significantly higher 
Plasma concentrations and expressions of TNF and resistin. Data expressed as mean \pm SEM.

\begin{tabular}{|c|c|c|c|c|c|c|c|}
\hline \multirow{2}{*}{$\begin{array}{l}\text { Group } \\
\text { Clamp / Time }\end{array}$} & & \multicolumn{3}{|c|}{ Type 2 diabetes } & \multicolumn{3}{|l|}{ Controls } \\
\hline & & $0 \mathrm{~min}$ & $30 \mathrm{~min}$ & $240 \mathrm{~min}$ & $0 \mathrm{~min}$ & $30 \mathrm{~min}$ & $240 \mathrm{~min}$ \\
\hline \multirow[t]{2}{*}{$\mathrm{p}-\mathrm{TNF}\left(\mathrm{pg} \cdot \mathrm{ml}^{-1}\right)$} & HEC & $7.45 \pm 0.41$ & $7.65 \pm 0.47$ & $8.05 \pm 0.44$ & $1.27 \pm 0.32$ & $1.19 \pm 0.20$ & $1.39 \pm 0.25$ \\
\hline & AT-HEC & $8.22 \pm 0.61$ & $8.49 \pm 0.67$ & $8.05 \pm 0.52$ & $1.19 \pm 0.22$ & $1.15 \pm 0.18$ & $1.03 \pm 0.19$ \\
\hline \multirow[t]{2}{*}{ TNF relative expression(AU) } & $\mathrm{HEC}$ & $18.59 \pm 2.33$ & $24.79 \pm 3.34$ & $26.43 \pm 5.59$ & $5.74 \pm 1.04$ & $5.44 \pm 1.00$ & $7.42 \pm 0.81$ \\
\hline & AT-HEC & $24.35 \pm 3.86$ & $21.41 \pm 3.89$ & $25.07 \pm 3.36$ & $7.30 \pm 0.75$ & $6.61 \pm 1.28$ & $7.92 \pm 0.96$ \\
\hline \multirow[t]{2}{*}{ p-Resistin (ng.ml-1) } & HEC & $3.99 \pm 0.32$ & $4.03 \pm 0.35$ & $4.26 \pm 0.30$ & $3.83 \pm 0.33$ & $3.50 \pm 0.32$ & $3.91 \pm 0.32$ \\
\hline & AT-HEC & $3.52 \pm 0.25$ & $4.30 \pm 0.42$ & $4.37 \pm 0.37$ & $3.57 \pm 0.29$ & $4.50 \pm 0.63$ & $3.88 \pm 0.40$ \\
\hline \multirow[t]{2}{*}{ Resistin relative expression (AU) } & $\mathrm{HEC}$ & $11.94 \pm 1.54$ & $14.59 \pm 1.94$ & $14.17 \pm 2.24$ & $6.59 \pm 1.33$ & $6.36 \pm 1.48$ & $8.38 \pm 1.59$ \\
\hline & AT-HEC & $14.84 \pm 2.21$ & $11.77 \pm 1.65$ & $17.87 \pm 2.69$ & $6.99 \pm 0.97$ & $6.71 \pm 1.05$ & $7.24 \pm 1.60$ \\
\hline
\end{tabular}

Supported by: grant IGA MH CZ NR 8821-3

in $\mathrm{D}$ ( $\mathrm{p}<0.05$ for plasma; $\mathrm{p}<0.01$ for expression levels). We have observed an increase in plasma resistin during both clamps $(\mathrm{p}<0.001$; ANOVA), that was comparable also between groups. On the contrary, the changes in resistin expression were only modest - a trend towards increase during HEC was detected in both groups.

Conclusion: TNF and resistin are associated with insulin resistance and type 2 diabetes, as it is documented by increased plasma concentrations and expressions in adipose tissue. In type 2 diabetes and healthy subjects, acute hyperinsulinaemia stimulates the expression of TNF, while ARB ameliorates this effect. Acute hyperinsulinaemia is associated with increase in plasma resistin in both type 2 diabetes and healthy subjects, independently on ARB. This effect can not be explained by stimulated expression in subcutaneous adipose tissue, which implies the involvement of other sources of circulating resistin. Our results suggest, that changes in TNF expression might be involved in the improvement of insulin sensitivity connected with ARB treatment.

\section{6}

The cytokines levels in subcutaneous adipose tissue in situ and in plasma; the effect of weight reduction

M. Vitkova ${ }^{1,2}$, J. Vrzalova ${ }^{3}$, E. Klimcakova ${ }^{1,2}$, M. Kovacikova ${ }^{1,2}$, Z.

Kováčová $^{1,2}$, J. Polak $^{1,2}$, M. Bajzova ${ }^{1,2}$, L. Rossmeislova ${ }^{2}$, Z. Hněvkovská ${ }^{1,2}$,

V. Stich ${ }^{1,2}$

${ }^{1}$ Department of Sport Medicine, 3rd Faculty of Medicine, Charles University in Prague, Czech Republic, ${ }^{2}$ Franco Czech Laboratory for the Clinical Research on Obesity, INSERM Toulouse and 3rd Faculty of Medicine, Prague, Czech Republic, ${ }^{3}$ Department of Immunology, Faculty of Medicine in Pilsen, Czech Republic

Background and aims: Cytokines (adipokines) produced by adipose tissue have been implicated in the development of insulin resistance (IR) and other metabolic disturbances linked to obesity. It has been shown that circulating adipokines are elevated in obese and diabetic subjects. The aim of our study was to investigate the levels of cytokines/adipokines in plasma and especially in situ in subcutaneous adipose tissue during the dietary intervention.

Materials and methods: 18 obese pre-menopausal women (BMI $35.8 \pm 4.8$ $\mathrm{kg} / \mathrm{m}^{2}$ ) followed 6 -months weight reduction program consisting of 3 phases: a 4 week $800 \mathrm{kcal} / \mathrm{d}$ very-low-caloric-diet (VLCD), a 2-month low-caloricdiet (LCD) with $600 \mathrm{kcal}$ less than the calculated daily energy requirements and a 3 to 4 months weight maintenance phase (WM). Anthropometric and biochemical parameters were determined before and at the end of each dietary phase. To evaluate insulin sensitivity, euglycemic-hyperinsulinemic clamp was done at each phase. Microdialysis of cytokines was performed in 8 women using $100 \mathrm{kDa}$ cut-off catheter. Interstitial concentration of cytokines was determined according to its recovery evaluated by "zero-flow" method. Adipokines, IL-6, IL-1 $\beta$, IL-8, IL-10, MCP-1, TNFa, leptin and PAI-1, were analyzed in plasma and dialysate using multiplex immunoassay at Luminex 100.

Results: The diet intervention resulted in the weight loss $(99.0 \pm 16.1 \mathrm{~kg}$ vs. $88.3 \pm 15.4 \mathrm{~kg}$ ), most prominent in the VLCD and the LCD phases. Body weight decrease was accompanied by reduction of insulin, triglycerides and leptin and significant improvement of insulin sensitivity. The levels of circulating cytokines remained unchanged during whole diet intervention except for TNF $\alpha$ and IL-8, which were increased after VLCD $(\mathrm{p}<0.001)$ and decreased subsequently in LCD and WM $(\mathrm{p}<0.01)$. Moreover, the relative changes of TNFa correlated with IL- 8 changes $(p=0.003)$. The interstitial levels of cytokines in adipose tissue were 10-1000 times higher than in plasma. Most of the observed factors had trend to increase during VLCD, and decreased to baseline levels during subsequent phases of the diet. Recovery of individual proteins in microdialysis measured by "zero flow" method ranged between $10-55 \%$.

Conclusion: During the long-term weight reducing dietary intervention in obese females, the time-course of levels of cytokines in plasma and in adipose tissue were not associated with indices of IR or body fatness. The results do not support hypothesis that insulin sensitivity is directly associated to cytokines production locally in adipose tissue or systematically in whole body. Supported by: grant GACR 303/07/0840 and by HEPADIP project supported by $E U$

\section{7}

Changes of inflammation markers and the effects of different hypoglycaemic agents in newly diagnosed type 2 diabetics

S.X. Lin, X.M. Tong, Z. Li, Y. Li, C. Hua

Department of Endocrinology, The Second Affiliated Hospital of SUN Yat-

Sen University, Guangzhou, China

Background and aims: In recent years chronic low-grade inflammation has been shown to be closely associated with obesity, type 2 diabetes and accelerated atherosclerosis. Recently many studies showed that insulin and metformin may have potential effort on anti-inflammation, but the evidence is not enough. To investigate the changes of serum inflammation marker levels including hs-CRP, TNF- $\alpha$ and IL- 6 and NF- $\kappa$ B activity in peripheral blood mononuclear cell (PBMNC) and the effects of different hypoglycaemic agents in patients with newly diagnosed Type 2 diabetes(T2DM).

Materials and methods: 67 normal individuals and 100 newly diagnosed T2DM patients were selected. The diabetic patients were divided into group A (with $7.0 \mathrm{mmol} / \mathrm{L}<\mathrm{FPG}<11.1 \mathrm{mmol} / \mathrm{L}$ ) and group B (with $\mathrm{FPG} \geq 11.1 \mathrm{mmol} /$ $\mathrm{L}$, insulin ). The patients of group A were randomised to group A1 [insulin] and group A2 (oral hypoglycemic agents). Group A2 were further divided into group $\mathrm{A} 2 \mathrm{a}[25 \mathrm{~kg} / \mathrm{m} 2<\mathrm{BMI} \leq 35 \mathrm{~kg} / \mathrm{m} 2$, metformin $]$ and group $\mathrm{A} 2 \mathrm{~b}[20 \mathrm{~kg} /$ $\mathrm{m} 2<\mathrm{BMI} \leq 25 \mathrm{~kg} / \mathrm{m} 2$, gliclazide].Serum hs-CRP, TNF- $\alpha$, IL- 6 were assayed by ELISA. Phosphorylation status of NF- $\kappa$ B p65 in PBMNC was determined by immunoblotting with phosphor-specific antibodies to NF- $\kappa \mathrm{B}$ p65(Ser536). Inflammation makers were assayed before treatment and at 12 weeks after glycemic control.

Results: Levels of serum hs-CRP, TNF- $\alpha$ and IL- 6 were higher in diabetics than that in control subjects $(\mathrm{P}<0.01)$. The levels of phosphorylation NF- $\kappa$ B P65 in PBMNC was increased in diabetics compared with control subjects $(\mathrm{P}<0.05)$. In whole group, the serum levels of hs-CRP, IL-6 and the levels of phosphorylation NF- $\kappa$ B P65 in PBMNC were decreased, TNF- $\alpha$ level did not change significantly after glycemic control comparing with baseline levels. In insulin group, the serum levels of hs-CRP, IL-6, and the levels of phosphorylation NF- $\kappa$ B P65 in PBMNC were decreased, TNF- $\alpha$ level did not change significantly after glycemic control. In metformin group, the serum levels of hs-CRP, IL-6, TNF- $\alpha$ and the levels of phosphorylation NF- $\kappa$ B P65 in PBMNC were decreased after glycemic control. In gliclazide group, there were no significant changes of these inflammation markers.

Conclusion: The levels of serum hs-CRP, IL- 6 and TNF- $\alpha$ and the levels of phosphorylation NF- $\kappa$ B P65 in PBMNC were increased in newly diagnosed T2DM patients, and the patients treated with insulin and metformin was associated with improvement of inflammation markers. 


\section{PS 069 Weight regulation}

\section{8}

FTO gene is associated not only with obesity-related quantitative traits but also with higher OGTT stimulated glycaemia and leptin levels

J. Vcelak, P. Lukasova, M. Vankova, D. Vejrazkova, H. Kvasnickova,

J. Vrbikova, K. Vondra, B. Bendlova

Institute of Endocrinology, Prague, Czech Republic

Background and aims: In 2007 several independent laboratories identified from broad genome-wide association scans the genetic variants in FTO (Fat mass and obesity associated) gene that are associated with obesity-related quantitative traits. Found associations were so strong, that they encouraged the genetic studies of polygenic diseases. FTO (16q12.2) is a member of one form of the non-heme dioxygenase superfamily. But physiological function of FTO in the organism is still not fully understood. The aim of the presented study is to compare the frequencies of four polymorphisms in the sets of DM2, polycystic ovary syndrome (PCOS) patients, gestational diabetics, offspring of DM2 and healthy controls without the family history of DM2 and to evaluate anthropometrical data, beta-cell secretion, insulin resistence and hormonal and lipid levels of the study subjects in relationship to the described polymorphisms.

Materials and methods: The study involved 1388 Czech Caucasian people: 383 patients with DM2 (M/F 134/249; age 61.0 $\pm 7.7 / 58.6 \pm 9.3$ years; BMI $\left.29.7 \pm 4.4 / 31.7 \pm 6.2 \mathrm{~kg} / \mathrm{m}^{2}\right) ; 286$ women with PCOS defined according to ESHRE consensus (age $27.2 \pm 6.6$ years; BMI $26.8 \pm 6.5 \mathrm{~kg} / \mathrm{m}^{2}$ ); 199 gestational diabetics (age $32.7 \pm 5.0$ years; BMI $23.9 \pm 4.3 \mathrm{~kg} / \mathrm{m}^{2}$ ); 165 offspring of DM2 (M/F 62/103; age 39.6 $\pm 10.5 / 36.6 \pm 12.8$ years; BMI $26.8 \pm 4.5 / 24.9 \pm 4.2$ $\mathrm{kg} / \mathrm{m}^{2}$ ) and 355 controls (M/F 115/240; age 29.1 $\pm 8.1 / 30.0 \pm 10.7$ years; BMI $23.9 \pm 3.0 / 23.5 \pm 4.6 \mathrm{~kg} / \mathrm{m}^{2}$ ) were collected. Oral glucose tolerance test (oGTT) with sampling for blood glucose, insulin, C-peptide and proinsulin was done (except of DM2 patients). Moreover, 16 anthropometrics parameters, 29 biochemical parameters and hormones, 42 oGTT-derived indices were evaluated. The SNPs: rs1121980; rs17817449; rs1421085; rs9939609 in the FTO gene were assessed by ABI TaqMan SNP Genotyping Assays. Statistics was done using NCSS 2004 and Statgraphics software.

Results: The genotypic distribution of alleles was not significantly different among groups and is independent on sex (chi-square test). These four SNPs are in strong Linkage Disequilibrium (pairwise $\mathrm{r}^{2}>0.97$ ). The homozygote carriers of risk and non-risk allele did not differ in age and body height within these groups. The association of FTO variants with BMI was apparent in all groups. Only the strongest associations with rs1421085 are presented (ANOVA).

Obesity-related quantitative traits in all subjects: (risk allele first)

Homozygotes CC vs. TT: BMI $(p<0.0000001 ;$ power $=0.9994)$, weight $(p=0.000045 ;$ power $=0.98)$, abdomen circumference $(p=0.000197$; power $=0.96)$.

Biochemical parameters in female controls:

Homozygotes CC vs. TT: Leptin ( $15.44 \pm 1.28$ vs. $10.56 \pm 0.96 \mathrm{ng} / \mathrm{ml} p=0.003$; power $=0.84)$, Glycemia(oGTT) $60 \mathrm{~min}(6.71 \pm 0.21$ vs. $5.81 \pm 0.18 \mathrm{mmol} / \mathrm{l}$ $p<0.005$; power $>0.80$ ), Glycemia(oGTT) $90 \mathrm{~min}$ ( $5.59 \pm 4.98$ vs. $4.98 \pm 0.14$ $\mathrm{mmol} / \mathrm{l} p<0.005$; power $>0.80$ ), Glycemia(oGTT) $120 \mathrm{~min}$ (4.99 \pm 0.15 vs. $4.59 \pm 0.12 \mathrm{mmol} / \mathrm{l} p<0.005$; power $>0.80)$, Glycemia(oGTT) AUC ( $690 \pm 16 \mathrm{vs.}$ $622 \pm 13 p=0.0011$; power $=0.912$ ).

Conclusion: Our study suggests that FTO gene is associated not only with obesity-related quantitative traits (particularly BMI, weight and abdomen circumference) but also with higher oGTT stimulated glycemia and leptin levels, especially in female controls.

Supported by: IGA MHCR NR/7809-5

\section{9}

Association of macronutrient intake with overweight and obesity among Bangladeshi population: a hospital based study

U.S. Munni, S. Shahid, S. Hossain, T. Khan, K. Fatema

Dept of Epidemiology and Biostatistics, BIHS, Dhaka, Bangladesh

Background and aims: Obesity is one of the most important risk factors for diabetes and other disorders of metabolic syndrome. Nutritional intake is a major determinant of obesity and it needs to be studied in various population groups based on their racial, socio-economic and cultural context. The information is highly required for designing evidence-based strategies to improve nutritional habits. In the present study we have investigated the nutritional intake of a group of Bangladeshi nondiabetic and diabetic subjects in relation to their demographic and anthropometric status.

Materials and methods: It was an observational exploratory study with 4063 subjects (1571 male and 2492 female; 1552 nondiabetic and 2511 diabetic) attending the health care centres and hospitals run by the Diabetic Association of Bangladesh in the capital and in northern Bangladesh. A pre-structured interviewer administered questionnaire was used to collect data. Anthropometric measurements (height, weight, waist and hip) were done by standard techniques. BMI and waist-hip ratio were calculated using appropriate formula. A BMI of 23 was taken as the cut-off point for overweight as defined by WHO for this population.

Results: The number of patients coming from rural, semi-urban and urban areas were 67, 699 and 3297 respectively. Fifty one percent subjects were illiterate and $27.7 \%$ and $21.2 \%$ had education up to secondary and graduate levels respectively. The mean ( \pm SD, years) age of the subjects was $46 \pm 14$; BMI $24.71 \pm 5.24$ and Waist-Hip ratio was $0.91 \pm 0.57$. The proportion of subjects with lower, middle and upper socioeconomic classes were $20.6 \%, 51 \%$ and $28.4 \%$ respectively. The proportion of calorie contributed by the three principal macronutrients was as follows: carbohydrate $58 \%$, fat $25 \%$, and protein $17 \%$. On group comparison between overweight plus obese (BMI>23) and normal weight individuals the intake of carbohydrate and protein did not vary between the groups, but fat intake was paradoxically lower in the overweight group. On logistic regression with normal weight and over weight (plus obesity) as dependent variables carbohydrate and protein intake was found to be associated with overweight when other confounders (age, waisthip ratio and diabetic state) were adjusted.

Conclusion: Weight gain in Bangladeshi subjects (both nondiabetic and diabetic) is associated with higher carbohydrate intake and a targeted approach to modify this nutritional behavior need to be taken in nondiabetic as well as diabetic population.

Supported by: World Diabetes Foundation and Diabetic Institute of Bangladesh

\section{0}

Association between the vitamin D-binding protein gene (DBP) and visceral obesity in obese children and adolescents

L.M. Johansson ${ }^{1}$, L.E. Johansson ${ }^{1}$, P. Danielsson ${ }^{2}$, S. Norgren ${ }^{2}$, C. Marcus ${ }^{2}$, M. Ridderstråle ${ }^{1}$

${ }^{1}$ Clinical sciences Malmö, Lund University, Malmö, ${ }^{2}$ Clinical science, Karolinska Institute, Stockholm, Sweden

Background and aims: A previous study has indicated that variants in the vitamin D-binding protein gene $(D B P)$ are associated with obesity and insulin resistance. Here we investigated if the $D B P$ single nucleotide polymorphism (SNP) rs17467825 has any impact on the development of obesity and/or obesity related traits in children and adolescents.

Materials and methods: rs17467825 was genotyped in 448 obese (age: 12.7 \pm 3.2 years, BMI: $34.3 \mathrm{~kg} / \mathrm{m} 2$ [30.9-38.3]) and 468 normal weight (age: $17.0 \pm$ 0.82 years, BMI: $20.7 \mathrm{~kg} / \mathrm{m} 2$ [19.5-22.3]) Swedish Caucasian children and adolescents, using the Sequenom platform (Matrix-Assisted Laser Desorption/ Ionization Time-of-Flight Mass Spectrometry - MALDI-TOF MS). Detailed phenotypic information including insulin sensitivity and body composition (DXA scan) was available for the obese subjects.

Results: We found no association between $D B P$ rs17467825 and obesity (minor allele frequencies were $4.0 \%$ and $5.8 \%$ for cases and controls, respectively, $p=0.37$ ). However, obese carriers of the minor allele (AA vs. AG+GG; $n=280$ and 168 , respectively) showed significantly decreased percent visceral fat mass (AA: 47.8\% [42.5-50.7], AG+GG: 45.9\% [40.0-49.7]; $p=0.013$ ), fasting plasma glucose (AA: $5.0 \mathrm{mmol} / \mathrm{l}$ [4.7-5.3], AG+GG: $4.8 \mathrm{mmol} / \mathrm{l}[4.5-$ 5.1], $p=0.001$ ) and insulin resistance (homeostasis model assessment index of insulin resistance - HOMA-IR; AA: 3.5 [2.6-4.8], AG+GG: 2.7 [2.0-4.5], $p=0.052$ ); all comparisons adjusted for age, gender, and BMI where appropriate. The difference in HOMA-IR was significant after adjusting for percent visceral fat mass $(p=0.045)$.

Conclusion: The DBP SNP rs17467825 has no major impact on the development of obesity in our cohort of Caucasian children and adolescents when expressed as BMI. It may be involved in the regulation of visceral fat mass and in addition seems to have an independent effect on insulin sensitivity. Supported by: Swedish Research Counsil, Novo Nordisk Foundation, Crafoord Foundation, Malmö University Hospital Foundation, the Albert Påhlsson Foundation, the Lundberg Foundation, the Diabetes Association in Malmö, Region Skåne, ALF, the Magnus Bergvall Foundation, the Fredrik and Ingrid Thurings Foundation, the Borgströms Foundation, and the Lars Hjerta Foundation 


\section{1}

Evaluation of the 6-minute walk test as a tool to estimate functional capacity in obese patients during weight loss

M. Ahlzén, M. Klintenberg, U. Björck, F. Norström, M. Ridderstråle Department of Clinical Sciences - Clinical obesity, Lund University, Malmö, Sweden

Background and aims: Obesity is associated with increased risk of several somatic conditions as well as decreased quality of life. However, physically fit obese subjects do not show excess mortality. Here we characterized obese patients before and after weight loss and evaluated the 6-minute walk test (6MWT) as a tool to estimate the functional capacity of obese subjects. In healthy subjects, the result of 6MWT is influenced by age, gender, height and weight.

Materials and methods: Subjects were recruited from the Obesity outpatient unit at the Department of Endocrinology, University Hospital MAS, Sweden. Weight loss was achieved by 14 group counseling sessions over the course of six to eight months preceded by 10 weeks on low calorie diet. For the $6 \mathrm{MWT}$ they were instructed to walk in there own maximal walking speed for 6 minutes. Heart rate was recorded before and after and maximal heart rate was estimated (220-age). Degree of pain (1-10) and localization as well as degree of effort (1-10) were reported. Differences between groups and correlations between variables were tested using standard non-parametric tests. Data are reported as median and interquartile range.

Results: 174 patients (46 men and 128 women, age 43 years [35-53], BMI $\left.40,4 \mathrm{~kg} / \mathrm{m}^{2}[37,6-44,6]\right)$ participated in the study. Walking distance $(534 \mathrm{~m}$ [485-568]) correlated negatively to age $(r=-0,15 ; p=0,04)$ and BMI $(r=-0,34$; $p=0,00003$ ). No correlation to height and no difference between genders were observed. Neither was there a correlation between distance and reported degree of effort. There was no correlation between heart rate before the test (78 bpm [70-88]) and measures of obesity. Heart rate after the test (130 pbm [119-141]) correlated to walking distance $(r=0,48 ; p<0,00001) .53 \%$ of the patients reported pain (graded 4 [3-5]) during the test (tibia $21,8 \%$, knee joint $7,5 \%$ and lower back $6,9 \%$ ). Reported pain did not significantly affect the walking distance but correlated to reported degree of effort $(r=0,22$, $p=0,0037$ ). 76 patients repeated the test after treatment (median weight loss being $19 \mathrm{~kg}[10-30])$. Walking distance increased significantly $(51,5 \mathrm{~m}$ [18,5$79], p<0,0001)$, whereas heart rate before $(-8,5 \mathrm{bpm}[-16-0,75], p<0,0001)$ and after the test decreased $(-4 \mathrm{bpm}[-14--4], p=0,002)$. The increase in walking distance correlated to the weight loss $(r=0,39 ; p=0,00059)$. The degree of reported effort $(p=0,0003)$ and pain $(p=0,00003)$ during the test decreased and fewer subjects reported associated pain (26\%).

Conclusion: The 6MWT is able to estimate physical capacity in obese patients based on walking distance and shows an improvement after weight loss. The test is easy and inexpensive and allows for self-evaluation and appreciation of treatment success beyond kilogram body weight loss. Activity-related pain was seen in more than half of the patients but responded promptly to weight loss. Further tests to validate these data are needed.

\section{2}

A genetic variant in $\mathrm{Zn}-\alpha_{2}$-glycoprotein is associated with obesity in Swedish men

E.-C. Långberg ${ }^{1}$, D. Lagarde ${ }^{2}$, H.F. Gu1 ${ }^{1}$, L. Essioux², G. Duchateau Nguyen ${ }^{2}$, R.G. Clerc ${ }^{2}$, C.-G. Östenson

${ }^{1}$ Molecular Medicine and Surgery, Karolinska Institutet, Stockholm, Sweden, ${ }^{2}$ F. Hoffmann-La Roche Ltd, Basel, Switzerland

Background and aims: In several animal studies $\mathrm{Zn}-\alpha_{2}$-glycoprotein (ZAG) has been demonstrated to be a novel adipokine involved in regulation of obesity. Our study hypothesis was to evaluate whether polymorphisms in this gene influences the development of obesity and/or type 2 diabetes (T2D) in humans.

Materials and methods: Genotyping of four SNPs was performed by dynamic allele specific hybridization (DASH) and TaqMan allelic discrimination in a total of 816 Swedish Caucasians including 290 non obese subjects with normal glucose tolerance (NGT), 199 obese subjects with NGT, 86 obese subjects with IGT and 241 patients with T2D.

Results: SNP rs2525554 showed significant allelic association with IGT obese subjects, where the minor allele $\mathrm{T}$ was present at a higher frequency in IGT obese subjects compared to non obese NGT controls ( 0.558 versus 0.465 , $P=0.032 ; \mathrm{OR}=1.453 ; 95 \% \mathrm{CI}=1.03-2.05)$. Quantitative trait analysis revealed a significant association for waist circumference (WC) within the obese NGT group; the subjects carrying TT and TC genotypes had an increased WC compared to the CC genotype carriers (105.6 \pm 0.6 versus $102.6 \pm 1.0$; $P=0.008$ ). Association with WC also showed significance when pooling the obese NGT and obese IGT groups together, comparing the same genotypes (106.9 \pm 0.5 versus $103.9 \pm 0.9 ; P=0.004)$. When analyzing the two NGT groups together there was also a significant difference in WC with regard to genotype (94.4 \pm 0.6 versus $91.6 \pm 1.0 ; P=0.014$ ). The same trends were also seen in non obese NGT subjects. Among obese subjects and non obese NGT controls significant associations with increased BMI, WC, WHR, $2 \mathrm{~h}$ glucose and systolic BP $(P=0.017 ; 0.003 ; 0.007 ; 0.038 ; 0.028$ respectively $)$ was found in the carriers with $\mathrm{T} / \mathrm{T}$ genotype. This effect was abolished when including the T2D patients.

Conclusions: This study provides evidence for association of genetic variation in the ZAG gene with obesity and suggests that SNP rs2525554 confers an increased risk for the development of abdominal obesity in Swedish men. Supported by: F. Hoffman-La Roche, Swedish Research Council, Swedish Diabetes Association, Novo Nordisk Scandinavia, GlaxoSmithKline, and Loo and Hans Osterman foundation

\section{3}

The association between depressive symptoms in childhood and overweight in adolescence. The TRAILS study

E.T. Liem ${ }^{1,2}$, P.J.J. Sauer ${ }^{1}$, A.J. Oldehinkel, ${ }^{3,4}$, R.P. Stolk ${ }^{2}$

${ }^{1}$ Department of Pediatrics, University Medical Center Groningen, ${ }^{2}$ Department of Epidemiology, University Medical Center Groningen, ${ }^{3}$ Department of Psychiatry and Graduate School of Behavioral and Cognitive Neurosciences, University Medical Center Groningen, ${ }^{4}$ Department of Child and Adolescent Psychiatry, Erasmus Medical Center - Sophia Children's Hospital, Rotterdam, Netherlands.

Background and aims: Prevention of overweight is a major public health concern. Targeted and tailored preventive strategies that take cost-effectiveness into account need to be developed and therefore, important predictors of overweight in childhood and adolescence need to be determined. Depressive symptoms have been described both as a predictor and as a complication of overweight. The aim of this study is to assess the association between depressive symptoms in childhood and overweight in adolescence.

Materials and methods: The TRacking Adolescents Individual Lives Survey (TRAILS) is a population-based cohort study among 2000 adolescents, presently aged 14-16 years. These adolescents have been assessed biennially from the age of 11 years to evaluate the development of psychosocial and somatic health from pre-adolescence into adulthood. At ages 11 and 15, we have assessed weight, height, skinfold thicknesses, and depressive symptoms through Youth Self Report and Child Behavior Checklist questionnaires. Body fat percentage and waist circumference have been obtained at age 15 .

Results: Results regarding the first 1037 participants showed that, at age 11 clinical depressive symptoms were present in $10.9 \%$ of girls and in $9.9 \%$ of boys. Mean BMI at age 15 was $21.28 \mathrm{~kg} / \mathrm{m}^{2}$ in girls and $20.18 \mathrm{~kg} / \mathrm{m}^{2}$ in boys. Results from linear regression analyses showed no significant results in boys.

Association between depressive symptoms at age 11 and BMI, skinfolds, BIA, and waist in girls.

\begin{tabular}{lll}
\hline Dependent variable & $\begin{array}{l}\text { Continuous predictor:B depressive } \\
\text { symptoms at age 11 }(95 \% \mathrm{CI}), \mathrm{P} \text {-value* }\end{array}$ & $\begin{array}{l}\text { Dichotomous predictor:Adjusted difference between depressed } \\
\text { and non-depressed ( at age 11) children }(95 \% \mathrm{CI}), \mathrm{P}-\mathrm{value}\end{array}$ \\
\hline BMI at age $15\left(\mathrm{~kg} / \mathrm{m}^{2}\right)$ & $0.18(-0.06-0.41), \mathrm{P}=0.14$ & $0.83(0.24-1.42), \mathrm{P}=0.01$ \\
\hline Sum of four skinfolds at age $15(\mathrm{~mm})$ & $2.01(-0.21-4.23), \mathrm{P}=0.08$ & $4.81(-0.94-10.55), \mathrm{P}=0.10$ \\
\hline Body fat at age 15 $(\%)$ & $0.44(0.05-0.83), \mathrm{P}=0.03$ & $1.27(0.28-2.25), \mathrm{P}=0.01$ \\
\hline Waist circumference at age $15(\mathrm{~cm})$ & $0.72(0.01-1.42), \mathrm{P}=0.05$ & $2.06(0.27-3.84), \mathrm{P}=0.02$ \\
\hline
\end{tabular}

* Adjusted for BMI at age 11 . 
The results regarding girls are listed in the table. After adjusting for body mass index (BMI) at age 11, depressive symptoms at age 11 are associated with higher body fat percentage and larger waist circumference at age 15 in girls. Dichotomizing depressive symptoms using a clinically relevant cut-off value, resulted in stronger associations.

Conclusion: In girls, clinically relevant depressive symptoms at age 11 are associated with an increased BMI, body fat percentage, and waist circumference at age 15.

Supported by: Hutchison Whampoa Ltd., Netherlands Organization for Scientific Research Medical Research Council and the "Geestkracht" Program of ZonMw

\section{4}

Expression of thyrotropin receptors in adipose tissue of obese subjects with type 2 diabetes

M. Nannipieri ${ }^{1}$, F. Cecchetti ${ }^{1}$, M. Anselmino ${ }^{2}$, E. Mancini ${ }^{1}$, F. Santini ${ }^{3}$, A. Pinchera ${ }^{3}$, E. Ferrannini ${ }^{1}$

${ }^{1}$ Internal Medicine, University of Pisa, ${ }^{2}$ Bariatric Surgery, Azienda Ospedaliera Universitaria of Pisa, ${ }^{3}$ Endocrinology, University of Pisa, Italy

Background and aims: Morbidly obese subjects may present abnormal thyroid function tests, probably as a result of a central reset of thyroid function. Information on the expression of TSH receptors (TSHR) in extra-thyroidal tissues in humans is scarse and conflicting. Whether TSHR and thyroid hormone receptors (ROTa1 and ROTa2 subunits) are expressed in subcutaneous (SAT) or visceral adipose tissue (VAT) is unknown.

Materials and methods: Total RNA was extracted from SAT and VAT tissue specimens obtained during abdominal surgery from 12 lean non-diabetic subjects $(\mathrm{C})\left(\mathrm{BMI}=24.1 \pm 2.9 \mathrm{kgm}^{-2}\right), 27$ obese subjects with type 2 diabetes $(\mathrm{T} 2 \mathrm{DM}-\mathrm{Ob})\left(\mathrm{BMI}=44.9 \pm 6.2 \mathrm{~kg} \mathrm{~m}^{-2}\right), 17$ obese subjects with impaired glucose tolerance (IGT-Ob) $\left(\mathrm{BMI}=47.2 \pm 7.1 \mathrm{~kg} \mathrm{~m}^{-2}\right)$ and 50 obese subjects with normal glucose tolerance $(\mathrm{Ob})\left(\mathrm{BMI}=47.5 \pm 9.6 \mathrm{~kg} \mathrm{~m}^{-2}\right)$. Relative quantification of TSHR, ROTa1 and ROTa2 mRNA (target gene) was performed by Real-Time PCR. Results were expressed as the target/reference (Hypoxantine phosphorybosyl-transferase) ratio for each sample.

Results: In SAT, TSHR expression was significantly higher in control than obese subjects regardless of glucose tolerance $(\mathrm{C}=0.68 \pm 0.4$ vs $\mathrm{Ob}=0.36 \pm 3$ vs IGT-Ob $=0.46 \pm 0.5 v s$ T2DM-Ob $=0.30 \pm 0.3, \mathrm{p}=0.005$ by Kruskall Wallis test), whereas no differences were found in ROTa1 or ROTa2 expression among groups. Similarly, in VAT TSHR was significantly more expressed in controls as compared to the other groups $(\mathrm{C}=0.65 \pm 0.5$ vs $\mathrm{Ob}=0.21 \pm 0.2 v s$ IGT$\mathrm{Ob}=0.19 \pm 0.2$ vs $\mathrm{T} 2 \mathrm{DM}-\mathrm{Ob}=0.25 \pm 0.28, \mathrm{p}=0.01)$; again, no differences were found in ROTa1 and ROTa2 among groups. TSHR in SAT and VAT were positively related to each other $(r h o=0.45, \mathrm{p}<0.0001)$. TSHR in both SAT and VAT were reciprocally associated with BMI ( $r h o=-0.20$ and -0.36 , respectively, $\mathrm{p} \leq 0.05$ for both); this association persisted after adjusting for sex, age and fasting glucose levels. While circulating thyroid hormones levels did not differ significantly across groups, plasma TSH levels were lower in $\mathrm{C}$ than in the other groups $(0.7 \pm 0.4 \mu \mathrm{U} / \mathrm{ml} v s 1.9 \pm 0.9,2.1 \pm 1.6,2.0 \pm 1.5$, C vs $\mathrm{Ob} v s$ IGT-Ob $v s$ DM2-Ob, $\mathrm{p}=0.0002)$ and were directly related to BMI $(r h o=0.35$, $\mathrm{p}=0.0002)$ and inversely related to VAT TSHR $(r h o=-0.28, \mathrm{p}=0.01)$.

Conclusion: TSHR gene is expressed in adipose tissue, to a similar extent in subcutaneous and visceral depots. In both depots, TSHR - but not thyroid hormone receptors - are reduced in obesity (and in proportion to the degree of obesity) regardless of the glucose tolerance status. The inverse association of TSHR in VAT and circulating TSH levels suggests that visceral fat may be the site of a feedback regulation.

Supported by: Italian Ministry of Research and Education

\section{5}

Is the favorable effect of type 2 diabetes mellitus on bone mineral density associated to increased body fat?

D.J. Hadjidakis, A. Mylonakis, I.I. Androulakis, M. Peppa, A.E. Raptis,

A. Papaefstathiou, T. Economopoulos, S.A. Raptis

2nd Department of Internal Medicine-Propaedeutic, Research Institute and

Diabetes Centre, «Attikon» and «Evgenidion» University Hospitals, Athens

University, Greece

Background and aims: In type 2 diabetes mellitus, insulin resistance has been associated with the presence of excessive fat mass and obesity. On the other hand, the observed positive effect of the disease on bone mineral densi- ty (BMD) has mostly been attributed to hyperinsulinemia. The present study investigates whether this positive effect is directly associated to excessive fat mass.

Materials and methods: In 117 postmenopausal women (age 63.1 \pm 5.7 yrs, mean $\pm 1 \mathrm{SD}$, range 51-70) suffering from diabetes mellitus type 2 for more than 5 years, never on insulin or glitazone treatment, whole body (WB) composition and BMD [L1-L4 vertebrae, total hip (TH) and femoral neck (FN)] measurements were performed by DXA. Somatometric parameters, such as Body Mass Index (BMI, $\mathrm{kg} / \mathrm{m}^{2}$ ), "Fat Mass Index" ("FMI", fat mass in $\mathrm{kg} / \mathrm{m}^{2}$ ), the proportion of whole body fat mass (WBFM\%) as well as the proportion of the trunk fat mass (TRFM\%) were evaluated. Diabetic women were twice separated in 2 subgroups according to the median value $[(+)$ above the median, (-) below the median] of either the "FMI" [FMI(-) and FMI(+)] or the BMI [BMI(-) and BMI(+)].

Results: Within the whole group of diabetic postmenopausal women only BMI but not "FMI" was significantly positively correlated to the BMD values of all anatomic sites (all $\mathrm{p}<0.01$, Pearson's correlation). Between the FMI(-) and $\mathrm{FMI}(+)$ subgroups, no significant differences were observed in the BMD values of any anatomic site (all p $>0.6$, t-test). Conversely, BMI $(+)$ subgroup presented significantly higher BMD values in L1-L4 vertebrae and TH compared to BMI $(-)(\mathrm{p}<0.01)$. In all subgroups only BMI but not "FMI" was significantly positively correlated with the BMD values of all anatomic sites ( $\mathrm{p}<0.01$ for BMI and $>0.4$ for "FMI" always). No significant correlation was observed in any subgroup between the WBFM\% or TRFM\% and any BMD value.

Conclusion: Mechanical loading due to increased whole body mass rather than the presence of excessive fat mass seems to be responsible for the observed positive effect of type 2 diabetes mellitus on bone mineral density of postmenopausal women. Adiposity, general or central, does not seem to be directly associated to bone density. 


\section{PS 070 Inflammation and fat}

\section{6}

Depot specific activity of the 15-lipoxygenase pathway in adipose tissue: the inflammatory response and metabolic disease

A.L. Harte ${ }^{1}$, M.J. Hill ${ }^{1}$, N.F. da Silva ${ }^{2}$, K.C. McGee ${ }^{1}$, A.R. Baker ${ }^{1}$,

J.C. Clapham ${ }^{2}$, S. Kumar' ${ }^{1}$, P.G. McTernan ${ }^{1}$

${ }^{1}$ Clinical Sciences Research Institute, University of Warwick Medical School, Coventry, United Kingdom, ${ }^{2}$ AstraZeneca, Alderley Park, United Kingdom

Background and aims: 15-Lipoxygenase (15-LO) is a key, highly regulated enzyme catalysing the oxygenation of arachidonic acid (AA) forming a variety of lipid metabolites that exert diverse pro- and anti-inflammatory properties. To date, no study has addressed the role of 15-LO in human adipose tissue (AT), whilst sub-clinical inflammation is known to represent a key player in the pathogenesis of obesity-mediated type 2 diabetes mellitus (T2DM). Therefore the aims of this study were to (1) investigate the mRNA expression profile of major pathways involved in AA metabolism in human abdominal subcutaneous (AbSc) and omental (Om) AT depots (2) assess the relative depot specific changes in mRNA and protein expression of the 15-LO pathway in inflammatory models (3) examine the role of adiposity.

Materials and methods: Paired AbSc and Om AT were collected from patients undergoing elective liposuction surgery (Age: $45.81 \pm 1.73$ yrs; lean BMI: $22.67 \pm 0.40 \mathrm{~kg} / \mathrm{m}^{2}$; obese BMI: $33.05 \pm 0.72 \mathrm{~kg} / \mathrm{m}^{2} ; \mathrm{n}=36$ ) for gene expression analysis by microarray and real time polymerase chain reaction (PCR). Protein expression and secretion were determined by Western blot and ELISA analysis.

Results: Microarray data showed that Om AT had significantly higher expression of $15-\mathrm{LO}$ than paired $\mathrm{AbSc} A \mathrm{~A}$, independent of $\mathrm{BMI}(\mathrm{p}=0.00016)$. In addition, other key elements of AA metabolism; prostaglandin D2 synthase $(\mathrm{p}=0.000008)$, leukotriene $\mathrm{A} 4$ hydrolase $(\mathrm{p}=0.00036)$, prostaglandin endoperoxide synthase $1(\mathrm{p}=0.000001)$ and cytochrome $\mathrm{p} 450(\mathrm{p}=0.000075)$ showed significantly higher expression in Om AT. PCR data further corroborated the 15-LO expression profile (AbSc AT: $\Delta$ Ct $17.10 \pm 0.86$ and Om AT: $\Delta$ Ct 6.48 $\pm 0.37(\mathrm{p}=<0.001))$ independent of BMI. Western blotting confirmed depot specific expression of 15- $\mathrm{LO}$ was still present at the protein level and continued to be independent of BMI. Lipoxin $\mathrm{A}_{4}$ secretion was observed by ELISA in isolated adipocytes.

Conclusion: These studies highlight, for the first time, that $15-\mathrm{LO}$ is clearly differentially expressed in human AT and that the pathway metabolite lipoxin $\mathrm{A}_{4}$ is secreted from adipocytes. Furthermore that omental AT, which is associated with increased inflammatory response, also has increased mRNA expression of the 15-LO pathway. Taken together, these findings suggest a previously undefined mechanism within human fat that may exacerbate the inflammatory response in obesity mediated T2DM.

\section{7}

Neutrophils transiently infiltrate intra-abdominal fat early in the course of high fat feeding

V. Elgazar-Carmon, A. Rudich, N. Hadad, R. Levy

Clinical Biochemistry, Ben-Gurion University, Beer-Sheva, Israel

Background and aims: It has been established that obesity is characterized by chronic inflammation of adipose tissue manifested by secretion of proinflammatory cytokines and macrophage infiltration. Yet, the initiating event(s) in adipose inflammation is still unknown. In various infectious and non-infectious inflammatory processes, chronic inflammatory infiltrate is characterized by predominant mononuclear cells and is usually preceded by acute infiltration of the tissue by circulating neutrophils. We aimed to study whether neutrophils infiltrate adipose tissue prior to macrophages, and to characterize adipocyte-neutrophil adherence in molecular terms.

Materials and methods: In-vivo: Male C57BL/6J mice were subjected to high fat feeding for 3 days to 16 weeks, after which subcutaneous and peri-epididymal fat tissues were analyzed by immunoblotting and immunohistochemistry. In-vitro: we determined the role of CD11b-ICAM-1 complex formation in mediating adherence between 3T3-L1 adipocytes and isolated mouse neutrophils using surface integrin exposure analyses by FACS, neutralizing antibodies and co-immunoprecipitation approaches.

Results: Early (3 and 7 days) after initiating high fat feeding of C57BL/6J mice, neutrophils transiently infiltrate the parenchyma of intra-abdominal, but not subcutaneous, adipose tissue. Mean periepdidymal fat myeloperoxidase ex- pression (representing neutrophils) was significantly increased 3.5-fold ( $\mathrm{p}<$ $0.01)$ and 2.9 -fold $(\mathrm{p}<0.03)$, at days 3 and 7 compared to day 0 . Immunohistochemistry analysis demonstrated physical binding between neutrophils and adipocytes. In an in-vitro adherence assay, mouse peritoneal neutrophils physically adhered to a monolayer of 3T3-L1 mouse adipocytes. The degree of adherence depended on neutrophils' activation state, being $41.9 \pm 3.7 \%$ or $29.5 \pm 2 \%$, when neutrophils were stimulated with PMA or the IL- 8 analog $\mathrm{KC}$, respectively, compared with $24.8 \pm 1.5 \%$ in unstimulated neutrophils. Activation-dependent adherence required $\mathrm{Ca}^{2+}$ and could be mimicked by $\mathrm{Mn}^{2+}$, suggesting the involvement of integrins, and corresponded to the degree of surface exposure of CD11b (Mac-1). The adherence was prevented by pre-incubating neutrophils or adipocytes, respectively, with neutralizing anti-CD11b or anti-ICAM-1 antibodies. Furthermore, immunoprecipitation of $\mathrm{CD} 11 \mathrm{~b}$ from lysates of a mixed neutrophil-adipocyte cell population resulted in co-immunoprecipitation of ICAM-1, indicating that the interaction is mediated by neutrophil CD11b and adipocyte ICAM-1.

Conclusion: The early and transient infiltration of neutrophils to intra-abdominal (but not subcutaneous) adipose tissue and the binding to adipocytes may provide a novel insight on the initiating events of the inflammatory process occurring in adipose tissue during development of obesity. Supported by: Israel Science Foundation, Israel Academy of Sciences

\section{8}

Glucose-dependent insulinotropic polypeptide (GIP) modulates inflammatory markers and regulates proliferation in subcutaneous adipose tissue

Ö. Gögebakan ${ }^{1}$, S. Mosig ${ }^{2}$, I. Middelbeck ${ }^{1}$, M.A. Osterhoff ${ }^{1}$, N. Rudovich ${ }^{3}$, D. Groth ${ }^{4}$, A.F.H. Pfeiffer ${ }^{1,3}$

${ }^{1}$ Clinical Nutrition, DIfE, Rehbrücke, ${ }^{2}$ Institute for Vaskular Medicine, Friedrich-Schiller-University Medicine, Jena, ${ }^{3}$ Endocrinology, Diabetes and Nutrition, Charité-University Medicine, Berlin, ${ }^{4}$ Bioinformatics, MaxPlanck-Institute, Potsdam Golm, Germany

Background and aims: Glucose-dependent insulinotropic polypeptide (GIP) is a gastrointestinal hormone that is secreted in response to food intake and modulates $\beta$-cell function. GIP acts on various tissues, including pancreatic $\beta$-cells, via interaction with its G-protein-coupled receptor. Some studies in rodents suggested that GIP directly links overnutrition to obesity. GIP exerts a physiological role on lipid uptake into adipocytes. Insulin release by GIP is contingent upon elevated blood glucose similar to the other important incretin hormone, glucagon like peptide 1 (GLP-1). This assures that insulin release is not stimulated under hypoglycaemic conditions. GIP looses its insulinomimetic effect in the presence of chronic hyperglycaemia and thus appears to be ineffective in poorly controlled T2DM at least with regard to insulin release. The mechanism by which this occurs is not clear and it is not known whether this loss of function is restricted to insulin release. To analyse the effect of GIP-Infusions on changes in adipose tissue gene expression at different blood-glucose levels in obese men and also detect different biomarkers and hormone interactions caused by GIP.

Materials and methods: Seventeen healthy overweight men (BMI: 28-40 $\mathrm{kg} / \mathrm{m} 2$; age: 30-65 years) with normal glucose tolerance underwent a singleblind intervention study at four different time points with euglycaemic or hyperglycaemic clamps in combination with GIP or saline infusions. Each solution was applied at physiological concentrations for four hours. Before and after the infusions biopsies from subcutaneous adipose tissue were taken. We isolated total RNA from all fat biopsies and hybridized the RNA to Agilent Whole Human Genome Microarrays (total 100 arrays). The results were verified by RT-PCR.

Results: Using Agilent Genespring Software and the MetaCore platform we identified several genes being involved in inflammatory and proliferative cell signalling. Under euglycaemic hyperinsulinaemic clamp conditions in combinations with a GIP-Infusion we find a significant upregulation of chemokine ligand 2 (CCL-2/MCP-1), interleukin-1 $\beta$ (IL-1 $\beta$ ), interleukin-6 (IL-6), fibroblast growth factor receptor 1 (FGFR-1) and oncostatin M (OSM). We also see an activation of cell proliferation by members of wnt signalling in adipose tissue after exposure with GIP mediated by cyclin D1 and several zinc finger proteins like ZNF 397 and ZNF 629.

Conclusion: GIP may play a role in inflammation and cell proliferation in adipose tissue of obese men. Depending on blood glucose levels GIP, is also able to upregulate lipid and glucose metabolism in adipose tissue. Supported by: Deutsche Forschungsgemeinschaft 


\section{9}

Regulatory effects of heat shock protein 60 on inflammatory adipocyte functions

V. Burkart, E. Gülden, S. Mollerus, J. Brüggemann, C. Habich

Institute for Clinical Diabetes Research, German Diabetes Center,

Düsseldorf, Germany

Background and aims: Adipose tissue has been characterized for a long time mainly by its energy storing capacity. Recent studies identified adipocytes as an important source of numerous inflammatory mediators including interleukin-6 (IL-6), IL-8 and monocyte chemoattractant protein-1 (MCP-1). Further investigations now provide increasing evidence that adipocyte-derived cytokines and chemokines sustain systemic low grade inflammation thereby promoting the development of metabolic disorders such as type 2 diabetes, and diabetes-specific complications. However, the physiological signals triggering the formation of inflammatory mediators in adipocytes are largely unknown. Analyses of the functional activities of adipocytes showed that this cell type shares essential features with innate immune cells. The stress protein heat shock protein (Hsp) 60, a putative beta-cell autoantigen, has been identified as a potent stimulator of pro-inflammatory activities of innate immune cells. These findings qualify Hsp60 as potential signal for the induction of pro-inflammatory mediators from adipocytes. Therefore, the present study was designed to analyse the regulatory effects of Hsp60 on adipocytes.

Materials and methods: Preadipocytes and mature adipocytes of the murine line 3T3-L1 were incubated with recombinant Hsp60 and the release of IL-6, KC and MCP-1 from these cells was determined by ELISA. The maturation state of the cells was determined by FACS, analysing the expression of the differentiation marker preadipocyte factor 1 (Pref-1). Binding of Hsp60 to the (pre-)adipocytes was studied by FACS analyses with fluorescent-labelled Hsp60.

Results: Analyses of the adipocyte maturation state by FACS confirmed a high expression level of Pref-1 in 3T3-L1 preadipocyte cultures (>90\%), whereas the expression in mature adipocyte cultures was strongly reduced $(<20 \%)$. Exposure of 3T3-L1 preadipocytes to increasing concentrations of Hsp60 $(1,10$ and $20 \mu \mathrm{g} / \mathrm{ml})$ dose-dependently induced a significant release of the cytokine IL- 6 (from $113 \pm 1$ to $459 \pm 45 \mathrm{pg} / \mathrm{ml}$ ) and the chemokines KC (from $2 \pm 1$ to $31 \pm 2 \mathrm{ng} / \mathrm{ml}$ ) and MCP-1 (from $16 \pm 1$ to $39 \pm 7 \mathrm{ng} / \mathrm{ml}$ ). Incubation of mature 3T3-L1 adipocytes with Hsp60 increased the levels of the inflammatory mediators IL-6 (from $259 \pm 13$ to $2208 \pm 202 \mathrm{pg} / \mathrm{ml}$ ), KC (from $11 \pm 1$ to $43 \pm 6 \mathrm{ng} / \mathrm{ml}$ ) and MCP-1 (from $37 \pm 2$ to $78 \pm 8 \mathrm{ng} / \mathrm{ml}$ ). Comparative analyses of mature 3T3-L1 adipocytes after different cultivation periods (14 versus 23 days) revealed no significant differences in the Hsp60-stimulated formation of IL- 6 and KC, whereas the Hsp60-induced release of MCP-1 decreased from $78 \pm 8$ to $35 \pm 1 \mathrm{ng} / \mathrm{ml}$. FACS analyses demonstrate specific Hsp60 binding to adipocytes with the characteristics of a receptor-ligand interaction. These findings implicate that Hsp60-stimulated formation of pro-inflammatory mediators in adipocytes is triggered by a receptor-mediated binding of the stress-protein.

Conclusion: Our results identified Hsp60 as important regulator of adipocyte functions supposed to contribute to the development of chronic, diabetes-associated inflammatory processes.

Suported by: Deutsche Forschungsgemeinschaft, Deutsche Diabetes-Gesellschaft, Bundesminister für Gesundheit and the Minister für Innovation, Wissenschaft, Forschung und Technologie des Landes Nordrhein-Westfalen.

\section{0}

Toll-like receptor 4 controls the inflammatory activity and maturation of adipocytes

E. Gülden, C. Habich, V. Burkart

Institute for Clinical Diabetes Research, German Diabetes-Center, Düsseldorf, Germany

Background and aims: Adipocytes have been identified as important cellular source of mediators which control a variety of metabolic and inflammatory processes. Recent findings show that adipocytes and their mediators do not only contribute to the development of type 2 diabetes and diabetes-associated complications but may also affect the autoimmune processes involved in the pathogenesis of type 1 diabetes. Depending on the maturation state of the adipocytes, their inflammatory activities share many features with innate immune cells. Innate immune cell functions are efficiently controlled by Tolllike receptors (TLR), in particular TLR4, the mammalian receptor for bacterial lipopolysaccharide (LPS). We therefore investigated whether TLR4 is able to control the release of inflammatory adipocyte mediators and to affect the differentiation of adipocytes to mature cells.

Materials and methods: Our studies were performed with adipocytes of the murine line 3T3-L1 and adipocytes of TLR4 deficient C57BL/10ScCr (TLR4 ${ }^{/-}$) and TLR4 expressing C57BL/10ScSn (TLR4 ${ }^{+/+}$) mice. Mature adipocytes were generated by cultivating preadipocytes in a defined differentiation medium. The purity and maturation state of the adipocytes were analysed with the lipid specific dye Oil Red O and by FACS-based determination of the macrophage specific marker CD11b and the differentiation marker Pref- 1 . The induction of inflammatory mediators by the TLR4 ligand LPS was quantified by ELISA.

Results: The purity of the isolated murine preadipocytes typically reached $>99.2 \%$ as determined by Oil Red O staining and CD11b expression. Analyses of the maturation state confirmed the presence of Pref- 1 in more than $95 \%$ of preadipocytes and in about $20 \%$ of mature adipocytes. LPS exposure $(24 \mathrm{~h})$ resulted in a dose-dependent increase of inflammatory mediators from preadipocytes of TLR $4^{+/+}$but not of TLR $4^{-/-}$mice. LPS $(1 \mathrm{ng} / \mathrm{ml})$ induced a significant increase of IL- 8 release $(1.5 \pm 0.1 \mathrm{ng} / \mathrm{ml}, \mathrm{p}<0.001)$ in $\mathrm{TLR}^{+/+}$preadipocytes but failed to induce IL- 8 release from TLR ${ }^{-/-}$cells. Studies on the impact of the maturation state on LPS-responsiveness showed that mature $\mathrm{TLR}^{+/+}$adipocytes exhibit an increased inducibility of inflammatory mediators which was most pronounced for the chemokine MCP-1. At $1 \mathrm{ng} / \mathrm{ml}$ LPS preadipocytes released $17.7 \pm 5.2 \mathrm{ng} / \mathrm{ml}$ MCP-1 whereas mature adipocytes released $55.3 \pm 5.9 \mathrm{ng} / \mathrm{ml} \mathrm{MCP}-1(\mathrm{p}<0.01)$. Complementary studies on the potential effect of chronic TLR4 activation during adipocyte maturation showed that differentiation of 3T3-L1 cells in the presence of LPS had neither an effect on TLR4- and Pref-1-expression nor on the Oil Red O staining pattern. The presence of LPS during differentiation also did not affect spontaneous MCP-1 release $(14.1 \pm 1.4 \mathrm{ng} / \mathrm{ml})$ by mature adipocytes; however, it significantly reduced LPS-inducible MCP-1 release. Mature adipocytes differentiated in the absence of LPS released $54.7 \pm .13 .7 \mathrm{ng} / \mathrm{ml} \mathrm{MCP}-1$ in response to $1 \mathrm{ng} / \mathrm{ml}$ LPS. In contrast, adipocytes differentiated in the presence of 1 or $100 \mathrm{ng} / \mathrm{ml}$ LPS released $35.7 \pm 6.7 \mathrm{ng} / \mathrm{ml}(\mathrm{p}<0.05)$ or $18.8 \pm 3.9 \mathrm{ng} / \mathrm{ml}$ MCP-1 $(\mathrm{p}<0.01)$ after LPS stimulation.

Conclusion: Our results point to important roles of TLR4 in the regulation of inflammatory adipocyte-activities and in the development of LPS-responsiveness during the maturation of adipocytes. Our findings further implicate that TLR4-dependent adipocyte functions may contribute to the inflammatory processes involved in the pathogenesis of diabetes and diabetes-associated complications.

Supported by: Deutsche Forschungsgemeinschaft, the Deutsche Diabetes-Gesellschaft, Bundesminister für Gesundheit and the Minister für Innovation, Wissenschaft, Forschung und Technologie des Lands Nordrhein-Westfalen

\section{1}

Adipose tissue derived SPARC and obesity related inflammation in healthy adults

K. Kos $^{1}$, S. Wong ${ }^{1}$, D. Kerrigan ${ }^{2}$, J.P.H. Wilding ${ }^{1}$, J.H. Pinkney ${ }^{3}$

${ }^{1}$ Obesity Research Group, Clinical Sciences, Liverpool, ${ }^{2}$ Department of Surgery, University Hospital Aintree, Liverpool, ${ }^{3}$ Unit for Diabetes, Peninsula Medical School and Royal Cornwall Hospital, Truro, United Kingdom

Background and aims: The extracellular matrix-associated- glycoprotein Secreted Protein Acidic and Rich in Cysteine (SPARC, osteonectin) is an adipokine with as yet poorly defined function. We have previously confirmed that adipose tissue (AT) expression of SPARC is upregulated in obesity and that its expression is associated leptin expression. However, little is known about a potential role of SPARC in inflammation and its contribution to cardiovascular risk.

Materials and methods: Morbidly obese non-diabetic subjects undergoing bariatric surgery (age $=44.5 \pm 1.4$ years (mean $\pm \mathrm{SE}$ ), $\mathrm{BMI}=46.8 \pm 1.9 \mathrm{~kg}$ / $\left.\mathrm{m}^{2}, \mathrm{n}=37\right)$ and age-matched lean controls undergoing elective surgery (age $=42.3 \pm 4.1$ years, BMI $=23.6 \pm 0.8 \mathrm{~kg} / \mathrm{m}^{2}, \mathrm{n}=18$ ) were recruited and consented. A blood sample, visceral-AT (VAT) and subcutaneous-abdominalAT (SCAT) biopsy were taken from each subject. Plasma cytokine levels were measured by ELISA and their AT-derived mRNA expression by real time-PCR using QuantiTect ${ }^{\circledast}$ Multiplex PCR (Qiagen, Crawley, UK), with prevalidated primers and Quantiprobes ${ }^{\oplus}$, on a ROTORGENE-2000 analyser (Corbett Research, Cambridge, UK).

Results: SPARC expression was higher in SCAT than VAT (1.18 \pm 0.02 Signal Units (SU) versus $0.073 \pm 0.001 S U, \mathrm{p}<0.001, \mathrm{n}=47$ ). VAT and SCAT expression were highly correlated $(\mathrm{r}=0.44, \mathrm{p}<0.01)$ and associated with increased 
fat mass. SCAT-SPARC was strongly correlated with hsCRP $(\mathrm{r}=0.44, \mathrm{p}<0.01)$; SCAT-retinol binding protein-4 (RBP-4; $r=0.36 ; \mathrm{p}<0.01)$, SCAT-adiponectin $(\mathrm{r}=-0.3, \mathrm{p}<0.05)$ and SCAT-IL6 $(\mathrm{r}=-0.3, \mathrm{p}<0.05)$ and SCAT- macrophage migration inhibitory factor (MMIF; $\mathrm{r}=0.3, \mathrm{p}<0.05$ ), but not TNFalpha, SCATmacrophage inflammatory protein-1 (MIP-1), SCAT-monocyte chemoattractant protein-1 (MCP-1) or RANTES. VAT-SPARC was also correlated with VAT-RBP-4 ( $\mathrm{r}=0.47, \mathrm{p}<0.001)$. After correction for fat mass, age, gender, HOMA-IR and GLUT-4, only the correlation with RBP-4 remained significant and this in both AT-depots. Stepwise multiple regression revealed RBP-4 as a key predictor of SPARC expression. In addition, SCAT- and VAT-SPARC mRNA was positively correlated with HOMA-IR $(r=0.3, p<0.05$ and $r=0.36$, $\mathrm{p}<0.05$ respectively).

Conclusion: SPARC does not appear to have a marked role as proinflammatory adipokine when correcting for fat mass. However, SPARC- AT-depot expression appears to have a marked correlation with local RBP-4 AT expression independent of fat mass and inflammation. RBP-4 and SPARC have been linked with lipid storage. A indirect influence on the pathogenesis of these two proteins on cardiovascular disease other than by inflammation cannot be excluded. Whilst AT-derived SPARC expression appears to be increased with insulin resistance, the close correlation of SPARC and RBP4 expression may suggest a disturbance of glucose metabolism by a common pathway. Supported by: Diabetes UK and University of Liverpool Research and Development Fund

\section{2}

The role of hyperglycaemia and hyperlipidaemia on inflammation in human abdominal subcutaneous adipose tissue and isolated adipocyte E.M.Y. Youssef ${ }^{1}$, A.L. Harte ${ }^{1}$, C.M. Kusminski ${ }^{1}$, N. da Silva ${ }^{1}$, E.A. Wahba ${ }^{2}$, A.A. Huseen ${ }^{3}$, A. Cerellio' ${ }^{1}$, P. O'Hare' ${ }^{1}$, S. Kumar', P.G. McTernan ${ }^{1}$ ${ }^{1}$ Diabetes and Metabolism Unit, Clinical Science Research Institute, Warwick , United Kingdom, ${ }^{2}$ Biochemistry Department, National Research Center, Cairo, Egypt, ${ }^{3}$ Clinical Pathology Department, National Institute of Diabetes and Endocrinology, Cairo, Egypt

Background and aims: Obesity-associated inflammation plays a major role in the pathogenesis of type 2 diabetes mellitus; however whether hyperglycaemia and hyperlipidaemia directly induce insulin resistance through inflammatory-based mechanisms is unclear. Therefore the key aims of this study were (1) to investigate the influence of chronic high-glucose concentration (H-Glc) and free fatty acids (FFAs) on the inflammatory pathways in human abdominal subcutaneous (AbSc) adipose tissue (AT) and AbSc adipocytes, and (2) to determine whether the effects induced by glucose and FFAs are mediated through NF- $\kappa$ B pathways

Materials and methods: AT was collected from patients undergoing elective liposuction surgery (age, $45 \pm 3.3 \mathrm{yrs}$; BMI, $21.9 \pm 2.4 \mathrm{~kg} / \mathrm{m}^{2} ; \mathrm{n}=4$ ). AbSc AT explants and adipocytes were treated with low-glucose (L-Glc): $5.6 \mathrm{mM}$ and H-Glc: $17.5 \mathrm{mM}$, with low $(0.2 \mathrm{mM})$ and high $(2 \mathrm{mM})$ doses of a palmitate: stearic mix for 48 hours. Conditioned media were collected and pro-inflammatory cytokine secretions were assessed by ELISA. Components of the NF$\kappa \mathrm{B}$ pathway were examined by Western blot analysis.

Results: Both L-Glc and high FFAs, H-Glc and high FFAs up-regulated key factors of the NF- $\kappa B$ pathway in both AbSc AT explants (NF- $\kappa B, p<0.05$; $\operatorname{IKK} \beta, \mathrm{p}<0.05, \mathrm{n}=5$ ) and isolated adipocytes (NF- $\mathrm{KB}, \mathrm{p}<0.05$; IKK $\beta, \mathrm{p}<0.05$, $\mathrm{n}=5$ ). Downstream both TNF- $\alpha$ and IL- 6 secretion was markedly increased from AbSc AT explants and isolated adipocytes treated with chronic LGlc combined with high FFAs and H-Glc in combination with high FFAs, compared to controls (L-Glc alone). (AbSc AT explants: TNF- $\alpha$ : Control $0.41 \pm 0.163 \mathrm{pg} / \mathrm{mL}$; L-Glc \& FFA $(2 \mathrm{mM}) 4.1 \pm 0.73 \mathrm{pg} / \mathrm{mL} ; \mathrm{H}-\mathrm{Glc} \&$ FFA $(2 \mathrm{mM}), 4.7 \pm 2.06 \mathrm{pg} / \mathrm{mL}$; $\mathrm{p}<0.05$; IL-6: Control, $1275 \pm 352.5 \mathrm{pg} / \mathrm{mL}$; L-Glc \& FFA $(2 \mathrm{mM}), 32389 \pm 19972 \mathrm{pg} / \mathrm{mL}$; H-Glc\&FFA $(2 \mathrm{mM}), 48741 \pm 23151 \mathrm{pg} /$ $\mathrm{mL}, \mathrm{p}<0.05, \mathrm{n}=4)$. Isolated adipocytes:TNF- $\alpha$ : Control, $0.9 \pm 0.23 \mathrm{pg} / \mathrm{mL} ; \mathrm{L}-$ Glc \& FFA $(2 \mathrm{mM}), 8.51 \pm 1.545 \mathrm{pg} / \mathrm{mL}$; H-Glc \& FFA $(2 \mathrm{mM}), 5.9 \pm 1.64 \mathrm{pg} / \mathrm{mL}$; p <0.05; IL-6:Control, $8734 \pm 2856.5 \mathrm{pg} / \mathrm{mL}$; L-Glc \& FFA(2mM), $39726 \pm 8345$ $\mathrm{pg} / \mathrm{mL} ; \mathrm{H}-\mathrm{Glc} \& \mathrm{FFA}(2 \mathrm{mM}), 42500 \pm 8277 \mathrm{pg} / \mathrm{mL}, \mathrm{p}<0.05, \mathrm{n}=4)$.

Conclusion: Principally, irrespective of hyperglycaemic conditions, hyperlipidaemia has a profound effect on the increased production of the inflammatory cytokines, ranging from a 10-25 fold increase in TNF $\alpha$ and IL- 6 production, respectively, with consistent upregulation of NF- $\mathrm{\kappa B}$ components in human AbSc AT explants and adipocytes. This study implicates elevated FFAs as a key instigator of the inflammatory response in both AT and adipocytes via NF- $\kappa \mathrm{B}$ with clear implications for the metabolic disease pathogenesis. Supported by: Egyptian Government

\section{3}

Does macrophage migration inhibitory factor precipitates obesityassociated type 2 diabetes development - an animal study

T. Cvjeticanin, S. Stosic-Grujicic, I. Stojanovic

Immunology, Institute for Biological Research "Sinisa Stankovic", Belgrade, Serbia

Background and aims: Hyperglycemia during type 2 diabetes (T2D) is a consequence of inappropriate insulin action. However, the final stage of this disease is characterized by $\beta$ cell apoptosis leading to actual insulin deficiency. Recently, T2D has been characterized as a state of persistent, low-grade inflammation, and various pro-inflammatory mediators along with fatty acids are implicated in $\beta$ cell apoptosis. Since some human studies discovered increased circulating levels of macrophage migration inhibitory factor (MIF) in patients with T2D and high MIF levels were associated with higher incidence of T2D, our aim was to further delineate the role of MIF in T2D.

Materials and methods: An animal model of obesity-associated T2D induced by high-fat (HF) food intake was used and applied on mice with MIF gene deletion (MIF-KO) and their wt counterparts C57BL/6 mice. Low-fat (LF) food was used as a control diet for both groups of mice. MIF secretion from pancreatic lymph node cells was determined by ELISA 5 weeks after the beginning of the experiment. Weight and basal non-fasting blood glucose levels were measured weekly. Intraperitoneal glucose tolerance test was performed every two weeks.

Results: The intake of HF diet increased body weight and impaired glucose tolerance in wt mice, while their blood glucose levels started to rise around week 9. Our preliminary data indicate that MIF secretion from pancreatic lymph node cells accompanied the weight gain in mice fed with HF diet in comparison to wt mice fed with low fat (LF) diet. Interestingly, MIF-KO animals on LF diet gained considerably more weight then wt $(21.3 \%$ and $6.0 \%$, respectively) after 4 weeks of feeding. At the same time MIF-KO on HF diet gained $30.2 \%$ of their original weight, while wt mice gained only $9.8 \%$. By the end of $8^{\text {th }}$ week, wt mice progressively gained weight and the difference between LF and HF group became significant, while MIF-KO mice stagnated. Although MIF-KO on HF diet mice became obese very early, they normally responded to glucose overload throughout the examination period and preserved normoglycemia.

Conclusion: Our results indicate that high levels of MIF are associated with development of obesity-associated T2D in C57BL/6 mice. Moreover, MIF is probably involved in the impairment of glucose homeostasis in mice on HF diet since its deletion lead to improved regulation of glycemia. Also, MIF inhibitors could be a valuable therapeutic approach in blocking $\beta$ cell failure, having in mind the normal response of obese MIF-KO mice to glucose overload. Our goal is to further investigate the precise mechanism of MIF action in T2D.

Supported by: EFSD/AstraZeneca research award

\section{4}

Co-culture of adipocytes with macrophages inhibits insulin action and promotes a pro inflammatory state in adipocytes which is modulated by long chain $\mathbf{n}-\mathbf{3}$ polyunsaturated fatty acids E. Oliver ${ }^{1,2}$, C. Phillips ${ }^{1}$, S. Toomey ${ }^{1}$, H.M. Roche

${ }^{1}$ Nutrigenomics, University College Dublin, Ireland, ${ }^{2}$ Department of Clinical Medicine, Trinity College Dublin, Institute of Molecular Medicine, Ireland

Background and aims: Obesity is the key aetiology factor that predisposes individuals to insulin resistance. Recent studies have shown that obese adipose tissue is characterised by increased infiltration of macrophages. These macrophages may release pro-inflammatory stressors, which may alter insulin sensitivity in adipocytes by modifying the insulin-signalling pathway, leading to type 2 diabetes mellitus (T2DM). There is strong evidence to suggest that long chain n-3 polyunsaturated fatty acids (LC n-3 PUFA) have anti-inflammatory as well as protective effects on T2DM risk. Cell culture studies have shown that pre-incubation with eicosapentaenoic (EPA) \& docosahexaenoic (DHA) can attenuate pro-inflammatory cytokine production and down-regulate NF- $\mathrm{\kappa B}$ activation in macrophages. This study investigated the molecular interactions between macrophages \& adipocytes and determined the effects on molecular markers of insulin sensitivity (GLUT4, IRS-1 $\&$ adiponectin) \& inflammation (IL-6, TNF- $\alpha$ \& IL-1 $\beta$ ) in adipocytes and to determine the therapeutic effects of anti-inflammatory n-3 LC-PUFA EPA \& DHA on these markers. 
Materials and methods: A co-culture of differentiated 3T3-L1 adipocytes and J774.2 macrophages was used as a model for obesity. In the contact system 3T3-L1 cells were cultured with unstimulated and LPS stimulated J774.2 cells $(0.1 \mu \mathrm{g} \mathrm{LPS} / \mathrm{ml})$ for 24 hours followed by EPA or DHA $(50 \mu \mathrm{mol} / \mathrm{L})$ treatment for a further 24 hours. The transwell system was identical except inserts kept cell types separate but allowed exchange of soluble molecules. Cultured cells and supernatants were harvested for RT-PCR, western blot, confocal and ELISA analysis.

Results: The presence of macrophages significantly reduced adipocyte GLUT4 ( $\mathrm{p}<0.05)$ and IRS-1 ( $\mathrm{p}$ 0.05) mRNA expression, classical markers of insulin sensitivity. In contrast, IL-6 $(\mathrm{p}<0.05)$ and TNF- $\alpha$ mRNA levels were up-regulated by the presence of macrophages. Also cell supernatant TNFa $(\mathrm{p}<0.05)$, IL-6 $(\mathrm{p}<0.05)$ and IL- $1 \beta(\mathrm{p}<0.05)$ concentrations were increased while adiponectin levels were reduced $(\mathrm{p}<0.05)$ on exposure to macrophages in both the contact and transwell systems. Adipocyte co-culture with LPS stimulated macrophages expressed significantly less GLUT4 protein $(\mathrm{p}<0.05)$. Interestingly, EPA and DHA modify these effects within the adipocytes, reducing the expression of pro-inflammatory mediators (TNF- $\alpha$, IL-6 and IL-1 $\beta$ ) and increasing markers of insulin sensitivity (GLUT4 and IRS-1).

Conclusion: These results suggest that obesity is associated with the generation of macrophage-derived soluble factors that promote a pro-inflammatory insulin-resistant adipocyte function. This work shows that pre-culturing macrophages with EPA and DHA can attenuate this effect. A longer pre-treatment of the macrophages with n-3 LC PUFA (Chronic pre-treatment) may be necessary to see a more defined attenuating effect. Further work is continuing to elucidate the molecular interactions between adipocytes, macrophages and dietary fatty acids.

Supported by: European Commission, (LIPGENE). Elizabeth Oliver is a recipient of the PhD in Molecular Medicine and the HRB PhD Training Site Programme at Trinity College Dublin

\section{5}

Metformin significantly improves immune activity of natural killer (nk) cells of type 2 diabetic patients by regulating the expression of glucotransporter 4 (glut4)

A. Czech ${ }^{1}$, P. Piatkiewicz 1 , M. Kniotek 2 , M. Nowaczyk ${ }^{2}$

${ }^{1}$ Chair and Department of Internal Medicine and Diabetology, ${ }^{2}$ Department

of Clinical Immunology, Warsaw Medical University, Warsaw, Poland

Background and aims: Abnormal metabolic conditions (hyperglycemia) may influence the function of peripheral blood NK cells which represent the first line defense in the immune system. This relationship can be particularly expressed by glucose transporter proteins in these cells. The earlier obtained results revealed significant differences in glucose transport between NK cells of healthy subjects and diabetic patients. The aim of this study was to evaluate the number and activity of NK cells obtained from Type 2 diabetic patients (T2DP) before and after the administration of metformin in comparison with healthy subjects and to determine the quantitative expression of insulin-dependent GLUT4 in these cells.

Materials and methods: Incubation tests were performed at baseline in 8 newly-diagnosed T2DP, naive to any hypoglycaemic drugs and 10 carefully matched control subjects; in the diabetic patients, the tests were repeated after 6 months of therapy with metformin accordingly to clinical indications. Peripheral blood mononuclear cells (PBMC) were isolated by Ficoll gradient centrifugation. The K562 human erythroleukemia cell line was used as the standard target for human NK cytotoxicity assay. K562 were labelled with $\mathrm{DIO}$ (3,3-dioctadecyloxacarbocyanine perchlorate). Target and effector cells were added to reach effector/target rations: 50:1 ,12:1. Dead target cells were stained with PI (propidium iodide). After 4 hours of incubation data were collected for analysis on the Becton-Dickinson FACScalibur flow cytometer. Immunofluorescent phenotyping of $\mathrm{NK}\left(\mathrm{CD} 16^{+}\right)$cells in PBMC was performed using specific murine anti-human CD16 PE-conjugated monoclonal antibodies. The expression of GLUT4 was investigated by flow cytometry. Cells were stained by using anti-human GLUT4 antibody and FITC-conjugated immunoglobulin. The data was analyzed using Cell Quest software.

Results: The T2DP at baseline in comparison to healthy subjects had an increased number $(13,35 \pm 6,0 \%$ vs $9,63 \pm 4,7 \%)$ but decreased activity $(3,1 \pm 2,2 \%$ vs $9,2 \pm 3,7 \%)$ of NK cells. These differences were statistically significant $(\mathrm{P}<0.01)$. Above abnormalities were associated with the higher level of GLUT4 protein in NK cells of T2DP versus control group $(43 \pm 4,8 \%$ vs $12,5 \pm 2,4 \%$ ), as shown in flow cytometry. Treatment with metformin resulted in a statistically significant reduction in fasting plasma glucose of $1.65 \mathrm{mmol} /$
$\mathrm{L}(P<0.001)$ and in $\mathrm{HbA}_{1 \mathrm{C}}$ of $0.82 \%(P<0.001)$ and also a significant decrease from $43 \%$ to $15.7 \%(P=0.002)$ in the level of GLUT4 protein presented by NK cells of type 2 diabetics. The T2DP after 6 month of metformin administration demonstrated substantially decreased number and increased activity of NK cells when compared to the baseline conditions $(10,83 \pm 4,6 \%$ and $5,7 \pm 2,8 \%$ respectively vs $13,35 \pm 6,0 \%$ and $3,1 \pm 2,2 \%$ ).

Conclusion: The high expression of GLUT4 in NK cells may be the cause of impaired immune response in T2DP. Increased number and decreased activity of NK cells may reflect incorrect metabolic conditions of the T2DP. Metformin therapy improves immune activity of NK cells in Type 2 diabetes. The influence of metformin on NK cells function constitutes a new pleiotropic action of this drug, and underlines the importance of immune response as a potential target of pharmacotherapy in type 2 diabetes mellitus. 


\section{PS 071 Adipocyte and related biology}

\section{6}

Insulin resistance caused by thiazide diuretics can be explained by adipose tissue alterations - visceral redistribution, inflammation and altered gene expression

J. Palming ${ }^{1}$, P.-A. Jansson ${ }^{1}$, F. Renström², I. Nagaev ${ }^{1}$, L. Johansson ${ }^{3,4}$, C. Karlsson ${ }^{4}$, L. Lind ${ }^{5}$ J.W. Eriksson ${ }^{1,4}$

${ }^{1}$ Department of Molecular and Clinical Medicine, Lundberg Laboratory for Diabetes Research, University of Gothenburg, ${ }^{2}$ Department of Public Health and Clinical Medicine, Umeå University, ${ }^{3}$ Department of Radiology, Uppsala University Hospital, ${ }^{4}$ AstraZeneca R\&D, Mölndal, ${ }^{5}$ Department of Medicine, Uppsala University Hospital, Sweden

Background and aims: Angiotensin II receptor blockers (ARBs) are associated with lower risk of developing T2DM compared to beta blockers and thiazide diuretics. The aim was to compare the effects of treatment with the ARB candesartan $(\mathrm{C})$, hydrochlorthiazide $(\mathrm{H})$ and placebo $(\mathrm{P})$ in adipose tissue and on adipokine levels.

Materials and methods: In a multicenter double-blind, cross-over trial, 26 non-diabetic, hypertensive patients with abdominal obesity (waist $>102 \mathrm{~cm}$, $\mathrm{M} ;>88 \mathrm{~cm}, \mathrm{~F}$ ) were randomized to three 12 week treatment periods with $\mathrm{C}$ (16-32 mg), H (25-50 mg) and P, respectively. Of the 22 patients ( $10 \mathrm{M}, 12$ $\mathrm{W}$ ) who completed the study was the mean age $54.8 \pm 10.4$ years and mean BMI $30.2 \pm 3.0 \mathrm{~kg} / \mathrm{m}^{2}$. After 8 weeks of each treatment period, subcutaneous adipose tissue biopsies were taken for analyzing the glucose uptake capacity and insulin signaling in adipocytes, adipocyte size and to isolate RNA. ${ }^{14} \mathrm{C}$-glucose uptake was measured after adipocyte incubation with insulin (50 $\mu \mathrm{U} / \mathrm{ml}, 1000 \mu \mathrm{U} / \mathrm{ml}$ ). Immunoblotting was used to analyze the insulin signaling in adipocytes after incubation with insulin $(50 \mu \mathrm{U} / \mathrm{ml}, 1000 \mu \mathrm{U} / \mathrm{ml})$. Gene expression was analyzed using real-time PCR. The insulin sensitivity was measured with euglycemic clamp. Abdominal subcutaneous and visceral adipose tissue (SAT and VAT, respectively) as well as liver fat content were measured using MRI and ${ }^{1} \mathrm{H}-\mathrm{MRS}$.

Results: The SAT/VAT ratio was reduced after treatment with $\mathrm{H}$ compared to $\mathrm{C}$ and $\mathrm{P}(\mathrm{p}<0.01)$. The three different treatments did not influence the adipocyte cell size or the basal or insulin stimulated glucose uptake capacity. Insulin stimulated phosphorylation of the insulin receptor, insulin receptor substrate 1 and protein kinase B in adipocytes displayed a similar pattern during the three treatment periods. In addition, the cellular content of these signaling proteins did not differ between the treatments. The serum level of the inflammation marker $\mathrm{C}$ reactive protein (hsCRP) were higher following $\mathrm{H}$ compared to the $\mathrm{C}$ and the $\mathrm{P}$ treatments ( $<<0.01$ for both). The serum levels of two other inflammation markers serum amyloid A (SAA) and E-selectin (SELE) were higher following $\mathrm{H}$ compared to the $\mathrm{C}$ treatment $(\mathrm{p}<0.05$, $\mathrm{p}<0.01$, respectively). The SAA mRNA expression in adipose tissue was increased after both the $\mathrm{H}(\mathrm{p}=0.016)$ and the $\mathrm{C}(\mathrm{p}=0.05)$ treatments compared to $\mathrm{P}$. As previously reported, the $\mathrm{H}$ treatment was followed by lower insulin sensitivity $(\mathrm{p}<0.01)$ and by increased liver fat content $(\mathrm{p}<0.05)$.

Conclusion: The thiazide $\mathrm{H}$ caused visceral fat redistribution and signs of low-grade inflammation with elevated serum levels of SAA, hsCRP and SELE compared to the $\mathrm{C}$ and the $\mathrm{P}$ treatments. In contrast, the $\mathrm{ARB} C \mathrm{C}$ had less pronounced adipocyte effects. Increased gene expression and secretion of inflammatory mediators from adipose tissue, e.g SAA, could contribute to the metabolic alterations exerted by thiazide treatment.

Supported by: Swedish Research Council, Swedish Diabetes Association, AstraZeneca Sweden and AstraZeneca R\&D

\section{7}

Cross-talk between interferon-gamma and hedgehog signalling restores adipogenesis in $3 \mathrm{~T} 3-\mathrm{L} 1$ cells

J. Todoric, O. Wagner, H. Esterbauer

Department of Medical and Chemical Laboratory Diagnostics, Medical University Vienna, Vienna, Austria

Background and aims: Hedgehog (Hh) signalling has been shown recently to inhibit adipocyte differentiation by preventing the induction of the proadipogenic master regulator PPAR $\gamma$. INF $\gamma$, a cytokine synthesized and secreted by T-lymphocytes, which production is increased in obesity, has also been demonstrated to inhibit the differentiation of adipocytes and to play a role in the development of insulin resistance. In this study we aimed to clarify the potential interaction between Hh signalling and IFN $\gamma$ in adipocyte differentiation.

Materials and methods: A synthetic hedgehog pathway activator (SAG) and recombinant INF $\gamma$ were used to stimulate $\mathrm{Hh}$ - and IFN $\gamma$-signalling, respectively. Luciferase and $\beta$-gal activity was measured in hedgehog reporter cell lines Shh-light II and Ptc1-/- LacZ to screen for potential pathway interactions. Relative mRNA levels of selected genes were analyzed by real-time PCR. 3T3-L1 cells were used to study adipocyte differentiation. Morphology of 3T3-L1 cells was judged by light microscopy and Oil-Red-O (OrO) staining. A two-tailed Student's $t$-test was performed to test for statistical differences.

Results: Luciferase activity of the Hh-target gene Gli1 was induced $>20$ fold by $100 \mathrm{ng} / \mathrm{ml} \mathrm{SAG}$ in Shh-light II cells. This induction was strongly inhibited in a dose dependent manner by simultaneous IFN $\gamma$ treatment (up to 7 fold reduction, $\mathrm{p}<0.01$ ). In Ptc1-/- cells, which exhibit constitutive Gli1 expression, Gli1 expression was reduced up to 2.5 fold in response to increasing concentrations of IFN $\gamma(\mathrm{p}<0.05)$. Next, 3T3-L1 cells were induced to form adipocytes and stimulated during 10 days of differentiation with $10 \mathrm{ng} / \mathrm{ml}$ of SAG and/or $50 \mathrm{ng} / \mathrm{ml} \mathrm{IFN} \gamma$. As expected, RNA levels of Hh-pathway target genes (Gli1, Gli2, Ptch1, Ptch2 and Hhip) were significantly increased after SAG-treatment relative to untreated cells (up to 3200 fold; $\mathrm{p}<0.001$ ). IFN $\gamma$ alone did not affect expression of Hh-target genes tested. However, co-stimulation (SAG plus IFN $\gamma$ ) of 3T3-L1 cells blunted the increase and resulted in decreased levels of this group of Hh-target genes (up to 60 fold inhibition; $\mathrm{p}<0.001)$. As published previously, SAG treatment also prevented induction of markers of adipocyte differentiation (Cebpa, Pparg, aP2/Fabp4, adiponectin, adipsin, Glut4, Lpl). Adipocytes incubated with SAG only showed an up to 20 fold decrease in mRNA levels of these genes as compared to untreated cells $(\mathrm{p}<0.001)$. Expression of adipocyte-marker genes was also inhibited by IFN $\gamma$ treatment, albeit to a lesser extent (up to 5 fold inhibition; $\mathrm{p}<0.001$ ). Interestingly, combined treatment (SAG plus IFN $\gamma$ ) efficiently abrogated inhibition of marker gene expression mediated by each of these stimuli and reverted RNA levels back up to $65 \%$ of those of untreated cells (SAG vs. SAG+IFN $\gamma$, $\mathrm{p}<0.001)$. This was associated with the reappearance of typical morphological changes associated with normal adipocyte differentiation (lipid droplets) that were completely blocked by SAG and partially inhibited by IFN $\gamma$. This reentry in the adipocyte differentiation program was also reflected by marked increase in OrO lipid-staining.

Conclusion: Co-activation of Hedgehog and Interferon-gamma signalling cascades in 3T3-L1 cells diminishes the pathways' individual anti-adipogenic actions, thus allowing precursor cells to re-enter the full adipocyte differentiation program.

\section{8}

Insulin resistance of cultured human preadipocytes is related to estimates of obesity of the donors

C.J.H. van der Kallen, M.M.J. van Greevenbroek, C.G. Schalkwijk, C.D.A. Stehouwer

Dept of Internal Medicine/Cardiovascular Research Institute Maastricht (CARIM), University Maastricht, Netherlands

Background: Obesity is a driving force behind the increasing prevalence of the metabolic syndrome and type 2 Diabetes Mellitus. Adipose tissue expands to accommodate increased lipid through hypertrophy of existing adipocytes and also by initiating differentiation of preadipocytes. It was suggested that it is not the absolute amount of adipose tissue but rather the capacity/flexibility of adipose tissue to expand that affects metabolic homeostasis. An important factor involved in these processes may be cellular insulin resistance of (pre)adipocytes since insulin plays an important role in adipocyte function e.g. lipid synthesis and lipolysis.

Aims: To identify whether insulin sensitivity of cultured human preadipocytes is related to measures of obesity in the original cell donors.

Methods: Preadipocytes were isolated from human volunteers via periumbilical liposuction biopsies $(n=20)$. Preadipocytes were cultured in DMEM medium containing $10 \%$ fetal calf serum and $1 \%$ glutamine/streptomycine/ peniciline (GSP). After 4-6 weeks in culture (between passage 4-8), uptake of palmitate by the preadipocytes was measured by addition of [14C]-palmitate $(0.3 \mu \mathrm{Ci}$, final concentration, $0.6 \mathrm{mM})$. The reaction was carried out at $37^{\circ} \mathrm{C}$ for 7 hours in absence or presence of $100 \mathrm{nM}$ insulin. Samples were applied to HP-TLC to separate triglycerides and phospholipids. Radioactivity was 
measured after exposure to an imaging screen with the Personal Molecular Imager FX (Bio-Rad).

Results: Insulin sensitivity of fatty acid incorporation into triglycerides was defined as; the difference between the insulin stimulated and the basal incorporation divided by the basal incorporation. A significant correlation was found between the insulin sensitivity of fatty acids incorporated into triglycerides and measures of obesity (waist circumference $(r=-0.567, P: 0.009)$ and waist-to-hip ratio (WHR, $r=-0.539, P: 0.006)$ ). These results remain significant after correction for age and sex. A more general measure of whole body obesity (BMI) did not reach significance $(r=-0.415, \mathrm{p}=0.069)$.

Conclusion: In this study we show that metabolic characteristics of primary cultured preadipocytes associate with obesity of the cell donor. More specifically, we show in primary cultured human preadipocytes that a larger waist circumference (or WHR) is associated with lower insulin sensitivity for fatty acid incorporation into cellular triglycerides. This suggests that adipose tissue of lean subjects has better metabolic flexibility than adipose tissue of obese individuals. The fact that these results were obtained with primary preadipocytes in culture suggests that this is either a direct consequence of a genetic defects or may result from environmental influences during life causing epigenetic imprinting. Environmental adversity may produce persistent epigenetic alterations that set the stage for insulin resistance, metabolic obesity and Type II diabetes. The observed data offer a possible explanation for mechanisms underlying clinical observations in obesity and insulin resistance.

\section{9}

Rates of glucose uptake in the adipose tissue in vivo after a mixed meal in morbid obesity

P. Mitrou ${ }^{1}$, G. Dimitriadis ${ }^{1}$, V. Lambadiari ${ }^{1}$, E. Maratou ${ }^{2}$, E. Boutati ${ }^{1}$,

A. Papakonstantinou ${ }^{3}$, V. Komesidou ${ }^{4}$, N. Katsilambros ${ }^{5}$,

T. Economopoulos ${ }^{1}$, S.A. Raptis ${ }^{1,2}$

${ }^{1}$ 2nd Department of Internal Medicine and Research Institute, Athens

University Medical School, Attikon University Hospital, ${ }^{2} \mathrm{Hellenic}$ National,

Diabetes Center, ${ }^{3} 1$ st Department of Surgery, Evangelismos Hospital,

${ }^{4}$ Department of Nutrition, Evangelismos Hospital, ${ }^{5}$ Eugenidion Hospital,

University of Athens, Greece

Background and aims: In obese subjects, hepatic glucose production is increased, and there is a defect in insulin-stimulated glucose uptake into muscle. This raises the question as to which tissue clears the abnormally large amounts of glucose entering the circulation in obese, non-diabetic subjects. This study was undertaken in subjects with morbid obesity to investigate insulin action on glucose disposal in adipose tissue (AD) after the ingestion of a mixed meal.

Materials and methods: A mixed meal was given to 30 obese (OB, age $34 \pm 1 \mathrm{yrs}, \mathrm{BMI} 47 \pm 1 \mathrm{~kg} / \mathrm{m}^{2}$, percentage of fat $\left.49.4 \pm 1.5 \%\right)$ and 10 non-obese subjects (NOB, age $39 \pm 4$ yrs, BMI $23 \pm 1 \mathrm{~kg} / \mathrm{m}^{2}$, percentage of fat $25.3 \pm 3 \%$ ). Plasma samples for measurements of glucose $(\mathrm{G})$ and insulin (I), were taken for $360 \mathrm{~min}$ from a vein $(\mathrm{V})$ draining the anterior abdominal subcutaneous $\mathrm{AD}$ depot and from the radial artery (A). With each sample $\mathrm{AD}$ blood flow (BF) was measured with ${ }^{133} \mathrm{Xe}$. Calculations: Glucose flux: $\left[\mathrm{G}_{(\mathrm{A}-\mathrm{V})}\right] \mathrm{x}[\mathrm{BF}]$; Glucose fractional extraction: $\left[\mathrm{G}_{(\mathrm{A}-\mathrm{V})}\right] /\left[\mathrm{G}_{(\mathrm{A})}\right]$;

Results: Postprandial changes (area under curve $0-360 \mathrm{~min}$ ) in OB vs NOB: (1) Arterial G was not different ( $2173 \pm 73$ vs $2040 \pm 40 \mathrm{mMmin})$, but I was increased $(23578 \pm 2372$ vs $10331 \pm 608 \mathrm{mU} / \mathrm{Lmin}, \mathrm{p}<0.05)$. (2) BF was decreased $(657 \pm 48$ vs $1345 \pm 102 \mathrm{ml} / 100 \mathrm{ml}$ tissue, $\mathrm{p}<0.05)$. (3) Although AD glucose flux (per $100 \mathrm{ml}$ tissue) was decreased $(164 \pm 45$ vs $320 \pm 91 \mu \mathrm{mol} / 100 \mathrm{ml}$ tissue, $\mathrm{p}<0.05$ ), fractional glucose uptake (which is independent of $\mathrm{BF}$ ) was not different (12 \pm 3 vs $13 \pm 5 \%$ per min). (4) Total AD glucose flux was increased $(125767 \pm 32056$ vs $51016 \pm 11998 \mu \mathrm{mol}, \mathrm{p}<0.05)$.

Conclusion: (1) In obesity, the sensitivity of glucose metabolism to insulin is impaired in the adipose tissue mainly due to a decrease in blood flow rates, (2) increased total fat mass compensates for insulin resistance.

\section{0}

Circulating lactoferrin is associated to insulin sensitivity in vivo and in vitro

J. Moreno-Navarrete ${ }^{1}$, J. Bassols ${ }^{1}$, F. Ortega ${ }^{1}$, J. Vendrell ${ }^{2}$, W. Ricart ${ }^{1}$, J. Fernandez-Real ${ }^{1}$

${ }^{1}$ Endocrinology, Hospital of Girona, ${ }^{2}$ Endocrinology, Hospital of Tarragona, Spain

Background and aims: There is a chronic low level inflammation associated with insulin resistance. Lactoferrin is a pleiotropic glycoprotein with anti-inflammatory activity. We aimed to investigate 1) the cross-sectional relationship between circulating lactoferrin, insulin resistance and innate immune system; and 2) the effects of LPS, interleukin-6 (IL-6) and rosiglitazone on lactoferrin secretion according to glucose tolerance status; 3 ) the effects of lactoferrin in insulin sensitivity under inflammatory conditions.

Materials and methods: Circulating non-stressed lactoferrin (ELISA), metabolic variables and inflammatory markers were measured in 229 men, 94 with normal (NGT) and 135 with altered glucose tolerance (AGT). Lactoferrin secretion and action were investigated in whole blood culture, and in the cell lines HepG2 and THP-1 (pAKT/AKT ratio after $100 \mathrm{nM}$ insulin).

Results: Circulating lactoferrin concentration was significantly higher in subjects with normal than in those with altered glucose tolerance $(\mathrm{p}<0.001)$, and was positively associated with insulin sensitivity $(\mathrm{r}=0.24, \mathrm{p}=0.002)$ and negatively with age $(r=-0.15, p=0.02)$, body mass index $(r=-0.15, p=0.02)$, waist to hip ratio $(r=-0.25, p=0.001)$, fasting glucose $(r=-0.20, p=0.005), \mathrm{HbA}_{1 c}(r=-$ $0.26, \mathrm{p}=0.001)$, HOMA value $(\mathrm{r}=-0.31, \mathrm{p}=0.001)$ and LBP $(\mathrm{r}=-0.43, \mathrm{p}<0.001)$ in the whole group of subjects (even after controlling for neutrophil count). In control subjects, lipopolysaccharide binding protein (LBP) contributed independently to $25 \%$ of circulating lactoferrin variance ( $\mathrm{p}=0.01$ ), after controlling for the effects of age, waist to hip ratio, $\mathrm{HbA}_{1}$, and HOMA in multiple lineal regression analysis. In subjects with altered glucose tolerance, age $(\mathrm{p}=0.03)$, waist to hip ratio $(\mathrm{p}=0.025)$ and HOMA value $(\mathrm{p}=0.01)$ contributed independently to $43 \%$ of circulating lactoferrin variance. LPS-induced lactoferrin secretion was higher in control men than in patients with type 2 diabetes $(4.92 \pm 1.3$ vs. $3.24 \pm 0.27, p=0.04)$. In the presence of IL-6, LPS-induced lactoferrin release decreased in non-diabetic subjects $(\mathrm{p}=0.001)$. Rosiglitazone treatment led to increased lactoferrin secretion in all subjects $(398.5 \pm$ 193.8 vs. $314.6 \pm 140.3, \mathrm{p}<0.0001)$. Lactoferrin treatment $(0.01,0.1,1,10 \mu \mathrm{M})$ increased insulin action dose-dependently in HepG2 under normal glucose conditions (1.1, 1.2, 1.6 and 2.2-fold). Lactoferrin $(0.01$ and $0.1 \mu \mathrm{M})$ rescued insulin resistance of HepG2 cells incubated in conditioned media from LPS stimulated THP-1 cells and increased insulin sensitivity by 2.9 and 1.6 -fold. Conclusion: The association between insulin action and lactoferrin is bi-directional. Insulin sensitizers led to increased lactoferrin concentration and lactoferrin rescued inflammation induced insulin resistance in vitro.

Supported by: Diabetes, Endocrinology and Nutrition Unit, Dr Josep Trueta Hospital; Girona Institute for Biomedical Research; and CIBER Fisiopatología de la Obesidad, Girona, Spain

\section{1}

Small heterodimer partner (SHP) modulates the transcriptional expression of LGK by LXR and PPAR

T.-H. Kim ${ }^{1,2}$, J.-M. Park ${ }^{1,2}$, M.-Y. Kim ${ }^{2,3}$, J.-S. Bae ${ }^{2,3}$, Y.-S. Park ${ }^{1,2}$, Y.-H. Ahn ${ }^{1,2}$ ${ }^{1}$ Brain Korea 21 Project for Medical Sciences, ${ }^{2}$ Center for Chronic Metabolic Disease Research, ${ }^{3}$ Dept. of Biochemistry and Molecular Biology, Yonsei University College of Medicine, Seoul, Republic of Korea

Background and aims: Small heterodimer partner (SHP) is an atypical nuclear receptor that lacks a conventional DNA binding domain. It is known to repress the transcriptional activity by either competing with coactivator in binding to transcriptional factor or recuiting corepressor directly to the transcriptional repression domain of SHP. SHP also inhibits the transcriptional activity of $\mathrm{LXR} \alpha$ and RXR $\alpha$ by direct interaction. In contrast to repressive role of SHP, the molecule is reported to augment PPAR $\gamma$ transcriptional activity by competing with the binding of other corepressors. Recently, we identified functional LXRE in the LGK promoter. LXRa directly increases LGK expression through the LXRE. These led us to explore the role of SHP in transcriptional regulation of LGK by LXR $\alpha$ and PPAR $\gamma$.

Materials and methods: pRGL-1448, LGK promoter into pGL3basic, was transfected into human hepatoma Alexander cells and the effect of SHP on LXR $\alpha$ or PPAR $\gamma$ dependent activation of LGK promoter was measured. To observe the inhibitory effect of SHP on PPAR $\gamma$ or LXR $\alpha$ dependent activa- 
tion of LGK, adenovirus expressing SHP was transduced into the primary cultured hepatocytes and measured mRNA level by real time PCR. To elucidate the repressive mechanism of SHP on LXRa or PPAR $\gamma$, we performed the mammalian two hybrid assay in Alexander cells and GST-pull down assay. Results: LXRa or PPAR $\gamma$ dependent activation of LGK promoter was decreased by SHP expression vector in a dose dependent manner in the Alexander cell lines. Adenoviral expression of SHP dramatically decreased LGK expression in the primary cultured hepatocytes. We observed that SHP directly interacts with RXR $\alpha$ rather than PPAR $\gamma$ or LXRa.

Conclusion: In this study, we demonstrated that LGK gene expression is down-regulated by SHP. The transcriptional activation of LGK by PPAR $\gamma$ and LXRa is controlled by SHP. The mechanism of LGK gene repression by SHP may occur through binding of RXR $\alpha$, which is a common partner for PPAR $\gamma$ and LXRa.

Supported by: Korea Science and Engineering Foundation grant funded by the Korea government and Brain Korea 21 project for Medical Science of Yonsei University College of Medicine

\section{PS 072 Fat distribution}

\section{2}

Abdominal obesity in type 1 and type 2 diabetes patients

R. Lichiardopol, L.D. Popescu, I. Ionescu, D. Dovan, C. Pencea

Clinic of Diabetes, Nutrition and Metabolic Diseases,

"N.C. Paulescu"Institute, Bucharest, Romania

Background and aims: Abdominal obesity is frequently associated with type 2 diabetes and is intensively investigated in this context.There are a few data on its prevalence and impact in type 1 diabetes.The aim of this study is to evaluate the prevalence of abdominal obesity and its possible relationships with macro and microvascular complications in type 1 and type 2 diabetes patients.

Materials and methods: Medical records data from 270 type 1 (136 M and $134 \mathrm{~W}$, median age of 42 ; range:20-84 yrs) and 1159 type 2 diabetes patients ( $518 \mathrm{M}$ and $641 \mathrm{~W}$, median age 59 ; range: $22-97 \mathrm{yrs}$ ), successively admitted to Diabetes Department during an entire year (1 Jan 2007-31 Dec 2007), were analyzed.We used waist circumference ( WC ) measurements and considered abdominal obesity at values exceeding $912 \mathrm{~mm}$ in women and $948 \mathrm{~mm}$ in men as these values were above the maximal level for the lower tertile of the distribution in this group.The same criterion was used to define ,increased levels"of some risk factors: Systolic Blood Pressure ( SBP ), HbA1c, Triglyceride/HDL-cholesterol (TG/HDL ) ratio.

Results: Sixty eight patients ( 25\%; 40 men,28 women) with type 1 diabetes had abdominal obesity(AO). The group with $\mathrm{AO}$ had greater mean (SD) age [49.3(12.4) vs 40.3(14.3) yrs; $\mathrm{p}<0.0001]$ and increased prevalence of coronary (CHD) heart disease (OR 2.80; 95\%CI: 1.49-5.27), diabetic (DR) retinopathy (OR 2.36; 95\% CI: 1.35-4.14) and diabetic peripheral (DPN) neuropathy (OR 2.89; 95\%CI: $1.62-5.16$ ) as compared to the group without AO. In type 1 patients, at similar levels of TG/HDL ratio $[(\geq 2.21)$ OR $5.25 ; 95 \% \mathrm{CI}$ : 1.93-14.25], HbAlc [(>8.5\%) OR 3.34; 95\% CI:1.65-6.74] and SBP [ $>120$ $\mathrm{mm} \mathrm{Hg}$ ) OR 2.28; 95\% CI: 1.23-6.75] the prevalence of CHD was greater in those with AO.The prevalence of DPN was increased, at each level of TG/ HDL ratio [ $<2.21$ OR 4.64; $95 \%$ CI: $1.57-13.69$ or $\geq 2.21$ OR 2.76; 95\% CI:1.166.60], SBP [<120 mm Hg OR 6.26; 95\% CI: 2.16-18.17 or $\geq 120 \mathrm{~mm} \mathrm{Hg} \mathrm{OR}$ 2.66; 95\% CI: 1.45-4.86] and at HbAlc >8.5\% OR 2.85;95\% CI:1.33-6.12 in people with AO. At similar increased $\mathrm{HbA1c}(>8.5 \%)$ levels, in patients with and without $\mathrm{AO}$, the prevalence of DR was greater (OR 4.15; 95\% CI: 1.65$10.44)$ in patients with AO.In logistic regression analysis, $\mathrm{CHD}(\mathrm{p}=0.0001)$, DR $(p=0.026)$ and DPN $(p=0.0018)$ were each significantly correlated with WC. Eight hundred and eighty (76\%) type 2 diabetes patients ( $407 \mathrm{M}, 473 \mathrm{~W}$ ) had abdominal obesity. As compared to $\mathrm{AO}$ group, the group of type 2 patients without AO had greater mean values for age [62.2(11.4) vs 59.5(10.1), but there vere no significant differences in prevalence of CHD, DR and DPN between groups. An increased TG/HDL ratio above 2.21 in people with ( OR 1.53; 95\% CI: 1.09- 2.14) and without (OR 1.83; 95\% CI: 1.08- 3.12) and HbA1c levels above $8.5 \%$ in people with (OR 1.37; 95\% CI: 1.04- 1.81) and without (OR 2.09 95\% CI: 1.24- 3.53) AO were all associated with an increase in CHD prevalence.An increase in SBP above $120 \mathrm{~mm} \mathrm{Hg}$ was not associated to variations in CHD prevalence. In logistic regression analysis CHD and DPN were correlated with $\mathrm{HbAlc}$ and with disease duration, while DR was correlated with SBP and disease duration. In simple linear regression analysis, the WC was directly correlated with age $(r=0.316 ; n=269 ; \mathrm{p}=0.000)$ in type 1 , while in type 2 diabetes patients an inverse correlation between WC and age $(\mathrm{r}=-0.141 ; \mathrm{n}=1158 ; \mathrm{p}=0.000)$ was found.

Conclusion: Our data suggest that abdominal obesity may have different comorbid associations and may reflect different physiopathogenic mechanisms in type 1 and type 2 diabetes.

\section{3}

Effect of fat distribution on several cardio-metabolic parameters in a healthy European population

E. Bobbioni-Harsch, V. Makoundou, V. Barthassat, Z. Pataky, A. Carpentier, F. Habicht, A. Golay

Service of Therapeutical Education for Chronic Diseases, Geneva University Hospital, Switzerland

Background and aims: Waist circumference and/or waist-to-hip ratio are used as indicators of fat distribution, but they depend on the extent of fat mass (FM). It is difficult to discriminate the effects of a preferential FM depo- 
sition from those due to FM enlargement. Furthermore, the specific influence of hip circumference is underestimated. Therefore, we evaluated the influence of waist and/or hip circumferences, after their adjustment for FM, on several cardio-metabolic parameters in a healthy European population (677 males, 829 females) participating in the RISC study.

Materials and methods: The population was recruited in 14 clinical centres of 9 European Countries. The effects of waist and hip circumferences were separately evaluated. For that, in a first analysis, we subdivided the participants into two sub-groups: subjects showing a waist circumference larger or smaller than that predicted from FM (respectively $\mathrm{W}+$ or $\mathrm{W}-$-). In a second, independent analysis, the participants were subdivided into two sub-groups: subjects showing a hip circumference larger or smaller than that predicted by FM (respectively $\mathrm{H}+$ or $\mathrm{H}-$ ). Factorial ANOVA was used to evaluate the differences between the groups.

Results: In men, the W+ in comparison to the W-group showed lower insulin sensitivity $(\mathrm{p}=0.0004)$, higher insulinemia $(\mathrm{p}=0.002)$, total $(\mathrm{p}=0.0008)$ and LDL cholesterol $(\mathrm{p}=0.002)$. W+ was not significantly associated with blood pressure, heart rate or carotid intima-media thickness (IMT). The $\mathrm{H}$ - group had a lower Fat Free Mass than the H+ group $(\mathrm{p}<0.0001)$. In women, the $\mathrm{W}+$ group showed higher insulinemia $(\mathrm{p}=0.01)$, total and LDL cholesterol $(\mathrm{p}=0.002)$ as well as a lower HDL cholesterol $(\mathrm{p}=0.005)$ than the $\mathrm{W}$ - group. The $\mathrm{H}+$ group had a smaller IMT $(\mathrm{p}=0.01)$ and lower circulating Free Fatty Acid $(\mathrm{p}=0.002)$ as well as a lower LDL cholesterol ( $\mathrm{p}=0.005)$.

Conclusion: Hip circumference exerts a protective effect on cardiometabolic risk factors. in women. Therefore, when evaluating risk factors in normal body weight or in moderately obese women, the effects of waist and hip circumference should be evaluated separately and after adjustment for the fat mass.

Supported by: EU

\section{4}

The TCF7L2 expression in subcutaneous and visceral fat tissues in women with gestational diabetes

N. Wawrusiewicz-Kurylonek, M. Kuzmicki, J. Szamatowicz, M. Gorska,

A.J. Kretowski

of Endocrinology, Diabetology and Internal Medicine, Medical University of Bialystok, Poland

Background and aims: Recent data have shown a strong association between the TCF7L2 gene variants and risk of type 2 diabetes. The TCF7L2 is a transcription factor which was found to play a role in glucose homeostasis and adipogenesis. In our study we aimed to examine if the expression of TCF7L2 mRNA in subcutaneous and visceral fat tissues in women with gestational diabetes mellitus (GDM) is different when compared to the healthy pregnant women.

Materials and methods: The subcutaneous (SAT), visceral (VAT) adipose tissues and placental tissue were obtained from 27 pregnant women (12 with normal glucose tolerance and 13 with GDM), during caesarian section. The mRNA levels of TCF7L2, adiponectin and PPAR $\gamma$ were quantified by RealTime PCR.

Results: TCF7L2 mRNA levels were 1.5 times lower in VAT and 2.4 times lower in SAT in women with GDM when compared to healthy pregnant controls. There were no significant differences in the placental TCF7L2 mRNA levels between the studied groups. We have observed a strong positive correlation between TCF7L2 gene expression and PPAR $\gamma$ or adiponectin mRNA levels in subcutaneous ( $R=0.57, \mathrm{p}=0.004 ; \mathrm{R}=0.85, \mathrm{p}=0.000000$ respectively) and visceral adipose tissue $(\mathrm{R}=0.7, \mathrm{p}=0.01 ; \mathrm{R}=0.79, \mathrm{p}=0.002$ respectively). There was also a negative correlation between the TCF7L2 expression in VAT and $\mathrm{BMI}(\mathrm{R}=-0.79, \mathrm{p}=001)$.

Conclusion: Our results show that TCF7L2 mRNA levels are decreased in fat tissues in women with GDM and may suggest the role of TCF7L2 in adipogenesis and pathogenesis of insulin resistance.

\section{5}

Lower cut-off point of waist circumference for central obesity and the metabolic syndrome in Korean

H.-K. Kim ${ }^{1}$, J.-Y. Park ${ }^{2}$, K.-U. Lee², J.-H. Kim ${ }^{1}$, C.-H. Kim ${ }^{3}$

${ }^{1}$ Health Promotion Center, Asan Medical Center, Seoul, ${ }^{2}$ Internal Medicine, University of Ulsan College of Medicine, Seoul, ${ }^{3}$ Internal Medicine, Soonchunhyang University College of Medicine, Bucheon, Republic of Korea

Background and aims: The waist circumference criterion for defining central obesity and the metabolic syndrome in Asian populations is still not fully established because there are ethnic differences in obesity prevalence and body fat distribution. We analyzed the cutoff value of waist circumference for Korean population in relation to ischemic ECG and compared it with that in relation to diabetes, hypertension, and multiple factors for metabolic syndrome.

Materials and methods: We examined the clinical and laboratory data of 31,399 subjects (age, $47.8 \pm 10.2$, range 20-89 years; 18,717 men and 12,682 women) who underwent medical check-ups in 2006 . We plotted the receiver operating characteristic (ROC) curve to determine the cutoff value of waist circumference in relation to ischemic ECG (signs suggesting myocardial infarction or ischemia). We also determined the cutoff values of waist circumference in relation to hypertension, diabetes, and multiple factors for metabolic syndrome other than central obesity using the 2005 revised NCEP-ATP III criteria.

Results: According to the ROC analyses, the cutoff values yielding the optimal sensitivity and specificity for predicting the presence of ischemic ECG were $87 \mathrm{~cm}$ for men (sensitivity $39 \%$, specificity $74 \%$ ) and $74 \mathrm{~cm}$ for women (sensitivity $66 \%$, specificity $55 \%$ ). The cutoff points of waist circumference in relation to hypertension, diabetes, and multiple factors for metabolic syndrome were $84,85,83 \mathrm{~cm}$ for men and $77,77,76 \mathrm{~cm}$ for women, respectively. The cutoff levels of waist circumference discriminating increased percent body fat (>15\%, which was associated with the presence of ischemic ECG) were $84.5 \mathrm{~cm}$ for men (sensitivity $85 \%$, specificity $71 \%$ ) and $74.5 \mathrm{~cm}$ for women (sensitivity $82 \%$, specificity $79 \%$ ).

Conclusion: The waist circumference cutoff values in relation to the cardio-metabolic diseases or risk factors were below $87 \mathrm{~cm}$ for men and $77 \mathrm{~cm}$ for women, which were lower than previously recommended Asian values of $90 \mathrm{~cm}$ for men and $80 \mathrm{~cm}$ for women. These results suggested that waist circumference criteria for defining central obesity and metabolic syndrome should be lowered in Korean population.

\section{6}

Abdominal adiposity is associated with increased plasma concentration of $\mathrm{C}$ reactive protein indipendent of body weight

E. Lapice, S. Maione, L. Patti, P. Cipriani, A.A. Rivellese, G. Riccardi, O. Vaccaro

Departement of Clinical and Experimental medicine, University of Naples Federico II, Naples, Italy

Background and aims: Abdominal adiposity provides additional information beyond overall adiposity in predicting cardiovascular (CV) risk. One likely mechanism linking visceral adiposity to $\mathrm{CV}$ disease is subclinical inflammation. $\mathrm{C}$ reactive protein (CRP) is an easily measured marker of systemic inflammation and has proved to be a strong predictor of CVD. Aim of the study is the evaluation of the relative importance of overall adiposity and abdominal obesity as correlates of plasma $\mathrm{C}$ reactive protein concentrations. Materials and methods: The design of the study is observational case-control. Participants were selected from a population of 3500 employees of a telephone company aged 35-65 years undergoing a health screening. We studied 350 people with abdominal adiposity (waist/hip ratio $\geq 0.9$ in males and $\geq 0.85$ in females) and 199 controls without abdominal obesity (waist/hip ratio $<0.9$ in males and $<0.85$ in females) matched on BMI, gender and age. Anthropometry, blood pressure, fasting lipids, glucose, insulin, fibrinogen and CRP (high sensitivity method) were measured.

Results: As a result of the selection criteria and successful matching waist/ hip ratio and waist girth were significantly and substantially higher in cases than control $(1.07 \pm 0.08$ vs $0.85 \pm 0.05 ; \mathrm{p}<0.001 ; 96.4 \pm 6.03$ vs $83.27 \pm 6.7$ $\mathrm{cm} ; \mathrm{p}<0.01)$ whereas no differences were observed in BMI $(24.81 \pm 2.46$ vs $\left.24.75 \pm 2.16 \mathrm{~kg} / \mathrm{m}^{2}\right)$, age $(44.74 \pm 6.17$ vs $44.91 \pm 6.46$ years $)$ and gender. CRP levels and the proportion of people with elevated CRP values $(>3 \mathrm{mg} / \mathrm{dl})$ was significantly higher in cases than controls $(1.96 \pm 2.6$ vs $1.53 \pm 1.74 \mathrm{mg} /$ 
$\mathrm{dl}, \mathrm{p}<0.01$; and $21.4 \%$ vs $10.1 \%, \mathrm{p}<0.05)$. In addition people with abdominal adiposity had significantly higher BP, triglycerides, insulin, HOMA-IR, and lower HDL cholesterol independent of BMI. The proportion of smokers was similar in the two groups. Multivariate analysis confirmed a significant, positive association of $\mathrm{W} / \mathrm{H}$ ratio with PCR values independent of BMI Conclusion: In healthy, non obese people abdominal adiposity is associated with subclinical inflammation beyond the effect of BMI, this may partly explain the excess CVD risk conferred by abdominal adiposity.

\section{7}

Relation of abdominal visceral and subcutaneous adipose tissue to markers of inflammation in type 2 diabetes

T. Mazzone ${ }^{1}$, S. Haffner ${ }^{2}$, M.H. Davidson ${ }^{3}$, R.B. D’Agostino ${ }^{4}$, S. Feinstein ${ }^{5}$, G. Kondos ${ }^{6}$, A. Perez ${ }^{7}$, S. Sam ${ }^{1}$

${ }^{1}$ Department of Medicine, Section of Endocrinology, Diabetes \& Metabolism, University of Illinois, Chicago, ${ }^{2}$ Division of Clinical Epidemiology, University of Texas Health Science Center, San Antonio, ${ }^{3}$ Clinical Development, Radiant Research, Chicago, ${ }^{4} \mathrm{CAS}$ Mathematics And Statistics, Boston University, Boston, ${ }^{5}$ Section of Cardiology, Rush University Medical Center, Chicago, ${ }^{6}$ Section of Cardiology, University of Illinois, Chicago, ${ }^{7}$ Clinical Research, Takeda Global Research \& Development Center, Inc., Deerfield, , United States

Background and aims: Inflammatory markers are increased in obesity and have been implicated in insulin resistance and linked with type 2 diabetes (DM2) and cardiovascular disease. Different fat depots may contribute differently to the increase in inflammatory markers. We investigated the relationship between visceral or subcutaneous fat depots to inflammatory markers in DM2.

Materials and methods: Three hundred eighty two subjects with DM2 underwent abdominal computed tomography to determine subcutaneous (SAT) and visceral (VAT) adipose tissue distribution. Fasting blood was obtained for inflammatory markers for all subjects. Each inflammatory marker was examined in relation to body mass index (BMI), SAT, and VAT areas using multivariable regression models adjusted for age, sex, duration of diabetes, smoking, statin use, and hemoglobin Alc levels; additional models included BMI or VAT and SAT.

Results: VAT was positively related to CRP, MCP, and PAI- 1 antigen before adjustment for BMI. After adjustment for BMI, the relationship to CRP was lost, but significant positive associations with MCP, PAI-1 antigen, ICAM1 , and VCAM were evident (Table 1). BMI was positively related to CRP $\left(\mathrm{R}^{2}=0.18, \mathrm{P}=0.000002\right)$ and remained related after adjustment for VAT and SAT. BMI was also positively related to fibrinogen $\left(\mathrm{R}^{2}=0.1, \mathrm{P}=0.002\right)$ and PAI-1 antigen $\left(\mathrm{R}^{2}=0.1, \mathrm{P}=0.01\right)$, but the significance for both was lost after adjustment for SAT and VAT. The only relation for SAT was a negative association with MMP9 after adjustment for $\mathrm{BMI}\left(\mathrm{R}^{2}=0.1, \mathrm{P}=0.01\right)$.

Conclusion: These results indicate that 1 ) VAT correlates positively with systemic inflammatory markers and some of these associations are independent of BMI, 2) BMI but not VAT strongly correlates with CRP independent of VAT and SAT, 3) SAT is negatively correlated with MMP9. Our results demonstrate a positive association between VAT and inflammatory markers in DM2. In contrast to previous studies, done predominantly on nondiabetic subjects, our results do not demonstrate an independent association between VAT and CRP or a positive association between SAT and any inflammatory marker.

Relation between VAT and Inflammatory Biomarkers

\begin{tabular}{|c|c|c|c|c|}
\hline Parameter & Multivariable Model & & Multivariable Model Plus BMI & \\
\hline & $\begin{array}{l}\text { Mean change }[\mathrm{CI}] \text { in biomarker } \\
\text { per } 10 \mathrm{~cm}^{3} \text { increase in VAT }\end{array}$ & P-value & $\begin{array}{l}\text { Mean change }[\mathrm{CI}] \text { in biomarker } \\
\text { per } 10 \mathrm{~cm}^{3} \text { increase in VAT }\end{array}$ & P-value \\
\hline CRP, mg/L & $2.84[1.39,5.79]$ & 0.004 & $1.29[-1.76,2.92]$ & 0.54 \\
\hline ICAM-1, pmol/L & $1.65[1.19,2.30]$ & 0.08 & $1.74[1.18,2.58]$ & 0.005 \\
\hline MCP, ng/L & $1.71[1.13,2.61]$ & 0.01 & $1.82[1.10,2.98]$ & 0.02 \\
\hline VCAM-1, pmol/L & $1.10[-1.17,1.42]$ & 0.45 & $1.41[1.05,1.89]$ & 0.02 \\
\hline Fibrinogen, $g / L$ & $1.22[-1.02,1.50]$ & 0.07 & $1.06[-1.21,1.36]$ & 0.65 \\
\hline MMP9, ng/mL & $1.35[-1.07,1.95]$ & 0.11 & $1.12[-1.38,1.72]$ & 0.62 \\
\hline PAI-1 antigen, $\mu \mathrm{g} / \mathrm{L}$ & $2.90[1.94,4.36]$ & $<0.0001$ & $2.79[1.73,4.50]$ & $<0.0001$ \\
\hline
\end{tabular}

$\mathrm{BMI}=$ body mass index; $\mathrm{VAT}=$ visceral adipose tissue; $\mathrm{CRP}=\mathrm{C}$-reactive protein; ICAM- $1=$ intercellular adhesion molecule $1 ; \mathrm{MCP}=$ monocyte chemoattractant protein; $\mathrm{VCAM}-1=$ vascular cell adhesion molecule $1 ; \mathrm{MMP} 9=$ matrix metallopeptidase 9; PAI-1 antigen=plasminogen activator inhibitor 1 antigen.

Supported by: Takeda Pharmaceuticals

\section{8}

Neck circumference is a powerful predictor of visceral adiposity and insulin resistance in morbid (WHO grade III) obesity

L.Y. Yang ${ }^{1}$, Y.P. Samarasinghe ${ }^{2}$, Y. Al-Zaman ${ }^{1}$, S.A. Amiel' ${ }^{2}$, S.J.B. Aylwin ${ }^{1}$ ${ }^{1}$ Department of Endocrinology, ${ }^{2}$ Diabetes Research Unit, King's College London, London, United Kingdom

Background and aims: There is large body of evidence suggesting that visceral adiposity (VA) is strongly implicated in the development of insulin resistance (IR), the metabolic syndrome and type 2 diabetes. There is however sparse data on the relationship between VA and IR in WHO grade III morbid obesity (BMI $\left.>40 \mathrm{~kg} / \mathrm{m}^{2}\right)$. Many studies investigating VA have used indirect measures of visceral adipose tissue (VAT) rather than gold standard imaging techniques. Waist circumference is widely used as one such measure. Although measuring waist circumference is a practical and transferable technique, anatomical landmarks required to obtain accurate readings are often difficult to locate in obese people, leading to high measurement variability. In addition there is little data on the value of waist circumference at BMIs beyond $34 \mathrm{~kg} / \mathrm{m}^{2}$. A small number of studies have shown that neck circumference is positively correlated with BMI, waist circumference and other factors associated with the metabolic syndrome in overweight and obese individuals with BMI $25-35 \mathrm{~kg} / \mathrm{m}^{2}$. The aims of this study were to assess the relationship between VA and IR in a WHO grade III obese cohort and ascertain whether correlations still exist between anthropometric measurements and CT quantified adipose tissue areas in these subjects.

Materials and methods: 13 non-diabetic patients with BMI $>40 \mathrm{~kg} / \mathrm{m}^{2}$ were recruited from King's College Hospital obesity clinic (10F; 3M, 7 Caucasian; 6 Afro-Caribbean, mean $(+/$-SD) age $47.8( \pm 11.4)$ years, weight $145.3( \pm 22.1)$ $\mathrm{kg}$, BMI $51.4( \pm 5.9) \mathrm{kg} / \mathrm{m}^{2}$. IR was calculated using HOMA-IR and a 2 hour $75 \mathrm{~g}$ OGTT was conducted. Measurements of BMI, waist, hip and neck circumference were taken at the same visit. A 2-slice CT scan at the L4 vertebral level and the greater trochanter was carried out. "Sliceomatic" (Tomovision, Montreal, Canada) image analysis software was used to measure VAT, abdominal subcutaneous adipose tissue (ASAT) and gluteal subcutaneous adipose tissue (GSAT) areas.

Results: Statistically significant correlations were demonstrated between VAT area and IR $\left(R^{2}=0.54, P=0.004\right)$. No relationships were established between ASAT, GSAT and IR. Neck circumference was the only anthropometric measurement to correlate significantly with VAT area $\left(R^{2}=0.57, P=0.003\right)$ and IR $\left(\mathrm{R}^{2}=0.45, \mathrm{P}=0.013\right)$. Waist circumference failed to show significant associations with VAT area $\left(\mathrm{R}^{2}=0.15, \mathrm{P}=0.19\right)$ or $\mathrm{IR}\left(\mathrm{R}^{2}=0.21, \mathrm{P}=0.12\right)$.

Conclusion: VA remains a strong and significant determinant of IR whilst subcutaneous fat shows no relationship with IR in WHO grade III obesity. Neck circumference surpasses all other anthropometric measurements as a powerful predictor of both VA and IR in this obese cohort. The ease of measurement and smaller likelihood for user errors make neck circumference a potentially useful adjunct to waist circumference in future. 


\section{9}

Gene expression profiling of the epicardial adipose tissue demonstrate a significant overexpression of the secretory phospholipase $A(2)$

A. Dutour ${ }^{1}$, C. Henegar ${ }^{2}$, A. Silaghi ${ }^{1}$, N. Naour ${ }^{2}$, B. Gaborit ${ }^{1}$, V. Achard ${ }^{1}$, K. Clement ${ }^{2}$

${ }^{1}$ Endocrinology, INSERM U626, Marseille, ${ }^{2}$ Les Cordeliers, INSERM UMRS 872, Paris, F-75006, France.

Background and aims: The epicardial adipose tissue (EAT) is a relatively neglected component of the heart in close contact with coronary vessels. This proximity suggests a potential pathophysiological role of the EAT in relation to the coronary disease. The objective of our study was to compare gene expression profile of paired biopsies of subcutaneous adipose tissue (SAT) and EAT, obtained from patients with coronary artery disease (CAD) or without CAD (NCAD), to identify target genes and functional pathways characterizing the transcriptomic signature of the EAT in CAD patients.

Materials and methods: We analyzed the transcriptomic signature of paired biopsies of SAT and EAT obtained from a series of 8 CAD and 8 NCAD patients. An integrative functional genomics approach was applied to identify relevant structural and functional themes annotating differentially expressed genes. Afterwards a co-expression networks analysis was performed to quantify relationships and relate co-regulated themes into a comprehensive map of transcriptional interactions characterizing the EAT. RT-PCR was used to confirm expression changes of some gene targets.

Results: Our analysis identified 2500 genes significantly overexpressed in EAT as compared to SAT of CAD subjects. The functional themes annotating these genes were mainly related to the cell cycle and the RNA processing and splicing. Surprisingly, themes related to the immune response and cell adhesion molecules were significantly overrepresented among those annotating genes up-related in the SAT, but not the EAT, of CAD patients. Among the genes annotated by significantly overrepresented functional themes, the secretory type II phospholipase A(2) (PLA2G2A) mRNA was highly increased in EAT as compared to SAT in both CAD (X13) and NCAD(X8) samples. RTPCR confirmed the significant overexpression of PLA2G2A mRNA in EAT $(\mathrm{p}=0.01)$ of both CAD and NCAD patients $(\mathrm{SAT}=10.8 \pm 4.6, \mathrm{EAT}=76.7$ \pm 17 ). Regarding a set of inflammatory genes, RT-PCR showed no difference in the expression levels of several pro-inflammatory cytokines (MCP 1, Il6, IL1 $\beta$ ) between SAT and EAT in each group, mostly confirming microarray results. However, they were increased in EAT of CAD compared to NCAD patients.

Conclusion: Our results demonstrate a significant over-expression of PLA2G2A in EAT, a phospholipase that was shown to be expressed in atherosclerotic lesions and in inflammatory conditions. These findings suggest a potentially important pathophysiological role of EAT in coronary atherogenesis. Supported by: PHRC

\section{PS 073 Lipid oriented therapies}

\section{0}

Specific metabolic effects of insulin detemir in human visceral adipocytes differentiated in vitro from precursor stromal cells S. Perrini ${ }^{1}$, A. Cignarelli ${ }^{1}$, L. Laviola ${ }^{1}$, M. Barbaro ${ }^{1}$, A. Conserva ${ }^{1}$, A. Peschechera ${ }^{1}$, A. Natalicchio ${ }^{1}$, A. Quagliara ${ }^{1}$, F. De Stefano ${ }^{1}$, M. Melchiorre ${ }^{1}$, M. De Fazio ${ }^{2}$, V. $\mathrm{Memeo}^{2}$, R. Giorgino', F. Giorgino ${ }^{1}$ ${ }^{1}$ D.E.T.O., Internal Medicine, Endocrinology and Metabolic Diseases, University of Bari, ${ }^{2}$ D.E.T.O., General Surgery, University of Bari, Italy

Background and aims: Clinical use of the acylated insulin analog detemir is associated with the stabilization of body weight, particularly in individuals with high BMI values. However, whether insulin detemir is able to directly modulate adipocyte metabolism, and if this insulin analog may exert peculiar metabolic effects on adipose tissue from different fat depots has not been determined. Therefore, the aim of this study was to evaluate metabolic responses induced by detemir, compared with regular human insulin, in human subcutaneous (SC) and visceral (V) adipocytes differentiated in vitro from precursor stromal cells.

Materials and methods: Paired biopsies from SC and V fat were obtained from 7 subjects $(3 \mathrm{~F} / 4 \mathrm{M}$, age $63 \pm 1$ years, BMI $25.9 \pm 1.4)$ with normal glucose tolerance and preserved insulin sensitivity (HOMA-IR=5.4 \pm 1.6 ). Preadipocytes were isolated from the stromal fraction of SC and V fat biopsies, respectively, and differentiated in vitro with dexamethasone, IBMX, insulin and rosiglitazone. Terminal differentiation of the adipocyte cultures was verified through morphological evaluation, Oil-O-Red staining, and assessment of gene expression of fat-specific molecules (PPARgamma, adiponectin, GLUT4). Glucose uptake was assessed through evaluation of $\left[{ }^{3} \mathrm{H}\right] 1,2$-deoxyD-glucose uptake rates.

Results: Under basal conditions, glucose uptake was $65 \%$ higher in V- as compared to SC-derived adipocytes $(165 \pm 13$ vs $99 \pm 12 \mathrm{pmol} / \mathrm{mcg} / \mathrm{min}$, $\mathrm{p}<0.05)$. In $\mathrm{SC}$ adipocytes the incubation with regular insulin induced a limited increase in glucose uptake $(+50 \%$ with regular insulin $100 \mathrm{nM}, \mathrm{p}=0.05$ vs. basal), while insulin detemir did not significantly modify glucose uptake rates ( $\mathrm{p}=0.40$ vs. basal). In $\mathrm{V}$ adipocytes, $100 \mathrm{nM}$ regular insulin induced a 3 -fold increase in glucose transport $(\mathrm{p}<0.05)$. However, insulin detemir appeared to be much more efficient, inducing 8 -fold and 5 -fold increases in glucose uptake at $100 \mathrm{nM}$ and $10 \mathrm{nM}$, respectively $(\mathrm{p}<0.05)$. The correlation of insulin-stimulated glucose uptake with clinical characteristics of the patients was then analyzed by regression analysis. Glucose uptake induced by insulin detemir was found to be inversely correlated with BMI $\left(\beta=-20.7, \mathrm{R}^{2}=0.87\right.$, $\mathrm{p}<0.05)$ and HOMA-IR $\left(\beta-70.7, \mathrm{R}^{2} 0.92, \mathrm{p}<0.05\right)$, whereas no correlation was found with regular insulin (BMI, $\beta=-3.6, \mathrm{R}^{2}=0.029, \mathrm{p}=$ n.s.; HOMA-IR, $\beta$ 20.6, $\mathrm{R}^{2} 0.08, \mathrm{p}=$ n.s.).

Conclusion: In conclusion, insulin detemir stimulates glucose uptake more efficiently than regular insulin in human visceral adipocytes, indicating that visceral adipose tissue is more important for insulin detemir than for regular insulin as a determinant of the effects on glucose metabolism. However, the inverse correlation between the effects on glucose uptake and the degree of adiposity/insulin resistance, which occurs for insulin detemir but not for regular insulin, suggests that the action of insulin detemir on visceral adipocytes is more prominently influenced by changes in total body fat, and this could be relevant to the weight-sparing effects of this insulin analog in obese type 2 diabetic patients.

Supported by: Novo Nordisk

\section{1}

Resistance training combined with a weight loss diet improves the lipid profile in adult hypercholesterolaemic obese women despite a significative decrease in circulating adiponectin

L. Forga ${ }^{1}$, F.J. Basterra-Gortari ${ }^{1}$, M. Izquierdo ${ }^{2}$, C. Martínez-Labari², M. García-Unciti ${ }^{3}$, M.J. Goñi ${ }^{1}$, E.M. Gorostiaga ${ }^{2}$

${ }^{1}$ Endocrinology, Hospital of Navarra, Pamplona, ${ }^{2}$ Research and Sports Medicine Center, Government of Navarra, Pamplona, ${ }^{3}$ Dietetic and Dietotherapy, University of Navarra, Pamplona, Spain

Background and aims: It has been shown that circulating adiponectin is positively associated with plasma HDL-C and negatively correlated with LDL-C and triglycerides. However, at present, both the physiological role of adiponectin on the improvement of lipid profile with body or fat mass loss and 
the effect of a progressive resistance training (RT) on circulating adiponectin are yet incompletely understood. The present study aimed to examine the relationships of plasma adiponectin and different body composition variables with lipid profile when a RT was applied to a group of adult hyperholesterolemic obese women with a concomitant weight-loss diet.

Materials and methods: The design was a 16 week randomized controlled trial. Thirty three women were assigned to 3 groups: a Control group (C; $\mathrm{n}=8$ ); a Diet group (WL; $\mathrm{n}=12$ ), with a caloric restriction of $500 \mathrm{Kcal} /$ day; and a Diet plus Resistance Training (WL+RT; $n=13$ ), with the same caloric restriction as WL group and a supervised resistance training program of 2 sessions/ week. Weight, energy intake, energy expenditure (acelerometry), volumes of different adipose tissue compartments and the muscle mass of thigh measured by magnetic resonance, and serum levels of estradiol, progesterone, adiponectin, total cholesterol (TC), HDL-cholesterol (HDL-C), measured LDL-cholesterol (LDL-C), VLDL-cholesterol (VLDL-C) and triglycerides (TG) were measured baseline and at week 16. ANOVA was used to determine differences among the three groups; Bonferroni was the post hoc procedure.

Results: Baseline characteristics were similar in the 3 groups. By week 16, no significant difference between $\mathrm{WL}$ and $\mathrm{WL}+\mathrm{RT}$ groups was observed neither in body mass, nor in abdominal fat or thigh fat. Compared to control group, body mass was significantly diminished in $\mathrm{WL}$ and $\mathrm{WL}+\mathrm{RT}$ groups by $-6.3 \%$ and $-7.7 \%(\mathrm{p}<0.001)$, respectively; whereas abdominal subcutaneous adipose tissue decreased by $-18.3 \%$ and $-21.4 \%(\mathrm{p}<0.001)$, and thigh subcutaneous fat by $-16.4 \%$ and $-18.9 \%(\mathrm{p}<0.001)$, respectively. Likewise, visceral fat mass was reduced in WL and WL+RT groups by $-19.9 \%$ and $-20.5 \%(\mathrm{p}<0.001)$, respectively. As expected, WL led to a significant lost in thigh muscle mass $(-3.5 \%)$; however, in the $\mathrm{WL}+\mathrm{RT}$ group, thigh muscle mass was maintained. Moreover, in the WL group no variation neither in lipid profile nor in adiponectin was observed. In turn, in WL+RT group we observed a decrease in: adiponectin $(-8.3 \%, \mathrm{P}<0.05)$, LDL-C $(-8.6 \%, \mathrm{P}<0.01)$, CT $(-13.6 \%, \mathrm{P}<0.01)$ and HDL-C $(-11.7 \%, \mathrm{P}<0.001)$, whereas TC/ HDL-C ratio was maintained unaltered. In a multiple linear regression analysis, the most significant factor impacting on total cholesterol in this WL+RT group (95\% of its variance) was LDL-cholesterol, even after accounting for the effects of age, BMI, and adiponectin.

Conclusion: In adult hypercholesterolemic obese women, resistance training ( 2 sessions/ week) seems to play an essential role in the improvement of the lipid profile, in spite of a significant decrease in circulating adiponectin. Supported by: a grant from the Spanish "Ministerio de Sanidad y Consumo" (in part)

\section{2}

Amelioration of glucotoxicity with insulin detemir alone or combined with premeal insulin aspart improves plasma lipoproteins, insulin secretion and hepatic steatosis in poorly controlled T2DM patients

K. Cusi ${ }^{1}$, S. Wang ${ }^{1}$, P. Kumar ${ }^{1}$, B. Balas ${ }^{1}$, R. Ali ${ }^{1}$, C. Darland ${ }^{1}$, A. Gastaldelli ${ }^{1}$, V. Kochunova ${ }^{2}$, Q. Peng ${ }^{2}$, J. Hardies ${ }^{2}$, M. Mathew ${ }^{1}$

${ }^{1}$ Medicine, UTHSCSA, ${ }^{2}$ Radiology, UTHSCSA, San Antonio, United States

Background and aims: Basal insulin therapy alone is commonly used in primary care although the addition of pre-meal insulin allows optimal control of pts with type 2 diabetes mellitus (T2DM). We examined the effects on glycemic and lipid control, insulin secretion, intramyocellular lipid and hepatic fat content, and subcutaneous and visceral fat of basal/premeal insulin therapy in poorly controlled pts with T2DM.

Materials and methods: 24 patients with T2DM $(59 \pm 3 \mathrm{yr}, \mathrm{BMI}: 31.4 \pm 1.4 \mathrm{~kg} /$ $\mathrm{m}^{2}$; reporting 17) were treated with a bedtime dose of insulin detemir for 3 months, followed by pre-meal insulin aspart for another 3 months. The insulin dose was adjusted every 3 to 5 days to target a fasting plasma glucose (FPG) of 70-100 mg/dl and 2-hour posprandial glucose $\leq 160 \mathrm{mg} / \mathrm{dl}$. Patients were admitted 3 times: before treatment, after 3 months of bedtime detemir insulin only, and after another 3 months of adding insulin aspart to the detemir insulin regimen. On each admission they had the following tests: 1) FPG, $A_{1 c}$, fructosamine and day-long plasma glucose, FFA, C-peptide and insulin profile (Day 1); 2) Lipoprotein particle number/size by NMR; 3) Glucose-stimulated insulin secretion (GSIS) during a hyperglycemic clamp (Day 2); 4) Total body fat by DXA; 5) Liver (LFAT) and muscle (IMCL) fat content by MRS, and abdominal fat by MRI.

Results: Insulin detemir significantly reduced FPG (from $178 \pm 18$ to $111 \pm 13 \mathrm{mg} / \mathrm{dl}$ ), fructosamine (from $325 \pm 10$ to $243 \pm 11 \mu \mathrm{mol} / \mathrm{l}$ ), $\mathrm{A}_{1 \mathrm{c}}$ (from $8.9 \pm 0.3 \%$ to $7.4 \pm 0.2 \%$ ), day-long glucose (from $240 \pm 2$ to $161 \pm 4 \mathrm{mg} / \mathrm{dl}$, all $\mathrm{p}<0.01$ ) and FFA (from $394 \pm 54$ to $328 \pm 29 \mathrm{nmol} / \mathrm{l}$ ). Detemir had a favora-

ble impact on the lipid profile as it reduced VLDL and LDL particle number (both $\mathrm{p}<0.02$ ) and increased LDL and HDL size (both $\mathrm{p}<0.05$ ). Insulin secretion improved as evidenced by a $\sim 2$ fold increase in day-long C-peptide and $\sim 2$-fold higher $2^{\text {nd }}$ phase GSIS (both $\mathrm{p}<0.01$ ). The combination of insulin detemir and aspart further improved glycemic control (FPG: 100 \pm 7 $\mathrm{mg} / \mathrm{dl}$, Alc: $6.9 \pm 0.2 \%$ and fructosamine: $226 \pm 6 \mu \mathrm{mol} / \mathrm{l}$; all $\mathrm{p}<0.01 \mathrm{vs}$. insulin detemir alone), increased $2^{\text {nd }}$ phase GSIS by $50 \%$ and HDL particle size $(\mathrm{p}<0.02)$. Total body fat $(\mathrm{DXA})$ did not increase significantly with either insulin regimen $(27.0 \pm 2.2 \%, 27.0 \pm 2.1 \%, 27.6 \pm 2.2 \%$, respectively, NS), nor did visceral fat. LFAT showed a trend to decrease at 3 and 6 months (from $17.9 \pm 7.1 \%$ to $7.2 \pm 2.1 \%$ and $10.5 .0 \pm 3.8 \%, \mathrm{p}=0.13$ and 0.19 vs. pretreatment, respectively) while IMCL was unchanged (from $0.77 \pm 0.38 \%$ to $0.41 \pm 0.11 \%$ and $0.83 \pm 0.20 \%$, both NS vs. pretreatment, respectively). Insulin sensitivity was unchanged. Intensified insulin therapy was not associated with severe hypoglycemia or any other adverse event.

Conclusion: In poorly controlled patients with T2DM, basal insulin detemir therapy aiming at a normal fasting plasma glucose concentration is a safe and effective way to achieve good glycemic control. Amelioration of glucotoxicity is associated with better glucose-stimulated insulin secretion, a less atherogenic apolipoprotein profile and a reduction in hepatic steatosis. Additional pre-meal rapid-acting insulin aspart allows to further optimize metabolic control.

Supported by: Novo Nordisk Inc. (investigator-initiated study)

\section{3}

Effect of ezetimibe/simvastatin (E/S) versus atorvastatin (A) on lipoprotein subclasses in type 2 diabetes (T2DM) patients with hypercholesterolaemia (HC)

J.E. Tomassini ${ }^{1}$, T. Mazzone ${ }^{2}$, R.B. Goldberg ${ }^{3}$, J.R. Guyton ${ }^{4}$, R.S. Weinstock ${ }^{5}$, A. Polis ${ }^{1}$, A.M. Tershakovec ${ }^{1}$

${ }^{1}$ Merck \& Co., Inc., N. Wales, ${ }^{2}$ Departments of Medicine and Pharmacology, University of Illinois at Chicago, ${ }^{3}$ Diabetes Research Institute, Miller School of Medicine, Univ. of Miami, ${ }^{4}$ Medical Center, Duke Univ., Durham, ${ }^{5}$ Division of Endocrinology, Diabetes and Metabolism, SUNY Upstate Medical University and VA Medical Center, Syracuse, United States

Background and aims: Patients with T2DM are at increased risk for cardiovascular disease, attributed partly to dyslipidemia characterized by elevated triglyerides (TG) levels and TG-rich proteins (VLDL, IDL), low HDL-C levels, and a predominance of small, dense, LDL particles. This analysis assessed the effect of the usual, recommended starting doses and next higher doses of $\mathrm{E} / \mathrm{S}$ and $\mathrm{A}$ on the cholesterol content of lipoprotein subclasses in patients with T2DM and HC.

Materials and methods: A post-hoc analysis of the data obtained from a multicenter, double-blind, randomized, $6 \mathrm{wk}$ trial which demonstrated the lipid-altering efficacy and safety of the usual, recommended starting doses of E/S (10/20mg) vs A (10 and $20 \mathrm{mg}$ ) and next higher doses of E/S (10/40 $\mathrm{mg}$ ) vs A (40 mg). The effect of E/S and A on the \% change from baseline in cholesterol content of lipoprotein subclasses was assessed by Vertical Auto Profile II (VAP II) and LDL subclass pattern by segmented gradient gel electrophoresis ( $\left.\mathrm{S}_{3} \mathrm{GGE}\right)$ in 1013 patients with T2DM and HC in the modified Intent-To-Treat population (ANCOVA model).

Results: E/S (10/20mg) vs A (10 and $20 \mathrm{mg})$ and E/S (10/40 mg) vs A (40 $\mathrm{mg}$ ), provided significantly greater reductions from baseline at $6 \mathrm{wks}$ in the cholesterol content of lipoprotein subfractions including $\mathrm{LDL}_{1}, \mathrm{LDL}_{2}, \mathrm{LDL}_{3}$, LDLC $^{r}$, total IDL, and total VLDL (see table) and in IDL , IDL $_{2}$ and VLDL . $_{3}$. Greater improvements were also observed in $\mathrm{HDL}_{3}$ at $\mathrm{E} / \mathrm{S} 20 \mathrm{mg}$ vs $\mathrm{A} 20 \mathrm{mg}$, in $\mathrm{VLDL}_{1+2}$ at $\mathrm{E} / \mathrm{S} 20 \mathrm{mg}$ vs $\mathrm{A} 10 \mathrm{mg}$, and in $\mathrm{VLDL}_{1+2}$ and $\mathrm{HDL}_{3}$ at $\mathrm{E} / \mathrm{S} 40$ $\mathrm{mg}$ vs $\mathrm{A} 40 \mathrm{mg}$. Changes in $\mathrm{LDL}_{4}$ and R-LDL subclass pattern $(\mathrm{A}, \mathrm{B}, \mathrm{I})$ were comparable for both treatments.

Conclusion: At 6 weeks, the usual, recommended, starting doses and next highest doses of E/S reduced the cholesterol content associated with most atherogenic lipoprotein subfractions significantly more than A in T2DM patients with $\mathrm{HC}$ at all dose comparisons. In addition to more effective reduction in true LDL-C level, our results demonstrate that E/S reduces TGrich lipoprotein fractions IDL and VLDL more than A. This suggests that the combination $\mathrm{E} / \mathrm{S}$ may provide benefit by improving both cholesterol-rich and triglyceride-rich atherogenic lipoprotein subfractions in these patients. The clinical significance of such effects awaits further assessment. 


\begin{tabular}{|c|c|c|c|c|c|c|}
\hline$[\mathrm{mg} / \mathrm{dL}]$ & & $\begin{array}{l}\text { A } 10 \\
\mathrm{~N}=205\end{array}$ & $\begin{array}{l}E / S 10 / 20 \\
N=206\end{array}$ & $\begin{array}{l}\text { A } 20 \\
\mathrm{~N}=205\end{array}$ & $\begin{array}{l}E / S 10 / 40 \\
N=193\end{array}$ & $\begin{array}{l}\text { A } 40 \\
N=204\end{array}$ \\
\hline $\mathrm{LDL}_{1}-\mathrm{C}$ & $\begin{array}{l}\text { Baseline† } \\
\% \text { change } \neq \\
\text { Differences }\end{array}$ & $\begin{array}{l}23.1 \\
-36.4 \\
-16.6^{\star * *}\end{array}$ & $\begin{array}{l}23.5 \\
-52.6 \\
- \\
\end{array}$ & $\begin{array}{l}24.0 \\
-44.4 \\
-8.2^{\star * *}\end{array}$ & $\begin{array}{l}24.0 \\
-57.2 \\
- \\
\end{array}$ & $\begin{array}{l}22.6 \\
-50.6 \\
-7.0^{\star * *}\end{array}$ \\
\hline $\mathrm{LDL}_{2}-\mathrm{C}$ & $\begin{array}{l}\text { Baseline } \dagger \\
\% \text { change } \neq \\
\text { Difference\$ }\end{array}$ & $\begin{array}{l}28.0 \\
-28.2 \\
-15.5^{\star * *} \\
\end{array}$ & $\begin{array}{l}30.8 \\
-44.1 \\
- \\
\end{array}$ & $\begin{array}{l}32.3 \\
-39.2 \\
-5.4^{* *} \\
\end{array}$ & $\begin{array}{l}31.5 \\
-51.6 \\
- \\
\end{array}$ & $\begin{array}{l}29.9 \\
-42.2 \\
-8.1^{\star * *} \\
\end{array}$ \\
\hline $\mathrm{LDL}_{3}-\mathrm{C}$ & $\begin{array}{l}\text { Baseline† } \\
\% \text { change } \neq \\
\text { Differences }\end{array}$ & $\begin{array}{l}53.6 \\
-35.0 \\
-13.1^{\star * *}\end{array}$ & $\begin{array}{l}51.8 \\
-48.2 \\
- \\
\end{array}$ & $\begin{array}{l}53.9 \\
-40.1 \\
-7.1^{\star * *}\end{array}$ & $\begin{array}{l}52.5 \\
-53.0 \\
- \\
\end{array}$ & $\begin{array}{l}55.3 \\
-45.8 \\
-5.7^{\star * *}\end{array}$ \\
\hline $\mathrm{LDL}-\mathrm{C}^{\mathrm{r}}$ & $\begin{array}{l}\text { Baseline } \dagger \\
\% \text { change } \neq \\
\text { Differences }\end{array}$ & $\begin{array}{l}117.0 \\
-32.8 \\
-14.3^{\star * *} \\
\end{array}$ & $\begin{array}{l}119.5 \\
-47.4 \\
- \\
\end{array}$ & $\begin{array}{l}120.0 \\
-40.4 \\
-6.8^{\star * *} \\
\end{array}$ & $\begin{array}{l}120.0 \\
-52.8 \\
- \\
\end{array}$ & $\begin{array}{l}119.0 \\
-46.6 \\
-6.2^{* * *} \\
\end{array}$ \\
\hline IDL & $\begin{array}{l}\text { Baseline } \dagger \\
\% \text { change } \neq \\
\text { Difference\$ }\end{array}$ & $\begin{array}{l}21.0 \\
-50.0 \\
-22.2^{\star * *}\end{array}$ & $\begin{array}{l}21.0 \\
-71.8 \\
- \\
\end{array}$ & $\begin{array}{l}20.0 \\
-60.9 \\
-11.8^{\star * *}\end{array}$ & $\begin{array}{l}21.0 \\
-75.0 \\
- \\
\end{array}$ & $\begin{array}{l}19.0 \\
-66.7 \\
-7.9^{* * *}\end{array}$ \\
\hline VLDL & $\begin{array}{l}\text { Baseline† } \\
\% \text { change } \neq \\
\text { Differences }\end{array}$ & $\begin{array}{l}29.0 \\
-26.5 \\
-7.3^{\star * *} \\
\end{array}$ & $\begin{array}{l}29.0 \\
-34.3 \\
- \\
\end{array}$ & $\begin{array}{l}29.0 \\
-29.7 \\
-3.7^{\star *} \\
\end{array}$ & $\begin{array}{l}30.0 \\
-36.7 \\
- \\
\end{array}$ & $\begin{array}{l}29.0 \\
-32.0 \\
-6.5^{\star * *}\end{array}$ \\
\hline $\mathrm{VLDL}_{3}$ & $\begin{array}{l}\text { Baseline } \dagger \\
\% \text { change } \neq \\
\text { Differences }\end{array}$ & $\begin{array}{l}16.0 \\
-25.0 \\
-8.3^{* * *}\end{array}$ & $\begin{array}{l}16.5 \\
-35.1 \\
-\end{array}$ & $\begin{array}{l}16.0 \\
-29.4 \\
-4.7^{\star * *}\end{array}$ & $\begin{array}{l}17.0 \\
-36.8 \\
-\end{array}$ & $\begin{array}{l}16.0 \\
-31.0 \\
-6.3^{* * *}\end{array}$ \\
\hline
\end{tabular}

$\dagger$ median; $¥ \%$ change from baseline

§Treatment differences= E/S 10/20mg minus A 10mg, E/S 10/20mg minus A 20mg, and E/S 10/40mg minus A 40mg

greal index of cholesterol associated with true LDL particle, excludes IDL and Lp(a)

${ }^{* *} \mathrm{P}<0.01 ;{ }^{* *} \mathrm{p}<0.001$ by ANCOVA model with terms for treatment, Visit $2 \mathrm{LDL}-\mathrm{C}$ stratum $(\geq 100-<130 \mathrm{mg} / \mathrm{dL}, \geq 130-<160 \mathrm{mg} / \mathrm{dL}, \geq 160-<190 \mathrm{mg} / \mathrm{dL}$,

$\geq 190 \mathrm{mg} / \mathrm{dL}$ ), and baseline value of the dependent variable

Supported by: Merck/Schering-Plough

Effect of ezetimibe/simvastatin (E/S) plus extended-release niacin (N) in type IIa and IIb hyperlipidaemia (HL) patients with diabetes (DM) or metabolic syndrome (MS)

J.R. Guyton ${ }^{1}$, B.G. Brown', S. Fazio ${ }^{3}$, J. Lin', A. Polis, ${ }^{4}$ J.E. Tomassini', A. Shah ${ }^{4}$, A.M. Tershakovec ${ }^{4}$

${ }^{1}$ Department of Medicine, Duke University Medical Center, Durham, ${ }^{2}$ Division of Cardiology, University of Washington, Seattle, ${ }^{3}$ Vanderbilt Lipid Laboratory, Vanderbilt University Medical Center, Nashville, ${ }^{4}$ Merck \& Co., Inc., N. Wales, United States

Background and aims: Patients with DM and MS are at increased risk for CVD attributed to high plasma triglycerides (TG), low HDL, and small, dense LDL. When LDL-C is also elevated, combination drug treatment should be considered. Combination $\mathrm{E} / \mathrm{S}+\mathrm{N}$ has been shown to substantially improve the lipid profile of patients with type IIa or IIb hyperlipidemia vs E/S and vs $\mathrm{N}$ alone. Here we assessed the effect of these treatments on lipid parameters in subgroups of patients with DM, MS without DM (MS/nonDM), or without either condition (nonDM/nonMS).

Materials and methods: In a double-blind, randomized 24 wk study (pre-defined initial phase of a $64 \mathrm{wk}$ study), $1220 \mathrm{HL}$ patients were treated with E/S $(10 / 20 \mathrm{mg})+\mathrm{N}(0.5-2 \mathrm{~g})$ vs $\mathrm{E} / \mathrm{S}(10 / 20 \mathrm{mg})$ or N $(0.5-2 \mathrm{~g})$. The effect of these treatments on various lipid parameters (LDL-C, TG, HDL-C, non-HDL-C, total cholesterol, Apo B, Apo A-I), lipid ratios (TC/HDL-C, LDL-C/HDL-C, Apo B/Apo A-I, non-HDL-C/HDL-C) and hsCRP were assessed within subgroups of patients with DM, MS/nonDM, or nonDM/nonMS by ANCOVA in the completers $(n=765)$ population. Clinical and laboratory adverse events (AEs) including fasting glucose (FG) increases were monitored in the modified Intent-To-Treat (mITT, $\mathrm{n}=757$ ) population for these subgroups.

\begin{tabular}{|c|c|c|c|c|c|c|}
\hline & \multicolumn{2}{|l|}{$\mathrm{DM}\left(\mathrm{n}=113^{\star}\right)$} & \multicolumn{2}{|c|}{ MS/nonDM $\left(n=301^{\star}\right)$} & \multicolumn{2}{|c|}{ nonDM/nonMS $\left(n=351^{\star}\right)$} \\
\hline & $\mathrm{E} / \mathrm{S}+\mathrm{N}$ vs $\mathrm{N}$ & $\mathrm{E} / \mathrm{S}+\mathrm{N}$ vs $\mathrm{E} / \mathrm{S}$ & $\mathrm{E} / \mathrm{S}+\mathrm{N}$ vs $\mathrm{N}$ & $\mathrm{E} / \mathrm{S}+\mathrm{N}$ vs $\mathrm{E} / \mathrm{S}$ & $\mathrm{E} / \mathrm{S}+\mathrm{N}$ vs $\mathrm{N}$ & $\mathrm{E} / \mathrm{S}+\mathrm{N}$ vs $\mathrm{E} / \mathrm{S}$ \\
\hline $\begin{array}{l}\text { LDL-C \% difference } \dagger \\
(95 \% \mathrm{CI})\end{array}$ & $\begin{array}{l}-37.0 \\
(-45.0,-29.1)\end{array}$ & $\begin{array}{l}0.4 \\
(-6.9,7.7) \\
\end{array}$ & $\begin{array}{l}-40.0 \\
(-45.1,-34.9)\end{array}$ & $\begin{array}{l}-2.9 \\
(-7.2,1.4) \\
\end{array}$ & $\begin{array}{l}-38.0 \\
(-42.3,-33.7)\end{array}$ & $\begin{array}{l}-8.5 \\
(-12.8,-4.2)\end{array}$ \\
\hline $\begin{array}{l}\text { Non-HDL-C \% difference } \dagger \\
(95 \% \mathrm{CI})\end{array}$ & $\begin{array}{l}-32.9 \\
(-40.6,-25.2)\end{array}$ & $\begin{array}{l}-3.1 \\
(-10.2,4.0)\end{array}$ & $\begin{array}{l}-32.3 \\
(-37.2,-27.3)\end{array}$ & $\begin{array}{l}-6.2 \\
(-10.4,-2.0)\end{array}$ & $\begin{array}{l}-35.0 \\
(-39.2,-30.8)\end{array}$ & $\begin{array}{l}-10.4 \\
(-14.6,-6.3) \\
\end{array}$ \\
\hline $\begin{array}{l}\text { HDL-C \% difference } \dagger \\
(95 \% \mathrm{CI})\end{array}$ & $\begin{array}{l}5.8 \\
(-3.4,15.0) \\
\end{array}$ & $\begin{array}{l}19.7 \\
(11.3,28.1) \\
\end{array}$ & $\begin{array}{l}-1.2 \\
(-7.1,4.7) \\
\end{array}$ & $\begin{array}{l}21.9 \\
(17.0,26.9) \\
\end{array}$ & $\begin{array}{l}3.3 \\
(-1.6,8.3) \\
\end{array}$ & $\begin{array}{l}23.1 \\
(18.2,28.1) \\
\end{array}$ \\
\hline $\begin{array}{l}\text { TG \% difference } \\
(95 \% \mathrm{CI}) \ddagger \\
\end{array}$ & $\begin{array}{l}-16.6 \\
(-27.7,-6.0) \\
\end{array}$ & $\begin{array}{l}-21.2 \\
(-30.2,-10.5) \\
\end{array}$ & $\begin{array}{l}-4.9 \\
(-11.0,1.5) \\
\end{array}$ & $\begin{array}{l}-18.0 \\
(-24.0,-12.1) \\
\end{array}$ & $\begin{array}{l}-16.2 \\
(-23.5,-9.1) \\
\end{array}$ & $\begin{array}{l}-19.2 \\
(-25.9,-12.9)\end{array}$ \\
\hline $\begin{array}{l}\text { FG \% difference } \$ \\
(95 \% \mathrm{CI})\end{array}$ & $\begin{array}{l}-0.2 \\
(-6.7,6.2)\end{array}$ & $\begin{array}{l}2.9 \\
(-3.2,8.9)\end{array}$ & $\begin{array}{l}-2.2 \\
(-6.3,1.9)\end{array}$ & $\begin{array}{l}3.7 \\
(0.2,7.2) \\
\end{array}$ & $\begin{array}{l}-0.1 \\
(-3.6,3.4)\end{array}$ & $\begin{array}{l}2.1 \\
(-1.4,5.5)\end{array}$ \\
\hline
\end{tabular}

$\mathrm{E} / \mathrm{S}=$ ezetimibe/simvastatin $(10 / 20 \mathrm{mg}) ; \mathrm{N}=$ extended-release niacin $(0.5-2 \mathrm{~g}), \mathrm{DM}=$ diabetes, $\mathrm{MS}=$ metabolic syndrome; FG = fasting glucose ${ }^{*}$ Completers include all patients with a baseline value who received at least $24 \mathrm{wk}$ of active study therapy and had an on-treatment value at the maximum titrated dose [i.e. $2 \mathrm{~g}$ of niacin (ER) for both $\mathrm{E} / \mathrm{S}+\mathrm{N}$ and $\mathrm{N}$ treatment groups]; $\mathrm{n}$ represents the number of patients in the analysis of the primary endpoint (percent change from baseline to wk 24 in LDL-C).

$\dagger$ LS means of treatment difference based on ANCOVA model with terms for treatment, DM/MS category, baseline LDL-C, baseline TG, gender, and treatment by DM/MS category interaction.

$\ddagger$ Hodges-Lehmann estimate of the median difference between treatments with a corresponding distributions free CI based on Wilcoxon“s rank sum test. $\$$ Analysis based on mITT population (all randomized patients with a baseline value and at least one post-baseline value with A Last Observation Carried Forward [LOCF] approach to impute any missing data for the wk 24 assessment), and included $n=112,299,346$ patients respectively in the DM, MS without DM and neither DM nor MS subgroups.

I LS means of treatment difference based on ANCOVA model with terms for treatment, DM/MS category, baseline fasting glucose, and treatment by DM/MS category interaction. 
Results: E/S+N decreased LDL-C and non-HDL-C significantly more than $\mathrm{N}$ in all subgroups and compared to $\mathrm{E} / \mathrm{S}$ in the nonDM/nonMS group (see table). Moreover, E/S+N treatment decreased non-HDL-C significantly compared to $\mathrm{E} / \mathrm{S}$ in the MS group. HDL-C increases were greater with $\mathrm{E} / \mathrm{S}+\mathrm{N}$ vs $\mathrm{E} / \mathrm{S}$ and similar vs $\mathrm{N}$ for all subgroups. $\mathrm{E} / \mathrm{S}+\mathrm{N}$ treatment reduced TG more than $\mathrm{N}$ and $\mathrm{E} / \mathrm{S}$ treatments in all subgroups, except for $\mathrm{E} / \mathrm{S}+\mathrm{N}$ vs $\mathrm{N}$ in $\mathrm{MS}$ patients. Mean FG trended higher in the N treatment groups vs E/S group, peaking at 8-12 wks and declining by 24 wks. New onset DM was identified as 2 consecutive $F G \geq 126 \mathrm{mg} / \mathrm{dL}$ or initiation of antidiabetic medication. Among MS patients, $8.8 \%$ met this criterion for $\mathrm{E} / \mathrm{S}+\mathrm{N}, 1.7 \%$ for $\mathrm{E} / \mathrm{S}$ and $5.1 \%$ for $\mathrm{N}$ groups. Among nonDM/nonMS patients the proportions were $1.2 \%(\mathrm{E} / \mathrm{S}+\mathrm{N}), 0 \%(\mathrm{E} / \mathrm{S})$ and $0 \%(\mathrm{~N})$.

Conclusion: The effects of $\mathrm{E} / \mathrm{S}+\mathrm{N}$ on lipid/lipoprotein profiles in subgroups of patients with or without DM or MS are consistent with the results obtained in the full HL cohort of the study. FG levels worsened more commonly with $\mathrm{MS}$ and with $\mathrm{N}$-containing therapy. Triple combination $\mathrm{E} / \mathrm{S}+\mathrm{N}$ is a treatment option for dyslipidemia in patients with DM and MS, but requires monitoring and possibly treating glucose changes as per the Niaspan label. Supported by: Merck/Schering-Plough

\section{5}

Rosuvastatin $20 \mathrm{mg}$ restores normal HDL apoA-I kinetics in type 2 diabetes

B. Vergès ${ }^{1,2}$, E. Florentin ${ }^{2}$, S. Baillot-Rudoni ${ }^{1}$, S. Monier ${ }^{2}$, J. Petit ${ }^{1,2}$, P. Gambert ${ }^{2}$, L. Duvillard ${ }^{2}$

${ }^{1}$ Endocrinology, Diabetology, Hôpital du Bocage, $\mathrm{CHU},{ }^{2}$ Biochemistry, INSERM CRI 866, Dijon, France

Background and aims: Patients with type 2 diabetes show an accelerated catabolism of HDL particles leading to a significant reduction of their plasma residence time, which may be detrimental. Rosuvastatin is the most powerful statin to reduce plasma LDL-cholesterol but its effects on the metabolism of HDL remain unknown, in type 2 diabetes.

Materials and methods: This prompted us to perform a randomised double blind cross-over trial of six-week treatment period with placebo or rosuvastatin $20 \mathrm{mg} / \mathrm{d}$ in 8 patients with type 2 diabetes treated with oral glucose lowering agents. In each patient, an in vivo kinetic study of HDL apoA-I with ${ }^{13} \mathrm{C}$ leucine was performed at the end of each treatment period. Moreover, a similar kinetic study was performed in 8 non-diabetic normolipidemic controls. Results: Rosuvastatin $20 \mathrm{mg}$ significantly reduced plasma LDL-cholesterol, triglycerides(TG) and HDL-TG. HDL apoA-I FCR was significantly reduced by rosuvastatin $(0.25 \pm 0.06$ vs $0.32 \pm 0.07 \mathrm{pool} / \mathrm{day}, \mathrm{p}=0.011)$ leading to a significant increase in plasma HDL apoA-I residence time ( $4.21 \pm 1.07$ vs $3.30 \pm 0.73$ day, $\mathrm{p}=0.011)$. Treatment with rosuvastatin was associated with a concomitant reduction in HDL apoA-I production rate (PR) $(12.79 \pm 3.98$ vs $16.19 \pm 4.31$ $\mathrm{mg} / \mathrm{kg}$.day, $\mathrm{p}=0.011$ ). The decrease in HDL apoA-I FCR, induced by rosuvastatin $20 \mathrm{mg}$ was correlated with the reduction in plasma $\mathrm{TG}(\mathrm{r}=0.75, \mathrm{p}=0.031)$ and in HDL-TG $(\mathrm{r}=0.84, \mathrm{p}=0.009)$. Treatment with rosuvastatin $20 \mathrm{mg} / \mathrm{d}$ restored, in patients with type 2 diabetes, HDL apoA-I FCR and PR values which were not significantly different from controls (apoA-I FCR: $0.25 \pm 0.06 \mathrm{vs} 0.20 \pm 0.06 \mathrm{pool} /$ day, $\mathrm{p}=0.16$ and apoA-I PR: $12.79 \pm 3.98$ vs $12.19 \pm 1.74 \mathrm{mg} / \mathrm{kg}$.day, $\mathrm{p}=0.75$ ).

Conclusion: Rosuvastatin induces significant modification of HDL apoA-I kinetics in patients with type 2 diabetes. Rosuvastatin $20 \mathrm{mg}$ is responsible for a $22 \%$ reduction in HDL apoA-I catabolism. Rosuvastatin restore to normal the increased HDL tun-over observed in type 2 diabetes. These kinetic modifications induced by rosuvastin may have beneficial effects by increasing HDL plasma residence time. Supported by: AstraZeneca

\section{6}

A molecular basis for the cell-specific activation of the peroxisome proliferator activator receptor PPAR gamma

P.C. Lefebvre ${ }^{1}$, B. Lefebvre ${ }^{1}$, A. Guédin ${ }^{1}$, N. Hennuyer ${ }^{1}$, C. Dacquet ${ }^{2}$, A. Ktorza ${ }^{2}$, B. Staels ${ }^{1}$

${ }^{1}$ Inserm U545, Atherosclerosis Dept- Pasteur Institute of Lille, ${ }^{2}$ SERVIER, Division of Metabolic Diseases, Suresnes, France

Background and aims: Thiazolidinediones (TZDs) are PPARgamma ligands highly efficient in gaining stable glycaemic control, in maintaining pancreatic beta cell functions and in improving insulin sensitivity of peripheral organs. Their clinical use is however hampered by side-effects such as oedema, os- teoporosis and decreased hemoglobin levels, the origin of which being supposedly linked to renal, bone and vascular PPARgamma-mediated effects. These unwanted effects may thus potentially be circumvented through the design of tissue-selective PPARgamma activators or SPPARMs. PPARgamma transcriptional activity is known to be regulated, as that of all other nuclear receptors, through protein-protein interactions, and most especially through the orchestrated dismissal and recruitment of transcriptional corepressors and coactivators respectively. We have therefore designed an experimental strategy aiming at identifying factor(s) that may regulate PPARgamma transcriptional activity in a cell-selective manner.

Materials and methods: Using PPARgamma as a bait, we have characterized its interaction with several cofactors in preadipocytes and adipocyte cell lines. We modulated the level of expression of these cofactors by RNA interference or by overexpression in these cell lines and studied the functional consequences of these altered expression levels on the transcriptional activity of PPARgamma-regulated chimaeric and endogenous genes. The interaction of these cofactors with PPARgamma was also monitored in intact cells using the one- and the two-hybrid technologies, as well as chromatin immunoprecipitation assays.

Results: We observed that the transcriptional corepressor SMRT is a major regulator of the transcriptional response of PPARgamma-containing heterodimers to agonist treatment. The ability of a reference PPARgamma agonist (Rosiglitazone) to trigger SMRT dismissal from the PPAR-containing heterodimer was strictly conditioned by the nature of the RXR isotype integrated into the heterodimer. The integration of a given RXR isotype in the heterodimer yielded either "SMRT-releasing" or "SMRT-tethered" heterodimers. In cellular backgrounds exhibiting an equivalent expression of RXR isotypes, we found that PPARgamma responsiveness to agonist stimulation was blunted compared to that observed in cells expressing preferentially the "SMRT-releasing" isotype. Similar results were obtained when manipulating RXR isotype expression levels through overexpression or RNA interference.

Conclusion: Our data show that PPARgamma transcriptional activity can be modulated in a tissue-specific manner through the variation of expression of RXR isotypes, opening new perspectives for the search of novel ligands which, upon the specific modulation of this interaction, will restrict or increase the transcriptional activity of PPARgamma.

Supported by: Servier

\section{7}

CB1- receptor blockade induces trans-differentiation towards an insulinsensitive brown fat phenotype in white adipocytes

N. Perwitz ${ }^{1}$, J. Wenzel ${ }^{1}$, I. Wagner ${ }^{1}$, J. Buening ${ }^{1}$, M. Drenckhan ${ }^{1}$, M. Ristow ${ }^{2}$, J. Klein ${ }^{1}$

${ }^{1}$ Department of Internal Medicine 1, University of Luebeck, ${ }^{2}$ Department of Human Nutrition, University of Jena, Germany

Background and aims: The endocannabinoid (EC) system is emerging as a key player in the control of energy homeostasis. It stimulates a positive energy balance via both central and peripheral pathways. CB1 cannabinoid receptor knockout mice are resistant to diet-induced obesity, and blockade of the $\mathrm{CB} 1$ receptor with the specific $\mathrm{CB} 1$ receptor antagonist rimonabant induces weight reduction and improves cardiovascular risk factors in overweight patients. The mechanisms responsible for these effects remain only partially elucidated. Here, we demonstrate a direct role of cannabinoid on thermogenesis and trans-differentiation in white adipocytes.

Results: Acute and chronic treatment of murine white adipocytes with rimonabant induced CB1 Receptor expression in a dose- and time dependent manner. Lipid accumulation and cell morphology were not effected by chronic CB1 Receptor inhibition as assessed by oil red O staining. As compared to control cells, pre-treatment with $100 \mathrm{nM}$ rimonabant for 5 days, increased insulin-induced glucose uptake significantly by $10 \%$. Furthermore, chronic treatment of white adipocytes with rimonabant strongly induced the thermogenic protein-1 (UCP-1) mRNA and protein expression by $125 \%$ and $300 \%(\mathrm{p}<0.05)$ in fully differentiated cells. This effect was dose dependent and peaked with a concentration at $1 \mu \mathrm{M}$. Moreover, we measured a $30 \%(\mathrm{p}<0.02)$ highly significant increase of the trans-differentiation factor PGC1 alpha after $1 \mu \mathrm{M}$ rimonabant treatment for 10 days. To verify these findings of a transdifferentiation process in white adipocytes during rimonabant stimulation we measured mitochondrial biogenesis and function. Indeed, we found that white adipocytes exposed to rimonabant during adipogenesis increased mitochondrial numbers compared to non-treated cells by $60 \%$ shown with electron microscopy images and FACS analyses using a mitochondrial specific 
MitoTracker Green Staining. Furthermore, pre-treatment with $1 \mu \mathrm{M}$ rimonabant for 10 days raised the cytochrom c oxidase activity by $30 \%(\mathrm{p}<0.05)$ and enhanced the oxygen consumption by $40 \%(\mathrm{p}<0.02)$.

Conclusion: Taken together, our data provide evidence for a trans-differentiation towards an insulin-sensitive brown fat phenotype in white adipocytes during $\mathrm{CB} 1$ receptor blockade. Thus, thermogenesis might be one mechanism responsible for the weight loss seen under rimonabant treatment in overweight patients.

Supported by: sanofi-aventis

\section{PS 074 GLP-1 based treatments: clinical and experimental}

\section{8}

Saxagliptin added to a sulfonylurea is safe and more efficacious than uptitrating a sulfonylurea in patients with type 2 diabetes

S. Ravichandran ${ }^{1}$, A.R. Chacra ${ }^{2}$, G.H. Tan ${ }^{3}$, A. Apanovitch ${ }^{1}$, R. Chen ${ }^{1}$

${ }^{1}$ Bristol-Myers Squibb, Princeton, United States, ${ }^{2}$ Department of Endocrinology, Universidade Federal de Sao Paulo, Brazil, ${ }^{3}$ Endocrinology,

Cebu Doctor's University Hospital, Cebu City, Philippines

Background and aims: Saxagliptin (SAXA) is a potent modulator of key hormones that regulate glucose homeostasis, specifically designed to provide selective, durable inhibition of the dipeptidyl peptidase-4 (DPP-4) enzyme. This 24-wk study (CV181-040) assessed the safety and efficacy of SAXA added to an intermediate dose of glyburide (GLY) versus up-titration of intermediate dose GLY monotherapy in patients with type 2 diabetes (T2D) and inadequate glycemic control $\left(\mathrm{HbA}_{1 \mathrm{c}} \geq 7.5 \%-\leq 10 \%\right)$ after $\geq 2$ mo of treatment with an intermediate dose of a sulfonylurea at enrollment.

Materials and methods: All patients received open-label GLY $7.5 \mathrm{mg}$ for a 4 -wk lead-in period. Then, 768 patients were randomized to receive blinded SAXA 2.5 or $5 \mathrm{mg}$ or GLY $2.5 \mathrm{mg}$, once daily, in addition to the open-label GLY. During the 24-wk study period, blinded up-titration of GLY was allowed in the GLY-only arm to a maximum total daily GLY dose of $15 \mathrm{mg}$ based on pre-defined protocol criteria. The primary end point was change from baseline in $\mathrm{HbA}_{1 \mathrm{c}}$. Decreases in fasting plasma glucose (FPG) and postprandial glucose (PPG) were also evaluated. Efficacy analyses were performed using an ANCOVA model utilizing LOCF methodology on randomized patients. Results: Treatment groups were well balanced at baseline (mean $\mathrm{HbA}_{1}, 8.4 \%$; mean age, $55 \mathrm{y}$; mean FPG, $173.3 \mathrm{mg} / \mathrm{dL}$ ). Approximately $92 \%$ of patients in the UP-GLY group had been up-titrated to a total GLY dose of $15 \mathrm{mg}$ per day by wk 24. SAXA 2.5 and $5 \mathrm{mg}$ demonstrated significant adjusted mean decreases from baseline to wk 24 compared with adjusted mean increases with UP-GLY, respectively, in $\mathrm{HbA}_{1 c}(-0.54 \%,-0.64 \%$ vs $0.08 \%$; both $P<.0001)$ FPG $(-7.1,-9.7 \mathrm{mg} / \mathrm{dL}$ vs $0.7 \mathrm{mg} / \mathrm{dL} ; P=.0218$ and $P=.002$, respectively), and PPG at 120 min during an OGTT $(-30.9,-34.2 \mathrm{mg} / \mathrm{dL}$ vs $7.6 \mathrm{mg} / \mathrm{dL}$; both $P<.0001)$. A greater proportion of patients in the UP-GLY treatment group were discontinued due to lack of glycemic control or rescued for failing to achieve glycemic targets (29.6\%) vs patients in the SAXA 2.5- and 5-mg groups ( $18.1 \%$ and $16.6 \%$, respectively). SAXA was well tolerated; the proportion of patients receiving SAXA 2.5 , SAXA $5 \mathrm{mg}$, and UP-GLY who had adverse events (AEs) were $75 \%, 72 \%$, and $77 \%$, respectively, despite longer exposure to study medication in the SAXA groups $(151,150$ vs $140 \mathrm{~d}$, respectively). The most common AEs with occurrence rates $\geq 5 \%$ (in all patients receiving SAXA vs UP-GLY) were urinary tract infection ( $8.0 \%$ vs $8.2 \%$ ), headache ( $7.6 \%$ vs $5.6 \%)$, nasopharyngitis ( $5.8 \%$ vs $6.7 \%)$, upper respiratory tract infection (5.4\% vs 6.7\%), back pain (5.4\% vs $4.5 \%)$, hypertension $(5.0 \%$ vs $2.2 \%$ ), diarrhea ( $4.8 \%$ vs $5.2 \%$ ), influenza ( $4.6 \%$ vs $6.0 \%$ ), and pain in the extremity ( $4.0 \%$ vs $5.6 \%)$. Reported hypoglycemic events were similar in patients treated with SAXA 2.5 and $5 \mathrm{mg}(13.3 \%$ [33/248] and 14.6\% [37/253]) vs UP-GLY (10.1\% [27/267]) ( $P=\mathrm{NS}$ for both).

Conclusion: For patients with T2D inadequately controlled with an intermediate dose of sulfonylurea, the combination of saxagliptin and glyburide provided improved glycemic control ( $\mathrm{HbA}$, FPG, PPG) without additional safety and tolerability concerns compared with up-titration of glyburide monotherapy. This improvement in glycemic control was achieved without any significant increase in hypoglycemia.

Supported by: Bristol-Myers Squibb and AstraZeneca

\section{9}

Saxagliptin added to a thiazolidinedione improves glycaemic control in patients with inadequately controlled type 2 diabetes

E. Allen ${ }^{1}$, P. Hollander ${ }^{2}$, J. Li ${ }^{1}$, R. Chen ${ }^{1}$

${ }^{1}$ Bristol-Myers Squibb, Princeton, ${ }^{2}$ Department of Endocrinology, Baylor University Medical Center, Dallas, United States

Background and aims: Saxagliptin is a potent modulator of key hormones that regulate glucose homeostasis, specifically designed to provide selective, durable inhibition of the dipeptidyl peptidase-4 (DPP-4) enzyme. Saxagliptin was added to ongoing thiazolidinedione (TZD) therapy to assess the safety 
and efficacy of this combination in patients with type 2 diabetes (T2D) and inadequate glycemic control on TZD alone.

Materials and methods: A multinational, randomized, placebo-controlled, parallel-group, double-blind study (CV181-013) was initiated among patients with T2D inadequately controlled ( $\mathrm{HbA}_{1 c} \geq 7.0 \%$ and $\left.\leq 10.5 \%\right)$, aged 18-77 y, receiving stable TZD monotherapy (pioglitazone 30 or $45 \mathrm{mg}$, or rosiglitazone 4 or $8 \mathrm{mg}$ ) for $\geq 12 \mathrm{wk}$ prior to screening. Following a 2 -wk dietary and exercise, placebo (PBO) and TZD lead-in period, 565 patients were randomized to receive saxagliptin $2.5 \mathrm{mg}$, saxagliptin $5 \mathrm{mg}$, or PBO, once daily, in addition to their stable TZD dose for 24 wks. Efficacy analyses for continuous variables were performed using an ANCOVA model with last observation carried forward (LOCF) methodology.

Results: Demographic and baseline diabetes characteristics were well balanced across treatment groups: mean age $54 \mathrm{y}$; mean duration of diabetes $5.2 \mathrm{y}$; mean $\mathrm{HbA}_{1 \mathrm{c}} 8.3 \%$; mean fasting plasma glucose (FPG) $161.8 \mathrm{mg} / \mathrm{dL}$. Relative to $\mathrm{PBO}$, patients randomized to saxagliptin 2.5 and $5 \mathrm{mg}$ demonstrated significant adjusted mean change from baseline in $\mathrm{HbA}_{1 \mathrm{c}}(\mathrm{PBO}-$ $0.30 \%$ vs saxagliptin $2.5 \mathrm{mg}-0.66 \%, P=.0007$, and saxagliptin $5 \mathrm{mg}-0.94 \%$, $P<.0001$ ); FPG (PBO - $2.8 \mathrm{mg} / \mathrm{dL}$ vs saxagliptin $2.5 \mathrm{mg}-14.3 \mathrm{mg} / \mathrm{dL}, P=.0053$, and saxagliptin $5 \mathrm{mg}-17.3 \mathrm{mg} / \mathrm{dL}, P=.0005$ ); and postprandial glucose (PPG) AUC (PBO $-2690 \mathrm{mg}^{*} \mathrm{~min} / \mathrm{dL}$ vs saxagliptin $2.5 \mathrm{mg}-7849 \mathrm{mg}^{\star} \mathrm{min} / \mathrm{dL}$, $P<.0001$, and saxagliptin $\left.5 \mathrm{mg}-9269 \mathrm{mg}^{\star} \mathrm{min} / \mathrm{dL}, P<.0001\right)$. Compared with PBO (25.6\%), a greater proportion of patients achieved target $\mathrm{HbA}_{1 c}<7 \%$ at wk 24 in the saxagliptin groups: saxagliptin $2.5 \mathrm{mg}(42.2 \%, P=.001)$ and saxagliptin $5 \mathrm{mg}(41.8 \%, P=.0013)$. The adjusted mean change from baseline PPG at 120 min during an OGTT was also significantly greater in patients treated with saxagliptin $2.5 \mathrm{mg}(-54.7 \mathrm{mg} / \mathrm{dL}, P<.0001)$ and saxagliptin $5 \mathrm{mg}$ $(-64.6 \mathrm{mg} / \mathrm{dL}, P<.0001)$, as compared with $\mathrm{PBO}(-14.6 \mathrm{mg} / \mathrm{dL})$. Saxagliptin treatment increased postprandial insulin and C-peptide AUC compared with PBO during OGTT. $\beta$-Cell function, as assessed using HOMA- $2 \beta$, improved in the saxagliptin 5 -mg group compared with $\mathrm{PBO}(P<.05)$. Overall, the rates of reported adverse events (AEs) and discontinuations due to AEs in the saxagliptin treatment groups were similar to $\mathrm{PBO}$. All reported hypoglycemic events were similar to PBO (saxagliptin $2.5 \mathrm{mg}, 4.1 \%$; saxagliptin $5 \mathrm{mg}, 2.7 \%$; PBO, 3.8\%).

Conclusion: In patients with T2D inadequately controlled on TZD monotherapy, the addition of saxagliptin was safe and well tolerated. The addition of saxagliptin to ongoing TZD therapy led to clinically meaningful and statistically significant improvements in glycemic control without increasing the risk of hypoglycemia.

Supported by: Bristol-Myers Squibb Company and AstraZeneca

\section{0}

Alogliptin added to pioglitazone therapy improves glycaemic control in patients with type 2 diabetes without increasing weight gain or hypoglycaemia

R. Pratley ${ }^{1}$, J. Reusch ${ }^{2}$, P. Fleck ${ }^{3}$, C. Wilson ${ }^{3}$, Q. Mekki ${ }^{3}$

${ }^{1}$ Medicine, University of Vermont, Burlington, ${ }^{2}$ Medicine, University of Colorado Denver and Health Sciences Center and Denver VAMC, Denver,

${ }^{3}$ Takeda Global Research \& Development Center Inc., Deerfield, United States

Background and aims: Alogliptin (ALO) is a potent and highly selective DPP-4 inhibitor being developed for the treatment of type 2 diabetes mellitus (T2DM). T2DM is a progressive disease and combination therapy is often required to adequately achieve and maintain glycemic control. This randomized, double-blind, placebo (PBO)-controlled study assessed the efficacy and safety of ALO added to pioglitazone (PIO) in patients with T2DM inadequately controlled on a thiazolidinedione alone or on a thiazolidinedione with metformin (MET) or a sulfonylurea (SU).

Materials and methods: Patients $(\mathrm{N}=493$; mean age $=55$ years; $74 \%$ white; $58 \%$ men; mean baseline $\mathrm{HbAlc}=8.0 \%$; mean duration of $\mathrm{T} 2 \mathrm{DM}=8$ years $)$ were assigned to receive ALO $12.5 \mathrm{mg}(\mathrm{n}=197)$, ALO $25 \mathrm{mg}(\mathrm{n}=199)$, or PBO $(\mathrm{n}=97)$ once daily in addition to their ongoing PIO regimen (mean dose $=35$ $\mathrm{mg}$ /day) for 26 weeks after a 4-week single-blind $\mathrm{PBO}$ run-in/stabilization period. Those patients already taking MET (56\%) or an SU (21\%) continued this therapy at a stable dose throughout the study. The primary endpoint was change from baseline in $\mathrm{HbAlc}$ at week 26 (or last observation) in the intentto-treat population.

Results: A total of 372 patients completed the study (ALO $12.5 \mathrm{mg}, 77.7 \%$; ALO $25 \mathrm{mg}$ 80.4\%; PBO, 73.2\%). At week 26, least-squares (LS) mean changes from baseline in $\mathrm{HbA1c}$ were significantly $(P<.001)$ greater for ALO $12.5 \mathrm{mg}(-0.66 \%)$ and ALO $25 \mathrm{mg}(-0.80 \%)$ vs PBO (-0.19\%) (Figure). The changes from baseline in $\mathrm{HbA1c}$ were similar regardless of age $(<65$ or $\geq 65$ years), BMI ( $<30$ or $\geq 30 \mathrm{mg} / \mathrm{kg}^{2}$ ), ethnicity (Hispanic or non-Hispanic), or background therapy (MET, SU, or none), and were greater in patients with higher baseline $\mathrm{HbAlc}$. At week 26, LS mean changes from baseline in fasting plasma glucose were significantly $(P=.003)$ greater for ALO $12.5 \mathrm{mg}(-1.09$ $\mathrm{mmol} / \mathrm{L})$ and ALO $25 \mathrm{mg}(-1.10 \mathrm{mmol} / \mathrm{L})$ vs $\mathrm{PBO}(-0.32 \mathrm{mmol} / \mathrm{L})$ (Figure). No significant differences from PBO in LS mean changes from baseline in weight and lipids were observed for either ALO dose at week 26 . The proportions of patients who experienced $\geq 1$ adverse event (ALO $12.5 \mathrm{mg}, 69.7 \%$; ALO $25 \mathrm{mg}, 72.4 \%$; PBO, 64.9\%) or $\geq 1$ serious adverse event (ALO $12.5 \mathrm{mg}$, $2.5 \%$; ALO $25 \mathrm{mg}, 6.5 \%$; $\mathrm{PBO}, 4.1 \%$ ) or who discontinued due to an adverse event (ALO $12.5 \mathrm{mg}, 3.0 \%$; ALO $25 \mathrm{mg}, 3.0 \%$; PBO, 3.1\%) were similar across groups. The proportion of patients who experienced hypoglycemia (ALO $12.5 \mathrm{mg}, 5.1 \%$; ALO $25 \mathrm{mg}, 7.0 \%$; PBO, 5.2\%) or edema (ALO $12.5 \mathrm{mg}, 6.1 \%$; ALO $25 \mathrm{mg}, 5.5 \%$; PBO, 7.2\%) were also similar across groups.

Conclusion: ALO added to ongoing PIO therapy (with or without MET or an $\mathrm{SU})$ significantly improved glycemic control in patients with T2DM, without increasing weight gain or hypoglycemia.

A.

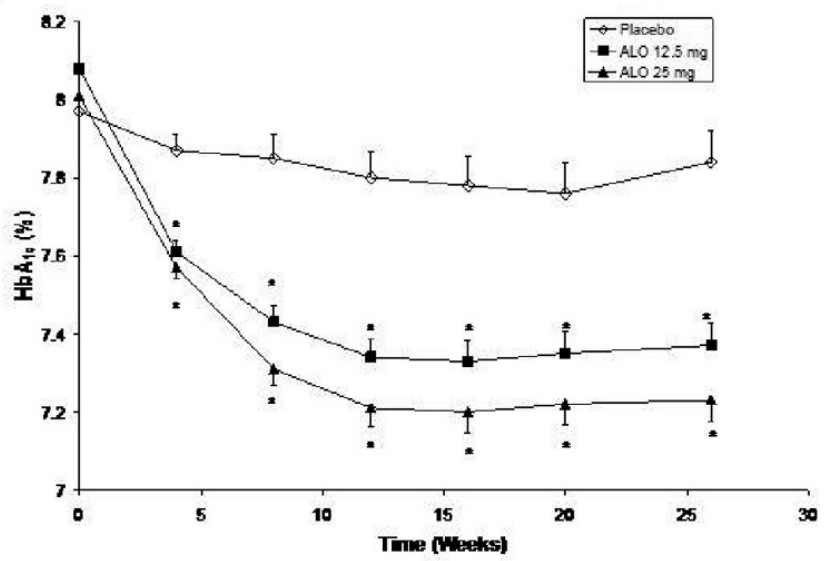

B.

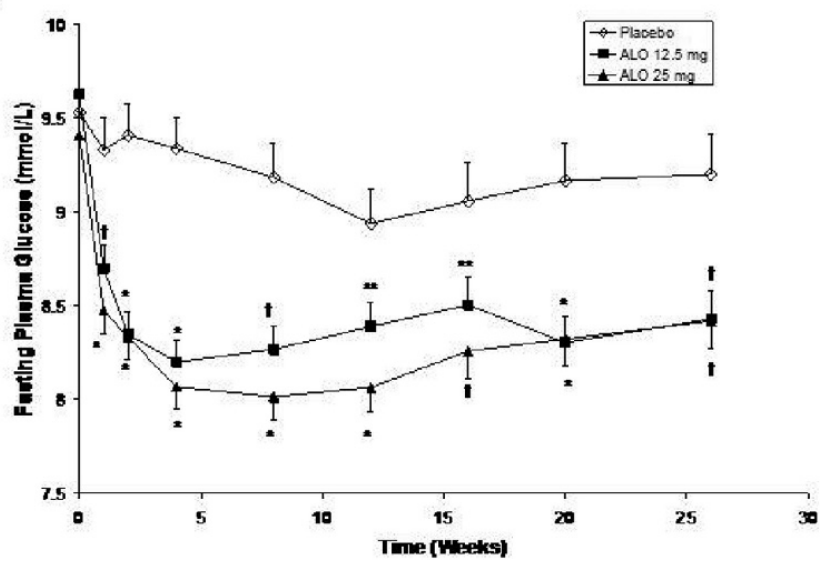

${ }^{*} \mathrm{P}<.001, \dagger \mathrm{P}<.01,{ }^{*} \mathrm{P}<.05$

Values are least-squares means (SE) except baseline values are mean

Supported by: Takeda Global Research \& Development Center

\section{1}

Alogliptin added to metformin therapy in patients with type 2 diabetes reduces $\mathrm{HbA}_{1 \mathrm{c}}$ without changing weight or increasing gastrointestinal symptoms or hypoglycaemia

G. Ellis ${ }^{1}$, P. Fleck ${ }^{2}$, C. Wilson ${ }^{2}$, Q. Mekki ${ }^{2}$, M. Nauck ${ }^{3}$

${ }^{1}$ Medicine, Helderberg Diabetes and Medical Centre, Cape Town, South Africa, ${ }^{2}$ Takeda Global Research \& Development Center Inc., Deerfield, IL, United States, ${ }^{3}$ Diabetes Clinic, Diabetes Center Bad Lauterberg, Germany

Background and aims: Alogliptin (ALO) is a potent and highly selective DPP-4 inhibitor being developed for the treatment of type 2 diabetes mellitus (T2DM). T2DM is a progressive disease and combination therapy is often 
required to adequately achieve and maintain glycemic control. This randomized, double-blind, placebo (PBO)-controlled study assessed the efficacy and safety of ALO in combination with metformin (MET) in patients with T2DM inadequately controlled on MET monotherapy.

Materials and methods: Patients $(\mathrm{N}=527$; mean age $=55$ years; mean $\mathrm{BMI}=32$ $\mathrm{kg} / \mathrm{m}^{2} ; 77 \%$ white; $50 \%$ men; mean baseline $\mathrm{HbAlc}=7.9 \%$; mean duration of T2DM $=6$ years) were assigned to receive ALO $12.5 \mathrm{mg}(\mathrm{n}=213)$, ALO $25 \mathrm{mg}$ $(n=210)$, or PBO $(n=104)$ once daily in addition to their ongoing MET regimen (mean dose $=1847 \mathrm{mg} /$ day) for 26 weeks after a 4-week single-blind PBO run-in/stabilization period. The primary endpoint was change from baseline in $\mathrm{HbAlc}$ at week 26 (or last observation) in the intent-to-treat population. Results: A total of 413 patients completed the study (ALO $12.5 \mathrm{mg}, 82.6 \%$; ALO $25 \mathrm{mg}$ 78.6\%; PBO, 69.2\%). Least-squares (LS) mean changes from baseline in $\mathrm{HbAlc}$ were significantly $(P<.001)$ greater for ALO $12.5 \mathrm{mg}$ $(-0.6 \%)$ and ALO $25 \mathrm{mg}(-0.6 \%)$ vs PBO $(-0.1 \%)$ at week 26 (Figure). The changes from baseline in $\mathrm{HbAlc}$ were similar regardless of age $(<65$ or $\geq 65$ years), BMI ( $<30$ or $\left.\geq 30 \mathrm{~kg} / \mathrm{m}^{2}\right)$, or ethnicity (Hispanic or non-Hispanic) and were greater in patients with higher baseline $\mathrm{HbAlc}$. At week 26 , significantly $(P<.001)$ greater proportions of patients achieved a $\geq 1 \%$ decrease from baseline for HbAlc levels (ALO $12.5 \mathrm{mg}, 28.6 \%$ and ALO $25 \mathrm{mg}, 30.0 \%$ vs PBO, $8.7 \%)$. LS mean changes from baseline in fasting plasma glucose were significantly $(P<.001)$ greater for ALO $12.5 \mathrm{mg}(-1.0 \mathrm{mmol} / \mathrm{L})$ and ALO $25 \mathrm{mg}(-1.0$ $\mathrm{mmol} / \mathrm{L})$ vs $\mathrm{PBO}(0 \mathrm{mmol} / \mathrm{L})$ at week 26 (Figure). LS mean changes from baseline in weight and lipids at week 26 were not clinically meaningful. The proportions of patients who experienced $\geq 1$ adverse event (ALO $12.5 \mathrm{mg}$, 62.9\%; ALO $25 \mathrm{mg}, 57.0 \%$; PBO, 66.3\%) or $\geq 1$ serious adverse event (ALO $12.5 \mathrm{mg}, 2.8 \%$; ALO $25 \mathrm{mg}, 3.9 \%$; PBO, 3.8\%) or who discontinued the study due to an adverse event (ALO $12.5 \mathrm{mg}, 3.3 \%$; ALO $25 \mathrm{mg}, 1.9 \%$; PBO, 1.0\%) were similar across groups. The proportion of patients with $\geq 1$ gastrointestinal symptom was also similar across groups (ALO $12.5 \mathrm{mg}, 10.3 \%$; ALO 25 $\mathrm{mg}, 12.6 \%$; PBO, 15.4\%). The incidence of hypoglycemia was low (ALO 12.5 mg, 2 patients; ALO 25 mg, 0 patients; $\mathrm{PBO}, 3$ patients).

Conclusion: ALO added to ongoing MET monotherapy improved glycemic control in patients with T2DM without changing weight or increasing the incidence of gastrointestinal symptoms or hypoglycemia.

A.

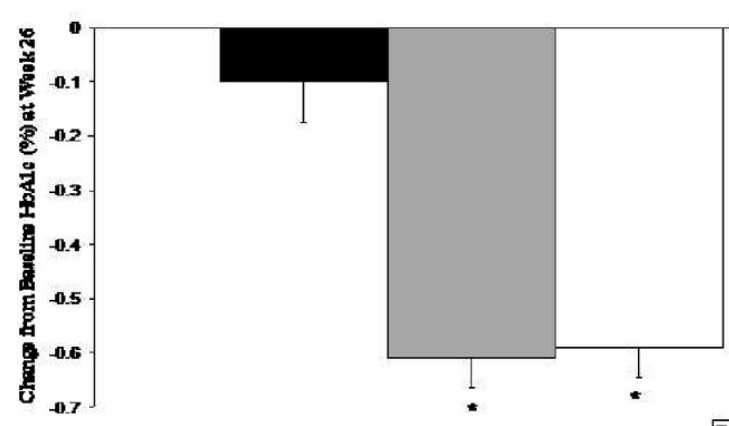

B.

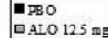
QALO $25 \mathrm{mg}$
QALO

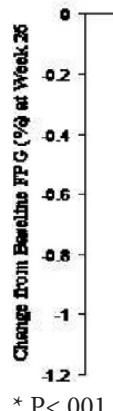

Values are least-squares mean (SE)

Supported by: Takeda Global Research \& Development Center

\section{2}

Efficacy and safety of alogliptin monotherapy in patients with type 2 diabetes inadequately controlled with diet and exercise

Q. Mekki ${ }^{1}$, P. Fleck ${ }^{1}$, C. Wilson ${ }^{1}$, R. DeFronzo ${ }^{2}$

${ }^{1}$ Takeda Global Research \& Development Center Inc., Deerfield, ${ }^{2}$ MedicineDiabetes, University of Texas Health Science Center, San Antonio, United States

Background and aims: Alogliptin (ALO) is a potent and highly selective DPP-4 inhibitor being developed for the treatment of type 2 diabetes mellitus (T2DM). DPP-4 inhibitors represent a new class of oral antihyperglycemic agents that improve glycemic control by preventing the degradation of the incretin hormones glucagon-like peptide-1 and glucose-dependent insulinotropic polypeptide. This randomized, double-blind, placebo (PBO)-controlled study was conducted to assess the efficacy and safety of ALO monotherapy in patients with T2DM inadequately controlled on diet and exercise alone. Materials and methods: Patients $(\mathrm{N}=329$; mean age $=53$ years; $53 \%$ men; $67 \%$ White; mean baseline $\mathrm{HbAlc}=7.9 \%$ ) were assigned to receive ALO 12.5 $\mathrm{mg}(\mathrm{n}=133)$, ALO $25 \mathrm{mg}(\mathrm{n}=131)$, or PBO $(\mathrm{n}=65)$ once daily for 26 weeks. The primary endpoint was change from baseline in HbAlc at week 26 (or last observation) in the intent-to-treat population.

Results: A total of 252 patients completed the study (ALO $12.5 \mathrm{mg}, 78.9 \%$; ALO $25 \mathrm{mg}$ 81.7\%; PBO, 61.5\%). At week 26, the least-squares (LS) mean changes from baseline in $\mathrm{HbA1c}$ were significantly $(P<.001)$ greater for ALO $12.5 \mathrm{mg}(-0.56 \%)$ and ALO $25 \mathrm{mg}(-0.59 \%)$ vs PBO (-0.02\%) (Figure). The changes from baseline in HbA1c were similar regardless of age $(<65$ or $\geq 65$ years), BMI ( $<30$ or $\geq 30 \mathrm{~kg} / \mathrm{m}^{2}$ ), or ethnicity (Hispanic or non-Hispanic), and were greater in patients with higher baseline $\mathrm{HbAlc}$ (Figure). A greater proportion of patients achieved HbAlc $\leq 7.0 \%$ for ALO $12.5 \mathrm{mg}(47 \% ; P=.001)$ and ALO $25 \mathrm{mg}(44 \% ; P=.008)$ vs PBO $(23 \%)$ at week 26 . The LS mean changes from baseline in fasting plasma glucose were significantly $(P<.001)$ greater for ALO $12.5 \mathrm{mg}(-0.57 \mathrm{mmol} / \mathrm{L})$ and ALO $25 \mathrm{mg}(-0.91 \mathrm{mmol} / \mathrm{L})$ vs PBO $(+0.63 \mathrm{mmol} / \mathrm{L})$ at week 26 . No significant differences from PBO in LS mean changes from baseline in weight were observed for either ALO dose at week 26. The proportions of patients who experienced $\geq 1$ adverse event (ALO 12.5 mg, 68.4\%; ALO $25 \mathrm{mg}, 67.4 \%$; PBO, 70.3\%), who discontinued the study due to an adverse event (ALO $12.5 \mathrm{mg}, 1.5 \%$; ALO $25 \mathrm{mg}, 1.5 \%$; PBO, 1.6\%), or experienced $\geq 1$ severe adverse event (ALO $12.5 \mathrm{mg}, 3.8 \%$; ALO $25 \mathrm{mg}, 0.8 \%$; PBO, 3.1\%) were similar across groups. The most common adverse events were nasopharyngitis (ALO $12.5 \mathrm{mg}, 9.0 \%$; ALO $25 \mathrm{mg}, 7.6 \%$; PBO, 7.8\%), headache (ALO $12.5 \mathrm{mg}, 7.5 \%$; ALO $25 \mathrm{mg}, 6.8 \%$; PBO, 4.7\%), and upper respiratory tract infection (ALO $12.5 \mathrm{mg}, 3.8 \%$; ALO $25 \mathrm{mg}$, 4.5\%; PBO, 9.4\%). The incidence of hypoglycemia was low and similar across groups (ALO 12.5 mg, 3.0\%; ALO 25 mg, 1.5\%; PBO, 1.6\%).

Conclusion: ALO monotherapy significantly improved glycemic control and was generally well tolerated in patients with T2DM inadequately controlled with diet and exercise.

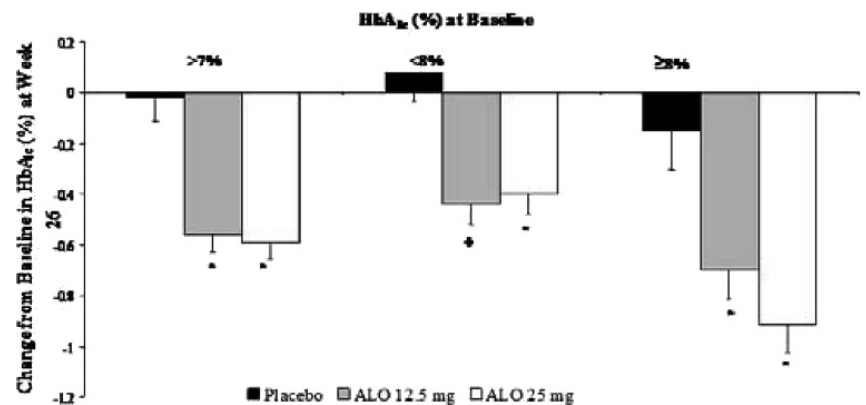

${ }^{*} \mathrm{P}<.001$ and $\dagger \mathrm{P}=.005$, vs placebo, ANCOVA.

Values are least-squares mean (SE)

Supported by: Takeda Global Research \& Development Center 


\section{3}

Exenatide improves acute phase insulin response to a meal in patients with type 2 diabetes

J. Han ${ }^{1}$, H. Dong ${ }^{1}$, L. Nielsen ${ }^{1}$, Y. Qu ${ }^{2}$, L. Glass ${ }^{2}$, D. Bhole ${ }^{1}$, D. Maggs ${ }^{1}$

${ }^{1}$ Amylin Pharmaceuticals, Inc., San Diego, ${ }^{2}$ Lilly Research Laboratories, Eli

Lilly and Company, Indianapolis, United States

Background and aims: The incretin mimetic exenatide can markedly lower postprandial glucose. However, conventional interpretations of insulin secretion during exenatide treatment can be confounded by small postprandial plasma glucose excursions, as well as difficulty in accounting for the accompanying glucoregulatory effects of glucagon suppression and slowed gastric emptying. To address this issue, we utilised an insulinogenic index of insulin secretion that calculates the insulin:glucose ratio (IGR) using peak postprandial glucose concentration, and corresponding insulin concentration, during a meal tolerance test:

$\mathrm{IGR}=\frac{\text { Insulin }(\mu U / \mathrm{mL}) \text { at peak glucose }- \text { Insulin at } t=0}{\text { Glucose }(\mathrm{mg} / \mathrm{dL}) \text { at peak }- \text { Glucose at } t=0}$

Materials and methods: Data were log-transformed and examined in an Analysis of Covariance (ANCOVA) model. Calculations were conducted in two separate cohort datasets where meal tolerance tests were performed in patients with type 2 diabetes at baseline and study end: (1) a pooled clinical database from three $30 \mathrm{wk}$ PBO-controlled trials (75M, 51F; age 55.1 $\pm 9.7 \mathrm{y}$; BMI $33.4 \pm 5.8 \mathrm{~kg} / \mathrm{m}^{2} ; \mathrm{HbA}_{1 \mathrm{C}} 8.3 \pm 1.1 \%$; duration of diabetes $7.7 \pm 5.7 \mathrm{y}$; mean $\pm \mathrm{SD}$ ); (2) a 26-wk insulin glargine comparator trial $(41 \mathrm{M}, 21 \mathrm{~F}$; age $56.5 \pm 8.3 \mathrm{y}$; BMI $32.0 \pm 5.0 \mathrm{~kg} / \mathrm{m}^{2} ; \mathrm{HbA}_{1 \mathrm{C}} 8.3 \pm 0.9 \%$; duration of diabetes $9.9 \pm 5.4 \mathrm{y} ;$ mean $\pm \mathrm{SD}$ ). Results: For cohort 1, IGR was significantly greater for the $5-\mu \mathrm{g}(\mathrm{n}=40)$ and $10-\mu \mathrm{g}(\mathrm{n}=43)$ exenatide-treated cohorts compared with the PBO $(n=43)$ cohort (geometric LS mean ratio of Wk 30 to baseline: $1.60 \pm 0.21,1.55 \pm 0.20$, and $0.75 \pm 0.09$, respectively; $\mathrm{p} \leq 0.0001$ exenatide vs. PBO). For cohort 2 , IGR was significantly greater for the exenatide cohort $(n=28)$ compared with the insulin glargine cohort $(\mathrm{n}=34$; geometric LS mean ratio of Wk 26 to baseline: $1.74 \pm 0.24$ vs. $1.13 \pm 0.14$, respectively; $\mathrm{p}=0.0274$ exenatide vs. glargine).

Conclusion: Exenatide treatment increases the acute phase insulin secretion response to a caloric challenge compared to $\mathrm{PBO}$-controlled patients and patients receiving insulin glargine, an effect that likely contributes to the improvement in postprandial glycaemia observed with exenatide treatment.

\section{4}

Exenatide sensitises insulin-mediated glucose uptake at liver and skeletal muscle, an effect amplified by hyperglycaemia

D. Zheng, V. Ionut, V. Mooradian, D. Stefanovski, R.N. Bergman

Physiology and Biophysics, University of Southern California, Los Angeles, United States

Background and aims: Exenatide (EX) is a long-acting mimetic of glucagonlike peptide-1 (GLP-1), which in addition to its currently recognized actions (enhancing glucose-dependent insulin secretion, suppressing glucagon secretion and GI motility), may have a glucose-lowering effect independent of an increased beta-cell response. The aim of our study was to elucidate whether exenatide stimulates insulin-independent glucose uptake directly or if it potentiates insulin-mediated glucose uptake.

Materials and methods: Protocol I: modified hyperinsulinemic euglycemic clamps in awake dogs $(n=6)$, with EX $(20 \mu \mathrm{g}$, sc) or saline (SAL). Plasma insulin was raised to $\sim 250 \mathrm{pM}$ (postprandial levels). Exogenous glucose was infused intraportally to maintain systemic euglycemia and simultaneously stimulate the putative portal GLP-1/glucose sensor together with exenatide. The rate of peripheral glucose disappearance (Rd) was calculated using Steele's equation. Net hepatic glucose balance (NHGB), the difference between endogenous glucose production (EGP) and 1st-pass hepatic glucose uptake (HGU), was calculated by subtracting intraportal glucose infusion (PoGinf) from the rate of glucose appearance $(\mathrm{Ra}) ; \mathrm{NHGB}=\mathrm{EGP}-\mathrm{HGU}=\mathrm{Ra}-$ PoGinf (positive values $=$ net production $[$ NHGP $]$, negative values $=$ net uptake $[$ NHGU $]$ ). To test the hypothesis that the potential enhancement of glucose disposal by exenatide is amplified at higher glycemia we performed Protocol II.

Protocol II: hyperinsulinemic hyperglycemic clamps $(n=8)$, was identical to Protocol I except that systemic glucose was raised to $150 \mathrm{mg} / \mathrm{dL}$ (postprandial levels). To investigate whether a rise in plasma insulin levels is necessary for exenatide's effect, we performed Protocol III.
Protocol III: euinsulinemic hyperglycemic clamps $(n=6)$ was identical to protocol II, except that plasma insulin was maintained at basal levels

Results: During the hyperinsulinemic euglycemic clamps with EX PoGinf was $18 \%$ higher than in SAL $(13.2 \pm 1.9 \mathrm{SAL}$ vs. $15.6 \pm 2.1 \mathrm{EX} \mathrm{mg} / \mathrm{kg} / \mathrm{min}, \mathrm{p}$ $=0.01)$, due to an $11 \%$ increase in Rd $(13.0 \pm 2.4 \mathrm{SAL}$ vs. $14.5 \pm 2.4 \mathrm{EX} \mathrm{mg} /$ $\mathrm{kg} / \mathrm{min}, \mathrm{p}=0.02)$ and a $46 \%$ increase in NHGU $(-1.1 \pm 0.1$ SAL vs. $-1.6 \pm 0.5$ $\mathrm{EX} \mathrm{mg} / \mathrm{kg} / \mathrm{min}, \mathrm{p}=0.3)$. When systemic glucose was elevated, EX enhanced PoGinf by $29 \%(15.9 \pm 1.6 \mathrm{SAL}$ vs. $20.4 \pm 2.1 \mathrm{EX} \mathrm{mg} / \mathrm{kg} / \mathrm{min}, \mathrm{p}<0.001), \mathrm{Rd}$

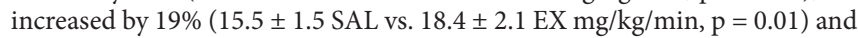
NHGU rose by $91 \%(-1.2 \pm 0.2 \mathrm{SAL}$ vs. $-2.3 \pm 0.3 \mathrm{EX} \mathrm{mg} / \mathrm{kg} / \mathrm{min}, \mathrm{p}=0.03)$. However, in the absence of hyperinsulinemia, despite elevated glucose, EX did not cause greater PoGinf $(2.7 \pm 0.7 \mathrm{SAL}$ vs. $2.3 \pm 0.4 \mathrm{EX} \mathrm{mg} / \mathrm{kg} / \mathrm{min})$. $\mathrm{Rd}$ increased slightly and similarly with or without exenatide (4.1 $\pm 0.5 \mathrm{SAL}$ vs. $4.0 \pm 0.3 \mathrm{EX} \mathrm{mg} / \mathrm{kg} / \mathrm{min}$ ) and NHGP was similarly suppressed (to $51 \%$ of basal SAL vs. $57 \%$ of basal EX).

Conclusion: We conclude that there exists a systemic glucose-lowering effect of exenatide as a result of the sensitization of insulin-mediated glucose uptake at liver and the periphery (presumably skeletal muscle). Therefore, along with its other known actions, exenatide represents an acute insulin sensitizer, acting effectively in postprandial conditions (high insulin and glucose levels) and possibly rescuing the impaired insulin sensitivity in type 2 diabetes. Supported by: Investigator Initiated Grant from Amylin

\section{5}

Relative contribution of splanchnic glucose metabolism and gastric emptying in exenatide attenuation of post-meal hyperglycaemia in T2DM subjects

E. Cersosimo, A. Cervera, E. Wajcberg, C. Triplitt, J. Joya, P. Zuo,

R. DeFronzo

Medicine/Diabetes, University of Texas, San Antonio, United States

Background and aims: Exenatide markedly attenuates postprandial and reduces fasting glucose, but the prevalent mechanisms of action during acute vs. chronic exposure are unknown.

Materials and methods: We examined 26 T2DM subjects $(45 \pm 10 \mathrm{y} ; 7 \mathrm{~F} / 19 \mathrm{M}$; $\mathrm{BMI}=33 \pm 6 \mathrm{~kg} / \mathrm{m}^{2}, \mathrm{FPG}=126-188 \mathrm{mg} / \mathrm{dl}$ range, $\left.\mathrm{HbA}=9.3 \pm 1.9 \%\right)$ treated with diet $(n=6)$, metformin $(n=6)$, sulfonylurea $(n=5)$ or both $(n=9)$. Subjects received: i) 6-h meal tolerance test (MTT) with iv saline or iv exenatide $(n=13)$ (ACUTE) or ii) 6-h MTT before and after $(n=13) 2$ weeks of exenatide (10 ug sc bid) (CHRONIC) with no exenatide on the study day. The meal had $600 \mathrm{cal}$ of fat/protein/glucose $(75 \mathrm{~g})$ with $100 \mu \mathrm{Ci}$ of $\left[1-{ }^{14} \mathrm{C}\right]$-glucose, and $1 \mathrm{~g}$ acetaminophen to monitor gastric emptying. Endogenous glucose production (EGP), rate of oral glucose appearance $(\mathrm{RaO})$ and splanchnic glucose uptake $\left[\mathrm{SGU}=75 \mathrm{~g}-\mathrm{RaO}_{(0-360)}\right.$ ] were measured with dual isotope technique. Results: IV Exenatide completely prevented the post-meal rise in glucose (126 [fasting] to $123 \mathrm{mg} \%$ [mean postprandial]), increased insulin (by $11 \pm 4$ $\mu \mathrm{IU} / \mathrm{ml}, \mathrm{p}<0.01$ ) and decreased glucagon ( (by $4 \pm 1 \mathrm{pg} / \mathrm{ml}, \mathrm{p}<0.05$ ) vs. iv saline. Two weeks exenatide sc decreased fasting glucose (by $43 \mathrm{mg} \%, \mathrm{p}<0.05$ ), reduced mean post-prandial glucose (by $45 \mathrm{mg} \%, \mathrm{p}<0.05$ ), increased insulin (by $12 \mu \mathrm{IU} / \mathrm{ml}, \mathrm{p}<0.01$ ) and reduced glucagon (by $6 \mathrm{pg} / \mathrm{ml}, \mathrm{p}<0.05$ ) vs. the pre-exenatide MTT. Both iv (ACUTE) and sc (CHRONIC) exenatide similarly suppressed EGP (by $15-20 \%, \mathrm{p}<0.05$ ), decreased RaO of glucose (129 to 82 vs. 101 to $63 \mathrm{mg} / \mathrm{min}$, respectively) and increased SGU ( $33 \pm 2$ to $56 \pm 4 \mathrm{~g}$ vs. $33 \pm 4$ to $52 \pm 5 \mathrm{~g}$, respectively) during the MMT. However, acetaminophen ${ }_{(\mathrm{AUC})}$ decreased by $47 \%$ (1761 to $831 \mu \mathrm{g} / \mathrm{ml} .360 \mathrm{~min}, \mathrm{p}<0.05)$ with iv exenatide, indicating delayed gastic emptying was responsible for the increase in splanchnic glucose retention. In contrast, acetaminophen ${ }_{(\mathrm{AUC})}$ was identical before and after 2 weeks of sc exenatide $(1481 \pm 44$ vs. $1487 \pm 83 \mu \mathrm{g} / \mathrm{ml} .360 \mathrm{~min})$. Thus, increase in splanchnic (hepatic) glucose uptake, not delayed gastric emptying, was responsible for decreaed $\mathrm{RaO}$ following sc exenatide. Plasma exenatide concentration during iv infusion was $96 \pm 17 \mathrm{pg} / \mathrm{ml}$ (therapeutic range) vs. undetectable levels during sc exenatide.

Conclusion: Acute elevation in plasma exenatide to clinical practice levels reduces post-meal glucose by slowing gastric emptying and inhibiting EGP, while chronic exenatide markedly augments hepatic glucose uptake and modestly reduces EGP.

Supported by: Amylin/Lilly Alliance 


\section{PS 075 Novel GLP-1 based treatments}

\section{6}

Intranasal administration of exenatide in patients with type 2 diabetes: pharmacokinetics, pharmacodynamics, safety and tolerability E. Blase, W. Deng, B. Walsh, M. Fineman, C. Rhodes

Amylin Pharmaceuticals, Inc., San Diego, United States

Background and aims: Exenatide, an incretin mimetic administered twice daily (BID) via subcutaneous (SC) injection, has been demonstrated to improve glycaemic control with associated weight reduction. The present study examined the pharmacokinetic and pharmacodynamic effects of intranasal (IN) exenatide administration in patients with type 2 diabetes using at least one oral antidiabetic agent $\left(13 \mathrm{M}, 7 \mathrm{~F}\right.$; age $55 \pm 9 \mathrm{y}$; BMI $31.3 \pm 3.2 \mathrm{~kg} / \mathrm{m}^{2}$; $\mathrm{HbA}_{1 \mathrm{c}} 8.13 \pm 1.28 \%$; FPG $8.68 \pm 1.92 \mathrm{mmol} / \mathrm{L}$; mean $\pm \mathrm{SD}$ ).

Materials and methods: Exenatide IN and placebo IN were proprietary formulations containing an absorption enhancer. Saline IN consisted of a $0.65 \%$ aqueous solution of sodium chloride. All IN administrations consisted of one to three $100-\mu \mathrm{L}$ nasal sprays. Each patient received exenatide $5 \mu \mathrm{g}$ SC $30 \mathrm{~min}$ after saline IN, and on a separate day, placebo IN. Thereafter, patients received on separate days either 60,200 , and $600 \mu$ g exenatide IN ( $n=11)$; or $600,800,1200$, and $1800 \mu \mathrm{g}$ exenatide IN $(\mathrm{n}=6)$. A standardised meal was given immediately after exenatide IN. Blood samples were collected prior to and during the 8 hours following study medication.

Results: Plasma exenatide concentration peaked at 15-30 min with exenatide IN compared to 2 hours with SC. Exenatide exposure (AUC, $C_{\text {max }}$ ) increased in a dose-related manner from $60 \mu \mathrm{g}$ to $1800 \mu \mathrm{g}$, and therapeutic plasma concentrations $(>50 \mathrm{pg} / \mathrm{mL})$ were maintained for 3 to 5 hours with exenatide IN doses greater than $600 \mu \mathrm{g}$. Near-maximal improvements in postprandial glucose were observed at $600 \mu \mathrm{g}$ (incremental $\mathrm{AUC}_{0-240 \text { min }}-148.8 \pm 105.2$ $\mathrm{mmol} \cdot \mathrm{min} / \mathrm{L}$ vs. $+656.6 \pm 66.8 \mathrm{mmol} \cdot \mathrm{min} / \mathrm{L}$ with placebo; mean $\pm \mathrm{SE}$ ), and these improvements were maintained at all higher doses. The most frequent adverse events (AEs) with exenatide IN were nausea (6 patients), vomiting (5 patients), and sneezing ( 2 patients); these AEs occurred only at doses of 600 $\mu \mathrm{g}$ and above. Nausea and sneezing also occurred with placebo IN (1 patient for each $\mathrm{AE}$ ). There were no serious AEs or hypoglycaemic events.

Conclusion: In this study, intranasal administration of exenatide in patients with type 2 diabetes resulted in therapeutic plasma exenatide concentrations and improved PPG control.

\section{7}

Pulmonary administration of GLP-1 (GLP-1 Technosphere powder) I: Kinetics

J. Cassidy ${ }^{1}$, R. Baughman ${ }^{1}$, D. Costello ${ }^{2}$, B. Levy ${ }^{3}$, A. van Vliet ${ }^{4}$, M. Diaz ${ }^{1}$, C. Damico ${ }^{5}$, P. Richardson ${ }^{6}$

${ }^{1}$ Experimental Pharmacology, MannKind Corporation, Paramus, United States, ${ }^{2}$ Clinical Research, MannKind Corporation, Paramus, United States, ${ }^{3}$ Metabolic Diseases, Johnson \& Johnson Pharmaceutical Services, LLC, Raritan, United States, ${ }^{4}$ PRA Group BV, PRA International, Zuidlaren, Netherlands, ${ }^{5}$ Program Management, MannKind Corporation, Paramus, United States, ${ }^{6}$ Research and Development, MannKind Corporation, Paramus, United States

Background and aims: GLP-1 enhances insulin release and regulates both glucagon release and gastrointestinal motility. MKC253 is GLP-1 (15\% by weight) adsorbed to Technosphere microparticles for administration using the MedTone Inhaler. Technosphere microparticles dissolve rapidly in the lung, providing a drug delivery platform for pulmonary administration of protein and peptide drugs.

Materials and methods: The safety, tolerability, pharmacokinetics, and pharmacodynamics of MKC253 were evaluated in a first-in-human, single-dose, open-label, dose-escalation trial in 26 healthy adult males. Five doses of MKC253 were tested in fasted subjects: 4 subjects received 0.05 and $0.45 \mathrm{mg}$ GLP-1 and 6 subjects received 0.75, 1.05, and $1.5 \mathrm{mg}$ of GLP-1.

Results: All doses of MKC253 were well tolerated. No nausea or vomiting was reported. GLP-1 was rapidly absorbed; peak concentrations were achieved at the first post-dose sampling time (3 minutes). Mean peak concentrations of active GLP- 1 were $>300 \mathrm{pmol} / \mathrm{L}$ at the $1.5 \mathrm{mg}$ dose, and 2 subjects achieved concentrations above the quantitation limit of the assay $(500 \mathrm{pmol} / \mathrm{L})$. The half-life of GLP-1 was about 2 minutes. Maximal plasma concentrations of active GLP- 1 at the $1.5 \mathrm{mg}$ dose were approximately $2 / 3$ those of total GLP1. Despite high levels of DPP-IV in the lungs, the levels of GLP-1 following MKC253 inhalation were equivalent to those achieved with intravenous bolus administration of $50 \mu \mathrm{g}$ and are more than 10X those reached following physiological postprandial release. GLP-1 plasma concentrations increased dramatically at doses above $0.75 \mathrm{mg}$, suggesting that DPP-IV degradation has been mitigated.

Conclusion: Pulmonary administration of MKC253 produces high circulating GLP-1 concentrations without nausea and vomiting.

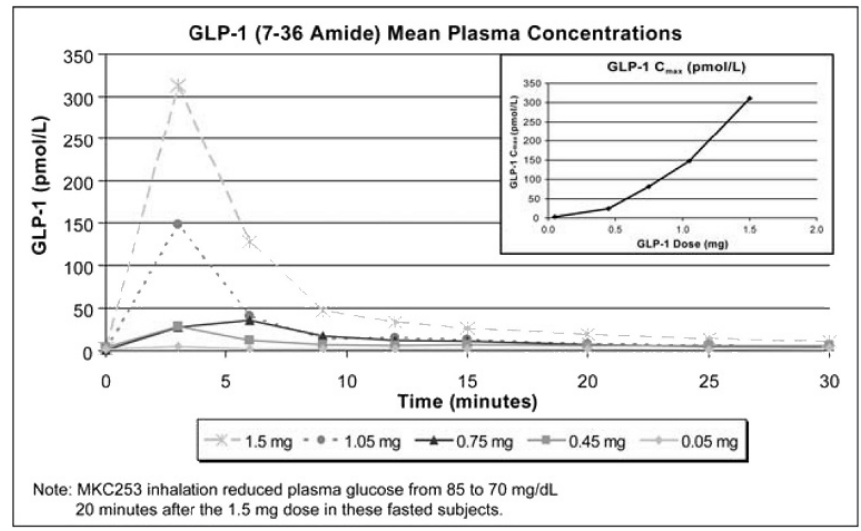

\section{8}

Pulmonary administration of GLP-1 (GLP-1 Technosphere powder) II: Dynamics

D. Costello ${ }^{1}$, R. Baughman ${ }^{2}$, B. Levy ${ }^{3}$, A. van Vliet ${ }^{4}$, A. Price ${ }^{2}$, M. Diaz 2 , C. Damico ${ }^{5}$, P. Richardson ${ }^{6}$

${ }^{1}$ Clinical Research, MannKind Corporation, Paramus, United States, ${ }^{2}$ Experimental Pharmacology, MannKind Corporation, Paramus, United States, ${ }^{3}$ Metabolic Diseases, Johnson \& Johnson Pharmaceutical Services, LLC, Raritan, United States, ${ }^{4}$ PRA Group BV, PRA International, Zuidlaren, Netherlands, ${ }^{5}$ Program Management, MannKind Corporation, Paramus, United States, ${ }^{6}$ Research and Development, MannKind Corporation, Paramus, United States

Background and aims: GLP-1 enhances insulin release and regulates both glucagon release and gastrointestinal motility. MKC253 is GLP-1 (15\% by weight) adsorbed to Technosphere microparticles for administration using the MedTone Inhaler. Technosphere microparticles dissolve rapidly in the lung, providing a drug delivery platform for pulmonary administration of protein and peptide drugs.

Materials and methods: The safety, tolerability, pharmacokinetics, and pharmacodynamics of MKC253 were evaluated in a first-in-human, single-dose, open-label, dose-escalation trial in 26 healthy adult males. Five doses of MKC253 were tested in fasted subjects: 4 subjects received 0.05 and $0.45 \mathrm{mg}$ GLP-1 and 6 subjects received $0.75,1.05$, and $1.5 \mathrm{mg}$ of GLP-1.

Results: Mean peak concentrations of active GLP- 1 were $>300 \mathrm{pmol} / \mathrm{L}$ at the $1.5 \mathrm{mg}$ dose. MKC253 was well tolerated at all doses tested. The most frequent adverse events were cough and headache. No subject vomited or reported nausea or profuse sweating, although the literature reports $>50 \%$ of subjects with GLP-1 concentrations $>100 \mathrm{pmol} / \mathrm{L}$ have these adverse events. Inhalation of MKC253 led to rapid increases in plasma insulin in fasting subjects. Maximum insulin concentrations were measured at 6 minutes after dosing, the first time point sampled. The insulin increase was dose dependent; the highest mean insulin level of $375 \mathrm{pmol} / \mathrm{L}$ was achieved with the $1.5 \mathrm{mg}$ dose of MKC253. The plasma C-peptide concentration profile matched the plasma insulin curve. Inhalation of MKC253 reduced plasma glucose; 20 minutes after the $1.5 \mathrm{mg}$ dose, mean fasting plasma glucose was reduced from 85 to $70 \mathrm{mg} / \mathrm{dL}$.

Conclusion: Pulmonary administration of GLP-1 releases endogenous insulin and reduces plasma glucose in fasting subjects without nausea and vomiting. 


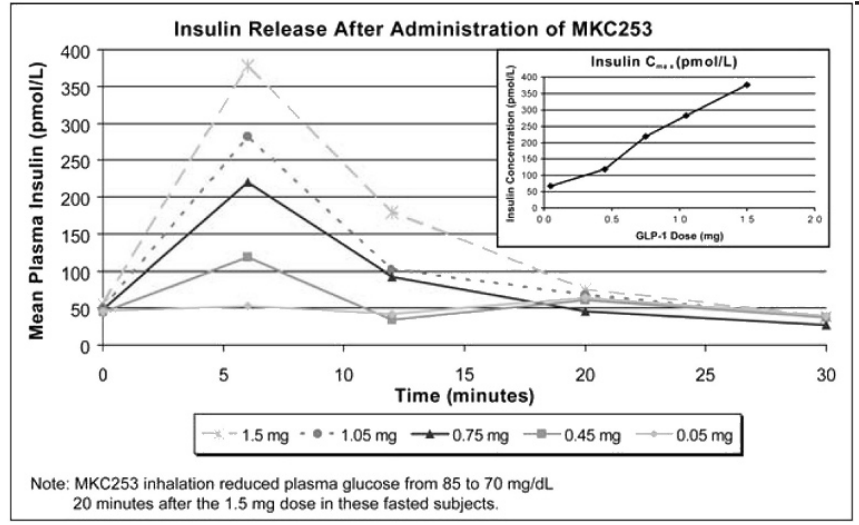

\section{9}

Pharmacodynamics of oral encapsulated analogues of GLP-1 in domestic pigs

R.R.C. New, T.P. Broke-Smith

Diabetology Limited, Cobham, United Kingdom

Background and aims: The pharmacodynamics of encapsulated analogues of GLP-1 (G001 and G002) using proprietary Axcess ${ }^{\mathrm{TM}}$ oral formulations were investigated in relation to subcutaneous injected delivery of the same GLP1 analogues in juvenile domestic pigs. The Axcess formulation has already been tried and tested in humans and proved successful in phase II studies with insulin.

Materials and methods: Six study arms used 5-8 juvenile domestic pigs each. The dosing groups were: placebo capsule (Axcess excipients only), no treatment, $0.4 \mathrm{mg}$ s.c. injected G001, $0.4 \mathrm{mg}$ s.c. injected G002, $4 \mathrm{mg}$ capsule Axcess-formulated G001, $4 \mathrm{mg}$ capsule Axcess-formulated G002. The Axcessformulated capsules where delivered via intrajejunal stoma. Treatments took place on days $1,3,7,9,11,14,16$ and 18 . Blood samples were taken at $t+0$, $10,15,30,60,120,180,240,300,360$ and 480 minutes. As this was the first test of these peptides in this model and using this oral delivery formulation, doses used were comparatively large in order to ensure that there were data generated for analysis.

Results: Falls in blood glucose were observed for all treatment arms which were greater than the controls. The oral formulations of each analogue generated glucose falls that were similar to those produced by the s.c. injection treatments. No advantage was gained by incorporating a protease inhibitor in the formulation (data not shown).

\begin{tabular}{lll}
\hline Treatment Arm & & $\begin{array}{l}\text { Mean AUC } \\
\text { plasma of olucose (st.dev.) }\end{array}$ \\
\hline No treatment & & $-42.9(108.5)$ \\
\hline Subcutaneous injection & G001 & $-61.3(49.1)$ \\
& G002 & $-81.1(41)$ \\
\hline Capsule i.j. delivered & Placebo & $-40.4(69.9)$ \\
& G001 & $-51.4(49.4)$ \\
& G002 & $-70.2(74.2)$ \\
\hline
\end{tabular}

Mean $\mathrm{AUC}_{480}$ of \% change in plasma glucose

Conclusion: This proof of concept study has confirmed that, using a standard pharmaceutical capsule, the Axcess delivery technology is capable of delivering oral encapsulated GLP-1 analogues via the jejunum. This supports the development of Axcess-formulated GLP-1 and/or its analogues as an oral alternative to the subcutaneous injections of these peptides for the treatment of type 2 diabetes in man.

\section{0}

Albiglutide, a long-acting GLP-1 mimetic: pharmacodynamics, pharmacokinetics, safety and tolerability in subjects with type 2 diabetes M.W. Stewart ${ }^{1}$, J. Matthews ${ }^{2}$, E.H. De Boever ${ }^{3}$, R.L. Dobbins ${ }^{2}$, R.J. Hodge ${ }^{4}$, S.E. Walker ${ }^{5}$, C.H. Holland ${ }^{6}$, M.A. Bush ${ }^{7}$

${ }^{1}$ Alternative Development Program, GlaxoSmithKline, King of Prussia, ${ }^{2}$ Clinical Pharmacology Discovery Medicine, GlaxoSmithKline, Research Triangle Park, ${ }^{3}$ BioPharm Drug Discovery, GlaxoSmithKline, King of Prussia, ${ }^{4} \mathrm{CSSO}$, GlaxoSmithKline, Research Triangle Park, ${ }^{5}$ Discovery Biometrics, GlaxoSmithKline, Research Triangle Park, ${ }^{6} \mathrm{Clinical}$ Immunology, GlaxoSmithKline, King of Prussia, ${ }^{7}$ Clinical Pharmokinetics, GlaxoSmithKline, Research Triangle Park, United States

Background and aims: Native GLP-1 is rapidly degraded, so a long-acting GLP-1 mimetic might be of greater benefit for the management of type 2 diabetes (T2D). Albiglutide (ALB) is a GLP-1 mimetic generated by genetic fusion of a DPP-IV-resistant GLP-1 dimer to human serum albumin. The pharmacodynamics $(\mathrm{PD})$, pharmacokinetics $(\mathrm{PK})$, safety and tolerability of ALB were assessed in subjects with T2D in two clinical studies.

Materials and methods: Study 1 evaluated fasting plasma glucose (FPG), 24h glucose profiles and PK of ALB vs placebo (PBO) in subjects with T2D. Subjects were diet-controlled or withdrawn from metformin (MET), a sulfonylurea (SU), or MET+SU for 2 weeks prior to the first dose. Subcutaneous (sc) injections of $\mathrm{PBO}$ or $\mathrm{ALB}(9,16$, or $32 \mathrm{mg})$ were administered on days 1 and 8.

Study 2 was non-PBO controlled and evaluated PD and PK of single doses (16 and $64 \mathrm{mg}$ ) of ALB after sc injection in the arm, thigh, or abdomen. Subjects with T2D were diet-controlled or remained on MET or a thiazolidinedione. On day 3 post-dose $\left(\mathrm{T}_{\max }\right)$, FPG was assessed prior to breakfast and post-prandial glucose (PPG) profiles were assessed after breakfast.

Results: In study 1, baseline-adjusted FPG and mean 24 h glucose (on day 9) were reduced relative to PBO (FPG: 1.32-2.81 mmol/l; AUC(0-24): 1.72-3.13 $\mathrm{mmol} / \mathrm{l}$ ) over the dose range evaluated.

In study 2, glucose reductions were consistent across injection sites. Mean FPG was reduced by $0.23-1.17 \mathrm{mmol} / \mathrm{l}$ and $1.19-1.94 \mathrm{mmol} / \mathrm{l}$ at the 16 and $64 \mathrm{mg}$ doses, respectively, and mean PPG by $0.34-0.89 \mathrm{mmol} / \mathrm{l}$ and $2.29-2.93$ $\mathrm{mmol} / \mathrm{l}$ at the 16 and $64 \mathrm{mg}$ doses, respectively. The PK of ALB was comparable across different sites following sc administration. Exposure was moderately greater than dose proportional for $\mathrm{AUC}(0-\infty)$ and $\mathrm{C}_{\max }$. T1/2 ranged from 4-6d; $\mathrm{T}_{\max }$ ranged from 3-4d.

In both studies, the majority of adverse events (AEs) were mild in intensity. No dose-related trends in the frequency of AEs or significant lab changes were reported for subjects receiving ALB.

Conclusion: ALB was well tolerated with a favorable safety profile and improved FPG and PPG with the potential for at least weekly dosing in subjects with T2D.

\section{1}

8 weeks treatment with R1583, a novel long-acting, human GLP-1 analogue improves beta cell function in metformin-treated diabetic subjects: a double-blind, placebo-controlled phase 2 study R. Berria ${ }^{1}$, A. Gastaldelli ${ }^{2}$, M. Nauck ${ }^{3}$, M. Boldrin ${ }^{1}$, V. Asnaghi ${ }^{4}$, R. Balena ${ }^{4}$ ${ }^{1}$ Roche Labs Inc, Nutley, United States, ${ }^{2} \mathrm{CNR}$, Institute of Clinical Physiology, Pisa, Italy, ${ }^{3}$ Fachklinik F. Diabetes und Stoffwechselkrankheiten, Diabeteszentrum, Bad Lauterberg im Harz, Germany, ${ }^{4}$ Hoffmann-La Roche, Basel, Switzerland

Background and aims: R1583 is a novel, long-acting 10\% formulation of $\left[\mathrm{Aib}^{8-35}\right]$ human GLP-1 (7-36 amide). We sought to evaluate the effects of R1583 on several parameters of $\beta$-cell function in patients with type 2 diabetes mellitus (T2DM), inadequately controlled with metformin, after 8 weeks of treatment with R1583 compared to placebo.

Materials and methods: As part of a larger, randomized, double-blind phase 2 trial, a subset of 118 T2DM subjects (age: $56 \pm 8 \mathrm{yrs}$; BMI: $33.3 \pm 5.1 \mathrm{~kg} / \mathrm{m}^{2}$; HbA1c: $7.9 \pm 0.6 \%$; duration of T2DM: $5 \pm 4$ yrs), inadequately controlled with metformin $\geq 1500 \mathrm{mg} /$ daily, were studied to assess the response to a mixed meal test (MMT). Volunteers were randomized to 8 weeks of treatment with placebo (PLO) or R1583, given either as 5, 10, $20 \mathrm{mg}$ weekly (QW), or 10 or $20 \mathrm{mg}$ once every two weeks (Q2W). Patients underwent a 240-min MMT, performed under post-absorptive conditions at baseline (PRE) and at week 9 (POST, one week after the last injection of active drug or PLO). PRE and POST treatment insulin secretion rate (ISR) was calculated by C-peptide de- 
convolution analysis, and beta cell glucose sensitivity ( $\beta$-GS) as the slope of the dose-response curve of ISR vs. glucose concentration. Changes in plasma glucose parameters are reported as $\%$ changes from baseline.

Results: After 8 weeks of treatment with R1583, fasting plasma glucose (FPG) decreased by $21 \%, 24 \%$ and $26 \%$ vs. PRE in the 5,10 and $20 \mathrm{mg}$ QW groups respectively, compared to $-8 \%$ in the PLO arm. In the Q2W arm FPG declined by $12 \%$ and $16 \%$ in the 10 and $20 \mathrm{mg}$ groups respectively. R1583 also decreased two-hour postprandial plasma glucose ( $2 \mathrm{~h}-\mathrm{PPG}$ ) by $17 \%, 25 \%$ and $22 \%$ in the 5,10 and $20 \mathrm{mg}$ groups respectively (vs. $-9 \%$ in PLO), as well as glucose-AUC, which decreased by $21 \%, 26 \%$ and $23 \%$ in the 5,10 and $20 \mathrm{mg}$ groups respectively (vs. - $8 \%$ in PLO). In the Q2W arm, $2 \mathrm{~h}-\mathrm{PPG}$ decreased by $13 \%$ and $16 \%$, while glucose-AUC declined by $11 \%$ and $15 \%$ in the 10 and $20 \mathrm{mg}$ groups respectively. ISR improved significantly during fasting (average in the weekly regimen: $+11 \mathrm{pmol} / \mathrm{min}^{2}, \mathrm{p}=0.03 \mathrm{vs}$. PLO), as well as total insulin response during the $240 \mathrm{~min}$ after the meal (average: $+9 \mathrm{nmol} / \mathrm{m}^{2}$, $\mathrm{p}=0.003$ vs. PLO). Both changes were directly proportional to the drug dose $\left(\mathrm{r}^{2}=0.91\right.$ and 0.93 respectively, $\left.\mathrm{p}<0.001\right)$. The $\triangle$ AUC-ISR/ $\Delta$ AUC-glucose calculated during the first 2 hours after the meal, clearly improved in all weekly regimens (Figure 1). Moreover, $\beta$-GS was enhanced in all weekly regimens $(\mathrm{p}<0.001$ vs. PRE; $\mathrm{p}=0.06$ vs. PLO).

Conclusion: These data suggest that R1583 significantly improves $\beta$-cell function in patients with T2DM treated with metformin, and is a promising long-acting, human GLP-1 analogue for the treatment of T2DM.

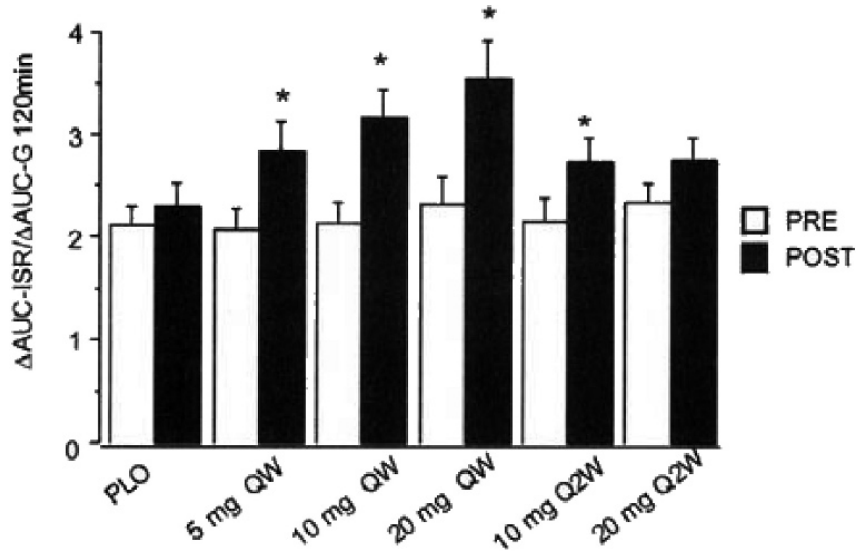

Figure 1: $\Delta \mathrm{AUC}$-ISR/ $\Delta \mathrm{AUC}$-glucose $(120 \mathrm{~min})$ at baseline and week nine Supported by: Hoffmann-La Roche

\section{PS 076 Exenatide: clinical trials}

\section{2}

Exenatide resulted in significantly greater improvements in postprandial glycaemic control compared to sitagliptin

L. MacConell ${ }^{1}$, X. Guan ${ }^{1}$, T. Okerson ${ }^{1}$, J. Holcombe ${ }^{2}$, R. DeFronzo ${ }^{3}$

${ }^{1}$ Amylin Pharmaceuticals, Inc., San Diego, ${ }^{2}$ Lilly Research Laboratories, Eli

Lilly and Company, Indianapolis, ${ }^{3}$ Medicine, University of Texas Health

Science Center, San Antonio, United States

Background and aims: Exenatide (Ex) and sitagliptin (Sita) are incretinbased therapies used to treat T2DM. Ex binds to the GLP-1 receptor, whereas Sita increases circulating levels of endogenous GLP-1.

Materials and methods: This double-blind crossover study compared their effects on postprandial (PP) measures of glucose control and gastric emptying in 61 (evaluable) patients with T2DM treated with MET (54\% F; age $54 \pm 9$ y; BMI $33 \pm 5 \mathrm{~kg} / \mathrm{m}^{2} ; \mathrm{HbA}_{1 \mathrm{c}} 8.5 \pm 1.2 \%$; 2-hr PPG 2-hr PPG 13.6 \pm 3.63 $\mathrm{mmol} / \mathrm{L}$ ). The evaluable population consisted of subjects meeting predefined requirements (including detectable Ex and Sita PK within the first 2-hrs of the meal test). Ad libitum food intake was measured in a subset of subjects $(\mathrm{N}=25)$. Patients received Ex $(5 \mu \mathrm{g}$ BID for $1 \mathrm{wk}$ followed by $10 \mu \mathrm{g}$ BID for 1 wk) or Sita (100 mg qAM for 2 wks). After 2 wks, subjects crossed over to the alternate therapy. All values are LS mean \pm SE, all ratios are Ex to Sita.

Results: After 2 wks of treatment, FPG was similar between Ex $(9.1 \pm 0.24$ $\mathrm{mmol} / \mathrm{L})$ and Sita $(8.8 \pm 0.24 \mathrm{mmol} / \mathrm{L})$ (baseline, $9.9 \pm 0.34 \mathrm{mmol} / \mathrm{L} ; \mathrm{P}=0.3234)$. In response to a standard meal, 2-hr PPG was significantly lower with Ex vs. Sita in both evaluable $(\mathrm{N}=61 ; 7.4 \pm 0.34 \mathrm{mmol} / \mathrm{L}$ vs. $11.5 \pm 0.35 \mathrm{mmol} / \mathrm{L}$, $\mathrm{P}<0.0001)$ and ITT populations $(\mathrm{N}=95 ; 9.2 \pm 0.41 \mathrm{mmol} / \mathrm{L}$ vs. $11.7 \pm 0.42 \mathrm{mmol} /$ L, P $<0.0001$ ). Ex reduced 2-hr PPG excursion compared with Sita (change from preprandial), $-1.7 \pm 0.29 \mathrm{mmol} / \mathrm{L}$ vs. $+2.6 \pm 0.30 \mathrm{mmol} / \mathrm{L}, \mathrm{P}<0.0001$. At crossover, Ex to Sita switch was associated with an increase in 2-hr PPG of $+4.05 \pm 0.61 \mathrm{mmol} / \mathrm{L}$, whereas switch from Sita to Ex had a further reduction of 2-hr PPG of $-4.22 \pm 0.56 \mathrm{mmol} / \mathrm{L}$. Ex improved the insulinogenic index relative to Sita (ratio: $1.50 \pm 0.26, \mathrm{P}=0.0239$ ) and reduced $\mathrm{PP}$ glucagon and triglyceride concentrations (ratio AUC: $0.88 \pm 0.03, \mathrm{P}=0.0011 ; 0.90 \pm 0.04, \mathrm{P}=0.0118$, respectively). Ex but not Sita reduced gastric emptying (acetaminophen ratio AUC: $0.56 \pm 0.05, \mathrm{P}<0.0001)$. After 2 wks, Ex reduced body weight compared to Sita $(-0.8 \pm 0.2 \mathrm{~kg}$ vs. $-0.3 \pm 0.2 \mathrm{~kg}, \mathrm{P}=0.0056)$. During the ad libitum meal, Ex reduced while Sita increased food intake from baseline (-134 $\pm 97 \mathrm{kcal}$ vs. $+130 \pm 97$ kcal, $\mathrm{P}=0.0227$ ). Nausea (Ex: $34 \%$; Sita: $12 \%$ ) and/or vomiting (Ex: $24 \%$; Sita: $3 \%$ ), were the most common adverse events.

Conclusion: This study demonstrates a greater decline in PPG with Ex vs. Sita, which may be due in part to more significant effects of Ex on slowing gastric emptying (Ex only), a more robust PP insulin response, and a greater reduction in PP glucagon. Furthermore, decreased ad libitum food intake was observed only with Ex. These data delineate key differences in the MOAs between exenatide and sitagliptin.

\section{3}

Comparison of glucose variability in patients with diabetes treated with exenatide or a biphasic insulin regimen L.C. Glass ${ }^{1}$, Y. $\mathrm{Qu}^{1}$, M.S. Lewis ${ }^{1}$, I.B. Hirsch ${ }^{2}$

${ }^{1}$ Eli Lilly, Indianapolis, United States, ${ }^{2}$ Metabolism, Endocrinology \& Nutrition, University of Washington Medical Center, Seattle, United States

Background and aims: High variability in glucose concentrations is associated with an increased production of reactive oxygen species, which leads to oxidative stress and has been associated with vascular complications in type 2 diabetes (T2D). This post-hoc analysis compared glucose variability in patients with T2D randomized to treatment with either exenatide (EXE) or biphasic insulin aspart (BASP).

Materials and methods: 478 patients with T2D suboptimally controlled on metformin and sulfonylurea, with a baseline and at least one post-baseline measurement for 7-point self monitored blood glucose profile (7-SMBG), were randomized in an open label study to add either EXE ( $\mathrm{n}=239 ; 5 \mu \mathrm{g}$ bid for 4 weeks, then $10 \mu \mathrm{g}$ bid through study end) or BASP bid before morning and evening meals $(n=239$; titrated to achieve fasting plasma glucose $<7 \mathrm{mM}$ and postprandial plasma glucose $<10 \mathrm{mM}$ ) for a total of 12 months. 7-SMBG measurements were collected before and after the morning, midday, and evening meals, and $3 \mathrm{AM}$, on two separate days within one week before each clinic visit. The daily mean, SD, M-value and mean amplitude of 
glycemic excursion (MAGE) of glucose for each patient was calculated using the 7 measurements in that day. The between-day standard deviation for each patient was calculated using the matching values of each 7-SMBG time point from two separate days. The mean of the between- and within-day SD, daily mean, M-value and MAGE of glucose were summarized and compared at baseline using ANOVA, and at the end of study using ANCOVA.

Results: Baseline values for age (58.7 years), BMI $\left(30.4 \mathrm{~kg} / \mathrm{m}^{2}\right)$, sex $(50.8 \%$ male), and A1C (8.6\%) were similar between EXE and BASP, as were all baseline values for the mean daily plasma glucose, the mean of the betweenand within-day SD, M-value, and MAGE. At study end, both treatments significantly reduced $\mathrm{AlC}$ to a similar extent (mean \pm SE change: BASP, -0.88 \pm 0.07 ; EXE, $-1.00 \pm 0.07 ; \mathrm{p}<0.001$ compared to baseline, $\mathrm{p}=0.20$ between treatments). Mean daily plasma glucose or M-value was not statistically significantly different between treatments, however EXE had significantly lower within-day plasma glucose SD (BASP $2.09 \mathrm{mM}$ vs. EXE $1.78 \mathrm{mM}, \mathrm{p}<0.001$ ) and MAGE (BASP $3.73 \mathrm{mM}$ vs. EXE $3.23 \mathrm{mM}$, p<0.001). Overall, patients treated with EXE also had significantly lower between-day plasma glucose SD than those on BASP (Table 1). The overall incidence of hypoglycemia for BASP and EXE was not different between groups ( $17.6 \%$ vs. $14.6 \%, \mathrm{p}=0.07)$, but the incidence of nocturnal hypoglycemia was significantly greater for BASP than for EXE ( $6.3 \%$ vs. $0.8 \%, \mathrm{p}=0.002)$.

Conclusion: EXE reduced both within- and between-day glucose variability significantly more than BASP, and had a lower incidence of nocturnal hypoglycemia than BASP. Thus, EXE resulted in a more favorable glucose profile, which may lead to less oxidative stress. This last possibility requires further study.

Table 1. Within-patient, between-day BG variability (mean SD from 2 $B G$ reading days)

\begin{tabular}{|c|c|c|c|c|c|}
\hline & \multicolumn{2}{|c|}{$\begin{array}{l}\text { Insulin Aspart } \\
(\mathrm{mM})\end{array}$} & \multicolumn{2}{|c|}{ Exenatide (mM) } & \multirow{2}{*}{$\begin{array}{l}\text { Between- } \\
\text { treatment } \\
\text { P-value } \\
52 \text { weeks }\end{array}$} \\
\hline & Baseline $\dagger$ & 52 weeks & Baseline $\dagger$ & 52 weeks & \\
\hline Before Breakfast & 1.10 & $0.92^{*}$ & 1.00 & $0.88^{*}$ & 0.59 \\
\hline After Breakfast & 1.50 & 1.46 & 1.39 & $1.04^{*}$ & $<0.001$ \\
\hline Before Lunch & 1.47 & $1.33^{*}$ & 1.54 & $1.13^{*}$ & 0.053 \\
\hline After Lunch & 1.81 & $1.54^{*}$ & 1.63 & $1.27^{*}$ & 0.02 \\
\hline Before Dinner & 1.70 & $1.47^{\star}$ & 1.79 & $1.21^{*}$ & 0.02 \\
\hline After Dinner & 1.70 & $1.46^{*}$ & 1.63 & $1.21^{*}$ & 0.03 \\
\hline $3 \mathrm{AM}$ & 1.28 & $1.11^{\star}$ & 1.22 & $1.00^{*}$ & 0.30 \\
\hline $\begin{array}{l}\text { Average of } \\
\text { 7-Point SMBG }\end{array}$ & 1.52 & $1.32^{\star}$ & 1.48 & $1.15^{*}$ & 0.004 \\
\hline
\end{tabular}

${ }^{*} \mathrm{p}<0.05$ compared to baseline, $\uparrow$ baseline values not significantly different between treatments

Supported by: Eli Lilly and Company

\section{4}

Exenatide and biphasic insulin aspart differentially affect postprandial glucose excursions in patients with type 2 diabetes

J.K. Malone ${ }^{1}$, R. Brodows ${ }^{1}$, Y. Qu ${ }^{1}$, D. Johns ${ }^{1}$, B. Calhoun ${ }^{1}$, T. Okerson ${ }^{2}$, J. Holcombe ${ }^{1}$

${ }^{1}$ Eli Lilly and Company, Indianapolis, ${ }^{2}$ Amylin Pharmaceuticals, San Diego, United States

Background and aims: In patients with type 2 diabetes (T2D) and mild to moderate elevations in $\mathrm{HbAlc}$, evidence suggests that postprandial glucose (PPG) excursion, compared with fasting glucose (FG), has a greater effect on overall glycemic control. Treatment of T2D with exenatide (EXE) or biphasic insulin aspart 30/70 (BASP) results in similar improvements in glycemic control when added to metformin and a sulfonylurea. In the current post hoc analysis we examined the relative contribution of PPG excursions to hyperglycemia in EXE and BASP-treated patients.

Materials and methods: A total of 463 patients [(EXE: 232, BASP: 231) mean \pm SD: age $58.6 \pm 8.9$ years; $\mathrm{HbAlc} 8.6 \pm 1.0 \%$; BMI $\left.30.4 \pm 4.1 \mathrm{~kg} / \mathrm{m}^{2}\right]$ with a baseline and at least one post-baseline self-monitored blood glucose (SMBG) (before and 2 hours after each meal) were included in the analysis. PPG area under the curve $\left(\mathrm{AUC}_{\mathrm{PPG}}\right)$ excursions, total glucose area under the curve but above $6.1 \mathrm{mmol} / \mathrm{L}\left(\mathrm{AUC}_{\mathrm{T}}\right)$ ) and the percentage of hyperglycemia due to PPG excursions $\left(\mathrm{AUC}_{\mathrm{PPG}} \div \mathrm{AUC} \mathrm{T}_{\text {Total }} \times 100\right)$ were calculated and compared between treatments.
Results: At baseline, $\mathrm{AUC}_{\mathrm{PPG}} \mathrm{AUC}_{\mathrm{Total}}$, and mean $\mathrm{FG}$ were similar between the EXE and BASP-treated patients. At 52 weeks, both EXE and BASP treatment significantly reduced overall hyperglycemia $\left[\mathrm{AUC}_{\text {Total }} \pm \mathrm{SE}\right](-22.1 \pm 2.0 \mathrm{mmol} /$ $\mathrm{L}^{*} \mathrm{~h}$ vs. $-25.3 \pm 2.1 \mathrm{mmol} / \mathrm{L}^{*} \mathrm{~h}, \mathrm{P}=0.70$ between treatments $)$ and $\mathrm{FG}(-1.6 \pm 0.2$ $\mathrm{mmol} / \mathrm{L}^{\star} \mathrm{h}$ for both groups; $\mathrm{P}=0.54$ between treatments). EXE-treatment reduced PPG excursions significantly more than did treatment with BASP $(-8.2 \pm$ $1.0 \mathrm{mmol} / \mathrm{L}^{\star} \mathrm{h}$ vs. $\left.-4.7 \pm 1.0 \mathrm{mmol} / \mathrm{L}^{\star} \mathrm{h}, \mathrm{P}=0.002\right)$. The greater reduction in PPG excursion with EXE-treatment resulted in significantly lower contribution of PPG to overall hyperglycemia compared to BASP ( $37 \%$ vs. $42 \%, \mathrm{P}=0.04$ ).

Conclusion: Although both EXE- and BASP-treatments significantly improved overall glycemic control, EXE reduces PPG excursions more than BASP. Therefore, EXE should be considered an important treatment option in helping patients with T2D achieve post-prandial, and thus, overall glycemic control.

Supported by: Eli Lilly and Company and Amylin Pharmaceuticals

\section{5}

Change in $\mathrm{HbA}_{1 \mathrm{c}}$, fasting serum glucose, weight, lipids, and vital signs by baseline BMI in 2 clinical trials of exenatide $v s$. insulin comparators M. Trautmann ${ }^{1}$, D. Johns ${ }^{1}$, J. Burger ${ }^{1}$, T. Okerson ${ }^{2}$, R. Brodows ${ }^{1}$

${ }^{1}$ Lilly Research Laboratories, Eli Lilly and Company, Indianapolis, ${ }^{2}$ Amylin Pharmaceuticals, Inc., San Diego, United States

Background and aims: Exenatide (EX) treatment is associated with weight reduction. Consequently, we examined the relationship between baseline $\mathrm{BMI}$ and change in $\mathrm{HbA}_{1}$, fasting serum glucose (FSG), weight, lipids, and vital signs in patients with type 2 diabetes treated with metformin (MET)/ sulphonylurea (SU) and either EX or an insulin comparator (insulin glargine [IG] or biphasic insulin aspart [IA]).

Materials and methods: Data from 2 clinical trials of patients with type 2 diabetes with suboptimally controlled glycaemia treated with MET/SU were examined. Patients were treated with EX ( $n=281$ vs. IG, $n=251$ vs. IA), IG ( $\mathrm{n}=267$, QD at bedtime for $26 \mathrm{wks}$ ), or IA ( $\mathrm{n}=248$, BID for $52 \mathrm{wks}$ ). Data collected at 28 wks from the EX vs. IA study were pooled with data from the EX vs. IG study at 26 wks. Insulin doses were titrated for optimal glucose control. EX was administered at $5 \mu \mathrm{g}$ BID for 4 wks and $10 \mu \mathrm{g}$ BID thereafter. All patients continued their pre-study MET/SU treatments. Patients were divided into baseline BMI quartiles $\left(\mathrm{kg} / \mathrm{m}^{2}\right.$ [min-max]; exenatide, low $[\geq 22.4-\leq 27.6]$, midlow [ $>27.6-\leq 30.4]$, midhigh $[>30.5-\leq 33.5]$, high $[>33.5-\leq 45.5]$; insulin comparators, low $[\geq 22.5-\leq 27.3]$, midlow $[>27.3-\leq 30.1]$, midhigh $[>30.1-$ $\leq 33.7]$, high [ $>33.7-\leq 44.2])$. Correlations were determined between baseline BMI and change in $\mathrm{HbA}_{1 c}$, FSG, weight, lipids (total cholesterol, HDL, LDL, triglycerides), heart rate, and systolic and diastolic blood pressure. From analysis of covariance, changes within each treatment and baseline BMI quartile and Least Square (LS) mean differences (exenatide minus insulin) and 95\% confidence intervals (CI) are presented.

Results: There were no significant between group differences in $\mathrm{HbA}_{1 \mathrm{c}}$ changes in all patients and across all BMI quartiles. Compared with the insulin group, the EX group had a significantly greater reduction in systolic blood pressure in all patients and across low, midlow, and high BMI quartiles and weight in all patients and across all BMI quartiles. Compared with the EX group, the insulin group had a significantly greater reduction in FSG in all patients and across low, midlow, and midhigh BMI quartiles (Table 1). There were no consistent significant between-group differences across BMI quartiles in changes in lipids, heart rate, and diastolic blood pressure. There were no significant correlations between baseline $\mathrm{BMI}$ and change in $\mathrm{HbA}_{1 \mathrm{c}}, \mathrm{FSG}$, weight, total cholesterol, HDL, LDL, triglycerides, heart rate, and systolic and diastolic blood pressure in either treatment group (all $|\mathrm{r}|<0.1, \mathrm{P} \geq 0.114$ ).

Conclusion: This post-hoc, meta-analysis shows that baseline BMI does not predict improvements in glycaemic control or lipid changes for either EX or insulin treatment. Regardless of initial BMI, there appears to be clinically meaningful effects on systolic blood pressure and weight with EX and larger decreases in FSG with insulin.

Supported by: Amylin Pharmaceuticals, Inc. and Eli Lilly and Company 


\section{6}

Exenatide is superior to insulin in attaining tight glycaemic control $\left(\mathrm{HbA}_{1 \mathrm{c}} \leq 6.5 \%\right)$ with a more favourable accompanying metabolic profile D. Maggs ${ }^{1}$, D. Bhole ${ }^{1}$, P. Yan ${ }^{1}$, A. Stonehouse ${ }^{1}$, R. Brodows ${ }^{2}$, T. Okerson ${ }^{1}$ ${ }^{1}$ Amylin Pharmaceuticals, Inc., San Diego, ${ }^{2}$ Lilly Research Laboratories, Eli Lilly and Company, Indianapolis, United States

Background and aims: Exenatide (Ex) and insulin (Ins) are injectable therapies used in the treatment of type 2 diabetes. In pooled datasets from two studies comparing $10 \mu \mathrm{g}$ exenatide BID to either Ins glargine or biphasic Ins aspart in patients treated with background therapy of metformin and sulphonylurea for approximately 6 months, we examined metabolic endpoints in those patients who achieved $\mathrm{HbA}_{1 \mathrm{c}}$ of $\leq 6.5 \%$.

Materials and methods: In this post-hoc analysis, 1015 patients comprised the pooled ITT population with entry $\mathrm{HbA}_{1 c}>6.5 \%$ and at least one postbaseline $\mathrm{HbA}_{1 \mathrm{c}}$ value $\left(547 \mathrm{M}, 468 \mathrm{~F}\right.$; age $58.8 \pm 9.1$; BMI $30.8 \pm 4.3 \mathrm{~kg} / \mathrm{m}^{2}$ $\left[\right.$ mean $\pm \mathrm{SD}$ ]; baseline $\mathrm{HbA}_{1 \mathrm{c}} 8.4 \pm 0.03 \%$; baseline FSG $10.7 \pm 0.09 \mathrm{mmol} / \mathrm{L}$ $[$ mean $\pm \mathrm{SE}]$ ).

Results: $\mathrm{HbA}_{1 \mathrm{c}}$ threshold, body weight change, and blood pressure data were as follows:

\begin{tabular}{|c|c|c|c|c|}
\hline & \multicolumn{2}{|l|}{ Exenatide } & \multicolumn{2}{|l|}{ Insulin } \\
\hline & $\begin{array}{l}\mathrm{HbA}_{1 \mathrm{c}} \\
\leq 6.5 \%\end{array}$ & $\begin{array}{l}\mathrm{HbA}_{1 \mathrm{c}} \\
>6.5 \%\end{array}$ & $\begin{array}{l}\mathrm{HbA}_{1 \mathrm{c}} \\
\leq 6.5 \%\end{array}$ & $\begin{array}{l}\mathrm{HbA}_{1 \mathrm{c}} \\
>6.5 \%\end{array}$ \\
\hline Dose & $10 \mu \mathrm{g}$ & $10 \mu \mathrm{g}$ & $24.2 \pm 2.1 \mathrm{U}^{¥}$ & $25.4 \pm 1.2 \mathrm{U}^{¥}$ \\
\hline $\mathrm{N}=$ & 133 & 383 & 85 & 414 \\
\hline$\%$ of patients & $26^{*}$ & 74 & 17 & 83 \\
\hline$\Delta$ Body weight $(\mathrm{kg})$ & $-3.7 \pm 0.3^{\star *}$ & $-1.6 \pm 0.1^{\star *}$ & $+0.19 \pm 0.3$ & $+2.0 \pm 0.1$ \\
\hline $\begin{array}{l}\Delta \text { Systolic blood } \\
\text { pressure }(\mathrm{mm} \mathrm{Hg})\end{array}$ & $-7.2 \pm 1.5^{\star}$ & $-3.6 \pm 0.8^{\dagger}$ & $-0.3 \pm 1.4$ & $-0.4 \pm 0.8$ \\
\hline $\begin{array}{l}\Delta \text { Diastolic blood } \\
\text { pressure }(\mathrm{mm} \mathrm{Hg})\end{array}$ & $-2.1 \pm 0.9^{\S}$ & $-1.1 \pm 0.5^{\S}$ & $-0.9 \pm 1.0$ & $-0.5 \pm 0.5$ \\
\hline
\end{tabular}

(mean \pm SE); ${ }^{*}$ Ins dose at approximately 6 months, ${ }^{\star} \mathrm{P}<0.005$,

${ }^{*} \mathrm{P}<0.0001,{ }^{\dagger} \mathrm{P}<0.05,{ }^{\circledR} \mathrm{P}=\mathrm{NS}$; Ex vs. Ins

In the patients who achieved $\mathrm{HbA}_{1 c}$ of $\leq 6.5 \%$, Ex induced favourable reductions from baseline compared to Ins in total cholesterol (Ex: $-0.221 \pm 0.050$ $\mathrm{mmol} / \mathrm{L}, \mathrm{P}<0.0001$; Ins: $+0.002 \pm 0.093 \mathrm{mmol} / \mathrm{L}, \mathrm{P}=\mathrm{NS} ; \Delta \mathrm{P}<0.005)$ and LDLcholesterol (Ex: $-0.162 \pm 0.045 \mathrm{mmol} / \mathrm{L}, \mathrm{P}<0.0005$; Ins: $+0.130 \pm 0.075 \mathrm{mmol} /$ $\mathrm{L}, \mathrm{P}=\mathrm{NS} ; \Delta \mathrm{P}<0.0005)$. Both Ex and Ins induced favourable changes compared to baseline in HDL-cholesterol (Ex: $+0.058 \pm 0.014 \mathrm{mmol} / \mathrm{L}, \mathrm{P}<0.0001$; Ins: $+0.089 \pm 0.018 \mathrm{mmol} / \mathrm{L}, \mathrm{P}<0.0001 ; \Delta \mathrm{P}=\mathrm{NS})$ and triglycerides $(\mathrm{Ex}:-$ $0.185 \pm 0.090 \mathrm{mmol} / \mathrm{L}, \mathrm{P}<0.05 ;$ Ins: $-0.421 \pm 0.164 \mathrm{mmol} / \mathrm{L}, \mathrm{P}<0.05 ; \Delta \mathrm{P}=\mathrm{NS})$. In patients achieving tight glycaemic control $\left(\mathrm{HbA}_{1 \mathrm{c}}\right.$ of $\left.\leq 6.5 \%\right)$, hypoglycaemia occurred more frequently with Ins treatment compared to exenatide [overall: Ins 60\%, Ex 52\% ( $\triangle \mathrm{P}=\mathrm{NS})$; nocturnal: Ins 36\%, Ex 20\% $(\Delta \mathrm{P}<0.01)]$.

Conclusion: In summary, patients are more likely to achieve tight glycaemic control $\left(\mathrm{HbA}_{1 c}\right.$ of $\left.\leq 6.5 \%\right)$ with exenatide than with basal or biphasic aspart Ins. The exenatide-treated patients able to achieve tight glycaemic control manifest with a more favourable overall metabolic profile than those treated with insulin. The implications of the differential effects observed with these two therapeutic approaches deserve further study.

\section{7}

Exenatide improved systolic blood pressure compared to insulin or placebo in patients with type 2 diabetes

T. Okerson ${ }^{1}$, P. Yan ${ }^{1}$, A. Stonehouse ${ }^{1}$, R. Brodows ${ }^{2}$, D. Bhole ${ }^{1}$

${ }^{1}$ Amylin Pharmaceuticals, Inc., San Diego, ${ }^{2}$ Lilly Research Laboratories, Eli

Lilly and Company, Indianapolis, United States

Background and aims: The incretin mimetic exenatide (Ex) is approved as an adjunctive treatment of type 2 diabetes (T2DM). In pooled datasets from clinical studies comparing $10 \mu \mathrm{g}$ Ex BID to insulin glargine, biphasic insulin aspart, or placebo, we examined the effects on systolic blood pressure (SBP) in patients with T2DM in the normal $(<130 \mathrm{~mm} \mathrm{Hg})$ or abnormal $(\geq 130 \mathrm{~mm}$ $\mathrm{Hg})$ range after approximately 6 months of treatment.

Materials and methods: In this post-hoc analysis, 1050 patients comprised the ITT insulin-comparator (I-C) population $(54 \% \mathrm{M}$; age $59 \pm 0.3$; baseline (BL) BMI $31 \pm 0.1 \mathrm{~kg} / \mathrm{m}^{2}$; $\mathrm{HbA}_{1 \mathrm{c}} 8.4 \pm 0.03 \%$; BL FPG $10.7 \pm 0.09 \mathrm{mmol} / \mathrm{L}$; mean \pm SE). 1121 patients comprised the ITT PBO-controlled (PBO-C) population (59\% M; age $55 \pm 0.3$; BL BMI $33 \pm 0.2 \mathrm{~kg} / \mathrm{m}^{2}$; $\mathrm{HbA}_{1 \mathrm{c}} 8.4 \pm 0.03 \%$; FPG $9.9 \pm 0.09 \mathrm{mmol} / \mathrm{L}$; mean $\pm \mathrm{SE}$ ).

Results: SBP data were as follows:

For each strata of abnormal SBP (130-139 mm Hg, 140-149 mm Hg, and $\geq 150$ $\mathrm{mm} \mathrm{Hg}$ ) Ex-treated patients experienced greater improvement in SBP compared to insulin or PBO-comparators. For I-C, change from BL for Ex in each strata was $-3.3 \pm 1.2 \mathrm{~mm} \mathrm{Hg}, \mathrm{P}=0.0114 ;-5.9 \pm 1.1 \mathrm{~mm} \mathrm{Hg}, \mathrm{P}=0.0275 ;-16.4 \pm 1.5 \mathrm{~mm} \mathrm{Hg}$, $\mathrm{P}=0.0210$, respectively, and for $\mathrm{PBO}-\mathrm{C}$, change from $\mathrm{BL}$ for $\mathrm{Ex}$ in each strata was $-4.6 \pm 1.0 \mathrm{~mm} \mathrm{Hg}, \mathrm{P}=0.0288 ;-8.0 \pm 1.3 \mathrm{~mm} \mathrm{Hg}, \mathrm{P}=0.1201 ;-20.0 \pm 2.4 \mathrm{~mm} \mathrm{Hg}$, $\mathrm{P}=0.0151$, respectively (P value: Ex vs. I-C or PBO-C). Significant changes in overall diastolic blood pressures (DBP) were observed in the I-C (Ex: - $1.5 \pm 0.4$ $\mathrm{mm} \mathrm{Hg}, \mathrm{P}<0.0005$; PBO: $-0.8 \pm 0.4 \mathrm{~mm} \mathrm{Hg}, \mathrm{P}=0.0481$ ) but not the PBO-C (Ex: $-0.6 \pm 0.4 \mathrm{~mm} \mathrm{Hg}, \mathrm{P}=0.0924$; PBO: $-0.4 \pm 0.4 \mathrm{~mm} \mathrm{Hg}, \mathrm{P}=0.3206$ ). However, pulse pressure (PP) effects followed a similar pattern of improvement as SBP. In the abnormal SBP baseline groups, including weight change as a covariate in the analysis of covariance model showed favourable differences when Ex was compared to either insulin $(-2.1 \mathrm{~mm} \mathrm{Hg}, \mathrm{P}=0.077)$ or $\mathrm{PBO}(-3.2 \mathrm{~mm} \mathrm{Hg}, \mathrm{P}=0.003)$. In patients who lost weight in each group and had abnormal SBP, Ex produced greater SBP reductions (I-C: Ex: $-3.25 \pm 0.15 \mathrm{~kg} ;-10.2 \pm 0.9 \mathrm{~mm} \mathrm{Hg}$ vs. Insulin: $-1.96 \pm 0.19$ $\mathrm{kg} ;-4.5 \pm 1.6 \mathrm{~mm} \mathrm{Hg}$; SBP, Ex vs. insulin, $\mathrm{P}=0.0007$. PBO-C: Ex: $-3.29 \pm 0.16 \mathrm{~kg}$; $-8.7 \pm 1.0 \mathrm{~mm} \mathrm{Hg}$ vs. PBO: $-2.66 \pm 0.19 \mathrm{~kg} ;-6.6 \pm 1.0 \mathrm{~mm} \mathrm{Hg}$; SBP, Ex vs. PBO, $\mathrm{P}=0.0291$ ). The percentage of patients who changed their anti-hypertensive drug regimen was similar between Ex vs. I-C and Ex vs. PBO-C. Adverse events were as previously reported, and included nausea and vomiting in Ex patients.

Conclusion: Exenatide treatment resulted in greater improvement in SBP and PP compared to insulin or PBO-treated patients. These clinically meaningful blood pressure improvements with Exenatide therapy are only partially explained by weight loss and deserve further investigation.

\begin{tabular}{|c|c|c|c|c|c|c|}
\hline \multicolumn{7}{|l|}{ Insulin-comparator } \\
\hline & \multicolumn{2}{|c|}{ BL SBP $<130 \mathrm{~mm} \mathrm{Hg}$} & \multicolumn{2}{|c|}{$B L S B P \geq 130 \mathrm{~mm} \mathrm{Hg}$} & \multicolumn{2}{|l|}{ SBP overall } \\
\hline & Exenatide & Insulin & Exenatide & Insulin & Exenatide & Insulin \\
\hline $\mathrm{N}(\%)$ & $144(14)$ & $148(14)$ & $391(37)$ & $367(35)$ & $535(51)$ & $515(49)$ \\
\hline Baseline SBP (mm Hg) & $118 \pm 0.6$ & $118 \pm 0.6$ & $145 \pm 0.7$ & $144 \pm 0.7$ & $138 \pm 0.7$ & $136 \pm 0.7$ \\
\hline$\Delta$ Systolic blood pressure (mm Hg) & $+5.1 \pm 1.2$ & $+8.2 \pm 1.2$ & $-8.5 \pm 0.8$ & $-4.0 \pm 0.8$ & $-4.9 \pm 0.7$ & $-0.5 \pm 0.7$ \\
\hline$P$ value (end point vs. baseline) & $<0.0001$ & $<0.0001$ & $<0.0001$ & $<0.0001$ & $<0.0001$ & 0.4858 \\
\hline$P$ value ( $\Delta$ Ex vs. $\Delta$ insulin) & 0.0814 & & $<0.0001$ & & $<0.0001$ & \\
\hline \multicolumn{7}{|l|}{ PBO-comparator } \\
\hline & \multicolumn{2}{|c|}{ BL SBP $<130 \mathrm{~mm} \mathrm{Hg}$} & \multicolumn{2}{|c|}{$B L S B P \geq 130 \mathrm{~mm} \mathrm{Hg}$} & \multicolumn{2}{|l|}{ SBP overall } \\
\hline & Exenatide & PBO & Exenatide & PBO & Exenatide & PBO \\
\hline N (\%) & $276(25)$ & $255(23)$ & $285(25)$ & $305(27)$ & $561(50)$ & $560(50)$ \\
\hline Baseline SBP (mm Hg) & $117 \pm 0.5$ & $118 \pm 0.5$ & $140 \pm 0.6$ & $140 \pm 0.6$ & $129 \pm 0.6$ & $130 \pm 0.6$ \\
\hline$\Delta$ Systolic blood pressure (mm Hg) & $+5.1 \pm 0.8$ & $+6.4 \pm 0.8$ & $-8.3 \pm 0.8$ & $-4.6 \pm 0.8$ & $-1.7 \pm 0.7$ & $+0.4 \pm 0.6$ \\
\hline$P$ value (end point vs. baseline) & $<0.0001$ & $<0.0001$ & $<0.0001$ & $<0.0001$ & 0.0085 & 0.4947 \\
\hline$P$ value ( $\Delta$ Ex vs. $\Delta$ PBO) & 0.1272 & & $<0.0005$ & & $<0.0005$ & \\
\hline
\end{tabular}

(mean \pm SE) 


\section{8}

Beta cell function and glycaemic control following one year exenatide therapy, and after 12 week wash-out, in patients with type 2 diabetes A. Corner ${ }^{1}$, M.C. Bunck ${ }^{2}$, M. Diamant ${ }^{2}$, B. Eliasson ${ }^{3}$, J.L. Malloy ${ }^{4}$, R.M. Shaginian ${ }^{5}$, W. Deng ${ }^{4}$, D.M. Kendall ${ }^{4}$, M.R. Taskinen ${ }^{1}$, U. Smith ${ }^{3}$, H. YkiJarvinen ${ }^{1}$, R.J. Heine ${ }^{2}$

${ }^{1}$ Department of Medicine, Helsinki University Hospital, Finland,

${ }^{2}$ Department of Endocrinology, VU University Medical Center, Amsterdam, Netherlands, ${ }^{3}$ Lundberg Laboratory for Diabetes Research, Sahlgrenska

University Hospital, Goteborg, Sweden, ${ }^{4}$ Amylin Pharmaceuticals, Inc., San

Diego, United States, ${ }^{5}$ Eli Lilly, Nederland, Houten, Netherlands

Background and aims: Traditional blood glucose lowering agents do not sustain adequate glycaemic control in most type 2 diabetic (T2D) patients. Preclinical and clinical studies with exenatide, an incretin mimetic, have shown improvements in beta-cell function.

Materials and methods: This study compared the effect of 1-year exenatide (Ex) vs. insulin glargine (IG) treatment on beta cell function, and following a 12 -week wash-out. 69 metformin treated patients with T2D (mean \pm SD: age $59 \pm 8$; $\mathrm{HbAlC} 7.5 \pm 0.8 \%$; BMI $31 \pm 4 \mathrm{~kg} / \mathrm{m} 2$; body weight $91.5 \pm 13.1 \mathrm{~kg}$ ) were randomised to exenatide $(n=36)$ or insulin glargine $(n=33)$. Beta cell function was measured during a hyperglycaemic $(15 \mathrm{mM})$ clamp with arginine stimulation at week 0 , week 52 and after a 4 -week wash-out.

Results: Following 1 year treatment both therapies reduced $\mathrm{HbA1C}$ similarly: by $0.8 \pm 0.1 \%$, and $0.7 \pm 0.2 \%$ for exenatide and insulin glargine, respectively $(\mathrm{P}=0.92)$. After 12 weeks of wash-out, glycaemic control returned to pre-treatment values in both treatment groups. Exenatide significantly reduced body weight compared to insulin glargine $(-3.6 \pm 0.8 \mathrm{~kg}$ vs $+1.0 \pm 0.8 \mathrm{~kg}(\mathrm{P}=0.0002)$ after 1-year intensified treatment. After cessation of exenatide, body weight trended back to pre-treatment values after 12-weeks wash-out, however a significant between group difference remained $[-2.4 \pm 0.7 \mathrm{~kg}(\mathrm{Ex})$ and $+0.2 \pm 0.8 \mathrm{~kg}$ $(\mathrm{IG}) ; \mathrm{P}<0.0001]$. Combined glucose and arginine stimulated C-peptide secretion increase in the exenatide $(\mathrm{n}=29)$ group by 1.46 fold $(\mathrm{P}<0.0001)$ greater compared to insulin glargine $(\mathrm{n}=30)$ and relative to pre-treatment after 1 year. The mean $( \pm$ SE) AUC of the C-peptide response showed a $2.19 \pm 0.24$ vs. $0.31 \pm 0.07$ fold increase from pre-treatment, respectively $(\mathrm{P}<0.0001)$. After the 4-week wash out all clamp derived beta cell function parameters were not significantly different from pre treatment values.

Conclusion: One year of exenatide therapy, compared to insulin glargine improved indices of beta cell function, glycaemic control and body weight. Following wash-out the beneficial effects on beta cell function and glycaemic control were not sustained.

Supported by: Amylin Pharmaceuticals, Inc. \& Eli Lilly and Company

\section{9}

Exenatide improves postprandial hyperglycaemia and dyslipidaemia in metformin treated patients with type 2 diabetes

M.C. Bunck ${ }^{1}$, A. Corner ${ }^{2}$, M. Diamant ${ }^{1}$, B. Eliasson ${ }^{3}$, J.L. Malloy ${ }^{4}$, R.M. Shaginian ${ }^{5}$, W. Deng ${ }^{4}$, D.M. Kendall ${ }^{4}$, U. Smith ${ }^{3}$, H. Yki-Jarvinen ${ }^{2}$, R.J. Heine ${ }^{1}$, M.R. Taskinen ${ }^{2}$

${ }^{1}$ Department of Endocrinology / Diabetes Center, VU University Medical Center, Amsterdam, Netherlands, ${ }^{2}$ Department of Medicine, Helsinki University Hospital, Finland, ${ }^{3}$ Lundberg Laboratory for Diabetes Research, Sahlgrenska University Hospital, Goteborg, Sweden, ${ }^{4}$ Amylin Pharmaceuticals, Inc., San Diego, United States, ${ }^{5}$ Eli Lilly, Nederland, Houten, Netherlands

Background and aims: Postprandial metabolic derangements may affect cardiovascular disease risk. Current therapies do not adequately correct these disturbances in patients with type 2 diabetes (T2D).

Materials and methods: In this investigator designed study, we compared the long term effects of exenatide vs. insulin glargine treatment on the plasma glucose, insulin and lipid responses to a standardised meal. Sixty-nine metformin treated patients with T2D (age \pm sd $59 \pm 8 \mathrm{yr}$; HbAlc $7.5 \pm 0.8 \%$; BMI $31 \pm 4 \mathrm{~kg} / \mathrm{m} 2)$ were randomised to exenatide $(\mathrm{n}=36)$ or insulin glargine $(\mathrm{n}=33)$. Before and after 51 weeks on treatment and again after 5 weeks off-treatment, 59 patients (exenatide: $n=30$; insulin glargine: $n=29$ ) received a breakfast and lunch both containing $50 \mathrm{~g}$ of fat, $75 \mathrm{~g}$ of carbohydrates, and $35 \mathrm{~g}$ protein.

Results: At 1 year the incremental postprandial glucose excursion $( \pm \mathrm{SE})$, compared to pre-treatment areas under the curve, were lower for exenatide $(0.43 \pm 0.07)$, and remained similar for insulin glargine $(1.06 \pm 0.14 ; \mathrm{P}<0.0001)$. One year treatment with either drug reduced postprandial triglyceride excur- sions from pre-treatment $( \pm \mathrm{SE})$; for exenatide: $15399 \pm 7065.34 \mathrm{mg}^{\star} \mathrm{min} / \mathrm{dL}$, and insulin glargine: $521.79 \pm 4147.515 \mathrm{mg}^{*} \mathrm{~min} / \mathrm{dL}(\mathrm{P}=0.0155)$. After 1 year of treatment exenatide reduced the postprandial HDL decline compared to insulin glargine $(\mathrm{P}=0.0133)$. No effects on LDL and total cholesterol were observed. After the 5 week off treatment period, all postprandial measures were similar to those at pre treatment.

Conclusion: 1-year of exenatide therapy, compared to insulin glargine, lowered postprandial glucose and triglyceride excursions. These beneficial effects were not sustained 5-weeks following wash-out.

Supported by: Amylin Pharmaceuticals, Inc. \& Eli Lilly and Company

\section{0}

Patient views of diabetes treatment experience comparing once weekly and twice daily exenatide

K.S. Boye ${ }^{1}$, T.H. Kim², M. Martin ${ }^{3}$, M. Wintle ${ }^{2}$

${ }^{1}$ Eli Lilly and Company, Indianapolis, ${ }^{2}$ Amylin Pharmaceuticals, San Diego,

${ }^{3}$ Health Outcomes, Health Research Associates, Seattle, United States

Background and aims: Qualitative interviews were conducted on a subset of the population in a 52-week randomized, multicenter, open-label study (DURATION-1) of 295 subjects with Type 2 diabetes who received either a once weekly (QW) formulation of exenatide ( $2 \mathrm{mg}$, QW) or exenatide twice daily (10 mcg, BID).

Materials and methods: Exenatide BID subjects were switched to exenatide QW at week 30 through the remainder of their study participation. A subset of 33 subjects was enrolled in each study arm for telephone or face to face interviews. The first interviews were conducted within two weeks of enrollment and final interviews at 52 weeks. Subjects were asked to discuss their feelings about injections, treatment benefits, and treatment satisfaction. Transcript extracts were organized by like responses to compare exenatide QW and exenatide BID groups.

Results: For both the overall study and the interview subgroup, exenatide QW and exenatide BID populations were demographically similar, and the interview subgroup demonstrated comparable changes in clinical parameters as with the overall study results. 51 subjects (24 exenatide BID and 27 exenatide $\mathrm{QW})$ remained in the interview sample at Week 52 . When the exenatide BID group (who experienced both treatments) was asked to indicate their most beneficial change during treatment, most of the group $(63 \% ; \mathrm{N}=15)$ reported it to be the convenience of a once weekly injection, followed by $25 \%(\mathrm{~N}=6)$ citing control of blood glucose. When the exenatide QW group (on exenatide QW for 52 weeks) was asked to state the most beneficial benefit during treatment, most of the group (48\%; $\mathrm{N}=13)$ reported it to be lower blood sugar levels and $19 \%(\mathrm{~N}=5)$ reported it to be the convenience of a once weekly injection. At the week 30 interviews, $75 \%$ of the exenatide BID group and $90 \%$ of the exenatide QW group indicated that the treatment regimen was easy. By the 52 week interviews, $83 \%$ of the exenatide BID group who had switched to exenatide QW at week 30 and $96 \%$ of the exenatide QW group reported that the treatment regimen was easy. Other benefits expressed by both groups included weight loss, better eating habits, more energy and feeling better.

Conclusion: In conclusion, the majority of exenatide QW group reported their most beneficial change at 52 weeks as having their blood glucose in better control, while the exenatide BID group reported the most beneficial change was the convenience of a once weekly injection.

Supported by: Amylin Pharmaceuticals and Eli Lilly \& Company 


\section{PS 077 Exenatide: real life clinical practice}

\section{1}

\section{Real-world six-month outcomes of patients initiating exenatide in a} primary care electronic medical record database

M. Wintle ${ }^{1}$, R. Fabunmi ${ }^{1}$, K. Boye ${ }^{2}$, C. McAdam-Marx ${ }^{3}, X$. Ye ${ }^{3}$, D. Brixner ${ }^{3}$ ${ }^{1}$ Amylin Pharmaceuticals, Inc., San Diego, ${ }^{2}$ Lilly Research Laboratories, Eli Lilly and Company, Indianapolis, ${ }^{3}$ Pharmacotherapy, University of Utah, Salt Lake City, United States

Background and aims: This study evaluated real-world outcomes of $\mathrm{HbA}_{1 \mathrm{C}}$ and weight in patients with type 2 diabetes (T2DM) initiating exenatide therapy.

Materials and methods: Patients extracted from the General Electric (GE) electronic medical record (EMR) research database from January 1, 2000 through June 30, 2007 were $\geq 18$ years old, and T2D defined by ICD- 9 codes, $\geq 2$ fasting blood glucose levels $\geq 7.0 \mathrm{mmol} / \mathrm{L}$, or glycosylated hemoglobin $\left(\mathrm{HbA}_{1 \mathrm{C}}\right)$ over $7.0 \%$. Patients had prescription orders in the previous $395 \mathrm{~d}$ for metformin (MET), a sulphonylurea (SU), or a thiazolidinedione (TZD) as monotherapy or in combination. Baseline $\mathrm{HbA}_{1 \mathrm{C}}$, weight and $\mathrm{BMI}$ were documented $45 \mathrm{~d}$ prior to $15 \mathrm{~d}$ post-exenatide initiation and $\pm 45 \mathrm{~d}$ at $6 \mathrm{mo}$ post-exenatide initiation.

Results: For the 2086 patients with 6 mo of follow-up data, baseline mean (SD) $\mathrm{HbA}_{1 \mathrm{C}}$ was $8.4 \%$ (1.2), weight was $110.6 \mathrm{~kg}(24.9 \mathrm{~kg})$, and BMI was 38.5 $\mathrm{kg} / \mathrm{m}^{2}$ (7.9). Upon initiation of exenatide, 363 (17.4\%), 147 (7.1\%), and 84 (4.0\%) were on MET, SU, or TZD monotherapy, respectively; 524 (25.1\%) and 257 (12.3\%) were on MET with TZD or SU, and 711 (34.1\%) were on all three. Overall $\mathrm{HbA}_{1 \mathrm{C}}$ change at $6 \mathrm{mo}(\mathrm{n}=878)$ was $-0.7 \%$. When exenatide was added to MET, SU, or TZD monotherapy, changes were $-0.9 \%(\mathrm{p}<0.0001)$, $1.0 \%(\mathrm{p}<0.0001)$, and $-0.8 \%(\mathrm{p}<0.0032)$, respectively. $\mathrm{HbA}$ lowering ranged from $-0.5 \%$ to $-0.8 \%$ with multiple oral antidiabetic drugs (OADs) $(\mathrm{p}<0.0001$ ). Overall weight loss $(\mathrm{n}=1784)$ was $-2.8 \mathrm{~kg}(\mathrm{p}<0.0001)$. In the 6 mo following addition of exenatide to prior monotherapy, $14 \%, 23 \%$, and $45 \%$ of patients discontinued MET, SU, and TZD, respectively. Similar discontinuations were seen in the multiple OAD groups.

Conclusion: The average baseline $\mathrm{HbA}_{1 \mathrm{C}}(8.4 \%)$ and $\mathrm{BMI}\left(38.5 \mathrm{~kg} / \mathrm{m}^{2}\right)$ is high in this primary care T2DM population, suggesting difficulty in the real world to achieve $\mathrm{HbA}_{1 \mathrm{C}}$ and body weight goals. Exenatide therapy demonstrated significant reductions in $\mathrm{HbA}_{1 \mathrm{C}}$ and weight over 6 mo with decreases in concomitant medications.

\section{2}

Exenatide utilisation and effectiveness in a health plan population

B. Schroeder ${ }^{1}$, D. Misurski ${ }^{2}$, R. Wade ${ }^{3}$, R. Quimbo ${ }^{3}$, L. Nielsen ${ }^{1}$,

R. Fabunmi ${ }^{1}$, M. Wintle ${ }^{1}$

${ }^{1}$ Amylin Pharmaceuticals, Inc., San Diego, ${ }^{2}$ Lilly Research Laboratories, Eli Lilly and Company, Indianapolis, ${ }^{3}$ Consulting and Strategies, HealthCore, Inc., Wilmington, United States

Background and aims: Numerous clinical outcomes trials have demonstrated the benefits of achieving glycaemic goals in patients with type 2 diabetes (T2D). In controlled clinical trials, the incretin mimetic exenatide improved glycaemic control in patients with T2D; 34 to $46 \%$ of patients achieved $\mathrm{HbA}_{1 \mathrm{C}}$ $\leq 7 \%$ and mean $\mathrm{HbA}_{1 \mathrm{C}}$ change from baseline was -0.8 to $-0.9 \%$ (baseline $\mathrm{HbA}_{1 \mathrm{C}}: 8.2$ to $\left.8.7 \%\right)$. To investigate the effects of exenatide in clinical practice, this retrospective cohort study used a large, US commercial health plan claims database to describe baseline characteristics, comorbidities, concomitant therapies, and clinical effectiveness in patients initiated on exenatide.

Materials and methods: A total of 4936 patients were identified having a new prescription claim for exenatide between May 1, 2005 and June 30, 2006 (first claim = index date), with $\geq 12$ months of pre- and post-index eligibility, and $\geq 18$ years old.

Results: Mean $( \pm \mathrm{SD})$ age was $54 \pm 10$ years $(10 \% \geq 65 \mathrm{y} ; 51 \%$ female $)$. The 12 month mean (SE) medication possession ratio (MPR = days of supply/365 days) in patients with $>1$ prescription claim was $66 \pm 30 \%$. Most patients analysed $(94 \%)$ were treated with at least one other antidiabetic medication at initiation (100d pre-index to $15 \mathrm{~d}$ post-index); $23 \%$ with $1 \mathrm{drug}, 34 \%$ with 2 drugs, and $37 \%$ with $\geq 3$ drugs. The mean number of antidiabetic drugs (including exenatide) per patient was similar at initiation (3.08) and post-index
(3.05). Clinical effectiveness was measured in all patients with an A1C $>7.0 \%$ at baseline ( $\leq 100 \mathrm{~d}$ pre-index) and having both baseline and post-index (60$365 \mathrm{~d}) \mathrm{A1C}$ data available $\left(\mathrm{n}=201\right.$; mean baseline $\left.\mathrm{HbA}_{1 \mathrm{C}}=8.9 \pm 1.5 \%\right)$. In this cohort, $30 \%$ achieved $\mathrm{HbA}_{1 \mathrm{C}} \leq 7 \%$ in the post-index period and mean $\mathrm{HbA}_{1 \mathrm{C}}$ change from baseline was $-0.8 \%$. In patients with an MPR $>50 \%(n=104), 41 \%$ achieved $\mathrm{HbA}_{1 \mathrm{C}} \leq 7 \%$, and mean $\mathrm{HbA}_{1 \mathrm{C}}$ change from baseline was $-1.1 \%$. Conclusion: The mean change in $\mathrm{HbA}_{1 \mathrm{C}}$ and percentage of patients achieving $\mathrm{HbA}_{1 \mathrm{C}} \leq 7 \%$ in this real-world analysis mirrored results of controlled clinical trials. Furthermore, glycaemic improvement was achieved without a further increase in concomitant antidiabetic drugs.

\section{3}

Medication compliance and persistency in patients with type 2 diabetes initiating exenatide or insulin glargine

R. Fabunmi ${ }^{1}$, L. Nielsen ${ }^{1}$, R. Wade ${ }^{2}$, R. Quimbo ${ }^{2}$, B. Schroeder ${ }^{1}$, M. Massoudi ${ }^{1}$, D. Misurski ${ }^{3}$, M. Wintle ${ }^{1}$

${ }^{1}$ Amylin Pharmaceuticals, Inc., San Diego, ${ }^{2}$ Consulting and Strategies, HealthCore, Inc., Wilmington, ${ }^{3}$ Lilly Research Laboratories, Eli Lilly and Company, Indianapolis, United States

Background and aims: The incretin mimetic, exenatide (EX), improves glycaemic control in patients with type 2 diabetes (T2D) and was noninferior to insulin glargine or aspart in clinical trials. In this retrospective analysis of a US commercial health plan claims database, we studied T2D patients initiating EX or insulin glargine (IG). Both drugs are injectables frequently used in T2D patients not achieving glycaemic control with oral antidiabetic medications (OAD).

Materials and methods: Patients were $\geq 18 \mathrm{y}$ with a pre-index T2D diagnosis and an initial prescription claim for EX $(n=3262)$ or IG $(n=3038)$ between May 1, 2005 and June 30, 2006; $\geq 6$ mo pre-index (1st claim=index date), and $\geq 12$ mo post-index data. Baseline diabetes therapies were defined as prescriptions filled $100 \mathrm{~d}$ pre-index through $15 \mathrm{~d}$ post-index. The EX cohort had no previous exposure to insulins and vice versa; no other insulin was started. Results: EX: age ( \pm SD) $53 \pm 10 y$; $54 \%$ female. IG: age $56 \pm 12 y ; 41 \%$ female. The Deyo-Charlson comorbidity index (DCI) score was 1.45 for EX vs 1.82 for IG $(\mathrm{p}<0.001)$. Baseline OAD for EX vs IG, respectively, were $77 \%$ vs $69 \%$ MET; $47 \%$ vs $65 \%$ SFU; $50 \%$ vs $49 \%$ TZD; $56 \%$ vs $60 \%$ multiple OAD. For patients with $>1$ prescription fill, $12 \mathrm{mo}$ medication possession ratio $(\mathrm{MPR}=$ days of supply/365 d) was $68 \pm 29 \%$ for EX and $58 \pm 28 \%$, for IG ( $<<0.0001$ ). The percentage of this cohort with MPR $\geq 80 \%$ was significantly higher for EX than for IG ( $47 \%$ vs $29 \%$; $\mathrm{p}<0.0001)$. More patients discontinued treatment (prescription refill gap $>60 \mathrm{~d})$ for IG than for EX $(75 \%$ v $56 \%$; $<<0.0001)$. The corresponding percentages for a refill gap $>90 \mathrm{~d}$ were $52 \%$ and $34 \%$, respectively $(\mathrm{p}<0.0001)$.

Conclusion: This study provides the first real-world comparison of T2D patient characteristics newly initiated on EX or IG. Patients initiating EX had fewer comorbidities and fewer were treated with SFU. Compliance and persistency were generally good on both injectable drugs, but significantly better for EX.

\section{4}

Exenatide therapy in insulin treated patients with type 2 diabetes and obesity J.P. Govindan, B. Healey, D.N. Kalupahana, B.M. Singh

The Wolverhampton Diabetes Centre, New Cross Hospital, Wolverhampton, United Kingdom

Background and aims: Those with type 2 diabetes and obesity who are on insulin therapy are a challenging clinical problem, especially so if glycaemic control is poor. This often requires up-titration of insulin doses, resulting in further weight gain. An advantage of exenatide therapy is that it improves glycaemic control with concomitant weight loss. There are very few studies of exenatide and insulin combination therapy in such patients. Our aim was to study efficacy of combined exenatide and insulin in obese type 2 diabetes (ObT2DM) patients with poor glycaemic control.

Materials and methods: The study was undertaken in an outpatient setting. In this prospective study, to date (October 2007 to February 2008), we have initiated exenatide therapy in $40 \mathrm{ObT} 2 \mathrm{DM}$ patients with progressive weight gain and poor glycaemic control. According to a set protocol, the patients were to have regular follow up over a 6 month period; exenatide was initiated at $5 \mu \mathrm{gm}$ bi daily, maximized to $10 \mu \mathrm{gm}$ bi daily at four weeks; total insulin 
doses were halved at initiation; further dose down-adjustment occurred according to self blood glucose monitoring but every effort was made to not increase insulin doses to the 3 month point so long as glycaemic control was safe. The study evaluated the following parameters: $\mathrm{HbAlc}$, weight, insulin requirement, side effects and tolerability.

Results: We present the results of 27 patients who have completed three months treatment. Three patients were withdrawn from the study due to intolerable gastrointestinal side effects. The 24 patients remaining were aged 54 (41-65) years and $19(80 \%)$ were males. The baseline weight (mean \pm SD) was $128 \pm 15 \mathrm{~kg}$ which decreased to $115 \pm 14 \mathrm{~kg}$ at three months $(\mathrm{P}<0.001)$, a weight loss of $8.7 \pm 4.9 \mathrm{~kg}$ (range $2.8-28 \mathrm{~kg}$ ). Body mass index fell from $43.0 \pm$ $4.7 \mathrm{~kg} / \mathrm{m}^{2}$ to $39.8 \pm 4.5 \mathrm{~kg} / \mathrm{m}^{2}(\mathrm{P}<0.001)$. HbAlc at baseline $8.8 \pm 1.7 \%$ showed no change at three months being $8.5 \pm 1.7 \%(\mathrm{P}=0.23)$. Total daily insulin doses at baseline was $170 \pm 94 \mathrm{U} /$ day (range 48-420 ) fell drastically to $36 \pm$ $40 \mathrm{U} /$ day $(\mathrm{P}<0.001)$. Of the 24 patients, insulin was completely stopped in 10 patients and in the remaining 14 the doses ranged between 10-120 U/day. In addition to the 3 discontinued, 10 patients had mild nausea which improved with time. There were no episodes of significant hypoglycemia.

Conclusion: Exenatide can be used effectively in obese type 2 diabetes patients established on insulin therapy leading to significant weight loss and reduction in insulin dosage requirements but with no improvement in glycaemic control. It is expected that either glycaemic control will improve as weight loss progresses or that insulin will require up titration.

\section{5}

The short term effects of exenatide therapy in insulin treated patients with type 2 diabetes mellitus - a new option to achieve weight loss Y.P. Samarasinghe, A. Shlomowitz, N. Munro, M.D. Feher

Beta Cell Diabetes Centre, Chelsea and Westminster Hospital, London, United Kingdom

Background and aims: Exenatide is the first drug in the class of glucagon like peptide-1 (GLP-1) analogues. Through nutrient mediated release in the small intestine Exenatide induces glucose dependent insulin secretion. It also slows gastric emptying and produces satiety through hypothalamic pathways. Weight loss has been consistently reported with Exenatide in key licensing trials. Exenatide is licensed in the UK for combination therapy with metformin and/or sulphonylurea. It is not yet licensed for treatment with thiazolidinediones or insulin. Weight gain is often seen in insulin treated patients with T2DM. This additional weight may adversely affect other vascular risk factors including hypertension and dyslipidaemia and thus worsen overall cardiovascular risk. We undertook an audited to ascertain the effects of Exenatide therapy in insulin treated T2DM to establish the effects of this treatment on weight and diabetes control.

Materials and methods: This audit was conducted in a central London teaching hospital in a dedicated diabetes centre. All patients commenced on Exenatide in outpatients were entered into a database and over a maximum three month period followed up closely by diabetes specialist nurses and doctors in the clinic. Regular assessments of the patients were made looking for adverse effects of the drug, glycaemic control and changes in weight. All changes were documented on a spreadsheet from which we obtained our analysis.
Results: 71 patients who had been commenced on Exenatide and had at least one follow up visit were analysed. 23 of these patients had been on insulin (mean(SD) dose 69.0(62.1)units) prior to commencing the Exenatide. Median(range) age of this group was 58(41-73)years. Median(range) duration of diabetes was $18.1(4-53)$ years. 8 of these patients had their insulin withdrawn and 9 had it decreased on starting Exenatide (5 remained on the same dose and 1 increased). In the group that stopped insulin, 6 had to have it restarted (but with a lower mean(SD) starting dose of 55.7(63.5)units), 3 because of poor glycaemic control and 3 due to Exenatide intolerance. Mean(SD) weight and $\mathrm{HbAlc}$ at the start of treatment of insulin treated patients who tolerated the Exenatide were 106.6(13.9) $\mathrm{kg}$ and 9.2(1.6)\%. After a mean of 6 weeks follow up mean(SD) weight reduced by $2.2(2.5) \mathrm{kg}$ and at 3 months $1.7(1.1) \mathrm{kg}$. There was however no change in HbAlc at 3 months $(0.0(1.0) \%)$. Duration of diabetes did not predetermine whether Exenatide would be effective as patients above the median duration of diabetes also achieved the same weight reduction without losing glycaemic control at 3 months $(1.2(0.8) \mathrm{kg}$ and $0.0(0.4) \%$ respectively).

Conclusion: There now appears to be a choice of therapy for weight reduction in insulin treated T2DM patients. Many patients can be safely treated with Exenatide without deterioration in their glycaemic control and with the added benefit of weight loss. In the short term insulin may be withdrawn or have a reduction in dose requirement. Even in those individuals where insulin was recommenced, there was a reduction in the total daily dose which may have lessen the insulin associated weight gain. Intolerance of Exenatide in a clinic based setting does not seem to be any greater than in previous clinical trials. Individuals with a long history of T2DM may also benefit from Exenatide therapy.

\section{6}

Metabolic improvement associated with temporal pattern of weight loss in exenatide-treated patients with type 2 diabetes

D. Kendall ${ }^{1}$, L. Nielsen ${ }^{1}$, J. Han ${ }^{1}$, R. Brodows ${ }^{2}$, D. Bhole

${ }^{1}$ Amylin Pharmaceuticals, Inc., San Diego, ${ }^{2}$ Lilly Research Laboratories,

Eli Lilly and Company, Indianapolis, United States

Background and aims: Exenatide (EX) therapy improves glycaemic control and cardiometabolic risk factors in patients with type 2 diabetes. In clinical trials, EX reduced body weight (BW) in the majority of patients. However, the temporal pattern of weight loss was not fully examined.

Materials and methods: This post hoc analysis studies the specific impact of the pattern of BW change on glycaemic control and other metabolic risk factors during $2 \mathrm{y}$ of continuous EX therapy. We analysed a pooled database of patients treated with EX in combination with $\pm M E T \pm S U \pm T Z D$ for $2 y$ $(n=457)$. Patients were stratified into 3 cohorts based on the pattern of BW change during treatment: Early ( $>3 \%$ BW loss by Wk12, not regained); Late ( $>3 \%$ BW loss after Wk12); and Neutral ( $<3 \%$ BW loss).

Results: Demographic characteristics were similar across cohorts (age 5558y; BMI $31-34 \mathrm{~kg} / \mathrm{m}^{2}$ ). BW and $\mathrm{HbA}_{1 \mathrm{C}}$ changes are shown below. Similar results were observed when stratifying by $3 \%$ or $5 \%$ BW loss in patients treated with EX from 1 to $3.5 y$.

\section{Two Year Completers Stratified by 3\% Weight Loss}
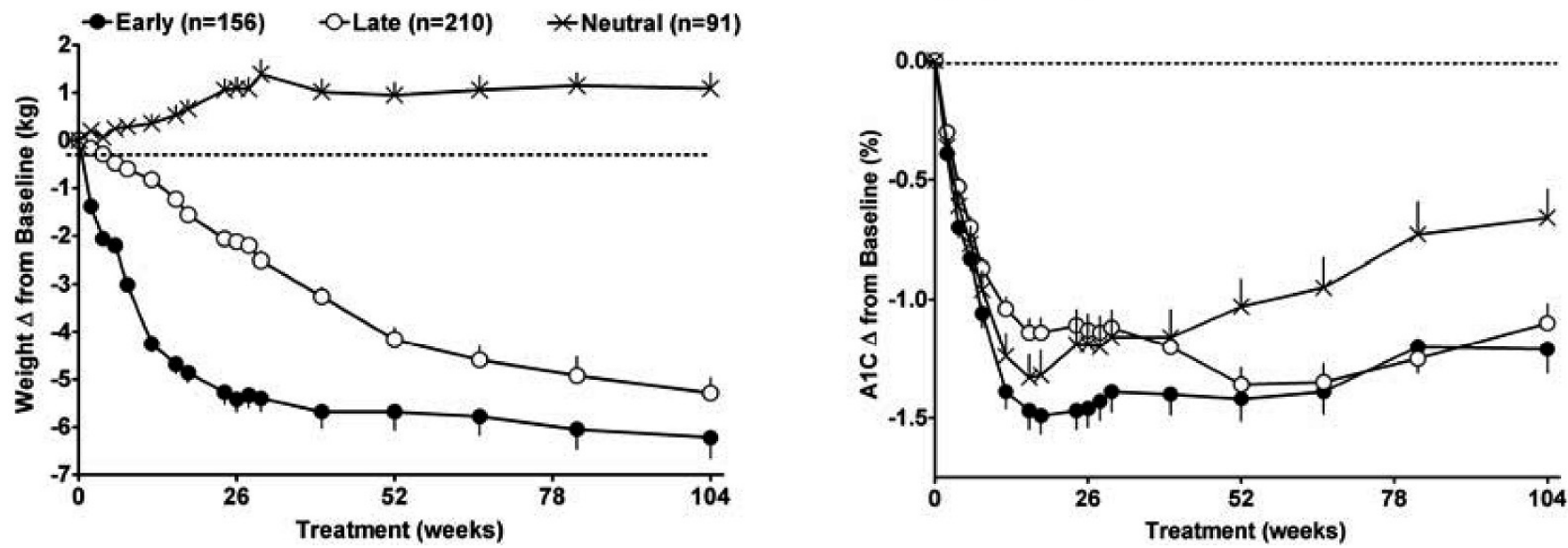
These data demonstrate that consistent BW loss was observed in a majority $(80 \%)$ of patients continuously treated with EX for $2 \mathrm{y}$. While one third (34\%) of EX-treated patients lost greater than 3\% BW during the first $12 \mathrm{wks}$ of therapy, an additional $46 \%$ had more gradual BW loss. Yet both groups achieved BW reductions of $\sim 5 \%$ over $2 \mathrm{y}$ and similar improvements in metabolic control. The neutral cohort also had significant $\mathrm{HbA}_{1 \mathrm{C}}$ reduction. EX therapy was associated with improvements in HDL-C, LDL-C, and triglycerides (TG), with larger reductions in TG in patients who achieved BW loss early.

Conclusion: Either early or later weight loss are observed in the majority of EX-treated patients, and this weight loss further enhances improvements in metabolic control.
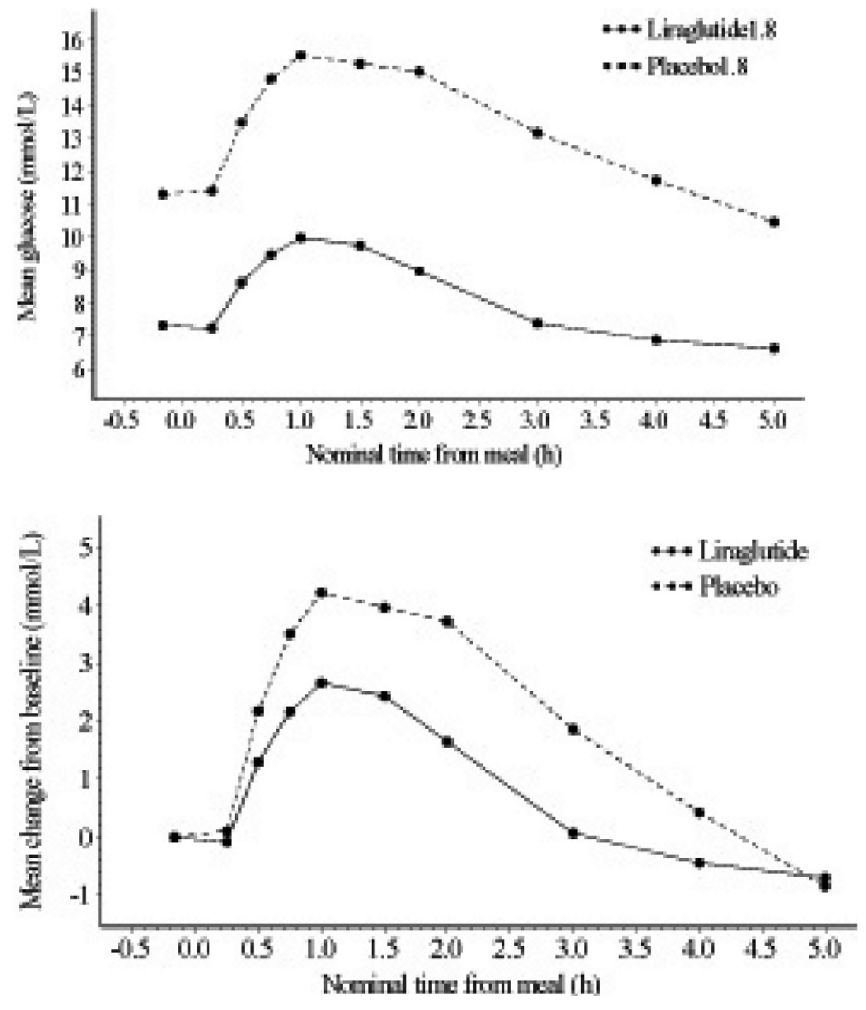

\section{PS 078 Liraglutide}

\section{7}

The once-daily human GLP-1 analogue liraglutide improves both absolute and baseline corrected postprandial glucose levels

A. Flint ${ }^{1}$, C. Kapitza ${ }^{2}$, C. Hindsberger ${ }^{1}$, M. Zdravkovic ${ }^{1}$

${ }^{1}$ Global Development, Novo Nordisk A/S, Bagsvaerd, Denmark, ${ }^{2}$ Profil Institut für Stoffwechselforschung GmbH, Neuss, Germany

Background and aims: GLP-1 is known to slow gastric emptying and decrease postprandial glucose.This sub-study investigated the effect of the oncedaily human GLP-1 analogue, liraglutide, on postprandial glucose, insulin and gastric emptying during a standard meal test.

Materials and methods: The trial was a randomised, placebo controlled, double-blind cross-over trial. Eighteen subjects with type 2 diabetes (diet treated or $\mathrm{OAD}$ monotherapy, mean $\mathrm{HbA}_{1 \mathrm{C}} 7.8 \pm 0.6 \%$ ) were enrolled. The dose of liraglutide was increased weekly by $0.6 \mathrm{mg}$ to $1.8 \mathrm{mg} /$ day (duration of each treatment period: 3 weeks) and meal tests were performed at each dose level. Before and after an energy fixed meal including $1.5 \mathrm{~g}$ paracetamol, blood samples for measurement of plasma glucose, insulin and paracetamol (gastric emptying) were drawn.

Results: At the highest dose tested, there was a significant lowering of fasting and peak postprandial glucose concentrations, together with a significant reduction in the incremental postprandial glycemic levels (on average $1.1 \mathrm{mmol} / \mathrm{L}$, see Figure). In spite of a lower glucose level, this was associated with a higher fasting and total insulin response compared to placebo. At the highest dose tested paracetamol kinetics was compatible with an early delay in gastric emptying $\left(\mathrm{AUC}_{0-60 \mathrm{~min}}\right)$, but no significant effect on $\mathrm{AUC}_{0-300 \mathrm{~min}}$. Figure: Mean absolute (left) and baseline corrected (right) postprandial plasma glucose concentrations after $1.8 \mathrm{mg} /$ day of liraglutide

Conclusion: Liraglutide $1.8 \mathrm{mg} /$ day improves not only fasting glucose levels, but also confers improvement in both absolute and incremental postprandial glucose levels. Based on this study, the postprandial effects on glycemic control of liraglutide, are most likely mediated through a combination of increased insulin secretion together with an early delay in gastric emptying.

\section{8}

Liraglutide, a human GLP-1 analogue, reduces bodyweight in subjects with type 2 diabetes, irrespective of body mass index at baseline O. Schmitz 1 , D. Russell-Jones ${ }^{2}$ J. Shaw ${ }^{3}$, M. Brandle ${ }^{4}$, D. Matthews ${ }^{5}$, A. Frid ${ }^{6}$, M. Zdravkovic ${ }^{7}$, A. Garber ${ }^{8}$, B. Zinman ${ }^{9}$

${ }^{1}$ Department of Endocrinology and Diabetes, University of Aarhus, Denmark, ${ }^{2}$ Endocrinology \& Metabolism, Royal Surrey County Hospital, Guildford, United Kingdom, ${ }^{3}$ International Diabetes Institute, Melbourne, Australia, ${ }^{4}$ Endocrinology, Kantonsspital, St Gallen, Switzerland, ${ }^{5}$ OCDEM, University of Oxford, United Kingdom, ${ }^{6}$ Endocrinology, Öresund Diabetes Team AB, Lund, Sweden, ${ }^{7}$ Novo Nordisk A/S, Bagsvaerd, Denmark, ${ }^{8}$ Baylor College of Medicine, Methodist Hospital, Houston, United States, ${ }^{9}$ Sinai Centre for Diabetes, Mount Sinai Hospital, Toronto, Canada

Background and aims: Oral antidiabetic drugs (OADs) prescribed for the treatment of subjects with type 2 diabetes are often associated with weight gain. Material and methods: This analysis examined the impact of baseline body mass index (BMI) on responses to liraglutide, the investigational once-daily human glucagon-like peptide (GLP)-1 analogue, added to various oral therapies. Mean (SE) changes in bodyweight (BW) from baseline to end-of-trial

\begin{tabular}{|c|c|c|c|c|c|}
\hline & \multicolumn{5}{|l|}{ Trial } \\
\hline & LEAD-1 & LEAD-2 & LEAD-3 & LEAD-4 & LEAD-5 \\
\hline Subjects (n) & 695 & 725 & 746 & 543 & 232 \\
\hline Concomitant OADs & glimepiride 2-4 mg & metformin $2 \mathrm{~g}$ & - & $\begin{array}{l}\text { metformin } 2 \mathrm{~g}+ \\
\text { rosiglitazone } 8 \mathrm{mg}\end{array}$ & $\begin{array}{l}\text { metformin } 2 \mathrm{~g}+ \\
\text { glimepiride } 2-4 \mathrm{mg}\end{array}$ \\
\hline Baseline $\mathrm{HbA}_{1 \mathrm{c}}(\%$, mean) & 8.4 & 8.3 & 8.3 & 8.3 & 8.6 \\
\hline Baseline BW (kg, mean) & 81.9 & 88.1 & 92.6 & 96.3 & 85.5 \\
\hline \multicolumn{6}{|c|}{$\Delta \mathrm{BW}($ mean, $\mathrm{kg}$ ) with liraglutide } \\
\hline $\mathrm{BMI}>35 \mathrm{~kg} / \mathrm{m}^{2}$ & -0.7 & -4.4 & -3.0 & -3.0 & -2.9 \\
\hline $30 \leq \mathrm{BMI}<35 \mathrm{~kg} / \mathrm{m}^{2}$ & -0.5 & -3.0 & -2.4 & -1.9 & -1.8 \\
\hline $25 \leq \mathrm{BMI}<30 \mathrm{~kg} / \mathrm{m}^{2}$ & -0.4 & -2.7 & -2.1 & -1.9 & -1.6 \\
\hline $\mathrm{BMI}<25 \mathrm{~kg} / \mathrm{m}^{2}$ & -0.1 & -1.5 & -1.2 & -2.5 & --1.3 \\
\hline
\end{tabular}


from four 26-week, and one 52-week phase 3 clinical trials (the Liraglutide Effect and Action in Diabetes [LEAD] studies) were divided according to screening BMI $\left(<25, \geq 25\right.$ to $<30, \geq 30$ to $\left.<35, \geq 35 \mathrm{~kg} / \mathrm{m}^{2}\right)$.

Results: In LEAD-1 approximately one-third of subjects were either slimmer Asians, and/or had previous metformin treatment discontinued at trial entry. Thus lower baseline weight, removal of metformin and addition of sulphonylureas (SU) could explain the absence of weight loss in that trial (Table 1). All BMI sub-groups experienced weight loss with liraglutide, with the greatest decrease in BW occurring in subjects with BMI $\geq 35 \mathrm{~kg} / \mathrm{m}^{2}$. In each subgroup, liraglutide + metformin (LEAD-2) produced a greater decrease in BW than liraglutide + glimepiride (LEAD-1), liraglutide monotherapy (LEAD-3), liraglutide + metformin + rosiglitazone (LEAD-4), or liraglutide + metformin + glimepiride (LEAD-5), except for the group with pre-trial BMI $<25 \mathrm{~kg} / \mathrm{m}^{2}$, in which liraglutide + metformin + rosiglitazone had the greatest effect.

Conclusion: Liraglutide added to OADs results in significant weight loss in subjects with type 2 diabetes, especially those with a high baseline BMI.

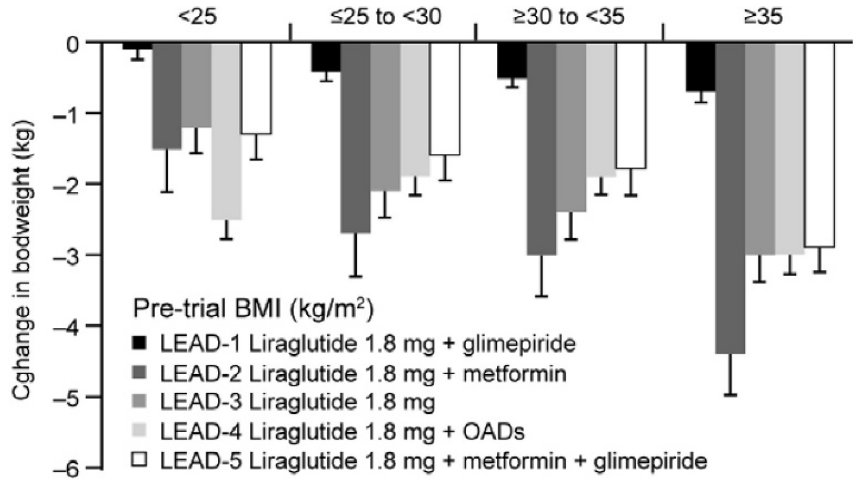

Supported by: Novo Nordisk A/S

\section{9}

Effects of the once-daily human GLP-1 analogue liraglutide on appetite and energy intake in type 2 diabetes

M. Horowitz ${ }^{1}$, A. Flint ${ }^{2}$, S. Doran ${ }^{1}$, M.F. Rasmussen ${ }^{2}$, C. Kapitza ${ }^{3}$, A.H. Andreasen $^{2}$, K.L. Jones ${ }^{1}$, M. Zdravkovic ${ }^{2}$, I.M. Chapman ${ }^{1}$

${ }^{1}$ Discipline of Medicine, University of Adelaide, Australia, ${ }^{2}$ Novo Nordisk A/S, Bagsvaerd, Denmark, ${ }^{3}$ Profil Institut für Stoffwechselforschung $\mathrm{GmbH}$, Neuss, Germany

Background and aims: Exogenous GLP-1 decreases appetite and energy intake. The aims of this study were to assess the effect of the once daily GLP-1 analogue, liraglutide, compared with glimepiride and placebo, on appetite, energy intake and meal duration at an ad libitum buffet meal and gastric emptying in type 2 diabetes.

Materials and methods: This trial was a double-dummy, randomised, double-blind two-centre study with a balanced, incomplete latin square design. Placebo and glimepiride were used as comparators. Forty-six subjects with type 2 diabetes (mean $\mathrm{HbA}_{1 \mathrm{C}} 7.4 \%$ ) treated by diet or oral antidiabetic drug monotherapy, were enrolled. The dose of liraglutide was increased weekly by $0.6 \mathrm{mg}$ to $1.8 \mathrm{mg} /$ day. Subjects were studied on two test days after two weeks' treatment at the highest liraglutide/glimepiride/placebo dose level. On Day 1 a yoghurt preload $(250 \mathrm{kcal} \sim 1047 \mathrm{~kJ})$, including $1.0 \mathrm{~g}$ paracetamol, was consumed, followed by a buffet meal one hour later. Gastric distension (by antral ultrasonography), gastric emptying (by plasma paracetamol concentrations), energy intake and its macronutrient distribution, duration of eating and sensations of appetite (hunger, fullness and satiety) and nausea (by visual analogue scales) were measured. On Day 2 resting energy expenditure was measured (by indirect calorimetry) before a buffet meal and energy intake, duration of eating and perceptions of appetite and nausea were measured again. Body weight was measured before and after each of the two treatment periods.

Results: The estimated reduction in ad libitum energy intake was $9 \%$ to $15 \%$ after liraglutide treatment compared to both placebo and glimepiride (NS) (meal with preload, estimated ratios: $0.91,95 \%$ CI $[0.78 ; 1.06]$ and $0.91,95 \%$ CI [0.78; 1.07]; meal without preload, estimated ratios: 0.91 [0.76; 1.09] and $0.85[0.70 ; 1.03])$. The energy intake was on average $3-4 \mathrm{MJ}$. At the meal with a preload the duration of eating was 4.3 minutes $95 \%$ CI [1.7; 6.9] less after liraglutide compared to placebo $(\mathrm{P}=0.002)$. Fasting hunger was lower after liraglutide compared to placebo or glimepiride on both days, however only significant on Day 2 (Day 1: $\mathrm{P}=0.117$ and Day 2: $\mathrm{P}<0.001$ ). The $\mathrm{AUC}_{0-3}$ (estimated ratio $0.88,95 \% \mathrm{CI}[0.80 ; 0.96]$ ) and $\mathrm{Cmax}$ (estimated ratio 0.80 , $95 \%$ CI $[0.72 ; 0.89])$ for paracetamol were significantly lower after liraglutide compared to placebo. Resting energy expenditure tended to be higher (not significantly) after liraglutide treatment compared to placebo and glimepiride (by $576 \mathrm{~kJ}$ and $270 \mathrm{~kJ}$ ). There were no treatment differences with respect to macronutrient composition, antral area or nausea (low ratings reported throughout). A significant weight loss of 1-2 $\mathrm{kg}$ was observed after liraglutide treatment compared to placebo and glimepiride (estimated differences -1.31 $\mathrm{kg}, 95 \%$ CI [-2.06; -0.56] and $-2.02 \mathrm{~kg}, 95 \%$ CI [-2.79; -1.24]).

Conclusion: In subjects with type 2 diabetes, four weeks' treatment with liraglutide decreased hunger, slowed gastric emptying and reduced body weight by $1-2 \mathrm{~kg}$. Treatment with liraglutide was also associated with a nonsignificant reduction in energy intake and a non-significant increase in resting energy expenditure.

\section{0}

The effect of liraglutide on the absorption pharmacokinetics of concomitant oral drugs with different solubility and permeability properties in healthy subjects

M. Zdravkovic ${ }^{1}$, M. Ekblom ${ }^{1}$, L. Brøndsted ${ }^{1}$, J. Vouis ${ }^{2}$, H. Lennernäs ${ }^{3}$, M. Malm-Erjefält ${ }^{1}$

${ }^{1}$ Novo Nordisk A/S, Bagsvaerd, Denmark, ${ }^{2}$ Phase 1 Services, Quintiles AB, Uppsala, Sweden, ${ }^{3}$ Department of Pharmacy, Uppsala University, Sweden

Background and aims: Liraglutide is a once-daily human GLP-1 analogue under development for the treatment of type 2 diabetes. It causes a minor delay in gastric emptying and hence may potentially change the absorption pattern of concomitant oral drugs. In particular, drugs with low solubility may increase their dissolution if retained in the stomach for a prolonged period and thus have the potential to show increased exposure (AUC) and $\mathrm{C}_{\max }$, and a delay in $t_{\max }$. In this trial, the effect of liraglutide on the absorption pharmacokinetics of four drugs with different solubility $(\mathrm{S})$ and permeability $(\mathrm{P})$ characteristics was investigated in a randomised, double-blind, two-period crossover trial. The four oral drugs were selected based on the Biopharmaceutical Classification System (BCS).

Materials and methods: This trial was conducted in two parts. In part A, single doses of $40 \mathrm{mg}$ atorvastatin and $500 \mathrm{mg}$ griseofulvin (Class II drugs; high $\mathrm{S} \&$ low $\mathrm{P})$ were given to healthy subjects $(\mathrm{n}=42)$. In part $\mathrm{B}$, separate subjects $(\mathrm{n}=28)$ received single doses of $20 \mathrm{mg}$ lisinopril (Class III drug; low $\mathrm{S} \&$ high $\mathrm{P}$ ) and $1 \mathrm{mg}$ digoxin (Class IV drug; low $\mathrm{S} \& \mathrm{P}$ ). The doses were given in each crossover period, separated by a wash-out period of 9 days, and the dosing times ensured the absorption peaks to correspond with the expected $\mathrm{C}_{\max }$ of $1.8 \mathrm{mg}$ liraglutide at steady state. Blood was collected up to $60-72 \mathrm{~h}$ after dosing and analysed for respective drug. For $\mathrm{AUC}$ and $\mathrm{C}_{\max }$, equivalence was declared if the $90 \%$ CI of the liraglutide/placebo ratio was within $[0.80 ; 1.25]$.

Results: During liraglutide treatment, the AUCs of griseofulvin and atorvastatin were equivalent to that after placebo treatment while the AUCs of lisinopril and digoxin $\left(\mathrm{AUC}_{0-72}\right.$ ) decreased by $15 \%$ and $16 \%$, respectively. Moreover, $\mathrm{C}_{\max }$ for atorvastatin, lisinopril and digoxin were decreased by $38 \%, 27 \%$ and $31 \%$ while $\mathrm{C}_{\max }$ for griseofulvin was increased by $37 \%$. In addition, griseofulvin $t_{\max }$ was not affected by liraglutide treatment, while $t_{\text {max }}$ for atorvastatin, lisinopril and digoxin were delayed by $1.25 \mathrm{~h}, 2.00 \mathrm{~h}$ and 1.12 $\mathrm{h}$. The unchanged overall exposure of both griseofulvin and atorvastatin support that liraglutide only induced a slight delay in gastric emptying which did not enhance the overall dissolution and absorption of the drugs to any major extent. The decreased $\mathrm{C}_{\max }$ for atorvastatin was most likely an effect of the delayed gastric emptying since the absorption is normally very rapid from the small intestine. The lower $\mathrm{C}_{\max }$ and delayed $\mathrm{t}_{\max }$ for lisinopril and digoxin was in accordance with a slight delay in gastric emptying induced by liraglutide. The minor decease in plasma AUC for lisinopril and digoxin suggested that the extent of absorption was not affected to any major degree.

Conclusion: The liraglutide-induced shift in the absorption kinetics of the oral drugs followed the expected kinetics according to the individual drugs absorption properties and supported the presence of a slight delay in gastric emptying. However, based on the drugs exposure levels the changes in absorption kinetics were considered minor and with no clinical significance. Further, a similar absorption pattern would be expected for other concomitantly taken BCS Class II, III and IV drugs. 


\section{1}

Metabolism and excretion of the once-daily human GLP-1 analogue liraglutide in healthy subject and its in vitro degradation by dipeptidyl peptidase IV and neutral endopeptidase

I. Bjørnsdottir ${ }^{1}$, A. Olsen ${ }^{1}$, U. Larsen ${ }^{2}$, H. Helleberg ${ }^{1}$, J. Vanggaard ${ }^{1}$,

B. Oosterhuis ${ }^{3}$, J. van Lier ${ }^{3}$, M. Zdravkovic ${ }^{4}$, M. Malm-Erjefält ${ }^{4}$

${ }^{1}$ DMPK, Novo Nordisk A/S, Måløv, Denmark, ${ }^{2}$ Isotope Chemistry, Novo

Nordisk A/S, Måløv, Denmark, ${ }^{3}$ Clinical Research, PRA Int., Zuidlaren,

Netherlands, ${ }^{4}$ GLP-1 Development, Novo Nordisk A/S, Bagsvaerd,

Denmark.

Background and aims: Liraglutide is a once-daily human GLP-1 analogue in clinical development for the treatment of type 2 diabetes. The present study investigated the elimination pathways of liraglutide and its metabolite profiles in plasma and excreta in humans. Furthermore, the in vitro degradation of liraglutide and GLP-1 by dipeptidyl peptidase IV (DPP-IV) and neutral endopeptidases (NEP) was studied to identify liraglutide metabolites and investigate if these could be related to the metabolites found in human plasma and to those of native GLP-1.

Materials and methods: Seven healthy males received a single dose of $\left[{ }^{3} \mathrm{H}\right]-$ liraglutide $(0.75 \mathrm{mg} / 385 \mu \mathrm{Ci})$. Blood, urine and faeces were sampled up to 14 days post dose and the recovered radioactivity measured by liquid scintillation counting and metabolites profiled by HPLC and radiochromatography. $\left[{ }^{3} \mathrm{H}\right]$-liraglutide was incubated in vitro for 4,8 and $24 \mathrm{~h}$, and $\left[{ }^{3} \mathrm{H}\right]$-GLP-1 for $0.5,1$ and $2 \mathrm{~h}$, with DPP-IV and NEP. Liraglutide metabolites were identified by liquid chromatography and mass spectrometry and compared to the GLP1 metabolites reported in the literature. The in vitro liraglutide and GLP-1 metabolite profiles, from incubation with DPP-IV and NEP, were compared to the metabolite profile observed in human plasma.

Results: The cumulative recovery of total radioactivity in urine and faeces was $20 \%$ and $6 \%$, respectively. The majority of the radioactivity excreted in urine was suggested to be tritiated water since the total recovered radioactivity in freezedried samples was only $6 \%$ in urine and $5 \%$ in faeces. Three minor liraglutiderelated metabolites were excreted in urine and faeces, respectively, during the first 6-8 days post dose but no intact liraglutide was detected. In plasma, intact liraglutide accounted for $\geq 89 \%$ of the total radioactivity exposure (AUC ${ }_{2-24}$ ). Simultaneously, two minor metabolites were detected (representing $\leq 9 \%$ and $\leq 5 \%$ of total plasma radioactivity). Liraglutide was cleaved by DPP-IV exclusively in the $\mathrm{Ala}_{8}$ - $\mathrm{Glu}_{9}$ position of the N-terminal of the peptide moiety, while NEP extensively degraded liraglutide into 11 different metabolites. Both DPPIV and NEP cleaved liraglutide at a much slower rate than observed for GLP-1. The cleavage sites of the observed in vitro liraglutide metabolites correlated well with the cleavage sites reported for native GLP-1. Furthermore, the chromatographic retention time of the primary human plasma metabolite correlated well with that of DPP-IV truncated liraglutide. However, both plasma metabolites eluted also very close to metabolites formed by NEP.

Conclusion: Liraglutide is metabolised in vitro by DPP-IV and NEP in a similar manner to native GLP-1 although at a much slower rate. The metabolite profiles suggest involvement of DPP-IV, and most likely NEP, also for degradation of liraglutide in vivo in human. Further, the low levels of metabolites in plasma, urine, and faeces and the lack of excreted intact liraglutide support that liraglutide is fully degraded within the body.

\section{2}

Liraglutide, a human GLP-1 analogue, significantly improves beta cell function in subjects with type 2 diabetes

D.R. Matthews ${ }^{1}$, M. Marre ${ }^{2}$, T.D. Le-Thi ${ }^{3}$, M. Zdravkovic ${ }^{3}$, R. Simo ${ }^{4}$,

A. Garber ${ }^{5}$, B. Zinman ${ }^{6}$

${ }^{1}$ The Oxford Centre for Diabetes Endocrinology \& Metabolism, United Kingdom, ${ }^{2}$ Service d'Endocrinologie-Diabétologie, Groupe Hospitalier Bichat - Claude Bernard, Paris, France, ${ }^{3}$ Novo Nordisk A/S, Bagsvaerd, Denmark, ${ }^{4}$ Endocrinology, Vall d'Hebron University Hospital, Barcelona, Spain, ${ }^{5}$ Baylor College of Medicine, Methodist Hospital, Houston, United States, ${ }^{6}$ Sinai Centre for Diabetes, Mount Sinai Hospital, Toronto, Canada

Background and aims: Type 2 diabetes is associated with a reduced $\beta$-cell function and liraglutide, a once-daily human GLP-1 analogue, has been shown to increase $\beta$-cell function. This analysis examined the impact of liraglutide treatment on $\beta$-cell function in subjects with type 2 diabetes from four 26-week trials and one 52-week trial from the LEAD ( Liraglutide Effect and Action in Diabetes) studies.

Materials and methods: The trials were randomised, double-blind, placebo controlled trials. Baseline data and results are presented in Table 1 for the highest dose of liraglutide $(1.8 \mathrm{mg})$. In the trials, liraglutide was used as monotherapy, or added to OADs, or to metformin (met) $2 \mathrm{~g}$ and/or glimepiride 4 $\mathrm{mg}$. The comparators and background therapies are shown in Table 1.

Results: An increase in homeostasis model assessment (HOMA) $\beta$-cell function (between $28 \%$ and $34 \%$ from baseline, vs comparator) was demonstrated with $1.8 \mathrm{mg}$ liraglutide in four of the five trials, and the changes were significant versus either comparator or background. The pro-insulin/insulin ratio decreased with liraglutide in all trials (between 0.05 and 0.12 from baseline) and the difference was significant in all trials versus the comparator (not applicable for LEAD 3), and also when compared to background therapy in LEAD 1.

Conclusion: Based on these data, liraglutide significantly improved $\beta$-cell function. However, further studies are needed to evaluate whether this will lead to delay of progression of type 2 diabetes.

Supported by: Novo Nordisk A/S

\section{3}

Liraglutide inhibits hyperglycaemia induced expression of PAI-1 and vascular adhesion molecules in an in vitro model of endothelial cell dysfunction

A.E. Dear ${ }^{1}$, H. Liu ${ }^{1}$, L.B. Knudsen ${ }^{2}$, R.W. Simpson ${ }^{3}$

${ }^{1}$ Medicine, Monash University, Melbourne, Australia, ${ }^{2}$ Novo Nordisk, Malov, Denmark, ${ }^{3}$ Endocrinology, Box Hill Hospital, Melbourne, Australia

Background and aims: Hyperglycemia in type 2 diabetes is associated with endothelial cell dysfunction one of the earliest detectable abnormalities in the

Baseline data and comparisons of beta-cell function in five trials investigating liraglutide

\begin{tabular}{|c|c|c|c|c|c|}
\hline $\begin{array}{l}\text { Trial ID } \\
\text { (number of subjects) }\end{array}$ & $\begin{array}{l}\text { LEAD 1 } \\
(\mathrm{n}=1041)\end{array}$ & $\begin{array}{l}\text { LEAD 2 } \\
(\mathrm{n}=1091)\end{array}$ & $\begin{array}{l}\text { LEAD } 3 \\
(\mathrm{n}=746)\end{array}$ & $\begin{array}{l}\text { LEAD } 4 \\
(\mathrm{n}=533)\end{array}$ & $\begin{array}{l}\text { LEAD } 5 \\
(\mathrm{n}=581)\end{array}$ \\
\hline $\begin{array}{l}\text { Treatment } \\
\text { Comparator }\end{array}$ & $\begin{array}{l}\text { liraglutide } \\
\text { rosiglitazone }\end{array}$ & $\begin{array}{l}\text { liraglutide } \\
\text { glimepiride }\end{array}$ & $\begin{array}{l}\text { liraglutide } \\
\text { glimepiride }\end{array}$ & $\begin{array}{l}\text { liraglutide } \\
-\end{array}$ & $\begin{array}{l}\text { liraglutide } \\
\text { glargine }\end{array}$ \\
\hline Background therapy & glimepiride & metformin & - & $\begin{array}{l}\text { metformin }+ \\
\text { rosiglitazone }\end{array}$ & $\begin{array}{l}\text { metformin }+ \\
\text { glimepiride }\end{array}$ \\
\hline $\begin{array}{l}\text { Baseline } \mathrm{HbA}_{1 \mathrm{c}}(\%) \\
\text { BMI }\left(\mathrm{kg} / \mathrm{m}^{2}\right) \\
\text { Diabetes' duration (years) }\end{array}$ & $\begin{array}{l}8.4 \pm 1.0 \\
29.8 \pm 5.0 \\
7.9 \pm 5.4 \\
\end{array}$ & $\begin{array}{l}8.4 \pm 1.0 \\
31.0 \pm 4.7 \\
7.4 \pm 5.2 \\
\end{array}$ & $\begin{array}{l}8.3 \pm 1.1 \\
33.1 \pm 5.8 \\
5.4 \pm 5.3 \\
\end{array}$ & $\begin{array}{l}8.3 \pm 1.1 \\
33.9 \pm 5.2 \\
8.9 \pm 5.9 \\
\end{array}$ & $\begin{array}{l}8.2 \pm 0.9 \\
30.5 \pm 5.3 \\
9.4 \pm 6.2 \\
\end{array}$ \\
\hline \multicolumn{6}{|c|}{ Pro-insulin/Insulin ratio (absolute values, $95 \%$ CI) } \\
\hline $\begin{array}{l}\text { Liraglutide vs background } \\
\text { Liraglutide vs C2 }\end{array}$ & $\begin{array}{l}-0.09 *[-0.15 ;-0.03] \\
-0.05 *[-0.10 ;-0.01]\end{array}$ & $\begin{array}{l}-0.12 *[-0.18 ;-0.06] \\
-0.03[-0.08 ; 0.02]\end{array}$ & $\begin{array}{l}- \\
-0.05[-0.11 ; 0.01]\end{array}$ & $\begin{array}{l}-0.12 *[-0.19 ;-0.06] \\
-\end{array}$ & $\begin{array}{l}-0.10 *[-0.15 ;-0.05] \\
\text { ND }\end{array}$ \\
\hline \multicolumn{6}{|c|}{ HOMA $\beta$-cell function $(\Delta \%, 95 \%$ CI) } \\
\hline $\begin{array}{l}\text { Liraglutide vs background } \\
\text { Liraglutide vs } \mathrm{C} 2\end{array}$ & $\begin{array}{l}34.15[-0.23 ; 68.54] \\
30.31 *[2.00 ; 58.62]\end{array}$ & $\begin{array}{l}28.20 *[8.29 ; 48.12] \\
1.41[-15.06 ; 17.88]\end{array}$ & $\begin{array}{l}- \\
-94.71[-231.28 ; 41.87]\end{array}$ & $\begin{array}{l}21.40 *[10.96 ; 31.83] \\
-\end{array}$ & $\begin{array}{l}34.00 *[20.66 ; 47.34] \\
\text { ND }\end{array}$ \\
\hline
\end{tabular}

$* p<0.03$.

$\mathrm{ND}=$ not determined due to cross-reactivity of glargine with assay. Data represent estimated mean differences between treatments (from baseline to end of treatment).

Supported by: Novo Nordisk Inc. 
pathogenesis of atherosclerosis. Accelerated atherosclerosis is a major contributor to morbidity and mortality in type 2 diabetes. Hyperglycemia induces expression of PAI-1 and vascular adhesion molecules including VCAM-1, ICAM-1 and E-Selectin. Liraglutide is a once daily human glucagon-like peptide-1(GLP-1) analogue. Liraglutide improves glycaemic control and lowers body weight in subjects with type 2 diabetes.

Our study aimed to explore the in vitro effect of liraglutide on vascular endothelial cell (EC) expression of PAI-1, VCAM-1, ICAM-1 and E-Selectin under high glucose conditions in the C11-STH human vascular EC system.

Methods: PAI-1protein levels were assayed in conditioned medium using ELISA kits (Biopool) whilst Reverse Transcriptase (RT) PCR was used to determine PAI-1, VCAM-1, ICAM-1 and E-Selectin mRNA expression in C11-STH vascular EC.

Results: Exposure to $10 \mathrm{mM}$ glucose over 48 hrs resulted in a $60 \%$ increase in PAI-1 mRNA expression $(\mathrm{n}=3, \mathrm{p}<0.05)$. In addition PAI-1 protein expression was increased $50 \%$ when exposed to $10 \mathrm{mM}$ glucose over $48 \mathrm{hrs}(\mathrm{n}=3, \mathrm{p}<0.05)$. Exposure to $10 \mathrm{mM}$ glucose over $48 \mathrm{hrs}$ increased VCAM-1 and ICAM-1 mRNA expression by $80 \%(\mathrm{n}=3, \mathrm{p}<0.01)$ and $60 \%(\mathrm{n}=3, \mathrm{p}<0.05)$ respectively. Exposure to $20 \mathrm{mM}$ glucose over $48 \mathrm{hrs}$ increased VCAM-1 and ICAM-1 mRNA expression by $90 \%(\mathrm{n}=3, \mathrm{p}<0.001)$ and $80 \%(\mathrm{n}=3, \mathrm{p}<0.001)$ respectively. A non-significant trend to induction of E-Selectin mRNA expression was observed in response to 10 or $20 \mathrm{mM}$ glucose exposure over $48 \mathrm{hrs}$. Earlier time points demonstrated no significant increase in PAI-1, VCAM-1, ICAM-1 and E-Selectin expression in response to high glucose exposure. Addition of liraglutide (100nM), at $5.5 \mathrm{mM}$ glucose, did not affect basal expression of PAI1, VCAM-1, ICAM-1 and E-Selectin at any time point. Addition of liraglutide $(100 \mathrm{nM})$, at the time of exposure to $10 \mathrm{mM}$ glucose, inhibited glucose-mediated induction of PAI- $1 \mathrm{mRNA}$ and protein by $90 \%$ at $48 \mathrm{hrs}(\mathrm{n}=3, \mathrm{p}<0.05)$. Addition of liraglutide $(100 \mathrm{nM})$ completely inhibited $10 \mathrm{mM}$ or $20 \mathrm{mM}$ glucose-mediated induction of VCAM- 1 and ICAM- 1 mRNA $(n=3, p<0.05)$ at $48 \mathrm{hrs}$.

Conclusion: Liraglutide inhibited glucose-mediated induction of PAI- 1 and the vascular adhesion molecules VCAM-1and ICAM-1 in our human cell system. These results indicate a potential role for liraglutide in inhibition of endothelial cell dysfunction in type 2 diabetes.

\section{PS 079 Liraglutide clinical studies}

\section{4}

Patient reported outcomes in subjects with type 2 diabetes treated with monotherapy regimens of once-daily human GLP-1 analogue liraglutide or glimepiride

B.W. Bode ${ }^{1}$, P.M. Hale 2 , M. Hammer ${ }^{3}$, M.A. Testa ${ }^{4}$, A. Garber ${ }^{5}$

${ }^{1}$ Endocrinology, Atlanta Diabetes Associates, Atlanta, United States, ${ }^{2}$ Novo Nordisk Inc, Princeton, United States, ${ }^{3}$ Novo Nordisk A/S, Bagsvaerd, Denmark, ${ }^{4}$ Biostatistics, Harvard School of Public Health, Boston, United States, ${ }^{5}$ Endocrinology, Baylor College of Medicine, Houston, United States

Background and aims: Improved glycemic control, weight loss and reduced risk of hypoglycemia may improve the Health-Related Quality of Life (HRQOL) in subjects with T2DM. Liraglutide, a once daily GLP-1 analog, improves glycemic control, has a low risk of hypoglycemia, and can result in weight loss. Patient Reported Outcomes (PRO) were assessed to establish whether treatment with liraglutide has an effect on HRQOL.

Materials and methods: In this 52-week, double dummy, placebo-controlled, blinded trial, 746 subjects were randomized to either once-daily liraglutide monotherapy [1.2 $\mathrm{mg}(\mathrm{n}=251)$ or $1.8 \mathrm{mg}(\mathrm{n}=247)$ ] or glimepiride monotherapy [active control, $8 \mathrm{mg} /$ day $(\mathrm{n}=248)$ ] to investigate changes in glycemic control, weight, body composition and PROs in subjects with T2DM previously treated with lifestyle or oral monotherapy. A self-administered questionnaire (77 items) assessed general perceived health, mental and emotional health, cognitive functioning, overall quality of life (QOL), weight image, weight concern, body size and body distress, and was completed at baseline and weeks 28 and 52. Age and baseline-adjusted treatment means were compared in 732 subjects using linear mixed models. The relationships among baseline to endpoint changes in $\mathrm{HbAlc}, \mathrm{BMI}$ and $\mathrm{PRO}$ scales were assessed using Pearson's correlation coefficient.

Results: Subjects were $50 \%$ male, with baseline mean \pm SD HbAlc $8.2 \pm 1.1 \%$ and BMI $33.1 \pm 5.8 \mathrm{~kg} / \mathrm{m}^{2}$. Decreases in HbAlc $($ mean $\pm \mathrm{SE})$ were greater for 1.2 $\mathrm{mg}[-0.73 \pm 0.09 \%, \mathrm{P}=0.013]$ and $1.8 \mathrm{mg}[-1.00 \pm 0.08 \%, \mathrm{P}<0.001]$ liraglutide doses compared to glimepiride [-0.44 $\pm 0.08 \%]$. BMI increased significantly for glimepiride $\left[0.39 \pm 0.09 \mathrm{~kg} / \mathrm{m}^{2}, \mathrm{P}<0.001\right]$, decreased for $1.2 \mathrm{mg}[-0.73 \pm 0.09$ $\left.\mathrm{kg} / \mathrm{m}^{2}, \mathrm{P}<0.001\right]$ and $1.8 \mathrm{mg}\left[-0.85 \pm 0.09 \mathrm{~kg} / \mathrm{m}^{2}, \mathrm{P}<0.001\right]$ liraglutide doses, and liraglutide changes were different from glimepiride $(\mathrm{P}<0.001)$.

Pooling all groups, decreases in BMI were associated with improvements in weight image and decreases in weight concern $(\mathrm{r}=0.19$ and 0.25 , both $\mathrm{P}<$ $0.0001)$. Decreases in weight concern were associated with increases in overall QOL $(r=-0.16, \mathrm{P}<0.0001)$, general perceived health $(\mathrm{r}=-0.19, \mathrm{P}<0.0001)$ and mental and emotional health $(\mathrm{r}=-0.12, \mathrm{P}=0.002)$. Decreases in HbAlc correlated with increases in general perceived health $(r=-0.14, \mathrm{P}=0.0002)$.

Conclusion: Treatment with either $1.2 \mathrm{mg}$ or $1.8 \mathrm{mg}$ liraglutide provided superior glycemic control as compared to glimepiride, and both doses resulted in significant decreases in BMI. For $1.8 \mathrm{mg}$ liraglutide, the superior efficacy and positive impact on weight and weight concern were associated with clinically important improvements in physical and emotional domains of quality of life.

\begin{tabular}{|c|c|c|c|c|}
\hline Patient Reported Outcomes Scale ${ }^{1}$ & $\begin{array}{l}\text { Baseline Covariate Score } \\
(\mathrm{N}=732)\end{array}$ & $\begin{array}{l}\text { Glimepiride } 8 \text { mg/day } \\
\text { Mean (SE) }\end{array}$ & $\begin{array}{l}\text { Liraglutide } 1.2 \mathrm{mg} / \text { day } \\
\text { Mean }(\mathrm{SE})^{2}\end{array}$ & $\begin{array}{l}\text { Liraglutide } 1.8 \mathrm{mg} / \text { day } \\
\text { Mean }(\mathrm{SE})^{2}\end{array}$ \\
\hline Overall QOL+ & 450.4 & $451.7(2.6)$ & $453.8(2.6)$ & $462.3(2.6)^{\star *}$ \\
\hline Mental \& Emotional+ & 464.6 & $466.3(2.7)$ & $464.4(2.7)$ & $476.1(2.8)^{\star}$ \\
\hline Psych Distress + & 496.6 & $498.4(2.9)$ & $497.7(2.9)$ & $507.3(2.9)^{\star}$ \\
\hline Psych Well-Being + & 411.5 & $412.5(3.1)$ & $409.5(3.1)$ & $424.2(3.1)^{\star \star}$ \\
\hline Perceived Health + & 430.3 & $430.3(3.2)$ & $441.4(3.2)$ & $444.2(3.2)^{\star}$ \\
\hline Weight Image -- & 50.6 & $48.7(2.0)$ & $47.6(2.0)$ & $40.0(2.0)^{\star *}$ \\
\hline Weight Concern -- & 37.8 & $38.8(1.2)$ & 30.0 (1.2)\# & $32.8(1.2)^{\star *}$ \\
\hline
\end{tabular}

1. + higher better; -- lower better.

2. vs. Glimeperide: ${ }^{\star} \mathrm{P}<0.05,{ }^{*}, \mathrm{P}<0.01, \# \mathrm{P}<0.001$

Supported by: Novo Nordisk 


\begin{tabular}{|c|c|c|c|c|c|}
\hline & $\begin{array}{l}\text { Liraglutide } 0.6 \mathrm{mg}+ \\
\text { metformin }\end{array}$ & $\begin{array}{l}\text { Liraglutide } 1.2 \mathrm{mg}+ \\
\text { metformin }\end{array}$ & $\begin{array}{l}\text { Liraglutide } 1.8 \mathrm{mg}+ \\
\text { metformin }\end{array}$ & $\begin{array}{l}\text { Placebo + } \\
\text { metformin }\end{array}$ & $\begin{array}{l}\text { Glimepiride + } \\
\text { metformin }\end{array}$ \\
\hline Final $\mathrm{HbA}_{1 \mathrm{c}} \%(\mathrm{SD})^{\mathrm{a}}$ & $7.8(1.1)$ & $7.5(1.1)$ & $7.5(1.2)$ & $8.6(1.4)$ & $7.5(1.1)$ \\
\hline Change $\mathrm{HbA}_{1 \mathrm{c}}, \%$ (SE) & $-0.7(0.1)^{*}$ & $-1.0(0.1)^{*}$ & $-1.0(0.1)^{*}$ & $+0.1(0.1)$ & $-1.0(0.1)$ \\
\hline Mono/combi change $\mathrm{HbA}_{1 \mathrm{c}}, \%(\mathrm{SD})^{\mathrm{a}}$ & $\begin{array}{l}-0.8(0.8) / \\
-0.5(1.1)\end{array}$ & $\begin{array}{l}-1.3(1.2) / \\
-0.6(0.9)\end{array}$ & $\begin{array}{l}-1.3(1.1) / \\
-0.7(1.1)\end{array}$ & $\begin{array}{l}-0.5(1.3) / \\
+0.5(1.0)\end{array}$ & $\begin{array}{l}-1.1(0.8) / \\
-0.8(1.0)\end{array}$ \\
\hline$\% \mathrm{HbA}_{1 \mathrm{c}}<7.0$ & $28.0^{*} \dagger$ & $35.3^{*}$ & $42.4^{*}$ & 10.8 & 36.3 \\
\hline$\% \mathrm{HbA}_{1 c} \leq 6.5$ & $11.3^{*} \dagger$ & $19.8^{*}$ & $24.6^{*}$ & 4.2 & 22.2 \\
\hline Final FPG, mmol/L (SD) ${ }^{\mathrm{a}}$ & $9.1(2.6)$ & $8.4(2.6)$ & $8.5(2.3)$ & $10.7(57)$ & $8.8(2.4)$ \\
\hline Change FPG, mmol/L (SE) & $-1.1(0.2)^{*}$ & $-1.6(0.2)^{*}$ & $-1.7(0.2)^{*}$ & $+0.4(0.2)$ & $-1.3(0.2)$ \\
\hline Weight change, kg(SE) & $-1.8(0.2) \dagger$ & $-2.6(0.2)^{\star} \dagger$ & $-2.8(0.2)^{*} \dagger$ & $-1.5(0.3)$ & $+1.0(0.2)$ \\
\hline \% reporting minor hypo events ${ }^{\mathrm{a}}$ & 3.3 & 0.8 & 2.5 & 2.5 & 16.9 \\
\hline
\end{tabular}

${ }^{*} p<0.05$ in comparison to placebo + metformin; $\uparrow p<0.05$ in comparison to glimepiride + metformin; ${ }^{a}$ No statistical analysis

\section{5}

Liraglutide, a once-daily human GLP-1 analogue, in type 2 diabetes provides similar glycaemic control with reduced body weight compared with glimepiride when added to metformin

K. Hermansen ${ }^{1}$, M.A. Nauck ${ }^{2}$, A. Frid ${ }^{3}$, N.S. Shah ${ }^{4}$, T. Tankova ${ }^{5}$, I.H. Mitha ${ }^{6}$, M. During ${ }^{7}$, M. Zdravkovic ${ }^{7}$, D. Matthews ${ }^{8}$

${ }^{1}$ Endocrinology and Metabolism, Aarhus University Hospital, Denmark, ${ }^{2}$ Diabeteszentrum, Bad Lauterberg im Harz, Germany, ${ }^{3}$ Öresund Diabetes Team AB, Lund, Sweden, ${ }^{4}$ Seth G.S. Medical College and KEM Hospital, Mumbai, India, ${ }^{5}$ Medical University, Clinic of Diabetology, Sofia, Bulgaria, ${ }^{6}$ BenMed Hospital, Benoni, South Africa, ${ }^{7}$ Statistics, Novo Nordisk A/S, Bagsvaerd, Denmark, ${ }^{8}$ OCDEM, University of Oxford, United Kingdom

Background and aims: This placebo-controlled, double-blind, randomised 26-week trial investigated the effect on glycaemic control $\left(\mathrm{HbA}_{1 \mathrm{c}}\right)$ of three liraglutide doses (0.6, 1.2 or $1.8 \mathrm{mg}$, subcutaneous; sc, once daily; OD) added to metformin (met; $1 \mathrm{~g}$ twice daily; $\mathrm{BD}$ ) compared with metformin monotherapy (placebo + metformin) or glimepiride (4 mg OD) added to metformin (glimepiride + metformin).

Materials and methods: The trial included 1091 subjects with type 2 diabetes previously treated with oral antidiabetic drug (OAD) monotherapy (35\%) or combination therapy (65\%), with mean \pm SD baseline $\mathrm{HbA}_{1 c} 8.4 \pm 1.0 \%$ and body weight (BW) $88.6 \pm 17.3 \mathrm{~kg}$.

Results: $\mathrm{HbA}_{1 \mathrm{c}}$ was significantly reduced in all liraglutide groups vs placebo + metformin. Reduction in $\mathrm{HbA}_{1}$ was greater in subjects previously treated with OAD mono vs combination therapy. The fraction of subjects achieving $\mathrm{HbA}_{1 \mathrm{c}}<7.0 \%$ or $\leq 6.5 \%$ was significantly higher in liraglutide + metformin vs placebo + metformin, with no difference between liraglutide + metformin vs glimepiride + metformin. Change in fasting plasma glucose followed the same pattern as for $\mathrm{HbA}_{1 \mathrm{c}}$. BW decreased significantly in all liraglutide groups vs glimepiride + metformin. Reduction in BW was significantly greater in liraglutide1.2 $\mathrm{mg}+$ metformin and liraglutide1.8 $\mathrm{mg}+$ metformin vs placebo + metformin. Presence of liraglutide antibodies was $0-4 \%$ (only subjects not entering the extension phase of the study were included, $n=25-32$ ). Minor hypoglycaemia $(<3.1 \mathrm{ml} / \mathrm{L})$ occurred in $<4 \%$ of subjects in liraglutide + met- formin and placebo + metformin, and in 16.9\% in glimepiride + metformin. There was no major hypoglycaemia. The most common adverse events in liraglutide + metformin were gastrointestinal disorders (mainly nausea). Nausea initially occurred in 6-12\% of subjects in liraglutide + metformin, declining to placebo + metformin levels $(2 \%)$ after $8-16$ weeks of treatment.

Conclusion: Liraglutide was well tolerated, produced a clinically significant reduction in $\mathrm{HbA}_{1 \mathrm{c}}$, and in contrast to glimepiride + metformin resulted in few hypoglycaemic episodes and weight loss in subjects with type 2 diabetes. Supported by: Novo Nordisk A/S

\section{6}

Significantly better glycaemic control and weight reduction with liraglutide, a once-daily human GLP-1 analogue, compared with glimepiride: all as monotherapy in type 2 diabetes

A. Garber ${ }^{1}$, R. Henry ${ }^{2}$, R. Ratner ${ }^{3}$, P. Garcia-Hernandez ${ }^{4}$, H.M. Rodriguez Pattzi $^{5}$, I. Olvera-Alvarez ${ }^{6}$, P.M. Hale ${ }^{7}$, M. Zdravkovic ${ }^{8}$, B. Bode ${ }^{9}$ ${ }^{1}$ Division of Endocrinology, Diabetes and Metabolism, Baylor College of Medicine, Houston, United States, ${ }^{2}$ Endocrinology, University of California, San Diego, United States, ${ }^{3}$ Endocrinology, MedStar Research Institute, Hyattsville, United States, ${ }^{4}$ Endocrinology, Hospital Universitario de Monterrey, Mexico, ${ }^{5}$ Endocrinology, Private Practice, Mexico City, Mexico, ${ }^{6}$ Internal Medicine, Hospital Ángeles México, Mexico City, Mexico, ${ }^{7}$ Novo Nordisk Inc, Princeton, United States, ${ }^{8}$ Novo Nordisk A/S, Bagsvaerd, Denmark, ${ }^{9}$ Endocrinology, Atlanta Diabetes Associates, Atlanta, United States

Background and aims: This 52-week randomised trial compared the efficacy and safety of two doses of liraglutide (1.2 and $1.8 \mathrm{mg}$, OD) to glimepiride ( 8 mg OD).

Materials and methods: Subjects were previously treated with diet and exercise (D/E) or previous OAD monotherapy (mono). In total, 746 subjects were randomised (mean age $53.0 \pm 10.9$ years); mean body mass index $33.1 \pm 5.8 \mathrm{~kg} /$ $\mathrm{m}^{2}$, mean $\left.\mathrm{HbA}_{1 \mathrm{c}} 8.3 \pm 1.1 \%\right)$.

Results: Liraglutide 1.2 and $1.8 \mathrm{mg}$ reduced $\mathrm{HbA}_{1 \mathrm{c}}$ more than glimepiride (ANCOVA, $p=0.0014$ and $p<0.0001$ ) and more of the subjects in the liraglu-

\begin{tabular}{|c|c|c|c|}
\hline & $\begin{array}{l}\text { Liraglutide } 1.2 \mathrm{mg} \\
\mathrm{N}=251\end{array}$ & $\begin{array}{l}\text { Liraglutide } 1.8 \mathrm{mg} \\
\mathrm{N}=247\end{array}$ & $\begin{array}{l}\text { Glimepiride } \\
\mathrm{N}=\mathbf{2 4 8}\end{array}$ \\
\hline $\begin{array}{l}\text { Final } \mathrm{HbA}_{1 \mathrm{c}} \%(\mathrm{SD}) \\
\text { Change } \mathrm{HbA}_{1 \mathrm{c}} \text {, all \% } \\
\text { Change } \mathrm{HbA}_{1 \mathrm{c}} \text { from D/E \% } \\
\text { Change } \mathrm{HbA}_{1 c} \text {, mono \% }\end{array}$ & $\begin{array}{l}7.5(1.3) \\
-0.84^{*} \\
-1.19 \\
-0.47^{\star} \\
\end{array}$ & $\begin{array}{l}7.2(1.2) \\
-1.14^{*} \dagger \\
-1.60^{*} \dagger \\
-0.71^{*} \dagger \\
\end{array}$ & $\begin{array}{l}7.8(1.2) \\
-0.51 \\
-0.88 \\
-0.17 \\
\end{array}$ \\
\hline$\% \mathrm{HbA}_{1 c}<7.0 \%$ & $43^{*}$ & $51^{*}$ & 28 \\
\hline$\% \mathrm{HbA}_{1 \mathrm{c}} \leq 6.5 \%$ & $28^{*}$ & $38^{*}$ & 16 \\
\hline Weight change, $\mathrm{kg}(\mathrm{SE})$ & $-2.05(0.28)^{\star}$ & $-2.45(0.28)^{*}$ & $1.12(0.27)$ \\
\hline $\begin{array}{l}\text { Final FPG, mmol/L (SD) } \\
\text { Change FPG, mmol/L }\end{array}$ & $\begin{array}{l}8.6(3.1) \\
-0.8^{*}\end{array}$ & $\begin{array}{l}8.3(2.7) \\
-1.42^{*} \dagger\end{array}$ & $\begin{array}{l}9.3(3) \\
-0.3\end{array}$ \\
\hline Change PPG, $\mathrm{mmol} / \mathrm{L}$ & -1.7 & $-2.1^{*}$ & -1.4 \\
\hline \% reporting minor hypo events/subject/year & $\begin{array}{ll}12 \\
0.30^{*}\end{array}$ & $\begin{array}{l}8 \\
0.25^{*}\end{array}$ & $\begin{array}{ll}24 \\
1.96 \\
\end{array}$ \\
\hline$\%$ reporting nausea & 27.5 & 29.3 & 8.5 \\
\hline
\end{tabular}

${ }^{*} p<0.05$ vs. glimepiride; $\dagger p<0.051 .8 \mathrm{mg}$ vs. $1.2 \mathrm{mg}$

FPG, fasting plasma glucose; PPG, post-prandial glucose 
tide groups reached $\mathrm{HbA}_{1 \mathrm{c}} \leq 6.5$ and $<7.0 \%$ ( $p<0.01$ vs. glimepiride). In addition, the decrease in $\mathrm{HbA}_{1 \mathrm{c}}$ with liraglutide $1.8 \mathrm{mg}$ was significantly greater than the decrease with liraglutide $1.2 \mathrm{mg}(p=0.0046)$. At the end of the study, there was significant weight decrease in the liraglutide groups, as compared to weight gain in the glimepiride group. The most common adverse events in the liraglutide groups were gastrointestinal disorders (mainly nausea). Nausea occurred in approximately $29 \%$ of subjects in the liraglutide groups, but was transient. The rates of minor hypoglycaemic episodes $(<3.1 \mathrm{mmol} / \mathrm{L})$ were significantly lower for the liraglutide groups, vs. glimepiride. No subjects reported major hypoglycaemic events.

Conclusion: Liraglutide monotherapy significantly lowered $\mathrm{HbA}_{1 c}$ versus glimepiride and, at the same time, resulted in weight loss and lower rates of hypoglycaemia.

Supported by: Novo Nordisk

\section{7}

Liraglutide added to a sulphonylurea (SU) offers significantly better glycaemic control and favourable weight change compared with rosiglitazone and SU combination therapy in T2D

M. Marre' ${ }^{1}$ J. Shaw ${ }^{2}$, M. Brandle ${ }^{3}$, W.M. Wan Bebakar', N.A. Kamaruddin', J. Strand ${ }^{6}$, M. Zdravkovic ${ }^{7}$, T.D. Le-Thi ${ }^{8}$, S. Colagiuri ${ }^{9}$

${ }^{1}$ Groupe Hospitalier Bichat - Claude Bernard, Service d'EndocrinologieDiabetologie, Paris, France, ${ }^{2}$ International Diabetes Institute, Melbourne, Australia, ${ }^{3}$ Endocrinology, Kantonsspital St Gallen, Switzerland,

${ }^{4}$ Endocrinology, Universiti Sains Malaysia, Kelantan, Malaysia, ${ }^{5} \mathrm{Hospital}$ Universiti Kebangsaan Malaysia, Kuala Lumpur, Malaysia, ${ }^{6}$ Oulun

Diakonissalaitos, Oulu, Finland, ${ }^{7}$ Novo Nordisk A/S, Bagsvaerd, Denmark, ${ }^{8}$ Novo Nordisk A/S, Bagsvaerd, Denmark, ${ }^{9}$ Metabolic Health, University of Sydney, Australia

Background and aims: This placebo-controlled 26-week, double-dummy, randomised, parallel group study investigated the effect on glycaemic control $\left(\mathrm{HbA}_{1 \mathrm{c}}\right)$ of three doses of the once-daily human GLP-1 analogue liraglutide $(0.6,1.2$ or $1.8 \mathrm{mg}$ once daily; OD) added to glimepiride (2-4 mg OD), versus glimepride alone (placebo), and versus rosiglitazone (4 mg) in combination with glimepiride.

Materials and methods: 1041 subjects (mean (SD) age 56 (10) years, body mass index $\left.30(5) \mathrm{kg} / \mathrm{m}^{2}, \mathrm{HbA}_{1 \mathrm{c}} 8.4(0.9)\right)$ were stratified based on previous oral antidiabetic drug (OAD) monotherapy $(30 \%)$ or combination therapy (70\%).

Results: All doses of liraglutide + glimipiride resulted in significantly greater reductions from baseline in $\mathrm{HbA}_{1 c}$ and fasting plasma glucose (FPG) compared with placebo $\left.{ }^{*}\right)(p<0.0001)$, and the differences between lira 1.2 and 1.8 mg were also significantly greater vs rosiglitazone + glimepiride for $\mathrm{HbA}_{4 c}(\dagger)$ $(p<0.0001)$. The $\mathrm{HbA}_{1 c}$ reduction was about $50 \%$ greater in subjects previously on OAD monotherapy. Significantly more subjects achieved $\mathrm{HbA}_{1 c}$ $<7 \%$ and $\leq 6.5 \%$ with liraglutide $1.2 \mathrm{mg}+$ glimepiride and liraglutide $1.8 \mathrm{mg}$ + glimepiride versus placebo and glimepiride + rosiglitazone $(p \leq 0.0003)$. Change in body weight was superior for all doses of liraglutide compared with rosiglitazone $(p<0.0001)$. Liraglutide antibodies were found in $9.3-12.7 \%$ of liraglutide-treated subjects. Main adverse event with liraglutide was nausea (all $\leq 11 \%$ ); mostly mild and transient in nature. Minor hypoglycaemic episodes $(<3.1 \mathrm{mmol} / \mathrm{L})$ occurred in $<10 \%$ of subjects with all treatments. One major hypoglycaemic episode occurred with liraglutide $1.8 \mathrm{mg}$; no medical assistance was required.
Conclusion: Liraglutide added to an SU was well tolerated and provided improved glycaemic control ( $\mathrm{HbA}_{1 c}$ decrease of $-1.4 \%$ vs placebo+ glimepiride) with a favourable weight profile compared with SU and thiazolidinedione combination therapy.

\section{8}

Effect of the GLP-1 analogue liraglutide on glycaemic control and weight reduction in patients on metformin and rosiglitazone: a randomized double-blind placebo-controlled trial

B. Zinman ${ }^{1}$, J. Gerich', J. Buse ${ }^{3}$, A. Lewin ${ }^{4}$, S.L. Schwartz ${ }^{5}$, P. Raskin ${ }^{6}$, P.M. Hale $^{7}$, M. Zdravkovic ${ }^{8}$, L. Blonde ${ }^{9}$

${ }^{1}$ Sinai Centre for Diabetes, Mount Sinai Hospital, Toronto, Canada, ${ }^{2}$ University of Rochester School of Medicine, Rochester, United States, ${ }^{3}$ University of North Carolina School of Medicine, Chapel Hill, United States, ${ }^{4}$ Clinical Trials, National Research Institute, Los Angeles, United States, ${ }^{5}$ Endocrinology, Diabetes \& Glandular Disease Clinic, San Antonio, United States, ${ }^{6}$ University of Texas Southwestern Medical, Dallas, United States, ${ }^{7}$ Novo Nordisk Inc, Princeton, United States, ${ }^{8}$ Novo Nordisk A/S, Bagsvaerd, Denmark, ${ }^{9}$ Endocrinology, Diabetes and Metabolism, Ochsner Diabetes Clinical Research Unit, New Orleans, United States

Background and aims: This 26-week randomized trial compared the efficacy and safety of two doses of liraglutide (1.2 and $1.8 \mathrm{mg}$, QD) to placebo, all added to metformin $2 \mathrm{~g}(1 \mathrm{~g}$, BID) and rosiglitizone $8 \mathrm{mg}$ (4 mg, BID).

Materials and methods: Subjects were previously treated with 1 or more OADs. In total, 533 subjects were randomized (mean age $55.1 \pm 10.2$ years, mean body mass index $33.5 \pm 5.2 \mathrm{~kg} / \mathrm{m}^{2}$, mean $\left.\mathrm{HbA}_{1 \mathrm{c}} 8.3 \pm 1.0 \%\right)$.

Results: Liraglutide $1.2+$ metformin + rosiglitazone and $1.8+$ metformin + rosiglitazone reduced $\mathrm{HbA}_{1 \mathrm{c}}$ more than placebo (ANCOVA, $p<0.0001$ ) and more of the subjects in the liraglutide groups reached $\mathrm{HbA}_{1 c} \leq 6.5$ and $7.0 \%$ $(p<0.01$ vs. placebo $)$. Body weight decreased significantly with liraglutide + metformin + rosiglitazone, as compared to placebo + metformin + rosiglitazone. Most common adverse events in the liraglutide groups were gastrointestinal disorders (mainly nausea) and were generally transient. The rates of minor hypoglycemic episodes $(<3.1 \mathrm{mmol} / \mathrm{L})$ were 0.38 and 0.64 events/year for liraglutide 1.2 and $1.8+$ metformin + rosiglitazone, respectively, vs. 0.17 events/year for placebo + metformin + rosiglitazone $(p=0.0968$ and 0.0040 , respectively). No subjects reported major hypoglycemic events.

Conclusion: Liraglutide added to metformin + rosiglitazone was generally well tolerated and significantly improved glycaemic control and reduced body weight in comparison placebo + metformin + rosiglitazone therapy with low rates of hypoglycemia.

\section{9}

\begin{tabular}{|c|c|c|c|c|c|}
\hline & $\begin{array}{l}\text { Liraglutide } 0.6 \mathrm{mg}+ \\
\text { glimepiride }\end{array}$ & $\begin{array}{l}\text { Liraglutide } 1.2 \mathrm{mg}+ \\
\text { glimepiride }\end{array}$ & $\begin{array}{l}\text { Liraglutide } 1.8 \mathrm{mg}+ \\
\text { glimepiride }\end{array}$ & Placebo & $\begin{array}{l}\text { Rosiglitazone + } \\
\text { glimepiride }\end{array}$ \\
\hline $\begin{array}{l}\text { Final } \mathrm{HbA}_{1 \mathrm{c}}, \%(\mathrm{SD}) \\
\text { change } \mathrm{HbA}_{1 \mathrm{c}}, \%\end{array}$ & $7.9(1.3)-0.60^{*}$ & $7.5(1.2)-1.08^{\star} \dagger$ & $7.5(1.3)-1.13^{\star} \dagger$ & $8.7(1.3) 0.23$ & $8.0(1.3)-0.44$ \\
\hline Mono/combi change $\mathrm{HbA}_{1 \mathrm{c}} \%$ & $-0.8 /-0.4$ & $-1.4 /-0.7$ & $-1.5 /-0.8$ & $-0.4 / 0.7$ & $-0.8 /-0.1$ \\
\hline $\begin{array}{l}\% \mathrm{HbA}_{1 c}<7 \% \\
\% \mathrm{HbA}_{1 c} \leq 6.5 \%\end{array}$ & $\begin{array}{l}23.2^{*} \\
12.0\end{array}$ & $\begin{array}{l}33.8^{\star} \dagger \\
21.1^{\star} \dagger\end{array}$ & $\begin{array}{l}40.2^{\star} \dagger \\
20.5^{\star} \dagger\end{array}$ & $\begin{array}{l}7.0 \\
3.5\end{array}$ & $\begin{array}{l}21.2 \\
9.5\end{array}$ \\
\hline Weight change, kg (SE) & $0.7(0.2) \dagger$ & $0.3(0.2) \dagger$ & $-0.2(0.2) \dagger$ & $-0.1(0.3)$ & $2.1(0.2)$ \\
\hline $\begin{array}{l}\text { Final FPG, mmol/L (SD) } \\
\text { Change FPG, mmol/L (SE) }\end{array}$ & $\begin{array}{l}9.2(2.5) \\
-0.7(0.2)^{*}\end{array}$ & $\begin{array}{l}8.4(2.7) \\
-1.6(0.2)^{*}\end{array}$ & $\begin{array}{l}8.3(2.6) \\
-1.6(0.2)^{*} \dagger\end{array}$ & $\begin{array}{l}10.7(2.8) \\
1.0(0.2)\end{array}$ & $\begin{array}{l}9.1(2.8) \\
-0.9(0.2)\end{array}$ \\
\hline Minor hypo events/year & 0.17 & 0.51 & 0.47 & 0.17 & 0.12 \\
\hline$\%$ reporting nausea & 5.2 & 10.5 & 6.8 & 1.7 & 2.6 \\
\hline
\end{tabular}

${ }^{*}$ Statistically significant in comparison to placebo + glimepiride, $p<0.0005$; $\uparrow$ Statistically significant in comparison to rosiglitazone + glimepiride, $p<0.0006$

Supported by: Novo Nordisk A/S 


\begin{tabular}{|c|c|c|c|}
\hline & $\begin{array}{l}\text { Liraglutide } 1.2+\text { metformin }+ \\
\text { rosiglitazone } \mathrm{N}=178\end{array}$ & $\begin{array}{l}\text { Liraglutide } 1.8+\text { metformin }+ \\
\text { rosiglitazone } \mathrm{N}=178\end{array}$ & $\begin{array}{l}\text { Placebo + metformin }+ \\
\text { rosiglitazone } \mathrm{N}=177\end{array}$ \\
\hline Final $\mathrm{HbA}_{1}, \%(\mathrm{SD})$ & $7.0(1.1)$ & $7.1(1.1)$ & $7.9(1.3)$ \\
\hline Change $\mathrm{HbA}_{1 \mathrm{c}}, \%$ & $-1.48^{\star}$ & $-1.48^{*}$ & -0.54 \\
\hline$\% \mathrm{HbA}_{1 c}<7.0 \%$ & $58^{*}$ & $54^{\star}$ & 28 \\
\hline$\% \mathrm{HbA}_{1 \mathrm{c}} \leq 6.5 \%$ & $36^{*}$ & $37^{\star}$ & 14 \\
\hline Weight change, kg (SE) & $-1.02(0.33)^{\star}$ & $-2.02(0.32)$ & $0.60(0.34)$ \\
\hline Final FPG, mmol/L (SD) & $7.7(2.6)$ & $7.6(2.3)$ & $9.5(3.1)$ \\
\hline Change FPG, mmol/L & $-2.2^{*}$ & $-2.4^{*}$ & -0.4 \\
\hline Change PPG, $\mathrm{mmol} / \mathrm{L}$ & -2.6 & $-2.7^{*}$ & -0.8 \\
\hline$\%$ reporting minor hypo & 9 & 8 & 5 \\
\hline events/subject/year & 0.38 & $0.64^{*}$ & 0.17 \\
\hline
\end{tabular}

${ }^{*} p<0.05$ vs. placebo.

FPG, fasting plasma glucose; OAD, oral antidiabetic drug; PPG, post-prandial glucose

Supported by: Novo Nordisk

Liraglutide, a human GLP-1 analogue, reduces systolic blood pressure in subjects with type 2 diabetes

S. Colagiuri ${ }^{1}$, A. Frid ${ }^{2}$, M. Zdravkovic ${ }^{3}$, T.D. Le-Thi ${ }^{3}$, A. Vaag ${ }^{4}$, A. Garber ${ }^{5}$,

B. Zinman ${ }^{6}$

${ }^{1}$ Metabolic Health, The University of Sydney, Australia, ${ }^{2}$ Öresund Diabetes

Team AB, Lund, Sweden, ${ }^{3}$ Novo Nordisk A/S, Bagsvaerd, Denmark,

${ }^{4}$ Steno Diabetes Center, Gentofte, Denmark, ${ }^{5}$ Baylor College of Medicine,

Methodist Hospital, Houston, United States, ${ }^{6}$ Sinai Centre for Diabetes,

Mount Sinai Hospital, Toronto, Canada

Background and aims: Type 2 diabetes is associated with an increased risk of cardiovascular disease (CVD). Increased blood pressure (BP) is also a risk factor for CVD and prevalent among subjects with type 2 diabetes.

Materials and methods: This analysis examined the impact of treatment with liraglutide, a once-daily human GLP-1 analogue, on systolic and diastolic BP in subjects with type 2 diabetes from four 26-week and one 52-week LEAD [Liraglutide Effect and Action in Diabetes] studies. The studies were randomised, double-blind, placebo-controlled trials.
Results: The baseline and study data are presented in Table 1 for the highest dose of liraglutide $(1.8 \mathrm{mg} /$ day). Liraglutide reduced systolic BP in all trials with a statistically significant difference in four of five trials (from -2.7 to -4.5 $\mathrm{mmHg}$ vs comparator treatments). The reductions in systolic BP occurred after 2 weeks of treatment and could not be explained by changes in body weight. No statistically significant decrease in diastolic BP was observed. The percentage of subjects reaching a $\mathrm{BP}$ target of below 130/80 $\mathrm{mmHg}$ in LEAD 5 was $24.3 \%, 18.4 \%$ and $21.6 \%$ for liraglutide $1.8 \mathrm{mg}$, placebo and glargine (NS), respectively. The percentages in LEAD 1 and 2 were similar across all treatments $(\sim 25 \%)$. There was a trend for pulse to increase slightly, by between 0.9 and $4.5 \mathrm{bpm}$ with liraglutide treatment versus both background and comparator treatments, and these reached statistical significance in LEAD 1, LEAD 4 and LEAD 5.

Conclusion: Liraglutide treatment reduced mean systolic blood pressure by between 2.7 to $4.5 \mathrm{mmHg}$ in subjects with type 2 diabetes. Liraglutide treatment may therefore improve the $\mathrm{CV}$ risk profile of subjects with type 2 diabetes by reducing systolic blood pressure.

\begin{tabular}{|c|c|c|c|c|c|c|}
\hline $\begin{array}{l}\text { Trial ID } \\
\text { (N subjects) } \\
\end{array}$ & & $\begin{array}{l}\text { LEAD } 1 \\
(1041) \\
\end{array}$ & $\begin{array}{l}\text { LEAD } 2 \\
(1091) \\
\end{array}$ & LEAD 3 (746) & LEAD 4 (533) & $\begin{array}{l}\text { LEAD } 5 \\
(581)\end{array}$ \\
\hline $\begin{array}{l}\text { Treatment } \\
\text { Comparator }(\mathrm{C})\end{array}$ & & $\begin{array}{l}\text { Liraglutide } 1.8 \mathrm{mg} \\
\text { Rosiglitazone } 4 \mathrm{mg}\end{array}$ & $\begin{array}{l}\text { Liraglutide } 1.8 \mathrm{mg} \\
\text { Glimepiride } 4 \mathrm{mg}\end{array}$ & $\begin{array}{l}\text { Liraglutide } 1.8 \mathrm{mg} \\
\text { Glimepiride } 4 \mathrm{mg}\end{array}$ & $\begin{array}{l}\text { Liraglutide } 1.8 \mathrm{mg} \\
\text { - }\end{array}$ & $\begin{array}{l}\text { Liraglutide } 1.8 \mathrm{mg} \\
\text { Glargine }\end{array}$ \\
\hline Background therapy & & Glimepiride $4 \mathrm{mg}$ & Metformin $2 \mathrm{~g}$ & - & $\begin{array}{l}\text { Metformin }+ \\
\text { Rosiglitazone }\end{array}$ & $\begin{array}{l}\text { Glimepiride } 4 \mathrm{mg}+ \\
\text { Metformin } 2 \mathrm{~g}\end{array}$ \\
\hline $\begin{array}{l}\frac{\text { Baseline }}{\mathrm{HbA}_{1}, \%}(\mathrm{SD}) \\
\mathrm{BMI}^{\mathrm{k}} \mathrm{kg} / \mathrm{m}^{2}(\mathrm{SD}) \\
\mathrm{BP}, \mathrm{mmHg}\end{array}$ & & $\begin{array}{l}8.4(1.0) \\
29.8(5.0) \\
132 / 81\end{array}$ & $\begin{array}{l}8.4(1.0) \\
31.0(4.7) \\
131 / 80\end{array}$ & $\begin{array}{l}8.3 \pm 1.1 \\
33.1 \pm 5.8 \\
5.4 \pm 5.3\end{array}$ & $\begin{array}{l}8.3 \pm 1.1 \\
33.9 \pm 5.2 \\
8.9 \pm 5.9\end{array}$ & $\begin{array}{l}8.2(0.9) \\
30.5(5.3) \\
135 / 81\end{array}$ \\
\hline Systolic BP (mmHg) & $\begin{array}{l}\text { liraglutide } 1.8 \mathrm{mg} \text { vs } \\
\text { background } \\
\text { liraglutide } 1.8 \mathrm{mg} \text { vs } \mathrm{C}\end{array}$ & $\begin{array}{l}-0.5[-3.8 ; 2.8] \\
-1.9[-4.7 ; 0.9] \\
\end{array}$ & $\begin{array}{l}-0.5[-3.7 ; 2.7] \\
-2.7[-5.4 ;-0.0]^{*} \\
\end{array}$ & $\begin{array}{l}- \\
-3.0[-5.2 ;-0.7]^{*} \\
\end{array}$ & $\begin{array}{l}-4.5[-7.2 ;-1.9]^{\star} \\
- \\
\end{array}$ & $\begin{array}{l}-2.5[-5.4 ; 0.3] \\
-4.5[-6.8 ;-2.2]^{*} \\
\end{array}$ \\
\hline $\begin{array}{l}\text { Diastolic BP } \\
(\mathrm{mmHg})\end{array}$ & $\begin{array}{l}\text { liraglutide } 1.8 \mathrm{mg} \text { vs } \\
\text { background } \\
\text { liraglutide } 1.8 \mathrm{mg} \text { vs } \mathrm{C}\end{array}$ & $\begin{array}{l}0.4[-1.6 ; 2.4] \\
0.7[-0.9 ; 2.4] \\
\end{array}$ & $\begin{array}{l}0.5[-1.5 ; 2.5] \\
-0.2[-1.8 ; 1.5]\end{array}$ & $\begin{array}{l}- \\
0.0[-1.3 ; 1.4]\end{array}$ & $\begin{array}{l}-1.1[-2.7 ; 0.5] \\
-\end{array}$ & $\begin{array}{l}0.0[-1.7 ; 1.6] \\
-0.4[-1.8 ; 0.9]\end{array}$ \\
\hline $\begin{array}{l}\text { Body weight } \\
(\mathrm{kg})\end{array}$ & $\begin{array}{l}\text { liraglutide } 1.8 \mathrm{mg} \text { vs } \\
\text { background } \\
\text { liraglutide } 1.8 \mathrm{mg} \text { vs C }\end{array}$ & $\begin{array}{l}0.1[-0.9 ; 0.6] \\
2.3[-3.0 ;-1.7]^{*}\end{array}$ & $\begin{array}{l}1.3[-2.2 ;-0.4]^{*} \\
3.7[-4.5 ;-3.0]^{*}\end{array}$ & $\begin{array}{l}- \\
-3.6[-4.3 ;-2.9]^{*}\end{array}$ & $\begin{array}{l}-2.6[-3.4 ;-1.8]^{*} \\
- \\
\end{array}$ & $\begin{array}{l}1.4[-2.1 ;-0.7]^{*} \\
3.4[-4.0 ;-2.9]^{*}\end{array}$ \\
\hline Pulse (beats/min) & $\begin{array}{l}\text { liraglutide } 1.8 \mathrm{mg} \text { vs } \\
\text { background } \\
\text { liraglutide } 1.8 \mathrm{mg} \text { vs } \mathrm{C}\end{array}$ & $\begin{array}{l}4.5[2.7 ; 6.4]^{*} \\
1.3[-0.2 ; 2.8]\end{array}$ & $\begin{array}{l}0.9[-0.9 ; 2.8] \\
1.3[-0.3 ; 2.8]\end{array}$ & $\begin{array}{l}- \\
1.1[-0.4 ; 2.6]\end{array}$ & $3.6[1.8 ; 5.4]^{*}$ & $\begin{array}{l}1.7[-0.1 ; 3.5] \\
2.5[1.1 ; 4.0] \text { * }\end{array}$ \\
\hline
\end{tabular}

${ }^{*} p<0.05$.

Supported by: Novo Nordisk A/S 


\section{PS 080 Mechanisms involved in treatment with DPP-IV inhibitors}

\section{0}

Action of exendin-4 treatment upon glucose transport in liver and muscle, in normal and type 2 diabetic state

L. Arnés, V. Sancho, I. Valverde, M.L. Villanueva-Peñacarrillo

Metabolism, Nutrition \&Hormones, Fundación Jiménez Díaz, Madrid, Spain

Background and aims: It is known that GLP-1 modulates the major glucotransporter expression in liver, muscle and fat, in normal and diabetic conditions. Exendin-4 (Ex-4), 53\% structurally GLP-1 homologous peptide, is also antidiabetic and has GLP-1-like effects upon glucose and/or lipid metabolism in extrapancreatic tissues where it is agonist of the GLP-1 specific receptors. We have studied the possible in vivo effect of Ex-4 upon glucose transport and Glut 4 and Glut 2 expression in muscle and liver, respectively, of a type 2 rat diabetic model, compared to normal animals.

Materials and methods: Type 2 diabetic rat model (T2D) was developed by streptozotocin injection $(100 \mu \mathrm{g} / \mathrm{g} \mathrm{bw})$ in neonatal Wistar rats. Adult rats, normal $(\mathrm{N})$ and $\mathrm{T} 2 \mathrm{D}$ were treated (3 days), through an osmotic pump, with saline solution (control: $\mathrm{n}=12-19$ rats) or $0.1 \mathrm{nmol} / \mathrm{kg} / \mathrm{h} \mathrm{Ex-4}(\mathrm{n}=12-14)$. In fed conditions, blood samples were taken before (basal) and by the end of the treatment, for plasma insulin and glucose measurements; then, the muscle and liver were collected. Glucose transport (GT) $-{ }^{3} \mathrm{H}-2 \mathrm{DOG}$ uptake- was studied in soleus muscle, during $60-\mathrm{min}$ at $37^{\circ} \mathrm{C}$, in KRB-HEPES containing $0.5 \mathrm{M}$ 2-deoxi-D- $\left[1,2-{ }^{3} \mathrm{H}\right]$ glucose $(\mathrm{Sp}$. Ac. $40 \mathrm{nCi} / \mu \mathrm{mol})$ and $2 \mathrm{M}\left[{ }^{14} \mathrm{C}\right]$ sorbitol (Sp. Ac. $5 \mathrm{nCi} / \mu \mathrm{mol}$ ). Glycogen content in the liver was determined by the anthrone reagent method. Glut2 and Glut4, mRNA and protein, were determined by RT-PCR and Western blot, respectively. Significance of the differences was analyzed by ANOVA or Student $t$-test.

Results: In both, N and T2D rats, after either control or Ex-4 treatment, no significant modifications were detected of their initial value in body weight (279.6 $\pm 6 \mathrm{~g}, \mathrm{n}=33$ and $297 \pm 7 \mathrm{~g}, \mathrm{n}=24, \mathrm{~N}$ and T2D, respectively), plasma glucose $(154 \pm 7 \mathrm{mg} / \mathrm{dl}, \mathrm{n}=19$ and $170 \pm 10 \mathrm{mg} / \mathrm{dl}, \mathrm{n}=17)$ or insulin $(0.75 \pm 0.10$ $\mathrm{ng} / \mathrm{ml}, \mathrm{n}=26$, and $0.57 \pm 0.10 \mathrm{ng} / \mathrm{ml}, \mathrm{n}=23)$. In the muscle: GT in T2D-control $(0.348 \pm 0.008 \mathrm{nmol} / \mathrm{mg}$ per $\mathrm{min}, \mathrm{n}=5)$ was apparently lower than that in $\mathrm{N}$-control rats $(0.435 \pm 0.046 \mathrm{nmol} / \mathrm{mg}$ per min, $\mathrm{n}=5)$; Ex- 4 treatment did not modify the control level in N, but increased that in T2D to a value $(0.578 \pm 0.063 \mathrm{nmol} / \mathrm{mg}$ per min, $\mathrm{n}=6, \mathrm{p}=0.03)$ even higher $(p<0.05)$ than that of N-control. Muscle Glut4-mRNA in T2D-control showed to be indistinguishable from that in N-control, while Glut4-protein was lower $(86 \pm 6 \%$ $\mathrm{N}$-control, $\mathrm{n}=8, p<0.05)$; Ex-4 in $\mathrm{N}$ did not change the Glut4-mRNA, but induced a small but clear stimulation of the protein $(124 \pm 4 \%$ control, $n=4$, $p<0.05)$; no modification was detected in either Glut4-protein or -mRNA in the T2D group after Ex-4. In the liver: control Glut2-mRNA in T2D was about 2-times higher $(p<0.001)$ than that in $\mathrm{N}$, while the protein was lower $(66 \pm 8 \% \mathrm{~N}$-control, $p<0.05)$; Ex-4 induced an increment of Glut2-mRNA in $\mathrm{N}(6.4 \pm 0.7$ times control, $\mathrm{n}=4, p<0.001)$ and in T2D $(2.5 \pm 0.2$ times control, $\mathrm{n}=5, p<0.005)$, and also of the protein $(\mathrm{N}+\mathrm{Ex}-4: 174 \pm 18 \%$ control, $\mathrm{n}=5$, $p<0.05 ; \mathrm{T} 2 \mathrm{D}+\mathrm{Ex}-4: 254 \pm 56 \%$ control, $\mathrm{n}=5, p<0.05)$. Liver glycogen content in T2D-control $(225 \pm 20 \mathrm{ng} / \mu \mathrm{g}$ protein, $\mathrm{n}=5)$ was lower $(p<0.001)$ than that in $\mathrm{N}(386 \pm 16 \mathrm{ng} / \mu \mathrm{g}$ protein, $\mathrm{n}=5) ; \mathrm{Ex}-4$ did not altered the value in $\mathrm{N}(342 \pm 26$ $\mathrm{ng} / \mu \mathrm{g}$ protein, $\mathrm{n}=5)$, but induced an increase in T2D (309 $\pm 22 \mathrm{ng} / \mu \mathrm{g}$ protein, $\mathrm{n}=5, p<0.02)$ showing that $\mathrm{Ex}-4$ has a trend to normalize the liver glycogen content ( $\mathrm{p}=0.06$ vs $\mathrm{N}$-control)

Conclusion: These data suggest that the antidiabetic action of Ex-4 may occur, at least in part, through its increasing effect upon glucose transport in the muscle and also that upon the glucotransporter expression and glycogen synthesis in the liver.

Supported by: Ministerio de Sanidad y Consumo

\section{1}

Exenatide enhanced pancreatic compensation in insulin-resistant diabetic mice

D. Bhole ${ }^{1}$, R. Gelling ${ }^{2}$, K. Tatarkiewicz' ${ }^{1}$ L. Nielsen ${ }^{1}$, M. Xiu Steele ${ }^{3}$, D. Hargrove $^{1}$, D. Maggs ${ }^{1}$, D. Parkes ${ }^{1}$, D. Kendall ${ }^{1}$, E. Murphy ${ }^{4}$

${ }^{1}$ Clinical, Amylin Pharmaceuticals, Inc., San Diego, ${ }^{2}$ KineMed, Inc., Emeryville, ${ }^{3}$ Gladstone Institute, University of California San Francisco, San Francisco, ${ }^{4}$ Medicine, University of California San Francisco, United States

Background and aims: The incretin mimetic, exenatide, improves glycaemic control, reduces weight and improves insulin secretion in patients with type 2 diabetes. To determine pancreatic compensation during exenatide treatment we performed a series of studies using a unique deuterated glucose disposal technique in a mouse model of diabetes. This model is characterised by both insulin-resistance and deficient insulin secretion.

Materials and methods: Four-wk-old male C57Bl6/J mice were fed a high fat (HF) diet ( $58 \%$ fat) for 5 wks, followed by low dose streptozotocin (STZ 100 $\mathrm{mg} / \mathrm{kg}$ ) treatment (IP weekly for 2 wks except in a HF-fed control population). STZ-treated mice with blood glucose $>220 \mathrm{mg} / \mathrm{dL}$ and $<500 \mathrm{mg} / \mathrm{dL}$ at wks 7 and 8 were studied. Mice were treated with exenatide $(20 \mu \mathrm{g} / \mathrm{kg} /$ day $)$ or vehicle administered by continuous subcutaneous infusion via osmotic minipump for 4 wks. Mice had a 30 min glucose challenge with ${ }^{2} \mathrm{H}$-glucose as part of the glucose disposal test to measure insulin sensitivity and pancreatic compensation (determined from the absolute rate of whole body glycolysis, i.e., the total production of ${ }^{2} \mathrm{H}_{2} \mathrm{O}$ from ${ }^{2} \mathrm{H}$-glucose).

Results: Exenatide therapy reduced blood glucose $\mathrm{AUC}_{0-30 \min }$ by $34 \%$ relative to HF-STZ/vehicle-treated mice $(\mathrm{p}<0.001)$ and by $24 \%$ relative to HF-fed animals not treated with STZ $(p<0.01)$. Insulin concentrations were $52 \%$ lower in both HF-STZ-treated groups vs. HF-only animals $(\mathrm{p}<0.05)$. Exenatide therapy also increased insulin sensitivity [by $209 \%$ vs HF-no STZ /vehicle mice $(\mathrm{p}<0.01)$ and by $89 \%$ vs HF-STZ-only mice $(\mathrm{p}<0.05)]$. Overall, exenatide therapy significantly increased pancreatic compensation compared with HF-STZ-treatment $(31 \%, \mathrm{p}<0.05)$, and this compensation was comparable to that observed in HF-fed mice not treated with STZ.

Conclusion: Exenatide treatment improved glycaemic control, insulin sensitivity, and pancreatic $\beta$-cell response in an animal model of type 2 diabetes characterised by insulin-resistance and deficient insulin secretion.

\section{2}

Exendin-4 restores rapamycin-induced apoptosis in pancreatic betacells by inhibiting JNK and p38 signalling pathways

Y. Kawasaki ${ }^{1}$, S.-I. Harashima ${ }^{1}$, E. Mukai ${ }^{1}$, Y. Nakamura ${ }^{1}$, N. Harada ${ }^{1}$, K. Toyoda ${ }^{1}$, Y. Hamamoto ${ }^{1}$, A. Hamasaki ${ }^{1}$, Y. Seino ${ }^{2}$, Y. Yamada ${ }^{1,3}$, N. Inagaki

${ }^{1}$ Department of Diabetes and Clinical Nutrition, Graduate School of Medicine, Kyoto University, ${ }^{2}$ Division of Diabetes and Clinical Nutrition, Kansai Electric Power Hospital, Osaka, ${ }^{3}$ Department of Endocrinology and Diabetes and Geriatric Medicine, Akita University School of Medicine, Japan

Background and aims: Rapamycin is a mTOR inhibitor used to prevent rejection in islet cell transplantation. However, recently, it was reported that rapamycin also impaired glucose-stimulated insulin secretion and decreased viability of islets, or induced fluminant diabetes by increasing insulin resistance and reducing beta cell function and mass. On the other hand, exendin-4, an analog of glucagon-like peptide-1, is known to promote insulin secretion, increases beta cells mass and insulin gene expression. Therefore, our purpose of the current study is to examine whether or not exendin-4 recovers rapamycin-induced beta cell cytotoxicity.

Materials and methods: The mouse beta cell line MIN6 cells were treated with rapamycin and/or exendin-4 for 6 - $48 \mathrm{~h}$ in DMEM containing $25 \mathrm{mM}$ glucose. Various inhibitors were added $30 \mathrm{~min}$ before the treatment with rapamycin and/or exendin-4. Dead cells were stained with PI and counted by flowcytometry. Apoptosis was detected by Annexin-V-FITC Apoptosis Detection Kit. Phosphorylation of proteins was detected by Western blot.

Results: At first, MIN6 cells were treated with $0.1 \mathrm{nM}-100 \mathrm{nM}$ rapamycin to confirm cytotoxic effect of rapamycin. G1 arrest and cell death were observed in $12 \mathrm{~h}$ by the treatment with $10 \mathrm{nM}$ and more rapamycin. $10 \mathrm{nM}$ rapamycin also induced apoptosis in MIN6 cells in $6 \mathrm{~h}$. Contrarily, $10 \mathrm{nM}$ exendin-4 completely restored rapamycin-induced apoptosis and G1 arrest when cells were treated together with both rapamycin and exendin-4. Next, we investigated what signaling pathway is major for exendin-4-blocked apoptosis in- 
duced by rapamycin. As PKA, ERK and PI3K are important for exendin- 4 signaling in beta cells, we at first examined involvement of these kinases. H89, a PKA inhibitor, partially blocked inhibition of rapamycin-induced apoptosis by exendin-4. By contrast, LY294002, a PI3K inhibitor, and PD98059, an ERK inhibitor, had little effect on exendin- 4 reversed phenomena, implying that an alternative signaling pathway is related to the inhibitory effect of exendin4 against rapamycin. Therefore, we examined JNK signaling pathway because it was recently reported that JNK is a key molecule of beta-cell apoptosis. As expected, SP600125, a JNK inhibitor, blocked rapamycin-induced apoptosis by about $60 \%$. Interestingly, SB203580, a p38 inhibitor, also inhibited rapamycin-induced cell death by about $40 \%$. Western blot analysis further confirmed that rapamycin increased phosphorylation of JNK and p38 in $30 \mathrm{~min}$, while exendin- 4 decreased phosphorylation of both JNK and p38 in $30 \mathrm{~min}$. However, exendin-4 did not alter phosphorylation of MKK4 and MKK3, an upper stream kinase of JNK and p38, respectively, indicating that an uncertain signaling pathway of exendin-4 interacts with JNK and p38.

Conclusion: Rapamycin induced apoptosis in beta cells in several hours through direct activation of JNK and p38 signaling pathways, while exendin-4 restored the rapamycin-induced apoptosis via regulating not only PKA but JNK and p38. In the patients who receive islet cell transplantation, exendin-4 might be useful to prevent rapamycin-induced apoptosis in pancreatic beta cells.

\section{3}

Is there a local "incretin system" in human pancreatic islets?

R. Lupi ${ }^{1}$, S. Del Guerra ${ }^{1}$, V. D’Aleo ${ }^{1}$, S. Torri ${ }^{1}$, M. Galli ${ }^{1}$, M. Bugliani ${ }^{1}$,

F. Filipponi ${ }^{2}$, U. Boggi ${ }^{2}$, F. Mosca 2 , S. Del Prato ${ }^{1}$, P. Marchetti ${ }^{1}$

${ }^{1}$ Endocrinology and Metabolism - Metabolic Unit, ${ }^{2}$ Department of

Oncology, Transplantation and Advanced Technologies in Medicine,

University of Pisa, Italy

Background and aims: Incretins are intestinal hormones released in response to nutrient ingestion and which potentiate glucose-stimulated insulin secretion. Glucagon-like peptide-1 (GLP-1) is an important physiological incretin and is secreted from the intestinal L-cells after pro-glucagon cleavage by pro-convertase (PC)-1/3. Recent evidence from cell lines and rodent studies suggests that pancreatic alpha-cells also may secrete GLP-1. We assessed whether a local incretin (GLP-1) system is present in human pancreatic islets and the possible role of type 2 diabetes.

Materials and methods: The expression of glucagon, GLP-1, PC-1/3 and DPP-IV was determined by immunohistochemistry; isolated islets were prepared by enzymatic digestion and gradient separation from the pancreas of non-diabetic (Ctrl) and matched type 2 diabetic (T2DM) multiorgan donors; islets GLP-1, PC- $1 / 3$ and DPP-IV gene expression was measured by quantitative RT-PCR; GLP-1 protein expression was assessed by Western-immunoblotting; the active form of GLP-1 in the islets incubation medium was measured by radioimmunoassay.

Results: Immunohistochemistry showed co-localization of glucagon and GLP-1; PC-1/3 was found in most islet cells and scattered DPP-IV positivity was also observed. GLP-1 mRNA and protein expression was found in isolated islets. Gene expression (gene/beta-actin ratio) of DPP-IV and PC-1/3 mRNA differed $(\mathrm{p}<0.01)$ between T2DM (respectively $0.60 \pm 0.02$ and $0.21 \pm 0.13$ ) and Ctrl $(0.20 \pm 0.02$ and $0.84 \pm 0.01)$ islets. Active GLP-1 was detected in islets incubation medium, with lower $(\mathrm{p}<0.01)$ values with $\mathrm{Ctrl}(143 \pm 84 . \mathrm{pmol} / \mathrm{l} / 4 \mathrm{~h})$ than T2DM $(568 \pm 123 \mathrm{pmol} / 1 / 4 \mathrm{~h})$ islets. In addition, exposure of Ctrl islets for $24 \mathrm{~h}$ to $1.0 \mathrm{mmol} / \mathrm{l} \mathrm{FFA} \mathrm{(oleic:palmitic,} 2: 1$ ) determined a significant increase $(\mathrm{p}<0.01)$ of GLP-1 release $(978 \pm 191 \mathrm{pmol} / \mathrm{l} / 4 \mathrm{~h})$.

Conclusion: These results suggest the presence of a local GLP-1 system in human islets, which could be altered in type 2 diabetes.

\section{4}

GLP-1 analogue treatment increases beta cell replication and improves metabolic outcome in streptozotocin-diabetic rats transplanted with an insufficient beta cell mass

G. Joanny, N. Téllez, J. Escoriza, J. Soler, E. Montanya

Lab. Diabetes and Experimental Endocrinology, University of Barcelona, Endocrine Unit, Hospital Universitari Bellvitge, IDIBELL, CIBERDEM, L'Hospitalet de Llobregat, Spain

Background and aims: We have previously shown that a new DPPIV stabilized GLP-1 analogue enhanced beta cell regeneration in partially pancreate- ctomized rats. The treatment increased beta cell replication and neogenesis, and decreased beta cell apoptosis resulting in an increased beta cell mass. Shortage of islets is a major limitation in human islet transplantation and usually determines the transplantation of an insufficient islet mass, and the requirement of two or more transplants to restore normoglycemia. Strategies to increase graft beta cell mass could alleviate the shortage of islets and improve the outcome of islet transplantation. The aim of this study was to determine the effects of this new DPPIV stabilized GLP-1 in rat transplanted islets. In this model, the contribution of neogenesis is negligible due to the almost complete absence of ductal or exocrine tissue in the grafted cells, allowing a direct analysis of the role of enhanced beta cell replication in the outcome of the graft.

Materials and methods: We used a DPPIV stabilized GLP-1 analogue which has been derivatised with a chemical moiety that binds to albumin in a reversible manner thereby extending plasma half-life to at least one day. Streptozotocin-diabetic Lewis rats were syngeneically transplanted with an insufficient beta cell mass of 800 islets and were treated with the GLP-1 analogue $(100 \mu \mathrm{g} / \mathrm{kg}$. day, SC once daily) or with vehicle from the day of transplantation to day 14. An additional control group of non-transplanted STZ-diabetic rats treated with the GLP-1 analogue was studied. Blood glucose and body weight were monitored on days $0,3,7,14$ and after graft removal. Beta cell mass was determined by morphometry, beta cell replication by BrdU incorporation and beta cell apoptosis by the TUNEL technique in insulin stained sections of harvested grafts.

Results: Blood glucose levels decreased in transplanted GLP-1-treated animals compared to control non-transplanted GLP-1-treated group at all time points (day14: $285 \pm 44 \mathrm{vs} 515 \pm 52 \mathrm{mg} / \mathrm{dl} ; \mathrm{p}<0.05$ ), but were not significantly reduced in transplanted vehicle-treated animals. Furthermore, $30 \%$ (3 of 10) of transplanted rats treated with GLP-1 achieved and maintained normoglycemia until the end of the study. In contrast, only $11 \%$ (1 of 9) of vehicle treated and none of non-transplanted GLP-1-treated animals achieved normoglycemia. All normoglycemic animals reverted to clear hyperglycemia after graft removal. Beta cell replication in islet grafts of GLP-1-treated group was higher than in grafts of vehicle-treated animals $(1.31 \pm 0.35 \%$ vs $0.57 \pm 0.10 \%$; $\mathrm{p}<0.01)$, and than in normal pancreas $(0.26 \pm 0.02 \%$; $<<0.01)$. Beta cell apoptosis was similar in grafts of animals treated with GLP-1 or vehicle, and higher than in control pancreas. Beta cell mass in islet grafts of GLP-1-treated rats was $44 \%$ higher than in vehicle-treated animals ( $1.24 \pm 0.32$ vs $0.86 \pm 0.22$ $\mathrm{mg}$ ) although the difference did not reach statistical significance.

Conclusion: Treatment with this new GLP-1 analogue increased beta cell replication and improved metabolic evolution in diabetic rats transplanted with an insufficient islet mass. These results suggest that the effect of the GLP1 analogue on replication of transplanted beta cells improved the outcome of the graft.

Supported by: FIS PI-060891, Novo Nordisk

\section{5}

GLP-1 receptor activation attenuates beta cell damage in Akita mice S. Yamane ${ }^{1}$, Y. Hamamoto $^{1}$, N. Harada ${ }^{1}$, K. Toyoda ${ }^{1}$, Y. Seino ${ }^{2}$, N. Inagaki ${ }^{1}$ ${ }^{1}$ Department of Diabetes and Clinical Nutrition, Kyoto University Graduate School of Medicine, Kyoto, ${ }^{2}$ Department of Diabetes and Clinical Nutrition, Kansai Electric Power Hospital, Osaka, Japan

Background and aims: Dysfunction of pancreatic $\beta$-cells and decreased $\beta$ cell mass plays an important role in the development of diabetes mellitus. Recent studies have revealed that the endoplasmic reticulum (ER) stress is one of the causes of $\beta$-cell damage in diabetes. On the other hand, a growing body of evidence suggests that incretin hormones,exemplified by glucagon-like peptide-1(GLP-1),not only increase insulin secretion and upregulate insulin biosynthesis but also enhance resistance to $\beta$-cell death. In this study,we examined in vivo effects of exendin-4, a potent GLP-1 receptor agonist,on pancreatic $\beta$-cell in Akita mice,an animal model of ER stress-mediated diabetes. Materials and methods: A point mutation of insulin 2 gene in Akita mice promotes ER stress in $\beta$-cells that leads to $\beta$-cell apoptosis and hyperglycemia. We examined the changes in blood glucose levels,islet morphology,and the expression of CHOP,a marker of ER stress,histologically in Akita mice treated with vehicle alone or with exendin- $4(24 \mathrm{nmol} / \mathrm{kg})$ that was injected intraperitoneally twice daily for 6 weeks. We also evaluated the effect of exendin- 4 treatment on $\beta$-cell replication and apoptosis by 5-bromo-2'-deoxyuridine (BrdU) staining and TdT-mediated dUTP-biotin nick-end labeling (TUNEL) staining,respectively.

Results: Exendin-4 treatment significantly reduced blood glucose levels $(p<0.05)$,but there was no significant difference in body weight between ex- 
endin-4 treated group and control group. Immunohistochemical examination showed an increase in insulin positive areas in the islets in exendin- 4 treated group compared with control group. Furthermore,treatment of Akita mice with exendin-4 resulted in significant decrease in the number of CHOPpositive $\beta$-cells even after an adjustment by $\beta$-cell area. In BrdU or TUNEL staining evaluated at 6 weeks of exendin- 4 treatment,we could not find any differences in proliferation or apoptosis of $\beta$-cell between exendin- 4 treated group and control group.

Conclusion: Our data suggest that GLP-1 receptor activation can attenuate ER stress-mediated $\beta$-cell damage through the reduction of ER stress. Mechanisms responsible for this beneficial effect of exendin- 4 are not fully accounted for by these results,therefore,further examination is required in the future.

\section{6}

Expression, storage and rapid regulated secretion of active glucagon like peptide-1 following muscle-targeted gene transfer

A. Mahmoud ${ }^{1}$, A. Aldibbiat ${ }^{1}$, S.J.M. Niessen ${ }^{2}$, S.C. Campbell ${ }^{1}$,

G.N. Holliman ${ }^{1}$, S. Joshi ${ }^{1}$, K.T. Scougall ${ }^{1}$, J.A.M. Shaw ${ }^{1}$

${ }^{1}$ Diabetes, Diabetes Research Group, Institute of Cellular Medicine,

Newcastle Upon Tyne, ${ }^{2}$ Department of Veterinary Clinical Sciences, Royal

Veterinary College, Hatfield, United Kingdom

Background and aims: Glucagon-like peptide-1 (GLP-1) is a potent insulinotropic hormone with an important role in enhancing post-prandial glucose-dependent insulin secretion. Short half-life necessitates regular self-injection limiting clinical utility for those with diabetes. Muscle-targeted GLP1 gene delivery may enable long-term therapeutic efficacy following simple infrequent intramuscular plasmid injection.

The aims of this study were to generate a plasmid construct enabling regulated secretion of GLP-1 and evaluate expression / intracellular localisation in addition to small molecule ligand-mediated post-translational processing / secretion following gene transfer to a murine muscle cell line, and intramuscular injection in mice.

Materials and methods: A novel approach was employed in which GLP-1(736) cDNA was expressed downstream of a growth hormone signal peptide sequence as a fusion peptide linked by a consensus furin cleavage sequence to four copies of a mutated FK506 binding protein (4FM) enabling conditional endoplasmic reticulum aggregation and rapid disaggregation / entry into the constitutive secretory pathway in presence of a non-immunosuppressant rapamycin-analogue ligand (AP21998). Following plasmid synthesis and sequence confirmation, transfections were undertaken in the $\mathrm{C} 2 \mathrm{C} 12$ mouse muscle cell line employing jetPEI or the constructs were intramuscularly injected in mice preinjected with hyaluronidase and followed by in situ electroporation.

Results: GLP-1 expression in vitro was confirmed by RT-PCR. GLP1 / 4FM biosynthesis and intracellular co-localisation was demonstrated by immunofluorescence staining / Western blotting. AP21998 ligand-induced secretion of bioactive GLP1 was quantified by specific ELISA ( $>140$ pmol l-1 $^{-1}$ over a 4 hour incubation). Dose responsivity within the range of $0-2 \mu \mathrm{M}$ AP21998 was confirmed. GLP1 expression following in vivo gene transfer to mouse muscle was evidenced by immunohistochemical staining with systemic secretion confirmed by immunoassay.

Conclusion: GLP-1 biosynthesis and storage following plasmid-mediated gene transfer to muscle has been attained in addition to rapid ligand-induced post-translational processing / secretion of bioactive hormone. Supported by: Aleppo University.Syrian Arab Republic

\section{7}

Effect of GLP-1 on the bone remodeling in insulin resistant state B. Nuche-Berenguer ${ }^{1}$, P. Moreno ${ }^{1}$, S. Dapía ${ }^{2}$, P. Esbrit ${ }^{3}$,

M.L. Villanueva-Peñacarrillo ${ }^{1}$

${ }^{1}$ Metabolism, Nutrition and Hormones, Fundación Jiménez Díaz, Madrid, ${ }^{2}$ Trabeculae, S.L, Parque Tecnolóxico de Galicia, Orense, ${ }^{3}$ Laboratory of Bone and Mineral Metabolism, Fundación Jiménez Díaz, Madrid, Spain

Background and aims: It is now well documented the insulin-independent antidiabetic action of GLP-1, its insulinotropic effect and positive action upon glucose metabolism in liver, muscle and fat, and its neurotrophic and anorectic among other properties. It has been suggested that hormones with incretin character could participate in the changes in bone turnover occur- ring after nutrient absorption. Here we have explored the possible in vivo modulating effect of GLP-1 on bone remodelling markers in normal and in an insulin-resistant model.

Materials and methods: Insulin resistance (IR) was induced in adult Wistar rats by chronic feeding during 8 weeks with standard chow combined with $D$ fructose (20\% in the drinking water). Groups of normal (N, $n=20$ rats) and IR $(n=20)$ were 3 -days treated, through an osmotic pump, with saline solution (control) or GLP-1 $(0.86 \mathrm{nmol} / \mathrm{kg} / \mathrm{h})$. In fed conditions, plasma samples were taken before (basal) and by ending the treatment; then, rats were sacrificed and one femur and one tibia from each were collected. In plasma, osteocalcin (OC) and tartrate-resistant acid phosphatase (TRAP) -by ELISA-, insulin -by RIA- and glucose concentrations were measured. In the tibia, after total RNA extraction, the OC, osteoprogeterin (OPG) and RANK ligand (RANKL) gene expression were determined by real time PCR. Femoral bone structure was evaluated -by $\mu \mathrm{CT}$ - in 3 rats per experimental group.

Results: In plasma: GLP-1 did not change the basal OC value in $\mathrm{N}$ rats $(407.87 \pm 16.1 \mathrm{ng} / \mathrm{ml}, \mathrm{n}=15)$, but decreased that in IR $(-22.2 \pm 4 \% \Delta$ of basal, $\mathrm{p}<0.03)$, which showed an initial lower $(\mathrm{p}<0.0001)$ level than $\mathrm{N}(289.40$ $\pm 16.64 \mathrm{ng} / \mathrm{ml}, \mathrm{n}=15)$; N-basal TRAP $(2.26 \pm 0.20 \mathrm{U} / \mathrm{l}, \mathrm{n}=16)$ was not modified by GLP-1, while in the IR, with a lower than normal $(\mathrm{p}<0.0001)$ basal value $(1.14 \pm 0.16 \mathrm{U} / \mathrm{l}, \mathrm{n}=15)$, GLP-1 induced a trend towards a normalization ( $48.3 \pm 20.3 \% \Delta$ basal); in IR, basal insulin and glucose were only slightly higher and lower, respectively, than in N, and neither control or GLP-1 treatment significantly modified the values in any group, $\mathrm{N}$ or IR. In the tibia of 7-11 rats: OC mRNA-control in IR was $0.49 \pm 0.09$ times that in the $\mathrm{N}$ group $(\mathrm{p}<0.001)$, in which, treatment with GLP-1 increased $4.22 \pm 0.83$ times its control value $(\mathrm{p}<0.01)$, as it did, although in a lower extent ( $\mathrm{p}<0.05$ vs $\mathrm{N}$ ), in IR $(1.82 \pm 0.18$ times control, $p<0.01)$; control OPG mRNA in IR was lower than in $\mathrm{N}(0.66 \pm 0.12$ times, $\mathrm{p}<0.02)$, and GLP- 1 treatment increased the value in $\mathrm{N}(2.29 \pm 0.23$ times control, $\mathrm{p}<0.01)$ and also in IR $(2.84 \pm 0.46$ times control, $\mathrm{p}<0.01)$; control RANKL mRNA was also lower $(\mathrm{p}<0.001)$ in IR $(0.56 \pm 0.09$ times N), and GLP-1 induced only a slight increase in N (1.28 \pm 0.22 times control) as well as in IR (1.76 \pm 0.44 times control). In IR rats, OPG/RANKL mRNA ratio was 1.18-fold of N, and GLP- 1 treatment raised the value in an equal magnitude in both groups (1.79- and 1.61-fold, $\mathrm{N}$ and IR, respectively). In the femur of IR rats, trabecular thickness and anisotropy were higher and lower, respectively, than those in $\mathrm{N}$ group, without any apparent change after GLP-1 treatment; femoral cortical thickness and the momentum of inertia were higher in the IR group than in $\mathrm{N}$ rats, and they further increased after GLP-1 treatment.

Conclusion: The insulin-resistance of this experimental model is associated to a low bone remodelling state, and GLP-1 treatment seems to favour both bone formation and bone resistance. Supported by: Ministerio de Sanidad y Consumo

\section{8}

Differential importance of GIP vs. GLP-1 receptor signalling vs. DPP-4 inhibition for beta cell survival and function in mice

A. Maida ${ }^{1}$, Y. Seino ${ }^{2}$, D.J. Drucker ${ }^{1,3}$

${ }^{1}$ Samuel Lunenfeld Research Institute, Toronto, Canada, ${ }^{2}$ Medicine, Kyoto University, Japan, ${ }^{3}$ Medicine, Banting and Best Diabetes Centre, Toronto, Canada

Background and aims: Glucagon-like peptide-1 (GLP-1) and glucose-dependent insulinotropic polypeptide (GIP) are incretin hormones secreted by gut endocrine cells that act on structurally distinct $\beta$-cell receptors to trigger glucose-dependent insulin secretion. Although exogenous GLP-1 receptor (GLP-1R) activation promotes cytoprotection and endogenous GLP-1R signaling is essential for $\beta$-cell function, whether the GIP receptor (GIPR) is critically important for $\beta$-cell survival remains unclear

Materials and methods: We administered the $\beta$-cell toxin streptozotocin (STZ) to wildtype (WT) mice with or without concomitant treatment with the GIPR agonist [D-Ala2]-GIP, the GLP-1R agonist exendin-4 (Ex-4) or the dipeptidyl peptidase-4 inhibitor sitagliptin. The importance of endogenous GIPR signaling for $\beta$-cell survival was assessed in Gipr-/- mice. The importance of DPP- 4 inhibition for $\beta$-cell function and survival was assessed in WT and DIRKO mice.

Results: Exogenous administration of Ex-4 for either 7 or 60 days significantly reduced the extent of STZ-mediated $\beta$-cell damage as indicated by improved blood glucose and insulin levels, decreased levels of HbAlc, and increased pancreatic insulin content and $\beta$-cell mass. In contrast, comparable administration of the potent GIPR agonist [D-Ala2]-GIP for 7 or 60 days did not improve parameters of $\beta$-cell function under identical experimental 
conditions. To assess the importance of basal GIPR signaling for $\beta$-cell survival we assessed STZ-induced cytotoxicity in Gipr-/- mice. Unlike previous results in Glp1r-/- mice, Gipr-/- mice did not exhibit enhanced sensitivity to STZ-induced diabetes or increased $\beta$-cell apoptosis. Potentiation of endogenous incretin action via prolonged continuous administration of the DPP4 inhibitor sitagliptin reduced HbAlc, and improved levels of plasma and pancreatic insulin after STZ administration. Furthermore sitagliptin reduced the levels of activated caspase-3 in WT mice, but had no effect on caspase-3 activation in dual incretin receptor knockout (DIRKO) mice.

Conclusion: These findings reveal important differences in the endogenous physiological roles and pharmacological importance of murine GIP vs GLP-1 receptors vs DPP-4 inhibition for the preservation of $\beta$-cell mass and function in vivo.

Supported by: JDRF, CIHR, and Merck Frosst Inc.

\section{PS 081 Sitagliptin and vildagliptin}

\section{9}

In patients with type 2 diabetes, sitagliptin added to metformin effectively lowers $\mathrm{HbA}_{1 \mathrm{c}}$ regardless of patient age, gender, body mass index, or baseline measure of beta cell function M. Alba, A.S. Swern, R.B. Langdon, D. Williams-Herman Merck Research Laboratories, Rahway, United States.

Background and aims: Sitagliptin, a DPP-4 inhibitor, improves glycemic control and is well tolerated when added to ongoing metformin therapy in patients with type 2 diabetes. The purpose of this pooled analysis was to evaluate consistency of glucose-lowering by sitagliptin across subgroups defined by baseline differences in age, gender, body mass index (BMI), HOMA- $\beta$, and proinsulin/insulin $[\mathrm{P} / \mathrm{I}]$ ratio.

Material and methods: Data were pooled from two randomized, placebocontrolled, double-blind, Phase III trials. In these studies, sitagliptin or placebo was added to ongoing treatment with metformin. Changes from baseline in $\mathrm{HbA}_{1 c}\left(\Delta \mathrm{HbA}_{1 c}\right.$, the primary endpoint $)$ and fasting plasma glucose $(\triangle \mathrm{FPG})$ were then assessed at 18 or 24 weeks. Overall, 891 patients were randomized to receive either sitagliptin $100 \mathrm{mg}$ once daily $(\mathrm{n}=560)$ or placebo $(\mathrm{n}=331)$ added on to metformin 1500-2550 mg/day. Treatment effects on $\Delta \mathrm{HbA}_{1 \mathrm{c}}$ and $\triangle F P G$ (differences between sitagliptin and placebo treatments) were evaluated using an ANCOVA model that included terms for treatment, trial, baseline subgroup, and treatment-by-subgroup interaction, with baseline efficacy value included as a covariate. Subgroups were defined as shown in the Table. Results: At baseline, the two treatment groups had similar demographic, anthropometric and disease characteristics. The overall averages at baseline were: age, 54.6 years; duration of known T2DM, 6.6 years; BMI, $31.0 \mathrm{~kg} / \mathrm{m}^{2}$; $\mathrm{HbA}_{1,}$, 8.2\%; FPG, 9.9 mmol/L; HOMA- $\beta$, 42.8; and P/I ratio, 0.4. Compared with placebo, treatment with sitagliptin reduced LS-mean $\mathrm{HbA}_{1 \mathrm{c}}$ in the overall cohort by $-0.7 \%(95 \% \mathrm{CI}-0.9$ to -0.6$)$ and FPG by $-1.4 \mathrm{mmol} / \mathrm{L}(95 \% \mathrm{CI}$ -1.7 to -1.1$)$. Compared with placebo, sitagliptin treatment lowered $\mathrm{HbA}_{1 c}$ significantly in all subgroups, with between-group differences in LS-mean $\Delta \mathrm{HbA}_{1 c}$ from $-0.5 \%$ to $-0.9 \%$ (Table). Differences in the placebo-subtracted outcome are likely due to differences in $\triangle \mathrm{HbA}_{1}$ in the placebo arm, with changes from baseline in the sitagliptin-treated groups ranging from $-0.7 \%$

\begin{tabular}{|c|c|c|c|c|c|}
\hline \multirow[b]{2}{*}{ Baseline characteristic } & \multicolumn{2}{|c|}{$\begin{array}{l}\text { Sitagliptin } \\
100 \text { mg q.d. }\end{array}$} & \multicolumn{2}{|c|}{ Placebo } & \multirow{2}{*}{$\begin{array}{l}\text { Between-group } \\
\text { difference in } \\
\text { LS-mean } \Delta \mathrm{HbA}_{1 \mathrm{c}} \\
(\%)(95 \% \mathrm{CI})\end{array}$} \\
\hline & $\mathrm{N}$ & $\begin{array}{c}\text { LS-mean } \\
\Delta \mathrm{HbA}_{\mathrm{cc}}(\%)\end{array}$ & $\mathrm{N}$ & \begin{tabular}{|c|} 
LS-mean \\
$\Delta \mathrm{HbA}_{1 \mathrm{c}}(\%)$
\end{tabular} & \\
\hline \multicolumn{6}{|l|}{ Age $(p=0.278)$} \\
\hline$<50$ years & 168 & -0.7 & 82 & 0.2 & $-0.9(-1.1,-0.7)$ \\
\hline$\geq 50$ and $<65$ years & 293 & -0.8 & 185 & -0.1 & $-0.7(-0.8,-0.5)$ \\
\hline$\geq 65$ years & 87 & -0.7 & 49 & 0.0 & $-0.8(-1.1,-0.4)$ \\
\hline \multicolumn{6}{|l|}{ Gender $(p=0.842)$} \\
\hline Female & 248 & -0.8 & 144 & -0.0 & $-0.7(-0.9,-0.6)$ \\
\hline Male & 300 & -0.8 & 172 & -0.0 & $-0.7(-0.9,-0.6)$ \\
\hline \multicolumn{6}{|l|}{ BMI $(p=0.246)$} \\
\hline$<25 \mathrm{~kg} / \mathrm{m}^{2}$ & 66 & -0.7 & 31 & -0.3 & $-0.5(-0.8,-0.1)$ \\
\hline$\geq 25$ and $<30 \mathrm{~kg} / \mathrm{m}^{2}$ & 181 & -0.9 & 96 & -0.2 & $-0.7(-0.9,-0.5)$ \\
\hline$\geq 30 \mathrm{~kg} / \mathrm{m}^{2}$ & 269 & -0.7 & 158 & 0.1 & $-0.8(-1.0,-0.6)$ \\
\hline \multicolumn{6}{|l|}{ HOMA- $\beta$ tertiles $(p=0.801)$} \\
\hline Lower $(\leq 23.9)$ & 161 & -0.8 & 89 & -0.1 & $-0.7(-0.9,-0.5)$ \\
\hline Middle $(>23.9$ and $\leq 45.7)$ & 168 & -0.8 & 84 & 0.0 & $-0.8(-1.0,-0.6)$ \\
\hline Upper $(>45.7)$ & 162 & -0.7 & 87 & -0.1 & $-0.7(-0.9,-0.5)$ \\
\hline \multicolumn{6}{|c|}{ Proinsulin-to-insulin ratio tertiles $(p=0.967)$} \\
\hline Lower $(\leq 0.27)$ & 159 & -0.8 & 72 & -0.1 & $-0.6(-0.9,-0.4)$ \\
\hline Middle $(>0.27$ and $\leq 0.45)$ & 159 & -0.7 & 72 & -0.1 & $-0.6(-0.9,-0.4)$ \\
\hline Upper $(>0.45)$ & 148 & -0.7 & 83 & -0.1 & $-0.6(-0.8,-0.4)$ \\
\hline
\end{tabular}


to $-0.9 \%$. Tests for the interaction between baseline characteristics and treatment groups for $\Delta \mathrm{HbA}_{1 \mathrm{c}}$ were uniformly non-significant $(\mathrm{p}=0.278-0.976)$. The same was observed for $\triangle \mathrm{FPG}(\mathrm{p}=0.270-0.743)$.

Conclusion: In patients with type 2 diabetes, sitagliptin provided effective and consistent glycemic control regardless of baseline differences in age, gender, BMI, HOMA- $\beta$, and $\mathrm{P} / \mathrm{I}$ ratio.

Table. By subgroup, change from baseline in least-squares (LS) mean $\mathrm{HbA}_{1 c}$ $\left(\triangle \mathrm{HbA}_{1}\right)$ at the predefined study endpoint times (18 or 24 weeks). Supported by: Merck \& Co.

\section{0}

\section{Sitagliptin added to ongoing treatment with pioglitazone study up to 52} weeks in Japanese patients with T2DM

H. Maegawa ${ }^{1}$, A. Kashiwagi ${ }^{1}$, N. Tajima ${ }^{2}$, T. Kadowaki ${ }^{3}, K^{2}$. Nonaka ${ }^{4}$, T. Okamoto $^{4}$, K. Okuyama ${ }^{4}$, M. Nishii ${ }^{5}$, J.M. Amatruda ${ }^{6}$

${ }^{1}$ Department of Medicine, Shiga University of Medical Science, Otsu, Japan, ${ }^{2}$ Department of Internal Medicine, Jikei University School of Medicine, Tokyo, Japan, ${ }^{3}$ Graduate School of Medicine, The University of Tokyo, Japan, ${ }^{4}$ Banyu Pharmaceutical Co., Ltd., Tokyo, Japan, ${ }^{5}$ Ono Pharmaceutical Co., Ltd., Osaka, Japan, ${ }^{6}$ Merck \& Co., Inc., Rahway, United States

Background and aims: The use of pioglitazone (PIO) for treating type 2 diabetes mellitus (T2DM) has been increasing in Japan. However, its monotherapy may not be sufficient to get patients to current glycemic goals, raising the importance of combination therapy. Sitagliptin (SITA) is an oral, highly selective DPP-4 inhibitor that lowers glucose in a glucose-dependent manner by enhancing the incretin axis. This 12 -wk, randomized, double-blind (DB), placebo (PBO)-controlled study with 40-wk open-label extension assessed the efficacy and tolerability of the addition of SITA to ongoing PIO in Japanese patients with T2DM.

Materials and methods: Patients on PIO alone or PIO in combination with other oral antihyperglycemic agents (OHA) entered the screening period. For patients on PIO plus other OHAs, the other OHAs were discontinued and patients entered a wash-out period of $\geq 6$ wks. Following this period, eligible patients with an A1C of 6.5-10.0\% completed a 2 -wk, single blind PBO runin. Patients on a stable PIO dose $\geq 16 \mathrm{wks}$ and on diet/exercise $\geq 8 \mathrm{wks}$, were then randomized (1:1) to the addition of once-daily SITA $50 \mathrm{mg}$ or PBO for a 12 -wk DB treatment period. The DB period was followed by a 40 -wk openlabel extension period where patients on PBO were re-allocated to SITA 50 $\mathrm{mg}$ and SITA could be titrated from $50 \mathrm{mg}$ to $100 \mathrm{mg}$. The primary endpoint was A1C change from baseline at Week 12 . The efficacy analysis was based on the full analysis set with last observation carried forward.

Results: A total of 134 patients were randomized to SITA $50 \mathrm{mg}(\mathrm{N}=66)$ or $\mathrm{PBO}(\mathrm{N}=68)$ with mean baseline $\mathrm{A} 1 \mathrm{C}$ of $7.7 \%$ and $7.6 \%$, respectively. In DB period, SITA significantly $(\mathrm{p}<0.001)$ reduced $\mathrm{A} 1 \mathrm{C}$ from baseline relative to $\mathrm{PBO}$ at Week 12 (between-treatment difference in least-squares [LS] mean A1C change from baseline $[95 \% \mathrm{CI}]=-0.8 \%[-1.0,-0.6]$; SITA $=-0.4 \%$ and $\mathrm{PBO}=0.4 \%)$. Similarly, SITA significantly $(\mathrm{p}<0.001)$ reduced fasting plasma glucose and 2-hr postprandial glucose, relative to PBO. In addition, $\beta$-cell function as assessed by HOMA- $\beta$ and insulinogenic index significantly improved with SITA relative to $\mathrm{PBO}$ ( $\mathrm{p}<0.001$ for both comparisons). The occurrences of overall adverse experiences (AEs), serious AEs, drug-related AEs, and gastrointestinal AEs were similar between the groups. Hypoglycemia was reported in 2 patients in each group.

In open-label extension period, of 66 patients allocated to SITA, 50 patients completed 52 -wk treatment. In the population, excellent durability was observed at Week 52; A1C change from baseline: $-0.7 \%$, and the proportions of patients with A1C level $<6.5 \%(<7.0 \%): 28.0 \%(62.0 \%)$, without an increase of hypoglycemia. There was an increase from baseline in body weight of $0.76 \mathrm{~kg}$ with SITA at Week 52. Similar results of efficacy and safety were also observed in the group where PBO was switched to SITA at Week 12.

Conclusion: In this study, up-to 52-wk treatment with SITA added to ongoing therapy with PIO in Japanese patients with T2DM, provided effective glycemic control and was well tolerated, with a low occurrence of hypoglycemia and without clinically meaningful change in body weight.

\section{1}

Triple combination therapy with sitagliptin, metformin, and rosiglitazone improves glycaemic control in patients with type 2 diabetes J.C. Arjona Ferreira ${ }^{1}$, A. Dobs ${ }^{2}$, B.J. Goldstein ${ }^{3}$, L. Wieczorek ${ }^{1}$, G. Golm², M.J. Davies ${ }^{1}$, D. Williams-Herman ${ }^{1}$, K.D. Kaufman ${ }^{1}$, J.M. Amatruda ${ }^{1}$ ${ }^{1}$ Merck Research Laboratories, Rahway, ${ }^{2}$ The Johns Hopkins University School of Medicine, Baltimore, ${ }^{3}$ Medicine, Jefferson Medical College, Philadelphia, United States

Background and aims: Efficacy and safety of sitagliptin, a selective DPP-4 inhibitor, were examined in patients with type 2 diabetes who had inadequate glycemic control (A1C $7.5 \%$ to $11 \%$ ) on the combination of metformin and rosiglitazone.

Materials and methods: After a dose-titration/stabilization period of up to 20 weeks with metformin $(\geq 1500 \mathrm{mg} / \mathrm{d})$ and rosiglitazone $(\geq 4 \mathrm{mg} / \mathrm{d})$ and a 2 -week placebo run-in, 278 patients (mean baseline $\mathrm{AlC}=8.8 \%$ ) were randomized in a 2:1 ratio to the addition of once-daily sitagliptin $100 \mathrm{mg}(\mathrm{n}=$ $181)$ or placebo $(n=97)$ for 18 weeks. The primary endpoint was A1C change from baseline at Week 18 .

Results: The addition of sitagliptin significantly $(\mathrm{p}<0.001)$ reduced A1C by $0.7 \%$ relative to placebo (change from baseline $-0.9 \%$ with sitagliptin vs. $-0.2 \%$ with placebo), with a greater response observed in patients with a baseline A1C $>9 \%$ (change from baseline with sitagliptin -1.3\%). From the mean baseline $\mathrm{A} 1 \mathrm{C}$ of $8.8 \%$, the proportion of patients achieving $\mathrm{A} 1 \mathrm{C}<7 \%$ was significantly $(\mathrm{p}=0.005)$ greater with the addition of sitagliptin $(22 \%)$ compared with placebo $(10 \%)$. Relative to placebo, sitagliptin significantly $(\mathrm{p}<0.001)$ reduced fasting plasma glucose by $1.0 \mathrm{mmol} / \mathrm{L}$ (change from baseline $-1.5 \mathrm{mmol} / \mathrm{L}$ with sitagliptin vs. $-0.5 \mathrm{mmol} / \mathrm{L}$ with placebo) and 2-hr postprandial glucose by $2.2 \mathrm{mmol} / \mathrm{L}$ (change from baseline $-2.9 \mathrm{mmol} / \mathrm{L}$ with sitagliptin vs. -0.8 $\mathrm{mmol} / \mathrm{L}$ with placebo). Measures related to $\beta$-cell function, such as HOMA$\beta$ (12.9 vs. 3.5$)$, proinsulin/insulin ratio ( -0.12 vs. -0.03$)$, and insulinogenic index $(-2.7$ vs. $-1.1 \mu \mathrm{IU} / \mathrm{mL} / \mathrm{mg} / \mathrm{dL})$, were significantly $(\mathrm{p} \leq 0.05)$ improved with sitagliptin compared with placebo, respectively. Sitagliptin was generally well tolerated compared with placebo, with similar incidences of hypoglycemia ( $2.2 \%$ vs. $0 \%$, respectively), gastrointestinal adverse experiences $(6.1 \%$ vs. $9.3 \%$, respectively), and edema ( $2.8 \%$ vs. $4.1 \%$, respectively). Mean body weight change from baseline was not significantly different between sitagliptin $(0.7 \mathrm{~kg})$ and placebo $(0.4 \mathrm{~kg})$.

Conclusion: In this study, triple therapy with sitagliptin added to the combination of metformin and rosiglitazone substantially improved glycemic control and was generally well tolerated in patients with type 2 diabetes. Supported by: Merck \& Co., Inc.

\section{2}

Safety and tolerability of sitagliptin, a selective DPP-4 inhibitor, in patients with type 2 diabetes: pooled analysis of 6139 patients in clinical trials for up to 2 years

D. Williams-Herman, E. Round, A. Swern, B. Musser, M.J. Davies, K.D. Kaufman, J.M. Amatruda

Merck Research Laboratories, Rahway, United States

Background and aims: The safety and tolerability of sitagliptin were assessed by pooling data from 12 large, double-blind, randomized, completed Phase IIb and III studies that included 6139 patients receiving either sitagliptin 100 $\mathrm{mg} /$ day $(\mathrm{N}=3415)$ or placebo or an active comparator $(\mathrm{N}=2724$; non-exposed group).

Materials and methods: The studies were 18 wks to 2 yrs in duration and assessed sitagliptin as monotherapy, initial combination therapy with metformin, or add-on to oral antihyperglycemic agents (metformin, pioglitazone, sulfonylurea, sulfonylurea + metformin, or metformin + rosiglitazone). Patients in the non-exposed group were taking placebo, pioglitazone, metformin, sulfonylurea, sulfonylurea + metformin, or metformin + rosiglitazone. Safety data included evaluation of clinical and laboratory adverse experiences (AEs).

Results: The mean exposure to drug was 307 dosing days in the sitagliptin group and 294 dosing days in the non-exposed group. For clinical AEs, the incidences of AEs overall, serious AEs, and discontinuations due to AEs were similar between groups. The number of deaths was $11(0.3 \%)$ in the sitagliptin group and $16(0.6 \%)$ in the non-exposed group. The incidences of drug-related AEs overall and discontinuations due to drug-related AEs were higher in the non-exposed group, primarily due to events of hypoglycemia in sulfonylurea-treated patients. For specific AEs reported more frequently in a 
treatment group (with $95 \% \mathrm{CI}$ around the between-group difference excluding "0"), 6 AEs were higher in the sitagliptin group and 11 were higher in the non-exposed group. The incidence of gastrointestinal AEs did not meaningfully differ between groups. In an analysis in which the proportion of patients with an ischemia-related $\mathrm{AE}$ was assessed, no meaningful difference was found between the sitagliptin $(2.0 \%)$ and non-exposed $(2.3 \%)$ groups. Three patients $(0.09 \%)$ in the sitagliptin group had a fatal ischemic event compared with $7(0.26 \%)$ in the non-exposed group. No meaningful differences in immune-related AEs, including the incidence, severity, and types of infections, were found. The number of incident angioedema-related AEs per $100 \mathrm{pa}-$ tient-years while on an ACE inhibitor was the same (0.9) in both groups. No meaningful differences in laboratory AEs, either summary measures or specific AEs, were observed.

Conclusion: In patients with type 2 diabetes, sitagliptin $100 \mathrm{mg} /$ day was well tolerated in clinical trials up to 2 years in duration.

Supported by: Merck \& Co., Inc.

\section{3}

Improved glycaemic control with vildagliptin $100 \mathrm{mg}$ administered either as a morning or evening dose as add-on therapy to metformin in type 2 diabetes mellitus

M. Goodman ${ }^{1}$, H. Thurston ${ }^{2}$, B. Minic ${ }^{3}$, T. Thuren ${ }^{1}$

${ }^{1}$ Cardiovascular \& Metabolism, Global CD\&MA, Novartis Pharmaceuticals Corporation, East Hanover, United States, ${ }^{2}$ Biostatistics, Global CD\&MA, Novartis Pharma AG, Basel, Switzerland, ${ }^{3}$ Cardiovascular \& Metabolism, Global CD\&MA, Novartis Pharma AG, Basel, Switzerland

Background and aims: Vildagliptin is a potent and selective dipeptidyl peptidase-IV inhibitor that improves glycemic control in patients with type 2 diabetes mellitus (T2DM) by increasing $\alpha$ - and $\beta$-cell responsiveness to glucose. A single $100 \mathrm{mg}$ qd dose given before the PM meal was previously shown to suppress hepatic glucose production throughout the overnight period, suggesting that qd PM dosing would be as effective as qd AM dosing. The present study therefore evaluated the efficacy and safety of vildagliptin $100 \mathrm{mg}$ qd as add-on to ongoing metformin therapy in patients whose T2DM was inadequately controlled with metformin alone, with an exploratory objective of comparing the efficacy of a morning or evening vildagliptin dose regimen.

Materials and methods: This 24-week, multicenter, double-blind, parallelgroup, placebo-controlled trial randomized patients whose T2DM $(N=370)$ was inadequately controlled ( $\mathrm{HbA}, 7.5-11.0 \%)$ on stable metformin monotherapy ( $\geq 1500 \mathrm{mg}$ daily) to receive either a morning dose (AM), or an evening dose (PM) of vildagliptin $100 \mathrm{mg}$ or placebo as add-on therapy. The primary efficacy variable was comparison of change in $\mathrm{HbA}_{1 \mathrm{c}}$ from baseline to endpoint between the vildagliptin $100 \mathrm{mg}$ qd AM dose and placebo. Amongst the secondary efficacy variables were comparison of the AM and PM dose in reduction of $\mathrm{HbA}_{1 c}$ levels and responder rate $(\geq 0.7 \%$ absolute reduction in $\mathrm{HbA}_{1}$ level from baseline at endpoint).

Results: The AM dose of vildagliptin $100 \mathrm{mg}$ qd significantly reduced $\mathrm{HbA}_{1 c}$ from baseline to endpoint compared to placebo (adjusted mean change $0.83 \%$; $95 \%$ CI $[-1.13,-0.54] ; p<0.001)$. A similar efficacy was observed irrespective of administration as an AM or PM dose (difference in adjusted mean change $-0.13 ; 95 \%$ CI $[-0.42,0.16] ; p=0.38$ ). The responder rate was similar irrespective of whether vildagliptin was administered in the morning $(53.8 \%)$ or evening (50.4\%), and this rate was significantly higher in the pooled vildagliptin $100 \mathrm{mg}$ group $(\mathrm{AM}+\mathrm{PM})$ compared to placebo $(52.1 \%$ vs. $21.7 \%$; $p<0.001)$. The incidence of adverse events $(34.7 \%$ and $34.4 \%)$ and serious adverse events (2.8\% and $2.5 \%)$ was similar in the pooled vildagliptin and placebo groups respectively, with a slightly higher incidence of AEs (39.0\%) and SAEs (3.3\%) in the PM group than the AM or placebo groups.

Conclusion: In T2DM patients inadequately controlled with metformin monotherapy, the addition of either a morning or evening dose of vildagliptin $100 \mathrm{mg}$ qd significantly improved glycemic control. The safety and tolerability profile of vildagliptin added to metformin was comparable to metformin monotherapy plus placebo.

Supported by: Goodman and Thuren are employees of Novartis Pharmaceuticals Corporation

\section{4}

Vildagliptin is as effective as TZDs in metformin failures: results of GALIANT - a primary care diabetes study

R. Braceras ${ }^{1}$, L. Blonde ${ }^{2}$, M. Banerji ${ }^{3}$, S. Dagogo-Jack ${ }^{4}$, R. Pratley ${ }^{5}$, A. Marcellari ${ }^{1}$, D. Purkayastha ${ }^{1}$, M. Baron ${ }^{1}$

${ }^{1}$ Novartis Pharmaceuticals, East Hanover, ${ }^{2}$ Department of Endocrinology, Diabetes and Metabolic Diseases, Ochsner Medical Center, New Orleans,

${ }^{3}$ Diabetes Treatment Center, SUNY Downstate Medical Center and Kings County Hospital, Brooklyn, ${ }^{4}$ Division of Endocrinology, Diabetes \& Metabolism, University of Tennessee Health Science Center, Memphis, ${ }^{5}$ Diabetes and Metabolism Translational Medicine Unit, University of Vermont College of Medicine, Burlington, United States

Background: Vildagliptin, a potent and selective dipeptidyl peptidase 4 inhibitor has demonstrated efficacy similar to TZDs without weight gain during phase III clinical trials in patients with type 2 diabetes (T2DM).

Materials and methods: GALIANT tested the hypothesis that the efficacy of vildagliptin would be non-inferior to a TZD as add-on therapy in T2DM patients not reaching goal on metformin monotherapy (HbAlc $>7 \%$ while taking $>/=1000 \mathrm{mg} /$ day). Patients were recruited from primary care sites to ensure they were representative of the US T2DM patient population. The choice of TZD, the initial dose and titration were all left to the discretion of investigator's to simulate TZD use in actual practice. Patients were randomized (2:1) to open-label vildagliptin (100 mg daily [qd]) or a TZD. The ITT population consisted of 2478 patients (vildagliptin, 1653; TZD, 825). The difference between treatment groups for mean change in $\mathrm{HbA}_{1 \mathrm{c}}$ after 12 weeks was the primary efficacy variable. Non-inferiority would be established if the upper limit of the two-sided $95 \%$ confidence interval did not exceed $0.4 \%$. Once non-inferiority was demonstrated, superiority would be tested.

Results: Adjusted mean change in $\mathrm{HbA}_{1 \mathrm{c}}$ is illustrated in Figure 1. Vildagliptin was non-inferior to a TZD for the overall population and for subgroups of race (Caucasian/non-Caucasian), age $(<65,>\mid=65)$ and $\mathrm{BMI}(<30,>\mid=30)$. The overall incidence of adverse events was similar for vildagliptin and TZDs (39.5\% and $36.3 \%$ respectively ). Body weight decreased with vildagliptin ($0.58 \pm 0.09 \mathrm{~kg})$ and increased with TZDs $(0.33 \pm 0.11 \mathrm{~kg})(P<0.001)$. This relationship was seen in all subsets tested.

Conclusion: These data demonstrated that vildagliptin has similar efficacy to a TZD as add-on therapy in patients not reaching goal on metformin and is associated with beneficial effects on weight regardless of race, BMI and age.

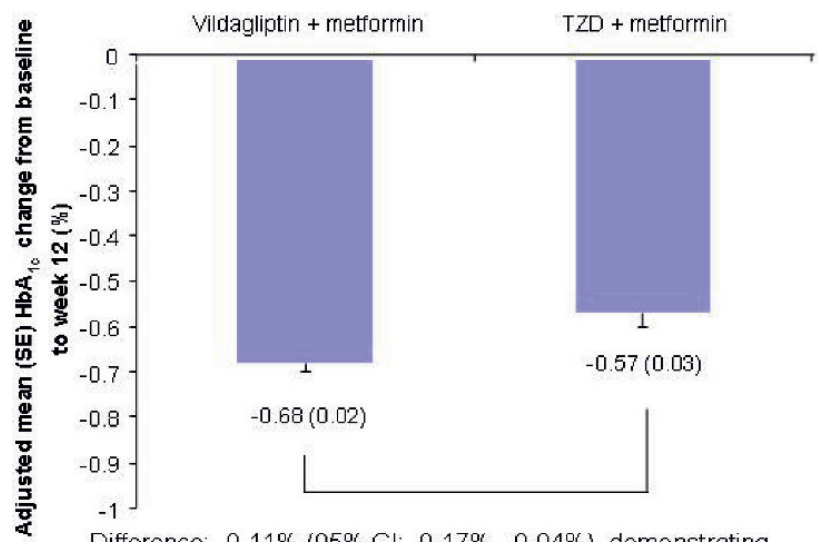

Difference: $-0.11 \%(95 \% \mathrm{Cl}:-0.17 \%,-0.04 \%)$, demonstrating equivalent glycemic control with vildagliptin versus $\mathrm{TZD}$

Furthermore, superiority of vildagliptin was demonstrated $(P=0.001)$

Figure 1. Adjusted mean (SE) change in HbAlc levels from baseline to week 12 vildagliptin and TZD

Supported by: Novartis Pharmaceuticals 


\section{5}

Cardiovascular safety profile of vildagliptin, a new DPP-4 inhibitor for the treatment of type 2 diabetes

W. Kothny ${ }^{1}$, C. Gimpelewicz ${ }^{2}$, S. Byiers ${ }^{2}$, D. Mills ${ }^{2}$, M. Fitchet

${ }^{1}$ Novartis Pharmaceuticals Corporation, East Hanover, United States,

${ }^{2}$ Novartis Pharma AG, Basel, Switzerland

Background: Vildagliptin, a potent and selective DPP-4 inhibitor, improves islet function by increasing $\alpha$ - and $\beta$-cell responsiveness to glucose.

The cardio- and cerebrovascular (CCV) safety profile of oral antidiabetics is currently subject to extensive debate. As diabetes per se is an established risk factor for atherosclerosis and ischemic events, an oral antidiabetic treatment should not increase the risk of these adverse events.

Aim: To characterize the cardio- and cerebrovascular safety profile of vildagliptin in diabetic patients.

Methods: The vildagliptin clinical trials database was analyzed for cardio- and cerebrovascular adverse events defined by preferred terms reflecting atherosclerotic or ischemic disease. Additionally, subcategories of pre-defined cardiovascular, cerebrovascular and peripheral vascular adverse events were analyzed. For these categories, the adverse event incidence rates and corresponding unadjusted odds ratios of each treatment group vs. placebo are presented. Results: In the treated patients (3784), overall incidences of cardio- and cerebrovascular events for vildagliptin $50 \mathrm{mg}$ qd $(0.7 \%)$ and $50 \mathrm{mg}$ bid $(0.6 \%)$ were less than placebo (1.3\%); the corresponding unadjusted odds ratios were less than 1 ( 0.55 and 0.45 respectively, $\mathrm{p}<0.05$ vildagliptin $50 \mathrm{mg}$ bid vs placebo). There were no predefined peripheral vascular adverse events on vildagliptin or placebo. The incidence rates of all cardio- and cerebrovascular serious adverse events observed for vildagliptin $50 \mathrm{mg}$ qd and $50 \mathrm{mg}$ bid were lower than placebo $(0.5 \%$ and $0.5 \%$ respectively vs. $0.9 \%)$; corresponding unadjusted odds ratios were below 1 ( 0.54 and 0.64 respectively). Analysis of cardio- and cerebrovascular (preferred terms) did not reveal clustering of any specific events.

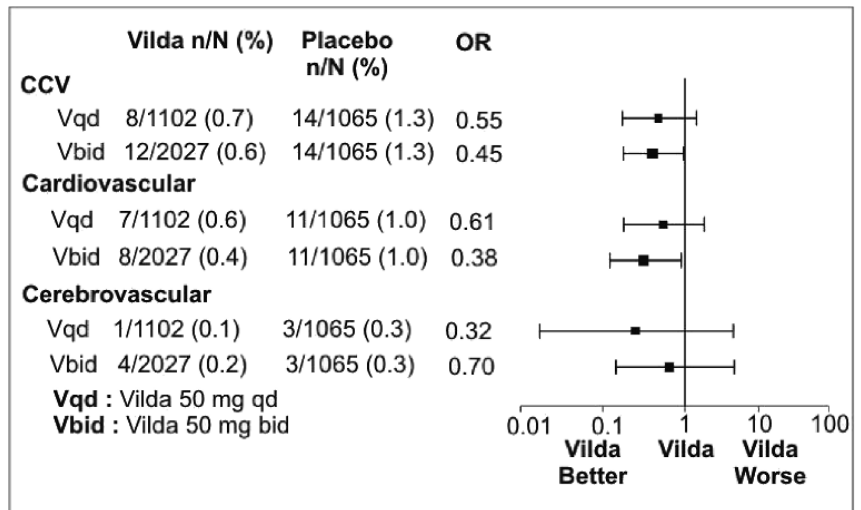

Conclusion: Adverse event data from vildagliptin clinical trials reveals a favorable cardiovascular safety profile

Supported by: Novartis Pharmaceuticals, USA

\section{6}

Dipeptidyl-peptidase 4 inhibition augments postprandial lipid mobilization and oxidation in type 2 diabetic patients

M. Boschmann ${ }^{1}$, S. Engeli ${ }^{2}$, P. Budziarek ${ }^{1}$, A. Strauss ${ }^{1}$, F. Sweep ${ }^{3}$, F.C. Luft ${ }^{1}$, H. Yan-Ling ${ }^{4}$, R. Szabo ${ }^{5}$, J. Jordan ${ }^{2}$

${ }^{1}$ Franz Volhard CRC, University Medicine Berlin, Germany, ${ }^{2}$ Institute of Clinical Pharmacology, Hannover Medical School, Germany, ${ }^{3}$ Department of Clinical Endocrinology, Radboud University Medical Center, Nijmegen, Netherlands, ${ }^{4}$ Exploratory Clinical Development, Novartis Institute for Biomedical Research Inc., Cambridge, United States, ${ }^{5}$ Exploratory Clinical Development, Novartis Pharmaceutical Corporation, East Hanover, United States

Background and aims: The metabolic response to dipeptidyl-peptidase-IV (DPP-4) inhibition has been primarily attributed to glucagon like peptide-1 (GLP-1) mediated improvement in glucose-dependent insulin and glucagon secretion. Based on recent studies with the selective DPP-4 inhibitor vildagliptin, we tested the hypothesis that there may be an additional direct effect on tissue metabolism.
Materials and methods: We included 20 patients with type 2 diabetes, body mass index between 28 and $40 \mathrm{~kg} / \mathrm{m} 2$, and an $\mathrm{HbAlc}$ of $<9 \%$ at screening. Within a randomized, double blind, cross-over study, we compared seven days treatment with $100 \mathrm{mg} /$ day vildagliptin vs. placebo. On the last day of each treatment period, patients underwent a thorough metabolic evaluation before and after ingestion of a mixed meal ( $5 \mathrm{kcal} / \mathrm{kg}$ body weight) including indirect calorimetry and microdialysis in abdominal subcutaneous adipose tissue and in the vastus lateralis muscle.

Results: Vildagliptin inhibited DPP-4 activity and substantially raised postprandial GLP-1 concentrations. Fasting and postprandial blood pressure, heart rate as well as venous glucose, insulin, glycerol, and free fatty acids were virtually identical with vildagliptin and with placebo. Vildagliptin augmented postprandial plasma norepinephrine $(\mathrm{p}<0.01)$. Furthermore, vildagliptin increased dialysate glycerol and lactate concentrations in adipose tissue and attenuated postprandial lactate concentrations in skeletal muscle $(\mathrm{p}<0.0001$ for all). Postprandial carbohydrate oxidation rate was reduced and lipid oxidation rate was increased with vildagliptin ( $\mathrm{p}<0.01$ for both).

Conclusion: Augmentation of endogenous GLP-1 through DPP-4 inhibition improves postprandial lipid mobilization and oxidation. The response may have been secondary to GLP-1 mediated sympathetic activation.

Supported by: Novartis Pharmaceutical Corp.

\section{7}

Inhibition of DPP-4 with vildagliptin improved insulin secretion in response to oral as well as "isoglycaemic" glucose without numerically changing the incretin effect in patients with type 2 diabetes

J.E. Foley ${ }^{1}$, L. Becker ${ }^{2}$, L. Köthe ${ }^{2}$, S. Dejager ${ }^{3}$, A. Schweizer ${ }^{4}$, M.A. Nauck ${ }^{2}$ ${ }^{1}$ Novartis, East Hanover, United States, ${ }^{2}$ Diabeteszentrum, Bad Lauterberg im Harz, Germany, ${ }^{3}$ Novartis, Paris, France, ${ }^{4}$ Novartis, Basel, Switzerland

Background and aims: The incretin effect is the higher insulin secretory response to oral as compared to intravenous glucose caused by gastrointestinal hormones like GIP and GLP-1. Typically, the incretin effect is reduced or lost in patients with Type 2 diabetes (T2DM). DPP-4 inhibitors block the degradation and inactivation of GLP-1 and GIP, thereby increasing nutrientstimulated plasma concentrations of these incretins. The aim of the present study was to assess insulin secretory responses to oral glucose as well as "isoglycaemic" intravenous glucose infusions in order to quantitatively assess the incretin effect after treatment of patients with T2DM previously on metformin after 12 or 13 days of additional treatment with the DPP-4 inhibitor vildagliptin or placebo.

Materials and methods: 21 patients with type 2 diabetes previously treated with metformin $(\sim 2 \mathrm{~g} /$ day $)$, including 3 women and 18 men with the mean \pm SE of age $(59.0 \pm 1.9$ years $)$, of BMI $\left(28.7 \pm 0.6 \mathrm{~kg} / \mathrm{m}^{2)}\right.$, of fasting plasma glucose $(9.6 \pm 0.5 \mathrm{mmol} / \mathrm{L})$, of $\mathrm{HbA}_{1 \mathrm{c}}(7.3 \pm 0.1 \%)$ and of duration of diabetes (5.9 \pm 0.7 years), were studied in a two-period crossover design. They received vildagliptin, $100 \mathrm{mg}$ once daily, or placebo, for 13 days, in randomized order. On day 12 , an oral glucose challenge ( $75 \mathrm{~g})$ was administered in the morning 30 min after the medication. On day 13, an "isoglycaemic" intravenous glucose infusion was guided by measuring capillary glucose every 5 min to match the glycaemic excursion after oral glucose. Blood was drawn over 240 min for the determination of glucose (capillary), insulin, and C-peptide (venous). The incretin effect was calculated as the difference in the insulin secretory responses between oral and "isoglycaemic" intravenous glucose stimulation relative to that after oral glucose $(=100 \%)$. Statistics: Repeated-measures analysis of variance.

Results: Glucose concentrations during "isoglycaemic" intravenous glucose infusions were well matched to those after oral glucose. Starting from similar baseline values, vildagliptin relative to placebo treatment significantly increased insulin secretory responses to both oral glucose and "isoglycaemic" intravenous glucose (e.g., $\mathrm{AUC}_{\mathrm{C} \text {-peptide oral }}$ by $31.4 \%, \mathrm{p}=0.0002$; $\mathrm{AUC}_{\mathrm{C}}$ by $33.7 \%, p=0.026$ ). The incretin effect (IE) was not changed significantly for vildagliptin versus placebo $\left(\mathrm{IE}_{\mathrm{C} \text {-peptide }}, 38.5 \pm 5.2\right.$ vs. $39.7 \pm 3.7 \%, \mathrm{p}=0.97$ ).

Conclusions: DPP-4 inhibition with vildagliptin improved insulin secretory responses after stimulation with both oral and intravenous glucose. As a result and against expectations, the incretin effect was not significantly improved. The vildagliptin effect on insulin secretion stimulated by intravenous glucose may be mediated by increased basal incretin levels, a change in the incretin-related environment of islets with beneficial effects persisting at least overnight, or influences on other, yet identified mediators of DPP-4 inhibition.

Supported by: Novartis Pharmaceuticals 


\section{PS 082 Treatment with PPARy agonists}

\section{8}

Impact of rosiglitazone discontinuation on glycaemic control P. Weissman ${ }^{1}$, B. Arondekar', L. Menditto ${ }^{2}$

${ }^{1}$ School of Medicine, University of Miami, ${ }^{2}$ US Health Outcomes, GSK, Philadelphia, United States

Background and aims: Because of the media controversy in 2007 regarding the association of thiazolidinediones and risk of ischemic cardiovascular events, this study aimed to evaluate the impact of discontinuation of rosiglitazone (RSG) therapy on glycemic control in patients (pts) with type 2 diabetes (T2D). Although, no prospective, randomized clinical trial has conclusively demonstrated a link between TZDs and increase in ischemic cardiovascular events, there is an established link between loss of glycemic control and increased risk of long term microvascular complications.

Materials and methods: Data from the General Electric (GE) Centricity electronic medical records database was used to identify T2D pts with 2 or more prescriptions (Rx's) for RSG (as monotherapy (mono) or combination (combo) therapy) in a 12 month period prior to 5/21/2007 who subsequently discontinued RSG. Discontinuation was defined as no new Rx for RSG (in a follow-up visit post 5/21/2007), or a stop date for RSG recorded in the database, or no new Rx for RSG in a 90 day period following last date of previous Rx for RSG prior to 5/21/2007. Data on these pts were available until 9/30/2007. Outcomes assessed included Rx patterns, FPG levels, HbAlc levels, and percentage of pts at goal post discontinuation. Lab values were assessed in patients irrespective of whether they received no new OAD, a new add-on agent or continued on the second agent (for combo pts.).

Results: A total of 6,871 patients (64\% aged $40-69$ years, $50 \%$ males) who were on RSG mono (41\%) or combo therapy( $28 \%$ RSG+Metformin (MET), $15 \%$ RSG+Sulfonylurea (SU), 6.5\% RSG+MET+SU, 5\% RSG+Insulin (INS), $5 \%$ RSG+others) discontinued RSG following 5/21/2007. Among those who discontinued, $33.4 \%$ did not receive a subsequent $\mathrm{Rx}$ for any OAD or INS, $20 \%$ received a Rx for MET mono, $15 \%$ for SU mono, $10 \%$ INS mono, $9 \%$ pioglitazone mono. Patients on RSG mono (and with lab values prior and post discontinuation - last FPG on RSG and first FPG post discontinuation) experienced an increase in FPG levels from $132.5 \mathrm{mg} / \mathrm{dL}$ to $140.4 \mathrm{mg} / \mathrm{dL}$ ( $\mathrm{p}=0.14$ ) with $53 \%$ of patients at goal $(\mathrm{FPG}<130 \mathrm{mg} / \mathrm{dL})$ prior to discontinuation compared to $49 \%$ post RSG discontinuation. Similarly, pts on combo RSG therapy who discontinued also experience a statistically significant increase in FPG levels post discontinuation $(134.5 \mathrm{mg} / \mathrm{dL}$ to $143.7 \mathrm{mg} / \mathrm{dL}, \mathrm{p}=0.025)$ and decrease in number of patients at goal from $43 \%$ to $39.8 \%$. There was no change in $\mathrm{HbAlc}$ levels, consistent with the short duration of follow-up.

Conclusion: A third of the pts who discontinued RSG post 5/21/2007 did not receive any $\mathrm{Rx}$ for an OAD or INS post discontinuation. There was an increase in FPG levels post RSG discontinuation with fewer patients at goal following discontinuation. The follow-up period was short and no difference in HbAlc levels was observed. The loss of glycemic control in the short-term could potentially impact the outcomes of these patients. This data highlights trends which suggest that discontinuation of RSG may be associated with undesirable trends in medication use and glycemic control.
919

Rosiglitazone treatment in non-alcoholic fatty liver disease E. Szymanska-Garbacz ${ }^{1}$, M. Saryusz-Wolska ${ }^{1}$, M. Jablkowski ${ }^{2}$, J. Bialkowska ${ }^{2}$, A. Borkowska ${ }^{1}$, A. Omulecka ${ }^{3}$, J. Loba ${ }^{1}$, L. Czupryniak $^{1}$ ${ }^{1}$ Diabetology \& Metabolic Disease Dept, ${ }^{2}$ Infectious Diseases Dept, ${ }^{3}$ Pathology Dept, Medical University of Lodz, Poland

Background and aims: Non-alcoholic fatty liver disease (NAFLD) is associated with insulin resistance, subclinical inflammation and increased risk of diabetes development. Glitazones as compounds directly improving insulin sensitivity have been suggested as potential agents for NAFLD treatment. We conducted a prospective study aiming at assessing the efficacy and safety of rosiglitazone (RSG) treatment in a group of individuals with NAFLD and normal glucose tolerance. Materials and methods: Twenty-seven non-diabetes and otherwise healthy subjects (mean $[ \pm$ SD] age $43.9 \pm 10.8$ years, BMI $29.2 \pm 3.1 \mathrm{~kg} / \mathrm{m} 2$ ) with biopsyconfirmed NAFLD were enrolled into an open-label controlled prospective study and were given RSG 4 mg once daily for 6 months. The control group comprised 13 age-, gender-, and BMI-matched healthy subjects, without any pharmacological treatment. Body weight, oral glucose tolerance test (OGTT) with plasma glucose and insulin measurements extended to $240 \mathrm{~min}, \mathrm{HbAlc}$, lipids, aspartate aminotransferase (AST) and alanine aminotraferase (ALT) activity, plasma subclinical inflammation and endothelial dysfunction markers (TNF-alfa, sVCAM, sICAM, sE-selectin, interleukins IL-1 $\beta$, IL-6, thrombomodulin $[\mathrm{TM}]$ ), adiponectin and hematocrit were assessed at baseline and after the treatment period.

Results: At baseline NAFLD subjects had significantly elevated AST and ALT activities, glucose at $60 \mathrm{~min}$ and insulin at all time points of OGTT, HOMA, triglycerides, sE-selectin, IL-6 and TM as well as lower HDL cholesterol and adiponectin as compared to the controls. After 6 months of RSG treatment ALT activity decreased significantly (from $104 \pm 60$ to $74 \pm 56 \mathrm{IU} / \mathrm{l}, \mathrm{p}<0.05$ ). No change in mean body weight, plasma glucose, HbAlc or lipids was noted. Insulin levels decreased significantly by $30-50 \%$ in each point of OGTT as well as HOMA index (from $3.71 \pm 1.95$ to $2.05 \pm 1.68, \mathrm{p}<0.001$ ). Adiponectin increased from $2178 \pm 1904$ to $5557 \pm 2164 \mathrm{ng} / \mathrm{ml}$ ( $\mathrm{p}<0.001)$, sE-selectin decreased from $75.8 \pm 33.5$ to $52.2 \pm 20.5 \mathrm{ng} / \mathrm{ml}(\mathrm{p}<0.01$ ) as did IL-6 (from $8.0 \pm 4.1$ to $5.0 \pm 1.9 \mathrm{pg} / \mathrm{ml}, \mathrm{p}<0.01$ ) and TM (from $1.82 \pm 0.59$ to $1.44 \pm 0.48 \mathrm{ng} / \mathrm{ml}, \mathrm{p}<0.05$ ). Other assessed parameters remained stable. No adverse events were noted during the study.

Conclusion: RSG treatment in non-diabetes individuals with NAFLD was associated with significant increase in insulin sensitivity. In addition, RSG use resulted in marked improvement of liver and endothelial function as well as decrease in subclinical inflammation, and these effects were apparently unrelated to changes in blood glucose or lipids levels. However, long-term beneficial effects of RSG treatment in NAFLD subjects remain to be confirmed. Supported by: Medical University of Lodz Grant No 502-18-557

\section{0}

Medication adherence to a fixed dose combination (FDC) of glimepiride/ rosiglitazone maleate in a managed health care plan

L. Menditto ${ }^{1}$, B. Arondekar ${ }^{1}$, S. Thayer ${ }^{2}$, C. Harley ${ }^{2}$

${ }^{1}$ GlaxoSmithKline, Philadelphia, United States, ${ }^{2}$ Health Economics and

Outcomes, i3 Innovus, Eden Prairie, United States

Background and aims: The purpose of this study was to assess changes in medication adherence rates associated with oral hypoglycemic agents in patients switching from monotherapy (mono) or dual therapy with sulfonylurea (SU) and/or rosiglitazone (RSG) to glimepiride-rosiglitazone FDC therapy. We hypothesized that switching to FDC therapy would lead to increased adherence to prescribed medications.

Materials and methods: This was a retrospective, claims-based analysis using data from a large, US managed care plan. Subjects with $\geq 1 \mathrm{Rx}$ fills for RSG,

Medication Possession Ratio

\begin{tabular}{|c|c|c|c|c|}
\hline & $\begin{array}{l}\text { Baseline MPRMean } \\
\text { (Std Dev) }\end{array}$ & $\begin{array}{l}\text { Baseline MPRMean } \\
\text { (Std Dev) }\end{array}$ & $\begin{array}{l}\text { Change in MPRMean } \\
\text { (Std Dev) }\end{array}$ & P-value \\
\hline Mono/Dual $(\mathrm{N}=2,518)$ & $0.91(0.12)$ & $0.81(0.13)$ & $-0.10(0.13)$ & \\
\hline Mono/FDC (N=543) & $0.89(0.13)$ & $0.87(0.14)$ & $-0.03(0.16)$ & $\begin{array}{l}\text { Mono/Dual vs. Mono/FDC } \\
\mathrm{P}<0.0001\end{array}$ \\
\hline Dual/Dual $(\mathrm{N}=13,145)$ & $0.84(0.16)$ & $0.89(0.12)$ & $0.05(0.14)$ & \\
\hline Dual/FDC $(\mathrm{N}=\mathbf{2 8 4})$ & $0.77(0.18)$ & $0.87(0.14)$ & $0.10(0.20)$ & $\begin{array}{l}\text { Dual/Dual vs. Dual/FDC } \\
\mathrm{P}<0.0001\end{array}$ \\
\hline
\end{tabular}


SU, or glimepiride-rosiglitazone(GL-RSG) FDC therapy during the identification period were included. Subjects who received either SU or RSG mono during the pre-index (pre-I) period and switched to dual therapy (SU+RSG) in the post-index (post-I) period (Mono/Dual cohort) were compared with those who switched to FDC therapy (Mono/FDC cohort). Subjects who received dual therapy with SU and RSG during the pre-I period and remained on dual therapy in the post-I period (Dual/Dual cohort) were compared with those who switched to FDC therapy (Dual/FDC cohort). Change in Medication Possession Ratio (MPR) was assessed.

Results: The study sample included 16,490 subjects with mean age of 57 years. $64 \%$ of the subjects were male. There was significantly less reduction in the mean MPR between pre-I period and the post-I period for the Mono/FDC cohort compared with the Mono/Dual cohort ( -0.03 vs. $-0.10 ; p<0.0001)$. There was significantly greater improvement in the MPR change for the Dual/FDC cohort compared with the Dual/Dual cohort (0.10 vs. $0.05 ; p<0.0001$ ).

Conclusion: This study identified a relationship between adherence to therapy and Rx drug regimens, namely significantly less reduction in mean MPR when mono users switch to FDC vs. dual therapies and greater improvement in mean MPR when dual therapy users switch to FDC vs. remaining on dual therapies.

\section{1}

Achieving glycaemic goal with initial combination therapy versus sequential augmentation therapy using metformin and pioglitazone B.J. Pandya ${ }^{1}, \mathrm{~A} . \mathrm{Yu}^{2}$, E. Wu ${ }^{2}, \mathrm{~K} . \mathrm{Chen}^{2}$, A. Kaltenboeck ${ }^{2}$, J. Seale², M. Mattson ${ }^{2}$, M. Bron ${ }^{1}$, R.W. Baran ${ }^{1}$

${ }^{1}$ Takeda Global Research \& Development Center, Inc., Deerfield,

${ }^{2}$ Consulting Services, Analysis Group, Inc., Boston, United States

Background and aims: Aggressive control of diabetes is recommended by clinical guidelines. This study aims to determine if early, simultaneous combination of oral antidiabetic (OAD) therapy offers a clinical outcomes benefit over sequential augmentation therapy in treated populations. Thus, we compared glycemic goal achievement (glycosylated hemoglobin $\left[\mathrm{HbA}_{1 \mathrm{c}}\right]<7 \%$ ) in patients with type 2 diabetes mellitus (T2DM) treated with an initial combination of metformin and pioglitazone (fixed-dose or loose-dose) (COMBO) vs those treated sequentially with initial metformin monotherapy and later augmented with pioglitazone (AUG).

Materials and methods: Adult T2DM patients treated with COMBO or AUG therapy were identified from the Integrated Healthcare Information Services (IHCIS) claims database (01/01/00 -03/31/07), which includes medical, pharmacy, and lab records. Patients were included with a baseline $\mathrm{HbA}_{1 c} \geq 7 \%$ and a second lab value within 9 months following therapy initiation. The proportion of patients achieving $\mathrm{HbA}_{1 c}<7 \%$ was examined at months $6,12,18$, and 24 ( \pm 3 months). Last observation carried forward (LOCF) was applied to impute missing $\mathrm{HbA}$, values. Goal achievement was studied in subgroups of patients with baseline $\mathrm{HbA}_{1 \mathrm{c}}$ between $7 \%$ and $9 \%$ and $\mathrm{HbA}_{1 \mathrm{c}}>9 \%$. Baseline differences were adjusted in the logistic regression by controlling for age, gender, baseline $\mathrm{HbA}_{1}$, comorbidities, prior resource utilization, and prior medications use.

Results: Of the 561 study patients, 213 received COMBO, and 348 received AUG. More patients in the COMBO than AUG groups achieved goal $(70.9 \%$ vs $49.4 \%, 68.5 \%$ vs $46.6 \%, 67.1 \%$ vs $46.0 \%$, and $67.1 \%$ vs $45.7 \%$ ) at months $6,12,18,24$, respectively (all $P<0.0001$ ). Logistic regression confirmed the findings, as AUG therapy exhibited odds ratios (OR) of 0.26-0.27 across all time points $(P<0.0001)$. Further subgroup analysis revealed that the clini$\mathrm{cal}$ advantage of COMBO therapy is more pronounced among patients with baseline $\mathrm{HbA}_{1 c}>9 \%$ (OR: 0.13-0.15; all $P<0.001$ ) than those with baseline $\mathrm{HbA}_{1 \mathrm{c}}$ between $7 \%$ and $9 \%$ (OR: $0.36-0.43$; all $P<0.0025$ ).

Conclusion: Patients treated with simultaneous combination therapy are more likely to achieve glycemic goal $\left(\mathrm{HbA}_{1 c}<7 \%\right)$ than those treated with sequential augmentation therapy. This benefit is especially more pronounced among those patients with higher baseline HbA1c levels.

Goal Achievement by Group

\begin{tabular}{llllll}
\hline & \multicolumn{3}{l}{ COMBO Therapy $(\mathbf{N}=\mathbf{2 1 3})$} & \multicolumn{3}{l}{ AUG Therapy $(\mathbf{N}=348)$} \\
\hline Month & $\mathbf{N}$ & \% & N & \% & P-Value \\
\hline 6 & 151 & 70.9 & 172 & 49.4 & $<0.0001$ \\
\hline 12 & 146 & 68.5 & 162 & 46.6 & $<0.0001$ \\
\hline 18 & 143 & 67.1 & 160 & 46.0 & $<0.0001$ \\
\hline 24 & 143 & 67.1 & 159 & 45.7 & $<0.0001$ \\
\hline
\end{tabular}

Supported by: Takeda Pharmaceuticals

\section{2}

INT131, a non-TZD selective PPAR $\gamma$ modulator (SPPARM), does not cause toxicities typical of TZD full PPAR $\gamma$ agonists following 6-month high dose treatment of rats or monkeys

A.M. DePaoli, L.S. Higgins

Development, InteKrin Therapeutics, Los Altos, United States

Background and aims: PPAR $\gamma$ is a validated target for treatment of insulin resistance, but the available thiazolidinedione (TZD) full agonists have significant side effects. INT131 (formerly T0903131, T131, and AMG 131) is a potent non-TZD selective PPAR $\gamma$ modulator (SPARRM) being developed for treatment of type 2 diabetes mellitus (T2DM). INT131 has demonstrated consistent unique properties in functional assays for receptor activation compared with multiple PPAR $\gamma$ full agonists. Studies with INT131 in rodent models of T2DM have demonstrated similar insulin sensitizing and glucose lowering activity with significantly less fluid retention, weight gain and cardiac hypertrophy than seen with currently available TZDs. A four week clinical study with INT131 in subjects with T2DM has similarly demonstrated significant dose responsive insulin sensitizing and glucose lowering actions, and suggested this efficacy can be separated from typical TZD side effects such as weight gain and fluid retention. Preclinical results for numerous PPAR ligands in rodent and monkey have shown time and dose dependent fluid retention, edema, cardiac hypertrophy, fatty replacement of bone marrow, and in some cases cardiac death. In order to assess the safety profile of INT131, six month safety studies were conducted in cynomolgus monkeys and in rats. Material and methods: Cynomolgus monkeys were treated with $0,0.3,3.0$, or $30 \mathrm{mg} / \mathrm{kg} / \mathrm{d}$ INT131 Besylate by nasogastric gavage once daily for 26 weeks. Groups included 4 animals/sex/dose, with 2 additional animals/sex for 0 and $30 \mathrm{mg} / \mathrm{kg} /$ day groups for a 4 week recovery period. Sprague-Dawley rats were treated with $0,30,100$, or $100 \mathrm{mg} / \mathrm{kg} / \mathrm{d}$ INT131 Besylate by gavage once daily for 26 weeks. Groups included 15 animals/sex/dose, with 5 additional animals/sex/ group which completed a 4 week recovery period after the dosing period. Measurements for both studies included TK, body weight, clinical observations, hematology, clinical chemistry, urinalysis, organ weight, gross necropsy and microscopic observations. The monkey study also included ECG, ophthalmology exam, echocardiograms, and careful observation for edema.

Results: INT131 was well tolerated with no clinical indications of edema, signs of plasma volume expansion, cardiac hypertrophy, significant weight gain, hepatic toxicity, or fatty replacement of marrow in either rats or monkeys at any dose. The NOAEL was identified in monkey as the high dose (30 $\mathrm{mg} / \mathrm{kg} / \mathrm{d}$ ), which translated to exposures of equal or greater than 70 fold the anticipated clinical exposure in the Phase $2 \mathrm{~b}$. Greater exposures were attained in the rat, with absence of typical PPAR $\gamma$ full agonist toxicities at 2-10 fold (males/ females) higher levels than in the high dose monkey group.

Conclusion: INT131 demonstrated no significant fluid retention, weight gain, cardiac hypertrophy, or hepatic toxicity in cynomolgus monkeys or in rats over 6 months at dose exposure multiples of equal or greater than 70 times the anticipated clinical exposure in the highest dose in an ongoing Phase $2 \mathrm{~b}$ study in subjects with T2DM. These results support in-vitro studies, rodent studies, and the clinical data demonstrating that INT131 is a SPPARM with unique PPAR $\gamma$ activating properties which minimize the commonly recognized side effects seen with full $\operatorname{PPAR} \gamma$ agonists. These data support further study of INT131 as a potent insulin sensitizer for the treatment of T2DM without the significant side effects of the TZDs. Supported by: InteKrin Therapeutics

\section{3}

Prevalence of stroke and transient ischaemic attack in diabetic medicare patients treated with oral antidiabetics in the US

F. Shaya ${ }^{1}$, C.M. Gbarayor ${ }^{1}$, D. Du ${ }^{1}$, B. Stuart ${ }^{2}$, T. Shaffer ${ }^{2}$, M. Bron ${ }^{3}$, B. Pandya ${ }^{3}$, R.W. Baran ${ }^{3}$

${ }^{1}$ Department of Pharmaceutical Health Services Research, University of Maryland School of Pharmacy, Baltimore, ${ }^{2}$ Peter Lamy Center for Drug Therapy and Aging, University of Maryland School of Pharmacy, Baltimore, ${ }^{3}$ Takeda Global Research \& Development Center, Inc., Deerfield, United States

Background and aims: Diabetes is a major risk factor for cardiovascular comorbidities. This study aims to determine the prevalence of stroke in elderly diabetic patients based on the Medicare Beneficiary Survey (MCBS) of type 2 diabetic patients in the US. Patients treated with background oral antidiabetic 
drugs (OADs), and on metformin (MET), sulfonylureas (SUs), pioglitazone (PIO), and rosiglitazone (ROSI) therapies were examined.

Materials and methods: All MCBS fee-for-service enrollees in 2001-03 with a diagnosis of type 2 diabetes (T2DM) were extracted for a cross-sectional analysis. Patients were included in the analysis if they were aged $\geq 65$ years, were residing in the community, were treated with OADs, and reported data in the Cost and Use portion of MCBS Part A and B. An analytic file was constructed for each patient, with demographics, historical diagnosis, drug utilization, comorbidities, and mortality. Regression analysis was used to assess the correlation between MET, SUs, PIO, and ROSI use and stroke, adjusting for age, race, gender, comorbidities, and functional status.

Results: Of 8,348 patients available for analysis in MCBS sample database, 1,826 were diagnosed with T2DM. Demographically, most were Caucasian (74\%), lived in urban areas (72\%), reported good health (60\%), and were not dependent on others for their activities of daily living (60\%). Additionally, most T2DM patients were treated with insulin or OADs $(60 \%)$, and a smaller cohort used at least PIO or ROSI (14\%). Of 1,826 Medicare T2DM patients in 2001, the prevalence of stroke with transient ischemic attack (TIA) was 5\% $(\mathrm{N}=98)$ and $4 \%(\mathrm{~N}=67)$ for stroke without TIA. Similar trends continued in 2002-03. Patient characteristics such as older age, higher comorbidities, and restricted activities of daily living were correlated with a higher prevalence of stroke in the analysis. When data were pooled into datasets with 3 years of follow-up and adjusted for disease severity (diabetic OAD users $\mathrm{N}=831$ ), the prevalence of stroke without TIA in 617 SU patients was $3.96 \%$, in 416 MET patients was $3.4 \%$, in 92 PIO patients was $3.94 \%$, and in 115 ROSI patients was $5.06 \%$. Prevalence of stroke with TIA in these respective groups was $6.2 \%, 5.3 \%, 6.25 \%$, and $7.0 \%$.

Conclusion: This is the first analysis of stroke occurrence and correlation to OAD use in the US type 2 diabetic Medicare population. In a largely Caucasian, urban, healthy, and independent elderly diabetic population with background $\mathrm{OAD}$ use, prevalence of stroke without TIA ranged from $3.4 \%$ to $5.06 \%$, while stroke with TIA ranged from $5.3 \%$ to $7.0 \%$. These results suggest a need for greater awareness of stroke rates in the elderly diabetic population receiving US Medicare benefits.

Supported by: Takeda Pharmaceuticals

\section{4}

Risk of cardiovascular disease and mortality in type 2 diabetes: a comparison between metformin, sulfonylureas and thiazolidinediones by meta-analysis

J.B. Ruige ${ }^{1}$, H. Vanhee ${ }^{1}$, D. De Bacquer ${ }^{2}$, J. Kaufman ${ }^{1}$, G. T'Sjoen ${ }^{1}$

${ }^{1}$ Endocrinology, University Hospital Ghent, ${ }^{2}$ Public Health, Ghent

University, Ghent, Belgium

Background and aims: Metformin, sulfonylureas and thiazolidinediones (TZDs) are commonly used for treatment of hyperglycemia in type 2 diabetes mellitus, which is strongly associated with cardiovascular disease (CVD) and mortality. Our purpose was to estimate possible class effects of oral antihyperglycemic agents on CVD and mortality.

Materials and methods: Articles were identified by means of a MEDLINE and Embase search and citation tracking. Eligible studies were cohort studies, nested case-control studies and RCTs on the relationship between a single class of agent and CVD or mortality. Data was extracted pertaining to class of oral agent used, type of outcome studied, study design, presence or absence of cardiac morbidity at baseline, sex, mean age of study population, BMI, follow-up period and total number of study subjects. Associations of classes of agents and CVD or mortality were re-expressed in a uniform manner, an estimate of the odds ratio (OR) and $95 \% \mathrm{CI}$, to be used in meta-regression analyses.

Results: Eleven out of 13 potentially eligible articles provided sufficient information (total number of events: 43289). The meta-analyses suggested a favourable outcome for metformin; compared to sulfonylureas a summary OR $(95 \% \mathrm{CI})$ of 0.59 (0.40 to 0.87$)$ was estimated, and, compared to TZDs a summary OR of 0.69 (0.55 to 0.86$)$ was estimated. Sulfonylureas might be preferable to TZDs with an OR of 0.79 (0.59 to 1.06) (all random-effects models; P value of heterogeneity test $<0.05$ ). Meta-regression analysis did not identify study-characteristics modifying the relationship between class of agents and CVD or mortality.

Conclusion: Metformin is associated with a favourable outcome with respect to CVD and mortality, if used as first-line anti-hyperglycemic agent in type 2 diabetes mellitus.

\section{5}

Rosiglitazone treatment and cardiovascular disease in the Veterans Affairs Diabetes Trial

H.J. Florez ${ }^{1,2}$, T. Moritz ${ }^{3}$, J. Marks ${ }^{1,2}$, D. Reda ${ }^{3}$, S. Warren ${ }^{4}$, W. Duckworth ${ }^{5}$, C. Abraira ${ }^{1,2}$

${ }^{1}$ Medicine, University of Miami, ${ }^{2}$ VADT Research Group, Miami VA Medical Center, Miami, ${ }^{3}$ VADT Research Group, Hines VA Cooperative Studies Program Coordinating Center, Hines, ${ }^{4}$ VADT Research Group, CSP - Research Pharmacy Coordinating Ctr, Albuquerque, ${ }^{5} \mathrm{VADT}$ Research Group, Phoenix VA Medical Center, Phoenix, United States

Background: Recent data suggest that the insulin sensitizer rosiglitazone may be associated with increased risk of cardiovascular disease (CVD) in patients with type 2 diabetes (T2D).

Methods: We evaluated the patterns of rosiglitazone use and its effect on CVD in 1792 patients age $60 \pm 8.7$ years and with T2D for $11.5 \pm 7.5$ years enrolled in the Veteran's Affairs Diabetes Trial (VADT). The VADT is assessing the effect of intensive glycemic control on a composite CVD endpoint that includes: myocardial infarction (MI), congestive heart failure (CHF), invasive revascularization, inoperable coronary artery disease (CAD), amputation for ischemia, stroke or cardiovascular death.

Results: The frequency of rosiglitazone use was greater in the intensive group than in the standard group $(\mathrm{p}<0.05)($ table). However, the use in both groups decreased over time according to safety prescription guidelines and as participants required increasing use of other agents to maintain appropriate separation in Alc values (median Alc after 6 months, 6.9\% intensive vs. 8.4\% standard), with the median insulin dose steadily $20 \%$ higher in the intensive arm during the study. After an interim average follow-up of 5.3 years, a total of 966 primary endpoints (in 540 patients) have been confirmed. The effect of dosage and duration of rosiglitazone use on CVD outcomes will be evaluated in the entire cohort with time-dependent covariate survival analysis, with data collected until April $30^{\text {th }}, 2008$.

Conclusion: These results suggest that in older patients with T2D the use of rosiglitazone may help sustain glycemic control. We will present a full report of the effect of rosiglitazone on CVD outcomes based on the complete data available at the end of the study.

\begin{tabular}{|c|c|c|c|c|c|}
\hline $\begin{array}{l}\text { Treatment } \\
\text { Group }\end{array}$ & Year 1 & Year 2 & Year 3 & Year 4 & Year 5 \\
\hline Standard & $\begin{array}{l}77.6 \% \\
(633 / 816)\end{array}$ & $\begin{array}{l}66.8 \% \\
(510 / 764)\end{array}$ & $\begin{array}{l}63.6 \% \\
(466 / 733)\end{array}$ & $\begin{array}{l}57.6 \% \\
(392 / 681)\end{array}$ & $\begin{array}{l}53.8 \% \\
(220 / 409)\end{array}$ \\
\hline Intensive & $\begin{array}{l}82.5 \% \\
(661 / 801)\end{array}$ & $\begin{array}{l}76.2 \% \\
(578 / 759)\end{array}$ & $\begin{array}{l}73.4 \% \\
(523 / 713)\end{array}$ & $\begin{array}{l}68.2 \% \\
(451 / 661)\end{array}$ & $\begin{array}{l}65.5 \% \\
(258 / 394)\end{array}$ \\
\hline
\end{tabular}

Supported by: CSP-Department of Veterans Affairs Office of Research \& Development, National Eye Institute, American Diabetes Association, Glaxo-SmithKline Pharm., Novo Nordisk Pharm., sanofi-aventis Pharm., Kos Pharm., and Amylin

\section{6}

Effect of thiazolidinediones on bone metabolism in diabetic patients B. Cartenì, M. D’Adamo, B. Micchelini, V. Guglielmi, G. Donadel, D. Lauro, M. Federici, R. Lauro, P. Sbraccia

Internal Medicine, University of Rome, Italy

Background and aims: Thiazolidinediones (TZDs) have been shown in vitro to have an effect on bone metabolism through the inhibition of osteoblasts differentiation and function. Studies in mice treated with rosiglitazone (ROSI) have confirmed this finding showing decreased bone mass. Furthermore recent studies have documented the effect of 12 weeks of ROSI treatment in post-menopausal women on bone metabolism showing reduced levels of bone formation.

The aim of our study was to assess the association between TZDs treatment and bone metabolism.

Materials and methods: To this end, we studied a group of 20 diabetic subjects treated with TZDs (18 pioglitazone, 2 ROSI; $12 \mathrm{M} / 8 \mathrm{~F}$; aged $62.3 \pm 1.9$ ) alone or in association with other oral hypoglycaemic agents (OHA) and/or insulin (TZD group), compared to18, age and sex -matched diabetics treated with other OHA and/or insulin (No-TZD group); both treatments lasted for at least 6 months. The two groups did not differ as regard to metabolic control (HbAlc), disease duration and BMI. 
All subjects underwent DEXA scan to evaluate bone mass at the lumbar and femoral site; evaluation of bone metabolism was performed by serum measurement of bone formation markers alkaline phosphatase, total and bone isoforms (ALP, B-ALP), and osteoprotegerin (OPG), and bone resorption marker C-telopeptide of collagen (CTX-I), whereas calcium metabolism was assessed by measurement of serum calcium and phosphate, plasma parathyroid hormone (PTH), 25-OH Vitamin $\mathrm{D}_{3}$.

Results: Bone mineral density (BMD) at the hip was similar in the two groups, whereas BMD at the lumbar spine was significantly lower in the TZD group vs. No-TZD (Z-score $0.05 \pm 0.34$ vs. $1.12 \pm 0.36, p=0.03$ ), although values were in the normal range for both groups. Serum calcium and phosphate levels, PTH, OPG and 25-OH VitD were not different in the two groups. CTX-I was higher in TZD vs. No-TZD $(0.27 \pm 0.03 \mathrm{ng} / \mathrm{ml}$ vs. $0.17 \pm 0.02 \mathrm{ng} /$ $\mathrm{ml}, \mathrm{p}=0.01$ ), whereas the difference between serum B-ALP levels in the two groups approached the statistical significance, with the TZD group showing lower levels ( $21.64 \pm 1.6 \mathrm{UI} / \mathrm{L}$ vs. $29.39 \pm 9.3 \mathrm{UI} / \mathrm{L})$. Moreover, bone metabolism markers levels did not correlate with $\mathrm{HbAlc}$, disease duration, age, and years since menopause (in females).

Conclusion: In summary our data show that TZD treated diabetic subjects have higher levels of bone resorption compared to a control group (No-TZD), and slightly, although not significant, lower levels of bone formation.

Our findings appear in agreement with recent in vitro study showing a novel role for PPAR $\gamma$ in osteoclast differentiation.

\section{PS 083 Novel oral agents}

\section{7}

Glucokinase activator RO4389620 improves beta cell function and plasma glucose fluxes in patients with type 2 diabetes

R.C. Bonadonna ${ }^{1}$, C. Kapitza ${ }^{2}$, T. Heise ${ }^{2}$, A. Avogaro ${ }^{3}$, M. Boldrin 4 , J. Grimsby $^{4}$, M.E. Mulligan ${ }^{4}$, C. Arbet-Engels ${ }^{4}$, R. Balena ${ }^{5}$

${ }^{1}$ Department of Biomedical and Surgical Sciences, University of Verona School of Medicine, Italy, ${ }^{2}$ Profil Institut für Stoffwechselforschung GmbH, Neuss, Germany, ${ }^{3}$ Department of Experimental Medicine, University of Padova School of Medicine, Italy, ${ }^{4}$ Hoffmann-La Roche Ltd, Nutley, United States, ${ }^{5}$ Hoffmann-La Roche Ltd, Basel, Switzerland

Background: Glucokinase (GK) plays a key role in glucose (G) regulation. Aims. We tested the acute G lowering effects of RO4389620, an allosteric GK activator (half-life: $~ 7.5$ hours), developed for type 2 diabetes treatment. Research Methods. Fifteen patients with diet-treated type 2 diabetes $(51 \pm 2$ years (mean \pm SEM); BMI $29.2 \pm 0.9 \mathrm{~kg} \mathrm{~m}^{-2}$ ) received a single oral dose of placebo (Plc), $25 \mathrm{mg}$ (D25) or $100 \mathrm{mg}$ (D100) RO4389620 $120 \mathrm{~min}$ before an oral $75 \mathrm{~g}$ G load labeled with $1-^{2} \mathrm{H}-\mathrm{G}$, in a randomized, double-blind, 3-period crossover study. $6,6-{ }^{2} \mathrm{H}-\mathrm{G}$ was infused to measure endogenous $\mathrm{G}$ output (EGO) and $\mathrm{G}$ utilization (GU). G fluxes units are presented as $\mu \mathrm{mol} \cdot \mathrm{min}^{-1} \cdot \mathrm{m}^{-2}$ BSA.

Results: Fasting G (see Figure) was lower with RO4389620 (7.5 \pm 0.3 vs. $6.7 \pm 0.4$ vs. $5.4 \pm 0.3 \mathrm{mM}$, Plc vs. $\mathrm{D} 25$ vs. $\mathrm{D} 100$, respectively; $\mathrm{p}<0.001)$ due to a decline in EGO $(314 \pm 12,266 \pm 15,117 \pm 20 ; \mathrm{p}<0.01)$ and a tiny increase in GU $(340 \pm 13,341 \pm 11,383 \pm 16 ; p<0.05)$. Plasma $G$ levels during the OGTT (see Figure) were lower with both D25 and D100 (mean difference over 5hours vs Plc, corrected for fasting G: $-1.03 \pm 0.28 \mathrm{mM}$ and $-2.34 \pm 0.28 \mathrm{mM} / \mathrm{L}$, respectively; $\mathrm{p}<0.001$ ), probably due to somewhat higher $\mathrm{GU}$ in the early phase of the OGTT (D100 vs. Plc. $0-120 \mathrm{~min}, 1182 \pm 63$ vs $1003 \pm 65, \mathrm{p}<0.03$ ). Analysis of OGTT plasma G and C-peptide concentration curves by state-ofart mathematical modeling showed that the sensitivity of $\beta$-cells to both the rate of increase of $\mathrm{G}$ (derivative or dynamic control) (1223 \pm 244 vs. $1781 \pm 542$ vs. $2666 \pm 500 \mathrm{pmolm}^{-2}$ per $\mathrm{mM} / \mathrm{min}$; $\mathrm{p}<0.02$ for D100 vs Plc) and to G concentration (proportional or static control) $(191 \pm 25$ vs $145 \pm 18,353 \pm 44$ vs $215 \pm 24,799 \pm 116$ vs $462 \pm 59,1257 \pm 197$ vs $723 \pm 100 \mathrm{pmolmin}^{-1} \mathrm{~m}^{-2}$, D100 vs $\mathrm{Plc}$ at $\mathrm{G}=5.0,7.5,11.7$ and $15.0 \mathrm{mM}$, respectively; $\mathrm{p}<0.01$ ) was higher with RO4389620.

Conclusion: Single dose administration of GK-activator RO4389620 lowers both fasting and post-challenge $\mathrm{G}$ in patients with type 2 diabetes, by improving $\beta$-cell function, EGO and GU. GK activation is a promising route to pursue in type 2 diabetes therapy.

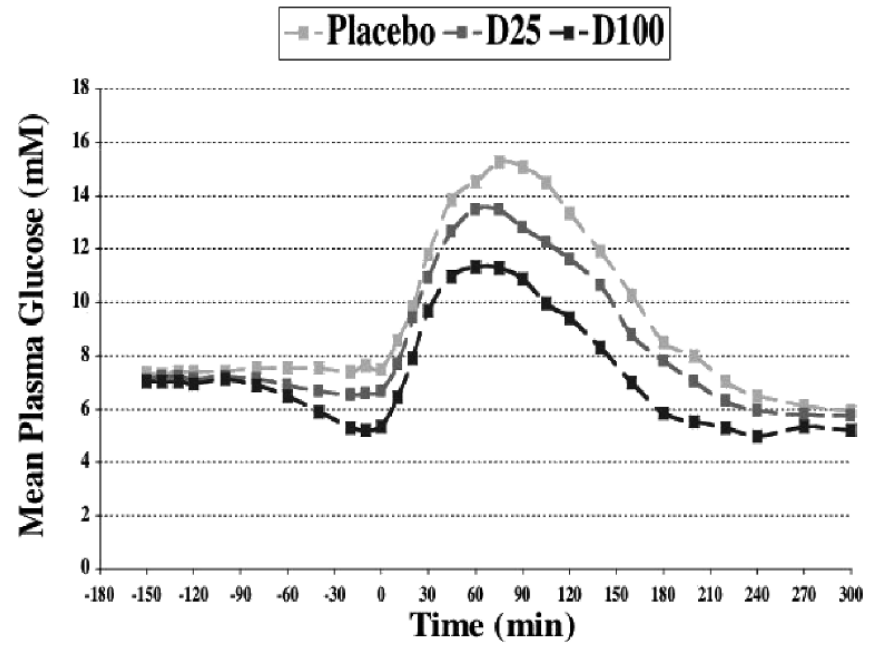

Supported by: Hoffmann-La Roche 


\section{8}

Phase I assessments of a novel glucokinase activator RO4389620 in healthy male volunteers

S. Zhai ${ }^{1}$, M.-E. Mulligan ${ }^{1}$, J. Grimsby ${ }^{1}$, C. Arbet-Engels ${ }^{1}$, M. Boldrin ${ }^{1}$, R. Balena' ${ }^{2}$ J. Zhi ${ }^{1}$

${ }^{1}$ Roche, Nutley, United States, ${ }^{2}$ F. Hoffmann-La Roche Ltd, Basel, Switzerland

Background and aims: RO4389620 is a nonessential, mixed-type activator of glucokinase (GK). It binds to an allosteric site on GK, which leads to an increase in the catalytic effectiveness of GK to metabolize glucose, and increases the glucose phosphorylation capacity of pancreatic $\beta$-cells and hepatocytes. Thus, RO4389620 is proposed as a novel treatment of type 2 diabetes. The aim of the present phase I studies was to assess the pharmacokinetics (PK) and pharmacodynamics (PD), tolerability, and, safety of single ascending doses (SAD) of RO4389620, as well as the potential food effect (FE) on RO4389620 PK.

Material and methods: SAD was a double-blind, randomized, placebo-controlled study and FE was an open-label, two-way crossover study; both were conducted in healthy male volunteers (HVs). In the SAD study, $24 \mathrm{HVs}$ received a single oral dose of RO4389620 or placebo; the dose escalation was a leap-frog design at doses of 5, 10, and, $25 \mathrm{mg}$. In the FE study $(\mathrm{N}=16)$, a single dose of $10 \mathrm{mg}$ was administered with and without a high fat, high calorie meal. Blood and urine samples were collected up to 48 hours for PK analysis. PD assessments included $4 \mathrm{~h}$ profile of plasma glucose, insulin, Cpeptide, and glucagon.

Results: RO4389620 was rapidly absorbed with an elimination half-life of 8 to 10 hours. $\mathrm{C}_{\max }$ and $\mathrm{AUC}_{\infty}$ (RO4389620 and its inactive metabolite M4) were proportional to doses. Approximately $13 \%$ of the dose was excreted in urine as unchanged drug. Administration of RO4389620 resulted in a dose dependent glucose reduction in 4-h fasting plasma glucose; the nadir in fasting glucose levels decreased with RO4389620 dose, ranging from a median change from baseline of $-13 \%$ at $5 \mathrm{mg}$ to $-22 \%$ at $25 \mathrm{mg}$. No clinically meaningful food-effect on RO4389620 PK was observed from the FE study. RO4389620 was well tolerated up to $25 \mathrm{mg}$ in HVs. The most common adverse event was headache, with no clear dose-related pattern. No clinically significant laboratory, vital sign, or ECG abnormalities were reported.

Conclusion: RO4389620 administered to healthy male subjects as a single oral dose of $5 \mathrm{mg}, 10 \mathrm{mg}$, or $25 \mathrm{mg}$ was well tolerated. Oral administration of RO4389620 consistently showed dose-dependent exposure and significant, dose-dependent reduction of fasting plasma glucose, demonstrating potential utility in the treatment of type 2 diabetes.

\section{9}

Liver selective glucokinase activation a new paradigm for treatment of type 2 diabetes

C. Valcarce ${ }^{1}$, T. Bödvarsdóttir², P. Wahl², M. Larsen², K. Fosgerau², N. Blume ${ }^{2}$, J. Selmer ${ }^{1}$, A. Mjalli ${ }^{1}$

${ }^{1}$ Transtech Pharma, High Point, United States, ${ }^{2}$ Novo Nordisk, Maaloev, Denmark

Background and aims: Data from transgenic animals suggest that selective activation of liver glucokinase(GK) will provide a mechanism that ensures reduction of blood glucose without increasing the risk for hypoglycemia.

The aim of the studies presented here was to demonstrate that TTP355, an oral, liver selective GK activator, regulates blood glucose without inducing hypoglycemia in multiple animal models of diabetes and has the potential to be a safe and effective first line therapy for the treatment of diabetes

Materials and methods: The effects of TTP355 on glucose metabolism and insulin secretion was evaluated: in vitro (isolated enzyme, hepatocytes and islets), and in vivo (normal rats as well as in rodent and non-rodent animal models of type 2 and type 1 diabetes).

Results: TTP355 activates recombinant GK in the presence of $15 \mathrm{mM}$ glucose. TTP355 increases glucose utilization in hepatocytes without affecting the interaction of GK with GKRP or its translocation to the nucleus. TTP355 does not increase insulin secretion by perifused islets or perfused pancreas at concentrations where a significant increase in glucose metabolism is observed in hepatocytes. Administration of TTP355 to normal rats causes a dose-dependent decrease in glucose excursion during OGTT ( $\mathrm{p}<0.01$ vs. vehicle) without an increase in insulin secretion. In mildly diabetic Goto Kakizaki-rats (GKrats), once daily oral dosing of TTP355, significantly reduces $\mathrm{HbA}_{1 \mathrm{C}}$ after only one week of treatment, $(\mathrm{p}<0.05)$, after 5 weeks of treatment,
$\mathrm{HbA}_{1 \mathrm{C}}$ levels were normalized ( $\left.3.1 \pm 0.05\right)$, as compared to normal Wistar rats. GKrats have a reduced number of $\beta$ cells from birth and throughout life. In TTP355 treated GKrats there was an improvement in $\beta$ cell morphology and an increase of approximately $33 \%$ (n.s.) in $\beta$ cell mass. In diabetic ob/ob mice diurnal glycemia is normalized after three weeks of dosing and $\mathrm{HbA}_{1 \mathrm{C}}$ levels are decreased by $-2.63 \pm 0.29, \mathrm{p}<0.001$. There was a significant progression in the effect of the treatment over time, as measured by the difference in the glucose AUC between the $1^{\text {st }}$ (after $1^{\text {st }}$ dose) and the $2^{\text {nd }}$ (after $18^{\text {th }}$ dose) $24 \mathrm{~h}$ blood glucose profiles $(\triangle \mathrm{AUC} \mathrm{p}<0.001)$. Chronic treatment with TTP355 does not increase lactate levels in blood, as observed in metformin treated animals. No significant effect on body-weight, food intake, liver or plasma lipids and insulin levels were observed. In Type 1 STZ-minipigs, chronic treatment with TTP355 reduces the exogenous insulin required to maintain glucose control, and reduces the daily glucose excursions in response to meal. The data indicate that TTP355 increases GLP-1 secretion and may preserve residual $\beta$ cell function in this model. TTP355 prevents the development of ketoacidosis when insulin is withdrawn. No episodes of hypoglycemia were observed when fed or fasted,normal or diabetic, animals were administered high doses of TTP355.

Conclusion: Evidence from studies in vitro and in vivo shows that liver GK is a suitable pharmaceutical target for the treatment of diabetes, and that the liver selective GK activator TTP355 provides the expected therapeutic effects. Because of TTP355's efficacy is independent of the severity or the etiology of the diabetes in the animal models tested, this therapeutic approach may be suitable for a broad range of diabetic patients, from glucose intolerant to $\beta$ cell deficient. Preliminary data in man indicate that TTP355 may have the same or better efficacy when administered to humans.

\section{0}

Quick release bromocriptine (Cycloset ${ }^{\mathrm{tm}}$ ) improves glycaemic control in patients with diabetes failing metformin/sulfonylurea combination therapy

R.E. Scranton ${ }^{1}$, W. Farwell ${ }^{1,2}$, M. Ezrokhi ${ }^{1}$, J.M. Gaziano ${ }^{2}$, A.H. Cincotta ${ }^{1}$ ${ }^{1}$ Veroscience, Tiverton, United States, ${ }^{2}$ Harvard Medical School, Boston, United States

Background and aims: Pre-clinical studies of insulin resistance indicate that administration of the dopamine D2 receptor agonist, bromocriptine, pulsed at the time of day of the circadian peak in hypothalamic dopaminergic tone corrects ("resets") aberrant hypothalamic signaling that in turn reduces elevated postprandial glucose, lipid excursions and insulin resistance. Clinical studies of insulin resistance also inidcate that timed (morning) administration of Cycloset ${ }^{\mathrm{TM}}$ (bromocriptine mesylate), improves postprandial glucose and lipid metabolism across the day while the drug is substantially removed from the circulation before lunch time. Post pranidal hyperglycemia is improved without increases in insulin levels. However, no studies have investigated the potential of Cycloset to improve glycemic control in subjects with type 2 diabetes (DM) inadequately controlled on the combination of metformin plus sulfonylurea. The aim of this study was to assess the impact, if any, of Cycloset on glycemic control in such subjects.

Materials and methods: The Cycloset Safety Trial was a one year, 2:1 randomized trial of the overall safety of Cycloset versus placebo among 3070 persons with DM. Within the study design, pre-specified six month efficacy analyses were conducted among a sub-group of subjects receiving metformin plus a sulfonylurea and having an $\mathrm{HbAlc}$ (A1C) of $\geq 7.5 \%$ and $\leq 10 \%$ at screening. Study investigators were instructed to alter the dose (or after 3 months in the study - the dose and regimen) of non-study (concomitant) DM therapies if needed to maintain or achieve ideal glycemic control. Two physicians blinded as to treatment group assignment assessed changes in concomitant DM therapies. Changes in DM therapies were classified as unchanged, intensified (increase dose of DM medication or addition of DM medication) or decreased. An ANCOVA model which included study center and baseline $\mathrm{A} 1 \mathrm{C}$ in the model was used to assess between group differences in the change from baseline A1C after 24 weeks of treatment. The proportion of subjects reaching an $\mathrm{A} 1 \mathrm{C}$ of $\leq 7.0 \%$ at Week 24 was compared between the two treatment groups using the Cochran-Mantel-Haenzsel test. The intent to treat population (ITT) was comprised of 267 subjects ( 177 Cycloset; 90 placebo). Results: The ITT study population had a mean A1C of 8.3, a mean age of 60 , was $66 \%$ male, and had a mean body index of $32 \mathrm{~kg} / \mathrm{m}^{2}$. After 24 weeks of treatment, \% A1C decreased by 0.67 among Cycloset treated subjects, and remained relatively unchanged $(+0.02)$ among the placebo subjects resulting in a between-treatment difference from baseline of $-0.7(\mathrm{P}=0.0002)$. More Cycloset versus placebo treated subjects reached a target A1C of $\leq 7.0(39 \%$ 
vs. $11 \%$, respectively; $\mathrm{p}=0.0004)$. In addition, placebo treated subjects were more likely to intensify concomitant diabetes therapy compared to Cycloset ( $22 \%$ vs. $16 \%$, respectively). Cycloset subjects were also twice as likely to decrease the intensity of concomitant diabetes therapies compared to placebo ( $6 \%$ vs. $3 \%$, respectively).

Conclusion: Cycloset represents an effective and novel treatment approach to the management of type 2 diabetes among subjects failing a standard and widely employed combination therapy of metfomin plus sulfonylurea. Supported by: Veroscience and S2 Therapeutics

\section{1}

Eight week treatment of $\mathrm{db} / \mathrm{db}$ mice with a small molecule antagonist of the glucagon receptor lowers $\mathrm{HbA}_{1 \mathrm{c}}$ and plasma triglyceride levels without $\alpha$-cell hyperplasia

S.C. Potter, P.D. van Poelje, J. Gomez-Galeno, M.L. McCaleb,

D.L. Linemeyer, M.D. Erion

Metabasis Therapeutics, La Jolla, United States

Objective: Impairment of hepatic glucagon signaling by genetic knockdown of the glucagon receptor has demonstrated marked improvements in glucose homeostasis in murine models of type 2 diabetes; however, significant $\alpha$-cell hyperplasia has been associated with this genetic manipulation. Compound $\mathrm{H}(\mathrm{Cmpd} \mathrm{H})$ is a small molecule glucagon receptor antagonist that displaces glucagon with $\mathrm{IC}_{50}$ values of 7 and $35 \mathrm{nM}$ in human and mouse receptor binding assays, respectively, and inhibits the downstream effects of glucagon stimulation on primary human hepatocytes. The aim of this study was to evaluate the effects of 8 weeks of Cmpd $\mathrm{H}$ treatment to $\mathrm{db} / \mathrm{db}$ mice on glycemic control, glucoregulatory hormones, and pancreatic histology.

Methods: Eight-week old $\mathrm{db} / \mathrm{db}$ mice were pre-screened and divided into matched groups based on blood glucose, glycosylated hemoglobin A1c (HbAlc), plasma triglycerides, body weight and body fat mass (by NMR). Mice were fed Purina 5008 chow (control) or chow supplemented with $0.1 \%$ Cmpd H (w/w) ad libitum. The daily Cmpd H dose was $~ 100 \mathrm{mg} / \mathrm{kg}$. Blood glucose and HbAlc were measured bi-weekly; body weight was monitored routinely throughout. After 8 weeks of drug treatment body composition was measured, mice were anesthetized, and plasma and liver were collected for biochemical analyses. Additionally, pancreata were excised, fixed, and weighed. Three sections of each pancreas were immunostained for glucagon and analyzed by digital microscopy.

Results: Cmpd H treatment for 8 weeks reduced fed blood glucose by 123 $\mathrm{mg} / \mathrm{dL}$ compared to control animals. HbAlc was significantly lower in the Cmpd H-treated group compared to the control group at all time points measured, with a $1.6 \%$ difference after 8 weeks of treatment. Cmpd $\mathrm{H}$ treatment was associated with elevated plasma insulin and glucagon compared to control animals. There were no effects of Cmpd $\mathrm{H}$ treatment on body weight, fat mass, plasma cholesterol, liver glycogen content, or pancreas weight. Plasma triglycerides were decreased $40 \%$. Pancreatic histology revealed more islets and a larger islet area in Cmpd H-treated mice compared to control mice. $\mathrm{Cmpd} \mathrm{H}$ treatment resulted in a greater total glucagon staining area; however, when normalized to islet area there was no difference compared to control mice. Furthermore, visual inspection did not reveal a treatment-related effect on $\alpha$-cell number per islet.

Conclusion: Eight week administration of Cmpd $\mathrm{H}$ dosed as a $0.1 \%$ food admixture significantly reduced HbAlc and fed blood glucose in $\mathrm{db} / \mathrm{db}$ mice without effects on weight gain or body fat composition. Moreover, potential $\beta$ cell sparing and improvements in plasma triglycerides were observed with $\mathrm{Cmpd} \mathrm{H}$ treatment. Importantly, these benefits were realized without the $\alpha$ cell hyperplasia previously associated with genetic manipulations of hepatic glucagon signaling.

\begin{tabular}{|c|c|c|c|c|c|c|}
\hline & $\begin{array}{l}\text { Blood Glucose, } \\
\mathrm{mg} / \mathrm{dL}\end{array}$ & $\mathrm{HbAlc}, \%$ & $\begin{array}{l}\text { Trigs, } \\
\mathrm{mg} / \mathrm{dL}\end{array}$ & $\begin{array}{l}\text { Insulin, } \\
\mathrm{ng} / \mathrm{mL}\end{array}$ & $\begin{array}{l}\text { Glucagon, } \\
\mathrm{pg} / \mathrm{mL}\end{array}$ & $\% \mathrm{G} / \mathrm{I}^{\dagger}$ \\
\hline Control & $500 \pm 20$ & $9.6 \pm 0.1$ & $241 \pm 31$ & $3.7 \pm 0.5$ & $175 \pm 23$ & $8.9 \pm 1.3$ \\
\hline Cmpd H & $377 \pm 21^{*}$ & $8.0 \pm 0.2^{*}$ & $141 \pm 12^{*}$ & $10.6 \pm 2.3^{*}$ & $717 \pm 90^{*}$ & $9.9 \pm 0.9$ \\
\hline
\end{tabular}

Pre-treatment blood glucose values were $388 \pm 18$ and $385 \pm 20 \mathrm{mg} / \mathrm{dL}$ for

control and $\mathrm{CpdH}$, respectively.

$\dagger$ glucagon staining area/islet area

${ }^{*} \mathrm{p}<0.05 \mathrm{v}$. control

\section{2}

A novel low-molecular-weight antagonist of glucose-dependent insulinotropic polypeptide receptor, SKL-14959, prevents obesity and insulin resistance

Y. Tsubamoto, T. Nakamura, H. Kinoshita, H. Tanimoto, H. Noda Drug Discovery Laboratories, Sanwa Kagaku Kenkyusho Co., Ltd., Mie, Japan

Background and aims: Besides the physiological roles such as potentiation of insulin secretion from pancreatic beta cells and control of blood glucose level, glucose-dependent insulinotropic polypeptide (GIP) promotes fat deposition in adipose tissue leading to pathophysiological conditions of obesity and insulin resistance. These functions of GIP in animals suggest that the antagonism of GIP signaling is efficacious as a treatment of obesity and insulin resistance in human. In fact, GIP receptor deficient mice developed neither obesity nor insulin resistance even when they were fed high fat diet. Furthermore, recent investigations showed that GIP receptor antagonist peptides ameliorated obesity and insulin resistance. To develop a treatment of obesity and insulin resistance with this new mechanism of action, we studied and found a novel low-molecular-weight antagonist for GIP receptor, SKL-14959. In this presentation, we show the efficacy of SKL-14959 in diet-induced obesity and type 2 diabetes models in mice.

Materials and methods: To evaluate the efficacy of SKL-14959 in obesity, male ICR mice received a high fat diet (40\% fat) with or without SKL-14959 at $0.14 \%$ for 14 weeks. Body weight and food intake were recorded throughout the experiment. At the end of the experiment, the white adipose tissue, liver and skeletal muscles were excised and weighed. Triglyceride contents in the liver and skeletal muscles were then analyzed. Efficacy of SKL-14959 in insulin resistance was evaluated by giving saline or SKL-14959.Na solution subcutaneously to female KKAy mice at a dose of 10 or $100 \mathrm{mg} / \mathrm{kg}$ once a day for 4 weeks. Blood samples were taken from tail vein once a week to measure plasma glucose, insulin and $\mathrm{HbA}_{1 c}$. After administration period, insulin sensitivity test was performed and liver was excised to measure triglyceride contents.

Results: In the diet-induced obesity mice, body weight increased with high fat diets and SKL-14959 reduced the gain by $38 \%(\mathrm{p}<0.05)$ without affecting daily food intake. Amount of white adipose tissue and weight of the liver increased in high fat fed group and SKL-14959 was efficacious in suppressing their increases. Also, SKL-14959 prevented triglyceride accumulation in the liver, soleus muscle and lateral vastus muscle by $45 \%$ ( $\mathrm{p}<0.05), 48 \%$ and $18 \%$, respectively. In the diabetic mice, SKL-14959 significantly decreased plasma glucose $(10 \mathrm{mg} / \mathrm{kg}: 210 \pm 10 \mathrm{mg} / \mathrm{dl}, 100 \mathrm{mg} / \mathrm{kg}: 195 \pm 7 \mathrm{mg} / \mathrm{dl})$, insulin $(10 \mathrm{mg} / \mathrm{kg}: 31 \pm 8 \mathrm{ng} / \mathrm{ml}, 100 \mathrm{mg} / \mathrm{kg}: 47 \pm 15 \mathrm{ng} / \mathrm{ml})$ and $\mathrm{HbA}_{1 \mathrm{c}}(10 \mathrm{mg} /$ $\mathrm{kg}: 3.7 \pm 0.2 \%, 100 \mathrm{mg} / \mathrm{kg}: 3.5 \pm 0.2 \%)$ from the control levels $(272 \pm 35 \mathrm{mg} / \mathrm{dl}$, $96 \pm 28 \mathrm{ng} / \mathrm{ml}$ and $4.3 \pm 0.4 \%$, respectively). In the insulin tolerance test, the daily dose of SKL-14959 improved insulin sensitivity in the type 2 diabetes model. Amount of accumulated triglyceride in the liver was decreased from the control level ( $48 \pm 6 \mu$ mole/g liver) by SKL- 14959 (10mg/kg: $37 \pm 5 \mu \mathrm{mole} / \mathrm{g}$ liver, $100 \mathrm{mg} / \mathrm{kg}$ : $32 \pm 6 \mu \mathrm{mole} / \mathrm{g}$ liver). In these experiments, data on serum chemistry suggested that SKL-14959 had very little adverse effects.

Conclusion: We have found SKL-14959, a novel low-molecular-weight GIP receptor antagonist, had quite favorable effects of suppressing weight gain without reduction of food intake in diet-induced obesity model as well as amelioration of insulin resistance and glycemic control in type 2 diabetes model. We expect that SKL-14959 could become an effective treatment of obesity and insulin resistance in human. 


\section{3}

Association of fetuin-A with decreased serum triglyceride after six weeks of extended-release niacin intervention in individuals with metabolic syndrome

S.T. Mathews ${ }^{1}$, S.V. Kaushik ${ }^{1}$, E.P. Plaisance ${ }^{2}$, T. Kim ${ }^{1}$, E. Huang ${ }^{1}$, A.J. Mahurin ${ }^{3}$, P.W. Grandjean ${ }^{4}$

${ }^{1}$ Nutrition and Food Sciences, Auburn University, ${ }^{2}$ Anatomy, Physiology and Pharmacology, Auburn University, ${ }^{3}$ Residency program, Baptist Health Family Medicine, Montgomery, ${ }^{4}$ Kinesiology, Exercise Technology Laboratory, Auburn University, United States

Background and aims: Fetuin-A (also known as alpha-2-HS glycoprotein, AHSG) has been increasingly shown to be associated with insulin resistance and metabolic syndrome (MetS) in humans. Further, several single nucleotide polymorphisms in the AHSG gene are associated with adipocyte insulin action and type 2 diabetes. At the molecular level, fetuin-A inhibits insulin receptor tyrosine kinase activity, insulin signaling and insulin action in skeletal muscle, liver, and fat cells. Phosphorylation of fetuin-A has been shown to be critical for its inhibitory activity. Niacin is one of the most effective pharmacological agents for the treatment of dyslipidemia and has been shown to reduce CVD morbidity and mortality presumably by improving blood lipid characteristics. However, recent evidence suggests that other factors may be involved. Our purpose was to determine the effect of niacin administration on serum total- and phosphorylated fetuin-A concentrations in individuals with metabolic syndrome and to correlate these with changes in blood lipids, insulin sensitivity, and vascular inflammation.

Materials and methods: Fifteen sedentary, obese, male participants, who met the NCEP ATP III criteria for metabolic syndrome, were treated with extended-release niacin (Niaspan) for 6 weeks. Serum fetuin-A levels were assayed by a sandwich ELISA method and Ser312-fetuin-A phosphorylation (phosphofetuin-A) status was estimated by Western blot analysis.

Results: Serum phosphofetuin-A concentrations were positively correlated with triglycerides in MetS, while C-reactive protein (CRP) was negatively correlated with fetuin-A in MetS. Serum total fetuin-A and phosphofetuinA concentrations decreased significantly following niaspan treatment $(p<$ 0.005). Changes in fetuin-A concentrations were correlated with changes in triglyceride concentrations after niaspan treatment $(r=0.58, p<0.05)$. We observed that the change in high density lipoprotein cholesterol following Niaspan intervention was negatively correlated with the change in serum fetuin-A and phosphorylated fetuin-A concentrations. Interestingly, serum cortisol levels were significantly elevated following niaspan intervention. The change in cortisol was significantly correlated with the change in serum nonesterified fatty acids (NEFA), suggesting that increased cortisol and NEFA concentrations may contribute to the mild hyperglycemia and insulin resistance observed in niacin-treated individuals.

Conclusion: This is the first report demonstrating that serum total- and phosphofetuin-A levels are amenable to intervention by extended-release niacin treatment and are correlated with changes in serum triglycerides. These findings are consistent with earlier findings that fetuin-A may be involved in lipid metabolism and modulation of inflammatory pathways. The increase in serum cortisol following niacin treatment may also provide further insight into potential mechanisms by which niacin reduces insulin sensitivity.

\section{4}

The flavonoid silibinin reverses metabolic alterations in insulin resistance model of high fructose-fed rats by decreasing hepatic gluconeogenesis and glycolysis in perifused liver cells

C. Sánchez ${ }^{1}$, D. Detaille ${ }^{1}$, N. Sanz ${ }^{1}$, G. Rodríguez-Villanueva ${ }^{1}$,

F. Gómez-Peralta ${ }^{2}$, J. Recio-Córdova ${ }^{2}$, J. López-Novoa ${ }^{1}$, M. El-Mir ${ }^{1}$

${ }^{1}$ Physiology and Pharmacology, ${ }^{2}$ Endocrinology Unit, University Hospital of Salamanca, Salamanca, Spain

Background and aims: Insulin resistance, hiperinsulinaemia and disturbances in glucose metabolism can be produced in normal rats by feeding a fructose-enriched diet. The flavonoid silibinin (SB), a natural antioxidant, has been recently proposed to be beneficial in type 2 diabetes. Previous "in vitro" studies of our group have shown that SB inhibits hepatic gluconeogenesis (GNG) and glycolysis in perifused rat hepatocytes. The aim of this study was to determine "in vivo" the capacity of silibinin to reverse the alteration of carbohydrate metabolism associated to insulin resistance induced in high fructose-fed rats.
Materials and methods: Male Wistar rats (160-180 g) were divided into two batches. One batch received a standard diet, and the other was fed with a fructose-enriched diet, containing $60 \%$ fructose. After 4 weeks of this dietary regimen, each batch of rats was subdivided into two groups. One group received $\mathrm{SB}(50 \mathrm{mg} / \mathrm{kg} /$ day, i.p. injection) for additional two weeks, and the other group received SB vehicle. At the end of the treatment, a sample of blood was taken from each rat for subsequent measurement of glucose, insulin, triglycerides and other plasma parameters. Hepatocytes were isolated from rat livers (all groups) according to the method of Berry and Friend, and they were perifused at $37^{\circ} \mathrm{C}$ with Krebs-bicarbonate-calcium saturated with $\mathrm{O}_{2} / \mathrm{CO}_{2}$ and titrated with increasing concentrations of dihydroxyacetone (DHA) as energetic substrate to get seven consecutive steady states. For each steady state, we measured, in cellular perifusate, hepatic glucose production (GNG) and glycolysis (lactate and pyruvate).

Results: Blood glucose, triglycerides and insulin, as expected, were significantly elevated in fructose-treated groups compared with the control. Treatment with SB lowered all these parameters till normal values. On the other hand, in perifused liver cells, fructose-treated rats show alterations of glucose hepatic metabolism typical to insulin resistance state by increasing both gluconeogenesis $(J)$ and glycolysis as compared to control by $34 \%$ and $45 \%$, respectively (DHA $2.4 \mathrm{mM} ; \mathrm{p}<0.001$ ). The most attractive data was that silibinin treatment completely reversed these metabolic alterations in fructose-fed animals by returning GNG $\left(J_{\text {Glucose }}\right)$ and glycolysis $\left(J_{L+P}\right)$ to normal values (DHA $2.4 \mathrm{mM} ; \mathrm{p}<0.001$ ). Concerning the global metabolism of DHA $\left(J_{2 G+L+P}\right)$, this parameter is activated in fructose-fed rats as compared to control rats (DHA $2.4 \mathrm{mM} ; \mathrm{p}<0.001)$. SB also completely normalizes this alteration (DHA $2.4 \mathrm{mM} ; \mathrm{p}<0.001$ ). Furthermore, the administration of fructose caused an inhibition of cytosolic NADH/NAD ${ }^{+}$ratio (estimated by lactate/ pyruvate ratio) by $22 \%$ compared with control rats (DHA $2.4 \mathrm{mM} ; \mathrm{p}<0.001$ ). Treatment with SB significantly lowered cytosolic NADH/NAD ${ }^{+}$ratio in all groups of rats (DHA $2.4 \mathrm{mM} ; \mathrm{p}<0.01$ ).

Conclusion: Silibinin "in vivo" reverses metabolic alterations in insulin resistance induced in fructose-fed rat by decreasing hepatic gluconeogenesis and glycolysis. These results strongly suggest that silibinin, beyond its whole antioxydant properties, could be useful as a complementary therapy to decrease hyperglycaemia and hyperinsulinaemia in pre-diabetes and type 2 diabetes.

\section{5}

Use of gene expression signature technology to identify VVP-808, a novel insulin sensitiser with efficacy in vitro and in vivo

K.R. Walder ${ }^{1}$, J.C. Molero ${ }^{1}$, N. Konstantopoulos ${ }^{1}$, D. Segal ${ }^{1}$, V. Foletta ${ }^{1}$, K. Windmill'1 J. Curran ${ }^{2}$, T.D. Dyer ${ }^{2}$, K. Shields ${ }^{3}$, J. Jowett ${ }^{3}$, G. Krippner ${ }^{4}$, J. Blangero ${ }^{2}$, G.R. Collier ${ }^{5}$

${ }^{1}$ School of Exercise and Nutrition Sciences, Deakin University, Waurn Ponds, Australia, ${ }^{2}$ Genetics, Southwest Foundation for Biomedical Research, San Antonio, United States, ${ }^{3}$ International Diabetes Institute, Caulfield, Australia, ${ }^{4}$ Verva Pharmaceuticals, Geelong, Australia, ${ }^{5} \mathrm{ChemGenex}$ Pharmaceuticals, Geelong, Australia

Background and aims: The aims of the current study were to develop a new small molecule library screening strategy based on gene expression signature (GES) technology, and to utilise this screen to identify new insulin sensitising agents.

Materials and methods: Differentiated 3T3-L1 adipocytes were rendered insulin-resistant using TNFa ( $3 \mathrm{ng} / \mathrm{ml}$ for $72 \mathrm{~h}$ ), and insulin sensitivity was restored by adding a cocktail of known antidiabetic agents for the last $24 \mathrm{~h}$. Mouse cDNA microarrays were used to establish gene expression profiles for the insulin resistant cells, and the cells with insulin sensitivity restored. Bayesian linear model selection was performed to find the optimal set of 11 genes (the GES) that best defined the difference between the two cell states. Insulin resistant 3T3-L1 cells were then treated with a library of $\sim 1500$ compounds, and analysed for similarity of expression of the 11 GES genes. Hit compounds were selected for further testing in vitro and in vivo.

Results: Using the GES, we identified a family of compounds that dose-dependently improved insulin stimulated glucose transport and incorporation into lipid in 3T3-L1 adipocytes $\left(\mathrm{p}=9 \times 10^{-7}\right)$. The lead compound, designated VVP808, was further tested in animal models of insulin resistance and diabetes. Treatment of $d b / d b$ mice with VVP808 for 7 days resulted in a dose-dependent reduction in fasting blood glucose concentration (up to $65 \%$ reduction at $50 \mathrm{mg} / \mathrm{kg} / \mathrm{d}$, Day 0: $24.7 \pm 1.8$ vs Day $7: 9.5 \pm 1.0 \mathrm{mmol} / \mathrm{L}, \mathrm{p}=0.00004)$. The improvement in glycemia was associated with a $7 \%$ reduction in body weight $(\mathrm{p}=0.002)$, however a subsequent pair-feeding study showed that the 
reduction in blood glucose concentration was independent of body weight loss. Treatment of diet-induced obese (DIO) C57Bl/6 mice with VVP808 for 21 days resulted in a dose-dependent improvement in glucose tolerance and reduction in fasting blood glucose concentration. The area under the blood glucose curve during an ipGTT was reduced by up to $28 \%(\mathrm{p}=0.037)$, while fasting blood glucose was significantly reduced at doses from $20-100 \mathrm{mg} / \mathrm{kg} / \mathrm{d}$ $(\mathrm{p}<0.05)$. These effects were accompanied by a dose-dependent decrease in body weight of $5-11 \%(\mathrm{p}<0.05)$.

Conclusion: The GES screening strategy successfully identified a family of compounds with insulin sensitising and antidiabetic effects in vitro and in vivo. VVP808 is a novel insulin sensitiser that also causes moderate loss of body weight in obese animals, and therefore has excellent potential for development as an antidiabetic agent. A clinical study evaluating the safety and efficacy of VVP808 in type 2 diabetic patients is planned for the second half of this year.

Supported by: A research grant from Verva Pharmaceuticals

\section{PS 084 Traditional oral therapies}

\section{6}

Association of $K C N J 11$ and $A B C C 8$ genetic polymorphisms with response to repaglinide in Chinese diabetic patients

Y. He, R. Zhang, X. Shao, C. Hu, C. Wang, Y. Bao, J. Lu, W. Jia

Endocrinology \& Metabolism, Shanghai Jiaotong University affiliated No.6

People Hospital, Shanghai Diabetes Institute, China

Background and aims: Repaglinide stimulates insulin secretion by inhibiting ATP-sensitive potassium ( $\mathrm{K}_{\text {ATP }}$ ) channels in pancreatic beta cells. Common variants of two genes encoding $\mathrm{K}_{\text {ATP }}$ channels (KCNJ11 E23K, ABCC8 exon16 -3T/C) have been reported to be associated with type 2 diabetes.To investigate whether there is an association between $K C N J 11$ E23K, ABCC8 exon16 -3T/C polymorphisms and the therapeutic effect of repaglinide in patients with type 2 diabetes.

Materials and methods: A total of 100 Chinese patients with newly diagnosed type 2 diabetes were treated with repaglinide for 24 weeks. Arginine stimulation tests were performed to evaluate beta cell function. Gene variations were detected with polymerase chain reaction-restriction fragment length polymorphism (PCR-RFLP). Responders were defined by a $>25 \%$ decrease in fasting plasma glucose (FPG) or a $>20 \%$ decrease in hemoglobin $\mathrm{A} 1 \mathrm{c}(\mathrm{HbA1c})$ values (or both) after the 24-week repaglinide treatment. Results: Both baseline Hemoglobin A1c (HbAlc) and the decrease of $\mathrm{HbAlc}$ $(\triangle \mathrm{HbAlc})$ were significantly higher in patients with $\mathrm{K}$ allele of KCNJ11 $\mathrm{E} 23 \mathrm{~K}$ variant when compared with $\mathrm{E} / \mathrm{E}$ homozygotes ( $\mathrm{p}=0.0103$ and 0.0221 respectively). The decrease in $2 \mathrm{~h}$ postprandial plasma glucose ( $2 \mathrm{hPG}$ ) was significantly greater in $\mathrm{E} / \mathrm{K}$ heterozygotes than $\mathrm{E} / \mathrm{E}$ homozygotes $(\mathrm{p}=0.0367)$. There was a significant difference in the response rate to repaglinide treatment between $\mathrm{E}$ allele carriers and $\mathrm{K}$ allele carriers ( $68 \%$ versus $79 \%$, $\mathrm{p}=0.03$ ). The changes in fasting insulin $(\Delta \mathrm{FIN})$ and homeostasis model assessment of insulin resistance $(\triangle \mathrm{HOMA}-\mathrm{IR})$ were significantly greater in patients with $A B C C 8$ exon16-3 C/C, vs. $\mathrm{T} / \mathrm{C}$ and $\mathrm{T} / \mathrm{T}$ variant $(\mathrm{p}=0.0372$ and $0.0274 \mathrm{re}-$ spectively).

Conclusion: Our results showed that KCNJ11 E23K variant was associated with the therapeutic effect of repaglinide. In addition, $A B C C 8$ exon $16-3 \mathrm{~T} / \mathrm{C}$ variant was related to insulin sensitivity after repaglinide treatment. Supported by: the Key Project from Science and Technology Commission of Shanghai Municipality, China, National Basic Research Program of China and Program for Shanghai Outstanding Medical Academic Leader

\section{7}

Effects of treatment with repaglinide or gliclazide on glucose control and early-phase insulin secretion in patients with newly diagnosed type 2 diabetes

H. Zhang ${ }^{1}$, P. Bu ${ }^{1}$, M. Lei ${ }^{2}$, Z. Mo ${ }^{3}$, E. Liao ${ }^{1}$

${ }^{1}$ Institute of Endocrinology and Metabolism, The Second Xiangya Hospital of Central South University, ${ }^{2}$ Department of Endocrinology, Xiangya Hospital of Central South University, ${ }^{3}$ Department of Endocrinology, The Third Xiangya Hospital of Central South University, Chang Sha, China

Background and aims: A loss of early-phase insulin secretion is an early marker of type 2 diabetes (T2D), and the restoration of it can improve insulin sensitivity, $\beta$ cell function, and lipid metabolism. The aim of this study was to evaluate the effects of treatment with Repaglinide (Rg) or Gliclazide (Gli) on glucose control, insulin secretion, and lipid profile in patients with T2D.

Materials and methods: In the 4-week open-labelled parallel study, 40 patients newly diagnosed with T2D were randomized 1:1 to Rg t.i.d or Gli qd treatment, and 34 patients completed the study. Both drugs were titrated to achieve a fasting blood glucose target of $\leq 7 \mathrm{mmol} / \mathrm{L}$. No lipid regulators were given during the study. A standard mixed meal tolerance test was performed at baseline and end of study. Plasma glucose (PG) and serum insulin concentration were measured at fasting and 5 points after meal $(30,60,90,120$, and $\left.180^{\prime}\right)$. Total insulin secretion was evaluated by the area under insulin concentration curve $\left(\mathrm{AUC}_{\mathrm{ins}}\right)$. The homeostasis model assessment of insulin resistance (HOMA-IR) was used to estimate insulin sensitivity. $\beta$ cell function was measured by early-phase insulin secretion index (the ratio of insulin change to glucose change over the first 30' after meal, $\Delta \mathrm{I}_{30} / \Delta \mathrm{G}_{30}$ ).

Results: Demographic and baseline characteristics were comparable between the two groups. At week 4, fasting and postprandial PG decreased significantly, and postprandial insulin concentrations increased significantly in 


\begin{tabular}{|c|c|c|c|c|}
\hline & & $\operatorname{Rg}(n=19)$ & Gli $(n=15)$ & Between groups $\mathrm{P}$ value \\
\hline$\overline{\mathrm{AUC}_{\mathrm{Ins}}(\mathrm{mU} / \mathrm{l} / \mathrm{h})}$ & $\begin{array}{l}\text { Baseline } \\
\text { Week } 4\end{array}$ & $\begin{array}{l}33.27(8.68,106.77) \\
77.14(32.59,185.31)^{*}\end{array}$ & $\begin{array}{l}44.91(9.82,102.55) \\
79.655(10.16,152.77) *\end{array}$ & $\begin{array}{l}1.000 \\
0.656\end{array}$ \\
\hline HOMA-IR & $\begin{array}{l}\text { Baseline } \\
\text { Week } 4\end{array}$ & $\begin{array}{l}0.46(0,10.43) \\
2.33(0,9.66)\end{array}$ & $\begin{array}{l}0.51(0,8.07) \\
1.29(0,8.28)\end{array}$ & $\begin{array}{l}0.837 \\
0.302\end{array}$ \\
\hline$\overline{\Delta \mathrm{I}_{30} / \Delta \mathrm{G}_{30}}$ & $\begin{array}{l}\text { Baseline } \\
\text { Week } 4\end{array}$ & $\begin{array}{l}2.64(0.08,71.06) \\
10.88(2.45,85.42)\end{array}$ & $\begin{array}{l}3.17(0.16,14.38) \\
6.32(0.87,19.96)^{*}\end{array}$ & $\begin{array}{l}0.716 \\
0.030^{\ddagger}\end{array}$ \\
\hline $\mathrm{TG}(\mathrm{mmol} / \mathrm{L})$ & $\begin{array}{l}\text { Baseline } \\
\text { Week } 4\end{array}$ & $\begin{array}{l}1.98(1.08) \\
1.34(0.70)^{*}\end{array}$ & $\begin{array}{l}1.79(0.93) \\
1.62(1.07) \\
\end{array}$ & $\begin{array}{l}0.741 \\
0.378 \\
\end{array}$ \\
\hline$\overline{\mathrm{CHOL}}(\mathrm{mmol} / \mathrm{L})$ & $\begin{array}{l}\text { Baseline } \\
\text { Week } 4\end{array}$ & $\begin{array}{l}4.90(0.69) \\
4.10(0.64)^{*}\end{array}$ & $\begin{array}{l}5.09(1.08) \\
4.92(1.47) \\
\end{array}$ & $\begin{array}{l}0.546 \\
0.035^{\text {* }}\end{array}$ \\
\hline
\end{tabular}

HOMA-IR $=($ FPG $\times$ FINS $) / 22.5$;

$\Delta \mathrm{I} 30=30$-min insulin - fasting insulin; $\Delta \mathrm{G} 30=30$-min plasma glucose - fasting plasma glucose.

Values of AUC ${ }_{\text {Ins }}$, HOMA-IR, and $\Delta \mathrm{I}_{30} / \Delta \mathrm{G}_{30}$ are presented as median (min, max); Value of TG and CHOL are presented as Mean (SD).

* vs. baseline, $\mathrm{p}<0.05$.

¥ Comparisons between groups were performed using analysis of variance (ANOVA) followed by post hoc testing with Bonferroni test, $\mathrm{p}<0.05$

both groups (all points, $\mathrm{p}<0.05$ ). The time to maximum insulin concentration occurred earlier in the Rg group than in the Gli group (90' vs.120') (Figure). $\mathrm{AUC}_{\text {ins }}$ increased significantly in both groups $(\mathrm{p}<0.05)$, and no difference was found between the two groups. $\Delta \mathrm{I}_{30} / \Delta \mathrm{G}_{30}$ increased significantly in both groups ( $\mathrm{p}<0.05)$, especially in the $\operatorname{Rg}$ group ( $\mathrm{p}<0.05 \mathrm{vs}$. Gli). There was no significant difference in HOMA-IR during the study. TG and CHOL decreased significantly in the Rg group $(\mathrm{p}<0.05)$, but not in the Gli group (Table).

Conclusion: No significant differences were observed in glucose control and total insulin secretion after treatment between the two groups. However, treatment with $\mathrm{Rg}$ was associated with significantly greater improvements in $\beta$ cell function and lipid metabolism.

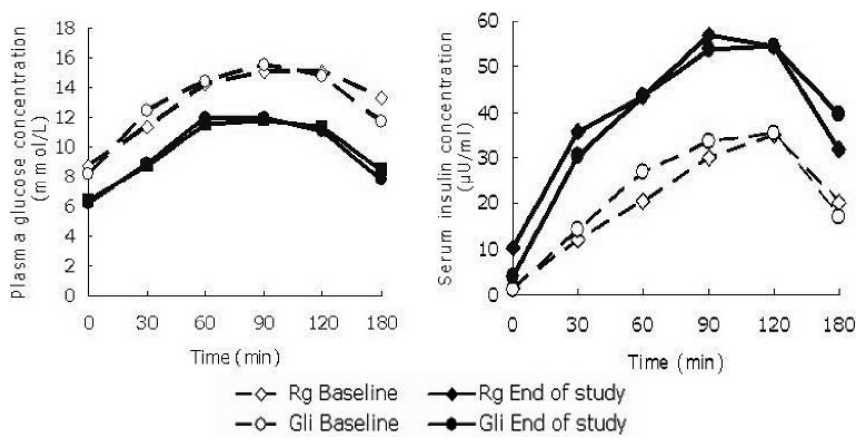

\section{8}

Postprandial C-peptide in type 2 diabetes in relation to $\mathrm{HbA}_{1 \mathrm{c}}$ and the use of sulphonylurea

Å. Nybäck-Nakell, P.-E. Lins, U. Adamson, L. Landstedt-Hallin

Department of Clinical Sciences, Karolinska Institutet, Danderyd Hospital, Stockholm, Sweden

Background and aims: Progressive decline in beta cell function is of importance for the deterioration of glycaemic control, over time, in patients with type 2 diabetes treated with oral glucose-lowering drugs (OGLD). Sulphonylureas have been implicated to be involved in this process via exhaustion of beta cell function. The aim of this observational study was to describe changes in glycaemic control and beta cell function, assessed as postprandial C-peptide, in relation to treatment with sulfonylurea over a period of 10 years.

Material and methods: 462 patients with a clinical diagnosis of type 2 diabetes attended a four day diabetes course at Danderyd Hospital in Stockholm 1997-1998. HbA1c, postprandial C-peptide (PP-CPT) and postprandial Pglucose were measured on day 1 (see Table).

After 10 years, 341 patients who were still alive, were invited to a follow-up visit, 171 of them accepted. Median age at follow-up was 68 years (range $40-$ 87), BMI $29.9 \mathrm{~kg} / \mathrm{m}^{2}(20-46)$ and known duration of diabetes 14 years (846).

Results: As expected blood glucose lowering therapies had been modified over time. The use of insulin had increased from 33 to $74 \%$ and OGLD + insulin from 18 to $52 \%$ while the use of insulin secretagogues remained similar 56 and $48 \%$ respectively. Over ten years there was a significant decrease in HbAlc $(p=0.002)$, PP CPT $(p<0.0001)$ and PP CPT/glucose ratio, a measurement correcting for the prevailing glucose level $(\mathrm{p}=0.03)$. There was a nega- tive correlation between HbAlc and PP CPT $(\mathrm{p}<0.0001, \mathrm{r}=-0.30)$ as well as between HbA1c and PP CPT/glucose ratio $(\mathrm{p}<0.0001, \mathrm{r}=-0.52)$.

In a stepwise multiple regression analysis with the long-term change in PP $\mathrm{CPT} /$ glucose ratio as the dependent variable, the baseline PP CPT/glucose ratio explained $44 \%$ of the change, $\mathrm{HbAl} \mathrm{c}$ at follow-up explained $14 \%$ while the use of a sulphonylurea only explained an additional $2 \%$ and BMI and diabetes duration explained $1 \%$ each.

Conclusion: We conclude that this observational study, does not support the concept, that sulphonylurea treatment is of major importance for the observed progressive decline in beta cell function.

\begin{tabular}{lll}
\hline Table & $\begin{array}{l}\mathbf{1 9 9 7 - 1 9 9 8} \\
\mathbf{n}=\mathbf{1 7 1}\end{array}$ & $\begin{array}{l}\mathbf{2 0 0 7} \\
\mathbf{n}=\mathbf{1 7 1}\end{array}$ \\
\hline HbA1c (median, range) & $7.3 \%(4.3-12.3)$ & $6.7 \%(4.5-13)$ \\
\hline PP CPT (median, range) & $\begin{array}{l}2.01 \mathrm{nmol} / \mathrm{l} \\
(0.36-8.3)\end{array}$ & $\begin{array}{l}1.4 \mathrm{nmol} / 1 \\
(0.04-3.8)\end{array}$ \\
\hline PP CPT/glucose ratio & $0.15(0.02-0.87)$ & $0.13(0.004-0.56)$ \\
(median, range) & & \\
\hline Diet only (\%) & 17 & 4 \\
\hline Metformin (\%) & 37 & 55 \\
\hline Secretagogues: SU*/glinide & & $42^{*} / 6^{* *}$ \\
\hline Insulin $(\%)$ & $56^{*}$ & 74 \\
\hline Acarbose $^{*} /$ glitazone $^{* *}(\%)$ & 33 & $2^{* *}$ \\
\hline
\end{tabular}

\section{9}

Effects of a one-year treatment with metformin on metabolic and cardiovascular risk factors in non-diabetic upper-body obese subjects with mild glucose anomalies: the BIGPRO1 trial

A. Fontbonne ${ }^{1}$, I. Diouf ${ }^{2}$, M. Baccara-Dinet ${ }^{3,4}$, E. Eschwege ${ }^{2}$, M.-A. Charles ${ }^{2}$ ${ }^{1}$ UR 024, IRD, Montpellier, ${ }^{2}$ U780, INSERM, Villejuif, ${ }^{3}$ Endocrinology, Lapeyronie Hospital, Montpellier, ${ }^{4}$ Merck Santé, Lyon, France

Background and aims: Metformin has recently been considered as a possible pharmacologic complement to lifestyle measures for type 2 diabetes prevention in high-risk ("pre-diabetic") subjects. As few trials of metformin have been conducted in non-diabetic subjects, little is known about its effects on metabolic and cardiovascular risk factors in this population. The aim of the present analysis was to study these effects, using the data of the BIGPRO1 trial, which was conducted in the early 1990s in non-diabetic upper-body obese subjects.

Material and methods: The BIGPRO1 trial was a multicenter randomized double-blind controlled clinical trial, metformin $850 \mathrm{mg}$ bid vs placebo tablets for one year, conducted in 324 (164 metformin, 160 placebo) non-diabetic subjects aged 35-60 (men), 40-65 (women), without cardiovascular diseases, with a high waist-to-hip ratio ( $\geq 0.95$, men; $\geq 0.80$, women), and no contraindications to metformin. This analysis was performed on two subsets of subjects who could qualify as "pre-diabetic": subset A, subjects with impaired glucose tolerance or impaired fasting glucose according to current WHO definition (49 metformin, 52 placebo); subset B, subjects fulfilling the inclusion criteria of the Diabetes Prevention Program (28 metformin, 23 placebo). In each subset, we compared the change between 1 year and baseline for variables of interest, between the metformin (M) and the placebo (P) groups. 
Results: At baseline, there was a significant difference in age between the two treatment groups in both subsets (subset A, $53 \pm 6($ mean \pm SD) [M] vs $49 \pm 7 \mathrm{yr}$ $[P], p<0.01$; subset $B, 53 \pm 6[\mathrm{M}]$ vs. $48 \pm 7 \mathrm{yr}[\mathrm{P}], \mathrm{p}<0.01)$, and a trend towards a difference in sex ratio (subset $\mathrm{A}, 25 \%$ men $[\mathrm{M}]$ vs $42 \%[\mathrm{P}], \mathrm{p}<0.06$; subset B, $29 \%[M]$ vs $48 \%[P], p<0.15$ ), hence subsequent comparisons were adjusted for age and sex. In subset A, 1-yr changes were significantly different for systolic blood pressure (SBP: $-13 \pm 18[\mathrm{M}]$ vs $-2 \pm 14 \mathrm{mmHg}[\mathrm{P}], \mathrm{p}<0.01$ ), fasting blood glucose (FBG: $-0.35 \pm 1.34[\mathrm{M}]$ vs $0.65 \pm 2.05 \mathrm{mmol} / \mathrm{L}[\mathrm{P}], \mathrm{p}<0.04)$, and total cholesterol (TC: $-0.15 \pm 0.89[\mathrm{M}]$ vs $0.36 \pm 0.77(\mathrm{mmol} / \mathrm{L})[\mathrm{P}], \mathrm{p}<0.03)$. Similar results were observed in subset B (SBP: $-16 \pm 16[\mathrm{M}]$ vs $3 \pm 12 \mathrm{mmHg}$ [P], p<0.01; FBG: $-0.75 \pm 1.58[\mathrm{M}]$ vs $1.74 \pm 3.02(\mathrm{mmol} / \mathrm{L})[\mathrm{P}], \mathrm{p}<0.02$; TC: $0.13 \pm 0.73[\mathrm{M}]$ vs $0.44 \pm 0.74(\mathrm{mmol} / \mathrm{L})[\mathrm{P}], \mathrm{p}<0.05)$, with the addition of a significant difference in 1-yr change for diastolic blood pressure (DBP: $-10 \pm 15$ $[\mathrm{M}]$ vs $2 \pm 10 \mathrm{mmHg}[\mathrm{P}], \mathrm{p}<0.02)$. There was no significant difference in $1-\mathrm{yr}$ change for weight, waist-to-hip ratio, $2 \mathrm{~h}$ post-load blood glucose, fasting and $2 \mathrm{~h}$ post-load insulin, and triglyceride, between the two treatment groups. Conclusion: Compared to placebo, metformin significantly reduced blood pressure, fasting blood glucose and total cholesterol in "pre-diabetic" upperbody obese subjects, with no significant effect on weight or plasma insulin. This brings support to some current recommendations to use metformin as a complement to lifestyle measures in subjects at high risk to develop diabetes or cardiovascular disease.

Supported by: Merck Santé, France

\section{0}

Long-term treatment with metformin in type 2 diabetes treated with insulin is associated with vitamin B12 deficiency, but not with increasing homocysteine levels: a randomised, controlled trial

A. Kooy ${ }^{1}$, J. de Jager ${ }^{1}$, P. Lehert ${ }^{2}$, D. Bets ${ }^{3}$, J. van der Kolk ${ }^{1}$,

C.D.A. Stehouwer ${ }^{4}$

${ }^{1}$ Bethesda Diabetes Research Center, Hoogeveen, Netherlands, ${ }^{2}$ Department of Statistics, FUCAM - Louvain Academy, Louvain, Belgium, ${ }^{3}$ E. Merck Nederland BV, Amsterdam, Netherlands, ${ }^{4}$ Internal Medicine, University Hospital Maastricht, Netherlands

Background and aims: Metformin is a key treatment option in type 2 diabetes. However, metformin may decrease vitamin B12 levels and increase levels of homocysteine, a cardiovascular risk factor. We investigated whether long term treatment with metformin during 52 months affects serum concentrations of homocysteine, folate and vitamin B12 in subjects with type 2 diabetes treated with insulin.

Materials and methods: In this placebo-controlled, randomised trial, 745 patients with type 2 diabetes, treated with insulin and not known with a contraindication for the use of metformin, were approached; 390 gave informed consent and entered the study. 277 subjects $(=72 \%)$ completed the trial. At the final visit, laboratory samples were available for 257 patients (127 M, 130 P).

Results: During placebo treatment, vitamin B12 increased by $0.2 \mathrm{pmol} / 1[0 \%$ $(-3$ to +4$)]$, folate increased by $1.01 \mathrm{nmol} / \mathrm{l}[+8 \%(+4$ to +12$)]$, and homocysteine increased by $1.60 \mu \mathrm{mol} / 1$ [ $+20 \%(95 \% \mathrm{CI}:+16$ to +25$)]$. During metformin treatment, vitamin B12 decreased by $89.8 \mathrm{pmol} / 1$ [-19\% (-22 to -15$)]$, folate increased by $0.21 \mathrm{nmol} / 1[+3 \%(-1$ to +6$)]$, and homocysteine increased by $3.26 \mu \mathrm{mol} / 1[+26 \%(+21$ to +31$)$. Compared with placebo, metformin treatment was associated with decreases in vitamin B12 of $-19 \%$ [ $(-24 \%$ to $-14) ; \mathrm{p}<0.001]$ and folate of $-5 \%$ [(-10 to -0.4$) ; \mathrm{p}=0.033]$, and an increase in homocysteine of $+5 \%$ [(-1 to +11$) ; \mathrm{p}=0.091]$. The effects of metformin on vitamin B12, folate, and homocysteine were not affected after adjustment for age, previous metformin treatment, duration of diabetes, gender, insulin dose, and smoking habits. At baseline, 3 patients (2.2\%) in the metformin group and 4 patients $(1.6 \%)$ in the placebo group had vitamin B12 values below the value of $150 \mathrm{pmol} / \mathrm{l}$. At the end of the study period 16 patients $(8.3 \%)$ in the metformin group and 3 patients $(1.6 \%)$ in the placebo group had vitamin $B 12$ values below the value of $150 \mathrm{pmol} / \mathrm{l}[\mathrm{p}=0.003]$. The hazard ratio for developing vitamin B12 deficiency during metformin therapy was 5.5 [(1.6 to 19.1); $\mathrm{p}=0.01$ ]. We used General Mixed Model with Generalised Estimation Equations (GEE) to explore the effects of metformin on vitamin $\mathrm{B} 12$, folate, and homocysteine and to investigate whether metformin-associated changes in homocysteine, if any, could be explained by changes in folate and (or) vitamin B12, and, if so, whether the changes were independent of age, gender, duration of diabetes, smoking, body mass index, insulin dose, serum creatinine, HDL cholesterol, or glycated haemoglobin. The goodness of fit between alternative models was compared using the maximum likelihood technique. P-values $<0.05$ were considered statistically significant.
Conclusion: In patients with type 2 diabetes, 52 months of treatment with metformin reduces levels of folate and vitamin B12, without a significant increase in homocysteine. The increased risk of developing a significant vitamin B12 deficiency (vitamin B12<150 pmol/l) in metformin therapy may support the strategy to determine vitamin B12 levels at the start and after 4 months of treatment.

Supported by: grants from Altana, Lifescan, E. Merck/Santé, Merck Sharpe \& Dohme and Novo Nordisk (this part of the HOME-trial)

\section{1}

Metformin lowers plasma asymmetric dimethylarginine (ADMA) levels of subjects with type 2 diabetes independent of glycaemic control J.G. Boyle, I.P. Salt, G.C. Jones, M. Small, J.M.C. Connell, N. Sattar, S.J. Cleland;

University of Glasgow, Division of Cardiovascular \& Medical Sciences, Glasgow, United Kingdom

Background and aims: Circulating levels of the endogenous competitive inhibitor of NO synthase, asymmetric dimethylarginine (ADMA) are elevated in patients with diabetes, and in several prospective studies, have been noted to predict higher risk of cardiovascular events. Metformin is widely used in the treatment of type 2 diabetes, in part because it lower MI risk, but its precise mode of action remains uncertain. Metformin's vascular protection in UKPDS was apparently independent of glucose lowering actions. We studied the effect of Metformin and Gliclazide on plasma ADMA and glycaemic control in subjects with type 2 diabetes.

Materials and methods: We randomised twenty men with type 2 diabetes aged between 50-70 years to Metformin (500 milligrams three times daily) and Gliclazide ( 80 milligrams twice daily) in randomised, double-blinded, glycaemia controlled, cross-over design over 10 weeks in each arm. There was a six week run in and washout period. At the end of each ten week phase of therapy blood sampling was performed. Plasma ADMA was measured by high performance liquid chromatography.

Results: The mean age 56.5 years and BMI was $31 \mathrm{~kg} / \mathrm{m}^{2}$. BMI was not different after each period of therapy $(\mathrm{p}=0.80)$ but mean $\mathrm{HbAl} \mathrm{c}$ was lower post gliclazide versus post metformin $[7.8 \%$ versus $8.3 \%, p<0.001]$. However, ADMA was significantly lower following metformin therapy $[0.48 \mathrm{mmol} / \mathrm{l}$ versus $0.50 \mathrm{mmol} / \mathrm{l}, \mathrm{p}=0.019]$.

Conclusion: To our knowledge our results provide the first evidence that metformin lowers ADMA in subjects with type 2 diabetes independent of its effects on glycaemia. The results therefore significantly extend prior supportive evidence of ADMA lowering from uncontrolled study of metformin in women with PCOS. We propose that beneficial effects of Metformin in subjects with type 2 diabetes are in part mediated by lowering ADMA levels in plasma.

Supported by: British Heart Foundation

\section{2}

Metformin action is mediated through reactive nitrogen species in primary mouse hepatocytes

Y. Fujita ${ }^{1}$, M. Hosokawa ${ }^{1}$, S. Fujimoto ${ }^{1}$, A. Abudukadier ${ }^{1}$, H. Fujiwara ${ }^{1}$, E. Mukai ${ }^{1}$, M. Sasaki ${ }^{1}$, E. Ogawa ${ }^{1}$, Y. Seino ${ }^{1,2}$, N. Inagaki ${ }^{1}$

${ }^{1}$ Department of Dabetes and Clinical Nutrition, Kyoto University,

${ }^{2}$ Department of Diabetes, Kansai Denryoku Hospital, Osaka, Japan

Background and aims: Metformin is one of the most commonly used antidiabetic drugs for type 2 diabetes. The effect of metformin is known to be mediated by activation of AMP-activated protein kinase (AMPK), but the mechanism remains controversial. Recent studies suggest that metformin activates AMPK via peroxynitrite, a reactive nitrogen species (RNS) in bovine aortic endothelial cells (BAECs). The aim of this study is to elucidate the relationship between metformin and RNS in primary mouse hepatocytes. Since metformin is known as a mild mitochondrial complex 1 inhibitor, we compared its action with rotenone, a typical mitochondrial complex 1 inhibitor, to investigate the mechanism of action.

Materials and methods: Hepatocytes were isolated from male 8-week-old C57/BL6J mice by collagenase digestion. Primary hepatocytes were maintained in DMEM with $10 \%$ fetal bovine serum. Protein expressions of AMPKa and phospho-AMPKa were analyzed by Western blot. For gluconeogenesis measurements, hepatocytes were isolated after overnight fast and incubated for 120 minutes in glucose-free DMEM with $1 \mathrm{mM}$ pyruvate. Glucose pro- 
duction was determined by glucose oxidase method. Immunohistochemical staining of anti-nitro tyrosine was performed for intracellular peroxynitrite detection. NO production was determined by Griese method. ATP, ADP and AMP content was assessed by luminometric method. In addition, we used endothelial nitric oxide synthase (eNOS) knockout mice to confirm the requirement for intracellular NO production in metformin action.

Results: Both 120-minute exposure of $2 \mathrm{mM}$ metformin and $100 \mathrm{nM}$ rotenone increased phosphorylation of AMPKa in mouse hepatocytes. Direct exposure of peroxynitrite increased phosphorylation of AMPKa. Both metformin and rotenone significantly decreased gluconeogenesis (metformin, $363 \pm 22$; rotenone, $174 \pm 33$; control, $422 \pm 19 \mathrm{nmol} / \mathrm{mg}$ protein; $P<0.05$ control vs. metformin and rotenone). On the other hand, metformin increased antinitro tyrosine staining but rotenone did not. Metformin increased NO production by $27 \%$ while rotenone dramatically reduced it to $47 \%$, compared to control. Rotenone significantly increased the AMP/ATP ratio $(P<0.05$ control vs. rotenone) but metformin did not. Exposure of metformin to hepatocytes of eNOS knockout mice did not phosphorylate AMPKa or suppress gluconeogenesis. Moreover, exposure of rotenone in eNOS knockout mice increased phosphorylation of AMPK $\alpha$ and suppressed gluconeogenesis similarly to that in C57/BL6J mice (metformin, 455 \pm 61 ; rotenone, 205 \pm 19 ; control, $480 \pm 57$ $\mathrm{nmol} / \mathrm{mg}$ protein; $P<0.05$ control vs. rotenone, not significant control vs. metformin).

Conclusion: Activation of AMPK by metformin is RNS-dependent in primary mouse hepatocytes. Differently from the action of rotenone, metformin action is AMP-independent, suggesting that metformin does not affect mitochondrial metabolism. Loss of NO synthesis in eNOS knockout mice attenuates the inhibitory effect of metformin on gluconeogenesis, indicating that intra-hepatocellular eNOS is required for the pharmacological effect of metformin in liver.

\section{3}

Metformin combined with exogenous glucagon-like peptide-1 gives added benefit by improving acute glycaemic control in type 2 diabetic subjects

J. Cuthbertson ${ }^{1}$, S. Patterson ${ }^{2}$, F.P.M. O ' Harte $^{2}$, P.M. Bell'1;

${ }^{1}$ Endocrinology and Diabetes Research Centre, Royal Group of Hospitals, Belfast, ${ }^{2}$ School of Biomedical Sciences, University of Ulster, Coleraine,

United Kingdom

Background and aims: Glucagon-like peptide-1 (GLP-1) and glucose-dependent insulinotropic polypeptide (GIP) constitute the incretin hormones that form a major element of the enteroinsular axis which promotes postprandial insulin secretion. The therapeutic potential of these hormones in diabetes is limited by their rapid inactivation by the enzyme dipeptidylpeptidase-IV (DPP-IV). Metformin has been reported to act as an inhibitor of DPP-IV. We investigated the acute effects of metformin and GLP-1 alone or in combination on plasma DPP-IV activity and active GLP- 1 concentrations in type 2 diabetes.

Materials and methods: Ten subjects with type 2 diabetes (9 M/1 F), age $69.0 \pm 2.7$ years (mean \pm SEM), body mass index $29.6 \pm 1.7 \mathrm{~kg} / \mathrm{m}^{2}, \mathrm{HbA}_{1 \mathrm{c}} 7.0$ $\pm 0.1 \%$ ) received one of three combinations after an overnight fast in a randomised crossover design; metformin $1 \mathrm{~g}$ orally plus subcutaneous injection (sc) saline (Met), GLP-1 (1.5 nmol/kg body weight sc) plus placebo tablet (GLP) or metformin $1 \mathrm{~g}$ plus GLP-1(Met+GLP-1). At 15 min glucose was raised to $15 \mathrm{mmol} \mathrm{l}^{-1}$ by rapid intravenous infusion and responses assessed over the next 3 hours. This stimulus does not activate the enteroinsular axis and secretion of endogenous GLP-1, enabling the effect of exogenously administered GLP-1 to be examined in isolation. The DPP-IV assay is a fluorimetric method to measure free AMC (7-amino-4-methyl-coumarin) liberated from the DPP-IV substrate, Gly-Pro-4-AMC. Mean sample fluorescence values obtained were converted to DPP-IV activity, equivalent to AMC concentration, from the standard curve. GLP-1(7-36)amide was measured by a GLP-1 (active) enzyme-linked immunoassay kit. The assay is based on the use of a monoclonal antibody that binds specifically to the $\mathrm{N}$-terminal region of active GLP-1 molecule.

Results: Mean area under curve (AUC) ${ }_{0-180 \text { min }}$ plasma glucose responses were lowest following Met+GLP $(1629 \pm 90)($ mean \pm SEM, student t-test, ANOVA) compared to GLP $(1885 \pm 86, \mathrm{p}<0.002)$ and $\operatorname{Met}(2045 \pm 115$, $\mathrm{p}<0.001) \mathrm{mmol} /$ $1 /$ min respectively. At $60 \mathrm{~min}$ mean glucose levels were also lowest following Met+GLP $(8.8 \pm 0.7$ compared to GIP $(10.9 \pm 0.5, \mathrm{p}<0.0001)$ and Met $(12.4 \pm$ $0.7, \mathrm{p}<0.0001) \mathrm{mmol} / \mathrm{l}$ respectively. Mean AUC serum insulin responses were similar following either Met+GLP $(5426 \pm 498)$ or GLP $(5655 \pm 854)$ treatment but both were higher than Met $(3521 \pm 410, \mathrm{p}<0.0001$ and $\mathrm{p}<0.04) \mathrm{mU} /$ 1/min respectively. Mean AUC for plasma DPP-IV activity was lower following Met+GLP $(1505 \pm 2, \mathrm{p}<0.001)$ or Met $(1508 \pm 2, \mathrm{p}<0.002)$ compared to GLP $(1587 \pm 3) \mu \mathrm{mol} / \mathrm{ml} / \mathrm{min}$ respectively. Mean AUC measures for plasma active GLP- 1 concentrations were higher following Met+GLP ( $820 \times 10^{4} \pm 51$ x $\left.10^{4}\right)$ compared to GLP $\left(484 \times 10^{4} \pm 31 \times 10^{4} \mathrm{p}<0.000\right)$ and Met $\left(419 \times 10^{4} \pm\right.$ $\left.34 \times 10^{4}, \mathrm{p}<0.001\right) \mathrm{pmol} / \mathrm{l} / \mathrm{min}$ respectively.

Conclusion: In patients with type 2 diabetes metformin inhibits DPP-IV activity and thus increases active GLP-1 concentrations. Metformin given in combination with GLP-1(sc) significantly lowers plasma glucose concentrations in type 2 diabetic subjects. Metformin may have additional beneficial effects in combination therapy with incretin hormones over and above its traditional insulin sensitising role.

Supported by: R\&D Fellowship 


\section{PS 085 Diabetes in childhood}

\section{4}

Transient neonatal diabetes with UPD6 mutation; one of the many faces of monogenic diabetes in the young

T. Dugal, A. Gossage

Diabetes and Endocrinology, East Surrey Hospital, Redhill, United Kingdom

Background and aims: $1-2 \%$ patients with diabetes have monogenic diabetes. It is important to make an early diagnosis as this can have a dramatic impact on the management and quality of life of the patient as well as having implications for the family. Transient neonatal diabetes(TNDM) is a rare but distinct form of monogenic diabetes( incidence 1:400,000). Neonates classically present in the first week of life with growth retardation and diabetes. Although remission occurs usually after three months, there is a tendency to develop diabetes later in life needing treatment.

Case: A female baby at 41 weeks gestation was delivered normally. She appeared small for dates and weighed only $2.25 \mathrm{kgs}$. She went home on the $14^{\text {th }}$ day of life weighing $2.4 \mathrm{kgs}$ and was fed on Cow\&Gate, taking fairly large quantities of $200 \mathrm{cc} / \mathrm{kg} /$ day. She was admitted at age 15 days, passing a lot of urine and few loose stools. She weighed only $1.8 \mathrm{kgs}$, after losing $25 \%$ of her body weight in 2 days. She was dehydrated with a blood glucose of $88 \mathrm{mmol} / \mathrm{L}$. There was no acidosis and no ketones.Family history was unremarkable. With rehydration her biochemistry returned to normal with 48 hrs without insulin. She recovered well with good weight gain. However was readmitted after one year with gastroenteritis, blood sugar was $2 \mathrm{mmol} / \mathrm{L}$ and OGTT was normal. Aged three, she represented with polydipsia. OGTT was impaired. She was managed on diet only till she was 28 years old and from then has manitained good blood sugar control on rather small doses of Mixtard 10U/5U. She was found to have uniparental disomy of chromosome 6 (UPD6). She subsequently gave birth to a male child who was genetically tested and not found to have the anomalies associated with TNDM, i.e UPD6, 6q24methylation or 6q24 duplication.

Conclusion: TNDM is a rare disorder and can be associated with syndromes, several with mental retardation. Evidence suggests it is the result of overexpression of an imprinted and paternally expressed gene in the $6 \mathrm{q} 24$ region.
Two imprinted genes(ZAC\&HYAMI) have been identified, Three genetic mechanisms have been shown to result in TNDM, paternal unipaternal isodisomy of chromosome 6, paternally inherited duplication of 6q 24 and a methylation defect.

It is different from permanent neonatal diabetes (PNDM) which is usually a disorder of ATP channels and their inability to close, hence requiring life long sulphonyurea therapy. This is mediated through mutations in KCNJ11 gene encoding Kir6.2 subunit of the ATPase sensitive potassium channel. Early recognition and appropriate treatment for all forms of neonatal diabetes is essential, for this awareness of the condition is needed.

\section{5}

Factors associated with obesity in new onset insulin treated diabetes in childhood

M. Cedillo ${ }^{1}$, A.K. Adeniji ${ }^{2}$, I.M. Libman ${ }^{1}$, V.C. Arena ${ }^{2}$, D.J. Becker ${ }^{1}$

${ }^{1}$ Pediatric Endocrinology, Children's Hospital of Pittsburgh, ${ }^{2}$ Biostatistics, University of Pittsburgh, United States

Background and aims: From the 1980s to 2000s, there has been a continual increase in body mass index (BMI) at onset in children diagnosed with insulin requiring diabetes. We investigated associations of demographic, metabolic and genetic factors with BMI at onset and after 3 months with the hypothesis that low risk HLA and higher C-peptide at onset predict higher BMI.

Materials and methods: All children age $<19$ years, diagnosed with insulinrequiring diabetes from January $1^{\text {st }}, 2004$ to December $31^{\text {st }}, 2006$ at Children's Hospital of Pittsburgh were recruited and 321 consented. All received a minimum of 3 insulin injections per day and dietary education from a nutritionist at onset. Clinical and research data were obtained from hospital and research medical records. Blood samples were drawn at diagnosis and 3 months including C-peptide levels, HbA1c, islet cell antibodies and HLA typing. Results are presented for Caucasians $(n=276)$ and African Americans $(n=19)$ who have BMI measures both at baseline and 3-months. Predictors of BMI at baseline, 3 months and change from baseline to 3 months were examined using multiple linear regression analyses.

Results: BMI percentile (mean \pm standard deviation, median and interquartile range) increased significantly from onset $52.2 \pm 34.2,53.07$, [18.7-86.9]

Table 1 Characteristics of Diabetes presenting in the first 6 months of life

\begin{tabular}{|c|c|c|c|c|c|c|}
\hline $\begin{array}{l}\text { Gene } \\
\text { Clinical syndrome } \\
\text { Inheritance }\end{array}$ & $\begin{array}{l}\text { PNDM/ } \\
\text { TNDM }\end{array}$ & $\begin{array}{l}\text { Number of cases } \\
\text { described } \\
\% \text { in } \\
\text { Consanguineous or } \\
\text { isolated populations }\end{array}$ & $\begin{array}{l}\text { Median } \\
\text { birth } \\
\text { weight } \\
\text { (SDS) }\end{array}$ & $\begin{array}{l}\text { Age of } \\
\text { diagnosis } \\
\text { in weeks. } \\
\text { Median } \\
\text { (range) }\end{array}$ & $\begin{array}{l}\text { Pancreatic } \\
\text { appearance }\end{array}$ & Other features \\
\hline $\begin{array}{l}\text { ZAC/HYAMI } \\
\text { imprinting defect } \\
\text { on } 6 \mathrm{q} 24\end{array}$ & TNDM & $\begin{array}{l}=150 \\
\text { rare }\end{array}$ & $\begin{array}{l}2,100 \mathrm{~g} \\
(-2.94)\end{array}$ & $0.5(0-4)$ & Normal & Macroglossia (23\%) \\
\hline $\begin{array}{l}\text { Kir6.2 (KCNJ11) } \\
\text { Spontaneous } \\
\text { dominant }(10 \%) \\
\end{array}$ & $\begin{array}{l}\text { PNDM } \\
\text { TNDM } \\
(10 \%)\end{array}$ & $\begin{array}{l}=100 \\
\text { rare }\end{array}$ & $\begin{array}{l}2.580 \mathrm{~g} \\
(-1.73)\end{array}$ & $6(0-260)$ & Normal & $\begin{array}{l}\text { Developmental delay (20\%) } \\
\text { Epilepsy }(6 \%) \\
\text { DKA }(30 \%)\end{array}$ \\
\hline $\begin{array}{l}\text { EIF2AK3 } \\
\text { Wolcott-Rallison } \\
\text { syndrome } \\
\text { recessive }\end{array}$ & PNDM & $\begin{array}{l}30 \\
90 \%\end{array}$ & $?$ & $13(6-65)$ & $\begin{array}{l}\text { Atrophy of } \\
\text { pancreas (?), } \\
\text { Exocrine } \\
\text { dysfunction } \\
(25 \%)\end{array}$ & $\begin{array}{l}\text { Epiphyseal dysplasia (90\%) } \\
\text { Osteopenia (50\%). acute liver } \\
\text { failure }(75 \%) \text {, developmental } \\
\text { delay ( } 80 \%) \text {, hypothyroidism } \\
(25 \%)\end{array}$ \\
\hline $\begin{array}{l}\text { FOXP3 } \\
\text { IPEX syndrome } \\
\text { X linked }\end{array}$ & PNDM & $\begin{array}{l}14 \\
\text { Rare }\end{array}$ & $\begin{array}{l}2860 \\
-1.2\end{array}$ & $6(0-30)$ & $?$ & $\begin{array}{l}\text { Only boys affected } \\
\text { Chronic diarrhoea with villous } \\
\text { atrophy (95\%); Pancreatic and } \\
\text { thyroid autoantibodies ( } 75 \%) \\
\text { Thyroiditis (20\%), } \\
\text { eczema (50\%); anaemia } \\
\text { (30\%)often die young (1st yr) }\end{array}$ \\
\hline $\begin{array}{l}\text { GCK } \\
\text { (glucokinase) } \\
\text { recessive }\end{array}$ & PNDM & $\begin{array}{l}6 \\
85 \%\end{array}$ & $\begin{array}{l}1720 \\
-2.75\end{array}$ & & Normal & $\begin{array}{l}\text { Parents have fasting } \\
\text { hyperglycaemia as heterozygotes }\end{array}$ \\
\hline $\begin{array}{l}\mathrm{IPF} 1 \\
\text { recessive }\end{array}$ & PNDM & $\begin{array}{l}2 \\
50 \% \\
\end{array}$ & $\begin{array}{r}2140 \\
-2.97 \\
\end{array}$ & & Absent & $\begin{array}{l}\text { Parents may have early-onset } \\
\text { diabetes as heterozygotes }\end{array}$ \\
\hline $\begin{array}{l}\text { HNF-1 } \beta \\
\text { dominant }(60 \%) \\
\text { spontaneous } \\
\end{array}$ & TNDM & $\begin{array}{l}2 \\
\text { Rare }\end{array}$ & $\begin{array}{l}1900 \\
-3.21\end{array}$ & & Atrophy & Renal developmental disorders \\
\hline $\begin{array}{l}\text { PTF1A } \\
\text { recessive }\end{array}$ & PNDM & $\begin{array}{l}3 \\
100 \% \\
\end{array}$ & $\begin{array}{l}1390 \\
-3.8 \\
\end{array}$ & & Atrophy & $\begin{array}{l}\text { Severe neurological dysfunction } \\
\text { and cerebellar hypoplasia }\end{array}$ \\
\hline
\end{tabular}


Distribution of subjects within BMI percentile by age group

\begin{tabular}{lllllll}
\hline \multicolumn{7}{c}{ Age at Diagnosis } \\
\hline Time & BMI \%ile & $\begin{array}{l}\mathbf{0} \text { to }<5 \\
(\mathbf{n}=\mathbf{4 4})\end{array}$ & $\begin{array}{l}\mathbf{5} \text { to }<\mathbf{1 0} \\
(\mathbf{n}=\mathbf{1 1 1})\end{array}$ & $\begin{array}{l}\mathbf{1 0} \text { to }<\mathbf{1 5} \\
(\mathbf{n}=\mathbf{1 1 6})\end{array}$ & $\begin{array}{l}\mathbf{1 5} \text { to }<\mathbf{1 9} \\
(\mathbf{n}=\mathbf{2 4})\end{array}$ & $\begin{array}{l}\text { Total \% } \\
(\mathbf{n}=\mathbf{2 9 5})\end{array}$ \\
\hline Baseline & $<25$ & $41 \%(18)$ & $34 \%(38)$ & $24 \%(28)$ & $16 \%(4)$ & $30 \%(88)$ \\
\hline 3 months & $\mathbf{2} 5$ & $14 \%(6)$ & $23 \%(26)$ & $32 \%(37)$ & $25 \%(6)$ & $25 \%(75)$ \\
\hline & $\geq \mathbf{8 5}$ & $9 \%(4)$ & $1.8 \%(2)$ & $5.2 \%(6)$ & $0 \%(0)$ & $4 \%(12)$ \\
\hline
\end{tabular}

to three months $(73.5 \pm 23.1,79.4$, [58.7-93.1]) $(\mathrm{p}<0.0001$; paired t-test). This change was similar between Caucasians $(20.4 \pm 23.8)$ and African Americans $(21.7 \pm 32.6)$. BMI percentile (\%ile) at diagnosis was significantly associated with the independent factors of African American race, higher baseline Cpeptide and older age. BMI \%ile at 3 months was independently associated with African American, higher baseline C-peptide, higher baseline HbAlc and male gender. Greater change in BMI \%ile from onset to 3 months was significantly associated with lower baseline c-peptide, higher baseline HbAlc, male gender and younger age at onset. There was no association between BMI and number of islet cell antibodies or HLA haplotype (only 10 had low risk haplotypes). Table 1 shows the prevalence of BMI $<25^{\text {th }} \%$ ile and $\geq 85^{\text {th }} \%$ ile at baseline and 3 months.

Conclusion: Consistent with our previous experience, higher BMI percentile at onset was associated with being African American, older age, and higher C-peptide. Those with the most severe insulin deficiency (lower C-peptide and higher $\mathrm{HbAlc}$ ) had a greater rise in BMI, likely representing catch up to prior levels so that after 3 months $43 \%$ of children age 0 - 9 years and $38 \%$ children $10-18$ years had a BMI $\geq 85^{\text {th }}$ percentile, with males being the most obese. As predicted, those with the highest $\mathrm{C}$-peptide were at greatest risk, but there was no support for an HLA relationship. Supported by: NIH

\section{6}

Increase in obesity after diagnosis of insulin-requiring diabetes in children: cause for concern

I.M. Libman ${ }^{1}$, C. Baldwin ${ }^{1}$, S. Brooke ${ }^{2}$, P. Balfour ${ }^{3}$, D. Becker ${ }^{1}$

${ }^{1}$ Pediatric Endocrinology and Diabetes, Children's Hospital of Pittsburgh,

${ }^{2}$ School of Medicine, Drexel University, Philadelphia, ${ }^{3}$ Internal Medicine,

University of Virginia Medical Center, Charlottesville, United States

Background and aims: Our group has shown a continual increase in obesity prevalence in pediatric cohorts at onset of insulin requiring diabetes over the last decades. Also, our adolescents, after a mean diabetes duration of 5 years, consume fewer carbohydrate calories but more fat, exceeding the recommended intake. We hypothesize that the prevalence of obesity increases after diagnosis, in spite of dietary recommendations and education.

Materials and methods: All children age $<19$ years, diagnosed with insulin-requiring diabetes from 1/1/1995 to 31/12/2002 at Children's Hospital of Pittsburgh, with residence in Allegheny County, Pennsylvania, were identified. Data were obtained from retrospective review of records at diagnosis and 4 clinic visits $(2-3,6,12$ and 36 months). Obesity was defined as a body mass index $(\mathrm{BMI}) \geq 85^{\text {th }} \%$ ile for age and sex. All were started on a minimum of 3 insulin injections per day and received dietary education from a nutritionist at onset.

Results: Complete data were available for 270 of 351 children (77\%).

Prevalence of obesity by visit and by BMI percentile gromp at onset

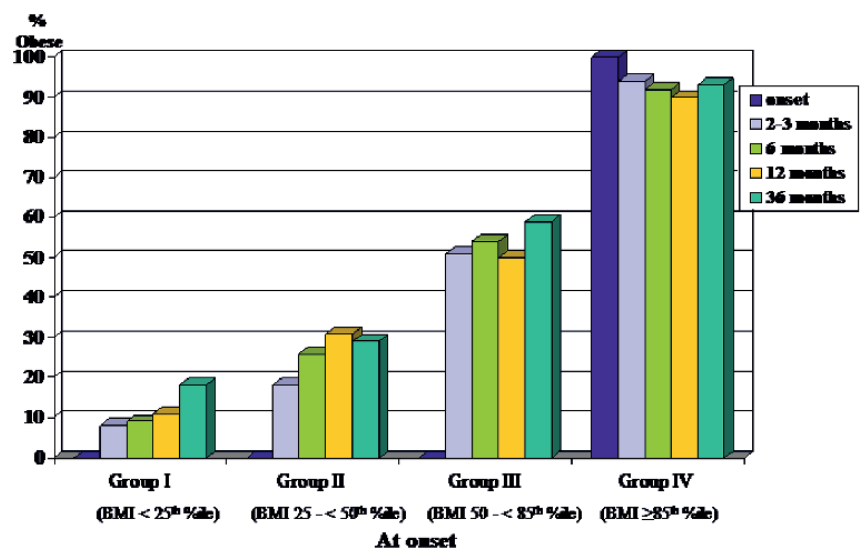

In group I, BMI \%ile (median and [interquartile range]) was 5.7 [1.2-15.3] while at 2-3 months this increased to 53.3 [36.6-68.2] $(\mathrm{p}=0.0005$; paired $\mathrm{t}$ test). A second significant increase was seen between 12 and 36 months (58.0 [35.2-74.9] vs 68.8 [46.6-81.5] $(\mathrm{p}=0.0005))$. In group II and III significant changes were seen between onset and 2-3 months (Group II: 33.5 [28.4$39.4]$ vs 68.9 [57.5-82.9] and group III: 66.6 [57.1-75.4] vs 85.2 [75.2-91.3] $(\mathrm{p}=0.0005))$.

Conclusion: 1) 17\% were obese at onset. After 3 years $\sim 1$ in 3 children age 0 - 9 years and 1 in 2 children $10-18$ years had a BMI $\geq 85^{\text {th }} \%$ ile 2 ) prevalence of obesity in those with BMI $<85^{\text {th }} \%$ ile at onset increased with time, while in those initially obese, it decreased minimally 3 ) non-obese children had a remarkable increase in BMI percentile during the first 2 to 3 months after diagnosis. The initial months after diagnosis are critical in preventing excessive weight gain. Despite education to provide recommended dietary allowances for age, these are exceeded or these recommendations are currently excessive. Recommendations to calorie restrict, if obese at onset, at best prevents further excessive weight gain. The relevance of this problem beyond our population and the implications long term should be explored.

Supported by: Cochrane Weber Endowed Fund

Prevalence of BMI $<25$ th \%ile and $\geq 85$ th $\%$ ile by age group and visit

\begin{tabular}{|c|c|c|c|c|c|c|}
\hline Visit & BMI \%ile & $\begin{array}{l}0-4 \text { years } \\
\mathrm{n}=57(\%)\end{array}$ & $\begin{array}{l}\text { 5-9 years } \\
\mathrm{n}=92(\%)\end{array}$ & $\begin{array}{l}10-14 \text { years } \\
\mathrm{n}=104(\%)\end{array}$ & $\begin{array}{l}15-18 \text { years } \\
\mathrm{n}=17(\%)\end{array}$ & $\begin{array}{l}\text { Total } \\
\mathrm{n}=270(\%)\end{array}$ \\
\hline Onset & $\begin{array}{l}<25 \\
\geq 85\end{array}$ & $\begin{array}{l}23(40) \\
2(4)\end{array}$ & $\begin{array}{l}37(40) \\
14(15)\end{array}$ & $\begin{array}{l}41(39) \\
26(25)\end{array}$ & $\begin{array}{l}3(18) \\
5(29)\end{array}$ & $\begin{array}{l}104(38) \\
47(17)\end{array}$ \\
\hline 2-3 months & $\begin{array}{l}<25 \\
\geq 85 \\
\end{array}$ & $\begin{array}{l}4(7) \\
16(28) \\
\end{array}$ & $\begin{array}{l}4(4) \\
36(39) \\
\end{array}$ & $\begin{array}{l}7(7) \\
\mathbf{3 8}(\mathbf{3 6}) \\
\end{array}$ & $\begin{array}{l}1(6) \\
8(47) \\
\end{array}$ & $\begin{array}{l}16(6) \\
98(37) \\
\end{array}$ \\
\hline 6 months & $\begin{array}{l}<25 \\
\geq 85 \\
\end{array}$ & $\begin{array}{l}4(7) \\
19(33) \\
\end{array}$ & $\begin{array}{l}4(4) \\
37(40) \\
\end{array}$ & $\begin{array}{l}7(7) \\
39(37) \\
\end{array}$ & $\begin{array}{l}1(6) \\
8(47) \\
\end{array}$ & $\begin{array}{l}16(6) \\
103(38)\end{array}$ \\
\hline 12 months & $\begin{array}{l}<25 \\
\geq 85 \\
\end{array}$ & $\begin{array}{l}4(7) \\
20(35) \\
\end{array}$ & $\begin{array}{l}4(4) \\
36(39) \\
\end{array}$ & $\begin{array}{l}9(8) \\
39(37) \\
\end{array}$ & $\begin{array}{l}1(6) \\
\mathbf{9 ( 5 3 )} \\
\end{array}$ & $\begin{array}{l}18(7) \\
104(\mathbf{3 8})\end{array}$ \\
\hline 36 months & $\begin{array}{l}<25 \\
\geq 85 \\
\end{array}$ & $\begin{array}{l}3(5) \\
18(32) \\
\end{array}$ & $\begin{array}{l}2(2) \\
38(41)\end{array}$ & $\begin{array}{l}1(1) \\
48(45) \\
\end{array}$ & $\begin{array}{l}1(6) \\
9(53)\end{array}$ & $\begin{array}{l}7(3) \\
113(42)\end{array}$ \\
\hline
\end{tabular}




\section{7}

Disposition modeling of blood glucose control in children: novel insights from a longitudinal study

A.N. Jeffery ${ }^{1}$, B.S. Metcalf ${ }^{1}$, J. Hosking ${ }^{1}$, L.D. Voss ${ }^{1}$, M.J. Murphy ${ }^{2}$,

T.J. Wilkin ${ }^{1}$

${ }^{1}$ Department of Endocrinology and Metabolism, Peninsula College of

Medicine and Dentistry, Plymouth, ${ }^{2}$ Department of Biochemical Medicine,

Ninewells Hospital, Dundee, United Kingdom

Background and aims: The hyperbolic relationship between insulin sensitivity $(\% \mathrm{~S})$ and insulin secretion (\%B) defines the behaviour of the glucose-insulin feed-back loop, and underpins the HOMA model. Disposition modeling - plotting the shifting relationship between $\% \mathrm{~S}$ and $\% \mathrm{~B}$ over time - offers new insights into the dynamic behaviour of the loop as it seeks constantly to optimize control of glucose while environmental pressures change.

Methods: Disposition is the point on a disposition curve defined by concurrent values of $\% \mathrm{~B}$ and $\% \mathrm{~S}$. Disposition index (DI) is the product of $\% \mathrm{~B}$ and $\% \mathrm{~S}$ which, being constant for a hyperbola, defines the disposition curve. DI determines blood glucose (inversely). We monitored 218 healthy children (126 boys) from the EarlyBird cohort annually from age 5 to $10 y$, measuring changes in BMI SDS, fasting glucose, HOMA-\%S, HOMA-\%B, disposition and DI. The relationship between $\% \mathrm{~B}$ and $\% \mathrm{~S}$ was plotted year-by-year for the cohort as a whole, and for tertiles BMI SDS at $5 y$ to examine the impact of BMI on disposition.

Results: Glucose rose $(4.3-4.8 \mathrm{mmol} / \mathrm{l}, \mathrm{p}<0.001)$ throughout the $5 \mathrm{y}$ period, consistent with the fall in DI $(186.3-115.6, \mathrm{p}<0.001)$ and a progressive weakening of loop control. BMI (SDS) did not change significantly from $5-7 y$ $(+0.02 \mathrm{SD}, \mathrm{p}=0.4)$ but rose substantially from $7-10 \mathrm{y}(+0.17 \mathrm{SD}, \mathrm{p}<0.001)$. The disposition map for the cohort as a whole (see figure) shows that, from $5-7 y$, the fall in DI was attributable to loss of $\% \mathrm{~B}$, with some compensatory increase in $\% \mathrm{~S}$. From $7 \mathrm{y}$, however, $\% \mathrm{~S}$ ceased to rise further, the trajectory reversed, and the continuing fall in DI became attributable to falling $\% \mathrm{~S}$ (rising insulin resistance) with a compensatory rise in $\% \mathrm{~B}$ but a higher glucose level. When analyzed by $\mathrm{BMI}$ tertile, \%B was higher and \%S lower in the heaviest compared with the lightest children at each age $(p<0.01$ at $5 y, 7 y, 8 y, 9 y, 10 y ; p=0.14$, 0.15 at $6 y)$. Furthermore, the switch in trajectory occurred earlier (6-7y), and at lower sensitivity $(221 \%)$, in the heaviest compared with the lightest children $(7-8 y, 331 \%)$.

Conclusion: The switch in disposition is a novel observation, demonstrating how the loop reverts to beta cell reserve once insulin sensitivity nears its limit. The inflexion may be a crucial prelude to beta cell insufficiency, and BMI appears to be a key determinant of the stage at which it occurs. Disposition mapping may help us better understand the changes that lead to childhood diabetes.

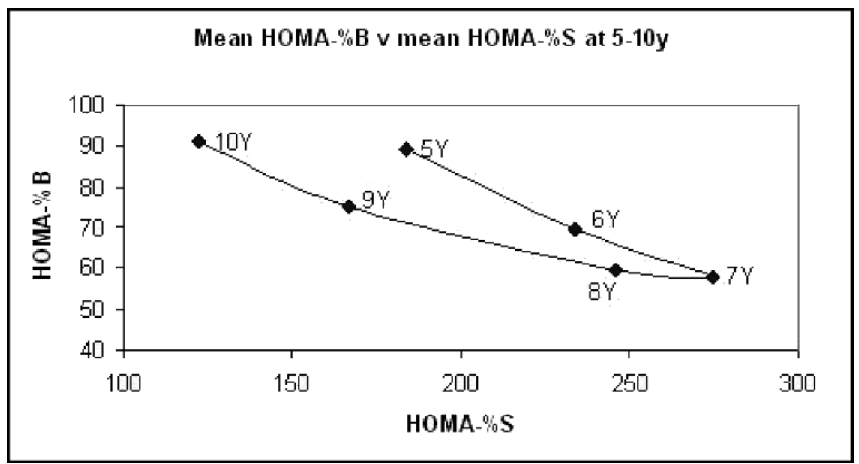

Supported by: Abbott, Astra-Zeneca, GSK, Ipsen, Novo Nordisk, Roche Pharmaceuticals, Unilever, Child Growth Foundation, Diabetes Foundation, Diabetes UK, EarlyBird Diabetes Trust, S\& SW NHS Executive R\&D.

\section{8}

Three kind of mealtime insulin administration and metabolic control in diabetic children on insulin pumps

E. Pankowska, A. Szypowska, M. Lipka, M. Szpotańska, M. Blazik, L. Groele, D. Golicka

Pediatric Diabetology and Birth Defects, Medical University, Warsaw, Poland

Background: Insulin pump is an advanced technology which offers a new option of bolus used for delivering meal time insulin. There are a possibilities to administrate normal bolus $(\mathrm{N})$, dual-wave (D-W) and square-wave (S-W) in which insulin is delivered in prolonged time.

Aims: of the study was to assess whether using of D-W boluses has an impact on metabolic control ( $\mathrm{HbAlc}$ ) and to estimate the compliance of pediatric patients in implementing this system in real-life.

Materials and methods: Cross-sectional study included 499 patients -pump records from outpatient clinic. We analyzed the number of D-W/S-W boluses per two weeks, insulin requirement $(\mathrm{U} / \mathrm{kg} / \mathrm{d})$ from records of downloaded insulin pumps, HbAlc and patients' weight measured at visit. Patients were educated to calculate a dose of meal-time insulin using a carbohydrate unit (CU) and fat-protein unit (FP). One CU unit was defined as 10 grams of carbohydrate and one FP as a 100 calories from fat and/or protein products. The same insulin-ratios (IR) was for CU and FPU which was implemented for calculation insulin's amount for $\mathrm{N}$ boluses and $\mathrm{S}-\mathrm{W}$ boluses, where dose for $\mathrm{N}$ bolus=IR x number of CU; S-W= IR x number of FPU.

Results: Averagely D-W/S-W bolus were used 16.6 SD 0.77/14 days (0-95) in group aged 10.6 SD4.1 years (1.1 -18.8) with HbA1c 7.42 SD 1.47\% (4.4-13.5) and insulin requirement $0.79 \mathrm{SD} 0.22 \mathrm{U} / \mathrm{kg} / \mathrm{d}$ (90.11-1.94). $18.8 \%$ of patients did not programmed $\mathrm{D}-\mathrm{W} / \mathrm{S}-\mathrm{W}$ boluses during a two weeks and had a higher HbA1c (7.82 SD1.69\% vs 7.32 SD1.4\%, p=0.0052). $47 \%$ of patients used at least one $1 \mathrm{D}-\mathrm{W} / \mathrm{S}-\mathrm{W}$ boluses per day. The significantly lowest $\mathrm{HbAlc}$ was found in group programmed from 1 to $2 \mathrm{D}-\mathrm{W} / \mathrm{S}-\mathrm{W}$ bolus/day $(\mathrm{p}=0.0001)$ compared with group less than $1 \mathrm{D}-\mathrm{W} / \mathrm{S}-\mathrm{W}$ bolus /day and more than $2 \mathrm{~S}$ W/S-W bolus/day. For patients with $\mathrm{HbAlc}<7.5 \%$ statistically relevant was higher number of D-W/S-W boluses 19.55 (95\%CI:17.44- 1.65 vs 12.42 (95\% CI:10.22-14.6), but there was no correlation between number of boluses and $\mathrm{HbAlc}$ in patients in remission phase $(<0.5 \mathrm{U} / \mathrm{kg} / \mathrm{d})(\mathrm{r}=0.012, \mathrm{p}=0.9)$.

Conclusion: The patients using at least one D-W/S-W bolus per day get a recommended level of HbAlc. The pediatric diabetes patients on CSII are able to use D-W/S-W boluses in daily practices.

\section{9}

The optimal type of bolus following a pizza meal in children and adolescents with type 1 diabetes

A.E. Scaramuzza ${ }^{1}$, D. Iafusco ${ }^{2}$, E. Giani ${ }^{1}$, D. Spiri ${ }^{1}$, A. De Palma ${ }^{1}$, A. Bosetti ${ }^{3}$, L. Santoro ${ }^{1}$, C. Mameli ${ }^{1}$, A. Gazzarri ${ }^{1}$, M. Macedoni ${ }^{1}$, S. Riboni ${ }^{1}$, G.V. Zuccotti ${ }^{1}$

${ }^{1}$ Paediatriacs, University of Milano, ${ }^{2}$ Paediatriacs, Second University of Naples, Italy, ${ }^{3}$ Nutrition and Dietetics, University of Milano, Italy

Background and aims: We attempted to identify an optimal insulin pump meal bolus by comparing postprandial blood sugar levels following 6 methods of insulin pump meal blousing for a consistent pizza meal.

Materials and methods: We evaluated 26 children and adolescents, aged 422 yrs (mean $14.81 \pm 4.28$ yrs) with type 1 diabetes mellitus (T1DM) from 1 to $19 \mathrm{yrs}(8.23 \pm 4.41 \mathrm{yrs}), \mathrm{BMI} 22.16 \pm 4.28 \mathrm{~kg} / \mathrm{m}^{2}$, in therapy with subcutaneous insulin infusion (insulin requirement $0.84 \pm 0.32 \mathrm{U} / \mathrm{kg} /$ day). Each patient participated in a study to compare postprandial glucose values following 6 meal bolus regimens for a consistent pizza meal. Each patient utilized the following aspart regimens on three consecutive days, and glucose values were recorded with SMBG: a) 6-h dual-wave bolus (30\% of insulin given immediately and $30 \%$ given over a 6 -h period) given 15 min prior a pizza "margherita" meal; b) 6-h dual-wave bolus (30\% of insulin given immediately and 30\% given over a 6-h period) given just before a pizza "margherita" meal; c) a single-wave bolus ( $100 \%$ of insulin given immediately) given $15 \mathrm{~min}$ before a pizza "margherita" meal; d) a single-wave bolus ( $100 \%$ of insulin given immediately) given just before a pizza "margherita" meal; e) 6-h dual-wave bolus (30\% of insulin given immediately and $30 \%$ given over a 6 -h period) given 15 min prior a pizza with vegetables meal; and f) a single-wave bolus ( $100 \%$ of insulin given immediately) given $15 \mathrm{~min}$ before a pizza with vegetables meal. Total $\mathrm{CHO}$ was kept constant for each meal; insulin dose was calculated according to 
Glycaemic values following various types of bolus in children with T1DM

\begin{tabular}{|c|c|c|c|c|c|c|c|c|c|}
\hline Type of bolus & T0 & T30 & T60 & T90 & T120 & T180 & $\mathrm{T} 240$ & T300 & T360 \\
\hline Double-wave 6-h bolus $15 \mathrm{~min}$ before & $\begin{array}{l}151 \pm 74 \\
\text { NS }\end{array}$ & $\begin{array}{l}157 \pm 75 \\
\text { NS }\end{array}$ & $\begin{array}{l}180 \pm 89 \\
\text { NS }\end{array}$ & $\begin{array}{l}176 \pm 93 \\
\text { NS }\end{array}$ & $\begin{array}{l}187 \pm 81 \\
0.056\end{array}$ & $\begin{array}{l}172 \pm 81 \\
\text { NS }\end{array}$ & $\begin{array}{l}144 \pm 92 \\
\text { NS }\end{array}$ & $\begin{array}{l}122 \pm 57 \\
\mathbf{0 . 0 1 9}\end{array}$ & $\begin{array}{l}97 \pm 41 \\
\mathbf{0 . 0 0 1}\end{array}$ \\
\hline Double-wave 6-h bolus just before & $\begin{array}{l}98 \pm 46 \\
\text { NS } \\
\mathbf{0 . 0 0 4} \text { VS bolus } 1\end{array}$ & $\begin{array}{l}139 \pm 52 \\
\mathbf{0 . 0 1 1}\end{array}$ & & & $\begin{array}{l}151 \pm 58 \\
\mathbf{0 . 0 0 1}\end{array}$ & $\begin{array}{l}149 \pm 66 \\
\mathbf{0 . 0 0 1}\end{array}$ & $\begin{array}{l}136 \pm 58 \\
\mathbf{0 . 0 0 3}\end{array}$ & $\begin{array}{l}148 \pm 67 \\
\mathbf{0 . 0 0 0 5}\end{array}$ & $\begin{array}{l}129 \pm 73 \\
\mathbf{0 . 0 1}\end{array}$ \\
\hline Single-wave bolus 15 min before & $\begin{array}{l}132 \pm 58 \\
\text { NS }\end{array}$ & $\begin{array}{l}136 \pm 54 \\
\text { NS }\end{array}$ & $\begin{array}{l}134 \pm 62 \\
\text { NS }\end{array}$ & $\begin{array}{l}148 \pm 72 \\
\text { NS }\end{array}$ & $\begin{array}{l}156 \pm 70 \\
\mathbf{0 . 0 3} \\
\end{array}$ & $\begin{array}{l}145 \pm 67 \\
\text { NS }\end{array}$ & $\begin{array}{l}141 \pm 50 \\
\text { NS }\end{array}$ & $\begin{array}{l}137 \pm 58 \\
\text { NS }\end{array}$ & $\begin{array}{l}144 \pm 68 \\
\text { NS }\end{array}$ \\
\hline ingle-n & $\begin{array}{l}129 \pm 65 \\
\text { NS }\end{array}$ & $\begin{array}{l}130 \pm 58 \\
\text { NS }\end{array}$ & $\begin{array}{l}118 \pm 68 \\
\text { NS }\end{array}$ & $\begin{array}{l}131 \pm 79 \\
\text { NS }\end{array}$ & $\begin{array}{l}152 \pm 77 \\
\text { NS }\end{array}$ & $\begin{array}{l}153 \pm 74 \\
\text { NS }\end{array}$ & $\begin{array}{l}174 \pm 63 \\
\mathbf{0 . 0 4 8} \\
\end{array}$ & $\begin{array}{l}176 \pm 76 \\
\mathbf{0 . 0 3 5} \\
\end{array}$ & $\begin{array}{l}176 \pm 68 \\
\mathbf{0 . 0 3 5} \\
\end{array}$ \\
\hline Double-wave 6-h bolus 15 min before (vegetables) & $\begin{array}{l}149 \pm 56 \\
\text { NS }\end{array}$ & $\begin{array}{l}159 \pm 62 \\
\text { NS }\end{array}$ & $\begin{array}{l}176 \pm 55 \\
\mathbf{0 . 0 0 5} \\
\end{array}$ & $\begin{array}{l}175 \pm 77 \\
\mathbf{0 . 0 6} \\
\end{array}$ & $\begin{array}{l}198 \pm 75 \\
\mathbf{0 . 0 0 0 5} \\
\end{array}$ & $\begin{array}{l}191 \pm 78 \\
\mathbf{0 . 0 0 1} \\
\end{array}$ & $\begin{array}{l}168 \pm 68 \\
\mathbf{0 . 0 1 5} \\
\end{array}$ & $\begin{array}{l}142 \pm 64 \\
\text { NS }\end{array}$ & $\begin{array}{l}119 \pm 67 \\
\mathbf{0 . 0 1} \\
\end{array}$ \\
\hline ngle-n & $\begin{array}{l}115 \pm 56 \\
\text { NS }\end{array}$ & $\begin{array}{l}109 \pm 58 \\
\text { NS }\end{array}$ & $\begin{array}{l}106 \pm 45 \\
\text { NS }\end{array}$ & $\begin{array}{l}109 \pm 49 \\
\text { NS }\end{array}$ & $\begin{array}{l}120 \pm 53 \\
\text { NS }\end{array}$ & $\begin{array}{l}141 \pm 58 \\
\text { NS }\end{array}$ & $\begin{array}{l}153 \pm 65 \\
\text { NS }\end{array}$ & $\begin{array}{l}168 \pm 69 \\
0.01\end{array}$ & $\begin{array}{l}160 \pm 79 \\
\mathbf{0 . 0 0 2}\end{array}$ \\
\hline
\end{tabular}

glycaemic value and $\mathrm{CHO}$, using ISF and INS:CHO ratio personalized for each patient.

Results: The results are shown in the table (significance are vs the time 0 for each type of bolus).

Conclusion: Use of a dual-wave bolus extended over a 6-h period following a pizza meal if given $15 \mathrm{~min}$ before provided significantly less postprandial hyperglycaemia during the $6-\mathrm{h}$ period. Single-wave bolus could be used only if given 15 min before meal and if the pizza is served with some vegetables, even if we observed a rise in glycaemic values in the last two hours of the study.kground and aims:

\section{0}

Efficacy and safety of insulin glulisine versus insulin lispro as part of a basal-bolus insulin regimen in children and adolescents with type 1 diabetes mellitus

A. Philotheou ${ }^{1}$, S. Arslanian ${ }^{2}$, L. Blatniczky ${ }^{3}$, V. Peterkova ${ }^{4}$, E. Souhami ${ }^{5}$, T. Danne ${ }^{6}$

${ }^{1}$ UCT Diabetes Clinical Trials Unit, New Groote Schuur Hospital, Cape Town, South Africa, ${ }^{2}$ Div. Pediatric Endocrinology, Metabolism and Diabetes Mellitus, Children's Hospital of Pittsburgh, University of Pittsburgh School of Medicine, United States, ${ }^{3}$ Buda Children's Hospital, Budapest, Hungary, ${ }^{4}$ Endocrinological Scientific Centre, Russian Academy of Science, Moscow, Russian Federation, ${ }^{5}$ sanofi-aventis, Antony, France, ${ }^{6}$ Children's Hospital auf der Bult, Hannover, Germany

Background and aims: The aim of the study was to assess efficacy and safety of insulin glulisine (glulisine) versus insulin lispro (lispro) in children and ad- olescents with type 1 diabetes mellitus (T1DM) treated with insulin glargine (glargine) once daily or NPH twice daily as basal insulin.

Materials and methods: A 26-week, multicentre, open, randomised, parallel-group, non-inferiority trial conducted in 572 patients with T1DM aged 4-17 years and $\mathrm{HbA}_{1 c} 6-11 \%$, treated with glargine once daily (evening) or $\mathrm{NPH}$ twice daily as basal insulin. Patients received glulisine $(\mathrm{n}=277)$ or lispro $(\mathrm{n}=295)$ 0-15 min pre-meal.

Results: At baseline, patients (glulisine versus lispro; mean \pm standard deviation) were: age $12.5 \pm 3.1$ versus $12.6 \pm 2.9$ years, body mass index $20.8 \pm 3.4$ versus $20.5 \pm 3.3 \mathrm{~kg} / \mathrm{m}^{2}, \mathrm{HbA}_{1 \mathrm{c}} 8.2 \pm 1.1$ versus $8.2 \pm 1.0 \%$ (33\% were at $\mathrm{ADA}$ age-specific $\mathrm{HbA}_{1 \mathrm{c}}$ targets) and T1DM duration $5.3 \pm 3.6$ versus $5.2 \pm 3.2$ years. Treatment at randomisation (baseline): NPH 30.3 versus $27.1 \%$, glargine 69.7 versus $72.9 \%$. 58.0\% (glulisine) and 60.5\% (lispro) of patients had 3-4 bolus insulin injections, remaining stable throughout. Glulisine versus lispro baseline to endpoint $\mathrm{HbA}_{1 \mathrm{c}}$ adjusted mean changes were similar (glulisine-lispro: $-0.06,95 \%$ CI: $[-0.24,0.12]$, pre-specified non-inferiority margin $=0.4 \%)$. Overall, for all age groups together, significantly more $(\mathrm{p}=0.0386)$ patients achieved ADA age-specific $\mathrm{HbA}_{1 \mathrm{c}}$ targets at endpoint with glulisine (38.4\%) versus lispro (32.0\%). This difference was most pronounced in 13-17-year olds, with 31.1 versus $21.1 \%$ of glulisine versus lispro patients achieving ADA age-specific $\mathrm{HbA}_{1 \mathrm{c}}$ target $<7.5 \%$ at endpoint $(\mathrm{p}=0.0251$ ). From Month 4 to endpoint, symptomatic hypoglycaemia rates were similar (see table). Data for 3-point self-monitored blood glucose profiles are also shown in the table. Conclusion: In conclusion, glulisine was as effective and safe as lispro in children and adolescents with T1DM. Compared with lispro, glulisine allowed higher numbers of patients to reach ADA age-specific $\mathrm{HbA}_{1 c}$ targets, particularly amongst adolescents aged 13-17 years.

Supported by: sanofi-aventis

\begin{tabular}{|c|c|c|c|c|}
\hline & & Glulisine $(n=277)$ & Lispro(n=295) & p-value \\
\hline $\mathrm{HbA}_{1 \mathrm{c}}(\%)$ mean $(\mathrm{SD})$ & $\begin{array}{l}\text { Baseline } \\
\text { Endpoint } \\
\text { Adjusted mean change }^{*}\end{array}$ & $\begin{array}{l}8.20 \pm 1.05 \\
8.31 \pm 1.37 \\
0.10 \pm 0.08 \\
\end{array}$ & $\begin{array}{l}8.17 \pm 1.02 \\
8.37 \pm 1.32 \\
0.16 \pm 0.07 \\
\end{array}$ & $\begin{array}{l}- \\
\text { NS } \\
\end{array}$ \\
\hline BG before breakfast $(\mathrm{mmol} / \mathrm{L})$ & $\begin{array}{l}\text { Baseline } \\
\text { Endpoint }\end{array}$ & $\begin{array}{l}9.49 \pm 0.21 \\
8.77 \pm 0.21 \\
\end{array}$ & $\begin{array}{l}9.55 \pm 0.20 \\
9.46 \pm 0.20 \\
\end{array}$ & $\begin{array}{l}- \\
0.0135 \\
\end{array}$ \\
\hline BG before main meal $(\mathrm{mmol} / \mathrm{L})$ & $\begin{array}{l}\text { Baseline } \\
\text { Endpoint }\end{array}$ & $\begin{array}{l}10.26 \pm 0.27 \\
9.76 \pm 0.24 \\
\end{array}$ & $\begin{array}{l}10.35 \pm 0.26 \\
9.80 \pm 0.24 \\
\end{array}$ & NS \\
\hline BG 2 hours after the start of the main meal $(\mathrm{mmol} / \mathrm{L})$ & $\begin{array}{l}\text { Baseline } \\
\text { Endpoint }\end{array}$ & $\begin{array}{l}8.87 \pm 0.24 \\
9.20 \pm 0.22 \\
\end{array}$ & $\begin{array}{l}9.08 \pm 0.23 \\
9.04 \pm 0.21 \\
\end{array}$ & NS \\
\hline $\begin{array}{l}\text { Monthly rate of hypoglycaemia per patient from Month } 4 \\
\text { to treatment end (events/patient-month) }\end{array}$ & $\begin{array}{l}\text { Symptomatic } \\
\text { Severe }\end{array}$ & $\begin{array}{l}3.10 \pm 4.33 \\
0.06 \pm 0.24 \\
\end{array}$ & $\begin{array}{l}2.91 \pm 4.35 \\
0.07 \pm 0.27 \\
\end{array}$ & $\begin{array}{l}\text { NS } \\
\text { NS } \\
\end{array}$ \\
\hline Patients with $\mathrm{AE}(\%)^{\dagger}$ & $\begin{array}{l}\text { Any } \mathrm{AE} \\
\text { Serious } \mathrm{AE}^{\ddagger} \\
\text { Hypoglycaemia reported } \\
\text { as AE }\end{array}$ & $\begin{array}{l}53.4 \\
10.8 \\
7.2\end{array}$ & $\begin{array}{l}58.6 \\
12.5 \\
8.1\end{array}$ & $\begin{array}{l}\text { NS } \\
\text { NS } \\
\text { NS }\end{array}$ \\
\hline
\end{tabular}

${ }^{*}$ Difference in adjusted mean between treatment groups: -0.06 (95\% CI: -0.24, 0.12); ${ }^{\dagger}$ Frequency and type of AE were similar between treatment groups; ${ }^{*}$ Frequency and type of serious $\mathrm{AE}$ were similar between the two groups; glulisine versus lispro (\%): diabetic ketoacidosis (2.2 vs 1.4$)$; hyperglycaemia (0.0 vs 0.3 ); and ketonuria ( 0.0 vs 0.7 ); $\mathrm{AE}=$ adverse event; $\mathrm{BG}=$ blood glucose; $\mathrm{NS}=$ not significant 


\section{1}

Glargine treatment can ameliorate clinical features in children and adolescents with cystic fibrosis and early glucose derangements

A. Franzese ${ }^{1}$, I. De Simone ${ }^{1}$, E. Mozzillo ${ }^{1}$, G. Valerio ${ }^{2}$, V. Raia ${ }^{1}$, R. Spadaro ${ }^{1}$, R. Nugnes ${ }^{1}$

${ }^{1}$ Paediatrics, University Federico II, ${ }^{2}$ School of Movement Sciences,

Parthenope University, Naples, Italy

Background and aims: A strict association of diabetes and increased morbidity and mortality in cystic fibrosis (CF) patients has been previously described. Since 1998, Moran et al suggested that pre-diabetic status may have adverse effects on nutrition and lung function in CF patients. Successively several studies confirmed a decline in nutritional and pulmonary status 2 to 4 years before the diagnosis of CF-Diabetes. Conversely insulin treatment can ameliorate clinical findings of the CF-diabetic patients. To evaluate the effect of glargine on Body Mass Index z-score (BMI z-score), lung function, as forced expiratory volume in the first second (FEV1\%), number of acute pulmonary infections and Hemoglobin Glycosilated Alc (HbAlc) in (CF) patients with early pre-diabetic glucose derangements.

Materials and methods: 17 CF patients $10-20$ years of age and 5 patients $\leq$ 10 years with glucose abnormalities in Oral Glucose Tolerance Test (OGTT: glucose peak $>11.1 \mathrm{mmol} / \mathrm{L}$ and/or T120 glucose value $>7.7$ ) and/or in Continuous Glucose Monitoring System (CGMS: recorded data $>7.7 \mathrm{mmol} / \mathrm{L}$ at every time) were enrolled in a preliminary open trial with glargine. BMI zscore, FEV1\%, number of acute lung infections and HbAlc in the first year of treatment were recorded.

Results: The results of the patients who completed 12 months of treatment are reported. BMI $\mathrm{z}$-score and $\mathrm{HbAlc}$ did not significantly change in the whole group, while a significant improvement in BMI z-score was found in patients with a baseline BMI- $z$ score $<-1(\mathrm{p}=0.017)$. On the contrary a $8.8 \%$ increase in $\mathrm{FEV}_{1}(\mathrm{p}=0.01)$ and $42 \%$ decrease in the number of lung infections $(\mathrm{p}=0.003)$ was found in the whole group.

Conclusion: One year treatment with Glargine improved the course of lung disease in CF young patients with early glucose derangements.

\section{2}

Glucose RapidSpray ${ }^{\mathrm{TM}}$ for a new management of hypoglycaemia in children up to 5 years of age with type 1 diabetes

E. Pronina ${ }^{1}$, H. Petraikina ${ }^{1}$, M. Antsiferov ${ }^{1}$, O. Duchareva ${ }^{1}$, C. Guglielmi ${ }^{2}$, M. Khazrai ${ }^{2}$, P. Pozzilli ${ }^{2}$

${ }^{1}$ Endocrinology, Morozovskaya Children City Clinical Hospital of Moscow, Russian Federation, ${ }^{2}$ Endocrinology and Diabetes, Università Campus BioMedico, Roma, Italy

Background and aims: Hypoglycaemia continues to be a complex condition to tackle especially in children up to 5 years of age because of difficulties in administering the correct dose of glucose as well as child compliance. Moreover a very rapid absorption of glucose is needed in these kids which may be not easy to achieve due to the inability of the child to swallow under particular circumstances such as severe hypoglycaemia. Finally, the small body weight of children requires only small amount of glucose to reverse hypoglycaemia. This may help to avoid hyperglycaemic rebound which is due to the counter regulatory hormone response as well as the usually high amount of glucose that the parents administer to their children. The aim of this study was to evaluate the effect of administering small amounts of glucose through the Glucose RapidSpray ${ }^{\mathrm{TM}}$ (GRS) during first signs of hypoglycaemia in children affected by type 1 diabetes (T1D) to improve the metabolic control as measured by HbAlc over a period of 6 months follow up. This device consists of a $19 \mathrm{ml}$ bottle containing $10 \mathrm{~g}$ glucose solution with the addition of artificial flavours and excipient to facilitate buccal absorption delivering by spray puffing glucose in quantities as small as 0.5 grams.

Materials and methods: We designed an open randomized trial in 27 children up to 5 years of age affected by T1D. Patients were randomly allocated into two groups: group A) GRS (10 to 20 puffs of GRS, $0.5 \mathrm{~g}$ to $1 \mathrm{~g}$ glucose) depending on hypoglycaemic symptoms; group B) traditional treatment of hypoglycaemia episodes (sugar, fruit juice, candies, etc). The following parameters were evaluated at entry into the trial, at 3 months follow-up and at the end of the study (6 months): HbAlc, number and characteristics of hypoglycaemic episodes, compliance to treatment and quality of life of patients and their parents.

Results: HbAlc at entry into the trial was $9.4 \% \pm 1.2$ in group A and $9.1 \%$ \pm 0.6 in group B (p NS). After 3 months both groups showed a significant improvement in $\mathrm{HbA1c}$ but not different between groups (group A: $8.7 \% \pm$ 1 , group B: $8.6 \% \pm 0.7 \mathrm{p} \mathrm{NS}$ ) which further improved at 6 months (group A: $8.4 \% \pm 0.9$, group $\mathrm{B}: 8.4 \% \pm 0.6$ ). There was a statistically significant difference in the improvement of the $\mathrm{HbAlc}$ in the GRS treated group vs. control group over the period of observation $(0.98 \%$ vs. $0.60 \%$ difference, respectively, $\mathrm{p}<0.02$, paired $\mathrm{T}$ test). Patients in group A showed a tendency for less mild and/or severe hypoglycaemic episodes during the day compared to group $B$ in the course of the 6 months observational period $(2.5 \pm 0.8$ vs. $3.1 \pm 0.9$, $\mathrm{p}=0.09$ ). Regarding compliance patients appeared to be keen to be sprayed in the mouth, appreciating the strawberry or orange taste of the GRS. Parents found easy the use of this device and felt more comfortable in managing hypoglycaemic episodes in their children. No adverse effects were observed with GRS treatment.

Conclusion: Hypoglycaemia remains a major hazard in toddlers and children with T1D. A more acceptable and practical tool to control and manage hypoglycaemia is required. The availability of GRS, in view of the small amount of glucose it delivers and the fine tuning allowed in glucose administration, represents an ideal tool in very young patients which can also improve the overall metabolic control.

Supported by: Generex 


\section{PS 086 Nutrition and diet}

\section{3}

Insulin resistance, hepatic glucose metabolism and mitochondrial function in offspring rats exposed to malnutrition during fetal and early life

J. Choi, M. Kang, S. Hong, J. Song

Division of Metabolic Disease, Center of Biomedical Science, National Institutes of Health, Seoul, Republic of Korea

Background and aims: Malnutrition, either a protein depletion or high fat intake, during prenatal and postnatal periods may predispose to chronic diseases including obesity and diabetes in later life. We investigated how the maternal and postnatal diets containing different level of dietary protein and fat affected the growth, insulin resistance, hepatic glucose metabolism and mitochondrial function in the adult offspring.

Materials and methods: Dams were fed one of the four diets during gestation and lactation: a low fat and low protein diet (5\% fat, $8 \%$ protein, LF-LP), a low fat and normal protein (5\% fat, $20 \%$ protein, LF-NP); a high fat and low protein (23\% fat, $8 \%$ protein, HF-LP); a high fat and normal protein ( $23 \%$ fat, $20 \%$ protein, HF-NP). Eight offspring per dam were allocated. After weaning, offspring were fed on the same diet as dam's diet by age 12 weeks. In dams and offspring, food intake, body weight, organ growth, glucose tolerance test, the plasma glucose, insulin and lipids levels were observed with the measurement of hepatic lipids and glycogen content, glucose metabolizing enzyme activities and mitochondrial functions.

Results: Maternal diet modification affected offspring growth with no change in litter size. Offsprings with LP diets showed lower body weight and organ weights such as liver, kidney and fat tissue with the smaller protein intakes by their dams and the smaller intakes of protein and energy by offspring themselves. At age 12 weeks, HF-NP offspring showed the worst glucose tolerance than the others. The levels of plasma insulin were significantly higher in the NP groups than LP groups. With low insulin in LP groups, glucose level was not increased in LF-LP but high in HF-LP. In the HF groups, the levels of hepatic total lipid and triglyceride were higher in HF-NP group, whereas no difference could be observed in LF groups. With no significant difference in glucose 6-phosphatase activity could be observed among groups, hepatic glycogen content was highest in HF-LP and glucokinase activity was significantly higher in LF-NP and HF-LP. High fat diet decreased hepatic mitochondrial succinate dehydrogenase and cytochrome $\mathrm{C}$ oxidase activity. Low fat and adequate protein intake secured the proper activity of cytochrome $\mathrm{C}$ oxidase activity since we observed the highest activity in LF-NP group.

Conclusion: Postnatal growth, insulin sensitivity and glucose and energy metabolism varied depending on the diets throughout prenatal and postnatal growth periods. With normal dietary protein level, a high fat diet exacerbated insulin resistance through increasing plasma insulin, fat deposit and decreasing energy metabolism. In contrast, a high fat and low protein diet reduced partly adverse effects on insulin resistance through enhancing hepatic glucose metabolism in the present study.

Supported by: a Korea National Institute of Health intramural research grant (in part)

\section{4}

The metabolic syndrome of $\omega 3$-deficient rats

W.J. Malaisse ${ }^{1}$, Y. Zhang ${ }^{1}$, N. Bulur ${ }^{1}$, E. Hupkens ${ }^{1}$, L. Portois ${ }^{2}$, M.

Hacquebard $^{2}$, K. Louchami ${ }^{1}$, Y.A. Carpentier ${ }^{2}$, A. Sener ${ }^{1}$

${ }^{1}$ Laboratory of Experimental Hormonology, ${ }^{2}$ Laboratory of Experimental Surgery, Université Libre de Bruxelles, Belgium

Background and aims: Extensive investigations conducted in $2^{\text {nd }}$-generation rats depleted in long-chain polyunsaturated $\omega 3$ fatty acids ( $\omega 3 \mathrm{D}$ rats) have documented that they represent a novel animal model for the metabolic syndrome. They indeed display visceral obesity, liver steatosis, insulin resistance, hypertension and consequent cardiac hypertrophy. The major aim of the present study was to find out whether a comparable situation may be found in rats deprived of dietary $\omega 3$ for only three months, i.e. under condition more likely to simulate the insufficient intake of $\omega 3$, as presently often prevailing in Western populations.

Materials and methods: Two groups of 6-week old female rats each were given free access for 3 months to either a control or $\omega 3$-depleted diet.

Results: Although the liver protein content was identical in control and $\omega 3 \mathrm{D}$ rats, its triglyceride content was increased $(\mathrm{p}<0.05)$ in $\omega 3 \mathrm{D}$ rats $(6.42 \pm 0.50$ vs $4.16 \pm 0.25 \mu \mathrm{mol} / \mathrm{g}$ wet $\mathrm{wt}$ ). This coincided, in liver phospholipids, with a lower total fatty acid content, the absence of detectable amounts of $\mathrm{C} 18: 3 \omega 3$, $\mathrm{C} 20: 5 \omega 3$ and $\mathrm{C} 22: 5 \omega 3$, a 4 -times lower content of C22:6 63 , a much higher

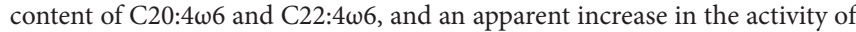

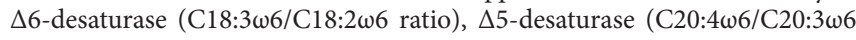
ratio) and elongase (C20:2 $\omega 6 / \mathrm{C} 18: 2 \omega 6$ and $\mathrm{C} 22: 4 \omega 6 / \mathrm{C} 20: 4 \omega 6)$. The weight percentages of C16:0, C18:0 and C24:0 were also significantly increased in the liver phospholipids of $\omega 3 \mathrm{D}$ rats, with increased C16:1 $\omega 7 / \mathrm{C} 16: 0$ ratio. Inversely, despite a higher weight percentage of C18:1 $\omega 9$ in the liver phospholipids of $\omega 3 \mathrm{D}$ rats, that of further precursors of nervonic acid (C20:1 $\omega 9$ and $\mathrm{C} 22: 1 \omega 9)$ only represented in the $\omega 3 \mathrm{D}$ rats $53.4 \pm 15.6 \%(\mathrm{p}<0.06)$ of the mean corresponding value recorded in control animals. An increase in the absolute or relative to body wt values for parametrial adipose tissue mass was initiated after 3 months of $\omega 3$ deprivation, but only became statistically significant in the $\omega 3 \mathrm{D}$ rats a few months later. Last, the $\omega 3 \mathrm{D}$ rats, displayed significantly increased plasma glucose concentration $(16.1 \pm 0.7 v s 13.4 \pm 0.7 \mathrm{mmol} / \mathrm{l})$ and insulin resistance index $(1.73 \pm 0.17 v s 1.18 \pm 0.12)$.

Conclusion: A dietary deficiency of $\omega 3$ in otherwise normal rats is sufficient to cause within 3 months insulin resistance, liver steatosis, and typical changes in the fatty acid pattern of hepatic phospholipids, with emphasis on the

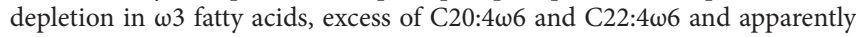
increased activity of $\Delta 5-, \Delta 6$ - and $\Delta 9$-desaturases. In conclusion, therefore, in the search of an animal model for the metabolic syndrome provoked by a dietary deficiency in $\omega 3$, the present study not only validates the prior use of $2^{\text {nd }}$-generation $\omega 3 \mathrm{D}$ rats, but also introduces a much more simple and realistic approach for the same purpose.

\section{5}

Effects of the dietary omega- 3 polyunsaturated fatty acids in the treatment of cardiomyopathy in type 2 diabetes mellitus patients A.A. Serhiyenko, V.A. Serhiyenko, A.M. Urbanovich Endocrinology, National, Lviv, Ukraine

Background and aims: The present study has examined the effect of simvastatin (SIM), $\omega-3$ polyunsaturated fatty acids ( $\omega$-3 PUFA) and their combinations on heart rate variability (HRV), QTc interval parameters; dynamics of biochemical parameters in type 2 diabetic patients with diabetic cardiomyopathy (DCMP).

Materials and methods: 86 patients with type 2 DM and DCMP $(51,2 \pm 4,3$ years), BMI $26,3 \pm 2,79 \mathrm{~kg} / \mathrm{m}^{2}$, DM duration $7,6 \pm 2,4$ years were observed. The following short-term HRV measures were assessed by a computer assisted technique: coefficient of variation $(\mathrm{CV})$, root mean square successive difference (RMSSD), spectral power in the low- (LF) and high-frequency (HF) bands. We investigated levels of total cholesterol (TC), low (LDL-C), high density lipoprotein-cholesterol (HDL-C), triglycerides (TG) concentrations, superoxide dismutase (SOD), gluthation peroxidase (GOP). Patients were allocated to three groups. Patients of group A $(n=25)$ received SIM $20 \mathrm{mg}$ tid; $\mathrm{B}(\mathrm{n}=37)$ - capsules of fish oil [ $(1,0 \mathrm{~g}$ eicosapentaenoic (EPA), 1,0 g docosahexaenoic (DHA)] acid and 0,1\% a-tocopherol acetate tid; C (n=24) - SIM 10 $\mathrm{mg}$ tid plus capsules of fish oil. All patients were on the same diet. Research joined patients with the diagnosed lipid abnormalities (NCEP ATP III). The duration of the study was 3 months. Statistics: ANOVA.

Results: Lipid disorders [high level of total TC, LDL-C, TG $(7,3 \pm 10,1$; $4,5 \pm 0,3 ; 2,87 \pm 0,2 \mathrm{mmol} / \mathrm{l}, \mathrm{p}<0,05)$ and decrease level of HDL-C] in the patients with DCMP is accompanied by a decrease of RMSSD, QTc interval parameters, SOD, GPO activities in the RBC's. It has been discovered that the monotherapy by SIM is accompanied by negative dynamics of activity of enzymes of a liver that proved by of ultrasonic scanning. After 3 months of treatment there was a more significant decrease in LDL-C, TC, TG concentration, positively influenced HRV, QTc interval parameters, some indexes of the oxidative stress were observed in the 3 -rd group (table). In particular, the increasing of RMSSD parameters $(4,72 \pm 0,53 \mathrm{~ms}$ was watched before the treatment; 6,81 $\pm 0,63 \mathrm{~ms}$-after treatment, Fisher's t-criterion $=59,56, \mathrm{p}<0,001$ ), LF, HF $\left(859,7 \pm 12,6 \mathrm{~ms}^{2}\right.$ and $\left.1134,6 \pm 17,4 \mathrm{~ms}^{2}, \mathrm{p}<0,001\right), \mathrm{CV}(8,7 \pm 0,3 \%$ and $6,2 \pm 0,2 \%, \mathrm{p}<0,001)$. The authentic decreasing of QTc interval parameters $(0,57 \pm 0,069$ and $0,52 \pm 0,057, \mathrm{p}<0,05)$, the increasing activity of SOD, GPO $(10,94 \pm 0,22 ; 321,7 \pm 19,2 \mathrm{mcmol} \mathrm{GSH} / \mathrm{min} 1 \mathrm{~g} \mathrm{Hb}, \mathrm{p}<0,001)$ were observed in the 3 -rd group (table).

Treatment difference are presented with $95 \%$ CI. ${ }^{*} \mathrm{p}<0,01$; ${ }^{* *} \mathrm{p}<0,001$ Conclusion: Usage of SIM and $\omega$-3-PUFA are accompanied by decreasing of clinical developments of DCMP, significantly improvement of the lipid profile, RMSSD, QTc interval parameters, some indexes of the oxidative stress. The combined purpose SIM and $\omega-3$ PUFA allows to lower a doze SIM, that 
allows to recommend their combination in the treatment of lipid disorders in patients with DCMP.

\begin{tabular}{llll}
\hline $\begin{array}{l}\text { Mean \% change from } \\
\text { baseline at 3 months }\end{array}$ & $\begin{array}{l}\text { SIM } \\
(\mathrm{n}=25)\end{array}$ & $\begin{array}{l}\omega-3 \text { PUFA } \\
(\mathrm{n}=37)\end{array}$ & $\begin{array}{l}\text { SIM / } \omega-3 \text { PUFA } \\
(\mathrm{n}=24)\end{array}$ \\
\hline RMSSD, ms & $+14,9$ & $+23,6^{*}$ & $+44,1^{* *}$ \\
\hline LF, ms2 & $+11,3$ & $+19,8^{*}$ & $+30,2^{* *}$ \\
\hline HF, ms2 & $+20,1^{*}$ & $+16,5$ & $+31,9^{* *}$ \\
\hline $\mathrm{CV}, \%$ & $-10,3$ & $-21,2^{*}$ & $-29,1^{* *}$ \\
\hline QTc, $s$ & $-9,5$ & $-11,4$ & $-19,3^{*}$ \\
\hline LDL-C & $-34,2^{*}$ & $-12,8$ & $-52,9^{* *}$ \\
\hline HDL-C & $+15,3$ & $+7,1$ & $+26,8^{*}$ \\
\hline TC & $-8,2$ & $-12,5$ & $-19,5$ \\
\hline TG & $-12,6$ & $-35,4^{*}$ & $-57,4^{* *}$ \\
\hline
\end{tabular}

\section{6}

Relationship of carbohydrate intake to the increase in fasting glucose levels with increasing age

A. Dzien ${ }^{1}$, C. Dzien-Bischinger ${ }^{1}$, M. Lechleitner ${ }^{2}$, E. Theurl ${ }^{3}$, H. Winner ${ }^{3}$ ${ }^{1}$ Internal Medicine, Medical Center Henschtelhof, Innsbruck, ${ }^{2}$ Internal Medicine, Hospital Hochzirl, Innsbruck, ${ }^{3}$ Economics and Statistics, University of Innsbruck, Innsbruck, Austria

Background and aims: Aging is associated with an increase in insulin resistance and an increased risk for type 2 diabetes. Various life-style recomendations, including dietary advice, are associated with a reduction in the diabetes risk. We were interested in the age- associated changes in reported food intake and the relationship with fasting glucose values.

Methods: 1492 non-diabetic patients (977 women, 515 men) attended our outdoor center for diagnostic and therapeutic procedures. Beside clinical and laboratory controls, all patients were asked to report about their food intake using a standardized questionnaire. Data are presented as mean $\pm \mathrm{SD}$. Twosample comparisons of normally distributed variables were analysed by the Student's $t$-test. The Wilcoxon-Mann-Whitney test was used for variables that did not have a normal distribution, and the Pearson correlation coefficient to evaluate the relationship between variables.

Results: Fasting glucose levels increased continuously with increasing age from $87.5 \pm 12.0 \mathrm{mg} / \mathrm{dL}$ in the age group up to 30 years to $101.8 \pm 16.2 \mathrm{mg} / \mathrm{dL}$ at an age of 90 years (Table 1), while the reported daily calory intake decreased from 2173 in the youngest to 1783 in the oldest subgroup. The carbohydrate consumption decreased from $197 \mathrm{~g}$ to $164 \mathrm{~g}$, the fat intake decreases, and the intake of dietary fibres remained stable (Table 1). The consumption of white bread products increased with increasing age. No significant differences could be observed between men and women. The carbohydrate intake revealed a stront realtionship with fasting glcuose levels $(\mathrm{R}=0.874$ in the age group up to 30 years and $\mathrm{R}=0.733$ in the oldest group up to 90 years.)

Conclusion: In our evaluation the intake of white bread products paralleled an increased in fasting glucose levels with advancing age. Dietary recommendation should thus focus on an intake of complex carbohydrates especailly in the elderly.

Table 1

\begin{tabular}{lllllllll}
\hline $\begin{array}{l}\text { Age } \\
\text { group } \\
\text { (years) }\end{array}$ & $\begin{array}{l}\text { Fasting } \\
\text { glucose } \\
(\mathrm{mg} / \mathrm{dL})\end{array}$ & $\begin{array}{l}\text { Total } \\
\text { calories }\end{array}$ & $\begin{array}{l}\text { Protein } \\
\text { intake } \\
(\mathrm{g})\end{array}$ & $\begin{array}{l}\text { Fat } \\
\text { intake } \\
(\mathrm{g})\end{array}$ & $\begin{array}{l}\text { Fibre } \\
\text { intake } \\
(\mathrm{g})\end{array}$ & $\begin{array}{l}\text { Carbo- } \\
\text { hydrate } \\
\text { intake }(\mathrm{g})\end{array}$ & $\begin{array}{l}\text { White } \\
\text { bread } \\
\text { portions } \\
\text { intake/ } \\
\text { month) }\end{array}$ \\
\hline$>30$ & 344 & $87.5 \pm 12.0$ & 2173 & 93 & 83 & 22.6 & 197.7 & 15 \\
\hline $30-50$ & 551 & $93.0 \pm 10.8$ & 2095 & 89 & 78 & 21.9 & 184.1 & 19 \\
\hline $50-70$ & 435 & $100.9 \pm 13.0$ & 1942 & 80 & 66 & 21.3 & 164.9 & 24 \\
\hline $70-90$ & 162 & $101.8 \pm 16.2$ & 1783 & 74 & 59 & 21.9 & 163.9 & 25 \\
\hline
\end{tabular}

\section{7}

Viscosity of fiber preloads affects food intake in adolescents A. Jenkins ${ }^{1}$, V. Vuksan ${ }^{2}$, S. Panahi ${ }^{2}$, P. Breitman ${ }^{2}$, E. Jovanovski ${ }^{2}$ ${ }^{1}$ Nutritional Sciences and Clinical Nutrition and Risk Factor Modification Centre, ${ }^{2}$ Department of Nutritional Sciences, Faculty of Medicine, University of Toronto, Canada

Background and aims: The prevalence of obesity is increasing most rapidly in adolescents and the inability to control food intake is one of the most challenging factors contributing to increases in body weight. Viscous dietary fiber that develops viscosity in the gastrointestinal tract is capable of addressing various aspects of food intake control, thus representing a promising treatment modality.

Our objective was to assess subsequent food intake and appetite in relation to the level of viscosity following three liquid preloads each containing $5 \mathrm{gr}$ of either a high (viscous polysaccharide blend; VPB), medium (glucomannan; GLM), or low (cellulose; CE) viscosity dietary fiber.

Materials and methods: In this acute, double-blind, randomized, controlled and crossover trial, 31 healthy weight adolescents (25:females; 6 :males; age: $16.1 \pm 0.6$ years; BMI: $22.2 \pm 3.7 \mathrm{~kg} / \mathrm{m}^{2}$ ) consumed one of the three preloads in random order 90 min prior to an ad libitum pizza meal. Preloads were identical in taste, appearance, nutrient content and quantity of fiber, but different in their viscosities $(10,410$ and 700 poise for CE, GLM, and VPB, respectively). Pizza intake (primary outcome), appetite ratings, 24-hour food intake after the pizza meal from the 24-hour diaries, and physical comfort (secondary outcomes) were assessed.

Results: Pizza intake was significantly lower $(\mathrm{p}<0.05)$ after consumption of the high viscosity VPB $(278 \pm 11 \mathrm{~g})$ compared to the medium viscosity GLM $(313 \pm 123 \mathrm{~g})$ and low viscosity CE $(316 \pm 138 \mathrm{~g})$ preloads, without difference between GLM and CE. Appetite scores, physical symptoms and 24-hour intake did not differ among treatment groups.

Conclusion: A highly viscous fiber preload leads to reduced subsequent food intake in healthy-weight adolescents and may represent a part of weight management strategy for adolescent population.

Supported by: Natural Factors

\section{8}

Differential effect of weight-loss diet strategies on spouses and effect of spouse support on the participant; The Dietary Intervention Randomized Clinical Trial (DIRECT)-Spouse Study

R. Golan ${ }^{1}$, D. Schwarzfuchs ${ }^{2}$, R. Zuk-Ramot ${ }^{2}$, O. Tangi ${ }^{1}$, I. Shai ${ }^{1}$

${ }^{1}$ Epidemiology, Ben Gurion University of the Negev, Beer Sheva, ${ }^{2}$ Epidemiology, Nuclear Research Center Negev, Dimona, Israel

Background and aims: The effect of diet strategy on spouses may be a key factor for long-term success of the participants' weight loss. We examined to what extent, attendance to a 2-years weight loss intervention study in one of 3 diet strategies affects weight, and nutritional patterns of the participant's spouse. We further evaluated the effect of spouse support on the participant. Materials and methods: Moderately obese participants with or without type 2 diabetes of the Dietary Intervention Randomized Controlled Trial (DIRECT) were randomly assigned to one of the three following 2-year intervention arms: low-fat, Mediterranean, and low-carbohydrate diets. Our study included 6 months of meetings with 74 spouses of the DIRECT participants and an additional 18 months of follow up among a sub-group of 59 spouses of this group.

Results: The spouses were all women, age 50.7 years, Mean BMI $26.66 \mathrm{~kg} /$ $\mathrm{m}^{2} \pm 4.72$. After 6 months, spouses of the DIRECT participants who followed the low-carb diet lost $4.62 \mathrm{~kg} \pm 6.56, \mathrm{P}=0.025$, spouses of DIRECT participants who followed the Mediterranean diet lost $2.30 \mathrm{~kg} \pm 2.39, \mathrm{P}=0.023$, spouses of DIRECT participants who followed the low-fat diet lost $1.48 \mathrm{~kg} \pm 2.72$, $\mathrm{P}=0.035$, as compared to baseline (Figure 1 ). The weight change correlation between DIRECT-participants and their spouses were significant in the low-carb diet $(\mathrm{r}=0.32, \mathrm{pv}=0.005)$. In overall, 74 DIRECT participants, whose spouses took part in group support meetings, lost significantly more weight $(-5.15 \mathrm{~kg} \pm 5.04))$ than 248 DIRECT participants whose spouses did not participate in group meetings $(-3.54 \mathrm{~kg} \pm 5.12)$ within 2 years $(\mathrm{Pv}=0.02)$. Spouses demonstrated changes in their nutritional patterns, with a decrease in the consumption of the following food groups; Saturated fat and Trans fat, meat and poultry, Bread and pastry, Grains, pasta and potato, Fruit, Sweets and Sweetened beverages. Spouses increased their intake of Fish and Vegetables. 
The changes fitted the principals of the three diet strategies the DIRECT participants were assigned to.

Conclusion: The low- carb diet may be considered as the strategy with the most successful halo-effect on the spouse in terms of weight loss and household dietary patterns. In each out of the three diet strategies, focusing on both man and wife as a 'unit' for designing weight loss, could provide a cost-effective approach to public health.

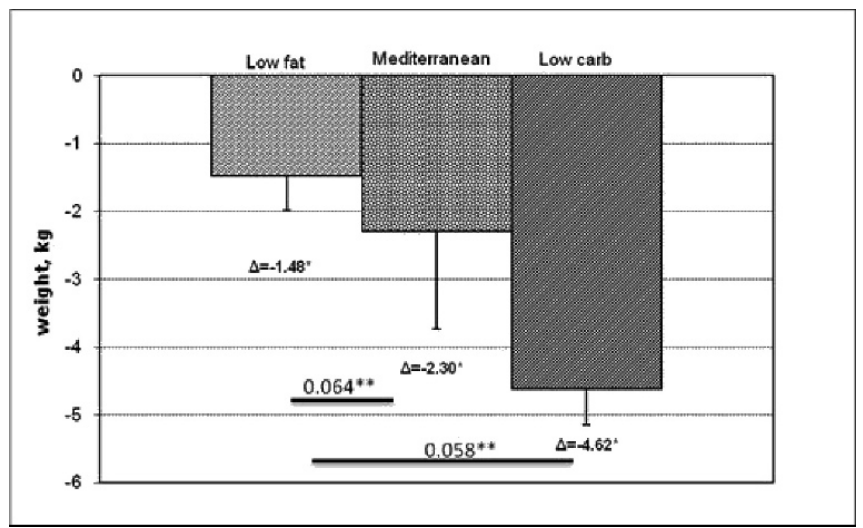

Figure 1. Changes in weight of 74 spouses after 6 months (maximal weightloss phase) of the DIRECT, across the randomized assigned dietary arm groups of the DIRECT-Participants; an indirect effect

\section{PS 087 Insulin therapy: acronym studies}

\section{9}

The DURABLE trial 24-week results: safety and efficacy of insulin lispro mix 25 vs. insulin glargine added to oral antihyperglycaemic agents (OHAs) in patients with type 2 diabetes (T2D)

B.H.R. Wolffenbuttel ${ }^{1}$, J. Buse ${ }^{2}$, W.H. Herman ${ }^{3}$, H. Jiang ${ }^{4}$, J. Fahrbach ${ }^{4}$, M. Palaisa ${ }^{5}$, S. Martin ${ }^{4}$

${ }^{1}$ Endocrinology \& Metabolism, University Medical Center, Groningen, Netherlands, ${ }^{2}$ Medicine and Clinical Epidemiology, University of North Carolina, Chapel Hill, United States, ${ }^{3}$ Internal Medicine and Epidemiology, University of Michigan, Ann Arbor, United States, ${ }^{4}$ Eli Lilly, Indianapolis, United States

Background and aims: In a clinical setting, when OHAs fail to achieve glycaemic control in patients (pts) with T2D, adding insulin is an appropriate next step. The DURABLE trial (assessing DURAbility of Basal vs Lispro Mix 25 [LM25] insulin Efficacy) compares ability of 2 common starter insulin regimens to achieve glycaemic control during a 24 -wk initiation phase and then maintain control during a subsequent 2 -yr maintenance phase. We report results of the 24 -wk initiation phase.

Materials and methods: A total of 2091 pts with T2D (63\% Caucasian, $6.3 \%$ African descent, $12.1 \%$ Hispanic, $15.4 \%$ Asian, and 3.3\% other race/ethnicities), aged $30-80$ yrs, $\mathrm{HbAlc}>7.0 \%$ on at least 2 OHAs (minimum dose: 1500 $\mathrm{mg}$ /day metformin, $1 / 2$ maximum dose sulfonylurea, and/or either $30 \mathrm{mg} /$ day pioglitazone or $4 \mathrm{mg}$ /day rosiglitazone) were randomized to either LM25 (25\% lispro, $75 \%$ insulin lispro protamine suspension) bid or glargine (GL) qd added to pre-study OHAs. Insulin was titrated with dosing algorithms to achieve $\mathrm{HbAlc} \leq 6.5 \%$ : fasting plasma glucose (FPG) and pre-evening meal target $<6.1 \mathrm{mmol} / 1$ for LM25, FPG target $\leq 5.5 \mathrm{mmol} / 1$ for GL.

Results: Baseline characteristics were similar between groups (mean): age 57 yrs, T2D duration 9.5 yrs, BMI $32 \mathrm{~kg} / \mathrm{m}^{2}$, and HbA1c (LM25 9.1\%, GL 9.0\%, $\mathrm{p}=0.414$ ). At 24 -wk endpoint, LM25 use resulted in lower HbA1c (mean \pm $\mathrm{SD})$ than GL $(7.2 \pm 1.1$ vs $7.3 \pm 1.1 \%, \mathrm{p}=0.005)$, greater $\mathrm{HbAlc}$ reduction from baseline ( $1.8 \pm 1.3$ vs $1.7 \pm 1.3 \%, \mathrm{p}=0.005)$, and a significantly higher $\%$ of pts reaching $\mathrm{HbAl}$ c target of $<7.0 \%$ ( $47.5 \mathrm{vs} 40.3 \%, \mathrm{p}<0.001$ ). FPG was lower with GL ( $6.8 \pm 1.9$ vs $7.4 \pm 1.9 \mathrm{mmol} / \mathrm{l}, \mathrm{p}<0.001)$. With LM25, 2 -hr postprandial glucose values were lower after morning and evening meals ( $9.3 \pm 2.8$ vs $9.5 \pm$ $2.7 \mathrm{mmol} / 1, \mathrm{p}=0.016$ and $9.1 \pm 2.6 \mathrm{vs} 9.8 \pm 2.7 \mathrm{mmol} / \mathrm{l}, \mathrm{p}<0.001$, respectively) but not significantly different between groups after midday meal. Midday premeal glucose was lower for pts on LM25 (7.2 \pm 2.4 vs $7.6 \pm 2.4 \mathrm{mmol} / \mathrm{l}, \mathrm{p}<0.001)$, while evening premeal glucose was not significantly different. LM25 was associated with a higher endpoint insulin dose $(0.47 \pm 0.23$ vs $0.40 \pm 0.23 \mathrm{U} / \mathrm{kg} / \mathrm{day}$, $\mathrm{p}<0.001)$ and more weight gain $(3.6 \pm 4.0$ vs $2.5 \pm 4.0 \mathrm{~kg}, \mathrm{p}<0.0001)$. At 24 wks, pts on LM25 experienced higher rates of hypoglycaemia: symptoms or self monitored plasma glucose $\leq 3.9 \mathrm{mmol} / \mathrm{l}$, (mean $28.0 \pm 41.6$ vs $23.1 \pm 40.7$ episodes/pt/yr, $\mathrm{p}=0.007$; median $8.7 \mathrm{vs} 7.0$ ), but lower rates of nocturnal hypoglycaemia (mean $8.9 \pm 19.3$ vs $11.4 \pm 25.3$ episodes/pt/yr, $\mathrm{p}=0.009$; median 0 vs 0 ). Rates of severe hypoglycaemia were low and similar between the two groups (LM25: $0.10 \pm 1.6$ vs GL: $0.03 \pm 0.3$ episodes/pt/yr; $\mathrm{p}=0.167$ ).

Conclusion: In a large multi-national T2D population, both LM25 and GL added to 2 or 3 OHAs demonstrated significant $\mathrm{HbAlc}$ reduction. Compared to GL, LM25 resulted in lower endpoint $\mathrm{HbA} 1 \mathrm{c}$ and a higher \% of pts reaching HbAlc target of $<7.0 \%$, but with slightly more weight gain and higher rates of hypoglycaemia. Nocturnal hypoglycaemia rates were lower with LM25. Durability of regimens (length of time glycaemic control is maintained) will be evaluated in the following 2-yr maintenance phase.

Supported by: Eli Lilly and Company

\section{0}

Real-world analysis of percent of patients with type 2 diabetes achieving glycaemic goal with insulin glargine

D. Misurski ${ }^{1}$, B. Schroeder ${ }^{2}$, R. Wade ${ }^{3}$, R. Quimbo 3 , L. Nielsen², R. Fabunmi' ${ }^{2}$ M. Wintle ${ }^{2}$

${ }^{1}$ Lilly Research Laboratories, Eli Lilly and Company, Indianapolis, ${ }^{2} \mathrm{Amylin}$ Pharmaceuticals, Inc., San Diego, ${ }^{3}$ Consulting and Strategies, HealthCore, Inc., Wilmington, United States

Background and aims: The primary aim of type 2 diabetes (T2D) therapy is helping patients achieve glycaemic goals $\left(\mathrm{HbA}_{1 \mathrm{C}}<7 \%\right)$ as recommended 
by the ADA. In the recent randomised controlled trials 4 -T and INITIATE, patients treated with basal insulin alone achieved goal $28 \%$ and $40 \%$ of the time, respectively, despite progressive insulin titration. In this retrospective cohort study using a large, US commercial health plan claims database, we describe the clinical effectiveness of newly prescribed basal insulin glargine (IG) in insulin-naive patients.

Materials and methods: A total of 13,154 insulin-naive (not prescribed insulin in previous $6 \mathrm{mo}$ ) patients were identified with a new prescription claim for IG between Jan 1, 2004 and June 30, 2006, $\geq 6$ mo of pre-index eligibility (first claim=index date), $\geq 12 \mathrm{mo}$ of post-index eligibility, and $\geq 18 \mathrm{y}$ old.

Results: From this cohort, 7730 (59\%) patients had no other insulin claims other than IG in the entire post-index period. All patients with baseline (100d pre-index) and post-index $(60-365 \mathrm{~d}) \mathrm{HbA}_{1 \mathrm{C}}$ data available and baseline $\mathrm{HbA}_{1 \mathrm{C}} \geq 7.0 \%$ were analysed $\left(\mathrm{n}=313\right.$; mean baseline $\left.\mathrm{HbA}_{1 \mathrm{C}}[ \pm \mathrm{SD}]=9.8 \pm 2.1 \%\right)$. Mean $( \pm S D)$ age was $52 \pm 8 \mathrm{y}$ ( $41 \%$ female; $3 \% \geq 65 \mathrm{y})$. In this cohort of patients who did not add any additional insulin $(\mathrm{n}=313), 27 \%$ achieved $\mathrm{HbA}_{1 \mathrm{C}}<7 \%$ in the post-index period (mean [min, max] time index to post-index $\left.\mathrm{HbA}_{1 \mathrm{C}}=238 \mathrm{~d}[63,365]\right)$. Mean $( \pm \mathrm{SD})$ post-index $\mathrm{HbA}_{1 \mathrm{C}}$ was $8.2 \pm 1.9 \%$.

Conclusion: In this real-world analysis of patients initiated on IG, the percentage of patients achieving $\mathrm{HbA}_{1 \mathrm{C}}<7.0 \%$ and mean post-index $\mathrm{HbA}_{1 \mathrm{C}}$ indicate that most patients do not achieve recommended glycaemic targets--results that mirror controlled clinical studies. In addition, more than half of patients initiating IG did not supplement with additional insulin therapy over the course of the first year of therapy, despite not reaching glycaemic goals. Because the contribution of postprandial glucose to $\mathrm{HbA}_{1 \mathrm{C}}$ increases as $\mathrm{HbA}_{1 \mathrm{C}}$ approaches goal, agents targeting fasting glucose alone, like IG, may be insufficient in helping patients with T2D achieve glycaemic goals.

\section{1}

Characteristics of patients needing basal insulin to be given twice-daily or a second insulin formulation during the first year of the 4-T Trial J.C. Levy ${ }^{1}$, A.J. Farmer ${ }^{2}$, K.I. Thorne ${ }^{3}$, S.K. Paul ${ }^{3}$, J.L. Darbyshire ${ }^{3}$,

M.J. Davies ${ }^{4}$, R.R. Holman

${ }^{1}$ Oxford Centre for Diabetes, Endocrinology and Metabolism, Churchill Hospital, Oxford, ${ }^{2}$ Department of Primary Health Care, Oxford University, ${ }^{3}$ Diabetes Trials Unit, Oxford University, ${ }^{4}$ Department of Cardiovascular Sciences, University of Leicester, Leicester, United Kingdom

Background and aims: 4-T (Treating to Target in Type 2 diabetes) is a three arm parallel group, open-label randomised trial comparing biphasic, prandial and basal analogue insulin regimens when added to the treatment of patients with type 2 diabetes who had sub-optimal glycaemic control on maximally tolerated doses of metformin and sulfonylureas. We have carried out an exploratory analysis to identify potential differences in the characteristics of trial patients failing to achieve or maintain glycaemic control within prespecified safety limits over one year on a single insulin formulation.

Materials and methods: Fifty-eight UK and Irish centres randomised 708 patients to twice-daily biphasic insulin $(\mathrm{n}=235$, NovoMix 30$)$, thrice-daily prandial insulin ( $\mathrm{n}=239$, NovoRapid), or once-daily basal insulin $(\mathrm{n}=234$, Levemir). Insulin doses were titrated using a predefined algorithm based on self-monitored capillary glucose measurements to achieve fasting and premeal targets of $4.0-5.5 \mathrm{mmol} / 1$. We compared the characteristics of patients requiring basal insulin twice-daily because of protocol-defined evening hyperglycaemia, with those achieving acceptable values on once-daily basal insulin. We also compared baseline characteristics of patients with $\mathrm{HbAlc}$ $>10 \%$ from 24 weeks (or $>8 \%$ on two subsequent occasions) up to one year who required a second insulin preparation per protocol, with those achieving lower values on a single insulin preparation.

Results: Of patients allocated to basal insulin, 79 (34\%) required it twice-daily. Compared with those requiring basal insulin once-daily, they were older (mean $( \pm \mathrm{SD}) 65.0 \pm 9.2$ vs. $60.4 \pm 10.0$ years, $\mathrm{p}=0.002)$, lighter $(80.7 \pm 15.7$ vs. $87.5 \pm 16.5 \mathrm{~kg}, \mathrm{p}=0.006)$ and had lower triglycerides (median (IQR) $1.2(1.0$ $1.7)$ vs. $1.6(1.2-2.2), p=0.002)$. Overall, $73(10.3 \%)$ patients required a second insulin preparation (biphasic insulin 21 (8.9\%), prandial insulin $10(4.2 \%)$ basal insulin $42(17.9 \%)$ ). Compared with those remaining on a single insulin preparation, they had higher mean $\mathrm{HbAlc}(9.0 \pm 0.7$ vs. $8.5 \pm 0.8 \%, \mathrm{p}<0.0001)$, BMI ( $31.2 \pm 4.5$ vs. $29.7 \pm 4.6 \mathrm{~kg} / \mathrm{m} 2, \mathrm{p}=0.0089)$ and higher median triglycerides (1.9 (1.4-2.6) vs. $1.5(1.1-2.1), \mathrm{p}=0.0012)$.

Conclusion: Patients who required Levemir insulin to be given twice a day were older, lighter and had lower triglycerides. In contrast, those who required two insulin preparations within the first year were more hyperglycaemic, heavier and had higher triglycerides. These differing characteristics may help guide therapy choices when adding insulin to metformin and sulfonylurea therapy in patients with sub-optimal glycaemic control.

Supported by: Novo Nordisk Ltd

\section{2}

Clinical and lifestyle characteristics in newly diagnosed type 2 diabetes: baseline data from the DESMOND randomised controlled trial H.M. Dallosso' ${ }^{1}$, M.E. Carey ${ }^{1}$, M.J. Davies ${ }^{2}$, S. Heller ${ }^{3}$, K. Khunti ${ }^{4}$, C. Skinner ${ }^{5}$

${ }^{1}$ Diabetes Research, University Hospitals Leicester, United Kingdom, ${ }^{2}$ Cardiovascular Sciences, University Leicester, United Kingdom, ${ }^{3}$ Academic Unit Diabetes, Endocrinology and Metabolism, University Sheffield, United Kingdom, ${ }^{4}$ Health Sciences, University Leicester, United Kingdom, ${ }^{5}$ Psychology, University Wollongong, Australia

Background and aims: Biomedical and psychosocial data on people newly diagnosed with type 2 diabetes (T2DM) are lacking. The aim of the study is to describe baseline characteristics of patients entering the randomised controlled trial of the DESMOND structured group education programme, in order to provide an up-to-date profile of newly diagnosed type 2 diabetes and identify areas of priority that need including in treatment and group education programmes.

Methods: Practices from 13 sites in the UK referred people newly diagnosed with T2DM between 2004 and 2005. Eligibility criteria were age $>18$ years, referral within 4 weeks of diagnosis and ability to participate in group education programme. Clinical data were collected at diagnosis by the practice and patients completed a questionnaire which included questions on lifestyle, diabetes related illness beliefs, and depression.

Results: 1109 patients were referred and 824 entered the study (74\%). Mean (SD) age was 59.5 (12.1) years, $54.7 \%$ were male and $96.6 \%$ were Caucasian. Mean (SD) HbAlc was 8.08 (2.11) \% and did not differ by sex. HbAlc was > $6.5 \%$ in $72.3 \%$. Mean (SD) total cholesterol was $5.35(1.31) \mathrm{mmol} / \mathrm{l}$ and significantly higher in men ( $\mathrm{p}<.001)$. Total cholesterol was $>5 \mathrm{mmol} / \mathrm{l}$ in $53.9 \%$. Mean (SD) blood pressure values were 140.6 (17.6) and 81.8 (10.5) $\mathrm{mmHg}$ with $45 \%>140 / 90 \mathrm{mmHg}$. Mean (SD) BMI was 32.36 (6.25) and was significantly higher in women $(\mathrm{p}<.001) .69 \%$ women and $54 \%$ men were obese (BMI $>30)$. There was a low level of physical activity ( $16 \%$ reporting vigorous activity $\geq 3$ times a week and $29.5 \%$ reporting moderate activity $\geq 5$ times a week). $27.8 \%$ reported smoking in previous week. Median (IQR) 10 year risk estimate of coronary heart disease or stroke (UKPDS Risk Engine) was $17.7 \%$ $(11.6 \%$ to $29.1 \%)$. Risks were higher in men ( $<<.001)$ and increased with age $(\mathrm{p}<.001)$. Scores for depressive symptoms were higher $(\mathrm{p}=.01)$ in women than men, with $16.1 \%$ women and $8.2 \%$ men reporting symptoms indicative of depression. These values reflect general population levels in the UK. Data on illness beliefs provided measures of whether patients understood their diabetes, perceptions of its duration, ability to affect its course, its impact and seriousness. For example $40.1 \%$ agreed "symptoms of their diabetes are puzzling" and 7.1\% agreed "life will be shorter because I have diabetes".

Conclusion: The high referral (equivalent to 0.96 per 1000 patient years) and consent rates and wide eligibility criteria mean the data provide an up-todate and representative picture of the newly diagnosed. HbAlc was lower than earlier studies on the newly diagnosed which may be the result of earlier diagnosis and more pro-active case finding. Mean BMI was higher than earlier studies and is indicative of the growing prevalence of obesity. Cardiovascular risk factors such as obesity, total cholesterol and physical activity are important lifestyle factors that need to be included in education programmes. Patients' misconceptions about their diabetes need addressing in education programmes in the newly diagnosed.

Supported by: Diabetes UK

\section{3}

Insulin detemir use amongst older patients: results from the 12-week, 26-week and 52-week cohorts of the PREDICTIVE ${ }^{\mathrm{TM}}$ study M. Pinget ${ }^{1}$, A. Dornhorst ${ }^{2}$, S. Gonbert ${ }^{3}$

${ }^{1}$ Service de Medicine E, Hopital Civil, Strasbourg, France, ${ }^{2}$ Department of Metabolic Medicine, Imperial College, London, United Kingdom, ${ }^{3}$ Novo Nordisk Pharmaceutique SAS, Paris, France

Background and aims: Many physicians are reluctant to initiate or intensify insulin treatment in older patients due to concerns over their ability to withstand side-effects. Increased frailty and/or impaired cognitive function 


\begin{tabular}{|c|c|c|c|c|c|c|}
\hline & Type 1 & & & Type 2 & & \\
\hline Parameter & $\begin{array}{l}12 \text { weeks' } \\
\text { treatment } \\
\mathrm{N}=588\end{array}$ & $\begin{array}{l}26 \text { weeks' } \\
\text { treatment } \\
\mathrm{N}=216\end{array}$ & $\begin{array}{l}52 \text { weeks' } \\
\text { treatment } \\
\mathrm{N}=60\end{array}$ & $\begin{array}{l}12 \text { weeks' } \\
\text { treatment } \\
\mathrm{N}=4102\end{array}$ & $\begin{array}{l}26 \text { weeks' } \\
\text { treatment } \\
\mathrm{N}=570\end{array}$ & $\begin{array}{l}52 \text { weeks' } \\
\text { treatment } \\
\mathrm{N}=278\end{array}$ \\
\hline $\begin{array}{l}\text { Serious adverse drug reactions } \dagger \\
{[\mathrm{n}(\%)]}\end{array}$ & $5(0.85)$ & $2(0.93)$ & $2(3.33)$ & $7(0.17)$ & $2(0.35)$ & $0(0)$ \\
\hline Total & $-36.4^{* * *}$ & $-27.3^{* * *}$ & $-86.1^{\star * *}$ & $-7.4^{* * *}$ & $-9.8^{\star * *}$ & $-11.7^{\star * *}$ \\
\hline Major & $-2.60^{* * *}$ & $-5.46^{\star * *}$ & $-14.56^{*}$ & $-0.65^{\star * *}$ & $-0.91^{\star * *}$ & $-0.78^{\star}$ \\
\hline Nocturnal & $-14.8^{* * *}$ & $-11.7^{\star * *}$ & $-22.2^{\star *}$ & $-3.3^{* * *}$ & $-3.3^{\star * *}$ & $-3.5^{\star *}$ \\
\hline HbAlc \% [mean change from baseline (SD)] & $-0.27(0.99)^{* * *}$ & $-0.53(1.28)^{\star * *}$ & $\begin{array}{l}-0.73 \\
(1.69)^{*} \\
\end{array}$ & $\begin{array}{l}-0.76 \\
(1.21)^{\star * *} \\
\end{array}$ & $\begin{array}{l}-0.68 \\
(1.55)^{\star * *} \\
\end{array}$ & $\begin{array}{l}-0.77 \\
(1.43)^{\star * *} \\
\end{array}$ \\
\hline FPG mmol/L [mean change from baseline (SD)] & $\begin{array}{l}-1.61 \\
(3.37)^{\star * *} \\
\end{array}$ & $-1.39(3.30)^{* * *}$ & $-2.21(3.80)^{* *}$ & $-2.36(2.90)^{\star * *}$ & $-1.83(2.99)^{\star * *}$ & $-1.66(3.05)^{\star * *}$ \\
\hline Weight kg [mean change from baseline (SD)] & $\begin{array}{l}-0.1 \\
(2.9) \mathrm{NS}\end{array}$ & $\begin{array}{l}-0.5 \\
(3.6) \mathrm{NS}\end{array}$ & $\begin{array}{l}-0.2 \\
(4.7) \mathrm{NS}\end{array}$ & $\begin{array}{l}-0.4 \\
(2.9)^{* * *}\end{array}$ & $\begin{array}{l}-0.3 \\
(5.7) \mathrm{NS}\end{array}$ & $\begin{array}{l}0.7 \\
(5.4)^{*}\end{array}$ \\
\hline
\end{tabular}

$\dagger=$ not including major hypoglycaemia; ${ }^{*} \mathrm{p}<0.05 ;{ }^{* *} \mathrm{p}<0.001 ;{ }^{* * *} \mathrm{p}<0.0001 ; \mathrm{NS}=$ non-significant.

may all contribute to a diminished awareness of hypoglycaemia, and so insulin regimens must be adapted to account for these issues. Clinical trials have shown insulin detemir to incur a lower risk of hypoglycaemia compared with $\mathrm{NPH}$ at equivalent levels of glycaemic control; this property might be particularly advantageous in elderly patients. PREDICTIVE ${ }^{\mathrm{TM}}$ is an open-label, cross-nation observational study designed to assess the safety and efficacy of insulin detemir (IDet) in everyday clinical practice, including in vulnerable patient groups.

Materials and methods: Older patients ( $>65$ years) from countries participating in PREDICTIVE ${ }^{\mathrm{TM}}$ were enrolled into the study for at least 12 weeks (11 countries), at least 26 weeks ( 6 countries) or for at least 52 weeks (France only) and were treated with IDet at their physician's discretion. Data were obtained for efficacy and safety endpoints and stratified by diabetes type 1 (T1DM, n=864) or type $2(\mathrm{~T} 2 \mathrm{DM}, \mathrm{n}=4950)$. The primary endpoint was the incidence of serious adverse drug reactions.

Results: A total of 588 patients with T1DM were recruited to the 12 -week cohort (37\% male, mean age 71.0 years, mean duration of diabetes 25.2 years; mean weight $73.9 \mathrm{~kg}$ ) and 4102 patients with T2DM ( $42 \%$ male, mean age 72.0 years, mean duration of diabetes 13.4 years; mean weight $83.5 \mathrm{~kg}$ ). In the 26-week cohort, $\mathrm{n}=216$ for patients with T1DM ( $41 \%$ male, mean age 71.3 years, mean duration of diabetes 25.2 years; mean weight $74.5 \mathrm{~kg}$ ) and $n=570$ for patients with T2DM ( $46 \%$ male, mean age 71.9 years, mean duration of diabetes 14.2 years; mean weight $83.0 \mathrm{~kg}$ ). Finally, the French (52-week) cohort comprised 60 patients with T1DM ( $50 \%$ male, mean age 72.3 years, mean duration of diabetes 21.9 years; mean weight $68.6 \mathrm{~kg}$ ) and 278 patients with T2DM ( $47 \%$ male, mean age 73.0 years, mean duration of diabetes; 17.7 years mean weight $78.2 \mathrm{~kg}$ ). The Table shows safety and efficacy data for all three cohorts. Serious adverse drug reactions were infrequent, with incidence under $1 \%$ in the 12 -week and 26-week cohorts, and less than $5 \%$ in the 52 -week cohort. Treatment with IDet reduced the frequency of total, major and nocturnal hypoglycaemia without compromising glycaemic control, which improved in all groups $(\mathrm{p}<0.05)$. IDet use incurred little or no weight gain, and patients in the 12-week and 26-week cohorts lost a modest amount of weight $(\mathrm{p}<0.05)$. Conclusions: IDet can be initiated in elderly patients, and represents a good choice of basal insulin, as it incurs a low risk of serious adverse drug reactions and a low risk of hypoglycaemia, while facilitating good glycaemic control. Supported by: Novo Nordisk

\section{4}

Comparison of in-patient insulin regimens: DEtemir plus Aspart $v$. $N \mathrm{PH}$ plus regular in medical patients with type 2 diabetes (DEAN Trial) G. Umpierrez ${ }^{1}$, T. Hor ${ }^{2}$, D. Smiley ${ }^{1}$, A. Temponi ${ }^{1}$, D. Umpierrez ${ }^{1}$, M. Ceron ${ }^{1}$, C. Muñoz ${ }^{2}$, C.A. Newton ${ }^{1}$, D. Baldwin ${ }^{2}$

${ }^{1}$ Division of Endocrinology, Metabolism and Lipids, Emory University School of Medicine, Atlanta, GA, United States, ${ }^{2}$ Section of Endocrinology, Rush University Medical Center, Chicago, United States

Background and aims: The association of inpatient hyperglycaemia and poor clinical outcome is well established. Few studies, however, have focused on the optimal management of hyperglycaemia in non-intensive care unit patients with type 2 diabetes mellitus (T2DM). We conducted a prospective, multi-centre, randomised trial to compare the efficacy and safety of a 'basal-bolus' regimen (insulin detemir/insulin aspart) vs. a standard split-mixed $(\mathrm{NPH} /$ regular insulin) regimen in patients with T2DM.
Materials and methods: A total of 130 non-surgical patients (M60/F70; age: $58 \pm 10$ yrs $\left( \pm\right.$ SEM), BMI: $\left.33 \pm 10 \mathrm{~kg} / \mathrm{m}^{2}\right)$ admitted to general medicine services with a blood glucose (BG) between 7.7-22.2 $\mathrm{mmol} / \mathrm{L}$ (mean admission BG: $13.2 \pm 4 \mathrm{mmol} / \mathrm{L}_{,} \mathrm{HbA}_{1 \mathrm{c}}: 8.3 \pm 2 \%$ ), and a history of $\mathrm{T} 2 \mathrm{DM}>3$ months were randomised to receive insulin detemir/insulin aspart $(\mathrm{n}=67)$ or NPH/regular insulin $(\mathrm{n}=63)$. Half the detemir total daily dose (TDD) was given once-daily and for aspart, $17 \%$ of TDD was given with each meal. NPH and regular insulin were given 3/5 of TDD before breakfast and 2/5 of TDD before dinner. The starting TDD was $0.4 \mathrm{U} / \mathrm{kg}$ for BG between 7.7-11.1 $\mathrm{mmol} / \mathrm{L}$ or $0.5 \mathrm{U} / \mathrm{kg}$ for BG between 11.2-22.2 mmol/L. Doses were adjusted daily, based on pre-meal and bedtime BGs. There were no differences in the pre-treatment $B G$, mean daily $B G$, or pre-meal BG levels between groups. Results: The mean daily BG level after the first day of detemir/aspart $v s$. $\mathrm{NPH} /$ regular insulin was $8.8 \pm 4 \mathrm{mmol} / \mathrm{L}$ and $8.6 \pm 4 \mathrm{mmol} / \mathrm{L}$, respectively, $p=\mathrm{NS}$. The BG target $<7.7 \mathrm{mmol} / \mathrm{L}$ before meals was achieved in $45 \%$ in the detemir/aspart group (breakfast 58\%) and in $48 \%$ of the NPH/regular group (breakfast 57\%), $p=$ NS. Insulin dose in the detemir/aspart group (detemir: 30 $\pm 29 \mathrm{U} / \mathrm{d}$, aspart: $27 \pm 20 \mathrm{U} / \mathrm{d}$ ) was not significantly different than that used in the NPH/regular insulin group (NPH: $27 \pm 20 \mathrm{U} / \mathrm{d}$, regular: $18 \pm 14 \mathrm{U} / \mathrm{d}$ ). Of the $1090 \mathrm{BG}$ readings in the detemir/aspart group, 19 readings (18 patients) were $<3.3 \mathrm{mmol} / \mathrm{L}(1.7 \%)$ and 2 readings were $<2.2 \mathrm{mmol} / \mathrm{L}(0.2 \%)$. Of the $1038 \mathrm{BG}$ readings in the NPH/regular insulin group, 13 readings (12 patients) were $<3.3 \mathrm{mmol} / \mathrm{L}(1.3 \%)$ and 2 were $<2.2 \mathrm{mmol} / \mathrm{L}(0.2 \%), p=\mathrm{NS}$. None of the episodes of hypoglycaemia were associated with adverse outcomes. There were no differences in length of hospital stay or mortality between groups. Conclusion: A basal/bolus insulin regimen with detemir once-daily and aspart before meals results in equivalent glycaemic control with minimal hypoglycaemia when compared with a split-mixed insulin regimen of NPH and regular twice-daily in hospitalised patients with T2DM.

Supported by: Novo Nordisk

\section{5}

Reducing fasting blood glucose variability is associated with a lower incidence of nocturnal hypoglycaemic events after starting insulin detemir: 3 month PREDICTIVE ${ }^{\text {mix }}$ data from 11 European countries L. Niskanen ${ }^{1}$, A. Virkamäki ${ }^{2}$, J.B. Hansen ${ }^{3}$, T. Saukkonen ${ }^{4}$

${ }^{1}$ Department of Medicine, Kuopio University Hospital, Finland, ${ }^{2}$ Mehiläinen Diabetes Clinic, Mehiläinen Oy, Helsinki, Finland, ${ }^{3}$ Novo Nordisk A/S, Bagsvaerd, Denmark, ${ }^{4}$ Novo Nordisk Farma, Espoo, Finland

Background and aims: It has been suggested that plasma glucose variability (FGV) is an independent risk factor for diabetes complications, and a marker of instability of diabetes. Its relation to the occurrence of hypoglycaemic episodes is, however, poorly studied.

Materials and methods: PREDICTIVE ${ }^{\mathrm{m}}$ is a global prospective multinational observational study of the empirical use of the basal analogue insulin detemir (IDet). This analysis includes pooled 3-month European follow-up data from patients with type 1 (DM1) or type 2 diabetes (DM2) who had any other long-acting basal insulin (NPH insulin, insulin glargine) as part of pre-study therapy and who switched to IDet.

Results: At baseline, the percentage of patients reporting at least one nocturnal hypoglycaemic episode (NOCT) during the preceding 4 weeks was $36.0 \%$ (2186 of 6135 ) for DM1 patients (DM1 NOCT+) and 13.4\% (832 of 6289) for DM2 patients (DM2 NOCT+). DM1 NOCT+ patients were on av- 
erage older $(43.6 \pm 15.9$ vs. $41.3 \pm 16.6 \mathrm{yrs}, \mathrm{p}<0.001)$, had a longer duration of diabetes $(18.7 \pm 12.6$ vs. $16.3 \pm 12.3$ yrs, $\mathrm{p}<0.001)$, lower $\mathrm{HbA}_{1 \mathrm{c}}(8.0 \pm 1.4$ vs. $8.2 \pm 1.6 \%, \mathrm{p}<0.001)$ and fasting plasma glucose (FPG; $9.1 \pm 3.0$ vs. $9.5 \pm 3.0$ $\mathrm{mmol} / \mathrm{L} ; \mathrm{p}<0.001)$ compared with DM1 patients without nocturnal hypoglycaemia (DM1 NOCT-). However, FGV assessed by the coefficient of variance of FPG was higher in DM1 NOCT+ than in DM1 NOCT- $(33.8 \%$ vs. $26.6 \%$, $\mathrm{p}<0.001)$. DM2 patients with nocturnal hypoglycaemia (DM2 NOCT + ) were of similar age, but had a longer duration of diabetes (13.7 \pm 8.3 vs. $12.6 \pm 7.6$ yrs, $\mathrm{p}<0.001)$, lower body weight $(85.4 \pm 17.3$ vs. $91.0 \pm 18.7 \mathrm{~kg}, \mathrm{p}<0.001)$, $\mathrm{HbA}_{1 \mathrm{c}}(7.9 \pm 1.5$ vs. $8.2 \pm 1.5 \%, \mathrm{p}<0.001)$ and $\mathrm{FPG}(9.0 \pm 2.5$ vs. $9.8 \pm 2.6$ $\mathrm{mmol} / \mathrm{L}, \mathrm{p}<0.001)$, yet $\mathrm{FGV}$ was higher $(21.8$ vs. $14.7 \%, \mathrm{p}<0.1001)$ than in DM2 patients without nocturnal hypoglycaemia (DM2 NOCT-). After 3 months' treatment of IDet, $\mathrm{HbA}_{1 \mathrm{c}}$ improved $(-0.4 \pm 1.1 \%$ in DM1, $\mathrm{p}<0.001$; $-0.5 \pm 1.1 \%, \mathrm{p}<0.001$ in $\mathrm{DM} 2)$, but the percentage of subjects with NOCT decreased (from 36.0 to $13.5 \%$ in DM1, $\mathrm{p}<0.001$, and from 13.4 to $2.8 \%$ in DM2, $\mathrm{p}<0.001)$. FGV was reduced also $(29.5$ to $24.5 \%$ in DM1, $\mathrm{p}<0.001,15.7$ to $12.9 \%$ in DM2, $\mathrm{p}<0.01)$. The change in FGV correlated with reduced number of NOCTs in both DM1 $(r=0.116, p<0.001)$ and DM2 $(r=0.145, p<0.001$; both adjusted for the change in $\mathrm{HbA}_{1 \mathrm{c}}$ ). At 3 months, the risk of NOCT increased with increasing FGV. Those with nocturnal hypoglycaemic events had a significantly higher FGV compared with those without (32.8 vs. $23.0 \%$ in DM1, $\mathrm{p}<0.001$, and 20.7 vs. $12.7 \%$ in $\mathrm{DM} 2, \mathrm{p}<0.001$ ).

Conclusion: Lowering FGV is associated with a lower incidence of nocturnal hypoglycaemia in both type 1 and type 2 diabetes after treatment with insulin detemir for 3 months, and this relationship is independent of the changes in metabolic control. Therefore, FGV can serve as a useful marker of nocturnal hypoglycaemia in a clinical setting. Reduced FGV may contribute to the simultaneous improvement of metabolic control and reduction of nocturnal hypoglycaemia frequency with insulin detemir therapy.

Supported by: Novo Nordisk A/S

\section{6}

A comparison of approaches to treatment intensification in patients with type 2 diabetes: insights from the PREDICTIVE ${ }^{\mathrm{TM}}$ Study

A. Dornhorst ${ }^{1}$, S. Sreenan ${ }^{2}$, M. Pinget ${ }^{3}$, S. Gonbert ${ }^{4}$

${ }^{1}$ Department of Metabolic Medicine, Imperial College, London, United

Kingdom, ${ }^{2}$ Department of Endocrinology, Connolly Hospital, Dublin,

Ireland, ${ }^{3}$ Service de Medicine E, Hopital Civil, Strasbourg, France, ${ }^{4}$ Novo

Nordisk Pharmaceutique SAS, Paris, France

Background and aims: Patients with type 2 diabetes who experience poor glycaemic control using an oral antidiabetic drug (OAD) combined with basal insulin (basal + oral therapy - BOT) require intensification of their treatment. Some physicians seek to achieve this by increasing basal insulin dose frequency, but, if prandial glucose levels are not addressed, this strategy may have limited effectiveness. The PREDICTIVE ${ }^{\mathrm{TM}}$ study is a prospective, multinational observational study of the use of insulin detemir (IDet) in a variety of treatment regimens. This analysis uses pooled 12-week data from PREDICTIVE ${ }^{\mathrm{TM}}$ to compare outcomes following different approaches to insulin intensification.

Materials and methods: Data were analysed from 685 participants with T2DM who had been in PREDICTIVE ${ }^{\text {TM }}$ for approximately 12 weeks. Patients on a BOT regimen at baseline were switched to once-daily (OD) IDet as the basal insulin. Of these, 456 patients (group 1) received OD IDet + OADs (50\% male, mean age 60.9 years; mean weight $86.9 \mathrm{~kg}$; mean duration of diabetes 10.3 years), and 166 had their treatment intensified at their physician's discretion: 40 patients (Group 2) received BD IDet + OADs (38\% male, mean age 62.0 years; mean weight $89.4 \mathrm{~kg}$; mean duration of diabetes 11.9 years) and 126 patients (Group 3) received the addition of a bolus insulin to OD IDet ( $45 \%$ male, mean age 61.5 years; mean weight $91.3 \mathrm{~kg}$; mean duration of diabetes 9.4 years).

Results: Switching to IDet incurred significant advantages in glycaemic control $\left(\mathrm{HbA}_{1 \mathrm{c}}\right.$, fasting blood glucose [FBG], FBG variability) in groups 1 and 3 , but significant benefits were not seen for patients switched to a BD + $\mathrm{OAD}$ regimen (table). Risk of hypoglycaemia was reduced in all three groups, significantly so in groups 1 and 2, with little or no weight gain for all three groups. Serious adverse drug reactions, the primary endpoint for the PREDICTIVE $^{\mathrm{TM}}$ study, were not observed for any patient (excluding major hypoglycaemia). Increase in basal insulin dose was greater in the $\mathrm{BD}$ group.

Conclusion: These data suggest that patients on a BOT regimen can benefit from a switch of their basal insulin to IDet. However, the data also suggest that if intensification of the regimen is required, then switching from an OD

to a $\mathrm{BD}$ basal insulin regimen will provide few benefits, despite an increase in the basal insulin dose. In contrast, the addition of prandial insulin to OD IDet can significantly improve $\mathrm{HbA}_{1 \mathrm{c}}$, $\mathrm{FBG}$ and $\mathrm{FBG}$ variability without increasing hypoglycaemia or body weight.

\begin{tabular}{|c|c|c|c|}
\hline Parameter & $\begin{array}{l}\text { Once-daily } \\
\text { IDet and OAD } \\
\text { regimen } \\
\mathrm{n}=456 \\
\end{array}$ & $\begin{array}{l}\text { Twice-daily } \\
\text { IDet and OAD } \\
\text { regimen } \\
\mathbf{n}=40\end{array}$ & $\begin{array}{l}\text { Once-daily IDet } \\
\text { as part of basal- } \\
\text { bolus regimen } \\
n=126\end{array}$ \\
\hline $\begin{array}{l}\text { Mean } \mathrm{HbA}_{1 \mathrm{c}} \%(\mathrm{SD}) \\
\text { Baseline } \\
12 \text { weeks } \\
\text { Mean change }\end{array}$ & $\begin{array}{l}8.0(1.3) \\
7.6(1.3) \\
-0.5(1.0)^{\star * *}\end{array}$ & $\begin{array}{l}8.3(1.2) \\
8.2(1.4) \\
-0.0(1.4)^{\mathrm{NS}}\end{array}$ & $\begin{array}{l}8.7(1.5) \\
7.4(1.0) \\
-1.3(1.5)^{* * *}\end{array}$ \\
\hline $\begin{array}{l}\text { Mean FBG, mmol/L (SD) } \\
\text { Baseline } \\
12 \text { weeks } \\
\text { Mean change }\end{array}$ & $\begin{array}{l}8.7(2.4) \\
7.4(1.8) \\
-1.3(2.3)^{\star * *}\end{array}$ & $\begin{array}{l}9.0(2.6) \\
9.1(3.5) \\
0.1(2.7)^{\mathrm{NS}}\end{array}$ & $\begin{array}{l}10.3(2.5) \\
7.3(1.4) \\
-3.0(2.7)^{\text {*** }}\end{array}$ \\
\hline $\begin{array}{l}\text { FBG variability, } \mathrm{mmol} / \mathrm{L} \\
(\mathrm{SD}) \\
\text { Baseline } \\
12 \text { weeks } \\
\text { Mean change }\end{array}$ & $\begin{array}{l}1.3(1.3) \\
0.9(0.8) \\
-0.4(1.2)^{* * *}\end{array}$ & $\begin{array}{l}1.7(1.3) \\
1.3(1.1) \\
-0.4(1.0)^{\mathrm{NS}}\end{array}$ & $\begin{array}{l}1.4(1.3) \\
1.0(0.9) \\
-0.4(1.4)^{*}\end{array}$ \\
\hline $\begin{array}{l}\text { Total hypoglycaemic } \\
\text { events/patient year } \\
\text { Baseline } \\
12 \text { weeks } \\
\text { Mean change }\end{array}$ & $\begin{array}{l}9.6 \\
2.2 \\
-7.3^{*}\end{array}$ & $\begin{array}{l}12.7 \\
1.0 \\
-11.7^{\star}\end{array}$ & $\begin{array}{l}5.9 \\
3.8 \\
-2.1^{\mathrm{NS}}\end{array}$ \\
\hline $\begin{array}{l}\text { Major hypoglycaemic } \\
\text { events/patient year } \\
\text { Baseline } \\
12 \text { weeks } \\
\text { Mean change }\end{array}$ & $\begin{array}{l}0.52 \\
0.00 \\
-0.52^{* *}\end{array}$ & $\begin{array}{l}1.04 \\
0.65 \\
-0.39^{*}\end{array}$ & $\begin{array}{l}0.78 \\
0.00 \\
-0.78^{\mathrm{NS}}\end{array}$ \\
\hline $\begin{array}{l}\text { Nocturnal hypoglycaemic } \\
\text { events/patient year } \\
\text { Baseline } \\
12 \text { weeks } \\
\text { Mean change } \\
\end{array}$ & $\begin{array}{l}4.3 \\
0.7 \\
-3.5^{\star * *}\end{array}$ & $\begin{array}{l}6.2 \\
0.0 \\
-6.2^{*}\end{array}$ & $\begin{array}{l}1.8 \\
0.3 \\
-1.6^{\mathrm{NS}}\end{array}$ \\
\hline $\begin{array}{l}\text { Weight, kg (SD) } \\
\text { Baseline } \\
12 \text { weeks } \\
\text { Mean change }\end{array}$ & $\begin{array}{l}86.3(16.4) \\
85.8(16.3) \\
-0.6(2.4)^{* * *}\end{array}$ & $\begin{array}{l}88.7(20.4) \\
89.1(21.8) \\
0.4(4.8)^{\mathrm{NS}}\end{array}$ & $\begin{array}{l}91.0(17.2) \\
90.8(17.6) \\
-0.3(3.2)^{\mathrm{NS}}\end{array}$ \\
\hline $\begin{array}{l}\text { Mean daily basal insulin } \\
\text { dose (pre-study basal and } \\
\text { detemir), U/kg (SD) } \\
\text { Pre-study basal } \\
\text { Baseline (new therapy) } \\
12 \text { weeks } \\
\text { Mean change } \\
\end{array}$ & $\begin{array}{l}0.26(0.14) \\
0.24(0.12) \\
0.30(0.17) \\
0.04(0.11)\end{array}$ & $\begin{array}{l}0.44(0.39) \\
0.35(0.22) \\
0.64(0.61) \\
0.21(0.43)\end{array}$ & $\begin{array}{l}0.36(0.22) \\
0.23(0.12) \\
0.31(0.16) \\
-0.05(0.20)\end{array}$ \\
\hline
\end{tabular}




\section{PS 088 Insulin therapy in type 1 diabetes}

\section{7}

Improved glycaemic control in subjects with type 1 diabetes using Accu-Check Advisor Insulin Guidance Software

S. Garg, C. Beatson, T. Bookout, K. McFann, S. Ellis

Barbara Davis Center, University of Colorado Health Sciences Center, Aurora, United States

Background and aims: Management of type 1 diabetes could be significantly improved with the availability of computerized insulin algorithms for home use.

Materials and methods: This was a one year open label randomized control trial involving 123 adult subjects with type 1 diabetes (A1c's 7.5-11\%) assigned to either the Insulin Guidance Software (Advisor) for personal data assistant (experimental group) or the control group.

Results: The primary end point was an improvement in Alc at 6 and 12 months by $\geq 0.4 \%$. Baseline demographis were similar in the two groups. Baseline mean Alc was $8.54 \pm 0.11$ in the control group and $8.42 \pm 0.11$ $(\mathrm{p}=.4265)$ in the experimental group. The mean Alc was significantly lower from 3 to 12 months in the experimental group $(p<0.02)$. Alc reduction of $\geq 0.6 \%$ was maintained at 12 months in the experimental group. Also, a significantly higher number of subjects achieved $\mathrm{A} 1 \mathrm{c}<7.5 \%$ in the experimental group from 3-9 months. Within target range (WTR) glycemia $(70-150 \mathrm{mg} /$ $\mathrm{dL}$ ) increased in the PDA group from 3 to 9 months without change in insulin dose or body weight. Above target range (ATR) glycemia was improved in the PDA group throughout the study as did the number of tests/subject/ day. Nocturnal hypoglycemia was not different between groups, however, the PDA group did have more severe hypoglycemic events.

Conclusion: This is the first report that shows improved glycemic control can be maintained over 12 months in patients with type 1 diabetes by using Advisor software with no change in insulin dose or weight.

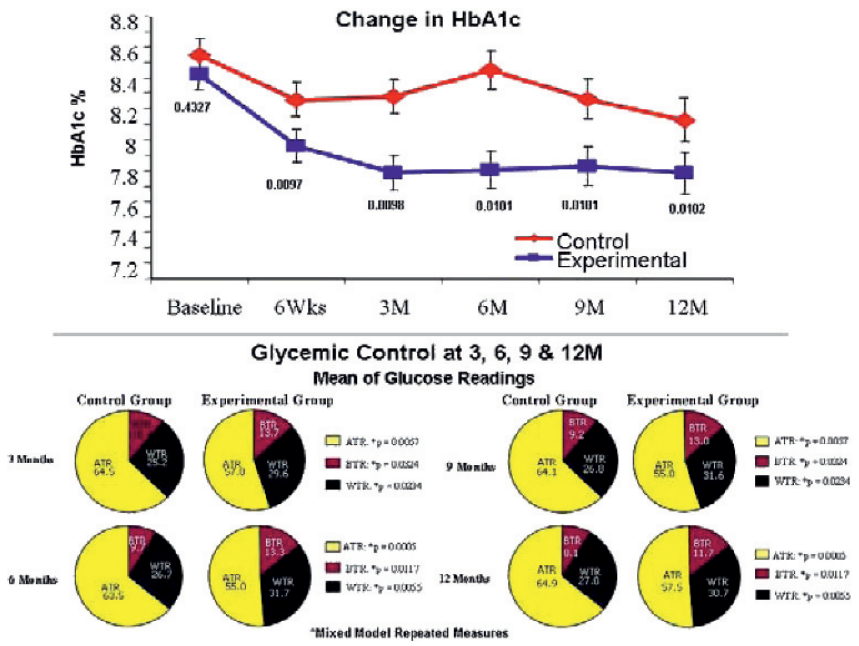

Supported by: Roche Diagnostics Corporation

\section{8}

Pharmacodynamics and pharmacokinetics of long-acting insulin analogues detemir and glargine after 7 days of use and after its first administration in subjects with type 1 diabetes

G. Bock, A. Wutte, G. Köhler, S. Korsatko, B. Semlitsch, T.R. Pieber Internal Medicine, Medical University Graz, Austria

Background and aims: To get further insight into the pharmacodynamic and pharmacokinetic properties of long-acting insulin analogues this study has been designed to compare the duration of action between the insulin analogues Detemir (Det) and Glargine (Glar) after a single dose (SD) and the steady state (SS) effect after 7 once daily injections in patients with Type 1 diabetes.
Materials and methods: 36 subjects $\left(37 \pm 10\right.$ years, BMI $24.7 \pm 2.6 \mathrm{~kg} / \mathrm{m}^{2}$, $\mathrm{HbAlc} 7.6 \pm 0.8$, mean $\pm \mathrm{SD}$ ) were randomised to receive either long acting insulin analogue Det or Glar and underwent 30 hours euglycemic clamp procedures twice, on one occasion after s.c. injection of the first dose of $0.4 \mathrm{U} / \mathrm{kg}$ Det or Glar and on the second occasion after multiple dosing of $0.4 \mathrm{U} / \mathrm{kg}$ Det or Glar in double-blind fashion.

Results: The primary endpoint "duration of action", defined as period from insulin injection to end of insulin action (plasma glucose $>8.3 \mathrm{mmol} / \mathrm{l}$ without glucose infusion) was 23.3 hours (10.5-29.0) for insulin Det and 27.1 hours (4.0-30.0) for insulin Glar after multiple dosing. 66\% of the subject with insulin Det had more than 24 hours of insulin action, whereas $89 \%$ in the glargine group had a longer duration than 24 hours. The duration of action after single dose administration was shorter for insulin Glar (19.8 hours; 0.0 - 30.0) than insulin Det (25.9 hours; 15.0 - 30.0). After single dose administration of insulin Glar 1/3 of subjects did not have an onset of action (defined as $50 \%$ reduction of the regular i.v. insulin from clamp onset to maintain euglycemia) within 10 hours after insulin injection. A larger variability in duration of action for insulin Glar was observed during both clamps in comparison to insulin Det.

Conclusion: The results of this study indicate that both basal insulin analogues have duration of action approximating 24 hours, and hence both are suitable for once daily dosing in patients with type 1 diabetes. In steady state insulin Glar demonstrates a longer duration, whereas the lower variability of insulin detemir would be expected to be beneficial for patients with insulin therapy due to less day-to-day fluctuations in blood glucose lowering effect.

\begin{tabular}{|c|c|c|c|}
\hline & $\begin{array}{l}\text { Detemir } \\
N \quad \text { mean( } S D) \text { range }\end{array}$ & $\mathrm{N}$ & $\begin{array}{l}\text { Glargine } \\
\text { mean(SD) range }\end{array}$ \\
\hline $\begin{array}{l}\text { Duration of } \\
\text { action SD (h) }\end{array}$ & $1825.9(4.6) 15.0-30.0$ & 18 & $19.8(14.4) 0.0-30.0$ \\
\hline $\begin{array}{l}\text { Duration of } \\
\text { action SS (h) }\end{array}$ & $1823.3(4.9) 10.5-29.0$ & 18 & $27.1(7.7) 4.0-30.0$ \\
\hline
\end{tabular}

\section{9}

Insulin detemir provides sustained glycaemic control over a 4-year period in type 1 diabetic subjects

B. Norberg, J. Jendle

Endocrine and Diabetes Center, University of Orebro, Karlstad, Sweden

Background and aims: The advantages of basal insulin analogs for the treatment of diabetes are being questioned. The present analysis sought to determine whether in a clinical setting a sustained improvement of diabetes control could be achieved when switching patients with type 1diabetes on a basal-bolus treatment regimen from NPH insulin or insulin glargine to basal insulin treatment with insulin detemir and continuing on the short acting insulin.

Materials and methods: Subject with type 1 diabetes $(n=102)$ inadequately controlled by a basal-bolus regimen with short acting insulin analogs (insulin aspart or lispro) in combination with a basal insulin where treated with insulin detemir in combination with the previous short acting insulin treatment. Insulin detemir was given sc OD or BID. 58 males and 44 females were included and followed during up to 48 months, 11 drop-outs (change therapy; 4 CSII, 2 glargine, 5 lost follow-up). Baseline data; $44 \pm 7$ years body weight of $77 \pm 15 \mathrm{~kg}$ with a BMI $25.3 \pm 3.9 \mathrm{~kg} / \mathrm{m}^{2}$. The diabetes duration was $20 \pm 8$ years. Results: Baseline HbAlc was reduced from $7.9 \pm 1.6 \%$ to $7.3 \pm 1.2 \%$, $\mathrm{p}<0.001$. The total daily insulin dose was increased from $51.8 \pm 18.4 \mathrm{U} / \mathrm{d}$ to $55.1 \pm 19.8$ $\mathrm{U} / \mathrm{d}, \mathrm{p}=0.019$. The basal insulin dose was not significantly changed from baseline $27.7 \pm 11.8 \mathrm{U} / \mathrm{d}$ to $29.1 \pm 11.9 \mathrm{U} / \mathrm{d}, \mathrm{p}=0.179$. The average bolus dose was increased from $23.9 \pm 9.8$ to $26.0 \pm 9.8 \mathrm{U} / \mathrm{d}, \mathrm{p}=0.028$. Neither weight gain, nor any significant change in BMI was observed during the study period. No serious hypoglycaemic episodes were registered.

Conclusion: Our study suggests a beneficial effect on the glycaemic control without any weight gain up to 4 year treatment when changing type 1 diabetics inadequately controlled on basal-bolus treatment to insulin detemir in combination with previous short acting insulin analog. 


\section{0}

Deleterious outcomes on abrupt transition from insulin glargine to detemir in type $1 \mathrm{DM}$

U.M. Kabadi ${ }^{1,2}$

${ }^{1}$ Endocrinology, VAMC, Des Moines, ${ }^{2}$ Internal Medicine, University of Iowa college of Medicine, Iowa City, United States

Background: Iowa Care (Iowa Medicaid), USA switched insulin glargine to detemir in subjects with Diabetes Mellitus (DM) without the knowledge or approval of healthcare providers begining 8/2006.

Objective: To examine the impact of this transition on various parameters of diabetes management in type $1 \mathrm{DM}$.

Study Design

A retrospective review of the records of subjects with type $1 \mathrm{DM}$ was conducted until 8/2007 in whom the transition had occurred. Only those subjects completing at least 6 months of therapy with insulin detemir are included in this report. Twenty four subjects with type $1 \mathrm{DM}$, duration $19 \pm$ 3 years with age $36 \pm 4$ years who were switched from GI to DI (Group 1) fulfilled 6 months' duration of therapy with DI. Glycemic control (HbAlc), body weight (BW), daily insulin dose (Units), total (T) as well as glargine (GI) or detemir (DI) and rapid acting insulin, Aspart (AI) as well as Hypoglycemic events during the last 4 weeks of the period, pre switch and again at the end of 6 months post switch were assessed. Records of 21 subjects matched for age (39 \pm 5 years) and continuing GI for 6 months (Group 2) during the same period were examined for comparison.

Methods: Initially, subjects were switched from GI to DI in the same daily dose in AM. The daily doses of DI and AI in Group 1 were adjusted by telephone at weekly intervals based on self blood glucose monitoring until stabilization was achieved. They were also followed in the outpatient clinic at intervals of 3 months similar to subjects continuing GI.

Results: All subjects in Group 1 were changed to DI twice a day because of significant rise in hypoglycemia when the whole daily dose was used once a day. Glycemic control remained stable on continuing GI once daily; $\mathrm{HbAlc}$ $7.6 \pm 0.3$ to $7.8 \pm 0.3 \%$, while it worsened on switching to DI being injected twice a day; $\mathrm{HbA1c}, 7.9 \pm 0.6$ to $8.8 \pm 0.8$ despite a higher daily dose; DI, $46 \pm 9$ units Vs Pre Switch GI, $36 \pm 8$ units and Group 2 GI, $35 \pm 6$ units; and greater total insulin dose; $80 \pm 12$ units Vs $68 \pm 10$ Pre Switch and Group 2 GI, $62 \pm 10$ units [P $<0.05$ for all comparisons]. Body weights and hypoglycemic events were not significantly different Pre and Post switch [p $>0.05$ for all groups]. Conclusion: Therefore, insulin glargine and detemir appear to be far from being bioequivalent. Moreover, switching to detemir insulin from glargine insulin is likely to result in lapse of glycemic control causing decreased quality of life as well as higher costs and undue hardship to both patients and providers because of the resulting higher daily insulin dose as well as an increased number of injections, and need for frequent recurrent evaluations during the initial period of switch.

\section{1}

Once-daily dosing of insulin detemir is associated with lower risk of hypoglycaemia at similar levels of $\mathrm{HbA}_{1 \mathrm{c}}$ compared to once-daily NPH insulin in subjects with type 1 diabetes mellitus

J.E. Shaw ${ }^{1}$, J. Larsen ${ }^{2}$, C.B. Pedersen ${ }^{2}$, S. Tang-Fui ${ }^{3}$

${ }^{1}$ Department of Medicine, International Diabetes Institute, Melbourne, Australia, ${ }^{2}$ Novo Nordisk, Bagsvaerd, Denmark, ${ }^{3}$ Endocrinology, Diabetes \& Specialist Centre, Melbourne, Australia

Background and aims: This is an explorative analysis comparing the efficacy and safety of once daily dosing with insulin detemir (IDet) and NPH insulin (NPH), in combination with mealtime insulin aspart (IAsp). The analysis is based on a 24-month, multi-national, open-label, parallel-group trial in subjects with type 1 diabetes mellitus (T1DM) using a treat-to-target concept.

Materials and methods: IDet or NPH was administered in the evening and titrated to a pre-breakfast plasma glucose (PG) target $\leq 6 \mathrm{mmol} / \mathrm{L}(108 \mathrm{mg} /$ $\mathrm{dL})$. If the pre-breakfast target was obtained, but the pre-dinner PG remained $>6 \mathrm{mmol} / \mathrm{L}$ a second dose could be added in the morning. A total of 497 subjects were randomised 2:1 to IDet $(n=331)$ or NPH $(n=166)$. Of these, 133 (40.2\%) subjects on IDet and 77 (46.4\%) on NPH, remained on a once-daily basal insulin regimen throughout the trial and the results reported here are based on this subset of subjects.

Results: The baseline characteristics of subjects remaining on a basal once daily regimen were generally comparable between IDet and NPH (men: 61\%, mean age: 33 years, duration of diabetes: 12 years, body mass index (BMI):
$24.8 \mathrm{~kg} / \mathrm{m}^{2}, \mathrm{HbA}_{1 \mathrm{c}}: 8.0 \%$ ), although weight, $\mathrm{BMI}$ and diabetes' duration were slightly higher in the NPH arm. After 24 months, $\mathrm{HbA}_{1 \mathrm{c}}$ was comparable between treatments (IDet: 7.25\%; NPH: 7.36\%, difference [IDet-NPH]: $-0.11 \%$ points, [95\% CI: $-0.43 ; 0.20]$ ) with reductions of $0.73 \%$ and $0.62 \%$ points, respectively. The risk of major hypoglycaemia (requiring assistance) was $82 \%$ lower with IDet than with NPH, while the risk of all nocturnal episodes as well as minor nocturnal episodes (PG $<3.1 \mathrm{mmol} / \mathrm{L}$ ) was $53 \%$ lower with IDet (all: $p<0.005$ ). The average number of all hypoglycaemic episodes per subject per year was 13.4 with IDet and 22.5 with NPH. A similar proportion (45\%) of subjects in both groups achieved an $\mathrm{HbA}_{1 c} \leq 7 \%$, but $38.2 \%$ of all patients using once-daily IDet reached this level of control in the absence of confirmed hypoglycaemia during the last month of treatment compared to $21.6 \%$ using NPH $(p=0.018)$. At 24 months, self-measured 9-point PG profiles were generally lower with IDet from bedtime to lunch next day, but higher between lunch and bedtime compared to NPH. The median daily dose of IDet and NPH was 30 and 28 units, respectively, with a median daily dose of IAsp of 36 units in both groups. Fasting PG tended to be lower with IDet than with NPH (7.80 vs. $8.86 \mathrm{mmol} / \mathrm{L}$; NS), resembling that observed with IDet relative to NPH in the analysis of the full intention-to-treat population of 495 subjects.

Conclusion: This study showed that once-daily administration of IDet can provide relatively good glycaemic control and a significantly lower risk of hypoglycaemia in a large proportion of T1DM subjects as compared to oncedaily dosing with NPH.

Supported by: Novo Nordisk

\section{2}

Early insulin treatment in latent autoimmune diabetes in adults (LADA) did not improve residual beta cell function or metabolic control M. Thunander ${ }^{1,2}$, H. Thorgeirsson ${ }^{2}$, C. Törn ${ }^{3}$, C. Petersson ${ }^{4}$,

M. Landin-Olsson ${ }^{2}$

${ }^{1}$ Endocrinology and Diabetes, Vaxjo Central Hospital, Vaxjo,

${ }^{2}$ Endocrinology and Diabetes, Lund University Hospital, Lund, ${ }^{3}$ Clinical Sciences, Diabetes Laboratory, Lund University, ${ }^{4}$ Primary Care, and R\&Dcenter, Kronoberg, Vaxjo, Sweden

Background and aims: Most adults with autoimmune diabetes not insulinrequiring at onset become so within 3-5 yrs. Latent autoimmune diabetes in adults (LADA) has been identified suitable for evaluating new therapies in autoimmune diabetes. We investigated the effect of early insulin treatment on residual beta-cell function, and metabolic control, during the first three years, compared to a group treated with diet only or oral hypoglycaemic agents (OHA).

Methods: During three yrs 38 of newly diagnosed patients in our regions, not insulin-requiring at diagnosis and positive for at least one of ICA or GADab, $(92 \%$ were positive for both) were included. Mean age was $53.0( \pm 16)$ (range 19-80)yrs, M/F ratio 19/17. No strict randomization was possible since many patients refused insulin treatment before it was clinically necessary. 20 patients started early insulin therapy, the remaining 18 served as controls, treated with diet only or OHA, and received insulin subsequently when/if necessary. Residual beta cell capacity was evaluated with glucagon-stimulated C-peptide at baseline, and after 12, 24 and 36 months. Metabolic control was assessed by HbAlc.

Results: At baseline age was 49 vs 58 yrs, $\mathrm{p}<0.04$, mean glucagon-stimulated C-peptide 1.2 vs $1.7 \mathrm{nmol} / \mathrm{l}$ (ns) and mean $\mathrm{HbAlc}$ was $6.5( \pm 1.5)$ vs 5.9 $( \pm 1.2) \%$ (ns) in the early insulin treated compared to the controls. During follow-up mean C-peptide was 0.75 $( \pm 0.51), 0.67( \pm 0.61), 0.61( \pm 0.59)$ among insulin treated and 2.04( \pm 1.05$), 1.46 \pm(0.71), 1.40( \pm 0.93)$ among controls. The annual absolute $-0.29( \pm .37),-0.51( \pm 0.33),-0.64( \pm 0.44)$ vs $0.47( \pm 1.20)$, $-0.17( \pm 0.63),-0.17( \pm 0.92)$ and the relative $0.39( \pm 0.29), 0.45( \pm 0.38)$, $0.39( \pm 0.29)$ vs $0.88 \pm(0.49), 0.95( \pm 0.34), 0.88( \pm 0.49)$ decline in C-peptide were not significantly different between the groups. In the control group insulin treatment was initiated due to clinical need in 7/18 (39\%) patients during the 3 years. By logistic regression the only factor determining a residual C-peptide level > 0,5 nmol/l after 3 years was initial level of C-peptide, but not age, HbAlc or early insulin treatment.

Conclusion: In this study when comparing both the absolute and the relative decline of C-peptide early insulin treatment did not result in superior preservation of beta cell function or better metabolic control. The study does, however, suffer from incomplete randomization leading to differences in baseline C-peptide levels. 


\section{PS 089 Insulin therapy: body weight and cardiovascular disease}

\section{3}

Body weight change associated with insulin therapy in type 2 diabetes patients - a five-year observational study

L. Czupryniak, M. Nieznaj, M. Saryusz-Wolska, M. Pawlowski, J. Loba Diabetology and Metabolic Diseases Dept, Medical University of Lodz, Poland

Background and aims: In type 2 diabetes patients body weight increase is a major unwanted effect of insulin treatment (IT), and many physicians are reluctant to initiate insulin therapy in poorly controlled but obese patients. Physicians' or patients' insulin therapy-associated fear of weight gain may cause a significant delay in IT introduction, even if it might be an only effective method of improving blood glucose control. We conducted a five-year observational study aiming at assessing longitudinal body weight change and its determinants after IT initiation in type 2 diabetes patients.

Materials and methods: The study group comprised 269 type 2 diabetes patients at IT initiation, with $\mathrm{HbAlc}>8.0 \%$ despite maximum oral treatment ( 155 women, 114 men, mean $[ \pm S D]$ age $60.3 \pm 11.6$ years, mean diabetes duration $4.9 \pm 3.2$ years); they were followed up for five years, with yearly assessments. Upon the results at one year of IT the study subjects were subdivided according to their body weight change: patients with body weight increase after 1 year of IT were included into Weight Gain (WG) group, and those in whom body weight decreased within this period - into Weight Reduction (WR) group.

Results: After 1 year of IT weight gain was noted in 177 (66\%) subjects (WG group), while in 92 (34\%) patients body weight decreased (WR group). There was no difference in age at diabetes diagnosis, time to IT initiation or HbAlc after 5-year follow-up between the groups, however at baseline WG subjects were significantly less obese than WR ones: mean body weight in WG Group was $79.0 \pm 13.4 \mathrm{~kg}$ (BMI $29.5 \pm 6.4 \mathrm{~kg} / \mathrm{m} 2$ ), and after 1,2 , and 5 years of IT it increased to $83.3 \pm 13.4 \mathrm{~kg}(31.2 \pm 6.7 \mathrm{~kg} / \mathrm{m} 2), 85.0 \pm 13.7 \mathrm{~kg}$ $(31.8 \pm 7.3 \mathrm{~kg} / \mathrm{m} 2)$, and $86.1 \pm 14.1 \mathrm{~kg}(33.0 \pm 10.0 \mathrm{~kg} / \mathrm{m} 2)$, respectively; in WR Group baseline body weight was $82.9 \pm 15.7 \mathrm{~kg}(30.4 \pm 5.3 \mathrm{~kg} / \mathrm{m} 2)(\mathrm{p}<0.05 \mathrm{vs}$ WG Group), and after 1,2 , and 5 years of IT it was $80.7 \pm 15.8 \mathrm{~kg}(29.6 \pm 5.2$ $\mathrm{kg} / \mathrm{m} 2), 81.6 \pm 15.2 \mathrm{~kg}(29.9 \pm 5.0 \mathrm{~kg} / \mathrm{m} 2)$, and $83.1 \pm 13.4 \mathrm{~kg}(30.8 \pm 5.1 \mathrm{~kg} / \mathrm{m} 2)$ ( $\mathrm{p}<0.05$ vs WG group), respectively. Absolute body weight change since the baseline at 1,2, and 5 years of IT in WG was $4.4 \pm 3.2,5.8 \pm 4.7,5.8 \pm 7.4 \mathrm{~kg}$, and in WR $-2.2 \pm 3.2 \mathrm{~kg},-2.1 \pm 4.8$, and $0.03 \pm 7.2 \mathrm{~kg}$ (all $\mathrm{p}<0.01$ between the groups), respectively. The patients who gained over $10 \mathrm{~kg}$ after 1 year of IT were significantly less overweight at baseline $(75.3 \pm 13.9$ vs $80.6 \pm 14.3 \mathrm{~kg}$; BMI $28.1 \pm 4.9$ vs $29.9 \pm 6.1 \mathrm{~kg} / \mathrm{m} 2$; respectively, $\mathrm{p}<0.05)$, younger at diabetes diagnosis ( $50.8 \pm 13.9$ vs $55.6 \pm 11.5$ years, $\mathrm{p}<0.05)$, and their time to IT was longer $(5.9 \pm 6.9$ vs $4.9 \pm 5.0$ years, $\mathrm{p}<0.05)$ than those who gained less than $10 \mathrm{~kg}$. Weight gain after 1 year of IT showed significant negative correlation with the baseline body weight $(r=-0.16 ; \mathrm{p}<0.01)$. Gender, concomitant antidiabetic oral therapy or the number of insulin injections per day were not related to the body weight change.

Conclusion: Insulin therapy initiation in type 2 diabetes is not associated with weight gain in all the subjects; one out of three patients actually loses weight within the first year of insulin treatment. The risk of weight gain is greater in younger, less overweight subjects than in older obese patients, who may actually experience weight loss following IT initiation. It might be also suggested that those patients in whom IT was delayed are at particularly high risk of weight gain. Overall, obesity, older age or the fact of a patient's having been recently diagnosed with diabetes should not preclude physicians from prescribing insulin for the treatment of type 2 diabetes.

\section{4}

Cardiovascular risk profile detoriates in patients with type 2 diabetes with pronounced weight gain on long-term insulin therapy

H.J. Jansen, C.J. Tack, M. van der Graaf, G.M. Vervoort

General Internal Medicine, Radboud University Nijmegen Medical Centre, Netherlands

Background and aims: Insulin therapy is frequently needed to achieve adequate glycaemic control in type 2 diabetes mellitus (T2DM), but often at the expense of weight gain.
The aim of the study was to compare two pre-defined subgroups (weight gainers and non-gainers). We hypothesized that gainers would have developed more trunk fat, and that a large weight gain would have resulted in an increased cardiovascular risk profile. To determine the underlying cause of excessive weight gain, we assessed physical activity and caloric intake.

Patients and methods: We studied 20 patients with T2DM who had been started on insulin therapy between 2001-2006. Two subgroups were defined: weight gainers ( $\geq 1 \mathrm{~kg}$ per 2 months within the first 18 months, and a weight gain of $>10 \%$ after total follow-up; $n=10$ ) and non-gainers (maximal weight gain $\leq 2 \mathrm{~kg}$ within the first 18 months, and thereafter stable body weight). Body mass index (BMI) was measured. DEXA was performed to assess trunk fat and percentage body fat. Magnetic resonance spectroscopy (MRS) was performed to determine liver fat content. Table 1 shows laboratory measurements. Energy expenditure (EE) was measured by wearing a Sensewear ${ }^{\circ}$ Body Monitoring System for five consecutive days. Standardised questionnaire survey for assessing patients dietary habits were taken for three consecutive days.

Results: After a mean of 4 years of insulin therapy, mean body weight increased from $79.1 \pm 14.6 \mathrm{~kg}$ to $92.7 \pm 13.5(\mathrm{SD})$ with a BMI of $33.0 \mathrm{~kg} / \mathrm{m}^{2}$ and from $88.0 \pm 15.2$ to $86.0 \pm 14.0 \mathrm{~kg}$ with a BMI $28.5 \mathrm{~kg} / \mathrm{m}^{2}$ for gainers $(0.8 \mathrm{U} / \mathrm{kg}$ insulin) and non-gainers ( $0.7 \mathrm{U} / \mathrm{kg}$ insulin), respectively. Blood pressure was similar between the two groups. Table 1 shows the laboratory results between gainers and non-gainers. Asterix denotes a significant difference. MRS: Percentage of liver fat content between the two groups $9.68 \pm 0.43 \%$ vs. 9.80 $\pm 0.98 \%$ (P=NS). DEXA: The percentage of total body fat was significantly higher in the gainers than in non-gainers $(35.9 \pm 9.0$ versus $27.0 \pm 8.5 \%$, $\mathrm{P}=0.04)$. Trunk fat mass was $18.2 \pm 5.9 \mathrm{~kg}$ compared to $13.5 \pm 4.8 \mathrm{~kg}$ in nongainers $(\mathrm{P}=\mathrm{NS})$. Gainers had a significantly lower $\mathrm{EE}$ than non-gainers (9911 \pm 3625 vs. $13184 \pm 3016 \mathrm{kcal}, \mathrm{P}=0.046$ ). The total number of steps taken by gainers was $27321 \pm 18634$ versus $29103 \pm 16552$ by non-gainers $(\mathrm{P}=\mathrm{NS})$. Gainers appeared to have higher caloric intake than non-gainers (1878 \pm 397 vs. $1706 \pm 424 \mathrm{kcal}, \mathrm{P}=\mathrm{NS}$ ).

Conclusion: The increase in body weight after start of insulin treatment in patients with type 2 diabetes shows large inter-individual variation despite a similar drop in $\mathrm{HbAlc}$, and a similar insulin need. Pronounced weight gain was associated with a differentiated change in metabolic and cardiovascular parameters. The increase in body weight in gainers seems mainly explained by lower physical activity levels. It may be important to assess lifestyle characteristics before onset of insulin treatment in order to predict and potentially intervene those at risk for extensive body weight increase.

Laboratory results gainers vs. non-gainers

\begin{tabular}{lll}
\hline decrease mean $\mathrm{HbA1c}(\%)$ & 10.1 to 7.13 & 9.2 to 7.59 \\
\hline fasting insulin $(\mathrm{pmol} / \mathrm{l})$ & $22.9 \pm 10.6$ & $32.2 \pm 19.7$ \\
\hline C-peptide $(\mathrm{pmol} / \mathrm{l})$ & $0.59 \pm 0.28$ & $0.67 \pm 0.30$ \\
\hline Total cholesterol $(\mathrm{mmol} / \mathrm{l})^{*}$ & $4.9 \pm 0.9$ & $3.8 \pm 0.6$ \\
\hline LDL-cholesterol $(\mathrm{mmol} / \mathrm{l})^{*}$ & $3.0 \pm 0.9$ & $2.0 \pm 0.5$ \\
\hline ALT $(\mathrm{U} / \mathrm{l})^{*}$ & $33.7 \pm 10.5$ & $24.0 \pm 10.8$ \\
\hline FFA $(\mathrm{umol} / \mathrm{l})$ & $0.7 \pm 0.14$ & $0.53 \pm 0.38$ \\
\hline Adiponectin $(\mathrm{ug} / \mathrm{ml})^{*}$ & $3.3 \pm 1.4$ & $1.9 \pm 1.0$ \\
\hline Leptin $(\mathrm{ng} / \mathrm{ml})^{*}$ & $47.0 \pm 26.5$ & $21.9 \pm 13.3$ \\
\hline hsCRP $(\mathrm{mg} / \mathrm{l})$ & $4.7 \pm 4.1$ & $2.5 \pm 1.9$ \\
\hline
\end{tabular}

\section{5}

Insulin detemir reduces gain in body weight and fat mass in ZDF rats when compared with both NPH and insulin glargine

C. Fledelius, J. Damgaard, A. Vinterby, U. Ribel, J.S. Petersen, J. Sturis Insulin Pharmacology, Novo Nordisk, Malov, Denmark

Background and aims: Studies have shown that even a modest body weight (BW) reduction can improve the cardiovascular risk profile in T2D patients. BW gain is a well-known consequence of insulin therapy in T1D and T2D patients. Compared with conventional basal insulin therapy, insulin detemir has been consistently shown to result in attenuated BW gain in both T1D and T2D patients at similar degrees of glycemic control. Furthermore, the benefit of detemir on BW has been shown to increase with increasing BMI. At present, it is not known how this difference in BW is associated with changes in fat mass. Therefore, in this study we compared the effects of detemir on glycemic control, BW and fat mass in the ZDF rat model of T2D with two other basal insulins, NPH and insulin glargine. 
Materials and methods: Diabetic ZDF rats received vehicle, NPH, detemir, or glargine subcutaneously twice daily for four weeks. Pilot studies were performed that identified doses of the three insulins that were equivalent on glucose lowering profiles and that documented duration of action compatible with twice daily dosing in these animals. BW, fat mass, and glycemic control were determined at baseline and at the end of study. HbAlc was used as an index of glycemic control. Fat mass was estimated using quantitative magnetic resonance.

Results: $\mathrm{HbAlc}$, BW, and fat mass did not differ significantly between groups at baseline, $\mathrm{HbAlc}$ averaging $7.4 \pm 0.3 \%$ (mean $\pm \mathrm{SD}$ ). Compared with vehicle, $\mathrm{NPH}$, detemir, and glargine treatment all resulted in decreased $\mathrm{HbAlc}$ and increased BW and fat mass. Four-week reductions in $\mathrm{HbAlc}$ did not differ significantly between groups (NPH: $1.6 \pm 0.4 \%$; detemir: $1.7 \pm 0.4 \%$; glargine: $1.7 \pm 0.4 \%$, NS ANOVA). Increases in BW (NPH: $138 \pm 16 \mathrm{~g}$; detemir: $125 \pm 12 \mathrm{~g}$; glargine: $138 \pm 16 \mathrm{~g}, \mathrm{p}<0.05$ ANOVA) and fat mass (NPH: $97 \pm 10 \mathrm{~g}$; detemir: $88 \pm 6 \mathrm{~g}$, glargine: $95 \pm 7 \mathrm{~g}, \mathrm{p}<0.01$ ANOVA) were both significantly smaller with detemir compared to NPH and glargine, whereas no significant differences were observed between NPH and glargine.

Conclusion: In conclusion, four weeks treatment with detemir resulted in significantly less increased BW and fat mass in comparison to NPH and glargine in the ZDF rat model of T2D, at equivalent levels of glycemic control. The results indicate that the attenuated weight gain observed with detemir in this model can largely be attributed to a reduced increase in adiposity.

\section{6}

Insulin-naïve patients with type 2 diabetes and higher BMI experience weight loss when initiated onto insulin detemir: 12-week, 26-week and 52-week follow-up data from PREDICTIVE ${ }^{\mathrm{TM}}$

H. Hanaire ${ }^{1}$, S. Sreenan ${ }^{2}$, A. Dornhorst ${ }^{3}$, S. Gonbert ${ }^{4}$, J.B. Hansen ${ }^{1}$ Service Diabetologie, Hopital Rangueil, Toulouse, France, ${ }^{2}$ Department of Endocrinology, Connolly Hospital, Dublin, Ireland, ${ }^{3}$ Department of Metabolic Medicine, Imperial College, London, United Kingdom, ${ }^{4}$ Novo Nordisk Pharmaceutique SAS, Paris, France, ${ }^{5}$ Novo Nordisk A/S, Bagsvaerd, Denmark

Background and aims: Weight gain is a well-recognised barrier to insulin initiation and compliance, and it can undermine patient quality of life. Evidence from clinical trials has consistently shown less weight gain for patients taking insulin detemir compared with NPH and insulin glargine. The multinational observational study, PREDICTIVE ${ }^{\mathrm{TM}}$, provides the opportunity to determine this trend in clinical practice. The aim of this subset analysis was to determine whether a weight sparing effect correlates with BMI in insulinnaïve patients with type 2 diabetes initiated onto insulin detemir and treated for over 12,26 or 52 weeks.

Materials and methods: This analysis concerned insulin-naïve patients with type 2 diabetes recruited into the PREDICTIVE ${ }^{\mathrm{TM}}$ study, and initiated onto insulin detemir with OADs. Data were stratified by patients' baseline BMI. Weight change $(\mathrm{kg})$ and BMI $\left(\mathrm{kg} / \mathrm{m}^{2}\right)$ were monitored at baseline and endpoint.

Results: A total of 1964 patients (47\% male; mean age 60.5 years; mean duration of diabetes 8 years; mean weight $84.3 \mathrm{~kg}$, and BMI $29.8 \mathrm{~kg} / \mathrm{m}^{2}$ ) from 11 countries were treated for 12 weeks. 277 patients ( $53 \%$ male; 60.2 years; mean duration of diabetes 9.1 years; mean weight $88.2 \mathrm{~kg}$, and BMI $31.1 \mathrm{~kg} / \mathrm{m}^{2}$ ) from 6 countries were treated for 26 weeks, and 159 French patients (56\% male; mean age 61.7 years; mean duration of diabetes 13.1 years; mean weight $82.1 \mathrm{~kg}$, and BMI $29.2 \mathrm{~kg} / \mathrm{m}^{2}$ ) were treated for 52 weeks. Patients treated for 12 weeks lost weight when initiated onto insulin detemir $\sim 0.6 \mathrm{~kg}$ of weight $(\mathrm{SD}=3.4 \mathrm{~kg}, p<0.0001)$, those treated for 26 weeks tended to lose a small (non-significant) amount of weight $\sim 0.4 \mathrm{~kg}(\mathrm{SD}=4.2 \mathrm{~kg}, p=0.20)$ and patients in the French cohort treated for 52 weeks had minimal (non-significant) weight gain $\sim 0.1 \mathrm{~kg}(\mathrm{SD}=5.8 \mathrm{~kg}, p=0.85)$.

Weight change data for each of the three treatment periods were then stratified by BMI, and reanalysed (table). The pattern of weight change was different in subjects with normal BMI and those who were overweight or obese; on average, patients in the highest BMI categories $\left(\geq 30 \mathrm{~kg} / \mathrm{m}^{2}\right)$ lost weight when initiated onto insulin detemir.

Conclusion: Data from the PREDICTIVE ${ }^{\mathrm{TM}}$ study show a modest but statistically significant mean weight loss for insulin-naïve patients initiated onto and treated with insulin detemir for 12 weeks. Subsets with longer treatment periods (26 weeks, 52 weeks) experienced no significant weight changes. Across groups, insulin detemir's weight sparing benefit was more noticeable in patients with high BMI $\left(>30 \mathrm{~kg} / \mathrm{m}^{2}\right)$ who tended to lose weight Supported by: Novo Nordisk

\section{7}

A specific patient population achieves glycaemic control with prandial premixed (lispro/ILPS) insulin therapy with lower insulin dose and less weight gain than basal bolus therapy

S. Martin, J. Scism-Bacon, H. Jiang

Eli Lilly and Company, Indianapolis, United States

Background and aims: After failure of oral antihyperglycemic agents (OHAs) + basal insulin to control hyperglycemia in T2D patients (pts), advancement of insulin therapy is required. In this post-hoc analysis of data from T2D pts previously treated with glargine + OHAs, we attempted to identify pts for whom prandial premixed therapy (PPT) would provide comparable safety and efficacy to basal bolus therapy (BBT) for insulin therapy advancement. Materials and methods: The original study was a 24 -wk, multicenter, randomized, parallel, open-label study evaluating the effectiveness of PPT [lispro mix 50; 50\% insulin lispro, 50\% insulin lispro protamine suspension (ILPS)] tid with meals or BBT [glargine at bedtime + mealtime lispro] in 374 T2D pts inadequately controlled $(7.5 \% \leq \mathrm{HbAlc} \leq 12 \%)$ on basal insulin glargine plus OHAs. In the current analysis, efficacy and safety were assessed in subgroup populations defined by incremental $0.5 \%$ ranges of baseline $\mathrm{HbAlc}$ from $7.5-12 \%$ inclusive. Results: We report data for one subset of pts from the original study $(n=269)$ with baseline $\mathrm{HbAlc}(7.5 \% \leq \mathrm{HbA} 1 \mathrm{c} \leq 9.5 \%)$. In this group, baseline values for age, BMI, percentage male, and HbAlc were similar between BBT and PPT regimens. At study end, $\mathrm{HbAlc}$, fasting plasma glucose (FPG), and postprandial glucose $(\mathrm{PPG})$ were reduced significantly $(\mathrm{P}<0.0001)$ from baseline for both insulin regimens with no significant treatment difference (Table). Weight gain and TDI dose were significantly lower with PPT than BBT. There was no significant difference between therapies in the number of serious adverse events (BBT 8; PPT 5, $\mathrm{P}=0.573$ ) or number of pts reporting severe hypoglycemia (BBT 4; PPT 5, P=0.744). Due to the small number of pts ( $\mathrm{n}=$ $\sim 40$ in each treatment arm), no conclusions could be drawn for the subset of pts with baseline $\mathrm{HbAlc}>9.5 \%$.

Conclusion: In T2D pts with HbAlc values of $7.5 \%$ to $9.5 \%$, PPT (3 daily injections) resulted in similar glycemic improvement as BBT (4 daily injections), but with less weight gain and less total daily insulin dose. Thus, PPT offers a suitable alternative to BBT for pts with $7.5 \% \leq \mathrm{HbAlc} \leq 9.5 \%$ who prefer 3 daily injections to 4 daily injections.

\begin{tabular}{|c|c|c|c|}
\hline & $\begin{array}{l}\text { BBT } \\
(\mathrm{n}=138)\end{array}$ & $\begin{array}{l}\text { PPT } \\
(\mathrm{n}=131)\end{array}$ & $\begin{array}{l}\text { P-value between } \\
\text { therapies }\end{array}$ \\
\hline Baseline HbAlc & $8.41 \pm 0.62$ & $8.29 \pm 0.51$ & 0.083 \\
\hline HbA1c change from baseline & $-1.77 \pm 0.86$ & $-1.55 \pm 0.87$ & 0.185 \\
\hline $\begin{array}{l}\text { Pts achieving HbAlc }<7 \% \\
{[\mathrm{n}(\%)]}\end{array}$ & $85(76)$ & $68(62)$ & 0.030 \\
\hline FPG change $(\mathrm{mmol} / \mathrm{L})$ & $-2.56 \pm 2.76$ & $-1.92 \pm 3.02$ & 0.254 \\
\hline PPG change $(\mathrm{mmol} / \mathrm{L})$ & $-5.35 \pm 3.15$ & $-4.37 \pm 3.59$ & 0.088 \\
\hline Weight gain $(\mathrm{kg})$ & $4.70 \pm 4.80$ & $3.13 \pm 3.92$ & 0.037 \\
\hline $\begin{array}{l}\text { Endpoint Insulin dose } \\
\text { (unit/kg) }\end{array}$ & $1.33 \pm 0.84$ & $1.08 \pm 0.50$ & 0.015 \\
\hline $\begin{array}{l}\text { Hypo rate with BG } \\
\leq 3.3 \mathrm{mmol} / \mathrm{L} \text { (episodes/pt/yr) }\end{array}$ & $21.35 \pm 26.24$ & $22.87 \pm 27.44$ & 0.739 \\
\hline $\begin{array}{l}\begin{array}{l}\text { Nocturnal hypo rate } \\
\text { (episodes/pt/yr) }\end{array} \\
\end{array}$ & $7.10 \pm 11.75$ & $5.20 \pm 7.74$ & 0.110 \\
\hline
\end{tabular}

Data shown are for the patient group with $7.5 \% \leq$ baseline HbAlc $\leq 9.5 \%$ Values are mean \pm SD

Supported by: Eli Lilly and Company

\section{8}

Better glycaemic control for 30,171 insulin-naïve patients with type 2 diabetes after starting biphasic insulin aspart 30/70 (BIAsp 30) therapy: IMPROVE $^{\text {mx }}$ study subgroup analysis

R. Kawamori ${ }^{1}$, M.V. Srishyla ${ }^{2}$, Y. Wenying ${ }^{3}$

${ }^{1}$ Department of Metabolism and Endocrinology, Juntendo University School of Medicine, Tokyo, Japan, ${ }^{2}$ Novo Nordisk A/S, Virum, Denmark, ${ }^{3}$ Department of Endocrinology, China-Japan Friendship Hospital, Beijing, China

Background and aims: The IMPROVE ${ }^{\text {tw }}$ study is an international, open-label, non-randomised, non-interventional, 26-week observational study of 
Table: Weight change stratified by BMI in insulin naïve patients with type 2 diabetes treated with insulin detemir in the PREDICTIVETM study

\begin{tabular}{|c|c|c|c|c|c|c|c|c|}
\hline \multicolumn{3}{|c|}{ 12-week treatment group } & \multicolumn{3}{|c|}{26 week treatment group } & \multicolumn{3}{|c|}{52 week treatment group } \\
\hline BMI category & $\begin{array}{l}\text { Mean change } \\
\text { (SD) kg }\end{array}$ & $\begin{array}{l}\text { Mean weight change } \\
\text { versus BMI: } \\
\text { p-value }\end{array}$ & BMI category & $\begin{array}{l}\text { Mean change } \\
\text { (SD) kg }\end{array}$ & $\begin{array}{l}\text { Mean weight } \\
\text { change versus } \\
\text { BMI: } \\
\text { p-value }\end{array}$ & BMI category & $\begin{array}{l}\text { Mean change } \\
\text { (SD kg) }\end{array}$ & $\begin{array}{l}\text { Mean weight } \\
\text { change versus } \\
\text { BMI: } \\
\text { p-value } \\
\end{array}$ \\
\hline$<25 \mathrm{~kg} / \mathrm{m}^{2}$ & $0.69(3.35)$ & & $<25 \mathrm{~kg} / \mathrm{m}^{2}$ & $1.52(3.19)$ & & $<25 \mathrm{~kg} / \mathrm{m}^{2}$ & $0.78(3.55)$ & \\
\hline $25-<30 \mathrm{~kg} / \mathrm{m}^{2}$ & $-0.40(2.76)$ & & $25-<30 \mathrm{~kg} / \mathrm{m}^{2}$ & $-0.42(3.66)$ & & $25-<30 \mathrm{~kg} / \mathrm{m}^{2}$ & $1.61(5.45)$ & \\
\hline $30-<35 \mathrm{~kg} / \mathrm{m}^{2}$ & $-1.02(3.62)$ & $<0.0001^{\star}$ & $30-<35 \mathrm{~kg} / \mathrm{m}^{2}$ & $-0.64(4.93)$ & $<0.01^{\star}$ & $30-<35 \mathrm{~kg} / \mathrm{m}^{2}$ & $-0.79(5.37)$ & $<0.01^{\star}$ \\
\hline$>35 \mathrm{~kg} / \mathrm{m}^{2}$ & $-1.52(4.67)$ & & $>35 \mathrm{~kg} / \mathrm{m}^{2}$ & $-1.20(4.45)$ & & $>35 \mathrm{~kg} / \mathrm{m}^{2}$ & $-3.46(8.19)$ & \\
\hline
\end{tabular}

* Analysed by ANCOVA - linear regression of weight change during study period by BMI at baseline.

biphasic insulin aspart 30/70 (BIAsp 30) in the treatment of type 2 diabetes. Here we present a subgroup analysis of the safety and effectiveness of BIAsp 30 in previously insulin-naïve patients.

Materials and methods: By 22 February 2008, 30,171 insulin-naïve patients had been recruited from Canada $(n=216)$, China $(n=18,800)$, India $(n=5,391)$, Japan ( $\mathrm{n}=1816)$, Poland ( $\mathrm{n}=3,901)$, and Russia $(\mathrm{n}=47)$; all had reported data at final visit and are included in this analysis. Of these, $25.6 \%(n=7717)$ had received no therapy prior to enrolment; $24.8 \%(\mathrm{n}=7470)$ received one oral antidiabetic drug $(\mathrm{OAD}) ; 36.9 \%(\mathrm{n}=11,139)$ two OADs, and $12.4 \%(\mathrm{n}=3727)$ had received more than two OADs. Sulphonylureas were the most commonly taken $\mathrm{OAD}(70.9 \%$ of patients), followed by biguanides $(69.1 \%)$ and alphaglucosidase inhibitors (19.8\%).

Results: The mean (SD) age of the insulin-naïve cohort was 55.0 years (12.3); diabetes duration 5.9 years (5.5); BMI $25.6(4.3) \mathrm{kg} / \mathrm{m}^{2}$; weight 70.5 (13.8) $\mathrm{kg}$ and $60.0 \%$ were male. At final visit, glycaemic control improved significantly from baseline in the total insulin-naive cohort and in all four subgroups (Table). Overall, $\mathrm{HbA}_{1 \mathrm{c}}$ decreased by $2.4 \%$ from $9.4 \%$ to $6.9 \%$, corresponding to $58.7 \%$ of patients reaching target $\mathrm{HbA}_{1 \mathrm{c}}<7.0 \%$. The greatest improvement was seen in patients who previously received 'no therapy' $\left(-3.2 \% \mathrm{HbA}_{1}\right)$. Not surprisingly, this subgroup had the shortest duration of diabetes and the highest baseline $\mathrm{HbA}_{1 c}$ (Table). Increasing numbers of OADs were associated with longer duration of diabetes and smaller reductions in $\mathrm{HbA}_{1 \mathrm{c}}$. Fasting plasma glucose was significantly reduced from baseline in all subgroups, as was postprandial plasma glucose after all three meals ( $<<0.001$ for all subgroups); the greatest reductions occurred after breakfast. There was no significant change in weight across subgroups. Major hypoglycaemia was reduced from baseline in all subgroups, while minor hypoglycaemia generally increased from baseline. At the end of the study, only $0.1 \%$ of insulin-naïve patients reported any major hypoglycaemia and $9.6 \%$ reported any minor hypoglycaemia. The overall mean BIAsp 30 dose at baseline was $0.4 \mathrm{U} / \mathrm{kg}$, which increased by 0.1 $\mathrm{U} / \mathrm{kg}$ over the 26 -week study.

Conclusion: Starting insulin therapy with BIAsp 30 significantly improved glycaemic control, regardless of whether patients had previously received no therapy or one or more OADs. Fewer OADs and shorter diabetes duration were associated with greater improvements in glycaemic control. BIAsp 30 also reduced major hypoglycaemia without significant weight change. BIAsp 30 is an effective first insulin treatment, even when used before any OAD therapy.

\section{9}

Initial dose of insulin glargine in type 2 diabetes based on $\mathrm{BMI}$ is safe D.R. Franco ${ }^{1,2}$, J. Baptista ${ }^{1}$, F.R.M. Abreu ${ }^{1}$, R.B. Baptista ${ }^{1}$, M.R.C. Alves ${ }^{3}$, F.G. Eliaschewitz ${ }^{1,4}$

${ }^{1}$ Clinical Research, CPCLIN, São Paulo, ${ }^{2}$ Endocrinology, Intermédica Saúde, ${ }^{3}$ Fac. Public Heath, Un. São Paulo, ${ }^{4}$ Internal Medicine, H. Heliópolis, São Paulo, Brazil

Background and aims: Basal Glargine insulin (GI) therapy is an efficient approach to treat type 2 diabetic patients that fail to achieve glycemic control on oral drugs $(\mathrm{OAD})$. Several algorithms have been developed and validated, most of them starting with a $10 \mathrm{UI}$ dose at bedtime for all patients. However, with this approach the titrating period can be quite long, affecting adherence to treatment. The aim of this study was to verify the safety, measured by frequency of hypoglycemic events (glycemia $<56 \mathrm{mg} \%$ ), of two algorithms (AG) that calculate the initial insulin dose according to BMI, in comparison with further two algorithms that use the standard $10 \mathrm{UI}$ as the initial dose.

Materials and methods: We conducted a single-center study with 125 type 2 diabetic patients screened for treatment failure with one or two OADs, naive for insulin therapy, with an $\mathrm{HbAlc}$ between $7 \%$ and $12 \% .92$ patients were randomized and received at least one dose of insulin (ITT population). A screening period of two weeks was followed by 12 weeks treatment with 10 visits plus a 4-week follow-up. 26 patients were randomized to LAMET AG with an initial insulin dose of $10 \mathrm{UI}$ and a 2/2 UI titration two times/week until the FPG reached the target of $100 \mathrm{mg} \% ; 23$ patients were grouped to DeGold AG with an initial dose varying form $0.2 \mathrm{UI}$ to $0.35 \mathrm{UI} / \mathrm{Kg}$ according to $\mathrm{BMI}$

Table. Changes from baseline in glycaemic measurements for insulin-naïve patients and the four constitutive subgroups

\begin{tabular}{|c|c|c|c|c|c|c|}
\hline \multirow[t]{2}{*}{ Measurement, mean (SD) } & & \multicolumn{5}{|l|}{$\underline{\text { Pre-study therapy }}$} \\
\hline & & $\begin{array}{l}\text { Insulin-naïve cohort } \\
(\mathrm{n}=30,171) \#\end{array}$ & $\begin{array}{l}\text { No therapy } \\
(\mathrm{n}=7717)\end{array}$ & $\begin{array}{l}1 \text { OAD } \\
(\mathrm{n}=7470)\end{array}$ & $\begin{array}{l}2 \text { OADs } \\
(\mathrm{n}=11,139)\end{array}$ & $\begin{array}{l}>2 \text { OADs } \\
(\mathrm{n}=3727)\end{array}$ \\
\hline Duration of diabetes, years $(\mathrm{SD})$ & & $5.9(5.5)$ & $1.9(3.5)$ & $5.5(4.9)$ & $7.6(5.1)$ & $10.1(5.7)$ \\
\hline $\mathrm{HbAlc} \% \mathrm{Hb}$ & $\begin{array}{l}\text { Baseline } \\
\text { Final visit } \\
\text { Change } \dagger\end{array}$ & $\begin{array}{l}9.4(1.9) \\
6.9(1.0) \\
-2.4(1.9)^{\star}\end{array}$ & $\begin{array}{l}9.9(2.2) \\
6.7(0.9) \\
-3.2(2.2)^{\star}\end{array}$ & $\begin{array}{l}9.2(1.9) \\
6.9(1.0) \\
-2.3(1.8)^{\star}\end{array}$ & $\begin{array}{l}9.2(1.8) \\
7.0(1.0) \\
-2.2(1.7)^{\star}\end{array}$ & $\begin{array}{l}9.3(1.8) \\
7.3(1.2) \\
-2.0(1.7)^{\star}\end{array}$ \\
\hline Fasting plasma glucose, $\mathrm{mmol} / \mathrm{L}$ & $\begin{array}{l}\text { Baseline } \\
\text { Final visit } \\
\text { Change }\end{array}$ & $\begin{array}{l}11.1(3.4) \\
6.5(1.2) \\
-4.6(3.4)^{*}\end{array}$ & $\begin{array}{l}12.4(3.8) \\
6.4(1.2) \\
-6.0(3.9)^{*}\end{array}$ & $\begin{array}{l}10.8(3.30) \\
6.6(1.2) \\
-4.2(3.2)^{*}\end{array}$ & $\begin{array}{l}10.7(3.1) \\
6.6(1.2) \\
-4.1(3.1)^{*}\end{array}$ & $\begin{array}{l}10.4(2.9) \\
6.6(1.4) \\
-3.8(2.9)^{*} \\
\end{array}$ \\
\hline Postprandial plasma glucose (breakfast), mmol/L & $\begin{array}{l}\text { Baseline } \\
\text { Final visit } \\
\text { Change }\end{array}$ & $\begin{array}{l}15.2(4.7) \\
8.3(1.8) \\
-6.9(4.8)^{*}\end{array}$ & $\begin{array}{l}17.2(5.3) \\
8.0(1.5) \\
-9.2(5.4)^{*}\end{array}$ & $\begin{array}{l}14.7(4.4) \\
8.2(1.7) \\
-6.5(4.4)^{*}\end{array}$ & $\begin{array}{l}14.5(4.3) \\
8.4(1.8) \\
-6.2(4.3)^{*}\end{array}$ & $\begin{array}{l}14.8(4.0) \\
9.0(2.1) \\
-5.8(4.0)^{*} \\
\end{array}$ \\
\hline Major hypoglycaemia, events/patient year & $\begin{array}{l}\text { Baseline } \\
\text { Final visit } \\
\text { Change }\end{array}$ & $\begin{array}{l}0.05 \\
0.01 \\
-0.05^{*}\end{array}$ & $\begin{array}{l}0.02 \\
0.00 \\
-0.01 \mathrm{NS}\end{array}$ & $\begin{array}{l}0.04 \\
0.00 \\
-0.04^{*}\end{array}$ & $\begin{array}{l}0.07 \\
0.01 \\
-0.06^{*}\end{array}$ & $\begin{array}{l}0.11 \\
0.01 \\
-0.10^{*}\end{array}$ \\
\hline Minor hypoglycaemia, events/patient year & $\begin{array}{l}\text { Baseline } \\
\text { Final visit } \\
\text { Change }\end{array}$ & $\begin{array}{l}1.66 \\
2.70 \\
1.03^{*}\end{array}$ & $\begin{array}{l}0.53 \\
2.76 \\
2.22^{*}\end{array}$ & $\begin{array}{l}1.55 \\
2.26 \\
0.72^{*} \\
\end{array}$ & $\begin{array}{l}2.09 \\
2.91 \\
0.82^{*}\end{array}$ & $\begin{array}{l}3.02 \\
2.89 \\
-0.13 \mathrm{NS} \\
\end{array}$ \\
\hline Weight, kg & $\begin{array}{l}\text { Baseline } \\
\text { Final visit } \\
\text { Change }\end{array}$ & $\begin{array}{l}70.6(13.7) \\
70.6(12.9) \\
0.0(4.0) \mathrm{NS}\end{array}$ & $\begin{array}{l}69.7(12.2) \\
69.7(11.2) \\
0.0(3.7) \mathrm{NS} \\
\end{array}$ & $\begin{array}{l}70.3(13.5) \\
70.2(12.6) \\
-0.1(3.9) \mathrm{NS} \\
\end{array}$ & $\begin{array}{l}71.5(14.6) \\
71.5(13.8) \\
0.0(4.2) \mathrm{NS}\end{array}$ & $\begin{array}{l}70.6(14.4) \\
70.5(13.7) \\
-0.1(3.9) \mathrm{NS} \\
\end{array}$ \\
\hline
\end{tabular}

${ }^{*} \mathrm{p}<0.001$; NS: not significant. \#Data on the number of OADs being taken were missing for 118 patients. $\dagger$ Values rounded to one decimal place.

Supported by: Novo Nordisk 
and with the same titration as above; 21 patients were grouped to FTT AG also with $10 \mathrm{UI}$ as the initial dose and titration varying from 2 to $8 \mathrm{UI}$ according to the FPG, and 22 patients were randomized to FAST AG with the initial dose varying according to $\mathrm{BMI}$ and the titration schedule varying according to FPG. Patients in the 4 groups were comparable in age, gender, weight, diabetes duration and previous medication. Comparisons used ANOVA/Kruskal-Wallis or Mann-Whitney tests. Other end points were time to target, variation in HbAlc, weight, FPG and insulin doses from basal to the end of the study.

Results: There were no significant differences in FPG variation, HbAlc, time to target, proportion of patients reaching $\mathrm{HbAlc}<7 \%$ and between $7.1-7.5 \%$, and hypoglycemic events ( $<55 \mathrm{mg} \%$ ) between the 4 groups, as shown in Table 1. Each group was also compared to the standard risk LAMET AG, again without evidencing statistical difference. DeGold $(0.17 \pm 0.38)$ was comparable to LAMET $(0.42 \pm 0.7)$ and was much lower than FTT $(1.5 \pm 3.3)$ and FAST $(1.23 \pm$ 2.21 ), though not reaching statistical significance. However, when LAMET and DeGold were jointly compared with the 2 fast titration groups (FTT and FAST), the increase in hypoglycemic events became evident $(\mathrm{p}<0.039)$.

Conclusion: Starting IG titration with an initial dose based on BMI is safe. We show that the hypoglycemic risk is greater with increasing titration rhythm rather than when a higher initial insulin dose is employed.

Table 1

\begin{tabular}{|c|c|c|c|c|}
\hline & \multicolumn{4}{|l|}{ Algorithms } \\
\hline & LAMET & DeGold & FAST & FTT \\
\hline Number of patients & 26 & 23 & 22 & 21 \\
\hline Initial FPG in mg/dl & $197.0 \pm 59.4$ & $196.6 \pm 54.8$ & $179.4 \pm 51.4$ & $196.1 \pm 53.4$ \\
\hline Final FPG in mg/dl & $119.4 \pm 36.2$ & $106.6 \pm 18.0$ & $109.0 \pm 28.7$ & $107.6 \pm 17.3$ \\
\hline Initial HbAlc in \% & $9.5 \pm 1.7$ & $9.2 \pm 1.5$ & $9.4 \pm 1.7$ & $9.4 \pm 1.3$ \\
\hline Final HbAlc in \% & $7.4 \pm 1.0$ & $6.8 \pm 0.7$ & $7.4 \pm 1.3$ & $7.3 \pm 0.6$ \\
\hline Time to traget in days & $28 \pm 31.3$ & $22 \pm 19.8$ & $15 \pm 19.1$ & $20 \pm 17.3$ \\
\hline $\begin{array}{l}\text { \% Patients with } \mathrm{HbAlc} \\
\leq 7 \%\end{array}$ & 34.6 & 17.4 & 33.3 & 25 \\
\hline $\begin{array}{l}\% \text { Patients with } \\
7.1 \%<\text { HbAlc }<7.5 \%\end{array}$ & 34.6 & 69.6 & 28.6 & 35 \\
\hline $\begin{array}{l}\text { Number of } \\
\text { Hypoglycemia events }\end{array}$ & 9 & 4 & 28 & 21 \\
\hline
\end{tabular}

Supported by: sanofi-aventis

\section{0}

Incidence rates of heart failure, stroke and acute myocardial infarction in type 2 diabetics patients using insulin glargine and other insulin J. Juhaeri ${ }^{1}$, S. Gao ${ }^{1}$, M.-P. Dain ${ }^{2}$

${ }^{1}$ Epidemiology Unit, Global Phamacovigilance and Epidemiology, sanofiaventis, Bridgewater, United States, ${ }^{2}$ Global Diabetes Franchise, sanofiaventis, Bridgewater, United States

Background and aims: Although the efficacy of insulin glargine in improving blood glucose profile is well known, its impact on cardiovascular diseases (CVD) risks remains unclear. The aim of this study were to examine and compare incidence rates of heart failure, stroke and acute myocardial infarction (AMI) in Type 2 diabetic patients using insulin glargine and other types of insulin.

Materials and methods: Using a retrospective cohort design, data from PharMetrics database in the US were used to examine risk of CVD across different types of insulin, insulin glargine and other long or intermediate acting insulins. Insulin was ascertained using the National Drug Code directory. International Classification of Diseases, Ninth Revision, Clinical Modification (ICD-9CM) codes were used to identify CVD events, including heart failure (ICD-9CM code of 428), stroke (ICD-9CM codes of 430-438), and AMI (ICD-9CM code of 410). Only new CVD events were analyzed and patients with previous history of CVD were excluded. We used Poisson regression to examine the associations between insulin treatment and CVD. Age, gender, history of hypertension, history of dyslipidemia, days of supply and duration of diabetes were the covariates adjusted in the multivariate analyses.

Results: The insulin glargine only group had larger proportions of patients aged 40-60 years, men and a history of hypertension or dyslipidemia, longer days of supply, and a longer duration of diabetes than other long/intermediate acting insulins group. The incidence rates of heart failure, stroke and AMI in the insulin glargine only group were 304.8 (95\% CI: [275.0, 334.6]), 158.5 (95\% CI: $[137.0,180.0])$ and 93.3 (95\% CI: $[76.8,109.8])$ cases per 10,000 person-years, respectively. After adjustment for the covariates, the incidence rates of CVD events in the insulin glargine only group were comparable to those in other long/intermediate acting insulins group (reference), except for AMI, which tended to be lower in the insulin glargine group than in other long/intermediate acting insulins group. The corresponding incidence rate ratios (RR) were 1.0 (95\% CI: [0.9, 1.2]) for heart failure, 1.0 (95\% CI: [0.8, $1.3])$ for stroke, and 0.8 (95\% CI: [0.62, 0.95]) for AMI, respectively.

Conclusion: Incidence rates of CVD events in the insulin glargine and in other long/intermediate acting insulins groups were comparable, except for AMI where the risk tended to be lower in the insulin glargine group. Insulin glargine use alone, or in combination with other insulins might be associated with a lower risk of CVD events, although the possible underlying mechanisms are unclear. Supported by: sanofi-aventis

\section{1}

Glycaemic control and cardiovascular risk in insulin-treated patients with type 2 diabetes in Germany and the UK

S. Gough ${ }^{1}$, K.B. Frandsen ${ }^{2}$, A.D. Toft ${ }^{2}$

${ }^{1}$ Institute of Biomedical Research, University of Birmingham, United

Kingdom, ${ }^{2}$ Novo Nordisk, Bagsvaerd, Denmark

Background and aims: One of the major aims of insulin initiation in type 2 diabetes is to improve glycaemia in patients not controlled by lifestyle and oral therapy and reduce an increased risk of future diabetes-related complications. Monitoring of the response to insulinisation in such patients may suggest strategies for improving the attainment of target levels in clinical practice. This analysis determined 'real-life' $\mathrm{HbA}$, values and other cardiovascular risk factors in patients in Germany and the UK in insulin-treated patients with type 2 diabetes.

Materials and methods: We analysed data from IMS - one of the biggest databases collating population-based data directly from computerised patient records in practices in the EU. Patients with type 2 diabetes, with a first insulin prescription between 1 August 2002 and 31 July 2006, and a recorded $\mathrm{HbA}_{1 \mathrm{c}}$ value at least 6 months after the initial dose, were included. For this analysis, patients' last recorded $\mathrm{HbA}_{1 c}$ value was used. Results were analysed separately for patients receiving insulin only or insulin + oral antidiabetic drugs (OADs).

Results: Data were obtained for 6625 patients treated by specialists $(\mathrm{n}=1108)$ or primary care practitioners (PCPs) $(\mathrm{n}=5517)$ in Germany, and 2118 patients treated by PCPs in the UK (table). Results are reported for insulin/insulin + OADs. Mean $\mathrm{HbA}_{1}$ values were $7.5 / 7.7 \%$ in Germany and $8.2 / 8.2 \%$ in the UK. Percentage of patients with $\mathrm{HbA}_{1 \mathrm{c}}>7.0 \%$ was $63 / 69$ in Germany and 77/82 in

\begin{tabular}{|c|c|c|c|c|}
\hline & \multicolumn{2}{|l|}{ Germany } & \multicolumn{2}{|l|}{ UK } \\
\hline & $\begin{array}{l}\text { Insulin } \\
\mathrm{n}=3702\end{array}$ & $\begin{array}{l}\text { Insulin + OADs } \\
\mathrm{n}=2923\end{array}$ & $\begin{array}{l}\text { Insulin } \\
\mathrm{n}=845\end{array}$ & $\begin{array}{l}\text { Insulin + OADs } \\
\mathrm{n}=1273\end{array}$ \\
\hline Mean age (years \pm SD) & $69.3 \pm 11.7$ & $67.3 \pm 10.9$ & $64.3 \pm 13.9$ & $63.2 \pm 12.0$ \\
\hline Mean weight $(\mathrm{kg} \pm \mathrm{SD})$ & $85.9 \pm 19.3$ & $90.1 \pm 20.2$ & $85.8 \pm 20.9$ & $92.6 \pm 19.8$ \\
\hline Mean HbA1c $(\% \pm$ SD) & $7.5 \pm 1.3$ & $7.7 \pm 1.3$ & $8.2 \pm 1.7$ & $8.2 \pm 1.5$ \\
\hline Mean duration of insulin therapy (years \pm SD) & $2.3 \pm 1.1$ & $2.2 \pm 1.1$ & $2.1 \pm 1.0$ & $2.0 \pm 1.0$ \\
\hline Mean duration of diabetes (years \pm SD) & $4.0 \pm 3.2$ & $4.8 \pm 3.4$ & $10.0 \pm 9.0$ & $10.5 \pm 7.7$ \\
\hline Mean BP (mmHg) & $141 / 80$ & $145 / 81$ & $135 / 75$ & $136 / 75$ \\
\hline Anti-hypertensive drugs (\% patients) & 71.9 & 79.8 & 74.4 & 81.6 \\
\hline Lipid-lowering drugs (\% patients) & 32.3 & 39.6 & 63.4 & 72.4 \\
\hline
\end{tabular}

Characteristics of patients who initiated insulin between August 2002 and July 2006 
the UK. For $\mathrm{HbA}_{1 \mathrm{c}}>10.0 \%$, percentages were $6 / 6$ in Germany and 13/13 in the UK. Mean duration of insulin therapy was just over 2 years in all groups, but patients in the UK had had diabetes for longer (10.0/10.5 years vs. 4.0/4.8 years in Germany). In both countries, most patients were receiving anti-hypertensive drugs, but mean systolic blood pressure was higher than the 130 $\mathrm{mmHg}$ target recommended for people with diabetes. A sizeable proportion of patients (greater in the UK) were on lipid-lowering medication. Results did not differ markedly for patients treated by specialists or PCPs in Germany. Conclusion: In patients who had been on insulin for around 2 years, approximately two-thirds in Germany and four-fifths in the UK did not meet the $\mathrm{HbA}_{1 \mathrm{c}}$ target of $7 \%$. Thus, despite widespread awareness of $\mathrm{HbA}_{1 \mathrm{c}}$ targets, better control is still needed. Insulin therapy was initiated earlier in the disease in Germany, and mean $\mathrm{HbA}_{1 \mathrm{c}}$ levels were lower, compared with the UK. Initiating patients on insulin sooner is one possible strategy for reducing the risk of longer-term diabetes-related complications.

Supported by: Novo Nordisk A/S

\section{2}

Effects of insulin treatment on myocardial function, perfusion and glucose metabolism in patients with primary left ventricular dysfunction and type 2 diabetes: a pilot study

S. Masi ${ }^{1}$, P. Iozzo ${ }^{1}$, C. Simi ${ }^{1}$, F. Frassi ${ }^{1}$, M. Emdin ${ }^{1}$, P. Pisani ${ }^{1}$, S. Pardini ${ }^{1}$, C. Porciello ${ }^{1}$, A. Giorgetti ${ }^{1}$, D. Petroni ${ }^{1}$, D. Giannessi ${ }^{1}$, P. Di Cecco ${ }^{1}$, P.A. Salvadori ${ }^{1}$, D. Neglia ${ }^{1}$, E. Ferrannini ${ }^{1,2}$

${ }^{1}$ Institute of Clinical Physiology, CNR National Research Council, Pisa,

${ }^{2}$ Department of Internal Medicine, University of Pisa School of Medicine, Italy

Background and aims: Chronic hyperglycaemia is a major risk factor in heart disease. The role of insulin in cardiac protection is controversial, as acute insulin stimulation increases myocardial perfusion and glucose disposal, but chronic insulin exposure is implicated in the development of sympathetic activation, arrhythmogenic risk, dyslipidaemia, insulin resistance and atherosclerosis. Direct effects of prolonged insulin treatment on myocardial metabolism and perfusion, especially in relation to changes in glucose control, are not known. We investigated myocardial and metabolic outcomes of insulin glargine (GLAR) treatment in a human model of non-ischaemic left ventricular dysfunction (LVD) and Type 2 diabetes (T2DM).

Materials and methods: The study included 9 patients (age range 55-72 years, BMI $27.7 \pm 2.9 \mathrm{~kg} / \mathrm{m}^{2}, \mathrm{HbA}_{1 \mathrm{c}} 6.2 \pm 0.8 \%$ ), undergoing a 1-month runin or wash-out period, as appropriate, followed by 6 months of GLAR treatment. After run-in and at the end of the treatment period, insulin-mediated myocardial perfusion (MBF) and glucose uptake (MGU) were evaluated by ${ }^{13} \mathrm{~N}$-ammonia or ${ }^{18} \mathrm{~F}$-fluoro-deoxy-glucose and positron emission tomography, during euglycaemic hyperinsulinaemia $\left(1 \mathrm{mU} \cdot \mathrm{min}^{-1} \cdot \mathrm{kg}^{-1}\right)$. On a different day, MBF was measured at rest and during pharmacologic stress with adenosine. Coronary flow reserve (CFR) was computed as the ratio of stress to rest MBF. Left ventricular (LV) ejection fraction (EF) and end-diastolic diameter (EDD) were determined by 2D-echocardiography. Safety measurements included the lipid profile, circulating noradrenaline levels, inflammatory markers, liver enzymes and serum creatinine.

Results: Fasting plasma glucose (PG) levels were reduced within 15 days (from $6.9 \pm 1.5$ to $5.9 \pm 1.1 \mathrm{mmol} / \mathrm{L}, \mathrm{p}<0.01$ ), and maintained throughout the study. Fasting insulin levels were almost doubled during the treatment period, but whole-body insulin-mediated glucose disposal was preserved (19.4 \pm 7.2 vs $\left.17.1 \pm 8.2 \mu \mathrm{mol} \cdot \mathrm{min}^{-1} \cdot \mathrm{kg}^{-1}, \mathrm{p}=\mathrm{ns}\right)$; other cardiac risk factors (lipid profile, neuroendocrine activation, $\mathrm{C}$-reactive protein, blood pressure, heart rate) were not affected, and body weight was stable. No change in liver or kidney function was noted. Baseline MGU and CFR were $0.39 \pm$ $0.28 \mu \mathrm{mol} \cdot \mathrm{min}^{-1} \cdot \mathrm{g}^{-1}$ and $1.82 \pm 0.49$ and did not change after treatment $(0.31$ $\pm 0.16 \mu \mathrm{mol} \cdot \mathrm{min}^{-1} \cdot \mathrm{g}^{-1}$ and $1.68 \pm 0.48$, respectively, $\mathrm{p}=\mathrm{ns}$ vs baseline). At entrance, LVD was characterized by EF and EDD of $42 \pm 11 \%$ and $66 \pm 8 \mathrm{~mm}$, respectively. The improvement in glucose control with insulin treatment was a significant predictor of positive changes in stress-MBF $(r=0.86, p=0.007)$, CFR $(r=0.75, p=0.032)$ and MGU $(r=0.74, p=0.022)$. Patients with a lower $\mathrm{EDD}$ at entrance were the best responders in terms of $\mathrm{CRF}(\mathrm{r}=0.67, \mathrm{p}=0.071)$ and insulin-mediated MBF ( $\mathrm{r}=0.70, \mathrm{p}=0.053)$.

Conclusion: We found that treatment with GLAR improves glucose control without affecting metabolic and cardiac risk factors or LV function in these patients with non-ischaemic LVD and T2DM. Changes in PG levels induced by GLAR appeared to predict a beneficial response in myocardial perfusion and metabolism. These results will need to be confirmed in a study with a larger number of patients.

Supported by: sanofi-aventis

\section{PS 090 Insulin pumps}

\section{3}

One year continuous use of real time glucose monitoring combined with insulin pump, as a mean to normalise glucose variability and $\mathrm{HbA}_{1 \mathrm{c}}$ in IDDM

C.S. Zoupas ${ }^{1}$, O. Kepaptzoglou ${ }^{1}$, N. Kefaloyannis ${ }^{2}$, I. Taraoune ${ }^{1}$, F. Giannakopoulos ${ }^{1}$, M. Sfakianaki ${ }^{2}$, P. Garoutsou ${ }^{1}$, H. Vasilopoulos ${ }^{3}$, E. Kyrlaki², A. Pappas ${ }^{2}$

${ }^{1}$ Diabetes Center, Hygeia General Hospital, Athens, ${ }^{2}$ Diabetes Center, Venizelio-Pananio General Hospital, Heraklion, Crete, ${ }^{3}$ Diabetes Center, Evaggelismos General Hospital, Athens, Greece

Background and aims: The usefulness of long term impact of continuous use of Paradigm REAL- Time System (PRT, Medtronic Minimed) on glycemic control is not yet known. PRT is an Insulin Infusion Pump combined with a Continuous Glucose Monitoring System (CGMS).The aim of this study was to evaluate the effect of the system on glycemic control, glucose fluctuations and insulin requirements for a long period of time.

Materials and methods: A total of 15 patients, mean age $34.0 \pm 20.3$ years and diabetes duration $16.7 \pm 9.1$ years $(\mathrm{X} \pm \mathrm{SD}$ ), wore the Sensor-Augmented Pump system continuously for 12 months. For educational purposes all subjects were on real time CGM for the 1st month without the Pump function (remained on MDI). The Pump therapy was added in a second stage. HbAlc, average daily and monthly glucose, hypo/hyperglycemic excursions and total daily dose (TDD) were evaluated at baseline, 4 and 6 and 12 months respectively.

Results: $\mathrm{HbAlc}(\%)$ decreased from $8.31 \pm 1.49$ at baseline, to $7.09 \pm 0.67$ after $4,7.15 \pm 0.73$ after 6 months and $6.87 \pm 0.69(\mathrm{X}+\mathrm{SD})$ after 12 months, $(\mathrm{p}=0.009)$. Glycemic control improved from the beginning and the average Daily and Monthly Glucose Values did not significantly differ after 1, 4, 6 and 12 months (Table 1). The average Area Under the Curve (AUC) above $180 \mathrm{mg} / \mathrm{dl}^{\star} 24 \mathrm{~h}$ was $13.78 \pm 6.77 \mathrm{mg} / \mathrm{dl}^{\star} 24 \mathrm{~h}$ after the 1 st month use of real time CGM without Pump. Tend to be reduced after the introduction of Pump and remained lower, $10.76 \pm 4.79,10.75 \pm 4.79$ and $9.83 \pm 3.15 \mathrm{mg} / \mathrm{dl}{ }^{*} 24 \mathrm{~h}$ at 4,6 and 12 months respectively. Average monthly AUC below $70 \mathrm{mg} / \mathrm{dl}$ remained constant throughout the study period (Table 1), and severe hypoglycemia occurred only once! No significant weight gain were noticed. There was a statistically significant decrease of Total Daily Insulin Dose from $54.1 \pm 16.1$ to $43.1 \pm 19.0,40.4 \pm 17.3$ and $39.8 \pm 16.8$ units $(\mathrm{X} \pm \mathrm{SD})$ at 4,6 and 12 months. In addition the Total Daily Bolus Insulin Dose decreased gradually despite the increased number of boluses given by the patients in order to avoid extreme high glucose excursions. (Table1).

Conclusion: Our results indicate that one year continuous use of PRT provide a useful tool to normalize glycemic control in IDDM. It decreases HbAlc and AUC of high glucose excursions. Patients were driven by the system to the intensification of insulin therapy with more efficient use of bolus. As the time pass the patient learns to react correctly to the predictive alarms of high and low excursions and remains motivated to achieve his targets for a long period of time. 


\begin{tabular}{|c|c|c|c|c|c|c|}
\hline MONTHS & BASELINE & 1 & $\mathrm{p}$ & 4 & 6 & 12 \\
\hline $\begin{array}{l}\text { MONTHLY AVERAGE } \\
\text { GLUCOSE (mg/dl) }\end{array}$ & & 149.3 & & 145.8 & 147.9 & 143.9 \\
\hline $\begin{array}{l}\text { AREA ABOVE HIGH } \\
\text { LIMITS }\left(\mathrm{mg} / \mathrm{dl}^{\star} 24 \mathrm{~h}\right)\end{array}$ & & 13.78 & & 10.76 & 10.75 & 9.73 \\
\hline $\begin{array}{l}\text { AREA BELOW LOW } \\
\text { LIMITS }\left(\mathrm{mg} / \mathrm{dl}^{*} 24 \mathrm{~h}\right. \\
\end{array}$ & & 0.5 & & 0.5 & 0.4 & 0.6 \\
\hline HbAlc (\%) & 8.31 & & & 7.09 & 7.15 & 6.87 \\
\hline DIF- WEIGHT (kg) & 0 & & & 1.4 & 0.5 & 0.6 \\
\hline $\mathrm{TDD}(\mathrm{U})$ & 54.1 & & & 43.1 & 40.4 & 39.8 \\
\hline BASAL/BOLUS (\%) & $54 / 46$ & & & $48 / 52$ & $50 / 50$ & $53 / 47$ \\
\hline No BOLUS & 2.9 & & & 5.8 & 6.1 & 6.8 \\
\hline BASAL TDD(U) & 29.2 & & & 20.7 & 20.2 & 21.1 \\
\hline BOLUS TDD $(\mathrm{U})$ & 24.2 & & & 23.4 & 20.2 & 18.7 \\
\hline
\end{tabular}

\section{4}

The natural history of residual beta cell function in patients with type 1 diabetes under continuous subcutaneous insulin infusion therapy

S. Cernea ${ }^{1}$, C. Moretti ${ }^{2}$, C. Tubili ${ }^{3}$, C. Suraci ${ }^{4}$, D. Pitocco ${ }^{5}$, I. Barchetta ${ }^{6}$, S. Manfrini ${ }^{1}$, N. Napoli ${ }^{1}$, R. Buzzetti ${ }^{2}$, P. Pozzilli ${ }^{1}$

${ }^{1}$ Endocrinology and Diabetes, University Campus Bio-Medico,

${ }^{2}$ Endocrinology and Diabetes, University, ${ }^{3}$ Endocrinology, San CamilloForlanini Hospital, ${ }^{4}$ Endocrinology, San Pertini Hospital, ${ }^{5}$ Endocrinology, Catholic University, ${ }^{6} \mathrm{Clinical}$ and Experimental Hepathology, University

"Sapienza", Roma, Italy

Background and aims: Evidence exists that preservation of even some $\beta$ cell function improves the metabolic control and impacts on long-term disease outcomes. Few data exist regarding the relationship between continuous subcutaneous insulin infusion (CSII) therapy with residual $\beta$ cell function. Small studies have suggested that implementation of intensive insulin therapy by CSII at diagnosis is effective in protecting $\beta$ cell function even 2 years after diagnosis. The aim of this study was to investigate the residual $\beta$ cell function as measured by $\mathrm{C}$ peptide levels in patients with type 1 diabetes (T1D) receiving CSII across different ages, duration of disease and of CSII therapy.

Materials and methods: This was a cross-sectional study in which patients with T1D on CSII were recruited by participating centers of the IMDIAB group. Clinical features and parameters of metabolic control were obtained. Patients had the basal C peptide levels measured and we calculated the "stimulated" C peptide values by using an empirical formula based on an extrapolation of stimulated C peptide values from the DCCT trial. We have used the cutoff level of $0.2 \mathrm{nM}$ as the clinically relevant "stimulated" C peptide value. According to the time of CSII initiation, patients were divided in 4 groups: A) <1 year; B) 2-5 years; C) 6-15 years; and D) 16-40 years after diagnosis. C peptide values were compared between groups by means of ANCOVA and $t$ tests and statistical significance assumed at $\mathrm{p}<0.05$.

Results: The study included 42 patients with T1D on CSII with a mean age of $37.5 \pm 11.3$ years and a mean duration of diabetes (DD) of $16.1 \pm 11.5$ years. Group A $(\mathrm{n}=5)$ had a mean DD of $4.4 \pm 3.1$ years, started pump therapy at $0.8 \pm 0.4$ years after diagnosis and has been on CSII for $3.4 \pm 3.1$ years. The $\mathrm{HbAlc}$ level was $7.1 \pm 0.8 \%$, BMI: $23.3 \pm 2.2 \mathrm{~kg} / \mathrm{m}^{2}$ and the insulin dosage: $0.47 \pm 0.02 \mathrm{IU} / \mathrm{kg} /$ day. In this group the basal C peptide level was $0.17 \pm 0.2 \mathrm{nM}$ and calculated $C$ peptide was $0.49 \pm 0.5 \mathrm{nM}$, more than twice above the clinical relevant threshold. Group B $(n=10)$ had a mean DD of $5.9 \pm 2.3$ years, but started CSII at $3.5 \pm 1$ years after disease onset and had been on a pump for $2.4 \pm 2$ years. The metabolic control (HbAlc: $7.4 \pm 1.5 \%$ ) and insulin requirement: $(0.52 \pm 0.1 \mathrm{IU} / \mathrm{kg} /$ day $)$ were similar to group $\mathrm{A}, \mathrm{p}=\mathrm{NS}$. The $\mathrm{C}$ peptide values were much lower than in group A (basal: $0.04 \pm 0.04 \mathrm{nM}$; calculated: $0.11 \pm 0.1 \mathrm{nM})(\mathrm{p}<0.05)$, already half of the significant threshold. As expected, the $C$ peptide levels in group $C(n=14)$ with mean time of CSII initiation of $10.5 \pm 3.2$ years was also low (basal: $0.05 \pm 0.07 \mathrm{nM}$; calculated: $0.14 \pm 0.2 \mathrm{nM}$ ) and even lower in group D ( $\mathrm{n}=13)$ (time of CSII initiation $25.1 \pm 6.6$ years) (basal: $0.02 \pm 0.01 \mathrm{nM}$; calculated: $0.07 \pm 0.05 \mathrm{nM}$ ).

Conclusion: Data from our study suggest that even as far as 4 years after diagnosis there is still some residual $\beta$ cell function, as evaluated by $\mathrm{C}$ peptide measurement in patients with T1D. The severe decrease in C peptide levels seen with delayed CSII startup suggests that early initiation of pump therapy is critical in preservation of residual $\beta$ cell function.

\section{5}

The hourly basal insulin dose of Japanese type 1 diabetic patients is flatter than that of European patients

M. Hirose, T. Kawamura, T. Hashimoto, T. Higashide, K. Kimura, H. Inada, S. Niihira, S. Aono, T. Yamano

Pediatrics, Osaka City University Graduate School of Medicine, Osaka, Japan

Background and aims: About CSII treatment of type 1 diabetes patients, some European studies have showed that the percentage of basal dose made up over the half of total daily insulin dose (TDD), and hourly basal dose was the highest in the dawn period(1.2-1.5 times from average basal insulin dose). In our experience of CSII in Japanese Type 1 diabetes patients, the basal insulin infusion rates were lower than that reported in these Europian studies. In the present study, we analyzed the hourly basal insulin dose of Japanese type 1 diabetes patients on CSII.

Materials and methods: Subjects are 51 ( male : female $=12: 39$ ) type 1 diabetic patients with CSII treatment of Minimed 508. The average age is 14.4 \pm 7.1 (from 1 to 33 years old ). And their average BMI is $20.9 \pm 3.7$, and their average $\mathrm{HbAlc}$ is $7.8 \pm 1.4 \%$.

The dose of bolus insulin was adjusted by carbohydrate counting. The hourly basal insulin dose was adjusted according to the stability of blood glucose level without meal and correction insulin, such as 30 rule.

We downloaded their all insulin infusion history for 4 weeks by Medtronic Com Station.

We have analyzed the various parameters which might affect on the basal insulin rate such as, age, puberty, sex, metabolic and metabolic control.

Results: TDD was $41.6 \pm 19.6 \mathrm{U} /$ day, and the basal insulin dose in TDD was 40.9 $\pm 11.9 \%$. The average hourly basal insulin dose of all patients was $0.63 \sim 0.76 \mathrm{U} / \mathrm{h}$ and almost constant all through the day, so called the flat pattern. In one third of the subjects have the two wave pattern of hourly basal doses which were high at early morning and early evening. However, the highest hourly basal dose was less than 1.1 times of the average dose. In the poor diabetic control group (HbAlc > $7.5 \%$ ), the percentage of basal insulin dose in TDD was significantly higher than that of good controlled group (HbAlc $<7.5)$ ( $44.9 \%$ vs. $37.3 \%)$. Adversely TDD in this group was lower than that of good control group ( $0.87 \mathrm{vs.} 0.92 \mathrm{U} / \mathrm{kg} /$ day). Furthermore in the poor control group, the hourly basal insulin dose was the highest in daytime. We speculated that they have increased daytime basal insulin dose because of the shortage of their bolus insulin dose. Neither sex nor puberty nor age was related to the patterns of hourly basal rate in this study.

Conclusion: From all data downloaded by CSII, the basal insulin dose and the changes of the hourly basal insulin dose of Japanese type 1 diabetes patient are less than that of European patients. Japanese patients may have little effect of insulin in dawn phenomenon. And we have speculated these results may be caused by Japanese dietary habit such as high-carbohydrate diet.

\section{6}

Drop out from continuous subcutaneous insulin infusion: an Italian survey

F. Lombardo ${ }^{1}$, D. Iafusco ${ }^{2}$, R. Bonfanti ${ }^{3}$, P. Buono ${ }^{4}$, V. Cherubini ${ }^{5}$, G. D’Annunzio ${ }^{6}$, P. Frongia ${ }^{7}$, D. Lo Presti ${ }^{8}$, F. Prisco ${ }^{9}$, I. Rabbone ${ }^{10}$, G. Salzano ${ }^{11}$, R. Schiaffini $i^{12}$, A. Scaramuzza ${ }^{13}$, S. Toni ${ }^{14}$, S. Tumini ${ }^{15}$ ${ }^{1}$ Department of Pediatrics, University of Messina, ${ }^{2}$ Ped., 2nd Univ.Naples, ${ }^{3}$ Ped., HSR, Milan, ${ }^{4}$ Ped., Federico II, Naples, ${ }^{5}$ Ped, Univ., Ancona, ${ }^{6}$ Ped., Univ, Genoa, ${ }^{7}$ Ped., G.Brotzu H., Cagliari, ${ }^{8}$ Ped, Univ, Catania, ${ }^{9}$ Ped, 2 nd Univ. Naples, ${ }^{10} \mathrm{Ped}$, OIRM, Turin, ${ }^{11} \mathrm{Ped}$, Univ, Messina, ${ }^{12} \mathrm{Ped}$, OPBG, Rome, ${ }^{13}$ Ped, L. Sacco H., Milan, ${ }^{14}$ Ped, Meyer H., Florence, ${ }^{15}$ Ped, Univ, Chieti, Italy

Background and aims: The continuous subcutaneous insulin infusion (CSII) is an efficacious option to the multiple daily injections (MDI) in the treatment of children and adolescents with type 1 diabetes mellitus (T1DM). Unfortunately, this is an expensive therapy and the identification of the most appropriate sub-population suitable for CSII treatment is crucial in order to avoid drop-out. For this reason, recently, a detailed guideline on CSII has been published by the Italian Society for Pediatric Endocrinology and Diabetology (ISPED) (Acta Bio Medica 2008). For the last three years we have been involved in a collaborative study aiming to characterize the reasons for the drop-out. Our results showed that the main reason was not only the patient's 
selection but also a time-based failure: in fact, the drop-out rate is higher in the first 6-months of CSII therapy (J Ped 2007).

Materials and methods: This is the first national study on behalf of the Italian Study Group on Diabetes of ISPED. We collected data from 22 Italian Pediatric Diabetes Centres during a 2-month period from January to February 2008. The overall study population consisted in 9314 children and adolescents with T1DM. We are analyzing all the data in order to calculate the prevalence of patients which chose to drop-out, the main reasons and the timing of drop-out, the metabolic control obtained during the CSII therapy. Results: This abstract presents only preliminary results of the collaborative study. We can assure that results will be completed at the time of the EASD Congress. Among the overall the study population with T1DM, 1165 children and adolescents are on CSII, representing a rate of $12.5 \% .83$ patients $(42 \mathrm{M}$, 41 F) dropped-out (7.1\%) after $20.8 \pm 17.2$ months treatment period. The main reasons of the drop-out were psychological issues and poor compliance. The rate of major adverse events, such as DKA or severe hypoglycaemic events, during the CSII treatment, does not statistically differ from the one reported in the MDI period treatment. No other adverse effects were reported during CSII therapy. The rate of drop out was respectively 16 patients (19.2\%), in the first 6 months, 16 patients $(19,2 \%)$, between 6 and 12 months, 23 patients (27.2\%) between 12 and 24 months, 12 patients (14\%) between 24 and 36 months and 16 patients $(19,2)>36$ months.

Conclusion: Our study represents, to our knowledge, the first national report on CSII-drop out rate. CSII is an useful tool, it is reasonable to use it when there are appropriate indications, especially in the pediatric age. The national data are in disagree with the results of a previous study performed in two pediatric diabetes centres of Southern Italy in which we found that the drop out rate was higher in the first six months of CSII therapy. In fact, on the basis of our national preliminary data, in Italy CSII drop-out incidence is likely to be homogeneously distributed over time.

Supported by: Diabetes Study Group of the Italian Society of Pediatric Endocrinology and Diabetology

\section{7}

Cross-sectional study: continuous subcutaneous insulin infusion or multiple daily injections for functional insulin treatment in type 1 diabetes?

J. Pumprla, K. Howorka, M. Puck, I. Mihaljevic, N. Howorka, E. Perneczky, M. Gabriel

Centre of Biomedical Engineering \& Physics, Res Group Functional Rehabilitation, Vienna, Austria

Background and aims: Continuous subcutaneous insulin infusion (CSII) in type 1 diabetes results usually in moderate improvement of glycaemic control and diabetes-related quality of life. Such improvement is dependent on diabetes education provided and inversely related to the initial glycaemic control on pre-pump treatment. Our aim was to investigate motifs and characteristics of FIT- (functional insulin treatment) educated patients with type 1 diabetes who later on have chosen CSII vs. those who have chosen multiple daily injections, MDI. Furthermore, we evaluated the impact of such freely chosen treatment strategy (CSII or MDI) on parameters of metabolic control, quality of life and treatment satisfaction.

Materials and methods: Cross-sectional study comparing the outcomes in FIT-educated patients with CSII and in MDI controls using functional insulin treatment with injections. Additionally, the impact of CSII and MDI on metabolic control was assessed in a longitudinal retrospective pilot investigation. Structured outpatient FIT UPDATE module (educational weekend specifically developed for FIT patients) including detailed information on insulin pumps and pens was used for the invitation to participate in the study. Eventually, 25 type 1 patients with CSII, and 112 with MDI $(6.3 \pm 1.4$ daily injections, insulin analogs in $93 \%$ ) comparable in diabetes (20 yrs) and FIT (11 yrs) duration, and associated diseases were included in the study, and 4 invited patients ( 2 with CSII and 2 with MDI) rejected such voluntary participation.

Results: Differences have been found for reported number of daily blood glucose self-measurements (CSII: $5.6 \pm 0.9$ vs MDI: $5.1 \pm 0.8, \mathrm{p}=0.01$ ), perceived dietary flexibility ( $\mathrm{p}=0.03)$, DTSQ for FIT subscore for basal insulin satisfaction ( $13 \pm 3$ vs $12 \pm 4, \mathrm{p}=0.02)$ but not for HbAlc $(7.1 \pm 0.9$ vs $7.3 \pm 1.0 \%$, $\mathrm{p}=0.4$ ), neither for frequency of daily dosage adjustment $(\mathrm{p}=0.3)$, treatment satisfaction total score (Bradley s et al. DTSQ extended for FIT: $90 \pm 20$ vs $86 \pm 20, p=0.4$ ) and its subscores (DTSQ classic total score, $\mathrm{p}=0.7$, subscores predictability $\mathrm{p}=0.8$, hypoglycaemia proneness $\mathrm{p}=0.5$, correctional insulin $\mathrm{p}=0.3$, prandial insulin $\mathrm{p}=0.5$ ) nor for diabetes-specific quality of life (Bradley s ADDQoL), and subscores (all $p>0.3$ : for working life, family life, friendship, sex, physical appearance, things to do, holidays and travel, self-confidence, society reaction, future, finances and dependence), with tendency towards significance for subscore in motivation showing higher motivation in pump patients $(\mathrm{p}=0.07)$.

Conclusion: Functional insulin use with in average more than 6 daily injections results in FIT-educated patients in similar outcomes independently of the mode of insulin delivery. Pump treatment is associated with slightly more "motivation" for treatment.

\section{8}

The access to insulin pump therapy in type 1 children and adolescents has largely increased in France since 2001

N. Tubiana-Rufi ${ }^{1}$, V. Sulmont ${ }^{2}$, V. Lassmann-Vague ${ }^{3}$, B. Guerci ${ }^{4}$, H. Hanaire ${ }^{5}$, H. Leblanc ${ }^{6}$, E. Leutenegger ${ }^{7}$, M. Mihaileanu ${ }^{8}$

${ }^{1}$ Endocrinology and Diabetology Department, Robert Debré Hospital, Paris, ${ }^{2}$ Endocrinology and Pediatry Department, American Memorial Hospital, Reims, ${ }^{3}$ Marseille, ${ }^{4}$ Diabetology and Endocrinology Department, Jeanne d'Arc Hospital, Nancy, ${ }^{5}$ Diabetology, metabolic diseases and nutrition department, Rangueil Hospital, Toulouse, ${ }^{6}$ Diabetology and Endocrinology Department, Saint-Louis Hospital, Paris, ${ }^{7}$ ABR Pharma, Paris, France, ${ }^{8}$ Medical department, VitalAire, Paris, France

Background and aims: Insulin pump therapy in paediatric patients with type 1 diabetes (T1D) has been developing in many countries in the last few years. The present study aims at updating assessment of the use of pump therapy in T1D children and teenagers aged $<18$ in France, in comparison with a previous survey performed in 2001 (one year after the treatment started to be reimbursed by the French health service).

Materials and methods: A national survey was performed in France in 2007. A questionnaire on insulin pump prescription in T1D children and teenagers was sent to all French paediatric centres and 113 adult diabetes centres.

Results: 60 paediatric centres (89\%) and 28 adult diabetes centres (25\%) answered the survey. The answering paediatric centres are localized in all the French regions and follow up 8,477 T1D children and adolescents (Med: 85 patients/centre. Min: 15. Max: 650). Only 4 centres do not use insulin pump, meaning that $93 \%$ of the answering paediatric centres use this therapy. The number of young patients treated with pump is 1,304, representing $15.4 \%$ of the total T1D patients ( $18 \pm 14 \%$ per centre on average). The insulin pump treatment was initiated during the preceding year in 491 young patients $(38 \%$ of patients treated with pump). Among the 28 adult diabetes centres answering the study, 22 effectively manage young diabetes patients. They follow up 596 T1D children and teenagers (Med: 23 patients/centre. Min: 1. Max: 60). 157 of these patients are treated with insulin pump $(26.3 \%$ of the total young patients managed) (32 $\pm 22 \%$ per centre). Compared to the results of the 2001 study in paediatric centres, these data indicate that the number of young T1D patients treated by insulin pump has increased almost 10 times within 6 years: in 2001, 13 paediatric centres were following only 140 children and adolescents treated by an insulin pump, versus 56 centres and 1,304 young patients under pump therapy in 2007.

Conclusion: This study, conducted in a large sample of French T1D children and adolescents, representing 60 to $75 \%$ of the total national estimated population, emphasizes the large increase within the last 6 years of the use of pumps in paediatric centres in all French regions. At the moment, $16 \%$ of a total of 9073 T1D paediatric patients benefit from this therapy (89\% in paediatric centres). The medical training programs, the implementation of pumps reimbursement and appropriate medical care organisation, have enabled to improve the access to insulin pump treatment for paediatric patients in France. The study is actually on going through an observational program, in order to evaluate more precisely the indications and the modalities of paediatric insulin pump management in France.

\section{9}

Insulin requirement in children under 6-year-old treated with insulin pump from the onset of type 1 diabetes mellitus: a 5-year follow-up L. Groele, A. Szypowska, M. Lipka, M. Błazik, D. Golicka, E. Pańkowska Department of Diabetology, Neonatology and Birth Defects, Medical University of Warsaw, Poland

Background and aims: Continous increase of type 1 diabetes (T1DM) incidence among young children is a challenge to establish an effective and safe 
method of insulin delivery. The aim is to assessment of insulin requirement of total daily dose (TDD) and basal insulin regarding to metabolic control in young children treated with insulin pump from the onset of T1DM

Materials and methods: 58 children (31 girls) under 6-year-old (mean age $3,3 \pm 1,5$ years) were analyzed for 175 patient-years of follow-up. Data was collected every six month:HbAlc, SDS BMI, diabetic ketoacidosis (DKA) and severe hypoglycaemia (SH), TDD and basal insulin were downoloaded from pump memory.

Results: $\mathrm{HbAlc}$ decreased from $9,6 \%$ at recognition to $6,7 \%$ at $3^{\text {rd }}$ month $(\mathrm{p}<0,0001)$. During 5 -year follow-up metabolic control was sustained (HbAlc:6, $8 v s 6,7 v s 6,9 v s 7,0 v s 7,1 v s 7,1 v s 7,3 v s 7,1 v s 6,9 v s 7,2 \%) p=0,002$. TDD increased from $0,4 \mathrm{U} / \mathrm{kg} / \mathrm{d}$ to $0,7 \mathrm{U} / \mathrm{kg} / \mathrm{d} \mathrm{p}<0,0001$. The insulin daily dose increased from $0,5 \mathrm{U} /$ $\mathrm{kg} / \mathrm{d}$ in the $6^{\text {th }}$ months to $0,6 \mathrm{U} / \mathrm{kg} / \mathrm{d}$ in the $12^{\text {th }}$ months and than was sustained $0,7 \mathrm{U} / \mathrm{kg} / \mathrm{d} \mathrm{p}<0,0001$. The basal insulin raised from $10 \%$ to $25 \%$ of TDD $\mathrm{p}<0,0001$, SDS BMI ranged from 0,0 to $0,4 \mathrm{p}=0,48$. There was one episod of DKA and one of SH. $66 \%$ children walked to kindergarden and 70\%mothers work profesionally Conclusion: The insulin requirement has been increased throughout the first year after T1DM recognition, than has been kept at $0,7 \mathrm{U} / \mathrm{kg} / \mathrm{d}$ with basal insulin below 30\% TDD.Initiating insulin pump therapy at onset of T1DM in young children provides sustained metabolic control, with minimal risk of $\mathrm{SH}$ and DKA and without increase of body mass

\section{0}

Achievement of good glycaemic control with increased C-peptide secretion in poorly controlled type 2 diabetic patients via long-term continuous subcutaneous insulin infusion

S.B. Choi ${ }^{1}$, J.H. Lee', J.H. Lee ${ }^{2}$, J.H. Park ${ }^{2}$, Y.I. Jo' ${ }^{1}$, Y.H. Noh ${ }^{2}$

${ }^{1}$ Internal Medicine, ${ }^{2}$ Biochemistry, Konkuk University, Seoul, Republic of Korea

Background and aims: Continuous subcutaneous insulin infusion (CSII) therapy is increasingly considered a promising therapeutic strategy in both T1DM and T2DM, however, little is known about the long-term beneficial effects of CSII in T2DM in terms of glycaemic control and possibility of the prevention and/or delay of the development of diabetic complications. To see whether hyperglycaemia is optimally controlled via CSII in patients with T2DM who had failed with OHA and/or insulin injection and the achievement of good glycaemic control is accompanied with improvement of other abnormal conditions, we compared $\mathrm{HbA}_{1 c}$ levels and laboratory findings including lipid profile and serum C-peptide [before (AC) and 2 hours after meal (PP2)] levels before and up to 24 months after CSII therapy.

Materials and methods: Among T2DM patients treated with CSII at the Diabetes Center of Konkuk University Hospital from 2005 to 2007 we selected 531 patients (male $53 \%, \mathrm{HbA}_{1 \mathrm{c}} 9.24 \pm 1.96 \%$, Table 1 ) with poorly controlled but without severe nephropathy at baseline $\left(\mathrm{HbA}_{1 \mathrm{c}} \geq 7.0 \%\right.$ and serum creatinine $\leq 1.5 \mathrm{mg} / \mathrm{dl}$ ). We analyzed $\mathrm{HbA}_{1 \mathrm{c}}$ and the other laboratory data examined at 6 month to 1 year interval rate.

Results: CSII therapy nearly normalized HbA level by $6.55 \pm 1.26 \%$ (29 $\%$ reduction compared to baseline level, $p<0.001$, Fig. $1 \mathrm{~A}$ ) at 6 month with gradual improvement over 24 months. The proportion of patients achieving $\mathrm{HbA}_{1 \mathrm{c}}$ target $<6.5 \%$ greatly increased ( $0 \%$ at baseline vs $71 \%$ at 24 month, Fig.1B). AC and PP2 C-peptide levels increased by $20 \%$ (at 12 month) and $27.6 \%$ (at 18 month), respectively ( $p<0.001$, for both) and were continuously improved. Patients with proteinuria decreased from $30 \%$ (at baseline) to $16 \%$ (at 24 month), however, creatinine clearance levels calculated by CockcroftGault equation remained unchanged. Diastolic but not systolic blood pressure level decreased throughout all the period $(78.6 \pm 11.6$ at baseline vs 70.5 $\pm 9.8 \mathrm{mmHg}$ at 24 month, $p<0.001)$, although the mean BMI increased by $7.7 \pm 1.0 \%$ compared to baseline level $(p<0.001)$. HDL, LDL, and TG levels increased 12,19 , and $7 \%$, respectively $(p<0.001$, for all), but the increased mean LDL and TG levels at 24 month were within normal range.

Conclusion: CSII achieved optimal glycaemic control and improved C-peptide secretion, proteinuria, and diastolic BP, suggesting resolution of glucotoxicity via CSII may be plausible even in T2DM patients with secondary failure.
Table 1. Clinical characteristics of the subjects at baseline

\begin{tabular}{llll}
\hline${ }^{*} \mathrm{p}<0.05$ vs Male & All subjects & Male & Female \\
\hline Number & 531 & 281 & 250 \\
\hline Age (years) & $56.6 \pm 11.6$ & $55.5 \pm 11.8$ & $57.9 \pm 11.2$ \\
\hline Known diabetes duration (years) & $11.1 \pm 7.4$ & $10.5 \pm 7.4$ & $11.8 \pm 7.4^{*}$ \\
\hline Blood pressure & $132.1 \pm 19.2$ & $133.6 \pm 21.3$ & $130.8 \pm 17.2$ \\
Systolic (mmHg) & $78.6 \pm 11.6$ & $78.9 \pm 12.5$ & $78.3 \pm 10.7$ \\
Diastolic (mmHg) & & & \\
\hline BMI (kg/m2) & $23.8 \pm 3.6$ & $23.4 \pm 3.2$ & $24.3 \pm 3.9^{*}$ \\
\hline Previous treatment & & & \\
\hline Diet only (\%) & 9.6 & 11.7 & 7.2 \\
\hline Oral hypoglycemic agent (\%) & 65.0 & 66.2 & 63.6 \\
\hline Intermediate-acting insulin (\%) & 20.0 & 17.8 & 22.4 \\
\hline Combination (\%) & 5.4 & 4.3 & 6.8 \\
\hline HbA1c (\%) & $9.24 \pm 1.96$ & $9.39 \pm 2.17$ & $9.08 \pm 1.69$ \\
\hline
\end{tabular}

(A) (B)

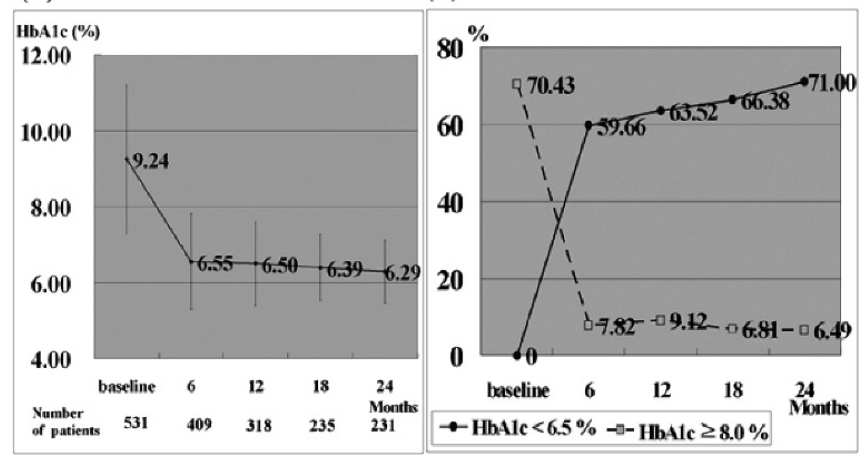




\section{PS 091 Insulin therapy: others}

\section{1}

Insulin glulisine improves glycaemic control in type 2 diabetic patients not optimally controlled with oral antidiabetic drugs: results of a randomised controlled study

J.-T. Woo' ${ }^{1}$, S.-W. Kim², S.-H. Baik³ , K.-H. Yoon ${ }^{4}$, R. Kawamori ${ }^{5}$,

Y. Iwamoto ${ }^{6}$, T. Kadowaki ${ }^{7}$, M. Iwasaki ${ }^{8}$

${ }^{1}$ Department of Endocrinology and Metabolism, Kyung Hee University Medical Center, Seoul, Republic of Korea, ${ }^{2}$ Department of Internal Medicine, Sungkyunkwan University School of Medicine, Kangbuk Samsung Hospital, Seoul, Republic of Korea, ${ }^{3}$ Department of Internal Medicine, Korea University Guro Hospital, Seoul, Republic of Korea, ${ }^{4}$ Department of Endocrinology and Metabolism, Kangnam Saint Mary's Hospital, Seoul, Republic of Korea, ${ }^{5}$ Department of Medicine, Metabolism and Endocrinology, School of Medicine, Juntendo University, Tokyo, Japan, ${ }^{6}$ Diabetes Center, Tokyo Women's Medical University, Japan, ${ }^{7}$ Department of Metabolic Diseases, Graduate School of Medicine, The University of Tokyo, Japan, ${ }^{8}$ Department of Computer and Information Science, Seikei University, Tokyo, Japan

Background and aims: The rapid-acting, insulin glulisine (GLU) with or without oral antidiabetic drugs (OAD) was compared with OAD therapy for efficacy and safety in Japanese and Korean type 2 diabetic (T2DM)patients inadequately controlled with $\mathrm{OAD}$ (sulfonylurea (SU) or $\mathrm{SU}+$ biguanide). Three treatment groups were assessed in this study: 3 daily injections of GLU in combination with OAD (GLU+OAD), GLU-only or OAD-only and the primary objective was the $\mathrm{HbA}_{1 \mathrm{c}}$ change from baseline to endpoint. Secondary objectives were plasma glucose parameters and safety.

Materials and methods: In an open, randomized, parallel group, comparative, 16-week, controlled trial, 393 patients were randomized and 387 patients (Japan: 198; Korea: 189) were treated with GLU+OAD ( $=130)$, GLU-only $(\mathrm{N}=127)$ or OAD-only $(\mathrm{N}=130)$. T2DM patients diagnosed for at least 1 year, $\mathrm{BMI}<30 \mathrm{~kg} / \mathrm{m}^{2}, \mathrm{HbA}_{1 \mathrm{c}} \geq 8.0 \%$ to $\leq 11.0 \%$, treated with a stable regimen of SU or SU + biguanide were eligible. GLU was titrated to achieve a 2-hour postprandial blood glucose value of 128 to $172 \mathrm{mg} / \mathrm{dL}$ using plasma-referenced blood glucose meter. In both GLU+OAD and OAD-only groups, prior OAD therapy was continued on a stable dose.

Results: Baseline $\mathrm{HbA}_{1 \mathrm{c}}$ values were similar among treatment groups. Both GLU groups had larger reductions in adjusted mean $\mathrm{HbA}_{1 \mathrm{c}}$ than the OAD group: (GLU+OAD - $2.07 \%$, GLU-only $-1.25 \%$, OAD-only - $0.61 \%$ ). Superiority of GLU+OAD and GLU-only to OAD-only was shown by a difference in adjusted mean $\mathrm{HbA}_{1 \mathrm{c}}$ change of $-1.46 \%(P<0.0001)$ and $-0.64 \%(P<0.0001)$, respectively (Table 1 ). Both GLU groups had better 2 -hour postprandial plasma glucose control than the OAD-only group. Mean daily GLU doses increased from baseline to endpoint: (GLU+OAD 13.3 to $22.5 \mathrm{U}$, GLU-only 14.2 to $38.0 \mathrm{U}$ ). The rate of all symptomatic hypoglycemia per patient year in the entire treatment phase was 11.9 in GLU+OAD, 8.8 in GLU-only and 1.7 in OAD-only. There was only 1 event of severe hypoglycemia (GLU+OAD). Efficacy and safety was similar in Japanese and Korean subpopulations. Conclusion: This study demonstrated that both GLU+OAD and GLU-only are well tolerated and effective in T2DM patients not optimally controlled with OAD therapy.

\section{2}

Initiation of insulin glargine versus intensified lifestyle management in people with type 2 diabetes and $\mathrm{HbA}_{1 \mathrm{c}} 7-8 \%$

J.-F. Blickle' ${ }^{1}$, N. Hancu², M. Piletic ${ }^{3}$, V. Profozic ${ }^{4}$, M. Shestakova ${ }^{5}$, M.-P. Dain ${ }^{6}$, S. Jacqueminet ${ }^{7}$, A. Grimaldi ${ }^{7}$

${ }^{1}$ Médecine Interne, Diabète et Maladies Métaboliques, Hôpitaux

Universitaires de Strasbourg, France, ${ }^{2}$ Clinical Center of Diabetes, Luliu Hatieganu University of Medicine, Cluj Napoca, Romania, ${ }^{3}$ Diabetes Department, General Hospital Novo Mesto, Novo Mesto, Slovenia, ${ }^{4}$ Department for Clinical Pharmacology, Vuk Vrhovac University Clinic, Zagreb, Croatia, ${ }^{5}$ Endocrinology Research Center, Russian Academy of Medical Sciences, Moscow, Russian Federation, ${ }^{6}$ sanofi-aventis, Paris, France, ${ }^{7}$ Service de Diabétologie, Hôpital de la Pitié, Paris, France

\begin{tabular}{|c|c|c|c|c|c|c|}
\hline & $\begin{array}{l}\text { Treatment an } \\
\text { GLU+OAD }\end{array}$ & GLU-only & OAD-only & $\begin{array}{c}\text { Difference, } \\
\text { GLU+OAD vs } \\
\text { OAD-only } \\
\text { [95\% Cl] }\end{array}$ & $\begin{array}{c}\text { Difference, } \\
\text { GLU-only vs } \\
\text { OAD-only } \\
\text { [95\% Cl] }\end{array}$ & P-value \\
\hline Total, N & 128 & 124 & 128 & & & \\
\hline Baseline & $8.99 \pm 0.08$ & $9.02 \pm 0.08$ & $9.04 \pm 0.08$ & $\begin{array}{l}-0.05 \pm 0.11 \\
{[-0.26 ; 0.16]}\end{array}$ & $\begin{array}{l}-0.02 \pm 0.11 \\
{[-0.23 ; 0.20]}\end{array}$ & $\begin{array}{l}- \\
-\end{array}$ \\
\hline Change & $-2.07 \pm 0.09$ & $-1.25 \pm 0.09$ & $-0.61 \pm 0.09$ & $\begin{array}{c}-1.46 \pm 0.12 \\
{[-1.69 ;-1.22]}\end{array}$ & $\begin{array}{c}-0.64 \pm 0.12 \\
{[-0.88 ;-0.41]}\end{array}$ & $\begin{array}{l}<0.0001^{\mathrm{b}} \\
<0.0001^{\mathrm{b}}\end{array}$ \\
\hline Japanese, $\mathrm{N}$ & 67 & 65 & 65 & & & \\
\hline Baseline & $8.96 \pm 0.10$ & $8.91 \pm 0.10$ & $9.01 \pm 0.10$ & $\begin{array}{l}-0.05 \pm 0.14 \\
{[-0.33 ; 0.23]}\end{array}$ & $\begin{array}{l}-0.10 \pm 0.14 \\
{[-0.39 ; 0.18]}\end{array}$ & - \\
\hline Change & $-2.21 \pm 0.10$ & $-1.31 \pm 0.10$ & $-0.45 \pm 0.10$ & $\begin{array}{c}-1.76 \pm 0.15 \\
{[-2.04 ;-1.47]}\end{array}$ & $\begin{array}{l}-0.86 \pm 0.15 \\
{[-1.15 ;-0.56]}\end{array}$ & $\begin{array}{l}<0.0001^{3} \\
<0.0001^{b}\end{array}$ \\
\hline Korean, $\mathrm{N}$ & 61 & 59 & 63 & & & \\
\hline Baseline & $9.02 \pm 0.12$ & $9.14 \pm 0.12$ & $9.07 \pm 0.11$ & $\begin{array}{l}-0.05 \pm 0.16 \\
{[-0.37 ; 0.27]}\end{array}$ & $\begin{array}{c}0.07 \pm 0.17 \\
{[-0.25 ; 0.40]}\end{array}$ & - \\
\hline Change & $-1.91 \pm 0.14$ & $-1.20 \pm 0.14$ & $-0.77 \pm 0.13$ & $\begin{array}{l}-1.14 \pm 0.19 \\
{[-1.52 ;-0.76]}\end{array}$ & $\begin{array}{l}-0.44 \pm 0.19 \\
{[-0.82 ;-0.06]}\end{array}$ & $\begin{array}{l}<0.0001^{a} \\
0.0248^{b}\end{array}$ \\
\hline
\end{tabular}

${ }^{a} \mathrm{GLU}+\mathrm{OAD}$ vs $\mathrm{OAD}$-only, ${ }^{\mathrm{b}} \mathrm{GLU}$-only vs $\mathrm{OAD}$-only. $\mathrm{GLU}=$ insulin glulisine, $\mathrm{OAD}=\mathrm{Oral}$ antidiabetic drugs

Supported by: sanofi-aventis 
HbAlc, FPG, body weight, insulin dose and hypoglycaemia

\begin{tabular}{|c|c|c|c|c|}
\hline & & Glargine $(\mathrm{n}=103)$ & $\mathbf{L M}(\mathrm{n}=108)$ & p-value \\
\hline$\%$ of patients with $\mathrm{HbA}_{1 \mathrm{c}}$ at endpoint & $\begin{array}{l}<7 \% \text { at endpoint } \\
<6.5 \% \text { at endpoint }\end{array}$ & $\begin{array}{l}66 \\
34 \\
\end{array}$ & $\begin{array}{l}38 \\
11 \\
\end{array}$ & $\begin{array}{l}<0.001 \\
0.0001\end{array}$ \\
\hline $\mathrm{HbA}_{1 \mathrm{c}}(\%)$ & $\begin{array}{l}\text { Baseline } \\
\text { Endpoint } \\
\text { Change } \\
\end{array}$ & $\begin{array}{l}7.6 \pm 0.3 \\
6.8 \pm 0.7 \\
-0.8 \pm 0.7 \\
\end{array}$ & $\begin{array}{l}7.5 \pm 0.4 \\
7.3 \pm 0.9 \\
-0.2 \pm 0.9 \\
\end{array}$ & $<0.0001$ \\
\hline FPG (mg/dL) & $\begin{array}{l}\text { Baseline } \\
\text { Endpoint } \\
\text { Change }\end{array}$ & $\begin{array}{l}170 \pm 34 \\
122 \pm 33 \\
-50 \pm 47 \\
\end{array}$ & $\begin{array}{l}167 \pm 27 \\
161 \pm 41 \\
-5 \pm 39 \\
\end{array}$ & $<0.0001$ \\
\hline Weight (kg) & $\begin{array}{l}\text { Baseline } \\
\text { Endpoint } \\
\text { Change }\end{array}$ & $\begin{array}{l}85.0 \pm 12.5 \\
86.0 \pm 13.4 \\
+0.91 \pm 2.9 \\
\end{array}$ & $\begin{array}{l}84.0 \pm 13.7 \\
81.5 \pm 13.5 \\
-2.5 \pm 3.2 \\
\end{array}$ & $<0.0001$ \\
\hline Daily insulin glargine dose (U/kg) & $\begin{array}{l}\text { Starting dose } \\
\text { Endpoint }\end{array}$ & $\begin{array}{l}0.12 \pm 0.05 \\
0.27 \pm 0.15 \\
\end{array}$ & $\begin{array}{l}\mathrm{n} / \mathrm{a} \\
\mathrm{n} / \mathrm{a}\end{array}$ & $\mathrm{n} / \mathrm{a}$ \\
\hline Hypoglycaemia (events patient per year) & $\begin{array}{l}\text { Symptomatic } \\
\text { Nocturnal } \\
\text { Severe }\end{array}$ & $\begin{array}{l}4.2 \pm 6.6 \\
0.7 \pm 2.1 \\
0.04 \pm 0.35\end{array}$ & $\begin{array}{l}2.0 \pm 7.8 \\
0.3 \pm 2.1 \\
0.00 \pm 0.00\end{array}$ & $\begin{array}{l}<0.0001 \\
0.0011 \\
0.147\end{array}$ \\
\hline
\end{tabular}

Results are given for all randomized and treated patients; 4 patients (insulin glargine, $3 ; \mathrm{LM}, 1$ ) were randomized, but not treated. Results are mean \pm standard deviation; $\mathrm{HbA}_{1 \mathrm{c}}=$ haemoglobin $\mathrm{A}_{1 \mathrm{c}} ; \mathrm{FPG}=$ fasting plasma glucose $\mathrm{LM}=$ lifestyle management; $\mathrm{n} / \mathrm{a}=$ not applicable

Background and aims: To determine whether earlier administration of insulin glargine versus intensified lifestyle management (LM) improves glycaemic control in Type 2 diabetes (T2DM) patients with haemoglobin $\mathrm{A}_{1 c}\left(\mathrm{HbA}_{1 c}\right)$ $7-8 \%$ treated with oral agents.

Materials and methods: TULIP (Testing the Usefulness of insulin gLargine [glargine] when Initiated Promptly in T2DM) was a 9-month, 12-visit, openlabel, multinational, multicentre, randomized study to evaluate starting glargine or intensifying LM in T2DM patients aged 40-75 years, body mass index (BMI) $24-35 \mathrm{~kg} / \mathrm{m}^{2}$ and $\mathrm{HbA}_{1 \mathrm{c}} 7-8 \%$, treated with maximum doses of metformin and sulphonylurea for $\geq 2$ years. Glargine was injected once daily (evening) and titrated to capillary fasting blood glucose (FBG) $70-100 \mathrm{mg} / \mathrm{dL}$. In the LM arm, dietary and physical activity counseling recommended stable weight for people with BMI $<27 \mathrm{~kg} / \mathrm{m}^{2}$ or weight loss of $3 \mathrm{~kg}$ for patients with BMI $\geq 27 \mathrm{~kg} / \mathrm{m}^{2}$. Daily calorie distribution could be maintained or reduced in patients whose BMI was $<27 \mathrm{~kg} / \mathrm{m}^{2}$ and reduced by one-third for patients whose BMI was $\geq 27 \mathrm{~kg} / \mathrm{m}^{2}$. Patients were recommended a diet composed of $50-55 \%$ carbohydrates, $30-35 \%$ lipids and $10-15 \%$ protein. Patients were recommended to undertake 2 hours of brisk walking or equivalent per week (in segments of 20 minutes) in addition to their daily activity. A total of 215 patients were randomized to glargine $(n=106)$ or LM $(n=109)$. The primary objective was to determine the percentage of patients achieving $\mathrm{HbA}_{1 c}<7 \%$ at endpoint. Secondary endpoints included changes in $\mathrm{HbA}_{1 \mathrm{c}}$ and fasting plasma glucose (FPG), and hypoglycaemia incidence.

Results: 211 (52.6\% male) patients were randomized and treated; mean $( \pm$ standard deviation) age $60.7 \pm 7.9$ years, weight $84.5 \pm 13.1 \mathrm{~kg}$, BMI $29.9 \pm 3.5$ $\mathrm{kg} / \mathrm{m}^{2}$ and $\mathrm{HbA}_{1 \mathrm{c}} 7.6 \pm 0.4 \%$. More patients reached $\mathrm{HbA}_{1 \mathrm{c}}<7 \%$ (66 vs $38 \%$; $\mathrm{p}<0.0001$ ) or $<6.5 \%$ (34 vs $11 \%$; $\mathrm{p}=0.0001$ ) with glargine versus LM. In contrast to the glargine-treated group, the LM-treated group showed a decrease in weight $(+0.9 \pm 2.9$ vs $-2.5 \pm 3.2 \mathrm{~kg} ; \mathrm{p}<0.0001)$ as well as the expected lower hypoglycaemia. Results for hypoglycaemia, $\mathrm{HbA}_{1 \mathrm{c}}$, FPG and weight are shown in the table.

Conclusion: In T2DM patients with $\mathrm{HbA}_{1 \mathrm{c}} 7-8 \%$, adding glargine to existing oral therapy resulted in greater improvements in glycaemic control versus intensified LM. Nevertheless, lifestyle management should be maintained by all patients with diabetes, including those using insulin.

Supported by: sanofi-aventis

\section{3}

Daily blood glucose profiles in subjects with type 2 diabetes in a randomised, crossover, double-blind comparison of insulin detemir and insulin glargine

A. King

Diabetes Department, Diabetes Care Center, Salinas, United States

Background and aims: Basal insulin analogues such as insulin detemir and insulin glargine are indicated for once-daily use as monotherapy or in combination with other antihyperglycaemic treatments in patients with type 2 diabetes (T2D). Both insulin detemir and insulin glargine have similar durations of action, as indicated by isoglycaemic clamp studies. The aim of this study was to compare 24 -hr glycaemic control with insulin detemir $v s$. insulin glargine using a continuous glucose monitoring system (CGMS).

Materials and methods: T2D patients using basal but not bolus insulin $(\mathrm{N}=35)$ were randomised to receive a single daily injection of insulin detemir or insulin glargine at $8 \mathrm{PM}$ for 1 week and switched to the other insulin the next week. Insulin was titrated to achieve blood glucose (BG) measurements $<120 \mathrm{mg} / \mathrm{dL}(<6.7 \mathrm{mmol} / \mathrm{L})$ but without $>5 \%$ of readings $<70 \mathrm{mg} / \mathrm{dL}$ $(<3.9 \mathrm{mmol} / \mathrm{L})$ during the basal period (12 PM to $6 \mathrm{AM})$. From $6 \mathrm{PM}$ until breakfast the following morning, no food was to be eaten, in order to obtain accurate basal BG readings. During both weeks, subjects wore a CGMS (Medtronic CGMS System Gold) until basal BG targets were achieved. CGMS readings for the last 24 -hr period were recorded and mean values were compared between treatments using a single-tail, paired t-test.

Results: All subjects achieved basal BG targets in a mean of 3.65 days. Mean BG values during the basal period and the entire $24-\mathrm{hr}$ period were not significantly different between insulin detemir and insulin glargine, except at 2 $\mathrm{AM}(p<0.05)$ (Figure).

Conclusion: There was no clinically significant difference between insulin detemir and insulin glargine in glycaemic control during the basal period or throughout the day and we can conclude that once-daily dosing of insulin detemir and insulin glargine provides comparable glycaemic efficacy over a 24-hour period in subjects with T2D.

\section{Daily blood glucose measured by CGMS (mean \pm SE):} insulin glargine vs. insulin detemir

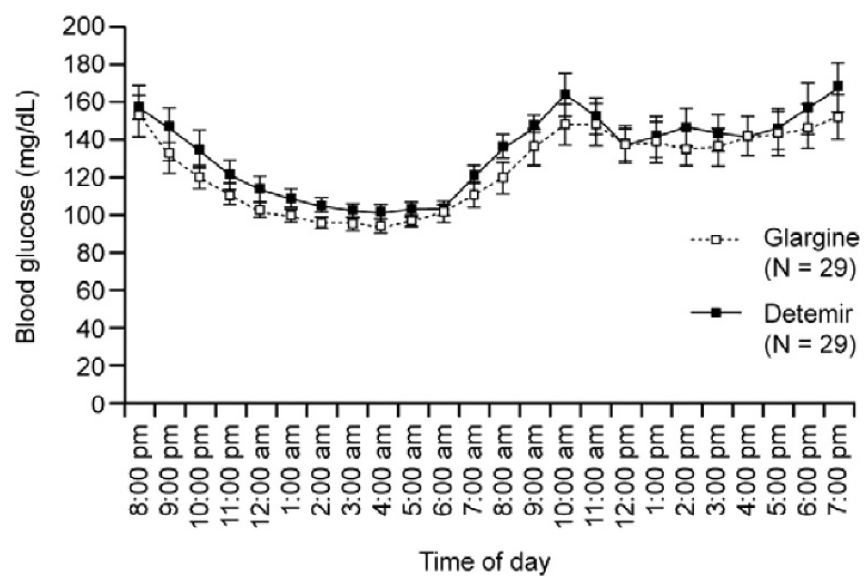

Supported by: Novo Nordisk 


\section{4}

Patient characteristics as predictors of treatment efficacy: premix analogue versus basal insulin in type 2 diabetes

V. Fonseca ${ }^{1}$, J. Davidson ${ }^{2}$, P. Home ${ }^{3}$, W. Duckworth ${ }^{4}$, J. Snyder ${ }^{5}$, C. Koenen ${ }^{6}$, A.D. Toft $^{6}$, A. Barnett ${ }^{7}$

${ }^{1}$ Tulane University Medical Center, Tulane University Health Sciences Center, New Orleans, United States, ${ }^{2}$ UT Southwestern Medical School \&, Medical City Dallas Endocrine \& Diabetes Assoc of Texas, Dallas, United States, ${ }^{3}$ School of Medical Sciences, University of Newcastle Upon Tyne, United Kingdom, ${ }^{4}$ Diabetes Research, Veterans Affairs Medical Center, Pheonix, United States, ${ }^{5}$ Endocrinology, Southwest Medical Associates, Las Vegas, United States, ${ }^{6}$ Novo Nordisk A/S, Bagsvaerd, Denmark, ${ }^{7}$ Diabetes and Obesity Group, University of Birmingham, United Kingdom

Background and aims: Insulin therapy for people with type 2 diabetes is often started with a basal insulin analogue or premix insulin analogue. This pooled analysis aimed to determine if baseline factors can predict improved glycaemic control with these regimens.

Materials and methods: Published studies performed by Novo Nordisk (as individual data were needed) were identified which met criteria of: treat-to-target design, insulin-naïve, type 2 diabetes, treated with biphasic insulin aspart 30 (BIAsp 30 ) once/twice-daily plus oral agents (OAs) or basal insulin analogue (insulin glargine or insulin detemir) plus OAs. Patient data were pooled and age, diabetes duration, gender, $\mathrm{BMI}, \mathrm{HbA}_{1 c}$, fasting plasma glucose (FPG), mean postprandial plasma glucose (PPG) and bedtime plasma glucose $(\mathrm{PG})$ at baseline were investigated for prediction of improved $\mathrm{HbA}_{1 \mathrm{c}}$, FPG or PPG with the two insulin types. Statistical analyses employed a linear mixed model with insulin type, OAs, time, and time ${ }^{2}$ as fixed effects, and patient and trial as random effects.

Results: Six trials met the criteria: $\mathrm{n}=288$ for BIAsp $30, \mathrm{n}=1101$ for basal analogue. Baseline age and bedtime PG were inter-related predictors of $\mathrm{HbA}_{1 c}$ : at higher age or higher PG, BIAsp 30 was more beneficial, and at lower age and PG, basal insulin was better (Figure). Thus, at bedtime PG of $9.9 \mathrm{mmol} / \mathrm{L}, \mathrm{BI}-$ Asp 30 was better for those over 35 years old, whereas at 45 years, basal insulin was better if PG was below $7.2 \mathrm{mmol} / \mathrm{L}$. Basal analogue had more effect than BIAsp 30 on FPG. For the two pairs of co-variates baseline $\mathrm{HbA}_{1 \mathrm{c}}$ and FPG, and baseline FPG and post-breakfast PG, it was not possible to define regions within which one insulin was predicted to give a greater improvement in $\mathrm{HbA}_{1 c}$ than the other. Mean PPG was similar between regimens for younger people, but for older people BIAsp 30 was better. Minor hypoglycaemia was reported by $56 \%$ of BIAsp 30 -treated people and $45 \%$ of basal analogue-treated people. Conclusion: The data suggest that people with low bedtime PG starting insulin therapy early should start with basal analogue, but older people and those with higher bedtime PG should start with BIAsp 30 to achieve greater $\mathrm{HbA}_{1 \mathrm{c}}$ reductions.

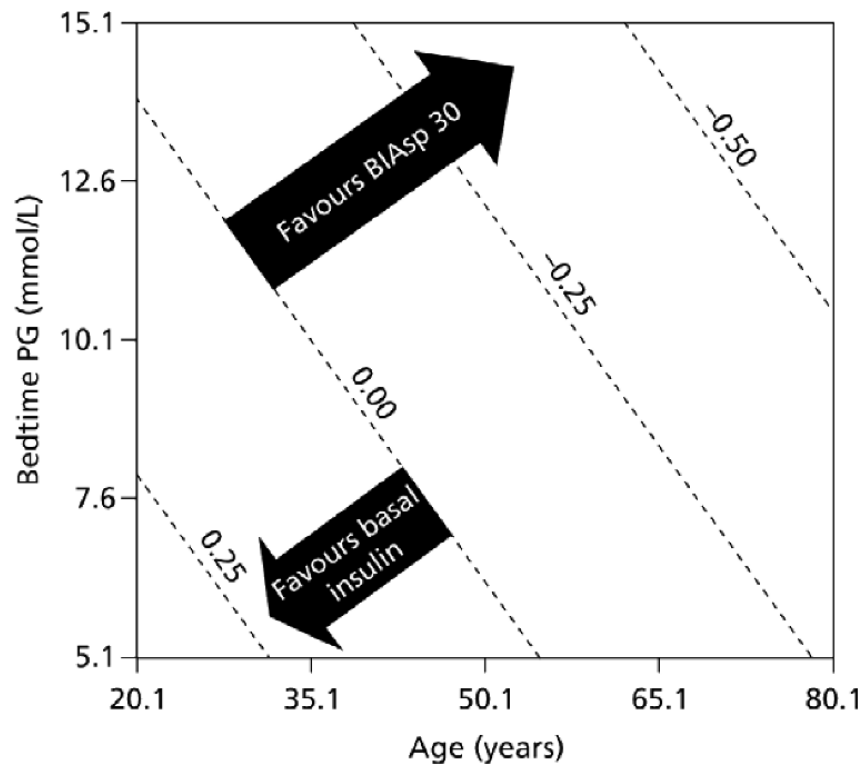

Figure. treatment difference in predicted $\mathrm{HbA}_{1 \mathrm{c}}$ (\%) (BIAsp 30 - basal) according to age and bedtime plasma glucose

Supported by: Novo Nordisk

\section{5}

Thrice-daily BIAsp 70 more closely mimics physiological insulin secretion compared with twice-daily BIAsp 30

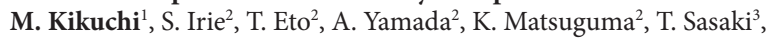
Y. Katayama ${ }^{3}$, K. Tsukuda ${ }^{1}$

${ }^{1}$ The Institute for Adult Diseases, Asahi Life Foundation, Marunouchi Hospital, Tokyo, ${ }^{2}$ Clinical Pharmacology Department, Kyushu Clinical Pharmacology Research Clinic, Fukuoka, ${ }^{3}$ Novo Nordisk Pharma Ltd, Tokyo, Japan

Background and aims: Biphasic insulin aspart (BIAsp) is a premixed insulin analogue, containing soluble, rapid-acting insulin (IAsp) and intermediateacting protamine-bound IAsp. We compared the insulin profiles of BIAsp 70 three-times-daily (TID) and BIAsp 30 twice-daily (BID) (containing 70 and $30 \%$ of the rapid-acting fraction, respectively) in Japanese type 2 diabetes patients with the insulin profile of healthy subjects. The aim of this study was to investigate how closely physiological insulin profiles are reproduced.

Materials and methods: This study was a two-site, randomised, open-label, treat-to-target, parallel group comparison. At the first site, type 2 diabetes patients were randomised into two treatment groups, BIAsp 70 TID $(n=20)$ and BIAsp 30 BID $(n=20)$ and at the second site, untreated healthy subjects $(n=20)$ were also included as a comparator (control group). Patient inclusion criteria included type 2 diabetes with current insulin treatment $\geq 12$ weeks, aged 20-69 years, $\mathrm{HbA}_{1 \mathrm{c}}<9.0 \%$, BMI $18.5-25.0 \mathrm{~kg} / \mathrm{m}^{2}$, and total daily insulin dose $0.2-1.0 \mathrm{U} / \mathrm{kg}$ body weight. Healthy subjects were defined as individuals with normal glucose tolerance (OGTT). Pharmacokinetic measurements (24 h) were performed at both sites. Serum insulin concentrations $(24 \mathrm{~h})$ were obtained with 3 standard meals. This included endogenous and exogenous insulin after 4 weeks of treatment in subjects with type 2 diabetes.

Results: Total serum insulin concentrations rapidly increased after BIAsp 70 administration at breakfast, lunch, and dinner until $\mathrm{C}_{\max }$ was reached with a median $t_{\max }$ of $1 \mathrm{~h}$. Significant differences in plasma insulin concentrations were noted between BIAsp 70 TID and BIAsp 30 BID after lunch when BIAsp 30 was not administered and during the early phase after injections at breakfast and dinner. The $24 \mathrm{~h}$ plasma insulin profile of the BIAsp 70 TID group was more similar to physiological insulin secretion in healthy subjects compared with the BIAsp 30 BID group. This was reflected in smaller differences in the insulin profile curve areas between the BIAsp 70 TID group and healthy subjects compared with the BIAsp 30 BID group ( $<<0.0001)$. The frequency of adverse events was similar between the BIAsp 70 and BIAsp 30 groups. All reported events were judged as unlikely to be related to the trial product.

Conclusion: BIAsp 70 TID provides a pharmacokinetic profile which more closely mimics physiological insulin secretion compared with BIAsp 30 BID. This can be attributed to the increased insulin concentration during the early phase after injection, reflecting the increased rapid-acting fraction. BIAsp 70 TID offers a useful treatment option in Japanese type 2 diabetes patients. With a range of premix ratios, individual treatment regimens may be tailored to achieve improved glycaemic control, while maintaining a limited number of daily injections.

Supported by: Novo Nordisk Pharma Ltd

\section{6}

A thrice-daily regimen of BIAsp 70 improves glycaemic control without increasing the risk of hypoglycaemia in Japanese patients with type 2 diabetes: a comparison of thrice-daily BIAsp 70 and twice-daily BIAsp 30 T. Kadowaki ${ }^{1}$, M. Kanai ${ }^{2}$, Y. Katayama ${ }^{2}$, K. Kaku ${ }^{3}$

${ }^{1}$ Department of Diabetes and Metabolic Diseases, The University of Tokyo, ${ }^{2}$ Novo Nordisk Pharma Ltd, Tokyo, Japan, ${ }^{3}$ Diabetes and Endocrine Division, Kawasaki Medical School, Okayama, Japan

Background and aims: Biphasic insulin aspart (BIAsp) is a premixed insulin analogue containing soluble, rapid-acting insulin aspart (IAsp) and intermediate-acting protamine bound IAsp. In this study, we compared the efficacy and safety of two different BIAsp formulations: BIAsp 30 and BIAsp 70, (containing $30 \%$ and $70 \%$ rapid-acting insulin, respectively), in a 28 -week, two-arm, open-label, treat-to-target clinical trial. Titration was based on a pre-determined algorithm.

Materials and methods: Type 2 diabetes patients $(n=296)$ treated with intermediate-acting insulin, long-acting insulin, or premixed human insulin (once- or twice-daily regimen for at least 24 weeks) were randomised 1:1 to treatment with BIAsp 30 twice daily (BID) or BIAsp 70 three-times-daily 
(TID). Insulin was injected immediately before meals. After 16 weeks of treatment, subjects in the TID regimen with a fasting plasma glucose (FPG) $>130 \mathrm{mg} / \mathrm{dL}(>7.2 \mathrm{mmol} / \mathrm{L})$ could be switched to BIAsp 30 at the pre-dinner dosing to complete the remaining 12 weeks of the study, resulting in $67 \%$ of the subjects being switched. The primary endpoint was $\mathrm{HbA}$ after 16 weeks of treatment. The secondary efficacy endpoints included $\mathrm{HbA}$ after 28 weeks of treatment and parameters derived from the 7-point plasma glucose (PG) profile after 16 weeks of treatment. The study was designed to test non-inferiority of the BIAsp 70 TID regimen compared with the BIAsp 30 BID regimen. If non-inferiority was shown, statistical superiority was evaluated.

Results: After 16 weeks of treatment, the $\mathrm{HbA}_{1 \mathrm{c}}$ of subjects on the BIAsp 70 TID regimen was significantly lower than with BIAsp 30 BID (7.22 vs. 7.57, $\mathrm{p}<0.0001)$. The $\mathrm{HbA}_{1 \mathrm{c}}$ of BIAsp 70 TID was shown superior to BIAsp 30 BID by $-0.35 \%(95 \% \mathrm{CI}[-0.51 ;-0.19], \mathrm{p}<0.0001)$. Similarly, after 28 weeks of treatment, the $\mathrm{HbA}_{1 \mathrm{c}}$ of BIAsp 70 TID (including switching to BIAsp 30 at dinner) was shown to be superior to BIAsp 30 BID by $-0.30 \%$ (95\% CI [-0.47, -0.14], $\mathrm{p}=0.0004)$. The mean PG of the BIAsp 70 TID regimen, derived from the 7-point PG profile, was significantly lower than BIAsp $30 \mathrm{BID}$ by $-8.2 \mathrm{mg} / \mathrm{dL}$ $(-0.46 \mathrm{mmol} / \mathrm{L})(95 \% \mathrm{CI}[-16.1,-0.4], \mathrm{p}=0.0402)$. The postprandial increment of BIAsp 70 TID was lower by $-34.4 \mathrm{mg} / \mathrm{dL}(-1.91 \mathrm{mmol} / \mathrm{L})(95 \% \mathrm{CI}[-45.1$, -23.7], $\mathrm{p}<0.0001)$. The insulin dose increased more with BIAsp 70 TID compared with BIAsp $30 \mathrm{BID}$. The incidence of adverse events was similar in the two treatment groups. Despite a lower $\mathrm{HbA}_{1 \mathrm{c}}$, the frequency of hypoglycaemic events was not increased in the BIAsp 70 TID regimen compared with BIAsp 30 BID (18.84 and 21.29 episodes/patient-year respectively, relative risk $0.88, \mathrm{p}=0.4077)$.

Conclusion: The glycaemic control of BIAsp 70 TID was demonstrated to be superior to BIAsp $30 \mathrm{BID}$, without increasing the risk of hypoglycaemia.

Supported by: Novo Nordisk Pharma Ltd

\section{7}

Effect of switching from insulin lispro mix 50 to lispro mix 25 at the evening meal during prandial premixed (Lispro/ILPS) therapy (PPT) in type 2 diabetes (T2D) patients

H. Jiang ${ }^{1}$, Z. Milicevic ${ }^{2}$ J. Scism-Bacon ${ }^{1}$, S. Martin ${ }^{1}$

${ }^{1}$ Eli Lilly and Company, Indianapolis, United States, ${ }^{2}$ Eli Lilly and Company, Vienna, Austria

Background and aims: This post-hoc analysis investigated the efficacy and safety of switching from lispro mix 50 (LM50) (50\% lispro, 50\% insulin lispro protamine suspension [ILPS]) to lispro mix 25 (LM25) (25\% lispro, 75\% ILPS) at the evening meal during PPT (LM50 tid with meals). The analysis was based on two 24-wk randomized, multicenter, parallel, open-label studies in T2D patients (pts) treated with 0-2 daily insulin injections (1 study) or glargine (1 study) and randomized to PPT tid vs once-daily glargine (+ metformin) or glargine + mealtime lispro (+ oral antihyperglycemic agents). Investigators could substitute LM25 for LM50 at the evening meal at any time during the studies to help achieve fasting plasma glucose (FPG) target of 6.1 $\mathrm{mmol} / \mathrm{L}$.

Materials and methods: A total of 138/314 pts (44\%) in the PPT arms were switched to LM25 at the evening meal. Analysis of continuous variables was based on ANCOVA, and percentages of pts reaching $\mathrm{HbAlc}$ target was based on logistic regression models, with baseline HbAlc, age, BMI and duration of T2D as covariates.

Results: Baseline HbAlc and postprandial glucose (PPG) were higher in pts switched to LM25 at the evening meal than those not switched (Table). Other characteristics were similar. After 24 weeks, improved glycemic control was shown in both groups with significant reductions from baseline in $\mathrm{HbAlc}$, FPG and PPG $(\mathrm{P}<0.0001)$. There was no significant difference between groups in endpoint total daily insulin dose, however pts switched to LM25 had a higher evening mealtime insulin dose than those not switched (Table). The switched group was associated with greater reduction in $\mathrm{HbAlc}$ and higher proportion of pts achieving $\mathrm{HbAlc}$ target $<7.0 \%$, but with greater risk of hypoglycemia. Four pts in the switched group and 5 in the non-switched group reported severe hypoglycemia.

Conclusion: PPT tid resulted in improved glycemic control for T2D pts who were insulin-naive or previously treated with insulin, with a large proportion achieving HbAlc target $<7.0 \%$. Patients not reaching FPG goals on LM50 tid who were switched to LM25 at the evening meal demonstrated better glycemic control, use of larger evening meal insulin dose, and greater risk of hypoglycemia.

\begin{tabular}{|c|c|c|c|}
\hline & $\begin{array}{l}\text { Switched } \\
\text { Group } \\
(\mathrm{n}=138)\end{array}$ & $\begin{array}{l}\text { Non-switched } \\
\text { Group } \\
(\mathrm{n}=176)\end{array}$ & P-value \\
\hline \multicolumn{4}{|l|}{ Baseline characteristics } \\
\hline Age (yrs) & $55.7 \pm 9.8$ & $57.0 \pm 9.2$ & 0.229 \\
\hline$\%$ male & 56 & 48 & 0.174 \\
\hline BMI & $32.8 \pm 6.3$ & $33.4 \pm 5.7$ & 0.371 \\
\hline Duration T2D (yrs) & $11.5 \pm 6.3$ & $10.6 \pm 5.9$ & 0.192 \\
\hline HbAlc (\%) & $8.5 \pm 1.2$ & $8.2 \pm 1.1$ & 0.025 \\
\hline FPG $(\mathrm{mmol} / \mathrm{L})$ & $9.7 \pm 2.6$ & $9.6 \pm 2.9$ & 0.714 \\
\hline $\mathrm{PPG}(\mathrm{mmol} / \mathrm{L})$ & $12.6 \pm 3.2$ & $11.7 \pm 3.4$ & 0.014 \\
\hline \multicolumn{4}{|l|}{24 weeks characteristics ${ }^{\mathrm{a}}$} \\
\hline HbAlc change & $-1.5 \pm 1.2$ & $-1.1 \pm 1.0$ & 0.005 \\
\hline $\mathrm{HbAlc}<7.0 \%[\mathrm{n}(\%)]$ & $77(58)$ & $77(49)$ & 0.009 \\
\hline Total daily insulin dose (U) & $98.7 \pm 63.6$ & $93.6 \pm 64.7$ & 0.367 \\
\hline Morning meal dose (U) & $28.4 \pm 18.0$ & $30.1 \pm 20.6$ & 0.438 \\
\hline Noon meal dose (U) & $25.6 \pm 19.6$ & $28.4 \pm 22.0$ & 0.247 \\
\hline Evening meal dose (U) & $44.4 \pm 31.4$ & $34.8 \pm 24.3$ & 0.0004 \\
\hline FPG change (mmol/L) & $-1.7 \pm 2.8$ & $-1.5 \pm 3.0$ & 0.302 \\
\hline Overall PPG change $(\mathrm{mmol} / \mathrm{L})$ & $-3.8 \pm 3.4$ & $-2.9 \pm 3.6$ & 0.487 \\
\hline $\begin{array}{l}\text { PPG change post-evening meal } \\
(\mathrm{mmol} / \mathrm{L})\end{array}$ & $-3.8 \pm 4.2$ & $-3.5 \pm 4.4$ & 0.1853 \\
\hline Weight gain $(\mathrm{kg})$ & $3.0 \pm 3.7$ & $2.0 \pm 4.2$ & 0.072 \\
\hline Hypo rate (episodes/pt/yr) & $57.2 \pm 53.4$ & $24.1 \pm 32.6$ & $<0.0001$ \\
\hline $\begin{array}{l}\text { Nocturnal hypo rate (episodes/ } \\
\mathrm{pt} / \mathrm{yr} \text { ) }\end{array}$ & $11.4 \pm 15.2$ & $4.2 \pm 7.5$ & $<0.0001$ \\
\hline
\end{tabular}

${ }^{\mathrm{a}}$ All reported changes at 24 weeks are from baseline.

Supported by: Eli Lilly and Company

\section{8}

Outcomes of initiating different insulin regimens in patients with type 2 diabetes at the Steno Diabetes Center 2004-2006

E. Eldrup ${ }^{1}$, A.D. Toft ${ }^{2}$

${ }^{1}$ Steno Diabetes Center, Gentofte, Denmark, ${ }^{2}$ Novo Nordisk, Bagsvaerd, Denmark

Background and aims: For patients initiating insulin therapy at the Steno Diabetes Center (SDC) from 2004-2006, standard procedure was starting a regimen of bedtime basal NPH insulin (basal-only therapy, BOT) in addition to oral antidiabetic drugs. Some patients were initiated with premixed insulin (biphasic insulin aspart 30/70 or biphasic human insulin 30/70). We retrospectively analysed insulin regimen and $\mathrm{HbA}_{1 c}$ measurements over 12 months after insulin initiation.

Materials and methods: During 2004-2006, a total of 621 people in the SDC Type 2 Diabetes Clinic initiated insulin treatment (approximately $50 \%$ of the 450 patients referred to the clinic annually). The majority initiated insulin as out-patients $(77 \%, n=479)$. After initiating insulin therapy with BOT or premix, some patients transferred to the alternative treatment (and in rare cases, transferred back again). Medical records at the SDC were used to assess

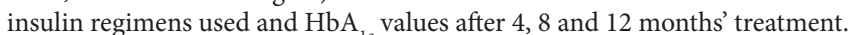
Results: The mean (SD) age of all 621 patients included was 59.4 (13.2) years; duration of diabetes 8.7 (6.4) years; weight 89.1 (21.0) kg and 58\% were male. Baseline $\mathrm{HbA}_{1 \mathrm{c}}$ was 9.0 (1.9)\% and complications were common: retinopathy $28 \%$ of patients, neuropathy $32 \%$ and microalbuminuria $35 \%$. The only difference at baseline between patients initiated with premix or BOT was BMI: $29.5(6.5)$ and $30.8(6.2) \mathrm{kg} / \mathrm{m}^{2}$, respectively $(p<0.05)$. Of the 192 patients initiated with premix, 61 (32\%) were in-patients and $131(68 \%)$ out-patients. Of the 429 patients initiated with BOT, $81(19 \%, p<0.001 v s$. premix) were inpatients and $348(81 \%)$ out-patients. The proportion of patients that stayed on their initial therapy was higher for premix ( $90 \%$ and $92 \%$ of those initiated as in-patients and out-patients, respectively) than for BOT ( $60 \%$ and $66 \%$ of those initiated as in-patients and out-patients, respectively; both $p<0.05 v s$. $\mathrm{BOT}$ ). For patients who switched from BOT to premix, mean (SD) $\mathrm{HbA}_{1 \mathrm{c}}$ at the time of switching was $8.9(1.6) \%$. In 557 patients followed for more than 3 months, the final mean $\mathrm{HbA}_{1 c}$ was $7.7(1.4) \%(\mathrm{n}=345)$ if premix was their final insulin regimen, compared with $7.6(1.3) \%(n=212)$ if BOT was their final regimen $(p>0.05)$. The proportion of patients reaching $\mathrm{HbA}_{1 c}<7.0 \%$ while attending the Type 2 Clinic was $35 v$ s. $29 \%$ for premix and BOT, respectively $(p>0.05)$. For patients initiated with premix or BOT, $\mathrm{HbA}_{1 \mathrm{c}}$ of out-patients was lower than that of in-patients at baseline and at 12 months (i.e. all pa- 
Table. $\mathrm{HbA}_{1 \mathrm{c}}$ values during 12 months for people with type 2 diabetes who initiated insulin treatment with premix or BOT as in- or out-patients during 2004-2006. Patient data only included until a switch away from original treatment.

\begin{tabular}{|c|c|c|c|c|c|}
\hline \multirow[t]{2}{*}{ Mean $\mathrm{HbA}_{1 \mathrm{c}}(\mathrm{SD}), \%$} & \multicolumn{2}{|l|}{ Premix } & \multicolumn{2}{|l|}{ BOT } & \multirow[t]{2}{*}{ All patients } \\
\hline & $\begin{array}{l}\text { Initiated as } \\
\text { in-patients }\end{array}$ & $\begin{array}{l}\text { Initiated as } \\
\text { out-patients }\end{array}$ & $\begin{array}{l}\text { Initiated as } \\
\text { in-patients }\end{array}$ & $\begin{array}{l}\text { Initiated as } \\
\text { out-patients }\end{array}$ & \\
\hline Baseline & $\begin{array}{l}10.0(1.9) \\
\mathrm{n}=61\end{array}$ & $\begin{array}{l}8.4(1.5) \\
n=131\end{array}$ & $\begin{array}{l}9.9(2.0) \\
\mathrm{n}=81\end{array}$ & $\begin{array}{l}8.9(1.8) \\
n=348\end{array}$ & $\begin{array}{l}9.0(1.9) \\
\mathrm{n}=621\end{array}$ \\
\hline 4 months & $\begin{array}{l}7.7(1.4) \\
\mathrm{n}=44\end{array}$ & $\begin{array}{l}7.5(1.3) \\
\mathrm{n}=94\end{array}$ & $\begin{array}{l}7.7(1.4) \\
\mathrm{n}=36\end{array}$ & $\begin{array}{l}7.5(1.2) \\
\mathrm{n}=196\end{array}$ & $\begin{array}{l}7.6(1.3) \\
\mathrm{n}=370\end{array}$ \\
\hline 8 months & $\begin{array}{l}8.1(1.7) \\
\mathrm{n}=43\end{array}$ & $\begin{array}{l}7.4(1.2) \\
\mathrm{n}=95\end{array}$ & $\begin{array}{l}7.8(1.2) \\
\mathrm{n}=34\end{array}$ & $\begin{array}{l}7.5(1.2) \\
\mathrm{n}=159\end{array}$ & $\begin{array}{l}7.6(1.3) \\
\mathrm{n}=331\end{array}$ \\
\hline 12 months & $\begin{array}{l}8.2(1.6) \\
n=33\end{array}$ & $\begin{array}{l}7.3(1.3) \\
n=65\end{array}$ & $\begin{array}{l}8.0(1.3) \\
n=28\end{array}$ & $\begin{array}{l}7.6(1.2) \\
\mathrm{n}=107\end{array}$ & $\begin{array}{l}7.6(1.4) \\
\mathrm{n}=233\end{array}$ \\
\hline
\end{tabular}

Premix: biphasic insulin aspart 30/70 or biphasic human insulin 30/70; BOT: basal-only insulin therapy

Supported by: Novo Nordisk

tients who finished on each regimen) (Table). A small amount of weight gain was seen in patients finishing on premix or NPH-based BOT after 12 months: $3.0(4.8) \mathrm{kg} v s .3 .2(6.3) \mathrm{kg}$.

Conclusion: If patients with type 2 diabetes initiate insulin with premixed insulin, approximately $30 \%$ more patients will stay on this regimen than if a BOT regimen is initiated, and equally good metabolic control may be obtained within 12 months.

\section{9}

Insulin therapy in type 2 diabetes; what is the evidence?

M.J.P. Van Avendonk, K.J. Gorter, M. Van den Donk, G.E.H. Rutten Julius Center for Health Sciences and Primary Care, University Medical Center Utrecht, Netherlands

Background and aims: There is no consensus about the insulin regimen to be chosen, in case maximal tolerated dosages of oral blood glucose lowering agents (OHA) are not sufficient to achieve good glycaemic control in type 2 diabetes. Our aim was to systematically review the recent literature regarding insulin therapy (human and analogue), in order to provide the best clinical evidence for prescribing insulin in type 2 diabetes patients.

Materials and methods: A MEDLINE search was performed to identify randomised controlled trials (RCT) published in English between January 1, 2000 and April 1, 2007, involving insulin use in adults with type 2 diabetes mellitus.

Results: The search resulted in 943 hits, 93 RCTs were selected by title. Eventually 67 trials met the inclusion criteria. The studies were very diverse and of different quality. They comprised all possible insulin regimes. We distinguished the following comparisons: 1) combination of insulin therapy with OHA versus insulin monotherapy, 2) long-acting insulin versus another long-acting insulin, 3) premix insulin versus OHA, other premixes or longacting insulin, 4) preprandial or basal-bolus therapy versus other basal-bolus therapies. Three trials could not be assigned to one of these comparisons. Continuing metformin and/or sulfonylurea after start of therapy with basal long-acting insulin results in better glycaemic control with less insulin requirements, less weight gain and less hypoglycaemic events compared to insulin monotherapy. Long-acting insulin analogues in combination with OHA are associated with similar glycaemic control but fewer hypoglycaemic episodes compared to NPH. Most of the trials demonstrated better glycaemic control with premix insulin therapy than with a long-acting insulin once daily, but premix insulin causes more hypoglycaemic episodes. Analogue premix provides similar improvements in $\mathrm{HbA} 1 \mathrm{c} \%$, but lower postprandial glucose levels compared to human premix, without increase in hypoglycaemic events or weight gain. Drawing conclusions from the limited number of studies concerning basal-bolus regimen is not possible, mainly because of its diversity. Conclusion: A once daily basal insulin regimen added to OHA is an ideal starting point for insulin therapy in type 2 diabetes. All next steps, from one to two or even more injections per day should be taken very carefully and in thorough deliberation with the patient, who has to comply with such a regimen for many years. The clinician should keep in mind that a positive balance between benefits and adverse events is not obvious at all. Insulin analogues may have some small advantages compared to conventional human insulins. However, long-term effects of treatment with these analogues have not been investigated until now. Before any final judgement about relevant effects of insulin therapy can be made large multi-centre trials are needed with a long follow-up and relevant outcome measures.

Supported by: sanofi-aventis 


\section{PS 092 Insulin and costs}

\section{0}

Cost advantages and improved patient treatment satisfaction with insulin glargine in type 2 diabetes patients. The long-acting insulin glargine $v s$. NPH insulin cost evaluation study in Germany (LIVE-DE) T. Scholten ${ }^{1}$, T. Kohlmann², J. Moock², R. Holle ${ }^{3}$, W. Landgraf ${ }^{4}$, O. Pirk ${ }^{5}$, H. Hauner ${ }^{6}$

${ }^{1}$ Allgemeines Krankenhaus Hagen, University Witten/Herdecke, ${ }^{2}$ Institute of Community Medicine, University of Greifswald, ${ }^{3}$ Institute of Health Economics \& Health Care Management, Helmholtz Center Munich, ${ }^{4}$ SanofiAventis, Berlin, ${ }^{5}$ IMS Health HEOR, Nürnberg, ${ }^{6}$ Center for Nutrition Medicine, TU Munich, Munich, Germany

Background and aims: Rational decisions for reimbursement of innovative drugs cannot be derived appropriately from randomized clinical trials alone but also require real-life data to demonstrate overall effectiveness and benefits of a drug. Since such data from countries with different healthcare systems cannot be easily transferred to German conditions, real-life data from Germany were collected.

Materials and methods: LIVE-DE was a non-interventional, cross-sectional, retrospective study performed between February and May 2007 in 199 randomly selected centers of primary care physicians in Germany. The study enrolled 1602 consecutive type 2 diabetes (T2D) patients with statutory health insurance status who were eligible for documentation when either treated with insulin glargine (GLA) or NPH insulin based regimens for at least 6 months prior of documentation. Primary objective was to assess total direct diabetes treatment costs (DTC) derived from antidiabetic medications (insulins, OADs), blood glucose self-monitoring (test strips, lancets), hypoglycemic treatment (glucagon use) and needles for a 6 months period under reallife conditions. Secondary objectives included patient reported outcomes (PROs) using established (DTSQs, SF-12, PAID) and a newly developed questionnaire (Insulin Treatment Experience Questionnaire, ITEQ).

Results: Mean total DTC were $658 \pm 258 €$ and $685 \pm 242 €$ per patient during 6 months in GLA and NPH patients, respectively (mean difference $-27 €, \mathrm{p}$ $=0.001$, Wilcoxon Test). Despite higher drug costs for GLA relevant savings were observed in consumption of short-acting insulins $(-62 €)$, test strips $(-52$ $€)$ and needles $(-6 €)$. GLA patients had also on average fewer basal insulin injections per day ( 1.09 vs 1.47$)$ and required less blood glucose self-measurements. Glucagon use for treatment of severe hypoglycemic events was only documented for NPH (4 patients). SF-12 and ITEQ results showed that GLA patients reported significantly better physical well-being and higher satisfaction with insulin treatment. In addition, DTSQs scores indicated slightly better treatment satisfaction in GLA vs NPH insulin users. General need of care, number of referrals, hospital admissions, glycemic control and demographics were similar in both groups, but NPH was mainly used in a basal-bolus (79\%) whereas GLA was frequently prescribed in a basal/OAD (BOT) regimen (43\%). After statistical adjustment of these factors, total DTC per patient during 6 months in GLA users were slightly increased compared to NPH (adjusted mean $+73 €, \mathrm{p}<0.001$, ANCOVA) but improved patient satisfaction with GLA remained significant.

Conclusion: Under real-life conditions total DTC are similar in GLA and NPH T2D patients while patient treatment satisfaction is higher with GLA. LIVE-DE provides new evidence to the German healthcare system demonstrating that treatment preferences and reimbursement decisions should adopt a comprehensive perspective including all relevant cost categories and PROs.

Supported by: sanofi-aventis Germany

\section{1}

2-year glycaemic control after initiation of insulin glargine or NPH insulin in type 2 diabetes

J.L. Leahy ${ }^{1}$, M.-P. Dain ${ }^{2}$, G.G. Rhoads ${ }^{3}$, L. Kennedy ${ }^{4}$

${ }^{1}$ Endocrinology, Diabetes and Metabolism, University of Vermont College of Medicine, Burlington, United States, ${ }^{2}$ sanofi-aventis, Paris, France, ${ }^{3}$ School of Public Health, University of Medicine and Dentistry of New Jersey, Piscataway, United States, ${ }^{4}$ Shands Hospital, University of Florida, Gainsville, United States

Background and aims: To compare 2-year glycemic control, hypoglycemia rates, and health care costs associated with insulin glargine versus NPH insulin in patients with T2DM in a real world setting.
Materials and methods: Retrospective observational analysis from a large US managed care database of patients diagnosed with T2DM between 2001 and 2005. Patients who had failed treatment with oral antidiabetic drugs (OADs) initiated glargine $(n=2,105)$ or $\mathrm{NPH}(\mathrm{n}=734)$ in addition to OADs, with continuous plan enrollment for $>18$ months ( 6 months before and at least 12 months after insulin initiation), and with available A1C values from a central laboratory were evaluated. The analysis controlled for baseline demographics, A1C, comorbidities, health care utilization, pharmacy copayment, and follow-up antidiabetic medications. Costs were evaluated using actual paid claims by health insurance, adjusting for inflation to the most current year value.

Results: Patients at baseline had the same mean age of 54 years, and no difference in medical insurance claims for hypoglycemia (3.2\% vs. $4.3 \%$ in glargine vs NPH initiators, respectively). There were differences between glargine and NPH groups at baseline between sex (44\% vs. 52\% female), baseline A1C (9.3\% vs. $8.9 \%$ ), access to endocrinologist (36\% vs $46 \%$ ), average number of OADs (2.3 vs. 2.0), Charlson comorbidity score for overall comorbidities ( 0.64 vs 0.82 ), and 6-month total healthcare costs $(\$ 8,797$ vs $\$ 12,924)$. Differences in baseline characteristics were controlled for in the analyses. Adjusted 1-year mean $\mathrm{AlC}$ was 8.05 vs $8.51 \%(\delta=-0.45, \mathrm{p}=0.0036)$ and 2 -year mean $\mathrm{AlC}$ was 8.03 vs $8.37 \%(\delta=-0.33, p=0.0099)$ for glargine and $\mathrm{NPH}$, respectively. In the last quarter of the 2-year follow-up period, $16.6 \%$ in the NPH group filled a prescription for insulin glargine, whereas $2.7 \%$ in the glargine group filled a prescription for NPH insulin. Adjusted rate of patients per quarter in the first year with medical claims for hypoglycemia was 1.7 vs. $2.9 \%(\delta=-1.2 \%$, $\mathrm{p}=0.0559)$ and 2 -year quarterly rate was 1.55 vs. $2.51 \%(\delta=-0.96 \%, \mathrm{p}=0.0139)$. Adjusted 1 -year total healthcare costs were $\$ 16,184$ vs. $\$ 21,104$ (quarterly $\delta=$ $\$ 1,034, p=0.0372$ ) and 2 -year costs was $\$ 30,032$ vs. $\$ 42,208$ (quarterly $\delta=-$ $\$ 1,522, \mathrm{p}=0.0029)$ (Table).

Conclusion: This analysis from real-world clinical practice indicates that initiation of insulin glargine, relative to NPH, was associated with sustained improvements in glycemic control with lower rate of medically claimed hypoglycemia and lower total healthcare expenditures in patients with T2DM.

Adjusted Total Health Care Costs in Patients Initiating Therapy With Insulin Glargine vs NPH Insulin

\begin{tabular}{lllll}
\hline $\begin{array}{l}\text { Health Care } \\
\text { Costs }\end{array}$ & $\begin{array}{l}\text { Insulin } \\
\text { Glargine } \\
(\mathbf{n}=\mathbf{2 1 0 5})\end{array}$ & $\begin{array}{l}\text { NPH } \\
\text { Insulin } \\
(\mathbf{n}=734)\end{array}$ & $\begin{array}{l}\text { Quarterly } \\
\text { Difference }\end{array}$ & $\boldsymbol{P}$ Value \\
\hline 1-year & $\$ 16,184$ & $\$ 21,104$ & $\$ 1034$ & 0.0372 \\
\hline 2 -year & $\$ 30,032$ & $\$ 42,208$ & $\$ 1522$ & 0.0029 \\
\hline
\end{tabular}

Supported by: sanofi-aventis U.S. Group

\section{2}

A cost-effectiveness analysis of continuous subcutaneous insulin injection $v s$. multiple daily injections in type 1 diabetes patients: an Italian perspective

P. Lynch ${ }^{1}$, S. Giardina ${ }^{2}$, A. Nicolucci ${ }^{3}$, P. Ponzi ${ }^{2}$, M. Orsini Federici², M.E. Minshall ${ }^{4}$

${ }^{1}$ Health Economics, Medtronic Diabetes, Northridge, United States, ${ }^{2}$ Medtronic Italia SPA, Sesto San Giovanni, Italy, ${ }^{3}$ Department of Clinical Pharmacology and Epidemiology, Consorzio Mario Negri Sud, S. Maria Imbaro, Italy, ${ }^{4} \mathrm{Health}$ Economics and Outcomes Research, IMS Health, Noblesville, United States

Background and aims: The objective of this study was to project long term costs and outcomes for continuous subcutaneous insulin infusion (CSII) compared with multiple daily injections (MDI) of insulin in adult type 1 diabetes mellitus (T1DM) patients in Italy from the National Health Service (NHS) perspective.

Materials and methods: The CORE Diabetes Model (CDM) was used to determine the incremental cost-effectiveness ratio (ICER) of CSII compared with MDI among adult patients with T1DM in Italy. The primary input variable was change in $\mathrm{HbA}_{1 \mathrm{c}}$ and was assumed to be a $1.2 \%$ improvement for CSII as compared to MDI in a cohort of Italian T1DM patients with an average $\mathrm{HbA}_{1}$ baseline value of $8.95 \%$. Based on existing evidence, it was also assumed that CSII patients had 50\% less hypoglycemic events compared with MDI patients. A series of Markov constructs simulated the progression of diabetes-related complications. The average annual cost for CSII and MDI were $€ 5,699.20$ and $€ 2,734.71$, respectively. The cost of pump, procedures, complications, and optimal drug therapy were derived from Italian-specific 
sources and other published data. A 60-year time horizon and a discount rate of $3.0 \%$ per annum on costs and clinical outcomes were used.

Results: Treatment with CSII was associated with improvements in life expectancy of 0.893 years vs. MDI and quality adjusted life expectancy (QALE) of 0.974 years vs. MDI with corresponding ICERs of $€ 36,803$ per life-year and $€ 33,751$ per quality-adjusted life year (QALY) gained for CSII compared with MDI. Improved glycemic control from CSII treatment led to a lower incidence of diabetes complications. The cumulative incidence of proliferative diabetic retinopathy $(\mathrm{PDR})$ was reduced by $27 \%$ (relative risk $[\mathrm{RR}]=0.728$ ) with a number needed to treat (NNT) of 11 patients to avoid one case of PDR. The cumulative incidence of end-stage renal disease (ESRD) was reduced by $18 \%(R R=0.816)$ with a NNT of 22 patients to avoid one case of ESRD. Finally, findings for cardiovascular complications showed that the cumulative incidence of peripheral vascular disease (PVD) was reduced by $14 \%(\mathrm{RR}=0.856)$; with a NNT of 46 patients to avoid one case of PVD.

Conclusion: Setting the willingness to pay at $€ 40,000$ /QALY (based on a $£ 30,000$ NICE threshold), the analysis demonstrated that CSII is a cost-effective treatment option when compared to MDI for adult patients with T1DM in Italy.

\section{3}

Glycaemic control and costs with insulin glargine plus glulisine versus premix - a randomized, prospective, observational study

F. Lee ${ }^{1}$, Q. Zhang ${ }^{2}$, J. Mersey ${ }^{1}$, L. Bromberger ${ }^{1}$, M. Bhushan ${ }^{3}$, R. Bhushan ${ }^{3}$,

P. Levin ${ }^{1}$

${ }^{1}$ Diabetes, Model Clinical Research, Baltimore, ${ }^{2}$ sanofi-aventis, United

States, Bridgewater, ${ }^{3}$ Diabetes, Metabolic Center Of Louisiana Research

Foundation, Baton Rouge, United States

Background and aims: Despite extensive use of basal-bolus and premixed analog insulin therapy, real-world comparative effectiveness of the regimens has not been determined.

Materials and methods: Patients with T2DM at 2 US endocrinology practice centers were randomized to GLAR/GLU $(n=106)$ or analog premix $(n=91)$. Subsequent to randomization, patients continued treatment following center's usual practice with no additional therapeutic protocols. Data collected at 0, 3, 6 and 9 months included A1C, hypoglycemia, insulin dose, concomitant medications, and therapy change. Medication costs were estimated using published average wholesale price.

Results: Treatment groups were comparable at baseline with mean age 56 years, $46 \%$ male, $59 \%$ Caucasian, and 38\% African-American, duration of diabetes 13 years, A1C $9.25 \%$, and BMI $35.8 \mathrm{~kg} / \mathrm{m}^{2}$. During 4 months before randomization, $88 \%$ used insulin with mean daily dose of $71 \mathrm{IU}$, about $70 \%$ of patients used oral hypoglycemic agent(s) and $29 \%$ had chart records for hypoglycemia. Mean follow-up time was 183 days. 1 patient (1\%) randomized to GLAR/GLU switched to premix therapy relative to $9(10 \%)$ randomized to premix switched to GLAR/GLU. In ITT analysis, adjusted mean follow-up A1C was $6.98 \%$ in GLAR/GLU vs. $7.57 \%$ in premix $(\delta=-0.59 \%, p=0.009)$ and A1C reduction was $2.27 \%$ (95\% CI: $1.63-2.91)$ vs. $1.68 \%$ (1.20-2.16). Mean number of concomitant oral anti-diabetic agents were 0.94 vs. $1.22(\delta=-0.28$, $\mathrm{p}=0.041)$. Mean daily insulin dose was $74 \mathrm{IU}$ vs. $85 \mathrm{IU}(\delta=-11, \mathrm{p}=0.267)$. Hypoglycemia was recorded in charts for $36 \%$ vs. $43 \%(\delta=-7 \%, \mathrm{p}=0.374)$ patients in GLAR/GLU vs. premix. Daily costs for all anti-diabetic medications were $\$ 10.82$ for GLAR/GLU vs. $\$ 12.06$ in premix $(\delta=-\$ 1.24, \mathrm{p}=0.209)$. For each additional $1 \%$ A1C reduction during the follow-up period (178 days), GLAR/GLU saved \$225.54 compared to diabetes treatment cost for premix (\$1933.20 vs. \$2158.74)

Conclusion: Glargine plus glulisine, compared to analog premix, produces improved glycemic control with no increased medication costs. Supported by: sanofi-aventis U.S. Group (editorial)

\section{4}

The relative cost effectiveness of insulin glargine versus detemir insulin using UK real life data in patients with type 2 diabetes mellitus A.P. Tetlow, P. McEwan, J. Gordon

CRC, Cardiff Research Consortium, United Kingdom

Aims: The purpose of this study was to evaluate the cost effectiveness of insulin glargine compared with insulin detemir in Type 2 diabetes mellitus (T2DM) patients newly initiating insulin treatment using observational data collected from general practices throughout the UK.
Methods: Data used for this study was the THIN (The Health Improvement Network) database, a UK primary care database including 2,335,667 active patients recorded over 15 years from 211 practices. Subjects were identified with T2DM, no prior insulin history, who had received at least 12 months of oral therapy, 35 years of age and older, with a baseline $\mathrm{HbA}_{1 \mathrm{c}}>=6.5 \%$ and initiated on either glargine $(n=2197)$ or detemir $(n=357)$. Effectiveness, measured by changes in $\mathrm{HbA}_{1 \mathrm{c}}$ over a 12 month period following initiation, was standardised to adjust for differences in insulin units per $\mathrm{kg}$ prescribed. Data analysis was undertaken using a hierarchical linear mixed-effects model with dose-adjusted change in $\mathrm{HbA}_{1 c}$ as the dependent variable adjusting for baseline $\mathrm{HbA}_{1 c}$, hypoglycaemia, number of oral antidiabetic drug (OAD) prescriptions, insulin type, mean increase in $\mathrm{HbA}_{1 c}$ in the year prior to insulinisation, prior co-morbidities, concurrent bolus usage and disease duration as fixed-effect covariates with general practice modelled as a grouping variable. A published discrete event simulation model based on the UKPDS 68 study was used to model macrovascular and microvascular complications over a 40 year time horizon. Direct costs and quality of life (EQ5D) were taken from published sources and the HODaR database respectively. Costs and benefits were discounted annually at $3.5 \%$.

Results: Overall baseline demographic and risk factor profiles were similar in the glargine and detemir groups. Mean age was 61 years versus 59 years; proportion male was $45 \%$ versus $47 \%$; mean weight was $85.8 \mathrm{~kg}$ versus $85.5 \mathrm{~kg}$; duration 6.2 years versus 5.7 years and baseline $\mathrm{HbA}_{1 \mathrm{c}} 9.66 \%$ versus $9.53 \%$ in the glargine and detemir cohorts respectively. Compared with detemir, glargine patients showed a superior reduction in $\mathrm{HbA}_{1 \mathrm{c}}$ by $0.29 \%(\mathrm{p}=0.021)$ over the 12 months following insulinisation. Over the 12 month period mean insulin units per kg per day were 0.53 for glargine and 0.56 for detemir. Insulin costs were the same per unit of effectiveness for glargine and detemir. Mean OAD prescriptions over the 12 months post insulinisation were identical for both insulin preparations. Incorporating this into a simulated cohort of 10,000 patients followed over a 40 year time horizon translated into 139 fewer cardiovascular events. For glargine and detemir discounted cost per

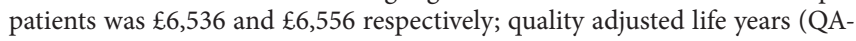
LYs) were 7.71 and 7.64 for glargine and detemir respectively. This resulted in a dominant (more effective and cost saving) discounted incremental cost effectiveness ratio.

Conclusion: This study sought to characterise the real life observational profile of glargine and detemir usage in insulin naive T2DM patients and to assess the relative cost-utility of the decision to either initiate patients on glargine or detemir. In this context, and after adjusting for available confounding variables, glargine was more effective with marginal cost savings.

Suppored by: sanofi-aventis

\section{5}

Comparative analysis of treatment outcomes of patients on insulin detemir and insulin glargine

B. Alemayehu ${ }^{1}$, B. Borah ${ }^{2}$, H. Henk ${ }^{2}$, M. Aagren ${ }^{3}$, F. Forma ${ }^{1}$

${ }^{1}$ Novo Nordisk Inc., Princeton, United States, ${ }^{2} \mathrm{i} 3$ innovus, Eden Prairie, United States, ${ }^{3}$ Novo Nordisk Inc., Virum, Denmark

Background and aims: The primary aim when prescribing insulin is to effectively lower blood glucose level, $\mathrm{HbA}_{1 c}$. The objective of reducing $\mathrm{HbA}_{1 \mathrm{c}}$ is limited by the threshold for acceptable hypoglycaemic event rates, and this tolerability level and the dose applied to achieve glycaemic control are key determinants of the costs related the medical treatment. Thus these parameters are important in decision of therapy. This study was undertaken to assess differences in glycaemic control, overall and diabetes-related costs of type 2 diabetes patients treated with basal analog insulins (i.e. insulin detemir, IDet, and insulin glargine, IGlar) using retrospective data of patients enrolled in a large US health plan with medical and pharmacy benefits.

Methods: Patients were identified if their first prescription claim (index) for IDet or IGlar occurred between 05/01/2006 and 12/31/2006. Eligible patients were required to be age 18 or above, have 6 months of continuous enrollment of the health plan pre- and post-index date, no evidence of any insulin use during pre-index and an $\mathrm{HbA}_{1 c}$ reading during both the pre- and post-index period. Patients were excluded from the analysis if they were treated with other insulins than basal analog insulin. Thus, only insulin naïve patients starting on IDet or IGlar ( \pm oral antidiabetics) are included. Primary outcomes include daily average consumption (DACON) of IDet or IGlar, change in $\mathrm{HbA}_{1 \mathrm{C}}$, and overall and diabetes-related costs. Differences in outcomes between IDet and IGlar users were adjusted for baseline characteristics through generalized linear modeling (GLM). Propensity score matching was used to reduce selection bias between the two groups. 
Results: There were 48 basal IDet patients and 258 IGlar patients in the study. There was no significant difference in adjusted DACON or HbAlc, between IDet and IGlar cohorts. However, adjusted diabetes-related costs and total medical costs for IDet cohort were lower than the IGlar cohort ( $\$ 2,262$ vs. $\$ 3,409 ; \mathrm{p}<0.03)$ and $(\$ 707$ vs. $\$ 1,510, \mathrm{p}<0.03)$ respectively. Adjusted overall medical costs were not statistically different, but the trend also suggested lower costs for the IDet cohort ( $\$ 2,319$ vs. $\$ 3,704 ; \mathrm{p}=0.07)$. No differences in overall or diabetes-related pharmacy costs were observed.

Conclusion: No significant difference in DACON or $\mathrm{HbA}_{1 \mathrm{c}}$ measures and no significant difference in adjusted pharmacy cost between insulin detemir and insulin glargine users were observed during the study period. However, insulin detemir patients experienced a significantly lower diabetes-related and overall adjusted medical cost during the follow up period.

Post-intervention adjusted treatment outcomes between insulin determir and Insulin glargine

\begin{tabular}{llll}
\hline Outcomes & $\begin{array}{l}\text { insulin detemir } \\
(\mathbf{N}=\mathbf{4 8})\end{array}$ & $\begin{array}{l}\text { insulin glargine } \\
(\mathbf{N}=\mathbf{2 5 8})\end{array}$ & P-Value \\
\hline $\begin{array}{l}\text { Average Daily Consumption } \\
\text { (DACON) }\end{array}$ & 29.31 & 29.59 & 0.937 \\
\hline HbA1C Level & 8.24 & 7.92 & 0.152 \\
\hline Follow-up total cost & $\$ 6,014$ & $\$ 7,023$ & 0.268 \\
\hline Follow-up medical cost & $\$ 2,319$ & $\$ 3,704$ & 0.071 \\
\hline $\begin{array}{l}\text { Follow-up pharmaceutical cost } \$ 3,074 \\
\begin{array}{l}\text { Follow-up diabetes-related } \\
\text { total cost }\end{array}\end{array} \quad \$ 2,262$ & $\$ 2,899$ & 0.452 \\
\hline $\begin{array}{l}\text { Follow-up diabetes-related } \\
\text { medical cost }\end{array}$ & $\$ 707$ & $\$ 1,510$ & 0.033 \\
\hline $\begin{array}{l}\text { Follow-up diabetes-related } \\
\text { pharmaceutical cost }\end{array}$ & $\$ 1,277$ & $\$ 1,149$ & 0.026 \\
\hline
\end{tabular}

Supported by: Novo Nordisk

\section{PS 093 New treatments}

\section{6}

Open label study to assess the saftey, pharmacokinetics (PK) and pharmacodynamics (PD) of five oral insulin formulations in healthy subjects

M. Kidron ${ }^{1,2}$, I. Raz ${ }^{1}$, M. Wolfensberger ${ }^{3}$, K. Kessler ${ }^{3}$, H. Shwob ${ }^{3}$,

C. Schruefer ${ }^{3}$

${ }^{1}$ Diabetes Unit, Hadassah Hebrew University Hospital, Jerusalem, Israel, ${ }^{2}$ Clinical Research, Oramed Pharmaceuticals, Jerusalem, Israel, ${ }^{3}$ R\&D, Swiss Caps AG, Kirchberg, Switzerland

Background and aims: To evaluate the difference of activity as well as safety, pharmacokinetics and pharmacodynamics of five oral insulin formulations Materials and methods: Eight subjects completed the study which consisted of 5 visits. During each of the visits, the subjects were given a different formulation of the oral insulin capsule. All of the formulations contained $8 \mathrm{mg}$ of insulin. Subjects received the drug in the morning after an overnight fast. The treatments were separated by a 72 to 96 hour washout period. Individual blood samples (29 totals) for PK/PD analysis were collected up to 5 hours post-dose. Glucose, insulin and c-peptide levels were measured.

Results: Administration of an oral form of insulin in the fasted state demonstrated a significant decrease in c-peptide levels in all formulations (16\%$92 \%)$ as well as reduction in blood glucose (7\%-32\%). All of the formulations were well tolerated by the volunteers, and no serious adverse events have been reported.

Conclusion: The results of this study gave a positive indication for the final formulation of oral insulin. This study showed that there was a biological active absorption of oral insulin in healthy volunteers. Phase IIa study, has commenced in order to assess the clinical potential of the oral insulin formulation, in type 2 diabetic volunteers

Supported by: Oramed Pharmaceuticals Inc.

\section{7}

Pramlintide or mealtime insulin added to basal insulin treatment in patients with type 2 diabetes

M. Riddle' 1 , R. Pencek ${ }^{2}$, S. Charenkavanich ${ }^{2}$, K. Lutz 2 , L. Porter ${ }^{2}$, K. Wilhelm ${ }^{2}$

${ }^{1}$ Internal Medicine, Oregon Science \& Health University, Portland, ${ }^{2}$ Amylin Pharmaceuticals, Inc., San Diego, United States

Background and aims: Intensification of basal insulin therapy with mealtime insulin can improve glycaemic control in patients with type 2 diabetes, but is often accompanied by an increased risk of hypoglycaemia and weight gain. The addition of pramlintide (PRAM) to basal insulin has also been demonstrated to improve glycaemic control and has been associated with reduced body weight and no increase in the risk of hypoglycaemia. This randomised, open-label, 24-week, multicenter study compared the efficacy and safety of the addition of mealtime PRAM or rapid-acting insulin (RAI) to basal insulin in patients with type 2 diabetes $(\mathrm{N}=112$; age $54 \pm 11 \mathrm{y}$, duration of diabetes $9 \pm 6$ y, $\mathrm{HbA}_{1 \mathrm{C}} 8.3 \% \pm 0.8 \%$, FPG $8.9 \pm 2.5 \mathrm{mmol} / \mathrm{L}$, BMI $36 \pm 6 \mathrm{~kg} / \mathrm{m}^{2}$; mean $\pm \mathrm{SD}$ ). Materials and methods: Patients were either insulin-naive (52\% PRAM, 57\% RAI) or on insulin therapy for $<6$ months and taking $<50$ U/day $(20 \pm 10 \mathrm{U} /$ day PRAM, $24 \pm 12$ U/day RAI); prior OADs were continued. Basal insulin (glargine or detemir) dosage was titrated from Day 1 seeking an FPG $\geq 3.9$ to $<5.5 \mathrm{mmol} / \mathrm{L}$ in both treatment groups. PRAM treatment (fixed dose; 120 $\mu \mathrm{g}$ ), which alone does not cause hypoglycaemia, was started at Day 1 , whereas RAI treatment (titrated to effect) was started at Week 4 to allow initial basal insulin dose titration without added risk of mealtime insulin-induced hypoglycaemia.

Results: PRAM and RAI resulted in similar and significant mean $( \pm \mathrm{SE}) \mathrm{HbA}_{1 \mathrm{C}}$ reductions $(-0.9 \pm 0.2 \%$ vs. $-1.1 \pm 0.2 \%)$ and similar FPG $(6.7 \pm 0.4 \mathrm{mmol} / \mathrm{L}$ vs. $6.8 \pm 0.3 \mathrm{mmol} / \mathrm{L})$ at Week 24 . The percent patients achieving an $\mathrm{HbA}_{1 \mathrm{C}}$ of $\leq 7 \%$ or $\leq 6.5 \%$ with PRAM was $45 \%$ and $29 \%$, respectively, compared to $55 \%$ and $32 \%$ with RAI. Basal insulin doses at Week 24 were $52 \pm 4 \mathrm{U}$ (PRAM) and $57 \pm 4 \mathrm{U}$ (RAI). RAI dose was titrated to $37 \pm 3 \mathrm{U}$ by Week 24 , resulting in a total daily insulin dose of $94 \pm 7 \mathrm{U}$. Weight gain was associated with RAI treatment $(+4.2 \pm 0.6 \mathrm{~kg})$, but not with PRAM treatment $(-0.3 \pm 0.7 \mathrm{~kg} ; \mathrm{p}<0.0001 \mathrm{vs}$. RAI). Incidence of hypoglycaemia was lower with PRAM compared to RAI ( $55 \%$ vs. $82 \%$ ); no severe hypoglycaemic events were reported. PRAM was associated with a higher incidence of nausea $(21 \%$ vs. $0 \%$; primarily mild in 
intensity). More patients treated with PRAM vs. RAI ( $30 \%$ vs. $11 \%$; $\mathrm{p}<0.05$ ) achieved the primary composite endpoint of $\mathrm{HbA}_{1 \mathrm{C}} \leq 7 \%$ with no increase in body weight and no severe hypoglycaemia.

Conclusion: A fixed dose of pramlintide resulted in similar improvements in glycaemic control to titrated rapid-acting insulin when added to basal insulin therapy, and compared to mealtime insulin, a lower risk of hypoglyacemia and no weight gain.

\section{8}

Continuous subcutaneous pramlintide infusion (CSPI) therapy in patients with poorly controlled type 1 diabetes: data from a Pilot Study D.M. Huffman, G.W. McLean

Diabetes, University Diabetes \& Endocrine Consultants, Chattanooga, United States

Background and aims: Pramlintide acetate, an amylin analog approved for premeal injection in patients with type 1 diabetes mellitus (T1DM), reduces postprandial blood glucose excursions, A1C and body weight. Since amylin is normally co-secreted from beta cells in a basal-bolus manner, we investigated the efficacy and safety of continuous subcutaneous (SC) pramlintide infusion (CSPI) in patients with T1DM.

Materials and methods: A 16-wk, open-label, single-arm pilot study enrolled patients $(\mathrm{n}=11$, mean $\pm \mathrm{SD}$; age $39.0 \pm 4.0 \mathrm{y}$, A1C $8.20 \pm 0.60 \%$, wt $92.3 \pm 18.4 \mathrm{~kg}$, BMI $29.7 \pm 5.1 \mathrm{~kg} / \mathrm{m} 2)$ with long-term T1DM $(20.7 \pm 1.3 \mathrm{y})$ and inadequate blood glucose (BG) control despite long-term insulin pump therapy (duration of pump therapy $9.5 \pm 6.0 \mathrm{y}$, total daily insulin dose $74.1 \pm 41.9 \mathrm{U}$, total basal $26.6 \pm 10.3 \mathrm{U}$; total bolus $47.5 \pm 31.9 \mathrm{U}$ ). Subjects initiated pramlintide using a separate continuous SC infusion (Animas 2020 insulin pump) at a basal rate of $9 \mu \mathrm{g} / \mathrm{hr}(1.5 \mathrm{U} / \mathrm{hr})$. After 3 days of basal pramlintide therapy, premeal boluses were initiated at $15 \mu \mathrm{g}(2.5 \mathrm{U})$ and titrated in $15 \mu \mathrm{g}$ increments to $60 \mu \mathrm{g}(10 \mathrm{U})$ per meal. Basal insulin doses were reduced $10 \%$ upon initiation of basal CSPI; insulin boluses were reduced $~ 10 \%$ upon beginning bolus pramlintide therapy. Basal and bolus insulin doses were adjusted weekly to safely improve glycemic control. A meal tolerance test (MTT) was performed at baseline and after $16 \mathrm{wks}$ of pramlintide therapy. Three days of continuous glucose monitoring (CGM), were performed at baseline and following 4 and 16 wks of pramlintide therapy.

Results: After 16 wks pramlintide therapy, mean \pm SD) A1C decreased to $7.85 \pm 0.74 \%$ ( $0.35 \%)$. Mean fasting glucose fell from $198.2 \pm 66.9$ to $135.8 \pm 63.9 \mathrm{mg} / \mathrm{dl}$ by wk 16 . Mean daily glucose measured by CGM decreased from $155.8 \pm 34.6 \mathrm{mg} / \mathrm{dl}$ pre-CSIP to $139.8 \pm 19.7$ at wk 16 of CSPI. Glucose variability measured by MAGE from CGMS data decreased from $95.7 \pm 26.7$ $\mathrm{mg} / \mathrm{dl}$ to $82.7 \pm 23.0$ by week 16 . Weight declined to $91.8 \pm 20.1 \mathrm{~kg}(-0.5 \mathrm{~kg})$ and waist circumference decreased from $99.9 \pm 13.4 \mathrm{~cm}$ to $93.8 \pm 14.6 \mathrm{~cm}$ by wk 16. Mean BMI was $29.5 \pm 5.61 \mathrm{~kg} / \mathrm{m} 2$ at wk 16 . After 16 wks of CSPI, mean insulin: $\mathrm{CHO}$ ratio was $20 \%$ lower than baseline, basal insulin doses remained unchanged $(27.7 \pm 11.7 \mathrm{U})$ and total daily insulin dose decreased $20 \%$ to $60.2 \pm 28.4$ U. Triglycerides fell from $172 \pm 154 \mathrm{mg} / \mathrm{dl}$ to $92 \pm 54 \mathrm{mg} / \mathrm{dl}$; total cholesterol decreased from $198.9 \pm 28.5$ to $155.82 \pm 36.0 \mathrm{mg} / \mathrm{dl}$; and LDL$\mathrm{C}$ decreased from $113.0 \pm 35.5$ to $91.3 \pm 30.1 \mathrm{mg} / \mathrm{dl}$ at wk 16. MTT after $16 \mathrm{wks}$ of CSPI demonstrated reduced and delayed postprandial glucose excursions and delayed triglyceride excursions. All subjects experienced mild postprandial hypoglycemia during the study period, but no severe hypoglycemia was reported. Three of eleven subjects experienced mild nausea upon beginning basal CSPI, but all subjects successfully titrated bolus doses to $60 \mu \mathrm{g}$ within three wks.

Conclusion: In this pilot study in subjects with T1DM treated with insulin pumps, CSPI was well tolerated, improved A1C and postprandial glucose excursions, and produced favorable changes in body weight and lipids. Subjects reported improved well-being during CSPI and a majority wished to continue CSPI after study end. Further investigation of CSPI will be needed to fully assess the advantages, optimal dosages and long-term efficacy of this therapy. Supported by: Amylin Pharmaceuticals Inc.

\section{9}

Pharmacokinetics, pharmacodynamics, safety and tolerability of pramlintide in adolescent subjects with type 1 diabetes

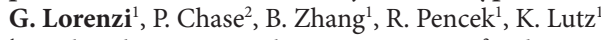

${ }^{1}$ Amylin Pharmaceuticals, Inc., San Diego, ${ }^{2}$ Pediatrics, University of

Colorado Health Science Center, Denver, CO, United States

Background and aims: Pramlintide improves glycaemic control in adult subjects with diabetes by reducing postprandial glucagon secretion, slowing gastric emptying, and reducing food intake. Pramlintide has not previously been investigated in adolescent subjects. The present randomised, singleblind, dose-rising, placebo-controlled, crossover study evaluated the pharmacokinetics, pharmacodynamics, safety and tolerability of pramlintide in adolescent subjects (12-17 y) with type 1 diabetes.

Materials and methods: Subjects (9F:3M; $15 \mathrm{y} ; \mathrm{HbA}_{1 \mathrm{c}} 8.4 \%$, BMI $25 \mathrm{~kg} / \mathrm{m}^{2}$ ) were randomised to 1 of 3 treatment sequences of study medication $(15 \mu \mathrm{g}$ pramlintide, $30 \mu \mathrm{g}$ pramlintide, or placebo), which was administered as a single SC dose at each of 3 study-site visits just before a standardised breakfast. Insulin lispro (50\% of usual mealtime dose) was injected separately. Acetaminophen $(1000 \mathrm{mg})$ was administered orally after the first bite of the meal to measure gastric emptying rates.

Results: In 9 evaluable subjects, plasma pramlintide concentrations increased dose-proportionately, mean $\mathrm{C}_{\max }$ was reached $\sim 15 \min \left(\right.$ median $\mathrm{T}_{\text {max }}$ ) after administration, and concentrations then declined in parallel. Incremental plasma glucagon $\mathrm{AUC}_{0-3 \mathrm{~h}}$ and glucose $\mathrm{AUC}_{0-3 \mathrm{~h}}$ were reduced with pramlintide vs. placebo. Acetaminophen $C_{\text {max }}$ was decreased with pramlintide vs. placebo, and pramlintide delayed the median $\mathrm{T}_{\max }$ by $\sim 2.5$-3.8-fold.

Conclusion: Pramlintide was well tolerated, and there were no treatmentrelated adverse events in this study.

Pharmacokinetic and Pharmacodynamic Results

\begin{tabular}{|c|c|c|c|}
\hline & Placebo & $\begin{array}{l}\text { Pramlintide } \\
15 \mu \mathrm{g}\end{array}$ & $\begin{array}{l}\text { Pramlintide } \\
30 \mu \mathrm{g}\end{array}$ \\
\hline \multicolumn{4}{|l|}{ Plasma Pramlintide } \\
\hline $\begin{array}{l}\mathrm{AUC}_{0-\infty} \\
\left(\mathrm{pg}^{*} \mathrm{hr} / \mathrm{mL}\right)\end{array}$ & & $98 \pm 31$ & $251 \pm 151$ \\
\hline $\begin{array}{l}\mathrm{C}_{\max } \\
(\mathrm{pg} / \mathrm{mL})\end{array}$ & & $100 \pm 28$ & $218 \pm 70$ \\
\hline $\begin{array}{l}\text { Median } \mathrm{T}_{\text {max }} \\
(\mathrm{min})\end{array}$ & & 17 & 15 \\
\hline $\begin{array}{l}\text { Plasma Glucagon Incremental } \\
\text { AUC } \\
\left(\text { ng }^{\star} h \mathrm{hh} / \mathrm{L}\right)(95 \% \text { CI difference })\end{array}$ & $34.5 \pm 27.9$ & $\begin{array}{l}3.7 \pm 19.8 \\
(-44.7,-8.1)\end{array}$ & $\begin{array}{l}5.1 \pm 21.1 \\
(-60.4,-21.9)\end{array}$ \\
\hline $\begin{array}{l}\text { Plasma Glucose Incremental } \\
\text { AUC }_{0-3 \mathrm{~h}} \\
\left(\mathrm{mmol}^{*} \mathrm{hr} / \mathrm{L}\right)(95 \% \mathrm{CI} \\
\text { difference })\end{array}$ & $12 \pm 9$ & $\begin{array}{l}7 \pm 7 \\
(-13 \pm 2)\end{array}$ & $\begin{array}{l}7 \pm 6 \\
(-12 \pm 4)\end{array}$ \\
\hline \multicolumn{4}{|l|}{ Plasma Acetaminophen } \\
\hline $\begin{array}{l}\mathrm{AUC}_{0-8 \mathrm{~h}} \\
\left(\mu \mathrm{mol}^{\star} \mathrm{hr} / \mathrm{L}\right)\end{array}$ & $326.3 \pm 78.6$ & $338.0 \pm 75.0$ & $316.5 \pm 67.8$ \\
\hline $\begin{array}{l}\mathrm{C}_{\max } \\
(\mu \mathrm{mol} / \mathrm{L}) \\
(95 \% \text { GCIR })\end{array}$ & $85.2 \pm 23.8$ & $\begin{array}{l}77.3 \pm 29.5 \\
(4.2 \pm 7.3)\end{array}$ & $\begin{array}{l}62.8 \pm 13.4 \\
(3.7 \pm 6.6)\end{array}$ \\
\hline $\begin{array}{l}\text { Median } \mathrm{T}_{\max } \\
(\mathrm{min})\end{array}$ & 47 & 120 & 180 \\
\hline
\end{tabular}

All data are mean \pm SD unless otherwise indicated; the $95 \%$ confidence interval (CI) difference for pramlintide vs. placebo is indicated for the plasma glucagon and glucose parameters; the geometric $95 \%$ confidence interval ratio (GCIR) for pramlintide vs. placebo is indicated for the acetaminophen parameter; each subject received one dose of study medication per visit (Visits 2, 3, 4); the time interval between Visits 2 and 4 was $\leq 5$ weeks; the time interval between Visits 2, 3, and 4 did not need to be evenly spaced; for any visits that occurred on consecutive days, the time interval between the two doses of study medication was $>24$ hours.

Pramlintide reduced postprandial glucagon and glucose excursions and slowed gastric emptying in adolescent subjects with type 1 diabetes, similar to what has been observed in adults. These effects were observed in the absence of treatment-related adverse events. Long-term studies evaluating the efficacy and safety of pramlintide in adolescents are warranted. 


\section{0}

Pramlintide, as an adjunct to premixed insulin, reduced $\mathrm{HbA}_{1 \mathrm{c}}$ and body weight in patients with type 2 diabetes

T. Darsow, G. Lorenzi, B. Zhang, H. Maier, K. Wilhelm

Amylin Pharmaceuticals, Inc., San Diego, United States

Background and aims: Pramlintide, as an adjunct to mealtime insulin, reduces $\mathrm{HbA}_{1 \mathrm{c}}$, glucose fluctuations and body weight in patients with type 2 and type 1 diabetes. A significant percentage of patients with diabetes choose a premixed insulin formulation over basal + bolus insulin therapy as a means of simplifying their treatment regimen.

Materials and methods: The present post-hoc analysis of an open-label study examined the effects of pramlintide over 26 weeks in a subset of patients with type 2 diabetes using premixed insulin $(70 / 30$ or $75 / 25)(\mathrm{N}=28$, age $56 \pm 9 \mathrm{y}$, $\mathrm{DM}$ duration $13 \pm 5 \mathrm{y}, \mathrm{HbA} 8.1 \pm 1.2 \%$, postprandial glucose [PPG] concentration $11.6 \pm 3.4 \mathrm{mmol} / \mathrm{L}, \mathrm{BMI} 39 \pm 6 \mathrm{~kg} / \mathrm{m}^{2}$, weight $112 \pm 23 \mathrm{~kg}$, daily insulin dose $88 \pm 47$ units; mean $\pm S D$ ). Outcome measures included changes from baseline in $\mathrm{HbA}_{1 \mathrm{c}}$, PPG concentrations, body weight, daily insulin doses, and safety.

Results: After 26 weeks of pramlintide therapy (120 $\mu$ g administered subcutaneously either two or three times daily with major meals), a mean $( \pm \mathrm{SE})$ change in $\mathrm{HbA}_{1 \mathrm{c}}$ of $-0.66 \pm 0.14 \%$ (95\% CI: $-0.97,-0.36$ ) was observed. Mean $( \pm \mathrm{SE})$ PPG concentrations were reduced from baseline by $1.2 \pm 0.8 \mathrm{mmol} / \mathrm{L}$ despite significant reductions in mean $( \pm$ SE) daily insulin doses $(-9.1 \pm 3.9 \%$ [95\% CI: -17.1, -1.2]). Pramlintide treatment also resulted in a significant and progressive reduction from baseline in mean $( \pm$ SE) body weight $(-4.1 \pm 0.8$ $\mathrm{kg}$ [95\% CI: $-5.7,-2.4]$ ) at Week 26. Pramlintide was generally well tolerated. Nausea $(29 \%)$ and hypoglycaemia $(11 \%)$ were mostly mild-to-moderate in intensity. No events of severe hypoglycaemia occurred.

Conclusion: In summary, pramlintide treatment resulted in significant reductions from baseline in $\mathrm{HbA}_{1 \mathrm{c}}$, daily insulin doses, and body weight in patients using premixed insulin. These results suggest that patients with type 2 diabetes who are not achieving optimal glycaemic control with premixed insulin may experience glycaemic improvements and weight loss with adjunctive pramlintide therapy.

\section{1}

Pramlintide improved glycaemic control and reduced weight in patients with type 2 diabetes treating to target with basal insulin

K. Wilhelm ${ }^{1}$, M. Riddle ${ }^{2}$, B. Zhang ${ }^{1}$, H. Maier ${ }^{1}$, O. Kolterman ${ }^{1}$

${ }^{1}$ Amylin Pharmaceuticals, Inc., San Diego, ${ }^{2}$ Internal Medicine, Oregon

Science \& Health University, Portland, United States

Background and aims: $\mathrm{As}_{\mathrm{HbA}}$ approaches $7 \%$, the relative contribution of fasting plasma glucose (FPG) to elevated glycaemia ( $\mathrm{HbA}_{1 \mathrm{c}}$ ) decreases and the contribution of postprandial glucose (PPG) increases. While basal insulin can effectively control FPG, pramlintide, an analog of the $\beta$-cell hormone amylin, reduces PPG.

Materials and methods: To explore the effect of pramlintide on glycaemia in patients achieving a FPG target, a post hoc analysis of a 16-week, doubleblind, placebo-controlled, randomised study was performed in a subset of patients achieving a FPG $<6 \mathrm{mmol} / \mathrm{L}$ at Week 16 with insulin glargine titration (target FPG 4-6 mmol/L) and pramlintide $(120 \mu \mathrm{g})$ or placebo with major meals.

Results: Patient (pramlintide, $n=28$; placebo, $n=33$ ) characteristics were well balanced between groups (age $56 \pm 8$ y, DM duration $11 \pm 6$ y, BMI $35 \pm 5 \mathrm{~kg} / \mathrm{m}^{2}$; mean \pm SD).

In the subgroup of patients achieving a FPG $<6 \mathrm{mmol} / \mathrm{L}$ at Week 16, FPG reductions were achieved with moderate increases in daily insulin doses in both groups. Pramlintide reduced PPG increments compared to placebo. Pramlintide reduced $\mathrm{HbA}_{1 \mathrm{c}}$ to a greater extent than placebo and to a greater extent than what was observed in the population as a whole. More patients in this subgroup achieved an $\mathrm{HbA}_{1 \mathrm{c}} \leq 7.0 \%$ with pramlintide vs. placebo ( $43 \%$ vs. $27 \%$ ). As was observed in the total population, improvements in glycaemia were accompanied by progressive weight loss with pramlintide and weight gain with placebo. Pramlintide was generally well tolerated. The most frequent adverse events were mild to moderate hypoglycaemia (pramlintide $57 \%$; placebo 55\%) and nausea (pramlintide 39\%; placebo $18 \%$ ). No severe hypoglycaemia occurred.

Conclusion: In patients attaining target FPG concentrations with basal insulin titration, pramlintide led to additional improvements in postprandial glucose increments and $\mathrm{HbA}_{1 c}$ compared with titration of basal insulin alone.
These effects were achieved with weight loss and without an increased risk of hypoglycaemia.

\begin{tabular}{|c|c|c|c|c|}
\hline & \multicolumn{2}{|c|}{ All patients } & \multicolumn{2}{|l|}{$\begin{array}{l}\text { Week 16 FPG } \\
<6 \mathrm{mmol} / \mathrm{L} \\
\end{array}$} \\
\hline & $\begin{array}{l}\text { Pramlinti } \\
+ \text { Insulin } \\
\text { Glargine } \\
\mathrm{N}=105\end{array}$ & $\begin{array}{l}\text { e Placebo } \\
\text { + Insulin } \\
\text { Glargine } \\
\mathrm{N}=106\end{array}$ & $\begin{array}{l}\text { Pramlintide } \\
\text { + Insulin } \\
\text { Glargine } \\
\mathrm{N}=\mathbf{2 8}\end{array}$ & $\begin{array}{l}\text { Placebo } \\
+ \text { Insulin } \\
\text { Glargine } \\
\mathrm{N}=33\end{array}$ \\
\hline \multicolumn{5}{|c|}{ Daily insulin dose (units) } \\
\hline $\begin{array}{l}\text { Baseline } \\
\text { Week } 16 \\
\Delta \text { at Week } 16\end{array}$ & $\begin{array}{l}48 \pm 2 \\
61 \pm 3 \\
12 \pm 2 \\
\end{array}$ & $\begin{array}{l}54 \pm 4 \\
70 \pm 5 \\
13 \pm 2 \\
\end{array}$ & $\begin{array}{l}51 \pm 4 \\
65 \pm 7 \\
14 \pm 4 \\
\end{array}$ & $\begin{array}{l}48 \pm 7 \\
57 \pm 7 \\
9 \pm 2 \\
\end{array}$ \\
\hline \multicolumn{5}{|c|}{ FPG $(\mathrm{mmol} / \mathrm{L})$} \\
\hline $\begin{array}{l}\text { Baseline } \\
\text { Week } 16 \\
\Delta \text { at Week } 16 \\
\end{array}$ & $\begin{array}{l}8 \pm 0.3 \\
7 \pm 0.2 \\
-2 \pm 0.4 \\
\end{array}$ & $\begin{array}{l}8 \pm 0.3 \\
7 \pm 0.2 \\
-0.7 \pm 0.3 \\
\end{array}$ & $\begin{array}{l}8 \pm 0.6 \\
5 \pm 0.1 \\
-4 \pm 0.6 \\
\end{array}$ & $\begin{array}{l}7 \pm 0.3 \\
5 \pm 0.1 \\
-2 \pm 0.3 \\
\end{array}$ \\
\hline \multicolumn{5}{|c|}{ PPG increments $(\mathrm{mmol} / \mathrm{L})$} \\
\hline $\begin{array}{l}\text { Baseline } \\
\text { Week } 16 \\
\Delta \text { at Week } 16 \\
\end{array}$ & $\begin{array}{l}3 \pm 0.2 \\
2 \pm 0.1 \\
-1 \pm 0.2 \\
\end{array}$ & $\begin{array}{l}3 \pm 0.1 \\
3 \pm 0.1 \\
0 \pm 0.2 \\
\end{array}$ & $\begin{array}{l}3 \pm 0.3 \\
2 \pm 0.2 \\
-1 \pm 0.3 \\
\end{array}$ & $\begin{array}{l}3 \pm 0.3 \\
3 \pm 0.2 \\
-0.3 \pm 0.2 \\
\end{array}$ \\
\hline \multicolumn{5}{|l|}{$\mathrm{HbA}_{1 \mathrm{c}}(\%)$} \\
\hline $\begin{array}{l}\text { Baseline } \\
\text { Week } 16 \\
\Delta \text { at Week } 16\end{array}$ & $\begin{array}{l}8.5 \pm 0.1 \\
7.8 \pm 0.1 \\
-0.7 \pm 0.1 \\
\end{array}$ & $\begin{array}{l}8.5 \pm 0.1 \\
8.1 \pm 0.1 \\
-0.4 \pm 0.1\end{array}$ & $\begin{array}{l}8.7 \pm 0.2 \\
7.4 \pm 0.2 \\
-1.2 \pm 0.2\end{array}$ & $\begin{array}{l}8.2 \pm 0.2 \\
7.6 \pm 0.2 \\
-0.5 \pm 0.2\end{array}$ \\
\hline \multicolumn{5}{|c|}{ Body weight (kg) } \\
\hline $\begin{array}{l}\text { Baseline } \\
\text { Week } 16 \\
\Delta \text { at Week } 16\end{array}$ & $\begin{array}{l}103 \pm 2 \\
102 \pm 2 \\
-1.6 \pm 0.3\end{array}$ & $\begin{array}{l}103 \pm 2 \\
104 \pm 2 \\
0.7 \pm 0.3\end{array}$ & $\begin{array}{l}103 \pm 3 \\
102 \pm 3 \\
-1.0 \pm 0.5\end{array}$ & $\begin{array}{l}105 \pm 4 \\
106 \pm 3 \\
0.8 \pm 0.6\end{array}$ \\
\hline
\end{tabular}

Data are mean \pm SE for the intent-to-treat last observation carried forward (ITT LOCF) population. Insulin data are mean \pm SE for the intent-to-treat observed population.

\section{2}

Inhaled insulin: safe and efficacious administration with a commercially available dry powder inhaler

L. Heinemann ${ }^{1}$, A. Brugger ${ }^{2}$, C. Cook ${ }^{2}$, U. Eckers ${ }^{1}$, A. Hutchcraft ${ }^{2}$,

L. Nosek ${ }^{1}$, K. Rave ${ }^{1}$, J. Troeger ${ }^{2}$, P. Valaitis ${ }^{2}$, T. Heise ${ }^{1}$

${ }^{1}$ Management, Profil Inst. für Stoffwechselforschung, Düsseldorf, Germany, ${ }^{2}$ Research, Baxter Healthcare Corporation, Round Lake, United States

Background and aims: Inhaled insulin is usually delivered with specifically developed inhalers, which are often large and not easy to handle. In this study we investigated the PK, PD and safety of recombinant human insulin inhalation power (RHIIP) manufactured using PROMAXX technology, which allows formation of uniform protein microspheres.

Methods: RHIIP was administered with a small commercially available dry powder inhaler (DPI). Thirty healthy male subjects $(30 \pm 1.1$ years (mean \pm SEM), BMI $\left.24.2 \pm 0.3 \mathrm{~kg} / \mathrm{m}^{2}\right)$ in a randomized crossover study received 10 IU human regular insulin sc (sc RHI) and $6.5 \mathrm{mg}$ of RHIIP (187 IU) delivered via the Cyclohaler $^{\mathrm{TM}}$ under euglycemic glucose clamp conditions. Subjects were trained to inhale RHIIP with an inhalation flow rate of $90 \pm 30 \mathrm{~L} / \mathrm{min}$ prior to dosing. Results: Inhalation of RHIIP was well tolerated with no episode of cough or shortness of breath. RHIIP showed a faster onset of action than sc RHI (time to reach $10 \%$ of total area under the glucose infusion rates (GIR) curves $73 \pm 2$ vs $95 \pm 3$ min; GIR-tmax $173 \pm 13$ vs $218 \pm 9$ min, $p<0.0001$ ) (s. Figure). Duration of action ( $371 \pm 11$ vs $366 \pm 7 \mathrm{~min}$ ) and total metabolic effect (GIR-AUC0$10 \mathrm{~h} 2734 \pm 274$ vs $2482 \pm 155 \mathrm{mg} / \mathrm{kg}$ ) were comparable. PK results were in accordance with these PD findings: RHIIP was absorbed faster (time to reach $10 \%$ of total area under the insulin curves $44 \pm 3$ vs $66 \pm 3 \mathrm{~min}, \mathrm{p}<0.0001$ ), and maximum insulin levels were reached earlier ( $86 \pm 10$ vs $141 \pm 12 \mathrm{~min}$, $\mathrm{p}=0.002$ ). Relative BA of RHIIP was $12 \pm 2 \%$; relative BP was $6 \pm 1 \%$.

Conclusion: In this study, PROMAXX technology allowed for safe and efficacious administration of human insulin powder to the deep lung with an off-the-shelf DPI designed for upper airway drug delivery. RHIIP showed a fast onset of action and BA comparable to that reported for other inhaled insulin formulations using specifically designed devices. Thus, RHIIP seems to have the potential to achieve even higher BA through further improvements in the insulin delivery technique. 


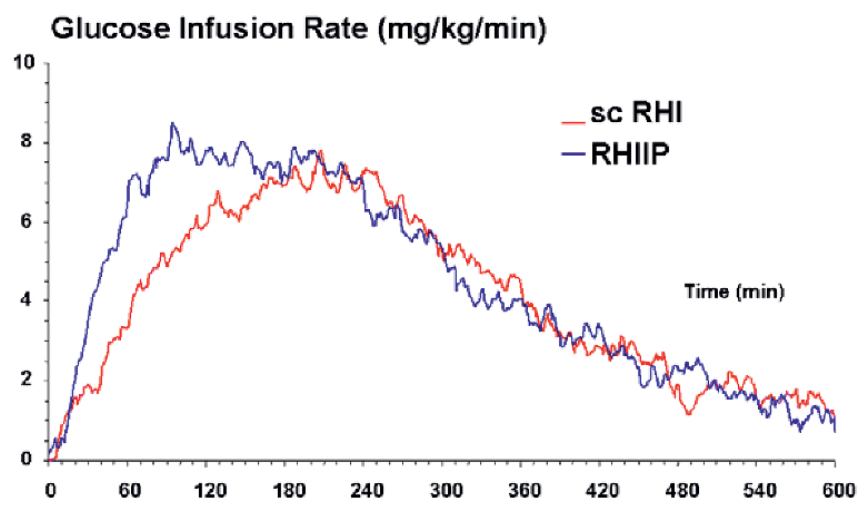

Supported by: Baxter Health Care, Il, US.

\section{3}

Technosphere insulin: defining the mechanism of action R. Angelo, K. Rousseau, M. Grant, A. Leone-Bay, P. Richardson MannKind Corporation, Paramus, United States

Background and aims: Technosphere Insulin is a novel dry powder formulation of insulin adsorbed to Technosphere microparticles for pulmonary delivery. Technosphere Insulin delivers insulin to the blood stream with a pharmacokinetic profile (time to maximum concentration, approximately $13-15$ minutes) that closely mimics early endogenous insulin release. Technosphere Insulin is composed of insulin and fumaryl diketopiperazine (FDKP), a proprietary excipient. To characterize the role of FDKP in insulin absorption, Calu-3 cells (bronchial cells) were exposed to either Technosphere Insulin solutions or a panel of known penetration enhancers. Materials and methods: Monolayers of Calu-3 cells were treated for up to 2 hours with Technosphere Insulin, capric acid, deoxycholate, and various surfactants. Exposure to capric acid, deoxycholate, and the surfactants disrupted the actin cytoskeleton of the cells and increased the permeability of Lucifer yellow, an accepted paracellular transport marker.

Results: Technosphere Insulin had no detectable impact on either the actin cytoskeleton or Lucifer yellow permeability. Additionally, Technosphere Insulin did not reduce monolayer integrity (measured by transepithelial electrical resistance) or promote cytotoxicity (lactate dehydrogenase assay). Incubation of both Calu-3 and A549 alveolar cells with fluorescently labeled insulin in the presence of FDKP revealed no visible intracellular insulin accumulation. In contrast, treatment with penetration enhancers capric acid $(17 \mathrm{mM})$ or digitonin caused significant accumulation of the fluorescently labeled drug. These experiments demonstrate that Technosphere Insulin does not compromise either tight junctions or the plasma membrane. Finally, cellular permeability of insulin and solutions of insulin formulated as Technosphere Insulin were comparable in Calu-3 monolayers, indicating that FDKP does not facilitate insulin absorption.

Conclusion: Together, these data suggest that FDKP is not a penetration enhancer and does not promote insulin absorption. Rather, it comprises particles that efficiently deliver insulin to the deep lung, where it is absorbed by natural processes.

\section{4}

Technosphere insulin pharmacokinetics unchanged in subjects who smoke

R. Baughman ${ }^{1}$, J. Cassidy ${ }^{1}$, B. Levy ${ }^{2}$, M. Hompesch ${ }^{3}$, M. Diaz, A. Boss ${ }^{1}$, P. Richardson ${ }^{1}$

${ }^{1}$ MannKind Corporation, Paramus, ${ }^{2}$ Johnson \& Johnson Pharmaceutical Services, LLC, Raritan, ${ }^{3}$ Profil Institute for Clinical Research, Inc., Chula Vista, United States

Background and aims: Smoking has been found to alter insulin absorption with some inhaled insulin formulations, increasing both maximum and overall insulin exposure 2- to 3-fold. This phase I, open-label, single-dose, euglycemic clamp study compared the pharmacokinetics and tolerability of Technosphere Insulin Inhalation Powder in subjects with type 2 diabetes who smoke ( $n=12)$ to subjects with type 2 diabetes who do not smoke (non-smoking $>2$ years; negative urine cotinine, $n=12$ ).

Materials and methods: Subjects (age range: 41-63 years) who met inclusion criteria, including extensive pulmonary function testing, were administered
$30 \mathrm{U}$ Technosphere Insulin on a single occasion while fasting, 4 to 6 hours after starting intravenous insulin infusion during a clamp procedure. Serial blood samples were drawn for insulin and C-peptide assay.

Results: Insulin concentrations following Technosphere Insulin dosing were adjusted to account for infused insulin and basal endogenous insulin. Confirmation of steady endogenous insulin was inferred from the absence of a measured change in the plasma C-peptide concentration after dosing. All values presented were determined from the baseline-adjusted concentrations. The mean maximum insulin concentration for smokers $\left(\mathrm{C}_{\max \text { smokers }}\right)$ was $22 \mu \mathrm{U} / \mathrm{mL}$ and the mean $\mathrm{C}_{\text {max non-smokers }}$ was $24 \mu \mathrm{U} / \mathrm{mL}, \mathrm{p}=0.905$ (see Figure). The median time of maximum insulin concentration $\left(\mathrm{T}_{\max }\right.$, smokers $)$ was 20 minutes and median $\mathrm{T}_{\text {max, non-smokers }}$ was 12 minutes, $\mathrm{p}=0.010$. The mean baseline adjusted $\mathrm{AUC}_{0.480}$ was $2100 \mu \mathrm{U} / \mathrm{mL}^{\star} \mathrm{min}$ for smokers and $1700 \mu \mathrm{U} / \mathrm{mL}^{*} \min$ for non-smokers, $\mathrm{p}=0.479$. Dosing was well tolerated with 1 adverse event in the smoker group and 4 adverse events in 2 subjects in the nonsmoker group, with no hypoglycemia or respiratory (other than cough) adverse events observed. No adverse event was attributed to the study drug.

Conclusion: The rapid absorption of insulin following Technosphere Insulin dosing does not appear to be significantly altered in this smoking population. This differs from what has been reported with other inhaled insulins.

\section{Baseline Corrected Plasma Mean Insulin Concentrations}

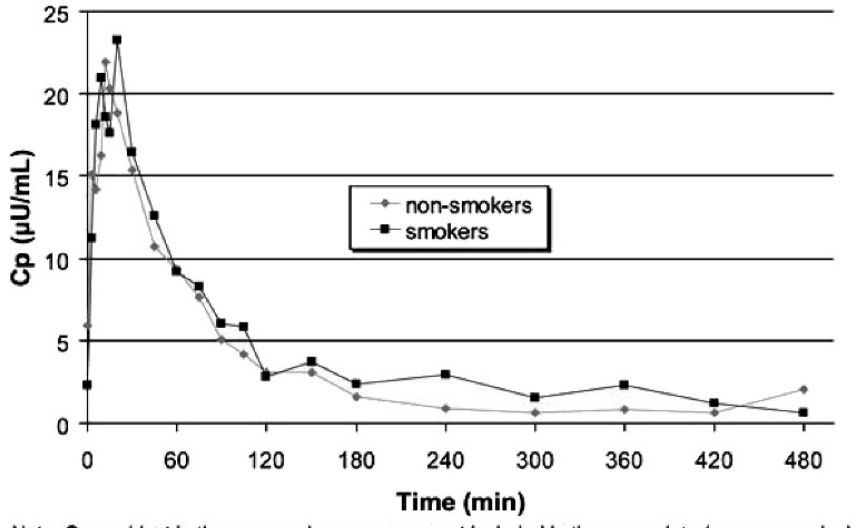

Note: One subject in the non-smoker group was not included in the mean data (non-responsive)

\section{5}

Pharmacokinetic and pharmacodynamic properties of a dry powder inhaled insulin administered via a novel dual-piezo inhalation device compared to sc administered regular human insulin M. Hompesch ${ }^{1}$, A. Kollmeier ${ }^{1}$, D. Becker², D. Byron'², E. Peers ${ }^{3,4}$, E. Reynolds ${ }^{2}$, A. Scrimgeour ${ }^{3}$, N. Whitfield ${ }^{3}$, L. Heinemann ${ }^{5}$ ${ }^{1}$ Profil Institute for Clinical Research, Inc., Chula Vista, CA, United States, ${ }^{2}$ MicroDose Technologies, Inc., Monmouth Junction, United States, ${ }^{3}$ Vectura Group Plc, Ruddington, United Kingdom, ${ }^{4}$ Sanofi-aventis operations, subsequently sanofi-aventis, Paris, France, ${ }^{5}$ Profil Institut fuer Stoffwechselforschung GmbH, Neuss, Germany

Background and aims: Primary objective of this study was to evaluate the pharmacokinetic (PK) and pharmacodynamic (PD) properties of a newly developed dry powder inhaled insulin (INH) formulation delivered by a novel electronic inhaler capable of delivering 2 discrete blister doses simultaneously.

Materials and methods: In this randomized, four way crossover study, euglycemic automated glucose-clamps (target blood glucose $90 \mathrm{mg} / \mathrm{dl}$ ) were completed in 14 out of 16 enrolled healthy volunteers (age 30.4 \pm 7.4 years $\left(\right.$ mean \pm SD); BMI $\left.24.4 \pm 2.1 \mathrm{~kg} / \mathrm{m}^{2}\right)$. Each volunteer received three different doses of INH on three different study days (see Table 1) and 10 IU Humulin $\mathrm{R}^{\prime}$ (RHI) by SC injection into the abdomen on a fourth day.

Results: PD parameters as shown in the table below reveal a more rapid onset of action with INH; Maximal metabolic effect $\left(\mathrm{GIR}_{\max }\right)$ and $\mathrm{AUC}_{\text {GIR } 0-480}$ were lower with the $1.5 \mathrm{mg}$ INH doses in comparison to the $3 \mathrm{mg}$ INH dose and the 10 IU SC RHI dose respectively. The metabolic effects of $3 \mathrm{mg}$ INH were equivalent to those of the $10 \mathrm{IU}$ SC. Overall relative biopotency was $11-17 \%$ for the INH doses. PK data reflect the PD results. No clinically relevant adverse events were observed.

Mean (SD); ${ }^{*}$ pair wise t-test, ${ }^{*}$ Wilcoxon Signed Rank test: $\mathrm{p}$ values referring to $3 \mathrm{mg}$ INH and 10 IU SC

Conclusion: Inhalation of this INH formulation by a novel inhalation device was well tolerated and shows a bioavailability and biopotency that is better 


\begin{tabular}{|c|c|c|c|c|c|c|}
\hline $\begin{array}{l}\text { Table } 1 \\
\text { PD Parameters - Completers }\end{array}$ & early $\mathrm{T}_{50 \%}(\mathrm{~min})$ & $\mathrm{T}_{\max }(\min )$ & late $\mathrm{T}_{50 \%}(\mathrm{~min})$ & $\mathrm{GIR}_{\max }(\mathrm{mg} / \mathrm{kg} / \mathrm{min})$ & $\begin{array}{l}\text { GIR-AUC } \\
0-480\end{array}$ & $\begin{array}{l}\text { Rel Bioavailability \% } \\
0-120 \text { min } \\
0-\infty\end{array}$ \\
\hline $\begin{array}{l}1.5 \mathrm{mg} \text { INH } \\
\left(2^{\star} 0.75 \mathrm{mg} \text { blisters }\right)\end{array}$ & $61(45)$ & $178(81)$ & $293(81)$ & $5.4(2.8)$ & $1515(818)$ & $\begin{array}{l}21.0(13.3) \\
16.6(12.1)\end{array}$ \\
\hline $\begin{array}{l}1.5 \mathrm{mg} \text { INH } \\
(1.5 \mathrm{mg} \text { blister })\end{array}$ & $86(84)$ & $183(83)$ & $350(82)$ & $5.6(2.4)$ & $1541(768)$ & $\begin{array}{l}23.3(11.9) \\
14.4(9.9)\end{array}$ \\
\hline $\begin{array}{l}3 \mathrm{mg} \text { INH } \\
\left(2^{\star} 1.5 \mathrm{mg} \text { blisters }\right)\end{array}$ & $58(31) ; \mathrm{p}=0.03^{\star *}$ & $172(55) \mathrm{p}=0.04^{\star *}$ & $304(95) \mathrm{p}=0.02^{\star *}$ & $7.1(2.8)$ & $1945(968) \mathrm{p}=0.05^{\star}$ & $\begin{array}{l}18.3(14.2) \\
12.0(8.6)\end{array}$ \\
\hline 10 IU Humulin R SC & $77(29)$ & $223(61)$ & $401(60)$ & $7.5(2.0)$ & $2328(618)$ & - \\
\hline
\end{tabular}

than that observed with other inhaled insulins. Together with the small size of the inhaler, this may provide superior effectiveness and compliance compared to other inhaled insulin preparations.

Supported by: Vectura Group Plc

\section{6}

Rimonabant reduces intra-abdominal and liver fat, and improves glycaemic control: the ADAGIO-LIPIDS substudy in patients with type 2 diabetes

J.-P. Despres ${ }^{1}$, R. Ross ${ }^{2}$, G. Boka ${ }^{3}$, N. Alméras ${ }^{1}$, I. Lemieux ${ }^{1}$

${ }^{1}$ Quebec Heart Institute, Laval Hospital Research Center, Quebec,

Canada, ${ }^{2}$ Department of Medicine, Queens University, Kingston, Canada,

${ }^{3}$ Department of Research and Development, sanofi-aventis, Antony, France

Background and aims: In overweight/obese patients with type 2 diabetes (T2DM) either naïve to antidiabetic therapy or on monotherapy with metformin or sulphonylurea or treated with insulin, rimonabant, the first selective cannabinoid receptor type- $1\left(\mathrm{CB}_{1}\right)$ antagonist, improves glycaemic control and associated cardiometabolical risk factors.

In the ADAGIO-Lipids study, the beneficial effect of rimonabant on a comprehensive set of cardiometabolical risk variables was demonstrated in 803 abdominally obese $(\mathrm{AO})$ patients with atherogenic dyslipidaemia (high triglycerides [TG] and/or low high-density lipoprotein cholesterol [HDL-C]). Furthermore, a computed tomographic (CT) imaging substudy showed a reduction in both intra-abdominal and liver fat with rimonabant treatment. Among the study patients, $17 \%$ had T2DM.

Materials and methods: We report the analyses from the ADAGIO-Lipids study conducted in a subsample of patients with T2DM, randomised to placebo $(n=66)$ or rimonabant $20 \mathrm{mg} /$ day $(\mathrm{n}=71)$ plus hypocaloric diet $(600 \mathrm{kcal} /$ day deficit) and treated for 1 year, with a 2-month safety follow-up. HDL-C and TG were co-primary endpoints. Intra-abdominal (visceral) fat and liver fat were measured in a total of 36 patients.

Results: At baseline the mean (SD) HbAlc was 7.0 (1.1)\% and 7.1 (1.5)\%, and the mean fasting plasma glucose (FPG) was $8.0(2.2) \mathrm{mmol} / \mathrm{L}$ and 7.9 (2.8) $\mathrm{mmol} / \mathrm{L}$, in the placebo and rimonabant groups, respectively. Significantly greater changes from baseline were observed with rimonabant $20 \mathrm{mg}$ vs placebo for HbAlc, FPG ( $\mathrm{p}<0.0001)$, HDL-C and TG levels $(\mathrm{p}<0.0001)$, intra-abdominal and liver fat, body weight and waist circumference $(\mathrm{p}<0.0001$, $\mathrm{p}=0.0008$, respectively) (Table). Gastrointestinal, nervous system, psychiatric and general adverse events were more common with rimonabant $20 \mathrm{mg}$.

Conclusion: In T2DM patients with $\mathrm{AO}$ and atherogenic dyslipidaemia, rimonabant $20 \mathrm{mg}$ significantly improved glycaemic control and cardiometabolical profile while significantly reducing the intra-abdominal and liver fat.

Changes (mean [SD]) from baseline in efficacy variables

\begin{tabular}{lll}
\hline & $\begin{array}{l}\text { Placebo } \\
(\mathbf{n}=66)\end{array}$ & $\begin{array}{l}\text { Rimonabant 20 mg } \\
(\mathbf{n}=71)\end{array}$ \\
\hline HbAlc, \% & $0.3(0.9)$ & $-0.5(1.2)$ \\
\hline FPG, mmol/L & $0.6(1.5)$ & $-0.7(2.2)$ \\
\hline HDL-C, \% & $-2.7(12.5)$ & $9.7(17.4)$ \\
\hline TG, \% & $12.4(70.5)$ & $-23.0(27.7)$ \\
\hline Visceral adipose tissue, \% & $-2.7(26.4)$ & $-18.1(16.8)$ \\
\hline Liver attenuation (HU) & $3.3(22.1)$ & $19.3(30.1)$ \\
\hline Weight, kg & $-1.4(4.5)$ & $-5.7(4.9)$ \\
\hline Waist, cm & $-2.7(4.0)$ & $-5.5(5.1)$ \\
\hline All & &
\end{tabular}

All $\mathrm{p}<0.05$ vs placebo.

Supported by: sanofi-aventis

\section{PS 094 Diabetes in daily practice}

\section{7}

Miscoded blood glucose meters: is this a problem in diabetes management?

S. Ozcan ${ }^{1}$, S. Celik², G. Bayrak ${ }^{3}$, A. Yuncu', B. Bektas ${ }^{5}$, N. Yildirim ${ }^{6}$, G. Cosansu' ${ }^{1}$, N. Olgun 7 , A. Kahraman ${ }^{8}$

${ }^{1}$ Florence Nightingale School of Nursing, Istanbul University, ${ }^{2}$ Istanbul Medical Faculty Hospital, ${ }^{3}$ Medical Faculty Hospital, Ondokuz Mayis University, Samsun, ${ }^{4}$ Medical Faculty Hospital, Akdeniz University, Antalya, ${ }^{5}$ Medical Faculty Hospital, Dokuz Eylul University, Izmir, ${ }^{6}$ Diabetes Outpatient Clinic, Dr Sami Ulus Children Hospital, Ankara, ${ }^{7}$ School of Nursing, Marmara University, Istanbul, ${ }^{8}$ Cerrahpasa Medical Faculty Hospital, Istanbul University, Turkey

Background and aims: This study aimed to evaluate the frequency of miscoding behaviour for blood glucose meters and the factors that are related to miscoding.

Materials and methods: Instrument: A questionnaire that prepared by researchers filled by checking and comparing the codes of patients' glucometers and stribs. Patients: 454 patients were involved in the study from 4 centers. Including criteria: Patients with Type 1, Type 2 who applied to diabetes unit for regular visit; who didn't have acute problems; who accepted to be involved in research study; and who brought their glucometers and stribs. Procedure: The study was carried out between July-September 2007. A questionnaire was filled by interviewing with patient and checking his glucometer and strips' codes. If patient didn't have his glucometer and strips he invited again by giving an appointment.

Ethical issues: Legal permissions from each hospitals and informed consent from patients were taken.

Results: Mean of diagnosis' duration in the subjects was $10.48 \pm 7.27$ yrs. Mean age of them was $53.03 \pm 15.13$ yrs. $50,2 \%$ of patients were female. $18 \%$ of the subjects were found miscoding their blood glucose meters in the study. Low literacy groups, patients who are in low economical status, patients with Type 2 diabetes and older patients had more coding errors in their glucometers. Patients who did not take "general diabetes education" and "education about coding of meters" had more coding errors $(\mathrm{p}<0.001)$. Patients who had miscoded meters had significantly high levels of fasting and postprandial blood glucose and HbAlc. Conclusion: Frequency of use miscoded meter was high in the study (18\%). According to the study results, use miscoded meters could effect negatively the blood glucose control. While patient education could help reduce the miscoding error, it is advisable that manually coded meters should be checked by HCPs regularly. 


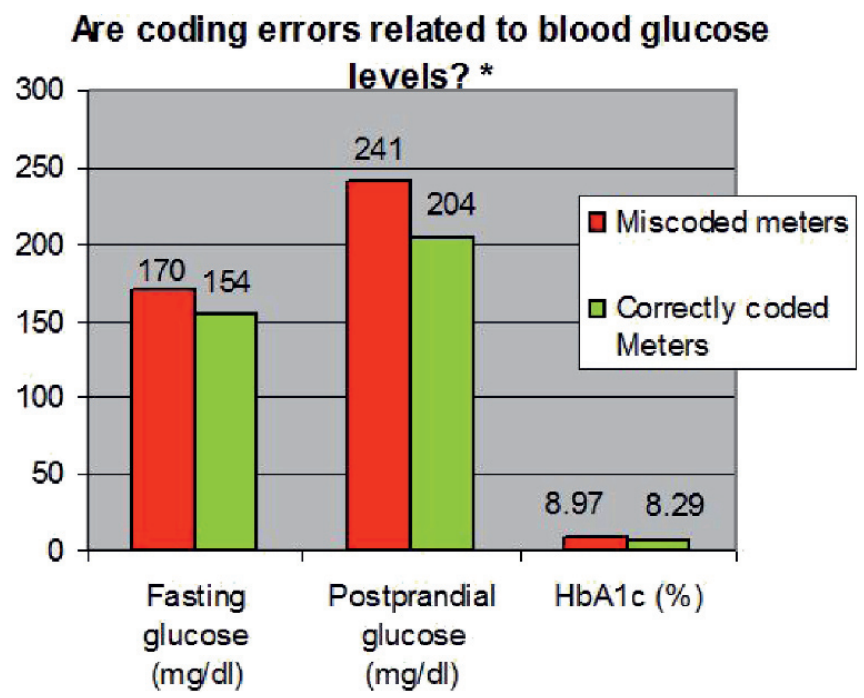

Supported by: Bayer HealthCare (logistical)

\section{8}

Maintenance of glycaemic control in type 2 diabetic patients during the Ramadan fast, with evening administration of once daily sulphonylurea

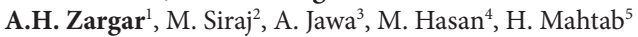

${ }^{1}$ Department of Endocrinology, Sher-i-Kashmir Institute of Medical Sciences, Srinagar, India, ${ }^{2}$ Medicine, OHRC, Hyderabad, India, ${ }^{3}$ Medicine, KEMC, Lahore, Pakistan, ${ }^{4}$ Endocrinology, Mayo Hosp., Lahore, Pakistan, ${ }^{5}$ Advisor, NHCN, Dhaka, Bangladesh

Background and aims: During Ramadan, adult Muslims are required to abstain from food, water, \& beverages from sunrise to sunset for 1 month. Most patients break their fast with a meal after sunset, followed by dinner about 3 hours later \& a breakfast like meal just before dawn the next day. The diurnal variation in plasma glucose is altered during Ramadan \& the postprandial surges in plasma glucose are shifted between $8 \mathrm{pm}$ to $6 \mathrm{am}$. In type 2 diabetic patients it is difficult to maintain glycemic control with multiple dose sulfonylureas because of the need to administer them during the non-fast overnight span of 10-16 hours. However, sulphonylureas with a sustained 24hour duration of action, administered in the evening with the first meal after the days' fast could, in theory, maintain glycemic control during Ramadan. The objective of this study was to assess whether type 2 diabetic patients under glycemic control with a once daily sulphonylurea taken at breakfast, were able to maintain the same degree of control by switching the timing of its administration to the evening during Ramadan \& reverting to the morning dose thereafter.

Materials and methods: In an open label prospective study, type 2 diabetic patients from Bangladesh, Pakistan, \& India, under glycemic control with gliclazide switched to its evening administration at the same dose during Ramadan, \& reverted to the morning schedule thereafter. The primary outcome was the difference in fasting plasma glucose before \& after Ramadan.

Results: In 136 patients, mean (95\% CI) FPG decreased by $1.1 \mathrm{mg} / \mathrm{dl}$ ( 0 to 3.4, $\mathrm{P}=0.3$ ) with evening medication by the end of 25 days fasting, \& increased by $3.1 \mathrm{mg} / \mathrm{dl}$ ( 1.1 to $6.3, \mathrm{P}=0.01)$ after reverting to morning medication 20 days later. There were $5(3.5 \%)$ hypoglycemic episodes before, 3 (2.2\%) during, \& 2 (1.5\%) after Ramadan. Mean HbAlc decreased by $0.8 \%$ ( 0.7 to $1.0, \mathrm{P}=0.00$ ), mean lipid ratio improved by $0.6(0.3$ to $0.9, \mathrm{P}=0.00)$, while mean body weight increased by $0.4 \mathrm{Kg}(0.2$ to $0.6, \mathrm{P}=0.00)$ between pre \& post Ramadan.

Conclusion: Against the background of misuse of hypoglycemic agents, alterations in diet \& risk of hypoglycemia, type 2 diabetic patients under glycemic control with a long acting sulphonylurea can maintain the same degree of control by switching to an evening administration of the same dose during Ramadan \& reverting to a morning schedule thereafter. The frequency of hypoglycemia is low \& weight gain minimal.

\section{9}

Unmet treatment needs among patients with type 2 diabetes uncontrolled on basal-only insulin therapy: implications of adding bolus insulin therapy

E. Durden ${ }^{1}$, E. Sarpong ${ }^{2}$, G. Lenhart ${ }^{1}$

${ }^{1}$ Medstat, Thomson, Boston, ${ }^{2}$ Eli Lilly and Company, Indianapolis, United States

Background and aims: Patients with type 2 diabetes mellitus (T2DM) tend to have difficulty achieving glycemic control. An abundance of research findings suggest that failure to achieve optimal glycemic control among T2DM patients has implications for diabetes related complications, health care re-

Table 1 Response of type 2 diabetic patients undertaking the Ramadan fast to monotherapy with long acting sulphonylurea

\begin{tabular}{|c|c|c|c|c|}
\hline & $\begin{array}{l}\text { Bangladesh } \\
\mathrm{N}=35\end{array}$ & $\begin{array}{l}\text { Pakistan } \\
\mathrm{N}=51\end{array}$ & $\begin{array}{l}\text { India } \\
\mathrm{N}=50\end{array}$ & $\begin{array}{l}\text { All } \\
N=136\end{array}$ \\
\hline \multicolumn{5}{|c|}{ Fasting plasma glucose (mg/dl) } \\
\hline Pre Ramadan ${ }^{\star}$ & $117.1+/-8.8$ & $120.2+/-12.1$ & $109.2+/-14.3$ & $115.4+/-13.1$ \\
\hline During Ramadan\# & $116.1+/-11.4$ & $119.3+/-9.5$ & $107.9+/-17.2$ & $114.3+/-14.1$ \\
\hline Post Ramadan* & $115.8+/-13.2$ & $120.8+/-13.2$ & $118.1+/-25.3$ & $118.5+/-18.6$ \\
\hline \multicolumn{5}{|c|}{ Glycosylated hemoglobin (\%) } \\
\hline Pre Ramadan & $7.8+/-0.8$ & $8.6+/-0.9$ & $6.3+/-1.3$ & $7.6+/-1.4$ \\
\hline Post Ramadan & $7.2+/-0.7$ & $7.2+/-0.5$ & $5.9+/-0.9$ & $6.8+/-1.0$ \\
\hline \multicolumn{5}{|c|}{ Hypoglycemic episodes } \\
\hline Pre Ramadan & $1(2.9)$ & $0(0)$ & $4(8.0)$ & $5(3.5)$ \\
\hline During Ramadan & $1(2.9)$ & $0(0)$ & $2(4.0)$ & $3(2.2)$ \\
\hline Post Ramadan & $1(2.9)$ & $0(0)$ & $1(2.0)$ & $2(1.5)$ \\
\hline \multicolumn{5}{|l|}{ Weight (kg) } \\
\hline Pre Ramadan & $63.3+/-6.3$ & $67.0+/-9.2$ & $69.7+/-9.8$ & $67.0+/-9.1$ \\
\hline Post Ramadan & $63.8+/-6.3$ & $67.9+/-9.1$ & $69.5+/-9.5$ & $67.4+/-8.9$ \\
\hline \multicolumn{5}{|c|}{ Lipid Ratio (TC/HDL) } \\
\hline Pre Ramadan & $4.3+/-1.6$ & $5.6+/-1.7$ & $4.3+/-1.7$ & $4.9+/-1.8$ \\
\hline Post Ramadan & $4.0+/-1.0$ & $4.5+/-1.1$ & $4.0+/-0.9$ & $4.3+/-1.1$ \\
\hline \multicolumn{5}{|c|}{ Triglycerides (mg/dL) } \\
\hline Pre Ramadan & $174.5+/-83.3$ & $175.1+/-60.8$ & $207.1+/-120.7$ & $186.4+/-92.5$ \\
\hline Post Ramadan & $164.2+/-62.0$ & $137.7+/-22.8$ & $159.9+/-60.7$ & $152.6+/-51.3$ \\
\hline
\end{tabular}

Medication with gliclazide modified release $60 \mathrm{mg} /$ day (Diamicron $M R$ ) * taken with breakfast, \& \# taken in the evening

with the first meal after the days' fast.

Plus/minus values are means $+/$ - standard deviation.

Other values are numbers of patients with percentages of the group in parenthesis. 
source utilization and costs. However, little is known about the characteristics of patients who remain uncontrolled on current basal monotherapy and the extent to which the addition of bolus therapy affects glycemic and economic burden. The objectives of this study are to evaluate the characteristics of T2DM patients and to quantify the impact of adding bolus therapy to previous basal-only insulin regimens on complications, resource utilization and costs.

Materials and methods: US health claims and clinical laboratory data from 2003-2005 were used to identify and stratify patients for analysis. The patient cohort was limited to T2DM patients receiving a basal-only insulin regimen at baseline with at least one $\mathrm{HbAlc}$ value in the database and six months continuous eligibility pre-post index HbAlc. Data on $\mathrm{HbAlc}$, complications, demographic characteristics and resource utilization of all patients were analyzed using descriptive statistics. Negative Binomial (NBR) and Multinomial Logit (MNL) models were estimated to evaluate the association between insulin therapy and complications, resource utilization and costs.

Results: Of 1,607 patients on basal monotherapy at baseline (mean age 53 years; female $63 \%$, glargine monotherapy $32 \%$ ), $8 \%$ added bolus insulin therapy during the follow-up period. Approximately $73 \%$ of the study cohort had $\mathrm{HbAlc}$ values greater than $7 \%$; a greater proportion of patients initiating bolus therapy had HbAlc values $>9 \%$ at baseline ( $45 \%$ vs $35 \%$; $\mathrm{p}<.05$ ). Those initiating bolus therapy were younger $(\mathrm{p}<.05)$, had more comorbidities (Charlson Comorbidity Index), and a higher proportion had diabetes-related complications relative to those who did not initiate bolus therapy. Our NBR estimates indicates that patients who initiated bolus insulin therapy had significantly greater number of inpatient admissions and higher total costs during the follow-up period than patients who did not initiate bolus therapy. Interestingly, our MNL regression revealed no significant differences in follow-up HbAlc between the two groups.

Conclusion: In our study, healthcare resource utilization among patients with T2DM who initiated bolus therapy was significantly higher as compared to patients who did not initiate bolus therapy during the follow-up period. A considerable proportion of T2DM patients who initiated bolus therapy show signs of complications in the pre-period, suggesting the urgency of therapy intensification. Ostensibly, the results are somewhat surprising. However, the results suggest what may be occurring in the "real world"; that is patients who add bolus therapy may have already been in poorer health due to already developed diabetes-related complications. Thus, not only should we expect these patients to incur more utilization and costs in the short term, but further postponement of therapy intensification may compromise the achievement of any meaningful reductions in patients' glycemic and economic burdens. Additional research that captures a longer follow-up period of observation is needed to assess concretely the potential beneficial impact of adding bolus therapy to basal monotherapy.

Supported by: Eli Lilly and Company

\section{0}

Influencing factors of metabolic control level in patients with diabetes mellitus type 2

J. Reviriego, J. Diago, P. Polavieja, F. Chacon

Lilly Spain, Alcobendas, Spain

Background and aims: To assess the association of several factors with metabolic control (MC) in non-insulin treated type 2 diabetes mellitus (DM2) patients in standard clinical practice in Spain. To estimate mean $\mathrm{HbAl}$ at the point when additional Oral Glucose Lowering Drugs (OGLD) are added to treatment.

Materials and methods: A retrospective, observational, naturalistic and multicentre study, that reviews clinical records of a 2 -year period previous to the inclusion of patients. A total of 216 physicians participated (endocrinologists, internal medicine specialists and general practitioners). A logistic regression model was carried out using poor MC as response variable, and doctor's specialty, sociodemographic data and clinical variables as covariables. MC was defined according to IDF and ADA (American Diabetes Association) criteria (good metabolic control $=\mathrm{HbAlc} \leq 6.5 ; \mathrm{HbAlc}<7 \%$, respectively).

Results: Clinical records from 2,266 patients were reviewed, $47.2 \%$ female. Mean age (SD) was 64.4 (11.1) years. Median time (IQR) of diagnosis was $5.9(2.9-10.1)$ years. According to IDF and ADA criteria, $41.8 \%$ and $59.9 \%$ of patients had good MC, respectively. Table 1 shows the variables influencing MC and their impact after applying a logistic regression model.
Odds Ratio Estimates

IDF Criteria

\begin{tabular}{|c|c|c|c|}
\hline \multirow{2}{*}{$\begin{array}{l}\text { Effect } \\
\text { No studies against Illiteracy }\end{array}$} & \multirow{2}{*}{$\begin{array}{l}\begin{array}{l}\text { Point } \\
\text { Estimate }\end{array} \\
0.5\end{array}$} & \multicolumn{2}{|c|}{$\begin{array}{l}\text { 95\% Wald } \\
\text { Confidence Limits }\end{array}$} \\
\hline & & 0.2 & 1.5 \\
\hline Primary studies against Illiteracy & 0.4 & 0.2 & 1.2 \\
\hline Secondary studies against Illiteracy & 0.3 & 0.1 & 0.9 \\
\hline University degree against Illiteracy & 0.3 & 0.1 & 0.8 \\
\hline $\begin{array}{l}\text { Other non-university studies against } \\
\text { Illiteracy }\end{array}$ & 0.5 & 0.0 & 9.8 \\
\hline Self-monitoring of blood glucose (number) & 1.4 & 1.1 & 1.9 \\
\hline Number of OGLD & 1.7 & 1.4 & 2.0 \\
\hline $\begin{array}{l}\text { Accomplishment of the following indicator: } \\
\text { "Begin oral glucose-lowering drugs when } \\
\text { lifestyle interventions alone are unable to } \\
\text { maintain blood glucose at target levels" }\end{array}$ & 1.5 & 1.1 & 1.9 \\
\hline \multicolumn{4}{|l|}{$\begin{array}{l}\text { Odds Ratio Estimates } \\
\text { ADA Criteria }\end{array}$} \\
\hline Effect & $\begin{array}{l}\text { Point } \\
\text { Estimate }\end{array}$ & \multicolumn{2}{|c|}{$\begin{array}{l}95 \% \text { Wald } \\
\text { Confidence Limits }\end{array}$} \\
\hline IbA1c valu & 0.9 & 0.8 & 0.9 \\
\hline Number of OGLD & 1.9 & 1.6 & 2.2 \\
\hline
\end{tabular}

Age, gender, time since diagnosis, presence of CVRF, or cholesterol values did not show any influence on MC. The first OGLD was administered with a HbAlc mean value (SD) of 7.8(1.4)\% for all patients. The second and third OGLD were added when HbAlc mean value (SD) was $8.0(1.3) \%$ and $8.1(1.1) \%$, respectively. Endocrinologists prescribed the first OGLD with a $\mathrm{HbAlc}$ mean value (SD) of $8.0(1.5) \%$, whereas internal medicine specialists and GPs began OGLD treatment with a $\mathrm{HbAlc}$ mean value $(\mathrm{SD})$ of $7.8(1.4) \%$ and 7.5(1.3), respectively. The same trend was observed for the second OGLD (8.1[1.2]\% for endocrinologists; $7.9[1.3] \%$ for internists and $7.8[1.3] \%$ for GPs), and for the third $(8.3[1.1] \%, 8.0[1.0] \%$ and $7.7[1.1] \%$, respectively.

Conclusion: Regarding IDF criteria, educational level is the only sociodemographic variable influencing DM2 MC, suggesting that there is a need to promote DM2 educational programs and improve follow-ups in low-educated patients. Nevertheless, blood glucose self-monitoring, OGLD treatment, and the number of OGLD and HBAlc controls in the last 2 years, appear to be associated with DM2 MC. However, these measures might be directly related to the treatment of those patients with worse disease evolution. According to the evolution of DM2 in non-insulin treated patients, every new OGLD is added when MC worsens. Endocrinologists prescribe OGLD with worse $\mathrm{HbA1c}$ levels than internists or GPs, probably because they care for patients in worse clinical status who are referred from other physicians. Supported by: Lilly Spain

\section{1}

Glycated albumin more accurately reflects glycaemic control than $\mathrm{HbA}_{1 \mathrm{c}}$ in diabetic subjects on peritoneal and hemodialysis

B.I. Freedman ${ }^{1}$, R.N. Shenoy ${ }^{1}$, J.A. Planer ${ }^{1}$, A.J. Bleyer ${ }^{1}$, C.Y. Cardona ${ }^{1}$, T.P. Peacock ${ }^{1}$, L. Andries ${ }^{1}$, J.R. Byers ${ }^{1}$, K.D. Clay ${ }^{1}$, G.B. Russell ${ }^{2}$,

Z.K. Shihabi ${ }^{3}$

${ }^{1}$ Internal Medicine/Nephrology, ${ }^{2}$ Biostatistical Sciences, ${ }^{3}$ Pathology, Wake Forest University School of Medicine, Winston-Salem, United States

Hemoglobin $(\mathrm{Hb}) \mathrm{A}_{1 \mathrm{c}}$ less accurately reflects glycemic control in diabetic hemodialysis (HD) patients, relative to the glycated albumin (GA) assay. The accuracy of the $\mathrm{HbA}_{1 \mathrm{c}}$ in diabetic patients on peritoneal dialysis (PD) is unknown. We measured a simultaneous $\mathrm{HbA}$ and GA in an expanded diabetic cohort of 472 cases with end-stage renal disease (ESRD; 55 on PD and 417 on HD) and in 49 non-nephropathy controls. Analyses were performed to determine factors associated with $\mathrm{HbA}_{1 \mathrm{c}}$ and $\mathrm{GA}$.

In the $417 \mathrm{HD}$ cases, $55 \mathrm{PD}$ cases, and 49 non-nephropathy controls, respectively, mean [SD] of 3 monthly serum glucose concentrations during the preceding 90 days were $169.6 \mathrm{mg} / \mathrm{dl}$ [62], $168.6 \mathrm{mg} / \mathrm{dl}$ [66] , and $146.1 \mathrm{mg} / \mathrm{dl}$ [66] (overall $\mathrm{p}=0.11$ ); with a corresponding $\mathrm{HbA}_{1 \mathrm{c}}$ of $6.78 \%$ [1.6], 6.87\% [1.4], and $7.30 \%$ [0.4] (overall $\mathrm{p}=0.08$ ). In HD cases, PD cases, and non-nephropathy controls, respectively, the mean \% GA using the bromocresol green serum albumin assay was 18.8\% [7.3], 16.0\% [4.9], and 15.3 [5.5]; and mean \% GA computed using bromocresol purple serum albumin assay was $21.1 \%$ [8.2], $19.0 \%$ [5.7], and $16.4 \%$ [5.7] ( $\mathrm{p}=0.02$ for comparison of PD vs non-neph- 
ropathy controls; $\mathrm{p}=0.0001$ for $\mathrm{HD}$ vs non-nephropathy controls). The mean $\left(\mathrm{GA} \div \mathrm{HbA}_{1 c}\right)$ ratio in diabetic ESRD cases on $\mathrm{HD}(3.12$ [0.84]) and PD (2.77 [0.53]) were both significantly higher compared to non-nephropathy controls (2.21 [0.49]; $\mathrm{p}=0.0001$ for PD and HD cases vs controls), implying a difference in the relationship between GA vs serum glucose and $\mathrm{HbA}_{1 c}$ vs serum glucose in dialysis patients. A best-fit linear model was then created with $\mathrm{HbA}_{1 \mathrm{c}}$ or $\mathrm{GA}$ as the outcome variable, and glucose concentration, race, dialysis modality, and serum albumin concentration as independent variables. After adjustment for serum glucose concentration, dialysis modality had significant effects in the model for $\mathrm{HbA}_{1 c}$, demonstrating that dialysis status affects the relationship between $\mathrm{HbA}_{1 c}$ and serum glucose concentration. However, dialysis modality was not significant in a similar model with GA as the outcome variable. Among patients on PD, the GA assay was not significantly impacted by serum albumin concentration.

In diabetic hemodialysis and peritoneal dialysis patients, the relationship between $\mathrm{HbA}_{1 c}$ and GA differs significantly from that in subjects without nephropathy. Relative to the GA assay, $\mathrm{HbA}_{1 c}$ underestimates glycemic control and is impacted by ESRD in patients on peritoneal dialysis and hemodialysis. The GA assay is not significantly impacted by either peritoneal or hemodialysis, allowing for more accurate determination of recent glycemic control among diabetic ESRD patients.

Supported by: Asahi Kasei Pharma Corporation Diagnostics Department

\section{2}

Measurement of serum fructosamine concentration is a low-cost, easyspecimen handling, and precise method to monitor glucose fluctuations in type 1 diabetes mellitus

M. Guevara-Aguirre ${ }^{1}$, E. Vidgen ${ }^{2}$, J. Guevara-Aguirre ${ }^{1}$, J. Saavedra ${ }^{1}$ A.L. Rosenbloom ${ }^{3}$, G. Bernstein ${ }^{4}$

${ }^{1}$ IEMYR, Institute of Endocrinology, Quito, Ecuador, ${ }^{2}$ University of Toronto, Canada, ${ }^{3}$ University of Florida, Gainesville, United States, ${ }^{4}$ Generex Biotechnology Corp., Toronto, Canada

Background and aims: Precise laboratory measurements are needed for proper monitoring of metabolic control of type 1 diabetes mellitus (T1DM) and determination of glycated hemoglobin concentrations (HbAlc) is considered the gold standard for monitoring; however, whole-blood specimen handling has inherent logistic difficulties in places such as developing countries with poor transportation and communication systems; moreover, obvious economic restraints coexist. It has been recently shown that glycated albumin is better related to fasting plasma glucose than is HbAlc (Clin Biochem 40: 1398-05, 2007); moreover, it is well known that fructosamine (Fruc) represents an accurate measure of glycated albumin. Measurement of Fruc has advantages over that of HbAlc in regard to specimen processing and handling; precision, reproducibility and cost of the test.

Materials and methods: 26 adult T1DM participated in a 3-phase, 2-cohort 585-day study. First Phase, was a 144-day stabilization period at which all subjects received basal twice daily (BID) subcutaneous (s.c.) isophane insulin $(\mathrm{NPH})+$ meal time (Thrice daily -TID-) s.c. regular insulin. It was followed by 99 -day Phase 2 , a first comparison phase where the 26 T1DM were allocated to two cohorts of 13 subjects each. Control subjects received BID s.c. NPH + TID s.c. regular insulin whereas Treated subjects received Basal twice daily sc-isophane insulin (NPH) + TID Meal time Oral insulin (Generex Oral$\left.\operatorname{lyn}^{\mathrm{m} x}\right)$. When this period ended, Phase 3 was initiated and the two treatment cohorts were maintained for a 372-day second comparison; however, Control subjects were switched to a schedule of Basal Once a Day s.c. glargine insulin-analogue and pre-meal TID s.c. lispro insulin analogue whereas Treated subjects continued receiving basal BID s.c. NPH and + TID meal time Generex Oral-lyn ${ }^{\mathrm{m}}$. During all phases, glucose readings were self-obtained by research subjects (pre-breakfast, pre-lunch and pre-dinner) (Mean-G), and downloaded every two weeks using an Accu-Check Software. Both analytes, Fruc and $\mathrm{HbAlc}$ were determined every 2 weeks. Self-measured glucose levels (Gluc) were measured before each major meal and meter readings were downloaded using an Accu-Check software. Study data for control and treated cohorts during the entire period were combined aiming to determine the relationship among these 3 variables.

Results: The 3-way Pearson product-moment correlation showed strong linear association between all three sets of measurements (Gluc-HbAlc $\mathrm{r}=0.43 \mathrm{p}<.0001 / /$ Gluc-Fruc $\mathrm{r}=0.45 \mathrm{p}<.0001 / / \mathrm{HbA1c}-$ Gluc $\mathrm{r}=0.43 \mathrm{p}<.0001$ // HbAlc-Fruc $r=0.76$ p $<.0001$ // Fruc-Gluc $r=0.45$ p $<.0001$ // Fruc-HbAlc $\mathrm{r}=0.76 \mathrm{p}<.0001)$. As it can be appreciated, both analytes, HbAlc and Fruc provide adequate assessment of glucose fluctuations and glycemic control when compared to daily glucose determinations during the 585-day period.
Conclusion: We conclude that Fruc is a valid alternative to HbAlc because its measurement is easier, less costly, precise, and adequately reflects glucose fluctuations in T1DM. These characteristics make it suitable especially in places where specimen handling, transportation and test costs are relevant. Supported by: Generex Biotechnology Corp

\section{3}

A study on the use of syringe, pen needle, lancet for self monitoring of blood glucose

E. Jung ${ }^{1}$, M. Hong 2 , J. Yoo ${ }^{3}$

${ }^{1}$ Nursing division, Severance Hospital, ${ }^{2}$ Nursing division, Seoul Paik Hospital, ${ }^{3}$ Nursing division, Seoul Boramae Hospital, Seoul, Republic of Korea

Background and aims: Active self-care behaviors such as that injected insulin and SMBG is required to reach the treatment target for diabetes. Insulin therapy is a medical necessity for all types of diabetes patients who cannot reach their glycemic goals without insulin therapy. Also SMBG is helpful in pattern recognition for the patient to note; concurrent doses of medicines for diabetes, meal plan and exercise. It is strongly emphasized in diabetes education that syringe, pen needle and lancet must be disposed after single use; however, many diabetic patients do not follow the principle. The reuse of syringe, pen needle used for insulin injection may make the tip of the syringe or pen needle break in the skin since it has become weaker and also cause pain, bleeding, or bruise. In addition, it would increase risk for infection and makes it difficult to perform the right dose administration of insulin. The purposes of this study are to research the status of the reuse of syringe and pen needle for insulin injection and lancet for SMBG and patients' perception of reuse. And we aim to find out actual reasons for reuse and factors that lead to reuse.

Materials and methods: The study subjects were 1,053 persons who are treated with injected insulin in general hospitals in the Republic of Korea. Systematic questionnaires were used, and one-to-one interviews were performed from January 22, 2007 to February 12, 2007. The research results were processed for statistics by using SPSS.

Results: 1 . Type 1 diabetic patients accounted for $11.0 \%$, and type 2 diabetic patients accounted for $81.0 \%$. The average prevalence period of the subjects was 13.2 years, and the average injected insulin period was 5.7 years. 2 . The re-users accounted for $34.0 \%$ and used them 4.4 times on average. 3 . As for reasons why syringe or pen needle were used again, the answer, 'I thought that there were nothing wrong with using syringe or pen needle several times' accounted for $69.0 \%$. The second most answer was 'For the reduction of cost'. 4. $94.0 \%$ of the subjects had been educated on how to inject insulin. $88 \%$ out of them answered that they had been educated on using insulin needle. 5. As for the subjects' perception for the side effects of reuse of syringe or pen needle, the answers were as follows: 'Reuse may increase pain' (48.0\%), 'A needle may be broken in the skin' (39.0\%). In general, the perception for the side effects of reuse was perceived negatively below $50 \%$. 6. $89 \%$ of the subjects had performed SMBG. 7. The re-users of lancet for SMBG accounted for $67.0 \%$. The reasons were as follows: 'I thought that there were no side effects' 'I had used them several times by habit'

Conclusion: The results of this research turned out that diabetic patients tended to use syringe, pen needle, or lancet again due to lack in the awareness of hazards from reuse even if they had been educated on how to inject insulin, SMBG, and to use needles. Thus, diabetes educators need to include the side effects of reuse in their teaching by providing concrete cases or proofs and educate diabetic patients repeatedly. Also, they should consciously make effort to eradicate reuse-induced harm by providing efficient education based on the age, educational background, and income level of the patients. In addition the application of insurance should encompass consumables for diabetic management such as insulin syringes, pen needles, and lancets to reduce a financial burden of diabetic patients before long. 


\section{PS 095 Cognitive and psychological aspects}

\section{4}

The structure of cognitive impairment in type 2 diabetes

I. Semenova ${ }^{1}$, L. Chugunova ${ }^{1}$, M. Shestackova ${ }^{1}$, A. Vorontsov ${ }^{2}$,

V. Vladimirova ${ }^{2}$, A. Ilin ${ }^{3}$, P. Kamchatnov ${ }^{4}$, A. Chugunov ${ }^{4}$

${ }^{1}$ Nephrology, National Research Center for Endocrinology, ${ }^{2}$ Radiology, National Research Center for Endocrinology, ${ }^{3}$ Biochemistry, National Research Center for Endocrinology, ${ }^{4}$ Neurology, Russian State Medical University, Moscow, Russian Federation

Background and aims: Diabetes mellitus is associated with cognitive deficit right up to dementia.Taking into account all possible factors the modest and mild cognitive impairment(MCI)are debated as early presentation of cerebrovascular disease in diabetic patients, but $\mathrm{MCI}$ is more severe impairment which often precedes dementia.The aim of the present study was to estimate frequency of early cognitive impairment and their metabolic,vascular and cerebral MRI scan (T1,T2weighted)characteristics in middle-aged patients whith type 2 diabetes.

Materials and methods: Our study included 126 (42 men and 84 women) patients 50-75years old with type 2 diabetes and 20 (3 men and 17 women) control subjects without diabetes and hypertension. Glycated hemoglobin(HbAlc), body mass index(BMI), systolic(SBP) and diastolic blood pressure, biochemistry assay, MAU, haemostatic factors, cerebral MRI scan, neuropsychological tests: Frontal Assesment Battery(FAB), Mini Mental State Examination(MMSE), Global Deterioration Scale(GDS), Clock Drawing test $(\mathrm{CDT})$ were measured.

Results: Mean age of patients was 60,5+- 7,5years. Mean duration of diabetes was $8,03+-6,23$ years. Median HbAlc was 7,8\%[7,1;8,7]. Mild cognitive impairment was detected in 64 cases $(52,8 \%)$ by MMSE tests and 29 patients $(23,9 \%)$ had modest cognitive impairment (MMSE 28-30scores,GDS=2). Only $23,3 \%$ patients didn't have cognitive impairment.Patient with MCI had significant more duration of diabetes $(\mathrm{p}<0,043)$, hypertension $(\mathrm{p}<0,003)$ and mean level of triglyceride $(p<0,015)$ comparing with patient wich have modest cognitive impairment. MCI patients had MRI leukoareosis and silent lacunar stroke significantly more frequent than other patients and control subjects $(\mathrm{p}<0,001)$. Mau exhibited a significant negative correlation with MMSE $(r=-0,264 \mathrm{p}<0,003), \mathrm{FAB}(\mathrm{r}=-0,216 \mathrm{p}<0,015)$ and CDT $(\mathrm{r}=-0,269 \mathrm{p}<0,002)$. Association between $\mathrm{HbAlc}$ and neuropsychological tests scores was not estimated.

Conclusion: High frequency of mild cognitive impairment in type 2 diabetes is strong associated with vascular MRI abnormalities, cerebrovascular risk factors and with MAU level as marker of endotelial dysfuncion.

Supported by: National Programme for Diabetes

\section{5}

A systematic review and meta-analysis of the prevalence of eating disorders in patients with type 2 diabetes mellitus

Y.S. Cheah ${ }^{1}$, D. Hopkins ${ }^{2}$, J. Treasure ${ }^{3}$, K. Ismail ${ }^{3}$

${ }^{1}$ Department of Endocrinology \& Diabetes, The Lewisham Hospital NHS

Trust, London, ${ }^{2}$ Department of Diabetic Medicine, King's College Hospital, London, ${ }^{3}$ Academic Department of Psychological Medicine, Institute of Psychiatry, King's College London, United Kingdom

Background and aims: Unlike type 1 diabetes mellitus, the relationship between type 2 diabetes mellitus and eating disorders is less well defined. To examine this further we performed a systematic literature review and metaanalysis of the pooled prevalence of all Eating Disorders (ED), comprising of anorexia nervosa (AN), bulimia nervosa (BN), binge eating disorder (BED) and other eating disorders not otherwise specified (EDNOS, excluding BED) in adults with type 2 diabetes mellitus.

Materials and methods: The Meta-analysis of Observational Studies in Epidemiology (MOOSE) guidelines were used on reporting observational data. Inclusion Criteria

1. Cross-sectional, cohort studies

2. Adults aged $>18$ years with type 2 diabetes mellitus

3. Documented prevalence of ED (AN, BN, BED, EDNOS) meeting the Diagnostic and Statistical Manual of Mental Disorders (DSM) III-Revised or IV, or the International Classification of Disease 10 (ICD-10) criteria.

\section{Search Strategy}

Searches of relevant electronic libraries for all studies on ED and type 2 diabetes mellitus to September 2007 were undertaken. The Cochrane Depression, Anxiety and Neurosis Group search strategy for Eating Disorders, and the Cochrane Metabolic and Endocrine Disorder Group search strategy for type 2 diabetes mellitus were used. Hand searches of the last 4 years' proceedings from the American Diabetes Association and Diabetes UK conferences were also undertaken. Full manuscripts of abstracts meeting the inclusion criteria were obtained. Experts in the fields of diabetes and ED identified from the literature were directly contacted to identify unpublished data.

\section{Data Analysis}

The prevalence of ED and its subtypes from each study meeting the inclusion criteria were extracted. A meta-analysis of the pooled data using a random effects model was performed.

Results: The search strategy identified 1873 abstracts. Thirteen abstracts fulfilled the inclusion criteria. Two studies were excluded: one for not specifying the type of diabetes mellitus and one for not specifying the diagnostic criteria for ED. The results of 3 articles reporting separate data from a single study were amalgamated. Therefore 9 studies with a total of 2291 patients were included for the meta-analysis, of which only 2 were case-controlled studies. There were no cases of AN. The weighted pooled prevalence of BN was $0.7 \%$ (95\% confidence interval 0.3-1.9\%), BED 6.5\% (3.5-11.7\%), EDNOS 5.9\% (3$11.2 \%)$ with all ED $13.2 \%(7.1-23 \%)$.

Conclusion: As there were only 2 case-controlled studies, a meta-analysis to compare ED in diabetic with non-diabetic adults was not possible. A recent population-based study of 9282 adults found a lifetime prevalence of AN, $\mathrm{BN}$ and $\mathrm{BED}$ to be $0.6 \%, 1.0 \%$ and $2.8 \%$ respectively. Although not a direct comparison, our review suggests that $\mathrm{ED}$ are more prevalent in the diabetic population, BED being the most prevalent subtype.

The small number of studies in this field have suggested that ED develop before the diagnosis of type 2 diabetes mellitus. Further research is required to establish the possible role ED may have in the pathogenesis and natural history of type 2 diabetes mellitus, obesity, and the metabolic syndrome, and the impact of ED on glycaemic control and diabetes complications.

\section{6}

Initial experience with Dose Adjustment for Normal Eating (DAFNE) training in routine clinical practice in Ireland: biomedical and psychosocial outcomes at 12 months L. Daly ${ }^{1}$, D. O'Shea ${ }^{2}$, D. Smith ${ }^{3}$, C.H. Courtney ${ }^{4}$, S.F. Dinneen ${ }^{1}$ ${ }^{1}$ Diabetes Day Centre, Galway University Hospital, ${ }^{2}$ Diabetes Day Centre, St. Vincent's University Hospital, Dublin, ${ }^{3}$ Diabetes Day Centre, Beaumont Hospital, Dublin, ${ }^{4}$ Diabetes Day Centre, Royal Victoria Hospital, Belfast, Ireland

Background and aims: Dose Adjustment for Normal Eating (DAFNE) is a 5-day training course in flexible, intensive insulin therapy for patients with Type 1 diabetes. In the UK DAFNE has been shown to improve glycaemic control without increasing severe hypoglycemia, while significantly reducing the negative impact of diabetes on quality of life. The aim of this study was to investigate the effect of DAFNE training on glycaemic control, hypoglycaemia and psychosocial outcomes in Irish DAFNE Centres.

Material and methods: Twenty-two DAFNE courses were delivered in 5 centres in Ireland between March 2004 and November 2006. Clinical outcomes measured at baseline and 12 months post-training included $\mathrm{HbAlc}$, weight and rates of severe hypoglycaemia. The burden of living with diabetes was assessed using the PAID (Problem Areas in Diabetes) and HADS (Hospital Anxiety and Depression Scale) questionnaires. EQ5D was used to assess health status.

Results: Complete data at baseline and 12 months were available for $70 \mathrm{pa}-$ tients $(48.6 \%$ male). The range of HbAlc among participants at baseline varied from 6.0 to 14.2 percent. For the whole cohort, HbAlc levels (mean+/$\mathrm{SD})$ after 1 year showed minimal change from baseline $(8.41+/-1.41 \%$ vs. $8.32+/-1.30 \%, \mathrm{p}=0.446)$. Weight reduced from $77.5+/-14.5 \mathrm{~kg}$ at baseline to $76.8+/-15.8 \mathrm{~kg}$ at 12 months $(\mathrm{p}=0.31$ ). Rates of severe hypoglycaemia were low at baseline and were not significantly different at 12 months. Mean PAID scores decreased from $22.45+/-17.88$ to $14.05+/-13.79, \mathrm{p}=0.001$. Mean HADS scores decreased from 4.10+/-3.33 to $3.34+/-2.77$ (anxiety), $\mathrm{p}=0.047$ and from $3.76+/-2.77$ to $3.14+/-2.96$ (depression), $\mathrm{p}=0.097$. Perceived health status as determined by the visual analogue scale of EQ5D increased from $79.73+/-12.99$ to $80.76+/-12.70, p=0.534$. For the sub-group of patients with baseline $\mathrm{HbAlc}>8.4 \%$ the level of $\mathrm{HbAlc}$ improved at 1 year post DAFNE (mean $9.47+/-1.19 \%$ vs $8.98+/-1.33 \%$; $\mathrm{p}=0.009$ ). For this sub- 
group there was no significant increase in severe hypoglycaemia and no significant change in weight at 12 months. For the cohort with baseline HbAlc $\leq 8.4 \%$ the level of HbAlc increased at 12 months (mean $7.46+/-0.75 \%$ to $7.72+/-0.93 \% ; p=0.081$ ). Concurrently there was no significant change in frequency of severe hypoglycaemia and weight decreased from mean 76.86 +/- $13.64 \mathrm{~kg}$ to $75.36+/-13.25 \mathrm{~kg}(\mathrm{p}=0.003)$.

Conclusion: These results demonstrate that DAFNE training in a routine clinical setting in Ireland is associated with significant improvement in measures of psychological distress and diabetes-related anxiety. The lack of a significant improvement in $\mathrm{HbAlc}$ at 12 months may have resulted from the very wide range of $\mathrm{HbAlc}$ among participants at baseline. This reflects the policy of the participating centres to offer DAFNE training to all patients with Type 1 diabetes. Further work is required to identify sub-groups of patients who derive maximum benefit from DAFNE training and to maintain these benefits for longer. These issues are currently being explored in the Irish DAFNE study.

Supported by: Health Research Board Ireland

\section{7}

High prevalence of alexithymia in type 1 diabetic patients

L. Chatzi ${ }^{1}$, E. Solidaki ${ }^{1}$, I. Christou ${ }^{2}$, E. Kyrlaki ${ }^{2}$, M. Sfakianaki ${ }^{2}$, P. Bitsios ${ }^{3}$, M. Kogevinas ${ }^{1,4}$, N. Kefalogiannis ${ }^{2}$, A. Pappas ${ }^{2}$

${ }^{1}$ Department of Social Medicine, Faculty of Medicine, University of Crete, Heraklion, Greece, ${ }^{2}$ Diabetic Clinic, Venizelio Hospital, Heraklion, Greece, ${ }^{3}$ Department of Psychiatry, Faculty of Medicine, University of Crete, Heraklion, Greece, ${ }^{4}$ Centre for Research in Environmental Epidemiology, (CREAL), Institut Municipal d'Investigacio Medica, Barcelona, Spain

Background and aims: Type 1 diabetes (T1D) has long been identified as a disease with increased comorbidity in mental health disorders such as anxiety and depression. Alexithymia is a psychological construct characterised by inability to express emotions verbally, poor imagination and operational thinking, leading to failure in psychological self-regulation. The aim of this case-control study was to measure the prevalence of alexithymia and low mood in T1D outpatients who did not meet the criteria for major depression, and to compare it with that of non-diabetic controls.

Materials and methods: The study population included 96 T1D outpatients followed in the Diabetic Clinic of Venizelio General Hospital in Heraklion, Greece and 105 age- and sex-matched healthy controls. Subjects diagnosed for clinical depression were excluded from the study. Alexithymia and low mood were assessed in both groups with the 20 -item Toronto Alexithymia Scale, (TAS-20) and the 21-item Beck Depression Inventory (BDI-21) respectively. Multivariate regression models were used to evaluate the association of alexythimia and low mood with the presence of diabetes, diabetes control (HbAlc, number of mild or severe hypoglycemia in the last year), parameters of treatment intensification (number of glucose measurements/week, number of insulin injections/day), and diabetic complications (retinopathy, coronary heart disease, nephropathy).

Results: The prevalence of alexithymia was $20 \%$ in T1D patients and $7 \%$ in the controls (OR, 4.1; 95\%CI, 1.6-10.6). The presence of low mood did not differ significantly between the two groups. Alexithymia was inversely associated with the number of glucose measurements per week $(\beta c-0.16, \mathrm{p}=0.074)$ and the number of insulin injections per day $(\beta c-2.06, \mathrm{p}=0.051)$. There were no associations between alexithymia and $\mathrm{HbAlc}$, or somatic complications. Conclusion: We found high prevalence of alexithymia but not low mood in diabetic patients who did not meet the criteria for major depression compared to healthy controls. While alexithymia may be an adaptive response to protect diabetic patients from developing depression, it may interfere with patients' ability to self-care and comply with treatment. Therefore, special attention should be paid in the medical care of diabetic patients with alexithymic characteristics.

Table: Differences between the intervention and control group at baseline, after 6 and 12 months.

\begin{tabular}{|c|c|c|c|c|c|c|c|c|c|}
\hline & Baseline & $\begin{array}{l}6 \\
\text { months }\end{array}$ & $\begin{array}{l}12 \\
\text { months }\end{array}$ & $\begin{array}{l}\Delta 0-6 \\
\text { months }\end{array}$ & $\begin{array}{l}\text { P value } \\
(95 \% \mathrm{CI})\end{array}$ & $\begin{array}{l}\Delta 6-12 \\
\text { months }\end{array}$ & $\begin{array}{l}\text { P value } \\
(95 \% \\
\text { CI })\end{array}$ & $\begin{array}{l}\Delta 0-12 \\
\text { months }\end{array}$ & $\begin{array}{l}\text { P value } \\
(95 \% \mathrm{CI})\end{array}$ \\
\hline $\begin{array}{l}\text { CHD risk } \\
\text { (\%, UKPDS) } \\
\text { Intervention }\end{array}$ & $10.6 \pm 7.2$ & NA & $9.9 \pm 5.9$ & \multirow[t]{2}{*}{ NA } & \multirow[t]{2}{*}{ NA } & \multirow[t]{2}{*}{ NA } & \multirow[t]{2}{*}{ NA } & \multirow[t]{2}{*}{-0.32} & \multirow[t]{2}{*}{$\begin{array}{l}\mathrm{P}=0.75 \\
(-2.27 ; 1.63)\end{array}$} \\
\hline $\begin{array}{l}\text { CHD risk } \\
\text { (\%, UKPDS) } \\
\text { Control }\end{array}$ & $11.1 \pm 8.8$ & NA & $11.2 \pm 7.0$ & & & & & & \\
\hline $\begin{array}{l}\text { Weight }(\mathrm{kg}) \\
\text { Intervention }\end{array}$ & $94.4 \pm 19.8$ & $95.3 \pm 20.8$ & $94.1 \pm 22.1$ & \multirow[t]{2}{*}{0.15} & \multirow[t]{2}{*}{$\begin{array}{l}\mathrm{P}=0.78 \\
(-0.90 ; 1.19)\end{array}$} & \multirow[t]{2}{*}{-0.18} & \multirow{2}{*}{$\begin{array}{l}\mathrm{P}=0.70 \\
(-1.14 \\
; 0.77)\end{array}$} & \multirow[t]{2}{*}{-0.20} & \multirow[t]{2}{*}{$\begin{array}{l}\mathrm{P}=0.73 \\
(-1.33 ; 0.93)\end{array}$} \\
\hline $\begin{array}{l}\text { Weight }(\mathrm{kg}) \\
\text { Control }\end{array}$ & $95.8 \pm 17.0$ & $96.0 \pm 17.2$ & $95.8 \pm 16.6$ & & & & & & \\
\hline $\begin{array}{l}\mathrm{HbAlc}(\%) \\
\text { Intervention }\end{array}$ & $6.8 \pm 1.0$ & $6.7 \pm 1.1$ & $6.8 \pm 1.1$ & \multirow[t]{2}{*}{-0.07} & \multirow[t]{2}{*}{$\begin{array}{l}P=0.65 \\
(-0.39 ; 0.24)\end{array}$} & \multirow[t]{2}{*}{0.04} & \multirow{2}{*}{$\begin{array}{l}\mathrm{P}=0.73 \\
(-0.17 \\
; 0.24)\end{array}$} & \multirow[t]{2}{*}{-0.05} & \multirow[t]{2}{*}{$\begin{array}{l}\mathrm{P}=0.71 \\
(-0.33 ; 0.22)\end{array}$} \\
\hline $\begin{array}{l}\text { HbAlc (\%) } \\
\text { Control }\end{array}$ & $6.7 \pm 1.0$ & $6.7 \pm 0.9$ & $6.8 \pm 0.9$ & & & & & & \\
\hline $\begin{array}{l}\text { Moderate } \\
\text { physical activity } \\
\text { (min/day) } \\
\text { Intervention }\end{array}$ & $85.3 \pm 11.7$ & $68.1 \pm 9.5$ & $87.4 \pm 12.6$ & \multirow[t]{2}{*}{-9.80} & \multirow[t]{2}{*}{$\begin{array}{l}P=0.47 \\
(-36.62 \\
; 17.03)\end{array}$} & \multirow[t]{2}{*}{9.8} & \multirow[t]{2}{*}{$\begin{array}{l}P=0.65 \\
(-33.10 \\
; 52.73)\end{array}$} & \multirow[t]{2}{*}{-0.70} & \multirow[t]{2}{*}{$\begin{array}{l}\mathrm{P}=0.97 \\
(-42.84 \\
; 41.45)\end{array}$} \\
\hline $\begin{array}{l}\text { Moderate } \\
\text { physical } \\
\text { activity } \\
\text { (min/day) } \\
\text { Control }\end{array}$ & $68.1 \pm 9.5$ & $72.3 \pm 12.9$ & $97.1 \pm 14.6$ & & & & & & \\
\hline $\begin{array}{l}\text { Heavy } \\
\text { physical activity } \\
\text { (min/day) } \\
\text { Intervention }\end{array}$ & $30.1 \pm 4.8$ & $41.0 \pm 7.4$ & $60.1 \pm 11.7$ & \multirow[t]{2}{*}{20.14} & \multirow[t]{2}{*}{$\begin{array}{l}\mathrm{P}=0.01 * \\
(4.6 ; 35.70)\end{array}$} & \multirow[t]{2}{*}{-6.46} & \multirow[t]{2}{*}{$\begin{array}{l}\mathrm{P}=0.66 \\
(-35.80 \\
; 22.88)\end{array}$} & \multirow[t]{2}{*}{11.68} & \multirow[t]{2}{*}{$\begin{array}{l}\mathrm{P}=0.38 \\
(-14.73 \\
; 36.08)\end{array}$} \\
\hline $\begin{array}{l}\text { Heavy } \\
\text { physical activity } \\
\text { (min/day) } \\
\text { Control }\end{array}$ & $39.5 \pm 6.4$ & $27.9 \pm 4.3$ & $55.2 \pm 11.2$ & & & & & & \\
\hline
\end{tabular}

Data are means $\pm \mathrm{SD}, \mathrm{CHD}=$ coronary heart disease, $\mathrm{NA}=$ not applicable, ${ }^{*} \mathrm{P}<0.05$ 


\section{8}

A cognitive behavioural treatment improves lifestyle in patients with type 2 diabetes when added to managed care: a randomised controlled trial

L.M.C. Welschen, P.C. van Oppen, P.J. Kostense, J.M. Dekker, G. Nijpels EMGO Institute, VU University Medical Center, Amsterdam, Netherlands

Background and aims: It has been shown that behavioural interventions lower risk factors of developing complications in patients with diabetes. However, study designs are often lacking a theoretical background and consequently it is difficult to identify successful factors in interventions. We have developed a theory-based cognitive behavioural intervention, which was added to an existing managed care system. Problem Solving Treatment (PST), a practical skill building treatment with goal setting as the main element, was used to guide the intervention. We hypothesised that PST might increase patient's attitude, social influences and self-efficacy, the determinants of behaviour in our theoretical framework, the ASE-model. The increase in the determinants might change patient's intention to change behaviour, resulting in improvement of clinical characteristics and a reduction in the risk of developing coronary heart disease (CHD). The aim of this study was to investigate the effect of adding a cognitive behavioural intervention to managed care on CHD risk.

Materials and methods: For this randomised controlled trial, patients were recruited from general practices $(n=14)$ who are participating in a Diabetes Management System. The intervention group $(n=76)$ received the cognitive behavioural intervention in addition to managed care. The control group $(n=78)$ received managed care only. The primary outcome measure was the risk of developing CHD, calculated with the UKPDS risk engine at baseline and after 12 months. Other outcome measures were clinical characteristics, lifestyle (physical activity, dietary behaviour, and smoking status), determinants of behaviour change (attitude, social influences and self-efficacy, based on the ASE-model), quality of life and depression. These outcome measures were all measured at baseline, after 6 and 12 months. Differences between the two groups after 6 and 12 months were analysed by two-way analysis of variance.

Results: The intervention reduced CHD risk but not significant (see Table). The amount of heavy physical activity at 6 months increased significantly in the intervention group compared with the control group but this effect disappeared after 12 months. More patients in the intervention group quit smoking, although not statistically significant. Quality of life and level of depression improved as well. No effects were found on clinical characteristics, dietary behaviour and determinants of behavioural change.

Conclusion: Adding a cognitive behavioural intervention to managed care improved physical activity at the short term and seems to have beneficial effects on cardiovascular risk profile. It remains a challenge to find behavioural interventions that have effects on the long term and on clinical outcome measures.

\section{9}

Bariatric surgery for patients with type 2 diabetes and morbid obesity: improving psychological status?

S. Jackson ${ }^{1}$, M. Morris ${ }^{1}$, K.T. Lilley ${ }^{1}$, A.B. Johnson ${ }^{2}$

${ }^{1}$ Psychology, University of the West of England, ${ }^{2}$ Diabetes \& Endocrinology, Southmead Hospital, Bristol, United Kingdom

Background and aims: Surgical treatment is increasingly recognised as the most effective means of achieving weight loss and potentially normalising glucose tolerance in morbidly obese patients with type 2 diabetes (T2DM). The psychological status of individuals presenting for such treatment is unclear. This study was undertaken to compare the psychological status of morbidly obese individuals with and without (T2DM) pre and six months post bariatric surgery.

Materials and methods: Two groups of participants were recruited: a preoperative group (T1: $n=41,27$ with T2DM, 14 without; age range: $25-58$; mean BMI $51.8 \mathrm{~kg} / \mathrm{m}^{2} \pm \mathrm{sd}=8.31$; mean $\left.\mathrm{HbAlc} 9.15 \pm 1.98\right)$ and post-operative group (T2: $\mathrm{n}=11,9$ with T2DM, 2 without; age range: $35-48$; mean BMI $42.18 \mathrm{~kg} / \mathrm{m}^{2} \pm \mathrm{sd}=8.36$; mean HbAlc $8.28 \pm 1.36$ ). Participants completed psychological scales measuring anxiety and depression (HADS); quality of life (WHOQoL-Bref); and social anxiety (DAS-24). All were considered suitable for surgery after comprehensive assessment. Participants completed psychological scales which measured anxiety and depression (HADS); quality of life (WHOQoL-Bref); and social anxiety (DAS-24).

Results: A comparison of study measures mean scores with clinically relevant normative populations revealed that the T1 group had significantly increased levels of social anxiety $(65.0 \pm 14.8$; norm $=29.54 \pm 12.39)$, general anxiety $(9.76 \pm 4.42$; norm $=6.14 \pm 3.76)$ and depression $(9.22 \pm 4.01$; norm $=3.68 \pm 3.07)$. For the T2 group $(n=11)$, levels of social anxiety $(56.18 \pm 18.7)$ were also significantly raised when compared to normative data. Levels of general anxiety were slightly raised $(6.45 \pm 3.75)$, while levels of depression were still significantly raised $(5.36 \pm 3.80)$. Non-parametric statistical testing revealed that BMI was significantly lower in the T2 group ( $<<.002)$, as were levels of anxiety and depression $(\mathrm{p}<.05)$, while ratings of quality of life were significantly higher $(\mathrm{p}<.05)$. Where participants in groups $\mathrm{T} 1$ and $\mathrm{T} 2$ could be matched an average percentage drop of $23.7 \%$ was found for $\mathrm{HbAlc}$ levels suggesting a clinically useful reduction.

Conclusion: These data suggest that morbid obesity significantly raises levels of social anxiety, general anxiety and depression and that bariatric surgery improves both physiological and psychological status to a certain extent.

\section{0}

The evaluation of the anxiety level and the cognitive functioning of the children and adolescents with type 1 diabetes in dependence on insulin therapy method

A. Grabowska, M. Urban, B. Florys, B. Glowinska-Olszewska

2nd Pediatric Clinic, Medical University, Bialystok, Poland

Background and aims: The aim of this study was the evaluation of emotional and cognitive functioning of the children and adolescents with type 1 diabetes in relation to insulin therapy method.

Materials and methods: 110 patients in the age range of 7.2 to 19.1 years suffering from type 1 diabetes since 6.17 years took part in the study. They were divided into two groups in dependence on insulin therapy: intensive vs personal insulin pump. The evaluation of anxiety level was performed on the base of the psychological methods: State-Trait Anxiety Inventory (STAI) and State-Trait Anxiety Inventory for Children (STAIC). The evaluation of cognitive functioning was measured with Wechsler Intelligence Scale for Children-Revised (WISC-R) and Wechsler Adult Intelligence Scale-Revised -Polish versions (WAIS-R(PL)).

Results: We have found higher anxiety level as trait than state in diabetic patients $(\mathrm{p}<0.00)$. We have observed higher level of anxiety in the group using the insulin pump than in the group on intensive method (not statistical significance). Patients with personal insulin pump gained higher scores in the subtest: Information $(p<0.04)$, Similarities $(p<0.00)$, Arithmetic $(p<0.01)$, Vocabulary ( $<<0.01)$, Comprehension $(\mathrm{p}<0.02)$, Picture Completion $(\mathrm{p}<0.01)$, Picture Arrangement $(\mathrm{p}<0.02)$. In this group we also observed higher level of the Intelligence (Verbal, $\mathrm{p}<0.00$; Nonverbal, $\mathrm{p}<0.03$; General, $\mathrm{p}<0.00$ ).

Conclusion: Higher level of anxiety as trait than as state occurs in all diabetic patients. 2. Higher level of anxiety was observed in group using insulin pomp than in group on intensive insulin therapy. 3 . The group treated with personal insulin pump had higher scores in all cognitive tests.

\section{1}

Cognitive function in 6 to 12 year old offspring of women with type 1 diabetes

R.C. Temple ${ }^{1}$, M. Hardiman ${ }^{2}$, M. Martinez ${ }^{2}$, M. Pellegrini ${ }^{2}$

${ }^{1}$ Elsie Bertram Diabetes Centre, Norfolk and Norwich University Hospital NHS Trust, Norwich, ${ }^{2}$ Learning Difficulty Service, Attleborough Day Services, Attleborough, United Kingdom

Background and aims: Recent studies of offspring of women with diabetes have suggested they perform less well academically at school and that impairment of cognitive function may be related to maternal glycaemic control during pregnancy. The aim of our study was to make detailed assessments of cognitive function in 6-12 year old offspring of women with type 1 diabetes and to assess whether there was any relationship between cognitive function and characteristics of the pregnancy.

Materials and methods: Women with type 1 diabetes, who had received care throughout their pregnancy at out hospital and had children aged between 6 and 12 years, were invited to participate in the study. Assessment of the cognitive function of offspring was tested using the WISC -IV test (Weschler Intelligence Scale for Children). Infant birthweight ratio was calculated by dividing infant birthweight by $50^{\text {th }}$ centile weight matched for gestation and sex.

Results: We present the results in 40 offspring. Mean maternal duration of diabetes was 15.1 (8.4) years and mean gestation at booking for antenatal care was 7.1 (2.8) weeks. HbA1c at booking, 12 weeks, 24 weeks and 34 weeks respectively were $6.7(1.0) \%, 6.0(0.8) \%, 5.6(0.7) \%$ and $5.7(0.8) \%$. Mean ges- 
tation at delivery was 37.2 weeks. We found no difference in overall Full Scale IQ compared to UK normative data or in letter-number sequencing, a test of working memory. However, the digit span, a further test of working memory, was poorer than other parts of the WISC-IV test and significantly lower compared to UK normative data. Digit span in offspring in the study was 8.37 (2.23) compared to UK normative of 10.05 (3.2), $\mathrm{p}<0.01$. We found no correlation between measurement of digit span or $\mathrm{HbAlc}$ at any stage during pregnancy $(r=-0.225$ to 0.002$)$, gestational age at delivery $(r=-0.178)$ or infant birthweight ratio $(r=-0.176)$. We divided offspring into 2 groups according to digit span test result. Comparing offspring with a digit span $\geq 9(n=20)$ with offspring with a digit span $<9(n=20)$, we found no significant difference in duration of diabetes $(16.0(7.8)$ vs $14.2(9.1)$ years, gestation at booking $(7.7$ (3.7) vs 6.5 (1.3) weeks, gestation at delivery $(37.2(0.9)$ vs $37.2(1.1)$ weeks or infant birthweight ratio $(1.2(0.1)$ vs $1.2(0.2)$. There was also no significant difference in HbAlc at booking (6.4(0.8) vs 7.0(1.1)\%, at 12 weeks $(6.0(0.7)$ vs $6.0(0.9) \%$, at 24 weeks $(5.6(0.7)$ vs $5.6(0.6) \%$ and 34 weeks $(5.6(0.8)$ vs $5.8(0.7) \%$ between groups. There was no difference in incidence of maternal severe hypoglycaemic episodes between groups. Comparing infants born before or after 37 weeks gestation, digit span was non-significantly lower (7.9 (1.8) vs $8.0(2.4)$.

Conclusion: These results, in 6-12 year old offspring of women with type 1 diabetes, suggest there is impairment of working memory but no overall impairment of cognitive function. We were unable to identify specific risk factors. These results suggest poor working memory may be a factor in the previously documented lower academic achievement in children of women with diabetes.

\section{2}

Nurse-led multidisciplinary management improves metabolic control, self-care practices and psychological well-being of type 2 diabetic patients

X.H. Wu, L.Y. Chen, Y. Cai, X.P. Wang, C.P. Yuan, J.Y. Gu, C. Liu

Department of Endocrinology, First Affiliated Hospital of Nanjing Medical University, China

Background and aims: Group-based structured education program and nurse-care system have recently been shown to improve the glycemic control and quality of care in diabetic patients in western countries. In china, along with the huge growing numbers of diabetic patients and limited diabetologists in clinic care, it is urgent to develop an effective and feasible disease management strategy based on the routine clinic practice. In the present study, we aimed to verify the effect of the nurse-led multidisciplinary management on metabolic control, self-care practices and psychological well-being of type 2 diabetic patients.

Materials and methods: Totally, 180 type 2 diabetic patients with more than one year duration and $\mathrm{HbAlc}$ higher than $6.5 \%$ were enrolled and divided randomly into intensive management group $(n=90)$ and usual care group $(n=90)$. The two groups were comparable regarding age, education level, waist circumference (WC), HbAlc, systolic blood pressure (SBP), diastolic blood pressure (DBP), body mass index (BMI), total cholesterol (TC), triglyceride (TG), high density lipoprotein cholesterol (HDL-C), chronic complications and psychological well-being score. The intensive management group (IMG) was under the instruction of a nurse-led multidisciplinary team including diabetes nurses, diabetologists, dieticians, and rehabilitation physicians. The participants were referred to a 14-week, 28-hour diabetes education program. Subsequent follow-up visits were arranged by the nurses quarterly and IMG offered the individual medication adjustments, meal planning, and exercise reinforcement. In contrast, patients in the usual care group (UCG) continued to follow habitual consultations in the diabetes clinic and received medication and general education. One year later, outcome of metabolic control, self-care practices and psychological well-being of the patients in both groups were evaluated.

Results: After one year intervention, the level of HbAlc, DBP and WC decreased significantly in IMG $(P<0.05)$, whereas UCG did not show any significant pre- and post-intervention change. The decline of DBP, HbAlc and TC were $(3.57 \pm 1.8) \mathrm{mmHg},(0.79 \pm 0.3) \%$ and $(0.22 \pm 0.2) \mathrm{mmol} / \mathrm{L}$, respectively, in IMG, which were significantly higher than that in UCP. The rate of HbAlc below $6.5 \%$ reached $30.8 \%$ one year later in IMG, which was significantly higher than $7.1 \%$ in UCG $(\mathrm{P}<0.05)$. However, there was no significant difference in the change of target-reaching rate of BMI, lipids profile and BP between the two groups. Concerning the self-care practice, the proportion of regular diet, exercise and self glucose monitoring (more than 5 times/month) were markedly increased in IMG whereas only the proportion of regular exercise significantly increased in UCG $(\mathrm{P}<0.05)$. The improvement of psychological well-being in IMG were significantly higher than that in UCG $(\mathrm{P}<0.01)$. Conclusion: The nurse-led multidisciplinary management could significantly improve the blood glucose control, self-care practices and psychological well-being of type 2 diabetic patients. It could be served as an effective and feasible disease management strategy in China.

\section{3}

Patient satisfaction among patients with glucose intolerance attending comprehensive cardiac rehabilitation - does cardiac follow-up treatment with an integrated diabetes care make a difference? Results from a randomized clinical trial

N.K. Nissen ${ }^{1}$, A.B. Soja ${ }^{2}$, A.-D. Zwisler ${ }^{2}$, S. Rasmussen ${ }^{1}$, T.M. Melchior ${ }^{3}$, E. Hommel ${ }^{4}$, J. Fischer Hansen ${ }^{5}$

${ }^{1}$ Department of Epidemiology, National Institute of Public Health, Copenhagen, ${ }^{2}$ Department of Cardiology, Rigshospitalet, Copenhagen, ${ }^{3}$ Department of Cardiology, Roskilde County Hospital, ${ }^{4}$ Steno Diabetes Center, Gentofte, ${ }^{5}$ Department of Cardiology, Bispebjerg University Hospital, Copenhagen, Denmark

Background and aims: Patient satisfaction with comprehensive cardiac rehabilitation (CR) is important in a user-oriented as well as an outcome-oriented perspective. Patients with glucose intolerance (GI) eligible for CR have to cope with both the cardiovascular disease and GI, and this might be the reason for increased level of depression and lack of adherence to treatment among these patients. Therefore treatment should consider these patients' situation and their special needs. We aimed to investigate the patients' satisfaction with CR with integrated diabetes care compared to usual care (UC) in a randomised clinical trial (DANSUK) using standardised methods.

Materials and methods: The DANSUK study was conducted as a sub study during the last year of a centrally randomised clinical trial of the value of a CR clinic. Of 201 patients eligible for CR, 104 were diagnosed with GI having either type 2 diabetes $(\mathrm{N}=68)$ or impaired glucose tolerance $(\mathrm{N}=36)$. The metabolic status was based on fasting plasma glucose values and an oral glucose tolerance test. Patients were randomized after being discharged from a cardiac care unit to $\mathrm{CR}(\mathrm{N}=51)$ and usual care $(\mathrm{N}=53)$. Mean age was 64 years; 34 $\%$ were women; $7 \%$ had congestive heart failure, $67 \%$ had ischemic heart disease, and $26 \%$ had high risk for ischemic heart disease. Patients randomized to CR received 6-12 weeks of an individually tailored, multidisciplinary programme with an integrated diabetes module and clinical control after 3, 6 and 12 months. Patient satisfaction was measured as a secondary outcome after 12 month using a standardised interview-questionnaire with closed answers to questions covering overall satisfaction and level of information. A total of 95 patients (91\%) answered the questionnaire about satisfaction.

Results: Among cardiac patients with GI receiving CR, 92\% reported a high level of overall satisfaction with follow up treatment compared with $30 \%$ among patients receiving usual care $(\mathrm{p}<0.00001)$. The CR group reported a higher level of satisfaction according to information about their disease (CR: $94 \%$, UC: $42 \%$; $<0.0001$ ), information on diagnose and treatment (CR: 91 $\%$, UC: $47 \%$; p $<0.00001$ ), self-management of life-style changes (CR: $84 \%$, UC: $43 \%$; $=0.01$ ), and self-management of medicine (CR: $36 \%$, UC: $17 \%$; $\mathrm{p}<0.05)$. Subgroup analysis showed no differences in degree of satisfaction according to gender, age or ethnic group.

Conclusion: We found that $\mathrm{CR}$ with an integrated diabetes module significantly improves overall satisfaction with follow-up treatment together with level of information and self-management among glucose intolerant patients receiving CR compared with patients receiving usual care. The results should be interpreted with caution due to the non-blinded design.

Supported by: Danish Pharmacy Foundation of 1991, Eva and Henry Fronkels Memorial Foundation, Danish Heart Foundation 


\section{PS 096 Diabetes treatment and exercise}

\section{4}

\section{Free time activities and blood glucose control in patients with type 1 diabetes \\ C. Bizzarri ${ }^{1}$, D. Benevento ${ }^{2}$, D. Pitocco ${ }^{3}$, A. Crinò ${ }^{4}$, S. Spera ${ }^{4}$, C. Tubili ${ }^{5}$ F. Costanza ${ }^{2}$, A. Maurizi ${ }^{2}$, L. Cipolloni ${ }^{2}$, R. Cassone Faldetta ${ }^{6}$, M. Cappa $^{1}$, P. Pozzilli², the IMDIAB group ${ }^{6}$ \\ ${ }^{1}$ Endocrinology and Diabetology, Bambino Gesù Children's Hospital, ${ }^{2}$ Endocrinology and Diabetology, Università Campus Bio-Medico, ${ }^{3}$ Diabetology, Catholic University, ${ }^{4}$ Pediatrics, Bambino Gesù Children's Hospital, ${ }^{5}$ Diabetology, San Camillo Hospital, ${ }^{6}$ Endocrinology and Diabetology, San Giovanni's Hospital, Roma, Italy}

Background and aims: Poor metabolic control is associated with the development of complications in patients with type 1 diabetes (DM1) and amongst factors contributing to it, sedentary life and lack of physical exercise play a major role. In the present study we investigated the influence of different leisure time behaviors (TV viewing, playing computer games and doing some form of sport) on blood glucose control in a group of young DM1 patients. Materials and methods: We evaluated, by direct interviews, 86 consecutive subjects (44 males), median age: 18.8 years, range: 6 - 32, 49 subjects younger than 18 years. Median disease duration was 15.3 years (range: 1 - 25), mean HbA1C: $8.1 \% \pm 1.4$, mean insulin requirements: $0.74 \pm 0.27 \mathrm{U} / \mathrm{kg} /$ day. Mean daily hours of watching TV were $2.02 \pm 0.89$ hours/day; 3 patients $(3.5 \%)$ watched TV $<1$ $\mathrm{h}$ /day, 48 patients watched TV $1-2 \mathrm{~h} /$ day (56\%), 23 subjects (26.6\%) watched TV $2-3 \mathrm{~h} /$ day, and 12 subjects (13.9\%) spent more than $3 \mathrm{~h} /$ day watching TV. Results: Daily hours of watching TV were significantly and positively related to DM1 duration (p: 0.0005) and not significantly different in patients younger or older than 18 years (younger: $2.1 \pm 1.0$, older: $1.93 \pm 0.7$ - p: 0.38 ). Mean time spent playing sports was $2.57 \pm 2.24$ hours/week (range: 0 - 8); it was not significantly different in patients younger or older than 18 years (younger: $2.76 \pm 2.1$, older: $2.33 \pm 2.2$ - p: 0.36 ) and 25 subjects (29\%) were completely sedentary. Mean time spent using a personal computer, excluding studying or working by the computer was significantly higher in patients $>18$ years ( younger: $1.37 \pm 1.27$, older: $2.49 \pm 1.97-\mathrm{p}$ : 0.003 ) and not correlated with DM1 duration (p: 0.34). HbA1C\%, daily insulin requirements and BMI were not related to the number of hours of TV watching ( $p$ : $0.38-0.21-0.20$ respectively), nor to the number of daily hours spent using the computer (p: $0.06-0.8-0.79$ respectively). Conversely, the time spent doing some form of sport (hours/week) was significantly and negatively related to $\mathrm{HbA1C}$ levels (p: 0.02). Patients who practiced more physical exercise tended to have lower daily insulin requirements and lower BMI, but the association did not reach statistical significance (p: 0.27 and p: 0.22 respectively).

Conclusion: Previous studies described a direct and significant correlation between excessive TV viewing and elevated $\mathrm{HbAlC}$ levels. We do not confirm these findings. On the contrary, physical activity remains one of the most significant determinant of blood glucose control in DM1 patients, and surprisingly increasing duration of the disease is associated with more TV watching (but not computer use), suggesting that DM1 induces a passive lifestyle behavior.

\section{5}

The PREPARE (Prediabetes Risk Education and Physical Activity Recommendation and Encouragement) study: results at 3 months T. Yates ${ }^{1}$, K. Khunti ${ }^{2}$, F. Bull ${ }^{1}$, T. Gorely ${ }^{1}$, D. Talbot ${ }^{3}$, M. Davies ${ }^{4}$ ${ }^{1}$ School of Sport and Execise Sciences, Loughborough University, ${ }^{2}$ Health Sciences, University of Leicester, ${ }^{3}$ Corporate Reserch, Unilever, Bedford, ${ }^{4}$ Cardiovascular Sciences, University of Leicester, United Kingdom

Background and aims: The PREPARE study is a randomized controlled trial designed to test the efficacy of a theory-driven, group-based, structured educational programme at increasing physical activity and improving glucose tolerance in individuals with impaired glucose tolerance (IGT); we also investigate whether the efficacy of the educational programme is enhanced by the inclusion of a pedometer and personalized steps/day goals. Three-month follow-up data is reported here.

Materials and methods: Overweight or obese individuals with IGT were recruited. At baseline and follow-up each participant was given an oral glucose tolerance test: outcomes included fasting and 2-hour glucose, fasting insu- lin, lipid profile and markers of chronic low-grade inflammation. Standard anthropometric measurements were also taken and participants were asked to wear a sealed piezoelectric pedometer for seven consecutive days and to complete a questionnaire measuring their perceptions of IGT. Participants were randomized to receive either usual care, structured education (E group) or structured education which incorporated pedometer use (EP group). The study was powered to detect a $1 \mathrm{mmol} / \mathrm{l}$ difference in 2-hour glucose at one year. Differences in change from baseline in the intervention groups compared to the control group were analysed using ANOVA modeling; Dunnett's adjustment for multiple comparisons was applied.

Results: 101 (male $\mathrm{n}=64$; female $\mathrm{n}=37$ ) participants were recruited; $30 \%$ were from a south Asian ethnic background. Three-month follow-up data was available for 93 (92\%) of participants. In the EP group at three months there was: an increase in ambulatory activity of $2611 \pm 2846$ steps per day ( $p<0.01$ vs. control); a decrease in 2-hour and fasting glucose of $-1.4 \pm 1.9 \mathrm{mmol} / \mathrm{l}(\mathrm{p}<0.01$ vs. control) and $-0.2 \pm 0.5 \mathrm{mmol} / \mathrm{l}(\mathrm{p}=0.04 \mathrm{vs}$. control) respectively; and a decrease in tumor necrosis factor- $\alpha$ of $-0.1 \pm 0.3 \mathrm{pg} / \mathrm{ml}$ ( $\mathrm{p}=0.04 \mathrm{vs}$.control). There was no significant change in any of these variables in the $\mathrm{E}$ group compared to the control group. There was no significant change in either intervention group compared to the control group in fasting insulin, interleukin-6, C-reactive protein, blood lipid profile or waist circumference. Compared to baseline, both intervention groups reported a significant $(\mathrm{p}<0.05)$ increase in perceived knowledge of prediabetes, although there was no significant difference compared to the control group. Change in ambulatory activity was correlated with change in perceived knowledge of prediabetes $(\rho=0.28, p=0.01)$.

Conclusion: This study suggests that structured education that enables participants to set realistic, personalized steps per day goals is effective at increasing ambulatory activity, improving glucose tolerance and reducing levels of inflammatory adipokines in individuals with IGT. A decrease in 2-hour glucose of this magnitude has been associated with around a $60 \%$ reduction in the relative risk of developing type 2 diabetes. This study also suggests that targeting illness perceptions may be important when promoting health behaviour change in those with IGT.

Supported by: Diabetes UK and Unilever Corporate Research

\section{6}

Long term effects of empowerment-based patient education on weight loss, diet and physical activity in patients with type 2 diabetes

L. Dencker ${ }^{1}$, H. Meden ${ }^{1}$, S. Molsted ${ }^{1}$, J. Tribler ${ }^{1}$, O. Snorgaard ${ }^{2}$

${ }^{1}$ Diabetes School, Nordsjællands Hospital, Hillerød, Denmark, ${ }^{2}$ Department of Endocrinology, Hvidovre Hospital, Denmark

Background and aims: Diet and physical activity (PA) are cornerstones in the treatment of type 2 diabetes (T2D). Patient education programmes are widely used to improve diet and level of PA. The aim of this study was to investigate the effect of an empowerment-based patient education programme on body weight, diet and PA in T2D patients.

Materials and methods: 702 T2D patients consecutively referred from general practice were included. $53 \%$ were women, age was $61 \pm 10($ mean \pm SD) years, duration of diabetes was $4.7 \pm 4.8$ years, and BMI was $31.3 \pm 6.0 \mathrm{~kg} / \mathrm{m}^{2}$. The intervention consisted of 3 periods of education during 12 months: Four days at baseline, 2 days after 3 months, and one day after 12 months. The education was based on the empowerment philosophy and was delivered by a physician, a nurse, a dietician, and a physiotherapist in groups of 10-12 patients. We aimed to improve the patients' self management capacity by identifying rather than solving problems, increasing knowledge and skills, and urging them to set up own goals. The education further included practical skills in preparing healthy food and a programme for PA.

Food intake and PA were assessed with The Danish Health and Morbidity Survey 2000 Questionnaire, which includes a seven-day record. Data for body weight and waist circumference were provided by the general practitioners. Results: At baseline, the Danish recommendations on diet were followed by $24 \%$ of the subjects according to the reported intake of fruit and cooked or raw vegetables ( $\geq 600$ gram per day), by $41 \%$ according to fish intake (eating fish several times per week), by $15 \%$ according to both fruit/vegetables and fish intake, and by $88 \%$ according to physical activity (30 minutes of moderate PA per day). $71 \%$ reported maintaining a healthy diet in order to stay healthy. Of those however, only $19 \%$ met the recommendations for fruit/vegetables and fish. The patients' goals were loss of weight (61\%), increased PA (57\%) and a more healthy diet (41\%).

During the intervention, the body weight of women decreased from $85.8 \pm 18.2$ to $83.2 \pm 17.3 \mathrm{~kg}$ at 3 months $(\mathrm{p}<0.001)$ and to $81.8 \pm 17.0 \mathrm{~kg}$ at 12 months $(\mathrm{p}<0.001)$. The body weight of men decreased from $96.7 \pm 18.8$ to $93.6 \pm 17.6 \mathrm{~kg}$ at 
3 months ( $\mathrm{p}<0.001)$ and to $93.2 \pm 17.4 \mathrm{~kg}$ at 12 months ( $\mathrm{p}=0.028)$. Waist circumference decreased in women from $103.4 \pm 13.9$ to $99.5 \pm 13.3 \mathrm{~cm}(\mathrm{p}=0.003)$ after 12 months and was unchanged in men. Compared to baseline, $9 \%$ increased intake of cooked vegetables ( $\mathrm{p}=0.024), 12 \%$ increased intake of raw vegetables $(\mathrm{p}=0.005)$, and $22 \%$ increased intake of fish $(\mathrm{p}<0.001)$. No changes were reported in the intake of fruit. The level of PA was increased in $9 \%(\mathrm{p}=0.001)$. The weight reduction was positively associated with the change of intake of fruit $(\mathrm{p}=0.011)$ and PA $(\mathrm{p}<0.001)$, and negatively associated with the change of intake of fish ( $\mathrm{p}=0.050)$.

Conclusion: An empowerment- and group-based patient education programme had long term (12 months) effects on weight loss, diet, and level of physical activity.

\section{7}

The role of combined physical therapy interventions and exercise on different aspects of health related patients with type 2 diabetes

I. Mokhtari ${ }^{1}$, K. Ezzaty ${ }^{2}$, N. Karimi ${ }^{2}$, M. Kamali fard ${ }^{3}$, K. Esmailzade ${ }^{4}$, M. Ojaghi ${ }^{5}$

${ }^{1}$ PM\&R Department, Diabetic Foot and Rehabilitation Clinic, Special Medical Center, ${ }^{2}$ Physiotherapy Department, University of Social Welfare \& Rehabilitation, ${ }^{3}$ Occupational Medicine, Health and Labour Research Center, ${ }^{4} \mathrm{PM} \& \mathrm{R}$ Department, University of Social Welfare \& Rehabilitation, ${ }^{5}$ Diabetic Foot and Rehabilitation Clinic, Special Medical Center, Tehran, Islamic Republic of Iran

Background and aims: The purpose of this Randomized Controlled Trial (RCT) study was to investigate the impact of a 4 month physical therapy interventions and exercise on different aspects of health related quality of life including physical and social functioning, bodily pain, general health, vitality, social functioning, role limitation- emotional, and total scores using SF36 scale in subjects with type 2 diabetes.

Materials and methods: All subjects met the American Diabetes Association diagnostic criteria for type 2 diabetes. Among 142 patients with type 2 diabetes, 95 subjects have the inclusion criteria for this study. They were randomized to control group $(\mathrm{n}=48$, age $=53 \pm 2 / 2$ mass $=73 \pm 3 / 1)$ and physical therapy interventions group $(n=47$, age $=52 \pm 1 / 7$; mass $=73 \pm 4 / 2)$. All patients in the latter group were given a training session and received 30 minute pain modalities (IF, IR and kneading massage) for lower extremity. Then stretching, strength and endurance exercises were trained to them during 3- 4 sessions. The patients did the supervised exercises 3 times per week during 4 month. The control group did not receive any treatment. All subjects were given an SF-36 scale to fulfill pretest and posttest.

Results: There were no significant differences in the main parameters of health related quality of life among groups at the baseline. At the end of the rehabilitation program, In a between group comparison, the results of independent sample t-test analysis showed significant changes in physical functioning, general health, physical component summary and total scores of SF36 scale $(\mathrm{P}<0.001)$. But social functioning, role limitation- emotional, mental component summary scores were not significance $(\mathrm{P}>0.05)$. Also, there were no significant changes in the control group.

Conclusion: Regular supervised integrated exercise and physical therapy is a safe and effective intervention in diabetic patients, which significantly improves health related quality of life especially physical components in patients with type 2 diabetes, which may favorably influence their long-term prognosis.

S= Significant, NS $=$ Non - significant

\begin{tabular}{|c|c|c|c|c|}
\hline \multirow[t]{2}{*}{ variables } & \multicolumn{2}{|c|}{ control group } & \multicolumn{2}{|c|}{ experimental group } \\
\hline & Pvalue & changes & P value & changes \\
\hline physical functioning & 0.81 & NS & 0.02 & $S$ \\
\hline role limitation-physical & 0.13 & NS & $<0.001$ & $\mathrm{~S}$ \\
\hline bodily pain & 0.25 & NS & $<0.001$ & $S$ \\
\hline general health & 0.54 & NS & 0.01 & S \\
\hline vitality & 0.89 & NS & 0.12 & $S$ \\
\hline role limitation- emotional & 0.14 & NS & 0.50 & NS \\
\hline mental health & 0.22 & NS & 0.11 & NS \\
\hline physical component summary & 0.89 & NS & $<0.001$ & S \\
\hline mental component summary & 0.55 & NS & 0.33 & NS \\
\hline total & 0.55 & NS & 0.002 & S \\
\hline
\end{tabular}

Supported by: Physiotherapy Department of University of Social Welfare \& Rehabilitation

\section{8}

A randomized controlled trial of nordic walking versus exercise on prescription to improve glycaemic control in type 2 diabetes

B. Gram ${ }^{1}$, C. Christiansen ${ }^{2}$ J. Gram ${ }^{2}$

${ }^{1}$ Institute of Sports Science and Clinical Biomechanics, University of Southern Denmark, Odense, ${ }^{2}$ Dept. of Endocrinology, Ribe County Hospital, Esbjerg, Denmark

Background and aims: The effectiveness of physical exercise in the treatment of type 2 diabetes has long been recognized. It is, however, unknown whether one specific type of training is more suitable than others with respect to the physical/metabolic effects as well as the ability to induce good compliance and change in physical life style.

Our aim was to evaluate the effect of two different physical exercise programs lasting 4 months aiming at introducing long term life style changes in physical activity with a follow-up period of 8 months in patients with type 2 diabetes. Materials and methods: A randomized, controlled study. 68 patients with type 2 diabetes ( 37 males, 31 females; mean age $60.7 \pm 1.2)$ were allocated in three groups: 1. Nordic Walking (NW) $(n=22), 2$. Exercise (Exercise on Prescription) (Ex) $(n=24)$ and 3. Control group (C) $(n=22)$.

During the first 2 months, the participants in the two intervention groups trained 2 times weekly and the next 2 months once a week. Each supervised training session lasted $45 \mathrm{~min}$ and the participants were instructed to exercise corresponding to moderate intensity. The controls received no supervised training but, at the inclusion they were advised to be physical active. All participants were tested at 0,4 , and 12 months (biochemistry, anthropometry, DEXA, indirect $\mathrm{VO}_{2}$ max test, SF36). The primary effect parameter was glucose metabolism evaluated by glycated hemoglobin $\left(\mathrm{HbA}_{1 \mathrm{c}}\right)$. The sample size calculation was based on a minimal relevant difference in $\mathrm{HbA}_{1 \mathrm{c}}$ of $0.8 \%$ point.

Results: The overall attendance rates were $63 \pm 7 \%$ in NW and $64 \pm 7 \%$ in Ex. $\mathrm{HbA}_{1 \mathrm{c}}$ did not change significantly in the intervention groups compared to Control $(\Delta \mathrm{NW}$ : $-0.4 \%(-0.9-0.1)(95 \% \mathrm{CI})$ and $\Delta \mathrm{Ex}:-0.2 \%(-0.6-0.2)$ after 4 months and $\Delta$ NW: $0.0 \%(-0.6-0.5)$ and $\Delta$ Ex: $0.3 \%(-0.3-0.9)$ after 12 months. Neither did Total HDL-cholesterol and triglyceride levels. Fat mass significantly decreased in the NW-group after 4 months $(-1.0 \mathrm{~kg}(-1.7-0.1))$ and after12 months both in NW (-1.8 $\mathrm{kg}(-3.2--0.4))$ and in Ex $(-1.5 \mathrm{~kg}(-2.9$ - -0.05). No significant changes in blood pressure, $\mathrm{VO}_{2} \max$, and in quality of life measured by SF36

Conclusion: Four months' physical exercise programs with low to moderate intensity of Nordic Walking or Exercise per prescription did not significantly improve $\mathrm{HbA}_{1 c}$ in patients with type 2 diabetes neither at end of programs nor at follow-up. Besides loss of fat mass in both intervention groups at 12 months there were no parameters indicating a significant change in physical lifestyle at follow-up.

Supported by: Danish Diabetes Association and the Association of Danish Physiotherapists 


\section{PS 097 Health care: national surveys}

\section{9}

Ecodia2 survey: improvement in the quality of diabetes care in France B. Detournay ${ }^{1}$, S. Bouee ${ }^{2}$, D. Simon ${ }^{3}$, C. Attali ${ }^{4}$, G. Brami ${ }^{5}$, B. Charbonnel ${ }^{6}$, E. Eschwege ${ }^{7}$, A. Fagot-Campagna ${ }^{8}$, A. Grimaldi ${ }^{3}$, B. Guerci ${ }^{9}$, S. Halimi ${ }^{10}$, F. Nachit-Ouinekh ${ }^{11}$, M. Varroud-Vial ${ }^{12}$;

${ }^{1}$ Health Economics, CEMKA-EVAL, Bourg-la-Reine, ${ }^{2}$ Epidemiology, CEMKA-EVAL, Bourg-la-Reine, ${ }^{3}$ Diabetology, Hôpital Pitié-Salpetrière, Paris, ${ }^{4}$ General Practice, Faculté de médecine, Créteil, ${ }^{5} \mathrm{Medical}$ Department, GlaxoSmithKline, Marly-le-Roi, ' ${ }^{\circ}$ iabetology, Hôpital Hotel-Dieu, Nantes, ${ }^{7}$ Unité 780, INSERM, Villejuif, ${ }^{8}$ Chronic Diseases, INVS, Saint-Maurice, ${ }^{9}$ Diabetology, Hôpital Jeanne d'Arc, Dommartin les Toul, ${ }^{10}$ Diabetology, Hôpital Michallon, Grenoble, ${ }^{11}$ Health Economics, GlaxoSmithKline, Marlyle-Roi, ${ }^{12}$ Diabetology, ANCRED, Villeneuve Saint-Georges, France.

Background and aims: Recent years have a seen a number of healthcare initiatives and developments aimed at improving the quality of diabetes care. The objective of this study was to assess care provision for diabetes in standard care in France and its evolution in the recent years.

Materials and methods: The ECODIA2 study was a descriptive cross-sectional epidemiological study of patients with type 2 diabetes in France performed in 2005. Data were collected using questionnaires completed by a representative sample of physicians treating diabetes. Data were compared with those from the 1999 ECODIA study which used a similar methodology.

Results: Questionnaires were available for 4071 patients with type 2 diabetes. Compared to the first 1999 ECODIA study, little change was observed for age (mean age: 65 years), gender (ratio: 5 men to 4 women), time since diagnosis (mean: 8.3 years) or the prevalence of risk factors $(80.4 \%$ were overweight, $10.4 \%$ were smokers and $87.3 \%$ had hypertension) for the diabetic patients in 2005 in France. In contrast, only 19.2\% of patients presented an HbAlc level $>8 \%$, which represents a marked improvement compared to $31.4 \%$ in 1999 $(\mathrm{p}<0.001)$. Between 1999 and 2005, the proportion of patients treated by diet and exercise alone decreased from $9.5 \%$ to $2.7 \%$, the proportion treated with oral antidiabetic drugs who received biguanides increased from $30.8 \%$ to $46.6 \%$ and the proportion treated with insulin rose from $5.6 \%$ to $14.1 \%$. Nonetheless, the improvement on glycaemic control has not yet been translated into a reduction in the incidence of micro- or macro-vascular diabetesrelated complications.

Conclusion: These results reveal a promising evolution in the quality and effectiveness of diabetes care in France over the last six years. Similar improvements in care have been observed in other countries. The results obtained reinforce the need to pursue and expand public health policies aimed at implementing best practice recommendations into standard care. It is also important to monitor regularly the impact of these policies in everyday clinical practice.

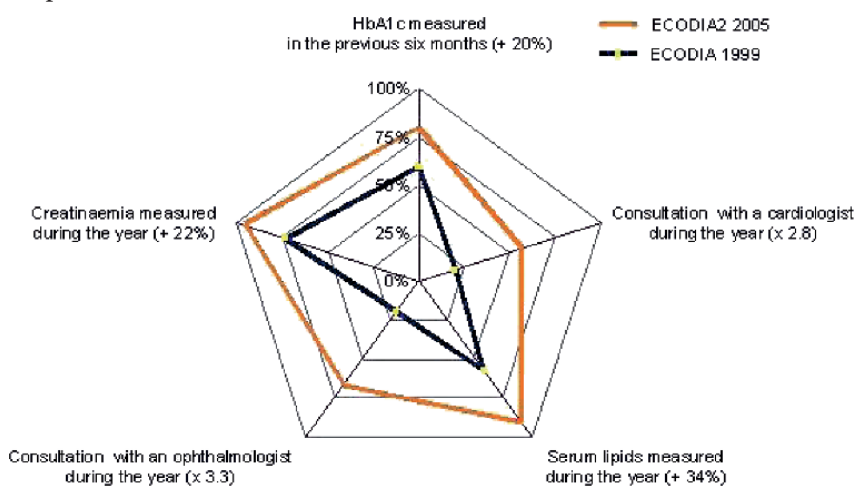

Figure 1. Improvement in management of type 2 diabetes in France between 1999 and $2005^{\star}$

* patients declared as having type 2 diabetes only

Supported by: GlaxoSmithKline
1040

Gender differences in the prevalence of undiagnosed type 2 diabetes and associated cardiovascular risk factors according to the education level among the employed residents of Chelyabinsk

I.A. Vaychulis ${ }^{1}$, I.I. Shaposhnik ${ }^{1}$, T.N. Vaychulis ${ }^{2}$

${ }^{1}$ Internal Medicine Department, Chelyabinsk State Medical Academy,

${ }^{2}$ Endocrinology Department, Chelyabinsk Municipal Hospital No 1,

Chelyabinsk, Russian Federation

Background and aims: The association between type 2 diabetes (DM2) and socioeconomic factors differs across various populations. The aim of this study was to estimate the prevalence of unknown DM2 and cardiovascular (CV) risk factors among working residents of Chelyabinsk (Russian Federation) according to the education level and gender to define target social groups for DM2 screening and prevention.

Materials and methods: Chelyabinsk is a big industrial city with the population aged $40 \mathrm{y}$ and older of 493963 (197533 m /296430 w). We examined a total of $4238(1661 \mathrm{~m} / 2577 \mathrm{w})$ randomly selected workers of 15 enterprises and institutions, located in all 7 administrative districts of the city, aged 40-70 $y($ mean \pm SD $52.0 \pm 6.8 \mathrm{y})$. After exclusion of the employees with known DM fasting capillary glucose (FCG) was analyzed with glucometer Accu-Chek Active (Roche Diagnostics) and in people with FCG 5.6-6.0 mmol/l standard OGTT was performed. Weight, height, waist circumference (WC), systolic (SBP) and diastolic blood pressure (DBP) were measured and body mass index (BMI) was calculated. General linear univariate model with age adjustment was used to compare sex-specific prevalence rates of unknown DM2 (WHO criteria, 1999), total obesity $\left(\mathrm{BMI} \geq 30 \mathrm{~kg} / \mathrm{m}^{2}\right)$, abdominal obesity (WC $>88 \mathrm{~cm}$ in $\mathrm{w}, \mathrm{WC}>102 \mathrm{~cm}$ in $\mathrm{m}$ ) and arterial hypertension $\mathrm{AH}(\mathrm{SBP} \geq 140$ and/or DBP $\geq 90 \mathrm{~mm} \mathrm{Hg}$ and/or medication use) in high vs secondary/professional education groups. Multinominal logistic regression analysis controlling for age, obesity, $\mathrm{AH}$ and family history of DM2 was used to estimate the association between undiagnosed DM 2 and level of education.

Results: Age-adjusted prevalence of unknown DM 2 and CV risk factors in men did not significantly differ across the education groups ( $p>0.05$, table). In multinominal logistic regression analysis having undiagnosed DM2 was not significantly associated with lower education level neither in men [OR 1.35 ; 95\%CI 0.86-2.12], nor in women [1.39; 0.89-2.17]. However, among women, unknown DM2, AH, total and abdominal obesity were consistently more prevalent in the employees with lower education (table). Age-adjusted prevalence of undiagnosed DM2 was higher in men (7.4 $\pm 0.6 \%)$ than in women $(5.7 \pm 0.8 \%$, $\mathrm{p}=0.029)$, but this difference turned out to be non-significant when compared men to women with lower education $(7.4 \pm 0.6 \%$ vs $6.4 \pm 1.0 \%$, respectively, $\mathrm{p}>0.05$ ).

Conclusion: Education level was not independently related to undiagnosed DM2, but women with lower education have demonstrated significantly higher prevalence of CV risk factors and unknown DM2. Level of education should be considered when selecting first-line target groups for DM2 screening and prevention among the employed women $40 \mathrm{y}$ and older.

Age-adjusted prevalence of unknown DM 2 and CV risk factors by educational level.

\begin{tabular}{|c|c|c|c|c|}
\hline \multirow{2}{*}{$\begin{array}{l}\text { Gender } \\
\text { Education } \\
\text { level }\end{array}$} & \multicolumn{2}{|l|}{ Men } & \multicolumn{2}{|l|}{ Women } \\
\hline & $\begin{array}{l}\text { Secondary / } \\
\text { professional }\end{array}$ & $\begin{array}{l}\text { High } \\
\text { education }\end{array}$ & $\begin{array}{l}\text { Secondary / } \\
\text { professional }\end{array}$ & $\begin{array}{l}\text { High } \\
\text { education }\end{array}$ \\
\hline $\begin{array}{l}\text { Education } \\
\text { level }\end{array}$ & 1133 & 492 & 1776 & 743 \\
\hline $\begin{array}{l}\text { Unknown } \\
\text { DM2 }\end{array}$ & $7.9 \pm 1.4$ & $6.2 \pm 1.2$ & $6.4 \pm 1.0$ & $3.8 \pm 0.8^{*}$ \\
\hline Total obesity & $21.0 \pm 2.2$ & $19.7 \pm 1.8$ & $41.5 \pm 2.1$ & $29.4 \pm 1.8^{* *}$ \\
\hline $\begin{array}{l}\text { Abdominal } \\
\text { obesity }\end{array}$ & $18.9 \pm 2.1$ & $18.3 \pm 1.7$ & $45.4 \pm 2.1$ & $32.0 \pm 1.8^{* *}$ \\
\hline $\begin{array}{l}\text { Arterial } \\
\text { hypertension }\end{array}$ & $59.9 \pm 2.6$ & $55.1 \pm 2.2$ & $52.3 \pm 2.2$ & $43.2 \pm 1.8^{* *}$ \\
\hline \multicolumn{5}{|c|}{$\begin{array}{l}{ }^{*} \mathrm{p}<0.05 \text { compared to the lower education group. } \\
{ }^{* *} \mathrm{p}<0.001 \text { compared to the lower education group. } \\
\text { Data are shown as mean } \% \pm S E \text {. }\end{array}$} \\
\hline \multicolumn{5}{|c|}{ Supported by: Servier and sanofi-aventis } \\
\hline
\end{tabular}




\section{1}

Patients opinion on the barriers to diabetes control in areas of conflicts: the Iraqi example

A.A. Mansour

Medicine, Basrah College of Medicine, Basrah, Iraq

Background: The health system in Iraq has undergone progressive decline since the embargo that followed the second gulf war in 1991. The aim of this study is to see barriers to glycemic control from the patient perspective, in a diabetic clinic in the south of Iraq.

Methods: A cross sectional study from the diabetes out-patient clinic in AlFaiha general hospital in Basrah, South Iraq for the period from January to December 2007. The study includes diabetics whether type 1 or 2 if they have at least one year of follow up in the same clinic. Those with $\mathrm{HbAlC} \geq 7 \%$ were interviewed by special questionnaire, that was filled in by the medical staff of the clinic. The subjects analyzed in this study were adults ( $\geq 18$ years old) with previously diagnosed diabetes $(n=3522)$. The duration of diabetes range from 1 to 30 years.

Results: Mean $\mathrm{HbAlC}$ was $8.4 \pm 2$ percent, with 835 (23.7\%) patients with HbAlC less than $7 \%$ and $2688(76.3 \%)$ equal to or more than $7 \%$. Of 3522 studied patients, $46.6 \%$ were men and $51.5 \%$ were women, with mean age of $53.78 \pm 12.81$ year and age range 18-97 years. Patient opinion for not achieving good glycemic control among 2688 patients with $\mathrm{HbA1C} \geq 7 \%$ included the following. No drug supply from primary health care center (PHC) or drug shortage is a cause in $50.8 \%$ of cases, while drugs and or laboratory expense were the cause in $50.2 \%$. Thirty point seven percent of patients said that they were unaware of diabetics complications and $20.9 \%$ think that diabetes is an untreatable disease. Thirty percent think that non-control of their diabetes is due to migration after the war. No electricity or erratic electricity, self-monitoring of blood glucose (SMBG) is not available, or strips were not available or could not be used, and illiteracy as a cause was seen in 15\%,10.8\% and $9.9 \%$ respectively.

Conclusion: Our patients with diabetes mellitus declared that of the causes for poor glycemic control most of them related to the current health situation in Iraq.

\section{2}

Improving the control of diabetes among Israeli Arabs

J. Khury, D. Kurzum, S. Abd elhadi, D. Besharat, I. Hilf

Family Medicie- Nazareth B' Clinic, Clalit Medical Services- Nortern district, Nazareth, Israel

Background and aims: The prevalence of Diabetes Mellitus (DM) among Israeli Arabs (about 12\%) is significantly higher than it is among Israeli Jews and other populations from other developed countries (6-8\%). On the other hand, the percentage of controlled diabetic patient $(\mathrm{HBA1C}<7)$ among this group $(28 \%)$ is less the in Israeli Jewish population and populations from other developed countries (55\%). It's also known that the incidence and severity of diabetic complication in Israeli Arabs is high especially among Arab women. Lastly DM was declared as a universal epidemic. It's wildly proved that controlling DM markly reduces the incidence of diabetic complications and death, what makes it worth to invest in the efforts to control this disease. The prevalence of DM among Nazareth B' Clinic patient is as high as $14 \%$ (1221 patients), only $38 \%$ of them were controlled. $21 \%$ of the patients were severely uncontrolled (HBA1C $>9$ ).

Materials and methods: We conducted a study which aimed to achieve the goal of $45 \%$ controlled patients at the first year of intervention. DM "control" included desirable values of Blood Lipids, Blood Pressure, Proteinurea, BMI and eye examination. We randomly choused 184 uncontrolled patients with HBA1C $>9$, age between 20-65 years from the Electronic Medical Records (EMR). They were divided into sub groups of 8-10 patients. Each sub group was invited for follow by Family Physician, Diabetic Nurse, Dietitian and Social Worker every 3 months. The multidiscipline crew met once weekly to discuss the problematic issues that arise throughout the day work. The intervention based on improving the

1- "Medication Adherence" and way of life which included encounter with dietitian, social worker, joining groups for physical activity and empowerment.
2- "Clinical Interia" which included encounter with especially trained nurse and physician and recheck up by pharmacist for acknowledgment of medication usage.

3- "Treatment failure" by treating the treatment resistance with proper and effective medications.

Results: The results of the first year of interventions show a significant improvement of most of the control criteria of DM as shown in the following table.

Conclusion: Follow up and treatment of problematic and hard to control Diabetic populations should be carried out by a multidiscipline team of family physicians, especially trained nurses, dietitians and social worker:

\section{3}

Lipid management in type 1 and type $\mathbf{2}$ diabetes patients in Belgium - subject for quality improvement

N. Debacker ${ }^{1}$, F. Nobels ${ }^{2}$, C. Mathieu ${ }^{3}$, A. Scheen ${ }^{4}$, P. Van Crombrugge ${ }^{2}$ ${ }^{1}$ Epidemiology, Scientific Institute of Public Health, Brussels,

${ }^{2}$ Endocrinology, OLV Ziekenhuis, Aalst, Belgium, ${ }^{3}$ Endocrinology, UZ

Gasthuisberg, Leuven, ${ }^{4}$ Endocrinology, Centre Hospitalier Universitaire Sart Tilman, Liege, Belgium

Background and aims: In Belgium the Initiative for Quality improvement and Epidemiology for Diabetes (IQED) organised in all hospital-based multidisciplinary diabetes centres aims to improve quality of care. As a 2008 quality initiative blood lipid management is highlighted.

Materials and methods: In 2007121 diabetes centres provided data on a random sample of $10 \%$ of their adult diabetes patients on $\geq 2$ daily insulin injections. By using the IQED-software data on patient characteristics, glycemic control, cardiovascular risk profile, diabetes complications, blood glucose self-monitoring, follow-up procedures and treatment were obtained. LDL-cholesterol was calculated using the Friedewald formula.

\begin{tabular}{lll}
\hline & $\begin{array}{l}\text { Type 1 } \\
\text { diabetes }\end{array}$ & $\begin{array}{l}\text { Type 2 } \\
\text { diabetes }\end{array}$ \\
\hline Number of patients & 3186 & 7174 \\
\hline Median age (years) & 44 & 69 \\
\hline Median duration (years) & 17 & 12 \\
\hline \% men & 56 & 49 \\
\hline Median HbA1c (\%) & 7.8 & 7.4 \\
\hline Statin treatment & 28 & \\
\hline \% treated with statins & 71 & 72 \\
\% treated with statins when presence of CVD & $29-100-100$ & $48-71-93$ \\
distribution across the centres (P10-P50-P90) & 35 & 56 \\
\% treated with statins when microalbuminuria & $0-31-100$ & $25-53-88$ \\
distribution across the centres (P10-P50-P90) & 58 \\
\% treated with statins when older than 40 years & 37 & \\
and $\geq 1$ CV risk factor & & \\
distribution across the centres (P10-P50-P90) & $0-25-56$ & $30-57-85$ \\
\hline Achievement of LDL-targets & & \\
\hline Statin treated patients reaching LDL-target & & \\
- No CVD: \% LDL $<100$ mg/dl & 46 & 66 \\
distribution across the centres (P10-P50-P90) & $0-25-67$ & $0-37-68$ \\
- CVD: \% LDL<70 mg/dl & 29 & 34 \\
distribution across the centres (P10-P50-P90) & $0-0-50$ & $0-17-43$ \\
\hline
\end{tabular}

Results: Table: population characteristics, statin treatment, achievement of treatment goals and distribution of the results across the centres (percentile 10,50 and 90 ) by type of diabetes .

Of DM1 and DM2 patients resp. 8 and $31 \%$ have cardiovascular disease (CVD: myocardial infarction, PTCA/CABG or stroke), 16 and $35 \%$ have microalbuminuria and 50 and $80 \%$ have at least one other CV risk factor (BMI $>30 \mathrm{~kg} /$ $\mathrm{m}^{2}$; blood pressure $>140 / 90 \mathrm{mmHg}$ or smoking). Where in case of CVD an equal proportion of DM1 and DM2 patients are treated with statins, more DM2 than DM1 patients are treated in case of another risk profile. Compared to 2006 , where $24 \%$ of DM1 and $50 \%$ of DM2 patients were treated, statin

\begin{tabular}{llllllllll}
\hline & fasting glucose & HBA1C & CHOL & LDL & HDL & TG & sys. BP & dias BP & BMI \\
\hline prior intervention & 257.2 & 11.7 & 174.6 & 112.1 & 44.1 & 215.2 & 129.7 & 75.9 & 34.3 \\
\hline after intervention & 158.9 & 8.5 & 171.3 & 102.5 & 45.3 & 153.2 & 125.6 & 74.9 & 30.6 \\
\hline desired goal & 110 & 7 & 160 & 100 & & & 120 & 80 & 25 \\
\hline
\end{tabular}


treatment increased significantly (irrespective of age and diabetes duration). For treatment goal achievement we looked at LDL-cholesterol levels, available for only $51 \%$ and $55 \%$ of resp. DM1 and DM2 patients. Treatment goals are reached significantly more often in type 2 than in type 1 diabetes patients without CVD. Compared to 2006, where $23 \%$ of DM2 patients with CVD and $52 \%$ of DM2 patients without CVD reach the LDL-target, significantly more patients reach the target in 2007. The variations between the centres are large. Also regarding the determination of fasting lipids the variation between the centres is high: $10 \%$ of the centres have nearly no fasting lipid determinations and $10 \%$ have for nearly all their patients a fasting lipid profile.

Conclusion: The studied population has an important $\mathrm{CV}$ risk requiring appropriate treatment. Compared to 2006 more patients are treated and reach treatment goals. However, a large part of the patients remains without treatment and does not reach the treatment goals and this varies considerably between the centres. Moreover a fasting blood lipid profile is not available for a large part of the population, hampering the calculation of LDL-cholesterol. So room for improvement remains. To induce this we aim to bring cardiovascular risk and treatment to the attention of the centres by providing more specific feedback (benchmarks) concerning this aspect.

\section{4}

IDF quality assistance indicators based on metabolic control level in patients with DM2 in Spain

J.I. Diago, J. Reviriego, P. Polavieja, F. Chacon

Medical Research, Lilly Spain, Alcobendas, Spain

Background and aims: To evaluate the compliance level of measures of care recommended by IDF (International Diabetes Federation) in relation to metabolic control in non-insulin treated type 2 diabetes mellitus (DM2) patients, in standard clinical practice in Spain.

Materials and methods: A retrospective, observational, naturalistic and multicentre study, that $\mathrm{w}$ reviews clinical records of a 2 -year period previous to the inclusion of patients in the study. A total of 216 physicians participated (endocrinologists, internal medicine specialists and general practitioners). Sociodemographic, socioeconomic, clinical and assistance variables were collected. Indicators of quality of assistance provided to patients with DM2 according to the IDF 2005 were assessed (see Table 1). The $6^{\text {th }}$ IDF indicator ("begin insulin therapy when optimized oral glucose-lowering drugs and lifestyle interventions are unable to maintain blood glucose control at target levels") was not applicable due to patients' inclusion criteria. Metabolic control was defined according to IDF and ADA (American Diabetes Association) criteria (good metabolic control $=\mathrm{HbAlc} \leq 6.5 ; \mathrm{HbAlc}<7 \%$, respectively). Group comparisons were done by means of Chi-square tests.

Results: Clinical records from a total of 2,266 patients were reviewed. Mean age (SD) was 64.4 (11.1) years. $47.2 \%$ were female; $7.7 \%$ of fertile age. Median time (IQR) of diagnosis was 5.9 (2.9-10.1) years. According to IDF and ADA criteria, $41.8 \%$ and $59.9 \%$ of patients had good metabolic control, respectively. Mean number (SD) of accomplished indicators was 6.7 (2.1), out of 12 selected for this population. There were no significant differences between good and poor metabolic control groups following IDF (6.6 (2.2) and 6.7 (2.1) respectively) or ADA criteria (6.7 [2.2] and 6.6 [2.1] respectively). Indicators number number $2,8,9,10$ and 12 were the least followed ones $(48.3 \%$, $34.3 \%, 22.6 \%, 35.2 \%$ and $48.6 \%$ respectively). Their monitoring was slightly higher in patients with good vs. poor metabolic control $(\mathrm{p}<0.05$ for indicators 2, 9 and 10, following ADA criteria) (see Table 1). Indicators 4 and 5 were monitored more frequently in patients with poor control $(\mathrm{p}<0.01)$.

Conclusion: Compliance of quality assistance indicators in clinical practice in Spain is low, although it is slightly higher in patients with good metabolic control. Some assistance indicators are more followed in patients with poor metabolic control, what probably indicates the more care that these patients need. Nevertheless, there is a need to improve both, number and frequency of these measurements of care, in daily clinical practice in Spain.

Supported by: Lilly Spain

\begin{tabular}{|c|c|c|c|c|c|}
\hline & \multicolumn{2}{|c|}{ Metabolic control according to IDF } & \multicolumn{2}{|c|}{ Metabolic control according to ADA } & \multirow{2}{*}{$\begin{array}{l}\text { Total } \\
\%\end{array}$} \\
\hline & $\begin{array}{l}\text { Poor control } \\
\%\end{array}$ & $\begin{array}{l}\text { Good control } \\
\%\end{array}$ & $\begin{array}{l}\text { Poor control } \\
\%\end{array}$ & $\begin{array}{l}\text { Good control } \\
\%\end{array}$ & \\
\hline \multicolumn{6}{|c|}{ Indicator 1: Provide education to patients about diabetes, lifestyle management and diet (IDF $\mathrm{p}=0.073)(\mathrm{ADA} p=0.085)$} \\
\hline Yes & 61.2 & 64.9 & 60.6 & 64.2 & 62.8 \\
\hline \multicolumn{6}{|c|}{ Indicator 2: Advise patients about the benefits of targeting HbA1c $<6,5 \%($ IDF $p=0.617)($ ADA $p=0.046)$} \\
\hline Yes & 47.9 & 48.9 & 45.8 & 50.0 & 48.3 \\
\hline \multicolumn{6}{|c|}{ Indicator 3: Monitor HBA1C every 2-6 months (IDF p=0.896) (ADA p=0.277) } \\
\hline Yes & 95.2 & 95.0 & 94.5 & 95.5 & 95.1 \\
\hline \multicolumn{6}{|c|}{ Indicator 4: Self-monitoring of blood glucose (IDF $\mathrm{p}<0.001)($ ADA $\mathrm{p}=0.009)$} \\
\hline Yes & 67.7 & 59.6 & 67.6 & 62.2 & 64.3 \\
\hline
\end{tabular}

Indicator 5: Begin oral glucose-lowering drugs when lifestyle interventions alone are unable to maintain blood glucose at target levels $($ IDF $\mathbf{p}<0.001)($ ADA $\mathbf{p}<0.001)$

\begin{tabular}{|c|c|c|c|c|c|}
\hline Yes & 91.2 & 80.6 & 92.6 & 82.8 & 86.8 \\
\hline \multicolumn{6}{|c|}{ Indicator 7: Measure blood pressure annually, and at every routine clinic visit if found to be above target levels (IDF p=0.051) (ADA p=0.082) } \\
\hline Yes & 96.2 & 97.7 & 96.0 & 97.4 & 96.8 \\
\hline \multicolumn{6}{|c|}{ Indicator 8: Assess cardiovascular risk protection at diagnosis and at least annually thereafter (BMI, smoking, lipids, etc) (IDF $p=0.578)(A D A p=0.712)$} \\
\hline Yes & 33.9 & 35.0 & 33.9 & 34.6 & 34.3 \\
\hline \multicolumn{6}{|c|}{ Indicator 9: Examination of the eyes at diagnosis and annually (IDF p=0.460) (ADA p=0.017) } \\
\hline Yes & 22.1 & 23.4 & 20.0 & 24.3 & 22.6 \\
\hline \multicolumn{6}{|c|}{ Indicator 10: Check annually for proteinuria in an early morning urine sample (or a random sample) using dipstick (IDF p=0.252) (ADA p=0.004) } \\
\hline Yes & 34.2 & 36.6 & 31.7 & 37.6 & 35.2 \\
\hline \multicolumn{6}{|c|}{ Indicator 11: Assess feet of the people with diabetes as part of an annual review (IDF $p=0.296)(A D A p=0.402)$} \\
\hline Yes & 68.6 & 70.6 & 68.4 & 70.1 & 69.4 \\
\hline \multicolumn{6}{|c|}{ Indicator 12: Diagnose sonsorimotor nerve damage (IDF $\mathrm{p}=0.888$ ) (ADA $\mathrm{p}=0.361$ ) } \\
\hline Yes & 48.7 & 48.4 & 47.4 & 49.4 & 48.6 \\
\hline \multicolumn{6}{|c|}{ Indicator 13: Identify possibility of pregnancy annually. Contraceptive advice (IDF p=0.253) (ADA p=0.182) } \\
\hline Yes & 55.8 & 69.0 & 52.6 & 67.4 & 60.5 \\
\hline
\end{tabular}




\section{5}

Improved $\mathrm{HbA}_{1 \mathrm{c}}$ and blood pressure values in 11752 patients with diabetes on the primary health care level ten years after initiation of a diabetes-check up programme by the "Deutsche BKK"

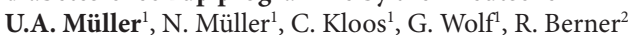

${ }^{1}$ Funktionsbereich Endokrinologie und Stoffwechsel, University Hospital Jena, ${ }^{2}$ Controlling und Versorgungsmanagement, Deutsche BKK Stuttgart, Wolfsburg, Germany

Background and aims: The Deutsche BKK, the health insurance for employees of the Volkswagen AG and Deutsche Post AG, initiated and sponsored a diabetes quality assurance programme ("Diabetes TÜV") in 1997. The intention of the programme was to improve the quality of primary care for insured patients with known diabetes by motivating local general physicians to perform standardized diabetes-related clinical examinations. $\mathrm{HbA}_{1 c}$ and blood pressure (BP) could be evaluated now over a period of ten years.

Patients and methods: Participants were informed about the programme by Deutsche BKK and their local general practitioners twice by a mailing. Physicians were motivated to support the programme by a additional remuneration of 25 Euro for every examination performed. In order to improve the reliability of clinical examinations and data recording, patients' and physicians' signatures on the documentation of the results was a prerequisite for the remuneration. All patients with known type 1 or type 2 diabetes were eligible for the programme. Participants who agreed to participate were recruited in consecutive order as they were referred. The presence of diabetic late complications, cardiovascular events, $\mathrm{HbA}_{1 \mathrm{c}}$, blood pressure, creatinine and microalbuminuria were documented once a year. 15111 documentation sheets of 11752 patients were provided by 351 practices in the periods 1997-2000 and 2002-2006. $\mathrm{Hba}_{1 \mathrm{c}}$ was adjusted to an upper normal range of $6.1 \%$ of total $\mathrm{Hb}$. Results: The mean systolic BP was 142.9, the mean diastolic BP pressure 81.0 $\mathrm{mmHg}$ and the mean $\mathrm{HbA}_{1 \mathrm{c}} 7.10 \%$ of total $\mathrm{Hb}$ over the whole period. There was a significant improvement of systolic $\mathrm{BP}(-10.5 \mathrm{mmHg})$, diastolic BP ($4.1 \mathrm{mmHg})$ and $\mathrm{HbA}_{1 \mathrm{c}}(-0,49 \%$ of total $\mathrm{Hb})$ over the decade (Table). The decrease was continuous over the whole period (Bonferroni).

Conclusion: The quality of care of patients with diabetes mellitus insured by the Deutsche BKK improved over the period from 1997 to 2006. The decrease of BP was comparable to the intervention group of the UKPDS (BP systolic -10; diastolic $-5 \mathrm{mmHg}$ ). The mean $\mathrm{HbA}_{1 \mathrm{c}}$ at the beginning of the programme was lower than the baseline $\mathrm{HbA}_{1 c}$ of the UKPDS. The mean $\mathrm{HbA}_{1 c}$ at the last observation year was comparable to the intervention group of UKPDS and DCCT. A yearly documentation was sufficient for the monitoring of changes in the quality of diabetes care.

Quality of care in the Diabetes-TÜV programme

\begin{tabular}{llllllllll}
\hline & 1997 & 1998 & 1999 & 2000 & 2002 & 2003 & 2004 & 2005 & 2006 \\
\hline $\mathrm{n}$ & 668 & 1317 & 1335 & 1070 & 871 & 2065 & 1322 & 876 & 2175 \\
\hline $\begin{array}{l}\text { BP syst. } \\
\text { (mmHg) }\end{array}$ & 149,0 & 147,2 & 146,0 & 144,4 & 141,1 & 142,6 & 140,8 & 140,9 & 138,6 \\
\hline $\begin{array}{l}\text { BP diast. } \\
\text { (mmHg) }\end{array}$ & 82,8 & 82,8 & 82,3 & 81,7 & 81,1 & 81,1 & 80,3 & 80,1 & 78,7 \\
\hline $\begin{array}{l}\text { HbAlc } \\
\text { (\% of total } \\
\text { Hb) }\end{array}$ & 7,44 & 7,56 & 7,50 & 7,32 & 6,95 & 6,83 & 6,77 & 6,95 & 6,95 \\
\hline
\end{tabular}

Supported by: Deutsche BKK Wolfsburg

\section{PS 098 Health care: others}

\section{6}

\author{
Addressing the gap for diabetes education services in a rural US \\ community \\ L.M. Siminerio ${ }^{1}$, A. Uhler ${ }^{2}$, A. Cook ${ }^{3}$, G. Piatt ${ }^{3}$, J. Zgibor ${ }^{4}$, K. Ruppert ${ }^{4}$ \\ ${ }^{1}$ Endocrinology, University of Pittsburgh Diabetes Institute, ${ }^{2} \mathrm{UPMC}$, \\ University of Pittsburgh Medical Center, ${ }^{3} \mathrm{UPMC}$, University of Pittsburgh \\ Diabetes Institute, ${ }^{4}$ Graduate School of Public Health, University of \\ Pittsburgh Diabetes Institute, United States
}

Background and aims: Healthy People 2010 is a comprehensive set of disease prevention and health objectives for the US. The goals are to increase quality within focused areas. Diabetes self-management education (DSME) is essential for the proper management of diabetes and ultimately prevention of complications. A Chronic Care Commission has been instituted in the US state of Pennsylvania (PA) to improve quality of care, specifically for diabetes. A Healthy People objective is to increase the proportion of persons with diabetes who receive education from 45 to $60 \%$. Citizens residing in PA are lagging behind the target for education (50\%). PA has the second largest senior population and largest rural population of any state accounting for $30 \%$ of its population. Evidence suggests that sub-optimal outcomes in people with diabetes in rural communities are due to limited preventive health services and access to specialists, like diabetes educators. Clinicians in rural settings also lack availability of clinical information systems and organizational support from larger health systems. Our aim was to use elements of the Chronic Care Model (health system, community, decision support, information systems, self-management, system redesign) as a framework to help clinicians meet Healthy People goals in rural PA.

Materials and methods: The Pittsburgh Regional Initiative for Diabetes Education (PRIDE) was developed to build a collaboration between the University of Pittsburgh Medical Center with outlying rural communities in an effort to gain organizational and community support for self-management services. ADA standards were used for decision support; an information system served as a registry for local primary care practices and educators to communicate data; and an education center was established to address system redesign. Our first objective was to examine all patients with a diagnosis of diabetes seen at the local primary care practices to determine characteristics of those who had not received DSME. A total of 844 patients were seen at these practices between 7/18/06 and 2/29/08. Of them, 566 (67\%) patients had a complete education history and were included in this analysis.

Results: Mean age was $61.4,48 \%$ were males, $82 \%$ had type 2 diabetes, with mean diabetes duration of 9 yrs. Risk factors for coronary artery disease (CAD) (hyperlipidemia, obesity BMI $>30$, reported smoking, and hypertension) were studied in this group. $49 \%$ had $\mathrm{LDL} \geq 100 \mathrm{mg} / \mathrm{dL}, 60 \%$ were obese, $25 \%$ were smokers, and $82 \%$ had some indication of hypertension. $94.5 \%$ of these patients had 2 or more risk factors for CAD. Co-morbidities included CAD (20\%), retinopathy (2.5\%), neuropathy (16\%) and nephropathy (15.5\%). $35 \%(n=200)$ had not received DSME.

Conclusion: Despite high rates of obesity and risk factors for CAD, a large proportion of patients had not received DSME that includes nutrition therapy. As we quickly approach the 2010 milestone, US systems, like PA, would be wise to consider a paradigm of systematic approaches in support of clinicians in high risk rural communities. A critical first step is the identification of those people who are in greatest need for DSME.

Supported by: United States Air Force administered by the U.S. Army Medical Research Acquisition Activity, Fort Detrick, Maryland 


\section{7}

Resource use associated with type 2 diabetes in 24 countries in Asia, Latin America, the Middle East and Africa

S. Mechiche ${ }^{1}$, A. Ringborg ${ }^{2,3}$, P. Lindgren ${ }^{2,3}$, J. Bengt $^{4}$, A. Ramachandran ${ }^{5}$, J.J. Gagliardino ${ }^{6}$

${ }^{1}$ sanofi-aventis, Paris, France, ${ }^{2}$ Cardiovascular and Metabolic Diseases, i3 Innovus, Stockholm, ${ }^{3}$ Institute of Environmental Medicine, Karolinska Institute, Stockholm, ${ }^{4}$ Centre for Heath Economics, Stockholm School of Economics, Sweden, ${ }^{5}$ Dr. A. Ramachandran's Diabetes Hospitals, India Diabetes Research Fondation, Chennai, India, ${ }^{6}$ Faculty of Medical Sciences, CENEXA (UNLP-CONICET), La Plata, Argentina

Background and aims: There is a paucity of data on the economic burden imposed by type 2 diabetes (T2DM) in the developing world. The aim of this study was to estimate average quantities of diabetes-related resource use among T2DM patients in 24 countries in Asia, Latin America, the Middle East and Africa and to investigate predictors of resource use in each region.

Materials and methods: Cross-sectional observational data on diabetes-related resource use were collected from 15,016 patients with T2DM within the second wave of the International Diabetes Management Practices Study (IDMPS). Mean (SD) and median (min-max) annual quantities were determined using a prevalence-based approach and predictors of diabetes-related resource use investigated using negative binomial regression. Analyses were conducted using available-case/complete-case methods as well as with missing values imputed.

Results: The mean (SD) age of study patients recruited in Asia ( $n=4678$ ), Latin America $(n=6090)$ and the Middle East \& Africa $(n=4248)$ was $57(12)$, 60 (12) and 57 (11) years, respectively, and mean diabetes duration was 8 (7), 10 (9) and 8 (7) years. On average, patients made 3.4, 5.4 and 2.5 GP visits per year while the average number of specialist visits was 8.2, 6.3 and 5.0 per year in Asia, Latin America and the Middle East \& Africa, respectively. Among patients visiting a GP during the 3-month recall period, the median (min-max) number of visits was 2 (1-20), 2 (1-24) and 1 (1-12) in the respective regions and among those visiting a specialist the median (min-max) number of visits per 3 months was 2 (1-30), 2 (1-12) and 1 (1-18). The frequency of hospitalisation was $8.5 \%, 6.5 \%$ and $8.8 \%$ and the mean (SD) number of inpatient days amounted to 3.8 (18.1), $2.2(13.9)$ and 2.6 (13.5) per year in the respective regions. Results of the negative binomial regression models pointed to the major influence of diabetes-related complications on resource use. The proportion of patients with microvascular complications was $51 \%$ in Asia, $40 \%$ in Latin America and 40\% in the Middle East \& Africa and the corresponding proportions with macrovascular complications were $20 \%, 22 \%$ and $23 \%$. In Asia, the presence of macrovascular complications was associated with a 5.5 times higher expected annual rate of inpatient days (incidence rate ratio (IRR) 5.5, 95\% CI 2.8-10.9, n=3243). In the Middle East \& Africa, the expected rate of inpatient days was 7.9 times greater for patients with macrovascular complications (IRR 7.9, 95\% CI 3.9-15.8, $\mathrm{n}=2622$ ) and 2.4 times greater for patients with microvascular complications (IRR 2.4, 95\% CI 1.5-3.5, $\mathrm{n}=2630$ ). The effect of complications on resource use was equally pronounced in Latin America, where inadequate glycemic control ( $\mathrm{HbAl} 1 \mathrm{c} \geq 7 \%$ ) was identified as an additional predictor of hospitalisation.

Conclusion: The status of micro- and macrovascular complications as major predictors of resource use in T2DM demonstrates the health economic importance of early diagnosis and treatment.

Supported by: sanofi-aventis

\section{8}

Cardiovascular risk targets in type $\mathbf{2}$ diabetes should be lower for patients of South Asian ethnicity: the United Kingdom Asian Diabetes Study (UKADS)

S. Bellary ${ }^{1,2}$, N.T. Raymond ${ }^{3}$, J.P. O’Hare ${ }^{3}$, S. Mughal ${ }^{1}$, K. Johal ${ }^{3}$, S. Kumar ${ }^{3}$, A.H. Barnett ${ }^{1,2}$

${ }^{1}$ Diabetes, Heart Of England NHS Trust, Birmingham, ${ }^{2}$ Medicine, University of Birmingham, ${ }^{3}$ Diabetes, Warwick Medical School, Coventry, United Kingdom

Background and aims: Management of cardiovascular risk in patients with diabetes in primary care is determined by targets set by national and international bodies. These targets are not ethnicity specific and may underestimate risk in certain population groups. We compared cardiovascular risk factors between patients of White European (WH) vs. South Asian (SA) ethnic background. Our study hypothesis was that patients of SA ethnicity would have higher cardiovascular risk, reflected in elevated individual and combined risk factors

Materials and methods: The study was cross-sectional, with all data collected from 25 general practices in East Birmingham and Coventry during 2004-5, the baseline assessment phase of the main UKADS study. Demographic and clinical data, plus blood and urine samples were collected at diabetes research clinics, by a trained team including specialist nurses and Asian link workers. Individual risk factors and CHD risk estimated by the Framingham equation and using the UKPDS risk engine were analysed.

Results: A total of 1978 patients were recruited to the study, 1486 (75\%) SA and $492(25 \%) \mathrm{WH}$. The SA group, were younger (mean age 57.0 vs. 64.8 yrs, $\mathrm{P}<0.01$ ), had longer duration of diabetes (mean 7.8 vs. $6.3, \mathrm{P}<0.01$ ) and included more women ( $48 \%$ vs. $41 \%, \mathrm{P}=0.02$ ).

Systolic blood pressure and HDL cholesterol were significantly lower in SA vs. WH: 140 vs. $144 \mathrm{mmHg}, \mathrm{p}<0.01$ and 1.29 vs. $1.48, \mathrm{P}<0.01$ respectively. Total cholesterol and $\mathrm{HbAlc}$ were both significantly higher for SA (4.67 vs. 4.32, $\mathrm{P}<0.01$ and 8.2 vs. $7.2, \mathrm{P}<0.01$ ). Diastolic $\mathrm{BP}$ was slightly but not significantly higher for SA. $15 \%$ of both groups were current smokers, but significantly more WH were ex-smokers $38 \%$ vs. $9 \% \mathrm{SA}, \chi^{2}=244.63,2 \mathrm{df}, \mathrm{P}<0.01$. Using both the Framingham equation and UKPDS risk engine to estimate 10 year CHD risk, no significant differences between the WH and SA groups were identified; Framingham $9.7 \%$ vs. $9.9 \%$ and UKPDS $17.9 \%$ vs.17.5\% respectively.

Conclusion: Significant differences in individual risk factor profiles comparing these two ethnic groups were identified in this study. No difference in CHD risk was found using one general and one diabetes specific method. Since SA with type 2 diabetes are widely acknowledged to have higher cardiovascular event rates, it is surprising that risk factor profiles compared to $\mathrm{WH}$ are not more clearly elevated. Lower and ethnicity group specific targets should be generated to address this imbalance.

Supported by: Pfizer, sanofi-aventis, Servier Laboratories UK, Merck Sharp \& Dohme/Schering-Plough, Takeda UK, Roche, Merck Pharma, Daiichi-Sankyo UK, Boehringer Ingleheim, Eli Lilly, NovoNordisk, Bristol Myers Squibb, Solvay Health Care and Assurance Medical Society, UK.

\section{9}

The impact of ethnicity on quality of care for diabetes in primary care measured by process indicators and intermediate outcomes

A.T. Tran ${ }^{1}$, A.K. Jenum ${ }^{1}$, J.G. Cooper ${ }^{2}$, T. Claudi ${ }^{3}$, M.F. Hausken ${ }^{4}$, W. Ingskog ${ }^{1}$, J. Straand ${ }^{5}$

${ }^{1}$ The Diabetes Research Center, Aker and Ullevål University Hospital, Oslo, ${ }^{2}$ Department of Medicine, Stavanger University Hospital, Stavanger, ${ }^{3}$ Department of Medicine, Nordland Hospital, Bodø, ${ }^{4}$ National Adult Diabetes Registry, Western Norway Regional Health Authority, Stavanger, ${ }^{5}$ General Practice Research Unit, Faculty of Medicine, University of Oslo, Norway

Background and aims: Norway has had updated guidelines for the care of diabetes in primary care since 1988. Surveys in 1995 (2 regions), 2000 (3 regions) and 2005 (4 regions) have shown that the quality of care has improved, measured both by process indicators and intermediate outcomes. The aim of the present study was, for the first time in Norway, to assess the impact on ethnicity on the quality of care for patients with type 2 diabetes (T2DM) treated in primary care. A list based system for general practitioners (GPs) has since 2001 provided the denominator of their population. Only data from the population in Oslo (the capital) reflects the recent immigration from nonwestern countries, as $<2 \%$ were immigrants in the other regions in 2005 .

Materials and methods: We used data from a retrospective cross-sectional study of electronic medical records of 58000 patients from 49 GPs in Oslo in 2005. 2064 patients with diagnosed diabetes were identified and the relevant data extracted by a computer program and manually validated by a research nurse. Ethnicity was self-reported from the medical records according to country of birth and categorized as follows: Westerners, South Asians, Middle East/North Africa (MENA), East Asians, South Sahara Africa (SSA), Eastern Europe (EE), Central and South America (CSA). All non-western (NW) groups were also merged. We excluded 128 patients with $<6$ months of follow-up, 264 with $\geq 2$ visits to specialists in diabetes care, 2 with ethnicity status missing and 17 with T1DM.

Results: Of the 1653 T2DM study patients 524 (32\%) were NW. Table 1 shows mean age for the ethnic groups, processes of care (\% with recommended procedures recorded) and mean values for major risk factors. Mean age was substantially lower for NW (53.5 vs. 66.4 years), but mean diabetes duration was identical (6.6 years for both groups). Mean $\mathrm{HbAlc}$ was significantly higher for 
Table 1. Basic characteristics, processes of care and risk factor levels by ethnicity

\begin{tabular}{|c|c|c|c|c|c|c|c|c|}
\hline & Westerners & South Asians & MENA & East Asians & SSA & EE & CSA & All NW \\
\hline $\mathrm{N}$ & 1129 & 322 & 81 & 54 & 30 & 29 & 8 & 524 \\
\hline$\overline{\text { Age (mean) }}$ & 66.4 & 52.3 & 53.1 & 57.7 & 50.3 & 63.5 & 55.1 & 53.5 \\
\hline SD & 12.7 & 9.5 & 9.9 & 10.5 & 8.2 & 13.5 & 5.5 & 10.3 \\
\hline $\operatorname{Men}(\%)$ & 50.0 & 49.7 & 51.9 & 38.9 & 73.3 & 48.3 & 62.5 & 50.4 \\
\hline HbAlc & 95.2 & 93.5 & 91.4 & 96.3 & 96.7 & 89.7 & 100 & 93.5 \\
\hline$\%$ measured & 7.0 & 7.7 & 7.2 & 7.2 & 7.5 & 7.6 & 6.8 & 7.5 \\
\hline $\operatorname{Mean}(\%)$ & 1.1 & 1.5 & 1.5 & 1.1 & 1.2 & 1.2 & 1.5 & 1.4 \\
\hline \multicolumn{9}{|l|}{$\mathrm{SD}$} \\
\hline Systolic BP & 92.5 & 85.7 & 87.7 & 85.2 & 86.7 & 96.6 & 87.5 & 86.6 \\
\hline$\%$ measured & 138.9 & 126.6 & 128.4 & 132.6 & 129.8 & 134.0 & 124.1 & 128.1 \\
\hline $\operatorname{Mean}(\mathrm{mmHg})$ & 17.0 & 16.6 & 18.2 & 19.2 & 17.7 & 21.0 & 13.7 & 17.5 \\
\hline \multicolumn{9}{|l|}{$\underline{\mathrm{SD}}$} \\
\hline Cholesterol & 92.3 & 96.9 & 95.1 & 94.4 & 100 & 93.1 & 100 & 96.4 \\
\hline$\%$ measured & 5.1 & 4.9 & 5.2 & 5.4 & 5.2 & 5.0 & 5.2 & 5.0 \\
\hline $\operatorname{Mean}(\mathrm{mmol} / \mathrm{l})$ & 1.1 & 1.0 & 0.9 & 1.0 & 1.1 & 0.9 & 0.7 & 1.0 \\
\hline \multicolumn{9}{|l|}{$\mathrm{SD}$} \\
\hline Ref.ophthalm.\% & 60.4 & 63.4 & 49.4 & 59.3 & 56.7 & 62.1 & 50.0 & 60.1 \\
\hline $\begin{array}{l}\text { U-microalb. } \\
\text { \%measured }\end{array}$ & 46.8 & 46.9 & 43.2 & 50.0 & 30.0 & 37.9 & 37.5 & 45.0 \\
\hline
\end{tabular}

NW (7.5 vs. 7.0\%, $\mathrm{p}<0.001$ ), and highest for South Asians (7.7\%), but blood pressure significantly lower for NW. Only minor ethnic differences were found for process indicators. NW were treated more intensely with glucose-lowering drugs ( 18 vs. $28 \%$ were non-users), but in all treatment groups they had significantly higher HbAlc (7.4 vs. $7.1 \%$ for oral drugs only, 8.4 vs. $7.9 \%$ for oral drugs and insulin combined, and 8.6 vs. $7.7 \%$ for insulin alone).

Conclusion: NW immigrants were younger than westerners, but mean diabetes duration was identical. When measured by process indicators, only minor ethnic differences in the quality of care for T2DM patients were found. However, metabolic control was poorer in NW, despite more intensive treatment with glucose-lowering drugs.

Supported by: Norwegian Medical Association

\section{0}

Does screening work for the common good? ADDITION- Leicester: design of a multi-factorial intervention trial in an ethnically diverse population with screen-detected type 2 diabetes

M.J. Davies ${ }^{1}$, D.R. Webb ${ }^{1}$, B.T. Srinivasan ${ }^{1}$, S. Hiles ${ }^{2}$, J. Hill' ${ }^{2}$, S. Campbell2 N.A. Taub $^{3}$, A. Farooqi ${ }^{4}$, N. Wareham ${ }^{5}$, S. Griffin ${ }^{5}$, K. Khunti ${ }^{3}$

${ }^{1}$ Department of Cardiovascular Sciences, University of Leicester,

${ }^{2}$ Department of Diabetes and Endocrinology, University Hospitals of

Leicester NHS Trust, ${ }^{3}$ Department of Health Sciences, University of

Leicester, ${ }^{4}$ East Leicester Medical Practice, Eastern Leicester Primary Care

Trust, ${ }^{5} \mathrm{MRC}$ Epidemiology Unit, University of Cambridge, United Kingdom

Background and aims: Incentive-driven opportunistic screening for type 2 Diabetes Mellitus (T2DM) is now common UK clinical practice despite lack of proven benefits. Doubts surrounding screening methodology, cost-effectiveness and cardio-protective efficacy remain. UK National Screening Committee guidelines advocate screening targeted to populations at risk. ADDITION-Leicester will evaluate the feasibility of screening a mixed ethnic UK population, quantify effectiveness of optimised treatments on modelled cardio-vascular risk and assess economic and psychological costs of screening. Methods: The study is a pragmatic cluster RCT of screening and intervention in 20 general practices (total practice population 65,792). An Intensive group (I) will receive multi-factorial treatment (structured education, aspirin, protocol-driven tight control of blood pressure, glucose and lipids) from a hospital-based diabetes team, whilst a conventional group (R) will receive routine care according to national guidelines. People aged 40-75yrs (25-75 South Asian) will be offered fasting and 2-hr post-challenge glucose assessments, anthropometric measurements, Quality of Life / well-being questionnaires and other cardiovascular parameters (BP, \%Body Fat, serum lipids, urinanalysis, ECG, smoking status, alcohol consumption, medical history). Diabetes will be diagnosed using WHO criteria. We will require 308 participants (154 per arm) with screen-detected diabetes to detect a 6\% difference $(80 \%$ power, alpha 0.05 ) in modelled cardiovascular outcome (UKPDS CHD risk) between routine and intensive groups at one-year assuming an intra cluster correlation coefficient of 0.14 and allowing for a loss to follow-up of $15 \%$. Assuming a prevalence of screen-detected diabetes of $4.5 \%$ in our mixed ethnic population, we will need to screen over 7000 (30\%- 2100 South Asian) individuals over 2 years.

Preliminary Results: Optimisation of intensive multi-factorial treatment is associated with clinically important improvements in intermediate outcomes at 12 months (Tabulated). Whether such a program will be a cost-effective means of reducing CVD events over five years is being addressed in the

12 month data $(\mathrm{n}=150)$

\begin{tabular}{|c|c|c|c|c|c|c|c|}
\hline & $\begin{array}{l}\text { Mean } \\
(\mathrm{sd}) \text { baseline }(\mathrm{R})\end{array}$ & $\begin{array}{l}\text { Mean } \\
\text { (sd) follow up (R) }\end{array}$ & $\begin{array}{l}\text { Mean } \\
\text { (sd) baseline (I) }\end{array}$ & $\begin{array}{l}\text { Mean } \\
\text { (sd) follow up (I) }\end{array}$ & $\Delta(\mathrm{IvsR})$ & $95 \% \mathrm{CI}$ & $\mathrm{P}$ \\
\hline HbAlc (\%) & $\begin{array}{l}7.15 \\
(1.6)\end{array}$ & $\begin{array}{l}6.71 \\
(1.0)\end{array}$ & $\begin{array}{l}7.1 \\
(1.42)\end{array}$ & $\begin{array}{l}6.35 \\
(0.46)\end{array}$ & -0.32 & $\begin{array}{l}-0.72 \\
\text { to } \\
+0.11\end{array}$ & 0.14 \\
\hline $\begin{array}{l}\text { Systolic BP } \\
\text { (mm Hg) }\end{array}$ & $\begin{array}{l}147.6 \\
(20.1)\end{array}$ & $\begin{array}{l}140.6 \\
(17.7)\end{array}$ & $\begin{array}{l}148.8 \\
(19.1)\end{array}$ & $\begin{array}{l}129.1 \\
(14.6)\end{array}$ & -12.7 & $\begin{array}{l}-18.9 \\
\text { to } \\
-6.45\end{array}$ & 0.0001 \\
\hline $\begin{array}{l}\text { Total Chol } \\
(\mathrm{mmol} / \mathrm{L})\end{array}$ & $\begin{array}{l}5.7 \\
(1.3)\end{array}$ & $\begin{array}{l}4.8 \\
(1.1)\end{array}$ & $\begin{array}{l}5.2 \\
(1.2)\end{array}$ & $\begin{array}{l}3.8 \\
(0.9)\end{array}$ & -0.52 & $\begin{array}{l}-0.92 \\
\text { to } \\
-0.12\end{array}$ & 0.01 \\
\hline $\begin{array}{l}\text { HDL Chol } \\
(\mathrm{mmol} / \mathrm{L})\end{array}$ & $\begin{array}{l}1.2 \\
(0.5)\end{array}$ & $\begin{array}{l}1.3 \\
(0.4)\end{array}$ & $\begin{array}{l}1.3 \\
(0.5)\end{array}$ & $\begin{array}{l}1.2 \\
(0.3)\end{array}$ & -0.09 & $\begin{array}{l}-0.26 \\
\text { to } \\
+0.08\end{array}$ & 0.3 \\
\hline $\begin{array}{l}\text { UKPDS CHD } \\
\text { risk }(\%)\end{array}$ & $\begin{array}{l}20.9 \\
(16.3)\end{array}$ & $\begin{array}{l}16.0 \\
(12.4)\end{array}$ & $\begin{array}{l}20.9 \\
(14.4\end{array}$ & $\begin{array}{l}11.3 \\
(6.1)\end{array}$ & -4.69 & $\begin{array}{l}-7.9 \\
\text { to } \\
-1.4\end{array}$ & 0.005 \\
\hline
\end{tabular}


multi-centre ADDITION-Europe study. The results of this study will inform approaches to screening and chronic disease management in primary care, uniquely describing the needs of a significant UK ethnic minority at risk of premature vascular morbidity.

\section{1}

Factors associated with receipt of appropriate care among patients with a prolonged and persistent period of hyperglycaemia: what matters when action is needed?

J.E. Lafata ${ }^{1}$, E.A. Dobie ${ }^{1}$, G. Divine ${ }^{2}$, M. Ulcickas Yood ${ }^{3}$, B.D. McCarthy ${ }^{4}$ ${ }^{1}$ Center for Health Services Research, Henry Ford Health System, Detroit, ${ }^{2}$ Biostatistics and Research Epidemiology, Henry Ford Health System, Detroit, ${ }^{3}$ Medicine, Yale University, New Haven, ${ }^{4}$ Medicine, Allina Medical Clinic, Minneapolis, United States

Background and aims: To estimate the prevalence of prolonged and persistent periods of hyperglycemia among patients with diabetes and the factors associated with receipt of appropriate care once Alcs are substantially and consistently above target levels.

Materials and methods: Using claims data, we identified insured patients with diabetes who initiated oral hyperglycemic monotherapy between 20002005 ( $\mathrm{N}=5082)$. Actuarial methods were used to estimate time to a period of prolonged and persistent hyperglycemia ( $2 \mathrm{Alc}$ results over $8 \%$ at least 90 days apart, not yet dispensed insulin with no medication change in the preceding 90 days). Among those experiencing prolonged hyperglycemia $(\mathrm{N}=1391)$ we estimate time to and factors associated with appropriate care defined as either a medication change (dose change, class change, or class addition) or achieving glycemic goal of $\mathrm{A} 1 \mathrm{c}<=7 \%$.

Results: At inception, mean age was 60.1 years, $48 \%$ were female, $37 \%$ African American, and mean A1c was 8.6\%. Eight percent of patients faced prolonged hyperglycemia within 1 year, and this proportion steady rises to $41 \%$ within 5 years. Among those with prolonged hyperglycemia, 35\% had been dispensed combination therapy, and $20 \%$ had no hyperglycemic medication on hand in the prior 90 days. Mean A1c was 9.7\% (range 8.1 -18.9). Mean time to appropriate care was 7.3 months, with $27 \%$ not receiving appropriate care within 1 year. Those with no medication on hand (hazard ratio $=0.67$, $95 \% \mathrm{CI}=0.55-0.82)$, or on combination therapy $(0.81,0.71-0.93)$ faced longer delays. Longer delays were also associated with increasing drug co-payments (0.96, 0.93-0.98), while shorter delays were associated with increasing income levels (1.04, 1.01-1.07), and higher A1c levels > 9\% (1.41, 1.08-1.83). Recent visits to primary care $(4.22,3.65-4.89)$ or endocrinology $(3.66,2.10-6.38)$ were associated with shorter delays.

Conclusion: Patients facing prolonged and persistent periods of hyperglycemia are at risk of further delays in appropriate management. Our results illustrate that when patients with diabetes face prolonged and persistent hyperglycemia, the likelihood of receiving appropriate care is related to a combination of patient, physician, and system factors many of which are amenable to intervention.

Supported by: sanofi-aventis

\section{2}

Association of waist circumference, cardiometabolic risk factors and health care utilization in a German internet-based cohort study

N. Cadi Soussi ${ }^{1}$, N. Finer ${ }^{2}$, H. Hauner ${ }^{3}$

${ }^{1}$ sanofi-aventis R\&D, Paris, France, ${ }^{2}$ Wellcome Clinical Research Facility, University of Cambridge, United Kingdom, ${ }^{3}$ Klinik für Ernährungsmedizin Klinikum rechts der Isar, Technical University of Munich, Germany

Background and aims: The association of obesity measured by Body Mass Index (BMI), and health care costs (HCC), is well established. Although abdominal obesity (AO) measured by waist circumference (WC) is an independent predictor for diabetes and cardio-vascular diseases, few real-life studies have evaluated its association with HCC. The association of WC, BMI and medical resource utilization of a large cohort of German adults were explored.

Materials and methods: PROCEED (Prospective Obesity Cohort of Economic Evaluation and Determinants) is an international longitudinal, observational cohort study of overweight/obese and control normal weight subjects recruited from an internet panel, stratified by BMI and gender. Past and current health conditions were collected through questions about several health disorders, symptoms, prescription drugs as well as resource utilization. Anthropometric data were validated in a 5\% random sample of subjects during in-home visits. The prevalences of cardiometabolic risk factors, the indicators of inpatient and outpatient health care utilization (at least one event) and the use of any prescription medications (at least one) in the past 3 months for subjects above and below the gender specific median WC for each BMI class were compared with Fisher's and Mann-Whitney-Wilcoxon tests. All the tests were two-sided with a global type I error a, equal to $5 \%$.

Results: 5,406 subjects (99\% Caucasian) were recruited in Germany. In the normal weight, overweight and obese categories respectively: mean age was $43( \pm 10), 45( \pm 10)$ and $46( \pm 9)$ years and gender specific median WC 88/79, $99 / 90$ and $111 / 101 \mathrm{~cm}$ respectively (male/female). In the diabetic population, the prevalences of hypertension and dyslipidemia (defined by low HDL and/or high TG) were $27 / 33 \%, 53 / 60 \%, 60 / 65 \%$ and $0 / 50 \%, 35 / 39 \%, 53 / 38 \%$ respectively (above/below the gender specific median WC) in the normal, overweight and obese categories. In the non-diabetic population, the prevalences of hypertension and dyslipidemia were $15 / 21 \%, 21 / 28 \%, 30 / 42 \%$ and $3 / 12 \%, 9 / 12 \%, 15 / 17 \%$ respectively. The table shows the health care utiliza-

\begin{tabular}{|c|c|c|c|c|c|c|c|}
\hline & & Cor & & Over & eight & & \\
\hline & & Blwl & -24 & EMII 2 & $5-29.9$ & & -30 \\
\hline & & $\begin{array}{c}\text { below } \\
\text { median WC }\end{array}$ & $\begin{array}{c}\text { above } \\
\text { median Wh }\end{array}$ & $\begin{array}{c}\text { below } \\
\text { median WC }\end{array}$ & $\begin{array}{l}\text { above } \\
\text { median WC }\end{array}$ & $\begin{array}{l}\text { below } \\
\text { median'wic }\end{array}$ & $\begin{array}{c}\text { above } \\
\text { median we }\end{array}$ \\
\hline & & $n(\%)$ & $n(\%)$ & $\mathrm{n}(\%)$ & $n(\%)$ & $n(\%)$ & $n(\%)$ \\
\hline & $\begin{array}{l}\text { Inpatient visits } \\
\text { (Hospital or ER) }\end{array}$ & $6(5.8 \%)$ & $8(82 \%)$ & $1396.7 \%) \mu$ & $190(9.9 \%)$ & $57(92 \pi)$ & $69(11.9 \%)$ \\
\hline Overall & $\begin{array}{l}\text { Outpatient consultations, } \\
\text { surgerie } s, \ldots\end{array}$ & $75(72.1 \%)$ & $74(05.5 \%)$ & $1559(4.6 \%) \mu$ & $1499(08.3 *)$ & $461 \eta(2 *) £$ & $476(62.1 \%)$ \\
\hline & $\begin{array}{l}\text { Use of } \geqq 1 \text { prescription } \\
\text { m edication }\end{array}$ & $49(47.15)$ & $47(18 *)$ & $1111 \xi 325) \mu$ & $1191(62.2 \%)$ & $376(60.5 \%) f$ & $421(02.5 \%)$ \\
\hline & $\begin{array}{l}\text { N } \\
\text { Inpatient visits } \\
\text { (Hospital or ER) }\end{array}$ & $\begin{array}{c}104 \\
19.1 \%)\end{array}$ & $\begin{array}{c}98 \\
0(0 \%)\end{array}$ & $\begin{array}{c}2089 \\
24(14.4 \%)\end{array}$ & $\begin{array}{c}1914 \\
36(146)\end{array}$ & $\begin{array}{c}621 \\
11(14.3 \%)\end{array}$ & $\begin{array}{c}580 \\
13(92 \%)\end{array}$ \\
\hline Diabetic & $\begin{array}{l}\text { Outpatient consultations, } \\
\text { surgerie } s, \ldots\end{array}$ & $11(100 \%)$ & $5(100 \%)$ & $152(91 \%)$ & $237(91.9 *)$ & $69(69.5 \%)$ & $13494.4 \%)$ \\
\hline sopulation & $\begin{array}{l}\text { Use of } z 1 \text { prescription } \\
\text { medication }\end{array}$ & $8(02.7 \%)$ & $5(83.3 \%)$ & $122(03.1 \%) \mu$ & $223(66.4 \%)$ & $67(87 \%)$ & $127(69.4 \%)$ \\
\hline & $\begin{array}{l}\text { w } \\
\text { Inpatient visits } \\
\text { (Hospital or ER) }\end{array}$ & $\begin{array}{c}11 \\
5(5.4 \%)\end{array}$ & $\begin{array}{c}6 \\
8(6.7 \%)\end{array}$ & $\begin{array}{c}167 \\
115 \text { (5\%) म }\end{array}$ & $\begin{array}{c}258 \\
154(9.3 \%)\end{array}$ & $\begin{array}{c}77 \\
458.5 \%) f\end{array}$ & $\begin{array}{c}142 \\
56(12.8 \times)\end{array}$ \\
\hline $\begin{array}{c}\text { Non } \\
\text { Diabetic }\end{array}$ & $\begin{array}{l}\text { Outpatient consultations, } \\
\text { surgerie } s, \ldots\end{array}$ & $64(688 \%)$ & $68(3.9 \%)$ & $1407(32 \%) \mu$ & $1252(06.2 \%)$ & $392(22.1 \%) f$ & $342(08.1 \%)$ \\
\hline sopulation & $\begin{array}{l}\text { Use of } \geq 1 \text { prescription } \\
\text { medication }\end{array}$ & $41(4 t .1 \%)$ & $42(45.7 \%)$ & $969(51.5 \%) \mu$ & $968(5854)$ & $309(568 \%) f$ & $294(67.1 \%)$ \\
\hline & $\mathrm{N}$ & 93 & 92 & 1922 & 1656 & 544 & 430 \\
\hline
\end{tabular}

$\$ p<0.05$ for WC median groups comparison in Control population $4 \mathrm{p} \leq 0.05$ for 'WC median groups comparison in Overweight population $f \mathrm{p} \leq 0.05$ for $\mathrm{WC}$ median groups comparison in obese population 
tion outcomes in the diabetic, non-diabetic and overall populations by BMI and WC categories.

Conclusion: The increased of medical resource utilization observed with increasing BMI is consistent with the literature. In addition, within each BMI group, a higher waist circumference was associated with increased health care resource utilization. This trend was still observed after stratification for diabetes. Thus, it seems relevant to account for both BMI and $\mathrm{AO}$ when evaluating the burden of cardiometabolic risk factors.

Supported by: sanofi-aventis

\section{3}

Reasons for diabetes clinical inertia in primary care practice P.J. O’Connor ${ }^{1}$, J.M. Sperl-Hillen ${ }^{1}$, W.A. Rush ${ }^{1}$, P.E. Johnson ${ }^{2}$, G. Biltz', H.L. Ekstrom ${ }^{1}$

${ }^{1}$ Research Foundation, HealthPartners Research Foundation, ${ }^{2}$ Carlson School of Management, University of Minnesota, Minneapolis, United States

Background and aims: Previous studies suggest clinical inertia, defined as lack of treatment intensification in a patient not at goal, is largely responsible for the slow improvement seen in population measures of diabetes care. It is controversial how much of the inertia problem is attributable to providers, care systems, or patient preference.

Materials and methods: An electronic visit-based decision support tool called Diabetes Wizard was successfully implemented in 6 HealthPartners Clinics from October 2006 through May 2007 as part of the NIDDK funded study entitled "Reducing Clinical Inertia in Diabetes Care (DK068314). The Wizard gave relevant clinical information and specific recommendations for treatment intensification for glycemia, BP, and lipids. In addition, 19 consented physicians in the clinics agreed to complete an electronic visit action resolution (VAR) form at each encounter if the patient was not at goal for glycemia, blood pressure, or lipids and treatment was not intensified. The VAR was completed by checking from a list of possible reasons or through free text.

Results: Out of 1639 encounters with patients not achieving A1C less than 7 percent, the WIZARD was used and VAR completed 831 times (51 percent). Inertia occurred in 258 (43 percent) of the VAR completed visits. Reasons that were specified by physicians for glycemia inertia were: (1) Choice of patient not to increase or add medications, 70 (19.6 percent), (2) Need for an updated A1C, 62 (17.3 percent), (3) Followed by endocrinology or diabetes nurse, 53 (14.8 percent), (4) Addressed dietary/lifestyle changes instead, 45 (12.6 percent), (5) Other problem/acute illness addressed, 17 (4.7 percent), (6) Not my patient, 13 (3.6 percent), (7) Addressed adherence problem, 13 (3.6 percent). Other reasons that were sited less frequentlly (less than 2.5 percent) were medication regimen already too complex, hypoglycemia concerns, drug intolerance, cost concerns, advanced age or severe co-morbidities, referred to endocrinology or diabetes educator, close to goal, and other miscellaneous reasons.

Conclusion: Only a relatively small amount, less than 20 percent, of total glycemia inertia at diabetes patient encounters is attributable to patient refusal or preference. The remaining reasons could potentially be addressed through care system changes, including point of care testing to assure up-to-date A1C test results are available at visits, provider training, and better coordination of care with the extended care team and primary provider colleagues.

Supported by: National Institute of Diabetes and Digestive and Kidney Diseases, National Institute of Health, Department of Health and Social Services

\section{4}

Diabetes increases recurrences of acute urinary tract infections but does not lead to a different prescription pattern in primary care

K.J. Gorter ${ }^{1}$, D. Van Oostveen ${ }^{1}$, E. Hak ${ }^{1}$, N.P.A. Zuithoff ${ }^{1}$,

A.I.M. Hoepelman ${ }^{2}$, G.E.H. Rutten ${ }^{1}$

${ }^{1}$ Julius Center for Health Sciences and Primary Care, ${ }^{2}$ Division of Internal

Medicine and Infectious Diseases, Utrecht, Netherlands

Background and aims: Women with diabetes have an increased risk of urinary tract infections, especially recurrences. The relation between characteristics of diabetes and the risk of recurrent UTIs is unclear. Most diabetes guidelines do not include a treatment advice in case of UTIs and most guidelines on UTIs do not offer a targeted approach for women with diabetes
Aim: To assess diabetes related risk of recurrent urinary tract infections (UTIs) in adult women with diabetes (DM) in primary care and to describe the pattern of prescribed antibiotics in case of UTIs in women. Retrospective cohort study

Materials and methods: Women of $\geq 30$ years in two primary health care centres $(n=7,063)$ in a large city in The Netherlands during a period of 43 months were the source population.

From these women with DM $(\mathrm{n}=340)$ and without DM $(\mathrm{n}=6,618)$ with uncomplicated UTI were studied. Main outcome measures were independent risk of diabetes and related characteristics for UTI recurrences (i.e. relapses and re-infections combined) [OR; 95\% CI] and the DM associated pattern of antibiotic prescriptions for first episodes and recurrences of UTI.

Results: In women with diabetes relapses and re-infections were reported in $7.1 \%$ and $15.9 \%$ versus $2.0 \%$ and $4.2 \%$ in women without diabetes. Multivariable logistic regression analysis showed a higher risk of a recurrent UTI in women with DM than in those without DM (OR 2.0; 95\% CI 1.4-2.9). Especially women who used oral hypoglycaemic medication (2.1; 1.2-3.5), insulin $(3.0 ; 1.7-5.1)$, who had $>5$ years diabetes $(2.9 ; 1.9-4.4)$ and who had retinopathy (4.1;1.9-9.1) were at risk. A different pattern of antibiotics was prescribed for recurrent versus first UTI episodes in women both with and without diabetes. However, diabetes did not influence this association.

Conclusion: Women with diabetes have an elevated risk of UTI recurrences, especially if their diabetes exists $>5$ years, is treated with tablets or insulin or complicated by retinopathy. Diabetes does not lead to a difference in the pattern of antibiotic prescriptions. Research focusing on antibiotic treatment of UTI's is needed in women with DM. 


\section{PS 099 Monitoring type 2 diabetes}

\section{5}

Which blood glucose samples of the day predicts $\mathrm{HbA}_{1 \mathrm{c}}$. Results from the ADAG (HbA Derived Average Glucose) study

R. Borg ${ }^{1}$, J.C. Kuenen ${ }^{2}$, B. Carstensen ${ }^{1}$, J. Nerup ${ }^{1}$, H. Zheng ${ }^{3}$, D. Schoenfeld ${ }^{3}$, R.J. Heine ${ }^{2}$, D.M. Nathan ${ }^{3}$

${ }^{1}$ Steno Diabetes Center, Gentofte, Denmark, ${ }^{2}$ Dept of Endocrinology,

VU Medical Center, Amsterdam, Netherlands, ${ }^{3}$ Harvard Medical School,

Massachusetts General Hospital, Boston, United States

Background and aims: Intensive self-monitoring of blood glucose (SMBG) is necessary in insulin-treated diabetic patients. The benefit of regular use of SMBG in non-insulin-using type 2 diabetes (T2DM) is less clear. Earlier studies have tried to establish which measurements of the day have greatest impact on mean blood glucose levels (MBG). The ADAG study demonstrated that MBG derived from seven-point profiles predicts $\mathrm{HbA}_{1 c}$ with a precision of $\pm 1.65 \%$. There was no difference in this relationship for the two types of diabetes. The aim of this analysis is to examine which SMBG value(s) of the day provides the best prediction of $\mathrm{MBG}$ as measured by $\mathrm{HbA}_{1 c}$.

Materials and methods: The ADAG study established the relationship between $\mathrm{MBG}$ and $\mathrm{HbA}$ levels by comparing the average glucose calculated from a combination of continuous glucose monitoring and frequent SMBG profiles during a 3-month period with the $\mathrm{HbA}_{1 \mathrm{c}}$ value at the end of the 3months. From this intensive blood glucose monitoring, we examine the laboratory-quality Hemocue SMBG measurements performed for 8-10 days during the 12 week study period for each of 441 subjects. Multiple linear regression models were constructed using measurements at different times of the day as separate explanatory variables. Models were fitted separately for the T1DM ( $\mathrm{n}=273)$ and T2DM ( $\mathrm{n}=168)$ diabetic subjects.

Results: SMBG at particular times of the day were stronger predictors of $\mathrm{HbA}_{1 \mathrm{c}}$ than others, and this differed between T1DM and T2DM. In general, the combined pre-meal values predict $\mathrm{HbA}_{1 \mathrm{c}}$ better than combined post-meal values for both diabetes types and, in particular, for T2DM.

Conclusion: Pre-prandial glucose monitoring is generally more closely associated with $\mathrm{HbA}_{1 \mathrm{c}}$ than post-pandial glucose levels. For T1DM, pre-breakfast, pre-lunch, and post-dinner values predict $\mathrm{HbA}_{1 \mathrm{c}}$ the best, and for T2DM, the combination of pre-breakfast and pre-dinner values provides the best prediction. These findings provide insight for the patient and clinician who seek the optimal blood glucose monitoring at minimal expense and discomfort.
Table 1. Parameter estimates (in \% HbAlc per mmol/l) from the statistical model with all glucose variables. The combined mean pre- and post-meal values were evaluated in a separate model.

\begin{tabular}{|c|c|c|c|c|}
\hline & \multicolumn{2}{|l|}{ T1DM } & \multicolumn{2}{|l|}{ T2DM } \\
\hline & Estimate $^{\star}$ (SE) & P-value & Estimate $^{\star}(\mathrm{SE})$ & $\mathrm{P}$-value \\
\hline Pre-breakfast & $0.1010(.0212)$ & $<0.0001$ & $0.1550(.0302)$ & $<0.0001$ \\
\hline Post-breakfast & $0.0059(.0194)$ & 0.759 & $0.0560(.0236)$ & 0.019 \\
\hline Pre-lunch & $0.1327(.0229)$ & $<0.0001$ & $0.0115(.0319)$ & 0.719 \\
\hline Post-lunch & $0.1145(.0230)$ & $<0.0001$ & $0.1064(.0329)$ & 0.002 \\
\hline Pre-dinner & $0.0488(.0221)$ & 0.0291 & $0.1615(.0313)$ & $<0.0001$ \\
\hline Post-dinner & $0.0792(.0214)$ & 0.0003 & $0.0106(.0317)$ & 0.738 \\
\hline Residual SD & $0.68 \% \mathrm{HbA}_{\mathrm{dc}}$ & & $0.56 \% \mathrm{HbA}_{\mathrm{lc}}$ & \\
\hline Pre-meal mean & $0.2729(.0310)$ & $<0.0001$ & $0.3283(.0387)$ & $<0.0001$ \\
\hline Post-meal mean & $0.1966(.0288)$ & $<0.0001$ & $0.1541(.0338)$ & $<0.0001$ \\
\hline Residual SD & $0.70 \% \mathrm{Hb} A_{c}$ & & $0.58 \% \mathrm{HbA}_{\mathrm{lc}}$ & \\
\hline
\end{tabular}

*\% per mmol/l. For T1DM, step-wise addition of the individual timed samples revealed that the prediction obtained by pre-breakfast, prelunch, and post-dinner values (residual $\mathrm{SD}=0.72 \%$ ) was not substantially improved by adding further variables. For T2DM, the combination of prebreakfast and pre-dinner values (residual $\mathrm{SD}=0.61 \%$ ) provide an adequate prediction, with little improvement by adding further glucose measures.

Supported by: ADA, EFSD/LifeScan grant, Abbott Diabetes Care, Merck \& Company, Bayer Healthcare, LifeScan, GlaxoSmithKline, Medtronic Minimed, sanofi-aventis Netherlands, Hemocue

\section{6}

Computer-guided versus standard column-based intravenous insulin regimens: a comparison of insulin infusion protocols in the medical intensive care unit

C.A. Newton ${ }^{1}$, D. Smiley ${ }^{1}$, P.C. Davidson ${ }^{2}$, B.W. Bode ${ }^{2}$, R.D. Steed ${ }^{2}$,

S. Jacobs ${ }^{1}$, A.E. Kitabchi ${ }^{3}$, F. Stentz ${ }^{3}$, P. Mulligan ${ }^{1}$, A. Cleveland ${ }^{2}$,

G.E. Umpierrez ${ }^{1}$

${ }^{1}$ Division of Endocrinology, Emory University School of Medicine, Atlanta, ${ }^{2}$ Endocrinology, Atlanta Diabetes Associates, ${ }^{3}$ Division of Endocrinology, University of Tennessee Health Science Center, Memphis, United States

A variety of continuous insulin infusion (CII) algorithms are available to improve and maintain strict glycemic control in the intensive care unit (ICU). To date, few have been prospectively evaluated against each other to determine if any differences exist. We conducted a multi-center, randomized trial (NCT\#00394524) comparing the safety and efficacy of a computer-guided (Glucommander) and a column-based paper protocol (Standard). 138 medical ICU patients with a mean age of 58.1 (range 19-80) years and a blood glucose $(\mathrm{BG})>7.8 \mathrm{mmol} / \mathrm{L}$ were enrolled at 4 hospital centers. Patients were excluded if they were admitted for diabetic ketoacidosis or hyperglycemic hyperosmolar state or if they were anticipated to need surgery. Both protocols

Patient characteristics and glycemic control achieved and maintained with the CII protocols.

\begin{tabular}{|c|c|c|c|c|}
\hline & & $\begin{array}{l}\text { Glucommander } \\
(\# \text { patients }=69) \\
(\text { Total \# BG tests }=2848)\end{array}$ & $\begin{array}{l}\text { Standard } \\
(\# \text { patients }=69) \\
(\text { Total \# BG tests }=2578)\end{array}$ & p-value \\
\hline \multicolumn{2}{|l|}{ Initial glucose $(\mathrm{mmol} / \mathrm{L})$} & $11.2 \pm 3.5$ & $10.3 \pm 2.3$ & NS \\
\hline \multicolumn{2}{|c|}{ Time to achieve target BG of $80-120 \mathrm{mg} / \mathrm{dL}$ (hours) } & $5.0 \pm 2.9$ & $8.0 \pm 9.4$ & $<0.01$ \\
\hline \multicolumn{2}{|c|}{ Mean BG maintained once target achieved $(\mathrm{mmol} / \mathrm{L})$} & $5.7 \pm 0.5$ & $6.7 \pm 0.9$ & $<0.0001$ \\
\hline \multicolumn{2}{|l|}{ Insulin infusion rate (units/Kg/day) } & $0.67 \pm 0.2$ & $0.48 \pm 0.16$ & 0.03 \\
\hline \multicolumn{2}{|l|}{ Median (range) duration of CII (days) } & $2.0(0.4-10)$ & $2.7(0.1-10)$ & NS \\
\hline \multicolumn{2}{|l|}{ Hemoglobin Alc (\%) } & $7.1 \pm 2.0$ & $6.8 \pm 1.3$ & NS \\
\hline \multicolumn{2}{|l|}{ Mortality } & $24.1 \%$ & $20.3 \%$ & NS \\
\hline \multicolumn{2}{|l|}{ \# BG tests $(\%)$ within target range } & $1943(68.2 \%)$ & $1223(47.4 \%)$ & $<0.0001$ \\
\hline Mild Hypoglycemia: $\mathrm{BG}<3.3 \mathrm{mmol} / \mathrm{L}$ & $\begin{array}{l}\text { \# BG tests (\%) } \\
\text { \# Patients (\%) }\end{array}$ & $\begin{array}{l}78(2.5 \%) \\
29(42.0 \%)\end{array}$ & $\begin{array}{l}36(1.2 \%) \\
21(30.4 \%)\end{array}$ & $\begin{array}{l}0.0002 \\
\text { NS }\end{array}$ \\
\hline Severe Hypoglycemia: $\mathrm{BG}<2.2 \mathrm{mmol} / \mathrm{L}$ & $\begin{array}{l}\text { \# BG tests (\%) } \\
\text { \# Patients (\%) }\end{array}$ & $\begin{array}{l}5(0.16 \%) \\
2(2.9 \%) \\
\end{array}$ & $\begin{array}{l}2(0.07 \%) \\
2(2.9 \%) \\
\end{array}$ & $\begin{array}{l}\text { NS } \\
\text { NS } \\
\end{array}$ \\
\hline Hyperglycemia (BG >11.1 mmol/L) & $\begin{array}{l}\text { \# BG tests (\%) } \\
\text { \# Patients (\%) }\end{array}$ & $\begin{array}{l}20(0.7 \%) \\
7(10.1 \%) \\
\end{array}$ & $\begin{array}{l}57(2.2 \%) \\
17(24.6 \%)\end{array}$ & $\begin{array}{l}<0.0001 \\
0.02\end{array}$ \\
\hline
\end{tabular}


used glulisine (Apidra) insulin and targeted BG to $4.4-6.7 \mathrm{mmol} / \mathrm{L}$ for up to 10 days. Assessments were made for differences in mean daily BG, number of hypoglycemic events ( $\mathrm{BG}<3.3 \mathrm{mmol} / \mathrm{L}$ and $<2.2 \mathrm{mmol} / \mathrm{L}$ ), number of hyperglycemic episodes (BG $>11.1 \mathrm{mmol} / \mathrm{L}$ ), and insulin infusion rates. Gender, ethnicity, diabetes history and admitting diagnoses were similar for the Glucommander and Standard groups. Efficacy and safety differences between the groups in terms of CII parameters and glycemic control are outlined in the table.

Ten (14.5\%) patients in the Standard group failed to achieve or to maintain glycemic targets despite progression to the column with maximum infusion rates. These patients remained on the standard protocol for 26.25 hours with average glucose levels of $9.1 \pm 2.7 \mathrm{mmol} / \mathrm{L}$ before being transitioned to an alternative treatment option with glucose levels at the time of withdrawal from the study of $9.5 \pm 3.3 \mathrm{mmol} / \mathrm{L}$. In conclusion, the computer-guided protocol achieved BG control faster and maintained BG within the targeted range of 4.4-6.7 $\mathrm{mmol} / \mathrm{L}$ better than the standard algorithm. The Glucommander was associated with higher insulin delivery and higher rate of mild hypoglycemic events. The higher insulin doses were achieved because the computer has no limitation on dosing increments, which was made more evident by the Standard protocol treatment failures. Further studies are needed to determine if the more consistent $\mathrm{BG}$ control from computer-based titration protocols may have benefit on clinical outcomes.

Supported by: sanofi-aventis

\section{7}

\section{Predictors of postprandial hyperglycaemia in patients with type 2}

diabetes

C.A. Nita ${ }^{1,2}$, A. Rusu' ${ }^{1}$, C.G. Bala ${ }^{1,2}$, N. Hancu ${ }^{1,2}$

${ }^{1}$ Diabetes, „Iuliu Hațieganu” University of Medicine and Pharmacy, ${ }^{2}$ Clinical Center of Diabetes, Emergency County Hospital, Cluj Napoca, Romania

Background and aims: Growing evidence suggests that all aspects of glucose metabolism - glycated hemoglobin (Alc), fasting plasma glucose and postprandial glycemia (PPG) - are clinically relevant parameters to be monitored. PPG, in particular, appears to be related to the cardiovascular disease. Although measurement of PPG has not yet been adopted as part of the routine diabetes management, it may be more accurate indication of overall glycemic control than FPG or Alc.

The objective of this study was to investigate the factors associated with postprandial glucose excursions in patients with type 2 diabetes (T2DM).

Materials and methods: We have evaluated the factors associated with postprandial hyperglycemia ( $>140 \mathrm{mg} / \mathrm{dl}$ at 2 hours after the meal), corrected for sex and treatment in 122 consecutive patients with T2DM attending the outpatient clinic from Clinical Center of Diabetes, Nutrition and Metabolic Diseases Cluj-Napoca, Romania. These patients were included in a larger epidemiological study aiming to assess the impact of postprandial hyperglycemia on cardiovascular risk in persons with type 2 diabetes. A complete medical history and physical examination were performed. Blood samples were collected in the overnight fasting state, and A1c, total cholesterol, HDLcholesterol and triglycerides were assessed. A six points blood glucose profile (before and $2 \mathrm{~h}$ after meals) measured by patients at home, together with a meal questionnaire was performed for each patient. To determine variables associated with higher postprandial glycemic levels, factor analysis followed by linear regression model was performed.

Results: The study group had a median age of 58 years ( $\min 29, \max 77$ years), $59.8 \%$ were males. The median duration of diabetes was 6 years $(\min 0$, max 37 years). Stratification for antidiabetic treatment was as follows: $10.7 \%$ were on diet alone, $13.9 \%$ on metformin, $9 \%$ on sulfonylurea, $23 \%$ on combined oral therapy, $30.3 \%$ on insulin alone and $13.1 \%$ on insulin plus oral therapy. By factor analysis we have extracted 4 factors that explained $73 \%$ of the variance of postprandial glycemia [Factor 1 with positive loadings of weight and body mass index, Factor 2 with positive loadings of total cholesterol and LDL-cholesterol, Factor 3 with positive loadings of diabetes duration and age, Factor 4 with positive loadings of triglycerides and HDL-cholesterol]. In an unadjusted linear regression analysis with mean postprandial glycemia as a dependent variable, the model which included the above mentioned factors identified was significantly associated with postprandial glycemia $(r=0.392$, $\mathrm{p}=0.002$ ). When individual regression coefficients were evaluated, that due to Factor 2 displayed a p value of 0.593 and was removed from the analysis. A second regression included Factor 1, 3 and 4, and the model remained statistically significant $(r=0.389, p=0.001, F$ change $=6.123$, significance of $F$ change $=0.001$ ). After adjustment for sex and treatment, only Factor 1 and Factor 4 remained significantly associated with postprandial glycemic values
( $\mathrm{p}=0.025$, and 0.004 ), indicating that postprandial glycemia is best explained by a multiple regression that included Factor 1 and Factor 4 as independent variables $(\mathrm{r}=0.422, \mathrm{p}<0.001)$.

Conclusion: The results of our study shows that weight, body mass index, triglycerides level and HDL-cholesterol are independently associated with postprandial glucose excursions.

\section{8}

Non-invasive monitoring of blood glucose in critically ill patients

A. Weinstein ${ }^{1}$, O. Herzenstein ${ }^{1}$, E. Gabis ${ }^{1}$, P. Singer ${ }^{2}$

${ }^{1}$ OrSense Ltd., Nes Ziona, ${ }^{2}$ ICU, Rabin Medical Center, Petach-Tikva, Israel

Background and aims: The purpose of this study is to evaluate the feasibility of the fully non-invasive NBM device (OrSense Ltd.) for continuous glucose monitoring in critically ill patients. Critically ill patients frequently experience abnormalities in carbohydrate metabolism and a severe insulin resistance state. Hyperglycemia is a negative predictor of outcome in these patients, as high blood glucose (BG) values are associated with an increased risk of morbidity and mortality. Current BG monitoring methods do not provide the continuous glucose monitoring (CGM) needed to implement tight glucose control protocols.

Materials and methods: The NBM uses a red/near-infrared sensor located at the finger's base. It performs a temporary occlusion of the blood flow, resulting in an enhanced, time dependent, optical signal which enables calculation of BG and hemoglobin concentrations.A study was conducted on 14 patients (7F, 7M, ages 34-92 years) in the intensive care unit (ICU) of the Rabin Medical Center, upon receipt of informed consent. The NBM probe was placed on patients' thumbs, where it performed non-invasive CGM for up to 24 hours, with readings every 10 minutes. The results obtained from the NBM device were compared to arterial blood samples taken through an arterial line every 30-60 minutes and analyzed with a blood gas machine (ABL 700, Radiometer, Copenhagen, Denmark).

Results: A total of 14 sessions were conducted, one from each subject. Patient compliance was good, and no adverse effects were identified. A prospective analysis based on a uniform model with personal calibration was performed on the NBM readings, for a total of 208 paired data points. The calibration phase lasted 3 hours, and utilized reference $B G$ values taken at $t_{0}+1, t+2, t+3$, and $t_{0}+4$. An additional reference point was supplied every 8 hours thereafter $\left(t_{0}+12\right.$ and $t_{0}+20$, if available), in accordance with the ICU's internal protocol whereby blood samples are taken at these regular intervals. Reference BG range was $62-369 \mathrm{mg} / \mathrm{dl}$. The resulting median relative absolute error was $7.3 \%$, and the median absolute error was $8.6 \mathrm{mg} / \mathrm{dl}$. A Clarke error grid analysis showed that $95.2 \%$ of the measurements fell within zones A $(74.5 \%)$ and B (20.7\%). Furthermore, we find that both the NBM and ABL 700 showed comparable estimates of time spent in hypoglycemia, mid-range, and hyperglycemia.

Conclusion: This study indicates the potential use of the non-invasive NBM for continual, accurate, safe, and easy-to-use BG evaluation in ICU. The device is of clinical utility for trend analysis, excursion alerts and closed-loop systems, enabling automatic glycemic control. It holds the promise of improving patients' care and survival, as well as reducing staff workload.

\section{9}

Blood glucose measurement techniques for hyperglycaemic clamp studies

T. Mura ${ }^{1}$, S. Lepape ${ }^{1}$, J.-L. Faillie ${ }^{1}$, H. Chevassus ${ }^{1}$, J. Cayuelas ${ }^{1}$, A. Chalançon ${ }^{1}$, I. Gabillaud ${ }^{1}$, P. Petit ${ }^{1,2}$, F. Galtier ${ }^{1}$

${ }^{1}$ Centre d'Investigation Clinique, CHRU Montpellier\&Inserm CIC0001, ${ }^{2}$ Centre de Pharmacologie et Innovation dans le Diabète, Université Montpellier1 \& CNRS UMR 5232, Montpellier, France

Background and aims: Because of the cost of glucose analyzers, glucose meters designed for self-monitoring are sometimes used to measure blood glucose during metabolic investigations.

However there have been debates on the analytical quality of glucose meters. For disease management, the ISO recommends less than $5 \%$ errors, an error being defined as more than $20 \%$ discrepancy with reference value. But when glucose meters are used to adjust infusion rates during hyperglycemic clamps, even variations $<20 \%$ may result in erroneous dextrose infusion rates. 
The aim of our study was to compare the accuracy of 2 methods of glucose measurement and the the frequency of errors in subsequent dextrose infusion rates, for blood glucose $>7 \mathrm{mmol} / \mathrm{l}$.

Materials and methods: 242 blood glucose values $>7 \mathrm{mmol} / \mathrm{l}$ were obtained during 32 hyperglycemic clamps performed on 15 healthy men (age 20 -34 years, mean BMI $22.3 \mathrm{~kg} / \mathrm{m} 2$ ). Reference blood glucose values were obtained in the Biochemistry Laboratory, Olympus analyser (glucose oxydase). During the clamps, blood glucose was assessed with 2 methods:

-blood glucose analyzer 2300 Stat Plus, System Industry

-self-blood glucose monitoring system (SBGMs) Medisense Optium Xceed ${ }^{\circ}$ with electrodes for veinous samples

During the $10 \mathrm{mmol} / \mathrm{l}$ hyperglycemic clamp, Stat Plus analyzer glucose values were entered on a spreadsheet using de Fronzo formulas to calculate the $30 \%$ dextrose infusion rate. Blood glucose values obtained by the reference method and by the SBGMs were entered a posteriori in the same spreadsheet to calculate the respective infusion rates. Quantitative variables were compared with non parametric tests for paired data (Wilcoxon signed-rank test). Medians of differences are presented with 5 th and 95 th percentiles. Error rates, defined as discrepancies of $\pm 10 \%, \pm 20 \%$ and $\pm 30 \%$ with reference values, were calculated for blood glucose values and dextrose infusion rates. Qualitative variables were compared using chi-2 test. Statistical analysis was performed using SAS 9.1 for Windows.

Results: For blood glucose, medians of differences between reference method and Stat Plus analyzer or SBGMs were $0.08 \mathrm{mmol} / \mathrm{l}(-1.19 ;+0.85 ; \mathrm{P}=0.21)$ and $0.27 \mathrm{mmol} / \mathrm{l}(-1.40 ;+1.62 ; \mathrm{P}=0.0004)$ respectively. For dextrose infusion rates, medians of differences between reference method and Stat Plus analyser or SBGMs were $-8 \mathrm{ml} / \mathrm{h}(-51 ;+62 ; \mathrm{P}=0.0004)$ and $-13 \mathrm{ml} / \mathrm{h}(-82 ;+68$; $\mathrm{P}=0.0004)$ Error rates are depicted in the table.

Conclusion: The frequency of errors in the dextrose infusion rates is high with the 2 methods. However, the accuracy of the Stat Plus is better, and errors due to Stat Plus analyzer are random and not systematic, as shown by the lack of significant difference between medians with reference method. Our results are in favor of the use of a Stat PLus analyser over SBGMs for high blood glucose measurements.

Comparison of error rates obtained with Stat Plus analyser and SBGMs

\begin{tabular}{|c|c|c|c|c|c|c|}
\hline & & variable & $\mathrm{N}$ & $\begin{array}{l}\text { errors } \\
\text { (n) }\end{array}$ & $\begin{array}{l}\text { error rate } \\
(\%)\end{array}$ & P value \\
\hline $\begin{array}{l}\text { blood } \\
\text { glucose }\end{array}$ & $\begin{array}{l}\text { error }>10 \% \\
\text { error }>20 \%\end{array}$ & $\begin{array}{l}\text { Stat Plus } \\
\text { analyser } \\
\text { SBGMs } \\
\text { Stat Plus } \\
\text { analyser } \\
\text { SBGMs }\end{array}$ & $\begin{array}{l}242 \\
242 \\
242 \\
242\end{array}$ & $\begin{array}{l}23 \\
71 \\
5 \\
11\end{array}$ & $\begin{array}{l}9.5 \\
29.3 \\
2.1 \\
4 . w 6\end{array}$ & $\begin{array}{l}<0.0001 \\
0.12\end{array}$ \\
\hline $\begin{array}{l}\text { dextrose } \\
\text { infusion }\end{array}$ & $\begin{array}{l}\text { error }>10 \% \\
\text { error }>20 \% \\
\text { error }>30 \%\end{array}$ & $\begin{array}{l}\text { Stat Plus } \\
\text { analyser } \\
\text { SBGMs } \\
\text { Stat Plus } \\
\text { analyser } \\
\text { SBGMs } \\
\text { Stat Plus } \\
\text { analyser } \\
\text { SBGMs }\end{array}$ & $\begin{array}{l}215 \\
215 \\
215 \\
215 \\
215 \\
215\end{array}$ & $\begin{array}{l}160 \\
199 \\
123 \\
171 \\
99 \\
141\end{array}$ & $\begin{array}{l}74.4 \\
92.6 \\
57.2 \\
79.3 \\
46.0 \\
65.6\end{array}$ & $\begin{array}{l}<0.0001 \\
<0.0001 \\
<0.0001\end{array}$ \\
\hline
\end{tabular}

\section{0}

Whole 24-hour continuous glucose monitoring profiles with insulin glargine or oral antidiabetic drug therapy in patients with type 2 diabetes mellitus

J.-F. Yale ${ }^{1}$, S. Harris ${ }^{2}$, J.A. Stewart ${ }^{3}$, H.C. Gerstein ${ }^{4}$

${ }^{1}$ McGill Nutrition Centre, McGill University Health Centre, Montreal,

Canada, ${ }^{2}$ Family Medicine, University of Western Ontario, London, Canada,

${ }^{3}$ Biometrics, sanofi-aventis, Bridgewater, United States, ${ }^{4}$ Medicine, McGill

University Health Centre, McMaster University, Canada

Background and aims: This analysis used data from the continuous glucose monitoring (CGM) portion of a randomized-controlled trial (RCT) in which adults with type 2 diabetes mellitus (T2DM) on stable therapy with 0,1 , or 2 oral antidiabetic drugs (OADs), $\mathrm{HbA}_{1 c} 7.5 \%-11.0 \%$, and not taking a thiazolidinedione were allocated to either the addition of evening insulin glargine (GLAR, initiated at 10 units/day and increased by 1 unit/day until targeted fasting plasma glucose [FPG] was achieved) or optimization of OAD, including the addition of metformin, sulphonylureas, repaglinide, nateglinide, or a thiazolidinedione, if required, for the next 24 weeks. Both groups targeted FPG $\leq 5.5 \mathrm{mM}$. The main objective of this RCT was to assess the change in A1C between conventional therapy with physician adjustment of OAD agents and addition of nighttime insulin glargine with self-titration.(Gerstein et al, 2006)[Gerstein 2006] The current analysis was performed on a subset of data to characterize whole 24-hour CGM profiles by comparing both the estimated mean glucose function at week 24 to baseline for each study arm $(n=37$, GLAR; $n=39, O A D)$ and the estimated mean glucose functions of the 2 arms at week 24 .

Materials and methods: This study used a Fourier approximation to each 24hour CGM curve as previously published.(Miller and Strange, 2007)[Miller 2007] Using model adjusted mean Fourier coefficient sets, continuous plots of blood glucose (BG) vs time were obtained by study arm and visit; comparisons with baseline and between study arms were made by plotting the difference curve and \pm 2 standard errors (SEs).

Results: In the whole study, GLAR was associated with a greater decrease in A1C during the 24 -week trial compared with adjustment of OADs $(1.55 \%$ vs $1.25 \%, P=0.005, \mathrm{~N}=405)$, without differences in overall hypoglycemia $(48.5 \%$ vs $42.2 \%, P=0.23$ ). In the current analysis, a subgroup of patients who had CGMS data was examined. Assessments of 24-hour BG curves at week 12 and week 24 were combined and compared to baseline as only 56 subjects (28 each arm) had $\geq 1$ complete 24-hour profile at baseline and week 12/24. GLAR mean BG profile at week 12/24 was lower than baseline by $>2$ SEs for most of the 24 hours; OAD arm had BG levels lower than baseline by 2 SEs except from 8-11 am and a short period around $7 \mathrm{pm}$. Both arms showed excellent reduction in mean $\mathrm{A} 1 \mathrm{C}$ level (8.3\% to $7.0 \%$ in the OAD arm, $8.4 \%$ to $6.8 \%$ for GLAR, $P=$ NS between groups in these subcohorts); however, only GLAR successfully controlled prandial breakfast BG levels from 8-11 am.

Conclusion: While in this subgroup analysis GLAR and OAD showed similar effects on mean $\mathrm{HbA}_{1 \mathrm{c}}$, 24-hour CGM profiles revealed greater reductions for GLAR between midnight and noon.

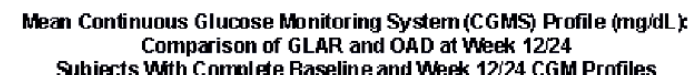
Subjects With Compl ete Baseline and Week 12/24 CGM Profiles
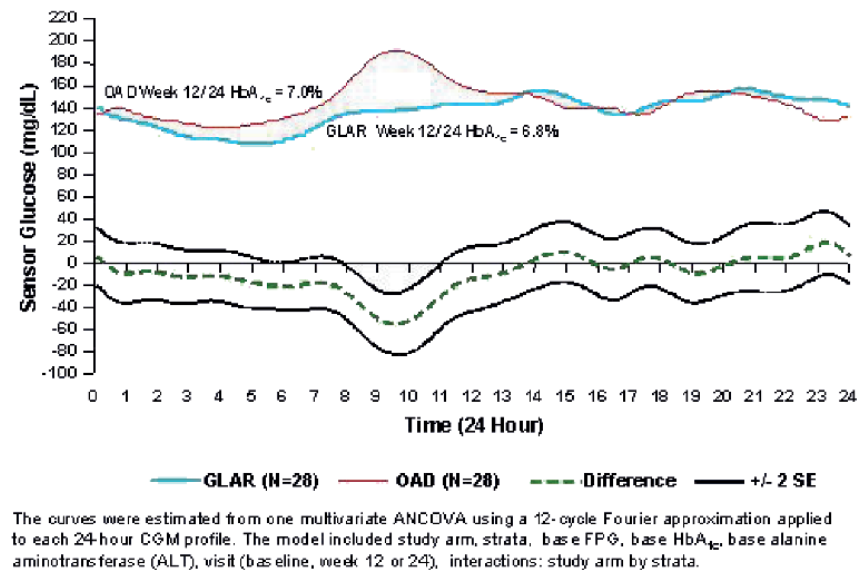
aminotrans ferase (ALT), visit (bas eline, wedk 12 or 24), inter actions: study arm by strata.

Supported by: sanofi-aventis U.S. Group (editorial)

\section{1}

Non-invasive system for continuous glucose monitoring

A. Karasik ${ }^{1,2}$, O. Herzenstein ${ }^{3}$, E. Gabis ${ }^{3}$, A. Weinstein ${ }^{3}$

${ }^{1}$ Endocrinology, Sheba Medical Center, Ramat Gan, ${ }^{2}$ Faculty of Medicine, Tel Aviv University, ${ }^{3}$ OrSense Ltd., Nes Ziona, Israel

Background and aims: Non-invasive glucose devices hold the promise of delivering painless and hassle-free continuous glucose monitoring (CGM). Such devices could assist subjects with diabetes in complying with the protocols known to prevent long and short-term complications; ultimately improving health and reducing the cost of diabetes. This study presents the use of the fully non-invasive NBM device (OrSense Ltd.) for CGM in subjects with type 1 and type 2 diabetes.

Materials and methods: The NBM uses a red/near-infrared sensor located at the finger's base. It performs a temporary occlusion of the blood flow, resulting in an enhanced, time dependent, optical signal which enables calculation of glucose concentration. The device was tested on 15 patients in sessions of 
24 hours, with NBM measurement every 10 minutes. Reference values were measured every 30-60 minutes using a self monitoring glucometer (Abbott Freestyle).

Results: A total of 1068 paired glucose values (NBM $v$ s. reference) from 25 sessions were produced, with a reference glucose range of $23-404 \mathrm{mg} / \mathrm{dL}$. In the paired data set, the median relative absolute difference (RAD) was $12.3 \%$ and a Clarke EGA showed that $95.1 \%$ of the measurements fell within the clinically acceptable A+B regions (zone A - 66.7\% and zone B - 28.4\%). Use of the device did not cause any discomfort for subjects, was safe and well tolerated.

Conclusion: The RAD values obtained in the study are comparable to values reported for currently approved CGM invasive apparatus. The information gained was of clinical utility for trend analysis as it detected both peaks and troughs in blood glucose levels. This work substantiates the potential use of OrSense's NBM as a non-invasive sensor for continuous, safe and easy-to-use blood glucose evaluation, which holds the promise of improving blood glucose control in both type 1 and type 2 diabetes.

\section{2}

iSense Mastery ${ }^{\text {tw }}$ CGM performance during in-clinic glucose clamping studies was found to track plasma glucose very closely

M.V. Rebec ${ }^{1}$, W.K. Ward ${ }^{1}$, J.L. House ${ }^{1}$, E.M. Anderson ${ }^{1}$, R. Bruce ${ }^{1}$,

H.M. Duman ${ }^{1}$, B. Hernandez $z^{2}$, M.S. Kipnes ${ }^{2}$

${ }^{1}$ iSense Corp., Wilsonville, ${ }^{2}$ Research, DGD Research, San Antonio, United States

Background and aims: There is an increasing effort to control blood glucose levels so that long-term complications associated with diabetes can be reduced. A concern with that therapeutic approach is the higher incidence of hypogycemic episodes. Continuous glucose monitoring (CGM) systems have the potential of reducing the frequency and severity of such hypoglycemic episodes. A key to the application of CGM technology in conrolling hypoglycemia is the performance of CGM in the hypoglycemic region. Quantifying such performance requires well-designed clinical study protocols, such as ones that incorporate glucose clamping. The aim of this study was to evaluate the performance of the iSense Mastery ${ }^{\mathrm{m}} \mathrm{CGM}$ during a glucose clamping protocol that includes extensive hypoglycemic episodes.

Materials and methods: Ten subjects (five type I and five type II) were recruited to participate in the study. Infusions of insulin and dextrose were carried out to clamp four to five glucose levels during an eight-hour glucose clamping protocol that was conducted in an in-clinic setting. Each clamping level was maintained for 35 - 45 minutes depending on the targeted level chosen. Venous blood was drawn every five minutes and the plasma glucose concetration was determined using an Alanox GM9. Three iSense biosensors were inserted in the abdominal area of the subjects using a trocar-based insertion process. The trocar was then removed, leaving the iSense biosensor in the subcutaneous tissue. The glucose sensors were attached to a potentiostat, and the glucose related current readings were digitized. The digital signals were transmitted via RF to a receiving station attached to a laptop PC, where the data was logged. Glucose related data was stored every minute. An algorithem was applied to the digitized data to obtain retrospective glucose values. Performance assesments were made on data pairs of CGM glucose values and the reference glucose values obtained on the Analox.

Results: A total of 30 sensors were inserted and 29 of them functioned for the duration of the study. A one point calibration was applied at the 300th minute of each study. All the digitized minute by minute current values were coverted to glucose values from the 300 to 600 minute point of each study. The overall Mean Absolute Relative Difference for all the glucose values above 75 $\mathrm{mg} / \mathrm{dl}$ (1087) was 14.4\% and Median Absolute Relative Difference was 11.0\%. The Absolute Differences were determined for all the glucose values that were $75 \mathrm{mg} / \mathrm{dl}$ or less (275). They had a mean of $17.3 \mathrm{mg}$ and median of $12.9 \mathrm{mg} / \mathrm{dl}$. The Clarke Error Grid analysis of all the data pairs (1354) showed $93.3 \%$ of the data points were in the $\mathrm{A} \& \mathrm{~B}$ regions.

Conclusion: The iSense Mastery ${ }^{\text {mic }}$ CGM demostrated robust perfomance robust performance when tested using a demanding glucose clamping protocol that incorporates extended hypo testing and rapid changes in the glucose concetrations. It can be concluded that the iSense glucose sensors show very reliable performance, even at this development stage. The significant beneficial potential of the CGM system is clearly demostrated by its perormance in the hypoglycemia region.

\section{3}

Correlations between plasma glucose (PG) measures and $\mathrm{HbA}_{1 \mathrm{c}}$ in insulin-treated patients (pts) with type 2 diabetes (T2D)

L. Ilag ${ }^{1}$, S. Choi ${ }^{1}$, M. Carey ${ }^{2}$, D. Shrom ${ }^{1}$, R. Heine ${ }^{1}$

${ }^{1}$ Lilly Research Labs, Eli Lilly \& Co., Indianapolis, ${ }^{2}$ Medical Writing, i3 Statprobe, Cary, United States

Background and aims: A strong correlation has been demonstrated between HbAlc and self-monitored PG values, especially fasting PG (FPG), in diettreated T2D pts. PG correlations are weaker for pts treated with oral agents or insulin. As data on the relationship of PG values to $\mathrm{HbAlc}$ in insulin-treated T2D pts are limited, the correlations between individual or mean of $\geq 2$ PG values and $\mathrm{HbAlc}$ were analysed in insulin-treated pts. The goal was to determine which PG values show strong correlation with HbAlc and whether different insulin regimens affect those correlations in this patient group.

Materials and methods: Data were pooled from 5 randomised clinical trials (RCTs) in T2D pts treated with bedtime basal insulin glargine (G) or insulin lispro mixes (LM) [25\% lispro/75\% insulin lispro protamine suspension (ILPS) BID (2 studies); 50\% lispro/50\% ILPS TID (2 studies); 50\% lispro/50\% ILPS BID and 25\% lispro/75\% ILPS QD (1 study)]. HbAlc data from the first 16-week period of crossover studies and the 24-week period of parallel studies were used as well as 7-point self-monitored PG profiles conducted in the 2 weeks prior to HbAlc collection and the means of fasting and evening postmeal, all premeal, all postmeal and all 7 PG values. Pearson's correlation coefficients were calculated for PG values and HbAlc. For combined analyses, partial correlation was used to remove treatment effect. Receiver-operating characteristic (ROC) curves were generated to analyse the sensitivity of PG values (FPG, pre- or postmeal PG) against HbAlc above or below 7\%. Results: The mean \pm SE HbAlc in combined dataset (LM, $n=317 ; \mathrm{G}, \mathrm{n}=306$ ) was $7.4 \pm 0.04 \%$. The table shows correlation coefficients between $\mathrm{HbAlc}$ and key PG values (individual or means). FPG had the lowest correlation with $\mathrm{HbA1c}$ over-all and particularly in the G-treated pts. Among individual PG values, evening premeal and evening postmeal PG values had the highest correlation with $\mathrm{HbAlc}$ in both G-treated and LM-treated pts. Higher correlations were generally seen between $\mathrm{HbAlc}$ and PG values that were means of $\geq 2$ PG measurements. The mean of all 7 values had the highest correlations in both groups and in the combined analysis. The areas under ROC curves were consistent with the correlation analyses.

Conclusion: The correlations between PG values and HbAlc in insulintreated pts in RCTs is consistent with previous studies, i.e. they were lower than reported in non-insulin treated T2D pts ( $\mathrm{r} \sim 0.75)$. Although correlations vary in each treatment group, FPG generally had a weaker correlation with HbAlc compared to other PG values while evening premeal and evening 2$\mathrm{hr}$ postmeal PG had stronger correlations. Means of $\geq 2 \mathrm{PG}$ values had higher correlations to $\mathrm{HbAlc}$. These data suggest that FPG is not a reliable glycaemic indicator in insulin-treated pts and that checking $\geq 2$ PG values during the day may better guide insulin intensification.

\begin{tabular}{|c|c|c|c|c|c|}
\hline \multirow[t]{2}{*}{ PG values } & \multicolumn{3}{|c|}{ Correlation Coefficients* } & \multicolumn{2}{|c|}{$\begin{array}{l}\text { Area under the } \\
\text { ROC }\end{array}$} \\
\hline & Combineda & G & LM & G & LM \\
\hline Fasting & 0.40 & 0.39 & 0.41 & 0.64 & 0.67 \\
\hline Noon $2 \mathrm{hr}$ postmeal & 0.44 & 0.47 & 0.41 & 0.70 & 0.69 \\
\hline Evening premeal & 0.46 & 0.47 & 0.45 & 0.71 & 0.70 \\
\hline Evening $2 \mathrm{hr}$ postmeal & 0.46 & 0.48 & 0.43 & 0.74 & 0.70 \\
\hline $\begin{array}{l}\text { Mean of FPG and } 2 \mathrm{hr} \\
\text { postmeal }\end{array}$ & 0.52 & 0.53 & 0.51 & 0.76 & 0.72 \\
\hline $\begin{array}{l}\text { Mean of } \\
\text { 7-point PG }\end{array}$ & 0.58 & 0.59 & 0.56 & 0.78 & 0.74 \\
\hline
\end{tabular}

apartial correlation coefficient

*all correlations significantly greater than $0(\mathrm{p}<0.001)$ 


\section{4}

Meal size estimation using continuous glucose monitoring: towards a hybrid closed-loop artificial pancreas

B.W. Bequette ${ }^{1}$, H. Lee ${ }^{1}$, K. Caswell ${ }^{2}$, E. Dassau ${ }^{3}$, F.J. Doyle III ${ }^{3}$,

B.A. Buckingham ${ }^{2}$

${ }^{1}$ Department of Chemical and Biological Engineering, Rensselaer Polytechnic Institute, Troy, ${ }^{2}$ Division of Pediatric Endocrinology and Diabetes, Stanford Medical Center, Stanford, ${ }^{3}$ Department of Chemical Engineering, University of California, Santa Barbara, United States

Background and aims: A hybrid closed-loop artificial pancreas, where an insulin bolus is delivered at mealtime, has the potential for significantly improved glucose regulation, compared to a feedback-only system. This "feedforward" control strategy requires that an individual with type 1 diabetes be vigilant in estimating the amount of carbohydrates in their meals, and remember to bolus at mealtime. Unfortunately, juveniles forget to bolus, so it is desirable to have an automated system that detects when a meal has been consumed, and estimates the amount of carbohydrates in the meal to provide an appropriate meal bolus.

Materials and methods: In this presentation we develop such a meal detection and meal size estimation algorithm based on a continuous glucose monitoring, optimal estimation theory and signal processing techniques. A set of threshold values for the first and second derivatives of glucose with respect to time are found to detect a meal within 15-45 minutes of actual meal consumption. When a meal is detected, a finite impulse response filter, based on the estimated second derivative of glucose with time, is used to estimate the meal size (grams of carbohydrate). Results are presented based on both in silico and clinical studies. The clinical studies are based on continuous monitoring data from 19 subjects with type 1 diabetes mellitus, consuming a total of 25 meals (mean meal size of 53 grams of carbohydrates; range of 14 to 103 grams).

Results: While 4 meals were not detected (16\%), the meal size estimation filter is trained using 5 meals from 4 individuals out of the 21 detected meals, with a median detection time of 42 minutes and a median absolute meal size estimation error of $3.7 \%$. The algorithm was validated against the remaining 16 meals: the median detection time was 32 minutes, and the median absolute error in estimating the meal size was $24 \%$ with a mean absolute error of $32 \%$. In silico closed-loop studies reveal a significant improvement glucose regulation performance using the proposed meal detection and meal size estimation algorithm in a hybrid closed-loop strategy, compared to feedbackonly control. The postprandial mean glucose levels are reduced from 217 to $145 \mathrm{mg} / \mathrm{dL}$ and the maximum postprandial glucose is reduced from 333 to $232 \mathrm{mg} / \mathrm{dL}$.

Conclusion: This meal detection and meal size estimation algorithm can be incorporated into: 1) a hybrid closed-loop algorithm allowing for a meal bolus even if the subject forgets to self-administer a meal bolus, and 2) a fully automated closed-loop system using model predictive control. Supported by: JDRF

\section{5}

Does glucose variability influence the relationship between average glucose and HbA levels?

J.C. Kuenen ${ }^{1}$, R. Borg ${ }^{2}$, H. Zheng ${ }^{3}$, D. Schoenfeld ${ }^{3}$, M. Diamant ${ }^{1}$, R. Heine ${ }^{1}$, D. Nathan ${ }^{3}$ on behalf of the ADAG Study Group

${ }^{1}$ Diabetes Center, VU University Medical Center, Amsterdam, Netherlands, ${ }^{2}$ Steno Diabetes Center, Gentofte, Denmark, ${ }^{3}$ Diabetes, Harvard Medical School, Boston, United States

Background and aims: The $\mathrm{HbA}_{1 \mathrm{c}}$-Derived Average Glucose (ADAG) study demonstrated a close linear relationship between average glucose (AG) during 3-months [derived from continuous glucose monitoring (CGM) and frequent self-monitored blood glucose (SMBG)] and $\mathrm{HbA}_{1 c}$ at the end of 3 months. In this ADAG sub study, we examined the influence of glycaemic variability on the relationship between $\mathrm{AG}$ and $\mathrm{HbA}$ levels.

Materials and methods: We analysed $\mathrm{AG}$ and $\mathrm{HbA}_{1 \mathrm{c}}$ data from 268 patients with diabetes mellitus type 1 (T1DM) and 159 with diabetes mellitus type 2 (T2DM) from 10 international centers. AG was calculated from 7-point SMBG collected during 3 days/week and CGM performed for 2-3 days on 4 occasions during a 12 week-period. From the CGM data we calculated 3 different measures of variability: the mean amplitude of glycaemic excursions (MAGE, measuring major swings in glucose), the standard deviation (SD) of all glucose levels, and the continuous overlapping net glycaemic action
(CONGA). The CONGA average or CONGA $\mathrm{n}=1-24 \mathrm{~h}$ is measuring intraday variability. The MAGE, SD and CONGA average were subdivided according to tertiles of low, moderate and high glucose variability. We tested for differences in the relationship between $\mathrm{AG}$ and $\mathrm{HbA}_{1 c}$ for all diabetic subjects pooled and for T1DM and T2DM separately, according to tertiles of the respective measures of glucose variability, adjusted for demographic variables. Results: Approximately 2700 glucose values were obtained for each subject during the 3-month monitoring period. Linear regression analysis showed a correlation between $\mathrm{HbA}_{1 c}$ and $\mathrm{AG}$ values $\left(\mathrm{AGmmol} / \mathrm{L}=1.59 \times \mathrm{HbA}_{1 c}-2.59\right.$, $\left.\mathrm{R}^{2}=0.84, \mathrm{P}<0.0001\right)$. There was a significant difference in the relationship between $\mathrm{AG}$ and $\mathrm{HbA}_{1 \mathrm{c}}$ comparing the highest variable tertile vs the lower tertile for SD, MAGE and CONGA average for all patients and for T1DM and T2DM separately. The differences between high and medium tertiles for all measures of variability were only significant for the pooled analysis and in the T1DM. For T2DM, the high vs the medium variability tertiles for $\mathrm{SD}$, and the medium vs the low variability for MAGE had a significant effect on the $\mathrm{HbA}_{1 c}-\mathrm{AG}$ relationship. (Table 1) The impact of this difference in the $\mathrm{HbA}_{1}$-AG relationship for high vs low glucose variability, defined by MAGE, in T1DM patients with HbA1c levels of $7 \%$ and $9 \%$ would be a calculated difference in mean glucose of $1.8 \mathrm{mmol} / \mathrm{L}$ and $3.7 \mathrm{mmol} / \mathrm{L}$, respectively.

Conclusion: Over most levels of glucose variability, but especially at higher variability levels, the relationship between $\mathrm{HbA}_{1}$ and $\mathrm{AG}$ appears to be altered for the entire group and for T1DM only. For the T2DM, HbA - -AG relationship depends on the variability measure used. Although the differences in "interpretation" of $\mathrm{HbA}_{1 c}$ are influenced by variability, the clinical impact is modest.

\begin{tabular}{|c|c|c|c|c|}
\hline & Variability measure & $\begin{array}{l}\text { High vs } \\
\text { Low }\end{array}$ & $\begin{array}{l}\text { Medium vs } \\
\text { Low }\end{array}$ & $\begin{array}{l}\text { High vs } \\
\text { Medium }\end{array}$ \\
\hline \multirow{3}{*}{$\begin{array}{l}\text { T1DM + T2DM } \\
\mathrm{N}=\mathbf{4 2 7}\end{array}$} & $\mathrm{SD}$ & $0.002^{*}$ & 0.29 & $0.001^{*}$ \\
\hline & CONGA average & $0.003^{*}$ & 0.11 & $0.04^{*}$ \\
\hline & MAGE & $<0.001^{*}$ & $0.005^{*}$ & $0.005^{\star}$ \\
\hline \multirow{3}{*}{$\begin{array}{l}\text { T1DM } \\
\mathrm{N}=268\end{array}$} & SD & $0.01^{*}$ & 0.39 & $<0.001^{*}$ \\
\hline & CONGA average & $0.02^{*}$ & 0.33 & $0.008^{*}$ \\
\hline & MAGE & $<0.001^{*}$ & 0.14 & $<0.0001^{*}$ \\
\hline \multirow{3}{*}{$\begin{array}{l}\text { T2DM } \\
N=159\end{array}$} & SD & $0.008^{\star}$ & 0.18 & $0.03^{*}$ \\
\hline & CONGA average & $0.02^{*}$ & 0.10 & 0.41 \\
\hline & MAGE & $0.006^{*}$ & $0.007^{*}$ & 0.24 \\
\hline
\end{tabular}

Tabel 1: Differences in the relationship between AG and $\mathrm{HbA1c}$ for all diabetic subjects pooled and for T1DM and T2DM separately, according to tertiles of the respective measures of glucose variability, adjusted for demographic variables. The reference range for the three tertiles of glucose variability for SD are: Low $<34$, Medium 34-61 and High $>61$; for CONGA average: $<45$, 45-80 and >80; and for MAGE: $<80,80-144$ and $>144$. $\mathrm{P}^{*}$ is statistic significant different between the groups.

Supported by: ADA, EFSD/LifeScan grant, Abbott Diabetes Care, Merck \& Company, Bayer Healthcare, LifeScan, GlaxoSmithKline, Medtronic Minimed, sanofi-aventis Netherlands, Hemocue 


\section{PS 100 Monitoring type 1 diabetes}

\section{6}

Significantly improved $\mathrm{HbA}_{1 \mathrm{c}}$ values with no increase in hypoglycaemia in pregnant women with type 1 diabetes using real-time continuous glucose monitors

M.K. Voelmle, P.J. Ritchie, K.K. McFann, R.G. Naik, S.K. Garg

University of Colorado Denver, Barbara Davis Center for Diabetes, Aurora, United States

Background and aims: Optimizing glucose control in pregnant patients with diabetes continues to be a challenge. Intensive insulin management that includes increased glucose monitoring improves glycemic control. The purpose of this retrospective pilot study was to evaluate the glycemic control of pregnant patients with type $1 \mathrm{DM}$ who used Real-time Continuous Glucose Monitors (RTCGM) in addition to self monitored blood glucose (SMBG) as compared to women who only performed SMBG. The secondary outcome was to evaluate hypoglycemia in patients with near-normal A1c.

Material and methods: A total of 13 patients used a DexCom STS or Medtronic Paradigm RT during pregnancy, initiating sensor use at 0-24 weeks gestation. A comparison group matched for age, duration of diabetes, ethnicity, and normal AERs $(<20 \mathrm{ug} / \mathrm{min})$ was used for data analysis. Mean $( \pm \mathrm{SD})$ age and duration of diabetes was $30.5 \pm 4.4$ yrs and $15.4 \pm 6.5$ for the RTCGM group. Baseline characteristics were similar in both groups. Baseline Alc values were $6.7 \pm 1.1$ and $6.9 \pm 0.7$ in the sensor and control groups respectively $(p>0.05)$.

Results: Reductions in mean A1c values were seen at all time points in the sensor group (Figure 1a). 8 women in the sensor group with available data significantly improved Alc over a three-month period from sensor initiation, without increasing percentage of time in hypoglycemic range. Of those 8 , Alc's were improved from $6.6 \pm 1.1$ to $6.1 \pm 1.0$ ( $\mathrm{p} \leq 0.05$ ) without any increase in low blood sugar (Figure 1b).

Conclusion: We conclude use of real-time continuous glucose monitors in pregnant mothers with diabetes can significantly improve A1c values without changing the amount of time spent in hypoglycemia. This pilot study supports the need for larger randomized control trials to evaluate clinical implications of RTCGM in the management of pregnant women with type 1 DM and their fetal outcomes.

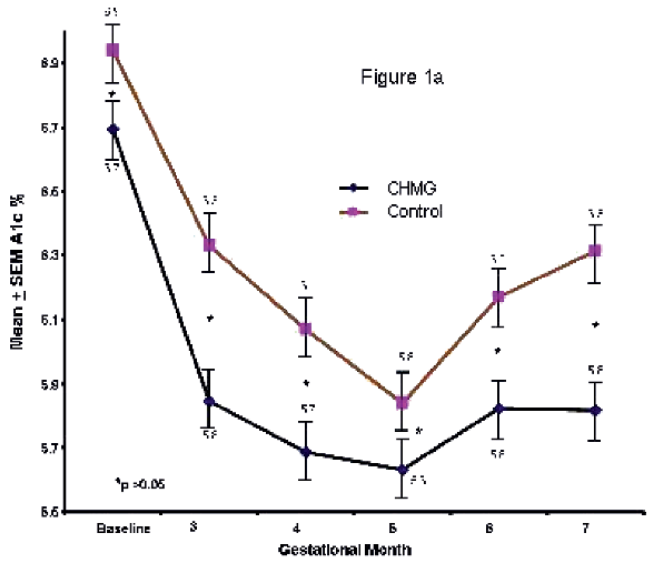

Figure 16

Percent of Glucose Readings at Baseline and Three Mlonths in Subjects Using Continuous Home Glucose Monitoring
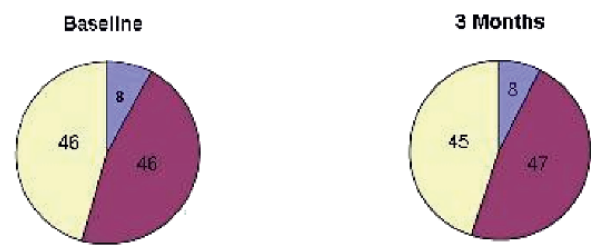

口 BTR $\square$ ATR $\square$ WTR
1067

Symptom attributions during hypoglycaemia and hyperglycaemia a continuous glucose monitoring study

T. Kubiak ${ }^{1}$, A. Wittig ${ }^{1}$, B. Mraz ${ }^{2}$, U. Herrmann ${ }^{3}$, W. Kerner ${ }^{3}$

${ }^{1}$ Institute of Psychology, University of Greifswald, ${ }^{2} \mathrm{~A}$. Menarini, Diagnostics, Berlin, ${ }^{3}$ Clinic for Diabetes and Metabolic Disorders, Klinikum Karlsburg, Germany

Background and aims: Symptoms of acute hypoglycaemia and hyperglycaemia often are ambiguous for people with diabetes, with incorrect symptom attributions and misinterpretations presumably being common. The aim of the present study was to investigate (1) the accuracy of detection of hypoglycaemia and hyperglycaemia in people with longstanding type 1 diabetes mellitus, and (2) determinants of correct and incorrect symptom attributions and attributional patterns depending on the type of symptoms, employing ambulatory assessment methodology (continuous glucose monitoring combined with electronic diaries).

Materials and methods: $\mathrm{N}=12$ type 1 diabetes patients (age $\mathrm{M} \pm \mathrm{SD}: 47.7 \pm 11.5$ years; duration of disease $>20$ years, gender female $58.3 \%$ ) completed electronic diaries (Psion Series 3a hand-held computers) for two consecutive days, assessing self-reports on (a) perceived symptoms, (b) symptom attributions, and (c) estimated blood glucose levels. In addition, the participants were equipped with a continuous glucose monitoring system (GlucoDay S). Results: A mean glucose monitoring time of $48.2 \pm 0.2$ hours was achieved (sensor vs. reference: $\mathrm{r}=.81$; ICC $=.84$; MAD $\%=22.0 \pm 0.4$; mean sensor glucose $7.8 \pm 1.5 \mathrm{mmol} / \mathrm{L}$; SD sensor glucose $3.1 \pm 0.9$ ). $7.6 \pm 6.5$ hypoglycaemic $(10.0 \mathrm{mmol} / \mathrm{L})$ were captured. On average, $1.5 \pm 1.9$ undetected hypoglycaemic events (blood glucose estimation vs. sensor glucose) and 7.5 \pm 7.0 undetected hyperglycaemic episodes occurred per participant. In case of hypoglycaemia, the most common incorrect attributions were of an unspecific nature $(46.7 \%$, e.g. being tired due to physical exhaustion); in case of hyperglycaemia cardiovascular attributions of symptoms were the most common misinterpretations with 35\%. Mixed logit regressions revealed associations between incorrect attributions and neuroglycopenic symptoms for hypoglycaemia $(\mathrm{b}=1.3, \mathrm{p}$ $<.05)$, and affective symptoms, such as depressed mood or irritability for hyperglycaemia $(\mathrm{b}=1.2, \mathrm{p}<.05)$.

Conclusion: Different types of symptoms are associated with different detection rates of hypoglycaemia and hyperglycaemia. Furthermore, this study provides initial evidence for distinct patterns of incorrect symptom attributions. As well, the findings are in line with clinical experience (e.g., ambiguity of neuroglycopenic symptoms). The results of this study could add new starting points to enhance established patient education interventions, such as programmes for improving hypoglycaemia awareness. Supported by: A. Menarini Diagnostics

\section{8}

Non-invasive glucose monitoring in patients with type 1 diabetes: repeatability in the same subjects

A. Caduff ${ }^{1}$, W.A. Stahel ${ }^{2}$, C. Bull ${ }^{3}$, M.S. Talary ${ }^{1}$, J. Klisic ${ }^{1}$, M. Donath ${ }^{4}$, L. Heinemann ${ }^{5}$

${ }^{1}$ Solianis Monitoring, Zurich, Switzerland, ${ }^{2}$ Seminar for Statistics, ETH Zurich, Switzerland, ${ }^{3}$ Centre for Clinical Research, University Hospital Zurich, Switzerland, ${ }^{4}$ Clinic for Endocrinology and Diabetes, University Hospital Zurich, Switzerland, ${ }^{5}$ Research, Profil Institute for Metabolic Research, Neuss, Germany

A non-invasive multisensor device (MD) for continuous glucose monitoring, based on dielectric spectroscopy, combined with additional sensors for optical, sweat/moisture and temperature measurement has been developed. The motivation for the MD is based on the understanding that various temporal fluctuations of the properties of skin, can introduce significant perturbations to the actual glucose related measurement. The aim of this study was to measure glucose excursions on different study days in the same subjects using an identical set of calibration parameters.

The multisensor system was worn on the upper right arm by 10 patients with Type 1 Diabetes (age $45 \pm 13$ y, BMI $25.5 \pm 2.2 \mathrm{~kg} / \mathrm{m} 2$, duration of diabetes $22.5 \pm 10.5$ y, HbAlc $7.0 \pm 0.5 \%$ ). Glucose was administered orally to induce two consecutive hyperglycemic excursions $(12-15 \mathrm{mmol} / \mathrm{L})$ within 8 hours. Euglycemia was re-established by i.v. insulin administration. Based on the sensor signals registered after the first study day, a global calibration model was derived, and the respective coefficients of the individual sensor signals were determined. The model included one coefficient specific to each run. 
On the second study day, 5 of these patients were again administered glucose orally to induce a glucose profile with one hyperglycemic excursion. The coefficients from the first study day were re-applied to the 5 test runs of the second study day in order to study the prediction power of the model. Both study days for all subjects were performed within a period of two months. Application of the calibration model from day one to day two yielded an R2= 0.68 and $\mathrm{MARD}=27.3 \%$. These data indicate that a global model can be derived using training runs and successfully applied to a set of test runs with a different glucose profile. The global parameterisation is shown to be transferable, leading to a successful tracking of glucose variations. Repeating this in a larger study group and under less experimentally controlled conditions, will be another important step towards non-invasive glucose monitoring.
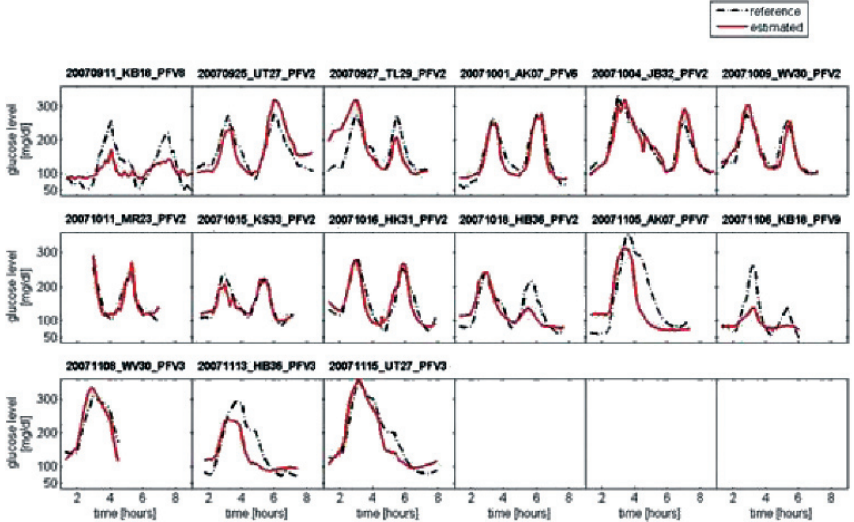

Supported by: Solianis Monitoring

\section{9}

Permanent use of a continuous glucose monitor significantly reduces hypoglycaemia and $\mathrm{HbA}_{1 \mathrm{c}}$ in type 1 diabetic patients treated by insulin pump with high occurrence of hypoglycaemia

R.P. Radermecker ${ }^{1,2}$, A. Saint Remy ${ }^{3}$, J. Bringer ${ }^{1,4}$, E. Renard ${ }^{1,4}$

${ }^{1}$ Endocrinology Department, CHU de Montpellier, Montpellier, France,

${ }^{2}$ Diabetes, Nutrition and Metabolic disorders Department, CHU Sart

Tilman, Liege, Belgium ${ }^{3}$ Nephrology Department, CHU Sart Tilman,

Liege, Belgium, ${ }^{4}$ Endocrinology Department, Université Montpellier 1,

Montpellier, France

Background and aims: High occurrence of hypoglycaemia in type 1 diabetic (T1D) patients is a debilitating condition and has been shown to hamper the improvement of $\mathrm{HbAlc}$. We performed a prospective, randomized, controlled, cross-over study to assess the effectiveness of the permanent use of a continuous glucose monitor (CGM) for 12 weeks on glucose control in T1D patients presenting frequent hypoglycaemia while treated by insulin pump.

Materials and methods: 13 patients $(5 \mathrm{M}, 8 \mathrm{~F})$, aged $47+/-10$ (SD), with T1D since $25+/-15$ years, under insulin pump since $5.5+/-7.0$ years, showing $>$ 6 recorded capillary blood glucose $(\mathrm{CBG})$ values $<60 \mathrm{mg} / \mathrm{dl}$ in their meter memory for the last 14 days while performing daily 6.2 CBG tests on average, were randomized for permanent use of a CGM (Guardian RT ${ }^{\circ}$, Medtronic, Northridge, CA, USA) or usual CBG testing (control arm) for 12 weeks, followed by a cross-over period for the next 12 weeks. A review of guidelines for adaptation of pump delivery according to CBG was performed in all patients at study inclusion, and a specific education for CGM management before CGM use. The sc glucose sensor, calibrated twice daily against CBG and replaced every 3 days, generates a continuous signal which is on line transmitted wirelessly to the monitor resulting in the presentation of blood glucose estimation every $5 \mathrm{~min}$. Hypo and hyper alarms were set at 80 and $240 \mathrm{mg} / \mathrm{dl}$, respectively. Patients were taught how to adapt insulin delivery and glucose intakes according to online CGM data while usual CBG testing was maintained. A CBG test was requested before action in case of hypo/ hyper alarm. Outpatient visits were scheduled after 2 and 12 weeks of each study period. Glucose control was assessed at baseline (BL) and after 12 and 24 weeks from recorded CBG values $<60 \mathrm{mg} / \mathrm{dl}$ for the last 2 weeks in the meter memory and HbAlc.

Results: From the 13 included patients, 4 dropped out during the first study period ( 3 while using CGM and 1 in the control arm). Among the 9 completers, while CBG testing remained similar during both study periods, occurrence of CBG values $<60 \mathrm{mg} / \mathrm{dl}$ per 14 days significantly decreased dur- ing CGM use from $15.3+/-8.6(\mathrm{BL})$ to $7.6+/-6.8(\mathrm{p}=0.0076)$, versus no significant change during the control period $(11.1+/-4.5)$. During CGM use, $\mathrm{HbAlc}(\%)$ significantly decreased from $8.3+/-0.4(\mathrm{BL})$ to $7.8+/-0.6$ $(\mathrm{p}=0.035)$ versus no significant modification during control period $(8.0+/$ 0.8 ). No 'period effect' was identified. Of note, the difference of CBG values $<60 \mathrm{mg} / \mathrm{dl}$ per 14 days between BL and end of study was significantly correlated with BL occurrence (Spearman $\mathrm{r}=0.95, \mathrm{p}<0.05$ ). No severe hypoglycaemia, ketosis or adverse event related to CGM use was reported.

Conclusion: Our data support the hypothesis that permanent use of a CGM for 12 weeks allows a significant reduction of hypoglycaemia in T1D patients treated by insulin pump who are prone to hypoglycaemia. Moreover, reduction of hypo occurrence is accompanied by a significant improvement of $\mathrm{HbA1c}$. Although these benefits should be confirmed on longer term, frequent occurrence of hypoglycaemia under insulin pump therapy may represent a sound indication for CGM. In such cases, permanent use appears as necessary.

Supported by: Fonds Léon Frédéricq Université de Liège

\section{0}

Evaluation of microdialysis-based glucose monitoring in blood and subcutaneous adipose tissue in type 1 diabetic patients

J.K. Mader ${ }^{1}$, F. Feichtner ${ }^{2}$, S. Korsatko ${ }^{1}$, G. Köhler ${ }^{1}$, R. Schaller ${ }^{2}$, J. Plank ${ }^{1}$, T.R. Pieber ${ }^{1,2}$, M. Ellmerer ${ }^{1}$

${ }^{1}$ Internal Medicine / Diabetes and Metabolism, Medical University Graz, ${ }^{2}$ Institute of Medical Technologies and Health Management, Joanneum Research GmbH, Graz, Austria

Background and aims: Tight glycaemic control (TGC) is beneficial for critically ill patients at a cardiac and medical ICU. In order to achieve TGC a continuous glucose signal could be helpful to decrease workload. Subcutaneous adipose tissue (SAT) has been suggested as an alternative site for continuous glucose monitoring and has extensively been tested in type-1-diabetic patients. In the critically ill patient, vascular access is granted, and thus, continuous blood glucose monitoring might be preferable. The aim of the present study was to test a microdialysis-based technique for peripheral venous glucose monitoring and compare the technique with SAT glucose monitoring in type-1-diabetic patients.

Materials and methods: 21 type-1-diabetic patients (age: $31.9 \pm 3.1$ years; BMI: $25.1 \pm 8.1 \mathrm{~kg} / \mathrm{m}^{2}$ ) were investigated over a period of 26 hours. SAT microdialysis (MDs) was performed using a standard CMA60 catheter (CMA microdialysis, Sweden), for vascular microdialysis (MDv), a conventional double lumen catheter (mtb Zier, Germany) with a planar flow-through microdialysis unit was used. Microdialysis samples were collected in 15 to 30 minutes intervals and analyzed for glucose with a standard laboratory analyzer. Microdialysis samples were prospectively calibrated to reference glucose by applying a one-point calibration. MDs and MDv glucose levels were compared against arterialized-venous reference blood measurements (Beckman Glucose Analyzer).

Results: Both microdialysis techniques could be applied without restrictions and all 21 patients completed the trial period of 26 hours. Mean glucose difference [2SD] between reference blood glucose and glucose readings obtained from microdialysis were as follows: MDs 5.0 [40.3] \%, and MDv 8.9 $[32.8] \%$, respectively. Mean absolute relative difference (MARD) $[ \pm S D]$ was $15.7 \pm 7.8 \%$ (MDs), and $15.9 \pm 10.8 \%$ (MDv), respectively. Clarke Error Grid analysis indicated $71.5 \%$ in $\mathrm{A}, 25.6 \%$ in $\mathrm{B}, 2.3 \%$ in $\mathrm{C}$ and $0.6 \%$ in D for MDs and $68.1 \%$ in $\mathrm{A}$ and $31.9 \%$ in B for MDv, respectively.

Conclusion: Glucose monitoring from SAT and blood using a microdialysisbased approach indicated similar performance when investigated in type 1 diabetic patients for a period of 26 hours.

Supported by: European Commission 


\section{1}

What is the best way to analyse glycaemic variability using continuous glucose monitoring data?

B.C. Whitelaw, P. Choudhary, D. Hopkins

Department of Diabetes, King's College Hospital, London, United Kingdom

Background and aims: Continuous glucose monitoring (CGM) provides detailed information about glucose fluctuations and is often used in the clinical and research setting to evaluate glycaemic variability. Glycaemic variability can contribute to risk of micro and macrovascular complications and also to the risk of severe hypoglycaemia. Most methods currently used to evaluate glycaemic variability were developed for use with frequent capillary glucose sampling but are now being applied to CGM data. There is currently no consensus as to the best method of assessing glycaemic variability from CGM data. The aim of this project is to apply the different methods of assessing glycaemic variability to a range of CGM data to assess both their validity and their correlation with a clinical assessment of glycaemic variability. A subsequent aim is to make an assessment as to which method has the strongest correlation with a clinical assessment.

Materials and methods: We analysed 48 continuous hours of CGM data from 22 subjects, 7 non-diabetic controls and 15 with type 1 diabetes. The subjects with diabetes were purposively sampled to reflect a wide range of duration of diabetes $(17+/-10 y r s)$ and hypoglycaemia experience. Mean age was $48+/-12$ yrs and mean $\mathrm{HbAlc}$ was $7.3 \%+/-1.1$. Replicating methodology used by Ryan et al in development of the lability index, we compared established measures of glycaemic variability with a clinical lability score derived from blinded review of CGMS traces by two independent clinicians. Several indices of glycaemic variation were calculated including: standard deviation (SD), Mean Amplitude of Glycaemic Excursion (MAGE), Average Daily Risk Range (ADRR), Glycaemic Risk Assessment Diabetes Equation (GRADE) and Continuous Overall Net Glycaemic Action (CONGA). CONGA was calculated using 1,2 and 4 hour intervals, giving three separate indices CONGA1, CONGA2 and CONGA4. Each method of assessing glycaemic variability was then correlated with the clinical lability score, using Spearman's rank correlation.

Results: Correlations of different measures of glycaemic variability are shown in table 1 . All measures of glycaemic variability gave a statistically significant correlation with clinical assessment, confirming their validity for use with CGM traces. CONGA2 and CONGA4 provide the strongest correlations (0.96 for both).

Conclusion: Our data shows that even though many of these indices of glycaemic variability were originally developed for use with capillary glucose values, they have a close correlation to a clinical assessment of variability when used with continuous data obtained from CGM. CONGA2 and CONGA4 have the strongest correlation with glycaemic variability. They are the standard deviations of the differences between the any individual glucose value and the value 2 or 4 hours previously respectively. This suggests that CONGA2 or CONGA4 should be used when analysing CGM traces to determine glycaemic variability.

Table 1: Correlation between clinical assessment and different indices of glycaemic variability

\begin{tabular}{lll}
\hline Index & Correlation Coefficients & p value \\
\hline ADRR & 0.57 & 0.007 \\
\hline GRADE & 0.88 & $<0.001$ \\
\hline MAGE & 0.91 & $<0.001$ \\
\hline SD & 0.95 & $<0.001$ \\
\hline CONGA1 & 0.91 & $<0.001$ \\
\hline CONGA2 & 0.96 & $<0.001$ \\
\hline CONGA4 & 0.96 & $<0.001$ \\
\hline
\end{tabular}

\section{2}

Impact of an algorithm guiding patient responses to real-time continuous glucose monitoring (CGM) in type I diabetes managed with continuous subcutaneous insulin infusion (CSII)

D.N. O'Neal ${ }^{1}$, B. Krishnamurthy' ${ }^{1}$, F.J. Cameron ${ }^{2}$, P.G. Colman ${ }^{3}$, S. Hamblin ${ }^{4}$, M.A. O'Connell ${ }^{2}$, C. Rodda ${ }^{5}$, H. Teede ${ }^{5}$, J.D. Best ${ }^{1}$, A. Jenkins ${ }^{1}$

${ }^{1}$ University of Melbourne Department of Medicine, St Vincent's Hospital, Fitzroy, ${ }^{2}$ Department of Endocrinology and Diabetes, Royal Children's Hospital, Melbourne, ${ }^{3}$ Department of Endocrinology and Diabetes, Royal Melbourne Hospital, Melbourne, ${ }^{4}$ Department of Endocrinology and Diabetes, Western Hospital, Footscray, ${ }^{5}$ Department of Endocrinology and Diabetes, Monash Medical Centre, Clayton, Australia

Background and aims: The Australian Sensor Augmented Pump Study demonstrated that use of Real-Time CGM reduced HbAlc by $0.4 \%$ in type I diabetes (T1D) CSII patients. This study may underestimate the potential benefit CSII-CGM technology as subjects were not advised how to respond to CGM data. Aims are to develop and test a decision support tool for T1D patients using CSII-CGM technology.

Materials and methods: Algorithms were developed providing guidelines for both reactive and proactive responses to Real-Time CGM information. Sixty (38 female/22 male) CSII using T1D patients were recruited in pairs matched for age, gender and HbAlc. All were commenced on Real-Time CGM (Paradigm MMT722, Medtronic, Minimed, Northbridge, CA) and randomised to Algorithm: (Baseline:Age 29.8(13.3)Y; HbAlc7.9(0.7)\%) or no Algorithm (Baseline:Age 27.4(16.2)Y; HbA1c 7.8(0.8)\% for 16 weeks. HbA1c was measured at baseline and completion. A 6-day masked CGM trace was performed pre-Real-Time CGM initiation and 1 week prior to study-end. Analysis was by paired t-test and ANOVA.

Results: Algorithms have been developed. As of 3/08 HbAlc of the $11 \mathrm{com}-$ pleted subjects fell: $7.5(0.5) \%$ to $7.0(0.5) \%$; $p=0.006$. For Algorithm-users $(n=6)$ : $7.6(0.4) \%$ to $6.9(0.7) \%$; mean change $0.7 \%$, range of change $-1.2 \%$ to $+0.1 \%$; $5 / 6$ reached $\mathrm{HbAlc}<7.1 \%$. For non-Algorithm users $(n=5)$ : HbAlc $7.4(0.5) \%$ to $7.2(0.3) \%$; mean change $0.2 \%$, range of change $-0.8 \%$ to $+0.4 \%$; $1 / 5$ achieved $\mathrm{HbAlc}<7.1 \%$. CGM data did not differ significantly between the two groups. In particular there was no difference in time spent in hypoglycaemia $(<4.0 \mathrm{mmol} / \mathrm{L})$. Conclusion: Preliminary results suggest that patient education may improve benefit of Real Time- CGM use in CSII treated T1D patients.

Supported by: Medtronic Australia, National Health and Medical Research Council (Australia) CCRE

\section{3}

The effect of preprandial vs. postprandial administration of rapid-acting insulin analogue on continuous glucose monitoring in type 1 diabetic patients

A. Gelber ${ }^{1}$, I.F. Liberty ${ }^{2}$, Z.H. Perry ${ }^{3}$, E. Botach ${ }^{2}$, I. Harman-Boehm ${ }^{2}$ ${ }^{1}$ Goldman Medical School, Ben-Gurion University Faculty of Health Sciences, ${ }^{2}$ Diabetes Unit, Soroka University Medical Center, ${ }^{3}$ Department of Surgery A, Soroka University Medical Center, Beer-Sheva, Israel

Background and aims: Rapid acting insulin analogs have an onset of action 15 minutes after injection and peak action at 30-120 minutes. It is recommended that they be injected 10-15 minutes prior to meals. Several studies have found that preprandial injection of analog is preferable but concluded that postprandial injection can provide adequate glucose control. The aim of our study was to examine whether postprandial administration of the mealtime insulin bolus is as effective as preprandial administration in controlling glycemia in type 1 diabetic patients using an insulin pump or multiple daily injections and to assess patient satisfaction of both methods.

Materials and methods: Twelve subjects with type 1 diabetes- 6 on multiple injections and 6 on insulin pumps- were studied in two separate time periods. During the first three day time period the patients injected the meal rapid analog insulin bolus within 15 minutes before the meal, and during the second three day period, immediately after the meal. The patients were monitored by a Continuous Glucose Monitoring System (CGMS) for both time periods. The data was derived from the Minimed CGMS Solutions software and analyzed using SPSS. Mean blood glucose, nadir and peak values, 2 hour postprandial glucose, 2 hour AUC, standard deviation of mean glucose and time spent above $180 \mathrm{mg} \%$ or below $70 \mathrm{mg} \%$ were calculated. Analytical statistics were done using parametric measures ( paired-sample $\mathrm{T}$ test $\mathrm{P}<0.05$ )

Results: Patients ranged in age from 23-71years with $\mathrm{HbAlc}$ values ranging from 5.7-7.6\%. Mean BMI 26.97 \pm 3.72 . Mean basal insulin dose18.86 \pm 11.48 units and mean daily bolus of $22.36 \pm 14.38$ units.In the postprandial injection period, there was a significantly higher percentage of time during which the 
subjects using an insulin pump compared to MDI were above the recommended upper glucose limit of $180 \mathrm{mg} / \mathrm{dl}(26.5 \pm 13.35$ vs $8.46 \pm 11.00 \%$ respectively $\mathrm{p}=0.037$ ). In addition, in the same time period there were significantly higher maximal glucose values two hours post prandial in the patients using an insulin pump $(139.74+26.12 \mathrm{vs} 119.91 \pm 17.99 \mathrm{mg} \%, \mathrm{p}=0.038)$. There were no other significant differences seen in any of the parameters used to assess glycemic control between the two time periods nor were there any other significant differences seen between patients on multiple injection or pump therapy. Patient satisfaction scores were similar in the two periods but more patients preferred post-prandial injections citing improved flexibility and decreased fear of hypoglycemia.

Conclusion: The current study which did not find a significant advantage for pre-prandial injection makes it is reasonable to instruct type 1 diabetes patients using multiple daily injections to administer the insulin bolus immediately after the meal, if this timing better suits their life style. Patients on pump therapy may need to increase their mealtime bolus when switching from pre to postprandial timing of the bolus. The greater flexibility in the timing of mealtime injections and decreased fear of hypoglycemia may improve adherence to treatment.

\section{4}

Continuous glucose monitoring system compared to self blood glucose monitoring in type 1 diabetics at haemodialysis

P.T. Monteagudo ${ }^{1}$, M.S.M. Marrocos ${ }^{2}$, C. Bittencourt ${ }^{1}$, M.D.F. Neves ${ }^{1}$, C. Stella ${ }^{1}$, E.B. Rangel ${ }^{3}$, C. Melaragno ${ }^{3}$, J.O.P. Medina ${ }^{3}$, S.A. Dib ${ }^{1}$,

A.R. Chacra ${ }^{1}$, J.R. Sa ${ }^{1}$

${ }^{1}$ Medicine, Division of Endocrinology, Federal University of Sao Paulo, ${ }^{2}$ Nephrology, Servidor Publico Estadual Hospital, ${ }^{3}$ Medicine, Division of Nephrology, Federal University of Sao Paulo, Sao Paulo, Brazil

Background and aims: The better glycemic control during dialysis periods, the better clinic conditions patients will have for an eventual transplantation. But diabetics with renal insufficiency are at risk for hyper and hypoglycemic episodes, and usually have instable diabetes. The continuous glucose monitoring (CGM) is a device that allows a better glycemic evaluation. The objective was to compare glycemic behavior at CGM and the auto monitoring results in 11 type 1 diabetics (DM1) (6 men) at renal replacement therapy. Materials and methods: They were submitted to CGM for 3 days. We analyzed the $24 \mathrm{~h}$ before hemodialysis and $24 \mathrm{~h}$ during and after dialysis, each 4 hour. Patients had $38.7 \pm 11.9$ y of age; $17.7 \pm 4$.0y of diabetes duration; $25.7 \pm 20.2$ months in dialysis; $22.2 \pm 3.5 \mathrm{~kg} / \mathrm{m}^{2}$ of body mass index. Basal insulin was glargine in 5, determir in 2 and NPH in 4 patients, $37.6 \pm 21.4$ UI in the dialysis day and $31.6 \pm 21.4 \mathrm{UI}$ in the non dialysis day (ns). Glycated hemoglobin was $9.0 \pm 1.4 \%$. They realized 4 capillary glycemias each day, and ultra rapid insulin replacements were performed by 10 patients, and 9 counted carbohydrates. All had advanced diabetic retinopathy ( 2 amaurotic) and peripheral neuropathy (2 with neuropathic pain), 9 had autonomic neuropathy ( 8 with postural hypotension) and 6 had macroangyopathic disease ( 2 with coronary insufficiency).

Results: Table shows CGM results, and in the 3 last lines the glucometer values. $T$ test was performed for continuous variables, and "compare two proportions" for statistical analyses; ${ }^{*} \mathrm{p}<0.005$ vs. $24 \mathrm{~h}$ Before dialysis and \&: $\mathrm{p}<0.03$ vs. total CGM value.

\begin{tabular}{llllllll}
\hline Periods & $\boldsymbol{n}$ & $\begin{array}{l}\text { Glycemias } \\
m g / d l\end{array}$ & \multicolumn{2}{l}{ \% Glycemias } \\
\cline { 5 - 8 } & & $\leq 70$ & $70<$ Glyc $<140$ & $\geq 140$ & $\geq 180$ \\
\hline 24h Before dyal & $\mathbf{3 0 7 0}$ & $207 \pm 97$ & 5.6 & 23.2 & 71.2 & 57.5 \\
\hline Day of dialysis & $\mathbf{2 8 3 1}$ & $234 \pm 100^{*}$ & $3.9^{*}$ & $16.4^{*}$ & $79.7^{*}$ & $71.1^{*}$ \\
\hline TOTAL-CGMS & $\mathbf{5 9 0 1}$ & $220 \pm 99$ & 4.7 & 19.8 & 75.5 & 64.0 \\
\hline Glucometer-pre & $\mathbf{4 7}$ & $203 \pm 118$ & 17.2 & 10.6 & 72.3 & 44.7 \\
\hline Glucometer-post & $\mathbf{4 4}$ & $226 \pm 114$ & 6.8 & 20.5 & 72.7 & 59.1 \\
\hline Glucometer-total & $\mathbf{9 1}$ & $214 \pm 116$ & $12.1 \&$ & 15.4 & 72.5 & $51.7 \&$ \\
\hline
\end{tabular}

Conclusion: Our type 1 diabetic patients in chronic hemodialysis, although using basal/bolus insulin treatment, are in bad glycemic control. Especially in the day of dialysis, when mean CGM glycemia was significantly greater compared to the day before dialysis $(234 \pm 100$ vs. $\pm 207 \pm 97 \mathrm{mg} / \mathrm{dL}$ ), with lesser hypoglycemia frequency ( 3.9 vs. $5.4 \%$ ), 16.4 vs. $23.2 \%$ within normal values and 71.1 vs. $57.5 \%$ with hyperglycemia $\geq 180 \mathrm{mg} / \mathrm{dl}$ ( $\mathrm{p}<0.05$ in all). When we compared the glucometer values with the CGM, the proportion of hypoglycemia at self-blood glucose monitoring was more prevalent $(12.1 \%$ vs.
$4.7 \%$ respectively), and hyperglycemias $\geq 180 \mathrm{mg} / \mathrm{dl}$ was less prevalent $(51.7 \%$ vs. $64.0 \%$ ). These findings suggest that a more frequent self blood glucose monitoring might improve glycemic control in type 1 diabetic patients in hemodialysis.

Supported by: Minimed Solutions

\section{5}

Evaluating the effect of the FreeStyle Navigator Continuous Glucose Monitoring System in diabetes self-management

T. Danne ${ }^{1}$, T. Kracht ${ }^{1}$, K. Walte ${ }^{1}$, B. Aschemeier ${ }^{1}$, R. Geldmacher ${ }^{2}$, L. Sölter ${ }^{2}$, W. von dem Berghe ${ }^{2}$, J.R. Bugler ${ }^{3}$, Z. Welsh ${ }^{3}$, C. Jones ${ }^{3}$, R. Hartmann ${ }^{1}$, O. Kordonouri ${ }^{1}$

${ }^{1}$ Diabetes Center for Children and Adolescents, Kinderkrankenhaus Auf der Bult, Hannover, Germany, ${ }^{2}$ Schwerpunktpraxis, Diabetes-Zentrum Hannover-Nord, Germany, ${ }^{3}$ Research, Abbott Diabetes Care, Oxon, United Kingdom

Background and aims: This study was designed to investigate the use and impact of the FreeStyle Navigator Continuous Glucose Monitoring System (FSN-CGM) under home use conditions in self-management of diabetes.

Materials and methods: The study was for 60 days of continuous sensor wear (arm or abdomen) in patients with type 1 diabetes at home. The patients performed sensor insertions and calibrations. Real-time data and alarms were not available for the first 20 days (masked phase). Real-time data, trend arrows and threshold alarms for hypo- and hyperglycaemia were un-masked for the next 40 days. For the masked phase, patients were instructed to make all treatment decisions based on capillary BG tests only and to perform BG tests with the built-in FreeStyle BG meter. In the un-masked phase, subjects were instructed to make treatment decisions based on FSN-CGM except during hypoglycaemia or rapidly changing glucose values when confirmatory capillary BG test was performed prior to self-management decisions. The primary endpoint was to demonstrate a reduction in time spent outside euglycaemia (70-180 mg/dL) between the first 20 days (phase A) and last 20 days (phase B) of the study. Both phases were compared for a number of specified measures of glycaemic variability by paired t-test. HbAlc was recorded by DCA 2000 at the start and end of the study.

Results: Twenty-seven adult patients with type 1 diabetes (male/female ratio $14 / 13$ ) were included and completed the study (mean age 33.0 \pm 10.3 , range 18-55 yrs; diabetes duration $18.2 \pm 10.9 \mathrm{yrs})$. The average baseline $\mathrm{HbAlc}$ was $7.3 \pm 1.0 \%$ with $21 / 27$ patients on CSII therapy, the remaining on MDI. Mean baseline glucose was $169 \pm 27 \mathrm{mg} / \mathrm{dL}$ for phase A. During the study, two adverse events occurred for an adhesive reaction and bleeding/pain on sensor insertion; no serious adverse events were reported. Comparing phase A and phase $B$, patients had a significant reduction of the time spent outside euglycaemia from 10.2 to $8.8 \mathrm{hrs} /$ day $(\mathrm{p}<0.05)$. Furthermore, there were significant reductions in glucose $\mathrm{SD}$ from 61 to $55 \mathrm{mg} / \mathrm{dL}(\mathrm{p}<0.01)$, hyperglycaemic time $(>180 \mathrm{mg} / \mathrm{dL})$ from 9.5 to $8.2 \mathrm{hrs} /$ day $(\mathrm{p}<0.05)$, and the High Blood Glucose Index 1 (HBGI; Kovatchev BP et al, Diabetes Technol Ther 4:295-303, 2002) risk score from 9.0 to $7.6(\mathrm{p}=0.025)$. Hypoglycaemic time $(<70 \mathrm{mg} / \mathrm{dL})$ decreased from 0.74 to $0.59 \mathrm{hrs} /$ day without statistical significance $(\mathrm{p}>0.05)$. Mean $\mathrm{HbAlc}$ fell from $7.3 \pm 1.0 \%$ at baseline to $6.7 \pm 0.7 \%$ at the end of the study $(\mathrm{p}<0.001)$.

Conclusion: Data of this study indicate that the use of the FreeStyle Navigator Continuous Glucose Monitoring System has a positive effect on self-management of diabetes concerning glycaemic control and glycaemic variability. These findings are encouraging and supportive of similar data reported by other continuous glucose monitoring utilization studies. Supported by: Abbott Diabetes Care, UK

\section{6}

Use of real time continuous glucose monitoring in type 1 diabetes patients on intraperitoneal insulin (CIPII). A feasibility study H.J.G. Bilo ${ }^{1,2}$, K.H. Groenier ${ }^{3}$, N. Kleefstra ${ }^{1,4}$, S.J.J. Logtenberg ${ }^{1}$ ${ }^{1}$ Diabetes Centre, Isala Clinics, Zwolle, ${ }^{2}$ Internal Medicine, UMCG, Groningen, ${ }^{3}$ General Practice, UMCG, Groningen, ${ }^{4}$ Medical Research Group, Langerhans, Zwolle, Netherlands

Background and aims: Continuous intraperitoneal insulin infusion (CIPII) is used in patients with poorly controlled diabetes. Real time continuous glucose monitoring (CGM) can provide continuous information about current glucose values without the need for multiple invasive measurements. A 
prospective CGM system might be a valuable addition to CIPII, especially in those patients still experiencing frequent and/or unexpected hyper- or hypoglycemic episodes. Our aim was to investigate the effectiveness and safety of real time CGM in patients treated with CIPII.

Materials and methods: Open label, cross-over, randomized, controlled trial. A baseline phase of 72 hours, in which blinded CGM data were collected, was followed by 2 study phases in which subjects used either blinded or open CGM for $2 \times 72$ hours with a one-week crossover period in between. In the open phase the CGM system would alert whenever a glucose level fell below or rose above preset values $(3.9$ and $11.1 \mathrm{mmol} / \mathrm{L}$, respectively). The primary efficacy endpoint was percentage of time spent in euglycemia. A secondary endpoints was patient satisfaction as measured with the User Acceptance Questionnaire (UAQ) for CGM. Device accuracy was evaluated using median relative absolute difference (RAD) of continuous glucose values to paired SMBG meter readings.

Results: Baseline characteristics: $6 \mathrm{~F} / 4 \mathrm{M}$ type 1 diabetes patients, median (IQR) age 43.8 (31.8-50.6) years, median (IQR) duration of diabetes 20.5 (14.7-35.3) years, mean (SD) $\mathrm{HbA}_{1 c} 8.5$ (1.4) \%. Outcomes: Median RAD of sensor values compared to SMBG values was $14.0 \%$.

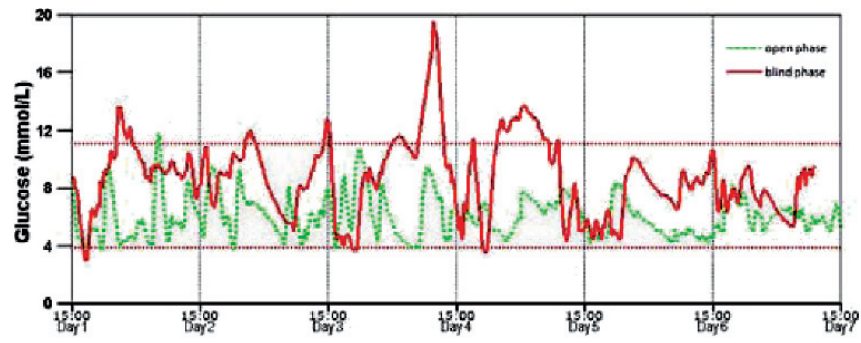

Figure 1. presents an example of CGM data in 1 subject.

Percentage of time in euglycemia $(4.0-10.0 \mathrm{mmol} / \mathrm{L})$ was $68 \%$ and $64 \%$ in the open and blind phase, respectively $(\mathrm{p}=0.41)$. Looking at a more narrow range $(4.0-8.7 \mathrm{mmol} / \mathrm{L})$ these percentages were $54 \%$ and $48 \%(\mathrm{p}=0.37)$. Percentage of time in hyperglycemia $(>10.7 \mathrm{mmol} / \mathrm{L})$ was $25 \%$ and $29 \%(p=0.25)$ in the open and blind phase, respectively. The mean (SD) UAQ scores regarding comfort, sensor operation, alarm and overall assessment (range 0-100) were 73.9 (14.4), 61.0 (18.9), 54.4 (7.8) and 60.8 (22.5), respectively, indicating satisfaction with CGM.

Conclusion: Patient satisfaction and user acceptance with the CGM system were generally high. However, efficacy on glycemic control should be evaluated in a sufficiently powered trail of longer duration. CIPII combined with CGM could serve as the next step to the development of a closed loop system.

Supported by: Mark Peyrot

\section{7}

Glycaemic profile by continuous glucose monitoring system in a cohort of adolescents treated with continuous subcutaneous insulin infusion or long acting insulin analogues

M. Anzellotti, A. Di Stefano, S. Carinci, F. Chiarelli, S. Tumini

Departemet of Pediatrics, University of Chieti, Italy.

Background and aims: The purpose of the study was to analyze daily glycemic trend in a cohort of adolescents on "basal-bolus" insulin regimen with continuous subcutaneous insulin infusion (CSII), glargine and detemir compared with a control group of healthy adolescents using continuous glucose monitoring system (CGMS). It was analyzed if CGMS could reveal different glycemic pattern in these groups of patients with similar metabolic control. Numbers of boluses, number of hypoglycemia and its duration, ketoacidosis and accuracy of CGMS were also evaluated.

Materials and methods: The 45 adolescents with type 1 diabetes who participated in the study had median age $17.08 \pm 3.21 \mathrm{yrs}$ old, mean duration of diabetes $8.77 \pm 4.82$ yrs, mean $\mathrm{HbAlc} 7.84 \pm 0.95 \%$, and mean BMI $25.69 \pm 4.71$ $\mathrm{Kg} / \mathrm{m}^{2}$. Fifteen patients were on CSII with rapid insulin analogue, 15 patients on glargine and rapid analogue at meals, 15 patients on detemir and regular insulin at breakfast and lunch and rapid analogue at dinner. Eligibility criteria for patients with T1DM included: treatment with basal bolus regimen al least from 6 months, duration of diabetes more than 2 years and similar BMI and $\mathrm{HbA1c}$. Ten healthy adolescents were enrolled as control group.

Results: The accuracy of CGMS was evaluated by linear regression of 704 data pairs recorded during the study. The correlation cofficient was $r=0,94$ $(p<0.0001)$. The glycemic profile was divided in daily glycemic profile from $8.00 \mathrm{am}$ to $24.00 \mathrm{pm}$ and in night glycemic profile from $00.00 \mathrm{am}$ to $8.00 \mathrm{am}$. During the night there weren't statistical significant difference between the 3 insulin regimens versus healthy subjects.During the day the glycemic profile revealed statistical significant difference between patients treated with CSII versus other insulin regimens (detemir from 11.00 to $12.00 \mathrm{am}(\mathrm{p}<0,001)$, from 12.00 to $1.00 \mathrm{pm}(\mathrm{p}<0,01)$, from 1.00 to $2.00 \mathrm{pm}(\mathrm{p}<0,0001)$, from 3.00 to $6.00 \mathrm{pm}(\mathrm{p}<0,05)$; glargine from $11.00 \mathrm{am}$ to $1.00 \mathrm{pm}(\mathrm{p}<0,01)$, from 1.00 to $2.00 \mathrm{pm}(\mathrm{p}<0,0001)$, from 3.00 to $4.00 \mathrm{pm}(\mathrm{p}<0,0001)$, from 4.00 to 5.00 $\mathrm{pm}(\mathrm{p}<0,001)$ and from 5.00 to $6.00 \mathrm{pm}(\mathrm{p}<0,01)$. It was also evaluated glycemic variability by the absolute value of measured glucose minus $5.5 \mathrm{mmol} / \mathrm{l}$ that revealed statistical significant difference between patients in treatment with CSII in comparison with patients in treatment with glargyne $(\mathrm{p}<0.37)$. The area under the curve (AUC) was calculated but it wasn't statistical significant difference between the 3 groups. The 45 patients with T1DM had 83 episodes of glucose level below $60 \mathrm{mg} / \mathrm{dl}(3.25 \mathrm{mmol} / \mathrm{l})$ measured by CGMS. The number of daily hypoglycemia were 52 episodes. The number of night hypoglycemias were 31 . There weren't statistical significant difference between groups in number and mean duration of hypoglycemia during the day and during the night. It was also noted that patients on CSII grant a mean of 5 boluses a day ( $43.5 \%$ ) versus 3.5 in patients on glargine (30.5\%) and 3 boluses in patients on detemir $(26 \%)$. No severe hypoglycemic or ketoacidosis events occurred in either groups.

Conclusion: The CGMS is useful to identify glycemic variability that are not evident with routine monitoring. Basal-bolus treatment with CSII seemed to be associated with better daily glycemic control. Patients on CSII grant more boluses and present less glycemic variability during daily time than other groups. 


\section{PS 101 Diabetes at the hospital}

\section{8}

The use of basal/bolus insulin regimen in the management of patients with diabetic ketoacidosis (DKA)

D. Smiley ${ }^{1}$, S. Jones ${ }^{2}$, P. Mulligan ${ }^{1}$, A. Temponi ${ }^{1}$, C. Semakula ${ }^{2}$, T. Keyler ${ }^{2}$, D. Umpierrez ${ }^{1}$, S. Nazarian ${ }^{2}$, N. Tesfaye ${ }^{2}$, M. Ceron ${ }^{1}$, G. Umpierrez'; ${ }^{1}$ Division of Endocrinology, Emory University, Atlanta, ${ }^{2}$ Division of Endocrinology, University of Minnesota, Minneapolis, United States

Background and aims: The traditional treatment of DKA includes the intravenous (IV) infusion of regular insulin until resolution of hyperglycemia/ ketosis, followed by subcutaneous (SC) injections of split-mixed or multidose regimen of NPH/regular insulin. The aim of this randomized controlled study (NCT00590044) was to compare the safety and efficacy of the traditional approach (NPH/regular) versus a basal/rapid-acting insulin analog (glargine/glulisine) regimen both during the acute IV phase and during the SC transition to maintenance period.

Materials and methods: A total of 60 patients with DKA were randomized to receive a continuous IV infusion of regular or glulisine insulin (starting dose $0.1 \mathrm{U} / \mathrm{kg} / \mathrm{hr}$ ) until the resolution of DKA. Following resolution of DKA, patients were transitioned to a split-mixed (NPH/regular) insulin $(n=30)$ or to glargine/glulisine $(\mathrm{n}=30)$ at a starting dose of $0.6 \mathrm{U} / \mathrm{kg} / \mathrm{day}$ for lean and 0.8 $\mathrm{U} / \mathrm{kg} /$ day for obese subjects. NPH and regular were given as $3 / 5$ of the total daily dose in the morning and $2 / 5$ in the evening. Glargine was given once daily as $1 / 2$ of the total daily dose and the remaining $1 / 2$ was given as glulisine in equally divided doses before meals.

Results: There were no differences in the admission BG: $29 \pm 7 \mathrm{mmol} / \mathrm{L}$ vs. $33 \pm 10 \mathrm{mmol} / \mathrm{L}, \mathrm{pH}: 7.18 \pm 0.1$ vs. $7.19 \pm 0.1, \mathrm{HCO} 313 \pm 5 \mathrm{mmol} / \mathrm{L}$ vs. $14 \pm 5$ $\mathrm{mmol} / \mathrm{L}, \mathrm{BOHB}:(768 \pm 288 \mathrm{umol} / \mathrm{L})$ vs. $(864 \pm 288 \mathrm{umol} / \mathrm{L})$ between groups $(\mathrm{p}=\mathrm{NS})$. The mean time to resolution of DKA was similar between patients treated with IV regular (11.4 $\pm 8 \mathrm{hrs})$ and those treated with IV glulisine insulin $(8.6 \pm 4 \mathrm{hrs}, \mathrm{p}=\mathrm{NS})$. There were no differences between groups in the total amount and/or rate of insulin infusion until resolution of DKA. There was no significant difference in mean BG level during the hospital stay between the $\mathrm{NPH} /$ regular $(11 \pm 3 \mathrm{mmol} / \mathrm{L})$ and the glargine/glulisine $(12 \pm 3 \mathrm{mmol} / \mathrm{L})$ groups, $\mathrm{p}=0.08$. NPH/regular insulin, however, was associated with a higher rate of mild $(\mathrm{BG}<3.3 \mathrm{mmol} / \mathrm{L})$ and severe hypoglycemia $(\mathrm{BG}<2.2 \mathrm{mmol} / \mathrm{L})$ during the hospital stay. In the NPH/regular group, a total of 8 patients $(27 \%)$ had 15 episodes of mild hypoglycemia, and 2 patients (6.6\%) had 2 episodes of severe hypoglycemia. In the glargine/glulisine group, only four patients (13\%) had a single episode of mild hypoglycemia and no patient in this group had severe hypoglycemia.

Conclusion: Our results indicate that the use of basal/bolus insulin regimen in the management of DKA resulted in improvement in glycemic control and a lower rate of hypoglycemia during the SC maintenance period. Our study indicates that the use of glargine and glulisine insulin regimen is as effective and safer compared to the traditional NPH and regular insulin for the management of diabetes following resolution of DKA.

Supported by: sanofi-aventis

\section{9}

Postprandial urinary glucose may be useful for preventing arteriosclerosis with PWV data

M. Kato ${ }^{1}$, N. Kato ${ }^{1}$, H. Fujii ${ }^{2}$, Y. Watanabe ${ }^{2}$, T. Miyakawa ${ }^{2}$

${ }^{1}$ Internal Medicine, Kato Clinic of Internal Medicine, Takasago, Katsushika, ${ }^{2}$ Internal Medicine, Tama-Center Mirai Clinic, Tokyo, Japan

Background and aims: Japanese diabetics are more likely to develop insulin deficiency compared with Caucasian type 2 diabetic patients. In this study, the usefulness of measuring postprandial urinary glucose as a marker of postprandial hyperglycemia was investigated by urinary glucose levels during the 75-g oral glucose tolerance test (OGTT). The aortic pulse wave velocity (PWV) is an independent marker of cardiovascular risk, so the relationship between PWV and blood or urine glucose levels was investigated in patients who underwent both the OGTT and PWV measurement.

Materials and methods: Japanese diabetics are more likely to develop insulin deficiency compared with Caucasian type 2 diabetic patients. In this study, the usefulness of measuring postprandial urinary glucose as a marker of postprandial hyperglycemia was investigated by urinary glucose levels during the 75-g oral glucose tolerance test (OGTT). The aortic pulse wave velocity
(PWV) is an independent marker of cardiovascular risk, so the relationship between PWV and blood or urine glucose levels was investigated in patients who underwent both the OGTT and PWV measurement.

Results: Among 306 patients, 160 had diabetes according to the result of the OGTT. However, a diagnosis of diabetes was missed in $33 \%$ of patients when judged from the FPG alone. When a 2-hr urinary glucose level $\geq 100 \mathrm{mg} / \mathrm{dL}$ was used as a screening criterion, it was found to be elevated in 156 out of 160 patients (98\%). In the subjects with a normal, borderline, and diabetic OGTT pattern, the mean PWV was 13.7, 15.8, and $15.9 \mathrm{~m} / \mathrm{S}$, respectively. It showed differences between the normal pattern and the borderline or diabetic patterns, but not significant. When the PWV was compared with the blood glucose level after OGTT, the mean PWV was 14.8, 15.9, and $16.1 \mathrm{~m} /$ $\mathrm{S}$ when the fasting blood glucose was less than 110, 110-125, and over 126 $\mathrm{mg} / \mathrm{dL}$, respectively. It showed no significant differences between the three groups. However, it was $13.7,15.9$, and $16.3 \mathrm{~m} / \mathrm{S}$ when the blood glucose level at $60 \mathrm{~min}$ after the OGTT was less than 180, 180-199, and over200 mg/ $\mathrm{dL}$, respectively, with a significant difference $(\mathrm{p}<0.05)$ between the first and third group. There was also a difference $(\mathrm{p}<0.1)$ between the groups in which urinary glucose was $100 \mathrm{mg} / \mathrm{dL}$ or more and less than $100 \mathrm{mg} / \mathrm{dL}$ from 60 min after the OGTT (the mean PWV was 14.2 and $16.0 \mathrm{~m} / \mathrm{S}$, respectively). Although the PWV is known to increase with age, there were no significant age differences between the groups for which these significant differences of PWV were noted.

Conclusion: Postprandial hyperglycemia is a factor that promotes the development of arteriosclerosis. In the present study, the PWV was also more closely related to the blood glucose level at $60 \mathrm{~min}$ after the OGTT than to the fasting or 120 min glucose levels. Urinary glucose is an important parameter for screening, because it reliably detects patients with postprandial hyperglycemia. The PWV showed a significant difference in relation to the time of measurement after the OGTT (from $60 \mathrm{~min}$ ), so measurement of postprandial urinary glucose may be useful for preventing arteriosclerosis, as is measurement of blood glucose, while also being more economical.

\section{0}

Impact of hyperglycaemia on morbimortality, length of hospitalisation and rates of rehospitalisations in a general hospital setting in Brazil S.A.O. Leite, S.B. Locatelli, A.R.F. Oliveira, D. Tockus, S.P. Niece, T. Tosin Internal Medicine, Universidade Positivo, Curitiba, Parana, Brazil

Background and aims: It is already known that hyperglycemia in hospitalized patients is related to a higher incidence of clinical and surgical complications and to worse outcomes. Adequate glycemic control and earlier diagnosis of type 2 diabetes during hospitalization are cost-effective measures.

Material and methods: This study was designed to determine the impact of hospital hyperglycemia on morbimortality in a university hospital setting during a 3 month period by reviewing patients' records. The primary purposes of this trial were to verify if hyperglycemia is properly and early diagnosed, how it is managed during the hospitalization and also to evaluate the relationship between control of hospital hyperglycemia and outcomes such as complications during hospital stay, extent of hospitalization, frequency of rehospitalizations, death rates and number of days at the ICU(Intensive Care Unit) after admission. The secondary objective was to evaluate the medical management of hyperglycemia. Statistical analysis utilized Kruskall-Wallis and "a posteriori" d.m.s. to complement, Spearman and Qui-square test with a level of significance of $5 \%(\mathrm{p}<0.05)$.

Results: 779 patient records fulfilling inclusion criteria were reviewed. They were divided into 5 groups: 1-)diabetics with normal blood glucose( $\mathrm{n}=123)$; 2-)diabetics with hyperglycemia $(\mathrm{n}=76) ; 3$-)non diabetics with hyperglycemia $(\mathrm{n}=225)$; 4 -)persistent hyperglycemia during 3 consecutive days $(n=57)$ and 5-)normal glucose control $(\mathrm{n}=298)$. Patients in groups 2, 3 and 4 compared groups 1 and 5 had significantly higher mortality rates ( $17.7 \%$ vs. $2.8 \%)$ and ICU admissions with complications ( $23.3 \%$ vs $4.5 \%$ ). Group 4 had longer hospitalization (mean 15.5 days) and group 5 had the lowest rate of rehospitalizations (mean of 1.28). Only 152 hyperglycemic patients were treated. Insulin "sliding-scale" alone was the most frequent treatment used with wide variations in glucose target medical prescriptions. Insulin IV infusion was used in 3,8\% of patients at the ICU. Alc was measured in 11 patients.

Conclusion: There is a correlation between hospital hyperglycemia and morbimortality, length of hospitalization and number of re-hospitalizations, among other parameters. Most of patients do not have their blood glucose assessed at hospital, despite of high number of patients with hyperglycemia without diagnosis of diabetes, Alc was not measured. Even when patients were assessed for hyperglycemia, they are not treated properly. 


\section{1}

Practical knowledge and confidence in the management of diabetes is lacking amongst hospital doctors

P. Muthusamy, E. Ejenavi, S.M. Rajbhandari

Diabetes Dept, Lancashire Teaching Hospital, Chorley, United Kingdom

Background and aims: One of the reasons why diabetic patients who are hospitalised have prolonged length of stay is due to the suboptimal management of their diabetes during admission. Day to day management of glycaemic control has been increasingly delegated to diabetes specialist (nurses or doctors) despite $20 \%$ of all in-patients having diabetes. In order to assess whether this is due to lack of knowledge or due to lack of confidence we conducted an audit amongst hospital doctors of all grades and specialities.

Materials and methods: This was a prospective study using anonymous multiple-choice questionnaires. Out of 16 questions, 6 assessed factual knowledge of oral hypoglycaemic agent, insulin and treatment targets, 5 assessed hypothetical case scenario such as adjusting insulin doses and oral hypoglycaemic agents and 5 questions assessed self-reported confidence. Questionnaires were sent by e-mail and letters to all grades of doctors working in 2 hospitals. A reminder was sent to all doctors after 3 weeks.

Results: Out of 115 questionnaires sent 44 (38.3\%) replies were obtained. All answers were anonymous so it was not possible to pursue individuals for reply. We found that basic knowledge of HbA1c and blood glucose targets were known to $80 \%$ of doctors. Regarding various insulin preparation available, only $60 \%$ could correctly identify if the particular insulin was long acting, short acting or mixture of insulin. When asked about how familiar were they with commonly prescribed insulin in the hospital, around 50\% felt that these were well known to them. In a hypothetical case scenario of persisting hyperglycaemia on insulin treated patient, $70 \%$ of replies correctly adjusted the dose of insulin but only $45 \%$ felt confident in doing so. On the other hand $80 \%$ had self-reported competency in managing metabolic emergencies such as ketoacidosis, hyperosmolar state or hypoglycaemia. The most common in-patient clinical scenario of uncomplicated hyperglycaemia was correctly managed only by $25 \%$ of subjects and hypoglycaemia by $79 \%)$. Most ( $80 \%)$ of the doctors stated that they would like further training in the management of diabetes for in-patient.

Conclusion: Our study suggests that there is a lack of practical knowledge amongst hospital doctors in the management of diabetes. Following this audit we have developed a case based teaching tool, which has been highly appreciated by trainee doctors.

\section{2}

In-patient prevalence of diabetes in an Irish teaching hospital: resource implications?

G. Moloney ${ }^{1}$, R. Connolly ${ }^{1}$, A. Egan ${ }^{1}$, E. Higgins ${ }^{1}$, F. Waldron-Lynch ${ }^{1}$, F. Dunne $e^{1,2}$

${ }^{1}$ Endocrinology, University College Hospital Galway, ${ }^{2}$ Department of Medicine, National University of Ireland, Galway, Ireland

Background and aims: Patients with diabetes mellitus are acknowledged to be at higher risk of an in-patient hospital admission and to experience a greater length of hospital stay.Several bodies of research have shown that good glycaemic control as an in-patient improves the clinical outcome.One of the key strategies in improving the quality of in-patient management of diabetes is the provision of specialist diabetes services. The aim of this study was to assess the point prevalence of diabetes in the general adult wards of a major teaching hospital, with comparison of the demographics of diabetic patients to those of the general patient population and analysis of the use of multidisciplinary diabetic services.

Materials and methods: A cross-sectional study at a tertiary centre was undertaken during a defined 24 hour period. A list of all in-patients was compiled and those with a diagnosis of diabetes identified using data from the Hospital In-Patient Enquiry scheme (HIPE). A chart review was undertaken to identify specific patient characteristics, and referral pattern to the diabetes services.

Results: There were 340 in-patients during the defined period, 19 (5.6\%) of whom had diabetes mellitus. 13(68\%) of these patients were male and 6 (32\%) were female. Type 2 diabetes was present in $13(68 \%)$. The mean age of the diabetic cohort was 68 years compared to 58 in the non-diabetic population. The median length of stay (LOS) was 18 days for diabetic patients compared to 11 days for the non-diabetic population. The mean LOS for patients $>66$ years was 28 days, compared to 15 days for patients $<66$ years. 15 patients
(78.9\%) were admitted to services other than diabetes, and 8 (42\%) were admitted because of a vascular complication. All diabetic patients survived to discharge. Of the non-diabetic patients, the mortality was $5 \%$ ( 16 out of 321 ). 10 of 15 patients on general wards were reviewed by diabetes services during their hospital admission.

Conclusion: Our study population followed established trends regarding age, gender and type of diabetes. The main reason for admission of diabetic patients was management of vascular complications. Diabetes services were utilised for two thirds of patients. The length of stay for patients with diabetes was greater compared to background patient population with significant resource implications.

\section{3}

Difference between insulin sensitivity expressed by ability to lower plasma glucose concentration and to keep plasma glucose concentration stable during medical treatment

M. Matsuda, Y. Hisano, N. Hayakawa, M. Shigeto, Y. Akiyama,

M. Masuzawa, T. Okabe

Diabetes and Endocrine Department, Kameda Medical Center, Kamogawa, Japan

Background and aims: Insulin sensitivity has been expressed mainly by two ways, ability to lower BG (blood glucose) and to keep BG constant. To understand the difference between these two kinds of parameters, we applied insulin infusion analysis which was used to stabilize plasma glucose conc. after acute phase of critically ill diabetic patients.

Materials and methods: Insulin sensitivity is defined as a decremental conc. of BG by a fixed increment of insulin (or delta insulin) (ISI-delta [mg/dl per $\mathrm{U}]$ ). On the other hand, whole body insulin resistance (IRI-abs [U/hr]) can be defined as an absolute amount of insulin to keep fixed glucose turnover and keep BG constant. These two parameters were analyzed by plotting insulin infusion rates on $\mathrm{x}$-axis, and the differences of BGs on $\mathrm{y}$-axis. BG was measured from 1 to $6 \mathrm{hrs}$ intervals. GIR (Glucose infusion rate [g/hr]) is converted to the theoretical increase of $\mathrm{BG}[\mathrm{mg} / \mathrm{dl}$ per $\mathrm{hr}]$ in glucose distribution space, and is added on the graph to estimate the next BG value, and insulin infusion rate was adjusted to achieve the desired BG. BG was measured by PCx (Abbott, USA), and human regular insulin (1 U/ml saline) and glucose were infused by infusion pumps for clinical use calibrated routinely. Data during 15115 hours from 139 subjects $(\mathrm{M} / \mathrm{F}=80 / 59$, age $=60 \pm 16$ y.o., BMI $=24 \pm 5 \mathrm{~kg} / \mathrm{m}^{2}$ ) were analyzed in this method. After connecting two consequent observed points, reading $\mathrm{X}(\mathrm{U} / \mathrm{hr})$ on $\mathrm{X}$-axis $(\mathrm{Y}=0$ : to cencel the effect of glucose infusion) was used to calculate IRI-abs. ISI-delta was the slope of the line, when IRI-abs became fixed. When IRI-abs was not stable, ISI-delta was calculated by the least square method to minimize the error between the expected points and actual observed points. An average IRI-abs and ISI-delta were calculated every 24 hours starting next day after initiation of insulin infusion. Only when GIR was more than $2.2 \mathrm{mg} / \mathrm{kg}$ per min and insulin infusion rate was more than $1 \mathrm{mU} / \mathrm{kg}$ per min, the calculation was made to avoid the influence of residual endogenous glucose production and insulin secretion. GIR during $1 \mathrm{mU}$ insulin clamp was simulated by IRI-abs and ISI-delta. Calculation was made by EXCEL application.

Results: Total number of sampling of BG was 6405. BG before insulin infusion was $220 \pm 187 \mathrm{mg} / \mathrm{dl}$, and the average BG was $154 \pm 57 \mathrm{mg} / \mathrm{dl}$, achieving final BG of $130 \pm 48 \mathrm{mg} / \mathrm{dl}$. During 15115 hours, BGs less than $50 \mathrm{mg} / \mathrm{dl}$ were occurred only 5 times; 3 incidental halts of glucose infusion, one incident of wrong insulin preparation, and one patient with a pancreatic tumor. ISI-delta and IRI-abs were calculated from data of 377 days. Average ISI-delta was 57 $\pm 10 \mathrm{mg} / \mathrm{dl}$ per $\mathrm{U}$ per hr. IRI-abs was shifted from $-0.8 \pm 2.6$ to $4.2 \pm 4.9$ $\mathrm{U} / \mathrm{hr}$ in one subject, and the average IRI-abs was $1.0 \pm 2.9 \mathrm{U} / \mathrm{hr}$. Significant correlation between ISI-delta and IRI-abs was not demonstrated. However, a simulated GIR during $1 \mathrm{mU}$ insulin clamp was correlated with ISI-delta $(\mathrm{r}=0.7, \mathrm{p}<0.05)$, and inverse of IRI-abs $(\mathrm{r}=0.7, \mathrm{p}<0.05)$.

Conclusion: We have calculated two types of insulin sensitivity indices, IRIabs and ISI-delta. Insulin clamp can detect the change of both IRI-delta and IRI-abs, while the ISI-delta cannot detect the change of IRI-abs. Since both IRI-abs and ISI-delta affect insulin requirement during real clinical settings, it is possible to conclude that insulin sensitivity is better approximated by insulin clamp technique, rather than the other methods which calculate indices like ISI-delta only. 
Table 1

\begin{tabular}{|c|c|c|c|c|c|c|c|c|}
\hline & \multicolumn{2}{|l|}{ IG Phase I } & \multicolumn{2}{|l|}{ CII Phase I } & \multicolumn{2}{|l|}{ IG Phase II } & \multicolumn{2}{|c|}{ NPH Phase II } \\
\hline & CGMS & CAPG & CGMS & CAPG & CGMS & CAPG & CGMS & CAPG \\
\hline mean glycemia in $\mathrm{mg} / \mathrm{dl}$ & $128 \pm 41$ & $134 \pm 44$ & $134 \pm 39$ & $138 \pm 37$ & $131 \pm 30$ & $127 \pm 37$ & $143 \pm 32$ & $144 \pm 37$ \\
\hline MAGE & $42(30-126)$ & $54(25-201)$ & $44(19-104)$ & $72(0-126)$ & $40(20-116)$ & $39(10-181)$ & $38(16-108)$ & $48(12-124)$ \\
\hline 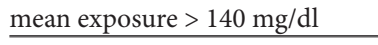 & $13(0-51)$ & $10(0-64)$ & $11(2-37)$ & $11(0-29)$ & $4(0-81)$ & $6(0-47)$ & $16(6-29)$ & $10(0-44)$ \\
\hline$\underline{p}$ & $0.7123(\mathrm{NS})$ & & $0.2411(\mathrm{NS})$ & & $0.1141(\mathrm{NS})$ & & 0.5147 (NS) & \\
\hline mean exposure $<70 \mathrm{mg} / \mathrm{dl}$ & $0(0-6)$ & $0(0-1)$ & $0(0-5)$ & $0(0-0)$ & $0(0-3)$ & $0(0-0)$ & $0(0-4)$ & $0(0-0)$ \\
\hline$\underline{p}$ & 0.0110 & & $0.0513(\mathrm{NS})$ & & 0.4260 (NS) & & $0.4728(\mathrm{NS})$ & \\
\hline excursion rate/time $>140 \mathrm{mg} / \mathrm{dl}$ & $4(1-8)$ & $1(0-2)$ & $5(3-7)$ & $2(0-4)$ & $3(0-5)$ & $1(0-2)$ & $4(1-8)$ & $1(0-2)$ \\
\hline$\underline{p}$ & 0.0069 & & 0.0051 & & 0.0218 & & 0.0209 & \\
\hline excursion rate $<70 \mathrm{mg} / \mathrm{dl}$ & $1(0-11)$ & $0(0-4)$ & $1(0-4)$ & $0(0-2)$ & $1(0-6)$ & $0(0-1)$ & $0(0-2)$ & $0(0-1)$ \\
\hline $\bar{p}$ & 0.0110 & & 0.1238 (NS) & & 0.0169 & & 0.4728 (NS) & \\
\hline
\end{tabular}

\section{4}

Glycaemia variability in acute myocardial infarction goes undetected despite intensive capillary glucose monitoring

J. Baptista ${ }^{1}$, D.R. Franco ${ }^{1,2}$, C.R. Sampaio ${ }^{3}$, M.C. Alves ${ }^{4}$, D.J. Goldberg ${ }^{1}$, F.G. Eliaschewitz ${ }^{1,5}$

${ }^{1}$ Clinical Research, CPCLIN, ${ }^{2}$ Endocrinology, Intermédica Saúde,

${ }^{3}$ Cardiology, H. SEPACO, ${ }^{4}$ Fac. Public Health, Un. São Paulo, ${ }^{5}$ Internal

Medicine, H. Heliópólis, São Paulo, Brazil

Background and aims: Acute changes in glycemia can cause oxidative stress and lead to endothelial dysfunction. Therefore, reducing the variability of glucose levels is increasingly recognized as an additional target in diabetes control. Variability can be quantified by mean amplitude of excursions, MAGE, or quantified above certain thresholds as AUC or rate of excursions per time. Our aim was to compare control and variability parameters obtained by intensive capillary glycemia (CAPG) measurements with continuous glucose monitoring systems (CGMS), in hyperglycemic acute myocardial infarct (AMI) patients followed 3-4 days in Intensive Care Unit (ICU) that were included in a randomized study comparing glucose control between insulin Glargine (IG) and standard care (StC) therapy.

Materials and methods: Hyperglycemic patients $(207 \pm 73 \mathrm{mg} / \mathrm{dl})$ admitted at the ICU within $24 \mathrm{~h}$ of AMI were randomized to receive basal-bolus regimen with IG and Regular Insulin (RI) $(\mathrm{N}=10)$, or StC $(\mathrm{N}=10)$, which comprises glycemia control by continuous insulin infusion (CII) for 24-48 $\mathrm{h}$ followed by control with NPH insulin and RI (Fig 1). After a period of stabilization with RI, CGMS was installed. Groups were comparable in onset of AMI, age, gender, weight, duration of diabetes, previous treatment, complications, and glycemia at the beginning of CGMS. CGMS data were analyzed in blind fashion and compared with CAPG parameters. Mann-Whitney and T tests were used.

Results: Mean glycemia by CGMS and CAPG was comparable. Mean exposure to hyperglycemia in the IG group was 4 times lower measured by CGMS and $60 \%$ less by CAPG, but without reaching significance. CGMS and CAPG data in the same treatment group show similar values for all parameters, with the exception of CGMS data on mean exposure and excursion rates $<70 \mathrm{mg} /$ $\mathrm{dl}$, higher for IG in the first $48 \mathrm{~h}$ compared to StC. The main difference was a consistently increased rate of excursions $>140 \mathrm{mg} / \mathrm{dl} /$ day measured by CGMS (Table 1).

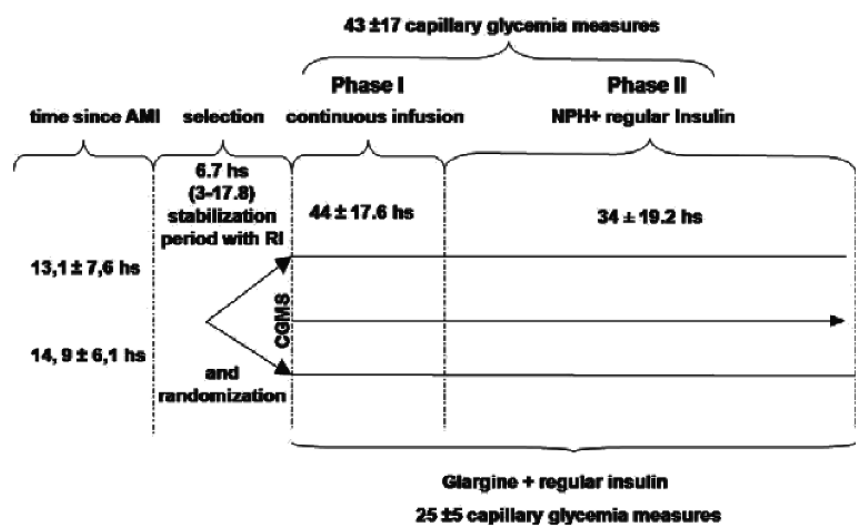

Conclusion: CAPG during ICU allows us to obtain glycemic control data similar to those measured by CGMS, excepting the rate of excursions to hyperglycemia. Capillary monitoring detects only $25-50 \%$ of excursions. Thus, if variability in glycemia levels is an important component of diabetes control, it is being missed even if intensive capillary glycemia control is employed. Supported by: sanofi-aventis

\section{5}

Do patients with diabetes and those without react differently to hyperglycaemia in the hospital?

R. Juneja ${ }^{1}$, Q. Zhang ${ }^{2}$, V. Abad ${ }^{3}$, A. Golas ${ }^{1}$, J. Carroll' ${ }^{1}$, S. Flanders ${ }^{1}$, C. Roudebush ${ }^{1}$

${ }^{1}$ Med, Indiana U, Indianapolis, ${ }^{2}$ sanofi-aventis, U.S., Bridgewater, ${ }^{3}$ Diabetes, Epsilon Grp Virginia, Charlottesville, United States.

Background: Inpatient hyperglycemia is a contributing factor to morbidity and mortality. Little is known about the levels of blood glucose (BG) at which mortality becomes most prevalent

Methods: We analyzed data from the SUGAR ${ }^{\mathrm{mi}}$ ( $\underline{\text { Systematic }}$ Utilization of Glucose $\underline{A}$ ssessment and $\underline{R}$ esponse) program implemented at two large academic medical centers. SUGAR is a process improvement program centered around Tight Glycemic Control (TGC) that includes the Clarian GlucoStabilizer $\left(\mathrm{CGS}^{\mathrm{m}}\right)$ computerized insulin administration systems, education on principles of TGC and its importance, and electronic data collection. This study focused on the relationship of mean BG for the entire hospital stay to inhospital mortality for patients with and without diabetes. The primary outcome measure was a standardized mortality ratio (SMR) calculated as the actual mortality rate divided by an expected mortality rate provided by the University HealthSystem Consortium. We examined 36,404 patient encounters from June 2003 - Sept 2007 (10,988 for patients with diabetes and 25,416 without preexistent diabetes). All encounters included at least one day in the Intensive Care Unit (ICU). We generated locally smoothed curves of the SMR versus mean BG for patients with and without diabetes to portray in a continuous way the dependence of the SMR on mean BG. The value of SMR at a $B G$ value is the $S M R$ for all patients whose mean $B G$ was within $0.3 \mathrm{mmol} / \mathrm{L}$ of that value.

Results: Table 1 presents actual mortality, expected mortality, and SMR for patients across discrete ranges of mean BG. Patients without diabetes appear to derive maximum benefit when their mean $B G$ is in the neighborhood of 5.0-7.2 mmol/L with a pronounced trend of increased SMR either above or below this range. Patients with diabetes appear to derive maximum benefit when their mean BG is in the $5.0-10.0 \mathrm{mmol} / \mathrm{L}$ range with worsening outcomes as BG level moves away from this range in either lower or higher direction.

Conclusion: Current treatment guidelines recommend a blood glucose of $<6.1 \mathrm{mmol} / 1$ for ICU patients and $<10 \mathrm{mmol} / \mathrm{l}$ for non-ICU patients. Our findings suggest that patients with diabetes appear to be tolerating a wider range of blood glucose in the hospital. Further analysis is planned to examine relationships between $B G$ variability and hospital outcomes by controlling for patient characteristics, antidiabetic medications prior to hospital admission and insulin therapy during hospital stay. 
Table 1. Actual mortality, expected mortality, and standardized mortality ratio for patients with and without diabetes by blood glucose range.

\begin{tabular}{|c|c|c|c|c|c|c|c|c|}
\hline \multirow[t]{2}{*}{ Mean BG (mmol/L) } & \multicolumn{4}{|c|}{ Patients with Diabetes } & \multicolumn{4}{|c|}{ Patients without Diabetes } \\
\hline & $\mathrm{n}$ & $\begin{array}{l}\text { Actual Mortality } \\
\text { Rate (\%) }\end{array}$ & $\begin{array}{l}\text { UHC Expected } \\
\text { Mortality Rate (\%) }\end{array}$ & SMR (\%) & $\mathrm{n}$ & $\begin{array}{l}\text { Actual Mortality } \\
\text { Rate (\%) }\end{array}$ & $\begin{array}{l}\text { UHC Expected } \\
\text { Mortality Rate (\%) }\end{array}$ & SMR (\%) \\
\hline$<5$ & 75 & 33.3 & 14.5 & 2.3 & 815 & 10.4 & 7.3 & 1.4 \\
\hline $5-7.2$ & 3607 & 12.8 & 14.2 & 0.9 & 16190 & 6.8 & 9.6 & 0.7 \\
\hline$>7.2-10$ & 5572 & 9.2 & 11.5 & 0.8 & 7612 & 13.6 & 11.3 & 1.2 \\
\hline$>10-12.2$ & 1246 & 11.0 & 10.4 & 1.1 & 574 & 33.1 & 14.8 & 2.2 \\
\hline$>12.2$ & 488 & 20.1 & 9.3 & 2.2 & 225 & 50.2 & 18.9 & 2.7 \\
\hline
\end{tabular}

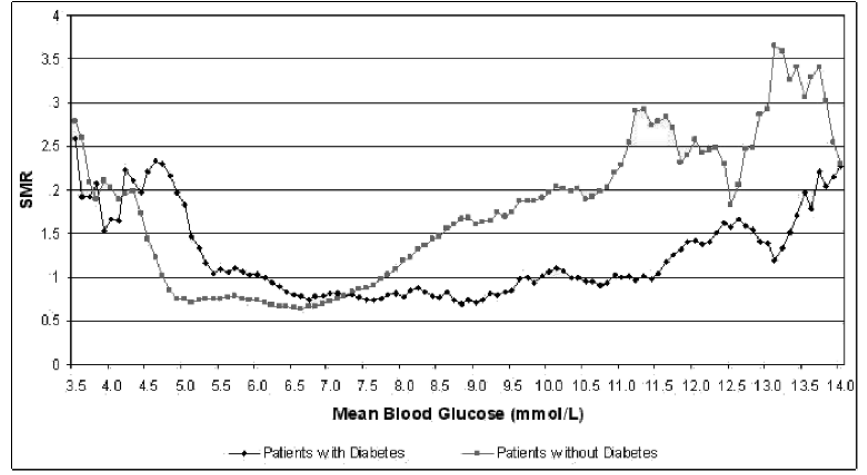

Supported by: sanofi-aventis U.S. Group (editorial)

\section{6}

A prospective audit of glucocorticoid-induced hyperglycaemia in nondiabetic hospital in-patients

G. Daniels, J. Kaye

Endocrinology and Diabetes, Sir Charles Gairdner Hospital, Perth, Australia

Background and aims: Glucocorticoids are commonly prescribed in a wide variety of clinical situations and in people with established diabetes, worsening of glycaemic control is a well-recognised side effect. Glucocorticoids administered to non-diabetic patients may be associated with transient hyperglycaemia or new-onset diabetes, however, there have been very few studies that have accurately described the prevalence on glucocorticoid-induced hyperglycaemia, its predictors or its impact or consequences on hospitalised patients not otherwise known to have diabetes.

Materials and methods: This study, over a 3 month period, examined glucocorticoid-induced hyperglycaemia in patients receiving $>25 \mathrm{mg}$ prednisolone; or $>8 \mathrm{mg}$ dexamethasone; or equivalent, for any indication (apart from palliative care use or use as part of an organ transplantation or chemotherapy regime). Two definitions of hyperglycaemia were considered. The first required 2 or more glucose levels within the diabetic range (fasting $\geq 7.0$ or random $\geq 11.1 \mathrm{mmol} / 1)$. The second required 2 or more glucose levels within the range considered appropriate for therapeutic intervention for hyperglycaemia in hospital in-patients (fasting $\geq 6.0$ or random $\geq 10.0 \mathrm{mmol} / \mathrm{l}$ ).

Results: 64 consecutive in-patients were studied. All had fasting and preprandial capillary glucose levels measured for 48 hours. Admission diagnoses were grouped as respiratory (53\%), intra-cranial pathology (19\%) or other (28\%). 14 had a known diagnosis of diabetes. Of the remaining 50, based on a diabetic range definition, $15(30 \%)$ were hyperglycaemic. These patients were older $(68.3+/-16.4$ vs $55.2+/-21.2$ years; $\mathrm{p}=0.04)$, were more likely to have respiratory disease ( $80 \%$ vs $46 \% ; p=0.04$ ) and less likely to have received dexamethasone ( $0 \%$ vs $37 \%$; $\mathrm{p}=0.005)$ therapy. There was a non-significant trend toward longer hospital length of stay however, only $2(13 \%)$ received any form of hypoglycaemic therapy. Prior glucocorticoid use did not influence risk of hyperglycaemia. Glucose levels peaked prior to lunch $(10.6 \pm 5.0$ vs 7.4 $\pm 2.7 ; \mathrm{p}<0.0001$ ), however, unexpectedly, fasting glucose levels were significantly elevated in the glucocorticoid treated group $(7.7 \pm 1.6$ vs $6.0 \pm 1.7$; $\mathrm{p}<0.001$ ) with $60 \%$ having both fasting levels greater than $7.0 \mathrm{mmol} / \mathrm{l}$. Nearly half $(47 \%)$ of patients admitted with a respiratory illness were hyperglycaemic. They had longer hospital stays $(11.5+/-9.2$ vs $6.1+/-5.1$ days; $\mathrm{p}=0.04)$, however age; gender and prior glucocorticoid use did not differ significantly between those with or without hyperglycaemia. Considering a therapeutic range definition, prevalence increased to $50 \%$.
Conclusion: In this prospective study, the prevalence of significant hyperglycaemia in glucocorticoid treated non-diabetic inpatients was $30 \%$ with up to $50 \%$ of patients having some degree of abnormal glucose regulation. Significant fasting hyperglycaemia was an unexpected feature. Glucocorticoid-induced hyperglycaemia was associated with an older age, prednisolone (as opposed to dexamethasone use), and admission with a respiratory illness but not with prior glucocorticoid use. It also predicted a longer length of hospital stay. Despite this, very few patients received any form of glucose lowering therapy.

\section{7}

Should the diabetes team be involved in the first line for hyperglycaemic crises?

S.M. Nguyen ${ }^{1}$, G. Waeber ${ }^{2}$, M.-D. Schaller ${ }^{3}$, L. Trueb ${ }^{4}$, R.C. Gaillard ${ }^{1}$, J. Ruiz ${ }^{1}$

${ }^{1}$ Endocrinology, Diabetology and Metabolism, CHUV, ${ }^{2}$ Internal Medicine, CHUV, ${ }^{3}$ Intensive Care, CHUB, ${ }^{4}$ Emergency Medicine, CHUV (Centre Hospitalier Universitaire Vaudois), Lausanne, Switzerland

Background and aims: Diabetes mellitus affects about $7.5 \%$ of the adult population. Diabetic keto-acidosis (DKA) and the hyperglycemic hyperosmolar state (HHS) are the most serious acute complications of diabetes. They are frequent within the diabetic population and tend to increase with the growing diabetes pandemic. These acute complications remain associated with a high mortality rate ( $<5 \%$ for DKA and $15 \%$ for HHS) despite an optimal care. The aim of the study was to analyze the precipitating factors, complications, treatment, length of stay (LOS) and mortality of these hyperglycemic crises in the university hospital of Lausanne.

Materials and methods: This study describes a retrospective analysis of 173 patients admitted for DKA, HHS or both in the department of Internal Medicine (Emergency Room, Intensive Care Unit, Ward) between 1995 and 2006. Demographic factors, admission diagnoses and laboratory data were examined. The acute hyperglycemic crises were defined according to the $2006 \mathrm{ADA}$ criteria, i.e. DKA as a plasma glucose $\geq 13,9 \mathrm{mmol} / \mathrm{L}$, an arterial $\mathrm{pH} \leq 7,30$ with the presence of either blood or urine ketones; HHS as a plasma glucose $\geq 33,3 \mathrm{mmol} / \mathrm{L}$ together with an effective serum osmolality $\geq 320 \mathrm{mOsm} / \mathrm{kg}$. We defined mixed DKA-HHS crises as the combination of both.

Results: Median age of the study population was $50(33,5-67,8)$ years, $61 \%$ were males. At the admission, $32 \%$ of cases presented ACD, 32\% HHS and 35\% mixed DKA-HHS. The main precipitating factors were non-adherence (49\%), infections (26\%), inaugural diabetes (24\%), idiopathic ( $8 \%$ ) and cardiovascular (4\%). The median LOS was 9 (6-15) days, type 2 diabetic patients had a longer LOS (14 vs. 6 days, $\mathrm{p}<0.0001)$. DKA patients had a shorter median hospital LOS than other patients ( 7 vs. 10 days, $p=0.022$ ). In-hospital mortality was the highest in mixed DKA-HHS (8.2\%), followed by DKA (5.4\%) and HHS (3.6\%). Older age was a predictive factor for mortality ( 77 vs. 53 years, $p=0.011$ ), as well as in multivariate analysis $(\mathrm{p}<0.0001)$. Consultation by a diabetes team was associated with much lower mortality rates $(1.8 \%$ vs. $58.3 \%, \mathrm{OR}=0.04, \mathrm{p}<0.0001)$. A consultation by a specialized diabetes team during the first 3 hospitalization (early) days significantly reduced LOS in both univariate (7 vs. 15,5 days, $\mathrm{OR}=0.35, \mathrm{p}<0.0001)$ and multivariate analyses $\left(\mathrm{R}^{2}=0.46, \mathrm{p}<00001\right)$.

Conclusion: Patient's non adherence with their diabetes treatment and inaugural diabetes are the major precipitating factor for DKA, HHS and mixed DKA-HHS crises, which was never previously described in HHS or mixed DKA-HHS crises. We observe a shift in the distribution of the precipitating factors, which were previously outclassed by infections. Mortality was highest in the mixed DKA-HHS crises group, a situation that can be accounted for by the greater metabolic unbalance in this group. We highlight that an early consultation by a diabetes team highly decreases the LOS. Thus, we suggest that diabetic patients' education be reinforced, the diabetes team be involved as soon as the first hospitalization days and patient's education program for emergency team could be useful. 


\section{PS 102 Costs of diabetes}

\section{8}

Long-term consequences on annual earnings of the onset of type 1 diabetes in young adults. Longitudinal analysis of annual data 1990-2005 for persons born 1949-1970

K. Steen Carlsson ${ }^{1}$, M. Landin-Olsson ${ }^{2}$, L. Nyström³ ${ }^{3}$ S. Gudbjörnsdottir ${ }^{4}$ for the DISS Group

${ }^{1}$ LUCHE, Lund University, ${ }^{2}$ Dpt Clinical Sciences in Lund, Lund University, ${ }^{3}$ Dpt Public Health and Clinical Medicine, Umeå University, ${ }^{4} \mathrm{NHV}$, Nordic School of Public Health, Göteborg, Sweden

Background and aims: The tools to study long-run labour-market consequences caused by the onset of diabetes have not been available earlier. The availability of high-quality register data for research and a unique civic registration number for each individual used in all Swedish administrative population registers have changed this. Our aim was to quantify the long-term consequences on annual earnings of the onset of diabetes for persons aged 15-34 years old using data from the national longitudinal database LISA at Statistics Sweden.

Materials: Based on the Diabetes Incidence Study in Sweden DISS which since 1983 prospectively registers all incident cases of diabetes in young adults aged 15-34 years old (400/year; total 9,298 for 1983-2005), and control persons $(\mathrm{N}=37,192)$ from LISA matched for age, sex and municipality of residence at the time of diabetes onset, we constructed a longitudinal socio-economic database, Econ-DISS. It contains annual information on demographics, education, profession, income, work place, unemployment, sick-leave and early retirement for the period 1990-2005. For persons with diabetes onset after 1990, data include also the pre-diagnosis phase. There is also annual socio-economic information on parents through record linkage using the Multiple Generations Register at Statistics Sweden. From Econ-DISS, we selected all individuals diagnosed with type 1 diabetes $(\mathrm{N}=3,650)$ and their controls $(\mathrm{N}=14,629)$ born $1949-1970$ as the youngest were potentially active in the labour market in 1990.

Methods: Non-parametric comparisons of cases and controls (Pearson's $\chi^{2}$ and Kruskal-Wallis tests) before and after onset of diabetes; and longitudinal regression methods accounting for known correlates of earnings: own and parent's education, sex, business cycle, region of residence, the presence of young children in household, age at onset and duration of diabetes.

Results: We found no statistical differences in annual earnings before diabetes onset. After onset, persons with diabetes gradually lag behind (Fig 1). Equality of median earnings was rejected for each year 1995-2005 $(p<0.01)$. Equality of populations was rejected for each year 1993-2005 $(p<0.01)$. In 2005, the median annual earnings were EUR 1,390 (5\%) lower for persons with diabetes. For the $1^{\text {st }}$ quartile, the difference was even greater, EUR 2,760 $(18 \%)$. The longitudinal regression analysis showed that differences were greatest for persons with compulsory level of education, all else equal. Each additional year of diabetes duration reduced earnings by $1 \%$ for women and by $0.4 \%$ for men, all else equal.

Conclusion: Persons with diabetes have significantly lower earnings, and these differences were most pronounced for persons with less education.

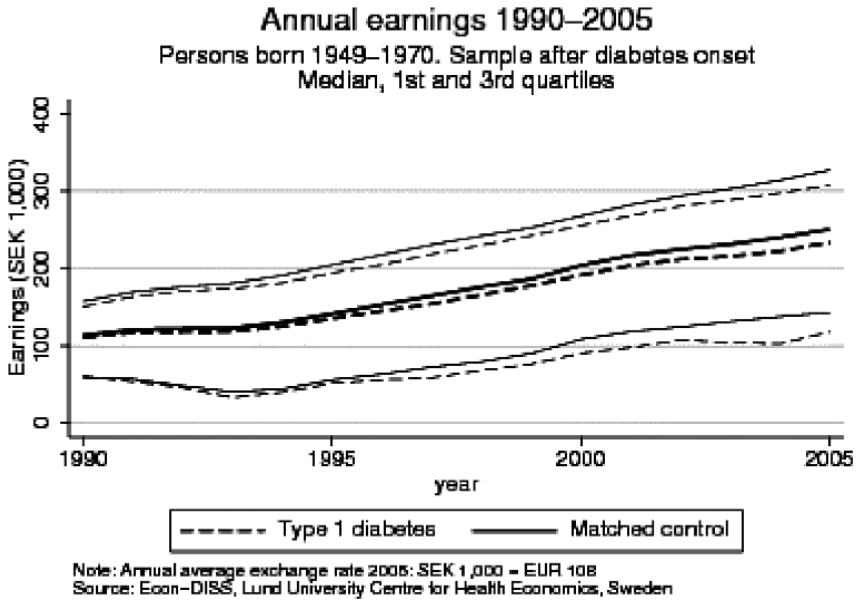

Supported by: Foundation for Research at the Swedish Society for Diabetes, Novo Nordisk $A / S$

\section{9}

Risk of diabetes is spreading to all socio-economic groups: a prospective childhood study

L.D. Voss, J. Hosking, B.S. Metcalf, A.N. Jeffery, T.J. Wilkin

Endocrinology and Metabolism, Peninsula Medical School, Plymouth,

United Kingdom

Background and aims: In today's rapidly changing society, the relationship between socio-economic status and diabetes merits re-examination. Lifestyle interventions to prevent type 2 diabetes continue to target areas of relative deprivation, based on the assumption that this is where the greatest prevalence of obesity and metabolic disturbance will be found. However, the evidence is largely historical. We have re-examined the question, crosssectionally and longitudinally, in a contemporary cohort of healthy young children to establish whether adiposity and metabolic risk are still linked to deprivation, and whether deprivation is associated dynamically with change in adiposity or metabolic risk from 5 to $8 \mathrm{y}$.

Materials and methods: Subjects: Earlybird is a prospective, non-intervention cohort study of 300 healthy UK children, recruited from randomly selected city schools representing a wide socio-economic range. Deprivation: a postcode-based Index of Multiple Deprivation (IMD) was used to assess material living conditions. The higher the score, the higher the deprivation. Mean IMD score of the total cohort at baseline was 21.7, close to the UK mean of 26.3, but covered a substantial range (6.5- 73.0). Measures of adiposity: body mass index (BMI) sds using 1990 UK norms, waist circumference, and sum of 5 skinfolds (biceps, triceps, subscapular, supra-iliac and umbilical) at 5y and 8y. Metabolic Risk: a composite metabolic risk z-score (MetRisk) was derived from mean arterial blood pressure, and HOMA-IR, triglycerides, cholesterol/HDL ratio from fasting venous blood. We report on 266 subjects ( 146 boys, 120 girls) with complete data sets.

Results: In boys, mean BMI sds was $0.21 \pm 1.08$ at 5y, increasing to $0.30 \pm$ 1.10 at $8 \mathrm{y}$. Unexpectedly, BMI was inversely related to IMD score at $5 \mathrm{y}(\mathrm{r}=$ $-0.18, \mathrm{p}=0.04)$ and again at $8 \mathrm{y}(\mathrm{r}=-0.17, \mathrm{p}=0.05)$. Waist circumference was also inversely related to IMD score at $5 \mathrm{y}(\mathrm{r}=-0.21, \mathrm{p}=0.01)$ and at $8 \mathrm{y}(\mathrm{r}=$ $-0.20, p=0.02$. Sum of skinfolds was not significantly related to IMD score at either age (both $p>0.12$ ). Importantly, changes in all measures of adiposity from $5-8 y$ were unrelated to IMD score (all $r<0.14, p>0.10$ ). There were no significant associations between MetRisk and IMD score at $5 y(r=-0.05$, $\mathrm{p}=0.59)$ nor at $8 \mathrm{y}(\mathrm{r}=-0.15, \mathrm{p}=0.08)$. Change in MetRisk from $5-8 \mathrm{y}$ was not significantly related to IMD score $(\mathrm{r}=-0.14, \mathrm{p}=0.10)$. In girls, mean BMI sds was $0.51 \pm 0.98$ at $5 y$, increasing to $0.55 \pm 1.10$ at $8 y$. No measure of adiposity was related to IMD score at any age (all $\mathrm{r}<0.10$, all $\mathrm{p}>0.31$ ). Again, changes in all measures of adiposity from 5-8y were unrelated to IMD score (all $\mathrm{r}<0.15$, $\mathrm{p}>0.11$ ), and there were no significant associations between MetRisk and IMD score at any age $(\mathrm{r}<0.1, \mathrm{p}>0.27)$. Change in MetRisk from 5-8y was unrelated to IMD score $(r<0.1, \mathrm{p}>0.27)$.

Conclusion: Our data do not support the popular assumption that obesity and metabolic distubance remain more prevalent among poorer children in an urbanised population. Indeed, we found evidence to the contrary in boys, making lack of power an unlikely cause. Findings were consistent when using three alternative measures of socio-economic status: entitlement to free school meals, parental income and occupation, suggesting robust data. Any relationship between deprivation, obesity and metabolic risk appears to have been lost. With all neighbourhoods affected by an increasingly obesogenic environment, all children may now be vulnerable, with population-wide implications for public health policy.

\section{0}

Cost and resource use associated with different insulin regimens initiated in 5 European countries in the $\mathbf{6}$ months following initiation of insulin H. Smith ${ }^{1}$, A. Simpson ${ }^{1}$, A. Tynan ${ }^{1}$, C. Nicolay ${ }^{2}$, A. Goday ${ }^{3}$, A. Liebl ${ }^{4}$, M. Benroubi ${ }^{5}$, S. Jones ${ }^{6}$

${ }^{1}$ Eli Lilly, Surrey, United Kingdom, ${ }^{2}$ Eli Lilly, Bad Homburg, Germany, ${ }^{3}$ Endocrinology, Hospital Del Mar, Barcelona, Spain, ${ }^{4}$ Centre for Diabetes and Metabolism, Fachklinik Bad Heilbrunn, Germany, ${ }^{5}$ Diabetes Centre, General Hospital, Athens, Greece, ${ }^{6}$ The Academic Centre, James Cook University Hospital, Middlesborough, United Kingdom

Background and aims: INSTIGATE is a prospective observational study in 5 European countries (France, Germany, Greece, Spain, UK) investigating patients with type 2 diabetes initiating insulin during usual care. Data are collected over 24 months following insulin initiation. The primary objective 
of the study is to assess the direct costs of care for type 2 diabetes before and after insulin initiation.

Materials and methods: Direct costs of diabetes care were calculated by collecting data on individual resource use and assigning local unit costs (from 2006). Reported costs and resource use are for the 6 months prior to insulin initiation at baseline (BL) and the 6 months after insulin initiation $(6 \mathrm{~m})$.

Results: Data were collected from 1153 patients at BL and 1051 patients at $6 \mathrm{~m}$. With the exception of France, patients had more frequent contact with health care professionals (HCP) at $6 \mathrm{~m}$ than BL (Figure 1), although the types of HCP and type of contact (visit or phonecall) varied between countries.

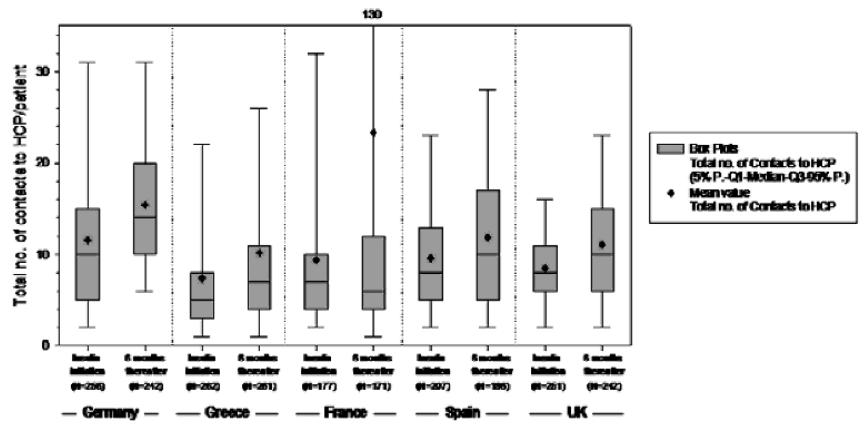

Figure 1. Total number of contact with HCPs per patient, by country

Median total costs of diabetes care were $€ 512 \mathrm{BL}$ and $€ 7256 \mathrm{~m}$. The total costs in the 5 countries are reported in table 1 .

Within regimens the largest differences in median costs were seen in basal bolus ( $€ 481 \mathrm{BL}$ and $€ 8886 \mathrm{~m}$ ) and short acting insulin regimens ( $€ 453 \mathrm{BL}$ and $€ 7716 \mathrm{~m}$ ). Further analysis is needed to assess the impact of insulin regimen changes on the cost of diabetes care.

There were some changes in type of resource use and associated costs from $\mathrm{BL}$ to $6 \mathrm{~m}$. Median cost of general practice care fell ( $€ 54 \mathrm{BL}, € 38 \mathrm{6m}$ ), while median cost of specialist care for glycaemic control increased ( $€ 53 \mathrm{BL}, € 105$ $6 \mathrm{~m})$. As expected the median cost of oral antidiabetic medications fell ( $€ 138$ $\mathrm{BL}, € 376 \mathrm{~m}$ ), and median cost of insulin medication increased ( $€ 1 \mathrm{BL}, € 189$ $6 \mathrm{~m}$ ). Median costs of blood glucose monitoring also increased (€60 BL, €184 $6 \mathrm{~m})$.

Table 1: Total cost (euros) of diabetes care by country

\begin{tabular}{|c|c|c|c|c|c|c|c|c|}
\hline Country & Visit & $\mathbf{N}$ & Min. & Q1 & Median & Q3 & Max. & Mean \\
\hline \multirow[t]{2}{*}{ Germany } & Baseline & 256 & 0 & 237.2 & 407.8 & 676.6 & 11532 & 605.0 \\
\hline & 6 month & 233 & 257 & 741.7 & 895.6 & 1114.7 & 3270 & 961.3 \\
\hline \multirow[t]{2}{*}{ France } & Baseline & 177 & 23 & 421.0 & 647.3 & 1372.5 & 6570 & 1317.5 \\
\hline & 6 month & 152 & 207 & 579.0 & 805.2 & 1213.3 & 22660 & 1449.0 \\
\hline \multirow{2}{*}{$\begin{array}{l}\text { United } \\
\text { Kingdom }\end{array}$} & Baseline & 251 & 101 & 431.7 & 593.6 & 812.0 & 5495 & 697.9 \\
\hline & 6 month & 222 & 156 & 528.3 & 712.0 & 893.3 & 6963 & 772.5 \\
\hline \multirow[t]{2}{*}{ Greece } & Baseline & 262 & 1 & 224.0 & 436.1 & 659.8 & 2549 & 497.2 \\
\hline & 6 month & 256 & 101 & 369.9 & 537.9 & 717.6 & 2124 & 577.2 \\
\hline \multirow[t]{2}{*}{ Spain } & Baseline & 207 & 1 & 323.9 & 514.7 & 730.2 & 8041 & 630.9 \\
\hline & 6 month & 188 & 84 & 518.0 & 698.9 & 1042.7 & 19341 & 1109.7 \\
\hline
\end{tabular}

Conclusion: After insulin initiation the costs of diabetes care increased. Except in France, contact with HCPs also increased. Costs associated with care from HCPs for diabetes shifted from primary care to specialist care after insulin initiation and the cost associated with blood glucose monitoring increased.

Supported by: Eli Lilly \& Co, Amylin Pharmaceuticals

\section{1}

The relationship between socioeconomic deprivation, educational attainment and clinical outcomes in type 2 diabetes: a cohort study M.J. O’Kane ${ }^{1}$, M. McMenamin'2 J. Innes ${ }^{3}$, A. Moore ${ }^{4}$, B. Bunting ${ }^{3}$, V. Coates ${ }^{5}$

${ }^{1}$ Clinical Chemistry Laboratory, Altnagelvin Hospital, Londonderry, ${ }^{2}$ Institute of Nursing Research, University of Ulster, Coleraine, ${ }^{3}$ School of Psychology, University of Ulster, Londonderry, ${ }^{4}$ Causeway Data Communications Ltd, Innovation Centre, Coleraine, ${ }^{5}$ Institute of Nursing Research, University of Ulster, Londonderry, United Kingdom

Background: Socioeconomic deprivation may be associated with worse outcomes in type 2 diabetes. An understanding of this relationship would allow more appropriate and targeted intervention to improve care.

Aim: To study the relationship between socioeconomic deprivation, educational attainment and clinical outcomes in type 2 diabetes.

Methods: A random sample of 457 patients with type 2 diabetes [ $57 \%$ male, age 64.7 (11.5), mean (SD) years, duration of diabetes 11.2(6.2) years] stratified by Noble Index of Deprivation measure was selected from a hospital diabetes service database. Information on patient specific socioeconomic status, educational attainment, behaviour and attitudes was gathered by patient interview. Clinical outcome data were extracted from the hospital diabetes database. Data analysis was by multivariate logistic and linear regression with correction for age, gender and duration of diabetes.

Results: Cohort Descriptive data Antidiabetic treatment: diet alone $7.4 \%$, oral agents alone $54.3 \%$ and insulin $38.3 \%$.Other treatments: antiplatelet agents $78 \%$, lipid lowering agents $90.1 \%$, antihypertensive agents $86 \%$. Metabolic indices: BMI $32.3(6.0) \mathrm{kg} / \mathrm{m}^{2}$, HbAlc 7.6 (1.4) \%, total cholesterol 4.0 (0.9) $\mathrm{mmol} / \mathrm{L}$, systolic BP 134.2 (20.6) and diastolic BP 72.7 (11.2) $\mathrm{mmHg}$. The breakdown by deprivation [in quintiles of the $\mathrm{N}$ Ireland population as a whole] was: Group 1 [least deprived] 8.7\%, group $218.6 \%$, group $320.9 \%$, group $422.4 \%$ and group 5 [most deprived] 29.6\%. Complication prevalence: hypertension $82 \%$, myocardial infarction $11 \%$, angina $18.2 \%$, claudication $7.2 \%$, stroke $2.3 \%$, any retinopathy $22 \%$, proteinuria $25.6 \%$, renal impairment $14.3 \%$.

Data analysis Hypertension [adjusted OR 1.2] and coronary angioplasty [adjusted OR 3.3] were more prevalent in the most deprived v. least deprived groups $[p<0.05]$. Higher educational attainment [A level equivalent or above] v. lower educational attainment [ no qualification and GCSE / trade] was associated with reduced BMI [adjusted slope $-0.13, \mathrm{p}=0.01$ ]

Conclusion: Although deprivation and low educational attainment were associated with adverse outcome indicators the effects were small. It is possible that any detriment associated with deprivation is minimised by aggressive management of modifiable risk factors.

Supported by: NIreland Research and Development Office

\section{2}

Economic cost associated with type 1 and type 2 diabetes in the United States

T. Dall ${ }^{1}$, S. Mann ${ }^{1}$, Y. Zhang ${ }^{1}$, W. Quick ${ }^{2}$

${ }^{1}$ The Lewin Group, The United HealthCare Service, Inc., Falls Church, ${ }^{2}{ }^{2} 3$ Research, The United HealthCare Service, Inc., Morristown, United States.

Background and aims: Estimate the economic cost associated with diagnosed type 1 diabetes (T1DM) and type 2 diabetes (T2DM) in the United States in 2007

Materials and methods: Medical claims and national surveys were analyzed to estimate the proportion of diagnosed diabetes cases and excess medical costs associated with T1DM and T2DM. The economic value of lost productivity associated with T1DM and T2DM were estimated using findings from the literature and presented as ranges based on alternate assumptions of productivity loss per case of T1DM and T2DM. The primary sources used to develop the model are the Census Bureau's population estimates for 2007; the 2004-2006 National Health Interview Survey (NHIS) to estimate diagnosed diabetes prevalence, work days absent, inability to work because of permanent disability, and rates of insulin and oral agent use; the 2003-2005 Medical Expenditure Panel Survey (MEPS) to estimate average medical cost per health care event (e.g., per visit) or per annum usage per person (e.g., annual cost for insulin, home health services, podiatry, ambulances, and other medical supplies ); the 2004-2005 Nationwide Inpatient Sample (NIS) to estimate total inpatient days and hospital costs by chronic condition category; the 2003-2005 National Ambulatory Medical Care Survey (NAMCS) and 
National Hospital Ambulatory Medical Care Survey (NHAMCS) to estimate total emergency and ambulatory visits; the 2000 National Home and Hospice Care Survey (NHHCS) to estimate hospice visits; and the 2004 National Nursing Home Survey (NNHS) to estimate total resident days in nursing facilities and the prevalence of diagnosed diabetes among the population in long-term care. The Ingenix MCURE database, containing medical claims for 16.2 million insured people under age 65 in 2006 (683,000 with a diagnosis of diabetes), was used to determine the proportion of diagnosed diabetes cases that are T1DM and how patterns of health care use for the population under age 65 differ by diabetes status (no diabetes diagnosis, diagnosed T1DM, or diagnosed T2DM or type unknown). A similar analysis for the 65 and older population was conducted with the 2006 Medicare 5\% Sample File (which contains claims records for approximately 17.95 million people age 65 and older, of which 1.03 million have a diagnosis of diabetes). Analysis of the NHIS shows that approximately $40 \%$ of diagnosed diabetes cases are under age 65 and commercially insured, and approximately $37 \%$ of cases are age 65 and older and insured by the government.Medical costs are estimated by demographic (age and sex), health care delivery setting, and complication/ comorbidity group. Non-medical costs are estimated by demographic, and include absenteeism, presenteeism, inability to work because of long term disability, and mortality.

Results: Previous research estimates that the economic burden of diagnosed diabetes in 2007 is US $\$ 174$ billion. The preliminary estimates from this study suggest that approximately $\$ 16.2$ billion (9\%) of this economic burden is associated with the T1DM. This estimate includes medical costs of $\$ 11.2$ billion for T1DM and $\$ 105.0$ billion for T2DM. Lost productivity is estimated at $\$ 5.0$ billion for T1DM and $\$ 53.1$ billion for T2DM.

Conclusion: The economic burden per case of diabetes is larger for T1DM, but the majority of cases and economic costs are associated with T2DM and therefore might be largely preventable.

Supported by: National Changing Diabetes Program, Novo Nordisk

\section{3}

Economic cost associated with pre-diabetes and undiagnosed diabetes in the United States

Y. Zhang ${ }^{1}$, A. Baldwin ${ }^{2}$, Y. Chen ${ }^{1}$, W. Yang ${ }^{1}$, W. Quick ${ }^{3}$, T. Dall ${ }^{1}$

${ }^{1}$ The Lewin Group, The United HealthCare Service, Inc., Falls Church,

${ }^{2} \mathrm{i} 3$ Pharma Informatics, The United HealthCare Service, Inc., Cary, ${ }^{3} \mathrm{i} 3$

Research, The United HealthCare Service, Inc., Morristown, United States.

Background and aims: Build on recent cost of diabetes study, this study estimates health care use and costs associated with undiagnosed diabetes mellitus and pre-diabetes mellitus

Materials and methods: We analyzed medical claims data for a commercially insured population (continuously insured during 2004, 2005 and 2006, $\mathrm{N}=3,232,134)$.

A population with pre-diabetes in 2004 was identified using existing test results from Fasting Plasma Glucose (FPG) and Oral Glucose Tolerance Test (OGTT). We used multivariate regression analyses to compare health care use patterns for this population during 2005 and 2006 with those of (1) people with a FPG or OGTT result of no pre-diabetes in 2004, and (2) all people for whom there is no evidence of diabetes or pre-diabetes (including people who did not receive a FPG or OGTT in 2004). A proxy for the population with undiagnosed diabetes was constructed by identifying people who are on the verge of first diagnosis for diabetes: those who had diagnoses for diabetes in 2006 medical claim records but none in 2004 and 2005. We use multivariate regression analyses to compare health care use patterns during the 2004 to 2005 period for this "undiagnosed" diabetes population to patterns for a population with no indication of diabetes between 2004 and 2006.Using nationally representative data sources, we estimate the prevalence of pre-diabetes and undiagnosed diabetes, and total physician office visits, outpatient visits, emergency visits, and hospital inpatient days for seven categories of complications typically associated with diabetes: neurological, peripheral vascular, cardiovascular, renal, endocrine, ophthalmic, and other complications. We combine pre-diabetes and undiagnosed diabetes prevalence rates with health care use patterns to calculate etiological fractions. Multiplying these fractions by total national healthcare use by complication group provides estimates of health care use associated with pre-diabetes and undiagnosed diabetes.

Results: Preliminary estimates suggest that approximately 36.8 million (12\%) Americans have pre-diabetes and 5.1 million (1.7\%) have undiagnosed diabetes. Compared to their counterparts with no diabetes diagnoses, those who had undiagnosed diabetes had increased use of health care services for hospital inpatients days (adjusted rate ratio, aka. $R R=1.50$ ), emergency room visits $(R R=1.30)$, outpatient visits $(R R=1.25)$ and physician office visits $(\mathrm{RR}=1.11)$; the increase in risk varies by complication categories. Compared to their counterparts with no indication of diabetes, undiagnosed or pre-diabetes, those who had pre-diabetes had increased outpatient visits $(\mathrm{RR}=1.30)$ and physician offices visits $(\mathrm{RR}=1.12)$ but no increase risk of emergency visits and hospital inpatient days. Sensitivity analyses suggest a consistent dose response in increased risk over the spectrum of diabetes prognosis. Full results will be available by May, 2008 .

Conclusion: Both pre-diabetes and undiagnosed diabetes are associated with increased use of health care services (and costs), and there is a consistent dose-response relationship between use of health care services and the presence of normal glucose levels, pre-diabetes, undiagnosed diabetes, and diagnosed diabetes.

Supported by: National Changing Diabetes Program, Novo Nordisk

\section{4}

Bedside monitoring of blood $\beta$-hydroxybutyrate shortens hospitalisation for diabetic ketoacidosis in children and lowers the cost

A. Rewers ${ }^{1}$, K. McFann ${ }^{2}$, M. Rewers ${ }^{3}$

${ }^{1}$ Department of Pediatrics, ${ }^{2}$ Department of Preventive Medicine, ${ }^{3}$ Barbara Davis Center for Childhood Diabetes, University of Colorado, Aurora, CO, United States

Background and aims: Bedside monitoring of blood $\beta$-hydroxybutyrate (B-OHB) has been used in treatment of diabetic ketoacidosis (DKA). Previously, we demonstrated that the levels of B-OHB correlate closely with timedependent levels of venous $\mathrm{pH}$, bicarbonate, and $\mathrm{pCO}_{2}$ The purpose of this study was to evaluate the effect of bedside monitoring of blood B-OHB levels on duration of hospital stay and the cost of admission for DKA.

Materials and methods: All children admitted to our hospital for DKA during a period of 20 months were treated using a standard treatment protocol. DKA and its severity were defined using the 2007 ISPAD criteria. Bedside monitoring of blood B-OHB, on average every 2 hours, using the Precision $\mathrm{Xtra}^{\text {tw }}$ (Abbott Diabetes Care, Abbott Park, IL) was added to the protocol in 53 randomly selected patients with type 1 diabetes admitted for DKA. Patients who received IV fluids or insulin prior to admission to our hospital were not included. The direct hospital cost and the length of admission in the $\mathrm{B}$-OHB group were compared to those of 53 DKA control patients treated concurrently but without B-OHB monitoring (no B-OHB). Controls were frequency matched on age (median $12 \mathrm{y}$ vs. $12 \mathrm{y}$ in the B-OHB group), severity of DKA (pH 7.17 vs. 7.16 ) gender (female $60 \%$ vs. $57 \%$ ) and duration of diabetes (newly diagnosed $57 \%$ vs. $51 \%$ ).

Results: The cost of hospitalization was bordeline lower in patients whose B-OHB levels were monitored at the bedside. The median cost of hospitalization was 10,657 US\$ (range 1,581-31,983) in the B-OHB group vs 14,358 US\$ (range 2,154-40,657) in the control group ( $p=0.11$ ) and the mean cost of hospitalization in the B-OHB group was 11,526 US\$(SD 6,301) vs 14,441US\$ $(\mathrm{SD} 8,738)(\mathbf{p}=\mathbf{0 . 0 5})$ in the control group. The length of hospital stay was slightly shorter in the B-OHB group, compared to the No B-OHB group, but the difference did not reach the level of statistical significance with the median hospital stay of $24 \mathrm{~h}$ (range 4-106) in the B-OHB group vs 26h (range $4-145)$ in the control group ( $\mathrm{p}=0.21)$ and the mean hospital stay of $29 \mathrm{~h}$ (SD $21)$ vs $36 \mathrm{~h}(\mathrm{SD} 31)(\mathrm{p}=0.17)$, respectively . While this preliminary evidence suggests benefit, the exact mechanisms of lower cost are unclear. The B-OHB results were not part of routine hospital documentation and sometimes unknown to the provider who ordered laboratory tests, treatment and the timing of discharge.

Conclusion: The results of this unmasked intervention suggest that addition of blood B-OHB monitoring to standard DKA treatment protocol may reduce the length and the cost of hospitalization. Future randomized trials should evaluate overall cost-effectiveness and outcomes of such a modification. Supported by: Abbott Laboratories 


\section{5}

Cost-effectiveness of adding sitagliptin vs. an sulfonylurea in Swiss patients with type 2 diabetes inadequately controlled on metformin monotherapy

M. Brandle ${ }^{1}$, J. Chen'2, E. Alemao ${ }^{3}$, D. Yin ${ }^{3}$, J. Cook², T. Szucs ${ }^{4}$

${ }^{1}$ Head of Endocrinology and Diabetology, Cantonal Hospital of St. Gallen, Switzerland, ${ }^{2}$ Health Statistics and Economics, Merck \& Co., Upper

Gwynedd, Unitzed States, ${ }^{3}$ Global Outcomes Research, Merck \& Co., Whitehouse Station, United States, ${ }^{4} \mathrm{Head}$ of Medical Economics, Institute of Social- and Preventive Medicine, University of Basel, Switzerland

Background and aims: Sitagliptin (Sita) is an oral antihyperglycemic agent that inhibits the dipeptidyl peptidase (DPP-4) enzyme. It is licensed in Europe as a treatment adjunct to metformin (Met), diet and exercise in the management of type 2 diabetes mellitus. The objective of the study was to determine the cost effectiveness of adding Sita vs adding sulfonylurea (SU) to drug regimens of patients inadequately controlled on Met monotherapy.

Materials and methods: A discrete event simulation model (JADE), which employed the United Kingdom Prospective Diabetes Study (UKPDS) Outcomes Model risk equations for predicting risks of diabetes related complication, was used. Lifetime costs and outcomes were projected for alternative treatment strategies of adding Sita, compared with adding $\mathrm{SU}$ to Met in patients not at hemoglobin AlC (HbAlc) goal on Met monotherapy. Changes in $\mathrm{HbAlc}$ as well as side effects associated with these different treatment strategies were based on clinical trial data. Mean baseline values for age, duration of diabetes, \% HbAlc, systolic blood pressure and other risk factors of diabetes complications were obtained from a local epidemiologic study involving patients with type 2 diabetes not at $\mathrm{HbAlc}$ goal on Met monotherapy. Direct medical costs associated with treatment regimens (based 2008 values), diabetes complications and adverse events were accounted in 2007 values based on Swiss-specific unit costs. UKPDS based utility weights associated with diabetes complications were incorporated. Utility decrements associated with medication side effects were based on published data. All future costs and outcomes were discounted at $3 \%$ according to local guidelines on cost-effectiveness analysis. One-way sensitivity analyses were conducted by varying key input parameters.

Results: Drug costs of adding Sita vs. adding SU to Met was CHF 15,325 and CHF 14,411 respectively over a lifetime horizon. However, diabetes complication costs and adverse event costs were lower with Sita compared to SU, resulting in a total incremental cost of CHF 204 per patient with Sita compared to SU over life time. The quality adjusted life years (QALYs) gained due to adding Sita vs. adding SU was 0.066 thus providing an incremental cost per QALY of CHF 3,091 (Table). In the one way sensitivity analysis, the results were robust to changes in the model input parameters.

Conclusion: This cost-effectiveness analysis demonstrates that adding Sita to Met compared to adding SU to Met is a cost-effective treatment option in patients failing Met therapy in the Swiss setting.

Table: Direct medical Costs, QALYs, and ICERs for Sita vs. SU in Patients Failing Met

\begin{tabular}{llll}
\hline & Sita & SU & Sita - SU \\
\hline Medication Costs (CHF) & 15,325 & 14,411 & +914 \\
\hline Diabetes Complication Costs (CHF) & 52,696 & 53,090 & -394 \\
\hline Adverse Event Costs (CHF) & 1,129 & 1,447 & -318 \\
\hline Total Costs (CHF) & 69,152 & 68,948 & 204 \\
\hline QALY (years) & 6.323 & 6.257 & +0.066 \\
\hline Cost per QALY (CHF) - & & - & 3,091 \\
\hline
\end{tabular}

$1 \mathrm{CHF}=0.643$ Euro

Supported by: Merck and Co

\section{6}

Short-term economic benefits of self monitoring blood glucose (SMBG) in type 2 diabetes for health maintenance organisations (HMOs)

F.D. Mataveli ${ }^{1,2}$, L. Turatti ${ }^{3}$, A. Pimazoni Netto ${ }^{4}$

${ }^{1}$ Internal Medicine, Federal University of São Paulo, ${ }^{2}$ Lifescan, Johnson \& Johnson, ${ }^{3}$ Endocrinology, State University of São Paulo, ${ }^{4}$ Hospital do Rim, Federal University of São Paulo, São Paulo, Brazil

Background and aims: Acceptable HbAlc levels may be associated with lower costs in the management of type 2 diabetes. This study was aimed to evaluate the association between SMBG and healthcare costs, according to $\mathrm{HbAlc}$ value, under the Brazilian Managed Care perspective.

Materials and methods: An economic model based on data published in 2006 by the Brazilian Regulatory Agency for Managed Care (ANS) was developed to evaluate the 3 years impact of SMBG in a hypothetical HMO cohort of 1,000 pharmacologically treated patients with diabetes type 2 . HbAlc changes due to SMBG were based on the results of a large cohort study of patients with type I and type II diabetes, designed to assess the correlation between SMBG frequency and HbAlc changes over a 1-year period. Daily SMBG frequency was associated with a $0.6 \% \mathrm{HbAlc}$ reduction in the pharmacologically treated type 2 diabetes patients group. Estimates for medical care charges were based on a study standardized 3-year estimate, in which successive $1 \%$ increases in $\mathrm{HbAlc}$ resulted in cumulative increases, starting from $\mathrm{HbAlc}$ value of $6 \%$, of 4, 10, 20 and 30\% in medical care charges. The average cost of an individual enrolled in a $\mathrm{HMO}$ was set as the value for a $6 \%$ $\mathrm{HbAlc}$ individual. Increases of $1 \%$ in $\mathrm{HbAlc}$ followed the criteria for cumulative costs, being the average cost multiplied by 1.04, 1.1, 1.2 and 1.3. Average annual costs per patient were calculated based on total medical charges and enrolled population for 3 different Brazilian HMOs: an Insurance Company (IC) - R \$2,858; a Medical Cooperative (MC) - R \$ 1,365 and a Medicine Group $(\mathrm{MG})$ - $\mathrm{R} \$ 1,134(\mathrm{R} \$ 1=0.37 €)$. HbAlc distribution in a HMO population in Brazil was based on literature and adapted locally by a board of specialists. Distribution of $\mathrm{HbAlc}$ range values was presented as follows: $30 \% \mathrm{HbAlc} 7-8 ; 30 \% \mathrm{HbAlc} 8-9 ; 30 \%$ 9-10; $10 \% \mathrm{HbAlc}>10$. Total costs in diabetes management were observed to increase up to $400 \%$ due to coexisting clinical complications and comorbidities in comparison to patients with diabetes only. A cost complication factor varying from 1.2 to 1.8 was then assigned to different levels of $\mathrm{HbAlc}(<7$ to $>10 \mathrm{HbAlc}$ ) and average costs to reflect this variation in costs. Individual changes of $0.6 \%$ in $\mathrm{HbAlc}$ obtained with 1-year SMBG resulted in change for the next lower HbAlc level at the end of the 3-year period. Patients not under SMBG change for the next higher level of HbAlc during the 3-year period. Costs were considered and calculated according to the individual $\mathrm{HbAlc}$ level at the end of the third year of the analysis. SBMG costs were based on 1 strip daily dispensation for 3 years. Strip average unit cost is $\mathrm{R} \$ 1.10$. Costs were discounted at $5 \%$ annual rate. Results: Over a 3-year period, daily SMBG frequency (1strip/day) was associated with significant savings compared to no SMBG at all in all three Brazilian HMOs: IC (R $\$ 3,265,561)$, MC (R \$ 884,077) and MG (R \$ 649,767). Sensitive analyses on complication cost factor varying from 1,4 to 2,0 and from 1,0 to 1,6 resulted in savings of: $\mathrm{R} \$ 3,499,280$ and $\mathrm{R} \$ 3,031,842$ (IC); $\mathrm{R} \$ 1,100,102$ and $\mathrm{R} \$ 772,451$ (MC); R \$ 743,232 and $\mathrm{R} \$ 556,302$ (MG), respectively. Conclusion: SMBG may have a positive economic impact in the management of patients with diabetes type 2 under the perspective of the Brazilian Managed Care System.

\section{7}

Cost-effectiveness of a computerised decision support diabetes management program that improves diabetes control

F.G.W. Cleveringa ${ }^{1}$, P.M.J. Welsing ${ }^{2}$, M. van den Donk ${ }^{1}$, K.J. Gorter ${ }^{1}$, L.W. Niessen ${ }^{3}$, G.E.H. Rutten ${ }^{1}$, K. Redekop ${ }^{3}$

${ }^{1}$ General Practice, Julius Center for Health Sciences and Primary Care, Utrecht, ${ }^{2}$ Public Affairs, Pfizer B.V., Capelle a/d IJssel, ${ }^{3}$ Institute for Medical Technology Assessment, Erasmus Medical Center, Rotterdam, Netherlands

Background and aims: Costs related to diabetes are high; countries in Europe spent $2.5-6.5 \%$ of their total health care budget on type 2 diabetes (DM2) and the United States 10\%. Improvements in diabetes health outcomes are possible by strict control of glucose, lipids and blood pressure, as recommended in the guidelines. Guidelines-based care can also reduce the costs of DM2 related complications. However it is difficult to implement and adhere to guidelines; at least $30 \%$ of DM2 patients do not achieve the strict targets for glycaemic and cardiovascular control. Information technology enabled 
diabetes management has the potential to improve care, delay diabetes complications and lower health care costs, but most evaluations of these programs have not included a proper cost effectiveness analysis.

The Diabetes Care Protocol (DCP), which consists of task delegation, decision support and benchmark information, has been shown to improve patient outcomes, but the cost-effectiveness of DCP is unknown. We therefore aim to study the cost effectiveness of DCP from a Dutch health care perspective.

Materials and methods: In a cluster randomised trial, 55 primary care practices were assigned to usual diabetes care (control group), or the DCP (intervention group). Baseline and one year follow-up measurements (i.e. HbAlc, blood pressure, total cholesterol, HDL cholesterol, medication etc) were performed by practice nurses (intervention) and research assistants (control). The one year follow-up data of individual patients in both groups are used to compute lifetime disease outcomes (cardiovascular disease and other DM2 related complications) and direct medical costs using a validated probabilistic Dutch diabetes model and the UKPDS risk engine. The costs of implementing and maintaining DCP and medication use are also included. Incremental costs per quality-adjusted life year will be calculated and probabilistic sensitivity analyses performed. Correction for clustering (usually neglected in cost-effectiveness analyses) is performed using generalized linear models.

Results: 3391 patients participated in the trial (intervention group, 1699; control group, 1692). Baseline characteristics of both groups were comparable. No statistically significant difference was seen in $\mathrm{HbAlc}$ reduction at 1 -year (intervention group vs. control group; $0.2 \%$ vs. $0.1 \%$ ). However, statistically significant between-group differences were seen for reduction in systolic blood pressure $(2.6 \mathrm{mmHg}$ vs. $0.5 \mathrm{mmHg})$, total cholesterol $(0.3 \mathrm{mmol} / \mathrm{L}$ vs. $0.1 \mathrm{mmol} / \mathrm{L})$, and 10 year UKPDS coronary heart disease risk $(1.9 \%$ vs. $0.3 \%)$. Overall costs and health outcomes and the incremental cost-effectiveness ratio of the DCP versus usual care will be presented, including the relative contribution of diabetes control and reduction in cardiovascular disease risk. Results of sensitivity analyses are expressed using a cost-effectiveness plane and acceptability curve.

Conclusion: Delegation of routine diabetes care to a practice nurse combined with computerised decision support and benchmark information improved patient outcome and reduced cardiovascular risk for DM2 patients. The longterm cost-effectiveness of DCP will be presented at the EASD.

Supported by: Pfizer

\section{PS 103 Quality of life}

\section{8}

\author{
The equivalency of diabetes distress \\ K. Glaister \\ Faculty of Health, Curtin University, Perth, Australia
}

Background and aims: The nature of diabetes is generally considered pervasive, such that many feel its impact on their quality of life. The emotional distress generated may be considered minor, presenting transient hassles, or significant, presenting major obstacles in life pursuits. The aim of the study was to determine the level of distress experienced by people with diabetes and to explore if gender differences of reported measures of distress existed.

Materials and methods: A cross-sectional survey involving a representative sample of people $(\mathrm{N}=940)$ with diabetes in Western Australia, was conducted in 2007. The inclusion criteria were: adults, over 18 years of age; diagnosis of diabetes and English literacy. To measure distress the relatively new Diabetes Distress Scale, developed by Polonsky and colleagues was used. This scale is a 17 -item scale, with four subscales representing emotional burden, physician related distress, regimen-related distress and interpersonal distress. Distress was measured using Likert ratings from 1 'serious problem' through to 4 'not a problem'.

Results: Findings suggest that the cohort did not perceived their condition to be causing significant levels of distress in their life $(M=3.4, S D \pm 0.21$, range $=0.63)$. Least problematic were physician related distress $(\mathrm{M}=3.6, \mathrm{SD} \pm$ $0.01)$, followed by interpersonal distress $(\mathrm{M}=3.4, \mathrm{SD} \pm 0.15)$, regimen-related distress $(\mathrm{M}=3.3, \mathrm{SD} \pm 0.17)$ and emotional burden $(\mathrm{M}=3.2, \mathrm{SD} \pm 0.12)$; a pattern consistent with that found by the instrument developers. Three of the four sub-scales confirmed a gender difference in responses. Females $(\mathrm{M}$ $=3.13, \mathrm{SD} \pm 0.77$ ) reported more difficulties with dealing with the emotional burden of having diabetes than males $(\mathrm{M}=3.27, \mathrm{SD} \pm 0.73), t(972)=2.82, \mathrm{p}$ $=0.005$. Furthermore, females $(\mathrm{M}=3.22, \mathrm{SD} \pm 0.74)$ reported more regimen related distress than males $(\mathrm{M}=3.35, \mathrm{SD} \pm 0.65), t(942.93)=2.84, \mathrm{p}=0.005$. Given the data for the interpersonal distress data violated the homogeneity assumption a Mann-Whitney $U$ test was used, which indicated that females (Mean Rank $=436.12, \mathrm{n}=482$ ) reported significantly more difficulties with interpersonal relationships as a result of having diabetes than that reported by male participants (Mean Rank $=507.63, \mathrm{n}=458),(\mathrm{U}=93806.50, \mathrm{z}=-4.22$, $\mathrm{p}<0.001$ ). Over half of the items in the DDS displayed a similar relationship with gender, the notable exceptions being the 4 Physician related distress items.

Conclusion: Distress associated with diabetes is undoubtedly present, but where support from a modern health care system exists it may be less pervasive. Distress eventuates more from the emotional stressors resulting from having the condition and the associated management regimen. However, this cannot be assumed to be a consistent reaction for all, clearly gender differences exist. The instrument used in this study is a comprehensive and relatively quick tool that could be used as a clinical assessment tool, however, it would be worth validating its construct further with other know groups. Supported by: Helen Bailey Scholarship, Department of Health, Western Australia

\section{9}

The influence of family cohesion and adaptability on type 1 diabetes mellitus patients

E. Liouri ${ }^{1}$, A. Koutsovasilis ${ }^{1}$, K. Kounenou ${ }^{1}$, N. Giannakopoulos ${ }^{1}$,

E. Foufopoulou ${ }^{1}$, E. Karagiaouri ${ }^{1}$, M.-P. Koukouli ${ }^{1}$, S. Iraklianou ${ }^{1}$,

C. Karagianni ${ }^{2}$, A. Melidonis ${ }^{1}$

${ }^{1}$ Diabetes Center, Tzanio General Hospital, Piraeus, ${ }^{2}$ Second Department of Pediatrics, Medical School, University of Athens, Greece

Background and aims: Type 1 diabetes mellitus appears in a young age and follows the patient changing not only his/her life, but also that of the family. With this study, we attempt to establish possible differences between families of children and adolescents with type 1 diabetes and those without it as to the adaptability, the cohesion and the type of family as defined by Olson.

Materials and methods: 66 patients with type 1 diabetes (Group A), and 44 without diabetes (Group B) of comparable age and gender filled out an approved questionnaire (FACES-III) of 40 questions determining the level of adaptability (rigid, structured, flexible, very flexible) and cohesion (disengaged, separated, connected, very connected) in a family, as well as its type 
according to international literature where 4 types of family are specified (extremely imbalanced, moderately imbalanced, moderately balanced, balanced). $\mathrm{HbAlc}$ was recorded in type 1 diabetics while somatometric characteristics were recorded in both groups. Statistical analysis was carried out using SPSS 15.0. A p-value $<0.05$ was considered significant.

Results: Concerning adaptability, a statistically significant difference was found between the two groups $(\mathrm{p}=0,044)$, with non-diabetics scoring better on the fourth level of adaptability $(59.3 \%$ vs $32,5 \%)$. There is also a difference in cohesion, with diabetics showing the highest rates $(38.5 \%$ vs $11.1 \%$ of non-diabetics) on the level of connected families $(\mathrm{p}=0,016)$, while non-diabetics show a higher level of cohesion ( $37 \%$ vs $23.1 \% \mathrm{p}=0.041)$. Non-diabetics outnumber diabetics in the fourth type of family $(81.5 \%$ vs $61.5 \%, p=0,034)$, without a statistically significant difference in other types of family. The level of adaptability, as well as the level of cohesion and the type of family, affect the regulation of diabetes mellitus. Children in families with the highest adaptability had $\mathrm{HbAlc}=7.17 \pm 0.88$ (statistically significant difference vs rigid adaptability $(8.2 \pm 0.91), \mathrm{p}=0.010)$; children in families with the highest cohesion had $\mathrm{HbAlc}=6.67 \pm 0.59$ (statistically significant difference vs disengaged families $(\mathrm{HbA} 1 \mathrm{c}=7.68 \pm 1.07), \mathrm{p}=0.014)$ and children in balanced families had $\mathrm{HbAlc}=7.16 \pm 1.08$ (statistically significant difference vs extremely imbalanced families $(7.7 \pm 1.04,) \mathrm{p}=0.026)$.

Conclusion: Families of children and adolescents with type 1 diabetes mellitus show a lower adaptability and cohesion compared to families of healthy controls of the same age. The findings are similar concerning the type of family. Family type, adaptability and cohesion play a crucial role in the regulation of diabetes mellitus during the first years of its emergence.

\section{0}

Depression, sense of coherence, and health related quality of life in patients with type 1 diabetes - association with diabetic nephropathy (The FinnDiane study)

A.J. Ahola ${ }^{1}$, M. Saraheimo ${ }^{1,2}$, C. Forsblom ${ }^{1,2}$, H. Sintonen ${ }^{3}$, P.-H. Groop ${ }^{1,2}$

${ }^{1}$ Department of Diabetes Genetics, Folkhälsan Research Center, ${ }^{2}$ Division of Nephrology, Department of Medicine, Helsinki University Central Hospital, ${ }^{3}$ Department of Public Health, University of Helsinki, Finland

Background and aims: Patients with diabetes have an increased risk of psychological disturbances. Our aim was to evaluate depression, sense of coherence, and health related quality of life in a large sample of Finnish patients with type 1 diabetes and to assess whether these outcome measures were associated with the presence of diabetic nephropathy.

Materials and methods: Patients ( $\mathrm{N}=956,45.6 \% \mathrm{men})$ were recruited from the Finnish Diabetic Nephropathy (FinnDiane) study. Beck Depression Inventory (BDI), Antonovsky's coherence test (SOC), and the 15D health-related quality of life (HRQoL) instrument were used as outcome measures. The $\chi^{2}-$ test and the Kruskal-Wallis test were used in statistical analyses when appropriate. Logistic regression analysis was used to study independent variables associated with diabetic nephropathy. Cross-sectional data are presented.

Results: The overall prevalence of depression (BDI $\geq 16$ ) was $12.4 \%$. Depression was found in $9.5 \%, 9.8 \%, 18.4 \%$ and $26.1 \%$ of patients with normo-, micro-, macroalbuminuria and ESRD, respectively $(p<0.0001)$. The median depression score increased from 4 to 8 with deteriorating renal status $(p<0.0001)$. Altogether, $21.3 \%$ of the patients had poor sense of coherence (SOC < 60). The prevalence of poor sense of coherence was 19.1\%, 19.7\%, $30.8 \%$ and $23.9 \%$ in patients with normo-, micro-, macroalbuminuria and ESRD, respectively $(p<0.05)$. In contrast, there was no difference in the median SOC score between the four groups. Median HRQoL score was highest in subjects with normoalbuminuria $(0.9451)$ and decreased with deteriorating renal status $(p<0.0001)$, reaching the value of 0.8527 in subjects with ESRD. In a logistic regression analysis, duration of diabetes (OR 1.025; 95\%CI 1.002-1.047), BMI (1.071; 1.008-1.138), smoking (2.709; 1.476-4.970), $\mathrm{HbA}_{1}$ $(1.538 ; 1.273-1.859)$, serum creatinine $(1.055 ; 1.042-1.068)$, and the HRQoL score $(0.020 ; 0.001-0.095)$ were independently associated with diabetic nephropathy. Of the other psychological measures, neither BDI nor SOC were associated with diabetic nephropathy.

Conclusion: Depression, poor sense of coherence, and poor health related quality of life are prevalent in type 1 diabetic patients with diabetic nephropathy. However, out of these psychological measures, only HRQoL was independently associated with diabetic neprhopathy when adjusted for other confounding factors. Whether psychological disturbances are a primary feature or a consequence of diabetic complications remains to be established. Supported by: Signe and Ane Gyllenberg Foundation, Karl and Else Stockmann Foundation, Folkhälsan Research Foundation

\section{1}

Characteristics associated with poor quality of life in adults with type 1 diabetes treated with continuous subcutaneous insulin injection

M. Franciosi ${ }^{1}$, A. Maione ${ }^{1}$, B. Pomili ${ }^{1}$, I. Otranto ${ }^{1}$, R. Amoretti ${ }^{2}$, E. Busetto ${ }^{3}$, F. Capani ${ }^{4}$, D. Bruttomesso ${ }^{5}$, P. Di Bartolo ${ }^{6}$, A. Girelli ${ }^{7}$, F. Leonetti ${ }^{8}$,

L. Morviducci ${ }^{9}$, P. Ponzi ${ }^{3}$, E. Vitacolonna ${ }^{4}$, A. Nicolucci ${ }^{1}$

${ }^{1}$ Dept. Clinical Pharmacology, Consorzio Mario Negri Sud, S. M. Imbaro

${ }^{2}$ D.U., A.O. S. G. Addolorata, Roma, ${ }^{3}$ Fondazione Medtronic Italia, Medtronic, Sesto S. Giovanni Italy, ${ }^{4}$ Dept. of Medicine and Ageing, University G. D'Annunzio, Chieti, ${ }^{5}$ Dept. of Clinical and Experimental Medicine, University of Padova, ${ }^{6}$ D.U., AUSL Ravenna, ${ }^{7}$ D.U., Spedali Civili di Brescia, ${ }^{8}$ Dept. of Clinical Sciences, La Sapienza University, Roma, ${ }^{9}$ D.U., A.O. S. Camillo, Roma, Italy

Background and aims: Aim of this study was to explore patient characteristics associated with poor QoL perception among adults with T1DM using continuous subcutaneous insulin infusion (CSII).

Materials and methods: The study involved 472 patients aged between 18-55 yrs and treated with CSII, recruited by 43 diabetes clinics. During a routine visit, physicians collected clinical and sociodemographic data and patients completed the Diabetes Specific Quality of Life Scale (DSQOLS). The summary score of DSQOLS summarizes the daily burden and restrictions related to diabetes, with lower scores indicating a worse QoL perception. The main results were obtained by using a regression-tree technique (RECPAM), that permitted to identify homogeneous and distinct subgroups expressing a different likelihood of scoring in the lower tertile of the DSQOLS score. All the analyses were adjusted for patient age and number of diabetes complications.

Results: Overall, $57.2 \%$ of the patients were female, $38.4 \%$ had $\geq 1$ diabetes complications, $25.2 \%$ reported $\geq 1$ hypoglycaemic episodes (HEs) and $11.2 \%$ reported $\geq 1$ ketoacidosis episodes (KEs) in the previous 12 months. The mean age was of $35.1 \pm 10.9 \mathrm{yrs}$ and the mean duration of diabetes was of $18.4 \pm 10.2$ yrs. Male patients not reporting KEs and using CSII for $>2$ yrs had the lowest likelihood of scoring in the lower tertile of DSQOLS and thus represented the reference category. Patients who reported $\geq 1 \mathrm{KEs}$, independently of other characteristics, showed a five-fold risk of poor QoL as compared to the reference category $(\mathrm{OR}=5.1 ; 95 \% \mathrm{CI} 2.3-11.6)$. These patients also showed the highest reported frequency of HEs. Among patients who didn't report KEs, females with a duration of diabetes of $<10$ yrs represented the group of patients with the highest likelihood of poor QoL (OR=6.2; 95\% CI 2.7-14.6), while for females with longer diabetes duration the likelihood of scoring in the lower tertile of the DSQOLS was two-fold as compared with the reference group ( $\mathrm{OR}=2.4$; $95 \% \mathrm{CI} 1.3-4.7)$. Among males, those treated with CSII for $\leq 2$ yrs showed a two-fold risk of poor QoL (OR=2.3; 95\% CI 1.1-4.7) as compared to those treated with CSII for $>2$ yrs. The extremely high risk of poor QoL among women with a diabetes duration $<10$ yrs as compared to those with longer diabetes duration was associated with a significantly higher likelihood of wearing the pump for $\leq 2$ yrs ( $75 \%$ vs. $45 \%)$.

Conclusion: Unstable diabetes (high frequency of KEs and HEs) is the most important clinical variable associated with poor QoL among patients treated with CSII. In both genders, being on treatment with CSII for $\leq 2$ yrs represented an important correlate of poor QoL, thus suggesting that specific strategies are needed to help the patient to cope with this therapeutic modality, especially during the initial phase of the treatment.

Supported by: Fondazione Medtronic Italia, Sesto San Giovanni (MI) Italy

\section{2}

\section{Assessment of lower limb risk factors for falls in those with diabetes mellitus}

C. MacGilchrist ${ }^{1}$, L. Paul ${ }^{2}$, B. Kennon ${ }^{3}$, A. McFadyen ${ }^{4}$, B. Ellis ${ }^{1}$, T. Howe ${ }^{1}$ ${ }^{1}$ School of Health and Social Care, Glasgow Caledonian University, ${ }^{2}$ Nursing and Health Care, Glasgow University, ${ }^{3}$ Centre for Diabetes and Metabolism, Southern General Hospital, ${ }^{4}$ School of Engineering and Computing, Glasgow Caledonian University, Glasgow, United Kingdom

Background and aims: People with Diabetes Mellitus (DM) are at an increased risk of falling with the incidence of falls within the elderly diabetic population estimated to be around 39\%. Falls constitute a significant public health burden in terms of patient morbidity and mortality as well as economic cost. Accurately predicting which patients are at risk of falling is challenging. The aims of this study were to establish lower limb risk factors for falls in patients with DM. 
Materials and methods: Sixty patients with DM, over 55 years old, were recruited from the Southern General Hospital, Glasgow, Scotland. Subjects were classified as fallers or non-fallers with 'fallers' defined as individuals who self reported at least one fall in the last year. The following risk factors, generally associated with falls, were assessed; presence of neuropathy [ $10 \mathrm{~g}$ monofilament screening on ten sites], neuropathy disability score (NDS), neuropathy symptom score (NSS) and vibration perception threshold (VPT) bilaterally to the hallux, foot problems [the foot deformity score (FDS)], balance and gait [Tinetti Performance-Oriented Assessment of Mobility], mean ankle dorsiflexion strength (DF) [hand-held dynamometer], footwear suitability [Menz \& Sherrington footwear assessment tool], spatial and temporal parameters - an average of 3 passes along a $61 \mathrm{~cm} \times 366 \mathrm{~cm}$ walkway [GAITRite ${ }^{\oplus}$ System]. Multivariate discriminant analysis was performed to identify the variables most predictive of falls in this group.

Results: Thirty five percent $(n=21)$ of the sample had fallen in the preceding year. The mean age of the 'fallers' (F) was 69.2yrs and the 'non-fallers' (NF) 65.9yrs. Forty percent were classified as neuropathic (diagnosis achieved using a combination of signs and symptoms of neuropathy). Eighty-six percent $(n=18)$ of 'fallers' but only 56\% $(n=22)$ of 'non-fallers' had peripheral neuropathy. Gait analysis demonstrated that fallers walked with a significantly lower velocity $(\mathrm{F}=78.46 \mathrm{~cm} / \mathrm{s}, \mathrm{NF}=108.46 \mathrm{~cm} / \mathrm{s} ; \mathrm{p}<0.001)$ and had smallers step length bilaterally (Right $\mathrm{F}=48.82 \mathrm{~cm} \mathrm{NF}=61.09 \mathrm{~cm}$, Left $\mathrm{F}=48.43 \mathrm{~cm}$ $\mathrm{NF}=60.88 \mathrm{~cm}$; both $\mathrm{p}<0.001)$. The prevelence of foot deformity was high in both groups; 'fallers' (58\%) and 'non-fallers' (54\%). The mean Tinetti score classified 'fallers' as being at moderate risk (22.4) and 'non-fallers' as low risk (25.6). The mean ankle dorsiflexor muscle strength was significantly lower in 'fallers' than 'non-fallers' (2.4kg and 3.9kg respectively; $\mathrm{p}<0.001)$. Multivariate discriminant analysis identified velocity and DF strength as the variables with the best discriminating power and together, with cross-classification validation, they correctly classified $\sim 76 \%$ of the 60 participants.

Conclusion: These novel findings highlight the lower limb risk factors in those with DM. Multivariate analysis suggests that walking velocity and dorsiflexor muscle strength may be useful in identifying those patients at risk of falling. Health Care Professionals could use these simple clinical measures to identify those at risk of falling and thus implement appropriate early preventative strategies.

Supported by: School of Health and Social Care of the Glasgow Caledonian University in conjunction with HealthQwest

\section{3}

Identifying depression in the diabetes clinic: how can we target this important co-morbidity?

L. Matone ${ }^{1}$, R.P. Raghavan ${ }^{2}$, T. Durston ${ }^{2}$, J. Phull', P. Singhal ${ }^{2}$

${ }^{1}$ Department of Psychiatry, ${ }^{2}$ Department of Diabetes, Weston General

Hospital, Weston-Super-Mare, United Kingdom

Background and aims: The prevalence of depression in patients with diabetes is higher than in the general population. Depression is associated with personal suffering, reduced social functioning and emerging evidence indicates that patients with mental health conditions may receive poor quality medical care. Active screening/surveillance for depression is not routine in diabetic outpatient clinics. We set out to identify depression within a diabetes secondary care outpatient population, using self-administered questionnaires in order to establish current care and improve service provision.

Materials and methods: A prospective study of 100 consecutive patients with diabetes attending secondary care outpatients clinics, between January and May 2007 was undertaken. Anonymised data regarding patient demographics, diabetes history and management were collected using a questionnaire and pathology reports. In addition, patients were requested to complete a general questionnaire once and the Beck Depression Inventory (BDI) at presentation (while waiting to see the health professional) and again 6 weeks later.

Results: Subjects who returned both BDI questionnaires (74/100) were included. The composition of the respondents was $46 \%(\mathrm{n}=34)$ female and $54 \%(n=41)$ male with a median BDI score of 7 (range $0-49)$. The median HbAlc was 7.95 (range 5.8 - 12.9). A target HbAlc $\leq 7$ was only seen in $7 \%$ $(n=1)$ with a BDI score $\geq 17(n=13$, indicating depression) versus $23 \%(n=14)$ out of 61 subjects with a BDI score $<17.24 \%$ of the study group had a past history of depression, but only $54.2 \%(n=13)$ were receiving drug treatment for depression. Using the BDI, $18 \%$ scored positive for depression (BDI score $\geq 17$ ), of whom $69 \%(n=9)$ were previously undiagnosed. Within the group that had known depression and were on treatment, The mean BDI score was 17.6. In this group $23.1 \%(n=3)$ had a depression score classed 'Severe / Extreme' (score $\geq 31$ ). Another concern was that $18.2 \%(\mathrm{n}=2)$ with a past his- tory of depression were scoring Severe / Extreme on the BDI and were not receiving pharmacological treatment for depression. The mean BDI score was 15.9 in this group. Subjects older than 65 years of age were less likely to be significantly depressed. People suffering from depression were less likely to be employed compared to non-depressed group (20\% vs. $37 \%$ ).

Conclusion: Within this study, $17 \%$ of outpatients scored positive for depression using the BDI: over half of this group had not been previously diagnosed with depression. We have demonstrated that there is a high prevalence of concomitant depression in diabetic patients, and it may impact on glycaemic control. There is a need to create awareness and address this important comorbidity in diabetes. The identification of depression in diabetic outpatients could be substantially improved by the knowledge and use of the BDI screening tool. When the BDI score is $>17$, it is suggested that a patient undergoes further evaluation to determine more precisely a diagnosis of depression. Depression is more likely in younger subjects a finding which has been noted in previous research on this subject. Our study shows that the BDI is very easy to use and could become part of the annual review process allowing health professionals to pinpoint those individuals who are in need of further psychological evaluation and support.

\section{4}

Social, psychological, and health care factors associated with patient selfcare success in the multi-national DAWN Youth Survey

M. Peyrot ${ }^{1}$, H.-J. Aanstoot ${ }^{2}$ on behalf of the DAWN Youth Survey Group ${ }^{1}$ Sociology, Loyola College, Baltimore, United States, ${ }^{2}$ Diabetes Center for Children and Youth, Diabeter, Rotterdam, Netherlands

Background and aims: This study assessed the social, psychological, and health care factors associated with self-reported self-care success among young adults with diabetes.

Materials and methods: Data are from a cross-sectional internet survey of independent national samples of patients with diabetes aged 18-25 ( $\mathrm{N}=1905)$ from the Diabetes Attitudes, Wishes and Needs (DAWN) Youth Survey. Respondents from the US, Japan, Brazil and five countries in Europe provided self-report data for all measures. Self-care success was a 7 -item scale measuring success in controlling blood glucose, taking medications, SMBG, diet and exercise (alpha $=.81)$. Hierarchical multiple regression was used to assess the associations of self-care success with country, respondent demographic and disease characteristics, aspects of healthcare provision and participation, perceived social support from family and friends, and patient psychological functioning (diabetes coping, subjective quality of life, and psychological well-being).

Results: All factors were significantly $(\mathrm{p}<0.05)$ associated with self-care success, and together accounted for $42 \%$ of the variance. Success was higher in Denmark and Germany and lower in Japan; after adjustment for all covariates, success was lower in Brazil and Japan, and higher in Denmark. Males and those using insulin pumps reported more success; these relationships were mediated by psychological functioning. After adjustment for all covariates, respondents with type 1 diabetes and those diagnosed at older ages reported more success. More information at diagnosis and more communication among health care team members were associated with more success. Greater perceived physician support and understanding of the difficulties of living with diabetes, and patient involvement in decision making were associated with more success, and the physician factors mediated the relationships of success with information at diagnosis and health care team communication. Health care factors accounted for one-quarter of the model's explanatory power. Family and friend support were both associated with more success. Diabetes coping success, perceived quality of life, and psychological well-being were associated with more success and accounted for just over half of the model's explanatory power; these factors mediated the relationship of selfcare success with family and friend support.

Conclusion: Self-care success is multi-factorially determined. Country of residence makes a contribution beyond the factors examined here. Some individual characteristics have indirect effects that are mediated by psychological factors, while others have effects that are masked by psychological factors. From the patient perspective, physician support and understanding of psychosocial issues are the most important aspects of health care. Social support contributes to good psychological functioning and in turn to more self-care success. Successful coping is the strongest correlate with self-care success, predicting both good quality of life and psychological well-being, and in turn more self-care success. Enhancing greater provider empathy, social support for the patient and patient psychological coping appear to be promising strategies for improving self-care outcomes.

Supported by: Novo Nordisk 


\section{5}

Contribution of psychological symptoms on immediate and prolonged effects of diabetes self-management education

M. Pibernik-Okanovic, D. Ajdukovic, M. Prasek, Z. Metelko

Outpatient Clinic, Vuk Vrhovac Institute, Zagreb, Croatia

Background and aims: Depressive symptoms and diabetes-related distress may adversely interact with patients' effectiveness in the learning process during diabetes self-management education. This study was aimed at comparing baseline and follow-up indicators of diabetes knowledge and glycaemic control in patients with and without these symptoms who attended an educational course.

Materials and methods: One hundred and sixty-six patients (aged $48 \pm 15$ years, $52 \%$ female, $38 \%$ type 1 , with diabetes duration of $10 \pm 8$ yrs, $78 \%$ insulin treated) who participated in a 5-day educational course were assessed for depression and diabetes-related distress using the Center for Epidemiological Studies-Depression scale (CES-D) and the Problem Areas in Diabetes (PAID) scale. The CES-D score $>15$ and the PAID score $>40$ were considered indicative of severe depressive symptoms and high distress caused by diabetes. Diabetes knowledge was assessed at the beginning and at the end of the course using parallel forms of the Diabetes Knowledge Test (DKT). Indicators of glycaemic control as measured by $\mathrm{HbAlC}$ were assessed at the beginning of the course and after 3,6 and 12 months. Baseline and follow-up indicators of diabetes knowledge and glycaemic control were compared in the subgroups of depressed-distressed, depressed, distressed and symptom-free individuals using the Kruskal-Wallis ANOVA.

Results: Thirty-two percent of patients attending the educational course reported elevated depressive symptoms and high diabetes-related distress, $9 \%$ expressed elevated depressive symptoms, $10 \%$ high diabetes distress and $49 \%$ reported none of these symptoms. The four subgroups differed with respect to diabetes knowledge assessed at the beginning of the course $(\mathrm{H}=11.2$ $\mathrm{p}=0.01$ ), with depressed-distressed individuals having poorer knowledge as compared to the symptom-free individuals $(\mathrm{p}=0.02)$. Diabetes knowledge did not differ across the subgroups after the course $(\mathrm{H}=6.2 \mathrm{p}=0.10)$, indicating comparable improvement in all participants. Depressed-distressed, depressed, distressed and symptom-free individuals had comparable baseline $\mathrm{HbA1C}$ values $(\mathrm{H}=3.6 \mathrm{p}=0.31)$, which improved correspondingly after 3 and 6 months $(\mathrm{H}=3.2 \mathrm{p}=0.36$ and $\mathrm{H}=6.7 \mathrm{p}=0.08$ respectively). Differences in $\mathrm{HbAlC}$ values across the subgroups were observed at 12 -month followup assessment $(\mathrm{H}=7.7 \mathrm{p}=0.05)$, indicating poorer control in depressed-distressed vs depressed individuals $(\mathrm{p}=0.04)$.

Conclusion: The benefits of a five-day educational course defined as improvements in diabetes knowledge and long-term diabetes control were shown to be comparable in patients reporting depressive symptoms and diabetes-related distress as compared to patients without these symptoms. Patients with double symptoms have greater difficulties in maintaining an acceptable longterm glycaemic control, suggesting a need for a more intensive follow-up. Supported by: Ministry of Science, Education and Sports of the Republic of Croatia

\section{6}

Quality of life, coping ability and metabolic control in patients with type 1 diabetes followed by group care with a carbohydrate counting programme

M.E. Basile ${ }^{1}$, M. Trento ${ }^{1}$, E. Borgo ${ }^{1}$, C. Kucich ${ }^{1}$, P. Passera ${ }^{1}$, A. Trinetta ${ }^{1}$, G. Grassi ${ }^{1}$, F. Cavallo ${ }^{2}$, M. Porta ${ }^{1}$

${ }^{1}$ Laboratory of Clinical Pedagogy, Internal Medicine, University of Turin,

${ }^{2}$ Department of Public Health and Microbiology, University of Turin, Italy

Background and aims: Patients with type 1 diabetes (DM1) must adapt their daily life to therapeutic changes and modify insulin dosages according to the Carbohydrate $(\mathrm{CHO})$ content of their meals. We have validated a process to train patients to carbohydrate counting (CCHO) within the clinical-pedagogic Group Care (GC) approach, in order to verify its additional effects on quality of life, knowledge of diabetes, coping ability (problem solving, social support seeking, avoidance) and metabolic control.

Materials and methods: Out of 56 patients with DM1 followed by GC, 27 were randomized to attend the $\mathrm{CCHO}$ programme and 29 controls continued $\mathrm{GC}$ without $\mathrm{CCHO}$. The two groups were similar by age, duration of diabetes, years of attendance in clinic, years in GC, schooling, occupation, family history of diabetes, $\mathrm{HbAlc}, \mathrm{BMI}$, mean insulin dosage and blood lipids. The $\mathrm{CCHO}$ programme included 8 sessions, lasting 1 hour each and held every
3 months. At the beginning and after 30 months 3 questionnaires were administered to verify quality of life (DQOL), knowledge and coping. Every 3 months $\mathrm{HbAlc}$, body weight, blood glucose, blood pressure, number of hypoglycaemic episodes and insulin dosage were measured. At the beginning, after 1 year and at the end of the study blood lipids were measured.

Results: DQOL improved significantly $(\mathrm{p}<0.0001)$ in both patients followed by CCHO $(88.7 \pm 9.2$ vs78.0 \pm 9.9$)$ and Controls $(88.7 \pm 12.5$ vs $80.4 \pm 1 ., 7)$ and remained so after adjustment for age $(\mathrm{p}<0.02)$. Knowledge $(9.3 \pm 1.7$ vs $10.6 \pm 0.6, \mathrm{p}<0.0001$ ) and the 3 coping areas (problem solving: $28.0 \pm 1.9$ vs $30.0 \pm 1.5, \mathrm{p}<0.0001$; social support seeking: $18.0 \pm 4.4$ vs $17.0 \pm 5.1, \mathrm{p}<0.02$; avoidance: $16.7 \pm 3.5$ vs $14.8 \pm 3.5, \mathrm{p}<0.001)$ improved only in the patients followed by $\mathrm{CCHO}$. The improvement in knowledge remained significant after adjusting for age $(\mathrm{p}<0.001)$, with a linear trend in favour of patients with poor schooling, and in the problem solving $(\mathrm{p}<0.0001)$ and avoidance $(\mathrm{p}<0.003)$ areas of coping. HbAlc showed a non significant divergent trend between patients followed by CCHO (from 7,56 $\pm 1,26$ to $7,33 \pm 1,03$ ) and controls (from $7,73 \pm 1,24$ to $7,89 \pm 1,42$ ). There were no modifications in insulin mean dosage, hypoglycaemic episodes or blood lipids.

Conclusion: This study confirms that GC improves quality of life in people with DM1 but suggests that, to modify adaptation to their disease, they need more specific educational and cognitive support. The carbohydrate counting programme we developed appears effective in promoting change especially in people with poor schooling.

\section{7}

Marked improvement of quality of life and treatment satisfaction with intraperitoneal insulin (CIPII) compared to CSII in type 1 diabetes N. Kleefstra ${ }^{1,2}$, S.J.J. Logtenberg ${ }^{1}$, S.T. Houweling ${ }^{1,2}$, K.H. Groenier ${ }^{3}$, H.J.G. Bilo ${ }^{1,4}$

${ }^{1}$ Diabetes Centre, Isala Clinics, Zwolle, ${ }^{2}$ Medical Research Group, Langerhans, Zwolle, ${ }^{3}$ General Practice, UMCG, Groningen, ${ }^{4}$ Internal Medicine, UMCG, Groningen, Netherlands

Background and aims: To report about the effects of intraperitoneal (IP) insulin delivered with an implantable pump on Health Related Quality of Life (HRQOL) and treatment satisfaction as compared to intensive subcutaneous (SC) insulin therapy in poorly controlled type 1 diabetes patients.

Materials and methods: Open-label, randomized controlled cross-over trial. After a 3-month qualification phase, during which optimization of insulin therapy was targeted, patients who had a $\mathrm{HbA}_{1 c}>7.5 \%$ and/or $\geq 5$ hypoglycemic events/week, were randomized to receive either 6 months IP insulin (CIPII) followed by 6 months SC insulin by CSII or vice versa, with a 4 -week washout phase in between. Treatment adjustments were done according to protocol. IP insulin was delivered through an implantable pump (MIP 2007C, Medtronic), SC insulin was delivered with continuous subcutaneous insulin infusion (CSII). HRQOL was assessed with the SF-36 and the WHO-5 (range 0-100); Treatment Satisfaction was assessed with the DTSQ (range 0-36) at baseline and at the end of both treatment phases. General linear models were used to test differences, taking treatment order into account.

Results: Baseline characteristics: 11 males, 13 females; mean (SD) age 43.6 (11.8) years; mean diabetes duration 22.6 (10.6) years; mean $\mathrm{HbA}_{1 c} 8.6$ (1.1) $\%$.After randomization groups were comparable regarding clinical characteristics. One subject was lost to follow-up (start IP insulin).

Outcomes: Scores at end of both treatment phases on the SF-36 subscales are shown in Figure 1; With IP insulin scores are significantly higher on 6 out of the 8 subscales. The Mental and the Physical component summary score of the SF-36 were also higher with IP ( $\mathrm{p}=0.002$ and $\mathrm{p}=0.006$, respectively). The total DTSQ score at the end of the IP-phase was 32.6 (3.9) as compared to $23.3(9.2)$ at the end of the SC treatment phase $(\mathrm{p}<0.001)$. The WHO-5 score at the end of the IP treatment phase 64.9 (22.7) compared to 45.2 (22.7) at the end of the SC treatment phase ( $\mathrm{p}<0.001)$.

Conclusion: In a selected group of patients, CIPII significantly improves HRQOL and Treatment Satisfaction as compared to CSII, despite having to undergo a surgical procedure to have the IP-pump implanted. 


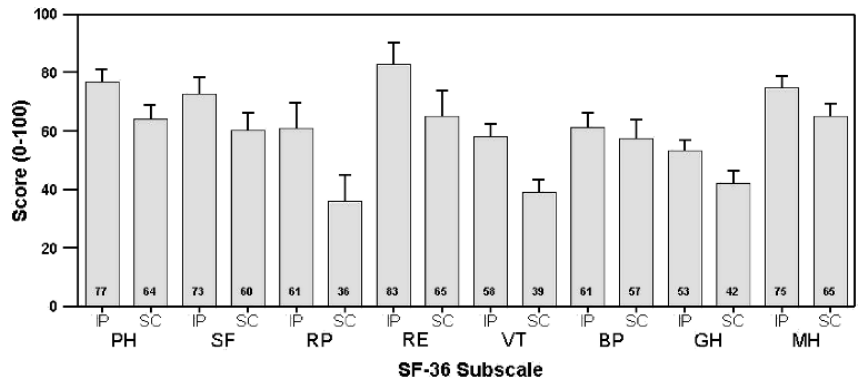

Figure 1: Scores on the SF-36 subscales after 6 months of CIPII and CSII in type 1 diabetes patients $(n=23)$.

Legend: $\mathrm{IP}=$ IntraPeritoneal, $\mathrm{SC}=$ Subcutaneous. $\mathrm{PH}=\mathrm{Physical}$ Functioning, $\mathrm{SF}=$ Social Functioning, $\mathrm{RP}=$ Role Physical, $\mathrm{RE}=$ Role Emotional, $\mathrm{VT}=$ Vitality, $\mathrm{BP}=$ Bodily Pain, $\mathrm{GH}=$ General Health, $\mathrm{MH}=$ Mental Health. Supported by: Medtronic Minimed

\section{PS 104 Education}

\section{8}

Clinical efficacy and cost-effectiveness of a brief educational intervention for self-efficacy in type 1 diabetes: results from the BITES randomised controlled trial

J.T. George ${ }^{1}$, A. Peña ${ }^{2}$, S. Lomax ${ }^{1}$, P. Droomgoole ${ }^{1}$, D.J. Torgerson ${ }^{3}$,

J.C. Thow ${ }^{1}$

${ }^{1}$ Diabetes and Endocrinology, York Hospital, United Kingdom,

${ }^{2}$ Coordinación de Políticas de Salud, Instituto Mexicano del Seguro Social, Mexico, ${ }^{3}$ York Trials Unit, University of York, United Kingdom

Background and aims: Educational interventions providing self-management skills for type 1 diabetes have been shown to improve glycaemic control. Although intensive 5-day educational interventions like have been shown to be effective in European setting (Düsseldorf, DAFNE and Bucharest- Düsseldorf studies), resource pressures demand brief interventions that are clinically and economically effective. We evaluated the biophysical and psychological effectiveness of a brief ( 2.5 day) psycho-educational intervention with integrated cost-effectiveness analysis.

Materials and methods: Our randomised controlled clinical trial in secondary-care setting had $\mathrm{HbAlc}$ and severe hypoglycaemia as primary endpoints with blood pressure, weight, height, lipid profile and composite psychometric scores as secondary outcomes. BITES curriculum was delivered in groups using pre-approved material. Assessments were done at baseline, 3, 6 and 12 months. We calculated incremental cost-effectiveness ratios (ICER) and confidence limits for the ICER were estimated using non-parametric approach of bootstrapping.

Results: 54 and 60 adult participants (type 1 diabetes for longer than 12 months, multiple injection therapy for at least 2 months) were allocated to intervention or control groups. HbAlc showed no statistically significant change at 3 months (difference $=0.0195 \%$ confidence interval (CI) $(-0.23$ to $0.26, \mathrm{p}=0.92$ ), 6 months (difference $=-0.0695 \% \mathrm{CI}-0.32$ to $0.20 \mathrm{p}=0.67$ ) and 12 months (difference $=0.0195 \%$ CI -0.30 to $0.32, p=0.94$ ). Incidence of severe hypoglycaemia (per patient per year) in the intervention group (0.41) and control group (0.48) was not statistically different. Lipids, blood pressure, use of insulin and body mass index demonstrated no statistical significance between the two groups.

Diabetes Knowledge Test, Illness Perception Questionnaire, Hypoglycaemia Fear Scale and Short Form 36 showed no change. Diabetes treatment satisfaction improved at 3 months (difference $=9.495 \%$ CI 5.2to 13.6) $\mathrm{p}=0$. 0005), 6 months (difference $=10.495 \%$ CI 6.0 to $14.8 \mathrm{p}=0.0005$ ) and 12 months (difference $=7.195 \%$ CI 2.1 to12.1 p $=0.006$ ). Significant improvement was seen in Diabetes Empowerment Scale, 3.6 points more on average in the intervention group (95\% CI: 0.65 to 6.6). There were no significant differences between groups in QALY at 3, 6 and 12 months. An ICER of $£ 38$, 400 (USD 76,000, Euro 38, 400) per QALY was obtained, including in loss of productivity.

Control group had more visits to primary-care physician (average: 6.3 visits in intervention group; 7.3 in control group) and specialist services (average: 1.9 visits in intervention group; 3.2 in control group). Intervention group reported less sick days (average: 0.85 days per patient in the intervention group vs 5.1 days in the control group) though statistically insignificant.

Conclusion: Dietary liberalisation did not lead to decline in $\mathrm{HbAlc}$ or increase in serious hypoglycaemic episodes. Although improvements in treatment satisfaction and empowerment were achieved even in absence of any statistically significant glycaemic improvement, cost-effectiveness has to be interpreted in context of the local health economy.

Trial Registration: Current Controlled Trials ISRCTN75807800

Supported by: York NHS Research and Development Innovation Fund

\section{9}

Predictors of insulin requirement in the early days after diagnosis of type 1 diabetes

S. Pilacinski, A. Gawrecki, D. Zozulinska-Ziolkiewicz,

B. Wierusz-Wysocka

Department of Internal Medicine and Diabetology, Poznan University of Medical Sciences, Poland

Background and aims: In the majority of patients with newly diagnosed type 1 diabetes dose of insulin is reduced shortly after introduction of treatment. 
Low insulin requirement in the initial course of diabetes reflects partially preserved insulin secretion and high insulin sensitivity, being associated with good metabolic control and prolonged clinical remission of diabetes. Data on direct predictors of insulin requirement in the early postinitial period of diabetes are inconclusive. Therefore, aim of this study was to determine predictors of insulin requirement at the end of initial hospitalization after diagnosis of type 1 diabetes.

Materials and methods: We followed prospectively 279 consecutive patients (111 women and 168 men, aged 16-34, mean age $24.8 \pm 5.0$ ), admitted to the Department with newly diagnosed type 1 diabetes. 34 patients presented with diabetic ketoacidosis. Mean glycaemia at diagnosis was $22.7 \pm 8.0 \mathrm{mmol} / \mathrm{l}$, $\mathrm{HbA} \quad 11.0 \pm 2.5 \%$ and BMI $21.7 \pm 3.1 \mathrm{~kg} / \mathrm{m}^{2}$. In all patients intensive functional insulin treatment in multiple injections was introduced. Mean hospitalization time was $7 \pm 2$ days. The group was divided according to the daily insulin dose at discharge from the hospital (median value $0.21 \mathrm{U} / \mathrm{kg} / 24 \mathrm{~h}$ ). Statistical analysis was performed using Statistica 5.1 software. Mann-Whitney $\mathrm{U}$ test and multivariate logistic regression were used.

Results: In the group with lower insulin requirement, lower initial glycaemia $(21.3 \pm 8.1$ vs. $24.1 \pm 7.7 \mathrm{mmol} / \mathrm{l}), \mathrm{HbA}_{1 \mathrm{c}}$ value $(9.9 \pm 2.3 \%$; vs. $12.0 \pm 2.2 \%$, $\mathrm{p}<0.0001)$, serum total cholesterol concentration $(4.42 \pm 1.11$ vs. $4.79 \pm 1.30$ $\mathrm{mmol} / \mathrm{l}, \mathrm{p}<0.05)$ and lower serum triglyceride concentration $(1.26 \pm 1.09$ vs. $1.79 \pm 1.50 \mathrm{mmol} / \mathrm{l}, \mathrm{p}<0.0001)$, higher serum HDL concentration $(1.27 \pm 0.35$ vs. $1.19 \pm 0.40 \mathrm{mmol} / \mathrm{l}, \mathrm{p}<0.05)$ and higher capillary blood bicarbonate concentration $(25.2 \pm 4.6$ vs. $20.7 \pm 6.9 \mathrm{mmol} / \mathrm{l}, \mathrm{p}<0.0001)$ were recorded, when compared with subjects with insulin requirement above $0.21 \mathrm{U} / \mathrm{kg} / 24 \mathrm{~h}$. There was also a tendency towards increased age $(25.3 \pm 5.0$ vs. $24.2 \pm 5.0$ years, $\mathrm{p}=0.053)$ and BMI $\left(22.0 \pm 2.9\right.$ vs. $\left.21.3 \pm 3.3 \mathrm{~kg} / \mathrm{m}^{2}, \mathrm{p}=0.068\right)$ in the group with lower insulin requirement. In the multivariate logistic regression model, lower initial $\mathrm{HbA}_{1 \mathrm{c}}$ (OR: 1.43; 95\%CI: 1.13-1.81, $\mathrm{p}<0.01$ ), lower white blood cell count (OR: 1.12; 95\%CI: 1.01-1.26, $\mathrm{p}<0.05)$, lower erythrocyte sedimentation rate (OR: 1.07; 95\%CI: 1.01-1.13, $\mathrm{p}<0.05$ ), absence of clinically evident infection (OR: 1.81; 95\%CI: 1.07-3.07, $\mathrm{p}<0.05$ ) and higher capillary blood bicarbonate concentration at diagnosis (OR: 1.18; 95\%CI: 1.07-1.30, $\mathrm{p}<0.001$ ) were predictors of lower $(<0.21 \mathrm{U} / \mathrm{kg} / 24 \mathrm{~h})$ insulin dose at discharge from hospital, independently from age, gender and BMI.

Conclusion: Mild initial metabolic decompensation and absence of acute inflammation may be considered as predictors of low insulin requirement in the early days after diagnosis of type 1 diabetes.

Supported by: Polish Ministry of Science and Higher Education

\section{0}

18 years after first ambulatory flexible intensified insulin therapy: current impact on metabolic control in 227 consecutive type 1 diabetic patients

D. Albrecht, U. Keller, H. Zulewski

Division of Endocrinology and Diabetes, University Hospital Basel, Switzerland

Background and aims: Good metabolic control in type 1 diabetic patients is crucial for prevention of diabetes complications. Achievement of HbAlc values below $7.0 \%$ is a very difficult task as shown by various studies including the recent DCCT update with mean $\mathrm{HbA1c}$ around $7.8 \%$. Flexible intensified insulin therapy was introduced in our institution in 1990 as standard of care for patients with type 1 diabetes. The ambulatory training program teaches the patient to apply long and short acting insulins according to certain rules and algorithms in order to cover the insulin needs in fasting state as well as in response to meals or during physical activity. Here we analyzed the impact of such therapeutic approach on blood glucose control in 227 consecutive type 1 diabetic patients followed routinely at our outpatient clinic.

Methods and results: Using a computerized database we screened in a crosssectional study 237 patients with type 1 diabetes of whom 227 were finally included because 10 patients missed follow up appointments. The median age was 46.3 years, median duration of diabetes 19.0 years, $56 \%$ were male, $33 \%$ smokers. In $58 \%$ of the patient flexible intensified insulin therapy was taught in a formal group-based ambulatory training program. The other patients were taught individually during the ambulatory visits. Insulin analogues were used in $96 \%$ of the patients. Metabolic control was similar in patients treated in a group-based program or individually. The median HbAlc (measured by DCA $2000+$ analyzer, Bayer Inc.) of the last available value was $7.1 \%(6.6 \%$ - 7.8\%, lower quartile - upper quartile). Patients with diabetes duration $<5$ years $(n=31)$ had a median HbAlc of $6.6 \%(5.8$ - 7.6) most likely due to preserved endogenous insulin secretion. In patients with diabetes duration of 5 years or more $(n=196)$ the median HbAlc value was $7.1 \%$ (6.7 - 7.8). $44.1 \%$ achieved the therapeutic target of $\mathrm{HbAlc}<7 \%, 64.8 \%<7.5 \%$ and $79.7 \%<8$ $\%$. Thus $20.3 \%$ showed HbAlc values $\geq 8 \%$. Similar to published data, smokers had worse HbAlc values: median HbAlc 7.4\% (6.7 - 8.3) in smokers vs. $7.1 \%$ (6.6 - 7.6) in non-smokers ( $\mathrm{p}=0.026$, Mann-Whitney U-Test). As compared to men, women had a higher HbAlc: women median HbAlc $7.1 \%(6.8$ - 7.8) vs. men $6.9 \%(6.5$ - 7.7) $(\mathrm{p}=0.014$, after exclusion of patients with eating or psychiatric disorders $(\mathrm{n}=15))$. Severe hypoglycaemia requiring assistance from another person was rare and observed in about 6 patients per year.

Conclusion: Ambulatory training in flexible intensified insulin therapy for diabetes mellitus type 1 is associated with a diabetes control that was equal or better as compared to published data. Such treatment strategy increases patient's responsibility for good metabolic control and may diminish the rate of complications.

Supported by: Stiftung Diabetes-Gesellschaft Region Basel

\section{1}

Do teenagers and young adults agree to clinical contact by text or email messaging?

T. Koko, J. Bodansky, R. Parslow

Diabetes Center, Leeds General Infirmary, United Kingdom

Background and aims: Many teenage and young adult patients have unacceptable levels of glycaemic control, evidenced by high HbAlc values. This usually emanates from poor adherence to insulin injections, self monitoring of blood glucose (SMBG) values, diet and exercise. However, patients are reluctant to admit to non-adherence. New technology could promote adherence by prompting patients to perform SMBG, exercise and take their insulin using mobile text and email messaging. SMBG results can be returned to clinicians by Bluetooth/mobile phone and email. Prior to evaluation of these technological innovations in a clinical setting, this study aimed at assessing their availability in a clinic cohort of 16-25 year old patients, and whether they were willing to use them for this purpose.

Materials and methods: Consecutive patients attending a teenage/young adult teaching hospital clinic were invited to self complete a structured questionnaire.

Results: 52 consecutive patients (29M, 21F, 2 unknown sex; mean age 18, range 14-24; mean duration of diabetes 12 , range 1-22 years) agreed to complete the questionnaire. All had a mobile phone, $87 \%$ of them Bluetooth enabled. $88 \%$ had computer access at work, school or college, and $92 \%$ had access at home. 58\% possessed a laptop or PDA (personal digital assistant).All but one patient had internet access; $87 \%$ at home, $27 \%$ at school and $38 \%$ at college. Only $58 \%$ were willing to have their SMBG results monitored, while $42 \%$ were either unwilling or unsure about this .Only $48 \%$ were agreeable to receive text messages to remind them to adhere to their treatment, but $46 \%$ would not agree to this and 2 were unsure. Regarding advice via email, $67 \%$ were in favour of this, but the remainder were against this or unsure.

Conclusion: The teenagers and young adults surveyed here had a high availability of access to communication by mobile phone and email, thus potentially enabling easy transfer of SMBG results to clinicians, advice to patients and messaging reminders to adhere to their treatment plan. However, about half the subjects were unwilling to have their SMBG values monitored or receive prompting to adhere to their therapy. This may reflect a reticence to be repeatedly reminded about their condition or provide information about their adherence to their treatment and their glycaemic control.

\section{2}

Optimising basal plus insulin therapy in type 2 diabetes by telecare assistance for self-monitoring of blood glucose - the ELEONOR study S. Del Prato ${ }^{1}$, A. Nicolucci ${ }^{2}$, G. Vespasiani ${ }^{3}$

${ }^{1}$ Department of Endocrinology and Metabolism, University of Pisa, ${ }^{2}$ Department of Clinical Pharmacology and Epidemiology, Consorzio Mario Negri Sud, Santa Maria Imbaro, ${ }^{3}$ Diabetology and Metabolic Disorders Center, Madonna del Soccorso Hospital, San Benedetto del Tronto, Italy

Background and aims: Evaluate whether a Telecare programme facilitates optimisation of basal (glargine; GLAR) followed by the addition of one insulin bolus injection (glulisine; GLU) in T2DM patients (pts) with secondary failure to oral hypoglycaemic agents (OHAs). We present preliminary results of 200 (out of 240) pts.

Materials and methods: 200 pts ( $54 \%$ male; mean \pm SD age $58.9 \pm 8.2$ yrs, BMI $29.9 \pm 4.3 \mathrm{~kg} / \mathrm{m}^{2}$, diabetes duration $10.9 \pm 6.9 \mathrm{yrs}$ ) with poor glycaemic 
HbA1c, fasting blood glucose and insulin doses

\begin{tabular}{|c|c|c|c|c|c|}
\hline & $\begin{array}{l}\text { Group } 1 \\
(\mathrm{n}=91)\end{array}$ & & $\begin{array}{l}\text { Group } 2 \\
(n=109)\end{array}$ & & $\begin{array}{l}\text { p-value for between-group } \\
\text { difference }\end{array}$ \\
\hline $\begin{array}{l}\mathbf{H b A}_{1 \mathbf{c}}, \% \\
\text { V2 (start GLAR) } \\
\text { V3 (start GLU) } \\
\text { V5 (endpoint) } \\
\end{array}$ & $\begin{array}{l}8.8 \pm 1.0 \\
7.8 \pm 0.9 \\
7.1 \pm 0.7\end{array}$ & $\begin{array}{l}- \\
\mathrm{p}<0.0001 \text { vs V2 } \\
\mathrm{p}<0.0001 \text { vs V3 }\end{array}$ & $\begin{array}{l}8.9 \pm 0.9 \\
7.8 \pm 0.9 \\
7.0 \pm 0.7\end{array}$ & $\begin{array}{l}- \\
\mathrm{p}<0.0001 \text { vs V2 } \\
\mathrm{p}<0.0001 \text { vs V3 }\end{array}$ & $\begin{array}{l}- \\
0.7082 \\
0.2461\end{array}$ \\
\hline $\mathrm{HbA}_{1 \mathrm{c}} \leq 7.0 \%$ at endpoint, $\mathrm{n}(\%)$ & $45(50.6)$ & & $59(54.6)$ & & 0.5692 \\
\hline $\begin{array}{l}\text { FBG, } \mathbf{m m o l} / \mathrm{L} \\
\mathrm{V} 2 \\
\mathrm{~V} 3 \\
\mathrm{~V} 5 \\
\end{array}$ & $\begin{array}{l}11.7 \pm 3.1 \\
6.0 \pm 1.0 \\
6.4 \pm 1.6\end{array}$ & $\begin{array}{l}- \\
\mathrm{p}<0.0001 \text { vs V2 } \\
\mathrm{p}=0.0412 \text { vs V3 }\end{array}$ & $\begin{array}{l}11.6 \pm 3.2 \\
6.1 \pm 1.1 \\
6.3 \pm 1.6\end{array}$ & $\begin{array}{l}- \\
\mathrm{p}<0.0001 \text { vs V2 } \\
\mathrm{p}=0.1546 \text { vs V3 }\end{array}$ & $\begin{array}{l}- \\
0.8015 \\
0.8290\end{array}$ \\
\hline $\begin{array}{l}\text { GLAR dose, U } \\
\text { V2 } \\
\text { V3 } \\
\text { V5 } \\
\end{array}$ & $\begin{array}{l}9.9 \pm 0.6 \\
26.9 \pm 15.5 \\
27.6 \pm 16.7\end{array}$ & $\begin{array}{l}- \\
\mathrm{p}<0.0001 \text { vs V2 } \\
\mathrm{p}=0.3460 \text { vs V3 }\end{array}$ & $\begin{array}{l}10.0 \pm 0.6 \\
28.1 \pm 15.8 \\
28.6 \pm 16.2\end{array}$ & $\begin{array}{l}- \\
\mathrm{p}<0.0001 \text { vs V2 } \\
\mathrm{p}=0.3748 \text { vs V3 }\end{array}$ & $\begin{array}{l}- \\
0.6340 \\
0.9360\end{array}$ \\
\hline $\begin{array}{l}\text { GLU dose, U } \\
\text { V3 } \\
\text { V4 } \\
\text { V5 } \\
\end{array}$ & $\begin{array}{l}3.6 \pm 1.7 \\
7.4 \pm 6.4 \\
8.1 \pm 8.1\end{array}$ & $\begin{array}{l}- \\
\mathrm{p}<0.0001 \text { vs V3 } \\
\mathrm{p}=0.0682 \text { vs V4 }\end{array}$ & $\begin{array}{l}3.6 \pm 1.7 \\
7.8 \pm 6.0 \\
8.3 \pm 7.1\end{array}$ & $\begin{array}{l}- \\
\mathrm{p}<0.0001 \text { vs V3 } \\
\mathrm{p}=0.0397 \text { vs V4 }\end{array}$ & $\begin{array}{l}- \\
0.5682 \\
0.7008\end{array}$ \\
\hline $\begin{array}{l}\text { Timing of GLU dose (V5), n (\%) } \\
\text { Breakfast } \\
\text { Lunch } \\
\text { Supper } \\
\text { No GLU injection }\end{array}$ & $\begin{array}{l}7(7.7) \\
35(38.5) \\
39(42.9) \\
10(11.0)\end{array}$ & & $\begin{array}{l}5(4.6) \\
42(38.5) \\
48(44.0) \\
14(12.8)\end{array}$ & & \\
\hline $\begin{array}{l}\text { Body weight, kg } \\
\text { V1 (screening) } \\
\text { V5 }\end{array}$ & $\begin{array}{l}79.0 \pm 13.2 \\
79.5 \pm 13.9\end{array}$ & $\begin{array}{l}- \\
\mathrm{p}\end{array}=0.1786$ vs $\mathrm{V} 1$ & $\begin{array}{l}82.4 \pm 14.2 \\
82.5 \pm 14.9\end{array}$ & $\begin{array}{l}- \\
\mathrm{p}\end{array}=0.8613$ vs $\mathrm{V} 1$ & \\
\hline
\end{tabular}

Active treatment phase and Telecare/SMBG was performed between V2 and V5; GLAR was started at V2 (baseline); GLU was started at V3. V2-V3: 8-16 weeks; V3-V4: 12 weeks; V4-V5: 12 weeks. Analysis of covariance or Chi square test were used as appropriate. Results are means \pm SD unless otherwise stated. FBG=fasting blood glucose; GLAR=insulin glargine; GLU=insulin glulisine; $\mathrm{SMBG=standard} \mathrm{blood} \mathrm{glucose} \mathrm{monitoring;} \mathrm{V=visit}$

control $\left(\mathrm{HbA}_{1 c} 8.9 \pm 0.9 \%\right)$ on one or more OHAs participated in this openlabel, multicentre, randomised, controlled, parallel-group study. After a 4-wk run-in, all pts were started on GLAR (Visit 2) and titrated to achieve FBG $\leq 5.5 \mathrm{mmol} / \mathrm{L}$. At GLAR initiation (baseline), pts were randomised to either Telecare (allowing electronic transfer of blood glucose [BG] readings, $n=91$ ) or standard BG monitoring (SMBG; $n=109$ ) to identify the highest postprandial peak and glargine dose adjustment by phone, as deemed necessary by the investigators, or dose adjustment at each visit, respectively. After 8-16 wks of GLAR, pts with FBG $\leq 7.0 \mathrm{mmol} / \mathrm{L}$ in both groups ( $\mathrm{n}=91$ and 109 , respectively) added one dose of GLU at the meal with the highest postprandial BG; this treatment was maintained for 24 wks. The primary objective was to compare Telecare with SMBG in terms of $\mathrm{HbA}_{1 c}$ change. The primary endpoint was change in $\mathrm{HbA}_{1 \mathrm{c}}$ from baseline to endpoint. Secondary endpoints included BG profiles, weight change, insulin doses and safety (including hypoglycaemia). Preliminary results are reported without lifting the study blinding. Results: During GLAR titration, $\mathrm{HbA}_{1 \mathrm{c}}$ improved with both $\mathrm{BG}$ monitoring approaches by $1 \%$ (Table). One GLU injection was added to $83 \%$ of pts reaching target FBG (Breakfast 12; Lunch 39; Supper 43\%), resulting in a further $0.7-0.8 \% \mathrm{HbA}_{1 \mathrm{c}}$ drop in both groups (endpoint $\mathrm{HbA}_{1 \mathrm{c}}: 7.1 \pm 0.7$ vs $7.0 \pm 0.7 \%$; both $\mathrm{p}<0.0001$ vs GLAR) with 50.6 and $54.6 \%$ of pts, respectively, reaching $\mathrm{HbA}_{1 \mathrm{c}} \leq 7.0 \%$ at endpoint. With GLAR, FBG decreased in both groups (from $11.7 \pm 3.1$ to $6.0 \pm 1.0$ and from $11.6 \pm 3.3$ to $6.1 \pm 1.1 \mathrm{mmol} / \mathrm{L}$, respectively; both $\mathrm{p}<0.0001)$ with no further change after GLU initiation $(6.4 \pm 1.6$ vs $6.3 \pm 1.6$ $\mathrm{mmol} / \mathrm{L})$. There was no difference in weight changes $(+0.4 \pm 3.0$ vs $+0.1 \pm 5.0$ $\mathrm{kg})$ and GLAR ( $29 \pm 16$ vs $28 \pm 17 \mathrm{U} / \mathrm{d})$ or GLU ( $8.3 \pm 7.1$ vs $8.1 \pm 8.1 \mathrm{U} / \mathrm{d})$ dose. 5 episodes of severe hypoglycaemia were experienced by 4 pts.

Conclusion: GLAR plus one bolus GLU injection at the time of the meal with the highest BG peak results in marked improvement of glycaemic control and achievement of glycaemic targets $\left(\mathrm{HbA}_{1 \mathrm{c}} \leq 7 \%\right)$, with low risk of severe hypoglycaemia, in a large proportion of pts with secondary failure to OHA. Telecare and SMBG are equally effective for initiation and titration of the basal plus strategy.

Supported by: sanofi-aventis

\section{3}

Diabetes knowledge among children and adolescents in Germany: unfamiliarity with risk factors and concealment of own illness K. Kempf ${ }^{1,2}$, R. Landgraf ${ }^{1}$, S. Martin ${ }^{1,2}$

${ }^{1}$ Nationales Aktionsforum Diabetes mellitus, NAFDM, ${ }^{2}$ Westdeutsches Diabetes- und Gesundheitszentrum, Sana Kliniken Düsseldorf GmbH, Düsseldorf, Germany

Background and aims: There is a growing epidemic of type 2 diabetes mellitus (T2DM) worldwide and it is estimated that 7-8 million people suffer from T2DM in Germany. Studies have shown that the manifestation of T2DM in high risk populations can be prevented by lifestyle modifications but prevention programs can only be promising if basics about, symptoms of and risks for T2DM are well known. Nevertheless, data about T2DM knowledge, especially among children and adolescents, are rare worldwide and so far only available from one cross-sectional study in Mexico. Therefore, it was the aim of this study to determine the knowledge about T2DM among students in Germany.

Materials and methods: We developed a questionnaire for students (class levels 5-13) including 12 questions about T2DM knowledge and 2 questions about awareness about and the attitude towards diabetes within the family and social environment. Differences between T2DM knowledge were analyzed by chi square test and odds ratios (OR) were determined using logistic regression with adjustment for school type, class level, sex, diabetes within family and social environment.

Results: Including complete age-groups within schools 4383 questionnaires have been filled out by students of grammar and secondary schools (e.g. German Gymnasium, Realschule and Hauptschule, 3 each) in the region of Düsseldorf. Comparable to the results from the Mexican study $92 \%$ of the students knew that in case of diabetes blood glucose levels are elevated but only $27 \%$ were able to define thirst and urgency (21\%) as typical symptoms for diabetes. More important, only $12 \%$ of the students were aware of the fact that overweight, lack of physical activity and fat-rich diet are risk factors for T2DM. In total, the chance for adequate diabetes knowledge was depending on class level (OR 2.5 [2.1-2.9] for class level 11-13 and 2.6 [2.0-3.2] for class level 8-10 vs. 5-7), school type (OR 1.4 [1.2-1.6]), female sex (OR 1.4 [1.11.7]) and diabetes within the family or social environment (OR 1.5 [1.2-1.9]). Indeed, $66 \%$ of the students were acquainted with someone with diabetes, 
nevertheless $12 \%$ would not inform classmates about their own illness $(8 \%$ from grammar school vs. $19 \%$ from secondary school, $\mathrm{p}<0.001$ ).

Conclusion: The data demonstrate that the knowledge, especially about risk factors for T2DM, is insufficient among children and adolescents in Germany. To enable effective diabetes prevention the knowledge about diabetes should be increased and diabetes concealment reduced. Education about healthy diet and risk of overweight and lack of physical activity in context with diabetes should not only be intensified in curricula but also implemented in schools. Supported by: Industrieforum Diabetes, Roche Diagnostics GmbH, B. Braun Melsungen $A G$

\section{4}

European implications of the READ (Ramadan focused Education and Awareness in Diabetes) programme

V. Bravis ${ }^{1,2}$, E. Hui ${ }^{1}$, S. Salih ${ }^{3}$, S. Mehar ${ }^{3}$, M. Hassanein ${ }^{4}$, D. Devendra ${ }^{1,3}$ ${ }^{1}$ Jeffrey Kelson Diabetes Centre, Central Middlesex Hospital, London, ${ }^{2}$ Investigative Sciences, Imperial College, London, ${ }^{3}$ Brent Integrated Diabetes Pathway, Brent tPCT, London, ${ }^{4}$ Diabetes, Glan Clwyd Hospital, North Wales, United Kingdom

Background and aims: Europe has one of the highest prevalence of diabetes, an estimated 53 million people according to IDF data in 2007. There are 14 million Muslims living in Europe and approximately 1 million suffer with diabetes. During Ramadan, Muslims fast from dawn to dusk for the duration of one lunar month. The majority of Muslim patients with diabetes are unaware of the complications associated with fasting, like hypoglycaemia, and are passionate about the Ramadan fast. The EPIDIAR study showed that $79 \%$ of patients with type 2 diabetes fast in 13 Muslim nations during Ramadan. Their risk of severe hypoglycaemia during this fasting period increased by five-fold. The safety of fasting has not been studied in the European Muslim population with Type 2 diabetes. Our Ramadan-focused, structured education programme aimed to meet the unfulfilled patient need for education to allow for safer fasting for people with diabetes who choose to fast during Ramadan. It was based on the ADA consensus statement for management of diabetes during Ramadan. The impact of the programme on weight and hypoglycaemic episodes (HE) during Ramadan in a UK Muslim population with Type 2 diabetes was evaluated.

Materials and methods: In the READ (Ramadan focused Education and Awareness in Diabetes) programme, 57 patients (Group A) took part in a structured education programme about meal planning, physical activity, glucose monitoring, hypoglycaemia, dosage and timing of medications. Group B did not ( 66 patients). HE (capillary blood glucose $\leq 3.5 \mathrm{mmol}^{-1}$ and/or symptoms) and mean weight were measured before and after Ramadan. Group A patients filled in standardised educational session feedback forms, where patients reported satisfaction levels by use of numbers (1 strongly agree, 5 strongly disagree).

Results: After Ramadan, there was a mean weight loss of $0.7 \mathrm{~kg}$ in group A $(p<0.001)$, compared to a $0.6 \mathrm{~kg}$ increase in group $B(p<0.001)$. The weight changes observed were independent of the class of hypoglycaemic agents used. There was a significant decrease in the total number of HE in group A, from 9 to 5 , compared to a four fold increase in group B from 9 to 36 $(p<0.001)$. The majority were in patients treated with short-acting sulphonylureas (group $\mathrm{A}=100 \%$, group $\mathrm{B}=94 \%$ ). Education feedback was very positive demonstrating that overall attendees had learnt a great deal and as a result would be likely to change their behaviour.

Conclusion: Ramadan-focused education in diabetes empowers patients to change their lifestyle during Ramadan. It minimises the risk of HE and prevents weight gain during this festive period for Muslims. We encourage all healthcare providers in Europe to implement this novel education programme, which is the first to study outcomes of fasting in the European Region. The intervention is pragmatically designed; hence it is generalisable and potentially replicable for the rest of Europe. Currently, there is no European or EASD consensus available for managing Muslims with diabetes during Ramadan. This study provides a template for a larger randomised control trial, the results of which could have significant impact for the Muslim population of Europe affected by diabetes.

Supported by: Daichii-Sankyo, Ash Healthcare

\section{5}

An intake of fat is a major factor which affects on basal insulin dose in type 1 diabetes patient

T. Hashimoto ${ }^{1}$, T. Kawamura ${ }^{1}$, M. Hirose ${ }^{1}$, H. Fujimoto ${ }^{2}$, K. Yoshida ${ }^{2}$, C. Takemura ${ }^{1}$, T. Higashide ${ }^{1}$, K. Kimura ${ }^{1}$, H. Inada ${ }^{1}$, S. Niihira ${ }^{1}$, S. Aono ${ }^{1}$, T. Yamano

${ }^{1}$ Pediatrics, Osaka City University Graduate School of Medicine, ${ }^{2}$ Nutrition/ Dietary Section, Osaka City University Hospital, Osaka, Japan

Background and aims: Recently, the rapid acting insulin analogues, the long-acting insulin analogues, and pump therapy have made the roles of basal insulin and bolus insulin clear in the insulin therapy of type 1 diabetes patients. The dose of bolus insulin is depended on carbohydrate counting. However, it is not known what is responsible for the dose of basal insulin. In this study, we have focused on the factors which might affect the dose of basal insulin in type 1 diabetes patients.

Materials and methods: Type 1 patients who were visiting to our clinic, had been on multiple daily insulin (MDI) injection using rapid-acting insulin and long-acting insulin ( $\mathrm{n}=111$ : male 50, female 61$)(7 \sim 34 \mathrm{yr}$, average $18.4 \mathrm{yr})$ or continuous subctaneous insulin infusion using rapid acting insulin $(n=46$ : male 10, female 36) (4 33 yr, average $15.2 \mathrm{yr}$ ) were enrolled as the subjects of the study. The enrollment criteria included the disease duration more than 2 years, a daily insulin requirement of 0.5 to $1.5 \mathrm{U} / \mathrm{kg} /$ day, and $\mathrm{HbA1c}$ less than $9 \%$. The dose of bolus insulin was adjusted by carbohydrate counting. The basal insulin dose was adjusted according to the stability of blood glucose level without meal and correction insulin. The various factors, including age, sex, body weight, BMI, HbAlc, disease duration, total daily dose of insulin (TDD), adipocytokines (adiponectin, resistin, and leptin), and the meal contents of daily food, have been analyzed their relationships with the dose of basal insulin. The meal contents were analyzed using the photographs which were taken with the digital cameras of daily foods on subjects.

Results: The ratios of basal insulin to TDD (B/T ratio) were $35 \%$ and $40 \%$ in MDI and CSII, respectively. The $\mathrm{B} / \mathrm{T}$ ratio and basal insulin dose $(\mathrm{U} / \mathrm{kg})$ were not related with age, sex, body weight, BMI, HbAlc, disease duration, TDD, adiponectin, leptin either. The level of serum resistin was weakly related with the $\mathrm{B} / \mathrm{T}$ ratio $(\mathrm{p}<0.05)$. The accuracy of our food analysis using the photographs was confirmed by three experienced dietitians and sample meals. The food analysis has revealed that an intake of fat in daily meals was strongly related to the $\mathrm{B} / \mathrm{T}$ ratio and basal insulin dose $(\mathrm{U} / \mathrm{kg})(\mathrm{p}<0.05)$.

Conclusion: The $\mathrm{B} / \mathrm{T}$ ratio of our Japanese patients was lower than that reported in the western countries. Neither age nor sex nor obesity nor puberty was related to the $\mathrm{B} / \mathrm{T}$ ratio and the basal insulin dose $(\mathrm{U} / \mathrm{kg})$ in this study. The serum resistin levels were related to the $\mathrm{B} / \mathrm{T}$ ratios. It is known that resistin is a maker of insulin resistance. However, the puberty was not related to resistin and the $\mathrm{B} / \mathrm{T}$ ratio in this study. The further investigation will be needed to know the relationships among them. The meal content, especially an intake of fat seemed to be a major factor of affecting on the basal insulin doses in type 1 diabetes. This evidence may explain the lower $\mathrm{B} / \mathrm{T}$ ratio of our Japanese type 1 diabetes patients.

\section{6}

Development of training and education for people with a diagnosis of pre-diabetes in culturally diverse communities

J. Troughton ${ }^{1}$, S. Amin ${ }^{2}$, H. Daly ${ }^{1}$, L. Martin- Stacey ${ }^{1}$, N. Patel ${ }^{3}$, M.A. Stone ${ }^{3}$ ${ }^{1}$ Diabetes Research Department, University Hospitals of Leicester NHS Trust, ${ }^{2}$ Diabetes, University Hospitals of Leicester NHS Trust, ${ }^{3}$ Department of Health Sciences, University of Leicester, United Kingdom

Background: The prevalence of pre-diabetes (impaired glucose tolerance, impaired fasting glucose or both) is increasing. This condition is associated with an increased risk of type 2 diabetes (2-10\% progression per annum) and cardio-vascular endpoints. Lifestyle modifications have been shown to be effective in delaying progression, suggesting the need for effective educational interventions to encourage and empower people with this condition to change their lifestyle behaviour. In an action research project based on diabetes education, we have previously developed a methodology for modifying education programmes to be fit-for-purpose for specific groups including people from black and minority (BME) ethnic groups.

Methods: In order to develop training and education programmes designed for people who have received a diagnosis of pre-diabetes, we have applied the methodology previously developed, using the newly diagnosed DESMOND (Diabetes Education and Self Management for Ongoing and Newly 
Diagnosed) education module and training programme for educators as our starting point. This methodology involves an iterative process similar to the 'audit cycle' and includes the use of data collection and analysis using rigorous qualitative methods to gather and consider the implications of feedback from key stakeholders including health care users (patients attending education sessions) and providers and facilitators (trainers, educators and interpreters). Each 'cycle' involves identification and implementation of modifications needed; piloting including data collection using observation, interviews and focus groups; collation and analysis of data collected; interpretation and reflection; and identification of further changes needed. The cycle is repeated until piloting suggests that a fit-for-purpose model has been developed. Our previous experience of modifying the DESMOND newly diagnosed programme for people from BME groups has enabled us to use our methodology to concurrently develop pre-diabetes training and education for people with language and cultural needs.

Results: During the first 'cycle' of modifications and qualitative data collection, feedback and observational data indicated good levels of acceptability and effectiveness for the training and education including, in patients, increased understanding of the nature and implications of their diagnosis and assimilation of messages about lifestyle changes. Some further modifications were also suggested, including resource and curriculum changes relating to methods of conveying key messages about physical activity and to information given about the differences between complications of diabetes and prediabetes. Following a further cycle, it was felt that, with very minor additional modifications, training and education schedules were ready for implementation and evaluation in a larger sample.

Conclusion: The methods used have enabled us to develop a fit-for-purpose model for the provision of training and education relevant to people with a diagnosis of pre-diabetes from culturally diverse populations. The effectiveness of the education module now requires formal evaluation. Supported by: NIHR

\section{7}

Efficacy of intensive multi-factorial intervention for cardiovascular disease prevention in high-risk type 2 diabetes: 2 -year interim analysis of the MIND.IT study

A.A. Rivellese ${ }^{1}$, O. Vaccaro ${ }^{1}$, D. Ardigò ${ }^{2}$, M. Trovati ${ }^{3}$, F. Cavalot ${ }^{3}$, R. Miccoli ${ }^{4}$, L. Franzini ${ }^{2}$, C. Di Loreto ${ }^{5}$, P. De Feo ${ }^{5}$, G. Derosa ${ }^{6}$, A. Damato, D. Zavaroni ${ }^{8}$, I. Zavaroni ${ }^{2}$

${ }^{1}$ Dept of Clinical/Experimental Medicine, Federico II University, Napoli, ${ }^{2}$ Dept of Internal Medicine, Parma University, ${ }^{3}$ Dept of Clinical/ Biological Sciences, Turin University,San Luigi Gonzaga Hospital, ${ }^{4}$ Dept of Endocrinology, Pisa University, ${ }^{5}$ Dept of Internal Medicine, Perugia University, ${ }^{6}$ Diabetes Center, Pavia University, ${ }^{7}$ Dept of Endocrinology, Bari University, ${ }^{8}$ Piacenza Hospital, Diabetes Unit, Italy

Background and aims: The Multiple INtervention in type 2 Diabetes. ITaly (MIND.IT) study is a multi-centric randomized, controlled trial in type 2 diabetic patients at high cardiovascular (CVD) risk(2 or more CVD risk factors). The study compares the Usual Care (UC) of CVD prevention with a multifactorial treat-to-target Intensive Care (IC) strategy as implemented according to national/international guidelines.The study has a planned follow-up duration of 5 years and a combined primary end-point of CVD morbidity and mortality. This report gives the results of the pre-planned ad-interim analysis after 2 years of follow-up to assess middle-term efficacy of the intervention on CVD risk factors.

Materials and methods: Ten large diabetes clinics were randomized to carry on their usual clinical practice for CVD primary prevention (UC group) or to perform an intensive program of lifestyle and pharmacological intervention aiming at the following targets: weight loss $>5 \%$ (if overweight), $\mathrm{HbAlc}$ $<7 \%$, LDL-cholesterol (LDL-c) $<100 \mathrm{mg} / \mathrm{dL}$, blood pressure $(\mathrm{BP})<130 / 80$ $\mathrm{mmHg}$, and anti-platelet treatment implementation. IC operators were asked to implement a pre-determined step-wise treat-to-target intervention program.Laboratory analyses were performed in each centre;an external quality control system was implemented and systematic differences between laboratories were corrected before statistical analysis .

Results: A total of 1461 type 2 diabetic patients (759M/702F, age $61 \pm 5$ years) free of previous CVD was enrolled. In two years the drop-out rate was $17.1 \%$ for the IC and $23.2 \%$ for the UC group.A statistically significant, and clinically relevant, decrease in HbAlc (mean \pm SD from $7.7 \pm 0.05 \%$ to $7.0 \pm 0.01$ $\%, \mathrm{p}<0.0001$ ) and BMI (from $30.4 \pm 0.3$ to $29.7 \pm 0.1, \mathrm{p}<0.0001$ ) was observed in the IC group, whereas no differences were detectable in the other. In addition, BP and LDL-c decreased significantly in both groups, but more in the
IC group (systolic BP from $144 \pm 13$ to $132 \pm 14$ vs $148 \pm 15$ to $147 \pm 19 \mathrm{mmHg}$, diastolic BP from $85 \pm 8$ to $79 \pm 7$ vs $86 \pm 8$ to $82 \pm 10 \mathrm{mmHg}$, LDL-c from $144 \pm 35$ to $108 \pm 31$ vs $142 \pm 28$ to $118 \pm 32 \mathrm{mg} / \mathrm{dL}$; p for interaction intervention arm ${ }^{\star}$ time" $<0.0001$ for all comparisons). Accordingly the proportion of patients achieving the treatment goals was significantly higher in the IC than in the UC group: $\mathrm{HbAlc} 51 \%$ vs $27 \%$, LDL-c $43 \%$ vs $27 \%$, systolic BP 41 vs $11 \%$, diastolic BP $41 \%$ vs $28 \%$ ( $<<0.001$ for all ).

Conclusion: The application of a multi-factorial treat-to-target intervention strategy is associated with a significant improvement in all major CVD risk factors and the efficacy of this approach after two years of treatment is far beyond what achieved in the usual practice in diabetes care. In addition, data demonstrate, for the first time, that a significant improvement in metabolic control (HbAlc) can be achieved without a concomitant body weight increase.

Supported by: FoRiSID

\section{8}

A diabetic foot screening programme in primary care

A. Macleod ${ }^{1}$, M. McCarthy ${ }^{1,2}$, A. Tahrani ${ }^{1}$, S. Wooton-Whiting ${ }^{3}$

${ }^{1}$ Hummingbird Centre, Royal Shrewsbury Hospital, ${ }^{2}$ Belvidere Medical Practice, Shropshire County PCT, ${ }^{3}$ Podiatry, Shropshire County PCT, Shrewsbury, United Kingdom

Background and aims: In 2002 a project was commenced to look at the feasibility of providing foot screening for people with diabetes in primary care. Chiropody services were restricted and there was the impression that diabetic foot lesions were referred late. We now describe the results of this project. Materials and methods: A local Primary Care Trust (PCT) agreed to fund a pilot study which could be expanded if found useful. The Podiatry Department and the Diabetic Clinic formulated a template that was downloaded onto primary care computer systems and which recorded peripheral pedal pulses, as measured by palpation and Doppler, and nerve function, measured by vibration threshold (Neurothesiometer) at the great toe pulp. Practices are visited in rotation by accredited podiatrists so that each patient is screened annually. Screening visits are also used to update patients regarding footcare. A recall system is run by the Podiatry Department and updated by practices. Results: Initially the scheme involved twelve practices within the county town and environs. With the advent of the new General Practice Contract in 2004 the Foot-Screening programme expanded and now serves two PCTs and all 66 practices within the county of Shropshire. Patients are categorised as low or high risk and the latter are referred into the hospital Diabetic Foot Clinic. Percentages of patients screened in the programme are given in the table. There has been a marked increase in screening from baseline in 2003 to 2007 , which is statistically significant ( $p<0.001$ for both neuropathy and peripheral circulation screening). Eighty six percent of the 18,614 patients with diabetes in Shropshire County have been screened over the last year, and the details and action documented on the computer system.

Conclusion: Since the start of the pilot programme, the community FootScreening programme has achieved near maximal coverage of people with diabetes in Shropshire. It is valued as much for its input into foot education as for its detection of high risk patients and their swift referral into the Diabetic Foot Clinic.

Foot screening of Diabetic Patients: Percentage of patients screened

\begin{tabular}{llllll}
\hline Year & 2003 & 2004 & 2005 & 2006 & 2007 \\
\hline $\begin{array}{l}\text { Total Number of Patients with } \\
\text { Diabetes }\end{array}$ & 14,203 & 15,628 & 16,121 & 16,867 & 17,853 \\
\hline Peripheral Pulses Recorded & $12 \%$ & $22 \%$ & $79 \%$ & $81 \%$ & $87 \%$ \\
\hline Neuropathy Testing Recorded & $12 \%$ & $22 \%$ & $78 \%$ & $81 \%$ & $86 \%$ \\
\hline
\end{tabular}

\section{9}

Factors that can influence adherence to an Education Program for Preventive Diabetic Foot Self-care

A.M. Caetano, A. Gonçalves, A. Valongo, I. Lessa, A. Recto, R. Oliveira, A. Castela, R. Duarte, J.-M. Boavida, J. Raposo

Diabetic Foot, APDP, Lisbon, Portugal

Background and aims: Therapeutic education represents a vital tool in the prevention of major complications of the diabetic foot. The aim of this study was to identify some factors that can influence adherence to an Education 
Program for Preventive Diabetic Foot Self-care in a Portuguese Diabetic Foot Clinic.

Materials and methods: A retrospective case-control study based on clinical data collected from diabetic patients that attended a Diabetic Foot Clinic between January and December 2007. From a total total population of 1048 diabetic patients who were invited to attend a structured and free Education Program for Preventive Diabetic Foot Self-care we have studied some clini$\mathrm{cal}$ and social-economic factors including demographic variables, metabolic control and foot ulcer risk factors in all the patients that attended the Education Program (134 Patients) - group A and in a random sample of those who did not attend the Education Programme $(n=134)$ - group B. Statistical analysis was performed through SPSS (Statistical Package for the Social for science).

Results: The comparative analysis between the two samples showed statistic significant differences in gender (group A: $70,90 \%$ vs group B: $45,52 \%$ ) for male and $(29,10 \%$ vs $54,48 \%)$ for female; $\mathrm{p}<0,001$; with mean age 49,30 vs 64,20 years old. The average duration of the disease: group A: 9 years vs group B: 12 years; $p<0,001$. The last $\mathrm{HbA1c}$ patients with $=<6,5 \%: 12,69 \%$ ( group A) vs $15,67 \%$ ( Group B) p $<0,05$. In Group A, the majority of patients lived in Lisbon metropolitan area where the Diabetic Clinic is located were unemployed (8,2\%), retired (38,1\%). $64,2 \%$ had high school degree. $14,2 \%$ of the patients were accompanied by another person. Type 2 Diabetes was less prevalent in group A $(70.1 \%)$ than in group B $(74.5 \%)$. There were no significant differences regarding foot ulcer risk between both groups

Conclusion: Factors that can positively influence adherence to an Education Diabetic Foot Prevention programme include: male gender, less duration of disease, worse metabolic control, higher school degree and professional inactivity. This study also shows a low adherence to the education program $(12 \%$ of the total population).We believe that this study provided some clues in order to improve the future adherence to the educational programs.

\section{PS 105 Pregnancy in type 1 diabetes mellitus}

\section{0}

Pregnancy outcomes in women with type 1 diabetes treated with longacting insulin analogues. A case control study

K. Todorova - Ananieva ${ }^{1}$, M. Genova ${ }^{2}$

${ }^{1}$ High-risk Pregnancy Department, University Hospital of Obstetrics and Gynaecology, ${ }^{2}$ Department of Clinical Laboratory and Immunology, Medical University, Sofia, Bulgaria

Background and aims: The aim was to evaluate and compare the pregnancy outcomes in women with type 1 diabetes (T1D) intensively treated with longacting human or analog insulin.

Materials and methods: A prospective two years case control study in ninety pregnant women with T1D. The intensified treatment consisted of insulin aspart as bolus insulin and long-acting insulin as basal. Women were divided into three groups according to the basal insulin: $\mathrm{n}_{1}=30$ treated with NPH insulin, $\mathrm{n}_{2}=30$ treated with insulin detemir and $\mathrm{n}_{3}=30$ treated with insulin glargine. Participants were matched for age, duration of diabetes, BMI, HbAlc before pregnancy, parity, number of previous pregnancies and abortions. Metabolic control, diabetic complications, severe hypoglycemic episodes and pregnancy induced hypertension and preeclampsia were registered. Perinatal mortality, stillbirth, macrosomia, weeks at and route of delivery were also recorded. Statistical methods: ANOVA - with multiple comparisons and chi square test have been used.

Results: No statistically significant difference in mean values for age, diabetes duration, BMI, parity and number of previous pregnancies. No differences were observed in pre-prandial, postprandial glucose and $\mathrm{HbAlc}$ levels in early pregnancy $\left(\mathrm{HbAlc} \mathrm{n}_{1}=7.3 \pm 0.8 \%, \mathrm{n}_{2}=6.9 \pm 0.7 \%, \mathrm{n}_{3}=7.1 \pm 0.8 \%, \mathrm{p}=0.7\right)$. No differences were observed in postprandial glucose and $\mathrm{HbAlc}$ levels in late pregnancy $\left(\mathrm{HbAlc} \mathrm{n}_{1}=7.8 \pm 0.3 \%, \mathrm{n}_{2}=7.1 \pm 0.6 \%, \mathrm{n}_{3}=7.3 \pm 0.7 \%, \mathrm{p}=0.06\right.$ ) as well. The level of pre-prandial glucose in late pregnancy was lowest in the first group $\left(\mathrm{n}_{1}=4.5 \pm 1.4 \mathrm{mmol} / \mathrm{l}, \mathrm{n}_{2}=6.6 \pm 1.1 \mathrm{mmol} / \mathrm{l}, \mathrm{n}_{3}=6.9 \pm 0.8 \mathrm{mmol} / \mathrm{l}\right.$, $\mathrm{p}=0.0001$ ). The dose of short-acting insulin was significantly higher in the first group in early $\left(\mathrm{n}_{1}=25.9 \pm 34.9 \mathrm{U} / \mathrm{kg}, \mathrm{n}_{2}=17.0 \pm 25.3 \mathrm{U} / \mathrm{kg}, \mathrm{n}_{3}=19.6 \pm 26.7\right.$ $\mathrm{U} / \mathrm{kg}, \mathrm{p}=0.004)$ and in late pregnancy $\left(\mathrm{n}_{1}=41.1 \pm 20.0 \mathrm{U} / \mathrm{kg}, \mathrm{n}_{2}=26.1 \pm 12.9\right.$ $\left.\mathrm{U} / \mathrm{kg}, \mathrm{n}_{3}=21.9 \pm 10.1 \mathrm{U} / \mathrm{kg}, \mathrm{p}=0.0001\right)$. There was no difference in the dose of long-acting insulins in early pregnancy between the groups, but in late pregnancy the dose in $\mathrm{n}_{1}$ group was statistically lower in comparison to the other two groups $\left(\mathrm{n}_{1}=16.0 \pm 6.8 \mathrm{IU} / \mathrm{kg}, \mathrm{n}_{2}=21.4 \pm 8.2 \mathrm{U} / \mathrm{kg}, \mathrm{n}_{3}=29.7 \pm 6.6 \mathrm{U} /\right.$ $\mathrm{kg}, \mathrm{p}=0.001)$. Severe hypoglycemic episodes in the first group was observed at rate of $16 \%(\mathrm{p}=0.03)$. There was no difference in the frequency of hypoglycaemic episodes between groups $n_{2}$ and $n_{3}$. Caesarean section was the main choice in all groups. There was statistically significant difference in the time of delivery in the third group compared to other two groups $\left(\mathrm{n}_{1}=36.7 \pm 1.1\right.$ week, $\mathrm{n}_{2}=36.2 \pm 1.2$ week, $\mathrm{n}_{3}=37.5 \pm 0.7$ week, $\mathrm{p}=0.001$ ). The newborn's body weight in the third group was statistically higher than the other two groups $\left(\mathrm{n}_{3}=3623.9 \pm 527.8 \mathrm{~g}, \mathrm{n}_{1}=3364.3 \pm 662.4 \mathrm{~g}, \mathrm{n}_{2}=3076.7 \pm 798.7 \mathrm{~g}, \mathrm{p}=0.02\right)$. The incidence of Respiratory Distress Syndrome was higher in the first group $(\mathrm{p}=0.04)$. The frequency of neonatal hypoglycaemia was higher in the third group ( $\mathrm{p}=0.003$ ). The incidence of spontaneous abortion and stillbirths was higher in the third group, but without statistical significance. The evaluation of risk factors could not establish relationship between the observed foetal complications and the insulin treatment.

Conclusion: The treatment with long-acting insulin analogs during pregancy has similar maternal efficacy and safety. In terms of foetal complications and safety the conduct of more long-treatment trials in larger groups are needed.

\section{1}

The efficacy of continuous subcutaneous insulin infusion and multiple daily injections of insulin in pregnant women with type 1 diabetes A. Hebda-Szydło ${ }^{1}$, K. Cyganek ${ }^{1}$, M.T. Małecki ${ }^{1}$, I. Trznadel-Morawska ${ }^{1}$, B. Katra ${ }^{1}$, T. Klupa ${ }^{1}$, I. Kaim² , A. Rerońn , J. Sieradzki ${ }^{1}$

${ }^{1}$ Department of Metabolic Disease, ${ }^{2}$ Department of Obstetrics and Perinatology, Jagiellonian University, Krakow, Poland

Background and aims: The risk of complications for the mother and infant in pregnancy complicated by type 1 diabetes mellitus (T1DM) can be reduced by a strict glycemic control. To achieve this goal two insulin regiments are 
commonly used: multiple daily injections (MDI) and continuous subcutaneous insulin infusion (CSII) via an insulin pump.

The purpose of this study was to assess metabolic control of pregnant women with T1DM treated by CSII and MDI and to compare the pregnancy outcomes in both groups.

Materials and methods: We included 216 pregnant women with T1DM in this observational study. Among them, there were 142 women on MDI, while 74 subjects were on CSII. We analysed HbAlc level and mean blood glycaemia (MBG) in each trimester of pregnancy, as well as the pregnancy outcomes for both groups.

Results: MDI and CSII groups did not differ in terms of age (mean 28.4 years \pm 6.0 vs. $29.0 \pm 4.0$, respectively) and duration of diabetes (mean $12.0 \pm 7.3$ years vs. $10.8 \pm 7.0$, respectively). They also had a similar degree of metabolic control at the study entry as measured by $\mathrm{HbAlc}(7.0 \pm 1.6$ vs. $6.9 \pm 1.2$; $\mathrm{p}=0.7$, respectively) and MBG (114.4 $\pm 38.0 \mathrm{mg} \%$ vs. $105.5 \pm 12.4 ; \mathrm{p}=0.07$, respectively). However, we observed a lower level of $\mathrm{HbAlc}$ in the second trimester in CSII than in MDI ( $5.7 \% \pm 0.7$ vs. $6.0 \% \pm 1.0 ; \mathrm{p}=0.03)$; no difference was seen for MBG $(105.6 \mathrm{mg} \% \pm 20.0$ vs. $102.6 \pm 20.0 ; \mathrm{p}=0.3)$. In the third trimester, we did not find differences between CSII and MDI groups in respect to the level of HbAlc (5.7 \pm 1.1 vs. $5.9 \% \pm 0.9 ; p=0.2)$ and MBG $(106.7 \pm 20.2$ vs. $106.2 \pm 48.9 ; p=0.9$, respectively). There was a tendency for slightly better pregnancy outcomes in CSII group as compared to MDI in respect to infant deaths ( $4.1 \%$ vs. $4.9 \%$ ), stillbirths ( $5.4 \%$ vs. $7.0 \%$ ), and congenital malformations ( $5.4 \%$ vs. $6.3 \%$ ), although at this sample size this was not significant ( $\mathrm{p}=0.7$ for combined outcomes). Unexpectedly, we found higher frequency of newborns with LGA (Large for Gestational Age) in CSII group ( $40.5 \%$ vs. $23.9 \%$; $\mathrm{p}=0.01$ respectively).

Conclusion: In this large observational study, the CSII model showed some evidence of superiority over an MDI regimen in respect to metabolic control in women with T1DM. The observation, concerning more frequent LGA births in CSII group warrants further confirmation.

\section{2}

Continuous subcutaneous insulin infusion and multiple dose insulin injection in type 1 diabetic pregnant women: a case control study

F. Pancani, C. Lencioni, M. Aragona, A. Bertolotto, A. Ghio, I. Cuccuru, V. Resi, S. Del Prato, L. Volpe, G. Di Cianni

Endocrinology-Metabolism, University of Pisa, Italy

Background and aims: Optimal glycemic control before and during pregnancy is essential to reduce the health risk for both the mother and the foetus in diabetic women. Recently, subcutaneous insulin infusion (CSII) has been used during pregnancy as an alternative to multiple dose insulin injection (MDI), but results concerning CSII effectiveness in pregnancy are not conclusive. The aim of this study was to evaluate the effects of CSII on glycaemic control, insulin requirement, maternal and foetal outcomes in type 1 diabetic pregnant patients.

Materials and Methods: We retrospectively evaluated 42 type 1 diabetic pregnancies, divided in two groups: CSII (including 20 women transferred on CSII therapy 3-6 months before pregnancy) and MDI (including 22 women with short insulin analogues before each meal and NPH insulin 2-3 times daily). Safety and efficiency of CSII versus MDI was evaluated by HbAlc, maternal hypoglycaemia, insulin requirement and pregnancy outcomes.

Results: The two groups were comparable for age (CSII: $31 \pm 3$ vs MDI $29.8 \pm 6.3$ years), diabetes duration (CSII: $16 \pm 8.2$ vs MDI: $12 \pm 7.7$ years), and pre-pregnancy BMI (CSII: $23 \pm 2.8$ vs MDI: $23.7 \pm 4.3 \mathrm{Kg} / \mathrm{m} 2$ ). CSII women (according to White's classification: 5 in B, 3 in C, 9 in D and 3 in F) planned pregnancy in $50 \%$ of cases, whereas only $18 \%$ of the pregnancies were planned in MDI women ( 9 in B, 7 in C, 6 in D of White's classification). At the first evaluation during pregnancy $(6 \pm 2.2$ weeks), $\mathrm{HbA1c}$ values (CSII: $6.98 \pm 0.7$ vs MDI: $7.4 \pm 1.3 \%$ ) and daily insulin doses (CSII $0.62 \pm 0.1$ vs MDI $0.69 \pm 0.21 \mathrm{IU} / \mathrm{kg}$ ) were similar in the two groups. Pregnancy was interrupted by miscarriage during first trimester in 4 CSII and 2 MDI patients. At the end of the pregnancy there were no differences for weight gain (CSII: $13.4 \pm 5.4$ vs MDI: $11.5 \pm 3.7 \mathrm{~kg}$ ) and $\mathrm{HbAlc}$ values (CSII: $6.3 \pm 0.6$ vs MDI: $5.8 \pm 2.1 \%)$. On the contrary, insulin requirement resulted significantly lower in CSII than in MDI $(0.76 \pm 0.3$ vs $1.1 \pm 0.3 \mathrm{IUI} / \mathrm{kg}, \mathrm{p}<0.009)$. We recorded only one severe hypoglycaemic episode in both groups. No ketoacidosis episodes were observed. The delivery occurred at $36.4 \pm 2.2$ weeks of gestation with caesarean section in $95 \%$ of cases; time of the delivery, rate of pre-term deliveries and caesarean section were similar between two groups. Birth weight was similar in both groups (CSII $3541 \pm 757 \mathrm{~g}$ vs MDI $3100 \pm 719 \mathrm{~g}$ ), and LGA were $53 \%$ in CSII, $27.5 \%$ in MDI ( $p=0.08)$. No differences concerning foetal morbidity were observed in either of two groups ( 10 newborns by CSII mothers and 8 by MDI had transitory neonatal hypoglicamia and/or hyperbilirubinemia ).
Conclusion: CSII and MDI insulin therapy are both effective in improving metabolic control, but CSII therapy is associated with a lower insulin daily requirement. However, no clinical advantages appear for maternal and foetal outcome

\section{3}

Reduced need for intensive care in newborns of women with type 1 diabetes - is routine admission to neonatal intensive care unit still necessary?

E. Stage ${ }^{1}$, P.B. Emmersen ${ }^{1}$, E.R. Mathiesen ${ }^{2}$, C. Mester ${ }^{3}$, G. Greisen ${ }^{3}$, P. Damm ${ }^{1}$

${ }^{1}$ Gynaecology and Obstetrics, ${ }^{2}$ Endocrinology, ${ }^{3}$ Neonatology, Rigshospitalet, Faculty of Health Sciences, Copenhagen, Denmark

Aims: Neonatal morbidity is increased in infants of women with type 1 diabetes (T1DM) compared with infants of healthy women, and the infants are routinely admitted to neonatal intensive care unit (NICU) after delivery in many centres. In our centre all infants of women with T1DM were previously admitted to the NICU for observation the first 24 hours after delivery, but on May $1^{\text {st }} 2006$ our routine was changed to improve the early mother-infant bonding. The newborns now follow their mothers to the maternity ward shortly after delivery. The outcome of this changed routine is evaluated.

Methods: The study included an old cohort of 102 consecutive infants born between May $1^{\text {st }} 2001$ and January $31^{\text {st }} 2003$ all admitted to the NICU immediately after delivery. The new cohort included 103 consecutive infants born between May $1^{\text {st }} 2006$ and October $31^{\text {st }} 2007$. Infants in the new cohort were routinely admitted to a specialised maternity ward together with their mothers and only transferred to NICU if requested by the pediatric team. All infants had their plasma glucose measured 2 hours after delivery and were treated with early feeding by cup with manufactured milk every third hour during the first 24 hours. Gastric tube was used when nessesary. In the maternity ward frequent skin-to-skin contact and breastfeeding were encouraged. Clinical data were drawn retrospectively from the medical records.

Results: After delivery 84 (82\%) infants from the new cohort were transferred directly together with their mothers to the maternity ward and 19 were transferred directly to the NICU mainly due to prematurity and/or respiratory problems. Ten (12\%) infants were later transferred from the maternity ward to the NICU for various reasons, only 4 of these due to hypoglycaemia. All infants survived in good health except one who died immediately after delivery in the NICU. In the new cohort the first nursing was initiated earlier and the percentage of neonatal hypoglycaemia reduced. Gastric tube feeding and CPAP treatment of respiratory distress were used less frequently (Table 1).

Table1. Pregnancy outcome and infant morbidity in the new cohort compared with the old cohort among infants born to women with type 1 diabetes.

New cohort Old cohort pvalue

Mainly admission Routine

to maternity ward admission to

2006-2007 NICU

2001-2003

\begin{tabular}{|c|c|c|c|}
\hline Number (\%) & 103 & 102 & - \\
\hline $\begin{array}{l}\text { Maternal HbAlc before } \\
\text { delivery }(\%)\end{array}$ & $5.9(0.5)$ & $6.3(0.6)$ & $<0.0001$ \\
\hline Gestational age (days) & $261(11)$ & $258(18)$ & NS \\
\hline $\begin{array}{l}\text { Preterm delivery ( }<37 \text { weeks } \\
\text { of gestation) }\end{array}$ & $28(27 \%)$ & $33(32 \%)$ & NS \\
\hline Weight (grams) & 3,487 & 3,540 & NS \\
\hline $\begin{array}{l}\text { First nursing }<2 \text { hours after } \\
\text { delivery }\end{array}$ & $78(76 \%)$ & $48(47 \%)$ & 0.0005 \\
\hline $\begin{array}{l}\text { Plasma glucose }<2,5 \mathrm{mmol} / 1 \\
\text { two hours after delivery }\end{array}$ & $42(41 \%)$ & $64(63 \%)$ & 0.0027 \\
\hline Gastric tube feeding & $33(32 \%)$ & $88(87 \%)$ & $<0.005$ \\
\hline Intravenous glucose therapy & $11(11 \%)$ & $31(30 \%)$ & 0.00053 \\
\hline Phototherapy of icterus & $12(12 \%)$ & $18(18 \%)$ & NS \\
\hline $\begin{array}{l}\text { Continuous positive airway } \\
\text { pressure (CPAP) }\end{array}$ & $16(16 \%)$ & $31(30 \%)$ & 0.014 \\
\hline
\end{tabular}

Data are given as numbers (\%) and mean (SD)

NICU: neonatal intensive care unit 
Conclusion: Observation in a maternity ward facilitating early mother-infant bonding seems safe in newborns of women with T1DM. These infants had less hypoglycaemia and received less treatment for respiratory problems compared with a historical cohort of infants routinely transferred to NICU. The demonstrated reduced need for neonatal intensive therapy in the new cohort may be related to early mother-child bonding, but improved metabolic control during pregnancy may also play a role.

\section{4}

Poor pregnancy outcome in women with type 1 diabetes is predicted by $\mathrm{HbA}_{1 \mathrm{c}}$ and spikes of high glucose values in the third trimester P. Damm ${ }^{1}$, H. Mersebach 2 , P. Brown' 2 , R. Kaaja ${ }^{3}$, M. Hod ${ }^{4}$, E. Mathiesen ${ }^{1}$ ${ }^{1}$ Center for Pregnant Women with Diabetes, Rigshospitalet, Copenhagen, Denmark, ${ }^{2}$ Novo Nordisk A/S, Bagsvaerd, Denmark, ${ }^{3}$ Department of Obstetrics and Gynaecology, Helsinki University Central Hospital, Helsinki, Finland, ${ }^{4}$ Division of Maternal Fetal Medicine, Tel Aviv University, PetahTiqva, Israel

Background and aims: Strict glycaemic control during pregnancy in type 1 diabetes is necessary to reduce the risk of adverse maternal and perinatal outcomes. Data from a randomised, controlled study of a rapid-acting insulin analogue (prandial insulin aspart versus human insulin) in pregnant women with type 1 diabetes $(n=322)$ were analysed for potential factors affecting outcome in late pregnancy. The trial included subjects who were pregnant or planning on becoming pregnant at enrollment. NPH was used as basal insulin.

Materials and methods: This post-hoc analysis included 259 subjects with known pregnancy outcome and excluded subjects with congenital malformations and/or foetal loss before gestational week 22. Two outcomes were examined: 1) composite outcome: poor outcome in late pregnancy $(n=78)$ including pre-eclampsia $(n=12)$, pre-term delivery $(n=53)$, both pre-term delivery and pre-eclampsia $(\mathrm{n}=10)$ and perinatal death $(\mathrm{n}=3) ; 2)$ excessive foetal growth (large for gestational age [LGA]/macrosomia) $(\mathrm{n}=88)$.

Results: $\mathrm{HbA}_{1}$ and at least one plasma glucose (PG) measurement $>11 \mathrm{mmol} /$ $\mathrm{L}(198 \mathrm{mg} / \mathrm{dL}$ ) (taken from 1 day's 8-point profile performed within 1 week prior to the third trimester visit) were found to be significant predictors for poor outcomes (see table). Other potential predictors (blood pressure, albuminuria and retinopathy in early pregnancy) did not predict any of the three outcomes $(p>0.10)$. Conclusions: These results suggest that both elevated $\mathrm{HbA}$ and spikes of high glucose levels impact on poor pregnancy outcomes; our findings therefore support the recommendation for achieving optimal glycaemic control also in late pregnancy in women with type 1 diabetes. Supported by: Novo Nordisk A/S

\section{5}

An audit of pregnancy outcomes in type 1 diabetes comparing continuous subcutaneous insulin infusion with multiple daily injections P.J. Hammond ${ }^{1}$, S. Ray ${ }^{1}$, J. Carling ${ }^{1}$, S. Dudley ${ }^{1}$, L. Dinning ${ }^{1}$, K. Johnson ${ }^{1}$, A. MacDonald ${ }^{2}$

${ }^{1}$ Diabetes Centre, Harrogate and District Foundation Trust, Harrogate, ${ }^{2}$ Medtronic Diabetes, Watford, United Kingdom

Background and aims: The benefits of continuous subcutaneous insulin infusion (CSII) over multiple daily injections (MDI) in terms of improvement in $\mathrm{HbAlc}$ and reduced hypoglycaemia frequency are now well-established in non-pregnant individuals with type 1 diabetes. However findings in pregnancy are inconsistent despite the fact that benefits of improved control would be expected to translate into improved fetal outcomes. The aim of this audit was to determine whether use of CSII was more effective than MDI at delivering target outcomes for mother and baby.

Materials and methods: We audited a total of 73 pregnancies in women with type 1 diabetes between 1999 and 2007, during which time all women were offered CSII as an alternative to MDI in pregnancy. Outcomes audited were $\mathrm{HbAlc}$ preconceptually, in each trimester and post-partum; intra-partum maternal glucose; subjective reporting of hypoglycaemia severity; fetal growth and birth weight; neonatal morbidity; maternal weight gain and insulin requirements.

Results: 39 used CSII, 9 switching from MDI in early pregnancy, with 34 live births; 34 used MDI alone, with 30 live births. HbAlc was significantly better in CSII users preconceptually ( $7.3 \pm 1.2$ vs $8.2 \pm 2.0 \%)$ and in the first trimester $(6.9 \pm 1.1$ vs $7.7 \pm 1.7 \%)$. However there were no significant differences through the remainder of the pregnancy, although mean $\mathrm{HbAlc}$ during pregnancy was lower in those women who switched to CSII during pregnancy than those who used either CSII or MDI alone $(6.6 \pm 0.9$ vs $6.8 \pm 0.8$ vs $7.0 \pm 1.0 \%$ ). Reported hypoglycaemia severity was significantly reduced in CSII users. There was no impact of CSII use on fetal growth or birth weight. Only one CSII user did not continue on CSII during the intrapartum phase, and those who continued CSII had a significantly lower intrapartum glucose than women on MDI who used intravenous insulin infusion (5.6 $\pm 2.3 \mathrm{vs} 7.0$ $\pm 2.8 \mathrm{mmol} / \mathrm{l})$. There was a corresponding reduction in frequency of neonatal hypoglycaemia, with a significantly higher mean neonatal glucose for those born to CSII users $(2.4 \pm 0.9$ vs $1.7 \pm 1.0 \mathrm{mmol} / \mathrm{l})$. There was significantly less weight gain, lower insulin requirements and better post-partum $\mathrm{HbAlc}$ for mothers who used CSII.

Conclusion: Pregnant women with type 1 diabetes using CSII rather than MDI can achieve better glycaemic control, particularly preconceptually and in early pregnancy, experience less severe hypoglycaemia, and realise benefits to themselves in reduced weight gain and to the neonate in reduced hypoglycaemia frequency.

\begin{tabular}{|c|c|c|c|c|c|}
\hline $\begin{array}{l}\text { Outcome 1: Composite endpoint: poor outc } \\
\text { in late pregnancy }\end{array}$ & & $\begin{array}{l}\text { Successful outcome in late } \\
\text { pregnancy } \\
(\mathbf{n}=181)\end{array}$ & $\begin{array}{l}\text { Poor outcome in late pregnancy } \\
(n=78)\end{array}$ & ${ }^{\star}$ Odds ratio $(95 \% \mathrm{CI})$ & $p$-value \\
\hline \multirow[t]{2}{*}{ One measurement of PG >11 mmol/L, n (\%) } & Yes & $35(19 \%)$ & $27(35 \%)$ & \multirow[t]{2}{*}{$2.17(1.18,3.98)$} & \multirow[t]{2}{*}{0.012} \\
\hline & No & $145(81 \%)$ & $51(65 \%)$ & & \\
\hline $\mathrm{HbA}_{1 c} \%$, mean $\pm(\mathrm{SD})$ & & $6.0(0.6)$ & $6.2(0.7)$ & $1.68(1.08,2.61)$ & 0.022 \\
\hline Outcome 2: LGA/macrosomia & & Normal weight $(\mathrm{n}=169)$ & LGA/macrosomic $(n=88)$ & ${ }^{\star}$ Odds ratio $(95 \% \mathrm{CI})$ & $p$-value \\
\hline \multirow[t]{2}{*}{ One measurement of PG >11 mmol/L, n (\%) } & Yes & $30(18 \%)$ & $31(35 \%)$ & \multirow[t]{2}{*}{$2.69(1.46,4.94)$} & \multirow[t]{2}{*}{0.001} \\
\hline & No & $138(82 \%)$ & $57(65 \%)$ & & \\
\hline $\mathrm{HbA}_{1 c} \%$, mean $\pm(\mathrm{SD})$ & & $6.0(0.6)$ & $6.3(0.6)$ & $2.74(1.73,4.35)$ & 0.000 \\
\hline
\end{tabular}

${ }^{*}$ Logistic regression analyses adjusted for body mass index, age, smoking status, randomised treatment and parity. 


\section{PS 106 Pregnancy: inflammation and pathogenetic mechanisms}

\section{6}

\begin{abstract}
Nitrosative stress and poly (ADP-ribose) polymerase activation in healthy and gestational diabetic pregnancies

E.M. Horvath ${ }^{1,2}$, R. Magenheim ${ }^{3,4}$, E. Kugler ${ }^{5}$, G. Vacz ${ }^{5}$, A. Szigethy ${ }^{4}$, C. Szabo ${ }^{1,2}$, Z. Lacza $^{5}$

${ }^{1}$ Department of Surgery, University of Medicine and Dentistry of New Jersey, Newark, United States, ${ }^{2}$ Cell Screen Center for Applied Research, Semmelweis University, Budapest, Hungary, ${ }^{3}$ School for Doctorial Studies, Semmelweis University, Budapest, Hungary, ${ }^{4}$ Department of Obstetrics and Gynecology, St. Margit Hospital, Budapest, Hungary, ${ }^{5}$ Institute of Human Physiology and Clinical Experimental Research, Semmelweis University, Budapest, Hungary
\end{abstract}

Background and aims: Oxidative stress increases during the course of normal pregnancy with a further elevation at the onset of labour and in various pathological conditions such as gestational diabetes (GDM) and preeclampsia. Hyperglycemia leads to increased generation of reactive oxygen and nitrogen species and subsequent DNA single-strand breakage, which in turn activate the nuclear enzyme poly (ADP-ribose) polymerase (PARP). PARP uses $\mathrm{NAD}^{+}$as substrate to form poly (ADP-ribose) (PAR). Its overactivation leads to the rapid depletion of $\mathrm{NAD}^{+}$and ATP causing cell dysfunction and necrotic cell death. Activation of PARP also leads to the translocation of apoptosis inducing factor (AIF) from the mitochondria to the nucleus, activating apoptotic pathways. Oxidative-nitrosative stress and PARP activation plays an important role in the formation of type 1 and type 2 diabetes and its complications. In the current study the presence and severity of these phenomena were examined in healthy and GDM pregnancies.

Materials and methods: Blood samples $\left(16-29^{\text {th }}, 36-40^{\text {th }}\right.$ week; $\left.n=25 ; 22 ; 5\right)$, placental and umbilical cord tissues $(n=6 ; 9 ; 5)$ were collected from healthy volunteers and diet or insulin treated GDM patients. Immunohistological staining was performed on leukocyte smears and tissue sections using antinitrotyrosine (NT), anti-PAR and anti-AIF antibody.

Results: In healthy pregnancies there is a linear correlation between the number of NT positive leukocytes and their PARP activation and gestational week ( $\left.b=1.22 \pm 0.35, R^{2}=0.43, b=0.12 \pm 0.01, p=0.0033 ; R^{2}=0.49, p=0.0008\right)$. In diet treated GDM patients the PARP staining was already strong on the $16-29^{\text {th }}$ week ( $\mathrm{p} \leq 0.001$ vs control) and did not increases further on. On the $16-29^{\text {th }}$ week there was correlation between PAR staining and the 2 hour value of the oGTT ( $\left.b=0.63 \pm 0.14, \mathrm{R}^{2}=0.49, \mathrm{p}=0.0002\right)$. While insulin therapy decreased the intensity of NT ( $\mathrm{p} \leq 0.05$ vs control (36- $40^{\text {th }}$ week)) and PAR staining ( $\mathrm{p} \leq 0.05$ vs $\operatorname{diet}\left(16-29^{\text {th }}\right.$ week)), in circulating leukocytes, it increased these parameters (NT: $\mathrm{p} \leq 0.001$ vs control and diet; PAR: $\mathrm{p} \leq 0.01$ vs diet, $\mathrm{p} \leq 0.05$ vs. control) and also AIF translocation ( $\mathrm{p} \leq 0.05 \mathrm{vs}$. control) in placental tissues.

Conclusion: While the continuous elevation of tyrosine nitration, PARP activation is physiological during pregnancy, the earlier elevation of PARP activity in GDM might contribute to the increased prevalence of pre-term deliveries and pre-eclampsia. The longer duration of the increased PARP activation may also explain the higher frequency of diabetes and cardiovascular diseases observed years after pregnancies complicated with carbohydrate intolerance. The highest PARP activity was observed in case of the most severe GDM, represented not only with the highest diagnostic oGTT results but also the later need of insulin treatment. Hence, PARP activation may be used as a potential marker to identify high-risk patients. While insulin has beneficial effects on the immune cells function, it may induce adverse reactions on the placenta.

\section{7}

Proinflammatory cytokines are up-regulated in gestational diabetes M. Kuzmicki ${ }^{1}$, J. Szamatowicz ${ }^{2}$, B. Telejko ${ }^{3}$, N. Wawrusiewicz-Kurylonek ${ }^{3}$, A. Nikolajuk ${ }^{3}$, A. Kretowski ${ }^{3}$, M. Gorska ${ }^{3}$

${ }^{1}$ Department of Pathophysiology of Pregnancy, ${ }^{2}$ Department of Gynecology, ${ }^{3}$ Department of Endocrinology, Diabetology and Internal Medicine, Medical University of Bialystok, Poland

Background and aims: Pro-inflammatory cytokines, including interleukin-6 (IL-6), interleukin-8 (IL-8) and interleukin-18 (IL-18) through their ability to interfere with insulin signaling, can contribute to the development of insulin resistance and the disturbances of glucose metabolism. In this study we examined whether serum IL-6, IL-8 and IL-18 levels, as well as their genes expression in adipose and placental tissue are elevated in patients with gestational diabetes (GDM) and related to the metabolic factors conferring insulin resistance Materials and methods: Serum IL-6, IL-8 and IL-18 concentrations were measured in 58 patients with GDM and 62 women with normal glucose tolerance (NGT) between 24 and 28 weeks of gestation, using immunoenzymatic method. IL-6, IL-8 and IL-18 mRNA expression in maternal subcutaneous adipose tissue (SAT), visceral adipose tissue (VAT) and placental tissue were evaluated in 15 women with GDM and 12 healthy pregnant women at term, using quantitative real time RT-PCR.

Results: Serum IL-6, IL-8 and IL-18 levels were significantly elevated in the GDM subjects as compared with the non-diabetic women [0.97 (0.66-1.49) $\mathrm{pg} / \mathrm{ml}$ vs $0.77(0.50-1.07) \mathrm{pg} / \mathrm{ml}, \mathrm{p}=0.006,2.03(1.15-4.17) \mathrm{pg} / \mathrm{ml}$ vs 0.73 $(0.39-0.94) \mathrm{pg} / \mathrm{ml}, \mathrm{p}<0.0001$ and $249.2(188.5-318.7) \mathrm{pg} / \mathrm{ml}$ vs 179.4 (133.7243.9) pg/ml, $\mathrm{p}<0.0001$, respectively]. Serum IL-6 concentrations correlated significantly with current $B M I(R=0.31, p=0.0007)$, fasting glucose $(R=0.33$, $\mathrm{p}=0.0003)$ and HOMA-IR $(\mathrm{R}=0.20, \mathrm{p}=0.02)$. Serum IL-8 levels were associated significantly with current $\mathrm{BMI}(\mathrm{R}=0.34, \mathrm{p}=0.0002)$, glucose $(\mathrm{R}=0.19$, $\mathrm{p}=0.04)$, insulin $(\mathrm{R}=0.44, \mathrm{p}<0.0001)$ and HOMA-IR $(\mathrm{R}=0.47, \mathrm{p}<0.0001)$, as well as with gestational age $(\mathrm{R}=0.34, \mathrm{p}=0.0002)$. Serum IL-18 concentrations correlated with insulin $(\mathrm{R}=0.24, \mathrm{p}=0.0091)$ and HOMA-IR values $(\mathrm{R}=0.24$, $\mathrm{p}=0.008)$. IL-8 mRNA expression in VAT was markedly higher in GDM than in NGT subjects [1.88 (0.61-2.24) vs $0.48(0.27-0.98) \mathrm{AU}, \mathrm{p}=0.004]$, whereas the expression of IL- 6 and IL-18 genes in adipose and placental tissue did not differ between the two groups of pregnant women. IL-8 mRNA expression in SAT correlated significantly with fasting glucose $(\mathrm{R}=0.47, \mathrm{p}=0.02)$. There were also significantly correlations between IL- 8 and IL-18 mRNA expression in SAT $(\mathrm{R}=0.63, \mathrm{p}=0.001)$ and placental tissue $(\mathrm{R}=0.43, \mathrm{p}=0.04)$.

Conclusion: Our results indicate that circulating proinflammatory cytokines are up-regulated in patients with GDM, potentially linking chronic subclinical inflammation with insulin resistance in GDM women.

\section{8}

Early pregnancy $\mathrm{C}$-reactive protein in the prediction of gestational diabetes mellitus

N. Fatema ${ }^{1}$, S. Akter ${ }^{2}$, F. Jebunnesa ${ }^{2}$, A. Akhter ${ }^{2}$, N. Sultana ${ }^{2}$, T. Wahed ${ }^{2}$, R. Helal ${ }^{2}, \mathrm{~L} . \mathrm{Ali}^{2}$

${ }^{1}$ Dept of Obstetrics and Gynecology, Square Hospital, ${ }^{2}$ Dept of Biochemistry and Cell Biology, BIRDEM, Dhaka, Bangladesh

Background and aims: Gestational diabetes mellitus (GDM) is a common medical disorder of pregnancy and is a leading cause of poor pregnancy outcome. The pathophysiology of GDM is complicated and still poorly understood. Low-grade systemic inflammation has been found to be associated with insulin resistance as well as with GDM, but the causal relationship between inflammation and GDM has not yet been established. The present study was undertaken to explore this relationship with C-reactive protein as a marker of chronic subclinical inflammation.

Materials and methods: A total 297 pregnant mothers of 6-16 weeks of pregnancy were enrolled in this study. All the patients were followed till delivery for the development of GDM. Fifty nine patients were lost in the follow-up and $31(21.4 \%)$ women developed GDM. By a nested case-control design the 28 GDM cases were compared with 71 Controls with non-GDM pregnancy. Controls and cases were matched with each other in respect of age and gestational weeks. Serum C-peptide was measured by chemiluminesence based ELISA and serum high sensitivity C-reactive protein (hsCRP) was measured by immunonephelometry. Insulin secretory capacity (HOMA\%B) and Insulin sensitivity (HOMA\%S) was assessed by Homeostasis Model assessment. McNemar test was done to calculate sensitivity, specificity, positive predictive value (PPV) and negative predictive value (NPV) of the parameters.

Results: The erythrocyte sedimentation rate (ESR) was highly significant in GDM as compared to Control (ESR, $\mathrm{mm}$ in $1^{\text {st }} \mathrm{hr}(\mathrm{wg}) 82.32 \pm 30.1$ vs $61.38 \pm 31.2, \mathrm{p}=0.003$ ). The high sensitivity $\mathrm{C}$-reactive protein (hsCRP) level of GDM was $6.82 \pm 2$ and that of the Control was $4.04 \pm 1.93$. The CRP level in the GDM group was significantly higher compared to the Control group $(\mathrm{p}<0.001)$. The GDM subjects had significantly higher serum c-peptide $(\mathrm{ng} / \mathrm{ml}$, $\mathrm{M} \pm \mathrm{SD}, 2.77 \pm 0.57$ vs $1.70 \pm 0.46, \mathrm{p}<0.001)$ higher HOMA\%B [median (range) $336(155-498)$ vs $312(59-474), \mathrm{p}=0.013]$ and lower HOMA\%S [22.2 (13.10$55.60)$ vs 31.8 (16.4-183.3), $\mathrm{p}<0.001]$ as compared to Control. On Pearson's correlation analysis hsCRP level had a highly significant positive correlation with BMI, gestational age, $\mathrm{C}$ peptide and $\mathrm{HOMA} \% \mathrm{~B}(\mathrm{p}<0.001)$ and it had an inverse correlation with HOMA\% $\mathrm{S}(\mathrm{p}<0.001)$. On binary logistic regression GDM was found to be associated with hsCRP after adjustment of the effects of the potential confounders. With a cut-off value at the $50^{\text {th }}$ percentile (de- 
termined from on ROC curve) fasting C-peptide level showed a sensitivity of $72 \%$, specificity $93 \%$, PPV $58 \%$ and NPV $96 \%$ for the prediction of GDM. The corresponding values for hsCRP were $61 \%, 83 \%, 59 \%$ and $84 \%$. Combination of C-peptide and hsCRP increased the PPV to $83 \%$ and NPV to $83 \%$.

Conclusion: Subclinical chronic inflammation seems to have a causal association with insulin resistance in GDM and early pregnancy hsCRP appear to have role in predicting the development of GDM at the later stages of pregnancy. Supported by: IPICS, DAB

\section{9}

\section{Association of high-sensitive C-reactive protein with the degree of} hyperglycaemia in gestational diabetes mellitus

N. Sultana ${ }^{1}$, N. Rosy ${ }^{2}$, F. Jebunnesa ${ }^{1}$, A. Akhter ${ }^{1}$, T. Wahed ${ }^{1}$, R. Helal ${ }^{1}$, L. $\mathrm{Ali}^{1}$

${ }^{1}$ Dept of Biochemistry \& Cell Biology, ${ }^{2}$ Dept of Obstetrics \& Gynecology, BIRDEM, Dhaka, Bangladesh

Background and aims: Gestational diabetes mellitus (GDM) complicates about $3-5 \%$ of pregnancies and is a risk for pregnancy related maternal and neonatal morbidities. GDM, like Type $2 \mathrm{DM}$, is now increasingly recognized as an inflammatory condition, but the characteristics and determinants of the inflammatory process in GDM still need further investigation. The present study was undertaken to explore the association of high-sensitive C-reactive Protein (hsCRP), a reliable marker of low grade systemic inflammation, with the degree of glycemic status in GDM.

Materials and methods: A total of $110 \mathrm{GDM}$ cases, along with 102 Controls, aged between 18-37 years, were studied for glycemic status and inflammatory markers. Erythrocyte sedimentation rate (ESR) was determined by standard technique, and serum hsCRP was measured by immunonephelometry. HOMA\%B and HOMA\%S were calculated by Homeostasis Model Assessment and those were used as markers of insulin secretory capacity and insulin sensitivity respectively.

Results: The age and gestational weeks of the study groups were found to be matched. $\mathrm{HbA}_{1 \mathrm{c}}$ level was significantly higher in GDM group (\%, $\mathrm{M} \pm \mathrm{SD}$; $5.96 \pm 0.78)$ as compared to Control $(5.25 \pm 0.43, \mathrm{p}<0.001)$. Serum C-peptide was also significantly higher in the GDM group ( $\mathrm{ng} / \mathrm{ml}, \mathrm{M} \pm \mathrm{SD} 3.82 \pm 0.95$ in GDM vs $1.93 \pm 0.46$ in control, $\mathrm{p}<0.001$ ), but the insulin secretory capacity (HOMA\% B) did not differ significantly between the two groups [HOMA\% B, Median (range), in GDM 141.4(34.0-464.7) and in Control 139.1(66.1339.4)]. HOMA\% S was significantly lower in GDM group [64.3(25.7-90.5) compared to control 124.5(49.03-299.5), $\mathrm{p}<0.001]$. There was significant difference in HOMA\% $\mathrm{S}$ level between the two groups in all trimesters of pregnancy $(\mathrm{p}<0.001)]$. ESR $\left(\mathrm{mm}\right.$ in $1^{\text {st }}$ hour, $\left.\mathrm{M} \pm \mathrm{SD}\right)$ was higher in GDM compared to control groups $(90.88 \pm 12.56$ vs $48.74 \pm 18.20, \mathrm{p}<0.001)$. The hsCRP was significantly higher in GDM group compared to control [hsCRP, mg/l, $\mathrm{M} \pm \mathrm{SD}, 9.56 \pm 1.99$ vs $2.19 \pm 0.95, \mathrm{p}<0.001]$. The hsCRP level was about 5 times higher in GDM group in all trimesters of pregnancy $(\mathrm{p}<0.001)$. The hsCRP levels of the GDM subjects having $\mathrm{HbA}_{1 \mathrm{c}}$ levels below and above $6.0 \%$ respectively were as follows: $\mathrm{HbA}_{1 \mathrm{c}}$ \%, median (range) 8.53(2.31-18.30) and 10.73(4.19-19.50). There was significant difference in hsCRP levels between the two groups $(p<0.001)$. hsCRP had a significant positive association with HbAlc $(p=0.005)$ in Pearson's correlation analysis. Multiple linear regression analysis showed significant independent association of $\mathrm{HbAlc}$ with hsCRP level $(\mathrm{P}=0.017)$.

Conclusion: Serum hsCRP is a highly sensitive marker for low grade systemic inflammation in GDM and its rise is strongly associated with the degree of hyperglycemia.

Supported by: IPICS, DAB

\section{0}

\section{Monocyte-chemoattractant-protein-1 is elevated in gestational diabetes} mellitus

J. Brix ${ }^{1}$, K. Klein ${ }^{2}$, M. Satler ${ }^{3}$, M. Elhenicky ${ }^{3}$, K. Krzyzanowska ${ }^{1}$,

G. Schernthaner ${ }^{1}$, P.W. Husslein ${ }^{2}$, G.-H. Schernthaner ${ }^{3}$

${ }^{1}$ Medicine I, Rudolfstiftung Hospital, ${ }^{2}$ Obstetrics and Fetomaternal

Medicine, Medical University of Vienna, ${ }^{3}$ Medicine II, Medical University of Vienna, Austria

Background and aims: Gestational diabetes mellitus (GDM) is defined as carbohydrate intolerance of variable severity, with onset/first recognition during pregnancy. GDM complicates about $7 \%$ of all pregnancies, and its prevalence is increasing. Experimental studies in animals and evidence from prospective and longitudinal studies in humans are consistent with an etiologic role of subclinical inflammation in the pathogenesis of T2DM. Inflammatory alterations, being involved in "type 2 diabetic" atherosclerosis, might be as well associated with GDM. Therefore we investigated whether GDM is associated with Monocyte-chemoattractant-protein-1 (MCP-1) and soluble CD40 Ligand $(\mathrm{sCD} 40 \mathrm{~L})$, functional relevant proteins in the inflammatory process.

Materials and methods: The study population consisted of 3 groups: Patients with GDM ( $n=32$; mean age $33 \pm 6$ years, mean $\mathrm{HbAlc:} 5.6 \pm 0.7 \%)$, pregnant women without GDM (non-GDM, $\mathrm{n}=18$; mean age $31 \pm 7$ years, mean HbA1c: $5.1 \pm 0.5 \%)$ and non pregnant controls $(\mathrm{CO}, \mathrm{n}=40$; mean age $30 \pm 8$ years; mean HbA1c: $5.1 \pm 0.3 \%)$ were included in a cross-sectional and longitudinal design. In the second trimester the diagnosis of gestational diabetes was secured by a $75 \mathrm{~g}$ oral glucose tolerance test. Blood samples for MCP-1 and sCD $40 \mathrm{~L}$ were obtained at $25 \pm 5$ and $33 \pm 4$ weeks of gestations, as well as $2 \pm 1$ months postpartum. HbAlc and Insulin levels were measured by standard laboratory procedures and HOMA was calculated.

Results: In controls $(n=40)$, MCP-1 levels were $157 \pm 63 \mathrm{pg} / \mathrm{ml}$ and sCD40L levels were $17.2 \pm 8.2 \mathrm{ng} / \mathrm{ml}$.Values of sCD40L did not differ between GDM, non-GDM at all time points investigated and controls. In contrast, MCP-1 was elevated in the third trimester in patients with GDM $(300 \pm 115 \mathrm{pg} / \mathrm{ml})$ compared to the second trimester $(227 \pm 89 \mathrm{pg} / \mathrm{ml} ; \mathrm{p}=0.003)$ and post partum $(218 \pm 111 \mathrm{pg} / \mathrm{ml} ; \mathrm{p}=0.001)$ to non-GDM in the second trimester, in the third trimester $(209 \pm 72 \mathrm{pg} / \mathrm{ml} ; \mathrm{p}=0.007)$ and post partum, and to controls $(\mathrm{p}<0.001)$. To study the associations three multivariate regression analysis were applied: first, we tried to identify predictors of MCP-1 in the third trimester in all participants. Multiple regression analysis in the third trimester revealed that only BMI was associated with $\mathrm{MCP}-1$ values (beta $=0.523$; $\mathrm{p}<0.001)$. In a separate analysis of pregnant women with GDM and without (non-GDM) we could not obtain a significant association of BMI prepregnancy and BMI at diagnosis of GDM (= time of OGTT) with MCP-1 values. Second, we investigated, which variables were able to predict MCP-1 values in the third trimester in pregnant women. Of all variables tested only the diagnosis of GDM was associated with MCP- 1 values (beta $=0.402, \mathrm{p}=0.007$ ). Third, we studied which variables that are associated with insulin resistance and sensitivity in T2DM, were related to MCP-1 values in the third trimester of our patients. Only glucose 1 hour was associated with MCP-1 values (beta $=0.307 ; \mathrm{p}=0.043$ )

Conclusion: The significant elevation of MCP-1 in the third trimester in GDM suggests an association between inflammation and GDM. Since MCP1 was elevated in all pregnant women compared to controls, inflammation may have a physiological role in the normal pregnancy but could have pathophysiological effect in GDM.

Supported by: Austrian Diabetes Association Research Prize

\section{1}

Amniotic fluid adipokines in diabetic pregnancy: a snap-shot of foetal enviroment

M. Stojkovic

School of Biological Sciences, Auckland University, New Zealand

Background and aims: A number of different circulating markers of inflammation are produced by different tissue and cells such as the endothelial cells, white blood cells, liver and adipose tissue. In pregnant individuals the placenta also synthesises a variety of signalling proteins, adding an additional level of complexity to the immune-metabolic network. Cytokine (and adipokine) patterns during normal pregnancy are still not fully understood, although a number of studies have reported elevations in cytokines in conjunction with normal labour onset. In pregnancies complicated by diabetes there is a further dysregulation of the metabolic, vascular and inflammatory pathways, supported by an increase in the circulation of inflammatory molecules. While the influence of direct infectious or immune stimuli on cytokine levels has been documented, it is unknown whether in pregnancy, adipokine levels in amniotic fluid (AF) vary and if they do, to what degree. Our aim was to compare $\mathrm{AF}$ concentrations of adipokines in pregnancies complicated by diabetes with the levels in healthy controls at comparable maternal and gestational age.

Materials and methods: AF samples were obtained by amniocentesis for fetal chromosome analysis performed between 16 and 20 weeks of gestation. Samples were matched for maternal age ( $\geq 35$ years) and gestational age at the time of amniocentesis. Analysis was conducted on 17 diabetic pregnancy samples ( $\mathrm{n}=7$ Type1 DM, $\mathrm{n}=8$ GDM and $\mathrm{n}=4$ Type2 DM) and 18 control 
samples. Using human serum adipokine kit (panel A and B; LINCOplex) we assayed for 11 different adipokines including IL-1 $\beta$, IL-6, IL-8, TNFa, MCP1, HGF, NGF, adiponectin, resistin, active PAI-1, leptin as well as insulin. Results: Except for IL-1 $\beta$ whose level was below the detection range, the Lincoplex kit generated data for 10 adipokines and insulin in AF of which 4 showed statistical differences between controls and diabetics (Table 1): Interleukin-8 (IL-8) $\mathrm{p}=0.03739$, Leptin $\mathrm{p}=0.01088$, MCP-1 $\mathrm{p}=0.07632$ and PAI-1 (active) $\mathrm{p}=0.04677$ (Figure 1). Post hoc analysis (Tukey test) show that two adipokines IL- 8 and MCP-1 are elevated in all the types of diabetes (GDM, Type 1 and Type 2 DM) compared to non diabetic controls, whereas PAI-1 (active) and leptin are observed to be significantly increased only in Type 1 DM compared to controls. Unexpectedly there was no significant difference in insulin AF levels between normal controls and diabetics.

Conclusion: We found that immunoregulatory mechanisms associated with normal pregnancy may be poorly developed in diabetics. Furthermore, we show that diabetes in pregnancy is characterized by a unique profile of AF adipokines. Whether this influence of fetus organs and if pancreatic b-cells are influence responsible for fetus development of diabetes is unknown and need to be elucidated.

Supported by: Maurice and Phylis Payker Trust, Auckland New Zealand, Faculty Research Development Fund, University of Auckland, Maurice Wikins Centre for Molecular Biodiscovery

\section{2}

Gestational diabetes has no impact on circulating apelin and apelin mRNA expression in fat and placental tissue

B. Telejko' ${ }^{1}$, M. Kuzmicki ${ }^{2}$, J. Szamatowicz ${ }^{3}$, A. Zonenberg ${ }^{1}$,

N. Wawrusiewicz-Kurylonek ${ }^{1}$, A. Nikolajuk ${ }^{1}$, A. Kretowski ${ }^{1}$, M. Gorska

${ }^{1}$ Department of Endocrinology, Diabetology and Internal Medicine,

${ }^{2}$ Department of Pathophysiology of Pregnancy, ${ }^{3}$ Department of Gynecology,

Medical University of Bialystok, Poland

Background and aims: Apelin is a 36-amino-acid bioactive peptide identified as the endogenous ligand of the orphan G-protein coupled receptor (APJ). Although its physiological functions have not yet been clarified, apelin has been recently described as a novel adipokine, produced and secreted by human mature adipoctes and up-regulated in various states of hyperinsulinemia/insulin resistance, such as obesity and type 2 diabetes. The aim of the study was to compare serum apelin concentrations and apelin gene expression in subcutaneous adipose tissue (SAT), visceral adipose tissue (VAT) and placental tissue from pregnant women with normal glucose tolerance (NGT) and gestational diabetes (GDM).

Materials and methods: Serum apelin concentration was measured in 101 patients with GDM and 101 women with NGT between 24 and 31 weeks of gestation, as well as in 15 women with GDM and 12 subjects with NGT at term (RIA). Apelin mRNA expression was measured in 12 women with NGT and 15 subjects with GDM, using quantitative real-time PCR.

Results: Median apelin concentrations did not differ significantly between the women with GDM and NGT, both - in the subjects between 24 and 31 weeks of gestation $(1656.5$ [1430.2-1852.1] vs 1555.6 [1281.2-1804.2] pg/ml) and at term (1607.9 [1453.4-1768.7] vs 1493.8 [1316.8-1956.7] pg/ml). No association between circulating apelin and glucose, insulin, HOMA-IR or BMI was found in both groups. Apelin gene expression did not differ significantly between SAT and VAT, but was markedly lower than in placental tissue $(\mathrm{p}<0.0001)$. There were no significant differences in apelin mRNA expression in SAT $(0.204[0.132-0.28]$ vs $0.112[0.036-0.184]$ AU), VAT $(0.233$ [0.151$0,42]$ vs $0.29[0.05-0.307] \mathrm{AU})$ and placental tissue $(1.93[0.522-3.18]$ vs 1.29 [0.495-2.44] AU) between the women with NGT and GDM. Apelin gene expression in placental tissue correlated with the expression of interleukin-6 $(\mathrm{R}=0.53, \mathrm{p}=0.007)$, interleukin -6 receptor $(\mathrm{R}=0.52, \mathrm{p}=0.007)$, tumour necrosis factor-alpha $(\mathrm{R}=0.51, \mathrm{p}=0.009)$ and visfatin $(\mathrm{R}=0.73, \mathrm{p}<0.0001)$ genes. There was also a correlation between apelin mRNA expression in SAT and $\operatorname{VAT}(\mathrm{R}=0.83, \mathrm{p}=0.04)$.

Conclusion: No association between apelin and GDM or the indices of insulin resistance was observed in our study. The placenta might be a substantial source of apelin in pregnant women, irrespective of their glucose tolerance status.

Supported by: State Committee for Scientific Research

\section{PS 107 Diabetic pregnancy: complications}

\section{3}

Foetal programming of hypertension and kidney disease in adult rats exposed in utero to maternal diabetes

M. Lelievre-Pegorier, C. Fassot, J.-P. Duong Van Huyen, M. Viltard, N. Freund, T. Nehiri, P. Bruneval, S. Laurent

Centre de Recherche des Cordeliers, INSERM U872, PARIS, France

Background and aims: Epidemiological and experimental studies have led to the hypothesis of fetal origin of adult diseases. We have previously demonstrated in the rat that in utero exposure to maternal diabetes impairs renal development leading to a $30 \%$ reduction in nephron number. The aim of the present study was to assess in the rat long-term effects of in utero exposure to maternal diabetes on arterial blood pressure (BP) and arterial and renal function.

Materials and methods: Diabetes was induced in pregnant rats by streptozotocin on day 0 of gestation. In the offsprings of control (CMO) and diabetic mothers (DMO), from 1 to 18 months of age BP, plasma renin activity (PRA), renal function, proteinuria, kidney histopathology and histomorphometry, arterial structure, elastic properties and gene expression profile (oligonucleotides chips, 22000 genes) were assessed on 3-month-old CMO and DMO rats.

Results: After 6 months of age, in DMO rats, BP was higher, creatinin clearance was impaired and associated with proteinuria. At the pre-hypertensive stage, PRA was similar in CMO and DMO, then after the onset of hypertension, PRA decreased in DMO. High salt intake given at the prehypertensive stage induced an increase in systolic BP in DMO.

In DMO, subunits of P450 (Cyp4f4, Cyp4f2, Cyp8b1) are over-expressed and prostacyclin receptor is under-expressed contributing to vasoconstriction. Surprisingly, there is no difference in arterial structure between the 2 groups. This could be due to the under-expression of genes coding for protein interact with myofibres (Evl, Ckap4, Dcamkl1).

Conclusion: In utero exposure to maternal diabetes induces salt-sensible hypertension and impairs kidney function at adulthood. This may be due to the nephron deficit resulting from the in utero exposure to maternal diabetes associated to an abnormal vascular fetal programming in these rats.

Supported by: INSERM, ALFEDIAM

\section{4}

Sex hormone binding globulin and urinary protein-creatinine ratio in the first trimester and subsequent development of pregnancy induced hypertension

R. Helal, F. Jebunnesa, N. Sultana, T. Wahed, L. Ali

Dept of Biochemistry \& Cell Biology, Bangladesh Institute of Research \& Rehabilitation in Diabetes, Endocrine and Metabolic Disorders, Dhaka, Bangladesh.

Background and aims: The causal association of insulin resistance and pregnancy induced hypertension is still uncertain due to lack of adequate prospective studies. Sex Hormone Binding Globulin (SHBG) has been shown to be a reliable marker for insulin sensitivity. We have used this marker to explore the association of PIH with insulin resistance in early pregnancy. Endothelial damage is another covariate of insulin resistance, and a marker of this damage, urinary protein-creatinine ratio ( $\mathrm{UPr} / \mathrm{Cr}$ ), has also been investigated in this study under the same design. This may help to clarify temporal aspects of insulin resistance and hypertension in pregnancy, as well as to find suitable predictive markers in PIH.

Materials and methods: A total of 404 pregnant subjects, from different hospitals of Bangladesh, were followed up for the development of PIH until delivery. PIH (and subgroups PE and GH) was diagnosed by standard criteria. By matching the age- and gestational weeks with PIH cases a control group was defined out of the Non-PIH subjects following a nested case-control design. Serum insulin level was measured by microparticle enzyme immunoassay (EIA), serum SHBG by chemiluminescent immunometric assay and Insulin was measured by ELISA technique. Insulin secretory capacity (HOMA\%B) and insulin sensitivity (HOMA\%S) were assessed by Homeostasis Model Assessment (HOMA). McNemar test was done to calculate sensitivity, specificity, positive predictive value (PPV) and negative predictive value (NPV) of the target parameters. 
Results: On case-control comparison SHBG was found to be significantly lower in the PIH group compared to the Control [SHBG, nmol/1, 170 (10$198)$ in PIH vs 180 (103-219) in Control, $\mathrm{p}=0.003]$. Among the covariates of insulin resistance BMI [23.7 \pm 4.1 in PIH vs $22.4 \pm 3.4$ in control, $\mathrm{p}=0.047$ ] and $\mathrm{UPr} / \mathrm{Cr}$ [9.6 (3.48-20.5) in PIH vs $4.39(1.1-15.5)$ in control, $\mathrm{p}=<0.001]$ were higher in PIH compared to control. Correlation analysis between $3^{\text {rd }}$ trimester blood pressure and $1^{\text {st }}$ trimester SHBG as well as UPr/Cr indicated that PIH is associated with early pregnancy insulin resistance and endothelial damage. SHBG and UPr/Cr were found to be strongly correlated to each other. Logistic regression analysis provided more confirmatory evidence on the association of PIH with SHBG $(\mathrm{p}=<0.001)$ and $\mathrm{UPr} / \mathrm{Cr}(\mathrm{p}=0.013)$ after adjusting the effect of the confounders. The value of the early pregnancy levels of these markers for predicting the development of PIH in the late pregnancy were explored. At an optimum cut-off value of $180 \mathrm{nmol} / \mathrm{l}$ (calculated from an ROC curve) the sensitivity, specificity, PPV and NPV of SHBG were found to be $63 \%, 80 \%, 39 \%$ and $80 \%$ respectively. At an optimum cut-off value of 6.79 the corresponding values for $\mathrm{UPr} / \mathrm{Cr}$ were $65 \%, 78 \%, 55 \%$ and $84 \%$. Conclusion: Insulin resistance and endothelial damage seem to be causally associated with PIH. The present data also suggest that early pregnancy SHBG and UPr/Cr can be clinically useful in predicting future development of PIH in late stages gestation. Among these two options, $\mathrm{UPr} / \mathrm{Cr}$ has a little better sensitivity and specificity and this can be preferred as it is technically much simpler (implementable even in rural settings) and cost-wise more economic.

Supported by: IPICS, DAB

\section{5}

Orosomucoid excretion in urine predicts preeclampsia in pregnant women with pregestational type 1 diabetes

M.S. Christiansen ${ }^{1}$, P. Ekbom², D. Hesse' ${ }^{1}$, U. Hesse ${ }^{3}$, P. Damm

E. Hommel ${ }^{5}$, B. Feldt-Rasmussen ${ }^{6}$, E.R. Mathiesen ${ }^{2}$

${ }^{1}$ Department of Clinical Biochemistry, Amager Hospital, Copenhagen,

${ }^{2}$ Department of Endocrinology, Rigshospitalet, Copenhagen, ${ }^{3}$ National Institute of Public Health, University of Southern Denmark, Copenhagen,

${ }^{4}$ Obstetric Clinic, Rigshospitalet, Copenhagen, ${ }^{5}$ Diabetes, Steno Diabetes Center, Gentofte, ${ }^{6}$ Department of Nephrology, Rigshospitalet, Copenhagen, Denmark

Background and aims: Increased prevalences of preecclampsia (4-fold) and preterm delivery (8-fold) is common in pregnancies complicated by type 1 diabetes. Furthermore these complications are associated with increased perinatal mortality and morbidity. Increased urinary excretion of the inflammatory protein orosomucoid independently predicts cardiovascular mortality in patients with type 2 diabetes. The aim of the present study was to evaluate the predictive value of urinary orosomucoid excretion on development of preeclampsia and preterm delivery in women with type 1 diabetes.

Materials and methods: Women with pregestational type 1 diabetes and singleton pregnancies were consecutively included, if they had at least one urine sample collected before 17 gestational weeks. Frozen urine samples were thawed and analysed for orosomucoid by immunoturbidimetry. Women with macroalbuminuria (urinary albumin excretion $>300 \mathrm{mg} / 24$ hours) or early abortion (before 22 gestational weeks) were excluded from the study. The clinical data were collected at the National University Hospital of Copenhagen from January 1996 to July 2002. The primary outcome measurements were development of preeclampsia (defined as blood pressure $>140 / 90$ (three measurements) and proteinuria $>0.3 \mathrm{~g} / 24$ hours in 2 urine samples later than 20 weeks of gestation) and preterm delivery (defined as delivery before 37 weeks of gestation).

Results: 173 pregnant women with type 1 diabetes were included before 17 gestational weeks. Mean (SD) age was 30 (3.9) years, HbA1c was $7.1(0.9)$ $\%$, and median (range) duration of diabetes was 13 (0-35) years. The median (range) value of urinary orosomucoid was increasing during pregnancy (< 17 weeks: $0.45 \mathrm{mg} / \mathrm{l}$ (0.06-27.12); $17-27$ weeks: $0.78 \mathrm{mg} / \mathrm{l}$ (0.06-14.46); > 27 weeks: $1.30 \mathrm{mg} / \mathrm{l}(0.09-28.90) ; \mathrm{p}<0.0001)$ by ANOVA). Development of preeclampsia was seen in $20(12 \%)$ women and $65(38 \%)$ women delivered preterm. Women developing preeclampsia had longer duration of diabetes, higher blood pressure, and higher excretion of urinary albumin and orosomucoid compared to women without preeclampsia. Using logistic regression analysis we found that urinary orosomucoid (collected $<17$ weeks) higher than the 90. percentile (OR: 6.85 (95\% CI: 1.97-23.88; p < 0.003)), nulliparity (3.88 (1.10-13.72); $\mathrm{p}<0.04)$, systolic BP > $120 \mathrm{~mm} \mathrm{Hg}(4.12$ (1.35-12.59); $\mathrm{p}<0.02)$ and duration of diabetes $>20$ years $(3.69(1.18-11.52) ; \mathrm{p}<0.03)$ independently predicted the development of preeclampsia. The analyses were adjusted for BMI, HbAlc, serum creatinine, smoking and microalbuminuria. Preterm delivery was independently predicted by duration of diabetes $>20$ years $(2.36(1.07-5.20) ; \mathrm{p}<0.04)$ and HbAlc $>7 \%$ at 10 weeks $(2.18(1.10$ $4.30) ; \mathrm{p}<0.03)$.

Conclusion: Increased urinary excretion of orosomucoid early in pregnancy predicts preeclampsia in women with pregestational type 1 diabetes. The pathophysiological link between increased urinary orosomucoid and preeclampsia is unknown but may be caused by increased inflammatory response and endothelial dysfunction.

Supported by: Johan and Lise Boserup Foundation

\section{6}

Early pregnancy maternal serum PAPP-A and urinary calcium-creatinine ratio as predictive markers of pregnancy induced hypertension B. Nasrin ${ }^{1}$, F. Jebunnesa ${ }^{2}$, A. Akhter ${ }^{2}$, N. Sultana ${ }^{2}$, T. Wahed ${ }^{2}$, R. Helal ${ }^{2}$, L. $\mathrm{Ali}^{2}$

${ }^{1}$ Dept of Obstetrics and Gynecology, BSMMU, ${ }^{2}$ Dept of Biochemistry and Cell Biology, BIRDEM, Dhaka, Bangladesh

Background and aims: Preeclampsia (PE) and gestational hypertensions (GH) are two hypertensive disorders of pregnancy (together termed as pregnancy induced hypertension or PIH) with involvement of both placental and maternal circulation. These are still called 'disease of theories' with considerable controversies, regarding causal agents and risk factors. The aim of this study was to investigate the possible causal association of PIH with serum PAPP-A and urinary calcium-creatinine ratio $(\mathrm{UCa} / \mathrm{Cr})$ as well as to evaluate the usefulness of maternal serum levels of these two variables as predictive markers of PIH.

Materials and methods: A total 200 women of 8-16 weeks of pregnancy were enrolled in this study. All the patients were followed till delivery for the development of PIH. Thirty patients were lost in the follow up, $3(1.76 \%)$ developed PE and $14(8.23 \%) \mathrm{GH}$. By a nested case-control design the $17 \mathrm{PIH}$ cases were compared with 48 Controls with normal pregnancy outcome. Controls and Cases were matched with each other in respect of age and gestational weeks. Maternal serum pregnancy associated plasma protein-A (PAPP-A) was assessed by enzyme-labeled chemiluminescent immunometric assay, urine calcium was measured by colorimetric method and urine creatinine was measured by alkaline-picrate methods. McNemar test was done to calculate sensitivity, specificity, positive predictive value (PPV) and negative predictive value (NPV) of the parameters.

Results: $\mathrm{UCa} / \mathrm{Cr}$ was significantly higher in the PIH group compared to Control [mg/mmol, median (range), 1.21(0.35-1.98) vs 0.72(0.21-5.07), $\mathrm{p}<0.001]$. Maternal serum PAPP-A was significantly lower in the PIH group compared to Control [mIU/ml, median (range) $1.8(0.70-4.1)$ vs $5.45(2.7-10), \mathrm{p}<0.001]$. On logistic regression analysis only PAPP-A was significantly associated with PIH ( $\mathrm{p}=0.044)$ when the effects of the confounders (BMI, TG, Cholesterol, $\mathrm{HDL}, \mathrm{UCa} / \mathrm{Cr}$ ) were adjusted. When tested as a predictive marker of PIH the sensitivity, specificity, PPV and NPV of maternal serum PAPP-A in the lowest $25^{\text {th }}$ percentile (selected from an ROC curve) were $82 \%, 95 \%, 87 \%$ and $93 \%$. At $75^{\text {th }}$ percentile the sensitivity, specificity, PPV and NPV of UCa/Cr were $41 \%, 79 \%, 41 \%$ and $79 \%$ respectively. An effort was made to see whether combination of other parameters (BMI, HDL and UPCr) with PAPP-A can increase the predictivity of PIH. The sensitivity, specificity, PPV and NPV of combined PAAP-A and BMI were 53\%, 98\%, 90\% and 85\% respectively; for PAPP-A and HDL the corresponding value were $47 \%, 100 \%, 100 \%, 84 \%$; and for PAPP-A and UCa/Cr those were $41 \%, 97 \%, 87 \%$ and $82 \%$ respectively. Conclusion: Maternal Serum PAPP-A has a causal association with the development of PIH and low PAPP-A in first trimester of pregnancy can be used as a predictive marker for later development of PIH. Supported by: Diabetic Association of Bangladesh 


\section{7}

Outcome of pregnancy in obese women after laparoscopic adjustable gastric banding

A. Lapolla ${ }^{1}$, M. Dalfrà ${ }^{1}$, F. Favretti ${ }^{2}$, G. Segato ${ }^{2}$, M. Forletto ${ }^{3}$, M. Marangon ${ }^{1}$, L. Busetto ${ }^{1}$

${ }^{1}$ Medical and Surgical Sciences, University of Padova, ${ }^{2}$ Surgery, Ospedale Regionale San Bortolo, Vicenza, ${ }^{3}$ Clinical Surgical 2, University of Padova, Italy

Background and aims: Obesity is considered a risk factor for poor pregnancy outcome. Aim of our study was to evaluate pregnancy outcome in obese women after Laparoscopic Adjustable Gastric Binding (P-LAGB) and in Normalweight Pregnant Women (NPW).

Materials and methods: Sixtyone pregnancies in obese women after LAGB compared to 858 in lean women, as control group, were retrospectively analysed.We took into consideration age, prepregnancy BMI, weight gain during pregnancy, maternal morbidity(gestational diabetes, gestational hypertension, preeclampsia/eclampsia),abortions, timing and mode of delivery, fetal outcome (neoanatal weight, adeguate growth for gestational age, NICU admission, perinatal death).

Results: Age was not different in both groups, BMI was respectively $35.5 \pm 7.3$ in P-LAGB vs $22.2 \pm 4$ in NPW $(\mathrm{p}<0.001)$ and weight gain $4.8 \pm 11.3 \mathrm{~kg}$ vs $11.6 \pm 5.2 \mathrm{~kg}(\mathrm{p}<0.001)$. As for maternal morbility, GDM was diagnosed in $6.6 \%$ of obese women and in $6 \%$ in NPW, gestational hypertension respectively in $14.8 \%$ vs $2.4 \%$ ( $\mathrm{p}<0.001)$, pre-eclamsia/ eclampsia in $11.5 \%$ vs $2.4 \%(\mathrm{p}<0.001)$.As for pregnancy outcome , abortion was $9.8 \%$ in P-LAGB vs $8.5 \%$ in NPW, caesarean section $43.6 \%$ vs $28.2 \%(\mathrm{p}<0.05)$, preterm delivery $14.5 \%$ vs $3.6 \%(\mathrm{p}<0.001)$, no difference in average gestation week of delivery was found. As for fetal outcome, no different rate of SGA and LGA babies was found, mean neonatal weight was lower in P-LAGB than in NPW ones $(3207 \pm 694 \mathrm{~g}$ vs $3354 \pm 443 \mathrm{~g} ; \mathrm{p}<0.05)$. In a multiple regression analysis in PLAGB prepregnancy BMI predicts gestational diabetes and LGA babies, furthermore weight gain predicts hypertensive disorders and LGA babies; when we considered separately obese women showing weight gain or weight loss during pregnancy, we found in weight loss group a reduction of GDM, hypertensive disorders and LGA babies rate, and an increase in preterm delivery frequency $(21.4 \%$ vs $12.5 \%)$ with respect to obese women with weight gain, this difference is however of no statistical significance.

Conclusion: Obese women also after surgical treatment show a poor pregnancy outcome with respect to normalweight women.

\section{PS 108 Gestational diabetes: metabolism}

\section{8}

Dysfunction of placental mitochondria in gestational diabetes P.G. Halvatsiotis ${ }^{1}$, K. Patsouras ${ }^{2}$, E. Delatola ${ }^{1}$, E. Boutati ${ }^{1}$, G. Salamalekis ${ }^{2}$, E. Salamalekis ${ }^{2}$, T. Economopoulos ${ }^{1}$, S.A. Raptis ${ }^{1,3}$

${ }^{1}$ 2nd Dept of Int Med Propaedeutic, Athens University Medical School, ${ }^{2} 3$ rd Dept of Obstetrics and Gynecology, Athens University Medical School, ${ }^{3}$ Hellenic National, Diabetes Center, Athens, Greece

Background and aims: Gestational diabetes mellitus (GDM) is a pathological state of carbohydrate intolerance beginning or first recognized during pregnancy, which when untreated increases the risk of adverse pregnancy outcome. Insulin resistance is widely accepted to be associated with increased morbidity, although its basis remains unclear. Decreasing activities of mitochondrial enzymes could be part of the pathogenesis of complications and thus the purpose of our study was to examine whether a decrease in placental mitochondrial function, is altered in GDM.

Materials and methods: Placental mitochondria from 6 women (GDM) $(30+2,46$ yo) and 5 matched to the BMI, race and age nondiabetic mothers (NGD) $(29,8+1,07$ yo) were isolated and gene transcripts were performed using a real-time QPCR technique for Citrate Synthase (CS), Cytochrome C oxidase I \& III (COXI \& COXIII), NADH and UCP2. All pregnancies were uncomplicated and normal newborns delivered through caesarean section. Statistical analysis performed with unpaired t-test.

Results: The GDM group demonstrated significantly higher serum insulin levels than NGD $(11,05+1,3$ vs $6,7+1,2 \mu \mathrm{IU} / \mathrm{ml})(\mathrm{p}=0,04)$, while at the same euglycemic levels indicating the presence of insulin resistance state for GDM mothers. GHbAlc levels of GDM were within normal limits $(5,6+0,5 \%)$ . There was no difference in the BMI of GDM vs NGD before pregnancy $(28,43+3,9$ vs $26,26+4,6)$ as well as for the weight gained during pregnancy between the groups $(12,75+1,5$ vs $17,5+1,9 \mathrm{~kg})$. Gene transcripts of CS $(0,26+0,06$ vs $0,5+0,08 \mathrm{AU})(\mathrm{p}=0,035), \operatorname{COX} 1(0,01+0,01$ vs $0,05+0,02 \mathrm{AU})$ $(\mathrm{p}=0,05)$, NADH $(7,49+4,1$ vs $28,51+0,3 \mathrm{AU})(\mathrm{p}=0,05)$ were significantly lower in the GDM group than NGD, while UCPs showed to be higher in $\operatorname{GDM}(0,22+0,04$ vs $0,11+0,01 \mathrm{AU})(\mathrm{p}=0,046)$. There was not detected any difference for COXIII.

Conclusion: In conclusion these data indicate the existence of a placental mitochondrial dysfunction which might contribute to restricted fetal growth by limiting energy availability in cells. This bioenergetic failure may be attributed to the increased production of oxidative species while the up-regulation of UCPs indicates an activation of the mitochondrial defence mechanism compensatory to the increased redox state. Our findings support the notion that the decreased mitochondrial function may play a crucial role in the development of complications of pregnancy such as preeclampsia.

\section{9}

Inverse correlation between vitamin D levels and glycosylated haemoglobin in a gestational diabetes clinic population S. Lau' ${ }^{1}$ J.E. Gunton ${ }^{1}$, N. Athayde ${ }^{2}$, N. Cheung ${ }^{1}$

${ }^{1}$ Diabetes \& Endocrinology, ${ }^{2}$ Obstetrics and Gynaecology, Westmead Hospital, Westmead, Australia

Background and aims: Vitamin D deficiency during pregnancy is increasingly recognised, particularly in dark-skinned or veiled women. Both short and long-term neonatal effects have been studied, but few have considered a potential maternal detriment. A role for Vitamin D has been implicated in $\beta$-cell function. Deficiency during pregnancy could therefore also contribute towards gestational diabetes (GDM).

Materials and methods: In 2007, we initiated routine third trimester testing of 25(OH)vitamin D (25OHD), collected simultaneously with the HbA1c, in women attending a GDM clinic with a diverse ethnic population. The diagnostic criteria used for GDM were a fasting plasma glucose $>/=5.5 \mathrm{mmol} / \mathrm{L}$ and/or 2 hour value $>/=8.0 \mathrm{mmol} / \mathrm{L}$ on $75 \mathrm{~g}$ oral glucose tolerance test. The IDS radioimmunoassay for measurement of $25 \mathrm{OHD}$ was used. We conducted a retrospective analysis to explore any relationship between vitamin D status and measures of glycaemia. Women with pre-gestational diabetes were excluded. Spearman Rank correlations were used to test the association between $25 \mathrm{OHD}, \mathrm{HbAlc}$ and blood glucose values. Mean values of ethnic groups were 
compared using ANOVA. A general linear model which included 25OHD, BMI at booking-in, ethnicity and age was tested for independent predictors of HbA1c. A p-value of $<0.05$ was considered significant for all tests.

Results: Results of the first 145 women are reported here. The average age was 32 yrs. $80 \%$ were insulin-requiring, reflecting the referral patterns to the clinic. The mean 25OHD levels for women from Caucasian $(n=27)$, East/SouthEast Asian $(n=43)$, Middle-Eastern $(n=15)$, Indian Subcontinental $(n=$ 47) and other/non-specified $(\mathrm{n}=13)$ backgrounds were 67.0, 66.5, 46.9, 49.1 and $41 \mathrm{nmol} / \mathrm{L}$ respectively (p-value for difference between groups $<0.001$ ). Overall, $40 \%$ of these women were classified as vitamin D insufficient / deficient, using a cut-off level of $50 \mathrm{nmol} / \mathrm{L}$. $9 \%$ had values $<25 \mathrm{nmol} / \mathrm{L}$. There was a highly significant inverse correlation between 25OHD and $\mathrm{HbAlc}(\mathrm{r}$ $=-0.316, p=0.001$ ). A weaker inverse correlation between 25OHD level and fasting blood glucose level at GDM diagnosis (on average 6-7 weeks before the time of collection of 25OHD) was also found $(r=-0.17, p=0.038)$. After adjusting for possible confounders, $25 \mathrm{OHD}$ was independently and inversely associated with $\mathrm{HbAlc}$.

Conclusion: Despite our sunny climate, Vitamin D deficiency is common in an Australian multi-ethnic clinic population and may contribute towards glycaemic control in GDM. This is the first report of an association between $25 \mathrm{OHD}$ level and HbAlc in pregnancy. The precise contribution of vitamin $\mathrm{D}$ status to $\beta$-cell function or insulin resistance should be tested in prospective studies and clinical trials.

\section{0}

1-hr glucose level after $100 \mathrm{~g}$ OGTT as marker of disturbance of glucose homeostasis in pregnant women

C. Lencioni ${ }^{1}$, G. Seghieri ${ }^{2}$, I. Cuccuru ${ }^{1}$, R. Anichini ${ }^{2}$, A. De Bellis ${ }^{2}$, A. Ghio ${ }^{1}$, V. Resi ${ }^{1}$, F. Tesi ${ }^{2}$, L. Volpe ${ }^{1}$, S. Del Prato ${ }^{1}$, G. Di Cianni ${ }^{1}$

${ }^{1}$ Endocrinology-Metabolism, University of Pisa, ${ }^{2}$ Internal Medicine, Spedali Riuniti, Pistoia, Italy

Background and aims: We have recently shown that pregnant women with one abnormal value (OAV) during OGTT not meeting criteria for gestational diabetes (GDM) and women with GDM are clinically indistinguishable, with both groups exhibiting impaired insulin secretion and insulin sensitivity. Moreover, among OAV women, greater impairment occurred with 1-h OAV suggesting this value could provide further information of metabolic status in non-GDM women. Therefore, in order to gain further insight on the significance of milder alterations of glucose tolerance we have investigated to which extent 1-h OGTT maternal glycemic value can reflect alteration in insulin secretion and action.

Methods: A 3-hr 100-g OGTT was performed at 27 \pm 3.2 week of gestation in 4053 pregnant women (GDM: 18\%; NGT: 63.3\%; OAV: 18.7\% - Fasting OAV: $28.8 \%$, 1-h OAV $41.6 \%$, and 2- or 3-h OAV: $29.5 \%$ ) for determination of plasma glucose and insulin concentration, calculation of incremental area under glucose (AUCgluc) and insulin curves (AUCins), 1st- and 2nd-phase insulin secretion (using Stumvoll's formulae), and insulin sensitivity (ISI Matsuda). The insulin secretion-sensitivity index (ISSI) was calculated as the product of 1st-phase index and ISI. Data have been, then, analyzed as a function of $20 \mathrm{mg} / \mathrm{dl}$ increments in 1-hr OGTT, resulting in 5 groups (G1: $<120, \mathrm{G} 2: 120$ 139, G3:140-159, G4:160-179, G5: $\geq 180 \mathrm{mg} / \mathrm{dl}$ )

Results: Prevalence of GDM $(0.3,1.8,3.9,13.7,80.3 \%$, respectively) and OAV $(4,8.7,19,26.7,41.6 \%$, respectively) increased with raising 1 -hr OGTT values. As expected, AUCgluc (from 8894 to $14493 \mathrm{mg} / \mathrm{dl} / \mathrm{min}$ ) and AUCins (from 6562 to $915010-3 \mathrm{pmol} / \mathrm{L} / \mathrm{min}$ ) both progressively increased over the 1-hr OGTT spectrum (both $\mathrm{p}<0.0001$ for trend). On the contrary, ISI decreased in a linear manner from G1 to G5 (from 6.853 to $4.222, \mathrm{p}<0.001$ ). In particular, even within normal values of 1 -hr OGTT (i.e. $<180 \mathrm{mg} / \mathrm{dl}$ ), ISI dropped by $29.3 \%(\mathrm{p}<0.001)$. Along with the decline in insulin sensitivity, both 1st- and 2nd-phase insulin secretion progressively declined (both $\mathrm{p}<0.001$ ) over the entire spectrum, as well as within non-diagnostic 1-hr OGTT values. Moreover, a linear reduction in ISSI was apparent (G1: 8950, G2: 6866, G3: 5619, G4: 4607, and G5: 3524; $<<0.001$ ). Of note, even within non-diagnostic 1-hr OGTT values ISSI declined by $48.5 \%$ ( $p<0.001$ ). Even when analysis was restricted to groups G1 - G3 with low GDM prevalence, the progressive nature of defects in insulin secretion (1st-phase: -10.9\%; 2ndphase: $-8.6 \%$ ), insulin sensitivity (ISI: $-19.1 \%$ ) was apparent accounting for a $-40.2 \%$ reduction in ISSI (all $\mathrm{p}<0.01$ ).

Conclusion: These results suggest, that in pregnant women, insulin secretion and insulin sensitivity progressively worsened on the basis of 1-hr glucose concentrations after a 100-g OGTT. Of note, a marked reduction in ISSI was already apparent even within non-diagnostic 1-hr OGTT values, suggesting that loss of glucose homeostasis is an early and continuous process. These results should draw attention to the need for better understanding of the risk associated to increased maternal glycemia even in pregnancies considered metabolically normal.

\section{1}

Indices of beta cell function and insulin sensitivity in normal pregnancy

E. Qvigstad ${ }^{1}$, N. Voldner ${ }^{2}$, K.F. Frøslie ${ }^{3}$, C.M. Hoff ${ }^{2}$, K. Godang ${ }^{1}$,

T. Henriksen ${ }^{2}$ J. Bollerslev ${ }^{1}$

${ }^{1}$ Department of Endocrinology, Rikshospitalet University Hospital, ${ }^{2}$ Department of Gynecology and Obstetrics, Rikshospitalet University Hospital, ${ }^{3}$ Department of Statistics, University of Oslo, Norway

Background and aims: Gestational diabetes (GDM) is associated with increased risk of maternal and fetal morbidity and complications on short and long term perspective. The increasing incidence of obesity in fertile age influences insulin sensitivity and places extra demands on beta-cell function during pregnancy. The STORK-study follows healthy pregnant Norwegian women to investigate effects of metabolism, anthropometry, nutrition and physical activity on pregnancy outcome and complications.

Materials and methods: Inclusions were done in week $14-16, n=553$. Oral glucose tolerance tests (OGTT) were performed at week 14-16 and 30-32, parallelled by anthropometry and obstetric data.

Results: Age 31,2 $\pm 1,2$ years (mean $\pm \mathrm{SD}$ ), weight gain 10,6 $\pm 3,8 \mathrm{~kg} .1,9$ $\%$ had early onset GDM (GDM1) at week 14-16 and 10,6 \% at week $30-32$ (GDM2).The participants were stratified according to the OGTT results.

BMI, b cell function and insulin sensitivity indices, median (25th; 75th quartiles).

\begin{tabular}{|c|c|c|c|}
\hline & $\begin{array}{l}\text { NGT } \\
(\mathrm{n}=460)\end{array}$ & $\begin{array}{l}\text { GDM1 } \\
(\mathrm{n}=11)\end{array}$ & $\begin{array}{l}\text { GDM2 } \\
(\mathrm{n}=49)\end{array}$ \\
\hline $\begin{array}{l}\mathrm{BMI} \\
(\mathrm{kg} / \mathrm{m} 2)\end{array}$ & $\begin{array}{l}24,1 \\
(22,0 ; 26,4)\end{array}$ & $\begin{array}{l}28,3 \\
(25,1 ; 30,5)^{*}\end{array}$ & $\begin{array}{l}26,1 \\
(22,0 ; 28,8)\end{array}$ \\
\hline $\begin{array}{l}\text { Insulin resistance week 14-16 } \\
\text { (HOMA-IR) }\end{array}$ & $\begin{array}{l}5,0 \\
(3,3 ; 7,2)\end{array}$ & $10,7(6,5 ; 19,2)$ & $\begin{array}{l}7,1 \\
(4,8 ; 10,5)^{*} \\
\end{array}$ \\
\hline $\begin{array}{l}\text { Insulin resistance week 30-32 } \\
\text { (HOMA-IR) }\end{array}$ & $\begin{array}{l}8,1 \\
(5,2 ; 11,9)\end{array}$ & $\begin{array}{l}13,8 \\
(7,5 ; 23,5)^{*}\end{array}$ & $\begin{array}{l}11,4 \\
(8,3 ; 16,2)^{*}\end{array}$ \\
\hline $\begin{array}{l}\text { Beta-cell function week 14-16 } \\
\text { (HOMA-B) }\end{array}$ & $\begin{array}{l}807 \\
(504 ; 1464) \\
\end{array}$ & $\begin{array}{l}977 \\
(600 ; 1487)\end{array}$ & $\begin{array}{l}874 \\
(624 ; 1233) \\
\end{array}$ \\
\hline $\begin{array}{l}\text { Beta-cell function week 30-32 } \\
\text { (HOMA-B) }\end{array}$ & $\begin{array}{l}952 \\
(640 ; 1505) \\
\end{array}$ & $\begin{array}{l}682 \\
(623 ; 1692) \\
\end{array}$ & $\begin{array}{l}877 \\
(693 ; 1554) \\
\end{array}$ \\
\hline $\begin{array}{l}\text { Fasting insulin week 14-16 } \\
\text { sensitivity (Quicki) }\end{array}$ & $\begin{array}{l}0,49 \\
(0,45 ; 0,53) \\
\end{array}$ & $\begin{array}{l}0,44 \\
(0,38 ; 0,46)^{*} \\
\end{array}$ & $\begin{array}{l}0,45 \\
(0,42 ; 0,49)^{*} \\
\end{array}$ \\
\hline $\begin{array}{l}\text { Fasting insulin week 30-32 } \\
\text { sensitivity (Quicki) }\end{array}$ & $\begin{array}{l}0,44 \\
(0,41 ; 0,48)\end{array}$ & $\begin{array}{l}0,40 \\
(0,37 ; 0,45)\end{array}$ & $\begin{array}{l}0,42 \\
(0,39 ; 0,44) \\
\end{array}$ \\
\hline $\begin{array}{l}\begin{array}{l}\text { Dynamic insulin week 14-16 } \\
\text { sensitivity (OGIS) }\end{array} \\
\end{array}$ & $\begin{array}{l}533 \\
(485 ; 593) \\
\end{array}$ & $\begin{array}{l}405 \\
(313 ; 429) * \$ \\
\end{array}$ & $\begin{array}{l}472 \\
(433 ; 524)^{*} \\
\end{array}$ \\
\hline $\begin{array}{l}\begin{array}{l}\text { Dynamic insulin week 30-32 } \\
\text { sensitivity (OGIS) }\end{array} \\
\end{array}$ & $\begin{array}{l}433 \\
(388 ; 486) \\
\end{array}$ & $\begin{array}{l}422 \\
(383 ; 466) \\
\end{array}$ & $\begin{array}{l}443 \\
(405 ; 487) \\
\end{array}$ \\
\hline $\begin{aligned} \text { Kruska-Wallis test } & { }^{*} \mathrm{p}<0.01 \text { for } \\
& \text { difference vs } \\
& \text { NGT }\end{aligned}$ & $\begin{array}{l}\$ \mathrm{p}<0.01 \text { for } \\
\text { difference vs } \\
\text { GDM2 }\end{array}$ & $\begin{array}{l}\text { Bonferroni } \\
\text { correction for } \\
\text { multiple tests. }\end{array}$ & \\
\hline
\end{tabular}

Subdivision of OGTT groups by BMI demonstrated a preponderance of overweight (BMI > 25) in glucose intolerant states (NGT 38\%, GDM2 58\% and GDM1 $82 \%$ respectively, Chi-squared test, $\mathrm{p}<0,005)$. OGIS decreased by 21 and $10 \%$ respectively in week $14-16$ in overweight GDM1 and GDM2 ( $<<0,01$ for comparision with NGT). At week 30-32 HOMA-IR increased by $32 \%$ in overweight GDM2 ( $<00,01$ vs NGT), and basal beta-cell function decreased by $7 \%$ in GDM 2 vs NGT, $p<0,01$. Similar effects were not found in individuals with $\mathrm{BMI} \leq 25$.

Conclusion: In this prospective study of normal pregnancies, BMI was increased in individuals with GDM1, insulin sensitivity indices decreased early in pregnancy, but the increase in insulin resistance was a later event. In GDM2 we found similar effects on insulin sensitivity. For individuals with overweight, the GDM rates were significantly increased, and the deterioration of indices of insulin sensitivity and later beta-cell function in this group exposes maternal and fetal metabolism for an increased risk of morbidity and complications. Supported by: Women's Health Centre, Rikshospitalet University Hospital Oslo, University of Oslo 


\section{2}

Deoxyfructosyl haemoglobin (beta) values in normal and gestational diabetic pregnancy

E. Anastasiou ${ }^{1}$, K. Economou ${ }^{1}$, G. Papageorgiou ${ }^{1}$, M. Alevizaki ${ }^{1}$, L. Spanou ${ }^{2}$, I. Drakopoulos ${ }^{2}$, K. Makris ${ }^{2}$

${ }^{1} 1$ st Endocrine Section - Diabetes Center, Alexandra Hospital, ${ }^{2} \mathrm{Clinical}$ Biochemistry Department, KAT General Hospital, Athens, Greece

Background and aims: According to the work of the National Glycohemoglobin Standardisation Program the current HbAlc assay has been harmonised on reference methods that measure a mixture of glycated haemoglobins The International Federation of Clinical Chemistry (IFCC) has recently approved a reference method for $\mathrm{HbA} 1 \mathrm{c}$ that specifically measures the concentration of only one molecular species of HbA1c. A new name, deoxyfructosyl haemoglobin (beta) $(\mathrm{DOFH})$ and measurement units $(\mathrm{mmol} / \mathrm{mol})$ were proposed to replace $\mathrm{HbAlc}$ (\%). DOFH represents the fraction of the b-chains of hemoglobin that has a stable hexose adduct on the N-terminal amino acid valine. The aim of the study is (i) to examine the relationship of DOFH values with the traditional HbAlc measurements in pregnant women who underwent an OGTT and (ii) to relate the levels of DOFH values with the glucemic status of pregnant women.

Materials and methods: In 181 successive pregnant women, at the third trimester of pregnancy, who underwent an OGTT-100g, blood samples were collected for HbAlc and DOFH measurements. HbA1c was measured by an HPLC (HA8160 Menarini-Arkray), while DOFH was measured in another lab, also using an HPLC (HA8160 Menarini-Arkray) which was calibrated and traceable to the IFCC reference method. Pregnant women were divided into four groups, according to OGTT glucose values (ADA criteria) and the way of treatment, once diagnosed as Gestational Diabetes (GDM): (i) Normals, (ii) One Abnormal Glucose Value, (iii) GDM treated with Diet (GDMD) and (iv) GDM treated with diet and Insulin(GDM-I).

Results: A very strong correlation was observed, between HbAlc (y) measured by the DCCT calibrated HPLC and HbAlc (x) calculated from DOFH which was measured by the IFCC calibrated instrument $(y=1,0218 x-0.3124$, $\mathrm{r}=0.935, \mathrm{p}<0.001)$. The DOFH values of the four groups are shown in the table. A positive correlation was observed between DOFH values and fasting glucose in the OGTT $(\mathrm{r}=0.47, \mathrm{p}<0.001)$. Correlations with glucose values at 60,120 and $180 \mathrm{~min}$ were also positive but weaker.

Conclusion: The excellent correlation which was found between $\mathrm{HbAlc}$ (measured by the DCCT calibrated instrument) and HbAlc (calculated from $\mathrm{DOFH}$ ), proves that both calibrations in instruments used in everyday practice can produce comparable results, although adoption of a new name and new measurement units requires education of both patients and health care providers. A further interesting finding of this study was that the DOFH values of pregnant women with one abnormal glucose value in the OGTT are similar to those of GDM women, an indirect indication that this group may need further attention, such as diet instructions.

\begin{tabular}{lcllll}
$\begin{array}{l}\text { DOFH Values Results } \\
\text { Groups }\end{array}$ & $\mathrm{N}$ & $\begin{array}{l}\text { Gest. Age } \\
\text { (Weeks) }\end{array}$ & $\begin{array}{l}\text { Age } \\
\text { (Years) }\end{array}$ & $\begin{array}{l}\text { BMI } \\
(\mathrm{kg} / \mathrm{m} 2)\end{array}$ & $\begin{array}{l}\text { DOFH } \\
(\mathrm{mmol} / \\
\mathrm{mol})\end{array}$ \\
\hline Normals & 59 & $28.2+4.63$ & $30.1+5.34$ & $23.9+3.56$ & $30.5+3.52$ \\
\hline $\begin{array}{l}\text { One abnormal } \\
\text { value }\end{array}$ & 21 & $28.0+5.58$ & $32.7+2.96$ & $24.5+3.84$ & $32.5+2.89$ \\
\hline GDM-D & 38 & $29.2+5.85$ & $32.0+4.08$ & $24.1+3.76$ & $32.8+2.69$ \\
\hline GDM-I & 63 & $28.8+6.14$ & $32.1+4.81$ & $24.8+3.62$ & $34.5+3.23$ \\
\hline ANOVA & & $\mathrm{NS}$ & $\mathrm{NS}$ & $\mathrm{NS}$ & $\mathrm{p}<0.001$ \\
\hline
\end{tabular}

\section{3}

The impact of recurrence of gestational diabetes (GDM) on glucose metabolism and subclinical inflammation

Y. Winhofer ${ }^{1}$, A. Tura ${ }^{2}$, T. Prikoszovich ${ }^{1}$, A. Luger ${ }^{1}$, G. Pacini ${ }^{2}$, A. Kautzky-Willer

${ }^{1}$ Department of Internal Medicine 3, Division of Endocrinology and Metabolism, Medical University of Vienna, Austria, ${ }^{2}$ National Research Council, Metabolic Modeling Unit, Institute of Biomedical Engineering, Padova, Italy.

Background and aims: GDM is often associated with obesity and subclinical inflammation and a major risk factor for the development of type 2 diabetes. When women do not regain normal glucose tolerance after delivery it is unclear, whether impaired glucose tolerance (IGT) or even type 2 diabetes already existed at pregestational state or they were caused by GDM itself. Therefore we aimed to compare parameters of glucose metabolism and subclinical inflammation before pregnancy $(\mathrm{bP})$, during pregnancy $(\mathrm{P})$ and postpartum (pp) in women with recurrence of GDM.

Materials and methods: Women with prior gestational diabetes (pGDM, $\mathrm{n}=25$ ) who participated in a prospective longitudinal observational study (Viennese Post Gestational Diabetes Project) and had recurrence of GDM in a subsequent pregnancy were included in this study. They were annually tested by an oral glucose tolerance test (oGTT), underwent an oGTT at 24 gestational weeks during pregnancy $(\mathrm{P})$ as well as 4 months postpartum (pp). Follow-up data of the last examination prior to subsequent pregnancy served as pregestational data (bP). Metabolic parameters derived from plasma concentrations of glucose, insulin and C-peptide during oGTT (i.e. OGIS=insulin sensitivity; HIE=hepatic insulin extraction), Leptin as well as markers of subclinical inflammation (CRP, usCRP and IL-6) were assessed at all time points.

Results: Pregnancy was associated with a significant increase in areas under the curve (AUC) of glucose $(+67 \%$; $\mathrm{p}=<.0001)$, insulin $(+42 \%$; $\mathrm{p}=0.03)$ and C-peptide ( $+22 \%$; $\mathrm{p}=0.03)$ under dynamic conditions while basal plasma concentrations did not rise significantly. Pregnancy induced insulin resist-

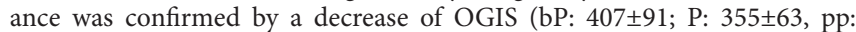
$\left.419 \pm 98 \mathrm{ml} / \mathrm{min}^{\star} \mathrm{m}^{2} ; \mathrm{p}=.0025\right)$, but not HOMA-R. Hepatic insulin extraction (HIE) was decreased, resulting in a higher amount of insulin reaching the peripheral circulation (HIE: bP: $68 \pm 13$; P: $62 \pm 10$, pp: $69 \pm 10 \%$; $p=0.04$ ). Disposition Index (OGIS*dyn_AUC_insulin), which characterizes the ability for compensating peripheral insulin resistance by modulating insulin release into systemic circulation, also increased during pregnancy (bP: $21 \pm 9$; $\mathrm{P}$ : $27 \pm 8 ; \mathrm{pp}: 20 \pm 8 \mathrm{nmol} / \mathrm{m}^{3} ; \mathrm{p}=0.03$ ). After pregnancy all parameters declined to values not different from those of pre-pregnancy, with the exception of BMI

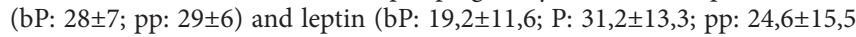
$\mathrm{ng} / \mathrm{ml}$ ) which remained increased (both $\mathrm{p}=0.03$ ). usCRP, but not CRP or IL6 , increased during pregnancy $(\mathrm{p}=0.02)$ and did not reach the pregestational value after delivery (bP: $0,51 \pm 0,53 ; \mathrm{P}: 0,69 \pm 0,45 ; \mathrm{pp}: 0,61 \pm 0,77 ; \mathrm{p}=0.02$ )

Conclusion: Since the metabolic parameters that changed during pregnancy in pGDM returned to the pregestational state within 4 months after delivery, we conclude, that pregnancy itself does not alter the natural course of the development of diabetes after GDM. On the other hand, because BMI and leptin, as marker of body fat content, remained increased after pregnancy, regulation of bodyweight and postpartum weight retention seem to be the major problems for long-term maintenance of normal glucose tolerance in pGDM.

\section{4}

Association of plasma individual NEFA with insulin sensitivity and /or insulin secretion in Bangladeshi GDM women

S. Murshed ${ }^{1}$, M. Khatun ${ }^{2}$, M. Rahman ${ }^{1}$, S. Akter ${ }^{1}$, T.A. Chowdhury ${ }^{2}$, A.K. Azad Khan ${ }^{3}$, L. Ali ${ }^{1}$

${ }^{1}$ Dept of Biochemistry \& Cell Biology, BIRDEM, ${ }^{2}$ Obstetrics and Gyneconology, BIRDEM, ${ }^{3}$ Dept of Gastroenterology, Diabetic Association of Bangladesh, Dhaka, Bangladesh

Background and aims: GDM is associated with elevated NEFA and insulin resistance, but the causal relationship between the two events is not yet established. Recently association of reduced membrane omega- 6 and omega-3 fatty acid with GDM has been reported in British and Korean population. The aim of the present study was to explore the relationship between plasma individual NEFA with insulin sensitivity and/or insulin secretion in Bangladeshi GDM women during 24-28 weeks of gestation ( $2^{\text {nd }}$ trimester $)$ and $29-36$ wks of gestation ( $3^{\text {rd }}$ trimester).

Materials and methods: A prospective study was performed with a total of 73 pregnant women. Among them 37 pregnant women with gestational diabetes mellitus (GDM) and 36 age and BMI matched nondiabetic healthy controls were collected from various hospitals during 24-28 wks of gestation $\left(2^{\text {nd }} \operatorname{tr}\right)$ and asked for a 2nd visit during their 29-36 wks of gestation $\left(3^{\text {rd }} \operatorname{tr}\right)$. Fasting Serum glucose, C-peptide, lipid profile, total NEFA were estimated by enzymatic-colorimetric method, $\mathrm{HbA}_{1 \mathrm{c}}$ was measured by HPLC method and individual NEFA were measured by gas chromatography.

Results: During $3^{\text {rd }}$ tr HOMA S (\%) in GDM [Median (range); 41.60 (14.6091)] was significantly decreased $(\mathrm{p}<0.006)$ than in control [58.45 $(12.30-$ 92.20)]. Serum TG $(\mathrm{mg} / \mathrm{dl})$ were significantly increased $(\mathrm{p}<0.001)$ during $2^{\text {nd }}$ and $3 \mathrm{rd}$ tr in GDM (Mean \pm SD; 222.65 \pm 16.36 and $255.24 \pm 9.69$ respectively) than in control ( $189.47 \pm 3.22$ and $237.86 \pm 18.45$ respectively). Total NEFA 
$(\mathrm{mmol} / \mathrm{l})$ was significantly increased $(\mathrm{p}<0.001)$ in GDM $(0.99 \pm 0.17)$ compared to control $(0.82 \pm 0.25)$ during $3^{\text {rd }}$ tr. Only plasma stearic acid (\%) in GDM was significantly increased $(\mathrm{p}<0.001)$ during $3^{\text {rd }} \operatorname{tr}(30.17 \pm 5.54)$ compared to $2^{\text {nd }} \operatorname{tr}(24.17 \pm 5.41)$. MUFA, palmitoleic acid, oleic acid were significantly decreased $\left(\mathrm{p}<0.001,0.01\right.$ and 0.001 respectively) in GDM during $3^{\text {rd }}$ $\operatorname{tr}\left(14.24 \pm 3.93,2.06 \pm 0.86\right.$ and $11.70 \pm 54.16$ respectively) compared to $2^{\text {nd }} \operatorname{tr}$ $(25.04 \pm 5.14,2.76 \pm 0.37$ and $20.53 \pm 6.07$ respectively). Among polyunsaturated fatty acids, arachidonic acid and docosahexaenoic acid were significantly decreased $(\mathrm{p}<0.001)$ in GDM at $3^{\text {rd }} \operatorname{tr}(2.20 \pm 0.09$ and $0.45 \pm 0.25$ respectively $)$ compared to $2^{\text {nd }} \operatorname{tr}\left(2.90 \pm 0.68\right.$ and $0.82 \pm 0.10$ respectively). During $2^{\text {nd }} t$ tr, arachidonic acid in GDM was positively correlated $(\mathrm{r}=0.53, \mathrm{p}=0.04)$ with HOMA B. During $3^{\text {rd }}$ tr, eicosapentaenoic acid in GDM was inversely correlated $(\mathrm{r}=-0.52, \mathrm{p}=0.05)$ and in control directly correlated $(\mathrm{r}=0.57, \mathrm{p}=0.02)$ with HOMA B. During $2^{\text {nd }} \mathrm{tr}$, eicosadienoic acid in GDM was positively correlated $(r=0.69, p=0.00)$ with HOMA S. During $3^{\text {rd }}$ tr, linoleic acid, linolenic acid, PUFA and $\omega 6$ FA in GDM were inversely correlated $(r=-0.66, p=0.01$; $\mathrm{r}=-0.66, \mathrm{p}=0.01$ and $\mathrm{r}=-0.65, \mathrm{p}=0.01$ respectively) and eicosadienoic acid eicosapentaenoic acid were directly correlated $(r=0.54, p=0.04$ and $r=0.60$, $\mathrm{p}=0.02$ ) with HOMA $\mathrm{S}$.

Conclusion: The data suggest that GDM is associated with hypersecretion of insulin which may be positively modulated by arachidonic acid and negatively modulated by eicosapentaenoic acid. GDM is also associated with decreased insulin sensitivity which may be positively modulated by eicosadienoic acid, eicosapentaenoic acid and negatively modulated by linoleic acid, linolenic acid, total PUFA and $\omega 6$ fatty acids.

Supported by: Diabetic Association of Bangladesh

\section{5}

Influence of pre-pregnancy body mass index, physical activity and dietary habits on glucose tolerance during pregnancy

A. Bertolotto, L. Volpe, S. Vignali, A. Calianno, M. Pugliese, C. Lencioni, M. Corfini, V. Resi, L. Benzi, G. Di Cianni

Endocrinology-Metabolism, University of Pisa, Italy

Background and aims: Abnormal glucose tolerance (AGT) during pregnancy, including all conditions from minor degree of glucose tolerance to overt gestational diabetes, increases the risk of maternal morbidity and adverse pregnancy outcome. Pre-pregnancy over-weight and obesity are considered the most important risk factors for AGT. To date, other factors that may influence glucose tolerance during pregnancy, such as physical activity and dietary habits are not considered as risk factors for AGT. The aim of the present study is to investigate the relative contribution of physical activity, dietary habits, pre-pregnancy body mass index (BMI) and other traditional risk factors on glucose tolerance during pregnancy.

Materials and methods: A 1-hr 50-g Glucose Challenge Test (GCT) was performed at $27 \pm 6.9$ week of gestation in a cohort of pregnant women. On the morning of the test, demographic, anthropometric and clinical data (age, family history of diabetes, obstetric history and pre-pregnancy BMI) were recorded. To assess physical activity, women were invited to fill the short form of the International Physical Activity Questionnaire (IPAQ) and we calculated the average amount of time per week spent during 7 days before the test (MET h/week). Moreover, to evaluate dietary habits, all women answered to a food frequency questionnaire; the energy intake and dietary nutrient content were calculated by a computerized program $\left(\right.$ MetaDieta $\left.^{\circ}\right)$.

Results: We studied 268 pregnant women (age $33.3 \pm 4$ years; primiparous $58 \%$; positive family history of diabetes mellitus $13 \%$; pre-pregnancy BMI $22.6 \pm 3.8 \mathrm{Kg} / \mathrm{m} 2$; weight gain $8 \pm 3.7 \mathrm{Kg}$ ). The mean physical activity was $18.5 \pm 14.8 \mathrm{MET} \mathrm{h} /$ week and the mean daily energy intake of the cohort was $2145 \pm 395 \mathrm{Kcal}$ (carbohydrates $48 \%$, total fats $36 \%$, proteins $16 \%$, cholesterol intake $243 \pm 82 \mathrm{mg} / \mathrm{d}$ and fibres $19.5 \pm 5.7 \mathrm{~g} / \mathrm{d}$ ). Women with GCT positive (GCT+: glycaemia $\geq 140 \mathrm{mg} / \mathrm{dl}$ ) were $105(39 \%)$ and GCT negative (GCT-) $163(61 \%)$. No difference between these two groups resulted in demographic and clinical data, excluding pre-pregnancy BMI (GCT $+23.5 \pm 4 \mathrm{Kg} / \mathrm{m} 2$ vs GCT $-22 \pm 3.6 \mathrm{Kg} / \mathrm{m} 2, \mathrm{p}=0.02)$ and weight gain $(\mathrm{GCT}+8.5 \pm 4.3$ vs GCT$7.6 \pm 3.3 \mathrm{Kg}, \mathrm{p}=0.03$ ). GCT+ was observed in $54 \%$ of overweight/obese (BMI $\geq 25 \mathrm{~kg} / \mathrm{m} 2)$ and $36.8 \%$ of normal weight $(B M I<25 \mathrm{~kg} / \mathrm{m} 2)(\mathrm{p}<0.002)$. All parameters referring to physical activity energy and diet (Kcal and diet components) were not statistically different between GCT + and GCT- groups. In joint analyses of BMI and physical activity, we divided the population into 4 groups: normal weight active (MET h/week $\geq 20$ ) and inactive (MET h/week $<3$ ) and overweight/obese active and inactive; we didn't observe any difference about 1-hr glucose values and GCT+ prevalence among the 4 groups. Finally, after a multivariate analyses including pre-pregnancy BMI, weight gain, MET h/week and Kcal/dayl, only pre-pregnancy BMI (F-value 9.264 $\mathrm{p}=0.002$ ) resulted an independent predictor of 1 -hr plasma glucose.

Conclusion: Our study confirms the importance of pre-pregnancy BMI as a risk factor for abnormal glucose tolerance during pregnancy. Although physical activity and diet are both well known factors to prevent glucose intolerance in general population, their contribution during pregnancy should be better evaluated.

\section{6}

Comparability of an European guideline and American Diabetes Association protocol in identifying high risk patients for gestational diabetes

L. Varadhan ${ }^{1}$, S. Bikmalla ${ }^{1}$, D. Radford ${ }^{2,3}$, D. Barton ${ }^{1,4}$

${ }^{1}$ Department of Diabetes and Endocrinology, Princess Royal Hospital NHS, Telford, ${ }^{2}$ Obstetrics and Gynaecology, Princess Royal Hospital NHS, Telford, United Kingdom, ${ }^{3}$ Obstetrics and Gynaecology, Royal Shrewsbury Hospital NHS, ${ }^{4}$ Department of Diabetes and Endocrinology, Royal Shrewsbury Hospital NHS, United Kingdom

Background and aims: Gestational diabetes (GDM) complicates about $4 \%$ of all pregnancies in the UK. Screening for this condition is essential in high risk patients. The recent NICE guidelines (National Institute of Clinical Excellence) released in March 2008 recommends screening of pregnant women at 24-28 weeks, only if they have one of the risk factors-BMI $>30 \mathrm{~kg} / \mathrm{m} 2$, previous macrosomia $>4.5 \mathrm{~kg}$, previous GDM, family history of diabetes or belonging to an ethnic group with high risk of diabetes. This guideline does not recommend using age as a factor as this would involve most pregnany patients. However the American Diabetes Association(ADA) guidelines suggest screening all pregnancies unless they have all of the following low risk features-Age $<25$, pre pregnancy weight normal, belonging to an ethnic group with low prevalence of diabetes, no family history, no history of abnormal glucose tolerance in past and no history of poor obstetric outcome. The aim of our study was to assess the comparability of the NICE and ADA criteria in risk-stratifying patients to subject to GTT, based on phenotypes of GDM patients at our hospital

Materials and methods: We conducted a retrospective analysis of case notes of all patients diagnosed and treated as GDM in the previous three years. The hospital protocol was to subject all pregnant women with glycosuria on more than 2 occasions to a random plasma glucose testing. Patients with values $\geq 6.5 \mathrm{mmol} / \mathrm{L}$ were then subjected to a standard $75 \mathrm{gm}$ GTT with plasma glucose taken at 0,1 and 2 hours. GTT was done ideally at 24-28 weeks, but earlier if risk factors existed. GDM was diagnosed based on WHO criteria (1999) if fasting plasma glucose(FPG) $\geq 7 \mathrm{mmol} / \mathrm{L}$ and/or $2 \mathrm{~h}$ post glucose $(2 \mathrm{~h}$ $\mathrm{PG}) \geq 7.8 \mathrm{mmol} / \mathrm{L}$. Comparative analysis was then made on the ability of the NICE and the ADA criteria to risk predict these patients.

Results: 172 patients were diagnosed with GDM in the past 3 years. Based on the NICE guidelines, 144 patients $(83.7 \%)$ had at least one risk factor for GDM, $83 \%$ of whom had more than one risk factor. Twenty eight patients (16.3\%) did not have any risk factors at all, and could have been missed if the screening was based on the current guideline. The sensitivity of the NICE risk assessment guidleline was $83.7 \%$. Screening based on ADA guidelines would have missed only 4 / 172 patients, this being more comprehensive due to the inclusion of age criteria. The sensitivity of the ADA risk assessment guideline was $97.7 \%$. However if the ADA guidelines for diagnosis of GDM had been used on these patients ( $F P G \geq 5.3 \mathrm{mmol} / \mathrm{L}, 1 \mathrm{hr}$ PG $\geq 10 \mathrm{mmol} / \mathrm{L}, 2 \mathrm{~h}$ PG $\geq 8.6 \mathrm{mmol} / \mathrm{L}$, 2 of these values being positive), the sensitivity will reduce significantly to $50.6 \%$

Conclusion: The ADA guidlelines are more sensitive in stratifying risk for GDM than the current NICE recommendations. This would proportionately increase the number of pregnant women undergoing GTT done. However this approach would be beneficial in decreasing morbidity perinatally due to fetal macrosomia and would potentially identify patients at risk of development of future diabetes or gestational diabetes. 


\section{PS 109 Gestational diabetes: outcomes}

\section{7}

Pregnancy outcomes in metformin-treated women with gestational diabetes

J. Balani, S. Hyer, A. Rodin, H. Shehata

Diabetes, St Helier University Hospital, Surrey, United Kingdom

Background: Preliminary evidence from on-going prospective studies suggests that Metformin may offer advantages over insulin in the management of gestational diabetes.

Objective: To compare maternal and neonatal outcomes in women with gestational diabetes treated in our institution with either Metformin or insulin Methods: We prospectively studied 100 consecutive women with gestational diabetes attending our Diabetes Antenatal clinic who were not adequately controlled by dietary measures. They were treated with Metformin $500 \mathrm{mg}$ bd and the dose was titrated up to a maximum of $2500 \mathrm{mg} /$ day to achieve target home blood glucose monitoring values (fasting $<6 \mathrm{mmol} / \mathrm{l}$, $1 \mathrm{~h}$ post-prandial $<8 \mathrm{mmol} / \mathrm{l}, 2 \mathrm{~h}$ post prandial $<7 \mathrm{mmol} / \mathrm{l})$. 11 women stopped taking Metformin (intolerance in 4, refusal to continue in 7) and 9 required additional insulin. Pregnancy outcomes in the 80 women who remained exclusively on Metformin until delivery were compared with a cohort of 80 women with gestational diabetes attending our clinic, matched for BMI and ethnicity, who were treated with basal-bolus human insulin.

Results: Women treated with Metformin gained less weight compared with the insulin group (mean (SEM) weight gain $(\mathrm{kg})$ : $0.3 \pm 0.03 \mathrm{v} 1.4 \pm 0.15$; $\mathrm{p}<0.01$ ). There were no significant differences in the occurrence of hypertension or pre-eclampsia. Neonatal morbidity was significantly improved in the Metformin group with less prematurity ( $0 \% \mathrm{v} 11 \%$; $<<0.01)$, less jaundice $(9 \%$ $\mathrm{v} 43 \% ; \mathrm{p}<0.01)$ and fewer admissions to the neonatal unit (5\% v $19 \% ; \mathrm{p}<0.01)$. No significant differences were found in the rate of Caesarean delivery, birth weight, number of babies with macrosomia, incidence of shoulder dystocia, congenital malformations or abnormalities in the post-natal glucose tolerance test.

Conclusion: These findings, taken together with accumulating evidence from larger trials, suggest that Metformin is beneficial in the management of gestational diabetes and may offer advantages over insulin.

\section{8}

Glycaemic control during labour in gestational diabetes: easy management with fair outcomes

J.A. Flores, J.J. Chillarón, J. Puig, M. Fernández Miró, D. Benaiges, M. Prados, J.F. Cano

Endocrinology, Hospital del Mar, Barcelona, Spain

Background and aims: Several studies in patients with type 1 diabetes have demonstrated the importance of tight glycemic control during labour on the development of neonatal hypoglycemia. Nevertheless, few studies have evaluated the importance of intrapartum glycemic control in patients with gestational diabetes $(\mathrm{GDM})$ and simple guidelines for its management are needed. The aims of our study were to evaluate metabolic control during labour using a simple protocol in women with GDM and to assess the impact of maternal factors during labour on the development of neonatal hypoglycemia.

Materials and methods: A prospective study was carried out in patients with GDM admitted for delivery between October 2006 and October 2007. A new protocol for glycemic control during labour was applied which included iv glucose and insulin infusions. Capillary blood glucose (CBG) and ketone were monitored every 2 hours. Glucose infusion was adjusted according to fasting hours and development of ketosis. Insulin infusion was started if CBG was $>130 \mathrm{mg} / \mathrm{dl}$. Ketosis was defined as ketonemia $>0,5 \mathrm{nmol} / \mathrm{l}$. Newborn management included serial CBG measurements during the first 24 hours of life. Hypoglycemia was defined as a CBG $<35 \mathrm{mg} / \mathrm{dl}$ for term babies of appropiated weight and $<20 \mathrm{mg} / \mathrm{dl}$ for preterm babies or small for gestational age (SGA).

Results: 73 women with GDM were included, with an average age of $31 \pm$ 5,3 years, a pre-gestational BMI of $27,5 \pm 5,47,1 \%$ were nuliparus, and $18 \%$ had previous GDM. 35\% required insulin treatment during pregnancy. Gestational age at delivery was $39,06 \pm 1,67$ weeks. Delivery was induced in $36 \%$ and $35 \%$ underwent caesarean section. Mean CBG during labour was 99,3
$23,6 \mathrm{mg} / \mathrm{dl}$ and $14,7 \%$ required insulin infusion (average dose 2,2ui/hour). $16,6 \%$ developed minor ketosis (mean ketonemia 0,83 $\pm 0,57 \mathrm{nmol} / \mathrm{l}$ ) which was corrected in less than four hours. There were no maternal hypoglycemic episodes. Mean newborn weight was $3372 \pm 537 \mathrm{gr}, 8 \%$ were macrosomic and $7 \%$ SGA. The incidence of neonatal hypoglycemia was 5,8\%. Development of neonatal hypoglycemia was unrelated to all maternal variables studied (mean CGB, maximal CBG, insulin use or dose, ketosis).

Conclusion: The use of a simple intrapartum management protocol achieved a satisfactory level of metabolic control, with only $15 \%$ of patients requiring insulin infusion. Further, there were no maternal hypoglycemic episodes and the incidence of minor ketosis was low. The incidence of neonatal hypoglycemia was $5,8 \%$ and it was unrelated to glycemic control during labour and to other maternal variables studied.

\section{9}

Obesity and caesarean delivery in gestational diabetes mellitus B. Barquiel, L. Herranz, P. Martín-Vaquero, I. Castro, J.A. Rosado, L.F. Pallardo

Department of Diabetes, Hospital Universitario La Paz, Madrid, Spain

Background and aims: Gestational diabetes mellitus (GDM) is associated with a higher incidence of Cesarean Delivery (CD). We aimed to assess the relative effect of obesity and glycemic control on CD in women with GDM. Materials and methods: Retrospective cohort study. A total of 1960 GDM patients were included during a follow-up period from 1987 through 2006 in the Diabetes Department. Their mean age was $33 \pm 4$ years old, their mean prepregnancy BMI $24.8 \pm 4.7 \mathrm{~kg} / \mathrm{m}^{2}$ and their mean gestational age at delivery $38.8 \pm 1.1$ weeks. Obesity was considered as a BMI $\geq 30 \mathrm{~kg} / \mathrm{m}^{2}$. Good glycemic control was defined as $\mathrm{A} 1 \mathrm{C}<5.4 \%$ during the third trimester of pregnancy (this cut-off value corresponded to the $\mathrm{p}_{70}$ of the A1C distribution of the sample). We compared the $\mathrm{CD}$ rate among non-obese and obese women, and between women with good and poor glycemic control. $\mathrm{X}^{2}$ test was used for statistical analysis. Subjects were divided by pregravid BMI into normal (BMI $<25 \mathrm{~kg} / \mathrm{m}^{2}$ ), overweight (BMI $25-30 \mathrm{~kg} / \mathrm{m}^{2}$ ) and obese subgroups. Logistic regression analysis was run to determine the risk of $\mathrm{CD}$ in overweight and obese compared to normal prepregnancy BMI. For CD, Attributable Risk Percent (AR\%) in overweight and obese women was calculated. This parameter estimates the excess of CDs that could be explained by overweight and obesity among GDM women exposed to these respective risk factors.

Results: 236 (12\%) women were obese and $584(29.8 \%)$ had poor glycemic control. Prevalence of CD was $22.1 \%$. The CD rate was higher in obese diabetic women compared to non-obese ( $32.6 \%$ vs $20.6 \%, p=0.000)$. In contrast, $\mathrm{CD}$ was no different between poor and good glycemic control groups $(23.7 \%$ vs $21.4 \%, p=0.263$ ). For the subgroups defined, we found an enhanced $C D$ risk in overweight [OR 1.61 (CI 95\%: 1.26 - 2.07)] and in obese [OR 2.03 (CI $95 \%: 1.49$ - 2.77)] compared to normal-weight women $(\mathrm{p}=0.000)$. The CD AR\% was $38.0 \%$ for being overweight and $50.8 \%$ for obesity.

Conclusion: In women with GDM, poor glycemic control is not associated with a higher $\mathrm{CD}$ rate. Pregravid BMI is confirmed as a risk factor for $\mathrm{CD}$, with an enhanced impact as prepregnancy BMI increases. In conclusion, overweight and obesity but not glycemic control could explain the higher $\mathrm{CD}$ rate in GDM.

\section{0}

Pregnancies in prediabetic women

U. Moll' ${ }^{1}$, M. Landin-Olsson ${ }^{1}$, H. Olsson ${ }^{2}$

${ }^{1}$ Department of Endocrinolgy, ${ }^{2}$ Department of Oncology, Institution of Clinical Science, Lund University, Sweden

Background and aims: Diabetes that occurs during pregnancy (gestational diabetes) can result in diabetic fetopathy, which is characterized by high birth weight and various postnatal complications. Elevated blood glucose during early pregnancy can lead to malformations and miscarriage. For the mother there is also an increased risk of developing type 2 diabetes later in life. We have studied characteristics during pregnancy in women who later in life developed diabetes.

Materials and methods: The Swedish birth registry (Medicinska födelseregistret MFR) started 1973 and contains data of the health of the mothers during pregnancy and peri- and postnatal status of the babies. In a population based large cohort study including 23524 women aged 35-75 yrs from the southern part of Sweden we have identified 802 women with diabetes. In the 
MFR we identified 30559 pregnancies related to the women in our cohort. By merging data from the Swedish birth registry and this cohort we have studied if pregnancies of diabetic women, women developing diabetes later in life (after their pregnancy) and women without diabetes differ from each other.

Results: In MFR we found that 110/30559 (0, 4\%) pregnancies of the women in the cohort was complicated by diabetes. Among the women who later developed diabetes we found that they had a higher weight $(70,2 \mathrm{vs} .63,2 \mathrm{~kg}$ $\mathrm{p}<0,001)$ at the beginning of the pregnancy and a higher weight at delivery $(82,7$, vs. $76,1 \mathrm{~kg} \mathrm{p}<0,001)$ but they had less weight-gain $(12,9$ vs. 14,1 $\mathrm{kg}, \mathrm{p}=0,005$ ) during pregnancy than non-diabetic women. The weight gain of the "prediabetic" women was negative correlated to the pre-pregnancy weight. No correlation was found in the other studygroups. The babies of the "prediabetic" women had a higher birth weight ( 3624 vs. $3506 \mathrm{~g} \mathrm{p}<0,001)$ and were more often large for gestational age, LGA. They also had a lower Apgar score after 1 minute $(8,7$ vs. $8,9 \mathrm{p}=0,001)$.

Conclusion: The pregnancies of "prediabetic" women differ regarding weight and weight gain during pregnancy. These women gave birth to larger babies, the deliveries were more complicated and the children had lower Apgar-score at 1 minute compared to non-diabetic pregnancies.

The cases of "prediabetic" pregnancies in this study are most likely undiagnosed cases of gestational diabetes, since the frequency of gestational diabetes in the study is low. Better diagnostics and better care of the women with gestational diabetes would probably improve pregnancy outcome in these cases.

\section{1}

Correlates of postpartum glucose testing after gestational diabetes mellitus in 14,486 racially/ethnically diverse women, 1999-2006 J.M. Lawrence ${ }^{1}$, J. Liu ${ }^{1}$, W. Chen ${ }^{1}$, D.A. Sacks ${ }^{2}$

${ }^{1}$ Research \& Evaluation, Kaiser Permanente, Pasadena, ${ }^{2}$ Obstetrics \& Gynecology, Kaiser Permanente, Bellflower, United States

Background and aims: While glucose testing is recommended at 6-12 weeks postpartum (PP) after gestational diabetes mellitus (GDM), data on the correlates of PP glucose testing in large diverse populations are limited. The aim of this study was to examine the prevalence and correlates of PP glucose testing from hospital discharge to 6 months PP.

Materials and methods: From 1999-2006, there were 14,486 singleton births to women with GDM based on the 100-g OGTT results using ADA criteria in a large managed care organization with GDM prevalence of 7.1-8.2/100 per year. Data were obtained from birth certificate (maternal/infant characteristics), pharmacy (GDM treatment; insulin or glyburide vs. neither), and laboratory (PP testing by fasting plasma glucose [FPG] test or a 75-g 2-hour OGTT) databases. Adjusted ORs and 95\% CI were calculated using multiple logistic regression analysis for the outcome glucose testing (yes/no).

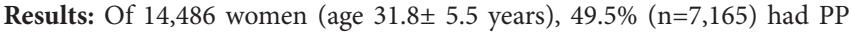
glucose testing from discharge- 6 months (87\% FPG, 13\% OGTT) and $23.4 \%$ $(\mathrm{n}=3,393)$ were tested from $6-12$ weeks PP (82\% FPG, $18 \%$ OGTT). Mean and median \# of days to testing were 53 and 42, respectively. After adjusting for the 11 delivery medical centers, age, race/ethnicity, parity, GDM treatment, any health care visit from discharge- 6 months, mode of delivery, infant birthweight (BW), and year of delivery were associated with PP testing (all $\mathrm{p}$ $<0.0001$ except BW, $\mathrm{p}<0.05)$ while maternal education, household income, and gestational age at delivery were not. Women age $34-39(3,629)$ and $\geq 40$ years $(1,137)$ were more than twice as likely to be tested as women $20-24$ years $(1,166)$; $13-19$ year olds $(186)$ were $45 \%$ less likely to be tested. Asian and Pacific Islander women $(2,790)$ were $29 \%$ more likely (aOR $1.29 ; 1.14-1.50$ ) and Hispanic women $(7,623)$ were $19 \%$ more likely (aOR $1.19 ; 1.08-1.32$ ) to be tested compared with non-Hispanic White (NHW) women $(2,982)$, while Black women (930) and other women (161) did not differ from NHWs. Compared to primiparas, women with one (aOR 0.82; 0.74-0.90) and $\geq 2$ two previous deliveries (aOR $0.67 ; 0.60-0.74$ ) were less likely to be tested. Women treated with insulin or glyburide while pregnant ( $24 \%$ of sample) were $23 \%$ more likely to be tested (aOR $1.23,1.12-1.36$ ) than those who were not. Women without a health care visit ( $25 \%$ of sample) in the 6-months after delivery were $60 \%$ less likely to be tested; those with a cesarean delivery were $16 \%$ less likely to be tested than those with a vaginal birth; and women whose infant weighed $<2500 \mathrm{~g}$ were $15 \%$ less likely to be tested than those with infants 2500-3999g; testing did not differ for mothers of macrosomic ( $>4000 \mathrm{~g}$ ) infants, which constituted $12 \%$ of these births. Women giving birth in 2006 being 3.84 (CI 3.26-4.53) times more likely to be tested than in 1999; $34 \%$ were tested in 1999 vs. $53 \%$ in 2006 . Of the women with PP glucose test results available $(7,014), 0.9 \%$ (61) had diabetes (DM) and $12.6 \%(881)$ had pre-DM based on ADA criteria.
Conclusion: Women at higher risk for DM, including minority women, older women, and those requiring pharmacological treatment for GDM, were more likely to be tested, although many were tested outside the 6-12 week window. As type $2 \mathrm{DM}$ is being diagnosed at younger ages and among women in all racial/ethnic groups, it has become increasingly important to conduct PP glucose testing to identify women with DM or pre-DM after a GDM pregnancy. Supported by: $A D A$

\section{2}

Glucose tolerance at follow up in women with previous gestational diabetes with or without metabolic syndrome

T. Prikoszovich ${ }^{1}$, G. Pacini ${ }^{2}$, A. Tura ${ }^{2}$, O. Wagner ${ }^{3}$, C. Winzer ${ }^{1}$, A. Luger ${ }^{4}$, A. Kautzky-Willer ${ }^{4}$

${ }^{1}$ Department of Internal Medicine III, Division of Nephrology and Metabolism, Medical University of Vienna, Austria, ${ }^{2}$ Institute of Biomedical Engineering, CNR, Padova, Italy, ${ }^{3}$ Department of Medical and Chemical Laboratory Diagnostics, Medical University of Vienna, Austria, ${ }^{4}$ Department of Internal Medicine III, Division of Endocrinology and Metabolism, Medical University of Vienna, Austria

Background and aims: Gestational Diabetes (GDM) is characterized by impaired glucose metabolism during pregnancy. After pregnancy these women do not completely normalise their metabolism and maintain an increased risk of later maternal Type 2 diabetes mellitus (T2DM) and vascular disease. Materials and methods: We investigated 119 women (Age: $33.76 \pm 0.73 \mathrm{yrs,}$ BMI: $27.65 \pm 0.52 \mathrm{~kg} / \mathrm{m}^{2}$ ) with a history of gestational diabetes (pGDM) 3 months after delivery with an intravenous (FSIGT) and an oral glucose tolerance test (75g-oGTT, $3 \mathrm{~h}$ ) and reinvestigated them annually up to 5 yrs post partum with an oGTT to evaluate their glucose tolerance. Furthermore parameters of endothelial dysfunction were evaluated. We defined the subgroup of women showing the metabolic syndrome (MS) according to WHO criteria early after delivery. Insulin resistance (Insulin sensitivity SI $<0.85$, hypertriglyceridemia ( $>=150 \mathrm{mg} / \mathrm{dl}$ ), low HDL-cholesterol $(<39 \mathrm{mg} / \mathrm{dl})$, high blood pressure $(>=140 / 90 \mathrm{mmHg}$ ) and impaired glucose metabolism (IFG, IGT, $\mathrm{T} 2 \mathrm{DM}$ ) as well as microalbuminuria (Albumin to creatinine ratio: $30 \mathrm{mg} / \mathrm{g}$ ) characterized the women with a MS.

Results: According to the WHO-criteria we identified 42 pGDM (35\%) with a metabolic syndrome (MS) at baseline. $90 \%$ of pGDM with MS already had impaired glucose metabolism 3 months after pregnancy, but no pGDM without MS $(\mathrm{p}<0.0001)$. Women with MS were older, had elevated concentrations of inflammation parameters (sCRP: $\mathrm{p}<0.01$, PAI- $1: \mathrm{p}<0.0001$ ) and higher levels of circulating adhesion molecules (ELAM: $\mathrm{p}=0.04$, ICAM-1: $\mathrm{p}<0.05$ ), leptin $(\mathrm{p}=0.008)$ and lower concentration of adiponectin $(\mathrm{p}<0.008)$ compared to $\mathrm{pGDM}$ without MS. pGDM with MS also developed or retained impaired glucose metabolism at the follow-up visits more frequently than those without metabolic syndrome at baseline ( $69 \%$ vs. $31 \%$; $p<0.0001)$. pGDM with MS showed a nearly 5-fold higher risk to develop diabetes or retain impaired glucose metabolism (OR: 4.9 [2.2-11.1]; $\mathrm{p}<0.0001$ ).

Conclusion: Women with a history of gestational diabetes who feature the metabolic syndrome (WHO) early after delivery are at a very high risk to develop overt diabetes or retain impaired glucose metabolism five years after delivery and to develop cardiovascular disease.

\section{3}

Postpartum reversibility of impaired incretin effect in patients with gestational diabetes mellitus

M. Kosinski ${ }^{1}$, F.K. Knop ${ }^{2,3}$, K. Cypryk ${ }^{1}$, J.J. Holst ${ }^{3}$, T. Vilsbøll ${ }^{2}$

${ }^{1}$ Department of Endocrinology and Metabolic Diseases, Polish Mother's Memorial Hospital, Lodz, Poland, ${ }^{2}$ Department of Internal Medicine, Gentofte Hospital, Copenhagen, Denmark, ${ }^{3}$ University of Copenhagen, Department of Biomedical Sciences, Denmark

Background and aims: The incretin effect accounts for approximately 50$60 \%$ of the beta-cell secretory response (as assessed by C-peptide responses) following oral ingestion of glucose in healthy individuals. The incretin effect is severely reduced in patients with diabetes mellitus (DM).

Materials and methods: To establish the reversibility of this pathophysiological phenomenon we investigated 13 women with gestational diabetes mellitus (GDM); fasting plasma glucose (FPG): 5.0 (3.6-6.4) $\mathrm{mM}$ (mean (range); $\mathrm{HbA}_{1} \mathrm{c}$ : 5.4 (5.3-5.9)\%; plasma glucose (PG) 120 min following 75-g oral glucose tolerance test (OGTT): $10.0(7.9-11.6) \mathrm{mM})$. The incretin effect 
was measured over 2 days by means of 4-hour 50-g OGTT and isoglycemic intravenous $(i v)$ glucose infusion during pregnancy (third trimester) and again 2-3 months post partum.

Results: In order to obtain isoglycemic conditions $33 \pm 1 \mathrm{~g}$ (mean \pm SEM) of glucose were infused intravenously during pregnancy as compared to $23 \pm 3$ $\mathrm{g}$ following delivery $(\mathrm{P}<0.05)$. The incretin effect $\left(100 \% \mathrm{x}\left(\mathrm{AUC}_{\mathrm{C} \text {-pep,OGTT }}{ }^{-}\right.\right.$ $\left.\left.\mathrm{AUC}_{\mathrm{C} \text {-pep,iv }}\right) / \mathrm{AUC}_{\mathrm{C} \text {-pep,OGTT }}\right)$ was reduced to 'diabetic' levels in women with GDM $(30 \pm 5 \%($ mean \pm SEM $))$ but significantly $(\mathrm{P}<0.05)$ increased to normal levels following delivery ( $54 \pm 9 \%)$.

Conclusion: Our results suggest that the reduced incretin effect in GDM is a consequence of the diabetic state and can be reversed as hyperglycemia ceases following delivery.

Supported by: Danish Medical Research Council

\section{4}

Influence of maternal obesity on insulin sensitivity and secretion in the offspring

G. Mingrone ${ }^{1}$, A. Mari ${ }^{2}$, C. Guidone ${ }^{1}$, A. Iaconelli ${ }^{1}$, L. Leccesi ${ }^{1}$, D. Gniuli ${ }^{1}$, C. Chiellini ${ }^{1}$, A. Favuzzi ${ }^{1}$, M. Manco ${ }^{3}$

${ }^{1}$ Internal Medicine, Università Cattolica S. Cuore, ${ }^{2}$ Institute of Biomedical Engineering, National Research Council, ${ }^{3}$ Liver Unit, Bambino Gesù Hospital and Reaserch Institute, Rome, Italy

Background and aims: To clarify the effects of maternal obesity on insulin sensitivity and secretion in the offspring.

Materials and methods: Fifty-one offspring of both sexes of obese $(\mathrm{Ob})$ and 15 offspring of normal weight (C) mothers were studied. Plasma glucose, insulin and C-peptide were measured during an OGTT. Insulin sensitivity was calculated using the Oral Glucose Insulin Sensitivity (OGIS) index and insulin secretion and beta-cell glucose sensitivity were computed by a mathematical model. Fasting leptin and adiponectin were also measured. Body composition was assessed by Dual-X-ray Absorptiometry.

Results: No birth weight statistical difference was observed in the two groups. Sixty-nine \% of the Ob offspring were obese and $19 \%$ were overweight. Ob were more insulin resistant than C subjects $(398.58 \pm 79.32$ vs. $513.81 \pm 70.70$ $\mathrm{ml} \cdot \mathrm{min}^{-1} \mathrm{~m}^{-2}$ in women, $\mathrm{P}<0.0001$ and $416.42 \pm 76.17$ vs. $484.242 \pm 45.76$ $\mathrm{ml} \cdot \mathrm{min}^{-1} \mathrm{~m}^{-2}$ in men $\left.\mathrm{P}<0.05\right)$. Insulin secretion after OGTT was higher in $\mathrm{Ob}$ than in $C$ men $\left(63.94 \pm 21.20\right.$ vs. $\left.35.71 \pm 10.02 \mathrm{nmol} \cdot \mathrm{m}^{-2}, \mathrm{P}<0.01\right)$, but did not differ significantly in women. Beta-cell glucose sensitivity was not statistically different between groups. A multivariate analysis of variance showed that maternal obesity and offspring gender concurred together with BMI and beta-cell glucose sensitivity to determine the differences in insulin sensitivity and secretion observed in the offspring.

Conclusion: Obese mothers can give birth to normal birth weight babies who later develop obesity and insulin resistance. The maternal genetic/epigenetic transmission shows a clear sexual dimorphism, with male having a higher value of insulin sensitivity (although not statistically significant) associated with a significantly higher insulin secretion than female offspring.

Supported by: Università Cattolica S. Cuore MIUR

\section{PS 110 Diabetic microangiopathy: pathogenetic mechanisms}

\section{5}

Inducible overexpression of sflt-1 in podocytes ameliorates glomerulopathy in diabetic mice

L. Gnudi ${ }^{1}$, C.-H. Ku ${ }^{1}$, K.E. White 2 , A. Dei Cas ${ }^{1}$, A. Hayward ${ }^{1}$, Z. Webster ${ }^{3}$, R. Bilous ${ }^{2}$, S. Marshall ${ }^{2}$, G. Viberti ${ }^{1}$

${ }^{1}$ Cardiovascular Division, King's College, London, ${ }^{2}$ Diabetes and Metabolism, University of Newcastle, ${ }^{3}$ MRC Clinical Sciences Centre, Imperial College, London, United Kingdom

Background and aims: In vivo podocyte-specific doxycycline (DOX)-inducible overexpression of the soluble VEGF receptor-1 (sFlt-1) in mice was used to gain insights into the role of VEGF-A upregulation in diabetic glomerulopathy.

Materials and methods: Experiments were conducted in non-diabetic and diabetic transgenic mice and wild type controls, treated with vehicle (VEH) or DOX for 10-weeks. Blood glucose was measured by a glucose-oxidase method and blood pressure by a non-invasive technique. sFlt-1, VEGF-A, VEGF receptor-2 (VEGFR2), and nephrin protein expression in renal cortex were determined by western immunoblotting; urine sFlt- 1 and free VEGF-A protein levels, and albuminuria by ELISA; glomerular ultrastructural morphology by electron microscopy, VEGFR2 cellular localization with immunogold techniques.

Results: Non-diabetic DOX-treated transgenic mice showed a 2-fold increased in renal cortex sFlt-1 expression and 4 -fold increase in sFlt-1 urine excretion $(\mathrm{p}<0.001)$, coupled with a $50 \%$ decrease in free urine VEGF-A and a $30 \%$ upregulation of renal cortex VEGF-A expression $(\mathrm{p}<0.04)$; albuminuria, glomerular ultrastructural morphology, and cortex VEGFR2 expression were similar among groups; VEGFR2 was localised on podocytes.

DOX-treated transgenic diabetic mice were characterized by a $60 \%$ increase in $24 \mathrm{~h}$ urine sFlt-1 excretion paralleled by a $\sim 70 \%$ decrease in urine free VEGF-A when compared to VEH-treated diabetic mice $(\mathrm{p}<0.04)$. DOXtreated diabetic animals had lower urine albumin excretion at 10-weeks than VEH-treated diabetic mice (D-VEH vs D-DOX: 117.5[69-199] vs 43[26.869] $\mu \mathrm{g} / 24 \mathrm{~h}$, geometric mean [CI], $\mathrm{p}=0.003)$. Diabetes-induced mesangial expansion, glomerular basement membrane thickening, and foot-process fusion were ameliorated in DOX-treated diabetic animals ( $\mathrm{p}<0.05$ for all). Diabetes-induced upregulation of VEGF-A and nephrin were not affected in DOX-treated mice.

Conclusion: In summary podocyte-specific sFlt-1 overexpression ameliorates diabetic glomerulopathy, presumably by modulating the autocrine/paracrine actions of VEGF-A on podocytes and other neighboring glomerular cells, and directly implicates VEGF-A in the pathogenesis of this complication. Supported by: Diabetes UK

\section{6}

Microvascular reactivity in painful and painless peripheral diabetic neuropathy

J. Doupis, T.E. Lyons, T. Dinh, A. Veves

Foot Center and Microcirculation Lab, Joslin - Beth Israel Deaconess Medical Center, Boston, United States

The pathogenesis of pain in diabetic neuropathy is not known. We investigated the differences in microvascular reactivity between DM patients with painful and painless neuropathy. We studied four groups matched for age and gender: healthy control subjects [C, $n=47$, age $54 \pm 14$ years (mean \pm sd), 21 $\mathrm{M}$ ], non-neuropathic DM patients [NND, $\mathrm{n}=97,56 \pm 13 \mathrm{yrs}, 48 \mathrm{M}$ ], patients with painless neuropathy [PDN, $\mathrm{n}=26,57 \pm 10 \mathrm{yrs}, 18 \mathrm{M}$ ] and patients with painful neuropathy [PLDN, $n=76$, age $59 \pm 9$ years, $48 \mathrm{M}$ ]. We measured the foot skin vasodilator responses to the iontophoresis of acetylcholine $(\mathrm{ACH}$, endothelium dependent) and sodium nitroprusside (SNP, endothelium independent) using a Laser Doppler Perfusion Imager and the nerve axon reflex-related vasodilation (NARV) that measures the C nociceptive fiber action using a single laser Doppler probe. The Neuropathy Disability Score was similar in PDN $(12 \pm 6)$ and PLDN $(11 \pm 7)$ but was higher comparing to NND $(1.2 \pm 1.5)$ and $C(0.3 \pm 0.8) \mathrm{p}<0.0001]$. The Neuropathy Symptom Score was similar in C $(0.2 \pm 0.5)$, NND $(1.5 \pm 1.8)$ and PDN $(1 \pm 0.9)$ but higher in PLDN $(6 \pm 2.7)(\mathrm{p}<0.0001)$. The response to $\mathrm{ACH}$-induced vasodilation was similar 
in PDN $(14.9 \pm 11.6 \%)$ and PLDN $(17.7 \pm 17.2 \%)$ and lower in both groups when compared to NND $(25.5 \pm 28.5 \%)$ and C $(25.6 \pm 20.9 \%)(\mathrm{p}<0.05)$. Similar differences were found in SNP induced vasodilation. The vibration perception threshold measurements (large myelinated fiber function) were equally elevated in both PDN $(42 \pm 11 \mathrm{~V})$ and PLDN $(34 \pm 15 \mathrm{~V})$ when compared to NND $(13 \pm 7 \mathrm{~V})$ and $C(10 \pm 7 \mathrm{~V})(\mathrm{p}<0.0001)$. Similar results were observed in Semmes-Weinstein monofilament measurements (small fiber function) [PDN (5.5 \pm 1$)$, PLDN $(5.4 \pm 1), \mathrm{NND}(3.9 \pm 0.5)$, and C (3.8 \pm 0.5$), \mathrm{p}<0.0001]$. NARV was higher in PLDN [29.6(10.6 \pm 58.5$) \%]$ (median Q1:Q3 percentiles)] when compared to PDN [8.9(-0.4:35.5)\%, $(\mathrm{p}<0.05)$. NARV was also lower in both PDN and PLDN compared to NND [50(9:118.1)\%] and C [43.6(9.2:133.3)\%] $(p<0.01)$. We conclude that both endothelium dependent and independent skin vasodilation are equally impaired in patients with painful and painless neuropathy. However, the c-nociceptive fiber action was less impaired in patients with painful neuropathy despite the fact that the function of large myelinated fibers and small A $\delta$ fibers were equally impaired.

Supported by: NIH

\section{7}

Abnormal retinal autoregulation during visual stimulation with flicker light in well-controlled type 1 diabetes without diabetic retinopathy A. Mandecka ${ }^{1}$, J. Dawczynski ${ }^{2}$, M. Blum ${ }^{3}$, N. Müller ${ }^{1}$, C. Kloos ${ }^{1}$, W. Vilser ${ }^{4}$, G. Wolf ${ }^{1}$, U.A. Müller ${ }^{1}$

${ }^{1}$ Internal Medicine III, Friedrich Schiller University Jena, ${ }^{2}$ Department of Ophthalmology, Friedrich Schiller University Jena, ${ }^{3}$ Department of Ophthalmology, Helios Klinikum, Erfurt, ${ }^{4}$ IMEDOS, Jena, Germany

Background and aims: Stimulation of the retina with flicker light increases retinal arterial and venous diameters in humans. Nitric oxide is a mediator of the retinal vasodilation to flicker light. The reduction of vasodilation is considered as a marker of endothelial dysfunction. We investigated the response of retinal vessels to flicker in type 1 diabetic patients without diabetic retinopathy (DR) and with well- controlled diabetes mellitus. Additionally the static vessel analysis was done by measuring of central retinal arterial equivalent (CRAE) und central retinal venous equivalent (CRVE).

Materials and methods: 18 normotensive patients with type 1 diabetes without retinopathy and 19 age and sex matched healthy subjects were included. DR was classified according to the Early Treatment Diabetic Retinopathy Study criteria. The diameter of retinal vessels was measured with a Dynamic Vessel Analyzer. Changes in vasodilation after stimulation with flickering light are expressed as percent change over baseline values. Parameters were compared between diabetic and control participants using the non-paired $t$ test after checking for normal distribution with the Kolmogorov-Smirnov test.

Results: $\mathrm{HbA}(1 \mathrm{c})$ was $7.5+/-1.0 \%$ and blood glucose $6.6+/-1.9 \mathrm{mmol} / \mathrm{l}$ in type 1 diabetic patients, indicating relatively good glycaemic control. In arteries, the response to flicker was significantly diminished in diabetic patients compared to healthy volunteers $(\mathrm{p}<0.023)$. In diabetic patients flicker stimulation increased arterial diameters by $+2.7 \%$ in contrast to $+4.4 \%$ in controls. The venous vasodilation was significantly reduced in diabetic patients compared to healthy controls. Venous vessel diameter increased by $+3.1 \%$ in diabetic individuals and by $+5.3 \%$ in the control group $(\mathrm{p}<0.002)$.

The static vessel analysis, showed greater central retinal artery equivalent (CRAE) in diabetic patients in comparison to controls, but the difference did not reach statistical significance $(204.7 \mu \mathrm{m}$ vs. $198.2 \mu \mathrm{m}, p=0.245)$. The central retinal vein equivalent (CRVE) did not differ in diabetic patients compared to healthy participants $(228.6 \mu \mathrm{m}$ vs. $229.2 \mu \mathrm{m}, p=0.943)$.

Conclusion: Type 1 diabetic patients without retinopathy with relatively good glycaemic control show reduced retinal vasodilation after stimulation with flicker indicating dysfunction in retinal autoregulation, likely endothelial dysfunction. There were no differences in static vessel analysis between healthy people and diabetic patients. Our study implies that the use of provocation test in conjunction with retinal vessel analysis together with the conventional methods used to detect retinopathy could lead additional information regarding abnormal retinal autoregulation.

\section{8}

Lower interphotoreceptor retinoid-binding protein is an early event in the human diabetic retina

M. García-Ramírez ${ }^{1}$, M. Villarroel ${ }^{1}$, J. Matesanz ${ }^{1}$, M.A. Alonso²,

R. Fortuna ${ }^{3}$, L. Masmiquel ${ }^{4}$, C. Hernández ${ }^{1}$, R. Simó ${ }^{1}$

${ }^{1}$ Diabetes and Metabolism Research Unit, Institut de Recerca Hospital Universitari Vall d'Hebron, Barcelona, ${ }^{2}$ Ophthalmology Department, Hospital Son Llàtzer, Palma de Mallorca, ${ }^{3}$ Biochemistry Department, Hospital Son Llàtzer, Palma de Mallorca, ${ }^{4}$ Endocrinology Division, Hospital Son Llàtzer, Palma de Mallorca, Spain

Background and aims: Interphotoreceptor retinoid-binding protein (IRBP) facilitates the transport of retinoids between the photoreceptor outer segments and retinal pigment epithelia (RPE) and, therefore, plays a major role in the visual cycle. In addition, it is essential to the maintenance of photoreceptors. A reduction in IRBP has been related to several degenerative retinal diseases in animal models. Recently we have reported a lower content of IRBP the vitreous fluid from patients with proliferative diabetic retinopathy (PDR), by means of proteomic analysis. The aims of the present study are: 1) To confirm that intravitreous levels of IRBP are decreased in PDR. 2) To determine whether the decrease in IRBP production (mRNA levels and protein content) is found in the early stages of diabetic retinopathy. 3 ) To evaluate whether the loss of IRBP expression is associated with photoreceptor damage.

Materials and methods: Samples of vitreous from 9 type 1 diabetic patients with PDR and 7 non-diabetic patients with macular hole (control group) were selected from our vitreous bank. Both groups were closely matched by age. Human postmortem eyes $(n=18)$ were obtained from 9 diabetic donors with mild non-PDR or even without clinically detectable retinopathy. Fourteen eye cups obtained from 7 non-diabetic donors matched by age were used as the control group. IRBP mRNA levels were quantified by quantitative real-time PCR (Q-RT-PCR). IRBP protein content in the neuroretina was determined by Western blot (WB) and confocal microscopy. IRBP in the vitreous fluid was assessed by WB. Neuroretinal rhodopsin content was measured by WB and confocal microscopy. The results of Q-RT-PCR and WB are expressed in arbitrary units after correction for beta-actin. Measurements of IRBP by confocal laser scanning are expressed as fluorescence intensity units.

Results: IRBP levels were significantly lower in the vitreous fluid of PDR patients in comparison with the control group $(6.29 \pm 2.48$ vs. $10.36 \pm 4.49 ; \mathrm{p}=0.04)$. Retinas from diabetic donors showed significantly lower IRBP mRNA levels $(1.15 \pm 0.93$ vs. $2.48 \pm 2.11 ; \mathrm{p}=0.04)$ and lower protein content in confocal laser scanning measurements ( $5788 \pm 4532$ vs. $10173 \pm 3459$; $\mathrm{p}=0.003$ ). IRBP levels were also lower in neuretinal extracts and in the vitreous fluid from diabetic donors than in non-diabetic donors, but the differences were not statistically significant. It should be noted that in all the retinas from diabetic donors (including those without vascular abnormalities in the preceding ophthalmologic examination) we found typical features of neurodegeneration (apoptosis and glial activation), thus proving that diabetic damage was present. Loss of IRBP production was neither associated with a significant decrease of rodopsin expression nor with a higher rate of apoptosis in the photoreceptor layer.

Conclusion: Lower production of IRBP not only exists in PDR but also in the early stage of diabetic retinopathy. At this stage, the loss of IRBP is not associated with a significant reduction of photoreceptors. The meaning and clinical consequences of low IRBP production observed in diabetic patents deserve further investigation.

\section{9}

Vitreous levels of tumour necrosis factor- $\alpha$, interleukin- 6 and interleukin- $1 \beta$ in patients with proliferative diabetic retinopathy C. Gustavsson ${ }^{1,2}$, C.-D. Agardh ${ }^{1}$, B. Beding-Barnekow ${ }^{2}$, E. Agardh ${ }^{1,2}$ ${ }^{1}$ Unit on Vascular Diabetic Complications, Clinical Sciences, ${ }^{2}$ Ophthalmology, Clinical Sciences, Malmö, Sweden

Background and aims: There is increasing evidence for an inflammatory contribution to the pathogenesis of diabetic retinopathy, with tumour necrosis factor- $\alpha$ (TNF- $\alpha$ ), interleukin-6 (IL-6) and IL-1 $\beta$ as important mediators. The purpose was to study associations between serum and intravitreal levels of these cytokines and proliferative diabetic retinopathy compared with non-diabetic patients. Materials and methods: Twenty-four patients with proliferative diabetic retinopathy (PDR) with vitreous bleeding and 27 non-diabetic subjects who underwent vitrectomy for other reasons (haemorrhage $n=1$, retinal detachment $n=16$, macular hole $n=7$, epiretinal membrane $n=2$, or cataract debris $\mathrm{n}=1$ ) were included in the study. Serum and vitreous levels of TNF- $\alpha$, IL-6 
and IL- $1 \beta$ were measured by an ELISA-mimicking method and analyzed by non-parametric testing and multivariate logistic regression analysis.

Results: Vitreous levels of TNF- $\alpha$ were high in both patient groups, although lower in PDR patients (18.0, (8.0-45.0) pg/ml and 22.0, (13.0-47.0) pg/ml, median, (min-max), respectively, $p=0.013$ ). TNF- $\alpha$ was detectable in serum from in all diabetic patients and 24/27 non-diabetic patients (88.9\%). Serum TNF levels were higher in diabetic than in non-diabetic patients (9.0, (5.0$53.0) \mathrm{pg} / \mathrm{ml}$ and $6.7,(3.0-11.0) \mathrm{pg} / \mathrm{ml}$, respectively, $p=0.001)$. Vitreous levels of IL-6 were higher in diabetic than in non-diabetic patients (173.5, (25.0$1401.0) \mathrm{pg} / \mathrm{ml}$ and $44.0,(5.0-4425.0) \mathrm{pg} / \mathrm{ml}$, respectively, $p=0.017)$, but serum levels did not differ $(p=0.982)$. IL-1 $\beta$ was undetectable in all samples. Cytokine levels were not dependent on co-morbidity or medication $(p \geq 0.180)$. Conclusion: Intravitreal cytokine levels were high both in diabetic and nondiabetic subjects with retinovascular diseases necessitating vitrectomy. The relationship between serum and intravitreal cytokine levels in proliferative diabetic retinopathy is probably complex. Comparatively higher cytokine levels in the vitreous may support an intraocular production, and TNF- $\alpha$ and IL-6 can contribute to an inflammatory process in diabetic retinopathy. Supported by: Lund University, Swedish Diabetes Federation, Järnhardt Foundation, Malmö University Hospital Foundation, Foundation for Visually Impaired in Former Malmöhus Län, Carmen and Bertil Regnér Foundation, Skane County Council for Research and Development, HemoCue

\section{0}

Glucotoxicity and lipotoxicity in kidney cells: role of oxidative and endoplasmic reticulum stress

E. Katsoulieris, J.G. Mabley, C.P.K. Chatterjee

Pharmacy and Biomolecular sciences, University of Brighton, United Kingdom

Background and aims: We are investigating glucotoxicity and lipotoxicity in vitro in NRK-52E proximal tubular cells. Our particular interest is in kidney cell killing by reactive oxygen species (ROS) and endoplasmic reticulum (ER) stress.

Materials and methods: Cytotoxicity assays used are MTT reduction, LDH release or Hoechst-propidium iodide (HPI) staining for apoptosis and necrosis determination. Reactive oxygen species were measured using nitroblue tetrazolium salt (NBT) reduction into blue formazan. ER stress was evaluated by Western blotting against the markers $\mathrm{CHOP}$ and protein chaperones glucose regulated protein 78 and 94 .

Results: Direct application of oxidative stress inducers $(0.5 \mathrm{mmol} / \mathrm{L}$ paraquat and $0.3 \mathrm{mmol} / \mathrm{L}$ hydrogen peroxide) was toxic to cells by both apoptotic and necrotic pathways. In a similar manner, high glucose and saturated fatty acid (palmitic) were toxic in a dose-dependent and time-dependent manner. Cell death due to $35 \mathrm{mmol} / \mathrm{L}$ glucose was by both necrosis and apoptosis, while $300 \mu \mathrm{mol} / \mathrm{L}$ palmitic acid caused apoptosis, predominantly. Oxidative stress (NBT reduction) due to $24 \mathrm{~h}$ treatment with palmitic acid was similar to that following $72 \mathrm{~h}$ treatment with $25 \mathrm{mmol} / \mathrm{l}$ glucose. The antioxidants EUK-134 and tempol inhibited NBT reduction due to high glucose by $30 \%$ and $60 \%$ respectively $(\mathrm{P}<0.01)$ and in case of palmitic acid, EUK-134 returned superoxide to basal levels, while reducing cell killing and ER stress protein levels. One day treatments with paraquat $(0.5 \mathrm{mmol} / \mathrm{L})$ and hydrogen peroxide $(1 \mathrm{mmol} /$ L) increased CHOP levels by $200 \%(\mathrm{P}<0.05)$ and $450 \%(\mathrm{P}<0.01)$ respectively. Treatment with high glucose $(25-35 \mathrm{mmol} / \mathrm{l})$ significantly increased GRP94 and phosphorylated CHOP, which was increased at 1, 3 and 7 days. Similarly, 24-hour treatments with palmitic acid caused significant concentrationdependent increases in both GRP78 $(135 \%, \mathrm{P}<0.05)$ and CHOP expression $(266 \%, \mathrm{P}<0.01)$.

Conclusion: We propose that rat kidney proximal tubular cells are subject to gluco and lipotoxicity. Cell death is mediated by oxidative stress, which, in part, induces ER stress.

Supported by: Diabetes UK, Kidney Research UK

\section{1}

Experimental diabetes induce changes in adenosine receptors expression and adenosine availability in rat kidney glomeruli

R. San Martin, C. Gajardo, T. Sotelo, E. Troncoso, C. Quezada, C. Escudero, L. Sobrevia

Instituto de Bioquímica, Universidad Austral de Chile, Valdivia, Chile

Background and aims: Glomerular expression and activity of vascular endothelial growth factor (VEGF) have been shown to be relevant for main- taining the filtration barrier and cellular functions, but the regulation of the pathologic VEGF over production has not been clearly recognized. Adenosine $A_{2 B}$ receptor mediates an increases in the expression and release of VEGF from glomeruli in response to high glucose concentration exposure ex vivo (Valladares et al. 2008). In contrast adenosine $A_{1}$ receptor inhibits VEGF release ex vivo. Our objectives are determine the effects of diabetes in the expression of adenosine receptors and adenosine extracellular bioavailability in rat glomeruli.

Materials and methods: Diabetes was induced in male rats (Sprague dawley) by intravenous streptozotocin injection $(65 \mathrm{mgr} / \mathrm{Kg})$. This study has been carried out along the "Principles of laboratory animal care" (NIH Publication no. 85-23, revised 1985). After 15 days post induction glomeruli were isolated by a differential sieving method. For determine extracellular adenosine contents, the glomeruli were incubated for 1 hour in tyrode buffer at $37^{\circ} \mathrm{C}$ and $5 \% \mathrm{CO}_{2}$. Adenosine concentrations were quantified in supernatants using High Performance Liquid Chromatography (HPLC). The adenosine A and $A_{2 B}$ receptors, equilibrative nucleosides transporters 1,2 and 3 (ENT1, ENT2 and ENT3), and 5'-ectonucleotidase (CD73) mRNA were quantified by real time PCR. The adenosine receptors expression was evaluated by immunohistochemistry in kidney section of rats.

Results: An increase in adenosine $\mathrm{A}_{2 \mathrm{~B}}$ receptor mRNA $(P<0.05, \mathrm{n}=5)$ and protein contents were detected in glomeruli from diabetic rats. While adenosine $A_{1}$ receptor was no changed by this experimental condition. The extracellular adenosine content in isolated glomeruli from diabetic rats was higher $(P<0.05, \mathrm{n}=3)$ than controls. The ENT1, ENT2 and ENT3 mRNA were no altered by diabetes but CD73 transcript level was increased $(P<0.05, n=5)$. Conclusion: Diabetes induces an increase in the low affinity adenosine $A_{2 B}$ receptor expression in kidney glomeruli which is accompanied for a rising in the extracellular nucleoside availability in this compartment, probably due to a higher activity of synthesis by CD73. Due to $A_{2 B}$ receptor increase the production of VEGF by podocytes, our finding may be relevant for explain a higher glomerular VEGF activity that occur as a remarkable feature at the onset of diabetic nephropathy.

Supported by: Fondecyt, DIUACHS

\section{2}

Orally administered microencapsulated lysozyme downregulates AGE formation and prevents the early stage of diabetic nephropathy

R. Candido ${ }^{1}$, B. Toffoli ${ }^{2}$, S. Bernardi ${ }^{2}$, M. Cocchietto ${ }^{3}$, L. Zorzin ${ }^{3}$, R. Carretta ${ }^{2}$, G. Sava ${ }^{3}$, B. Fabris ${ }^{2}$

${ }^{1}$ Diabetic Centre D3, A.S.S. 1 Triestina, Trieste, ${ }^{2}$ Department of Clinical Medicine and Neurology, University of Trieste, ${ }^{3}$ Callerio Foundation Onlus, Institutes of Biological Researches, Trieste, Italy

Background and aims: Diabetic nephropathy is the leading cause of endstage kidney disease in the developed countries and is correlated to chronic hyperglycaemia. An increased production and deposition of advanced glycated end-products (AGEs) is known to play a major role in the pathogenesis of diabetic kidney damage. This study was undertaken to determine if lysozyme (LZ), microencapsulated in orally administrable chitosan-coated alginate microspheres (MS), is effective in preventing the early stage of diabetic nephropathy.

Materials and methods: Four groups of Wistar rats $(n=10)$ were studied for 7 weeks: 1 . Non-diabetic control rats (C), 2. Untreated streptozotocin-induced $(50 \mathrm{mg} / \mathrm{kg})$ diabetic rats (D), 3. Diabetic rats treated orally with microencapsulated LZ (MSLZ), and 4. Diabetic rats treated with non-encapsulated LZ. Serum and kidney AGEs levels, microalbuminuria, glomerular and tubular area and receptor for AGE (RAGE) gene expression were evaluated.

Results: Microencapsulated LZ treatment significantly reduces the concentration of serum AGEs in the diabetic animals (MSLZ: $128 \pm 37 \mathrm{ng} / \mathrm{ml}$ vs D: $296 \pm 64 \mathrm{ng} / \mathrm{ml} ; \mathrm{p}<0.01)$ and their kidney deposition. Correspondingly, MSLZ significantly prevented the development of microalbuminuria as compared to untreated diabetics rats (MSLZ: $3.47 \pm 1.12 \mathrm{mg} / 24$ hours vs D: $0.95 \pm 0.54$ $\mathrm{mg} / 24$ hours; $\mathrm{p}<0.01)$. Furthermore, MSLZ significantly prevented the development of glomerular (MSLZ: $6401 \pm 591 \mu^{2}$ vs D: $7448 \pm 273 \mu \mathrm{m}^{2}$; $\mathrm{p}<0.01$ ) and renal hypertrophy (kidney-to-body weight ratio MSLZ: $8.9 \pm 0.9 \mathrm{~g} / \mathrm{mg}$ vs D: $10.5 \pm 0.8 \mathrm{~g} / \mathrm{mg} ; \mathrm{p}<0.01$ ) as well as RAGE gene overexpression (MSLZ: $1.21 \pm 0.12$ vs $\mathrm{D}: 1.89 \pm 0.26$ arbitrary units). An equidose of free LZ was less effective or totally ineffective.

Conclusion: The present study shows that orally administered LZ, and, even more, microencapsulated LZ, exerts a renoprotective effect in experimental diabetic nephropathy by inhibition of AGEs production and deposition. 


\section{PS 111 Genetics of complications}

\section{3}

Association of single nucleotide polymorphisms within intron 2 of vascular endothelial growth factor gene with early progression of retinopathy in type 1 diabetes

K. Nakanishi ${ }^{1,2}$, C. Watanabe ${ }^{1,2}$

${ }^{1}$ Dept. of General Internal Medicine and Metabolism, Toranomon Hospital, Kawasaki, ${ }^{2}$ Dept. of Metabolism, Okinaka Memorial Institute for Medical Research, Tokyo, Japan

Background and aims: Vascular endothelial growth factor-A (VEGFA) is an endothelial cell-specific mitogen and implicated in the progression to proliferative diabetic retinopathy (PDR). Association of polymorphism in VEGFA gene and retinopathy has been investigated in several studies. However, because almost all studies were designed as case-control cross-sectional study, time to the development and progression of retinopathy was not addressed. We have recently done longitudinal observation of optic fundus in the cohort of type 1 diabetes [Diabetologia 50 (Suppl. 1) S448]. Using this cohort, we evaluated the influence of multiple single nucleotide polymorphisms (SNPs) across VEGFA gene on the development and progression of retinopathy.

Materials and methods: Subjects were 175 patients with type 1 diabetes (men/women: 94/81, age at onset: $34 \pm 14$ years, mean \pm SD). Seven SNPs across VEGFA gene (rs699947 in 5' promoter region; rs833068, rs833070, and rs2146323 in intron 2; rs3025010 in intron 5; rs3025021 in intron6; rs3025035 in intron7) were genotyped using TaqMan assays. Optic fundi were evaluated longitudinally $22 \pm 18$ times during $18 \pm 9$ years. Outcomes were mild nonproliferative diabetic retinopathy (NPDR), severe NPDR, and PDR. Mean $\mathrm{HbA}_{1 c}$ value during entire illness was calculated as index of longterm glycemic control.

Results: Although cumulative incidence of mild NPDR did not differ among genotypes in any SNP, that of severe NPDR differed among genotypes in rs833070 [ $\mathrm{p}=0.037$; incidence rate (IR): $2.25,2.68$, and $4.30 / 100$ patientyears in CC $(n=93)$, CT $(n=65)$ and TT $(n=11)$ genotype] and rs2146323 [ $\mathrm{p}=0.036$; IR: $4.14,2.69,2.22 / 100$ patient-years in AA $(\mathrm{n}=12), \mathrm{AC}(\mathrm{n}=62)$, and CC $(\mathrm{n}=94)$ genotype]. Cumulative incidence of severe NPDR was higher in AA $(n=14)$ than other genotypes $(n=154)$ in $r 699947$ ( $p=0.031$; IR: 3.87 vs. $2.41 / 100$ patient-years), TT $(n=11)$ than other genotypes $(n=158)$ in rs833070 ( $\mathrm{p}=0.010$; IR: 4.30 vs. $2.44 / 100$ patient-years), AA $(\mathrm{n}=12)$ than other genotypes ( $\mathrm{n}=155)$ in $\mathrm{rs} 2146323$ ( $\mathrm{p}=0.011$; IR: 4.14 vs. $2.40 / 100$ patientyears). Although mean $\mathrm{HbA}_{1 \mathrm{c}}$ value was higher in $\mathrm{AA}(9.3 \pm 1.6 \%)$ than other genotypes $(8.1 \pm 1.2 \%, \mathrm{p}=0.015)$ in rs699947, it did not differ between TT $(9.2 \pm 1.8 \%)$ and other genotypes $(8.2 \pm 1.2 \%, \mathrm{p}=0.09)$ in rs833070, and between AA $(9.1 \pm 1.8 \%)$ and other genotypes $(8.2 \pm 1.2 \%, \mathrm{p}=0.14)$ in rs2146323. All patients who had TT in rs833070 also had AA in rs69947 and AA in rs2146323. Cumulative incidence of PDR did not differ among genotypes in any SNP. After adjusting possible confounders (sex, mean $\mathrm{HbA}_{1 c}$ value, hypertension, age and BMI at the onset) by Cox's proportional hazards model, TT genotype in rs833070 and AA genotype in rs2146323 were marginally insignificant as risk factors for severe NPDR [HR (95\%CI): 1.66 (0.93-2.73), $\mathrm{p}=0.084$; and 1.65 (0.92-2.74), $\mathrm{p}=0.088$, respectively], whereas, when mean $\mathrm{HbA}_{1 c}$ value was withdrawn from this model, TT genotype in rs833070 and AA genotype in rs2146323 were significant risk factors for severe NPDR [HR (95\%CI): 1.67 (1.01-2.54), $\mathrm{p}=0.047$; and $1.67(1.01-2.74)$, $\mathrm{p}=0.047$, respectively].

Conclusion: Our longitudinal study of optic fundus in type 1 diabetes demonstrated that SNPs in VEGFA genes, especially within intron 2, associates with early progression of retinopathy in type 1 diabetes under the influence of glycemic exposure.

\section{4}

Genetic factors have a major impact on nephropathy leading to haemodialysis than drinking alcohol, hypertension and smoking in Japanese type 2 diabetic patients M. Hattori ${ }^{1}$, H. Ueshima ${ }^{2}$, T. Okamura ${ }^{2}$, T. Fujiii ${ }^{1}$, S. Kono ${ }^{1}$, Y. Furuichi ${ }^{1}$, M. Imura ${ }^{3}$, Y. Noto, K. Koyama ${ }^{5}$, K. Yamada ${ }^{6}$, T. Oura ${ }^{7}$, K. Kaneko ${ }^{8}$, A. Shimatsu ${ }^{1}$, H. Kuzuya ${ }^{1}$, K. Kogawa ${ }^{1}$

${ }^{1}$ Diabetes, National Hospital Organization Kyoto Medical Center, Kyoto, ${ }^{2}$ Health Science, Shiga University of Medical Science, Otsu, ${ }^{3}$ Diabetes and Endocrinology, Yaizu City General Hospital, Shizuoka, ${ }^{4}$ Diabetes, Kanazawa Medical Center, Kanazawa, ${ }^{5}$ Diabetes, Tokyo Medical Center, ${ }^{6}$ Diabetes, Yamada Kennichi Naika Clinic, Tagajyo Miyagi, ${ }^{7}$ Diabetes and Kidney, Oura Clinic, Naha Okinawa, ${ }^{8}$ Medicine, Kasumigaura Medical Center, Tsuchiura, Japan

Background and aims: Diabetic nephropathy is the primary cause leading to hemodialysis in Japan. Genetic factors and daily habits (drinking alcohol, smoking cigarettes, excessive intake of salts, greasy food and carbohydrates are considered as risk factors for developing diabetic nephropathy. Our study aims to examine which factors are more influential for the development of the end stage of renal diseases resulting in hemodialysis.

Materials and methods: We recruited age- and sex-matched: group 1) 618 type 2 diabetic patients with nephropathy (273 on hemodialysis) (duration of diabetes $\geq 10 y e a r s$, presence of retinopathy and neuropathy, urine alb/cre $\geq 30 \mathrm{mg} /$ gCre, alb $\geq 30 \mathrm{mg} / 24 \mathrm{~h}$ ), group 2) 280 type 2 diabetic patients without nephropathy (duration of diabetes $\geq 10$ years, negative microalbuminuria), and group 3 ) 312 healthy subjects (absence of diabetes and kidney diseases in the past and family history). The average age was $61 \pm 12($ mean $\pm S D)$ years old. Individual subjects in the three groups signed the informed consents, answered questionnaires on daily habits and provided blood samples for genetic analysis. We performed the whole genome mapping on using microsatellite markers at $1 \sim 20 \mathrm{cM}$ intervals. The data were evaluated by analysis of variance among the three groups, and multivariate logistic analysis for relative risk for hemodialysis, and Chi-square test for association study of the genetic mapping analysis.

Results: The analysis of variance among the three groups showed that blood pressure (both systolic and diastolic), the number of smoking cigarettes and alcohol consumption were risk factors for diabetic nephropathy in addition to hyperglycemia. The genome scanning revealed that five markers were associated with diabetic nephropathy. Of the five, marker D showed a prominent significance in association with diabetic nephropathy $\left(P=10^{-16}\right)$. In the multivariate logistic analysis of the marker $\mathrm{D}$ and the above risk factors for hemodialysis in the type 2 diabetic patients (vs. healthy control subjects), the relative risk was 8.51 for marker D-homozygote $(P=0.000$, $95 \%$ Confidence Interval: 3.63 to 19.97$), 6.63$ for marker D-heterozygote $(P=0.000,95 \% \mathrm{CI}$ : 3.57 to 12.32$), 1.01$ for drinking $1 \mathrm{~g}$ of alcohol ( $P=0.006,95 \% \mathrm{CI}: 1.00$ to 1.03$)$, 1.01 for smoking 1 cigarette/day ( $P=0.665,95 \%$ CI: 0.98 to 1.03$), 1.07$ for 2 $\mathrm{mmHg}$ increase of systolic blood pressure $(P=0.000,95 \% \mathrm{CI}$ : 1.05 to 1.09$)$, and 0.87 for BMI ( $P=0.009,95 \% \mathrm{CI}: 0.79$ to 0.97$)$. Age and sex difference were insignificant factors. The total cholesterol and HDL-C levels remained unclear for risk factors in the present study.

Conclusion: Genetic factors have a major impact on diabetic nephropathy leading to hemodialysis in Japanese type 2 diabetic patients despite a significant contribution of daily habits, especially drinking alcohol.

Supported by: Millennium Projects of the Ministry of Education and Science of Japan, National Hospital Organisation of Japan

\section{5}

Contribution of PARP-1 polymorphisms in nephropathy in type 2 diabetic patients

S. Hadjadj ${ }^{1}$, X. Piguel ${ }^{1}$, G. Guilloteau ${ }^{2}$, D. Dardari ${ }^{3}$, B. Guerci ${ }^{4}$, M. Matta ${ }^{5}$, J.-M. Halimi ${ }^{6}$, O. Verier-Mine ${ }^{7}$, R. Roussel ${ }^{8}$, S. Leroux ${ }^{9}$, F. Bridoux ${ }^{10}$, M. Marre ${ }^{8}$, R. Marechaud ${ }^{1}$

${ }^{1}$ Endocrinology-Diabetology, CHU Poitiers, ${ }^{2}$ Endocrinology-Diabetology, CHU Angers, ${ }^{3}$ Endocrinology-Diabetology, $\mathrm{CH}$ Sud Francilien, Corbeil Essonnes, ${ }^{4}$ Diabetology - Nutrition, CHU Nancy, ${ }^{5}$ EndocrinologyDiabetology, CHU Toulouse, ${ }^{6}$ Nephrology, CHU Tours, ${ }^{7}$ EndocrinologyDiabetology, CHR Valenciennes, ${ }^{8}$ Endocrinology-Diabetology, CHU Bichat AP-HP, Paris, ${ }^{9}$ Inserm u927, CHU Poitiers, ${ }^{10}$ Nephrology, CHU Poitiers, France

Background and aims: Poly (ADP) ribose polymerase (PARP-1) is a key enzyme in diabetic complications activated by oxidative stress-induced DNA 
damage. We hypothesised that PARP-1 polymorphism is linked to the presence of diabetic nephropathy in patients with type 2 diabetes.

Materials and methods: We studied 3 different SNP, tagging the haplotypic structure of the PARP-1 gene. The genetic variants were studied according to the presence/absence of diabetic nephropathy in a case-control design. Setting: French multicentre collaborative study (15 diabetics clinic and 5 nephrology clinics). Patients: Diabetic nephropathy status was classified by an adjudication committee: nephropathic cases had retinopathy associated with microalbuminuria or proteinuria, regardless estimated glomerular filtration rate (eGFR), non nephropathic controls had normoalbuminuria assessed in the absence of ACE-I or ARB prescription, with eGFR higher than or equal to $30 \mathrm{ml} / \mathrm{min}$, and either diabetic retinopathy regardless diabetes duration or long-term diabetes duration (20 years or more).

Results: A total of 1200 caucasian patients were included: 797 cases $(528$ men/279 women) and 403 non-nephropathic controls (221 men/182 women). The PARP-1 genetic variants were in Hardy-Weinberg equilibrium. The association between PARP-1 polymorphism and diabetic nephropathy is summarized in table regarding genotypic and haplotypic analyses.

Conclusion: PARP-1 genetic variants seem to play a weak role in the genetic susceptibility to diabetic nephropathy in type 2 diabetic people.

PARP-1 polymorphisms and diabetic nephropathy

\begin{tabular}{|c|c|c|c|c|c|}
\hline genetic variants & cases & controls & $\mathrm{p}$ & $\begin{array}{l}\mathrm{p} \text { (domi- } \\
\text { nant) }\end{array}$ & $\begin{array}{l}\mathrm{p} \text { (reces- } \\
\text { sive) }\end{array}$ \\
\hline $\begin{array}{l}\text { rs } 8679 \\
\text { (AA/AG/GG): n }\end{array}$ & $765 / 285 / 41$ & $209 / 165 / 25$ & 0.107 & 0.441 & 0.031 \\
\hline $\begin{array}{l}\text { rs } 747658 \\
\text { (AA/AG/GG): n }\end{array}$ & $605 / 243 / 26$ & $287 / 124 / 13$ & 0.854 & 0.928 & 0.576 \\
\hline $\begin{array}{l}\text { rs } 3219090 \\
\text { (CC/CT/TT): n }\end{array}$ & $370 / 343 / 79$ & $189 / 182 / 28$ & 0.231 & 0.092 & 0.832 \\
\hline haplotype freque & & & $\begin{array}{l}\text { Odds-ratio } \\
(95 \% \mathrm{CI})\end{array}$ & $\mathrm{p}$ value & \\
\hline haplotype AAC & 0.443 & 0.427 & 1 & & \\
\hline haplotype GAC & 0.231 & 0.269 & $\begin{array}{l}0.809 \\
(0.650-1.006) \\
\end{array}$ & 0.056 & \\
\hline haplotype AGT & 0.156 & 0.165 & $\begin{array}{l}0.898 \\
(0.698-1.156)\end{array}$ & 0.404 & \\
\hline haplotype AAT & 0.158 & 0.132 & $\begin{array}{l}1.135 \\
(0.864-1.490)\end{array}$ & 0.362 & \\
\hline
\end{tabular}

Supported by: AFD Recherche, PHRC 2004 (French Ministry of Health), GEMMS Poitiers

\section{6}

The Q121 variant of ENPP1 (PC-1) gene is associated with chronic kidney disease among patients with type 2 diabetes

S. De Cosmo ${ }^{1}$, A. Minenna ${ }^{1}$, Y.-Y. Zhang ${ }^{2}$, R. Thompson ${ }^{2}$, G. Miscio' ${ }^{1}$, M. Vedovato ${ }^{3}$, A. Rauseo ${ }^{1}$, A. Saller ${ }^{4}$, S. Mastroianno ${ }^{1}$, F. Pellegrini ${ }^{5}$, R. Trevisan ${ }^{6}$, P. Fioretto ${ }^{4}$, A. Doria ${ }^{2}$, V. Trischitta ${ }^{1,7}$

${ }^{1}$ Endocrinology, Scientific Institute "Casa Sollievo della Sofferenza", San Giovanni Rotondo (FG), Italy, ${ }^{2}$ Research Division, Joslin Diabetes Center, Boston, United States, ${ }^{3}$ Chair of Metabolic Diseases, University of Padova, Italy, ${ }^{4}$ Department of Medical and Surgical Sciences, University of Padova, Italy, ${ }^{5}$ Department of Clinical Pharmacology and Epidemiology, Consorzio Mario Negri Sud, S. Maria Imbaro, Italy, ${ }^{6}$ Unit of Diabetes, Ospedali Riunit di Bergamo, Italy, ${ }^{7}$ Department of Clinical Sciences, "Sapienza" University, Rome, Italy

Background and aims: Insulin resistance plays a role in diabetic kidney complications. The ENPP1 K121Q polymorphism has been repeatedly associated with insulin resistance, type 2 diabetes (T2D) and related vascular complications. This study investigated whether the ENPP1 Q121 variant is associated with chronic kidney disease (CKD) in T2D

Materials and methods: Three sets of patients with T2D were studied: one from Gargano (360M/324F), one from Boston (M361/F222) and one from Padua (M100/F25). In all sets glomerular filtration rate (GFR) was estimated (e-GFR) with the abbreviated MDRD formula

Results: In the Gargano and Boston populations, according to dominant model of inheritance, Q121 carriers (i.e. individual with either KQ or QQ genotype) had an increased risk of having CKD: ORs=1.69 (95\%CI 1.1-2.6) and 1.50 (95\% CI 1.0-2.2), respectively. In the Padua set the association was in the same direction though not reaching a formal statistical significance
$(\mathrm{OR}=1.77,95 \% \mathrm{CI} 0.7-4.5)$. When the three studies were pooled, the risk of $\mathrm{CKD}$ in $\mathrm{Q} 121$ carriers was highly significant $(\mathrm{OR}=1.58,95 \% \mathrm{CI} 1.2-2.1)$. Also pooled mean differences of absolute GFR values were different across genotype groups with Q121 carriers showing lower GFR, as compared to KK individuals ( $74 \pm 20$ vs $71 \pm 21 \mathrm{ml} / \mathrm{min} / 1.73 \mathrm{~m}^{2} \mathrm{KK}$ vs Q121, respectively; $\mathrm{p}=0.04$ ). Conclusion: Our data indicate that patients with T2D carrying the ENPP1 Q121 variant are at increased risk of CKD. If this finding is confirmed in prospective studies, the Q121 variant might be useful to identify patients at high risk of developing renal dysfunction, who can be targeted with specific preventive and therapeutic strategies.

\section{7}

The influence of the human leukocyte antigen locus on the development, progression, and mortality of diabetic nephropathy

J.M. Söderlund ${ }^{1,2}$, J. Ilonen ${ }^{3,4}$, C. Forsblom ${ }^{1,2}$, O. Heikkilä ${ }^{1,2}$, K. Hietala ${ }^{1,2}$, M. Parkkonen ${ }^{1,2}$, M. Rosengård-Bärlund ${ }^{1,2}$, M. Saraheimo ${ }^{1,2}$, L. Thorn ${ }^{1,2}$, J. Wadén ${ }^{1,2}$, P.-H. Groop ${ }^{1,2}$

${ }^{1}$ Department of Diabetes Genetics, Folkhälsan Research Center, Helsinki, ${ }^{2}$ Department of Medicine, Helsinki University Central Hospital, ${ }^{3}$ Department of Clinical Microbiology, University of Kuopio, ${ }^{4}$ Immunogenetics Laboratory, University of Turku, Finland

Background and aims: The human leukocyte antigen (HLA) class II locus on chromosome 6p21.3 is a known susceptibility locus for type 1 diabetes (T1D) and other autoimmune diseases. However, its potential role in the development and progression of diabetic nephropathy (DN) is still poorly understood. The locus contains numerous genes with multiple alleles and recently there have been suggestions that HLA DRB1*04 alleles would protect from nephropathy.

Materials and methods: 1935 well-characterised Finnish patients with T1D were grouped into patients with normoalbuminuria $(n=674)$, microalbuminuria $(n=459)$, macroalbuminuria $(n=567)$, and end-stage renal disease (ESRD; $\mathrm{n}=235$ ) based on their urinary albumin excretion rate in at least two out of three timed urine collections. Of the 1935 patients there were 1434 with prospective data and of these 206 progressed. The HLA genotyping was performed with commercial kits and haplotypes were constructed using known linkage disequilibrium patterns. The statistical analyses were performed with SPSS 13.0 using $\chi^{2}$ tests for categorical variables and Kaplan-Meier and/or Cox-regression for progression and survival analyses.

Results: We identified 26 HLA haplotypes and 101 different HLA genotypes. The most common genotype was the T1D susceptibility genotype (DR3)-DQA1 ${ }^{*} 05-\mathrm{DQB} 1{ }^{*} 02 / \mathrm{DRB} 1^{*} 0401-\mathrm{DQB1}{ }^{*} 0302$ with more than $20 \%$ of patients carrying it, however $80.9 \%(n=1565)$ of the patients carried one of the two haplotypes. In cross-sectional analyses (DR11/12/13)-DQA1*05$\mathrm{DQB1} 1^{*} 0301$ was enriched in patients without $\mathrm{DN}(58.8 \%$ vs. $41.5 \%, \mathrm{p}=0.056$, $\mathrm{n}=53$ ). The uncommon (DR7)-DQA1*0201-DQB1 ${ }^{*} 02$ haplotype was enriched in macroalbuminuric patients compared to ESRD patients ( $86.8 \%$ vs. $13.2 \%, \mathrm{p}=0.025, \mathrm{n}=38$ ). (DR3)-DQA1 ${ }^{*} 05-\mathrm{DQBB}{ }^{*} 02$ was found to be protective against the progression from macroalbuminuria to $\operatorname{ESRD}(\mathrm{p}=0.01)$. Patients with at least one copy of the DRB1*0401-DQB1 ${ }^{*} 0302$ haplotype showed a trend for enhanced survival compared to patients without this haplotype. There were, however, no trends for the progression of kidney disease with DRB1*0401-DQB1*0302.

Conclusion: We found suggestive enrichment of certain HLA haplotypes regarding renal status. The effect of HLA haplotypes was more evident on the progression of DN as well as on patient survival. Interestingly, the haplotypes which showed a trend for enhanced survival or protection against the progression of DN, were the two major risk haplotypes for developing T1D.

\section{8}

Haptoglobin genotype and renal disease progression in type 1 diabetes T. Costacou ${ }^{1}$, R.E. Ferrell ${ }^{2}$, T.J. Orchard ${ }^{1}$

${ }^{1}$ Department of Epidemiology, ${ }^{2}$ Department of Human Genetics, University of Pittsburgh, United States

Background and aims: Haptoglobin (Hp) is a plasma protein that binds free hemoglobin, thereby inhibiting hemoglobin-induced oxidative damage. Hp genotype influences the risk of vascular complications in type 2 diabetes and we have recently demonstrated an association with cardiovascular disease among individuals with childhood-onset type 1 diabetes. Here, we investigated whether Hp genotype is associated with renal disease progression in type 1 diabetes. 
Materials and methods: Participants from the Epidemiology of Diabetes Complications Study with DNA available were studied for the incidence of microalbuminuria (MA), overt nephropathy $(\mathrm{ON})$, end-stage renal disease (ESRD) and low estimated glomerular filtration rate (eGFR). MA was defined as albumin excretion rate $>20 \mu \mathrm{g} / \mathrm{min}, \mathrm{ON}$ as albumin excretion rate $>200 \mu \mathrm{g} /$ min in multiple timed urine specimen, ESRD as renal dialysis or transplantation, and low eGFR as $<60 \mathrm{~mL} / \mathrm{min} / 1.73 \mathrm{~m}^{2}$ by the Cockcroft-Gault equation. Results: The proportions of the cohort with the Hp 2/2, 2/1 and 1/1 genotypes were $47.2 \%, 41.3 \%$ and $11.5 \%$, respectively. During 18 years of followup, there were 111 incident MA cases (41.1\%), 61 incident ON cases (16.8\%), 59 incident ESRD cases (12.4\%), and 82 incident cases of low eGFR (16.2\%). Univariately, no significant differences were observed in the proportion of cases by Hp genotype for MA and ON. However, the proportion of events was higher among those with the Hp 2/2 compared to Hp 2/1 or $1 / 1$ for ESRD ( 17.0 vs. 7.7 and $10.5 \%$, respectively, $\mathrm{p}=0.01$ ). Cumulative incidence (including 9 prevalent cases) was also higher in the $\mathrm{Hp} 2 / 2$ (19.1\%) than the Hp $2 / 1(8.6 \%)$ or Hp $1 / 1(12.1 \%)(\mathrm{p}=0.007)$. In multivariable Cox proportional hazard models, adjusting for known predictors in this population (diabetes duration, HbA1c, lipids, hypertension, eGFR) the Hp 2/2 genotype remained associated with increased ESRD incidence compared to the Hp1/1 genotype $(\mathrm{HR}=3.53,95 \% \mathrm{CI}=1.41-8.82)$ whereas the Hp $2 / 1$ genotype risk, though increased, did not reach statistical significance ( $\mathrm{HR}=2.37,95 \% \mathrm{CI}=0.88-6.40)$. Associations were similar for progression to ESRD from MA (HR for Hp 2/2 to $1 / 1=3.29,95 \% \mathrm{CI}=1.34-8.09$ ) and to ESRD from ON (HR for Hp $2 / 2$ to $1 / 1=3.53,95 \% \mathrm{CI}=1.43-8.72$ ), as well as for the incidence of low eGFR (HR for $\mathrm{Hp} 2 / 2$ to $1 / 1=2.66,95 \% \mathrm{CI}=1.38-5.13)$. However, the Hp genotype was not a determinant of either MA (HR for Hp $2 / 2$ versus Hp 1/1=1.31, 95\% $\mathrm{CI}=0.58-2.95$ ) or ON (HR for $\mathrm{Hp} 2 / 2$ versus $\mathrm{Hp} 1 / 1=0.72,95 \% \mathrm{CI}=0.33-1.58$ ) incidence in multivariable models. Adjustment for cardiovascular disease status did not alter these findings.

Conclusion: These data suggest that although Hp genotype is not related in this cohort of type 1 diabetes to the incidence of renal damage (micro- or macroalbuminuria), contrary to findings in type 2 diabetes, it may be a determinant of worsening of renal function (eGFR) and progression to ESRD. Supported by: NIH

\section{9}

Implication of superoxide dismutase 1 gene in the type 1 diabetic nephropathy: the SURGENE Study

K. Mohammedi ${ }^{1,2}$, N. Bellilii ${ }^{2}$, S. Maimaitiming ${ }^{2}$, C. Abi Khalii ${ }^{1,2}$, F. Travert ${ }^{1}$, N. Emery ${ }^{2}$, F. Fumeron ${ }^{2}$, G. Velho ${ }^{2}$, R. Roussel ${ }^{1,2}$, M. Marre ${ }^{1,2}$

${ }^{1}$ Endocrinologie Diabétologie et Nutrition, Assistance Publique des Hôpitaux de Paris, ${ }^{2}$ Xavier Bichat School of Medicine, INSERM, U695, Paris, France,

Background and aims: Oxidative stress has been involved in the pathophysiology of diabetic nephropathy. Antioxidant enzymes counteract oxidative stress. Several polymorphisms in antioxidant enzymes genes have been associated with diabetic nephropathy. In the DCCT/EDIC study, rs17880135 polymorphism of the superoxide dismutase 1 (SOD1) gene was associated with persistent microalbuminuria and severe nephropathy. We studied the impact of rs17880135 in SOD1 gene on the development and progression of diabetic nephropathy.

Materials and methods: We studied 310 type 1 diabetic (T1D) subjects included in the Survival Genetic Nephropathy (SURGENE), a prospective observational study designed to analyse the genetic determinants of diabetic nephropathy. At inclusion, there were $56 \%$ of males and $44 \%$ of females, mean age was 34 years, $81 \%$ were normoalbuminuric. Patients were compared to 310 non-diabetic age- and sex - matched subjects. The genotypes for rs17880135 were determined by the Taqman process. Renal events were defined as a change to the next nephropathy stage (absence, microalbuminuria, macroalbuminuria, renal failure, end-stage renal failure). We tested the association of genetic variations with albuminuria, hemodynamic parameters and renal events.

Results: Minor allele frequency was $11 \%$ for cases and $8 \%$ for controls $(\mathrm{p}=0.27)$. Genotypes frequencies were in Hardy-Weinberg equilibrium. In T1D subjects, 53 renal events occurred during follow-up. There was no significant association between this polymorphism and albuminuria (TT $64.5 \pm 12.6$ vs $\mathrm{G}+29.9 \pm 26.1 \mathrm{mg} / \mathrm{l}, \mathrm{p}=0.45$ ), blood pressures or progression of nephropathy (renal events). In healthy controls, allele $\mathrm{G}$ carriers had higher SBP levels $(\mathrm{G}+136.8 \pm 2.5 \mathrm{mmHg}$, TT $129.3 \pm 1.0, \mathrm{p}=0.04)$. In the pooled populations, rs17880135 $\mathrm{G}$ allele carriers had higher risk for hypertension [OR=2.29 (1.14-4.57), $\mathrm{p}=0.02]$.
Conclusion: rs17880135 polymorphism in SOD1 gene was not associated with type 1 diabetic nephropathy in SURGENE study. However, this gene could be a candidate gene for hypertension.

\section{0}

ADMA and chronic renal failure in type 2 diabetic subjects: role of DDAH -1151 A/C polymorphism

I. Testa ${ }^{1}$, M. Marra ${ }^{1}$, A. Ceriello ${ }^{1}$, F. Marchegiani ${ }^{2}$, C. Sirolla ${ }^{3}$, M. Boemi ${ }^{1}$, A.R. Bonfigli ${ }^{1}$, M. Cucchi ${ }^{1}$, R. Testa ${ }^{1}$

${ }^{1}$ Diabetology Unit, ${ }^{2}$ Center of Molecular Biology and Genetics, ${ }^{3}$ Center of Epidemiology and Biostatistic, INRCA, Ancona, Italy

Background and aims: To investigate whether the genetic polymorphism of Dimethylarginine Dimethylamino hydrolase (DDAH2) gene at the position $-1151 \mathrm{~A} / \mathrm{C}$, an enzyme which degrades asymmetric dimethylarginine (ADMA) to citrulline and dimethylamine, regulates ADMA levels in type 2 diabetic patients (T2DM) with chronic renal failure.

Materials and methods: Two groups of carefully selected subjects of both sexes were enrolled and compared. The first group (control group) comprised 120 T2DM subjects without complications (mean age 70.0 \pm 8.0 years) whereas the second (CRF group) comprised $50 \mathrm{~T} 2 \mathrm{DM}$ patients (mean age 75.2 \pm 7.0 years) with chronic renal failure. The -1151 A/C DDAH2 promoter polymorphism was determined by a polymerase chain reaction-restriction fragment length polymorphism approach.

Results: Significant differences between control and CRF group in plasma levels of ADMA $(0.35 \times / \div 1.86$ versus $0.42 \times / \div 1.94 \mu \mathrm{mol} / \mathrm{l}, \mathrm{p}=0.048$, respectively), symmetric dimethylarginine (SDMA) $(0.32 \times / \div 1.62$ versus $0.42 \times / \div 1.81$ $\mu \mathrm{mol} / \mathrm{l} \mathrm{p}=0.002$, respectively) was found. Whereas no differences in L-arginine plasma levels was found. No differences in the frequency of DDAH2 $1151 \mathrm{~A} / \mathrm{C}$ polymorphism between groups was found. Analysis of variance showed that ADMA plasma levels was only dependent on DDAH2 $1151 \mathrm{~A} /$ $\mathrm{C}$ polymorphism $(\mathrm{F}=3.15 ; \mathrm{p}=0.04)$ and no interaction between groups and DDAH2 $1151 \mathrm{~A} / \mathrm{C}$ polymorphism was found $(\mathrm{F}=0.35 ; \mathrm{p}=0.70)$.

Conclusion: The finding that DDAH2 1151 polymorphism regulates ADMA levels in type 2 diabetic patients with chronic renal failure allow to open a new insight on the pathway involving ADMA/DDAH/Nitric Oxide Synthase in the development and progression of chronic renal failure in type 2 diabetic patients. Determination of the DDAH2 $1151 \mathrm{~A} / \mathrm{C}$ polymorphism may help to identify those individuals at high risk to develop cardiovascular diseases at an early stage, suggesting a close monitoring of cardiovascular risk factors before the onset of cardiovascular disease.

\section{1}

Paraoxonase genotypes and activity and oxidized LDL in type 2 diabetes mellitus: relationship with vascular complications

D. Lucchesi ${ }^{1}$, L. Pucci ${ }^{1}$, V. Longo ${ }^{2}$, S. Triscornia ${ }^{1}$, C. Fotino ${ }^{1}$, G. Penno ${ }^{1}$, S. Del Prato ${ }^{1}$, R. Miccoli ${ }^{1}$

${ }^{1}$ Endocrinology and Metabolism, University of Pisa, ${ }^{2}$ Clinical Physiology Institute, National Research Council, Pisa, Italy

Background and aims: Atherogenic dyslipidemia and the unbalance between oxidative and anti-oxidative mechanisms in lipoprotein metabolism might be major determinants not only in the development of atherosclerosis, but also in the induction and progression of microvascular damage in diabetes mellitus.

Materials and methods: Serum enzymatic activity and Q192R and L55M gene polymorphisms of paraoxonase-1 (PON1), an esterase associated with apolipoprotein A-I (apoA-I) to which is largely attributed the antioxidant capacity of HDL, have been assessed in 306 type 2 diabetic subjects to investigate the relationship, eventually mediated through circulating oxidized LDL (oxLDL) concentrations, with vascular complications.

Results: PON1 activity, slightly lower in diabetic subjects than in 100 controls $(66.1+/-41.3$ vs $76.4+/-40.0 \mathrm{nmol} / \mathrm{ml} / \mathrm{min} ; \mathrm{p}=0.03)$, was strongly affected by both gene variants, but also significantly modulated by apoA-I and HbA1c levels. Neither PON1 activity nor genotypes or their combination contributed to circulating oxLDL variability. OxLDL/apoB ratio $(0.56+/-0.27$ vs $0.51+/-0.18, \mathrm{p}=0.036$ ), an index of the proportion of oxidatively modified LDL, was higher in diabetics with cardiovascular disease (CVD) when compared with those with no CVD. No differences emerged in PON1 activity and genotypes distributions in subjects with CVD when compared with those without CVD. OxLDL and oxLDL/apoB ratio were also higher 
in subjects with microalbuminuria or overt nephropathy and oxLDL/apoB ratio was increased in subjects with retinopathy when compared with those without microvascular complications. PON1 genotypes were equally distributed in subjects with and without retinopathy, while the LL genotype (L55M) resulted more frequent in those with raised albuminuria $(\mathrm{p}=0.007)$. Systolic blood pressure (sBP, $p=0.002), \mathrm{HbAlc}(\mathrm{p}=0.01)$, apoB $(\mathrm{p}=0.02)$, LL genotype $(\mathrm{p}=0.002)$, and mainly oxLDL $(\mathrm{p}<0.0001)$ independently affected nephropathy. Duration of diabetes $(p<0.0001)$, HbAlc $(p=0.006), s B P(p=0.003)$, but also oxLDL $(\mathrm{p}=0.01)$ contributed independently to retinopathy.

Conclusion: In subjects with type 2 diabetes mellitus, gene polymorphisms in the coding and regulatory regions of PON1 largely affect serum activity of the enzyme but show no relationship with CVD or retinopathy, while the weak direct association of L55M variant with diabetic renal damage is of uncertain relevance. Our study does not support a causal relationship between PON1 activity and oxidative modification of circulating LDL. However, high oxLDL levels are strongly associated with albuminuria and with retinal lesions suggesting that oxLDL might play an independent role in initiation and progression of microvascular damage, mainly of nephropathy.

\section{PS 112 Diabetic retinopathy screening: clinical aspects}

\section{2}

English national screening programme for diabetic retinopathy F.K.M. O'Leary, I.M. Stratton, D.M. Prentis, P.H. Scanlon National Screening Programme for Diabetic Retinopathy, Cheltenham, United Kingdom

Background and aims: The aim of the National Programme is to set up a screening system for two million people with diabetes in England. The purpose is to reduce the risk of vision loss by the prompt identification (and effective treatment if necessary) of sight threatening diabetic retinopathy at the appropriate stage during the disease process.

Materials and methods: The delivery of systematic care is done by 101 local programmes which identify patients and call them in for 2 field retinal photography with mydriasis. Grading is carried out and all image sets with pathology and $10 \%$ of normal image sets are at least double graded, with arbitration if needed. The National Programme organises quality assurance on these programmes and sets national standards by which performance is assessed. All programmes should submit an annual report using a standard pro forma to improve consistency of data collection. A national grading standard has been approved and it is now mandatory for software to support this.

Results: Initial data from 74 programmes have been received. Size of each programme is 17595 (12262 to 23589$)$ median $\left(25^{\text {th }}\right.$ to $75^{\text {th }}$ centile). The proportion of eligible people invited (target $80 \%$ ) is $70.5 \%$ (43.8\% to $86.7 \%$ ). The proportion of people with no discernable retinopathy was $69.3 \%$ (62.6\% to $76.3 \%$ ). Backgroound retinopathy was seen in $25.1 \%$ (18.3 to $30.9 \%$ ) of people and preproliferative and proliferative retinopathy in $4.6 \%$ (3.2\% to $7.4 \%)$ Conclusion: Despite careful drafting of grading criteria there is considerable variation in interpretation and application. Some of the variation may be due to differences in the populations screened, however it is likely that some of the variability is due to variation in the implementation of the grading criteria.

Initial data from 74 local programmes, grading data available from 66

\begin{tabular}{llll}
\hline & Median & 25th centile & 75th centile \\
\hline People with diabetes & 17595 & 12263 & 23590 \\
\hline Proportion invited to screening & $70.5 \%$ & $43.8 \%$ & $86.7 \%$ \\
\hline Inappropriate referrals & $0.6 \%$ & $0.0 \%$ & $2.0 \%$ \\
\hline \% initial screens & $56.5 \%$ & $25.8 \%$ & $99.0 \%$ \\
\hline No retinopathy & $69.3 \%$ & $62.6 \%$ & $76.3 \%$ \\
\hline Background retinopathy & $25.1 \%$ & $18.3 \%$ & $30.9 \%$ \\
\hline $\begin{array}{l}\text { Preproliferative and proliferative } \\
\text { retinopathy }\end{array}$ & $4.6 \%$ & $3.2 \%$ & $7.4 \%$ \\
\hline Assessable image sets & $96.3 \%$ & $90.2 \%$ & $97.5 \%$ \\
\hline Results notified within 6 weeks & $77.5 \%$ & $38.0 \%$ & $90.0 \%$ \\
\hline Cataract referrals & $8.2 \%$ & $0.0 \%$ & $14.4 \%$ \\
\hline
\end{tabular}

\section{3}

A multi-disciplinary team meeting as part of a diabetes eye screening programme: effects on referral to hospital eye clinics C. Frickey ${ }^{1}$, B. Jennings ${ }^{2}$, L. Lacey ${ }^{3}$, W. Gatling ${ }^{1,3}$, D.V. Coppini ${ }^{1}$, M.G. Masding ${ }^{1,3}$

${ }^{1}$ Diabetes Centre, Poole Hospital NHS Foundation Trust, ${ }^{2}$ Medical Photography, Poole Hospital NHS Foundation Trust, ${ }^{3}$ Dorset Diabetic Eye Screening Programme, Dorset Primary Care Trust, Ferndown, United Kingdom

Background: Our county has a well-established optometrist-based screening programme for diabetic eye disease, previously using slit-lamp biomicroscopy. We have recently implemented an optometrist-based programme using mydriatic dual-view digital fundal photography. After initial capture and grading by optometrists and trained retinal screeners, the images of patients graded as having significant diabetic eye disease are discussed at hour-long weekly multi-disciplinary team (MDT) meetings in each of the secondary care centres involved in the programme, involving ophthalmologists, diabetologists and retinal screeners. The MDT options are for onward referral to the hospital ophthalmology department, recall to a specialist hospital diabe- 
tes eye clinic involving ophthalmology and diabetology review, or return to the screening programme. We have reviewed the outcomes of our local MDT to assess referral rates for hospital care.

Material and methods: Records were kept of patients reviewed in the MDT between 1 February 2007 and 31 January 2008, and the outcome of that review.

Results: Of 11,600 patients screened in the Poole area, 516 patient images (4.4\%) were reviewed in the MDT, with 276 (2.4\%) of these eventually requiring hospital care. Of the patients reviewed in the MDT, 114 (22.1\%) were referred for direct ophthalmology care (including laser treatment), 162 (31.4\%) were recalled to specialist diabetes eye clinics, and 240 (46.5\%) were referred back to the screening programme for digital photography by either optometrists or retinal screeners.

Conclusion: An image based optometric screening programme helps prevent unnecessary clinic referrals in nearly half of patients graded as having significant diabetic eye disease in a digital photography-based screening system. This helps ophthalmologists to use their time more productively to treat patients and reduces the stress and anxiety for patients who may previously have been referred for hospital care.

\section{4}

Functional brain connectivity changes and neurocognitive functioning in T1DM patients with proliferative retinopathy: preliminary findings using magnetoencephalography

E. van Duinkerken ${ }^{1}$, N.S.M. Schoonenboom ${ }^{2}$, M. Klein ${ }^{1}$, M. Diamant ${ }^{3}$, C.J. Stam ${ }^{2}$, F.J. Snoek ${ }^{1}$

${ }^{1}$ Medical Psychology, ${ }^{2}$ Clinical Neurophysiology, ${ }^{3}$ Endocrinology, VU University Medical Centre, Amsterdam, Netherlands

Background and aims: Type 1 diabetes mellitus (T1DM) is associated with mild, specific cognitive deficits. Earlier research from our group in patients with and without proliferative retinopathy suggests that cognitive impairment is due to microvascular damage in the brain. Magnetoencephalography (MEG) is a well-established method to detect subtle changes in brain activation and is widely used in brain research. Using the synchronization likelihood (SL), correlations in brain activation over time between different brain regions can be computed, indicating functional connectivity. The aim of the present study was to use MEG to detect differences in functional connectivity comparing T1DM patients with and without proliferative retinopathy and healthy controls. Furthermore, neuropsychological assessment was used to compare cognitive functioning between groups in relation to MEG outcomes.

Materials and methods: Eight T1DM patients with proliferative retinopathy or previous lasercoagulation (DPR), $14 \mathrm{~T} 1 \mathrm{DM}$ patients without complications (NDPR) and 14 healthy controls underwent an MEG and neuropsychological assessment. With MEG brain magnetic field was recorded for various frequency bands during eyes-closed condition, measuring default brain activity. SL was computed and intra-, inter- and local hemispheric locations were created. Neuropsychological assessment covered memory, executive functions, attention, motor speed and information processing speed. Repeated measures ANOVA was used to test for hemispheric differences in SL. MANOVA was used to test for differences in cognitive performance. Post-hoc testing was used to differentiate between groups.

Results: There were no differences between DPR patients (mean age (SD): 43 (8) yrs; median disease duration (range): 31 (18) yrs; mean age of disease onset (SD): 10 (3.12) yrs; mean $\mathrm{HbA}_{1 c}$ (SD): 7.5 (0.88) \%; education level: 5), NDPR patients (mean age (SD): 40 (10) yrs; median disease duration (range): 18 (30) yrs; mean disease onset (SD): 21 (9) yrs; mean $\mathrm{HbA}_{1 \mathrm{c}}$ (SD): 8.1 (1) \%; education level: 5.5) and healthy controls (mean age (SD): 41 (10) yrs; education level: 5.6) with respect to age, sex and education level. DPR patients had significantly longer disease duration $(\mathrm{p}=0.001)$ and earlier disease onset $(p=0.001)$ than NDPR patients. Lower SL was found for DPR compared to healthy controls for alfa2 frequency band inter hemispheric parietal region $\left(\mathrm{p}=0.008 ; \eta^{2}=0.251\right)$, local hemispheric left parietal region $\left(\mathrm{p}=0.046 ; \eta^{2}=\right.$ $0.170)$ and right frontal region $\left(\mathrm{p}=0.017 ; \eta^{2}=0.220\right)$. Neuropsychological performance did not differ between groups $(\mathrm{p}=0.551)$.

Conclusion: The lowered SL found in DPR compared to NDPR and healthy controls suggests significant changes in functional brain connectivity. It is unclear whether these abnormalities are related to earlier disease onset, as suggested from previous studies, using structural brain imaging, disease duration or the presence of microangiopathy in the brain. The unaffected neuropsychological performance found in the DPR group, despite MEG ab- normalities, confirms earlier findings indicating that functional changes are diffuse and subtle, warranting longitudinal follow-up in larger samples. Supported by: Dutch Diabetes Research Foundation

\section{5}

Effects of blood pressure lowering on incidence and progression of retinopathy in patients with type 2 diabetes mellitus: a randomised controlled trial

J.W. Beulens ${ }^{1}$, J.R. Vingerling ${ }^{2}$, J.K. Cruickshank ${ }^{3}$, A.D. Hughes ${ }^{4}$,

A. Stanton ${ }^{5}$, J. Lu' ${ }^{6}$, A. Patel 7 , S.A.M. Thom ${ }^{4}$, D.E. Grobbee ${ }^{1}$, R.P. Stolk ${ }^{8,1}$ on behalf of the AdRem Project Team and ADVANCE Management Committee

${ }^{1}$ Julius Center for Health Sciences and Primary Care, University Medical Center Utrecht, Utrecht, Netherlands, ${ }^{2}$ Departments of Ophthalmology \& Epidemiology, Erasmus Medical Center Rotterdam, Rotterdam, Netherlands, ${ }^{3}$ Cardiovascular Sciences Research Group, University of Manchester \& Royal Infirmary, Manchester, United Kingdom, ${ }^{4}$ International Centre for Circulatory Health, National Heart \& Lung Institute, Imperial College London, United Kingdom, ${ }^{5}$ Molecular and Cellular Therapeutics and RCSI Research Institute, Royal College of Surgeons in Ireland, Dublin, Ireland, ${ }^{6}$ Department of Endocrinology, Chinese PLA General Hospital, Beijing, China, ${ }^{7}$ The George Institute for International Health, University of Sydney, Australia, ${ }^{8}$ Department of Epidemiology, University Medical Center Groningen, Netherlands

Background and aims: The risk of vascular retinal disorders is closely and continuously related to systolic blood pressure level, but data from long-term randomised controlled trials of blood pressure lowering on incidence or progression of retinal disorders in type 2 diabetes are scarce.

Materials and methods: The ADVANCE Retinal Measurements (AdRem) Study is a sub-study of ADVANCE, a randomised controlled double-blind trial of routine blood pressure lowering with a fixed combination of perindopril $(4 \mathrm{mg})$ and indapamide $(1.25 \mathrm{mg})$ in patients with type 2 diabetes. There were no blood pressure criteria for study entry. After a 6-week active run-in period, 1602 patients were randomised to active treatment or matching placebo, in addition to current therapy. At baseline and the final visit, sevenfield stereoscopic retinal photographs of both eyes were taken and graded in a central reading center. New or worsening retinopathy defined as progression of $\geq 2$ steps in ETDRS classification was the primary outcome. Gradable retinal images of both baseline and final photograph were received from 1241 patients.

Results: At baseline, retinopathy was present among $40.8 \%$ of patients. Mean \pm sd systolic and diastolic blood pressure were $141.8 \pm 21.0$ and $79.1 \pm 10.7 \mathrm{~mm}$ Hg. Compared with patients on placebo, those on active therapy had a mean reduction in systolic and diastolic blood pressure of $6.1 \pm 1.2$ and $2.3 \pm 0.6$ $\mathrm{mm} \mathrm{Hg}(\mathrm{p}<0.001)$. We documented progression of retinopathy in 59 (4.8 $\%)$ patients and incident retinopathy in $128(10.3 \%)$ patients during a median follow-up of 4.1 (3.7- 4.3) years. Fewer patients on active therapy experienced new or worsening retinopathy compared with those on placebo (OR 0.78 (95\%-CI: $0.57-1.06)$, but this difference was not significant $(\mathrm{p}=0.12)$. Occurrence of any vascular lesion was similar in both groups $(\mathrm{p}=0.34)$, but active therapy significantly reduced the occurrence of arteriovenous nicking compared to placebo (OR 0.60; 95\%-CI: 0.38- 0.94; $\mathrm{p}=0.025$ ).

Conclusion: Routine blood pressure lowering with a fixed perindopril/indapamide combination may reduce incidence or progression of retinal disorders among patients with established type 2 diabetes. The reduction in AdRem is similar to the 4.5-year results of UKPDS.

Supported by: Institut de Recherches Internationales Servier, National Health Medical Research Council of Australia

\section{6}

Diabetic retinopathy is associated with increased IMT and endothelial dysfunction in type 2 diabetes

J. Sieradzki ${ }^{1}$, M.T. Malecki ${ }^{1}$, G. Osmenda ${ }^{2}$, M. Walus-Miarka ${ }^{1}$, J. Skupien ${ }^{1}$, T.J. Guzik ${ }^{2}$, K. Cyganek ${ }^{1}$, T. Adamek-Guzik ${ }^{2}$, B. Mirkiewicz-Sieradzka ${ }^{1}$ ${ }^{1}$ Department of Metabolic Diseases, ${ }^{2}$ Department of Internal Medicine and Agricultural Medicine, Jagiellonian University, Medical College, Krakow, Poland

Background and aims: Microangioathy and macroangiopathy in type 2 diabetes (T2DM) frequently coexist. Both types of vascular diabetic complica- 
tions share traditional risk factors, for example hyperglycemia and arterial hypertension. It is not clear whether the presence of microangiopathy, such as diabetic retinopathy (DR), constitutes a predictor of atherosclerosis in T2DM and what could be a putative mechanism of this association. The aims were: 1) To search in cross-sectional analysis for the association between DR and intima-media thickness (IMT) of carotid artery in T2M patients; 2) To compare endothelial function in T2DM patients with and without DR.

Materials and methods: We examined 182 consecutive patients with T2DM for at least 5 years, mean age at examination $56.3 \pm 6,52$ yrs. We assessed in these individuals: 1) IMT of carotid artery by ultrasound;2) endothelial function by flow-mediated dilatation (FMD) method and measurement of concentrations of two biochemical markers: von Willebrand factor (vWF) and s-ICAM-1. All patients had ophthalmological examination with fundus photography. Statistical analysis included Student, Mann-Whitney, chi-square and Fisher tests and multiple regression analysis.

Results: DR was found in 71 (39.0\%) diabetic patients. IMT was higher in patients with DR than in patients without $\mathrm{DR}(0.87 \mathrm{~mm}$ vs. $0.79 \mathrm{~mm}, \mathrm{p}=0.0001$, respectively). Additionally, FMD was lower in the complication group than in non-DR subjects ( $8.38 \%$ vs. $10.45 \%, p=0.0023$, respectively). However, concentrations of circulating endothelial function markers s-ICAM-1 and vWF were not different between the groups. In multiple regression analysis DR was among the predictors of increased IMT $(\mathrm{p}=0.016)$ and decreased FMD $(\mathrm{p}=0.002)$. In addition, this analysis showed borderline significance for the association of DR with vWF and s-ICAM-1 levels ( $p=0.07$ and $p=0.09$, respectively).

Conclusion: DR is associated with increased IMT thickness and endothelial dysfunction in T2DM. Therefore, diabetic patients with DR need particularly intensive cardiovascular screening. Impaired endothelial function may be a common denominator of pathogenesis of microvascular complications and atherosclerosis in T2DM.

Supported by: Jagiellonian University

\section{7}

Thickness changes of the retinal layers in diffuse diabetic macular oedema assessed by optical coherence tomography

G. Somfai ${ }^{1}$, E. Tátrai ${ }^{1}$, M. Ferencz ${ }^{1}$, D. Cabrera ${ }^{2}$

${ }^{1}$ Department of Ophthalmology, Mária street, Semmelweis University, Faculty of Medicine, Budapest, Hungary, ${ }^{2}$ Bascom Palmer Eye Institute, University of Miami, United States

Background and aims: Diabetic macular edema (DME) is the leading cause of visual impairment among patients with diabetes. There are several patterns of DME. In our study we aimed to assess which retinal layers show thickness changes in diffuse diabetic macular edema (dDME).

Materials and methods: 11 eyes with background retinopathy and dDME on optical coherence tomography (OCT) examination and 8 control healthy eyes were enrolled in our study with 1.0 best corrected visual acuity (mean age 56 [42-64] years and 54 [47-59] years, respectively). We performed standard macular mapping in all eyes using a Stratus OCT ${ }^{\text {m }}$ device. All OCT images were segmented using an algorithm of our own design, average thickness data were obtained for the following cellular layers: retinal nerve fiber layer (RNFL), ganglion cell layer and internal plexiform layer complex (GCL+IPL), inner nuclear layer (INL), outer plexiform layer (OPL) and outer nuclear layer (ONL). Mann-Whitney U test was performed for the pairwise comparison of the two groups.

Results: Average layer thickness significantly increased in all layers in the dDME group except for the RNFL. (RNFL: $43.83 \pm 2.00$ vs. $44.06 \pm 2.64$ [ $\mathrm{p}=0.934$ ], GCL+IPL: $73.10 \pm 2.47$ vs. $78.96 \pm 3.01$ [ $\mathrm{p}=0.003$ ], INL: $35.34 \pm 1.69$ vs. $38.06 \pm 1.60$ [ $\mathrm{p}=0.005]$, OPL: $35.45 \pm 2.01$ vs. $37.55 \pm 2.00$ [ $\mathrm{p}=0.048]$, ONL: $83.33 \pm 3.61$ vs. $95.02 \pm 11.87$ [0.016], results are mean $\mu \mathrm{m} \pm \mathrm{SD}$ )

Conclusion: Our results show that all cellular layers of the retina but the RNFL become thicker in eyes with diffuse diabetic macular edema. It is still unknown how various cellular layers of the retina are involved in dDME formation, however OCT image segmentation may yield a closer look at the pathopysiology of the retina.

Supported by: Zsigmond Diabetes Fund of the Hungarian Academy of Sciences and Research to Prevent Blindness

\section{8}

Low 5-year incidence of retinopathy in subjects with impaired fasting glucose (IFG), the NANSY-Eye Study

M. Tyrberg ${ }^{1}$, A. Melander ${ }^{2}$, U. Lindblad ${ }^{2}$

${ }^{1}$ Department of Ophthalmology, Lund University, Helsingborg, ${ }^{2}$ Clinical

Sciences, Lund University, Malmö, Sweden

Background and aims: Retinopathy is present early in type 2 diabetes. At baseline in the NANSY-Eye Study the prevalence of retinopathy was 10\% in subjects with IFG. NANSY-Eye is a randomized placebo-controlled Swedish study investigating if treatment with sulfonylurea, in addition to dietary regulation and increased exercise, prevents the development of retinopathy in subjects with IFG.

Materials and methods: Subjects were surveyed in primary care with repeated fasting blood glucose measurements. Those with a mean of two consecutive values $\geq 5.6$ and $<6.9 \mathrm{mmol} / 1$ were invited to participate. At baseline 154 had retinal photographs taken. After 5 years retinal photos from 75 subjects have been graded. Fundusphoto (dia) was taken in two fields after dilation of the pupil. The photos were graded according to the alternative classification of the Wisconsin Epidemiologic Study of Diabetic Retinopathy.

Results: 41 men and 34 women have been photographed. At baseline ten had retinopathy, and out of these three had retinopathy after 5 years. Five of these ten had been treated with sulfonylurea. At study term after 5 years four of the 75 had developed retinopathy, and out of these one had been treated with sulfonylurea. Those with retinopathy at study term had higher systolic (154 vs. $142 \mathrm{mmHg}, \mathrm{p}=0.083$ ) and diastolic ( $87 \mathrm{vs} 77 \mathrm{mmHg}, \mathrm{p}=0.005$ ) blood pressure compared with those without retinopathy.

Conclusion: The main finding in this study was the very low incidence of retinopathy in subjects with IFG who had received dietary and exercise advices. Larger studies are warranted to measure the incidence more accurately. Retinopathy in this narrow glucose interval is associated with high blood pressure.

Supported by: Gorthon and Zoega Foundation Helsingborg, Council for Medical Health Care Research in South Sweden, County Council's Research and Development Foundation, Committee for the Blind in Malmohus County, NEPI Foundation in Malmö and Stockholm, Health \& Medical Care Committee of the Regional Executive Board of the Region Västra Götaland

\section{9}

$\mathrm{HbA}_{1 c}$ as predictor of diabetic retinopathy in non-diabetic subjects: the Hoorn Study

H. Zavrelova ${ }^{1,2}$, E. van 't Riet ${ }^{1}$, B.C.P. Polak ${ }^{1,2}$, R.J. Heine ${ }^{1}$,

C.D.A. Stehouwer ${ }^{3}$, G. Nijpels ${ }^{1}$, J.M. Dekker ${ }^{1}$

${ }^{1}$ EMGO Institute, VU University Medical Center, Amsterdam, ${ }^{2}$ Department of Ophthalmology, VU University Medical Center, Amsterdam,

${ }^{3}$ Department of Internal Medicine, Academic Hospital Maastricht, Netherlands.

Background: $\mathrm{HbAlc}$ is used to estimate mean blood glucose levels in known type 2 diabetes mellitus patients (KDM). For subjects with non-diabetic glucose levels $\mathrm{HbAlc}$ is not in use as indicator of mean blood glucose, because of limited agreement on the correlation of fasting and postload glucose with $\mathrm{HbAlc}$ in non-diabetic subjects. The aim of the present study was to address the question whether elevated $\mathrm{HbAlc}$ predicts future diabetes complications in subjects with normal glucose metabolism (NGM) and impaired hyperglycaemia (IH).

Methods: In the Hoorn Study, a sex and glucose status stratified sample (n $=631$, age 50-75) of a population-based cohort study underwent duplicate OGTT at baseline. At baseline and after 10 years a physical examination was performed. The 10 year incidence of diabetic retinopathy (DR) could be determined in 233 subjects. DR was assessed by fundus photography. WHO '06 criteria based on the mean of duplicate measurements were used to define glucose status. High HbAlc was defined by the clinically used cut-off point of $\geq 6.0 \%$. Logistic regression analysis with adjustment for age and gender was used. Odds ratios were computed in categories of glucose status with either low or high HbA1c, all relative to NGM subjects with low HbA1c.

Results: The study population of 233 subjects consisted of $110 \mathrm{NGM}, 66 \mathrm{IH}$, $41 \mathrm{NDM}$ and 16 KDM subjects. A total of 27 out of 233 subjects developed DR. Only $2.7 \%$ of NGM and $16.7 \%$ of IH subjects had high HbAlc as opposed to $68.3 \%$ of NDM and $81.3 \%$ of KDM subjects. Subjects with HbAlc $\geq 6.0 \%$ had a strongly elevated risk of developing DR compared to subjects with low HbAlc. This was observed, not only in diabetic patients, but also in 
NGM and IH subjects (see table 1). IH and NDM subjects with HbA1c $<6 \%$ had moderately elevated risk of developing DR.

Conclusion: Elevated $\mathrm{HbA1c}$ predicts diabetic retinopathy, also in subjects without diabetes mellitus.

Table 1. Age and sex adjusted 10-year risk of developing diabetic retinopathy relative to NGM subjects with $\mathrm{HbA1c}<6 \%$. (Odds Ratio (95\% CI interval).

\begin{tabular}{lllll}
\hline & NGM & IH & NDM & KDM \\
\hline $\begin{array}{l}\text { HbA1c } \\
<\mathbf{6 . 0 \%}\end{array}$ & 1 ; reference & $1.31(.39-4.38)$ & $1.19(.13-10.70)$ & - \\
\hline HbA1c & 10.14 & 7.19 & 1.71 & 8.71 \\
$>=\mathbf{6 . 0 \%}$ & $(.75-136.24)$ & $(1.63-31.77)$ & $(.44-6.62)$ & $(2.20-34.55)$
\end{tabular}

\section{0}

The impact of different type and severity of hypertension on retinopathy, nephropathy and the ECG abnormalities in diabetic patients

Z. Xü ${ }^{1}$, Z. Wang ${ }^{1}$, Y. Wang ${ }^{1}$, Z. $\mathrm{Mo}^{2}, \mathrm{X}^{\mathrm{Li}}{ }^{1}$

${ }^{1306 t h ~ H o s p i t a l ~ o f ~ P L A, ~ D i a b e t e s ~ C e n t e r, ~ B e i j i n g, ~}{ }^{2}$ The Third Xiangya

Hospital, Endocrinology Department, Changsha, China

Background: There are 180 million people with hypertension and 40 million people with diabetes in China. About $50 \%$ of Chinese diabetic patients with hypertension. The awareness, treatment and control of hypertension were $30.2 \%, 24.7 \%$ and $6.1 \%$ respectively, based on the nationwide survey in 2002 . Hypertension aggravates diabetic complications development.

Objective. To investigate the impact of different type of hypertension on diabetic retinopathy nephropathy and abnormal ECG.

Methods: 5841 patients from the diabetic complication assessment database from 1994 to 2006 were divided into the different groups based on their blood pressure level and the type of hypertension, group A ( $\mathrm{n}=3580, \mathrm{BP}<140 /<90$ $\mathrm{mmHg}$ ), group $\mathrm{B}(\mathrm{n}=456, \mathrm{BP}<140 /<90 \mathrm{mmHg}$, with hypotensive agents ), group $\mathrm{C}(\mathrm{n}=1271, \mathrm{BP}=140-159 / 90-99 \mathrm{mmHg})$, group $\mathrm{D}(\mathrm{n}=396, \mathrm{BP}=160$ $179 / 100-109 \mathrm{mmHg})$, group $\mathrm{E}(\mathrm{n}=138, \mathrm{BP} \geq 180 / 110 \mathrm{mmHg})$, group 3 ( $\mathrm{n}=1262$, systolic hypertension only ), group $4(\mathrm{n}=103$, diastolic hypertension only) and group $5(\mathrm{n}=440$, both systolic and diastolic hypertension), and analyzed for their retinopathy, nephropathy and abnormal ECG results.

Results: Of these patients, 1006 patients with retinopathy, including 898 (15.37\%) with background and $108(1.85 \%)$ with proliferative retinopathy; 1145 with abnormal urine albumin excretion, 1077 with abnormal ECG. The prevalence of retinopathy, nephropathy and the abnormal ECG was increased from $15.0 \%, 17.2 \%, 29.9 \%$ in Group A to $34.8 \%, 54.5 \%, 66.7 \%$ in Group E. No differences in the prevalence of retinopathy and nephropathy between Group A and Group B (15.0 \% vs. $17.8 \% ; 17.2 \%$ vs. $16.3 \%)$. There were significant differences of the ECG abnormalities between different groups (29.9\% for Group A, 37.4\% for Group B, 40.5\% for Group C, $49.4 \%$ for Group D and $66.7 \%$ for Group E). There were significantly differences of the prevalence of retinopathy, nephropathy and abnormalities among Group 3 (21.6\%, $46.7 \%, 42.3 \%)$, Group 4 (11.0\%, 35.6\%, 45.0\%), and Group 5 (22.8\%, 47.3\%, 49.5\%).

Conclusion: Hypertension aggravates the retinopathy, nephropathy and abnormalities in the diabetic patients. No significant difference of the prevalence of retinopathy and nephropathy, except ECG abnormalities between the diabetic patients with normal blood pressure and those diabetic patients with taking the hypotensive agents and with satisfactorily controlled blood pressure.

Supported by: Beijing Capital Medical Development Foundation

\section{1}

Low prevalence of retinopathy and ocular lesions in patients with diabetes mellitus - , "Diabetes TÜV", a continuous, biennial, cross-sectional analysis of diabetes metabolic control and diabetic complications - data of the years 2004 to 2006

C.S. Kloos ${ }^{1}$, N. Müller ${ }^{1}$, J. Dawczynski ${ }^{2}$, R. Berner ${ }^{3}$, G. Wolf ${ }^{1}$, U.A. Müller ${ }^{1}$ ${ }^{1}$ University Hospital, Klinikum für Innere Medizin III, Jena, ${ }^{2}$ University Hospital, Jena, ${ }^{3}$ Controlling, Deutsche BKK, Deutsche BKK, Stuttgart, Germany

Background and aims: Since 1997, first as a pilot project, then on a nationwide scale, a health insurance company in Germany ("Deutsche BKK") intiated an evaluation of treatment quality and prevalence of diabetic complications ("Diabetes TÜV") for all insured patients with diabetes mellitus. Results of the ophthalmologic examinations are presented here.

Materials and methods: A total of 7166 patients from 2004 to 2006 participated in the "Diabetes TÜV". 5777 patients (81\%) were screened by 110 ophthalmologists (9076 examinations documented, multiple examinations $36.3 \%$ ). An standardised documentation protocol was used (initiative group for early diagnosis of diabetic eye diseases, Prof. Kroll, Marburg, Germany). Years since diabetes diagnosis was documented in $100 \%$, the type of diabetes in $88 \%, \mathrm{HbA}_{1 \mathrm{c}}$, blood pressure (RR) and age in $32 \%$. Patients' characteristics were as follows: diabetes mellitus type 1 (DM1): $7.4 \%$, age $47.0 \pm 20.34$ y., years since diabetes diagnosis $13.7 \pm 12.0 \mathrm{y}$., $\mathrm{HbA}_{1 \mathrm{c}} 7.21 \pm 1.23 \%$, prevalence of arterial hypertension $31 \%$, diabetes mellitus type 2 (DM2): age $66.3 \pm 10.2 y$., years since diabetes diagnosis $8.6 \pm 8,0 \mathrm{y}$.; $\mathrm{HbA}_{1 \mathrm{c}} 6.91 \pm 1.16 \%$, prevalence of arterial hypertension $65 \%$.

Results: $87.1 \%$ had no sign of diabetic retinopathy (DR) (years sínce diabetes diagnosis $7.4 \mathrm{y}, \mathrm{HbA}_{1 \mathrm{c}} 7 \%$ ). In $15.2 \% \mathrm{DR}$ was detected, mostly early stages (grading of $\mathrm{RD} /$ diabetes diagnosis/HbA 1 : mild $\mathrm{DR}$ 12.9\%/12.6y./HbA 7.03\%; severe non-proliferative $\mathrm{RD} 2.2 \% / 12.6 \mathrm{y} . / \mathrm{HbA}_{1 c} 8.2 \%$; proliferative $\mathrm{RD}$ $\left.0.5 \% / 15.5 \mathrm{y} . / \mathrm{HbA}_{1 c} 7.6 \%\right)$. Prevalence of $\mathrm{RD}$ did not differ significantly between DM1 and 2 (no RD in DM1 82\% vs. DM2 87\%, mild RD 95.7/97.5\%, non proliferative $\mathrm{RD} 2.8 / 1.6 \%$, proliferative $\mathrm{RD} 1.3 / 0.4 \%)$. Regression analysis revealed association of $\mathrm{RD}$ to duration of diabetes, $\mathrm{HbA}_{1 \mathrm{c}}$ and blood pressure (decreasing ranking, $\mathrm{p}<0.05$ ), but not for diabetes type and age. Macular edema was present in $0.7 \%$, vitreous hemorrhage in $0.3 \%$, retinal detachment with or without macular involvement in less than $0.1 \%(n=8)$, cataract or secondary cataract in $31.6 \%$, an artificial lens in $14.7 \%$, iris neovascularisation in less than $0.1 \%(n=4)$. As diagnostic or therapeutic measures were recommended: fluorescent angiography in $0.2 \%$, focal laser coagulation in $0.4 \%$, cryocoagulation in $0,1 \%$ and vitrectomy in less than $0.1 \%(n=4)$ of the patients. The frequency of ophthalmologic visits was once per year for $56.6 \%$ of the patients, twice for $31.1 \%$, thrice for $11.3 \%$ and more in $1 \%$ (4 to 9 visits). Conclusion: The quality of documentation in the check-up programme "Diabetes TÜV" of Deutsche BKK was satisfying. No lateralisation of the findings occurred. The prevalence of RD in the primary care level in diabetic patients is low, and mostly mild and comparable to the last evaluation in 2002 to 2004 (2002-04 11.6\%, 2004-06 12.6\%). Longer duration of diabetes, elevated $\mathrm{HbA}_{1 \mathrm{c}}$ and blood pressure are associated with the prevalence of $\mathrm{RD}$, well in accordance with randomised studies (e.g. UKPDS). Attempts to further prevent diabetic eye complications should focus on improving insufficiently controlled blood pressure or glycemic control. The "Diabetes TÜV" is a successful tool to monitor the quality of care in diabetes on the primary care level. 


\section{PS 113 Experimental nephropathy: inflammation, oxidative stress and growth factors}

\author{
1182 \\ Rosiglitazone inhibit the upregulation of monocyte chemoattractant \\ protein-1 induced by advanced glycosylation end products in human \\ renal mesangial cells \\ Y. Yuan, Z. Sun \\ Department of Endocrinology, ZhongDa Hospital, South East University, \\ Nanjing, China
}

Background and aims: Previous work shown advanced glycosylation end products (AGEs) increased the expression of monocyte chemoattractant protein-1 (MCP-1) in human renal mesangial cells (HRMCs). The aim of this study was to further investigate the effect of rosiglitazone on the upregulation of MCP-1 induced by AGEs

Materials and methods: HRMC were incubated with AGE-bovine serum albumin (AGE-BSA)in the presence or absence of rosiglitazone. MCP-1 mRNA and protein expression in HRMC were analyzed by reverse transcription-polymerase chain reaction (RT-PCR) and Western blotting. The MCP-1 protein in supernatant was also quantified by ELISA.

Results: About a $50 \%$ increase in the MCP- 1 mRNA $(0.635 \pm 0.030$ vs $0.326 \pm 0.025, \mathrm{P}<0.01)$, protein $(0.347 \pm 0.041$ vs $0.110 \pm 0.015, \mathrm{P}<0.01)$ and production was observed in HRMCs incubated with AGE-BSA $\left(80 \mu \mathrm{g} \cdot \mathrm{ml}^{-1}\right)$ as compared to BSA ( $\left.80 \mu \mathrm{g} \cdot \mathrm{ml}^{-1}\right)$ for $24 \mathrm{~h}$. Furthermore, the MCP-1 protein levels in lysates increased with the elevated AGE-BSA of 80, 160, 320 and $640 \mu \mathrm{g} . \mathrm{ml}^{-1}$ (361.80134.96 vs $723.34 \pm 21.61 ; 368.16 \pm 32.49$ vs $862.95 \pm 21.28$; $356.94 \pm 28.14$ vs $1016.63 \pm 35.67 ; 367.61 \pm 24.32$ vs $1022.96 \pm 37.15$ pg.ml ${ }^{-1}$ respectively, $\mathrm{P}<0.01 \mathrm{n}=3$ ). MCP-1 protein were increased significantly with prolongation of experimental time ( $1 \mathrm{~h}, 4 \mathrm{~h}, 8 \mathrm{~h}, 24 \mathrm{~h}, 48 \mathrm{~h})$ as well $(0.212 \pm 0.011$, $0.389 \pm 0.032,0.356 \pm 0.032,0.254 \pm 0.033,0.213 \pm 0.021)$ when HRMCs exposed to AGE-BSA $\left(160 \mu \mathrm{g} \cdot \mathrm{ml}^{-1}\right)$. MCP-1 mRNA expression increased significantly at first hour $(0.647 \pm 0.045$ vs $0.327 \pm 0.0141, \mathrm{P}<0.01)$, but the peak at $4 \mathrm{~h}(1.071 \pm 0.067)$. Rosiglitazone $\left(1 \mu \mathrm{mol} . \mathrm{L}^{-1}\right)$ show the significant role of inhibition for MCP-1 in HRMCs incubated with AGE-BSA (160 $\mu \mathrm{g} \cdot \mathrm{ml}^{-1}$ ) overexpression ( mRNA: $0.348 \pm 0.031$ vs $0.635 \pm 0.053, \mathrm{P}<0.001 \mathrm{n}=3$; protein: $0.271 \pm 0.028$ vs $0.342 \pm 0.031, \mathrm{P}<0.001, \mathrm{n}=3$; protein in lysates: $463.85 \pm 34.48$ vs $962.95 \pm 51.28$ pg. $\mathrm{ml}^{-1}, \mathrm{P}<0.01, \mathrm{n}=3$ ).

Conclusion: The present study suggest that AGEs have the potency of concentration- and time-dependently upregulation the expression of MCP-1 in cultured human mesangial cells. Rosiglitazone can markedly reduce the upregulation of MCP-1 induced by AGE.

\section{3}

Antioxidant SOD-mimetic prevents NADPH oxidase-induced oxidative stress and renal damage in the early stage of experimental diabetes and hypertension

E.B.M. Peixoto, B.S. Pessôa, S.K. Biswas, J.B. Lopes de Faria Internal Medicine, University of Campinas, Brazil

Background and aims: Hypertension is common in patients with diabetes mellitus (DM), and their combination increases the frequency and severity of nephropathy in an additive manner. It has been demonstrated that the presence of hypertension increases renal oxidative stress by increasing NADPH oxidase dependent superoxide production and by decreasing antioxidant defense in the early stage of experimental DM. In the present study, we investigated whether the administration of tempol, an antioxidant that is a mimetic of the superoxide dismutase (SOD), corrects the oxidative imbalance and oxidative stress-induced renal injuries in the presence of DM and hypertension. Methods: DM was induced in spontaneously hypertensive rats (SHR) by streptozotocin $(60 \mathrm{mg} / \mathrm{kg})$ at 4 weeks of age. Blood glucose levels of $\geq 270$ $\mathrm{mg} / \mathrm{L}$ were considered diabetic. The diabetic rats were randomly assigned to receive intraperitoneally no treatment or treatment with tempol at a dose of $250 \mathrm{mg} / \mathrm{kg}$, mean of 5 rats per group. After 20 days, rats were sacrificed and kidneys were collected. Renal extracelullar antioxidant EC-SOD expression was assessed by Western Blot. Oxidative stress-induced DNA base modification was evaluated by immunohistochemistry for 8-hydroxy-2'-deoxyguanosine (8-OHdG). NADPH-induced superoxide generation was assessed by lucigenin-enhanced chemiluminescence. Urinary albumin was assessed by single radial immunodiffusion. Renal collagen IV protein expression was evaluated by imunohistochemistry and Western Blot analysis.

Results: Plasma glucose levels were higher in untreated and treated diabetic than in control SHR ( $482 \pm 52$ vs $444 \pm 276 v s 169 \pm 24 \mathrm{mg} / \mathrm{dl}$, respectively, $\mathrm{p}<0.0001)$. Systolic blood pressure was unaltered by diabetes or by treatment with tempol, control $=159 \pm 11$, untreated diabetic $=156 \pm 7$, tempol diabetic $=152 \pm 9 \mathrm{mmHg}$. Tempol treatment reestablished the imbalance in redox status induced by DM. Renal antioxidant SOD, in arbitrary units, that was 1.274 \pm 0.823 in control rats dimished to $0.41 \pm 0.60$ in diabetic and raised to 2.45 \pm 1.01 with treatment with tempol, $\mathrm{p}<0.0001$. Diabetes increases the renal $\mathrm{NADPH}$-dependent superoxide production (RLU/20s/mg) from $11.84 \pm 0.73$ to $15.01 \pm 2.01$, and it was markedly reduces ( $\mathrm{p}=0.0495$ ) by tempol treatment to $4.58 \pm 1.89 .8$-OHdG, a marker of oxidative stress-induced DNA damage in the kidney was elevated in diabetic rats (695 (642-754) vs 159 (130-206) positive cells/50 high field) and it diminished $(\mathrm{p}=0.016)$ to $595(555-659)$ with tempol treatment. Reduction of oxidative stress markers was associated with reduction in renal injuries parameters associated with diabetic nephropathy. Tempol reduced $(\mathrm{p}=0.052) \mathrm{DM}$-induced albuminúria from 680 $(180$ - 1815) to $366(109-1342) \mu \mathrm{g} / 24$ hour; and DM-induced elevation in glomerulus and tubulointersticial expression of collagen IV was reduced by tempol $(\mathrm{p}=0.042)$ to the level observed in control rats $(4.24 \pm 2.22$ vs $1.55 \pm$ 1.51 , arbitrary units).

Conclusion: In the context of these and previous findings by our group, we conclude that an imbalance in renal redox status is associated with markers of renal injury in the early stage of DM and hypertension. Antioxidant treatment not only reestablished the redox status but also prevented oxidative stress-induced renal damage.

Supported by: FAPESP, CAPES, CNPq

\section{4}

Effectiveness of resveratrol treatment in the improvement of oxidative stress and protection against diabetic nephropathy via normalisation of Mn-SOD dysfunction

D. Koya, M. Kitada

Endocrinology \& Metabolism, Kanazawa Medical University, Kahokugun, Ishikawa, Japan

Background and aims: Resveratrol (RSV), a one of the polyphenols found in red wines, is known as an antioxidants as well as an enhancer of mitochondrial biogenesis, and has the beneficial effects for several diseases including cardiovascular disease, insulin resistance, and extension of lifespan. However it remains unclear whether RSV has the protected effect against diabetic nephropathy.

Materials and methods: Male diabetic $\mathrm{db} / \mathrm{db}$ mice and age-matched nondiabetic $\mathrm{db} / \mathrm{m}$ mice, at 9 weeks of age, were divided into four groups; 1 ) nondiabetic $\mathrm{db} / \mathrm{m}$ mice, 2) diabetic $\mathrm{db} / \mathrm{db}$ mice, 3) $\mathrm{db} / \mathrm{m}$ mice treated with RSV, 4) $\mathrm{db} / \mathrm{db}$ mice treated with RSV. RSV was given orally mixed $(0.3 \%)$ in chow. After 8 weeks, we performed following experiments. Mn-SOD (manganesesuperoxide anion dismutase) activity in the kidney was measured by inhibiting extra cellular and $\mathrm{Cu} / \mathrm{Zn}$ SOD activity with $\mathrm{KCN}(1 \mathrm{mM})$ by using SOD assay kit. Urinary albumin and 8-OHdG (8-hydroxydeoxyguanosine) excretion were measured using ELISA kit. The mRNA expression of Mn-SOD, PGC-1a (peroxisome proliferative activated receptor $\gamma$ coactivator- $1 \alpha$ ), and NRF-1 (nuclear respiratory factors-1), cytochrome c oxidoreductase were estimated by real time PCR. Mitochondrial DNA contents were calculated as the ratio of cytochrome $c$ oxidase subunit 2 DNA to uncoupling protein 2 DNA as mitochondrial DNA per nuclear genome. The tyrosine nitration of immunoprecipitated Mn-SOD was estimated by western blotting. The staining for nitrotyrosine was estimated by immunohistochemistry. Mesangial expansion was quantified as a PAS-stained positive area. The blood glucose levels were measured every two weeks, and HbAlc was measured by HPLC at the end of experiments.

Results: Urinary 8-OHdG excretion and the staining for nitrotyrosine in the kidney of $\mathrm{db} / \mathrm{db}$ mice were increased compared with $\mathrm{db} / \mathrm{m}$ mice. The alterations were improved on treatment with RSV. Reduced Mn-SOD activity and increased Mn-SOD nitrosation was observed in $\mathrm{db} / \mathrm{db}$ mice compared with $\mathrm{db} / \mathrm{m}$ mice. On the other hand, mitochondrial biogenesis indicated by overexpression of PGC-1 $\alpha$, NRF-1, cytochrome c oxidoreductase and mitochondrial DNA contents, was significantly enhanced in $\mathrm{db} / \mathrm{db}$ mice compared with $\mathrm{db} / \mathrm{m}$ mice, accompanied by the Mn-SOD overexpression. Urinary albumin excretion and mesangial expansion increased in $\mathrm{db} / \mathrm{db}$ mice significantly compared with $\mathrm{db} / \mathrm{m}$ mice, and these abnormalities in $\mathrm{db} / \mathrm{db}$ mice 
improved on treatment with RSV. The blood glucose level was significantly elevated in $\mathrm{db} / \mathrm{db}$ mice compared with $\mathrm{db} / \mathrm{m}$ mice throughout the period of experiments. RSV significantly ameliorated the elevated blood glucose levels in $\mathrm{db} / \mathrm{db}$ mice at the end of experiments; however, there was no difference in $\mathrm{HbAlc}$ between $\mathrm{db} / \mathrm{db}$ mice and $\mathrm{db} / \mathrm{db}$ mice treated with RSV.

Conclusion: These findings suggest that altered mitochondrial biogenesis with Mn-SOD dysfunction caused by oxidative stress occurs in the kidney of $\mathrm{db} / \mathrm{db}$ mice. Therefore, reduction of oxidative stress by RSV, especially via normalization of Mn-SOD dysfunction, but not via enhancement of mitochondrial biogenesis, might be effective in protecting against diabetic nephropathy.

\section{5}

TGF- $\beta 1$ upregulates insulin-like growth factor binding protein (IGFBP)7 expression in human renal proximal tubular epithelial cell (HRPTEC) J. Watanabe, Y. Takiyama, J. Honjyo, T. Isoe, H. Sakagami, Y. Makino,

Y. Fujita, M. Haneda

Department of Medicine, Asahikawa Medical College, Japan

Background and aims: Tubulointerstitial fibrosis is presently considered to be a common pathway of various progressive kidney diseases including diabetic nephropathy (DN) leading to end-stage renal disease, and TGF- $\beta$ plays an important roles in these pathological mechanisms. In order to identify novel molecules derived from human renal tubular cell regulated by TGF- $\beta$, we used LC-MS/MS-based proteomic technique. Several proteins in cultured media of HRPTEC were identified to be upregulated by TGF- $\beta$, one of which was $33 \mathrm{kDa}$ IGFBP-7. IGFBP-7, also named as IGFBP-related protein 1, MAC 25 or prostacyclin-stimulating factor is a recently described member of the low-affinity IGFBP family, which binds IGF-I, IGF-II and insulin by inhibiting insulin signaling. Recently, the serum concentrations of IGFBP-7 have been reported to be associated with insulin resistance. To clarify the potential effects of IGFBP-7 in DN, we assessed the regulation of IGFBP-7 expression in HRPTEC.

Materials and methods: First, we studied the time- (1-24 hrs) or dose-(0.625$10 \mathrm{ng} / \mathrm{ml}$ ) dependent effect of TGF- $\beta_{1}$ on the expressions of IGFBP-7 protein and mRNA, using Western blotting analysis and quantitative real-time RTPCR. We also examined the expression of IGFBP-7 in human kidney tissue with type 2 diabetes and overt nephropathy. Renal biopsy specimens were obtained with the informed consent of the patients with diabetic nephropathy, according to the regulations of the local ethics committee.

Results: HRPTEC barely expressed and secreted IGFBP-7 into cultured media. The secretion and intracellular expression of IGFBP-7 were also increased by the treatment of high glucose ( $30 \mathrm{mM}_{\mathrm{D}}$-glucose) overnight. Interestingly the biguanide metformin, known as an insulin sensitizer, decreased the secretion and intracellular expression of IGFBP-7, and furthermore inhibited TGF $\beta$-enhanced the secretion and intracellular expression of IGFBP-7. The stimulatory effects of TGF- $\beta_{1}$ on the secretion and intracellular expression of IGFBP-7 protein were in a dose- and time-dependent fashion. We next examined the effect of TGF- $\beta_{1}$ on IGFBP-7 gene expression by quantitative realtime RT-PCR. The treatment with TGF- $\beta_{1}$ at the concentration of $2.5 \mathrm{ng} / \mathrm{ml}$ overnight significantly $(\mathrm{p}<0.01)$ increased approximately two fold IGFBP-7 mRNA expression compared to control. Finally, in the immunohistochemical analysis, the positive staining for IGFBP-7 was observed in tubular cells but not in glomerular cells.

Conclusion: In conclusion, TGF- $\beta_{1}$ or high glucose enhances IGFBP-7 expression in HRPTEC, suggesting that IGFBP-7 may play a role in the renal tubular cell damage, leading to the progression of DN. Furthermore, we found that IGFBP-7 is expressed by human renal tubular cells in vitro and in human kidney tissue with overt DN accompanied with type 2 diabetes. Taken together, these data suggest that IGFBP-7 might be one of candidate biomarker for the diagnosis of DN, especially tubular injury.

\section{6}

The effect of $\alpha$-lipoic acid on proteinuria and renal TGF $\beta$ expression in OLETF rat

S. Lee ${ }^{1}$, S. Kang 2 , J. Kang ${ }^{1}$, O. Ryu ${ }^{1}$, C. Kim ${ }^{1}$, B. Lee ${ }^{1}$, E. Hong ${ }^{1}$, H. Kim ${ }^{1}$,

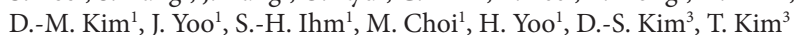
${ }^{1}$ Division of Endocrinology and Metabolism, Hallym University Sacred Heart Hospital, Anyang-Si, Gyenggi-Do, ${ }^{2}$ Division of Endocrinology and Metabolism, Seoul Red Cross Hospital, Seoul, ${ }^{3}$ Division of Endocrinology and Metabolism, College of Medicine, Hanyang University, Seoul, Republic of Korea

Background and aims: This study was undertaken to investigate whether $\alpha$ lipoic acid (ALA) could reduce urinary protein excretion and renal TGF $\beta$ protein expression in obese type 2 diabetes mellitus animal model, Otsuka Long Evans Tokushima Fatty (OLETF) rat.

Materials and methods: Obese 30 male OLETF rats were randomly divided to 3 groups at the age of 30 weeks. The rats in the Control group fed normal rat chow while the rats in the ALA group were fed with rat chow containing ALA ( $0.5 \%$ of food weight). Ten rats in the Pair-fed group were fed with normal rat chow, but were given the same amount of food as consumed by the ALA group. During 5 weeks of ALA feeding, food intake and body weight were checked in metabolic chamber. Blood glucose levels, HbAlc and urinary protein excretion were measured at 30 weeks and 35 weeks of age, and renal TGF $\beta$ protein expression at 35 weeks of age was measured by Western blot and represented by relative unit (RU). Immunohistochemical staining for TGF $\beta$ protein in renal tissue was also examined at 35 weeks of age.

Results: Food intake, body weight, blood glucose levels, HbAlc and urinary protein excretion among the Control, ALA and Pair-fed groups at 30 weeks of age were not different. At 35 weeks of age, food intake was significantly decreased in the ALA group than the Control group (Control group vs. ALA group, $27.7 \pm 1.1 \mathrm{~g} /$ day vs. $22.4 \pm 1.4 \mathrm{~g} / \mathrm{day}, \mathrm{P}<0.001$ ), and body weight was significantly decreased in the ALA group than the Control and Pair-fed groups (Control group: $694.4 \pm 10.3$ g, ALA group: $600.4 \pm 7.4$ g, Pair-fed group: $685.4 \pm 11.6 \mathrm{~g}, \mathrm{P}<0.001)$. Blood glucose levels were significantly decreased in the ALA group than the Control and Pair-fed groups (Control group: $157.7 \pm$ $4.6 \mathrm{mg} / \mathrm{dL}$, ALA group: $130.7 \pm 4.8 \mathrm{mg} / \mathrm{dL}$, Pair-fed group: $153.7 \pm 3.3 \mathrm{mg} / \mathrm{dL}$, $\mathrm{P}<0.001$ ) although blood glucose levels from 30 weeks to 34 weeks of age and $\mathrm{HbAlc}$ at 35 weeks of age were not different among the groups. Urinary protein excretion and renal TGF $\beta$ protein expression were significantly decreased in the ALA group than the Control and Pair-fed groups (urinary protein excretion, Control group: $5.033 \pm 0.254 \mathrm{mg} / \mathrm{mg}$.Cr, ALA group: 3.633 $\pm 0.303 \mathrm{mg} / \mathrm{mg}$.Cr, Pair-fed group: $4.977 \pm 0.339 \mathrm{mg} / \mathrm{mg} \cdot \mathrm{Cr}, \mathrm{P}<0.001$; renal TGF $\beta$ protein expression, Control group: $7.09 \pm 0.17$ RU, ALA group: 4.14 $\pm 0.26 \mathrm{RU}$, Pair-fed group: $7.00 \pm 0.29 \mathrm{RU}, \mathrm{P}<0.001)$. In the ALA group at 35 weeks of age, urinary protein excretion and renal TGF $\beta$ protein expression were positively related in the Control, ALA and Pair-fed groups (Control group, $\mathrm{r}=0.847, \mathrm{P}=0.002$; ALA group, $\mathrm{r}=0.954, \mathrm{P}<0.001$; Pair-fed group, $\mathrm{r}=0.858, \mathrm{P}=0.002$ ). TGF $\beta$ staining in glomeruli was observed in all groups but was decreased in the ALA group at 35 weeks of age.

Conclusion: These results suggest that ALA may prevent the increase of food intake, body weight, blood glucose, urinary protein excretion and renal TGF $\beta$ protein expression in obese type 2 diabetic rat model. The effect of ALA on diabetic nephropathy presented as proteinuria and renal TGF $\beta$ expression in diabetic patients needs to be further clarified.

\section{7}

Involvement of matrix metalloproteinase-2 in down-regulation of pigment epithelium-derived factor in experimental diabetic nephropathy K. Fukami ${ }^{1}$, S.-I. Yamagishi ${ }^{2}$, Y. Takamiya ${ }^{1}$, S. Ueda ${ }^{1}$, Y. Kaida ${ }^{1}$,

A. Hayashida ${ }^{1}$, T. Kusumoto ${ }^{1}, K_{\text {K. Koike }}^{1}$, S. Okuda ${ }^{1}$

${ }^{1}$ Department of Medicine, Division of Nephrology, ${ }^{2}$ Department of Medicine, Division of Cardiovascular Medicine, Kurume University School of Medicine, Kurume city, Fukuoka, Japan

Background and aims: Pigment epithelium-derived factor (PEDF), a glycoprotein that belongs to the superfamily of serine protease inhibitors, was first purified from the conditioned media of human retinal pigment epithelial cells as a factor which possesses potent neuronal differentiating activity. Recently, we, along with others, have found that PEDF has anti-oxidative and anti-inflammatory properties in vivo, both of which could potentially be exploited as a therapeutic option for the treatment of vascular complications in diabetes. Indeed, overexpression of PEDF was reported to allevi- 
ate microalbuminuria, prevent the expression of fibrogenic factors, and to significantly reduce the production of an extracellular matrix protein in the diabetic kidney. However, the kinetics and regulation of PEDF in the diabetic kidney are not fully understood. Since PEDF is known to be a substrate for matrix metalloproteinnase-2 (MMP-2), we investigated its implications for down-regulation of PEDF in the diabetic kidney.

Materials and methods: Nine wk-old Sprague-Dawley rats received single 50 $\mathrm{mg} / \mathrm{kg}$ intravenous injection of streptozotocin. Control non-diabetic rats received citrate buffer alone. Animals with blood glucose levels greater than 250 $\mathrm{mg} / \mathrm{dl} 48 \mathrm{~h}$ later were considered diabetic. After 16 weeks, MMP-2 expressions in the glomeruli and urine were evaluated by zymography. Urinary albumin excretion (UAE) was measured by an enzyme-linked immunosorbent assay. PEDF expression in the kidney was evaluated by western blot analysis and immnohistochemistry.

Results: Plasma level of glucose, $\mathrm{HbAlc}$ and kidney to total body weight ratio were increased by about 2-3-folds in diabetic rats, compared with non-diabetic control rats (plasma glucose; $589+/-38 \mathrm{mg} / \mathrm{dl}, \mathrm{HbAlc} ; 8.8+/-0.3 \%$ in diabetic rats). Zymography revealed that both pro- and active-MMP-2 expressions in the glomeruli and urine were increased to about 4-5-folds in diabetic rats, which were associated with the increase in UAE. PEDF expression in the glomeruli was down-regulated in experimental diabetes. Further, when proteins extracted from glomeruli of control rats were exposed to MMP-2, PEDF levels were found to be decreased in an MMP-2-dose-dependent manner. Conclusion: The present study demonstrated for the first time that MMP-2 overexpression at early phase of diabetic nephropathy was involved in PEDF down-regulation in the diabetic kidney. Our observations suggest that restoration of PEDF via MMP-2 suppression may offer a promising strategy for halting the development and progression of diabetic nephropathy.

\section{8}

Effects of 1,25 dihydroxyvitamin D3 on the early diabetic glomerulopathy

L. Wogensen ${ }^{1}$, J. Nyengaard ${ }^{2}$, S. Krag ${ }^{1}$

${ }^{1}$ Research Laboratory for Biochemical Pathology, ${ }^{2}$ Dept of Stereology,

Aarhus University, Denmark

Background and aims: Diabetes is a common cause of renal disease. TGF- $\beta 1$ is known to be a key mediator in the pathogenesis of diabetic nephropathy. Recent evidence suggests that vitamin D can interact with the TGF-beta signaling pathway, and vitamin $\mathrm{D}$ has positive effects on different models of renal disease

Materials and methods: As a model of type 2 diabetes, diabetic $\mathrm{db} / \mathrm{db}$ mice and non-diabetic $\mathrm{db} /+$ control animals were divided into two groups each. One group was injected with 1,25 dihydroxyvitamin D3 (VitD3) 3 times weekly for 8 weeks, while the other half only received vehicle (Veh). After 8 weeks the animals were euthanized and renal morphology and collagen content evaluated.

Results: The diabetic mice treated with VitD3 had lower mean blood glucose compared with Veh controls $(15.8+/-6.1 \mathrm{mM}$ vs. $22.0+/-4.5 \mathrm{mM})(\mathrm{p}=0.016)$ ). At the same time the plasma insulin level was higher in the $\mathrm{db} / \mathrm{db}$ vitD3 than the Veh group $(29.7+/-16.6 \mu \mathrm{g} / \mathrm{l}$ vs $14.1+/-8.1 \mu \mathrm{g} / \mathrm{l})(\mathrm{p}<0.05)$. To eliminate the effect of different blood glucose levels, subpopulations with comparable glucose levels were used in the morphological analysis. The basement membrane thickness (BMT) was increased in db/db VEH animals $(151+/-11 \mathrm{~nm}, \mathrm{n}=10)$. This increase was eliminated by VitD3 $(136+/-13 \mathrm{~nm} n=9 ; \mathrm{p}=0.012)$. Diabetes increased the volume fraction of the mesangium from $0.197+/-0.045$ in the $\mathrm{db} /+$ Veh control group to $0.273+/-0.035$ in the $\mathrm{db} / \mathrm{db}$ Veh group. However, VitD3 had no effect on the accumulation of mesangial matrix (db/db VitD3 $0.258+/-0.030$ ). Finally measurement of hydroxyproline revealed no differences among the four groups in total collagen content, and thus no sign of interstitial fibrosis. We have conducted mesangial cell culture experiments that support these findings since vitD3 further stimulates TGF- $\beta 1$-induced fibronectin synthesis $(\mathrm{p}<0.05)$.

Conclusion: VitD eliminates the basement membrane thickening in $\mathrm{db} / \mathrm{db}$ diabetic mice without effect on diabetes-induced mesangial expansion. Supported by: Danish Research Agency

\section{PS 114 Screening and investigation of kidney disease}

\section{9}

Screening for kidney disease in diabetic patients - how useful are renal ultrasonography and serum immunology?

S.J.C. Sammut, T.S. Purewal

Diabetes and Endocrinology, Royal Liverpool University Hospital, United Kingdom

Background and aims: Kidney disease in diabetes is common. However, the incidence of non-diabetic kidney disease in diabetic patients is also as high as $30 \%$. Identifying these potentially treatable conditions is important. The primary aim of this study was to evaluate the usefulness of performing immunological blood tests and renal ultrasound to screen for alternative renal pathology in patients with presumed diabetic nephropathy.

Materials and methods: Retrospective review and correlation of results of immunological blood testing and renal ultrasonography in diabetic clinics at the Royal Liverpool University University Hospital over a three-year period. Results: A total of 1186 immunology screens were performed over the time period (from a total of 24000 patient visits). 754 of these patients also had renal ultrasonography performed. There were a total of 232 positive immunology screens (20\%). 454 patients had normal renal appearances on ultrasonography (60\%); in 300 some abnormality was detected (40\%), of which 154 were deemed of significance (20\%). Of these 154 patients, 39 had a positive immunology screen (25\%). Of the 232 patients with positive immunology: 88 had normal renal appearances on ultrasonography (38\%); 78 did not have a renal ultrasound performed (34\%); and 66 had some abnormality visualised (28\%), of which 37 were deemed significant abnormalities (16\%). The cost per case for picking up abnormal results at UK NHS costings was $£ 190$ per abnormal ultrasound scan (at $£ 40$ per scan) and $£ 475$ per positive immunology screen (at $£ 121$ each).

Conclusions: There was a striking similarity between the group of patients as a whole and the subgroup of patients with positive immunology, in terms of whether a renal ultrasound had been performed and if it showed a significant abnormality. This suggests that there is a poor correlation between abnormalities seen on ultrasound and abnormalities picked up on serum immunological screening. However, $20 \%$ of both immunological and renal ultrasound tests found significantly abnormal results. The abnormality pickup rate is high enough to justify continuation of both tests in screening of diabetic patients for nondiabetes related nephropathy. It should be noted, however, that these cases will often require further diagnostic workup. Ultrasound scanning should generally be performed first. Further work is needed to evaluate if these costs are truly justified, by showing that the tests lead to a final diagnosis (i.e. of non-diabetic nephropathy) and interventions that result in improved outcomes for patients.

\section{0}

Cystatin C formulas for prediction of GFR in patients with DM type 2 and renal dysfunction M. Zavrsnik ${ }^{1}$, S. Bevc ${ }^{2}$, R. Hojs ${ }^{2}$, E. Robert ${ }^{2}$, M. Gorenjak ${ }^{3}$, L. Puklavec ${ }^{4}$ ${ }^{1}$ Department of Diabetology and Endocrinology, ${ }^{2}$ Department of Nephrology, ${ }^{3}$ Department of Clinical Chemistry, ${ }^{4}$ Department of Nuclear Medicine, University Medical Centre Maribor, Slovenia

Background and aims: Estimation of the glomerular filtration rate (GFR) is essential for the evaluation of patients with chronic kidney disease (CKD). Recently, serum cystatin C-based equations were proposed as markers for estimation of GFR. Present study compared serum cystatin C-based equations in patients with diabetes mellitus type 2 and CKD.

Materials and methods: GFR was estimated using six equations (Larsson, LeBricon, Hoek, Grubb, simple cystatin C formula (GFR = 100/cystatin C) and our own cystatin $C$ formula $\left(\mathrm{GFR}=90.63 \times\right.$ cystatin $\mathrm{C}^{-1.192}$ ) that are based on serum cystatin $\mathrm{C}$ in 133 adult patients with diabetes mellitus type 2 and CKD. The serum cystatin $\mathrm{C}$ was determined by an immunonephelometric method. GFR was measured using ${ }^{51}$ CrEDTA clearance (gold standard), and the correlation, accuracy, bias and precision were determined. Ability to correctly estimate patient's GFR with different equations compare to gold standard below and above $60 \mathrm{ml} / \mathrm{min} / 1.73 \mathrm{~m}^{2}$ was analyzed.

Results: The mean ${ }^{51} \mathrm{CrEDTA}$ clearance was $42 \mathrm{ml} / \mathrm{min} / 1.73 \mathrm{~m}^{2}$, the mean serum cystatin C was $2.58 \mathrm{mg} / \mathrm{l}$. Statistically significant correlation between ${ }^{51} \mathrm{CrEDTA}$ clearance with all six serum cystatin C-based equations $(\mathrm{r}=0.872$ 0.901 ) was found. The Receiver Operating Characteristic (ROC) curve analysis 
(cut-off for GFR $60 \mathrm{ml} / \mathrm{min} / 1.73 \mathrm{~m}^{2}$ ) showed no difference of diagnostic accuracy between serum cystatin C-based equations. The serum cystatin C-based equation showed different bias. All equations underestimated measured GFR (Larsson -50.9; Hoek -22.8; LeBricon -16.6; Grubb -17.8; our cystatin C formula -10.6; simple cystatin C formula $-1.1 \mathrm{ml} / \mathrm{min} / 1.73 \mathrm{~m}^{2}$ ). According to bias the simple cystatin $\mathrm{C}$ formula turned out as most precise method for calculating GFR compare to measured GFR by gold standard. Analysis of the SD of the mean difference between estimated and measured GFR showed that all equations lacked precision (minimal Larsson 16.3; maximal our cystatin C formula $\left.19.6 \mathrm{ml} / \mathrm{min} / 1.73 \mathrm{~m}^{2}\right)$. Ability to correctly estimate patient's GFR was high for all equations (LeBricon 92.5\%, Hoek $88.0 \%$, Grubb $88.0 \%$, simple cystatin C formula $88.7 \%$, our cystatin C formula 91.7\%), except for Larsson formula where $21.1 \%$ of subjects were misclassified. Percentage of correct estimation of patient's GFR was significantly higher for simple cystatin $\mathrm{C}$ formula, our cystatin $\mathrm{C}$ formula and LeBricon formula compared to Larsson formula ( $88.7 \%$ versus $78.9 \%$, $\mathrm{P}<0.046 ; 91.7 \%$ versus $78.9 \%, \mathrm{P}<0.0057 ; 92.5 \%$ versus $78.9 \%, \mathrm{P}=0.035)$. No difference in ability to correctly estimate patient's GFR between simple cystatin C, our cystatin C, LeBricon, Hoek and Grubb formulas was found.

Conclusion: Our results indicate that all serum cystatin C-based equations, excluding Larsson formula, are reliable markers of GFR in patients with diabetes mellitus type 2 and CKD and for daily clinical practice most simple formula (100/cystatin C) could be appropriate enough for estimation of GFR. Supported by: University Medical Centre Maribor

\section{1}

Efficacy of serum cystatin $\mathrm{C}$ and parathyroid hormone as renal function markers in Japanese patients with type 2 diabetes mellitus, and the correlation between elevated serum cystatin $\mathrm{C}$ level and diabetic complications

T. Kimura ${ }^{1}$, H. Ikeda ${ }^{1}$, J. Fujikawa ${ }^{2}$, K. Nomura ${ }^{1}$, T. Aoyama ${ }^{1}$, Y. Wada ${ }^{1}$,

K. Nabe ${ }^{1}$, Y. Hamamoto ${ }^{3}$, S. Honjo ${ }^{1}$, H. Koshiyama ${ }^{1}$

${ }^{1}$ Center for Diabetes \& Endocrinology, Tazuke Kofukai Foundation Medical Research Institute Kitano Hospital, Osaka, ${ }^{2}$ Department of Laboratory Medicine, Tazuke Kofukai Foundation Medical Research Institute Kitano Hospital, Osaka, ${ }^{3}$ Division of Diabetes \& Clinical Nutrition, Kyoto University, Japan

Background and aims: There have been few reports about usefulness of serum cystatin $\mathrm{C}$ in Japanese diabetic patients. We examined the efficacy of serum cystatin $\mathrm{C}$ and serum intact parathyroid hormone (iPTH) as diagnostic markers for early diabetic nephropathy in Japanese patients with type 2 diabetes. We also investigated the prevalence of diabetic complications in the patients with elevated serum cystatin C level.

Subjects and methods: A total of 231 consecutive Japanese with type 2 diabetes (mean age 66 years; 147 male and 84 female) were included. Estimated GFR (eGFR) was calculated by the formula of Modification of Diet in Renal Disease. Correlation analysis and receiver operator characteristics analysis were used for examining the accuracy of serum cystatin $\mathrm{C}$ and that of iPTH to detect chronic kidney stage (CKD) stage 2 and 3 . The subjects were divided into two groups: those with serum cystatin $\mathrm{C}$ equal or more than $0.85 \mathrm{mg} / \mathrm{l}$ ( $\mathrm{H}-\mathrm{Cys} \mathrm{C}$ group) and those with serum cystatin C less than $0.85 \mathrm{mg} / \mathrm{l}$ (L-CysC group). The differences of the prevalence of each diabetic complication between the two groups were analyzed by chi-square test.

Results: There was a significant positive correlation between the reciprocal of serum cystatin $\mathrm{C}$ and eGFR $(\mathrm{r}=0.86, \mathrm{p}<0.01)$, although not between serum $\mathrm{iPTH}$ and eGFR ( $r=0.0059$, not significant). To detect CKD stage 3 , the area under the curve (AUC) of cystatin C was high (0.965), whereas that of $\mathrm{PPTH}$ was low (0.567). Targeting CKD stage 2, the AUC of cystatin C was 0.873 ; that of $\mathrm{PTTH}$ was 0.452 . To screen CKD stage 3 , serum cystatin Chad the best test characteristics at the cutoff level of $0.85 \mathrm{mg} / \mathrm{L}$ (sensitivity of $86 \%$ and specificity of $94 \%$ ); and that of intact PTH was $37.5 \mathrm{pg} / \mathrm{ml}$ (sensitivity of $54 \%$ and specificity of $38 \%$ ). To screen CKD stage 2, the best cut-off of cystatin C was $0.60 \mathrm{mg} / \mathrm{L}$ (sensitivity of $88 \%$ and specificity of $80 \%$ ), and that of iPTH was $40 \mathrm{pg} / \mathrm{ml}$ (sensitivity of $61 \%$ and specificity of $59 \%)$. H-CysC group showed significantly higher prevalence of diabetic retinopathy, positive treadmill test, compared to L-CysC group. There was no significant difference in the prevalence of diabetic polyneuropathy, thickened intima-media of carotid arteries, and stroke between $\mathrm{H}-\mathrm{Cys} \mathrm{C}$ and L-CysC groups. Urinary protein excretion per 24-hour was significantly better correlated with serum cystatin C than with eGFR, serum creatinine, and creatinine clearance.

Conclusion: These results suggest that serum cystatin C, not serum iPTH, is a useful marker for detecting mild decrease in GFR in Japanese patients with type 2 diabetes. Elevated serum cystatin $\mathrm{C}$ level was correlated with higher prevalence of diabetic retinopathy and positive treadmill test. Cystatin $\mathrm{C}$ showed better correlation with urinary protein excretion than creatinine-based renal function markers. The present study suggests that diabetic patient with elevated serum cystatin C level should be carefully examined for systemic complications such as diabetic retinopathy, CKD, and asymptomatic ischemic heart disease.

\section{2}

Estimating renal function in (obese) diabetic patients; gaining renal function by gaining weight?

H. Joosten ${ }^{1}$, L. Santing ${ }^{2}$, L.A.G. Lieverse ${ }^{3}$, S.J.J. Logtenberg ${ }^{2}$, N. Kleefstra ${ }^{2,4}$, H.J.G. Bilo ${ }^{2,5}$

${ }^{1}$ Internal Medicine, Isala Clinics, Zwolle, ${ }^{2}$ Diabetes Centre, Isala Clinics, Zwolle, ${ }^{3}$ Internal Medicine, Maxima Medisch Centrum, Eindhoven, ${ }^{4}$ Medical Research Group, Langerhans, Zwolle, ${ }^{5}$ Internal Medicine, UMCG, Groningen, Netherlands

Background and aims: Guidelines recommend the use of equations like the Cockcroft-Gault (CG) and the Modification of Diet in Renal Disease (MDRD-4) formula to estimate renal function in patients with diabetes mellitus (DM). The use of elaborate 24-hours urines sampling is receded to the background. Patients with diabetes are at risk for renal impairment. Early detection of renal function loss will help to alleviate risk on endstage renal and cardiovascular disease. Accurate assessment of renal function in DM is therefore of great importance. However, both the performance and generalizability of equation formulas in DM patients are debatable, as is the influence of body mass index (BMI) on the accuracy of these formulas. Our aim was to analyze the accuracy of renal function estimations and the influence of BMI on estimating renal function in DM patients.

Materials and methods: In this retrospective cohort study data from 849 consecutive subjects with DM, collected between 2005 and 2006 at the outpatient diabetes clinic of Maxima Medical centre in Eindhoven, were evaluated. Creatinine clearance was calculated from 24-hours urine samples $(\mathrm{ml} / \mathrm{min})$. Serum creatinine was measured using the enzymatic method. We compared creatinine clearance of 24-hours urine sampling $(\mathrm{CrCl})$ with the individual estimations of renal function (eGFR) calculated with CG ( $\mathrm{ml} / \mathrm{min}$ ) and MDRD-4 (ml/min/1.73 m2). Patients were subdivided into 2 groups; BMI in $<30.0 \mathrm{~kg} / \mathrm{m}^{2}$ and $\mathrm{BMI} \geq 30.0 \mathrm{~kg} / \mathrm{m}^{2}$. Accuracy was determined by mean difference and precision by standard deviation (SD).

Results: The cohort consisted of 380 females and 469 males. Patient characteristics (median (interquartile range)): age 63 (20-91) years, HbAlc 6.7 (6.0-7.5)\% and BMI 27.7 (24.7-32.0) kg/m2. Median plasma creatinine level was 79.0 (67.097.0) $\mu \mathrm{mol} / \mathrm{l}$, with a mean (SD) $\mathrm{CrCl}$ of $112.6( \pm 44.7) \mathrm{ml} / \mathrm{min}$, mean CG of 97.5 $\mathrm{ml} / \mathrm{min}( \pm 41.3)$ and mean MDRD-4 $76.4( \pm 24.6) \mathrm{ml} / \mathrm{min} / 1,73 \mathrm{~m}^{2}$. Of all patients, $33.3 \%$ had a $\mathrm{CrCl}<90 \mathrm{ml} / \mathrm{min}$, which doubled using the MDRD-4. In all subjects compared to $\mathrm{CrCl}$, the $\mathrm{CG}$ had the smallest differences in both BMI groups. In the BMI group $<30$ the $\mathrm{CrCl}$ was 114.6 and $96.8 \mathrm{ml} / \mathrm{min}$, for man and woman respectively, compared to 133.9 and $110.7 \mathrm{ml} / \mathrm{min}$ in $\mathrm{BMI} \geq 30$. In both BMI groups, the MDRD-4 estimates were significantly lower $(\mathrm{p}<0.01)$ than the creatinine clearance, with increasing inaccuracy at increasing BMI. Conclusion: Formulas to estimate renal function are very useful in clinical practice. Nowadays the MDRD-4 seems to dominate the CG and $\mathrm{CrCl}$. In our study however, eGFR markedly differs when formulas are used compared to $\mathrm{CrCl}$. In our DM patients, the CG is a more accurate formula to estimate renal function compared to the MDRD-4. MDRD-4 did not show accuracy untill CrCl decreased below $30 \mathrm{ml} / \mathrm{min}$. Precision of all renal function prediction equations increased with decreasing renal function. Despite the direct influence of weight (as a variable) in the CG formula, the CG does not overestimate $\mathrm{CrCl}$ in obese patients $\left(\mathrm{BMI} \geq 30 \mathrm{~kg} / \mathrm{m}^{2}\right.$ ). In contrast to earlier reports, we conclude that CG is especially more accurate to estimate renal function in the higher BMI-group $\left(\geq 30 \mathrm{~kg} / \mathrm{m}^{2}\right)$.

\section{3}

Risk factors and prognostic value of a renal artery stenosis in type 2 diabetic patients

I.I. Klefortova ${ }^{1}$, M. Shamkhalova ${ }^{1}$, O. Remizov ${ }^{2}$, A. Bukhman ${ }^{2}$, M. Shestakova ${ }^{1}$, M. Sharia ${ }^{3}$, D. Ustyuzhanin ${ }^{3}$, E. Tugeeva ${ }^{4}$, U. Buziashvili $^{4}$ ${ }^{1}$ Diabetic Nephropathy, Endocrinology Research Center, ${ }^{2}$ Tomography, Endocrinology Research Center, ${ }^{3}$ Tomography, Cardiology Research Center, ${ }^{4}$ Diagnostics, Bakoulev Scientific Center of Cardiovascular Surgery, Moscow, Russian Federation

Background and aims: To determine independent predictors and determine in prognostic value of RAS in subjects with type 2 diabetes and coexistent hypertension. 
Materials and methods: We studied 157 patients (63 males, 94 female). All patients underwent duplex sonography (DS). Patients with RAS detected by DS underwent magnetic resonance angiography (MRA) or multislice computed tomography (MSCT) of the renal arteries. We used 1.5 T MR scanner and 16 slices MSCT.

Results: We found that DS, MRA and MSCT detected RAS in 58 type 2 diabetics $(36.9 \% ; 29.9 \%$ unilateral, $7.0 \%$ bilateral). RAS was associated with smoking $(\mathrm{RR}=1,84$, CI 95\% $(1,23 ; 2,75), \mathrm{P}<0.05)$, incidence of previous myocardial infarction $(\mathrm{RR}=1,63, \mathrm{CI} 95 \%(1,09 ; 2,45), \mathrm{P}<0.05)$, coronary arteries atherosclerosis $(\mathrm{RR}=2,06, \mathrm{CI} 95 \%(1,41 ; 3), \mathrm{P}<0.05)$, femoral artery atherosclerosis $(\mathrm{RR}=2,87$, CI $95 \%(2,31 ; 3,57), \mathrm{P}<0.05)$, carotid artery atherosclerosis $(\mathrm{RR}=1,89$, CI 95\% $(1,25 ; 2,87), \mathrm{P}<0.05)$ and isolated systolic hypertension $(\mathrm{RR}=1,89, \mathrm{CI} 95 \%(1,25 ; 2,87), \mathrm{P}<0.05)$. RAS was determined as a significant predictor of death, new heart failure episodes, myocardial infarction, doubling of serum creatinine, ESRD within 24 months of follow up according to the Kaplan-Meier survival method by log rank test $(\mathrm{p}=0,00089)$.

Conclusion: RAS was associated with smoking, coronary, carotid, femoral artery atherosclerosis, incidence of previous myocardial infarction and isolated systolic hypertension. The patients with RAS had poor prognosis. Supported by: Federal Programme "Diabetus mellitus"

\section{4}

Clinical performance of the CLINITEK Microalbumin 9 reagent strip: a new urinalysis dry reagent strip

A.H.B. Wu ${ }^{1}$, E.P. Jortani ${ }^{2}$, E.P. Womack ${ }^{2}$, M. Vallen-Thompson ${ }^{3}$, J. Fan ${ }^{3}$, S.P. Volkir ${ }^{3}$, K. Schultz ${ }^{1}$

${ }^{1}$ Laboratory Medicine, University of California, San Francisco, ${ }^{2}$ Laboratory Medicine, University of Louisville School of Medicine, ${ }^{3}$ Siemens Healthcare Diagnostics Inc., Deerfield, United States

Background and aims: The CLINITEK Microalbumin 9 strip is a new urinalysis dry reagent strip developed for the CLINITEK family of instruments. When read on the CLINITEK 50, Status" or Advantus ${ }^{\text {mat }}$ analyzers, the Microalbumin 9 strip reports semiquantitative results for nine urine tests: albumin, blood (occult), creatinine, glucose, ketone, leukocyte, nitrite, $\mathrm{pH}$, and total protein, as well as calculated results for albumin-to-creatinine (A:C) and protein-to-creatinine $(\mathrm{P}: \mathrm{C})$ ratios. Results are automatically displayed in approximately 1 minute. The use of analyzers reduces inter- and intrareader variability caused by such factors as timing variation, visual acuity, and variable lighting conditions. The objective of this study was to evaluate the clinical performance of the new urinalysis dry reagent strip.

Materials and methods: Evaluations of this strip were conducted at two clinical laboratory sites using 689 urine specimens over an 8-week period. One site tested four CLINITEK Advantus and the other tested four CLINITEK Status analyzers. Both sites also tested four CLINITEK 50 analyzers. Each site analyzed $\geq 338$ urine specimens in singlicate, testing a minimum of 105 positive and negative specimens for each analyte. Three reagent strip types, two lots each, were used during the study.

Results: Positive, negative, exact, and \pm one-level agreement rates were determined as compared to existing MULTISTIX or Microalbumin 2 reagent strips. The all-site combined results ( $\mathrm{n} \geq 426$, range 426-2727) covering all tests and comparative strips across the complete set of analyzers are shown herein. The mean positive agreement rate for all reagents was $94.1 \%$ (range 79.9\%-99.8\%). The mean negative agreement rate was $94.9 \%$ (85\%-99.4\%). Mean exact agreement was $89.3 \%$ (80.5\%-99.5\%) and the mean " \pm one-level" agreement rate was $99.7 \%$ (98.2\%-100.0\%).

Conclusion: There was excellent agreement for all analytes between the CLINITEK Microalbumin 9 strip and existing MULTISTIX and Microalbumin 2 strips. Therefore, the CLINITEK Microalbumin 9 strip is clinically suitable for providing physicians with a single strip to monitor microalbuminuria, glucose, and traditional urinalysis tests to assist in the diagnosis and assessment of kidney function, urinary tract infections, and renal complications of metabolic disorders (e.g., diabetic nephropathy).

Supported by: Siemens Healthcare Diagnostics

\section{PS 115 Human diabetic nephropathy: risk factors, cardiovascular disease and anaemia}

\section{5}

Normoalbuminuric CKD: its prevalence, clinical features, and impact on cardiovascular disease independent of microalbuminuria H. Yokoyama ${ }^{1}$, M. Oishi ${ }^{2}$, K. Kawai ${ }^{3}$, Y. Fukumoto ${ }^{4}$, H. Sone ${ }^{5}$, M. Kobayashi ${ }^{6}$, G. JDDM-study ${ }^{1}$

${ }^{1}$ Internal Medicine, Jiyugaoka Clinic, Obihiro, ${ }^{2}$ Internal Medicine, Oishi Clinic, Kyoto, ${ }^{3}$ Internal Medicine, Kawai Clinic, Tsukuba, ${ }^{4}$ Internal

Medicine, Fukumoto Clinic, Ibusuki, ${ }^{5}$ Dept. of Lifestyle Medicine \& Applied Nutrition, Ochanomizu University, Tokyo, ${ }^{6}$ Internal Medicine, Toyama University, Japan

Background and aims: Existence of type 2 diabetic patients with normoalbuminuria and with chronic kidney disease (CKD), as defined by estimated glomerular filtration rate $\left(\mathrm{eGFR}, \mathrm{ml} / \mathrm{min} / 1.73 \mathrm{~m}^{2}\right)<60$, has been reported but the prevalence and associated clinical factors remain elucidated. Recent studies indicated that reduced eGFR is an independent risk factor for cardiovascular disease (CVD). However, tt is uncertain whether reduced eGFR is associated with prevalent CVD independent of the effect of microalbuminuria (MA).

Materials and methods: We investigated what proportions of patients are distributed when stratified by nephropathy stages and CKD stages in a largescale population of Japanese type 2 diabetes $(\mathrm{N}=3297)$, and the common and independent factors associated for increase in albuminuria and decrease in eGFR. We further investigated whether reduced eGFR is associated with CVD independent of MA.

Results: 1 ) The proportion of subjects with an eGFR $<60$ was $16.6 \%$ among those with normoalbuminuria ( $\mathrm{N}=2298), 20.6 \%$ among those with microalbuminuria $(\mathrm{N}=705)$, and $52.7 \%$ among those with macroalbuminuria $(\mathrm{N}=294)$. There were 381 patients with normoalbuminuria and with an eGFR $<60$, and $63.3 \%$ of them had neither diabetic retinopathy nor neuropathy. They were older, had more proportion of women, hypertension, hyperlipidemia, and CVD, and less smokers as compared with those with normoalbuminuria and with an eGFR $>=60$. Clinical factors that increased commonly to nephropathy stages and CKD stages were duration of diabetes, pulse pressure, hypertension, cardiovascular disease, retinopathy, and neuropathy. Age and female were only associated with decrease in eGFR. Male, BMI, A1c, systemic blood pressure, triglyceride, and smoking were only associated with increase in albuminuria. 2) Among patients without macroalbuminuria, those with reduced eGFR had a twofold, and those with MA also had a twofold higher prevalence of CVD. Compared with patients without reduced eGFR and without MA (OR 1.0), OR was significantly higher in those with reduced eGFR and without MA (OR 2.03, 95\% CI 1.41-2.92) and was similarly higher in those with MA and without reduced eGFR (OR 1.92 95\% CI 1.39-2.65). It was highest in those with reduced eGFR and MA (OR 4.09, 95\% CI 2.646.35). The OR remained significant after adjustment for age, sex, hypertension, hyperlipidemia, smoking, BMI, and urinary albumin excretion rate. Conclusion: Our finding indicates that a significant proportion of type 2 diabetic patients have non-albuminuric renal insufficiency. Renal disease in type 2 diabetes could be heterogeneous, implying a possibility of involvement of renal atherosclerosis and lipid toxicity. Reduced eGFR and MA were independently associated with increasing the prevalence of CVD, independent of the effect of traditional cardiovascular risk factors and urinary albumin excretion rate in Japanese patients with type 2 diabetes.

\section{6}

Increased cardiovascular risk in normoalbuminuric type 2 diabetic patients with reduced renal function

A. Cignarelli, L. Laviola, S. Di Leo, S. Perrini, A. Leonardini, J. Rinaldi, G. Garruti, A. Bellomo Damato, G. Stefanelli, R. Giorgino, F. Giorgino D.E.T.O., Internal Medicine, Endocrinology and Metabolic Diseases, University of Bari, Italy

Background and aims: Reduced kidney function, estimated by the eGFR, predicts all-cause mortality and cardiovascular $(\mathrm{CV})$ disease in the general population. In type 2 diabetic patients, albuminuria represents the most important marker of kidney damage, and is considered an independent indi- 
cator of endothelial dysfunction and atherogenic risk. However, limited information is available on the association of reduced eGFR, albuminuria and $\mathrm{CV}$ risk in type 2 diabetes. The objective of this cross-sectional study was to evaluate the distribution of eGFR and of albuminuria in a cohort of type 2 diabetic patients, and the pattern of conventional CV risk factors and the estimated CV risk in type 2 diabetic patients with normal (eGFR $>60 \mathrm{ml} / \mathrm{min}$ ) or reduced (eGFR $<60 \mathrm{ml} / \mathrm{min}$ ) kidney function.

Materials and methods: 406 type 2 diabetic patients (227M, 179F) were consecutively enrolled in this cross-sectional study. eGFR was calculated by the Cockroft-Gault equation; albuminuria was measured by a RIA assay and expressed as AER; the 10-y CV risk was estimated with the UKPDS risk engine.

Results: $42 \%$ of the patients showed eGFR $>90 \mathrm{ml} / \mathrm{min}, 46 \%$ eGFR $60-90$ $\mathrm{ml} / \mathrm{min}$, and $12 \%$ eGFR $<60 \mathrm{ml} / \mathrm{min} ; 77 \%$ were normo-, $21 \%$ micro-, and $2 \%$ macroalbuminuric. However, AER and eGFR appeared to be independently distributed in the study group, since $77 \%$ of the 64 patients with reduced kidney function (eGFR $<60 \mathrm{ml} / \mathrm{min}$ ) had AER in the normal range. Patients were then sub-grouped according to an eGFR value $>60 \quad(n=352)$ or $<60 \quad(n=55)$, respectively, and paired by glycemic control ( $\mathrm{HbA}_{1 \mathrm{c}}=7.8 \%$ in both groups). Patients with reduced eGFR were older and more often female, and showed lower body weight ( $67.0 \mathrm{vs} 80.2 \mathrm{~kg}, \mathrm{p}<0.01)$, longer diabetes duration (13.7 vs $9.7 \mathrm{y}, \mathrm{p}<0.01$ ), but no difference in lipid profile, statin use, or anti-diabetic therapy. Diastolic blood pressure was lower in patients with reduced kidney function as compared with patients with normal eGFR $(75.1 \mathrm{vs} 79.3 \mathrm{mmHg}$, $\mathrm{p}<0.01)$, and this was associated with higher use of hypotensive medications (69\% vs $57 \%)$. Interestingly, waist circumference showed a bi-modal distribution: it appeared to be positively correlated with eGFR in the type 2 diabetic population with normal kidney function, and not associated with eGFR in patients with eGFR $<60 \mathrm{ml} / \mathrm{min}$. The 10 -year CHD risk, estimated by the UKPDS risk engine, was higher in diabetic patients with low eGFR $(27.7 \%$ vs $19.5 \%, \mathrm{p}<0.01)$. The OR for a high CHD risk was $6.62(2.56-17.15, \mathrm{p}<0.001)$ when adjusted for $\mathrm{HbA}_{1 \mathrm{c}}$, diabetes duration, gender, smoking, systolic blood pressure, HDL, albuminuria, and waist. Finally, in the subgroup of normoalbuminuric patients, subjects with low eGFR $(n=42)$ showed a higher $\mathrm{CV}$ risk as compared with subjects with normal eGFR $(\mathrm{n}=269)(23.9 \%$ vs $18.0 \%, \mathrm{p}=0.046$ ).

Conclusion: A significant fraction of type 2 diabetic patients shows a reduced kidney function in the presence of normoalbuminuria. The evaluation of eGFR may contribute to identify type 2 diabetic patients with higher CV risk.

\section{7}

Carotid atherosclerosis and progression of diabetic nephropathy in patients with type 2 diabetes

D.-J. Chung, D.-H. Cho, J.-O. Chung, J.-Y. Yoon, M.-Y. Chung

Department of Endocrinology and Metabolism, Chonnam National

University Medical School, Gwangju, Republic of Korea

Background and aims: Cardiovascular risk is higher among people with chronic kidney disease (CKD) than among those with normal renal function. This association is probably due to systemic atherosclerosis and reduced renal function. Carotid intima-media thickness (IMT) is an independent predictor of cardiovascular mortality in the type 2 diabetic patients. It was reported that carotid IMT was significantly higher in subjects with chronic kidney disease (CKD) in general population, but the relationship between carotid IMT and diabetic CKD was not well known. The aim of our study was to elucidate whether carotid IMT is associated with progression of diabetic nephropathy in type 2 diabetic patients.

Materials and methods: We recruited a total of 177 type 2 diabetic patients ( 89 men and 88 women) with CKD ( $\geq$ stage 2 ) by diabetic nephropathy and followed for $3.2 \pm 2.4$ years. Renal function was evaluated by serum creatinine levels, estimated glomerular filtration rate (eGFR) (calculated by the Cockcroft-Gault equation) and urinary albumin/creatinine ratio (ACR). The carotid IMT was assessed using B-mode ultrasound by measuring generally used parameters. Baseline-to-study end changes in eGFR were calculated, and yearly change of eGFR $(\mathrm{mL} / \mathrm{min} /$ year) was computed.

Results: Overall, the mean age was $60.5 \pm 11.2$ years, duration of diabetes $15.6 \pm 8.2$ years, $\mathrm{HbA}_{1 \mathrm{C}} 7.8 \pm 2.2 \%$, ACR 1,327.4 $\pm 623.1 \mathrm{mg} / \mathrm{gCr}$, serum creatinine $2.1 \pm 1.1 \mathrm{mg} / \mathrm{dL}$, and systemic blood pressure (BP) $133.2 \pm 13.6 / 86.3 \pm 10.9$ $\mathrm{mmHg}$. Mean calculated GFR was $62.4 \pm 18.4 \mathrm{~mL} / \mathrm{min} / 1.73 \mathrm{~m}^{2}$. Of the study population, 68 patients $(38.4 \%)$ were smokers or exsmokers, 165 patients (93.2\%) were having hypertension, and 159 patients (89.8\%) were taking angiotensin converting enzyme inhibitor or angiotensin receptor blocker. Age, waist circumference, duration of diabetes, serum creatinine, ACR and eGFR were significantly correlated with mean or maximum carotid IMT (Table 1). But lipid profiles, $\mathrm{HbA}_{1 \mathrm{C}}$, and systolic and diastolic $\mathrm{BP}$ were not correlated with carotid IMT. Mean yearly change of eGFR was $5.3 \pm 4.3 \mathrm{~mL} / \mathrm{min} /$ year. Yearly change of eGFR was positively correlated with mean and maximum carotid IMT (Figure 1). Changes of ACR or serum creatinine were not significantly correlated with carotid IMT.

Conclusion: Our data suggests that chronic kidney disease is associated with an increased prevalence of carotid atherosclerosis and carotid atherosclerosis may be predictor of progression of diabetic nephropathy in patients with type 2 diabetes.

Table 1. Correlations between carotid IMT and clinical characteristics

\begin{tabular}{lllll}
\hline Variables & $\begin{array}{l}\text { Rt. mean } \\
\text { IMT }\end{array}$ & $\begin{array}{l}\text { Lt. mean } \\
\text { IMT }\end{array}$ & $\begin{array}{l}\text { Mean } \\
\text { IMT }\end{array}$ & $\begin{array}{l}\text { Maximum } \\
\text { IMT }\end{array}$ \\
\hline Age & $0.350^{* *}$ & $0.361^{* *}$ & $0.394^{\star *}$ & $0.369^{* *}$ \\
\hline Waist circumference & $0.162^{*}$ & 0.121 & $0.159^{*}$ & 0.153 \\
\hline Duration of diabetes & $0.382^{* *}$ & $0.359^{* *}$ & $0.360^{* *}$ & $0.401^{* *}$ \\
\hline Total cholesterol & 0.173 & 0.138 & 0.127 & 0.102 \\
\hline HDL-cholesterol & -0.211 & -0.183 & -0.104 & -0.156 \\
\hline Triglycerides & 0.200 & 0.174 & 0.198 & 0.165 \\
\hline Serum creatinine & $0.283^{*}$ & 0.218 & $0.244^{*}$ & 0.217 \\
\hline ACR & $0.259^{*}$ & 0.233 & $0.270^{*}$ & $0.228^{*}$ \\
\hline eGFR & $-0.296^{\star}$ & $-0.286^{*}$ & $-0.265^{*}$ & $-0.282^{*}$ \\
\hline${ }^{*} \mathrm{p}<0.05,{ }^{* *} \mathrm{p}<0.01$ & & & &
\end{tabular}

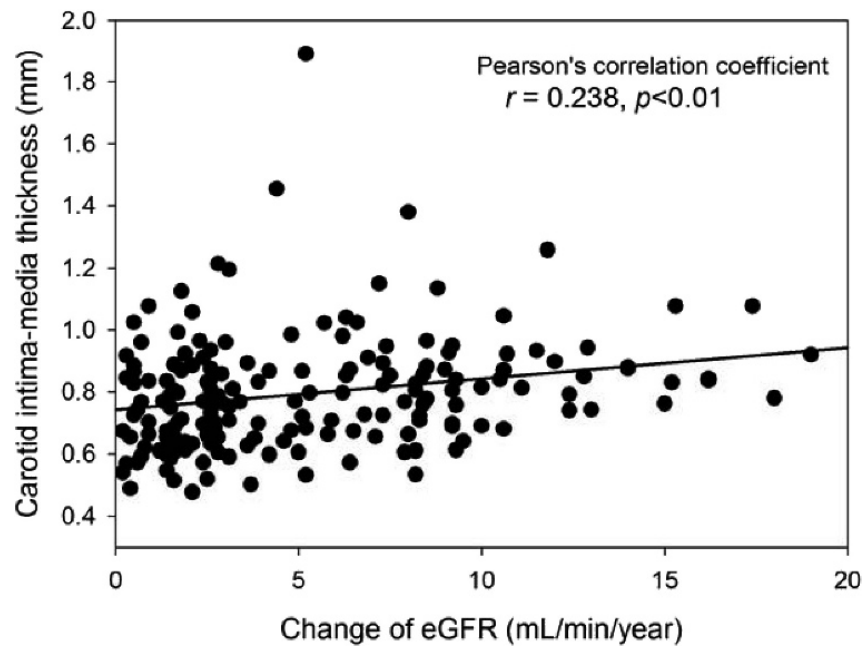

\section{8}

Plasma hs-troponin $\mathrm{T}$ predicts cardiovascular and all cause mortality as well as deterioration in kidney function in type 1 diabetic patients with nephropathy

P. Rossing ${ }^{1}$, A. Jorsal ${ }^{1}$, L. Tarnow ${ }^{1}$, H.-H. Parving ${ }^{2,3}$

${ }^{1}$ Steno Diabetes Center, Gentofte, ${ }^{2}$ Medical Endocrinology, Rigshospitalet, University of Copenhagen, ${ }^{3}$ Faculty of Health Science, University of Aarhus, Denmark

Background and aims: Patients with type 1 diabetes have an elevated risk for early death due to cardiovascular disease and development of end stage renal disease. Plasma high sensitive Troponin T (TnT) is a marker of myocardial injury and micronecrosis. The aim of this study was to investigate the predictive value of plasma levels of plasma hs-TnT in relation to mortality, cardiovascular (CVD) events and progression of diabetic nephropathy in patients with type 1 diabetes.

Materials and methods: A prospective observational follow-up study; including 442 type 1 diabetic patients with overt diabetic nephropathy (234 men; age $42.1 \pm 10.5$ years mean \pm SD, duration of diabetes $28.2 \pm 8.8$ years, GFR $76 \pm 34$ $\mathrm{ml} / \mathrm{min} / 1.73 \mathrm{~m}^{2}$ ) and a group of 458 patients with longstanding type 1 diabetes and persistent normoalbuminuria ( $278 \mathrm{men}$; age $45.4 \pm 11.5$ years, duration of diabetes $27.8 \pm 10.1$ years). Plasma hs TroponinT was measured at baseline. 
Results: Follow-up: 8.2 (0.0-12.9) years (median (range)). During follow up 178 patients died (109 deaths due to CVD), and 89 patients developed end stage renal disease (ESRD). Individuals with high TnT levels (4 ${ }^{\text {th }}$ quartile) had significantly higher all-cause mortality than patients with low levels $\left(1^{\text {st }}\right.$ quartile) (hazard ratio (HR) 8.00 (3.38 to 18.9) adjusted for sex, age, smoking, systolic blood pressure, HbAlc, eGFR, cholesterol, and history of CVD). For patients with diabetic nephropathy the HRadj. for high vs low TnT level was 12.2 (2.80 to 53.5). High TnT levels also predicted CVD mortality (covariate-adjusted HR 17.4 (3.83 to 79.5). Further adjustment for baseline levels of hsCRP and NTproBNP did not change the significant impact of TnT. For CVD fatal and non fatal events the HR adj was 3.52 (1.80 to 6.87). Furthermore, patients with high TnT levels had significantly higher risk for progression to ESRD than patients with low levels (covariate-adjusted HR 1.48 (1.01 to 2.16)). In addition, we found that patients with high levels of plasma hsTnT had an elevated rate of decline in glomerular filtration rate.

Conclusion: High levels of plasma hsTroponin T predict all-cause and CVD mortality in patients with diabetic nephropathy. Furthermore, high levels of hsTnT predicted deterioration of kidney function towards end-stage renal disease.

Supported by: Roche Diagnostics Germany

\section{9}

Diabetes mellitus and anaemia in patients with chronic kidney disease A. Lejnieks ${ }^{1}$, V. Kuzema ${ }^{2}$, A. Petersons ${ }^{2}$

${ }^{1}$ Riga Stradins University, Department of Internal Medicine, Riga Eastern Hospital, Hospital "Linezers", ${ }^{2}$ Riga Stradins University, Department of Internal Medicine, Paul Stradins Clinical University Hospital, Centre of Nephrology, Latvia

Background and aims: Diabetic kidney disease is the leading cause of endstage renal disease (ESRD) in USA and Europe. In Latvia, 23\% of the ESRD patients suffered from diabetes mellitus. Anaemia is a most common complication of chronic kidney disease (CKD). In diabetic nephropathy (DN) anaemia develops at an earlier stage of CKD and tends to be more severe than in non-diabetic kidney disease. The aim of this study was to analyze the prevalence and severity of anaemia in diabetic and non-diabetic subjects with glomerular filtration rate (GFR) below $60 \mathrm{ml} / \mathrm{min}$.

Materials and methods: This is a retrospective analysis of 2147 consultations in CKD patients with GFR $<60 \mathrm{ml} / \mathrm{min}$ during five years (2002-2006). The data was collected from the archive of Latvian Nephrology Centre. According to the guidelines by the Dialysis Outcomes Quality Initiative in 2006, the evidence of anaemia in subjects with CKD was considered in men with level of $\mathrm{Hb}<13.5 \mathrm{~g} / \mathrm{dl}$ and $<12 \mathrm{~g} / \mathrm{dl}$ for woman. We used $T$-tests, ANOVA for comparisons between parameters in independent groups with the appropriation. The Pearson Chi - square or Mann Whitney U tests were used to compare qualitative variables. Survival times for decrease of haemoglobin $(\mathrm{Hb})$ level below $11 \mathrm{~g} / \mathrm{dl}$ were estimated using Kaplan-Meier method and log-rank test for comparison. The criterion for statistical significance was taken as a twotailed $P$ value $<0.05$

Results: A total of 641 patients with CKD III-V were analyzed. We found that $69.6 \%$ of diabetic patients and $52.1 \%$ of non-diabetic patients with CKD III-V had an anaemia (Chi-Square value 10.6; $P=0.001$ ). Mean age in diabetics and non-diabetics were $63.9 \pm 14.2$ years and $60.4 \pm 15.0$ years $(P<0.001)$. Mean haemoglobin levels for diabetic versus non-diabetic patients were $12.5 \pm 1.8$ $\mathrm{g} / \mathrm{dl} v s 13.0 \pm 1.7 \mathrm{~g} / \mathrm{dl}(P=0.027) ; 10.3 \pm 1.5 \mathrm{~g} / \mathrm{dl} v s 11.1 \pm 1.8 \mathrm{~g} / \mathrm{dl}(P=0.016) ;$ $9.3 \pm 1.5 \mathrm{~g} / \mathrm{dl} v s 9.5 \pm 1.9 \mathrm{~g} / \mathrm{dl}(P=0.780)$ in CKD stage III, IV, V, respectively. We noted that the patients with diabetic kidney disease and GFR $<60 \mathrm{ml} /$ min had lower median serum ferritin level than non-diabetics $(98.2 \mathrm{ng} / \mathrm{ml} v \mathrm{~s}$ $105.5 \mathrm{ng} / \mathrm{ml}, P=0.044)$. In addition, patients with diabetes and CKD III-V had a shorter median survival time to decrease of haemoglobin level below $11 \mathrm{~g} / \mathrm{dl}$ compared to non-diabetic patients (12 months $v s 21$ months; log-rank 43.7, $P<0.001)$. The subjects with $\mathrm{DN}$ had higher mean systolic blood pressure $(153.4 \pm 23.7 \mathrm{mmHg} v s \quad 147.7 \pm 23.8 \mathrm{mmHg}, P<0.001)$. The diastolic blood pressure records were similar in the both groups. Patients with diabetes and GFR $<60 \mathrm{ml} / \mathrm{min}$ had significantly lower albumin level $(38.9 \pm 6.5 \mathrm{~g} / \mathrm{l} v \mathrm{~s}$ $40.8 \pm 7.1 \mathrm{~g} / \mathrm{l}, P=0.021)$. Diabetic patients with CKD III-V had significantly lower mean Ca/P product than in non-diabetic patients $\left(43.4 \pm 14.1 \mathrm{mg}^{2} / \mathrm{dl}^{2}\right.$ vs $39.6 \pm 11.5 \mathrm{mg}^{2} / \mathrm{dl}^{2}, P=0.010$ ). Median serum PTH levels were similar in analysed patients groups. The mean $\mathrm{HbAlc}$ in patients with diabetes was $7.6 \pm 1.4 \%$

Conclusion: Anaemia in patients with diabetic kidney disease and GFR < $60 \mathrm{ml} / \mathrm{min}$ is more prevalent and severe than in non-diabetic kidney disease. Anaemia constitutes an additional burden in diabetic kidney disease with advancing age and comorbidities (hypoalbuminemia, arterial hypertension, metabolic disorders).

\section{0}

Insulin analogues mitigate the decline of haemoglobin in diabetic patients with nephropahy

C. Hasslacher ${ }^{1}$, E. Collenberg ${ }^{1}$, D. Raupp ${ }^{1}$, J. Dreyhaupt ${ }^{2}$

${ }^{1}$ Internal Medicine, St. Josephkrankenhaus, ${ }^{2}$ Medical Biometry, Institute of Biometry and Informatics, University of Heidelberg, Germany

Background and aims: Decrease of hemoglobin occurs in diabetic patients with nephropathy earlier than in nondiabetic patients, probably due to impaired synthesis of erythropoietin (EPO). Apart from EPO, insulin also stimulates erythropoiesis. We investigate whether there are differences between human insulin and insulin analogs in respect of their erythropoiesis-stimulating effect.

Materials and methods: Hemoglobin concentration and other factors which may influence hemoglobin levels ( metabolic control (HbAlc), inflammation (hsCRP), antihypertensive medication (RAAS-blocking drugs, beta blocker) were analyzed in 142 type 1 diabetic patients with various degrees of kidney function. Sixty-eight patients were treated with human insulin and $74 \mathrm{pa}-$ tients received an insulin analog.

Results: Hemoglobin concentration did not differ in patients with normal renal function (creatinine clearance (CCL) $>90 \mathrm{ml} / \mathrm{min}$ ) treated with human insulin or insulin analogs. In patients with impaired renal function $(\mathrm{CCL}<90$ $\mathrm{ml} / \mathrm{min}$ ) there was a significant decrease of hemoglobin with declining kidney function in patients treated with human insulin $(r=0.469 ; \mathrm{p}<0.01)$ but not in patients treated with insulin analog $(r=-0.012 ; \mathrm{p}=0.94)$. The difference between the respective correlations was significant $(p=0.03)$. Patients with moderately reduced kidney function (CCL $30-60 \mathrm{ml} / \mathrm{min}$ ) who were treated with insulin analogs showed a significantly higher hemoglobin concentration than those treated with human insulin $(142+12.4$ vs $125+13.1 \mathrm{~g} / \mathrm{l}$; $\mathrm{p}=0.006$ ). There was no difference between the human and analog insulin treated groups with respect to gender (ratio male/female 0,9 vs 1,0 ), age ( 56 vs 57 years), diabetes duration ( 24 vs 25 years), metabolic control ( $\mathrm{HbAlc}$ 7,8 vs $7,9 \%$ ), kind of antihypertensive therapy (RAAS blocking drugs $74 \%$ vs $80 \%$ ) and inflammation status (hs CRP 3,42 vs $3,12 \mathrm{mg} / \mathrm{l}$ )

Conclusion: Insulin analogs mitigate the decline of hemoglobin in diabetic patients with impaired renal function. This may be because these insulins stimulate erythropoiesis via IGF receptor or a sustained activation of the insulin receptor.

Supported by: Manfred Lautenschläger Stiftung Diabetes Germany

\section{1}

Efficacy of the biosimilar erythropoiesis-stimulating agent epoetin zeta to correct and/or maintain haemoglobin concentration in patients with diabetic nephropathy

P. Scigalla ${ }^{1}$, R. Koytchev ${ }^{2}$

${ }^{1}$ Clinical Research, CRC-International Clinical Research Consulting, Berlin, ${ }^{2}$ Clinical Research, CCDRD AG, Neuenhagen, Germany

Background and aims: Reports in the literature suggest more pronounced anaemia and reduced erythropoietin responsiveness in diabetic patients with chronic renal failure. We report efficacy data from 2 clinical trials of epoetin zeta versus epoetin alfa to correct and/or maintain haemoglobin $(\mathrm{Hb})$ concentrations in a subgroup of end-stage renal failure patients with diabetic nephropathy.

Materials and methods: Two randomized, double-blind, multicentre, Phase III trials were conducted. In the correction phase study (Trial 1), patients had anaemia at baseline $(\mathrm{Hb}<9.0 \mathrm{~g} / \mathrm{dL})$, individually corrected up to a target $\mathrm{Hb}$ of 11.0-12.0 g/dL; the equivalence acceptance range for mean $\mathrm{Hb}$ concentration for the whole study population was $\pm 1.0 \mathrm{~g} / \mathrm{dL}$ during the last 4 weeks of the trial. In the maintenance study (Trial 2), patients had stable Hb of 10.5$12.5 \mathrm{~g} / \mathrm{dL}$ at entry, with no change in $\mathrm{Hb}>0.6 \mathrm{~g} / \mathrm{dL}$ in the previous 4 weeks; the equivalence acceptance range for mean $\mathrm{Hb}$ concentration was $\pm 0.6 \mathrm{~g} / \mathrm{dL}$ for the whole study population. In both trials, patients were randomly assigned to receive either epoetin zeta or epoetin alfa intravenously, 1-3 times weekly, for 24 weeks.

Results: Trial $1(\mathrm{n}=609)$ : in the last 4 weeks of treatment, 27 patients with diabetic nephropathy were receiving epoetin zeta. The $\mathrm{Hb} 95 \% \mathrm{CI}$ for the complete haemodialysis population was -0.25 to $0.20 \mathrm{~g} / \mathrm{dL}$ versus -0.81 to 
$0.67 \mathrm{~g} / \mathrm{dL}$ for the diabetic nephropathy population. Trial $2(\mathrm{n}=313)$ : in this crossover trial, 37 patients had diabetic nephropathy. The 95\% CI for the complete haemodialysis population was 0.09 to $0.28 \mathrm{~g} / \mathrm{dL}$ versus -0.03 to 0.36 $\mathrm{g} / \mathrm{dL}$ for the diabetic nephropathy population. The dosage of epoetin zeta and epoetin alfa was equivalent for the entire population of both studies and no significant difference in dose requirements was observed in the subgroup of patients with diabetic nephropathy.

Conclusion: For the diabetic nephropathy population in Trial 1, the 95\% CI was within the $\pm 1 \mathrm{~g} / \mathrm{dL}$ acceptance range, despite this being wider than in the general haemodialysis population. The $95 \%$ CIs for the Trial 2 diabetic population was also wider than in the general population, but again remained well within the $\pm 0.6 \mathrm{~g} / \mathrm{dL}$ acceptance range. These data suggest that epoetin zeta can effectively maintain $\mathrm{Hb}$ levels in this hard-to-treat subgroup when compared to the overall haemodialysis patient population.

Supported by: STADA R\&D GmbH Germany

\section{2}

Increased risk of chronic kidney disease among type 2 diabetic patients with nonalcoholic liver disease

G. Zoppini ${ }^{1}$, L. Bertolini ${ }^{2}$, S. Rodella ${ }^{3}$, M. Chonchol ${ }^{4}$, E. Bonora ${ }^{1}$, M. Muggeo ${ }^{1}$, G. Targher ${ }^{1,2}$

${ }^{1}$ Endocrinology, Ospedale Civile Maggiore, University of Verona, Italy, ${ }^{2}$ Diabetology, Ospedale Sacro Cuore, Negrar, Italy, ${ }^{3}$ Radiology, Ospedale Sacro Cuore, Negrar, Italy, ${ }^{4}$ Renal Diseases and Hypertension, University of Colorado Health Sciences Center, Denver, United States

Background and aims: It is unknown whether chronic kidney disease (CKD) is associated with the presence of non-alcoholic fatty liver disease (NAFLD) among patients with type 2 diabetes. We assessed whether NAFLD predicts the development of CKD in type 2 diabetic patients participating in the Valpolicella Heart Diabetes Study.

Materials and methods: We followed 1,760 outpatients with type 2 diabetes and normal or near-normal kidney function and without overt proteinuria for 6.5 years for the occurrence of CKD (defined as overt proteinuria and/ or estimated glomerular filtration rate [GFR] $<60 \mathrm{ml} / \mathrm{min} / 1.73 \mathrm{~m}^{2}$ ). NAFLD was diagnosed by liver ultrasound and exclusion of other common causes of chronic liver disease.

Results: During follow-up, 547 participants developed incident CKD (i.e., $\sim 4.5 \%$ of participants progressed every year to estimated GFR $<60 \mathrm{ml} /$ $\min / 1.73 \mathrm{~m}^{2}$ and/or overt proteinuria). The presence of NAFLD was associated with a moderately increased risk for CKD (hazard ratio 1.69; 95\% confidence interval 1.3 to $2.6 ; p<0.001$ ). Adjustments for gender, age, BMI, waist circumference, blood pressure, smoking, diabetes duration, HbAlc, lipids, baseline estimated GFR, microalbuminuria, and medications (hypoglycemic, lipid-lowering, antihypertensive or anti-platelet drugs) did not appreciably attenuate this association (hazard ratio 1.49; $95 \%$ confidence interval 1.1 to $2.2 ; p<0.01)$.

Conclusion: Our findings suggest that NAFLD is associated with an increased incidence of CKD in patients with type 2 diabetes independent of numerous confounding factors. Further prospective studies are required to confirm the reproducibility of these results.

\section{PS 116 Human diabetic nephropathy: markers and associated factors}

\section{3}

Endothelial progenitor cells are decreased in type 2 diabetic patients depending on the urinary albumin excretion G. Schernthaner ${ }^{1}$, J. Brix ${ }^{1}$, F. Hoellerl' ${ }^{2}$, R. Koppensteiner ${ }^{2}$, G.-H. Schernthaner';

${ }^{1}$ Medicine I, Rudolfstiftung Hospital, ${ }^{2}$ Medicine II, Medical University of Vienna, Vienna, Austria

Background and aims: Patients with Type 2 Diabetes Mellitus (T2DM) and microalbuminuria (MIA) or macroalbuminuria (MAA) have an increased risk for cardiovascular disease (CVD) morbidity and mortality. The mechanisms responsible for this increased risk are not completely understood. Endothelial progenitor cells (EPC) are (supposedly) bone marrow derived cells circulating in the peripheral blood. They have been demonstrated to be involved in animal adult neovascularisation and (animal and human) endothelial homeostasis and may predict macrovascular disease and mortality (in humans). Thus, it was of interest to investigate the number of EPC in T2DM patients presenting with MIA or MAA in comparison with normoalbuminuric (NOA) patients.

Materials and methods: In total, 138 patients with T2DM were included: 72 NOA, 42 MIA and 24 MAA. The patients in the three groups were carefully matched and did not differ (by ANOVA) for age ( $65 \pm 11$ years), diabetes duration ( $13 \pm 10$ years), $\mathrm{HbA} 1 \mathrm{c}(7.8 \pm 1.6 \%), \mathrm{BMI}(30 \pm 5 \mathrm{~kg} / \mathrm{m} 2)$, systolic and diastolic blood pressure, total cholesterol, LDL-cholesterol, triglyceride and serum creatinine $(1.2 \pm 0.4 \mathrm{mg} / \mathrm{dl})$ (given are mean of all patients). Circulating progenitor cells (CPC; CD34+/CD133+) and EPC (CD34+/CD133+/ $\mathrm{CD} 309+)$ were enumerated by fluorescence activated cell sorting out of peripheral blood specimen.

Results: EPC were significantly reduced in T2DM patients with MIA compared with patients with NOA $(102 \pm 54$ vs $144 \pm 84, \mathrm{p}=0.01)$. In patients with MAA the number of EPC was even more decreased ( $53 \pm 29$ vs $144 \pm 84$; $\mathrm{p}<0.001)$. Patients with MIA or MAA were also significantly different for the number of EPC ( $102 \pm 54$ vs $53 \pm 29, \mathrm{p}<0.001)$. By contrast, total circulating progenitor cells, were not significantly different among the 3 groups of patients with T2DM with NOA, MIA or MAA ( $\mathrm{p}=0.16)$. Multivariate linear regression showed that EPC were independently associated with diabetes duration (Beta $=-0.165, \mathrm{p}=0.036)$ and history of cardiovascular disease (Beta $=-0.203$, $\mathrm{p}=0.01$ ) but strongest with status of albuminuria (Beta $=-0.380, \mathrm{p}<0.001$ ).

Conclusion: In conclusion, this is the first study demonstrating decreased numbers of endothelial progenitor cells in diabetic patients with microalbuminuria or macroalbuminuria. Since low endothelial progenitor cells are important predictors of future cardiovascular morbidity and mortality in nondiabetic high risk patients, these new findings could be relevant for the understanding of the high cardiovascular risk of type 2 diabetic patients with microalbuminuria or macroalbuminuria.

Supported by: Austrian Diabetes Association Research Prize

\section{4}

Urine matrix metalloproteinase- $2,-8$ and -9 activities in type 2 diabetic nephropathy

N.J. van der Zijl1' ${ }^{1}$ R. Hanemaaijer ${ }^{2}$, M.E. Tushuizen ${ }^{1}$, R.K. Schindhelm³

J. Boerop ${ }^{1}$, C. Rustemeijer ${ }^{4}$, H.J. Bilo ${ }^{5}$, J.H. Verheijen ${ }^{2}$, M. Diamant ${ }^{1}$

${ }^{1}$ Endocrinology / Diabetes Centre, VU University Medical Centre,

Amsterdam, ${ }^{2}$ BioSciences, TNO Quality of Life, Leiden, ${ }^{3} \mathrm{Clinical}$ Chemistry, Isala Clinics, Zwolle, ${ }^{4}$ Internal Medicine, Amstelland Hospital, Amstelveen,

${ }^{5}$ Internal Medicine, Isala Clinics, Zwolle, Netherlands

Background and aims: Matrix metalloproteinases (MMPs), including the gelatinases MMP-2 and -9 and the collagenase MMP-8, are involved in extracellular proteolysis. Since the development of diabetic nephropathy (DN) involves an increased synthesis and decreased degradation of the extracellular matrix (ECM) in the glomerulus, MMPs may play a pathophysiological role. Recently, we found increased urinary MMP-2 and -9 activities, but not plasma MMP antigen levels, in type 1 diabetic patients with nephropathy. However, no data are available on urinary MMPs in relation to DN in patients 
with type 2 diabetes mellitus (T2DM). The objective of this study was to determine whether urinary MMPs might be early markers of DN.

Materials and methods: Active and total levels (active and pro-active forms) of MMP-2, -8 and -9 were measured in $24 \mathrm{~h}$ urine collections from 75 T2DM patients and 28 age-matched healthy controls. T2DM patients were stratified according to albuminuria, defined as albumin-to-creatinine ratio (ACR) $>2.5$ $\mathrm{mg} / \mathrm{mmol}$ for males and $>3.5 \mathrm{mg} / \mathrm{mmol}$ for females (albuminuria (UA), $\mathrm{n}=28$; without albuminuria (NA), $\mathrm{n}=47$ ). Additionally, serum creatinine, fasting plasma glucose (FPG) and $\mathrm{HbA}_{1 c}$ were measured. Urinary MMPs were adjusted for urinary creatinine. Only levels of active MMPs are presented, given their biological significance. The relation between clinical and biochemical variables and MMPs and the determinants of renal function, calculated according to the MDRD equation ( $\mathrm{eGFR}_{\mathrm{MDRD}}$ ), were assessed by univariate and multivariable regression analyses.

Results: In the T2DM group (63\% males; (median (IQR)) age 62 (54-70) yrs; diabetes duration 10 (4-17) yrs), 28 subjects had UA (ACR: 10.6 vs. $0.6 \mathrm{mg}$ / mmol in NA). Urinary MMP-2, -8 and -9 levels were significantly elevated in T2DM subjects, irrespective of the presence of albuminuria, as compared to controls (MMP-2: 0.5 vs. $0.0 \mu \mathrm{g} / \mathrm{mmol}$; MMP-8: 4.1 vs. $2.4 \mu \mathrm{g} / \mathrm{mmol}$; MMP-9: 11.1 vs. $1.7 \mu \mathrm{g} / \mathrm{mmol}$, all $P<0.001)$. Similarly, T2DM UA subjects had higher urinary MMPs than T2DM NA subjects (MMP-2: 0.5 vs. $0.0 \mu \mathrm{g} / \mathrm{mmol}$; MMP8: 4.8 vs. $3.4 \mu \mathrm{g} / \mathrm{mmol}$; MMP-9: 13.0 vs. $3.0 \mu \mathrm{g} / \mathrm{mmol}$, all $P<0.05)$. T2DM NA subjects with elevated MMPs scored worse on known risk factors for DN (systolic $\mathrm{BP}$, diabetes duration, $\mathrm{BMI}, \mathrm{HbA}_{1 c}$ and $\mathrm{FPG}$, all $P<0.01$ ). Univariate linear regression analyses identified age, sex, systolic $\mathrm{BP}$, diabetes duration, BMI, $\mathrm{HbA}_{1 c}$ and FPG as determinants of MMP-2, -8 and -9 , all $P<0.03$ ). In multivariable regression analyses age, sex, diabetes duration and MMP-9 were independent determinants of eGFR $\mathrm{MDRD}\left(\mathrm{R}^{2}=0.457\right.$, all $\left.P<0.05\right)$. Of UA patients, $93 \%$ had at least one urinary MMP activity increased, as compared to $71 \%$ of NA and $25 \%$ of Controls $(P<0.05)$.

Conclusion: Although urinary MMP-2, -8 and -9 activities are highest in T2DM UA patients, their elevated levels are already present in some T2DM patients without albuminuria who scored worse on the known risk factors for DN. We hypothesize that urinary MMP activity may reflect incipient diabetes-related renal disease and may be used as an early marker of DN. However, the predictive value of urinary MMP activities in DN should be addressed in large-scaled longitudinal studies.

\section{5}

Serum lipopolysaccharide (LPS) activities are associated with early progression of nephropathy in patients with type 1 diabetes (The FinnDiane Study)

M.J. Lehto ${ }^{1}$, P.J. Pussinen ${ }^{2}$, A.M. Tuomainen ${ }^{2}$, C. Forsblom ${ }^{1,3}$, P.-H. Groop ${ }^{1,3}$ on behalf of the FinnDiane Study Group;

${ }^{1}$ Folkhälsan Research Centre, Folkhälsan Institute of Genetics, Helsinki, ${ }^{2}$ Institute of Dentistry, University of Helsinki, ${ }^{3}$ Department of Medicine, Helsinki University Central Hospital, Finland

Background and aims: Based on prospective studies about one third of patients with type 1 diabetes develop diabetic nephropathy during their lifetime. Many of these patients suffer from low grade chronic inflammation, which might be caused by viral or bacterial pathogens. Lipopolysaccharide (LPS), which is an important component of the outer membrane of gram-negative bacteria, has been shown to induce systemic inflammation. High serum LPS concentrations, commonly found in e.g. patients with periodontitis, increase the risk of atherosclerosis and cardiovascular diseases. The aim of the current study is to investigate whether serum LPS activities are associated with progression of kidney disease in patients with type 1 diabetes.

Materials and methods: Type 1 diabetic patients were examined as a part of the nationwide Finnish Diabetic Nephropathy Study (FinnDiane). Totally 477 patients were selected for this study. The average follow-up time was 5.9 (SD 2.1) years. At the basal visit, 239 patients were normoalbuminuric [NA] (albumin excretion rate - AER $<20 \mu \mathrm{g} / \mathrm{min}$ ) and 238 patients had macroalbuminuria $[\mathrm{MA}]$ (AER $>200 \mu \mathrm{g} / \mathrm{min}$ ). Subjects were further divided into non-progressors and progressors based on the AER and kidney function at the time of re-examination. Eighty out of 239 normoalbuminuric patients had progressed to microalbuminuria [MI] $(20 \mu \mathrm{g} / \mathrm{min}<$ AER $<200 \mu \mathrm{g} / \mathrm{min})$, while 79 out of 238 macroalbuminuric patients had developed end-stage renal disease [ESRD]. All groups were matched for sex and smoking. Serum LPS activities were determined with Limulus Amoebocyte Lysate (LAL) chromogenic endpoint assay (Hycult Biotechnology).

Results: The relationship between serum LPS activities and various clinical parameters was assessed. The strongest correlation was found with serum triglyceride concentrations $(\mathrm{r}=0.61, \mathrm{p}<0.001)$. MA-patients had significantly higher serum LPS activity than NA-patients at baseline [53 (IQR 38-74) vs. 42 (IQR 31-60) EU/ml; p <0.001]. Patients who progressed from NA to MI had higher LPS activity [49 (IQR 34-87) vs. 39 (IQR 29-54) EU/ml; p=0.001), $\mathrm{HbA}_{1 \mathrm{c}}$, cholesterol, triglycerides, as well as lower estimated glucose disposal rate than non-progressors. When these variables were analyzed with Coxregression, only $\mathrm{HbA}_{1 c}$ (HR 1.30 95\%CI 1.13-1.50) was an independent predictor of progression. When non-smokers were analyzed separately with the same model, both $\mathrm{HbA}_{1 \mathrm{c}}$ (HR 1.28 95\%CI 1.10-1.49) and lnLPS (HR 1.92 95\%CI 1.04-3.55) predicted progression. Serum LPS was not associated with progression from MA to ESRD [ 58 (IQR 44-73) vs. 50 (IQR 35-75) EU/ml; $\mathrm{p}=\mathrm{ns}]$.

Conclusion: Serum LPS is an independent predictor of early progression of diabetic kidney disease from normal AER to microalbuminuria in nonsmoking patients with Type 1 diabetes.

Supported by: Stockmann Foundation, Folkhälsan Research Foundation

\section{6}

Type 1 diabetic nephropathy is associated with increased circulating activated platelets and platelet hyperreactivity

L. Tarnow ${ }^{1}$, A.D. Michelson ${ }^{2}$, M.R. Barnard ${ }^{2}$, A.L. Frelinger ${ }^{2}$, B.R. Jensen ${ }^{1}$, B. Aasted ${ }^{3}$, H.-H. Parving ${ }^{4,5}$, P. Rossing ${ }^{1}$, I. Tarnow ${ }^{6}$

${ }^{1}$ Steno Diabetes Center, Gentofte, Denmark, ${ }^{2}$ Center for Platelet Function Studies, University of Massachusetts Medical School, Worcester, United States, ${ }^{3}$ Department of Veterinary Pathobiology, Faculty of Life Sciences, Frederiksberg, Denmark, ${ }^{4}$ Department of Endocrinology, Rigshospitalet, Copenhagen, Denmark, ${ }^{5}$ Faculty of Health Sciences, Aarhus University, Denmark, ${ }^{6}$ Department of Small Animal Clinical Sciences, Faculty of Life Sciences, Frederiksberg, Denmark

Background and aims: Patients with diabetes (DM) have increased platelet activation and platelet reactivity compared to non-diabetic controls. High platelet reactivity has been associated with cardiovascular outcomes in type 2 DM. Patients with diabetic nephropathy are at increased risk of cardiovascular disease. We investigated the relationship between platelet activation/reactivity and the presence of nephropathy in patients with type $1 \mathrm{DM}$.

Materials and methods: Type 1 diabetic patients with persistent normoalbuminuria $(n=53)$ or overt diabetic nephropathy $(n=34)$, and healthy age- and sex-matched controls $(n=30)$ were studied. Platelet surface P-selectin expression, activation of platelet surface GPIIb-IIIa (PAC1 binding), monocyteplatelet (MPAs) and neutrophil-platelet aggregates (NPAs) were measured by whole blood flow cytometry as markers of platelet activation. Platelet reactivity was assessed in response to exogenously added adenosine diphosphate (ADP) and thrombin receptor activating peptide (TRAP). Associations were explored using a 2-way analysis of variance for repeated measurements.

Results: Platelet P-selectin expression (mean flourescent intensity; basal and in response to 0.5 or $20 \mu \mathrm{M}$ ADP) was higher in nephropathy patients compared with patients with normoalbuminuria $(p=0.019)$, and nondiabetic controls $(p=0.0059)$. NPAs were higher in nephropathy patients $(\mathrm{n}=20)$ compared to patients with normoalbuminuria $(\mathrm{n}=26)(p=0.0088)$. MPAs were significantly higher in nephropathy patients compared to nondiabetic controls only $(p=0.0075)$. There were no differences between groups in PAC1 binding or in response to activation with TRAP in any end-point. A significantly higher proportion of patients with nephropathy received aspirin (23 out of 34) compared to patients with normoalbuminuria (13 out of 53) $(\mathrm{p}<0.0001)$.

Conclusions: Type 1 diabetic nephropathy is associated with circulating activated platelets and platelet hyperreactivity to ADP, despite a higher number of these patients receiving anti-platelet treatment with aspirin. This platelet hyperreactivity may be pathogenically related to the known increased risk of cardiovascular events in patients with nephropathy.

Supported by: Danish Diabetes Association, Poul and Erna Sehested Hansens Foundation, Murermester Lauritz Peter Christensen og hustru Kirsten Sigrid Christensens Foundation 


\section{7}

Insulin resistance and C-reactive protein are associated with highnormal urinary albumin excretion in normoalbuminuric type 1 diabetic patients

L. Duvnjak, T. Bulum, N. Car, Z. Metelko

Clinical Department, VUK Vrhovac University Clinic, Zagreb, Croatia

Background and aims: Higher insulin resistance and low-grade inflammatory markers were found to be associated with increased risk of diabetic nephropathy in type 1 diabetic patients. As it is well known that the degree of albuminuria is closely related to the progression of nephropathy, we aimed to determine whether estimated glucose disposal rate (eGDR) and C- reactive protein significantly differ between type 1 diabetic patients with high normal and low normal urinary albumin excretion (UAE).

Materials and methods: Two hundred and 65 normoalbuminuric (UAE < $30 \mathrm{mg} / 24 \mathrm{~h}$ at three occasions) patients were included and divided upon the UAE median $(13,2 \mathrm{mg} / 24 \mathrm{~h})$ into low-normoalbuminuric $(\mathrm{n}=132)$ and highnormoalbuminuric group $(n=133)$. None showed signs of renal or cardiovascular disease and received antihypertensive or other drugs that could attenuate low-grade inflammation. Estimated glucose disposal rate was calculated using the equation: $\mathrm{eGDR}=24.31-(12.22 \mathrm{xWHR})-(3.29 \mathrm{xHT})-(0.57 \mathrm{xHbA1c})$. The units were $\mathrm{mg} \mathrm{kg}^{-1} \mathrm{x} \mathrm{min}^{-1}$, WHR=waist-to-hip ratio, $\mathrm{HT}=$ hypertension. This equation was derived from a substudy of 24 participants in the EDC Study who underwent euglycemic-hyperinsulinemic clamp studies. UAE and C-reactive protein were determined by an immunoturbidimetric assay.

Results: The low-normoalbuminuric $(\mathrm{UAE}=7,15 \pm 3,29)$ and high-normoalbuminuric group (UAE $=21,27 \pm 5,94, \mathrm{p}=0.0001)$ did not differ regarding age, gender, BMI, HbAlc, HDL cholesterol, triglycerides, systolic and diastolic blood pressure, smoking status. High-normoalbuminuric in comparison to low-normoalbuminuric group showed significantly higher diabetes duration $(22 \pm 10,42 ; 18 \pm 9,31 ; \mathrm{p}=0.003)$, C-reactive protein $(2,39 \pm 4,09 ; 1,12 \pm 2,03 \mathrm{mg} / \mathrm{l}$; $\mathrm{p}=0.04)$, and lower eGDR $(5,75 \pm 1,74 ; 8,96 \pm 1,9 ; \mathrm{p}=0.02)$. UAE was positively correlated with diabetes duration, BMI, triglycerides, C-reactive protein, $\mathrm{HbAlc}$, and inversely with eGDR and HDL. In a multiple regression analysis with diabetes duration, eGDR, C-reactive protein, BMI, HDL, triglycerides, $\mathrm{UAE}$ and $\mathrm{HbA1c}$ in a model, eGDR was independently associated with diabetes duration $(\beta=-0,20, p=0.01)$, C-reactive protein $(\beta=-0,106 ; p=0.04)$ and UAE $(\beta=-0,49, p=0.04)$; $C$-reactive protein was independently associated with eGDR $(-\beta=-0,20, p=0.04)$ and diabetes duration $(\beta=0,77 ; \mathrm{p}=0.04)$;

Conclusion: Insulin resistance and $C$ reactive protein are associated with high-normal UAE in type 1 diabetic patients. The independent relation of diabetes duration with eGDR and C-reactive protein suggest that chronic exposure to glucose may stimulate an increase in insulin resistance and induce inflammatory activity already at the normoalbuminuric stage. Whether the detection of increased eGDR and C-reactive protein value in normoalbuminuric type 1 diabetics have predictive value for development of microalbuminuria needs to be assessed.

Supported by: Croatian Ministry of Science

\section{8}

Changes in $\mathrm{O}$-linked $\mathrm{N}$-acetylglucosamine is associated with diabetic nephropathy in the human kidney

N. Fülöp ${ }^{1}$, J. Cseh ${ }^{1}$, P. Degrell ${ }^{1}$, L. Pajor ${ }^{2}$, J.C. Chatham ${ }^{3}$, I. Wittmann ${ }^{1}$ ${ }^{1}$ 2nd Department of Internal Medicine, University of Pécs, Pécs, Hungary, ${ }^{2}$ Department of Pathology, University of Pécs, Hungary, ${ }^{3}$ Department of Medicine/Cardiovascular Disease, University of Alabama at Birmingham, United States

Background and aims: There is increasing evidence that the attachment of a single $\mathrm{N}$-acetylglucosamine to the serine and threonine residues of nuclear and cytoplasmic proteins (O-GlcNAc) plays an important role in cell signaling pathways. It has also been reported that increases in O-GlcNAc contributes to the development of diabetes and diabetic complications; however, little is known about O-GlcNAc levels in diabetic nephropathy. Therefore the goal of this study was to determine whether O-GlcNAc could be detected in human kidney biopsy specimens, and if so to examine whether O-GlcNAc levels were increased in the kidneys of patients with diabetic nephropathy compared to the non-diabetic individuals.

Materials and methods: Using immunohistochemistry biopsy specimens of type- 2 diabetic patients $(n=6)$ and patients with previously diagnosed thin basement membrane nephropathy $(n=7)$ serving as controls, O-GlcNAc was assessed by using CTD110.6 antibody.
Results: Protein O-GlcNAc was detected for the first time in the human kidney. In diabetic patients, we found significantly increased number of CTD110.6 positive podocytes in the glomeruli and significantly elevated staining in the tubuli (both in the nucleus and in the cytosol). Furthermore we detected an intense, granular staining in diabetic tubuli.

Conclusion: Therefore in conclusion we propose that increased O-GlcNAc levels may play a role in the development of diabetic nephropathy.

CTD110.6 positive tubuli and glomeruli in the control and diabetic groups $\left({ }^{*}: \mathrm{p}<0,05\right.$ vs control)

\begin{tabular}{lllll}
\hline & \multicolumn{2}{l}{ Tubuli } & & Glomeruli \\
\cline { 2 - 3 } & \multicolumn{2}{l}{ Cytoplasm } & Nucleus & \\
\cline { 2 - 3 } & Diffuse & Granular & & \\
\hline Control & $31.72 \%$ & $1.00 \%$ & $39.17 \%$ & $38.24 \%$ \\
\hline Diabetic & $51.49 \%^{*}$ & $13.62 \%^{*}$ & $64.54 \%^{*}$ & $63.15 \%^{*}$ \\
\hline
\end{tabular}

\section{9}

Reduced levels of 1,25 $(\mathrm{OH}) 2 \mathrm{D} 3$ and osteoprotegerin occur before the onset of microalbuminuria in diabetes

D.K. Singh ${ }^{1}$, K. Farrington ${ }^{2}$, B. Summerhayes ${ }^{3}$, A. Viljoen ${ }^{4}$, G. Sivakumar ${ }^{4}$, P. Winocour ${ }^{5}$

${ }^{1}$ Diabetes and Renal, Lister Hospital, Stevenage, ${ }^{2}$ Renal Unit, Lister Hospital, Stevenage, ${ }^{3}$ Diabetes and Endocrinology, QEII Hospital, Welwyn Garden City, ${ }^{4}$ Chemical Pathology, Lister Hospital, Stevenage, ${ }^{5}$ Diabetes and Endocrinology, QEII Hospital, Welwyn Garden City, United Kingdom

Background: Derangements of mineral metabolism and associated bone disturbances along with accelerated vascular calcification may occur early in the progression of chronic kidney disease (CKD) in diabetes mellitus (DM). Abnormalities of vitamin D metabolism, and disturbances of the osteoprotegerin (OPG)/RANKL (Receptor activator of nuclear factor kappa B ligand) axis may be implicated. PTH and $1,25(\mathrm{OH})_{2} \mathrm{D}_{3}$ influence bone metabolism by their action on the OPG/RANKL axis. In the vessel wall, RANKL promotes, whilst OPG tends to protect against calcification.

Aims: To study the relationship between $1,25(\mathrm{OH}){ }_{2} \mathrm{D}_{3}$ and the OPG/RAN$\mathrm{KL}$ axis in diabetic patients with normal estimated glomerular filtration rate (eGFR) and without microalbuminuria and in controls.

Materials and methods: We studied 41 non-diabetics, 40 type 1 diabetics and 41 type 2 diabetics with normal eGFR ( $>90 \mathrm{ml} / \mathrm{min}$ ). We measured serum levels of 1,25 $(\mathrm{OH}) \mathrm{D} 3$ and other determinants of bone metabolism in addition to routine haematology and biochemistry.

Results: Median 1,25 $(\mathrm{OH})_{2} \mathrm{D}_{3}$ levels were lower in type $1 \mathrm{DM}(41.0 \mathrm{pmol} / \mathrm{l}$ : $\mathrm{p}<0.001)$ and type $2(41.0 \mathrm{pmol} / \mathrm{l}: \mathrm{p}=0.024)$ than in non-diabetics $(56.1$ $\mathrm{pmol} / \mathrm{l})$, though the groups did not differ with respect to calcium, phosphate, iPTH, and Ntx (cross-linked N-telopeptides of type 1 collagen) levels. However, the levels of total alkaline phosphatase were higher in patients with Type $1 \mathrm{DM}($ median $=88.0 \mathrm{IU} / \mathrm{L})$ than in controls $($ median $=69.0 \mathrm{IU} / \mathrm{L}: \mathrm{p}<0.001)$ and those with Type $2 \mathrm{DM}$ (median $=81.0 \mathrm{IU} / \mathrm{L}: \mathrm{p}=0.008$ ).

OPG levels were lower $($ median $=1.97 \mathrm{pmol} / \mathrm{L})$ in patients with Type $1 \mathrm{DM}$ than in controls $($ median $=2.57 \mathrm{pmol} / \mathrm{L}: \mathrm{p}=0.002)$ and in those with Type $2 \mathrm{DM}$ (median level $=2.91 \mathrm{pmol} / \mathrm{L}: \mathrm{p}<0.001$ ). RANKL levels and OPG/ RANKL ratio did not differ significantly between the groups. There were no correlations between levels of $1,25(\mathrm{OH})_{2} \mathrm{D}_{3}$ and OPG and RANKL, however $1,25(\mathrm{OH})_{2} \mathrm{D}_{3}$ did correlate with alkaline phosphatase levels $(\mathrm{r}=-.208, \mathrm{p}=$ $0.021)$ across the whole study population.

Conclusion: Disturbances of $1,25(\mathrm{OH})_{2} \mathrm{D}_{3}$ and OPG levels occur in patients with diabetes before the onset of microalbuminuria. These abnormalities may play a vital role in the pathogenesis and progression of bone and vascular pathology.

Supported by: Eli Lilly, Roche

\section{0}

Microvascular complications and risk factors in type 2 diabetic patients F.J. Cañizo-Gómez ${ }^{1}$, C. Fernandez-Perez ${ }^{2}$, F.J. Pajuelo-Fernandez ${ }^{3}$ ${ }^{1}$ Endocrinology, Hospital Infanta Leonor, ${ }^{2}$ Preventative Medicine, Hospital Clinico Universitario, ${ }^{3}$ Clinical Research, MSD, Madrid, Spain

Background and aims: Various risk factors such as poor glycaemic control, dyslipidaemia, hypertension, obesity and cigarette smoking, act synergistically for the development of micro and macrovascular complications in type 
2 diabetes mellitus (T2DM) subjects. Our aim was to perform a prospective study of T2DM patients without microvascular complications (nephropathy and retinopathy), analysing the association between various risk factors at baseline and the development of microvascular complications at follow-up, with data obtained from patients records.

Materials and methods: The prospective observational study consisted of 376 T2DM patients enrolled in 2004. The inclusion criteria at baseline in 2004 were normoalbuminuria (urine albumin $<30 \mathrm{mg} / 24 \mathrm{~h}$.) and absence of retinopathy (assessed by fundoscopy). The clinical end-point was microalbuminuria (urine albumin $>30 \mathrm{mg} / 24 \mathrm{~h}$.) and/or presence of retinopathy at follow-up in 2007 . The mean study period was 3.5 years. The following variables were extracted from over diet dose at baseline in 2004: age, gender, diabetes duration, fasting plasma glucose, $\mathrm{HbAlC}$, systolic (SBP) and diastolic blood pressure (DBP), weight, height, body mass index (BMI), waist circumference (WC), total cholesterol, triglycerides (TG), HDL-cholesterol, LDL-cholesterol, C-reactive protein (CRP), fibrinogen, microalbuminuria, creatinine, smoking status, exercise, alcohol consumption, use of hypoglycaemic and lipid-reducing drugs, antihypertensive medications, and other data such family history of diabetes and risk factors. Comparisons of mean levels were performed with the Student's $t$-test for unpaired samples, and comparison of proportions with the chi-square test. Logistic regression analyses were performed with development of microvascular complications at follow up as dependent variable, and age, gender, diabetes duration, and other risk factors included at baseline in 2004 as independent variables. Odds ratios (O.R.) were estimated, with an O.R. of $>1.0$ signifying a positive association. $\mathrm{P}<0.05$ was considered statistically significant (SPSS, 13.0).

Results: Ninety five patients (25\%) developed a microvascular complication at follow-up (46 nephropathy, 49 retinopathy). Compared to those who did not develop microvascular complications at follow-up, the ones who developed microvascular complications were predominantly males $(\mathrm{P}<0.05)$, tended to be older than 60 years $(\mathrm{P}=0.011)$, to have higher percentage of hypertension $(\mathrm{P}=0.008), \mathrm{CRP}>3 \mathrm{mg} / \mathrm{l}(\mathrm{P}=0.007)$, to be taking more insulin $(\mathrm{P}=0.000)$ and less oral hypoglycaemic agents $(\mathrm{P}=0.003)$; and more likely to have a higher mean diabetes duration, WC, SBP, DBP, creatinine, microalbuminuria, and CRP $(\mathrm{P}<0.05$ for all). In the logistic regression analyses of baseline risk factors for the development of microvascular complications at follow-up, the principal independent risk factors were microalbuminuria $>12 \mathrm{mg} / 24 \mathrm{~h}$. (O.R.:6.12; $\mathrm{P}=0.000)$, CRP $>3 \mathrm{mg} / \mathrm{l}(\mathrm{O} . \mathrm{R} .:$ 3,00; $\mathrm{P}=0.004)$ and hypertension (O.R.:2,43; $\mathrm{P}=0.023$ ).

Conclusion: In the T2DM patients studied, levels of microalbuminuria higher than $12 \mathrm{mg} / 24 \mathrm{~h}$., more than $3 \mathrm{mg} / \mathrm{l}$ of CRP and the presence of hypertension were all independent risk factors for the development of microvascular complications.

\section{1}

Renal impairment among type 2 diabetic subjects

C.E. Koro ${ }^{1,2}$, B. Lee ${ }^{1}$

${ }^{1}$ GlaxoSmithKline, Collegeville, ${ }^{2}$ Pharmaceutical Health Services Research, University of Maryland, Baltimore, United States

Background and aims: Type 2 diabetes is a major risk factor for chronic kidney disease (CKD). However, there are limited data on the epidemiology of renal impairment among type 2 diabetic subjects. This study aims to estimate the proportion of type 2 diabetic patients who have different degrees of renal impairment severity, quantified using CKD stages. The CKD stages were categorized based on the classification system established by the National Kidney Foundation Kidney Disease Outcomes Quality Initiative. Further, this study describes the patterns of antidiabetic drug use among subjects with various stages of CKD.

Materials and methods: Using data from the Fourth National Health and Nutrition Examination Survey (1999-2004 NHANES IV), which is representative of non-institutionalized adults in the United States, we performed a cross-sectional analysis on diabetic subjects who were 20 years old or older at the time of interview. To limit the analysis to adults with diagnosed type 2 diabetes, we excluded subjects who met both of the following criteria: age at the time of diagnosis was less than 30 years old and started insulin within one year of their diagnosis.

Results: Descriptive statistical analysis was performed for the study. About forty percent of diabetic subjects captured in this study had CKD. The majority of CKD patients (71.6\%) were at stages 2 and 3 . The proportion of CKD patients using one antidiabetic medication increased from $36.3 \%$ to $78.2 \%$ as CKD progressed from stage 1 to stage 5. On the other hand, the proportion of patients using two or more medications decreased from $36.2 \%$ at stage 1 to
$1.3 \%$ at stage 5 . The proportion of drug naive patients decreased from $18.0 \%$ at stage 1 to $6.6 \%$ at stage 4 . The proportion of CKD patients using sulfonylurea decreased steadily from $45.1 \%$ at stage 1 to $11.7 \%$ at stage 5 . Similarly, the proportion of CKD patients using metformin decreased from $45.6 \%$ at stage 1 to $23.8 \%$ at stage 3 . Metformin was not used in subjects with CKD stages 4 and 5. The proportion of CKD patients on insulin only steadily increased from $6.7 \%$ at stage 1 to $58.3 \%$ at stage 5 . On the other hand, the proportion of patients on OAD only steadily decreased from $69.0 \%$ to $27.8 \%$.

Conclusion: The number of antidiabetic agents used trended towards a decrease with the increase in CKD stage. As the stage of CKD increased, subjects were more likely to be on insulin and less likely to be on OADs. Metformin was not used in stages 4 and 5 , consistent with its labelled contraindication.

The distribution of renal impairment among type 2 diabetics by CKD stage

\begin{tabular}{llll}
\hline CKD Stage & $\mathbf{n}$ & $\mathbf{N}$ & $\mathbf{\%}$ \\
\hline No CKD & 618 & $6,783,138$ & 50.8 \\
\hline Stage 1 & 121 & $1,153,761$ & 8.6 \\
\hline Stage 2 & 173 & $1,488,358$ & 11.1 \\
\hline Stage 3 & 254 & $2,365,299$ & 17.7 \\
\hline Stage 4 & 32 & 236,397 & 1.8 \\
\hline Stage 5 & 16 & 70,621 & 0.5 \\
\hline Missing & 248 & $1,263,854$ & 9.5 \\
\hline Total & 1,462 & $13,361,428$ & 100.0 \\
\hline
\end{tabular}




\section{PS 117 Treating diabetic nephropathy}

\section{2}

The decrease in urinary albumin excretion induced by lifestyle intervention is mediated by the abdominal fat reduction in obese nondiabetic women

C. Invitti, L. Gilardini, M. Croci, G. Redaelli, C. Antonio

Unit of Metabolic Diseases and Diabetes, Istituto Auxologico Italiano, Milan, Italy

Background and aims: Microalbuminuria is a modifiable early marker of generalized endothelial dysfunction and atherosclerosis. Weight loss may reduce urinary albumin excretion (UAE); however it is unclear what weight loss-induced changes are responsible for UAE decrease. We therefore investigated the effect of 3-month lifestyle modifications on UAE in 123 non diabetic obese women $\left(49.4 \pm 13.5 \mathrm{yrs}\right.$, BMI $36.8 \pm 4.9 \mathrm{~kg} / \mathrm{m}^{2}$, waist circumference $111.5 \pm 10.7 \mathrm{~cm})$.

Materials and methods: Before and after lifestyle modifications, all women underwent an oral glucose tolerance test and a bioimpedance analysis. A blood sample was taken for lipids and C-reactive protein (CRP) measurement. Microalbuminuria was defined by albumin/creatinine ratio $(\mathrm{A} / \mathrm{C})$ of $3.5-25 \mathrm{mg} / \mathrm{mmol}$ and metabolic syndrome (MS) defined by ATPIII criteria. Glomerular filtration rate (GFR) was estimated with Cockroft- Gault formula.

Results: Seven percent of women had microalbuminuria. Women in the III tertile of $\mathrm{A} / \mathrm{C}$ ratio had significantly higher BMI ( $38.2 \pm 5.6$ vs $36.7 \pm 3.8$ vs $35.9 \pm 5.1 \mathrm{~kg} / \mathrm{m}^{2}, \mathrm{p}<0.05$ in the III, II and I tertile of $\mathrm{A} / \mathrm{C}$ ratio), insulin $(12.6 \pm 6.0$ vs $10.9 \pm 5.9$ vs $9.3 \pm 4.6 \mu \mathrm{U} / \mathrm{ml}, \mathrm{p}<0.01)$, HOMA-IR $(2.9 \pm 1.5$ vs $2.5 \pm 1.6$ vs $2.0 \pm 1.1, \mathrm{p}<0.01$ ) levels, had a higher prevalence of MS ( $43 \%$ vs $38 \%$ vs $29 \%$ ) and have higher levels of all the other MS components compared to those in the II and I tertile of $\mathrm{A} / \mathrm{C}$ ratio. GFR, fat mass and C- reactive protein levels did not influence $\mathrm{A} / \mathrm{C}$ ratio. The lifestyle intervention induced a mean weight decrease of $5.2 \pm 4.0 \%$. Microalbuminuria reverted to normoalbuminuria in $80 \%$ of cases. Despite women in the I, II and III tertile of A/C ratio showed similar weight loss, A/C ratio decreased only in women in the III tertile (from $3.2 \pm 4.7$ to $2.4 \pm 3.8 \mathrm{mg} / \mathrm{mmol}, \mathrm{p}<0.01$ ) who showed the greatest decrease in waist circumference $(-4.4 \pm 2.5 \%$ vs $3.7 \pm 3.9 \%$ vs $2.3 \pm 3.0 \%, \mathrm{p}<0.01$ in the III vs II vs I tertile of $\mathrm{A} / \mathrm{C}$ ratio) and a reduction in post-load insulin excursion ( $+20 \% \pm 11.0 \%$ vs $+15 \pm 10.7 \%$ vs $-7.0 \pm 4.8 \%)$. Changes in GFR, lipids, blood pressure and glucose did not influence changes in $\mathrm{A} / \mathrm{C}$ ratio.

Conclusion: In non-diabetic obese women, lifestyle modifications induce a decrease in UAE which is mediated by the reduction in abdominal fat but not by changes in the others MS components.

\section{3}

Obesity predicts changes in urine albumin/creatinine ratio in patients with type 2 diabetes: the DEMAND study

M. Comaschi ${ }^{1}$, M.C. Rossi ${ }^{2}$, A. Nicolucci ${ }^{2}$, A. Ceriello ${ }^{3}$, D. Cucinotta ${ }^{4}$,

C. Giorda ${ }^{5}$, I. Otranto ${ }^{2}$, F. Pellegrini ${ }^{2}$, B. Pomili², U. Valentini ${ }^{6}$,

G. Vespasiani ${ }^{7}$, S. De Cosmo ${ }^{8}$

${ }^{1}$ Internal Medicine Unit, Emergency Department, University Hospital S. Martino, Genova, Italy, ${ }^{2}$ Department of Clinical Pharmacology and Epidemiology, Consorzio Mario Negri Sud, S. Maria Imbaro, Italy, ${ }^{3}$ University of Warwick, Coventry, United Kingdom, ${ }^{4}$ Department of Internal Medicine, Policlinico Universitario, Messina, Italy, ${ }^{5}$ Metabolism and Diabetes Unit, Chieri, Italy, ${ }^{6}$ Diabetes Unit, Spedali Civili, Brescia, Italy, ${ }^{7}$ Diabetes and Metabolism Unit, Madonna del Soccorso Hospital, S. Benedetto del Tronto, Italy, ${ }^{8}$ Unit of Endocrinology, Casa Sollievo della Sofferenza, S. Giovanni Rotondo, Italy

Background and aims: While central obesity has recently received attention as a potential risk factor for renal disease in nondiabetic subjects, its role in individuals with T2DM has been seldom investigated. Aim of this study was to evaluate whether waist circumference and BMI predicted the progression or regression of albumin/creatinine ratio (ACR) in a cohort of individuals with T2DM.

Materials and methods: A random sample of 1289 patients aged $18-80$ years was recruited by 50 Italian diabetes clinics affiliated to the Associazione Medici Diabetologi. A morning spot urine sample was collected to centrally measure ACR and to detect urinary infections. Patients were re-evaluated after one year. Progression of ACR was defined as a $50 \%$ increase in levels from baseline to follow-up, while regression was defined as a $50 \%$ reduction. A multivariate logistic regression was performed to evaluate factors associated with progression and regression of ACR.

Results: Overall, 1019 were included in the analyses, while 211 were excluded for urinary infections and 59 because of incomplete information. After one year, 170 (16.7\%) patients had a progression of ACR levels, while $241(23.7 \%)$ showed a regression. The likelihood of ACR progression increased by $14 \%$ for every five-years increase in diabetes duration $(\mathrm{OR}=1.14$; 95\%CI 1.03 1.27 ) and by $8 \%$ for each five- $\mathrm{cm}$ increase in waist circumference measured at baseline ( $\mathrm{OR}=1.08 ; 95 \% \mathrm{CI} 1.00-1.16)$. Furthermore, the risk of progression increased by $28 \%$ for every one-unit increase in BMI during the follow-up $(\mathrm{OR}=1.28 ; 95 \% \mathrm{CI} 1.12-1.46)$. The likelihood of ACR regression increased by $3 \%$ (OR=1.03; IC95\% 1.01-1.05) for every one-cm reduction of waist circumference during the study. No other variables were independently associated with regression of ACR.

Conclusion: In individuals with T2DM obesity is a factor for progression of ACR, independent of blood glucose, blood pressure, and baseline ACR levels. This independent effect could be mediated primarily by adipogenic inflammation and endothelial dysfunction which accompany central obesity. Reducing waist circumference can favor the regression of albuminuria, probably by improving the mechanism of insulin resistance.

\section{4}

The SURE Study: effects of structured versus usual care on renal endpoint in type 2 diabetes: a randomized multi-centre study W.Y. So ${ }^{1}$, C.-Y. Yeung ${ }^{1}$, G.T.C. $\mathrm{Ko}^{2}$, K.W. Chan ${ }^{3}$, C.H. Chung ${ }^{3}$, G. Kum ${ }^{4}$, L.M. Fung ${ }^{5}$, T.P. Ip 6 , I.T. Lau', J.K.Y. Li ${ }^{8}$, S.C. Siu', M.W. Tsang, V.T.F. Yeung ${ }^{9}$, W.Y.S. Leung ${ }^{10}$, J.C.N. Chan ${ }^{1}$

${ }^{1}$ Department of Medicine and Therapeutics, The Chinese University of Hong Kong, ${ }^{2}$ Department of Medicine, Alice Ho Miu Ling Nethersole Hospital, ${ }^{3}$ Department of Medicine, The Princess Margaret Hospital, ${ }^{4}$ Department of Medicine, The United Christian Hospital, ${ }^{5}$ Department of Medicine, Caritas Medical Centre, ${ }^{6}$ Department of Medicine, Tung Wah Eastern Diabetes Center, ${ }^{7}$ Department of Medicine, The Tseung Kwan O Hospital, ${ }^{8}$ Department of Medicine, Yan Chai Hospital, ${ }^{9}$ Department of Medicine, Our Lady of Maryknoll Hospital, ${ }^{10}$ Department of Pharmacy, The Queen Elizabeth Hospital, Hong Kong SAR, Hong Kong

Background and aims: Multifaceted care reduced mortality, micro and macrovascular complications in type 2 diabetes. We hypothesize that structured care (SC) reduces end stage renal disease (ERSD) in type 2 diabetes.

Materials and methods: Chinese Type 2 diabetic patients with plasma creatinine between 150 and $350 \mu \mathrm{mol} / \mathrm{L}$ were recruited from 9 public hospitals in Hong Kong. The SC group was managed by a diabetes team using a protocol with predefined clinic and assessment schedules and treatment goals: a) blood pressure $<130 / 80 \mathrm{mmHg}$ b) $\mathrm{HbA}_{1 c}<7 \%$ c) LDL-C $<2.6 \mathrm{mmol} / \mathrm{L} \mathrm{d}$ ) triglyceride $<2 \mathrm{mmol} / \mathrm{L}$ and e) persistence with blockers of renin angiotensin system. Main outcome measures were composite primary endpoints of death, ESRD defined as serum creatinine $>500 \mu \mathrm{mol} / \mathrm{L}$ or need for dialysis.

Results: 205 patients [mean (SD) age: $65 \pm 7.2$ years; disease duration: $14 \pm 7.9$ years; plasma creatinine: $197 \pm 39.9 \mu \mathrm{mol} / \mathrm{L}$ ] were randomized to receive either SC $(n=104)$ or UC $(n=101)$. Of these, 167 patients (SC: 84 and UC: 83) completed the 2-year study. The SC group had better metabolic control than the UC group $\left(\mathrm{HbA}_{1 \mathrm{c}}: 7.3 \pm 1.3 \%\right.$ vs $8.0 \pm 1.6 \%$, p<0.01; LDL-C: $2.49 \pm 0.81$ vs $2.84 \pm 1.05 \mathrm{mmol} / \mathrm{L}, \mathrm{p}=0.01)$ with $61 \%(\mathrm{n}=63)$ in the SC group and $28 \%$ $(\mathrm{n}=28)$ in the UC group $(\mathrm{p}<0.001)$ attaining $\geq 3$ treatment goals. Primary composite endpoint occurred in 24 patients in the SC group and 24 patients in the UC group. Patients who attained $\geq 3$ treatment targets $(n=91)$ had reduced risk of reaching composite endpoint [14 vs 34; RR: 0.43 (95\% CI: 0.21 - 0.86)] and death [4 vs 15 deaths; RR 0.30 (95\% CI 0.10 - 0.95)] than those who attained 2 or fewer targets $(\mathrm{n}=114)$.

Conclusion: Attainment of multiple treatment targets reduced ESRD and death rates in type 2 diabetes. In addition to protocol and specialist care, clinical audits and feedback are needed to improve clinical outcomes. Supported by: Health Service Rearch Fund 


\section{5}

Effect of dietary salt supplementation on albumin excretion rate (AER) response to angiotensin receptor blockade $+/$ - thiazide therapy in hypertensive patients with type 2 diabetes and increased AER E.I. Ekinci, G. Thomas, B. Chappell, R. MacIsaac, C. Johnson, C. Houlihan, H. Hao, S. Finch, S. Panagiotopoulos, C. O'Callaghan, G. Jerums Department of Endocrinology, Austin Health and The University of Melbourne, Australia

Background and aims: This was a double blind, crossover, randomised placebo controlled study to assess the effect of sodium chloride $(\mathrm{NaCl})$ supplementation on the actions of angiotensin receptor blockade (ARB) alone +/- thiazide on AER in hypertensive patients with type 2 diabetes mellitus (T2DM) and elevated AER (10-200 $\mathrm{mcg} / \mathrm{min})$. Participants had either habitual low dietary sodium (LDS), 2/3 24 hour urine sodium (Na) excretion of $<100 \mathrm{mmol} /$ day or high dietary sodium (HDS) intake, 2/3 24 hour urine $\mathrm{Na}$ excretion of $>200 \mathrm{mmol} /$ day.

Materials and methods: During a washout period (week -6 to 0), usual antihypertensives were replaced by verapamil, prazosin, methyldopa or hydralazine in LDS (mean age 60, median AER $48 \mathrm{mcg} / \mathrm{min}$, mean BMI 32.9 ) and HDS (mean age 65, median AER $23 \mathrm{mcg} / \mathrm{min}$, mean BMI 32.0) to target blood pressure $\leq 140 / 90$. There was no statistically significant difference in baseline AER in HDS and LDS groups. Patients then received telmisartan $40 \mathrm{mg} /$ day (T) for 4 weeks. In the next 4 weeks hydrochlorothiazide $12.5 \mathrm{mg}$ /day (Hct) was added to T. For the second 2 weeks of each treatment period, patients randomly received capsules containing either $100 \mathrm{mmol} /$ day $\mathrm{NaCl}$ or placebo (P). After a second 6 week washout period, median AER was unchanged from baseline in the LDS $(53 \mathrm{mcg} / \mathrm{min})$ and HDS $(28 \mathrm{mcg} / \mathrm{min})$ groups. The regimen was repeated but $\mathrm{NaCl}$ supplementation and $\mathrm{P}$ were reversed. $24 \mathrm{hr}$ urinary AER and Na excretion were measured at weeks $0,4,8$, 14,18 and 22.

Results: Mean Na excretion (mmol/24 hr) in the LDS group with $\mathrm{T}+\mathrm{P}$ was $124 \pm 7$ and $126 \pm 13$ with $\mathrm{T}+\mathrm{Hct}+\mathrm{P}$. Mean Na excretion in the HDS group with $\mathrm{T}+\mathrm{P}$ was $264 \pm 24$ and $249 \pm 15$ with $\mathrm{T}+\mathrm{Hct}+\mathrm{P}$. An increase in mean urine $\mathrm{Na}$ excretion of $56 \mathrm{mmol} /$ day was seen in both groups during $\mathrm{NaCl}$ supplementation. The statistical comparison of the various interventions on AER are shown in the table and the figure shows the AER response as treatment / baseline ratio.

Conclusion: This study shows that in diabetic subjects with habitually low salt intake, salt supplementation markedly reduces AER response to ARB with or without the addition of diuretic. By contrast, in subjects with habitually high salt intake, the response to ARB with or without diuretic, is already reduced and is not influenced any further by salt supplementation. In this analysis, the effects of the various interventions on AER were independent of changes in blood pressure.

\section{Analysis of variance results from a 3 way analysis of covariance}

\begin{tabular}{lll}
\hline Explanatory variable & $\underline{\text { F }}$ & P value \\
\hline Salt supplement (Salt vs placebo) & 5.18 & 0.026 \\
\hline Treatment (T vs T+Hct) & 4.99 & 0.028 \\
\hline Habitual sodium intake group (High vs Low) & 0.01 & 0.9 \\
\hline Salt supplement by Habitual sodium intake group & 4.68 & 0.034 \\
\hline Change in Mean Arterial Pressure (covariate) & 0.86 & 0.4 \\
\hline
\end{tabular}

\section{AER RESPONSE}

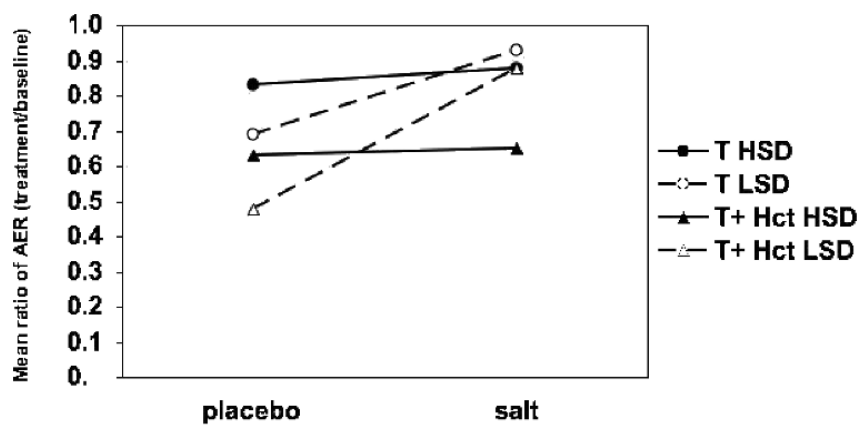

Supported by: NHMRC, Pfizer
Irbesartan delays progression of nephropathy as measured by estimated glomerular filtration rate: post-hoc analysis of the Irbesartan Diabetic Nephropathy Trial

R.W. Bilous ${ }^{1}$, S.C. Bain², C. Beckham³ , S. Hogan ${ }^{4}$, M. Evans ${ }^{5}$

${ }^{1}$ Academic Centre, James Cook University Hospital, Middlesbrough, ${ }^{2}$ Diabetes and Endocrine Unit, Swansea University, ${ }^{3}$ Bristol-Myers Squibb, Uxbridge, ${ }^{4}$ sanofi-aventis, Guildford, ${ }^{5}$ Department of Metabolic Medicine, Diabetes and Endocrinology, University of Wales College of Medicine, Cardiff, United Kingdom

Background and aims: Results from the Irbesartan Diabetic Nephropathy Trial (IDNT) showed that irbesartan slowed progression of type 2 diabetic nephropathy compared with amlodipine and placebo; this effect was independent of blood pressure (BP) reduction. IDNT had a primary composite endpoint of doubling serum creatinine, end-stage renal disease (characterized by need for dialysis or transplantation) or death. Based upon updated guidelines from the US Kidney Disease Outcomes Quality Initiative group, which are being implemented throughout Europe, estimated glomerular filtration rate (eGFR) is a more accurate and convenient measure for estimating kidney function than the previous standard measure, serum creatinine. eGFR is calculated using an equation that corrects serum creatinine measurements for patient variability in age, sex, and race. A post-hoc analysis to assess nephropathy as measured by eGFR in IDNT was performed.

Materials and methods: IDNT was a phase 3, randomized controlled trial in 1715 type 2 diabetic patients with nephropathy who received irbesartan (300 $\mathrm{mg} /$ day), amlodipine (10 mg/day), or placebo (non-angiotensin II receptor blocker, -angiotensin-converting enzyme inhibitor, or -calcium channel blocker antihypertensives). Median follow up was 2.6 years ( 31 months). The Modification of Diet in Renal Disease equation was used to calculate eGFR using baseline data for age, sex and race, and baseline and quarterly-measured serum creatinine levels.

Results: Similar reductions in BP (mean arterial pressure; MAP) were achieved in the irbesartan and amlodipine arms; MAP was reduced more effectively in both these arms vs placebo $(p=0.001)$. There was a smaller mean change in eGFR from baseline ( $\triangle \mathrm{eGFR}$ ) in the irbesartan arm compared with the placebo and amlodipine arms towards the end of the study (irbesartan vs placebo, -10.99 vs $-14.81 \mathrm{ml} / \mathrm{min} / 1.73 \mathrm{~m}^{2}, \mathrm{p}=0.011$ at month 39 ; irbesartan vs amlodipine, $-11.67 \mathrm{vs}-16.11 \mathrm{ml} / \mathrm{min} / 1.73 \mathrm{~m}^{2}, \mathrm{p}=0.004$ at month 42 , and -12.72 vs $-16.46 \mathrm{ml} / \mathrm{min} / 1.73 \mathrm{~m}^{2}, \mathrm{p}=0.043$ at month 45$)$. Rate of $\Delta \mathrm{eGFR}$ from months 21-48 was significantly lower for irbesartan vs placebo and amlodipine ($1.66,-3.07$, and $-3.45 \mathrm{ml} / \mathrm{min} / 1.73 \mathrm{~m}^{2}$ per year, respectively; $\mathrm{p}=0.0002$ and $p=0.00006$ for irbesartan vs placebo and irbesartan vs amlodipine; $p=0.75$ for amlodipine vs placebo).

Conclusion: In patients with type 2 diabetes and nephropathy, irbesartan, compared to amlodipine or placebo, significantly reduced the rate of eGFR decline after 20 months of treatment.

Supported by: Bristol-Myers Squibb, sanofi-aventis

\section{7}

The effect of direct renin inhibition alone or in combination with angiotensin II receptor blockade on markers of the renin-angiotensin aldosterone system

E. Frandsen ${ }^{1}$, F. Persson ${ }^{2}$, F. Boomsma ${ }^{3}$, M. Prescott ${ }^{4}$, W.P. Dole ${ }^{5}$, P. Rossing ${ }^{2}$, H.-H. Parving ${ }^{6,7}$

${ }^{1}$ Dept of Clinical Physiology and Nuclear Medicine, Glostrup University Hospital, Denmark, ${ }^{2}$ Steno Diabetes Center, Gentofte, Denmark,

${ }^{3}$ Department of Medicine, Erasmus MC, Rotterdam, Denmark, ${ }^{4}$ Novartis, East Hanover, United States, ${ }^{5}$ Novartis, Cambridge, United States, ${ }^{6}$ Department of Medical Endocrinology, University Hospital, Copenhagen, Denmark, ${ }^{7}$ Faculty of Health Science, Aarhus University, Denmark

Background and aims: Blockade of the $\mathrm{AT}_{1}$ receptor by irbesartan reduces albuminuria, but increases plasma renin activity (PRA) and angiotensin (Ang) I and II levels. Direct renin inhibition with aliskiren further reduces albuminuria when added to irbesartan; this may be due to reduction of PRA and more effective renin system blockade. We report the effect of aliskiren alone and in combination with irbesartan on renin system biomarkers in patients with type 2 diabetes, hypertension and albuminuria.

Materials and methods: In a double-blind, randomized, cross-over study, 24 patients were treated in 2-month periods with placebo, aliskiren $300 \mathrm{mg}$, irbesartan $300 \mathrm{mg}$ or the combination. High-sensitivity PRA (using sheep angiotensinogen as substrate, hsPRA), plasma concentrations of renin (PRC), prorenin (Pro), Ang I, Ang II, angiotensinogen, aldosterone and ACE activity 
were measured at the end of each treatment period. Treatment effects were assessed in a mixed model analysis. Results are geometric mean change vs placebo.

Results: Aliskiren significantly reduced hsPRA, Ang I and Ang II by $87 \%$, $75 \%$ and $52 \%$ respectively; irbesartan had the opposite effect (Table). In combination, the stimulatory effect of irbesartan was inhibited by aliskiren to a similar degree $(88 \%, 78 \%$ and $56 \%$ for hsPRA, Ang I and Ang II respectively). Renin specific activity (renin bioactivity/total renin) was $3 \%$ and $4 \%$ (i.e. $97 \%$ and $96 \%$ renin inactivation) after aliskiren and combination therapy respectively. Combination treatment caused a synergistic effect on the degree of RAAS blockade. Overall, active treatment-induced increases in PRC correlated significantly with reductions in UAER $(\mathrm{r}=-0.339, \mathrm{p}=0.005)$. Similar correlations were observed for each treatment (aliskiren, $\mathrm{r}=-0.420, \mathrm{p}=0.052$; irbesartan, $\mathrm{r}=-0.486, \mathrm{p}=0.022$; combination, $\mathrm{r}=-0.319, \mathrm{p}=0.148$ ). Irbesartan reduced angiotensinogen by $26 \%(p<0.001)$; changes after aliskiren or the combination were not significant. There were no significant changes in ACE activity or aldosterone levels.

Conclusion: Each monotherapy reduced albuminuria and affected renin system biomarkers in the expected fashion. Combining aliskiren with an $\mathrm{AT}_{1}$ receptor blocker provided a larger reduction in albuminuria that correlated with more complete intra-renal renin system blockade (reflected by increases in PRC), and thus improves renoprotection.

\begin{tabular}{llllll}
\hline & hsPRA \% & Pro \% & PRC \% & Ang I \% & Ang II \% \\
\hline Aliskiren & $\downarrow 87$ & $\uparrow 14$ & $\uparrow 279$ & $\downarrow 75$ & $\downarrow 52$ \\
& $\mathrm{p}<0.001 \uparrow$ & $\mathrm{p}=0.06$ & $\mathrm{p}<0.001$ & $\mathrm{p}<0.001$ & $\mathrm{p}<0.001$ \\
\hline \multirow{2}{*}{ rbesartan } & $\uparrow 321$ & $\uparrow 39$ & $\uparrow 178$ & $\uparrow 207$ & $\uparrow 237$ \\
& $\mathrm{p}<0.001$ & $\mathrm{p}<0.001$ & $\mathrm{p}<0.001$ & $\mathrm{p}<0.001$ & $\mathrm{p}<0.001$ \\
\hline Combination & $\downarrow 47$ & $\uparrow 34$ & $\uparrow 1068$ & $\downarrow 30$ & $\uparrow 52$ \\
& $\mathrm{p}=0.006$ & $\mathrm{p}<0.001$ & $\mathrm{p}<0.001$ & $\mathrm{p}=0.058$ & $\mathrm{p}=0.041$ \\
\hline
\end{tabular}

Supported by: Novartis

\section{8}

Renoprotective effects of direct renin inhibition compared to and in combination with maximum recommended dose of irbesartan in patients with type 2 diabetes and albuminuria

F. Persson ${ }^{1}$, P. Rossing ${ }^{1}$, H. Reinhard ${ }^{1}$, T. Juhl ${ }^{1}$, C.D.A. Stehouwer ${ }^{2}$, C. Schalkwijk ${ }^{2}$, A.H.J. Danser ${ }^{3}$, F. Boomsma ${ }^{3}$, E. Frandsen ${ }^{4}$, H.-A. Dieterich ${ }^{5}$, M. Prescott ${ }^{6}$, W.P. Dole ${ }^{7}$, H.-H. Parving ${ }^{8,9}$

${ }^{1}$ Steno Diabetes Center, Gentofte, Denmark, ${ }^{2}$ Dept of Medicine, University Hospital, Maastricht, Netherlands, ${ }^{3}$ Dept of Medicine, Erasmus MC, Rotterdam, Netherlands, ${ }^{4}$ Dept of Clinical Physiology and Nuclear Medicine, University Hospital, Glostrup, Denmark, ${ }^{5}$ Novartis, Basel, Switzerland, ${ }^{6}$ Novartis, East Hanover, United States, ${ }^{7}$ Novartis, Cambridge, United States, ${ }^{8}$ Department of Medical Endocrinology, University Hospital, Copenhagen, Denmark, ${ }^{9}$ Faculty of Health Science, Aarhus University, Denmark

Background and aims: Like other pharmacological agents that block the renin-angiotensin-aldosterone system (RAAS), the direct renin inhibitor aliskiren has antiproteinuric and antihypertensive effects in patients with type 2 diabetes. We investigated if aliskiren ( $300 \mathrm{mg}$ OD) has an antiproteinuric effect comparable to the maximum recommended dose of irbesartan $(300 \mathrm{mg}$ OD), and also if there is any additional benefit from the combination. In addition, treatment effects on cardiovascular biomarkers were investigated.

Materials and methods: Double-blind, randomised, cross-over trial. After 1 month washout 24 patients with type 2 diabetes, hypertension and albuminuria ( $>30 \mathrm{mg} /$ day) were randomised to four 2 -month treatment periods, receiving treatment with placebo (baseline), aliskiren $300 \mathrm{mg}$ once daily, irbesartan $300 \mathrm{mg}$ once daily or the combination of the two active treatments, in random order. During washout and all treatment periods, patients also received long-acting furosemide in a stable dose to control sodium retention. The primary endpoint was change in urinary albumin excretion rate (UAER). Secondary measures included change in arterial blood pressure (BP).

Results: Placebo (baseline) geometric mean UAER was $258 \mathrm{mg} /$ day (range 841265), seated office BP was $135 / 78 \mathrm{mmHg}$, GFR was 90 (SD 27) $\mathrm{ml} / \mathrm{min} / 1.73$ $\mathrm{m}^{2}$. Aliskiren treatment showed a significant reduction in UAER by $48 \%$ (95\% CI 27-62) compared to placebo $(\mathrm{p}<0.001)$, not significantly different from irbesartan treatment, which reduced UAER by $58 \%(42-70)$ ( $\mathrm{p}<0.001$ vs. placebo). Dual RAAS blockade reduced UAER by $71 \%$ (59-79) which was significantly more than with either monotherapy $(\mathrm{p}<0.001$ and $\mathrm{p}=0.028)$. Seated office $\mathrm{BP}$ was reduced $7 / 4 \mathrm{mmHg}$ by aliskiren, $6 / 4 \mathrm{mmHg}$ by irbesartan and $12 / 8 \mathrm{mmHg}$ by the combination, all statistically significant compared to pla- cebo, except for irbesartan on diastolic BP. GFR was significantly reduced $4.6(8.8-0.3) \mathrm{ml} / \mathrm{min}$ by aliskiren, $8.0(12.3-3.6) \mathrm{ml} / \mathrm{min}$ by irbesartan and $11.7(15.9-7.4) \mathrm{ml} / \mathrm{min}$ by the combination. Combination treatment showed a slight increase in plasma potassium by $0.2 \mathrm{mmol} / \mathrm{l}(\mathrm{p}=0.036)$. Both monotherapies achieved a reduction in hsCRP levels compared to placebo (from $2.5 \mathrm{mg} / \mathrm{l}$ to $1.6 \mathrm{mg} / \mathrm{l}$ for both, aliskiren $\mathrm{p}=0.047$, irbesartan $\mathrm{p}=0.043$ ), sICAM1 was reduced compared to placebo by combination therapy ( $636 \mu \mathrm{g} / \mathrm{l}$ to 596 $\mu \mathrm{g} / \mathrm{l}, \mathrm{p}=0.017$ ) while sVCAM-1, vWf, fibrinogen and NT-proBNP levels were unchanged through all treatments.

Conclusion: Direct renin inhibition with aliskiren showed similar beneficial effects on UAER and hsCRP as the maximum recommended dose of irbesartan. The combination showed significantly improved renoprotective (UAER) effects, compared to either monotherapy in type 2 diabetic patients with albuminuria.

Supported by: Novartis

\section{PS 118 Hypertension: treatment}




\section{9}

A high saturated fat diet increased and low fat diets decreased pulse pressure and systolic blood pressure in men with metabolic syndrome in the LIPGENE dietary intervention study

I.M.F. Gjelstad ${ }^{1,2}$, H.L. Gulseth ${ }^{1,2}$, J. Lovegrove ${ }^{3}$, C. Defoort ${ }^{4}$, E. Blaak ${ }^{5}$, J. López Miranda ${ }^{6}$, L. Partyka ${ }^{7}$, B. Karlström ${ }^{8}$, H.M. Roche ${ }^{9}$, K.I. Birkeland ${ }^{2}$, C.A. Drevon ${ }^{1}$;

${ }^{1}$ Dept Nutrition, University of Oslo, Norway, ${ }^{2}$ Dept Endocrinology, Aker University Hospital / University of Oslo, Norway, ${ }^{3} \mathrm{Hugh}$ Sinclair Unit of Human Nutrition, University of Reading, United Kingdom, ${ }^{4}$ Nutrition Humaine et Lipids, INSERM, Marseilles, France, ${ }^{5}$ Dept Human Biology, Maastricht University, Netherlands, ${ }^{6}$ Lipid and Atherosclerosis Unit, Reina Sofia University Hospital, University of Cordoba, Spain, ${ }^{7}$ Dept Clinical Biochemistry, Jagiellonian University Medical College, Krakow, Poland, ${ }^{8}$ Dept Public Health and Caring Sciences / Clinical Nutrition and Metabolism, Uppsala University, Sweden, ${ }^{9}$ Conway Institute, University College Dublin, Ireland

Background and aims: Subjects with metabolic syndrome (MS) have increased risk for type 2 diabetes (T2D) and cardiovascular disease (CVD). Elevated blood pressure (BP) is a key feature of MS and contributes to the increased CVD risk in T2D. High pulse pressure (PP) is an independent risk factor of CVD and often coexists with MS and T2D. Dietary fatty acids affect plasma lipids and lipoproteins, but the impact of dietary fat on BP is controversial. We have evaluated the effects of amount and type of dietary fatty acids on BP in subjects with MS.

Materials and methods: Free-living subjects with MS from 8 European centres were randomized to follow one of four isoenergetic diets for 3 months: High fat (38 energy percent, E\%) diet rich in saturated fatty acids (16 E\%) (HF-SFA), high fat (38 E\%) diet rich in monounsaturated fatty acids (20 E\%) (HF-MUFA), and two low fat diets (28 E\%) with or without supplement of $1.2 \mathrm{~g} / \mathrm{d}$ marine omega-3 fatty acids (LF-n3 and LF-P). A food exchange model was used to achieve the dietary aims. 349 subjects (159 males, 190 females) aged 35-70 completed the study per protocol (HF-SFA: $n=84$, HF-MUFA: $n=91$, LF-n3: $n=89$ and LF-P: $n=85$ ).

Results: There were no significant effects of the dietary intervention on BP in the total LIPGENE cohort. However, in males the change in PP was significantly different between the four diets in an one-way ANOVA $(\triangle \mathrm{PP}$ : HFSAFA $3.8 \mathrm{mmHg}$, HF-MUFA -1.7 mmHg, LF-n3 -2.0 mmHg and LF-P -3.1 $\mathrm{mmHg}, \mathrm{p}=0.005)$. After Bonferroni correction the change in PP remained statistically significant between the HF-SFA group and the LF-n3 and the LF-P groups; $\mathrm{p}=0.032$ and 0.006 , respectively. In drug naïve males $(n=124)$ systolic blood pressure (SBP) was significantly changed ( $\triangle$ SBP: HF-SFA 4.6 mmHg, HF-MUFA - $0.5 \mathrm{mmHg}$, LF-n $3-2.7 \mathrm{mmHg}$ and LF-P $-2.6 \mathrm{mmHg}$, $\mathrm{p}=0.009$ ). In females there were no effects of the dietary intervention on BP. Conclusion: Altered quantity and quality of dietary fatty acids significantly affected PP and SBP in males with MS, but not in females.

Supported by: LIPGENE, an EU Sixth Framework Program Integrated Project, Norwegian Foundation for Health and Rehabilitation, South-Eastern Norway Regional Health Authority, Johan Throne Holst Foundation single-pill amlodipine/atorvastatin: a pooled analysis of patients with and without diabetes

T.G. Jenssen ${ }^{1}$, C.A. Aguilar-Salinas ${ }^{2}$, F.D.R. Hobbs ${ }^{3}$, R.D. Feldman ${ }^{4}$, R.C. Blank ${ }^{5}$, R.A. Moller ${ }^{6}$, I.M. Schou ${ }^{7}$, H. Shi ${ }^{7}$

${ }^{1}$ Department of Medicine, Rikshospitalet, Oslo, Norway, ${ }^{2}$ Department of Endocrinology and Metabolism, Instituto Nacional de Ciencias Medicas y Nutricion, Mexico City, Mexico, ${ }^{3}$ General Practice Primary Care Clinical Sciences Building, University of Birmingham, United Kingdom, ${ }^{4}$ Robarts Research Institute, University of Western Ontario, London, Canada, ${ }^{5}$ Department of Cardiology, Presbyterian Hospital, Charlotte, United States, ${ }^{6}$ Pfizer Inc, New York, United States, ${ }^{7}$ Pfizer Australia, Sydney, Australia

Background and aims: Patients with type 2 diabetes (DM) are at increased risk of cardiovascular (CV) events and have difficulty reaching their blood pressure (BP) goal. This pooled analysis of the GEMINI-US, GEMINI-AALA, and JEWEL $1 / 2$ studies compared the utility of single-pill amlodipine besylate/atorvastatin calcium (amlo/atorva) for BP and low-density lipoprotein cholesterol (LDL-C) control in patients with hypertension (uncontrolled) and dyslipidemia (controlled/uncontrolled), with or without DM.

Materials and methods: In these 14-16-week, open-label studies, amlo/atorva (5/10-10/80 mg) was electively titrated to achieve therapeutic goals. Changes in BP and LDL-C from baseline, and goal attainment, were evaluated, using study-specific targets (DM patients: $\mathrm{BP}$ goals $=130 / 80-140 / 80 \mathrm{mmHg}$, LDL-C goals $=2.5-3.0 \mathrm{mmol} / \mathrm{L} ;$ non $-\mathrm{DM}$ patients: $\mathrm{BP}$ goals $=130 / 80-150 / 90$ $\mathrm{mmHg}$, LDL-C goals $=2.5-4.5 \mathrm{mmol} / \mathrm{L}$ ).

Results: Of 5114 patients in the pooled population, 1637 had DM (mean baseline HbAlc was $7.0 \%$ for DM vs. $5.7 \%$ for non-DM). Mean age of DM and non-DM patients was 61.1 and 60.0 years, respectively, and $47.8 \%$ and $44.5 \%$ were female. Mean dose of amlo/atorva at study end was 7.4/23.4 mg for DM patients, and 6.9/24.0 mg for non-DM patients. BP reductions from baseline to study end were comparable for DM and non-DM patients, but the percentage reaching BP/both goals was lower for DM patients (47\%/39\% compared with $72 \% / 60 \%$ for non-DM patients), contributed to by stricter BP goals for DM patients (Table). Among DM patients taking no add-on antihypertensive therapy $(\mathrm{n}=815), 50.8 \%$ reached their BP goal. Mean LDL$\mathrm{C}$ reduction from baseline was slightly higher in non-DM patients. Adverse events-related discontinuations were similar for patients with and without DM (3.7\% and $4.4 \%)$.

Conclusion: Single-pill amlo/atorva helps to control 2 major risk factors for CV disease by simultaneously producing large decreases in BP and LDL-C, and is well tolerated, in patients with and without DM. The differences in BP and LDL-C goal attainment between DM and non-DM patients, are mainly due to DM patients having lower targets.

Supported by: Pfizer Inc.

\section{1}

Aliskiren in combination with valsartan lowers blood pressure more effectively than either agent alone in patients with hypertension,

\section{0}

Control of blood pressure and low-density lipoprotein cholesterol with

Change in BP and lipids and goal attainment for patients with and without DM

\begin{tabular}{lll}
\hline & Patients with DM $(\mathrm{n}=1637)$ & Patients without DM $(\mathrm{n}=3477)$ \\
\hline Baseline BP, mean (SD), mmHg & $147.0(13.0) / 86.2(8.7)$ & $150.1(12.6) / 90.4(8.3)$ \\
\hline Change in BP from baseline to study end, mean (SD), mmHg & $-18.1(13.5) /-10.0(8.5)$ & $-20.8(13.9) /-11.6(8.3)$ \\
\hline Baseline LDL-C, mean (SD), mmol/L & $3.0(1.0)$ & $3.5(1.0)$ \\
\hline Change in LDL-C from baseline to study end, mean (SD), mmol/L & $-0.9(0.9)^{*}$ & $-1.1(1.0)$ \\
\hline BP goal attainment at study end (\%) & $47.2^{*}$ & 71.6 \\
\hline LDL-C goal attainment at study end (\%) & 77.6 & 79.8 \\
\hline Both BP and LDL-C goal attainment at study end (\%) & $38.9^{*}$ & 60.0 \\
\hline
\end{tabular}

${ }^{*} \mathrm{p}<0.001$ versus non-DM 


\begin{tabular}{|c|c|c|c|c|}
\hline & $\begin{array}{l}\text { Aliskiren/ } \\
\text { valsartan }\end{array}$ & Aliskiren & Valsartan & Placebo \\
\hline \multicolumn{5}{|c|}{ Patients with metabolic syndrome } \\
\hline Number of patients & 204 & 205 & 216 & 202 \\
\hline Change in $\mathrm{msSBP}, \mathrm{mmHg}$ & $-17.1 \pm 1.0^{*}$ & $-13.4 \pm 1.0^{*} \ddagger$ & $-13.8 \pm 1.0^{*} \dagger$ & $-5.1 \pm 1.0$ \\
\hline Change in $\mathrm{msDBP}, \mathrm{mmHg}$ & $-12.4 \pm 0.6^{*}$ & $-9.3 \pm 0.6^{\star} \ddagger$ & $-10.0 \pm 0.6^{*} \ddagger$ & $-4.7 \pm 0.6$ \\
\hline Responder rate, \% & $68.6^{*}$ & $53.7^{*} \ddagger$ & $55.6^{*} \ddagger$ & 29.7 \\
\hline \multicolumn{5}{|c|}{ Patients without metabolic syndrome } \\
\hline Number of patients & 232 & 223 & 237 & 252 \\
\hline Change in msSBP, mmHg & $-16.8 \pm 0.9^{*}$ & $-12.8 \pm 1.0^{*} \ddagger$ & $-12.3 \pm 0.9^{*} \ddagger$ & $-4.4 \pm 0.9$ \\
\hline Change in $\mathrm{msDBP}, \mathrm{mmHg}$ & $-12.1 \pm 0.6^{*}$ & $-8.9 \pm 0.6^{\star} \ddagger$ & $-9.8 \pm 0.6^{*}+$ & $-3.8 \pm 0.5$ \\
\hline Responder rates, $\%$ & $62.9^{*}$ & $53.4^{*} \dagger$ & $54.9^{*}$ & 29.8 \\
\hline
\end{tabular}

Least-squares mean changes \pm SEM were from an ANCOVA model. Responder rates were analyzed by logistic regression.

${ }^{*} \mathrm{p}<0.0001$ vs placebo; $\uparrow \mathrm{p}<0.05, \ddagger \mathrm{p}<0.01$ vs combination therapy.

regardless of metabolic status: a post-hoc analysis of an 8-week doubleblind study

S.A. Yarows ${ }^{1}$, S. Oparil ${ }^{2}$, S. Patel ${ }^{3}$, V. Arora ${ }^{3}$, J. Zhang ${ }^{3}$

${ }^{1}$ Chelsea Internal Medicine/IHA, Chelsea, ${ }^{2} \mathrm{UAB}$ Vascular Biology

and Hypertension Program, Birmingham, ${ }^{3}$ Novartis Pharmaceuticals

Corporation, East Hanover, United States

Background and aims: More than one-third of patients with hypertension also have metabolic syndrome and are thus at increased cardiovascular risk. This post-hoc analysis in a double-blind study in patients with hypertension assessed the antihypertensive efficacy of the direct renin inhibitor (DRI) aliskiren in combination with valsartan compared with either agent alone in the subgroup of patients with metabolic syndrome.

Materials and methods: In all, 1797 patients with hypertension (mean sitting diastolic BP [msDBP] $95-<110 \mathrm{mmHg}$ ) were randomized to aliskiren/ valsartan 150/160 mg, aliskiren $150 \mathrm{mg}$, valsartan $160 \mathrm{mg}$ or placebo once daily for 4 weeks, followed by forced titration to double the initial doses for 4 weeks. Metabolic syndrome was defined according to NCEP ATP III criteria. Changes from baseline in mean sitting systolic BP (msSBP), msDBP, and responder rates ( $\mathrm{msDBP}<90 \mathrm{mmHg}$ and/or $\geq 10 \mathrm{mmHg}$ reduction) were assessed at week 8 endpoint for the intent-to-treat population.

Results: Baseline msSBP/msDBP was similar in the subgroups of patients with metabolic syndrome $(154.0 / 100.3 \mathrm{mmHg} ; \mathrm{n}=827)$ and without metabolic syndrome (153.6/100.3 mmHg; $\mathrm{n}=944)$. At week 8 endpoint, aliskiren/valsartan 300/320 mg provided highly effective BP lowering, regardless of metabolic status (Table). BP reductions and responder rates in the aliskiren/valsar$\tan$ treatment group were significantly greater than for either monotherapy or placebo in both patient subgroups. There were no significant differences in the BP-lowering effects of study treatments between patients with or without metabolic syndrome $(\mathrm{p}>0.2)$. Overall rates of AEs were similar across treatment groups and were not notably affected by the presence of metabolic syndrome (with, 31.7-36.4\%; without, 33.0-38.6\%). The most common AEs were headache (overall, 5.5\%) and nasopharyngitis (3.2\%).

Conclusion: Combination therapy with aliskiren and valsartan provided significant additional BP-lowering effects compared with either monotherapy in patients with metabolic syndrome, similar to those in patients without metabolic syndrome. Aliskiren/valsartan is thus a highly effective and generally well-tolerated treatment option for patients with hypertension, regardless of metabolic status. Supported by: Novartis Pharmaceuticals Corporation

\section{2}

Aliskiren-based therapy lowers blood pressure more effectively than hydrochlorothiazide-based therapy in obese hypertensive patients: subgroup analysis of a 52-week, randomized, double-blind trial R.E. Schmieder ${ }^{1}$, T. Philipp ${ }^{2}$, J. Guerediaga ${ }^{3}$, M. Gorostidi ${ }^{3}$, V. Arora ${ }^{4}$, M.F. Prescott ${ }^{4}$, D.L. Keefe ${ }^{4}$

${ }^{1}$ University of Erlangen-Nürnberg, Erlangen, Germany, ${ }^{2}$ University

Hospital, Essen, Germany, ${ }^{3}$ Hospital San Agustin, Aviles, Spain, ${ }^{4}$ Novartis

Pharmaceuticals Corporation, East Hanover, United States

Background and aims: To compare long-term blood pressure (BP) reduction and safety of the direct renin inhibitor aliskiren (ALI) and hydrochlorothiazide (HCT) in obese patients (body mass index $[\mathrm{BMI}] \geq 30 \mathrm{~kg} / \mathrm{m}^{2}$ ) with hypertension.

Materials and methods: Post-hoc analysis of the obese subgroup $(\mathrm{n}=396)$ in a double-blind, multicenter study in which, after a 6-wk placebo-controlled phase, 1124 patients with sitting diastolic BP (DBP) 95-109 $\mathrm{mmHg}$ received ALI $300 \mathrm{mg}(\mathrm{n}=567)$ or HCT $25 \mathrm{mg}(\mathrm{n}=557)$ once daily for $46 \mathrm{wks}$. Amlodipine (AMLO) $5 \mathrm{mg}$ was added from wk 12 and titrated to $10 \mathrm{mg}$ from wk 18 if $\mathrm{BP}$ was $\geq 140 / 90 \mathrm{mmHg}$.

Results: Patients in the obese subgroup $(\mathrm{n}=396)$ had a mean body weight of $96.0 \mathrm{~kg}$ and BMI of $34.0 \mathrm{~kg} / \mathrm{m}^{2}$ (vs $75.4 \mathrm{~kg}$ and $26.3 \mathrm{~kg} / \mathrm{m}^{2}$ respectively in nonobese patients $[\mathrm{n}=721])$. Compared with non-obese patients, the obese subgroup showed a higher incidence of metabolic syndrome (66.7\% vs $29.5 \%$ ) and diabetes (17.4\% vs $7.4 \%$ ) but similar mean baseline BP (154.5/99.2 vs $154.2 / 98.8 \mathrm{mmHg}$ ). Within the obese subgroup, ALI and HCT arms were well matched for demographics and baseline BP (155.0/99.4 vs 154.0/99.0 $\mathrm{mmHg}$ ). At wk 12 endpoint (Table), ALI $300 \mathrm{mg}$ monotherapy provided significantly larger reductions in mean sitting systolic BP (SBP) and DBP, and BP response (DBP $<90$ or $\geq 10 \mathrm{mmHg}$ reduction) and control $(<140 / 90 \mathrm{mmHg}$ [pre-specified for the overall population]) rates than HCT $25 \mathrm{mg}$ monotherapy. At wk 52 endpoint, ALI-based therapy (87/183 patients [47.5\%] with add-on AMLO) provided significantly larger DBP reduction and response rates than HCT-based therapy (78/150 [52.0\%] with add-on AMLO) and a trend towards larger SBP reductions and control rates. ALI lowered BP effectively in obese (Table) and non-obese patients (non-obese: wk 12, 17.1/12.3 $\mathrm{mmHg}$; wk 52, 21.1/15.6 mmHg; all comparisons $\mathrm{p}=\mathrm{NS}$ vs obese patients). By contrast, HCT was significantly less effective in obese (Table) than nonobese patients (non-obese: wk 12, 15.7/11.3 mmHg; wk 52, 20.8/15.4 mmHg; all comparisons $\mathrm{p}<0.05$ vs obese patients). Larger BP reductions with ALI relative to HCT were also observed at wk 52 endpoint in obese patients who received only monotherapy throughout the study $(19.9 / 16.4 \mathrm{mmHg}$ with ALI monotherapy [ $\mathrm{n}=96]$ vs $16.7 / 12.6 \mathrm{mmHg}$ with HCT monotherapy [ $\mathrm{n}=72]$; $\mathrm{p}=0.007$ for DBP). AE rates in obese patients were similar with ALI- and HCT-based regimens $(72.1 \%$ vs $68.1 \%)$.

Conclusion: In this post-hoc analysis of obese patients with hypertension, ALI lowered BP more effectively than HCT as monotherapy (300 mg and 25 $\mathrm{mg}$ respectively) or with add-on AMLO (5-10 mg).

\section{Efficacy in the obese subgroup}

\begin{tabular}{llllll}
\hline Parameter & \multicolumn{2}{l}{$\begin{array}{l}\text { Week 12 endpoint } \\
\text { (monotherapy) }\end{array}$} & & \multicolumn{2}{l}{$\begin{array}{l}\text { Week 52 endpoint (with } \\
\text { add-on AMLO) }\end{array}$} \\
\cline { 2 - 3 } \cline { 5 - 6 } $\begin{array}{llllll}\text { ALI 300 mg } \\
(\mathrm{n}=208)\end{array}$ & $\begin{array}{l}\text { HCT 25 mg } \\
(\mathrm{n}=185)\end{array}$ & & $\begin{array}{l}\text { ALI-based } \\
\text { regimen } \\
(\mathrm{n}=183)\end{array}$ & $\begin{array}{l}\text { HCT-based } \\
\text { regimen } \\
(\mathrm{n}=150)\end{array}$ \\
\hline SBP, $\mathrm{mmHg}$ & $-16.7 \pm 1.0^{* *}$ & $-12.2 \pm 1.0$ & & $-19.9 \pm 1.0$ & $-17.6 \pm 1.1$ \\
\hline DBP, $\mathrm{mmHg}$ & $-12.3 \pm 0.5^{* * *}$ & $-9.1 \pm 0.6$ & & $-15.5 \pm 0.5^{* *}$ & $-13.3 \pm 0.6$ \\
\hline Response rate & $71.2 \%^{*}$ & $59.5 \%$ & & $86.3 \%^{*}$ & $78.0 \%$ \\
\hline BP control rate & $60.6 \%^{* *}$ & $44.3 \%$ & $65.0 \%$ & $58.7 \%$ \\
\hline
\end{tabular}

Least-squares mean \pm SEM changes were from an analysis of covariance model containing treatment, region and baseline BP; responder and control rates were analyzed by logistic regression (all ITT population). ${ }^{*} \mathrm{p}<0.05$, ${ }^{* *} \mathrm{p}<0.01,{ }^{* * *} \mathrm{p}<0.001$ vs HCT.

Supported by: Novartis Pharmaceuticals Corporation 


\section{3}

Does salt sensitivity influence the magnitude of microalbuminuria in hypertensive type 2 diabetic patients?

S.D. Jelic ${ }^{1}$, M. Sumarac-Dumanovic ${ }^{2}$, Z. Caparevic ${ }^{1}$

${ }^{1}$ University Clinic for Internal Medicine, CHC"Dr Dragisa Misovic",

${ }^{2}$ Institute for Endocrinology, Diabetes and Metabolic Diseases, School of

Medicine, University of Belgrade, Serbia.

Background and aims: Objective of this investigation was to assess the influence of the salt sensitivity of blood pressure on the magnitude of microalbuminuria in hypertensive type 2 diabetic patients.

Materials and methods: The study included 66 ( 35 female $/ 31$ male) hypertensive non-proteinuric type 2 diabetic patients (mean age: $49.51+6.29$ years; duration of diabetes: $11.29+5.02$ years and duration of hypertension: $11.86+6.02$ years). After obtaining an informed consent and three-week wash-out period (without any anti-hypertensive drug), normal ( $150 \mathrm{mmoL}$ of sodium daily), low-(40 mmoL of sodium daily) and high-salt ( $300 \mathrm{mmoL}$ of sodium daily) diet was sequentially applied in every patient for 3, 9 and 9 days respectfully. The increment of mean arterial pressure $(\triangle \mathrm{MAP})$ on high- vs. low-salt diet exceeding $10 \mathrm{mmHg}$ was used as the criterion for salt sensitivity. Sodium sensitivity index (SSI) as given by Imanishi M et al. (Diabetes Care 2001; 24: 111-116) is also calculated for every subject. During the last 5 days of low- as well as high-salt diet, inhibitor of the angiotensin-converting enzyme, captopril, was given in a daily dose of $50 \mathrm{mg}$ in order to assess the influence of the dietary salt intake on the renoprotective effects of this group of anti-hypertensive drugs. Urinary albumin excretion (UAE) during salt restriction and salt-load was compared with values during normal salt intake in both salt-sensitive and salt-resistant subjects. The effects of acute administration of captopril on UAE, during different levels of dietary salt intake, in these two groups of patients were also compared. Statistical analysis was performed using the STATISTICA 4.5 program (StatSoft, Tulsa, OK, USA).

Results: According to the given criterion 34 (51.51\%) of studied hypertensive type 2 diabetic patients were salt-sensitive. Mean UAE during the normal salt intake, were higher in salt-sensitive (SS) than in salt-resistant (SR) subjects $(117.44+81.33$ vs $83.31+60.23 \mathrm{mg} /$ day, $\mathrm{P}=0.0564)$, but this difference was of borderline significance. Salt restriction significantly reduced UAE only in SS (113.35+77.55 mg/day, P0.1). During low-salt intake, captopril significantly reduced UAE in both groups (SS: $109.73+76.64 \mathrm{mg} /$ day, $\mathrm{P}=0.000001$; SR: $80.75+50.24 \mathrm{mg} /$ day, $\mathrm{P}=0.000018)$. Salt load significantly rised UAE in SS $(121.68+81.57 \mathrm{mg} /$ day, $\mathrm{P}=0.000001)$, but not in SR subjects $(84.28+60.31$ $\mathrm{mg}$ /day, $\mathrm{P}=0.067)$. Again, captopril significantly reduced UAE in both groups (SS: $117.15+80.69 \mathrm{mg} /$ day, $\mathrm{P}<0.00001$; SR: $81.58+60.51 \mathrm{mg} /$ day, $\mathrm{P}<0.00001$ ) during high-salt intake.

Conclusion: This study demonstrated that dietary salt intake influenced UAE only in salt-sensitive subjects with hypertension and type 2 diabetes. However, angiotensin-converting enzyme inhibitor, captopril, has reduced UAE in salt-sensitive as well as in salt-resistant subjects, independently of dietary salt intake.

\section{4}

Diurnal changes in urinary excretions of immunoglobulin G, transferrin, and ceruloplasmin depend on diurnal changes in blood pressure in normoalbuminuric type 2 diabetic patient

M. Hosoba, T. Narita, H. Fujita, T. Morii, T. Shimotomai, J. Koshimura, Y. Yamada

Department of Endocrinology, Diabetes and Geriatric Medicine, Akita Unibersity School of Medicine, Japan

Background and aims: We reported that urinary excretions of immunoglobulin G (IgG), transferrin and ceruloplasmin (molecular radii<55 $\AA$ ) selectively increased in parallel with enhanced glomerular filtration rate after acute protein loading (APL), which cause increased glomerular capillary pressure (GCP) due to afferent arterioles dilatation, mimicking diabetic intra-renal hemodynamic changes. However, increased urinary excretions of albumin and a2-macroglobulin were not detected. Furthermore, we found that increased urinary excretions of IgG and ceruloplasmin were present in normoalbuminuric type 2 diabetic patients were stabilized after strict glycemic control without change in albuminuria. Taken together, we hypothesized that changes in urinary excretions of IgG, transferrin and ceruloplasmin may be more sensitive clinical markers of changes in GCP, especially in normoalbuminuric state than urinary excretion of albumin. Aim of this study is to clarify whether changes in blood pressure influence urinary excretions of these plasma proteins (molecular radii $<55 \AA$ ), which may reflect impaired renal hemodynamics in diabetic state.

Materials and methods: Ambulatory blood pressure monitoring was used to examine diurnal changes in blood pressure. Urinary excretions of IgG, transferrin, ceruloplasmin, a2-macroglobulin $(88 \AA)$ and albumin $(36 \AA)$ were measured in urine samples collected separately during day and night times in 20 healthy subjects and 26 normotensive- normoalbuminuric diabetic patients. Diurnal index (DI) as \% of (nighttime mean - daytime mean) / daytime mean was used for the evaluations of blood pressure levels and urinary excretions of above mentioned plasma proteins. U-IgG/Cr, U-CRL/Cr, $\mathrm{U}-\mathrm{Tf} / \mathrm{Cr}, \mathrm{U}-\mathrm{Alb} / \mathrm{Cr}$, and $\mathrm{U}-\mathrm{A} 2 / \mathrm{Cr}$ represent ratios of urinary immunoglobulin $\mathrm{G}$, ceruloplasmin, transferrin, albumin, and $\alpha-2$ macroglobulin to urinary creatinine in daytime and nighttime urine samples, respectively.

Results: Statistically significant strong correlations between DI of SBP and those of U-IgG/Cr, U-Tf/Cr and U-CRL/Cr $(r=0.613, \mathrm{p}=0.0009 ; \mathrm{r}=0.415$ $\mathrm{p}=0.0352$, and $\mathrm{r}=0.506, \mathrm{p}=0.0083$, respectively) were found in diabetic patients, whereas these close relationships were not found in control subjects. In contrast with these results, the correlation between $\mathrm{DI}$ of $\mathrm{U}-\mathrm{Alb} / \mathrm{Cr}$ and that of SBP was not found $(r=0.306, p=0.1285)$ even in diabetic patients. Furthermore, DI of U-A2/Cr did not reveal significant correlations with DI of SBP in both control subjects and diabetic patients $(r=0.049, p=0.836$ and $\mathrm{r}=0.227, \mathrm{p}=0.2655$, respectively).

Conclusion: Labile tendency of GCP following systemic blood pressure changes in normotensive- normoalbuminuric diabetic patients was clinically detected by measurement of urinary excretions of IgG, transferrin and ceruloplasmin. Considering the relation between increased GCP and progression of diabetic nephropathy, the present finding supports an importance of early introduction of blood pressure control even in normotensive- normoalbuminuric diabetic patients.

Supported by: Japanese Ministry of Science, Education, Sports, Culture, and Technology

\section{5}

The effect of achieving the goal for antihypertensive treatment in type 2 diabetic patients. Comparing office and $24 \mathrm{~h}$ blood pressure levels O. Torffvit

Nephrology, Clinical Sciences, Lund, Sweden

Background and aims: Antihypertensive treatments in studies are effective in arresting diabetic complications. How is it in daily practice? Do 24 hour measurements offer an advantage beyond office pressure?

Materials and methods: 109 type 2 diabetic patients (22 females), 65 (35-89) years old, with micro- $(n=46)$ and macroalbuminuria $(n=63)$ were followed for median 5 years ( $1-9$ years) with yearly measurements of renal function (GFR), night urine collections and 24 hours ambulatory blood pressure (ABP) measurements. The aim for antihypertensive treatment was $140 / 80 \mathrm{mmHg}$. Results: $51 \%(\mathrm{n}=55)$ developed one or more of the following cardiovascular complications: $16 \%(\mathrm{n}=17)$ died, $6 \%(\mathrm{n}=7)$ had stroke, $11 \%(\mathrm{n}=12)$ myocardial infarction, $13 \%(\mathrm{n}=14)$ heart failure, $34 \%(\mathrm{n}=37)$ uraemia $(\mathrm{GFR} \leq 30$ $\left.\mathrm{ml} / \mathrm{min} / 1.73 \mathrm{~m}^{2}\right), 16 \%(\mathrm{n}=17)$ needed dialysis, and $20 \%(\mathrm{n}=22)$ angina pectoris. Dependent on the type of measurements different number of patients achieved the aim of BP below 140/80: Office blood pressure (BP) (n=11/28) was compared with daytime (26/44) and night-time (49/85) and 24 hour ABP $(36 / 57)$ measurements, in parenthesis (number of patients who achieved aim for systolic/diastolic BP). Achieving the goal for treatment of night BP levels resulted in reduction of the frequency of stroke ( $0 \%$ vs. $12 \%, \mathrm{p}=0.013)$ and uraemia ( $20 \%$ vs. $44 \%, \mathrm{p}=0.009$ ). Mean of $24 \mathrm{~h} \mathrm{ABP}$ below $140 / 80 \mathrm{mmHg}$ resulted in reduction of dialysis treatment ( $6 \%$ vs. $21 \%, \mathrm{p}=0.042)$. In contrast, the development of angina pectoris ( $24 \mathrm{~h}$ diastolic ABP, $28 \%$ vs. $12 \%, \mathrm{p}=$ 0.032; night diastolic $\mathrm{ABP}, 25 \%$ vs. $4 \%, \mathrm{p}=0.032)$ and heart failure increased (office BP, $36 \%$ vs. $10 \%$, p=0.014; 24 h diastolic ABP, $21 \%$ vs. $4 \%, \mathrm{p}=0.007$ ) when the aim of $140 / 80 \mathrm{mmHg}$ was achieved.

Conclusion: Achieving the goal of $140 / 80 \mathrm{mmHg}$ for $24 \mathrm{~h} \mathrm{ABP}$ significant reductions in renal complications beyond the effect of treating office BP. The increase in cardiac complications was probably not the results of good antihypertensive treatment but of poor function of the ischemic heart. 


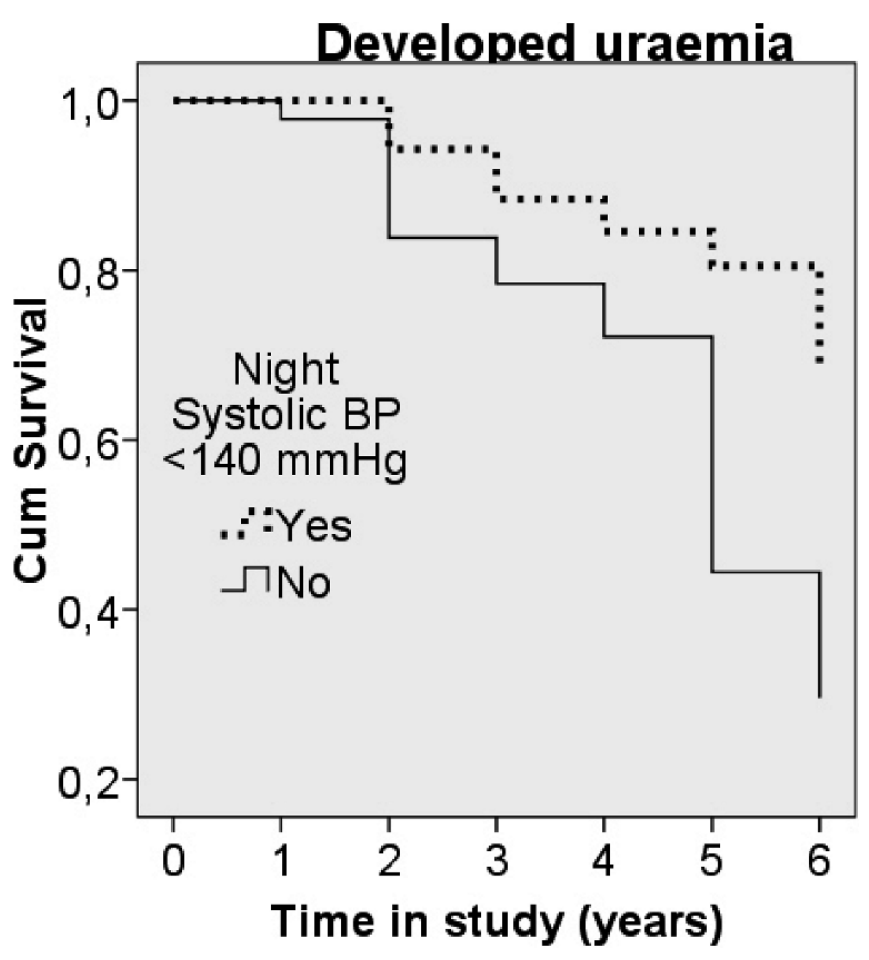

Supported by: Region Skane

\section{PS 119 Hypertension and cardiovascular risk}

\section{6}

Insulin inhibits secretion of $\mathrm{N}$-terminal-proANP ${ }_{53-90}$ : novel mechanism in obesity associated hypertension

N.N. Rudovich ${ }^{1,2}$, O. Pivovarova ${ }^{1,2}$, W. Doehner ${ }^{3}$, S.D. Anker ${ }^{3}$, A. Ernst ${ }^{4}$, N.G. Morgenthaler ${ }^{5}$, A. Bergmann ${ }^{5}$, A.F.H. Pfeiffer ${ }^{1,2}$

${ }^{1}$ Clinical Nutrition, DIFE Potsdam-Rehbrücke, Nuthetal, ${ }^{2}$ Endocrinology, Diabetes and Clinical Nutrition, Campus B. Franklin, Berlin, ${ }^{3}$ Cardiology, Campus Virchow-Klinikum, Berlin, ${ }^{4}$ SphingoTec GmbH, Borgsdorf, ${ }^{5}$ B.R.A.H.M.S AG, Berlin, Germany

Background and aims: Central obesity is highly associated with hypertension. Previous studies have shown that obesity and obesity associated hypertension is favoured by low levels of atrial natriuretic peptide. We aimed to assess the ANP levels and their relationship with insulin and other factors of the metabolic syndrome in normotensive obese and non obese subjects and in patients with hypertension.

Materials and methods: One hundred and twenty-one non diabetic, non hypertensive subjects ( 63 non obese and 58 with central obesity) and 58 patients with essential hypertension (ET) were examined with an oral glucose tolerance test ( $75 \mathrm{~g}$ glucose, $180 \mathrm{~min}$ ); a subgroup of these patients (17 non hypertensive and 14 subjects with ET, all subjects without type 2 diabetes) underwent 2-h euglycemic hyperinsulinemic glucose clamps (100 $\mathrm{mU}$ of human insulin $\mathrm{x} \mathrm{m}^{2}$ body surface $/ \mathrm{min}$ ). Clinical, biochemical parameters, and levels of N-terminal (NT)-proANP ${ }_{53-90}$ were assessed.

Results: Normotensive subjects with central obesity had lower NT-proANP ${ }_{90}$ levels compared with non obese subjects and patients with hypertension ( $\mathrm{p}<0.05 ; \mathrm{p}<0.01$, respectively). In non hypertensive subjects, fasting NTproANP ${ }_{53-90}$ levels were significantly inversely correlated with BMI, diastolic blood pressure, fasting insulin and HOMA-IR and positively with age and HDL-cholesterol. In all study groups, NT-proANP ${ }_{53-90}$ levels decreased rapidly at $30 \mathrm{~min}$ after oral glucose loading and remained suppressed during the tests $(p<0.0001$; for basal levels vs. levels at 30, 60, 90, 120 and $180 \mathrm{~min}$ of the oGTT). Post-loading concentrations of NT-proANP ${ }_{53-90}$ were significantly lower in obese subjects. Fasting and post-loading NT-proANP ${ }_{53-90}$ levels correlated inversely with insulin levels during oGTT. In the euglycemic hyperinsulinemic clamps, NT-proANP ${ }_{53-90}$ levels decreased at $120 \mathrm{~min}$ of the test under continuous insulin infusion in hypertensive and non hypertensive subjects (mean \pm sem; $61.0 \pm 8.4$ vs. $54.2 \pm 8.8, \mathrm{p}=0.026 ; 59.5 \pm 4.5$ vs. 49.3 $\pm 3.6, \mathrm{p}=0.001$; respectively).

Conclusion: The present study demonstrates that endogenous and exogenous insulin unexpectedly suppressed secretion of NT-proANP ${ }_{53-90}$ in humans. Subjects with central obesity had significantly reduced basal and postprandial NT-proANP ${ }_{53-90}$ levels which were significantly inversely related to the BMI, diastolic blood pressure, fasting insulin and HOMA-IR. Our findings provide a novel and causal link between hyperinsulinemia and nutrition with elevated blood pressure in central obesity and the Metabolic Syndrome.

\section{7}

Obesity and blood pressure: explaining the relationship A. Casolaro, A. Natali, E. Muscelli, E. Ferrannini

Department of Internal Medicine, University of Pisa, Italy

Background and aims: Obesity and hypertension are strongly associated in the population both cross-sectionally and longitudinally. The mechanisms that link blood pressure to fat depots, however, are still not entirely clear, particularly within the physiologic domain of fat mass and blood pressure levels (BP). The aims of this study were: a) to evaluate which index of obesity is more strongly associated with blood pressure and whether there are gender differences; b) to verify whether aging, sedentary life style, insulin resistance/hyperinsulinaemia or plasma adiponectin concentrations play any role in mediating the effect of obesity on BP; c) to verify whether this effect of obesity is amplified by the genetic predisposition to develop hypertension. Materials and methods: In 19 European recruiting centres, waist, hip and thigh circumference, body fat (by electrical bioimpedance), insulin sensitivity (euglycaemic clamp) and BP (multiple automatic measurements) were measured by standardised procedures in 1,476 ( 808 women) non-diabetic, non-hypertensive subjects aged 30-60 yrs and covering a wide BMI range $\left(18-44 \mathrm{~kg} \mathrm{~m}^{-2}\right)$. Insulin exposure (as the plasma insulin area under the OGTT 
curve), plasma total adiponectin, and the ACE $D / I$ genotype were measured in centralised laboratories. Information on family history of hypertension and alcohol consumption was collected through validated questionnaires. Physical activity was estimated by a portable accelerometer (Actigraph) carried for 4-7 days.

Results: a) Of the anthropometric indices, waist, percent body fat and BMI, in that order, were the strongest correlated of $\mathrm{BP}$, with $\mathrm{r}$ values ranging from $0.37-0.33$ in women and from $0.28-0.23$ in men. Systolic, diastolic and mean BP all showed a similar pattern of associations. b) Age, physical activity, alcohol consumption, insulin resistance (as the $\mathrm{M} / \mathrm{I}$ value), insulin exposure and adiponectin levels did not account for these associations as their strength was reduced by less than $20 \%$ when they were all introduced as confounders. c) Unexpectedly, the sensitivity of BP to the effect of obesity was lower in subjects with family history of hypertension ( $\mathrm{n}=$, age-adjusted $\mathrm{r}=0.33$ vs 0.54 and 0.26 vs 0.37 , in women and men, respectively) and was significantly (p) attenuated by the presence of the ACE $D$ allele (age-adjusted r's in $I I, I D$ and $D D=0.54,0.47,0.33$ and $0.40,0.30,0.26$, in women and men, respectively). Conclusion: In non-hypertensive subjects, waist circumference and percent body fat, are strong determinants of blood pressure, particularly in women. This association is not explained by age, lifestyle or metabolic traits and is weaker in the individuals predisposed to develop hypertension or carrying the ACE $D$ allele.

Supported by: EU, AstraZeneca Sweden

\section{8}

\section{Alterations of vasorelaxation after oral glucose intake in obese patients with hypertension}

Y. Jaber, Z. Nehar, M. Nguyen, M. Brahimi, S. Chiheb, E. Cosson, P. Valensi Department of Endocrinology, Diabetology, Nutrition, Paris Nord University - CRNH IdF, Paris, France

Background and aims: Artery stiffness is increased in diabetic, hypertensive and obese patients. The vasorelaxing effect induced by plasma insulin increase after a carbohydrate-rich meal has been shown to be reduced in insulin resistant subjects. The aim of this study was to examine the influence of hypertension on the changes in artery stiffness after an oral glucose load (OGTT) in nondiabetic obese patients.

Materials and methods: We included 35 obese patients, aged $41.9 \pm 13.0 \mathrm{yrs}$, free of known dysglycaemia and with no diabetes according to OGTT, 19 of them with mild or moderate hypertension. Peripheral (radial artery) and central (aorta) blood pressure (BP) and artery stiffness (amplification index, AIx) were measured repeatedly at 30-min intervals during $180 \mathrm{~min}$ after glucose intake by aplanation tonometry (SphygmoCor $\left.{ }^{\circledR}\right)$.

Results: During OGTT, there was a trend to higher blood glucose and plasma insulin responses in hypertensive patients. Peripheral and central BP decreased significantly only in hypertensive patients while remaining higher than in normotensive patients. Peripheral and central AIx decreased significantly after glucose in both hypertensive and normotensive patients. However the decrease in central AIx was significantly delayed in hypertensives as compared to normotensives. The decrease in peripheral and central AIx at 30 minutes was significantly lower in the hypertensive patients with central pulse pressure $\geq 40 \mathrm{mmHg}(\mathrm{n}=9)$ than in those with pulse pressure $<40 \mathrm{mmHg}$ ( $\mathrm{n}=7$ patients $)(\mathrm{p}<0.05$ for both $)$.

Conclusion: In obese patients, 1- oral glucose intake induces a vasorelaxation both at peripheral and central levels, but this effect occurs later in hypertensives, in particular in those with a more severe artery stiffness, than in normotensives; 2- vasorelaxation is likely to result from insulin response; 3 - the abnormal vascular response occurs despite BP decrease and is likely to result from a more severe vascular insulin resistance.

\section{9}

The metabolic correlates of nondiabetic hyperglycaemia in subjects with essential hypertension

G. Penno ${ }^{1}$, G. Dell'Omo ${ }^{2}$, L. Pucci ${ }^{1}$, D. Lucchesi ${ }^{1}$, E. Russo ${ }^{1}$, G. Daniele ${ }^{1}$, R. Miccoli ${ }^{1}$, S. Del Prato ${ }^{1}$, R. Pedrinelli ${ }^{2}$

${ }^{1}$ Endocrinology and Metabolism, ${ }^{2}$ Cardio-Thorax Disease, University of Pisa, Italy

Background and aims: Non-diabetic hyperglycemia (impaired fasting glucose, IFG), a slightly increased fasting plasma glucose (FPG) below the diabetic threshold, constitutes a relatively new nosologic category aimed at the early identification of people at risk for diabetes. IFG is a clinically and pathophysiologically relevant parameter in non-diabetic hypertensive patients but the knowledge about its metabolic and clinical correlates is limited. IFG also concurs to diagnose Metabolic Syndrome (MetS), a frequent and prognostically negative phenotype in essential hypertension, but the impact of its not univocal definition (either $110-125$ or $100-125 \mathrm{mg} / \mathrm{dL}$ by the NCEP-ATPIII and AHA-NHLBI definitions, respectively) on the identification of patients with MetS is unclear.

Materials and methods: A group of 983 non-diabetic, referred essential hypertensive patients without history of cardiovascular disease have been recruited. They were stratified by normal fasting plasma glucose (FPG, $<100$ $\mathrm{mg} / \mathrm{dL} ; \mathrm{n} .652)$, milder (100-109 mg/dL; n. 196) and more advanced (110$125 \mathrm{mg} / \mathrm{dL} ; \mathrm{n}$. 135) non-diabetic hyperglycemia. MetS was screened by using either 110 or $100 \mathrm{mg} / \mathrm{dL}$ as a FPG cutoff for IFG. Furthermore, the 10-year predicted coronary heart disease (CHD) risk was calculated by Framingham Risk Score, a conventional risk factor stratification system.

Results: Fasting plasma glucose $>/=100 \mathrm{mg} / \mathrm{dL}$ was associated with older age, male gender, atherogenic dyslipidemia, obesity, higher white blood cell (WBC) count and worse CHD risk profile without differences between the 100-109 and 110-125 mg/dL categories except for older age and higher WBC count in the latter. IFG increased markedly (from 13\% to 33\%) by the 110 and $100 \mathrm{mg} / \mathrm{dL}$ thresholds $(\mathrm{p}<0.001)$. Abdominal obesity, triglycerides at or above $150 \mathrm{mg} / \mathrm{dl}$, and HDL below the gender-adjusted cut-offs were present in $38 \%, 33 \%$ and $29 \%$ of the overall sample. MetS prevalence augmented only marginally (from $34 \%$ to $42 \%$ ) and mainly by inclusion of less metabolically compromised patients. Indeed, the $8 \%$ increase in MetS was due to the inclusion of 76 additional subjects with lower triglycerides, BMI, WBC count, higher HDL-C, less frequent atherogenic dyslipidemia and obesity.

Conclusion: The metabolic abnormalities and negative CHD risk profile are largely prevalent and homogeneously distributed in referred hypertensive patients with either mild or more advanced nondiabetic hyperglycemia. Use of a lower FPG threshold increased markedly the prevalence of IFG but had a largely less than proportional impact on MetS and mainly because of the inclusion of patients with slightly metabolically compromised profile. Thus, lowering the FPG threshold seems to have a relatively limited impact on Metabolic Syndrome diagnosis.

\section{0}

Independent impact of blood pressure on definition of metabolic syndrome in African American women

T. Gaillard, K. Osei;

Division of Endocrinology, Diabetes and Metabolism, The Ohio State University, Columbus, United States

Background: African American women (AAW) suffer disproportionately from hypertension and its consequences. Metabolic Syndrome (MetS), defined by The Adult Treatment Panel (ATP) III criteria include systolic (SBP) $(\geq 130 \mathrm{mmHg})$ and diastolic (DBP) $(\geq 85 \mathrm{mmHg})$ blood pressure. However, whether BP is associated with the other 4 components of ATP III parameters as well as other cardiovascular disease (CVD) risk factors in AAW is unknown. Therefore, we investigated CVD risk clusters, prevalence of various components of MetS as well as MetS associated with both SBP and DBP in non-diabetic adult, overweight and obese AAW.

Methods: We studied the clinical and metabolic CVD risk factors of 258 AAW (mean age $42.0 \pm 7.7$ yrs and mean BMI of $33.3 \pm 8\left(\mathrm{~kg} / \mathrm{m}^{2}\right)$. Fasting serum insulin, glucose and c-peptide levels were obtained in each subject. Waist circumference (WC), SBP and DBP were measured. Insulin sensitivity (Si), (Bergman's minimal model method) and insulin resistance (IR) (homeostasis model assessment (HOMA) were calculated. We measured 1) metabolic CVD risk factors associated with each tertile of the BP (i.e. serum glucose, insulin resistance, LDL-C, HDL-C, triglyceride and 2) examined the prevalence of various components of MetS and prevalence of MetS as defined by ATP III according to the tertiles of BP.

Results: Mean age and BMI did not differ with the worsening of SBP and DBP tertiles. MetS was found in $35.5 \%$ of our AAW. Percent of MetS did not differ per SBP and DBP tertiles. Worsening of SBP and DBP was associated with worsening of WC, but had only minimal effects on serum glucose, HOMA-IR, SI and serum triglyceride (albeit within normal limits). Comparing SBP and DPB tertiles, we found similar percent of the components and prevalence of MetS. The prevalence of MetS was not significantly associated with the tertiles BP.

Conclusion: Both SBP and DBP are associated with MetS independent of glucose, IR and lipids and lipoproteins. The $3^{\text {rd }}$ tertile of $\mathrm{BP}$ was not associated 
with greater prevalence of various components of MetS nor the prevalence of MetS. Our study suggests that both SBP and DBP are equally associated with MetS in AAW. We conclude that obese/overweight AAW with family history of type 2 diabetes, BP appears to have an independent impact on the definition of MetS as defined by ATP III. Thus, nondiabetic, obese/overweight AAW meeting ATP III criteria for systolic or diastolic BP should be targeted for screening for MetS.

Supported by: General Clinical Research Center

\section{1}

Morning blood pressure surge in diabetic patients - relationship with metabolic syndrome

I. Pietrzak, A. Szadkowska, B. Mianowska, E. Czerniawska, J. Bodalski, W. Młynarski

Department of Pediatrics, Medical University, Lodz, Poland

Background and aims: The elevated morning blood pressure surge is regarded as one of risk factor $\mathrm{s}$ of diabetic micro- and macroangiopathy. The pathogenetic background of this relationship is still unknown.

To estimate the relationship between morning blood pressure surge and components of the metabolic syndrome in children and adolescents with type 1 diabetes mellitus.

Materials and methods: 146 patients (F-54, M-92) aged 9.8-19.5 years, with duration of diabetes 0.1-15.6 years, without evidence of arterial hypertension were recruited. In all patients 24 -hour automatic blood pressure monitoring was performed with an oscillometric device. We defined morning blood pressure surge (MBPS) as the average of systolic/diastolic BP during the first 2 hours upon wakening minus the average of systolic/diastolic BP during the first hour that included the lowest sleep BP (MBPS-SBP/MBPS-DBP). The height, weight, waist circumference, adipose tissue mass were measured. $\mathrm{HbAlc}$ and plasma lipids concentrations were examined. Euglycemic-hyperinsulinemic clamp was performed to establish insulin resistance. Glucose disposal rate was calculated as M-index in $\mathrm{mg} / \mathrm{kg} / \mathrm{min}$. Body mass index and daily dose of insulin were calculated.

Results: We diagnosed 16 hypertensive and 32 prehypertensive patients according to ABPM results. In hypertensive and prehypertensive patients the MBPS-SBP was higher than in normotensive individuals (respectively MBPS-SBP - $27.3 \pm 2.1$ vs $23.2 \pm 1.4$ vs $18.4 \pm 1, \mathrm{p}<0.001$, MBPS-DBP $-21.1 \pm 2.4$ vs $18.6 \pm 1.6$ vs $15.0 \pm 1.2, \mathrm{p}=0.054)$. We found the positive correlation between MBPS-SBP and adipose tissue mass $(r=0.42, p=0.01)$. There were no significant relationships between MBPS and BMI-SDS, waist circumference, daily dose of insulin, HbAlc, lipids and M-index.

Conclusion: 1) Diabetic children and adolescents with elevated blood pressure have higher morning blood pressure surge than normotensive patients. 2) There is the positive correlation between systolic MBPS and adipose tissue mass.

\section{2}

Relation of pulse pressure and ambulatory arterial stiffness index with clinical characteristics and cardiovascular risk factors in type $\mathbf{2}$ diabetic patients

A. Januszewicz ${ }^{1}$, S. Ito' 2 J.L. Izzo Jr. , S. Katayama ${ }^{4}$, A. Mimran ${ }^{5}$, A.J. Rabelink ${ }^{6}$, E. Ritz ${ }^{7}$, L.M. Ruilope ${ }^{8}$, L.C. Rump ${ }^{9}$, G. Viberti ${ }^{10}$, H. Haller ${ }^{11}$ ${ }^{1}$ Department of Hypertension, Institute of Cardiology, Warsaw, Poland, ${ }^{2}$ Department of Clinical Medicine, Division of Nephrology, Endocrinology, and Vascular Medicine, Tohoku University Graduate School of Medicine, Sendai, Japan, ${ }^{3}$ Department of Medicine, Erie County Medical Center, Buffalo, United States, ${ }^{4}$ The Fourth Department of Medicine, Saitama Medical School, Iruma, Japan, ${ }^{5}$ Department of Internal Medicine, Hospital Lapeyronie, Montpellier, France, ${ }^{6}$ Department of Nephrology and Hypertension, Leiden University Medical Center, Netherlands, ${ }^{7}$ Department of Nephrology, University of Heidelberg, Germany, ${ }^{8}$ Division of Hypertension, Hospital 12 de Octubre, Madrid, Spain, ${ }^{9}$ Medical Clinic, Marienhospital Herne, University Clinic Ruhr-University-Bochum, Herne, Germany, ${ }^{10}$ Unit for Metabolic Medicine, KCL Guy'sHospital, London, United Kingdom, ${ }^{11}$ Nephrology Section, Hannover Medical School, Germany

Background and aims: Whether ambulatory arterial stiffness index (AASI) outperforms pulse pressure (PP) in cardiovascular risk prediction in Type 2 diabetic patients (T2DM) is controversial.

Materials and methods: We evaluated relationship between office PP and cardiovascular risk factor in $4449(46 \% \mathrm{M})$ T2DM patients with normoalbu- minuria and one or more additional cardiovascular risk factors enrolled in the ROADMAP study at baseline. We also evaluated relationship between ABPM - mean 24hr systolic BP (SBP), diastolic BP (DBP), PP and AASI - in a subgroup of 1137 patients at baseline.

Results: In the whole group mean (SD) age was 57.7 (8.7) yrs, duration of diabetes 6.1 (6.0) yrs. Office PP level correlated significantly (with $\mathrm{p}<0,001$, if not stated otherwise) with age $(\mathrm{r}=0,389)$, known duration of $\mathrm{DM}(\mathrm{r}=0,173), 10 \mathrm{yr}$ risk of CHD ( $r=0,377)$, metabolic syndrome (MS) ( $r=0,158)$, history of cardiovascular disease (CVD) $(r=0,209)$, GFR $(r=-0,094)$, fasting plasma glucose (FPG) level ( $r=0,063)$, albumin to creatinine ratio (ACR) $(r=0,080)$, and HDL cholesterol level $(r=0,038 ; p<0,05)$. When adjusted for age and systolic BP only correlations with known duration of DM $(\mathrm{r}=0,092)$, 10yr risk of CHD $(r=0,058)$ and MS $(r=-0,084)$ were significant (with $\mathrm{p}<0,001)$. In the subgroup of 1137 patients with ABPM performed, after adjusting for $24 \mathrm{~h} \mathrm{SBP}$ there were significant correlation between PP and AASI and: age $(r=0,349 ; \mathrm{p}<0,0001$ and $r=0,074 ; p<0,05$ ), female gender (only for PP: $r=0,129 ; p<0,0001$ ), 10yr risk of CHD ( $r=0,218 ; p<0,0001$ and $r=0,081 ; p<0,01)$, known duration of diabetes $(r=0,164 ; p<0,0001$ and $r=0,061 ; p<0,05$ ), BMI (only for AASI: $r=-0,059$; $\mathrm{p}<0,05)$, creatinine clearance $(r=-0,232 ; \mathrm{p}<0,0001$ and $\mathrm{r}=-0,064 ; \mathrm{p}<0,05)$ and GFR (only for PP: $r=-0,164 ; \mathrm{p}<0,0001)$. Adjusted for AASI, PP correlated significantly (with $\mathrm{p}<0,0001$, if not stated otherwise) with: age $(\mathrm{r}=0,184)$, female gender $(r=0,070 ; p<0,05)$, BMI $(r=0,173), 10 y r$ risk of $\mathrm{CHD}(r=0,217)$, history of CVD $(r=0,181)$, known duration of diabetes $(r=0,126)$, MS $(r=0,179)$, FPG level $(r=0,120)$, ACR $(r=0,146)$ and $\mathrm{HbAlC}$ level $(r=0,076 ; \mathrm{p}<0,05)$. Adjusted for PP, AASI correlated significantly only with BMI $(r=-0,069 ; \mathrm{p}<0,05)$.

Conclusion: In this group of T2DM with normoalbuminuria office PP, adjusted for age and SBP levels, correlated significantly with duration of diabetes and $10 \mathrm{yr}$ risk of CHD. The relation between ambulatory $24 \mathrm{~h}$ PP and cardiovascular risk factors as compared with AASI was more pronounced. Supported by: Daiichi-Sankyo 


\section{PS 120 Autonomic neuropathy: clinical features}

\section{3}

\begin{abstract}
Autonomic neuropathy is related to hypertension in type $\mathbf{1}$ and even in newly diagnosed type 1 diabetes mellitus

K. Keresztes, I. Istenes, Z. Putz, N. Tóth, N. Németh, P. Vargha, P. Kempler Semmelweis University Budapest, I.st. Dept. of Medicine, Hungary
\end{abstract}

Background and aims: Autonomic neuropathy is a serious complication of diabetes mellitus associated with poor prognosis. However, factors leading to increased mortality have not been clarified up to now. The aim of our study was to evaluate a possible connection between autonomic neuropathy and hypertension in type 1 and in newly diagnosed type 1 diabetic patients. Materials and methods: We examined 32 patients with Type 1 diabetes (mean age: $37.8 \pm 12.7$ years), 40 patients with newly diagnosed Type 1 diabetes (mean age: $34.7 \pm 11.3$ years) and 25 healthy control subjects (mean age: $38.3 \pm 12.8$ years). The five standard cardiovascular reflex tests of cardiovascular autonomic function were applied; the severity of autonomic neuropathy was characterized by the autonomic score. Ambulatory 24-hour blood pressure monitoring (ABPM) was performed by MEDITECH ABPM 04 device. Results: Autonomic dysfunction was found in 29/32 Type 1, and in 12/40 newly diagnosed Type 1 diabetic patients. There was a significant correlation between the severity of autonomic neuropathy and the blood pressure load (systolic: $\mathrm{p}<0.001$; diastolic: $\mathrm{p}<0.01$ ), the hyperbaric impact values (systolic: $\mathrm{p}<0.01$; diastolic: $\mathrm{p}<0.05)$ and microalbuminuria $(\mathrm{p}<0.001)$ in Type 1 diabetic patients. Autonomic neuropathy as well as microalbuminuria, were independently associated with hypertension among Type 1 diabetic patients. Decrease of systolic blood pressure after standing correlated positively with systolic $(\mathrm{p}<0.01)$ and diastolic $(\mathrm{p}<0.05)$ diurnal blood pressure indices. Analysing the relationship between ABPM parameters and the five cardiovascular reflex tests separately, the blood pressure load and the hyperbaric impact values correlated negatively with the $30 / 15$ ratio and Valsalva ratio - both tests reflect mainly parasympathetic function. The autonomic score was higher in newly diagnosed diabetic patients than in controls $(p=0.005)$. Among patients with newly diagnosed Type 1 diabetes autonomic dysfunction was independently associated with smoking $(\mathrm{p}<0.01)$, serum cholesterol and hypertension (both:p $<0.05$ ). In the newly diagnosed diabetic group also a predominantly parasympathetic test - the heart rate variation after standing - was inversely related to the diastolic blood pressure values $(\mathrm{p}<0.05)$. These associations remained significant even after adjustment for other cardiovascular risk factors.

Conclusion: Autonomic dysfunction is quite frequent complication and seems to be related to hypertension in both Type 1 and newly diagnosed Type 1 diabetes mellitus. Our data suggest that a relative sympathetic overactivity due to predominantly parasympathetic neuropathy might be responsible for the higher blood pressure values observed. ABPM is suggested being performed for the early assessment of hypertension in both type 1 and newly diagnosed type 1 diabetic patients with autonomic neuropathy, while nondippers should be screened for postural hypotension. Our data suggest that traditional cardiovascular risk factors should be considered as potential risk factors for the development of nerve dysfunction even in newly diagnosed Type 1 diabetic patients. Hypertension itself as well as orthostatic hypotension and diminished diurnal indices may contribute to poor prognosis of autonomic neuropathy.

\section{4}

Cardiac autonomic neuropathy in type 1 diabetes - association with metabolic syndrome and diabetic nephropathy

L. Duarte, J. Costa, P. Matos, A. Pereira, I. Almeida, A. Gralho, R. Duarte, J.F. Raposo, J.M. Boavida

Portuguese Diabetes Association, Lisbon, Portugal

Background and aims: Cardiac autonomic neuropathy has been associated with increased morbidity and mortality in patients with diabetes. The prevalence and importance of metabolic syndrome in type 1 diabetes has been gaining growing evidence in the last years. The aim of this study was to estimate the prevalence of cardiac autonomic neuropathy in a cohort of patients with type 1 diabetes and evaluate whether it is associated with metabolic syndrome, with individual cardiovascular risk factors and with diabetic nephropathy.
Materials and methods: A cohort of 209 type 1 diabetic patients with more than 10 years of duration of diabetes, randomly selected from our clinical files, was studied. Cardiac autonomic neuropathy was assessed by three tests of heart rate variation (maximum-minimum heart rate during deep breathing, Valsalva and lying-standing change) and one of blood pressure variation (lying-standing change). Metabolic syndrome was defined according to the International Diabetes Federation criteria and diabetic nephropathy was assessed by determination of albumin excretion rate. Statistical analysis was performed using SPSS - version 11.0. Descriptive statistics are presented and comparison between groups was performed using Student's $t$ test, Mann-Withney test and $\chi^{2}$ test. Results: The cohort was constituted by 209 type 1 diabetic patients (58.9\% men), mean age $37 \pm 11$ years and duration of diabetes $23.8 \pm 7.6$ years. There was no significant difference according to gender in any of the analysed parameters. The overall prevalence of cardiac autonomic neuropathy was $42.1 \%$ and of metabolic syndrome was $32.1 \%$. Analyzing the subgroups of patients with and without cardiac autonomic neuropathy we found statistically significant differences in duration of diabetes $(26 \pm 7$ vs $22 \pm 8$ years, $\mathrm{p}<0.001)$, HbAlc $(9.1 \pm 1.4$ vs $8.6 \pm 1.4 \%, p=0.007)$, systolic blood pressure $(138 \pm 19$ vs $129 \pm 14 \mathrm{mmHg}, \mathrm{p}<0.001)$, diastolic blood pressure $(82 \pm 13 \mathrm{vs} 79 \pm 10 \mathrm{mmHg}$, $\mathrm{p}=0.024)$, triglycerides [95.3 $(24.8-608.1)$ vs $74.5(28-290) \mathrm{mg} / \mathrm{dl}, \mathrm{p}=0.001]$, serum creatinine [1.08 (0.74-3.9) vs $0.97(0.6-2.9) \mathrm{mg} / \mathrm{dl}, \mathrm{p}=0.001]$, and creatinine clearance $\left(70.7 \pm 21.7\right.$ vs $\left.83.8 \pm 16.7 \mathrm{ml} / \mathrm{min} / 1.73 \mathrm{~m}^{2}, \mathrm{p}<0.001\right)$. The prevalence of metabolic syndrome in patients with cardiac autonomic neuropathy (43.2\%) was significantly higher than in patients without cardiac autonomic neuropathy $(24 \%), p=0.004$. Although the difference in microalbuminuria and proteinuria individually was not statistically significant, the prevalence of diabetic nephropathy (microalbuminuria and/or proteinuria) in patients with cardiac autonomic neuropathy (50\%) was significantly higher than in patients without cardiac autonomic neuropathy (15.1\%), $\mathrm{p}<0.001$.

Conclusion: Our study suggests that cardiac autonomic neuropathy is associated with cardiovascular risk, with increased prevalence of metabolic syndrome and with increased prevalence of diabetic nephropathy. These findings may have implications in more aggressive medical intervention to improve prognosis and survival in this population.

Supported by: Portuguese Diabetes Association

\section{5}

Cardiac autonomic neuropathy, arterial hypertension and microcirculation in type 2 diabetic patients

D. Roula ${ }^{1}$, A. Belhadj-Mostefa ${ }^{1}$, M.K. Bourahli ${ }^{2}$, A. Bouguerba ${ }^{1}$, F. Touati ${ }^{1}$, J.R. Attali ${ }^{3}$, P. Valensi ${ }^{3}$

${ }^{1}$ Medicine, Department of Internal Medicine, Constantine, Algeria,

${ }^{2}$ Department of Physiology, Constantine, Algeria, ${ }^{3}$ Department of

Endocrinology Diabetology Nutrition Jean Verdier Hospital, Bondy, France

Background and aims: Several data suggest the role of hypertension and cardiac autonomic neuropathy (CAN) in diabetic microangiopathic complications. The aim of this study was to evaluate the influence of hypertension and CAN on peripheral microcirculation, endothelial dysfunction and artery stiffness in patients with type 2 diabetes (T2D).

Materials and methods: 98 patients ( 48 men), mean age $54.7 \pm 8.4$ years, with T2D known since $7.8 \pm 6.5$ years and poor glycemic control (HbAlc $8.1 \pm 1.9 \%$ ), with hypertension for 60 of them, were included. CAN was assessed by 3 heart rate variabilitry tests. A post-ischemia reactive hyperaemic test was performed, and changes in blood flow (F2/F1) and diameter (D2/D1) of the brachial artery which account mostly for the reactivity of downstream microvasculature and for endothelium function, respectively, were evaluated by pulsed Doppler velocimetry. Artery stiffness was assessed by measuring pulse wave velocity (PWV) between the carotid and femoral arteries using the Complior device.

Results: F2/F1 was not correlated with $\mathrm{HbA1c}$ and did not differ significantly in patients with or without hypertension, microangiopathic complications or both. F2/F1 was significantly lower in CAN+ patients (n:73, $4.4 \pm 1.6$ vs $5.7 \pm 1.7$ in $\mathrm{CAN}-; \mathrm{p}<0.005)$. In hypertensive patients and in those with microangiopathic complications, F2/F1 was lower in those with CAN (respectively, $4.5 \pm 1.9$ vs $6.1 \pm 1.7 ; \mathrm{p}<0.002$ and $4.3 \pm 1.6$ vs $5.7 \pm 1.5 ; \mathrm{p}<0.005$ ) while there was no influence of CAN in normotensive patients and in patients without microangiopathic complications. D2/D1 did not differ significantly in patients with or without CAN, hypertension or microangiopathic complications. In CAN+ patients, PWV was higher in hypertensive than in normotensive patients $(12.6 \pm 3.0 \mathrm{~m} / \mathrm{s}$ vs $10.6 \pm 2.2 \mathrm{~m} / \mathrm{s} ; \mathrm{p}<0.005)$ while hypertension had no significant influence on PWV in CAN-patients $(12.2 \pm 1.7 \mathrm{~m} / \mathrm{s}$ and $11.3 \pm 2.1 \mathrm{~m} / \mathrm{s}$, respectively). 
Conclusion: Our results suggest, in T2D, the role of nerve control impairment in microcirculation dysfunction, particularly in patients with hypertension or microangiopathic complications, and the role of hypertension on artery stiffness only when CAN is present. Supported by: ANDRS Algeria

\section{6}

The spatial QRS-T angle is increased in patients with type 2 diabetes mellitus and cardiac autonomic neuropathy

C. Voulgari ${ }^{1}$, N. Tentolouris ${ }^{1}$, I. Moyssakis ${ }^{2}$, D. Perrea ${ }^{3}$, D. Kyriaki ${ }^{1}$, N. Katsilambros ${ }^{1}$

${ }^{1} 1$ st Department of Propaedeutic Medicine, Athens University Medical School, Laiko General Hospital, ${ }^{2}$ Department of Cardiology, Laiko General Hospital, ${ }^{3}$ Department of Experimental Surgery and Surgical Research, Athens University Medical School, Greece

Background and aims: Cardiac autonomic neuropathy (CAN) is a common complication of diabetes and it has been associated with dispersion of ventricular repolarization and arrhythmia provocation. QT prolongation has long been used as an indicator of ventricular repolarization heterogeneity in patients with diabetes but it has been criticized as being a poor indicator of ventricular arrhythmogenicity. The spatial QRS-T angle (sQRS-Ta) is a novel vectorcardiographic marker akin to the concept of the ventricular gradient and a robust index of ventricular repolarization heterogeneity. Recent data suggest that it is a dominant predictor of cardiovascular morbidity and mortality in the general population. We have previously shown that the sQRS-Ta is increased in uncomplicated subjects with type 2 diabetes (T2DM). However, no data exists on the effect of CAN on the SQRS-Ta. In this cross-sectional study we examined differences in the sQRS-Ta between diabetic patients with and without CAN. Additionally, potential relationships between the sQRS-Ta and demographic, clinical and metabolic variables were studied.

Materials and methods: A total of 138 patients (mean age $57.3 \pm 9.3$ years) with T2DM and free of macrovascular complications and 138 healthy control subjects, strictly matched with the diabetic subjects in terms of gender and age were studied. Diagnosis of CAN was based on the battery of the classical autonomic function tests. All subjects underwent a digital 15 min ECG and 30 min heart rate recordings. ECG parameters were measured and analyzed using the MEANS program. Indices of the spectral analysis of the heart rate variability (HRV) were obtained using the VariaCardio system. Ankle-brachial-pressure index (ABI), LV mass index (LVMi) and myocardial performance index (Tei index) were assessed by ultrasonography.

Results: Among participants with T2DM there were 66 subjects with CAN, and 72 without CAN. The SQRS-Ta values were higher in the diabetic in comparison with the control subjects $\left(24.5 \pm 10.7^{\circ}\right.$ versus $9.7 \pm 4.5^{\circ}$, respectively, $P<0.001)$. Additionally, the sQRS-Ta values were higher in the patients with CAN than in those without CAN $\left(30.1 \pm 11.3^{\circ}\right.$ versus $19.5 \pm 7.1^{\circ}$, respectively, $P<0.001)$. On the contrary, no significant differences were found in the values of the QT interval between diabetic and control subjects or between those with and without CAN. Multivariate linear regression analysis in the patients with T2DM, after adjustment for age, sex, BMI, waist circumference, fasting glucose and lipids, $\mathrm{ABI}, \mathrm{LVMi}$, glomerular filtration rate and presence of microalbuminuria, demonstrated independent associations between the sQRS-Ta and the presence as well as the severity of CAN $(P<0.001), \mathrm{HbAlc}$ $(P<0.001)$, duration of diabetes $(P=0.011),(P=0.027)$, Tei index $(P=0.02)$ and all indices of HRV $(P<0.001)$.

Conclusion: The sQRS-Ta is increased in patients with T2DM with CAN, suggesting ventricular repolarization heterogeneity. Additionally, it is strongly associated with duration and degree of hyperglycaemia as well as myocardial performance. Increased sQRS-Ta values warrants consideration for risk stratification and prevention strategies in patients with T2DM.

\section{7}

Can spectral analysis of heart rate variability detect subclinical autonomic dysfunction as reliably as spontaneous baroreceptor sensitivity?

E.J. Robinson, J.L.B. Marques, S.R. Heller

Academic Unit of Diabetes, Endocrinology \& Metabolism, University of Sheffield, United Kingdom

Background and aims: Cardiovascular autonomic neuropathy is one of the most serious complications of diabetes. However it is often detected late, when treatment options are limited. Of the tools that have been used to detect early autonomic neuropathy, spontaneous baroreceptor sensitivity (BRS) has emerged as the gold standard. However, this is expensive to perform and relatively operator dependent. Spectral analysis of heart rate variability (HRV) is cheaper, easier to perform and therefore is more suitable as a screening test. We aimed to identify whether HRV was able to identify subclinical autonomic neuropathy with the same accuracy as BRS.

Materials and methods: Fifty young patients with Type 1 diabetes (age 18-40 years) underwent BRS analysed in the sequence method and spectral analysis of heart rate variability (Total Power-TP, Low frequency power-LF and high frequency power-HF). Patients were divided into two groups, normal (BRS+) and abnormal (BRS-) baroreceptor sensitivity. 14 age matched controls underwent HRV.

Results: Total BRS (mean \pm SD) was $19 \pm 8$ and $8 \pm 3$ in the BRS+ and BRSgroups respectively. All logarithmically transformed HRV measures were significantly lower $(\mathrm{p}<0.0001)$ in the BRS- group when compared to both BRS+ group and healthy volunteers. TP- $5.4 \pm 0.72,6.7 \pm 0.97$ and $7 \pm 1.12$, LF$4.2 \pm 0.83,5.6 \pm 1.02,5.8 \pm 1.32$ and HF $3.7 \pm 1.08,5.4 \pm 1.23,5.7 \pm 1.5$ for each group respectively. There was no significant difference between BRS+ group and healthy volunteers in any of the spectral parameters analysed. Pearson's correlation coefficient was $>0.74$ for Total BRS and each of the spectral parameters $(\mathrm{p}<0.001)$.

Conclusion: Spontaneous baroreceptor sensitivity testing and spectral analysis of heart rate variability are equally able to differentiate degrees of autonomic dysfunction in young people with Type 1 diabetes. HRV is a robust tool which should now be used in a prospective study to determine the natural history of subclinical autonomic neuropathy.

Supported by: Diabetes UK \& Sheffield Teaching Hospitals Charitable Trustees

\section{8}

Reproducibility of power spectral analysis of heart rate variability in acute and chronic phase of Charcot's neuroarthropathy

R. Bem, A. Jirkovská, M. Dubský, V. Fejfarova, J. Lupinkova, J. Skibova Diabetes Centre, Institute for Clinical and Experimental Medicine, Prague, Czech Republic

Background and aims: Autonomic neuropathy increases blood flow of the foot and may play a crucial role in the pathogenesis of Charcot's neuroarthropathy (CNO), but its diagnosis is applied very rarely in daily practice. According to our previous studies, the power spectral analysis of heart rate variability (PSA of HRV) may be preferable to the traditional Ewing's test for detecting autonomic neuropathy because of its minimal requirements for patient collaboration and time expenditure. The aim of our study was to evaluate the differences of autonomic neuropathy assessed by PSA of HRV in acute and chronic phase of $\mathrm{CNO}$ and their potential association with parameters of $\mathrm{CNO}$ activity and bone turnover.

Materials and methods: Thirty-four patients with acute CNO treated consecutively in our foot clinic during a 16-month period were enrolled in this study. Inclusion criteria consisted of the clinical diagnosis of acute $\mathrm{CNO}$ and the ability to tolerate at least two Ewing's tests. The clinical diagnosis of CNO was reinforced by positive bone scans. Bone turnover was monitored by 1CTP (COOH-terminal telopeptide region of type 1 collagen) and by scintigraphic parameters. Autonomic neuropathy was indicated by abnormal results on at least three of the four Ewing's tests. The PSA cumpower LFHF $1+2+3$ is the cumulative parameter of low-frequency (LF) and high-frequency (HF) bands in all three supine-standing-supine positions, for diagnosis of severe autonomic neuropathy, we selected the fifth percentile of the results obtained from the 123 healthy controls with normal Ewing 's tests. PSA of HRV and Ewing's test were assessed in the acute phase immediately after diagnosis of $\mathrm{CNO}$ and in chronic phase which was defined by clinical signs and as decrease of temperature differences, laboratorial and scintigrafic parameters one year from the inclusion.

Results: No significant correlations between PSA cumpower LFHF $1+2+3$ and markers of CNO activity and bone remodeling (1CTP and scintigraphic parameters) were seen. Severe autonomic neuropathy was seen in 31/34 (91.2\%) of the patients by Ewing's tests and in 34/34 (100\%) of the patients by PSA of HRV. There was no significant difference in the assessment of autonomic neuropathy by PSA of HRV in acute and chronic phase of CNO in the same patient (mean cumpower LFHF $1+2+3$ : $156.8 \pm 151.1$ vs. $175,5 \pm 178,7$; NS); a strong correlation of this parameter in acute and chronic phase of $\mathrm{CNO}$ was seen $(\mathrm{r}=0.828, \mathrm{p}<0.0001)$. In addition, there were no significant differences in Ewing's tests between acute and chronic phase of CNO. 
Conclusion: This study suggests that PSA of HRV may be used for diagnosis of autonomic neuropathy both in acute and chronic phase of CNO; the parameters of PSA of HRV were not influenced by the activity of CNO. Supported by: MZO 00023001

\section{9}

Cardiac autonomic dysfunction and score of risk of diabetes

I. Banu, M. Nguyen, I. Pinal, S. Chiheb, E. Cosson, P. Valensi

Department of Endocrinology, Diabetology, Nutrition, Paris Nord University, France

Background and aims: The Finnish risk score (Findrisk) has been developed to evaluate the risk of incident diabetes. An association has also been reported between this score and glycemic disorders and metabolic syndrome. Cardiac autonomic dysfunction (CAD) is often found in nondiabetic obese patients. The aim of this study was to look for a relationship between the Findrisk score and CAD in overweight or obese patients.

Materials and methods: We included 267 patients, free of known dysglycaemia and referred consecutively to our department (Jan 1998-Oct 2005) for overweight or obesity (BMI $38 \pm 7 \mathrm{~kg} / \mathrm{m}^{2}$ ). Findrisk score was calculated, an oral glucose tolerance test was performed with prediabetes and diabetes defined according to WHO 1999, metabolic syndrome was defined according to IDF criteria, and CAD was assessed on heart rate variability during three standard tests (deep-breathing, lying-to-standing and Valsalva) which were interpreted according to age.

Results: Findrisk score was found to be $\geq 15$ in 88 patients. The score correlated significantly with BMI, prediabetes and diabetes, metabolic syndrome, HOMA insulin resistance index, systolic and pulse blood pressure $(\mathrm{p}<0.04$ to $<0.001$ ). In 230 patients the $3 \mathrm{CAD}$ tests were normal or only one of them was abnormal (mild CAD) while 2 or 3 tests were abnormal (confirmed or severe $\mathrm{CAD}$ ) in the remainder. In the patients with a score $\geq 15$, the rate of confirmed or severe CAD was significantly higher than in those with a score $<15(22.7 \%$ vs $9.5 \% ; \mathrm{p}=0.003)$. This difference was still significant after age adjustment (OR 2.424 [95\% CI: 1.08-5.44]; $\mathrm{p}=0.03$ ). In multivariate analyses the association between the score and confirmed and severe CAD was independent of age, BMI, glycemic disorders and metabolic syndrome.

Conclusion: In overweight or obese patients, 1- a higher Findrisk score is associated significantly with current glycemic and metabolic disorders, and with confirmed or severe CAD; 2- CAD might contribute to insulin resistance and increased blood pressure.

\section{0}

Small fiber neuropathy and impaired autonomic function characterise neuropathy in subjects with impaired glucose tolerance

Z. Putz ${ }^{1}$ Á. Tabák ${ }^{1}$, N. Tóth ${ }^{1}$, I. Istenes ${ }^{1}$, R. Gandhi ${ }^{2}$ Z. Hermányi ${ }^{3}$, N. Németh ${ }^{1}$, K. Keresztes ${ }^{1}$, G. Jermendy ${ }^{3}$, S. Tesfaye ${ }^{2}$, P. Kempler ${ }^{1}$;

${ }^{1} 1$ st Deparment of Medicine, Semmelweis University, Budapest, Hungary,

${ }^{2}$ Diabetic Research Unit, Royal Hallamshire Hospital, Sheffield, United

Kingdom, ${ }^{3}$ rrd Deparment of Internal Medicine, Bajcsy Zsilinszky Hospital, Budapest, Hungary

Background and aims: Impaired glucose tolerance (IGT) is currently identified in about $40 \%$ of subjects with neuropathy of unknown origin. The clinical characteristics of IGT associated neuropathy are not well understood.

Materials and methods: We examined 46 patients with IGT (age: $53.04 \pm 11.10$ years, fasting blood glucose $5.40 \pm 0.57 \mathrm{mmol} / \mathrm{l} ; 120 \mathrm{~min}$ blood glucose: $8.61 \pm 1.01 \mathrm{mmol} / \mathrm{l} ; \mathrm{HbA}_{1 \mathrm{c}}: 5.97 \pm 0.38 \%$; $\mathrm{x} \pm \mathrm{SD}$ ) and 45 healthy controls subjects (age: $55.84 \pm 11.41$ years). Cardiovascular autonomic neuropathy was assessed by the five standard cardiovascular reflex tests and heart rate variability $(\mathrm{HRV})$ characterized by the triangle index $\left(\mathrm{HRV}_{\mathrm{ti}}\right)$. Sensory nerve function (current perception threshold [CPT]) was assessed by the Neurometer $\mathrm{R}$ device, Medoc equipment and the Rydel-Seiffer calibrated tuning fork. Peak plantar pressure was measured by dynamic pedobarograph. The frequency and intensity of symptoms were graded using Neuropathy Total Symptom Score (NTSS).

Results: Significant differences were found between IGT and control subjects regarding the following parameters: beat-to-beat variation: $11.9 \pm 5.7$ vs. $19.5 \pm 4.1$ beats $/ \mathrm{min} ; \mathrm{p}=0.0001$; Valsalva ratio: $1.23 \pm 0.25$ vs. $1.46 \pm 0.22$; $\mathrm{p}=0.0001$; postural systolic blood pressure changes: $-4.37 \pm 5.7$ vs. $-0.6 \pm 2.0$ mmHg; $\mathrm{p}=0.0001$; sustained handgrip: $19.9 \pm 7.9$ vs. $23.7 \pm 6.1 \mathrm{mmHg} ; \mathrm{p}=0.012$; and $\mathrm{HRV}_{\mathrm{ti}}: 26.7 \pm 7.8$ vs. $39.3 \pm 9.0 ; \mathrm{p}=0.0001$. Both hyperaesthesia representing the early neuropathy (median nerve $\mathrm{p}<0.001$, peroneal nerve $\mathrm{p}<0.01$ ) just as hypaesthesia (both median and peroneal nerves $\mathrm{p}<0.005$ ) were detected more frequently at $5 \mathrm{~Hz}$ Neurometer CPT testing among IGT subject compared to controls. Heating detection thresholds were increased among IGT subjects compared to controls at both upper and lower limbs while a significant increase was found only at lower limb for cooling detection threshold. There were no significant differences between the two groups at Rydel-Seiffer tuning fork assessment. It is of particular importance that peak plantar pressure measured by the dynamic pedobarograph was significantly higher in the IGT group versus controls $(\mathrm{p}<0.005)$. Mild to moderate symptoms of neuropathy (NTTS) were present in 5 subject with IGT while none of them was detected in healthy controls.

Conclusion: Small fiber neuropathy including widespread impairment of autonomic function represents the key clinical characteristics of IGT associated nerve dysfunction. Our data indicate that neural impairment could be non-invasively detected in these subjects and non-invasive methods may serve as an alternetive to skin punch biopsy. Cardiovascular autonomic neuropathy may contribute to the increased cardiovascular risk among subjects with IGT. 


\section{PS 121 Neuropathy: experimental and clinical treatments}

\section{1}

Diabetes induces hyperactivity in the spinal dorsal horn neurons: a timecourse study in streptozotocin- induced diabetic rats C. Morgado, I. Tavares

Institute of Histology and Embryology, Faculty of Medicine of Porto and IBMC, University of Porto, Portugal

Spontaneous pain is a common sign of neuropathy in diabetic patients. Several studies showed an increase in the background activity of dorsal horn spinal neurons during diabetes, which may explain that abnormal pain condition. The spinal hyperactivity is probably related to the constant barrage of peripheral input, since peripheral fibres of diabetic rats show ectopic activity. It may also reflect plastic changes at the spinal cord and, more interestingly, of brainstem centres devoted to descending pain modulation.

In the present study, we performed a time-course evaluation of spinal dorsal horn neurons activity in streptozotocin diabetic rats, using Fos expression as a marker of neuronal activity. Furthermore we evaluated the contribution of peripheral input to the spinal neuronal activity by blocking the primary afferent input. Diabetes was induced in adult male Wistar rats by i.p injection of streptozotocin (STZ; $50 \mathrm{mg} / \mathrm{kg}$ ). One group of rats was sacrificed prior to the injection (time-point 0 ) and the others at 1, 2, 4, 6 and 10 weeks post-injection ( $\mathrm{n}=6$ rats per time-point). Spinal segments T13-L5 were removed and immunoreacted against Fos protein (Ab5; Oncogene). At 4 weeks post-injection an additional group was submitted to peripheral nerve block by applying a $5 \%$ lidocaine patch to the internal surface of the left tight. The patch was daily replaced during 3 days. At this time the animals were sacrificed, the spinal segments L2-L3 were removed and immunohistochemically processed for Fos as in the others experimental groups. Fos-immunoreactive (Fos-IR) neurons in the superficial (laminae I-II) or deep (laminae III-V) dorsal horn were counted bilaterally in the time-course study and ipsilateral in the lidocaine treated rats. Data were compared by ANOVA, followed by the Tukey post-hoc test. The numbers of Fos-IR neurons increased moderately at 1 and 2 weeks after STZ injection. A significant increase of Fos level was observed at 4 weeks, followed by a slight decrease at 6 weeks and an even higher increase at 10 weeks post-injection. Peripheral nerve block did not change spinal dorsal horn Fos expression when compared with baseline level at 4 weeks. These findings show that diabetes induces a gradual increase in spontaneous activity of spinal dorsal horn neurons, which is more pronounced at 4 and 10 weeks after STZ injection, and may underlie the spontaneous pain associated to diabetic neuropathy. Since peripheral nerve block did not affect spinal Fos expression it seems that spinal hyperactivity is not exclusively due to the constant input from the periphery but seems to involve plastic changes at spinal and, probably, supraspinal levels.

Supported by: AstraZeneca Foundation, Sociedade Portuguesa de Diabetologia/Novo Nordisk

\section{2}

Effects of rimonabant on painful diabetic neuropathy in male STZ rats. Comparison with duloxetine and gabapentine

P. Chamiot-Clerc, M.-A. Chabanaud, F. Gillot, L. Noah, M.-P. Pruniaux,

A.-M. Galzin

Pharmacology Metabolism, sanofi-aventis, Rueil-Malmaison, France

Background and aims: Recent clinical studies evidenced beneficial effects of Rimonabant (RIMO) in type 2 diabetic patients. Furthermore, RIMO has been shown to display anti-nociceptive effects in both inflammatory and neuropathic pain models (1-2). We aimed at assessing the effects of RIMO on streptozotocin (STZ)-induced painful diabetic neuropathy (PDN) in rats, in comparison with Duloxetine (DULOX) and Gabapentine (GABA) that are the two reference treatments used against PDN in humans.

Materials and methods: Male Sprague Dawley rats (200-225g) were administered i.p. with STZ $65 \mathrm{mg} / \mathrm{kg}$ (STZ-rats) or solvent (Sham-rats) on day 0 (D0). Body weights (BW) were recorded daily. From D14, STZ-rats were treated orally with RIMO (10 mg/kg/d, n=10), DULOX (30 mg/kg, n=10), GABA (100 mg/kg, $\mathrm{n}=10$ ) or vehicle (VHC) (Tween $0.1 \%, \mathrm{n}=10$ ), whereas Shamrats received VHC only. Mechanical allodynia was assessed at D14 and D28, using the electronic Von Frey system and spontaneous horizontal locomotor activity was monitored from D24 to D27 using an infra-red lighted open field and videorecording system (Videotrack, Viewpoint). At the end of the study, blood was collected for the determination of plasma metabolites.

Results: Before any treatment (D14), a significant increase in blood glucose levels was observed in STZ-rats in comparison to Sham-rats (586.4 \pm 10.2 $\mathrm{mg} / \mathrm{dl}$ vs. $109.9 \pm 4.9 \mathrm{mg} / \mathrm{dl}$ respectively; $\mathrm{p}<0.005)$ associated with a decrease in BW (-27\% vs Sham-rats, $\mathrm{p}<0.005)$. Furthermore, STZ-rats showed a significant decrease in the nociceptive threshold, as compared to Sham-rats (49.6 \pm $1.5 \mathrm{~g}$ vs $75.8 \pm 1.6 \mathrm{~g}$ respectively; $\mathrm{p}<0.005)$. At $\mathrm{D} 28$, this threshold was further decreased in VHC-treated STZ rats vs Sham-rats $(46.2 \pm 1.5 \mathrm{~g}$ vs. $77.7 \pm 1.2 \mathrm{~g}$ respectively; $\mathrm{p}<0.005$ ) whereas it was increased by RIMO treatment $+27 \%$ vs VHC-treated STZ rats; $\mathrm{p}<0.05)$. DULOX and GABA treatment also increased the nociceptive threshold in a similar manner $(+28 \%$ and $+27 \%$ vs VHC respectively, $\mathrm{p}<0.05$ ). Nocturnal locomotor activity (total distance) of VHCtreated STZ rats was significantly decreased compared to Sham-rats (-57\%; $\mathrm{p}<0.01)$. Locomotor activity in STZ animals was not affected by treatment except by DULOX during the first three hours of observation (-52\% vs. VHCtreated STZ rats; $\mathrm{p}<0.05$ ), Blood glucose levels at D28 were still significantly higher in VHC-treated STZ-rats than in Sham-rats $(520.4 \pm 17.2 \mathrm{mg} / \mathrm{dl}$ vs. $135.7 \pm 2.7 \mathrm{mg} / \mathrm{dl}$ respectively, $\mathrm{p}<0.005)$. No significant difference was observed between the different treated groups at D28 $(481.8 \pm 15.6 \mathrm{mg} / \mathrm{dl} ; 484.2$ $\pm 12.7 \mathrm{mg} / \mathrm{dl}$ and $482.9 \pm 20.9 \mathrm{mg} / \mathrm{dl}$ in RIMO-, DULOX- and GABA-treated STZ-animals respectively) and vs. VHC-treated STZ-rats. Whereas VHC-, DULOX- GABA-treated STZ rats lost BW by $47 \%, 18 \%$ and $9 \%$ respectively between D14 and D28, RIMO-treated rats lost $8 \%$ only during the same period ( $<<0.01$ vs. VHC-treated STZ rats). Although non significantly, RIMO and GABA improved insulinemia by $+41 \%$ and $+121 \%$ respectively vs VHCtreated STZ rats, whereas DULOX did not (-2\% vs. VHC).

Conclusion: These results show that Rimonabant prevents the development of allodynia in STZ-induced diabetic rats, similarly to the reference compounds Duloxetine and Gabapentine but without significantly altering spontaneous locomotor activity. Furthermore, Rimonabant prevents BW loss and increases insulinemia indicating a reduced severity of diabetes symptoms in this model.

\section{3}

The effect of transplantation of adipose tissue-derived mesenchymal stem cells on the established stage of experimental diabetic neuropathy

H. Mizukami ${ }^{1}$, S.-I. Yamagishi ${ }^{1}$, A. Sugawara ${ }^{1}$, S. Ogasawara ${ }^{1}$, K. Sugimoto ${ }^{2}$, S. Yagihashi ${ }^{1}$

${ }^{1}$ Pathology and Molecular Medicine, ${ }^{2}$ Laboratory Medicine, Hirosaki University, Hirosaki, Japan

Background and aims: Diabetic neuropathy is the most common complication of diabetic patients. There is no curative therapy for the advanced stage of this disorder and stem cell transplantation has now been experimentally applied. We investigated the effects of adipose tissue-derived mesenchymal stem cells (ADMSC) on the established stage of experimental diabetic neuropathy in rats since ADMSC are easily accessible and have a greater potential of the production of growth factors with high proliferative activity compared to other stem cells derived from bone marrow or peripheral blood.

Materials and methods: ADMSC were isolated from subcutaneous fatty tissue of male Wistar rats and confirmed to have diverse differentiation. As recipients of ADMSC, female rats were made diabetic at 8 weeks of age by streptozotocin and followed for 16 weeks until they developed a constant delay of motor nerve conduction velocity (MNCV) and altered pain sensation. Then, ADMSC $\left(2.0 \times 10^{6}\right)$ were transplanted to the right soleus muscle in these diabetic rats. The medium was injected to the contralateral side. The mRNA expression of various growth factors in ADMSC as well as in transplanted tissues was evaluated by RT-PCR. The survival of ADMSC in transplanted sites was examined by SRY gene detection of Y-chromosome and capillary density of the muscles was morphometrically evaluated.

Results: ADMSC were CD90+,CD117-, CD34-, CD45- by FACS and Sca1+, CD90+ by RT-PCR, and differentiated into adipocytes, osteocytes and neuronal cells in a specific medium. Two weeks after transplantation, MNCV in the transplanted side was nearly normalized whereas that in contralateral side was still significantly slow. At 4 weeks, MNCV in the treated side was declined but still faster than the contralateral side. Pain sensation threshold was reduced in diabetic rats and there was a trend to improve in the treated side. The quantitation of capillary density did not find significant difference by the treatment at 4 weeks. Expression of mRNA of growth factors in ADSC was high and augmented expression of TGF-beta in diabetic muscle was normalized in the treated side. Conclusion: These results suggest that ADSC transplantation was promising for the treatment of advanced stage of diabetic neuropathy, but still needs to be well designed for the longer survival of transplanted cells. 
Supported by: Japanese Ministry of Education, Science, Culture and Sports, Japanese Ministry of Health and Welfare

\section{4}

Glucocorticoid receptor antagonist metyrapone reduces neuronal loss in hippocampus and protects beta cells during streptozotocin diabetes development

M.A. Orlovsky, Y.V. Lebed, G.G. Skibo

Cytology, Bogomoletz Institute of Physiology, Kyiv, Ukraine

Recent studies had shown that the first signs of diabetic encephalopathy (hippocampal neuronal loss, astrogliosis etc) are already observed at the early terms of diabetes development. Several lines of evidences suggests that glucocorticoids (GC), whose levels are elevated in diabetes, can cause neuronal damage and death in hippocampus, giving possible explanation for the mechanisms of diabetic encephalopathy. In the present study we had tried to elucidate whether or not blockade of GC synthesis can protect hippocampal neurons during the development of streptozotocin diabetes mellitus. Experiments were accomplished on Wistar-line male adult rats kept under the standard conditions. Single i.p. streptozotocin injection $(45 \mathrm{mg} / \mathrm{kg}$ in citrate buffer) were used to model diabetes type I. Metyrapone was given s.c. in dose of $100 \mathrm{mg} / \mathrm{kg}$ each $12 \mathrm{hr}$ starting from the streptozotocin injection. Control rats received vehicle s.c. in the same manner. The total duration of diabetes was 7 and 14 days: according to our previous findings a significant neuronal loss is observed in the pyramidal layer of the hippocampus at these terms. Immunofluorescent confocal microscopy with antibodies to specific neuronal marker NeuN was used to measure the amount of neurons in different regions of the hippocampus. Islet's beta-cells were studied in pancreatic sections using immunofluorescent microscopy with insulin antibodies. It was established, that at the day 7 metyrapone led to a significant reduction in fasted glucemia level down to $7.97 \pm 1.40 \mathrm{mM}$ as compared $16.43 \pm 2.29$ $\mathrm{mM}$ in vehicle-treated animals; the similar difference in glucemia level was preserved till the end of the experiments. Metyrapone administrations also inhibited neuronal loss in CA1, CA2 and CA3 areas of hippocampus with the most prominent changes observed in CA2 area (see table). Additionally, metyrapone protected pancreatic beta-cells: there were $22 \pm 4$ beta-cells/islet in metyrapone-treated group (day 14 ) in respect to $8 \pm 2$ beta-cells/islet in vehicle-treated group and $36 \pm 8$ in non-diabetic control. Thus, it can be concluded, that metyrapone can protect pancreatic beta-cells and hippocampal neurons during diabetes development, however further studies needed to find out the primary mechanism of metyrapone-induced neuroprotection (inhibition of GC synthesis, glucemia reduction or normalization of insulin production).

Effects of metyrapone on the neuronal count in CA2 area during the diabetes development

\begin{tabular}{lll}
\hline $\begin{array}{l}\text { neurons per } \mathrm{mm} \text { of pyramidal layer } \\
\text { length }\end{array}$ & Vehicle & Metyrapone \\
\hline Control & $220.19 \pm 13.98$ & - \\
\hline Diabetes, day 7 & $168.13 \pm 10.12$ & $215.36 \pm 16.12 *$ \\
\hline Diabetes, day 14 & $160.80 \pm 9.97$ & $183.54 \pm 7.70^{*}$ \\
\hline
\end{tabular}

Supported by: Ukrainian State Foundation for Fundamental Researches, GENOM programme of National Academy of Sciences Ukraine

\section{5}

Effects of all-trans retinoic acid on treatment of type 2 diabetic neuropathy

J. Park, M. Cho, J. Nam, J. Han, J. Yoo, C. Ahn, B. Cha, E. Lee, S. Lim, K. Kim, K. Kim, H. Lee

Internal medicine, Yonsei University College of Medicine, Seoul, Republic of Korea

Background and aims: Peripheral neuropathy is one of the most common complications in diabetes mellitus. Nerve growth factors (NGF) are believed to regulate peripheral and central nervous system, neuronal differentiation, and regeneration of damaged nerves, and their role in diabetic neuropathy is being emphasized these days. Retinoic acid increases the endogenous expression of NGF. The aim of the present study was to investigate the effects of All Trans Retinoic Acid on diabetic neuropathy in OLETF rats, an animal model of type 2 diabetes
Materials and methods: In order to evaluate the effect of ATRA (All-Trans Retinoic Acid, Promega, Medison, WI, USA), we orally administered of 10 $\mathrm{mg} / \mathrm{kg}$ body weight ATRA dissolved in cellulose to 20 OLETF (Otsuka LongEvans Tokushima Fatty) rats for 16 weeks, and we administered cellulose as a vehicle to 20 OLETF rats and 10 LETO(Long-Evans Tokushima Otsuka) rats. At the end of the treatment period, we measured blood glucose levels and contents of NGF (nerve growth factor) in serum and sciatic nerve. Neurometer CPT tests were conducted to examine improvements of the current stimulus thresholds in rats.

Results: Fasting glucose levels were significantly lower in the LETO rats $(105.4 \pm 14.4 \mathrm{mg} / \mathrm{dL})$ than in the non treated OLETF rats $(178.5 \pm 38.3 \mathrm{mg} / \mathrm{dL}$, $\mathrm{P}<0.01)$ or in the ATRA treated OLETF rats $(151.0 \pm 25.1 \mathrm{mg} / \mathrm{dL}, \mathrm{P}<0.01)$. A decrease in serum glucose was observed in the ATRA treated OLETF rats when compared with the non treated OLETF rats. Contents of NGF in LETO rats were $1423.50 \pm 503.21 \mathrm{pg} / \mathrm{mL}$ in serum and $150.0 \pm 28.8 \mathrm{pg} / \mathrm{g}$ in nerve; in non treated OLETF rats the values were $683.71 \pm 169.89 \mathrm{pg} / \mathrm{mL}$ in serum and $243.2 \pm 41.9 \mathrm{pg} / \mathrm{g}$ in nerve; and in ATRA treated OLETF rats the values were $1855.17 \pm 667.34 \mathrm{pg} / \mathrm{mL}$ in serum and $377.9 \pm 61.5 \mathrm{pg} / \mathrm{g}$ in nerve $(\mathrm{P}<0.05)$. The non treated OLETF rats significant increase $(\mathrm{P}<0.01)$ in the thresholds for current stimulus. No differences were found between LETO rats and ATRA treated OLETF rats

Conclusion: Our results have shown that ATRA treatment increases serum and nerve contents of NGF in OLETF rats and suggest that ATRA has a potential therapeutic role for diabetic neuropathy.

Supported by: Seoul R \& BD programme Republic of Korea

\section{6}

Long-term safety and efficacy of pregabalin as treatment of painful diabetic neuropathy: findings from 7 open-label extension studies K. Murphy, B. Emir

Pfizer Inc, New York, United States

Background and aims: Pregabalin has consistently demonstrated robust efficacy for treating painful DPN in randomized clinical trials (RCTs) of 5-13 weeks' duration. We report safety and efficacy findings from pooling 7 longterm, open-label (OL) extensions of pregabalin RCTs in painful DPN.

Materials and methods: Pregabalin dosage was adjusted between 150 and $600 \mathrm{mg} / \mathrm{d}$ based on response and tolerability. Mean daily dosage was monitored and pain evaluated quarterly (100-mm visual analog scale [VAS]).

Results: The pooled OL population included 1426 patients. At baseline, mean age was 58.8 years $(\mathrm{SD}=10.7)$; mean weight, $92.5 \mathrm{~kg}(\mathrm{SD}=19.2)$; mean duration of diabetes, 11.02 years $(\mathrm{SD}=9.0)$; mean $\mathrm{HbA}$ level, $8.01 \%(\mathrm{SD}=7.33)$; and mean pain VAS $(\mathrm{n}=1321), 60.5(\mathrm{SD}=23.59)$. The most common dosage range throughout the OL studies was $300-449 \mathrm{mg} / \mathrm{d}$ pregabalin $(40.7 \%$ of patients), followed by $600 \mathrm{mg} / \mathrm{d}(32.0 \%) .349$ pregabalin patients completed 2 years of treatment. 12 weeks into the OL extensions, mean pain VAS $(\mathrm{n}=770)$ had decreased to $30.6(\mathrm{SD}=23.81)$. Throughout the remainder of the studies, mean pain VAS remained stable at this level through endpoint, 32.9 $(\mathrm{SD}=26.52 ; \mathrm{n}=1246)$. Most AEs were of mild-to-moderate intensity and generally transient. No clinically meaningful changes in $\mathrm{HbA}_{1 \mathrm{c}}$ levels were noted (mean change $0.14 \%$ ), and mean weight change for all patients was $+3.4 \mathrm{~kg}$ at endpoint.

Conclusion: Data from these 7, long-term, OL extension studies suggest that, in patients with painful DPN, the pain-relieving effect of pregabalin is durable over at least a 2-year period. Long-term use of pregabalin is generally well-tolerated and is not associated with clinically meaningful changes in $\mathrm{HbA}_{1 c}$ levels. Supported by: Pfizer Inc.

\section{7}

Effect of duloxetine $60 \mathrm{mg}$ once daily in patients with diabetic peripheral neuropathic pain in long-term open-label study

V. Skljarevski ${ }^{1}$, D. Desaiah ${ }^{1}$, Q. Zhang ${ }^{1}$, A.S. Chappell ${ }^{1}$, M.J. Detke ${ }^{1}$, J.L. Gross ${ }^{2}$

${ }^{1}$ Lilly Research Laboratories, Eli Lilly and Company, Indianapolis, United States, ${ }^{2}$ Centre De Pesquisas Em Diabetes, Centre De Pesquisas Em Diabetes, Porto Alegre, Brazil

Background and aims: This open-label study was designed to evaluate the maintenance of effect of duloxetine $60 \mathrm{mg}$ once daily over 26 weeks of therapy in patients with diabetic peripheral neuropathic pain (DPNP) following 8 weeks of acute therapy. 
Materials and methods: Patients ( $\geq 18$ years of age) who had daily pain for $\geq 6$ months due to symmetric sensorimotor diabetic peripheral neuropathy (confirmed by a score of $\geq 3$ on Michigan Neuropathy Screening Instrument), and had Brief Pain Inventory (BPI) 24-hour average pain score $\geq 4$, were first treated with duloxetine $60 \mathrm{mg}$ once daily for 8 weeks. Responders (i.e., those who had $\geq 30 \%$ reduction in BPI 24 -hr average pain score) at the end of acute therapy continued on duloxetine $60 \mathrm{mg}$ once daily (maintenance treatment arm), and nonresponders were escalated to duloxetine $120 \mathrm{mg}$ once daily (rescue treatment arm) for 26 weeks. The primary efficacy measure was change from baseline (Week 8) to endpoint in the patient-rated BPI 24-hr average pain score for patients entering maintenance phase only. Secondary efficacy measures included BPI Severity (BPI-S) and Interference (BPI-I), Sensory Portion of the Short-Form McGill Pain Questionnaire (SF-MPQ), Patient's Global Impression of Improvement (PGI-I), and Clinical Global Impression of Severity (CGI-S). These variables were assessed for the change from baseline using a within-group t-test with $95 \%$ confidence interval (CI). Vital signs and treatment-emergent adverse events (TEAEs) were assessed.

Results: Of the 216 patients enrolled, $53 \%$ were responders, $32 \%$ were nonresponders, and $15 \%$ discontinued at the end of acute therapy. The BPI $24-\mathrm{hr}$ average pain score (mean change, -2.49 ) and scores on all the secondary efficacy measures significantly $(P<.001)$ improved at the end of acute therapy. During the maintenance therapy, the change of BPI 24-hr average pain score from baseline to endpoint is 0.35 with 0.79 as the upper bound of the $97.5 \%$ $\mathrm{CI}$ which is less than the prespecified noninferiority margin of $1.5(P<.001)$. At the same time, most BPI-S and BPI-I scores slightly increased, with no significant difference from zero except for the pain right now (mean change, $0.48, P=.026)$ and normal work $(0.63, P=.016)$ scores. In the rescue treatment arm, BPI-S scores achieved statistically significant pain reduction (average pain, $P<.001$; worst pain, $P<.001$; least pain, $P=.016$; and pain right now, $P=.011$ ); BPI-I scores were improved significantly for general activity, walking ability, and normal work. The PGI-I score at endpoint was 2.32 in the maintenance and 3.04 in the rescue therapy. Also in the rescue therapy, CGI$S$ and SF-MPQ scores did not change significantly except for CGI-S (-0.46, $P=.003)$. TEAEs and vital signs in this study were similar to those reported in randomized DPNP studies of duloxetine.

Conclusion: Following 8 weeks of acute treatment, duloxetine maintained its efficacy in the management of DPNP for 26 weeks and was well tolerated. Supported by: Eli Lilly and Company

\section{8}

Safety and efficacy of duloxetine in the treatment of diabetic peripheral pain in elderly patients

J.F. Wernicke ${ }^{1}$, A.D. Wasan ${ }^{2}$, M.J. Ossanna ${ }^{1}$, J. Raskin ${ }^{3}$, M.J. Robinson ${ }^{1}$, J.A. Hall ${ }^{1}$, S.E. Edwards ${ }^{1}$, S. Lipsius ${ }^{4}$, A.L. Meyers ${ }^{1}$, B.H. McCarberg ${ }^{5}$ ${ }^{1}$ Eli Lilly, Indianapolis, United States, ${ }^{2}$ Dept of Anesthesiology, Brigham and Women's Pain Management Center, Chestnut Hill, United States, ${ }^{3}$ Eli Lilly Canada, Toronto, Canada, ${ }^{4} \mathrm{i} 3$ Statprobe, Ann Arbor, United States, ${ }^{5}$ Department of Family Medicine, Kaiser Permanente, Escondido, United States

Background and aims: Treatment of diabetic peripheral neuropathic pain (DPNP) in elderly patients ( $\geq 65$ years of age) can be challenging due to frequent use of concomitant medications and presence of coexisting illnesses in this population. Tricyclic antidepressants are often used to treat DPNP, but in elderly patients, tolerability and safety issues often result in insufficient treatment. Duloxetine (DLX) is a selective serotonin and norepinephrine reuptake inhibitor approved for management of DPNP. We present a post-hoc analysis of the safety and efficacy of duloxetine in the elderly.

Material and methods: Data from 3 double-blind, placebo-controlled trials in adult patients with DPNP were pooled and stratified by age $(<65$ and $\geq 65$ years). Patients were randomized to DLX $60 \mathrm{mg}$ once daily (QD; $\mathrm{n}=105$ elderly, 239 nonelderly $[<65]$ ), $60 \mathrm{mg}$ twice daily (BID; $\mathrm{n}=109$ elderly, 232 nonelderly), or placebo (PBO; n=109 elderly, 230 nonelderly) for 12 weeks of acute treatment. Each acute study was followed by an extension phase, with acute phase completers re-randomized to routine care or DLX 60 BID for 52 weeks. The primary efficacy measure was the weekly mean 24-hour average pain score measured by a patient-rated 11-point Likert scale. Safety and efficacy of DLX in elderly patients were assessed using both acute and extension phase data. Safety assessments included spontaneously reported treatmentemergent adverse events (TEAEs) and discontinuation rates due to TEAEs. All analyses were done on an intent-to-treat basis. Fisher's exact test was used for pairwise comparisons within each age group and logistic regression was used for age and therapy-by-age subgroup analyses.
Results: In the acute phase, the age subgroups did not significantly differ in overall rates of TEAEs. A significant relationship between therapy and age was observed for nasopharyngitis ( $\mathrm{p}=.047$; higher incidence in nonelderly [9.5\%] vs elderly [1.8\%] for DLX 60 BID) as well as for abdominal pain ( $\mathrm{p}=.037$; higher incidence in elderly [5.7\%] vs nonelderly [0.8\%] for DLX 60 QD). A greater percentage of elderly patients discontinued due to TEAEs $(\mathrm{p}<.001)$. The greater discontinuation rate for the elderly was seen in all treatment groups (therapy-by-age interaction $\mathrm{p}=.926$ ). In the extension phase of the trials, a therapy-by-age interaction was observed in overall rate of TEAEs due to age subgroup differences in the routine care group $(\geq 1$ TEAE: elderly [86.6\%], nonelderly [74.6\%]); in the DLX group, TEAE rate was $77.3 \%$ in elderly and $77.9 \%$ in nonelderly. DLX showed improvement in weekly mean 24-hour average pain vs $\mathrm{PBO}$ in both age groups $(\mathrm{p}<.01)$.

Conclusion: Duloxetine was well tolerated and efficacious in the treatment of DPNP regardless of age strata. There were few significant differences between age groups that were dependent on therapy received, and there was no consistent signal indicating that safety and efficacy of duloxetine might differ significantly in elderly vs nonelderly patients. This suggests that in patients 65 years of age and older, duloxetine may be a valuable option for treatment of DPNP, in particular for those who have difficulty tolerating other DPNP medications.

Supported by: Eli Lilly and Company

\section{9}

Evidence of neuroregeneration using vascular endothelial growth factor zinc finger protein activator (SB-509) in diabetic neuropathy: a chronic degenerative polyneuropathy

D.G. Ando ${ }^{1}$, M. Merren ${ }^{2}$, W. Douglas ${ }^{3}$, S. Hamilton ${ }^{4}$, Y.-L. Lee ${ }^{4}$, K. Spratt ${ }^{4}$, E. Benaim ${ }^{4}$, M. Kipnes ${ }^{3}$

${ }^{1}$ Therapeutic Development, Sangamo BioSciences, Richmond, ${ }^{2}$ Neurology, Diabetes and Glandular Disease Research Associates, San Antonio,

${ }^{3}$ Clinical, Diabetes and Glandular Disease Research Associates, San Antonio, ${ }^{4}$ Development, Sangamo BioSciences, Richmond, United States

Background and aims: Diabetic Neuropathy (DN) is a progressive neurodegenerative disease with an incidence up to $50 \%$ in long-term patients. Although the pathogenesis is related to hyperglycemia, the specific mechanisms are not clear. The pathophysiology includes severe nerve damage with demyelination and alteration in axonal transport. Vascular Endothelial Growth Factor (VEGF) has protective and regenerative effects on neuronal cells in culture.

Materials and methods: Using VEGF zinc finger protein activator (SB-509) we have treated twenty-four patients with a single treatment of sixty mgs of SB-509, intramuscularly in the lower extremities, in a placebo-control clinical trial. Patients were treated in both legs with either SB-509 or placebo and evaluated by quantitative sensory testing (QST) using the Vibratron 2 instrument, and motor/sensory nerve conduction velocities (NCV) in the lower extremities.

Results: In 24 evaluated patients, improvements in motor NCVs and sensory NCVs were seen at the six-month time-point in the SB-509 group when the average of all motor NCV (2 peroneal and 2 tibial NCV per patient) and the average of two sural NCV from baseline was compared to placebo. The mean value of motor NCV improvement in the SB-509 group (twelve patients) was $0.78 \mathrm{M} / \mathrm{sec}$ compared to $-0.93 \mathrm{M} / \mathrm{sec}$ in the placebo group (twelve patients) $(\mathrm{p}=0.21)$. The mean value of sural NCV improvement in the SB-509 group was $1.18 \mathrm{M} / \mathrm{sec}$ compared to $-0.43 \mathrm{M} / \mathrm{sec}$ in the placebo group ( $\mathrm{p}=0.29$ ). QST testing at six months showed a $25 \%$ improvement in vibration units from baseline in the SB-509 group compared to 5\% worsening in the placebo group $(\mathrm{p}<0.02)$ Improvements in NCV included three patients with unmeasurable NCVs who, after treatment with SB-509, showed improved NCV over the six-month follow-up.

Conclusion: These improvements in neurologic endpoints suggest that SB509 has novel regenerative potential for the reversal of nerve damage in diabetic neuropathy. This single treatment data showing improvement in sensory and motor neuropathic deficits are the basis on which two multi-dosing clinical trials in mild to moderate and severe diabetic neuropathy are currently in progress. 


\section{PS 122 Neuropathy: screening and associated features}

\section{0}

\section{LDIflare demonstrates early functional changes in painful diabetic neuropathy \\ S.T.M. Krishnan ${ }^{1}$, C. Quattrini ${ }^{2}$, M. Jeziorska ${ }^{3}$, R. Malik ${ }^{2}$, G. Rayman ${ }^{1}$ ${ }^{1}$ Diabetes Centre, Ipswich Hospital NHS Trust, ${ }^{2}$ Division of Cardiovascular Medicine, The University of Manchester, ${ }^{3}$ Division of Regenerative Medicine, The University of Manchester, United Kingdom}

Background and aims: Structural and functional changes in small unmyelinated $\mathrm{C}$ fibres appear to precede large fibre pathology and have been implicated in foot ulceration in people diabetes. Furthermore, $\mathrm{C}$ fibre dysfunction is considered to be involved in the genesis of neuropathic pain. The present study has 2 aims a) to compare 3 methods of assessing small fibre function, 2 non-invasive and functional, the LDIflare and Quantitative Sensory Testing (QST) and one invasive and structural, the measurement of dermal nerve fibre density (NFD) and b) to examine the hypothesis that painful neuropathy is associated with functional defects in C-fibre function whereas painless neuropathy with more advanced structural pathology reflected by reduction in NFD.

Methods: Fifteen healthy controls (HC), 10 painful (PFN) and 12 painless (PLN) neuropaths were studied. The LDIflare was assessed by heating dorsal foot skin to 44 degree $\mathrm{C}$ to induce axon reflex vasodilatation and measuring the flare size by laser Doppler imaging. NFD per $\mathrm{mm}^{2}$ was assessed in $3 \mathrm{~mm}$ skin biopsies taken from the same site, immunostained with PGP 9.5. QST was measured using Computer Aided Sensory Evaluator (CASE IV).

Results: Compared with controls, mean NFD and all the QST measures were significantly abnormal in the PLN group but not in the PFN group (Table 1). In contrast, the LDIflare in $\mathrm{cm}^{2}$ was reduced in both neuropathic groups (PFN 1.59+/-0.41; PLN 1.51+/-0.56) compared to HC 4.38+/-1.4 (p < 0.001). Across the groups NFD correlated well with LDIflare $(\mathrm{r}=0.57, \mathrm{p}<0.0001)$.

Table 1

\begin{tabular}{llll}
\hline & HC & PFN & PLN \\
\hline LDIflare $\left(\mathrm{cm}^{2}\right)$ & $4.38+/-1.4$ & $1.59+/-0.4 \dagger$ & $1.51+/-0.56 \dagger$ \\
\hline NFD $\left(\mathrm{mm}^{2}\right)$ & $424+/-176.3$ & $307.6+/-164.5$ & $205.8+/-165.3^{*}$ \\
\hline VPT (Volts) & $7.0+/-2.8$ & $8.7+/-2.2$ & $37.0+/-12.9 \dagger$ \\
\hline VDT (JND) & $18.4+/-3.2$ & $19.5+/-3.2$ & $23.0+/-3.7 \ddagger$ \\
\hline CDT (JND) & $10.4+/-5.0$ & $13.8+/-5.1$ & $19.5+/-4.6 \dagger$ \\
\hline WDT (JND) & $17.5+/-2.0$ & $18.3+/-6.1$ & $25.2+/-1.8 \dagger$ \\
\hline HPO (JND) & $21.6+/-1.8$ & $21.3+/-3.0$ & $25.3+/-1.6 \dagger$ \\
\hline
\end{tabular}

$(\dagger-\mathrm{p}<0.0001) ;(*-\mathrm{p}=0.006) ;(\ddagger-\mathrm{p}=0.004)$ Mean $+/-\mathrm{SD}$

Symbols indicate $\mathrm{p}$ value when compared to HC

Conclusion: Thus, the LDIflare demonstrates early functional abnormalities in painful neuropathy whereas skin nerve fibre density and QST were only abnormal in those with more advanced painless neuropathy. As the LDIflare correlates with NFD, is non invasive and has excellent reproducibility, it may be valuable in detecting early small fibre dysfunction. Further, bigger studies are warranted to confirm the findings.

\section{1}

Current perception threshold determined with a novel method, the Neurometer is clinically useful in detecting functional abnormalities of large and medium-size myelinated nerve fibres in patients with type 2 diabetes mellitus

T. Inukai, R. Matsutomo, K. Hara, T. Nakano, M. Suetsugu, S. Matsumoto, T. Nakamachi, K. Takebayashi, Y. Aso

Department of Internal Medicine, Dokkyo Medical University Koshigaya Hospital, Koshigaya, Japan

Background and aims: Diabetic neuropathy is a common and important complication of diabetes mellitus, however there are few methods to evaluate objectively and quantitatively diabetic peripheral neuropathy. The measurement of the current perception threshold (CPT) using the Neurometer (Neurotron Inc. Baltimore, USA), a novel method, could elucidate the functional status of three distinct nerve fibre types - large myelinated $(A \beta)$ fibres, medium-size myelinated $(\mathrm{A} \delta$ ) fibres and unmyelinated $(\mathrm{C})$ fibres, at setting of $2000 \mathrm{~Hz}, 250 \mathrm{~Hz}$ and $5 \mathrm{~Hz}$, respectively. We compared the Neurometer method and several conventional methods in type 2 diabetic patients.

Materials and methods: The current studies were conducted in 116 patients with type 2 diabetes mellitus and age-matched healthy controls. At each frequency, a CPT below the normal range measured by the Neuromater is considered as hyperaesthesia, while a CPT above the normal range is considered as hypoaesthesia. As the conventional methods, the measurement of the motor and sensory nerve conduction velocities (MNCV, SNCV), of the coefficient of variation of the R-R interval on ECG (CVr-r), of vibration perception threshold (VPT) by SMV5 vibrometer, of $\triangle \mathrm{sBP}$ in Schellong test and the status of Achilles tendon reflexes were applied.

Results: The CPT at $2000 \mathrm{~Hz}$ was significantly higher in diabetic patients than in controls $(\mathrm{p}<0.05)$ and showed significantly negative correlation with MNCV and SNCV $(\mathrm{r}=-0.279, \mathrm{p}<0.01, \mathrm{r}=-0.350, \mathrm{p}<0.01$, respectively). At $2000 \mathrm{~Hz}$ and $250 \mathrm{~Hz}$, the CPTs exhibited positive correlations with VPT $(\mathrm{r}=0.288, \mathrm{p}<0.01, \mathrm{r}=0.207, \mathrm{p}<0.05$, respectively). At $5 \mathrm{~Hz}, \Delta \mathrm{sBP}$ in patients with hypoaesthesia (higher $\mathrm{CPT}$ ) tended to be less than in those with normoaesthesia (normal CPT) and hyperaesthesia (lower CPT). There were no significant differences in CVr-r among normoaesthesia, hyperaesthesia and hypoaesthesia groups classified by CPTs at $5 \mathrm{~Hz}$. Significantly higher CPT values at $2000 \mathrm{~Hz}$ were obtained in patients with proliferative diabetic retinopathy and with macroalbuminuria, compared with those wirhout complications ( $\mathrm{p}<0.01, \mathrm{p}<0.05$, respectively).

Conclusion: The present investigations suggest that the $\mathrm{CPT}$ measurement by the Neurometer is clinically useful in detecting functional abnormalities of large and medium-size myelinated nerve fibres, rather than unmyelinated nerve fibres in patients with type 2 diabetes.

\section{2}

Investigation into the role of human bias in $10 \mathrm{~g}$ monofilament force delivery

M.C. Spruce ${ }^{1}$, C.H. Lewis ${ }^{2}$, M. Griffin ${ }^{2}$

${ }^{1}$ School of Health Professions, ${ }^{2}$ Institute of Sound and Vibration Research, University of Southampton, Southampton, United Kingdom.

Background and aims: To verify the dynamic effects of multiple testers on $10 \mathrm{~g}$ monofilament reliability

Previous studies advocate that the $10 \mathrm{~g}$ monofilament is capable of providing repeatable force for $\leq 100$ compressions, after which, a 24 hour rest period is required. However, these studies are based upon automated force delivery equipment. Whilst this approach tests fibre integrity, it fails to account for the potential non-linearity in human application. Consequently, the rate of mechanical degradation under real-time dynamic conditions, as would be the case in a busy clinical environment, remains unknown. Similarly, there have been no investigations into the inter-reliability and repeatability of the $10 \mathrm{~g}$ monofilament between multiple users.

Materials and methods: Twenty new CE approved $10 \mathrm{~g}$ monofilaments (same brand) were tested by 20 trained male subjects for peak force. Devices were applied in five runs of ten cycles against a research grade strain gauge at a controlled rate. All data was captured using an oscilloscope recording device permitting statistical handling.

Results: Significant differences in both mean and median peak force were found between all subjects $(\mathrm{p}=0.021)$. Further post hoc analysis revealed highly significant differences in the above modalities on Run 3 and 5 ( $p=0.013, p=0.03$ ) respectively, in comparison to Run 1 . Finally, a highly significant difference was observed between subjects for the total range of peak force $(\mathrm{p}=0.001)$.

Conclusion: This is the first study to demonstrate that when used by a human, rather than a machine, the $10 \mathrm{~g}$ monofilament suffers premature mechanical decay at $50 \%$ of the recommended number of applications. Similarly, the total range of peak force varies significantly between subjects. Hence, the clinical reliability of the $10 \mathrm{~g}$ monofilament appears unstable, once the human variable is factored in, requiring a fresh approach to both method and equipment. 


\section{3}

Same day intra-subject reproducibility of vibration perception threshold in type 1 diabetes

C.L. Martin ${ }^{1}$, B.H. Waberski ${ }^{2}$, R. Pop-Busui ${ }^{3}$, J.W. Albers ${ }^{4}$, W.H. Herman ${ }^{5}$, P. Cleary ${ }^{2}$

${ }^{1}$ Michigan Diabetes Research and Training Center, University of Michigan, Ann Arbor, ${ }^{2}$ The Biostatistics Center, The George Washington University,

Rockville, ${ }^{3}$ Division of Metabolism Endocrinology and Diabetes, University of Michigan, Ann Arbor, ${ }^{4}$ University of Michigan Medical School,

University of Michigan, Ann Arbor, ${ }^{5}$ University of Michigan Health

Systems, University of Michigan, Ann Arbor, United States

Background and aims: All surviving Diabetes Control and Complications Trial (DCCT) participants were invited to enroll in the Epidemiology of Diabetes Interventions and Complications (EDIC) study in 1994. Of these 1,375 subjects (96\%) agreed to participate; 687 and 688 from the former intensive (INT) and conventional (CONV) treatment groups, respectively. Beginning 13 years after DCCT completion quantitative sensory testing was added to the EDIC annual test battery, and vibration perception threshold (VPT) was assessed for the first time among former DCCT subjects. To evaluate VPT reproducibility, same-day, same examiner repeated tests were preformed on a random subset of participants.

Materials and methods: Study coordinators from the 28 EDIC clinical sites were centrally trained and certified to perform VPT using the Vibratron II (Physitemp, Inc., Clifton NJ). Unilateral, dominant side VPTs for the index finger and great toe were determined using a forced choice algorithm. Vibration units (VU) were adjusted up or down in $10 \%$ increments following incorrect or correct responses, respectively. Testing continued until five errors were recorded over a minimum of 18 trials. VUs for the first five errors and five lowest correct responses were rank ordered, the highest and lowest values discarded, and the remaining 8 values averaged to determine the VPT. To evaluate test reproducibility 2 to 6 subjects per site were randomly assigned to repeat testing of the dominant side great toe. Examiners were instructed to allow at least 30 minutes between the primary and repeat test during which time the subject was to wear the same footwear worn prior to the primary test. The same examiner performed both tests and was not masked to primary test results. Tests were reviewed and scored centrally. Intraclass correlation was performed to determine the coefficient of reliability for primary versus repeated tests.

Results: The 94 subjects identified for repeat testing were representative of the EDIC cohort in age, (mean $46.1+7.5$ years) gender ( $53 \%$ male), diabetes duration (mean years $24.2+4.5$ ) and treatment assignment (50\% INT). Repeat VPT of the dominant great toe has been completed in 81 (86\%) subjects. Completion of repeat testing among sites ranged from $50 \%$ (3 sites) to $100 \%$ (19 sites). The mean primary VPT was $3.74 \pm 2.73 \mathrm{VU}$ and the mean repeat VPT was $4.01 \pm 2.77 \mathrm{VU}$ and the difference was not statistically significant $(\mathrm{p}=0.10)$. The calculated test-retest coefficient of reliability was 0.85 .

Conclusion: VPT among this sample of EDIC subjects demonstrated good overall reproducibility. These results were not obtained with any masking of examiners, and, aside from instructions to wait at least 30 minutes between the initial and repeated test and to otherwise reproduce the EDIC VPT protocol, were not performed under rigidly controlled circumstances. We plan to relate VPT to other measures of peripheral neuropathy collected concurrently in EDIC subjects.

Supported by: National Institute of Diabetes and Digestive and Kidney Diseases and the General Clinical Research Centers Program, National Center for Research Resources

\section{4}

Transforming growth factor 1 (TGF $\beta 1$ ) and aminoterminal pro-brain natriuretic peptide (NT-proBNP) levels could be associated to diabetic neuropathy

J.M. Pou ${ }^{1}$, J. Jurado 2 , M. Ferrandiz ${ }^{3}$, J. Merce', L. Comerma 2 , J. Freixa ${ }^{4}$,

J. Ybarra ${ }^{5}$

${ }^{1}$ Dpt. Endocrinology and Nutrition, Autonomous University of Barcelona. Hospital Sta. Creu i S. Pau, Barcelona, ${ }^{2}$ Primary Care Center, Institut Catala de la Salut, Olot, Girona, ${ }^{3}$ Dept. Neurophysiology, Institut Calatala de la Salut, Hospital Josep Trueta, Girona, ${ }^{4}$ Dpt. Biochemistry, Autonomous University of Barcelona, Hospital Sta. Creu i S. Pau, Barcelona, ${ }^{5}$ Instituto de Cardiología Avanzada (ICAMED), Centro Medico Teknon, Barcelona, Spain

Background and aims: The plasma levels of the N-terminal fragment of brain natriuretic peptide (NT-proBNP) recently gained extreme importance as marker of myocardial dysfunction in type 2 diabetes mellitus (T2DM). In a previous work, we observed that NT-proBNP levels also display a significant association with the Diabetic Neuropathy (DPN), even after correction for age and cardiovascular disease (CVD). Scarce data is available regarding the association between diabetic polyneuropathy (DPN) and other peptides as Transforming Growth Factor Beta 1 (TGF $\beta$ 1). TGF $\beta 1$ manifested several important roles in diabetic nephropathy but its relationship to DPN and other microangiopathy is not quite understood.

Materials and methods: Hence, we set forth a cross-sectional pilot study, to assess the hypothetical relationships between NT-proBNP and TGF $\beta 1$ levels, CVD and DPN in a group of T2DM patients (N=190; W:46 \%) randomly selected from the North Catalonia Diabetes Study (NCDS). CVD and DPN (clinical and neurophysiology evaluation) prevalence were assessed. NT-proBNP was measured by immunoassay (Elecsys ${ }^{\circledR}$, Roche Diagnostics, Indianapolis, Inc.) and total TGF $\beta 1$ by ELISA (ProOmega ${ }^{\circledR}$ )

Results: Patients' age: $65.6 \pm 7.2$, T2DM duration: $11.2 \pm 4.5$ years, HbAlc: 7.3 \pm 1.7 ; DPN: $48.6 \%$. Log-transformed NT-proBNP levels (logNT-proBNP) were significanlly higher in T2DM patients with CVD $(P<0.004)$ and DPN $(P<0.000)$ and also log-transformed TGF $\beta 1(\log$ TGF $\beta 1)$ was higher in CVD $(p<0.001)$ and DPN $(p<0.04)$ patients. LogNT-proBNP manifested an important correlation with $\log$ TGF $\beta 1(\mathrm{p}<0.0001)$. Log NT-proBNP correlated with: age $(P<0.001)$, microalbuminuria $(\mathrm{p}<0.03)$, CVD $(P<0.000)$ and DPN $(P<0.000)$-even after correction for age and CVD $(\mathrm{p}<0.001)$-Log TGF $\beta 1$ correlated with creatinine $(\mathrm{p}<0.001)$, microalbuminuria $(\mathrm{p}<0.001)$, C-reative protein plasma levels $(\mathrm{CRP})(\mathrm{p}<0.02), \mathrm{CVD}(P<0.006)$ and DPN $(P<0.000)$-even after correction for microalbuminuria, creatinine and CVD $(\mathrm{p}<0.014)$

Conclusion: Noteworthy, TGF $\beta 1$ displays a new and significant association with DPN independent of microalbuminuria and renal function. TGF $\beta 1$ and NT-proBNP plasma levels appear as important molecules for diabetic microangiopathic complications' development and screening. Further prospective trials are warranted.

Supported by: Fondo de Investigación Sanitaria and Spanish Network CO3/08: Instituto Carlos III

\section{5}

Epidemiology of neuropathic pain in pre-diabetes and diabetes. The MONICA/KORA Augsburg Surveys and Myocardial Infarction Registry (KORA-A Study)

D. Ziegler' ${ }^{1}$, W. Rathmann' ${ }^{2}$, T. Dickhaus ${ }^{2}$, C. Meisinger ${ }^{3,4}$, A. Mielck ${ }^{5}$ ${ }^{1}$ Institute for Clinical Diabetes Research, German Diabetes Center, Düsseldorf, ${ }^{2}$ Institute of Biometrics and Epidemiology, German Diabetes Center, Düsseldorf, Institute of Epidemiology, Helmholtz Zentrum München - German Research Center for Environmental Health, Neuherberg, ${ }^{4} \mathrm{MONICA} / \mathrm{KORA}$ Myocardial Infarction Registry, Central Hospital of Augsburg, ${ }^{5}$ Institute of Health Economics and Health Care Management, Helmholtz Zentrum München - German Research Center for Environmental Health, Neuherberg, Germany

Background and aims: The lowest glycaemic threshold and risk factors for the development of neuropathic pain have not been established. The aim of this study was to determine the prevalence and risk factors of neuropathic pain in two different populations in relation to the degree of hyperglycaemia.

Materials and methods: KORA-A is based on participants from the population-based MONICA/KORA Augsburg Surveys $(\mathrm{S} 2+\mathrm{S} 3)$ and the Augsburg Myocardial Infarction Registry (MIR). Subjects aged 25-74 years with diabetes (S2+S3: $n=195$; MIR: $n=214)$ and controls matched for age and sex (S2+S3: $n=198$; MIR: $n=212$ ) were assessed for neuropathic pain by the Michigan Neuropathy Screening Instrument using its pain-relevant questions in conjunction with an examination score cutpoint $>2$. An oral glucose tolerance test was performed in the controls.

Results: Among the control subjects from the $\$ 2+3$ population, $46(23.2 \%)$ had impaired glucose tolerance (IGT), 71 (35.9\%) had impaired fasting glucose (IFG), and 81 had normal glucose tolerance (NGT). The corresponding numbers among the MIR control subjects were 61 (28.8\%), 70 (33.0\%), and 81. Among the $\mathrm{S} 2+\mathrm{S} 3$ participants the prevalence of neuropathic pain was $13.3 \%$ in the diabetic subjects, $8.7 \%$ in those with IGT, $4.2 \%$ in those with IFG, and $1.2 \%$ in those with NGT ( $\mathrm{p}=0.003$ for diabetic vs NGT, IFG, and IGT). In the MIR subjects the prevalence of neuropathic pain was higher in each group, reaching $21.0 \%$ in the diabetic subjects, $14.8 \%$ in those with IGT, $5.7 \%$ in those with IFG, and $3.7 \%$ in those with NGT ( $<<0.001$ for diabetes vs NGT, IFG, and IGT). Multivariate analyses showed that in the entire 
S2 + S3 population studied $(n=393)$, age, weight, peripheral arterial disease $(\mathrm{PAD})$, and diabetes were independent factors significantly associated with neuropathic pain, while in the diabetic group it was age, weight, and PAD (all $\mathrm{p}<0.05)$. In the entire MIR group studied ( $\mathrm{n}=426)$, age, waist circumference, peripheral arterial disease (PAD), and diabetes were independent factors significantly associated with neuropathic pain, while in the diabetic group neuropathic pain was associated with waist circumference, physical activity, and PAD (all $\mathrm{p}<0.05)$.

Conclusion: The prevalence of neuropathic pain is 2-3fold increased in subjects with IGT and diabetes compared to those with IFG, but even higher among survivors of myocardial infarction with diabetes and IGT. Neuropathic pain is predominantly associated with cardiovascular risk factors and disease including obesity, low physical activity, and peripheral arterial disease which may constitute important prevention targets.

KORA research platform and the MONICA Augsburg were financed by the Helmholtz Zentrum München - German Research Center for Environmental Health, funded by the German Federal Ministry of Education, Science, Research and Technology and by the State of Bavaria

\section{6}

Investigation of the impact of painful diabetic peripheral neuropathy on sleep quality and sleep architecture in type 1 and 2 diabetic patients using polysomnography

M.E.V. Eriksson ${ }^{1}$, R. Gouni², L.C. Gribble ${ }^{1}$, D.D. Coppini ${ }^{3}$, D. Kerr²,

J. Boyle

${ }^{1}$ Faculty of Health \& Medical Sciences, University of Surrey, Guildford,

United Kingdom, ${ }^{2}$ Bournemouth Diabetes and Endocrine Centre, The royal

Bournemouth and Christchurch Hospitals, Bournemouth, United Kingdom,

${ }^{3}$ Poole Diabetes Centre, Poole General Hospital, Poole, United Kingdom

Background and aims: Painful diabetic peripheral neuropathy (DPN) is a common complication of diabetes mellitus (DM). Diabetic neuropathic pain is known to be more severe at night and patients with painful DPN often report that their pain has a severe impact on their sleep. Loss of sleep and poor sleep quality may have a great impact on patients' psychological and physical well being and the aim of this study was to investigate how neuropathic pain may interfere with sleep in these patients.

Materials and methods: Sleep architecture and pain interference for 45 type 1 and type 2 diabetic patients (males $=29$, females $=16$, mean age of 65.0 \pm SD 9.7 years) with painful DPN was studied. Inclusion criteria required neuropathic pain of diabetic origin and a score of above 12 on the Leeds Assessment of Neuropathic Symptoms and Signs (LANSS). Patients had a mean of $16.7 \pm$ SD 13.6 years since diagnosis of diabetes, established neuropathy for a mean of $7.9 \pm$ SD 9.5 years and had a mean $\mathrm{HbAlc}$ of $8.0 \pm$ SD $1.6 \%$. Patients were randomised and have completed the baseline period of a 36 day double-blind parallel groups investigation into the effects of pregabalin, duloxetine and amitriptyline on aspects of pain and sleep. Overnight sleep was measured between $23.00 \mathrm{~h}$ to $07.00 \mathrm{~h}$ using polysomnography (PSG) and subjective sleep was assessed using sleep diaries. Daytime sleepiness and pain interference were measured using the Karolinska Sleepiness Scale (KSS) and the Short Form Brief Pain Inventory (BPI), respectively.

Results: Increased pain interference correlated with increased PSG number of awakenings (NAW) $(r=0.40, p=0.0133)$ and increased duration of stage 1 $(\mathrm{r}=0.34, \mathrm{p}<0.05)$. Mean PSG sleep efficiency (SE \%) was $77.7 \pm \mathrm{SD} 11.8 \%$, indicating poor sleep. In addition, pain interference correlated significantly with subjective quality of sleep $(\mathrm{sQOS})(\mathrm{r}=0.43, \mathrm{p}<0.01)$ and subjective daytime sleepiness $(r=0.32, p<0.05)$. Both pain interference and sQOS correlated with years since neuropathy diagnosis $(r=0.33, p<0.05$ and $r=0.34$, $\mathrm{p}<0.05$, respectively). Pearson correlations were used on all above baseline data.

Conclusion: These important findings provide objective evidence that diabetic patients with painful DPN have poor sleep. Pain is associated with increased number of PSG awakenings and increased amount of stage 1 sleep, resulting in more fragmented and lighter sleep. Observations of poor sleep and day time sleepiness adds further important information to the better understanding of painful PDN, enabling possible improvements in treatment strategies for affected patients.

Supported by: Pfizer Ltd and sponsored by The University of Surrey

\section{7}

Truncal pruritus of unknown origin may be a symptom of diabetic polyneuropathy

H. Sasaki ${ }^{1}$, H. Yamaoka ${ }^{1}$, K. Ogawa ${ }^{1}$, M. Nakatani ${ }^{1}$, J. Ibata ${ }^{1}$, H. Yamasaki ${ }^{1}$, H. Furuta ${ }^{1}$, M. Nishi ${ }^{1}$, K. Naka ${ }^{2}$, Y. Yamamoto ${ }^{2}$, K. Nanjo ${ }^{1}$

${ }^{1}$ First Department of Medicine, Wakayama University of Medical Science, ${ }^{2}$ Department of Internal Medicine, Wakayama Rosai Hospital, Wakayama, Japan

Background and aims: To know the clinical significance of the pruritus in diabetic (DM) patients, we investigated the prevalence of pruritus in DM and non-diabetic (NDM) subjects, and the relevance to the signs and symptoms of diabetic polyneuroapthy (DPN) of pruritus. Furthermore, the relationships between pruritus and quantitatively assessed nerve functions were examined.

Materials and methods: I. Prevalence and relevance of pruritus to signs and symptoms of DPN A large scale survey questionnaire for 2,656 DM outpatients was performed by 48 medical practitioners and hospital physicians in Wakayama Prefecture, Japan. The relations between pruritus and following parameters were investigated: age, sex, duration of DM, hemoglobin Alc (HbA1c), Achilles tendon reflex (ATR), vibration perception time at the ankles measured by $\mathrm{C} 128$ tuning fork (VPT) and subjective symptoms including numbness in toes and soles, pain in toes and soles, painful leg cramp and pruritus. The similar questionnaire was also administered to 499 NDM subjects. II. Nerve functions associated with pruritus. The relationships between pruritus and several nerve functions: motor and sensory nerve conduction velocities (MCV, SCV), sensory nerve action potential, heart rate variability, quantitatively accessed VPT, a fall of systolic blood pressure at head-up tilt test $(\mathrm{dBP})$ were investigated in $66 \mathrm{DM}$ patients. Statistical analyses were performed by chi-squared test, unpaired t-test and multiple logistic regression analysis.

Results: I. Prevalence and relevance of pruritus to signs and symptoms of DPN Prevalence of numbness, pain, and pruritus in DM $(27.5 \%, 9.6 \%$, $26.3 \%)$ is significantly higher than those in $\mathrm{NDM}(8.0 \%, 4.4 \%, 14.6 \%)$. Although the truncal pruritus of unknown origin (TPUO)was more high-frequent in DM than NDM ( $11.3 \%$, vs $2.9 \%, \mathrm{p}=0.0001)$, the prevalence of limb pruritus or pruritus of known origin was similar between the both groups. The prevalence of numbness, pain, leg cramp, bilateral areflexia of ATR and decreased VPT $(V P T<10 \mathrm{sec})$ in the patients with TPUO was significantly higher than those in the patients without TPUO. Duration of diabetes in the patients with TPUO was significantly longer than that in the patients without TPUO. Multiple logistic regression analysis revealed that numbness and ATR areflexia were independent risk factors of TPUO from age, sex, duration and $\mathrm{HbA1c}$. II. Nerve functions associated with pruritus. MCV and dBP in the patients with TPUO were significantly impaired than those without TPUO. Slow MCV and large dBP were also extracted as significant risk factors of TPUO by multiple logistic regression analysis.

Conclusion: The prevalence of TPUO in DM was significantly higher than that in NDM. TPUO was significantly associated with symptoms and signs of DPN, orthostatic hypotension and decreased motor nerve conduction velocity. Therefore TPUO seems to be a newly recognized symptom of DPN.

\section{8}

Gait alterations of diabetic patients while walking on different surfaces L. Allet ${ }^{1,2}$, S. Armand ${ }^{2}$, R.A. de Bie ${ }^{1}$, Z. Pataky ${ }^{3}$, A. Golay ${ }^{3}$, K. Aminian ${ }^{4}$, E.D. de Bruin ${ }^{5}$

${ }^{1}$ Department of Epidemiology, Maastricht University, Netherlands, ${ }^{2}$ Neurosciences, Geneva University Hospital and University of Geneva, Switzerland, ${ }^{3}$ Service of Education for Chronic Disease, Geneva University Hospitals and University of Geneva, Switzerland, ${ }^{4}$ Laboratory of Movement Analysis and Measurement, EPFL, Lausanne, Switzerland, ${ }^{5}$ Institute of Human Movement Sciences and Sport, ETH, Zurich, Switzerland

Background and aims: Diabetic patients have been shown to suffer from increased risk of injurious falls. Since peripheral neuropathy (PN) affects both sensory and motor function, the neuromuscular damage it implies, may result in altered lower extremity biomechanics. This could lead to associating gait abnormalities with the aforementioned heightened fall risk. However, to date, the cause of increased fall risk in diabetic patients is still under debate. In most existing studies gait was assessed in specialized kinesiology laboratories. Activities in daily life however, require us to move about in challenging environments. We aimed to evaluate gait parameters in diabetic patients 
under various real life conditions and compared them to those recorded for healthy controls.

Materials and methods: This clinical observation has been reviewed by the Local Ethics Committee. The gait of 45 subjects was assessed on three different surfaces (tar, grass, stones) with the Physilog (BioAGM, CH), a system containing accelerometers and gyroscopes. Temporal and spatial gait parameters as well as stride-to-stride variability of 30 patients with type 2 diabetes, 15 with and 15 without clinical neuropathy were compared to 15 healthy controls.

Results: The effect of diabetic neuropathy and the effect of surfaces were calculated with an ANOVA for repeated measures and a Bonferroni post hoc analysis. Diabetic patients' gait parameters differed significantly $(\mathrm{p}<0.05)$ from those of healthy controls. Post hoc analysis revealed a significant difference between healthy individuals and patients with $\mathrm{PN}$ for all evaluated gait parameters (e.g.: velocity $\mathrm{p}=0.000$, stride $\mathrm{p}=0.008$ and coefficient of variation of stride as a gait instability index $p=0.006)$ except for shank $(p$ $=0.09)$ and knee $(p=1)$ range. It further showed a significant differences between diabetic patients without PN and healthy controls in all evaluated gait parameters (e.g.: for velocity $\mathrm{p}=0.001$ and for coefficient of variation of stride $p=0.002)$ except for stride $(p=0.4)$ and lower limb movements $(p>$ 0.06 ). No difference was observed between diabetic patients with and without PN. Patients with and those without PN adjusted to the variety of different surfaces using identical strategies. These strategies were also similar to those used by healthy controls. Neverthless, the surface effect was most important in patients with PN followed by patients without PN and healthy controls. Conclusion: Walking in real life conditions revealed gait difficulties in patients with type 2 diabetes before neuropathy was clinically detectable. The strategies patients used while changing from a flat (tarred walkway) to a more complex surface (grass or stones) remain the same between patients with and those without sensory loss. Although the change in velocity is not important enough to really influence patients' quality of life, clinicians should be aware that diabetic individuals' gait capacity decreases and fall risk increases in an already early stage of the disease.

\section{9}

Subclinical hearing impairment: an earliest complication of type 1 diabetes mellitus?

M. Dabrowski ${ }^{1}$, G. Niedzielska ${ }^{2}$, A. Nowakowski ${ }^{3}$

${ }^{1}$ Diabetology, NZOZ Beta-Med, Rzeszow, ${ }^{2}$ Otolaryngology, Medical

University, Lublin, ${ }^{3}$ Endocrinology, Medical University, Lublin, Poland

Background and aims: The data regarding hearing function in type 1 diabetes are inconsistent. The aim of this study was to evaluate hearing threshold, cochlear micromechanics and central auditory pathway in relatively young type 1 diabetic patients, with short duration of the disease.

Materials and methods: 31 patients with type 1 diabetes, aged below 45 years (mean $29.1 \pm 7.1$ years), with diabetes duration $<10$ years (mean $4.6 \pm 2.7$ years) and no evident hearing impairment, were compared with age-matched (30.3 \pm 7.8 years) control group of 26 healthy volunteers. In all subjects puretone audiometry, otoacoustic emissions and auditory brainstem responses were evaluated. Metabolic control of diabetes was determined by $\mathrm{HbA}_{1 \mathrm{C}}$ assessment.

Results: Pure-tone audiometry was performed in frequency range 125-12000 Hz. 25 diabetics had normal hearing, 5 had mild and 1 moderate hearing loss. In control group 23 subjects had normal hearing, and 3 had mild hearing loss. The hearing threshold in frequencies $3-12 \mathrm{kHz}$ was significantly higher in diabetic group than in control group (fig. 1), and was correlated with age, but no association was found with metabolic control and duration of diabetes. Transient evoked otoacoustic emissions (TEOAEs) in diabetic group were absent bilaterally in 6 and unilaterally in 2 subjects. In control group there were 2 and 1 subject respectively. The TEOAE amplitude was significantly lower in diabetic group when compared with controls (table 1$)$, and in diabetics showed negative linear correlation with age $(\mathrm{p}<0.001)$, but not with $\mathrm{HbA}_{1 \mathrm{C}}$ or duration of diabetes. Auditory brainstem responses (ABR) in diabetic group revealed significantly longer latency time of wave V (5.78 \pm 0.25 vs $5.69 \pm 0.18 \mathrm{~ms}, \mathrm{p}=0.04)$ and interval I-V $(4.03 \pm 0.20$ vs $3.95 \pm 0.17 \mathrm{~ms}$, $\mathrm{p}=0.03$ ) when compared with control group. The negative linear correlation between diabetes duration and latency time of wave $V(p=0.015)$, intervals IIII ( $<<0.05)$, III-V $(p=0.03)$, and I-V $(p=0.002)$ was revealed. No association between $\mathrm{ABR}$ and age or metabolic control was found.

Conclusion: The results of this study confirmed existence of subtle but significant hearing impairment in type 1 diabetic subjects. Both cochlear micromechanics, and central auditory pathway function are altered in diabetes. The abnormalities in audiological evaluations can occur before development of diabetic microvascular complications, but the prognostic value of this finding remains unclear, due to lack of long-term prospective observations.

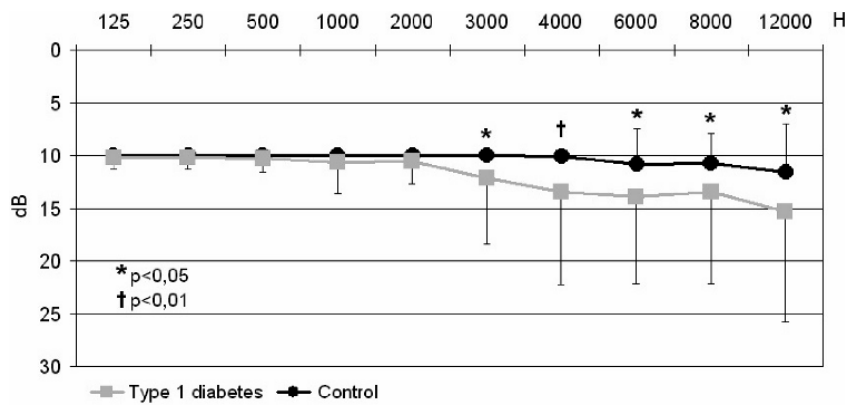

Fig. 1. Hearing threshold in diabetic and control groups (mean \pm SD)

Table 1. TEOAE amplitude and ABR responses in diabetic and control group

TEOAE amplitude in diabetic and control groups $(\mathrm{dB})($ mean $\pm \mathrm{SD})$

\begin{tabular}{llll}
\hline Band $(\mathrm{kHz})$ & Diabetes & Control & $\mathrm{p}$ value \\
\hline $1.2-3.5$ & $7.75 \pm 4,43$ & $10.00 \pm 4.01$ & 0.006 \\
\hline Frequency $(\mathrm{kHz})$ & & & \\
\hline 1 & $3.67 \pm 4,02$ & $4.81 \pm 4.74$ & NS \\
1.5 & $7.12 \pm 5,49$ & $10.57 \pm 5.77$ & 0.002 \\
2 & $7.21 \pm 4,75$ & $10.73 \pm 4.81$ & 0.0002 \\
3 & $7.18 \pm 5,16$ & $8.16 \pm 4.05$ & NS \\
4 & $4.75 \pm 3,79$ & $6.45 \pm 3.08$ & 0.01 \\
\hline
\end{tabular}




\section{PS 123 Diabetic foot: pathogenetic mechanisms}

\section{0}

The involvment of insulin in skin complications under pathological conditions

H. Lederman ${ }^{1}$, G. Weingarten ${ }^{1}$, M. Hochberg ${ }^{2}$, D. Enk ${ }^{2}$, E. Wertheimer ${ }^{1}$ Pathology, Tel Aviv University, ${ }^{2}$ Dermatology, Hadassah University Hospital, Jerusalem, Israel.

Background and aims: Maintaining skin integrity is essential for life. This is achieved by continuous skin cell proliferation, differentiation and programmed cell death. These processes are impaired in diabetes, leading to delayed wound healing that might result in limb amputations and even death. In our previous studies we have demonstrated that insulin directly regulates skin turnover, regulating skin proliferation and differentiation. Furthermore we have found that impaired insulin signaling leads to abnormal skin turnover. In the current work we investigated the effects of insulin and its downstream signaling on the additional process participating in skin turnover, skin apoptosis.

Materials and methods: We studied genetically manipulated primary skin epidermal cells which were genetically manipulated to under- or over-express various proteins involved in mediating insulin signaling, including insulin receptor (IR), insulin receptor substrates 1 and 2 (IRS- 1 and IRS- 2 respectively), PI-3-Kinase (PI3K) or Ras. Apoptosis was induced in these cells by UVB assault, and the effect of the genetic manipulations on induction of apoptosis was measured.

Results: We found that lack of IR caused a dramatic increase of keratinocyte cell death following UVB assault. We next studied the IRS family which play a role as docking proteins and are the first to be phosphorylated by the IR. Surprisingly, while manipulating IRS-2 expression had no significant effects on skin cell death, over-expression of IRS-1 resulted in a marked increase in the number of apoptotic skin cells after UVB irradiation. Lack of IRS-1 expression had no significant effect, perhaps due to redundancy between the members of the IRS family.

Over or under-expression of the downstream insulin signaling protein PI3K had no effect on keratinocytes apoptosis induced by UVB. In contrast, it was found that Ras, which mediates the signal to the Raf-MEK-MAPK cascade is involved in cell death after UVB. Over expression of constitutively active Ras caused a significant increase in apoptosis in response to cellular assault.

Conclusion: This data provides evidence that insulin signaling participates in programmed cell death in skin cells under pathological conditions. This signal in keratinocytes is mediated by IRS-1 via the Ras pathway. IR inactivation tilts the balance towards increased apoptosis, perhaps by delaying cell differentiation thus exposing the cells to a greater assault.

Unveiling the mechanism of insulin involvement in skin turnover is of major importance for our understanding of normal skin physiology and the process of skin wound healing and hopefully will lead to new insights on the pathogenesis of skin diseases.

\section{1}

One of the possible pathogenetic mechanisms of foot problems in diabetic patients on dialysis therapy is haemodynamic changes during dialysis procedure

E.V. Bublik ${ }^{1}$, G. Galstyan ${ }^{2}$, G. Melnichenko ${ }^{1}$, V. Safonov ${ }^{3}$

${ }^{1}$ Endocrinology, Moscow Sechenov Medical Academy, ${ }^{2}$ Diabetic foot, National Research Centre of Endocrinology, ${ }^{3}$ Nephrology, Moscow Sechenov Medical Academy, Russian Federation

Background and aims: The cause of high prevalence of neuroischemic ulcers in diabetic patients on dialysis therapy is still not well understood. The aim is to study prevalence of diabetic foot complications in diabetic patients on dialysis therapy and evaluate the role of the haemodynamic changes during dialysis procedure on arterial blood flow in lower extremities.

Materials and methods: We studied 109 dialysis patients. Follow-up period - 18 months. Mean age 49 years. 60 had diabetes mellitus (29 on haemodialysis, 31 on peritoneal dialysis) and 49 were non-diabetic patients (matched by sex, age and dialysis duration to diabetic group) (24 on haemodialysis, 25 on peritoneal dialysis). Mean duration of diabetes was 24 years; $60 \%$ of patients had type 2 diabetes. Distal polyneuropathy was diagnosed according to Neurological Disability Score. Vascular status was assessed by Doppler and toe pressure measurements. Criteria of transient intradialysis hypotension is increasing of systolic BP more than $30 \mathrm{mmHg}$ more than 10 cases during 10 months). We monitored systolic BP in brachial, ankle and toe arteries during dialysis procedure.

Results: Peripheral Vascular Disease - 26,6\% (17/60) in diabetic group and $4 \%(2 / 49)$ in non-diabetic group. Ulcers were diagnosed in diabetic patients only - 13,3\% (8/60) (5 of them were neuroischemic). The number of patients with neuroischemic ulcers had increased after starting dialysis therapy and it was statistically significant in haemodialysis group only $\left(p=0,023, \chi^{2}=4,17\right)$. The possible explanation is changes in BP during dialysis procedure. Changes in BP were significant during haemodialysis procedure only. The lowest systolic BP in brachial, ankle and toe arteries was registered in the middle of haemodialysis procedure. In this point decreasing of BP (\%) was the most strongly pronounced in toe than in brachial and ankle arteries (19\% vs $8,4 \%$ vs $4,7 \%$ ). The intradialysis hypotension is the most clinically significant decrease in systolic BP. Among patients with Peripheral Vascular Diseases the neuroischemic ulcers were frequently diagnosed in the group with intradialysis hypotension than without $(\mathrm{p}=0,001)$.

Conclusion: Prevalence of neuroischemic ulcers increases after starting of haemodialysis. It may be due to decrease in systolic BP in brachial, ankle and toe arteries during haemodialysis procedure. Evident decrease of BP might induce or make worse limb ischemia especially in diabetic patients with Peripheral Vascular Disease.

\section{2}

Connexin mimetic peptide Gap27 accelerates wound healing in ex-vivo wound healing models, enhances the proliferation and migration of human fibroblasts and keratinocytes but has no effect on the migration of human keratinocytes from diabetic origin

R. Lobmann ${ }^{1,2}$, S. Pollok ${ }^{3}$, A. Pfeiffer ${ }^{3}$, P. Houdek ${ }^{3}$, I. Moll' ${ }^{3}$ J.M. Brandner ${ }^{3}$ ${ }^{1}$ Endocrinology and Metabolism, University Hospital of Magdeburg, ${ }^{2}$ Endocrinology, Diabetology and Geriatrics, General Hospital Stuttgart, ${ }^{3}$ Dermatology and Venerology, University Hospital Hamburg-Eppendorf, Germany

Background and aims: Connexins are transmembrane proteins that form Gap Junctions (GJ), communicating channels that allow the exchange of small molecules between adjacent cells. GJ are important for migration, differentiation and proliferation of cells. Connexin 43 (Cx43) has been shown to be ubiquitously expressed in human epidermis and to be downregulated during early wound healing at the wound margins and in regenerating epidermis. The fact that $\mathrm{Cx} 43$ is still present at the margins of chronic wounds implicates that the downregulation is important for an effective wound closure. Phoshorylation of Cx43 on serine368 (S368) has been shown to decrease gap junctional intercellular communication (GJIC).

Materials and methods: For the evaluation of $\mathrm{Cx} 43$ phosporylation in the course of wound healing and of the influence of inhibition of $\mathrm{Cx} 43$ by a mimetic peptide (Gap 27) our well established porcine ex-vivo wound healing model was used. Cell migration was investigated by life cell imaging of cultured human keratinocytes and fibroblasts

Results: We could observe an even distribution of pS368 throughout the epidermal layers of native porcine skin. After wounding, pS368 was downregulated in parallel to Cx43. To further elucidate the role of $\mathrm{Cx} 43$ we investigated the influence of a Cx43 mimetic peptide (Gap27) which results in a disruption of GJIC. Treatment of porcine ex-vivo wound healing models with Gap27 resulted in a significantly accelerated wound healing compared to the controls. The treated models showed a higher number of proliferative keratinocytes in the regenerating epidermis and at the wound margins. No difference concerning Cx43 phosphorylation (pS368) was observed. Confluent keratinocyte and fibroblast cultures that were treated with Gap27 prior to a scratch wound assay showed an enhanced migration that resulted in a faster wound closure. Interestingly, Gap27 treatment had no effect on the migration of human keratinocytes from diabetic origin.

Conclusion: These data suggest a different influence of Gap27 on wound healing in diabetic and non-diabetic keratinocytes.

Supported by: Buerger Buesing donation 


\section{3}

Stabilisation of HIF- 1 alpha is sufficient to reverse the negative effect of hyperglycaemia on HIF function and to markedly improve wound healing in diabetic mice

S.-B. Catrina ${ }^{1}$, I. Botusan ${ }^{1}$, V.G. Sunkari ${ }^{1}$, O. Savu ${ }^{1,2}$, A. Catrina ${ }^{3}$, J. Grünler ${ }^{1}$, S. Lindberg ${ }^{1}$, T. Pereira ${ }^{4}$, L. Poellinger ${ }^{4}$, K. Brismar ${ }^{1}$

${ }^{1}$ Molecular Medicine and Surgery, Karolinska Institute, Stockholm, Sweden, ${ }^{2}$ National Institute for Diabetes "C. Paulescu", Bucharest, Romania, ${ }^{3}$ Rheumatology, Karolinska Institute, Stockholm, Sweden, ${ }^{4}$ Cellular and Molecular Biology, Karolinska Institute, Stockholm, Sweden

Background and aims: Hypoxia is essential in wound healing since it plays a pivotal role in regulation of all the critical processes involved in tissue repair, i.e. cell motility, recruitment of circulating angiogenic cells, and angiogenesis. Hypoxia inducible factor alpha (HIF-1alpha) is the critical transcription factor that regulates the adaptive responses to hypoxia. HIF-1alpha stability and function is regulated by oxygen-dependent soluble hydroxylase enzymes targeting critical proline and aspariginyl residues in two different transactivation domains. We and others showed that hyperglycemia complexly affects both HIF-1alpha stability and activation.

We proposed to further investigate the mechanisms which lie behind the repressive effect of hyperglycemia on HIF function and their consequence on diabetic wound healing.

Materials and methods: The effect of the different oxygen-dependent hydroxylase inhibitors on the HIF-1alpha stability and their effect on HIF critical target genes was studied in primary mouse fibroblasts by western blot, reporter gene, QRT-PCR. Transient transfections using Gal4-reporter system together with constitutively or siRNA induced VHL deficient cells were used for studying the molecular mechanisms of the HIF repression in hyperglycemia. Gain of function studies with different adenovirus carried HIF constructs were performed in a wound model in $\mathrm{db} / \mathrm{db}$ mice.

Results: Hyperglycemia affects by a VHL dependent mechanism HIF stability and modulates both its transactivation domains. Consecutively it suppresses expression of HIF-1 target genes essential for wound healing both in vitro and in vivo. However, by blocking HIF-1alpha hydroxylation through chemical inhibition it is possible to reverse this negative regulatory effect of hyperglycemia and to improve the wound healing process (granulation, vascularisation, epidermal regeneration, and recruitment of endothelial precursors). Local adenovirus-mediated transfer of two stable HIF constructs demonstrated that stabilisation of HIF-1alpha is necessary and sufficient for promoting wound healing in a diabetic environment. Our findings outline the necessity to develop specific hydroxylase inhibitors as therapeutic agents for chronic diabetes wounds.

Conclusion: We demonstrate for the first time that negative regulation of HIF-1alpha is essential for development of diabetic wounds and we provide mechanistic rationale that stabilization of HIF-1alpha is sufficient to reverse the pathological process.

Supported by: Family Stefan Persson Foundation, Juvenile Diabetes Research Fund, von Kantzow Foundation, Thurings Foundation, Swedish Diabetes Association, and Swedish Research Council

\section{4}

High glucose causes direct impairment of cellular activities associated with wound healing

E. Yefet, G. Weingarten, E. Wertheimer

Pathology, Sackler Faculty of Medicine Tel Aviv University, Israel

Background and aims: Diabetes mellitus is one of the most severe metabolic diseases affecting people worldwide. One major complication of diabetes mellitus is impaired wound healing leading to amputations and even death. Therefore, it is crucial to understand the mechanisms which are involved in the pathogenesis of this complication. In the present study we examined how the high glucose affects cellular activities that participate in the wound healing process.

Materials and methods: The studies were carried on primary skin epidermal keratinocytes, which are central players in the wound healing process. We chose to focus on keratinocytes adhesion due to its important role in the wound healing process. In addition, we explored some of the molecular pathways involved in adhesion. Finally, we examined whether glucose has any effect on skin organization in a three dimensional skin co-culture model. This model allowed us to examine skin organization and differentiation in conditions that are in close proximity to those of the whole animal.
Results: The results demonstrate that high glucose has a negative effect on keratinocytes adhesion when both cells were pre-incubated and extracellular matrix (ECM) was produced in high glucose. Adhesion is also decreased when cells were plated onto ECM produced by cells grown in high glucose. Moreover, adhesion is also decreased when cells were plated on ECM proteins-collagen, laminin and fibronectin that were directly exposed to high glucose environment. This last finding is suggestive of a possible direct reaction between glucose and those ECM proteins. On the other hand, cells that were pre-incubated in high glucose attached more rapidly to the various ECM proteins surfaces. Molecular analysis of cells-ECM interactions has demonstrated that in ECM produced under high glucose there was a lower expression of laminin 5, but a higher expression of integrin alpha 3 - the receptor for laminin, collagen and fibronectin. These changes could explain the results in keratinocytes adhesion. Our studies further showed that insulin increases keratinocytes attachment ability, but only in the control group and not in the high glucose environment, suggesting that under conditions of hyperglycemia, insulin effects on cell adhesion is impaired. Finally, we followed skin organization using the 3D skin organotypic coculture. Our results demonstrated that high glucose leads to abnormal skin organization that includes abnormal epidermal appearance, several dividing layers instead of only one and thinner suprabasal layers.

Conclusion: In summary, our results demonstrate that high glucose might cause direct impairment in wound healing process regardless of secondary impairment due to neuropathy and angiopathy that were previously described. Therefore, new therapeutic approaches for diabetes should focus on the pathophysiology of the skin.

\section{5}

Serum proinflammatory cytokines TNF-alpha and IL-6 are raised in acute Charcot osteoarthropathy and correlate with a marker of bone resorption

M. Edmonds ${ }^{1}$, T. Dew ${ }^{2}$, R. Musto 2 , S. Thomson 2 , R. Sherwood ${ }^{2}$, C. Moniz 2 , N. Petrova

${ }^{1}$ Diabetic Foot Clinic, King's College Hospital NHS Foundation Trust, London, ${ }^{2}$ Department of Clinical Biochemistry, King's College Hospital NHS Foundation Trust, London, United Kingdom

Background and aims: The Charcot foot is characterised by severe inflammation but its role in pathogenesis is poorly understood. The aim of this study was to investigate serum proinflammatory cytokines and bone turnover in acute Charcot osteoarthropathy.

Materials and methods: We studied 3 groups of patients: 27 presenting with acute Charcot osteoarthropathy, 14 with chronic Charcot osteoarthropathy and 24 diabetic control patients. We measured markers of inflammation, namely serum proinflammatory cytokines, tumour necrosis factor-alpha (TNF-alpha) and interleukin-6 (IL-6), as well as serum high sensitivity Creactive protein (hsCRP) - an acute phase protein, in the three groups of patients. We also measured serum C-telopeptide of type I collagen (CTx), a marker of bone resorption, serum bone specific alkaline phosphatase (BAP), a marker of bone formation and serum osteoprotegerin (OPG), a cytokine that modulates bone resorption and osteoclastic activity.

Results: In acute Charcot patients, there was a significant increase in the serum levels of IL-6 $(3.8 \pm 3.6 \mathrm{pg} / \mathrm{ml})$ compared with chronic Charcot patients $(1.7 \pm 1.2 \mathrm{pg} / \mathrm{ml})$ and diabetic controls $(1.9 \pm 1.5 \mathrm{pg} / \mathrm{ml}), \mathrm{p}=0.025$. Furthermore, there was a significant rise in serum TNF-alpha in acute Charcot patients $(1.7 \pm 0.9 \mathrm{pg} / \mathrm{ml})$ compared with chronic Charcot patients $(1.3 \pm 0.4$ $\mathrm{pg} / \mathrm{ml})$ and diabetic control patients $(1.1 \pm 0.4 \mathrm{pg} / \mathrm{ml}), \mathrm{p}=0.008$. Similarly, serum hsCRP was significantly increased in acute Charcot patients $(10.1 \pm 13.4$ $\mathrm{mg} / \mathrm{l})$ compared with chronic Charcot patients $(2.6 \pm 2.2 \mathrm{mg} / \mathrm{l})$ and diabetic control patients $(4.8 \pm 4.3 \mathrm{mg} / \mathrm{l}), \mathrm{p}=0.013$. Serum CTx, a breakdown product of type 1 collagen, was significantly raised in patients with acute Charcot foot $(0.41 \pm 0.39 \mathrm{ng} / \mathrm{ml})$, compared with patients with chronic Charcot foot $(0.10 \pm 0.04 \mathrm{ng} / \mathrm{ml})$ and diabetic patients $(0.11 \pm 0.68 \mathrm{ng} / \mathrm{ml}), \mathrm{p}=0.001$. Serum BAP was also significantly raised in acute Charcot patients $(18.5 \pm 9.2 \mu \mathrm{g} / \mathrm{L})$ compared with chronic Charcot patients $(12.7 \pm 4.4 \mu \mathrm{g} / \mathrm{L})$ and diabetic controls $(14.7 \pm 5.1 \mu \mathrm{g} / \mathrm{L}), \mathrm{p}=0.041$. Serum OPG was significantly raised in acute Charcot patients $(5.5 \pm 1.7 \mathrm{pmol} / \mathrm{l})$ compared with chronic Charcot patients $(4.9 \pm 1.4 \mathrm{pmol} / \mathrm{l})$ and diabetic patients $(4.4 \pm 1.4 \mathrm{pmol} / \mathrm{l}), \mathrm{p}=0.048$. Serum CTx was significantly correlated with serum IL-6 $(r=0.472, p<0.001)$ and with serum TNF-alpha $(r=0.556, p<0.001)$. Similarly, serum CTx was significantly correlated with serum hsCRP $(r=0.561, \mathrm{p}<0.001)$.

Conclusion: This study has shown that the proinflammatory cytokines, TNFalpha and IL-6, as well as hsCRP are raised in acute Charcot osteoarthropathy 
and are significantly associated with CTx. These findings indicate that inflammation may play an important role in the pathological bone resorption of the acute Charcot foot.

Supported by: Diabetes UK

\section{6}

Increased percentage of CD14+ monocytes in acute Charcot osteoarthropathy

N.L. Petrova ${ }^{1}$, M. Edmonds ${ }^{1}$, A. Sabokbar ${ }^{2}$, G. Mabilleau ${ }^{2}$

${ }^{1}$ King's College Hospital NHS Fouandation Trust, Diabetic Foot Clinic, London, ${ }^{2}$ University of Oxford, Botnar Research Centre, Oxford, United Kingdom

Background and aims: Recently, we have demonstrated that osteoclasts generated in vitro from patients with acute Charcot osteoarthropathy were functionally more aggressive and exhibited a considerable increase in the extent of their resorbing activity in response to the cytokine receptor activator of nuclear factor $\kappa \beta$ ligand (RANKL). It is possible that this increased osteoclastic activity may be a result of a relative increase in the proportion of $\mathrm{CD} 14+$ monocytes (osteoclast precursors) in peripheral blood mononuclear cells (PBMCs). Thus the aim of this study was to measure the percentage of CD14+monocytes in acute Charcot osteoarthropathy.

Materials and methods: We studied 11 diabetic patients with recent onset of acute Charcot osteoarthropathy and these were matched for age, gender and type of diabetes with 10 diabetic patients with no previous history of Charcot osteoarthropathy. We also studied 6 aged and sex-matched healthy controls. Peripheral blood mononuclear cells were isolated from EDTA treated whole blood and were labelled with an anti-CD14 monoclonal antibody labelled with phycoerythrin. Data was acquired using FACSCalibur-BD and analysed by CellQuest software.

Results: The mean percentage of CD14+ monocytes in PBMCs was significantly greater in the patients with acute Charcot osteoarthropathy $(8.7 \pm 3.4$ $\%)$ compared with diabetic patients $(5.2 \pm 3.3 \%)$ and healthy controls $(4.0 \pm$ $2.5 \%),(\mathrm{p}=0.013)$. Furthermore, the percentage of $\mathrm{CD} 14+$ monocytes in acute Charcot osteoarthropathy was 1.7 times and 2.1 times greater when compared with the percentage in diabetic patients $(\mathrm{p}=0.028)$ and healthy controls $(\mathrm{p}=0.009)$.

Conclusion: This study has shown that in acute Charcot osteoarthropathy there is a relative increase in the percentage of CD14+ monocytes, acting as osteoclast precursors, and these findings may explain the increased bone resorption of the acute Charcot foot.

Supported by: Diabetes UK

\section{PS 124 Diabetic foot: assessment}

\section{7}

Evaluation of a laser Doppler device O2C" for the measurement of microvascular skin blood flow and skin oxygenation in diabetic and nondiabetic subjects

C. Hohberg ${ }^{1}$, E. Tarakci ${ }^{1}$, S. Forst ${ }^{1}$, A. Pfützner ${ }^{1}$, T. Forst ${ }^{1,2}$

${ }^{1}$ Medical, Institute for Clinical Research and Development, ${ }^{2}$ Endocrinology, Johannes Gutenberg University, Mainz, Germany

Background and aims: Laserdopplerfluxmetry of skin capillary blood flow is an important feature for the evaluation of microvascular function in patients with diabetes mellitus. This study was done to evaluate a new device for the measurement of skin microvascular function and $\mathrm{Hb}$ oxygenation.

Materials and methods: Twenty non-diabetic subjects (age $46.6 \pm 14.8$ years; mean \pm SD) and twenty diabetic patients (age $59.4 \pm 8.4$ years) participated in two repeated microvascular measurements using micro-lightguide spectrophotometry (O2C; LEA Medizintechnik, Giessen, Germany). This technique allows simultaneous, non-invasive measurement of microvascular blood flow by laserdopplerfluxmetry (LDF) and the $\mathrm{Hb}$ oxygenation $\left(\mathrm{SO}_{2}\right)$ at the same anatomical area. A skin probe was placed on non-hairy skin at the hypothenar side of the left hand for the measurement of $\mathrm{SO}_{2}$, and the postischaemic microvascular response was measured in two different laser penetration depths of $2 \mathrm{~mm}\left(\mathrm{LDF}_{\text {skin }}\right)$ and $8 \mathrm{~mm}\left(\mathrm{LDF}_{\text {muscle }}\right)$.

Results: As shown in the table a high correlation could be observed between the repeated measurements of $\mathrm{LDF}_{\text {skin. }} \mathrm{LDF}_{\text {muscle }}$ and $\mathrm{SO}_{2}$ in non-diabetic and in diabetic subjects. The coefficient of variation for repeated measurements were found at $15.0 \pm 19.6 \%$ for LDFskin, $7.5 \pm 5.6 \%$ for LDF muscle, and 43.4 $\pm 73.8 \%$ for SO2 in non-diabetic patients and at $18.2 \pm 19.9 \%$ for LDFskin, $7.9 \pm 9.1 \%$ for LDF muscle, and $34.2 \pm 46.7 \%$ for $\mathrm{SO} 2$ in diabetic patients.

Conclusion: Lightguide spectrophotometry is an easy, non-invasive and reliable method for the measurement of superficial microvascular blood flow and $\mathrm{Hb}$ oxygenation in non-diabetic and in diabetic subjects. Further studies are required to clarify the pathology of these measures in diabetic populations with specific complications like diabetic microangiopathy or neuropathy.

\section{Correlation of repeated measurements}

\begin{tabular}{llll}
\hline & & Coefficient of correlation $(\mathrm{r})$ & Significance $(\mathrm{p})$ \\
\hline Control & LDFskin & 0.97 & $<0.0001$ \\
\hline Control & LDFmuscle & 0.93 & $<0.0001$ \\
\hline Control & SO2 & 0.74 & $=0.0002$ \\
\hline Diabetic & LDFskin & 0.95 & $<0.0001$ \\
\hline Diabetic & LDFmuscle & 0.89 & $<0.0001$ \\
\hline Diabetic & SO2 & 0.93 & $<0.0001$ \\
\hline
\end{tabular}

\section{8}

Increased QT time is associated with mortality in patients with diabetes mellitus, foot ulcer and lower extremity arterial disease

M. Löndahl ${ }^{1}$, C. Johansson ${ }^{2}$, P. Katzman ${ }^{1}$

${ }^{1}$ Department of Endocrinology, Lund University Hospital, Lund,

${ }^{2}$ Department of Anesthesiology, Helsingborg Hospital, Helsingborg, Sweden

Background and aims: Long QT-time as well is known risk factors for mortality in patients with or without diabetes mellitus. The magnitude of long QT-time as a risk factor for mortality in a high risk population such as diabetic patients with lower extremity arterial disease and foot ulcer is however unknown. The aim of this study was to evaluate the significance of increased QT-time on long-term mortality in patients with diabetes and arterial disease in the lower limb.

Materials and methods: A retrospective chart study was performed including all patients with a foot ulcer and critical ischemia visiting the Diabetic Foot Clinic during one year. Peripheral ischemia was defined as previous performed vascular surgery including PTA, or present systolic arterial toe blood pressure below $50 \mathrm{~mm} \mathrm{Hg}$. Patients were dived into groups according to QTc-time $<$ or $\geq 440 \mathrm{~ms}$.

Results: 102 patients (64 M / 38 F, 22 with type 1 diabetes / 80 with type diabetes 2) were included. Hypertension was seen in 86 patients and a history of myocardial infarction was present in 36 patients. QTc was $<440 \mathrm{~ms}$ in 61 patients and $\geq 440 \mathrm{~ms}$ in 41 . The 6-year mortality rate was significantly higher in the group of patients with longer QTc $(\mathrm{p}<0,0001$, figure 1$)$. 
Conclusion: Even in a high risk population as diabetic patients with foot ulcer and lower extremity arterial disease QTc-time seems to be a significant additional risk factor for long-term mortality.

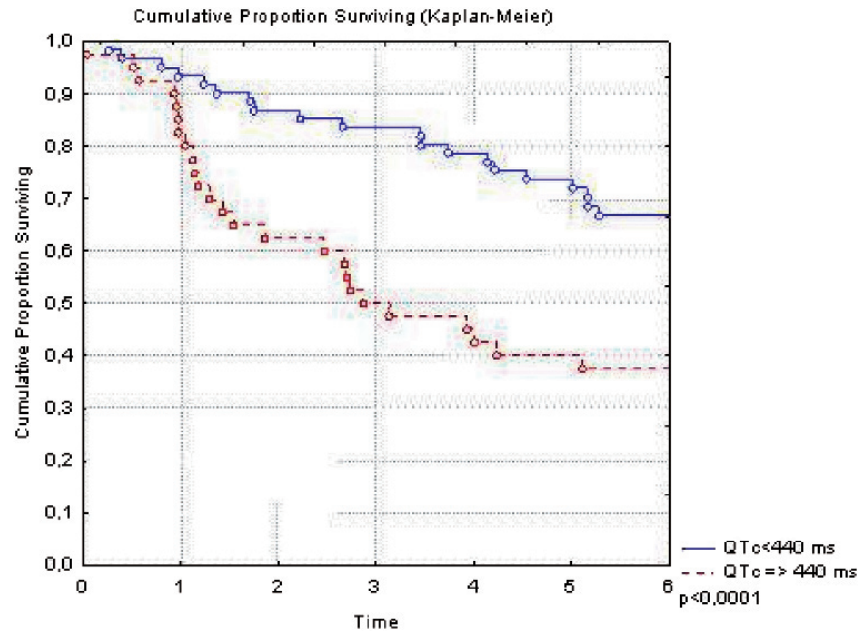

Supported by: Mrs Thelma Zoegas Foundation, Region Skåne Foundation

\section{9}

An evaluation of the accuracy and usability of a novel optical wound measurement system

F.L. Bowling ${ }^{1}$, L.D. King ${ }^{2}$, J.A. Paterson ${ }^{3}$, H. Fadavi ${ }^{1}$, R.W. Daniel ${ }^{4}$,

D.R. Matthews 5 , A.J.M. Boulton ${ }^{1}$

${ }^{1}$ Dept of Diabetes and Endocrinology, University of Manchester,

${ }^{2}$ Diabetic Foot Clinic, Oxford Centre for Diabetes, Endocrinology and Metabolism, ${ }^{3}$ Wound Imaging Group, Eykona Technologies Ltd, Oxford, ${ }^{4}$ Dept of Engineering, University of Oxford, ${ }^{5}$ Oxford Centre for Diabetes, Endocrinology and Metabolism, University of Oxford, United Kingdom

Background and aims: We present initial results from a novel optical wound imaging system which may be beneficial to remote diagnosis and telemedicine.

Materials and methods: Accuracy and performance of a prototype imaging system was evaluated in a two-centre study. 36 foot ulcers from $31 \mathrm{pa}-$ tients (age 45-93) were inspected across 12 weeks. 25 ulcers were reviewed at consecutive visits, resulting in a total of 76 wound inspections. Mean ulcer size was $1097 \pm 1094 \mathrm{~mm}^{2}$. Digital Elliptical Measurement (DEM) and hand-measured Elliptical Measurement (EM) were taken. Image processing produced calibrated-colour three-dimensional (3D) models of the ulcers and surrounding region. Off-line DEM was then performed on the 3D models using interactive tools. Absolute system accuracy was estimated by imaging a fabricated wound of known-size from five viewpoints and performing six independent sessions of Digital Area Measurement (DAM). Repeatability of DEM was estimated by repeating digital measurement five times on six representative wounds and observing variation.

Results: Pearson, Spearman and Kendall correlations between Length, Width and Elliptical Area measurement of DEM under clinician control against traditional hand-measured EM are presented in Table 1. In all cases $\mathrm{P}<0.001$ $(\mathrm{N}=76)$.

Table 1 - Correlation of DEM to EM measurement

\begin{tabular}{llllll}
\hline Measurement & Pearson & Spearman & Kendall & Min Error & Max Error \\
\hline Length & 0.958 & 0.922 & 0.801 & $0.01 \mathrm{~mm}$ & $14.95 \mathrm{~mm}$ \\
\hline Width & 0.948 & 0.906 & 0.755 & $0.02 \mathrm{~mm}$ & $14.96 \mathrm{~mm}$ \\
\hline Elliptical Area & 0.961 & 0.929 & 0.791 & $0.51 \mathrm{~mm}^{2}$ & $886.7 \mathrm{~mm}^{2}$ \\
\hline
\end{tabular}

DAM consisted of marking the boundary in the 3D model allowing an area $\left(\mathrm{mm}^{2}\right)$ to be calculated. Variation from the standard known-size wound area was $<8 \%$ across 30 trials. Intra-observer variation of wound Length and Width using DEM gave a coefficient of variation of $<3 \%$. 3D wound images are ideal for electronic transmission and can provide reliable monitoring of wounds over time.

Conclusion: Correlation of DEM against traditional EM was excellent. Side tests into absolute accuracy (DAM) and measurement repeatability (DEM) are promising. Early clinician feedback and empirical results suggest this has great potential due to simplicity and speed of use in clinical settings. The interactive $3 \mathrm{D}$ images are particularly appropriate for telemedicine and remote diagnosis.

\section{0}

\section{CRP as a marker for infection in diabetic foot ulcers}

P.L.H. van Battum, L. Prompers, N.C. Schaper, M.S.P. Huijberts

Medicine, Division of Endocrinology, Maastricht University Medical Center, Netherlands

Introduction: Diabetic foot disease is a feared complication of diabetes and is often accompanied by infection. Infection in diabetic foot disease can be limb threatening and is the mean reason for (major) amputation. However, classical clinical signs of infection often lack in this situation and markers of infection like c-reactive protein (CRP) may be of additional value. The aim of this study was to investigate whether CRP is a reliable marker of infection and/or its severity in patients with diabetic foot ulcers.

Methods and patients: In the Eurodiale study, a prospective cohort study of 1232 patients presenting with a new diabetic foot ulcer, patients were followed on a monthly basis until healing, death, major amputation or up to a maximum of 1 year. Ulcers were treated according to international guidelines. The presence of infection was defined according to the PEDIS classification and the clinical impression of the treating physician.

Results: In 669 subjects data were obtained on both PEDIS classification and CRP. In patients with infection $(n=416)$ CRP was normal in $33 \%$, elevated $<\mathrm{x} 3$ the upper limit of normal (ULN) in 23\% and > $3 \mathrm{x}$ ULN in $44 \%$. In patients without infection these numbers were: $58 \%, 22 \%$ and $20 \%$ respectively. The positive predictive value (PPV) and negative predictive value (NPV) for CRP were $72.4 \%$ and $51.6 \%$ respectively.

In patients with limb threatening infection according to the clinician $(\mathrm{n}=$ 106) CRP was normal in $24 \%,<3 \times$ ULN in $20 \%$ and $>3 \times$ ULN in $56 \%$. The PPV and NPV of CRP for the diagnosis of limb threatening infection were $31.9 \%$ and $90.9 \%$ respectively. In case of osteomyelitis(diagnosed by X-ray) (n $=144) \mathrm{CRP}$ was normal in $27 \%,<3 \times$ ULN in $26 \%$ and $>3 \times$ ULN in $47 \%$. The PPV and NPV of CRP were $39.6 \%$ and $85.2 \%$ respectively.

Conclusion: CRP is a poor diagnostic marker for the presence of infection, moreover CRP seems not to be of additional value in separating mild from severe infection. These data suggest that for the diagnosis of infection, and its severity, the clinician has to rely on clinical examination and, if necessary, imaging procedures.

\section{1}

Health status and quality of life of patients affected by Charcot neuroarthropathy

L. Vileikyte ${ }^{1}$, J.S. Gonzalez ${ }^{2}$, M. Peyrot ${ }^{3,4}$, J.S. Ulbrecht ${ }^{5}$, R.R. Rubin ${ }^{4}$, A.P. Garrow ${ }^{1}$, P.R. Cavanagh ${ }^{6}$, A.J.M. Boulton ${ }^{1}$

${ }^{1}$ Medicine, University of Manchester, Manchester, United Kingdom, ${ }^{2}$ Psychiatry, Harvard Medical School, Boston, United States, ${ }^{3}$ Sociology, Loyola College, Baltimore, United States, ${ }^{4}$ Medicine, Johns Hopkins University, Baltimore, United States, ${ }^{5}$ Medicine, Pen State University, State College, United States, ${ }^{6}$ Diabetic Foot Clinic, The Cleveland Clinic, Cleveland, United States

Background and aims: While it is recognized that Charcot Neuroarthropathy $(\mathrm{CN})$ is associated with high ulceration and amputation rates, little is known about the impact of $\mathrm{CN}$ on an individual's health status and quality of life (QoL).

Materials and methods: This UK and USA study of 489 high-risk patients for neuropathic foot ulceration ( $71 \%$ male; mean age 62 years; $71 \%$ type 2 $\mathrm{DM}$, diabetes duration 17 years; $16 \%$ active ulcers; $36 \%$ past ulceration) identified 67 patients who were diagnosed both clinically and radiologically with mid-foot deformity characteristic of CN. Patient-reported measures of health status and QoL included - both generic (SF-12 and Hospital Anxiety and Depression Scale, HADS) and foot problem-specific (NeuroQoL) instruments. Neuropathy severity was assessed by the Neuropathy Disability Score (NDS) and the Vibration Perception Threshold (VPT).

Results: Compared to high-risk patients with no $\mathrm{CN}$, the $\mathrm{CN}$ patients were younger (59 vs 62 years, $\mathrm{p}=.04$ ), reported more co-morbidities ( 1.3 vs 0.9 , $\mathrm{p}<.001$ ), had more severe neuropathy (NDS: 8.6 vs 7.2 , $\mathrm{p}<.001$; VPT: 47 vs 40 volts, $\mathrm{p}<.001)$ and more past $(84 \%$ vs $36 \%, \mathrm{p}<.001)$ and active foot ulceration 
( $40 \%$ vs $16 \%, \mathrm{p}<.001$ ). Further, CN patients reported more severe NeuroQoL symptoms of reduced feeling ( 3.8 vs $2.7, \mathrm{p}<.001)$ and unsteadiness ( 2.8 vs $2.3 ; \mathrm{p}=.007)$; more impaired activities of daily living, NeuroQoL-ADL (3.2 vs 2.4; $\mathrm{p}<.001$ ); and more NeuroQoL-emotional burden (2.8 vs $2.0 ; \mathrm{p}<.001$ ). However, no differences in symptoms of pain, anxiety or depression were observed between those with and without $\mathrm{CN}$. In multivariate regression analyses, controlling for disease, demographic characteristics, neuropathy severity and active foot ulceration, $\mathrm{CN}$ was a significant independent predictor of NeuroQoL-emotional burden ( $\mathrm{beta}=.10, \mathrm{p}<.05$ ), poorer foot problem-related QoL (beta $=.17, \mathrm{p}<.001$ ) and diminished SF12 physical functioning (beta=.08 , $\mathrm{p}<.05$ ), but not mental functioning. Furthermore, no significant association was found between $\mathrm{CN}$ and HADS-depression or anxiety.

Conclusion: These data illustrate the detrimental effect Charcot Neuroarthropathy has on individuals' functioning, as indicated by generic and condition-specific measures. However, only condition-specific measures were able to identify emotional effects. The latter finding emphasizes the importance of condition-specific measures when assessing the emotional state of patients with CN.

Supported by: Diabetes UK, ADA

\section{2}

Successful conservative management of forefoot osteomyelitis in diabetes using magnetic resonance imaging to establish diagnosis and determine antibiotic duration

J. Valabhji ${ }^{1}$, N. Oliver ${ }^{1}$, D. Samarasinghe ${ }^{2}$, L. Bloomfield ${ }^{3}$, T. Mali ${ }^{3}$, D. Hogg ${ }^{4}$, W.M.W. Gedroyc ${ }^{5}$, N.J.W. Cheshire ${ }^{6}$, R.G.J. Gibbs ${ }^{6}$

${ }^{1}$ Department of Endocrinology, ${ }^{2}$ Department of Microbiology, ${ }^{3}$ Department of Podiatry, ${ }^{4}$ Department of Orthotics, ${ }^{5}$ Department of Radiology,

${ }^{6}$ Department of Vascular Surgery, Imperial College Healthcare NHS Trust, London, United Kingdom

Background and aims: To assess the effectiveness over 5 years of a clinical algorithm for the conservative management in diabetes of forefoot osteomyelitis diagnosed by magnetic resonance imaging (MRI), in which interval MRI was used to determine antibiotic duration.

Materials and methods: Subjects presenting with neuropathic forefoot ulcers and MRI suggesting associated osteomyelitis were treated for a minimum 3 months with augmentin (clindamycin and ciprofloxacin if penicillin allergic; rifampicin and doxycycline if known methicillin-resistant staphylococcus aureus carriage). Antibiotics were modified if isolates were resistant. MRI was repeated at 3 months: if the lesion had healed with resolution of, or significant improvement in, underlying bone signal change, then antibiotics were discontinued. If healing had not yet been achieved or if there was no significant improvement in underlying bone signal change on MRI, then antibiotics were continued for a further 3-month cycle with interval MRI. If there was clinical or radiological deterioration, endoluminal or open vascular surgical intervention was undertaken where appropriate, or the subject underwent digital or more proximal amputation.

Results: Thirty-one out of 42 episodes of forefoot osteomyelitis in 36 subjects were treated successfully without amputation $(74 \%)$. A second episode in the same subject was at an anatomically distant site. Median (range) duration of antibiotics was $6(3-12)$ months and of follow-up post-cessation of antibiotics was $15(3-48)$ months. Of the 11 episodes in which treatment failed, 8 deteriorated on treatment, and 3 relapsed at 2, 17 and 39 months post-cessation of antibiotics. Of the 36 subjects (mean (standard deviation) age 64 (13) years, duration of diabetes 16 (11) years and HbAlc 8.3 (1.8) \%; 5 with Type 1 diabetes), all had clinical evidence of a peripheral neuropathy; $89 \%$ had retinopathy; $47 \%$ had chronic kidney disease at stages 3, 4 or 5 (equivalent to a glomerular filtration rate less than $30 \mathrm{ml} / \mathrm{min}$ ). Staphylococcus aureus was identified in 27 episodes, 6 of which were methicillin resistant and betahaemolytic streptococcus in 9; other isolates included coliforms, proteus and enterococcus. Age, $\mathrm{HbAlc}$, renal function and presence of peripheral vascular disease were not significant predictors of successful outcome. Three subjects died at 12, 22 and 28 months post-cessation of antibiotics of cardiovascular disease without foot lesions at time of death.

Conclusion: The clinical algorithm for the conservative management of forefoot osteomyelitis in diabetes successfully avoided any form of amputation in $74 \%$ of subjects. Determination of antibiotic duration using interval MRI may have contributed to the low rate of relapse.

\section{PS 125 Diabetic foot: treatment}

\section{3}

Safety and effectiveness of a novel super-oxidized solution (sos) in managing post-surgical lesions of the diabetic foot: a prospective, randomized clinical trial

C. Goretti, S. Mazzurco, A. Scatena, A. Tedeschi, L. Rizzo, A. Piaggesi

Department of Endocrinology and Metabolism, University of Pisa, Italy

Background and aims: To evaluate the efficacy and safety of SOS (Dermacyn', Oculus Innovative Sciences, Petaluma, CA) in the management of infected lesions of the diabetic foot after surgical debridement.

Materials and methods: 40 patients with surgical outcomes $>5 \mathrm{~cm}^{2}$ on account of an infected foot lesion, an ankle-brachial pressure index $\geq 0.9$ and the presence of at least two arteries in the ankle documented by palpable pulses or Doppler CW have been randomized to receive local SOS treatment (Group A, n. 20) or standard local treatment with povidone iodine (Group B, n. 20) in addition to metabolic control, systemic antibiotics and off loading, as necessary. At baseline and at weekly control visits area of lesions, clinical signs of infection, microbiological sampling eventual new debridement procedures and adverse events were recorded blindly to the local treatment. Endpoints at week 24 included healing rate (HR), healing time (HT), time for sterilization of the lesions (ST), number of debridement procedures (ND) and number of adverse events NA).

Results: $85 \%$ of Group A patients healed in 24 weeks compared to $53 \%$ of group B ones $(\mathrm{p}<.01)$; HT was $10.5 \pm 1.3$ wks in Group A vs $16.5 \pm 1.7$ wks in Group B ( $\mathrm{p}<.01)$, ST was $5.5 \pm 2.1$ wks in Group A compared to $16.2 \pm 6.6$ wks in Group B $(\mathrm{p}<.01)$, ND were carried out in 3 patients of group A and in 9 of group $\mathrm{B}(\mathrm{p}<.05)$, no differences were observed in NA (4 in group A vs 9 in Group B).

Conclusion: SOS local treatment proved to be as safe as and more effective than diluted povidone iodine in the management of wide post-surgical infected lesions of the diabetic foot left to heal for secondary intent. Supported by: Oculus Innovative Sciences, Petaluma, CA

\section{4}

Physical and psychological determinants of adherence to foot care in patients at high risk for foot ulceration

J.S. Gonzalez ${ }^{1,2}$, L. Vileikyte ${ }^{3}$, M. Peyrot ${ }^{4,5}$, R.R. Rubin ${ }^{5}$, J. Ulbrecht ${ }^{6}$, P.R. Cavanagh ${ }^{7}$, A.J. Boulton ${ }^{3}$

${ }^{1}$ Psychiatry, Harvard Medical School, Boston, United States, ${ }^{2}$ Psychiatry, Massachusetts General Hospital, Boston, United States, ${ }^{3}$ Manchester Diabetes Centre, University of Manchester, United Kingdom, ${ }^{4}$ Sociology, Loyola College, Baltimore, United States, ${ }^{5}$ Johns Hopkins University, Baltimore, United States, ${ }^{6}$ Pen State University, State College, United States, ${ }^{7}$ Diabetic Foot Clinic, The Cleveland Clinic, Cleveland, United States

Background and aims: Educational interventions to enhance preventive foot care (PFC) have met with limited success, and factors shaping PFC are seldom addressed.

Materials and methods: This cross-sectional study of 376 UK and US patients with diabetic neuropathy (DN) (67\% male, 76\% type 2 diabetes, $25 \%$ with past foot ulceration (PFU), mean Vibration Perception Threshold (VPT) 40 volts) employed hierarchical multivariate regression analyses to examine the relationships between objective DN severity (VPT, PFU), subjective DN severity (NeuroQoL-symptoms of pain and reduced feeling), psychological factors (personality: Big Five Inventory, BFI- conscientiousness; Hospital Anxiety and Depression, HADS-depression scale; Patient Interpretation of Neuropathy instrument (PIN) assessing DN-specific cognitions and emotions), and 9-item PFC scale, based on ADA guidelines (Cronbach's alpha $=.58$ ).

Results: After controlling for demographic and disease variables, independent predictors of better PFC in the total sample were: PFU $(\beta=.22 ; \mathrm{p}<.001)$; VPT $(\beta=.10 ; p=.044)$; NeuroQoL-pain $(\beta=.15 ; p=.008)$; BFI-conscientiousness $(\beta=.17 ; \mathrm{p}<.001)$; and PIN measures - understanding of foot ulcer causes $(\beta=.10 ; p=.03)$ and anticipated consequences $(\beta=.09 ; p=037)$. While PIN-anger at health care providers was associated with less PFC $(\beta=-.13 ; \mathrm{p}=.004)$, the relationship between HADS-depression and PFC was non-significant. The total model accounted for $36 \%$ of variance in PFC with psychological factors accounting for $9 \%$ of incremental variance. There were more predictors of PFC in patients without PFU than in those with PFU. Specifically, in 
those without PFU, DN severity (VPT: $\beta=.13 ; \mathrm{p}=.021$ ), BFI-conscientiousness ( $\beta=.19 ; \mathrm{p}=.001)$, PIN-anticipated consequences $(\beta=.13 ; \mathrm{p}=02)$ and PIN-foot ulcer unpredictability beliefs $(\beta=.12 ; \mathrm{p}=.03)$ were predictive of better PFC. In contrast, in patients with PFU only PIN-anger at health care providers ( $\beta=-$ $.27 ; \mathrm{p}=.013)$ was predictive of less PFC.

Conclusion: These data suggest that although PFU is the strongest independent predictor of PFC, psychological factors such as personality, DN-specific cognitions and emotions are also important determinants of PFC and should be addressed when designing future interventions. In addition, addressing psychological factors prior to developing foot ulcers would seem to be more effective than addressing them after a foot ulcer has developed. Supported by: Diabetes UK, ADA

\section{5}

MRSA infection can be reduced in diabetic foot clinic

S. Neupane ${ }^{1}$, S.T. Lo ${ }^{1}$, A. Arosi ${ }^{1}$, D. Orr ${ }^{2}$, S.M. Rajbhandari ${ }^{1}$

${ }^{1}$ Diabetes Dept, ${ }^{2}$ Microbiology Dept, Lancashire Teaching Hospital, Chorley, United Kingdom

Background and aims: MRSA infection is common in diabetic foot ulcer and is associated with increased morbidity and mortality. In our previous audit in 2002 the prevalence of MRSA was $33.3 \%$ so we implemented changes such as active screening for MRSA from foot wound, restrict the use of antibiotics only in clinically infected cases and developed close liaison with microbiologist on choice of antibiotics. In addition we also used disposable gloves and apron and washed hand with alcohol gel after each patient contact in foot clinic. The aim of this audit was to study if these measures reduced the prevalance of MRSA. The secondary aim was to evaluate outcome of these patients and their foot ulcers.

Materials and methods: This was a retrospective re-audit conducted on patients attending foot clinic between January 2006 to December 2007. The list of subjects attending foot clinic in 2 local hospitals was obtained from clinical coding department. Their clinical, biochemical and microbiology record were studied and relevant information was collected.

Results: We found that the overall prevalence of MRSA has reduced from $33.3 \%$ in 2002 to $15 \%$ of subjects with ulcers and to $18.7 \%$ of total positive cultures by 2006/07. Of the 133 patients with foot ulcers seen in 2006/07, 19 did not have diabetes and were excluded from further analysis. Of the remaining 114 subjects ( 71 male \& 16 type $1 \mathrm{DM}$ ), MRSA was isolated from $19(14.3 \%)$ subjects. There were no difference in age, sex, type of diabetes, HbAlc, presence of cardiovascular disease, serum creatinine and total cholesterol between subjects with and without MRSA. Serum albumin was significantly lower in subjects with MRSA $(39.6 \pm 6.4$ vs $32.9 \pm 8.6 \mathrm{gm} / \mathrm{L} ; \mathrm{p}$ $<0.001$ ). We found that only $42.9 \%$ of ulcers with MRSA infection healed by 6 months in comparison to $80.9 \%$ of ulcers without MRSA infection $(\mathrm{p}<0.05)$. There was higher overall mortality $(31.6 \%$ vs $11.6 \%$; $<<0.05)$ and amputation (35.7\% vs $11.3 \%$; $\mathrm{p}<0.05)$ in subjects with MRSA infection.

Conclusion: Our observation shows that incidence of MRSA can be reduced in foot clinic with strict hygiene but its presence is still associated with higher morbidity and mortality.

\section{6}

A structured follow-up reduces the incidence of ulcerative recurrences in high risk diabetic foot patients: a prospective study

A. Piaggesi, L. Rizzo, E. Fallani, A. Tedeschi, S. Del Prato

Department of Endocrinology and Metabolism, University of Pisa, Italy

Background and aims: A retrospective analysis showed an incidence of foot ulceration in high risk patients of our clinic of $39.7 \%$ in one year, $60.6 \%$ in three years and $70 \%$ in five years. We did this study to test if a structured follow-up program would be effective in reducing the incidence of ulceration in high risk patients.

Materials and methods: All patients presenting at our clinic for the prevention of lower limb complication were screened for ulcerative risk according to the score proposed by the International Consensus on Diabetic foot. All patients with a score $\geq 2$ received, as a part of a structured prevention program, custom orthesis and shoes, in relation with the severity of their risk condition according to the protocol described by Dahmen et al. Patients were then followed for the incidence of ulceration for one year. A subset of patients was followed up for three and five years.

Results: 1423 patients were screened for ulceration risk in 2002 and among

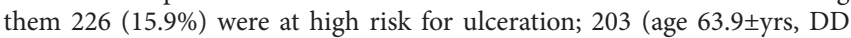

$18.3 \pm 9.3 \mathrm{yrs}, \mathrm{HbAlc} 8.9 \pm 1.4 \%$ ) accepted to participate and were enrolled in the study. Among them $44 \%$ had neuropathy and deformity, $22 \%$ had previous ulceration, $27 \%$ previous minor amputation and $7 \%$ had neuro-osteoarthropathy.

During the first 12 -month follow-up, $12.7 \%$ of patients which received orthesis and shoes developed an ulcer, thus showing a reduction of $-27 \%$ compared to the expected figures. This group had a more severely impaired VPT at baseline compared to non-developers $(37.1 \pm 15.1$ vs $26.5 \pm 8.2$ volt, $\mathrm{p}<0.01)$ and reported to use the orthesis and shoes for a shorter time during the day ( $5.7 \pm 3.4$ vs $7.8 \pm 1.6 \mathrm{hrs}, \mathrm{p}<0.05$ ). Among the subgroup of patients (N.55) with a longer follow-up, the observed cumulated incidence of ulceration was $17.3 \%(-43.3 \%)$ and $21.2 \%(-48.8 \%)$ in three and five years, respectively. Conclusion: A structured follow-up prevention program, with the use of orthesis and shoes, is effective in reducing the incidence of re-ulceration in diabetic patients at high-risk for lower limb complications.

\section{7}

Resource utilisation and costs for management of patients with diabetic foot ulcers. Experiences from 10 European countries in the EURODIALE trial

G. Ragnarson Tennvall on behalf of the EURODIALE Group

The Swedish Institute for Health Economics, IHE, Lund, Sweden

Background and aims: As part of the EURODIALE trial the aim of the study was to investigate the resource utilisation and cost for management of the diabetic foot in 10 European countries and to analyse the costs associated with diabetic foot disease.

Materials and methods: Information about resource utilisation was collected in the trial. For analysis of resource use 1088 patients were available and out of these 821 patients were included in the costing analysis based on country specific unit cost data. Costs were calculated individually for each patient by multiplying the unit cost for every type of resource by the number of resources used from inclusion into the trial and until a defined endpoint. The endpoints were when the foot was healed, the patient had deceased, a lower leg amputation was performed, or when 12 months follow-up had ended. The cost calculation covered direct costs for diagnostic procedures, off-loading techniques, interventional procedures, hospitalisations, antibiotic therapy, specialist consultations, topical treatment, and indirect costs. Indirect costs are related to loss of production for patients who had an employment and who had been on sickness leave because of the foot ulcer.

Results: Resource use as well as costs varied between outcome groups and between disease severity groups. The total direct and indirect costs were 7700 Euro for patients who healed and 25200 Euro for patients who underwent major amputation. The total costs per patient in the group with the most severe disease (both infection and peripheral arterial disease) were about four times higher (16800 Euro) than for patients in the least severe group (4500 Euro) without infection or peripheral arterial disease. For patients with infection only the total costs were 9300 Euro. The total costs for patients with peripheral arterial disease but without infection were 9850 Euro. The average number of procedures used was generally low with the exception of antibiotic therapy, topical treatment, hospitalisation, and consultations. The distribution of different types of costs within each outcome group differed in comparison with previously published studies.

Conclusion: The costs of diabetic foot ulcers are highly related to the outcome and the severity of the disease and the cost increases sharply in patients with both peripheral arterial disease and infection. Given these high costs there is an urgent need for efforts to prevent these conditions and for new strategies to treat them more effectively, at an earlier stage. The results show substantial differences in resource use and cost between different patient groups treated in Europe. The information is important in illustrating the magnitude of the problem in economical terms for decision makers both within and outside the health care system.

Supported by: European Commission 


\section{8}

Healing of diabetic foot ulcers may be modified by high dose atorvastatin - a 6 month randomised controlled pilot trial

O.E. Johansen ${ }^{1}$, K.I. Birkeland ${ }^{2}$, A.P. Jørgensen ${ }^{1}$, E. Orvik ${ }^{1}$, B. Sørgård ${ }^{1}$, B.R Torjussen $^{3}$, T. Ueland ${ }^{4}$, P. Aukrust ${ }^{4}$, L. Gullestad ${ }^{5}$;

${ }^{1}$ Medical department, Asker and Baerum Hospital, RUD, ${ }^{2}$ Dept of Endocrinology, Aker University Hospital, Oslo, ${ }^{3}$ Medical department, Østfold County Hospital, Sarpsborg, ${ }^{4}$ Research Institute for Internal Medicine, Rikshospitalet-Radiumhospitalet University Hospital, Oslo, ${ }^{5}$ Department of Cardiology, Rikshospitalet-Radiumhospitalet University Hospital, Oslo, Norway.

Background and aims: Diabetic foot ulcers (DFUs) are common complications of diabetes mellitus, with a complex pathogenesis involving mechanical, vascular, inflammatory, oxidative, endothelial and nutritive factors. Given the beneficial "pleiotrophic" effects of statins on several aspects of cardiovascular disease, including modulation of inflammation and endothelial function in addition to their lipid-lowering effects, possibly even so in a dose dependent manner, we explored whether low $(10 \mathrm{mg})$ or high $(80 \mathrm{mg})$ dose atorvastatin could modulate the DFU burden.

Materials and methods: Thirteen diabetic patients ( 10 men, 11 type 2 diabetets, mean age/diabetes duration $64 / 18$ years) with neuropathic DFUs $<4$ months from two centres were randomised to treatment with 10 or $80 \mathrm{mg}$ atorvastatin for six months in addition to conventional DFU care (prompt debriedment of contaminated and necrotic tissue, management of any underlying infection and provision of pressure relief measures).

Results: Six patients with six ulcers received $10 \mathrm{mg}$, and seven patients with nine ulcers received $80 \mathrm{mg}$ atorvastatin. Background (micro- and macrovascular complications, medications) and DFU characteristics (duration [median 33 days], University of Texas DFU grading [I-A (46\%), I-B (46\%) and II-B $(8 \%)]$ ) were similar. All ulcers in the $10 \mathrm{mg}$ and six of nine in the $80 \mathrm{mg}$ group healed. However, two previously healed DFUs recurred and six novel ulcers developed in the $10 \mathrm{mg}$ group, as compared to none and one, respectively, in the $80 \mathrm{mg}$ group ( $\mathrm{p}=0.048$ ) (Fig 1). There was a significant between-group change in CRP $(-1.5 \mathrm{mg} / \mathrm{l}, \mathrm{p}=0.044)$ and a trend towards greater betweengroup reductions in lipid parameters and a tendency towards an increase in ankle-brachial pressure index $(0.15, \mathrm{p}=0.201)$, favouring the $80 \mathrm{mg}$ group (Table 1). No significant impact was found on liver enzymes.

Conclusion: The observed beneficial impact on reducing recurrent and novel DFUs of 6-months high-dose atorvastatin treatment could be related to it's pleiotrophic effects. Larger studies are needed to explore this therapeutic option further.
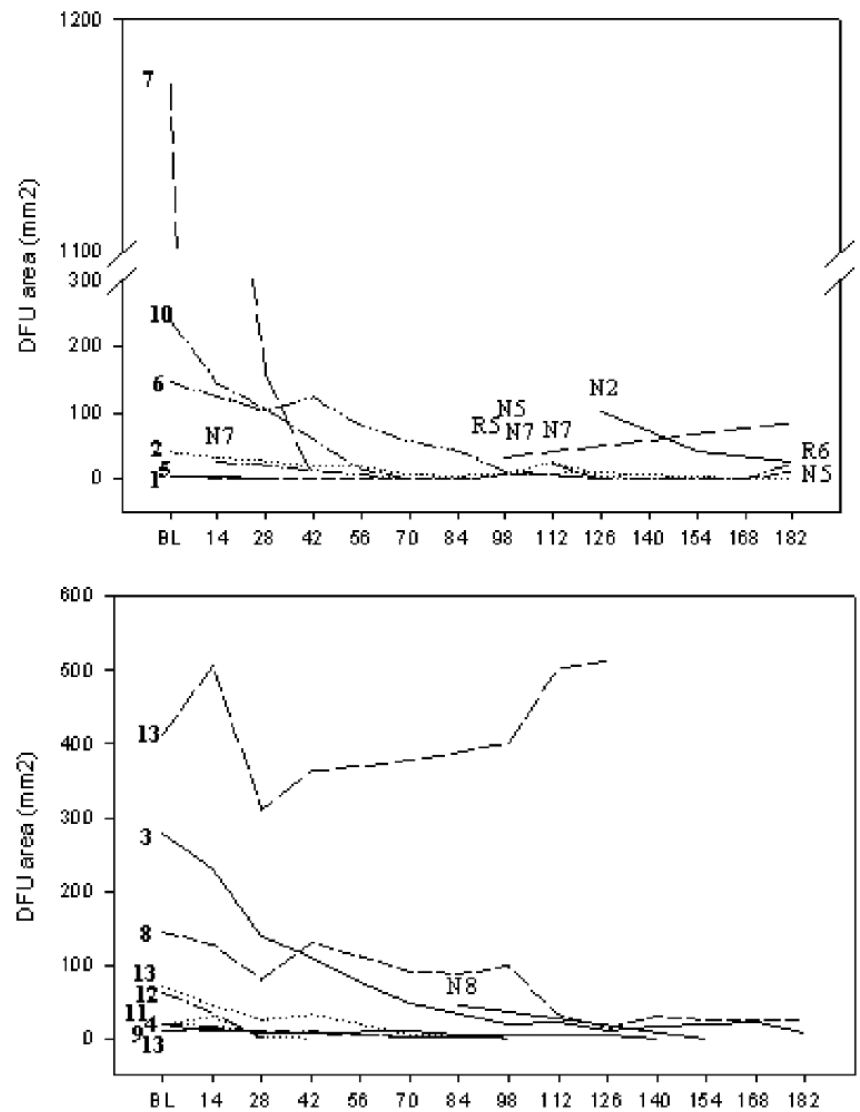

Figure 1. Surface area development of baseline and new (recurrent or noval) DFU during 6 months with atovastatin $10 \mathrm{mg}$ (upper) or $80 \mathrm{mg}$ (lower). Numbers to the left indicates patient number. Abbreviations: DFU - diabetic foot ulcer, $\mathrm{N}$ - new ulcer, $\mathrm{R}$ - recurrent ulcer

Table 1. Impact of 10 or $80 \mathrm{mg}$ atorvastatin added to conventional DFU treatment for six months.

\begin{tabular}{llllll}
\hline & $10 \mathrm{mg}$ & atorvastatin & $80 \mathrm{mg}$ & atorvastatin \\
\cline { 2 - 6 } & Study start $(n=6)$ & Study end $(n=6)$ & Study start $(n=7)$ & Study end $(n=7)$ & $\begin{array}{l}\text { Mean between-group changes } \\
(Z \text {-value })\end{array}$ \\
\hline HbAlc $(\%)$ & $8.5(7.3,10.2)$ & $8.4(7.2,9.8)$ & $9.2(7.1,11.0)$ & $9.8(7.1,11.8)$ & $0.4(-1.146), \mathrm{p}=0.252$ \\
\hline Total cholesterol $(\mathrm{mmol} / \mathrm{l})$ & $4.3(2.4,5.3)$ & $4.2(2.8,5.4)$ & $4.7(2.9,7.3)$ & $3.9(3.3,5.0)$ & $-0.7(-1.077), \mathrm{p}=0.281$ \\
\hline LDL cholesterol $(\mathrm{mmol} / \mathrm{l})$ & $2.4(1.3,3.2)$ & $2.2(1.2,3.2)$ & $2.3(1.3,3.9)$ & $1.6(0.5,2.3)$ & $-0.2(-1.287), \mathrm{p}=0.198$ \\
\hline HDL cholesterol $(\mathrm{mmol} / \mathrm{l})$ & $1.3(0.9,1.8)$ & $1.5(1.1,2.0)$ & $1.4(0.9,2.6)$ & $1.5(0.9,2.7)$ & $-0.1(0.108), \mathrm{p}=0.138$ \\
\hline Triglycerides $(\mathrm{mmol} / \mathrm{l})$ & $1.5(0.5,2.4)$ & $1.1(0.7,2.1)$ & $2.1(1.2,4.3)$ & $1.6(0.9,3.6)$ & $-0.2(0.000), \mathrm{p}=1.000$ \\
\hline Total leukocyte count $\left(10^{9}\right)$ & $7.9(4.3,11.9)$ & $6.9(4.1,10.1)$ & $7.2(4.1,9.2)$ & $6.7(4.5,10.8)$ & $0.5(-0.143), \mathrm{p}=0.886$ \\
\hline CRP $(\mathrm{mg} / \mathrm{l})$ & $2.0(1.0,3.3)$ & $2.1(0.4,4.4)$ & $2.9(0.5,6.0)$ & $1.6(0.5,3.0)$ & $-1.5(-2.014), \mathrm{p}=0.044$ \\
\hline Ankle brachial pressure index & $1.14(1.00,1.28)$ & $1.06(0.92,1.28)$ & $1.14(0.95,1.30)$ & $1.22(1.03,1.45)$ & $0.15(-1.278), \mathrm{p}=0.201$ \\
\hline
\end{tabular}

\section{9}

The improvement of diabetic foot care in Tuscany: results in amputation reduction

R. Anichini ${ }^{1}$, A. De Bellis ${ }^{1}$, C. Seghieri ${ }^{2}$, C. Baggiore ${ }^{3}$, S. Nuti ${ }^{2}$, A. Piaggesi ${ }^{4}$ ${ }^{1}$ Diabetology, Diabetes Foot Unit, Pistoia, ${ }^{2}$ Epidemiology University of Pisa, Lab MES, ${ }^{3}$ Internal Medicine, Diabetes UNIT, Florence, ${ }^{4}$ Malattie del Metabolismo e Diabetologia, Diabetes Foot Unit, Pisa, Italy

Background and aims: In order to improve the organization of diabetic foot care in Tuscany and to standardize diagnostic and therapeutic protocols, in years 1999-2002 the implementation of international consensus of diabetic foot (ICDF) has been performed. In 2004 the Official Regional Health Authority established a new model of organization of diabetic foot care in Tuscany in three different levels, on the basis of the complexity of foot care serv- 
surgery procedures). All diabetic foot units were able to perform screening, education and preventive programme, according to the international guidelines. One of the most important objectives was defined as a epidemiological surveillance based on clinical outcomes as a high percentage of population screened, reduction in hospital admissions and reduction in lower extremity amputations.

Methods: We studied a relatively stable population 3536392 inhabitants in 2000 and 3638211 in 2007 (with increase in the years minor of 3\%). The data base for this study was extracted from the DRG of Health Regional System database (Tuscany) for diagnosis of diabetes, for foot lesions, procedures, number and duration of hospitalization and type of amputation.

Results: Here we show the following preliminary data: in years 2000-2007 there were in Tuscany 4374 major lower extremity amputations,(LEA) of which 1232 were diabetes related (about 28\%). Among individuals with diabetes LEA rates went from 4,2/100,000 inhabitants in the first year (148) to $3,3 / 100,000$ inhabitants in the eighth year (120). Over the same period non diabetic related LEA decreased from 11,8 to $10,5 / 100,000$ inhabitants. Moreover, hospitalization rate for diabetic foot was significantly reduced from 4,3 to $3,1 / 1000$ patients.

Conclusions: Our data demonstrate that the introduction of a better organized diabetes foot care is able to improve in outcomes one of the most feared diabetic complications.

\section{PS 126 Oxidative stress and endothelial function}

\section{0}

Low-grade inflammatory markers and oxidative stress in patients with type 1 diabetes mellitus

M.B. Gomes ${ }^{1}$, R.A. Cobas ${ }^{1}$, E. Nunes ${ }^{2}$, H.C. Castro-Faria-Neto ${ }^{2}$, M.N. Silva ${ }^{1}$ M.F. Bevilacqua ${ }^{1}$, E. Tibiriçá 2

${ }^{1}$ Diabetes Unit, State University of Rio de Janeiro, ${ }^{2}$ Immunopharmacology laboratory, FIOCRUZ, Rio de Janeiro, Brazil

Background and aims: Patients with type 1 diabetes present a high mortality and morbidity rate from cardiovascular disease. The underlying mechanisms that cause accelerated atherosclerosis in these patients are poorly understood. An association with glucose control, oxidative stress, markers of insulin resistance and of low-grade inflammation have been suggested. Few studies have been conducted on patients with type 1 diabetes with and without chronic complications or on subjects without diabetes to assess whether these factors could exist independently of the presence of diabetes. The present study was designed to evaluate if type 1 diabetic patients matched to subjects without diabetes (controls) have altered levels of low-grade inflammatory markers (Platelet-activating factor acetyhydrolase activity (PAF-AH), hs-CRP, white blood cells count(WBC) and sedimentation rate, and markers of oxidative stress (Oxidized LDL) and whether their levels are related to clinical complications and metabolic variables.

Materials and methods: We studied cross-sectionally 118 type 1 diabetic outpatients ( $45.8 \%$ males) aged $27.1 \pm 11.3$ years with a duration of diabetes of $12.3 \pm 8.5$ years, with $(n=38)$ and without $(n=80)$ clinical chronic complications and 110 controls subjects ( $49 . \%$ males) aged $26.1+7.2$ years. Clinical complications were evaluated by ECG, direct ophthalmoscopy, microalbuminuria .PAF-AH activity was measured with PAF-AH assay kit (Cayman Chemical, Ann Arbor, MI, USA). Analysis of the LDL oxidation coefficient (OxC) was performed using spectrophotometric readings at 205, 232 and $280 \mathrm{~nm}$ at different time points : 0, 1 hour, 3 hours, 6 hours and 24 hours after CuSO 4 addition of copper sulfate to purified LDL fractions. Laboratory analysis included lipid profile, $\mathrm{WBC}$, sedimentation rate, uric acid, $\mathrm{HbAlc}$ (HPLC), FBG, 2 h glucose, hs-CRP.

Results: Diabetic patients had higher PAF-AH activity [ $(0.015 \pm 0.005)$ vs $(0.013 \pm 0.005-\mu \mathrm{mol} / \mathrm{min} / \mathrm{mL} \mathrm{p}=0.02]$, hs_CRP [ 0.12(0.01-1.02) vs 0.04 (0.01-1.02 mg/dL p=0.001)] ,WBC ( $7.2 \pm 1.7$ vs $6.1 \pm 1.4 * 10^{3} \mu \mathrm{l} \mathrm{p}=0.004$ ) and sedimentation rate [14 (1-50) vs $7(1-40) \mathrm{mm} / \mathrm{h} \mathrm{p}=0.0001]$ levels than controls. No difference was noted between patients with and without chronic complications. Patients had a lower level of uric acid than controls, respectively $(3.5 \pm 1.1$ vs $4.8 \pm 1.3 \mathrm{mg} / \mathrm{dL} \mathrm{p}=0.000)$. Basal OxC values were similar in the two groups. Patients with type 1 diabetes had earlier LDL oxidation with lower coefficient $(\mathrm{OxC})$ than controls, one $(6.9 \pm 2.6$ vs $8.0 \pm 2.6, \mathrm{p}=0.008)$ and three hours ( $6.2 \pm 1.9$ vs $7.1 \pm 2.1, \mathrm{p}=0.01)$ after the addition of CuSO4. In patients, a correlation was found between PAF-AH and $\mathrm{OxC} 1 \mathrm{~h}(\mathrm{r}-0.26 \mathrm{p}=0.01)$ and $\mathrm{OxC} 3 \mathrm{~h}(\mathrm{r}=-0.25 \mathrm{p}=0.02)$. Separated stepwise multiple regression with PAF$\mathrm{AH}$ or hs-CRP or $\mathrm{OxC} 1 \mathrm{~h}$ or $\mathrm{OxC} 3 \mathrm{~h}$ as a dependent variables showed that diabetes was the most important significant independent variable.

Conclusion: According to our results, markers of low-grade inflammation and oxidative stress are increased in patients with type 1 diabetes probably independent of the presence of clinical microvascular disease. The natural anti-oxidant defense represented by uric acid is decreased in these patients. The influence of low-grade inflammatory and oxidative markers on the development of cardiovascular disease in patients with type 1 diabetes must be addressed in prospective studies.

Supported by: FAPERJ E-26/170.128/2004 CNPQ 471014/2004-4

\section{1}

FORT and FORD two novel assays for the assessment of oxidative stress in patients with newly diagnosed patients with diabetes mellitus type 2 I. Papassotiriou ${ }^{1}$, M. Pavlatou ${ }^{1,2}$, M. Papastamataki ${ }^{1}$, F. Apostolakou ${ }^{1}$, N. Katsilambros ${ }^{2}, \mathrm{~N}$. Tentolouris ${ }^{2}$

${ }^{1}$ Clinical Biochemistry, Aghia Sophia Children's Hospital, ${ }^{2}$ First Department of Propaedeutic Medicine, University of Athens Medical School, Athens, Greece

Background and aims: To evaluate the levels of free oxygen radicals (free oxygen radicals test, FORT) and free oxygen radicals defense (FORD) in pa- 
tients with newly diagnosed patients with diabetes mellitus type 2 (DM2). The disease seems to be involved strongly in the production of reactive oxygen species (ROS).

Materials and methods: Forty-five patients with newly diagnosed DM2 and 20 apparently healthy individuals (control group) were included in the study. ROS were determined using the FORT test (Callegari, Parma, Italy), which is based on the Fenton reaction. When a $20 \mu \mathrm{L}$ blood sample was dissolved in an acidic buffer, the hydroperoxides reacted with the transition metal ions liberated from the proteins in the acidic medium and were converted to alkoxyand peroxy-radicals. The radical species produced by the reaction, which are directly proportional to the quantity of lipid peroxides present in the sample, interact with an additive (phenylendiamine derivative) that forms a radical molecule evaluable by spectrophotometer at $505 \mathrm{~nm}$ (Form CR 2000; Callegari, Parma, Italy). Results are expressed as FORT U (Fort units) whereby 1 FORT $\mathrm{U}$ corresponds to $0.26 \mathrm{mg} / \mathrm{L} \mathrm{H}_{2} \mathrm{O}_{2}$. Similarly, the FORD test uses, preformed stable and colored radicals and determines the decrease in absorbance that is proportional to the blood antioxidant concentration of the sample according to the Lambert Beer's law. In the presence of an acidic buffer $(\mathrm{pH}=5.2)$ and a suitable oxidant $\left(\mathrm{FeCl}_{3}\right)$ the chromogen, which contains 4Amino-N,N-diethylaniline sulfate forms a stable and colored radical cation photometrically detectable at $505 \mathrm{~nm}$. Antioxidant compounds in the sample reduce the radical cation of the chromogen quenching the color and producing a decoloration of the solution, which is proportional to their concentration. The absorbance values obtained for the samples are compared with a standard curve obtained using Trolox (6-Hydroxy-2,5,7,8-tetramethylchroman-2-carboxylic acid), a permeable cell derivative of vitamin E commonly employed as an antioxidant.

Results: The main results of the study showed that: a) free oxygen radical levels (FORT) were increased in diabetic patients $\left(2.86 \pm 0.56 \mathrm{mmol} / \mathrm{L} \mathrm{H}_{2} \mathrm{O}_{2}\right)$ compared to controls $\left(1.87 \pm 0.26 \mathrm{mmol} / \mathrm{L} \mathrm{H}_{2} \mathrm{O}_{2}\right),(\mathrm{p}<0.0001)$ and $\left.\mathrm{b}\right)$ FORD levels were lower in diabetic patients $(1.23 \pm 0.18 \mathrm{mmol} / \mathrm{L}$ Trolox $)$ compared to controls $(1.34 \pm 0.14 \mathrm{mmol} / \mathrm{L}$ Trolox $),(\mathrm{p}<0.01)$. The intraassay and interassay coefficients of variation were $3.7 \%$ and $6.2 \%$, respectively for FORT, while the intraassay and interassay coefficients of variation were $4.2 \%$ and $6.6 \%$, respectively for FORD.

Conclusion: The determination of free oxygen radicals (FORT) and free oxygen radicals defense (FORD) seem to play an important role in the generation of oxidative stress, an imbalance between oxidants and antioxidants that can lead to oxidative damage and is involved in the pathogenesis of several diseases, such as DM2. The methodology described above is simple, reliable, rapid and reproducible.

\section{2}

Hyperglycaemia, both chronic and in short-term peaks, results in rapid increase of expression of monocyte inducible ciclooxygenase (COX-2) in subjects with type 2 diabetes mellitus

P. Rossetti, P. Candeloro, T. Corazzi, C.G. Fanelli, F. Porcellati, P. Cioli,

L. Cecchetti, P. Gresele, G.B. Bolli

Internal Medicine, University of Perugia, Italy

T2DM is associated with a two-to-four fold increase of vascular complications as result of both chronic hyperglycemia (elevated A1C) and isolated spikes of (post-prandial) hyperglycemia. Monocyte inducible COX-2 has been advocated as a player in the pathogenesis of ischemic events. Aim of our study was to establish if both chronic and acute, short-term hyperglycemia affect COX-2 expression in monocytes of subjects with T2DM. Peripheral blood monocyte COX-2 was measured (Western Blotting) in 120 subjects with T2DM (A1C $7.9 \pm 1.0 \%$; fasting BG (FBG) $180 \pm 9 \mathrm{mg} / \mathrm{dl}$; age $67 \pm 9$ years) and 50 age and sex-matched healthy controls (A1C 5.9 $\pm 0.3 \%$; FBG $94 \pm 9 \mathrm{mg} / \mathrm{dl}$; age $62 \pm 14$ years). COX-2 was expressed in monocytes of $84 \%$ of T2DM subjects and $4 \%$ of normal volunteers $(\mathrm{p}<0.01)$. Average monocyte COX-2 was $0.31 \pm 0.05 \mathrm{ng} / \mu \mathrm{g}$ total proteins in T2DM and correlated with FBG $(\mathrm{r}=0.52 ; \mathrm{p}=0.05)$ and $\mathrm{A} 1 \mathrm{C}(\mathrm{r}=0.52 ; \mathrm{p}=0.03)$. In $5 \mathrm{~T} 2 \mathrm{DM}$ subjects with poor control at baseline in whom BG was optimally improved for 3 months (A1C decreased from $8.1 \pm 0.4 \%$ to $7 \pm 0.6 \%$; $p<0.01$ ), monocytes COX-2 decreased from $0.18 \pm 0.06$ to $0.12 \pm 0.05 \mathrm{ng} / \mu \mathrm{g}$ total proteins, $\mathrm{p}<0.05$. Following these observations, monocytes were isolated from healthy volunteers and cultured in $22 \mathrm{mM}$ glucose-containing medium for 4 hours, showing increase of COX2 ( 0 to $0.6 \pm 0.15 \mathrm{ng} / \mu \mathrm{g}$ total protein, $\mathrm{p}<0.001)$. After 4 -h additional incubation at $5.5 \mathrm{mM}$ glucose-containing medium, COX-2 reduced to $0.3 \pm 0.11 \mathrm{ng} / \mu \mathrm{g}$ total protein, $\mathrm{p}<0.01$, and incubation for additional $20-\mathrm{h}$ abolished it $(\mathrm{p}<0.01)$. Five T2DM subjects with good baseline glycemic control (A1C $6.4 \pm 0.3 \%$, FBG $110 \pm 6 \mathrm{mg} / \mathrm{d}$ ), underwent two subsequent differential steps (4h-each) of hyperglycemia (clamp, BG $13.9 \mathrm{mM}$ ) and euglycaemia (clamp, BG $5.5 \mathrm{mM}$ ). After acute hyperglycemia monocyte COX-2 expression increased by $56 \%$. However a prompt normalization of BG reached after $30 \mathrm{~min}$ and maintained thereafter (i.v. insulin bolus of $100 \mathrm{mU} / \mathrm{kg}$, folllowed by a constant infusion of $0.5 \mathrm{mU} / \mathrm{Kg} / \mathrm{min}$ ) decreased COX-2 expression by $71 \%$ after 4 -h.

Conclusion: Hyperglycemia is associates with COX-2 induction both in chronic as well as acute states. Short-term hyperglycemia mimicking postprandial spikes, induces a rapid upregulation of monocyte COX-2. This phenomenon is rapidly reversible after BG normalization. These observations may have practical implications for clinical management of T2DM.

\section{3}

Role of $\mathrm{p}^{\mathrm{Shc}}$ in the modulation of oxidative stress responses in human liver cells

F. De Stefano, F. Losurdo, A. Quagliara, S. Perrini, A. Natalicchio, A. Peschechera, M. Melchiorre, A. Cignarelli, A. Leonardini, M. Barbaro, A. Conserva, L. Laviola, F. Giorgino

D.E.T.O., Internal Medicine, Endocrinology and Metabolic Diseases, University of Bari, Italy

Background and aims: Oxidative stress plays an important role in the pathogenesis of nonalcoholic fatty liver disease (NAFLD). However, the molecular determinants of oxidative stress-induced liver damage remain poorly understood. The protein $\mathrm{p} 66^{\mathrm{shc}}$ has been proposed as a cellular sensor of the mitochondrial redox balance. Specifically, $\mathrm{p} 66^{\text {shc }}$, through its phosphorylation on $\mathrm{Ser}^{36}$, has been shown to modulate various cellular responses and apoptotic rates in cells exposed to oxidative stress. The objective of our study was to investigate the role of $\mathrm{p} 66^{\text {shc }}$ in the regulation of intracellular responses to oxidative stress in human liver cells.

Materials and methods: Human HepG2 hepatoma cells with adenovirusmediated overexpression of $\mathrm{p} 66^{\text {shc }}$ (Adp66 ${ }^{\text {shc }}$ ) were exposed to $\mathrm{H}_{2} \mathrm{O}_{2}$, a commonly used oxidative stress inducer. Cellular responses to oxidative stress were assessed by gene expression and immunoblotting techniques.

Results: HepG2-Adp66 $6^{\text {Shc }}$ cells were characterized, under basal conditions, by increased $\mathrm{p} 66^{\text {Shc }}$ phosphorylation on $\mathrm{Ser}^{36}$ (approximately 50-fold; $\mathrm{p}<0.005$ ) vs. control cells (wild type and Mock HepG2 cells). Moreover, increased $\mathrm{p} 66^{\text {Shc }}$ protein levels were associated to increased mRNA expression levels of the antioxidant enzyme MnSOD as compared to controls, suggesting a potential biochemical compensation to the increased oxidative stress associated with the overexpression of $\mathrm{p} 66^{\text {Shc }}$. HepG2-Adp $66^{\text {Shc }}$ exposed to 0.5 $\mathrm{mM} \mathrm{H}_{2} \mathrm{O}_{2}$ showed a further increase in the level of $\mathrm{Ser}^{36}$ phosphorylation of p $66^{\text {Shc }}(2$-fold vs. control cells; $p<0.005)$, associated with increased Erk- 1 phosphorylation, peaking at 15 min ( 7 -fold vs controls; $\mathrm{p}<0.005)$. Additionally, $\mathrm{H}_{2} \mathrm{O}_{2}$-treated $\mathrm{HepG} 2$-Adp66 $6^{\text {Shc }}$ showed increased Akt phosphorylation on $\operatorname{Ser}^{473}$ (approximately 2 -fold; $\mathrm{p}<0.005$ ) as well as increased $\mathrm{Thr}^{32}$-phosphorylation of FOXO3a, a member of the Forkhead family of transcription factors. Following $\mathrm{Thr}^{32}$-phosphorylation, the ability of FOXO3a to induce gene expression of anti-oxidants is diminished. Indeed, treatment of HepG2-Adp$66^{\text {shc }}$ with $\mathrm{H}_{2} \mathrm{O}_{2}$ resulted in reduced expression of MnSOD, a gene controlled by FOXO3a.

Conclusion: Overexpression of the $\mathrm{p} 66^{\mathrm{Shc}}$ protein isoform in human hepatoma cells induces an increase of the intracellular oxidative stress under basal conditions, and a reduced cellular ability to react against oxidative stress inducers via an Akt/Foxo3a signalling pathway. Thus, the $\mathrm{p} 66^{\text {shc }}$ protein activates a specific intracellular signaling pathway that modulates the response to oxidative stress in liver cells. This may be relevant to the patogenesis of the hepatic dysfunction associated with the metabolic syndrome and type 2 diabetes.

\section{4}

Changes in serum corticosterone levels and adrenal cortex nitric oxide synthase activity in streptozotocin-diabetic rats: involvement of oxidative stress

E.M. Repetto ${ }^{1,2}$, J.M. Cipelli ${ }^{1}$, R. Sanchez ${ }^{1}$, F. Astort ${ }^{1,2}$, C. Martinez Calejman $^{1,2}$, J.M. Di Gruccio ${ }^{1}$, G.G. Piroli ${ }^{1}$, P. Arias ${ }^{3}$, C.B. Cymeryng ${ }^{1,2}$ ${ }^{1}$ Human Biochemistry, University of Buenos Aires, ${ }^{2}$ Conicet, CEFYBO, ${ }^{3}$ Physiology, University of Buenos Aires, Argentina

Background and aims: Increased oxidative stress, arising as a consequence of excess fuel supply, has been associated with the pathogenesis of chronic diabetic complications. The aim of the present study was to evaluate different 
parameters of oxidative stress and its role in the induction of nitric oxide synthase (NOS) activity in the adrenal cortex of streptozotocin (STZ)-diabetic rats. The impact of STZ-induced diabetes and of antioxidant treatment on corticosterone secretion was also evaluated.

Materials and methods: Male Wistar rats were treated with STZ $(40 \mathrm{mg} / \mathrm{kg}$ i.p. for three consecutive days) or citrate buffer (controls). After instauration of hyperglycemia, different groups of STZ-treated and control animals received antioxidant treatment (melatonin $7.5 \mathrm{mg} / \mathrm{kg}$ p.o. or $\alpha$-tocopherol 100 $\mathrm{mg} / \mathrm{kg}$, p.o.). Thiobarbituric acid-reactive substances (TBARS, an index of lipid peroxidation), $\mathrm{HO}-1$ expression, superoxide dismutase (SOD), catalase and NOS activities, and reduced glutathione (GSH) levels were determined in adrenal cortex homogenates at different time points after the induction of the diabetic state. Serum corticosterone levels were measured by RIA. Statistic evaluation was performed using an ANOVA followed by Tukey's post hoc test.

Results: After 4 weeks of treatment STZ-diabetic animals showed a significant increase in basal corticosterone levels (101 \pm 8.7 versus $49.7 \pm 2.0 \mathrm{ng} / \mathrm{ml}$; mean \pm SEM) and in tissue TBARS concentrations, as compared to controls. NOS activity was increased from the second to the eighth week of treatment. GSH and antioxidant enzyme activities (HO-1, SOD and catalase) were increased by 4-8 weeks. Antioxidant treatment reduced TBARS levels and prevented the increase in HO-1 and NOS activity. Corticosterone levels were clearly reduced in melatonin and $\alpha$-tocopherol-treated diabetic animals (43.4 \pm 6.1 and $45.6 \pm 10.4 \mathrm{ng} / \mathrm{ml}$, respectively).

Conclusion: Our results suggest that, in the adrenal cortex of STZ-treated rats, increased oxidative stress is associated with an increase in NOS activity. Upregulation of HO-1, SOD and catalase activities is probably involved in cytoprotective mechanisms triggered by the production of reactive oxygen or nitrogen species, or by other parameters of cell damage associated with the diabetic state. As described previously, STZ-induced diabetes resulted in elevated corticosterone levels; this increase was not present in diabetic animals receiving antioxidant treatment. Accordingly, the involvement of oxidative stress-induced mechanisms in the dysregulation of the hypothalamic-pituitary-adrenal axis observed in STZ-treated animals is postulated.

Supported by: University of Buenos Aires, CONICET and Agencia Nacional de Promoción Científica y Tecnológica

\section{5}

Induction of stearoyl-CoA desaturase (SCD-1) protects human arterial endothelial cells against lipotoxicity

A. Peter, C. Weigert, H. Staiger, K. Rittig, A. Cegan, P. Lutz, F. Machicao, H.-U. Häring, E. Schleicher

Department of Internal Medicine, Devision of Endocrinology, Diabetes,

Vascular Medicine, Nephrology, University of Tübingen, Germany

Endothelial lipotoxicity has been implicated in the pathogenesis of multiple stages of cardiovascular disease from early endothelial dysfunction to manifest atherosclerosis and its complications. Saturated free fatty acids are the major inducers of endothelial cell apoptosis and inflammatory cytokines. In humans, the enzyme stearoyl-CoA desaturase-1 (hSCD-1) is the limiting step of the desaturation of saturated to monounsaturated fatty acids. Since we could demonstrate the expression of SCD-1 in primary human arterial endothelial cells (HAECs) we aimed to prove a beneficial role of upregulated hSCD-1 expression. In contrast to other cells which are less susceptible to lipotoxicity, hSCD-1 was not upregulated in HAECs upon palmitate treatment. Following that, we could show that upregulation of hSCD-1 using the LXR activator TO901317 in HAECs protects the cells against palmitate-induced lipotoxicity, cell apoptosis and expression of inflammatory cytokines IL-6 and IL-8. Increased hSCD-1 activity was determined as C16:1/16:0-ratio and enhanced triglyceride storage in palmitate treated cells. The beneficial effect was clearly attributed to enhanced hSCD-1 activity. Overexpression of hSCD-1 blocked palmitate-induced cytotoxicity and knockdown of hSCD-1 using si-RNA abolished the protective effect of TO901317 in HEK293 cells. Additionally, inhibition of hSCD-1 with 10/12 CLA blocked the effect of TO901317 on palmitate-induced lipotoxicity, cell apoptosis and inflammatory cytokine induction in HAECs. We conclude that upregulation of hSCD-1 led to a desaturation of saturated fatty acids and facilitates their esterification and storage thereby preventing downstream effects of lipotoxicity in HAECs. These findings add a novel aspect to the atheroprotective actions of LXR activators in cardiovascular disease.

Supported by: German Research Foundation

\section{6}

Protective effects of a prescribed traditional Chinese medicine preparation on oxidative stress induced by high glucose in bEnd. 3 cells X. Gao ${ }^{1}$, Y. Kang ${ }^{1}$, L. Zhou ${ }^{2}$, M. Wang ${ }^{2}$

${ }^{1}$ Endocrinology \& Metabolism, Zhongshan Hospital, ${ }^{2}$ The National Center for Drug Screening, Shanghai Institute of Materia Medica, Chinese Academy of Science, Shanghai, China

Background and aims: Endothelial dysfunction is a critical and initiating factor in the pathogenesis of diabetic vascular complications. An underlying mechanism for such abnormality is the enhanced generation of endothelial mitochondrial reactive oxygen species (ROS). We previously observed that a prescribed traditional Chinese medicine preparation "Qing Huo Yi Hao" (QHYH) could decrease urinary microalbumin excretion in type 2 diabetic patients and improve endothelial cell growth in high glucose medium. This study was designed to gain some insights into the antioxidant effects of QHYH on oxidative stress induced by high glucose in endothelial cells. Since a recent report suggests that genetic ablation of uncoupling protein 2 (UCP2) produces more ROS in endothelial cells, we therefore investigated the effect of QHYH on UCP2 expression, and whether it is accounted for the antioxidant action of QHYH in endothelial cells.

Materials and methods: Mice brain microvascular cells (bEnd. 3 cells) were cultured in normal glucose (NG, $5.6 \mathrm{mM}$ ), high glucose (HG) or HG in the presence of QHYH (1:100 dilution). High glucose concentration was $35 \mathrm{mM}$ except for ROS detection experiments in which $25 \mathrm{mM}$ glucose was applied. ROS and nitric oxide (NO) production were measured by the fluorescent probes $\mathrm{H}_{2}$ DCF-DA and DAF-FM DA, respectively. UCP2 mRNA was detected by real-time PCR. Phosphorylation of endothelial nitric oxide synthase (eNOS) and protein kinase B (Akt), and UCP2 protein expression were determined by Western blotting. UCP2 siRNA was used to evaluate the role of $\mathrm{UCP} 2$ in the antioxidant effect elicited by QHYH.

Results: High glucose $(25 \mathrm{mM})$ increased ROS production $(142.27 \pm 11.44 \%$ vs. $100.00 \pm 1.92 \%$ in NG, $P<0.01)$ in bEnd. 3 cells while UCP2 mRNA and protein expression were decreased at the same time. UCP2 mRNA was reduced by glucose in a concentration- and time-dependent manner, with the maximum effect observed at $35 \mathrm{mM}(69.90 \pm 4.84 \%, P<0.01 v s$. NG) occurring after 48 hours of incubation $(66.93 \pm 4.73 \%, P<0.001 v s .0$ h). High glucose $(35 \mathrm{mM})$ also down-regulated phosphorylation of eNOS at Ser1177 and Akt at Ser 473, and diminished NO generation in bEnd.3 cells compared to NG treatment. Addition of QHYH reversed the high glucose induced ROS production ( $53.65 \pm 8.11 \%$ vs. $142.27 \pm 11.44 \%$ in HG group, $P<0.001)$, eNOS and Akt phosphorylation(72.81 $\pm 7.02 \%$ vs. $45.49 \pm 2.51 \%$ in HG group, $P<0.05$ and $76.71 \pm 4.00 \%$ vs. $120.98 \pm 1.20 \%$ in HG group, $P<0.001$, respectively), $\mathrm{NO}$ generation $(118.82 \pm 2.95 \% v s .53 .83 \pm 3.98 \%$ in $\mathrm{HG}, \mathrm{P}<0.001$, and UCP2 protein expression ( $94.09 \pm 6.30 \%$ vs. $53.83 \pm 3.98 \%$ in HG, $P<0.001)$. When UCP2 expression was blocked by UCP2 siRNA, the antioxidant action of QHYH against high glucose was attenuated, and had little effect on ROS production and NO generation in high glucose environment.

Conclusion: QHYH is capable of protecting endothelial cells from high glucose induced ROS production, down-regulation of eNOS and Akt phosphorylation and reduction of $\mathrm{NO}$ generation. The protective action of $\mathrm{QHYH}$ was partially related to UCP2 protein expression, since deletion of UCP2 by siRNA led to its abolishment. These results suggest a therapeutic potential of QHYH in the treatment of diabetic vascular complications.

\section{7}

Protective effects of berberine against vascular dysfunctions: molecular mechanisms and therapeutic implications

Y. Wang ${ }^{1}$, Y. Huang ${ }^{2}$, K.S.L. Lam ${ }^{1}$, C.W. Lau $^{2}$, A. Xu ${ }^{1}$

${ }^{1}$ Department of Medicine and Research Centre of Heart,Brain Hormone and Healthy aging, Faculty of Medicine, The University of Hong Kong, ${ }^{2}$ Department of Physiology, The Chinese University of Hong Kong, Hong Kong, China

Background and aims: Vascular disease is one of the most common complications in type 2 diabetic patients. Endothelial dysfunction, characterized by impaired vasodilation, is a key early event of vascular disease as well as diabetes. Endothelium-derived nitric oxide (NO) has multiple beneficial effects such as enhancing vasodilatation, suppressing platelet aggregation and leucocytes migration, decreasing cellular adhesion to endothelium and attenuating vascular smooth muscle cell proliferation and migration. Berberine, an isoquinoline alkaloid purified from traditional Chinese Medicine, has a 
wide spectrum of clinical applications such as in bacterial diarrhea, intestinal parasitic infection, cardiovascular diseases, inflammation and hypercholesterolemia. This study evaluated the effect of berberine on endothelial functions, and investigated the cellular mechanisms involved.

Materials and methods: The effects of berberine on the production of nitric oxide (NO) and activation of eNOS and AMP-activated protein kinase (AMPK) were examined in human umbilical vein endothelial cells (HUVEC). Aortic rings isolated from Sprague-Dawley rats were used for evaulation of vascular reactivity.

Results: Berberine stimulated phosphorylation of eNOS at ser1177 and the NO production in HUVEC in a time- and concentration-dependent manner. In addition, berberine induced endothelium-dependent vasodilatation in rat aortic rings. These effects were largely inhibited by Compound C, a selective inhibitor of AMPK. Further analysis revealed that berberine activated AMPK through its upstream kinase LKB1. In Hela cells (which lack LKB1), the ability of berberine to stimulate phosphorylation of AMPK was significantly attenuated. Berberine stimulated phosphorylation of LKB1 at Ser428, and enhanced the physical interaction between LKB1 and AMPK. On the other hand, STO609, a selective inhibitor of calmodulin-dependent kinase kinase $\beta$, had no obvious effect on berberine-induced activation of AMPK and eNOS.

Conclusion: Berberine stimulates endothelium-dependent vasodilatation through the activation of the AMPK/eNOS/NO signaling cascade. Berberine and its derivatives might represent a new class of therapeutic agents for the treatment and/or prevention of endothelial dysfunctions associated with obesity and diabetes.

Supported by: Hong Kong Research Grant Council

\section{8}

Glucose-potentiated aortic smooth muscle cell migration to oxidised low-density lipoprotein: statin and angiotensin-converting enzyme inhibitor sensitivity

M. Campbell ${ }^{1}$, D.W. Harkin ${ }^{2,3}$, E.R. Trimble ${ }^{1,4}$, I.S. Young ${ }^{1,4}$

${ }^{1}$ Nutrition and Metabolism Group, Queen's University, ${ }^{2}$ Clinical Vascular

Research, Queen's University, ${ }^{3}$ Regional Vascular Surgery, Belfast Hospitals

Trust, ${ }^{4}$ Clinical Biochemistry, Belfast Hospitals Trust, Belfast, United

Kingdom

Background and aims: In diabetes there is premature onset and increased severity of atherosclerosis. Glucose-potentiated signalling events, including extracellular signal-regulated kinase (ERK) activation, are implicated in cellular changes associated with atheroma formation. In atherosclerosis vascular smooth muscle cells (VSMC) migrate to factors, such as oxidised (ox)LDL, which are found at raised levels in plaques. Statins and ACE inhibitors, used to treat dyslipidaemia and hypertension, respectively, have additional (pleiotropic) benefits in atherosclerosis therapy. The aims of this work are to assess the interactions of high glucose (15 mmol/l: HG) and oxLDL on explantderived aortic VSMC migration and to identify if clinically relevant statin or ACE inhibitor concentrations can inhibit migration.

Materials and methods: Migration was recorded over $18 \mathrm{~h}$ using the Dunn chamber and analysed by cyclical statistics (Rayleigh test; $\mathrm{n} \geq 30$ ). Total and phosphorylated ERK cellular protein levels were assessed by western blot (data presented as mean \pm SEM, $n=4$ ) and analysed using ANOVA and Fisher's LSD ( $\mathrm{p}<0.01$ considered significant). ERK activation was confirmed by activity assay.

Results: Migration, absent in a native LDL gradient $(\leq 100 \mu \mathrm{g} / \mathrm{ml})$, was detected in a gradient of oxLDL ( $\geq 50 \mu \mathrm{g} / \mathrm{ml}, \mathrm{p}<0.01)$ for VSMC exposed to 5 $\mathrm{mmol} / \mathrm{l} \mathrm{D}$-glucose (normal glucose: NG). In $\mathrm{HG}$ the threshold for migration to oxLDL was lowered to $25 \mu \mathrm{g} / \mathrm{ml}$ ( $\mathrm{p}<0.01$, detected after $6 \mathrm{~h}$ ). In NG supplemented with the non-metabolisable analogue L-glucose $(10 \mathrm{mmol} / \mathrm{l})$ the threshold did not differ from that for NG alone (osmotic control). Irrespective of glucose level pre-incubation of the cells $(2 \mathrm{~h})$ with CD36 antibody ( 0.5 $\mu \mathrm{g} / \mathrm{ml}$ ) prevented migration to $50 \mu \mathrm{g} / \mathrm{ml}$ oxLDL. Under similar conditions clinically relevant (circulating) levels of ACE inhibitor (enalapril, $1 \mu \mathrm{mol} / \mathrm{l}$ ) or statin (pravastatin, $10 \mathrm{nmol} / \mathrm{l}$ ) also prevented migration. These antibody and inhibitor effects were specific since migration occurred to $50 \mu \mathrm{g} / \mathrm{ml}$ oxLDL in NG and HG in the presence of non-specific antibody or solvent (dimethylsulphoxide) controls ( $<<0.01$ for all). A significant increase in phospho-ERK occurred (western blot), compared to basal ( $0.5 \%$ bovine serum albumin), for cells exposed for $18 \mathrm{~h}$ to $50 \mu \mathrm{g} / \mathrm{ml}$ oxLDL in $\mathrm{NG}(+170 \pm 20 \%, \mathrm{p}<0.01)$ or $25 \mu \mathrm{g} / \mathrm{ml}$ oxLDL in HG $(+260 \pm 30 \%, \mathrm{p}<0.01)$. No changes in total ERK protein levels were detected and solvent did not significantly alter phosphoERK levels. Pravastatin decreased phospho-ERK to basal levels for $50 \mu \mathrm{g} / \mathrm{ml}$
oxLDL in NG $(+20 \pm 25 \%, \mathrm{p}<0.01$ vs solvent alone), and $25 \mu \mathrm{g} / \mathrm{ml}$ oxLDL in HG $(+5 \pm 15 \%, \mathrm{p}<0.01 v s$ solvent alone). Enalapril also significantly decreased phospho-ERK but to a lesser degree for $50 \mu \mathrm{g} / \mathrm{ml}$ oxLDL in NG $(+50 \pm 20 \%$, $\mathrm{p}<0.01 v s$ solvent alone) and $25 \mu \mathrm{g} / \mathrm{ml}$ oxLDL in $\mathrm{HG}(+40 \pm 15 \%, \mathrm{p}<0.01 v s$ solvent alone). Similar results to those for phospho-ERK were found when an ERK activity assay was employed under matched conditions $(n=3)$.

Conclusion: Aortic VSMC migration, a key event in atheroma formation, occurs to lower levels of oxLDL in the presence of raised glucose. Migration to oxLDL depends on ERK signalling which can be blocked by clinically relevant levels of pravastatin or enalapril. 


\section{PS 127 Diabetes related changes in endothelial gene expression}

\section{9}

Resistin and high glucose induce ICAM-1, P-selectin and fractalkine in human endothelial cells

I.I. Manduteanu, E. Dragomir, M. Calin, A. Gan, D. Stan, M. Pirvulescu, M. Simionescu

Cell Adhesion and Thrombosis, ICBP "N.Simionescu", Bucharest, Romania

Background and aims: Atherosclerosis is accelerated in diabetic conditions. Clinical data, and in vivo and in vitro studies suggest that endothelial dysfunction play a key role in this process and that cell adhesion molecules, cytokines and chemokines are actively involved. Resistin, a recently described cytokine, has an established role in mice as a link between obesity and diabetes but an unclear function in humans were it is assumed to be primarily involved in inflammation. Recent clinical data indicate that plasma resistin levels are increased in patients with diabetes complicated with atherosclerosis, but the involved mechanisms are not very well known. To better understand resistin role in these conditions, we performed studies on resistin and high glucose (as inducers of accelerated atherosclerosis in diabetic conditions) direct action on human endothelial cells (HEC). The aims of our studies were: i) to investigate the effects of resistin and high glucose on ICAM-1, P-selectin and fractalkine exprssion in HEC; ii) to reveal the signaling pathways possible involved.

Materials and methods: Human endothelial cells (Eahy926 cells) in culture were exposed to resistin $(50$ or $100 \mathrm{ng} / \mathrm{ml})$, high glucose $(33 \mathrm{mM})$ or resistin and high glucose for 6,18, 24 and 48 hours. The expression of cell adhesion molecules ICAM-1, P-selectin and fractalkine was detected by RT-PCR (gene expression) and by Elisa and Western blot (protein expression). To identify resistin and high glucose downstream signaling pathways the activation of p38, and JNK MAPK and of transcription factors NF-kB and AP1 was determined (western blot). In addition, intracellular accumulation of reactive oxygen species (ROS) in HEC was monitored by fluorimetry using 2',7'-dichlorofluorescein diacetate (DCFH-DA)

Results: Our data show that resistin or high glucose induced: i) an increase in gene and protein expression of ICAM-1, P-selectin and fractalkine; ii) an enhancement of the nuclear level of p65 and pc-Jun proteins; iii) an increase in the intracellular level of ROS; iv) an increased activation of JNK and p38 MAPK. Co stimulation with resistin and high glucose: I) induced ICAM1, P-selectin and fractalkine at levels that do not exceed the levels of every inducer alone; ii) activated p38 and JNK MAPK at similar levels as every inducer alone and iii) increased intracellular ROS levels at similar levels as every single inducer.

Conclusion: Our results suggest that resistin, high glucose or resistin and high glucose activate human endothelial cells by inducing the cell adhesion molecules ICAM-1, P-selectin and fractalkine and MAPK, ROS and the transcription factors NF-kB and AP-1 may be involved in the activation process. Supported by: Romanian Academy and Ministry of Research and EducationNational Center of Grants Management

\section{0}

Effect of consumption of conjugated linoleic acids on the LDL induced expression of adhesion molecules on endothelial cells J. Schrezenmeir ${ }^{1}$, K. Pfafferodt ${ }^{2}$, P. Winkler ${ }^{3}$, C. Laue ${ }^{3}$, U. Helwig $^{2}$, M. Pfeuffer ${ }^{2}$

${ }^{1}$ Physiology and Biochemistry of Nutrition, Max Rubner-Institut (MRI), Karlsruhe, ${ }^{2}$ Physiology and Biochemistry of Nutrition, Max Rubner-Institut (MRI), Kiel, ${ }^{3}$ Tecura GmbH Medizin \& Biotechnik, Kiel, Germany

Background and aims: Increased expression of adhesion molecules, such as the intercellular adhesion molecule (ICAM-1), is associated with an activated endothelium. Adhesion molecules promote the migration of leucocytes into the intima, an early step in atherogenesis. Further, elevated levels of soluble adhesion molecules are frequently observed to be a risk marker for the development of type 2 diabetes. This study focused on conjugated linoleic acids (CLA) and their effect on the LDL-induced expression of ICAM-1 on endothelial cells.

Materials and methods: In a randomized, double blind, crossover study, 20 healthy males, age 50 to 75 years, with an increased body mass index
(BMI: $25-30 \mathrm{~kg} / \mathrm{m} 2$ ) consumed $4.25 \mathrm{~g} / \mathrm{d}$ of either linoleic acid (LA) or cis9, trans-11 CLA isomer or the trans 10, cis-12 CLA isomer or a 50:50 mix of both isomers for 4 weeks. 4 -week treatments were separated by 4-6 week washout periods. Blood samples were drawn in the fasting state and $5 \mathrm{~h}$ after ingestion of a liquid fat- and sucrose-rich test meal plus 3 capsules of the respective supplement $(2.125 \mathrm{~g})$. Fasting and postprandial LDL were isolated by ultracentrifugation and adjusted to a protein concentration of $0.3 \mathrm{mg} / \mathrm{ml}$. Aliquots were oxidized with copper $(2$ or $5 \mu \mathrm{M} \mathrm{Cu}, 18 \mathrm{~h})$. Human umbilical vein endothelial cells (HUVECs) were incubated for $16 \mathrm{~h}$ with native LDL as well as oxidized LDL. The expression of ICAM-1 was determined by an enzyme-linked immunosorbent assay (ELISA). Cytotoxicity induced by the incubation of HUVECs with LDL was assessed from the release of lactate dehydrogenase (LDH) into the culture media. Statistical analysis of the results was carried out by a multi-factor analysis of variance (MANOVA) followed by post hoc Bonferroni. Data given are means \pm SEM.

Results: Oxidized LDL induced expression of ICAM-1 more than native LDL, and expression was higher with higher degree of oxidation (2 or $5 \mu \mathrm{M}$ $\mathrm{Cu}$ ). LDL isolated from subjects after consumption of trans-10, cis-12 CLA stimulated ICAM-1 less than LDL after LA intervention (Bonferroni, $\mathrm{P}<0.05$ ). ICAM expression after consumption of the CLA mix and cis-9, trans-10 CLA intervention tended to be lower than after LA consumption. Interestingly, postprandial LDL did not increase the ICAM-1 expression beyond that of fasting LDL, but they were more cytotoxic (Bonferroni, $\mathrm{P}<0.05)$. LDL obtained after CLA mix intervention were more cytotoxic than after all other interventions (Bonferroni, $\mathrm{P}<0.05$ ). But overall the cytotoxic effects were low. Conclusion: LDL obtained after CLA-enriched diets, in particular after consumption of the trans-10, cis-12 CLA isomer, decreased expression of ICAM1 in endothelial cells compared to LA. This indicates that CLA may attenuate inflammatory processes and thus act in a beneficial manner.

Supported by: German Federal Ministry of Education and Research; Cognis GmbH, Monheim

\section{1}

Centella Asiatica inhibits TNFa-induced adhesion molecule expression in endothelial cells of umbilical cords from gestational diabetic women N. Di Pietro ${ }^{1}$, P. Di Tomo ${ }^{1}$, A. Giardinelli ${ }^{1}$, P. Di Fulvio ${ }^{2}$, S. Di Silvestre ${ }^{1}$, G. Formoso ${ }^{2}$, P. Lanuti ${ }^{3}$, M. Marchisio ${ }^{3}$, A. Arduini ${ }^{4}$, A. Consoli ${ }^{2}$, A. Pandolfi ${ }^{1}$

${ }^{1}$ Dept. Biomedical Sciences, Ce.S.I. Institute, University G d'Annunzio, Chieti, ${ }^{2}$ DMSI, Ce.S.I. Institute, University G d'Annunzio, Chieti, ${ }^{3}$ Dept. Biomorphology, Ce.S.I. Institute, University G d'Annunzio, Chieti, ${ }^{4}$ Dept., Iperboreal Pharma, Chieti, Italy

Background and aims: Diabetes mellitus is associated with inflammatory endothelial activation and increased vascular leukocyte adhesion molecules expression, both playing a prominent role in the development of vascular complications. Centella Asiatica (CA) has shown anti-inflammatory properties in a variety of experimental models: however, its actions on vascular adhesion molecule synthesis and exposure have not yet been tested. Thus, we evaluated the effect of $C A$ on TNFa-stimulated adhesion molecule expression in cultured endothelial cells obtained from umbilical cords of gestational diabetic and control women.

Materials and methods: Human Umbilical Vein Endothelial Cells (HUVEC) obtained at delivery form umbilical cords of 10 healthy women (C) and 10 women with gestational diabetes (GD) were stimulated with TNF $\alpha(1 \mathrm{ng} / \mathrm{mL})$ after a 48 hours pre-incubation with $C A(25 \mu \mathrm{g} / \mathrm{mL})$ or with buffer. After 12 and 16 hours, vascular cell adhesion molecules (VCAM-1), intercellular cell adhesion molecules (ICAM-1) and E-Selectin protein levels (Western Blot) and their surface expression (biparametric flow cytometry analysis) were assessed. The functional consequences of C-and GD-HUVEC treatment with $C A$ on VCAM-1 membrane exposure were also evaluated by human monocytoid cell (U937 line) adhesion assay.

Results: After a 12 hours TNFa stimulation, VCAM-1, ICAM-1 and E-Selectin protein levels were significantly higher in GD- as compared in C-HUVEC ( $\mathrm{p}<0.05$, Western Blot analysis). Preincubation with $C A$ significantly decreased the effects of 12 hours TNF $\alpha$-stimulation on VCAM-1 protein levels in GD-HUVEC (25-30\%), while no effect was observed on C-HUVEC. Flow cytometry analysis demonstrated that, following $C A$ pre incubation, the percentage of cells positive for surface VCAM- 1 and ICAM-1 expression was modestly but significantly lower both in C- and GD-HUVEC after 12 and 16 hours TNFa stimulation. In addition, as compared to cells not pre-exposed to $C A$, both VCAM-1 and ICAM-1 MFI ratio (Mean Fluorescence Intensity) was lower in both CA-preincubated C- and GD-HUVEC after 12 (MFI R 
for VCAM-1: 0.85 and 0.65, respectively; MFI R for ICAM-1: 0.80 and 0.70, respectively) and 16 hours (MFI R for VCAM-1: 0.85 and 0.65 , respectively; MFI R for ICAM-1: 0.88 and 0.90 , respectively) TNF $\alpha$-stimulation. We also examined the functional consequences of $\mathrm{C}$ - and GD-HUVEC treatment with $C A$ in terms of U937 cell adhesion to cells. In agreement with data on TNFa-increased VCAM-1 expression, treatment of C- and GD-HUVEC with $C A$ for 16 hours produced a significant decrease in U937 cell adhesion $(p<0.001)$.

Conclusion: In conclusion, our in vitro study demonstrates a role for Centella Asiatica in mitigating the potentially dangerous effects on endothelium of chronic exposure to hyperglycemia in vivo.

Supported by: Peter Italia, Rome

\section{2}

VEGF induces von Willebrand Factor (VWF) secretion from endothelial cells via activation of PKC-delta

O.M. Lorenzi, M. Frieden, P. Villemin, M. Fournier, M. Foti, U.M. Vischer

PHYM, Centre Médical Universitaire, Geneva, Switzerland

Background and aims: VEGF plays a significant role in diabetic retinopathy and atherosclerosis, in part via the activation of protein kinase C (PKC). Von Willebrand Factor, secreted from endothelial cells, is a key mediator of primary haemostasis. It may play a role in retinal capillary occlusion and in the progression of atherosclerosis. In the present study we investigated the mechanisms of VEGF-induced VWF secretion from cultured endothelial cells. In particular, we sought to identify the PKC isoenzyme(s) involved in endothelial secretion.

Materials and methods: VWF secretion from human umbilical vein endothelial cells (HUVEC) in response to VEGF and histamine was measured by ELISA, and the effect of various inhibitors (including siRNA to inactivate $\mathrm{PKC}$ ) was tested. We also measured the effect of the secretion agonists on cytosolic free calcium $\left(\left[\mathrm{Ca}^{2+}\right]_{\mathrm{i}}\right.$ ) by single cell imaging using fura-2 fluorescence.

Results: VEGF and histamine rapidly induced VWF secretion, with a similar time course. Several PKC inhibitors (staurosporine, Ro31-8220, Myristoylated PKC peptide inhibitor and Go6983) blocked VEGF-but not histamine-induced VWF secretion in cultured HUVEC. PKC- $\alpha$ and novel PKCs $(\delta, \varepsilon$, and $\eta)$, but not PKC- $\beta$, were expressed in these cells. Both VEGF and histamine activated PKC- $\delta$, as judged from Western Blots using antibodies against the activated/phosphorylated isoenzyme. However, gene inactivation experiments using siRNA indicated that PKC- $\delta$ (but not PKC- $\alpha$ ) is involved in the regulation of VEGF- but not histamine-induced secretion. Both VEGF and histamine induce a rise in $\left[\mathrm{Ca}^{2+}\right]_{\mathrm{i}}$, but the response to VEGF is weaker and even absent in a significant subset of cells. Further, VEGF-induced secretion is largely preserved when the rise in $\left[\mathrm{Ca}^{2+}\right]_{\mathrm{i}}$ was blocked by BAPTA- AM.

Conclusion: Our data firmly establish the key role of PKC- $\delta$ in VEGF-induced endothelial VWF secretion. They also identify striking agonist specificities in signal-secretion coupling. Both VEGF and histamine activate phospholipase $\mathrm{C}$, leading to a rise in $\left[\mathrm{Ca}^{2+}\right]_{i}$ and $\mathrm{PKC}$ activation. However, histamine-induced VWF secretion is dependent on $\left[\mathrm{Ca}^{2+}\right]_{i}$ but not PKC, whereas VEGF-induced secretion is largely dependent on PKC- $\delta$ and only to a smaller extent on $\left[\mathrm{Ca}^{2+}\right]_{i}$. These discrepancies indicate that a third, VEGFspecific signalling intermediate - which remains to be identified - is required as a PKC- $\delta$ co-activator. Our data also argue against the notion of redundancy among PKC isoenzymes. PKC- $\delta$ is a possible target for agonist-specific inhibition of endothelial VWF secretion.

Supported by: Swiss National Science Foundation, and from the Carlos and Elsie de Reuter Foundation

\section{3}

Ursodeoxycholic acid inhibits endothelial cell plasminogen activator inhibitor-1 expression induced by high glucose depend on transforming growth factor signalling

T. Yokoi ${ }^{1}$, Y. Wada ${ }^{2}$, H. Koshiyama ${ }^{2}$, H. Masaki ${ }^{1}$, H. Takahashi ${ }^{1}$

${ }^{1}$ Department of Clinical Sciences and Laboratory Medicine, Kansai Medical University, Moriguchi, Osaka, ${ }^{2}$ Center for Diabetes and Endocrinology, The Tazuke Kofukai Medical Research Institute Kitano Hospital, Osaka, Japan

Background and aims: Plasminogen activator inhibitor-1 (PAI-1) is an independent risk factor for ischemic cardiovascular events. PAI-1 levels are elevated in the plasma of diabetes mellitus (DM) patients and this elevation correlates with vascular complications of DM. Although PAI-1 expression is upregurated depend on transforming growth factor (TGF) signaling, the molecular mechanism to prevent the PAI-1 elevation in DM remain unclear. Here, we show that Ursodeoxycholic acid (UDCA), known as a drug for chronic liver disease, inhibit endothelial cell PAI-1 expression induced by high glucose depend on TGF signaling.

Materials and methods: Cell culture and reagents: Human umbilical vein endothelial cells (HUVECs) were purchased from Sanko Junyaku (Tokyo, Japan) and cultured in endothelial basal medium-2 (Sanko Junyaku) supplemented with $2 \%$ FCS. Western blot analysis: Protein extract $(10 \mu \mathrm{g})$ was fractionated on SDS-PAGE gels and transferred to a polyvinylidine difluoride membrane (Immobilon-P; Millipore). The membrane was blocked with T-phosphate-buffered saline (1x PBS, $0.3 \%$ Tween 20) containing 3\% dry milk and incubated with primary antibody (anti-PAI-1, anti- -tubulin, and anti-TGF-beta1, anti-phosphorylated endothelial nitric oxide synthase at Ser1177) overnight at $4^{\circ} \mathrm{C}$. The immune complexes were detected by chemiluminescence methods (ECL; Amersham International). Measurement of PAI-1 levels by enzyme-linked immunosorbent assay: Total secreted PAI-1 and urokinase type plasminogen activator antigen levels (free plus complex forms) were measured (in duplicate) in conditioned culture media and plasma by using a commercial PAI-1 activity assay kit (CHEMICON).

Result: Western blot analysis showed that high glucose (glucose at 5.5 to $33 \mathrm{mM}$ ) increased the levels of PAI-1 expression in endothelial cells (ECs) in a time- and dose-dependent manner. High glucose also increased PAI-1 concentration of its supernatant, whereas mannitol as an osmotic negative control had no effect. Interestingly, first cultured with UDCA (at 100 to 1000 microM), high glucose did not increased PAI-1 expression in ECs and concentration of its supernatant. We next examine the mechanism that UDCA suppress the PAI-1 expression induced by high glucose in ECs. Although high glucose decreased the levels of eNOS expression in ECs, first cultured with UDCA high glucose did not decrease the levels of eNOS. N-nitro-Larginine-mythel-ester (L-NAME), a nitric oxide synthase (NOS) inhibitor, suppressed the UDCA-induced eNOS expression, but it did not antagonize the inhibitory effects of ursodeoxycholic acid on PAI-1 reduction. It has only partially effect on PAI-1 reduction. The conjugates of UDCA have no effect of PAI-1 reduction. We next paid attention to TGF expression in ECs. Although UDCA suppressed TGF-beta1 expression induced by high glucose, L-NAME did not inhibit this suppressive effect. SiRNA targeting of TGF-betal markedly suppressed PAI-1 upregulation induced by high glucose.

Conclusion: These data suggest that UDCA inhibits PAI-1 expression in ECs induced by high glucose under the pathway of TGF signaling.

\section{4}

Elevated long pentraxin 3 relates with the endothelial apoptosis due to glucose fluctuation

K. Konno, G. Fukuda, M. Kumekawa, A. Kumakura, R. Ito, T. Takahashi, T. Miwa, M. Odawara

The 3rd Department of Internal Medicine, Tokyo Medical University, Japan

Background and aims: Progression of atherosclerosis is a critical disorder for the patient with diabetes mellitus, hypertension or obesity. Continuous high concentration of glucose leads to systematic atherogenic changes. Recent studies revealed that glucose fluctuation also contributes to develop the atherosclerosis in macro vasculature. The long pentraxin 3 (PTX3) originally produced by endothelial cells is increased in patients with the acute coronary syndrome and independent from liver function, but its involvement in diabetic atherogenic changes is unclear. In this study, we investigated the association between PTX3 and fluctuated glucose concentration concerning apoptosis in endothelial cells.

Materials and methods: Human umbilical vein endothelial cells (HUVEC) were seeded on $6 \mathrm{~cm}$ Petri dishes or glass slides set in Petri dishes for imaging. Culture medium was changed every 24 hours and continued for 14 days. Experiments were classified into 4 groups including (1) a control group with $5 \mathrm{mM}$ glucose (L group), (2) an experimental group with $5 \mathrm{mM}$ glucose and $20 \mathrm{mM}$ mannitol (M group), (3) an experimental group with $25 \mathrm{mM}$ glucose (H group), and (4) an experimental group with $5 \mathrm{mM}$ or $25 \mathrm{mM}$ glucose (fluctuated glucose concentration group; LH group). Total RNA was isolated and real-time PCR (Bio-Rad, USA) was carried out for 18S, PTX3, Bcl-2 mRNA quantification. Each mRNA level was compared by arbitrary ratio to $18 \mathrm{~S}$ ribosomal RNA. The protein level of the PTX3 in culture medium was measured by ELISA-based assay (Perseus Proteomics Inc. Japan). Cell apoptosis was detected with transferase mediated dUTP biotin nick end labeling (TUNEL) method for the glass slides by TUNEL assay kit (Promega, 
USA). Images were observed by confocal microscopy (Zeiss, Germany). Caspase 3/7 activity in treated HUVEC was measured by use of Caspase-Glo 3/7 Assay kit (Promega). One-way ANOVA and post hoc test were used for the statistical analysis.

Results: The level of PTX3 mRNA in HUVEC was significantly increased in the $\mathrm{H}$ (2.4 folds) and the LH group (2.7 folds), which glucose concentration was fluctuated every 24 hours, as compared to the L group. There was not significant difference of the PTX3 mRNA level between the $\mathrm{H}$ and the LH group. In contrast, the level of Bcl-2 mRNA showed a decreasing tendency in the $\mathrm{H}$ and the LH group as compared to the L group. The PTX3 protein in the conditioned medium was significantly increased in the $\mathrm{H}$ and the LH group as compared to the L group. There was no significant difference of PTX3 level between the $\mathrm{H}$ and the $\mathrm{LH}$ group. Observing the apoptosis, the $\mathrm{H}$ group cells show a 1.2 folds significant increase of caspase $3 / 7$ activity as compared to the $\mathrm{L}$ or the $\mathrm{M}$ group cells. The LH group cells show a 1.6 folds significant increase of caspase 3/7 activity as compared to the $\mathrm{L}$ or the $\mathrm{M}$ group cells. TUNEL assay showed that glucose fluctuation in the LH group induced high apoptosis of HUVEC than continuous normal concentration of glucose in the L group. The apoptosis rate was increased in the LH group but there was no significant difference between the $\mathrm{H}$ group and the $\mathrm{LH}$ group.

Conclusion: These data suggest that glucose fluctuation may be related with PTX3 expression which contributes to early diagnosis of atherogenic disorder in diabetes mellitus. PTX3 would be a useful marker to help clarifying the endothelial damage.

\section{5}

Oxidised LDL decreases the expression of scavenger receptor class BI in vascular endothelial cells

H. Imachi, K. Murao, T. Muraoka, M. Fujiwara, R.A.M. Ahmed, J. Li, T. Ishida

Division of Endocrinology and Metabolism, Department of Internal Medicine, Kagawa University, Kita-gun, Kagawa, Japan

Background and aims: HDL mediates a normal physiological process called reverse cholesterol transport. In this process, a scavenger receptor of the class BI (SR-BI) facilitates the cellular uptake of cholesterol from HDL. Our previous report shows that human homologue of SR-BI/CD36 and LIMPII analogous-1 (hSR-BI/CLA-1), like mouse SR-BI functions as a receptor for HDL. In endothelial cells, HDL activates endothelial nitric-oxide synthase (eNOS) and produces nitric oxide via hSR-BI. On the other hand, oxidized LDL (OxLDL), a atherogenic lipoprotein, suppresses eNOS expression on endothelial cells and associates with development of atherosclerosis. In this present study, we have examined the role of OxLDL on hSR-BI expression in human endothelial cells.

Materials and methods: Total RNA and protein were extracted from human umbilical vein endothelial cells (HUVECs). HSR-BI expression was tested by real-time PCR and western blot analysis. HSR-BI promoter activity was detected using the reporter construct contained the hSR-BI gene sequences spanning the region from -1200 to +2 , as determined from the published sequences.

Results: The results of our studies showed that hSR-BI protein expression in HUVECs was suppressed by exposure to OxLDL in a dose-dependent manner. OxLDL decreased endogenous mRNA expression of hSR-BI. Transcriptional activity of hSR-BI promoter in HUVECs paralleled endogenous mRNA expression, and this activity was dependent on OxLDL. Using several pharmacological inhibitors for the signal transduction pathways, the inhibitory effect of OxLDL on hSR-BI promoter activity was abrogated by a specific inhibitor of p38 mitogen-activated protein (MAP) kinase. A dominant negative p38 MAP kinase also abrogated the inhibitory effect of OxLDL on hSR$\mathrm{BI}$ promoter. Co-transfection with the transcriptional factor, liver $\mathrm{X}$ receptor (LXR), increased hSR-BI promoter activity. However, OxLDL decreased LXR expression in HUVECs.

Conclusion: In conclusion, p38 MAP kinase pathways participate in OxLDLsuppression of SR-BI expression and LXR may affect this pathway. These results suggest one of the mechanisms of atherogenesis via OxLDL.

Supported by: Alumni Association of Faculty of Medicine, Kagawa University NO.2

\section{6}

Inhibitory effect of adiponectin on palmitate-induced apoptosis: crosstalk between cAMP and AMPK pathway

S.-Y. Park ${ }^{1,2}$, J.-E. Kim ${ }^{3}$, Y.-W. Kim³ ${ }^{3}$ J.-Y. Kim ${ }^{3}$, J.-M. Dan ${ }^{4}$, K.-C. Won ${ }^{5}$, Y.-B. Ahn ${ }^{6}$ K.-H. Song ${ }^{6}$

${ }^{1}$ Physiology, College of Medicine, Yeungnam University, Daegu, ${ }^{2}$ Agingassociated vascular research center, College of Medicine, Yeungnam

University, Daegu, ${ }^{3}$ Physiology, College of Medicine, Yeungnam Uni, Daegu, ${ }^{4}$ Orthopedics, College of Medicine, Pochun Cha University, Gumi, ${ }^{5}$ Internal Medicine, College of Medicine, Yeungnam University, Daegu, ${ }^{6}$ Internal Medicine, The Catholic University of Seoul, Seoul, Republic of Korea

Background and aims: Adiponectin has anti-inflammatory and anti-apoptotic effects in endothelial cells. However, it is unclear whether adiponectin prevents palmitate-induced apoptosis in endothelial cells. In the present study, we elucidated the effects of adiponectin on palmitate-induced apoptosis in human umbilical vein endothelial cells (HUVEC).

Materials and methods: HUVEC isolated from human umbilical cord vein were cultured in M199 and the passage of 3 to 5 were used in this experiment. Apoptosis was measured by annexin V staining and caspase- 3 activity and reactive oxygen species (ROS) was measured by live microscopy and flow cytometry using $\mathrm{H}_{2} \mathrm{DCFDA}$. Western blotting was used for protein expression. Results: Palmitate induced apoptosis and increased ROS generation. Antioxidant $\mathrm{N}$-acetyl cysteine inhibited ROS generation and apoptosis in HUVEC. Treatment of $5 \mu \mathrm{g} / \mathrm{ml}$ globular adiponectin inhibited palmitate-induced apoptosis, which was followed by reduced ROS generation. Adiponectin increased phosphorylated AMP activated protein kinase (AMPK), pAKT, and intracellular cAMP level in HUVEC. Treatment of AMPK inhibitor compound C (40 $\mu \mathrm{M})$ prevented inhibitory effects of adiponecin on palmitate-induced apoptosis, whereas AMPK activator AICAR $(1 \mathrm{mM})$ inhibited palmitate-induced apoptosis. PI-3 kinase inhibitor LY294002 $(50 \mu \mathrm{M})$ also prevented inhibitory effects of adiponecin on apoptosis. PKA inhibitor H-89 $(10 \mu \mathrm{M})$ and cAMP inhibitor SQ22536 $(50 \mu \mathrm{M})$ reversed inhibitory effects of adiponecin, whereas cAMP activator cholera toxin $(100 \mathrm{ng} / \mathrm{ml})$ and forskolin $(20 \mu \mathrm{M})$ inhibited palmitate-induced apoptosis. Suppressed palmitate-induced ROS generation by adiponectin was also reversed by compound C, LY294002, and H-89. Cotreatment of forskolin with compound C or forskolin with LY294002 did not inhibit palmitate-induced apoptosis, whereas co-treatment of AICAR with SQ22536 or AICAR with H-89 inhibited palmitate-induced apoptosis. Forskolin increased pAMPK level in HUVEC.

Conclusion: These results suggest that inhibitory effect of adiponectin on palmitate-induced apoptosis is mediated by the activation of AMPK and cAMP and cross-talk between cAMP and AMPK pathway may involve. Supported by: Korea Science and Engineering Foundation

\section{7}

Effects of pravastatin on akt-survival pathway in human pancreatic islet endothelial cells in hyperglycaemic condition

E. Favaro, I. Miceli, I. Ossola, I. Masi, E. Dellavalle, L. Semperboni, P. Cavallo Perin, M.M. Zanone

Department of Internal Medicine, University of Turin, Italy

Background and aims: The 3-hydroxy-3-methylglutaryl Coenzyme A (HMG-CoA) inhibitors, statins, are known to modulate the phosphatidylinositol 3-kinase (PI3K)/Akt pathways and improve vascular function, independently from their lipid-lowering effects. In endothelial cells, statins rapidly promote the activation of PI3K/Akt pathways, mediating cell survival, NO synthesis and migration. In the present study, we aimed to analyse whether the HMG-CoA inhibitor pravastatin may reverse the sensitivity to apoptosis of human islet microvascular endothelial cells (MECs) in high glucose conditions or after incubation with oxidatively modified LDL (oxLDL), and whether this may depend on activation of the PI3K/Akt pathway.

Materials and methods: Purified islet MECs were cultured in $28 \mathrm{mM}$ glucose, and, in time course experiments, apoptosis assessed by enzyme immunoassay measuring DNA fragmentation, phosphorylated Akt (p-Akt) and Akt detected by WB analysis and by ELISA assay. Cells were incubated with oxLDL or treated with increasing doses of pravastatin. Three different conditions were assayed: pravastatin was given daily, or started after four days of high glucose culture, or just overnight before cell collection.

Results: In high glucose culture, apoptosis progressively increased, from $7 \%$ after 2 days to $79 \%$ after 30 days ( $<0.05$ compared to normal glucose), with early increase of caspase- 3 activity. Apoptosis was accompanied by re- 
duced p-Akt/Akt ratio (mean ratio $1.9 \pm 0.6$ for normal glucose, $0.8 \pm 0.3$ for high glucose cultures, $\mathrm{p}<0.05$ ). The $\mathrm{p}$-Akt/Akt ratio exhibited a trend of progressive decrease with the duration of high glucose culture. Similarly, oxLDL induced dephosphorylation of Akt. Exposure to pravastatin significantly inhibited apoptosis of islet MECs chronically exposed to high glucose, and apoptosis induced by oxLDL $(\mathrm{p}<0.05)$. The effect of pravastin was evident at the daily dose of $1 \mathrm{micromol} / \mathrm{L}$, with lower concentration having not significant effect. The reduced apoptosis detected with pravastatin treatment was accompanied by prevention of the reduction of p-Akt/Akt ratio. This effect increased with treatment duration, with an increase of the p-Akt/Akt ratio of $12 \%$ for 1 day pravastatin treatment, $70 \%$ increase for 3 day treatment, and $130 \%$ increase for daily treatment when the cells were cultured for 8 days in high glucose (mean ratio $2.35 \pm 0.12$ for normal and $1.17 \pm 0.2$ for high glucose culture respectively, and $2.1 \pm 0.7$ for all statin treatment regimes, $\mathrm{p}<$ $0.05)$. These results were further confirmed by an Akt ELISA assay, detecting a similar trend. The protective effect of pravastatin on Akt phosphorylation was reduced by overnight treatment of islet MECs with pharmacological inhibitors of PI3K, wortmannin and LY294002, suggesting that this effect was at least in part dependent on the activation of PI3K.

Conclusion: Chronic hyperglycemia reduced islet MECs survival inhibiting the serine-threonine kinase Akt pathway, possibly contributing to the progressive reduction of beta cell function and mass characterising the natural history of the diabetic condition. Pravastatin by affecting this pathway represents a potential tool to improve islet vascularisation and, indirectly, islet function, being pancreatic islet microendothelium and beta cells linked in an interdependent physical and functional relationship.

\section{PS 128 Glycotoxic metabolites}

\section{8}

High serum levels of methylglyoxal-derived hydroimidazolone predict increased cardiovascular mortality in non-diabetic women, but not in diabetic subjects

B.K. Kilhovd ${ }^{1}$, A. Juutilainen ${ }^{2}$, S. Lehto ${ }^{2}$, T. Ronnemaa ${ }^{3}$, P. Torjesen ${ }^{1}$, K. Hanssen ${ }^{1}$, M. Laakso 2

${ }^{1}$ Endocrinology, Aker University Hospital, Oslo, Norway, ${ }^{2}$ Medicine, Kuopio University Hospital, Finland, ${ }^{3}$ Medicine, Turku University Hospital, Finland

Background: Advanced glycation end products (AGEs), glycation and glycoxidation modification products of proteins and lipids, have been linked to premature atherosclerosis in patients with diabetes, and in non-diabetic subjects. Biologically reactive dicarbonyl methylglyoxal (MG) is the major intracellular substrate in the formation of AGEs. The arginine-modification $\mathrm{N} \delta$-(5-hydro-5-methyl-4-imidazolon-2-yl)-ornithine (MG-H1) represents the most abundant protein modification by glycation in diabetes, and occurs both in extra- and intra-cellular proteins. We have previously reported that high levels of serum AGEs, measured with a polyclonal anti-AGE antibody, predict increased coronary heart disease mortality in diabetic and non-diabetic women.

Aims: To investigate the association of the levels of MG-H1 type of AGEmodified proteins (MG-H1-AGE) with cardiovascular disease (CVD) mortality in an 18 year follow-up study in Finnish diabetic and non-diabetic subjects.

Methods: The study design was a nested case-control study. Serum MG-H1AGE levels in samples drawn at baseline were measured with a DELFIA type immunoassay in 220 diabetic subjects (108 men and 112 women) and 61 non-diabetic subjects (48 men and 13 women) who died from CVD during the follow-up, and age- and gender-matched 157 diabetic subjects ( 85 men and 72 women) and 159 non-diabetic subjects (122 men and 37 women) who did not die from CVD.

Results: In type 2 diabetic subjects serum MG-H1-AGE levels were similar in subjects who died from CVD and in subjects who did not, $32.6(24.6 ; 42.1)$ (median and the interquartile range) vs. $31.3(22.5 ; 40.7) \mathrm{U} / \mathrm{ml}(\mathrm{p}=0.281)$. In non-diabetic subjects serum MG-H1 levels were significantly higher in subjects who died from CVD than in subjects who did not, $35.4(28.1 ; 44.7)$ vs. $31.3(24.2 ; 38.6) \mathrm{U} / \mathrm{ml}(\mathrm{p}=0.025)$. Corresponding MG-H1 levels were 41.2 $(35.6 ; 58.7)$ vs. $31.1(26.7 ; 35.7) \mathrm{U} / \mathrm{ml}, \mathrm{p}=0.003$ in women, and $34.4(26.3$; $41.2)$ vs. $32.0(22.8 ; 40.3) \mathrm{U} / \mathrm{ml}, \mathrm{p}=0.270$ in men. Multivariate logistic regression analysis after the adjustment for confounding factors showed a significant association of serum levels of MG-H1-AGE with CVD mortality in nondiabetic women $(\mathrm{p}=0.018)$, but not in non-diabetic men.

Conclusion: High baseline serum levels of MG-H1 type of AGE-modified proteins were associated with CVD mortality during an 18 year follow-up study in non-diabetic women, but not in non-diabetic men or in diabetic subjects.

Supported by: Aker University Hospital

\section{9}

Methylglyoxal modifies predominantly heat shock protein 27 in human diabetic hearts

T. Gawlowski ${ }^{1}$, B. Stratmann ${ }^{1}$, I. Stork ${ }^{1}$, A. Brodehl², R. Körfer², H. Milting², D. Tschoepe ${ }^{1}$

${ }^{1}$ Diabeteszentrum, Ruhr-Universität Bochum, Herz- und Diabeteszentrum NRW, Bad Oeynhausen, Germany, ${ }^{2}$ E.\&H. Klessmann-Institut für Kardiovaskuläre Forschung und Entwicklung, Ruhr-Universität Bochum, Herz- und Diabeteszentrum NRW, Bad Oeynhausen, Germany

Background and aims: The existence of "diabetic cardiomyopathy" is controversly discussed although it is well know that patients with diabetes mellitus (DM) have an increased risk of developing heart failure. However, the definition of diabetic cardiomyopathy suggests that structural and functional abnormalities of the myocardium are independent of other risk factors, such as hypertension and coronary artery disease. This clearly indicates the need for studies specifically in diabetic patients with cardiomyopathy to better understand the mechanisms which initiate and lead to the progression of diabetic cardiomyopathy. In patients with DM hyperglycemia plays an important role in the pathogenesis of diabetic complications including accumulation of methylglyoxal (MG), a highly reactive a-dicarbonyl metabolite of 
glucose degradation pathways. MG reacts with arginine residues of proteins to form the posttranslational modification argpyrimidine. However, little is know on the contribution of MG and argpyrimidine in the development of a cardiomyopathy.

Materials and methods: Left ventricular myocardial samples from explanted hearts of patients with cardiomyopathy with $(\mathrm{CM} / \mathrm{DM} ; \mathrm{n}=8)$ or without $(\mathrm{CM} ; \mathrm{n}=8) \mathrm{DM}$ and from non-failing hearts $(\mathrm{NF} ; \mathrm{n}=6)$ were investigated for the MG-modification argpyrimidine by western blot and immunohistology. Moreover, rat cardiomyoblast cell line H9c2 was treated with glucose or MG for the investigation of the modification argpyrimidine.

Results: HSP27 reveals to be the most argpyrimidine containing protein in cardiac tissue. It is highly expressed and co-localized with plakophilin-2 in intercalated discs of human myocardium. In relation to the total amount of HSP27 argpyrimidine modifications in diabetic hearts were significantly increased 3-fold $(\mathrm{p}<0.05)$ compared to CM and 9-fold $(\mathrm{p}<0.05)$ compared to NF. Moreover, we observed a 3.4-fold increase of phosphorylation in CM/ DM compared to NF and a 1.8-fold increase compared to CM. Experiments using the rat cardiomyoblast cell line H9c2 show that hyperglycemia $(25 \mathrm{mM}$ glucose) increases argpyrimidine content $(8.8$-fold vs $5 \mathrm{mM}$ glucose; $\mathrm{p}<0.01)$ and phosphorylation (3.8-fold vs $5 \mathrm{mM}$ glucose; $\mathrm{p}<0.01$ ) of HSP27. Treatment of $\mathrm{H} 9 \mathrm{c} 2$ cells with high amounts of MG $[50-400 \mu \mathrm{M}]$ revealed that argpyrimidine modification was not restricted to HSP27 but were also detectable in additional high molecular weight proteins.

Conclusion: These observations show that altered cardiac metabolism associated with DM leads to covalent modifications of human myocardial HSP27. However, up to now the functional and pathophysiological consequences of HSP27 modification remain to be elucidated.

Supported by: Ruhr-Universität Bochum and of the Erich \& Hanna KlessmannStiftung, Guetersloh, Germany

\section{0}

Elevated intracellular levels of methylglyoxal impair endothelium dependent vasodilatation in rat mesenteric arteries by an oxidative stress dependent pathway

O. Brouwers ${ }^{1}$, P.M.G. Niessen ${ }^{1}$, J.G.R. De $\mathrm{Mey}^{2}$, G.R.M. Haenen ${ }^{2}$, T. Miyata ${ }^{3}$, M. Brownlee ${ }^{4}$, C.D.A. Stehouwer ${ }^{1}$, C.G. Schalkwijk ${ }^{1}$

${ }^{1}$ Internal Medicine, Maastricht University, Netherlands, ${ }^{2}$ Pharmacology and Toxicology, Maastricht University, Netherlands, ${ }^{3}$ Centre for Translational and Advanced Research, Tohoku University, Japan, ${ }^{4}$ Medicine and Pathology, Albert Einstein College of Medicine, New York, United States

Background and aims: Increased formation of the glycolysis-derived reactive dicarbonyl compound methylglyoxal (MGO) and MGO-derived advanced glycation endproducts (AGEs) is thought to be implicated in endothelial dysfunction and, subsequently, in the development of diabetic vascular complications. A common feature of endothelial dysfunction is an impaired nitric-oxide (NO)-dependent vasodilatation. We now investigated the effect of MGO on vasoreactivity, the effect of glyoxalase-I (GLO-I), i.e. the enzyme detoxifying MGO, and the direct effect of free MGO and MGO-modified human albumin on NO bioavailability.

Materials and methods: Isolated mesenteric arteries of healthy and diabetic wild type and transgenic GLO-I rats were mounted in a myograph. The effect of MGO on NO-dependent vasodilatation was tested with acetylcholine (Ach; $0.01-10 \mu \mathrm{M})$ in the presence of indometacin $(10 \mu \mathrm{M})$ during contraction with potassium $(65 \mathrm{mM})$. GLO-I transgenic animals are developed by overexpression of the full-length human GLO-I cDNA under control a $\beta$ actin promotor system. Rats were made diabetes by a $65-\mathrm{mg} / \mathrm{kg}$ intra-venous streptozotocin injection. The reaction of MGO and MGO-modified human albumin with $\mathrm{NO}$ gas in deoxygenated water was measured with an Iso-NO electrode.

Results: Treatment of healthy mesenteric arteries with $0,0.1,0.33$ and $1.0 \mathrm{mM}$ MGO significantly $(\mathrm{p}<0.05)$ reduced the acetylcholine-induced vasodilation, as indicated by decreased potency $\left(\log \mathrm{EC}_{50}\right.$ was $-6.33,-6.21,-6.17$ and -5.94 $\mathrm{M}$, respectively) and efficacy ( $\mathrm{E}_{\max }$ was $71 ; 58 ; 51$ and 49 percentage dilatation, respectively). This effect was endothelium dependent as indicated by unaltered relaxing responses to the NO donor sodium nitroprusside. Impairment of dilatation by MGO was not observed in mesenteric arteries of GLO-I transgenic rats compared with their wild type littermates, indicating an intracellular MGO effect. In addition, the impaired dilatation of mesenteric arteries of diabetic rats was reduced by GLO-I overexpression. A pre-incubation of healthy mesenteric arteries with MGO increased staining of the major MGO-adduct 5-hydro-5-methylimidazolone (MG-H1) in endothelial cells and adventitia accompanied by an increase in the oxidative stress marker ni- tro-tyrosine. The MGO-induced impaired vasoreactivity was normalized by a pre-incubation of the vessels with the antioxidant $\mathrm{N}$-Acetyl Cysteine. Free MGO, but not MGO-modified albumin, reduced the half-life of NO, albeit with a very low potency (constant rate of $1.02 \log \mathrm{M} / \mathrm{sec}$ ).

Conclusion: We found that MGO inhibits endothelium-dependent NO mediated vasodilatation in rat mesenteric arteries. The MGO-induced impairment of NO-mediated dilatation depends on an intracellular effect and is probably due to an increase in oxidative stress and not to a direct quenching of NO. Diabetes induced impaired dilatation can be improved by overexpression of GLO-I. These data implicate that MGO leads to impaired endothelial function and that GLO-I may prevent this.

Supported by: Dutch Diabetes Research Foundation

\section{1}

Prevention of dicarbonyl stress induced by hyperglycaemia in microvascular endoethelial cells by sulforaphane P.J. Thornalley ${ }^{1,2}$, Q. Qian ${ }^{2}$, N. Rabbani ${ }^{1,2}$

${ }^{1}$ Clinical Sciences Research Institute, University of Warwick, Coventry, ${ }^{2}$ Department of Biological Sciences, University of Essex, Colchester, United Kingdom

Background and aims: Increased formation of dicarbonyl metabolites, glyoxal, methylglyoxal and 3-deoxyglucosone (3-DG), by microvascular endothelial cells during hyperglycaemia leads to increased formation of advanced glycation endproduct (AGE) residues in cellular and extracellular matrix proteins. Dicarbonyl glycation of the extracellular matrix is linked to endothelial cell detachment, anoikis and other dysfunction - correlating with increased circulating endothelial cells and risk of vascular disease in diabetes. A strategy to prevent increased levels of dicarbonyl metabolites in hyperglycaemia is to induce the expression of transketolase (TK) and other antioxidant response element (ARE) linked gene expression by activation of transcription factor of NF-E2-related factor-2 (nrf2) with small molecular activators such as the dietary bioactive compound sulforaphane (SFN). The aim of this study is to assess the involvement of nrf2 and TK in the countering of hyperglycaemia-induced accumulation of dicarbonyl metabolites by endothelial cells with SFN.

Materials and methods: Human microvascular HMEC-1 endothelial cells were cultured in MCDB-131 medium with $10 \%$ serum, $10 \mathrm{mM} \mathrm{L}$-glutamate, $10 \mathrm{ng} / \mathrm{ml}$ epidermal growth factor, and $1 \mu \mathrm{g} / \mathrm{ml}$ hydrocortisone. Incubations also contained low glucose $(5 \mathrm{mM})$ or high glucose $(30 \mathrm{mM})$ in the absence and presence of $4 \mu \mathrm{M}$ SFN. Knockdown of nrf2 and TK was achieved with nrf2 siRNA and TK siRNA - confirmed by PCR and real time RT-PCR. The concentration of dicarbonyls, glyoxal, methylglyoxal and 3-DG, in the culture medium was determined by derivatisation with 1,2-diaminobenzene and quantitation of the adducts by stable isotopic dilution analysis with liquid chromatography and tandem mass spectrometric detection. Data are mean $\pm \mathrm{SD}(\mathrm{n}=3)$.

Results: Incubation of HMEC-1 endothelial cells with high glucose for $24 \mathrm{~h}$ increased the concentration of dicarbonyls in the culture medium: glyoxal concentration was increased 3 -fold $(123 \pm 18 \mathrm{nM}$ versus $46 \pm 14 \mathrm{nM} ; \mathrm{P}<0.01)$, methylglyoxal was increased 2 -fold $(96 \pm 4 \mathrm{nM}$ versus $53 \pm 5 \mathrm{nM} ; \mathrm{P}<0.001)$, and 3 -DG was increased 4 -fold $(369 \pm 38 \mathrm{nM}$ versus $99 \pm 7 \mathrm{nM}: \mathrm{P}<0.001)$. Incubation with SFN $(4 \mu \mathrm{M})$ reversed the increases induced by high glucose concentration completely for glyoxal and methylglyoxal $(\mathrm{P}<0.01$ and $\mathrm{P}<0.001$, with respect to high glucose control, respectively). The high glucose concentration-induced increase in 3-DG concentration was only partially reversed by SFN: $3-D G 223 \pm 16 \mathrm{nM}(-54 \% ; \mathrm{P}<0.001$ and $\mathrm{P}<0.01$ with respect to low and high glucose concentration controls). Knockdown of nrf2 and TK abolished the decrease of dicarbonyl concentrations by SFN in incubations with high glucose; it also increased methylglyoxal concentration in incubations with low glucose concentration.

Conclusion: This shows that high glucose concentrations increase the synthesis and secretion of dicarbonyl glycating agents by microvascular endothelial cells and these increases may be reversed totally for glyoxal and methylglyoxal and partially for 3-DG by activation of nrf2 with SFN. The effects of SFN were mediated by nrf2-linked increased expression of TK. Both nrf2 and TK are, therefore, influential regulators of dicarbonyl metabolism in hyperglycaemia. Small molecule activators of nrf2 and inducers of ARE-linked gene expression such as SFN may prevent endothelial cell dysfunction in hyperglycaemia.

Supported by: JDRF 


\section{2}

Glyceraldehyde-derived advanced glycation end products (ages): a novel biomarker of postprandial hyperglycaemia in diabetic rats

Y. Jinnouchi ${ }^{1}$, S.-I. Yamagishi ${ }^{1}$, T. Matsui ${ }^{1}$, K. Nakamura ${ }^{1}$, T. Imaizumi ${ }^{1}$, M. Takeuchi' ${ }^{2}$ K. Miura ${ }^{3}$, T. Mine ${ }^{3}$, Y. Kitahara ${ }^{3}$

${ }^{1}$ Department of Internal Medicine, Kurume University School of Medicine, Kurume, ${ }^{2}$ Department of Pathophysiological Science, Hokuriku University, Kanazawa, ${ }^{3}$ Pharmaceutical Research Laboratories, Ajinomoto, Kawasaki, Japan

Background and aims: There is accumulating evidence that postprandial hyperglycemia is an independent risk factor for cardiovascular disease (CVD) in diabetic patients. Moreover, postprandial hyperglycemia is associated with endothelial dysfunction and increased intima-media thickness as well as a higher prevalence of atherosclerotic plaques of the common carotid arteries. These findings suggest that postprandial hyperglycemia is a therapeutic target for preventing CVD in diabetes. However, there is no convenient biomarker that could reflect cumulative postprandial hyperglycemia in diabetes. We have recently found that glyceraldehyde can rapidly react with amino groups of proteins to form glyceraldehyde-derived advanced glycation end products (AGEs), which evoke vascular inflammation and endothelial dysfunction, thereby being implicated in accelerated atherosclerosis in diabetes. In this study, we examined whether glyceraldehyde-derived AGEs were a biomarker that could reflect cumulative postprandial hyperglycemia in a type 2 diabetic animal model, Goto-Kakizaki (GK) rats fed twice a day.

Materials and methods: Male GK rats at 6 weeks of age were trained to consume their chow within 1 hour that was provided twice a day during the dark period. After 2 weeks, the GK rats were divided into 2 groups; either the vehicle (GK/VEH) or $50 \mathrm{mg} / \mathrm{kg}$ of nateglinide (GK/NAT) was administered twice daily just before each meal for 6 weeks. At the end of the treatments, daily blood glucose was monitored, and HbAlc, glucose-derived AGEs and glyceraldehyde-derived AGEs were measured.

Results: There was no significant difference of body weight and food intake between the two groups. The daily blood glucose profile revealed that postprandial blood glucose levels were significantly higher in GK/VEH rats than those in GK/NAT rats (Fig.1). HbA1c, glucose-derived AGEs or glyceraldehyde-derived AGEs levels in GK/VEH at the end of the experiments were not different from those at baseline. Further, although $\mathrm{HbAlc}$ or glucose-derived AGEs levels were not different between GK/VEH and GK/NAT (HbAlc; 3.93+/-0.07 \% vs. $3.93+/-0.16 \%$, glucose-derived AGEs; $11.07+/-5.55 \mathrm{U} / \mathrm{ml}$ vs. $7.67+/-4.45 \mathrm{U} / \mathrm{ml}$ ), glyceraldehyde-derived AGEs levels were significantly reduced in GK/NAT rats, compared with GK/VEH rats $(1.93+/-0.60 \mathrm{U} / \mathrm{ml}$ vs. $5.70+/-1.47 \mathrm{U} / \mathrm{ml}, \mathrm{p}<0.05)$. Conclusion: The present study demonstrated for the first time that glyceraldehyde-derived AGEs, but not HbAlc or glucose-derived AGEs, were a biomarker that could reflect cumulative postprandial hyperglycemia in diabetic rats. Glyceraldehyde-derived AGEs may a novel therapeutic target for preventing CVD in diabetes.

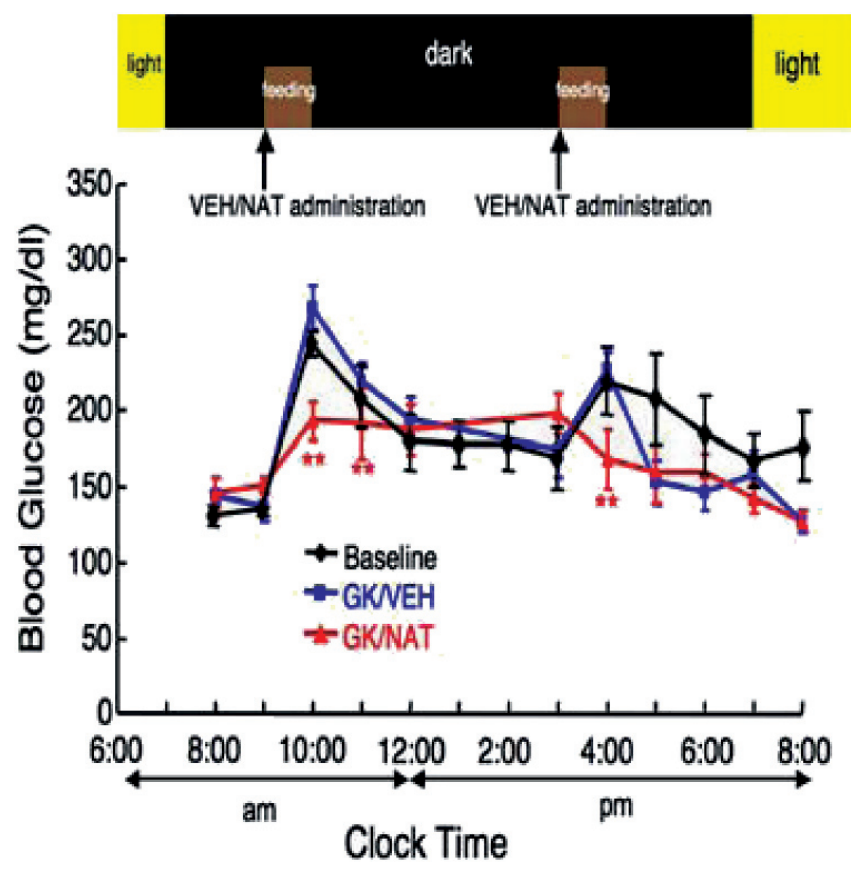

\section{3}

Increased protein damage by advanced glycation endproducts and oxidation adducts in low density lipoprotein of type 2 diabetic patients N. Rabbani, P.J. Thornalley Clinical Sciences Research Institute, University of Warwick, Coventry, United Kingdom.

Background and aims: Formation of advanced glycation endproducts (AGEs) and oxidation and nitration on apolipoprotein B100 - the major protein of low density lipoprotein (LDL) - have been implicated in increase of the atherogenic risk of LDL in type 2 diabetes. The gold standard method of quantifying markers of protein damage is stable isotope dilution analysis with liquid chromatography and tandem mass spectrometric analysis (LCMS/MS). The aim of this study was to quantify the content of AGEs, oxidation and nitration markers in apolipoprotein B100 of type 2 diabetic patients and normal healthy control subjects by LC-MS/MS.

Materials and methods: Normal healthy human subjects $(n=7)$ and subjects with type 2 diabetes $(\mathrm{n}=25)$ were recruited for this study. Glycated haemoglobin $\mathrm{HbA}_{1}$ of the diabetic patients was $8.5 \pm 1.6 \%$. LDL was isolated from venous plasma, stabilised with aprotinin and collected in the fasted state, by density gradient ultracentrifugation using Iodixanol. Purified LDL was washed with argon-purged water by ultrafiltration over a $100 \mathrm{kDa}$ microspin filter. The content of AGE, oxidation and nitration adduct residues in apolipoprotein B100 was analysed after de-lipidation and exhaustive enzymatic hydrolysis by stable isotope dilution analysis with LC-MS/MS. The following markers of protein damage were quantified: AGEs - $\mathrm{N}_{\varepsilon}$-carboxymethyl-lysine (CML), $\mathrm{N}_{\varepsilon}$-carboxyethyl-lysine (CEL), $\mathrm{N}_{\varepsilon}$-carboxymethyl-arginine (CMA), glyoxal-derived hydroimidazolone G-H1, methylglyoxal-derived hydroimidazolone MG-H1, 3-deoxyglucosone-derived hydroimidazolone 3DG-H, methylglyoxal lysine dimer (MOLD) and pentosidine; oxidation markers - methionine sulfoxide MetSO, dityrosine and $\mathrm{N}$-formylkynurenine (NFK); and the nitration marker 3-nitrotyrosine (3-NT).

Results: Methylglyoxal-derived hydroimidazolone MG-H1 and glyoxal-derived AGEs G-H1 and CMA were AGEs of apolipoprotein B100 increased significantly in type 2 diabetes. AGE residue contents ( $\mathrm{pmol} / \mathrm{mg}$ protein): MG-H1 - controls $107 \pm 73$, diabetics $270 \pm 117$ ( $<<0.01)$; CMA - controls 16 \pm 15 , diabetics $51 \pm 33(\mathrm{P}<0.01)$; and G-H1 - controls $0.6 \pm 0.5$, diabetics 5.5 $\pm 4.1(\mathrm{P}<0.01)$. Protein oxidation markers were also major markers of damage to apolipoprotein B100 and were increased in type 2 diabetes. Protein oxidation adduct residue contents ( $\mathrm{pmol} / \mathrm{mg}$ protein): MetSO (median; minimum - maximum) - controls 644 (426 - 2876), diabetics 6078 (753 - 24,706), $\mathrm{P}<0.001$; dityrosine - controls $10 \pm 9$, diabetics $19 \pm 10(\mathrm{P}<0.05)$. Other markers of protein damage were not increased significantly in apolipoprotein B100 of type 2 diabetic patients with respect to normal control values.

Conclusion: This study indicates that apolipoprotein B100 of LDL is highly susceptible to oxidation on methionine residues and this is markedly increased in type 2 diabetes. Major AGE residues of apolipoprotein B100 increased in type 2 diabetes are formed by glyoxal and methylglyoxal glycation, possibly from nascent dicarbonyls generated within the lipid particle. Supported by: British Heart Foundation

\section{4}

Follow-up skin autofluorescence as a marker of altered tissue AGE accumulation and oxidative stress in type 2 diabetes mellitus E.G. Gerrits ${ }^{1}$, H.L. Lutgers ${ }^{2}$, A.J. Smit ${ }^{2}$, H.J.G. Bilo ${ }^{1,2}$ ${ }^{1}$ Internal Medicine, Diabetes Centre, Isala Clinics, Zwolle, ${ }^{2}$ Internal Medicine, University Medical Center Groningen, Netherlands

Background and aims: Advanced Glycation Endproducts (AGEs) accumulate with age on long-lived proteins, but at a more rapid rate in conditions like diabetes mellitus, renal failure, and during high oxidative stress levels in general. Measuring AGEs by skin autofluorescence (AF) is a practically applicable and non-invasive method, which can be used for measuring skin AGE levels over time. The aim of this prospective study was to investigate the changes in skin autofluorescence, as a marker of AGE-accumulation and oxidative stress, in patients with type 2 diabetes mellitus.

Material and methods: Skin AF levels were measured in 452 well-controlled type 2 diabetic patients at baseline and after follow-up. Skin AF was assessed by an autofluorescence reader (AFR) at the ventral side of the lower arm. Results: After a mean follow-up period of $3.3 \pm 0.4$ years $22 \%$ of the study population showed a decrease in skin AF levels compared to baseline, $43 \%$ had stable follow-up skin AF levels, and 35\% had an increase in skin AF lev-

Fig. 1 
els. Minor associations were seen between the progression of AGE formation and a longer diabetes duration, less well controlled lipid levels, a lower estimated glomerular filtration rate, the use of ACE-inhibitors, and/or previous macrovascular events.

Conclusion: These data suggest that in a well-controlled type 2 diabetes mellitus population, progression of AGE accumulation is limited. A higher degree of continuous oxidative stress based on various factors may play a role in the progression of AGE accumulation, at least when monitored by skin autofluorescence.

Supported by: Dutch Diabetes Research Foundation

\section{5}

Polymorphisms in glyoxalase 1 gene are not associated with markers of atherosclerosis and arterial stiffness: the Hoorn and CoDAM studies

L. Engelen ${ }^{1}$, I. Ferreira ${ }^{2}$, O. Brouwers ${ }^{1}$, J.M. Dekker ${ }^{3}$, G. Nijpels ${ }^{3}$, R.J. Heine ${ }^{3}$, M.M.J. van Greevenbroek ${ }^{1}$, C.J. van der Kallen ${ }^{1}$, E.E. Blaak ${ }^{4}$, E.J.M. Feskens ${ }^{5}$, H. ten Cate ${ }^{2}$, C.D.A. Stehouwer ${ }^{2}$, C.G. Schalkwijk ${ }^{1}$

${ }^{1}$ Department of Internal Medicine, Maastricht University, Netherlands, ${ }^{2} \mathrm{De}-$ partment of Internal Medicine, University Hospital Maastricht, Netherlands, ${ }^{3}$ Institute for Research in Extramural Medicine, VU Medical Center, Amsterdam, Netherlands, ${ }^{4}$ Department of Human Biology, Maastricht University, Netherlands, ${ }^{5}$ Division of Human Nutrition, Wageningen University, Netherlands

Background and aims: The formation and accumulation of advanced glycation endproducts (AGEs) has gained increasing acceptance as a significant mechanism contributing to cardiovascular complications. Methylglyoxal (MGO), a reactive glycolysis derived dicarbonyl metabolite, is the most reactive precursor in the formation of AGEs. MGO can be detoxified by glyoxalase (GLO)-1 to D-lactate and therefore, the activity of GLO-1 may play an essential role in the formation of AGEs. Because genetic variation in the GLO-1 gene may alter the expression and/or the activity of GLO-1, we investigated whether single nucleotide polymorphisms (SNPs) in the GLO-1 gene are associated with vascular complications in a Dutch cohort.

Materials and methods: The study entailed cross-sectional analyses of data from two joint observational studies, the CoDAM Study and the Hoorn Study, comprising a total of 1278 subjects, aged $64.5 \pm 8.58$ years; $43.5 \%$ had normal glucose tolerance status, $23.2 \%$ had impaired glucose tolerance status and $33.3 \%$ had type 2 diabetes. Using HapMap and Haploview database, all potential SNPs of the GLO-1 gene that have a minor allele frequency $>5 \%$ were identified. Nine tag SNPs that cover the common variation of the GLO1 gene were selected and genotyped using TaqMan 7900HT. Plasma levels of the AGEs $\mathrm{N}^{\varepsilon}$-(carboxymethyl) lysine (CML) and $\mathrm{N}^{\varepsilon}$-(carboxyethyl) lysine (CEL) were measured by tandem mass spectrometry. Carotid intima-media thickness (IMT) - a marker of atherosclerosis, ankle-arm index (AAIx) - a marker of peripheral arterial disease, and large artery stiffness (compiled in a z-score of seven stiffness parameters, Hoorn Study only) were compared across genotypes with the use of ANCOVA (dominant, recessive and additive models). In addition, associations between the nine SNPs and blood pressure, glomerular filtration rate (GFR) - a marker of renal function and plasma AGE levels (CoDAM Study only) were performed. All analyses were adjusted for age, sex and glucose tolerance status.

Results: All genotyped SNPs were in Hardy Weinberg equilibrium. In the entire study population, no associations were found between all GLO-1 SNPs and IMT, AAIx and arterial stiffness. In addition, no associations were found with blood pressure, GFR and plasma levels of the AGEs CML and CEL. For example, in SNP rs2736654, carotid IMT in mm (mean value \pm SE) was $0.83 \pm 0.01,0.82 \pm 0.01$ and $0.82 \pm 0.01$ for GG, GT and TT respectively; the AAIx was $1.16 \pm 0.01,1.16 \pm 0.01$ and $1.16 \pm 0.01$ for GG, GT and TT respectively; and plasma CML in mmol/L was $1.78 \pm 0.05,1.76 \pm 0.03$ and $1.79 \pm 0.04$ for GG, GT and TT respectively. Similar results were found when stratified analyses according to glucose tolerance status and study population were performed.

Conclusion: No associations were found between GLO-1 gene polymorphisms and markers of atherosclerosis, arterial stiffness and renal function and AGEs in normal-, impaired glucose tolerant, and type 2 diabetic subjects.

\section{PS 129 AGE-receptors}

\section{6}

Functional analysis of the susceptibility haplotype in the receptor for advanced glycation end-products gene and its role in the hyperglycaemia-driven pathology K. Kankova ${ }^{1}$, P. Benes ${ }^{2}$, S. Kuchtickova ${ }^{1}$

${ }^{1}$ Dept. of Pathophysiology, ${ }^{2}$ Dept. of Experimental Biology/Laboratory of Cell Differentiation, Masaryk University, Brno, Czech Republic

Background and aims: Receptor for Advanced Glycation End-products (RAGE) belongs to the class of transmembrane receptors recognizing AGEs produced in accelerated rate during hyperglycemia and uremia and contributing to the development of diabetic complications. Our previous studies have shown that second most frequent haplotype in the RAGE gene (denoted RAGE $_{2}$ ) conferred susceptibility to the development and accelerated onset of diabetic nephropathy in T2DM (frequency $21.7 \%$ in nephropathy vs. $12.8 \%$ in control diabetic, $\mathrm{P}=0.015, \mathrm{OR}=1.65$ [95\% CI 1.08-2.5]). The aim of the study was to elucidate in vitro the functional role of the RAGE susceptibility $_{2}$ haplotype in the modulation of RAGE gene transcriptional activity.

Materials and methods: The two promotor and one SNP in intron 8 were identified as tagging variants for the RAGE 2 haplotype. A 788bp PCR fragment of the RAGE promoter region ( -738 to +49 relative to the transcription start containing the $-429 \mathrm{~T} / \mathrm{C}$ and $-374 \mathrm{~T} / \mathrm{A}$ polymorphic variants) was amplified using genomic DNA of homozygous subjects to create all possible haplotypes present in our population (i.e. T/T, T/A, C/T) and cloned into reporter vector pGL3-Basic (Promega). Additionally, a 303bp sequence of the RAGE intron 8 was amplified from homozygotes for the $2184 \mathrm{~A} / \mathrm{G}$ and cloned into the enhancer site of the same vector in the combination with the promoter variants. All constructs were propagated in the competent E. coli and subsequently used for transfection of BAEC cell line by Lipofectamine (all experiments were run in triplicates). After incubation for $48 \mathrm{hrs}$ at $37^{\circ} \mathrm{C}$ at $5 \% \mathrm{CO}_{2}$ at varying concentration of $\mathrm{D}$-glucose ( 5 and $30 \mathrm{mmol} / \mathrm{L}$ ) luciferase level was determined using Dual Luciferase Reporter Assay System (Promega).

Results: Luciferase activity (expressed in RLU) significantly differed between the three promotor haplotypes ( $\mathrm{p}<0.05$, ANOVA) with $-429 \mathrm{C} /-374 \mathrm{~T}$ giving the highest activity in both normo- and hyperglycemic conditions. Intron variant did not significantly influenced observed transcriptional activity of promoter variants in this assay system ( $\mathrm{p}>0.05$, ANOVA).

Conclusion: Using reporter gene assays we were able to elucidate functional impact of the RAGE promoter variants constituting previously identified risk haplotype for diabetic nephropathy. Speculated additional effect of the intron 8 variant as an enhancer factor/site was not proved. Functional effect of the RAGE variants further supports its significance in the pathogenesis of diabetic complications. Supported by: Agency of the Czech Academy of Sciences

\section{7}

Advanced glycation end-products accelerate in vitro calcification of vascular cell via the AGE-RAGE pathway

X.M. Ren ${ }^{1}$, H. Shao ${ }^{2}$, N.F. Liu ${ }^{1}$, Q. Wei ${ }^{1}$, Z.1. Sun ${ }^{3}$

${ }^{1}$ Institute for Cardiovascular Medicine, School of Clinical Medicine, ${ }^{2}$ Department of Clinical Pharmacology, Zhongda Hospital, ${ }^{3}$ Department of Endocrinology, Zhongda Hospital, Southeast University, NanJing, China

Background and aims: Vascular calcification is common in people with diabetes and its presence predicts premature mortality. There is a growing body of evidence that advanced glycation end products (AGEs), senescent macroprotein derivatives formed at an accelerated rate in diabetes, play an important role in the pathogenesis of accelerated atherosclerosis in diabetes. Moreover, results of previous studies suggest that AGEs may modulate vascular calcification. Therefore, we hypothesized that AGEs may have a direct impact on the calcium-regulating system of vascular smooth muscle cells (VSMCs), resulting in deposition of calcium in vascular wall.

Materials and methods: In this study, we investigated the effect of glycated bovine serum albumin (AGE-BSA) and the receptor for AGEs (RAGE) on in vitro calcification by rat VSMCs. AGE-BSA was prepared by incubating BSA with high-concentration glucose. To induce calcification, VSMCs were incubated with AGE-BSA or non-glycated BSA in DMEM containing $10 \mathrm{mmol} / \mathrm{L}$ ß-glycerophosphate. Calcium deposition, Von Kossa staining, alkaline phosphatase (ALP) activity and mRNA, the osteoblast transcription factor core binding factor 1 (Cbfa-1), and its downstream protein osteopontin were used 
to identify osteoblastic differentiation and mineralization in VSMCs induced by AGE-BSA. RAGE mRNA and protein were also detected by Real-time Polymerase Chain Reaction and Western blot analysis.

Results: AGE-BSA induced calcium accumulation in VSMCs in time- and dosedependent manners. Treatment of VSMCs with $200 \mu \mathrm{g} / \mathrm{ml}$ AGE-BSA for 3 days caused a significant increase of calcium depositionin as compared to non-glycated BSA culture $(1.11 \pm 0.1 \mathrm{vs} 0.61 \pm 0.06 \mu \mathrm{mol} / \mathrm{mg}$; $p<0.001)$. After 10 days of AGE-BSA treatment, calcification in VSMCs was also dramatically increased, as shown by von Kossa staining. Mechanistic studies revealed that elevated AGEBSA treatment of VSMCs enhanced ALP activity in a time-dependent manner $(91.1 \pm 11.4$ vs $50.5 \pm 12.3 \mathrm{U} / g$ protein, day $3 ; 179.2 \pm 11.4$ vs $111.5 \pm 15.1 \mathrm{U} / g$ protein, day $6 ; p<0.01$ ). Similarly, 200 $\mu \mathrm{g} / \mathrm{ml}$ AGE-BSA significantly increased ALP mRNA by 3.4 -fold at $24 \mathrm{~h}$, whereas in non-glycated BSA culture, VSMCs accumulated a slightly increase in ALP mRNA. AGE-BSA also increased the gene expression of Cbfa-1 and osteopontin by 4.1-fold and 1.8-fold, respectively, and osteopontin protein by 1.4-fold. We also showed that AGE-BSA increased RAGE mRNA and protein. Pretreatment of VSMCs with recombinant rat RAGE attenuated calcium deposition as well as ALP, osteopontin and Cbfa-1 mRNA induced by AGE-BSA. Conclusion: These results suggest that AGEs enhances in vitro vascular alcification by promoting osteoblastic differentiation of vascular smooth muscle cells in part through AGE-RAGE pathway. Blockade of AGE-RAGE signaling may be a promising target for therapeutic intervention to prevent diabetic vascular calcification.

\section{8}

Linoleic acid upregulates the expression of the receptor for advanced glycation endproducts in glucose-treated human aortic endothelial cells B.M. Haberberger ${ }^{1}$, R. Graydon ${ }^{2}$, J.M. Ames ${ }^{2}$, A. Stitt ${ }^{3}$, L.A. Powell ${ }^{1}$

${ }^{1}$ Diabetes Research Group, United Kingdom, ${ }^{2}$ School of Biological Sciences, United Kingdom, ${ }^{3}$ Centre for Vision Science, Queen's University Belfast, United Kingdom

Background and aims: Diabetes is associated with changes in the blood vessel wall that lead to accelerated atherosclerosis, the main cause of increased mortality and morbidity in diabetes. Atherosclerosis is an inflammatory process initiated by endothelial dysfunction that involves the recruitment of circulating monocytes to the endothelium. This process is promoted by reactive oxygen species (ROS) and regulated by inflammatory mediators including RAGE, MAPK 1 and the cytokine TNF- $a$. In diabetes, in addition to disturbances in glucose homeostasis, there is impairment of fatty acid metabolism which results in increased free fatty acids in the bloodstream. The aim of this work was to analyze the effects of linoleic acid (the main dietary fatty acid in a Western diet) on the expression of inflammatory mediators in glucose-treated endothelial cells.

Materials and methods: Human aortic endothelial cells (HAEC) were cultured in $5 \mathrm{mM}$ (NG) or $25 \mathrm{mM}$ (HG) D-glucose (5\% FCS) for 3 days prior to the addition of linoleic acid $(0.2 \mathrm{mM})$ using fatty acid free-bovine serum albumin as a carrier (6:1). The cells were extracted after $24 \mathrm{~h}$ and $76 \mathrm{~h}$ and the relative mRNA expression analysed via quantitative RT-PCR. The cell permeable fluorescent probes 2, 7'-dichlorodihydrofluorescein diacetate (DCFH$\mathrm{DA}, 10 \mu \mathrm{M})$ and dihydroethidium (DHE, $5 \mathrm{mM}$ ) were used to measure intracellular $\mathrm{H}_{2} \mathrm{O}_{2}$ and superoxide production respectively.

Results: After $24 \mathrm{~h}$, linoleic acid treatment increased RAGE expression significantly in HG $(\mathrm{p}<0.05)$ compared to the NG control. Further increases were observed in NG $(p<0.001)$ and HG $(p<0.001)$ after 76 h. TNF- $\alpha$ also increased significantly in $\mathrm{HG}$ after $76 \mathrm{~h}(\mathrm{p}<0.05)$ whereas maximal MAPK 1 expression was observed in HG after $24 \mathrm{~h}(\mathrm{p}<0.01)$ and remained constant until $76 \mathrm{~h}$. These linoleic acid-induced changes in mRNA expression were preceded by increased intracellular superoxide and $\mathrm{H}_{2} \mathrm{O}_{2}$ generation $(\mathrm{p}<0.01)$.

Conclusion: These results suggest that cellular ROS and AGEs generated by linoleic acid may mediate the upregulation of the inflammatory mediators MAPK1, TNF- $\alpha$ and RAGE.

\section{9}

RAGE deficiency induces a proinflammatory phenotype in bones and osteoblasts through PPAR- $a$ suppression

S.K. Biswas ${ }^{1}$, H. Li ${ }^{1}$, F. Duttenhoefer ${ }^{1}$, P.M. Humpert ${ }^{1}$, C. Kasperk ${ }^{1}$, M. Brownlee ${ }^{2}$, P.P. Nawroth ${ }^{1}$, A. Bierhaus ${ }^{1}$

${ }^{1}$ Department of Medicine I and Clinical Chemistry, University of Heidelberg, Germany, ${ }^{2}$ Department of Medicine and Pathology, Albert Einstein College of Medicine, New York, United States

Background and aims: AGE-RAGE interaction sustains inflammation and has been implicated in the pathogenesis of bone disease in diabetes. Remarkably, RAGE deficiency induces a proinflammatory phenotype in bones and osteoblasts, however, the physiologic role of RAGE in bone tissue is not clear. Therefore, we aimed to study the mechanism underlying inflammatory processes in RAGE deficient osteoblasts as well as signaling events that link RAGE deficiency and inflammation in bones from RAGE knock-out (RAGE-/-) mice.

Materials and methods: Osteoblasts from wild type (WT, C57BL/6) and RAGE-/- mice were transiently transfected with different plasmids and siRNA. Reporter gene assay, western blotting, PCR, immunoprecipitation and chromatin immunoprecipitation techniques were employed to study the expression and interactions of transcription factors and coactivators involved in the regulation of PPAR- $\alpha$ expression and nuclear translocation.

Results: The mild proinflammatory phenotype in bones and osteoblasts observed in RAGE-/- mice was associated with a reduced gene expression and intranuclear translocation of the anti-inflammatory nuclear receptor PPAR- $\alpha$. Moreover, PPAR- $\alpha$ activation by fibrates reduced inflammation in osteoblasts from RAGE-/- mice, implying that the lack of PPAR- $\alpha$ in RAGE deficiency might contribute to the observed proinflammatory phenotype. We therefore analyzed the PPAR- $\alpha$ promoter which contains a series of Sp1 binding sites. PPAR- $\alpha$ promoter driven reporter gene expression was reduced in RAGE-/- osteoblasts compared to WT osteoblasts. In addition, Sp1 overexpression significantly increased PPAR- $\alpha$ promoter activity in WT osteoblasts, but had only marginal effects in RAGE-/- osteoblasts. Sequential promoter deletions confirmed a strong Sp1 dependency of PPAR- $\alpha$ expression in WT osteoblasts, that was not observed in RAGE-/- osteoblasts. Suppression of Sp1 by siRNA transfection, however, decreased PPAR- $\alpha$ promoter expression by around 50\% in both WT and RAGE-/- osteoblasts. Remarkably, endogenous Sp1 gene and protein expression was similar in osteoblasts from WT and RAGE-/- mice, suggesting that a qualitative or functional defect of Sp1 in RAGE-/- osteoblasts might underlie the reduced PPAR- $\alpha$ gene expression in RAGE-/- mice. Consistently, protein and chromatin immunoprecipitation demonstrated reduced Sp1 phosphorylation and a decreased Sp1 binding to the PPAR- $\alpha$ promoter in RAGE-/- osteoblasts compared to WT osteoblasts. Conclusion: The proinflammatory phenotype observed in bones and osteoblasts of RAGE-/- mice is associated with a significant decrease in PPARa most likely due to dysfunctional Sp1 signaling and subsequent reduced PPAR-a expression.

Supported by: JDRF and Deutsche Forschungsgemeinschaft 


\section{0}

Accelerated atheroscerosis in mice knockout for galectin-3 fed a high fat diet

S. Menini ${ }^{1}$, C. Iacobini ${ }^{1}$, C. Ricci ${ }^{1}$, A. Scipioni ${ }^{1}$, V. Sansoni ${ }^{1}$, G. Mazzitelli ${ }^{1}$, P. Simonelli ${ }^{1}$, S. Cordone ${ }^{1}$, M. Serino ${ }^{2}$, M. Federici ${ }^{2}$, F. Pricci ${ }^{3}$, G. Pugliese ${ }^{1}$ ${ }^{1}$ Department of Clinical Sciences, La Sapienza University, ${ }^{2}$ Department of Internal Medicine, Tor Vergata University, ${ }^{3}$ Department of Cell Biology and Neurosciences, National Institute of Health of Italy, Rome, Italy

Background and aims: Galectin-3, a multifunctional lectin with (anti)adhesive and growth-regulating properties, was recently identified as a receptor for advanced glycation endproducts (AGEs)/advanced lipoxidation endproducts (ALEs) and modified LDLs promoting the uptake and removal of these harmful compounds. Clearance of AGEs/ALEs and modified LDLs via other receptors, including RAGE and scavenger receptors (SRs), is coupled to signaling pathways triggering macrophage activation and inflammation, ultimately leading to tissue injury. Galectin-3 expression was found to be increased in kidney and atherosclerotic lesions from both human patients and experimental animals. We previously showed that mice knockout (KO) for galectin-3 are more susceptible to develop glomerular disease induced by diabetes, AGEs and aging, as compared with the wild type (WT) mice.

Materials and methods: Coeval female galectin-3 KO and WT mice were fed for 8 months a high fat diet (HFD, $15 \%$ saturated fat, $1.25 \%$ cholesterol and $0.5 \% \mathrm{Na}$-cholate) or normal fat diet (NFD, $4 \%$ saturated fat). Metabolic parameters, morphometry of aortic lesions, apoptosis, inflammatory markers, AGEs/AGE-receptors and oxidative stress were assessed in these animals.

Results: Though serum cholesterol and triglyceride were increased in the 2 genotypes to the same extent, the HOMA-IR index was significantly increased only in WT-HFD mice $(1.62 \pm 0.37$ vs. $0.88 \pm 0.26$ nei WT-NFD, $p<0.01)$ and the i.p. insulin tolerance test showed a lower reduction in blood glucose in WT-HFD vs. KO-HFD mice. Conversely, circulating AGE and isoprostane 8-epi-prostaglandin F2a levels were higher in KO-HFD vs. WT-HFD mice. Blood pressure, as measured by arterial catheterism, was the same in the 2 genotypes and was unaffected by HFD. KO-HFD mice showed more extensive aortic lesions vs. WT-HFD mice, with significantly higher values $(\mathrm{p}<0.001)$ of lesion area $\left(66347 \pm 6180\right.$ vs. $\left.42973 \pm 8076 \mu \mathrm{m}^{2}\right)$ and length $(583 \pm 58$ vs. $450 \pm 50 \mu \mathrm{m})$. Aortic lesions of KO-HFD mice were also more complex, as shown by extensive lympho-monocyte infiltration (with higher F4/80 and exclusive CD3 staining), migration of smooth muscle cells from the media, and presence of cholesterol clefts and necrotic material surrounded by a fibrous cap, than those of WT-HFD, consisting only of fatty streaks. The gene and/or protein expression for activated caspase-3 (an index of apoptosis), monocyte chemoattractant protein, cycloxygenase-2, vascular cell adhesion molecule1, RAGE, SR-A1, SRB-1 e CD36 as well as the content of oxidized LDL, the glycoxidation and lipoxidation products $\mathrm{N}^{\varepsilon}$-carboxymethyllysine and 4-hydroxy-2-nonenal, and nitrothyrosine were significantly higher in KO-HFD than in WT-HFD (1.5-15x).

Conclusion: These data indicate that galectin-3 ablation favours the development of atherosclerotic lesions with a distinct inflammatory phenotype in the rodent model of lipid-induced atherogenesis. This suggests a protective role of galectin- 3 in vascular injury associated with hyperlipidemia, which is related to a more efficient removal of AGEs/ALEs and modified LDLs and/or a blunted activation of pro-inflammatory pathways, at variance with RAGE. Supported by: La Sapienza University, Rome, Italy

\section{1}

Galectin-3 ablation protects from nonalcoholic steatohepatitis induced by high fat diet

C. Iacobini ${ }^{1}$, C. Ricci $^{1}$, A. Scipioni ${ }^{1}$, V. Sansoni ${ }^{1}$, G. Mazzitelli ${ }^{1}$, A. Frasheri ${ }^{1}$, M. Serino ${ }^{2}$, M. Federici ${ }^{2}$, F. Pricci ${ }^{3}$, S. Menini ${ }^{1}$, G. Pugliese ${ }^{1}$

${ }^{1}$ Department of Clinical Sciences, La Sapienza University, ${ }^{2}$ Department of Internal Medicine, Tor Vergata University, ${ }^{3}$ Department of Cell Biology and Neurosciences, National Institute of Health of Italy, Rome, Italy

Background and aims: Nonalcoholic steatohepatitis (NASH) is caused by lipid accumulation resulting in steatosis (non-alcoholic fatty liver disease, NAFLD), followed by an inflammatory and fibrotic response possibly leading to cirrhosis. Advanced glycation endproducts (AGEs) and advanced lipoxidation endproducts (ALEs), which are cleared mainly by the liver through receptors such as receptor for AGEs (RAGE) and galectin-3, may play a role in this response. Galectin-3 ablation was shown to accelerate renal injury induced by diabetes, aging and AGEs injection, but also to attenuate cirrho- sis induced by $\mathrm{CCl}_{4}$. This study was aimed at evaluating the role of gal-3 in the development of NASH in an animal model of insulin-resistance without obesity and diabetes.

Materials and methods: Mice knockout (KO) for galectin-3 and the corresponding wild type (WT) animals were fed for 8 months a high fat diet (HFD, containing $15 \%$ saturated fat, $1.25 \%$ cholesterol and $0.5 \%$ Na-cholate) or normal fat diet (NFD, containing $4 \%$ saturated fat). The presence of NASH was assessed in hematoxylin/eosin stained sections using the NAFLD score and its extent was then graded according to Brunt's criteria using Masson's trichromic staining for fibrosis. Metabolic parameters were assessed in these animals, together with inflammatory markers, AGEs/AGE-receptors and oxidative stress. Results: Morphologic analysis showed NASH only in 3 out of 8 KO-HFD mice (class 3 NAFLD: micro/macrovescicular steatosis, with modest lobular inflammation and ballooning degeneration). Conversely, NASH (class 4 NAFLD: macrovescicular steatosis, portal and lobular inflammation, ballooning degeneration, Mallory's and acidophil bodies, fibrosis) was observed in all WT-HFD mice. NASH grading and staging showed grade/stage 1 NASH in the 3 KO-HFD and grade/stage 2 or 3 NASH in all the WT-HFD mice. Hepatic expression of $\mathrm{F} 4 / 80, \mathrm{CD} 3, \mathrm{~N}^{\varepsilon}$-carboxymethyllysine, RAGE, monocyte chemoattractant protein- 1 and tumour necrosis factor- $\alpha$ was significantly higher in WT-HFD vs. KO-HFD mice. At the gene expression level, sterol regulatory element binding protein 1c (SREBP-1c) was up-regulated in WT-HFD, but not KO-HFD mice, which showed a down-regulation of both HMG-CoA reductase and fatty acid synthase, whereas only the former was down-regulated in WT-HFD mice. Though serum cholesterol and triglyceride levels were increased in the 2 genotypes to the same extent, the HOMAIR index was significantly increased only in WT-HFD mice $(1.62 \pm 0.37$ vs. $0.88 \pm 0.26$ in WT-NFD, $\mathrm{p}<0.01$ ) and the intraperitoneal insulin tolerance test showed a lower reduction in blood glucose in WT-HFD vs. KO-HFD mice. Conversely, circulating AGE and isoprostane 8-epi-prostaglandin F2a levels were higher in KO-HFD vs. WT-HFD mice. Adipocyte diameter was higher and their number was lower in WT-HFD vs. KO-HFD mice; this difference between the 2 genotypes was observed also in the NFD-fed mice.

Conclusion: These data indicate that galectin-3 $\mathrm{KO}$ mice are protected from $\mathrm{NASH}$, possibly due to a lower degree of insulin-resistance, resulting in a less marked steatosis (or viceversa) and impairment of removal of AGEs/ALEs, thus limiting pro-inflammatory and pro-fibrotic effects.

Supported by: La Sapienza University, Rome, Italy

\section{2}

Atorvastatin downregulates monocyte CD36 scavenger receptor expression and nuclear NFkB levels in type 2 diabetic patients

E. Mandosi ${ }^{1}$, M. Fallarino ${ }^{1}$, A. Gatti ${ }^{1}$, A. Carnovale ${ }^{1}$, E. Lococo ${ }^{2}$, B. Buchetti ${ }^{2}$, S. Filetti ${ }^{1}$, L. Lenti ${ }^{2}$, S. Morano ${ }^{1}$

${ }^{1}$ Department of Clinical Sciences, ${ }^{2}$ Department of Experimental Medicine, "Sapienza" University, Rome, Italy

Background and aims: Type 2 diabetes increases the risk for cardiovascular disease and the 3-hydroxy-3-methylglutaryl coenzyme A (HMG-CoA) reductase inhibitors or statins reduce cardiovascular events in these patients. The benefits from statin therapy cannot be explained only by their lipid-lowering effect, but also by their anti-inflammatory properties. CD36 is a scavenger receptor expressed by many cell-types including monocytes; it is able to uptake oxidized low density lipoproteins (Ox-LDL) leading to foam cell formation and probably to Nuclear Factor-kB (NFkB) activation. As known inflammatory processes play a major role in atherogenesis and NFkB regulates different genes involved in this condition. Given that monocytes play a major role in the atherogenesis processes, the aim of this study was to test the effect of atorvastatin therapy on CD36 scavenger receptor expression and NFkB levels in circulating monocytes from type 2 diabetic patients.

Materials and methods: Twenty-two type 2 Caucasian diabetic patients (17 male and 5 female, age $60.8 \pm 7.1$ years, BMI $28.9 \pm 4.8 \mathrm{Kg} / \mathrm{m}^{2}$, $\mathrm{HbAlc}$ $7.6 \pm 1.1 \%)$, were recruited and treated for 8 weeks with atorvastatin $(20 \mathrm{mg} /$ die). At baseline and after treatment a blood sample was collected for measurement of glucose, lipid profile (total cholesterol, HDL and LDL cholesterol, triglycerides), glycated hemoglobin (HbAlc), C-reactive protein (CRP) and for isolation of monocytes. CD36 surface protein expression (anti- CD36 fluorescein isothiocyanate-FITC), expressed as the mean of the median fluorescence, Arbitrary Units (A.U.), NFkB levels in monocyte lysate (Western blot analysis), nuclear and cytoplasm NFkB levels (immunoblot analysis), expressed as Relative Units (R.U.), were assessed in these cells.

Results: Atorvastatin therapy significantly decreased total $(4.1 \pm 1.0$ vs $5.2 \pm 0.9 \mathrm{mmol} / \mathrm{L}, \mathrm{p}<0.0001)$ and LDL cholesterol $(2.4 \pm 0.9 \mathrm{vs} 3.2 \pm 0.7 \mathrm{mmol} / \mathrm{L}$, 
$\mathrm{p}<0.002)$ levels and incresased HDL cholesterol $(1.2 \pm 0.2$ vs $1.0 \pm 0.2 \mathrm{mmol} / \mathrm{L}$, $\mathrm{p}=0.02)$ levels. CD36 surface protein expression was reduced (104.6 \pm 20.1 vs $116.6 \pm 16.0 \mathrm{~A}: \mathrm{U} ., \mathrm{p}<0.02)$ in circulating monocytes after atorvastatin therapy while in monocyte lysates NFkB levels were increased $(22.9 \pm 6.8$ vs $18.4 \pm 8.4$ R.U., p $<0.01$ ) in diabetic patients after statin treatment. To test whether the increase of NFkB levels was located into the nucleus or into the cytoplasm of monocytes, an immunoblot analysis of the nuclear and cytoplasmic fractions was performed. The immunoblot analysis showed a reduced nuclear NFkB $(0.7 \pm 0.1$ vs $9.2 \pm 0.2$ R..U., $\mathrm{p}<0.05)$ and an increased cytoplasm NFkB levels $(26.5 \pm 4.0$ vs $17.9 \pm 0.6$ R.U., $p<0.05)$ after atorvastatin therapy.

Conclusion: These results suggest that, in addition to lowering serum cholesterol levels, atorvastatin therapy reduces the cardiovascular risk in treated patients by its anti-atherogenic and anti-inflammatory actions, through the decrease of the levels of molecules involved in endothelial dysfunction.

\section{PS 130 Impairment and repair of endothelial function}

\section{3}

Forearm muscle blood flow after a mixed meal is impaired at all stages of type 2 diabetes

V. Lambadiari ${ }^{1}$, G. Dimitriadis ${ }^{1}$, P. Mitrou ${ }^{1}$, E. Boutati ${ }^{1}$, E. Maratou ${ }^{2}$, T. Economopoulos ${ }^{1}$, S.A. Raptis ${ }^{1,2}$;

${ }^{1}$ 2nd Dpt Internal Medicine and Research Institute, Athens University Medical School, "Attikon" University Hospital, ${ }^{2}$ Hellenic National Diabetes Center, Athens University Medical School, "Attikon" University Hospital, Athens, Greece.

Background and aims: Endothelial dysfunction is an important determinant of altered vascular reactivity and plays a decisive role in the pathogenesis of cardiovascular complications in diabetes. Microvascular disturbances can be present long before overt diabetes develops. This study was undertaken to investigate the existence of a de novo impairment in forearm muscle (FM) endothelial function, in the various stages of type 2 diabetes.

Materials and methods: A standard mixed meal was given to five groups of subjects, whose features are shown in Table 1. Blood samples were drawn from the radial artery (A) and from a vein (V) draining the FM at 30-60min intervals for $6 \mathrm{~h}$ for the measurement of glucose and insulin. Simultaneous measurements of blood flow in FM were done with strain-gauge plethysmography. Glucose Flux (GFLUX) was calculated ([G in (A-V)] x [BF]).

Results: (a) GFLUX was higher in controls vs all other groups, (b) HOMA Index in controls was lower vs all other groups, (c) ISI Index was higher in controls vs all other groups, (d) peak-baseline BF was higher in controls vs all other groups (Table1), e) ISI was positively associated with peak-baseline $\mathrm{BF}(\beta$-coefficient $4,962 \pm 2,147, \mathrm{p}=0,026), \mathrm{f})$ GFLUX was positively associated with peak-baseline $\mathrm{BF}$ ( $\beta$-coefficient $66,120 \pm 29,3, \mathrm{p}=0,03$ ).

Conclusion: After meal ingestion, no response of insulin-stimulated blood flow was detected in the forearm muscles of relatives and patients with IGT, DM2-A, DM2-B. Consequently, postprandial glucose uptake was impaired, reinforcing glucose intolerance. Endothelial dysfunction combined with overt hyperglycemia will favor the development of microangiopathy.

\section{4}

Effects of mild hyperinsulinaemia on conduit vessel endothelial function: role of noradrenergic activation

C. Morgantini, F. Stea, B. Boldrini, E. Duranti, L. Ghiadoni, E. Ferrannini, A. Natali

Department of Internal Medicine, University of Pisa, Italy

Background and aims: A recent study has shown that in healthy subjects a low-dose insulin infusion severely impairs conductance arteries endothelial function suggesting a proatherogenic effect of hyperinsulinemia. This finding is in contrast with a wealth of in vitro and in vivo evidence showing that insulin facilitates both nitric oxide release and endothelium-dependent dilatation. The aim of this study was to try to replicate the above mentioned observation and evaluate to what extent the insulin effects on large arteries reactivity are modulated by the enhancement of adrenergic tone that typically occurs during the exogenous infusion of the hormone.

Materials and methods: In 20 healthy male volunteers (age: $27 \pm 5$ yrs), endothelium-dependent (FMD) and endothelium-independent (GTN) dilatation were evaluated by high-resolution ultrasound of the brachial artery with automatic edge detection system. FMD and GTN were expressed as percent maximal dilatation in response to $5 \mathrm{~min}$ of distal ischaemia or to the sublingual administration of $25 \mu \mathrm{g}$ of glyceryl trinitrate, respectively. These meas-

\begin{tabular}{|c|c|c|c|c|c|}
\hline Table & CONTROLS & RELATIVES & IGT & DM2-A & DM2-B \\
\hline & $\mathrm{N}=10$ & $\mathrm{~N}=11$ & $\mathrm{~N}=6$ & $\mathrm{~N}=6$ & $\mathrm{~N}=13$ \\
\hline Age(yrs) & $36 \pm 3$ & $41 \pm 3$ & $43,5 \pm 3$ & $53 \pm 4$ & $56 \pm 2$ \\
\hline $\mathrm{BMI}\left(\mathrm{kg} / \mathrm{m}^{2}\right)$ & $23,5 \pm 1$ & $25 \pm 1$ & $26 \pm 1,5$ & $24,5 \pm 1,5$ & $26 \pm 0,7$ \\
\hline GFLUX $_{0-360}\left(\mu \mathrm{mol} / 100 \mathrm{ml}\right.$ tissue $\left.{ }^{*} \min \right)$ & $928 \pm 157$ & $549 \pm 82^{*}$ & $556 \pm 105^{*}$ & $440 \pm 86^{*}$ & $532 \pm 65^{\star}$ \\
\hline HOMA-Index & $0,98 \pm 0,1$ & $1,43 \pm 0,1^{*}$ & $1,7 \pm 0,1^{*}$ & $1,8 \pm 0,2^{*}$ & $2,2 \pm 0,2^{\star}$ \\
\hline ISI-Index $\left(\mathrm{mg}^{\star} \mathbf{l}^{2} / \mathrm{mmol}^{\star} \mathrm{mU}^{\star} \mathrm{min}\right)$ & $82 \pm 3$ & $60 \pm 3^{*}$ & $45 \pm 1^{*}$ & $40 \pm 6^{*}$ & $29 \pm 4^{*}$ \\
\hline Peak-Baseline Blood-Flow (ml/100cctissue ${ }^{\star}$ min) & $3,3 \pm 0,5$ & $0,53 \pm 0,2^{*}$ & $0,8 \pm 0,1^{*}$ & $1,3 \pm 0,34^{*}$ & $1,3 \pm 0,3^{\star}$ \\
\hline
\end{tabular}


Table - FMD and GTN during saline (SALINE), Insulin (INS) and insulin+clonidine (INS+CLN) infusion

\begin{tabular}{llllllll}
\hline FMD (\%) & SALINE & INS & INS+CLN & GTN (\%) & SALINE & INSULIN & INS+CLN \\
\hline $\mathbf{- 6 0} \mathbf{~ m i n}$ & $7.3 \pm 0.6$ & $7.2 \pm 0.5$ & $7.6 \pm 1.8$ & $\mathbf{- 6 0 ~} \mathbf{~ i n}$ & $9.4 \pm 1.1$ & $9.1 \pm 1.0$ & $9.4 \pm 1.9$ \\
\hline $\mathbf{- 1 0} \mathbf{~ m i n}$ & $7.2 \pm 0.5$ & $7.1 \pm 0.3$ & $6.1 \pm 2.1$ & $\mathbf{- 1 0} \mathbf{~} \mathbf{~}$ & $9.8 \pm 1.2$ & $9.2 \pm 1.0$ & $10.5 \pm 1.3$ \\
\hline $\mathbf{1 2 0} \mathbf{~} i n$ & $7.4 \pm 0.6$ & $7.1 \pm 0.7$ & $5.9 \pm 1.0$ & $\mathbf{1 2 0} \mathbf{~ i n}$ & $9.6 \pm 1.3$ & $8.3 \pm 0.9$ & $12.4 \pm 1.9$ \\
\hline $\mathbf{2 4 0} \mathbf{~}$ in & $7.2 \pm 0.9$ & $7.2 \pm 0.7$ & $6.7 \pm 2.9$ & $\mathbf{2 4 0} \mathbf{~} \mathbf{n}$ & $9.2 \pm 1.8$ & $6.8 \pm 0.8$ & $12.2 \pm 0.7$ \\
\hline
\end{tabular}

urements were serially taken at times -60 and -10 min and again after 120 and $240 \mathrm{~min}$ of either insulin $\left(0.25 \mathrm{mU}^{\mathrm{min}}{ }^{-1} \mathrm{~kg}^{-1}, \mathrm{n}=10\right)$ or saline $(\mathrm{n}=5)$ intravenous infusion. Plasma glucose was maintained stable by means of a variable $20 \%$ glucose solution infusion according to the clamp technique. In 5 subjects, insulin infusion was preceded by an intravenous infusion of clonidine $\left(0.0052 \mu \mathrm{g} \mathrm{min}^{-1} \mathrm{~kg}^{-1}\right)$ started at time $-40 \mathrm{~min}$ and continued throughout the $240 \mathrm{~min}$ of the study. Before each vascular test plasma samples were collected for the determination of insulin, glucose, adrenaline and noradrenaline. Results: Insulin infusion raised plasma hormone concentrations from $63 \pm 4$ to $210 \pm 22 \mathrm{pmol} / \mathrm{l}$. Blood pressure, heart rate, brachial artery blood flow and plasma adrenaline remained stable in all studies. Basal artery diameter and post-ischemic flow increments were affected neither by insulin nor by clonidine. Plasma noradrenaline increased during insulin infusion from $260 \pm 40$ to $333 \pm 62 \mathrm{pg} / \mathrm{ml}(\mathrm{p}<0.05)$, while remained unchanged during combined insulin/clonidine infusion. As reported in the table, neither FMD nor GTN were affected by saline infusion. During insulin infusion no change in FMD was observed while the response to GTN showed a $25 \%$ decrease $(p<0.05$ by ANOVA). Infusion of clonidine alone resulted in a slight reduction in FMD ( $\mathrm{p}=\mathrm{ns}$ ), which was not further changed by adding insulin. GTN was slightly increased by clonidine and further enhanced by insulin $(\mathrm{p}<0.05$ by ANOVA).

Conclusion: A mild hyperinsulinaemia of 4-hour duration does not affect endothelium-dependent vasodilatation of conduit vessels but causes a decline in endothelium-independent vasodilatation that is entirely explained by the insulin-induced noradrenergic activation.

\section{5}

Fenofibrate protects microvascular endothelial cells through AMPactivated protein kinase activation and upregulation of eNOS

T. Atsuko, Y. Hattori, T. Okayasu, K. Suzuki, S. Hattori, K. Kasai Endocrinology and Metabolism, Dokkyo University School of Medicine, Mibu, Japan

FIELD (Fenofibrate Interventon \& Event Lowerig in Diabetes) showed that treatment with fenofibrate in individuals with type 2 diabetes mellitus not only reduced nonfatal coronary events but also reduced the need for laser treatment for diabetic retinopathy and inhibited the progress of diabetic nephropathy. The mechanism of the effect on this microangiopathy does not seem to be related to plasma concentrations of lipids. We hypothesized that AMP-activated protein kinase (AMPK) activity is a downstream mediator of the beneficial effects of fenofibrate on microvascular endothelial cells. Treatment of human glomerular microvascular endothelial cells (HGMEC) with fenofibrate resulted in transient activation of AMPK, as monitored by phosphorylation of AMPK and its down-stream target, acetyl-CoA carboxylase. Fenofibrate caused phosphorylation of Akt and eNOS, leading to increased production of NO, and also caused inhibition of cytokine-induced NF- $\mathrm{kB}$ activation, leading to suppression of expression of adhesion molecule genes. Significant decreases in eNOS activity and NO production in response to fenofibrate were observed in cells treated with AMPK siRNA or with AraA, a pharmacological inhibitor of AMPK. The attenuation of fenofibrate-induced inhibition of NF- $\kappa B$ activation was observed in cells treated with AMPK siRNA or with AraA. We demonstrated that TNF $\alpha$ stimulates IкB- $\alpha$ phosphorylation through induction of IKK activity, and that fenofibrate inhibits IKK activity and TNF $\alpha$-induced IкB- $\alpha$ phosphorylation. Furthermore, fenofibrate upregulates eNOS expression, mainly through mechanism of stabilizing eNOS mRNA. Our findings suggest that the beneficial effects of fenofibarate on microvascular endothelial cells might be attributed to the induction of AMPK activation and upregulation of eNOS expression beyond its lipid-lowering actions.

Supported by: Japan Private School Promotion Foundation

\section{6}

Adiponectin stimulate endothelial nitric oxide synthase activation and nitric oxide production by activating AMP-activated protein kinase Y. Long ${ }^{1}$, Y. $\mathrm{Yu}^{2}$

${ }^{1}$ Lab. of Endocrinology and metabolism, ${ }^{2}$ Division of Endocrinology and metabolism, West China Hospital of Sichuan University, Chengdu, China

Background and aims: Adiponection, a hormone secreted by adipocytes, is involved in regulating energy homeostasis and glucose and lipid metabolism, and possesses potent protective effects against endothelial dysfunction. The current study investigated the action of globular adiponectin on endothelial nitric oxide synthase ( eNOS ), nitric oxide ( $\mathrm{NO}$ ) production, and tested the role of AMP-activated protein kinase in $\operatorname{ser}^{1177}$ phosphorylation of eNOS.

Materials and methods: Human umbilical vein endothelial cells (HUVEC) were cultured by DMEM of low glucose with $10 \%$ NBS. After $4 \mu \mathrm{g} / \mathrm{ml} \mathrm{recom-}$ binant human globular adiponectin stimulated for 15 minutes, phospharylation of eNOS at ser ${ }^{1177}$, AMPK at thr ${ }^{176}$ and AKT at ser ${ }^{473}$ were tested by immunocytochemistry. And the activity of eNOS were evaluated by measuring the production of nitrite and nitrate using high performance liquid chromatography ( HPLC).

Results: The level of $\operatorname{ser}^{1177}$-eNOS Phospharylation were increased significantly after globular adiponectin compared with control ( IOD 16240.8 vs. $9159.5 \mathrm{p}=0.048$ ), but not as high as which stimulated by $200 \mathrm{nmol} / \mathrm{l}$ insulin ( IOD $23848.3 \mathrm{p}=0.034$ ) and $20 \mathrm{mmol} / \mathrm{l}$ metformin ( IOD $41472.91 \mathrm{p}=$ $0.005)$. And globular adiponectin caused AMPK $\left(\mathrm{thr}^{172}\right)$ phosphorylation ( IOD $26320.9 \mathrm{p}=0.003$ ) but not AKT $\left(\operatorname{ser}^{473}\right)$ ( IOD $10671.24 \mathrm{p}=0.98$ ). Globular adiponectin can stimulate more nitric oxide production althought no statistical significance when compared with control (nitrite / nitrate 0.0285 vs. $0.0045, \mathrm{p}=0.077)$.

Conclusion: Globular adiponectin can stimulated activation of eNOS and AMPK(thr172 ) phospharylation plays a crucial role in globular adiponectineNOS signal pathway.

Supported by: National Natural Science Foundation of China

\section{7}

Serum- and glucocorticoid-inducible kinase (SGK)-1 decreases human endothelial cell senescence

K. Basello ${ }^{1}$, M.F. Lombardo ${ }^{1}$, F. Ferrelli ${ }^{2}$, D. Pastore ${ }^{1}$, B. Capuani ${ }^{1}$,

A. Coppola ${ }^{1}$, P. Sbraccia ${ }^{1}$, M. Federici ${ }^{1}$, M. Tesauro ${ }^{1}$, G. Donadel ${ }^{1}$, D. Lauro ${ }^{1}$ ${ }^{1}$ Internal medicine, University of Rome Tor Vergata, ${ }^{2}$ IRCCS, San Raffaele, Pisana, Rome, Italy

Background and aims: In vitro culture of vascular cell lines have a finite lifespan and, after several cell divisions, vascular cells enter in an irreversible growth arrest, called "cellular senescence". This process is accompanied by a specific set of phenotypic changes in morphology, gene and proteins expression and activity. Interestingly, the presence of senescent vascular cells has been demonstrated in human atherosclerotic lesions but not in non-atherosclerotic lesions. An increasing body of evidences have established a critical role for the telomere length and telomerase activiy in inducing vascular cell senescence. Aging free radical theory suggests that reactive oxygen species (ROS) contribute significantly to senescent phenotype inducing molecular damages to lipids, proteins, and nucleic acids. It has been demonstrated that ROS production in human umbilical veins endothelial cell lines (HUVEC) is increased with increasing numbers of population doublings (PDL). SGK-1 is a serine/threonine protein kinase that is known to induce cell survival after different stress stimuli; SGK-1 transcription is induced by serum and glucocorticoid stimulation. The present study has been conducted to investigate the potential role of SGK-1 to delay the onset of senescence by counteracting the adverse effect of increased oxidative stress and/or other mechanisms involved in endothelial cell aging process.

Materials and methods: HUVEC have been used as experimental model. HUVEC have been infected with retroviral virus using PLPCX plasmid with 
SGK-1wt or SGK-1D60 (deleted N 60 terminal amino acids) constructs. ROS production has been evaluated by FACS analysis using DHR123 probe. SGK1 and hTERT expression have been measured by western blot and immunofluorescence analysis. SA-beta-galactosidase in situ staining has been used to determine the senescent status. OxyBlot technique has been used to detect and quantify carbonylated proteins that are induced by ROS. Telomerase activity has been quantified by immunofluorescence TRAP assay analysis. Results: SGK-1 had a nuclear localization in SGK-1wt infected HUVEC cells, differently, there was a cytoplasmatic localization in SGK-1D60 infected cells and a barely detectable SGK-1 nuclear localization in HUVEC cells infected with mock (pLPCX). SGK-1 expression levels were higher in SGK-1D60 than SGK-1wt cell lines $(p<0.05)$. Cells infected with SGK-1wt had a reduction of ROS production correlated to the increased numbers of PDL compared to mock $(\mathrm{p}<0.05)$. This result has been confirmed by a reduction in ROSinduced protein damage in SGK-1wt infected cells compared to mock and SGK-1D60 $(\mathrm{p}<0.05)$. It has been showed that cellular ageing decreased telomerase activity both in mock and SGK-1D60, differently, telomerase activity raised with increased numbers of PDL in SGK-1wt cell line $(p<0.05)$. Finally, SGK-1wt infected cells had a reduced levels of SA-beta-Gal staining compared to mock and SGK-1D60, even in cell lines with higher PDL $(\mathrm{p}<0.01)$. Conclusion: 1) In senescent endothelial cell lines increased expression of SGK-1 can reduce ROS production; 2) SGK-1 increased the telomerase activity in cell lines with higher PDL; 3) SGK-1 can decrease endothelial cell senescence.

\section{8}

Circulating endothelial progenitor cell number in long-standing type 1 diabetic patients

H. Reinhard ${ }^{1}$, P.K. Jacobsen ${ }^{1}$, L. Tarnov ${ }^{1}$, A.-S. Astrup ${ }^{1}$, M. Lajer ${ }^{1}$, N. Pedersen ${ }^{1}$, N. Billestrup ${ }^{1}$, T. Mandrup-Poulsen ${ }^{1,2}$, J. Kastrup ${ }^{3}$,

H.-H. Parving ${ }^{4}$, P. Rossing 1

${ }^{1}$ Research, Steno Diabetes Centre, Gentofte, ${ }^{2}$ Department of Biomedical Sciences, Core Unit for Medical Research Methodology, ${ }^{3}$ The Heart Centre, Rigshospitalet, Denmark, ${ }^{4}$ Medical Endocrinology, Rigshospitalet, Denmark

Background and aims: Circulating endothelial progenitor cells (EPC) are involved in neovascularisation and maintenance of vascular integrity. Decrease in number and dysfunction of EPC play a role in the progression of cardiovascular disease. Type 1 diabetic patients suffer from an increased risk of cardiovascular disease; underlying mechanisms are not well understood. The aim of our study was to evaluate EPC number in asymptomatic type 1 diabetic patients with and without diabetic nephropathy.

Materials and methods: We examined EPC number in 72 patients (M/F: 41/31) with long-standing type 1 diabetes without symptoms or history of cardiovascular disease (35 with normoalbuminuria and 37 with diabetic nephropathy). All patients were characterized regarding cardiovascular disease, including cardiac MR imaging. The number of EPC was assessed by in vitro cultures: Mononuclear cells were isolated from peripheral blood, using a Ficoll density gradient centrifugation. Cells were seeded on plates coated with human fibronectin in endothelial basal medium. After 7 days in culture, we counted double-stained cells for both UEA-1 and acLDL-DiI as EPC in 10 randomly selected fields in each patient.

Results: There was no difference in EPC number between patients with DN (120 SE (8)) and normoalbuminuric patients (108 (7)) or age-matched healthy controls (113 (5)). The 41 patients receiving simvastatin had higher number of EPC assessed by in vitro cultures, (133 (9)) cells per field compared to patients not receiving simvastatin, $(100(6))$ cells per field, $\mathrm{p}<0.02$. Similarly, the 17 patients treated with calcium-channel blocker (CCB), used as a third or fourth antihypertensive drug, also had more EPC, (138 (12)) cells per field compared to patients not treated with CCB (106 (6)) cells per field, $\mathrm{p}<0.009$. Patients with systolic 24 -hour blood pressure above the mean of $132 \mathrm{mmHg}$ tended to have higher EPC number (125 (50)) cells per field compared to patients below the mean (106 (41)) cells per field, $\mathrm{p}=0.078$. A multiple regression analysis confirmed these significant findings with estimates: 11 cells per field per $1 \%$ inverse change in $\mathrm{HbAlc}, 35$ cells per field and 24 cells per field regarding therapy with simvastatin and calcium-channel blocker, respectively. $(\mathrm{R}=0.507)$. EPC number was not related to treatment with RAAS blockers or aspirin therapy, however patients with EPC number below the median (114, (SD 45) were treated with a median of one antihypertensive drug compared to two in patients with EPC above the median, $\mathrm{p}<0.02$. Only $4.2 \%$ of patients had coronary artery stenosis. The EPC number was not correlated to lipids, age, gender or coronary plaque burden.
Conclusion: In asymptomatic patients with longstanding type 1 diabetes, low EPC number is associated with poor metabolic control. Treatment with simvastatin is associated with increased number of EPC, independently of lipid levels. Treatment with calcium-channel blockers, reflecting higher blood pressure, is also associated with increased number of EPC. However in these patients with long diabetes duration but low level of coronary artery disease, EPC was not related to diabetic nephropathy and coronary plaque burden.

\section{9}

Endothelial progenitor cells and endothelial dysfunction in prediabetic and newly diagnosed type 2 diabetic subjects

L. Pucci ${ }^{1}$, D. Lucchesi ${ }^{1}$, R. Vanacore ${ }^{2}$, M.C. Iorio ${ }^{2}$, L. Ghiadoni ${ }^{3}$, R. Di Stefano ${ }^{4}$, M.C. Barsotti ${ }^{4}$, E. Storti ${ }^{1}$, A. Balbarini ${ }^{4}$, S. Taddei ${ }^{3}$, R. Miccoli ${ }^{1}$, G. Penno ${ }^{1}$, S. Del Prato ${ }^{1}$

${ }^{1}$ Endocrinology and Metabolism, University of Pisa, ${ }^{2}$ Blood Bank, University of Pisa, ${ }^{3}$ Internal Medicine, University of Pisa, ${ }^{4}$ Cardio-Thorax Disease,

University of Pisa, Italy

Background and aims: Circulating progenitor cells with endothelial phenotype (EPCs) contribute to functional integrity of endothelial monolayer. This integrity is essential to prevent local injures-induced vascular leakage and to protect against initiation of atherogenesis. The concept of repair of damaged dysfunctional endothelial cell by EPCs has recently emerged. Reduction of circulating EPCs has been, indeed, associated with vascular dysfunction.

Materials and methods: We now investigated whether endothelial dysfunction is accompanied by quantitative alterations of the EPC pool in 34 subjects with impaired glucose regulation (IGR) or newly diagnosed (OGTT) type 2 diabetics (n. 18, naiveT2D) as compared to 26 normoglycaemic (NGT) individuals with (n. 13, NGT/Fam+) and without (n. 13, NGT/Fam-) firstdegree family history for type 2 diabetes. Endothelial function (flow-mediated dilation, FMD) of brachial artery was assessed by ultrasound; number of circulating EPCs (CD34/KDR double positive cells) was determined by flow cytometry.

Results: IGR, naiveT2D and NGT did not differ for gender, BMI, waist circumference, diastolic BP, total- and LDL-cholesterol, apoA1 and apoB, serum creatinine, cistatin $\mathrm{C}$, gammaGT, fibrinogen, hs-CRP, and SAA concentrations. However, IGR and naiveT2D were older $(55+/-5$ and $58+/-8$ vs $45+/-10$ yrs, $\mathrm{p}=0.001)$, had higher systolic $\mathrm{BP}(\mathrm{p}=0.001)$ and $\mathrm{HbAlc}$ values $(6.0+/-0.4$ and $65+/-0.6$ vs $5.5+/-0.4 \%, \mathrm{p}<0.0001)$, with not significant trend for lower HDL cholesterol $(p=0.07)$ and higher triglycerides $(p=0.08)$. Endothelium independent dilation (glycerol trinitrate, GTN) was not different, while FMD was lower in naiveT2D (Delta\% 4.4+/-2.3 M+/-SD) compared with IGR (Delta\% $6.0+/-2.8$ ) and in both naiveT2D and IGR compared to NGT (Delta\% 7.9+/-3.6; Kruskal-Wallis $\mathrm{p}=0.0017$ ) with no differences between Fam+ and Fam-. The same pattern was observed for the FMD/GTN ratio, an expression of the selective endothelial function impairment. Both patterns are confirmed between genders and both in young (age $50 \mathrm{yrs}, \mathrm{n} .51$, $\mathrm{p}=0.04)$ subjects. Interestingly, EPCs pool was higher in NGT/Fam- $(608+/$ 87 cells $/ \mathrm{ml}, \mathrm{M}+/$-SE) and similarly reduced in NGT/Fam $+(457+/-68$ cells/ $\mathrm{ml})$ and in IGR $(479+/-65$ cells $/ \mathrm{ml})$, and even more reduced in naiveT2D $(254+/-51$ cells $/ \mathrm{ml}$, Kruskal-Wallis, $\mathrm{p}=0.01)$. There were no gender-related or age-related differences.

Conclusion: Naive type 2 diabetic subjects showed depletion in the circulating EPC pool as well as impaired endothelium-dependent vasodilation. In subjects with non-diabetic hyperglycemia, reduction in FMD is paralleled by an intermediate depauperation of EPC pool. This impoverishment is already apparent in normoglycemic subjects with positive family history for diabetes suggesting that reduction in circulating EPCs may precede impairment in endothelial function.

\section{0}

Kinin-mediated recruitment of progenitor cells is altered in diabetes N. Kraenkel ${ }^{1}$, V. Adams ${ }^{2}$, M. Schoenauer ${ }^{3}$, R. Katare ${ }^{1}$, C. Emanueli ${ }^{1}$, P. Madeddu ${ }^{1}$

${ }^{1}$ Bristol Heart Center, University of Bristol, United Kingdom, ${ }^{2}$ Heart Center Leipzig, University of Leipzig, Germany, ${ }^{3}$ Diabetologische Schwerpunktpraxis und Lehrpraxis der Universitaet Leipzig, Germany

Background and aims: Reduced recruitment of vasculogenic (VPC) and endothelial progenitor cells (EPC) is associated with an increased incidence of ischaemic complications in diabetic patients. In the past we and others 
demonstrated an implication of the kallikrein-kinin-system (KKS) in reparative angiogenesis, mediated by mature endothelial cells. Here we investigated the hypothesis that bradykinin (BK) furthermore serves as a chemoattractant for VPC and EPC and that BK mediated recruitment of VPC is impaired in diabetic patients.

Materials and methods: EPC were cultured from peripheral blood mononuclear cells $(\mathrm{PB}-\mathrm{MNC})$ of non-diabetic $(\mathrm{C}, \mathrm{n}=10)$ and type 2 diabetic donors $(\mathrm{D}, \mathrm{n}=10) . \mathrm{CD}_{133^{+}}$and $\mathrm{CD} 34^{+} \mathrm{VPC}$ were isolated from PB-MNC by magnetic sorting. Expression of components of the KKS were analysed by qRTPCR and flow cytometry. Migration of VPC and EPC towards BK was studied in the presence or absence of BK-receptor antagonists. BK-responsive cells were isolated from $\mathrm{PB}-\mathrm{MNC}$ and the ability to give rise to EPC was studied in the BK-responsive $\left(\mathrm{BK}^{\mathrm{mig}}\right)$ and non-responsive $\left(\mathrm{BK}^{\mathrm{non}}\right)$ cell fractions. In vitro pro-angiogenic effects of $\mathrm{BK}^{\mathrm{mig}}$ - and $\mathrm{BK}^{\mathrm{non}}$-derived $\mathrm{EPC}$ were studied by matrigel assay. Homing of systemically injected $\mathrm{B}_{2} \mathrm{R}$-deficient or wild type MNC to the ischaemic limb of the recipient and support of blood flow recovery in the ischaemic leg was studied in a mouse hindlimb ischemia model. The abundance of $\mathrm{CD}_{133^{+}}$and $\mathrm{CD} 34^{+} \mathrm{VPC}$ co-expressing kinin receptors was analysed by flow cytometry in $\mathrm{PB}$ of healthy and diabetic donors.

Results: $\mathrm{CD} 133^{+}$and $\mathrm{CD} 34^{+}$VPC as well as cultured EPC expressed kininogens, tissue kallikrein and the constitutive $\mathrm{B}_{2} \mathrm{R}$. The inducible kinin $\mathrm{B} 1$ receptor $\left(\mathrm{B}_{1} \mathrm{R}\right)$ was expressed only at low levels. $\mathrm{CD} 133^{+} \mathrm{VPC}$ and cultured EPC migrated dose-dependently towards $\mathrm{BK}$. BK-induced migration was significantly reduced in the presence of $\mathrm{B}_{2} \mathrm{R}$ antagonist HOE140 (reduced by $43.3 \pm 9.9 \%$ vs. BK, $\mathrm{p}=0.002$ ) and PI3-kinase inhibitor LY294002 (reduced by $62.9 \pm 14.8 \%$ vs. $\mathrm{BK}, \mathrm{p}=0.03$ ). MNC migrating towards $\mathrm{BK}$ harboured a 3.9 fold higher number of $\mathrm{CD}_{133^{+}}(\mathrm{p}=0.01)$ and a 2 -fold higher number of $\mathrm{CD} 34^{+}$ VPC $(p<0.05)$ and gave rise to 2.5 -fold more EPC $(p=0.01)$ as compared to $\mathrm{BK}^{\text {non }}$. In contrast to $\mathrm{BK}^{\text {non }}, \mathrm{BK}^{\text {mig }}$-derived $\mathrm{EPC}$ facilitated tube formation in matrigel. $\mathrm{B}_{2} \mathrm{R}$-deficient $\left(\mathrm{B}_{2} \mathrm{R}^{-/}\right)$EPC homed less well to ischaemic adductors as compared to WT MNC and blood flow recovery was significantly reduced when $\mathrm{B}_{2} \mathrm{R}^{-1-}$ cells were injected. In diabetic subjects, the abundance of $\mathrm{CD} 34^{+}$ and $\mathrm{CD} 133^{+}$VPC among kinin receptor expressing cells was decreased as compared to non-diabetic subjects.

Conclusion: BK induced migration of VPC might represent a novel alternative signalling pathway mediating circulating stem cell recruitment to facilitate vasculogenesis. Reduction of VPC and EPC among BK sensitive cells in diabetic patients suggests an imbalance among the spectrum of cells recruited by kinins.

Supported by: EFSD/JDRF/NovoNordisk grant

\section{PS 131 Predictors of macrovascular disease}

\section{1}

Osteoprotegerin correlates with severity of peripheral artery disease in individuals with type 2 diabetes

E.P. O'Sullivan ${ }^{1}$, D.T. Ashley ${ }^{2}$, J. Kelly ${ }^{3}$, R.K. Crowley ${ }^{1}$, A. Agha ${ }^{1}$, C.J. Thompson ${ }^{1}$, A.L. Leahy ${ }^{4}$, C.J. Kelly ${ }^{4}$, D. O’Gorman ${ }^{2}$, P. Fitzgerald ${ }^{3}$, D. Smith ${ }^{1}$

${ }^{1}$ Diabetes and Endocrinology/RCSI Medical School, Beaumont Hospital, Dublin, ${ }^{2}$ School of Health and Human Performance, Dublin City University, ${ }^{3}$ Non-Invasive Vascular Lab/RCSI Medical School, Beaumont Hospital, Dublin, ${ }^{4}$ Department of Surgery/RCSI Medical School, Beaumont Hospital, Dublin, Ireland

Background and aims: Osteoprotegerin (OPG) is a member of the tumour necrosis factor receptor family and acts as an anti-resorptive agent in bone. Following the discovery that OPG knock-out mice develop severe vascular calcification in addition to osteoporosis, serum OPG has subsequently been shown to be associated with coronary artery calcification and cardiovascular mortality. Peripheral arterial disease (PAD) is also associated with vascular calcification and cardiovascular disease, yet the relationship between OPG and PAD in patients with diabetes has not been clarified. The aim of the study was to determine whether serum OPG correlates to severity of PAD, particularly in diabetes patients with non-compressible vessels, a clinical indicator of severe vascular calcification.

Materials and methods: 35 patients with type 2 diabetes and an age, gender and BMI-matched group of 29 without diabetes attending the vascular surgeons were enrolled into the study. All underwent assessment of PAD by means of ankle-brachial index (ABI). $63.9 \%$ had PAD, as defined by a resting $\mathrm{ABI}$ of $<0.7$, a drop in $\mathrm{ABI}$ post-exercise of $>0.2$, or toe pressures $<0.6$. ABIs were defined as non-compressible if the pulse could not be obliterated by inflation of a blood pressure cuff to $>220 \mathrm{mmHg}$. OPG was measured using a commercial ELISA assay. Statistical analysis was performed using the JMP IN 5.1 package.

Results: In the group as a whole, OPG was higher in those with PAD $(7.8 \pm 0.3 \mathrm{pmol} / \mathrm{l})$, compared to those with normal ABIs $(5.6 \pm 0.4 \mathrm{pmol} / \mathrm{l})$, $\mathrm{p}<0.0001$; a relationship which remained statistically significant after correction for the fact that there were more patients with diabetes in the nonPAD group ( $81.5 \%$ vs. $50 \%, \mathrm{p}=0.01)$. There was a negative correlation between OPG and result of ABI; $r=-0.41, p=0.003$. All but 1 of the 8 patients with non-compressible vessels had diabetes, and mean OPG was higher in these patients $(8.1 \pm 0.8 \mathrm{pmol} / \mathrm{l})$ compared to those with compressible vessels $(6.3 \pm 0.4 \mathrm{pmol} / \mathrm{l}), \mathrm{p}=0.04$.

Conclusion: OPG is higher and correlates with the severity of PAD as defined by ABI. Whether OPG is simply a marker of underlying atherosclerotic disease or is directly involved in the pathogenesis of vascular calcification requires further research but the significant association of OPG and severe vascular calcification in patients with non-compressible vessels suggests that OPG plays a key role in pathogenesis.

Supported by: sanofi-aventis, Pfizer Pharmaceuticals

\section{2}

Coronary atherosclerosis is characterised by more severe metabolic, inflammatory and endothelial alterations when compared to carotid atherosclerosis

E. Setola ${ }^{1}$, L.D. Monti ${ }^{1}$, P.C.G. Lucotti ${ }^{1}$, E. Galluccio ${ }^{1}$, M. Oldani ${ }^{1}$, M.G. Pala ${ }^{2}$, A. Rossodivita ${ }^{2}$, M. Comola ${ }^{3}$, A. Poggi ${ }^{3}$,

M. Marrocco-Trischitta ${ }^{2}$, G. Comi ${ }^{3}$, R. Chiesa ${ }^{2}$, E. Bosi ${ }^{1}$, O. Alfieri², P.M. Piatti ${ }^{1}$

${ }^{1}$ Internal Medicine and Specialty Department, Scientific Institute San Raffaele, ${ }^{2}$ Cardiothoracovascular Department, Scientific Institute San Raffaele, ${ }^{3}$ Neurology Department, Scientific Institute San Raffaele, Milan, Italy

Background and aims: Several studies have demonstrated that patients affected by coronary or carotid atherosclerosis are at high risk of developing atherosclerotic lesions in other vascular districts, but there are no data available on the specific differences between coronary and carotid atherosclerosis. Therefore, aim of the present study was to evaluate which metabolic, hor- 
monal and inflammatory alterations characterize coronary atherosclerosis compared to carotid atherosclerosis.

Materials and methods: We studied 66 patients affected by asymptomatic ischemic cardiopathy (Group 1) and 88 patients affected by carotid vasculopathy (Group 2). Group 1 included patients with a medical history of negative vascular events in other districts, in particular these patients were submitted to a carotid ultrasound that ruled out the presence of atherosclerotic lesions. In Group 2 patients presented a negative medical history for coronary events and a negative ECG for previous ischemic lesions. All patients did not have any known diabetes and fasting plasma glucose was below $126 \mathrm{mg} / \mathrm{dl}$. The two groups were comparable by age, BMI, systolic and diastolic blood pressure, total cholesterol, HDL cholesterol and triglycerides. Plasma glucose, serum insulin and FFA levels were evaluated during an oral glucose load in all subjects and inflammatory and endothelial markers were evaluated in the fasting state.

Results: Fasting plasma glucose $(107.1 \pm 3.6$ vs $96.1 \pm 2.0 \mathrm{mg} / \mathrm{dl} ; \mathrm{p}<0.01)$, HOMA index ( $2.90 \pm 0.4$ vs $1.93 \pm 0.3 ; \mathrm{p}<0.003), \mathrm{NOx}(18.7 \pm 1.60$ vs $11.7 \pm 0.86$ $\mu \mathrm{mol} / \mathrm{l} ; \mathrm{p}<0.001)$, IL-6 levels $(4.04 \pm 0.64 \mathrm{vs} 2.79 \pm 0.43 \mathrm{pg} / \mathrm{ml} ; \mathrm{p}<0.02)$ and TNF-alpha levels $(19.4 \pm 0.1 .3$ vs $15.7 \pm 1.7 \mathrm{pg} / \mathrm{ml} ; \mathrm{p}<0.05)$ were significantly higher in Group 1 when compared to Group 2. After the oral glucose load, $26.5 \%$ of Group 1 patients presented normal glucose tolerance compared to $44.5 \%$ in Group $2(\mathrm{p}<0.05)$. An altered glucose homeostasis, i.e. impaired glucose tolerance was found in $40.5 \%$ of Group 1 patients, while $33 \%$ evidenced type 2 diabetes mellitus. In Group 2, 35\% of patients presented impaired glucose tolerance and $20 \%$ type 2 diabetes mellitus. FFA levels during the oral glucose load were higher in Group 1 than in Group 2 (60 min: $0.38 \pm 0.03$ vs $0.23 \pm 0.01 \mu \mathrm{mol} / \mathrm{l}, \mathrm{p}<0.01 ; 120 \mathrm{~min}: 0.19 \pm 0.02$ vs $0.15 \pm 0.01 \mathrm{mmol} / \mathrm{l}, \mathrm{p}<0.03)$. Furthermore, NOx levels during the oral glucose load were significantly lower in Group 1 compared to Group 2 ( $\triangle$ AUC NOx: $-58.4 \pm 28.2$ vs $-13.3 \pm 12.1$ $\mu \mathrm{mol} / 1(0-120 \mathrm{~min}) ; \mathrm{p}<0.0001)$. Performing a multiple regression analysis, $2 \mathrm{~h}$ post load plasma glucose was independently related to vascular district $(p<0.001)$, AUC of FFA $(p<0.01)$, HDL cholesterol $(p<0.003)$, AUC of insulin $(\mathrm{p}<0.01)$ and basal NOx $(\mathrm{p}<0.02)$.

Conclusion: Coronary atherosclerosis is characterized by a higher prevalence of abnormal glucose tolerance and insulin resistance, a decreased antilipolytic activity of insulin, and a more proatherogenetic profile than carotid atherosclerosis.

\section{3}

Role of ectodomain shedding activity from metabolic syndrome to carotid and coronary artery disease

M. Cardellini ${ }^{1}$, S. Rizza ${ }^{1}$, R. Menghini ${ }^{1}$, E. Martelli ${ }^{2}$, F. Clementi ${ }^{1}$,

A. Ippoliti ${ }^{3}$, F. Romeo ${ }^{1}$, F. Folli ${ }^{4}$, M. Federici ${ }^{1}$;

${ }^{1}$ Department of Internal Medicine, University of Rome Tor Vergata,

Italy, ${ }^{2}$ Department of Cardiovascular Surgery, University of Rome, Italy,

${ }^{3}$ Department of Cardiovascular Surgery, University of Rome Tor Vergata,

Italy, ${ }^{4}$ Internal Medicine, University of Texas Health Science Center SA, San Antonio, United States

Background and aims: The process of ectodomain shedding of transmembrane proteins has been recently involved in the regulation of inflammation in relation to atherosclerosis and diabetes vascular complications. Several studies suggested that soluble factors including sICAM-1, sVCAM-1, sIL6R and sTNFR1 are increased in various settings of metabolic diseases. All these factors are substrates of A Disintegrin and Metalloprotease Domain 17 (ADAM17).

Materials and methods: To elucidate the involvement of this system in the progression of atherosclerosis, we analyzed four cohorts including: 1 . first degree relatives of type 2 diabetes patients (FDRs, $n=35$ ); 2. patients with chronic carotid artery stenosis (CTA, $n=32)$; 3 . patients with stable Coronary Artery Disease (CAD, $n=170$ ) all subjected to coronary angiography and/or cardio-TC and divided in monovessel and multivessel groups (CADmono $n=60$; CADmulti $n=110$ ) and 4. patient with carotid atherosclerosis who underwent to carotid endoarteriectomy ( $\mathrm{n}=71$ : NGT $=39, \mathrm{IGT}=19$ and $\mathrm{DM} 2=13$ ). All the subjects underwent an OGTT at the beginning of the study and analysis of lipids and inflammatory markers. Ectodomain shedding process activity was performed by analysis of sICAM-1, sVCAM-1, sIL6R, sTNFR1 and sCXCL16, which is also shed by ADAM10, in cohorts 1,2 and 3. In the fourth cohort we also evaluated ADAM17 and TIMP1/2/3/4 mRNA expression levels by Real Time PCR and ADAM17 activity in the atherosclerotic plaques obtained from surgery.

Results: Results showed that SCXCL16 did not differ among groups although in CADmulti showed increased levels compared to CADmono group $(\mathrm{p}=0.01)$. sTNFR1 resulted increased only in CADmulti compared with FDR, CTA and CADmono $(\mathrm{p}<0.001)$. sIL6R was increased in CTA and CAD compared with FDR $(p<0.001)$ while sICAM-1 and sVCAM- 1 significantly increased according to severity of vascular disease (FDRs $<\mathrm{CTA}<\mathrm{CAD}$ monovessel $<$ CAD multivessel, $\mathrm{p}<0.001$ Anova). Correlation of substrates with metabolic parameters in the different groups suggested that sICAM-1 was correlated with HOMA-IR in CAD ( $\mathrm{p}=0.002, \mathrm{r}=0.24)$ while sIL6R was correlated with HOMA-IR ( $\mathrm{p}=0.03, \mathrm{r}=0.37)$ and IMT $(\mathrm{p}=0.003, \mathrm{r}=0.54)$ in FDRs. Our data suggest that ADAM17 activity is gradually increased in the progression of the atherosclerotic disease. To elucidate the mechanism that underlie this observation, we analyzed ADAM17 and TIMP1/2/3/4 RNA expression levels by Real Time PCR and ADAM17 activity in the 71 atherosclerotic plaques. In this subjects RNA expression levels of TIMP3 is significantly reduced in DM2 compared with NGT $(\mathrm{p}<0,01)$ and significantly correlated with $\mathrm{HbAlc}$ $(\mathrm{R}=-0.31, \mathrm{p}<0.02)$ and $\mathrm{LDL}$ cholesterol $(\mathrm{R}=-0.28, \mathrm{p}<0,03)$. ADAM17 activity is increased in DM2 compared with NGT (NGT vs DM2 $\mathrm{p}<0,001$ ).

Conclusion: In conclusion our data suggest that the ectodomain shedding process is gradually involved in the progression of atherosclerosis from preclinical state to asymptomatic disease to clinically manifested disease, in relationship with the expression of TIMP3, which seems to be linked to factors correlated with glucotoxicity and hypercholesterolemia.

\section{4}

Chronic low-grade inflammation mediates the association between body mass index and pulse pressure in women with type 1 diabetes: a 20-yr longitudinal study P. Hovind ${ }^{1,2}$, I. Ferreira ${ }^{3,4}$, H.-H. Parving ${ }^{5,6}$, C.G. Schalkwijk ${ }^{3,7}$, C.D.A. Stehouwer ${ }^{3,7}$, P. Rossing ${ }^{1}$

${ }^{1}$ Steno Diabetes Center, Gentofte, Denmark, ${ }^{2}$ Department of Clinical Physiology and Nuclear Medicine, Bispebjerg Hospital, Copenhagen, Denmark, ${ }^{3}$ Department of Medicine, University Hospital Maastricht, Netherlands, ${ }^{4}$ Department of Clinical Epidemiology and MTA, University Hospital Maastricht, Netherlands, ${ }^{5}$ Department of Medicinal Endocrinology, Rigshospitalet, Copenhagen, Denmark, ${ }^{6}$ Faculty of Health Science, University of Aarhus, Denmark, ${ }^{7}$ Cardiovascular Research Institute, University of Maastricht, Netherlands

Background and aims: With ageing, diabetic patients show a steeper increase in pulse pressure (PP; a marker of arterial stiffening) than non-diabetics, which may partially explain their increased cardiovascular risk. Increases in body fatness, and associated low-grade inflammation, may be one of the mechanisms underlying these increases in pulse pressure, but this has not been thoroughly examined among type 1 diabetic patients. We have therefore investigated, in a 20 -yr prospective study, 1) whether the longitudinal development of BMI was associated with increases in PP and increases in a marker of low-grade inflammation (CRP); and 2) the extent to which CRP could explain any association between BMI and PP.

Materials and methods: Study population consisted of 277 type 1 diabetic patients (114 women) from an outpatient diabetic clinic (Gentofte, Denmark), who were consecutively admitted, upon diagnosis, between Sep'79-Aug'84 (mean age at diagnosis $27.5 \pm 13.8$ yrs; 216 adults). These patients attended the outpatient clinic every 3 or 4 months, throughout a total follow-up period of $>20$ yrs [median follow-up duration $=18$ yrs (range 1.0-21.5)], as part of their routine evaluation. This included the assessments of BMI and blood pressure, as well as $\mathrm{HbAlc}$, urinary albumin excretion rate (UAE), creatinine, cholesterol, and smoking status. PP was calculated as systolic - diastolic pressure. CRP was recently measured in blood samples collected throughout the whole longitudinal period. We used generalized estimating equations (GEEs) to investigate, longitudinally, the associations hypothesized and results hereby obtained are expressed in longitudinal regression coefficients (respective 95\% CI). All analyses were adjusted for age and the other risk factors assessed (listed above).

Results: BMI and PP increased significantly throughout the longitudinal period: by 0.22 and $0.23\left(\mathrm{~kg} / \mathrm{m}^{2}\right.$ per year) and by 0.45 and $0.59(\mathrm{mmHg} /$ year), in men and women, respectively $(\mathrm{P}<0.001$ for all). BMI was positively associated with PP in women $[\beta=0.52(0.15 ; 0.89)]$, but not in men $[\beta=-0.04(-0.48$; $0.40)]$; BMI was positively associated with $\log \mathrm{CRP}[\beta=0.030(0.008 ; 0.053)$; $\beta=0.041(0.015 ; 0.046)]$, in both men and women, respectively. Finally, the association between BMI and PP observed in women was attenuated by about $29 \%$ for $\log C R P[\beta=0.37(-0.10 ; 0.85)]$.

Conclusion: Life-course increases in body fatness are associated with increases in pulse pressure, a marker of arterial stiffness, in type 1 diabetic women (but not men). This association is, at least in part, mediated by the obesity-related increase in low-grade inflammation.

Supportd by: Danish Diabetes Association 


\section{5}

Gender difference in risk factors and mortality in patients with type 2 diabetes and myocardial infarction. A report from the DIGAMI 2 trial A. Norhammar, K. Malmberg, L. Rydén

Unit of Cardiology, Institution of medicine, Karolinska University Hospital, Stockholm, Sweden

Background and aims: Diabetic women are in general at increased risk for cardiovascular disease compared to men. The impact of gender after myocardial infarction is, however, less well explored among such patients.

Materials and methods: The DIGAMI 2 trial included 837 men and 416 women with type 2 diabetes and myocardial infarction. Risk factor profile and outcome were analysed by gender. Predictors for outcome (previous myocardial infarction, heart failure, ventricular fibrillation, cardiogenic shock, smoking, age, creatinine, complications to diabetes, admission blood glucose, HbAlc and diabetes duration) were analysed by a stepwise logistic regression model. Results: Women were older, had more frequently a history of heart failure and hypertension and were more often treated with diuretics at admission and discharge. Beta-blockers were given less often to women while ACE-inhibitors were used in a similar proportion of men and women. The combined end point (death, myocardial infarction and stroke) were more common in women (Figure). This difference disappeared after adjusting for age. Predictors for an event were previous heart failure and myocardial infarction, cardiogenic shock, creatinine, diabetes complications and blood glucose at admission.

Conclusion: The risk factor profile differs in men and women with type 2 diabetes and acute myocardial infarction with more previous heart failure and hypertension in women. Improved risk factor control may have a potential to improve the prognosis among female patients.

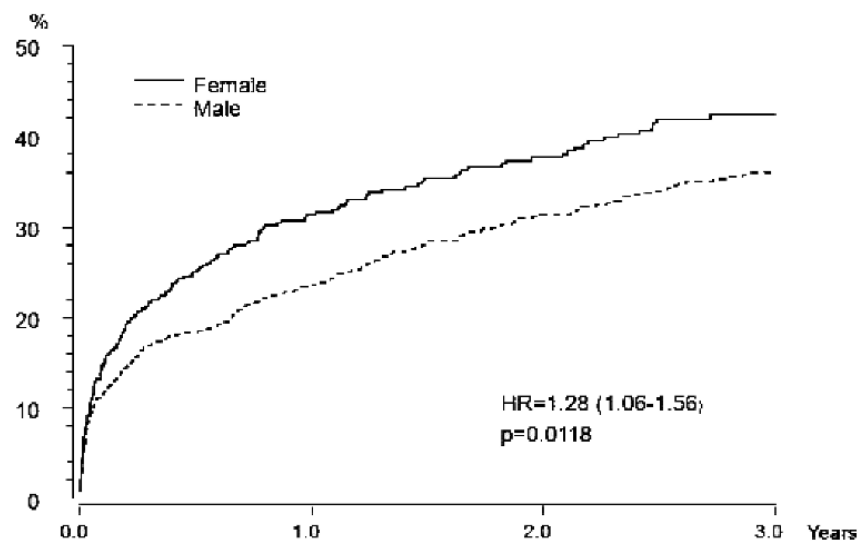

\section{6}

Apolipoprotein $\mathrm{B} / \mathrm{A1}$ ratio is independently associated with carotid intima media thickness in Korean diabetic patients with low LDL-cholesterol Y. Choi ${ }^{1}$, S. Choi ${ }^{2}$, S. Park ${ }^{3}$, D. Kim ${ }^{4}$, C. Kim ${ }^{5}$, S.-H. Jee ${ }^{6}$, E.-J. Lee ${ }^{7}$, K. Huh ${ }^{1}$ ${ }^{1}$ Endocrinology, Huh's Diabetes Center, Seoul, Republic of Korea,

${ }^{2}$ Endocrinology, Seoul National University Bundang Hospital, Seongnam, ${ }^{3}$ Endocrinology, Pochon Cha University, Seongnam, ${ }^{4}$ Endocrinology, Aju University, Suwon, ${ }^{5}$ Endocrinology, Hallym University, Anyang, Republic of Korea, ${ }^{6}$ Epidemiology, Yonsei University, Seoul, ${ }^{7}$ Endocrinology, Yonsei University, Seoul, Republic of Korea

Background and aims: The Apo B/Apo A1 ratio has been regarded as a useful predictor for cardiovascular events. Apo B measurement yields additional information on presence of atherogenic VLDL or small dense LDL, the type of dyslipidemia that is common in type 2 diabetes. In this study, we investigated whether Apo B/Apo A1 was associated with subclinical atherosclerosis in type 2 diabetic subjects with low LDL-cholesterol.

Materials and methods: Of 3777 patients with type 2 diabetes, who visited Huh's Diabetes Center from 2003 to 2007, we enrolled 1316 patient (mean age $57 \pm 10 \mathrm{yr}$, Male 55\%) with low LDL cholesterol ( $<100 \mathrm{mg} / \mathrm{dl})$. The Apo $\mathrm{B} / \mathrm{Apo} \mathrm{A} 1$ ratio was measured in all the subjects. Carotid artery intima media thickness (C-IMT) was measured bilaterally by high-resolution B-mode ultrasound and carotid atherosclerosis was defined as having a clearly isolated focal plaque or diffuse thickening of C-IMT $\geq 1.1 \mathrm{~mm}$.
Results: The Apo B/Apo A1 ratio was positively correlated with total cholesterol $(\mathrm{r}=0.122, \mathrm{p}<0.001)$, triglyceride $(\mathrm{r}=0.384, \mathrm{p}<0.001)$, LDL-cholesterol $(\mathrm{r}=0.271, \mathrm{p}<0.001)$, fasting glucose $(\mathrm{r}=0.059, \mathrm{p}<0.05)$, blood pressure $(\mathrm{r}=0.055, \mathrm{p}<0.05), \mathrm{BMI}(\mathrm{r}=0.173, \mathrm{p}<0.001)$ and negatively correlated with HDL-cholesterol $(\mathrm{r}=-0.537, \mathrm{p}<0.001)$. The Apo B/Apo A1 represents high LDL/low HDL cholesterol in our study subjects. In multiple regression model, the Apo B/Apo A-1 ratio was independently associated with C-IMT after adjusting for age, duration of diabetes, BMI, triglyceride, HDL cholesterol, systolic blood pressure, smoking in both sexes (men: $\beta=0.150, p<0.001$, women: $\beta=0.078, p<0.05)$. The relative risk for carotid artery atherosclerosis was 1.76 (95\% CI: 1.08,2.88) in men and 2.18 (95\% CI: 1.38,3.44) in women with highest tertile of the Apo $\mathrm{B} / \mathrm{Apo} \mathrm{A} 1$ ratio compared with men and women with lowest tertile of the Apo B/Apo A1 ratio.

Conclusion: The Apo B/Apo A1 ratio could be a good predictor for carotid atherosclerosis in type 2 diabetics with low LDL-cholesterol.

\section{7}

Combination of resistin (RETN) polymorphism and serum resistin level may be a good risk marker for susceptibility of stroke in Japanese type 2 diabetic patients

E. Nakashima ${ }^{1}$, A. Watarai ${ }^{1}$, M. Tomita ${ }^{2}$, T. Matsuki ${ }^{1}$, T. Tsukahara ${ }^{1}$, K. Naruse ${ }^{1}$, H. Kamiya ${ }^{1}$, T. Shibata ${ }^{1}$, Y. Oiso ${ }^{1}$, J. Nakamura ${ }^{1}$

${ }^{1}$ Endocrinology and Diabetes, Nagoya University, ${ }^{2}$ Information Systems and Mathematical Sciences, Nanzan University School, Nagoya, Japan

Background and aims: Diabetic macroangiopathy such as myocardial infarction, angina pectoris and cerebrovascular diseases are the major causes of mortality in diabetic patients. Resistin, an inflammatory cytokines, expressed in human monocytes and macrophages has reported to be elevated in the subjects with obesity and insulin resistance. Resistin has recently been also reported to have direct actions on arterial wall, and may contribute to the development of macroangiopathy. We previously reported that blood resistin levels were correlated with the inflammatory status and be associated with the prevalence of stroke in Japanese type 2 diabetic patients. But it also reported that the specific recognition of $-420 \mathrm{G}$ allele by $\mathrm{Sp} 1 / 3$ transcription factor was reported to increase resistin gene (RETN) promoter activity, leading to increased serum resistin levels in human. In this study, therefore, we explored its other SNPs in correlation with its blood levels and examined the combination of resistin SNPs and its blood levels for the possibility as a risk marker of stroke in Japanese type 2 diabetic patients.

Methods: A total of 349 type 2 diabetic outpatients were enrolled in this study. Resistin were measured by an enzyme-linked immunoassay kit. Genotyping of polymorphisms at position $-420,-358,188$, and 299 were performed by polymerase chain reaction restriction fragment length polymorphism method. Statistical analyses were performed using the program SPSS. Data are presented as means \pm SD. We also studied an association analysis of the model by diplotype-based QTL phenotype that is used estimated haplotypes.

Results: Serum resistin levels were significantly higher in patients with stroke than in those without stroke $(19.6 \pm 12.6$ vs. $12.4 \pm 7.8 \mathrm{ng} / \mathrm{ml}, \mathrm{P}<0.001)$. The serum resistin levels were increased in a genotype-dependent fashion in all 4 genotypes. There was strong linkage disequilibrium among these 4 SNPs. The enrolled patients were divided by their serum resistin concentrations (high or low group) and their genotypes (CC, CG, GG at -420) into 6 groups. After adjustment for age, gender, body mass index, systolic blood pressure, and serum levels of triglycerides and creatinine, the multivariate logistic-regression analysis were done and each odds ratio against CC_Low group was calculated.

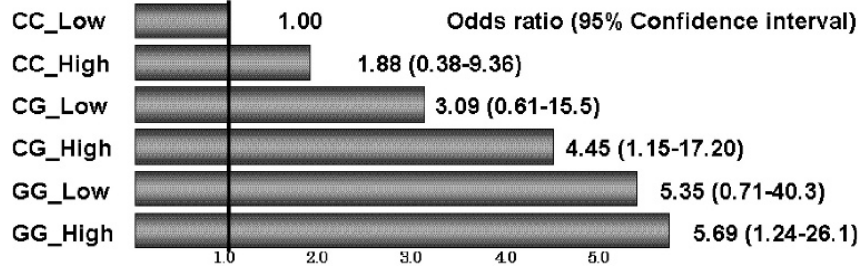

Figure

Conclusion: The serum resistin levels and its genotype were associated with the prevalence of diabetic macroangiopathy in Japanese type 2 diabetic patients. Our results suggest that the combination of RETN gene polymorphism and serum resistin levels might be a strong marker of susceptibility to stroke among Japanese type 2 diabetic patients.

Supported by: National Institute of Biomedical Innovation in Japan 


\section{8}

Partial tissue kallikrein deficiency does not have a major impact on macrovascular complications in type 2 diabetic patients from the DIABHYCAR study

M. Fysekidis ${ }^{1,2}$, N. Bellili ${ }^{3}$, R. Roussel ${ }^{1,2}$, S. Madani-Maimaitiming ${ }^{3}$, F. Fumeron ${ }^{3}$, K. Mohammedi ${ }^{1,2}$, M. Marre ${ }^{1,2}$

${ }^{1}$ Diabetology,Nutrition and Endocrinology, Bichat Hospital, APHP, ${ }^{2}$ Inserm, U695,INSERM, ${ }^{3}$ INSERM, U695,INSERM, Paris, France

Background and aims: Tissue kallikrein known to cleave kininogen thus releasing kinins that have local vasodilator and diuretic effects is implicated in the regulation of renal hemodynamics and blood pressure. Subjects affected with the $\mathrm{R} 53 \mathrm{H}$ polymorphism (5-7\% of the caucasian population) were reported to have decreased enzymatic activity leading to eutrophic inward remodeling of the arterial wall and reduced arterial ability to adapt to shear stress.We have tested the impact of $\mathrm{R} 53 \mathrm{H}$ on the risk for the occurrence of primary cardiovascular events such as myocardial infraction, stroke, cardiovascular death, heart failure, end stage renal disease or all of them combined in type 2 diabetic patients from the Non-Insulin-Dependent Diabetes, Hypertension, Microalbuminuria, Cardiovascular Events, and Ramipril (DIABHYCAR) study.

Materials and methods: The DIABHYCAR Study was a 6-year double-blind multicenter multinational clinical trial with 4,912 type 2 diabetic men and women selected from 1995 to 2001 from 16 European countries, aged 50 years or older with urinary albumin concentration (UAC) $>20 \mathrm{mg} / \mathrm{l}$. In the present study DNA samples were available for 3122 French type 2 diabetic patients. The tested hypothesis was that the $258 \mathrm{R} 53 \mathrm{H}$ and the $3 \mathrm{H} 53 \mathrm{H}$ would display a higher odds ratio (OR) for primary cardiovascular events than the 2861 R53R. Survival analysis was performed and adjustements were made using the Cox model.

Results: At baseline 44 from the $261 \mathrm{RH} / \mathrm{HH}$ subjects had a history of cardiovascular events and 502 from the 2861 RR group with no significant difference between the two groups ( $\chi^{2} \mathrm{RR}$ vs RH/HH:0,078. $p=0,778$ ). Age, sex ratio, $\mathrm{HbAlc}$, blood pressure, smoking and diabetes duration were similar between groups and they were variables in the Cox model. During follow up 495 combined primary events occurred with 46 of them in the $\mathrm{RH} / \mathrm{HH}$ group and the adjusted OR in the Cox model for participants with $\mathrm{RH} / \mathrm{HH}$ genotypes vs RR was 1,3 (95\% CI 0,94-1,79) with an attributable population risk at $2,3 \%(95 \% \mathrm{CI} 0,0-8,0 \%)$. There were 13 congestive heart failure events OR 1,56(95\% CI 0,83-2,93), 11 strokes OR 1,45 (95\% CI 0,77-2,72), 4 myocardial infarctions OR 0,54 (95\% CI 0,17-1,70), 18 deaths of cardiovascular origin OR 1,54 (95\% CI 0,91-2,60) and no end stage renal disease in the $\mathrm{RH} /$ $\mathrm{HH}$ group. No significant interactions with Angiotensin Converting Enzyme Insertion/Deletion polymorphism were found. Secondary event analysis for 37 amputations with 7 in the R53H group showed an OR of 2,66 (95\% CI $1,01-6,55)$ with a $\log$ rank $\mathrm{RH} / \mathrm{HH}$ vs RR: $6,63(p=0,018)$ and a population attributable risk of $1,65 \%$ (95\% CI 0,0-4,0\%).

Conclusion: Partial tissue kallikrein deficiency in type 2 diabetic patients with the $\mathrm{R} 53 \mathrm{H}$ polymorphism does not appear to have a major impact in the occurrence of mortality or macrovascular complications. The possible role in diabetic foot needs to be confirmed by further studies due to a small number of events and the lack of statistical power.

\section{9}

The effects of adiponectin gene polymorphisms on the progression of carotid intima-media thickness

H.J. Kim ${ }^{1}$, E.S. Kang ${ }^{2}$, H.C. Lee ${ }^{2}$

${ }^{1}$ Internal Medicine, Medical College, Kwandong University, Goyang, ${ }^{2}$ Internal Medicine, Medical College, Yonsei University, Seoul, Republic of Korea

Background and aims: Previous studies have documented environmental risk factors for the progression of carotid intima-media thickness (IMT); however, few have examined genetic risk factors for the progression of carotid IMT and none has specifically examined subjects with type 2 diabetes. This study examined the association between single nucleotide polymorphisms in the adiponectin gene and the progression of carotid IMT in patients with type 2 diabetes.

Materials and methods: We performed common carotid ultrasonography at baseline and 33-39 months later in 324 consecutive subjects with type 2 diabetes. The genotypes of the adiponectin gene were determined using a single base primer extension method.
Results: The A allele of $-11426 \mathrm{~A} / \mathrm{G}(P<0.05)$, G allele of $-10066 \mathrm{C} / \mathrm{G}(P=0.001)$, A allele of $-8564 \mathrm{G} / \mathrm{A}(P<0.01)$, A allele of $-450 \mathrm{~A} / \mathrm{G}(P<0.05)$, and $\mathrm{G}$ allele of $276 \mathrm{G} / \mathrm{T}(P<0.01)$ were associated with progressive carotid IMT. In the haplotype analysis, the $\mathrm{AC} / \mathrm{AC}$ haplotype combination in block 1, GAA/GAA haplotype combination in block 2 , and GGA/GAG and GGA/TAG haplotype combinations in block 4 of the adiponectin gene were associated with progressive carotid IMT $(P<0.05)$.

Conclusion: Variation in the adiponectin gene is associated with the rate of carotid IMT progression and is an important genetic factor for the progression of atherosclerosis in subjects with type 2 diabetes.

Supported by: Brain Korea 21 Project for Medical Science, Yonsei University

\section{0}

Prognostic value of osteoprotegerin for prediction of all-cause mortality in asymptomatic type 2 diabetic patients

A. Avignon ${ }^{1}$, A. Sultan ${ }^{1}$, J. Pochic ${ }^{1}$, C. Boegner ${ }^{1}$, C. Piot ${ }^{2}$,

D. Mariano-Goulard ${ }^{3}$, E. Renard ${ }^{4}$, A.-M. Dupuy ${ }^{5}$, J.-P. Cristol ${ }^{5}$

${ }^{1}$ Metabolic disease, CHU Montpellier \& Université Montpellier1,

Montpellier CEDEX 5, France, ${ }^{2}$ Cardiology, ${ }^{3}$ Nuclear Medicine,

${ }^{4}$ Endocrinology, ${ }^{5}$ Biochemistry, CHU Montpellier \& Université Montpellier1, France

Background and aims: Although intensive control of cardiovascular (CV) risk factors reduces mortality in diabetic patients (DP), this population still suffers from an excess mortality from CV diseases. The identification of DP likely to present an event could allow both to better target screening strategies and to further strengthen preventive measures, highlighting the need for improved methods to stratify residual risk within diabetic populations. In this regard, newer biomarkers like C-reactive protein, homocystein and fibrinogen among others have attracted attention but their value and appropriate use remain a source of debate. Very recently, osteoprotegerin (OPG) has emerged as a possible potent risk marker for CV disease. In DP, it has been shown to be associated with coronary atheroma, silent myocardial ischemia and CV events. However, no studies have evaluated usefulness of OPG for predicting all-cause death in DP which is the topic of the current study.

Materials and methods: A total of 356 consecutive type 2 DP (61 \pm 9 years; $61 \%$ male) free of symptoms of CV disease were evaluated by stress (exercise + dipyridamole) myocardial perfusion imaging. Risk factors, hs-CRP, homocystein, adiponectine, resistine and OPG levels were measured. Patients were followed up for death using telephone interview.

Results: Fourteen deaths occurred during a median follow-up of 34.3 [IQR 23.9-49.9] months. OPG at inclusion was higher in those patients who died compared to those who were alive at follow-up $(11.7 \pm 4.2 v s 7.2 \pm 4.1 \mathrm{pmol} /$ $1, \mathrm{P}<0.0001)$. After adjustment for age, predictors of mortality from the Cox proportional hazards model were smoking status $(\mathrm{P}=0.04)$, impaired renal function $(\mathrm{P}=0.02)$, peripheral arterial disease $(\mathrm{P}=0.002)$ and an $\mathrm{OPG}$ plasma value in the highest quartile, i.e. $>8.7 \mathrm{pmol} / \mathrm{l}(\mathrm{P}=0.02)$. None of the other classical or novel risk factors were significantly associated with time to death. In a multivariate model including all variables associated with time to death, an OPG plasma value in the highest quartile was the only independent predictor of death (HR, 4.39; 95\% CI, 1.14-19.65; $\mathrm{P}=0.03$ ). This remained true when further correcting for conventional risk factors. Predictive accuracy of OPG was evaluated using receiver-operator characteristic curve. Comparing the patients who were alive at the time of follow-up with those who had died gave an AUC $(95 \% \mathrm{CI})$ of $0.82(0.77-0.91 ; \mathrm{P}<0.0001)$. The cut-off value of 8.7 $\mathrm{pmol} / \mathrm{L}$ for plasma OPG corresponded to a negative predictive value of $99 \%$ (95\% CI 97-100\%) and a corresponding positive predictive value of $13 \%$ (6$22 \%$ ) for the 34.3 months risk of death.

Conclusion: In asymptomatic type $2 \mathrm{DP}$, an OPG value in the highest quartile $(>8.7 \mathrm{pmol} / \mathrm{l})$ identifies increased risk for all-cause death. Importantly, an OPG plasma level below that threshold portends an extremely low risk of death. Hence, the addition of the measurement of OPG to conventional screening strategies may provide an improved method of identifying asymptomatic DP at risk for death.

Supported by: sanofi-aventis France 


\section{PS 132 Macrovascular disease: risk factors, treatment and costs}

\section{1}

\section{Cigarette smoking and high LDL-cholesterol concentrations are associated with two different but additive proinflammatory gene expression signatures in circulating mononuclear cells \\ I. Zavaroni ${ }^{1}$, D. Ardigò ${ }^{1}$, I. Steinfeld ${ }^{2}$, E. Derlindati ${ }^{1}$, R. Navon ${ }^{2}$, L. Franzini ${ }^{1}$, A. Dei Cas ${ }^{1}$, V. Spigoni ${ }^{1}$, T. Quertermous ${ }^{3}$, P. Tsao ${ }^{3}$, Z. Yakhini ${ }^{2}$ ${ }^{1}$ Internal Medicine, University of Parma, Italy, ${ }^{2}$ Agilent Technologies, Tel Aviv, Israel, ${ }^{3}$ Cardiovascular Medicine, Stanford University, Stanford, United States}

Background and aims: Preliminary evidence indicates that the gene expression profile (GEP) of circulating white blood cells is significantly affected by the exposure to cardiovascular (CV) risk factors. However the few available studies have several limitations, including small sample size and narrow range of genes tested. Moreover, the interaction of multiple risk factors has not been investigated. The aim of the present study is to investigate GEP at whole-genome level in peripheral blood mononuclear cells (PBMC) of healthy volunteers in presence of cigarette smoking and/ or high LDL-cholesterol (LDL-c).

Materials and methods: To address this issue, PBMC's GEP was evaluated using Agilent whole genome oligonucleotide mRNA microarrays in 48 apparently healthy young-adults $(20 \mathrm{M} / 28 \mathrm{~F})$ with a median age of 35 years (IQR 7), all volunteers in a CV risk assessment study. Enrolled subjects were free of diabetes, CV and inflammatory diseases, and did not take pharmacological medications. Data analysis was performed using methods developed to address differential expression for quantitative phenotypes and enrichment of gene sets in ranked lists of genes.

Results: Despite the fact that regular smokers $(\mathrm{N}=8)$ had a similar clinical profile and comparable C-reactive protein levels to subjects who never smoked $(\mathrm{N}=28)$, their GEP was enriched with GO terms associated with the inflammatory response [Defense response, $\mathrm{p}=10^{-9}$; Response to biotic stimulus, $\mathrm{p}=10^{-9}$; Immune response, $\left.\mathrm{p}=10^{-9}\right]$ and the over-expression of genes related to innate immunity. "B-cell receptor signaling" was the single molecular pathway most over-represented $\left(\mathrm{p}=1.6^{*} 10^{-4}\right)$ in genes differentially expressed in smokers. In addition, this signature was also correlated with weekly cigarette consumption.

In parallel, the presence of high LDL-c (140-197 mg/dL; $3^{\text {rd }}$ LDL-c tertile) was also associated with a pro-inflammatory GEP compared to low LDL-c (40-104 mg/dL, $1^{\text {st }}$ tertile) in absence of clinical differences between the two groups. However, the high LDL-c expression-signature was more characterized by the over-expression of genes associated with cell-mediated immunity [Antigen presentation $\mathrm{p}=10^{-5}$, Antigen processing $\mathrm{p}=10^{-5}$ ] and the most enriched cellular pathway found was the "antigen processing and presentation" through MHC class II $\left(\mathrm{p}=1.8^{\star} 10^{-4}\right)$, whose key genes were all significantly up-regulated. Interestingly, in subjects who smoked and had high LDL-c compared to low LDL-c non-smokers, the transcriptomic profile was characterized by the co-presence of the two bio-signatures and the enrichment of both innate and cell-mediated immunity.

Conclusion: In conclusion, in apparently healthy young adults, both cigarette smoking and high LDL-c are associated with the over-expression of genes related to the inflammatory response. However, the two risk factors are characterized by two different transcriptomic "signatures", representative of innate and cell-mediated immunity respectively. The presence of both risk factors induces the expression of both these bio-profiles, suggesting an additive effect between the two in elevating the chronic sub-clinic inflammation associated with CV diseases.

Supported by: EU commission as part of the "Multi-Knowledge project"
1332

Elevated adiponectin levels are independent of sex, adiposity, renal function and diabetes characteristics in normoalbuminuric type 1 diabetic patients

C. Abi Khalil ${ }^{1,2}$, R. Aubert ${ }^{2}$, R. Roussel ${ }^{1,2}$, F. Travert $^{1}$, N. Emery ${ }^{2}$, N. Bellili' ${ }^{2}$, G. Velho' , F. Fumeron ${ }^{2}$, S. Hadjadj ${ }^{3}$, M. Marre ${ }^{1,2}$

${ }^{1}$ Diabétologie-endocrinologie-nutrition, Hopital Bichat, Paris, ${ }^{2}$ Faculté de médecine Xavier-Bichat, INSERM U695, Paris, ${ }^{3}$ Diabétologieendocrinologie-nutrition, $\mathrm{CHU}$ Poitiers, France

Background and aims: Total adiponectin levels were reported elevated in type I diabetes T1D and related to nephropathy. As these patients are traditionally known to be "lean" individuals, we studied if specific adiponectin isoforms were elevated in normoalbuminuric T1D patients, and if their determinants differed from those in general population, especially adiposity and kidney function.

Materials and methods: Total, High-, Medium-, and Low-Molecular Weight (HMW, MMW, LMW) adiponectin levels were measured by ELISA in 33 consecutive normoalbuminuric, normotensive T1D subjects (19 M and $14 \mathrm{~F}$, aged $35 \pm 2$, T1D duration $13 \pm 2$ years) and in 33 age and sex matched controls. Body Mass Index (BMI), fat mass (impedancemetry), Glomerular Filtration Rate (GFR, ${ }^{51} \mathrm{Cr}$-EDTA clearance), and $\mathrm{HbAlc}$ were also measured.

Results: Total adiponectin was elevated in T1D vs controls ( $8.4 \pm 0.8$ vs $6.9 \pm 0.7$ $\mu \mathrm{g} / \mathrm{L} / \mathrm{p}$ < 0.05$)$ with high HMW ( $4.1 \pm 0.6$ vs $3.1 \pm 0.5 \mu \mathrm{g} / \mathrm{L})$, MMW $(1.8 \pm 0.2$ vs $1.7 \pm 0.2 \mu \mathrm{g} / \mathrm{L})$ and LMW $(2.4 \pm 0.2$ vs $2.1 \pm 0.2 \mu \mathrm{g} / \mathrm{L})$. The T1D subjects had similar BMI (23.6 \pm 0.9 vs $24.0 \pm 0.7 \mathrm{~kg} / \mathrm{m} 2)$, waist circumference $(89 \pm 2$ vs $90 \pm 2 \mathrm{~cm})$, fat mass $(23.1 \pm 1.9$ vs $23.4 \pm 21.5 \%$ of weight), but higher GFR $(127 \pm 3$ vs $106 \pm 4 \mathrm{~mL} / \mathrm{min} / 1.73 \mathrm{~m} 2)$, than controls. In T1D, total adiponectin levels were not different according to sex (M 7.7 \pm 0.8 vs F $9.5 \pm 1.4 \mu \mathrm{g} / \mathrm{L})$, and not related to BMI ( $\rho=-0.06$; NS), waist circumference ( $\rho=0.02$; NS), HbAlc ( $\rho=-0.05$; NS), GFR $(\rho=0.13$; NS), or diabetes duration $(\rho=0.30$; NS). In controls, total adiponectin was indeed different according to sex (M $4.9 \pm 0.6$ vs F $9.7 \pm 1.0 \mu \mathrm{g} / \mathrm{L}, \mathrm{p}<0.001)$, and inversely related to BMI $(\rho=-0.44, \mathrm{p}<0.01)$ and waist circumference $(\rho=-0.40, p<0.02)$. Results were similar for all adiponectin isoforms.

Conclusion: The higher levels of all forms of ADPN in normoalbuminuric type 1 diabetic patients than in control patients were not explained by the difference in established determinants of adiponectin (adiposity, sex); neither by the T1DM characteristics (kidney function, diabetes duration). The nature of T1D effect on adiponectin levels remains unclear.

\section{3}

The inflammation marker YKL-40 is elevated in patients with type 1 diabetes and increases with levels of albuminuria

C.N. Rathcke ${ }^{1}$, F. Persson ${ }^{2}$, L. Tarnow ${ }^{2}$, P. Rossing ${ }^{2}$, H. Vestergaard ${ }^{1,3}$ ${ }^{1}$ Dept. of Endocrinology, Herlev Hospital, Herlev, Denmark, ${ }^{3}$ Faculty of Health Sciences, University of Copenhagen, Denmark

Background and aims: The inflammation marker YKL-40 is independently associated with an increased cardiovascular (CV) mortality in individuals without diabetes and CV disease. It is associated with atherosclerosis and is also elevated in patients with type 2 diabetes (T2D) without diabetic complications where it correlates with insulin resistance. In the present study and for the first time, levels of YKL-40 are examined in patients with type 1 diabetes (T1D) with increasing levels of albuminuria, associated with an increased risk of $\mathrm{CV}$ disease.

Materials and methods: The study population consisted of 3 groups of Caucasian patients with T1D attending Steno Diabetes Center in 2004: 58 with normoalbuminuria $(\mathrm{N})(\mathrm{u}$-albumin $<30 \mathrm{mg} / 24 \mathrm{~h}), 46$ with persistent microalbuminuria (MA)(30-300 $\mathrm{mg} / 24 \mathrm{~h})$ and 45 with persistent macroalbuminuria/diabetic nephropathy $(\mathrm{DMN})(\geq 300 \mathrm{mg} / 24 \mathrm{~h})$. Control group consisted of 55 healthy individuals $(\mathrm{C})$. The groups were matched by gender and duration of diabetes, aged $>30$ years.

Results: Median levels (range) of serum YKL-40 were significantly higher in $\mathrm{N}$ vs. C (C: $37(14-100) \mathrm{ng} / \mathrm{ml} ; \mathrm{N}: 53(14-255) \mathrm{ng} / \mathrm{ml}, \mathrm{p}<0.01)$ and were increasing with increasing levels of albuminuria: MA, 74 (24-874) ng/ml; DMN: 117 (28-1106) ng/ml, $\mathrm{p}<0.001$ for all comparisons). Levels of YKL-40 were correlated with urinary albumin/creatinine ratio (UACR) in the total group of participants $\left(\mathrm{r}^{2}=0.25, \mathrm{p}<0.001\right)$. Significant, but weak, intercorrelations of YKL- 40 were found with age, duration of diabetes, hemoglobin Alc and s-creatinine. After adjustment for significant covariates, albuminuria was significantly associated with levels of YKL-40, $\mathrm{p}<0.001$. 
Conclusion: Serum YKL-40 is elevated in patients with T1D with an independent association between increasing serum YKL-40 and increasing albuminuria. The present study is the first to suggest a role of YKL-40 in the gradually progressing vascular complications in patients with T1D.

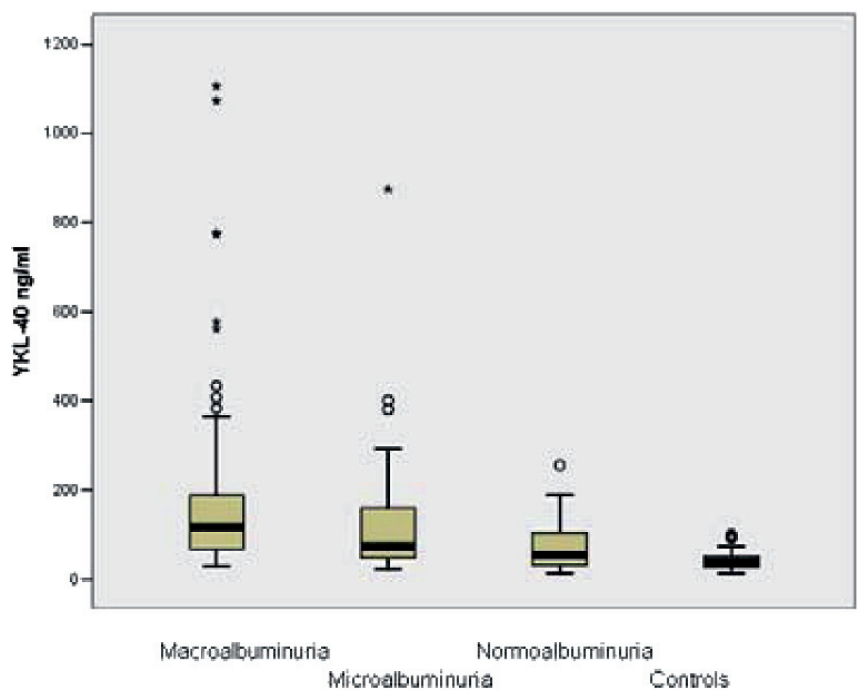

\section{4}

Exercise restores coronary vascular function independent of myogenic tone or hyperglycaemic status in $\mathrm{db} / \mathrm{db}$ mice

S. Ghosh ${ }^{1}$, F.M. Afsari ${ }^{2}$, M. Khazaei ${ }^{3}$, C.B. Verchere ${ }^{4}$, I. Laher

${ }^{1}$ Department of Pediatrics, Child and Family Research Institute, Vancouver, ${ }^{2}$ Department of Anesthesiolgy, Pharmacology and Therapeutics, University of British Columbia, Vancouver, ${ }^{3}$ Department of Anesthesiology,

Pharmacology and Therapeutics, University of British Columbia, Vanvouver, ${ }^{4}$ Department of Pathology and Laboratory Medicine, University of British Columbia, Vancouver, ${ }^{5}$ Department of Anesthesiology, Pharmacology and Therapeutics, University of British Columbia, Vancouver, Canada

Background and aims: Endothelial dysfunction is an early event in the development of diabetic vascular disease. Regulation of coronary function is important component in prevention of ischemic cardiac events, but remains poorly studied. Exercise is routinely recommended in the management of diabetes but the effects of this lifestyle modification on diabetic coronary function is relatively unknown. In this study, we investigated coronary artery myogenic tone and examined endothelial function, both of which are important determinants of maintaining vascular fluid dynamics in the myocardium. We hypothesized that exercise reduces pressure induced myogenic constriction of coronary arteries while at the same time also improving endothelial function $d b / d b$ mice, a frequently used animal model of type 2 diabetes.

Materials and methods: We used pressurized mouse coronary arteries isolated from the hearts of control and $d b / d b$ mice that were either sedentary or exercised for an hour a day on a motorized exercise wheel system (set at 5.2 meters/day, 5 days a week).

Results: Exercise caused a $\sim 10 \%$ weight loss in $d b / d b$ mice and decreased whole body oxidative stress as measured by plasma 8 -isoprostane levels, but failed to improve hyperglycemia or plasma insulin levels. Exercise failed to alter myogenic regulation of arterial diameter stimulated by increased transmural pressure and did not alter smooth muscle responses to U-46619 (a thromboxane agonist) or sodium nitroprusside (endothelial independent dilator). However, exercise restored acetylcholine-simulated, endothelial dependent coronary artery vasodilatation in $d b / d b$ mice, accompanied by increased expression of manganese superoxide dismutase (MnSOD) and decreased nitrotyrosine levels in the hearts of $d b / d b$ mice.

Conclusion: We conclude that the vascular benefits conferred by moderate levels of exercise were independent of changes in myogenic tone or hyperglycemic status, and primarily involved increased NO bioavailability in the coronary microcirculation.

Supported by: Canadian Heart and Stroke Foundation of British Columbia and Yukon, Canadian Institutes of Health Research; Li Tze Fong Memorial Fellowship, Michael Smith Foundation for Health Research and the Canadian Diabetes Association

\section{5}

e-Tracking (eT) as a new tool in the evaluation of early functional vascular changes in subjects without structural remodeling of carotid arteries in diabetes

Z. Bociaga ${ }^{1}$, K. Loboz-Grudzien ${ }^{2}$, M. Wilczynska ${ }^{3}$, J. Jaroch ${ }^{1}$, A. Kowalska ${ }^{1}$, A. Woszwillo ${ }^{1}$, E. Kruszynska ${ }^{1}$, M. Woda ${ }^{1}$

${ }^{1}$ Department of Cardiology, T.Marciniak Hospital, ${ }^{2}$ Public Health

Department, Medical Academy Wroclaw, ${ }^{3}$ Centre of Diabetic-

Endocrinologic Care, Diabetic-Endocrinologic Care, Wroclaw, Poland

Background and aims: Little is known about clinical application of e-Tracking (eT) in evaluation of early functional vascular changes in diabetes (D) without structural remodeling of carotid arteries (c.a.). We tested the hypothesis if $\mathrm{eT}$ indices of arterial stiffness (a.s.), with novel one-point pulse wave velocity-PWV among them, are an early marker of functional vascular abnormalities in D. The aim of the study was to compare eT indices in $\mathrm{D}$ and in healthy subjects $(\mathrm{N})$. We also examined relationship between $\mathrm{eT}$ indices and clinical data (age, mean systolic and diastolic blood pressure (MSBP,MSDP)), c.a. intima-media complex (IMT) and echocardiographic left ventricular systolic and diastolic indices (l.v.s.i., l.v.d.i.).

Materials and methods: The study group consisted of 93 subjects $(40 \mathrm{D}$ and $53 \mathrm{~N}$ ), mean age 52,5 years, $38 \mathrm{M}$ and $55 \mathrm{~F}$. eTracking of c.a. (a new builtin echo walltracking ALOKA system), B-Mode c.a. Doppler ultrasound, echocardiography and 24-ABPM were performed in each patient. From eT the following a.s. parameters (with blood pressure values introduced into formulas during examination) were calculated: Beta index (beta), Epsilon index (pressure-strain elasticity modulus,Ep), arterial compliance (AC), one-point pulse wave velocity $(\mathrm{PWV} \beta)$, augmentation index (AI). From ECHO-Doppler and Tissue Doppler (T.D.): ejection fraction (EF) and l.v.d.i. were evaluated. From 24-ABPM: MSBP and MDBP were calculated. Glycated haemoglobin (HbAlc) was measured in each patient.

Results: All pts had normal IMT values and preserved LV systolic function. In $\mathrm{D}$ mean values of beta $(10,7+/-3,3)$ were significantly higher $(\mathrm{p}<0,05)$ than in $\mathrm{N}(8,3+/-2,61)$.

Mean values of Ep and PWV were also significantly higher in D than in $\mathrm{N}$ ( 146,8 vs 118,$2 ; 6,86$ vs 6,39 respectively). In D group $66 \%$ pts had higher than normal values of beta and $59 \%$ had higher than normal values of Ep and PWV ( established as 9, 130 and 6,5 respectively, using ROC curve procedure in our normal population) while all of them had normal IMT value.Significant correlations between age and beta $(r=0,33, p<0,05)$ were observed. MSBP and MDBP correlated with $\mathrm{eT}$ indices except AI. Among the l.v.d.i. only E' (early wave filling from T.D.) correleted with AC, AI, PWV. Significant correlations between HbAlc and beta index $(r=0,39, p<0,05)$, Ep $(r=0,46 ; p<0,05), A C$ $(\mathrm{r}=-0,35, \mathrm{p}<007)$ but not with IMT were noted.

Conclusion: e-Tracking carotid arterial stiffness indices can be useful in detection of early functional vascular changes that precede arterial structural remodeling in patients with diabetes which is supported by correlation between $\mathrm{HbAlc}$ and $\mathrm{eT}$ indices in diabetic patients with normal IMT.

\section{6}

Arrhythmogenesis and sympathetic activation in human obesity

F. Paleari, P. Gamba, F. Quarti-Trevano, R. Dell'Oro, L. Magni, M. Volpe, M. Colombo, G. Grassi, G. Mancia

Clinica Medica, Università Milano-Bicocca, Ospedale San Gerardo, Monza, Milan, Italy

Background and aims: Human obesity, particularly when complicated by obstructive sleep apnea is characterized by an increased risk of ventricular arrhythmias. Although this has been ascribed to neuroadrenergic mechanisms, no direct evidence is available on this issue in humans.

Materials and methods: We studied 51 obese normotensives (age: $49.7 \pm 1.2$ years, mean \pm SEM), of which 28 without $(\mathrm{O}-)$ and 23 with $(\mathrm{O}+)$ monofocal premature ventricular beats in Lown class < II. A polysomnographic evaluation allowed the $\mathrm{O}$ - subjects to be further classified as without $(\mathrm{O}-\mathrm{OSA}-, \mathrm{n}=12)$ and with (O-OSA+, $\mathrm{n}=16)$ sleep apnea. A similar procedure was done in $\mathrm{O}+(\mathrm{O}+\mathrm{OSA}-, \mathrm{n}=10$ and $\mathrm{O}+\mathrm{OSA}+, \mathrm{n}=13)$. In each patient we measured, along with anthropometric and echocardiographic variables, beatto-beat mean blood pressure (BP, Finapres), heart rate (HR, EKG) and muscle sympathetic nerve activity (MSNA, microneurography) at rest and during baroreflex stimulation and deactivation (vasoactive technique).

Results: $\mathrm{O}$ - and $\mathrm{O}+$ subjects were age-matched and characterized by $\mathrm{BP}$ and echocardiographic values, which were in the normal range and superimpos- 
able in the 2 groups. Anthropometric values were similarly altered in the 2 groups. When compared to O-, $\mathrm{O}+$ showed a slight increase in HR but a significant elevation in MSNA ( $45.8 \pm 1.9$ vs. $68.2 \pm 1.5 \mathrm{bs} / 100 \mathrm{hb}, \mathrm{p}<0.001)$. This increase was potentiated in presence of OSA. This was accompanied by a significant $\mathrm{HR}$ and MSNA baroreflex impairment which was evident in $\mathrm{O}+$ $(-24.5 \pm 3.1$ and $-19.4 \pm 2.9 \%)$ and potentiated in O+OSA+ $(-35.1 \pm 3.8$ and $28.7 \pm 3.1 \%)$.

Conclusion: Thus in human obesity neurogenic and reflex mechanisms participate at the development of ventricular arrhythmias. This participation, however, appears to be more pronounced when obesity is associated with OSA, indicating that in obesity not only baroreflex but also chemoreflex dysfunction may represent an important factor in the genesis of cardiac rhythm disturbances

\section{7}

Early detection of macrovascular complications in subjects with associated type 1 diabetes mellitus and coeliac disease

F. Martini ${ }^{1}$, D. Pitocco ${ }^{1}$, C. Smaldone ${ }^{2}$, S. Giubilato ${ }^{1}$, F. Zaccardi ${ }^{1}$,

V. Pezzano ${ }^{2}$, A. Manto ${ }^{1}$, L.M. Biasucci ${ }^{2}$, G. Liuzzo ${ }^{2}$, F. Crea $^{2}$, G. Cammarota ${ }^{1}$, G. Ghirlanda ${ }^{1}$

${ }^{1}$ Internal Medicine, ${ }^{2}$ Cardiology, Catholic University, Rome, Italy

Background and aims: Immune system and inflammation seem to play a key role in the pathogenesis of type 1 diabetes (DM) and its complications. DM is often associated with other autoimmune diseases, like coeliac disease (CD). The aim of this study was: 1) To assess the effect of associated CD and Diabetes on early appearance of macrovascular complications, assessed through Carotid Intima-Media Tickness (CIMT), a well-known surrogate of cardiovascular disease; 2 ) To evaluate the role of inflammation ( $\mathrm{C}$ reactive protein-CRP), immunity $\left(\mathrm{CD} 4{ }^{+} \mathrm{CD} 28^{\text {null }} \mathrm{T}\right.$-cells and $\mathrm{CD} 4^{+} \mathrm{CD} 25^{+} \mathrm{T}$-cells $)$ and glicometabolic parameters on its pathogenesis.

Materials and methods: We enrolled 29 consecutive patients with DM and CD (12 males, mean age $35,72 \pm 10,81)$; CD was diagnosed by intestinal biopsy and every patient with $\mathrm{CD}$ was on a gluten-free diet. We also enrolled patients with only DM $(34,21$ males, mean age $34,50 \pm 7,29)$ or $\mathrm{CD}(26,6$ males, mean age $32,64 \pm 8,26$ ) as control groups. Demographic and clinical data were collected. Blood samples were taken at time of enrollment. Serum level of CRP was measured using a high sensitivity immuno-nephelometric assay. $\mathrm{CD} 4{ }^{+} \mathrm{CD} 28^{\text {null }} \mathrm{T}$-cells and $\mathrm{CD} 4^{+} \mathrm{CD} 25^{+} \mathrm{T}$-cells frequency was measured by flow cytometry.

Results: CIMT was significantly higher in patients with DM and CD $(\mathrm{DM}+\mathrm{DC}=0,60 \pm 0,16 ; \mathrm{DM}=0,48 \pm 0,12 ; \mathrm{CD}=0,48 \pm 0,17 ; \mathrm{p}=0.008)$, as Total cholesterol $(\mathrm{DM}+\mathrm{DC}=190,41 \pm 35,73 ; \mathrm{DM}=188,97 \pm 34,68 ; \mathrm{CD}=163,89 \pm$ $32,37 ; \mathrm{p}=0.047), \mathrm{LDL}$ cholesterol $(\mathrm{DM}+\mathrm{DC}=120,12 \pm 29,14 ; \mathrm{DM}=111,35 \pm$ $27,14 ; \mathrm{CD}=93,77 \pm 28,94 ; \mathrm{p}=0.039)$, Triglycerides $(\mathrm{DM}+\mathrm{DC}=80,59 \pm 32,09$; $\mathrm{DM}=63,15 \pm 21,09 ; \mathrm{CD}=87,74 \pm 51,65 ; \mathrm{p}=0.035)$ and Glycated Hemoglobin $(\mathrm{DM}+\mathrm{DC}=7,88 \pm 1,79 ; \mathrm{DM}=7,19 \pm 0,52 ; \mathrm{CD}=4,83 \pm 0,46 ; \mathrm{p}<0.0001)$.Serum levels of CRP were significantly higher in patients with T1DM and CD than in patients of control groups. No significant differences in $\mathrm{CD} 4^{+} \mathrm{CD} 28^{\text {null }} \mathrm{T}$-cells $(2,195 \pm 2,94$ vs $2,565 \pm 2,05 \%)$ and $\mathrm{CD} 4^{+} \mathrm{CD} 25^{+}$T-cells frequency $(1,56 \pm 0,64$ vs $1,43 \pm 0,73 \%$ ) were found.

Conclusion: Our study showed for the first time a higher CIMT in patients with $\mathrm{DM}$ and $\mathrm{CD}$ than in patients with $\mathrm{DM}$ or $\mathrm{CD}$ alone; furthermore, serum levels of CRP, marker of innate immune system activation, were higher in patients with T1DM and CD than in those with only one disease. It's also remarkable the different glicometabolic profile between $\mathrm{DM}+\mathrm{CD}$ vs $\mathrm{DM}$ or $\mathrm{CD}$. These results showed a possible enhanced activation of innate immune system and a worse glicometabolic pattern in T1DM and CD that can lead to a more severe CIMT- an inflammatory disease, whereas it seems that cellularmediated immune system isn't involved.

\section{8}

Long-term effect of anti-TNF treatment on atherosclerosis risk factors in rheumatoid arthritis patients

Y. Sayo ${ }^{1}$, H. Dobashi ${ }^{2}$, S. Matsubara ${ }^{2}$, T. Ishida ${ }^{2}$;

${ }^{1}$ Internal medicine, Social Insurance Ritsurin Hospital, Takamatsu-city Kagawa, ${ }^{2}$ First Internal medicine, Faculty of Medicine, Kagawa University, 1750-1 miki-cho Kita-gun Kagawa,

Background and aims: The tumor necrosis factor alpha (TNF-alpha) is one of the adipocytokines, which are well known as the physiological activity substances of the fat cell origin. TNF-alpha interferes the phosphorylation of intra-cellular domain in the insulin receptor on target organs, such as skeletal muscle and liver, which results in enhancement of insulin resistance. The expression of TNF-alpha on adipose tissue or skeletal muscle correlates with BMI in obese subjects. On the other hand, Rheumatoid arthritis (RA) is a chronic inflammatory disease induced by several inflammatory cytokines including TNF-alpha. TNF-alpha is considered as the most important cytokine in pathogenesis of RA. By using soluble TNF-alpha receptor for an insulin resistance model rat, TNF-alpha in blood can be neutralized and the insulin resistance of skeletal muscle can be decreased. TNF-alpha has been implicated in the progression of atherosclerosis through modulation of insulin resistance (IR) or other adipocytokines expression. We have already reported that anti-TNF treatment using Infliximab (IFX) or Etanercept (ETA) improved IR in RA patients. In both Annual European congress Rheumatology 2007 and annual meeting of the American College of Rheumatology 2007, the many reports that anti-TNF treatment decreased the risk of myocardial infarction, anti-TNF treatment might reduce the risk of cerebrovascular events in RA patients. There also reported increase of adiponectin might reduce the risk of arteriosclerosis RA patients. In this study, we examined the effect of antiTNF treatment using IFX or ETA on IR or adipocytokine expression in RA patients during one year. We also investigated the change of visceral fat in anti-TNF treatment before and after.

Materials and methods: We prospectively followed 50 previously anti-TNF naive RA patients who received the anti-TNF treatment for one year or more. We analyzed the IR using the Homeostasis Model Assessment Insulin Resistance (HOMA-IR) at 0, 6, 22, 56 weeks. Serum IL-6, TNF-alpha, adiponectin, resistin and CRP were also examined to evaluate the change of RA activity. We used the Homeostasis Model Assessment Insulin Secretion (HOMAbeta) as for insulin secretion. We measured visceral fat area of all subjects before and after anti-TNF treatment using CT scanning and Fat scan (N2 system, Japan).

Results: The insulin resistance was decreased significantly in the first 6 weeks by both ant-TNF treatments. ETA could reduce IR continuously till the 22 weeks, however, IFX could not sustain the improvement. The enhancement of adiponectin and reduction of resistin were observed in both treatments. Additionally, the reduction of visceral fat area was indicated in RA patients treated with anti-TNF agents.

Conclusion: Anti-TNF treatment could reduce the risk of atherosclerosis progression in RA. However, this effect may be different between ETA and IFX treatment.

\section{9}

Direct medical costs for type 2 diabetes mellitus complications in the U.S. commercial payer setting: a resource for economic research E.M. Pelletier ${ }^{1}$, P. Smith ${ }^{1}$, K.S. Boye ${ }^{2}$, D.A. Misurski ${ }^{2}$, S.L. Tunis ${ }^{3}$, M.E. Minshall ${ }^{3}$

${ }^{1}$ Health Economics and Outcomes Research, IMS Consulting Services, Watertown, United States, ${ }^{2}$ Eli Lilly and Company, Indianapolis, United States, ${ }^{3}$ Health Economics and Outcomes Research, IMS Consulting Services, Noblesville, United States

Background and aims: The large and growing humanistic and economic burden of type 2 diabetes mellitus (T2DM) in the US has been widely documented. Medical complications are key drivers of costs among T2DM patients. Detailed information on the clinical and economic impacts of T2DM treatments remains in high demand for US healthcare payers and other stakeholders. An important unmet need for the accurate assessment of the economic impact of T2DM complications is a set of unit costs that are empirically based, clearly and consistently defined, and applicable to a large segment of US managed care patients. This study used a large, multi-payer US database to examine direct medical costs of T2DM complications.

Materials and methods: Health insurance claims were extracted from the PharMetrics Patient-Centric Database, which includes adjudicated medical and pharmaceutical claims from over 90 US health plans. Patients experiencing at least 1 of 24 T2DM complications from 1/2003 through 12/2004 and with a T2DM diagnosis prior to or on the date of the first (index) complication during the study window were identified. Complication cost data were extracted for 44,021 patients who had a minimum of 12 months follow-up enrollment; cost data for months 13-24 were extracted for a subset of patients with at least 24 months of follow-up $(n=32,991)$. Direct medical costs included both the amounts charged by medical providers for treating the complication and the health plan contracted allowed amounts. Costs were expressed as \$US 2007. 
Results: The average age of the aggregate sample was 56 years and $52 \%$ were male; $75 \%$ were insured under a commercial plan and $17 \%$ had coverage from a public payer. During the first 12 months following the index complication, $74 \%$ of patients were on oral antidiabetics, $26 \%$ on insulins, $43 \%$ on ACE inhibitors, and $50 \%$ on antihyperlipidemics/statins. Table 1 shows the proportion of patients experiencing T2DM complications during the 12-month follow-up period and the corresponding average charged and allowed amounts; cost data for months 13-24 following the initial complication were provided for the patient subset. Allowed amounts for most complications were $30 \%$ $45 \%$ of charges. Congestive heart failure and myocardial infarction had the greatest fiscal impact because of the percentage of patients experiencing them and their associated costs.

Conclusion: These unit costing results provide an important resource for economic modeling and other types of costing research related to treating T2DM complications within the US managed care system.

Table 1. T2DM Complication Charged and Allowed Amounts in \$US 2007

\begin{tabular}{|c|c|c|c|c|c|}
\hline \multirow[t]{2}{*}{ T2DM Complication } & \multirow[t]{2}{*}{$\%$} & \multicolumn{2}{|c|}{ First 12 Months } & \multicolumn{2}{|c|}{ Months 13-24 } \\
\hline & & $\begin{array}{l}\text { Mean } \\
\text { Charged }\end{array}$ & $\begin{array}{l}\text { Mean } \\
\text { Allowed }\end{array}$ & $\begin{array}{l}\text { Mean } \\
\text { Charged }\end{array}$ & $\begin{array}{l}\text { Mean } \\
\text { Allowed }\end{array}$ \\
\hline Myocardial infarction & 7.2 & 41,695 & 14,853 & 3,797 & 1,363 \\
\hline Angina pectoris & 12.0 & 9,850 & 4,132 & 1,127 & 488 \\
\hline Congestive heart failure & 14.0 & 30,066 & 11,257 & 10,802 & 3,905 \\
\hline Ischemic stroke & 5.0 & 14,104 & 6,331 & 1,437 & 518 \\
\hline $\begin{array}{l}\text { Peripheral vascular } \\
\text { disease }\end{array}$ & 10.6 & 9,800 & 3,623 & 3,460 & 1,289 \\
\hline Gangrene & 1.0 & 25,125 & 10,094 & 3,582 & 1,057 \\
\hline Leg amputation & 0.5 & 27,320 & 14,750 & 7,777 & 4,620 \\
\hline Foot ulcer & 8.5 & 11,042 & 4,743 & 4,219 & 1,701 \\
\hline Kidney transplant & 0.6 & 22,465 & 9,890 & 11,497 & 5,399 \\
\hline Hemodialysis & 1.0 & 25,150 & 10,603 & 18,140 & 7,716 \\
\hline Peritoneal dialysis & 0.2 & 29,609 & 15,893 & 26,667 & 13,694 \\
\hline Cataracts & 0.3 & 1,094 & 558 & 71 & 60 \\
\hline Blindness & 0.9 & 3,357 & 997 & 106 & 71 \\
\hline Neuropathy & 25.4 & 3,657 & 1,568 & 999 & 401 \\
\hline Hypoglycemic event & 0.2 & 454 & 345 & 8 & 4 \\
\hline Lactic acidosis & 1.2 & 24,344 & 9,929 & 1,627 & 198 \\
\hline Keto-acidosis & 0.2 & 239 & 152 & 3 & 2 \\
\hline
\end{tabular}

Supported by: Eli Lilly and Company, USA

\section{0}

Comprehensive cardiac rehabilitation reduces length of stay in acute re-admissions for patients with diabetes. Three years results of the DANREHAB trial

A.B. Soja ${ }^{1}$, A. Zwisler ${ }^{1}$, S. Rasmussen ${ }^{2}$, T.M. Melchior ${ }^{3}$, E. Hommel ${ }^{4}$, M. Madsen ${ }^{5}$ J. Fischer Hansen ${ }^{6}$

${ }^{1}$ Department of Cardiology, Rigshospitalet, Copenhagen, ${ }^{2}$ Department of Epidemiology, National Institute of Public Health, University of Southern Denmark, Copenhagen, ${ }^{3}$ Department of Cardiology, Roskilde County Hospital, ${ }^{4}$ Steno Diabetes Center, Gentofte, ${ }^{5}$ Institute of Public Health Research, University of Copenhagen., ${ }^{6}$ Department of Cardiology, Bispebjerg University Hospital, Copenhagen, Denmark

Background and aims: Mortality and readmissions rates for ischemic events are high in patients with type 2 diabetes (T2DM). The Steno- 2 study has shown an outstanding beneficial effect of multifactoriel intervention in patients with T2DM and microalbuminuria. Although studies have reported beneficial impact of comprehensive cardiac rehabilitation (CR) on outcome in the overall population, little is known of the effect of CR in patients with T2DM. In this kind of population, preliminary results from the ACCORD study have given rise to som dispute, but we still hypothesize that patients with T2DM and macrovascular disease will benefit from CR receiving multifactorial intensified intervention. In a randomized clinical trial (The DANREHAB trial) on CR we found improvement in lifestyle and risk factor after 12 months compared to usual care (UC) among patients with T2DM. We now report if these effects are translated into clinical endpoints after 3 years.
Materials and methods: We used a centrally randomized clinical trial comparing CR with UC. Of 1614 eligible patients, 770 (47\%) were randomized in ways of 390 patients receiving $\mathrm{UC}$ and 380 patients CR. A total of 151 patients (20\%) had known diabetes at randomization and 75 patients were allocated to CR and 76 patients to UC. Patients randomized to CR received 6-12 weeks of an individually tailored, multi-disciplinary programme and clinical control after 3, 6 and 12 months. A composite blinded administrative register-based primary outcome measure included total mortality, myocardial infarction or first acute readmissions due to heart disease. For secondary outcomes we used acute re-admissions and acute length of stay. We used Cox-regression for analysing time-to-event and Poisson regression for analysing length of stay and number of re-admissions. The follow-up time was 3 years after randomisation.

Results: We find no difference in the composite outcome (CR versus UC; Hazard ratio: 0.87 95\%CI: 0.75-1.13). There were no significant differences when looking separately at the components in the primary outcome. Amongst the CR and UC patients $57 \%$ and $62 \%$ were acute re-admitted at least once $(\mathrm{P}=0.80)$. There were 238 and 355 acute re-admissions for $\mathrm{CR}$ patients and UC patients $(\mathrm{P}=0.17)$ with mean length of stay of 5.8 days and 7.7 days respectively $(\mathrm{P}<0.05)$.

Conclusion: Although the DANREHAB study earlier has demonstrated significantly greater reduction in blood pressure as well as improvement in glycemic control for patients with T2DM in CR compared to UC, these effects do not yet translate into the primary clinical endpoint at 3 years follow-up. Nevertheless there is a marginally significant reduction in acute length of stay for patients with diabetes in CR.

Supported by: Danish Pharmacy Foundation of 1991, Eva and Henry Fronkels Memorial Foundation, Danish Heart Foundation 


\section{PS 133 Cardiac complications}

\section{1}

Effect of desflurane on phosphorylation of Akt and GSK 3 beta proteins in the diabetic versus non diabetic human myocardium

S. Lemoine ${ }^{1}$, G. Beauchef ${ }^{2}$, L. Zhu ${ }^{1}$, P. Galera ${ }^{1}$, J.L. Gérard ${ }^{1}$, J.L. Hanouz ${ }^{1}$ ${ }^{1}$ Pole Anesthésie-Réanimation, ${ }^{2}$ Laboratoire de matrice extracellulaire et pathologie, CHU de Caen, France

Background and aims: The increasing incidence of diabete mellitus worldwide results in an increase perioperative care of diabetic patients who are at high risk for perioperative ischemia reperfusion injury. Thus developing perioperative cardioprotective strategies are of importance in diabetic patients in Cardiovascular surgery. The aim of this study was to quantify the phosphorylation of Akt and GSK 3 beta (proteins which are known to mediate the cardioprotection) after exposure to desflurane (a volatile halogenated anesthetic which has capacity to protect non diabetic myocardium against ischemia reperfusion injury) in human myocardium with non insulin dependant diabetes mellitus (NIDDM).

Materials and methods: After the approval of local medical ethics committee, right atrial appendages were obtained during cannulation for cardiopulmonary bypass from patients scheduled for routine coronary artery bypass surgery and aortic valve replacement. Right atria diabetic NIDDM and non diabetic (ND) were pinned in a chamber $(5 \mathrm{~mL})$ containing Tyrode's modified solution, oxygenated with $95 \% \mathrm{O}_{2}$ and $5 \% \mathrm{CO}_{2}$ and maintained at $37 \pm$ $0.5^{\circ} \mathrm{C}$. Preparations were stimulated at a frequency of $1 \mathrm{~Hz}$. In all groups, equilibration period was performed by oxygenated by $95 \% \mathrm{O}_{2}-5 \% \mathrm{CO}_{2}$ during 60-min (Control groups); and followed by a 5 -min exposure to desflurane $6 \%$ in Des groups.

After oxygenation (Control group) and after exposure to desflurane 6\% (Des group), atria were frozen and stored at $-80^{\circ} \mathrm{C}$ prior to protein extraction and western blot analysis. Frozen tissue samples were extracted into extraction buffer and protein concentration was determined using the BCA protein assay. Extracted protein samples from human atrial tissues were submitted to the western blot protocol. Then were incubated with antibodies recognizing phospho-Akt and phospho-GSK 3 beta. The western blots of each group were stripped and probed again with an antibody against beta-Tubulin to ensure equivalent loading. All band densities for protein of interest were then normalized to that of the band for beta-Tubulin in the same sample, then normalized to the mean of control tissues was defined as 1 arbitrary unit \pm SD. Statistical comparaison was made by analysis of variance.

Results: Brief administration of desflurane 6\% significantly increased Akt phosphorylation in Des.ND et Des.NIDDM groups $(2.37 \pm 0.45$ and 2.6 \pm 0.12 -fold increase vs. Cont. ND et NIDDM, P>0,0001); and GSK 3 beta phosphorylation in Des.ND and Des. NIDDM groups $(1.98 \pm 0.25$ et $2.05 \pm$ 0.12 -fold increase vs. Cont. ND and NIDDM, P<0.0001). Phosphorylation of Akt and GSK 3 beta are equivalent in Des.ND and Des. NIDDM groups (no significant).

Conclusion: Brief administration of desflurane induced phosphorylation of Akt and GSK 3 beta, two proteins kinases of the Reperfusion Injury Salvage Kinase pathway, mediate the cardioprotective effect. Interestingly this effect is equivalent in diabetic and non diabetic myocardium. These results suggest that desflurane could be used in clinical practice as a cardioprotector agent of patients with non insulin dependant diabetes mellitus.

\section{2}

MMP-9, inflammatory process activity and endothelial dysfunction in patients with STEMI and type 2 diabetes

D. Milosz ${ }^{1}$, L. Czupryniak ${ }^{1}$, M. Saryusz-Wolska ${ }^{1}$, G. Zasadzinska ${ }^{1}$,

A. Borkowska ${ }^{1}$, E. Cieplucha ${ }^{2}$, K. Chizynski², J. Loba ${ }^{1}$

${ }^{1}$ Diabetology and Metabolic Diseases Dept, ${ }^{2}$ Cardiology Dept, Medical

University of Lodz, Poland

Background and aims: The type 2 diabetes is an independent risk factor for coronary artery disease. Myocardial infarction has its onset as a result of the rupture of a vulnerable plaque and subsequent thrombus formation. Exogenous risk factors for rupture of a vulnerable plague leading to an acute coronary syndrome include: the sudden increase in arterial blood-pressure, vasospasmus reaction and inflammatory reaction, however the main "destabilizers of atherosclerosis plaque" are metaloproteinases. The aim of the study was to assess a relationship between MMP-9 and selected inflammatory markers, endothelial dysfunction, and pronouncement STEMI in patients with type 2 diabetes.

Materials and methods: This study comprised 60 patients (27 women, 33 men, mean age $58 \pm 8.5$ years) with STEMI, who underwent percutaneous coronary angioplasty Patients were divided into two groups. Study group consisted of 30 persons with type 2 diabetes treated with sulphonylureas derivatives, the control group were age-, gender- and body weight-matched 30 non-diabetes patients. Within 12 hours after ACS MMP-9, CRP, fibrinogen, leucocytosis, sVCAM, sICAM were evaluated in the patients. Lipids profiles were obtained after overnight fast condition. Severity of lesion in coronary arteries were obtained by Index Gensini Score. No significant differences in smoking status or non-diabetes medication were noted between the groups. Results: Patients in the study group demonstrated more advanced lesions in the coronary vessels (Gensini Score 60,9 \pm 33 ) as compared to the control group (Gensini Score 41 \pm 21 ). Patients with STEMI and type 2 diabetes demonstrated higher concentrations of MMP-9 $(1080 \pm 351$ vs $322.6 \pm 209 \mathrm{ng} / \mathrm{ml})$, CRP $(5.9 \pm 4.2$ vs $3.9 \pm 2.3 \mathrm{mg} / \mathrm{l})$, fibrinogen ( $5.1 \pm 1.9$ vs $4 \pm 1 \mathrm{~g} / \mathrm{l})$, leucocytosis $(12100 \pm 4200$ vs $9800 \pm 3400$ per mcl), VCAM $(1367 \pm 1025 \mathrm{vs} 930 \pm 295 \mathrm{ng} / \mathrm{ml})$, ICAM $(848 \pm 271$ vs $722 \pm 160 \mathrm{ng} / \mathrm{ml})$ in comparison to the control group.

Conclusion: The study results suggest that diabetes is most important factor intensifying inflammatory process in the coronary vessels and arrangement of MMP-9 may play a significant role in destabilisation of atherosclerotic plaque and development of ACS in this group of patients. Supported by: Medical University of Lodz

\section{3}

Is there a role for PDGF-BB in in-stent restenosis in diabetes?

T. Poletti, A. Guildford, M. Santin, M. Harrison

School of Pharmacy \& Biomolecular Sciences, University of Brighton, United Kingdom

Background and aims: Cardiovascular disease (CVD) represents a major complication and cause of death and is exacerbated in diabetes as a consequence of macrovascular changes. Formation of glycation products leads to atherosclerotic plaque deposition and structural changes in the vessel wall. Balloon angioplasty followed by stent deployment is the gold standard treatment. The outcome is adversely affected by in-stent restenosis (ISR) in $30 \%$ of cases. In diabetes, incidence increases to $60 \%$. The growth factor PDGF-BB has been implicated as a major participant in the development and progression of ISR. The effective but costly use of drug-eluting stents (DES) can reduce the incidence of ISR to $~ 3 \%$. There is an increased risk of late thrombosis and heart attack in patients treated with DES. Stent deployment is associated with tissue damage and protracted inflammatory response, which may be enhanced by the reported heightened inflammatory state in diabetes. Our established in vitro model of bare metal stent induced ISR was used to mimic the post-stent implantation environment and its effect on isolated mononuclear cell behaviour and phenotype. The isolated mononuclear cells (MC) from 3 cohorts [type 1 (T1), type 2 (T2) diabetes and controls (C)] were compared to characterize PDGF-BB production and MC population in the presence of the stent material (stainless steel-St.).

Materials and methods: Subjects were recruited at Royal Sussex County Hospital (Brighton, UK). Clinical data HbA1c, total cholesterol, BMI, HDL and age were recorded. MCs from peripheral blood were seeded onto pre-conditioned St discs for $24 \mathrm{hrs}, 37^{\circ} \mathrm{C}$, in physiological glucose. Supernatants from the incubation assay described above were analyzed for PDGF-BB by ELISA method. Cells were analyzed with Flow Cytometry for the following antibodies: CD45 (L; Leukocytes Common Antigen), CD3 (TL: T Lymphocytes), CD19 (BL: B Lymphocytes), CD14 (M: Monocytes), CD68 (AM: Activated Macrophages) and CD34 (HSC: Hematopoietic Stem Cells).

Results: Table 1: There was a significant difference in BMI observed between the groups. T2 were clinically obese whereas $\mathrm{T} 1$ and $\mathrm{C}$ had a normal BMI. No differences were observed in total cholesterol, HDL or HbA1c. CD14 (M) and CD19 (BL) were both elevated in diabetes. There were also differences in the proportion of cell populations between the T1 and T2 cohorts; There was a higher percentage of CD3 (TL) and CD45 (L) in the T2 cohort vs both T1 and $\mathrm{C}$ cohorts. CD68 (AM) percentage was higher in the T1 cohort than in the T2 and C. CD3 (TL) was lowest in C and increased in T1 but increased further in T2. Hematopoietic stem cells (CD34) were low in all groups. PDGF-BB was elevated in response to St. in control cells $(0.59 \mathrm{pg} / \mathrm{ml})$ but was unchanged in both diabetes cohorts.

Conclusion: The higher percentage of AM in both of the diabetes cohorts indicates a default pro-inflammatory state not witnessed in control cell popu- 
Patients Data and cells population distribution.

\begin{tabular}{|c|c|c|c|c|c|c|}
\hline & $\begin{array}{l}\text { Age } \\
\text { (years) }\end{array}$ & $\begin{array}{l}\text { BMI } \\
(\mathrm{kg} / \mathrm{m} 2)\end{array}$ & $\begin{array}{l}\text { HbA1c } \\
(\%)\end{array}$ & $\begin{array}{l}\text { Total Chol } \\
(\mathrm{mmol} / \mathrm{l})\end{array}$ & $\begin{array}{l}\text { HDL } \\
(\mathrm{mmol} / \mathrm{l})\end{array}$ & \\
\hline $\mathrm{T} 1 \mathrm{D}$ & $42.14(+/-12.37)$ & $24.43(+/-3.49)$ & $7.97(+/-2.15)$ & $4.35(+/-0.61)$ & $1.77(+/-0.34)$ & \\
\hline $\mathrm{T} 2 \mathrm{D}$ & $60.6(+/-6.95)$ & $31.85(+/-4.17)$ & $7.63(+/-1.78)$ & $3.84(+/-0.87)$ & $1.44(+/-0.79)$ & \\
\hline \multirow[t]{2}{*}{ Cells \% } & CD45 & CD3 & CD19 & CD14 & CD68 & CD34 \\
\hline & $\mathrm{L}$ & $\mathrm{TL}$ & $\mathrm{BL}$ & M & $\mathrm{AM}$ & HSC \\
\hline $\mathrm{T} 1 \mathrm{D}$ & $28.7(+/-4.61)$ & $6.45(+/-4.27)$ & $1.33(+/-1.57)$ & $15.98(+/-14.63)$ & $15.34(+/-15.6)$ & $0.51(+/-0.18)$ \\
\hline $\mathrm{T} 2 \mathrm{D}$ & $55.92(+/-35.24)$ & $12.07(+/-13.73)$ & $1.78(+/-1.49)$ & $15.19(+/-4.11)$ & $4.42(+/-4.04)$ & $0.9(+/-0.51)$ \\
\hline $\mathrm{C}$ & $24.3(+/-3.95)$ & $1.38(+/-0.87)$ & $0.4(+/-0.17)$ & $8.49(+/-6.49)$ & $0.98(+/-0.66)$ & $0.79(+/-0.44)$ \\
\hline
\end{tabular}

lations. In conclusion, it is highly unlikely that PDGF-BB plays a significant role in ISR in diabetes.

\section{4}

Assessment of the response to exercise of platelet reactivity in diabetic patients with angina and angiographically normal or stenotic coronary arteries

P. Lamendola ${ }^{1}$, D. Pitocco ${ }^{2}$, F. Zaccardi ${ }^{2}$, A. Sestito ${ }^{1}$, S. Caputo ${ }^{2}$, G.A. Lanza ${ }^{1}$, F. Crea ${ }^{1}$, G. Ghirlanda ${ }^{2}$

${ }^{1}$ Cardiology, ${ }^{2}$ Internal Medicine, Catholic University, Rome, Italy

Background and aims: We previously demonstrated that in patients with cardiac syndrome $\mathrm{X}$ (effort angina, positive exercise stress test and normal coronary arteries at angiography [NCA] ) platelet reactivity is reduced by exercise, in contrast with the increase observed in patients with obstructive coronary artery disease (CAD). However, patients with diabetes were excluded from these previous studies. Aim of this study was to investigate the effect of exercise on platelet reactivity in diabetic patients with angina and NCA at angiography.

Materials and methods: The study group included 17 patients (61 \pm 6 years; 6 men) with type 2 diabetes who referred because of effort angina and were found to have positive exercise stress test, but totally NCA at angiography. Two different comparison groups were studied: 1) 17 type-2 diabetic patients with effort angina and obstructive CAD at angiography (64 \pm 6 years, 15 men), and 2) 11 type- 2 diabetic patients (controls, $61 \pm 9$ years, 5 men) without any history of chest pain and no evidence or suspect of myocardial ischemia on maximal exercise stress test. All patients underwent, symptom-limited exercise stress test according to Bruce protocol. Platelet reactivity was measured before and 5 minutes after peak exercise by the platelet function analyzer (PFA)-100 system as the time for flowing whole blood to occlude a collagenadenosine diphosphate ring (closure time).

Results: There were no differences among groups in exercise stress test capacity. ST-segment depression was induced during exercise test in 9 (54\%) and in $8(47 \%)$ patients in the NCA group and in the CAD group, respectively $(\mathrm{P}=1.0)$. At baseline, there was no difference among groups in closure time (NCA $108 \pm 24 \mathrm{~s}$; CAD $124 \pm 47 \mathrm{~s}$; controls $113 \pm 35 \mathrm{~s} ; \mathrm{P}=0.44$ ). After exercise closure time was significantly reduced in CAD patients (to $93 \pm 27 \mathrm{~s} ; \mathrm{P}<0.01$ ), whereas no significant changes were observed in NCA patients $(103 \pm 21 \mathrm{~s})$ and in controls $(123 \pm 77 \mathrm{~s})$. The group-exercise interaction was statistically significant $(\mathrm{P}=0.033)$.

Conclusion: In contrast to classical CSX patients, in type- 2 diabetic patients with angina and NCA we found no significant changes in platelet reactivity with exercise, thus suggesting that the mechanisms involved in the improved platelet reactivity profile shown after exercise in non diabetic CSX patients are absent in diabetic patients with microvascular angina. Our data also suggest that diabetes by itself does not have any significant effect on basal and post-exercise platelet reactivity as assessed by the PFA-100 method.

\section{5}

Does post-prandial glycaemia in non-diabetic patients with acute myocardial infarction give additional information for short-term prognosis?

M.-C. Brindisi ${ }^{1}$, C. Richard ${ }^{2}$, L. Lorgis ${ }^{2}$, P. Sicard ${ }^{2}$, M. Zeller ${ }^{2}$, B. Vergès ${ }^{1}$,

Y. Cottin ${ }^{2}$

${ }^{1}$ Diabetology, ${ }^{2}$ Cardiology, CHU, Dijon, France

Background and aims: Admission and fasting glycemia (FG) are both risk markers in patients with acute myocardial infarction (AMI). Over the last few years, elevated postprandial glycemia (PPG) has emerged as a powerful predictor of cardiovascular disease in the population at large. However, the prognostic value of PPG in non-diabetic patients after AMI remains unknown. Materials and methods: Between the $1^{\text {st }}$ November 2005 and the $30^{\text {th }}$ April 2007, 193 patients admitted to the coronary care unit for AMI were included. Patients with known diabetes or admission glycemia $\geq 11.1 \mathrm{mmol} / \mathrm{l}$ were excluded from the study. Blood samples were taken on admission, and to determine overnight fasting and PP (2h after a standardized meal) glucose levels on days 1 (FG1 and PPG1) and 3 (FG3 and PPG3) following admission. Six-month follow-up data were collected. The primary outcome was 6-month mortality or in-hospital heart failure.

Results: Median (IQR) admission glycemia was 6.9 (5.7-8.1) mmol/l. Median FG1 was 5.65 (5.02-6.37) $\mathrm{mmol} / \mathrm{l}$ and FG3 was $5.20(4.79-5.78) \mathrm{mmol} / \mathrm{l}$. Median PPG1 was 7.41 (6.1-8.52) mmol/l and PPG3 was 7.39 (6.08-9.06) mmol/l. Median HbAlc taken on admission was 5.7 (5.5-5.9) \%. Only FG3 and PPG3 were significantly related to the outcomes (OR (95\% CI): 1.83 (1.29-2.61), p $=0.001$ and 1.25 (1.08-1.44), $\mathrm{p}=0.003$, respectively). After adjustment for the GRACE (Global Registry of Acute Coronary Events) risk score and left ventricular ejection fraction, FG3 or PPG3 remained strong predictors of outcomes (OR (95\%CI): 1.27 (1.07-1.51), p = 0.005, and 1.70 (1.14-2.54), p $=0.010$, respectively). When both FG3 and PPG3 were introduced into the multivariate model, only FG3, but not PPG3, tended to be associated with the prognosis ((OR (95\%CI): 1.19 (0.99-1.44), $\mathrm{p}=0.065$ and $1.43(0.92-2.22), \mathrm{p}$ $=0.116$, respectively).

Conclusion: Our results show that in non-diabetic patients, both FG and PPG measured 3 days after admission for AMI are predictors of a poor shortterm prognosis. However, our findings strongly suggest that the addition of PPG to FG does not significantly improve the prediction of risk.

\section{6}

QTc interval and adiponectin in patients with type 2 diabetes mellitus R.Z. Timar, V. Serban, L. Diaconu, A. Vlad, B. Timar

Diabetes Clinic, University of Medicine "Victor Babes", Timisoara, Romania

Background and aims: QT prolongation has been shown to predict cardiac death in diabetic patients. QTc was used as a marker of atherosclerosis because previous studies have shown that QTc is affected by cardiac ischemia and correlates stronger with the carotid intima-media thickening than with cardiac autonomic neuropathy in patients with type $2 \mathrm{DM}$. Adiponectin, the only adipocytokine known to increase insulin sensitivity, also has anti-inflammatory and anti-atherogenic properties. The aim of our study were to evaluate the prevalence of QTc (QT corrected for heart rate) prolongation in patients with type $2 \mathrm{DM}$ and to assess the relationship between QTc and plasma levels of adiponectin. We also evaluated the relation between QTc and HbAlc, HOMA-IR, inflammatory markers, plasma lipids, systolic blood pressure (SBP), diastolic blood pressure (DBP), body mass index (BMI), ischemic heart disease (IHD), microangiopathy, heart rate variability(HRV) and somatic neuropathy.

Materials and methods: The study enrolled 118 patients with type 2 DM. For each subject 100 consecutive heart beats were recorded on the resting ECG tracing. QT and RR intervals were measured with a ruler for 5 consecutive beats on V5 lead on the resting ECG tracing. QTc was calculated according to Bazett's formula. QTc for each subject was considered the mean value of 5 calculated intervals. QTc $>0.44 \mathrm{~s}$ was considered abnormally prolonged. After measuring RR intervals for all 100 consecutive beats on V5 lead, the index HRV was calculated according to the formula $=$ (standard deviation of $\mathrm{RR} /$ mean RR) X 100.

Results: The prevalence of prolonged QTc interval was $27.1 \%$ in patients with type $2 \mathrm{DM}$. 
Table 1. Study subjects characteristics by the presence or absence of QTc prolongation

\begin{tabular}{|c|c|c|c|}
\hline Parameter & $\begin{array}{l}\text { QTc in normal } \\
\text { ranges }\end{array}$ & $\begin{array}{l}\text { QTc prolonged > } \\
0.44 \mathrm{~s}\end{array}$ & $\mathrm{p}$ \\
\hline $\mathrm{N}(\%)$ & $86(72.9)$ & $32(27.1)$ & - \\
\hline Age (years) & $54.2 \pm 12.1$ & $59.14 \pm 11.3$ & 0.047 \\
\hline Sex M n $(\%)$ & $42(48.8)$ & $15(53.1)$ & NS \\
\hline Duration of DM (years) & $12.1 \pm 4.4$ & $15.0 \pm 5.4$ & 0.003 \\
\hline HbAlc (\%) & $7.5 \pm 1.4$ & $7.9 \pm 2.8$ & NS \\
\hline BMI $(\mathrm{kg} / \mathrm{m} 2)$ & $27.3 \pm 3.1$ & $29.24 \pm 4.5$ & 0.012 \\
\hline$\underline{\mathrm{DBP}}(\mathrm{mmHg})$ & $76.2 \pm 7.2$ & $85.14 \pm 7.5$ & $<0.001$ \\
\hline $\mathrm{SBP}(\mathrm{mmHg})$ & $138.5 \pm 11.0$ & $143.5 \pm 11.1$ & 0.031 \\
\hline Triglycerides (mg/dL) & $217.1 \pm 20.2$ & $228.3 \pm 19.4$ & $<0.001$ \\
\hline Cholesterol (mg/dL) & $204.2 \pm 24.3$ & $210.3 \pm 31.4$ & NS \\
\hline $\mathrm{HDLc}(\mathrm{mg} / \mathrm{dL})$ & $45.26 \pm 5.42$ & $38.6 \pm 6.6$ & $<0.01$ \\
\hline HOMA IR & $3.50 \pm 1.12$ & $4.92 \pm 1.81$ & $<0.001$ \\
\hline Adiponectin $(\mu \mathrm{g} / \mathrm{mL})$ & $13.6 \pm 1.6$ & $7.1 \pm 0.8$ & $<0.001$ \\
\hline Leptin (ng/mL) & $7.4 \pm 3.5$ & $12.6 \pm 2.4$ & $<0.001$ \\
\hline TNF- $\alpha(\mathrm{pg} / \mathrm{mL})$ & $6.5 \pm 1.2$ & $8.4 \pm 1.4$ & $<0.001$ \\
\hline IL-6 (pg/mL) & $3.2 \pm 0.5$ & $4.5 \pm 0.6$ & $<0.001$ \\
\hline hs-CRP (mg/L) & $5.4 \pm 0.6$ & $7.4 \pm 0.9$ & $<0.001$ \\
\hline Fibrinogen $(\mathrm{mg} / \mathrm{dL})$ & $232.9 \pm 40.7$ & $345.2 \pm 44.2$ & $<0.001$ \\
\hline
\end{tabular}

Data are mean \pm SD or $\mathrm{n}(\%)$. P was calculated with Student's t test or with chi-square test.

Patients with prolonged QTc had significantly higher age, diabetes duration, BMI, waist circumference, SBP, DBP, higher plasma tryglicerides, lower HDLc plasma levels and increased prevalence of IHD than patients without QT abnormalities. Serum adiponectin was strongly and significantly negatively correlated with QTc $(r=-0.76, \mathrm{p}<0.0001)$, after adjusting for age, sex, diabetes duration, BMI, waist circumference, HbAlc, plasma lipids. QTc was also correlated with HOMA-IR $(\mathrm{r}=0.58, \mathrm{p}<0.001)$, BMI $(\mathrm{r}=0.52, \mathrm{p}<0.05)$, waist circumference $(r=0.56, p<0.001)$, the presence of IHD $(r=0.63, p$ $<0.001$ ). A significant, but weak negative relationship was found between QTc and HRV $(\mathrm{r}=-0.36, \mathrm{p}<0.01)$. Also, QT prolongation was associated with increased plasma levels of leptin and of inflammatory markers. No relationship between was observed between QTc and gender, HbA1c, diabetic retinopathy, diabetic nephropathy, somatic neuropathy.

Conclusion: Low plasma levels of adiponectin were strongly and independently correlated with prolonged QTc and the presence of IHD. Our findings suggest that both hypoadiponectinemia and prolonged QTc could be costbenefit advantageous methods of identifying diabetic patients with increased risc for atherosclerotic cardiovascular diseases.

\section{7}

\section{Effect of glucose-insulin-potassium infusion (GIK) on stress-induced myocardial ischaemia \\ B. Boldrini ${ }^{1}$, S. Di Marco ${ }^{2}$, U. Conti ${ }^{2}$, F. Lattanzi ${ }^{2}$, C. Morgantini ${ }^{1}$, \\ E. Ferrannini ${ }^{1}$, A. Natali \\ ${ }^{1}$ Department of Internal Medicine, University of Pisa, Italy, ${ }^{2}$ Division of Cardiovascular Medicine, Hospital of Pisa and Pescia, Italy}

Background and aims: Clinical trials using Insulin and GIK in the treatment of acute coronary syndrome have given conflicting results. In experimental animal models, however, pre-treatment with insulin or GIK has been shown to consistently improve left ventricular perfusion and function during both acute and chronic ischaemia through metabolic and vascular preconditioning of the myocardial tissue. The aim of this study is to verify whether in order to exert its protective effect, insulin must be present before the ischemic event occurs. Data in vivo in man in this setting are lacking.

Materials and methods: Eleven non-diabetic patients with clinical and ECG evidence of inducible myocardial ischaemia and normal left ventricular function underwent a dipyridamole-atropine echocardiography test (stress echo). Another 11 non-diabetic patients with regional contractility defects and evidence of inducible ischaemia (on exercise ECG test) were submitted to a ${ }^{201} \mathrm{Tl}$ myocardial perfusion exercise scintigraphy. Each test (stress echo and ${ }^{201} \mathrm{Tl}$ scan) was done twice (within 14 days), once preceded by 60 minutes of normal saline, the other by 60 minutes of GIK infusion $(500 \mathrm{ml}$ of a $20 \%$ glucose solution with $\mathrm{KCl} 30 \mathrm{mEq}$ and insulin $15 \mathrm{U}$ ) at the rate of $2 \mathrm{ml} / \mathrm{kg} / \mathrm{h}$. Hyperglycaemia was accurately avoided either by adding extra insulin or by shifting on a $10 \%$ glucose solution. For the stress echo the extent of ischemia was evaluated by using an ischemia score (IS) calculated as the number of left ventricle segments multiplied by their wall motion score change divided by the duration (proportional to the dose) of the dipyridamole/atropine infusion. For the ${ }^{201} \mathrm{Tl}$ scan the analysis was done on the difference in perfusion score in the 20 segments of the left ventricle among the 3 conditions of stress, rest and reinjection. A global perfusion score was calculated as the sum of all the 16 segments perfusion score of all patients.

Results: GIK infusion in both tests was well tolerated and resulted in a plateau of plasma insulin concentration of $1,120 \pm 84 \mathrm{pmol} / \mathrm{l}$ and in a stable plasma potassium and glucose concentration throughout the tests $(3.7 \pm 0.5$ $\mathrm{mEq} / \mathrm{l}$ and $6.1 \pm 1.7 \mathrm{mmol} / \mathrm{l}$, respectively). On the stress echo, GIK infusion was associated with a $35 \%$ reduction in the severity of ischaemia (IS Saline: $0.55 \pm 0.09$, IS GIK: $0.36 \pm 0.09$ ). On ECG ergometry, GIK infusion slightly increased the time to ischaemia $(+0.6 \mathrm{~min}, \mathrm{p}=0.07)$ but the higher workload $(+8 \%)$ was achieved at a similar rate-pressure plateau (Saline: 22,250 $\pm 1,055$ vs GIK 22,539 $\pm 1,194 \mathrm{mmHg}$ x bpm). On the ${ }^{201} \mathrm{Tl}$ scan, an increase in number of ischaemic segments $(+48 \%, \mathrm{p}<0.05)$ was observed mainly to the detriment of viable (but non-ischaemic) and non-viable segments, which were reduced by $60 \%(p<0.01)$. When the perfusion pattern was analysed using the global perfusion score, the main effect of GIK was to enhance the reversibility of acute post-stress perfusion defects $(+94 \%, \mathrm{p}<0.01)$, while stress-induced left ventricular underperfusion was only marginally affected $(+13 \%, \mathrm{p}=\mathrm{ns})$.

Conclusion: In non-diabetic patients with inducible myocardial ischaemia acute GIK pre-treatment directly attenuates the effects of ischaemia on myocardial contractility, slightly improves exercise tolerance through systemic heamodynamics and causes a more rapid and diffuse recovery of exerciseinduced myocardial perfusion defects.

\section{8}

A low-dose treatment with atorvastatin for one year improves arterial stiffness in patients with type 2 diabetes mellitus

P. Grigoropoulou ${ }^{1}$, N. Tentolouris ${ }^{1}$, D. Perrea ${ }^{2}$, C. Zoupas ${ }^{1}$,

I. Eleftheriadou ${ }^{1}$, K. Alexiadou ${ }^{1}$, N. Katsilambros ${ }^{1}$

${ }^{1} 1$ st Department of Propaeudetic Medicine, Athens University Medical School, Laiko Hospital, ${ }^{2}$ Laboratory of Experimental Surgery and Surgical Research, Athens University Medical School, Athens, Greece

Background and aims: Type 2 diabetes mellitus (T2DM) is associated with a two to four-fold Aincreased in risk for coronary heart disease. Dyslipidaemia is a modifiable atherogenic risk factor in patients with T2DM, and low LDL cholesterol (LDLc) levels with the use of statins have demonstrated beneficial effects in the reduction of cardiovascular diseases in large primary and secondary prevention clinical trials. Increased arterial stiffness, assessed by pulse wave velocity (PWV), has also been emerged as an independent predictor for cardiovascular events. The aim of this prospective, open, intervention, parallel group study was to evaluate the effect of atorvastatin administration for 1 year on arterial stiffness, assessed by determination of PWV at the carotid- femoral (PWVcf) and the carotid-radial (PWVcr) arteries, in patients with T2DM.

Material and methods: A total of 54 patients with T2DM were recruited, 31 patients ( 11 men, 20 women, mean age $60.77 \pm 8.752$ years, diabetes duration $6.94 \pm 6.175$ years) were assigned to 12 -months atorvastatin therapy (10 mg daily) and a low fat diet, and 23 patients ( 8 men, 15 women, mean age $58.55 \pm 8.445$, diabetes duration $5.58 \pm 5.004$ ) to low-fat diet only. The two groups were comparable at baseline in terms of total cholesterol, LDLc, body mass index, and HbAlc. The main inclusion criteria were age between 40 and 75 years, LDLc $\geq 100 \mathrm{mg} / \mathrm{dl}$, and absence of clinically apparent macrovascular disease. PVW was determined by applanation tonometry (SphygmoCor Vx, AtCor Medical, Sydney, Australia). Plasma lipids, HbAlc, and PVW were evaluated prospectively at baseline, 3, 6, and 12 months in both groups.

Results: Total cholesterol and LDLc declined significantly in the atorvastatintreated group at the examined time intervals. In the same group a significant reduction in PWVcr and PWVcf was observed 3 months post-intervention ( $4.59 \%, \mathrm{p}<0.001$, and $9.18 \%, \mathrm{p}<0.001)$, which was maintained at $6(6.55 \%$, $\mathrm{p}=0.007$, and $12.99 \%, \mathrm{p}<0.001)$ and 12 months $(9.9 \%, \mathrm{p}=0.01$, and $12.43 \%$, $\mathrm{p}=0.003$, respectively). In the untreated group, a small non-significant improvement in lipid profile was observed; however, no significant changes in PWVcr and PWVcf were found.

Conclusion: Beyond the lipid-lowering effects, a low dose of atorvastatin improves arterial stiffness in patients with T2DM. The improvement starts 3 months after intervention and continuous for 1 year. 


\section{9}

Does multiple injection therapy with a rapid-acting insulin analogue prevent cardiovascular disease in type 2 diabetes? The NICE-study: a prospective, randomised, open-label, blinded endpoint study H. Nishimura ${ }^{1}$, M. Shintani ${ }^{1}$, K. Maeda ${ }^{1}$, I. Hanaoka ${ }^{2}$, H. Kuzuya ${ }^{3}$; ${ }^{1}$ Division of Diabetes and Endocrinology, Osaka Saiseikai Nakatsu Hospital, Osaka, ${ }^{2}$ Division of Diabetes, Wakayama Red Cross Center, Wakayama, ${ }^{3}$ Division of Diabetes and Kidney Disease, National Hospital Organization, Kyoto Medical Center, Kyoto, Japan

Background and aims: Diabetes mellitus is recognized to be one of the major risk factors for macrovascular disease. However, the effect of postprandial metabolic control by rapid-acting insulin analogues on macrovascular disease has not been investigated in type 2 diabetes. We, therefore, investigated whether multiple injection therapy with a rapid-acting analogue insulin aspart prevents cardiovascular disease in type 2 diabetes, and conducted the "Nippon ultrarapid Insulin and diabetic Complication Evaluation-study (NICE-study)".

Materials and methods: Three hundred and seventy-four type 2 diabetes patients on multiple injection therapy with insulin from 11 medical institutions (aged 20-84 years) were randomised into 2 treatment groups, (ultra)rapidacting analogue insulin aspart (U) and short-acting human regular insulin (R). As necessary, intermediate-acting or long-acting insulin was used $(40 \%$ of the $\mathbf{U}$ group and $42 \%$ of the $\mathbf{R}$ group). The primary endpoint was cardiovascular events (myocardial infarction, angina pectoris, PCI/CABG and TIA/cerebral infarction). During the observation period, aspirin, ARBs, and statins were administered to patients according to guidelines with usual daily dosing. Statistical analyses were based on intention to treat. The study was registered at ClinicalTrials.gov with the identifier NCT00575172 and conducted from January 2003 to December 2007.

Results: There was no difference in the baseline characteristics between the 2 groups (age, BMI, gender, duration of disease, $\mathrm{HbA}_{1 \mathrm{c}}$, blood pressure or LDL- cholesterol). After a median follow-up of 4.5 years (range 3.5-5.0), hazard ratios were calculated and adjusted for age, BMI, gender, blood pressure, LDL- cholesterol, and smoking. A significant decrease of $43 \%$ was found in the cumulative cardiovascular event rate between the $\mathrm{U}$ group, $6.4 \%$ (12.8/1000/year), and the $\mathbf{R}$ group, $11.1 \%(22.2 / 1000 /$ year) ( $\mathrm{HR}=0.57$, CI: $0.34-0.95, \mathrm{P}<0.02)$. However, there were no differences in cardiovascular and overall mortality. Total dosages of basal and bolus insulin in the two groups was not different at the start point (Bolus: $27.7 \pm 6.2$ in the $\mathbf{U}$ group vs. 26.8 \pm 8.2 in the $\mathbf{R}$ group, Basal: $12.2 \pm 4.0$ vs. $12.6 \pm 4.5 \mathrm{U} /$ day, respectively). At the end point, however, the bolus dosage was increased significantly in the U group (Bolus: $34.8 \pm 8.3$ vs. $28.3 \pm 4.8$, $\mathrm{p}<0.01$, Basal: $14.6 \pm 3.4$ vs. 14.2 $\pm 4.1 \mathrm{U} /$ day, $\mathrm{p}>0.05$, respectively). A significant decrease was seen in the 90 min. postprandial plasma glucose levels of the $\mathbf{U}$ group $v s$. the $\mathbf{R}$ group, (142 \pm 58 vs. $226 \pm 48 \mathrm{mg} / \mathrm{dl}$, respectively, $\mathrm{P}<0.02)$. A statistically significant difference was not found between the groups in fasting plasma glucose levels (128 \pm 42 vs. $133 \pm 54 \mathrm{mg} / \mathrm{dl}$, respectively), $\mathrm{HbA}_{1 c}$ ( $7.5 \pm 0.7$ vs. $7.5 \pm 0.7 \%$, respectively), or in the use of concomitant hypertensive/dyslipidemia/anticoagulation treatments.

Conclusion: Treatment with rapid-acting insulin analogue showed an improvement in postprandial plasma glucose and a reduction in the risk of cardiovascular events. The results may be mediated not only by the improvement in postprandial glucose levels but also by unknown mechanisms of the rapid-acting insulin analogue.

\section{0}

Effects of a rehabilitation programme on large and small artery elasticity, in cardiac patients. Comparison between diabetic and non-diabetic patients

P.R. Blanc ${ }^{1}$, A. Boussuges ${ }^{2}$, S. Maunier ${ }^{1}$, S. Chopra ${ }^{1}$, X. Debussche ${ }^{3}$ ${ }^{1}$ Cardiac rehabilitation, Sainte Clotilde center, Sainte Clotilde, Reunion, ${ }^{2}$ Université de la Méditerranée et IMNSSA, Faculté de Médecine Nord et IMNSSA, Marseilles, France, ${ }^{3}$ Service de diabétologie, CHD Félix Guyon, Sainte Denis, Reunion

Background and aims: Impairment in arterial compliance is associated with diabetes. Arterial stiffness is related to the cardiovascular disease risk. Treatments allowing arterial compliance increase should be relevant to improve prognosis. Previous studies have demonstrated that rehabilitation programs were able to improve arterial compliance in cardiac patients. In the present study, we have assessed the effect of a 6-week rehabilitation program on arte- rial compliance in diabetic patients (DP) in comparison with non-diabetic patients (NDP) with heart disease.

Materials and methods: 134 type 2 DP and 160 NDP engaged in aerobic training and multidisciplinary educational interventions five days per week. DP and NDP were matched appropriately for age (respectively 57.5 vs 56.5 years), sex ratio ( $29 \%$ vs $25 \%$ women) and type of heart disease. Sixty one percent of DP was treated by insulin. Baseline $\mathrm{HbA}(1 \mathrm{c})$ was greater than $6.6 \%$ in $64 \%$ of cases. Retinopathy and microalbuminuria were detected in respectively $31 \%$ and $36 \%$ of subjects. Large (C1) and small artery (C2) elasticity indices were measured using the HDI/PulseWaveTM CR-2000 Research CardioVascular Profiling System (Hypertension Diagnostics TM, Inc) before and after rehabilitation.

Results: Before exercise training C2 was lower in DP in comparison with $\operatorname{NDP}(3.3$ vs $3.6-\mathrm{p}<0.02)$ whereas $\mathrm{C} 1$ was similar $(15.7$ vs $15.3-\mathrm{p}=0.6)$. After training, significant increases in $\mathrm{C} 1$ and $\mathrm{C} 2$ were found in the two groups. In diabetic patient a $+9 \%$ increase in $\mathrm{C} 1(16.6-\mathrm{p}=0.01)$ and a $21 \%$ increase in $\mathrm{C} 2(4.2-\mathrm{p}<0.001)$ were found. This increase was assessed to $+7 \%$ for $\mathrm{C} 1$ $(16.7-p=0.002)$ and to $+18 \%$ for $\mathrm{C} 2(4.3-\mathrm{p}<0.001)$ in NDP. The percentage of increase in C1 and C2 was not statistically significant between DP and NDP. Conclusion: Small artery elasticity is lower in diabetics compared with non diabetics. A 6-week aerobic training and multidisciplinary educational program improves both large and small artery elasticity in DP and NDP. 


\section{PS 134 Metabolic risk and cardiovascular complications}

\section{1}

The metabolic syndrome, angiographically determined stable coronary artery disease, and subclinical inflammation

M. Woess ${ }^{1}$, C. Saely ${ }^{1,2}$, S. Beer ${ }^{1}$, P. Rein ${ }^{1}$, A. Vonbank ${ }^{1}$, C. Boehnel ${ }^{1}$, V. Jankovic ${ }^{1}, H$. Drexel ${ }^{1,2}$

${ }^{1}$ VIVIT Institute, Feldkirch, Austria, ${ }^{2}$ Medicine, University for Human Sciences, Triesen, Liechtenstein

Background and aims: The metabolic syndrome (MetS) and stable coronary artery disease (CAD) frequently coincide; the individual contributions of these entities to subclinical inflammation have not been investigated yet. We therefore aimed at investing markers of inflammation in patients with the MetS, in patients with CAD, and in patients who had both, the MetS and CAD.

Materials and methods: We enrolled 935 consecutive patients undergoing coronary angiography for the evaluation of suspected or established stable CAD. The MetS was defined according to National Cholesterol Education Programme Adult Treatment Panel III criteria; coronary stenoses with lumen narrowing $\geq 50 \%$ were considered significant.

Results: From our patients 520 (55.6\%) had significant coronary stenoses; the prevalence of the MetS was higher in our patients with significant stenoses than in those without such lesions ( $39.0 \%$ vs. $32.8 \%$; $p=0.048)$. The inflammatory markers hsCRP and white blood cell count (WBC) were significantly higher in MetS patients than in those without the MetS both among patients with significant coronary stenoses $(0.49 \pm 0.71$ vs. $0.42 \pm 0.88 \mathrm{mg} / \mathrm{dl} ; \mathrm{p}=$ 0.004 and $7.0 \pm 1.8$ vs. $6.5 \pm 1.8 \mathrm{G} / \mathrm{l} ; \mathrm{p}=0.003$, respectively) and in subjects who did not have such lesions $(0.44 \pm 0.51$ vs. $0.37 \pm 0.54 \mathrm{mg} / \mathrm{dl} ; \mathrm{p}=0.004$ and $7.1 \pm 1.8$ vs. $6.4 \pm 1.8 \mathrm{G} / \mathrm{l} ; \mathrm{p}<0.001$, respectively). In contrast, these inflammatory markers were not significantly elevated in patients with significant stenoses among subjects with the MetS $(p=0.776$ and $p=0.713$, respectively) nor among those who did not have the MetS $(\mathrm{p}=0.882$ and $\mathrm{p}=0.119$, respectively). Similar results were obtained with the International Diabetes Federation definition of the MetS.

Conclusion: We conclude that subclinical inflammation is strongly and significantly associated with the MetS but not with angiographically determined stable CAD.

Supported by: Innovationsstiftung of the Liechtenstein Global Trust Bank Peter Goop Stiftung; Vorarlberger Landesregierung; Medizinisches Zentrallabor Feldkirch; Vorarlberger Industriellenvereinigung; Vorarlberger Aerztekammer; Vorarlberger Landeskrankenhaus-Betriebsgesellschaft

\section{2}

Cardio-vascular risk factors and mortality in a geriatric population; is there a "reverse metabolic syndrome"?

U.M. Vischer' ${ }^{1}$, F. Herrmann ${ }^{1}$, M. Safar', J. Blacher ${ }^{2}$

${ }^{1}$ Dept of Rehabilitation and Geriatrics, Geneva University Hospitals,

Thônex/Geneva, Switzerland, ${ }^{2}$ Diagnosis and Therapeutic Center, Hôtel-

Dieu Hospital, Paris, France

Background and aims: Diabetes or insulin resistance, overweight, arterial hypertension and dyslipidemia are strong risk factors for cardio-vascular (CV) disorders in young adults. We investigated the predictive value and hierarchy of these risk factors in elderly high-risk subjects.

Materials and methods: Two-year total mortality was determined in a prospective cohort study of 331 elderly (mean age $[ \pm \mathrm{SD}]: 85 \pm 7$ years) high-risk patients admitted to two geriatric wards.

Results: Two-year total mortality was predicted by age, diabetes, low BMI, low diastolic blood pressure (DBP), low total and HDL-cholesterol, and previous $\mathrm{CV}$ events. The effect of diabetes was accounted for by previous $\mathrm{CV}$ events. In non-diabetic patients, total mortality was also predicted by high insulin sensitivity (low HOMA-IR and high QUICKI indexes). In multivariate analysis, the strongest predictors of mortality were a low BMI, low HDL-cholesterol and previous myocardial infarction. Low serum albumin, a marker of malnutrition, was associated with low blood pressure, total and HDL-cholesterol and HOMA-IR index. CRP, a marker of inflammation, was associated with low total and HDL-cholesterol values and high HOMA-IR index.

Conclusion: In elderly patients, malnutrition and/or chronic disorders generate a "reverse metabolic syndrome" in which, paradoxically, low BMI, low
DBP, low total and HDL-cholesterol and high insulin sensitivity predict total mortality. These inverse associations limit the value of conventional risk factors in elderly patients. Along with previous CV events, HDL-cholesterol remains a strong, coherent mortality risk factor. Future intervention studies should determine whether and when the prevention and treatment of malnutrition in the elderly should be incorporated into conventional cardiovascular prevention.

\section{3}

The association of serum adipocyte fatty acid-binding protein with coronary artery severity in Korean adults

E.-J. Rhee ${ }^{1}$, E.-S. Choi ${ }^{1}$, J.-H. Kim ${ }^{1}$, J.-C. Won ${ }^{1}$, C.-Y. Park ${ }^{1}$, W.-Y. Lee ${ }^{1}$, K.-W. Oh${ }^{1}$, B.-J. Kim², K.-C. Sung ${ }^{2}$, B.-S. Kim², J.-H. Kang ${ }^{2}$, S.-W. Park ${ }^{1}$, M.-H. Lee' ${ }^{2}$ S.-W. Kim ${ }^{1}$

${ }^{1}$ Endocrinology and metabolism, ${ }^{2}$ Cardiology, Kangbuk Samsung Hospital, Sungkyunkwan University School of Medicine, Seoul, Republic of Korea

Background and aims: Adipocyte fatty acid-binding protein (A-FABP), also known as aP2 or FABP4, is abundantly expressed in adipocytes and plays a role in glucose homeostasis. As there are increasing evidences that FABP4 might play an important role in inflammation and metabolic derangements in human body, we analyzed the relationship between the coronary artery disease and serum FABP4 levels in Korean adults.

Material and methods: In total 234 Korean adults (mean age 58.2 years) in whom coronary angiograms were performed due to chest pain, anthropometric measurements were done and fasting glucose and lipid profiles were measured. The severities of coronary artery disease were classified in 4 groups according to the number of stenotic vessels, that is, normal, 1-vessel, 2-vessel and 3-vessel disease. Serum FABP4 levels were measured with enzyme-linked immunosorbent assay. The presence of metabolic syndrome was diagnosed according to AHA/NHBL criteria with body mass index substituted for waist circumference.

Results: Among the subjects, 137 subjects (58.5\%) were male and 97 subjects $(41.5 \%)$ were female. 74 subjects $(31.6 \%)$ had diabetes, and 110 subjects $(47 \%)$ had metabolic syndrome. Regarding the severity of coronary artery disease, 99 subjects $(42.4 \%)$ were normal, 81 subjects (34.6\%) had 1-vessel disease, 32 subjects (13.7\%) had 2-vessel disease, and 22 subjects (9.4\%) had 3-vessel disease. Mean serum FABP4 levels were not different between the subjects with or without metabolic syndrome, but showed significantly higher levels in subjects with diabetes $(16.44 \pm 13.3$ vs. $12.78 \pm 11.0 \mathrm{ng} / \mathrm{mL} ; \mathrm{p}=0.042)$. When the metabolic parameters were compared according to each groups, age, fasting glucose and serum FABP4 levels increased significantly as the numbers of stenotic vessel increased from normal to 3 -vessel disease (Mean values for FABP4, $12.81 \pm 9.4,12.84 \pm 12.8,14.39 \pm 8.8$ and $22.40 \pm 18.1 \mathrm{ng} / \mathrm{mL}$ for normal, 1 -vessel, 2-vessel and 3-vessel groups; $\mathrm{p}=0.005)$. In post-hoc analysis, mean values for serum FABP4 levels showed significant differences between normal, 1 -vessel and 3 -vessel groups $(\mathrm{p}<0.05)$. However, these significances were attenuated after adjustment for age and fasting glucose $(\mathrm{p}=0.060)$.

Conclusion: Serum FABP4 levels increased as the numbers of stenotic coronary artery increased, but these differences were lost after adjustment for age and fasting glucose levels, suggesting that the relationship of serum FABP4 levels with the severity of coronary artery disease might be affected strongly by age and fasting glucose levels.

\section{4}

Do different definitions of the metabolic syndrome equally identify patients at risk for cardiovascular disease in type 1 diabetes?

L.M. Thorn ${ }^{1,2}$, C.M. Forsblom ${ }^{1,2}$, J. Wadén ${ }^{1,2}$, M. Saraheimo ${ }^{1,2}$, N. Tolonen ${ }^{1,2}$, D. Gordin ${ }^{1,2}$, O. Heikkilä ${ }^{1,2}$, K. Hietala ${ }^{1,2}$, J. Kytö ${ }^{1,2}$, M. Rosengård-Bärlund ${ }^{1,2}$, P.-H. Groop ${ }^{1,2}$

${ }^{1}$ Department of Medicine, Division of Nephrology, Helsinki University Central Hospital, ${ }^{2}$ Department of Diabetes Genetics, Folkhälsan Research Center, Helsinki, Finland

Background and aims: The prevalence of the metabolic syndrome varies between $15-70 \%$ in patients with type 1 diabetes, depending on age, glycemic control, presence of diabetic complications, and diagnostic criteria. The role of the syndrome as a predictor for cardiovascular disease and diabetes-related mortality in type 1 diabetes is not clear. The aim was therefore to assess its predictive value regarding new cardiovascular events and mortality in patients with type 1 diabetes using different definitions of the syndrome. 
Materials and methods: 3,743 adult patients with type 1 diabetes attending the prospective, nation-wide Finnish Diabetic Nephropathy Study were assessed. At baseline, mean age was $37.3 \pm 11.8$ yrs, duration of diabetes $22.6 \pm 12.1$ yrs, $52 \%$ were male, and $20 \%$ had diabetic nephropathy. The metabolic syndrome was defined according to the WHO, NCEP, and IDF criteria. Complete data on mortality were retrieved from death certificates and data on cardiovascular end-points from medical files (available in 64\%, $\mathrm{n}=2,392$ ). A cardiovascular event was defined as myocardial infarction, coronary revascularization, or stroke during follow-up.

Results: The prevalence of the metabolic syndrome at baseline was $44 \%, 34 \%$, and $36 \%$ with WHO, NCEP, and IDF criteria, respectively. After a median follow-up of 5.8 (IQR 4.1-6.9) years, 219 patients suffered a new cardiovascular event and 245 died, of whom 199 (81\%) died of cardiovascular or diabetesrelated causes.

The metabolic syndrome according to WHO and NCEP criteria independently predicted new cardiovascular events [HR $3.52(2.45-5.06)$ and 1.80 (1.36-2.39), respectively], adjusted for age, sex, LDL cholesterol, smoking, and $\mathrm{HbA}_{1}$. After further adjustment for previous cardiovascular events, WHO and NCEP were still independent predictors [HR 2.84 (1.96-4.12) and 1.59 (1.20-2.12)], while after adjustment for diabetic nephropathy only WHO remained significant [HR 2.08 (1.47-2.95)]. For cardiovascular and diabetesrelated mortality, both WHO and NCEP criteria were independent predictors [HR 7.37 (4.51-12.05) and 2.02 (1.49-2.74)], adjusted for age, gender, LDL cholesterol, smoking, and $\mathrm{HbA}_{1}$. After further adjustment for diabetic nephropathy, only WHO was an independent predictor [HR 2.71 (1.56-4.69)]. IDF criteria neither predicted new cardiovascular events nor diabetes-related mortality.

Conclusion: In type 1 diabetes, the metabolic syndrome independently predicts, not only cardiovascular morbidity, but also cardiovascular and diabetes-related mortality. Notably, the WHO criteria, which include microalbuminuria, was the strongest predictor followed by NCEP, while IDF was not a significant predictor in type 1 diabetes.

Supported by: Folkhälsan Research Foundation, Wilhelm and Else Stockmann Foundation, Liv och Hälsa Foundation, Finnish Medical Society, Perklén Foundation, and European Commission

\section{5}

Alanine aminotransferase and gamma glutamyl transferase are associated with the metabolic syndrome but not with angiographically determined coronary atherosclerosis

L. Risch ${ }^{1}$, C.H. Saely ${ }^{1,2}$, A. Vonbank ${ }^{1}$, P. Rein ${ }^{1}$, M. Woess ${ }^{1}$, S. Beer ${ }^{1}$,

C. Boehnel ${ }^{1}$, V. Jankovic ${ }^{1}$, S. Aczel ${ }^{1}$, H. Drexel ${ }^{1,2}$

${ }^{1}$ VIVIT Institute, Feldkirch, Austria, ${ }^{2}$ Medicine, University for Human Sciences, Triesen, Liechtenstein

Background and aims: We aimed at investigating the respective associations of the metabolic syndrome (MetS) and of angiographically determined coronary atherosclerosis with liver enzymes.

Materials and methods: We enrolled 1000 consecutive patients undergoing coronary angiography for the evaluation of suspected or established stable coronary artery disease (CAD). The MetS was defined according to Adult Treatment Panel III criteria; significant CAD was diagnosed in the presence of coronary stenoses with lumen narrowing $\geq 50 \%$.

Results: Serum alanine aminotransferase (ALT), the ALT / aspartate aminotransferase (AST) ratio, and serum gamma-glutamyl transferase (GGT) were significantly higher in patients with the MetS than in subjects without the MetS $(34 \pm 21$ vs. $29 \pm 20 \mathrm{U} / \mathrm{l} ; \mathrm{p}<0.001,1.16 \pm 0.39$ vs. $1.00 \pm 0.36$, p $<0.001$; and $53 \pm 88$ vs. $43 \pm 57 \mathrm{U} / \mathrm{l}, \mathrm{p}=0.001$, respectively). In contrast, these parameters of liver function were similar in patients with significant CAD and in those who did not have significant CAD ( $\mathrm{p}=0.592 ; \mathrm{p}=0.731$, and $\mathrm{p}$ $=0.716$, respectively). Analysis of covariance after multivariate adjustment including alcohol consumption confirmed that ALT, the ALT/AST ratio, and GGT were significantly and independently associated with the MetS (F = 13.87, $\mathrm{p}<0.001 ; \mathrm{F}=19.94, \mathrm{p}<0.001$; and $\mathrm{F}=8.55, \mathrm{p}=0.004$, respectively) but not with coronary athersoclerosis $(\mathrm{p}=0.317, \mathrm{p}=0.741$, and $\mathrm{p}=0.151$, respectively).

Conclusion: ALT, the ALT / AST ratio, and GGT are associated with the MetS but not with angiographically determined coronary atherosclerosis.

Supported by: Innovationsstiftung of the Liechtenstein Global Trust Bank; Peter Goop Stiftung; Vorarlberger Landesregierung; Medizinisches Zentrallabor Feldkirch; Vorarlberger Industriellenvereinigung; Vorarlberger Aerztekammer; Vorarlberger Landeskrankenhaus-Betriebsgesellschaft

\section{6}

Effects of noninvasive ventilation on cardiovascular risk profile in patients with metabolic syndrome and obstructive sleep apnea

R. Tkacova ${ }^{1}$, Z. Dorkova ${ }^{2}$, D. Petrasova ${ }^{3}$, A. Molcanyiova ${ }^{4}$, I. Tkac $^{5}$ ${ }^{1}$ Dept. of Respiratory Disorders, Medical Faculty, PJ Safarik University, ${ }^{2}$ Dept. for Respiratory Disorders, Medical Faculty, PJ Safarik University, ${ }^{3}$ Institute of Experimental Medicine, Medical Faculty, PJ Safarik University, ${ }^{4}$ Dept. of Biochemistry, Labmed, ${ }^{5}$ Dept. of Internal Medicine 4, Medical Faculty, PJ Safarik University, Kosice, Slovakia

Background and aims: The increased risk of atherosclerotic morbidity and mortality in patients with the metabolic syndrome and obstructive sleep apnea (OSA) has been linked to arterial hypertension, insulin resistance, systemic inflammation, and oxidative stress. In these patients, intermittent hypoxia is the strongest predictor of the nuclear factor- $\mathrm{\kappa B}$ dependent pathways activation resulting in increased serum tumor necrosis factor- $\alpha$ (TNFa) levels. In contrast, obesity rather than OSA drive raised serum C-reactive protein (CRP), interleukin 6 (IL-6), or leptin concentrations. We aimed to determine the effects of 8 -weeks therapy using noninvasive ventilation with continuous positive airway pressure (CPAP) on glucose and lipid profile, systemic inflammation, oxidative stress, and the global cardiovascular disease (CVD) risk in patients with severe OSA and metabolic syndrome.

Materials and methods: In 32 patients, serum cholesterol, triglycerides, high density lipoprotein (HDL) cholesterol, fibrinogen, apolipoprotein A-I (ApoA-I), apolipoprotein B (ApoB), high sensitivity CRP, IL-6, TNF- $\alpha$, leptin, malondialdehyde (MDA) and erythrocytic glutathione peroxidase (GPx) activity were measured at baseline and after 8 weeks of CPAP. Insulin resistance index (HOMA-IR) was based on the homeostasis model assessment method, the CVD risk was calculated using the multivariable risk factor algorithm. No patient changed hypolipidemic and/or antihypertensive medication, or changed weight $(>5 \%)$ during the follow-up.

Results: In patients who used CPAP for $\geq 4$ h.night $^{-1}(n=16)$, noninvasive ventilation reduced the number of apneas and hypopneas during sleep (from $64.7 \pm 23.3$ to $3.8 \pm 2.1$ events.hr ${ }^{-1}, \mathrm{p}<0.001$ ), and increased the lowest nocturnal $\mathrm{SaO}_{2}$ (from $66.5 \pm 15.6$ to $89.5 \pm 12.6 \%, \mathrm{p}<0.001$ ). Abolition of OSA was associated with reductions in systolic and diastolic blood pressure (from 148.6 \pm 19.6 to $129.4 \pm 15.6 \mathrm{~mm} \mathrm{Hg}, \mathrm{p}=0.001$; from $91.7 \pm 13.5$ to $79.5 \pm 8.2 \mathrm{~mm} \mathrm{Hg}, \mathrm{p}=0.006$, respectively), reductions in total cholesterol (from $8.80 \pm 1.55$ to $7.75 \pm 1.63$ $\mathrm{mmol} / \mathrm{l}, \mathrm{p}=0.002$ ), ApoB (from $1.15 \pm 0.29$ to $0.99 \pm 0.20 \mathrm{~g} / \mathrm{l}, \mathrm{p}=0.009$ ), and HOMA-IR (from $4.73 \pm 3.18$ to $2.93 \pm 1.77$, $\mathrm{p}=0.031$ ). In addition, serum MDA and TNF- $\alpha$ were reduced (from $1.73 \pm 0.26$ to $1.49 \pm 0.22 \mathrm{nmol} / \mathrm{ml}, \mathrm{p}=0.004$; from $2.13 \pm 0.98$ to $1.79 \pm 0.69 \mathrm{pg} / \mathrm{ml}, \mathrm{p}=0.037$, respectively), while erythrocytic GPx activity increased (from $29.2 \pm 8.1$ to $36.3 \pm 12.5 \mathrm{U} / \mathrm{g} \mathrm{Hb}, \mathrm{p}=0.015$ ). Serum high sensitivity CRP, IL- 6 and leptin concentrations remained unchanged $(\mathrm{p}=0.196, \mathrm{p}=0.984, \mathrm{p}=0.526$, respectively). Importantly, the calculated global CVD risk was reduced from $18.8 \pm 9.8$ to $13.9 \pm 9.7 \%(\mathrm{p}=0.001)$. In contrast, no significant changes in any parameter under investigation were observed in 16 patients whose OSA was not abolished by CPAP (use for $<4$ h.night $^{-1}$ ). Conclusion: In patients with the metabolic syndrome and severe OSA, abolition of sleep apnea by effective therapy using noninvasive ventilation improved insulin sensitivity, reduced oxidative stress markers and serum TNF- $\alpha$ concentrations, and reduced the global CVD risk. Supported by: Slovak Research and Development Agency

\section{7}

High-dose atorvastatin provides sustained benefit in reducing risk of cardiovascular disease among patients with diabetes or metabolic syndrome

P. Deedwania ${ }^{1}$, J.C. LaRosa ${ }^{2}$, J. Shepherd ${ }^{3}$

${ }^{1}$ Division of Cardiology, VA Central California Healthcare System, Fresno, United States, ${ }^{2}$ Department of Medicine, State University of New York Health Science Center, New York, United States, ${ }^{3}$ Department of Biochemistry, University of Glasgow, United Kingdom

Background and aims: Randomized clinical trials generally restrict the analyses to occurrence of first endpoint event. However, this approach precludes much potentially useful clinical and health economic information because a significant number of patients in long-term trials experience multiple subsequent events.Based on analyses of time to first event, the TNT trial showed that intensive lipid lowering with atorvastatin (ATV) $80 \mathrm{mg}$ significantly reduced the risk of CV events compared with ATV $10 \mathrm{mg}$ both among stable CHD patients with type 2 diabetes (T2D) and among those with metabolic 
syndrome (MetS). In this analysis, we evaluated the effect of treatment with ATV $80 \mathrm{mg}$ vs $10 \mathrm{mg}$ among patients with T2D or MetS during the follow up period after the occurrence of a first CV event.

Materials and methods: In TNT, 5854 stable CHD patients with either diabetes or MetS (270 T2D/no MetS; 4352 MetS/no T2D; 1232 T2D \& MetS) were randomized to double-blind therapy with ATV 10 or $80 \mathrm{mg}$ and followed for 4.9 years. Time-to-event analysis was used to estimate the treatment hazard ratios separately for time to first, second, third, fourth, and fifth occurrences of any CV event (defined as: any coronary event [CHD death, nonfatal MI, resuscitated cardiac arrest, revascularization procedure, procedure-related MI or documented angina]; a cerebrovascular event [fatal or nonfatal stroke, TIA]; PAD; hospitalization with primary diagnosis of CHF).

Results: A total of 2002 patients with T2D or MetS experienced the first CV event. The numbers with second, third, fourth, and fifth CV events were 1004, 478, 237 and 141, respectively. Among patients with T2D or MetS receiving ATV $80 \mathrm{mg}$ the relative risk of a first as well as subsequent CV events was significantly reduced compared with those receiving ATV $10 \mathrm{mg}$. (Fig).

Conclusion: In patients with either T2D or MetS who had survived the first CV eventthe treatment with ATV $80 \mathrm{mg}$ (compared to ATV $10 \mathrm{mg}$ ) significantly reduced the risk of subsequent $\mathrm{CV}$ events during the entire follow-up period.

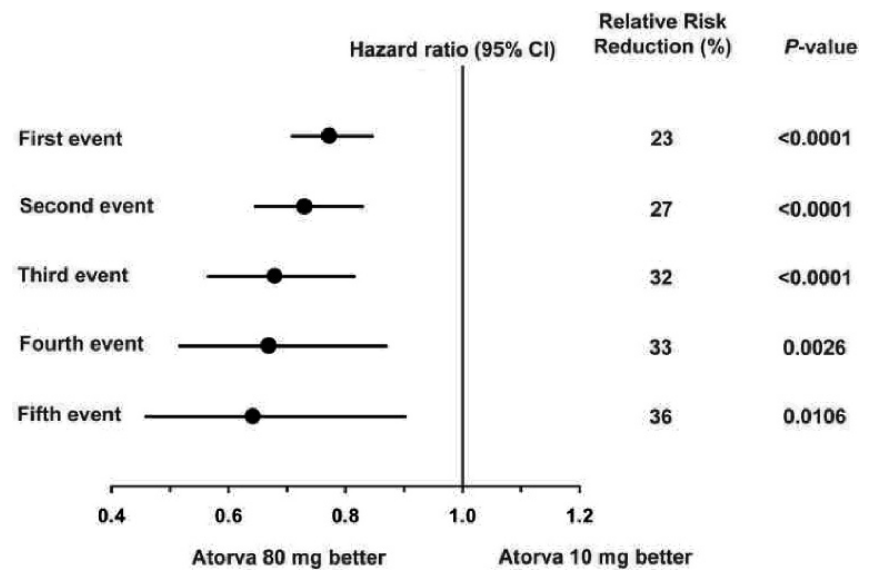

Supported by: Pfizer Inc

\section{8}

The clinical value of metabolic syndrome and its components in established type 2 diabetes mellitus: the FIELD trial

R. O’Brien ${ }^{1}$, R. Scott ${ }^{2}$, J. Best ${ }^{2}$, M.-R. Taskinen², C. Pardy ${ }^{2}$, C. Ehnholm²,

A. Keech $^{2}$

${ }^{1}$ NHMRC Clinical Trials Centre, University of Sydney, Australia, ${ }^{2}$ on behalf of the FIELD study investigators

Background and aims: In the randomised placebo-controlled FIELD trial of long-term fenofibrate among 9795 patients with type 2 diabetes mellitus, almost 1300 first cardiovascular (CVD) events accrued over 5 years. This provides an opportunity to assess whether the constellation of characteristics comprising metabolic syndrome (MS) have clinical value in the setting of type 2 diabetes mellitus.

Materials and methods: All subjects were classified in pre-specified analyses according to the presence of each individual criterion of metabolic syndrome (i) elevated blood pressure or treated hypertension, (ii) gender-specific low HDL cholesterol (HDLc) levels, (iii) elevated triglycerides (TG), (iv) increased waist circumference, and (v) impaired glucose tolerance or diabetes, according to the modified ATPIII definitions, and whether metabolic syndrome was present (at least 3 criteria present). Marked hypertriglyceridaemia (TG levels $>=2.3 \mathrm{mmol} / \mathrm{L}$ ) was also examined. Total cardiovascular event rates were estimated among those with and without each feature.

Results: $84 \%$ of subjects fulfilled the criteria for having MS: $84 \%$ had elevated blood pressure, $68 \%$ increased waist, 59\% low HDLc and 52\% high TG. Marked hypertriglyceridaemia was present in $25 \%$, and in $20 \%$ combined with low HDLc. In those with MS, or any individual feature of MS (except for increased waist; $p=n s)$, the cardiovascular event rates were at least $3 \%$ higher in absolute terms than among those without MS $(\mathrm{p}<0.001)$ or without the same feature. The highest event rates were seen in those with marked hypertriglyceridaemia plus low HDLc. The CVD event reductions with fenofibrate over 5 years in those with and without MS were proportionally (11\% versus $12 \%$ respectively) and in absolute terms (1.4\%; number needed to treat [NNT] $=71$ versus $1.6 \%$;NT $=63$ ) very similar. In contrast, the proportional risk reduction was $27 \%(\mathrm{p}=0.005)$ when marked dyslipidaemia (marked high TG plus low HDLc) was present (absolute reduction $4.3 \%$, NNT $=23$ ) compared with $6 \%(\mathrm{p}=0.32)$ among all others (absolute reduction $0.8 \%, \mathrm{NNT}=143 ; \mathrm{p}$ value for treatment by group interaction $=0.053$ ). In risk modelling adjusted for age, gender, prior CVD and HbAlc, low HDLc and elevated blood pressure were independent risk contributors (both $\mathrm{p}<0.001$ ), whereas increased waist $(\mathrm{p}=0.52)$ and elevated TG $(\mathrm{p}=0.069)$ were not.

Conclusion: Even in the presence of established type 2 diabetes mellitus, the presence of MS or any of its features, except increased waist, increases risk for CVD events significantly. The effects of fenofibrate were similar with or without MS being present, but markedly greater among those at greatest risk, with marked hypertriglyceridaemia plus low HDLc. Marked diabetic dyslipidaemia is an especially important marker of CVD risk and benefit from fenofibrate therapy.

Supported by: Laboratoires Fournier SCA, France 


\section{PS 135 Predictors of vascular complications}

\section{9}

Hyperglycaemia and its effect after acute myocardial infarction on cardiovascular outcomes in patients with type 2 diabetes mellitus (HEART2D)

I. Raz ${ }^{1}$, P. Wilson' 2 K. Strojek ${ }^{3}$, I. Kowalska ${ }^{4}$, V. Bozikov', A. Gitt ${ }^{6}$, G. Jermendy ${ }^{7}$, B. Campaigne ${ }^{8}$, L. Kerr ${ }^{8}$, Z. Milicevic ${ }^{8}$, S. Jacober ${ }^{8}$ ${ }^{1}$ Internal Medicine, Hadassah Hospital, Jerusalem, Israel, ${ }^{2}$ Endocrinology, Emory Univ, Atlanta, United States, ${ }^{3}$ Internal Diseases, Diabetology and Nephrology, Silesian Medical Univ, Zabrze, Poland, ${ }^{4}$ Endocrinology, Medical Univ of Bialystok, Bialystok, Poland, ${ }^{5}$ Endocrinology, Diabetes and Metabolic Diseases, Univ Hospital Dubrava, Zagreb, Croatia, ${ }^{6}$ Ludwigshafen Institut, Heidelberg Univ, Germany, ${ }^{7}$ Medical Dept, Bajcsy-Zsilinszky Teaching Hospital, Budapest, Hungary, ${ }^{8}$ Eli Lilly \& Co, Indianapolis, United States

Background and aims: Outcomes of clinical trials on patients with type 2 diabetes (T2D) have shown reduced microvascular complications but inconsistent results regarding macrovascular complications. HEART2D was a multinational, randomized, controlled trial designed to compare the impact of a prandial insulin strategy (PRANDIAL) vs a basal insulin strategy (BASAL) on cardiovascular (CV) outcomes in T2D patients after acute myocardial infarction (AMI). Primary aim was to determine the difference between insulin regimens on time from randomization to first combined CV event (CV death, nonfatal MI, nonfatal stroke, hospitalization for acute coronary syndromes, coronary revascularization procedures planned after randomization).

Materials and methods: Patients ( $\geq 30$ yrs of age) diagnosed with T2D at least 3 months before study admission were randomly assigned (21 days post AMI) to achieve targeted $\mathrm{HbA}_{1 \mathrm{C}}<7.0 \%$ by either (1) PRANDIAL of 3 premeal doses of insulin lispro and targeted 2-h postprandial (PP) blood glucose (BG) $<7.5 \mathrm{mM}$ or (2) BASAL of NPH BID or insulin glargine once-daily and targeted fasting/premeal $\mathrm{BG}<6.7 \mathrm{mM}$. Investigators intensified insulin regimen if $\mathrm{HbA}_{\mathrm{c}}>8.0 \%$ by: addition of bedtime NPH for PRANDIAL and conversion to human insulin 30/70 twice-daily for BASAL. Patients were studied initially for 18 months followed by an extended period until patient discontinued or the study was stopped by the sponsor. The study was stopped for futility after implementation at 4 th interim analysis.

Results: 1115 patients were randomized to either PRANDIAL (N=557) or BASAL $(\mathrm{N}=558)$ and took at least one study drug dose (intent to treat). Average days patients were on study drug was 963 (2.7 yrs [ 1 day minimum, 1078 days (3.0 yrs) maximum]). Patients in the PRANDIAL experiencing a combined adjudicated $\mathrm{CV}$ event was similar to the BASAL ( $\mathrm{N}=174,31.2 \%$ vs $\mathrm{N}=181,32.4 \%$; $\mathrm{HR}=0.98$; 95\% CI $[0.8,1.21])$. Mean $( \pm \mathrm{SD}) \mathrm{HbA}_{1 \mathrm{C}}$ did not differ between strategies ( $7.6 \pm 2.5$ vs $7.6 \pm 2.4 \%$; $\mathrm{p}=0.48$ ) during the study. Regimen intensification occurred in both groups (prandial 28 vs basal $21 \%$; $\mathrm{p}=0.005$ ). Approximately $47 \%$ of patients in PRANDIAL achieved BG target of $<7.5 \mathrm{mM}$ for daily mean PP BG and $46 \%$ in BASAL achieved daily mean FBG/pre-meal BG $<6.7 \mathrm{mM}$. Endpoint BG profiles in PRANDIAL showed lower daily mean PP BG $(8.1 \pm 2.2$ vs $8.7 \pm 2.2 \mathrm{mM}$; $\mathrm{p}<0.001)$, and mean 2 -h PP BG excursion $(0.1 \pm 1.1$ vs $1.2 \pm 1.3 \mathrm{mM}$; $\mathrm{p}<0.0001)$ compared to BASAL. Endpoint fasting BG (FBG) and daily mean of FBG/pre-meal BG were significantly lower for BASAL $(8.4 \pm 2.3$ vs $6.9 \pm 1.9 \mathrm{mM} ; \mathrm{p}<0.0001$ and $8.0 \pm 2.0$ vs $7.4 \pm 2.0 \mathrm{mM} ; \mathrm{p}<0.0001$, respectively).

Conclusion: Differences in PP and fasting BG were achieved between insulin treatments that targeted fasting/premeal or PP BG; however, rate of combined $\mathrm{CV}$ outcomes and $\mathrm{HbA}_{1 \mathrm{C}}$ were similar in these T2DM patients who recently experienced an AMI.

Supported by: Eli Lilly and Company
1360

Impaired glucose tolerance significantly increases the risk for cardiovascular events in patients undergoing elective coronary angiography

H. Sourij ${ }^{1}$, C.H. Saely ${ }^{2}$, S. Aczel ${ }^{2}$, R. Zweiker ${ }^{3}$, P. Langer ${ }^{2}$, P. Passler ${ }^{1}$, T. Marte ${ }^{2}$, G. Hoefle ${ }^{2}$, W. Benzer ${ }^{2}$, T.C. Wascher ${ }^{1,4}$, H. Drexel $^{2,5}$ ${ }^{1}$ Internal Medicine, Metabolism and vascular biology research group, Medical University of Graz, Austria, ${ }^{2}$ Department of Medicine, Academic Teaching Hospital Feldkirch, Vorarlberg Institue For Vascular Investigation and Treatment (VIVIT), Feldkirch, Austria, ${ }^{3}$ Department of Internal Medicine, Div. of Cardiology, Medical University of Graz, Austria, ${ }^{4}$ Department of Internal Medicine, Hanusch Krankenhaus, Vienna, Austria, ${ }^{5}$ University of Human Science, Triesen, Liechtenstein

Background and aims: Prevalence of impaired glucose tolerance (IGT) in patients undergoing coronary angiography for suspected coronary artery disease has been shown to be high. Until now it is unclear whether IGT is associated with increased risk for future cardiovascular events in this population or not.

Materials and methods: We enrolled 1040 patients (374 female, 666 male, mean age $63.7 \pm 10.2$ years) undergoing elective coronary angiography for suspected coronary artery disease. In patients without previously known diabetes mellitus, an oral glucose tolerance test (oGTT) was performed. Cardiovascular events were recorded prospectively over 3 years.

Results: 394 patients had normal glucose tolerance, 190 suffered from IGT and 456 were diabetics ( 244 previously known and 212 newly diagnosed by the oGTT). The incidence of vascular events was significantly higher in patients with IGT $(14.9 \%, \mathrm{p}=0.029)$ and patients with diabetes mellitus type 2 (13.4\%, $\mathrm{p}=0.004)$ compared to normal glucose tolerant patients $(8.9 \%)$. No difference between IGT and diabetes mellitus type 2 regarding cardiovascular event rate could be observed $(\mathrm{p}=0.778)$.

Hazard ratios in multivariate adjusted Cox-Regression analysis were 1.89 ([95\%-CI 1.11-3.24], $\mathrm{p}=0.020)$ for patients with IGT and 1.73 ([1.102.74], $\mathrm{p}=0.019$ ) for diabetic patients.

Conclusion: IGT and diabetes mellitus type 2 are associated with the same risk for future cardiovascular events in patients undergoing elective coronary angiography. We therefore strongly recommend performing an oGTT in these patients to access the real cardiovascular risk.

Supported by: Innovationsstiftung of the Liechtenstein Global Trust Bank; Fachhochschule Dornbirn; Vorarlberger Industriellenvereinigung; Vorarlberger Landeskrankenhaus-Betriebsgesellschaft

\section{1}

The prognostic ability of troponin after the introduction of hs-CRP and HOMA index in different groups of abnormal glucose regulation patients with acute coronary syndrome

A. Koutsovasilis ${ }^{1}$, A. Melidonis ${ }^{1}$, D. Levisianou ${ }^{1}$, G. Koukoulis ${ }^{2}$, I. Skoularigis ${ }^{2}$, M. Zairis ${ }^{3}$, S. Foussas ${ }^{3}$, F. Triposkiadis ${ }^{2}$ ${ }^{1}$ Diabetes Center, Tzanio General Hospital, Piraeus, ${ }^{2}$ Cardiology Department, Larissa University Hospital, Larissa, ${ }^{3}$ Cardiology Department, Tzanio General Hospital, Piraeus, Greece.

Background and aims: Cardiac troponins are considered the "gold standard" measurements for diagnosing myocardial damage in patients with or without chest pain. The presence of elevated levels of serum high-sensitivity C-reactive protein (hs-CRP) is widely accepted as a significant risk factor of cardiovascular event patients while insulin resistance with hyperinsulinemia is an independent risk factor for ischemic heart disease. The aim of this study was to examine the prognostic ability of Tnl combined with hs-CRP and insulin resistance HOMA index as to the end points during the first year after hospitalization for Acute Coronary Syndrome (STEMI or NSTEMI) in patients with abnormal glucose regulation.

Materials and methods: Longitudinal, prospective, observational unicenter study. 448 consecutive patients who were admitted to the coronary care unit (CCU) and discharged from February 2006 through January 2007 were included in this study. First-year end points were: death (of cardiovascular cause), myocardial infarction, cardiac failure (clinical and echocardiographic determination) and unstable angina after hospitalization. Non-diabetic patients went through an Oral Glucose Tolerance Test (OGTT) three months after discharge and IGTs were categorized.

Results: Diabetes was previously diagnosed (Group A) in 133 (29,7\%) patients, newly diagnosed (Group B) in $43(9,6 \%)$ patients, an IGT (Group C) was ob- 
served in $91(20,3 \%)$ patients while $181(40,4 \%)$ were patients with normal glucose regulation (Group D). The incidence of one-year complications was $22.6 \%$, $16.3 \%, 12.1 \%$ and $11.6 \%$ in groups $\mathrm{A}, \mathrm{B}, \mathrm{C}$ and $\mathrm{D}$ respectively ( $\mathrm{p}=0.022$ ). Between hs-CRP and HOMA, hs-CRP had the strongest association with the oneyear outcome, since HR of patients with both $\mathrm{TnI}$ and hs-CRP in the top quartile was 2.85 (95\%CI: 1.28-5.41, p<0.001), 2.62 (95\%CI: 1.34-4.81, $\mathrm{p}<0.001), 2.18$ (95\%CI: $1.30-3.96, \mathrm{p}<0.001)$ and 2.05 (95\%CI: 1.41-4.66, $\mathrm{p}<0.001)$ compared with those with TnI and hs-CRP in the bottom quartile while patients' $\mathrm{HR}$ with $\mathrm{TnI}$ and HOMA in the top quartile was 2.59 (95\%CI: 1.33-6.31, $\mathrm{p}=0.012$ ), 2.36 (95\%CI: 1.29-5.99, $\mathrm{p}=0.016), 1.84$ (95\%CI: $1.31-6.44, \mathrm{p}=0.033)$ and $1.92(95 \% \mathrm{CI}$ : 1.23-6.13, $\mathrm{p}=0.036$ ) compared with those in the bottom quartile for $\mathrm{A}, \mathrm{B}, \mathrm{C}, \mathrm{D}$ Group respectively (after adjustment to age, sex, waist circumference, triglycerides, HDL, total cholesterol and smoking). Individuals with all these three biomarkers in the top quartile had a HR 3.56 (95\%CI: 1.49-6.36, $\mathrm{p}<0.0001)$, 3.02 (95\%CI: 1.55-5.98, p<0.0001), 2.48 (95\%CI: 1.38-6.44, $\mathrm{p}<0.001$ ), and 2.49 $(95 \% \mathrm{CI}: 1.32-6.16, \mathrm{p}<0.001)$ compared with those with all these biomarkers in the bottom quartile for A, B, C and D Group respectively.

Conclusion: In this population of patients with Coronary Artery Disease, patients with known diabetes are at high risk for complications during hospitalization while newly diagnosed diabetic patients are at intermediate risk. The main finding is that the introduction of hs-CRP modifies more strongly the prognostic value of Tnl, compared to the introduction of HOMA, in all patient groups.

\section{2}

Lipid predictors of cardiovascular events in statin-treated coronary patients with diabetes mellitus

H. Drexel ${ }^{1,2}$, S. Aczel ${ }^{1}$, T. Marte ${ }^{1}$, A. Vonbank ${ }^{1}$, C.H. Saely ${ }^{1,2}$

${ }^{1}$ VIVIT Institute, Feldkirch, Austria, ${ }^{2}$ Medicine, University for Human Sciences, Triesen, Liechtenstein

Background and aims: Statins are a cornerstone in the treatment of diabetic patients with coronary artery disease (CAD), but vascular risk in these patients remains high. We therefore aimed at identifying which lipid parameters drive vascular risk in this important patient population despite statin treatment.

Materials and methods: We recorded vascular events over 5.6 years in 491 consecutive statin-treated patients with angiographically proven stable CAD, covering 2750 patient-years.

Results: Low HDL cholesterol (standardized adjusted hazard ratio (HR) 0.73 [0.60-0.89]; $\mathrm{p}=0.001$ ), low apolipoprotein A1 (HR 0.77 [0.65-0.92]; $\mathrm{p}$ $=0.003)$, a small LDL particle diameter $(0.76[0.64-0.91] ; \mathrm{p}=0.002)$, and high triglycerides $(1.20$ [1.05-1.38]; $\mathrm{p}=0.007)$ significantly predicted vascular events, but not total cholesterol $(\mathrm{p}=0.995)$, LDL cholesterol $(\mathrm{p}=0.961)$, or apolipoprotein B $(\mathrm{p}=0.077)$. Patients with T2DM $(\mathrm{n}=116)$ were at a higher vascular risk than non-diabetic subjects $(38.6 \%$ vs. $24.1 \%$; $\mathrm{p}<0.001)$. Importantly, like in the total population, low HDL cholesterol $(\mathrm{HR}=0.58$ [0.41-0.82]; $\mathrm{p}=0.002)$, low apolipoprotein A1 ( $\mathrm{HR}=0.70$ [0.51-0.95]; $\mathrm{p}=$ $0.022)$, a small LDL particle diameter $(0.67$ [0.50-0.91]; $\mathrm{p}=0.010)$, and high triglycerides (1.30 [1.11-1.53]; $\mathrm{p}=0.001)$ predicted vascular risk in statintreated coronary patients with T2DM, whereas total cholesterol $(\mathrm{p}=0.822)$, LDL cholesterol $(\mathrm{p}=0.235)$, and apolipoprotein $\mathrm{B}(\mathrm{p}=0.366)$ did not.

Conclusion: The pattern of low HDL cholesterol, low apolipoprotein A1, small LDL particles, and high triglycerides is the main lipid risk factor in statin-treated coronary patients with T2DM.

Supported by: Innovationsstiftung of the Liechtenstein Global Trust Bank; Peter Goop Stiftung; Vorarlberger Landesregierung; Medizinisches Zentrallabor Feldkirch; Vorarlberger Industriellenvereinigung; Vorarlberger Aerztekammer; Vorarlberger Landeskrankenhaus-Betriebsgesellschaft

\section{3}

Multivariate biochemical basis of vascular complications and mortality in 4,197 patients with type 1 diabetes

V.-P. Mäkinen ${ }^{1,2}$, C. Forsblom ${ }^{1}$, L.M. Thorn ${ }^{1}$, J. Wadén ${ }^{1}$, D. Gordin ${ }^{1}$ O. Heikkilä ${ }^{1}$, K. Hietala ${ }^{1}$, J. Kytö ${ }^{1}$, M. Rosengård-Bärlund ${ }^{1}$, M. Saraheimo ${ }^{1}$, M. Parkkonen ${ }^{1}$, K. Kaski ${ }^{2}$, M. Ala-Korpela ${ }^{1,2}$, P.-H. Groop, FinnDiane Study Group ${ }^{1}$

${ }^{1}$ Folkhälsan Research Center, Folkhälsan Insititute of Genetics, Helsinki, ${ }^{2}$ Department of Biomedical Engineering and Computational Science, Helsinki University of Technology, Espoo, Finland

Background and aims: Risk assessment of diabetic complications is challenging due to the gradual disease processes and the lack of accurate metabolic phenotypes. Here the focus is on the latter problem: to reveal the multi-variate biochemical features behind the clinical outcomes and premature deaths in a set of Finnish patients with type 1 diabetes.

Materials and methods: Baseline biochemical and clinical data were collected for 2173 men and 2024 women with type 1 diabetes (age at onset below 35 years, insulin treatment within a year of onset) from the Finnish Diabetic Nephropathy Study. The vitality status was obtained after an average of 6.5 years of follow-up (25,714 patient years, 295 deaths). Clinical records and measurements of 20 biochemical variables (lipoprotein subfractions, apolipoproteins, serum and urine creatinine, inflammatory markers and 24h-urine albumin) were collected by standardized questionnaires and laboratory methods. The data were analysed by a self-organizing map (SOM), and visualized separately for males and females with computational significance estimates and confidence intervals. The metabolic phenotypes revealed by the SOMs were then compared against the observed all-cause mortality.

Results: Two sides of the diabetic complications cluster were revealed: a metabolic syndrome profile of insulin resistance, low HDL2C, and abdominal obesity, and a high-adiponectin, high-LDLC, high-HDL2C profile associated with microvascular complications. A 10.1-fold [CI95\%: 7.3-13.1] population adjusted risk of death in males, and a 10.7-fold [CI95\%: 7.9-13.7] risk in females was observed at the intersection of the two metabolic states.

Conclusion: The SOM approach offers a visual way of incorporating multivariate biochemical datasets into clinical decision making, as demonstrated in this study by the dissection of diabetic complications into a metabolic continuum between two characteristic phenotypes. We expect this type of computational medicine to be crucial not only for cultivating the scientific understanding of multifactorial metabolic disorders, but also for enabling tailored treatments of complex diseases.

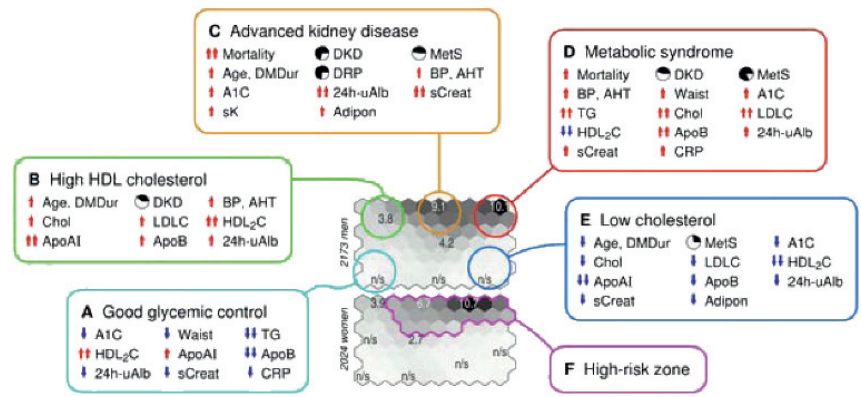

Supported by: Stockmann Foundation, Folkhälsan Research Foundation, Jenny and Antti Wihuri Foundation

\section{4}

Discovery of biomedical markers of early diagnosis of diabetic complications with proteomic analysis

T. Takada ${ }^{1}$, T. Hosaka ${ }^{1}$, K. Tanaka ${ }^{1}$, T. Moriya ${ }^{1}$, M. Oh-Ishi ${ }^{2}$, Y. Kodera ${ }^{2}$, T. Maeda ${ }^{2}$, Y. Fujita ${ }^{1}$

${ }^{1}$ Department of Endocrinology, Diabetes \& Metabolism, ${ }^{2}$ Department of Physics, Kitasato University, Kanagawa, Japan

Microangiopathy of diabetes represents one of the most serious consequences of the disease. Particularly, retinopathy, nephropathy, and possibly peripheral neuropathy exhibit significant pathology. To find diabetic complications in the early stage of the disease, we need biomedical markers high in specificity and sensitivity. The only available biomedical marker in recent years has been microalbuminuria for the diagnosis of diabetic nephropathy. Proteomic analysis is a useful tool for the identification of altered protein expressions in various pathologic conditions. Although tissue biopsy and cytology is an ideal sample for analyses, it is invasive and costly to patients. Therefore, we analyzed serum samples to find the biomedical markers for early diagnosis of diabetic complications. Serum samples were taken from 17 type 1 diabetic patients (male/female, 6/11; mean age: $52.0 \pm 19.7$ ), 41 type 2 diabetic patients (male/female, 22/19; mean age: $56.8 \pm 14.5$ ), and 8 healthy subjects (male/female, $5 / 3$; mean age: $28.7 \pm 0.8$ ) as a control. Mean $\mathrm{HbAlc}$ value was $8.9 \pm 2.3 \%$ in type 1 and $9.6 \pm 2.3 \%$ in type 2 diabetic patients. The serum samples were analyzed with sodium dodecyl sulfate polyacrylamide gel electrophoresis (SDS-PAGE) and two-dimensional electrophoresis. Fractions of albumin and globulin that interfere with proteomic analysis were removed. Low molecular weight proteins defined as less than $20 \mathrm{KDa}$ (peptide) was analyzed with the method developed by three of our authors (Oh-ishi, Kodera, Maeda). Pro- 
tein and peptide were identified with mass spectrometry and tandem mass spectrometry.

As a result of SDS-PAGE, a protein band was increased in 36 diabetic patients (10 in type 1, 26 in type 2). Among these patients, glycemic controls of 26 patients were poor regardless of diabetic type. As a result of peptide analysis, peptide band of approximately $10 \mathrm{KDa}$ was increased in 6 diabetic patients (2 in type 1, 4 in type 2). We identified the apolipoprotein C-III in this peptide band. In addition, a peptide band of approximately $14 \mathrm{KDa}$ was increased in 6 patients ( 3 in type 1, 3 in type 2 ). We also identified a preplatelet basic protein in this peptide band. We found several altered protein expressions in the serum of diabetic patients with hyperglycemia regardless of the type of diabetes. Our results suggest that these protein expressions may become biomarkers for early diagnoses of diabetic complications. Further studies are warranted to clarify the relationship between these protein expressions and the development of diabetic complications.

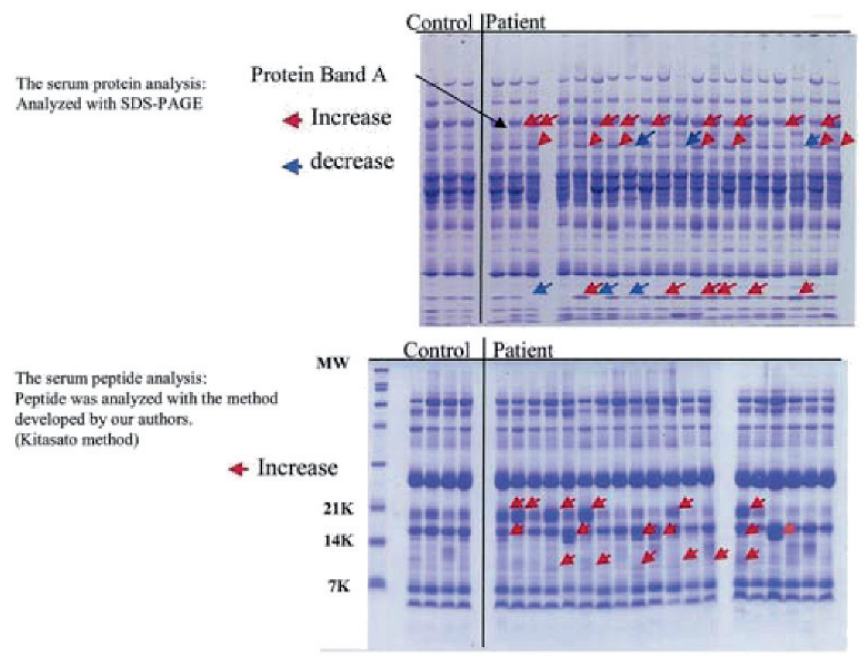

\section{5}

Performance of the UKPDS Risk Engine and the Framingham risk equations in estimating cardiovascular disease in the EPIC-Norfolk cohort

R.K. Simmons ${ }^{1}$, R.L. Coleman ${ }^{2}$, H.C. Price ${ }^{2}$, R.R. Holman², K.-T. Khaw ${ }^{3}$, N.J. Wareham ${ }^{1}$, S.J. Griffin ${ }^{1}$

${ }^{1}$ MRC Epidemiology Unit, Institute of Metabolic Science, Cambridge,

${ }^{2}$ Diabetes Trial Unit, Oxford University, ${ }^{3} \mathrm{Clinical}$ Gerontology, University of Cambridge, United Kingdom

Background and aims: Individuals with type 2 diabetes have a 2 to 4 fold increased risk of cardiovascular disease (CVD) compared to those without diabetes. Multifactorial treatment can significantly reduce this risk. Multivariate equations such as Framingham are used to estimate CVD risk in order to target therapy to those at highest absolute risk, and to provide patients and practitioners with prognostic information. This study examines the performance of the UKPDS Risk Engine (version 3) and the Framingham risk equations in estimating CVD incidence in three population sub-groups: (i) individuals with known diabetes (DM); (ii) individuals with non-diabetic hyperglycaemia, defined as $\mathrm{HbA}_{1 \mathrm{c}} \geq 6.0 \%$ (HG); and, (iii) individuals with $\mathrm{HbA}_{1 c}<6.0 \%$ (normoglycaemia) (NG).

Materials and methods: The data are from a population-based prospective cohort (EPIC-Norfolk). Participants aged 40-79 years recruited from UK general practices attended a health examination (1993-1998) and were followed for CVD events/death until April 2007. CVD risk estimates were calculated for 10,138 individuals with complete data on age, sex, ethnicity, smoking status, diabetes, total cholesterol, HDL-cholesterol, systolic blood pressure and $\mathrm{HbA}_{1 \mathrm{c}}$ using the UKPDS Risk Engine and Framingham CVD risk equation. Estimation of events by each score was compared using the area under the receiver operating characteristic curve (aROC) and the Net Reclassification Improvement (NRI) statistic.

Results: Over 10.5 years of follow-up there were 69 CVD events in the 272 individuals with diabetes, 160 in the 906 with non-diabetic hyperglycaemia and 732 in the 8,960 with normoglycaemia (Table 1). The estimated CVD 10year risk in individuals with diabetes was $26 \%$ and $28 \%$ using the UKPDS and Framingham scores respectively. In the HG group, estimated CVD risk was $28 \%$ and $31 \%$ respectively, and for the NG group, $19 \%$ and $23 \%$ respectively. In the DM and HG groups, there was no significant difference in the ability of the two risk scores to either discriminate between or correctly reclassify individuals at different risk of CVD events (Table 1). The discrimination of both scores was poor in the NG group.

Conclusion: The UKPDS and Framingham risk equations perform reasonably well at estimating CVD risk in individuals with diabetes in EPIC-Norfolk. These scores can therefore assist with targeting of therapy to those at highest absolute risk. The UKPDS Risk Engine unsurprisingly overestimates risk in those without diabetes. The overestimates of risk in those without diabetes by the Framingham score confirm previous findings. Our results highlight that care is still needed when using scores to communicate risk information to individuals.

\section{6}

Benefits of long-term fenofibrate therapy on amputations in type 2 diabetes mellitus in the FIELD trial

P. Colman ${ }^{1}$, K. Rajamani', L.-P. Li ${ }^{2}$, M. D’Emden ${ }^{2}$, M. Voysey ${ }^{2}$, A. Keech'; ${ }^{1}$ NHMRC Clinical Trials Centre, University of Sydney, Australia, ${ }^{2}$ on behalf of the FIELD study investigators.

Background and aims: In the FIELD trial in 9795 patients with type 2 diabetes mellitus, fenofibrate reduced total cardiovascular events and retinopathy requiring laser therapy, suggesting effects on both macrovascular and microvascular complications. The study provided an opportunity to explore in detail the nature and predictors of amputations, which were also significantly reduced by treatment.

Materials and methods: All on-study non-traumatic amputations were reviewed in blinded fashion by 2 clinicians. Amputations of digits and forefoot were classified as having a presumed microvascular basis provided that no prior or concurrent large vessel disease (including angioplasty and bypass surgery) in that same limb, or evidence of causative embolism, was present. Larger amputations or those with evidence of embolism or existing vascular disease in the affected limb were classified as macrovascular or mixed. All analyses of the effects of treatment were by intention-to-treat.

Results: Compared with patients suffering any other cardiovascular event or no event during 5 years of follow-up, those with an amputation had a significantly longer duration of diabetes, higher systolic blood pressure, more current smoking, more prior vascular disease, more male gender, more microvascular disease and more baseline insulin use (all $\mathrm{p}<0.0001$ ). Com-

Table 1: Actual and estimated CVD risk using UKPDS and Framingham scores in EPIC-Norfolk

\begin{tabular}{llll}
\hline & $\begin{array}{l}\text { Individuals with prevalent } \\
\text { diabetes }(\mathrm{n}=272)\end{array}$ & $\begin{array}{l}\text { Individuals with non-diabetic } \\
\text { hyperglycaemia }(\mathrm{n}=906)\end{array}$ & $\begin{array}{l}\text { Normoglycaemic individuals } \\
(\mathrm{n}=8,960)\end{array}$ \\
\hline Actual mean CVD risk, \% & 25.4 & 17.7 & 8.2 \\
\hline Estimated CVD 10-yr risk: UKPDS score, \% (95\% CI) & $26.4(25.5-27.3)$ & $27.5(27.0-28.0)$ & $19.2(19.1-19.3)$ \\
\hline Estimated CVD 10-yr risk: Framingham score, \% (95\% CI) & $27.6(26.8-28.4)$ & $31.4(30.9-31.9)$ & $23.1(23.0-23.2)$ \\
\hline aROC* $(95 \%$ CI) for the UKPDS score & $0.61(0.53-0.69)$ & $0.65(0.60-0.70)$ & $0.53(0.51-0.55)$ \\
\hline aROC (95\% CI) for the Framingham score & $0.60(0.52-0.68)$ & $0.67(0.62-0.71)$ & $0.52(0.49-0.54)$ \\
\hline NRI $^{* *}(\%), \mathrm{p}$-value comparing UKPDS and Framingham models & $-7.0 \%, \mathrm{p}=0.290$ & $-6.9 \%, \mathrm{p}=0.111$ & $-9.7 \%, \mathrm{p}<0.001$ \\
\hline
\end{tabular}

${ }^{*}$ Area under the receiver operating characteristic curve

${ }^{*}$ The NRI refers to the net gain in correct reclassification. A positive NRI indicates an improvement in classification, while a negative NRI corresponds to a worsening in classification. The negative NRIs in the table above indicate that the UKPDS risk engine correctly reclassified risk of CVD events in more individuals than the Framingham score 
pared with subjects classified as having a macrovascular amputation, those with presumed microvascular amputations were slightly younger $(\mathrm{p}=0.03)$, heavier $(\mathrm{p}<0.001)$, had slightly higher HbAlc $(\mathrm{p}=0.07)$ and with more other microvascular disease at baseline, including known retinopathy, peripheral neuropathy and albuminuria $(\mathrm{p}=0.002)$.

\section{Effects of fenofibrate on amputations}

\begin{tabular}{llll}
\hline & $\begin{array}{l}\text { Relative risk } \\
\text { reduction }\end{array}$ & $\begin{array}{l}\text { 95\% confidence } \\
\text { interval }\end{array}$ & p value \\
\hline Microvascular amputations & $47 \%$ & $8-70 \%$ & 0.025 \\
\hline Macrovascular amputations & $23 \%$ & $-21-51 \%$ & 0.26 \\
\hline All first amputations & $38 \%$ & $10-57 \%$ & 0.011 \\
\hline
\end{tabular}

Conclusion: Compared with amputations associated with large vessel limb disease, small digit (presumed microvascular) amputations occur in a population with higher levels of established microvascular complications of diabetes. The measured effects of fenofibrate on amputations were particularly striking in this group, further supporting important clinical benefits with fenofibrate treatment on microvascular disease in type 2 diabetes mellitus. Supported by: Laboratoires Fournier SCA, France

\section{PS 136 Complications: animal models and pathogenic mechanisms}

\section{7}

ERM proteins mediate the effects of $\mathrm{Na} / \mathrm{H}$ exchanger activation in diabetic rat hearts

A. Darmellah, C. Rucker-Martin, D. Feuvray

Hôpital Marie Lannelongue, UMR CNRS 8078, Université Paris-Sud XI, Le Plessis Robinson, France

Background and aims: To further investigate molecular linkers between NHE1 and Akt activity previously identified in hypertrophied left ventricular (LV) myocytes from diabetic GK rats.

Materials and methods: Individual myocytes were isolated from the LV wall of perfused hearts. Intracellular acidification (by the ammonium pulsing method) was induced to activate NHE1. Fluorescence microscopy was used to detect immunocytochemical localisation of phosphorylated (activated) ezrin/radixin/moesin (ERM) proteins and whole-cell lysates were used to measured their levels, as well as levels of several phosphorylated kinases.

Results: ERM proteins were localised predominantly at the intercalated disk regions under basal conditions. Profound changed in activated ERM were observed in acidified cells, with immunolabelling detected in regions corresponding to the transverse tubules. This correlated with a marked increase in phospho-ERM levels, which was blocked by NHE1 inhibitor treatment. Levels of phospho-Akt paralleled those of phospho-ERM under the various experimental conditions used. Moreover, the pattern of GSK-3beta phosphorylation was strikingly similar to that observed for Akt activity under the conditions used.

Conclusion: Activated ERM proteins mediate the effects of acid-induced NHE1 activation in LV myocytes. Akt is a downstream effector in the cascade activated by NHE1/ERM interaction. in addition, GSK-3beta phosphorylation is required for downstream effects of Akt-NHE1/ERM signalling. Our findings provide insight into mechanisms explaining the role of NHE1 activation in cardiac hypertrophy in the GK model of type 2 diabetes.

Supported by: French Ministry for Education and Research

\section{8}

Rat diabetic heart does not respond to ischaemic preconditioning: the role of methionine-centered redox cycle

V. Vinokur ${ }^{1}$, B. Bulvik ${ }^{1}$, E. Berenshtein ${ }^{1}$, R. Eliashar ${ }^{2}$, M. Chevion ${ }^{1}$ ${ }^{1}$ Cellular Biochemistry and Human Genetics, Hebrew University of Jerusalem, ${ }^{2}$ Department of Otolaryngology, Hadassah Hospital, Jerusalem, Israel

Background and aims: Diabetes is associated with increased risk of cardiovascular morbidity and mortality. While the non-diabetic heart responds to ischemic preconditioning (IPC) and acquires protection against subsequent prolongued ischemia, the diabetic heart often fails to get protected by IPC. The role of methionine-centered redox cycle (MCRC) in IPC response of diabetic heart compared to the normal one was analyzed. The aims of the current study was to characterize the effect of IPC on heart function in diabetic hearts as compared to non-diabetic ones, and monitor the changes in MCRC proteins relate to cardioprotection.

Materials and methods: Rats were made diabetic by streptozotocin injection $(70 \mathrm{mg} / \mathrm{kg}$, i.p.). After 4 weeks, hearts were isolated and retrogradely perfused according to Langendorff. The hearts were subjected to IPC (2 min. ischemia and $3 \mathrm{~min}$. (re)perfusion, 3 cycles) followed by prolongued ischemia (35 min.). Heart hemodynamics, contractile function and MCRC proteins e.g. thioredoxin $1(\operatorname{Trx})$, thioredoxin reductase $1(\operatorname{TrxR})$ and methionine sulfoxide reductase $\mathrm{A}$ and $\mathrm{B}$ isoforms concentrations were measured.

Results: The post-ischemic recovery of function in diabetic hearts was markedly better than that in controls. However, unlike the control ones, the diabetic hearts did not improve their post-ischemic recovery following the IPC procedure. No alterations in the Trx concentration were detected in the control hearts during IPC, as well as during ischemia or reperfusion. On the other hand, the diabetic hearts have demonstrated a significant 2.3 fold decrease at the end of ischemia preceded by IPC. During the reperfusion the Trx concentration has restored. The hearts from the control and diabetic groups have demonstrated a significant increase in MsrA concentration at the end of post-IPC ischemia, 2.5 and 1.9 fold respectively. This increase was 
followed by down-regulation to the initial level during the reperfusion. In TrxR and MsrB concentrations no changes were observed in any stage of the experiment neither in control hearts nor in diabetic ones.

Conclusion: We hypothesize that the inability of the diabetic heart to maintain the required level of thioredoxin, one of the most important proteins in cellular redox balance regulation, underlies the heart inability to be protected by IPC. Moreover, the MsrA concentration in control hearts has shown much more considerable increase in response to IPC followed by ischemia than diabetic ones, demonstrating better protection. Studies aimed at bringing the light on MCRC involvement in better post-ischemic recovery of diabetic hearts are in progress.

Supported by: Israel Science Foundation

\section{9}

Ethanol exposure decreases the expression of AMP-activated protein kinase and GLUT4 in rat cardiac myocytes

X.L. Sun 1 , X.B. Wei ${ }^{2}$, H. Ding ${ }^{2}$, X.J. Zhang ${ }^{1}$, L. Feng', L.M. Tian' ${ }^{1}$, L. Gao ${ }^{3}$, J.J. Zhao ${ }^{1}$

${ }^{1}$ Endocrinology, Provincial Hospital affiliated to Shandong University, ${ }^{2}$ Department of Pharmacology, Shandong University School of Medicine, ${ }^{3}$ Scientific Center, Shandong Provincial Hospital, Jinan, China

Background and aims: We and other studies found that chronic ethanol intake may cause lower expression of Glucose transporter 4 (GLUT4) in skeletal muscles, adipocytes and livers. It is well known that GLUT4, a signal at the end of insulin signaling pathway, is regulated by insulin signal and contributes to glucose metabolism. Recently, a few studies have addressed that AMP-activated protein kinase (AMPK) is necessary for GLUT4, and this effect may be occurred in a independent - insulin signaling pathway manner. Studies on skeletal muscles showed that effect of AMPK on GLUT4 was mediated by myocyte enhancer factor-2 (MEF2), a muscle-specific regulator for GLUT4 gene transcription. GLUT4 promoter has a MEF2 binding element located between -473 and -464 . Our aims of the current study were to investigate whether there existed the effects of AMPK on MEF2 and GLUT4 in rat cardiac muscles. If so, whether this event was involved in ethanol action.

Materials and methods: (1) In vivo, male Wistar rats weighing $180 \pm 5 \mathrm{~g}$ were randomly divided into two groups: AICAR (an AMPK activator) group and control group. AICAR-treated rats were received a subcutaneously injection with $0.8 \mathrm{mg} \mathrm{AICAR} / \mathrm{g}$ body weight. Control animals were injected with a corresponding volume of $0.9 \% \mathrm{NaCl}$. Treatment for 2 hours, all rats were anesthetized and the heart ventricles were dissected out for an analysis of the mRNA levels of MEF2A and 2D subtypes as well as GLUT4 by RT-PCR (normalized to GAPDH). (2) In vitro, cardiomyocytes were prepared from ventricles of neonatal Wistar rats (1-3-day-old). After culture, cardiomyocyte specific proteins, $\alpha$-cardiac muscle actinin, were identified by immunofluorescence. The cells were treated with $100 \mathrm{mM}$ edible ethanol or $1 \mathrm{mM}$ AICAR. Treatment for 4 hours, total proteins were gained for analyzing the expression of phospho-AMPK alpha (the activated AMPK) and GLUT4 by Western blotting (normalized to $\beta$ actin) and total RNA was extracted for determining the mRNA levels of GLUT4 by RT-PCR (normalized to GAPDH), respectively.

Results: (1) Compared with control rats, AMPK activator, AICAR, obviously up-regulated the mRNA levels of MEF2A and GLUT4 $(p<0.05)$. We, however, failed to detect the significant change of MEF2D mRNA level, which is in agreement with a previous report of strong MEF2A while weak MEF2D expression in heart tissues. (2) In the cardiomyocyte experiments in vitro, AICAR increased significantly the expression of phospho-AMPK alpha protein $(\mathrm{p}<0.05)$, while ethanol exposure decreased markedly the activated AMPK levels $(\mathrm{p}<0.05)$. Like the experiment above in vivo, the AICAR-mediate AMPK activity increase was associated with the up-regulation of GLUT4 expression at both mRNA and protein levels $(p<0.05)$. However, ethanol exposure to rat cardiac myocytes could decrease the GLUT4 expression not only at the mRNA but also at the protein levels relative to control $(\mathrm{p}<0.05)$. Conclusion: AICAR may enhance AMPK activity and increase the expression of MEF2A and GLUT4 in rat cardiac myocytes. While ethanol is able to decline AMPK activity associated with a decrease in GLUT4 expression in rat cardiac myocytes. This maybe partly indicate that ethanol impairs insulin sensitivity in rat heart muscle via, at a certain extent, decreasing the expression of AMPK activity, MEF2A and GLUT4.

Supported by: Natural Science Foundation of Shandong Province

\section{0}

Changes of adrenergic and cholinergic coronary circulation regulation in dogs induced by multiple insulin administration

\section{A. Nescheret}

Laboratory of Diabetes Mellitus Epidemiology, Institute of Endocrinology and Metabolism, Kiev, Ukraine

Background and aims: Recently we have shown that in experiments on dogs both acute hyperinsulinemia and insulin deficiency (alloxane diabetes) modulate adrenergic and cholinergic heart function regulation and circulation reactions to acute myocardial ischemia. Nevertheless the mechanisms of the coronary tone physiological and pathophysiological effects of insulin in the heart circulation have not been fully elucidated. The purpose of this study was to assess whether multiple high dose insulin administrations may be involved in the pathophysiology of diabetic neuropathy and deterioration of coronary circulation and heart function regulation.

Materials and methods: During 30 days of chronic experiments on healthy conscious dogs (ID) insulin administrations (1.0 IU $/ \mathrm{kg}$ in muscle of hind leg) were carried out. Every morning animals were injected with insulin 4 hours before feeding. Final investigations of neurohormonal coronary artery (CA) tone and heart function regulation were fulfilled in acute experiments on 20 anesthetized dogs (alpha-chloralose, 50-100 mg/kg, i.v.). Control physiological investigations of circulation were performed on 10 clinically healthy dogs (HD). Catheterization and programmed autoperfusion of left CA, catheterization and continuous drainage of coronary sinus, heart and main vessels catheterization were performed without opening the chest. Cardiohaemodynamics, CA perfusion pressure (CAPP) with another clinical and biochemical parameters were registered. Test-reactions of CA and femoral artery (FA) resistance were provoked by bolus injection of epinephrine (E, $5.0 \mathrm{mkg}$ ), norepinephrine (NE, $5.0 \mathrm{mkg}$ ) and acetylcholine (Ach, $1.0 \mathrm{mkg}$ ) into arterial blood stream.

Results: Due to multiple insulin administrations the dog's body masses and venous blood glucose levels augmented (from $15.1 \pm 1.4$ to $18.0 \pm 1.5 \mathrm{~kg}$, $\mathrm{P}<0.05$, and from $3.36 \pm 0.6$ to $4.7 \pm 0.8 \mathrm{mmol} / \mathrm{l}, \mathrm{P}<0.05$, resp.). But the levels of venous blood $\mathrm{pH}, \mathrm{pCO}_{2}, \mathrm{pO}_{2}$, as well as blood pressure and other cardiohaemodynamics parameters did not change significantly. In ID group $\mathrm{E}$ and Ach induced more pronounced decrease of CAPP in comparison with HD group $(-5.7 \pm 0.4 \mathrm{kPa} ;-5.6 \pm 0.4 \mathrm{kPa} ; \mathrm{P}<0.05$; and $-4.5 \pm 0.3 \mathrm{kPa} ;-4.3 \pm 0.3 \mathrm{kPa}$; $\mathrm{P}<0.05$, resp.). Meantime no differences between ID and HD groups were observed in circulation reactions to $\mathrm{NE}(-4.6 \pm 0.5 \mathrm{kPa}$ and $-4.5 \pm 0.4 \mathrm{kPa} ; \mathrm{P}>0.5)$. Also in ID group after bolus injection of $\mathrm{E}$ into FA stream but not after NE the elevation of FA perfusion pressure (FAPP) was significantly augmented in comparison with HD group $(+8.0 \pm 0.6 \mathrm{kPa} ;+6.1 \pm 0.4 \mathrm{kPa} ; \mathrm{P}<0.05$ and $6.3 \pm 0.8$ $\mathrm{kPa} ;+6.3 \pm 0.7 \mathrm{kPa} ; \mathrm{P}>0.05$, resp.). Besides that the degree of decrease of FAPP to Ach in ID group was increased in comparison with $\mathrm{HD}(-7.3 \pm 0.2 \mathrm{kPa}$ and $6.6 \pm 0.1 \mathrm{kPa} ; \mathrm{P}<0.05)$.

Conclusion: These findings suggest that multiple high dose insulin administrations to healthy animals can provoke the development of typical for our previously observed early diabetic states in experiments on dogs changes of coronary circulation and heart function regulation deterioration: augmentation of coronary vessels and heart reactivity to adrenergic and cholinergic nervous systems stimulation.

\section{1}

High-fat diet-induced atherosclerosis was alleviated by lithium chloride, a glycogen synthase kinase-3 (GSK3) inhibitor, in apolipoprotein Edeficient mice

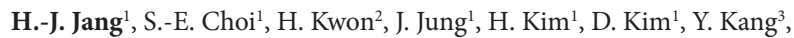
K. Lee ${ }^{1}$

${ }^{1}$ Endocrinology and metabolism, Ajou University, ${ }^{2}$ Endocrinology and metabolism, Kwon's Endo Im Clinic, ${ }^{3}$ Institutes for Medical Sciences, Ajou University, Suwon, Republic of Korea

Background and aims: Glycogen synthase kinase-3 (GSK-3) is a serine/ threonine kinase with roles in multiple biological functions, including cell fate determination, metabolism, proliferation, and carcinogenesis. However, the role of GSK-3 in atherosclerosis has not been elucidated. The present study was performed to investigate the role of GSK-3 in atherosclerosis using lithium chloride ( $\mathrm{LiCl}$ ), an inhibitor of GSK-3, in apolipoprotein (apo) E-deficient mice.

Materials and methods: Ten-week-old male mice were divided randomly into baseline, vehicle, and treatment groups. Mice in the vehicle and treat- 
ment groups were fed a typical western diet (i.e., $20 \%$ fat, $0.5 \%$ cholesterol) for 14 weeks without or with $\mathrm{LiCl}(10 \mathrm{mg} / \mathrm{kg} /$ day).

Results: $\mathrm{LiCl}$ treatment had no effect on glucose tolerance but improved insulin tolerance and body weight. Examination of plasma lipid profiles indicated that total cholesterol levels were significantly decreased by $\mathrm{LiCl}$ treatment as compared with the vehicle group $(P<0.05)$. However, there were no differences in triglycerol, HDL-cholesterol, or free fatty acid levels between the groups. In comparison with vehicle-treated controls, LiCl-treated apolipoprotein (apo) E-deficient mice showed $60 \%$ and $40 \%$ reductions in atherosclerotic lesion areas in the aorta and aortic valves, respectively $(P<0.05) . \mathrm{LiCl}$ treatment also decreased macrophage infiltration into atherosclerotic lesion areas of the aortic valves.

Conclusion: These results indicated that $\mathrm{LiCl}$, an inhibitor of GSK-3, reduces total plasma cholesterol levels, atherosclerotic lesion areas in the aorta and aortic valves, and macrophage infiltration into atherosclerotic lesions in apolipoprotein (apo) E-deficient mice. The results of the present study provided experimental evidence for a novel strategy of atherosclerosis treatment using GSK-3 inhibitors.

Supported by: GRRC project of the Gyeonggi Provincial Government, Republic of Korea

\section{2}

The influence of the presence of retinopathy on the circulating endothelial progenitor cell counts, endothelial function and carotid intima-media thickness in type 1 diabetes

L. Sibal ${ }^{1,2}$, A. Jones ${ }^{2}$, A. Aldibbiat ${ }^{1}$, G. Mitchell ${ }^{3}$, C. Oates ${ }^{3}$, J. Weaver ${ }^{1}$, J.A.M. Shaw ${ }^{1,2}$, P.D. Home ${ }^{1,2}$;

${ }^{1}$ School of Clinical Medical Sciences-Diabetes, The Medical

School,Newcastle University ${ }^{2}$ Department of Diabetes, Newcastle upon

Tyne Hospitals, ${ }^{3}$ Medical Physics, Newcastle upon Tyne Hospitals, United

Kingdom

Background: The relationship of retinopathy to cardiovascular (CV) risk is controversial. Endothelial dysfunction (measured as flow mediated dilatation, FMD) and increased carotid intima-media thickness (CIMT) predate clinical CV disease, while circulating endothelial progenitor cells (EPCs) are reduced in the presence of CV risk factors. We have determined how abnormalities of FMD, CIMT and EPCs in type 1 diabetes (T1DM) are affected by the presence of diabetic retinopathy.

Methods: FMD, CIMT and EPCs were measured in 74 young adults with T1DM without microalbuminuria or arterial disease and 80 healthy controls. EPCs were quantified in the lymphocyte gate and CD14-positive gate by flowcytometry with matching isotype control antibodies to reduce background noise. Retinopathy was graded from digital photography.

Results: FMD was comparable in those with TIDM with $(4.8 \pm 3.0 \%)$ and without $(5.3 \pm 2.9 \%)$ retinopathy, though significantly reduced compared to controls $(9.0 \pm 3.4 \%$; $\mathrm{p}<0.001)$. CIMT was higher in T1DM people $(0.62 \pm 0.00$ vs controls $0.50 \pm 0.00 \mathrm{~mm} ; \mathrm{p}<0.001)$ and in those with retinopathy compared with those without $(0.64 \pm 0.01$ vs $0.59 \pm 0.01 \mathrm{~mm}, \mathrm{p}=0.009)$. The median EPC and CV progenitor counts (cells $/ 10^{6}$-events) in T1DM without and with retinopathy and controls were: $\mathrm{CD} 34+$ cells $(108,291,418, \mathrm{p}=0.018$ to 0.002 respectively); $C D 133+$ cells $(49,159,213$, NS to $\mathrm{p}=0.012$ respectively) and CD34+/VE-Cadherin $(67,155,216, \mathrm{p}=0.008$ to 0.001 respectively). Within the T1DM group CD34+/VEGFR2 count was lower in those without retinopathy ( 3 vs $10, \mathrm{p}=0.042$ ).

Conclusion: CIMT was higher in those with retinopathy suggesting possible association with macrovascular pathology while the reduced number of circulating EPCs in those with T1DM compared to healthy controls was significantly attenuated in the presence of retinopathy. Further studies of the mechanisms influencing EPC number and function will be required to fully elucidate their role in the pathogenesis of microvascular and macrovascular complications of diabetes and their value as a marker of CV risk in this complex heterogeneous population.

\section{3}

Relationship between post-occlusive forearm skin reactive hyperaemia and diabetic microangiopathy or macroangiopathy in patients with type 2 diabetes - a novel index for detecting microvascular dysfunction using laser Doppler flowmetry

R. Suganuma, Y. Aso, T. Inukai

Internal Medicine, Dokkyo Medical University, Koshigaya Hospital,

Koshigaya, Japan

Background and aims: To investigate the relationship between post-occlusive forearm skin reactive hyperemia (PORH) and macro- and microangiopathy, or serum high molecular weight (HMW) adiponectin.

Materials and methods: We studied 20 age-matched healthy control subjects and 104 patients with type 2 diabetes. Cutaneous blood flow (flux) in response to 5-min arterial occlusion in the forearm was measured using laser Doppler flowmetry. We defined the PORH-index as the ratio of the area under the curve (AUC) $1 \mathrm{~min}$ after the release of the cuff relative to the AUC of a 1-min period before the cuff inflation. The flux in response to arterial occlusion was divided morphologically into three patterns: normal peak, early non-dominant peak, and early dominant peak. Brachial-ankle pulse wave velocity (baPWV), an index of arterial stiffness, was measured.

Results: The PORH-index was markedly lower in diabetic patients than in control subjects. The prevalence of an early dominant peak pattern was significantly higher in diabetic patients than in control subjects. The PORH-index correlated negatively with urinary albumin and baPWV in diabetic patients. We found no significant relationship between serum HMW adiponectin and PORH-index or the three morphologic patterns of flux in diabetic patients. The PORH-index was an independent determinant of cardiovascular disease in diabetic patients.

Conclusion: The PORH-index may be the most sensitive indicator for microvascular and macrovascular function among the various measures of PORH in patients with type 2 diabetes. However, the measures of PORH, including the PORH-index, were not associated with the serum HMW adiponectin concentrations in patients with type 2 diabetes.

\section{4}

Microvascular reactivity in acute hyperglycaemia in type 1 diabetes M. Prázný, E. Horová, J. Škrha

3rd Dept. of Internal Medicine, 1st Faculty of Medicine, Prague, Czech Republic

Background and aims: High plasma glucose variability and excursions are considered as a risk factor for macrovascular complications in diabetes. Less is known about microvascular damage. The aim of the study was to evaluate skin microvascular reactivity (MVR) in type 1 diabetic patients during isoglycemic and hyperglycemic hyperinsulinemic clamps to test the effect of acute hyperglycemia. Lipid parameters, fibrinogen and the cell adhesion molecules were evaluated, too.

Materials and methods: 20 type 1 diabetic patients (mean age $51 \pm 7$ years, BMI $25 \pm 3 \mathrm{~kg} . \mathrm{m}^{-2}$, diabetes duration $24 \pm 7$ years, $\mathrm{HbA}_{1 \mathrm{C}} 7.8 \pm 0.9 \%$ ) were studied initially during isoglycemic ( 2 hours) and consequently hyperglycemic ( 2 hours) hyperinsulinemic clamps and after following next two weeks during appropriate intravenous volume load as control examination to exclude non-specific factors, especially hemodilution. Skin MVR was measured by laser Doppler flowmetry continually during clamps and after post-occlusive $(\mathrm{PORH})$ and thermal hyperemia (TH) at the end of isoglycemic and hyperglycemic phases. Maximal perfusion (max), time to maximal perfusion $(\operatorname{tmax})$, velocity of perfusion increase $(\max / \mathrm{t})$ and percentage of baseline increase (\%) were calculated. Paired t-test, ANOVA, Wilcoxon test and Pearson correlation were used for statistical analysis of data using Statistica for Windows software.

Results: Venous blood glucose was $6.5 \pm 1.9 \mathrm{mmol}^{-1} \mathrm{l}^{-1}$ in isoglycemic and $12.0 \pm 1.6 \mathrm{mmol} . \mathrm{l}^{-1}$ in hyperglycemic phase of the clamp, respectively. Maximal perfusion in PORH increased in hyperglycemic phase compared to basal value and the same was true for the velocity of perfusion increase which was increased in isoglycemic phase, too. Negative relationship was found between plasma insulin concentration and time to maximal flow during PORH ( $r=-$ $\left.0.7, \mathrm{p}=0.007 ; \mathrm{y}=193.6-2 \cdot 5^{\star} \mathrm{x}\right)$. No other changes in microvascular reactivity were observed during clamps. No changes in microvascular reactivity were observed in control test. Generally, the cell adhesion molecules concentration declined at the end of both clamps and in the control test. Similar was true for lipid parameters. Results of post-occlusive reactive hyperemia and cell adhe- 
Microvascular reactivity and cell adhesion molecules. a- $\mathbf{p}<0.001, \mathrm{~b}-\mathrm{p}<0.01, \mathrm{c}-\mathrm{p}<0.05$

\begin{tabular}{|c|c|c|c|c|c|}
\hline & \multicolumn{3}{|c|}{ CLAMP $(n=20)$} & \multicolumn{2}{|c|}{ CONTROL TEST $(n=20)$} \\
\hline & baseline & ISO & HYPER & baseline & end \\
\hline PORHmax (PU) & $40 \pm 16$ & $39 \pm 19$ & $47 \pm 16^{\mathrm{b}}$ & $32 \pm 12$ & $35 \pm 12$ \\
\hline PORHtmax (s) & $13.5 \pm 5.1$ & $6.1 \pm 4.7^{\mathrm{a}}$ & $10.4 \pm 8.4$ & $11.8 \pm 6.0$ & $11.3 \pm 9.0$ \\
\hline PORHmax/t (PU/s) & $2.6 \pm 1.5$ & $11.2 \pm 14.9^{c}$ & $10.4 \pm 16.5^{\mathrm{c}}$ & $2.7 \pm 2.4$ & $3.7 \pm 3.0$ \\
\hline PORH\% (\%) & $385 \pm 165$ & $328 \pm 309$ & $392 \pm 219$ & $303 \pm 104$ & $394 \pm 28$ \\
\hline E-selectin (ng.ml $\left.{ }^{-1}\right)$ & $29 \pm 12$ & - & $27 \pm 11^{\mathrm{a}}$ & $29 \pm 15$ & $28 \pm 14^{\mathrm{b}}$ \\
\hline P-selectin (ng. $\left.\mathrm{ml}^{-1}\right)$ & $132 \pm 50$ & - & $93 \pm 34^{\mathrm{a}}$ & $126 \pm 50$ & $111 \pm 27$ \\
\hline ICAM-1 (ng.ml-1) & $251 \pm 48$ & - & $215 \pm 41^{\mathrm{a}}$ & $237 \pm 40$ & $212 \pm 33^{b}$ \\
\hline VCAM (ng. $\mathrm{ml}^{-1}$ ) & $812 \pm 183$ & - & $673 \pm 117^{a}$ & $815 \pm 203$ & $665 \pm 165^{\mathrm{a}}$ \\
\hline
\end{tabular}

sion molecules are shown in the table. Fibrinogen was slightly increased at

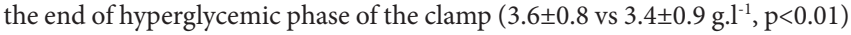
but was declined in control test $\left(3.2 \pm 0.9\right.$ vs $\left.3.4 \pm 1.0 \mathrm{~g}^{-1} \mathrm{l}^{-1}, \mathrm{p}<0.05\right)$. No relationship was observed between parameters measured during both clamps and control tests.

Conclusion: Microvascular reactivity was probably modulated by vasodilatory effect of hyperinsulinemia during clamps. The fibrinogen increase at the end of the clamp documents hypercoagulability in hyperglycemia. Decline in the cell adhesion molecules concentration was probably caused by nonspecific effect (hemodilution). No relationship was observed between acute hyperglycemia $\left(+5.5 \mathrm{mmol}^{-1} \mathrm{l}^{-1}\right)$ and microvascular reactivity in type 1 diabetic patients.

Supported by: Ministry of Education of Czech Republic

\section{5}

Addition of aggregation to modeling of viscosity improves accuracy of the calculation of wall shear stress

H.J.M. Mutsaerts ${ }^{1}$, M. Out ${ }^{2}$, P.T. Goedhart ${ }^{3}$, C. Ince ${ }^{4}$, J.A. Romijn ${ }^{2}$,

T.J. Rabelink ${ }^{5}$ J.H.C. Reiber ${ }^{1}$, F.M.A. Box ${ }^{1}$

${ }^{1}$ Div. of Image Processing, Dept. of Radiology, Leiden University Medical

Center, ${ }^{2}$ Dept. of Endocrinology, Leiden University Medical Center,

${ }^{3}$ Laboratory for Clinical Hemorheology, Academic Medical Center,

Amsterdam, ${ }^{4}$ Dept. of Physiology, Academic Medical Center, Amsterdam,

${ }^{5}$ Dept. of Nephrology, Leiden University Medical Center, Netherlands

Background and aims: Low wall shear stress (WSS) modulates the endothelium and in the long-term contributes to the development of atherosclerotic lesions. Viscosity is an important determinant of WSS. In order to take into account the non-Newtonian viscosity of blood in computational fluid dynamics, one needs a constitutive equation which defines the relationship between viscosity and shear rate. Previous studies showed that for high shear rates a model with hematocrit (hct) in the exponent is able to approximate real viscosity. However, at lower shear rates this model becomes inadequate, as red blood cell (RBC) aggregation differs between individuals. Especially patients diagnosed with type 2 diabetes mellitus (T2DM) are known for their heightened RBC aggregation. The aim of this study was to test improvement of viscosity modeling by addition of aggregation parameters.

Materials and methods: Hct, aggregation- and viscosity measurements were performed in duplicate in subjects diagnosed with an asymptomatic plaque in the carotid artery (intima media thickness $>0,08 \mathrm{~cm}$ and stenosis $<70 \%$, measured by duplex ultrasonography) ( $\mathrm{n}=56,43-79 \mathrm{yrs}, 63 \%$ male, $50 \%$ T2DM). Aggregation parameters include amplitude, half time $\left(t_{1 / 2}\right)$, aggregation index (AI), fit error, Tfast, Tslow and threshold shear rate $\left(\gamma_{\text {thr }}\right)$. Viscosity was measured on following shear rates: $0,87 \mathrm{~s}^{-1}, 2,19 \mathrm{~s}^{-1}, 5,49 \mathrm{~s}^{-1}, 10,15 \mathrm{~s}^{-1}$, $47,1 \mathrm{~s}^{-1}$ and $87 \mathrm{~s}^{-1}$. A forward linear regression analysis (inclusion criterion $p<0,05)$ was performed to investigate improvements with various aggregation parameters and hct for modeling of viscosity on the six mentioned shear rates. Influence from gender and age was tested. A linear regression analysis was performed to test correlations of $\mathrm{HbA}_{1 c}$ with aggregation parameters. Results: Hct was significantly correlated with viscosity in all instances. Gender was correlated with viscosity, but not after adjustment for hct. Age was not correlated with viscosity. Both were not correlated with $\mathrm{HbA}_{1 \mathrm{c}}$ or any of the aggregation parameters. Viscosity modeling on $0,87 \mathrm{~s}^{-1}$ was improved by addition of only $t_{1 / 2}$ (standard error decreased with $8 \%$ ). Viscosity modeling on 2,19 $\mathrm{s}^{-1}$ was improved by addition of both $\mathrm{t}_{1 / 2}$ and $\mathrm{y}_{\mathrm{thr}}$ (standard error decreased with 14\%). Viscosity modeling on $5,49 \mathrm{~s}^{-1}, 10,19 \mathrm{~s}^{-1}, 47,1 \mathrm{~s}^{-1}$ and $87 \mathrm{~s}^{-1}$ was improved by addition of only $y_{\text {thr }}$ (standard error decreased with respectively $7 \%, 8 \%, 17 \%$ and $18 \%)$. HbAlc was significantly correlated with $\operatorname{AI}(p=0,007), \mathrm{t}_{1 / 2}(p=0,037)$ and $\mathrm{y}_{\text {thr }}(p=0,002)$.

Conclusion: The combination of het and $t_{1 / 2}$ is able to model viscosity in lowest shear rates, whereas the combination of hct and $y_{\text {thr }}$ approximates real viscosity in higher shear rates. A cross-over point exists around 2,19 $\mathrm{s}^{-1}$. This difference is probably due to the fact that $y_{\text {thr }}$ reflects the tendency for aggregation whereas $t_{1 / 2}$ displays the aggregation kinetics. Furthermore both parameters are associated with $\mathrm{HbAlc}$. Our findings indicate the relevance of aggregation in WSS calculations, which increases with increasing $\mathrm{HbA}_{1 c}$. Supported by: Dutch Diabetes Research Foundation 


\section{PS 137 Screening methods for diabetic complications}

\section{6}

Comprehensive evaluation of coronary arteries by multidetector-row cardiac computed tomography according to the glucose tolerance status of asymptomatic subjects

S. Lim 1 , S. Choi 1 , K. Park², Y. Koo², H. Lee², H. Jang ${ }^{1}$

${ }^{1}$ Internal Medicine, Endocrinology and Metabolism, Seoul National

University Bundang Hospital, Seongnam, ${ }^{2}$ Internal Medicine,

Endocrinology and Metabolism, Seoul National University College of

Medicine, Seoul, Republic of Korea

Objective: Cardiovascular disease is a major cause of mortality in subjects with diabetes. There is an increasing need to detect early atherosclerosis before cardiac events occur through noninvasive techniques. We performed multidetector-row computed tomography (MDCT) to investigate the status of coronary artery stenosis (CAS) and plaque characteristics, which has been limited in asymptomatic subjects.

Research design and methods: The number of diseased vessels, the extent of stenosis ( $\geq 50 \%$ luminal narrowing), the composition of plaques (noncalcified, mixed, or calcified), and the coronary artery calcium score (CACS) were measured by MDCT in 1043 asymptomatic subjects (mean $[ \pm$ SD] age $=49.2 \pm 9.5$ years). Anthropometric parameters and metabolic profiles were also measured. Results: $20.8 \%$ of subjects had plaques and $5.1 \%$ had significant CAS. The mean $( \pm$ SD) of CACS was $17.2 \pm 81.2$ in this study group. Subjects with impaired fasting glucose (IFG, $\mathrm{n}=215,20.6 \%)$ or diabetes $(\mathrm{n}=112,10.7 \%)$ had more CAS, a greater plaque burden, and higher CACS than those of normal subjects (all $P<0.01$ ). More vulnerable (noncalcified and mixed) plaque was observed in subjects with diabetes (19.4\%) and IFG (11.3\%) than in normal (6.6\%). After adjustment for confounding factors, impaired glucose metabolism was strongly associated with significant CAS and a greater vulnerable plaque burden.

Conclusion: Considering the relatively high prevalence of vulnerable-plaque in IFG or diabetes, even when they are asymptomatic, MDCT could be considered as a useful diagnostic tool for detecting early coronary atherosclerosis in prediabetes and diabetes.

Supported by: Korea Science and Engineering Foundation, Ministry of Science and Technology, IT R\&D programme, and Seoul R\&BD programme, Republic of Korea

\section{7}

Lower bone mineral density is associated with higher coronary calcification by multi-detector row coronary computed tomography in women independently from cardiovascular risk factors

S. Choi ${ }^{1}$, J. An ${ }^{1}$, S. Lim ${ }^{1}$, K. Park ${ }^{2}$, H. Lee ${ }^{2}$, H. Jang 1 , C. Shin ${ }^{2}$

${ }^{1}$ Internal Medicine, Endocrinology and Metabolism, Seoul National

University Bundang Hospital, Seongnam, ${ }^{2}$ Internal Medicine,

Endocrinology and Metabolism, Seoul National University College of

Medicine, Seoul, Republic of Korea

Background and aims: There are growing concerns about the direct association between bone mineral density (BMD) and vascular calcification, which is related to cardiovascular disease. Coronary multi-detector row computed tomography (MDCT) is recently developed as a non-invasive tool for coronary status and evaluate coronary calcification more precisely. To evaluate the relationship between the BMD and coronary calcification by using MDCT Design: We had enrolled 815 subjects who received their routine check up in health care center. After excluding subject who had factors affecting altered bone mechanism and cardiovascular disease, the data of 462 subjects were finally analyzed. We measured the coronary calcification with MDCT, BMD, anthropometric, and metabolic parameters.

Results: The BMD of femur area and lumber spine are negatively associated with coronary calcium score in both premenopausal and postmenopausal women, but not in men after adjustment for age and BMI. This inverse correlation was getting stronger with longer year since menopause, in femur area than lumbar spine $(r=-0.37, P<0.05)$, and in postmenopausal women with osteoporosis and osteopenia than normal BMD $(r=-0.49, P<0.05)$.

Conclusion: The increased coronary calcium score by MDCT is associated with a low BMD in pre- and postmenopausal women, independently from cardiovascular risk factors, age, and BMI .

Supported by: Seoul R\&BD programme, Republic of Korea

\section{8}

Noninvasively measured subendocardial viability ratio and ejection duration were impaired in newly diagnosed type 2 diabetes H.-J. Kim, K. Han, B. Koo, H.-J. Kim, K. Pak, K. Min, E. Kim Internal medicine, Eulji University School of Medicine, Seoul, Republic of Korea

Background and aims: Heart failure of ischemic or non-ischemic origin is a significant source of morbidity and mortality in diabetes. With an increase in aortic or ventricular chamber pressure, especially during diastole, endocardial perfusion can be compromised, which will reduce in myocardial contractility. This study was conducted to investigate whether the propensity for myocardial ischemia expressed as subendocardial viability ratio (SEVR, $\%$ ) and ejection duration (ED, \%) from central artery pressure analysis is increased in prediabetic stage or newly diagnosed type 2 diabetic patients.

Materials and methods: Three hundred and forty-three subjects were included [30 80 years, (M:F 45.8\%:54.2\%)] and divided into three groups (control, $\mathrm{n}=131$; prediabetes, $\mathrm{n}=93$; newly diagnosed diabetes, $\mathrm{n}=119$ ). Diabetes was diagnosed by ADA criteria and prediabetes was defined as having impaired fasting glucose or impaired glucose tolerance after 75g OGTT. Augmentation index (AIx, \%) by pulse-wave reflection, SEVR, and ED were calculated from the wave pattern of the central artery by applanation tonometry using SphygmoCor ${ }^{\circ}$. Other cardiovascular risk factors such as obesity, lipid profiles and smoking pack-years were also evaluated. General linear model (GLM) analysis was used to identify the determining factors of AIx, SEVR, and ED from multivariate.

Results: $\mathrm{HbAlc}$ and fasting plasma glucose (FPG) were significantly higher and HDL-cholesterol significantly lower in newly diagnosed diabetic patients than other two groups $(P<0.05$, respectively). BMI and triglyceride were significantly higher in both of prediabetic and diabetic subjects than control $(P<0.05$, respectively). While AIx was not different (control, $25 \pm 11 \%$; prediabetes, $23 \pm 10 \%$; newly diagnosed diabetes, $24 \pm 12 \% ; P=n s$ ), SEVR was decreased and ED was increased significantly in diabetic patients compared to control or prediabetes (SEVR- $151 \pm 26 \%$ vs. $160 \pm 27 \%$ or $158 \pm 24 \%, P<0.05$; ED- $37 \pm 4 \%$ vs. $35 \pm 4 \%$ or $35 \pm 4 \%, P<0.05$, respectively). From GLM analysis with group and sex as factors, AIx had a significant association with age, height and LDL cholesterol $(P<0.001, P=0.043$ and $P=0.005$, respectively), but not with glucose level or blood pressure. By contrast, in ED, only age, $\mathrm{BMI}$ and $\mathrm{HbAlc}$ but not lipid profile showed a significant association with ED $(P=0.015, P=0.001$ and $P<0.001$, respectively). While BMI and HbAlc were also significantly associated with SEVR $(P<0.001$ in both), age showed no significant association with it.

Conclusion: Even though aortic stiffness was not significantly increased in prediabetes or newly diagnosed diabetic patients, SEVR and ED were significantly impaired in newly diagnosed diabetic patients, but not in prediabetic condition. These results imply that the propensity for myocardial ischemia is increased in newly diagnosed type 2 diabetic patients.

\section{9}

Screening unrecognised peripheral arterial disease using ankle-brachial index: ELLIPSE STUDY diabetics group results

D. Huet ${ }^{1}$, Investigators of the ELLIPSE Study

${ }^{1}$ Diabetology, Endocrinology, Groupe hospitalier Paris Saint Joseph, Paris, France

Aims: Peripheral arterial disease (PAD) is often underdiagnosed, in lack of symptoms and increase the major cardio vascular (CV) event risk. ELLIPSE is a multicentric, epidemiological, french study to investigate prevalence of undiagnosed $\mathrm{PAD}$ in high risk $\mathrm{CV}$ patients including diabetics.

Materials and methods: From April to November 2007: 2444 inpatients, age $>55 \mathrm{y}$, without PAD but with coronary or cerebrovascular disease or at least one of CV risk factors (diabetes, smoking, hypertension, dyslipidemia,) where enrolled by 244 trained investigators for measurement of ankle brachial index (ABI). Diabetes + was defined as patient taking insulin or any oral hypoglycemic agents. $\mathrm{PAD}+$ patients was defined by an $\mathrm{ABI}<0.9$.

Results: 2129 patients investigated by (22\% cardiologists, $22 \%$ diabetologists, 23 internists, $14 \%$ neurologists and $15 \%$ geriatrists) are included in the primary analysis (all ABI values available). In this population, 942 diabetes + patients was younger than the 1187 diabetes - patients 69.8 years $( \pm 9.5)$ vs $74.8( \pm 10.6) \mathrm{p}<0.05$, and had less frequently symptomatic arterial disease (coronary $21.5 \%$ vs $30.3 \%$ - cerebrovascular $11.8 \%$ vs $28.2 \%$ or both $5.5 \%$ vs $8.1 \%) \mathrm{p}<0.05$ but more frequently only CV risk factors $(61.1 \%$ vs $35.4 \%)$. 
Diabetes + patients present significantly more hyperlipemia ( $54.2 \%$ vs $38.5 \%)$ and hypertension $(77.1 \%$ vs $73.1 \%$ ). The mean ABI was $0.92 \pm 0.22$ (range $0.13-2.75$ ) in diabetics patients and did not differed from the global population PAD was diagnosed in $41.7 \%$ for diabetes + vs $40.9 \%$ for diabetes - NS $4.9 \% \mathrm{ABI}<0.53 .3 \% \mathrm{ABI}>1.3$

Conclusion: Prevalence of undiagnosed PAD is important in PAD asymptomatic diabetic's patients: $\mathbf{4 3 . 1} \%$. The extend of systematic ABI (examination simple, rapid, reproductible) in diabetics patients will increase support aggressive secondary prevention care in the event of PAD +, especially in patients without coronary or cerebro-vascular disease.

Supported by: BMS, sanofi-aventis

\section{0}

\section{Screening of peripheral arterial disease in asymptomatic diabetic} patients

R. Da $\operatorname{Ros}^{1}$, R. Assaloni ${ }^{2}$, G. Zuodar ${ }^{3}$, S. Pressoni ${ }^{3}$, M. Deotto ${ }^{3}$, A. Ceriello ${ }^{4}$ ${ }^{1}$ Distretto 4, Ass1 Triestina, Trieste, Italy, ${ }^{2}$ Casa Di Cura, Foot and Ankle Clinic, Abano Terme, Italy, ${ }^{3}$ University of Udine, Internal Medicine, Italy, ${ }^{4}$ University Hospital of Coventry and Warwickshire, Centre of Excellence in Diabetes and Endocrinology, Coventry, United Kingdom

Background and aims: Peripheral arterial disease (PAD) is an important complication of diabetes, with consequences on quality of life and survival. PAD is a risk factor for increasing mortality and cardiovascular events. $\mathrm{ADA}$ and AHA indicate every year screening for PAD in patients with type 2 diabetes and over 40 years old, but literature data show that screening is underutilized and PAD underdiagnosed. The most used screening test is ankle brachial index (ABI), it requires about 15 minutes to be performed and it has demonstrate low sensitivity in asymptomatic diabetic patients. Moreover alternative tests like pulse oximetry and toe pressure failed to demonstrate better efficacy and usefulness compared to ABI. Aim of the study: to compare accuracy of screening tests in asymptomatic diabetic patients and to evaluate efficacy of arterial sound interpretation like a new proposed diagnostic test. Materials and methods: 324 limbs, in 162 diabetic asymptomatic consecutive outpatients presented in a primary care service, were evaluated. We investigate all the limbs with: transcutaneous oximetry; pulse oximetry at rest and after uplift of the limb, ABI at rest and after walking test, temperature and a new test: the arterial sound interpretation at ankle level and Doppler waveform analysis. We defined PAD as monophasic waveforms on waveform analysis.

Results: Prevalence of PAD was $18 \%$. Patients with peripheral arterial disease were older than patients without, with a longer history of diabetes, and higher prevalence of hypertension, cardiovascular disease and renal failure (all $\mathrm{p}<0.01$ ). Diagnostic test specificity, sensitivity and $\mathrm{K}$ test values for agreement with color-duplex, are reported in table 1.

\begin{tabular}{lccclccc} 
Test & Sens & Spec & K-test & Test & Sens & Spec & K-test \\
\hline ABI & 58 & 82 & 0.35 & $\begin{array}{l}\text { TcPO2 }< \\
50 \mathrm{mmHg}\end{array}$ & 62 & 68 & 0.21 \\
\hline $\begin{array}{l}\text { ABI after } \\
\text { walking test }\end{array}$ & 38 & 91 & 0.31 & $\begin{array}{l}\text { TcPO2 }< \\
40 \mathrm{mmHg}\end{array}$ & 48 & 82 & 0.20 \\
\hline $\begin{array}{l}\text { Pulse-oximetry } \\
\text { hand/foot }\end{array}$ & 24 & 75 & 0.01 & $\begin{array}{l}\text { Temperature } \\
\text { difference hand/ } \\
\text { foot }>2\end{array}$ & 83 & 36 & 0.09 \\
$\begin{array}{l}\text { Pulse-oximetry } \\
\text { after elevation }\end{array}$ & 37 & 84 & 0.20 & $\begin{array}{l}\text { Arterial sound } \\
\text { interpretation }\end{array}$ & 90 & 95 & 0.81 \\
\hline
\end{tabular}

Conclusion: This study, according to literature data, confirm low ABI sensitivity for PAD screening in diabetic asymptomatic patients and found fair agreement with color duplex. Transcutaneous oximetry, pulse oximetry and $\mathrm{ABI}$ after walking test are not effective for PAD screening. Arterial sound interpretation presents good sensitivity and sensibility, very good agreement with color duplex and short performing time. This test, if data will be confirmed in a larger number of people, can be proposed for screening of diabetic patients.

\section{1}

Clinically relevant peripheral arterial occlusion is associated with a high as well as a low-pressure ankle-to-brachial ration index (ABI)

L. Potier ${ }^{1}$, F. Bouilloud ${ }^{1}$, J.-L. Golmard², M. Dadon' ${ }^{3}$ J. Le Doeuff',

C. Deybach ${ }^{1}$, M. Halbron' ${ }^{1}$, G. Ha Van ${ }^{1}$, A. Grimaldi ${ }^{1}$,

A. Hartemann-Heurtier ${ }^{1}$

${ }^{1}$ Diabetes department, ${ }^{2}$ Biostatistics Unit, ${ }^{3}$ Vascular Surgery Department,

University Pierre et Marie Curie-Paris 6, School of Medicine, Paris, France

Background and aims: The importance of peripheral vascular calcification in causing luminal narrowing remains uncertain. Defining clinically relevant peripheral arterial occlusion on an ABI threshold of $<0.9$ may misclassify patients with an increased artery rigidity if the latter results in "falsely" high pressure and obscures underlying occlusion. The aim of this study was to compare the severity of peripheral arterial occlusive disease (PAOD) between diabetic patients with a high, normal or low-pressure ABI.

Materials and methods: Observations were undertaken in a single diabetes department as part of routine assessment of all patients admitted for further investigation and treatment of suspected PAOD, with or without an associated foot ulcer. Patients with previous revascularisation procedure were excluded from this study. 160 lower limbs from 81 diabetic patients had TcPO2 and $\mathrm{ABI}$ measurements performed as well as colour duplex imaging (CDI). $\mathrm{ABI}$ was measured using a hand-held Doppler probe with the systolic pressure being recorded in the dorsalis pedis or, if undetectable, in the posterior tibial artery. The severity of arterial occlusion was scored on CDI according to an adapted version of a validated published angiographic score $(0=$ no significant arterial lesion, $1=$ at least one significant stenosis to $21=$ maximal severity). The relationship between $\mathrm{ABI}$ and time to healing was studied as an indicator of PAOD severity in the subset of $35 \%$ limbs that had foot ulcer.

Results: $\mathrm{ABI}$ was $>1.3$ in $13.8 \%$ limbs (incompressible arteries=group 1), between 0.9 and 1.3 in $37.6 \%$ (group 2), and $<0.9$ in 37\% (group 3). CDI showed no significant arterial lesion in $15 \%$ patients in group 3 (false positive $\mathrm{ABI}$ ), and significant lesion in $57 \%$ patients in group 2 (false normal $\mathrm{ABI})$ and in $60 \%$ patients in group $1 . \mathrm{TcPO} 2$ from group $1(36 \pm 19 \mathrm{~mm} \mathrm{Hg})$ was not different from group $3(36 \pm 20, p=0.28)$ but significantly lower than $\mathrm{TcPO} 2$ from group $2(42 \pm 16, \mathrm{p}<0.02)$. Duplex score in presence of PAOD from group $1(8.0 \pm 3.9)$ was significantly higher than duplex score from group $2(4.8 \pm 2.7, \mathrm{p}<0.05)$ and even than duplex score from group 3 (7.4 \pm 3.7 , $\mathrm{p}<0.0001)$. Wound healing time was not different between group 1 and 3 , but significantly shorter in group $2(\mathrm{p}<0.003)$.

Conclusion: In presence of clinical suspicion of PAOD, lower limbs with incompressible arteries show a lower prevalence of confirmed PAOD than IPS $<0.9$, but when present indicators of PAOD severity are comparable or even higher.

\section{2}

The performance of pulse wave velocity for the diagnosis of peripheral vascular disease in patients with type $\mathbf{2}$ diabetes mellitus

I. Eleftheriadou ${ }^{1}$, V. Danias ${ }^{1}$, P. Grigoropoulou ${ }^{1}$, C. Arapostathi ${ }^{1}$, D. Perrea ${ }^{2}$, N. Katsilambros ${ }^{1}$, N. Tentolouris ${ }^{1}$

${ }^{1} 1$ st Department of Propaedeutic Medicine, Athens University Medical School, Laiko General Hospital, ${ }^{2}$ Laboratory of Experimental Surgery and Surgical Research, Athens University Medical School, Athens, Greece

Background and aims: Pulse wave velocity (PWV) of the carotid-femoral (cf) arteries has emerged as a new method by which total cardiovascular risk can be assessed. Increased PWVcf reflects arterial stiffening and is an important predictor of cardiovascular events. In the present study we examined the validity of PWV for the diagnosis of peripheral vascular disease (PVD) in patients without and with type 2 diabetes mellitus (T2DM). In addition, we examined whether the presence of either peripheral (PN) or cardiac autonomic neuropathy (CAN) alters the validity of PWV for the diagnosis of PVD in patients with T2DM.

Materials and methods: A total of 155 patients ( 82 with T2DM and 73 without diabetes, mean age $65.8 \pm 8.8$ and $62.4 \pm 8.5$ years; male/female $48 / 34$ and 27/46, respectively) were examined. Diagnosis of PVD was based on the presence of either biphasic, monophasic or blunted waveforms in the artery posterior tibial. PWV was measured at the carotid-femoral (cf) and femoral-posterior tibial (ft) segments. Diagnosis of PN was based on neuropathy symptom score (NSS), neuropathy disability score (NDS) and vibration perception threshold (VPT), while of CAN on the battery of the autonomic function tests. 
Results: Higher values of PWVcf and PWVft have high specificity but low sensitivity for the diagnosis of PVD in both diabetic and nondiabetic patients. Presence of PN does not affect the performance of PWV, but presence of CAN reduces its specificity for the diagnosis of PVD in subjects with T2DM (table).

Conclusion: High values of PWV almost confirm the presence of PVD both in diabetic and nondiabetic patients, irrespective of the presence of PN. However, presence of CAN reduces the specificity of PWV in the subjects with T2DM.

\begin{tabular}{|c|c|c|c|c|}
\hline & \multicolumn{2}{|l|}{ Diabetics } & \multicolumn{2}{|c|}{ Nondiabetics } \\
\hline & sensitivity & specificity & sensitivity & specificity \\
\hline $\mathrm{PWVcf}>14 \mathrm{~m} / \mathrm{sec}$ & 0.12 & 0.91 & 0.00 & 0.95 \\
\hline \multirow[t]{3}{*}{ PWVft $>12 \mathrm{~m} / \mathrm{sec}$} & 0.09 & 0.97 & 0.09 & 0.98 \\
\hline & \multicolumn{2}{|l|}{$\begin{array}{l}\text { Diabetics } \\
\text { with PN }\end{array}$} & \multicolumn{2}{|l|}{$\begin{array}{l}\begin{array}{l}\text { Diabetics } \\
\text { without PN }\end{array} \\
\end{array}$} \\
\hline & sensitivity & specificity & sensitivity & specificity \\
\hline$\underline{P W V c f}>14 \mathrm{~m} / \mathrm{sec}$ & 0.08 & 0.94 & 0.22 & 0.89 \\
\hline \multirow[t]{3}{*}{ PWVft $>12 \mathrm{~m} / \mathrm{sec}$} & 0.12 & 0.94 & 0.00 & 0.99 \\
\hline & \multicolumn{2}{|l|}{$\begin{array}{l}\text { Diabetics } \\
\text { with CAN }\end{array}$} & \multicolumn{2}{|c|}{$\begin{array}{l}\text { Diabetics } \\
\text { without CAN }\end{array}$} \\
\hline & sensitivity & specificity & sensitivity & specificity \\
\hline PWVcf $>14 \mathrm{~m} / \mathrm{sec}$ & 0.22 & 0.80 & 0.08 & 0.92 \\
\hline $\mathrm{PWVft}>12 \mathrm{~m} / \mathrm{sec}$ & 0.11 & 0.80 & 0.08 & 0.99 \\
\hline
\end{tabular}

\section{3}

Short-term QT interval variability in patients with long-standing type-1 diabetes mellitus

C. Lengyel ${ }^{1}$, A. Orosz ${ }^{2}$, R. Takács ${ }^{1}$, T.T. Várkonyi ${ }^{1}$, I. Baczkó ${ }^{2}$, T. Wittmann ${ }^{1}$, J.G. Papp ${ }^{3}$, A. Varró ${ }^{2,3}$

${ }^{1} 1$ st Department of Medicine, University of Szeged, Hungary, ${ }^{2}$ Department of Pharmacology and Pharmacotherapy, University of Szeged, Hungary, ${ }^{3}$ Division for Cardiovascular Pharmacology, Hungarian Academy of Sciences, Szeged, Hungary

Background and aims: Determination of short-term QT-interval variability (QTV) is an intensively investigated new and non-invasive method for assessment of proarrhythmic risk. Several studies have demonstrated that temporal QTV is a more sensitive predictor of ventricular arrhythmias than the parameters of conventional QT-measurements. The aim of the present study was to evaluate the QTV in patients with long-standing type 1 diabetes mellitus (DM).

Patients and methods: $21 \mathrm{DM}$ patients (age: $33.7 \pm 2.7$ years, male/female ratio: $11 / 10$, duration of DM: $16.9 \pm 2.8$ years, HbAlc: $8.6 \pm 0.4 \%$, BMI: $25.1 \pm 0.9$ $\mathrm{kg} / \mathrm{m}^{2}$; mean \pm SEM) and 21 healthy controls (age: $33.3 \pm 2.0$ years, male/female ratio: $11 / 10$, HbAlc: $5.2 \pm 0.1 \%$, BMI: $\left.24.4 \pm 0.9 \mathrm{~kg} / \mathrm{m}^{2}\right)$ were enrolled into the study. ECGs were continuously recorded and all leads acquired by an ECG signal processing system. After analogue-to-digital conversion, the data were stored to hard disk and analyzed off-line using SPEL Advanced Haemosys software (v3.0, Experimetria Ltd.). The RR and QT intervals were measured as the average of 31 consecutive beats. To characterize the temporal instability of beat-to-beat repolarization, Poincare' plots of the QT intervals were constructed, where each QT value is plotted against its former value. QTV was calculated using the following formula: $\mathrm{QTV}=\Sigma\left|\mathrm{QT} \mathrm{T}_{\mathrm{n}+1}-\mathrm{QT}_{\mathrm{n}}\right|(30 \mathrm{x} \sqrt{2})^{-1}$. Autonomic function was assessed by means of five standard cardiovascular reflex tests.

Results: DM patients have an increased heart rate compared to the controls (78.1 $\pm 3.1 / \mathrm{min} v s 69.2 \pm 1.4 / \mathrm{min}, \mathrm{p}<0.05$, respectively). Comparison of the two groups (DM $v s$ control) revealed no difference in the QTc interval $(433 \pm 7.2$ $\mathrm{ms} v s 437 \pm 4.1 \mathrm{~ms}$ ). The QTV, however, was increased in the DM patients relative to those for the control group $(4.61 \pm 0.29 \mathrm{~ms}$ vs $3.19 \pm 0.22, \mathrm{p}<0.05)$. In the DM group, the mean autonomic score was $1.6 \pm 0.3$, and there were no significant correlations between the QTV values and the parameters of the five cardiovascular reflex tests.

Conclusion: The short-term QT variability may be elevated in long-standing type 1 diabetes mellitus despite of unchanged QTc interval. This enhanced temporal QT variability may early indicate the increased instability of cardiac repolarization.

Supported by: Hungarian National Research Foundation, the János Bolyai Research Scholarship, József Öveges Programme by the National Office for Research and Technology and Hungarian Ministry of Health

\section{4}

Endothelial function, artery stiffness and coronary artery disease in type 2 diabetic patients

A. Belhadj-Mostefa ${ }^{1,2}$, M. Henouda ${ }^{3}$, F. Touati ${ }^{1}$, J.R. Attali ${ }^{4}$, D. Roula ${ }^{1}$, P. Valensi ${ }^{4}$

${ }^{1}$ Medicine, Department of Internal Medicine, Constantine, Algeria, ${ }^{2}$ Health, ANDRS, Oran, Algeria, ${ }^{3}$ Medicine, Department of Cardiology, Constantine, Algeria, ${ }^{4}$ Medicine, Department of Endocrinology Diabetology Nutrition Jean Verdier Hospital, Bondy, France

Background and aims: Impairment of endothelium function and artery stiffness have been shown to be predictive of cardiovascular events. The aim of this study was to determine whether these disorders might be considered as markers of silent myocardial ischemia (SMI) or silent coronary stenoses (SCS) in type 2 diabetic patients.

Materials and methods: One hundred patients, $54.6 \pm 8.1$ years, with type 2 diabetes since $8 \pm 6.5$ years, were assessed for SMI by an ECG stress test. Coronary angiography was performed in patients with SMI. A post-ischemia reactive hyperaemic test was performed, and changes in blood flow (F2/F1) and diameter (D2/D1) of the brachial artery which account mostly for the reactivity of downstream microvasculature and for endothelium function, respectively, were evaluated by pulsed Doppler velocimetry. Artery stiffness was assessed by measuring pulse wave velocity (PWV) between the carotid and femoral arteries using the Complior device.

Results: SMI was found in 27 patients and SCS in 16 of them. D2/D1 (SMI-: $1.06 \pm 0.05$ and SMI+: $1.05 \pm 0.04$ ), F2/F1 (SMI-: $4.72 \pm 1.65$ and SMI+: $4.5 \pm 2.03$ ) and PWV (SMI-: $11.75 \pm 2.76$ and SMI+: $11.73 \pm 2.06$ ) did not differ significantly between patients with or without SMI. Compared with patients without SCS, patients with SCS had significantly lower D2/D1 (1.04 \pm 0.04 vs $1.07 \pm 0.06 ; \mathrm{p}<0.05)$, lower $\mathrm{F} 2 / \mathrm{F} 1$ only in the normotensive subgroup $(2.96 \pm 0.59$ vs $4.25 \pm 1.5 ; \mathrm{p}=0.06$ NS? dommage) and not in the hypertensive one $(4.85 \pm 2.28$ vs $4.78 \pm 1.48 ; \mathrm{p}=0.56)$, and significantly higher PWV ( $11.7 \pm 1.89$ vs $10.2 \pm 1.4 ; \mathrm{p}=0.03)$.

Conclusion: This study suggests that in type 2 diabetic patients more severe impairments in peripheral endothelium function and artery stiffness, and microcirculation function in normotensive patients, might be considered as markers for silent coronary stenoses.

Supported by: ANDRS ALGERIA

\section{5}

Strong association between capillary recruitment and forearm glucose uptake following acute per oral phosphodiesterase-5 inhibition in type 2 diabetes patients

P.-A. Jansson ${ }^{1}$, G. Murdolo ${ }^{1,2}$, L. Strindberg ${ }^{1}$, B. Nyström ${ }^{3}$, M. Sjöstrand ${ }^{1}$, P. Lönnroth ${ }^{1}$

${ }^{1}$ The Lundberg Laboratory for Diabetes Research, Dpt of Molecular and Clinical Medicine, University of Gothenburg, Sweden, ${ }^{2}$ Dpt of Internal Medicine, Perugia University, Italy, ${ }^{3} \mathrm{Dpt}$ of Cardiology, University of Gothenburg, Sweden

Background and aims: Impaired blood flow in skeletal muscle may contribute to a dysregulated metabolism in the insulin resistant state. The aim of this open non-randomized study was to test whether phosphodiesterase- 5 inhibition (PDE5Inh), first line therapy against erectile dysfunction, could improve recruitment of nutritive capillaries and ameliorate forearm glucose uptake (FGU) in insulin resistant subjects.

Materials and methods: Eight non-smoking postmenopausal female type 2 diabetic patients (T2D) without significant complications (Diabetes duration: $12 \pm 9$ yrs (Mean \pm SD); Medication: Life style treatment: $2 / 8$; Oral Hypoglycemic Agents: $3 / 8$; Insulin treatment: $3 / 8$; Blockers of the type 1 angiotensin-II receptor : 2/8; Statins: $3 / 8$ ) and eight age-matched, medication and nicotine free, non-diabetic, healthy control subjects (C) (Age: $62 \pm 2$ vs $59 \pm 4$ yrs (Mean \pm SD), $\mathrm{p}=\mathrm{ns}$; BMI: $31.9 \pm 4.6$ vs $25.1 \pm 4.7 \mathrm{~kg} / \mathrm{m}^{2}, \mathrm{p}<0.001$; Waist/Hip-ratio: $0.94 \pm 0.04$ vs $0.80 \pm 0.10, \mathrm{p}<0.001$; HbA1c: $6,2 \pm 1.4$ vs $4.3 \pm 0.2 \%, \mathrm{p}<0.003$; fP-Glucose: $8.8 \pm 2.2$ vs $5.2 \pm 0.4 \mathrm{mmol} / \mathrm{l}, \mathrm{p}<0.001$; fS-Insulin: $15 \pm 9$ vs $10 \pm 11 \mathrm{mU} / \mathrm{l}, \mathrm{p}=\mathrm{ns}$, respectively) were studied after fasting overnight. Microdialysis measurements in $\mathrm{m}$ brachioradialis in situ, recording of muscle blood flow (MBF) by plethysmography and concomitant sampling of forearm arterial and venous blood was performed before and $3 \mathrm{hrs}$ after oral intake of $10 \mathrm{mg}$ tadalafil. Substrate balance studies and equations according to Fick and Renkin enabled us to estimate FGU and capillary recruitment (PS glucose: permeability surface area, $\mathrm{ml} / 100 \mathrm{~g} \mathrm{x} \mathrm{min)} \mathrm{in}$ response of acute per oral selective PDE5Inh in the fasting state. 
Results: At baseline, PS for glucose was lower in T2D compared to C (median $\left(25^{\text {th}} ; 75^{\text {th }}\right.$ percentiles $):(0.09(0.04 ; 0.25)$ vs $0.28(0.18 ; 0.51) \mathrm{ml} / 100 \mathrm{~g} \mathrm{x}$ min, $\mathrm{p}<0.0001)$, and after PDE5Inh PS increased 4.4-fold in T2D $(\mathrm{p}<0.05)$ and 1.3-fold in C subjects ( $\mathrm{p}=\mathrm{ns})$. FGU was lower in T2D $0.39(0.19 ; 0.55)$ vs $0.63(0.43 ; 0.94) \mu \mathrm{mol} / 100 \mathrm{~g}$ x min, $\mathrm{p}<0.001)$ and increased 2.4 -fold $(\mathrm{p}=\mathrm{ns})$ and 1.7-fold $(\mathrm{p}=\mathrm{ns})$ after PDE5Inh in T2D and $\mathrm{C}$, respectively. Moreover, PDE5Inh induced a 1.3-fold increase of MBF after 90-150 $\min (\mathrm{p}<0.05)$ in both groups and, interestingly, a strong association revealed between AUC PS glucose and AUC forearm glucose uptake in T2D $\left(\mathrm{r}_{\mathrm{s}}: 0.96, \mathrm{p}<0.002\right)$, but this was not seen in $\mathrm{C}\left(\mathrm{r}_{\mathrm{s}}: 0.33, \mathrm{p}=0.15\right)$. During the study period circulating levels of glucose and insulin did not change in $\mathrm{C}$, whereas glucose decreased ca $1.2 \mathrm{mmol} / \mathrm{l}(\mathrm{p}<0.05)$ and insulin tended to go down in T2D subjects. S-FFA remained stable in both groups.

Conclusion: This open non-randomized study implies for the first time acute positive effects of PDE5Inh on capillary recruitment and its close relationship with FGU in insulin resistant skeletal muscle. We suggest further studies to elucidate whether PDE-5 inhibitors has the potential to become novel antihyperglycemic agents in type 2 diabetes.

Supported by: Swedish Medical Research Council, Swedish Diabetes Association and Region Västra Götaland

\section{PS 138 Dyslipidaemia mediated complications}

\section{6}

Postprandial modifications of HDL particle characteristics and endothelial function following 3 consecutive meals in men with and without type 2 diabetes

P.G. Scheffer ${ }^{1}$, M.E. Tushuizen ${ }^{2}$, R.P. Vermue ${ }^{1}$, C. Rustemeijer ${ }^{2}$, R.J. Heine ${ }^{2}$, M. Diamant ${ }^{2}$

${ }^{1}$ Clinical Chemistry, ${ }^{2}$ Endocrinology / Diabetes Centre, VU University Medical Centre, Amsterdam, Netherlands

Background and aims: Type 2 diabetes (T2DM) is associated with low-grade inflammation and oxidative stress, leading to cardiovascular damage. Postprandial dyslipidaemia, which is exaggerated and prolonged in T2DM, has also been associated with cardiovascular disease. We studied the effect of 3 consecutive meals on the composition and function of HDL in men with and without T2DM during a 24-h period.

Materials and methods: Fourteen men with T2DM (mean \pm SD: age $55.5 \pm 4.0$ yrs; BMI $32.7 \pm 4.1 \mathrm{~kg} / \mathrm{m}^{2}$; HbAlc $7.2 \pm 1.1 \%$;), and 14 age-matched healthy men were given 3 standardized high-fat mixed meals $(900 \mathrm{kcal} ; 50 \mathrm{~g}$ fat, $75 \mathrm{~g}$ carbohydrate, $35 \mathrm{~g}$ protein) as breakfast $(\mathrm{t}=0)$, lunch $(\mathrm{t}=4 \mathrm{~h})$ and dinner $(\mathrm{t}=8 \mathrm{~h})$. Blood sampling was performed just before the meals, and $12 \mathrm{~h}$ and $16 \mathrm{~h}$ after breakfast. Flow-mediated dilation (FMD) of the brachial artery was measured by ultrasound before each blood collection. The triglyceride (TG), cholesterol, and protein content were determined in HDL, which was isolated by ultracentrifugation. HDL particle size was measured by high performance gel-filtration chromatography. The anti-oxidative function of HDL was assessed by monitoring its capacity to inhibit the oxidation of dichlorodihydrofluorescein by oxidized 1-palmitoyl-2-arachidonoyl-phosphathidylcholine.

Results: Postprandial glucose and TG responses were increased in T2DM patients compared with controls. In T2DM, glucose peaked at $\mathrm{t}=2 \mathrm{~h}(11.1 \pm 3.2$ $\mathrm{mmol} / \mathrm{L})$ and TG peaked at $\mathrm{t}=6 \mathrm{~h}(3.5 \pm 2.0 \mathrm{mmol} / \mathrm{L})$. At baseline, T2DM, relative to controls tended to smaller HDL particles $(8.87 \pm 0.28$ versus $9.04 \pm 0.35 \mathrm{~nm}$; $P=0.18)$. In the fasting state, HDL from T2DM versus controls contained more TG $(94.2 \pm 27.5$ vs. $65.0 \pm 18.3 \mathrm{nmol} / \mathrm{g} ; P<0.01)$ and less cholesterol $(0.67 \pm 0.11$ vs. $0.92 \pm 0.15 \mathrm{mmol} / \mathrm{g} ; P<0.01)$. HDL-TG content increased postprandially in both groups reaching the highest level after two consecutive meals $(123.3 \pm 41.0$ $\mathrm{nmol} / \mathrm{g}$ for T2DM and $97.5 \pm 24.0 \mathrm{nmol} / \mathrm{g}$ for controls, both $P<0.001)$. The cholesterol content of HDL derived from T2DM was significantly decreased in the postprandial state at $\mathrm{t}=4 \mathrm{~h}(0.63 \pm 0.12 \mathrm{mmol} / \mathrm{g} ; P<0.05)$ and $\mathrm{t}=8 \mathrm{~h}(0.62 \pm 0.10$ $\mathrm{mmol} / \mathrm{g} ; P<0.05)$, but not in controls. FMD at baseline was lower in T2DM compared to controls $(5.0 \%$ vs. $7.6 \% ; P<0.01)$, and decreased postprandially in both groups after breakfast and only in T2DM after dinner (all $P<0.05$ ). At baseline, the anti-oxidative function of HDL was similar in patients and controls. In both groups, a non-statistically significant decrease in the antioxidative capacity of HDL occurred postprandially. In controls, this effect occurred only after the first meal whereas in T2DM it was observed especially after the third meal which was paralleled by a decreased FMD. Subsequently, HDL functionality returned to baseline levels in both groups.

Conclusion: The real-life exposure to 3 consecutive meals and the prolonged study-period allowed us to uncover the full scope of alterations in HDL composition and function in T2DM. Fasting HDL from T2DM had a higher TG-content, whereas HDL from both groups showed a relatively stronger postprandial rise in TG- than cholesterol-content. Postprandially, HDL from T2DM showed a delayed decline in anti-oxidative capacity relative to control HDL, paralleled by endothelial dysfunction.

\section{7}

Treat-to-target strategy for LDL-cholesterol improvement in type 2 diabetes: post-hoc analysis of data from the 2-year follow-up of the MIND.IT study

D. Ardigò ${ }^{1}$, M. Trovati ${ }^{2}$, A.A. Rivellese ${ }^{3}$, O. Vaccaro ${ }^{3}$, F. Cavalot ${ }^{2}$, R. Miccoli ${ }^{4}$, P.P. De Feo ${ }^{5}$, L. Franzini ${ }^{1}$, C. Di Loreto ${ }^{5}$, M. Boemi ${ }^{6}$, L. Patti ${ }^{3}$, I. Zavaroni ${ }^{1}$ ${ }^{1}$ Internal Medicine, Parma University, ${ }^{2}$ Internal Medicine, University of Torino, Italy, ${ }^{3} \mathrm{Clinical}$ and Experimental Medicine, University Federico II, Napoli, ${ }^{4}$ Endocrinology, University of Pisa, ${ }^{5}$ Internal Medicine, University of Perugia, Italy, ${ }^{6}$ Internal Medicine, INRCA-IRCCS, Ancona, Italy

Background and aims: The Multiple INtervention in type 2 diabetes. ITaly (MIND.IT) study is a multi-centric, randomized, nationwide, two-parallel 
arm trial involving 1461 type 2 diabetic patients $(759 \mathrm{M} / 702 \mathrm{~F}$, age $61 \pm 5$ years) at high cardiovascular (CVD) risk, as defined by 2 or more CVD risk factors. The study compares the Usual Care (UC) of CVD prevention with a multifactorial treat-to-target Intensive Care (IC) strategy, following the indications of national and international guidelines. The pre-planned ad-interim analysis after the completion of the first 2 years of follow-up showed a consistent improvement of all major CVD risk factors in the IC arm compared to UC, including LDL-cholesterol (LDL-c). The present work investigates the characteristics of the statin treatment operated by the two groups.

Materials and methods: Ten Italian centers for treatment of diabetes were randomized to the UC or IC arm. As far as the LDL-c is concerned, IC investigators were requested to achieve values $<100 \mathrm{mg} / \mathrm{dL}$ with a step-wise treat-to-target approach, but leaving the freedom to choose drug's molecule and dose. To compare results observed with different statins, daily dose was converted to the Atorvastatin-equivalent dose adopting the following proportion: $10 \mathrm{mg}$ Atorva $=40 \mathrm{mg}$ Prava $=20 \mathrm{mg}$ Simva $=5 \mathrm{mg}$ Rosuva. Lipid profile was assessed at peripheral level, but laboratory performance was certified by an external quality control system and systematic differences among laboratories were corrected before statistical analysis.

Results: Average LDL-c decreased from $144 \pm 35$ to $108 \pm 31 \mathrm{mg} / \mathrm{dL}$ in IC and from $142 \pm 28$ to $118 \pm 32 \mathrm{mg} / \mathrm{dL}$ in UC (p for interaction "intervention*time" $<0.0001$ ). This striking difference between the two groups was associated with a significant increase of statin prescription in out-of-target patient in IC compared to UC ( $45 \%$ vs $14 \%$ within the $1^{\text {st }}$ year, $\left.\mathrm{p}<0.001\right)$, and with a significantly lower withdrawal from treatment ( 28 vs $61 \%$ respectively, $\mathrm{p}<0.001$ ). However, in the out-of-target patients who started statin treatment in the first year, the average prescribed dose was similar between the two groups ( mode $=10 \mathrm{mg} /$ day in $45 \%$ of UC and $49 \%$ of IC, $\mathrm{p}=\mathrm{NS}$ ), resulting in an equivalent target achievement in treated patients (33\% UC and $27 \%$ IC, $p=N S$ ). In addition, IC investigators did not tend to increase dose nor to change drug in patients out-of-target during treatment.

Conclusion: The application of a multi-factorial treat-to-target intervention strategy is associated with a significant improvement in LDL-c beyond what achieved by the usual practice in diabetes care. However, at least in our cohort, the change in LDL-c appears to be more related to an increased number of treated patients and a decreased treatment withdrawal, than to a true treat-to-target approach. The general attitude of physicians to start treatment without increasing the dose or changing active principle when the target is not reached may account for the high percent of patients who remain out of target during "intensive" treatment.

Supported by: FoRiSID

\section{8}

Lipid-altering efficacy of extended-release niacin/laropiprant in dyslipidaemic patients with metabolic syndrome

H.E. Bays ${ }^{1}$, D.L. Maccubbin ${ }^{2}$, A.K. Shah ${ }^{2}$, J. Linn', C.M. Sisk², J.F. Paolini ${ }^{2}$ ${ }^{1}$ Endocrinology \& Metabolism, Louisville Metabolic and Atherosclerosis Research Center Inc. (L-MARC), Louisville, United States, ${ }^{2}$ Merck \& Co., Inc., Rahway, United States

Background and aims: Patients with metabolic syndrome (MetS) are at increased risk for atherosclerotic coronary heart disease (CHD). Current guidelines recommend reducing LDL-C as the primary lipid target to reduce CHD risk. Improvement in other dyslipidemias may further reduce $\mathrm{CHD}$ risk, such as increasing HDL-C and lowering triglyceride (TG) levels. Niacin is a lipid-altering drug that is underutilized due to flushing. Laropiprant (LRPT) reduces niacin-induced flushing, and in combination with extended-release niacin (ERN/LRPT), significantly improves multiple lipid parameters in dyslipidemic patients. This post-hoc subgroup analysis evaluated the efficacy and safety of ERN/LRPT in dyslipidemic patients with MetS, vs. those without MetS, in a Phase III randomized, double-blind, placebo-controlled, 24-week study $(\mathrm{n}=1613)$.

Materials and methods: Patients were randomized 3:2:1 to ERN/LRPT 1g, ERN 1g, or placebo. After 4 weeks, active treatment doses were doubled to $2 \mathrm{~g}$ ( 2 tablets) for the remaining 20 weeks.

Results: Relative to placebo, ERN/LRPT significantly lowered LDL-C and raised HDL-C to a similar degree in MetS vs. non-MetS cohorts (Table). ERN/ LRPT also significantly lowered TG vs. placebo (Table). The placebo-adjusted differences in percent change from baseline in TG were slightly greater in patients with MetS $(-30.2 \%[-36.0,-24.6])$ vs. those without MetS $(-22.2 \%$ [$27.1,-17.4]$ ), which may be due to the higher baseline median TG levels in the MetS group. For all lipid parameters, similar magnitude changes were observed with ERN/LRPT and ERN. ERN/LRPT produced less flushing than ERN during initiation and maintenance of therapy; this effect appeared to be consistent across MetS and non-MetS subgroups. ERN/LRPT was well-tolerated and had a safety profile similar to ERN. ERN/LRPT and ERN similarly resulted in small median increases in fasting blood glucose levels in MetS (2.0 and $4.0 \mathrm{mg} / \mathrm{dL}$, respectively) and non-MetS $(4.0 \mathrm{mg} / \mathrm{dL}$ for both treatment groups) patients, consistent with the known effects of niacin.

Conclusion: In conclusion, ERN/LRPT effectively improves LDL-C, HDL-C and TG levels in patients with and without MetS.

Supported by: Merck \& Co., Inc.

\section{9}

Significance of metabolic components of metabolic syndrome in African American women: HDL-C/Triglyceride ratio and insulin resistance paradox

K. Osei, T. Gaillard

Division of Endocrinology, Diabetes and Metabolism, The Ohio State University, Columbus, United States

Background: Metabolic Syndrome (MetS), defined by The Adult Treatment Panel (ATP) III criteria includes 5 factors that are associated with increased cardiovascular risks. However, whether the 3 metabolic components (serum glucose, triglyceride and HDL-C) carry similar CVD risk burden is unknown. Therefore, we investigated CVD risk factor clusters, prevalence of the components of MetS as well as the prevalence of MetS associated with each of the 3 metabolic components of MetS in nondiabetic, overweight and obese African American women (AAW).

Methods: We studied the clinical and metabolic CVD risk factors of 258 AAW (mean age $42.0 \pm 7.7 \mathrm{yrs}$ and mean BMI, $33.3 \pm 8\left(\mathrm{~kg} / \mathrm{m}^{2}\right)$. Fasting serum insulin, glucose and c-peptide levels were obtained in each subject. Waist circumference (WC), systolic (SBP) and diastolic (DBP) blood pressure were measured. Insulin sensitivity ( $\mathrm{Si}$ ), and insulin resistance (IR) (homeostasis model assessment (HOMA) were calculated. We examined the clusters of CVD risk factors of each tertile of the 3 metabolic components of MetS.

Results: As a group, MetS was $35.5 \%$ in our AAW. Mean age and BMI did not differ with the worsening of any of the metabolic parameters. Increased WC was associated with increased metabolic risks irrespective of metabolic parameters. The prevalence of MetS increased 3 fold from $1^{\text {st }}$ to $3^{\text {rd }}$ tertile of serum glucose, $14.1 \%$ and $42.3 \%$, respectively. The $1^{\text {st }} \mathrm{HDL}-\mathrm{C}$ tertile was associated with increased MetS vs $3^{\text {rd }} \mathrm{HDL}-\mathrm{C}$ tertile ( $42.3 \%$ vs $1.2 \%$, respectively). Comparing $3^{\text {rd }}$ with $1^{\text {st }}$ tertile of triglyceride ( $17 \%$ vs $7 \%$, respectively), there was no significant increase in MetS in our AAW. In contrast, when compar-

Table. Baseline $\rightarrow$ On Treatment $\mathrm{mg} / \mathrm{dL}$ (Percent Change ${ }^{\S}$ from Baseline in Lipid Parameters) by MetS Subgroup

\begin{tabular}{|c|c|c|c|c|c|c|}
\hline \multirow[t]{2}{*}{ Parameter } & \multicolumn{3}{|l|}{ Patients with MetS ${ }^{*}$} & \multicolumn{3}{|c|}{ Patients without MetS } \\
\hline & ERN/LRPT $(n=304)$ & ERN $(n=190)$ & Placebo(n=106) & ERN/LRPT $(n=392)$ & ERN $(n=244)$ & Placebo $(\mathrm{n}=151)$ \\
\hline${\mathrm{LDL}-\mathrm{C}^{* *}}^{*}$ & $\begin{array}{l}102 \rightarrow 83 \\
(-17.5 \%)\end{array}$ & $\begin{array}{l}100 \rightarrow 84 \\
(-17.0 \%)\end{array}$ & $\begin{array}{l}103 \rightarrow 102 \\
(0.3 \%)\end{array}$ & $\begin{array}{l}123 \rightarrow 97 \\
(-19.9 \%)\end{array}$ & $\begin{array}{l}116 \rightarrow 93 \\
(-18.7 \%)\end{array}$ & $\begin{array}{l}122 \rightarrow 119 \\
(-0.7 \%)\end{array}$ \\
\hline $\mathrm{HDL}-\mathrm{C}^{* *}$ & $\begin{array}{l}45 \rightarrow 54 \\
(22.2 \%)\end{array}$ & $\begin{array}{l}43 \rightarrow 53 \\
(22.7 \%)\end{array}$ & $\begin{array}{l}46 \rightarrow 46 \\
(1.1 \%)\end{array}$ & $\begin{array}{l}56 \rightarrow 65 \\
(17.1 \%)\end{array}$ & $\begin{array}{l}55 \rightarrow 65 \\
(18.7 \%)\end{array}$ & $\begin{array}{l}56 \rightarrow 54 \\
(-1.9 \%)\end{array}$ \\
\hline $\mathrm{TG}^{\dagger}$ & $\begin{array}{l}172 \rightarrow 122 \\
(-28.1 \%)\end{array}$ & $\begin{array}{l}171 \rightarrow 120 \\
(-28.0 \%)\end{array}$ & $\begin{array}{l}160 \rightarrow 149 \\
(1.8 \%)\end{array}$ & $\begin{array}{l}107 \rightarrow 89 \\
(-16.9 \%)\end{array}$ & $\begin{array}{l}112 \rightarrow 85 \\
(-18.7 \%)\end{array}$ & $\begin{array}{l}107 \rightarrow 115 \\
(3.7 \%)\end{array}$ \\
\hline
\end{tabular}

"Patients were classified as having MetS if they met $\geq 3$ of the following criteria: waist circumference $>102 \mathrm{~cm}$ for men and $>88 \mathrm{~cm}$ for women; fasting glucose $\geq 100 \mathrm{mg} / \mathrm{dL}$ or history of type 2 diabetes; TGs $\geq 150 \mathrm{mg} / \mathrm{dL} ; \mathrm{HDL}-\mathrm{C}<40 \mathrm{mg} / \mathrm{dL}$ for men and $<50 \mathrm{mg} / \mathrm{dL}$ for women; and systolic blood pressure $\geq 130 \mathrm{mmHg}$ and/or diastolic blood pressure $\geq 85 \mathrm{mmHg}$ or currently taking antihypertensive medication.

${ }^{s}$ Least squares mean for LDL-C and HDL-C and median for TG; ${ }^{* *}$ Mean; ${ }^{\dagger}$ Median 
ing the 3 metabolic components, the prevalence of MetS was higher in the $3^{\text {rd }}$ tertile for glucose (43.2\%), $1^{\text {st }}$ tertile of HDL-C $(42.3 \%)$ and least with the $3^{\text {rd }}$ tertile of triglyceride (17\%). While WC was increased with worsening of the 3 metabolic components, BMI, Si and HOMA-IR as well as BP did not differ among the tertiles.

Conclusion: We found that serum glucose and HDL-C were more predictive of MetS, each yielding at least $40 \%$ of MetS, while triglyceride, BP and insulin resistant indices had the least impact on MetS in our overweight/obese AAW. We propose 1) that the 3 metabolic parameters for MetS defined by ATP III should be weighted differently with respect to their potential for CVD risks and 2) that nondiabetic overweight/obese AAW in our $3^{\text {rd }}$ tertile of serum glucose $(>100 \mathrm{mg} / \mathrm{dl})$ and/or $1^{\text {st }}$ tertile of HDL-C $(<40 \mathrm{mg} / \mathrm{dl})$ should be targeted for screening for MetS.

Supported by: NIDDK DK-48127 and the General Clinical Research Center

\section{0}

Inadequate attainment of lipid levels in patients with or without type 2 diabetes and/or cardiovascular disease

G.A. Nichols ${ }^{1}$, B.M. Ambegaonkar ${ }^{2}$, V. Sazonov ${ }^{2}$, J.B. Brown ${ }^{1}$

${ }^{1}$ Center for Health Research, Kaiser Permanente, Portland, ${ }^{2}$ Merck \& Co., Inc., Whitehouse Station, United States

Background and aims: High density lipoprotein cholesterol (HDL-C) and triglycerides (TG) are secondary treatment targets for reduction of coronary heart disease risk in certain patient populations. However, these targets are often not addressed. Our objective was to document lipid recommended level attainment before and after initiation of dyslipidemia pharmacotherapy in patients with vs. without type 2 diabetes (T2DM) and/or cardiovasculat disease (CVD).

Materials and methods: We identified 5158 patients who initiated lipid modifying therapy (LMT) between July 2004-June 2006, were age $\geq 35$ years, continued LMT for $\geq 1$ year, and had full lipid panels 12 months pre- and 9-15 months post LMT initiation date. LDL-C goals and recommended levels for TG and HDL-C were defined according to updated NCEP ATP III guidelines. We classified patients into 4 groups: $T 2 D M+C V D, \mathrm{n}=261 ; T 2 D M$ only, $\mathrm{n}=1661 ; C V D$ only, $\mathrm{n}=698 ;$ NEITHER condition, $\mathrm{n}=2538$. We calculated the proportion in each group that met LDL-C, HDL-C and TG goals/recommended levels pre and post LMT initiation. Logistic regressions predicted lipid target attainment adjusting for age, sex, BMI, hypertension, mean statin dose, and baseline lipid levels.

Results: Overall, mean age was 62.5 years and $50 \%$ were women. Prior to LMT initiation, $33 \%$ of T2DM+CVD met LDL-C goals, compared to $20 \%$, $22 \%$ and $24 \%$ of T2DM, CVD , and NEITHER, respectively ( $<<0.001$ for all 3 comparisons). About $60 \%$ of all T2DM patients were at recommended TG level compared to $77 \%$ of $C V D$ and $68 \%$ of NEITHER ( $\mathrm{p}<0.001)$. Only $36 \%$ of T2DM+CVD, $40 \%$ of T2DM, $54 \%$ of $C V D$, and $59 \%$ of NEITHER were at recommended HDL-C levels ( $\mathrm{p}<0.001$ between all groups). A majority of the patients (96\% in each group) initiated statin monotherapy. Following 1 year of LMT, $89 \%$ of $T 2 D M+C V D, 75 \%$ of $T 2 D M, 72 \%$ of $C V D$, and $81 \%$ of NEITHER had attained LDL-C goal $(\mathrm{p}<0.001$ all comparisons except T2DM vs. $C V D$ ). After multivariate adjustment, $T 2 D M+C V D$ were significantly less likely to attain LDL-C goal relative to NEITHER (odds ratio $0.60,95 \% \mathrm{CI}$ $0.38-0.94)$, as were the T2DM $(0.27,0.22-0.33)$ and CVD $(0.26,0.20-0.33)$ groups. Attainment of recommended TG level improved among all patients (66\% pre-LMT vs. $80 \%$ post-LMT), with little difference between groups. Neither T2DM nor CVD status predicted TG recommended level attainment. Attainment of recommended HDL-C levels did not improve with LMT. Only about $33 \%$ of $T 2 D M+C V D, 39 \%$ of T2DM, $51 \%$ of $C V D$, and $58 \%$ of $N E I-$ THER had HDL-C above recommended levels post-LMT ( $\mathrm{p}<0.001$ except $T 2 D M+C V D$ vs. $T 2 D M)$. Relative to NEITHER patients, T2DM+CVD $(0.58$, $0.39-0.86)$ and $T 2 D M$ patients $(0.82,0.68-0.99)$ were less likely to attain recommended HDL-C levels.

Conclusion: Patients initiating dyslipidemia pharmacotherapy often achieved LDL-C goals, probably due to emphasis on statin therapy. However, HDL-C was largely unaddressed, especially among patients with T2DM and known CVD.
Goal/Recommended Level Attainment Before and After Lipid Modifying Therapy (LMT)

\begin{tabular}{llllll}
\hline & $\begin{array}{l}\text { T2DM+ } \\
\text { CVD } \\
(\mathrm{n}=261)\end{array}$ & $\begin{array}{l}\text { T2DM } \\
\text { Only } \\
(\mathrm{n}=1,661)\end{array}$ & $\begin{array}{l}\text { CVD } \\
\text { Only } \\
(\mathrm{n}=698)\end{array}$ & $\begin{array}{l}\text { Neither } \\
\text { Condition } \\
(\mathrm{n}=2,538)\end{array}$ & $\begin{array}{l}\text { Total } \\
(\mathrm{n}=5,158)\end{array}$ \\
\hline \% Attainment Before LMT & & & & \\
\hline LDL-C & $32.6 \%$ & $20.2 \%$ & $22.3 \%$ & $23.7 \%$ & $22.8 \%$ \\
Triglycerides & $60.5 \%$ & $59.7 \%$ & $76.9 \%$ & $68.2 \%$ & $66.3 \%$ \\
HDL-C & $36.0 \%$ & $40.2 \%$ & $54.9 \%$ & $58.8 \%$ & $51.1 \%$ \\
\hline \% Attainment After LMT & & & & \\
\hline LDL-C & $88.9 \%$ & $74.5 \%$ & $71.9 \%$ & $80.6 \%$ & $77.9 \%$ \\
Triglycerides & $77.0 \%$ & $76.3 \%$ & $87.4 \%$ & $81.0 \%$ & $80.1 \%$ \\
HDL-C & $32.6 \%$ & $39.0 \%$ & $50.9 \%$ & $58.0 \%$ & $49.7 \%$ \\
\hline
\end{tabular}

Supported by: Merck \& Co., Inc.

\section{1}

Cholesterol does not predict mortality in patients with type 2 diabetes of old age (ZODIAC-12)

S.T. Houweling ${ }^{1,2}$, N. Kleefstra ${ }^{1,2}$, S.J.J. Logtenberg ${ }^{1}$, G.W.D. Landman ${ }^{3}$, A.M. Kamper ${ }^{3}$, K.H. Groenier ${ }^{4}$, H.J.G. Bilo ${ }^{1,5}$

${ }^{1}$ Diabetes Centre, Isala clinics, Zwolle, ${ }^{2}$ Medical Research Group, Langerhans, Zwolle, ${ }^{3}$ Internal Medicine, Isala clinics, Zwolle, ${ }^{4}$ General Practice, UMCG, Groningen, ${ }^{5}$ Internal Medicine, UMCG, Groningen, Netherlands

Background and aims: Large intervention studies have been undertaken to study the effects of lipid lowering drugs in patients with type 2 diabetes (T2DM). In many of these studies 75 years (yrs) is the maximum age of inclusion. In daily diabetes practice we treat a lot of patients above $75 \mathrm{yrs}$ of age and often the questions arises whether a poor lipid profile is associated with increased risk in these higher age categories, and whether there is a benefit of statins. Therefore, we analyzed mortality risk in association with total cholesterol to HDL-cholesterol ratio (Chol/HDL) in patients with T2DM both below and above the age of 75 yrs.

Materials and methods: The ZODIAC study started in 1998 as part of a large shared care diabetes project in the Netherlands. Data on mortality were assessed after 5.8 yrs. We used Cox proportional hazard modeling to investigate the association between Chol/HDL and (cardiovascular and all-cause) mortality. The following variables were included in the model as confounders: Gender, smoking (yes, previous or no), duration of diabetes, estimated glomerular filtration rate, BMI, systolic blood pressure, macrovascular complications (yes or no), use of statins (yes or no) and albuminuria (yes or no). Results: Our study population consisted of 653 women and 490 men. Median (interquartile range (IR)) $\mathrm{HbA}_{1 c}$ at baseline was 7.3\% (6.6-8.3), 7.3\% (6.6-8.3) and $7.2 \%$ (6.5-8.2) for the total group, group $\leq 75 \mathrm{yrs}$ and group $>75 \mathrm{yrs}$, respectively. Mean (SD) Chol/HDL was 5.24 (1.59), 5.34 (1.56) and 4.93 (1.64) for the total group, group $\leq 75$ yrs and group $>75$ yrs, respectively. During follow up, 330 patients died. There were 851 patients aged $\leq 75 \mathrm{yrs}$ at baseline of whom 153 died (18\%) during follow up. There were 292 patients aged $>75 \mathrm{yrs}$ at baseline of whom 177 died (61\%) during follow up. Below or above the age of $75,13.2 \%$ and $4.5 \%$ of patients were on lipid lowering therapy, respectively. Hazard ratios (HRs) with $95 \%$ confidence intervals (95\% CI) of Chol/HDL as a continuous variable for all-cause and cardiovascular mortality, without adjustment for confounders were 1.09 (1.03-1.14) and 1.14 (0.99-1.32), respectively at age $\leq 75 \mathrm{yrs}$. For subjects aged $>75$ yrs, HRs $(95 \% \mathrm{CI})$ were 0.99 (0.88-1.11) and 1.07 (0.95-1.21) for all-cause and cardiovascular mortality, respectively. When adjusted for selected confounders HRs (95\%CI) were 1.10 (1.04-1.16) and 1.17 (0.99-1.38) for all-cause and cardiovascular mortality, respectively at age $\leq 75$. For those aged $>75$ yrs, these HRs $(95 \% \mathrm{CI})$ were 0.95 (0.83-1.09) and 0.98 (0.84-1.14), respectively. HRs (95\%CI) for having a lipid lowering drug were $1.25(0.99-1.59)$ and $2.00(0.83-4.78)$ for all-cause and cardiovascular mortality, respectively, at age $\leq 75$. For age $>75 \mathrm{yrs}$, these HRs (95\%CI) were $0.69(0.43-1.74)$ and 0.38 (0.09-1.64), respectively.

Conclusion: In patients with T2DM above the age of $75 \mathrm{yrs}$, Chol/HDL does not seem to predict (cardiovascular) mortality. There were too little patients in this study on lipid lowering treatment to draw definite conclusions about long-term effect of statins in this age category. Intervention trials with statins, especially in elderly patients with T2DM, are needed to further evaluate the efficacy of statins in this specific patient category. 


\section{2}

Genetic interaction between CETP and LIPC genes in risk of type 2 diabetes in the canary population: the Telde study

L. López Ríos ${ }^{1}$, A.M. Wägner ${ }^{1}$, M. Boronat ${ }^{1}$, F. Varillas ${ }^{1}$, D. Marrero ${ }^{1}$, N. Pérez ${ }^{1}$, Y. García ${ }^{1}$, R. Chirino² ${ }^{2}$ J. Nóvoa ${ }^{1}$

${ }^{1}$ Endocrinology, Hospital Universitario Insular de Gran Canaria,

${ }^{2}$ Physiology, Universidad de Las Palmas de Gran Canaria, Spain

Background and aims: Diabetic dyslipidaemia is common in type 2 diabetes (T2D) and insulin resistance (IR) and often precedes the onset of T2D. It includes elevated triglycerides (TG), low high-density lipoprotein (HDL) cholesterol (c) and small, dense low-density lipoprotein (LDL) particles. In addition, an increase in free-fatty acids is associated with IR and lipotoxicity, which may play a role in the development of T2D. Thus, genes involved in lipoprotein metabolism may be good candidates for the risk of T2D. In normotriglyceridaemia Cholesteryl Ester Transfer Protein (CETP) balances cholesterol and TG content in LDL, VLDL and HDL particles. In hypertriglyceridaemic conditions, CETP leads to an enrichment of LDL and HDL particles in TG. This makes them more prone to hydrolysis by hepatic lipase (HL), leading to the production of small, dense particles. LDL particles become more atherogenic and HDL, less protective. The Taq1B polymorphism in CETP is associated with increased enzyme activity and reduced HDLc. In addition, the G-250A polymorphism in LIPC (the gene encoding HL) is associated with changes in enzyme activity and lipid concentrations. Our aim was to asses the effects of Taq1B CETP and G-250A LIPC polymorphisms on changes of lipid profile and their influence on T2D.

Materials and methods: A population-based sample of 1030 people from the city of Telde (Gran Canaria, Canary Islands, Spain) was classified according to the results of a glucose tolerance test and assessed for the presence of cardiovascular risk factors. A total of 116 patients with T2D, 115 patients with impaired glucose regulation (impaired glucose tolerance and/or impaired fasting glucose) (IGR) and 226 controls matched for gender and age were genotyped. The Taq1B polymorphism was determined using RFLP-PCR while the G-250A LIPC was analysed with ARMS-PCR. For statistical analysis, we used chi-square test and logistic regression.

Results: The frequency of the B1B1 genotype increases with worsening glucose metabolism ( $42.5 \%$ controls, $46.1 \%$ in IGR and $54.3 \%$ diabetic population; $\mathrm{p}=0.042$ ). Although the GG genotype from LIPC was not significantly different between the groups, its frequency tended to increase with worsening glucose metabolism ( $47.13 \%$ control, $49.5 \%$ in IGR and $53.1 \%$ diabetic population; $\mathrm{p}>0.1$ ). To analyse the independent effect of each polymorphisms on diabetes prevalence, multinomial logistic regressions were used. After adjustment for age, sex, BMI, waist and B1B1 genotype of Taq1B CETP were associated with an increased risk of diabetes (OR: 1.828; IC 95\%: 1.12-2.99; $\mathrm{p}=0.016$ ), but not of IGR (OR: 0.525; IC95\%: 0.68-1.74; $\mathrm{p}=\mathrm{ns}$ ). The LIPC polymorphism was not associated with diabetes. Regarding genetic interaction, LIPC polymorphism did not influence the risk of diabetes in non-B1B1 carriers. However, in B1B1 genotype carriers, the presence of the minor allele in the LIPC polymorphism (A allele) reduced the risk of having diabetes (OR: 1.32; IC95\%: 0.71-2.44), while its absence increased this risk (O.R: 2.42; IC95\%: 1.30-4.50) $(\mathrm{P}=0.036)$.

Conclusion: The presence of the B1B1 Taq1B CETP genotype contributes to the risk of T2D, independently of age, sex, BMI and waist. However, among carriers of B1B1, allele A of the -250 LIPC polymorphism reduces this risk. Supported by: Fundación Canaria de Investigación y Salud (FUNCIS)

\section{3}

Increased hippocampal neurogenesis in a spontaneous model of type 2 diabetes (T2D), the Goto-Kakizaki (GK) rat

F.E. Saravia ${ }^{1}$, J. Beauquis ${ }^{1}$, J. Coulaud ${ }^{2}$, P. Roig $^{1}$, B. Portha ${ }^{2}$,

F. Homo-Delarche ${ }^{2}$, A.F. De Nicola ${ }^{1}$

${ }^{1}$ Neuroendocrine Biochemistry, Institute of Biology and Experimental

Medicine, Buenos Aires, Argentina, ${ }^{2} \mathrm{Cnrs}$ umr 7059, Paris-Diderot

University, Paris, France

Background and aims: Diabetes, regardless of the type, is associated with central nervous system (CNS) complications. Indeed, metabolic and vascular consequences of diabetes induce cognitive impairment and increase the risk of depression, stroke, dementia and Alzheimer disease. The hippocampus, part of the limbic system, is a particular CNS target of diabetic alterations, both in patients and animal models. More precisely, the dentate gyrus (DG) of the hippocampus is one of the rare brain areas, where new neurons -associ- ated to memory and learning process- are generated during adulthood. We previously showed that adult hippocampal neurogenesis is altered in animal models of type 1 diabetes. Here, we explored hippocampal neurogenesis, and vascularisation, as a key component of the neurogenic microenvironment in a spontaneous model of T2D, the GK rat.

Materials and methods: Four-month-old GK and control male Wistar (W) rats were used, $\mathrm{n}=4-5$. Bromodeoxyuridine (BrdU, $250 \mathrm{mg} / \mathrm{k} \mathrm{BW}$ ) was administered i.p. 21 days before cardiac perfusion with $3 \%$ paraformaldehyde. DG containing brain sections (vibratome) were used for immunohistochemistry with the following markers: 1) Ki67 (cellular proliferation); 2) BrdU (cell survival); 3) doublecortin (DCX, neuronal differentiation); and 4) von Willebrand factor (vWF, endothelium). Data were evaluated by computer-assisted image analysis.

Results: The number of proliferative Ki67 ${ }^{+}$DG cells was twice in GK than in W rats: $3309 \pm 362$ and $1496 \pm 227$, respectively, $\mathrm{p}<0.02$. BrdU ${ }^{+}$cell number -indicator of cell survival- was slightly increased in GK vs W. Neuronal maturation of new DG cells followed the same pattern: $\mathrm{DCX}^{+}$cells were twice as much in diabetic animals as compared to controls: $15776 \pm 745.3$ and 9923 \pm 1356 respectively, $\mathrm{p}<0.01$. Finally, the ratio $\mathrm{vWF}^{+}$vessel area $/ \mathrm{DG}$ area was decreased by more than $50 \%$ in diabetic animals: GK: $0.033 \pm 0.004$, W: 0.078 $\pm 0.013, \mathrm{p}<0.05$.

Conclusion: A net increase in hippocampal neurogenesis, together with increased cell proliferation and survival, was observed in type 2 diabetic GK rats. Interestingly, high neurogenesis rates have been observed in pathological situations, such as brain trauma, seizures and ischemia and diabetes is correlated with augmented risk of cerebrovascular disease. Macro- and microangiopathy have been described in GK rats in the cerebrovasculature and the islet of Langerhans, respectively. Therefore, cerebrovascular remodelling induced by chronic hyperglycemia/hyperlipidemia in GK rats might be responsible for their high DG neurogenesis, as corroborated by our data showing a markedly diminished blood vessel area.

Supported by: Universidad de Buenos Aires, Agencia Nacional de Promoción Científica y Tecnológica, Naturalia et Biologia and INSERM-CONICET

\section{4}

Effect of oral glucose load on triglyceridaemia and cholesterolaemia: does insulin sensitivity make a difference and is increasing post oral glucose load insulinaemia beneficial?

A. Major-Pedersen ${ }^{1}$, H. Dominguez ${ }^{2}$, T.S. Hermann ${ }^{3}$, N. Ihlemann ${ }^{4}$, B. Kveiborg ${ }^{5}$, B. Christiansen ${ }^{6}$, L. Køber ${ }^{4}$, C. Torp-Pedersen ${ }^{6}$

${ }^{1}$ Endocrinology M, Odense University Hospital, Denmark, ${ }^{2}$ Cardiology, Gentofte University Hospital, Denmark, ${ }^{3}$ Cardiology Clinic Y, Bispebjerg Hospital, Bispebjerg, Denmark, ${ }^{4}$ Cardiology, Rigshospital University Hospital, Copenhagen, Denmark, ${ }^{5}$ Cardiology, Gentofte University Hospital, Odense C, Denmark, ${ }^{6}$ Cardiology, Gentofte University Hospital, Copenhagen, Denmark

Background and aims: Dyslipidemia is an independent risk factor for cardiovascular disease, even before the development of overt diabetes. We investigated the action of oral glucose load on triglyceridemia in both insulin sensitive and insulin resistant individuals, and whether acute and chronic stimulation of first phase insulin secretion (with nateglinide) modified the postprandial triglyceride response. We also investigated the effect of chronic insulin secretion stimulation on total choleserol and sub-classes.

Materials and methods: We assessed post oral glucose load triglyceridemia (at $0,15,30,60,120,180,240$ minutes) in 56 insulin resistant subjects randomized to intervention/ control groups [Homeostasis Model Assessment (HOMA) $6.41 \pm 0.7]$, at baseline, "BL day", and after 3 months natelginide treatment/observation, "Closing day". Postprandial trygliceridemia was assessed on two additional days in the intervention group: On "Acute day" where an initial dose of nateglinide was administered before oral glucose load, and on "Acute+chronic day" where the intervention group met for a last nateglinide dose. We examined post-oral glucose triglyceridemia in 9 healthy, insulin-sensitive subjects [HOMA $0.65 \pm 0.13$ ]. Total cholesterol and sub-classes were measured in the healthy group and on $\mathrm{BL}$ and Closing days in intervention and control groups. Change in triglyceride concentrations between the different time points, within the same day, was analyzed with paired Student's t-test.

Results: Glucose load in the healthy group provoked a sharp fall in triglyceridemia at 2 hours post oral glucose load [ $(1.11 \pm 0.07)$ vs $(0.65 \pm 0.08), \mathrm{p}=0.0001]$. We found no difference in triglyceridemia when we compared post oral glucose load values to pre glucose load values, in the intervention group on $\mathrm{BL}$ and Closing days. On Acute day, triglyceridemia at 2 hours post glucose load 
decreased compared to pre-load values.[(1.59 \pm 0.15$)$ vs $(1.49 \pm 0.15), \mathrm{p}=0.05]$. On Acute+chronic day, 2 hour post load triglyceride values were increased $[(1.38 \pm 0.11)$ vs $(1.47 \pm 0.20) \mathrm{p}=0.05$. In the control group, there was no change in post load vs pre load triglyceridemia on Closing day. High density lipoprotein cholesterolemia decreased in the intervention group: BL day vs Closing day respectively $[(1.14 \pm 0.04)$ vs $(1.09 \pm 0.04), \mathrm{p}=0.04]$. No other cholesterol changes were observed.

Conclusion: Our findings suggest that acute stimulation of first phase insulin secretion decreases triglyceridemia in insulin resistant individuals, mimicking the beneficial glucose load effect on triglyceridemia we observed in insulin-sensitive individuals. Suprisingly, however, repeated insulin stimulation in insulin resistant individuals not only seems to augment triglyceridemia, but seems to reduce high density lipoproteinemia. These findings could impact the choice of blood glucose regulating therapeutic agents in the presence of insulin resistance, especially in light of the increased cardiovascular risk that accompanies this state.

Supported by: Novartis and the Danish Diabetes Association

\section{PS 139 Non-classical risk factors and markers of inflammation}

\section{5}

Nonclassical cardiovascular risk factors in young and apparently healthy, nonobese subjects with low insulin sensitivity

M. Straczkowski, I. Kowalska, M. Karolczuk-Zarachowicz, A. Adamska, M. Karczewska-Kupczewska, A. Nikolajuk, M. Gorska

Department of Endocrinology, Diabetology and Internal Medicine, Medical University of Bialystok, Poland

Background and aims: Insulin resistance is an important risk factor for type 2 diabetes and cardiovascular disease. Both conditions are associated with chronic low-grade inflammation and endothelial dysfunction. Proinflammatory cytokines, like IL-18, and adhesion molecules, like soluble intercellular adhesion molecule 1 (sICAM-1), are predictors of type 2 diabetes and are considered as 'nonclassical' risk factors for cardiovascular disease. The aim of the present study was to estimate serum concentrations of IL-18, sICAM-1, monocyte chemoattractant protein 1 (MCP-1) and high-sensitive C-reactive protein (hsCRP) in young and apparently healthy nonobese subjects with low insulin sensitivity.

Materials and methods: The study group consisted of 102 apparently healthy, young (age at examination between 18 and 30 years), nonobese (BMI $<30 \mathrm{~kg}$ $\mathrm{x} \mathrm{m}^{-2}$ ) subjects, 51 men and 51 women. All participants had fasting plasma glucose below $100 \mathrm{mg} / \mathrm{dl}$ and all had normal glucose tolerance in the OGTT according to the WHO criteria. Anthropometric and biochemical parameters and serum concentrations of IL-18, sICAM-1, MCP- 1 and hsCRP were measured. Insulin sensitivity was estimated with the euglycemic hyperinsulinemic clamp technique. Then, subjects were divided into 3 groups according to their insulin sensitivity (high, medium and low insulin sensitivity). The tertiles of insulin sensitivity were calculated for men and women separately. In statistical analysis, we used one-way ANOVA to assess differences between the groups and Pearson product-moment correlation and multiple regression analysis to assess the relationships between variables. Parameters, which did not have normal distribution, were log-transformed before analyses.

Results: The study groups did not differ in anthropometric and serum lipid parameters. In the entire group, we observed significant differences in se-

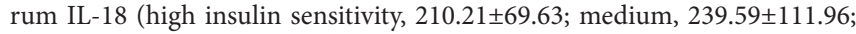
low, $295.52 \pm 106.28 \mathrm{pg} / \mathrm{ml} ; \mathrm{p}=0.0008$ ), sICAM-1 (high insulin sensitivity, 235.66 \pm 39.15 ; medium, $258.15 \pm 57.29$; low, $277.10 \pm 38.55 \mathrm{ng} / \mathrm{ml} ; \mathrm{p}=0.0015$ ) and hsCRP (high insulin sensitivity, $1.37 \pm 0.39$; medium, $1.60 \pm 0.68$; low $1.86 \pm 0.55 \mathrm{mg} / \mathrm{l} ; \mathrm{p}=0.002)$ concentrations between the groups. Additionally, we found differences in serum IL-18 $(\mathrm{p}=0.008)$ in the male group and in serum sICAM-1 $(\mathrm{p}=0.01)$ and hsCRP $(\mathrm{p}=0.0038)$ in the female group, with the highest values in subjects with low insulin sensitivity. Serum MCP-1 did not differ between the study groups. Serum IL-18, sICAM-1 and hsCRP were inversely related to insulin sensitivity in the entire study group $(\mathrm{r}=-0.33$, $\mathrm{p}=0.001 ; \mathrm{r}=-0.32, \mathrm{p}=0.001$ and $\mathrm{r}=-0.28, \mathrm{p}=0.005$, respectively). These associations were independent of potential confounding factors. No correlation between serum MCP-1 and insulin sensitivity was found.

Conclusion: Our data indicate that in young, nonobese subjects with low insulin sensitivity, there is an accumulation of nonclassical cardiovascular risk factors. Early detection of insulin resistance might be useful in identifying subjects at risk of cardiovascular disease.

Supported by: Medical University of Bialystok, Poland

\section{6}

Insulin resistance and metabolic disturbances are inversely related to lung function in Japanese

S. Jimba ${ }^{1}$, T. Nakagami ${ }^{1}$, J. Wada ${ }^{1}$, S. Fukushima ${ }^{1}$, M. Hasegawa ${ }^{1}$,

T. Wasada ${ }^{2}$, Y. Iwamoto ${ }^{1}$

${ }^{1}$ Diabetes Center, Tokyo Women's Medical University, Tokyo, ${ }^{2}$ Department of Internal Medicine, Saitama-ken Saiseikai Kurihashi Hospital, Saitamaken, Japan

Background and aims: Recently, it has been reported that lung dysfunction expressed by declined forced vital capacity (FVC) or forced expiratory volume in one second (FEV1) is a risk factor for cardiovascular events and death. Some prospective studies show that middle-aged individuals with lung dysfunction have increased risk of developing hypertension (HT), insulin resistance (IR) and diabetes (DM). However, there are very few studies showing 
the relationship between lung function with IR and metabolic disturbances in a large general population. Thus, our aim of this study is to examine whether lung dysfunction is independently associated with IR and several metabolic disorders among healthy Japanese adults.

Methods: This is a cross-sectional study in a general health examination programme at S.S.K Hospital from Oct '06 to Sep '07. We examined the data of 2,195 subjects (men: $67 \%$, mean age: $51 \pm 9$ years) who did not have previous $\mathrm{DM}$, cancer, endocrine, respiratory and congestive heart disease or were not under pharmacological treatments for HT or dyslipidemia. FVC and FEV1 were measured by spirometer. The homeostasis model assessment (HOMA) was calculated as: fasting immuno reactive insulin (F-IRI) $(\mu \mathrm{U} / \mathrm{mL}) \times$ fasting plasma glucose $(\mathrm{FPG})(\mathrm{mmol} / \mathrm{L}) / 22.5$ among those with fasting plasma glucose $\leq 6.10 \mathrm{mmol} / \mathrm{l}$. Smoking status and physical activity were asked by questionnaires. The multivariate linear regression models were used to evaluate whether lung function (FVC, FEV1) is independently associated with metabolic variables, F-IRI and HOMA.

Results: The average FVC and FEV1 were $109 \pm 14 \%$ and $2.87 \pm 0.65 \mathrm{~L}$. FVC was correlated with age (Spearman's correlation coefficients: -0.142), BMI $(-0.129)$, waist circumference $(\mathrm{Wc})(-0.117)$, systolic blood pressure (sBP) $(-0.124)$, diastolic blood pressure (dBP) $(-0.095)$, FPG $(-0.081)$, HbAlc ($0.130)$, triglyceride (TG) (-0.143), high density lipoprotein cholesterol (HDLC)(0.128). FEV1 was also significantly correlated with those variables. The multiple linear regression analysis showed that $\mathrm{FVC}$ was related with $\mathrm{HbAlc}$ $(\beta=-0.002, p=0.001), \mathrm{TG}(\beta=-0.332, p=0.001), \mathrm{sBP}(\beta=-0.077, p=0.001)$, HDL-C $(\beta=0.078, p=0.000)$, F-IRI $(\beta=-0.030, p=0.000)$ and HOMA $(\beta=-$ $0.007, p=0.000)$ after adjusting for age, sex, Wc, smoking status and physical activity. Further additional analysis adjusting for F-IRI showed that these relations were not changed in $\mathrm{HbAlc}, \mathrm{sBP}, \mathrm{HDL}-\mathrm{C}$. FEV1 was also associated with HbAlc $(\beta=-0.080, p=0.000)$, TG $(\beta=-11.937, p=0.000), \mathrm{sBP}(\beta=-2.609$, $p=0.001), \mathrm{dBP}(\beta=-1.323, p=0.027)$, HDL-C $(\beta=3.381, p=0.000)$, F-IRI $(\beta=$ $-0.823, p=0.000)$ and HOMA $(\beta=-0.183, p=0.000)$ after adjusting for age, sex, Wc, smoking status and physical activity. These associations were not changed in TG, sBP, dBP, and HDL-C, even after additional adjusting for F-IRI.

Conclusion: Decline in FVC or FEV1 was related with the deterioration of blood pressure, lipids and glucose metabolism, independently of IR. This may suggest that hypoxia and inflammation induced by lung dysfunction cause metabolic disorders with or without mediation of IR.

Supported by: Japan Medical Women's Association, Tokyo Women's Medical University Association, and the Ministry of Health, Labour and Warfare, Japan

\section{7}

Chronic obstructive pulmonary disease prevalence and severity in type 2 diabetic patients correlate to glycometabolic control and the presence of complications and co-morbidities

M.E. Lunati ${ }^{1}$, T. Cappelletti ${ }^{2}$, B. Masserini ${ }^{1}$, L.F. Sburlati ${ }^{1}$, V. Valenti ${ }^{3}$,

F.B. Blasi ${ }^{2}$, L. Allegra ${ }^{2}$, P. Beck-Peccoz ${ }^{1}$, E. Orsi ${ }^{1}$

${ }^{1}$ Department of Medical Science, Endocrinology and Diabetes Unit, IRCCS, Foundation Policlinico, Mangiagalli e Regina Elena, ${ }^{2}$ Institute of Respiratory Disease, IRCCS, Foundation Policlinico, Mangiagalli e Regina Elena, ${ }^{3}$ Institute of Respiratory Disease, IRCCS, Policlinico San Donato Milanese, Milan, Italy

Background and aims: Chronic Obstructive Pulmonary Disease (COPD) and diabetes mellitus are worldwide chronic diseases that represent leading causes of morbidity and mortality related to cardiovascular diseases. COPD is associated with low-grade systemic inflammation with increased levels of pro-inflammatory cytochines (TNF- $\alpha$, IL-6, IL-8). Increasing evidence points toward a role of these cytochines in the pathogenesis of insulin resistance, type 2 diabetes and its correlated complications. The aim of this study was to determine the prevalence of COPD in outpatients with type 2 diabetes and to assess a possible correlation with glycometabolic control, micro/macrovascular complications and co-morbidities such as dyslipidemia and obesity.

Materials and methods: We enrolled 152 consecutive type 2 diabetic outpatients attending the Endocrinology and Diabetes Unit (M:F 78:74, (mean \pm SD) age $65 \pm 10$, BMI $29 \pm 5$, fasting glycaemia $139 \pm 40$, HbAlc $7.04 \pm 0.99$ ). Spirometry was performed in all patients and COPD severity was classified according to GOLD guidelines. Patients were divided in subgroups depending on the degree of glycometabolic control ( $\mathrm{HbAlc} \leq 1>7 \%)$ and the presence of long-term complications and co-morbidities (yes/no).

Results: We identified 18 patients (prevalence $11.8 \%$, which is much higher than that reported in the general population in Italy, i.e. 3.6\%) with COPD (FVC $72 \pm 15 \%$, FEV1 55 $\pm 17 \%$ ). In patients with good glycometabolic con- trol COPD was lower than in those with less tight control ( $8.4 \%$ vs $15.9 \%$, $\mathrm{p}=0.008$ ). COPD prevalence in the subgroup of patients with complications was $15.8 \%$ compared to $7.9 \%$ in the subgroup without complications $(\mathrm{p}=0.017)$. In particular, COPD prevalence was significantly increased in diabetics with microalbuminuria, isolated or associated with cardiovascular events such as acute myocardial infarction and/or stroke, versus non- complicated subjects ( $18 \%$ vs $7.9 \%, \mathrm{p}<0.001$ and $31 \%$ vs $7.9 \%, \mathrm{p}<0.05$ respectively). Moreover, the subgroup with complications showed a worse respiratory function than that without (FVC: $66 \pm 12 \%$ vs $82 \pm 15 \%, \mathrm{p}=0.028$ and FEV1: $49 \pm 16 \%$ vs $68 \pm 12 \%, p=0.023$ ). Conversely, diabetic patients with dyslipidemia showed a lower COPD prevalence than non- dyslipidemic subjects $(10 \%$ vs $20 \%, p<0.001)$ and the percentage of COPD was also lower in obese patients, as compared to normal-weight subjects ( $7 \%$ vs $28 \%, \mathrm{p}<0.01$ ). The severity of obstruction did not appeared to be significantly affected by the presence of these co-morbidities.

Conclusion: Our data show an increased prevalence of COPD in type 2 diabetic patients with less tight glycometabolic control, long-term vascular complications and possibly worse nutritional status. We also found a correlation between the severity of obstruction and the presence of chronic complications. These findings might be related to the higher oxidative stress and low-grade inflammatory state which play an important role in both disease conditions.

\section{8}

Adenovirus induced decorin transfection inhibits pulmonary fibrosis in diabetic rats

H. Li, F.P. Zheng, F. Wu, W.N. Lu, Y. Ruan, F.-B. Zhang

Endocrinology and Metabolism, Sir Run Run Shaw Hospital, College of

Medicine, Zhejiang University, Hangzhou, China

Background and aims: To investigate the relation between proteoglycan II (Decorin, DCN) and transforming growth factor- $\beta 1$ (TGF- $\beta 1$ ) in the lung of diabetic rats and to evaluate the effect of adenovirus induced DCN transfection on diabetic pulmonary fibrosis in different stages.

Materials and methods: 84 SD rats were divided into 4 groups at random: the diabetic group (diabetes was induced by a single intraperitoneal injection of $65 \mathrm{mg} / \mathrm{kg}$ streptozotocin (STZ), group D), the decorin recombinant adenovirus transfection group (diabetic rats treated with a intraperitoneal injection of decorin recombinant adenovirus every other week starting 4 weeks after the diabetic model established, group AD-DCN), the Lac- $\mathrm{Z}$ recombinant adenovirus transfection group (diabetic rats treated with $\mathrm{AD}$-LacZ as above, group $\mathrm{AD}-\mathrm{LacZ}$ ) and the normal control group (group N). The blood glucose variation and the pulmonary pathological changes were observed at $8,12,16$ and 20 weeks after the induction of diabetes models. The mRNA and protein expressions of DCN, TGF- $\beta 1$ and type IV collagen were examined by RTPCR and immunohistochemistry.

Results: (1) The blood glucose levels of AD-DCN, AD-LacZ and D groups were increased significantly $(p<0.01)$. There were no statistical differences in the blood glucose levels among the groups of AD-DCN, AD-LacZ and $\mathrm{D}$ ( $p>0.05)$. (2) Compared with $\mathrm{N}$ and AD-DCN groups, the D and AD-LacZ groups showed increased thicknesses of alveolar septum and capillary basement membrane by HE staining and increased expressions of collagen fibers in alveolar septum, capillary basement membrane and bronchiole by Masson staining. (3) The mRNA and protein expressions of DCN, TGF- $\beta 1$ and type IV collagen in lung tissues of the AD-DCN, AD-LacZ and D groups were increased significantly compared with those in the $\mathrm{N}$ group at all observed time points $(\mathrm{p}<0.01$ or $\mathrm{p}<0.05)$. (4) The mRNA and protein expressions of TGF- $\beta 1$ and type IV collagen in lung tissures were increased along with the diabetic course in the AD-LacZ and D groups $\left(12^{\text {th }}, 16^{\text {th }}, 20^{\text {th }}\right.$ week vs $8^{\text {th }}$ week, $p<0.01$ or $\mathrm{p}<0.05)$. Adenovirus mediated DCN transfection downregulated the overexpression of TGF- $\beta 1$ and type IV collagen (compared with D and AD-LacZ groupsd, $\mathrm{p}<0.01$ or $\mathrm{p}<0.05$ ), but did not reached to the normol levels.

Conclusion: (1) The mRNA and protein expressions of TGF- $\beta 1$ and type IV collagen in the lung of diabetic rats were increased with the progress of diabetes. (2) AD-DCN transfection could significantly decrease the expression of TGF- $\beta 1$ and type IV collagen, thus improves the lung fibrosis changes in streptozotocin-induced diabetic rats.

Supported by: National Natural Science Foundation and Zhejiang Provincial Natural Science Foundation, P.R.China 


\section{9}

Haemodynamic alteration and lung injury induced by endotoxin in streptozotocin-induced diabetic rats

C.-H. Hung ${ }^{1}$, J.T. Cheng ${ }^{2}$, C.N. Chang ${ }^{1}$, Y.W. Chen ${ }^{3}$

${ }^{1}$ Department of Physical Therapy, National Cheng Kung University, Tainan,

${ }^{2}$ Department of Pharmacology, National Cheng Kung University, Tainan,

${ }^{3}$ Department of Physical Therapy, China Medical University, Taichung,

Taiwan

Background and aims: Wound healing is difficult in type I diabetic patients, which may easily contribute to further threatening infections. As a systemic infection, endotoxemia frequently leads to septic shock, including cardiac depression and multiple organ failures. However, the severity of cardiopulmonary dysfunctions during endotoxemia in type I diabetes remains unclear. The objective of this study is designed to know this change using type I-like diabetic rats under endotoxin-induced endotoxemia.

Materials and methods: Adult male Wistar rats were randomly assigned to control group and the group treated with streptozotocin to induce type I diabetic animal model. The survival time and cardiovascular parameters, including mean arterial pressure, heart rate, cardiac output, and stroke volume were continually monitored in animals receiving the intravenous infusion of endotoxin (lipopolysaccharide; LPS) or saline. At the 3rd hour after LPS injection, we determined arterial blood gas, Tumor necrosis factor-alpha (TNF- $\alpha$ ) level in serum and bronchoalveolar lavage fluid, histopathology of lung tissue, albumin content in lavage, and wet/dry weight ratio of lung.

Results: Before LPS administration, the mean arterial pressure of diabetic rats was significantly higher than normal control $(93.22 \pm 4.96 \mathrm{mmHg}$ versus $74.38 \pm 6.08 \mathrm{mmHg}, \mathrm{P}=0.015)$ although other hemodynamic parameters were no difference between two groups. Since the body weights of streptozotocin-induced diabetic rats were significantly lower than that of normal rats

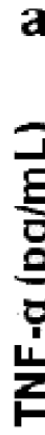

a

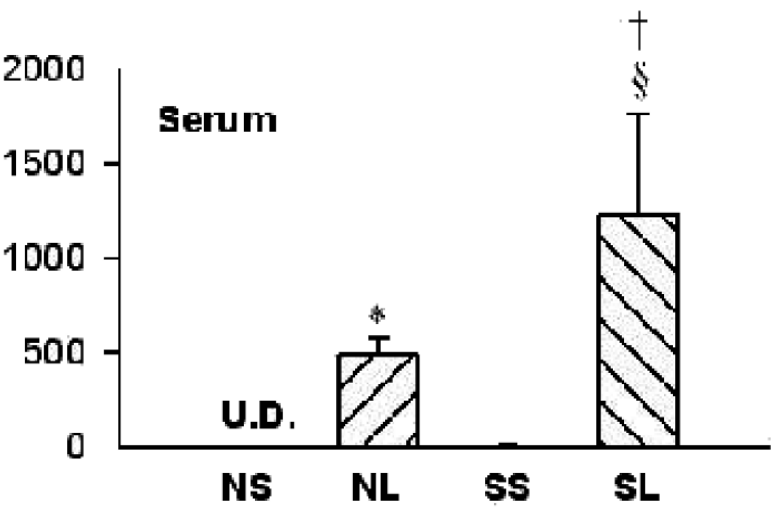

b

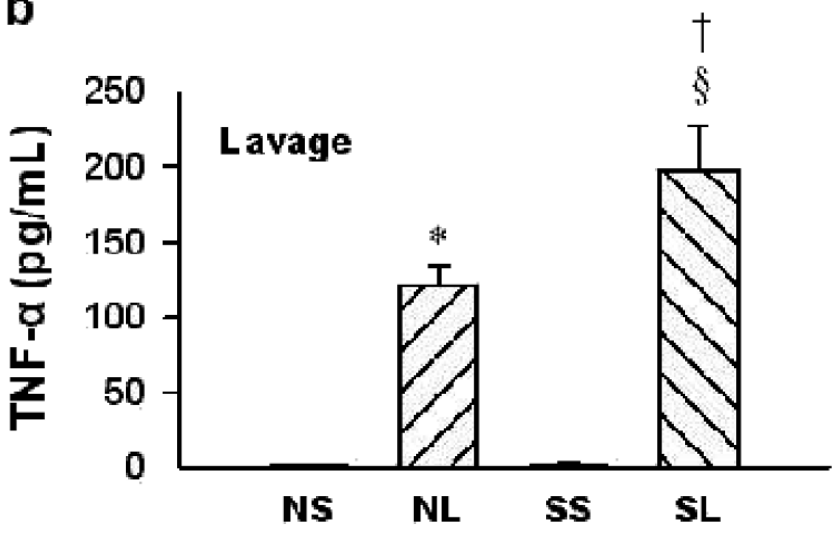

Fig. 1. The serum and lavage TNF- $\alpha$ levels in different groups of rats. NS, normal rats injected with saline; NL, normal rats injected with LPS; SS, STZ-induced diabetic rats ijected with saline; SL, STZ-induced diabetic rats injected with LPS; U.D., undetectable. Data are expressed as mean \pm S.E.M. $(\mathrm{n}=7)$. ${ }^{\star} P<0.05$, compared with the NS group; ${ }^{\dagger} P<0.05$, compared with the NL group; ${ }^{5} P<0.05$, compared with the SS group (one-way ANOVA)
( $376 \pm 11$ gm versus $262 \pm 10$ gm, $\mathrm{P}<0.05$ ), cardiac output index and stroke volume index of diabetic rats were significantly higher than those of normal rats in the controlled situation of saline administration (cardiac output index $=14.34 \pm 0.87 \mathrm{ml} \cdot \mathrm{min}^{-1} \cdot 100 \mathrm{~g}^{-1}$ versus $10.81 \pm 0.81 \mathrm{ml} \cdot \mathrm{min}^{-1} \cdot 100 \mathrm{~g}^{-1}, \mathrm{P}=0.004$; stroke volume index $=0.04 \pm 0.0033 \mathrm{ml} \cdot$ beat $^{-1} \cdot 100 \mathrm{~g}^{-1}$ versus $0.031 \pm 0.0025$ $\mathrm{ml} \cdot$ beat $\left.^{-1} \cdot 100 \mathrm{~g}^{-1}, \mathrm{P}=0.004\right)$. After administration of LPS, the heart rate, cardiac index and stroke volume index dropped in diabetic rats were more marked than that in normal group (all $\mathrm{P}<0.05$ ). Also, the increased level of TNF- $\alpha$ in serum and lavage by LPS treatment were significantly higher in diabetic rats than those in normal rats (Fig. 1). However, the arterial blood gas, albumin content, and wet/dry weight ratio of lung were indistinguishable between diabetic and normal rats. Then, the survival time in diabetic rats was significantly shorter than that in normal rats after LPS application (303.22 \pm $28.58 \mathrm{~min}$ versus $420.78 \pm 38.99 \mathrm{~min}, \mathrm{P}=0.027$ ).

Conclusion: These results indicate that the cardiopulmonary dysfunction during endotoxemia is more marked in type I diabetic rats as compared to normal rats. The greater increase of TNF- $\alpha$ in diabetic rat seems related to the higher mortality rate.

Supported by: National Science Council of Taiwan

\section{0}

Soluble CD40 ligand in essential hypertensive men: relationship with the metabolic derangement

E. Storti ${ }^{1}$, L. Pucci ${ }^{1}$, D. Lucchesi ${ }^{1}$, G. Dell'Omo ${ }^{2}$, E. Russo ${ }^{1}$, F. Caricato ${ }^{1}$, R. Miccoli ${ }^{1}$, G. Penno 1 , S. Del Prato ${ }^{1}$, R. Pedrinelli ${ }^{2}$, A. Solini ${ }^{3}$

${ }^{1}$ Endocrinology and Metabolism, ${ }^{2}$ Cardio-Thorax Disease, ${ }^{3}$ Internal Medicine, University of Pisa, Italy

Background and aims: Elevated levels of sCD40L have been consistently reported in subjects with diabetes. Furthermore, soluble CD40L levels were also higher in subjects with impaired glucose tolerance (IGT), metabolic syndrome, obesity, and insulin resistance. Patients with hypertension showed higher sCD40L levels with no significant differences between hypertension grades and in absence of any additional cardiovascular risk factors. It is therefore conceivable that metabolic abnormalities highly frequent in subjects with essential hypertension might account for the increased levels of sCD40L.

Materials and methods: To address this issue, we analyzed the metabolic correlates of sCD40L levels (ELISA assay) in a group of non-diabetic hypertensive patients. Albumin excretion rate, hs-CRP (ELISA), ambulatory blood pressure monitoring and carotid ultrasonography to measure intima-media thickness (IMT) were also performed.

Results: In the 90 never-treated essential hypertensive men, mean sCD40L level was $8.8+/-4.9 \mathrm{ng} / \mathrm{ml}(95 \% \mathrm{CI}$ for the mean: $7.7-9.8 \mathrm{ng} / \mathrm{ml})$. On the basis of SCD40L, subjects were divided by tertiles (thresholds at 6.6 and 11.0 $\mathrm{ng} / \mathrm{ml})$. The three groups did not differ for age, BMI, percentage of current smokers, systolic, diastolic and pulse BP as well prevalence of non-dippers (39\%, 33\%, and $39 \%)$ as assessed by ambulatory blood pressure monitoring, serum lipid and lipoprotein pattern, and fibrinogen concentration. Furthermore, the three groups had similar clinical and laboratory characteristics as far as creatinine and cystatin-C serum levels, eGFR, AER and distribution of microalbuminuric subjects. Finally, mean IMT $(0.79+/-0.22,0.83+/-0.29$ and $0.85+/-0.30 \mathrm{~mm}$ ) and percentage of subjects with wall thickening (IMT $>0.9$ to $<1.3 \mathrm{~mm}: 23 \%, 27 \%$, and $27 \%$, respectively) were superimposable in the three groups. No differences were observed in hs-CRP levels and no correlation emerged between $\mathrm{sCD} 40 \mathrm{~L}$ and hs-CRP $(\mathrm{r}=0.01, \mathrm{p}=0.93)$. Area under the curve (AUC) for glucose $(122+/-30,134+/-38$ and $139+/-37 \mathrm{mg} /$ min; ANOVA $p=0.16)$ and insulin $(61+/-35,77+/-33$ and $76+/-34$ microU/ min, $\mathrm{p}=0.27$ ) showed a trend, although not significant, towards an increase with sCD40L tertiles. A similar trend emerged for basal insulin (11.2+/-5.6, $14.7+/-7.7$ and $16.8+/-13.5 \mathrm{microU} / \mathrm{ml}, \mathrm{p}=0.10)$, while significant was the increase in fasting glucose $(95+/-11,103+/-16$, and $105+/-14 \mathrm{mg} / \mathrm{dl}, \mathrm{p}=0.028)$, Consistently, along the increase in $\mathrm{SCD} 40 \mathrm{~L}$, it was observed a worsening in insulin sensitivity expressed as HOMA\%S $(99+/-52,77+/-43$, and $72+/-35$, $\mathrm{p}<0.05$ ), ISIcomp (Matsuda index: $5.11+/-2.65,3.61+/-1.98$, and $3.28+/-1.87$, $\mathrm{p}=0.025$ ) or OGIS (oral glucose insulin sensitivity index: $421+/-67,386+/-90$, and $362+/-72, \mathrm{p}=0.004$ ).

Conclusion: In male subjects with newly diagnosed essential hypertension, sCD40L levels increase as insulin sensitivity decreases, with no correlation with blood pressure levels, other cardiovascular risk factors, nor the degree of sub-clinical atherosclerosis as assessed by carotid IMT. Caution is necessary in attributing to $\mathrm{SCD} 40 \mathrm{~L}$ an independent role as a cardiovascular risk factor in hypertensive subjects. 


\section{1}

MCP-1 reduction by Krüppel-like factor 5 siRNA is associated with NFKB p50 in endothelial cells

G. Fukuda, M. Kumekawa, K. Konno, S. Shimizu, T. Miwa, A. Kanazawa, Y. Notoya, M. Odawara

The 3rd department of Internal Medicine, Tokyo Medical University, Japan

Background and aims: Diabetic macroangiopathy is first initiated by the endothelial disorder through hyperglycemia and cytokines. Damaged endothelial cells produce endothelin (ET)-1, monocyte chemoattractant protein (MCP) -1 and begin to develop atherosclerotic changes. Vascular smooth muscle cells are also influenced and transformed their shape from contractile phenotype to synthesizing phenotype modulation. We have already reported that an embryonic form of myosin heavy chain (SMemb) increased in human umbilical vein endothelial cells (HUVECs) cultured by high concentration of glucose with parallel association of ET-1 expression. Krüppel-like factor (KLF) 5 is one of the important transcriptional factors and known to regulate SMemb expression. We investigated whether KLF5 inhibition may lead a beneficial effect on the prevention from atherosclerosis in HUVECs.

Materials and methods: The control siRNA and KLF5 siRNA were designed without showing any off-target effect, and determined the appropriate concentration of siRNA. HUVECs were transfected with $2.5 \mathrm{nM}$ control siRNA or $2.5 \mathrm{nM}$ KLF5 siRNA. The experiments were classified into 4 groups including (1) a control group with control siRNA, (2) an experimental group with KLF5 siRNA, (3) an experimental group with $10 \mathrm{ng} / \mathrm{ml}$ tissue necrosis factor (TNF) $-\alpha$ and control siRNA, and (4) an experimental group with $10 \mathrm{ng} / \mathrm{ml}$ TNF- $\alpha$ and KLF5 siRNA. Total RNA, culture medium and nuclear proteins were isolated from HUVECs. Real-time PCR was carried out for 18S, MCP1, ET-1, SMemb mRNA quantification. Each mRNA level was compared by arbitrary ratio to $18 \mathrm{~S}$ ribosomal RNA. The protein level of the MCP-1 in culture medium was measured by ELISA-based assay. The extracted nuclear protein was used to observe the effect of KLF5 siRNA on nuclear factor kappa $\mathrm{B}(\mathrm{NF \kappa B})$. The binding activity of p50 subunit was measured by EZ-Detect NFkB p50 Transcription Factor Kit (Pierce). One-way ANOVA was used for the statistical analysis.

Results: HUVECs grew linearly in either control siRNA or KLF5 siRNA without cytotoxic effect. The KLF5 mRNA level was reduced extensively in HUVECs when the cells were treated with KLF5 siRNA for 24 hours. The MCP-1 mRNA level was not altered when cells were treated with KLF5 siRNA alone. However, the MCP-1 mRNA level was increased 10 times when treated with TNF- $\alpha(\mathrm{p}<0.01)$. This augmentation was decreased in the presence of KLF5 siRNA $(\mathrm{p}<0.05)$. KLF5 siRNA did not significantly affect the ET-1 mRNA expression but significantly decreased the SMemb mRNA expression. SMemb mRNA level was further decreased when treated with TNF- $\alpha$. The MCP-1 protein was extensively increased by the treatment with TNF- $\alpha$ in the conditioned medium $(\mathrm{p}<0.05)$. It was attenuated significantly in the presence of KLF5 siRNA ( $\mathrm{p}<0.05)$. The binding activity of NFkB p50 subunit was 2.4 times significantly increased in the nuclear protein with TNF- $\alpha$ treatment $(\mathrm{p}<0.01)$ as compared to that without TNF- $\alpha$. This augmentation was attenuated by KLF5 siRNA as low as control siRNA without TNF- $\alpha(\mathrm{p}<0.01)$.

Conclusion: Present results suggest that KLF5 may work as a regulator of cytokines related to develop atherosclerotic changes observed in diabetes mellitus through NFKB regulation. It would be important to consider KLF5 as a new target for the treatment of diabetes as well as metabolic syndrome where TNF- $\alpha$ plays a trigger of inflammation intervened by MCP-1 and cardiovascular remodeling.

\section{2}

Human C-peptide exerts anti-inflammatory activity in LPS-stimulated monocytes through stimulation of PPAR- $\gamma$

V. Cifarelli, J. He, M. Trucco, P. Luppi

Pediatrics, University of Pittsburgh Medical School, Pittsburgh, United States

Background and aims: Inflammatory changes at the level of both the endothelium and the circulating monocytes are important factors in the pathogenesis of vascular complications in type 1 diabetes (T1D). Monocytes of T1D patients are activated, secrete inflammatory cytokines and interact with vascular lesions, leading to atherosclerotic plaque development. In recent years, the biological activity of C-peptide has been revisited by showing that this peptide displays anti-inflammatory activities and improves endothelial dysfunction in conditions of hyperglycemia. The potential anti-inflammatory effect of C-peptide on activated monocytes, however, has not been investigated. The purpose of this study, therefore, was to investigate whether C-peptide may afford anti-inflammatory effects in lipopolisaccaride (LPS)-activated monocytes and whether these effects are associated with activation of peroxisome proliferators-activated receptor- $\gamma$ (PPAR- $\gamma$ ), a nuclear transcription factor that plays a regulatory role in inflammation and atherosclerosis. We also tested whether activation of PPAR- $\gamma$ was associated with modulation of extracellular signal regulated kinases(ERK)-1 and 2 activation.

Materials and methods: The human monocytic cell line U-937 was exposed to high glucose $(30 \mathrm{mM})$ and lipopolisaccaride (LPS; $1 \mathrm{ng} / \mu \mathrm{L})$ in the presence or absence of human C-peptide for 4 hours. Expression of PPAR- $\gamma$ and phosphorylated-ERK1/2 in cellular extracts of U-937 was determined by Western blot analyses. Secretion of inflammatory cytokines in the culture supernatants was determined by Luminex. Flow cytometry was used to evaluate expression of the PPAR- $\gamma$-regulated CD36 scavenger receptor on U-937.

Results: We found that C-peptide per se stimulated PPAR- $\gamma$ expression in U937 under high glucose for 4 hours (see Figure of Western blot). This C-peptide effect was associated with up-regulation of the specific PPAR- $\gamma$-monocyte marker CD36. When cells were treated with LPS in the presence of Cpeptide (1-10nM), PPAR- $\gamma$ activity was also stimulated as compared to LPStreated U-937 cells in the absence of C-peptide (see Figure of Western blot). Furthermore, C-peptide significantly inhibited secretion of the inflammatory cytokines IL- $1 \beta$, IL-6, IL- 8 , MCP-1, MIP- $1 \alpha$, MIP- $1 \beta$ by LPS-stimulated U937 cells. A similar effect of C-peptide was also observed on LPS-stimulated monocytes isolated from T1D patients. Finally, C-peptide stimulatory effect on PPAR $-\gamma$ was associated with a modulatory activity in the phosphorylated, active form of ERK1/2.

Conclusion: C-peptide exerts anti-inflammatory (and potentially anti-atherogenic) effects on LPS-activated monocytes under conditions of hyperglycemia. These effects are associated with activation of the nuclear factor PPAR$\gamma$, which is considered a major controller of susceptibility to vascular injuries and diseases.

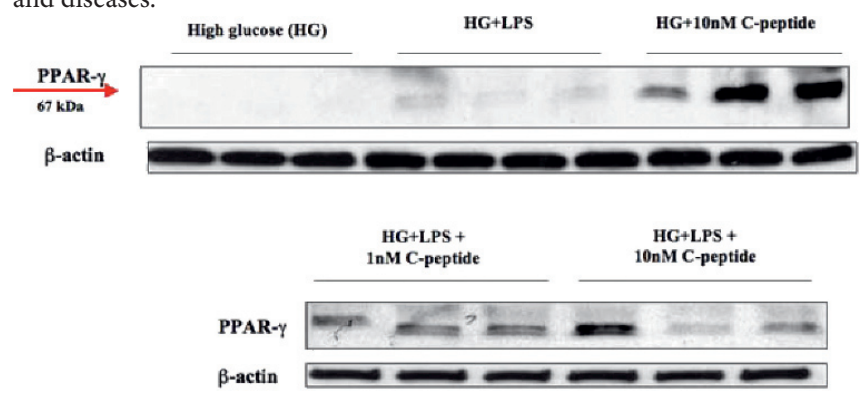

Supported by: USA Department of Defense 


\section{Author Index}

Aaboe, K. 637

Aagren, M. 1005

Aanstoot, H. 1104

Aarnisalo, J. 342

Aasted, B. 1206

Abad, V. 1085

Abd elhadi, S. 1042

Abdella, N. 731

Abderrahmani, A. 515

Abdul-Ghani, M. 620

Abdulkader, F. 115

Abhyankar, A. 494

Abi Khalil, C. 1169,1332

Abiru, N. 582

Abou-Samra, A. 727

AbouMrad, E. 236

Abraira, C. 925

Abreu, F. R. M. 979

Absalom, N. L. 362

Abudukadier, A. 942

Acerini, C. L. 181

Achard, V. 849

Achenbach, P. 330, 335, 344

Aczel, S. 404, 1355, 1360, 1362

Adaikalakoteswari, A. 220

Adamec, M. 596

Adamek-Guzik, T. 1176

Adams, V. 1320

Adamska, A. 1395

Adamson, U. 938

Adeniji, A. K. 945

Ader, M. 775

Afonso, G. 236

Afsari, F. M. 1334

Aftring, R. Paul. 24

Agardh, C. 565, 1159

Agardh, E. 1159

Agbalika, F. 34

Agha, A. 1321

Agostini, C. 21

Aguilar-Bryan, L. 544

Aguilar-Diosdado, M. 538

Aguilar-Salinas, C. A. $\quad 385,1220$

Aharon-Hananel, G. 776

Ahlzén, M. 821

Ahmed, A. Uddin. 420

Ahmed, M. 682

Ahmed, R. A. M. 1295

Ahn, C. 293, 1245

Ahn, Y. -B. 1296

Ahn, Y. -H. $\quad 614,615,841$

Ahola, A. J. 1100

Ahrén, B. 75, 611

Ahui, M. 235

Ait El Mkadem, S. 393, 641

Ait-Lounis, A. 25

Aitken, R. J. 366

Ajdukovic, D. 1105

Akan, P. 291

Åkerfeldt, M. 134

Akhter, A. $1128,1129,1136$

Akiyama, Y. 1083

Akkerman, J. N. 199

Akter, S. $323,1128,1144$

Al Arouj, M. 731

Al-Khalili, L. 618

Al-Turaifi, H. 439

Al-Turaifi, H. R. H. 437

Al-Zaman, Y. 848
Ala-Korpela, M. 1363

Alauddin, M. 648

Alba, M. 909

Alberobello, A. T. 305

Albers, J. W. 241, 1253

Alberti, G. $\quad 422,424,425$

Albrecht, D. 1110

Albrechtsen, A. 63,66

Albu, A. I.. 351

Aldibbiat, A. $439,600,906,1372$

AldibbiaT, A. I. M. 437

Aledo, J. C. 285

Alejandro, E. U. $\quad 451,550$

Alemao, E. 1095

Alemayehu, B. 1005

Alevizaki, M. 1142

Alexander, C. M. $\quad 417,432,680$

Alexiadou, K. 1348

Alexopoulos, C. 348

Alfieri, O. 1322

Ali, L. $323,648,729,1128,1129$,

$1134,1136,1144$

Ali, R. $\quad 647,852$

Ali, T. 486

Alibegovic, A. C. 310,630

Allagnat, F. 214

Allard, J. 646

Allegra, L. 1397

Allemann, S. 23

Allen, E. $\quad 78,859$

Allen, J. 181

Allen, T. J. 93, 96

Allet, L. 1258

Allred, N. D. 304

Almeida, I. 1234

Alméras, N. 1016

Alonso, M. A. 1158

Alonso, N. 578

Altirriba, J. 258

Altkrueger, A. $\quad 454,461$

Aluoch, A. 805

Álvarez, C. 452

Alves, M. C. 1084

Alves, M. R. C. 979

Alviggi, L. 384

Alzugaray, M. E. 483

Åman, J. 265

Amatruda, J. M. 73, 910, 911, 912

Ambegaonkar, B. M. 1390

Ames, J. M. 1308

Amiel, S. A. $\quad 227,848$

Amiel, S. G. C. 437

Amin, S. 1116

Aminian, K. 1258

Amisten, S. 476

Amod, A. 3

Amoretti, R. 1101

Amoruso, R. 67, 68

Ampudia, F. J. $\quad 230,275$

Amrani, A. 558

An, J. 1377

An, R. 26

An, Z. 665

Anand, A. 5

Anastasiou, E. 1142

Anavi, S. 787

Anchors, K. 142

Andersen, G. $\quad 63,324,328$

Andersen, L. B. 61
Anderson, E. M. 1062

Andersson, C. X. 810

Andersson, D. K. G. 419

Anderwald, C. 593

Anderwald-Stadler, M. 593

Ando, A. 770

Ando, D. G. 1249

Andrassy, M. 110

Andreasen, A. H. 889

Andreelli, F. 607

Andren Aronsson, C. 373

Andreozzi, F. 325, 691

Andries, L. 1021

Androulakis, I. I. 825

Anfossi, G. 58, 626, 793

Angel, B. 266, 273

Angelo, R. 1013

Anichini, R. 384, 1140, 1279

Anker, S. D. 1226

Annuzzi, G. 741, 753

Anselmino, M. 608, 794, 824

Anthonsen, S. 324

Antic, S. 148

Antonelli, A. 172

Antonio, C. 1212

Antsiferov, M. 952

Anzellotti, M. 1077

Aono, S. 985, 1115

Aoyama, T. 363, 414, 1191

Apanovitch, A. 858

Apelqvist, J. 429

Apostolakou, F. 1281

Aragona, M. 1122

Arakaki, R. F.. 326

Arantes, V. C. 457

Arantes, V. Cristina. 746

Arapostathi, C. 1382

Arbet-Engels, C. 42, 927, 928

Arcangeli, A. 156

Archimandritis, A. J. 339

Ardigò, D. 19, 1117, 1331, 1387

Arduini, A. 1291

Arena, V. C. 945

Argente, J. 275

Argoud, K. 361

Arias, P. 1284

Arjona Ferreira, J. C. 911

Armand, S. 1258

Armoni, M. 249

Arnal, J. 235

Arndt, T. 278

Arnés, L. 900

Arnkvist, H. J. 269

Arola, J. 33

Aron-Wisnewsky, J. 595

Arondekar, B. 918, 920

Aronne, L. 798

Arora, V. 1221, 1222

Arosi, A. 1275

Arranz, C. 345

Arredondo, M. 273

Arslanian, S. 950

Artmann, A. 736

Arvind, A. S. 5

Arvind, S. R. 5

Asai, K. 281

Asano, M. 769

Aschemeier, B. 1075

Asghar, S. 712
Ashcroft, F. M. 115, 239, 265

Ashley, D. T. 722,1321

Asmar, M. 632

Asnaghi, V. 871

Aso, Y. $\quad 808,1251,1373$

Assaloni, R. 1380

Astiarraga, B. D. 150

Astorri, E. 446, 561

Astort, F. 1284

Astrup, A. S. 46, 1318

Athayde, N. 1139

Atsuko, T. 1315

Attali, C. 1039

Attali, J. R. $\quad 1235,1384$

Attali, V. 684

Attaoua, R. 393, 641

Aubert, R. 607, 1332

Auer, V. J. 605

Augstein, P. 232

Aukrust, P. 1278

Aulchenko, Y. 12

Auleley, G. 153

Aulinger, B. A. 121,635

Austin, M. 290

Autier, V. 481

Avezum, A. 385

Avignon, A. 1330

Avogaro, A. 21, 694, 927

Avram, D. 138,547

Awaji, T. 464

Axelsson, S. 568

Aylwin, S. J. B. 848

Azab, Y. 116

Azad Khan, A. K. 420, 1144

B

Błazik, M. 989

Ba, H. 221

Baba, T. 46

Baccara-Dinet, M. 939

Bach, V. 710

Bachem, M. G.. 447

Bachou, T. 389

Backlund, J. C. 106

Bacos, K. 494

Baczkó, I. 1383

Bae, J. $\quad 614,615,841$

Bae, Y. 60

Baggiore, C. 1279

Baiget, M. 710

Baik, S. Hyun. 718, 991

Bailbé, D. 358, 481, 774

Baillot-Rudoni, S. 855

Bain, S. C. 1216

Bajpeyi, S. $\quad 757,759$

Bajza, A. 714

Bajzova, M. $723,730,816$

Baker, A. R. 826

Bakhtadze, E. 269

Bala, C. G. 1057

Balani, J. 1147

Balas, B. $\quad 619,852$

Balbarini, A. 1319

Baldi, S. 112, 161

Baldwin, A. 1093

Baldwin, C. 946

Baldwin, D. 964

Baldwin, J. 101 
Baldwin, M. 152

Baldwin, S. A. 101

Balena, R. $\quad 42,871,927,928$

Balfour, P. 946

Balkau, B. 297, 311, 313

Baltrusch, S. 175, 529

Banas, S. M. 774

Banerjee, M. 444

Banerji, M. 914

Banerji, M. A. 224, 650

Bang-Berthelsen, C. H. 521

Banu, A. 420

Banu, I. 221, 1239

Bao, Y. 936

Baptista, J. 979, 1084

Baptista, R. Brito. 979

Baquié, M. 238

Bar, Y. 436

Barak, L. 279

Baran, R. W. 430, 921, 923

Barbaro, M. 850, 1283

Barber, T. M. 327

Barberà, A. 258

Barbetti, F. 282

Barbu, C. G.. 351

Barbuta, M. 180

Barchetta, I. 984

Baren, J. 743

Bargero, G. 402

Barit, D. 93

Barker, C. J. 491

Barker, D. 53

Barnard, M. R. 1206

Barnett, A. H. 401, 994, 1048

Baron, A. 82,83

Baron, M. 914

Baroni, M. G. 284

Barquiel, B. 1149

Barras, E. 25

Barres, R. 90

Barrett, A. 361

Barreyro, F. J. 767

Barriga, K. 336

Barroso, I. 283

Barrou, B. 595

Barrow, B. A. 279,327

Barsotti, E. 172, 794

Barsotti, M. C. 1319

Barsukov, I. A. 355

Barthassat, V. 843

Bartolome, A. 534

Barton, D. 1146

Basello, K. 441, 1317

Bashan, N. 86, 801

Basile, M. Elisa. 1106

Bassols, J. 709, 840

Basterra-Gortari, F. J. $\quad 354,851$

Basu, A. 658,700

Basu, R. $122,658,700$

Bauer, R. J. 1

Baughman, R. $\quad 867,868,1014$

Bax, J. J. $\quad 113,114,166$

Bayard, F. 235

Bayrak, G. 1017

Bays, H. E. 1388

Bazzigaluppi, E. 584

Beatson, C. 967

Beauchef, G. 1341

Beauquis, J. 1393

Beauvieux, M. 705

Beauwens, R. 473

Becattini, B. 739

Beck-Nielsen, H. 225

Beck-Peccoz, P. 1397
Becker, D. 1015

Becker, D. J. $\quad 945,946$

Becker, L. 917

Becker, T. 462

Beckers, M. 333

Beckham, C. 1216

Beding-Barnekow, B. 1159

Beer, S. 404, 1351, 1355

Beguinot, F. 305, 683, 691

Beijnen, J. H. 120

Beisswenger, P. J. 43

Beitiger, W. 591

Bektas, B. 1017

Belardinelli, L. 686

Belcourt, A. 591, 603, 606

Belhadj-Mostefa, A. $\quad 1235,1384$

Belharbi Krimi, R. 609

Belinchón Sz.-Somoza, B. M. 375

Bell, G. I. 464

Bell, J. Tzenova. 9

Bell, P. M. 943

Bellary, S. $\quad 401,1048$

Bellia, A. 204

Bellili, N. 1169, 1328, 1332

Bellmann, K. 180

Bellomo, E. A. 101

Bellomo Damato, A. 1196

Beltramo, E. $157,217,218$

Beltrand, J. 81

Bem, R. 1238

Ben Nakhi, A. 731

Ben Yosef, D. 249

Ben-Romano, R. 86

Benaiges, D. 1148

Benaim, E. 1249

Benani, A. 132

Bendlova, B. 818

Benes, P. 1306

Benevento, D. 1034

Bengt, J. 1047

Benhamed, F. 742

Benito, M. 534

Benke, I. 409

Bennett, A. J. 299, 309

Benroubi, M. 154, 1090

Bensellam, M. 212

Bentley, L. 115

Benyo, Z. 256

Benzer, W. 1360

Benzi, L. 1145

Bequette, B. Wayne. 1064

Berclaz, P. 410

Bereczkova, E. 279

Berenshtein, E. 1368

Berge, K. 735

Berger, F. 605

Berggren, P. 257, 465, 479, 491

Bergholdt, R. 139

Berglund, G. 65

Berglund, L. G. T. 314

Bergman, B. C. $\quad 35,756$

Bergman, R. N. $732,775,792$, 864

Bergmann, A. 1226

Bergsten, P. 539, 552, 649, 789

Berkova, Z. 596, 604

Bernard, S. 22

Bernardi, S. 1162

Bernath, S. 714

Berndt, C. 14

Berndt, J. 289

Berne, C. 314, 403

Berner, R. 1045, 1181

Berney, T. 170,540
Bernstein, G. 1022

Berria, R. 871

Berrone, E. $157,198,217,218$

Bertelli, E. 443

Berthou, F. 131, 774

Bertolini, L. 1202

Bertolotto, A. 1122,1145

Berton, A. 333

Bertrand, G. 179

Bes-Rastrollo, M. 354

Besharat, D. 1042

Besin, G. 558

Bessenyei, S. 528

Best, J. 1358

Best, J. D. 1072

Best, L. C. 473

Bets, D. 940

Betteridge, D. J. 566

Beulens, J. W. 1175

Bevc, S. 1190

Bevilacqua, M. F. 1280

Beyan, H. 274, 570

Bezzi, P. 472

Bhole, D. $\quad 863,876,877,886,901$

Bhushan, M. 1003, 147

Bhushan, R. 1003, 147

Bialkowska, J. 919

Bianchetti, M. 41

Bianchi, C. 318

Biasucci, L. M. 1337

Biden, T. J. 134,490

Bieghs, V. 802

Bierhaus, A. 93, 243, 110, 1309

Bietiger, W. 603, 606

Bikmalla, S. 1146

Billestrup, N. 1318

Bilo, H. J. G. $\quad 182,434,1076$,

1107, 1192, 1204, 1304, 1391

Bilous, R. 1155

Bilous, R. W. 1216

Biltz, G. 1053

Bimmler, D. 447

Bingham, S. 349

Bingley, P. J. 335

Birk, J. B. 613

Birkeland, K. I. $\quad 294,423,1219$, 1278

Bischof, M. 593

Biskaeva, N. 659

Biswas, S. K. 194, 1183, 1309

Bitsios, P. 1027

Bittencourt, C. 1074

Bitzur, R. 407

Bizzarri, C. 1034

Bjerregaard, P. 400

Björck, U. 821

Björklund, A. 498, 551

Björnholm, M. 130

Bjørnsdottir, I. 891

Bjørnvold, M. 267, 365

Blaak, E. E. $\quad 36,189,247,352$, $386,758,1219,1305$

Blacher, J. 1352

Blanc, P. R. 1350

Blanco-Vaca, F. 710

Bland, R. 94

Blandino-Rosano, M. 538

Blangero, J. 935

Blank, R. C. 1220

Blanquart, C. 693

Blase, E. 866

Blasi, F. B. 1397

Blatniczky, L. 950

Blazik, M. 948

Blazquez, A. G. 610

Bleyer, A. J. 1021

Blickle, J. 992

Blomstedt, P. A. 105

Blonde, L. $\quad 898,914$

Blondeau, B. 341, 450

Bloomfield, L. 1272

Blouin, C. 85

Blüher, M. 163, 246, 289, 396

Blum, M. 1157

Blume, N. 929

Boavida, J. M. 1119, 1234

Bobbioni-Harsch, E. 721, 843

Bobek, V. 596

Bociaga, Z. 1335

Bock, G. 122, 968

Bockaert, J. 179

Bodalski, J. 1231

Bodansky, J. 1111, 118

Boddenberg, P. 232

Bode, B. W. 894, 896, 1056

Bödvarsdóttir, T. B. 216, 734, 929

Boegner, C. 1330

Boehm, B. O. 559

Boehnel, C. 1351, 1355

Boemi, M. 321, 1170, 1387

Boerop, J. 1204

Boesch, C. 671

Boesgaard, T. W. 328

Boetker, H. 676

Bogani, D. 362

Bogardus, C. 301

Boggi, U. 136, 315, 597, 656, 903

Böhler, S. 186

Boit, M. K. 426

Boka, G. 145, 1016

Boldrin, M. 42, 871, 927, 928

Boldrini, B. 1314,1347

Bolinder, J. 269

Boller, S. B. 176

Bollerslev, J. 1141

Bolli, G. B. 183,1282

Bolo, N. 228

Bombardieri, M. 446, 561

Bombelli, M. 47

Bona, G. 374

Bonadonna, R. C. $\quad 298,318,652$, 927

Bonal, C. 25

Bonato, V. 367,576

Bondinas, G. 556

Bonetti, S. 298

Bonfanti, R. 282, 986

Bonfigli, A. R. 321,1170

Bonifacio, E. 330, 344, 584

Bonnefoy, E. 22

Bonomo, K. 793

Bonora, E. 298, 652, 1202

Bontemps, S. 751

Bookout, T. 967

Boomsma, D. I. 387

Boomsma, F. 1217,1218

Boon, H. 36

Boonyasrisawat, W. 689

Boothby, M. 762

Borah, B. 1005

Boraska, V. 10

Borch-Johnsen, K. 54, 63, 66, 
Borok, E. 262

Boronat, M. 1392

Borra, R. 760

Bosch, F. 138

Boschero, A. C. $457,506,746$

Boschmann, M. 916

Boselli, L. 298

Bosetti, A. 949

Bosi, E. $575,584,1322$

Boss, A. 1014

Bosselaar, M. 36

Bost, F. 640

Botach, E. 1073

Bøtker, H. E. 109

Botta, G. 683

Böttcher, Y. 289, 396

Bottomley, W. 283

Botusan, I. 1263

Botusan, I. 763

Bouatia-Naji, N. 297

Boucek, P. 577, 592

Boucher, J. 716

Boudou, P. 664

Bouee, S. 1039

Bouguerba, A. 1235

Bouilloud, F. 1381

Boulton, A. J. M. 1269,1271 , 1274

Bourahli, M. K. 1235

Boussel, L. 22

Boussuges, A. 1350

Boutati, E. $\quad 839,1138,1313$

Bouwens, L. 29, 438

Bouzakri, K. 540

Bowden, D. W. 304

Bower, L. 288

Bowling, F. L. 1269

Box, F. M. A. 1375

Boye, K. 392, 881

Boye, K. S. $\quad 880,1339$

Boyer, P. 772

Boyle, J. 1256

Boyle, J. G. 941

Bozikov, V. 1359

Bozzetto, L. 741

Braceras, R. 914

Bradfield, J. P. 276

Bradley, E. A. 627,628

Brage, S. 61

Brahimi, M. 1228

Brajkovic, S. 515

Brami, G. 1039

Brandjes, D. P. M. 120

Brandle, M. 149, 888, 897, 1095

Brandner, J. M. 1262

Brangani, C. 652

Bränström, R. 654

Braumüller, H. 138

Brauner, P. 738

Bravis, V. 1114

Bray, G. A. 224, 650

Bréant, B. 450

Breitman, P. 957

Brenner, G. M. 313

Brichard, S. M. 200, 641, 721

Bridoux, F. 1165

Briefel, R. 392

Brindisi, M. 1345

Bringer, J. 1069

Brion, J. 133

Brismar, K. 52, 408, 429, 551, 763, 1263

Brix, J. 1130, 1203

Brixner, D. 881
Broca, C. 179

Brock, B. $\quad 466,502,521,676$

Brodehl, A. 1299

Brodie, C. 684

Brodows, R. $874,875,876,877$, 886

Broholm, C. 707

Broke-Smith, T. P. $\quad 4,869$

Bromberger, L. 1003

Bron, M. 430, 921, 923

Brøndsted, L. 890

Brooke, S. 946

Brophy, S. 369

Brorsson, C. A. 139

Brosi, H. 559

Brot-Laroche, E. 610

Brouwers, M. C. G. 809

Brouwers, O. 1300, 1305

Brown, A. E. 250

Brown, B. Greg. 854

Brown, J. B. 1390

Brown, J. E. P. 514

Brown, P. 1124

Brown, S. N. 226

Brownlee, M. 1300, 1309

Broz, J. 723

Brubaker, P. L. 125, 126, 458

Bruce, R. 1062

Bruckert, E. 385

Brudin, L. 52

Bruening, J. 262

Brüggemann, J. 829

Brugger, A. 1012

Brun, J. 393

Brun, M. 102

Brunak, S. 139

Bruneval, P. 1133

Bruning, J. C. 115

Brunner, E. J. $\quad 350,779$

Bruno, G. 374, 402

Brutman-Barazani, T. 684

Bruttomesso, D. 230, 1101

Bryan, J. 544

Bryhn, M. 735

Bu, P. 937

Bublik, E. V. 1261

Bucci, M. 760

Buchanan, T. A. 224, 650

Bucher, J. N.. 605

Buchetti, B. 1312

Buckingham, B. A. 1064

Budziarek, P. 916

Buening, J. 857

Buffier, P. 340

Bugler, J. R. 1075

Bugliani, M. 136, 172, 315, 597, 649,903

Bukhman, A. 1193

Bulat, N. 469

Buleon, M. 716

Bulhak, A. 206

Bull, C. 1068

Bull, F. 1035

Bulum, T. 1207

Bulur, N. 473, 954

Bulvik, B. 1368

Bunck, M. C. $663,878,879$

Bunghez, R. 351

Bunting, B. 1091

Buono, P. 986

Buonomo, R. 305

Burcelin, R. 640, 716

Burchfield, J. G. 490

Burger, J. 875
Burkart, V. $\quad 829,830$

Burnol, A. 356

Burns, C. 798

Burns, N. 382, 704

Burt, D. 198

Bury, A. 116

Buse, J. 146, 898, 959

Busetto, E. 1101

Busetto, L. 1137

Bush, K. 401

Bush, M. A. 870

Buss, J. L. 598

Butler, A. E. 30

Butler, P. C. 30

Butty, A. 512

Buurman, W. 802

Buziashvili, U. 1193

Buzzetti, R. 370, 575, 584, 984

Buzzi, F. 176

Byers, J. R. 1021

Byiers, S. 74, 915

Byron, D. 1015

\section{C}

Cabistañ, C. 710

Cabrera, D. 1177

Cabrera Rodríguez, R. 375

Cabrera-Rode, E. 345

Cadario, F. 374

Cadi Soussi, N. 1052

Caduff, A. 1068

Caetano, A. M. 1119

Cahova, M. 728

Cai, Y. 1032

Caille, D. 455

Caixàs, A. 710, 783

Cakır, M. 579

Calabrese, M. 367

Calderari, S. 208, 771

Calhoun, B. 874

Calianno, A. 1145

Calin, M. 1289

Calvo, F. $\quad 341,664$

Camastra, S. 608

Cambuli, V. M. 284

Cameron, A. J. $\quad 422,424,427$

Cameron, F. J. 1072

Cammarota, G. 1337

Campaigne, B. 1359

Campani, D. 136

Campbell, M. 1288

Campbell, R. L. 428

Campbell, S. 1050

Campbell, S. C. $\quad$ 437, 439, 906

Candeloro, P. 1282, 183

Candido, R. 1162

Cani, P. D. $\quad 70,716$

Cañizo-Gómez, F. J. 1210

Cano, J. F. 1148

Cantley, J. 490

Cao, L. 357

Capani, F. 1101

Caparevic, Z. 1223

Capizzi, M. 575, 584

Cappa, M. 1034

Cappelletti, T. 1397

Cappuccio, F. P. 350

Capuani, B. $\quad 441,1317$

Caputo, S. 1344

Car, N. 1207

Carani, S. B. 383

Cardellini, M. 317, 1323

Cardona, C. Y. 1021

Cardozo, A. K. $\quad 214,549$

Carey, M. 1063

Carey, M. E. 962

Caricato, F. 1400

Carinci, S. 1077

Carling, J. 1125

Carmellini, M. 367

Carmienke, S. 289

Carminati, P. 41, 765

Carneiro, E. M. 457,746

Carneiro, L. 132

Carnovale, A. 1312

Carobbio, S. 127

Carpene, C. 716

Carpentier, A. 721, 843

Carpentier, Y. A. 954

Carrasco, E. 266, 273

Carreira, E. 263

Carreras, M. C. 767

Carretta, R. 1162

Carroll, J. 1085

Carstensen, B. 49, 108, 397, 1055

Cartenì, B. 204, 926

Casas, R. 567, 568

Casas, S. 205, 258

Casimir, M. 497

Casini, A. 343

Casolaro, A. 1227

Cassidy, J. 867, 1014

Cassone Faldetta, R. 1034

Castan-Laurell, I. 716

Castaner, O. 599

Castaño, L. 275

Casteilla, L. 132

Castel, J. 102

Castela, A. 1119

Castro, I. 1149

Castro-Faria-Neto, H. C. 1280

Castro-Santiago, M. J. 285

Caswell, K. 1064

Catalano, K. J. $\quad 732$, 775, 792

Cataldo, D. 367

Catrina, A. 1263

Catrina, S. -B. 763,1263

Cattan, P. 341

Causso-Lariena, C. 354

Cavalera, M. 67

Cavallo, F. 1106

Cavallo, G. 318

Cavallo, M. G. 284

Cavallo Perin, P. 198, 402, 1297

Cavalot, F. $\quad 318,1117,1387$

Cavanagh, P. R. $\quad 1271,1274$

Cayuelas, J. 1059

Cebada-Aleu, A. 538

Cecchetti, F. 824

Cecchetti, L. 1282

Cederholm, J. 191, 412

Cederholm, T. 245

Cedillo, M. 945

Cegan, A. 1285

Celik, S. 1017

Ceolotto, G. 694

Ceperuelo-Mallafré, V. 814

Cerellio, A. 832

Ceriello, A. 57, 59, 210, 230, $1170,1213,1380$

Cernea, S. 984

Ceron, M. 964, 1078 
Chabanaud, M. 1242

Chabanon, H. 791

Chacon, F. 1020, 1044

Chacon, M. R. 814

Chacra, A. R. $\quad 858,1074$

Chadbourn, I. 554

Chaim, E. 150

Chalançon, A. 1059

Chamiot-Clerc, P. 1242

Chan, J. Y. 134

Chan, J. C. N. 144, 174, 385, 634, 708,1214

Chan, J. 301

Chan, K. W. 1214

Chandra, S. 806

Chang, C. N. 1399

Chang, Z. 531

Chantry, M. 153

Chao, L. 546

Chapman, I. M. 889

Chappell, A. S. 1247

Chappell, B. 1215

Charbonnel, B. 1039

Charenkavanich, S. 1007

Charles, M. 939

Charles, M. Aline. 154

Charpentier, G. 701

Chartoumpekis, D. V. 807

Chase, P. 1009

Chassin, L. J. 181

Chatham, J. C. 1208

Chatterjee, C. P. K. 1160

Chatterjee, P. 554

Chatterjee, R. 347

Chaturvedi, N. 23, 254, 421

Chatzi, L. 1027

Chaves, A. 620

Chavey, A. 358

Chavez, A. O. 531, 733

Che, Z. 719

Cheah, Y. S. 1025

Cheeseman, J. 32

Chen, B. 438

Chen, H. 501

Chen, J. 1095

Chen, K. 921

Chen, L. Yun. 1032

Chen, R. 78, 858, 859

Chen, S. 28

Chen, W. 1151

Chen, W. 140

Chen, W. 171

Chen, Y. 1093

Chen, Y. W. 1399

Cheng, J. T. 1399

Cheng, K. 456

Chéramy, M. 567

Cherrington, A. D. 665

Cherubini, V. 986

Cheshire, N. J. W. 1272

Cheung, N. 1139

Cheurfa, N. 313

Chevassus, H. 1059

Chevenne, D. 81

Chevion, M. 1368

Chiaramonte, F. 292

Chiarelli, F. 1077

Chiariello, L. 204

Chiasson, J. 223

Chiatamone Ranieri, S. 695

Chiavacci, R. M. 276

Chiellini, C. 1154

Chiesa, R. 1322

Chiheb, S. 1228, 1239
Chillarón, J. J. 1148

Chimenti, D. 161

Chimienti, F. 101, 521

Chirino, R. 1392

Chiu, J. D. 732

Chiumello, G. 282

Chizynski, K. 1342

Chmelik, M. 34, 621

Cho, D. 745,1197

Cho, M. 1245

Cho, N. 622

Cho, Y. M. 390, 692

Cho, Y. 399

Choi, D. Seop. 718

Choi, E. 1353

Choi, J. 953

Choi, K. M. 718

Choi, M. 1186

Choi, M. 527

Choi, S. 1063

Choi, S. 1326

Choi, S. 1371

Choi, S. 390, 692, 1376, 1377

Choi, S. B. 990

Choi, Y. 1326

Choi, Y. 399

Choi-Lundberg, D. 627

Chonchol, M. 1202

Chopra, S. 1350

Choudhary, P. 227, 1071

Chougnet, C. N. 208

Choukem, S. P. $\quad 341,664$

Chow, F. C. C. 708

Chowdhury, T. A. 1144

Christ, E. 671

Christ-Crain, M. 785

Christensen, D. L. 426

Christiansen, B. 1394

Christiansen, C. 1038

Christiansen, C. 225

Christiansen, M. S. 1135

Christopher, S. 383

Christou, I. 1027

Christulia, F. 214

Chugunov, A. 1024

Chugunova, L. 1024

Chung, C. H. 1214

Chung, D. $\quad 745,1197$

Chung, J. 745, 1197

Chung, M. 745, 1197

Church, C. 362

Church, D. B. 600

Ciccarelli, M. 305

Cieplucha, E. 1342

Cifarelli, V. 1402

Cignarelli, A. $850,1196,1283$

Cilio, C. 523

Cimino, A. 156

Cincotta, A. H. $39,128,685$, 930

Cincotta, J. 685

Cinek, O. 371

Ciociaro, D. 112

Cioli, P. 1282

Cipelli, J. M. 1284

Cipolloni, L. 1034

Cipponeri, E. 369

Cipriani, P. 846

Clapham, J. C. 826

Clark, A. 115,445

Clark, M. G. 627,628

Claudi, T. 1049

Claussen, C. D.. 395

Clay, K. D. 1021
Cleary, P. A. $\quad$ 106, 241, 1253

Cleland, S. J. 941

Clement, K. 849

Clement, S. 224, 650

Clementi, F. 1323

Clerc, R. G. 822

Cleveland, A. 1056

Cleveringa, F. G. W. 1097

Cnop, I. 445

Cnop, M. 133, 445, 509

Coates, V. 1091

Cobas, R. A. 1280

Cobelli, C. 30, 122

Cobo-Vuilleumier, N. 285

Cocchietto, M. 1162

Codner, E. 266, 273

Coffey, T. 82

Cohen, G. 524

Cohen, I. 761

Colagiuri, S. $\quad 149,897,899$

Colby, M. 392

Colca, J. R. 38

Colclough, K. 279

Cole, R. 82

Coleman, R. L. 1365

Colhoun, H. M. 566

Collenberg, E. 1200

Collette, J. 713

Collier, D. 369

Collier, G. 37

Collier, G. R. 935

Collins, C. C. 222

Collins, S. 115, 361

Colman, P. 1366

Colman, P. G. $\quad$ 141, 1072

Colombani, A. 132

Colombo, C. 282

Colombo, M. 1336

Colomé, E. 578

Comaschi, M. 1213

Comerma, L. 1254

Comi, G. 1322

Comola, M. 1322

Comuzzie, A. 531, 733

Concannon, P. 140

Congiu, T. 284

Connell, J. M. C. 89,941

Connolly, R. 1082

Connor, T. 624

Conserva, A. $\quad 850,1283$

Consoli, A. 318, 325, 629, 1291

Constantin-Marino, S. A. 655

Conti, R. 765

Conti, U. 1347

Conway, B. 406

Cook, A. 1046

Cook, C. 1012

Cook, J. 1095

Cooney, G. J. 612

Cooper, J. G. 1049

Cooper, M. E. 93, 96, 160, 193

Coppini, D. D. 1256

Coppini, D. V. 1173

Coppola, A. 441, 1317

Corazzi, T. 1282

Corcelle, V. 640

Cordina, C. 283

Cordone, S. 1310

Corfini, M. 1145

Corkey, B. E. 510, 803

Corkey, R. F. 803

Corner, A. $\quad 878,879$

Cornut, M. 771

Corpeleijn, E. $\quad 352,758$

Corripio, R. 275

Corvol, P. 208

Cosansu, G. 1017

Cosson, E. 221, 1228, 1239

Cossu, E. 284

Costa, J. 1234

Costa, P. F. 688

Costa, S. 204

Costacou, T. 1168

Costanza, F. 1034

Costello, D. $\quad 867,868$

Costes, S. 179

Cottin, Y. 1345

Couck, P. 332

Coulaud, J. 771, 778, 1393

Courtney, C. H. 1026

Cowan, P. 743

Cox, R. 115

Cox, R. D. 362

Craig, D. W. 142

Craig, T. J. 239

Crapo, R. 410

Crawford, R. 486

Crea, F. 1337, 1344

Crinò, A. 1034

Cristol, J. 1330

Croci, M. 1212

Cronister, C. 82

Crowley, R. K. 1321

Cruickshank, J. K. 1175

Cruz, A. 531

Cseh, J. 1208

Cselenyak, A. 623

Cuba, I. 345

Cucchi, M. 1170

Cuccuru, I. 1122, 1140

Cucinotta, D. 1213

Cuesta-Muñoz, A. L. 285

Cummings, M. H. 6

Cunha, D. A. 133,509

Cunningham, J. 554

Cuppen, E. 278

Cupples, L. A. 326

Curkendall, S. M. 680

Curran, J. 935

Cusi, K. 647, 852

Cuthbertson, J. 943

Cvjeticanin, T. 833

Cyganek, K. 1121, 1176

Cymeryng, C. B. 1284

Cypryk, K. 1153

Czech, A. 835

Czerniawska, E. 1231

Czupryniak, L. 919, 973, 1342

D

D’Adamo, M. 204, 926

D’Agostino, R. B. 847

D'Aleo, V. 136, 315, 597, 656, 772, 903

D'Alessio, D. A. 121, 635

D'Alfonso, R. 317

D’Annunzio, G. 986

D’Emden, M. 1366

D'Esposito, V. 683

D’Hertog, W. 525, 549

D’Agostino Jr., R. B. 119

Da Ros, R. 1380

da Silva, N. 832 
Dacquet, C. 856

Dadon, M. 1381

Dagogo-Jack, S. 914

Dahl, K. 734, 800

Dahlquist, G. 104

Daimon, M. 379

Dain, M. 980, 992, 1001

Dal Pos, M. 230

Dale, R. 202

Dalfrà, M. 1137

Dalgaard, L. T. 324

Dall, T. 1092, 1093

Dalla Man, C. 122

Dalle, S. 179

Dallosso, H. M. 962

Daly, H. 1116

Daly, L. 1026

Daly, M. 11

Damato, A. 1117

Dambrova, M. 243

Damdindorj, B. 475

Damgaard, J. 975

Damico, C. $\quad 867,868$

Damm, P. $\quad 1123,1124,1135$

Damotte, D. 235

Dan, J. 1296

Danias, V. 1382

Daniel, R. W. 1269

Daniele, G. 1229

Daniels, G. 1086

Daniels, S. R. 119

Danielsson, P. 820

Danne, T. 1075, 950

Danser, A. H. J. 1218

Dapía, S. 907

Darbyshire, J. L. 961

Dardari, D. 701, 1165

Darimont, C. 811

Darland, C. 852

Darmellah, A. 1367

Darsow, T. 1010

Dassau, E. 1064

Davalli, A. 531

Davey Smith, G. 299

Daviaud, D. 716

Davidson, H. W. 233, 485, 583, 585

Davidson, J. 994

Davidson, M. H. 847

Davidson, P. C. 1056

Davies, M. J. 73, 911, 912

Davies, M. J. $\quad 961,962,1035,1050$

Dawczynski, J. 1157, 1181

De Ambrogio, P. 402

De Bacquer, D. 924

De Bellis, A. 384, 1140, 1279

de Bie, R. A. 1258

De Bigontina, G. 156

De Block, C. E. 155

De Boever, E. H. 870

de Bruin, E. D. 1258

de Cabo, R. 646

De Caterina, A. 112

De Cosmo, S. 1166, 1213

De Courten, B. 190

De Fazio, M. 850

De Feo, P. P. 1117, 1387

De Feyter, H. M. M. 31

de Geus, E. J. C. 387

De Grijse, J. 332

De Hert, M. 711, 713

de Jager, J. 940

De Kerdanet, M. 81

de Kreutzenberg, S. 21
De la Questa, C. M. 230

de Leiva, A. 275

De Marinis, Y. Z. 631

De Mattheis, A. 47

De Medts, N. 29

De Mey, J. G. R. 1300

de Miguel, L. 452

De Natale, C. 741

De Nicola, A. F. 1393

De Palma, A. $\quad 181,949$

de Roos, A. 113, 114, 166

De Simone, I. 951

De Stefano, F. 850, 1283

De Tata, V. 343

De Vogel, J. 167

de Wet, H. 265

De Witt, H. J. 274

Deacon, C. 611

Dear, A. E. 893

Deary, I. J. 675

Debacker, N. 1043

Deblon, N. 674, 697

Debussche, X. 1350

Decochez, K. 332

Deedwania, P. 1357

Deeney, J. T. 510, 803

Defoort, C. 247, 1219

DeFronzo, R. A. $224,531,619$, $620,650,733,862,865,872$

Deghmoun, S. 263

Degrell, P. 1208

Dehghan, A. 12

Dei Cas, A. 19, 1155, 1331

Dejager, S. 75, 917

Dekker, J. M. 320, 387, 391, 1028, 1179,1305

Del Giudice, C. 204

Del Guerra, S. 136, 315, 597, 656, 903

Del Mese, P. $\quad$ 58, 626, 793

Del Prato, S. 315, 318, 597, 903, $1112,1122,1140,1171,1229$, $1276,1319,1400$

Del Zotto, H. H. 483

Dela, F. 310, 630

Delafontaine, P. 806

Delatola, E. 1138

Dell'Omo, G. 1229,1400

Dell'Oro, R. 1336

Dellavalle, E. 1297

Deloukas, P. 291

Delzenne, N. 70

Dembinska-Kiec, A. 247, 394

DeMicco, D. 566

Dencker, L. 1036

Denechaud, P. 742

Deng, W. $\quad 866,878,879$

Dennis, L. 32

Dentelli, P. 218

Deotto, M. 1380

DePaoli, A. M. 922

Der, K. 1

Derlindati, E. 19, 1331

Derosa, G. 1117

Desaiah, D. 1247

Despres, J. 1016

Detaille, D. 934

Detke, M. J. 1247

Detournay, B. $\quad 153,1039$

Devendra, D. 1114

Devlin, N. 722

Dew, T. 1265

Dey, D. 654

Deybach, C. 1381
Dezaki, K. $\quad 475,770$

Dhalla, A. K. 686

Di Bartolo, P. 230, 1101

Di Bella, A. 443

Di Cecco, P. 982

Di Cianni, G. 1122, 1140, 1145

Di Donna, S. 765

Di Fulvio, P. 629, 1291

Di Gruccio, J. M. 1284

Di Jeso, B. 691

Di Leo, S. 1196

Di Loreto, C. 1117,1387

Di Marco, S. 1347

Di Martino, V. 340

Di Paola, R. 689

Di Pietro, N. 629, 1291

Di Pietro, S. 575

Di Silvestre, S. 325, 629, 1291

Di Stasi, A. M. 195

Di Stefano, A. 1077

Di Stefano, R. 1319

Di Tomo, P. $\quad 629,1291$

Diaconu, L. 1346

Diago, J. I. $\quad 1020,1044$

Diakoff, E. 598

Diakogiannaki, E. 508

Diamant, M. $113,114,120,166$, $260,387,636,663,751,878,879$, $1065,1174,1204,1386$

Diaz, M. 867, 868, 1014

Diaz-Horta, O. 345

Diaz-Ricart, M. 205

Dib, S. A. 1074

Dick, E. 733

Dick, Jr., E. 531

Dickhaus, T. 1255

Didac, M. 369

Diep, L. M. 423

Dieterich, H. 1218

Dietrich, J. 723

Dimitriadis, G. $\quad 839,1313$

Dinarello, C. A. 1

Ding, H. 1369

Dinh, T. 1156

Dinneen, S. F. 1026

Dinning, L. 1125

Diouf, I. 939

Disse, E. 674

DiStefano, J. K. 142

Dittmann, J. 792

Divers, J. 304

Divine, G. 1051

Djiane, J. 131

Djuricic, B. 586,588

Dobashi, H. 1338

Dobbins, R. L. 870

Dobie, E. A. 1051

Dobransky, R. 279

Dobs, A. 911

Doehner, W. 1226

Dokter, W. H. A. $\quad 662,663$

Dole, W. P. $\quad 1217,1218$

Domin, J. 491

Dominguez, E. 345

Dominguez, H. 1394

Donadel, G. 204, 441, 926, 1317

Donath, M. 1068, 540

Donath, M. Y. $1,170,459,518$, 778

Donchenko, G. 56

Dong, B. R. 546

Dong, H. 863

Donic, V. 18

Donkó, Á. 256

Doran, S. 889

Doria, A. $\quad 689,1166$

Döring, A. 188

Döring, F. 468

Dorkova, Z. 1356

Dornhorst, A. 963, 966, 976

Doronzo, G. 58, 626, 793

Dotta, F. 136, 273, 367, 571, 575, 576

Douek, P. 22

Douglas, W. 1249

Doupis, J. 1156

Dova, L. 587

Dovan, D. 842

Dovolilova, E. 596, 604

Doyle III, F. J. 1064

Dragomir, E. 1289

Drain, P. 282

Drakopoulos, I. 389, 1142

Dray, C. 716

Drel, V. R. 95

Drenckhan, M. 857

Dreval, A. V. 355

Drevon, C. A. $\quad 247,1219$

Drews, G. 504

Drexel, H. $\quad 404,1351,1355,1360$, 1362

Drexhage, H. A. 274

Dreyhaupt, J. 1200

Droomgoole, P. 1108

Drucker, D. 146

Drucker, D. J. 908

Du, D. 923

Duarte, L. 1234

Duarte, R. 1119, 1234

Dubois, R. W. 680

Dubois, S. 624

Dubský, M. 1238

Duchareva, O. 952

Duchateau Nguyen, G. 822

Duckworth, W. 925, 994

Duderstadt, S. 188

Dudley, S. 1125

Düfer, M. 504, 544

Duffield, K. 13

Dugail, I. 85

Dugal, T. 944

Dulloo, A. G. 739

Duman, H. M. 1062

Dunger, D. B. 181

Dunmore, S. J. 514

Dunn, J. T. 227

Dunne, F. 1082

Dunning, B. E. 75

Duong Van Huyen, J. 1133

Dupont, O. 178

Dupuis, G. 558

Dupuis, J. 326

Dupuy, A. 1330

Durand, C. 88

Duranti, E. 1314

Durden, E. 1019

During, M. 797, 895

Durrington, P. N. 566

Durston, T. 1103

Duskova, M. 723

Dutour, A. 849

Duttenhoefer, F. 1309 
E

Ebbesson, S. 753

Ebel, N. 603

Eberle, H. 15

Eckel, R. H.. $\quad 35,756$

Eckers, U. 1012

Economopoulos, T. 825, 839, 1138,1313

Economou, K. 1142

Edghill, E. L. 237

Edmonds, M. 1265,1266

Edwards, S. E. 1248

Eeg-Olofsson, K. $\quad$ 191, 412

Eekhoff, E. M. W. 387

Efrat, S. 436

Egan, A. 1082

Egefjord, L. 502, 521

Egger, A. 671

Egido, E. M. $\quad 123,499$

Eguchi, K. 582

Ehehalt, F. $\quad 454,461$

Ehehalt, S. 376

Ehnholm, C. 1358

Ehrlich, S. F. 398

Ehses, J. A. 170, 771, 778

Eisenbarth, G. S. $\quad 336,574,583$, 585

Eising, S. $\quad 49,572$

Eizirik, D. L. 133, 214, 333, 509, 517, 548, 549

Ejenavi, E. 1081

Ek, J. 286

Ekblom, M. 890

Ekbom, P. 1135

Ekelund, U. 61

Ekinci, E. I. 1215

Ekstrom, H. L. 1053

El Bawab, S. 481

El-Mir, M. 934

Elbein, S. C. 301

Eldrup, E. 998

Eleftheriadou, I. $\quad 1348,1382$

Elfers, C. T. 635

Elfers, E. E. 121

Elgazar-Carmon, V. 827

Elgzyri, T. 669

Elhenicky, M. 1130, 253

Eliaschewitz, F. G. 1084, 979

Eliashar, R. 1368

Eliasson, B. $\quad 191,412,878,879$

Eliasson, L. 631

Eliasson, M. 415, 424

ElJaafari, A. 236

Elkind-Hirsch, K. E. 147

Ellard, S. 237, 239, 279, 280, 283

Ellingsgaard, H. $\quad 170,518$

Elliott, K. S. $\quad 300,302$

Elliott, P. 11, 12, 299

Ellis, B. 1102

Ellis, G. 861

Ellis, K. 121

Ellis, S. 967

Ellmerer, M. 1070

Elonheimo, O. M. 679

Elsborg, R. 225

Elsner, M. 480, 513

Emamaullee, J. A. 601

Emanueli, C. 1320

Emdin, M. 982

Emery, C. 244

Emery, C. J. 242

Emery, N. 311, 1169, 1332

Emir, B. 1246
Emmersen, P. B. 1123

Engberg, S. 364

Engelen, L. 1305

Engeli, S. 916

Engström, G. 724

Enigk, B. 396

Enk, D. 1260

Erbel, S. 555

Erben, R. G.. 532

Erdmann, K. S. 460

Eriksson, J. G. $\quad$ 53, 105

Eriksson, J. W. 252, 269, 836

Eriksson, K. 669

Eriksson, M. E. V. 1256

Erion, M. D. 931

Erlich, H. A. 140

Erlinge, D. 476

Ernst, A. 1226

Esbrit, P. 907

Eschwege, E. $\quad 153,416,939,1039$

Escoriza, J. 599, 904

Escrivá, F. 452

Escudero, C. 1161

Eshpeter, A. 448

Eshwaraiah, A. 5

Esmailzade, K. 1037

Esposito, I. 683

Essioux, L. 822

Esterbauer, H. 837

Esteve, E. 173

Estil.les, E. 599

Eto, K. 770

Eto, T. 995

\section{F}

Evans, M. $\quad 152,1216$

Ezrokhi, M. $\quad 128,39,685,930$

Ezzaty, K. 1037

Fabbri, G. 384

Fabris, B. 1162

Fabunmi, R. $\quad 881,882,883,960$

Facchetti, R. 47

Fadavi, H. 1269

Fadini, G. 21

Færch, K. 192

Fagot-Campagna, A. 153, 187, 416, 1039

Fahlman, C. 392

Fahrbach, J. 959

Faillie, J. 1059

Faivre-Defrance, F. 594

Falissard, B. 711

Fallahi, P. 172

Fallani, E. 1276

Fallarino, M. 1312

Faloia, E. 318

Falorni, A. 575, 576

Fan, J. 1194

Fan, R. 174, 634

Fanelli, C. G. 1282, 183

Fantus, I. George. 655

Farmer, A. J. 961

Farmer, B. 665

Farooqi, A. 1050

Farrington, K. 1209

Faruque, M. O. 323,648

Farwell, W. 930

Fassot, C. 1133

Fatema, K. 819

Fatema, N. 1128

Faubert, B. 696

Fauveau, V. 356, 607, 742

Favaro, E. 1297
Favre, D. 515

Favretti, F. 1137

Favuzzi, A. 1154

Fazio, S. 854

Federici, M. 67, 68, 204, 317, 441, $926,1310,1311,1317,1323$

Fedetz, M. 270

Fedetz, O. 270

Feher, M. D. 885

Feichtner, F. 1070

Feinstein, S. 847

Fejfarova, V. 1238

Feldmam, E. L. 241

Feldman, R. D. 1220

Feldt-Rasmussen, B. 1135

Felez, J. 710

Felici, A. C. 150

Feltbower, R. G. 118

Feng, L. 346, 1369

Ferencz, M. 1177

Ferlinz, K. 185

Fernandez, C. 361

Fernández, E. 452

Fernández Miró, M. 1148

Fernandez-Cadenas, I. 10

Fernandez-Perez, C. 1210

Fernandez-Real, J. M. 173, 709, 814,840

Fernandez-Veledo, S. 784

Ferrandiz, M. 1254

Ferrannini, E. 112, 161, 172, 608, $651,794,824,982,1227,1314$, 1347

Ferrante, T. C. 510

Ferrara, A. 398

Ferrarezi, D. A. F. 313

Ferrari, S. 172

Ferre, P. 341

Ferré, P. 791

Ferreira, I. 189, 254, 255, 386, 1305,1324

Ferrell, R. E. 1168

Ferrelli, F. 1317, 441

Ferrucci, L. 309

Feskens, E. J. M. $\quad$ 189, 352, 386, 1305

Fetita, L. 341, 664

Feuvray, D. 1367

Fica, S. V. 351

Fiedler, G. M. 246

Fiedorek, F. T. $\quad 40,796$

Fielding, B. 32

Filetti, S. 284, 1312

Filippi, C. 743

Filipponi, F. 136, 315, 597, 656, 903

Finch, S. 1215

Fineman, M. 866

Finer, N. 1052

Finocchietto, P. V. 767

Fioramonti, X. 607, 610

Fiorentino, L. 67, 68

Fioretto, P. 1166

Fiori, C. 150

Firneisz, G. 589

Fischer, C. P. $\quad 707,780$

Fischer, R. 667

Fischer Hansen, J. 1033, 1340

Fisher, R. M. 408

Fitchet, M. 915

Fite, A. 727

Fitzgerald, P. 1321

Fjeld, K. 286

Flachs, P. 738

Flacke, F. M. $\quad 677,678$

Flamez, D. 333, 517

Flanagan, S. 239, 286

Flanagan, S. E. 237

Flanders, S. 1085

Flatt, P. R. 124

Fleck, P. $\quad 76,77,860,861,862$

Fledelius, C. 975

Flint, A. 887,889

Florentin, E. 855

Flores, J. A. 1148

Flores, L. E. 483

Florez, H. J. 925

Florez, J. C. $\quad 326,689$

Florys, B. 1030

Flyvbjerg, A. 724,788

Fodri, D. 47

Foletta, V. 935

Foley, J. E. 75, 917

Folli, F. B. 531, 733, 1323

Fonseca, M. 345

Fonseca, V. A. $\quad 806,994$

Fontbonne, A. 939

Forbes, J. M. 160, 190

Foretz, M. 607

Forga, L. 354, 851

Forletto, M. 1137

Forma, F. 1005

Formisano, P. $\quad 305,683,691$

Formoso, G. 325, 629, 1291

Fornengo, P. 402

Forouhi, N. G. $\quad 349,421$

Forsberg, L. 654

Forsblom, C. M. 143, 1100, 1167, $1205,1354,1363$

Forst, S. 1267

Forst, T. $677,678,1267$

Fortuna, R. 1158

Fosgerau, K. 929

Foti, M. 697, 811, 1292

Fotino, C. 1171

Foufelle, F. 341, 791

Foufopoulou, E. 1099

Fourlanos, S. 141

Fournier, C. 153

Fournier, M. 697, 1292

Foussas, S. 1361

Fowler, D. 13

Fowler, K. T. 583

Fox, C. S. 326

Fradet, M. 481

Franc, S. 701

Franceschi, C. 321

Francini, F. 644

Franciosi, M. 1101

Franco, D. R. 979, 1084

Franconi, F. 384

Frandsen, E. 1217,1218

Frandsen, K. B. 981

Frandsen, M. 295

Fransson, L. 535

Fransson, U. 474, 487

Franzese, A. 951

Franzini, L. 19, 1117, 1331, 1387

Frascerra, S. 112

Fraser, D. 246

Frasheri, A. 1311

Frassi, F. 982

Frayling, T. M. 10, 64, 291, 299, 300, 302, 309 
Freimer, N. 11, 12

Freixa, J. 1254

Freixenet, N. 710

Frelinger, A. L. 1206

Freund, N. 1133

Friberg, J. 522

Frickey, C. 1173

Frid, A. $\quad 797,888,895,899$

Frieden, M. 1292

Friedlander, Y. 418

Friedrichsen, M. 613

Frier, B. M. 675,681

Frigerio, F. 127

Friis, H. 426

Frisch, L. M. 583

Fritsche, A. 186, 319, 320, 322, 395

Frizelle, P. A. 124

Froguel, P. 297, 301

Fröjdö, S. 88

Frongia, P. 986

Frontoni, S. 317, 318

Frøslie, K. F. 1141

Frystyk, J. 724

Fu, S. 28

Fu, Z. 17

Fujii, H. 1079

Fujii, T. 1164

Fujikawa, J. 363, 414, 1191

Fujimoto, H. 1115

Fujimoto, S. 942

Fujioka, K. 660, 726

Fujishima, K. 582

Fujita, H. 1224

Fujita, Y. 1185

Fujita, Y. 1364

Fujita, Y. 942

Fujiwara, H. 942

Fujiwara, M. 1295

Fukami, K. 1187

Fukuda, G. 1294, 1401

Fukumoto, Y. 1195

Fukushima, K. 582

Fukushima, S. 1396

Fuller, J. H. 254, 566

Fülöp, N. 1208

Fumeron, F. $311,313,1169,1328$, 1332

Fumey, G. 761

Funel, N. 136

Fung, L. M. 1214

Fung, Y. M. E. 649

Furuichi, Y. 1164

Furuta, H. 1257

Fusco, S. 695

Fysekidis, M. 1328

\section{G}

Gabillaud, I. 1059

Gabis, E. 1058,1061

Gable, D. 290

Gaborit, B. 849

Gabriel, M. 987

Gaddini, L. 195

Gaens, K. H. J. 809

Gagliardino, J. J. $\quad 482,483,644$, 1047

Gaillard, R. C. 1087

Gaillard, T. 1230, 1389

Gaisano, H. Y. 169

Gajardo, C. 1161

Galasso, R. 30

Galera, P. 1341
Galinier, A. 132

Gallart, L. 783

Galli, M. 136, 315, 597, 903

Gallis, J. 705

Gallo, R. 576

Galluccio, E. 1322

Galstyan, G. 1261

Galtier, F. 1059

Galvanovskis, J. 115

Galzin, A. 1242

Gamba, P. 47, 1336

Gambelunghe, G. 576

Gambert, P. 855

Gan, A. 1289

Gandhi, R. A. 244, 1240

Gangnerau, M. 358, 771

Ganz, F. 47

Gao, H. 410

Gao, L. $\quad 467,505,1369$

Gao, S. 980

Gao, X. 203, 1286

Gao, X. 262

Garaulet, M. 205

Garay-Malpartida, M. H. 215

Garber, A. 149, 797, 888, 892, $894,896,899$

Garbi, C. 691

García, B. 275

García, M. 158

García, M. E. 483

García, Y. 1392

García-Gimeno, M. A. 285

Garcia-Guerra, L. 784

Garcia-Hernandez, P. 896

García-Martínez, C. 646

Garcia-Ocana, A. 85

García-Ramírez, M. 1158

García-Rodríguez, L. A. 433

García-Unciti, M. 851

Garg, S. K. 967, 1066

Garmo, H. 314

Garoutsou, P. 983

Garren, H. 574

Garrow, A. P. 1271

Garruti, G. 1196

Gasa, R. 258

Gasc, J. 208

Gasperikova, D. 279

Gastaldelli, A. $112,531,647,702$, $733,852,871$

Gaster, M. 758

Gathercole, L. L. 762

Gatling, W. 1173

Gattesco, S. 211

Gatti, A. 1312

Gaudreau, S. 558

Gauguier, D. 361

Gauthier, B. 238

Gautier, J. 341

Gautier, J. 664

Gautier-Stein, A. 642

Gawlowski, T. 1299

Gawrecki, A. 1109

Gayet, C. 750

Gaziano, J. M. 39, 930

Gazzarri, A. 949

Gbarayor, C. M. 923

Geant, A. 102

Geddes, J. 681

Gedroyc, W. M. W. 1272

Gehrmann, W. 513

Gelber, A. 1073

Gelber, C. 360

Geldmacher, R. 1075
Gelling, R. 901

Geloneze, B. 150

Geloneze, S. R. 150

Genders, A. J. $\quad 625,628$

Geng, X. 282

Genova, M. 1120

Genovese, S. 370

George, J. T. 1108

Gérard, J. L. 1341

Gerasimidi-Vaseou, A. 389

Gerich, J. 898

German, J. 129

Gerozissis, K. $\quad 131,774$

Gerrits, A. J. 199

Gerrits, E. G. 1304

Gerstein, H. C. 1060

Gerstl, E. 117

Gertler, A. 131

Gerwien, R. 54

Gesualdo, P. 373

Geubtner, K. 569

Geurts, L. 70

Geyeregger, R. 737

Ghanaat-Pour, H. 137

Ghiadoni, L. 1314, 1319

Ghigo, E. 183

Ghio, A. 1122,1140

Ghirlanda, G. 1337, 1344

Ghosal, R. 44

Ghosh, S. 1334

Giacca, A. 655

Giaccari, A. 619

Giacco, F. 305

Giacco, R. 741

Giani, E. 949

Giannakopoulos, F. 983

Giannakopoulos, N. 1099

Giannessi, D. 982

Giannessi, F. 765

Giardina, S. 1002

Giardinelli, A. $\quad 629,1291$

Gibbs, R. G. J. 1272

Gier, B. 504, 544

Giesler, P. D. 122

Gilardini, L. 1212

Gilbert, M. 130

Gillespie, K. M. 366

Gillot, F. 1242

Giménez-Palop, O. 710, 783

Gimpelewicz, C. 915

Gin, H. 705

Ginsberg, H. N. 750

Giorda, C. 156, 1213

Giordanino, C. 58,793

Giordano, C. 370

Giorgetti, A. 982

Giorgino, F. 318, 584, 850, 1196, 1283

Giorgino, R. 850, 1196

Giotaki, E. 587

Girard, C. A. 115

Girard, J. 356, 742, 761

Girelli, A. 230, 1101

Girman, P. 596, 604

Giroix, M. 481, 771, 778

Gitt, A. 1359

Gitz, E. 199

Giubilato, S. 1337

Giunti, S. 93

Giusti, V. 811

Gjedde, A. 676

Gjelstad, I. M. F. 1219

Gjerlaugsen, N. 294

Gjørup, T. 673

Glaister, K. 1098

Glaser, B. 328

Glass, L. C. 863,873

Glowinska-Olszewska, B. 1030

Gloyn, A. L. $\quad 64,279,327$

Glümer, C. $\quad$ 192, 364

Glund, S. 90, 130

Gniuli, D. 1154

Gnudi, L. 19, 1155

Godang, K. 1141

Goday, A. 154, 1090

Godfredsen, C. 788

Godsland, I. 421

Goede, D. L. 636

Goedhart, P. T. 1375

Gögebakan, Ö. $\quad$ 528, 828

Gogg, S. 209

Goglia, F. 172

Golan, R. 246, 958

Golas, A. 1085

Golay, A. 721, 843, 1258

Goldberg, D. J. 1084

Goldberg, R. B. 853

Goldman, S. 333

Goldstein, B. J. 326, 911

Goldsworthy, M. 362

Golicka, D. 948, 989

Golla, S. 231

Gollinger, K. 737

Golm, G. 911

Golmard, J. 1381

Gombos, Z. 334

Gomes, M. B. 1280

Gomez, P. 205

Gómez-Foix, A. M. 646

Gomez-Galeno, J. 931

Gómez-Peralta, F. 934

Gomis, R. 205, 258

Gonbert, S. 963, 966, 976

Gonçalves, A. 1119

Goncharov, N. 659

Goñi, M. J. 851

Gonzalez, J. S. 1271, 1274

González, P. 345

González-Clemente, J. 710, 783

González-Yanes, C. 87, 693

Goodall, G. 184

Goodman, M. 74, 913

Goodman, N. 333

Gordin, D. 1354, 1363

Gordon, J. 1004

Gorely, T. 1035

Gorenjak, M. 1190

Goretti, C. 1273

Gorostiaga, E. M. 851

Gorostidi, M. 1222

Gorska, M. 337, 844, 1127, 1132, 1395

Gorter, K. J. $\quad 353,431,999,1054$, 1097

Gorus, F. 332

Gossage, A. 944

Gottlieb, P. A. $\quad 233,574$

Gough, S. 981

Gould, G. W. 89

Gouni, R. 1256

Gourdy, P. 235

Govindan, J. P. 884

Grabert, M. 117 
Gragnoli, C. 292

Gralho, A. 1234

Gram, B. 1038

Gram, J. 1038

Grammatikou, S. 348

Granada, M. 578

Grandjean, P. W. 933

Grankvist, N. 535, 537

Grant, M. 1013

Grant, S. F. A. 276

Granvik, M. 462

Grapengiesser, E. 471

Grarup, N. 63, 66, 324

Grassi, G. 1106

Grassi, G. $\quad 47,1336$

Graves, D. 197

Gray, D. W. R. 445

Graydon, R. 1308

Greenberg, I. 246

Grefhorst, A. 662

Greisen, G. 1123

Grémeaux, T. 640

Gresele, P. 1282

Gribble, L. C. 1256

Grieco, F. A. 576

Griffin, M. 1252

Griffin, S. J. $\quad 1050,1365$

Griffiths, P. D. 242

Grigorescu, F. 393, 641

Grigoropoulou, P. 1348, 1382

Grill, V. 271, 368, 498, 551

Grimaldi, A. 992, 1039, 1381

Grimsby, J. 42, 927, 928

Grippaldi, F. 652

Gris, J. 393

Grisouard, J. 785

Grobbee, D. E. 1175

Gröbe, H. 117

Groele, L. 948, 989

Groenewoud, M. J. 320

Groenier, K. H. $\quad$ 182, 434, 1076, 1107, 1391

Groop, L. C. 53, 62, 65, 269, 306, $312,318,669$

Groop, P.-H. $143,706,1100$, $1167,1205,1354,1363$

Gross, J. L. 77, 1247

Gross, R. 484

Groth, D. 828

Group, P. 405

Groves, C. J. $\quad 64,301,302$

Gruber, S. 593

Gruden, G. 198

Grudzien, M. 658

Gruetter, R. 127

Grünler, J. $\quad 763,1263$

Gu, H. F. 822

Gu, J. Y. 1032

Gu, Q. 203

Gu, X. $\quad 174,634$

Guan, Q. B. 346, 467

Guan, X. 872

Guardardo Mendoza, R. 531, 733

Guarino, M. P. 688

Gudbjörnsdottir, S. 191, 269, 412,1088

Guédin, A. 856

Guerci, B. 2, 988, 1039, 1165

Guerediaga, J. 1222

Guevara-Aguirre, J. 1022

Guevara-Aguirre, M. 1022

Guglielmi, C. 446, 561, 952

Guglielmi, V. 204, 926

Guidone, C. 1154
Guigas, B. 260

Guigne, C. 716

Guildford, A. 1343

Guillen, C. 534

Guilloteau, G. 1165

Guindi, C. 558

Guinez-Lelong, C. 356

Guiot, Y. 70

Gulde, G. 15

Gülden, E. $\quad 829,830$

Gullestad, L. 1278

Gulseth, H. L. 247, 1219

Gumenyuk, V. 55

Gunter, J. H. 445

Gunton, J. E. 456, 1139

Guo, H. 467

Guo, W. 803

Guoxian, D. 164

Gurgul-Convey, E. 520

Gurzov, E. N. 548

Gustavsson, C. 1159

Gusto, G. 754

Gutiérrez, A. 258

Gutjahr, G. 378

Gutt, B. 667

Guyton, J. R. 853, 854

Guzik, T. J. 1176

Gwiazda, K. S. 553

Gylfe, E. 492, 493

Gylling, H. 795

Gyulkhandanyan, A. 764

Gyürüs, E. 104

\section{H}

Ha Van, G. 1381

Haak, T. 15, 229

Haapala, L. 377

Habeos, I. G. 807

Haberberger, B. M. 1308

Habich, C. 829,830

Habicht, F. 843

Hackl, S. I. 485

Hacquebard, M. 954

Hadad, N. 827

Hadjadj, S. 1165,1332

Hadjidakis, D. J. 825

Haefliger, J. 455

Haenen, G. R. M. 1300

Haffner, S. M. 24, 847

Hafizur, R. M. 323

Hahn-Obercyger, M. 645

Haidar, T. 680

Hainault, I. 791

Hajduch, E. 85

Hak, E. 1054

Hakonarson, H. 276

Hakonen, E. 29

Halban, P. A. $170,459,540$

Halbron, M. 1381

Hale, P. M. $\quad 894,896,898$

Halff, G. 531

Halimi, J. 1165

Halimi, S. $\quad 153,1039$

Hall, J. A. 1248

Haller, H. $\quad 48,1232$

Halseth, A. 798

Halsne, Å. 712

Halvatsiotis, P. G. 1138

Hamamoto, Y. $363,414,440,902$, 905, 1191

Hamann, A. 2

Hamasaki, A. 902

Hamblin, S. 1072
Hamilton, B. 717

Hamilton, S. 1249

Hamman, R. F. 119

Hammar, E. 170, 459

Hammar, N. 415

Hammer, M. 894

Hammond, P. J. 1125

Hamo Tchatchouang, E. 221

Hamsten, A. 408

Han, J. 1245

Han, J. $\quad 863,886$

Han, K. $\quad 668,1378$

Han, P. 655

Hanaire, H. 976, 988

Hanaoka, I. 1349

Hancu, N. 992, 1057

Haneda, M. 1185

Hanefeld, M. 223, 409

Hanemaaijer, R. 1204

Hanouz, J. L. 1341

Hansen, A. K. 216

Hansen, B. C. 445

Hansen, J. B. 965, 976

Hansen, L. 265

Hansen, T. 54, 63, 66, 286, 295, $310,324,328$

Hansen, T. 79

Hanson, R. L. 301

Hanssen, K. 1298

Hanssens, L. 713

Hansson, O. 669

Hanzu, F. 205

Hao, H. 1215

Hara, K. 1251

Harada, N. 902, 905

Harashima, S. 902

Harcourt, B. E. 160

Hardies, J. 852

Hardiman, M. 1031

Hare, K. J. 661

Harel, C. 249

Hargrove, D. 901

Häring, H. 138, 186, 319, 320, $322,395,547,1285$

Harjutsalo, V. 103, 377

Harkin, D. W. 1288

Harley, C. 920

Harman-Boehm, I. 1073

Harries, L. W. 237

Harris, S. 1060

Harrison, L. C. 141

Harrison, L. N. $\quad 732,792$

Harrison, M. 486, 1343

Harte, A. L. $\quad 210,762,790,826$, 832

Harteman-Heurtier, A. 595

Hartemann-Heurtier, A. 1381

Hartikainen, A. 11

Hartmann, R. 1075

Hasan, K. 323

Hasan, M. 1018

Hasegawa, M. 1396

Hashimoto, T. 985,1115

Hashiramoto, M. 135, 511

Hassan, S. N. 648

Hassan, Z. 648, 729

Hassanein, M. 1114

Hasslacher, C. 1200

Hatlapatka, K. $\quad 98,478$

Hattersley, A. T. $10,64,237,239$, 280, 283, 286, 291, 299, 300, 302, 309

Hattori, M. 1164

Hattori, S. 1315

Hattori, Y. 1315

Haugaard, S. B. 755

Hauner, H. 1000, 1052

Hausken, M. F. 1049

Havrdova, T. 577, 592

Hawa, M. I. $\quad 369,566,580$

Hayakawa, N. 1083

Hayashida, A. 1187

Hayes, P. C. 743

Hayes, R. 184

Hayward, A. 1155

Hazzan, M. 594

He, J. 1402

He, N. 666

He, T. 530

He, Y. 936

Healey, B. 884

Hebda-Szydło, A. 1121

Hecker, C. 616

Hedderson, M. M. 398

Hedley-Dodd, A. 392

Hedman, M. 111

Hedrich, H. J. 278

Heid, I. M. 7

Heikkilä, O. $\quad 706,1167,1354$, 1363

Heikkinen, S. 706

Heine, R. J. 320, 387, 415, 636, 663 , $751,878,879,1055,1063,1065$, $1179,1305,1386$

Heinemann, L. 677, 1012, 1015, 1068

Heinonen, S. 111

Heinsen, F. 468

Heinzelmann, I. 138

Heise, T. 927, 1012

Hekkala, A. 51

Helal, R. $\quad 1128,1129,1134,1136$

Helleberg, H. 891

Hellenius, M. 408

Heller, R. 216

Heller, S. R. $\quad 962,1237$

Hellman, B. 471

Helwig, U. 1290

Hemmings, B. A. 176

Henegar, C. 849

Heng-Hung, L. 345

Hénique, C. 761

Henk, H. 1005

Henkin, Y. 246

Hennige, A. M. 138, 547

Hennuyer, N. 856

Henouda, M. 1384

Henquin, J. 99

Henriksen, T. 1141

Henrion, U. 463

Henry, R. 896

Henry, R. R. 224, 650

Hense, H. W. 188

Hensler, M. 735,738

Herbelin, A. 235

Herder, C. 779

Herman, W. H. 24, 241, 959, 1253

Hermann, R. 334, 342

Hermann, T. S. 1394

Hermanns, N. 15, 229

Hermansen, K. $\quad 149,797,895$

Hermányi, Z. 1240

Hernandez, B. 1062 


\begin{tabular}{|c|c|c|c|}
\hline Hernández-Fisac, I. 495 & Howorka, K. 987 & Im, S. 615 & Jang, H. 1371 \\
\hline Herranz, L. 1149 & Howorka, N. 987 & Imachi, H. 1295 & Jang, H. $\quad 1376,1377,390,692$ \\
\hline Herrera, B. M. $\quad 64,361$ & Hribal, M. L. 317,325 & Imaizumi, T. 1302 & Jani, R. 620 \\
\hline Herrera, P. 25 & Hsu, B. R. S. 28 & Imes, S. 590 & Jankovic, V. 1351,1355 \\
\hline Herrmann, F. 1352 & Hsu, I. R. 732,792 & Imura, M. 1164 & Jänne, J. 111 \\
\hline Herrmann, U. 1067 & Hu, C. 307,936 & Inada, H. $\quad 1115,985$ & Janovska, P. 738 \\
\hline Hertel, J. K. 303 & $\mathrm{Hu}, \mathrm{F} . \quad 80$ & Inagaki, N. $359,440,602,902$, & Jansen, H. J. 974 \\
\hline Hertenberger, C. 616 & Hu, L. 803 & 905,942 & Jansky, G. L.. 555 \\
\hline Herynek, V. 604 & Hua, C. 817 & Incani, M. 284 & Janssen, P. G. H. 353 \\
\hline Herzenstein, O. $\quad 1058,1061$ & Huang, E. 933 & Ince, C. 1375 & Jansson, P. $\quad 1385,209,836$ \\
\hline Hesse, D. 1135 & Huang, G. G. C. 437,439 & Ingskog, W. 1049 & Jansson, S. P. O. 419 \\
\hline Hesse, U. 1135 & Huang, Y. 1287 & Innes, J. 1091 & Januszewicz, A. $\quad 48,1232$ \\
\hline Hesselink, M. K. C. $\quad 167,168$ & Huang, Y. 169 & Inouye, K. E. 501 & Jaroch, J. 1335 \\
\hline Hessvik, N. P. 758 & Hub, R. 376 & Inukai, T. $808,1251,1373$ & Jarosz-Chobot, P. 268 \\
\hline Hollenberg, N. K. 45 & Hubbard, G. $\quad 531,733$ & Invitti, C. 1212 & Jarvelin, M. 11, 12, 297, 299, 309 \\
\hline Holliman, G. N. $\quad 437,439,906$ & Huber, P. M. 671 & Ionescu, I. 842 & Jaszlits, L. 714 \\
\hline Hollingsworth, K. G. 766 & Hubert, T. 594 & Ionut, V. $\quad 732,775,792,864$ & Jawa, A. 1018 \\
\hline Holman, R. R. $\quad 24,1365,961$ & Hucking, K. 775 & Iorio, M. C. 1319 & Jebunnesa, F. $1128,1129,1134$, \\
\hline Holmboe-Ottesen, G. 423 & Huckova, M. 279 & Iovino, S. 683 & 1136 \\
\hline Holmeide, A. Kristin. 735 & Huet, D. 1379 & Iozzo, P. $\quad 240,760,799,982$ & Jedinakova, T. 592 \\
\hline Holmen, J. 294 & Huet, F. 81 & Ip, T. P. 1214 & Jee, S. 1326 \\
\hline Holmkvist, J. 324 & Huffman, D. M. 1008 & Ippoliti, A. 1323 & Jeffery, A. 670 \\
\hline Holod, S. A. 767 & Huggins, C. 600 & Iraklianou, S. 1099 & Jeffery, A. N. 947,1089 \\
\hline Holst, J. J. 192, 216, 403, 608, 632, & Hughes, A. D. $\quad 23,421,1175$ & Irie, S. 995 & Jeffrey, K. D. 550 \\
\hline $636,637,661,676,1153$ & Hughes, S. J. 445 & Irminger, J. $\quad 771,778$ & Jelenik, T. 735 \\
\hline Home, P. D. 994,1372 & Huh, K. 1326 & Ishibashi, S. 770 & Jelic, S. D. 1223 \\
\hline Hommel, E. $\quad 1033,1135,1340$ & Hui, E. 1114 & Ishida, T. 1295,1338 & Jelinek, R. 687 \\
\hline Homo-Delarche, F. $\quad 771,778$, & Huijberts, M. S. P. 1270 & Ishizuka, T. 660,726 & Jendle, J. $\quad 724,797,969$ \\
\hline 1393 & Hummel, M. 264 & Islam, N. M. 323 & Jenkins, A. 957,1072 \\
\hline Hompesch, M. 1014,1015 & Hummel, S. $\quad 264,373$ & Islam, S. 323 & Jennings, B. 1173 \\
\hline Honeyman, M. C. 141 & Humpert, P. M. 243, 1309 & Ismail, K. 1025 & Jensen, B. R. 1206 \\
\hline Hong, E. 1186 & Humphreys, S. M. 32 & Iso, K. 744 & Jensen, J. 252 \\
\hline Hong, M. 1023 & Humphries, S. 290 & Isoe, T. 1185 & Jensen, R. 410 \\
\hline Hong, O. 804 & Hung, C. 1399 & Isomaa, B. 62 & Jenssen, T. G. 1220 \\
\hline Hong, S. 953 & Hunter, D. 80 & Issad, T. 693 & Jenum, A. K. $\quad 423,1049$ \\
\hline Honjo, S. $363,414,1191$ & Hunter, S. 369 & Istenes, I. 1233,1240 & Jermendy, G. $\quad 1240,1359$ \\
\hline Honjyo, J. 1185 & Hupkens, E. $\quad 473,954$ & Ith, M. 671 & Jerums, G. 1215 \\
\hline Honkanen, H. 342 & Hurtevent, J. 594 & Ito, H. 159 & Jeziorska, M. 1250 \\
\hline Honkanen, R. E. 535,537 & Huseen, A. A. 832 & Ito, R. 1294 & Jia, W. $301,307,936$ \\
\hline Honkasalo, M. T. 679 & Hussain, A. 712 & Ito, S. $\quad 48,1232$ & Jiang, H. $\quad 959,977,997$ \\
\hline Hopkins, D. 1025,1071 & Husslein, P. Wolf. 1130 & Itoh, M. 84 & Jiang, M. 73 \\
\hline Hopp, S. 547 & Hutchcraft, A. 1012 & Iwamoto, Y. $\quad 281,464,991,1396$ & Jianlei, L. 725 \\
\hline Hor, T. 964 & Hutton, J. 336 & Iwasaki, M. 477 & Jiaze, L. 93 \\
\hline Horová, E. 1374 & Hutton, J. C. $\quad 233,485,583,585$ & Iwasaki, M. 991 & Jilkova, Z. 735,738 \\
\hline Horovitz-Fried, M. 684 & Hyer, S. 1147 & Iwasaki, N. 281, 464 & Jimba, S. 1396 \\
\hline Horowitz, M. 889 & Hynx, D. 176 & Iyer, H. 5 & Jinnouchi, Y. 1302 \\
\hline Horsdal, H. T. 411 & Hyöty, H. 342 & Iyer, M. S. 732 & Jirkovská, A. 1238 \\
\hline Horváth, B. 256 & Hypponen, E. 299 & Izquierdo, M. 851 & Jo, S. 60 \\
\hline Horvath, E. M. 1126 & & Izzo Jr., J. L. $\quad 48,1232$ & Jo, Y. I. 990 \\
\hline Horvath, T. 262 & I & & Joanny, G. $\quad 599,904$ \\
\hline Horwitz, D. 230 & & $\mathbf{J}$ & Johal, B. 13 \\
\hline Hosaka, T. 1364 & Iacobini, C. 1310,1311 & & Johal, K. 1048 \\
\hline Hosking, J. $\quad 670,947,1089$ & Iaconelli, A. 1154 & Jaap, A. 743 & Johannesen, J. 572 \\
\hline Hosoba, M. 1224 & Iadicicco, C. 691 & Jaber, Y. 1228 & Johansen, A. 286 \\
\hline Hosokawa, M. 942 & Iafusco, D. $282,949,986$ & Jablkowski, M. 919 & Johansen, J. S. 780 \\
\hline Hosokawa, R. 414 & Iakoubov, R. 125 & Jablonski, K. A.. 326 & Johansen, O. E. 1278 \\
\hline Hossain, M. M. 323 & Ibata, J. 1257 & Jackson, S. 1029 & Johansson, B. B. 287 \\
\hline Hossain, S. 819 & Ibrahim, S. 213 & Jacob, K. J. 451 & Johansson, C. 1268 \\
\hline Houdek, P. 1262 & Idevall, O. 493 & Jacober, S. 1359 & Johansson, L. 836 \\
\hline Hougaard, D. M. 49 & Idevall-Hagren, O. 492 & Jacobs, M. 386 & Johansson, L. E. 820 \\
\hline Hougaard, P. 265 & Igoillo-Esteve, M. 133, 509 & Jacobs, S. 1056 & Johansson, L. M. 820 \\
\hline Houlihan, C. 1215 & Ihlemann, N. 1394 & Jacobsen, P. K. 1318 & Johansson, S. $\quad 286,287,303$ \\
\hline House, J. L. 1062 & Ihm, J. 527 & Jacobson, A. M. 228 & Johansson, S. 433 \\
\hline Houten, S. M. 167 & Ihm, S. 527, 1186 & Jacobson, P. 658 & Johns, D. 874,875 \\
\hline Houweling, S. T. $182,434,1107$, & Ikeda, H. $\quad 363,414,1191$ & Jacqueminet, S. 992 & Johnsen, S. P. 411 \\
\hline 1391 & Ikeda, T. $\quad 660,726$ & Jadzinsky, M. 78 & Johnson, A. B. 1029 \\
\hline Hove, K. 673 & Ikeda, T. 769 & Jafar Mohammadi, B. 64 & Johnson, C. 1215 \\
\hline Hovind, P. $\quad 255,1324$ & Ikonomi, P. 360 & Jager, J. 640 & Johnson, J. A. 428 \\
\hline Hovorka, R. 181,752 & Ikonomov, O. 251 & Jahan, S. 729 & Johnson, J. D. $\quad 451,533,550,553$ \\
\hline Hovsepyan, M. 789 & Ilag, L. 1063 & Jahannault, C. 484 & Johnson, K. 1125 \\
\hline Howard, B. V. 753 & Ilan, E. 787 & James, D. E. 37 & Johnson, P. E. 1053 \\
\hline Howe, T. 1102 & Ilin, A. 1024 & Janciauskiene, S. 523 & Johnson, P. R. V. 445 \\
\hline Howell, S. K. 43 & Ilonen, J. $\quad 51,569,277,334,342$, & Jandeleit-Dahm, K. A. M. 93 & Joly, E. 772 \\
\hline Howie, F. 743 & 1167 & Jang, D. Sik. 196 & Jonas, J. 212,526 \\
\hline
\end{tabular}




\begin{tabular}{|c|c|c|c|}
\hline Jonassen, T. E. N. 736 & Kallis, M. 523 & Katsilambros, N. $348,839,1236$, & Kiens, B. 673 \\
\hline Joner, G. $\quad 267,365$ & Kaltenboeck, A. 921 & $1281,1348,1382$ & Kikuchi, M. 995 \\
\hline Jones, A. 1372 & Kalupahana, D. N. 884 & Katsorida, M. 348 & Kilhovd, B. K. 1298 \\
\hline Jones, C. 1075 & Kalynyak, T. B. 550 & Katsoulieris, E. $\quad 554,1160$ & Kilonzo, B. 426 \\
\hline Jones, G. C. 941 & Kamal, M. A. 202 & Katsura, M. 259 & Kim, B. 1353 \\
\hline Jones, K. L. 889 & Kamali, M. 1037 & Katus, H. 110 & Kim, B. 1353 \\
\hline Jones, N. P. 24 & Kamaruddin, N. A. 897 & Katzman, P. 1268 & Kim, C. 1186 \\
\hline Jones, S. 1078 & Kamchatnov, P. 1024 & Kaufman, J. 924 & Kim, C. 1326 \\
\hline Jones, S. $\quad 1090,154$ & Kaminski, M. T. 175 & Kaufman, K. D. $73,911,912$ & Kim, C. 196 \\
\hline Jong, H. W. A. 114 & Kamiya, H. 1327 & Kaushik, S. V. 933 & Kim, C. 60 \\
\hline Jonsson, A. $53,62,65$ & Kammoun, H. 791 & Kautzky-Willer, A. $\quad 34,1143,1152$ & Kim, C. 845 \\
\hline Joosten, H. 1192 & Kamp, O. 114 & Kawai, K. 1195 & Kim, C. E. 276 \\
\hline Jordan, J. 916 & Kamper, A. M. 1391 & Kawamori, R. $329,978,991$ & Kim, D. 1186 \\
\hline Jørgensen, A. P. 1278 & Kanai, M. 996 & Kawamura, T. $\quad 985,1115$ & Kim, D. 1186 \\
\hline Jørgensen, M. E. 400 & Kanazawa, A. 1401 & Kawano, H. 84 & Kim, D. 1326 \\
\hline Jørgensen, T. $324,328,364,397$ & Kanda, Y. $\quad 135,511$ & Kawasaki, E. 582 & Kim, D. 1371 \\
\hline Jorgensen, T. 54 & Kane, A. 510 & Kawasaki, F. 135 & Kim, D. 399 \\
\hline Jørgensen, T. 63,66 & Kaneko, K. 1164 & Kawasaki, Y. 902 & Kim, D. 804 \\
\hline Jörns, A. $278,478,513,557,776$ & Kaneko, S. 768 & Kawashima, H. 359 & Kim, D. 804 \\
\hline Jorsal, A. 1198 & Kang, E. 293 & Kaya, Z. 110 & Kim, E. 1378,668 \\
\hline Jortani, E. P. 1194 & Kang, E. Seok. 1329 & Kaye, J. 1086 & Kim, E. 740 \\
\hline Jortay, J. 200 & Kang, J. 1186 & Kazdová, L. $71,728,812,815$ & Kim, F. 129 \\
\hline Joshi, S. 906 & Kang, J. 1353 & Keech, A. 1358,1366 & Kim, H. 1186 \\
\hline Jotic, A. $573,586,588$ & Kang, J. 527 & Keefe, D. L. 1222 & Kim, H. 1371 \\
\hline Jougla, E. 416 & Kang, M. 804 & Kefalogiannis, N. 1027 & Kim, H. 1378 \\
\hline Jovanovski, E. 957 & Kang, M. 953 & Kefaloyannis, N. 983 & Kim, H. 1378 \\
\hline Jowett, J. 935 & Kang, S. 1186 & Keil, U. 188 & Kim, H. 293 \\
\hline Joya, J. 865 & Kang, S. 804 & Keith, L. 625 & Kim, H. 668 \\
\hline Juge-Aubry, C. 811 & Kang, Y. 1286 & Keller, U. $\quad 785,1110$ & Kim, H. 668 \\
\hline Juhaeri, J. 980 & Kang, Y. 1371 & Kelly, C. J. 1321 & Kim, H. 845 \\
\hline Juhl, C. B. 225 & Kang, Y. 381 & Kelly, J. 1321 & Kim, H. J. 1329 \\
\hline Juhl, K. 734 & Kankova, K. 1306 & Kelly, S. 13 & Kim, H. Y. 718 \\
\hline Juhl, T. 1218 & Kantartzis, K. 322 & Kelsey, G. 470 & Kim, I. 381 \\
\hline Julià, M. 258 & Kanzleiter, T. 612 & Kempf, H. 208 & Kim, J. 1296 \\
\hline Julier, C. 140 & Kaouache, S. 393 & Kempf, K. 1113 & Kim, J. 1296 \\
\hline Julius, U. 95 & Kapitza, C. $\quad 887,889,927$ & Kempler, P. $\quad 1233,1240$ & Kim, J. 1353 \\
\hline Juncà, J. 578 & Kar, P. 6 & Kendall, D. $\quad 878,879,146,886$ & Kim, J. 196 \\
\hline Juneja, R. 1085 & Karaca, M. 102 & 901 & Kim, J. 622 \\
\hline Jung, C. 60 & Karagianni, C. 1099 & Kennedy, L. 1001 & Kim, J. 740 \\
\hline Jung, E. 1023 & Karagiaouri, E. 1099 & Kennon, B. 1102 & Kim, J. 845 \\
\hline Jung, H. $\quad 390,622$ & Karagüzel, G. 579 & Kepaptzoglou, O. 983 & Kim, J. S. 196 \\
\hline Jung, J. 1371 & Karalliedde, J. 44 & Keresztes, K. $\quad 1233,1240$ & Kim, K. 1245,1245 \\
\hline Jung, P. 658 & Karamanos, B. G. 339 & Kergoat, M. 481 & Kim, K. 622 \\
\hline Jurado, J. 1254 & Karanam, S. 350 & Kerner, W. 1067 & Kim, M. 293 \\
\hline Juutilainen, A. 1298 & Karasik, A. $\quad 223,1061$ & Kerr, D. $\quad 230,1256$ & Kim, M. $\quad 614,615$ \\
\hline & Karczewska-Kupczewska, & Kerr, L. 1359 & Kim, M. 692 \\
\hline $\mathbf{K}$ & M. 1395 & Kerr-Conte, J. $\quad 170,594$ & Kim, M. 841 \\
\hline & Kargar, C. 102 & Kerrigan, D. 201,831 & Kim, N. H. 718 \\
\hline Kaaja, R. 377,1124 & Karimi, N. 1037 & Keskinen, P. 51 & Kim, S. 1353 \\
\hline Kaakinen, M. 11,12 & Karkour, A. 463 & Kessels, M. M. 257 & Kim, S. 293 \\
\hline Kabadi, U. M. 970 & Karlsen, A. E. $216,521,522$ & Kessler, K. 1006 & Kim, S. 381 \\
\hline Kabir, M. $\quad 732,775$ & Karlsson, C. 836 & Kessler, L. $\quad 603,606$ & Kim, S. 381 \\
\hline Kabir, M. G. 648 & Karlström, B. 1219 & Kesty, N. 83 & Kim, S. 390 \\
\hline Kacheva, S. A. 520 & Karmi, A. C. 799 & Keyler, T. 1078 & Kim, S. 392 \\
\hline Kachko, I. 86 & Karnieli, E. 249 & Keymeulen, B. 332 & Kim, S. 399 \\
\hline Kachko - Chernetsky, I. 687 & Karolczuk-Zarachowicz, M. 1395 & Khalili, P. 724 & Kim, S. 60 \\
\hline Kadowaki, T. $910,991,996$ & Karpe, F. $\quad 32,682$ & Khan, F. M. 729 & Kim, S. 60 \\
\hline Kadowitz, P. J. 806 & Karvonen, M. $103,105,372$ & Khan, I. $\quad 648,729$ & Kim, S. 622 \\
\hline Kaduka, L. 426 & Kasai, K. 1315 & Khan, T. 819 & Kim, S. 672 \\
\hline Kahn, C. 690 & Kashiwagi, A. 329,910 & Khanam, P. A. 420 & Kim, S. 804 \\
\hline Kahn, S. E. 24,326 & Kaski, K. 1363 & Kharitonov, A. V. 563 & Kim, S. 804 \\
\hline Kahraman, A. 1017 & Kasperk, C. 1309 & Khatun, K. 420 & Kim, S. 991 \\
\hline Kaida, Y. 1187 & Kassis, N. 778 & Khatun, M. 1144 & Kim, S. G. 718 \\
\hline Kaim, I. 1121 & Kastendieck, C. 117 & Khaw, K. 349,1365 & Kim, S. P. 732 \\
\hline Kaisaki, P. J. 361 & Kastrup, J. 1318 & Khazaei, M. 1334 & Kim, S. P. 775 \\
\hline Kaiser, D. 465,479 & Katajamaa, M. 51 & Khazrai, M. 952 & Kim, S. P. 792 \\
\hline Kaiser, N. 524 & Kataoka, H. 91, 92 & Khedkar, A. P. 5 & Kim, T. 1186 \\
\hline Kaizik, S. 115 & Katare, R. 1320 & Khijnyak, O. 270 & Kim, T. 146 \\
\hline Kajantie, E. 53 & Katayama, S. $\quad 48,1232$ & Khoo, C. P. 560 & Kim, T. $\quad 614,615,841$ \\
\hline Kajiata, K. 660 & Katayama, Y. 995,996 & Khunti, K. $\quad 962,1035,1050$ & Kim, T. 933 \\
\hline Kajita, K. 726 & Kato, M. 1079 & Khury, J. 1042 & Kim, T. H. 880 \\
\hline Kajitani, N. $\quad 69,91,92,162$ & Kato, N. 1079 & Kidron, M. 1006 & Kim, T. N. 718 \\
\hline Kakei, M. 475 & Katra, B. 1121 & Kiefer, F. W. 737 & Kim, Y. 1296 \\
\hline Kaku, K. $\quad 135,259,329,511,996$ & Katrukha, A. G. 563 & Kiely, A. M. 653 & Kim, Y. 381 \\
\hline
\end{tabular}


Kim, Y. 399

Kim, Y. 43

Kim, Y. 672

Kim, Y. 740

Kim, Y. H. 718

Kim, Y. S. 370

Kimura, H. 359

Kimura, K. 985, 115

Kimura, T. $\quad 363,414,1191$

King, A. 993

King, L. D. 1269

King, S. 360

Kinoshita, H. 932

Kipnes, M. 76, 574, 1249

Kipnes, M. S. 1062

Kirkpatrick, C. 238

Kirmani, S. 657

Kirsch, S. E. 276

Kiss, E. 623

Kitabchi, A. 224, 650

Kitabchi, A. E. 1056

Kitabchi, A. E. 326

Kitabchi, A. E. 805

Kitada, M. 1184

Kitahara, Y. 1302

Kiviniemi, M. 334

Kleefstra, N. 182, 434, 1076, $1107,1192,1391$

Klefortova, I. I. 1193

Klein, J. 857

Klein, K. 1130

Klein, M. 1174

Kleiss, C. 591

Kletzien, R. F.. 38

Klimcakova, E. $\quad 723,730,816$

Klimenko, A. 55, 56

Klimes, I. 279

Klintenberg, M. 821

Klisic, J. 1068

Klonoff, D. 677,678

Kloos, C. S. $1045,1157,1181$

Klöting, I. 331

Klöting, N. 163, 289, 331, 396

Klovins, J. 243

Klupa, T. 280,1121

Knauf, C. 716

Kniotek, M. 835

Knip, M. 51, 265, 277, 334, 342

Knoch, K. $\quad 454,461$

Knoche, E. 480

Knop, F. K. $\quad 637,661,1153$

Knowler, W. C. 326

Knudsen, L. B. $\quad 518,601,800,893$

Knuuti, J. 760

Ko, G. T. C. 1214

Kobayashi, M. 1195

Køber, L. 1394

Koblas, T. 596, 604

Koch, C. 213

Kochunova, V. 852

Koczwara, K. 330, 344

Koda, J. 82,83

Kodera, R. 162, 69, 91, 92

Kodera, Y. 1364

Koehler, C. 223, 409

Koekman, A. C. 199

Koenen, C. 994

Kofinis, A. J. 339

Kogawa, K. 1164

Kogevinas, M. 1027

Koh, A. 590

Koh, D. 316

Koh, P. 96

Köhler, G. $\quad 968,1070$
Kohlmann, T. 1000

Kohnert, K. D. 232

Koike, K. 1187

Koitka, A. 96, 160

Koko, T. 1111

Kola, B. 785

Kolaitis, N. 587

Kolb, H. 234

Kolberg, J. A. 54

Kolehmainen, M. 795

Kolka, C. M. 792

Kollmeier, A. 1015

Kolm, P. 347

Kolonics, A. 623, 714

Kolostova, K. 723

Kolterman, O. 1011

Komesidou, V. 839

Komu, M. 760

Kon, W. 316

Kondos, G. 847

Kong, A. P. S. 144,708

Konno, K. 1294

Konno, K. 1401

Kono, S. 1164

Konrade, I. 243

Konstantopoulos, N. 37, 624, 935

Konstantopoulou, P. 348

Kontogianni, M. 308

Koo, B. $\quad 1378,668$

Koo, Y. 1376

Kooi, E. M. 168

Kooy, A. 940

Kopecký jr., J. $\quad 735,738,812,815$

Koppensteiner, R. 1203

Korbonits, M. 785

Korbutt, G. S. 448

Kordonouri, O. 1075

Körfer, R. 1299

Koro, C. E. 1211

Korpisalo, P. 111

Korsatko, S. 968, 1070

Korsgren, O. 444

Kos, K. 201, 831

Koshimura, J. 1224

Koshiyama, H. 363, 414, 1191, 1293

Koshkin, V. 764

Kosinski, M. 1153

Koskinas, J. S. 339

Kost, J. 687

Kostense, P. J. 1028

Köthe, L. 14, 917

Kothny, W. 915

Kotova, O. V. 487

Kotronen, A. 33

Koukouli, M. 1099

Koukoulis, G. 1361

Kounenou, K. 1099

Koutsovasilis, A. 1099, 1361

Kováčová, Z. 816

Kovacikova, M. $723,730,816$

Kovacova, Z. 723,730

Kovacs, P. $\quad 163,289,396$

Kowalska, A. 1335

Kowalska, I. 1359

Kowalska, I. $\quad 1395,280$

Kowlessur, S. 425

Koya, D. 1184

Koyama, K. 1164

Koytchev, R. 1201

Koznarova, R. 592, 596

Kracht, T. 1075

Kraenkel, N. 1320

Krafft, M. 591
Kraft, P. 80

Krag, S. 1188

Krarup, N. T. 63,66

Krarup, T. 637, 661

Kratz, K. 461

Kraus-Stojanowic, I. 468

Krause, S. 330, 344

Kravchun, N. 270

Kravitz, B. G. 24

Krebs, M. 593

Kreichauf, S. 264

Kreis, R. 671

Kretowski, A. J. $\quad 844,1127,1132$

Krichbaum, M. 229

Kriek, J. 260

Krippeit-Drews, P. 504, 544

Krippner, G. 935

Krishnamurthy, B. 1072

Krishnamurthy, S. 5

Krishnan, S. T. M. 1250

Kristensen, L. 734

Kristiansen, S. B. 109

Krogh-Madsen, R. 780

Krolewski, A. S. 220

Krssak, M. 34

Krušinová, E. 815

Krull, I. 671

Krusinova, E. 812

Kruszynska, E. 1335

Krzyzanowska, K. 1130

Ktorza, A. $\quad 102,856$

$\mathrm{Ku}, \mathrm{C} .1155$

Ku, Y. 390

Kubiak, T. 229, 1067

Kuboki, K. 744

Kuchmerovska, T. 55, 56

Kuchtickova, S. 1306

Kucich, C. 1106

Kuda, O. 738

Kuenen, J. C. 1055,1065

Kugler, E. 1126

Kuipers, F. 662

Kuklisova, L. 279

Kulaksızoglu, M. 638

Kulaksizoglu, S. 638

Kulich, M. 371

Kulzer, B. 15, 229

Kum, G. 1214

Kumakura, A. 1294

Kumar, B. N. 423

Kumar, P. 647, 852

Kumar, R. 523

Kumar, S. $\quad 401,762,790,826$, 832, 1048

Kumath, S. 733

Kumekawa, M. 1294, 1401

Kurata, H. 413

Kuriya, G. 582

Kurland, I. Jack. 773

Kurzum, D. 1042

Kus, V. 738

Kusminski, C. M. 790, 832

Kusumoto, T. 1187

Kutlu, B. 333

Kuusisto, J. 111

Kuzema, V. 1199

Kuzmicki, M. 844, 1127, 1132

Kuzuya, H. 1164, 1349

Kvaløy, K. 271

Kvasnickova, H. 818

Kveiborg, B. 1394

Kwon, H. 1371

Kwon, H. 804

Kyriaki, D. 1236

Kyriakopoulos, K. 348

Kyriazopoulou, V. 807

Kyrlaki, E. $\quad 983,1027$

Kytö, J. 1354, 1363

L

Laakso, M. 111, 1298

Laaksonen, D. E. 795

Laborie, L. 265

Labriola, L. 215

Lacey, L. 1173

Lachin, J. M. 24

Lacombe, D. 81

Lacraz, G. $\quad 771,778$

Lacza, Z. 1126

Ladrière, L. C. L. $\quad$ 133, 509

Lafata, J. E. 1051

Lagarde, D. 822

Lage, K. 139

Laher, I. 1334

Lahlou, N. 81

Lai, Y. 252

Laight, D. 6

Laine, A. 277, 334

Lajer, M. 1318

Lajoix, A. 484

Lajus, S. 496

Lalic, K. $\quad 573,586,588$

Lalic, N. M. $\quad 148,573,586,588$

Lam, C. WK. 708

Lam, K. S. L. 1287

Lam, L. 655

Lam, W. W. M. 708

Lamb, H. J. $\quad 113,114,166$

Lambadiari, V. $\quad 839,1313$

Lambert, J. 294

Lamendola, P. 1344

Lamina, C. 7

Lammers, R. 138

Lammertsma, A. A. 114,166

Lammi, N. M. 103, 105, 372

Lampasona, V. 330, 584

Landgraf, R. 1113

Landgraf, W. 1000

Landherr, U. 330

Landin-Olsson, M. 269, 972, 1088,1150

Landman, G. W. D. $\quad 434,1391$

Landstedt-Hallin, L. 938

Landy, C. 486

Lang, F. 547

Långberg, E. 822

Langdon, R. B. 909

Langefeld, C. D. 304

Langenberg, C. 61

Langer, P. 1360

Langin, D. 759

Langlois, A. $\quad 591,603,606$

Lango, H. 291

Langone, F. 506

Lankisch, M. 185

Lanska, V. 577

Lantieri, O. 754

Lanuti, P. 1291

Lanza, G. A. 1344

Lapice, E. 846

Lapolla, A. 1137

Lappas, M. 782

Larbig, M. 186 


\begin{tabular}{|c|c|c|c|}
\hline Larsen, A. F. 181 & Lee, P. 743 & Li, H. 1398 & Liu, N. F. 1307 \\
\hline Larsen, J. 971 & Lee, S. 1186 & Li, J. 1295 & Liu, X. 602 \\
\hline Larsen, M. 929 & Lee, S. 381 & Li, J. 410 & Liu, X. H. 357 \\
\hline Larsen, S. 673 & Lee, S. 399 & Li, J. 546 & Liu, Y. 583,585 \\
\hline Larsen, U. 891 & Lee, S. 804 & Li, J. 643 & Liu, Z. 262 \\
\hline Larsson-Nyrén, G. 498 & Lee, W. 1353 & Li, J. 859 & Liuzzo, G. 1337 \\
\hline Laskewitz, A. J. 662 & Lee, W. 60 & Li, J. K. Y. 1214 & Ljubicic, S. 472 \\
\hline Lassmann-Vague, V. 988 & Lee, W. J. 720 & Li, K. 171 & Llauradó, G. 783 \\
\hline Latif, Z. Ahmed. 323,420 & Lee, Y. 1249 & $\mathrm{Li}, \mathrm{K} . \quad 174,634$ & Lo, S. T. 1275 \\
\hline Latorraca, M. Q. 457,746 & Lee, Y. 399 & $\mathrm{Li}, \mathrm{K} . \quad 715,725,813$ & Lo Presti, D. 986 \\
\hline Latour, M. 772 & Lee, Y. J. 718 & Li, L. 1366 & Loba, J. $919,973,1342$ \\
\hline Lattanzi, F. 1347 & Lee, Y. Mi. 196 & Li, L. $\quad 171,447,666,715,725,813$ & Lobmann, R. 1262 \\
\hline Lau, C. 397 & Lefebvre, B. 856 & Li, N. 536 & Loboz-Grudzien, K. 1335 \\
\hline Lau, C. Wai. 1287 & Lefebvre, P. C. 856 & Li, Q. 346 & Locatelli, M. 575 \\
\hline Lau, I. T. 1214 & LeGall, M. 610 & Li, R. 715 & Locatelli, S. B. 1080 \\
\hline Lau, S. 1139 & Lehert, P. 940 & Li, S. 8 & Loche, S. 284 \\
\hline Lau, S. M. 456 & Lehmann, M. 562 & Li, X. $\quad 1180$ & Locke, J. 237 \\
\hline Laue, C. 1290 & Lehto, M. J. $\quad 143,1205$ & Li, X. 174 & Lockett, A. 4 \\
\hline Lauffer, L. M.. 125 & Lehto, S. 1298 & Li, X. 203 & Lockhart, B. 102 \\
\hline Laugen, J. M. 122 & Lei, M. 937 & Li, X. 634 & Lockman, K. A. 743 \\
\hline Laurent, K. 640 & Leibiger, B. $\quad 465,479,491$ & Li, X. J. $\quad 546,643$ & Lockstone, H. E. 361 \\
\hline Laurent, S. 1133 & Leibiger, I. B. $\quad 465,479,491$ & Li, Y. $\quad 17,817$ & Lococo, E. 1312 \\
\hline Lauritzen, T. $66,324,328$ & Leite, S. A. O. 1080 & Li, Y. T. 505 & Loder, M. K.. 26 \\
\hline Lauro, D. $\quad 204,441,926,1317$ & Lejnieks, A. 243,1199 & Li, Z. 817 & Loffroy, R. 22 \\
\hline Lauro, R. $\quad 67,68,204,317,926$ & Lelievre-Pegorier, M. 1133 & Liao, E. 937 & Logtenberg, S. J. J. $\quad 182,434,1076$, \\
\hline Laviola, L. $850,1196,1283$ & Leloup, C. 100,132 & Liatis, S. 348 & $1107,1192,1391$ \\
\hline Lawrence, J. M. 1151 & Lemaire, K. $\quad 297,462$ & Liberati, R. 765 & Loh, Y. 369 \\
\hline Lawrence, R. 300 & Lemieux, I. 1016 & Liberty, I. F. 1073 & Lomax, S. 1108 \\
\hline Lawson, M. L. 276 & Lemoine, S. 1341 & Libman, I. M. $\quad 945,946$ & Lombardi, A. 691 \\
\hline Laybutt, R. $134,174,634$ & Lencioni, C. $1122,1140,1145$ & Lichiardopol, R. 842 & Lombardo, F. 986 \\
\hline Le Doeuff, J. 1381 & Lengyel, C. 1383 & Lieb, W. 188 & Lombardo, M. F. $\quad 441,1317$ \\
\hline Le Marchand-Brustel, Y. 640 & Lenhart, G. 1019 & Liebisch, G. 759 & Löndahl, M. 1268 \\
\hline Le Stunff, H. 545 & Lennernäs, H. 890 & Liebl, A. 154,1090 & Long, Y. 1316 \\
\hline Le-Thi, T. D. $\quad 892,897,899$ & Lenter, M. 717 & Liem, E. T. 823 & Long, Y. Chau. 90 \\
\hline Leahy, A. L. 1321 & Lenti, L. 1312 & Liese, A. D. 119 & Longo, V. 1171 \\
\hline Leahy, J. L. 1001 & Lenzen, S. $\quad 175,278,442,480$ & Lieverse, L. A. G. 1192 & Longuet, C. 179 \\
\hline Lebbe, C. 341 & $513,516,520,529,557,776$ & Lilja, M. 424 & Lönnroth, P. 1385 \\
\hline Lebed, Y. V. 1244 & Leonardini, A. 1196,1283 & Lilley, K. T. 1029 & Loos, R. J. F. 61 \\
\hline Leblanc, H. 988 & Leone-Bay, A. 1013 & Lim, E. 766 & Lopes de Faria, J. B. 194,1183 \\
\hline Lebouvier, M. 340 & Leonetti, F. $\quad 318,101$ & Lim, G. E. 126 & Lopes de Faria, J. M. 194 \\
\hline Leccesi, L. 1154 & Leong, H. 316 & Lim, S. 1245 & Lopez, V. A. 417 \\
\hline Lechleitner, M. 956 & Lepape, S. 1059 & Lim, S. $\quad 390,1376,1377$ & López Miranda, J. 1219 \\
\hline Leclerc, I. 489 & Lepetukhin, A. 659 & Lin, J. 854,1388 & López Ríos, L. 1392 \\
\hline Leclerc, J. 607 & Lerche, S. 676 & Lin, S. X. 817 & Lopez-Jimenez, F. 408 \\
\hline Lecomte, P. J. $\quad 153,754$ & Lerin, C. 646 & Lin, Y. 553 & Lopez-Miranda, J. 247 \\
\hline Lederman, H. 1260 & Lernmark, Å. $\quad 565,572$ & Linck, N. 179 & López-Novoa, J. 934 \\
\hline Lee, B. 1186 & LeRoith, D. 773 & Lind, L. 836 & Lorenzi, G. 1009,1010 \\
\hline Lee, B. 1211 & Leroux, S. 1165 & Lindberg, S. 1263 & Lorenzi, O. M. 1292 \\
\hline Lee, B. 527 & Leslie, R. D. G. $\quad 274,369,566$, & Lindblad, U. 1178 & Lorenzo, M. 784 \\
\hline Lee, E. 1245 & 570,580 & Lindgren, C. M. $7,8,10,11,361$, & Lorgis, L. 1345 \\
\hline Lee, E. 1326 & Lessa, I. 1119 & Lindgren, O. 611 & Lortz, S. 214,516 \\
\hline Lee, E. 60 & Lettéron, P. 609 & Lindgren, P. 1047 & Losurdo, F. 1283 \\
\hline Lee, F. 1003 & Leturque, A. 610 & Lindholm, E. 269 & Lottati, M. $\quad 732,792$ \\
\hline Lee, H. 1064 & Leung, W. Y. S. 1214 & Lindroos, M. M. 240 & Louchami, K. $\quad 473,954$ \\
\hline Lee, H. 1245 & Leutenegger, E. 988 & Linemeyer, D. L. 931 & Lovegrove, J. A. 247,1219 \\
\hline Lee, $H_{.} \quad 1376,1377$ & Levchenko, T. 270 & Ling, C. 306,312 & Lövgren, T. 334 \\
\hline Lee, H. 293 & Leveringhaus, J. 547 & Lingvay, I. 747 & Low, P. A. 241 \\
\hline Lee, H. 390 & Levin, P. 1003 & Link, K. 565 & Lowe, J. M. 222 \\
\hline Lee, H. 530 & Levisianou, D. 1361 & Lins, P. 938 & Loyens, E. 802 \\
\hline Lee, H. 622 & Levy, B. $1014,867,868$ & Linssen, M. M. L. 260 & Lu, H. 764 \\
\hline Lee, H. 692 & Levy, J. C. 961 & Liouri, E. 1099 & Lu, J. 1175 \\
\hline Lee, H. 692 & Levy, R. 827 & Lipar, K. $\quad 592,596$ & Lu, J. 936 \\
\hline Lee, H. C. 1329 & Lévy Marchal, C. 81,263 & Lipka, M. 948, 989 & Lu, M. 654 \\
\hline Lee, I. T. 720 & Lewin, A. 898 & Lipsius, S. 1248 & Lu, T. 530 \\
\hline Lee, J. H. 990 & Lewin, E. 232 & List, J. F. $\quad 40,796$ & Lu, W. N. 1398 \\
\hline Lee, J. H. 990 & Lewis, C. H. 1252 & Literati, P. N. 623 & Lubberink, M. 114,166 \\
\hline Lee, K. 1371 & Lewis, E. J. 45 & Literati-Nagy, B. 714 & Luben, R. 349 \\
\hline Lee, K. 290 & Lewis, G. F. 655 & Literati-Nagy, P. 714 & Lucantoni, F. 571 \\
\hline Lee, K. 804 & Lewis, J. B. 45 & Literati-Nagy, Z. 714 & Lucchesi, D. $1171,1229,1319$, \\
\hline Lee, K. 845 & Lewis, J. P. 304 & Litwak, L. E. 3 & 1400 \\
\hline Lee, K. W. 370 & Lewis, K. 13 & Liu, C. 1032 & Luchner, A. 188 \\
\hline Lee, M. 1353 & Lewis, M. S. 873 & Liu, H. 893 & Luciani, D. S. $\quad 550,533$ \\
\hline Lee, M. 622 & Li, G. 435 & Liu, J. 1151 & Luciani, D. S. 553 \\
\hline Lee, M. 622 & Li, H. 1309 & Liu, M. 203 & Lucotti, P. C. G. 1322 \\
\hline
\end{tabular}


Ludvigsson, J. $\quad 567,568$

Ludvik, B. 2, 378

Luft, F. C. 916

Luger, A. 593, 1143, 1152

Lukasova, P. 818

Lukic, L. 573, 586, 588

Lunati, M. E. 1397

Lund, S. $\quad 109,788$

Lund, S. S. 295

Lund Nilsen, T. I. 368

Lundbom, N. 706

Lundin, F. 724

Luo, S. 128,685

Lupi, R. $133,136,161,315,597$, 656, 903

Lupinkova, J. 1238

Luppi, P. 1402

Lussier, R. 772

Lutgers, H. L. 1304

Luthman, H. 494

Lutz, K. 1007, 1009

Lutz, P. 1285

Lutz, S. Z. 138

Luzio, S. D. 4

Lv, X. Xia. 467

Lybaert, P. 473

Lykkegaard, D. N. E. 216

Lynch, K. 565

Lynch, P. 1002

Lyons, T. E. 1156

Lyssenko, V. 269

Lyssenko, V. 53, 62, 65

\section{M}

Ma, R. 634

Ma, R. C. W. 144,708

Ma, X. 307

Ma, Z. 498,551

Małecki, M. T. 1121

Maassen, J. Antonie. 320

Mabilleau, G. 1266

Mabley, J. G. $\quad 554,1160$

MacConell, L. 872

Maccubbin, D. L. 1388

MacDonald, A. 1125

Macedoni, M. 949

Macesic, M. 586, 588

Macfarlane, W. M. 486

MacGilchrist, C. 1102

Machann, J. 322, 395

Machicao, F. 319, 320, 322, 395, 1285

MacIsaac, R. 1215

Macleod, A. 1118

Madani-Maimaitiming, S. 1328

Madar, Z. $\quad 645,787$

Madec, A. 236

Madec, S. 161

Madeddu, P. 1320

Mader, J. K. 1070

Madsbad, S. $\quad 328,632,637,661$, 755

Madsen, K. S. 736

Madsen, M. 1340

Maechler, P. 127, 497, 536

Maeda, K. 1349

Maeda, S. 329

Maeda, T. 1364

Maeder, C. 697

Maedler, K. 777,781

Maegawa, H. 329, 910

Maekawa, F. 770

Magenheim, R. 1126
Mager, U. 795

Maggio, M. 309

Maggio, R. 760

Maggs, D. $\quad 863,876,901$

Magliano, D. J. $\quad 190,425,427$

Magnan, C. $\quad 102,132,607,610$

Magni, L. $\quad 47,1336$

Magnusson, A. 712

Magnusson, N. E. $109,466,502$, 521

Magyar, A. 256

Mahmoud, A. $\quad 600,906$

Mahtab, H. 1018

Mahtab, H. 420

Mahurin, A. J. 933

Maida, A. 908

Maier, H. $\quad 1010,1011$

Maillard, E. 591, 606

Maimaitiming, S. $\quad 311,1169$

Maione, A. 1101

Maione, S. 846

Mairal, A. 759

Maissel, A. 86

Maiztegui, B. 482

Majamaa, K. 240

Major-Pedersen, A. 1394

Mäkelä, M. 342

Mäkimattila, S. 706

Mäkinen, V. 1363

Makino, H. $\quad 69,91,92,162$

Makino, Y. 1185

Makoundou, V. 843

Makrilakis, K. 348

Makris, K. 389, 1142

Malagoli, R. 384

Malaisse, W. J. $\quad 473,954$

Malapaka, R. 38

Malarstig, A. 408

Malchiodi-Albedi, F. 195

Maldonado, R. 500, 503

Malecki, M. T. 280, 394, 1176

Malerba, G. 298

Mali, T. 1272

Malik, R. 1250

Malik, S. 417

Malinska, H. 71

Mallone, R. 236

Malloy, J. L. $\quad 878,879$

Mallozzi, C. 195

Malm-Erjefält, M. $\quad 890,891$

Malmberg, K. 1325

Malone, J. K. 874

Mameli, C. 949

Mancarella, R. 656

Mancia, G. 47, 1336

Mancini, E. $\quad 794,824$

Mancini, M. 741

Manco, M. 1154

Mandecka, A. 1157

Mandes, K. 606

Mandl, M. 593

Mandosi, E. 1312

Mandrup-Poulsen, T. 1318

Manduteanu, I. I. 1289

Manesis, E. K. 339

Manesso, E. 30

Manfrini, S. 984

Mankovsky, B. 50

Mann, S. 1092

Manning, A. K. 326

Mansour, A. A. 1041

Manteghetti, M. 484

Manto, A. 1337

Mantzoros, C. S. 308
Many, M. 200

Maragou, C. 389

Marangon, M. 1137

Maratou, E. $\quad 839,1313$

Marcellari, A. 914

Marchand, L. 276

Marchegiani, F. 1170

Marchesini, G. 318

Marchetti, P. 133, 136, 172, 315, $576,597,649,656,903$

Marchisio, M. 629, 1291

Marco, J. 123, 499

Marcovina, S. 119

Marcus, C. 820

Maréchal, D. 333

Marechaud, R. 1165

Mares-Guia, T. R. 215

Marette, A. 180

Mari, A. 240, 608, 651, 663, 702, 794, 1154

Mariano-Goulard, D. 1330

Marie, J. 609

Maringa, M. 285

Marini, M. A. 317,325

Maris, M. 525

Markaki, E. M. 774

Markova, I. 728

Markovic, I. 586, 588

Marks, J. 925

Markus, M. R. P. 188

Marone, R. 739

Marques, J. L. B. 1237

Marra, M. 321, 1170

Marraccini, P. 112

Marre, M. 297, 311, 313, 892, $897,1165,1169,1328,1332$

Marrero, D. 1392

Marrioneaux, O. S. 147

Marriott, C. E. 486

Marrocco-Trischitta, M. 1322

Marrocos, M. S. M. 1074

Marsden, P. K. 227

Marselli, L. 136

Marshall, S. 1155

Marte, T. 404, 1360, 1362

Martelli, E. 1323

Martens, G. 170

Martin, C. L. 241, 1253

Martin, D. 455

Martin, M. 880

Martín, M. A. 452

Martín, M. A. 500

Martin, S. 1113

Martin, S. 959, 977, 997

Martin- Stacey, L. 1116

Martín-del-Río, R. 495

Martín-Vaquero, P. 1149

Martínez, J. A. 354

Martinez, M. 1031

Martinez Calejman, C. 1284

Martinez de Ubago, M. 87

Martínez-Cáceres, E. 578

Martínez-González, M. A. 354

Martínez-Labari, C. 851

Martini, F. 1337

Martino, L. 343

Marttila, J. 342

Marucci, A. 689

Marzano, V. 68

Masaki, H. 1293

Masding, M. G. 1173

Masi, I. 1297

Masi, S. 982

Masiello, P. 343, 772

Masini, M. 136, 343, 597

Masmiquel, L. 1158

Masotti, D. 571

Massa, L. M. 644

Massa, O. 282

Masserini, B. 1397

Massó González, E. L. 433

Massoudi, M. 883

Mastroianno, S. 1166

Masulli, M. 753

Masuzawa, M. 1083

Mataveli, F. D. 1096

Matesanz, J. 158, 1158

Mathew, M. 647, 852

Mathews, S. T. 933

Mathiesen, E. R. $1123,1124,1135$

Mathieu, C. 525, 549, 557, 1043

Matikainen, N. 751

Matone, L. 1103

Matos, P. 1234

Matsubara, S. 1338

Matsuda, M. 259, 1083

Matsuguma, K. 995

Matsui, T. 1302

Matsuki, M. 135, 511

Matsuki, T. 1327

Matsumoto, S. $\quad 808,1251$

Matsumoto, T. 744

Matsuzawa, Y. 159

Matta, M. 1165

Matter, N. 406

Matteucci, A. 195

Matthews, D. R. $\quad 797,888,892$, 895,1269

Matthews, H. C. 362

Matthews, J. 870

Mattiello, L. 58, 626, 793

Mattila, I. 51

Mattson, M. 921

Mauer, M. 43

Maulny, L. 358

Maunier, S. 1350

Mauri, M. 711

Mauricio, D. 275

Maurizi, A. 1034

Maury, E. 721

Mavrogiannaki, A. N. 339

Mavros, P. 432

Maxova, M. 71, 728

Mayer-Davis, E. J. 119

Maynard, J. D. 406

Mazor, L. 86

Mazzitelli, G. 1310, 1311

Mazzone, T. 847,853

Mazzurco, S. 1273

Mc Gee, K. C. 210

McAdam-Marx, C. 881

McAteer, J. B. 326

McCaleb, M. L. 931

McCallum, J. 418

McCarberg, B. H. 1248

McCarthy, B. D. 1051

McCarthy, M. 1118

McCarthy, M. I. 9, 10, 11, 12, 64, 291, 299, 300, 301, 302 309, 361

McCrimmon, R. J. 226

McCulloch, L. 327

McDonald, A. 26

McDonald, W. G. 38 
McGee, S. L. 36

McIntosh, C. 533

McKeigue, P. 421

McKenna, M. P. 54

Mckinney, P. A. 118

McLean, G. W. 1008

McLeod, W. 373

McMenamin, M. 1091

McNamara, D. B. 806

McTernan, P. G. 57, 210, 350, $762,790,826,832$

Meas, T. 263

Mechiche, S. 1047

Meda, P. 455

Meden, H. 1036

Medina, J. O. P. 1074

Medrikova, D. 738

Megia, A. $\quad 783,814$

Mehar, S. 1114

Mehmeti, I. 516

Meidute-Abaravecien, S. 476

Meier, C. A. 811

Meier, J. J. 541

Meierhoff, G. 234

Meigs, J. B. 326

Meirhaeghe, A. 294

Meisinger, C. 380,1255

Meissner, T. 285

Meisterfeld, R. 454

Mekki, Q. $\quad 76,77,860,861,862$

Melander, A. 1178

Melaragno, C. 1074

Melchior, T. M. $\quad 1033,1340$

Melchiorre, M. $\quad 850,1283$

Melidonis, A. 1099, 1361

Melistas, L. 308

Mello, V. D. F. 795

Melnichenko, G. 1261

Meloncelli, I. 156

Melzer, D. 309

Memeo, V. 850

Menditto, L. 918, 920

Menge, B. A. 541

Menghini, R. $67,68,1323$

Menini, S. 68, 1310, 1311

Merani, S. 601

Merce, J. 1254

Merentie, M. 111

Merren, M. 1249

Mersebach, H. 1124

Mersey, J. 1003

Meschi, F. 282

Mester, C. 1123

Metcalf, B. S. $\quad 670,947,1089$

Metelko, Z. 1105, 1207

Meyer zu Vilsendorf, A. 557

Meyers, A. L. 1248

Mezghenna, K. 484

Mfopou, J. K. 438

Mianowska, B. 1231

Miao Jonasson, J. 429

Mibus, A. L. 96, 160

Micchelini, B. 204, 926

Miccoli, R. 318, 1117, 1171, 1229, $1319,1387,1400$

Miceli, I. 198, 1297

Michalska, M. 543

Michelson, A. D. 1206

Middelbeck, I. 828

Midthjell, K. 294, 303, 368

Mielck, A. 1255

Miele, C. 691

Miettinen, P. J. 29

Migrenne, S. 607
Mihaileanu, M. 988

Mihaljevic, I. 987

Miki, T. 477

Miki, T. 99

Milicevic, Z. 997, 1359

Milicic, T. 573, 586, 588

Miller, A. 193

Miller, C. B. 633

Miller, M. A. 350

Miller, R. 106

Miller, S. C. M. 89

Millis, M. P. 142

Mills, D. 915

Milosz, D. 1342

Milting, H. 1299

Mimran, A. 48, 1232

Min, K. $\quad 668,1378$

Minami, K. 27, 477

Mindlova, M. 596

Mine, T. 1302

Minenna, A. 1166

Mingrone, G. 1154

Minic, B. 913

Minkina-Pedras, M. 268

Minor, R. 646

Minshall, M. E. $\quad$ 1002, 1339

Miranda, M. 814

Mirkiewicz-Sieradzka, B. 1176

Mirra, P. 683

Miscio, G. $\quad 689,1166$

Misnikova, I. V. 355

Misovicova, N. 279

Misra, A. 385

Misu, H. 768

Misurski, D. A. $882,883,960$, 1339

Mitchell, B. D. 301

Mitchell, G. 1372

Mitchell, J. 83

Mitha, I. H. 895

Mithieux, G. $607,639,642$

Mitrou, P. $\quad 839,1313$

Mitton, A. 782

Miura, K. 1302

Miwa, T. $\quad 1294,1401$

Miyakawa, T. 1079

Miyamoto, S. $\quad 69,91,92,162$

Miyata, T. 1300

Miyazaki, J. 477

Miyazaki, S. 464

Mizuguchi, K. 84

Mizukami, H. 1243

Mizuno, K. 281

Mjalli, A. 929

Mlejnek, P. $\quad 812,815$

Mlynarik, V. 127

Mlynarski, W. 280

Mo, Z. 937, 1180

Moede, T. $\quad 465,479,491$

Moeller, N. 676

Mohamed-Ali, V. 735

Mohammedi, K. $\quad 311,1169,1328$

Mohideen, P. 74

Möhlig, M. 528

Mojiminiyi, O. 731

Mok, J. 60

Mokhtari, I. 1037

Molcanyiova, A. 1356

Moldes, M. 742

Moler, E. J. 54

Molero, J. C. 37, 935

Molina, G. 345

Molina, M. 620

Moll, I. 1262
Moll, U. 1150

Moller, R. A. 1220

Mollerus, S. 829

Moloney, G. 1082

Molsted, S. 1036

Moltchanova, E. V. $\quad 105,372$

Molven, A. 265, 286, 287, 303

Momim, S. 266

Monier, S. 855

Moniz, C. 1265

Monnier, L. 641

Monos, D. S. 276

Montanya, E. 599, 904

Monteagudo, P. T. 1074

Montet, X. 697

Monti, L. D. 19, 1322

Montori-Grau, M. 646

Moock, J. 1000

Moon, M. 390, 692

Mooradian, V. 792, 864

Moore, A. 1091

Moore, A. F. 326

Moore, F. 517, 548

Moore, M. C. 665

Morahan, G. $\quad$ 140, 141

Morales, E. $\quad 40,796$

Morano, S. 571, 1312

Mordik, A. 659

Moreno, P. 907

Moreno-Navarrete, J. M. 173, 709,840

Moretti, C. 984

Morgado, C. 1241

Morgan, N. G. $\quad 460,508$

Morgantini, C. 1314,1347

Morgenthaler, N. G. 1226

Mori, I. $\quad 660,726$

Morii, T. 1224

Morita, H. $\quad 660,726$

Moritz, T. 925

Moriuchi, A. 582

Moriya, T. 1364

Moro, C. 757,759

Morris, A. P. 9, 291

Morris, J. F. 445

Morris, M. 1029

Morrison, M. K. 222

Mortensen, B. 613

Mortensen, H. B. $\quad$ 265, 572

Mortensen, O. H. 707

Morton, G. J. 129

Morviducci, L. 1101

Mosaraf, M. H. 648

Mosca, F. 597, 903

Moser, E. 34

Mosig, S. 828

Mota-Carmo, M. 688

Moua, O. 583

Moulin, P. 22

Moustakas, A. K. 556

Movassat, J. 358, 545

Moyna, N. M. 722

Moyssakis, I. 1236

Mozzillo, E. 951

Mraz, B. 14,1067

$\mathrm{Mu}, \mathrm{H} . \quad 755$

Mudaliar, S. 224,650

Mueller, D. 344

Mueller, P. W. 335

Muggeo, M. 298, 652, 1202

Mughal, S. 1048

Mukai, E. $\quad 359,902,942$

Mulder, H. $\quad 487,494$

Müller, B. 785

Müller, N. $\quad$ 1045, 1157, 1181

Müller, U. A. $\quad 1045,1157,1181$

Mulligan, M. E. $\quad 42,927,928$

Mulligan, P. $\quad 1056,1078$

Mullooly, N. 488

Mundt, D. 181

Munni, U. S. 819

Muñoz, C. 964

Munoz, N. 311

Munro, N. 885

Muntoni, S. 376

Mura, T. 1059

Murao, K. 1295

Muraoka, T. 1295

Murdolo, G. 1385

Murphy, E. 901

Murphy, E. M. 694

Murphy, H. R. 13

Murphy, K. 1246

Murphy, M. J. 947

Murshed, S. 1144

Murthy, S. N. 806

Muscelli, E. $\quad 608,1227$

Muscogiuri, G. 619

Musen, G. 228

Musi, N. 224, 619, 650

Musiu, M. C. 284

Musser, B. 912

Müssig, K. 395

Musto, R. 1265

Mutel, E. 639

Muthusamy, P. 1081

Mutsaerts, H. J. M. 1375

Mwaniki, D. L. 426

Myers Jr, M. G. 130

Mygind, H. 79

Mylonakis, A. 825

N

Naamane, N. 517

Nabe, K. 363, 414, 1191

Nachit-Ouinekh, F. 1039

Nagaev, I. 836

Nagakawa, K. 359

Nagasaka, S. 770

Nagase, R. 162

Nagata, N. 768

Nagel-Reuper, C. 231

Nagorny, C. L. F. 494

Någren, K. $\quad 760$

Nagy, G. 296, 589

Nagy, R. 296

Nahar, J. S. 420

Nahar, Q. $\quad 323,648$

Naik, R. G. 1066

Najib, S. $\quad 87,693$

Naka, K. 1257

Nakagami, T. 379,1396

Nakakuki, M. 84

Nakamachi, T. 1251

Nakamura, J. 1327

Nakamura, K. 1302

Nakamura, S. 768

Nakamura, T. 932

Nakamura, Y. 329

Nakamura, Y. 902

Nakanishi, K. 1163

Nakano, S. 744

Nakano, T. 1251 
Nam, C. 293

Nam, J. 1245

Nam, M. 672

Nam, M. S. 370

Nam-Goong, I. 740

Nanjo, K. 1257

Nannipieri, M. 794, 824

Nantö-Salonen, K. 51

Naour, N. 849

Napoli, N. 984

Narayan, K. M. V. 347

Narita, T. 1224

Naruse, K. 1327

Nasrin, B. 1136

Nässander, U. 663

Nasser, S. 197

Nata, K. 769

Natali, A. 112, 1227, 1314, 1347

Natalicchio, A. $\quad 850,1283$

Natasa, B. 512

Nathan, D. 1065

Nathan, D. M. $\quad 106,1055$

Nathanson, D. 206, 207, 403

Natoli, J. L. 680

Nauck, M. A. 14, 149, 231, 797, 861, 871, 895, 917

Naujok, O. 442

Navon, R. 1331

Nawrodt, B. 14

Nawroth, P. P. $\quad 243,555,1309$

Nazaret, C. 609

Nazarian, S. 1078

Neal, D. 665

Nebesarova, J. 604

Neglia, D. $\quad 112,982$

Negri, C. 652

Negro, A. 506

Nehar, Z. 1228

Nehiri, T. 1133

Neil, H. A. W. 566

Németh, N. 296, 1233, 1240

Nemoto, M. 453

Nenquin, M. 99

Nerup, J. $\quad 49,140,1055$

Nescheret, A. 1370

Netea, M. G. 165

Neu for the DIARY Group Baden-

Wuerttemberg, A. 376

Neubauer, H. 717

Neupane, S. 1275

Neves, M. D. F. 1074

New, R. R. C. 4,869

Newgard, C. 462

Newsholme, P. $\quad 488,543,653$

Newton, C. A. $\quad 964,1056$

Neyrinck, A. 70

Ng, D. P. K. 316

Ng, M. $\quad 301,708$

Ng, M. C. Y. 144

Nguyen, M. 221, 1228, 1239

Nguyen, S. M. 1087

Nichols, G. A. 1390

Nicholson, T. J. 101

Nicolay, C. $\quad 1090,154$

Nicolay, K. 31

Nicoletti, C. 443

Nicolucci, A. 156, 230, 1002, $1101,1112,1213$

Niece, S. P. 1080

Niederhauser, G. 515

Niedzielska, G. 1259

Nielsen, A. R. $\quad 707,780$

Nielsen, L. 265
Nielsen, L. $\quad 863,882,883,886$, 901, 960

Nielsen, S. 46

Nielsen, T. 324, 66

Nielsen, T. T. 109

Niessen, H. G. 616

Niessen, L. W. 1097

Niessen, M. 176

Niessen, P. M. G. 1300

Niessen, S. J. M. $\quad 600,906$

Nieto-Vazquez, I. 784

Nieznaj, M. 973

Niihira, S. 985,1115

Nijhuis, J. 809

Nijpels, G. 320, 391, 1028, 1179, 1305

Nikolajuk, A. 337, 1127, 1132, 1395

Nikosjkov, A. 763

Nilsson, A. 572

Nilsson, C. 734

Nilsson, P. M. 52, 65, 191, 412, 724

Nin, J. W. M. 254

Nishi, M. 1257

Nishii, M. 910

Nishikawa, T. 159

Nishimura, H. 1349

Nishimura, R. 379

Nishishita, S. $\quad 69,91,92,162$

Niskanen, L. 965

Nissen, N. K. 1033

Nita, C. A. 1057

Nizze, H. 562

Njølstad, P. R. $\quad 265,286,287$, 303, 365

Noah, L. 1242

Nobels, F. 1043

Nocerino, V. 282

Noczynska, A. 280

Noda, H. 932

Noel, C. 594

Noël, L. 200, 721

Nofrate, V. 651,663

Noguchi, N. 769

Noh, Y. H. 990

Nolan, C. J. 772

Nolan, J. $\quad 382,704$

Nomura, K. $\quad 363,414,1191$

Nonaka, K. 910

Norberg, B. 969

Norgaard Pedersen, B. 49

Norgren, S. 820

Norhammar, A. 1325

Norris, J. M. 336, 373

Norström, F. 821

Nosek, L. 1012

Noto, Y. 1164

Notoya, Y. 1401

Noutsou, M. M. 339

Novelli, G. $\quad 374,402$

Novelli, M. 343, 772

Nóvoa, F. J. 275, 1392

Nowaczyk, M. 835

Nowakowski, A. 1259

Nowotny, P. 34, 593, 621

Nuche-Berenguer, B. 907

Nugnes, R. 951

Nunes, E. 1280

Nurbaya, S. 316

Nurmi, J. 334

Nuti, S. 1279

Nuutila, P. $\quad 240,760,799$

Nybäck-Nakell, Å. 938
Nyblom, H. K. 649

Nyengaard, J. 1188

Nyrén, O. 429

Nyström, B. 1385

Nyström, L. 269, 1088

Nyström, T. 206, 207, 403

\section{O}

O'Brien, R. 1358

O'Brien, R. M. 485

O'Callaghan, C. 1215

O'Connell, M. A. 1072

O'Connor, P. J. 1053

O'Gorman, D. J. $\quad 722,1321$

O'Hare, J. P. 401, 1048

O'Hare, P. 832

O'Hare, P. J. 790

O'Harte, F. P. M. 124, 943

O'Kane, M. J. 1091

O'Leary, F. K. M. 1172

O'Neal, D. N. 1072

O'Shea, D. 1026

O'Sullivan, E. P. $\quad 722,1321$

Oates, C. 1372

Oberauer, R. 717

Oberholzer, J. 603

Obermayer, R. 378

Obrosova, I. G. 95

Odawara, M. 1294, 1401

Ogasawara, S. 1243

Ogata, M. 281, 464

Ogawa, E. 942

Ogawa, K. 1257

Ogawa, Y. 84

Oh, K. $\quad 60,1353$

Oh, Y. 672

Oh-Ishi, M. 1364

Ohashi, T. 453

Ohkuma, S. 259

Ohta, M. 84

Oishi, M. 1195

Oiso, Y. 1327

Oizumi, T. 379

Ojaghi, M. 1037

Oka, Y. 542

Okabe, T. 1083

Okafor, L. 554

Okamoto, H. 769

Okamoto, T. 910

Okamura, T. 1164

Okano, H. 27

Okayasu, T. 1315

Okerson, T. $872,874,875,876$, 877

Okitsu, T. 602

Oknianska, A. R. 460

Ökten, A. 579

Okuda, S. 1187

Okuyama, K. 910

Ola, T. 566

Oldani, M. 1322

Oldehinkel, A. J. 823

Olgun, N. 1017

Öling, V. 569

Oliveira, A. R. F. 1080

Oliveira, R. 1119

Oliver, E. 834

Oliver, N. 1272

Olivieri, F. 321

Oliyarnyk, O. 71

Olsen, A. 891

Olsson, A. H. 306

Olsson, H. 1150

Olsson, T. 424

Olvera-Alvarez, I. 896

Omondi, B. O. 426

Omori, S. 329

Omulecka, A. 919

Omura, M. 159

Onyango, D. 514

Oosterhuis, B. 891

Oparil, S. 1221

Oprescu, A. I. 655

Orchard, T. J. $\quad 106,107,406,1168$

Ordovas, J. M. 308

Orešič, M. 33, 51

Orhan, F. 579

Orho-Melander, M. 65

Oriente, F. 683

Orlovsky, M. A. 1244

Ormio, P. 29

Orosz, A. 1383

Orr, D. 1275

Orsi, E. 1397

Orsini Federici, M. 1002

Örsy, P. 256

Ortega, F.J. $\quad 173,709,840$

Ortis, F. 509, 517, 548

Ortsäter, H. 535, 537

Orvik, E. 1278

Osei, K. $\quad 598,1230,1389$

Osmenda, G. 1176

Osmond, C. 53

Ossanna, M. J. 1248

Ossola, I. 1297

Östenson, C. 822

Osterhoff, M. A. $\quad 528,828$

Östgren, C. 52

Ota, T. 750

Otonkoski, T. 29,444

Otranto, I. 156, 1101, 1213

Ott, P. 409

Ottosen, P. 108

Oura, T. 1164

Out, M. 1375

Ouwens, D. M. 260

Ovadia, H. 801

Overbergh, L. 525, 549, 557

Owens, D. R. 4

Ozaki, M. 582

Ozcan, S. 1017

$\mathbf{P}$

Pańkowska, E. 989

Pace, S. 41

Pacini, G. 34, 593, 698, 702, 1143, 1152

Padovano, L. 689

Pagel, R. 562

Pages, G. 640

Paik, S. H. 370

Paisey, R. B. 288

Pajor, L. 1208

Pajuelo-Fernandez, F. J. 1210

Pak, K. $\quad 668,1378$

Pal, A. 327

Pala, M. G. 1322

Palaisa, M. 959

Paleari, F. 47, 1336

Pallardo, L. F. 1149

Pallayova, M. 18

Palmer, C. 309

Palmér, M. 565 
Panagiotopoulos, S. 1215

Panahi, S. 957

Pancani, F. 1122

Pandolfi, A. $\quad 325,629,1291$

Pandya, B. J. $\quad 430,921,923$

Panero, F. 374, 402

Pani, G. 695

Panicker, V. 309

Pankowska, E. 948

Paolini, J. F. 1388

Papadatos, D. 389

Papadopoulos, G. K. $\quad$ 556, 587

Papaefstathiou, A. 825

Papageorgiou, G. 1142

Papakonstantinou, A. 839

Papassotiriou, I. 1281

Papastamataki, M. 1281

Papatheodoridis, G. V. 339

Papavassiliou, A. G. 807

Papp, J. G. 1383

Pappas, A. 983, 1027

Paquot, N. 155

Parandeh, F. 471

Pardini, S. 982

Pardo, G. 709

Pardy, C. 1358

Pareja, J. C. 150

Parikh, H. 669

Parikka, V. 51

Park, C. 1353

Park, J. 1245

Park, J. $\quad 614,615,841$

Park, J. 845

Park, J. H. 990

Park, J. R. 718

Park, K. $\quad 390,692,1376,1377$

Park, K. 178

Park, S. 1296

Park, S. 399,1326

Park, S. 60,1353

Park, W. 399

Park, Y. 370

Park, Y. $\quad 614,615,841$

Park, Y. 692

Parkes, D. 82, 901

Pärkka, J. 760

Parkkola, R. 760

Parkkonen, M. 1167, 1363

Parkkonen, M. 143

Parnaud, G. 459

Parslow, R. $\quad 118,1111$

Partyka, L. 1219

Parving, H. $\quad 45,46,255,295$, $1198,1206,1217,1218,1318$, 1324

Pascal, S. M. A. 526

Paschou, S. A. 587

Passera, P. 1106

Passler, P. 1360

Pastore, D. $\quad 441,1317$

Pataky, Z. $\quad 721,843,1258$

Patch, A. 237, 280

Patel, A. 1175

Patel, C. 585

Patel, N. 1116

Patel, S. 1221

Paterson, A. D. 276

Paterson, J. A. 1269

Patsouras, K. 1138

Patterson, C. C. 104

Patterson, S. 943

Patti, L. $\quad 753,846,1387$

Pattou, F. 170, 594

Patzelt-Bath, A. $\quad 14,15$
Paul, G. 24

Paul, L. 1102

Paul, S. K. 961

Paulus, W. J. 114

Pauvaday, K. 424, 425

Pavlatou, M. 1281

Pawlowski, M. 973

Paz-Pacheco, E. 78

Peacock, T. P. 1021

Pearson, E. R. 786

Pearson, G. L. 490

Pedersen, B. K. $79,707,780$

Pedersen, C. B. 971

Pedersen, N. 1318

Pedersen, O. $\quad 54,63,66,192,286$, $295,310,324,328,364,397$

Pedras, J. 268

Pedrazzi, G. 19

Pedrinelli, R. $\quad 1229,1400$

Pedro, T. 275

Peers, E. 1015

Peitl, B. 714

Peixoto, E. B. M. 1183

Pektorova, L. 596, 604

Pelikánová, T. $\quad 812,815$

Pellegrini, F. $\quad 156,1166,1213$

Pellegrini, M. 1031

Pelletier, E. M. 1339

Peltonen, L. $\quad 11,12$

Pembleton, C. 743

Peña, A. 1108

Pencea, C. 842

Pencek, R. 1007, 1009

Penfornis, A. 153,340

Peng, Q. 852

Peng, T. Y. 398

Pénicaud, L. 132

Penno, G. $1171,1229,1319,1400$

Peppa, M. 825

Perabo, M. 121

Pereira, A. 1234

Pereira, T. 1263

Perez, A. 847

Perez, C. 531

Pérez, N. 1392

Pérez-Arana, G. 538

Pérez-Bravo, F. 266, 273

Perfetti, A. 305

Perino, A. 374

Perkins, B. 220

Permana, P. 72

Permezel, M. 782

Permutt, M. A. 328

Perneczky, E. 987

Pernow, J. 206

Perotto, M. 374, 402

Perrea, D. $\quad 348,1236,1348,1382$

Perrea, K. 348

Perreault, L. $\quad 35,756$

Perrini, S. 850, 1196, 1283

Perry, J. R. B. 309

Perry, Z. H. 1073

Persson, F. 45, 1217, 1218, 1333

Persson, M. 75

Perwitz, N. 857

Peschechera, A. $\quad 850,1283$

Pessin, J. 86

Pessôa, B. S. 1183

Peter, A. 1285

Peterkova, V. 950

Peters, A. 380

Petersen, A. B. $\quad 466,502,521$

Petersen, J. S. 975

Petersons, A. 1199
Petersson, C. 972

Petersson, H. 245

Petersson, U. 52

Petit, J. 340, 855

Petit, P. 1059, 484

Petraikina, H. 952

Petrasova, D. 1356

Petremand, J. 512

Petrone, A. 370, 575, 584

Petroni, D. 982

Petrova, N. 1265

Petrova, N. L. 1266

Petrucci, T. C. 195

Pettersen, E. 271

Pettersson, J. 537

Peuskens, J. 713

Peyot, M. 772

Peyrot, M. 1104, 1271, 1274

Pezzano, V. 1337

Pfafferodt, K. 1290

Pfeiffer, A. 1262

Pfeiffer, A. F. H. 528, 617, 828, 1226

Pfeuffer, M. 1290

Pfleger, C. 234

Pflüger, M. 264

Pfützner, A. 78, 677, 678, 1267

Pham Van Linh, L. 235

Phatak, H. M. 417

Phielix, E. 168

Philipp, T. 1222

Phillips, C. 834

Phillips, L. S. 347

Philotheou, A. 950

Phull, J. 1103

Piaggesi, A. $1273,1276,1279$

Piatkiewicz, P. 835

Piatt, G. 1046

Piatti, P. M. 1322

Pibernik-Okanovic, M. 1105

Pichiri, I. 652

Pieber, T. R. $\quad 968,1070$

Piekarski, R. 116

Pierce, B. 290

Pietris, C. 348

Pietrzak, I. 1231

Pignatti, P. 298

Piguel, X. 1165

Pilacinski, S. 1109

Piletic, M. 992

Pillot, B. 642

Pimazoni Netto, A. 1096

Pinach, S. 198

Pinal, I. 1239

Pinchera, A. 824

Pinget, M. 591, 603, 606, 963, 966

Pinkney, J. H. 201, 831

Piot, C. 1330

Pipeleers, D. 170, 332

Pipeleers-Marichal, M. 445

Pirinen, E. 111

Pirk, O. 1000

Pirola, L. 88

Piroli, G. G. 1284

Pirot, P. 214

Pirot, P. G. 485

Pirvulescu, M. 1289

Pisani, P. 982

Pitkäniemi, J. 377

Pitocco, D. 984, 1034, 1337, 1344

Pitzalis, C. 446

Pivovarova, O. 617, 1226

Pizarro-Delgado, J. 495

Plaisance, E. P. 933

Planer, J. A. 1021

Plank, J. 1070

Playdon, M. C. 35,756

Plevris, J. N. 743

Plomgaard, P. 707, 780

Pochic, J. 1330

Pociot, F. $49,139,140,521,522$, 572

Poderoso, J. J. 767

Poellinger, L. 1263

Poggi, A. 1322

Pohl, R. 678

Polak, B. C. P. 1179

Polak, J. 723, 730, 816

Polak, M. 81

Polanska, J. 268

Polavieja, P. 1020, 1044

Pold, R. 788

Poletti, T. 1343

Polis, A. 853,854

Pollok, S. 1262

Polychronakos, C. 276

Pomili, B. 156, 230, 1101, 1213

Pontiroli, A. E. 370

Ponzi, P. 1002, 1101

Pop-Busui, R. 241, 1253

Popescu, L. D. 842

Popova, V. 50

Popovic, P. 376

Porcellati, F. 183,1282

Porcheron, G. 697

Porciello, C. 982

Pörksen, S. 265

Porta, M. 157, 198, 217, 218, 1106

Porter, L. $\quad 146,798,1007$

Portha, B. $358,481,545,778$, 1393

Portois, L. 954

Portuesi, R. 561

Postic, C. 356, 742

Potier, L. 1381

Potter, S. C. 931

Pou, J. M. 1254

Poulsen, M. K. 225

Poulsen, P. 306, 312, 613

Poussier, A. 340

Poussin, C. 512

Pouta, A. 11, 299

Poutignat, N. 153

Pouwels, P. J. W. 751

Pouyssegur, J. 640

Powell, L. A. 1308

Power, C. 299

Pozzan, T. 178

Pozzilli, P. $369,370,446,560$, 561, 580, 952, 984, 1034

Pradler, E. 15

Prados, M. 1148

Praet, S. F. E. 36

Prager, R. 2, 593

Prasanna Kumar, K. M. 5

Prasek, M. 1105

Pratley, R. 76, 860, 914

Pratoomsoot, C. 184

Pravenec, M. 71, 815

Prazak, R. 518

Prazak, T. 738 
Pricci, F. $\quad 195,1310,1311$

Price, A. 868

Price, H. C. 1365

Prikoszovich, T. 34, 1143, 1152

Primitz, L. 47

Prins, M. H. 254

Prinster, A. 741

Prip-Buus, C. 761

Prisco, F. 986

Profozic, V. 992

Prokopenko, I. 10, 11, 12, 291, 301

Proks, P. 115

Promintzer, M. 593

Prompers, J. J. 31

Prompers, L. 1270

Pronina, E. 952

Prudente, S. 325

Pruniaux, M. 1242

Pryde, A. 743

Psyrogiannis, A. 807

Pucci, L. $\quad 1171,1229,1319,1400$

Puck, M. 987

Pugliese, G. $\quad 68,1310,1311$

Pugliese, M. 1145

Puig, J. 1148

Puklavec, L. 1190

Pulizzi, N. 53, 318

Pulkkinen, L. 795

Pullen, T. J. 470

Pumprla, J. 987

Purewal, T. S. 1189

Purkayastha, D. 914

Pussinen, P. J. 1205

Putz, Z. 1233,1240

Pye, S. 703

Q

Qi, D. S. 73

Qi, L. 80

Qian, Q. 1301

Qiao, Q. 415

Qiu, L. 501

Qu, H. 276

Qu, X. 202

Qu, Y. $863,873,874$

Quagliara, A. 850, 1283

Qualmann, B. 257

Quarti-Trevano, F. 47, 1336

Quattrini, C. 1250

Quertermous, T. 1331

Quezada, C. 1161

Quick, W. 1092, 1093

Quimbo, R. 882, 883, 960

Quintens, R. 170

Quoyer, J. 179

Qvigstad, E. 1141

$\mathbf{R}$

Rabbani, N. 220, 748, 1301, 1303

Rabbone, I. 986

Rabelink, A. J. 48, 1232, 1375

Rabl, W. 117

Raciti, G. A. 691

Radermecker, R. P. 1069

Radford, C. R. $\quad$ 57, 210

Radford, D. 1146

Radimerski, T. 785

Radtke, M. 368

Radziuk, J. 703

Ræder, H. 286

Raggi, M. C. 605
Raghavan, R. P. 1103

Ragnarson Tennvall, G. 1277

Rahman, M. 1144

Raia, V. 951

Rajab, A. 598

Rajamani, K. 1366

Rajas, F. 639

Rajasalu, T. 559

Rajbhandari, S. M. $\quad 1081,1275$

Raji, A. 228

Rajkovic, N. 573, 586, 588

Rajotte, R. V. 448

Ramachandran, A. 1047

Rami, B. 20, 253

Ramírez, M. Jose. 258

Ran, J. 17

Ran, X. W. 643

Randall, J. C. $7,8,361$

Rangasamy, S. 583

Rangel, E. B. 1074

Ranke, M. B. 376

Ranta, F. 138, 547

Raposo, J. F. 1119,1234

Raptis, A. E. 825,839

Raptis, S. A. 1138,1313

Raskin, J. 1248

Raskin, P. 898

Rasmussen, M. F. 889

Rasmussen, S. 1033,1340

Rathcke, C. N. 1333

Rathmann, W. 380, 1255

Ratner, R. 224, 574, 650, 896

Ratner, R. E. 145

Rattigan, S. $\quad 625,627,628$

Raun, K. 734, 800

Raupp, D. 1200

Rauseo, A. 1166

Ravassard, P. 436

Rave, K. 1012

Raverdy, V. 594

Ravichandran, S. 858

Ravier, M. A. 99

Ravn, G. M. 148

Ravussin, E. 83

Ray, S. 1125

Rayman, G. 13, 1250

Raymond, N. T. $\quad 401,1048$

Rayner, N. 9, 10, 301, 302

Rayner, W. N. 11

Raz, I. 360, 385, 776, 1006, 1359

Razavi, L. 805

Reaven, P. 224, 650

Reaven, P. D. 72

Rebec, M. V. 1062

Recchia, F. 112

Recio-Córdova, J. 934

Recto, A. 1119

Reda, D. 925

Redaelli, G. 1212

Redekop, K. 1097

Reed, L. J. 227

Reers, C. 555

Regazzi, R. 211, 472

Reghina, A. D. 351

Regoli, M. 443

Reiber, J. H. C. 1375

Reijngoud, D. J. 662

Reijonen, H. 569

Reiling, E. 320

Rein, P. 404, 1351, 1355

Reinbothe, T. M. 474

Reinhard, H. $\quad 1218,1318$

Reis, A. F. 313

Reis, M. A. B. 457,746
Reis, S. R. L. 457

Reiser, M. 605

Reith, W. 25

Remacha, À. 710

Remacle, C. 261

Remizov, O. 1193

Ren, X. M. 1307

Renard, E. 2, 393, 1069, 1330

Renard, J. 358

Rendas, A. B. 688

Rendell, M. 77

Rensen, S. 802

Renshaw, P. 228

Renström, E. 474

Renström, F. 836

Repetto, E. M. 1284

Reroń, A. 1121

Resi, V. 1122, 1140, 1145

Reusch, J. 860

Reusens, B. 261

Revel, D. 22

Reviriego, J. 1020, 1044

Rewers, A. 1094

Rewers, M. J. 336

Reyna, S. 619

Reynold, D. 401

Reynolds, E. 1015

Rezende, L. F. 506

Rhee, E. 1353

Rhee, M. K. 347

Rhoads, G. G. 1001

Rhodes, C. 866

Rhodes, C. J. 129

Ribas, V. 173

Ribaux, P. 540

Ribeiro, L. 607

Ribel, U. 975

Ribel-Madsen, R. 613

Riboni, S. 949

Riboulet-Chavey, A. 489

Ricart, W. $\quad 173,709,840$

Riccardi, G. $\quad 741,753,846$

Ricci, C. 1310,1311

Ricci Busciantella, N. 183

Rich, S. S. $\quad 43,140$

Richard, C. 1345

Richards, S. M. $625,627,628$

Richardson, P. $867,868,1013$, 1014

Richey, J. M. 732, 775, 792

Ridderstråle, M. 820, 821

Riddle, M. 1007, 1011

Rigalleau, V. 705

Rigazio, S. 760

Rijkelijkhuizen, J. M. 391

Rijzewijk, L. J. $113,114,166$

Rilo, H. L. 635

Rinaldi, J. 1196

Ring, S. M. 299

Ringborg, A. 1047

Risch, L. 1355

Risérus, U. 245

Rist, W. 717

Ristow, M. 857

Ritchie, P. J. 1066

Rittig, K. 1285

Ritz, E. $\quad 48,1232$

Ritzel, R. A. 555

Riveline, J. 701

Rivellese, A. A. $741,753,846$, 1117,1387

Rizza, R. A. $122,658,700$

Rizza, S. 317,1323

Rizzo, L. 1273,1276

Robert, E. 1190

Roberts, M. S. 107

Robichon, C. 742

Robinson, E. J. 1237

Robinson, M. J. 1248

Roccasecca, R. M. 283

Roche, H. M. $\quad 247,834,1219$

Rocheleau, G. 297

Rockell, J. 233, 574

Rodda, C. 1072

Rodella, S. 1202

Roden, M. 34, 621

Rodewald, T. 665

Rodin, A. 1147

Rodriguez, M. 102

Rodríguez, M. J. 275

Rodriguez Pattzi, H. M. 896

Rodríguez-Villanueva, G. 934

Roggli, E. 211

Rohleder, S. 532

Rohner-Jeanrenaud, F. $\quad 674,697$, 811

Roig, P. 1393

Roix, J. J. 501

Roland, B. 82

Roland, I. 333

Romeo, F. 1323

Romeo, S. 284

Romero-Corral, A. 408

Romijn, J. A. 113, 114, 166, 1375

Romon, I. 153,416

Ronco, P. M. 94

Rönn, T. S. $\quad 306,312$

Ronnemaa, T. 1298

Rønningen, K. S. 267

Rorsman, P. 115, 631

Ros, S. 258

Rosado, J. A. 1149

Rosales, M. A. B. 194

Rose, C. S. 66

Rosenbauer, J. 117

Rosenbloom, A. L. 1022

Rosengård-Bärlund, M. 1167, 1354,1363

Rosenstock, J. 77, 145

Ross, R. 1016

Rossetti, P. 183, 1282

Rossi, J. P. F. 483

Rossi, M. C. E. $156,230,1213$

Rossing, P. 46, 255, 1198, 1206, $1217,1218,1318,1324,1333$

Rossmeisl, M. 735, 738

Rossmeislova, L. 730,816

Rossodivita, A. 1322

Rosy, N. 1129

Roth, J. 82

Rottier, O. 70

Rouch, C. 131, 774

Roudebush, C. 1085

Roula, D. 1235,1384

Roumen, C. 352

Round, E. 912

Rousseau, K. 1013

Roussel, R. 311, 1165, 1169, 1328, 1332

Rouvre, M. 754

Rowe, M. W. 54

Roy, S. 197

Ruan, Y, 1398

Rubi, B. $\quad 500,503$ 
Rudovich, N. N. $\quad 617,828,1226$

Ruge, T. 32, 252

Ruige, J. B. 332,924

Ruilope, L. M. 48, 1232

Ruiz, J. 1087

Ruiz de Villa, M. C. 258

Rump, L. C. 48,1232

Rundle, J. K. 786

Rune, A. 618

Rungby, J. 411, 466, 502, 521, 676

Ruokonen, A. 11, 299, 309

Ruotolo, G. 415

Rupnik, M. 169

Ruppert, K. 107, 1046

Ruschke, K. 163, 289, 396

Rush, W. A. 1053

Ruskeepää, A. 33

Russ, H. A. 436

Russ, J. 690

Russell, G. B. 43, 1021

Russell-Jones, D. 148, 888

Russo, E. 1229,1400

Russo, I. 58, 626, 793

Rustan, A. C. 758

Rustemeijer, C. $\quad 751,1204,1386$

Rustenbeck, I. 98, 478

Rusu, A. 1057

Ruth, P. 504

Rutten, G. E. H. 353, 431, 999, 1054, 1097

Rutter, G. A. 26, 101, 470, 489

Rütti, S. 518

Rydén, L. 1325

Ryffel, G. U. 460

Ryhänen, M. 111

Ryu, O. 1186

\section{S}

Sa, J. R. 1074

Saadeh, M. 510

Saavedra, J. 1022

Sabatti, C. 11

Sabokbar, A. 1266

Sacks, D. A. 1151

Saeger, H. 454

Saely, C. H. 404, 1351, 1355, 1360 , 1362

Safar, M. 1352

Safonov, V. 1261

Saint Remy, A. 1069

Saisho, Y. 30

Saito, J. 159

Saji, H. 359

Sakagami, H. 1185

Salamalekis, E. 1138

Salamalekis, G. 1138

Salehi, A. $471,476,523,631$

Salehzadeh, F. 618

Salih, S. 1114

Salinas, I. 578

Saller, A. 1166

Salmon, I. 333

Salt, I. P. 941

Salvadori, P. A. 982

Salvatore, M. 741

Salvioli, S. 321

Salzano, G. 986

Salzsieder, E. 232

Sam, S. 847

Samarasinghe, D. 1272

Samarasinghe, Y. P. $\quad 227,848,885$

Samek, A. M. 756

Sammut, S. J. C. 1189
Sampaio, C. R. 1084

Sampson, S. R. 684

San Martin, R. 1161

Sánchez, C. 934

Sanchez, R. 1284

Sánchez, Y. 710

Sanchez Dominguez, M. 591

Sánchez-Margalet, V. 87, 693

Sancho, V. 900

Sandal, T. 265

Sandbæk, A. $\quad 66,324,328$

Sane, T. A. 679

Sang, Z. 226

Sanjeevi, C. B. 272

Sankaran, S. 401

Sanli, T. 696

Sanmartí, A. 578

Sansoni, V. 1310, 1311

Santana, A. 275

Santikul, M. 686

Santin, M. 1343

Santing, L. 1192

Santini, E. $\quad 161,172$

Santini, F. $\quad 608,824$

Santoro, L. 949

Santos, A. I. 688

Sanz, N. 934

Sanz, P. 285

Saraheimo, M. 1100, 1167, 1354, 1363

Saraste, J. 287

Saravia, F. E. 1393

Sardinha, L. B. 61

Sardu, C. 230

Sargsyan, E. 539

Saris, W. H. M. 36, 352

Sarkar, S. A. 485

Sarman, B. 262

Sarmiento, L. 345

Sarpong, E. 184, 1019

Sarr, M. G. 658

Saryusz-Wolska, M. 919, 973, 1342

Sasaki, H. 1257

Sasaki, M. $\quad 69,91,92,162,942$

Sasaki, T. 453

Sasaki, T. 995

Sasson, S. 524

Sasvári-Székely, M. 296

Satler, M. 20, 1130

Sato, A. 84

Sato, C. $\quad 69,91,92,162$

Satoh, J. 542

Satoh, T. 582

Sattar, N. 405, 941

Sattler, C. 564

Saudek, F. 577, 592, 596, 604

Sauer, P. J. J. 823

Saukkonen, T. 965

Saunders, G. 223

Sauter, N. S. $\quad 777,781$

Sava, G. 1162

Savale, S. 5

Savu, O. $\quad 763,1263$

Sayeed, F. 445

Sayeed, M. A. 420

Sayo, Y. 1338

Sazonov, V. 1390

Sbraccia, P. 204, 441, 926, 1317

Sbrignadello, S. 698

Sbrissa, D. 251

Sburlati, L. F. 1397

Scanlon, P. H. 1172

Scannon, P. J. 1
Scaramuzza, A. E. $\quad 986,949$

Scatena, A. 1273

Schäfer, S. A. 320

Schalkwijk, C. G. $\quad 189,254,255$, $295,386,809,838,1218,1300$, 1305,1324

Schaller, M. 1087

Schaller, R. 1070

Schantz, N. 605

Schaper, N. C. 1270

Scheen, A. J. $\quad 155,713,1043$

Scheffer, P. G. 1386

Scheijen, J. 809

Scherbaum, W. A. $\quad 185,580$

Schernthaner, G. 20, 253, 1130, 1203

Schernthaner, G. H. 20, 253, 1130,1203

Schiaffini, R. 986

Schick, F. 322, 395

Schindhelm, R. K. $\quad$ 120, 1204

Schirmbeck, R. 559

Schisano, B. 57, 210

Schjoedt, K. J. 46

Schleicher, E. 1285

Schleinitz, D. 289, 396

Schloot, N. C. $\quad 234,580,779$

Schlosser, M. 335

Schmid, A. I. $\quad 34,621$

Schmid, J. 671

Schmidlin, F. $\quad 771,778$

Schmidt, K. 521

Schmidt, W. E. 541

Schmieder, R. E. 1222

Schmitt, H. 529

Schmitz, G. 759

Schmitz, O. $109,148,149,466$, $502,521,63,676,788,888$

Schmitz-Peiffer, C. 490

Schneider, A. 380

Schneider, S. 541

Schneider-Muntau, A. 468

Schneiter, P. 705

Schober, E. 20, 253

Schoen, S. 373

Schoenauer, M. 1320

Schoenfeld, D. $\quad 1055,1065$

Schölch, C. 616

Scholten, T. 1000

Scholz, G. H. 289, 396

Schön, M. R. 163

Schoonenboom, N. S. M. 1174

Schopman, J. E. 681

Schou, I. Manjula. 1220

Schrader, H. 541

Schrauwen, P. 167,168

Schrauwen-Hinderling, V. B. 168

Schreier, N. 372

Schremmer-Danninger, E. 605

Schrezenmeir, J. $\quad 468,1290$

Schroeder, B. $\quad 882,883,960$

Schruefer, C. 1006

Schuit, F. 170, 297, 462

Schulthess, F. T. 777,781

Schultz, K. 1194

Schultz, V. 803

Schultz, W. J. 458

Schulze, H. 231

Schumm-Draeger, P. 59, 667

Schunkert, H. 188

Schvarcz, E. 265

Schwab, U. 795

Schwartz, E. 72

Schwartz, M. W. 129

Schwartz, S. L. 898

Schwartz, Z. 246

Schwarzfuchs, D. 246, 958

Schweizer, A. 75, 917

Schwenk, W. 657

Schwenke, D. 224, 650

Scigalla, P. 1201

Scipioni, A. 1310, 1311

Scism-Bacon, J. $\quad$ 977, 997

Scott, F. W. 507, 581

Scott, R. 1358

Scougall, K. T. 906

Scranton, R. E. $\quad 39,930$

Scrimgeour, A. 1015

Seale, J. 921

Sebastiani, G. 576

Seboek Kinter, D. 785

Sedimbi, S. K. 272

Seeberger, K. L. 448

Seeley, R. J. 121

Sega, R. 47

Segal, D. 37, 935

Segato, G. 1137

Seghieri, C. 1279

Seghieri, G. $\quad 384,1140$

Segre, O. 402

Segundo, C. 538

Seino, S. 27, 97, 99, 477

Seino, Y. $\quad 359,602,902,905,908$, 942

Seissler, J. 564, 580

Selmar, P. 225

Selmeci, L. 589

Selmer, J. 929

Selvarajah, D. 242, 244

Semakula, C. 1078

Semenova, I. 1024

Semlitsch, B. 968

Semperboni, L. 1297

Sencier, M. 591

Sener, A. 473, 954

Senior, P. 590

Senou, M. 200

Sentinelli, F. 284

Seo, J. A. 718

Seppänen-Laakso, T. $\quad 33,51$

Serban, V. 1346

Serhiyenko, A. A. 955

Serhiyenko, V. A. 955

Serino, M. 1310, 1311

Sermon, K. 438

Serradas, P. $\quad 610,771$

Sérusclat, A. 22

Service, S. 12

Sesti, G. 317, 325

Sestito, A. 1344

Sethi, B. $\quad 148,677,678$

Setola, E. 1322

Seyfritz, E. 606

Seyoum, B. 727

Sfakianaki, M. 983, 1027

Shaffer, T. 923

Shaginian, R. M. $\quad 878,879$

Shah, A. K. $\quad 854,1388$

Shah, N. S. 895

Shah, S. 382,704

Shahar, D. 246

Shahid, S. 819

Shahmanesh, M. 762 
Shao, S. 435

Shao, X. 936

Shapiro, A. M.J. 601

Shapiro, J. 590

Shaposhnik, I. I. 1040

Sharia, M. 1193

Sharma, A. 528

Sharma, A. M. 385

Sharoyko, V. V. 494

Sharplin, P. 152

Shashaj, B. 571

Shaw, J. 439

Shaw, J. 888,897

Shaw, J. A. M. 437, 600, 906, 1372

Shaw, J. E. $190,422,424,425$, 427,971

Shaw, K. M. 6,711

Shaya, F. T. 430,923

Shefin, S. M. 323

Shehata, H. 1147

Sheiner, E. 246

Shen, J. 17

Shen, L. 798

Shenoy, R. N. 1021

Shepherd, J. 1357

Shervani, N. J. 769

Sherwood, R. 1265

Shestackova, M. 1024

Shestakova, M. 659, 992, 1193

Sheu, W. H. 720

Shi, H. 1220

Shi, S. 813

Shibasaki, T. 97, 477

Shibata, T. 1327

Shield, J. P. H. 366

Shields, K. 935

Shigeto, M. 259, 1083

Shihabi, Z. K. 1021

Shikata, K. 69, 91, 92, 162,

Shimada, T. 281

Shimatsu, A. 1164

Shimizu, S. 1401

Shimoda, M. 135, 511

Shimomura, K. $\quad 115,239$

Shimotomai, T. 1224

Shin, C. $\quad 390,1377$

Shin, D. Hyun. 718

Shintani, M. 1349

Shiri-Sverdlov, R. 802

Shirihai, O. S. 803

Shisheva, A. 251

Shlomowitz, A. 885

Shochat, T. 407

Shojaee-Moradie, F. 752

Shrom, D. 1063

Shtandel, S. 270

Shuldiner, A. R. 301

Shwob, H. 1006

Shymanskyy, I. 56

Si, H. 803

Si, Y. 803

Sibal, L. 1372

Sicard, P. 1345

Sicree, R. A. 422,427

Siegmund, T. $\quad 59,667$

Siekiera, U. 268

Siepert, A. 562

Sieradzki, J. 280, 394, 1121, 1176

Sierra-Johnson, J. 408

Siewko, K. 337

Signore, A. 560

Sigrist, S. 591, 603, 606

Silaghi, A. 849

Silva, K. C. 194
Silva, M. N. 1280

Silvestre, R. A. $\quad 123,499$

Simell, O. $277,342,51$

Simell, S. 51

Simell, T. 51

Simi, C. 982

Siminerio, L. M. 1046

Simionescu, M. 1289

Simmons, R. K. 1365

Simms, P. $\quad 677,678$

Simo, R. $\quad 148,158,892,1158$

Simon, D. $153,416,1039$

Simón, I. 783

Simonelli, P. 1310

Simonis-Bik, A. M. C. 387

Simons, J. 418

Simons, L. A. 418

Simonson, D. 228

Simpson, A. $\quad 154,1090$

Simpson, R. W. 893

Singer, P. 1058

Singh, B. M. 884

Singh, D. K. 1209

Singh-Estivalet, A. 450

Singhal, P. 1103

Singleton, A. 309

Sintonen, H. 1100

Siraj, M. 1018

Sirolla, C. 321,1170

Sisk, C. M. 1388

Siu, S. C. 1214

Sivakumar, G. 1209

Sivakumaran, P. 96

Sjöberg, L. 377

Sjögren, M. 65

Sjöholm, Å. 137, 206, 207, 403, $535,537,633,654$

Sjöstrand, M. 1385

Skapare, E. 243

Skibo, G. G. 1244

Skibova, J. 592, 1238

Skinner, C. 962

Skljarevski, V. 1247

Skogstrand, K. 49

Skorpen, F. 271

Skoularigis, I. 1361

Škrha, J. 1374

Skrivarhaug, T. 365

Skupien, J. 280, 394, 1176

Skyler, J. 651

Sladek, R. 101, 297

Slusher, B. 95

Smaldone, C. 1337

Small, M. 941

Smidt, K. 466, 502

Smidt, U. M. 295

Smih, H. 154

Smiley, D. 964, 1056, 1078

Smit, A. J. 1304

Smit, J. W. A. $113,114,166$

Smith, D. $\quad 488,722,1026,1321$

Smith, F. E. 766

Smith, H. 1090

Smith, P. 1339

Smith, R. 327

Smith, S. R. $\quad 83,757,759$

Smith, U. $\quad 209,810,878,879$

Smits, P. 36

Snead, W. 665

Snoek, F. J. 1174

Snorgaard, O. 1036

Snyder, J. 994

So, W. Y. $\quad 144,1214$

Sobngwi, E. $\quad 341,664$
Sobrevia, L. 1161

Söderberg, S. 424, 425

Söderlund, J. M. 1167

Soedamah-Muthu, S. 107

Sogayar, M. C. 215

Sohn, E. Jin. 196

Soja, A. B. 1033,1340

Sol, E. M. $\quad 539,552$

Soldevila, B. 578

Soler, J. 904

Solidaki, E. 1027

Solimena, M. 454, 461

Solinas, G. 739

Solinger, A. M. 1

Solini, A. $\quad 161,1400$

Solskov, L. $\quad 109,788$

Sölter, L. 1075

Soltész, G. 104

Somasegeram, A. 96

Somers, V. K. 408

Somfai, G. 1177

Somigyi, A. 296

Sommer, C. L. 241

Somogyi, A. 589

Son, H. 804

Son, S. 381

Søndergaard, F. 411

Sone, H. 1195

Song, J. 953

Song, K. 1296

Song, M. 417

Songini, M. 584

Sonier, B. 581

Sonne, M. P. $\quad 310,630$

Sørensen, S. M. 673

Sørgård, B. 1278

Sörhede Winzell, M. 611

Soro-Paavonen, A. 93

Sotelo, T. 1161

Soty, M. 607, 642

Souhami, E. 950

Sourij, H. 1360

Sourris, K. C. 160

Souza, D. F. I. 457

Souza, L. M. I. 457

Souza, S. C. 501

Spadaro, R. 951

Spanou, L. $\quad 389,1142$

Sparén, P. 429

Sparsø, T. 328

Spazzafumo, L. 321

Speier, S. 479

Speliotes, E. K. $\quad$ 7, 8

Spera, S. 1034

Sperl-Hillen, J. M. 1053

Spigoni, V. 19, 1331

Spinas, G. A. 176

Spiri, D. 949

Spoletini, M. 575

Spratt, K. 1249

Spruce, M. C. 1252

Spyrantis, A. 559

Squires, P. E. 94

Sreenan, S. 966, 976

Srikanta, S. S. 5

Srinivasan, B. T. 1050

Srishyla, M. V. 978

St-Pierre, P. 625

Stabe, C. 150

Staels, B. 856

Stage, E. 1123

Stahel, W. A. 1068

Stahl, H. 717

Staiger, H. $\quad 319,395,1285$

Stallknecht, B. $\quad 310,630$

Stals, K. 283

Stam, C. J. 1174

Stamoyannou, L. 389

Stampfer, M. J. 246

Stan, D. 1289

Stangl, M. J. 605

Stanik, J. 279

Stanley, C. A. 276

Stanton, A. 23, 1175

Stea, F. 1314

Steck, A. 336

Steed, R. D. 1056

Steen Carlsson, K. 1088

Stefan, N. $\quad 319,322,395$

Stefanelli, G. 1196

Stefanovski, D. $732,792,864$

Stefanutti, A. 639

Stefl, B. 738

Stegmayr, B. 424

Stehouwer, C. D. A. $\quad 189,254$, $255,295,386,415,809,838,940$, $1179,1218,1300,1305,1324$,

Steiner, S. $\quad 677,678$

Steinfeld, I. 1331

Steinsdoerfer, M. 564

Stella, C. 1074

Stelzer, J. 409

Stene, L. C. 267

Stentz, F. 224, 805, 1056

Stettler, C. 23

Stevenson, E. 248

Stewart, J. A. 1060

Stewart, M. W. 870

Stich, V. $\quad 723,730,816$

Stienstra, R. 165

Stiller, D. 616

Stitt, A. 1308

Stoico, V. 652

Stojanovic, I. 833

Stojkovic, M. 1131

Stokes, R. 456

Stolarczyk, E. 610

Stolerman, E. S. 326

Stolinski, M. 752

Stolk, R. P. $\quad 353,823,1175$

Stone, M. A. 1116

Stonehouse, A. 876,877

Stoppiglia, L. F. 746

Stork, I. 1299

Storms, F. 187

Storti, E. 1319,1400

Stosic-Grujicic, S. 833

Stoyanov, S. B. 243

Straand, J. 1049

Strachan, D. P. 299

Straczkowski, M. 1395

Strand, J. 897

Stratmann, B. 1299

Stratton, I. M. 1172

Strauss, A. 916

Strauss, B. 797

Strauss, F. 734

Strindberg, L. 1385

Stritzke, J. 188

Strojek, K. 1359

Strom, A. 507, 581

Stuart, B. 923 
Suganuma, R. 1373

Sugawara, A. 1243

Sugawara, A. 769

Sugaya, T. 46

Sugden, M. C. 487

Sugihara, K. 769

Sugimori, M. 768

Sugimoto, K. 1243

Sugino, I. 744

Sui, L. 438

Sukhanov, S. 806

Sulli, N. 571

Sulmont, V. 988

Sultan, A. 1330

Sultana, N. 1128, 1129, 1134, 1136

Suma, M. 571

Sumarac-Dumanovic, M. 1223

Summanen, P. 143

Summerhayes, B. 1209

Sumnik, Z. 371

Sun, F. 752

Sun, G. 489

Sun, L. 202

Sun, X. Lan. 1369

Sun, Z. 447, 1182, 1307

Sundsten, T. 552

Sung, K. 1353

Sunkari, V. $\quad 763,1263$

Suraci, C. 984

Suryanarayan, S. 5

Sutherland, C. 786

Suzuki, K. 1315

Svärdsudd, K. $\quad 314,419$

Svenson, L. W. 428

Svensson, J. 572

Svensson, M. 65

Svensson, M. K. 252

Svetlova-Kovalenko, H. 270

Swanson, D. 410

Sweep, F. 916

Swern, A. S. $\quad 909,912$

Swift, P. 265

Swinton, C. 624

Sysi-Aho, M. 51

Szabo, C. 1126

Szabo, R. 916

Szadkowska, A. 1231

Szamatowicz, J. $\quad 1127,1132,844$

Szczepaniak, L. S. 747

Székely, A. 296

Szelachowska, M. 337

Szendroedi, J. M. 34, 621

Szewczyk, L. 116

Szigethy, A. 1126

Szilvássy, Z. 714

Szopa, M. 394

Szpotańska, M. 948

Szucs, T. 1095

Szwergold, B. S. 43

Szymanska-Garbacz, E. 919

Szypowska, A. 948, 989

\section{$\mathrm{T}$}

T'Sjoen, G. 924

Taback, S. P. 276

Tabák, Á. 1240

Tabak, A. 779

Tabák, A. G. 350

Tack, C. J. $\quad 36,165,974$

Taddei, S. 1319

Tahrani, A. 1118

Tai, E. 316
Tait, B. D. 141

Taittonen, M. 760

Taivankhuu, T. 557

Tajima, N. $\quad 379,413,453,910$

Takács, R. 1383

Takada, T. 1364

Takahashi, H. 1293

Takahashi, H. 97

Takahashi, I. 769

Takahashi, K. 542

Takahashi, T. 1294

Takamiya, Y. 1187

Takamura, T. 768

Takane, K. K. 85

Takasawa, S. 769

Takayama, H. 768

Takebayashi, K. 251, 808

Takeda, J. 690

Takemura, C. 1115

Takeuchi, M. 1302

Takiyama, Y. 1185

Takizawa, M. 281

Talary, M. S. 1068

Talbot, D. 1035

Talmud, P. 290

Tamarit-Rodriguez, J. 495

Tambascia, M. 150

Tan, A. L. Y. 160

Tan, G. 193

Tan, G. H. 858

Tan, M. 316

Tan, Y. 202

Tan, Y. 719

Tanaka, K. 1364

Tanaka, Y. 329

Taneja, T. $\quad 101,489$

Tang, W. $\quad 40,796$

Tang-Christensen, M. 736

Tang-Fui, S. 971

Tangi, O. 958

Tangi-Rozental, O. 246

Tanguy, M. 595

Tanimoto, H. 932

Tankova, T. 895

Tannapfel, A. 541

Tanti, J. 640

Taouis, M. 131, 774

Tapp, R. 23

Tappy, L. 705

Tarakci, E. 1267

Tarallo, S. 157, 217, 218

Taraoune, I. 983

Tarasov, A. I. 101

Targher, G. 1202

Tarnov, L. 1318

Tarnow, I. 1206

Tarnow, L. $\quad 46,295,1198,1206$, 1333

Taskinen, M. $\quad 751,878,879,1358$

Tatarkiewicz, K. 901

Tátrai, E. 1177

Taub, N. A. 1050

Taudorf, S. 707

Tauriainen, S. 342

Tavares, I. 1241

Tawaramoto, K. 135, 511

Taylor, J. M. 361

Taylor, K. 146

Taylor, R. 766

Tecilazich, F. 652

Tedeschi, A. 1273,1276

Teede, H. 1072

Teh, M. M. 227

Tejero, E. 531
Telejko, B. 1127, 1132

Téllez, N. 599, 904

Temma, T. 359

Temml, C. 378

Temple, R. C. 13,1031

Temponi, A. $\quad 964,1078$

ten Cate, H. 1305

Teng, R. 73

Tengholm, A. 177, 492, 493

Tentolouris, N. 1236, 1281, 1348, 1382

Teperino, R. 305

Terra, L. F. 215

Tershakovec, A. M. $\quad 853,854$

Tesauro, M. 441, 1317

Tesfaye, N. 1078

Tesfaye, S. 242, 244, 1240

Tesi, F. 1140

Testa, I. $\quad 321,1170$

Testa, M. A. 894

Testa, R. $\quad 321,1170$

Tetens, I. 397, 426

Tetlow, A. P. 1004

Thabit, H. $\quad 382,704$

Thamer, C. 395

Thammavong, N. 153

Thanopoulou, A. C. 339

't Hart, L. M. 320

Thayer, S. 920

Thelwall, P. 248

Thelwell, P. E. 766

Theurl, E. 956

Thévenon, C. 22

Theys, N. H. 261

Thiam, D. 341

Thiery, J. 246

Thivolet, C. $\quad 236,369$

Thom, S. 23

Thom, S. A. McG. 1175

Thomanek, P. 616

Thomas, G. 1215

Thomas, K. 248

Thomas, M. C. 96

Thomas, N. 383

Thomas, S. 759

Thompson, A. 283

Thompson, C. J. 1321

Thompson, R. 1166

Thomson, S. 1265

Thorand, B. 380

Thorens, B. 512, 607

Thoresen, G. H. 758

Thorgeirsson, H. 972

Thörn, K. 789

Thorn, L. M. $\quad 1167,1354,1363$

Thornalley, P. J. $\quad 219,220,748$, 1301, 1303

Thorne, K. I. 961

Thorsby, P. M. 294, 712

Thow, J. C. 1108

Thunander, M. 972

Thuren, T. $\quad 74,913$

Thurston, H. 913

Tian, L. M. 1369

Tiberti, C. $571,575,584$

Tibiriçá, E. 1280

Tichet, J. 311, 313, 754

Tiedge, M. 213, 562

Tiengo, A. 21

Tierney, A. C. 247

Tikhonova, T. 270

Tillin, T. 23, 421

Timar, B. 1346

Timar, R. Z. 1346

Timmers, S. 167

Timonen, M. 706

Timpson, N. 9, 10, 11, 299

Tirado, R. 783

Tirosh, A. 407

Tirosh, O. 787

Tkac, I. 1356

Tkacova, R. 1356

Tobian, J. 410

Tobin, V. 610

Tockus, D. 1080

Todd, J. A. 140

Todoric, J. $\quad 737,837$

Todorova-Ananieva, K. 1120

Toffoli, B. 1162

Toffolo, G. M. 30,122

Toft, A. D. $\quad 981,994,998$

Toft, U. 397

Tolonen, N. 1354

Tom, R. Z. 130

Tomassini, J. E. $\quad 853,854$

Tominaga, M. 379

Tomita, M. 1327

Tomlinson, J. 762

Tommasi, C. 652

Tomori, Z. 18

Tondi, C. 367

Tong, N. Wei. 357

Tong, P. 634

Tong, P. C. Y. $\quad 144,708$

Tong, X. M. 817

Toni, S. 986

Tönjes, A. 289, 396

Tonnesen, M. 522

Toomey, S. 834

Toräng, S. 661

Torffvit, O. 1225

Torgerson, D. J. 1108

Torjesen, P. 1298

Torjussen, B. R. 1278

Törn, C. 972

Tornei, F. 67,68

Torp-Pedersen, C. 1394

Torri, S. $\quad 136,315,903$

Torsvik, J. 286, 287

Tory, K. $\quad 623,714$

Tosin, T. 1080

Toso, C. 601

Tóth, N. 1233,1240

Touati, F. $\quad 1235,1384$

Touriño, C. 500

Tourrel-Cuzin, C. 102

Toyoda, K. 359, 602, 902, 905

Trabetti, E. 298

Traitel, T. 687

Tran, A. T. 1049

Trattnig, S. 34

Trautmann, M. $\quad 146,875$

Travert, F. 1169, 1332

Treasure, J. 1025

Treebak, J. T. 90

Trenell, M. I. $\quad 248,766$

Trento, M. 151, 1106

Trevisan, R. 1166

Tribble, N. D. 279

Tribler, J. 1036

Trikash, I. 55

Trimble, E. R. 1288

Trinetta, A. 1106 


\begin{tabular}{|c|c|c|c|}
\hline Triscornia, S. 1171 & Urdea, M. 54 & Van Schravendijk, C. 332 & Vilsbøll, T. $\quad 637,661,1153$ \\
\hline Troeger, J. 1012 & Uruno, A. 769 & van Vliet, A. 867,868 & Vilser, W. 1157 \\
\hline Trombetta, M. $298,318,652$ & Ustinov, J. 29 & van Vliet, M. 120 & Viltard, M. 1133 \\
\hline Troncoso, E. 1161 & Ustyuzhanin, D. 1193 & van Winkel, R. 713 & Vincent, M. A. 625 \\
\hline Troughton, J. 1116 & Uusitupa, M. 795 & Vanacore, R. 1319 & Vinciguerra, M. 697,811 \\
\hline $\begin{array}{l}\text { Trovati, M. } \quad 58,626,793,1117, \\
\quad 1387\end{array}$ & V & $\begin{array}{l}\text { Vancova, M. } 604 \\
\text { Vandenberghe, H. } 155\end{array}$ & $\begin{array}{l}\text { Vingerling, J. R. } 1175 \\
\text { Vinje, G. } 712\end{array}$ \\
\hline Troy, S. 607 & & Vang, K. 676 & Vinokur, V. 1368 \\
\hline Trubitsyna, Y. 128 & Vaag, A. $148,149,192,295,306$ & Vanggaard, J. 891 & Vinterby, A. 975 \\
\hline Trucco, M. 1402 & $310,312,613,630,673,755,899$ & Vanhee, H. 924 & Viollet, B. 90,607 \\
\hline Trueb, L. 1087 & Vaarala, O. 342 & Vankova, M. 818 & Viretto, M. $58,626,793$ \\
\hline Truong, W. 601 & Vaca-Sanchez, P. 465 & Vantyghem, M. 594 & Virkamäki, A. 111,965 \\
\hline Truyen, I. 332 & Vaccarino, V. 347 & Varadhan, L. 401,1146 & Virtanen, I. 444 \\
\hline Trznadel-Morawska, I. 1121 & Vaccaro, O. $\quad 753,846,1117,1387$ & Varadi, A. 496 & Virtanen, K. 799 \\
\hline Tsang, M. W. 1214 & Vacz, G. 1126 & Varga, T. 589 & Virtanen, S. M. 373 \\
\hline Tsao, P. 1331 & Vaitheesvaran, B. 773 & Vargha, P. 1233 & Viscardi, M. 282 \\
\hline Tsatsoulis, A. 587 & Valabhji, J. 1272 & Varillas, F. 1392 & Vischer, U. M. 1292,1352 \\
\hline Tschoepe, D. 1299 & Valaitis, P. 1012 & Várkonyi, T. T. 1383 & Vistisen, D. 388 \\
\hline Tschopp, O. 176 & Valcarce, C. 929 & Varney, M. D. 141 & Vitacolonna, E. 1101 \\
\hline Tseng, Y. 396 & Valensi, P. $\quad 221,1228,1235,1239$ & Varró, A. 1383 & Vitkova, M. $723,730,816$ \\
\hline Tsiani, E. 696 & 1384 & Varroud-Vial, M. $\quad 153,416,1039$ & Vlad, A. 1346 \\
\hline Tsubamoto, Y. 932 & Valenti, V. 1397 & Vartholomatos, G. 587 & Vladimirova, V. 1024 \\
\hline Tsukahara, T. 1327 & Valentine, W. J. 184 & Vasan, S. 383 & Vodouhe, C. 591, 603 \\
\hline Tsukuda, K. 995 & Valentini, G. 41 & Vasilopoulos, H. 983 & Voelmle, M. K. 1066 \\
\hline Tsurumi, M. 281 & Valentini, U. $\quad 156,1213$ & Vavrova, E. 596 & Vogt, L. 232 \\
\hline Tubiana-Rufi, N. 81,988 & Valentino, R. 305 & Vaychulis, I. A. 1040 & Vol, S. 754 \\
\hline Tubili, C. $\quad 1034,984$ & Valerio, G. 951 & Vaychulis, T. N. 1040 & Voldner, N. 1141 \\
\hline Tue Hansen, N. 139 & Valet, P. 716 & Vázquez, F. 275 & Volkir, S. P. 1194 \\
\hline Tugeeva, E. 1193 & Vallen-Thompson, M. 1194 & Vcelak, J. 818 & Volpe, L. $\quad 1122,1140,1145$ \\
\hline Tulassay, Z. 589 & Valone, F. 574 & Vecci, E. G. 571 & Volpe, M. 47,1336 \\
\hline Tumini, S. $\quad 1077,986$ & Valongo, A. 1119 & Vedala, A. 5 & Volz, C. 110 \\
\hline Tunis, S. L. 1339 & Valorani, M. $\quad 560,561$ & Vedovato, M. 1166 & von Bibra, H. 59 \\
\hline Tuomainen, A. M. 1205 & Valtat, B. 450 & Veiga-da-Cunha, M. 526 & von dem Berghe, W. 1075 \\
\hline Tuomi, T. 62 & Valverde, I. 900 & Veijola, R. $51,277,342$ & Von Hofe, E. 570 \\
\hline Tuomilehto, J. $103,105,377,415$, & van 't Riet, E. 391,1179 & Vejrazkova, D. 818 & von Rosenstiel, I. 120 \\
\hline $422,424,425$ & Van Avendonk, M. J. P. 999 & Vekens, E. 332 & Vonbank, A. $\quad 404,1351,1355$ \\
\hline Tura, A. $1143,1152,240,698$ & van Battum, P. L. H. 1270 & Velho, G. $311,313,1169,1332$ & 1362 \\
\hline Turatti, L. 1096 & Van Crombrugge, P. 1043 & Velikikh, N. 270 & Vondra, K. 818 \\
\hline Turner, N. 612 & van de Bunt, M. 279 & Vella, A. 122 & Vorontsov, A. 1024 \\
\hline Tushuizen, M. E. $\quad 636,751,1204$ & van de Laar, L. 445 & Veloso, R. V. 457 & Voss, L. 670 \\
\hline 1386 & van de Waarenburg, M. P. H. 809 & Vendrell, J. $\quad 173,709,783,814$ & Voss, L. D. 947,1089 \\
\hline Tutuncu, N. B. 638 & van den Broek, N. M. A. 31 & 840 & Vouis, J. 890 \\
\hline Tütüncü, Ö. 206, 207 & van den Donk, M. $\quad 431,999,1097$ & Verchere, C. B. 1334 & Voulgari, C. 1236 \\
\hline Tuunanen, H. 760 & Van der Elst, J. 438 & Veret, J. 545 & Voysey, M. 1366 \\
\hline Tynan, A. $\quad 154,1090$ & van der Graaf, M. 974 & Vergès, B. $\quad 340,1345,855$ & Vrbikova, J. 818 \\
\hline Tyrberg, M. 1178 & van der Kallen, C. J. $\quad 189,386$, & Verheijen, J. H. 1204 & Vrzalova, J. 816 \\
\hline & 838,1305 & Verier-Mine, O. 1165 & Vuksan, V. 957 \\
\hline $\mathrm{U}$ & van der Kolk, J. 940 & Vermeulen, I. 332 & \\
\hline & van der Meer, R. W. $113,114,166$ & Vermue, R. P. 1386 & W \\
\hline Ueda, M. 359 & Van der Merwe, M. 385 & Vernor, D. C. 147 & \\
\hline Ueda, S. 1187 & van der Zijl, N. J. 1204 & Verrienti, A. 571 & Waberski, B. H. 241,1253 \\
\hline Ueland, T. 1278 & van der Zon, G. C. M. 260 & Vervoort, G. M. 974 & Wada, J. 1396 \\
\hline Ueshiba, H. 744 & van Dijk, T. H. 662 & Vespasiani, G. $\quad 156,230,1112$, & Wada, J. 162 \\
\hline Ueshima, H. 1164 & van Duijn, C. M. 12 & 1213 & Wada, Y. $363,414,1191,1293$ \\
\hline Uga, M. 582 & van Duinkerken, E. 1174 & Vessby, B. 245,247 & Wade, R. $882,883,960$ \\
\hline Uhl, W. 541 & Van Eyck, D. 713 & Vestergaard, H. 1333 & Wadén, J. $1167,1354,1363$ \\
\hline Uhler, A. 1046 & Van Gaal, L. F. 155 & Veves, A. 1156 & Waeber, G. $\quad 512,515,1087$ \\
\hline Uhles, S. 491 & van Genderen, F. 332 & Vexiau, P. $\quad 341,664$ & Waelkens, E. $\quad 462,525,549$ \\
\hline Ulbrecht, J. S. 1271,1274 & van Greevenbroek, M. M. J. 189 , & Veyrat-Durebex, C. $674,697,811$ & Wagenmakers, A. J. M. 36 \\
\hline Ulcickas Yood, M. 1051 & $386,838,1305$ & Viberti, G. $24,44,48,1155,1232$, & Waget, A. 716 \\
\hline Ulianich, L. 691 & van Haeften, T. W. 320 & Vidal, H. 88 & Wägner, A. M. 275,1392 \\
\hline Ullrich, S. J. $\quad 138,547$ & van Hall, G. 79 & Vidgen, E. 1022 & Wagner, I. 857 \\
\hline Umpierrez, D. $\quad 964,1056,1078$ & Van Huffel, C. 333 & Vieira, E. 130 & Wagner, O. 837,1152 \\
\hline Umpierrez, G. $\quad 964,1078$ & van Leeuwen, M. 387 & Vignali, S. 1145 & Wagner, R. $\quad 233,574$ \\
\hline Umpleby, A. M. 752 & van Lier, J. 891 & Vila-Bedmar, R. 784 & Wahba, E. A. 832 \\
\hline Unbehauen, H. 396 & van Lommel, L. 170 & Vileikyte, L. 1271,1274 & Wahed, T. $1128,1129,1134,1136$ \\
\hline Undlien, D. E. 267,365 & Van Lommel, L. 297 & Viljanen, A. 799 & Wahl, P. 929 \\
\hline Ungaro, P. $\quad 305,683$ & van Loon, L. J. C. 36 & Viljanen, T. 760 & Wajcberg, E. 865 \\
\hline Unno, M. 769 & Van Oostveen, D. 1054 & Viljoen, A. 1209 & Walder, K. 37,624 \\
\hline Uno, Y. 660,726 & van Oppen, P. C. 1028 & Villa, M. 195 & Walder, K. R. 935 \\
\hline Uonaga, T. 602 & van Poelje, P. D. 931 & Villanueva-Peñacarrillo, M. & Waldhäusl, W. 34 \\
\hline Urban, M. 1030 & van Raalte, D. H. 663 & L. 900,907 & Waldron-Lynch, F. 1082 \\
\hline Urbani, A. 68 & Van Remmen, H. 619 & Villarroel, M. $\quad 158,1158$ & Wale, A. 554 \\
\hline Urbanovich, A. M. 955 & Van Schaftingen, E. 526 & Villemin, P. 1292 & Walker, L. 327 \\
\hline
\end{tabular}


Walker, M. 250

Walker, S. E. 870

Wallander, M. 433

Wallerstedt, E. 810

Walsh, B. 866

Walte, K. 1075

Walther, R. 463,543

Walus-Miarka, M. 1176

Wampers, M. 713

Wan Bebakar, W. M. 897

Wang, C. 307, 936

Wang, G. 507, 581

Wang, H. 238

Wang, H. 753

Wang, J. 349

Wang, J. L. 643

Wang, M. 1286

Wang, M. 17

Wang, S. 546

Wang, S. $\quad 647,852$

Wang, T. 803

Wang, X. P. 1032

Wang, Y. 1180

Wang, Y. 1287

Wang, Y. 144

Wang, Y. C. 643

Wang, Z. 1180

Ward, W. K. 1062

Wareham, N. J. $\quad 61,349,1365$, 1050

Warren, A. B. 805

Warren, S. 925

Wasada, T. 1396

Wasan, A. D. 1248

Wascher, T. C. 1360

Wasson, J. 328

Watanabe, A. 281

Watanabe, C. 1163

Watanabe, J. 1185

Watanabe, Y. 1079

Watarai, A. 1327

Watson, A. 93, 96

Watson, R. 86

Wawrusiewicz-Kurylonek, N. $844,1127,1132$

Weaver, J. 1372

Webb, D. 410

Webb, D. R. 1050

Weber, J. 285

Webster, Z. 1155

Wedekind, D. 278

Weder, C. 1

Weets, I. 332

Wegbrod, C. $\quad 454,461$

Wegner, L. 324

Wei, Q. 1307

Wei, X. Bing. 1369

Weichhart, T. 737

Weigert, C. 1285

Weill, A. 153,416

Weingarten, G. 690, 1260, 1264

Weinger, K. 228

Weinstein, A. 1058, 1061

Weinstock, R. S. 853

Weintraub, W. S. 347

Weiss, H. 213, 278, 562

Weissman, P. 918

Wellman, J. 188

Welschen, L. M. C. 1028

Welsh, Z. 1075

Welsing, P. M. J. 1097

Welters, H. J. 460

Wenying, Y. 978

Wenzel, J. 857
Wenzlau, J. M. 233, 583, 585

Wernicke, J. F. 1248

Wertheimer, E. $\quad 690,1260,1264$

West, N. A. 119

Wester Rosenlöf, L. 213

Westerbacka, J. 33

Westermeier, T. 223

Wetterling, T. 711

Weyer, C. 82, 83

Wheeler, M. B. $\quad 101,655,764$

White, K. E. 1155

Whitelaw, B. C. 1071

Whitfield, N. 1015

Whitmore, J. 1

Wichmann, H. 380

Widenmaier, S. 533

Widmann, C. 469,512

Wieczorek, L. 911

Wiederkehr, A. C. 178

Wienbergen, A. 478

Wierusz-Wysocka, B. 1109

Wiesmeth, A. 667

Wijesekara, N. 101

Wilczynska, M. 1335

Wilding, J. P. H. 201, 831

Wilhelm, K. 146, 1007, 1010, 1011

Wilinska, M. E. 181

Wilke, B. 331

Wilkin, T. J. $\quad 670,947,1089$

Wilkinson, I. D. 242, 244

Wilkinson-Berka, J. L. 193

Wilks, D. 612

Willasch, A. 376

Willenborg, M. 98, 478

Williams-Herman, D. 73, 909, 911,912

Willson, K. 627

Wilson, C. $\quad 76,77,860,861,862$

Wilson, P. 1359

Windmill, K. 935

Winhofer, Y. 1143

Winkler, C. 264,338

Winkler, P. 1290

Winner, H. 956

Winnick, J. J. 665

Winocour, P. 1209

Wintle, M. $880,881,882,883$, 960

Winzer, C. 34,1152

Witt, N. 23

Witte, D. R. $\quad 350,779$

Wittig, A. 1067

Wittmann, I. 1208

Wittmann, T. 1383

Woda, M. 1335

Woess, M. 1351, 1355

Wogensen, L. 1188

Wohl, P. 812, 815

Wojtaszewski, J. F. 90, 613

Wolf, G. 1045, 1157, 1181

Wolf, G. $\quad 463,543$

Wolf, S. 396

Wolfensberger, M. 1006

Wolffenbuttel, B. H. R. 959

Wolkow, P. 394

Wollheim, C. B. $\quad 178,238$

Wolpers, D. 504

Wolsk-Jackson, E. 79

Wolzt, M. 621

Womack, E. P. 1194

Won, J. 1353

Won, K. 1296

Wong, N. D. 417
Wong, S. 831

Wong, S. P. Y. 201

Wong, V. S. C. 458

Woo, J. 991

Woo, J. T. 370

Woo, V. $\quad 40,796$

Woolcott, O. O. $\quad 732,792$

Woollhead, A. 486

Wooton-Whiting, S. 1118

Woszwillo, A. 1335

Wouters, K. 802

Wright, R. J. 675

Wu, A. H. B. 1194

Wu, C. M. 720

Wu, C. S. 720

Wu, E. 921

Wu, F. 1398

Wu, X. Hong. 1032

Wu, Y. H. 643

Wuendrich, E. 454

Wun, C. 566

Wunderlich, T. F. 115

Wutte, A. 968

Wuttke, A. 177

Wyka, K. 280

Wymann, M. P. 739

\section{$\mathbf{X}$}

Xiang, K. 307

Xie, F. 435

Xie, Y. 699

Xin, W. 505

Xiu Steele, M. 901

$\mathrm{Xu}$, A. $\quad 719,1287$

Xu, G. 174,634

Xu, H. E. 38

$\mathrm{Xu}, \mathrm{L} . \quad 17$

Xu, L. 176

Xu, W. 95

Xu, Z. 1180

$\mathrm{Xu}, \mathrm{Z} .78$

Xue, M. 748

Y

Yada, T. 475,770

Yae, S. 792

Yagihashi, S. 1243

Yakhini, Z. 1331

Yale, J. 1060

Yamada, A. 995

Yamada, K. 1164

Yamada, Y. 902, 1224

Yamagishi, S. $\quad 1187,1302$

Yamagishi, S. 1243

Yamamoto, T. 281

Yamamoto, Y. 1257

Yamane, S. 602, 905

Yamano, T. 985, 1115

Yamaoka, H. 1257

Yamasaki, H. 1257

Yamasaki, H. 582

Yamauchi, A. 769

Yan, H. 203

Yan, L. 17

Yan, P. $\quad 876,877$

Yan, X. 430

Yan-Ling, H. 916

Yang, G. 257

Yang, G. $171,666,715,725,813$

Yang, J. 512

Yang, L. Y. 848

Yang, M. 171

Yang, M. 666

Yang, S. 257

Yang, S. J. 718

Yang, T. 449

Yang, T. B. 553

Yang, W. 1093

Yang, X. L. 144

Yang, Y. 725

Yarows, S. A. 1221

Yates, A. P. 473

Yates, T. 1035

Ybarra, J. 1254

Ye, J. 37

Ye, S. 316

Ye, W. 429

Ye, X. 881

Yeaman, S. J. 250

Yefet, E. 1264

Yeung, C. 1214

Yeung, C. Y. 708

Yeung, J. 533

Yeung, V. T. F. 1214

Yiannakouris, N. 308

Yildirim, N. 1017

Yin, D. 1095

Yki-Jarvinen, H. $\quad 33,878,879$

Ylä-Herttuala, S. 111

Yokoi, T. 1293

Yokoyama, H. 1195

Yong, J. 93

Yoo, H. 1186

Yoo, H. 527

Yoo, H. J. 718

Yoo, J. 1023

Yoo, J. 1186

Yoo, J. 1245

Yoo, S. 390

Yoo, S. 804

Yoon, J. 745, 1197

Yoon, K. 804, 991

Yoshida, K. 1115

Yoshiike, N. 379

Yoshikawa, T. 769

Yoshino, G. 744

Young, I. S. 1288

Youssef, E. M. Y. 832

Yu, A. 921

Yu, J. 257

Yu, L. 257

Yu, L. $\quad 583,585$

Yu, X. 213

Yu, Y. 1316

Yuan, C. P. 1032

Yuan, Y. 1182

Yulan, C. 715

Yuncu, A. 1017

Z

Zaccardi, F. 1337,1344

Zacharovova, K. 577, 596, 604

Zairis, M. 1361

Zak, K. 50

Zamaklar, M. 573, 586, 588

Zangen, S. W. $\quad 360,776$

Zani, F. 739

Zanone, M. M. 1297

Zargar, A. H. 1018

Zarra, E. 230

Zasadzinska, G. 1342

Zavaroni, D. 1117

Zavaroni, I. 19, 1117, 1331, 1387

Zavrelova, H. 1179 
Zbyn, S. 593

Zdravkovic, M. 148, 149, 797, $887,888,889,890,891,892,895$, $896,897,898,899$

Zeggini, E. $\quad 9,10,291,300,301$, 302

Zeitler, P. S. 119

Zeitz, U. 532

Zeller, M. 1345

Zethelius, B. $191,314,403,412$, 415

Zeyda, M. 737

Zgibor, J. 1046

Zgibor, J. C. 107

Zhai, S. $\quad 42,928$

Zhang, B. 1009, 1010, 1011

Zhang, B. 203

Zhang, F. 1398

Zhang, F. 654

Zhang, H. 719

Zhang, H. 937

Zhang, J. 1221

Zhang, J. 95

Zhang, L. 415

Zhang, Q. 1003, 1085

Zhang, Q. 1247

Zhang, Q. 207

Zhang, Q. 417

Zhang, Q. 631

Zhang, Q. 633, 654

Zhang, R. 307, 936

Zhang, W. 336

Zhang, W. 72

Zhang, X. J. $\quad 467,505,1369$

Zhang, X. X. 643

Zhang, Y. 1092, 1093

Zhang, Y. 1166

Zhang, Y. 17

Zhang, Y. 473, 954

Zhao, C. 432

Zhao, H. 634

Zhao, J. J. $\quad 346,467,505,1369$

Zheng, D. 732, 792, 864

Zheng, F. P. 1398

Zheng, H. 1055, 1065

Zhi, J. $\quad 42,928$

Zhou, L. 1286

Zhou, L. 226

Zhou, P. 719

Zhou, S. 447

Zhou, W. 719

Zhou, Z. 719

Zhu, L. 1341

Zhuang, D. 146

Ziegler, A. G. $\quad 264,330,344,338$

Ziegler, D. 1255

Ziemer, D. C. 347

Zierath, J. R. 90

Zimmet, P. 424

Zimmet, P. Z. $\quad 190,422,425,427$

Zinman, B. 24, 106, 149, 888, 892, 898, 899

Zipris, D. 233

Ziros, P. G. 807

Zitoun, C. $\quad 607,642$

Zolochevska, O. 56

Zonenberg, A. 1132

Zoppini, G. 1202

Zorzano, A. 173

Zorzin, L. 1162

Zouaoui, S. 641

Zoupas, C. S. 983,1348

Zozulinska-Ziolkiewicz, D. 1109

Zubarev, R. A. 649
Zucco, C. 374, 402

Zuccotti, G. V. 949

Zuellig, R. A. 176

Zuithoff, N. P. A. 1054

Zuk-Ramot, R. 958

Zulewski, H. 785,1110

Zuo, P. 531, 865

Zuodar, G. 1380

Zweiker, R. 1360

Zwilling, M. 338

Zwisler, A. 1033, 1340 


\section{European Association for the Study of Diabetes}

EASD Executive Committee

President:

Vice-President:

Vice-President:

Honorary Secretary:

Honorary Treasurer:

Chairman of the Postgraduate Education Sub-Committee:

Editor-in-Chief, DIABETOLOGIA:
E. Ferrannini, Pisa (retires 2008)

U. Smith, Göteborg (retires 2008)

C. Boitard, Paris (retires 2009)

M. Stumvoll, Leipzig (retires 2010)

G. Spinas, Zurich (retires 2009)

J. Nolan, Dublin (retires 2009)

E. Gale, Bristol (retires 2009)

EASD Council comprises of the Officers above and the following members:

Term Expiring 2008

K. Dahl Jørgensen, Oslo

B. Mankovsky, Kiev

S. Sasson, Jerusalem

\section{Term Expiring 2009}

V. Pirags, Riga

F. Pociot, Gentofte

M. Porta, Turin

M. Stumvoll, Leipzig
Term Expiring 2010

F. Ashcroft, Oxford

F. Bosch, Barcelona

V. Petrenko, Kaunas

A. Siebenhofer, Graz

The Past President, P.A. Halban, and the Secretary of the Postgraduate Education Sub-Committee, L. Czupryniak, are members of the Council ex officio.

\section{HONORARY AUDITORS}

N. Katsilambros, Athens and M. Tiedge, Rostock

\section{HONORARY MEMBERS}

G. Alberti, Newcastle; D. Andreani, Rome; E. Cerasi, Jerusalem; A. Czyzyk, Warsaw; J.K. Davidson, Atlanta; T. Deckert, Hellerup;

P. Freychet, Nice; L.G. Heding, Copenhagen; H. Keen, London; E. Kohner, London; P. Lefèbvre, Liège; R. Luft, Stockholm; C. Lurie, New York; J. Pirart, Brussels; S. Rahbar, Duarte; J. Roth, Whitestone; E. Shafrir, Jerusalem; D. Steiner, Chicago; E. von Wasielewski, Munich

\section{GOLD MEMBERS}

Astra Zeneca, Macclesfield, UK; Bayer HealthCare, Leverkusen, Germany; Eli Lilly \& Co., Indiana, USA; LifeScan, Inc., California, USA; Merck \& Co. Inc., New Jersey, USA; Merck KGaA, Darmstadt, Germany; Novartis Pharma AG, Basel, Switzerland; Novo Nordisk A/S, Bagsvaerd, Denmark; sanofi-aventis, Paris, France; Servier, Neuilly-sur-Seine, France; Johnson \& Johnson, New Jersey, USA

\section{SILVER MEMBERS}

Arkray Europe, Amstelveen, The Netherlands; Daiichi Sankyo Europe GmbH, Munich, Germany; Roche Diagnostics GmbH, Mannheim, Germany; Sooil Development Co., Ltd., Seoul, Korea

\section{ASSOCIATE MEMBERS}

Amylin Europe Ltd., Oxford, UK; A. Menarini Diagnostics, Grassina, Italy; Becton Dickinson Consumer Healthcare Europe, Pont-de-Claix, France; Berlin Chemie AG, Berlin, Germany; Boehringer Ingelheim GmbH, Ingelheim, Germany; HemoCue AB, Angelholm, Sweden; Medtronic Europe, Tolochenaz, Switzerland; Owen Mumford Ltd., Oxford, UK; Pelikan Technologies GmbH, Muenster, Germany; Ypsomed GmbH, Sulzbach, Germany

The EASD Executive Committee is also the Executive Committee of the European Foundation for the Study of Diabetes (EFSD).

EASD/EFSD office : Rheindorfer Weg 3, 40591 Düsseldorf, Germany - www.easd.org 